DOE/EIA-0512(91)

Distribution Category UC-950

\title{
Manufacturing Consumption of Energy 1991
}

\section{December 1994}

\author{
Energy Information Administration \\ Office of Energy Markets and End Use \\ U.S. Department of Energy \\ Washington, DC 20585
}

\section{MASTER}

DISTRADUTION OF THIS DCCUNENT BS UNLSATED: 


\section{Contacts}

General information about Energy Information Administration data on energy consumption can be obtained from Lynda T. Carlson, Director of the Energy End Use and Integrated Statistics Division (EEUISD) (202-586-1112).

General information regarding the contents of this publication can be obtained from Dwight $\mathrm{K}$. French, Chief of the Transportation and Industrial Branch (202-586-1126), or John L. Preston, Survey Manager for the Manufacturing Energy Consumption Survey (202-586-1128). Robert K. Adler (202-586-1134; Internet BADLER@ELA.DOE.GOV), Mark A. Schipper (202-586-1136; Internet MSCHIPPE@EIA.DOE.GOV), and Michael J. Margreta (202-586-2.327; Internet MMARGRET@EIA.DOE.GOV) are the contacts for estimation, analysis, and table preparation. Hattie Ramseur (202-586-1124) is the contact for related energy consumption publications. The FAX number for all EEUISD personnel is 202-586-0018.

EIA maintains the Electronic Publishing System (EPUB) to allow the general public to electronically access the tables found in this report and many of EIA's statistical reports. EPUB can be accessed on (202) 586-2557 with communication settings: baud rate of 300-2400, no parity, 8 data bits, 1 stop bit, full duplex, and terminal type (ANSI, ANSI-BBS, VT-100, etc.). 


\section{DISCLAIMER}

This report was prepared as an account of work sponsored by an agency of the United States Government. Neither the United States Government nor any agency thereof, nor any of their employees, make any warranty, express or implied, or assumes any legal liability or responsibility for the accuracy, completeness, or usefulness of any information, apparatus, product, or process disclosed, or represents that its use would not infringe privately owned rights. Reference herein to any specific commercial product, process, or service by trade name, trademark, manufacturer, or otherwise does not necessarily constitute or imply its endorsement, recommendation, or favoring by the United States Government or any agency thereof. The views and opinions of authors expressed herein do not necessarily state or reflect those of the United States Government or any agency thereof. 


\section{DISCLAIMER}

Portions of this document may be illegible in electronic image products. Images are produced from the best available original document. 
As part of the Energy Information Administration's (EIA) mission to provide meaningful data, the consumption surveys welcome comments from our customers. If you have any suggestions to make the data in this report more useful to your needs, please contact John L. Preston, MECS Manager, at 202-586-1128, FAX or Internet at the address below.

If you have any data or report-related requirements or suggestions for any of the other ELA consumption surveys, please contact the appropriate survey manager directly, or use the address below.

Commercial Buildings Energy Consumption Survey (CBECS): Martha Johnson, Survey Manager, at 202586-1135 (Internet: MJOHNSON@EIA.DOE.GOV).

Residential Energy Consumption Survey (RECS): Wendel Thompson, Survey Manager, at 202-586-1119 (Internet: WTHOMPSO@EIA.DOE.GOV).

Residential Transportation Energy Consumption Survey (RTECS): Ron Lambrecht, Survey Manager, at 202586-4962 (Internet: RLAMBREC@EIA.DOE.GOV).

You are encouraged to provide your comments to the survey managers. Your feedback is important to us.

EI-63, Mail Stop 2G-090

1000 Independence Avenue, SW

Washington, DC 20585

FAX: 202-586-0018

Internet: JPRESTON@EIA.DOE.GOV 


\section{Contents}

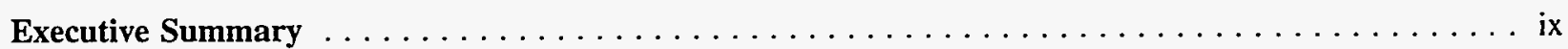

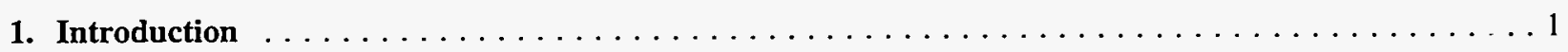

Manufacturing Energy Consumption Surveys $\ldots \ldots \ldots \ldots \ldots \ldots \ldots \ldots \ldots \ldots \ldots \ldots \ldots \ldots \ldots \ldots \ldots \ldots \ldots \ldots$

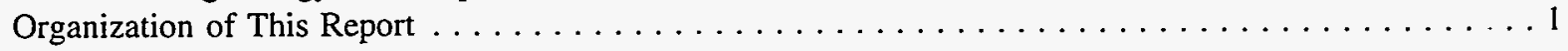

2. Energy Consumption in the Manufacturing Sector: An Overview $\ldots \ldots \ldots \ldots \ldots \ldots \ldots \ldots \ldots$

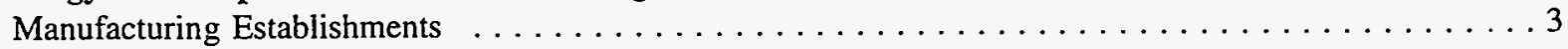

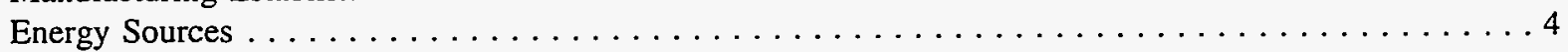

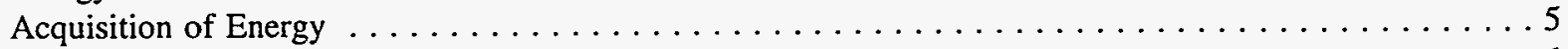

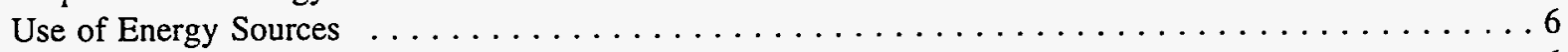

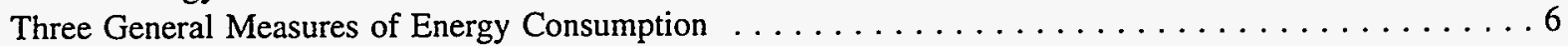

Specific Measures of Energy Consumption . . . . . . . . . . . . . . . . . . . . .

Summary of Manufacturing Energy Throughput, $1991 \ldots \ldots \ldots \ldots \ldots \ldots \ldots \ldots \ldots \ldots \ldots$

3. Energy Consumption in the Manufacturing Sector, $1991 \ldots \ldots \ldots \ldots \ldots \ldots \ldots \ldots \ldots \ldots$

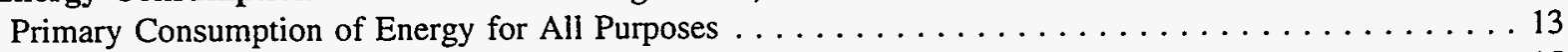

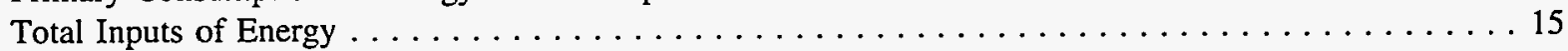

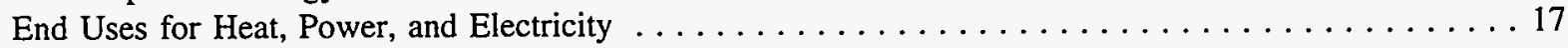

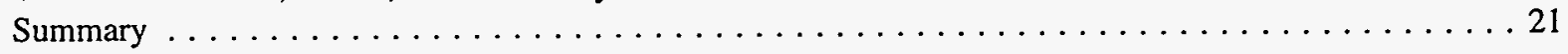

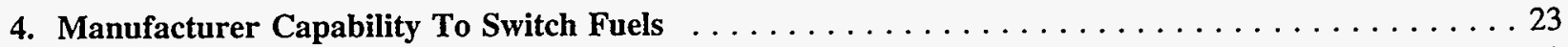

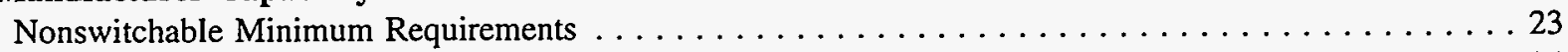

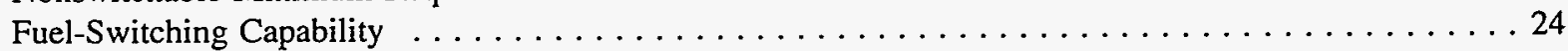

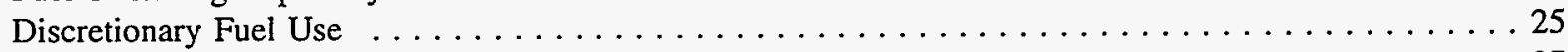

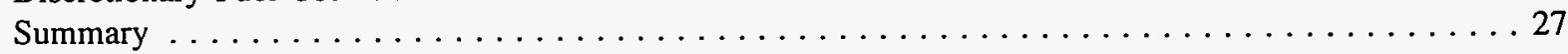

Appendices

A. Detailed Tables . . . . . . . . . . . . . . . . . . . . . . . . . 29

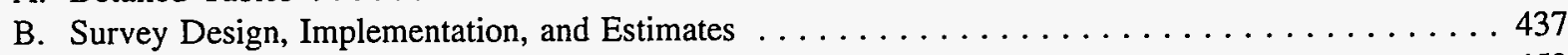

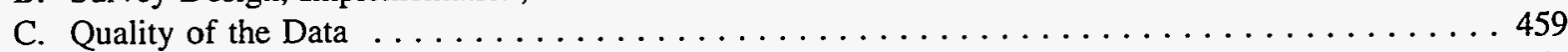

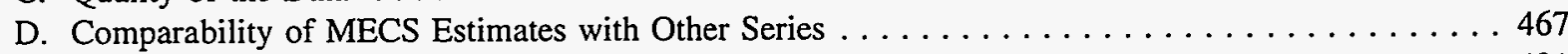

E. MECS Estimates by International Standard Industrial Classification Codes $\ldots \ldots \ldots \ldots \ldots .481$

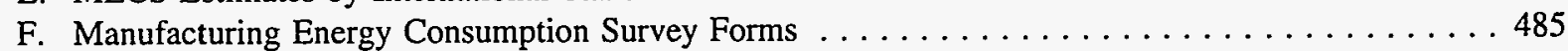

G. Descriptions of Major Industrial Groups and Selected Industries $\ldots \ldots \ldots \ldots \ldots \ldots \ldots \ldots \ldots 11$

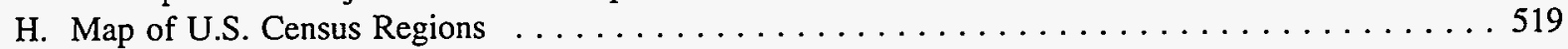

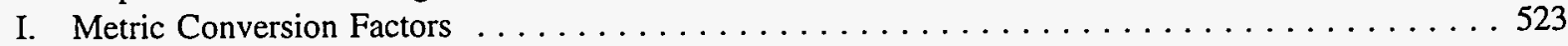

J. Related EIA Publications on Energy Consumption $\ldots \ldots \ldots \ldots \ldots \ldots \ldots \ldots \ldots \ldots \ldots \ldots \ldots \ldots \ldots \ldots$

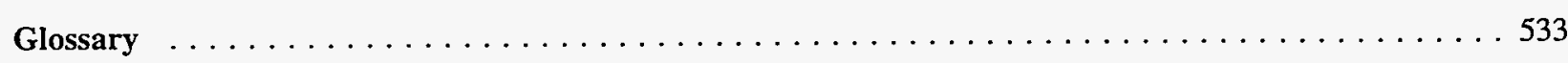




\section{Tables}

Page

A1. Total Primary Consumption of Energy for All Purposes by Census Region, Industry Group, and Selected

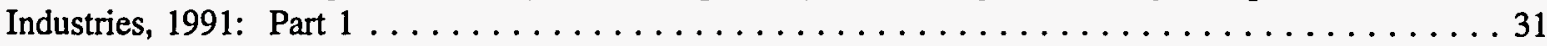

A1. Total Primary Consumption of Energy for All Purposes by Census Region, Industry Group, and Selected

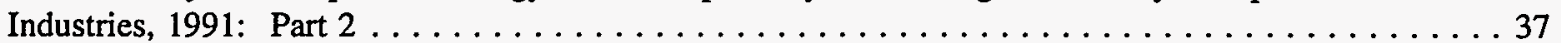

A2. Total Consumption of LPG, Distillate Fuel Oil, and Residual Fuel Oil for Selected Purposes by Census Region, Industry Group, and Selected Industries, $1991 \ldots \ldots \ldots \ldots \ldots \ldots \ldots \ldots \ldots \ldots \ldots$

A3. Total Primary Consumption of Combustible Energy for Nonfuel Purposes by Census Region, Industry Group, and Selected Industries, 1991: Part $1 \ldots \ldots \ldots \ldots \ldots \ldots \ldots \ldots \ldots \ldots \ldots \ldots \ldots$

A3. Total Primary Consumption of Combustible Energy for Nonfuel Purposes by Census Region, Industry

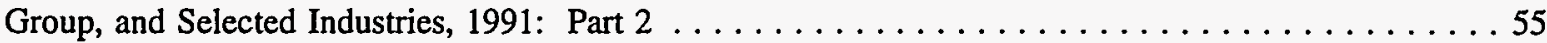

A4. Total Inputs of Energy for Heat, Power, and Electricity Generation by Census Region, Industry Group,

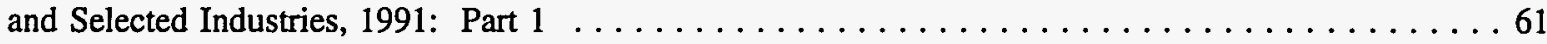

A4. Total Inputs of Energy for Heat, Power, and Electricity Generation by Census Region, Industry Group,

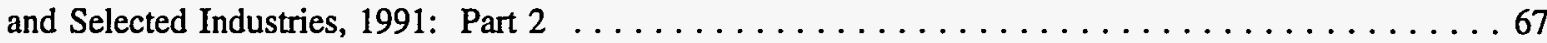

A5. Total Consumption of Offsite-Produced Energy for Heat, Power, and Electricity Generation by Census Region, Industry Group, and Selected Industries, 1991: Part $1 \ldots \ldots \ldots \ldots \ldots \ldots \ldots \ldots$

A5. Total Consumption of Offsite-Produced Energy for Heat, Power, and Electricity Generation by Census Region, Industry Group, and Selected Industries, 1991: Part $2 \ldots \ldots \ldots \ldots \ldots \ldots \ldots$

A6. Total Inputs of Selected Byproduct Energy for Heat, Power, and Electricity Generation by Census

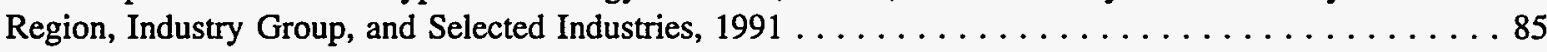

A7. Shell Storage Capacity of Selected Petroleum Products by Census Region, Industry Group, and Selected

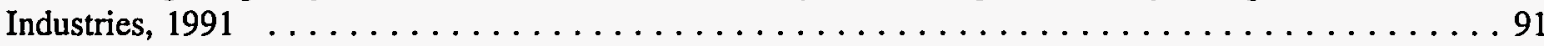

A8. Selected Energy Operating Ratios for Total Energy Consumption for Heat, Power, and Electricity Generation by Census Region, Industry Group, and Selected Industries, $1991 \ldots \ldots \ldots \ldots \ldots$

A9. Total Primary Consumption of Energy for All Purposes by Census Region and Economic Characteristics

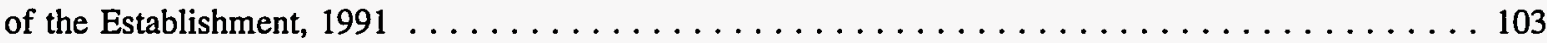

A10. Total Consumption of LPG, Distillate Fuel Oil, and Residual Fuel Oil for Selected Purposes by Census Region and Economic Characteristics of the Establishment, $1991 \ldots \ldots \ldots \ldots \ldots \ldots \ldots \ldots$

A11. Total Primary Consumption of Combustible Energy for Nonfuel Purposes by Census Region and Economic Characteristics of the Establishment, 1991 . . . . . . . . . . . . . . . . . . 109

A12. Total Inputs of Energy for Heat, Power, and Electricity Generation by Census Region and Economic

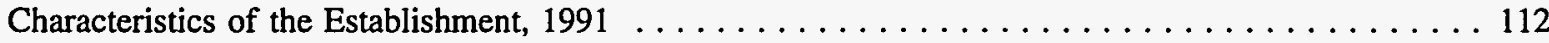

A13. Total Consumption of Offsite-Produced Energy for Heat, Power, and Electricity Generation by Census Region and Economic Characteristics of the Establishment, $1991 \ldots \ldots \ldots \ldots \ldots \ldots \ldots \ldots$

A14. Shell Storage Capacity of Petroleum Products by Census Region and Economic Characteristics of the

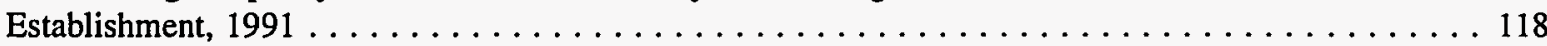

A15. Selected Energy Operating Ratios for Total Energy Consumption for Heat, Power, and Electricity Generation by Census Region and Economic Characteristics of the Establishment, 1991 . . . . . . 121

A16. Components of Total Electricity Demand by Census Region, Industry Group, and Selected Industries,

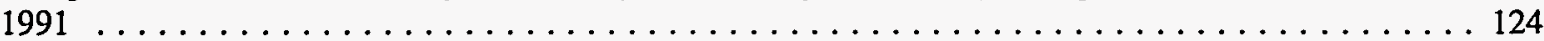

A17. Components of Onsite Electricity Generation by Census Region, Industry Group, and Selected Industries,

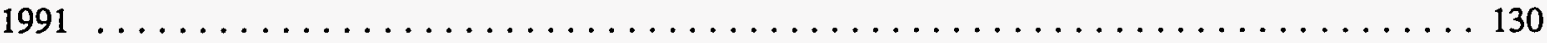

A18. Quantity of Electricity Sold to Utility and Nonutility Purchasers by Census Region, Industry Group, and

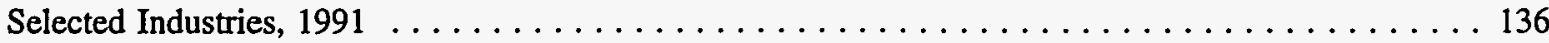

A19. Components of Total Electricity Demand by Census Region and Economic Characteristics of the

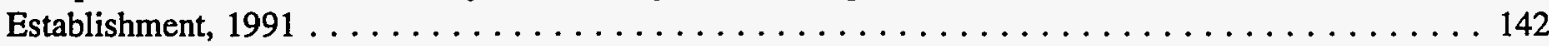

A20. Components of Onsite Electricity Generation by Census Region and Economic Characteristics of the

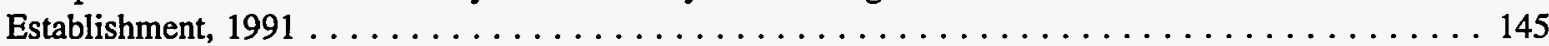

A21. Quantity of Electricity Sold to Utility and Nonutility Purchasers by Census Region and Economic

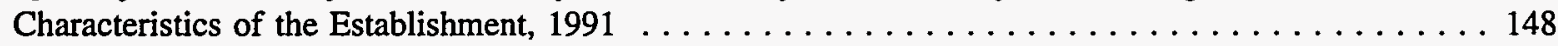




\section{Tables (Continued)}

A22. Total Quantity of Purchased Energy Sources by Census Region, Industry Group, and Selected Industries, 1991

A23. Quantity of Purchased Electricity, Steam, and Natural Gas by Type of Supplier, Census Region, Industry

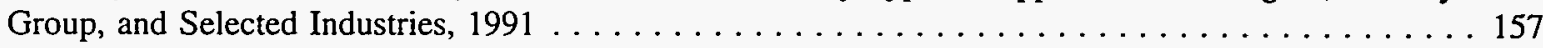

A24. Total Expenditures for Purchased Energy Sources by Census Region, Industry Group, and Selected

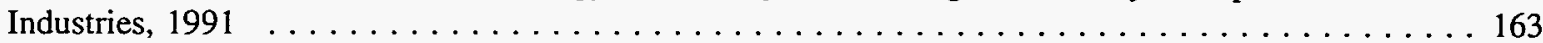

A25. Average Prices of Selected Purchased Energy Sources by Census Region, Industry Group, and Selected Industries, 1991: Part 1 . . . . . . . . . . . . . . . . . . . . . . . . . . . . . . . . . 169

A25. Average Prices of Selected Pürchased Energy Sources by Census Region, Industry Group, and Selected

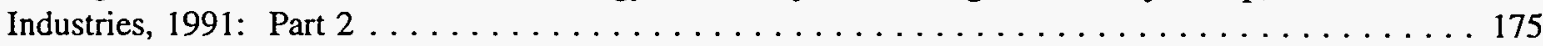

A26. Total Quantity of Purchased Energy Sources by Census Region and Economic Characteristics of the

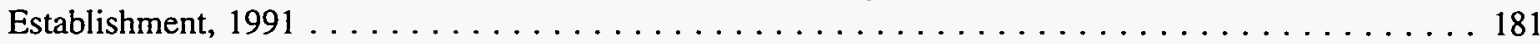

A27. Quantity of Purchased Electricity, Steam, and Natural Gas by Type of Supplier, Census Region, and Economic Characteristics of the Establishment, $1991 \ldots \ldots \ldots \ldots \ldots \ldots \ldots \ldots \ldots \ldots \ldots \ldots$

A28. Total Expenditures for Purchased Energy Sources by Census Region and Economic Characteristics of

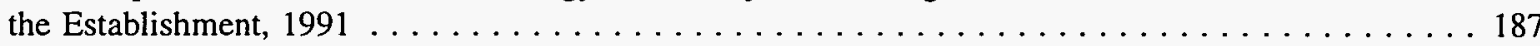

A29. Average Prices of Selected Purchased Energy Sources by Census Region and Economic Characteristics

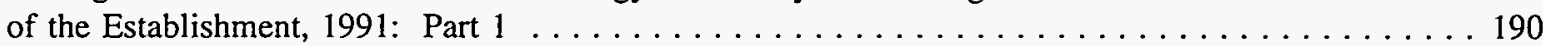

A29. Average Prices of Selected Purchased Energy Sources by Census Region and Economic Characteristics

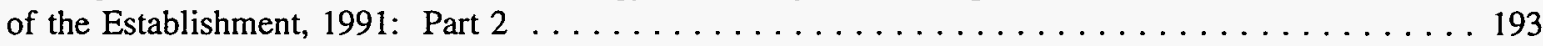

A30. Total Primary Consumption of Energy for All Purposes by Value of Shipment Categories, Industry

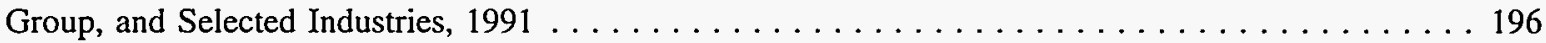

A31. Total Inputs of Energy for Heat, Power, and Electricity Generation by Value of Shipment Categories,

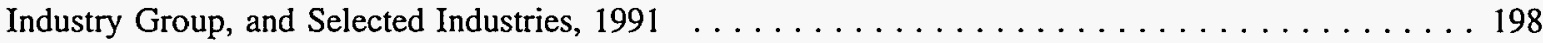

A32. Total Consumption of Offsite-Produced Energy for Heat, Power, and Electricity Generation by Value of Shipment Categories, Industry Group, and Selected Industries, $1991 \ldots \ldots \ldots \ldots \ldots \ldots$

A33. Total Primary Consumption of Energy for All Purposes by Employment Size Categories, Industry Group,

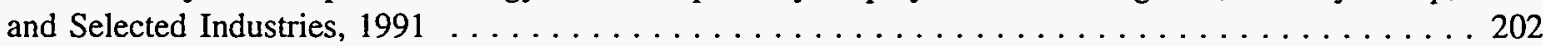

A34. Total Inputs of Energy for Heat, Power, and Electricity Generation by Employment Size Categories,

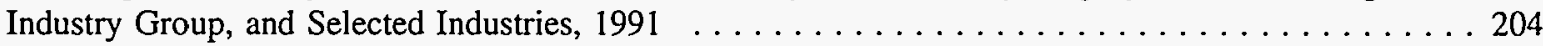

A35. Total Consumption of Offsite-Produced Energy for Heat, Power, and Electricity Generation by Employment Size Categories, Industry Group, and Selected Industries, 1991 . . . . . . . . . 206

A36. Total Inputs of Energy for Heat, Power, and Electricity Generation by Fuel Type, Industry Group,

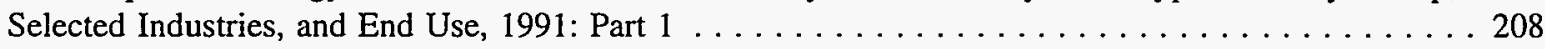

A36. Total Inputs of Energy for Heat, Power, and Electricity Generation by Fuel Type, Industry Group,

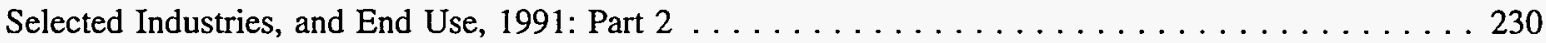

A37. Total Inputs of Energy for Heat, Power, and Electricity Generation by Fuel Type, Census Region, and

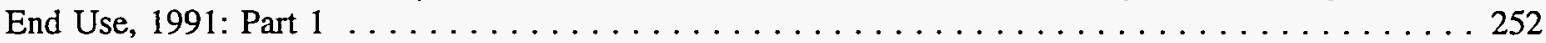

A37. Total Inputs of Energy for Heat, Power, and Electricity Generation by Fuel Type, Census Region, and

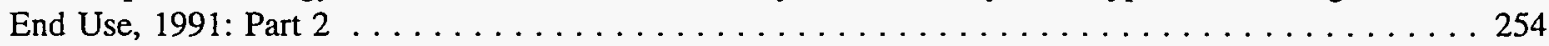

A38. Selected Combustible Inputs of Energy for Heat, Power, and Electricity Generation and Net Demand

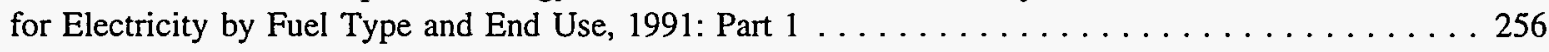

A38. Selected Combustible Inputs of Energy for Heat, Power, and Electricity Generation and Net Demand for Electricity by Fuel Type and End Use, 1991: Part $2 \ldots \ldots \ldots \ldots \ldots \ldots \ldots \ldots \ldots$

A39. Selected Combustible Inputs of Energy for Heat, Power, and Electricity Generation and Net Demand for Electricity by Fuel Type, Census Region, and End Use, 1991: Part $1 \ldots \ldots \ldots \ldots \ldots$

A39. Selected Combustible Inputs of Energy for Heat, Power, and Electricity Generation and Net Demand for Electricity by Fuel Type, Census Region, and End Use, 1991: Part $2 \ldots \ldots \ldots \ldots \ldots$ 


\section{Tables (Continued)}

A40. Total Inputs of Energy for Heat, Power, and Electricity Generation by Energy Management Program Sponsorship, Industry Group, Selected Industries, and Type of Energy Management Program, 1991 . . . . . . . . . . . . . . . . . . . . . . . . . . . . . . 304

A41. Total Inputs of Energy for Heat, Power, and Electricity Generation by Census Region, Industry Group, Selected Industries, and Type of Energy Management Program, 1991 . . . . . . . . . 326

A42. Total Inputs of Energy for Heat, Power, and Electricity Generation by Employment Size and Presence

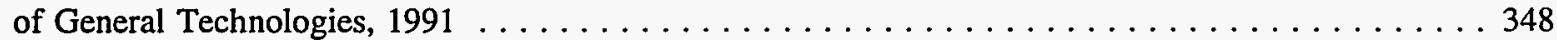

A43. Total Inputs of Energy for Heat, Power, and Electricity Generation by Value of Shipments and Presence

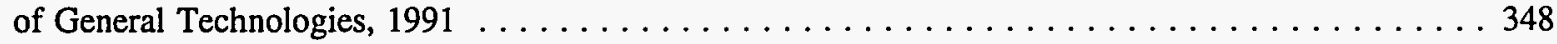

A44. Total Inputs of Energy for Heat, Power, and Electricity Generation by Census Region, Industry Group, Selected Industries, Presence of General Technologies, and Industry-Specific Technologies for Selected

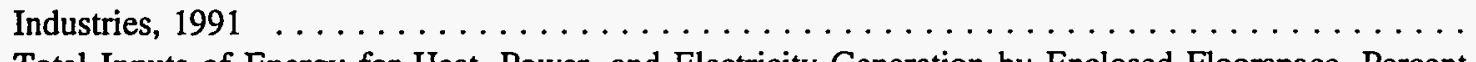

A45. Total Inputs of Energy for Heat, Power, and Electricity Generation by Enclosed Floorspace, Percent Conditioned Floorspace, and Presence of Computer Controls for Building Environment, 1991 . . . 376

A46. Total Expenditures for Purchased Electricity, Steam, and Natural Gas by Type of Supplier, Census

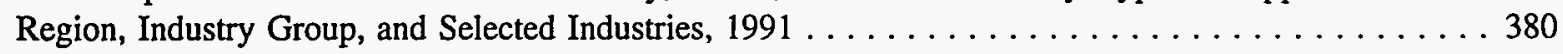

A47. Average Prices of Purchased Electricity, Steam, and Natural Gas by Type of Supplier, Census Region,

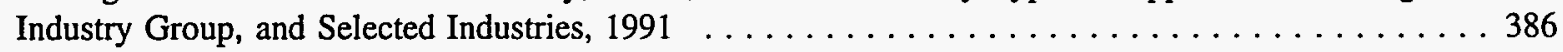

A48. Total Expenditures for Purchased Electricity, Steam, and Natural Gas by Type of Supplier, Census Region, and Economic Characteristics of the Establishment, 1991 . . . . . . . . . . . . . . 392

A49. Average Prices of Purchased Electricity, Steam, and Natural Gas by Type of Supplier, Census Region,

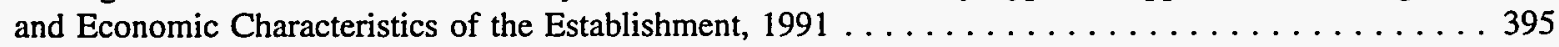

A50. Selected Energy Operating Ratios for Total Energy Consumption for Heat, Power, and Electricity Generation by Census Region and Economic Characteristics of the Establishment, 1991 . . . . . 398

A51. Selected Energy Operating Ratios for Total Energy Consumption for Heat, Power, and Electricity Generation by Census Region and Economic Characteristics of the Establishment, 1991 . . . . . 411

A52. Nonswitchable Minimum Requirements and Maximum Consumption Potential by Census Region,

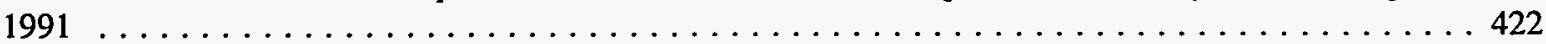

A53. Capability to Switch from Electricity to Alternative Energy Sources by Industry Group, Selected

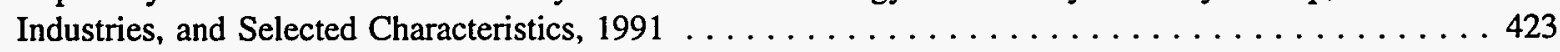

A54. Capability to Switch from Natural Gas to Alternative Energy Sources by Industry Group, Selected

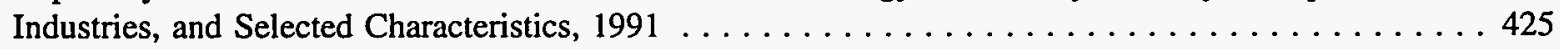

A55. Capability to Switch from Distillate Fuel Oil to Alternative Energy Sources by Industry Group, Selected

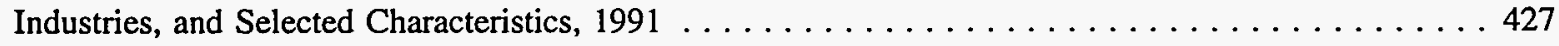

A56. Capability to Switch from Residual Fuel Oil to Alternative Energy Sources by Industry Group, Selected

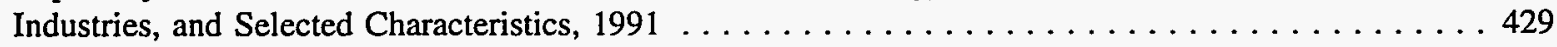

A57. Capability to Switch from Coal to Alternative Energy Sources by Industry Group, Selected Industries,

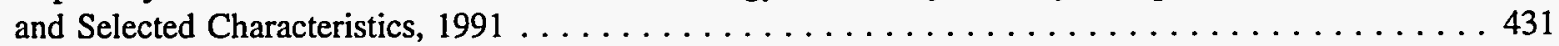

A58. Capability to Switch from LPG to Alternative Energy Sources by Industry Group, Selected Industries,

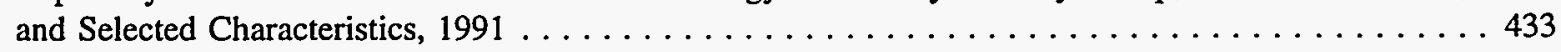

B1. Total Shipments of Energy Sources Produced Onsite from the Nonfuel Use of Other Energy Sources, by Industry Group and Selected Industries, $1991 \ldots \ldots \ldots \ldots \ldots \ldots \ldots \ldots \ldots \ldots \ldots \ldots \ldots \ldots$

B2. Conversion of Physical Units to British Thermal Units $\ldots \ldots \ldots \ldots \ldots \ldots \ldots \ldots \ldots \ldots \ldots$

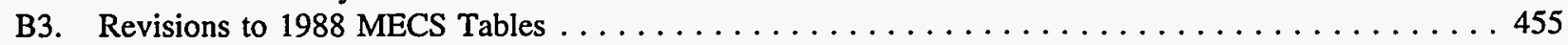

D1. Combined Industrial Energy Consumption Estimates and Sources of Information, $1991 \ldots \ldots \ldots$. 469

D2. A Comparison of the Components of MECS and SEDR Estimates of Energy Consumption . . . . . 471

E1. Manufacturing Consumption of Energy for Purposes of Heat, Power, and Electricity Generation by International Standard Industrial Classification Codes, $1991 \ldots \ldots \ldots \ldots \ldots \ldots \ldots \ldots \ldots \ldots \ldots$

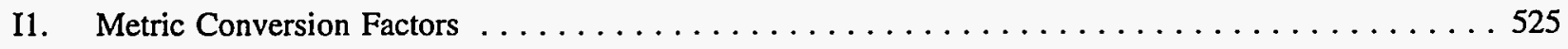




\section{Illustrations}

Figure 2.1. Largest Energy Consumers in the Manufacturing Sector, $1991 \ldots \ldots \ldots \ldots \ldots \ldots \ldots$

Figure 2.2. Summary of Manufacturing Energy Throughput, $1991 \ldots \ldots \ldots \ldots \ldots \ldots \ldots \ldots \ldots$

Figure 3.1. Primary Consumption of Energy for All Purposes by Major Industry Group, $1991 \ldots \ldots \ldots \ldots$

Figure 3.2. Primary Consumption of Energy for All Purposes by Energy Source, $1991 \ldots \ldots \ldots \ldots \ldots 14$

Figure 3.3 Shares of Primary Consumption in Terms of Site and Embodied Electricity, $1991 \ldots \ldots \ldots . . .14$

Figure 3.4 Total Input Energy for Heat, Power, and Electricity Generation, $1991 \ldots \ldots \ldots \ldots \ldots \ldots$

Figure 3.5 Total Input Energy for Heat, Power, and Electricity Generation

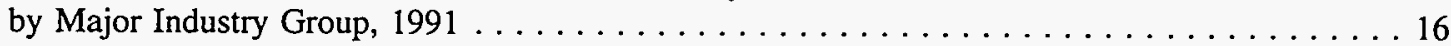

Figure 3.6 Byproduct Fuels Consumed by Manufacturers by Energy Source, $1991 \ldots \ldots \ldots \ldots \ldots \ldots$

Figure 3.7 Consumption of Energy for Heat, Power, and Electricity Generation

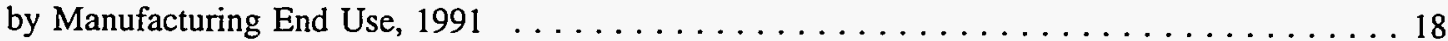

Figure 3.8 Consumption of Energy for Heat, Power, and Electricity Generation

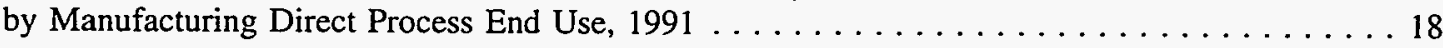

Figure 3.9 Consumption of Energy for Heat, Power, and Electricity Generation by Manufacturing Direct Non-Process End Use, 1991 . . . . . . . . . . . . . . . . 19

Figure 3.10 Consumption of Energy for Heat, Power, and Electricity Generation

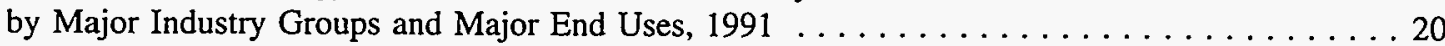

Figure 3.11 Unreported End-Use Fuels Consumed for Heat, Power, and Electricity Generation, 1991 . . . . . 20

Figure 4.1 Nonswitchable Minimum Requirements for Selected Fuels, $1991 \ldots \ldots \ldots \ldots \ldots \ldots \ldots$

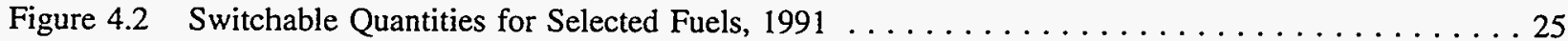

Figure 4.3 Manufacturers' Discretionary Ranges of Consumption

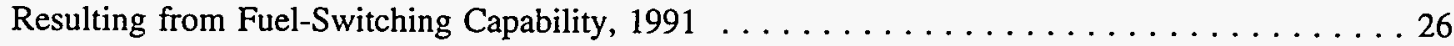

Figure 4.4. Discretionary Use Rates by Selected Fuels, $1991 \ldots \ldots \ldots \ldots \ldots \ldots \ldots \ldots \ldots \ldots \ldots \ldots$

Figure 4.5. Average National Prices of Selected Purchased Fuels, $1991 \ldots \ldots \ldots \ldots \ldots \ldots \ldots \ldots$ 


\section{Executive Summary}

The Manufacturing Consumption of Energy 1991 report presents statistics about the energy consumption of the manufacturing sector, based on the 1991 Manufacturing Energy Consumption Survey (MECS). The MECS is the only comprehensive source of national-level data on U.S. manufacturing energy use. The 1991 MECS is the third in an ongoing series of surveys conducted at 3-year intervals beginning in 1985. Pursuant to a provision of the Energy Policy Act of 1992, the MECS will be conducted biennially beginning in 1994.

The MECS surveys a nationally representative sample of manufacturing establishments by means of mailed questionnaires. The 1991 sample represented 98 percent of the U.S. manufacturing sector universe, which consists of all manufacturing establishments in the 50 States and the District of Columbia. Compared with the 1988 MECS, the designed sample size for 1991 was increased from 12,065 manufacturing establishments to 16,054 establishments.

The following are some of the key findings of the survey:

- The Primary Consumption of Energy for All Purposes in the manufacturing sector was 20.3 quadrillion Btu of energy in 1991, compared with 20.1 quadrillion Btu in 1988. About two-thirds of this amount was used to produce heat and power and to generate electricity, with about one-third being consumed as raw material input.

- The most common energy sources-natural gas, net electricity, coal, liquefied petroleum gas (LPG), residual and distillate fuel oil, and coke-made up 64 percent of Primary Consumption.

- The amount of Total Inputs of Energy for Heat, Power, and Electricity Generation was 15.0 quadrillion Btu, compared with 15.5 quadrillion Btu in 1988.

- The byproduct fuels-blast furnace gas, waste gas, petroleum coke, pulping liquor, wood byproducts, and waste oils/materials-made up 28 percent of Total Inputs.

- End-use allocation was collected for about 65 percent of Total Inputs ( 9.7 quadrillion Btu). This consumption was allocated to three groups of end uses: indirect uses, mainly boiler fuel (3.3 quadrillion Btu); direct process uses, including use in motors, ovens, strip heaters, and kilns (5.0 quadrillion Btu); and direct non-process uses, including facility space-conditioning, facility lighting, other facility support, onsite transportation, electricity generation, and other miscellaneous uses (1.2 quadrillion Btu). Manufacturers consumed an additional 5.3 quadrillion Btu of energy for which end uses were not assigned. (The 1991 MECS collected data on end-use allocation for the first time.)

- Manufacturers had the capability to switch 2.8 quadrillion Btu of all the electricity, natural gas, distillate and residual fuel oils, coal, and LPG they used, representing 28 percent of their actual consumption. (Manufacturers are said to have a fuel-switching capability if they are able to meet their requirements for heat, power, and electricity generation by substituting one energy source for another within 30 days without modifying the equipment that consumes the fuel and if they can resume the same level of production following the switch.)

- Manufacturers required an nonswitchable minimum of 7.0 quadrillion Btu of these energy sources, which was 72 percent of their actual consumption of these fuels. The fuels having the highest nonswitchable minimum requirements were, in order, natural gas, electricity, and coal.

- Manufacturers often used coal and natural gas whenever possible, even though they could have used another energy source instead. Price was apparently the primary reason that manufacturers switched energy sources whenever they were able to do so. 


\section{Introduction}

This report, Manufacturing Consumption of Energy 1991, provides estimates on energy consumption in the manufacturing sector of the U.S. economy. These estimates are based on data from the 1991 Manufacturing Energy Consumption Survey (MECS). This survey_administered by the Energy End Use and Integrated Statistics Division, Office of Energy Markets and End Use, Energy Information Administration (EIA)-is the most comprehensive source of national-level data on energy-related information for the manufacturing industries.

\section{Manufacturing Energy Consumption Surveys}

To determine how energy is being used in the manufacturing sector, EIA mails Forms $846 \mathrm{~A}$ through $\mathrm{C}$ to a nationally representative sample of the establishments in the 50 States and the District of Columbia that transform input materials or substances into new products, assemble components, or perform blending operations. ${ }^{1}$ In 1991, these establishments numbered approximately 350 thousand, and the MECS sample represented 98 percent of energy use in the U.S. manufacturing sector.

The 1991 MECS is EIA's third survey of the manufacturing sector. Previous manufacturing surveys were conducted in 1986 and 1989 (for reporting years 1985 and 1988, respectively). The next manufacturing survey will be conducted for reporting year 1994, with subsequent MECS's being conducted every 2 years thereafter.

The sample design of the 1991 and 1985 surveys differed somewhat from that of the 1988 survey, which necessitates that care be exercised when comparing estimates from the three surveys. The 1988 sample represented 100 percent of manufacturing energy use, but it included statistical adjustments to account for 2 percent of the population, namely the smallest manufacturing establishments. The 1991 and 1985 samples represented 98 percent of the population, without any statistical adjustment.

EIA conducts the MECS under the authority of the Federal Energy Administration Act of 1974, Public Law 93-275, as amended, and Section 205 of the Department of Energy Organization Act, Public Law 95-91, as amended by Section 3102 of the Omnibus Budget Reconciliation Act of 1986, Public Law 99-509. ${ }^{2}$ Section 171 of the Energy Policy Act of 1992 mandates the MECS as a biennial collection.

The Industry Division of the Bureau of the Census collects the data and compiles it for EIA. All data reported to the Bureau of the Census are confidential under the provisions of Section 9, Title 13, of the U.S. Code. EIA gratefully acknowledges the cooperation of the respondents in supplying the information used to produce the estimates in this report.

\section{Organization of This Report}

This introductory chapter is followed by a chapter giving an overall understanding of energy consumption in the manufacturing sector, a chapter on some of the more important results of the 1991 MECS on energy consumption and on manufacturing end uses, and a chapter on fuel-switching in the manufacturing sector. Data on manufacturing energy consumption by end use were collected for the first time in the 1991 MECS. This MECS report is the first to include fuel-switching data, which were published under separate cover for previous survey years.

\footnotetext{
'The manufacturing sector is composed of establishments classified in SIC 20 through SIC 39 of the U.S. economy as defined by the Office of Management and Budget (OMB). The manufacturing sector is a part of the industrial division, which also includes mining; construction; and agriculture, forestry, and fishing.

${ }^{2}$ The EIA also conducts energy consumption surveys in the residential, residential transportation, and commercial buildings sectors. These surveys are the Residential Energy Consumption Survey (RECS), the Residential Transportation Energy Consumption Survey (RTECS), and the Commercial Buildings Energy Consumption Survey (CBECS).
} 
The four chapters are followed by several technical appendices:

- Appendix A presents detailed statistical tables, providing measures of data reliability (Relative Standard Errors) as factors in the rows and columns of the data tables rather than separately as in previous reports. To better serve the user community, these tables were released to the public in May 1994 through EIA's electronic bulletin board system.

- Appendix B explains the sample design of and the estimation and implementation procedures for the MECS.

- Appendix $\mathrm{C}$ discusses the quality of the data.

- Appendix D compares MECS estimates with those provided by other sources.

- Appendix E presents one of the MECS consumption measures according to international classification codes.

- Appendix F reproduces Forms EIA 846 A through $C$, which were used to collect the data on which all estimates in this report are based, unless otherwise noted.

- Appendix $G$ describes the major industrial groups and selected industries.

- Appendix H provides a map of the U.S. Census Regions.

A Guide to the Tables in This Report

Energy Consumption

Storage Capacity

Energy Operating Ratios

Electricity Demand

Electricity Generation

Electricity Sales

Purchased Energy

Prices and Expenditures

Consumption by:

End Use

Energy Management

Technology

Fuel-Switching
Tables A1-A6, A9-A13, A30-A36

Tables A7, A14

Tables A8, A15, A50, A51

Tables A16, A19

Tables A17, A20

Tables A18, A21

Tables A22, A23, A26, A27

Tables A24, A25, A28, A29, A46-A49

Tables A37-A39

Tables A40, A41

Tables A42-A45

Tables A52-A58

- Appendix I presents metric conversion factors.

- Appendix $J$ lists related energy consumption publications for readers interested in earlier MECS publications or consumption reports for other sectors.

These appendices are followed by a glossary of statistical and engineering terms used in this report. 


\section{Energy Consumption in the Manufacturing Sector: An Overview}

The manufacturing sector of the U.S. economy is composed of approximately 350 thousand establishments. However, the number of establishments is of less significance for the MECS than their share of total energy consumption because these establishments differ so dramatically from each other in their processes and products.

The energy-consuming characteristics of the manufacturing sector differ from those of the residential, commercial building, and residential vehicle sectors in the energy sources manufacturers use, the ways in which they acquire that energy, and the ways in which they use that energy. These complexities require complex measurement techniques.

\section{Manufacturing Establishments}

A manufacturing establishment is an economic unit at a single physical location where the mechanical or chemical transformation of materials or substances into new products is performed. ${ }^{3}$ These operations are generally conducted in facilities described as plants, factories, or mills and characteristically use power-driven machines and materialhandling equipment. Manufacturing also includes such activities as the assembly of components of manufactured products and the blending of materials such as lubricating oil, plastics, resins, or liquors.

An establishment is not necessarily identical to a business concern or firm, either of which may consist of more than one establishment. For example, an automobile manufacturer may be a firm having over 200 establishments, such as stamping plants, auto assembly factories, and gear and axle plants. On the other hand, an automobile manufacturer may be a firm having one establishment, with components coming from several other firms.

Manufacturing establishments fall into the industrial categories listed in the 1987 Standard Industrial Classification (SIC) system, developed by the Office of Management and Budget. ${ }^{4}$ That system underlies all establishment-based Federal statistics classified by industry. The SIC system is used to promote the comparability of establishment data describing various facets of the U.S. economy. The levels of classification in the SIC are division, major group, industry group, and industry. The manufacturing sector contains 20 major groups (SIC 20 through SIC 39), 139 industry groups, and 459 industries. Manufacturing establishments are classified into industries based on the value of production of their primary products. See Appendix $G$ for a full description of manufacturing groups and industries used in this report.

Of the 10 largest energy consumers in the manufacturing sector, the two largest were the: (1) petroleum and coal and (2) chemicals and allied products major industry groups (Figure 2.1). Figure 2.1. Largest Energy Consumers in the
Manufacturing Sector, 1991

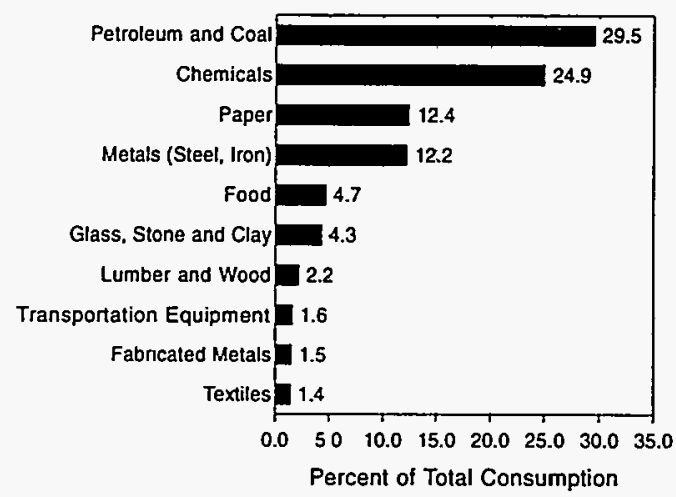

Sourse: Tato Al of this repont.

\footnotetext{
${ }^{3}$ Excluded from this definition of manufacturing establishment are construction, electric utilities, mining operations, agricultural production, and forestry and fishery operations.

${ }^{4}$ Office of Management and Budget, Standard Industrial Classification Manual, 1987 (Washington, DC, 1987).
} 


\section{Energy Sources}

Manufacturers consume large quantities of combustible and noncombustible energy sources. These energy sources include such commonly known ones as natural gas, electricity, coal, residual and distillate fuel oils, and liquefied petroleum gases (LPG), as well as a wide variety of uncommon energy sources. Moreover, some materials, which might be thought of as energy sources, are not counted as energy sources in the manufacturing sector.

Examples of energy sources consumed in the manufacturing sector but not in other sectors of the economy include:

- Pulping liquor, or "black liquor," produced during the transformation of wood to pulp in the Pulp and Paper Industry

- Petroleum coke and still gas resulting from the production of petroleum products in refineries

- Agricultural waste (like rice hulls and orchard prunings), packing crates, discarded tires, and other byproducts and waste materials

- Energy sources that manufacturers produce themselves, such as electricity generated from hydropower, wind power, solar power, and geothermal sources.

On the other hand, materials that are often thought of as energy sources in other sectors are not counted as such in the manufacturing sector. For example, wood consumed to produce paper, build furniture, or manufacture lumber is excluded as an energy source in the MECS. (However, wood that is consumed to produce heat and power is counted as an energy source.)

A further anomaly occurs in petroleum refining in accounting for crude oil. Petroleum refiners are treated differently from other manufacturing establishments in the MECS because their major economic activity is transforming crude oil and other inputs into a wide variety of products. Most of the major products, such as distillate and residual fuel oils, motor gasoline, kerosene, and LPG, are universally recognized and accounted for by consumers as energy sources. However, some of these products, such as asphalt, road oils, wax, lubricating oils, solvents, and specialized petrochemical feedstocks, are not considered to be energy sources.

\section{Energy Sources Used in the Manufacturing Sector}

Combustible

Solids

Anthracite

Bituminous and

subbituminous coal

Coal coke

Lignite

Agricultural waste

Petroleum coke

Roundwood (wood cut specifically for fuel use)

Waste materials (wastepaper, packing materials, etc.)

Wood chips, bark, and waste

Other solids

Gases

Natural gas

Acetylene

Blast furnace gas

Coke oven gas

Hydrogen

Waste and byproduct gases (refinery gas, offgas, fuel gas, vent gas, plant gas, and still gas)

Other gases

\section{Liquids}

Distillate fuel oil

(numbers 1, 2, and 4 fuel oils and diesel)

Kerosene

LPG (ethane, ethylene, propane, propylene, butane, and butylene)

Motor gasoline

Pulping or black liquor

Residual fuel oil (numbers 5, 6, navy special, and bunker $c$ )

Waste oils and tars

Other liquids 
The MECS does not count crude oil inputs as consumption in refineries because doing so would duplicate the consumption of the refinery energy products (like gasoline and LPG) that other sectors report. However, the MECS does count the crude oil input to nonenergy products (like asphalt and road oil) because they will not be reported by other sectors. For more information, see Appendix B, "Survey Design, Implementation, and Estimates."

\section{Acquisition of Energy}

Energy sources may arrive at an establishment from offsite, or they may be produced onsite. Energy sources produced offsite arrive at an establishment in one of several ways. They may be:

- Purchased directly from an energy supplier and paid for by the consuming establishment

- Purchased and paid for by a central purchasing entity separate from the establishment but delivered directly to the establishment

- Purchased by and transferred from another establishment within the consuming establishment's company

- Delivered from another establishment within a consuming establishment's company, with the consuming establishment being charged for the energy consistent with the company's accounting policy

- Paid for in-kind.

For example, manufacturing establishments usually purchase electricity directly from utilities as other consumers do. In some situations, however, several establishments of the same corporation and physically located in the same geographic area may pool their electricity purchases by having one of the establishments serve as the "central purchaser." In this case, the electricity is transferred from the central purchasing authority to the consuming establishment. Some manufacturers also purchase steam and hot water from a nearby manufacturer or transfer it from an establishment within the same corporation.

Manufacturers produce some of the energy they use onsite, as a byproduct of a manufacturing process, from onsite coal mines or gas or oil wells, and through the generation of electricity. Coke oven gas, which is produced as a byproduct when manufacturing coke from coal, is one example of energy being produced as a byproduct of a manufacturing process. A second example is hydrogen, which is produced as a byproduct when the electrolysis of brine (salt water, a nonenergy material) produces chlorine and caustic soda as the main products. Both coke oven gas and hydrogen are used as energy sources.

Manufacturers generate electricity onsite in three ways:

- Cogeneration (the production of electrical energy and another form of useful energy, such as steam, through the sequential use of energy)

- Conventional generation using combustible fuels

- Generation using solar power, wind power, hydropower, and geothermal sources. 


\section{Use of Energy Sources}

Manufacturers use energy sources in two major ways. The most widely understood use is to produce heat and power and to generate electricity. Heat, power, and electricity consumption can be subdivided into three major end uses: indirect use (boiler fuel), direct process uses, and direct non-process uses. ${ }^{5}$ Boiler fuel is used to transform one source of energy into another (like steam). Direct process uses include energy for motors, ovens, strip heaters, and kilns. Direct non-process uses include facility lighting and space-conditioning equipment.

The second way in which manufacturers use energy is as a raw material input to the manufacturing process or for some other purpose other than for heat, power, and electricity generation. ${ }^{6}$ (These purposes are referred to as "nonfuel uses" in this report.) For example, coal is consumed to produce coal coke. Also, natural gas is processed to extract ethane, propane, and butane. These gases, in turn, are frequently used as raw material input to produce fertilizers, pharmaceutical preparations, and other products.

\section{Three General Measures of Energy Consumption}

The MECS uses three general measures of energy consumption in the manufacturing sector: (1) Primary Consumption of Energy for All Purposes, (2) Total Inputs of Energy for Heat, Power, and Electricity Generation, and (3) Consumption of Offsite-Produced Energy for Heat, Power, and Electricity Generation. These measures are used for different purposes:

- The Primary Consumption measure is most appropriate in discussions of the amount and impact of total U.S. energy consumption.

- The Total Inputs measure is most useful in discussions of how energy use in the manufacturing sector compares with energy use in the residential and commercial sectors.

- The Consumption of Offsite-Produced Energy measure is used to continue the data series, "Purchased Fuels and Electric Energy," which was previously collected for EIA by the Bureau of the Census as a supplement to its Annual Survey of Manufactures. These data were published for the reporting years 1974 through 1981.

Primary Consumption of Energy for All Purposes is the most comprehensive measure of energy consumption and represents the first use of energy sources, no matter whether they are consumed as a fuel or as a nonfuel (mainly raw material):

- It includes all energy produced offsite.

- It includes all energy produced onsite from nonenergy materials (such as sawdust from furniture production, hydrogen from electrolysis of brine, and peanut shells from peanut processing).

- It includes net electricity, net steam, and net hot water.

- It excludes all energy sources produced onsite as a result of the use of another energy source.

For example, the Primary Consumption measure includes the coal (an energy source purchased offsite) used at steel works as a raw material to produce coal coke. However, the measure excludes the coal coke (an energy source produced onsite as a result of the use of another energy source).

\footnotetext{
SThe 1991 MECS was the first collection of national-level end-use data.

${ }^{6}$ Energy consumed as a raw material is frequently called a feedstock, although that terminology most often refers to petroleum-based inputs and natural gas.

${ }^{7}$ Net electricity, net steam, and net hot water are the sum of purchases, transfers in, and onsite production from renewables minus the quaritities sold and transferred out.
} 
Total Inputs of Energy for Heat, Power, and Electricity Generation is a less comprehensive measure of energy consumption because it measures only the energy used for its energy content:

- It includes all energy sources used to produce heat and power and to generate electricity, whether produced offsite or onsite.

- It includes net electricity, net steam, and net hot water.

- It excludes all energy sources used as raw material or other nonfuel uses.

For example, the Total Inputs measure includes coal coke consumed onsite as a fuel, but it excludes the coal that was consumed as a raw material input to produce the coke.

Consumption of Offsite-Produced Energy for Heat, Power, and Electricity Generation is the most restrictive measure of the three:

- It includes all energy sources purchased or transferred from offsite to produce heat and power and to generate electricity.

- It excludes all energy produced onsite.

- It excludes all energy sources used as raw materials or for other nonfuel purposes.

\section{Components and Measures of Combustible Energy Consumption}

Each of the three general measures of energy consumption covers different components of combustible energy consumption, which has six components:

1. Energy produced offsite and consumed as a fuel

2. Energy produced offsite and consumed for nonfuel purposes

3. Energy produced onsite from nonenergy inputs and consumed as a fuel

4. Energy produced onsite from nonenergy inputs and consumed for nonfuel purposes

5. Energy produced onsite from energy products and consumed as a fuel

6. Energy produced onsite from energy products and consumed for nonfuel purposes.

Primary Consumption includes Components 1, 2, 3, and 4. Total Inputs includes Components 1, 3, and 5. Total Consumption of Offsite-Produced Energy includes only Component 1. (None of the three measures explicitly includes Component 6 to avoid double counting.)

\section{Specific Measures of Energy Consumption}

In addition to the three general measures of energy consumption, this report provides specific measures related to electricity, steam, and hot water consumption.

Embodied energy for electricity is the energy electricity suppliers use to generate the electricity consumed at the site. $^{8}$ During the generation process, large energy losses occur:

- When heat is converted into mechanical energy for turning electric generators

- When the power plant uses electricity for its own purposes, such as pumping water into elevated reservoirs in pumped-storage hydroelectric plants

- When electricity is transmitted and distributed from the power plant to the consumer. ${ }^{9}$

\footnotetext{
"Other products from EIA commonly use the term "primary energy" to denote energy measures that incorporate input energy used to generate electricity. The MECS uses "embodied energy for electricity" for this concept to avoid confusion with the term "Primary Consumption of Energy."

${ }^{9}$ Although energy losses also occur during the production of natural gas, fuel oil, and district heat, they are so small compared with those occurring during the production of electricity that they are not considered in measuring primary energy consumption in this report.
} 
However, measuring the amount of the energy losses is complicated because their amount varies from year to year and from utility plant to utility plant, depending on the conversion process, the particular mix of input energy sources used in generation, and the efficiency of the utility plant. Since collecting data on these factors for each utility plant would be unreasonable within the framework of EIA consumption surveys, the amount of energy consumed to produce the electricity consumed onsite in any given year can only be estimated.

\section{Evaluating the Energy Value of Electricity Consumption}

Electricity consumption can be expressed in terms of either

- physical units, most commonly kilowatthours

- thermal units, most commonly British thermal units (Btu).

The physical unit is meant to give a clear understanding of the amount of a particular energy source being used, while the thermal unit is a measure of convenience used to aggregate or compare various energy sources measured in different physical units. Converting kilowatthours of electricity to Btu is not a trivial issue, because the amount of input energy needed to create a kilowatthour of electricity is far greater than the amount of useful energy in the kilowatthours at its point of use. Therefore, meaningful conversions of electricity use from kilowatthours to Btu can be given in terms of:

- Site (point-of-use) electricity, at the universal value of 3,412 Btu per kilowatthour. This value is useful to engineers, energy managers, and others trying to improve energy efficiency.

- Embodied (primary) electricity, at a value that reflects the content of the energy inputs used to produce the electricity. This rate is most useful to policymakers and analysts who are considering global resources and environmental issues.

Estimates of embodied electricity should be treated with caution. They should be considered rough alternative measures to site energy as indicators of the importance of electricity as a manufacturing energy source.

EIA bases this estimate on the approximate annual amount of combustible fuels (coal, natural gas, and petroleum products) used by steam-electric generating plants, which generate most of the Nation's electricity. ${ }^{10}$ In 1991, U.S. steam-electric utility plants are estimated to have used approximately 10,352 Btu of conventional-fuel energy to generate 1 kilowatthour of electricity-or approximately $3.03 \mathrm{Btu}$ of conventional-fuel energy to generate $1 \mathrm{Btu}$ of electricity, since 3,412 Btu equals 1 kilowatthour of electricity." Accordingly, in this report, estimates of site electricity consumption in kilowathours can be converted to estimates of embodied energy consumption by using 10,352 as the conversion factor. For example, in Table A1, Part 1, the Food and Kindred Products Industry group consumed 49,536 million kilowathours of electricity. Multiplying this estimate by 10,352 Btu per kilowatthour would yield 513 trillion Btu, the amount of embodied energy used to generate that amount of electricity.

Estimates of embodied electricity can only be approximations of the amount of energy actually used to generate electricity, especially in the manufacturing sector, because many manufacturers use energy sources other than the combustible fuels. For example, the electricity-intensive industries, such as aluminum, are concentrated in areas where electricity can be generated from energy sources other than conventional fuels, usually hydropower. Thus, the accuracy of the conversion factor varies across industry groups.

Electricity demand is the amount of electricity actually consumed onsite, regardless of where or how it was produced. It is a useful measure of electricity consumption without regard to the consumption of other energy sources. Electricity demand is estimated in this report as the sum of electricity purchases, transfers in, and total onsite generation minus the quantities of electricity sold or transferred offsite.

\footnotetext{
${ }^{10}$ The fossil fuels, especially coal, provide the principal energy sources for the generation of electricity. Nuclear and hydroelectric power are used to a lesser extent, with wood/waste, wind, geothermal, and solar energy supplying only a small amount of energy for electricity generation.

I"Table A8. Approximate Heat Rates for Electricity," Monthly Energy Review, August 1994, p. 165.
} 
Net electricity is estimated for each manufacturing establishment as the sum of purchased electricity, transfers in, and generation from noncombustible renewable resources minus the quantities of electricity sold and transferred offsite. Thus net electricity excludes the quantities of electricity generated or cogenerated onsite from combustible energy sources. The estimates of net electricity appear in the estimates of Total Primary Consumption for All Purposes (Table A1) and Total Inputs of Energy for Heat, Power, and Electricity Generation (Table A4).

Electricity receipts is the amount of electricity purchased and transferred into the establishment. This measure is used so that the MECS estimates of offsite-produced energy will be consistent with the Census Bureau's definition of "purchased fuels and electricity," on which the Bureau of the Census collected and published data from 1974 through 1981. The estimates for Electricity Receipts appear in the estimates of Total Consumption of OffsiteProduced Energy for Heat, Power, and Electricity Generation (Table A5).

Net steam is the sum of purchases, transfers in, and generation from renewable energy sources minus the quantities sold and transferred out.

Net hot water is the sum of purchases and transfers in, minus the quantities of hot water sold and transferred out.

Estimates of net steam and net hot water are not shown separately in the MECS but are included in the "Other" column of tables that show Primary Consumption and Total Inputs. The estimates of Offsite-Produced Energy for Heat, Power, and Electricity Generation include the steam and hot water purchased by and transferred to a manufacturing establishment in order to be consistent with the Census Bureau's definition of "purchased fuels and electricity." 


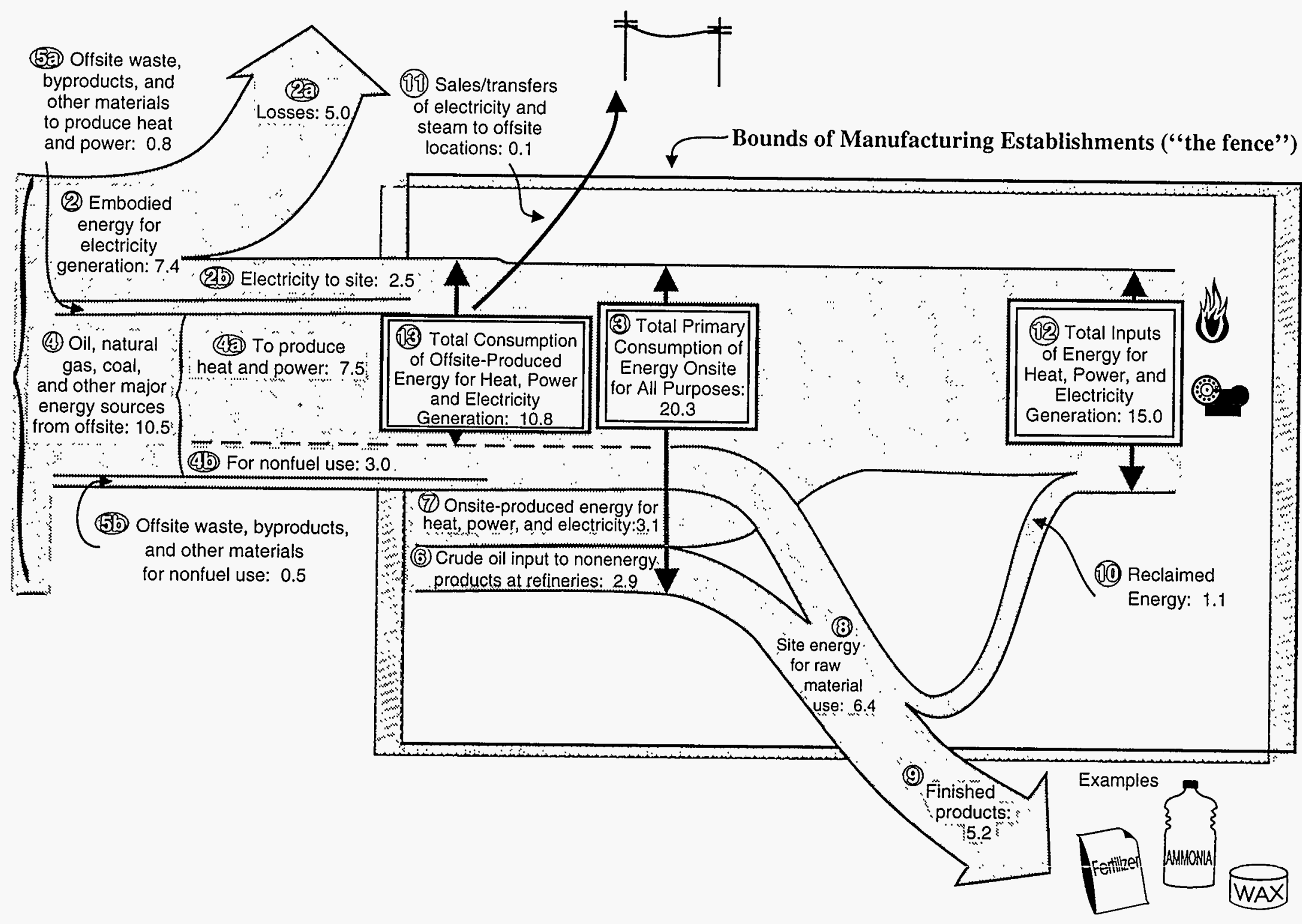




\section{Summary of Manufacturing Energy Throughput, 1991}

In 1991, 25.3 quadrillion Btu of energy can be attributed to the manufacturing sector (1 in Figure 2.2). This amount included 7.4 quadrillion Btu of embodied energy for electricity (2) and 20.3 quadrillion Btu of Primary Consumption of Energy for All Purposes (3).* Of the 7.4 quadrillion Btu of embodied energy for electricity, 5.0 quadrillion Btu was lost during the generation of the electricity offsite (2a), and 2.5 quadrillion Btu of electricity was delivered to the manufacturing site (2b).

The 20.3 quadrillion Btu of Primary Consumption of Energy for All Purposes (3) included:

- The 2.5 quadrillion Btu of electricity delivered to the manufacturing site (2b)

- 10.5 quadrillion Btu of natural gas, coal, fuel oil, and other major energy sources (4), with 7.5 quadrillion Btu of this amount being used inside the fence to produce heat and power (4a) and 3.0 quadrillion Btu being used for nonfuel uses (4b)

- 1.4 quadrillion Btu of offsite waste, byproducts, and other materials (5a and $5 \mathrm{~b}$ ); with 0.8 quadrillion Btu of this amount being used for heat and power (5a) and 0.5 quadrillion Btu being used for nonfuel uses (5b)

- 2.9 quadrillion Btu of crude oil input to nonenergy products (such as asphalt and road oil) at refineries (6)

- 3.1 quadrillion Btu of onsite-produced energy for heat, power, and electricity (waste and byproducts from onsite processing; energy from mines and wells onsite; and electricity and steam generated onsite from wind, solar, hydropower, and geothermal sources) (7).

The feedstock components of the Primary Consumption of Energy for All Purposes measure (8) included 3.0 quadrillion Btu of natural gas, coal, fuel oil, and other major energy sources (4b); 0.5 quadrillion Btu of offsite waste, byproducts, and other materials (5b); and 2.9 quadrillion Btu of crude oil input to nonenergy products (6). Of the 6.4 quadrillion Btu of site energy used for feedstock/raw material (8), 5.2 quadrillion Btu was used in finished products such as fertilizer, ammonia, and wax (9), and 1.1 quadrillion Btu was

onsite (10).

The 20.3 quadrillion Btu of Primary Consumption of Energy for All Purposes excluded 0.1 quadrillion Btu of electricity and steam that was transferred to offsite locations (11)

Manufacturers consumed 15.0 quadrillion Btu of Total Inputs of Energy for Heat, Power, and Electricity Generation. This amount included:

- 0.8 quadrillion Btu from offsite waste, byproducts, and other materials (5a)

- 2.5 quadrillion Btu of electricity delivered to the manufacturing site (2b)

- 7.5 quadrillion Btu of oil, natural gas, coal, and other major energy sources produced offsite and used to produce heat and power (4a)

- $\quad 3.1$ quadrillion Btu of onsite-produced energy for heat, power, and electricity (waste and byproducts from onsite processing energy from mines and wells onsite; and electricity and steam generated onsite from wind, solar, hydropower, and geothermal sources) (7)

- 1.1 quadrillion Btu of energy reclaimed from the byproducts and waste of raw materials produced onsite and used for heat and power and electricity generation (10).

The 20.3 quadrillion Btu of Total Inputs of Energy for Heat, Power, and Electricity Generation also excluded the 0.1 quadrillion Btu of electricity and steam that was transferred to offsite locations (11).

The 10.8 quadrillion Btu of Total Consumption of Offsite-Produced Energy for Heat, Power, and Electricity Generation (13) included:

- 2.5 quadrillion Btu of electricity (2b)

- 0.8 quadrillion Btu of offsite waste, byproducts, and other materials (5a)

- 7.5 quadrillion Btu of oil, natural gas, coal, and other major energy sources from offsite (4a).

"Components may not sum to totals due to independent rounding. 
$\because$ 


\section{Energy Consumption in the Manufacturing Sector, 1991}

In 1991, the amount of energy consumed in the manufacturing sector was as follows:

- Primary Consumption of Energy for All Purposes was 20.3 quadrillion Btu of energy, or about one-third of the total end-use energy consumed by the U.S. economy.

- Total Inputs of Energy for Heat, Power, and Electricity Generation were 15.0 quadrillion Btu.

The end uses for which manufacturing establishments consumed most of this energy were boiler fuel, direct processing, and direct non-processing (facility support).

\section{Primary Consumption of Energy for All Purposes}

The amount of Primary Consumption of Energy for All Purposes in 1991 was 20.3 quadrillion Btu, compared with 20.1 quadrillion Btu in 1988 and 17.5 quadrillion Btu in 1985. The shares of consumption for each of the commonly known energy sources remained stable during the 6-year period:

- The share for natural gas was 30 percent in 1991, 29 percent in 1988, and 30 percent in 1985.

- The share for net electricity was 12 percent in all 3 years. ${ }^{12}$

- The share for coal was 10 percent in 1991, 12 percent in 1988, and 14 percent in 1985.

- The share for liquefied petroleum gas (LPG) was 8 percent in 1991 and 5 percent in 1985 and 1988.

- The share for other energy sources was 41 percent in 1991,42 percent in 1988 , and 40 percent in $1985 .^{13}$

Six industry groups accounted for 88 percent (17.8 quadrillion Btu) of Primary Consumption of Energy for All Purposes: food and kindred products; paper and allied products; chemicals and allied products; petroleum and coal products; stone, clay, and glass products; and primary metals (Figure 3.1).

About two-thirds of Primary Consumption of Energy for All Purposes (13.9 quadrillion Btu) was used to produce heat and power and to generate electricity, with about onethird (6.4 quadrillion Btu) being consumed as raw material input (Table A3).

\section{Figure 3.1. Primary Consumption of Energy for All Purposes by Major Industry Group, 1991}

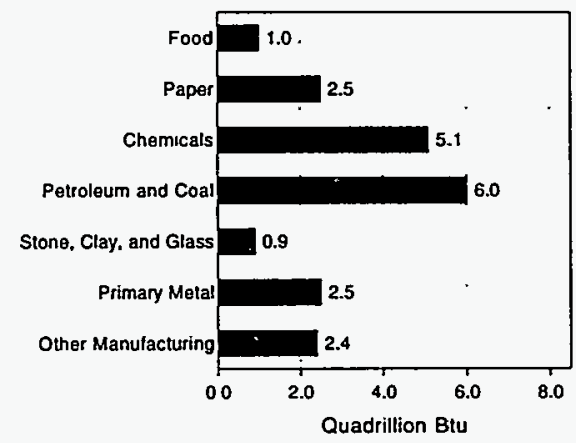

Soutce. Tablo AI of this repon.

\footnotetext{
12"Net electricity" is the sum of purchases, transfers in, and generation from noncombustible renewable resources, minus quantities sold and transferred out.

${ }^{13}$ Changes in shares for coal and LPG from 1988 to 1991 were statistically significant at the 0.05 level. Changes in shares for natural gas, net electricity, and other energy sources were nonsignificant.
} 
The most common energy sources-natural gas, net electricity, coal, liquefied petroleum gas (LPG), residual and distillate fuel oil, and coke-made up 64 percent of Primary Consumption. Making up the remaining 36 percent of Primary Consumption were still gas, waste gas, petroleum coke, pulping liquor, and other energy that respondents indicated they had used as Primary Energy, as well as crude oil inputs to such materials as asphalt and road oil (Figure 3.2).

Natural Gas. Natural gas was the energy source most heavily consumed at the manufacturing site in 1991, accounting for 30 percent of Primary Consumption. Natural gas consumption is heavily concentrated in the chemicals and allied products major industry group, where it is consumed as a fuel and as a feedstock. This major industry group accounted for about 36 percent $(2.2$ quadrillion Btu) of the Primary Consumption of natural gas (Table A1). About one-fourth of the natural gas consumed by the chemicals and allied products major industry group (0.6 quadrillion Btu) was used as an energy raw material (Table A3). Natural gas is used as a raw material in the preparation of numerous products, but one of the heaviest uses is for manufacturing nitrogenous fertilizers.

Electricity. Net electricity was the second most heavily consumed single energy source by manufacturers in 1991, accounting for 12 percent of site Primary Consumption (2.4 quadrillion Btu). ${ }^{14}$ However, taking into account the amount of energy used to generate the site net electricity (the embodied energy consumption for net electricity), net electricity accounted for 29 percent of site energy consumption (Figure 3.3).

Four major industry groups consumed 54 percent (1.3 quadrillion Btu) of Primary Consumption of net electricity: food and kindred products, paper and allied products, chemicals and allied products, and primary metal industries. Of these, the primary metal major industry group, which includes primary aluminum, was the heaviest consumer. The primary aluminum industry uses large quantities of electricity to produce aluminum ingots from alumina. In 1991, net electricity provided 77 percent of the aluminum industry's site energy consumption. (Taking into account the energy used to generate that site electricity, it accounted for 91 percent of the industry's embodied energy consumption.)

\section{Figure 3.2. Primary Consumption of Energy for All Purposes by Energy Source, 1991}

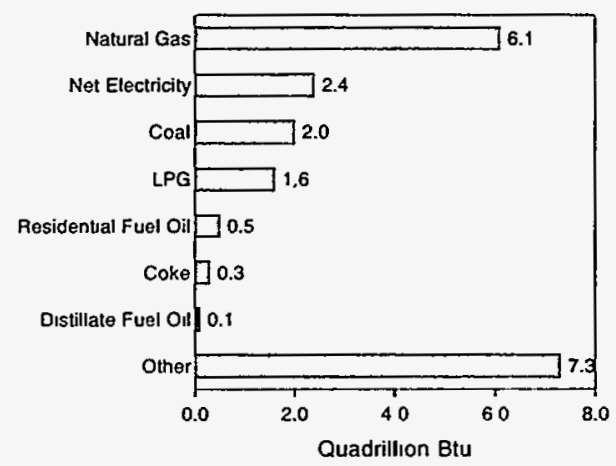

Source: Table Al of this report.

Coal. The 1991 Primary Consumption of coal was 2.0 quadrillion Btu. Coal is heavily consumed by the blast furnaces and steel mills industry to produce coke. Coking operations accounted for 0.8 quadrillion Btu, or 40 percent of Primary Consumption of coal.

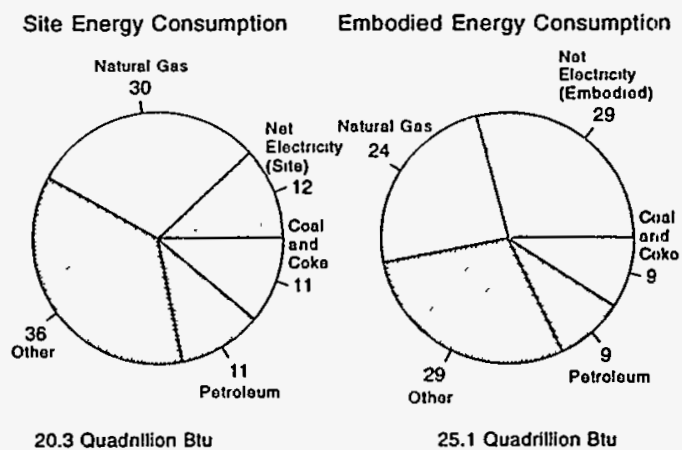

Source: Tablo A1 of this report.

${ }^{14}$ Unless otherwise specified, all estimates of electricity consumption shown in this report are given in terms of site energy. 
LPG. Liquefied petroleum gases (LPG) are consumed mostly as a raw material by manufacturers. The largest users are chemical industries that use LPG as petrochemical feedstock. An example is the production of ethylene from ethane. Of the total 1991 Primary Consumption of 1.6 quadrillion Btu, 1.5 quadrillion Btu (94 percent) was used as a raw material.

Distillate and Residual Fuel Oils. The Primary Consumption of distillate and residual fuel oils continued at a relatively low level in 1991. Residual fuel oil accounted for only 0.5 quadrillion Btu and distillate fuel oil, 0.1 quadrillion Btu. Total fuel oil consumption accounted for only about 3 percent of Primary Consumption, which also was the case in both the 1985 and 1988 MECS.

Other Energy Sources. Other energy sources accounted for 36 percent (7.3 quadrillion Btu) of Primary Consumption. Those energy sources included net steam and other energy that respondents indicated was used as a fuel and raw material input ( 4.4 quadrillion Btu). In addition, the estimate has been supplemented by the quantity of energy consumed by the petroleum refining industry to produce final products that are not normally considered to be energy sources (asphalt, road oil, solvents, lubricants, and waxes). Those products accounted for 40 percent (2.9 quadrillion Btu) of the other energy sources. (See Appendix B for a discussion of the rationale of this procedure.)

\section{Total Inputs of Energy}

The amount of Total Inputs of Energy for Heat, Power, and Electricity Generation in 1991 was 15.0 quadrillion Btu, compared with 15.5 quadrillion Btu in 1988 and 13.6 quadrillion Btu in 1985. Between 1988 and 1991, the shares of consumption for each of the commonly known energy sources remained stable:

- The share for natural gas was 34 percent in 1988 compared with 37 percent in 1991.

Figure 3.4. Total Input Energy for Heat, Power, and Electricity Generation, 1991

- The share for net electricity was 15 percent in 1988 compared with 16 percent in 1991.

- The share for coal and coke was 13 percent in 1988 compared with 12 percent in 1991.

- The share for petroleum products was 6 percent in 1988 compared with 4 percent in 1991.

- The share for byproducts was 28 percent in 1988 and 1991.

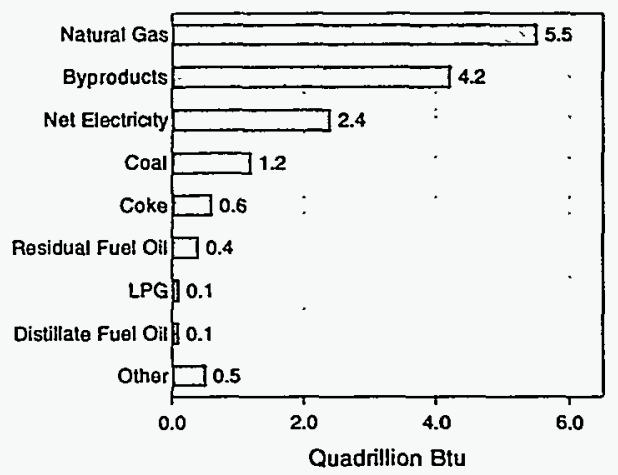

- The share for other energy sources was 3 percent Source: Tables A4 and A6 of this repont. in 1988 compared with 4 percent in $1991 .^{15}$

The shares for the various energy sources in 1985 cannot be compared with shares for 1988 and 1991 because data on the amount of byproduct fuels consumed in 1985 were published only in aggregate form.

Of the eight specific types of energy measured by Total Inputs, natural gas was the dominant energy source, followed by byproduct fuels (Figure 3.4). Energy sources produced offsite made up 72 percent of Total Inputs of Energy for Heat, Power, and Electricity Generation. Fuels produced onsite made up the remaining 28 percent.

\footnotetext{
${ }^{15}$ Changes in shares for natural gas, coal and coke, petroleum products, and other energy sources from 1988 to 1991 were statistically significant at the 0.05 level. Changes in shares for net electricity and byproducts were nonsignificant.
} 


\section{Consumption by Major Industry Group}

The major industry groups consuming the most energy for heat, power, and electricity generation were food and kindred products, paper and allied products, chemicals and allied products, petroleum and coal products, and primary metal industries (Figure 3.5). Industries vary in the proportion of energy they use for heat, power, and electricity generation and the proportion they use for raw materials. Some industries, like the food and kindred products and the paper and allied products industries, consume large amounts of energy for heat, power, and electricity generation and small amounts of energy for raw material. Other industries, such as the chemicals and petroleum industries, consume large amounts of energy as raw material.
Figure 3.5. Total Input Energy for Heat, Power, and Electricity Generation by Major Industry Group, 1991

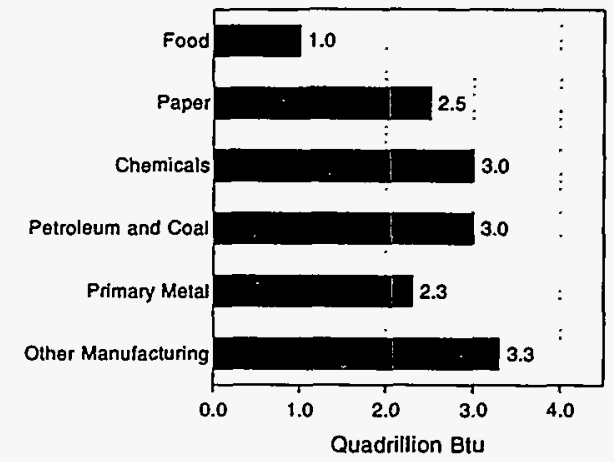

Source: Table A4 of this report.

Food and Kindred Products. In the food and kindred products major industry group, the 1991 estimates of Primary Consumption and Total Inputs are nearly identical at 1.0 quadrillion Btu. This close correspondence is because the food industry consumes virtually all its energy as a fuel to process food for distribution and consumes negligible amounts of energy as a raw material.

Paper and Allied Products. Estimates of Primary Consumption and Total Inputs are also similar for the paper and allied products major industry group (about 2.5 quadrillion Btu for each) because this group, too, consumes only small amounts of energy sources as energy raw material. It should be noted that the primary raw material input for the production of pulp and paper is wood. However, pulping wood is not counted as an energy source in the MIECS because it is considered by manufacturers to be a nonenergy raw material input. Therefore, both Primary Consumption and Total Inputs include wood byproducts.

Chemicals and Petroleum Industries. The chemicals and allied products and the petroleum and coal products major industry groups clearly illustrate these industries' heavy use of energy as a raw material. The 1991 Primary Consumption for chemicals and allied products was 5.1 quadrillion Btu, of which 2.4 quadrillion Btu was consumed as an energy raw material. Total Inputs for chemicals and allied products ( 3.0 quadrillion Btu) excludes the energy consumed as a raw material. The petroleum and coal products major industry group was quite similar, with a Primary Consumption of 6.0 quadrillion Btu, of which 3.0 quadrillion Btu was consumed for nonfuel use, as raw material input to nonenergy products.

\section{Byproduct Fuels}

Byproduct fuels-blast furnace gas, waste gas, petroleum coke, pulping liquor, wood byproducts, and waste oils/materials - made up 28 percent of Total Inputs of Energy for Heat, Power, and Electricity Generation (Figure 3.6). Industries differ in the amount of byproduct energy they use, with the blast furnace, petroleum refining, and paper and pulp industries being the largest consumers of byproduct fuels.

Blast Furnace Industry. The blast furnace industry consumed 1.6 quadrillion Btu of input energy in 1991. This industry commonly produces two byproduct fuels-blast furnace and coke oven gas. Blast furnace gas is a combustible waste gas generated in a blast furnace when iron ore is being reduced with coke to metallic iron. In addition, most steel works produce much of their own coke. Coke oven gas is a combustible mixture of gases produced by the carbonization of coal in a coke oven. These gases are "recycled" and consumed onsite as a fuel.

Of the total input energy consumption of this industry, 27 percent ( 0.4 quadrillion Btu) was provided by these byproduct energy sources (Table A6). 
Petroleum Refineries. The 1991 input energy requirement for the petroleum refining industry was 2.9 quadrillion Btu. That energy was consumed as a fuel to refine crude oil into useful products. Waste gas (still gas) and petroleum coke are byproducts of the petroleum refining process. Waste gas is any form or mixture of gas produced in refineries by distillation, cracking, reforming, and other refining processes. The principal constituents of waste gas are methane, hydrogen, ethane, ethylene, propane, propylene, butane, and butylene. Petroleum coke is a solid residue that is high in carbon content and low in hydrogen. It is the final product of thermal decomposition in the condensation process in cracking crude oil. Some of these byproducts are sold, but most are recycled into the refining process and consumed as a fuel. In 1991, these byproducts accounted for 1.8 quadrillion Btu, or 62 percent of the total input energy requirement of the petroleum refining industry.
Figure 3.6. Byproduct Fuels Consumed by Manufacturers by Energy Source, 1991

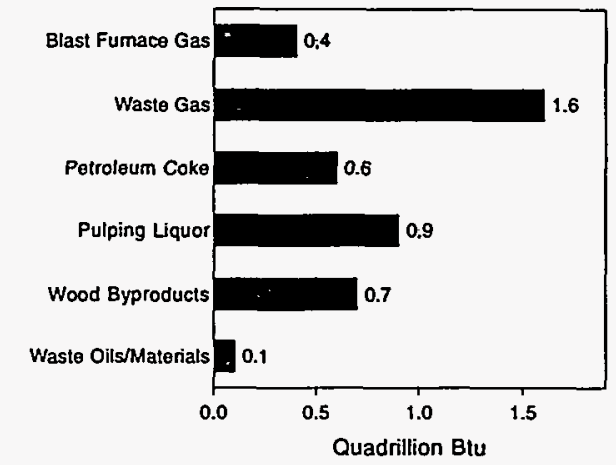

Source. Table A6 of this report.

Paper and Allied Products Industries. The paper and allied products industries use enormous amounts of wood to produce paper. Byproducts of the wood used to produce paper consist of pulping liquor and wood chips, bark, and wood waste. Pulping liquor is the alkaline spent liquor removed from the digester in the process of chemically pulping wood. After evaporation, the liquor is burned in a recovery furnace that provides heat and permits the recovery of certain basic chemicals from the liquor. Nearly all pulping liquor is consumed onsite, as are most of the wood byproducts. Total 1991 input energy for this major industry group was 2.5 quadrillion Btu. Of that amount, pulping liquor accounted for 0.9 quadrillion Btu and wood chips, bark and wood waste, for 0.3 quadrillion Btu. Thus, 48 percent of the total input energy requirement of the paper and allied products major industry group was met by these byproduct energy sources.

\section{End Uses for Heat, Power, and Electricity}

In 1991, 65 percent of the Total Inputs of Energy for Heat, Power, and Electricity Generation in the manufacturing sector was allocated to three groups of end uses: indirect uses (mainly boiler fuel), direct process uses, and direct non-process uses. The remaining 35 percent of energy inputs was unallocated. The 1991 MECS was the first collection of national-level end-use data.

\section{End-Use Consumption of Energy}

The manufacturing sector used 9.7 quadrillion Btu of natural gas, electricity, coal, residual and distillate fuel oils, and LPG to produce the heat, power, and electricity needed for:

- Boiler fuel, which is used to transform one source of energy into another (like steam)

- Direct process uses, including use in motors, ovens, strip heaters, and kilns

- Direct non-process uses, including facility heating, ventilation, and air-conditioning (HVAC); facility lighting, other facility support; onsite transportation; electricity generation; and other miscellaneous uses.

The manufacturing sector as a whole used the most energy for direct process uses (Figure 3.7). 


\section{Boiler Fuel}

Approximately one-third (3.3 quadrillion Btu) of reported 1991 end-use consumption was consumed as boiler fuel, energy that was then transformed into steam or some other energy source. This transformation of energy describes the largest specific end use of reported energy consumed by manufacturing establishments.

Transformation of energy is not usually considered an energy use because steam has subsequent end uses. Steam, the predominant output from industrial boilers, has multiple uses in most manufacturing establishments and is, therefore, difficult for manufacturers to measure and quantify since the energy content depends upon both temperature and pressure. In the surgical and medical instruments industry, for example, steam can be used to sterilize medical products such as sutures and syringes. Meanwhile, high-pressure steam can also be used to produce electricity or can be used by other industrial processes or non-processes that require thermal energy.

\section{Direct Process End Uses}

In 1991, over half (5.0 quadrillion Btu) of reported enduse consumption was used to transform raw material inputs into production outputs. The MECS identified four distinct direct processes-process heating, process cooling/refrigeration, machine drive (motors), ${ }^{16}$ electrochemical processes-and a group of other unspecified processes. The three most energy-intensive direct process end uses were process heating, machine drive, and electrochemical processes (Figure 3.8 ).

Process heating, which accounted for 62 percent (3.1 quadrillion Btu) of all reported direct process consumption, encompasses numerous uses. For example:

- The aluminum industry uses process heating to hold molten aluminum in crucibles after the electro-chemical process (the reduction process) of splitting alumina into molten aluminum metal and oxygen.
Figure 3.7. Consumption of Energy for Heat, Power, and Electricity Generation by Manufacturing End Use, 1991

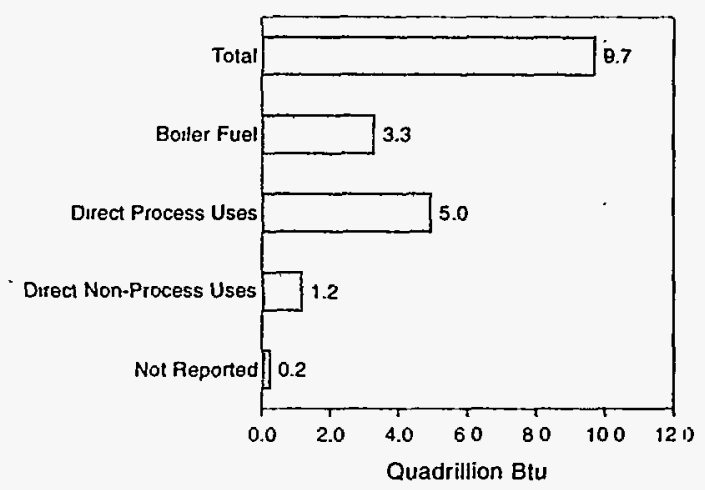

Source: Table A36 of this report.

Figure 3.8. Consumption of Energy for Heat, Power, and Electricity Generation by Manufacturing Direct Process End Use, 1991

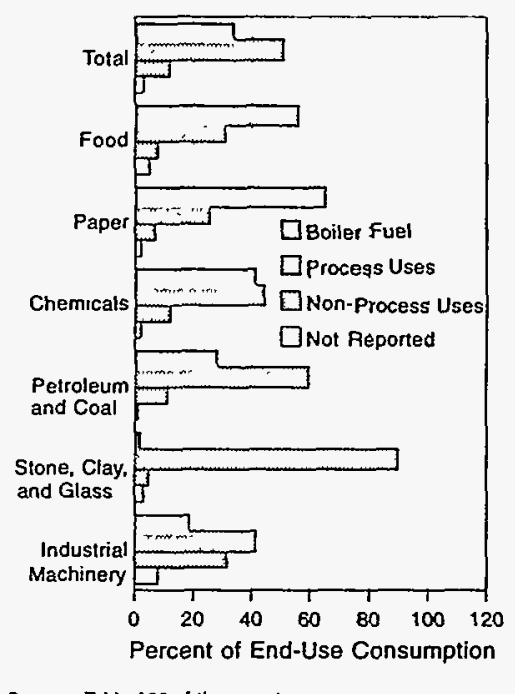

Source: Table A36 of this report.

- The steel industry uses process heating to melt scrap metal in electric-arc furnaces.

- The food industry uses process heating to dry food residual (such as yeast extract) for resale as livestock feed.

Machine drive, used for equipment such as industrial air compressors and pumps, consumed 1.3 quadrillion Btu (27 percent) of reported direct process consumption and represented the second largest reported direct process use of energy. Of that 1.3 quadrillion Btu, electricity accounted for 1.2 quadrillion Btu ( 88 percent) of consumption.

${ }^{16}$ Machine drive transforms thermal or electrical energy into mechanical energy. 
Electro-chemical use of energy ranked third in the reported consumption of energy for direct process uses. Over 0.3 quadrillion Btu of electricity, concentrated in the primary metal and chemicals and allied products industries, were consumed in that process. Of that amount of purchased and generated electricity, the primary metal industries accounted for 229 trillion Btu (63 percent) and the chemicals and allied products industries consumed 114 trillion Btu (32 percent). Within the primary metal industries, the primary aluminum industry consumed 216 trillion Btu (60 percent) of electro-chemical use. This industry uses the electro-chemical process to reduce alumina to molten aluminum metal and oxygen (Table A38).

To improve the energy efficiency of their direct process operations, manufacturing establishments participated in various demand-side management (DSM) and other energy management programs (Table A40). From 1989 through 1991, their activities and percent of participation, expressed in terms of the total input energy use represented by participating establishments, were as follows: installing or retrofitting motors to achieve better energy efficiency ( 36 percent), improving the efficiency of process heating ( 34 percent), and improving the efficiency of process cooling (15 percent).

\section{Direct Non-Process End Uses}

Of the 9.7 quadrillion Btu of consumption for which end uses were reported, only 1.2 quadrillion Btu was designated for direct non-process uses. Less than 8 percent ( 0.7 quadrillion Btu) of energy consumption in manufacturing establishments was directly related to facility use (Figure 3.9). Process uses of energy affect some facility-related use of energy. Heat that radiates from manufacturing processes may negate, or restrict, facility HVAC use of energy. For example, glass melters found in the glass industries create a large amount of heat energy that is then transferred to the surrounding working environment. Since cooling that environment is not usually considered economically viable, the proximity of the manufacturing process may reduce the likelihood that the enclosed manufacturing floorspace is conditioned. In addition, those indirect transfers of radiant heat from processing uses to the surrounding environment are not measured.
Figure 3.9. Consumption of Energy for Heat, Power, and Electricity Generation by Manufacturing Direct NonProcess End Use, 1991

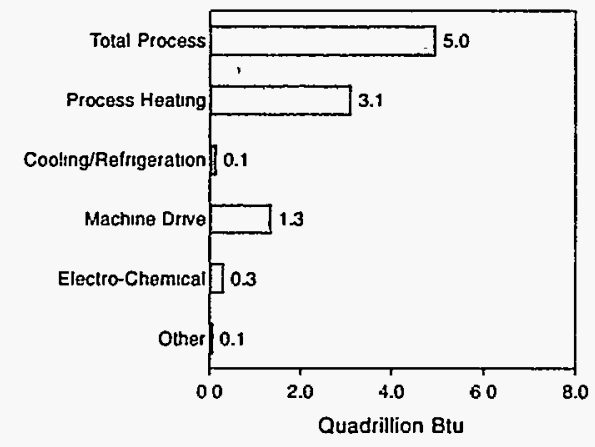

Source: Table A36 of thus roport.

To improve the energy efficiency of their direct non-process operations, manufacturing establishments participated in various DSM and other energy management programs (Table A40). From 1989 through 1991, their activities and percent of participation, expressed in terms of the total input energy use represented by participating establishments, were as follows: improving the efficiency of facility lighting ( 36 percent) and installing equipment to improve facility HVAC systems (23 percent).

\section{Industry Patterns of Energy Consumption for Different End Uses}

Since the production process is commonly considered the driving force behind the manufacturing sector, most industries consume most of their energy for direct process uses and consume relatively little for non-process uses. In 1991, direct non-process uses accounted for roughly 1.2 quadrillion Btu, or 12 percent of reported energy consumption. One of the heaviest users of energy for direct processes was the stone, clay, and glass major industry group, which consumed 0.7 quadrillion Btu ( 90 percent of all the energy it consumed) for direct process uses. This industry group is a heavy user of process heating, which is an energy-intensive end use. 
Other industries, however, used most of the energy they consumed for heat, power, and electricity for boiler fuel, such as the paper and allied products industries and the pulping and paper-making processes industries. The paper and allied products industries, which reported 1.2 quadrillion Btu for end-use consumption, reported consuming 66 percent of its reported inputs as boiler fuel, and only 26 percent in direct process uses and 7 percent in direct nonprocess uses, with the remaining 1 percent undiscerned. That configuration of consumption was also characteristic of the pulping and paper-making processes, where large quantities of steam are required to remove water from paper sheets (Figure 3.10).

Most industries use only a small percentage of the energy they consume for non-process end uses. However, in those industries where assembly-type manufacturing takes place in large, environmentally controlled buildings, it can be quite a large percentage. Examples are the furniture and fixtures industries (32 percent), transportation equipment industries, such as automobile plants (25 percent), or computer and office equipment industries (43 percent).

\section{Unallocated End-Use Consumption of Energy}

In addition to the 9.7 quadrillion Btu of the most common energy sources that manufacturers consumed for boiler fuel, direct process uses, and direct non-process uses, manufacturers consumed 5.3 quadrillion Btu of energy for which end uses were not assigned. Eight byproduct energy sources formed the major components of the 5.3 quadrillion Btu of unallocated end-use consumption: waste gas, pulping liquor (black liquor), blast furnace/coke oven gases, petroleum coke, coal coke, wood byproducts, waste oils/materials, and net steam (Figure 3.11).

While consumption of byproducts was not allocated to a specific end use, those energy sources generally have a finite number of uses at a manufacturing establishment. For example:

- Coal coke is generally consumed as a fuel for its heat content and as a reducing agent for smelting iron ore in the blast furnaces and steel mills industry, accounting for 0.5 quadrillion Btu (92 percent) of the unallocated coal coke.

- Pulping liquor, generated onsite at paper industry establishments, is generally consumed in recovery boilers to generate heat and recover catalysts for use in the pulping process.

\section{Figure 3.10. Consumption of Energy for Heat, Power, and Electricity Generation by Major Industry Groups and Major End Uses, 1991}

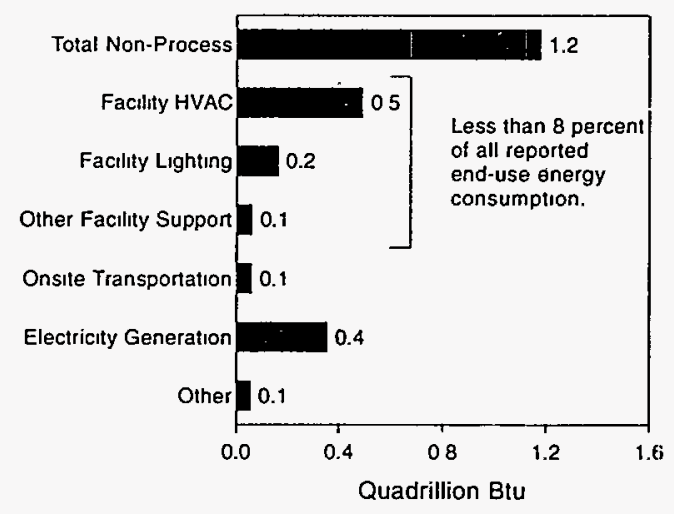

Source Table A36 of this report

Figure 3.11. Unreported End-Use Fuels Consumed for Heat, Power, and Electricity Generation, 1991

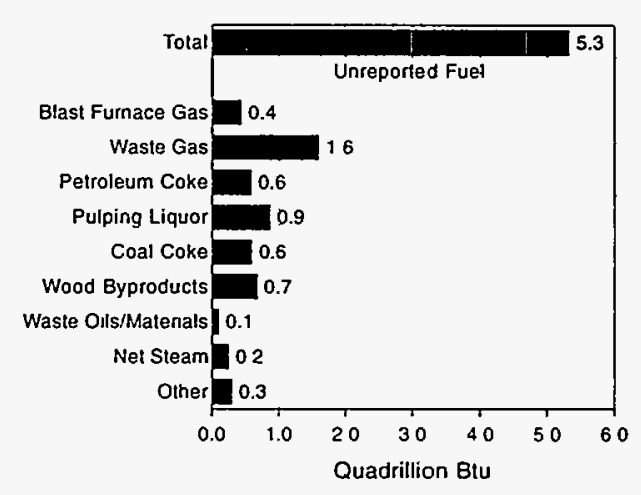

Source Tables A4, A6, A36 and D2 of this repon

- Waste gas is generally consumed for process heating in petroleum refineries, according to a 1984 American Petroleum Institute study. ${ }^{17}$

\footnotetext{
${ }^{17}$ Chemical and Engineering News, Energy Use by Petroleum Industry, June 9, 1986.
} 


\section{Summary}

In 1991, the Primary Consumption of Energy for All Purposes in the manufacturing sector was 20.3 quadrillion Btu of energy, compared with 20.1 quadrillion Btu in 1988. About two-thirds of this amount was used to produce heat and power and to generate electricity, with about one-third being consumed as raw material input to produce nonenergy products. The most common energy sources-natural gas, net electricity, coal, LPG, residual and distillate fuel oil, and coke-made up 64 percent of Primary Consumption.

The amount of Total Inputs of Energy for Heat, Power, and Electricity Generation was 15.0 quadrillion Btu in 1991, compared with 15.5 quadrillion Btu in 1988. The byproduct fuels-blast furnace gas, waste gas, petroleum coke, pulping liquor, wood byproducts, and waste oils/materials-made up 28 percent of Total Inputs.

End-use allocation was collected for about 65 percent of Total Inputs (9.7 quadrillion Btu). This consumption was allocated to three groups of end uses: indirect uses, mainly boiler fuel (3.3 quadrillion Btu); direct process uses, including use in motors, ovens, strip heaters, and kilns (5.0 quadrillion Btu); and direct non-process uses, including facility HVAC, facility lighting, other facility support, onsite transportation, electricity generation, and other miscellaneous uses (1.2 quadrillion Btu). Manufacturers consumed an additional 5.3 quadrillion Btu of energy for which end uses were not assigned. 



\section{Manufacturer Capability To Switch Fuels}

Manufacturers can often best adapt to changes in economic conditions, energy supply interruptions, and other constraints on energy use by switching fuels. For the MECS, manufacturers are said to have a fuel-switching capability if they are able to meet their requirements for heat, power, and electricity generation by substituting one energy source for another within 30 days without modifying the equipment that consumes the fuel and resuming the same level of production following the switch.

Although all manufacturers have certain minimum requirements for energy sources that cannot be replaced by another energy source, many manufacturers have some fuel-switching capability. The degree of a manufacturer's capability depends only on the characteristics of the equipment and practical limitations to switching, such as binding take-orpay agreements with energy suppliers and environmental regulations that limit the amounts of potential replacements that could have been consumed. Much of the fuel-burning equipment that manufacturers use is capable of burning more than one energy source.

The 1991 MECS determined fuel-switching capability for six commonly known energy sources and looked at the extent to which manufacturers exercised their ability to choose the mix of energy sources at their discretion.

\section{Nonswitchable Minimum Requirements}

Generally, a manufacturing establishment uses a certain quantity of an energy source that cannot be replaced by any other energy source, at least not within a 30-day period. The reasons for nonswitchable quantities are as varied as the types of production environments themselves. Among the practical limitations to switching are the characteristics of the establishment and the equipment itself, which would require, at the very least, significant modifications disruptive to production. Other switching deterrents are existing energy-supply contracts and environmental restrictions. In addition, the physical properties of certain energy sources could produce a desirable or harmful effect on the product.

For example, consider a manufacturing plant that consumed 2.0 million cubic feet of natural gas to produce automobile bodies. Of that amount, 500 thousand $(0.5$ million $)$ cubic feet of natural gas was nonswitchable because it was consumed in paint-drying ovens that required natural gas. Using any other energy source would have changed the tint of the pigments in the paint, rendering the products unsalable. The remaining 1.5 million cubic feet of natural gas was switchable because it was consumed in a boiler that could burn either natural gas or residual fuel oil, and the switch between those two energy sources could have taken place within a few hours.

In 1991, manufacturers required an nonswitchable minimum of 7.0 quadrillion Btu of electricity receipts, ${ }^{18}$ natural gas, distillate and residual fuel oils, coal, and liquefied petroleum gas (LPG), 72 percent of actual consumption of these fuels (Figure 4.1). This amount represents actual consumption decreased by the quantity of the energy sources that would no longer have been required if all ascertained switching from that energy source had occurred. This amount also includes the quantity of 1991 consumption for which switching capability was not ascertained.

The following are manufacturers' nonswitchable requirements for the most common energy sources, stated as a percentage of the actual consumption of each energy source:

- Electricity: 98 percent of actual consumption

- Natural gas: 65 percent of actual consumption

\footnotetext{
${ }^{18}$ The estimate of electricity receipts represents those quantities of electricity that were produced offsite and available onsite for consumption, but it does not include electricity generated onsite, nor has it been adjusted to account for any quantities that might have been resold or transferred to another establishment.
} 
- Distillate fuel oil: 80 percent of actual consumption

- Residual fuel oil: 55 percent of actual consumption

- Coal: 55 percent of actual consumption

- LPG: 53 percent of actual consumption.

\section{Fuel-Switching Capability}

In 1991, manufacturers had the capability to switch 2.8 quadrillion Btu of the most common energy sources, representing 28 percent of actual consumption. The energy sources for which fuel-switching capability is most desirable are the petroleum-based fuels. Since the mid1970's, concern about U.S. dependency on crude oil imports and adherence to environmental restrictions have prompted manufacturers to concentrate on reducing their consumption of petroleum-based fuels.

According to the Bureau of the Census, manufacturers' 1978 consumption of residual and distillate fuel oils accounted for 17 percent of total offsite-produced energy for heat, power, and electricity generation. ${ }^{19}$ By 1991 , those same petroleum-based energy sources accounted for only 4 percent of the total. This substantial decrease was likely due to manufacturers' replacement of petroleum-based fuels with other energy sources. By 1991, manufacturers had little capability remaining to replace petroleum-based fuels with other energy sources.

Natural gas has long been viewed as a prime alternative to petroleum-based fuels because of its widespread availability, supply reliability, clean-burning quality, and reduced storage capacity. However, a fuel-switching capability for natural gas is also necessary because of occasional supply disruptions. For example, natural gas production at the wellhead may be disrupted or the supplier may not be able to deliver the required amount of natural gas. Moreover, the natural gas supply may be interrupted during periods of extreme cold weather, when residential and commercial customers receive priority service at the sacrifice of manufacturers. Finally, the demand for natural gas sometimes exceeds supply in certain parts of the country, especially in the Northeast and Midwest, forcing manufacturers to use some other energy source for awhile.

The energy sources that had the greatest potential for switching were natural gas, with coal a distant second (Figure 4.2). The following were manufacturers' fuel-switching capabilities for the most common energy sources:

- About 17.0 billion kilowatthours of electricity receipts could have been replaced by other energy sources, 2 percent of actual consumption (Table A53). The switching potential for electricity is quite limited because most manufacturing equipment is not designed to permit switching between electricity and a combustible energy source. Most of the capability to replace electricity by combustible energy sources is in the form of redundant combustors that can be used in place of electric-powered equivalents.

- About 1.9 trillion cubic feet of natural gas was switchable, 35 percent of actual consumption. The primary replacement energy sources for natural gas were distillate fuel oil, coal coke, and residual fuel oil (Table A54). No single energy source could have decreased the consumption of natural gas even halfway to its minimum required level of 3.5 trillion cubic feet, but the dominant single replacement source, distillate fuel oil, could have replaced 0.7 trillion cubic feet ( 40 percent) of the natural gas that was switchable.

\footnotetext{
${ }^{19}$ Before 1985, Total Inputs for Heat, Power, and Electricity Generation were not collected. However, for those earlier years, the Bureat of the Census did tabulate total consumption of of fsite-produced energy for heat, power, and electricity generation, which is a key part of Total Inputs because most petroleum-based fuels are produced offsite.
} 
- About 4.8 million barrels of distillate fuel oil was switchable, 20 percent of actual consumption. Natural gas was the dominant replacement source for distillate fuel, capable of replacing 3.2 million barrels (Table A55).

- About 29.3 million barrels of residual fuel oil was switchable, 45 percent of actual consumption, with natural gas capable of replacing 19.5 million barrels (Table A56).

- About 23.6 million short tons of coal (45 percent of actual consumption) could have been replaced by other energy sources (Table A57).

- About 13.3 million barrels of LPG (47 percent of actual consumption) was switchable, with natural gas capable of replacing 11.4 million barrels (Table A58).

\section{Figure 4.2. Switchable Quantities for Selected Fuels, 1991}

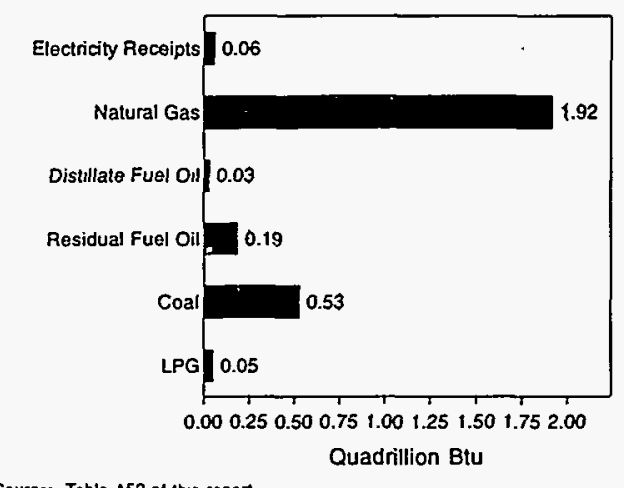

Source: Table A52 of this report.

\section{Discretionary Fuel Use}

Fuel-switching capability allows manufacturers substantial flexibility in choosing their mix of energy sources. The consumption of a given energy source can be maximized if all possible switching into that energy source were to take place. At the other extreme, consumption can be minimized if all possible switching from that energy source were to take place. (This value is the nonswitchable minimum requirement discussed earlier.) The interval defined by these maximum and minimum limits represents the discretionary range of consumption for an energy source, resulting from fuel-switching capability. Actual consumption lies within that discretionary range. A preference for a given energy source is indicated when its actual consumption approaches its maximum consumption potential, as in the case of both natural gas and coal (Figure 4.3). Conversely, a preference for other energy sources is indicated when actual consumption approaches its minimum required level.

For example, one manufacturer in the 1991 MECS used 3.0 million Btu of a given energy source, 1.0 million Btu of which could have been replaced by some other energy source and 2.0 million Btu of which could not have been replaced. In addition, the manufacturer could have used the given energy source to replace 2.0 million Btu of other energy sources. If the manufacturer had chosen to use the given energy source whenever possible, consumption of the energy source would have been at the maximum consumption level of 5.0 million Btu. If the manufacturer had chosen to use a substitute energy source whenever possible, the manufacturer's consumption of the given energy source would have been at the minimum consumption level of 2.0 million Btu. Thus, the maximum potential is 3.0 million Btu above the minimum level, and this amount (3.0 million Btu) represents the discretionary range of consumption. Since the actual consumption is 1.0 million Btu above the minimum level, the depth into the discretionary range is $1.0 / 3.0$ or 33 percent, which is the discretionary use rate of the given energy source.

One of the more interesting summary statistics that can be developed from the estimates of actual consumption, minimum consumption, and maximum consumption is the discretionary use rate. The discretionary use rate is a measure of the extent to which manufacturers elected to consume a given energy source when they could have consumed some other energy source, given their fuel-switching capabilities and production levels of 1991. (See Appendix B for an explanation and formula of the discretionary use rate.)

If manufacturers always choose to use an energy source whenever possible, the discretionary use rate for that energy source would be 100 percent. On the other hand, if manufacturers choose to minimize their consumption of a given energy source by always using other energy sources whenever possible, the discretionary use rate would be 0 percent. 


\section{Figure 4.3. Manufacturers' Discretionary Ranges of Consumption Resulting from Fuel-Switching Capability, 1991}

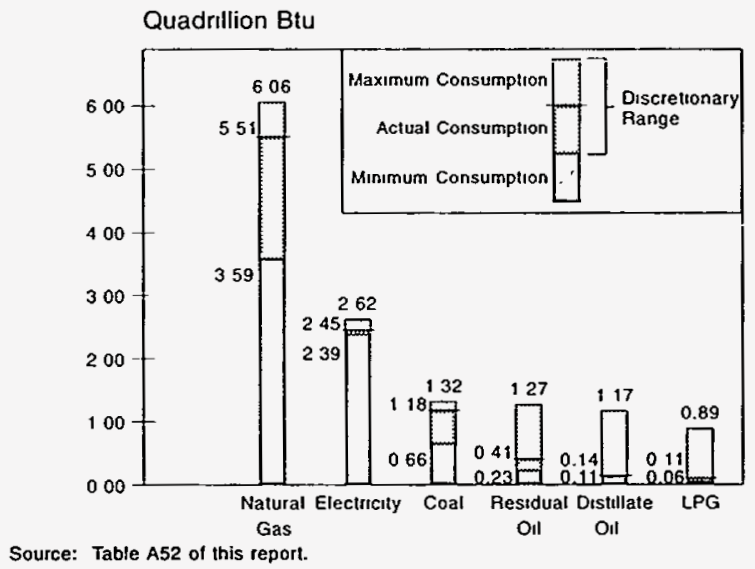

A look at the discretionary use rates for the commonly known fuels shows that manufacturers often used natural gas and coal whenever possible in 1991. In contrast, they seldom used distillate fuel oil and LPG, even when they could have done so (Figure 4.4). The discretionary use rates for the commonly known energy sources are as follows:

- Coal ranked highest in discretionary use with a robust rate of 80 percent. The consumption of coal was close to its short-term maximum level, given the levels of production in 1991 (Table A52).

- Natural gas ranked second in discretionary use with a rate of 77 percent.

- Electricity had a relatively low use rate of 26 percent, indicating that manufacturers preferred other energy sources when available. Electricity receipts rated a distant second to natural gas in terms of fuel demand by manufacturers, as they totaled 2.5 quadrillion Btu ( 0.7 trillion kilowatthours).

- The extremely low discretionary use rates for distillate fuel oil ( 3 percent) and LPG (6 percent) indicate that manufacturers generally avoided consuming these energy sources whenever possible.

- The discretionary use rate for residual fuel oil was 18 percent. Although that use rate was considerably higher than those for distillate fuel oil and LPG, it was still low enough to indicate a preference for other energy sources over residual fuel oil.

Price would appear to be the primary reason that manufacturers exercised their option to switch fuels (Figure 4.5). For example, the discretionary use of coal was due in part to the fact that manufacturers had no price incentive to switch to any other energy sources. At $\$ 1.78$ per million Btu, the national price of coal was substantially below that of any competing energy source.

\section{Figure 4.4. Discretionary Use Rates by Selected Fuels, 1991}

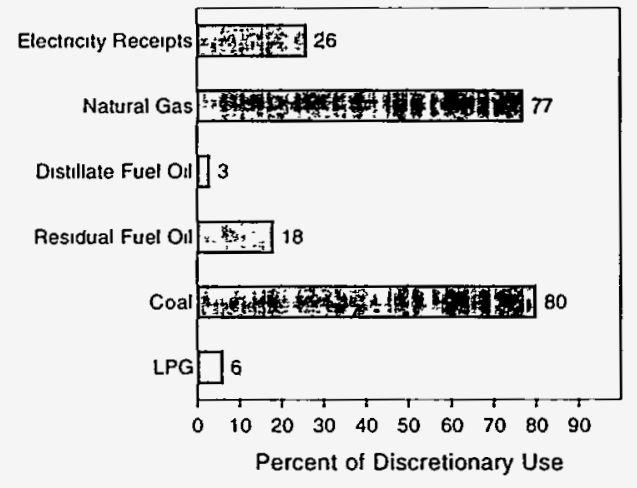

Source- Table A52 of this report. 
Furthermore, manufacturers had no price incentive to replace natural gas with distillate fuel, even though that switching capability existed in 1991 . Nationally, the price of natural gas stood at $\$ 2.30$ per million Btu, while the price of distillate fuel oil was $\$ 5.63$ per million Btu (Table A25). The price of distillate fuel was uniformly higher than the price of natural gas in the four Census Regions, with the difference never being less than $\$ 2.00$ per million Btu.

In certain geographic regions of the United States, however, price incentives existed to replace natural gas with residual fuel oil. Residual fuel oil could have replaced 0.6 trillion cubic feet ( 34 percent) of the 1.9 trillion cubic feet of natural gas that was switchable. At $\$ 2.54$ per million Btu, the national price of residual fuel oil was only marginally higher than that of natural gas. However, in the Northeast Census Region, the reported average price of natural gas hit $\$ 3.39$, compared with $\$ 2.90$ for residual fuel oil, thereby yielding a gas-oil price ratio of 1.17. The gas-oil price ratios were $1.10,0.87$, and 0.90 for the Midwest, South, and West Census Regions, respectively. Although price incentives existed in the Northeast and Midwest to switch from natural gas to residual fuel oil, these two regions consumed 0.7 trillion cubic feet of switchable natural gas, of which 0.3 trillion cubic feet ( 35 percent) could have been replaced by less expensive residual fuel oil in 1991 (Table A54). In these regions, manufacturers chose natural gas over residual fuel oil despite favorable price incentives.

\section{Figure 4.5. Average National Prices of Selected Purchased Fuels, 1991}

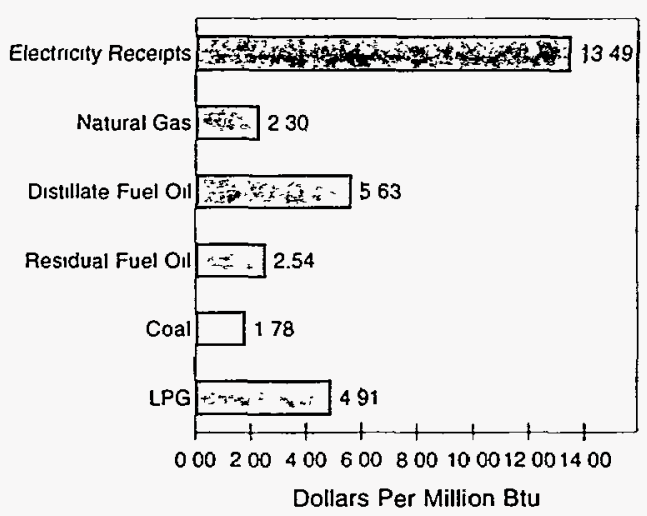

Souree: Table A25 of this report.

\section{Summary}

For the MECS, manufacturers are said to have a fuel-switching capability if they are able to meet their requirements for heat, power, and electricity generation by substituting one energy source for another within 30 days without modifying the equipment that consumes the fuel and if they can resume the same level of production following the switch.

In 1991, manufacturers required an nonswitchable minimum of 7.0 quadrillion Btu of electricity, natural gas, distillate and residual fuel oils, coal, and LPG, which was 72 percent of their actual consumption of these fuels. The fuels having the highest nonswitchable minimum requirements were, in order, natural gas, electricity, and coal.

Manufacturers had the capability to switch 2.8 quadrillion Btu of these energy sources, representing 28 percent of their actual consumption. Natural gas was the energy source having the greatest potential for switching. Manufacturers often used coal and natural gas whenever possible, even though they could have used another energy source instead. Price would seem to be the primary reason that manufacturers exercised their option to switch fuels. 

Appendix A

Detailed Tables 

Table A1. Total Primary Consumption of Energy for All Purposes by Census Region, Industry Group, and Selected Industries, 1991: Part 1

(Estimates in Btu or Physical Units)

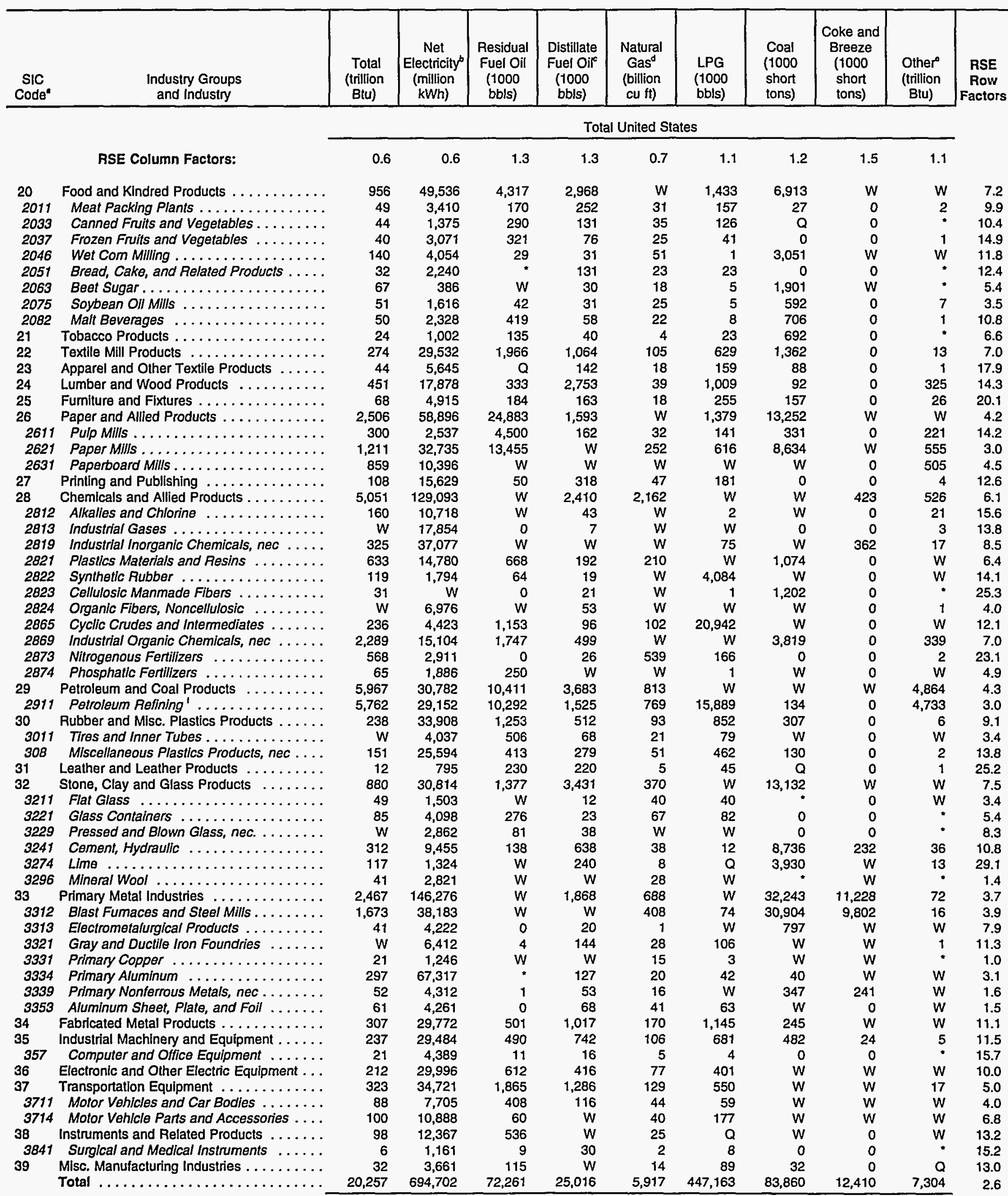

See footnotes at end of table.

Energy Information Administration/Manufacturing Consumption of Energy 1991 
Table A1. Total Primary Consumption of Energy for All Purposes by Census Region, Industry Group, and Selected Industries, 1991: Part 1 (Continued) (Estimates in Btu or Physical Units)

\begin{tabular}{|c|c|c|c|c|c|c|c|c|c|c|c|}
\hline $\begin{array}{c}\text { SIC } \\
\text { Code" }\end{array}$ & $\begin{array}{l}\text { Industry Groups } \\
\text { and Industry }\end{array}$ & $\begin{array}{c}\text { Total } \\
\text { (trillion } \\
\text { Btu) }\end{array}$ & $\begin{array}{c}\text { Net } \\
\text { Electricity } \\
\text { (million } \\
\text { kWh) }\end{array}$ & $\begin{array}{c}\text { Residual } \\
\text { Fuel Oil } \\
\text { (1000 } \\
\text { bbls) }\end{array}$ & $\begin{array}{c}\text { Distillate } \\
\text { Fuel Oile } \\
(1000 \\
\text { bbls })\end{array}$ & $\begin{array}{c}\text { Natural } \\
\text { Gas }^{d} \\
\text { (billion } \\
\text { cu ft) }\end{array}$ & $\begin{array}{c}\text { LPG } \\
(1000 \\
\text { bbls })\end{array}$ & $\begin{array}{l}\text { Coal } \\
\text { (1000 } \\
\text { short } \\
\text { tons) }\end{array}$ & $\begin{array}{c}\text { Coke and } \\
\text { Breeze } \\
(1000 \\
\text { short } \\
\text { tons) }\end{array}$ & $\begin{array}{c}\text { Other } \\
\text { (trillion } \\
\text { Etu) }\end{array}$ & $\begin{array}{c}\text { RSE } \\
\text { Row } \\
\text { Factors }\end{array}$ \\
\hline & & \multicolumn{9}{|c|}{ Northeast Census Region } & \\
\hline & RSE Column Factors: & 0.7 & 0.7 & 1.1 & 1.2 & 0.8 & 1.2 & 1.3 & 1.2 & 1.1 & \\
\hline 20 & Food and Kindred Products . . . . . . . . & 79 & 5,385 & 1,164 & 889 & 40 & 222 & 99 & 0 & 3 & 13.6 \\
\hline 2011 & Meat Packing Plants . . . . . . . . . . & 1 & 141 & $W$ & 34 & 1 & $Q$ & 0 & 0 & 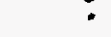 & 24.1 \\
\hline 2033 & Canned Fruits and Vegetables . . . . . . . . & 6 & 292 & 146 & 22 & 4 & 13 & 0 & 0 & * & 17.0 \\
\hline 2037 & Frozen Fruits and Vegetables . . . . . . . & 1 & 140 & 128 & 3 & $:$ & $\mathbf{Q}$ & 0 & 0 & $\cdot$ & 32.3 \\
\hline 2046 & Wet Corn Milling . . . . . . . . . . & * & 15 & W & $w$ & * & * & 0 & 0 & * & 23.3 \\
\hline 2051 & Bread, Cake, and Related Products . . . . & 7 & 382 & $\cdot$ & W & $W$ & 8 & 0 & 0 & • & 17.7 \\
\hline 2063 & Beet Sugar . . . . . . . . . . . . . . & 0 & 0 & 0 & 0 & 0 & 0 & 0 & 0 & 0 & NF \\
\hline 2075 & Soybean Oil Mills . . . . . . . . . . . & 0 & 0 & 0 & 0 & 0 & 0 & 0 & 0 & 0 & NF \\
\hline 2082 & Malt Beverages. & 8 & 496 & $w$ & 9 & 3 & 4 & $w$ & 0 & : & 16.2 \\
\hline 21 & Tobacco Products .............. & NA & NA & NA & NA & NA & NA & NA & NA & NA & 25.1 \\
\hline 22 & Textile Mill Products . . . . . . . . . . & 27 & 1,340 & 774 & 556 & 10 & 163 & 17 & 0 & 3 & 19.4 \\
\hline 23 & Apparel and Other Textile Products ..... & 5 & 497 & 44 & 49 & 2 & $\mathrm{Q}$ & 0 & 0 & 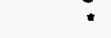 & 29.4 \\
\hline 24 & Lumber and Wood Products . . . . . . . & NA & NA & NA & NA & NA & NA & NA & NA & NA & 36.6 \\
\hline 25 & Furniture and Fixtures $\ldots \ldots \ldots \ldots \ldots$ & 7 & 449 & $Q$ & 49 & 2 & 60 & 0 & 0 & 2 & 29.9 \\
\hline 26 & Paper and Allied Products . . . . . . . . . & W & 8,054 & 11,439 & 625 & 36 & W & W & 0 & $11 \overline{1}$ & 6.0 \\
\hline 2611 & Pulp Mills . . . . . . . . . . . . . & 12 & 158 & 290 & 7 & 0 & 13 & 0 & 0 & 9 & 36.2 \\
\hline 2621 & Paper Mills .................. & 228 & 4,573 & 9,702 & $w$ & 19 & 284 & $w$ & 0 & 96 & 4.2 \\
\hline 2631 & Paperboard Mills . . . . . . . . . . . . . . & $w$ & 487 & W & $\mathbf{Q}$ & 6 & 5 & w & 0 & w & 15.0 \\
\hline 27 & Printing and Publishing $\ldots \ldots \ldots \ldots$ & 23 & 3,167 & 36 & 240 & 9 & 30 & 0 & 0 & 1 & 25.1 \\
\hline 28 & Chemicals and Allied Products . . . . . . . . & $W$ & 9,303 & 3,072 & 574 & 58 & 864 & $w$ & 0 & $\dot{w}$ & 8.5 \\
\hline 2812 & 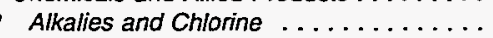 & $*$ & W & 0 & 0 & 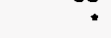 & 0 & 0 & 0 & 0 & 36.3 \\
\hline 2813 & Industrial Gases . . . . . . . . . . . . & 6 & 1,399 & 0 & 1 & $\bullet$ & * & 0 & 0 & 1 & 13.2 \\
\hline 2819 & Industrial Inorganic Chemicals, nec .... & 10 & 494 & 255 & 78 & 6 & 14 & 0 & 0 & 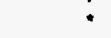 & 21.8 \\
\hline 2821 & Plastics Materials and Resins ........ & $w$ & 1,120 & 478 & 109 & 8 & $w$ & $w$ & 0 & $w$ & 9.9 \\
\hline 2822 & Synthetic Rubber . . . . . . . . . . . & $w$ & W & W & - & W & : & 0 & 0 & $\bullet$ & 25.7 \\
\hline 2823 & Cellulosic Manmade Fibers . . . . . . . . & 0 & 0 & 0 & 0 & 0 & 0 & 0 & 0 & 0 & NF \\
\hline 2824 & Organic Fibers, Noncellulosic ........ & * & 95 & $\mathbf{Q}$ & W & * & . & 0 & 0 & 0 & 14.7 \\
\hline 2865 & Cyclic Crudes and Intermediates . . . . . . & 12 & 406 & W & $w$ & 7 & 2 & $w$ & 0 & 1 & 20.4 \\
\hline 2869 & Industrial Organic Chemicals, nec ..... & W & 3,070 & 1,399 & W & $w$ & $\bar{W}$ & 0 & 0 & $w$ & 9.0 \\
\hline 2873 & Nitrogenous Fentilizers ........... & 1 & 29 & 0 & 3 & 1 & 1 & 0 & 0 & 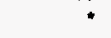 & 47.6 \\
\hline 2874 & Phosphatic Fertilizers . . . . . . . . & 0 & 0 & 0 & 0 & 0 & 0 & 0 & 0 & 0 & NF \\
\hline 29 & Petroleum and Coal Products ........ & 501 & 2,632 & 4,275 & $w$ & 28 & 853 & $w$ & 0 & 421 & 8.1 \\
\hline 2911 & Petroleum Refining ${ }^{\prime} \ldots \ldots \ldots \ldots$ & 484 & 2,093 & 4,275 & $w$ & 24 & 459 & W & 0 & 421 & 4.2 \\
\hline 30 & Rubber and Misc. Plastics Products ..... & 37 & 5,480 & 456 & 206 & W & W & 86 & 0 & 1 & 19.6 \\
\hline 3011 & Tires and Inner Tubes . . . . . . . . . . . & 2 & 125 & 63 & Q & 1 & 5 & 0 & 0 & : & 12.5 \\
\hline 308 & Miscellaneous Plastics Products, nec . . . & 28 & 4,807 & 251 & $\vec{w}$ & 7 & 180 & w & 0 & $w$ & 24.1 \\
\hline 31 & Leather and Leather Products . ....... & 4 & 205 & 142 & 197 & 1 & 29 & $\ddot{Q}$ & 0 & 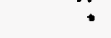 & 28.0 \\
\hline 32 & Stone, Clay and Glass Products ...... & 170 & 5,599 & 432 & 722 & 58 & $W$ & 3,451 & $w$ & $W$ & 17.6 \\
\hline 3211 & Flat Glass $\ldots \ldots \ldots \ldots \ldots \ldots$ & W & W & 0 & 1 & W & $w$ & 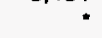 & 0 & 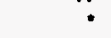 & 4.5 \\
\hline 3221 & Glass Containers ............. & 19 & 834 & 193 & 14 & 14 & 24 & 0 & 0 & $\cdot$ & 8.6 \\
\hline 3229 & Pressed and Blown Glass, nec. . . . . . & w & W & 80 & $w$ & W & 8 & 0 & 0 & $*$ & 10.1 \\
\hline 3241 & Cement, Hydraulic . . . . . . . . . . & 43 & 1,334 & 14 & W & $\because$ & 1 & 1,482 & $\mathbf{w}$ & $w$ & 18.1 \\
\hline 3274 & Lime . . . . . . . . . . . . . & $Q$ & $Q$ & 0 & $Q$ & $\bullet$ & $Q$ & $Q$ & 0 & . & NF \\
\hline 3296 & 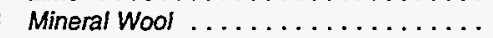 & 4 & 304 & 0 & W & $w$ & $w$ & 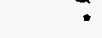 & $w$ & • & 1.7 \\
\hline 33 & Primary Metal Industries . . . . . . . . . & 471 & 18,002 & 852 & 326 & 102 & 310 & 10,016 & 738 & 12 & 9.1 \\
\hline 3312 & Blast Fumaces and Steel Mills . . . . . . . . & 376 & 7,828 & W & 156 & 65 & 30 & W & 522 & $w$ & 7.1 \\
\hline 3313 & Electrometalurgical Products . . . . . . . . & W & W & 0 & 1 & 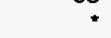 & 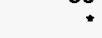 & W & $W$ & 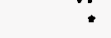 & 13.0 \\
\hline 3321 & Gray and Ductile Iron Foundries ...... & 5 & 350 & 0 & 14 & 2 & 18 & 1 & 74 & - & 16.6 \\
\hline 3331 & Primary Copper $\ldots \ldots \ldots \ldots \ldots$ & * & W & 0 & $w$ & 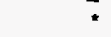 & 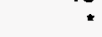 & 0 & 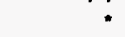 & * & 1.1 \\
\hline 3334 & Primary Aluminum . . . . . . . . . & $w$ & $w$ & * & W & W & W & $w$ & 0 & W & 4.8 \\
\hline 3339 & Primary Nonferrous Metals, nec . . . . . . . & W & 640 & 1 & W & 1 & 1 & $w$ & $w$ & 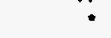 & 1.9 \\
\hline 3353 & Aluminum Sheet, Plate, and Foil ...... & $W$ & 451 & 0 & W & W & 23 & 0 & 0 & • & 2.2 \\
\hline 34 & Fabricated Metal Products . . . . . . . . . . & 57 & 5,074 & 368 & 377 & 32 & 155 & 9 & 37 & 1 & 15.7 \\
\hline 35 & Industrial Machinery and Equipment ..... & 41 & 5,202 & 408 & $w$ & 15 & $W$ & 0 & 0 & 2 & 19.2 \\
\hline 357 & Computer and Office Equipment . . . . . & 4 & 819 & 8 & 10 & 1 & 2 & 0 & 0 & 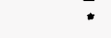 & 20.5 \\
\hline 36 & Electronic and Other Electric Equipment .. & 43 & 6,544 & 504 & 291 & 14 & 167 & 4 & 2 & 1 & 16.1 \\
\hline 37 & Transportation Equipment $\ldots \ldots \ldots \ldots$. & $w$ & 3,069 & 1,070 & $w$ & 10 & 60 & W & $\overrightarrow{0}$ & $w$ & 10.9 \\
\hline 3711 & Motor Vehicles and Car Bodies . ...... & $w$ & W & W & $w$ & 1 & 1 & 0 & 0 & $\because$ & 7.4 \\
\hline 3714 & Motor Vehicle Parts and Accessories .... & 8 & 887 & W & 6 & $w$ & $w$ & $w$ & 0 & * & 11.6 \\
\hline 38 & Instruments and Related Products ...... & 52 & 4,032 & 513 & $w$ & $\ddot{w}$ & $Q$ & w & 0 & $w$ & 17.2 \\
\hline 3841 & Surgical and Medical Instruments ..... & 2 & 332 & 9 & 16 & * & 2 & 0 & 0 & 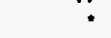 & 18.4 \\
\hline 39 & Misc. Manufacturing Industries . . . . . . . & $w$ & 1,187 & 84 & $w$ & W & 40 & 22 & 0 & - & 17.6 \\
\hline & Total $\ldots \ldots \ldots \ldots \ldots \ldots$ & 2,011 & 86,041 & 25,794 & 7,875 & 447 & 4,263 & W & $w$ & 588 & 5.0 \\
\hline
\end{tabular}


Table A1. Total Primary Consumption of Energy for All Purposes by Census Region, Industry Group, and Selected Industries, 1991: Part 1 (Continued) (Estimates in Btu or Physical Units)

\begin{tabular}{|c|c|c|c|c|c|c|c|c|c|c|c|}
\hline $\begin{array}{l}\text { SIC } \\
\text { Code }^{a}\end{array}$ & $\begin{array}{l}\text { Industry Groups } \\
\text { and Industry }\end{array}$ & $\begin{array}{c}\text { Total } \\
\text { (trillion } \\
\text { Btu) }\end{array}$ & $\begin{array}{c}\text { Net } \\
\text { Electricity } \\
\text { (million } \\
\text { kWh) }\end{array}$ & $\begin{array}{c}\text { Residual } \\
\text { Fuel Oil } \\
\text { (1000 } \\
\text { bbls) }\end{array}$ & $\begin{array}{c}\text { Distillate } \\
\text { Fuel Oil" } \\
\text { (1000 } \\
\text { bbls) }\end{array}$ & $\begin{array}{c}\text { Natural } \\
\text { Gas }^{\mathrm{a}} \\
\text { (billion } \\
\text { cu fit) }\end{array}$ & $\begin{array}{l}\text { LPG } \\
\text { (1000 } \\
\text { bbls) }\end{array}$ & $\begin{array}{l}\text { Coal } \\
\text { (1000 } \\
\text { short } \\
\text { tons) }\end{array}$ & \begin{tabular}{|c} 
Coke and \\
Breeze \\
(1000 \\
short \\
tons)
\end{tabular} & $\begin{array}{l}\text { Othere } \\
\text { (trillion } \\
\text { Btu) }\end{array}$ & $\begin{array}{c}\text { RSE } \\
\text { Row } \\
\text { Factors }\end{array}$ \\
\hline
\end{tabular}

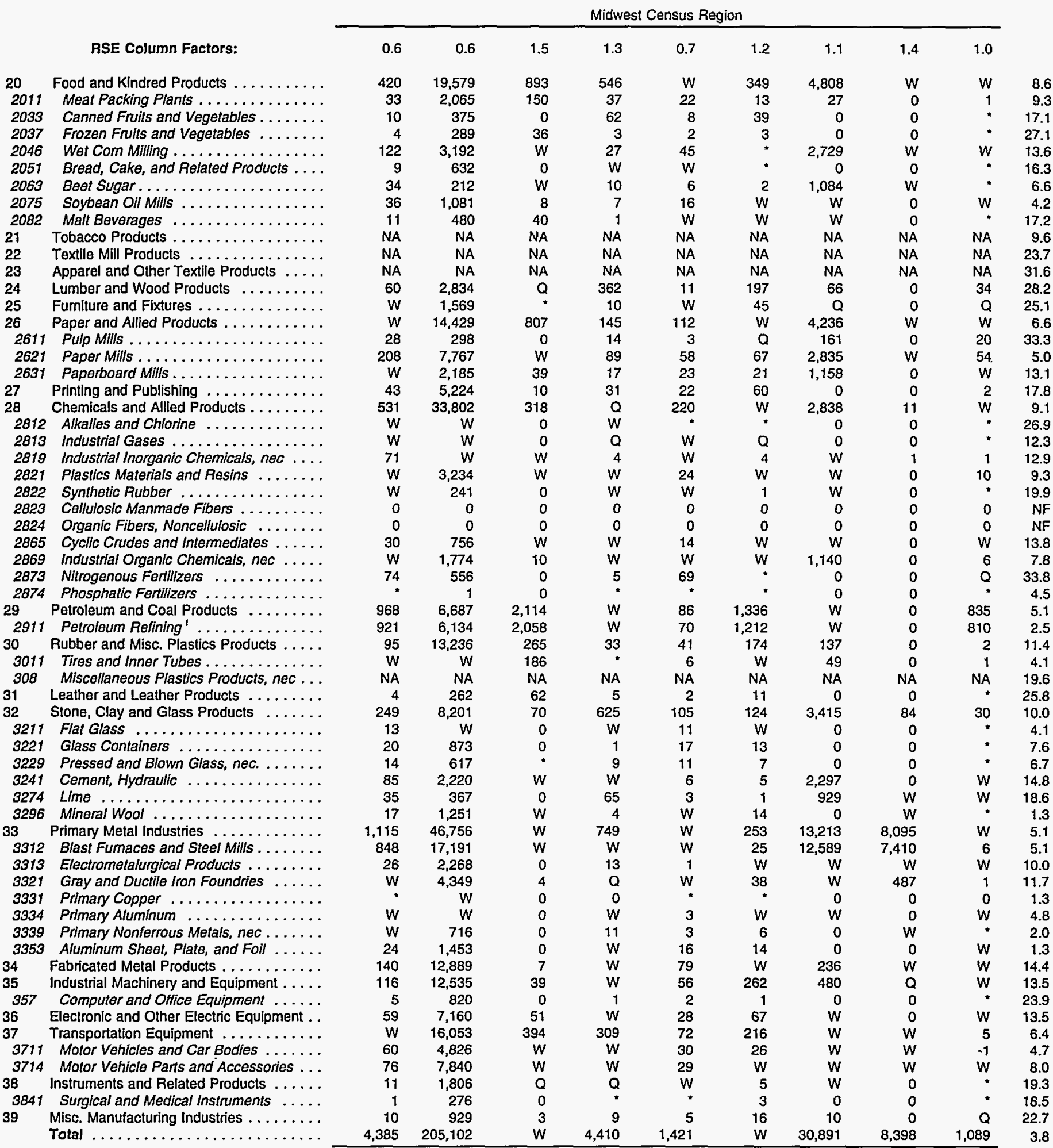

See footnotes at end of table. 
Table A1. Total Primary Consumption of Energy for All Purposes by Census Region, Industry Group, and Selected Industries, 1991: Part 1 (Continued)

(Estimates in Btu or Physical Units)

\begin{tabular}{|c|c|c|c|c|c|c|c|c|c|c|c|}
\hline $\begin{array}{l}\text { SIC } \\
\text { Coden }\end{array}$ & $\begin{array}{l}\text { Industry Groups } \\
\text { and Industry }\end{array}$ & $\begin{array}{c}\text { Total } \\
\text { (trillion } \\
\text { Btu) }\end{array}$ & $\begin{array}{c}\text { Net } \\
\text { Electricity } \\
\text { (million } \\
\text { kWh) }\end{array}$ & $\begin{array}{c}\text { Residual } \\
\text { Fuel Oil } \\
\text { (1000 } \\
\text { bbls) }\end{array}$ & $\begin{array}{c}\text { Distillate } \\
\text { Fuel Oife } \\
(1000 \\
\text { bbls })\end{array}$ & $\begin{array}{c}\text { Natural } \\
\text { Gas }^{d} \\
\text { (billion } \\
\text { cu fi) }\end{array}$ & $\begin{array}{c}\text { LPG } \\
\text { (1000 } \\
\text { bbls) }\end{array}$ & $\begin{array}{l}\text { Coal } \\
(1000 \\
\text { short } \\
\text { tons })\end{array}$ & $\begin{array}{l}\text { Coke and } \\
\text { Breeze } \\
(1000 \\
\text { short } \\
\text { tons) }\end{array}$ & $\begin{array}{c}\text { Other } \\
\text { (trillion } \\
\text { Btu) }\end{array}$ & \begin{tabular}{|c} 
RSE \\
Row \\
Factors
\end{tabular} \\
\hline
\end{tabular}

\begin{tabular}{|c|c|c|c|c|c|c|c|c|c|c|c|}
\hline & & & & & South & nsus $\mathrm{Re}$ & jion & & & & \\
\hline & RSE Column Factors: & 0.6 & 0.6 & 1.4 & 1.2 & 0.8 & 1.1 & 1.1 & 1.6 & 1.0 & \\
\hline 20 & Food and Kindred Products . & 255 & 15,937 & 1,572 & 859 & 131 & 623 & 766 & w & $\mathbf{Q}$ & 11.0 \\
\hline 2011 & Meat Packing Plants ...... & 10 & 892 & 14 & 167 & 5 & 112 & 0 & 0 & * & 16.9 \\
\hline 2033 & Canned Fruits and Vegetables . . . . . . . & 7 & 172 & 26 & 9 & 6 & 14 & 0 & 0 & - & 19.7 \\
\hline 2037 & Frozen Fruits and Vegetables & 8 & 551 & 122 & 12 & 5 & 6 & 0 & 0 & - & 17.6 \\
\hline 2046 & Wet Com Milling . & w & 755 & 0 & 2 & 4 & • & 322 & 0 & w & 23.0 \\
\hline 2051 & Bread, Cake, and Related Products & 11 & 837 & 0 & 19 & 7 & 8 & 0 & 0 & • & 14.2 \\
\hline 2063 & Beet Sugar . . . . . . . . . & w & w & 0 & 1 & w & * & 0 & w & - & 17.0 \\
\hline 2075 & Soybean Oil Mills & 15 & 535 & 34 & 24 & 8 & w & w & 0 & w & 4.9 \\
\hline 2082 & Malt Beverages. & 14 & 863 & W & $w$ & 8 & 1 & w & 0 & : & 14.0 \\
\hline 21 & Tobacco Products & 24 & 985 & 135 & 40 & 4 & 23 & 692 & 0 & - & 6.8 \\
\hline 22 & Textile Mill Products & 236 & 27,431 & 1,192 & 506 & 87 & 455 & 1,344 & 0 & 11 & 6.5 \\
\hline 23 & Apparel and Other Textile Products & 31 & 4,165 & Q & 71 & 12 & 124 & 83 & 0 & * & 22.8 \\
\hline 24 & Lumber and Wood Products & 209 & 9,218 & $\mathbf{Q}$ & 1,075 & 15 & 340 & 26 & 0 & 153 & 20.2 \\
\hline 25 & Furniture and Fixtures . & w & 2,592 & 63 & 99 & $w$ & 122 & $w$ & 0 & $w$ & 19.5 \\
\hline 26 & Paper and Allied Products ..... & 1,447 & 25,288 & 10,918 & 716 & w & 494 & 6,904 & 0 & w & 4.5 \\
\hline 2611 & Pulp Mills & 219 & 1,249 & 3,367 & 116 & 21 & 120 & 171 & 0 & 166 & 17.2 \\
\hline 2621 & Paper Mills & $w$ & 13,863 & 2,801 & $w$ & 122 & 149 & 3,910 & 0 & $w$ & 3.4 \\
\hline 2631 & Paperboard Mills ...... . & W & 5,650 & 4,660 & $w$ & W & 41 & 2,786 & 0 & 349 & 5.3 \\
\hline 27 & Printing and Publishing & 28 & 4,798 & $Q$ & 35 & 10 & 47 & 0 & 0 & 1 & 21.4 \\
\hline 28 & Chemicals and Allied Products ..... & 4,195 & 75,072 & $\bar{W}$ & 1,132 & 1,759 & 391,192 & w & 124 & 470 & 6.8 \\
\hline 2812 & Alkalies and Chlorine ... & 147 & 8,378 & 0 & 35 & W & 1 & w & 0 & 20 & 17.4 \\
\hline 2813 & Industrial Gases . . & w & 8,633 & 0 & 1 & $w$ & w & 0 & 0 & 1 & 12.8 \\
\hline 2819 & Industrial Inorganic Chemicals, nec . . . . & w & w & 325 & $w$ & w & 49 & w & 73 & 13 & 11.1 \\
\hline 2821 & Plastics Materials and Resins & w & 10,213 & w & w & 177 & 57,834 & w & 0 & w & 5.6 \\
\hline 2822 & Synthetic Rubber ........ & 111 & w & w & w & w & 4,083 & 0 & 0 & $w$ & 13.2 \\
\hline 2823 & Cellulosic Manmade Fibers & 31 & w & 0 & 21 & $w$ & 1 & 1,202 & 0 & $\because$ & 25.3 \\
\hline 2824 & Organic Fibers, Noncellulosic & w & 6,881 & w & w & W & w & w & 0 & 1 & 4.1 \\
\hline 2865 & Cyclic Crudes and Intermediates .. & 193 & 3,250 & 907 & 18 & 81 & w & w & 0 & w & 12.0 \\
\hline 2869 & Industrial Organic Chemicals, nec & 2,144 & 10,242 & 338 & w & w & w & 2,679 & 0 & 322 & 8.4 \\
\hline 2873 & Nitrogenous Fertilizers . . . . . . & 414 & 1,865 & 0 & 14 & 395 & 123 & 0 & 0 & 1 & 28.9 \\
\hline 2874 & Phosphatic Fertilizers & $w$ & 1,441 & 250 & w & w & 1 & w & 0 & 21 & 3.3 \\
\hline 29 & Petroleum and Coal Products & 3,479 & 13,878 & 2,084 & $w$ & 573 & w & w & w & 2,814 & 4.2 \\
\hline 2911 & Petroleum Refining' & 3,394 & 13,395 & 2,021 & $w$ & 561 & 2,450 & $w$ & 0 & 2,746 & 2.7 \\
\hline 30 & Rubber and Misc. Plastics Products & 88 & 12,327 & 532 & 241 & 35 & 253 & 61 & 0 & 3 & 10.4 \\
\hline 3011 & Tires and Inner Tubes. & w & 2,855 & W & $w$ & 13 & 53 & w & 0 & w & 4.1 \\
\hline 308 & Miscellaneous Plastics Products, nec... & 46 & 7,801 & 156 & $\ddot{w}$ & 16 & 123 & $w$ & 0 & $\because$ & 21.0 \\
\hline 31 & Leather and Leather Products. & 2 & 258 & 26 & 9 & . & 3 & 0 & 0 & $\mathbf{Q}$ & 24.5 \\
\hline 32 & Stone, Clay and Glass Products & 324 & 11,866 & 201 & 1,441 & 156 & w & 3,899 & $w$ & $\bar{w}$ & 11.4 \\
\hline 3211 & Flat Glass . . . . . . . . . & 23 & 771 & 0 & 7 & 19 & 9 & 0 & 0 & $\because$ & 4.0 \\
\hline 3221 & Glass Containers & 29 & 1,262 & w & $w$ & w & 23 & 0 & 0 & - & 8.3 \\
\hline 3229 & Pressed and Blown Glass, nec. & $w$ & 1,598 & 1 & $w$ & $w$ & W & 0 & 0 & . & 8.6 \\
\hline 3241 & Cement, Hydraulic . . . . . . . & 108 & 3,518 & 65 & 189 & 21 & 4 & 2,589 & 150 & 11 & 15.5 \\
\hline 3274 & Lime & 37 & 405 & 0 & 70 & $w$ & : & 1,155 & $w$ & $w$ & 21.8 \\
\hline 3296 & Mineral Wool & 16 & 936 & w & 1 & 11 & 16 & 0 & $\ddot{w}$ & $\because$ & 1.4 \\
\hline 33 & Primary Metal Industries . . . . & 630 & 47,291 & 1,442 & $w$ & w & W & w & 2,277 & 16 & 4.4 \\
\hline 3312 & Blast Furnaces and Steel Mills. & 381 & 10,744 & 1,437 & 207 & $w$ & 15 & 7,262 & 1,851 & w & 6.1 \\
\hline 3313 & Electrometalurgical Products & w & w & 0 & 7 & * & 0 & w & w & 1 & 11.1 \\
\hline 3321 & Gray and Ductile Iron Foundries & 21 & 1,622 & * & 56 & w & 46 & $\mathrm{a}$ & $w$ & : & 11.5 \\
\hline 3331 & Primary Copper & w & 200 & w & 5 & w & 1 & 0 & 0 & . & 1.1 \\
\hline 3334 & Primary Aluminum & 105 & 24,240 & 0 & $w$ & $w$ & 9 & w & $w$ & w & 4.2 \\
\hline 3339 & Primary Nonferrous Metals, nec. & w & 1,694 & 0 & 9 & w & w & W & 0 & W & 3.7 \\
\hline 3353 & Aluminum Sheet, Plate, and Foil & 25 & w & 0 & 26 & 17 & 22 & w & 0 & : & 1.8 \\
\hline 34 & Fabricated Metal Products . . . . . & 82 & 8,886 & Q & $w$ & 42 & $w$ & 0 & 23 & 3 & 21.0 \\
\hline 35 & Industrial Machinery and Equipment & 58 & 8,129 & 42 & 125 & 26 & $w$ & $\mathbf{Q}$ & $\mathbf{Q}$ & w & 15.7 \\
\hline 357 & Computer and Office Equipment & 4 & 800 & 3 & $\mathbf{Q}$ & 1 & * & 0 & 0 & $\because$ & 22.3 \\
\hline 36 & Electronic and Other Electric Equipment & 81 & 10,742 & 58 & 44 & 26 & 153 & w & w & w & 14.4 \\
\hline 37 & Transportation Equipment & 71 & 8,503 & W & 330 & 27 & 133 & w & 1 & 8 & 8.9 \\
\hline 3711 & Motor Vehicles and Car Bodies & 24 & 2,362 & 73 & 35 & 12 & 28 & w & 0 & w & 4.5 \\
\hline 3714 & Motor Vehicle Parts and Accessories. & 13 & 1,919 & . & 8 & w & 41 & w & 1 & * & 11.6 \\
\hline 38 & Instruments and Related Products & 17 & 3,285 & Q & 45 & 5 & 6 & 0 & 0 & * & 18.6 \\
\hline 3841 & Surgical and Medical Instruments & 2 & 331 & $\overline{0}$ & 11 & : & 1 & 0 & 0 & - & 21.5 \\
\hline 39 & Misc. Manufacturing Industries . & w & 1,168 & 28 & $\mathbf{Q}$ & w & 25 & 0 & 0 & w & 23.5 \\
\hline & Total & 11,296 & 291,819 & $w$ & 8,481 & 3,368 & w & 29,974 & 2,677 & 4,407 & 3.6 \\
\hline
\end{tabular}


Table A1. Total Primary Consumption of Energy for All Purposes by Census Region, Industry Group, and Selected Industries, 1991: Part 1 (Continued) (Estimates in Btu or Physical Units)

\begin{tabular}{|c|c|c|c|c|c|c|c|c|c|c|c|}
\hline \multirow[t]{3}{*}{$\underset{\text { Code }}{\operatorname{SIC}}$} & $\begin{array}{c}\text { Industry Groups } \\
\text { and Industry }\end{array}$ & $\begin{array}{c}\text { Total } \\
\text { (trillion } \\
\text { Btu) }\end{array}$ & $\begin{array}{c}\text { Net } \\
\text { Electricity } \\
\text { (million }^{\circ} \\
\mathrm{kWh} \text { ) }\end{array}$ & $\begin{array}{c}\text { Residual } \\
\text { Fuel Oil } \\
\text { (1000 } \\
\text { bbls) } \\
\end{array}$ & $\begin{array}{c}\text { Distillate } \\
\text { Fuel Oif } \\
\text { (1000 } \\
\text { bbls) }\end{array}$ & $\begin{array}{c}\text { Natural } \\
\text { Gas }^{d} \\
\text { (billion } \\
\text { cu ft) } \\
\end{array}$ & $\begin{array}{l}\text { LPG } \\
\text { (1000 } \\
\text { bbls) }\end{array}$ & $\begin{array}{l}\text { Coal } \\
(1000 \\
\text { short } \\
\text { tons }) \\
\end{array}$ & $\begin{array}{c}\text { Coke and } \\
\text { Breeze } \\
(1000 \\
\text { short } \\
\text { tons) } \\
\end{array}$ & $\begin{array}{l}\text { Other } \\
\text { (trillion } \\
\text { Btu) }\end{array}$ & \begin{tabular}{|c} 
RSE \\
Row \\
Factors
\end{tabular} \\
\hline & & \multicolumn{10}{|c|}{ West Census Region } \\
\hline & RSE Column Factors: & 0.7 & 0.7 & 1.2 & 1.1 & 0.8 & 1.1 & 1.3 & 1.2 & 1.0 & \\
\hline 20 & Food and Kindred Products . & 202 & 8,635 & 688 & 673 & 116 & 240 & 1,241 & w & w & 9.5 \\
\hline 2011 & Meat Packing Plants ..... & 5 & 311 & w & 14 & 3 & $\mathbf{Q}$ & 0 & 0 & * & 17.5 \\
\hline 2033 & Canned Fruits and Vegetables. & 20 & 537 & 119 & 38 & 16 & 60 & $\mathbf{0}$ & 0 & - & 12.4 \\
\hline 2037 & Frozen Frults and Vegetables & 26 & 2,091 & $\mathbf{Q}$ & 57 & 17 & 31 & 0 & 0 & 1 & 17.3 \\
\hline 2046 & Wet Com Milling . .......... & w & 93 & 0 & w & 2 & * & 0 & 0 & w & 20.5 \\
\hline 2051 & Bread, Cake, and Related Products .... & 6 & 388 & 0 & 2 & 5 & 7 & 0 & 0 & * & 26.8 \\
\hline 2063 & Beet Sugar $\ldots \ldots \ldots \ldots \ldots \ldots \ldots$ & W & w & w & 19 & w & 3 & 817 & 51 & - & 8.5 \\
\hline 2075 & Soybean Oll Mills $\ldots \ldots \ldots \ldots \ldots \ldots$ & 0 & 0 & 0 & 0 & 0 & 0 & 0 & 0 & 0 & NF \\
\hline 2082 & Malt Beverages. & 17 & 489 & w & w & w & W & w & 0 & * & 16.4 \\
\hline 21 & Tobacco Products & 0 & 0 & 0 & 0 & 0 & 0 & 0 & 0 & 0 & NF \\
\hline 22 & Textile Mill Products & 6 & 274 & 0 & 2 & 4 & $\mathbf{Q}$ & 0 & 0 & - & 30.1 \\
\hline 23 & Apparel and Other Textile Products ..... & NA & NA & NA & NA & NA & NA & NA & NA & NA & 29.9 \\
\hline 24 & Lumber and Wood Products $\ldots \ldots \ldots \ldots$ & 166 & 5,017 & 131 & 1,172 & 10 & 348 & 0 & 0 & 130 & 20.0 \\
\hline 25 & Furniture and Fixtures. & 3 & 304 & 0 & $Q$ & 1 & 28 & 0 & 0 & * & 32.8 \\
\hline 26 & Paper and Allied Products & w & 11,126 & 1,718 & 108 & 108 & w & w & 0 & w & 6.2 \\
\hline 2611 & Pulp MIIls . . . . . . . . . & 41 & 832 & 842 & 25 & 7 & 5 & 0 & 0 & 26 & 19.4 \\
\hline 2621 & Paper MIIIs . & W & 6,532 & W & 39 & 53 & 116 & W & 0 & w & 5.6 \\
\hline 2631 & Paperboard Mills ..... . & w & 2,074 & w & w & 39 & W & W & 0 & w & 8.1 \\
\hline 27 & Printing and Publishing .. & NA & NA & NA & NA & NA & NA & NA & NA & NA & 22.0 \\
\hline 28 & Chemicals and Allied Products .. & W & 10,916 & w & 186 & 125 & 68 & w & 288 & w & 13.4 \\
\hline 2812 & Alkalles and Chlorine ............. & w & w & $w$ & $w$ & 4 & . & 0 & 0 & 1 & 19.8 \\
\hline 2813 & Industrial Gases ... & $w$ & w & 0 & $w$ & 6 & w & 0 & 0 & * & 14.8 \\
\hline 2819 & Industrial Inorganic Chemicals, nec & w & 3,961 & w & w & w & $\mathbf{Q}$ & w & 288 & 3 & 11.0 \\
\hline 2821 & Plastles Materials and Resins . . . . & 3 & 212 & 0 & * & 2 & 2 & 0 & 0 & • & 17.2 \\
\hline 2822 & Synthetic Rubber .......... & * & * & 0 & • & * & • & 0 & 0 & * & 24.5 \\
\hline 2823 & Cellulosic Manmade Fibers . . & 0 & 0 & 0 & 0 & 0 & 0 & 0 & 0 & 0 & NF \\
\hline 2824 & Organic Fibers, Noncellulosic $\ldots \ldots \ldots$ & 0 & 0 & 0 & 0 & 0 & 0 & 0 & 0 & 0 & NF \\
\hline 2865 & Cyclic Crudes and Intermediates. & - & 11 & 0 & • & - & 1 & 0 & 0 & * & 25.4 \\
\hline 2869 & Industrial Organic Chemicals, nec & 5 & $\mathbf{Q}$ & 0 & $Q$ & w & Q & 0 & 0 & w & 13.6 \\
\hline 2873 & Nitrogenous Fortillzers $\ldots \ldots \ldots \ldots$ & 79 & 461 & 0 & 3 & 74 & 42 & 0 & 0 & * & 40.7 \\
\hline 2874 & Phosphatic Fertilizers ..... & $w$ & 444 & 0 & 7 & w & 0 & 0 & 0 & w & 22.7 \\
\hline 29 & Petroleum and Coal Products & 1,019 & 7,586 & 1,938 & w & 125 & 11,796 & w & 0 & 794 & 4.1 \\
\hline 2911 & Petroleum Refining ' ..... & 962 & 7,530 & 1,938 & 780 & 114 & 11,768 & 0 & 0 & 756 & 2.2 \\
\hline 30 & Rubber and Misc. Plastics Products . & 19 & 2,865 & 1 & $\mathbf{Q}$ & W & w & $\mathbf{Q}$ & 0 & - & 18.3 \\
\hline 3011 & Tres and Inner Tubes ............ & * & W & w & w & . & W & 0 & 0 & * & 7.2 \\
\hline 308 & Miscellanoous Plastics Products, nec . . . & 14 & 2,573 & 0 & $Q$ & 5 & 37 & 0 & 0 & * & 22.0 \\
\hline 31 & Leather and Leather Products . . . . & $\mathbf{Q}$ & 70 & 0 & $\mathbf{Q}$ & $\mathbf{Q}$ & $\mathbf{Q}$ & 0 & 0 & * & 42.3 \\
\hline 32 & Stone, Clay and Glass Products & 137 & 5,147 & 674 & 642 & 51 & 123 & 2,367 & 51 & 5 & 14.8 \\
\hline 3211 & $\ldots \ldots \ldots \ldots \ldots$ & w & 148 & w & w & w & w & 0 & 0 & • & 4.6 \\
\hline 3221 & Glass Containers & 18 & 1,129 & w & w & w & 22 & 0 & 0 & . & 9.5 \\
\hline 3229 & Pressed and Blown Glass, nec. .. & $w$ & w & 0 & * & w & 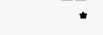 & 0 & 0 & . & 13.3 \\
\hline 3241 & Cement, Hydraulle $\ldots \ldots \ldots \ldots \ldots$. & 77 & 2,384 & W & 140 & 11 & 1 & 2,367 & $\mathbf{Q}$ & 3 & 20.8 \\
\hline 3274 & $\ldots \ldots \ldots \ldots \ldots \ldots$ & w & w & w & w & w & * & 0 & 0 & * & 21.3 \\
\hline 3296 & Mineral Wool ... & 3 & 330 & 0 & w & w & w & 0 & w & • & 2.1 \\
\hline 33 & Primary Metal Industries $\ldots \ldots \ldots$. & 251 & 34,227 & 87 & w & w & w & W & 118 & w & 5.8 \\
\hline 3312 & Blast Fumaces and Steel Mills. & 69 & 2,420 & w & w & w & 4 & w & 20 & 1 & 7.6 \\
\hline 3313 & Eloctrometalurgical Products & 0 & 0 & 0 & 0 & 0 & 0 & 0 & 0 & 0 & NF \\
\hline 3321 & Gray and Ductile Iron Foundries. & 3 & 92 & 0 & 3 & w & 3 & 0 & w & • & 37.2 \\
\hline 3331 & Primary Copper $\ldots \ldots \ldots \ldots \ldots \ldots$ & w & 1,027 & w & w & w & 1 & w & w & * & 1.0 \\
\hline 3334 & Primary Aluminum & 118 & 26,391 & $\cdot$ & 17 & 6 & 28 & 5 & 0 & 22 & 3.5 \\
\hline 3339 & Primary Nonferrous Metals, nec.. & w & 1,263 & 0 & $w$ & w & $w$ & $w$ & w & * & 1.0 \\
\hline 3353 & Aluminum Sheet, Plate, and Foil & w & w & 0 & w & W & w & 0 & 0 & - & 1.1 \\
\hline 34 & Fabricated Metal Products . . . . . . . . . & 28 & 2,922 & * & 78 & 16 & 103 & 0 & 0 & - & 19.6 \\
\hline 35 & Industrial Machinery and Equipment. & 22 & 3,619 & 0 & 14 & 8 & 72 & 0 & 0 & - & 27.0 \\
\hline 357 & Computer and Office Equipment & 9 & 1,950 & 0 & * & 2 & $\mathbf{Q}$ & 0 & 0 & • & 15.9 \\
\hline 36 & Electronic and Other Electric Equipment . & 29 & 5,550 & 0 & $\mathbf{Q}$ & 9 & 13 & 0 & 0 & w & 18.0 \\
\hline
\end{tabular}

See footnotes at end of table. 


\section{Table A1. Total Primary Consumption of Energy for All Purposes by Census Region, Industry Group, and Selected Industries, 1991: Part 1 (Continued) (Estimates in Btu or Physical Units)}

\begin{tabular}{|c|c|c|c|c|c|c|c|c|c|c|c|}
\hline $\begin{array}{c}\text { SIC } \\
\text { Code }\end{array}$ & $\begin{array}{l}\text { Industry Groups } \\
\text { and Industry }\end{array}$ & $\begin{array}{c}\text { Total } \\
\text { (trillion } \\
\text { Btu) }\end{array}$ & $\begin{array}{c}\text { Net } \\
\text { Electricity } \\
\text { (million } \\
\text { kWh) }\end{array}$ & $\begin{array}{c}\text { Residual } \\
\text { Fuel Oil } \\
\text { (1000 } \\
\text { bbls) } \\
\end{array}$ & $\begin{array}{c}\text { Distillate } \\
\text { Fuel Oif } \\
(1000 \\
\text { bbls) }\end{array}$ & $\begin{array}{c}\text { Natural } \\
\text { Gas }^{d} \\
\text { (billion } \\
\text { cu ft) } \\
\end{array}$ & $\begin{array}{l}\text { LPG } \\
\text { (1000 } \\
\text { bbls) }\end{array}$ & $\begin{array}{l}\text { Coal } \\
\text { (1000 } \\
\text { short } \\
\text { tons) } \\
\end{array}$ & $\begin{array}{c}\text { Coke and } \\
\text { Breeze } \\
\text { (1000 } \\
\text { short } \\
\text { tons) }\end{array}$ & $\begin{array}{c}\text { Other } \\
\text { (trillion } \\
\text { Btu) }\end{array}$ & $\begin{array}{c}\text { RSE } \\
\text { Row } \\
\text { Factors }\end{array}$ \\
\hline & & \multicolumn{10}{|c|}{ West Census Region } \\
\hline \multicolumn{2}{|r|}{ RSE Column Factors: } & 0.7 & 0.7 & 1.2 & 1.1 & 0.8 & 1.1 & 1.3 & 1.2 & 1.0 & \\
\hline 37 & Transportation Equipment & $w$ & 7,096 & $w$ & W & 20 & 141 & 0 & 0 & $W$ & 11.8 \\
\hline 3711 & Motor Vehicles and Car Bodies & $w$ & $W$ & 0 & 19 & 1 & 3 & 0 & 0 & 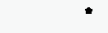 & 6.9 \\
\hline 3714 & Motor Vehicle Parts and Accessories ... & 3 & 243 & $\mathbf{Q}$ & 2 & 2 & 7 & 0 & 0 & • & 21.6 \\
\hline 38 & Instruments and Related Products . . . . . & 17 & 3,244 & 4 & 9 & 5 & 6 & 0 & 0 & • & 15.0 \\
\hline 3841 & Surgical and Medical Instruments ..... & 1 & 222 & 0 & 3 & * & 2 & 0 & 0 & - & 27.5 \\
\hline 39 & Misc. Manufacturing Industries . . . . . . . . & 2 & 376 & 0 & 1 & 1 & 8 & 0 & 0 & : & 26.4 \\
\hline & Total $\ldots \ldots \ldots \ldots \ldots \ldots$ & 2,564 & 111,741 & 5,344 & 4,250 & 681 & 13,381 & $w$ & $w$ & 1,220 & 4.6 \\
\hline
\end{tabular}

- See Appendices B and F for descriptions of the Standard Industrial Classilication system.

- "Net Electricity" is obtained by summing purchases, transfers in, and generation from noncombustible renewable resources, minus quantities sold and transferred out. It does not include electricity inputs from onsite cogeneration or generation from combustible fuels because that energy has already been included as generating fuel (for example, coal).

"Distillate Fuel Oil" includes Nos. 1, 2, and 4 fuel oils and Nos. 1, 2, and 4 diesel fuels.

d "Natural Gas" includes natural gas obtained from utilities, transmission pipelines, and any other supplier(s) such as brokers and producers.

- "Other" includes net steam (the sum of purchases, generation from renewables, and net transfers), and other energy that respondents indicated was used to produce heat and power or as feedstock/raw material inputs. See also Footnote "f".

'For the petroleum refining industry only, the feedstocks and raw material inputs for the production of nonenergy products (i.e., asphalt, waxes, lubricants, and

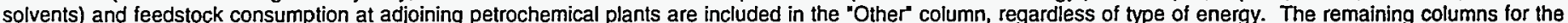

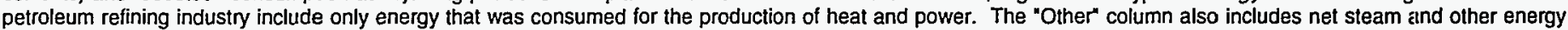
that respondents indicated was used in the production of heat and power. Those inputs and feedstocks that were converted to other energy products (t.g., crude oil converted to residual and distillate fuel oils) are excluded. See Appendix $B$ for more information.

NF=No applicable RSE row/column factor.

- Estimate less than 0.5. Data are included in higher level totals.

$W=$ Withheld to avoid disclosing data for individual establishments. Data are included in higher level totals.

$Q=$ Withheld because Relative Standard Error is greater than 50 percent. Data are included in higher level totals.

$N A=$ Not available. Data are included in higher level totals.

Notes: - To obtain a RSE percentage for any table cell, multiply the cell's corresponding RSE column and RSE row factors. - Totals may not equal sum of components because of independent rounding. - The derived estimates presented in this table are for the primary consumption of energy for heat and power and as feedstocks or raw material inputs. Primary consumption is defined as the consumption of the energy that was originally produced offsite or was produced onsite from input materials not classified as energy. Examples of the latter are hydrogen produced from the electrolysis of brine; the output of captive (onsite) mines or wells;

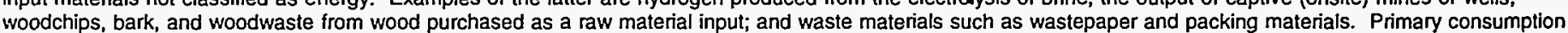
excludes quantities of energy that are produced from other energy inputs and, therefore, avoids double counting.

Source: Energy Information Administration, Office of Energy Markets and End Use, Energy End Use and Integrated Statistics Division, Form ElA-846, "1991 Manufacturing Energy Consumption Survey," and Office of Oil and Gas, Petroleum Supply Division, Form EIA-810, "Monthly Refinery Report" for 1991. 
Table A1. Total Primary Consumption of Energy for All Purposes by Census Region, Industry Group, and Selected Industries, 1991: Part 2 (Estimates in Trillion Btu)

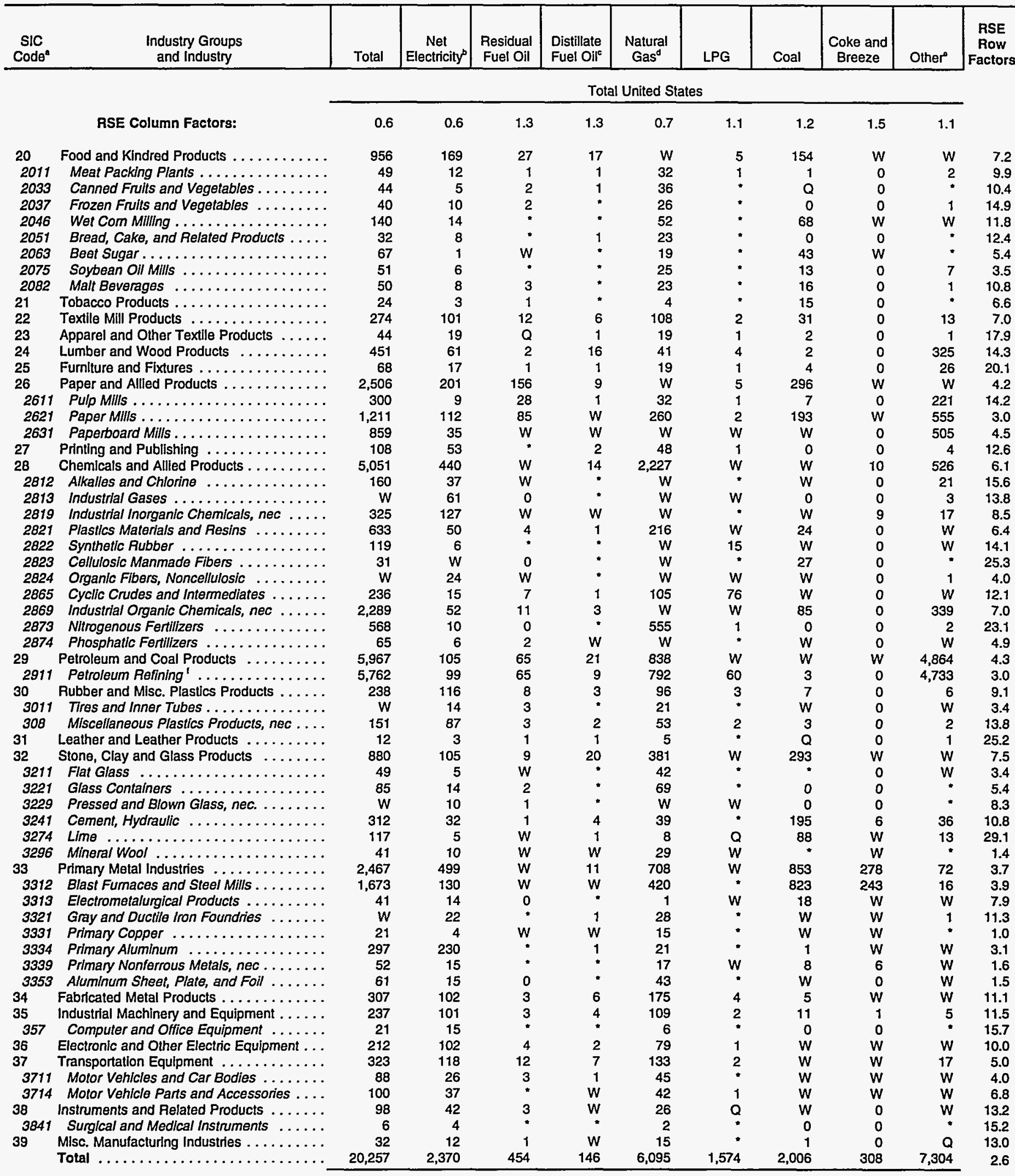

See footnotes at end of table. 


\section{Table A1. Total Primary Consumption of Energy for All Purposes by Census Region, Industry Group, and Selected Industries, 1991: Part 2 (Continued) (Estimates in Trillion Btu)}

\begin{tabular}{|c|c|c|c|c|c|c|c|c|c|c|c|}
\hline $\begin{array}{l}\text { SIC } \\
\text { Code" }\end{array}$ & $\begin{array}{l}\text { Industry Groups } \\
\text { and Indusiry }\end{array}$ & Total & $\begin{array}{c}\text { Net } \\
\text { Electricity }\end{array}$ & $\begin{array}{l}\text { Residual } \\
\text { Fuel Oil }\end{array}$ & $\begin{array}{l}\text { Distillate } \\
\text { Fuel Oif }\end{array}$ & $\begin{array}{l}\text { Natural } \\
\text { Gas }^{\AA}\end{array}$ & LPG & Coal & $\begin{array}{c}\text { Coke and } \\
\text { Breeze }\end{array}$ & Other & $\begin{array}{c}\text { RSE } \\
\text { Row } \\
\text { Factors }\end{array}$ \\
\hline & & \multicolumn{10}{|c|}{ Northeast Census Region } \\
\hline & RSE Column Factors: & 0.7 & 0.7 & 1.1 & 1.2 & 0.8 & 1.2 & 1.3 & 1.2 & 1.1 & \\
\hline 20 & Food and Kindred Products . & 79 & 18 & 7 & 5 & 42 & 1 & 2 & 0 & 3 & 13.6 \\
\hline 2011 & Meat Packing Plants ..... & 1 & * & w & * & 1 & $\mathbf{Q}$ & 0 & 0 & * & 24.1 \\
\hline 2033 & Canned Fruits and Vegetables. . & 6 & 1 & 1 & * & 4 & $*$ & $\mathbf{Q}$ & 0 & - & 17.0 \\
\hline 2037 & Frozen Fruits and Vegetables & 1 & - & 1 & - & - & $\mathbf{Q}$ & 0 & 0 & - & 32.3 \\
\hline 2046 & Wet Com Milling . . . . . . . & - & * & w & w & - & * & 0 & 0 & - & 23.3 \\
\hline 2051 & Bread, Cake, and Related Products .... & 7 & 1 & * & w & w & * & 0 & 0 & - & 17.7 \\
\hline 2063 & Beet Sugar $\ldots \ldots \ldots \ldots \ldots \ldots \ldots$ & 0 & 0 & 0 & 0 & 0 & 0 & 0 & 0 & 0 & NF \\
\hline 2075 & Soybean Oil Mills $\ldots \ldots \ldots \ldots \ldots \ldots$ & 0 & 0 & 0 & 0 & 0 & 0 & 0 & 0 & 0 & NF \\
\hline 2082 & Malt Beverages & 8 & 2 & w & * & 4 & * & w & 0 & - & 16.2 \\
\hline 21 & Tobacco Products ... & NA & NA & NA & NA & NA & NA & NA & NA & NA & 25.1 \\
\hline 22 & Textile Mill Products $\ldots \ldots \ldots \ldots \ldots \ldots$ & 27 & 5 & 5 & 3 & 11 & 1 & * & 0 & 3 & 19.4 \\
\hline 23 & Apparel and Other Textile Products & 5 & 2 & * & * & 2 & $\mathbf{Q}$ & 0 & 0 & - & 29.4 \\
\hline 24 & Lumber and Wood Products $\ldots \ldots \ldots \ldots$ & NA & NA & NA & NA & NA & NA & NA & NA & NA & 36.6 \\
\hline 25 & Furniture and Fixtures .... & 7 & 2 & $\mathbf{Q}$ & * & 2 & * & 0 & 0 & 2 & 29.9 \\
\hline 26 & Paper and Allied Products & w & 27 & 72 & 4 & 37 & w & w & 0 & 111 & 6.0 \\
\hline 2611 & Pulp Mills $\ldots \ldots \ldots \ldots \ldots \ldots \ldots$ & 12 & 1 & 2 & - & 0 & * & 0 & 0 & 9 & 36.2 \\
\hline 2621 & Paper Mills $\ldots \ldots \ldots \ldots \ldots \ldots \ldots$ & 228 & 16 & 61 & W & 19 & 1 & w & 0 & 96 & 4.2 \\
\hline 2631 & Paperboard Mills . . . . . . . . . . & w & 2 & w & $\mathrm{Q}$ & 6 & * & W & 0 & w & 15.0 \\
\hline 27 & Printing and Publishing $\ldots \ldots \ldots \ldots \ldots$ & 23 & 11 & * & 1 & 9 & * & 0 & 0 & 1 & 25.1 \\
\hline 28 & Chemicals and Allied Products. & w & 32 & 19 & 3 & 60 & 3 & w & 0 & w & 8.5 \\
\hline 2812 & Alkalies and Chlorine ...... & * & w & 0 & 0 & * & 0 & 0 & $\mathbf{0}$ & 0 & 36.3 \\
\hline 2813 & Industrial Gases ........ & 6 & 5 & 0 & * & - & * & 0 & 0 & 1 & 13.2 \\
\hline 2819 & Industrial Inorganic Chemicals, nec .... & 10 & 2 & 2 & * & 6 & * & $\mathbf{0}$ & 0 & * & 21.8 \\
\hline 2821 & Plastics Materials and Resins $\ldots \ldots \ldots$ & w & 4 & 3 & 1 & 8 & w & w & 0 & w & 9.9 \\
\hline 2822 & Synthetic Rubber $\ldots \ldots \ldots \ldots$ & w & w & w & * & $w$ & $*$ & 0 & 0 & - & 25.7 \\
\hline 2823 & Cellulosic Manmade Fibers . . . . . . . . . & 0 & 0 & 0 & 0 & 0 & 0 & 0 & 0 & 0 & NF \\
\hline 2824 & Organic Fibers, Noncellulosic ... & * & * & $\mathrm{Q}$ & w & * & * & 0 & 0 & 0 & 14.7 \\
\hline 2865 & Cyclic Crudes and Intermediates. & 12 & 1 & w & w & 7 & * & w & 0 & 1 & 20.4 \\
\hline 2869 & Industrial Organic Chemicals, nec. & w & 10 & 9 & w & $w$ & w & 0 & 0 & w & 9.0 \\
\hline 2873 & Nitrogenous Fentilizers $\ldots \ldots \ldots$ & 1 & • & 0 & * & 1 & * & 0 & 0 & * & 47.6 \\
\hline 2874 & Phosphatic Fertilizers & 0 & 0 & 0 & 0 & 0 & 0 & 0 & 0 & 0 & NF \\
\hline 29 & Petroleum and Coal Products & 501 & 9 & 27 & W & 29 & 3 & w & 0 & 421 & 8.1 \\
\hline 2911 & Petroleum Refining ${ }^{\prime} . . .$. & 484 & 7 & 27 & W & 25 & 2 & w & 0 & 421 & 4.2 \\
\hline 30 & Rubber and Misc. Plastics Products ..... & 37 & 19 & 3 & 1 & $w$ & $\bar{w}$ & 2 & 0 & 1 & 19.6 \\
\hline 3011 & Tires and Inner Tubes. . & 2 & - & * & $\mathrm{Q}$ & 1 & * & 0 & 0 & - & 12.5 \\
\hline 308 & Miscellaneous Plastics Products, nec . . . & 28 & 16 & 2 & w & 7 & 1 & w & 0 & w & 24.1 \\
\hline 31 & Leather and Leather Products $\ldots \ldots \ldots$ & 4 & 1 & 1 & 1 & 1 & $*$ & $\mathbf{Q}$ & 0 & * & 28.0 \\
\hline 32 & Stone, Clay and Glass Products & 170 & 19 & 3 & 4 & 60 & w & 77 & W & w & 17.6 \\
\hline 3211 & Flat Glass $\ldots \ldots \ldots \ldots \ldots$ & w & w & 0 & : & $w$ & W & * & 0 & $\because$ & 4.5 \\
\hline 3221 & Glass Containers .......... & 19 & 3 & 1 & * & 14 & $\because$ & 0 & 0 & • & 8.6 \\
\hline 3229 & Pressed and Blown Glass, nec. & w & w & 1 & w & w & • & 0 & 0 & - & 10.1 \\
\hline 3241 & Cement, Hydraulic . . . . . . . . & 43 & 5 & * & w & - & - & 33 & w & w & 18.1 \\
\hline 3274 & Lime & $\mathrm{Q}$ & $\mathrm{Q}$ & 0 & $Q$ & $*$ & $\mathrm{Q}$ & $Q$ & 0 & - & NF \\
\hline 3296 & Mineral Wool & 4 & 1 & 0 & w & w & w & * & w & . & 1.7 \\
\hline 33 & Primary Metal Industries & 471 & 61 & 5 & 2 & 105 & 1 & 267 & 18 & 12 & 9.1 \\
\hline 3312 & Blast Fumaces and Steel Mills. & 376 & 27 & w & 1 & 67 & * & w & 13 & w & 7.1 \\
\hline 3313 & Electrometalurgical Products & w & w & 0 & - & * & * & w & w & - & 13.0 \\
\hline 3321 & Gray and Ductile Iron Foundries & 5 & 1 & 0 & - & 2 & - & $\because$ & 2 & - & 16.6 \\
\hline 3331 & Primary Copper & * & w & 0 & w & * & . & 0 & * & - & 1.1 \\
\hline 3334 & Primary Aluminum & w & w & * & w & w & w & w & 0 & w & 4.8 \\
\hline 3339 & Primary Nonferrous Metals, nec. & w & 2 & * & w & 1 & * & w & w & - & 1.9 \\
\hline 3353 & Aluminum Sheet, Plate, and Foil & w & 2 & 0 & w & w & * & 0 & 0 & * & 2.2 \\
\hline 34 & Fabricated Metal Products . . . . . . & 57 & 17 & 2 & 2 & 33 & 1 & * & 1 & 1 & 15.7 \\
\hline 35 & Industrial Machinery and Equipment ..... & 41 & 18 & 3 & w & 16 & w & 0 & 0 & 2 & 19.2 \\
\hline 357 & Computer and Office Equipment & 4 & 3 & * & - & 1 & * & 0 & 0 & * & 20.5 \\
\hline 36 & Electronic and Other Electric Equipment & 43 & 22 & 3 & 2 & 14 & 1 & • & * & 1 & 16.1 \\
\hline 37 & Transportation Equipment $\ldots \ldots \ldots \ldots$ & w & 10 & 7 & W & 11 & * & w & 0 & w & 10.9 \\
\hline 3711 & Motor Vehicles and Car Bodies ... & w & w & W & w & 1 & * & 0 & 0 & * & 7.4 \\
\hline 3714 & Motor Vehicle Parts and Accessories ... & 8 & 3 & W & * & w & w & w & 0 & - & 11.6 \\
\hline 38 & Instruments and Related Products . . . . . & 52 & 14 & 3 & w & w & $\mathrm{Q}$ & w & 0 & $w$ & 17.2 \\
\hline 3841 & Surgical and Medical Instruments & 2 & 1 & * & * & * & * & 0 & 0 & - & 18.4 \\
\hline 39 & Misc. Manufacturing Industries & w & 4 & 1 & w & $w$ & * & 1 & 0 & - & 17.6 \\
\hline & Total & 2,011 & 294 & 162 & 46 & 461 & 16 & $w$ & $w$ & 588 & 5.0 \\
\hline
\end{tabular}

See footnotes at end of table. 
Table A1. Total Primary Consumption of Energy for All Purposes by Census Region, Industry Group, and Selected Industries, 1991: Part 2 (Continued)

(Estimates in Trillion Btu)

\begin{tabular}{cc|c|c|c|c|c|c|c|c|c|}
\hline $\begin{array}{c}\text { SIC } \\
\text { Code* }\end{array}$ & $\begin{array}{c}\text { Industry Groups } \\
\text { and Industry }\end{array}$ & $\begin{array}{c}\text { Net } \\
\text { Electricity }\end{array}$ & $\begin{array}{c}\text { Residual } \\
\text { Fuel Oil }\end{array}$ & $\begin{array}{c}\text { Distillate } \\
\text { Fuel Oif }\end{array}$ & $\begin{array}{c}\text { Natural } \\
\text { Gas }^{d}\end{array}$ & LPG & $\begin{array}{c}\text { RSE } \\
\text { Row } \\
\text { Lactors }\end{array}$ \\
\hline
\end{tabular}

\begin{tabular}{|c|c|c|c|c|c|c|c|c|c|c|c|}
\hline & & & & & Midwe & ensus $F$ & & & & & \\
\hline & RSE Column Factors: & 0.6 & 0.6 & 1.5 & 1.3 & 0.7 & 1.2 & 1.1 & 1.4 & 1.0 & \\
\hline 20 & Food and Kindred Products . & 420 & 67 & 6 & 3 & w & 1 & 107 & w & w & 8.6 \\
\hline 2011 & Meat Packing Plants ................ & 33 & 7 & 1 & - & 23 & $\bullet$ & 1 & 0 & 1 & 9.3 \\
\hline 2033 & Canned Frults and Vegetables . . . . . . . & 10 & 1 & 0 & * & 9 & $\cdot$ & 0 & 0 & : & 17.1 \\
\hline 2037 & Frozen Frults and Vegetables ....... & 4 & 1 & $\cdot$ & * & 2 & - & 0 & 0 & $\therefore$ & 27.1 \\
\hline 2046 & Wet Com Milling $\ldots \ldots \ldots \ldots \ldots \ldots$ & 122 & 11 & $w$ & * & 46 & - & 61 & w & w & 13.6 \\
\hline 2051 & Bread, Cake, and Related Products .... & 9 & 2 & 0 & $W$ & $w$ & $\cdot$ & 0 & 0 & $\because$ & 16.3 \\
\hline 2063 & 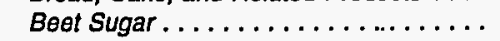 & 34 & 1 & W & $*$ & 6 & $\therefore$ & 24 & w & 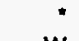 & 6.6 \\
\hline 2075 & Soybean Oll Mills $\ldots \ldots \ldots \ldots \ldots$. & 36 & 4 & $\because$ & • & 17 & w & w & 0 & w & 4.2 \\
\hline 2082 & Malt Beverages $\ldots \ldots \ldots \ldots \ldots \ldots$ & 11 & 2 & * & $*$ & w & $w$ & W & 0 & $\bullet$ & 17.2 \\
\hline 21 & Tobacco Products ... & NA & NA & NA & NA & NA & NA & NA & NA & NA & 9.6 \\
\hline 22 & Textile Mill Products . . . . . . . . . . . & NA & NA & NA & NA & NA & NA & NA & NA & NA & 23.7 \\
\hline 23 & Apparel and Other Textlie Products ..... & NA & NA & NA & NA & NA & NA & NA & NA & NA & 31.6 \\
\hline 24 & Lumber and Wood Products $\ldots \ldots \ldots \ldots$ & 60 & 10 & $Q$ & 2 & 11 & 1 & 1 & 0 & 34 & 28.2 \\
\hline 25 & Furniture and Fixtures $\ldots \ldots \ldots \ldots \ldots$ & w & 5 & 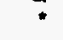 & 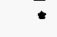 & w & - & 0 & 0 & $Q$ & 25.1 \\
\hline 26 & Paper and Allied Products ........... & w & 49 & 5 & 1 & 116 & $w$ & 94 & W & $\bar{w}$ & 6.6 \\
\hline 2611 & Pulp Mills ........... & 28 & 1 & 0 & • & 4 & $Q$ & 4 & 0 & 20 & 33.3 \\
\hline 2621 & 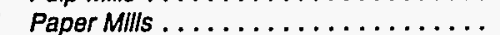 & 208 & 27 & w & 1 & 59 & * & 63 & w & 54 & 5.0 \\
\hline 2631 & Paperboard Mills ........................ & $w$ & 7 & $\because$ & $\cdot$ & 24 & $\cdot$ & 26 & 0 & w & 13.1 \\
\hline 27 & Printing and Publishing . & 43 & 18 & - & * & 23 & - & 0 & 0 & 2 & 17.8 \\
\hline 28 & Chemlcals and Alled Products . . . . . . & 531 & 115 & 2 & $\mathbf{Q}$ & 227 & w & 63 & 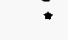 & $\bar{w}$ & 9.1 \\
\hline 2812 & Alkalles and Chlorine ............... & $w$ & W & 0 & W & * & • & 0 & 0 & - & 26.9 \\
\hline 2813 & Industrial Gasas $\ldots \ldots \ldots \ldots \ldots \ldots$ & $\mathbf{w}$ & w & 0 & $\mathbf{Q}$ & w & $\mathbf{Q}$ & 0 & 0 & • & 12.3 \\
\hline 2819 & Industrial Inorganic Chemicals, nec ..... & 71 & $w$ & w & * & w & $\div$ & $w$ & 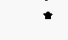 & 1 & 12.9 \\
\hline 2821 & Plastlics Materials and Resins ........ & w & 11 & w & w & 24 & w & w & 0 & 10 & 9.3 \\
\hline 2822 & Synthottc Rubbor $\ldots \ldots \ldots \ldots \ldots \ldots$ & $w$ & 1 & 0 & W & w & : & W & 0 & - & 19.9 \\
\hline 2823 & Celluloslc Manmado Fibers ........... & 0 & 0 & 0 & 0 & 0 & 0 & 0 & 0 & 0 & NF \\
\hline 2824 & Organlc Fibors, Noncellulosic ......... & 0 & 0 & 0 & 0 & 0 & 0 & 0 & 0 & 0 & NF \\
\hline 2865 & Cycllc Crudes and Intermedlates ....... & 30 & 3 & w & w & 14 & w & w & 0 & w & 13.8 \\
\hline 2869 & Industrial Organic Chemicals, nec ..... & $w$ & 6 & 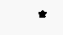 & w & w & w & 25 & 0 & 6 & 7.8 \\
\hline 2873 & Nitrogenous Fertllizers . . . . . . . . & 74 & 2 & 0 & * & 71 & • & 0 & 0 & Q & 33.8 \\
\hline 2874 & Phosphatlc Fentilizers ........... & : & * & 0 & • & $*$ & - & 0 & 0 & - & 4.5 \\
\hline 29 & Petroleum and Coal Products ......... & 968 & 23 & 13 & w & 89 & 5 & $\mathbf{w}$ & 0 & 835 & 5.1 \\
\hline 2911 & Petroleum Refining ${ }^{\prime}, \ldots \ldots \ldots \ldots$ & 921 & 21 & 13 & w & 73 & 5 & w & 0 & 810 & 2.5 \\
\hline 30 & Rubber and Mlsc. Plastics Products ..... & 95 & 45 & 2 & $\because$ & 42 & 1 & 3 & 0 & 2 & 11.4 \\
\hline 3011 & Tires and Inner Tubes ............... & $w$ & w & 1 & - & 7 & w & 1 & 0 & 1 & 4.1 \\
\hline 308 & Miscellaneous Plastics Products, nec ... & NA & NA & NA & NA & NA & $\mathrm{NA}$ & NA & $\mathrm{NA}$ & NA & 19.6 \\
\hline 31 & Leather and Leather Products ......... & 4 & 1 & * & $*$ & 2 & • & 0 & 0 & - & 25.8 \\
\hline 32 & Stone, Clay and Glass Products ........ & 249 & 28 & * & 4 & 108 & * & 76 & 2 & 30 & 10.0 \\
\hline 3211 & Flat Glass . . . . . . . . . . . & 13 & $w$ & 0 & w & 11 & $w$ & 0 & $\overline{0}$ & $\cdot$ & 4.1 \\
\hline 3221 & Glass Containers ............... & 20 & 3 & 0 & • & 17 & • & 0 & 0 & * & 7.6 \\
\hline 3229 & Pressed and Blown Glass, nec. ........ & 14 & 2 & 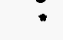 & * & 12 & - & 0 & 0 & * & 6.7 \\
\hline 3241 & Cement, Hydraulic ............... & 85 & 8 & w & w & 6 & • & 51 & 0 & w & 14.8 \\
\hline 3274 & Lime $\ldots \ldots \ldots \ldots \ldots \ldots \ldots \ldots \ldots$ & 35 & 1 & 0 & - & $\mathbf{3}$ & • & 21 & w & W & 18.6 \\
\hline 3296 & Mineral Wool ... & 17 & 4 & w & * & w & • & 0 & w & - & 1.3 \\
\hline 33 & Primary Metal Industries . & 1,115 & 160 & w & 4 & $w$ & 1 & 348 & 201 & w & 5.1 \\
\hline 3312 & Blast Fumaces and Steel Mills ......... & 848 & 59 & w & w & $w$ & : & 334 & 184 & 6 & 5.1 \\
\hline 3313 & Electrometalurglcal Products . . . . . . . & 26 & 8 & 0 & * & 1 & w & $w$ & w & w & 10.0 \\
\hline 3321 & Gray and Ductlle Iron Foundries ....... & W & 15 & • & Q & w & - & w & 12 & 1 & 11.7 \\
\hline 3331 & Primary Copper $\ldots \ldots \ldots \ldots \ldots$ & $\because$ & w & 0 & 0 & $\because$ & * & 0 & 0 & 0 & 1.3 \\
\hline 3334 & Primary Aluminum .............. & w & w & 0 & $w$ & 3 & w & w & 0 & w & 4.8 \\
\hline 3339 & Primary Nonferrous Metals, nec ........ & $w$ & 2 & 0 & * & $\mathbf{3}$ & $\because$ & 0 & w & * & 2.0 \\
\hline 3353 & Aluminum Sheot, Plate, and Foll ...... & 24 & $\mathbf{5}$ & 0 & w & 17 & - & 0 & 0 & w & 1.3 \\
\hline 34 & Fabricated Metal Products . . . . . . . . . & 140 & 44 & - & w & 82 & w & 5 & w & w & 14.4 \\
\hline 35 & Industrial Machinery and Equipment........ & 116 & 43 & • & $W$ & 58 & 1 & 11 & $\mathbf{Q}$ & $\ddot{w}$ & 13.5 \\
\hline 357 & Computer and Office Equlpment ........ & 5 & 3 & 0 & $\because$ & 2 & $\cdot$ & 0 & 0 & $\because$ & 23.9 \\
\hline 36 & Electronic and Other Electric Equipment. . & 59 & 24 & * & $\mathbf{w}$ & 29 & • & w & 0 & w & 13.5 \\
\hline 37 & Transportation Equipment ........... & w & 55 & 2 & 2 & 74 & 1 & w & w & 5 & 6.4 \\
\hline 3711 & Motor Vehicles and Car Bodies ....... & 60 & 16 & $\bar{w}$ & $\vec{w}$ & 31 & • & w & W & -1 & 4.7 \\
\hline 3714 & Motor Vehlcle Parts and Accessories ... & 76 & 27 & w & w & 30 & w & $w$ & w & w & 8.0 \\
\hline 38 & Instruments and Related Products ....... & 11 & 6 & $\mathbf{Q}$ & Q & w & $\because$ & w & 0 & $\because$ & 19.3 \\
\hline 3841 & Surglcal and Medical Instruments ..... & 1 & 1 & 0 & - & - & - & 0 & 0 & * & 18.5 \\
\hline 39 & Misc. Manufacturing Industries .... . & 10 & 3 & 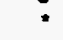 & - & 5 & $\bullet$ & 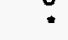 & 0 & Q & 22.7 \\
\hline & Total......$\ldots \ldots \ldots$ & 4,385 & 700 & $w$ & 26 & 1,464 & w & 742 & 208 & 1,089 & 3.8 \\
\hline
\end{tabular}

See footnotes at end of table. 
Table A1. Total Primary Consumption of Energy for All Purposes by Census Region, Industry Group, and Selected Industries, 1991: Part 2 (Continued) (Estimates in Trillion Btu)

\begin{tabular}{|c|c|c|c|c|c|c|c|c|c|c|c|}
\hline \multirow[t]{3}{*}{$\begin{array}{l}\text { SIC } \\
\text { Code }\end{array}$} & $\begin{array}{l}\text { Industry Groups } \\
\text { and Industry }\end{array}$ & Total & $\begin{array}{c}\text { Net } \\
\text { Electricity" }\end{array}$ & $\begin{array}{l}\text { Residual } \\
\text { Fuel Oil }\end{array}$ & $\begin{array}{l}\text { Distillate } \\
\text { Fuel Oir }\end{array}$ & $\begin{array}{l}\text { Natural } \\
\text { Gas }^{\circ}\end{array}$ & LPG & Coal & $\begin{array}{c}\text { Coke and } \\
\text { Breeze }\end{array}$ & Other & $\begin{array}{l}\text { RSE } \\
\text { Row } \\
\text { Factors }\end{array}$ \\
\hline & & \multicolumn{10}{|c|}{ South Census Region } \\
\hline & RSE Column Factors: & 0.6 & 0.6 & 1.4 & 1.2 & 0.8 & 1.1 & 1.1 & 1.6 & 1.0 & \\
\hline 20 & Food and Kindred Products & 255 & 54 & 10 & 5 & 135 & 2 & 17 & $w$ & $\mathbf{Q}$ & 11.0 \\
\hline 2011 & Meat Packing Plants . . . . . & 10 & 3 & 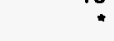 & 1 & 5 & 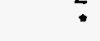 & 0 & 0 & 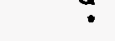 & 16.9 \\
\hline 2033 & Canned Fruits and Vegetables . & 7 & 1 & - & * & 6 & * & 0 & 0 & - & 19.7 \\
\hline 2037 & Frozen Fruits and Vegetables. & 8 & 2 & 1 & * & 6 & * & 0 & 0 & - & 17.6 \\
\hline 2046 & Wet Com Milling . ......... & w & 3 & 0 & • & 5 & - & 7 & 0 & w & 23.0 \\
\hline 2051 & Bread, Cake, and Related Products . . . . & 11 & 3 & 0 & * & 7 & - & 0 & 0 & $\because$ & 14.2 \\
\hline 2063 & 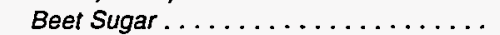 & w & w & 0 & * & w & * & 0 & w & - & 17.0 \\
\hline 2075 & Soybean Oil Mills .. & 15 & 2 & : & * & 9 & w & $w$ & 0 & w & 4.9 \\
\hline 2082 & Malt Beverages & 14 & 3 & w & w & 8 & * & $w$ & 0 & • & 14.0 \\
\hline 21 & Tobacco Products & 24 & 3 & 1 & 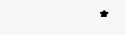 & 4 & * & 15 & 0 & - & 6.8 \\
\hline 22 & Textile Mill Products & 236 & 94 & 7 & 3 & 89 & 2 & 30 & 0 & 11 & 6.5 \\
\hline 23 & Apparel and Other Textile Products ..... & 31 & 14 & 0 & * & 12 & 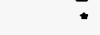 & 2 & 0 & $\because$ & 22.8 \\
\hline 24 & Lumber and Wood Products & 209 & 31 & $Q$ & 6 & 15 & 1 & 1 & 0 & 153 & 20.2 \\
\hline 25 & Furniture and Fixtures & w & 9 & - & 1 & w & - & $w$ & 0 & $w$ & 19.5 \\
\hline 26 & Paper and Allied Products & 1,447 & 86 & 69 & 4 & $w$ & 2 & 154 & 0 & w & 4.5 \\
\hline 2611 & Pulp Mills . . . . . . . . . & 219 & 4 & 21 & 1 & 22 & * & 4 & 0 & 166 & 17.2 \\
\hline 2621 & Paper Mills . & W & 47 & 18 & w & 126 & 1 & 87 & 0 & $w$ & 3.4 \\
\hline 2631 & I Paperboard Mills ..... & $w$ & 19 & 29 & $w$ & W & * & 62 & 0 & 349 & 5.3 \\
\hline 27 & Printing and Publishing .. & 28 & 16 & $\mathrm{Q}$ & $\because$ & 10 & * & 0 & 0 & 1 & 21.4 \\
\hline 28 & Chemicals and Allied Products & 4,195 & 256 & w & 7 & 1,812 & 1,377 & w & 3 & 470 & 6.8 \\
\hline 2812 & Alkalies and Chlorine $\ldots \ldots$ & 147 & 29 & 0 & * & w & • & w & 0 & 20 & 17.4 \\
\hline 2813 & Industrial Gases ........ & w & 29 & 0 & * & w & w & 0 & 0 & 1 & 12.8 \\
\hline 2819 & Industrial Inorganic Chemicals, nec .... & w & $w$ & 2 & w & w & * & $\mathbf{w}$ & 2 & 13 & 11.1 \\
\hline 2821 & Plastics Materials and Resins . . . . . . & w & 35 & $\bar{w}$ & $w$ & 182 & 209 & $w$ & 0 & $w$ & 5.6 \\
\hline 2822 & Synthetic Rubber ........ & 111 & w & w & w & w & 15 & 0 & 0 & w & 13.2 \\
\hline 2823 & Cellulosic Manmade Fibers & 31 & w & 0 & $\because$ & w & • & 27 & 0 & $\because$ & 25.3 \\
\hline 2824 & Organic Fibers, Noncellulosic & $w$ & 23 & w & w & w & w & $w$ & 0 & 1 & 4.1 \\
\hline 2865 & Cyclic Crudes and Intermediates . . . . . & 193 & 11 & 6 & * & 84 & $w$ & w & 0 & $w$ & 12.0 \\
\hline 2869 & Industrial Organic Chemicals, nec ..... & 2,144 & 35 & 2 & w & w & $w$ & 60 & 0 & 322 & 8.4 \\
\hline 2873 & Nitrogenous Fertilizers . . . . . . . . & 414 & 6 & 0 & $\because$ & 407 & $\because$ & 0 & 0 & 1 & 28.9 \\
\hline 2874 & Phosphatic Fertilizers $\ldots \ldots \ldots \ldots \ldots$ & w & 5 & 2 & W & w & * & W & 0 & 21 & 3.3 \\
\hline 29 & Petroleum and Coal Products $\ldots$ & 3,479 & 47 & 13 & w & 591 & w & w & w & 2,814 & 4.2 \\
\hline 2911 & Petroleum Refining ' ..... & 3,394 & 46 & 13 & w & 578 & 8 & $w$ & 0 & 2,746 & 2.7 \\
\hline 30 & Rubber and Misc. Plastics Products & 88 & 42 & 3 & 1 & 36 & 1 & 1 & 0 & 3 & 10.4 \\
\hline 3011 & Tires and Inner Tubes. . & w & 10 & w & w & 13 & • & w & 0 & w & 4.1 \\
\hline 308 & Miscellaneous Plastics Products, nec... & 46 & 27 & 1 & w & 16 & * & w & 0 & $\because$ & 21.0 \\
\hline 31 & Leather and Leather Products . . . . . . . & 2 & 1 & : & * & $\because$ & * & 0 & 0 & Q & 24.5 \\
\hline 32 & Stone, Clay and Glass Products .. & 324 & 40 & 1 & 8 & 161 & $w$ & 87 & w & w & 11.4 \\
\hline 3211 & Flat Glass $\ldots \ldots \ldots \ldots \ldots \ldots \ldots$ & 23 & 3 & 0 & * & 20 & • & 0 & 0 & $\because$ & 4.0 \\
\hline 3221 & 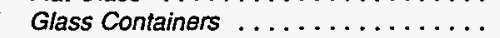 & 29 & 4 & w & w & w & * & 0 & 0 & - & 8.3 \\
\hline 3229 & Pressed and Blown Glass, nec. . . & w & 5 & * & w & w & w & 0 & 0 & . & 8.6 \\
\hline 3241 & Cement, Hydraulic . . . . . . . . & 108 & 12 & * & 1 & 22 & * & 58 & 4 & 11 & 15.5 \\
\hline 3274 & Lime... & 37 & 1 & 0 & • & w & • & 26 & w & w & 21.8 \\
\hline 3296 & Mineral Wool & 16 & 3 & $w$ & * & 12 & • & 0 & $w$ & $:$ & 1.4 \\
\hline 33 & Primary Metal Industries . & 630 & 161 & 9 & w & W & $w$ & w & 56 & 16 & 4.4 \\
\hline 3312 & Blast Fumaces and Steel Mills. & 381 & 37 & 9 & 1 & w & - & 193 & 46 & w & 6.1 \\
\hline 3313 & Electrometalurgical Products ... & w & w & 0 & * & * & 0 & w & $w$ & 1 & 11.1 \\
\hline 3321 & Gray and Ductile Iron Foundries ....... & 21 & 6 & - & - & w & * & $Q$ & $w$ & . & 11.5 \\
\hline 3331 & Primary Copper $\ldots \ldots \ldots \ldots \ldots \ldots$ & w & 1 & w & * & w & - & 0 & 0 & • & 1.1 \\
\hline 3334 & Primary Aluminum & 105 & 83 & 0 & w & w & • & w & w & w & 4.2 \\
\hline 3339 & Primary Nonferrous Metals, nec.. & w & 6 & 0 & * & w & $w$ & $w$ & 0 & w & 3.7 \\
\hline 3353 & Aluminum Sheet, Plate, and Foil & 25 & w & 0 & * & 18 & - & w & 0 & * & 1.8 \\
\hline 34 & Fabricated Metal Products . . . . . . . & 82 & 30 & Q & W & 44 & w & 0 & 1 & 3 & 21.0 \\
\hline 35 & Industrial Machinery and Equipment. & 58 & 28 & * & 1 & 27 & $w$ & $\mathbf{Q}$ & $\mathbf{Q}$ & $w$ & 15.7 \\
\hline 357 & Computer and Office Equipment & 4 & 3 & - & $\mathbf{Q}$ & 1 & - & 0 & 0 & • & 22.3 \\
\hline 36 & Electronic and Other Electric Equipment .. & 81 & 37 & * & " & 27 & 1 & w & $w$ & w & 14.4 \\
\hline 37 & Transportation Equipment & 71 & 29 & w & 2 & 28 & - & w & * & 8 & 8.9 \\
\hline 3711 & Motor Vehicles and Car Bodies & 24 & 8 & * & * & 12 & - & w & 0 & w & 4.5 \\
\hline 3714 & Motor Vehicle Parts and Accessories & 13 & 7 & * & • & w & * & w & * & * & 11.6 \\
\hline & Instruments and Related Products . & 17 & 11 & Q & * & 6 & - & 0 & 0 & • & 18.6 \\
\hline 3841 & Surgical and Medical Instruments & 2 & 1 & 0 & • & - & - & 0 & 0 & - & 21.5 \\
\hline 39 & Misc. Manufacturing Industries .. & w & 4 & * & $\mathbf{Q}$ & w & * & 0 & 0 & w & 23.5 \\
\hline & Total & $\$ 1,296$ & 996 & w & 49 & 3,469 & w & 700 & 66 & 4,407 & 3.6 \\
\hline
\end{tabular}

See footnotes at end of table. 
Table A1. Total Primary Consumption of Energy for All Purposes by Census Region, Industry Group, and Selected Industries, 1991: Part 2 (Continued)

(Estimates in Trillion Btu)

\begin{tabular}{|c|c|c|c|c|c|c|c|c|c|c|c|}
\hline \multirow[t]{3}{*}{$\begin{array}{l}\text { SIC } \\
\text { Coden }\end{array}$} & $\begin{array}{l}\text { Industry Groups } \\
\text { and Industry }\end{array}$ & Total & $\begin{array}{c}\text { Net } \\
\text { Electricity }\end{array}$ & $\begin{array}{c}\text { Residual } \\
\text { Fuel Oil }\end{array}$ & $\begin{array}{l}\text { Distillate } \\
\text { Fuel Oif }\end{array}$ & $\begin{array}{l}\text { Natural } \\
\text { Gas }^{\delta}\end{array}$ & LPG & Coal & $\begin{array}{c}\text { Coke and } \\
\text { Breeze }\end{array}$ & Other & \multirow[t]{2}{*}{$\begin{array}{l}\text { RSE } \\
\text { Row } \\
\text { Factors }\end{array}$} \\
\hline & & \multicolumn{9}{|c|}{ West Census Region } & \\
\hline & RSE Column Factors: & 0.7 & 0.7 & 1.2 & 1.1 & 0.8 & 1.1 & 1.3 & 1.2 & 1.0 & \\
\hline 20 & Food and Kindred Products & 202 & 29 & 4 & 4 & 119 & 1 & 28 & w & $w$ & 9.5 \\
\hline 2011 & Meat Packing Plants . . . . . . . . . & 5 & 1 & $w$ & . & 3 & $\mathbf{Q}$ & 0 & 0 & $\because$ & 17.5 \\
\hline 2033 & Canned Frults and Vegetables . . . . . . . . & 20 & 2 & 1 & - & 17 & - & 0 & 0 & - & 12.4 \\
\hline 2037 & Frozen Frults and Vegetables .. & 26 & 7 & $\mathbf{Q}$ & - & 17 & - & 0 & 0 & 1 & 17.3 \\
\hline 2046 & Wot Com Milling . . . . . . . & w & * & 0 & w & 2 & - & 0 & 0 & $w$ & 20.5 \\
\hline 2051 & Bread, Cake, and Related Products .... & 6 & 1 & 0 & $*$ & 5 & - & 0 & 0 & $\because$ & 26.8 \\
\hline 2063 & Beot Sugar $\ldots \ldots \ldots \ldots \ldots \ldots \ldots$ & w & w & w & • & W & - & 18 & 1 & * & 8.5 \\
\hline 2075 & Soybean Oll Mills $\ldots \ldots \ldots \ldots \ldots \ldots$ & 0 & 0 & 0 & 0 & 0 & 0 & 0 & 0 & 0 & NF \\
\hline 2082 & Malt Beverages $\ldots \ldots \ldots \ldots \ldots \ldots$ & 17 & 2 & w & w & w & w & w & 0 & * & 16.4 \\
\hline 21 & Tobacco Products $\ldots \ldots \ldots \ldots \ldots \ldots$ & 0 & 0 & 0 & 0 & 0 & 0 & 0 & 0 & 0 & $\mathrm{NF}$ \\
\hline 22 & Textlle Mill Products.$\ldots \ldots \ldots \ldots \ldots$ & 6 & 1 & 0 & - & 5 & $Q$ & 0 & 0 & * & 30.1 \\
\hline 23 & Apparel and Other Textile Products ..... & NA & NA & NA & NA & NA & NA & NA & NA & NA & 29.9 \\
\hline 24 & Lumber and Wood Products..$\ldots \ldots \ldots$. & 166 & 17 & 1 & 7 & 10 & 1 & 0 & 0 & 130 & 20.0 \\
\hline 25 & Fumiture and Fixtures $\ldots \ldots \ldots \ldots \ldots$ & 3 & 1 & 0 & $\mathbf{Q}$ & 1 & : & 0 & 0 & * & 32.8 \\
\hline 26 & Paper and Allied Products.$\ldots \ldots \ldots \ldots$ & $w$ & 38 & 11 & 1 & 111 & w & w & 0 & w & 6.2 \\
\hline 2611 & Pulp Mills $\ldots \ldots \ldots \ldots \ldots \ldots \ldots$ & 41 & 3 & 5 & * & 7 & - & 0 & 0 & 26 & 19.4 \\
\hline 2621 & 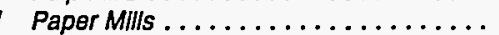 & w & 22 & w & • & 55 & - & $w$ & 0 & w & 5.6 \\
\hline 2631 & Paperboard Mills . . . . . . . . . . & $w$ & 7 & $w$ & w & 40 & w & $w$ & 0 & $w$ & 8.1 \\
\hline 27 & Printing and Publishing.... & NA & NA & NA & NA & NA & NA & NA & NA & NA & 22.0 \\
\hline 28 & Chemicals and Alled Products . & w & 37 & w & 1 & 129 & - & w & 7 & W & 13.4 \\
\hline 2812 & Alkalles and Chlorino $\ldots \ldots \ldots \ldots \ldots$ & $w$ & w & w & W & 4 & . & 0 & 0 & 1 & 19.8 \\
\hline 2813 & 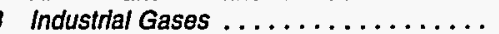 & $w$ & w & 0 & w & 6 & w & 0 & 0 & * & 14.8 \\
\hline 2819 & Industrial Inorganic Chemicals, nec .... & $w$ & 14 & $w$ & $w$ & $\mathbf{w}$ & a & w & 7 & 3 & 11.0 \\
\hline 2821 & Plastics Materlals and Resins . . . . . . & 3 & 1 & 0 & - & 2 & - & 0 & 0 & • & 17.2 \\
\hline 2822 & Synthetlc Rubber ......... & - & - & 0 & - & • & - & 0 & 0 & • & 24.5 \\
\hline 2823 & Cellulosic Manmade Fibers . . . . . . . . & 0 & 0 & 0 & 0 & 0 & 0 & 0 & 0 & 0 & NF \\
\hline 2824 & Organic Fibers, Noncellulosic . . . . . . . & 0 & 0 & 0 & 0 & 0 & 0 & 0 & 0 & 0 & NF \\
\hline 2865 & Cyclle Crudes and Intermediates ....... & : & * & 0 & • & * & - & 0 & 0 & . & 25.4 \\
\hline 2869 & Industrial Organic Chemicals, nec ..... & 5 & $\mathbf{Q}$ & 0 & $\mathbf{Q}$ & w & $\mathbf{Q}$ & 0 & 0 & w & 13.6 \\
\hline 2873 & Nitrogenous Fertilizers . . . . . . . & 79 & 2 & 0 & . & 77 & - & 0 & 0 & : & 40.7 \\
\hline 2874 & Phosphatic Fertilizers $\ldots \ldots \ldots \ldots \ldots$ & w & 2 & 0 & • & w & 0 & 0 & 0 & w & 22.7 \\
\hline 29 & Petroleum and Coal Products & 1,019 & 26 & 12 & w & 129 & 46 & w & 0 & 794 & 4.1 \\
\hline 2911 & Petroleum Refining '.... & 962 & 26 & 12 & 5 & 118 & 46 & 0 & 0 & 756 & 2.2 \\
\hline 30 & Rubber and Misc. Plastics Products . & 19 & 10 & * & $\mathbf{Q}$ & $w$ & $w$ & $\mathbf{Q}$ & 0 & * & 18.3 \\
\hline 3011 & Thres and Inner Tubes . . . . . . . . . . . & * & $w$ & $w$ & w & • & w & 0 & 0 & • & 7.2 \\
\hline 308 & Miscellaneous Plastics Products, nec ... & 14 & 9 & 0 & $Q$ & 5 & : & 0 & 0 & - & 22.0 \\
\hline 31 & Leather and Leather Products . . . . . & $\mathbf{Q}$ & • & 0 & $\mathbf{Q}$ & a & $\mathbf{Q}$ & 0 & 0 & - & 42.3 \\
\hline 32 & Stone, Clay and Glass Products & 137 & 18 & 4 & 4 & 53 & * & 53 & 1 & 5 & 14.8 \\
\hline 3211 & Flat Glass . . . . . . . . . . & $w$ & 1 & w & w & w & w & 0 & 0 & • & 4.6 \\
\hline 3221 & Glass Containers & 18 & 4 & $w$ & w & $\mathbf{w}$ & - & 0 & 0 & * & 9.5 \\
\hline 3229 & Pressed and Blown Glass, nec. & w & w & 0 & - & w & * & 0 & 0 & * & 13.3 \\
\hline 3241 & Cement, Hydraullic .......... & 77 & 8 & $w$ & 1 & 12 & * & 53 & $\mathbf{Q}$ & 3 & 20.8 \\
\hline 3274 & LIme .............. & w & $w$ & w & W & w & * & 0 & 0 & - & 21.3 \\
\hline 3296 & Mineral Wool .......... & 3 & 1 & 0 & $w$ & $w$ & w & 0 & $w$ & - & 2.1 \\
\hline 33 & Primary Metal Industries & 251 & 117 & 1 & $\mathbf{w}$ & $\mathbf{w}$ & w & w & 3 & w & 5.8 \\
\hline 3312 & Blast Fumaces and Steol Mills... & 69 & 8 & w & w & w & * & w & • & 1 & 7.6 \\
\hline 3313 & Electrometalurgical Products ... & 0 & 0 & 0 & 0 & 0 & $\mathbf{0}$ & 0 & 0 & 0 & NF \\
\hline 3321 & Gray and Ductile Iron Foundries ....... & 3 & . & 0 & - & w & - & 0 & w & • & 37.2 \\
\hline 3331 & Primary Copper $\ldots \ldots \ldots \ldots \ldots \ldots$ & w & 4 & $w$ & $w$ & $w$ & - & w & w & * & 1.0 \\
\hline 3334 & Primary Aluminum $\ldots \ldots \ldots \ldots \ldots$ & 118 & 90 & 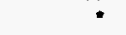 & • & 6 & - & $\bullet$ & 0 & 22 & 3.5 \\
\hline 3339 & Primary Nonferrous Metals, nec. . & $w$ & 4 & 0 & w & w & w & w & w & • & 1.0 \\
\hline 3353 & Aluminum Sheet, Plate, and Foil & w & w & 0 & W & w & w & 0 & 0 & - & 1.1 \\
\hline 34 & Fabricated Metal Products . . . . . . & 28 & 10 & : & * & 17 & - & 0 & 0 & • & 19.6 \\
\hline 35 & Industrial Machinery and Equipment . & 22 & 12 & 0 & - & 9 & * & 0 & 0 & - & 27.0 \\
\hline 357 & Computer and Office Equipment & 9 & 7 & 0 & - & 2 & $\mathbf{Q}$ & 0 & 0 & - & 15.9 \\
\hline 36 & Electronic and Other Electric Equipment . . & 29 & 19 & 0 & $\mathbf{Q}$ & 9 & * & 0 & 0 & w & 18.0 \\
\hline
\end{tabular}

See footnotes at end of table. 
Table A1. Total Primary Consumption of Energy for All Purposes by Census Region, Industry Group, and Selected Industries, 1991: Part 2 (Continued) (Estimates in Trillion Btu)

\begin{tabular}{|c|c|c|c|c|c|c|c|c|c|c|c|}
\hline $\begin{array}{c}\text { SIC } \\
\text { Code }\end{array}$ & $\begin{array}{l}\text { Industry Groups } \\
\text { and Industry }\end{array}$ & Total & $\begin{array}{c}\text { Net } \\
\text { Electricity }\end{array}$ & $\begin{array}{l}\text { Residual } \\
\text { Fuel Oil }\end{array}$ & $\begin{array}{l}\text { Distillate } \\
\text { Fuel Oif }\end{array}$ & $\begin{array}{l}\text { Natural } \\
\text { Gas }^{d}\end{array}$ & LPG & Coal & $\begin{array}{c}\text { Coke and } \\
\text { Breeze }\end{array}$ & Othere & $\begin{array}{c}\text { RSE } \\
\text { Row } \\
\text { Factors }\end{array}$ \\
\hline & & \multicolumn{10}{|c|}{ West Census Region } \\
\hline \multicolumn{2}{|r|}{ RSE Column Factors: } & 0.7 & 0.7 & 1.2 & 1.1 & 0.8 & 1.1 & 1.3 & 1.2 & 1.0 & \\
\hline 37 & Transportation Equipment $\ldots . . \ldots \ldots$ & $w$ & 24 & W & W & 20 & 1 & 0 & 0 & W & 11.8 \\
\hline 3711 & Motor Vehicles and Car Bodles . . . . . . . & $w$ & W & 0 & * & 1 & * & 0 & 0 & $\bullet$ & 6.9 \\
\hline 3714 & Motor Vehicle Parts and Accessories ... & 3 & 1 & Q & * & 2 & * & 0 & 0 & - & 21.6 \\
\hline 38 & Instruments and Related Products ..... & 17 & 11 & $\bullet$ & * & 6 & * & 0 & 0 & - & 15.0 \\
\hline 3841 & Surgical and Medical instruments $\ldots .$. & 1 & 1 & 0 & - & * & * & 0 & 0 & - & 27.5 \\
\hline 39 & Misc. Manufacturing Industries . . . . . . . & 2 & 1 & 0 & - & 1 & $\bullet$ & 0 & 0 & - & 26.4 \\
\hline & Total $\ldots \ldots \ldots \ldots \ldots \ldots \ldots$ & 2,564 & 381 & 34 & 25 & 701 & 52 & $W$ & $w$ & 1,220 & 4.6 \\
\hline
\end{tabular}

- See Appendices B and F for descriptions of the Standard Industrial Classification system.

" "Net Electricity" is obtained by summing purchases, transfers in, and generation from noncombustible renewable resources, minus quantities sold and transferred out. It does not include electricity inputs from onsite cogeneration or generation from combustible fuels because that energy has already been included als generating fuel (for example, coal).

"Distillate Fuel Oil" includes Nos. 1, 2, and 4 fuel oils and Nos. 1, 2, and 4 diesel fuels.

- "Natural Gas" includes natural gas obtained from utilities, transmission pipelines, and any other supplier(s) such as brokers and producers.

- "Other" includes net steam (the sum of purchases, generation from renewables, and net transfers), and other energy that respondents indicated was used to produce heat and power or as feedstock/raw material inputs. See also Footnote "f".

'For the petroleum refining industry only, the feedstocks and raw material inputs for the production of nonenergy products (i.e., asphalt, waxes, lubricants, and

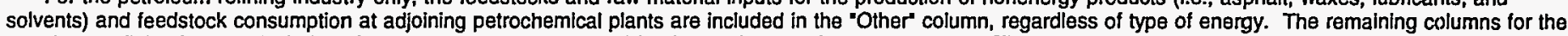

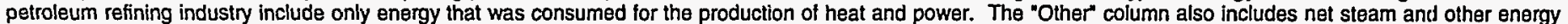
that respondents indicated was used in the production of heat and power. Those inputs and feedstocks that were converted to other energy products (e.g., crude oll converted to residual and distillate fuel oils) are excluded. See Appendix B for more information.

NF=No applicable RSE row/column factor.

- Estimate less than 0.5. Data are included in higher level totals.

$W=$ Withheld to avoid disclosing data for individual establishments. Data are included in higher level totals.

$\mathrm{Q}=$ Withheld because Relative Standard Error is greater than 50 percent. Data are included in higher level totals.

NA=Not available. Data are included in higher level totals.

Notes: - To obtain a RSE percentage for any table cell, multiply the cell's corresponding RSE column and RSE row factors. - Totals may not equal surn of components because of independent rounding. - The derived estimates presented in this table are for the primary consumption of energy for heat and power and as feedstocks or raw material inputs. Primary consumption is defined as the consumption of the energy that was originally produced offsite or was produced onsite from input materials not classified as energy. Examples of the latter are hydrogen produced from the electrolysis of brine; the output of captive (onsite) mines or wells;

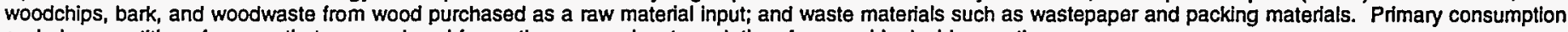
excludes quantities of energy that are produced from other energy inputs and, therefore, avoids double counting.

Source: Energy Information Administration, Office of Energy Markets and End Use, Energy End Use and Integrated Statistics Division, Form ElA-846, "I991 Manufacturing Energy Consumption Survey," and Office of Oil and Gas, Petroleum Supply Division, Form EIA-810, "Monthly Refinery Report" for 1991. 
Table A2. Total Consumption of LPG, Distillate Fuel Oil, and Residual Fuel Oil for Selected Purposes by Census Region, Industry Group, and Selected Industries, 1991

(Estimates in Barrels per Day)

\begin{tabular}{|c|c|c|c|c|c|c|c|c|c|c|c|}
\hline \multirow[b]{2}{*}{$\underset{\operatorname{Cod} \theta^{a}}{\operatorname{SIC}}$} & \multirow{2}{*}{$\begin{array}{l}\text { Industry Groups } \\
\text { and Industry }\end{array}$} & \multicolumn{3}{|c|}{$\begin{array}{l}\text { Primary Consumption for All } \\
\text { Purposes }\end{array}$} & \multicolumn{3}{|c|}{$\begin{array}{l}\text { Inputs for Heat, Power, and } \\
\text { Generation of Electricity }\end{array}$} & \multicolumn{3}{|c|}{$\begin{array}{l}\text { Primary Consumption for Nonfuel } \\
\text { Purposes }\end{array}$} & \multirow{2}{*}{$\begin{array}{c}\text { RSE } \\
\text { Row } \\
\text { Factors }\end{array}$} \\
\hline & & LPG & Distillate & Residual & LPG & Distillate ${ }^{\circ}$ & Residual & LPG & Distillate ${ }^{\circ}$ & Residual & \\
\hline
\end{tabular}

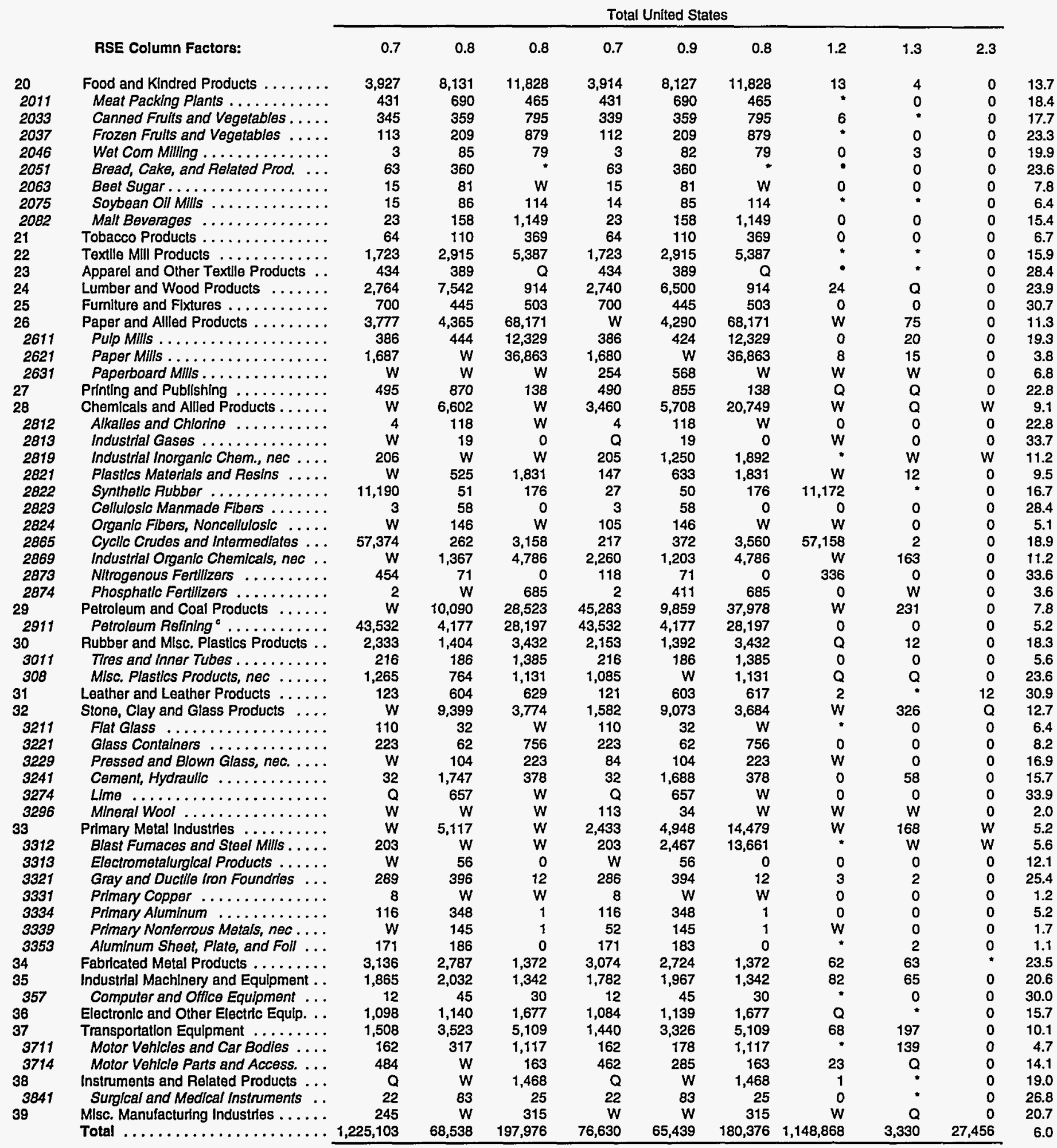

See footnotes at end of table.

Energy Information Administration/Manufacturing Consumption of Energy 1991 


\section{Table A2. Total Consumption of LPG, Distillate Fuel Oil, and Residual Fuel Oil for Selected Purposes by Census Region, Industry Group, and Selected Industries, 1991 (Continued) \\ (Estimates in Barrels per Day)}

\begin{tabular}{|c|c|c|c|c|c|c|c|c|c|c|c|}
\hline \multirow[b]{2}{*}{$\begin{array}{c}\text { SIC } \\
\text { Code }\end{array}$} & \multirow[b]{2}{*}{$\begin{array}{l}\text { Industry Groups } \\
\text { and Industry }\end{array}$} & \multicolumn{3}{|c|}{$\begin{array}{l}\text { Primary Consumption for All } \\
\text { Purposes }\end{array}$} & \multicolumn{3}{|c|}{$\begin{array}{l}\text { Inputs for Heat, Power, and } \\
\text { Generation of Electricity }\end{array}$} & \multicolumn{3}{|c|}{$\begin{array}{c}\text { Primary Consumption for Nonfuel } \\
\text { Pupposes }\end{array}$} & \multirow{2}{*}{$\begin{array}{c}\text { RSE } \\
\text { Row } \\
\text { Factors }\end{array}$} \\
\hline & & LPG & Distillate $^{b}$ & Residual & LPG & Distillate ${ }^{b}$ & Residual & LPG & Distillate $^{\circ}$ & Residual & \\
\hline & & \multicolumn{9}{|c|}{ Northeast Census Region } & \\
\hline & RSE Column Factors: & 1.0 & 1.0 & 0.8 & 1.0 & 1.0 & 0.8 & 1.1 & 1.4 & NF & \\
\hline 20 & Food and Kindred Products . . . . . . . & 609 & 2,437 & 3,190 & 609 & 2,437 & 3,190 & 0 & 0 & 0 & 18.6 \\
\hline 2011 & Meat Packing Plants . . . . . . . . & $\mathbf{Q}$ & 92 & W & $Q$ & 92 & W & 0 & 0 & 0 & 30.8 \\
\hline 2033 & Canned Fruits and Vegetables ..... & 36 & 60 & 400 & 36 & 60 & 400 & 0 & 0 & 0 & 15.9 \\
\hline 2037 & Frozen Fruits and Vegetables . . . . & $\mathbf{Q}$ & 9 & 350 & $\mathbf{Q}$ & 9 & 350 & 0 & 0 & 0 & 40.0 \\
\hline 2046 & Wet Com Milling . . . . . . . . . . . & $=$ & $w$ & $w$ & - & $w$ & $W$ & 0 & 0 & 0 & 22.6 \\
\hline 2051 & Bread, Cake, and Related Prod. . . . & 22 & W & - & 22 & $w$ & $\cdot$ & 0 & 0 & 0 & 25.9 \\
\hline 2063 & Beet Sugar . . . . . . . . . . . . & 0 & 0 & 0 & 0 & 0 & 0 & 0 & 0 & 0 & NF \\
\hline 2075 & Soybean Oil Mills . . . . . . . . . . & 0 & 0 & 0 & 0 & 0 & 0 & 0 & 0 & 0 & NF \\
\hline 2082 & Malt Beverages .......... & 11 & 25 & W & 11 & 25 & $w$ & 0 & 0 & 0 & 18.3 \\
\hline 21 & Tobacco Products . . . . . . . . . . & NA & NA & NA & NA & NA & NA & NA & NA & NA & 7.9 \\
\hline 22 & Textile Mill Products . . . . . . . . . & 446 & 1,523 & 2,122 & 446 & 1,523 & 2,122 & 0 & 0 & 0 & 22.1 \\
\hline 23 & Apparel and Other Textile Products .. & $\mathbf{Q}$ & 136 & 122 & $\mathbf{Q}$ & 136 & 122 & 0 & 0 & 0 & 41.7 \\
\hline 24 & Lumber and Wood Products ....... & NA & NA & NA & NA & NA & NA & NA & NA & NA & 38.3 \\
\hline 25 & Fumiture and Fixtures $\ldots \ldots \ldots \ldots$ & 164 & 135 & $Q$ & 164 & 135 & $Q$ & 0 & 0 & 0 & 38.5 \\
\hline 26 & Paper and Allied Products . . . . . . . & W & 1,711 & 31,340 & W & 1,709 & 31,340 & 0 & 2 & 0 & 10.1 \\
\hline 2611 & Pulp Mills . . . . . . . . . . . . & 37 & 20 & 795 & 37 & 20 & 795 & 0 & 0 & 0 & 38.3 \\
\hline 2621 & $\ldots \ldots \ldots \ldots$ & 777 & W & 26,582 & 777 & $w$ & 26,582 & 0 & $w$ & 0 & 4.4 \\
\hline 2631 & Paperboard Mills . . . . . . . . . . . & 13 & $\mathbf{Q}$ & W & 13 & Q & $W$ & 0 & 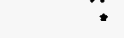 & 0 & 20.3 \\
\hline 27 & Printing and Publishing $\ldots \ldots \ldots \ldots$ & 83 & 656 & 98 & 82 & 641 & 98 & 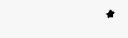 & $Q$ & 0 & 32.0 \\
\hline 28 & Chemicals and Allied Products . . . . . & 2,368 & 1,573 & 8,416 & 276 & W & 8,416 & 2,093 & $\vec{w}$ & 0 & 10.5 \\
\hline 2812 & Alkalies and Chlorine .......... & 0 & 0 & 0 & 0 & 0 & 0 & 0 & 0 & 0 & NF \\
\hline 2813 & Industrial Gases . . . . . . . . . . . & * & 3 & 0 & $*$ & 3 & 0 & 0 & 0 & 0 & 18.7 \\
\hline 2819 & Industrial Inorganic Chem., nec .... & 38 & 214 & 699 & 38 & 214 & 699 & 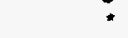 & 0 & 0 & 22.2 \\
\hline 2821 & Plastics Materials and Resins . . . . & $w$ & 298 & 1,310 & 39 & 418 & 1,310 & $w$ & 0 & 0 & 13.5 \\
\hline 2822 & Synthetic Rubber . . . . . . . . & 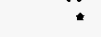 & 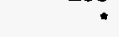 & W & 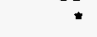 & 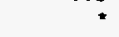 & W & 0 & 0 & 0 & 29.7 \\
\hline 2823 & Cellulosic Manmade Fibers . . . . . . & 0 & 0 & 0 & 0 & 0 & 0 & 0 & 0 & 0 & NF \\
\hline 2824 & Organic Fibers, Noncellulosic ..... & 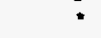 & $w$ & $\mathbf{Q}$ & * & $w$ & $\mathbf{Q}$ & 0 & 0 & 0 & 6.9 \\
\hline 2865 & Cyclic Crudes and Intermediates ... & 6 & $w$ & $\vec{w}$ & 5 & $w$ & $\vec{w}$ & . & : & 0 & 21.9 \\
\hline 2869 & Industrial Organic Chemicals, nec .. & $w$ & $\dddot{w}$ & 3,834 & 115 & 208 & 3,834 & $W$ & $w$ & 0 & 9.8 \\
\hline 2873 & Nitrogenous Fertilizers $\ldots \ldots \ldots$ & 2 & 8 & 0 & 2 & 8 & 0 & 0 & 0 & 0 & 47.5 \\
\hline 2874 & Phosphatic Fertilizers . . . . . . . & 0 & 0 & 0 & 0 & 0 & 0 & 0 & 0 & 0 & NF \\
\hline 29 & Petroleum and Coal Products ...... & 2,338 & W & 11,713 & 2,333 & 3,771 & 21,168 & $\mathbf{Q}$ & $\mathbf{Q}$ & 0 & 23.3 \\
\hline 2911 & Petroleum Refining ${ }^{e} \ldots \ldots \ldots \ldots$ & 1,257 & $w$ & 11,713 & 1,257 & $W$ & 11,713 & 0 & 0 & 0 & 6.3 \\
\hline 30 & Rubber and Misc. Plastics Products .. & W & 563 & 1,248 & Q & 553 & 1,248 & $\mathbf{Q}$ & 10 & 0 & 27.4 \\
\hline 3011 & Tires and Inner Tubes. . . . . . . . . . & 15 & $Q$ & 172 & 15 & $\mathbf{Q}$ & 172 & $\overline{0}$ & 0 & 0 & 27.7 \\
\hline 308 & Misc. Plastics Products, nec . . . . . & 494 & $\vec{w}$ & 688 & 349 & $\vec{w}$ & 688 & 0 & $\mathbf{Q}$ & 0 & 32.8 \\
\hline 31 & Leather and Leather Products . . . . . & 79 & 539 & 389 & 77 & 538 & 389 & 2 & $\cdot$ & 0 & 36.5 \\
\hline 32 & Stone, Clay and Glass Products .... & $w$ & 1,978 & 1,185 & 383 & 1,955 & 1,185 & $\mathbf{Q}$ & 23 & 0 & 18.5 \\
\hline 3211 & Flat Glass ............... & $w$ & 2 & 0 & $W$ & 2 & 0 & 0 & 0 & 0 & 4.0 \\
\hline 3221 & Glass Containers ........... & 65 & 38 & 529 & 65 & 38 & 529 & 0 & 0 & 0 & 10.1 \\
\hline 3229 & Pressed and Blown Glass, nec. . . . & 22 & $W$ & 218 & 22 & $W$ & 218 & 0 & 0 & 0 & 17.2 \\
\hline 3241 & Cement, Hydraulic . . . . . . . . & 3 & W & 38 & 3 & W & 38 & 0 & 0 & 0 & 17.5 \\
\hline 3274 & Lime $\ldots \ldots \ldots \ldots \ldots \ldots$ & $\mathbf{Q}$ & $\ddot{Q}$ & 0 & $\mathbf{Q}$ & $\ddot{Q}$ & 0 & 0 & 0 & 0 & NF \\
\hline 3296 & Mineral Wool . . . . . . . . . . . . & 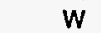 & $\bar{W}$ & 0 & $\vec{W}$ & $\bar{W}$ & 0 & 0 & 0 & 0 & 2.0 \\
\hline 33 & Primary Metal Industries . . . . . . . & 850 & 892 & 2,335 & 841 & 887 & 2,335 & $\mathbf{Q}$ & 5 & 0 & 18.2 \\
\hline 3312 & Blast Fumaces and Steel Mills . . . . . & 83 & 428 & $W$ & 83 & 428 & $W$ & 0 & 0 & 0 & 6.6 \\
\hline 3313 & Electrometalurgical Products ...... & 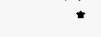 & 3 & 0 & * & 3 & 0 & 0 & 0 & 0 & 13.8 \\
\hline 3321 & Gray and Ductile Iron Foundries ... & 49 & 37 & 0 & 49 & 37 & 0 & 0 & 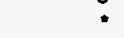 & 0 & 22.9 \\
\hline 3331 & Primary Copper ............ & 1 & $W$ & 0 & 1 & $w$ & 0 & 0 & 0 & 0 & 1.0 \\
\hline 3334 & Primary Aluminum .......... & $w$ & w & 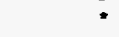 & $w$ & $w$ & 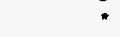 & 0 & 0 & 0 & 6.0 \\
\hline 3339 & Primary Nonferrous Metals, nec.... & 4 & w & 1 & 4 & $w$ & 1 & 0 & 0 & 0 & 1.9 \\
\hline 3353 & Aluminum Sheet, Plate, and Foil ... & 63 & $w$ & 0 & 63 & $w$ & 0 & 0 & 0 & 0 & 1.0 \\
\hline 34 & Fabricated Metal Products . . . . . . . . & 424 & 1,033 & 1,009 & 419 & 1,002 & 1,009 & $\mathbf{Q}$ & 30 & 0 & 21.4 \\
\hline 35 & industrial Machinery and Equipment .. & $W$ & $W$ & 1,118 & 482 & 965 & 1,118 & $\vec{Q}$ & $w$ & 0 & 29.1 \\
\hline 357 & Computer and Office Equipment ... & 5 & 28 & 21 & 5 & 28 & 21 & 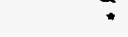 & 0 & 0 & 20.2 \\
\hline 36 & Electronic and Other Electric Equip. . . & 457 & 798 & 1,380 & 445 & 798 & 1,380 & $Q$ & • & 0 & 19.5 \\
\hline 37 & Transportation Equipment $\ldots \ldots \ldots$ & 164 & $w$ & 2,933 & $w$ & 1,389 & 2,933 & $w$ & w & 0 & 15.6 \\
\hline 3711 & Motor Vehicles and Car Bodies .... & 3 & $w$ & $W$ & 3 & $W$ & $W$ & 0 & 0 & 0 & 8.5 \\
\hline 3714 & Motor Vehicle Parts and Access. . . . & $W$ & 16 & w & 27 & 16 & $w$ & $w$ & 0 & 0 & 17.7 \\
\hline 38 & Instruments and Related Products ... & $Q$ & $w$ & 1,405 & $\mathbf{Q}$ & $W$ & 1,405 & 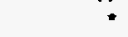 & $\star$ & 0 & 19.7 \\
\hline 3841 & Surgical and Medical Instruments .. & 6 & 44 & 25 & 6 & 44 & 25 & 0 & $*$ & 0 & 27.1 \\
\hline 39 & Misc. Manufacturing Industries . . . . . . & 110 & $w$ & 231 & W & W & 231 & $w$ & * & 0 & 21.8 \\
\hline & $\ldots \ldots \ldots \ldots \ldots \ldots$ & 11,681 & 21,576 & 70,668 & $w$ & 21,384 & 80,123 & $w$ & 315 & 0 & 9.3 \\
\hline
\end{tabular}

See footnotes at end of table. 


\section{Table A2. Total Consumption of LPG, Distillate Fuel Oil, and Residual Fuel Oil for Selected Purposes by Census Region, Industry Group, and Selected Industries, 1991 (Continued) \\ (Estimates in Barrels per Day)}

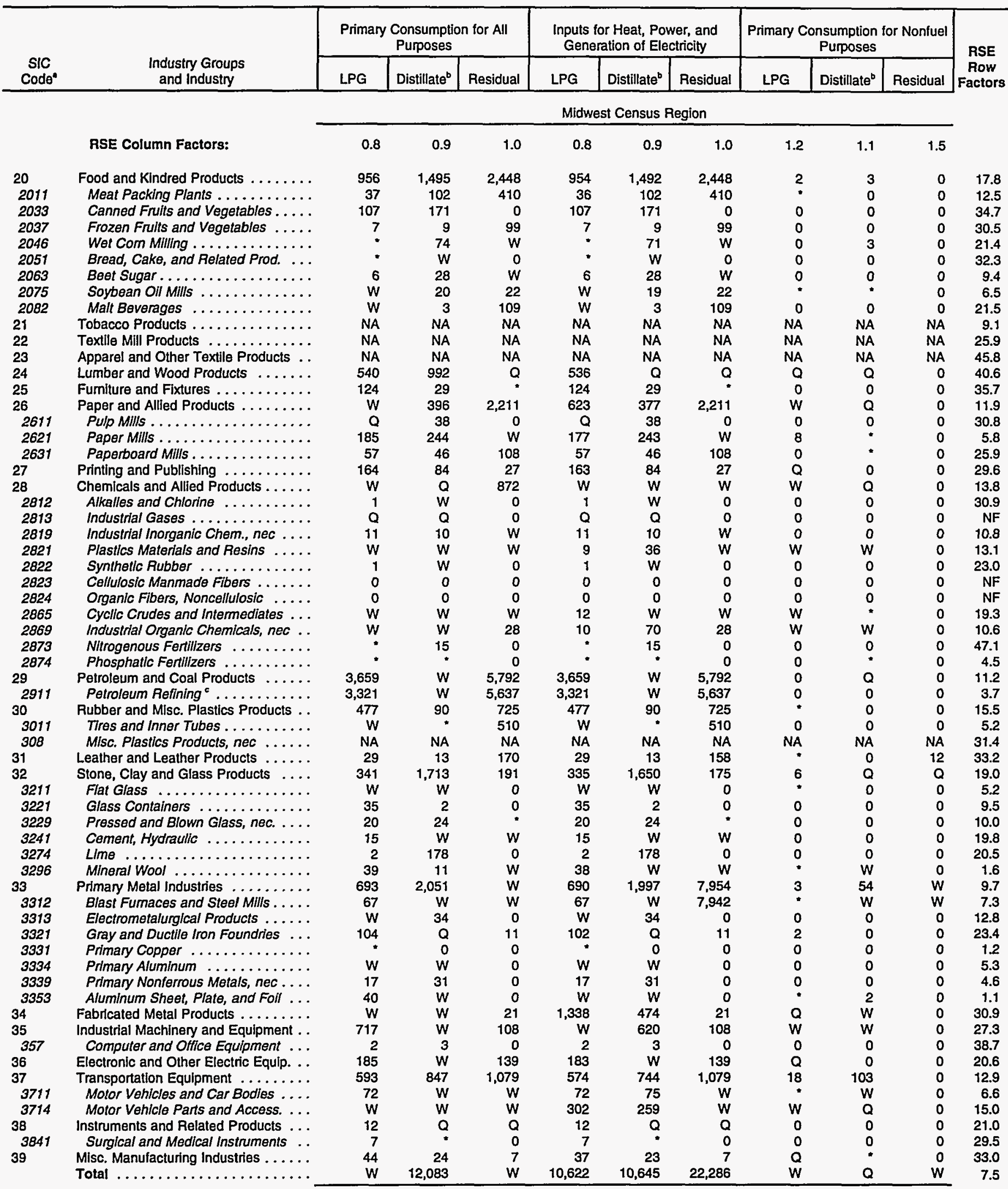

See footnotes at end of table. 


\section{Table A2. Total Consumption of LPG, Distillate Fuel Oil, and Residual Fuel Oil for Selected Purposes by Census Region, Industry Group, and Selected Industries, 1991 (Continued) (Estimates in Barrels per Day)}

\begin{tabular}{|c|c|c|c|c|c|c|c|c|c|c|c|}
\hline \multirow[b]{2}{*}{$\begin{array}{c}\text { SiC } \\
\text { Code" }\end{array}$} & \multirow[b]{2}{*}{$\begin{array}{l}\text { Industry Groups } \\
\text { and Industry }\end{array}$} & \multicolumn{3}{|c|}{$\begin{array}{c}\text { Primary Consumption for All } \\
\text { Purposes }\end{array}$} & \multicolumn{3}{|c|}{$\begin{array}{l}\text { Inputs for Heat, Power, and } \\
\text { Generation of Electricity }\end{array}$} & \multicolumn{3}{|c|}{$\begin{array}{c}\text { Primary Consumption for Nonfuel } \\
\text { Purposes }\end{array}$} & \multirow{2}{*}{$\begin{array}{c}\text { RSE } \\
\text { Row } \\
\text { Factors }\end{array}$} \\
\hline & & LPG & Distillate $^{b}$ & Residual & LPG & Distillate $^{b}$ & Residual & LPG & Distillate $^{b}$ & Residual & \\
\hline & & \multicolumn{9}{|c|}{ South Census Region } & \\
\hline & RSE Column Factors: & 0.8 & 0.8 & 1.0 & 0.8 & 0.8 & 0.9 & 1.0 & 1.2 & 2.4 & \\
\hline 20 & Food and Kindred Products & 9,706 & 2,354 & 4,307 & 1,706 & 2,354 & 4,307 & * & 0 & 0 & 21.1 \\
\hline 2011 & Meat Packing Plants . . . . . . . . . & 308 & 458 & 39 & 308 & 458 & 39 & 0 & 0 & 0 & 24.3 \\
\hline 2033 & Canned Fruits and Vegetables . . . . . & 37 & 24 & 70 & 37 & 24 & 70 & 0 & $\mathbf{0}$ & 0 & 22.1 \\
\hline 2037 & Frozen Fruits and Vegetables . . . . . & 17 & 34 & 334 & 17 & 34 & 334 & 0 & 0 & 0 & 24.2 \\
\hline 2046 & Wet Com Milling . . . . . . . . & 1 & 4 & 0 & 1 & 4 & 0 & 0 & 0 & 0 & 38.1 \\
\hline 2051 & Bread, Cake, and Related Prod. . . . & 21 & 51 & $\mathbf{0}$ & 20 & 51 & 0 & * & $\mathbf{0}$ & 0 & 20.4 \\
\hline 2063 & Beet Sugar . . . . . . . . . . . & 1 & 2 & 0 & 1 & 2 & 0 & 0 & 0 & 0 & 19.8 \\
\hline 2075 & Soybean Oil Mills . . . . . . . . . . & W & 66 & 93 & W & 66 & 93 & 0 & 0 & 0 & 7.5 \\
\hline 2082 & Malt Beverages ............ & 3 & $W$ & $\mathbf{W}$ & 3 & $w$ & W & 0 & 0 & 0 & 17.7 \\
\hline 21 & Tobacco Products ............. & 62 & 110 & 369 & 62 & 110 & 369 & 0 & 0 & 0 & 6.4 \\
\hline 22 & Textile Mill Products . . . . . . . . . & 1,246 & 1,386 & 3,265 & 1,246 & 1,386 & 3,265 & * & : & 0 & 13.4 \\
\hline 23 & Apparel and Other Textile Products .. & 340 & 196 & $\mathbf{Q}$ & 340 & 196 & Q & 0 & * & 0 & 37.9 \\
\hline 24 & Lumber and Wood Products $\ldots \ldots \ldots$ & 931 & 2,945 & $\mathbf{Q}$ & 911 & W & $\mathbf{Q}$ & 21 & $\mathbf{Q}$ & 0 & 31.8 \\
\hline 25 & Fumiture and Fixtures $\ldots \ldots \ldots \ldots$. & 334 & 272 & 173 & 334 & 272 & 173 & 0 & 0 & 0 & 28.7 \\
\hline 26 & Paper and Allied Products . . . . . . . . & 1,355 & 1,961 & 29,913 & 1,355 & 1,944 & 29,913 & • & 17 & 0 & 8.3 \\
\hline 2611 & Pulp Mills . . . . . . . . . . . . & 330 & 317 & 9,225 & 330 & 317 & 9,225 & 0 & 0 & 0 & 21.8 \\
\hline 2621 & Paper Mills . . . . . . . . . . . & 407 & $w$ & 7,675 & 407 & 974 & 7,675 & 0 & W & 0 & 4.8 \\
\hline 2631 & Paperboard Mills . . . . . . . . . & 113 & $w$ & 12,768 & 113 & 417 & 12,768 & $\cdot$ & $w$ & 0 & 8.0 \\
\hline 27 & Printing and Publishing $\ldots \ldots \ldots \ldots$ & 129 & 96 & $\mathbf{Q}$ & 129 & 96 & Q & 0 & 0 & 0 & 39.2 \\
\hline 28 & Chemicals and Allied Products . . . . . . & $1,071,758$ & 3,102 & $W$ & W & 2,878 & 10,968 & $1,069,272$ & 225 & $w$ & 9.7 \\
\hline 2812 & Alkalies and Chlorine ......... & 2 & 95 & 0 & 2 & 95 & 0 & 0 & 0 & 0 & 25.3 \\
\hline 2813 & Industrial Gases . . . . . . . . . . . & $\bar{W}$ & 2 & 0 & 2 & 2 & 0 & $w$ & 0 & 0 & 18.3 \\
\hline 2819 & Industrial Inorganic Chem., nec . . . . & 135 & w & 890 & 134 & 594 & $w$ & * & $W$ & $w$ & 13.4 \\
\hline 2821 & Plastics Materials and Resins . . . . . & 158,448 & w, & W & 93 & 178 & $w$ & 158,355 & $w$ & 0 & 6.7 \\
\hline 2822 & Synthetic Rubber . . . . . . . . & 11,188 & $w$ & W & 25 & $W$ & $W$ & 11,172 & $\cdot$ & 0 & 14.7 \\
\hline 2823 & Cellulosic Manmade Fibers ....... & 3 & 58 & 0 & 3 & 58 & 0 & 0 & 0 & 0 & 27.8 \\
\hline 2824 & Organic Fibers, Noncellulosic . . . . . & $w$ & $w$ & $w$ & 105 & W & $w$ & $w$ & 0 & 0 & 5.0 \\
\hline 2865 & Cyclic Crudes and Intermediates ... & W & 48 & 2,485 & 198 & 49 & $w$ & W & 1 & 0 & 17.1 \\
\hline 2869 & Industrial Organic Chemicals, nec .. & w & W & 925 & 2,126 & $Q$ & 925 & $w$ & $w$ & 0 & 14.8 \\
\hline 2873 & Nitrogenous Fertilizers ........ & 336 & 39 & 0 & * & 39 & 0 & 336 & 0 & 0 & 39.8 \\
\hline 2874 & Phosphatic Fentilizers ......... & 2 & $w$ & 685 & 2 & 390 & 685 & 0 & $w$ & 0 & 3.3 \\
\hline 29 & Petroleum and Coal Products ...... & $w$ & $w$ & 5,709 & 6,972 & $w$ & 5,709 & $\mathbf{Q}$ & $Q$ & 0 & 8.3 \\
\hline 2911 & Petroleum Refining ${ }^{e} \ldots \ldots \ldots \ldots$ & 6,713 & W & 5,537 & 6,713 & $w$ & 5,537 & $\overrightarrow{0}$ & 0 & 0 & 5.1 \\
\hline 30 & Rubber and Misc. Plastics Products .. & 692 & 661 & 1,457 & 658 & 659 & 1,457 & $\mathbf{Q}$ & 2 & 0 & 18.8 \\
\hline 3011 & Tires and Inner Tubes . . . . . . . . . & 145 & W & $W$ & 145 & W & $W$ & 0 & 0 & 0 & 4.9 \\
\hline 308 & Misc. Plastics Products, nec . . . . . & 338 & W & 428 & 304 & $w$ & 428 & $\mathbf{Q}$ & $*$ & 0 & 34.7 \\
\hline 31 & Leather and Leather Products . . . . . . & 8 & 26 & 70 & 8 & 26 & 70 & $*$ & 0 & 0 & 35.6 \\
\hline 32 & Stone, Clay and Glass Products .... & w & 3,948 & 552 & $w$ & 3,761 & 478 & $w$ & 187 & $\mathbf{Q}$ & 24.4 \\
\hline 3211 & Flat Glass $\ldots \ldots \ldots \ldots \ldots \ldots$ & 24 & 19 & 0 & 24 & 19 & 0 & * & 0 & 0 & 7.8 \\
\hline 3221 & Glass Containers ............ & 64 & W & W & 64 & $w$ & $w$ & 0 & 0 & 0 & 10.9 \\
\hline 3229 & Pressed and Blown Glass, nec. . . . . & W & $\ddot{w}$ & 4 & 40 & w & 4 & $w$ & 0 & 0 & 13.2 \\
\hline 3241 & Cement, Hydraulic ........... & 12 & 517 & 177 & 12 & 459 & 177 & 0 & 58 & 0 & 24.2 \\
\hline 3274 & Lime $\ldots \ldots \ldots \ldots \ldots \ldots$ & 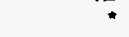 & 193 & 0 & $*$ & 193 & 0 & 0 & 0 & 0 & 22.0 \\
\hline 3296 & Mineral Wool . . . . . . . . . . . & 43 & 3 & $W$ & 43 & 3 & $W$ & 0 & 0 & 0 & 1.9 \\
\hline 33 & Primary Metal Industries . . . . . . . & $W$ & $w$ & 3,951 & 644 & 1,329 & 3,951 & W & $w$ & 0 & 6.2 \\
\hline 3312 & Blast Fumaces and Steel Mills . . . . . & 41 & 567 & 3,937 & 41 & $W$ & 3,937 & 0 & $W$ & 0 & 8.0 \\
\hline 3313 & Electrometalurgical Products . . . . . . & 0 & 19 & 0 & 0 & 19 & 0 & 0 & 0 & 0 & 11.1 \\
\hline 3321 & Gray and Ductile Iron Foundries ... & 127 & 153 & * & 127 & 152 & * & . & 1 & 0 & 22.8 \\
\hline 3331 & Primary Copper ............. & 4 & 15 & W & 4 & 15 & $w$ & 0 & 0 & 0 & 1.2 \\
\hline 3334 & Primary Aluminum $\ldots \ldots \ldots \ldots$ & 25 & $\mathbf{W}$ & 0 & 25 & $w$ & 0 & 0 & 0 & 0 & 5.5 \\
\hline 3339 & Primary Nonferrous Metals, nec . . . . & W & 25 & 0 & $W$ & 25 & 0 & w & 0 & 0 & 3.4 \\
\hline 3353 & Aluminum Sheet, Plate, and Foil ... & 61 & 70 & 0 & 61 & 70 & 0 & 0 & 0 & 0 & 1.2 \\
\hline 34 & Fabricated Metal Products . . . . . . . & W & $W$ & $\mathbf{Q}$ & 1,041 & 1,033 & $\mathbf{Q}$ & $\mathbf{Q}$ & $\mathbf{Q}$ & 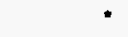 & 31.5 \\
\hline 35 & Industrial Machinery and Equipment . . & $w$ & 344 & 116 & $W$ & 343 & 116 & 58 & $\mathbf{Q}$ & 0 & 27.4 \\
\hline 357 & Computer and Office Equipment ... & $\because$ & Q & 9 & $*$ & Q & 9 & 0 & 0 & 0 & 42.4 \\
\hline 36 & Electronic and Other Electric Equip. . . & 420 & 121 & 159 & 419 & 121 & 159 & * & 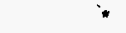 & 0 & 21.8 \\
\hline 37 & Transportation Equipment ........ & 365 & 903 & $w$ & 362 & 840 & W & $\mathbf{Q}$ & 64 & 0 & 12.5 \\
\hline 3711 & Motor Vehicles and Car Bodies . . . . & 78 & 97 & 199 & 78 & 53 & 199 & $\mathbf{0}$ & 44 & 0 & 5.6 \\
\hline 3714 & Motor Vehicle Parts and Access. . . . & 113 & 22 & • & 113 & 4 & $\cdot$ & 0 & 18 & 0 & 14.7 \\
\hline 38 & Instruments and Related Products ... & 17 & 123 & $\mathbf{Q}$ & 17 & 123 & $\mathbf{Q}$ & 0 & 0 & 0 & 31.3 \\
\hline 3841 & Surgical and Medical Instruments . . & 3 & 31 & 0 & 3 & 31 & $\mathbf{0}$ & 0 & 0 & 0 & 37.7 \\
\hline 39 & Misc. Manufacturing Industries . . . . . . & 68 & Q & 77 & 68 & $\mathbf{Q}$ & 77 & - & $\mathbf{Q}$ & 0 & 38.0 \\
\hline & Total $\ldots \ldots \ldots \ldots \ldots \ldots \ldots$ & $W$ & 23,236 & W & W & 21,957 & 63,327 & $w$ & 1,280 & $w$ & 7.5 \\
\hline
\end{tabular}

See footnotes at end of table. 
Table A2. Total Consumption of LPG, Distillate Fuel Oil, and Residual Fuel Oil for Selected Purposes by Census Region, Industry Group, and Selected Industries, 1991 (Continued) (Estimates in Barrels per Day)

\begin{tabular}{|c|c|c|c|c|c|c|c|c|c|c|c|}
\hline \multirow[b]{2}{*}{$\begin{array}{c}\text { SIC } \\
\text { Codea }\end{array}$} & \multirow[b]{2}{*}{$\begin{array}{l}\text { Industry Groups } \\
\text { and Industry }\end{array}$} & \multicolumn{3}{|c|}{$\begin{array}{l}\text { Primary Consumption for All } \\
\text { Purposes }\end{array}$} & \multicolumn{3}{|c|}{$\begin{array}{l}\text { Inputs for Heat, Power, and } \\
\text { Generation of Electricity }\end{array}$} & \multicolumn{3}{|c|}{$\begin{array}{c}\text { Primary Consumption for Nonfuel } \\
\text { Purposes }\end{array}$} & \multirow{2}{*}{$\begin{array}{l}\text { RSE } \\
\text { Row } \\
\text { Factors }\end{array}$} \\
\hline & & LPG & Distillate $^{b}$ & Residual & LPG & Distillate ${ }^{b}$ & Residual & LPG & Distillate ${ }^{\circ}$ & Residual & \\
\hline & & \multicolumn{9}{|c|}{ West Census Region } & \\
\hline & RSE Column Factors: & 0.9 & 0.9 & 1.0 & 0.9 & 0.9 & 1.0 & 1.3 & 1.5 & NF & \\
\hline 20 & Food and Kindred Products. & 656 & 1,845 & 1,884 & 645 & 1,845 & 1,884 & 11 & * & 0 & 17.8 \\
\hline 2011 & Meat Packing Plants . . . . . . . . . . & Q & 38 & W & Q & 38 & W & 0 & 0 & 0 & 22.7 \\
\hline 2033 & Canned Fruits and Vegetables . . . . . & 164 & 104 & 325 & 158 & 103 & 325 & 6 & $\star$ & 0 & 18.1 \\
\hline 2037 & Frozen Fruits and Vegetables ..... & 85 & 156 & $\mathbf{Q}$ & 85 & 156 & $\mathbf{Q}$ & * & 0 & 0 & 25.8 \\
\hline 2046 & Wet Com Milling . . . . . . . . . . & * & W & 0 & $\bullet$ & W & 0 & 0 & 0 & 0 & 24.1 \\
\hline 2051 & Bread, Cake, and Related Prod. . . . & 20 & 5 & 0 & 20 & 5 & 0 & 0 & 0 & 0 & 46.4 \\
\hline 2063 & Beot Sugar. . . . . . . . . . . . & 7 & 51 & W & 7 & 51 & W & 0 & 0 & 0 & 10.6 \\
\hline 2075 & Soybran Oll Mills . . . . . . . . . . & 0 & 0 & 0 & 0 & 0 & 0 & 0 & 0 & 0 & NF \\
\hline 2082 & Malt Boverages ........... & w & W & $w$ & W & W & W & 0 & 0 & 0 & 19.0 \\
\hline 21 & Tobacco Products $\ldots \ldots \ldots \ldots \ldots$ & 0 & 0 & 0 & 0 & 0 & 0 & 0 & 0 & 0 & NF \\
\hline 22 & Textile Mill Products . . . . . . . . . . & $\mathbf{Q}$ & 6 & 0 & $Q$ & 6 & 0 & 0 & 0 & 0 & 33.4 \\
\hline 23 & Apparel and Other Textile Products .. & NA & NA & NA & NA & NA & NA & NA & NA & NA & NF \\
\hline 24 & Lumber and Wood Products $\ldots . .$. & 953 & 3,210 & 360 & 953 & 3,171 & 360 & 0 & Q & 0 & 24.3 \\
\hline 25 & Furniture and Fixtures $\ldots \ldots \ldots \ldots$ & 78 & $\mathbf{Q}$ & 0 & 78 & $\mathbf{Q}$ & 0 & 0 & 0 & 0 & 53.5 \\
\hline 26 & Paper and Allied Products . . . . . . . . & W & 297 & 4,708 & 625 & 259 & 4,708 & $w$ & 37 & 0 & 10.6 \\
\hline 2611 & Pulp Mills . . . . . . . . . . . . & 15 & 69 & 2,308 & 15 & 49 & 2,308 & 0 & 20 & 0 & 19.6 \\
\hline 2621 & Paper MIlls . . . . . . . . . . . & 318 & 106 & $w$ & 318 & 106 & $W$ & 0 & 0 & 0 & 5.9 \\
\hline 2631 & Paperboard Mills . . . . . . . . . . & $W$ & $W$ & $W$ & 72 & 96 & W & W & W & 0 & 8.1 \\
\hline 27 & Printing and Publishing $\ldots \ldots \ldots \ldots$ & NA & NA & NA & NA & NA & NA & NA & NA & NA & 32.4 \\
\hline 28 & Chemicals and Alled Products . . . . . . & 188 & 509 & $W$ & $W$ & $W$ & W & W & $W$ & 0 & 17.6 \\
\hline 2812 & Alkalles and Chlorine .......... & $\cdot$ & W & $W$ & * & W & W & 0 & 0 & 0 & 25.0 \\
\hline 2813 & Industrial Gases $\ldots \ldots \ldots \ldots \ldots$ & W & W & 0 & $\cdot$ & W & 0 & $w$ & 0 & 0 & 19.9 \\
\hline 2819 & Industrial Inorganic Chem., nec . . . . & $\mathbf{Q}$ & W & W & $\mathbf{Q}$ & 431 & W & 0 & $W$ & 0 & 14.5 \\
\hline 2821 & Plastics Materials and Resins .... & 6 & $\bullet$ & 0 & 6 & * & 0 & 0 & 0 & 0 & 8.9 \\
\hline 2822 & Synthetic Rubber . . . . . . . . . & $\bullet$ & * & 0 & * & * & 0 & 0 & 0 & 0 & 25.2 \\
\hline 2823 & Collulosic Manmade Fibers . . . . . . . & 0 & 0 & 0 & 0 & 0 & 0 & 0 & 0 & 0 & NF \\
\hline 2824 & Organic Fibers, Noncellulosic . . . . & 0 & 0 & 0 & 0 & 0 & 0 & 0 & 0 & 0 & NF \\
\hline 2865 & Cycllc Crudes and Intermediates ... & 1 & 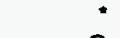 & 0 & 1 & $\bullet$ & 0 & 0 & 0 & 0 & 25.2 \\
\hline 2869 & Industrial Organic Chemlcals, nec . . & $\mathbf{Q}$ & $\mathbf{Q}$ & 0 & $\mathbf{Q}$ & $\mathbf{Q}$ & 0 & 0 & 0 & 0 & NF \\
\hline 2873 & Nitrogenous Fertilizers $\ldots \ldots \ldots$ & 115 & 9 & 0 & 115 & 9 & 0 & 0 & 0 & 0 & 42.8 \\
\hline 2874 & Phosphatic Fertilizers . . . . . . . . & 0 & 20 & 0 & 0 & 20 & 0 & 0 & 0 & 0 & 32.4 \\
\hline 29 & Petroleum and Coal Products ...... & 32,318 & W & 5,309 & 32,318 & 2,378 & 5,309 & 0 & 0 & 0 & 4.6 \\
\hline 2911 & Petroleum Reflining ${ }^{c} \ldots \ldots \ldots \ldots$ & 32,241 & 2,138 & 5,309 & 32,241 & 2,138 & 5,309 & 0 & 0 & 0 & 3.9 \\
\hline 30 & Rubber and Misc. Plastics Products .. & W & Q & 2 & W & $\mathbf{Q}$ & 2 & 0 & $\star$ & 0 & 14.3 \\
\hline 3011 & Tires and Inner Tubes . . . . . . . . . . & W & W & $W$ & $W$ & W & W & 0 & 0 & 0 & 7.4 \\
\hline 308 & Mlsc. Plastics Products, nec . . . . . & 101 & a & 0 & 101 & $\mathbf{Q}$ & 0 & 0 & 0 & 0 & 31.9 \\
\hline 31 & Leather and Leather Products . . . . . & $\mathbf{Q}$ & Q & 0 & $\mathbf{Q}$ & $\mathbf{Q}$ & 0 & 0 & 0 & 0 & NF \\
\hline 32 & Stone, Clay and Glass Products .... & 337 & 1,760 & 1,846 & 336 & 1,707 & 1,846 & 1 & $\mathbf{Q}$ & 0 & 12.7 \\
\hline 3211 & Flat Glass $\ldots \ldots \ldots \ldots \ldots$ & W & W & w & $W$ & W & W & 0 & 0 & 0 & 5.8 \\
\hline 3221 & Glass Containers . . . . . . . . . & 59 & W & W & 59 & W & W & 0 & 0 & 0 & 12.7 \\
\hline 3229 & Pressed and Blown Glass, nec. . . . . & 1 & $*$ & 0 & 1 & $\star$ & 0 & 0 & 0 & 0 & 14.9 \\
\hline 3241 & Cement, Hydraulic . . . . . . . & 3 & 384 & W & 3 & 384 & W & 0 & 0 & 0 & 21.7 \\
\hline 3274 & $L I m s \ldots \ldots \ldots \ldots \ldots \ldots$ & $\cdot$ & W & $w$ & • & $W$ & W & 0 & 0 & 0 & 24.4 \\
\hline 3296 & Mineral Wool . . . . . . . . . . & $W$ & W & 0 & $W$ & * & 0 & 1 & $w$ & 0 & 2.0 \\
\hline 33 & Primary Metal Industries . . . . . . . . & $w$ & W & 239 & 257 & 736 & 239 & $\mathbf{Q}$ & $w$ & 0 & 7.4 \\
\hline 3312 & Blast Fumaces and Steol Mills . . . . . & 11 & W & W & 11 & 92 & W & 0 & $W$ & 0 & 8.3 \\
\hline 3313 & Electrometalurgical Products ..... . . & 0 & 0 & 0 & 0 & 0 & 0 & 0 & 0 & 0 & NF \\
\hline 3321 & Gray and Ductlle Iron Foundries ... & 8 & 8 & 0 & 8 & 8 & 0 & 0 & 0 & 0 & 40.5 \\
\hline 3331 & Primary Copper . . . . . . . . . . & 3 & W & $W$ & 3 & W & W & 0 & 0 & 0 & 1.1 \\
\hline 3334 & Primary Aluminum $\ldots \ldots \ldots \ldots$ & 78 & 47 & • & 78 & 47 & $\cdot$ & 0 & 0 & 0 & 4.2 \\
\hline 3339 & Primary Nonferrous Metals, nec . . . . & $w$ & W & 0 & $W$ & W & 0 & $W$ & 0 & 0 & 1.1 \\
\hline 3353 & Aluminum Sheet, Plate, and Foil ... & W & W & 0 & $W$ & W & 0 & 0 & 0 & 0 & 1.1 \\
\hline 34 & Fabricated Metal Products . . . . . . . . & 283 & 214 & 1 & 276 & 214 & 1 & $Q$ & $*$ & 0 & 30.7 \\
\hline 35 & Industrial Machinery and Equipment .. & 198 & 39 & 0 & 197 & 39 & 0 & $\bullet$ & * & 0 & 39.0 \\
\hline 357 & Computer and Ofice Equipment ... & $Q$ & - & 0 & $\mathbf{Q}$ & 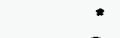 & 0 & 0 & 0 & 0 & 19.6 \\
\hline 36 & Electronic and Other Electric Equip. . . & 37 & a & 0 & 37 & $\mathbf{Q}$ & 0 & 0 & 0 & 0 & 36.4 \\
\hline
\end{tabular}

See footnotes at end of table. 


\section{Table A2. Total Consumption of LPG, Distillate Fuel Oil, and Residual Fuel Oil for Selected Purposes by Census Region, Industry Group, and Selected Industries, 1991 (Continued) (Estimates in Barrels per Day)}

\begin{tabular}{|c|c|c|c|c|c|c|c|c|c|c|c|}
\hline \multirow[b]{2}{*}{$\begin{array}{l}\text { SIC } \\
\text { Code" }\end{array}$} & \multirow[b]{2}{*}{$\begin{array}{l}\text { Industry Groups } \\
\text { and Industry }\end{array}$} & \multicolumn{3}{|c|}{$\begin{array}{l}\text { Primary Consumption for All } \\
\text { Purposes }\end{array}$} & \multicolumn{3}{|c|}{$\begin{array}{l}\text { Inputs for Heat, Power, and } \\
\text { Generation of Electricity }\end{array}$} & \multicolumn{3}{|c|}{$\begin{array}{c}\text { Primary Consumption for Nonfuel } \\
\text { Pumposes }\end{array}$} & \multirow{2}{*}{$\begin{array}{c}\text { RSE } \\
\text { Row } \\
\text { Factors }\end{array}$} \\
\hline & & LPG & Distillate & Residual & LPG & Distillate $^{\mathrm{b}}$ & Residual & LPG & Distillate $^{b}$ & Residual & \\
\hline & & \multicolumn{10}{|c|}{ West Census Region } \\
\hline & RSE Column Factors: & 0.9 & 0.9 & 1.0 & 0.9 & 0.9 & 1.0 & 1.3 & 1.5 & NF & \\
\hline 37 & Transportation Equipment & 386 & $w$ & $w$ & w & 353 & $w$ & $\mathbf{Q}$ & $w$ & 0 & 15.6 \\
\hline 3711 & Motor Vehicles and Car Bodies .... & 9 & 51 & 0 & 9 & W & 0 & 0 & $W$ & 0 & 6.9 \\
\hline 3714 & Motor Vehicle Parts and Access. . . . & 19 & 5 & Q & 19 & 5 & $\mathbf{Q}$ & 0 & 0 & 0 & 28.5 \\
\hline 38 & Instruments and Related Products ... & 17 & 23 & 10 & 16 & 23 & 10 & 1 & 0 & 0 & 23.4 \\
\hline 3841 & Surgical and Medical Instruments .. & 6 & 7 & 0 & 6 & 7 & 0 & 0 & 0 & 0 & 46.5 \\
\hline \multirow[t]{2}{*}{39} & Misc. Manufacturing Industries . . . . . . & 22 & 2 & 0 & 22 & 2 & 0 & 0 & 0 & 0 & 33.7 \\
\hline & Total $\ldots \ldots \ldots \ldots \ldots \ldots$ & 36,660 & 11,643 & 14,640 & 36,561 & 11,452 & 14,640 & 100 & 191 & 0 & 7.3 \\
\hline
\end{tabular}

See Appendices $B$ and $F$ for descriptions of the Standard Industrial Classification system.

- "Distillate" includes Nos. 1,2, and 4 fuel oils and Nos. 1,2, and 4 diesel fuels.

'For the petroleum refining industry only, the column of "Primary Consumption for All Purposes" includes only energy consumed for heat, power, and generation of electricity. See Appendix $B$ for more information.

NF=No applicable RSE row/column factor.

- Estimate less than 0.5. Data are included in higher level totals.

$W=$ Withheld to avoid disclosing data for individual establishments. Data are included in higher level totals.

$\mathrm{Q}=$ Withheid because Relative Standard Error is greater than 50 percent. Data are included in higher level totals.

$N A=N o t$ available. Data are included in higher level totals.

Notes: - To obtain a RSE percentage for any table cell, multiply the cell's corresponding RSE column and RSE row factors. - Totals may not equal sum of

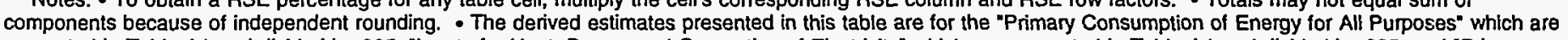
presented in Table A1 and divided by 365; "Inputs for Heat, Power, and Generation of Electricity" which are presented in Table A4 and divided by 365; and "Primary Consumption for Nonfuel Purposes" which are presented in Table A3 and divided by 365 . Primary consumption is defined as the consumption of the energy that was originally produced offsite or was produced onsite from input materials not classified as energy. Primary consumption excludes quantities of energy that are produced from other energy inputs and, therefore, avoids double counting.

Source: Energy Information Administration, Office of Energy Markets and End Use, Energy End Use and Integrated Statistics Division, Form ElA-846, "1991 Manufacturing Energy Consumption Survey." 
Table A3. Total Primary Consumption of Combustible Energy for Nonfuel Purposes by Census Region, Industry Group, and Selected Industries, 1991: Part 1 (Estimates in Btu or Physical Units)

\begin{tabular}{|c|c|c|c|c|c|c|c|c|c|c|}
\hline $\begin{array}{l}\text { SIC } \\
\text { Code" }\end{array}$ & $\begin{array}{l}\text { Industry Groups } \\
\text { and Industry }\end{array}$ & $\begin{array}{c}\text { Total } \\
\text { (trillion Btu) }\end{array}$ & $\begin{array}{c}\text { Residual } \\
\text { Fuel Oil } \\
(1000 \mathrm{bbls})\end{array}$ & $\begin{array}{c}\text { Distillate } \\
\text { Fuel Oip } \\
(1000 \mathrm{bbls})\end{array}$ & $\begin{array}{c}\text { Natural } \\
\text { Gas }^{c} \\
\text { (billion } \\
\text { cu ft) }\end{array}$ & $\begin{array}{c}\text { LPG } \\
\text { (1000 bbls) }\end{array}$ & $\begin{array}{c}\text { Coal } \\
\text { (1000 short } \\
\text { tons) }\end{array}$ & $\begin{array}{c}\text { Coke and } \\
\text { Breeze } \\
\left(\begin{array}{c}1000 \text { short } \\
\text { tons) }\end{array}\right.\end{array}$ & $\begin{array}{c}\text { Other' } \\
\text { (trillion Btu) }\end{array}$ & $\begin{array}{c}\text { RSE } \\
\text { Row } \\
\text { Factors }\end{array}$ \\
\hline
\end{tabular}

Total United States

\begin{tabular}{|c|c|c|c|c|c|c|c|c|c|c|}
\hline & RSE Column Factors: & 0.7 & 2.1 & 1.0 & 0.9 & 0.9 & 1.2 & 0.9 & 0.8 & \\
\hline 20 & Food and Kindred Products . ......... & 3 & 0 & 1 & w & 5 & 0 & w & 1 & 19.3 \\
\hline 2011 & Meat Packing Plants $\ldots \ldots \ldots \ldots \ldots$ & * & 0 & 0 & - & * & 0 & 0 & - & 30.3 \\
\hline 2033 & Canned Frults and Vegetables . . . . . . . & . & 0 & • & • & 2 & 0 & 0 & 0 & 41.3 \\
\hline 2037 & Frozen Fruits and Vegetables . . . . . . . & - & 0 & 0 & 0 & * & 0 & 0 & • & 49.6 \\
\hline 2046 & Wet Com Milling $\ldots \ldots \ldots \ldots \ldots \ldots$ & * & 0 & 1 & 0 & 0 & 0 & 0 & - & 28.7 \\
\hline 2051 & Bread, Cake, and Related Products .... & * & 0 & 0 & - & • & 0 & 0 & - & 31.5 \\
\hline 2063 & Boet Sugar $\ldots \ldots \ldots \ldots \ldots \ldots \ldots$ & . & 0 & 0 & 0 & 0 & 0 & $\mathbf{w}$ & - & 18.7 \\
\hline 2075 & Soybean Oll Mills $\ldots \ldots \ldots \ldots \ldots \ldots$ & 1 & 0 & • & 1 & • & 0 & 0 & - & 8.2 \\
\hline 2082 & Malt Boverages $\ldots \ldots \ldots \ldots \ldots \ldots$ & •" & 0 & 0 & 0 & 0 & 0 & 0 & * & 31.7 \\
\hline 21 & Tobacco Products $\ldots \ldots \ldots \ldots \ldots \ldots$ & 0 & 0 & 0 & 0 & 0 & 0 & 0 & 0 & NF \\
\hline 22 & Textlle Mill Products $\ldots \ldots \ldots \ldots \ldots \ldots$ & * & 0 & • & - & • & 0 & 0 & - & 32.7 \\
\hline 23 & Apparel and Other Textile Products $\ldots .$. & - & 0 & : & 0 & * & 0 & 0 & - & 62.6 \\
\hline 24 & Lumber and Wood Products $\ldots \ldots \ldots \ldots$ & 27 & 0 & Q & • & 9 & 0 & 0 & 25 & 44.3 \\
\hline 25 & Furniture and Fixtures $\ldots \ldots \ldots \ldots \ldots$ & $Q$ & 0 & 0 & • & 0 & 0 & 0 & $\mathbf{Q}$ & NF \\
\hline 26 & Paper and Allied Products . . . . . . . . . & 35 & 0 & 28 & w & w & * & 0 & W & 15.8 \\
\hline 2611 & Pulp Mills $\ldots \ldots \ldots \ldots \ldots \ldots \ldots \ldots$ & • & 0 & 7 & 0 & 0 & 0 & 0 & * & 36.2 \\
\hline 2621 & Paper Mills $\ldots \ldots \ldots \ldots \ldots \ldots \ldots$ & 8 & 0 & 5 & * & 3 & * & 0 & 7 & 6.5 \\
\hline 2631 & Paperboard Mills $\ldots \ldots \ldots \ldots \ldots \ldots$ & 27 & 0 & w & w & w & 0 & 0 & 26 & 14.6 \\
\hline 27 & Printing and Publlshing $\ldots \ldots \ldots \ldots \ldots$ & • & 0 & Q & • & $\mathbf{Q}$ & 0 & 0 & - & 59.1 \\
\hline 28 & Chemicals and Allied Products . . . . . . . . & 2,358 & W & a & 542 & w & $w$ & 291 & 259 & 8.5 \\
\hline 2812 & Alkalles and Chlorine ............ & 1 & 0 & 0 & 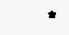 & 0 & 0 & 0 & 1 & 31.5 \\
\hline 2813 & Industrial Gases . ................ & w & 0 & 0 & w & w & 0 & 0 & w & 18.0 \\
\hline 2819 & Industrial Inorganic Chemicals, nec .... & 20 & w & w & w & * & $\mathbf{w}$ & 241 & 4 & 15.9 \\
\hline 2821 & Plastics Materials and Resins . . . . . . & 357 & 0 & 4 & 64 & w & 0 & 0 & w & 8.5 \\
\hline 2822 & Synthetic Rubber . ............ & w & 0 & - & w & 4,078 & 0 & 0 & - & 23.4 \\
\hline 2823 & Cellulosic Manmade Fibers . . . . . . . . . & 0 & 0 & 0 & 0 & 0 & 0 & 0 & 0 & NF \\
\hline 2824 & Organic Fibers, Noncellulosic $\ldots \ldots \ldots$ & $w$ & 0 & 0 & w & $\mathbf{w}$ & w & 0 & - & 6.9 \\
\hline 2865 & Cycllc Crudes and Intermediates ...... & 98 & 0 & 1 & 8 & 20,863 & 1 & 0 & 14 & 17.4 \\
\hline 2869 & Industrial Organlc Chemicals, nec ..... & 1,345 & 0 & 60 & $\mathbf{w}$ & w & 0 & 0 & 192 & 6.6 \\
\hline 2873 & Nitrogenous Fentilizers $\ldots \ldots \ldots \ldots \ldots$ & 290 & 0 & 0 & 281 & 122 & 0 & 0 & * & 26.4 \\
\hline 2874 & Phosphatic Fertilizers $\ldots \ldots \ldots \ldots \ldots$ & 31 & 0 & w & w & 0 & 0 & 0 & w & 3.5 \\
\hline 29 & Petroleum and Coal Products & 3,004 & 0 & 84 & • & w & $w$ & 0 & w & 3.5 \\
\hline 2911 & Petroleum Refining ${ }^{\circ} \ldots \ldots \ldots \ldots \ldots$ & 2,868 & 0 & 0 & 0 & 0 & 0 & 0 & 2,868 & 1.4 \\
\hline 30 & Rubber and Misc. Plastics Products ..... & 3 & 0 & 4 & • & $\mathbf{Q}$ & $\mathbf{Q}$ & 0 & 2 & 26.4 \\
\hline 3011 & Tires and Inner Tubes . . . . . . . . . . . . & w & 0 & 0 & * & 0 & $\mathbf{w}$ & 0 & w & 5.4 \\
\hline 308 & Miscellaneous Plastics Products, nec... & 1 & 0 & $\mathbf{Q}$ & • & $\mathbf{Q}$ & 0 & 0 & 1 & 44.6 \\
\hline 31 & Leather and Leather Products . . . . . . . & * & 4 & - & 0 & 1 & 0 & 0 & • & 40.5 \\
\hline 32 & Stone, Clay and Glass Products...$\ldots$. & 3 & $\mathbf{Q}$ & 119 & 1 & w & $\mathbf{Q}$ & $w$ & $w$ & 20.1 \\
\hline 3211 & Flat Glass $\ldots \ldots \ldots \ldots \ldots \ldots \ldots$ & •" & 0 & 0 & 0 & * & 0 & 0 & : & 8.0 \\
\hline 3221 & Glass Contalners $\ldots \ldots \ldots \ldots \ldots \ldots$ & • & 0 & 0 & 0 & 0 & 0 & 0 & - & 24.8 \\
\hline 3229 & Pressed and Blown Glass, nec. . . . . . . . & $w$ & 0 & 0 & $\mathbf{w}$ & $\mathbf{w}$ & 0 & 0 & * & 13.8 \\
\hline 3241 & Cement, Hydraullic $\ldots \ldots \ldots \ldots \ldots$. & - & 0 & 21 & • & 0 & 0 & 0 & • & 32.5 \\
\hline 3274 & Lime $\ldots \ldots \ldots \ldots \ldots \ldots \ldots \ldots$ & • & 0 & 0 & * & 0 & $\mathbf{Q}$ & w & - & 31.9 \\
\hline 3296 & Mineral Wool $\ldots \ldots \ldots \ldots \ldots$ & * & 0 & W & • & w & 0 & 0 & - & 2.0 \\
\hline 33 & Primary Metal Industries $\ldots \ldots \ldots \ldots \ldots$ & 909 & W & 61 & 22 & w & 30,190 & 671 & 54 & 4.4 \\
\hline 3312 & Blast Fumaces and Steel Mills . . . . . . . . & 838 & W & w & 20 & * & 29,829 & 250 & w & 7.6 \\
\hline 3313 & Electrometalurgical Products $\ldots \ldots \ldots \ldots$ & 12 & 0 & 0 & - & 0 & $\mathbf{w}$ & $\mathbf{w}$ & w & 9.9 \\
\hline 3321 & Gray and Ductile iron Foundries ...... & $w$ & 0 & 1 & - & 1 & $w$ & $\mathbf{w}$ & • & 18.5 \\
\hline 3331 & Primary Copper $\ldots \ldots \ldots \ldots \ldots \ldots$ & * & 0 & 0 & 0 & 0 & 0 & $\mathbf{w}$ & - & 1.3 \\
\hline 3334 & Primary Aluminum $\ldots \ldots \ldots \ldots \ldots$ & 44 & 0 & 0 & 0 & 0 & 40 & $\mathbf{w}$ & w & 3.6 \\
\hline 3339 & Primary Nonferrous Metals, nec........ & 10 & 0 & 0 & 0 & w & $\mathbf{w}$ & w & w & 1.6 \\
\hline 3353 & Aluminum Sheet, Plate, and Foil . . . . . & * & 0 & 1 & 0 & • & 0 & 0 & - & 2.7 \\
\hline 34 & Fabricated Metal Products $\ldots \ldots \ldots \ldots \ldots$ & 3 & - & 23 & 1 & 23 & • & $\mathbf{w}$ & w & 37.8 \\
\hline 35 & Industrial Machinery and Equipment ...... & 1 & 0 & 24 & 1 & 30 & 1 & 0 & * & 31.7 \\
\hline 357 & Computer and Office Equipment $\ldots \ldots$. & - & $\mathbf{0}$ & 0 & 0 & • & 0 & 0 & - & 45.4 \\
\hline 36 & Electronic and Other Electric Equipment .. & 15 & $\mathbf{0}$ & - & • & $\mathbf{Q}$ & $w$ & w & 13 & 30.5 \\
\hline 37 & Transportation Equipment $\ldots \ldots \ldots \ldots \ldots$ & 9 & 0 & 72 & w & 25 & $\mathbf{w}$ & W & 7 & 16.0 \\
\hline 3711 & Motor Vehicles and Car Bodies . . . . . . . & 2 & 0 & 51 & - & - & $\mathbf{w}$ & 0 & $\mathbf{w}$ & 7.3 \\
\hline 3714 & Motor Vehicle Parts and Accessories ... & 1 & 0 & a & - & 8 & - & 1 & 1 & 14.1 \\
\hline 38 & Instruments and Related Products $\ldots \ldots \ldots$ & . & 0 & - & • & - & 0 & 0 & * & 37.8 \\
\hline 3841 & Surglcal and Medical Instruments ..... & * & 0 & * & 0 & 0 & 0 & 0 & • & 35.9 \\
\hline \multirow[t]{2}{*}{39} & Misc. Manufacturing Industries . . . . . . . . & $\mathbf{Q}$ & 0 & $\mathbf{Q}$ & • & w & 0 & 0 & - & 38.6 \\
\hline & Total $\ldots \ldots \ldots \ldots \ldots \ldots \ldots$ & 6,373 & 10,022 & 1,216 & 573 & 419,337 & 30,869 & 1,028 & 3,394 & 5.8 \\
\hline
\end{tabular}


Table A3. Total Primary Consumption of Combustible Energy for Nonfuel Purposses by Census Region, Industry Group, and Selected Industries, 1991: Part 1 (Continued)

(Estimates in Btu or Physical Units)

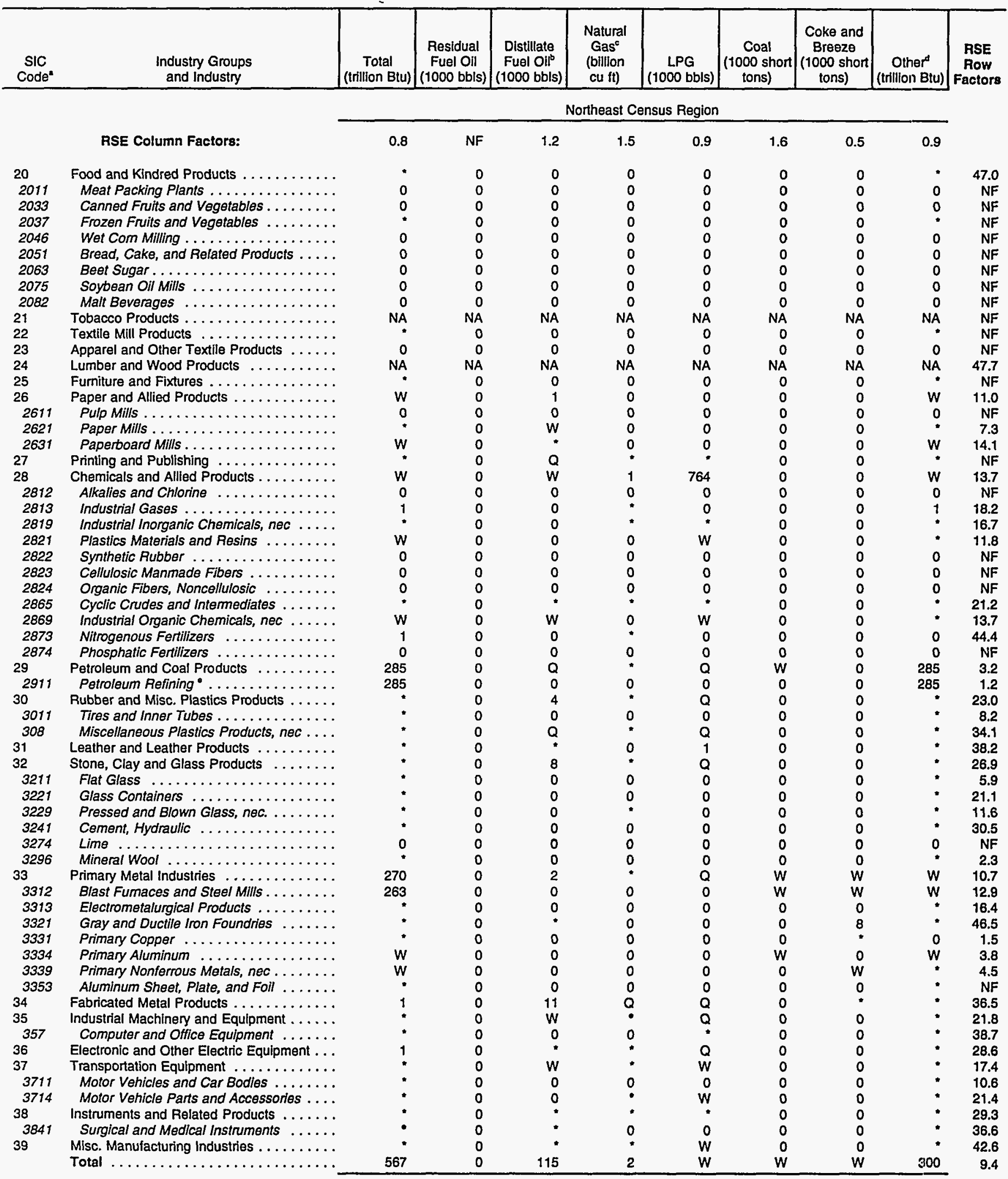

See footnotes at end of table. 


\section{Table A3. Total Primary Consumption of Combustible Energy for Nonfuel Purposes by Census Region, Industry Group, and Selected Industries, 1991: Part 1 (Continued) \\ (Estimates in Btu or Physical Units)}

\begin{tabular}{|c|c|c|c|c|c|c|c|c|c|c|}
\hline $\begin{array}{l}\text { SIC } \\
\operatorname{cod}^{*}\end{array}$ & $\begin{array}{l}\text { Industry Groups } \\
\text { and Industry }\end{array}$ & $\begin{array}{c}\text { Total } \\
\text { (trillion Btu) }\end{array}$ & $\begin{array}{c}\text { Residual } \\
\text { Fuel Oil } \\
(1000 \text { bbls })\end{array}$ & $\begin{array}{c}\text { Distillate } \\
\text { Fuel OIf } \\
(1000 \text { bbls })\end{array}$ & $\begin{array}{c}\text { Natural } \\
\text { Gas } \\
\text { (billion } \\
\text { cu ft) }\end{array}$ & $\begin{array}{c}\text { LPG } \\
\text { (1000 bbls) }\end{array}$ & $\begin{array}{c}\text { Coal } \\
(1000 \text { short } \\
\text { tons })\end{array}$ & $\begin{array}{c}\text { Coke and } \\
\text { Breeze } \\
(1000 \text { short } \\
\text { tons })\end{array}$ & $\begin{array}{c}\text { Othere } \\
\text { (trillion Btu) }\end{array}$ & $\begin{array}{c}\text { RSE } \\
\text { Row } \\
\text { Factors }\end{array}$ \\
\hline
\end{tabular}

RSE Column Factors:

Food and Kindred Products .............

20

2011 Meat Packing Plants................

2033 Canned Fruits and Vegetables ..........

2037 Frozen Frults and Vegetables .........

2046 Wot Com Milling .................

2051 Bread, Cake, and Related Products .....

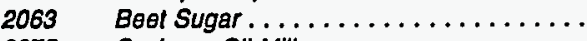

2075 Soybean Oll MIlls ...............

2082 Malt Beverages ..................

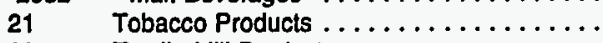

22 Textlle Mill Products ................

23 Apparel and Other Textlle Products ......

24 Lumber and Wood Products .............

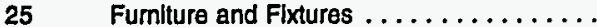

26 Paper and Allied Products ............

2611 Pulp Mills $\ldots \ldots \ldots \ldots \ldots \ldots \ldots \ldots$

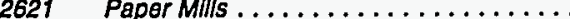

2631 Paperboard MIIIs ................

$27 \quad$ Printing and Publishing . ................

28 Chemlcals and Alled Products ..........

2812 Alkalles and Chlorine .............

2813 Industrial Gases .................

2819 Industrial Inorganic Chemicals, nec .....

2821 Plastics Materials and Resins .........

2822 Synthetlc Rubber ................

2823 Cellulosic Manmade Fibers ...........

2824 Organic Fibers, Noncellulosic .........

2865 Cyclle Crudes and Intermedlates ........

2869 Industrial Organic Chemicals, nec ......

2873 Nitrogenous Fertlizers . . . . . . . . .

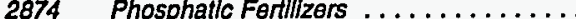

29 Petroleum and Coal Products ...........

2911 Petroleum Refining ${ }^{\circ} . \ldots \ldots \ldots \ldots \ldots$

30 Rubber and Misc. Plastles Products ......

3011 Tires and Inner Tubes ...............

308 Miscellaneous Plastlcs Products, nec ....

31 Leather and Leather Products .........

32 Stone, Clay and Glass Products .........

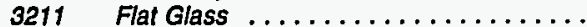

3221 Glass Contalners ..............

3229 Pressed and Blown Glass, nec. .......

3241 Cement, Hydraulic . . . . . . . . . . . . .

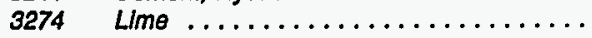

3296 Mineral Wool ..................

3312

3313

3321

3331

3334

3339

3353

34

35

357

38

37

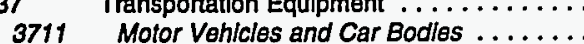

3714 Motor Vehicle Parts and Accessorles ....

38 Instruments and Related Products ........

3841 Surgical and Medical instruments ......

39 Mlsc. Manufacturing Industrtes .........

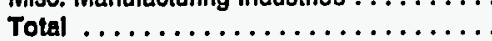

\section{Midwest Census Reglon}

Factors

\begin{tabular}{|c|c|c|c|c|c|c|c|c|}
\hline 0.8 & 1.5 & 1.0 & 1.0 & 1.1 & 0.9 & 1.0 & 0.8 & \\
\hline 2 & 0 & 1 & $w$ & 1 & 0 & $w$ & , & 12.5 \\
\hline 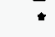 & 0 & 0 & 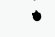 & : & 0 & 0 & • & 26.9 \\
\hline 0 & 0 & 0 & 0 & 0 & 0 & 0 & 0 & NF \\
\hline 0 & 0 & 0 & 0 & 0 & 0 & 0 & 0 & NF \\
\hline 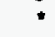 & 0 & 1 & 0 & 0 & 0 & 0 & . & 26.0 \\
\hline - & 0 & 0 & • & 0 & 0 & 0 & 0 & 28.3 \\
\hline * & 0 & 0 & 0 & 0 & 0 & $w$ & 0 & 18.3 \\
\hline 1 & 0 & 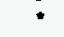 & 1 & 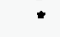 & 0 & 0 & " & 7.5 \\
\hline 0 & 0 & 0 & 0 & 0 & 0 & 0 & 0 & NF \\
\hline NA & NA & NA & NA & NA & NA & NA & NA & NF \\
\hline NA & NA & NA & NA & NA & NA & NA & NA & NF \\
\hline NA & NA & NA & NA & NA & NA & NA & NA & NF \\
\hline 3 & 0 & $Q$ & • & $\mathbf{Q}$ & 0 & 0 & $a$ & 60.8 \\
\hline " & 0 & 0 & • & 0 & 0 & 0 & 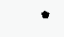 & 35.6 \\
\hline$w$ & 0 & $\mathbf{Q}$ & 0 & $w$ & 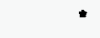 & 0 & $w$ & 20.1 \\
\hline 0 & 0 & 0 & 0 & 0 & 0 & 0 & 0 & NF \\
\hline 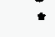 & 0 & 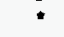 & 0 & 3 & • & 0 & • & 6.8 \\
\hline$w$ & 0 & • & 0 & 0 & 0 & 0 & $w$ & 20.8 \\
\hline 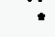 & 0 & 0 & 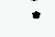 & $\mathbf{Q}$ & 0 & 0 & 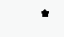 & NF \\
\hline 141 & 0 & $\mathbf{Q}$ & 39 & $w$ & $Q$ & 0 & $w$ & 12.1 \\
\hline 0 & 0 & 0 & 0 & 0 & 0 & 0 & 0 & NF \\
\hline - & 0 & 0 & • & 0 & 0 & 0 & • & 21.9 \\
\hline 1 & 0 & 0 & 0 & 0 & 0 & 0 & 1 & 40.4 \\
\hline$w$ & 0 & $w$ & 0 & $w$ & 0 & 0 & • & 12.3 \\
\hline - & 0 & 0 & • & 0 & 0 & 0 & " & 24.5 \\
\hline 0 & 0 & 0 & 0 & 0 & 0 & 0 & 0 & NF \\
\hline 0 & 0 & 0 & 0 & 0 & 0 & 0 & 0 & NF \\
\hline 9 & 0 & $\star$ & 0 & $W$ & 0 & 0 & $w$ & 20.4 \\
\hline$w$ & 0 & $w$ & $w$ & $w$ & 0 & 0 & 1 & 11.3 \\
\hline 29 & 0 & 0 & 28 & 0 & 0 & 0 & 0 & 28.3 \\
\hline 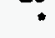 & 0 & 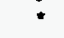 & 0 & 0 & 0 & 0 & 0 & 4.4 \\
\hline 467 & 0 & $\mathbf{Q}$ & - & 0 & 0 & 0 & 467 & 4.9 \\
\hline 445 & 0 & 0 & 0 & 0 & 0 & 0 & 445 & 1.2 \\
\hline 2 & 0 & 0 & * & • & $\mathbf{Q}$ & 0 & 1 & 24.4 \\
\hline $\bar{w}$ & 0 & 0 & 0 & 0 & $w$ & 0 & - & 6.7 \\
\hline NA & NA & NA & NA & NA & NA & NA & NA & 44.7 \\
\hline • & 4 & 0 & 0 & " & 0 & 0 & 0 & 40.6 \\
\hline 1 & $a$ & $Q$ & - & 2 & $\mathbf{Q}$ & " & • & 27.9 \\
\hline 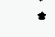 & $\overline{0}$ & 0 & 0 & - & 0 & 0 & • & 5.8 \\
\hline$*$ & 0 & 0 & 0 & 0 & 0 & 0 & • & 22.0 \\
\hline - & 0 & 0 & 0 & 0 & 0 & 0 & • & 14.7 \\
\hline - & 0 & 0 & 0 & 0 & 0 & 0 & $\bullet$ & NF \\
\hline • & 0 & 0 & 0 & 0 & $\mathbf{Q}$ & 0 & * & 57.6 \\
\hline - & 0 & $w$ & - & 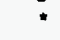 & $\overrightarrow{0}$ & 0 & • & 2.1 \\
\hline 359 & $w$ & 20 & $W$ & 1 & 12,024 & 286 & 10 & 8.0 \\
\hline$w$ & $w$ & $w$ & $w$ & - & $w$ & 167 & 1 & 8.5 \\
\hline 7 & 0 & 0 & $\because$ & 0 & $w$ & $w$ & $w$ & 12.0 \\
\hline$w$ & 0 & 0 & 0 & 1 & $w$ & $w$ & * & 23.2 \\
\hline 0 & 0 & 0 & 0 & 0 & 0 & 0 & 0 & NF \\
\hline$w$ & 0 & 0 & 0 & 0 & $w$ & 0 & $w$ & 6.4 \\
\hline 0 & 0 & 0 & 0 & 0 & 0 & 0 & 0 & NF \\
\hline * & 0 & 1 & 0 & • & 0 & 0 & • & 1.1 \\
\hline 1 & 0 & $w$ & $\cdot$ & Q & $\cdot$ & $w$ & $\mathbf{Q}$ & 37.3 \\
\hline 1 & 0 & $w$ & • & $w$ & 1 & 0 & $\bullet$ & 34.4 \\
\hline 0 & 0 & 0 & 0 & 0 & 0 & 0 & 0 & NF \\
\hline $\mathbf{Q}$ & 0 & 0 & $\bullet$ & $Q$ & 0 & 0 & $Q$ & 32.5 \\
\hline$w$ & 0 & 38 & - & 7 & $w$ & $w$ & $w$ & 9.9 \\
\hline 1 & 0 & $w$ & - & $\bullet$ & $w$ & 0 & $w$ & 8.4 \\
\hline$\Rightarrow$ & 0 & $Q$ & - & $w$ & 0 & 0 & • & 10.8 \\
\hline - & 0 & 0 & 0 & 0 & 0 & 0 & - & 42.9 \\
\hline - & 0 & 0 & 0 & 0 & 0 & 0 & • & NF \\
\hline Q & 0 & 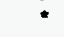 & - & $\mathbf{Q}$ & 0 & 0 & • & 39.6 \\
\hline 983 & $w$ & $Q$ & 58 & $w$ & 12,064 & 297 & 498 & 6.4 \\
\hline
\end{tabular}

See footnotes at end of table. 
Table A3. Total Primary Consumption of Combustible Energy for Nonfuel Purposes by Census Region, Industry Group, and Selected Industries, 1991: Part 1 (Continued)

(Estimates in Btu or Physical Units)

\begin{tabular}{cc}
\hline SIC & $\begin{array}{c}\text { Industry Groups } \\
\text { and Industry }\end{array}$ \\
Code & \\
\hline
\end{tabular}
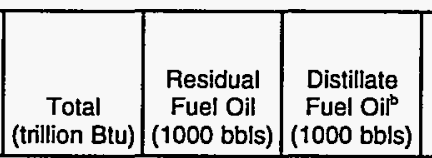

Natural
Gas $^{\circ}$
(billion
cu ft)

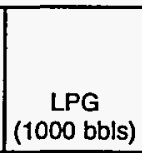

\begin{tabular}{|c|c|c|c|}
\hline $\begin{array}{c}\text { Coal } \\
\text { (1000 short } \\
\text { tons) }\end{array}$ & $\begin{array}{c}\text { Coke and } \\
\text { Breeze } \\
\text { (1000 short } \\
\text { tons) }\end{array}$ & $\begin{array}{c}\text { Other' } \\
\text { (trillion Btu) }\end{array}$ & $\begin{array}{c}\text { RSE } \\
\text { Row } \\
\text { Factors }\end{array}$ \\
\hline
\end{tabular}

South Census Region

RSE Column Factors:

Food and Kindred Products . . . . . . . . . .

Meat Packing Plants . . . . . . . . . .

Canned Fruits and Vegetables . . . . . . . .

Frozen Fruits and Vegetables . . . . . . . . .

Wet Com Milling . . . . . . . . . . . . .

Bread, Cake, and Related Products .....

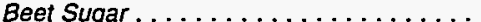

Soybean Oil Mills . . . . . . . . . . .

Malt Beverages ...............

Tobacco Products . .....................

Textile Mill Products . . . . . . . . . . . . .

Apparel and Other Textile Products ......

Lumber and Wood Products . . . . . . . . .

Fumiture and Fixtures . . . . . . . . .

Paper and Allied Products . . . . . . . . .

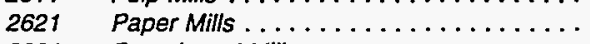

2631 Paperboard Mills ..............

27

28

2812

2813

2819

2821

2822

2823

2824

2865

2869

2873

2874

29

2911

30

3011

308

31

32

321

322

3241

3274

3296

33

3312

3313

3321

3331

3334

3339

3353

34

35

36
37

3711

3714

38

3841

39

Printing and Publishing .............

Chemicals and Allied Products . . . . . . . .

Alkalles and Chlorine ............

Industrial Gases . . . . . . . . . . . . . . . .

Industrial Inorganic Chemicals, nec . . . .

Plastics Materials and Resins . . . . . . .

Synthetic Rubber . . . . . . . . . . . . .

Cellulosic Manmade Fibers . . . . . . . . .

Onganic Fibers, Noncellulosic ........

Cyclic Crudes and intermediates .......

Industrial Organic Chemicals, nec . . . . . .

Nitrogenous Fertilizers $\ldots \ldots \ldots \ldots \ldots$

Phosphatic Fertilizers .............

Petroleum and Coal Products . ......

Petroleum Refining ${ }^{*} . . \ldots \ldots \ldots \ldots$

Rubber and Misc. Plastics Products . . . . .

Tires and Inner Tubes . . . . . . . . . . . . .

Miscellaneous Plastics Products, nec ....

Leather and Leather Products . . . . . . . .

Stone, Clay and Glass Products .......

Flat Glass .................

Glass Containers . . . . . . . . . . .

Pressed and Blown Glass, nec. . . . . . . .

Cement, Hydraulic . . . . . . . . . . . .

Lime ..................

Mineral Wool . . . . . . . . . . . . .

Primary Metal Industries .............. .

Blast Fumaces and Steel Mills . . . . . . . .

Electrometalurgical Products .........

Gray and Ductile Iron Foundries .......

Primary Copper . . . . . . . . . . . .

Primary Aluminum . . . . . . . . . .

Primary Nonferrous Metals, nec . . . . . . . .

Aluminum Sheet, Plate, and Foll . . . . . . .

Fabricated Metal Products ............

Industrial Machinery and Equipment ......

Computer and Office Equipment .......

Electronic and Other Electric Equipment . . .

Transportation Equipment ............

Motor Vehicles and Car Bodies ........

Motor Vehicle Parts and Accessories ....

Instruments and Related Products .......

Surgical and Medical Instruments ......

Misc. Manufacturing Industries . . . . . . . .

Total . . . . . . . . . . . . . . .

$\begin{array}{lll}0.7 & 2.3 & 1.0\end{array}$

0.9

$\begin{array}{ll}1 & 0 \\ 0 & 0 \\ 0 & 0 \\ 0 & 0\end{array}$

0

1

0.8

0
0
0

$\begin{array}{lll}0 & 0 & 0 \\ - & 0 & 0\end{array}$

$\begin{array}{lll}* & 0 & 0 \\ 0 & 0 & 0\end{array}$

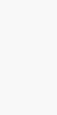

0

$\begin{array}{lllllll}0 & 0 & 0 & 0 & 0 & 0 & 0 \\ 5 & 0 & 6 & w & 0 & 0 & 0 \\ 0 & 0 & 0 & 0 & 0 & 0 & 0 \\ w & 0 & w & 0 & 0 & 0 & 0\end{array}$

2,164
1
$W$$$
1,853
$$

1,853
1,786

$\begin{array}{rrrr}1 & 0 & 1 & * \\ W & 0 & 0 & * \\ : & 0 & * & * \\ 2 & 0 & 0 & 0 \\ * & 0 & 68 & 1\end{array}$

$\begin{array}{ll}0 & 0 \\ w & 0 \\ . & 0\end{array}$

219
194

194
5

0
12
$W$

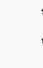

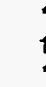

13

6

$+$

$\begin{array}{rr}* & 0 \\ * & 0 \\ 271 & 0\end{array}$

4,271

$\begin{array}{lr}\text { W } & \text { Q67 }\end{array}$

390,284

462

W

57,800

4,078

0
$W$

W

122

.

w

Q

See footnotes at end of table. 
Table A3. Total Primary Consumption of Combustible Energy for Nonfuel Purposes by Census Region, Industry Group, and Selected Industries, 1991: Part 1 (Continued) (Estimates in Btu or Physical Units)

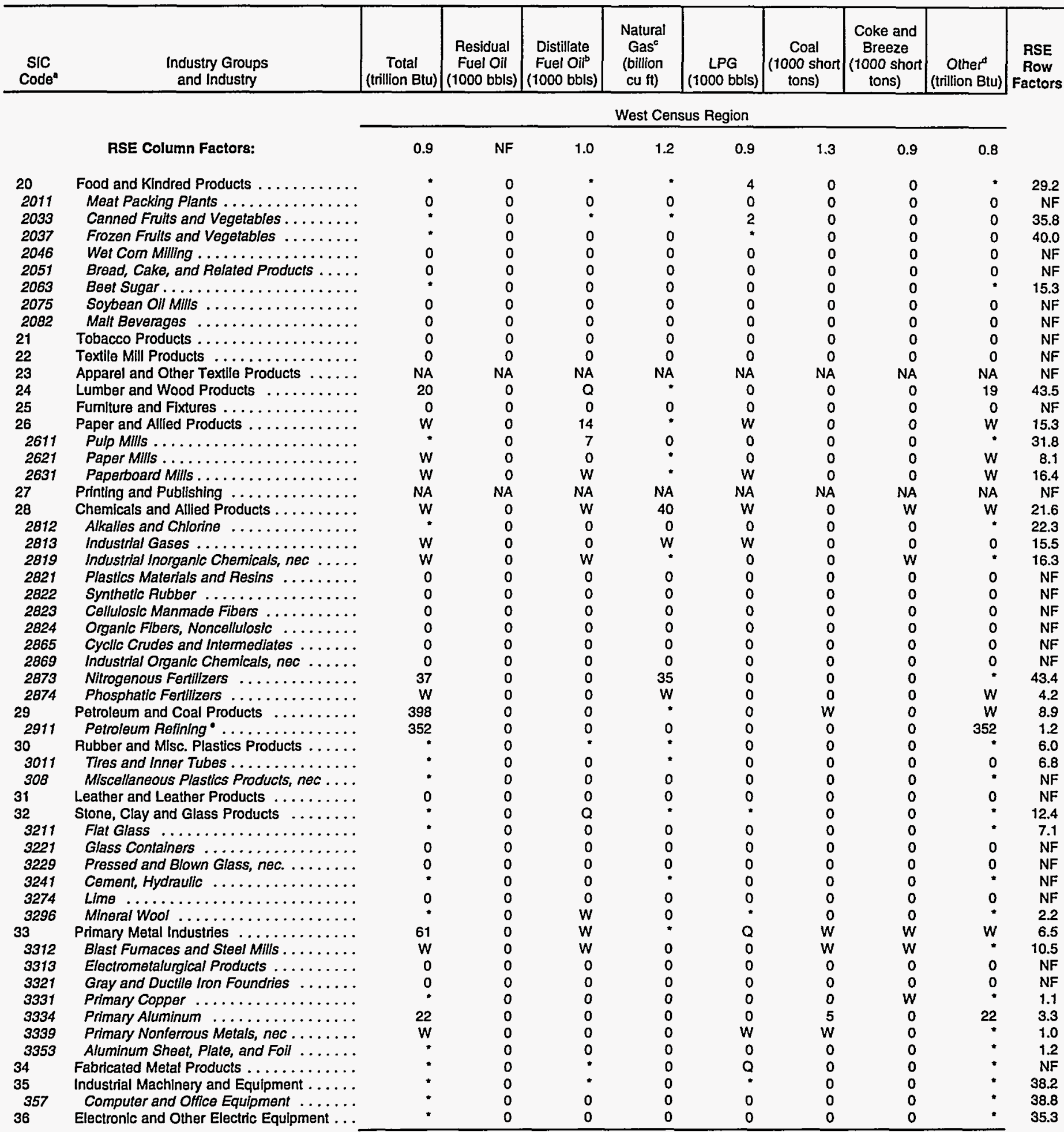

See footnotes at end of table. 


\section{Table A3. Total Primary Consumption of Combustible Energy for Nonfuel Purposes by Census Region, Industry Group, and Selected Industries, 1991: Part 1 (Continued) \\ (Estimates in Btu or Physical Units)}

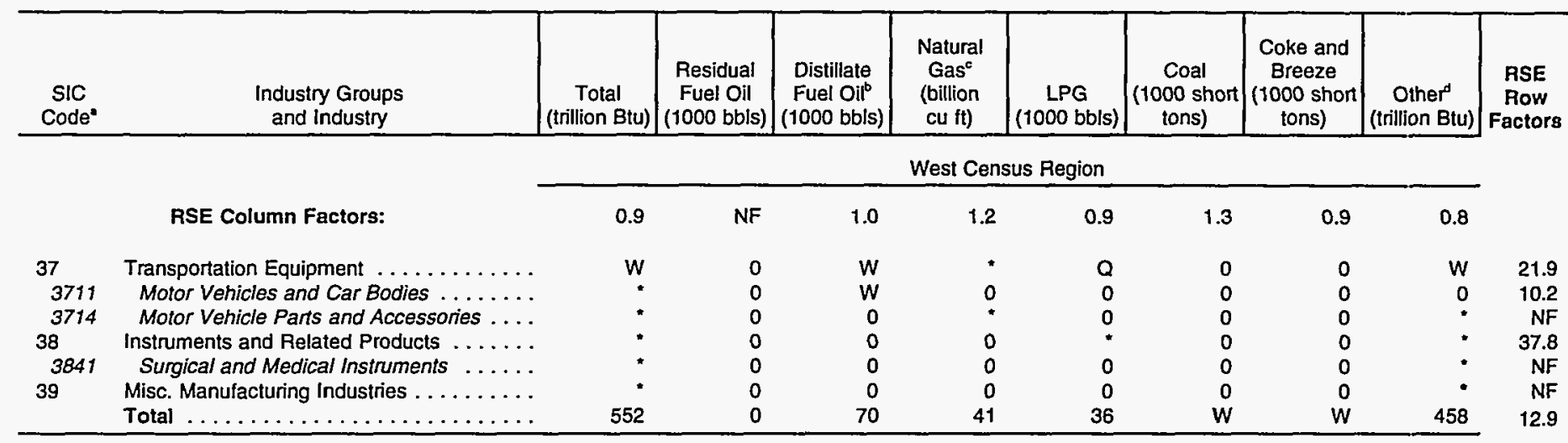

- See Appendices B and F for descriptions of the Standard Industrial Classification system.

- "Distillate Fuel Oil" includes Nos. 1, 2, and 4 fuel oils and Nos. 1, 2, and 4 diesel fuels.

- "Natural Gas" includes natural gas obtained from utilities, transmission pipelines, and any other supplier(s) such as brokers and producers.

"Other" includes energy that respondents indicated was used as feedstock/raw material inputs. See also Footnote "e".

- For the petroleum refining industry only, the feedstocks and raw material inputs for the production of nonenergy products (i.e., asphalt, waxes, lubricants, and solvents) and feedstock consumption at adjoining petrochemical plants are included in the "Other" column, regardless of type of energy. Those inputs and feedstocks that were converted to other energy products (e.g., crude oil converted to residual and distillate fuel oils) are excluded. See Appendix $B$ for more information.

NF=No applicable RSE row/column factor.

- Estimate less than 0.5. Data are included in higher level totals.

$W=$ Withheld to avoid disclosing data for individual establishments. Data are included in higher level totals.

$\mathrm{Q}=$ Withheld because Relative Standard Error is greater than $\mathbf{5 0}$ percent. Data are included in higher level totals.

NA=Not available. Data are included in higher level totals.

Notes: - To obtain a RSE percentage for any table cell, multiply the cell's corresponding RSE column and RSE row factors. - Totals may not equal sum of components because of independent rounding. - The derived estimates presented in this table are for the primary consumption of energy as feedstocks or raw material inputs. Primary consumption is defined as the consumption of the energy that was originally produced offsite or was produced onsite from input materials not classified as energy. Examples of the latter are hydrogen produced from the electrolysis of brine; the output of captive (onsite) mines or wells; woodchips, bark, and

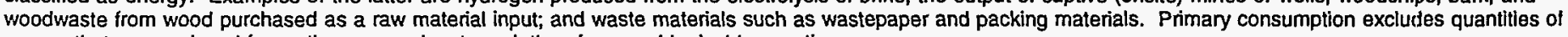
energy that are produced from other energy inputs and, therefore, avoids double counting.

Source: Energy Information Administration, Office of Energy Markets and End Use, Energy End Use and Integrated Statistics Division, Form ElA-846, "1991 Manufacturing Energy Consumption Survey," and Office of Oil and Gas, Petroleum Supply Division, Form ElA-810, "Monthly Refinery Report" for 1991. 
Table A3. Total Primary Consumption of Combustible Energy for Nonfuel Purposes by Census Region, Industry Group, and Selected Industries, 1991: Part 2

(Estimates in Trillion Btu)

\begin{tabular}{|c|c|c|c|c|c|c|c|c|c|c|}
\hline $\begin{array}{l}\text { SIC } \\
\text { Code }^{\circ}\end{array}$ & $\begin{array}{l}\text { Industry Groups } \\
\text { and Industry }\end{array}$ & Total & $\begin{array}{l}\text { Residual } \\
\text { Fuel Oil } \\
\end{array}$ & $\begin{array}{l}\text { Distillate } \\
\text { Fuel Oil } \\
\end{array}$ & $\begin{array}{c}\text { Natural } \\
\text { Gas }^{\circ}\end{array}$ & LPG & Coal & $\begin{array}{c}\text { Coke and } \\
\text { Breeze }\end{array}$ & Other & $\begin{array}{c}\text { RSE } \\
\text { Row } \\
\text { Factors }\end{array}$ \\
\hline & & \multicolumn{9}{|c|}{ Total United States } \\
\hline & RSE Column Factors: & 0.7 & 2.1 & 1.0 & 0.9 & 0.9 & 1.2 & 0.9 & 0.8 & \\
\hline 20 & Food and KIndred Products & 3 & 0 & - & w & " & 0 & w & 1 & 19.3 \\
\hline 2011 & Meat Packing Plants . . . . . . . . . . . & . & 0 & 0 & * & • & 0 & 0 & - & 30.3 \\
\hline 2033 & Canned Frults and Vegetables ......... & * & 0 & 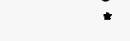 & • & $*$ & 0 & 0 & 0 & 41.3 \\
\hline 2037 & Frozen Fruits and Vegetables ........ & • & 0 & 0 & 0 & $*$ & 0 & 0 & • & 49.6 \\
\hline 2046 & Wet Com MIIIIng ................. & - & 0 & • & 0 & 0 & 0 & 0 & - & 28.7 \\
\hline 2051 & Bread, Cake, and Related Products ..... & $\cdot$ & 0 & 0 & 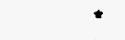 & * & 0 & 0 & • & 31.5 \\
\hline 2063 & 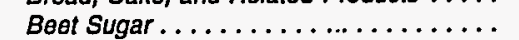 & * & 0 & 0 & 0 & 0 & 0 & w & - & 18.7 \\
\hline 2075 & Soybean Oll Mills ................ & 1 & 0 & * & 1 & * & 0 & 0 & • & 8.2 \\
\hline 2082 & Malt Beverages .. & * & 0 & 0 & 0 & 0 & 0 & 0 & * & 31.7 \\
\hline 21 & Tobacco Products . & 0 & 0 & 0 & 0 & 0 & 0 & 0 & 0 & NF \\
\hline 22 & Textile Mill Products & • & 0 & • & • & • & 0 & 0 & * & 32.7 \\
\hline 23 & Apparel and Other Textile Products ..... & - & 0 & - & 0 & 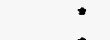 & 0 & 0 & * & 62.6 \\
\hline 24 & Lumber and Wood Products .......... & 27 & 0 & Q & $\cdot$ & • & 0 & 0 & 25 & 44.3 \\
\hline 25 & Furnlture and Fixtures $\ldots \ldots \ldots \ldots \ldots$ & $Q$ & 0 & 0 & • & 0 & 0 & 0 & $\mathbf{Q}$ & NF \\
\hline 26 & Paper and Allied Products . . . . . . . . & 35 & 0 & - & w & w & • & 0 & W & 15.8 \\
\hline 2611 & Pulp Mills $\ldots \ldots \ldots \ldots$ & $\because$ & 0 & • & 0 & 0 & 0 & 0 & • & 36.2 \\
\hline 2621 & Paper Mills .... & 8 & 0 & * & 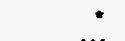 & * & • & 0 & 7 & 6.5 \\
\hline 2631 & Paperboard Mills . . & 27 & 0 & $w$ & w & w & 0 & 0 & 26 & 14.6 \\
\hline 27 & Printing and Publishing ... & $\because$ & 0 & Q & $\because$ & Q & 0 & 0 & . & 59.1 \\
\hline 28 & Chemicals and Allied Products. & 2,358 & W & $\bar{Q}$ & 558 & $\mathbf{w}$ & w & 7 & 259 & 8.5 \\
\hline 2812 & 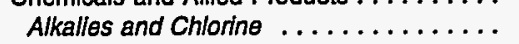 & 1 & 0 & $\overline{0}$ & : & 0 & 0 & 0 & 1 & 31.5 \\
\hline 2813 & Industrial Gases ................... & w & 0 & 0 & $w$ & $w$ & 0 & 0 & w & 18.0 \\
\hline 2819 & Industrial Inorganic Chemicals, nec ..... & 20 & W & $w$ & w & 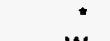 & w & 6 & 4 & 15.9 \\
\hline 2821 & Plastlos Materials and Resins . . . . . . . & 357 & 0 & $\because$ & 66 & w & 0 & 0 & w & 8.5 \\
\hline 2822 & Synthetlc Rubber ............... & w & 0 & * & w & 15 & 0 & 0 & - & 23.4 \\
\hline 2823 & Cellulosic Manmade Fibers . . . . . . . . . & 0 & 0 & 0 & 0 & 0 & 0 & 0 & 0 & $\mathrm{NF}$ \\
\hline 2824 & Organlc Fibers, Noncellulosic .......... & w & 0 & 0 & w & w & w & 0 & * & 6.9 \\
\hline 2865 & Cycllc Crudes and Intermedlates ....... & 98 & 0 & • & 9 & 75 & $\cdot$ & 0 & 14 & 17.4 \\
\hline 2869 & Industrial Organic Chemicals, nec . . . . . & 1,345 & 0 & * & w & w & 0 & 0 & 192 & 6.6 \\
\hline 2873 & Nitrogenous Fertilizers ............. & 290 & 0 & 0 & 289 & 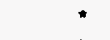 & 0 & 0 & * & 26.4 \\
\hline 2874 & Phosphatlc Fertilizers $\ldots \ldots \ldots \ldots$ & 31 & 0 & W & w & 0 & 0 & 0 & w & 3.5 \\
\hline 29 & Petroleum and Coal Products ......... & 3,004 & 0 & - & • & w & w & 0 & w & 3.5 \\
\hline 2911 & Petroleum Refining ${ }^{\circ} . \ldots \ldots \ldots \ldots$ & 2,868 & 0 & 0 & 0 & 0 & 0 & 0 & 2,868 & 1.4 \\
\hline 30 & Rubber and Misc. Plastics Products ...... & 3 & 0 & $*$ & * & $\mathbf{Q}$ & $\mathbf{Q}$ & 0 & 2 & 26.4 \\
\hline 3011 & Tires and Inner Tubes ............. & w & 0 & 0 & • & $\overrightarrow{0}$ & $\bar{w}$ & 0 & w & 5.4 \\
\hline 308 & MIscellaneous Plastics Products, nec .... & 1 & 0 & $\mathbf{Q}$ & • & $\mathbf{Q}$ & 0 & 0 & 1 & 44.6 \\
\hline 31 & Leather and Leather Products ......... & • & * & • & 0 & •" & 0 & 0 & • & 40.5 \\
\hline 32 & Stone, Clay and Glass Products ....... & 3 & $\mathbf{Q}$ & 1 & 1 & w & $\mathbf{Q}$ & $w$ & $w$ & 20.1 \\
\hline 3211 & Flat Glass $\ldots \ldots \ldots \ldots \ldots \ldots \ldots$ & 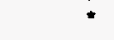 & $\overline{0}$ & 0 & 0 & $\because$ & 0 & 0 & : & 8.0 \\
\hline 3221 & Glass Containers ................ & * & 0 & 0 & 0 & 0 & 0 & 0 & • & 24.8 \\
\hline 3229 & Pressed and Blown Glass, nec. .... & w & 0 & 0 & w & w & 0 & 0 & • & 13.8 \\
\hline 3241 & Cement, Hydraulls ................ & $\cdot$ & 0 & 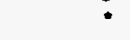 & $*$ & 0 & 0 & 0 & * & 32.5 \\
\hline 3274 & Lime $\ldots \ldots \ldots \ldots \ldots \ldots \ldots \ldots$ & • & 0 & 0 & • & 0 & $\mathbf{Q}$ & w & • & 31.9 \\
\hline 3296 & 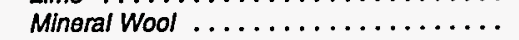 & • & 0 & W & • & w & 0 & 0 & •" & 2.0 \\
\hline & Primary Metal Industries . . . . . . . . & 909 & w & • & 22 & w & 808 & 17 & 54 & 4.4 \\
\hline 3312 & Blast Fumaces and Steol Milis . . . . . . . . & 838 & w & w & 21 & * & 799 & 6 & W & 7.6 \\
\hline 3313 & Electrometalurgical Products ......... & 12 & 0 & 0 & 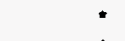 & 0 & $w$ & $w$ & w & 9.9 \\
\hline 3321 & Gray and Ductlle Iron Foundries ... & $\bar{w}$ & 0 & $*$ & • & 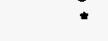 & w & $w$ & • & 18.5 \\
\hline 3331 & Primary Copper $\ldots \ldots \ldots \ldots \ldots$ & • & 0 & 0 & 0 & 0 & 0 & w & * & 1.3 \\
\hline 3334 & Primary Aluminum $\ldots \ldots \ldots \ldots \ldots$ & 44 & 0 & 0 & 0 & 0 & 1 & w & w & 3.6 \\
\hline 3339 & Primary Nonferrous Metals, nec . . . . . . & 10 & 0 & 0 & 0 & w & w & w & w & 1.6 \\
\hline 3353 & Aluminum Sheet, Plate, and Foll ... & • & 0 & : & 0 & • & 0 & 0 & - & 2.7 \\
\hline 34 & Fabricated Metal Products . . . . . . . . . & 3 & * & • & 1 & - & * & W & w & 37.8 \\
\hline 35 & Industrial Machinery and Equipment . . . . . . & 1 & 0 & •" & 1 & • & • & 0 & • & 31.7 \\
\hline 357 & Computer and Office Equipment ....... & : & 0 & 0 & 0 & • & 0 & 0 & $\cdot$ & 45.4 \\
\hline 36 & Electronic and Other Electric Equipment ... & 15 & 0 & $\cdot$ & 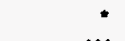 & Q & w & $w$ & 13 & 30.5 \\
\hline & Transportation Equipment . ........... & 9 & 0 & • & $w$ & - & w & $w$ & 7 & 16.0 \\
\hline 3711 & Motor Vehicles and Car Bodles ........ & 2 & 0 & • & • & • & $w$ & 0 & w & 7.3 \\
\hline 3714 & Motor Vehiclo Parts and Accessories .... & 1 & 0 & $\mathbf{Q}$ & - & - & - & * & 1 & 14.1 \\
\hline 38 & Instruments and Related Products ....... & : & 0 & •" & - & • & 0 & 0 & • & 37.8 \\
\hline 3841 & Surglcal and Medical Instruments ...... & * & 0 & * & 0 & 0 & 0 & 0 & • & 35.9 \\
\hline 39 & Mlsc. Manufacturing Industries .. & $\mathbf{Q}$ & 0 & $\mathbf{Q}$ & * & w & 0 & 0 & • & 38.6 \\
\hline & Total ................ & 6,373 & 63 & 7 & 590 & 1,470 & 823 & 25 & 3,394 & 5.8 \\
\hline
\end{tabular}

See footnotes at end of table. 


\section{Table A3. Total Primary Consumption of Combustible Energy for Nonfuel Purposes by Census Region, Industry Group, and Selected Industries, 1991: Part 2 (Continued) \\ (Estimates in Trillion Btu)}

\begin{tabular}{|c|c|c|c|c|c|c|c|c|c|c|}
\hline $\begin{array}{c}\text { SIC } \\
\text { Code }\end{array}$ & $\begin{array}{l}\text { Industry Groups } \\
\text { and Industry }\end{array}$ & Total & $\begin{array}{l}\text { Residual } \\
\text { Fuel Oil } \\
\end{array}$ & $\begin{array}{l}\text { Distillate } \\
\text { Fuel Oil } \\
\end{array}$ & $\begin{array}{c}\text { Natural } \\
\text { Gas }^{\circ}\end{array}$ & LPG & Coal & $\begin{array}{c}\text { Coke and } \\
\text { Breeze }\end{array}$ & Other & $\begin{array}{c}\text { RSE } \\
\text { Row } \\
\text { Factors }\end{array}$ \\
\hline & & \multicolumn{9}{|c|}{ Northeast Census Region } \\
\hline & RSE Column Factors: & 0.8 & NF & 1.2 & 1.5 & 0.9 & 1.6 & 0.5 & 0.9 & \\
\hline 20 & Food and Kindred Producls & - & 0 & 0 & 0 & 0 & 0 & 0 & - & 47.0 \\
\hline 2011 & Meat Packing Plants ..... & 0 & 0 & 0 & 0 & 0 & 0 & 0 & 0 & NF \\
\hline 2033 & Canned Fruits and Vegetables. & 0 & 0 & 0 & 0 & 0 & 0 & 0 & 0 & NF \\
\hline 2037 & Frozen Fruits and Vegetables. & * & 0 & 0 & 0 & 0 & 0 & 0 & - & $\mathrm{NF}$ \\
\hline 2046 & Wet Com Milling . ......... & 0 & 0 & 0 & 0 & 0 & 0 & 0 & 0 & NF \\
\hline 2051 & Bread, Cake, and Related Products & 0 & 0 & 0 & 0 & 0 & 0 & 0 & 0 & NF \\
\hline 2063 & Beet Sugar . . . . . . . . . . . & 0 & 0 & 0 & 0 & 0 & 0 & 0 & 0 & NF \\
\hline 2075 & Soybean Oil Mills ............ & 0 & 0 & 0 & 0 & 0 & 0 & 0 & 0 & NF \\
\hline 2082 & Malt Beverages. & 0 & 0 & 0 & 0 & 0 & 0 & 0 & 0 & NF \\
\hline 21 & Tobacco Products & NA & NA & NA & NA & NA & NA & NA & NA & NF \\
\hline 22 & Textile Mill Products & • & 0 & 0 & 0 & 0 & 0 & 0 & 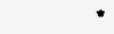 & NF \\
\hline 23 & Apparel and Other Textile Products . & 0 & 0 & 0 & 0 & 0 & 0 & 0 & 0 & $\mathrm{NF}$ \\
\hline 24 & Lumber and Wood Products $\ldots \ldots$ & NA & NA & NA & NA & NA & NA & NA & NA & 47.7 \\
\hline 25 & Furniture and Fixtures . ..... & * & 0 & 0 & 0 & 0 & 0 & 0 & • & NF \\
\hline 26 & Paper and Allied Products . & w & 0 & • & 0 & 0 & 0 & 0 & w & 11.0 \\
\hline 2611 & Pulp Mills $\ldots \ldots \ldots \ldots \ldots$ & 0 & 0 & 0 & 0 & 0 & 0 & 0 & 0 & $\mathrm{NF}$ \\
\hline 2621 & Paper Mills . . . . . . . . . . & * & 0 & W & 0 & 0 & 0 & 0 & * & 7.3 \\
\hline 2631 & Paperboard Mills . & w & 0 & • & 0 & 0 & 0 & 0 & w & 14.1 \\
\hline 27 & Printing and Publishing .... & • & 0 & Q & - & * & 0 & 0 & $*$ & NF \\
\hline 28 & Chemicals and Allied Products . & w & 0 & w & 1 & 3 & 0 & 0 & w & 13.7 \\
\hline 2812 & Alkalies and Chlorine ....... & 0 & 0 & 0 & 0 & 0 & 0 & 0 & 0 & NF \\
\hline 2813 & Industrial Gases ......... & 1 & 0 & 0 & : & 0 & 0 & 0 & 1 & 18.2 \\
\hline 2819 & Industrial Inorganic Chemicals, nec . . . . . & - & 0 & 0 & * & * & 0 & 0 & * & 16.7 \\
\hline 2821 & Plastics Materials and Resins ..... & w & 0 & 0 & 0 & w & 0 & 0 & * & 11.8 \\
\hline 2822 & Synthetic Rubber ............ & 0 & 0 & 0 & 0 & 0 & 0 & 0 & 0 & NF \\
\hline 2823 & Cellulosic Manmade Fibers & 0 & 0 & 0 & 0 & 0 & 0 & 0 & 0 & NF \\
\hline 2824 & Organic Fibers, Noncellulosic ... & 0 & 0 & 0 & 0 & 0 & 0 & 0 & 0 & NF \\
\hline 2865 & Cyclic Crudes and Intermediates ... & - & 0 & * & * & * & 0 & 0 & * & 21.2 \\
\hline 2869 & Industrial Organic Chemicals, nec & w & 0 & w & 0 & w & 0 & 0 & * & 13.7 \\
\hline 2873 & Nitrogenous Fertilizers . ........ & 1 & 0 & 0 & $*$ & 0 & 0 & 0 & 0 & 44.4 \\
\hline 2874 & Phosphatic Fentilizers . . . . . . & 0 & 0 & 0 & 0 & 0 & 0 & 0 & 0 & NF \\
\hline 29 & Petroleum and Coal Products & 285 & 0 & Q & * & Q & w & 0 & 285 & 3.2 \\
\hline 2911 & Petroleum Refining ${ }^{\circ} . . .$. & 285 & 0 & 0 & 0 & 0 & 0 & 0 & 285 & 1.2 \\
\hline 30 & Rubber and Misc. Plastics Products .. & * & 0 & • & - & Q & 0 & 0 & • & 23.0 \\
\hline 3011 & Tires and Inner Tubes . . . . . . . . . . & $=$ & 0 & 0 & 0 & 0 & 0 & 0 & * & 8.2 \\
\hline 308 & Miscellaneous Plastics Products, nec .... & - & 0 & $Q$ & . & $\mathbf{Q}$ & 0 & 0 & - & 34.1 \\
\hline 31 & Leather and Leather Products & - & 0 & * & 0 & * & 0 & 0 & - & 38.2 \\
\hline 32 & Stone, Clay and Glass Products & - & 0 & - & * & $\mathbf{Q}$ & 0 & 0 & * & 26.9 \\
\hline 3211 & $\ldots \ldots \ldots \ldots \ldots$ & * & 0 & 0 & 0 & 0 & 0 & 0 & * & 5.9 \\
\hline 3221 & Glass Containers $\ldots \ldots \ldots \ldots \ldots$ & * & 0 & 0 & 0 & 0 & 0 & 0 & - & 21.1 \\
\hline 3229 & Pressed and Blown Glass, nec. . . . & * & 0 & 0 & * & 0 & 0 & 0 & - & 11.6 \\
\hline 3241 & Cement, Hydraulic $\ldots \ldots \ldots \ldots$. & * & 0 & 0 & 0 & 0 & 0 & 0 & - & 30.5 \\
\hline 3274 & Lime $\ldots \ldots \ldots \ldots \ldots \ldots \ldots \ldots$ & 0 & 0 & 0 & 0 & 0 & 0 & 0 & 0 & NF \\
\hline 3296 & Mineral Wool & - & 0 & 0 & 0 & 0 & 0 & 0 & - & 2.3 \\
\hline 33 & Primary Metal Industries $\ldots \ldots \ldots \ldots$ & 270 & 0 & * & * & $\mathbf{Q}$ & w & w & w & 10.7 \\
\hline 3312 & Blast Fumaces and Steel Mills . . . . . & 263 & 0 & 0 & 0 & 0 & w & W & W & 12.9 \\
\hline 3313 & Electrometalurgical Products ..... & . & 0 & 0 & 0 & 0 & 0 & 0 & : & 16.4 \\
\hline 3321 & Gray and Ductile Iron Foundries ... . & • & 0 & • & 0 & 0 & 0 & • & • & 46.5 \\
\hline 3331 & Primary Copper $\ldots \ldots \ldots \ldots \ldots$ & * & 0 & 0 & 0 & 0 & 0 & - & 0 & 1.5 \\
\hline 3334 & Primary Aluminum $\ldots \ldots \ldots \ldots$ & w & 0 & 0 & 0 & 0 & w & 0 & w & 3.8 \\
\hline 3339 & Primary Nonferrous Metals, nec. & W & 0 & 0 & 0 & 0 & 0 & w & $\because$ & 4.5 \\
\hline 3353 & Aluminum Sheet, Plate, and Foil & • & 0 & 0 & 0 & 0 & 0 & 0 & - & NF \\
\hline 34 & Fabricated Metal Products $\ldots \ldots \ldots \ldots \ldots$ & 1 & 0 & - & Q & Q & 0 & • & - & 36.5 \\
\hline 35 & Industrial Machinery and Equipment . . . . . . & * & 0 & w & * & $\mathrm{Q}$ & 0 & 0 & - & 21.8 \\
\hline 357 & Computer and Office Equipment ....... & - & 0 & 0 & 0 & * & 0 & 0 & * & 38.7 \\
\hline 36 & Electronic and Other Electric Equipment . & 1 & 0 & : & : & Q & 0 & 0 & * & 28.6 \\
\hline 37 & Transportation Equipment .... & * & 0 & w & * & w & 0 & 0 & - & 17.4 \\
\hline 3711 & Motor Vehicles and Car Bodies . . . . . . . & * & 0 & 0 & 0 & 0 & 0 & 0 & * & 10.6 \\
\hline 3714 & Motor Vehicle Parts and Accessories. & - & 0 & 0 & * & w & 0 & 0 & - & 21.4 \\
\hline 38 & Instruments and Related Products ... & - & 0 & : & * & - & 0 & 0 & - & 29.3 \\
\hline 3841 & Surgical and Medical Instruments & * & 0 & . & 0 & 0 & 0 & 0 & . & 36.6 \\
\hline \multirow[t]{2}{*}{39} & Misc. Manufacturing Industries .. & * & 0 & * & - & w & 0 & 0 & - & 42.6 \\
\hline & Total $\ldots \ldots \ldots \ldots \ldots \ldots$ & 567 & 0 & 1 & 2 & w & w & w & 300 & 9.4 \\
\hline
\end{tabular}

See footnotes at end of table. 
Table A3. Total Primary Consumption of Combustible Energy for Nonfuel Purposes by Census Region, Industry Group, and Selected Industries, 1991: Part 2 (Continued)

(Estimates in Trillion Btu)

\begin{tabular}{|c|c|c|c|c|c|c|c|c|c|c|}
\hline $\begin{array}{c}\text { SIC } \\
\text { Code" }\end{array}$ & $\begin{array}{c}\text { Industry Groups } \\
\text { and Industry }\end{array}$ & Total & $\begin{array}{c}\text { Residual } \\
\text { Fuel Oil } \\
\end{array}$ & $\begin{array}{l}\text { Distillate } \\
\text { Fuel Oil } \\
\end{array}$ & $\begin{array}{c}\text { Natural } \\
\text { Gas }^{c}\end{array}$ & LPG & Coal & $\begin{array}{c}\text { Coke and } \\
\text { Breeze }\end{array}$ & Other & $\begin{array}{c}\text { RSE } \\
\text { Row } \\
\text { Factors }\end{array}$ \\
\hline & & \multicolumn{9}{|c|}{ Midwest Census Region } \\
\hline & RSE Column Factors: & 0.8 & 1.5 & 1.0 & 1.0 & 1.1 & 0.9 & 1.0 & 0.8 & \\
\hline 20 & Food and Kindred Products & 2 & 0 & * & w & * & 0 & $w$ & * & 12.5 \\
\hline 2011 & Meat Packing Plants . . . . . . . . . . & * & 0 & 0 & $\cdot$ & $\cdot$ & 0 & 0 & * & 26.9 \\
\hline 2033 & Canned Frults and Vegetables . . . . . . . . & 0 & 0 & 0 & 0 & 0 & 0 & $\mathbf{0}$ & 0 & NF \\
\hline 2037 & Frozen Fruits and Vegetables ........ & 0 & 0 & 0 & 0 & 0 & 0 & 0 & 0 & NF \\
\hline 2046 & Wot Com Milling ................ & * & 0 & : & 0 & 0 & 0 & 0 & : & 26.0 \\
\hline 2051 & Bread, Cake, and Related Products . . . . . & * & 0 & 0 & : & 0 & 0 & 0 & 0 & 28.3 \\
\hline 2063 & Beet Sugar . . . . . . . . . . . . . . . & - & 0 & 0 & 0 & 0 & 0 & w & 0 & 18.3 \\
\hline 2075 & Soybean Oll Mills $\ldots \ldots \ldots \ldots \ldots$. & 1 & 0 & * & 1 & • & 0 & 0 & : & 7.5 \\
\hline 2082 & Malt Beverages ............. & 0 & 0 & 0 & 0 & 0 & 0 & 0 & 0 & NF \\
\hline 21 & Tobacco Products .... & NA & NA & NA & NA & NA & NA & NA & NA & NF \\
\hline 22 & Textile Mill Products . & NA & NA & NA & NA & NA & NA & NA & NA & NF \\
\hline 23 & Apparel and Other Textile Products .. & NA & NA & NA & NA & NA & NA & NA & NA & NF \\
\hline 24 & Lumber and Wood Products $\ldots \ldots$. & $\mathbf{3}$ & $\mathbf{0}$ & $\mathbf{Q}$ & - & a & 0 & 0 & $\mathbf{Q}$ & 60.8 \\
\hline 25 & Fumiture and Fixtures .... & * & 0 & 0 & * & 0 & 0 & 0 & - & 35.6 \\
\hline 26 & Paper and Alled Products . & $w$ & 0 & a & 0 & w & - & 0 & w & 20.1 \\
\hline 2611 & Pulp Mills ............. & 0 & 0 & 0 & 0 & 0 & 0 & 0 & 0 & NF \\
\hline 2621 & Paper Mills . & * & 0 & • & 0 & • & - & 0 & - & 6.8 \\
\hline 2631 & Paperboard Mills ..... & $w$ & 0 & - & 0 & 0 & 0 & 0 & w & 20.8 \\
\hline 27 & Printing and Publlshing ... & * & 0 & 0 & * & Q & 0 & 0 & $\because$ & NF \\
\hline 28 & Chemicals and Allied Products $\ldots \ldots \ldots$. & 141 & 0 & Q & 40 & w & Q & 0 & w & 12.1 \\
\hline 2812 & Alkalles and Chlorine $\ldots \ldots \ldots \ldots \ldots$ & 0 & 0 & 0 & 0 & 0 & 0 & 0 & 0 & NF \\
\hline 2813 & Industrial Gases ....... & * & 0 & 0 & * & 0 & 0 & 0 & - & 21.9 \\
\hline 2819 & Industral Inorganic Chemicals, nec . . . . . & 1 & 0 & 0 & 0 & 0 & 0 & 0 & 1 & 40.4 \\
\hline 2821 & Plastles Materials and Resins . . . . . . . & w & 0 & w & 0 & w & 0 & 0 & * & 12.3 \\
\hline 2822 & Synthetic Rubber $\ldots \ldots \ldots \ldots \ldots$ & • & 0 & 0 & - & 0 & 0 & 0 & - & 24.5 \\
\hline 2823 & Cellulosic Manmade Fibers . . . . . . . . & 0 & 0 & 0 & 0 & 0 & 0 & 0 & 0 & NF \\
\hline 2824 & Organic Fibers, Noncellulosic . . . . . . . & 0 & 0 & 0 & 0 & 0 & 0 & 0 & 0 & $\mathrm{NF}$ \\
\hline 2865 & Cyclic Crudes and Intermediates & 9 & 0 & - & 0 & w & 0 & 0 & w & 20.4 \\
\hline 2869 & Industral Organic Chemicals, nec . . . . . . & w & 0 & w & w & w & 0 & 0 & 1 & 11.3 \\
\hline 2873 & Nitrogenous Fertillzers $\ldots \ldots \ldots \ldots \ldots$ & 29 & 0 & 0 & 29 & 0 & 0 & 0 & 0 & 28.3 \\
\hline 2874 & Phosphatic Fertilizers . . . . . . . . . & * & 0 & * & 0 & 0 & 0 & 0 & 0 & 4.4 \\
\hline 29 & Petroleum and Coal Products $\ldots \ldots \ldots \ldots$ & 467 & 0 & $\mathbf{Q}$ & * & 0 & 0 & 0 & 467 & 4.9 \\
\hline 2911 & Petroleum Refining ${ }^{\circ} \ldots \ldots \ldots \ldots$ & 445 & 0 & 0 & 0 & 0 & 0 & 0 & 445 & 1.2 \\
\hline 30 & Rubber and Misc. Plastics Products . . . . . & 2 & 0 & 0 & - & - & $\mathbf{Q}$ & 0 & 1 & 24.4 \\
\hline 3011 & Tires and Inner Tubes . . . . . . . . . . & w & 0 & 0 & 0 & 0 & $w$ & 0 & - & 6.7 \\
\hline 308 & Miscellaneous Plastics Products, nec . . . . & NA & NA & NA & NA & NA & NA & NA & NA & 44.7 \\
\hline 31 & Leather and Leather Products . . . . . . . . & * & * & 0 & 0 & * & 0 & 0 & 0 & 40.6 \\
\hline 32 & Stone, Clay and Glass Products .. & 1 & $\mathrm{Q}$ & $\mathbf{Q}$ & * & - & $\mathbf{Q}$ & - & - & 27.9 \\
\hline 3211 & Flat Glass $\ldots \ldots \ldots \ldots \ldots \ldots \ldots$ & . & 0 & 0 & 0 & • & 0 & 0 & - & 5.8 \\
\hline 3221 & Glass Contalners $\ldots \ldots \ldots \ldots \ldots \ldots$ & • & 0 & 0 & 0 & 0 & 0 & 0 & - & 22.0 \\
\hline 3229 & Prossed and Blown Glass, nec. . . . . . . & - & 0 & 0 & 0 & 0 & 0 & 0 & - & 14.7 \\
\hline 3241 & Cement, Hydraullic . . . . . . . . . . & - & 0 & 0 & 0 & $\mathbf{0}$ & $\mathbf{0}$ & 0 & - & NF \\
\hline 3274 & Lime $\ldots \ldots \ldots \ldots \ldots \ldots$ & - & 0 & 0 & 0 & 0 & $\mathbf{Q}$ & 0 & - & 57.6 \\
\hline 3296 & Mineral Wool ........ & * & 0 & w & * & - & 0 & 0 & - & 2.1 \\
\hline 33 & Primary Metal Industries .. & 359 & w & * & w & * & 322 & 7 & 10 & 8.0 \\
\hline 3312 & Blast Fumaces and Steel Mills . . . . . . . . & w & w & w & w & - & W & 4 & 1 & 8.5 \\
\hline 3313 & Electromelalurglcal Products . . . . . . . . & 7 & 0 & 0 & $\because$ & 0 & $w$ & $w$ & $w$ & 12.0 \\
\hline 3321 & Gray and Ductile Iron Foundries ....... & W & 0 & 0 & 0 & - & w & w & * & 23.2 \\
\hline 3331 & Primary Copper.$\ldots \ldots \ldots \ldots \ldots$ & 0 & 0 & 0 & 0 & 0 & 0 & 0 & 0 & NF \\
\hline 3334 & Primary Aluminum $\ldots \ldots \ldots \ldots \ldots$ & w & 0 & 0 & 0 & 0 & w & 0 & w & 6.4 \\
\hline 3339 & Primary Nonferrous Metals, nec . . . . . . . & 0 & 0 & 0 & 0 & 0 & 0 & 0 & 0 & NF \\
\hline 3353 & Aluminum Sheet, Plate, and Foll ... & • & 0 & • & 0 & - & 0 & 0 & * & 1.1 \\
\hline 34 & Fabricated Metal Products $\ldots \ldots \ldots \ldots \ldots$ & 1 & 0 & w & - & 0 & - & w & Q & 37.3 \\
\hline 35 & Industral Machinery and Equipment . . . . . . & 1 & 0 & w & • & w & * & 0 & - & 34.4 \\
\hline 357 & Computer and Office Equipment ....... & 0 & 0 & 0 & 0 & 0 & 0 & 0 & 0 & $\mathrm{NF}$ \\
\hline 36 & Electronlc and Other Electric Equipment ... & $\mathbf{Q}$ & 0 & 0 & : & $\mathbf{Q}$ & 0 & 0 & $\mathbf{Q}$ & 32.5 \\
\hline 37 & Transportation Equipment $\ldots \ldots \ldots \ldots$ & W & 0 & * & • & * & w & w & w & 9.9 \\
\hline 3711 & Motor Vehicles and Car Bodles . . . . . . . . & 1 & 0 & w & - & - & W & 0 & w & 8.4 \\
\hline 3714 & Motor Vehlcle Parts and Accessories .... & - & 0 & $\mathbf{Q}$ & - & w & $\mathbf{0}$ & 0 & * & 10.8 \\
\hline 38 & Instruments and Related Products ....... & - & 0 & 0 & 0 & 0 & 0 & 0 & - & 42.9 \\
\hline 3841 & Surglcal and Medical Instruments ...... & - & 0 & 0 & 0 & 0 & 0 & 0 & - & $\mathrm{NF}$ \\
\hline \multirow[t]{2}{*}{39} & Misc. Manufacturing Industries $\ldots \ldots \ldots \ldots$ & $\mathbf{Q}$ & 0 & * & • & $\mathbf{Q}$ & 0 & 0 & • & 39.6 \\
\hline & Total $\ldots \ldots \ldots \ldots$. & 983 & w & $\mathbf{Q}$ & 60 & w & 323 & 7 & 498 & 6.4 \\
\hline
\end{tabular}

See footnotes at end of table. 
Table A3. Total Primary Consumption of Combustible Energy for Nonfuel Purposies by Census Region, Industry Group, and Selected Industries, 1991: Part 2 (Continued)

(Estimates in Trillion Btu)

\begin{tabular}{|c|c|c|c|c|c|c|c|c|c|c|}
\hline $\begin{array}{c}\text { SIC } \\
\text { Code }\end{array}$ & $\begin{array}{l}\text { Industry Groups } \\
\text { and Industry }\end{array}$ & Total & $\begin{array}{l}\text { Residual } \\
\text { Fuel Oil } \\
\end{array}$ & $\begin{array}{l}\text { Distillate } \\
\text { Fuel Oil }^{\mathrm{b}} \\
\end{array}$ & $\begin{array}{c}\text { Natural } \\
\text { Gas }^{c}\end{array}$ & LPG & Coal & $\begin{array}{c}\text { Coke and } \\
\text { Breeze }\end{array}$ & Other' & $\begin{array}{l}\text { RSE } \\
\text { Row } \\
\text { Factors }\end{array}$ \\
\hline & & \multicolumn{9}{|c|}{ South Census Region } \\
\hline & RSE Column Factors: & 0.7 & 2.3 & 1.0 & 0.9 & 0.8 & 1.1 & 1.1 & 0.8 & \\
\hline 20 & Food and Kindred Products & 1 & 0 & 0 & 1 & * & 0 & 0 & $*$ & 38.1 \\
\hline 2011 & Meat Packing Plants . . . . . . . . & • & 0 & 0 & 0 & 0 & 0 & 0 & - & 37.4 \\
\hline 2033 & Canned Fruits and Vegetables. & 0 & 0 & 0 & 0 & 0 & o & 0 & 0 & NF \\
\hline 2037 & Frozen Fruits and Vegetables .... & 0 & 0 & 0 & 0 & 0 & 0 & 0 & 0 & NF \\
\hline 2046 & Wet Com Milling .. & 0 & 0 & 0 & 0 & 0 & 0 & 0 & 0 & NF \\
\hline 2051 & Bread, Cake, and Related Products .... . & . & 0 & 0 & * & : & 0 & 0 & 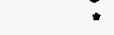 & 32.5 \\
\hline 2063 & Beet Sugar $\ldots \ldots \ldots \ldots \ldots \ldots \ldots$ & • & 0 & 0 & 0 & 0 & 0 & 0 & - & 22.1 \\
\hline 2075 & Soybean Oil Mills ............... & - & 0 & 0 & : & 0 & 0 & 0 & - & 11.1 \\
\hline 2082 & Malt Beverages ............... & - & 0 & 0 & 0 & 0 & 0 & 0 & - & 31.8 \\
\hline 21 & Tobacco Products ........ & 0 & 0 & 0 & 0 & 0 & 0 & 0 & 0 & NF \\
\hline 22 & Textile Mill Products . . . . & * & 0 & * & * & * & 0 & 0 & : & 34.7 \\
\hline 23 & Apparel and Other Textile Products & * & 0 & * & 0 & 0 & 0 & 0 & - & 56.1 \\
\hline 24 & Lumber and Wood Products . . . . . . . . & Q & 0 & 0 & 0 & - & 0 & 0 & Q & 62.5 \\
\hline 25 & Fumiture and Fixtures..$\ldots \ldots$. & $\bar{Q}$ & 0 & $\overline{0}$ & 0 & 0 & 0 & 0 & $\bar{a}$ & $\mathrm{NF}$ \\
\hline 26 & Paper and Allied Products . . . . . . . . & 5 & 0 & . & $\mathbf{w}$ & : & 0 & 0 & $\mathbf{w}$ & 10.9 \\
\hline 2611 & Pulp Mills $\ldots \ldots \ldots \ldots \ldots \ldots \ldots \ldots$ & 0 & 0 & 0 & 0 & 0 & 0 & 0 & 0 & NF \\
\hline 2621 & Paper Mills $\ldots \ldots \ldots \ldots \ldots \ldots \ldots$ & $\mathbf{w}$ & 0 & $w$ & 0 & 0 & 0 & 0 & w & 7.4 \\
\hline 2631 & Paperboard Mills . . . . . . & $w$ & 0 & $w$ & $w$ & * & 0 & 0 & $\because$ & 18.1 \\
\hline 27 & Printing and Publishing $\ldots \ldots \ldots \ldots \ldots \ldots$ & $\because$ & 0 & 0 & 0 & 0 & 0 & 0 & - & NF \\
\hline 28 & Chemicals and Allied Products . . . . . . & 2,164 & $w$ & : & 476 & 1,373 & $\mathbf{w}$ & $w$ & 244 & 8.7 \\
\hline 2812 & Alkalies and Chlorine ............. & 1 & 0 & 0 & : & 0 & 0 & 0 & 1 & 33.4 \\
\hline 2813 & Industrial Gases ....... & w & 0 & 0 & $w$ & $w$ & 0 & 0 & w & 21.0 \\
\hline 2819 & Industrial Inorganic Chemicals, nec ..... & $w$ & $w$ & w & $w$ & : & $w$ & w & 3 & 18.7 \\
\hline 2821 & Plastics Materials and Resins ..... & w & 0 & $w$ & 66 & 209 & 0 & 0 & w & 9.4 \\
\hline 2822 & Synthetic Rubber . . . . . . . . . . . & $w$ & 0 & * & $w$ & 15 & 0 & 0 & - & 24.2 \\
\hline 2823 & Cellulosic Manmade Fibers . . . . . . . . . & 0 & 0 & 0 & 0 & 0 & 0 & 0 & 0 & NF \\
\hline 2824 & Organic Fibers, Noncellulosic ......... & w & 0 & 0 & $\mathbf{w}$ & $w$ & w & 0 & : & 7.4 \\
\hline 2865 & Cyclic Crudes and intermediates ........ & 88 & 0 & : & 9 & $w$ & $\because$ & 0 & $w$ & 20.5 \\
\hline 2869 & Industrial Organic Chemicals, nec ...... & W & 0 & w & w & w & 0 & 0 & 191 & 7.1 \\
\hline 2873 & Nitrogenous Fertilizers $\ldots \ldots \ldots \ldots \ldots$ & 224 & 0 & 0 & 224 & $:$ & 0 & 0 & $\because$ & 38.3 \\
\hline 2874 & Phosphatic Fentilizers. & w & 0 & $w$ & $w$ & 0 & 0 & 0 & w & 4.1 \\
\hline 29 & Petroleum and coal Products $\ldots \ldots \ldots \ldots$ & 1,853 & 0 & $\ddot{w}$ & 0 & w & 0 & 0 & 1,853 & 2.8 \\
\hline 2911 & Petroleum Refining ${ }^{\circ} . \ldots \ldots \ldots$ & 1,786 & 0 & 0 & 0 & 0 & 0 & 0 & 1,786 & 1.4 \\
\hline 30 & Rubber and Misc. Plastics Products . . & 1 & 0 & : & 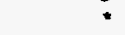 & $\mathbf{Q}$ & 0 & 0 & 1 & 22.8 \\
\hline 3011 & Tires and Inner Tubes .............. & w & 0 & 0 & * & 0 & 0 & 0 & w & 6.2 \\
\hline 308 & Miscellaneous Plastics Products, noc .... & $\because$ & 0 & : & . & $\mathbf{Q}$ & 0 & 0 & $\because$ & 29.9 \\
\hline 31 & Leather and Leather Products . . . . . . . . & • & 0 & 0 & 0 & : & 0 & 0 & - & 52.1 \\
\hline 32 & Stone, Clay and Glass Products .. & 2 & $\mathbf{Q}$ & * & 1 & $w$ & " & $w$ & • & 21.8 \\
\hline 3211 & Flat Glass $\ldots \ldots \ldots \ldots \ldots \ldots$ & * & 0 & 0 & 0 & $\because$ & 0 & 0 & - & 10.5 \\
\hline 3221 & Glass Containers $\ldots \ldots \ldots \ldots \ldots \ldots$ & 0 & 0 & 0 & 0 & 0 & 0 & 0 & 0 & NF \\
\hline 3229 & Pressed and Blown Glass, nec. . . . . . . . & w & 0 & 0 & w & w & 0 & 0 & : & 16.4 \\
\hline 3241 & Cement, Hydraulic . .............. & $\because$ & 0 & 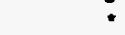 & 0 & 0 & 0 & 0 & - & 30.1 \\
\hline 3274 & Lime $\ldots \ldots \ldots \ldots \ldots \ldots \ldots \ldots$ & * & 0 & 0 & * & 0 & 0 & $w$ & - & 26.3 \\
\hline 3296 & Mineral Wool & • & 0 & 0 & 0 & 0 & 0 & 0 & • & 2.8 \\
\hline 33 & Primary Metal Industries . . . . . . . & 219 & 0 & $\mathbf{w}$ & $\mathbf{w}$ & $\mathbf{w}$ & 191 & 5 & 12 & 5.5 \\
\hline 3312 & Blast Fumaces and Steol Mills . . . . . . . & 194 & 0 & $w$ & w & 0 & $w$ & w & $\mathbf{w}$ & 9.2 \\
\hline 3313 & Electrometalurgical Products .... & 5 & 0 & 0 & 0 & 0 & $\ddot{w}$ & $\ddot{w}$ & $\because$ & 12.8 \\
\hline 3321 & Gray and Ductile Iron Foundries & 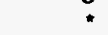 & 0 & 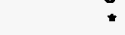 & 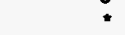 & 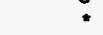 & 0 & $\ddot{w}$ & - & 21.2 \\
\hline 3331 & Primary Copper ............ & 0 & 0 & 0 & 0 & 0 & 0 & 0 & 0 & NF \\
\hline 3334 & Primary Aluminum ............. & 12 & 0 & 0 & 0 & 0 & $\mathbf{w}$ & $w$ & $\mathbf{w}$ & 4.9 \\
\hline 3339 & Primary Nonferrous Metals, nec........ & $\mathbf{w}$ & 0 & 0 & 0 & $\mathbf{w}$ & $\mathbf{w}$ & 0 & w & 2.1 \\
\hline 3353 & Aluminum Sheet, Plate, and Foil ... & $\because$ & 0 & 0 & 0 & 0 & 0 & 0 & : & 1.4 \\
\hline 34 & Fabricated Metal Products . . . . . . . & * & * & $\mathbf{Q}$ & . & $\mathbf{Q}$ & 0 & 0 & - & 69.4 \\
\hline 35 & Industrial Machinery and Equipment . & 1 & 0 & * & * & * & 0 & 0 & - & 43.3 \\
\hline 357 & Computer and Office Equipment .. & : & 0 & 0 & 0 & 0 & 0 & 0 & - & 45.7 \\
\hline 36 & Electronic and Other Electric Equipment ... & 13 & 0 & * & 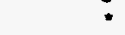 & 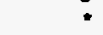 & $\mathbf{w}$ & $w$ & 12 & 30.3 \\
\hline 37 & Transportation Equipment $\ldots \ldots \ldots \ldots \ldots$ & 6 & 0 & - & * & $\mathbf{Q}$ & * & : & 6 & 15.2 \\
\hline 3711 & Motor Vehicles and Car Bodies ........ & 1 & 0 & - & 0 & 0 & 0 & 0 & 1 & 6.5 \\
\hline 3714 & Motor Vehicle Parts and Accessorios .... & : & 0 & • & : & 0 & : & : & : & 15.3 \\
\hline 38 & Instruments and Related Products ... & * & 0 & 0 & * & 0 & 0 & 0 & • & 73.9 \\
\hline 3841 & Surgical and Medical Instruments ...... & 0 & 0 & 0 & 0 & 0 & 0 & 0 & 0 & NF \\
\hline \multirow[t]{2}{*}{39} & Misc. Manufacturing Industries $\ldots \ldots \ldots \ldots$ & * & 0 & $Q$ & 0 & - & 0 & 0 & . & 48.0 \\
\hline & Total ..... & 4,271 & $w$ & 3 & 486 & w & 198 & 9 & 2,137 & 6.9 \\
\hline
\end{tabular}

See footnotes at end of table. 


\section{Table A3. Total Primary Consumption of Combustible Energy for Nonfuel Purposes by Census Region, Industry Group, and Selected Industries, 1991: Part 2 (Continued) (Estimates in Trillion Btu)}

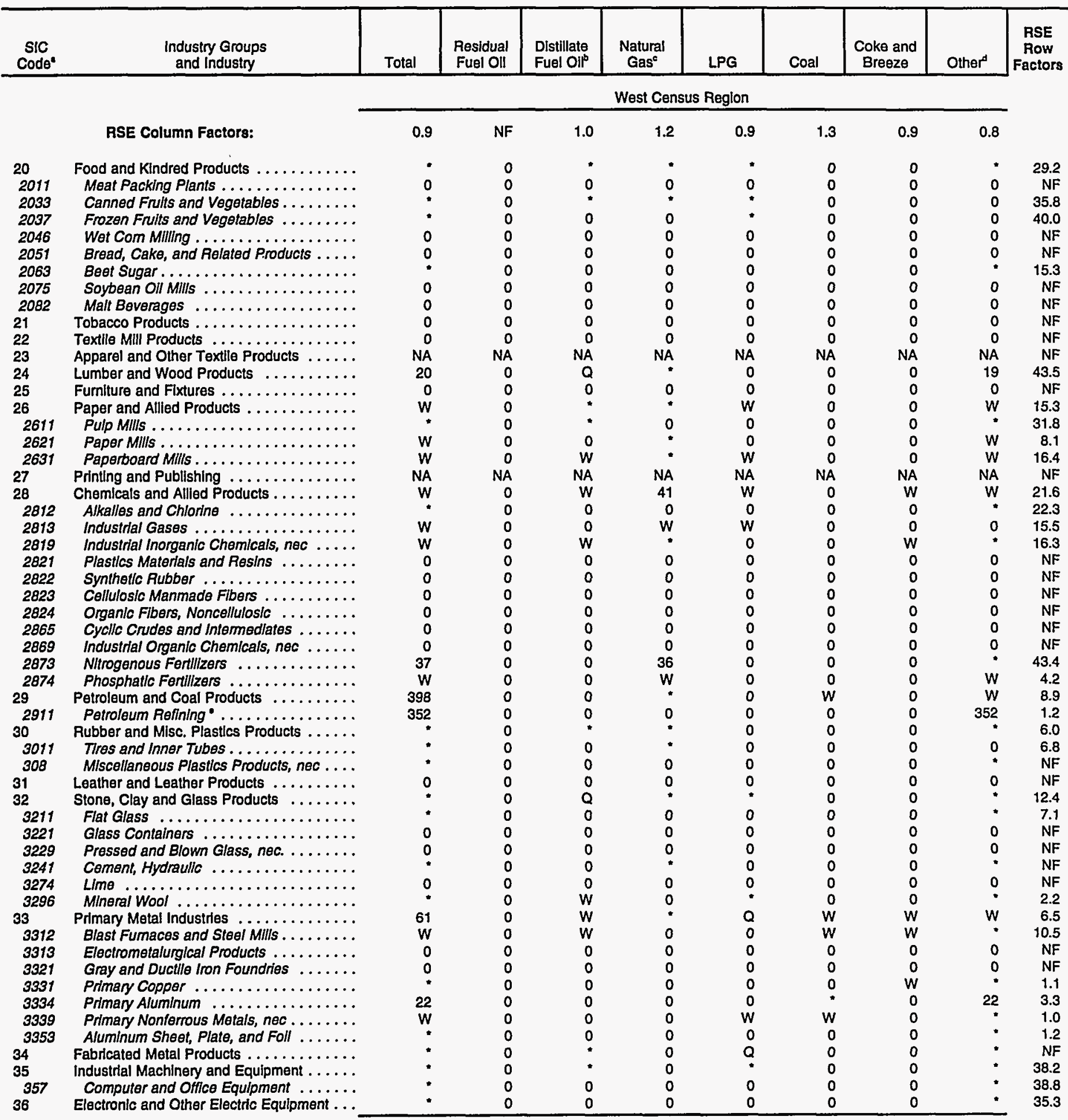

See lootnotes at end of table. 


\section{Table A3. Total Primary Consumption of Combustible Energy for Nonfuel Purposes by Census Region, Industry Group, and Selected Industries, 1991: Part 2? (Continued) (Estimates in Trillion Btu)}

\begin{tabular}{|c|c|c|c|c|c|c|c|c|c|c|}
\hline $\begin{array}{c}\text { SIC } \\
\text { Code }^{a}\end{array}$ & $\begin{array}{l}\text { Industry Groups } \\
\text { and Industry }\end{array}$ & Total & $\begin{array}{c}\text { Residual } \\
\text { Fuel OiI }\end{array}$ & $\begin{array}{l}\text { Distillate } \\
\text { Fuel Oil } \\
\end{array}$ & $\begin{array}{c}\text { Natural } \\
\text { Gas }^{c}\end{array}$ & LPG & Coal & $\begin{array}{c}\text { Coke and } \\
\text { Breeze }\end{array}$ & Other & $\begin{array}{l}\text { RSE } \\
\text { Row } \\
\text { Factors }\end{array}$ \\
\hline & \multirow[b]{2}{*}{ RSE Column Factors: } & \multicolumn{9}{|c|}{ West Census Region } \\
\hline & & 0.9 & NF & 1.0 & 1.2 & 0.9 & 1.3 & 0.9 & 0.8 & \\
\hline 37 & Transportation Equipment $\ldots \ldots \ldots \ldots$ & $w$ & 0 & $\mathbf{W}$ & * & $Q$ & 0 & 0 & $w$ & 21.9 \\
\hline 3711 & Motor Vehicles and Car Bodies . . . . . . . & $\cdot$ & $\mathbf{0}$ & W & 0 & 0 & 0 & 0 & 0 & 10.2 \\
\hline 3714 & Motor Vehicle Parts and Accessories . . . . & * & 0 & 0 & - & 0 & 0 & 0 & $\cdot$ & NF \\
\hline 38 & Instruments and Related Products . . . . . . & • & 0 & 0 & 0 & - & 0 & 0 & * & 37.8 \\
\hline 3841 & Surgical and Medical Instruments ...... & • & 0 & 0 & 0 & 0 & 0 & 0 & • & NF \\
\hline 39 & Misc. Manufacturing Industries . . . . . . . . & - & 0 & 0 & 0 & 0 & 0 & 0 & - & NF \\
\hline & Total $\ldots \ldots \ldots \ldots \ldots \ldots \ldots \ldots$ & 552 & 0 & $\cdot$ & 42 & - & W & $W$ & 458 & 12.9 \\
\hline
\end{tabular}

- See Appendices B and $\mathrm{F}$ for descriptions of the Standard Industrial Classification system.

- "Distillate Fuel Oil" includes Nos. 1, 2, and 4 fuel oils and Nos. 1, 2, and 4 diesel fuels.

c "Natural Gas" includes natural gas obtained from utilities, transmission pipelines, and any other supplier(s) such as brokers and producers.

- "Other" includes energy that respondents indicated was used as feedstock/raw material inputs. See also Footnote "e".

- For the petroleum refining industry only, the feedstocks and raw material inputs for the production of nonenergy products (i.e., asphalt, waxes, lubricants, and solvents) and feedstock consumption at adjoining petrochemical plants are included in the "Other" column, regardless of type of energy. Those inputs and feedstocks that were converted to other energy products (e.g., crude oil converted to residual and distillate fuel oils) are excluded. See Appendix B for more information.

$\mathrm{NF}=$ No applicable RSE row/column factor.

- Estimate less than 0.5. Data are included in higher level totals.

$W=$ Withheld to avoid disclosing data for individual establishments. Data are included in higher level totals.

$\mathrm{Q}=$ Withheld because Relative Standard Error is greater than 50 percent. Data are included in higher level totals.

$N A=$ Not available. Data are included in higher level totals.

Notes: - To obtain a RSE percentage for any table cell, multiply the cell's corresponding RSE column and RSE row factors. - Totals may not equal sum of components because of independent rounding. - The derived estimates presented in this table are for the primary consumption of energy as feedstocks or raw material inputs. Primary consumption is delined as the consumption of the energy that was originally produced offsite or was produced onsite from input materials not classified as energy. Examples of the latter are hydrogen produced from the electrolysis of brine; the output of captive (onsite) mines or wells; woodchips, bark, and woodwaste from wood purchased as a raw material input; and waste materials such as wastepaper and packing materials. Primary consumption excludes quantities of energy that are produced from other energy inputs and, therefore, avoids double counting.

Source: Energy Information Administration, Olfice of Energy Markets and End Use, Energy End Use and Integrated Statistics Division, Form ElA-846, "1991 Manufacturing Energy Consumption Survey," and Office of Oil and Gas, Petroleum Supply Division, Form EIA-810, "Monthly Refinery Report" for 1991. 
Table A4. Total Inputs of Energy for Heat, Power, and Electricity Generation by Census Region, Industry Group, and Selected Industries, 1991: Part 1 (Estimates in Btu or Physical Units)

\begin{tabular}{|c|c|c|c|c|c|c|c|c|c|c|c|}
\hline $\begin{array}{c}\text { SIC } \\
\text { Codea }\end{array}$ & $\begin{array}{l}\text { Industry Groups } \\
\text { and Industry }\end{array}$ & $\begin{array}{c}\text { Total } \\
\text { (trillion } \\
\text { Btu) }\end{array}$ & $\begin{array}{c}\text { Net } \\
\text { Electricity } \\
\text { (million } \\
\mathrm{kWh} \text { ) }\end{array}$ & $\begin{array}{c}\text { Residual } \\
\text { Fuel Oil } \\
\text { (1000 } \\
\text { bbls) }\end{array}$ & $\begin{array}{c}\text { Distillate } \\
\text { Fuel Oile } \\
(1000 \\
\text { bbls })\end{array}$ & $\begin{array}{l}\text { Natural } \\
\text { Gas }^{d} \\
\text { (billion } \\
\text { cu ft) }\end{array}$ & $\begin{array}{l}\text { LPG } \\
\text { (1000 } \\
\text { bbls })\end{array}$ & $\begin{array}{l}\text { Coal } \\
(1000 \\
\text { short } \\
\text { tons) }\end{array}$ & $\begin{array}{c}\text { Coke and } \\
\text { Breeze } \\
\text { (1000 } \\
\text { short } \\
\text { tons) }\end{array}$ & $\begin{array}{c}\text { Other" } \\
\text { (trillion } \\
\text { Btu) }\end{array}$ & $\begin{array}{c}\text { RSE } \\
\text { Row } \\
\text { Factors }\end{array}$ \\
\hline
\end{tabular}

\begin{tabular}{|c|c|c|c|c|c|c|c|c|c|c|c|}
\hline & \multirow[b]{2}{*}{ RSE Column Factors: } & \multicolumn{9}{|c|}{ Total United States } & \\
\hline & & 0.6 & 0.6 & $i .3$ & 1.3 & 0.7 & 1.2 & 1.2 & 1.6 & 1.2 & \\
\hline 20 & Food and Klndred Producls . . . . . . . . & 953 & 49,536 & 4,317 & 2,966 & 497 & 1,429 & 6,913 & W & w & 7.2 \\
\hline 2011 & Meat Packing Plants . . . . . . . . . . & 49 & 3,410 & 170 & 252 & 31 & 157 & 27 & 0 & 2 & 9.8 \\
\hline 2033 & Canned Fruits and Vegetables . . . . . . . & 44 & 1,375 & 290 & 131 & 35 & 124 & $\mathbf{Q}$ & 0 & $*$ & 10.4 \\
\hline 2037 & Frozen Fruits and Vegetables . . . . . . . & 40 & 3,071 & 321 & 76 & 25 & 41 & 0 & 0 & 1 & 14.7 \\
\hline 2046 & Wet Corn Milling . . . . . . . . . . & 140 & 4,054 & 29 & 30 & 51 & 1 & 3,051 & $w$ & $w$ & 11.7 \\
\hline 2051 & Bread, Cake, and Related Products .... & 32 & 2,240 & $\because$ & 131 & 22 & 23 & 0 & 0 & * & 12.4 \\
\hline 2063 & Beet Sugar . . . . . . . . . . . . & 67 & 386 & $\mathbf{W}$ & 30 & 18 & 5 & 1,901 & W & - & 5.5 \\
\hline 2075 & Soybean Oll Mills ............... & 50 & 1,616 & 42 & 31 & 24 & 5 & 592 & 0 & 7 & 3.5 \\
\hline 2082 & Malt Beverages $\ldots \ldots \ldots \ldots \ldots$ & 50 & 2,328 & 419 & 58 & 22 & 8 & 706 & 0 & 1 & 10.7 \\
\hline 21 & Tobacco Products . . . . . . . . . . . . & 24 & 1,002 & 135 & 40 & 4 & 23 & 692 & 0 & $*$ & 6.7 \\
\hline 22 & Textile Mill Products $\ldots \ldots \ldots \ldots \ldots \ldots$ & 273 & 29,532 & 1,966 & 1,064 & 105 & 629 & 1,362 & 0 & 13 & 7.2 \\
\hline 23 & Apparel and Other Textile Products .... & 44 & 5,645 & $\mathbf{Q}$ & 142 & 18 & 158 & 88 & 0 & 1 & 17.9 \\
\hline 24 & Lumber and Wood Products $\ldots . . . \ldots$ & 423 & 17,878 & 333 & 2,373 & 39 & 1,000 & 92 & 0 & 300 & 14.3 \\
\hline 25 & Furniture and Fixtures $\ldots \ldots \ldots \ldots \ldots$ & 67 & 4,915 & 184 & 162 & 18 & 255 & 157 & 0 & 25 & 20.3 \\
\hline 26 & Paper and Allied Products .......... & 2,472 & 58,896 & 24,883 & 1,566 & 532 & w & 13,252 & W & 1,257 & 4.3 \\
\hline 2611 & Pulp Mills $\ldots \ldots \ldots \ldots \ldots \ldots \ldots$ & 300 & 2,537 & 4,500 & 155 & 32 & 141 & 331 & 0 & 221 & 14.3 \\
\hline 2621 & Paper MIIIs ................. & 1,204 & 32,735 & 13,455 & $w$ & 252 & 613 & 8,634 & W & 548 & 3.0 \\
\hline 2631 & Paperboard MIlls . . . . . . . . . . . & 832 & 10,396 & W & 207 & 180 & 93 & W & 0 & 480 & 4.6 \\
\hline 27 & Printing and Publishing $\ldots \ldots \ldots \ldots$ & 108 & 15,629 & 50 & 312 & 47 & 179 & 0 & 0 & 4 & 12.8 \\
\hline 28 & Chemicals and Allied Products . . . . . . . & 3,040 & 129,093 & 7,573 & 2,083 & 1,620 & 1,263 & 11,345 & 132 & 611 & 5.2 \\
\hline 2812 & Alkalles and Chlorine $\ldots \ldots \ldots \ldots$ & 160 & 10,718 & W & 43 & W & 2 & $w$ & 0 & 21 & 15.7 \\
\hline 2813 & Industrial Gases . . . . . . . . . . . . & 91 & 17,854 & 0 & 7 & 24 & $\mathbf{Q}$ & 0 & 0 & 5 & 12.2 \\
\hline 2819 & Industrial Inorganic Chemicals, nec .... & 311 & 37,077 & 691 & 456 & 136 & 75 & 743 & 122 & 17 & 8.4 \\
\hline 2821 & Plastics Materials and Resins . . . . . . & 288 & 14,780 & 668 & 231 & 146 & 54 & 1,074 & 0 & 57 & 5.6 \\
\hline 2822 & Synthatic Rubber . . . . . . . . . . & 112 & 1,794 & 64 & 18 & 43 & 10 & W & 0 & W & 14.0 \\
\hline 2823 & Celluloslc Manmade Fibers . . . . . . . . . & 31 & W & 0 & 21 & W & 1 & 1,202 & 0 & $\cdot$ & 27.3 \\
\hline 2824 & Organic Flbers, Noncellulosic . . . . . . . & 98 & 6,976 & $W$ & 53 & W & 38 & 1,558 & $\mathbf{0}$ & W & 3.9 \\
\hline 2865 & Cyclle Crudes and Intermedlates ...... & 159 & 4,423 & 1,299 & 136 & 94 & 79 & W & $\mathbf{0}$ & W & 11.8 \\
\hline 2869 & Industrial Organic Chemicals, nec ..... & 1,191 & 15,104 & 1,747 & 439 & 625 & 825 & 3,819 & 0 & 394 & 7.6 \\
\hline 2873 & Nilrogenous Fentilizers $\ldots \ldots \ldots \ldots$ & 280 & 2,911 & 0 & 26 & 258 & 43 & 0 & 0 & 4 & 21.7 \\
\hline 2874 & Phosphatic Fertilizers . . . . . . . . . & 34 & 1,886 & 250 & 150 & 18 & 1 & $W$ & 0 & $W$ & 5.7 \\
\hline 29 & Petroleum and Coal Products $\ldots \ldots \ldots$ & 2,987 & 30,782 & 13,862 & 3,599 & 813 & 16,528 & W & W & 1,869 & 4.6 \\
\hline 2911 & Petroleum Rellning $\ldots \ldots \ldots \ldots \ldots \ldots$ & 2,893 & 29,152 & 10,292 & 1,525 & 769 & 15,889 & 134 & 0 & 1,864 & 3.5 \\
\hline 30 & Rubber and Mlsc. Plastics Products ..... & 237 & 33,908 & 1,253 & 508 & 93 & 786 & 295 & 0 & 5 & 10.3 \\
\hline 3011 & Tires and Inner Tubes . . . . . . . . . . . & 42 & 4,037 & 506 & 68 & 21 & 79 & 75 & $\mathbf{0}$ & 1 & 3.6 \\
\hline 308 & Mlscellaneous Plastics Products, nec... & 152 & 25,594 & 413 & W & 51 & 396 & 130 & 0 & W & 14.5 \\
\hline 31 & Leather and Leather Products . . . . . . . & 12 & 795 & 225 & 220 & 5 & 44 & $\mathbf{Q}$ & 0 & 1 & 25.2 \\
\hline 32 & Stone, Clay and Glass Products $\ldots \ldots$ & 894 & 30,814 & 1,345 & 3,312 & 369 & 577 & 13,127 & 374 & 76 & 7.6 \\
\hline 3211 & Flat Glass $\ldots \ldots \ldots \ldots \ldots \ldots$ & 49 & 1,503 & W & 12 & 40 & 40 & • & 0 & W & 3.4 \\
\hline 3221 & Glass Containers . . . . . . . . . . & 85 & 4,098 & 276 & 23 & 67 & 82 & 0 & 0 & * & 5.4 \\
\hline 3229 & Pressed and Blown Glass, nec. . . . . . . & W & 2,862 & 81 & 38 & W & 31 & 0 & 0 & - & 8.3 \\
\hline 3241 & Cement, Hydraullo . . . . . . . . . . . & 329 & 9,455 & 138 & 616 & 38 & 12 & 8,736 & 232 & 52 & 11.2 \\
\hline 3274 & Lime $\ldots \ldots \ldots \ldots \ldots \ldots \ldots \ldots$ & 117 & 1,324 & $w$ & 240 & 8 & $\mathbf{Q}$ & 3,926 & W & 13 & 29.4 \\
\hline 3296 & Mineral Wool . . . . . . . . . . . . . & 41 & 2,821 & $W$ & 12 & 28 & 41 & $\bullet$ & $W$ & $\bullet$ & 1.5 \\
\hline 33 & Primary Metal Industries $\ldots \ldots \ldots \ldots$ & 2,292 & 146,276 & 5,285 & 1,806 & 666 & 888 & 2,054 & 22,695 & 451 & 4.3 \\
\hline 3312 & Blast Fumaces and Stool Mills . . . . . . . . & 1,569 & 38,183 & 4,986 & 901 & 387 & 74 & 1,075 & 21,690 & 440 & 3.9 \\
\hline 3313 & Electromelalurgical Products . . . . . . . . & 31 & 4,222 & 0 & 20 & 1 & $W$ & $\mathbf{W}$ & $\mathbf{W}$ & W & 8.7 \\
\hline 3321 & Gray and Ductile Iron Foundries ...... & 74 & 6,412 & 4 & 144 & 28 & 105 & 5 & 859 & 1 & 11.4 \\
\hline 3331 & Primary Copper . . . . . . . . . . . . & 22 & 1,246 & $W$ & $W$ & 15 & 3 & W & 0 & 1 & 1.1 \\
\hline 3334 & Primary Aluminum $\ldots \ldots \ldots \ldots \ldots$ & 252 & 67,317 & 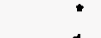 & 127 & 20 & 42 & 0 & 0 & 1 & 3.3 \\
\hline 3339 & Primary Nonferrous Metals, nec . . . . . . . & 42 & 4,312 & 1 & 53 & 16 & 19 & $\mathbf{W}$ & W & W & 1.7 \\
\hline 3353 & Aluminum Sheet, Plate, and Foil ...... & 60 & 4,261 & 0 & 67 & 41 & 62 & W & 0 & W & 1.4 \\
\hline 34 & Fabricated Metal Products . . . . . . . . . & 305 & 29,772 & 501 & 994 & 169 & 1,122 & 245 & $W$ & $W$ & 11.4 \\
\hline 35 & Industrial Machinery and Equipment . . . . . & 235 & 29,484 & 490 & 718 & 106 & 651 & 480 & 24 & 5 & 11.5 \\
\hline 357 & Computer and Office Equipment ..... . & 21 & 4,389 & 11 & 16 & 5 & 4 & 0 & 0 & $\star$ & 15.9 \\
\hline 36 & Electronic and Other Electric Equipment .. & 196 & 29,996 & 612 & 416 & 76 & 396 & $\mathbf{W}$ & 2 & w & 10.2 \\
\hline 37 & Transportation Equipment $\ldots \ldots \ldots \ldots$ & 333 & 34,721 & 1,865 & 1,214 & 129 & 526 & 1,464 & 40 & 27 & 4.9 \\
\hline 3711 & Motor Vehicles and Car Bodies ....... & 105 & 7,705 & 408 & 65 & 44 & 59 & W & W & 18 & 3.8 \\
\hline 3714 & Motor Vehicle Parts and Accessories ... & 99 & 10,888 & 60 & 104 & 40 & 168 & W & W & $w$ & 7.1 \\
\hline 38 & Instruments and Related Products . . . . . & 98 & 12,367 & 536 & $W$ & 25 & $\mathbf{Q}$ & $\mathbf{W}$ & 0 & W & 13.4 \\
\hline 3841 & Surgical and Medical Instruments .... . & 6 & 1,161 & 9 & 30 & 2 & 8 & 0 & 0 & 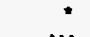 & 15.4 \\
\hline 39 & Misc. Manufacturing Industries . . . . . . . & 31 & 3,661 & 115 & $W$ & 14 & W & 32 & 0 & $W$ & 15.4 \\
\hline & Total $\ldots \ldots \ldots \ldots \ldots \ldots \ldots$ & 15,027 & 694,702 & 65,837 & 23,885 & 5,345 & 27,970 & 53,035 & 23,520 & 4,726 & 2.8 \\
\hline
\end{tabular}

See footnotes at end of table. 
Table A4. Total Inputs of Energy for Heat, Power, and Electricity Generation by Census Region, Industry Group, and Selected Industries, 1991: Part 1 (Continued) (Estimates in Btu or Physical Units)

\begin{tabular}{|c|c|c|c|c|c|c|c|c|c|c|c|}
\hline $\begin{array}{l}\text { SIC } \\
\text { Code }^{\mathrm{a}}\end{array}$ & $\begin{array}{l}\text { Industry Groups } \\
\text { and Industry }\end{array}$ & $\begin{array}{c}\text { Total } \\
\text { (trillion } \\
\text { Btu) }\end{array}$ & $\begin{array}{c}\text { Net } \\
\text { Electricity } \\
\text { (million } \\
\text { kWh) }\end{array}$ & $\begin{array}{c}\text { Residual } \\
\text { Fuel Oil } \\
\text { (1000 } \\
\text { bbls) } \\
\end{array}$ & $\begin{array}{c}\text { Distillate } \\
\text { Fuel Oif } \\
(1000 \\
\text { bbls) }\end{array}$ & $\begin{array}{c}\text { Natural } \\
\text { Gas } \\
\text { (billion } \\
\text { cu } t \text { ) }\end{array}$ & $\begin{array}{c}\text { LPG } \\
(1000 \\
\text { bbls })\end{array}$ & $\begin{array}{c}\text { Coal } \\
\text { (1000 } \\
\text { short } \\
\text { tons) } \\
\end{array}$ & $\begin{array}{c}\text { Coke and } \\
\text { Breeze } \\
\text { (1000 } \\
\text { short } \\
\text { tons) } \\
\end{array}$ & $\begin{array}{c}\text { Other } \\
\text { (trillion } \\
\text { Btu) }\end{array}$ & $\begin{array}{c}\text { RSE } \\
\text { Row } \\
\text { Factors }\end{array}$ \\
\hline & & \multicolumn{10}{|c|}{ Northeast Census Region } \\
\hline & RSE Column Factors: & 0.7 & 0.7 & 1.0 & 1.2 & 0.8 & 1.2 & 1.3 & 1.3 & 1.1 & \\
\hline 20 & Food and Kindred Products . . . . . . . . . & 79 & 5,385 & 1,164 & 889 & 40 & 222 & 99 & 0 & 3 & 13.8 \\
\hline 2011 & Meat Packing Plants . . . . . . . . . . . & 1 & 141 & W & 34 & 1 & Q & 0 & 0 & 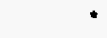 & 23.8 \\
\hline 2033 & Canned Fruits and Vegetables . . . . . . . & 6 & 292 & 146 & 22 & 4 & 13 & Q & 0 & $\cdot$ & 17.2 \\
\hline 2037 & Frozen Fruits and Vegetables . . . . . . . & 1 & 140 & 128 & 3 & * & $Q$ & 0 & 0 & $\cdot$ & 32.8 \\
\hline 2046 & Wet Com Milling . . . . . . . . . & $*$ & 15 & W & $W$ & - & * & 0 & 0 & $\cdot$ & 23.6 \\
\hline 2051 & Bread, Cake, and Related Products . . . . & 7 & 382 & $\bullet$ & W & $\mathbf{W}$ & 8 & 0 & 0 & * & 17.9 \\
\hline 2063 & Beet Sugar . . . . . . . . . . . & 0 & 0 & 0 & 0 & 0 & 0 & 0 & 0 & 0 & NF \\
\hline 2075 & Soybean Oil Mills ............... & 0 & 0 & 0 & 0 & 0 & 0 & 0 & 0 & 0 & NF \\
\hline 2082 & Malt Beverages ............ & 8 & 496 & $w$ & 9 & 3 & 4 & W & 0 & $\cdot$ & 16.4 \\
\hline 21 & Tobacco Products . . . . . . . . . . . . & NA & NA & NA & NA & NA & NA & NA & NA & NA & 25.3 \\
\hline 22 & Textile Mill Products . . . . . . . . . . . & 27 & 1,340 & 774 & 556 & 10 & 163 & 17 & 0 & 3 & 19.5 \\
\hline 23 & Apparel and Other Textile Products .... & 5 & 497 & 44 & 49 & 2 & $\mathbf{Q}$ & 0 & 0 & * & 29.7 \\
\hline 24 & Lumber and Wood Products $\ldots . \ldots \ldots$ & NA & NA & NA & NA & NA & NA & NA & NA & NA & 36.8 \\
\hline 25 & Fumiture and Fixtures $\ldots \ldots \ldots \ldots$ & 7 & 449 & a & 49 & 2 & 60 & 0 & 0 & 2 & 30.2 \\
\hline 26 & Paper and Allied Products . . . . . . . . & W & 8,054 & 11,439 & 624 & 36 & w & w & 0 & W & 6.1 \\
\hline 2611 & Pulp Mills . . . . . . . . . . . . . & 12 & 158 & 290 & 7 & 0 & 13 & 0 & 0 & 9 & 36.7 \\
\hline 2621 & Paper Mills . . . . . . . . . . . . . . & 228 & 4,573 & 9,702 & W & 19 & 284 & w & 0 & 96 & 4.3 \\
\hline 2631 & Paperboard Mills . . . . . . . . . . . . & 16 & 487 & W & $\mathbf{Q}$ & 6 & 5 & W & 0 & 1 & 15.4 \\
\hline 27 & Printing and Publishing $\ldots \ldots \ldots \ldots$ & 23 & 3,167 & 36 & 234 & 9 & 30 & 0 & 0 & 1 & 25.5 \\
\hline 28 & Chemicals and Allied Products . . . . . . . . & 135 & 9,303 & 3,072 & W & 57 & 101 & w & 0 & 15 & 9.0 \\
\hline 2812 & Alkalies and Chlorine .............. & 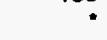 & $w$ & 0 & 0 & * & 0 & 0 & 0 & 0 & 36.5 \\
\hline 2813 & 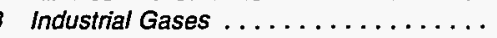 & 5 & 1,399 & 0 & 1 & • & 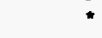 & 0 & 0 & 0 & 11.9 \\
\hline 2819 & Industrial Inorganic Chemicals, nec ... & 10 & 494 & 255 & 78 & 6 & 14 & 0 & 0 & $\cdot$ & 22.7 \\
\hline 2821 & Plastics Materials and Resins . . . . . . . & 20 & 1,120 & 478 & 153 & 8 & 14 & $\mathbf{w}$ & $\mathbf{0}$ & $\mathbf{w}$ & 9.2 \\
\hline 2822 & Synthetic Rubber . . . . . . . . . . . & $W$ & W & W & $\bullet$ & $w$ & * & 0 & 0 & $\bullet$ & 26.0 \\
\hline 2823 & Cellulosic Manmade Fibers . . . . . . . . . & 0 & 0 & 0 & 0 & 0 & 0 & 0 & 0 & 0 & NF \\
\hline 2824 & Organic Fibers, Noncellulosic ........ & $\cdot$ & 95 & $\mathbf{Q}$ & $\mathbf{w}$ & * & * & 0 & 0 & 0 & 14.8 \\
\hline 2865 & Cyclic Crudes and Intermediates ...... & 12 & 406 & W & W & 7 & 2 & W & 0 & 1 & 21.0 \\
\hline 2869 & Industrial Organic Chemicals, nec . . . . . & 38 & 3,070 & 1,399 & 76 & $W$ & 42 & 0 & 0 & $\mathbf{W}$ & 8.7 \\
\hline 2873 & Nitrogenous Fertilizers $\ldots \ldots \ldots \ldots$ & * & 29 & 0 & 3 & $\bullet$ & 1 & 0 & 0 & $\cdot$ & 46.4 \\
\hline 2874 & Phosphatic Fertilizers . . . . . . . . . . & 0 & 0 & 0 & 0 & 0 & 0 & 0 & 0 & 0 & NF \\
\hline 29 & Petroleum and Coal Products ........ & 238 & 2,632 & 7,726 & 1,376 & 29 & 852 & 145 & 0 & 136 & 9.3 \\
\hline 2911 & Petroleum Refining . . . . . . . . . . & 199 & 2,093 & 4,275 & W & 24 & 459 & W & 0 & 136 & 5.3 \\
\hline 30 & Rubber and Misc. Plastics Products . . . . . & 37 & 5,480 & 456 & 202 & $w$ & $\mathbf{Q}$ & 86 & 0 & 1 & 17.9 \\
\hline 3011 & Tires and Inner Tubes . . . . . . . . . . . & 2 & 125 & 63 & $\mathbf{Q}$ & 1 & 5 & 0 & 0 & * & 13.3 \\
\hline 308 & Miscellaneous Plastics Products, nec . . . & 28 & 4,807 & 251 & W & 7 & 127 & W & 0 & W & 23.6 \\
\hline 31 & Leather and Leather Products . . . . . . . & 4 & 205 & 142 & 196 & 1 & 28 & $\mathbf{Q}$ & 0 & $\bullet$ & 28.5 \\
\hline 32 & Stone, Clay and Glass Products . . . . . . & 181 & 5,599 & 432 & 714 & 58 & W & 3,451 & $W$ & W & 17.3 \\
\hline 3211 & Flat Glass $\ldots \ldots \ldots \ldots \ldots \ldots$ & W & $W$ & 0 & 1 & $W$ & w & 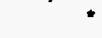 & 0 & $\cdot$ & 4.6 \\
\hline 3221 & Glass Containers .............. & 19 & 834 & 193 & 14 & 14 & 24 & $\mathbf{0}$ & 0 & * & 8.7 \\
\hline 3229 & Pressed and Blown Glass, nec. . . . . . . & W & W & 80 & $\mathbf{W}$ & W & 8 & 0 & 0 & * & 10.3 \\
\hline 3241 & Cement, Hydraulic . . . . . . . . . . & 54 & 1,334 & 14 & $w$ & $\bullet$ & 1 & 1,482 & $w$ & 14 & 17.8 \\
\hline 3274 & Lime . . . . . . . . . . . . & $Q$ & Q & 0 & $Q$ & * & $\mathbf{Q}$ & $\mathbf{Q}$ & 0 & 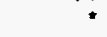 & NF \\
\hline 3296 & Mineral Wool . . . . . . . . . . . . . . & 4 & 304 & 0 & W & W & W & $*$ & $w$ & * & 1.7 \\
\hline 33 & Primary Metal Industries $\ldots \ldots \ldots \ldots$ & 358 & 18,002 & 852 & 324 & 102 & 307 & W & $w$ & W & 8.0 \\
\hline 3312 & Blast Fumaces and Steel Mills . . . . . . . . & 270 & 7,828 & W & 156 & 65 & 30 & 52 & $w$ & 124 & 6.8 \\
\hline 3313 & Electrometalurgical Products . . . . . . . & $W$ & $w$ & 0 & 1 & $\cdot$ & 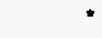 & W & W & - & 12.9 \\
\hline 3321 & Gray and Ductile Iron Foundries ...... & 5 & 350 & 0 & 13 & 2 & 18 & 1 & 67 & - & 16.5 \\
\hline 3331 & Primary Copper . . . . . . . . . . . . . & * & W & 0 & $\mathbf{W}$ & $\bullet$ & 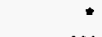 & $\mathbf{0}$ & 0 & * & 1.1 \\
\hline 3334 & Primary Aluminum $\ldots \ldots \ldots \ldots \ldots$ & $W$ & $w$ & * & W & $W$ & $w$ & $\mathbf{0}$ & 0 & - & 5.5 \\
\hline 3339 & Primary Nonferrous Metals, nec. . . . . . . & $W$ & 640 & 1 & W & 1 & 1 & $W$ & 0 & * & 1.8 \\
\hline 3353 & Aluminum Sheet, Plate, and Foil ..... & $W$ & 451 & 0 & W & W & 23 & 0 & 0 & * & 1.4 \\
\hline 34 & Fabricated Metal Products . . . . . . . . & 56 & 5,074 & 368 & 366 & 31 & 153 & 9 & 37 & 1 & 15.5 \\
\hline 35 & Industrial Machinery and Equipment ..... & 41 & 5,202 & 408 & 352 & 15 & 176 & $\mathbf{0}$ & 0 & 2 & 19.1 \\
\hline 357 & Computer and Office Equipment ...... & 4 & 819 & 8 & 10 & 1 & 2 & 0 & 0 & $\cdot$ & 20.7 \\
\hline 36 & Electronic and Other Electric Equipment .. & 42 & 6,544 & 504 & 291 & 14 & 162 & 4 & 2 & - & 16.2 \\
\hline 37 & Transportation Equipment & $W$ & 3,069 & 1,070 & 507 & 10 & W & $w$ & 0 & $W$ & 11.1 \\
\hline 3711 & Motor Vehicles and Car Bodies . ....... & $W$ & $W$ & W & $w$ & 1 & 1 & 0 & 0 & 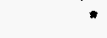 & 7.8 \\
\hline 3714 & Motor Vehicle Parts and Accessories .... & 8 & 887 & W & 6 & $\mathbf{W}$ & 10 & $w$ & 0 & • & 11.8 \\
\hline 38 & Instruments and Related Products ..... & 52 & 4,032 & 513 & $w$ & $\mathbf{W}$ & $\mathbf{Q}$ & W & 0 & W & 17.4 \\
\hline 3841 & Surgical and Medical instruments ..... & 2 & 332 & 9 & 16 & ? & 2 & 0 & 0 & $*$ & 19.2 \\
\hline 39 & Misc. Manufacturing Industries . . . . . . . & W & 1,187 & 84 & W & W & $W$ & 22 & $\mathbf{0}$ & - & 18.0 \\
\hline & Total $\ldots \ldots \ldots \ldots \ldots \ldots \ldots \ldots$ & 1,635 & 86,041 & 29,245 & 7,805 & 446 & $w$ & 7,420 & $W$ & 423 & 6.1 \\
\hline
\end{tabular}

See footnotes at end of table. 
Table A4. Total Inputs of Energy for Heat, Power, and Electricity Generation by Census Region, Industry Group, and Selected Industries, 1991: Part 1 (Continued) (Estimates in Btu or Physical Units)

\begin{tabular}{|c|c|c|c|c|c|c|c|c|c|c|c|}
\hline $\begin{array}{l}\text { SIC } \\
\text { Code }^{a}\end{array}$ & $\begin{array}{l}\text { Industry Groups } \\
\text { and Industry }\end{array}$ & $\begin{array}{c}\text { Total } \\
\text { (trillion } \\
\text { Btu) }\end{array}$ & \begin{tabular}{|c} 
Net \\
Electricity" \\
(million \\
KWh)
\end{tabular} & $\begin{array}{c}\text { Residual } \\
\text { Fusl Oil } \\
\text { (1000 } \\
\text { bbls) }\end{array}$ & $\begin{array}{c}\text { Distillate } \\
\text { Fuel OIfe } \\
(1000 \\
\text { bbls })\end{array}$ & $\begin{array}{l}\text { Natural } \\
\text { Gas } \\
\text { (billion } \\
\text { cu ft) }\end{array}$ & $\begin{array}{l}\text { LPG } \\
\text { (1000 } \\
\text { bbls) }\end{array}$ & $\begin{array}{l}\text { Coal } \\
\text { (1000 } \\
\text { short } \\
\text { tons) }\end{array}$ & $\begin{array}{c}\text { Coke and } \\
\text { Breeze } \\
\text { (1000 } \\
\text { short } \\
\text { tons) }\end{array}$ & $\begin{array}{c}\text { Other" } \\
\text { (trillion } \\
\text { Btu) }\end{array}$ & $\begin{array}{c}\text { RSE } \\
\text { Row } \\
\text { Factors }\end{array}$ \\
\hline
\end{tabular}

\begin{tabular}{|c|c|c|c|c|c|c|c|c|c|c|c|}
\hline & & & & & Midwe & Census : & & & & & \\
\hline & RSE Column Factors: & 0.6 & 0.6 & 1.5 & 1.3 & 0.7 & 1.2 & 1.1 & 1.4 & 1.0 & \\
\hline 20 & Food and Kindred Products & 419 & 19,579 & 893 & 544 & 211 & 348 & 4,808 & w & w & \\
\hline 2011 & Meat Packing Plants .. & 33 & 2,065 & 150 & 37 & 22 & 13 & 27 & 0 & 1 & 9.3 \\
\hline 2033 & Canned Fruits and Vegetables & 10 & 375 & 0 & 62 & 8 & 39 & 0 & 0 & : & 17.3 \\
\hline 2037 & Frozen Fruils and Vegetables & 4 & 289 & 36 & 3 & 2 & 3 & 0 & 0 & - & 27.2 \\
\hline 2046 & Wet Corn Milling $\ldots \ldots \ldots \ldots$ & 122 & 3,192 & w & 26 & 45 & 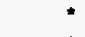 & 2,729 & w & w & 13.5 \\
\hline 2051 & Bread, Cake, and Related Products & 9 & 632 & 0 & w & w & * & 0 & 0 & - & 16.4 \\
\hline 2063 & Beet Sugar .............. & 34 & 212 & w & 10 & 6 & 2 & 1,084 & w & * & 6.6 \\
\hline 2075 & Soybean Oll Mills & 36 & 1,081 & 8 & 7 & 15 & w & w & 0 & w & 4.4 \\
\hline 2082 & Malt Beverages & 11 & 480 & 40 & 1 & $w$ & w & w & 0 & & 17.3 \\
\hline 21 & Tobacco Products . & NA & NA & NA & NÁ & NA & NA & NA & NA & NA & 9.7 \\
\hline 22 & Textile Mill Products & NA & NA & NA & NA & NA & NA & NA & NA & NA & 23.9 \\
\hline 23 & Apparel and Other Textlle Products & NA & NA & NA & NA & NA & NA & NA & NA & NA & 31.7 \\
\hline 24 & Lumber and Wood Products & 57 & 2,834 & Q & a & 11 & 196 & 66 & 0 & 32 & 27.3 \\
\hline 25 & Furniture and Fixtures ... & w & 1,569 & & 10 & w & 45 & Q & 0 & a & \\
\hline 26 & Paper and Allled Products & $\ddot{w}$ & 14,429 & 807 & 138 & 112 & 227 & 4,236 & w & $w$ & 6.5 \\
\hline 2611 & Pulp Mills & 28 & 298 & 0 & 14 & 3 & Q & 161 & 0 & 20 & 33.4 \\
\hline 2621 & Paper MIIIs . & 209 & 7,767 & w & 89 & 58 & 65 & 2,835 & w & 54 & 5.0 \\
\hline 2631 & Paperboard Mills . & 70 & 2,185 & 39 & 17 & 23 & 21 & 1,158 & 0 & 13 & 13.3 \\
\hline 27 & Prinling and Publlshing & 43 & 5,224 & 10 & 31 & 22 & 59 & 0 & 0 & 2 & 17.9 \\
\hline 28 & Chemlcals and Alled Products. & 398 & 33,802 & w & $w$ & 181 & $w$ & 2,822 & 11 & 29 & 10.1 \\
\hline 2812 & Alkalles and Chlorine & w & w & 0 & $\ddot{w}$ & & $\because$ & 0 & 0 & 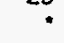 & 27.1 \\
\hline 2813 & Industral Gases & $w$ & $w$ & 0 & a & w & $Q$ & 0 & 0 & - & 13.6 \\
\hline 2819 & Industrial Inorganlc Chemicals, nac & 71 & $w$ & w & 4 & $w$ & 4 & w & 1 & 列 & 11.6 \\
\hline 2821 & Plastlics Materlals and Resins .. & 58 & 3,234 & w & 13 & 24 & 3 & $\ddot{w}$ & 0 & w & 8.5 \\
\hline 2822 & Synthetic Rubber & w & 241 & 0 & $w$ & $w$ & 1 & w & 0 & $\because$ & 19.7 \\
\hline 2823 & Cellulosic Manmado Fibers & 0 & 0 & 0 & 0 & 0 & 0 & 0 & 0 & 0 & $\mathrm{NF}$ \\
\hline 2824 & Organic Fibers, Noncellulosic & 0 & 0 & 0 & 0 & 0 & 0 & 0 & 0 & 0 & $\mathrm{NF}$ \\
\hline 2865 & Cyclle Crudes and Intermedlates & 22 & 756 & w & w & 14 & 5 & w & 0 & & 14.6 \\
\hline 2869 & Industrlal Organle Chemicals, nec . & 71 & 1,774 & 10 & 26 & 33 & 4 & 1,140 & 0 & 6 & 8.2 \\
\hline 2873 & 3 Nitrogenous Fentilizers & 46 & 556 & 0 & 5 & 41 & : & 0 & 0 & 2 & 35.7 \\
\hline 2874 & Phosphatic Fertilfzers & & 1 & 0 & 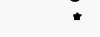 & $\because$ & - & 0 & 0 & & 4.6 \\
\hline 29 & Petroleum and Coal Products .. & 501 & 6,687 & 2,114 & $w$ & 86 & 1,336 & $w$ & 0 & 368 & 5.4 \\
\hline 2911 & Pelroleum Reffining ....... & 476 & 6,134 & 2,058 & $w$ & 70 & 1,212 & w & 0 & 364 & 3.0 \\
\hline 30 & Rubber and Misc. Plastics Products & 93 & 13,236 & 265 & 33 & 41 & 174 & 125 & 0 & 1 & 11.9 \\
\hline 3011 & Tres and Inner Tubes............ & w & w & 186 & 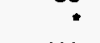 & 6 & w & w & 0 & & 4.1 \\
\hline 308 & Mlscollaneous Plastics Products, nec & NA & NA & NA & NA & NA & NA & NA & NA & NA & 18.4 \\
\hline 31 & Leather and Leather Products & 4 & 262 & 58 & 5 & 2 & 11 & 0 & 0 & & 25.9 \\
\hline 32 & Stone, Clay and Glass Products & 252 & 8,201 & 64 & 602 & 105 & 122 & 3,411 & 84 & 34 & 10.1 \\
\hline 3211 & Flat Glass & 13 & $w$ & 0 & w & 11 & $w$ & 0 & 0 & ד & 4.1 \\
\hline 3221 & Glass Containers & 20 & 873 & 0 & 1 & 17 & 13 & 0 & 0 & . & 7.6 \\
\hline 3229 & 9 Pressed and Blown Glass, nec. & 14 & 617 & - & 9 & 11 & 7 & 0 & 0 & 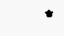 & 6.7 \\
\hline 3241 & Cement, Hydraullic & 89 & 2,220 & w & w & 6 & 5 & 2,297 & 0 & 23 & 15.0 \\
\hline 3274 & & 34 & 367 & 0 & 65 & 3 & 1 & 925 & w & w & 18.6 \\
\hline 3296 & Mineral Wool & 17 & 1,251 & w & $w$ & $w$ & 14 & 0 & $\ddot{w}$ & $\because$ & 1.2 \\
\hline 33 & Primary Metal Industries & 1,168 & 46,756 & 2,903 & 729 & 333 & 252 & 1,190 & 15,497 & 231 & 5.7 \\
\hline 3312 & Blast Fumaces and Steel Mills. & 917 & 17,191 & 2,899 & w & 218 & 25 & $w$ & 14,932 & 226 & 5.2 \\
\hline 3313 & Electrometalurglcal Products & 19 & 2,268 & 0 & 13 & 1 & w & $w$ & . & $w$ & 11.3 \\
\hline 3321 & Ductlle Iron Foundries & 45 & 4,349 & 4 & $\mathbf{Q}$ & $w$ & 37 & $\ddot{w}$ & w & 1 & 11.7 \\
\hline 3331 & Primary & & w & 0 & 0 & $\because$ & r & 0 & 0 & 0 & 3 \\
\hline 3334 & Priman & w & w & 0 & $w$ & 3 & w & 0 & 0 & * & 1 \\
\hline 3339 & s Metals, nec. & $w$ & 716 & 0 & 11 & 3 & 6 & 0 & $w$ & - & 2.0 \\
\hline 3353 & Aluminum Sheot, & 24 & 1,453 & 0 & w & 16 & w & 0 & 0 & w & 1.3 \\
\hline 34 & Fabricat & 139 & 12,889 & 7 & 173 & 79 & 489 & 236 & w & w & 15.0 \\
\hline 35 & hinery and Equlpment ..... & 115 & 12,535 & 39 & 226 & 56 & w & 479 & $\mathbf{Q}$ & w & 13.8 \\
\hline 357 & Computer and Office Equipment & 5 & 820 & 0 & 1 & 2 & 1 & 0 & 0 & 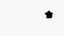 & 24.0 \\
\hline 36 & Electronic and Other Electric Equipment. . & 59 & 7,160 & 51 & $w$ & 28 & 67 & $w$ & 0 & w & 13.4 \\
\hline & Transportation Equipment ........... & 171 & 16,053 & 394 & 272 & 72 & 210 & 1,212 & 40 & $\ddot{9}$ & 6.1 \\
\hline 3711 & Motor Vehicles and Car Bodles. & 64 & 4,826 & w & 28 & 30 & 26 & w & w & w & 4.7 \\
\hline 3714 & Motor Vehiclo Parts and Accessorios & 76 & 7,840 & w & 95 & 29 & 110 & $w$ & $w$ & $w$ & 8.1 \\
\hline 38 & Instruments and Related Products. & 11 & 1,806 & a & $\mathbf{Q}$ & $w$ & 5 & w & 0 & $\cdots$ & 19.4 \\
\hline 38 & Surgical and Modical Instruments & j & 276 & 0 & ? & $\because$ & 3 & 0 & 0 & 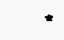 & 18.7 \\
\hline 39 & & 10 & 929 & & & & 13 & & & $\mathbf{Q}$ & 22.0 \\
\hline & To & 3,833 & 205,102 & 8,134 & 3,885 & 1,363 & 3,877 & 18,828 & 15,789 & 829 & 3.7 \\
\hline
\end{tabular}

See footnotes at end of table. 
Table A4. Total Inputs of Energy for Heat, Power, and Electricity Generation by Census Region, Industry Group, and Selected Industries, 1991: Part 1 (Continued) (Estimates in Btu or Physical Units)

\begin{tabular}{|c|c|c|c|c|c|c|c|c|c|c|c|}
\hline $\begin{array}{l}\text { SIC } \\
\text { Code }\end{array}$ & $\begin{array}{l}\text { Industry Groups } \\
\text { and Industry }\end{array}$ & $\begin{array}{c}\text { Total } \\
\text { (trillion } \\
\text { Blu) }\end{array}$ & $\begin{array}{c}\text { Net } \\
\text { Electricity } \\
\text { (million } \\
\text { kWh) }\end{array}$ & $\begin{array}{c}\text { Residual } \\
\text { Fuel Oil } \\
\text { (1000 } \\
\text { bbls) }\end{array}$ & $\begin{array}{c}\text { Distillate } \\
\text { Fuel Oife } \\
(1000 \\
\text { bbls })\end{array}$ & $\begin{array}{l}\text { Natural } \\
\text { Gas }^{d} \\
\text { (billion } \\
\text { cu ft) }\end{array}$ & $\begin{array}{c}\text { LPG } \\
(1000 \\
\text { bbls })\end{array}$ & $\begin{array}{l}\text { Coal } \\
\text { (1000 } \\
\text { short } \\
\text { tons) }\end{array}$ & $\begin{array}{c}\text { Coke and } \\
\text { Breeze } \\
\text { (1000 } \\
\text { shon } \\
\text { tons) }\end{array}$ & $\begin{array}{l}\text { Other } \\
\text { (triliton } \\
\text { Btu) }\end{array}$ & $\begin{array}{c}\text { RSE } \\
\text { Row } \\
\text { Factors }\end{array}$ \\
\hline
\end{tabular}

\begin{tabular}{|c|c|c|c|c|c|c|c|c|c|c|c|}
\hline & & & & & Sout & ensus $F$ & & & & & \\
\hline & RSE Column Factors: & 0.6 & 0.6 & 1.3 & 1.1 & 0.7 & 1.2 & 1.1 & 1.6 & 1.1 & \\
\hline 20 & Food and Kindred Products . . . . . . . & 254 & 15,937 & 1,572 & 859 & 130 & 623 & 766 & W & $Q$ & 11.1 \\
\hline 2011 & Meat Packing Plants . . . . . . . . . . & 10 & 892 & 14 & 167 & 5 & 112 & 0 & 0 & $\because$ & 16.9 \\
\hline 2033 & Canned Fruits and Vegetables . . . . . . . & 7 & 172 & 26 & 9 & 6 & 14 & 0 & 0 & $*$ & 19.7 \\
\hline 2037 & Frozen Fruits and Vegetables . . . . . . . & 8 & 551 & 122 & 12 & 5 & 6 & 0 & 0 & + & 17.6 \\
\hline 2046 & Wet Com Milling . . . . . . . . . . . . & $W$ & 755 & 0 & 2 & 4 & - & 322 & 0 & $w$ & 22.8 \\
\hline 2051 & Bread, Cake, and Related Products .... & 11 & 837 & 0 & 19 & 7 & 7 & 0 & 0 & $\bullet$ & 14.1 \\
\hline 2063 & Beet Sugar . . . . . . . . . . . . & W & $W$ & 0 & 1 & $w$ & * & 0 & W & $\cdot$ & 16.8 \\
\hline 2075 & Soybean Oil Mills ............... & 15 & 535 & 34 & 24 & 8 & $w$ & $\mathbf{w}$ & 0 & $w$ & 4.9 \\
\hline 2082 & Malt Beverages . . . . . . . . . . & 14 & 863 & W & W & 8 & 1 & W & 0 & $\cdot$ & 14.0 \\
\hline 21 & Tobacco Products $\ldots \ldots \ldots \ldots \ldots \ldots$ & 24 & 985 & 135 & 40 & 4 & 23 & 692 & 0 & - & 6.8 \\
\hline 22 & Textile Mill Products ............. & 235 & 27,431 & 1,192 & 506 & 87 & 455 & 1,344 & 0 & 10 & 6.5 \\
\hline 23 & Apparel and Other Textile Products ..... & 31 & 4,165 & $\mathbf{Q}$ & 71 & 12 & 124 & 83 & 0 & 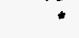 & 22.7 \\
\hline 24 & Lumber and Wood Products ......... & 204 & 9,218 & $\mathbf{Q}$ & W & 15 & 332 & 26 & 0 & 150 & 19.6 \\
\hline 25 & Fumiture and Fixtures $\ldots \ldots \ldots \ldots \ldots$ & W & 2,592 & 63 & 99 & $W$ & 122 & W & 0 & W & 19.4 \\
\hline 26 & Paper and Allied Products . . . . . . . . . . & 1,442 & 25,288 & 10,918 & 710 & 275 & 494 & 6,904 & 0 & 844 & 4.5 \\
\hline 2611 & Pulp Mills . . . . . . . . . . . . & 219 & 1,249 & 3,367 & 116 & 21 & 120 & 171 & 0 & 167 & 17.2 \\
\hline 2621 & Paper Mills . . . . . . . . . . . . . . & 606 & 13,863 & 2,801 & 355 & 122 & 149 & 3,910 & 0 & 325 & 3.4 \\
\hline 2631 & Paperboard Mills . . . . . . . . . . . . . . & 575 & 5,650 & 4,660 & 152 & 111 & 41 & 2.786 & 0 & 349 & 5.4 \\
\hline 27 & Printing and Publishing $\ldots \ldots \ldots \ldots$ & 28 & 4,798 & Q & 35 & 10 & 47 & 0 & 0 & 1 & 21.1 \\
\hline 28 & Chemicals and Allied Products . . . . . . . & 2,368 & 75,072 & 4,003 & 1,051 & 1,297 & W & 8,078 & $W$ & 561 & 6.2 \\
\hline 2812 & Alkalies and Chlorine ............. & 147 & 8,378 & 0 & 35 & W & 1 & $W$ & 0 & 20 & 17.3 \\
\hline 2813 & 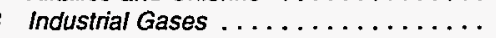 & 51 & 8,633 & 0 & 1 & 16 & 1 & 0 & 0 & 5 & 11.6 \\
\hline 2819 & Industrial Inorganic Chemicals, nec .... & 189 & W & w & 217 & 103 & 49 & 558 & $w$ & 12 & 10.0 \\
\hline 2821 & Plastics Materials and Resins ........ & 207 & 10,213 & $w$ & 65 & 113 & 34 & W & 0 & 38 & 5.5 \\
\hline 2822 & Synthetic Aubber ............... & 104 & W & $w$ & W & 40 & 9 & 0 & 0 & W & 13.2 \\
\hline 2823 & Cellulosic Manmade Fibers . . . . . . . . . & 31 & $W$ & 0 & 21 & $W$ & 1 & 1,202 & 0 & 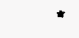 & 27.4 \\
\hline 2824 & Organic Fibers, Noncellulosic . . . . . . . & 98 & 6,881 & $w$ & W & $w$ & 38 & 1,558 & 0 & $W$ & 3.9 \\
\hline 2865 & Cyclic Crudes and Intermediates ....... & 125 & 3,250 & w & 18 & 73 & 72 & W & 0 & W & 12.8 \\
\hline 2869 & Industrial Organic Chemicals, nec ..... & 1,076 & 10,242 & 338 & Q & 580 & 776 & 2,679 & 0 & 378 & 9.3 \\
\hline 2873 & Nitrogenous Fertilizers . . . . . . . . . & 192 & 1,865 & 0 & 14 & 178 & 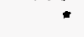 & 0 & 0 & 2 & 25.7 \\
\hline 2874 & Phosphatic Fertilizers . . . . . . . . . & 29 & 1,441 & 250 & 142 & 15 & 1 & $w$ & 0 & $W$ & 3.2 \\
\hline 29 & Petroleum and Coal Products ........ & 1,626 & 13,878 & 2,084 & W & 573 & 2,545 & W & W & 961 & 4.9 \\
\hline 2911 & Petroleum Refining . . . . . . . . . . . . & 1,608 & 13,395 & 2,021 & W & 561 & 2,450 & $w$ & 0 & 961 & 3.3 \\
\hline 30 & Rubber and Misc. Plastics Products . . . . . & 88 & 12,327 & 532 & 241 & 35 & 240 & 61 & 0 & 3 & 12.8 \\
\hline 3011 & Tires and inner Tubes . . . . . . . . . . . . & $W$ & 2,855 & $W$ & $w$ & 13 & 53 & W & 0 & 1 & 4.3 \\
\hline 308 & Miscellaneous Plastics Products, nec . . . & 48 & 7,801 & 156 & W & 16 & 111 & W & 0 & $\mathbf{Q}$ & 21.6 \\
\hline 31 & Leather and Leather Products ........ & 2 & 258 & 26 & 9 & $*$ & 3 & 0 & 0 & $\overline{\mathbf{Q}}$ & 25.1 \\
\hline 32 & Stone, Clay and Glass Products . . . . . & 323 & 11,866 & 174 & 1,373 & 155 & $W$ & 3,899 & W & $\vec{W}$ & 11.4 \\
\hline 3211 & Flat Glass $\ldots \ldots \ldots \ldots \ldots \ldots$ & 22 & 771 & 0 & 7 & 19 & 9 & 0 & 0 & * & 4.1 \\
\hline 3221 & Glass Containers . . . . . . . . . & 29 & 1,262 & $w$ & W & $w$ & 23 & 0 & 0 & * & 8.3 \\
\hline 3229 & Pressed and Blown Glass, nec. . . . . . . & $W$ & 1,598 & 1 & $w$ & $w$ & 15 & 0 & 0 & * & 8.9 \\
\hline 3241 & Cement, Hydraulic .............. & 109 & 3,518 & 65 & 167 & 21 & 4 & 2,589 & 150 & 12 & 15.6 \\
\hline 3274 & Lime $\ldots \ldots \ldots \ldots \ldots \ldots \ldots$ & 37 & 405 & 0 & 70 & W & - & 1,155 & 0 & W & 23.0 \\
\hline 3296 & Mineral Wool .................... & 16 & 936 & W & 1 & 11 & 16 & 0 & $w$ & $\because$ & 1.4 \\
\hline 33 & Primary Metal Industries . . . . . . . . . & 543 & 47,291 & 1,442 & 485 & $w$ & 235 & 433 & 4,418 & W & 5.7 \\
\hline 3312 & Blast Fumaces and Steel Mills . . . . . . . . & 317 & 10,744 & 1,437 & W & $w$ & 15 & W & 4,137 & w & 6.1 \\
\hline 3313 & Electrometalurgical Products .......... & W & W & 0 & 7 & * & 0 & w & 0 & 1 & 12.8 \\
\hline 3321 & Gray and Ductile Iron Foundries ..... & 21 & 1,622 & * & 56 & $w$ & 46 & $\mathbf{Q}$ & $w$ & $\cdot$ & 11.7 \\
\hline 3331 & Primary Copper . . . . . . . . . . . . & W & 200 & W & 5 & $w$ & 1 & $\overline{0}$ & 0 & * & 1.1 \\
\hline 3334 & Primary Aluminum ............. & 93 & 24,240 & 0 & W & $w$ & 9 & 0 & 0 & * & 4.1 \\
\hline 3339 & Primary Nonferrous Metals, nec . . . . . . . & 15 & 1,694 & 0 & 9 & $w$ & $W$ & $W$ & 0 & $w$ & 3.6 \\
\hline 3353 & Aluminum Sheet, Plate, and Foil ....... & 25 & W & 0 & 26 & 17 & 22 & W & 0 & $\because$ & 1.8 \\
\hline 34 & Fabricated Metal Products . . . . . . . . . & 81 & 8,886 & $Q$ & 377 & 42 & 380 & 0 & 23 & 2 & 21.1 \\
\hline 35 & Industrial Machinery and Equipment ..... & 57 & 8,129 & 42 & 125 & 26 & $W$ & $\mathbf{Q}$ & $\mathbf{Q}$ & $\vec{w}$ & 15.8 \\
\hline 357 & Computer and Office Equipment ...... & 4 & 800 & 3 & Q & 1 & $*$ & 0 & 0 & $*$ & 22.2 \\
\hline 36 & Electronic and Other Electric Equipment .. & 67 & 10,742 & 58 & 44 & 26 & 153 & 76 & 0 & * & 13.9 \\
\hline 37 & Transportation Equipment . . . . . . . . & w & 8,503 & W & 307 & 27 & 132 & W & 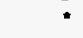 & w & 8.9 \\
\hline 3711 & Motor Vehicles and Car Bodies ....... & 35 & 2,362 & 73 & 19 & 12 & 28 & $w$ & 0 & $W$ & 5.3 \\
\hline 3714 & Motor Vehicle Parts and Accessories ... & 13 & 1,919 & 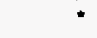 & 2 & $w$ & 41 & $w$ & 0 & $\cdot$ & 13.8 \\
\hline 38 & Instruments and Related Products ...... & 17 & 3,285 & $Q$ & 45 & 5 & 6 & 0 & 0 & $*$ & 18.4 \\
\hline 3841 & Surgical and Medical Instruments .. & 2 & 331 & 0 & 11 & * & 1 & 0 & 0 & $\bullet$ & 21.3 \\
\hline 39 & Misc. Manufacturing Industries . . . . . . . . & $\bar{W}$ & 1,168 & 28 & $Q$ & $w$ & 25 & 0 & 0 & W & 23.4 \\
\hline & Total $\ldots \ldots \ldots \ldots \ldots \ldots \ldots$ & 7,507 & 291,819 & 23,114 & 8,014 & 2,896 & W & 22,514 & W & 2,693 & 3.3 \\
\hline
\end{tabular}

See footnotes at end of table. 
Table A4. Total Inputs of Energy for Heat, Power, and Electricity Generation by Census Region, Industry Group, and Selected Industries, 1991: Part 1 (Continued) (Estimates in Btu or Physical Units)

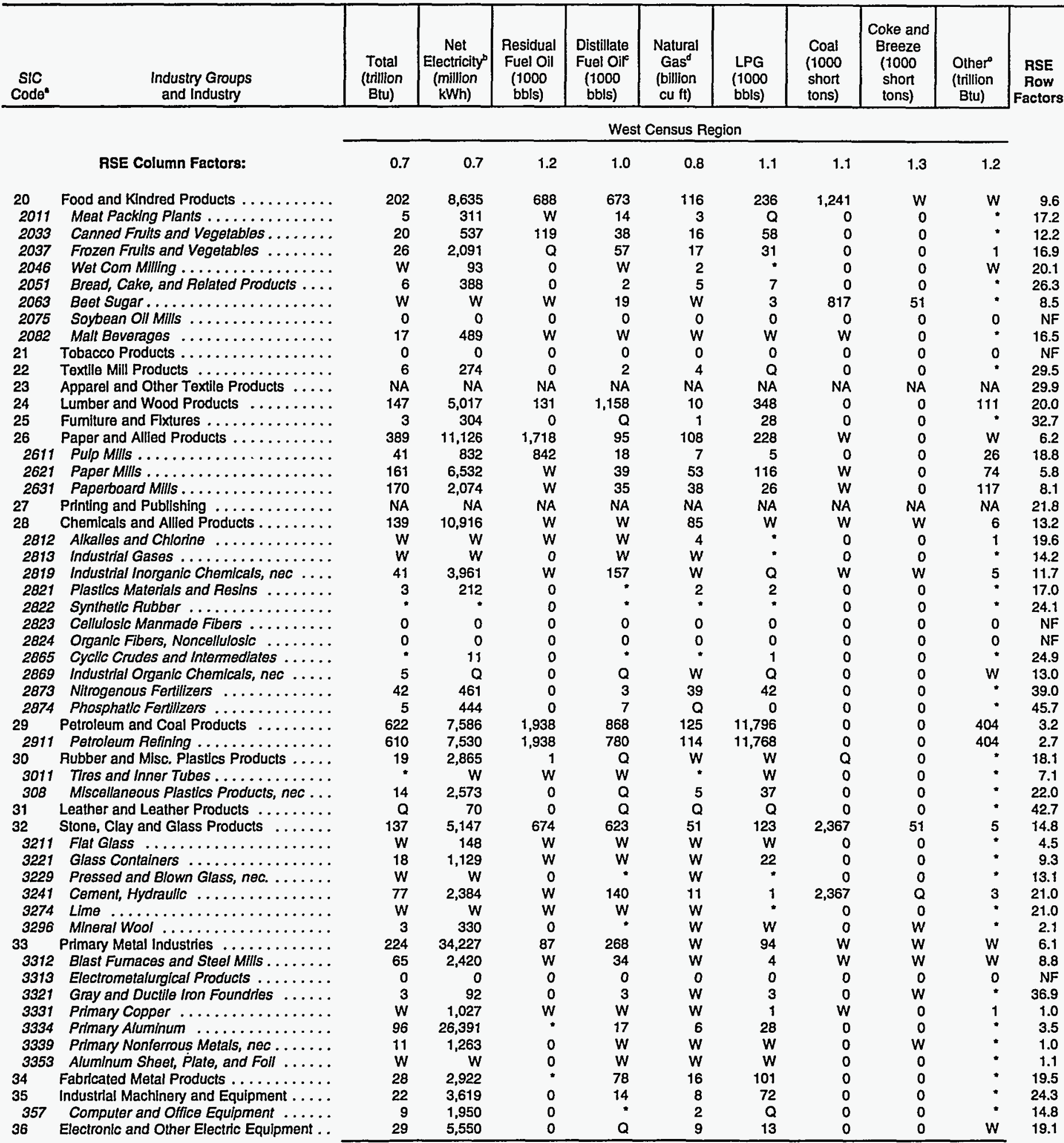

See footnotes at end of table. 
Table A4. Total Inputs of Energy for Heat, Power, and Electricity Generation by Census Region, Industry Group, and Selected Industries, 1991: Part 1 (Continued) (Estimates in Btu or Physical Units)

\begin{tabular}{|c|c|c|c|c|c|c|c|c|c|c|c|}
\hline \multirow[t]{3}{*}{$\begin{array}{c}\text { SIC } \\
\text { Code* }\end{array}$} & $\begin{array}{l}\text { Industry Groups } \\
\text { and Industry }\end{array}$ & $\begin{array}{c}\text { Total } \\
\text { (trillion } \\
\text { Btu) }\end{array}$ & $\begin{array}{c}\text { Net } \\
\text { Electricity } \\
\text { (million } \\
\mathrm{kWh} \text { ) }\end{array}$ & $\begin{array}{c}\text { Residual } \\
\text { Fuel Oil } \\
\text { (1000 } \\
\text { bbls) }\end{array}$ & $\begin{array}{c}\text { Distillate } \\
\text { Fuel Oif } \\
\text { (1000 } \\
\text { bbls) }\end{array}$ & $\begin{array}{c}\text { Natural } \\
\text { Gas }^{d} \\
\text { (billion } \\
\text { cu ft) }\end{array}$ & $\begin{array}{c}\text { LPG } \\
(1000 \\
\text { bbls })\end{array}$ & $\begin{array}{l}\text { Coal } \\
(1000 \\
\text { short } \\
\text { tons) }\end{array}$ & $\begin{array}{c}\text { Coke and } \\
\text { Breeze } \\
(1000 \\
\text { short } \\
\text { tons) }\end{array}$ & $\begin{array}{c}\text { Other } \\
\text { (trillion } \\
\text { Btu) }\end{array}$ & $\begin{array}{c}\text { RSE } \\
\text { Row } \\
\text { Factors }\end{array}$ \\
\hline & \multirow[b]{2}{*}{ RSE Column Factors: } & \multicolumn{10}{|c|}{ West Census Region } \\
\hline & & 0.7 & 0.7 & 1.2 & 1.0 & 0.8 & 1.1 & 1.1 & 1.3 & 1.2 & \\
\hline 37 & Transportation Equipment . . . . . . . . . & 47 & 7,096 & W & 129 & 19 & $W$ & 0 & 0 & $w$ & 11.9 \\
\hline 3711 & Motor Vehicles and Car Bodies . . . . . . & $w$ & $W$ & 0 & $W$ & 1 & 3 & 0 & 0 & $\bullet$ & 6.8 \\
\hline 3714 & Motor Vehicle Parts and Accessories ... & 3 & 243 & $\mathbf{Q}$ & 2 & 2 & 7 & 0 & 0 & - & 21.6 \\
\hline 38 & Instruments and Related Products . . . . . & 17 & 3,244 & 4 & 9 & 5 & 6 & 0 & 0 & - & 14.9 \\
\hline 3841 & Surgical and Medical lnstruments ..... & 1 & 222 & 0 & 3 & $\cdot$ & 2 & 0 & 0 & - & 27.4 \\
\hline 39 & Misc. Manufacturing Industries . . . . . . . . & 2 & 376 & 0 & 1 & 1 & 8 & 0 & 0 & - & 26.0 \\
\hline & Total $\ldots \ldots \ldots \ldots \ldots \ldots \ldots$ & 2,052 & 111,741 & 5,344 & 4,180 & 640 & 13,345 & 4,274 & 1,053 & 780 & 4.9 \\
\hline
\end{tabular}

- See Appendices B and $F$ for descriptions of the Standard Industrial Classification system.

- "Net Electricity" is obtained by summing purchases, transfers in, and generation from noncombustible renewable resources, minus quantitles sold and transferred

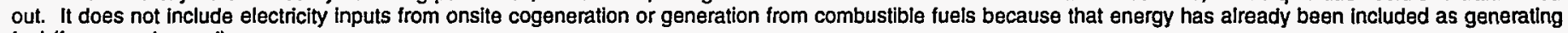
fuel (for example, coal).

c "Distillate Fuel OIl" includes Nos. 1, 2, and 4 fuel oils and Nos. 1, 2, and 4 diesel fuels.

"Natural Gas" includes natural gas obtained from utilities, transmission pipelines, and any other supplier(s) such as brokers and producers.

- "Other" includes net steam (the sum of purchases, generation from renewables, and net transfers), and other energy that respondents indicated was used to produce heat and power.

NF=No applicable RSE row/column factor.

- Estimate less than 0.5 . Data are included in higher level totals.

$W=$ Withheid to avoid disclosing data for individual establishments. Data are included in higher level totals.

$Q=$ Withheld because Relative Standard Error is greater than 50 percent. Data are included in higher level tolals.

NA=Not available. Data are included in higher level totals.

Notes: - To obtain a RSE percentage for any table cell, multiply the cell's corresponding RSE column and RSE row factors. - Totals may not equal sum of components because of independent rounding. - The estimates presented in this table are for the total consumption of energy for the production of heat and power, regardless of where the energy was produced. Specifically, the estimates include the quantities of energy that were originally produced offsite and purchased by or transferred to the establishment, plus those that were produced onsite from other energy or input materials not classified as energy, or were extracted froin captlive (onsite) mines or wells.

Source: Energy Information Administration, Office of Energy Markets and End Use, Energy End Use and Integrated Statistics Division, Form EIA-846, "1991 Manufacturing Energy Consumption Survey," and Office of Oil and Gas, Petroleum Supply Division, Form ElA-810, "Monthly Refinery Report" for 1991. 
Table A4. Total Inputs of Energy for Heat, Power, and Electricity Generation by Census Region, Industry Group, and Selected Industries, 1991: Part 2 (Estimates in Trillion Btu)

\begin{tabular}{cc|c|c|c|c|c|c|c|c|c|c|}
\hline $\begin{array}{c}\text { SIC } \\
\text { Code }\end{array}$ & $\begin{array}{c}\text { Industry Groups } \\
\text { and Industry }\end{array}$ & Total & $\begin{array}{c}\text { Net } \\
\text { Electricity }\end{array}$ & $\begin{array}{l}\text { Residual } \\
\text { Fuel Oll }\end{array}$ & $\begin{array}{c}\text { Distillate } \\
\text { Fuel Oif }\end{array}$ & $\begin{array}{c}\text { Natural } \\
\text { Gas }^{\circ}\end{array}$ & LPG & $\begin{array}{c}\text { RSE } \\
\text { Row } \\
\text { Coal }\end{array}$ & $\begin{array}{c}\text { Coke and } \\
\text { Breeze }\end{array}$ & $\begin{array}{c}\text { Factors } \\
\text { Othere }\end{array}$ \\
\hline
\end{tabular}

Total United States

\begin{tabular}{|c|c|c|c|c|c|c|c|c|c|c|c|}
\hline & & & & & & & & & & & \\
\hline & RSE Column Factors: & 0.6 & 0.6 & 1.3 & 1.3 & 0.7 & 1.2 & 1.2 & 1.6 & 1.2 & \\
\hline 20 & Food and Kindred Products . . . . . . . . . & 953 & 169 & 27 & 17 & 512 & 5 & 154 & w & w & 7.2 \\
\hline 2011 & Meat Packing Plants . . . . . . . . . . & 49 & 12 & 1 & 1 & 32 & 1 & 1 & 0 & 2 & 9.8 \\
\hline 2033 & Canned Frults and Vegetables . . . . . . . . & 44 & 5 & 2 & 1 & 36 & $\bullet$ & Q & 0 & $\bullet$ & 10.4 \\
\hline 2037 & Frozen Fruits and Vegetables . . . . . . & 40 & 10 & 2 & * & 26 & $\star$ & 0 & 0 & 1 & 14.7 \\
\hline 2046 & Wot Com Milling . . . . . . . . . . . & 140 & 14 & $\bullet$ & $*$ & 52 & * & 68 & W & W & 11.7 \\
\hline 2051 & Bread, Cake, and Rolated Products . . . . & 32 & 8 & $\bullet$ & 1 & 23 & - & 0 & 0 & * & 12.4 \\
\hline 2063 & Boot Sugar . . . . . . . . . . . . . & 67 & 1 & $W$ & $\bullet$ & 19 & - & 43 & $w$ & $\bullet$ & 5.5 \\
\hline 2075 & Soyboan Oll Mills . . . . . . . . . . . . . & 50 & 6 & 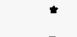 & $\bullet$ & 24 & $\cdot$ & 13 & 0 & 7 & 3.5 \\
\hline 2082 & Mall Beverages . . . . . . . . . . . & 50 & 8 & 3 & $\cdot$ & 23 & $:$ & 16 & 0 & 1 & 10.7 \\
\hline 21 & Tobacco Products . . . . . . . . . . . . . . & 24 & 3 & 1 & $*$ & 4 & * & 15 & 0 & $\bullet$ & 6.7 \\
\hline 22 & Textlle Mill Products $\ldots \ldots \ldots \ldots \ldots \ldots$ & 273 & 101 & 12 & 6 & 108 & 2 & 31 & 0 & 13 & 7.2 \\
\hline 23 & Apparel and Other Textlle Products ..... & 44 & 19 & $\mathbf{Q}$ & 1 & 19 & 1 & 2 & 0 & 1 & 17.9 \\
\hline 24 & Lumber and Wood Products ......... & 423 & 61 & 2 & 14 & 41 & 4 & 2 & 0 & 300 & 14.3 \\
\hline 25 & Furniture and Fixtures $\ldots \ldots \ldots \ldots \ldots$ & 67 & 17 & 1 & 1 & 19 & 1 & 4 & 0 & 25 & 20.3 \\
\hline 26 & Paper and Allled Products ........... & 2,472 & 201 & 156 & 9 & 548 & W & 296 & $W$ & 1,257 & 4.3 \\
\hline 2611 & Pulp MIlls $\ldots \ldots \ldots \ldots \ldots \ldots \ldots$ & 300 & 9 & 28 & 1 & 32 & 1 & 7 & 0 & 221 & 14.3 \\
\hline 2621 & Paper Mlls ................. & 1,204 & 112 & 85 & W & 260 & 2 & 193 & $W$ & 548 & 3.0 \\
\hline 2631 & Paperboard MIlls ............. & 832 & 35 & $w$ & 1 & 185 & - & W & 0 & 480 & 4.6 \\
\hline 27 & Printing and Publlshing $\ldots \ldots \ldots \ldots$ & 108 & 53 & $\cdot$ & 2 & 48 & 1 & 0 & 0 & 4 & 12.8 \\
\hline 28 & Chemicals and Alled Products . . . . . . . . & 3,040 & 440 & 48 & 12 & 1,669 & 4 & 253 & 3 & 611 & 5.2 \\
\hline 2812 & Alkalles and Chlorine ............ & 160 & 37 & W & $\bullet$ & W & $\bullet$ & W & 0 & 21 & 15.7 \\
\hline 2813 & Industrial Gases $\ldots \ldots \ldots \ldots \ldots \ldots$ & 91 & 61 & 0 & " & 25 & $\mathbf{Q}$ & 0 & 0 & 5 & 12.2 \\
\hline 2819 & Industrial Inorganic Chemicals, nec . . . . & 311 & 127 & 4 & 3 & 140 & $\bullet$ & 17 & 3 & 17 & 8.4 \\
\hline 2821 & Plastics Materials and Resins ....... & 288 & 50 & 4 & 1 & 151 & * & 24 & 0 & 57 & 5.6 \\
\hline 2822 & Synthollc Rubber $\ldots \ldots \ldots \ldots \ldots$ & 112 & 6 & $*$ & 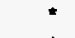 & 44 & * & W & 0 & W & 14.0 \\
\hline 2823 & Colluloslc Manmade Fibers . . . . . . . . & 31 & $W$ & 0 & $\bullet$ & $W$ & - & 27 & 0 & * & 27.3 \\
\hline 2824 & Organic Fibers, Noncellulosic ....... & 98 & 24 & W & 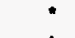 & $\mathbf{W}$ & $\bullet$ & 35 & 0 & $W$ & 3.9 \\
\hline 2865 & Cycllc Crudes and Intermediates ...... & 159 & 15 & 8 & 1 & 97 & - & W & 0 & $W$ & 11.8 \\
\hline 2869 & Industrial Organic Chemicals, nec ..... & 1,191 & 52 & 11 & 3 & 644 & 3 & 85 & 0 & 394 & 7.6 \\
\hline 2873 & Nitrogenous Fertilizers $\ldots . . . \ldots \ldots$ & 280 & 10 & 0 & * & 266 & $\bullet$ & $\mathbf{0}$ & 0 & 4 & 21.7 \\
\hline 2874 & Phosphatlc Fertllizers $\ldots \ldots \ldots \ldots \ldots$ & 34 & 6 & 2 & 1 & 19 & $*$ & W & 0 & W & 5.7 \\
\hline 29 & Petroleum and Coal Products $\ldots \ldots \ldots$ & 2,987 & 105 & 87 & 21 & 838 & 63 & $W$ & $w$ & 1,869 & 4.6 \\
\hline 2911 & Petroleum Rellning . . . . . . . . . . . & 2,893 & 99 & 65 & 9 & 792 & 60 & 3 & 0 & 1,864 & 3.5 \\
\hline 30 & Rubber and Mlsc. Plastics Products ..... & 237 & 116 & 8 & 3 & 96 & 3 & 7 & 0 & 5 & 10.3 \\
\hline 3011 & Tires and Inner Tubes ............. & 42 & 14 & 3 & $\because$ & 21 & $\bullet$ & 2 & 0 & 1 & 3.6 \\
\hline 308 & Miscellaneous Plastics Products, nec ... & 152 & 87 & 3 & W & 53 & 1 & 3 & 0 & $W$ & 14.5 \\
\hline 31 & Leather and Leather Products ........ & 12 & 3 & 1 & 1 & 5 & $\bullet$ & $\mathbf{Q}$ & 0 & 1 & 25.2 \\
\hline 32 & Stone, Clay and Glass Products $\ldots \ldots \ldots$ & 894 & 105 & 8 & 19 & 380 & 2 & 293 & 9 & 76 & 7.6 \\
\hline 3211 & Flat Glass $\ldots \ldots \ldots \ldots \ldots \ldots$ & 49 & 5 & W & 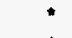 & 42 & $*$ & $\bullet$ & 0 & $W$ & 3.4 \\
\hline 3221 & Glass Containers ............ & 85 & 14 & 2 & $\star$ & 69 & * & 0 & 0 & * & 5.4 \\
\hline 3229 & Pressed and Blown Glass, nec. . . . . . & $W$ & 10 & 1 & 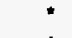 & W & • & 0 & 0 & - & 8.3 \\
\hline 3241 & Cement, Hydraulle $\ldots \ldots \ldots \ldots$ & 329 & 32 & 1 & 4 & 39 & $\bullet$ & 195 & 6 & 52 & 11.2 \\
\hline 3274 & $\operatorname{LIm\theta } \ldots \ldots \ldots \ldots \ldots \ldots \ldots \ldots$ & 117 & 5 & W & 1 & 8 & $\mathbf{Q}$ & 88 & $W$ & 13 & 29.4 \\
\hline 3296 & Mineral Wool $\ldots \ldots \ldots \ldots \ldots \ldots$ & 41 & 10 & $W$ & - & 29 & $\cdot$ & $\cdot$ & W & " & 1.5 \\
\hline 33 & Primary Metal Industries $\ldots \ldots \ldots \ldots$ & 2,292 & 499 & 33 & 11 & 686 & 3 & 46 & 563 & 451 & 4.3 \\
\hline 3312 & Blast Fumaces and Steol Mills . . . . . . . & 1,569 & 130 & 31 & 5 & 399 & $*$ & 24 & 538 & 440 & 3.9 \\
\hline 3313 & Electrometalurgical Products . . . . . . . . & 31 & 14 & 0 & $\star$ & 1 & W & $W$ & $W$ & w & 8.7 \\
\hline 3321 & Gray and Ducille Iron Foundries ...... & 74 & 22 & $\therefore$ & 1 & 28 & * & $\star$ & 21 & 1 & 11.4 \\
\hline 3331 & Primary Copper . . . . . . . . . . & 22 & 4 & W & W & 15 & - & $W$ & 0 & 1 & 1.1 \\
\hline 3334 & Primary Aluminum $\ldots \ldots \ldots \ldots$ & 252 & 230 & $*$ & 1 & 21 & - & 0 & 0 & 1 & 3.3 \\
\hline 3339 & Primary Nonferrous Metals, nec . . . . . . & 42 & 15 & $*$ & * & 17 & $\bullet$ & $W$ & w & $w$ & 1.7 \\
\hline 3353 & Aluminum Sheet, Plate, and Foll ...... & 60 & 15 & 0 & $\star$ & 43 & $\bullet$ & W & 0 & W & 1.4 \\
\hline 34 & Fabrlcated Metal Products . . . . . . . . . . & 305 & 102 & 3 & 6 & 174 & 4 & 5 & W & W & 11.4 \\
\hline 35 & Industrial Machinery and Equipment . . . . . & 235 & 101 & 3 & 4 & 109 & 2 & 11 & 1 & 5 & 11.5 \\
\hline 357 & Computer and Office Equipment . . . . . & 21 & 15 & $\cdot$ & $\cdot$ & 6 & - & 0 & 0 & • & 15.9 \\
\hline 38 & Electronic and Other Electric Equlpment .. & 196 & 102 & 4 & 2 & 79 & 1 & $W$ & $\cdot$ & W & 10.2 \\
\hline 37 & Transportation Equlpment $\ldots \ldots \ldots \ldots$ & 333 & 118 & 12 & 7 & 132 & 2 & 33 & 1 & 27 & 4.9 \\
\hline 3711 & Motor Vehicles and Car Bodles . ..... . & 105 & 26 & 3 & $\bullet$ & 45 & $\bullet$ & W & W & 18 & 3.8 \\
\hline 3714 & Motor Vehicle Parts and Accessories ... & 99 & 37 & $\star$ & 1 & 41 & 1 & W & W & $w$ & 7.1 \\
\hline 38 & Instruments and Related Products . . . . . & 98 & 42 & 3 & W & 25 & $\mathbf{Q}$ & W & 0 & W & 13.4 \\
\hline 3841 & Surgical and Medlcal Instruments .... & 6 & 4 & $\bullet$ & $*$ & 2 & $\bullet$ & 0 & 0 & 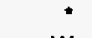 & 15.4 \\
\hline 39 & Mlsc. Manufacturing Industries . . . . . . . & 31 & 12 & 1 & $W$ & 15 & W & 1 & 0 & W & 15.4 \\
\hline & $\ldots \ldots \ldots \ldots \ldots$ & 15,027 & 2,370 & 414 & 139 & 5,506 & 105 & 1,184 & 583 & 4,726 & 2.8 \\
\hline
\end{tabular}

See tootnotes at end of table. 
Table A4. Total Inputs of Energy for Heat, Power, and Electricity Generation by Census Region, Industry Group, and Selected Industries, 1991: Part 2 (Continued) (Estimates in Trillion Btu)

\begin{tabular}{|c|c|c|c|c|c|c|c|c|c|c|c|}
\hline \multirow[t]{3}{*}{$\begin{array}{l}\text { SIC } \\
\text { Code }^{\circ}\end{array}$} & $\begin{array}{l}\text { Industry Groups } \\
\text { and Industry }\end{array}$ & Total & $\mid \begin{array}{c}\text { Net } \\
\text { Electricity }\end{array}$ & $\begin{array}{c}\text { Residual } \\
\text { Fuel Oil }\end{array}$ & $\begin{array}{l}\text { Distillate } \\
\text { Fuel Oif }\end{array}$ & $\begin{array}{l}\text { Natural } \\
\text { Gas }^{d}\end{array}$ & LPG & Coal & $\begin{array}{c}\text { Coke and } \\
\text { Breeze }\end{array}$ & Other & $\begin{array}{c}\text { RSE } \\
\text { Row } \\
\text { Factors }\end{array}$ \\
\hline & & \multicolumn{10}{|c|}{ Northeast Census Region } \\
\hline & RSE Column Factors: & 0.7 & 0.7 & 1.0 & 1.2 & 0.8 & 1.2 & 1.3 & 1.3 & 1.1 & \\
\hline 20 & Food and Kindred Products & 79 & 18 & 7 & 5 & 42 & 1 & 2 & 0 & 3 & 13.8 \\
\hline 2011 & Meat Packing Plants ..... & 1 & 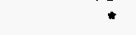 & w & * & 1 & $\mathbf{Q}$ & 0 & 0 & : & 23.8 \\
\hline 2033 & Canned Fruits and Vegetables ......... & 6 & 1 & 1 & - & 4 & 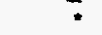 & $\mathbf{Q}$ & 0 & • & 17.2 \\
\hline 2037 & Frozen Fruits and Vegetables ...... & 1 & : & 1 & * & * & $\mathbf{Q}$ & 0 & 0 & • & 32.8 \\
\hline 2046 & Wet Corn Milling . ....... & • & - & w & w & - & - & 0 & 0 & - & 23.6 \\
\hline 2051 & Bread, Cake, and Related Products. & 7 & 1 & $\because$ & $w$ & $w$ & $\cdot$ & 0 & 0 & • & 17.9 \\
\hline 2063 & Beet Sugar $\ldots \ldots \ldots \ldots \ldots \ldots \ldots$ & 0 & 0 & 0 & 0 & 0 & 0 & 0 & 0 & 0 & NF \\
\hline 2075 & Soybean Oil Mills ............. & 0 & 0 & 0 & 0 & 0 & 0 & 0 & 0 & 0 & NF \\
\hline 2082 & Malt Beverages .... & 8 & 2 & $w$ & : & 4 & : & $\mathbf{w}$ & 0 & : & 16.4 \\
\hline 21 & Tobacco Products ... & NA & NA & NA & NA & NA & NA & NA & NA & NA & 25.3 \\
\hline 22 & Textile Mill Products & 27 & 5 & 5 & 3 & 11 & 1 & •* & 0 & 3 & 19.5 \\
\hline 23 & Apparel and Other Textile Products .. & 5 & 2 & : & - & 2 & $\mathbf{Q}$ & 0 & 0 & : & 29.7 \\
\hline 24 & Lumber and Wood Products . . . . . . . & NA & $\mathrm{NA}$ & NA & NA & NA & NA & NA & NA & NA & 36.8 \\
\hline 25 & Fumiture and Fixtures ..... & 7 & 2 & $\mathrm{Q}$ & $\because$ & 2 & * & 0 & 0 & 2 & 30.2 \\
\hline 26 & Paper and Allied Products . & w & 27 & 72 & 4 & 37 & $w$ & w & 0 & $w$ & 6.1 \\
\hline 2611 & Pulp Mills .......... & 12 & 1 & 2 & - & 0 & - & 0 & 0 & 9 & 36.7 \\
\hline 2621 & Paper Mills .... & 228 & 16 & 61 & w & 19 & 1 & w & 0 & 96 & 4.3 \\
\hline 2631 & Paperboard Mills. & 16 & 2 & w & $Q$ & 6 & • & $w$ & 0 & 1 & 15.4 \\
\hline 27 & Printing and Publishing & 23 & 11 & $\because$ & $\overline{1}$ & 9 & • & 0 & 0 & $i$ & 25.5 \\
\hline 28 & Chemicals and Allied Products . . . . . . . & 135 & 32 & 19 & w & 59 & • & w & 0 & 15 & 9.0 \\
\hline 2812 & Alkalies and Chlorine ............. & * & W & 0 & 0 & 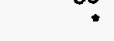 & 0 & 0 & 0 & 0 & 36.5 \\
\hline 2813 & Industrial Gases ................... & 5 & 5 & 0 & * & - & * & 0 & 0 & 0 & 11.9 \\
\hline 2819 & Industrial Inorganic Chemicals, nec ..... & 10 & 2 & 2 & - & 6 & * & 0 & 0 & * & 22.7 \\
\hline 2821 & Plastics Materials and Resins ......... & 20 & 4 & 3 & 1 & 8 & * & $w$ & 0 & w & 9.2 \\
\hline 2822 & Synthetic Rubber ............... & $w$ & $w$ & $w$ & * & $w$ & * & 0 & 0 & $\because$ & 26.0 \\
\hline 2823 & Cellulosic Manmade Fibers . . . . . . . & 0 & 0 & 0 & 0 & 0 & 0 & 0 & 0 & 0 & $\mathrm{NF}$ \\
\hline 2824 & Organic Fibers, Noncellulosic . . . . . . . & • & * & $\mathbf{Q}$ & w & • & • & 0 & 0 & 0 & 14.8 \\
\hline $28<4$ & Cyclic Crudes and Intemediates .... & 12 & 1 & $w$ & $w$ & 7 & * & $w$ & 0 & 1 & 21.0 \\
\hline 2869 & Industrial Organic Chemicals, nec ...... & 38 & 10 & 9 & $\because$ & w & • & 0 & 0 & $\dot{w}$ & 8.7 \\
\hline 2873 & Nitrogenous Fertilizers .............. & . & 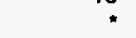 & 0 & * & $\because$ & • & 0 & 0 & $\because$ & 46.4 \\
\hline 2874 & Phosphatic Fertilizers . .......... & 0 & 0 & 0 & 0 & 0 & 0 & 0 & 0 & 0 & NF \\
\hline 29 & Petroleum and Coal Products . . . . . . . & 238 & 9 & 49 & 8 & 30 & 3 & 3 & 0 & 136 & 9.3 \\
\hline 2911 & Petroleum Refining .......... & 199 & 7 & 27 & w & 25 & 2 & w & 0 & 136 & 5.3 \\
\hline 30 & Rubber and Misc. Plastics Products ..... & 37 & 19 & 3 & 1 & w & $\bar{Q}$ & 2 & 0 & 1 & 17.9 \\
\hline 3011 & 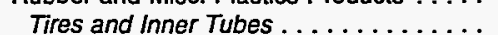 & 2 & 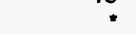 & * & $Q$ & 1 & 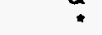 & 0 & 0 & : & 13.3 \\
\hline 308 & Miscellaneous Plastics Products, nec .... & 28 & 16 & 2 & $\mathrm{w}$ & 7 & - & $w$ & 0 & w & 23.6 \\
\hline 31 & Leather and Leather Products ......... & 4 & 1 & 1 & 1 & 1 & - & $\mathbf{a}$ & 0 & $\because$ & 28.5 \\
\hline 32 & Stone, Clay and Glass Products & 181 & 19 & 3 & 4 & 60 & w & 77 & w & w & 17.3 \\
\hline 3211 & Flat Glass ............ & w & $w$ & 0 & : & w & $w$ & * & 0 & $\because$ & 4.6 \\
\hline 3221 & Glass Containers... & 19 & 3 & 1 & * & 14 & $\because$ & 0 & 0 & - & 8.7 \\
\hline 3229 & Pressed and Blown Glass, nec. . . . . . . & $w$ & w & 1 & w & w & • & 0 & 0 & - & 10.3 \\
\hline 3241 & Cement, Hydraulic $\ldots \ldots \ldots \ldots \ldots \ldots$ & 54 & 5 & . & w & * & • & 33 & w & 14 & 17.8 \\
\hline 3274 & Lime $\ldots \ldots \ldots \ldots \ldots \ldots \ldots$ & $Q$ & $\mathbf{Q}$ & 0 & Q & - & $Q$ & $\mathbf{Q}$ & 0 & : & NF \\
\hline 3296 & Mineral Wool . . $\ldots \ldots \ldots \ldots \ldots$ & 4 & 1 & 0 & $\bar{w}$ & w & $\bar{w}$ & 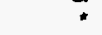 & W & • & 1.7 \\
\hline 33 & Primary Metal Industries & 358 & 61 & 5 & 2 & 105 & 1 & w & w & $w$ & 8.0 \\
\hline 3312 & Blast Fumaces and Steel Mills. & 270 & 27 & $w$ & 1 & 67 & * & 1 & $\ddot{w}$ & 124 & 6.8 \\
\hline 3313 & Electrometalumical Products . . . . . . . & w & $w$ & 0 & * & . & * & $w$ & $w$ & • & 12.9 \\
\hline 3321 & Gray and Ductile Iron Foundries & 5 & 1 & 0 & . & 2 & - & $\because$ & 2 & • & 16.5 \\
\hline 3331 & Primary Copper ............ & * & $\dot{w}$ & 0 & w & * & * & 0 & 0 & • & 1.1 \\
\hline 3334 & Primary Aluminum & w & $\ddot{w}$ & * & $\ddot{w}$ & w & w & 0 & 0 & - & 5.5 \\
\hline 3339 & Primary Nonferrous Metals, nec.. & w & 2 & * & w & 1 & • & $\mathbf{w}$ & 0 & - & 1.8 \\
\hline 3353 & Aluminum Sheet, Plate, and Foil ....... & w & 2 & 0 & w & $\mathbf{w}$ & - & 0 & 0 & • & 1.4 \\
\hline 34 & Fabricated Metal Products . . . . . . . . & 56 & 17 & 2 & 2 & 32 & 1 & : & 1 & 1 & 15.5 \\
\hline 35 & Industrial Machinery and Equipment . & 41 & 18 & 3 & 2 & 16 & 1 & 0 & 0 & 2 & 19.1 \\
\hline 357 & Computer and Office Equipment & 4 & 3 & 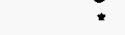 & 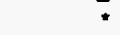 & 1 & : & 0 & 0 & • & 20.7 \\
\hline 36 & Electronic and Other Electric Equipment. & 42 & 22 & 3 & 2 & 14 & 1 & . & . & • & 16.2 \\
\hline 37 & Transportation Equipment . . . . . . . . . & W & 10 & 7 & 3 & 11 & w & w & 0 & w & 11.1 \\
\hline 3711 & Motor Vehicles and Car Bodies & $\dddot{w}$ & w & w & w & 1 & ? & 0 & 0 & $\because$ & 7.8 \\
\hline 3714 & Motor Vehicle Parts and Accessories & 8 & 3 & $w$ & $\because$ & w & - & $w$ & 0 & • & 11.8 \\
\hline & Instruments and Related Products ...... & 52 & 14 & 3 & w & w & Q & w & 0 & $w$ & 17.4 \\
\hline 3841 & Surgical and Medical Instruments & 2 & 1 & * & * & : & ? & 0 & 0 & : & 19.2 \\
\hline 39 & Misc. Manufacturing Industries . . . . & $w$ & 4 & 1 & w & w & w & 1 & 0 & - & 18.0 \\
\hline & Total ............... & 1,635 & 294 & 184 & 45 & 459 & $w$ & 167 & $w$ & 423 & 6.1 \\
\hline
\end{tabular}

See footnotes at end of table. 
Table A4. Total Inputs of Energy for Heat, Power, and Electricity Generation by Census Region, Industry Group, and Selected Industries, 1991: Part 2 (Continued) (Estimates in Trillion Btu)

\begin{tabular}{|c|c|c|c|c|c|c|c|c|c|c|c|}
\hline $\begin{array}{l}\text { SIC } \\
\text { Code }\end{array}$ & $\begin{array}{l}\text { Industry Groups } \\
\text { and Industry }\end{array}$ & Total & $\begin{array}{c}\text { Net } \\
\text { Electricity }\end{array}$ & $\begin{array}{l}\text { Residual } \\
\text { Fuel Oil }\end{array}$ & $\begin{array}{l}\text { Distillate } \\
\text { Fuel Oil }\end{array}$ & $\begin{array}{l}\text { Natural } \\
\text { Gas }^{\triangleleft}\end{array}$ & LPG & Coal & $\begin{array}{c}\text { Coke and } \\
\text { Breeze }\end{array}$ & Other & $\begin{array}{c}\text { RSE } \\
\text { Row } \\
\text { Factors }\end{array}$ \\
\hline
\end{tabular}

Midwest Census Region

\begin{tabular}{|c|c|c|c|c|c|c|c|c|c|c|c|}
\hline & & & & & & & & & & & \\
\hline & RSE Column Factors: & 0.6 & 0.6 & 1.5 & 1.3 & 0.7 & 1.2 & 1.1 & 1.4 & 1.0 & \\
\hline 20 & Food and Kindred Products . . . . . . . . . & 419 & 67 & 6 & 3 & 217 & 1 & 107 & w & $w$ & 8.6 \\
\hline 2011 & Meat Packing Plants . . . . . . . . . . . & 33 & 7 & 1 & $\star$ & 23 & $\bullet$ & 1 & 0 & 1 & 9.3 \\
\hline 2033 & Canned Fruils and Vegetables . . . . . . . . & 10 & 1 & 0 & * & 9 & • & 0 & 0 & - & 17.3 \\
\hline 2037 & Frozen Fruils and Vegetables . . . . . . & 4 & 1 & 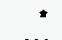 & • & 2 & $\cdot$ & 0 & 0 & $\cdot$ & 27.2 \\
\hline 2046 & Wet Com Mlling . . . . . . . . . . . & 122 & 11 & W & * & 46 & • & 61 & $W$ & $w$ & 13.5 \\
\hline 2051 & Bread, Cake, and Related Products .... & 9 & 2 & 0 & W & W & $\bullet$ & 0 & 0 & * & 16.4 \\
\hline 2063 & Beot Sugar . . . . . . . . . . . . & 34 & 1 & W & $\bullet$ & 6 & $\bullet$ & 24 & $w$ & $\cdot$ & 6.6 \\
\hline 2075 & Soybean Oll MIlls .............. & 36 & 4 & $*$ & $\bullet$ & 16 & $W$ & $W$ & 0 & W & 4.4 \\
\hline 2082 & Malt Beverages $\ldots \ldots \ldots \ldots \ldots \ldots$ & 11 & 2 & $\bullet$ & - & $W$ & $W$ & $W$ & 0 & $\cdot$ & 17.3 \\
\hline 21 & Tobacco Products . . . . . . . . . . . . & NA & NA & NA & NA & NA & NA & NA & NA & NA & 9.7 \\
\hline 22 & Textlle Mill Products $\ldots \ldots \ldots \ldots \ldots$ & NA & NA & NA & NA & NA & NA & NA & NA & NA & 23.9 \\
\hline 23 & Apparel and Other Textile Products .... . & NA & NA & NA & NA & NA & NA & NA & NA & NA & 31.7 \\
\hline 24 & Lumber and Wood Products $\ldots . . . \ldots$. & 57 & 10 & $\mathbf{Q}$ & $\mathbf{Q}$ & 11 & 1 & 1 & 0 & 32 & 27.3 \\
\hline 25 & Fumiture and Fixtures $\ldots \ldots \ldots \ldots \ldots$ & $W$ & 5 & $\cdot$ & * & W & $\cdot$ & $\mathbf{Q}$ & 0 & $\mathbf{Q}$ & 25.3 \\
\hline 26 & Paper and Allied Products . . . . . . . . . & W & 49 & 5 & 1 & 116 & 1 & 94 & W & W & 6.5 \\
\hline 2611 & Pulp Mllls $\ldots \ldots \ldots \ldots \ldots \ldots \ldots$ & 28 & 1 & 0 & * & 4 & $\mathbf{Q}$ & 4 & 0 & 20 & 33.4 \\
\hline 2621 & Paper MIlls . . . . . . . . . . . . & 209 & 27 & W & 1 & 59 & $\bullet$ & 63 & W & 54 & 5.0 \\
\hline 2631 & Paperboard Mills .............. & 70 & 7 & * & $\bullet$ & 24 & $\cdot$ & 26 & 0 & 13 & 13.3 \\
\hline 27 & Prlnting and Publishing $\ldots \ldots \ldots \ldots$ & 43 & 18 & $*$ & $\bullet$ & 23 & $\bullet$ & 0 & 0 & 2 & 17.9 \\
\hline 28 & Chemlcals and Allied Products . . . . . . . . & 398 & 115 & $W$ & $w$ & 186 & W & 63 & $\bullet$ & 29 & 10.1 \\
\hline 2812 & Alkalles and Chlorino ............ & W & $W$ & 0 & $w$ & $\cdot$ & $\bullet$ & 0 & 0 & $\bullet$ & 27.1 \\
\hline 2813 & Industral Gases . . . . . . . . . . & W & $W$ & 0 & $\mathbf{Q}$ & $W$ & $\mathbf{Q}$ & 0 & 0 & * & 13.6 \\
\hline 2819 & Industrlal Inorganic Chemicals, nec . . . . & 71 & $W$ & $W$ & 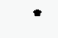 & $w$ & $\cdot$ & $W$ & $\cdot$ & - & 11.6 \\
\hline 2821 & Plastics Materials and Resins . . . . . . . & 58 & 11 & $W$ & $\cdot$ & 24 & • & $W$ & 0 & $w$ & 8.5 \\
\hline 2822 & Synthotic Rubber ............. & $\mathbf{W}$ & 1 & 0 & $W$ & W & 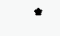 & $w$ & 0 & * & 19.7 \\
\hline 2823 & Cellulosic Manmado Fibers . . . . . . . . & 0 & 0 & 0 & 0 & 0 & 0 & 0 & 0 & 0 & NF \\
\hline 2824 & Organic Fibers, Noncellulosic . . . . . . & 0 & 0 & 0 & 0 & 0 & 0 & 0 & 0 & 0 & NF \\
\hline 2865 & Cyclic Crudes and Intermedlates . . . . . & 22 & 3 & $W$ & $w$ & 14 & $\cdot$ & $w$ & 0 & $\cdot$ & 14.6 \\
\hline 2869 & Industrial Organic Chemicals, nec . . . . . & 71 & 6 & $\bullet$ & $\bullet$ & 34 & $\cdot$ & 25 & 0 & 6 & 8.2 \\
\hline 2873 & Nitrogenous Fertillzers . . . . . . . . . & 46 & 2 & 0 & • & 42 & * & 0 & 0 & 2 & 35.7 \\
\hline 2874 & Phosphatic Fertilizers . . . . . . . . . . & • & * & 0 & $\bullet$ & $\bullet$ & • & 0 & 0 & * & 4.6 \\
\hline 29 & Petroleum and Coal Products ........ & 501 & 23 & 13 & W & 88 & 5 & $W$ & 0 & 368 & 5.4 \\
\hline 2911 & Petroleum Refining . ............ & 476 & 21 & 13 & W & 73 & 5 & W & 0 & 364 & 3.0 \\
\hline 30 & Rubber and Misc. Plastics Products .... . & 93 & 45 & 2 & $\cdot$ & 42 & 1 & 3 & 0 & 1 & 11.9 \\
\hline 3011 & Tres and Inner Tubes .............. & W & W & 1 & $\cdot$ & 7 & W & $W$ & 0 & $=$ & 4.1 \\
\hline 308 & Mlscellaneous Plastics Products, nec... & NA & NA & NA & NA & NA & NA & NA & NA & NA & 18.4 \\
\hline 31 & Leather and Leather Products . . . . . . . & 4 & 1 & $\bullet$ & $\bullet$ & 2 & $\bullet$ & 0 & 0 & 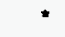 & 25.9 \\
\hline 32 & Stone, Clay and Glass Products ...... & 252 & 28 & $\bullet$ & 4 & 108 & - & 76 & 2 & 34 & 10.1 \\
\hline 3211 & Flat Glass $\ldots \ldots \ldots \ldots \ldots \ldots \ldots$ & 13 & W & 0 & W & 11 & W & 0 & 0 & $\cdot$ & 4.1 \\
\hline 3221 & Glass Contalners ............ & 20 & 3 & 0 & $\cdot$ & 17 & $\bullet$ & 0 & 0 & $\cdot$ & 7.6 \\
\hline 3229 & Pressed and Blown Glass, nec. . . . . . . & 14 & 2 & $\cdot$ & * & 12 & • & 0 & 0 & $\cdot$ & 6.7 \\
\hline 3241 & Cement, Hydraulic . . . . . . . . . . . & 89 & 8 & W & $w$ & 6 & * & 51 & 0 & 23 & 15.0 \\
\hline 3274 & $\operatorname{Lime} \ldots \ldots \ldots \ldots \ldots \ldots \ldots \ldots$ & 34 & 1 & 0 & $\star$ & 3 & * & 21 & $W$ & $W$ & 18.6 \\
\hline 3296 & Mineral Wool . . . . . . . . . . . . . . & 17 & 4 & $w$ & $W$ & $\mathbf{W}$ & * & 0 & $W$ & $\cdot$ & 1.2 \\
\hline 33 & Primary Metal Industries . . . . . . . . . . & 1,168 & 160 & 18 & 4 & 343 & 1 & 27 & 384 & 231 & 5.7 \\
\hline 3312 & Blast Fumaces and Steel Mills . . . . . . . . & 917 & 59 & 18 & $W$ & 224 & $\bullet$ & $W$ & 370 & 226 & 5.2 \\
\hline 3313 & Electrometalurglcal Products . . . . . . . & 19 & 8 & 0 & $\cdot$ & 1 & W & $W$ & $\bullet$ & W & 11.3 \\
\hline 3321 & Gray and Ductlle Iron Foundries ..... & 45 & 15 & * & $\mathbf{Q}$ & $\mathbf{W}$ & $\bullet$ & $W$ & W & 1 & 11.7 \\
\hline 3331 & Primary Copper . . . . . . . . . . & * & W & 0 & 0 & 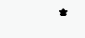 & 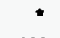 & 0 & 0 & 0 & 1.3 \\
\hline 3334 & Primary Aluminum $\ldots \ldots \ldots \ldots \ldots$ & $W$ & $W$ & 0 & W & 3 & $W$ & 0 & 0 & $\star$ & 5.1 \\
\hline 3339 & Primary Nonferrous Motals, nec . . . . . . . & W & 2 & 0 & $\bullet$ & 3 & $\bullet$ & 0 & $\mathbf{W}$ & $\star$ & 2.0 \\
\hline 3353 & Aluminum Shoet, Plate, and Foll . . . . . . & 24 & 5 & 0 & $W$ & 17 & $\mathbf{W}$ & 0 & 0 & W & 1.3 \\
\hline 34 & Fabricated Metal Products . . . . . . . . . & 139 & 44 & $\bullet$ & 1 & 81 & 2 & 5 & W & $W$ & 15.0 \\
\hline 35 & Industrlal Machinery and Equipment . . . . . & 115 & 43 & * & 1 & 58 & $\mathbf{W}$ & 11 & $\mathbf{Q}$ & W & 13.8 \\
\hline 357 & Computer and Offlce Equipment ...... & 5 & 3 & 0 & $\bullet$ & 2 & $\cdot$ & 0 & 0 & $\bullet$ & 24.0 \\
\hline 36 & Electronic and Other Electric Equipment .. & 59 & 24 & $\star$ & $\mathbf{W}$ & 29 & * & $W$ & 0 & $\mathbf{W}$ & 13.4 \\
\hline 37 & Transportation Equipment $\ldots \ldots \ldots \ldots$ & 171 & 55 & 2 & 2 & 74 & 1 & 27 & 1 & 9 & 6.1 \\
\hline 3711 & Motor Vehicles and Car Bodles . . . . . . . & 64 & 16 & $W$ & $*$ & 31 & * & $\mathbf{W}$ & $W$ & $W$ & 4.7 \\
\hline 3714 & Molor Vehicle Parts and Accessories ... & 76 & 27 & $w$ & 1 & 30 & $\bullet$ & $W$ & W & $\mathbf{W}$ & 8.1 \\
\hline 38 & Instruments and Related Products ...... & 11 & 6 & $\mathbf{Q}$ & $\mathbf{Q}$ & $W$ & $\bullet$ & $W$ & 0 & - & 19.4 \\
\hline 3841 & Surgical and Medlcal Instruments ..... & 1 & 1 & 0 & $*$ & - & $\bullet$ & 0 & 0 & $\bullet$ & 18.7 \\
\hline 39 & Misc. Manufacturing Industries . . . . . . . & 10 & 3 & $*$ & $\bullet$ & 5 & $*$ & * & 0 & $\mathbf{Q}$ & 22.0 \\
\hline & Total $\ldots \ldots \ldots \ldots \ldots \ldots \ldots$ & 3,833 & 700 & 51 & 23 & 1,404 & 14 & 420 & 392 & 829 & 3.7 \\
\hline
\end{tabular}

See footnotes at end of table. 
Table A4. Total Inputs of Energy for Heat, Power, and Electricity Generation by Census Region, Industry Group, and Selected Industries, 1991: Part 2 (Continued) (Estimates in Trillion Btu)

\begin{tabular}{|c|c|c|c|c|c|c|c|c|c|c|c|}
\hline $\begin{array}{c}\text { SIC } \\
\text { Code }\end{array}$ & $\begin{array}{l}\text { Industry Groups } \\
\text { and Industry }\end{array}$ & Total & $\begin{array}{c}\text { Net } \\
\text { Electricity }\end{array}$ & $\begin{array}{c}\text { Residual } \\
\text { Fuel Oil }\end{array}$ & $\begin{array}{l}\text { Distillate } \\
\text { Fuel Oif }\end{array}$ & $\begin{array}{c}\text { Natural } \\
\text { Gas }^{\circ}\end{array}$ & LPG & Coal & $\begin{array}{c}\text { Coke and } \\
\text { Breeze }\end{array}$ & Other & $\begin{array}{c}\text { RSE } \\
\text { Row } \\
\text { Factors }\end{array}$ \\
\hline & & \multicolumn{10}{|c|}{ South Census Region } \\
\hline & RSE Column Factors: & 0.6 & 0.6 & 1.3 & 1.1 & 0.7 & 1.2 & 1.1 & 1.6 & 1.1 & \\
\hline 20 & Food and Kindred Products & 254 & 54 & 10 & 5 & 134 & 2 & 17 & w & Q & 11.1 \\
\hline 2011 & Meat Packing Plants . & 10 & 3 & * & 1 & 5 & • & 0 & 0 & - & 16.9 \\
\hline 2033 & Canned Fruits and Vegetables . . . . . . . . & 7 & 1 & • & $*$ & 6 & * & 0 & 0 & * & 19.7 \\
\hline 2037 & Frozen Fruits and Vegetables . . . . . . . & 8 & 2 & 1 & * & 6 & • & 0 & 0 & - & 17.6 \\
\hline 2046 & Wet Com Milling .......... & w & 3 & 0 & - & 5 & * & 7 & 0 & w & 22.8 \\
\hline 2051 & Bread, Cake, and Related Products . . . . & 11 & 3 & 0 & - & 7 & * & 0 & 0 & - & 14.1 \\
\hline 2063 & Beet Sugar $\ldots \ldots \ldots \ldots \ldots \ldots$ & w & w & 0 & - & w & - & 0 & w & - & 16.8 \\
\hline 2075 & Soybean Oil Mills . . . . . . . . . . & 15 & 2 & * & * & 9 & $w$ & w & 0 & w & 4.9 \\
\hline 2082 & Malt Beverages $\ldots \ldots \ldots$ & 14 & 3 & w & w & 8 & - & w & 0 & * & 14.0 \\
\hline 21 & Tobacco Products . & 24 & 3 & 1 & * & 4 & • & 15 & 0 & - & 6.8 \\
\hline 22 & Textile Mill Products & 235 & 94 & 7 & 3 & 89 & 2 & 30 & 0 & 10 & 6.5 \\
\hline 23 & Apparel and Other Textile Products ..... & 31 & 14 & $\mathbf{Q}$ & * & 12 & • & 2 & 0 & - & 22.7 \\
\hline 24 & Lumber and Wood Products $\ldots \ldots \ldots \ldots$ & 204 & 31 & $\mathbf{Q}$ & w & 15 & 1 & 1 & 0 & 150 & 19.6 \\
\hline 25 & Fumiture and Fixtures .... & w & 9 & * & 1 & w & * & w & 0 & w & 19.4 \\
\hline 26 & Paper and Allied Products .. & 1,442 & 86 & 69 & 4 & 284 & 2 & 154 & 0 & 844 & 4.5 \\
\hline 2611 & Pulp Mills . . . . . . . . . & 219 & 4 & 21 & 1 & 22 & • & 4 & 0 & 167 & 17.2 \\
\hline 2621 & Paper Mills & 606 & 47 & 18 & 2 & 126 & 1 & 87 & 0 & 325 & 3.4 \\
\hline 2631 & Paperboard Mills & 575 & 19 & 29 & 1 & 115 & * & 62 & 0 & 349 & 5.4 \\
\hline 27 & Printing and Publishing ... & 28 & 16 & $\mathbf{Q}$ & - & 10 & * & 0 & 0 & 1 & 21.1 \\
\hline 28 & Chemicals and Allied Products .... & 2,368 & 256 & 25 & 6 & 1,335 & w & 180 & w & 561 & 6.2 \\
\hline 2812 & Alkalies and Chlorine $\ldots \ldots \ldots$ & 147 & 29 & 0 & - & w & * & w & 0 & 20 & 17.3 \\
\hline 2813 & Industrial Gases $\ldots \ldots \ldots \ldots \ldots \ldots$ & 51 & 29 & 0 & * & 17 & * & 0 & 0 & 5 & 11.6 \\
\hline 2819 & Industrial Inorganic Chemicals, nec .... & 189 & w & w & 1 & 106 & * & 12 & w & 12 & 10.0 \\
\hline 2821 & Plastics Materials and Resins . . . . . . & 207 & 35 & w & * & 117 & • & w & 0 & 38 & 5.5 \\
\hline 2822 & Synthetic Rubber $\ldots \ldots \ldots \ldots \ldots$ & 104 & w & w & w & 41 & * & 0 & 0 & w & 13.2 \\
\hline 2823 & Cellulosic Manmade Fibers ........ & 31 & w & 0 & * & w & • & 27 & 0 & * & 27.4 \\
\hline 2824 & Organic Fibers, Noncellulosic . . . . . . . . & 98 & 23 & W & w & w & * & 35 & 0 & w & 3.9 \\
\hline 2865 & Cyclic Crudes and Intermediates . . . . . . & 125 & 11 & w & - & 75 & • & w & 0 & w & 12.8 \\
\hline 2869 & Industrial Organic Chemicals, nec . . . . . & 1,076 & 35 & 2 & Q & 597 & 3 & 60 & 0 & 378 & 9.3 \\
\hline 2873 & Nitrogenous Fertilizers $\ldots \ldots \ldots \ldots$ & 192 & 6 & 0 & * & 183 & . & 0 & 0 & 2 & 25.7 \\
\hline 2874 & Phosphatic Fertilizers & 29 & 5 & 2 & 1 & 16 & * & w & 0 & w & 3.2 \\
\hline 29 & Petroleum and Coal Products & 1,626 & 47 & 13 & w & 591 & 9 & w & w & 961 & 4.9 \\
\hline 2911 & Petroleum Refining . . . . . . . . & 1,608 & 46 & 13 & W & 578 & 8 & w & 0 & 961 & 3.3 \\
\hline 30 & Rubber and Misc. Plastics Products & 88 & 42 & 3 & 1 & 36 & 1 & 1 & 0 & 3 & 12.8 \\
\hline 3011 & Tires and Inner Tubes . . . . . . . . . . & w & 10 & W & w & 13 & * & $w$ & 0 & 1 & 4.3 \\
\hline 308 & Miscellaneous Plastics Products, nec... & 48 & 27 & 1 & w & 16 & - & w & 0 & $\mathbf{Q}$ & 21.6 \\
\hline 31 & Leather and Leather Products & 2 & 1 & * & * & • & * & 0 & 0 & Q & 25.1 \\
\hline 32 & Stone, Clay and Glass Products & 323 & 40 & 1 & 8 & 160 & w & 87 & w & w & 11.4 \\
\hline 3211 & Flat Glass $\ldots \ldots \ldots \ldots \ldots$ & 22 & 3 & 0 & • & 20 & * & 0 & 0 & • & 4.1 \\
\hline 3221 & Glass Containers & 29 & 4 & w & w & w & * & 0 & 0 & * & 8.3 \\
\hline 3229 & Pressed and Blown Glass, nec. ....... & W & 5 & $*$ & W & w & * & 0 & 0 & - & 8.9 \\
\hline 3241 & Cement, Hydraulic . . . . . . . . . & 109 & 12 & * & 1 & 22 & - & 58 & 4 & 12 & 15.6 \\
\hline 3274 & Lime ...... & 37 & 1 & 0 & - & w & • & 26 & 0 & W & 23.0 \\
\hline 3296 & Mineral Wool & 16 & 3 & w & * & 12 & * & 0 & w & * & 1.4 \\
\hline 33 & Primary Metal Industries & 543 & 161 & 9 & 3 & w & 1 & 10 & 110 & w & 5.7 \\
\hline 3312 & Blast Fumaces and Steel Mills . . . . . . . . & 317 & 37 & 9 & w & w & * & w & 103 & W & 6.1 \\
\hline 3313 & Electrometalurgical Products .... & w & $w$ & 0 & 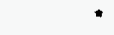 & * & 0 & W & 0 & 1 & 12.8 \\
\hline 3321 & Gray and Ductile Iron Foundries & 21 & 6 & • & * & w & * & $\mathbf{Q}$ & W & * & 11.7 \\
\hline 3331 & Primary Copper $\ldots \ldots \ldots \ldots$ & w & 1 & w & - & $\mathbf{w}$ & - & 0 & 0 & • & 1.1 \\
\hline 3334 & Primary Aluminum & 93 & 83 & 0 & w & w & • & 0 & 0 & - & 4.1 \\
\hline 3339 & Primary Nonferrous Metals, nec........ & 15 & 6 & 0 & - & w & w & w & 0 & w & 3.6 \\
\hline 3353 & Aluminum Sheet, Plate, and Foil & 25 & W & 0 & * & 18 & * & w & 0 & - & 1.8 \\
\hline 34 & Fabricated Metal Products . . . . . . . & 81 & 30 & $\mathbf{Q}$ & 2 & 43 & 1 & 0 & 1 & 2 & 21.1 \\
\hline 35 & Industrial Machinery and Equipment ..... & 57 & 28 & * & 1 & 27 & w & $\mathbf{Q}$ & $\mathbf{Q}$ & W & 15.8 \\
\hline 357 & Computer and Office Equipment .. & 4 & 3 & * & $\mathbf{Q}$ & 1 & * & 0 & 0 & • & 22.2 \\
\hline 36 & Electronic and Other Electric Equipment .. & 67 & 37 & • & - & 27 & 1 & 2 & 0 & " & 13.9 \\
\hline & Transportation Equipment & w & 29 & $w$ & 2 & 28 & * & $\mathbf{w}$ & * & w & 8.9 \\
\hline 3711 & Motor Vehicles and Car Bodies ........ & 35 & 8 & * & - & 12 & * & w & 0 & w & 5.3 \\
\hline 3714 & Motor Vehicle Parts and Accessories ... & 13 & 7 & * & - & w & * & w & 0 & $\bullet$ & 13.8 \\
\hline 38 & Instruments and Related Products . & 17 & 11 & Q & * & 6 & - & 0 & 0 & * & 18.4 \\
\hline 3841 & Surgical and Medical Instruments & 2 & 1 & 0 & * & . & - & 0 & 0 & * & 21.3 \\
\hline 39 & Misc. Manufacturing Industries $\ldots \ldots \ldots$. & w & 4 & * & $\mathbf{Q}$ & w & • & 0 & 0 & w & 23.4 \\
\hline & Total . & 7,507 & 996 & 145 & 47 & 2,983 & w & 502 & w & 2,693 & 3.3 \\
\hline
\end{tabular}

See footnotes at end of table. 
Table A4. Total Inputs of Energy for Heat, Power, and Electricity Generation by Census Region, Industry Group, and Selected Industries, 1991: Part 2 (Continued) (Estimates in Trillion Btu)

\begin{tabular}{|c|c|c|c|c|c|c|c|c|c|c|c|}
\hline $\begin{array}{l}\text { SIC } \\
\text { Code }^{a}\end{array}$ & $\begin{array}{l}\text { Industry Groups } \\
\text { and Industry }\end{array}$ & Total & $\begin{array}{c}\text { Net } \\
\text { Electricity }\end{array}$ & $\begin{array}{l}\text { Residual } \\
\text { Fuel Oil }\end{array}$ & $\begin{array}{l}\text { Distillate } \\
\text { Fuel Oife }\end{array}$ & $\begin{array}{c}\text { Natural } \\
\text { Gas }^{d}\end{array}$ & LPG & Coal & $\begin{array}{c}\text { Coke and } \\
\text { Breeze }\end{array}$ & Other & $\begin{array}{c}\text { RSE } \\
\text { Row } \\
\text { Factors }\end{array}$ \\
\hline & & \multicolumn{10}{|c|}{ West Census Region } \\
\hline & RSE Column Factors: & 0.7 & 0.7 & 1.2 & 1.0 & 0.8 & 1.1 & 1.1 & 1.3 & 1.2 & \\
\hline 20 & Food and Kindred Products $\ldots \ldots \ldots \ldots$ & 202 & 29 & 4 & 4 & 119 & 1 & 28 & w & w & 9.6 \\
\hline 2011 & Meat Packing Plants . . . . . . . . . & 5 & 1 & $w$ & : & 3 & Q & 0 & 0 & $\because$ & 17.2 \\
\hline 2033 & Canned Fruits and Vegetables . . . . . . . & 20 & 2 & 1 & - & 17 & - & 0 & 0 & * & 12.2 \\
\hline 2037 & Frozen Fruils and Vegetables ........ & 26 & 7 & $\mathbf{Q}$ & * & 17 & * & 0 & 0 & 1 & 16.9 \\
\hline 2046 & 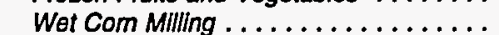 & $w$ & : & $\overline{0}$ & w & 2 & - & 0 & 0 & $w$ & 20.1 \\
\hline 2051 & Bread, Cake, and Related Products .... & 6 & 1 & 0 & - & 5 & • & 0 & 0 & • & 26.3 \\
\hline 2063 & Beot Sugar . . . . . . . . . . . . . & $w$ & w & w & - & w & • & 18 & 1 & * & 8.5 \\
\hline 2075 & Soybean Oll MIlls . . . . . . . . . . . . & 0 & 0 & 0 & 0 & 0 & 0 & 0 & 0 & 0 & NF \\
\hline 2082 & Malt Beverages ............... & 17 & 2 & w & w & w & w & w & 0 & • & 16.5 \\
\hline 21 & Tobacco Products $\ldots \ldots \ldots \ldots \ldots \ldots$ & 0 & 0 & 0 & 0 & 0 & 0 & 0 & 0 & 0 & NF \\
\hline 22 & Textlle Mill Products . . . . . . . . . . . & 6 & 1 & 0 & - & 5 & $\mathbf{Q}$ & 0 & 0 & • & 29.5 \\
\hline 23 & Apparel and Other Textlle Products ..... & NA & NA & NA & NA & NA & NA & NA & NA & NA & 29.9 \\
\hline 24 & Lumber and Wood Products $\ldots \ldots \ldots \ldots$ & 147 & 17 & 1 & 7 & 10 & 1 & 0 & 0 & 111 & 20.0 \\
\hline 25 & Furniture and Fixtures $\ldots \ldots \ldots \ldots \ldots$. & 3 & 1 & 0 & $\mathbf{Q}$ & 1 & - & 0 & 0 & • & 32.7 \\
\hline 26 & Paper and Allled Products . . . . . . . . . . & 389 & 38 & 11 & 1 & 111 & 1 & w & 0 & w & 6.2 \\
\hline 2611 & Pulp Mills $\ldots \ldots \ldots \ldots \ldots \ldots \ldots$ & 41 & 3 & 5 & - & 7 & * & 0 & 0 & 26 & 18.8 \\
\hline 2621 & 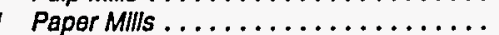 & 161 & 22 & w & * & 55 & - & w & 0 & 74 & 5.8 \\
\hline 2631 & Paperboard Mills . . . . . . . . . . & 170 & 7 & $w$ & * & 40 & • & w & 0 & 117 & 8.1 \\
\hline 27 & Printing and Publishing . . . . . . . . & NA & NA & NA & NA & NA & NA & NA & NA & NA & 21.8 \\
\hline 28 & Chemicals and Alled Products . . . . . . . . & 139 & 37 & $w$ & w & 88 & w & W & w & 6 & 13.2 \\
\hline 2812 & Alkalles and Chlorine ............. & w & $w$ & $w$ & w & 4 & $*$ & 0 & 0 & 1 & 19.6 \\
\hline 2813 & Industrial Gases . . . . . . . . . . . . & w & $w$ & 0 & W & w & - & 0 & 0 & - & 14.2 \\
\hline 2819 & Industrial Inorganic Chemicals, nec .... & 41 & 14 & w & 1 & w & $\mathbf{Q}$ & w & $w$ & 5 & 11.7 \\
\hline 2821 & Plastles Materlals and Resins . . . . . . . & 3 & 1 & 0 & - & 2 & - & 0 & 0 & • & 17.0 \\
\hline 2822 & Synthetlc Rubber ............... & : & " & 0 & * & $*$ & * & 0 & 0 & - & 24.1 \\
\hline 2823 & Colluloslc Manmade Fibers ........... & 0 & 0 & 0 & 0 & 0 & 0 & 0 & 0 & 0 & NF \\
\hline 2824 & Organic Flbers, Noncellulosic . . . . . . & 0 & 0 & 0 & 0 & 0 & 0 & 0 & 0 & 0 & NF \\
\hline 2865 & Cyclic Crudes and Intermediatos ....... & - & * & 0 & * & * & * & 0 & 0 & - & 24.9 \\
\hline 2869 & Industrlal Organic Chemicals, nec ....... & 5 & a & 0 & 0 & w & Q & 0 & 0 & w & 13.0 \\
\hline 2873 & Nitrogenous Fertilizers . . . . . . . . & 42 & 2 & 0 & - & 40 & " & 0 & 0 & $\because$ & 39.0 \\
\hline 2874 & Phosphatic Fortillzers ... & 5 & 2 & 0 & - & $\mathbf{Q}$ & 0 & 0 & $\mathbf{0}$ & * & 45.7 \\
\hline 29 & Petroleum and Coal Products ... & 622 & 26 & 12 & 5 & 129 & 46 & 0 & 0 & 404 & 3.2 \\
\hline 2911 & Petroleum Refining .............. & 610 & 26 & 12 & 5 & 118 & 46 & 0 & 0 & 404 & 2.7 \\
\hline 30 & Rubber and Misc. Plastics Products ..... & 19 & 10 & - & Q & $w$ & w & $\mathbf{Q}$ & 0 & * & 18.1 \\
\hline 3011 & Tires and Inner Tubes . . . . . . . . . & - & w & w & w & • & w & 0 & 0 & - & 7.1 \\
\hline 308 & Miscellaneous Plastlas Products, nec . . . & 14 & 9 & 0 & Q & 5 & * & 0 & 0 & - & 22.0 \\
\hline 31 & Leather and Leather Products . . . . . . . . & $Q$ & 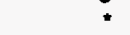 & 0 & $\mathbf{Q}$ & $\mathbf{Q}$ & $\mathbf{Q}$ & 0 & 0 & - & 42.7 \\
\hline 32 & Stone, Clay and Glass Products . . . . . & 137 & 18 & 4 & 4 & 53 & * & 53 & 1 & 5 & 14.8 \\
\hline 3211 & Flat Glass $\ldots \ldots \ldots \ldots \ldots \ldots \ldots$ & w & 1 & $w$ & w & $w$ & w & 0 & 0 & * & 4.5 \\
\hline 3221 & Glass Containers ............... & 18 & 4 & $w$ & w & w & $\because$ & 0 & 0 & * & 9.3 \\
\hline 3229 & Prossed and Blown Glass, noc. ........ & $\mathbf{w}$ & $w$ & 0 & $\because$ & $\ddot{w}$ & * & 0 & 0 & • & 13.1 \\
\hline 3241 & Cement, Hydraullc . . . . . . . . . . & 77 & 8 & W & 1 & 12 & - & 53 & $\mathbf{Q}$ & 3 & 21.0 \\
\hline 3274 & LIme ........... & w & $w$ & w & w & w & - & 0 & 0 & • & 21.0 \\
\hline 3296 & ; Mnoral Wool .... & 3 & 1 & 0 & - & $\mathbf{w}$ & w & 0 & w & - & 2.1 \\
\hline 33 & Primary Metal Industries & 224 & 117 & 1 & 2 & $w$ & : & w & $w$ & w & 6.1 \\
\hline 3312 & Blast Fumaces and Steol Mills . . . . . . . & 65 & 8 & w & " & $w$ & - & $w$ & $w$ & $w$ & 8.8 \\
\hline 3313 & Electrometalurgical Products . . . . . . . & 0 & 0 & 0 & 0 & 0 & 0 & 0 & 0 & 0 & NF \\
\hline 3321 & Gray and Ducllle Iron Foundries ....... & 3 & * & 0 & - & w & . & 0 & $w$ & * & 36.9 \\
\hline 3331 & Primary Copper .................. & $w$ & 4 & w & w & w & * & w & 0 & 1 & 1.0 \\
\hline 3334 & Primary Aluminum & 96 & 90 & * & - & 6 & - & 0 & 0 & • & 3.5 \\
\hline 3339 & Primary Nonferrous Metals, nec . . . . . . . & 11 & 4 & 0 & w & w & w & 0 & w & - & 1.0 \\
\hline 3353 & Aluminum Sheet, Plate, and Foll .. & w & w & 0 & w & w & w & 0 & 0 & • & 1.1 \\
\hline 34 & Fabricated Metal Products . . . . . . . . . & 28 & 10 & 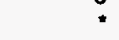 & $\because$ & 17 & $\because$ & 0 & 0 & " & 19.5 \\
\hline 35 & Industral Machinery and Equipment . . . . . & 22 & 12 & 0 & - & 9 & - & 0 & 0 & * & 24.3 \\
\hline 357 & Computer and Office Equipment ........ & 9 & 7 & 0 & - & 2 & $\mathbf{Q}$ & 0 & 0 & * & 14.8 \\
\hline 36 & Electronic and Other Electric Equipment .. & 29 & 19 & 0 & $\mathbf{Q}$ & 9 & $\because$ & 0 & 0 & $w$ & 19.1 \\
\hline
\end{tabular}

See footnotes at end of table. 
Table A4. Total Inputs of Energy for Heat, Power, and Electricity Generation by Census Region, Industry Group, and Selected Industries, 1991: Part 2 (Continued) (Estimates in Trillion Btu)

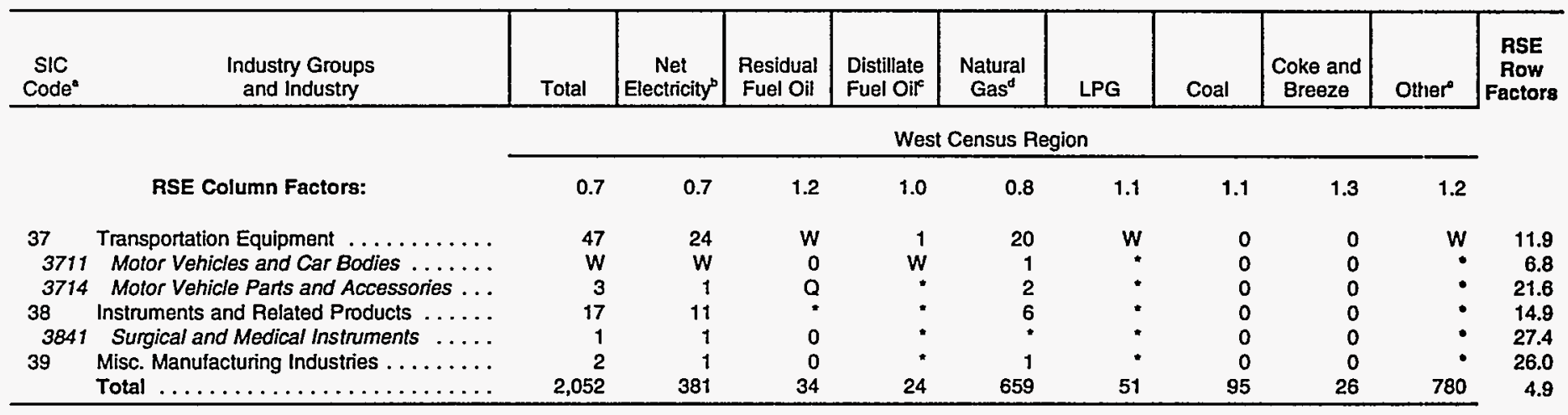

\footnotetext{
- See Appendices B and F for descriptions of the Standard Industrial Classification system.

- "Net Electricity" is obtained by summing purchases, transfers in, and generation from noncombustible renewable resources, minus quantities sold and transferred

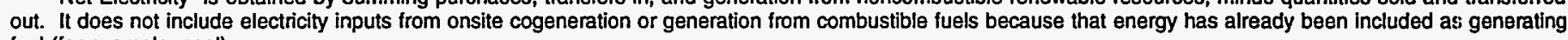
fuel (for example, coal).

c "Distillate Fuel Oil" includes Nos. 1, 2, and 4 fuel oils and Nos. 1, 2, and 4 diesel fuels.

"Natural Gas" includes natural gas obtained from utilities, transmission pipelines, and any other supplier(s) such as brokers and producers.

- "Other" includes net steam (the sum of purchases, generation from renewables, and net transfers), and other energy that respondents indicated was used to produce heat and power.

NF=No applicable RSE row/column factor.

- Estimate less than 0.5. Data are included in higher level totals.

$W=$ Withheld to avoid disclosing data for individual establishments. Data are included in higher level totals.

$\mathrm{Q}=$ Withheld because Relative Standard Error is greater than 50 percent. Data are included in higher level totals.

NA=Not available. Data are included in higher level totals.

Notes: - To obtain a RSE percentage for any table cell, multiply the cell's corresponding RSE column and RSE row factors. - Totals may not equal sum of

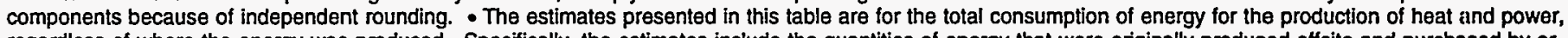
regardless of where the energy was produced. Specifically, the estimates include the quantities of energy that were originally produced offsite and purchased by or transferred to the establishment, plus those that were produced onsite from other energy or input materials not classified as energy, or were extracted from captive (onsite) mines or wells.

Source: Energy information Administration, Office of Energy Markets and End Use, Energy End Use and Integrated Statistics Division, Form ElA-846, "1991 Manufacturing Energy Consumption Survey," and Office of Oil and Gas, Petroleum Supply Division, Form ElA-810, "Monthly Refinery Report" for 1991.
} 
Table A5. Total Consumption of Offsite-Produced Energy for Heat, Power, and Electricity Generation by Census Region, Industry Group, and Selected Industries, 1991: Part 1 (Estimates in Btu or Physical Units)

\begin{tabular}{|c|c|c|c|c|c|c|c|c|c|c|c|}
\hline $\begin{array}{c}\text { SIC } \\
\text { Code" }\end{array}$ & $\begin{array}{l}\text { Industry Groups } \\
\text { and Industry }\end{array}$ & $\begin{array}{c}\text { Total } \\
\text { (trillion } \\
\text { Btu) }\end{array}$ & $\begin{array}{c}\text { Electricity } \\
\text { (million } \\
\text { kWh) }\end{array}$ & $\begin{array}{c}\text { Residual } \\
\text { Fuel Oi] } \\
\text { (1000 } \\
\text { bbls) }\end{array}$ & $\begin{array}{c}\text { Distillate } \\
\text { Fuel Oil } \\
\text { (1000 } \\
\text { bbls) }\end{array}$ & $\begin{array}{l}\text { Natural } \\
\text { Gas }^{d} \\
\text { (billion } \\
\text { cu ft) }\end{array}$ & $\begin{array}{l}\text { LPG } \\
\text { (1000 } \\
\text { bbls) }\end{array}$ & $\begin{array}{l}\text { Coal } \\
\text { (1000 } \\
\text { short } \\
\text { tons) }\end{array}$ & $\begin{array}{c}\text { Coke and } \\
\text { Breeze } \\
(1000 \\
\text { short } \\
\text { tons) }\end{array}$ & $\begin{array}{c}\text { Other } \\
\text { (trillion } \\
\text { Btu) }\end{array}$ & $\begin{array}{c}\text { RSE } \\
\text { Row } \\
\text { Factors }\end{array}$ \\
\hline
\end{tabular}

\begin{tabular}{|c|c|c|c|c|c|c|c|c|c|c|c|}
\hline & & & & & & nited 5 & & & & & \\
\hline & RSE Column Factors: & 0.6 & 0.6 & 1.3 & 1.3 & 0.7 & 1.2 & 1.2 & 1.6 & 1.2 & \\
\hline 20 & Food and Kindred Products . . . . . . . . & 922 & 50,518 & 4,317 & 2,966 & 497 & 1,429 & 6,913 & $w$ & W & 5.9 \\
\hline 2011 & Meat Packlng Plants . . . . . . . . . . . & 48 & 3,410 & 170 & 252 & 31 & 157 & 27 & 0 & 1 & 10.2 \\
\hline 2033 & Canned Frults and Vegotables . . . . . . . & 44 & 1,415 & 290 & 131 & 35 & 124 & $Q$ & 0 & : & 9.1 \\
\hline 2037 & Frozen Frults and Vegetables . . . . . . & 40 & 3,097 & 321 & 76 & 25 & 41 & $\overrightarrow{0}$ & 0 & 1 & 14.9 \\
\hline 2046 & Wet Com Mlling . . . . . . . . . . . . & 141 & 4,143 & 29 & 30 & 51 & 1 & 3,051 & $W$ & $w$ & 11.7 \\
\hline 2051 & Bread, Cake, and Related Products .... & 32 & 2,240 & * & 131 & 22 & 23 & 0 & 0 & $\cdot$ & 12.7 \\
\hline 2063 & Bet Sugar . . . . . . . . . . . . . . & 67 & 407 & $W$ & 30 & 18 & 5 & 1,901 & $w$ & - & 5.5 \\
\hline 2075 & Soybean Oll Mlls . . . . . . . . . . . . . & 50 & 1,632 & 42 & 31 & 24 & 5 & 592 & 0 & 6 & 3.5 \\
\hline 2082 & Malt Beverages $\ldots \ldots \ldots \ldots \ldots \ldots$ & 50 & 2,371 & 419 & 58 & 22 & 8 & 706 & 0 & $"$ & $\$ 1.1$ \\
\hline 21 & Tobacco Products $\ldots \ldots \ldots \ldots \ldots \ldots \ldots$ & 26 & 1,468 & 135 & 40 & 4 & 23 & 692 & 0 & * & 6.4 \\
\hline 22 & Textlle Mill Products . . . . . . . . . . . & 272 & 29,522 & 1,966 & 1,064 & 105 & 629 & 1,362 & 0 & 12 & 7.2 \\
\hline 23 & Apparel and Other Textile Products ..... & 44 & 5,645 & $\mathbf{Q}$ & 142 & 18 & 158 & 88 & 0 & 1 & 18.0 \\
\hline 24 & Lumber and Wood Products $\ldots . . . \ldots$. & 197 & 19,575 & 333 & 2,373 & 39 & 1,000 & 92 & 0 & 68 & 14.9 \\
\hline 25 & Fumiture and Fixtures $\ldots \ldots \ldots \ldots \ldots$ & 46 & 4,916 & 184 & 163 & 18 & 255 & 157 & 0 & 4 & 18.1 \\
\hline 26 & Paper and Alled Products .......... & 1,540 & 65,052 & 24,883 & 1,566 & 532 & $W$ & 13,063 & $\vec{w}$ & 307 & 4.2 \\
\hline 2611 & Pulp MIIIs $\ldots \ldots \ldots \ldots \ldots \ldots$ & 103 & 2,877 & 4,500 & 155 & 32 & 141 & 331 & 0 & 24 & 14.9 \\
\hline 2621 & Papor Mils . . . . . . . . . . . & 774 & 36,317 & 13,455 & W & 252 & 613 & 8,634 & $W$ & 106 & 2.9 \\
\hline 2631 & Paperboard Mills . . . . . . . . . . . & 527 & 12,611 & $W$ & 207 & 180 & 93 & W & 0 & 171 & 4.7 \\
\hline 27 & Printing and Publishing $\ldots \ldots \ldots \ldots$ & 108 & 15,629 & 50 & 311 & 47 & 179 & 0 & 0 & 4 & 13.0 \\
\hline 28 & Chemicals and Allied Products . . . . . . . & 2,674 & 139,059 & 7,427 & 1,999 & 1,616 & 1,119 & 11,153 & 132 & 221 & 5.6 \\
\hline 2812 & Alkalles and Chlorine ............ & 159 & 12,629 & $w$ & 43 & $W$ & 2 & $W$ & 0 & W & 16.1 \\
\hline 2813 & Industrial Gases ................ & 86 & 17,894 & 0 & 7 & 24 & $\mathbf{Q}$ & 0 & 0 & $\bullet$ & 12.0 \\
\hline 2819 & Industrial Inorganic Chemicals, nec . . . . & 303 & 38,176 & 691 & 456 & 136 & 75 & 551 & 122 & 10 & 8.4 \\
\hline 2821 & Plastlos Materials and Resins ....... & 262 & 15,027 & 668 & 187 & 146 & 54 & 1,074 & 0 & 31 & 5.9 \\
\hline 2822 & Synthetlc Rubber .............. & 68 & 1,946 & 64 & 18 & 43 & 7 & $W$ & 0 & $w$ & 12.6 \\
\hline 2823 & Cellulosic Manmado Fibers . . . . . . . . & 31 & $w$ & 0 & 21 & W & 1 & 1,202 & 0 & 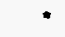 & 25.1 \\
\hline 2824 & Organic Flbers, Noncellulosic . . . . . . & 97 & 6,976 & $\mathbf{W}$ & 53 & $w$ & 38 & 1,558 & 0 & * & 3.7 \\
\hline 2865 & Cycllc Crudes and Intermedlates ...... & 136 & 4,432 & 1,153 & 95 & 94 & 79 & W & 0 & $w$ & 11.8 \\
\hline 2869 & Industral Organic Chemicals, nec ..... & 935 & 20,143 & 1,747 & 439 & 622 & 684 & 3,819 & 0 & 124 & 8.0 \\
\hline 2873 & Nitrogenous Fertilizers . . . . . . . . & 278 & 2,918 & 0 & 26 & 258 & 43 & 0 & 0 & $\mathbf{Q}$ & 22.5 \\
\hline 2874 & Phosphatic Fertllizers ............ & 36 & 2,419 & 250 & 150 & 18 & 1 & $W$ & 0 & $\bar{W}$ & 5.5 \\
\hline 29 & Petroleum and Coal Products $\ldots \ldots \ldots$. & 1,138 & 33,480 & 3,814 & 2,900 & 806 & 6,874 & $W$ & $\mathbf{W}$ & 123 & 5.4 \\
\hline 2911 & Petroleum Refining . . . . . . . . . & 1,065 & 31,562 & 3,695 & 826 & 762 & 6,235 & 134 & 0 & 118 & 3.5 \\
\hline 30 & Rubber and Misc. Plastics Products . . . . . & 235 & 33,913 & 1,253 & 508 & 93 & 786 & 295 & 0 & 3 & 9.6 \\
\hline 3011 & Tres and Inner Tubes . . . . . . . . . . & 42 & 4,037 & 506 & 68 & 20 & 79 & 75 & 0 & 1 & 3.6 \\
\hline 308 & Miscellaneous Plastlcs Products, nec ... & 150 & 25,597 & 413 & W & 51 & 396 & 130 & 0 & $W$ & 13.9 \\
\hline 31 & Leather and Leather Products . . . . . . . & 12 & 795 & 225 & 220 & 5 & 44 & $Q$ & 0 & 1 & 25.2 \\
\hline 32 & Stone, Clay and Glass Products ...... & 877 & 30,885 & 1,345 & 3,312 & 369 & 577 & 13,127 & 374 & 60 & 7.6 \\
\hline 3211 & Flat Glass $\ldots \ldots \ldots \ldots \ldots \ldots$ & 49 & 1,512 & $W$ & 12 & 40 & 40 & $\cdot$ & 0 & W & 3.4 \\
\hline 3221 & Glass Containers $\ldots \ldots \ldots \ldots \ldots$ & 85 & 4,098 & 276 & 23 & 67 & 82 & 0 & 0 & $\bullet$ & 5.5 \\
\hline 3229 & Pressed and Blown Glass, nec. . . . . . . & $w$ & 2,862 & 81 & 38 & W & 31 & 0 & 0 & $\cdot$ & 8.1 \\
\hline 3241 & Cement, Hydraulle .............. & 312 & 9,490 & 138 & 616 & 38 & 12 & 8,736 & 232 & 35 & 11.0 \\
\hline 3274 & Lime $\ldots \ldots \ldots \ldots \ldots \ldots \ldots \ldots$ & 117 & 1,324 & W & 240 & 8 & $\mathbf{Q}$ & 3,926 & $w$ & 13 & 29.4 \\
\hline 3296 & 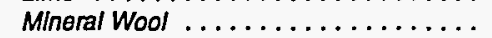 & 40 & 2,821 & W & 12 & 28 & 41 & 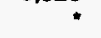 & $\ddot{W}$ & 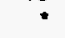 & 1.5 \\
\hline 33 & Primary Metal Industries . . . . . . . . . & 1,563 & 147,078 & 5,285 & 1,806 & 666 & 888 & 2,054 & 10,557 & 20 & 4.3 \\
\hline 3312 & Blast Fumaces and Steel Mills . . . . . . . & 842 & 39,480 & 4,986 & 901 & 387 & 74 & 1,075 & 9,553 & 10 & 4.3 \\
\hline 3313 & Electrometalurgical Products ......... & 30 & 3,796 & 0 & 20 & 1 & $w$ & $w$ & $\mathbf{W}$ & $W$ & 8.7 \\
\hline 3321 & Gray and Ductile Iron Foundries ..... & 74 & 6,414 & 4 & 144 & 28 & 105 & 5 & 858 & 1 & 11.4 \\
\hline 3331 & Primary Copper .............. & 21 & 1,246 & $w$ & $w$ & 15 & 3 & $\mathbf{w}$ & 0 & : & 1.1 \\
\hline 3334 & Primary Aluminum $\ldots \ldots \ldots \ldots$ & 254 & 67,707 & * & 127 & 20 & 42 & 0 & 0 & 1 & 3.3 \\
\hline 3339 & Primary Nonforrous Metals, nec . . . . . . & 40 & 3,784 & 1 & 53 & 16 & 19 & $w$ & $w$ & : & 2.1 \\
\hline 3353 & Aluminum Sheet, Plate, and Foll ...... & 60 & 4,261 & 0 & 67 & 41 & 62 & $W$ & 0 & $W$ & 1.4 \\
\hline 34 & Fabricated Metal Products ........... & 305 & 29,830 & 501 & 994 & 169 & 1,122 & 245 & $w$ & $w$ & 11.4 \\
\hline 35 & Industrial Machinery and Equlpment . . . . . & 236 & 29,658 & 490 & 718 & 105 & 651 & 480 & 24 & $\mathbf{5}$ & $\mathbf{1 1 . 5}$ \\
\hline 357 & Computer and Office Equlpment . ..... & 21 & 4,398 & 11 & 16 & 5 & 4 & 0 & 0 & 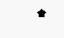 & 15.9 \\
\hline 36 & Electronic and Other Electric Equipment .. & 196 & 30,046 & 612 & 416 & 76 & 396 & $w$ & 2 & $W$ & 10.2 \\
\hline 37 & Transportatlon Equipment & 318 & 35,401 & 1,865 & 1,214 & 127 & 526 & 1,464 & 40 & 12 & 5.1 \\
\hline 3711 & Motor Vehicles and Car Bodles ....... & 90 & 8,285 & 408 & 65 & 42 & 59 & $W$ & $\mathbf{W}$ & 4 & 3.4 \\
\hline 3714 & Motor Vehicle Parts and Accessories ... & 99 & 10,918 & 60 & 104 & 40 & 168 & $w$ & $w$ & $w$ & 7.2 \\
\hline 38 & Instruments and Related Products ..... & 97 & 12,349 & 536 & w & 25 & $\mathbf{Q}$ & $\mathbf{W}$ & 0 & $W$ & 13.7 \\
\hline 3841 & Surglcal and Medical Instruments ..... & 6 & 1,161 & 9 & 30 & 2 & 8 & 0 & 0 & 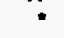 & 15.4 \\
\hline 39 & Misc. Manufacturing Industries . . . . . . . . & 31 & 3,661 & 115 & $w$ & 14 & $W$ & 32 & 0 & $W$ & 14.6 \\
\hline & Total $\ldots \ldots \ldots \ldots \ldots \ldots \ldots$ & 10,837 & 718,480 & 55,643 & 23,102 & 5,332 & 18,171 & 52,653 & 11,382 & 884 & 3.1 \\
\hline
\end{tabular}

See footnotes at end of table. 
Table A5. Total Consumption of Offsite-Produced Energy for Heat, Power, and Electricity Generation by Census Region, Industry Group, and Selected Industries, 1991: Part 1 (Continued)

(Estimates in Btu or Physical Units)

\begin{tabular}{|c|c|c|c|c|c|c|c|c|c|c|c|}
\hline $\begin{array}{c}\text { SIC } \\
\text { Code } \\
\end{array}$ & $\begin{array}{c}\text { Industry Groups } \\
\text { and Industry }\end{array}$ & $\begin{array}{c}\begin{array}{c}\text { Total } \\
\text { (trillion } \\
\text { Btu) }\end{array} \\
\end{array}$ & $\begin{array}{c}\text { Electricity" } \\
\text { (million } \\
\text { kWh) }\end{array}$ & $\begin{array}{c}\text { Residual } \\
\text { Fuel Oil } \\
\text { (1000 } \\
\text { bbls) }\end{array}$ & $\begin{array}{c}\text { Distillate } \\
\text { Fuel Oilc } \\
\text { (1000 } \\
\text { bbls) }\end{array}$ & $\begin{array}{c}\text { Natural } \\
\text { Gas } \\
\text { (billion } \\
\text { cu ft) } \\
\end{array}$ & $\begin{array}{c}\text { LPG } \\
\text { (1000 } \\
\text { bbls) } \\
\end{array}$ & $\begin{array}{l}\text { Coal } \\
(1000 \\
\text { short } \\
\text { tons) } \\
\end{array}$ & $\begin{array}{c}\text { Coke and } \\
\text { Breeze } \\
(1000 \\
\text { short } \\
\text { tons) } \\
\end{array}$ & $\begin{array}{c}\text { Other } \\
\text { (trillion } \\
\text { Btu) }\end{array}$ & $\begin{array}{c}\text { RSE } \\
\text { Row } \\
\text { Factors }\end{array}$ \\
\hline & & \multicolumn{10}{|c|}{ Northeast Census Region } \\
\hline & RSE Column Factors: & 0.7 & 0.7 & 1.0 & 1.2 & 0.8 & 1.2 & 1.3 & 1.4 & 1.1 & \\
\hline 20 & Food and Kindred Products & 79 & 5,443 & 1,164 & 889 & 40 & 222 & 99 & 0 & 3 & 13.9 \\
\hline 2011 & Meat Packing Plants .... & 1 & 141 & W & 34 & 1 & $\mathbf{Q}$ & 0 & 0 & - & 24.4 \\
\hline 2033 & Canned Fruits and Vegetables. & 6 & 292 & 146 & 22 & 4 & 13 & Q & $\mathbf{0}$ & - & 17.1 \\
\hline 2037 & Frozen Fruits and Vegetables ... & 1 & 140 & 128 & 3 & * & $\mathbf{Q}$ & 0 & $\mathbf{0}$ & - & 32.9 \\
\hline 2046 & Wet Com Milling ........ & * & 15 & w & w & * & - & 0 & 0 & • & 23.6 \\
\hline 2051 & Bread, Cake, and Related Products .... & 7 & 382 & - & w & w & 8 & 0 & 0 & • & 19.2 \\
\hline 2063 & Beet Sugar $\ldots \ldots \ldots \ldots \ldots \ldots \ldots$ & 0 & 0 & 0 & 0 & 0 & 0 & 0 & 0 & 0 & NF \\
\hline 2075 & Soybean Oil Mills . ........... & 0 & 0 & 0 & 0 & 0 & 0 & 0 & 0 & 0 & NF \\
\hline 2082 & Malt Beverages $\ldots \ldots \ldots \ldots \ldots \ldots$ & 8 & 521 & w & 9 & 3 & 4 & w & 0 & • & 16.4 \\
\hline 21 & Tobacco Products..$\ldots \ldots \ldots$ & NA & NA & NA & NA & NA & NA & NA & NA & NA & 25.3 \\
\hline 22 & Textile Mill Products & 27 & 1,372 & 774 & 556 & 10 & 163 & 17 & 0 & 3 & 19.4 \\
\hline 23 & Apparel and Other Textile Products & 5 & 497 & 44 & 49 & 2 & $\mathbf{Q}$ & 0 & 0 & - & 29.8 \\
\hline 24 & Lumber and Wood Products $\ldots \ldots \ldots \ldots$ & NA & NA & NA & NA & NA & NA & NA & NA & NA & 34.9 \\
\hline 25 & Furniture and Fixtures $\ldots \ldots \ldots \ldots \ldots$ & 5 & 449 & $\mathbf{Q}$ & 49 & 2 & 60 & 0 & 0 & 1 & 28.9 \\
\hline 26 & Paper and Allied Products & W & 9,289 & 11,439 & 624 & 36 & W & w & o & w & 6.1 \\
\hline 2611 & Pulp Mills . . . . . . . . . . & 5 & $\mathbf{Q}$ & 290 & 7 & 0 & 13 & 0 & 0 & 3 & 33.9 \\
\hline 2621 & Paper Mills & 166 & 5,878 & 9,702 & W & 19 & 284 & w & 0 & 29 & 4.2 \\
\hline 2631 & Paperboard Mills & 16 & 510 & w & Q & 6 & 5 & w & 0 & 1 & 15.5 \\
\hline 27 & Printing and Publishing & 23 & 3,167 & 36 & 233 & 9 & 30 & 0 & 0 & 1 & 26.0 \\
\hline 28 & Chemicals and Allied Products $\ldots \ldots \ldots$ & 135 & 9,511 & 3,072 & w & 57 & 101 & w & 0 & 14 & 9.1 \\
\hline 2812 & Alkalies and Chlorine ..... & * & w & 0 & 0 & • & 0 & 0 & 0 & 0 & 36.9 \\
\hline 2813 & Industrial Gases . . . . . . . . & 5 & 1,438 & 0 & 1 & • & • & 0 & 0 & 0 & 12.0 \\
\hline 2819 & Industrial Inorganic Chemicals, nec .... & 10 & 494 & 255 & 78 & 6 & 14 & 0 & 0 & - & 22.7 \\
\hline 2821 & Plastics Materials and Resins $\ldots \ldots \ldots$ & 19 & 1,122 & 478 & 109 & 8 & 14 & w & 0 & w & 9.6 \\
\hline 2822 & Synthetic Rubber & W & w & w & • & w & • & 0 & 0 & - & 26.1 \\
\hline 2823 & Cellulosic Manmade Fibers & 0 & 0 & 0 & 0 & 0 & 0 & 0 & 0 & 0 & NF \\
\hline 2824 & Organic Fibers, Noncellulosic . . . . . . . & * & 95 & $\mathbf{Q}$ & w & * & * & 0 & 0 & 0 & 15.0 \\
\hline 2865 & Cyclic Crudes and Intermediates ....... & 12 & 406 & w & w & 7 & 2 & w & 0 & 1 & 21.1 \\
\hline 2869 & Industrial Organic Chemicals, nec . . . . . & 38 & 3,076 & 1,399 & 76 & w & 42 & 0 & 0 & w & 8.7 \\
\hline 2873 & Nitrogenous Fertilizers $\ldots \ldots \ldots \ldots$ & • & 29 & 0 & 3 & * & 1 & 0 & 0 & • & 47.8 \\
\hline 2874 & Phosphatic Fentilizers & 0 & 0 & 0 & 0 & $\mathbf{0}$ & 0 & 0 & 0 & 0 & NF \\
\hline 29 & Petroleum and Coal Products $\ldots \ldots \ldots$ & 74 & 3,054 & 1,535 & 1,308 & 28 & 603 & 145 & 0 & 11 & 12.0 \\
\hline 2911 & Petroleum Refining . & 57 & 2,515 & 1,535 & W & 24 & 210 & W & 0 & 11 & 5.7 \\
\hline 30 & Rubber and Misc. Plastics Products ..... & 37 & 5,484 & 456 & 202 & W & $\mathbf{Q}$ & 86 & 0 & 1 & 18.3 \\
\hline 3011 & Tires and Inner Tubes . . . . . . . . . . . . & 2 & 125 & 63 & Q & 1 & 5 & 0 & 0 & - & 13.4 \\
\hline 308 & Miscellaneous Plastics Products, nec . . . & 28 & 4,810 & 251 & $\mathbf{w}$ & 7 & 127 & w & 0 & w & 24.2 \\
\hline 31 & Leather and Leather Products & 4 & 205 & 142 & 196 & 1 & 28 & $\mathbf{Q}$ & 0 & • & 28.3 \\
\hline 32 & Stone, Clay and Glass Products & 170 & 5,624 & 432 & 714 & 58 & $\mathbf{w}$ & 3,451 & w & w & 17.6 \\
\hline 3211 & Flat Glass $\ldots \ldots \ldots \ldots \ldots$ & w & w & 0 & 1 & w & w & - & 0 & - & 4.6 \\
\hline 3221 & Glass Containers $\ldots \ldots \ldots \ldots \ldots \ldots$ & 19 & 834 & 193 & 14 & 14 & 24 & 0 & 0 & - & 9.0 \\
\hline 3229 & Pressed and Blown Glass, nec. . . . . . . . & w & w & 80 & w & w & 8 & 0 & 0 & - & 10.3 \\
\hline 3241 & Cement, Hydraulic & 43 & 1,334 & 14 & $\mathbf{w}$ & - & 1 & 1,482 & w & w & 18.1 \\
\hline 3274 & Lime & $\mathbf{Q}$ & $\mathbf{Q}$ & 0 & Q & - & Q & $\mathbf{Q}$ & 0 & - & NF \\
\hline 3296 & Mineral Wool & 4 & 304 & 0 & $\mathbf{w}$ & w & $\mathbf{w}$ & - & $\mathbf{w}$ & - & 1.7 \\
\hline 33 & Primary Metal Industries & 200 & 17,771 & 852 & 324 & 102 & 307 & w & w & w & 9.0 \\
\hline 3312 & Blast Fumaces and Steol Mills .. & 114 & 8,136 & w & 156 & 65 & 30 & 52 & $w$ & 1 & 6.6 \\
\hline 3313 & Electrometalurgical Products . . . . . . . & w & w & 0 & 1 & * & • & w & w & - & 12.9 \\
\hline 3321 & Gray and Ductile Iron Foundries & 5 & 350 & 0 & 13 & 2 & 18 & 1 & 66 & - & 16.5 \\
\hline 3331 & Primary Copper & • & w & 0 & w & • & - & 0 & 0 & - & 1.1 \\
\hline 3334 & Primary Aluminum & w & w & * & $\mathbf{w}$ & w & w & 0 & 0 & - & 5.5 \\
\hline 3339 & Primary Nonferrous Metals, nec... & w & 100 & 1 & w & 1 & 1 & w & 0 & - & 2.1 \\
\hline 3353 & Aluminum Sheet, Plate, and Foil . . . . . & w & 451 & 0 & W & W & 23 & 0 & 0 & - & 1.2 \\
\hline 34 & Fabricated Metal Products . . . . . . & 56 & 5,074 & 368 & 366 & 31 & 153 & 9 & 37 & 1 & 15.6 \\
\hline 35 & Industrial Machinery and Equipment & 41 & 5,213 & 408 & 352 & 15 & 176 & 0 & 0 & 2 & 19.2 \\
\hline 357 & Computer and Office Equipment & 4 & 819 & 8 & 10 & 1 & 2 & 0 & 0 & • & 20.8 \\
\hline 36 & Electronic and Other Electric Equipment & 42 & 6,555 & 504 & 291 & 14 & 162 & 4 & 2 & - & 16.2 \\
\hline 37 & Transportation Equipment & w & 3,114 & 1,070 & 507 & 10 & w & w & 0 & w & 11.2 \\
\hline 3711 & Motor Vehicles and Car Bodies & w & w & w & w & 1 & 1 & 0 & 0 & • & 7.8 \\
\hline 3714 & Motor Vehicle Parts and Accessories & 8 & 887 & w & 6 & w & 10 & w & 0 & - & 11.9 \\
\hline 38 & Instruments and Related Products $\ldots$ & 52 & 4,015 & 513 & w & w & $\mathbf{Q}$ & $w$ & 0 & $w$ & 18.1 \\
\hline 3841 & Surgical and Medical Instruments . & 2 & 332 & 9 & 16 & * & 2 & 0 & 0 & - & 19.3 \\
\hline 39 & Misc. Manufacturing Industries .. & $\mathbf{w}$ & 1,187 & 84 & w & w & w & 22 & 0 & - & 18.4 \\
\hline & Total $\ldots \ldots \ldots \ldots \ldots$ & 1,226 & 87,851 & 23,054 & 7,692 & 445 & 3,168 & 7,420 & 680 & 83 & 6.0 \\
\hline
\end{tabular}

See footnotes at end of table. 
Table A5. Total Consumption of Offsite-Produced Energy for Heat, Power, and Electricity Generation by Census Region, Industry Group, and Selected Industries, 1991: Part 1 (Continued) (Estimates in Btu or Physical Units)

\begin{tabular}{|c|c|c|c|c|c|c|c|c|c|c|c|}
\hline$\underset{\operatorname{Cod\theta }^{a}}{\text { SIC }}$ & $\begin{array}{c}\text { Industry Groups } \\
\text { and Industry }\end{array}$ & $\begin{array}{c}\text { Total } \\
\text { (trillion } \\
\text { Btu) }\end{array}$ & $\begin{array}{c}\text { Electricity } \\
\text { (million } \\
\text { kWh) }\end{array}$ & $\begin{array}{c}\text { Residual } \\
\text { Fual Oil } \\
(1000 \\
\text { bbls) }\end{array}$ & $\begin{array}{c}\text { Distillate } \\
\text { Fuel Oile } \\
(1000 \\
\text { bbls })\end{array}$ & $\begin{array}{c}\text { Natural } \\
\text { Gas }^{d} \\
\text { (billion } \\
\text { cu ft) }\end{array}$ & $\begin{array}{c}\text { LPG } \\
(1000 \\
\text { bbls) }\end{array}$ & $\begin{array}{l}\text { Coal } \\
(1000 \\
\text { short } \\
\text { tons) }\end{array}$ & $\begin{array}{c}\text { Coke and } \\
\text { Breeze } \\
\text { (1000 } \\
\text { short } \\
\text { tons) }\end{array}$ & $\begin{array}{c}\text { Other } \\
\text { (trillion } \\
\text { Btu) }\end{array}$ & $\begin{array}{c}\text { RSE } \\
\text { Row } \\
\text { Factors }\end{array}$ \\
\hline & & \multicolumn{10}{|c|}{ Midwest Census Region } \\
\hline & RSE Column Factors: & 0.6 & 0.6 & 1.5 & 1.2 & 0.7 & 1.2 & 1.1 & 1.4 & 1.1 & \\
\hline 20 & Food and Kindred Products . . . . . . . . & 418 & 19,640 & 893 & 544 & 211 & 348 & 4,808 & W & $w$ & 8.6 \\
\hline 2011 & Meat Packing Plants ............ & 32 & 2,065 & 150 & 37 & 22 & 13 & 27 & 0 & 1 & 9.5 \\
\hline 2033 & Canned Fruits and Vegetables . . . . . . . . & 10 & 375 & 0 & 62 & 8 & 39 & 0 & 0 & $\star$ & 17.2 \\
\hline 2037 & Frozen Fruits and Vegetables . . . . . . & 4 & 289 & 36 & 3 & 2 & 3 & 0 & 0 & $*$ & 27.2 \\
\hline 2046 & Wot Com Mlling . . . . . . . . . . . & 122 & 3,199 & W & 26 & $4 \overline{5}$ & 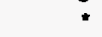 & 2,729 & $W$ & $w$ & 13.2 \\
\hline 2051 & Bread, Cake, and Related Products .... & 9 & 632 & 0 & W & $w$ & $\because$ & 0 & 0 & $\because$ & 16.4 \\
\hline 2063 & Beet sugar . . . . . . . . . . . . . & 34 & 234 & $W$ & 10 & 6 & 2 & 1,084 & $W$ & * & 6.6 \\
\hline 2075 & 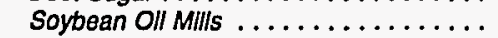 & 35 & 1,093 & 8 & 7 & 15 & $\vec{w}$ & W & 0 & $w$ & 4.4 \\
\hline 2082 & Malt Beverages $\ldots \ldots \ldots \ldots \ldots \ldots$ & 11 & 480 & 40 & 1 & $W$ & $W$ & $w$ & 0 & 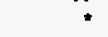 & 17.3 \\
\hline 21 & Tobacco Products . . . . . . . . . . . . & NA & NA & NA & NA & NA & NA & NA & NA & NA & 9.7 \\
\hline 22 & Textile MIII Products . . . . . . . . . . . . & NA & NA & NA & NA & NA & NA & NA & NA & NA & 24.1 \\
\hline 23 & Apparel and Other Textlle Products ..... & NA & NA & NA & NA & NA & NA & NA & NA & NA & 31.0 \\
\hline 24 & Lumber and Wood Products .......... & 29 & 2,834 & $\mathbf{Q}$ & $Q$ & 11 & 196 & 66 & 0 & 5 & 29.7 \\
\hline 25 & Furniture and Fixtures $\ldots \ldots \ldots \ldots \ldots$ & W & 1,571 & 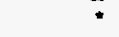 & 10 & $w$ & 45 & $\mathbf{Q}$ & 0 & $\star$ & 21.6 \\
\hline 26 & Paper and Allled Products . . . . . . . . . . & $W$ & 14,520 & 807 & 138 & 112 & 227 & 4,048 & $\vec{w}$ & $W$ & 6.2 \\
\hline 2611 & Pulp MIIIs ................. & 10 & 556 & 0 & 14 & 3 & $Q$ & 161 & 0 & 1 & 33.9 \\
\hline 2621 & Papor MIIls . . . . . . . . . . . . . & 172 & 7,730 & $w$ & 89 & 58 & 65 & 2,835 & $w$ & 17 & 5.1 \\
\hline 2631 & Paporboard Mills . . . . . . . . . . . . . . & 63 & 2,059 & 39 & 17 & 23 & 21 & 969 & 0 & 10 & 13.4 \\
\hline 27 & Printthg and Publishing $\ldots \ldots \ldots \ldots \ldots$ & 43 & 5,224 & 10 & 31 & 22 & 59 & 0 & 0 & 2 & 17.8 \\
\hline 28 & Chemlcals and Allied Products . . . . . . . . & 390 & 33,957 & $W$ & W & 181 & $w$ & 2,822 & 11 & 22 & 10.7 \\
\hline 2812 & Alkalles and Chlorine ............. & W & W & 0 & $w$ & 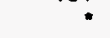 & $\because$ & 0 & 0 & 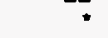 & 27.1 \\
\hline 2813 & Industrlal Gases . . . . . . . . . . . . . . & $w$ & w & 0 & $\mathbf{Q}$ & $w$ & $\mathbf{Q}$ & 0 & 0 & $\bullet$ & 13.5 \\
\hline 2819 & Industrlal Inorganic Chemicais, nec .... & 71 & $\ddot{w}$ & $W$ & 4 & $w$ & 4 & $w$ & 1 & 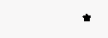 & 11.5 \\
\hline 2821 & Plastles Materials and Resins ......... & 52 & 3,248 & $\ddot{w}$ & 13 & 23 & 3 & $W$ & 0 & $w$ & 8.4 \\
\hline 2822 & 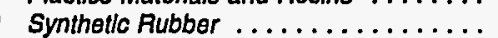 & $w$ & 241 & 0 & $w$ & $w$ & 1 & $w$ & 0 & 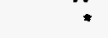 & 18.7 \\
\hline 2823 & Cellulosic Manmado Fibers . . . . . . . . . . & 0 & 0 & 0 & 0 & 0 & 0 & 0 & 0 & 0 & NF \\
\hline 2824 & Organic Flbers, Noncellulosic ........ & 0 & 0 & 0 & 0 & 0 & 0 & 0 & 0 & 0 & NF \\
\hline 2865 & Cycllc Crudes and Intemedlates ........ & 21 & 756 & $W$ & $w$ & 14 & 5 & $w$ & 0 & $Q$ & 13.3 \\
\hline 2869 & Industrial Organic Chemlcals, nec ..... & 71 & 1,822 & 10 & 26 & 33 & 4 & 1,140 & 0 & $\overline{6}$ & 8.2 \\
\hline 2873 & Nitrogenous Fertilizers $\ldots \ldots \ldots \ldots$ & 45 & 556 & 0 & 5 & 41 & 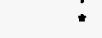 & 0 & 0 & $\mathbf{Q}$ & 36.4 \\
\hline 2874 & Phosphatlc Fertilizers . . . . . . . . . . & 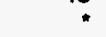 & 1 & 0 & - & $\because$ & $*$ & 0 & 0 & 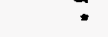 & 4.6 \\
\hline 29 & Petroleum and Coal Products ......... & 138 & 6,762 & 795 & $w$ & 86 & 647 & $w$ & 0 & 16 & 8.1 \\
\hline 2911 & Petroleum Refining . . . . . . . . . . . . . . & 113 & 6,168 & 739 & $W$ & 70 & 523 & $\mathbf{W}$ & 0 & 12 & 3.4 \\
\hline 30 & Rubber and Mlsc. Plastics Products ..... & 93 & 13,236 & 265 & 33 & 41 & 174 & 125 & 0 & 1 & 12.3 \\
\hline 3011 & Tres and Inner Tubes .............. & $W$ & $W$ & 186 & 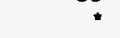 & 6 & $w$ & $w$ & 0 & : & 4.2 \\
\hline 308 & Miscellaneous Plasilcs Products, noc ... & NA & NA & NA & NA & NA & NA & NA & NA & NA & 18.5 \\
\hline 31 & Leather and Leather Products . . . . . . . . & 4 & 262 & 58 & 5 & 2 & 11 & 0 & 0 & 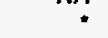 & 25.9 \\
\hline 32 & Stone, Clay and Glass Products ....... & 248 & 8,229 & 64 & 602 & 105 & 122 & 3,411 & 84 & 30 & 10.0 \\
\hline 3211 & Flat Glass $\ldots \ldots \ldots \ldots \ldots \ldots$ & 13 & $W$ & 0 & $W$ & 11 & $\mathbf{W}$ & 0 & 0 & $\cdot$ & 4.1 \\
\hline 3221 & Glass Contalners $\ldots \ldots \ldots \ldots \ldots \ldots$ & 20 & 873 & 0 & 1 & 17 & 13 & 0 & 0 & - & 7.6 \\
\hline 3229 & Pressed and Blown Glass, nec. . . . . . . . & 14 & 617 & 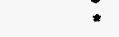 & 9 & 11 & 7 & 0 & 0 & • & 6.7 \\
\hline 3241 & Cement, Hydraulic .............. & 85 & 2,247 & W & $w$ & 6 & 5 & 2,297 & 0 & $w$ & 14.9 \\
\hline 3274 & 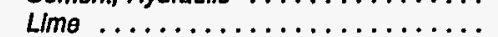 & 34 & 367 & 0 & 65 & 3 & 1 & 925 & $w$ & $w$ & 18.6 \\
\hline 3296 & 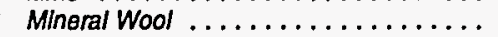 & 17 & 1,251 & $w$ & $W$ & $w$ & 14 & 0 & $W$ & 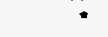 & 1.2 \\
\hline 33 & Primary Metal Industries . . . . . . . . . . & 762 & 47,532 & 2,903 & 729 & 333 & 252 & 1,190 & 7,808 & 13 & 5.6 \\
\hline 3312 & Blast Fumaces and Steel Mills . . . . . . . . & 510 & 17,777 & 2,899 & $w$ & 218 & 25 & $W$ & 7,243 & 7 & 5.4 \\
\hline 3313 & Eloctrometalurglcal Products .......... & 20 & 2,378 & 0 & 13 & 1 & $W$ & $\ddot{W}$ & * & $w$ & 11.5 \\
\hline 3321 & Gray and Ductile Iron Foundries ....... & 45 & 4,350 & 4 & $\mathbf{Q}$ & $\dot{w}$ & 37 & $w$ & $w$ & 1 & 11.7 \\
\hline 3331 & Primary Copper $\ldots \ldots \ldots \ldots \ldots$ & 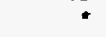 & W & 0 & 0 & 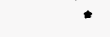 & $\cdot$ & 0 & 0 & 0 & 1.3 \\
\hline 3334 & Primany Aluminum $\ldots \ldots \ldots \ldots \ldots \ldots$ & $W$ & w & 0 & $W$ & 3 & $w$ & 0 & 0 & 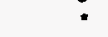 & 5.1 \\
\hline 3339 & Primary Nonferrous Metals, nec . . . . . . . & $W$ & 727 & 0 & 11 & 3 & 6 & 0 & $W$ & • & 2.0 \\
\hline 3353 & Aluminum Shest, Plate, and Foll ....... & 24 & 1,453 & 0 & $W$ & 16 & $w$ & 0 & 0 & $w$ & 1.3 \\
\hline 34 & Fabricated Metal Products . . . . . . . . . . . & 139 & 12,948 & 7 & 173 & 79 & 489 & 236 & $W$ & $w$ & 15.0 \\
\hline 35 & Industrial Machinery and Equipment ...... & 116 & 12,688 & 39 & 226 & 56 & W & 479 & $\mathbf{Q}$ & w & 13.6 \\
\hline 357 & Computer and Offlce Equipment ....... & 5 & 820 & 0 & 1 & 2 & 1 & 0 & 0 & $\because$ & 24.0 \\
\hline 36 & Electronic and Other Electric Equipment .. & 59 & 7,166 & 51 & $w$ & 28 & 67 & $w$ & 0 & $w$ & 13.4 \\
\hline 37 & Transportation Equjpment . . . . . . . . . . & 168 & 16,649 & 394 & 272 & 70 & 210 & 1,212 & 40 & 6 & 6.1 \\
\hline 3711 & Motor Vehicles and Car Bodles ........ & 63 & 5,392 & $w$ & 28 & 29 & 26 & $W$ & $W$ & $w$ & 4.5 \\
\hline 3714 & Motor Vehicle Parts and Accessories ... & 75 & 7,870 & $\dddot{W}$ & 95 & 29 & 110 & $w$ & $w$ & $w$ & 8.2 \\
\hline 38 & Instruments and Related Products ....... & 11 & 1,806 & $\mathrm{Q}$ & $Q$ & $W$ & 5 & $w$ & 0 & $\because$ & 19.5 \\
\hline 3841 & Surglcal and Medical instruments ..... & 1 & 276 & 0 & ? & 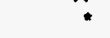 & 3 & 0 & 0 & - & 18.8 \\
\hline 39 & Misc, Manufacturing Industries . . . . . . . . & 9 & 929 & 3 & 8 & 5 & 13 & 10 & 0 & $\cdot$ & 21.4 \\
\hline & Total $\ldots \ldots \ldots \ldots \ldots \ldots \ldots$ & 2,948 & 207,104 & 6,709 & 3,806 & 1,362 & 3,188 & 18,639 & 8,101 & 147 & 3.9 \\
\hline
\end{tabular}

See footnotes at end of table. 
Table A5. Total Consumption of Offsite-Produced Energy for Heat, Power, and Ellectricity Generation by Census Region, Industry Group, and Selected Industries, 1991: Part 1 (Continued) (Estimates in Btu or Physical Units)

\begin{tabular}{|c|c|c|c|c|c|c|c|c|c|c|c|}
\hline $\begin{array}{c}\text { SIC } \\
\text { Code }^{a}\end{array}$ & $\begin{array}{c}\text { Industry Groups } \\
\text { and Industry }\end{array}$ & $\begin{array}{c}\text { Total } \\
\text { (trillion } \\
\text { Btu) }\end{array}$ & $\begin{array}{c}\text { Electricity } \\
\text { (million } \\
\text { kWh) }\end{array}$ & $\begin{array}{c}\text { Residual } \\
\text { Fuel Oil } \\
(1000 \\
\text { bbls })\end{array}$ & $\begin{array}{c}\text { Distillate } \\
\text { Fuel Oile }^{*} \\
(1000 \\
\text { bbls) }\end{array}$ & $\begin{array}{c}\text { Natural } \\
\text { Gas }^{d} \\
\text { (billion } \\
\text { cu ft) }\end{array}$ & $\begin{array}{l}\text { LPG } \\
(1000 \\
\text { bbls) }\end{array}$ & $\begin{array}{l}\text { Coal } \\
(1000 \\
\text { short } \\
\text { tons) }\end{array}$ & $\begin{array}{c}\text { Coke and } \\
\text { Breeze } \\
\text { (1000 } \\
\text { short } \\
\text { tons) }\end{array}$ & $\begin{array}{c}\text { Other } \\
\text { (Irillion } \\
\text { Btu) }\end{array}$ & $\begin{array}{c}\text { RSE } \\
\text { Row } \\
\text { Factors }\end{array}$ \\
\hline & & \multicolumn{10}{|c|}{ South Census Region } \\
\hline & RSE Column Factors: & 0.6 & 0.6 & 1.3 & 1.1 & 0.7 & 1.2 & 1.1 & 1.7 & 1.2 & \\
\hline 20 & Food and Kindred Products . . . . . . . . & 230 & 16,182 & 1,572 & 859 & 130 & 623 & 766 & $W$ & $w$ & 9.9 \\
\hline 2011 & Meat Packing Plants . . . . . . . . . . . & 10 & 892 & 14 & 167 & 5 & 112 & 0 & 0 & $*$ & 17.0 \\
\hline 2033 & Canned Fruits and Vegetables . . . . . . . & 7 & 200 & 26 & 9 & 6 & 14 & 0 & 0 & * & 19.4 \\
\hline 2037 & Frozen Fruits and Vegetables . . . . . . & 8 & 551 & 122 & 12 & 5 & 6 & 0 & 0 & . & 17.8 \\
\hline 2046 & Wet Com Milling . . . . . . . . . . . & $w$ & 837 & 0 & 2 & 4 & * & 322 & 0 & $W$ & 22.7 \\
\hline 2051 & Bread, Cake, and Related Products . . . . & 11 & 838 & 0 & 19 & 7 & 7 & 0 & 0 & $\bullet$ & 14.2 \\
\hline 2063 & Beet Sugar . . . . . . . . . . . . . & w & $W$ & 0 & 1 & 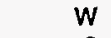 & $*$ & 0 & $W$ & - & 16.8 \\
\hline 2075 & Soybean Oil Mills. & 15 & 539 & 34 & 24 & 8 & $W$ & $w$ & 0 & $W$ & 4.9 \\
\hline 2082 & Malt Beverages ............ & 14 & 863 & W & W & 8 & 1 & W & 0 & $\bullet$ & 14.0 \\
\hline 21 & Tobacco Products . . . . . . . . . . . . & 26 & 1,452 & 135 & 40 & 4 & 23 & 692 & 0 & - & 6.4 \\
\hline 22 & Textile Mill Products . . . . . . . . . . . & 234 & 27,389 & 1,192 & 506 & 87 & 455 & 1,344 & 0 & 9 & 6.5 \\
\hline 23 & Apparel and Other Textile Products .... & 31 & 4,165 & $\mathbf{Q}$ & 71 & 12 & 124 & 83 & 0 & $\cdot$ & 22.8 \\
\hline 24 & Lumber and Wood Products ......... & 73 & 9,253 & $\mathbf{Q}$ & $W$ & 15 & 332 & 26 & 0 & 19 & 20.2 \\
\hline 25 & Fumiture and Fixtures $\ldots \ldots \ldots \ldots \ldots$ & W & 2,592 & 63 & 99 & $W$ & 122 & $W$ & 0 & 3 & 18.9 \\
\hline 26 & Paper and Allied Products . . . . . . . . . & 769 & 27,355 & 10,918 & 710 & 275 & 494 & 6,904 & 0 & 163 & 4.5 \\
\hline 2611 & Pulp Mills . . . . . . . . . . . . . & 71 & 1,421 & 3,367 & 116 & 21 & 120 & 171 & 0 & 18 & 17.7 \\
\hline 2621 & Paper Mills . . . . . . . . . . . . . & 325 & 14,513 & 2,801 & 355 & 122 & 149 & 3,910 & 0 & 42 & 3.4 \\
\hline 2631 & Paperboard Mills. & 331 & 6,887 & 4,660 & 152 & 111 & 41 & 2,786 & 0 & 101 & 5.4 \\
\hline 27 & Printing and Publishing $\ldots \ldots \ldots \ldots$ & 28 & 4,798 & Q & 35 & 10 & 47 & 0 & $\mathbf{0}$ & 1 & 21.2 \\
\hline 28 & Chemicals and Allied Products . . . . . . . & 2,009 & 83,551 & 3,964 & 1,050 & 1,293 & $W$ & 7,885 & $W$ & 182 & 6.7 \\
\hline 2812 & Alkalies and Chlorine $\ldots \ldots \ldots \ldots$ & 147 & 10,289 & 0 & 35 & $w$ & 1 & W & 0 & W & 17.9 \\
\hline 2813 & Industrial Gases . . . . . . . . . . . . . & 46 & 8,634 & 0 & 1 & 16 & 1 & 0 & 0 & $\cdot$ & 11.3 \\
\hline 2819 & Industrial Inorganic Chemicals, nec . . . . & 182 & W & W & 217 & 103 & 49 & 366 & $W$ & 7 & 10.7 \\
\hline 2821 & Plastics Materials and Resins . . . . . . & 188 & 10,445 & w & 65 & 113 & 34 & $\mathbf{W}$ & 0 & 18 & 5.8 \\
\hline 2822 & Synthetic Rubber . . . . . . . . . . . & 60 & W & $w$ & $W$ & 40 & 6 & 0 & 0 & $W$ & 11.8 \\
\hline 2823 & Cellulosic Manmade Fibers . . . . . . . . . & 31 & $W$ & 0 & 21 & $\mathbf{W}$ & 1 & 1,202 & 0 & * & 25.3 \\
\hline 2824 & Organic Fibers, Noncellulosic . . . . . . & 96 & 6,881 & w & W & W & 38 & 1,558 & 0 & • & 3.7 \\
\hline 2865 & Cyclic Crudes and Intermediates ..... & 102 & 3,259 & $W$ & 17 & 73 & 72 & W & 0 & W & 12.4 \\
\hline 2869 & Industrial Organic Chemicals, nec ..... & 820 & 15,110 & 338 & $Q$ & 576 & 636 & 2,679 & 0 & 109 & 10.0 \\
\hline 2873 & Nitrogenous Fertilizers $\ldots \ldots \ldots \ldots$ & 190 & 1,872 & 0 & 14 & 178 & $\bullet$ & 0 & 0 & $\bullet$ & 26.1 \\
\hline 2874 & Phosphatic Fertilizers . . . . . . . . & 31 & 1,908 & 250 & 142 & 15 & 1 & w & 0 & $W$ & 3.2 \\
\hline 29 & Petroleum and Coal Products $\ldots \ldots \ldots$ & 721 & 15,699 & 788 & W & 566 & 1,415 & $\mathbf{w}$ & $W$ & 70 & 5.8 \\
\hline 2911 & Petroleum Refining . . . . . . . . . . . & 703 & 15,216 & 726 & $W$ & 554 & 1,320 & $w$ & $\mathbf{0}$ & 70 & 3.4 \\
\hline 30 & Rubber and Misc. Plastics Products . . . . . & 87 & 12,328 & 532 & 241 & 35 & 240 & 61 & 0 & 2 & 10.7 \\
\hline 3011 & Tires and Inner Tubes . . . . . . . . . . . & W & 2,855 & W & $W$ & 13 & 53 & $\mathbf{W}$ & 0 & 1 & 4.3 \\
\hline 308 & Miscellaneous Plastics Products, nec ... & 46 & 7,801 & 156 & $W$ & 16 & 111 & $w$ & 0 & - & 21.0 \\
\hline 31 & Leather and Leather Products . . . . . . . & 2 & 258 & 26 & 9 & * & 3 & 0 & 0 & Q & 25.3 \\
\hline 32 & Stone, Clay and Glass Products . . . . . & 322 & 11,876 & 174 & 1,373 & 155 & $W$ & 3,899 & $W$ & $W$ & 11.5 \\
\hline 3211 & Flat Glass $\ldots \ldots \ldots \ldots \ldots \ldots \ldots$ & 23 & 781 & 0 & 7 & 19 & 9 & 0 & 0 & $\cdot$ & 4.1 \\
\hline 3221 & Glass Containers . . . . . . . . . . & 29 & 1,262 & W & $W$ & W & 23 & 0 & 0 & - & 8.3 \\
\hline 3229 & Pressed and Blown Glass, nec. . . . . . . & $\mathbf{W}$ & 1,598 & 1 & $W$ & W & 15 & 0 & 0 & $\cdot$ & 8.9 \\
\hline 3241 & Cement, Hydraulic . . . . . . . . . . . & 108 & 3,519 & 65 & 167 & 21 & 4 & 2,589 & 150 & 11 & 15.5 \\
\hline 3274 & Lime $\ldots \ldots \ldots \ldots \ldots \ldots \ldots$ & 37 & 405 & 0 & 70 & W & $\star$ & 1,155 & 0 & $W$ & 23.1 \\
\hline 3296 & Mineral Wool . . . . . . . . . . . . . . & 16 & 936 & W & 1 & 11 & 16 & 0 & $W$ & • & 1.4 \\
\hline 33 & Primary Metal Industries $\ldots \ldots \ldots \ldots$ & 411 & 47,437 & 1,442 & 485 & $w$ & 235 & 433 & 2,072 & W & 6.1 \\
\hline 3312 & Blast Fumaces and Steel Mills . . . . . . . & 187 & 11,037 & 1,437 & $\mathbf{W}$ & $\mathbf{W}$ & 15 & W & 1,792 & $W$ & 6.9 \\
\hline 3313 & Electrometalurgical Products .......... & W & W & 0 & 7 & $\cdot$ & 0 & $\mathbf{W}$ & 0 & 1 & 12.7 \\
\hline 3321 & Gray and Ductile Iron Foundries ..... & 21 & 1,622 & * & 56 & $w$ & 46 & $\mathbf{Q}$ & $W$ & $\cdot$ & 11.7 \\
\hline 3331 & Primary Copper . . . . . . . . . . & W & 200 & $w$ & 5 & W & 1 & 0 & 0 & • & 1.1 \\
\hline 3334 & Primary Aluminum $\ldots \ldots \ldots \ldots$ & 94 & 24,630 & 0 & $W$ & W & 9 & 0 & 0 & - & 3.9 \\
\hline 3339 & Primary Nonferrous Metals, nec . . . . . . & 14 & 1,694 & 0 & 9 & W & W & W & 0 & $\cdot$ & 4.6 \\
\hline 3353 & Aluminum Sheet, Plate, and Foil ...... & 25 & $W$ & 0 & 26 & 17 & 22 & $\mathbf{W}$ & 0 & $\cdot$ & 1.8 \\
\hline 34 & Fabricated Metal Products . . . . . . . . & 81 & 8,886 & $\mathbf{Q}$ & 377 & 42 & 380 & 0 & 23 & 2 & 21.2 \\
\hline 35 & Industrial Machinery and Equipment ..... & 57 & 8,138 & 42 & 125 & 26 & $w$ & $\mathbf{Q}$ & $\mathbf{Q}$ & W & 16.1 \\
\hline 357 & Computer and Office Equipment . . . . . & 4 & 809 & 3 & $\mathbf{Q}$ & 1 & $\cdot$ & 0 & 0 & $\cdot$ & 22.3 \\
\hline 36 & Electronic and Other Electric Equipment .. & 67 & 10,775 & 58 & 44 & 26 & 153 & 76 & 0 & $\bullet$ & 14.1 \\
\hline 37 & Transportation Equipment $\ldots \ldots \ldots \ldots$ & W & 8,542 & W & 307 & 27 & 132 & W & $\star$ & 2 & 8.8 \\
\hline 3711 & Motor Vehicles and Car Bodies . . . . . . & 23 & 2,375 & 73 & 19 & 12 & 28 & $w$ & 0 & W & 4.8 \\
\hline 3714 & Motor Vehicle Parts and Accessories . . . & 13 & 1,919 & $\cdot$ & 2 & W & 41 & W & 0 & * & 14.1 \\
\hline 38 & Instruments and Related Products . . . . . & 17 & 3,285 & $\mathbf{Q}$ & 45 & 5 & 6 & 0 & 0 & * & 18.4 \\
\hline 3841 & Surgical and Medical instruments ..... & 2 & 331 & $\mathbf{0}$ & 11 & $*$ & 1 & 0 & 0 & * & 21.4 \\
\hline 39 & Misc. Manufacturing Industries . . . . . . . & $\bar{W}$ & 1,168 & 28 & $\mathbf{Q}$ & $W$ & 25 & 0 & 0 & - & 21.4 \\
\hline & Total $\ldots \ldots \ldots \ldots \ldots \ldots \ldots \ldots$ & 5,258 & 305,128 & 21,779 & 7,783 & 2,885 & 6,057 & 22,321 & 2,306 & 487 & 3.5 \\
\hline
\end{tabular}

See footnotes at end of table. 
Table A5. Total Consumption of Offsite-Produced Energy for Heat, Power, and Electricity Generation by Census Region, Industry Group, and Selected Industries, 1991: Part 1 (Continued) (Estimates in Btu or Physical Units)

\begin{tabular}{|c|c|c|c|c|c|c|c|c|c|c|c|}
\hline $\begin{array}{c}\text { SIC } \\
\text { Code }\end{array}$ & $\begin{array}{c}\text { Industry Groups } \\
\text { and Industry }\end{array}$ & $\begin{array}{c}\text { Total } \\
\text { (trillion } \\
\text { Btu) }\end{array}$ & {$\left[\begin{array}{c}\text { Electricity } \\
(\text { million } \\
\mathrm{kWh})\end{array}\right.$} & $\begin{array}{c}\text { Residual } \\
\text { Fuel Oil } \\
\text { (1000 } \\
\text { bbls) }\end{array}$ & $\begin{array}{c}\text { Distillate } \\
\text { Fuel Oif } \\
(1000 \\
\text { bbls })\end{array}$ & $\begin{array}{c}\text { Natural } \\
\text { Gas }^{d} \\
\text { (billion } \\
\text { cu ft) }\end{array}$ & $\begin{array}{c}\text { LPG } \\
(1000 \\
\text { bbis) }\end{array}$ & $\begin{array}{l}\text { Coal } \\
\text { (1000 } \\
\text { short } \\
\text { tons) }\end{array}$ & $\begin{array}{c}\text { Coke and } \\
\text { Breeze } \\
\text { (1000 } \\
\text { short } \\
\text { tons) }\end{array}$ & $\begin{array}{c}\text { Other } \\
\text { (trillion } \\
\text { Btu) }\end{array}$ & $\begin{array}{c}\text { RSE } \\
\text { Row } \\
\text { Factors }\end{array}$ \\
\hline & & \multicolumn{10}{|c|}{ West Census Region } \\
\hline & RSE Column Factors: & 0.7 & 0.7 & 1.2 & 1.1 & 0.8 & 1.1 & 1.1 & 1.3 & 1.2 & \\
\hline 20 & Food and Kindred Products . . . . . . . . . & 196 & 9,253 & 688 & 673 & 116 & 236 & 1,241 & $w$ & $W$ & 10.1 \\
\hline 2011 & Meat Packing Plants . . . . . . . . . & 5 & 311 & W & 14 & 3 & $\mathbf{Q}$ & 0 & 0 & * & 17.3 \\
\hline 2033 & Canned Fruits and Vegetables . . . . . . . & 20 & 547 & 119 & 38 & 16 & 58 & 0 & 0 & * & 10.7 \\
\hline 2037 & Frozen Fruits and Vegetables . . . . . . & 26 & 2,118 & $Q$ & 57 & 17 & 31 & 0 & 0 & 1 & 17.1 \\
\hline 2046 & Wot Com Milling . . . . . . . . . . . & W & 93 & 0 & W & 2 & $\bullet$ & 0 & 0 & W & 20.3 \\
\hline 2051 & Bread, Cake, and Related Products .... & 6 & 388 & $\mathbf{0}$ & 2 & 5 & 7 & 0 & 0 & $*$ & 26.5 \\
\hline 2063 & Beot Sugar . . . . . . . . . . . . . . & W & W & $W$ & 19 & w & 3 & 817 & 51 & - & 8.5 \\
\hline 2075 & Soybean Oll MIlls $\ldots \ldots \ldots \ldots \ldots$ & 0 & 0 & 0 & 0 & 0 & 0 & 0 & 0 & 0 & NF \\
\hline 2082 & Malt Boverages .............. & 17 & 506 & W & W & $W$ & W & $\mathbf{W}$ & 0 & - & 16.4 \\
\hline 21 & Tobacco Products $\ldots \ldots \ldots \ldots \ldots \ldots$ & 0 & 0 & 0 & 0 & 0 & 0 & 0 & 0 & 0 & NF \\
\hline 22 & Textile Mill Products $\ldots \ldots \ldots \ldots \ldots$ & 6 & 274 & 0 & 2 & 4 & $\mathbf{Q}$ & 0 & 0 & - & 29.7 \\
\hline 23 & Apparel and Other Textile Products ..... & NA & NA & NA & NA & NA & NA & NA & NA & NA & 30.6 \\
\hline 24 & Lumber and Wood Products ......... & 83 & 6,670 & 131 & 1,158 & 10 & 348 & 0 & 0 & 41 & 19.7 \\
\hline 25 & Fumilure and Fixtures $\ldots \ldots \ldots \ldots \ldots$ & 3 & 304 & 0 & $Q$ & 1 & 28 & 0 & 0 & $\bullet$ & 33.4 \\
\hline 26 & Paper and Allied Products . . . . . . . . . & 262 & 13,888 & 1,718 & 95 & 108 & 228 & W & 0 & W & 6.7 \\
\hline 2611 & 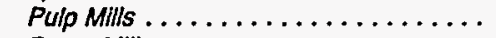 & 17 & 849 & 842 & 18 & 7 & 5 & 0 & 0 & 2 & 19.9 \\
\hline 2621 & 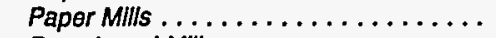 & 112 & 8,196 & W & 39 & 53 & 116 & W & 0 & 18 & 5.2 \\
\hline 2631 & 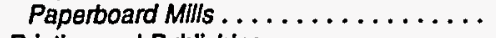 & 116 & 3,155 & W & 35 & 38 & 26 & $\mathbf{W}$ & 0 & 59 & 8.3 \\
\hline 27 & Printing and Publishing $\ldots \ldots \ldots \ldots$ & NA & NA & NA & NA & NA & NA & NA & NA & NA & 22.0 \\
\hline 28 & Chemicals and Allied Products ........ & 140 & 12,041 & W & W & 85 & W & W & $W$ & 4 & 12.6 \\
\hline 2812 & Alkalles and Chlorine ............ & $w$ & W & W & $W$ & 4 & $\cdot$ & 0 & 0 & • & 20.4 \\
\hline 2813 & Industrial Gases ................ & W & W & 0 & $W$ & $W$ & * & 0 & 0 & * & 15.1 \\
\hline 2819 & Industrial Inorganic Chemicals, nec .... & 40 & 4,479 & W & 157 & W & Q & W & W & 3 & 10.9 \\
\hline 2821 & Plastics Materials and Resins . . . . . . & 3 & 212 & 0 & $\dot{*}$ & 2 & 2 & 0 & 0 & 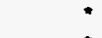 & 17.0 \\
\hline 2822 & Synthotlc Rubbor . . . . . . . . . . . . . & $\bullet$ & $\cdot$ & 0 & - & $\cdot$ & $\cdot$ & 0 & 0 & * & 24.2 \\
\hline 2823 & Cellulosle Manmade Fibers . . . . . . . . . & 0 & 0 & 0 & 0 & 0 & 0 & 0 & 0 & 0 & NF \\
\hline 2824 & Organic Fibers, Noncellulosic $\ldots . . .$. & 0 & 0 & 0 & 0 & 0 & 0 & 0 & 0 & 0 & NF \\
\hline 2865 & Cycllc Crudes and Intermedlates ...... & 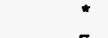 & 11 & 0 & $\therefore$ & 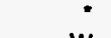 & 1 & 0 & 0 & $\because$ & 25.1 \\
\hline 2869 & Industrial Organic Chemicals, nec ..... & 5 & $\mathbf{Q}$ & 0 & a & $w$ & $Q$ & 0 & 0 & $W$ & 13.0 \\
\hline 2873 & Nitrogenous Fertilizers $\ldots \ldots \ldots$ & 42 & 461 & 0 & 3 & 39 & 42 & 0 & 0 & • & 39.6 \\
\hline 2874 & Phosphallc Fortllizers ........... & 5 & 510 & 0 & 7 & $\mathbf{Q}$ & 0 & 0 & 0 & $\bullet$ & 43.6 \\
\hline 29 & Petroleum and Coal Products $\ldots \ldots \ldots$ & 205 & 7,965 & 696 & 511 & 125 & 4,210 & 0 & 0 & 25 & 3.7 \\
\hline 2911 & Potroleum Refining $\ldots \ldots \ldots \ldots \ldots$ & 191 & 7,663 & 696 & 423 & 114 & 4,181 & 0 & 0 & 24 & 2.9 \\
\hline 30 & Rubber and Misc. Plastics Products ..... & 19 & 2,865 & 1 & $Q$ & W & $W$ & $\mathbf{Q}$ & 0 & $\cdot$ & 18.3 \\
\hline 3011 & Tires and Inner Tubes . . . . . . . . . . . & $\bullet$ & $W$ & W & $W$ & $\cdot$ & w & 0 & 0 & • & 7.1 \\
\hline 308 & Miscellaneous Plastlcs Products, nec ... & 14 & 2,573 & 0 & $\mathbf{Q}$ & 5 & 37 & $\mathbf{0}$ & 0 & - & 22.4 \\
\hline 31 & Leather and Leather Products . . . . . . . & $\mathbf{Q}$ & 70 & 0 & $\mathbf{Q}$ & $\mathbf{Q}$ & Q & 0 & 0 & - & 45.0 \\
\hline 32 & Stone, Clay and Glass Products $\ldots \ldots \ldots$ & 137 & 5,156 & 674 & 623 & 51 & 123 & 2,367 & 51 & 5 & 14.8 \\
\hline 3211 & Flat Glass $\ldots \ldots \ldots \ldots \ldots \ldots \ldots$ & $W$ & 148 & $w$ & $W$ & $W$ & $\mathbf{W}$ & 0 & 0 & - & 4.5 \\
\hline 3221 & Glass Containers $\ldots \ldots \ldots \ldots \ldots$ & 18 & 1,129 & W & $\mathbf{W}$ & $W$ & 22 & 0 & 0 & • & 9.4 \\
\hline 3229 & Pressed and Blown Glass, nec. . . . . . . & W & W & 0 & $\bullet$ & $W$ & $\cdot$ & 0 & 0 & • & 13.2 \\
\hline 3241 & Cement, Hydraullc . . . . . . . . . . & 77 & 2,390 & $W$ & 140 & 11 & 1 & 2,367 & Q & 3 & 21.1 \\
\hline 3274 & $\operatorname{LIm}{ }^{\prime} \ldots \ldots \ldots \ldots \ldots \ldots \ldots \ldots$ & W & $W$ & W & W & $W$ & 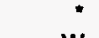 & 0 & 0 & * & 21.2 \\
\hline 3296 & Mineral Wool $\ldots \ldots \ldots \ldots \ldots \ldots$ & 3 & 330 & 0 & $\bullet$ & $w$ & $w$ & 0 & $w$ & * & 2.1 \\
\hline 33 & Primary Metal Industries . . . . . . . . . & 190 & 34,338 & 87 & 268 & $w$ & 94 & W & W & $w$ & 5.8 \\
\hline 3312 & Blast Fumaces and Steol Mills . . . . . . . & 31 & 2,531 & W & 34 & W & 4 & W & W & w & 8.3 \\
\hline 3313 & Electrometalurgical Products ........ & 0 & 0 & 0 & 0 & 0 & 0 & 0 & 0 & 0 & NF \\
\hline 3321 & Gray and Ducllle Iron Foundries . . . . . & 3 & 92 & 0 & 3 & $W$ & 3 & 0 & W & * & 37.1 \\
\hline 3331 & Primary Copper . . . . . . . . . . . . & $w$ & 1,027 & $w$ & $w^{*}$ & W & 1 & W & 0 & * & 1.0 \\
\hline 3334 & Primary Aluminum $\ldots \ldots \ldots \ldots$ & 96 & 26,391 & $\bullet$ & 17 & 6 & 28 & 0 & 0 & - & 3.5 \\
\hline 3339 & Primary Nonferrous Metals, nec . . . . . . & 11 & 1,263 & 0 & W & W & $\mathbf{W}$ & 0 & w & - & 1.0 \\
\hline 3353 & Aluminum Sheet, Plate, and Foll . . . . . . & $w$ & W & 0 & $W$ & W & W & 0 & 0 & - & 1.1 \\
\hline 34 & Fabricated Metal Products . . . . . . . & 28 & 2,922 & 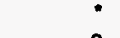 & 78 & 16 & 101 & 0 & 0 & : & 19.6 \\
\hline 35 & Industrial Machinery and Equipment . . . . . & 22 & 3,619 & 0 & 14 & 8 & 72 & 0 & 0 & * & 24.7 \\
\hline 357 & Computer and Office Equipment ...... & 9 & 1,950 & 0 & $*$ & 2 & $\mathbf{Q}$ & 0 & 0 & $\cdot$ & 14.9 \\
\hline 36 & Electronic and Other Electric Equipment .. & 29 & 5,550 & 0 & $\mathbf{Q}$ & 9 & 13 & 0 & 0 & w & 19.3 \\
\hline
\end{tabular}

See footnotes at end of table. 


\section{Table A5. Total Consumption of Offsite-Produced Energy for Heat, Power, and Electricity Generation by Census Region, Industry Group, and Selected Industries, 1991: Part 1 (Continued) (Estimates in Btu or Physical Units)}

\begin{tabular}{|c|c|c|c|c|c|c|c|c|c|c|c|}
\hline $\begin{array}{l}\text { SIC } \\
\text { Code }\end{array}$ & $\begin{array}{l}\text { Industry Groups } \\
\text { and Industry }\end{array}$ & $\begin{array}{c}\text { Total } \\
\text { (tritlion } \\
\text { Btu) }\end{array}$ & $\begin{array}{c}\text { Electricity } \\
\text { (million } \\
\text { kWh) }\end{array}$ & $\begin{array}{c}\text { Residual } \\
\text { Fuel Oil } \\
\text { (1000 } \\
\text { bbls) }\end{array}$ & $\begin{array}{c}\text { Distillate } \\
\text { Fuel Oif } \\
(1000 \\
\text { bbls) }\end{array}$ & $\begin{array}{l}\text { Natural } \\
\text { Gas }^{\mathbf{d}} \\
\text { (billion } \\
\text { cu ft) }\end{array}$ & $\begin{array}{c}\text { LPG } \\
(1000 \\
\text { bbis) }\end{array}$ & $\begin{array}{l}\text { Coal } \\
(1000 \\
\text { short } \\
\text { tons) }\end{array}$ & $\begin{array}{c}\text { Coke and } \\
\text { Breeze } \\
\text { (1000 } \\
\text { short } \\
\text { tons) }\end{array}$ & $\begin{array}{c}\text { Other } \\
\text { (trillion } \\
\text { Btu) }\end{array}$ & $\begin{array}{c}\text { RSE } \\
\text { Row } \\
\text { Factors }\end{array}$ \\
\hline & & \multicolumn{10}{|c|}{ West Census Region } \\
\hline \multicolumn{2}{|r|}{ RSE Column Factors: } & 0.7 & 0.7 & 1.2 & 1.1 & 0.8 & 1.1 & 1.1 & 1.3 & 1.2 & \\
\hline \multirow{7}{*}{$\begin{array}{c}37 \\
3711 \\
3714 \\
38 \\
3841 \\
39\end{array}$} & Transportation Equipment . . . . . & 47 & 7,096 & $w$ & 129 & 19 & $w$ & 0 & 0 & $w$ & 12.0 \\
\hline & Motor Vehicles and Car Bodies ....... & w & W & 0 & $W$ & 1 & 3 & 0 & 0 & - & 6.9 \\
\hline & Motor Vehicle Parts and Accessories ... & 3 & 243 & $\mathbf{Q}$ & 2 & 2 & 7 & $\mathbf{0}$ & 0 & * & 21.9 \\
\hline & Instruments and Related Products . . . . . & 17 & 3,244 & 4 & 9 & 5 & 6 & 0 & 0 & • & 14.9 \\
\hline & Surgical and Medical Instruments ..... & 1 & 222 & $\mathbf{0}$ & 3 & * & 2 & $\mathbf{0}$ & 0 & 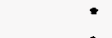 & 27.6 \\
\hline & Misc. Manufacturing Industries . . . . . . . . & 2 & 376 & & & 1 & 8 & 0 & 0 & • & 26.1 \\
\hline & Total $\ldots \ldots \ldots \ldots \ldots \ldots \ldots$ & 1,404 & 118,398 & 4,101 & 3,822 & 640 & 5,758 & 4,274 & 295 & 168 & 5.3 \\
\hline
\end{tabular}

- See Appendices B and $F$ for descriptions of the Standard Industrial Classification system.

b "Electricity" consists of quantities of electricity that were purchased or transferred in, and is equivalent to "purchased electricity" as defined in the Annual Survey of Manufactures.

"Distillate Fuel Oil" includes Nos. 1, 2, and 4 fuel olls and Nos. 1, 2, and 4 diesel fuels.

- "Natural Gas" includes natural gas obtained from utilities, transmission pipelines, and any other supplier(s) such as brokers and producers.

- "Other" includes all other energy that was purchased or transferred in and not shown elsewhere.

NF=No applicable RSE row/column factor.

- Estimate less than 0.5. Data are included in higher level totals.

$W=$ Withheld to avoid disclosing data for individual establishments. Data are included in higher level totals.

$\mathrm{Q}=$ Withheld because Relative Standard Error is greater than 50 percent. Data are included in higher level totals.

$\mathrm{NA}=$ Not available. Data are included in higher level totals.

Notes: - To obtain a RSE percentage for any table cell, multiply the cell's corresponding RSE column and RSE row factors. - Totals may not equal sum of components because of independent rounding. - The derived estimates presented in this table represent the consumption of energy originally produced offsite, acquired as a result of a purchase or transfer and consumed onsite for the production of heat and power. This definition is consistent with the definition of "purchased" fuels and electric energy used by the Bureau of the Census in the preparation of "Fuels and Electric Energy Consumed," of the Annual Survey of Manufactures, for 1974 through 1981. See Appendix B.

Source: Energy Information Administration, Office of Energy Markets and End Use, Energy End Use and Integrated Statistics Division, Form ElA-846, "1991 Manufacturing Energy Consumption Survey," and Office of Oil and Gas, Petroleum Supply Division, Form EIA-810, "Monthly Refinery Report" for 1991. 
Table A5. Total Consumption of Offsite-Produced Energy for Heat, Power, and Electricity Generation by Census Region, Industry Group, and Selected Industries, 1991:

Part 2

(Estimates in Trillion Btu)

\begin{tabular}{|c|c|c|c|c|c|c|c|c|c|c|c|}
\hline $\begin{array}{c}\text { SIC } \\
\operatorname{Cod}^{*}\end{array}$ & $\begin{array}{l}\text { Industry Groups } \\
\text { and Industry }\end{array}$ & Total & |Electricity & $\begin{array}{l}\text { Residual } \\
\text { Fuel Oil } \\
\end{array}$ & $\begin{array}{l}\text { Distillate } \\
\text { Fuel OII } \\
\end{array}$ & $\begin{array}{l}\text { Natural } \\
\text { Gas }^{\circ}\end{array}$ & LPG & Coal & $\begin{array}{c}\text { Coke and } \\
\text { Breeze }\end{array}$ & Other & $\begin{array}{c}\text { RSE } \\
\text { Row } \\
\text { Factors }\end{array}$ \\
\hline
\end{tabular}

Total United States

\begin{tabular}{|c|c|c|c|c|c|c|c|c|c|c|c|}
\hline & & & & & & & & & & & \\
\hline & RSE Column Factors: & 0.6 & 0.6 & 1.3 & 1.3 & 0.7 & 1.2 & 1.2 & 1.6 & 1.2 & \\
\hline 20 & Food and Klndred Products . . . . . . . & 922 & 172 & 27 & 17 & 512 & 5 & 154 & $w$ & $W$ & 5.9 \\
\hline 2011 & Meat Packing Plants . . . . . . . . . & 48 & 12 & 1 & 1 & 32 & 1 & 1 & 0 & 1 & 10.2 \\
\hline 2033 & Canned Fruits and Vegetables . . . . . . . . & 44 & 5 & 2 & 1 & 36 & $\bullet$ & Q & 0 & $\cdot$ & 9.1 \\
\hline 2037 & Frozen Fruits and Vegetables . . . . . . . & 40 & 11 & 2 & $*$ & 26 & $\cdot$ & 0 & 0 & 1 & 14.9 \\
\hline 2046 & Wet Com Milling . . . . . . . . . . & 141 & 14 & $\cdot$ & * & 52 & $*$ & 68 & $w$ & $W$ & 11.7 \\
\hline 2051 & Bread, Cake, and Related Products . . . . & 32 & 8 & * & 1 & 23 & - & 0 & 0 & $*$ & 12.7 \\
\hline 2063 & Bet Sugar . . . . . . . . . . . . . . & 67 & 1 & W & * & 19 & * & 43 & $w$ & $\cdot$ & 5.5 \\
\hline 2075 & Soybean Oll Mills .............. & 50 & 6 & $\cdot$ & - & 24 & $\star$ & 13 & 0 & 6 & 3.5 \\
\hline 2082 & Malt Boverages ............. & 50 & 8 & 3 & * & 23 & 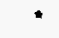 & 16 & 0 & 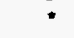 & 11.1 \\
\hline 21 & Tobacco Products . . . . . . . . . . . & 26 & 5 & 1 & - & 4 & $\star$ & 15 & 0 & $\bullet$ & 6.4 \\
\hline 22 & Textile Mijl Products $\ldots \ldots \ldots \ldots \ldots \ldots$ & 272 & 101 & 12 & 6 & 108 & 2 & 31 & 0 & 12 & 7.2 \\
\hline 23 & Apparel and Other Textile Products ..... & 44 & 19 & $\mathbf{Q}$ & 1 & 19 & 1 & 2 & 0 & 1 & 18.0 \\
\hline 24 & Lumber and Wood Products ......... & 197 & 67 & 2 & 14 & 41 & 4 & 2 & 0 & 68 & 14.9 \\
\hline 25 & Fumiture and Fixtures $\ldots \ldots \ldots \ldots \ldots$ & 46 & 17 & 1 & 1 & 19 & 1 & 4 & 0 & 4 & 18.1 \\
\hline 26 & Paper and Allied Products . . . . . . . . & 1,540 & 222 & 156 & 9 & 548 & $w$ & 292 & $W$ & 307 & 4.2 \\
\hline 2611 & Pulp MIIIs . . . . . . . . . . . . & 103 & 10 & 28 & 1 & 32 & 1 & 7 & 0 & 24 & 14.9 \\
\hline 2621 & Paper Mills . . . . . . . . . . . . & 774 & 124 & 85 & $W$ & 260 & 2 & 193 & $W$ & 106 & 2.9 \\
\hline 2631 & Paperboard Mills . . . . . . . . . . . . . . & 527 & 43 & $W$ & 1 & 185 & $*$ & W & 0 & 171 & 4.7 \\
\hline 27 & Printing and Publishing $\ldots \ldots \ldots \ldots \ldots$ & 108 & 53 & $\cdot$ & 2 & 48 & 1 & 0 & 0 & 4 & 13.0 \\
\hline 28 & Chemicals and Allied Products . . . . . . . & 2,674 & 474 & 47 & 12 & 1,665 & 4 & 249 & 3 & 221 & 5.6 \\
\hline 2812 & Alkalles and Chlorine .............. & 159 & 43 & W & $\bullet$ & $W$ & : & W & 0 & $w$ & 16.1 \\
\hline 2813 & Industrial Gases $\ldots \ldots \ldots \ldots \ldots \ldots$ & 86 & 61 & 0 & $\cdot$ & 25 & $\mathbf{Q}$ & 0 & 0 & $\cdot$ & 12.0 \\
\hline 2819 & Industrial Inorganic Chemicals, nec .... & 303 & 130 & 4 & 3 & 140 & 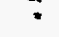 & 12 & 3 & 10 & 8.4 \\
\hline 2821 & Plastics Materials and Resins . . . . . . . & 262 & 51 & 4 & 1 & 151 & - & 24 & 0 & 31 & 5.9 \\
\hline 2822 & Synthetlc Rubber . . . . . . . . . . . & 68 & 7 & - & $*$ & 44 & $*$ & $w$ & 0 & $W$ & 12.6 \\
\hline 2823 & Cellulosic Manmade Fibers . . . . . . . . . & 31 & W & 0 & * & $W$ & - & 27 & 0 & - & 25.1 \\
\hline 2824 & Organlc Flbers, Noncellulosic ........ & 97 & 24 & W & • & $W$ & • & 35 & 0 & * & 3.7 \\
\hline 2865 & Cycllc Crudes and Intermedlates . ..... & 136 & 15 & 7 & 1 & 97 & • & W & 0 & $w$ & 11.8 \\
\hline 2869 & Industrial Organic Chemicals, nec ..... & 935 & 69 & 11 & 3 & 641 & 2 & 85 & 0 & 124 & 8.0 \\
\hline 2873 & Nitrogenous Fertilizers $\ldots \ldots \ldots \ldots$ & 278 & 10 & 0 & $*$ & 266 & $\cdot$ & 0 & 0 & $Q$ & 22.5 \\
\hline 2874 & Phosphatlc Fertilizers . . . . . . . . . . & 36 & 8 & 2 & 1 & 19 & $\cdot$ & $W$ & 0 & $w$ & 5.5 \\
\hline 29 & Petroleum and Coal Products ........ & 1,138 & 114 & 24 & 17 & 830 & 26 & $W$ & $w$ & 123 & 5.4 \\
\hline 2911 & Petroleum Reflning . . . . . . . . . . . . & 1,065 & 108 & 23 & 5 & 785 & 23 & 3 & 0 & 118 & 3.5 \\
\hline 30 & Rubber and Mlsc. Plastics Products . . . . . & 235 & 116 & 8 & 3 & 96 & 3 & 7 & 0 & 3 & 9.6 \\
\hline 3011 & Tres and Inner Tubes . . . . . . . . . . . & 42 & 14 & 3 & * & 21 & * & 2 & 0 & 1 & 3.6 \\
\hline 308 & Miscellaneous Plastics Products, nec ... & 150 & 87 & 3 & $\mathbf{w}$ & 53 & 1 & 3 & 0 & $w$ & 13.9 \\
\hline 31 & Leather and Leather Products . . . . . . . & 12 & 3 & 1 & 1 & 5 & : & $\mathbf{Q}$ & 0 & 1 & 25.2 \\
\hline 32 & Stone, Clay and Glass Products ...... & 877 & 105 & 8 & 19 & 380 & 2 & 293 & 9 & 60 & 7.6 \\
\hline 3211 & Flat Glass $\ldots \ldots \ldots \ldots \ldots$ & 49 & 5 & $W$ & * & 42 & - & * & 0 & $w$ & 3.4 \\
\hline 3221 & 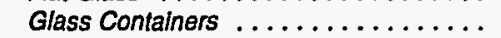 & 85 & 14 & 2 & 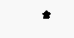 & 69 & - & 0 & 0 & * & 5.5 \\
\hline 3229 & Pressed and Blown Glass, nec. . . . . . . & $w$ & 10 & 1 & - & W & - & 0 & 0 & $\cdot$ & 8.1 \\
\hline 3241 & Cement, Hydraullc ............... & 312 & 32 & 1 & 4 & 39 & - & 195 & 6 & 35 & 11.0 \\
\hline 3274 & 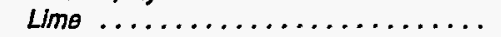 & 117 & 5 & $w$ & 1 & 8 & Q & 88 & $w$ & 13 & 29.4 \\
\hline 3296 & Mineral Wool $\ldots \ldots \ldots \ldots \ldots \ldots$ & 40 & 10 & w & - & 29 & 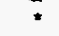 & 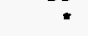 & W & 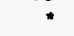 & 1.5 \\
\hline 33 & Primary Metal Industries . . . . . . . . . . & 1,563 & 502 & 33 & 11 & 686 & 3 & 46 & 262 & 20 & 4.3 \\
\hline 3312 & Blast Fumaces and Steel Mills . . . . . . . . & 842 & 135 & 31 & 5 & 399 & - & 24 & 237 & 10 & 4.3 \\
\hline 3313 & Electrometalurgical Products . . . . . . . . & 30 & 13 & 0 & - & 1 & W & $w$ & W & $w$ & 8.7 \\
\hline 3321 & Gray and Ductle Iron Foundries ...... & 74 & 22 & $\bullet$ & 1 & 28 & * & 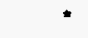 & 21 & 1 & 11.4 \\
\hline 3331 & 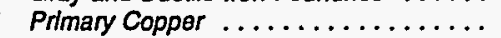 & 21 & 4 & $w$ & $w$ & 15 & • & $w$ & 0 & $*$ & 1.1 \\
\hline 3334 & Primary Aluminum $\ldots \ldots \ldots \ldots$ & 254 & 231 & 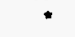 & 1 & 21 & $\cdot$ & 0 & 0 & 1 & 3.3 \\
\hline 3339 & Primany Nonferrous Metals, nec . . . . . . . & 40 & 13 & - & - & 17 & * & $W$ & W & • & 2.1 \\
\hline 3353 & Aluminum Shoet, Plate, and Foll ....... & 60 & 15 & 0 & * & 43 & - & $W$ & 0 & $w$ & 1.4 \\
\hline 34 & Fabricated Metal Products . . . . . . . . . . & 305 & 102 & 3 & 6 & 174 & 4 & 5 & $w$ & $w$ & 11.4 \\
\hline 35 & Industrial Machinery and Equlpment ..... . & 236 & 101 & 3 & 4 & 109 & 2 & 11 & 1 & 5 & 11.5 \\
\hline 357 & Computer and Office Equipment ....... & 21 & 15 & * & : & 6 & 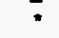 & 0 & 0 & * & 15.9 \\
\hline 36 & Electronic and Other Electric Equipment .. & 196 & 103 & 4 & 2 & 79 & 1 & W & * & $w$ & 10.2 \\
\hline 37 & Transportation Equlpment . . . . . . . . & 318 & 121 & 12 & 7 & 131 & 2 & 33 & 1 & 12 & 5.1 \\
\hline 3711 & Motor Vehicles and Car Bodles ......... & 90 & 28 & 3 & * & 44 & 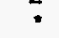 & $w$ & $\mathbf{w}$ & 4 & 3.4 \\
\hline 3714 & Motor Vehlclo Parts and Accessories ... & 99 & 37 & " & 1 & 41 & 1 & $W$ & W & $w$ & 7.2 \\
\hline 38 & Instruments and Related Products ...... & 97 & 42 & 3 & $w$ & 25 & $Q$ & $W$ & 0 & $w$ & 13.7 \\
\hline 3841 & Surgical and Medical Instruments ..... & 6 & 4 & 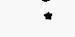 & * & 2 & 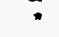 & 0 & 0 & $\because$ & 15.4 \\
\hline 39 & Misc. Manufacturing Industries . . . . . . . . & 31 & 12 & 1 & $\mathbf{w}$ & 15 & $w$ & 1 & 0 & $w$ & 14.6 \\
\hline & Total $\ldots \ldots \ldots \ldots \ldots \ldots \ldots \ldots$ & 10,837 & 2,451 & 350 & 135 & 5,492 & 67 & 1,175 & 282 & 884 & 3.1 \\
\hline
\end{tabular}

See footnotes at end of table. 
Table A5. Total Consumption of Offsite-Produced Energy for Heat, Power, and Electricity Generation by Census Region, Industry Group, and Selected Industries, 1991: Part 2 (Continued)

(Estimates in Trillion Btu)

\begin{tabular}{|c|c|c|c|c|c|c|c|c|c|c|c|}
\hline & RSE Column Factors: & 0.7 & 0.7 & 1.0 & 1.2 & 0.8 & 1.2 & 1.3 & 1.4 & 1.1 & \\
\hline 20 & Food and Kindred Products & 79 & 19 & 7 & 5 & 42 & 1 & 2 & 0 & 3 & 13.9 \\
\hline 2011 & Meat Packing Plants ..... & 1 & - & w & * & 1 & a & 0 & 0 & - & 24.4 \\
\hline 2046 & Wet Com Milling .......... & - & - & w & w & • & * & 0 & 0 & • & 23.6 \\
\hline 2051 & Bread, Cake, and Related Products . . . . & 7 & 1 & • & w & w & • & $\mathbf{0}$ & 0 & - & 19.2 \\
\hline 2063 & 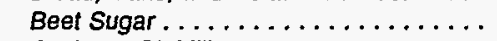 & 0 & 0 & 0 & 0 & 0 & 0 & 0 & 0 & 0 & NF \\
\hline 2075 & Soybean Oil Mills $\ldots \ldots \ldots \ldots \ldots \ldots$ & 0 & 0 & 0 & 0 & 0 & 0 & 0 & 0 & 0 & NF \\
\hline 2082 & Malt Beverages. & 8 & 2 & w & - & 4 & - & w & 0 & - & 16.4 \\
\hline 21 & Tobacco Products & NA & NA & NA & NA & NA & NA & NA & NA & NA & 25.3 \\
\hline 22 & Textile Mill Products & 27 & 5 & 5 & 3 & 11 & 1 & $*$ & 0 & 3 & 19.4 \\
\hline 23 & Apparel and Other Textile Products ..... & 5 & 2 & - & * & 2 & $\mathbf{Q}$ & 0 & 0 & * & 29.8 \\
\hline 2631 & Paperboard Mills ........ & 16 & 2 & w & $\mathbf{Q}$ & 6 & - & $w$ & 0 & 1 & 15.5 \\
\hline 27 & Printing and Publishing $\ldots \ldots \ldots \ldots \ldots$ & 23 & 11 & • & 1 & 9 & - & 0 & 0 & 1 & 26.0 \\
\hline 28 & Chemicals and Allied Products..$\ldots \ldots \ldots$ & 135 & 32 & 19 & w & 59 & • & $\mathbf{w}$ & 0 & 14 & 9.1 \\
\hline 2812 & Alkalies and Chlorine ..... & • & w & 0 & 0 & • & 0 & 0 & 0 & 0 & 36.9 \\
\hline 2813 & Industrial Gases . . . . . . . & 5 & 5 & 0 & * & • & • & 0 & 0 & 0 & 12.0 \\
\hline 2819 & Industrial Inorganic Chemicals, nec & 10 & 2 & 2 & • & 6 & • & 0 & 0 & - & 22.7 \\
\hline 2821 & Plastics Materials and Resins . . . . . . . & 19 & 4 & 3 & 1 & 8 & - & $w$ & 0 & w & 9.6 \\
\hline 2822 & Synthetic Rubber $\ldots \ldots \ldots \ldots \ldots \ldots$ & w & w & w & - & w & - & 0 & 0 & - & 26.1 \\
\hline 2823 & Celluiosic Manmade Fibers . . . . . . . . . & 0 & 0 & 0 & 0 & 0 & 0 & 0 & 0 & 0 & NF \\
\hline 2824 & Organic Fibers, Noncellulosic . . . . . . . & * & * & Q & w & * & - & 0 & 0 & 0 & 15.0 \\
\hline 2865 & Cyclic Crudes and Intermediates & 12 & 1 & w & w & 7 & - & w & 0 & 1 & 21.1 \\
\hline 2869 & Industrial Organic Chemicals, nec ..... & 38 & 10 & 9 & - & $\mathbf{w}$ & - & 0 & 0 & w & 8.7 \\
\hline 2873 & Nitrogenous Fertilizers & * & • & 0 & - & • & - & 0 & 0 & - & 47.8 \\
\hline 2874 & Phosphatic Fertilizers & 0 & 0 & 0 & 0 & 0 & 0 & 0 & 0 & 0 & NF \\
\hline 29 & Petroleum and Coal Products .... & 74 & 10 & 10 & 8 & 29 & 2 & 3 & 0 & 11 & 12.0 \\
\hline 3274 & Lime & $\mathbf{Q}$ & a & 0 & $\mathbf{Q}$ & * & Q & Q & 0 & * & NF \\
\hline 3296 & Mineral Wool & 4 & 1 & 0 & w & w & w & • & w & - & 1.7 \\
\hline 33 & Primary Metal Industries $\ldots \ldots \ldots$. & 200 & 61 & 5 & 2 & 105 & 1 & w & w & w & 9.0 \\
\hline 3312 & Blast Fumaces and Steel Mills . . . . . . . . & 114 & 28 & w & 1 & 67 & . & 1 & w & 1 & 6.6 \\
\hline 3313 & Electrometalurgical Products . . . . . . . . & w & w & 0 & * & * & - & $\mathbf{w}$ & w & • & 12.9 \\
\hline 3321 & Gray and Ductile Iron Foundries ...... & 5 & 1 & 0 & * & 2 & - & • & 2 & - & 16.5 \\
\hline 3331 & Primary Copper & - & w & 0 & w & - & - & 0 & 0 & * & 1.1 \\
\hline 3334 & I Primary Aluminum ... & w & w & • & w & $\mathbf{w}$ & w & 0 & 0 & - & 5.5 \\
\hline 3339 & Primary Nonferrous Metals, nec. & $\mathbf{w}$ & * & * & $w$ & 1 & * & w & 0 & • & 2.1 \\
\hline 3353 & Aluminum Sheet, Plate, and Foil & w & 2 & 0 & w & w & • & 0 & 0 & • & 1.2 \\
\hline 34 & Fabricated Metal Products . . . . . . & 56 & 17 & 2 & 2 & 32 & 1 & * & 1 & 1 & 15.6 \\
\hline 35 & Industrial Machinery and Equipment . & 41 & 18 & 3 & 2 & 16 & 1 & 0 & 0 & 2 & 19.2 \\
\hline 357 & Computer and Office Equipment . . & 4 & 3 & * & - & 1 & * & 0 & 0 & * & 20.8 \\
\hline 36 & Electronic and Other Electric Equipment .. & 42 & 22 & 3 & 2 & 14 & 1 & * & • & - & 16.2 \\
\hline 37 & Transportation Equipment & w & 11 & 7 & 3 & 11 & w & w & 0 & w & 11.2 \\
\hline 3711 & Motor Vehicles and Car Bodies & w & w & w & w & 1 & • & 0 & 0 & - & 7.8 \\
\hline 3714 & Motor Vehicle Parts and Accessories & 8 & 3 & w & - & w & - & w & 0 & - & 11.9 \\
\hline & Instruments and Related Products .... & 52 & 14 & 3 & W & w & $\mathbf{Q}$ & $w$ & 0 & w & 18.1 \\
\hline 3841 & Surgical and Medical Instruments & 2 & 1 & - & * & - & * & 0 & 0 & - & 19.3 \\
\hline 39 & Misc. Manufacturing Industries . . . . & $\bar{w}$ & 4 & 1 & $w$ & $w$ & w & 1 & 0 & - & 18.4 \\
\hline & Total & 1,226 & 300 & 145 & 45 & 458 & 12 & 167 & 17 & 83 & 6.0 \\
\hline
\end{tabular}

See footnotes at end of table. 
Table A5. Total Consumption of Offsite-Produced Energy for Heat, Power, and Electricity Generation by Census Region, Industry Group, and Selected Industries, 1991: Part 2 (Continued) (Estimates in Trillion Btu)

\begin{tabular}{|c|c|c|c|c|c|c|c|c|c|c|c|}
\hline $\begin{array}{c}\text { SIC } \\
\text { Code日 }\end{array}$ & $\begin{array}{l}\text { Industry Groups } \\
\text { and Industry }\end{array}$ & Total & Electricity & $\begin{array}{l}\text { Residual } \\
\text { Fuel Oil }\end{array}$ & $\begin{array}{l}\text { Distillate } \\
\text { Fuel Oile }\end{array}$ & $\begin{array}{l}\text { Natural } \\
\text { Gas }^{d}\end{array}$ & LPG & Coal & $\begin{array}{c}\text { Coke and } \\
\text { Breeze }\end{array}$ & Other & $\begin{array}{c}\text { RSE } \\
\text { Row } \\
\text { Factors }\end{array}$ \\
\hline
\end{tabular}

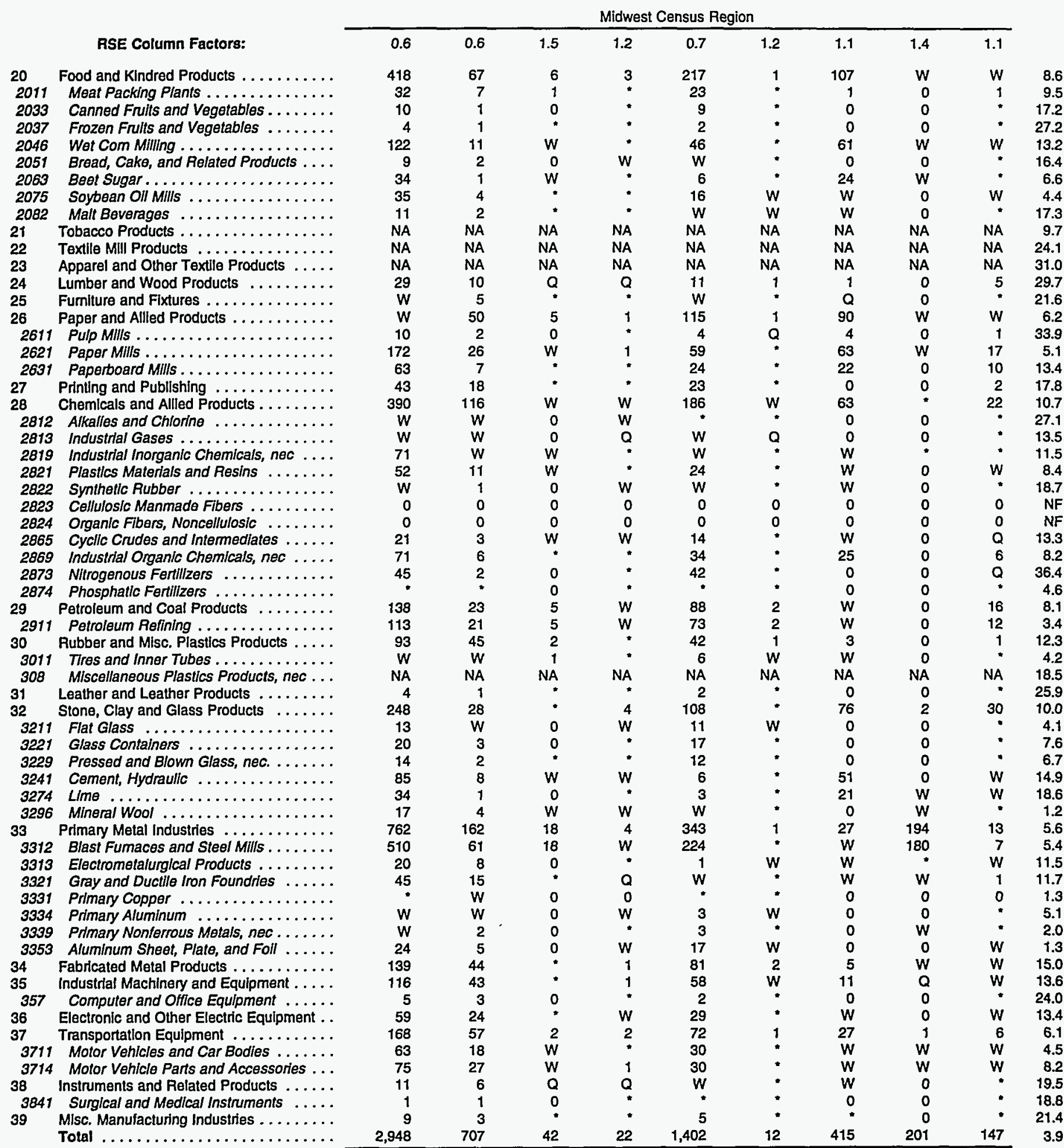

See footnotes at end of table. 
Table A5. Total Consumption of Offsite-Produced Energy for Heat, Power, and Electricity Generation by Census Region, Industry Group, and Selected Industries, 1991: Part 2 (Continued) (Estimates in Trillion Btu)

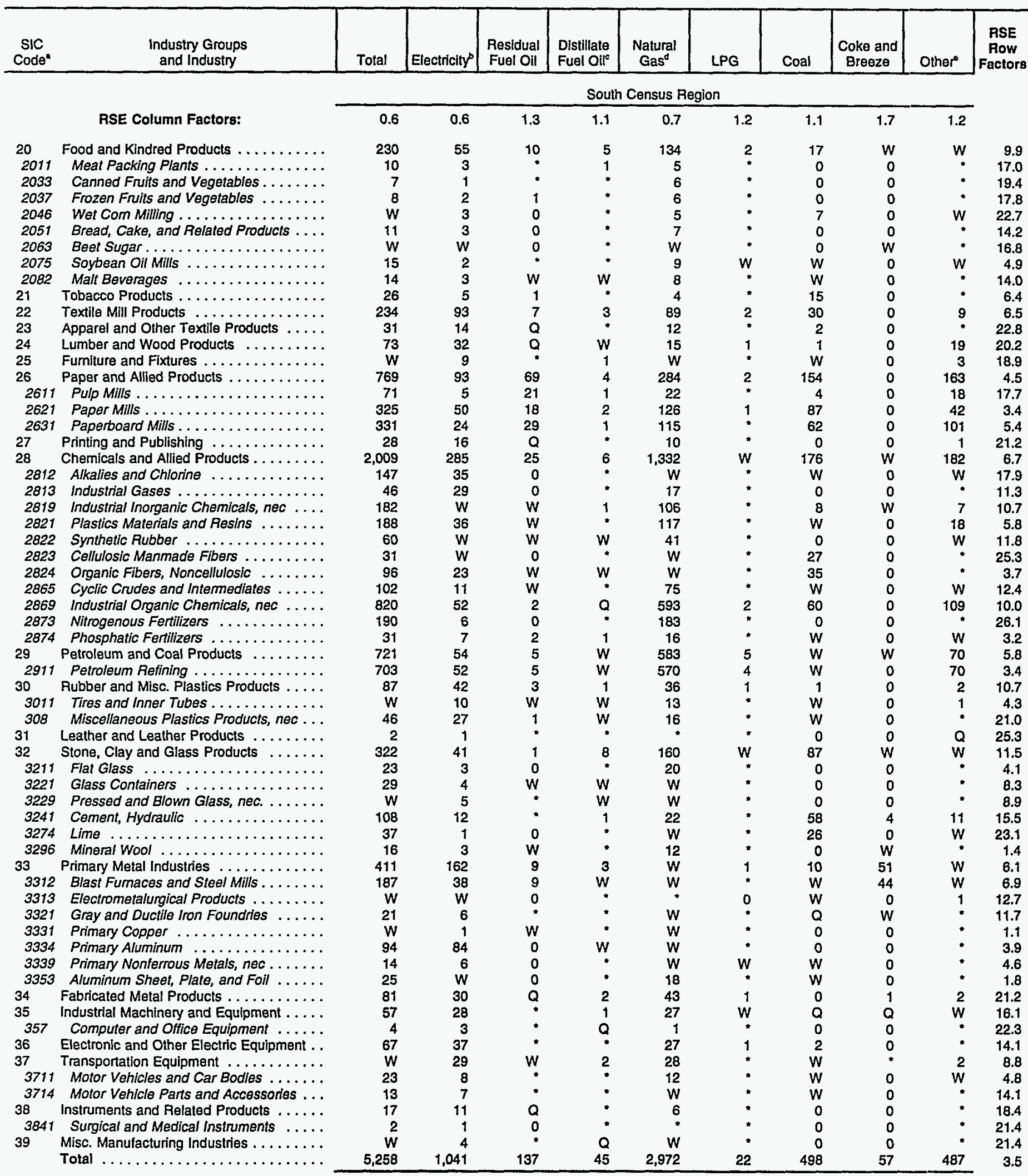

See footnotes at end of table. 
Table A5. Total Consumption of Offsite-Produced Energy for Heat, Power, and Electricity Generation by Census Region, Industry Group, and Selected Industries, 1991: Part 2 (Continued)

(Estimates in Trillion Btu)

\begin{tabular}{|c|c|c|c|c|c|c|c|c|c|c|c|}
\hline $\begin{array}{c}\text { SIC } \\
\text { Code }\end{array}$ & $\begin{array}{l}\text { Industry Groups } \\
\text { and Industry }\end{array}$ & Total & Electricity" & $\begin{array}{l}\text { Residual } \\
\text { Fuel OIl }\end{array}$ & $\begin{array}{l}\text { Distlllate } \\
\text { Fuel Olf }\end{array}$ & $\begin{array}{l}\text { Natural } \\
\text { Gas }^{d}\end{array}$ & LPG & Coal & $\begin{array}{c}\text { Coke and } \\
\text { Breeze }\end{array}$ & Other" & $\begin{array}{c}\text { RSE } \\
\text { Row } \\
\text { Factors }\end{array}$ \\
\hline
\end{tabular}

\begin{tabular}{|c|c|c|c|c|c|c|c|c|c|c|c|}
\hline & & & & & Wes & sus $R$ & & & & & \\
\hline & RSE Column Factors: & 0.7 & 0.7 & 1.2 & 1.1 & 0.8 & 1.1 & 1.1 & 1.3 & 1.2 & \\
\hline 20 & Food and Kindred Products $\ldots \ldots \ldots \ldots$ & 196 & 32 & 4 & 4 & 119 & 1 & 28 & w & w & 10.1 \\
\hline 2037 & Frozen Frults and Vegetables . . . . . . & 26 & & Q & • & 17 & • & 0 & 0 & 1 & 17.1 \\
\hline 2046 & Wet Com Mlling $\ldots \ldots \ldots \ldots \ldots \ldots$ & w & * & 0 & w & 2 & - & 0 & 0 & w & 20.3 \\
\hline 2051 & Bread, Cake, and Related Products .... & 6 & 1 & 0 & * & 5 & * & 0 & 0 & - & 26.5 \\
\hline 2063 & Beot Sugar $\ldots \ldots \ldots \ldots \ldots \ldots \ldots$ & w & W & $\mathbf{w}$ & * & w & - & 18 & 1 & - & 8.5 \\
\hline 21 & Tobacco Products $\ldots \ldots \ldots \ldots \ldots \ldots$ & 0 & 0 & 0 & 0 & 0 & 0 & 0 & 0 & 0 & NF \\
\hline 22 & Textlle Mill Products $\ldots \ldots \ldots \ldots \ldots$. & 6 & 1 & 0 & . & 5 & $\mathbf{Q}$ & 0 & 0 & - & 29.7 \\
\hline 23 & Apparel and Other Textile Products ..... & NA & NA & NA & NA & NA & NA & NA & NA & NA & 30.6 \\
\hline 24 & Lumber and Wood Products $\ldots \ldots \ldots$ & 83 & 23 & 1 & 7 & 10 & 1 & 0 & 0 & 41 & 19.7 \\
\hline 25 & Furniture and Fixtures $\ldots \ldots \ldots \ldots \ldots$ & 3 & 1 & 0 & $\mathbf{Q}$ & 1 & • & 0 & 0 & - & 33.4 \\
\hline 26 & Paper and Allied Products $\ldots \ldots \ldots \ldots$ & 262 & 47 & 11 & 1 & 111 & 1 & $\mathbf{w}$ & 0 & w & 6.7 \\
\hline 2611 & Pulp MIIIs $\ldots \ldots \ldots \ldots \ldots \ldots \ldots$ & 17 & 3 & 5 & - & 7 & - & 0 & 0 & 2 & 19.9 \\
\hline 2813 & Industrial Gases $\ldots \ldots \ldots \ldots \ldots$ & w & w & 0 & w & w & • & 0 & 0 & - & 15.1 \\
\hline 2819 & Industrtal Inorganic Chemicals, nec .... & 40 & 15 & w & 1 & w & Q & w & w & 3 & 10.9 \\
\hline 2821 & Plastlos Materials and Resins . . . . . . . & 3 & 1 & 0 & • & 2 & • & 0 & 0 & • & 17.0 \\
\hline 2822 & Synthelle Rubber $\ldots \ldots \ldots \ldots \ldots \ldots$ & * & - & 0 & • & - & - & 0 & 0 & * & 24.2 \\
\hline 2823 & Cellulosic Manmado Fibers . . . . . . . . . & 0 & 0 & 0 & 0 & 0 & 0 & 0 & 0 & 0 & NF \\
\hline 2824 & Organic Fibers, Noncellulosic $\ldots \ldots \ldots$ & 0 & 0 & 0 & 0 & 0 & 0 & 0 & 0 & 0 & NF \\
\hline 2865 & Cyclla Crudes and Intermediates ...... & " & - & 0 & • & - & - & 0 & 0 & • & 25.1 \\
\hline 2869 & Industrlal Organic Chemicals, nec ..... & 5 & $\mathbf{Q}$ & 0 & Q & w & Q & 0 & 0 & w & 13.0 \\
\hline 2873 & Nitrogenous Fentlizers $\ldots \ldots \ldots \ldots \ldots$ & 42 & 2 & 0 & , & 40 & • & 0 & 0 & - & 39.6 \\
\hline 2874 & Phosphatlc Fertilizers $\ldots \ldots \ldots \ldots \ldots$ & 5 & 2 & 0 & - & $\mathbf{Q}$ & 0 & 0 & 0 & - & 43.6 \\
\hline 29 & Petroleum and Coal Products $\ldots \ldots \ldots$ & 205 & 27 & 4 & 3 & 129 & 16 & 0 & 0 & 25 & 3.7 \\
\hline 2911 & Petroleum Reffining $\ldots \ldots \ldots \ldots$ & 191 & 26 & 4 & 2 & 118 & 16 & 0 & 0 & 24 & 2.9 \\
\hline 30 & Rubber and Mlsc. Plastics Products ..... . & 19 & 10 & • & $\mathbf{Q}$ & w & w & $\mathbf{Q}$ & 0 & - & 18.3 \\
\hline 3011 & Tres and Inner Tubes . . . . . . . . . . . . & • & W & W & w & - & $\mathbf{w}$ & 0 & 0 & • & 7.1 \\
\hline 308 & Mlscellaneous Plastles Products, nec... & 14 & 9 & 0 & Q & 5 & - & 0 & 0 & • & 22.4 \\
\hline 3313 & Electrometalurgical Products . . . . . . . . & 0 & 0 & 0 & 0 & 0 & 0 & 0 & 0 & 0 & NF \\
\hline 3321 & Gray and Ducille Iron Foundries ....... & 3 & - & 0 & - & w & • & 0 & w & - & 37.1 \\
\hline 3331 & Primary Copper $\ldots \ldots \ldots \ldots \ldots \ldots$ & w & 4 & W & $w$ & w & - & w & 0 & • & 1.0 \\
\hline 3334 & Primary Aluminum & 96 & 90 & * & - & 6 & - & 0 & 0 & - & 3.5 \\
\hline 3339 & Primary Nonforrous Metals, nec . . . . . . . & 11 & 4 & 0 & $\mathbf{w}$ & w & $\mathbf{w}$ & 0 & w & - & 1.0 \\
\hline 3353 & Aluminum Sheet, Plate, and Foll ....... & $\mathbf{w}$ & $\mathbf{w}$ & 0 & $\mathbf{w}$ & W & $\mathbf{w}$ & 0 & 0 & • & 1.1 \\
\hline 34 & Fabricated Metal Products $\ldots \ldots \ldots \ldots \ldots$ & 28 & 10 & • & • & 17 & - & 0 & 0 & - & 19.6 \\
\hline 35 & Industrial Machinery and Equipment ..... . & 22 & 12 & 0 & * & 9 & • & 0 & 0 & * & 24.7 \\
\hline 357 & Computer and Office Equipment ...... & 9 & 7 & 0 & * & 2 & Q & 0 & 0 & * & 14.9 \\
\hline 36 & Electronlc and Other Electric Equipment .. & 29 & 19 & 0 & $\mathbf{Q}$ & 9 & - & 0 & 0 & w & 19.3 \\
\hline
\end{tabular}

See footnotes at end of table. 
Table A5. Total Consumption of Offsite-Produced Energy for Heat, Power, and Electricity Generation by Census Region, Industry Group, and Selected Industries, 1991: Part 2 (Continued)

(Estimates in Trillion Btu)

\begin{tabular}{|c|c|c|c|c|c|c|c|c|c|c|c|}
\hline $\begin{array}{c}\text { SIC } \\
\text { Code }\end{array}$ & $\begin{array}{l}\text { Industry Groups } \\
\text { and Industry }\end{array}$ & Total & Electricity ${ }^{b}$ & $\begin{array}{l}\text { Residual } \\
\text { Fuel Oil }\end{array}$ & $\begin{array}{l}\text { Distillate } \\
\text { Fuel Oile } \\
\end{array}$ & $\begin{array}{c}\text { Natural } \\
\text { Gas }^{d}\end{array}$ & LPG & Coal & $\begin{array}{c}\text { Coke and } \\
\text { Breeze }\end{array}$ & Other & $\begin{array}{c}\text { RSE } \\
\text { Row } \\
\text { Factors }\end{array}$ \\
\hline & & \multicolumn{10}{|c|}{ West Census Region } \\
\hline \multicolumn{2}{|r|}{ ASE Column Factors: } & 0.7 & 0.7 & 1.2 & 1.1 & 0.8 & 1.1 & 1.1 & 1.3 & 1.2 & \\
\hline 37 & Transportation Equipment & 47 & 24 & $w$ & 1 & 20 & $w$ & 0 & 0 & $w$ & 12.0 \\
\hline 3711 & Motor Vehicles and Car Bodies ....... & $W$ & W & 0 & W & 1 & 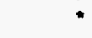 & 0 & 0 & $\bullet$ & 6.9 \\
\hline 3714 & Motor Vehicle Parts and Accessories ... & 3 & 1 & $Q$ & $\cdot$ & 2 & $*$ & 0 & 0 & - & 21.9 \\
\hline 38 & Instruments and Related Products . . . . . & 17 & 11 & $\bullet$ & $\cdot$ & 6 & * & 0 & 0 & - & 14.9 \\
\hline 3841 & Surgical and Medical Instruments ..... & 1 & 1 & 0 & $*$ & $\cdot$ & * & 0 & 0 & - & 27.6 \\
\hline \multirow[t]{2}{*}{39} & Misc. Manufacturing Industries . . . . . . . & 2 & 1 & 0 & $\bullet$ & 1 & * & 0 & 0 & - & 26.1 \\
\hline & Total $\ldots \ldots \ldots \ldots \ldots \ldots \ldots$ & 1,404 & 404 & 26 & 22 & 659 & 22 & 95 & 7 & 168 & 5.3 \\
\hline
\end{tabular}

See Appendices B and F for descriptions of the Standard Industrial Classification system.

- "Electricity" consists of quantities of electricity that were purchased or transferred in, and is equivalent to "purchased electricity" as defined in the Annual Survey of Manufactures.

c "Distillate Fuel Oil" includes Nos. 1, 2, and 4 fuel oils and Nos. 1, 2, and 4 diesel fuels.

"Natural Gas" includes natural gas obtained from utilities, transmission pipelines, and any other supplier(s) such as brokers and producers.

- "Other" includes all other energy that was purchased or transferred in and not shown elsewhere.

NF=No applicable RSE row/column tactor.

Estimate less than 0.5. Data are included in higher level totals.

$W=$ Withheld to avoid disclosing data for individual establishments. Data are included in higher level totals.

$\mathrm{Q}=$ Withheld because Relative Standard Error is greater than $\mathbf{5 0}$ percent. Data are included in higher level totals.

$\mathrm{NA}=$ Not available. Data are included in higher level totals.

Notes: - To obtain a RSE percentage for any table cell, multiply the cell's corresponding RSE column and RSE row factors. - Totals may not equal sum of components because of independent rounding. - The derived estimates presented in this table represent the consumption of energy originally produced offsite,

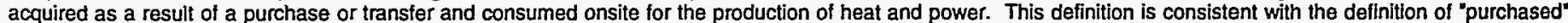
fuels and electric energy used by the Bureau of the Census in the preparation of "Fuels and Electric Energy Consumed," of the Annual Survey of Manufactures, for 1974 through 1981. See Appendix B.

Source: Energy Information Administration, Office of Energy Markets and End Use, Energy End Use and Integrated Statistics Division, Form ElA-846, "1991 Manufacturing Energy Consumption Survey," and Office of Oil and Gas, Petroleum Supply Division, Form ElA-810, "Monthly Refinery Report" for 1991. 
Table A6. Total Inputs of Selected Byproduct Energy for Heat, Power, and Electricity Generation by Census Region, Industry Group, and Selected Industries, 1991 (Estimates in Trillion Btu)

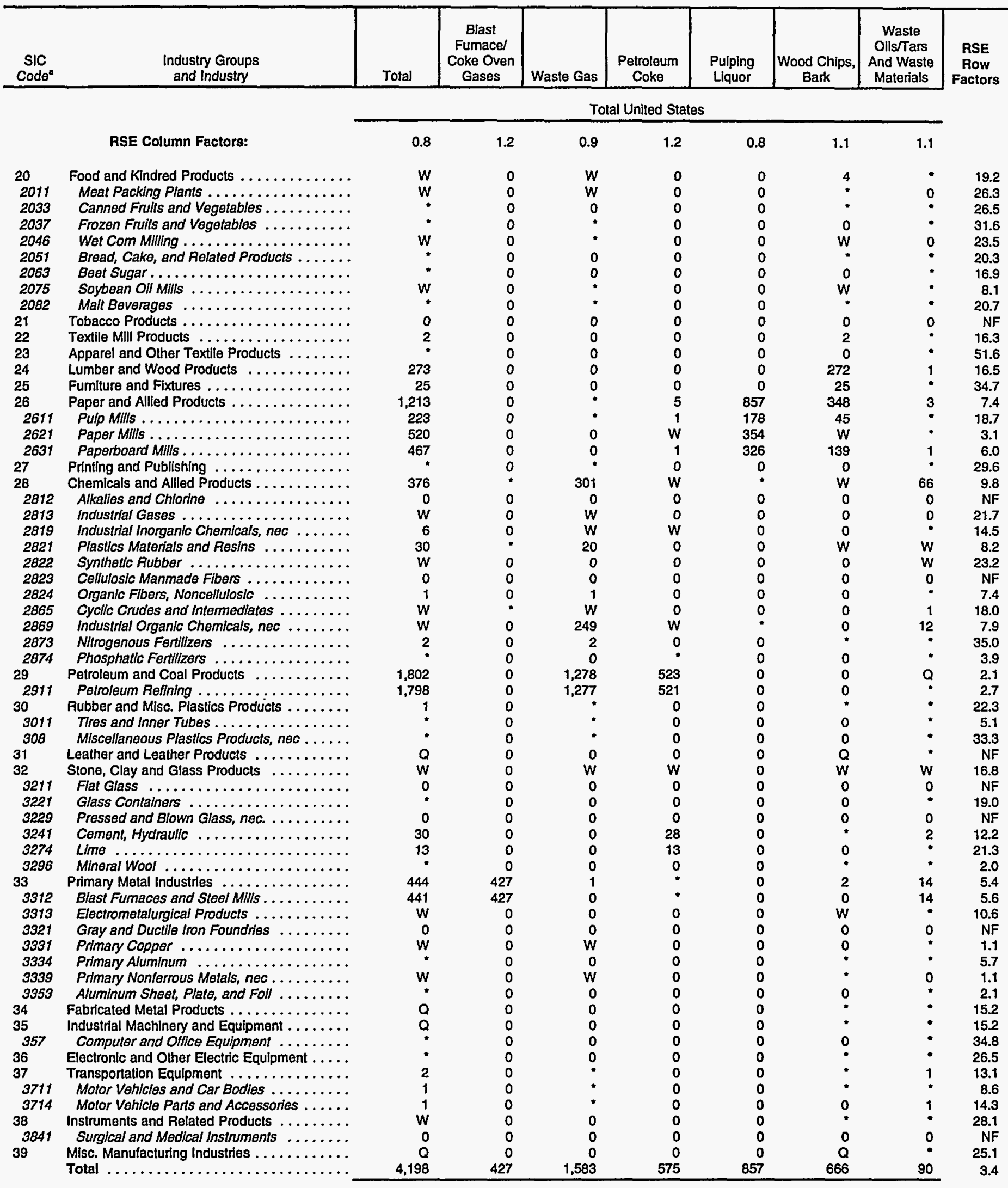

See footnotes at end of table. 


\section{Table A6. Total Inputs of Selected Byproduct Energy for Heat, Power, and Electricity Generation by Census Region, Industry Group, and Selected Industries, 1991 (Continued)}

(Estimates in Trillion Btu)

\begin{tabular}{|c|c|c|c|c|c|c|c|c|c|}
\hline $\begin{array}{c}\text { SIC } \\
\text { Code }\end{array}$ & $\begin{array}{l}\text { Industry Groups } \\
\text { and Industry }\end{array}$ & Total & $\begin{array}{c}\text { Blast } \\
\text { Fumace/ } \\
\text { Coke Oven } \\
\text { Gases }\end{array}$ & Waste Gas & $\begin{array}{c}\text { Petroleum } \\
\text { Coke }\end{array}$ & $\begin{array}{l}\text { Pulping } \\
\text { Liquor }\end{array}$ & $\begin{array}{c}\text { Wood Chips, } \\
\text { Bark }\end{array}$ & $\begin{array}{c}\text { Waste } \\
\text { Oils/Tars } \\
\text { And Waste } \\
\text { Materials }\end{array}$ & $\begin{array}{c}\text { RSE } \\
\text { Row } \\
\text { Factors }\end{array}$ \\
\hline
\end{tabular}

Northeast Census Region

\begin{tabular}{|c|c|c|c|c|c|c|c|c|c|}
\hline & & & & & & & & \\
\hline & RSE Column Factors: & 0.9 & 1.2 & 0.9 & 1.3 & 0.9 & 0.9 & 1.0 & \\
\hline 20 & Food and Kindred Products $\ldots \ldots \ldots \ldots \ldots$ & 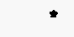 & 0 & $\bullet$ & 0 & 0 & $\bullet$ & $*$ & 22.3 \\
\hline 2011 & Meat Packing Plants . . . . . . . . . . . . & - & 0 & $\bullet$ & 0 & 0 & 0 & 0 & 29.5 \\
\hline 2033 & Canned Fruits and Vegetables . . . . . . . . . & 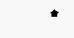 & 0 & 0 & 0 & 0 & $\star$ & * & 31.1 \\
\hline 2037 & Frozen Fruits and Vegetables . . . . . . . . & 0 & 0 & 0 & 0 & 0 & 0 & 0 & NF \\
\hline 2046 & Wet Com Milling . . . . . . . . . . . . & $=$ & 0 & 0 & 0 & 0 & $\cdot$ & 0 & 25.4 \\
\hline 2051 & Bread, Cake, and Related Products . . . . . . & * & 0 & 0 & 0 & 0 & $\cdot$ & 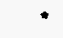 & 21.6 \\
\hline 2063 & Beet Sugar . . . . . . . . . . . . & 0 & 0 & 0 & 0 & 0 & 0 & $\mathbf{0}$ & NF \\
\hline 2075 & Soybean Oil Mills . . . . . . . . . . . . . & 0 & 0 & 0 & 0 & 0 & 0 & 0 & NF \\
\hline 2082 & Malt Beverages . . . . . . . . . . . & * & 0 & 0 & 0 & 0 & $\cdot$ & * & 24.7 \\
\hline 21 & Tobacco Products . . . . . . . . . . . . & NA & NA & NA & NA & NA & NA & NA & NF \\
\hline 22 & Textile Mill Products . . . . . . . . . . . . & $\bullet$ & 0 & 0 & 0 & 0 & $*$ & 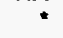 & 37.6 \\
\hline 23 & Apparel and Other Textile Products ...... & $\cdot$ & 0 & 0 & 0 & 0 & 0 & * & NF \\
\hline 24 & Lumber and Wood Products $\ldots \ldots \ldots \ldots$ & NA & NA & NA & NA & NA & NA & NA & 53.0 \\
\hline 25 & Fumiture and Fixtures $\ldots \ldots \ldots \ldots \ldots$ & 2 & 0 & 0 & 0 & 0 & 2 & $\cdot$ & 50.8 \\
\hline 26 & Paper and Allied Products . . . . . . . . . . & 98 & 0 & 0 & 1 & 58 & 39 & $\bullet$ & 5.9 \\
\hline 2611 & Pulp Mills . . . . . . . . . . . . . . & 9 & 0 & 0 & 0 & 7 & 2 & 0 & 39.8 \\
\hline 2621 & Paper Mills . . . . . . . . . . . . . & 89 & 0 & 0 & 1 & 51 & 37 & $\cdot$ & 4.3 \\
\hline 2631 & Paperboard Mills . . . . . . . . . . . . & $\cdot$ & 0 & 0 & 0 & 0 & $\mathbf{0}$ & • & 18.0 \\
\hline 27 & Printing and Publishing $\ldots \ldots \ldots \ldots \ldots$ & $\bullet$ & 0 & 0 & 0 & 0 & 0 & • & NF \\
\hline 28 & Chemicals and Allied Products . . . . . . . . & 1 & 0 & $\cdot$ & 0 & $\star$ & * & 1 & 13.3 \\
\hline 2812 & Alkalies and Chlorine ............. & 0 & 0 & 0 & 0 & 0 & 0 & 0 & NF \\
\hline 2813 & Industrial Gases . . . . . . . . . . . . & 0 & 0 & 0 & 0 & 0 & 0 & 0 & NF \\
\hline 2819 & Industrial Inorganic Chemicals, nec . . . . . & 0 & 0 & 0 & 0 & 0 & 0 & 0 & NF \\
\hline 2821 & Plastics Materials and Resins . . . . . . . & 1 & 0 & $\cdot$ & 0 & 0 & 0 & 1 & 10.7 \\
\hline 2822 & Synthetic Rubber . . . . . . . . . . & 0 & 0 & 0 & 0 & 0 & 0 & 0 & NF \\
\hline 2823 & Cellulosic Manmade Fibers . . . . . . . . . . & 0 & 0 & 0 & 0 & 0 & 0 & 0 & NF \\
\hline 2824 & Organic Fibers, Noncellulosic . . . . . . . . & 0 & 0 & 0 & 0 & 0 & 0 & 0 & NF \\
\hline 2865 & Cyclic Crudes and Intermediates . . . . . . . & 0 & 0 & 0 & 0 & 0 & 0 & 0 & NF \\
\hline 2869 & Industrial Organic Chemicals, nec ....... & $\cdot$ & 0 & * & 0 & 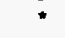 & 0 & $\star$ & 19.6 \\
\hline 2873 & Nitrogenous Fertilizers $\ldots \ldots \ldots \ldots \ldots$ & * & 0 & 0 & 0 & 0 & $\cdot$ & $\cdot$ & 52.6 \\
\hline 2874 & Phosphatic Fertilizers ............. & 0 & 0 & 0 & 0 & 0 & 0 & 0 & NF \\
\hline 29 & Petroleum and Coal Products $\ldots \ldots \ldots \ldots$ & $\mathbf{W}$ & 0 & 86 & $w$ & 0 & 0 & $\mathbf{0}$ & 2.9 \\
\hline 2911 & Petroleum Refining . . . . . . . . . . . & $w$ & 0 & 86 & $W$ & 0 & 0 & 0 & 2.9 \\
\hline 30 & Rubber and Misc. Plastics Products . . . . . . . & $\bullet$ & 0 & $\bullet$ & 0 & 0 & * & • & 24.0 \\
\hline 3011 & Tires and Inner Tubes . . . . . . . . . . . . . & 0 & 0 & 0 & 0 & 0 & 0 & 0 & NF \\
\hline 308 & Miscellaneous Plastics Products, nec ...... & 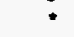 & 0 & 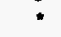 & 0 & 0 & 0 & 0 & 28.4 \\
\hline 31 & Leather and Leather Products . . . . . . . . & $*$ & 0 & 0 & 0 & 0 & 0 & $\cdot$ & NF \\
\hline 32 & Stone, Clay and Glass Products $\ldots \ldots \ldots \ldots$ & $w$ & 0 & 0 & $w$ & 0 & * & * & 10.8 \\
\hline 3211 & Flat Glass $\ldots \ldots \ldots \ldots \ldots \ldots \ldots$ & 0 & 0 & 0 & 0 & 0 & 0 & 0 & NF \\
\hline 3221 & 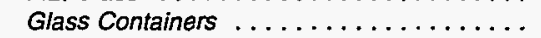 & * & 0 & 0 & 0 & 0 & 0 & - & 19.0 \\
\hline 3229 & Pressed and Blown Glass, nec. . . . . . . . . . & 0 & 0 & 0 & 0 & 0 & 0 & 0 & NF \\
\hline 3241 & Cement, Hydraulic . . . . . . . . . . . . & $w$ & 0 & 0 & $w$ & 0 & 0 & • & 21.9 \\
\hline 3274 & Lime $\ldots \ldots \ldots \ldots \ldots \ldots \ldots \ldots$ & 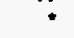 & 0 & 0 & 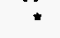 & 0 & 0 & 0 & NF \\
\hline 3296 & 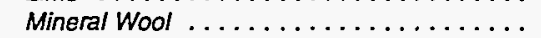 & $\cdot$ & 0 & 0 & 0 & 0 & 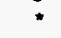 & $\cdot$ & 2.1 \\
\hline 33 & Primary Metal Industries $\ldots \ldots \ldots \ldots \ldots$ & $W$ & $w$ & 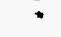 & 0 & 0 & 1 & $w$ & 9.1 \\
\hline 3312 & Blast Fumaces and Steol Mills . . . . . . . . . & 123 & $\mathbf{W}$ & 0 & 0 & 0 & 0 & $w$ & B.8 \\
\hline 3313 & Electrometalurgical Products . . . . . . . . . & 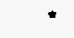 & 0 & 0 & 0 & 0 & $*$ & $*$ & 15.0 \\
\hline 3321 & Gray and Ductile Iron Foundries ........ & 0 & 0 & 0 & 0 & 0 & 0 & 0 & NF \\
\hline 3331 & Primary Copper . . . . . . . . . . . & 0 & 0 & 0 & 0 & 0 & 0 & 0 & NF \\
\hline 3334 & Primary Aluminum $\ldots \ldots \ldots \ldots \ldots$ & $\cdot$ & 0 & 0 & 0 & 0 & * & 0 & 6.6 \\
\hline 3339 & Primany Nonferrous Matals, nec . . . . . . . . & 0 & 0 & 0 & 0 & 0 & 0 & 0 & NF \\
\hline 3353 & Aluminum Sheet, Plate, and Foil . . . . . . . & * & 0 & 0 & 0 & 0 & 0 & * & 2.1 \\
\hline 34 & Fabricated Metal Products . . . . . . . . . . . & $*$ & 0 & 0 & 0 & 0 & 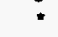 & * & 51.8 \\
\hline 35 & Industrial Machinery and Equipment . . . . . . & $\star$ & 0 & 0 & 0 & 0 & $\stackrel{*}{*}$ & * & 31.5 \\
\hline 357 & Computer and Office Equipment ......... & $\bullet$ & 0 & 0 & 0 & 0 & 0 & * & 34.9 \\
\hline 36 & Electronic and Other Electric Equipment ..... & 0 & 0 & 0 & 0 & 0 & 0 & 0 & NF \\
\hline 37 & Transportation Equipment $\ldots \ldots \ldots \ldots \ldots$ & 0 & 0 & 0 & 0 & 0 & 0 & 0 & NF \\
\hline 3711 & Motor Vehicles and Car Bodies . . . . . . . . . & 0 & 0 & 0 & 0 & 0 & 0 & 0 & NF \\
\hline 3714 & Motor Vehicle Parts and Accessories . . . . . . & 0 & 0 & 0 & 0 & 0 & 0 & 0 & NF \\
\hline 38 & Instruments and Related Products . . . . . . . & $W$ & 0 & 0 & 0 & 0 & * & $\cdot$ & 30.1 \\
\hline 3841 & Surgical and Medical Instruments ........ & 0 & 0 & 0 & 0 & 0 & 0 & 0 & NF \\
\hline 39 & Misc. Manufacturing Industries . . . . . . . . . & $\cdot$ & 0 & 0 & 0 & 0 & " & 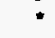 & 34.8 \\
\hline & Total $\ldots \ldots \ldots \ldots \ldots \ldots \ldots$ & 368 & $\mathbf{w}$ & 87 & 48 & 58 & 50 & $w$ & 5.5 \\
\hline
\end{tabular}

See footnotes at end of table. 


\section{Table A6. Total Inputs of Selected Byproduct Energy for Heat, Power, and Electricity Generation by Census Region, Industry Group, and Selected Industries, 1991 (Continued)}

(Estimates in Trillion Btu)

\begin{tabular}{|c|c|c|c|c|c|c|c|c|c|}
\hline $\begin{array}{l}\text { SIC } \\
\text { Code" }\end{array}$ & $\begin{array}{l}\text { Industry Groups } \\
\text { and Industry }\end{array}$ & Total & $\begin{array}{c}\text { Blast } \\
\text { Furnace/ } \\
\text { Coke Oven } \\
\text { Gases }\end{array}$ & Waste Gas & $\begin{array}{c}\text { Petroleum } \\
\text { Coke }\end{array}$ & $\begin{array}{l}\text { Pulping } \\
\text { Liquor }\end{array}$ & $\begin{array}{c}\text { Wood Chips, } \\
\text { Bark }\end{array}$ & $\begin{array}{c}\text { Waste } \\
\text { Oils/Tars } \\
\text { And Waste } \\
\text { Materials }\end{array}$ & $\begin{array}{c}\text { RSE } \\
\text { Row } \\
\text { Factors }\end{array}$ \\
\hline & & \multicolumn{7}{|c|}{ Midwest Census Region } & \\
\hline & RSE Column Factors: & 0.9 & 0.8 & 0.9 & 0.9 & 1.2 & 1.2 & 1.1 & \\
\hline 20 & Food and KIndred Products & $w$ & 0 & $w$ & 0 & 0 & * & - & 16.4 \\
\hline 2011 & Meat Packing Plants . . . . . . . . . . . & w & 0 & W & 0 & 0 & * & 0 & 21.4 \\
\hline 2033 & Canned Fruits and Vegetables . . . . . . . . . & $\because$ & 0 & 0 & 0 & 0 & 0 & - & 40.2 \\
\hline 2037 & Frozen Fruits and Vegetables $\ldots \ldots \ldots \ldots$ & - & 0 & - & 0 & 0 & 0 & 0 & 41.1 \\
\hline 2046 & 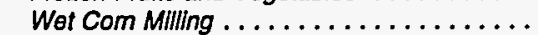 & - & 0 & - & 0 & 0 & 0 & 0 & 25.5 \\
\hline 2051 & Bread, Cake, and Related Products . . . . . . & 0 & 0 & 0 & 0 & 0 & 0 & 0 & NF \\
\hline 2063 & Beet Sugar $\ldots \ldots \ldots \ldots \ldots \ldots \ldots \ldots$ & 0 & 0 & 0 & 0 & 0 & 0 & 0 & NF \\
\hline 2075 & Soybean Oll MIlls . . . . . . . . . . . . . & * & 0 & - & 0 & 0 & 0 & • & 8.9 \\
\hline 2082 & Malt Beverages . . . . . . . . . . . . . & 0 & 0 & 0 & 0 & 0 & 0 & 0 & NF \\
\hline 21 & Tobacco Products $\ldots \ldots \ldots \ldots \ldots \ldots$ & NA & NA & NA & NA & NA & NA & NA & NF \\
\hline 22 & Textile Mill Products & NA & NA & NA & NA & NA & NA & NA & NF \\
\hline 23 & Apparel and Other Textile Products . . . . . . & NA & NA & NA & NA & NA & NA & NA & 49.2 \\
\hline 24 & Lumber and Wood Products . . . . . . . . . & 32 & 0 & 0 & 0 & 0 & 31 & $Q$ & 24.2 \\
\hline 25 & Furniture and Fixtures ..... & $\mathbf{Q}$ & 0 & 0 & 0 & 0 & $Q$ & " & 35.8 \\
\hline 26 & Paper and Allied Products. & 82 & 0 & 0 & 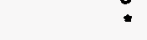 & 49 & 31 & $Q$ & 7.6 \\
\hline 2611 & Pulp Mills .......... & 23 & 0 & 0 & 0 & 18 & 5 & 0 & 25.2 \\
\hline 2621 & Paper Mills . & 51 & 0 & 0 & - & 30 & 20 & : & 4.4 \\
\hline 2631 & Paperboard Mills $\ldots \ldots \ldots \ldots \ldots \ldots$ & w & 0 & 0 & 0 & 1 & w & - & 14.5 \\
\hline 27 & Printing and Publlshing . . . . . . . . . . & $*$ & 0 & • & 0 & 0 & 0 & - & 35.2 \\
\hline 28 & Chemicals and Allied Products . . . . . . . . & w & 0 & w & 0 & 0 & $w$ & w & 13.5 \\
\hline 2812 & Alkalles and Chlorine $\ldots \ldots \ldots \ldots \ldots$ & 0 & 0 & 0 & 0 & 0 & 0 & 0 & NF \\
\hline 2813 & Industrlal Gases ........ & 0 & 0 & 0 & 0 & 0 & 0 & 0 & NF \\
\hline 2819 & Industrial Inorganic Chemicals, nec. & 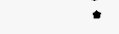 & 0 & - & 0 & 0 & 0 & 0 & 20.0 \\
\hline 2821 & Plastlos Materials and Resins ........... & w & 0 & - & 0 & 0 & w & $\mathbf{w}$ & 12.5 \\
\hline 2822 & Synthetic Rubber ............. & $\because$ & 0 & 0 & 0 & 0 & 0 & $\because$ & 22.1 \\
\hline 2823 & Cellulosic Manmado Fibers . . . . . . . . & 0 & 0 & 0 & 0 & 0 & 0 & 0 & NF \\
\hline 2824 & Organlc Flbers, Noncellulosic .......... & 0 & 0 & 0 & 0 & 0 & 0 & 0 & NF \\
\hline 2865 & Cycllc Crudes and Intermediates ......... & 0 & 0 & 0 & 0 & 0 & 0 & 0 & NF \\
\hline 2869 & Industrial Organlc Chemicals, nec ......... & $w$ & 0 & : & 0 & 0 & 0 & . & 8.2 \\
\hline 2873 & Nitrogenous Fentilizers $\ldots \ldots \ldots \ldots \ldots \ldots$ & 1 & 0 & 1 & 0 & 0 & 0 & * & 41.6 \\
\hline 2874 & Phosphatic Fertilizers . . . . . . . . . . & 0 & 0 & 0 & 0 & 0 & 0 & 0 & NF \\
\hline & Petroleum and Coal Products ..... & $w$ & 0 & 214 & w & 0 & 0 & $Q$ & 2.5 \\
\hline 2911 & 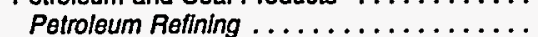 & $\ddot{w}$ & 0 & 213 & $w$ & 0 & 0 & - & 3.2 \\
\hline & Rubber and Misc. Plastics Products . . . . . . . & • & 0 & 0 & 0 & 0 & 0 & • & 8.0 \\
\hline 3011 & 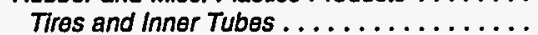 & - & 0 & 0 & 0 & 0 & 0 & * & 7.0 \\
\hline 308 & Mlscellaneous Plastics Products, nec ...... & NA & NA & NA & NA & NA & NA & NA & NF \\
\hline 31 & Leather and Leather Products . ...... & 0 & 0 & 0 & 0 & 0 & 0 & 0 & NF \\
\hline & Stone, Clay and Glass Products . . . . . . . & 28 & 0 & 0 & w & 0 & Q & $\mathbf{w}$ & 14.8 \\
\hline 3211 & Flat Glass $\ldots \ldots \ldots \ldots \ldots \ldots \ldots$ & 0 & 0 & 0 & 0 & 0 & 0 & 0 & NF \\
\hline 3221 & Glass Containers & 0 & 0 & 0 & 0 & 0 & 0 & 0 & NF \\
\hline 3229 & Pressed and Blown Glass, nec. ..... . & 0 & 0 & 0 & 0 & 0 & 0 & 0 & NF \\
\hline 3241 & Cement, Hydraulle ............... & 18 & 0 & 0 & 17 & 0 & 0 & 1 & 17.1 \\
\hline 3274 & LIme $\ldots \ldots \ldots \ldots \ldots \ldots \ldots \ldots \ldots$ & $\mathbf{w}$ & 0 & 0 & $\ddot{w}$ & 0 & 0 & 0 & 18.8 \\
\hline 3296 & MIneral Wool $\ldots \ldots \ldots \ldots \ldots \ldots \ldots \ldots$ & 0 & 0 & 0 & 0 & 0 & 0 & 0 & NF \\
\hline 33 & Primary Metal Industries & 228 & 222 & 0 & 0 & 0 & - & 6 & 7.3 \\
\hline 3312 & Blast Fumaces and Steol Mills . . . . . . . . . & 227 & 222 & 0 & 0 & 0 & 0 & 6 & 6.3 \\
\hline 3313 & Electrometalurgical Products . . . . . . . . & * & 0 & 0 & 0 & 0 & " & 0 & 13.6 \\
\hline 3321 & Gray and Ductlle Iron Foundries ........... & 0 & 0 & 0 & 0 & 0 & 0 & 0 & NF \\
\hline 3331 & Primary Copper ................. & 0 & 0 & 0 & 0 & 0 & 0 & 0 & NF \\
\hline 3334 & Primary Aluminum .............. & 0 & 0 & 0 & 0 & 0 & 0 & 0 & NF \\
\hline 3339 & Primary Nonferrous Motals, nec . . . . . . . . & 0 & 0 & 0 & 0 & 0 & 0 & 0 & NF \\
\hline 3353 & Aluminum Sheet, Plate, and Foll ......... & 0 & 0 & 0 & 0 & 0 & 0 & 0 & $\mathrm{NF}$ \\
\hline 34 & Fabricated Metal Products . . . . . . . . . & * & 0 & 0 & 0 & 0 & * & * & 21.1 \\
\hline 35 & Industrial Machinery and Equipment . . . . . . . & * & 0 & 0 & 0 & 0 & * & - & 27.6 \\
\hline 357 & Computer and Office Equipment ........ & 0 & 0 & 0 & 0 & 0 & 0 & 0 & NF \\
\hline 36 & Electronic and Other Electric Equlpment ...... & 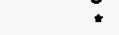 & 0 & 0 & 0 & 0 & : & * & 26.2 \\
\hline 37 & Transportation Equipment $\ldots \ldots \ldots \ldots \ldots \ldots$ & 2 & 0 & • & 0 & 0 & • & 1 & 8.9 \\
\hline 3711 & Motor Vehicles and Car Bodles .......... & : & 0 & . & 0 & 0 & • & : & 8.4 \\
\hline 3714 & Motor Vehicle Parts and Accessories ...... & 1 & 0 & . & 0 & 0 & 0 & 1 & 14.2 \\
\hline & Instruments and Related Products . . . . . . . & * & 0 & 0 & 0 & 0 & : & 0 & $\mathrm{NF}$ \\
\hline 3841 & Surgical and Medical Instruments . . . . . . . & 0 & 0 & 0 & 0 & 0 & 0 & 0 & NF \\
\hline 39 & Misc. Manufacturing Industries . . . $\ldots \ldots \ldots$ & $\mathbf{Q}$ & 0 & 0 & 0 & 0 & $\mathbf{Q}$ & 0 & NF \\
\hline & Total .. & 755 & 222 & 217 & 177 & 49 & 77 & 13 & 5.3 \\
\hline
\end{tabular}

See footnotes at end of table. 
Table A6. Total Inputs of Selected Byproduct Energy for Heat, Power, and Electricity Generation by Census Region, Industry Group, and Selected Industries, 1991 (Continued) (Estimates in Trillion Btu)

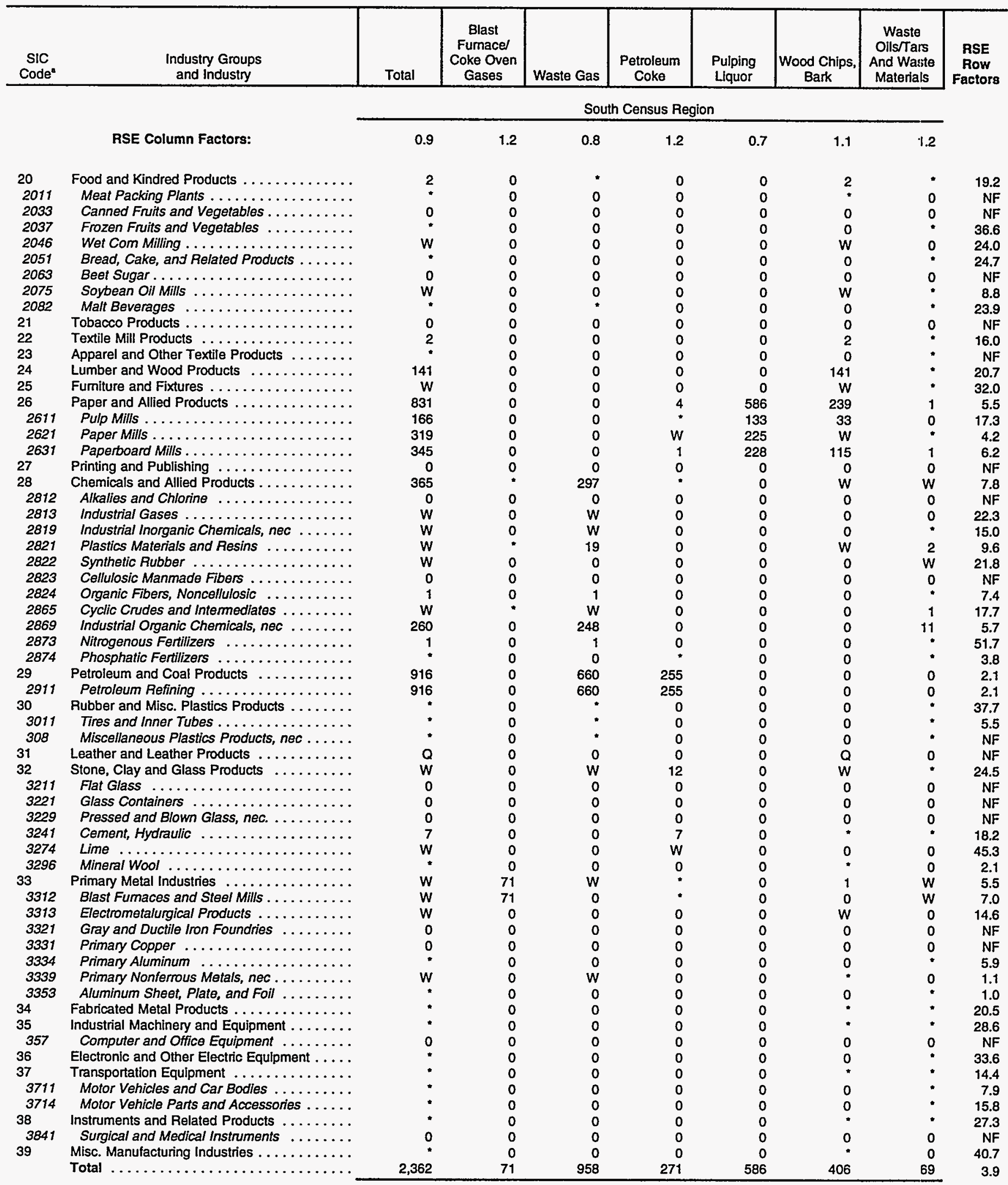

See footnotes at end of table. 
Table A6. Total Inputs of Selected Byproduct Energy for Heat, Power, and Electricity Generation by Census Region, Industry Group, and Selected Industries, 1991 (Continued) (Estimates in Trillion Btu)

\begin{tabular}{cc|c|c|c|c|c|c|c}
\hline SIC & Industry Groups \\
Code* & Tolal Industry & $\begin{array}{c}\text { Blast } \\
\text { Fumace/ } \\
\text { Coke Oven } \\
\text { Gases }\end{array}$ & Waste Gas & $\begin{array}{c}\text { Petroleum } \\
\text { Coke }\end{array}$ & $\begin{array}{c}\text { Wulping } \\
\text { Liquor }\end{array}$ & $\begin{array}{c}\text { Wood Chips, } \\
\text { Bark }\end{array}$ \\
$\begin{array}{c}\text { Oils } / T a r s \\
\text { And Waste } \\
\text { Materials }\end{array}$ & $\begin{array}{c}\text { RSE } \\
\text { Row } \\
\text { Factors }\end{array}$ \\
\hline
\end{tabular}

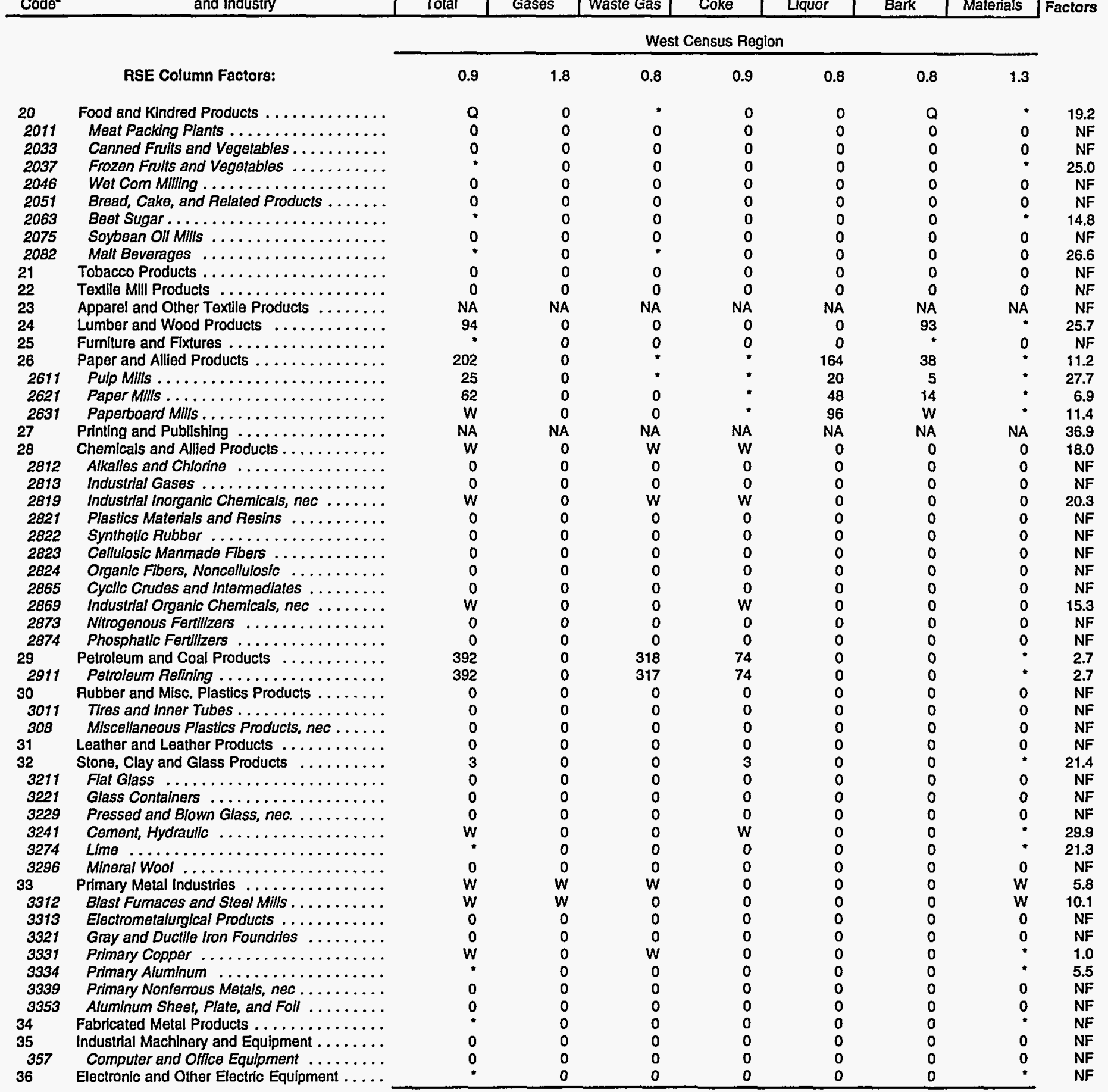

See footnotes at end of table. 


\section{Table A6. Total Inputs of Selected Byproduct Energy for Heat, Power, and Electricity Generation by Census Region, Industry Group, and Selected Industries, 1991 (Continued) (Estimates in Trillion Btu)}

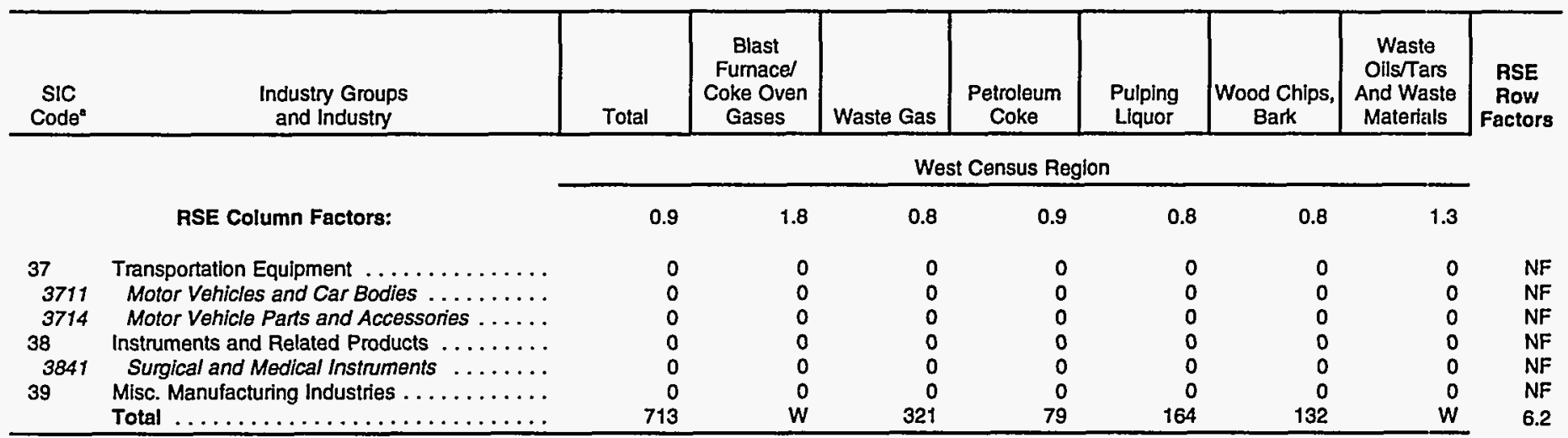

- See Appendices B and F for descriptions of the Standard Industrial Classification system.

NF=No applicable RSE row/column factor.

- Estimate less than 0.5. Data are included in higher level totais.

$W=$ Withheld to avoid disclosing data for individual establishments. Data are included in higher level totals.

$\mathrm{Q}=$ Withheld because Relative Standard Error is greater than $\mathbf{5 0}$ percent. Data are included in higher level totals.

NA=Not avallable. Data are included in higher level totals.

Notes: - To obtain a RSE percentage for any table cell, multiply the cell's corresponding RSE column and RSE row factors. - Totals may not equal sum of components because of independent rounding. - The estimates presented in this table are for the total consumption of energy for the production of heat and power, regardless of where the energy was produced. Specifically, the estimates include the quantities of energy that were originally produced offsite and purchased by or transferred to the establishment, plus those that were produced onsite from other energy or input materials not classified as energy, or were extracted frorn captive (onsite) mines or wells.

Source: Energy Information Administration, Office of Energy Markets and End Use, Energy End Use and Integrated Statistics Division, Form EIA-846, "1991 Manufacturing Energy Consumption Survey." 
Table A7. Shell Storage Capacity of Selected Petroleum Products by Census Region, Industry Group, and Selected Industries, 1991

(Estimates in Thousand Barrels)

\begin{tabular}{|c|c|c|c|c|c|c|}
\hline $\begin{array}{l}\text { SIC } \\
\text { Code }^{a}\end{array}$ & $\begin{array}{l}\text { Industry Groups } \\
\text { and Industry }\end{array}$ & Motor Gasoline & Residual Fuel Oil & Diesel & $\begin{array}{l}\text { Other Distillate } \\
\text { Fuel Oil }\end{array}$ & $\begin{array}{c}\text { RSE } \\
\text { Row } \\
\text { Factors }\end{array}$ \\
\hline & & \multicolumn{4}{|c|}{ Total Unlted States } & \\
\hline & RSE Column Factors: & 1.0 & 0.9 & 1.0 & 1.1 & \\
\hline 20 & Food and KIndred Products. & 38 & 1,448 & 306 & 531 & 12.1 \\
\hline 2011 & Meat Packing Plants $\ldots \ldots \ldots \ldots \ldots \ldots \ldots \ldots \ldots \ldots \ldots$ & 1 & 229 & 40 & 13 & 13.2 \\
\hline 2033 & Canned Frulls and Vegetables $\ldots \ldots \ldots \ldots \ldots \ldots \ldots \ldots$ & 1 & 43 & 22 & 12 & 18.2 \\
\hline 2037 & Frozen Frults and Vegetables $\ldots \ldots \ldots \ldots \ldots \ldots \ldots$ & 1 & 64 & 8 & 11 & 25.3 \\
\hline 2046 & Wet Com Milling $\ldots \ldots \ldots \ldots \ldots \ldots \ldots \ldots \ldots \ldots \ldots$ & - & 7 & 1 & 37 & 29.0 \\
\hline 2051 & Bread, Cake, and Related Products $\ldots \ldots \ldots \ldots \ldots \ldots \ldots$ & 2 & 1 & 7 & 12 & 16.6 \\
\hline 2063 & 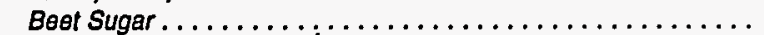 & 1 & w & 3 & 6 & 8.7 \\
\hline 2075 & Soybean Oll Mills $\ldots \ldots \ldots \ldots \ldots \ldots \ldots \ldots \ldots \ldots \ldots \ldots$ & 1 & 108 & w & $\mathbf{w}$ & 5.6 \\
\hline 2082 & $\ldots \ldots \ldots \ldots \ldots \ldots$ & • & 318 & 1 & 14 & 12.8 \\
\hline 21 & Tobacco Products $\ldots \ldots \ldots \ldots \ldots \ldots \ldots \ldots \ldots \ldots \ldots$ & 1 & 163 & 3 & 5 & 4.7 \\
\hline 22 & Textile Mill Products $\ldots \ldots \ldots \ldots \ldots \ldots \ldots \ldots \ldots$ & 5 & 725 & 29 & 329 & 11.1 \\
\hline 23 & Apparel and Other Textile Products.$\ldots \ldots \ldots \ldots \ldots \ldots \ldots$ & * & 78 & 5 & 43 & 35.2 \\
\hline 24 & Lumber and Wood Products $\ldots \ldots \ldots$ & 24 & 125 & 438 & 117 & 23.3 \\
\hline 25 & Fumiture and Fixtures $\ldots \ldots \ldots \ldots \ldots \ldots \ldots \ldots \ldots \ldots$ & $\mathbf{3}$ & 44 & 37 & 63 & 29.2 \\
\hline 26 & Paper and Allled Products $\ldots \ldots \ldots \ldots \ldots \ldots \ldots \ldots \ldots \ldots$ & 8 & 4,085 & 83 & 360 & 5.5 \\
\hline 2611 & Pulp Mills $\ldots \ldots \ldots \ldots \ldots \ldots \ldots \ldots \ldots \ldots \ldots \ldots \ldots \ldots$ & 1 & 865 & 20 & 33 & 16.5 \\
\hline 2621 & 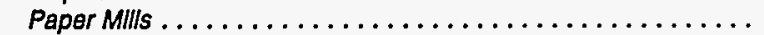 & 3 & 1,833 & 33 & 134 & 3.2 \\
\hline 2631 & 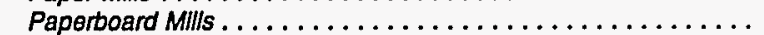 & 4 & 1,249 & 17 & 32 & 6.2 \\
\hline 27 & Printing and Publishing $\ldots \ldots \ldots \ldots \ldots \ldots \ldots \ldots \ldots \ldots$ & 24 & 11 & 24 & 80 & 25.5 \\
\hline 28 & Chemlcals and Alled Products $\ldots \ldots \ldots \ldots \ldots \ldots \ldots \ldots$ & 32 & 4,201 & 476 & 1,044 & 11.7 \\
\hline 2812 & 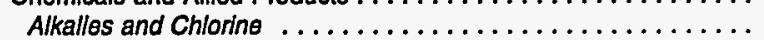 & 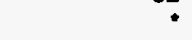 & 1 & 5 & 4 & 17.5 \\
\hline 2813 & Industrlal Gases ............... & * & 0 & $\mathbf{Q}$ & 1 & 13.9 \\
\hline 2819 & Industrlal Inorganic Chemicals, nec ... & 8 & 258 & 177 & 338 & 11.2 \\
\hline 2821 & Plastlos Materials and Resins ........ & 4 & 233 & 23 & 66 & 7.4 \\
\hline 2822 & 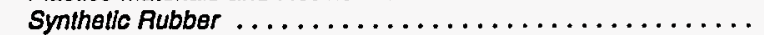 & 1 & 120 & 1 & w & 12.8 \\
\hline 2823 & Cellulosic Manmade Fibers ......... & - & 0 & * & 12 & 28.6 \\
\hline 2824 & Organic Fibers, Noncellulosic ..... & * & 488 & 4 & 5 & 3.9 \\
\hline 2865 & Cycllc Crudes and Intermediates ... & 2 & 584 & 28 & 41 & 12.2 \\
\hline 2869 & Industrlal Organlc Chemicals, nec $\ldots \ldots \ldots \ldots \ldots \ldots \ldots \ldots$ & 7 & 477 & 27 & 224 & 8.5 \\
\hline 2873 & Nitrogonous Fortillzers $\ldots \ldots \ldots \ldots \ldots \ldots \ldots \ldots \ldots \ldots$ & 1 & 0 & 3 & 14 & 22.6 \\
\hline 2874 & Phosphatlc Fertilizers . . . . . . . . . . . . . . . . . & 1 & 90 & 19 & 11 & 3.4 \\
\hline 29 & Petroleum and Coal Products ........ & 5 & 1,703 & 172 & 274 & 23.0 \\
\hline 2911 & Petroleum Refining $\ldots \ldots \ldots \ldots \ldots \ldots \ldots \ldots \ldots \ldots \ldots$ & • & 885 & 1 & w & 9.2 \\
\hline 30 & Rubber and Misc. Plastics Products ..... & 6 & 701 & $\mathbf{Q}$ & 176 & 12.8 \\
\hline 3011 & Tres and Inner Tubes . . . . . . . . & * & 448 & 3 & 21 & 4.2 \\
\hline 308 & Mlscellaneous Plastics Products, nec. & $\mathbf{Q}$ & 156 & $\mathbf{Q}$ & 114 & 15.7 \\
\hline 31 & Leather and Leather Products ....... & • & 14 & $\mathbf{Q}$ & 43 & 26.8 \\
\hline 32 & Stone, Clay and Glass Products ...... & 35 & 474 & 815 & 889 & 10.2 \\
\hline 3211 & Flat Glass $\ldots \ldots \ldots \ldots \ldots \ldots \ldots$ & 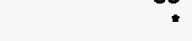 & $w$ & $w$ & 448 & 4.4 \\
\hline 3221 & Glass Containars ..... & - & 92 & w & 82 & 8.9 \\
\hline 3229 & Pressed and Blown Glass, nec $\ldots \ldots \ldots \ldots \ldots \ldots \ldots \ldots$ & • & 23 & 16 & w & 12.4 \\
\hline 3241 & Cement, Hydraulle . . . . . . . . . . . & 5 & 192 & 168 & 39 & 16.6 \\
\hline 3274 & Lime . . . . . . . . . . . & 2 & w & 54 & Q & 21.3 \\
\hline 3296 & Mineral Wool ............... & 1 & w & 19 & $\boldsymbol{9}$ & 1.7 \\
\hline 33 & Primary Metal Industries & 25 & 2,870 & 318 & 454 & 5.6 \\
\hline 3312 & Blast Fumaces and Steel Mills . . . . . . . . . . . . & 7 & 2,790 & 149 & 277 & 5.1 \\
\hline 3313 & Electrometalurgical Products . . . . . . . & 1 & 0 & 6 & • & 8.5 \\
\hline 3321 & Gray and Ductile Iron Foundries ... & 3 & 1 & 12 & 32 & 17.5 \\
\hline 3331 & Primary Copper $\ldots \ldots \ldots \ldots \ldots \ldots \ldots \ldots \ldots \ldots$ & 1 & w & $\mathbf{w}$ & 3 & 1.0 \\
\hline 3334 & Primary Aluminum $\ldots \ldots \ldots \ldots \ldots$ & 3 & w & 21 & 13 & 3.9 \\
\hline 3339 & Primary Nonferrous Metals, nec..... & 1 & $\mathbf{w}$ & 13 & 4 & 7.2 \\
\hline 3353 & Aluminum Sheet, Plate, and Foll ..... & 2 & w & 31 & 10 & 1.0 \\
\hline 34 & Fabricated Metal Products $\ldots \ldots \ldots \ldots \ldots \ldots \ldots \ldots$ & 29 & 125 & 132 & 207 & 22.2 \\
\hline 35 & Industrlal Machinery and Equipment $\ldots \ldots \ldots \ldots \ldots \ldots$ & 17 & 286 & 79 & 171 & 16.4 \\
\hline 357 & Computer and Office Equipment ... & 1 & 3 & 3 & 10 & 16.7 \\
\hline 36 & Electronlc and Other Electric Equipment .... & 5 & 239 & 47 & 158 & 16.7 \\
\hline 37 & Transportation Equipment ............. & 75 & 491 & 165 & 266 & 11.9 \\
\hline 3711 & Motor Vehicles and Car Bodles $\ldots \ldots \ldots \ldots \ldots \ldots \ldots \ldots$ & 20 & 109 & 25 & 49 & 4.1 \\
\hline 3714 & Motor Vehicle Parts and Accessories .... & 13 & 37 & 13 & 56 & 9.3 \\
\hline & Instruments and Related Products . . . . . . . . & 6 & 232 & 15 & 96 & 13.1 \\
\hline 3841 & Surgical and Medlcal Instruments .... & - & 2 & 2 & 8 & 22.9 \\
\hline 39 & Mlsc. Manufacturing Industries . . . . . . . & 1 & 23 & 6 & 57 & 21.5 \\
\hline & Total & 339 & 18,036 & 3,365 & 5,364 & 5.3 \\
\hline
\end{tabular}

See footnotes at end of table. 
Table A7. Shell Storage Capacity of Selected Petroleum Products by Census Region, Industry Group, and Selected Industries, 1991 (Continued) (Estimates in Thousand Barrels)

\begin{tabular}{|c|c|c|c|c|c|c|}
\hline $\begin{array}{c}\text { SIC } \\
\text { Code }\end{array}$ & $\begin{array}{c}\text { Industry Groups } \\
\text { and Industry }\end{array}$ & Motor Gasoline & Residual Fuel Oil & Diesel & $\begin{array}{c}\text { Other Distillate } \\
\text { Fuel Oil }\end{array}$ & $\begin{array}{c}\text { RSE } \\
\text { Row } \\
\text { Factore }\end{array}$ \\
\hline & & \multicolumn{4}{|c|}{ Northeast Census Region } & \\
\hline & RSE Column Factors: & 1.1 & 0.8 & 1.1 & 1.0 & \\
\hline 20 & Food and Kindred Products $\ldots \ldots \ldots \ldots \ldots \ldots$ & 3 & 196 & 59 & 142 & 20.2 \\
\hline 2011 & Meat Packing Planis . . . . . . . . . . . . . . . . & * & 1 & 2 & 3 & 22.6 \\
\hline 2033 & Canned Fruits and Vegetables . . . . . . . . . . . . . & • & 12 & 2 & 3 & 23.0 \\
\hline 2037 & Frozen Fruits and Vegotables $\ldots \ldots \ldots \ldots \ldots \ldots$ & • & $\mathbf{Q}$ & $?$ & 1 & 39.5 \\
\hline 2046 & Wet Com Milling . . . . . . . . . . . . . . . & 0 & $\bar{W}$ & 0 & : & 21.0 \\
\hline 2051 & Bread, Cake, and Related Products . . . . . . . . . . . . . & $\cdot$ & 1 & 4 & 10 & 21.4 \\
\hline 2063 & Beet Sugar . . . . . . . . . . . . . . . . . . . . & $\mathbf{0}$ & 0 & 0 & 0 & NF \\
\hline 2075 & 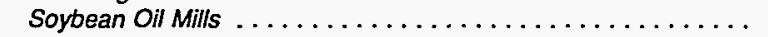 & 0 & 0 & 0 & 0 & NF \\
\hline 2082 & 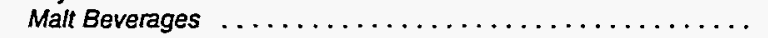 & 0 & W & * & $w$ & 16.5 \\
\hline 21 & Tobacco Products $\ldots \ldots \ldots \ldots \ldots \ldots \ldots \ldots \ldots \ldots$ & NA & NA & NA & NA & NF \\
\hline 22 & Textile Mill Products $\ldots \ldots \ldots \ldots \ldots \ldots \ldots \ldots$ & 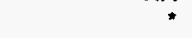 & 139 & a & Q & 25.8 \\
\hline 23 & Apparel and Other Textile Products $\ldots \ldots \ldots \ldots \ldots \ldots$ & 0 & $\mathbf{Q}$ & 0 & 14 & 35.6 \\
\hline 24 & Lumber and Wood Products $\ldots \ldots \ldots \ldots \ldots \ldots \ldots$ & NA & NA & NA & NA & 47.8 \\
\hline 25 & Fumiture and Fixtures $\ldots \ldots \ldots \ldots \ldots \ldots \ldots \ldots$ & * & $\mathbf{Q}$ & Q & $w$ & 30.8 \\
\hline 26 & Paper and Allied Products $\ldots \ldots \ldots \ldots \ldots \ldots \ldots$ & 2 & 1,075 & 14 & 81 & 10.9 \\
\hline 2611 & Pulp Mills $\ldots \ldots \ldots \ldots \ldots \ldots \ldots \ldots \ldots \ldots \ldots$ & ? & 1 & 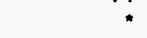 & 0 & 36.5 \\
\hline 2621 & Paper Mills . . . . . . . . . . . . . . . . . & 1 & 926 & 6 & 26 & 4.0 \\
\hline 2631 & 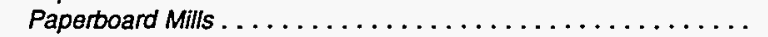 & $\cdot$ & 61 & 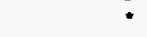 & 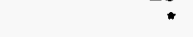 & 11.1 \\
\hline 27 & 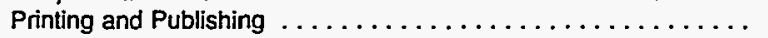 & $\mathbf{Q}$ & 4 & $\mathbf{Q}$ & 42 & 37.3 \\
\hline 28 & Chemicals and Allied Products $\ldots \ldots \ldots \ldots \ldots \ldots \ldots$ & 5 & 580 & 12 & 238 & 14.3 \\
\hline 2812 & 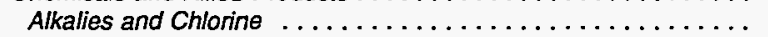 & 0 & 0 & 0 & 0 & NF \\
\hline 2813 & 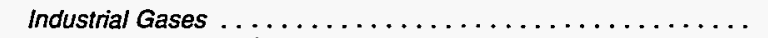 & 0 & 0 & 0 & - & 18.3 \\
\hline 2819 & 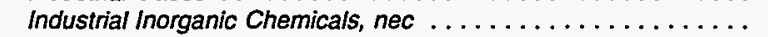 & * & 28 & $w$ & $w$ & 17.6 \\
\hline 2821 & Plastics Materials and Resins $\ldots \ldots \ldots \ldots \ldots \ldots$ & * & 97 & 6 & 47 & 11.0 \\
\hline 2822 & Synthetic Rubber . . . . . . . . . . . . . . . . . & 0 & W & 0 & $\cdot$ & 23.9 \\
\hline 2823 & Cellulosic Manmade Fibers . . . . . . . . . . . . . . . & 0 & 0 & 0 & 0 & NF \\
\hline 2824 & Organic Fibers, Noncellulosic $\ldots \ldots \ldots \ldots \ldots \ldots \ldots$ & 0 & $\cdot$ & 0 & $\cdot$ & 6.7 \\
\hline 2865 & Cyclic Crudes and Intermediates . . . . . . . . . . . & 1 & $W$ & W & 11 & 17.8 \\
\hline 2869 & Industrial Organic Chemicals, nec $\ldots \ldots \ldots \ldots \ldots \ldots$ & 1 & $W$ & 1 & 16 & 6.7 \\
\hline 2873 & Nitrogenous Fertilizers $\ldots \ldots \ldots \ldots \ldots \ldots \ldots \ldots$ & * & 0 & 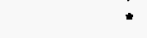 & 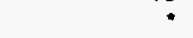 & 45.7 \\
\hline 2874 & 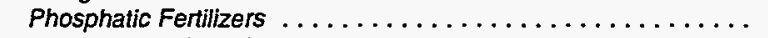 & 0 & 0 & 0 & 0 & NF \\
\hline 29 & Petroleum and Coal Products $\ldots \ldots \ldots \ldots \ldots \ldots \ldots$ & $\mathbf{Q}$ & 1,361 & $\mathbf{Q}$ & 41 & 22.5 \\
\hline 2911 & Petroleum Refining . . . . . . . . . . . . . . . . & 0 & 670 & 0 & 0 & 22.1 \\
\hline 30 & Rubber and Misc. Plastics Products $\ldots \ldots \ldots \ldots \ldots \ldots$ & . & 64 & 3 & 81 & 27.1 \\
\hline 3011 & Tires and Inner Tubes . . . . . . . . . . . . . . . . . . . & 0 & 3 & 1 & $\mathbf{Q}$ & 17.1 \\
\hline 308 & Miscellaneous Plastics Products, nec . . . . . . . . . . & * & 43 & 1 & 59 & 30.9 \\
\hline 31 & Leather and Leather Products $\ldots \ldots \ldots \ldots \ldots \ldots \ldots$ & * & 8 & 1 & 35 & 31.6 \\
\hline 32 & Stone, Clay and Glass Products $\ldots \ldots \ldots \ldots \ldots \ldots$ & 3 & 135 & 176 & 306 & 16.9 \\
\hline 3211 & Flat Glass $\ldots \ldots \ldots \ldots \ldots \ldots \ldots \ldots \ldots$ & * & 0 & 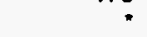 & W & 5.3 \\
\hline 3221 & 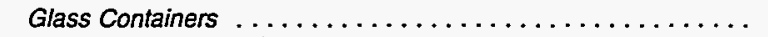 & * & $w$ & * & 45 & 12.7 \\
\hline 3229 & Pressed and Blown Glass, nec . . . . . . . . . . . . . . & * & $\mathbf{Q}$ & w & $W$ & 12.6 \\
\hline 3241 & Cement, Hydraulic . . . . . . . . . . . . . . . . . . & 1 & 12 & Q & 10 & 20.9 \\
\hline 3274 & 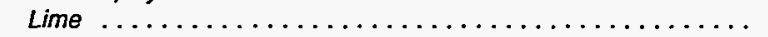 & * & 0 & $\widehat{Q}$ & $\mathbf{Q}$ & NF \\
\hline 3296 & Mineral Wool $\ldots \ldots \ldots \ldots \ldots \ldots \ldots \ldots \ldots \ldots$ & * & 0 & $w$ & $*$ & 1.9 \\
\hline 33 & Primary Metal Industries $\ldots \ldots \ldots \ldots \ldots \ldots \ldots \ldots$ & 6 & 544 & 48 & 129 & 8.4 \\
\hline 3312 & Blast Fumaces and Steel Mills . . . . . . . . . . . . . . . & 3 & $w$ & 16 & 93 & 8.7 \\
\hline 3313 & Electrometalurgical Products . . . . . . . . . . . . . . . & $\cdot$ & 0 & 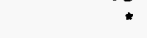 & 0 & 12.4 \\
\hline 3321 & Gray and Ductile Iron Foundries $\ldots \ldots \ldots \ldots \ldots \ldots$ & 1 & 0 & 2 & 4 & 29.2 \\
\hline 3331 & 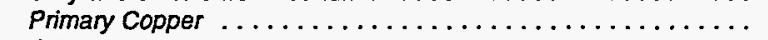 & 0 & 0 & 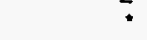 & 1 & 1.0 \\
\hline 3334 & 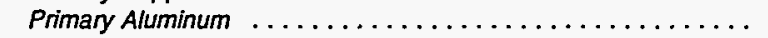 & * & 1 & - & 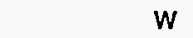 & 5.2 \\
\hline 3339 & Primary Nonferrous Motals, nec . . . . . . . . . . . . . . . & * & $W$ & W & $\cdot$ & 8.0 \\
\hline 3353 & Aluminum Sheet, Plate, and Foil $\ldots \ldots \ldots \ldots \ldots$ & * & $\mathbf{W}$ & W & $w$ & 1.0 \\
\hline 34 & Fabricated Metal Products $\ldots \ldots \ldots \ldots \ldots \ldots \ldots \ldots$ & 1 & 66 & 10 & 89 & 25.5 \\
\hline 35 & Industrial Machinery and Equipment $\ldots \ldots \ldots \ldots \ldots \ldots$ & 3 & 198 & 12 & 113 & 29.9 \\
\hline 357 & Computer and Office Equipment $\ldots \ldots \ldots \ldots \ldots \ldots$ & 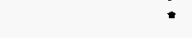 & 2 & 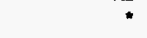 & 4 & 23.2 \\
\hline 36 & Electronic and Other Electric Equipment $\ldots \ldots \ldots \ldots \ldots \ldots$ & 1 & 117 & 3 & 97 & 19.0 \\
\hline 37 & Transportation Equipment & 8 & 281 & 16 & 89 & 13.3 \\
\hline 3711 & Motor Vehicles and Car Bodies . . . . . . . . . . . . . . . & 2 & $w$ & 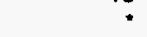 & $W$ & 7.8 \\
\hline 3714 & Motor Vehicle Parts and Accessories . . . . . . . . . . . . & 2 & 1 & 2 & 1 & 16.6 \\
\hline 38 & Instruments and Related Products $\ldots \ldots \ldots \ldots \ldots \ldots \ldots$ & 1 & 145 & 4 & 56 & 20.4 \\
\hline 3841 & Surgical and Medical Instruments $\ldots \ldots \ldots \ldots \ldots \ldots \ldots$ & 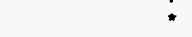 & 2 & 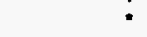 & 4 & 26.2 \\
\hline 39 & Misc. Manufacturing Industries $\ldots \ldots \ldots \ldots \ldots \ldots \ldots$ & - & 19 & 4 & 53 & 26.0 \\
\hline & 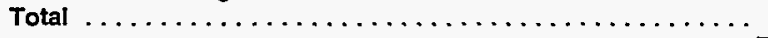 & 39 & 4,959 & 500 & 1,751 & 8.4 \\
\hline
\end{tabular}

See footnotes at end of table. 
Table A7. Shell Storage Capacity of Selected Petroleum Products by Census Region, Industry Group, and Selected Industries, 1991 (Continued) (Estimates in Thousand Barrels)

\begin{tabular}{|c|c|c|c|c|c|c|}
\hline $\begin{array}{c}\text { SIC } \\
\text { Code }\end{array}$ & $\begin{array}{l}\text { Industry Groups } \\
\text { and Industry }\end{array}$ & Motor Gasoline & Residual Fuel Oil & Diesel & $\begin{array}{c}\text { Other Distillate } \\
\text { Fuel Oil } \\
\end{array}$ & $\begin{array}{c}\text { RSE } \\
\text { Row } \\
\text { Factors }\end{array}$ \\
\hline & \multirow[b]{2}{*}{ RSE Column Factors: } & \multicolumn{4}{|c|}{ Midwest Census Region } & \\
\hline & & 0.9 & 1.0 & 1.0 & 1.0 & \\
\hline 20 & Food and Kindred Products & $\mathbf{Q}$ & 558 & 64 & 82 & 14.3 \\
\hline 2011 & Meat Packing Plants .... & * & 228 & 7 & 5 & 11.8 \\
\hline 2033 & Canned Fruits and Vegelables . . . . . . . . . . . . . . . & : & 0 & 7 & $\mathbf{Q}$ & 28.4 \\
\hline 2037 & Frozen Fruits and Vegetables $\ldots \ldots \ldots \ldots \ldots \ldots \ldots \ldots$ & 0 & 14 & 1 & 1 & 35.9 \\
\hline 2046 & 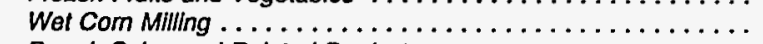 & " & w & $:$ & 4 & 16.4 \\
\hline 2051 & Bread, Cake, and Related Products . . ............... & $*$ & 0 & * & w & 26.4 \\
\hline 2063 & Beet Sugar $\ldots \ldots \ldots \ldots \ldots \ldots \ldots \ldots \ldots \ldots \ldots \ldots$ & $\cdot$ & w & 1 & w & 10.0 \\
\hline 2075 & 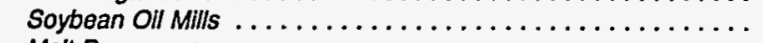 & • & w & $w$ & W & 5.8 \\
\hline 2082 & Mall Beverages $\ldots \ldots \ldots \ldots \ldots \ldots \ldots \ldots \ldots \ldots \ldots \ldots$ & 0 & 68 & • & 0 & 18.3 \\
\hline 21 & Tobacco Products ................ & NA & NA & NA & NA & 7.7 \\
\hline 22 & Textlle Mill Products..$\ldots \ldots \ldots \ldots \ldots$ & NA & NA & NA & NA & 28.0 \\
\hline 23 & Apparel and Other Textile Products . . . . . . . & NA & NA & NA & NA & 47.3 \\
\hline 24 & 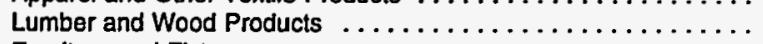 & 1 & Q & $\mathbf{Q}$ & $\mathbf{Q}$ & 50.1 \\
\hline 25 & Furnlture and Fixtures $\ldots \ldots \ldots \ldots \ldots \ldots \ldots \ldots \ldots \ldots$ & $\mathbf{Q}$ & - & 4 & 5 & 38.4 \\
\hline 26 & Paper and Allied Products $\ldots \ldots \ldots \ldots \ldots \ldots \ldots \ldots \ldots$ & 1 & 279 & 15 & 90 & 10.8 \\
\hline 2611 & Pulp Mills $\ldots \ldots \ldots \ldots \ldots \ldots \ldots \ldots \ldots \ldots \ldots \ldots$ & • & 0 & 3 & 0 & 27.0 \\
\hline 2621 & 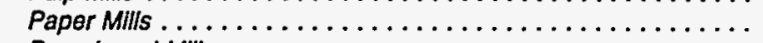 & • & 220 & 8 & 55 & 5.9 \\
\hline 2631 & Paperboard Mills. . & • & 20 & 1 & 9 & 16.9 \\
\hline 27 & Printing and Publishing $\ldots \ldots \ldots \ldots \ldots \ldots \ldots \ldots \ldots \ldots$ & $\mathbf{Q}$ & 6 & 5 & $\mathbf{Q}$ & 32.8 \\
\hline 28 & Chemicals and Allied Products . . . . . . . . & 4 & w & $\mathbf{Q}$ & 165 & 14.3 \\
\hline 2812 & Alkalles and Chlorine $\ldots \ldots \ldots \ldots$ & • & 0 & $\mathbf{w}$ & 0 & 22.9 \\
\hline 2813 & Industrial Gases $\ldots \ldots \ldots \ldots \ldots \ldots \ldots \ldots \ldots \ldots \ldots$ & 0 & 0 & $\mathbf{Q}$ & 0 & NF \\
\hline 2819 & Industrlal Inorganic Chemicals, nec $\ldots \ldots \ldots \ldots \ldots \ldots \ldots$ & • & $\mathbf{w}$ & $\mathbf{w}$ & 7 & 12.3 \\
\hline 2821 & Plastles Materials and Resins $\ldots \ldots \ldots \ldots \ldots \ldots \ldots$ & 1 & 36 & 3 & 2 & 9.4 \\
\hline 2822 & Synthetic Rubber $\ldots \ldots \ldots \ldots \ldots \ldots \ldots \ldots \ldots \ldots$ & • & 0 & 0 & $\bar{w}$ & 22.3 \\
\hline 2823 & Cellulosic Manmade Fibers . . . . . . . . & 0 & 0 & 0 & 0 & NF \\
\hline 2824 & Organic Fibers, Noncellulosic $\ldots \ldots \ldots \ldots \ldots \ldots \ldots \ldots$ & 0 & 0 & 0 & 0 & $\mathrm{NF}$ \\
\hline 2865 & Cyclle Crudes and Intermediates ....... & • & w & $\mathbf{w}$ & 21 & 14.3 \\
\hline 2869 & Industrial Organic Chemicals, nec $\ldots \ldots \ldots \ldots \ldots \ldots \ldots$ & • & w & 4 & 32 & 9.7 \\
\hline 2873 & Nitrogenous Fertilizers $\ldots \ldots \ldots \ldots \ldots \ldots \ldots \ldots \ldots \ldots$ & • & 0 & . & * & 34.1 \\
\hline 2874 & Phosphatic Fertilizers $\ldots \ldots \ldots \ldots \ldots \ldots \ldots \ldots \ldots$ & • & 0 & • & 0 & 4.1 \\
\hline 29 & Petroleum and Coal Products $\ldots \ldots \ldots \ldots$ & •" & w & $\mathbf{Q}$ & 98 & 43.1 \\
\hline 2911 & Petroleum Refining . . . . . . . . . & 0 & 53 & 0 & 0 & 4.9 \\
\hline & Rubber and Misc. Plastics Products ....... & $\mathbf{Q}$ & 227 & 6 & 23 & 15.2 \\
\hline 3011 & Tres and Inner Tubes . . . . . . . . . . . . . & $\bullet$ & 174 & . & w & 5.1 \\
\hline 308 & Mlscellaneous Plastics Products, nec . . . . . . . . . . . & NA & NA & NA & NA & 30.2 \\
\hline 31 & Leather and Leather Products $\ldots \ldots \ldots \ldots \ldots \ldots \ldots \ldots$ & • & 4 & 1 & 4 & 36.3 \\
\hline 32 & Stone, Clay and Glass Products $\ldots \ldots \ldots \ldots$ & 7 & 46 & 159 & 109 & 19.2 \\
\hline 3211 & 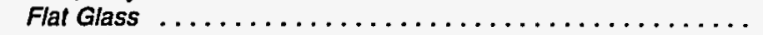 & • & 0 & 1 & W & 5.0 \\
\hline 3221 & Glass Containers $\ldots \ldots \ldots \ldots \ldots \ldots \ldots \ldots \ldots \ldots \ldots$ & - & 0 & $\mathbf{w}$ & w & 14.0 \\
\hline 3229 & Pressed and Blown Glass, nec $\ldots \ldots \ldots \ldots \ldots \ldots \ldots$ & • & • & w & W & 10.7 \\
\hline 3241 & Cement, Hydraulic $\ldots \ldots \ldots \ldots \ldots \ldots \ldots \ldots \ldots \ldots$ & 1 & w & 28 & 16 & 18.8 \\
\hline 3274 & Lime...$\ldots \ldots \ldots$ & 1 & • & w & 1 & 20.9 \\
\hline 3296 & Mineral Wool ........... & • & $w$ & • & w & 1.4 \\
\hline 33 & Primary Metal Industries & 8 & 991 & 102 & 221 & 7.3 \\
\hline 3312 & Blast Fumaces and Steel Mills . . . . . . . . & 2 & 985 & 74 & 170 & 6.8 \\
\hline 3313 & Electrometalurgical Products $\ldots \ldots \ldots \ldots \ldots \ldots \ldots \ldots \ldots$ & • & 0 & w & . & 11.3 \\
\hline 3321 & Gray and Ductile Iron Foundries $\ldots \ldots \ldots \ldots \ldots \ldots \ldots$ & 1 & 1 & 5 & 21 & 22.3 \\
\hline 3331 & Primary Copper $\ldots \ldots \ldots \ldots \ldots \ldots \ldots \ldots \ldots \ldots \ldots$ & 0 & 0 & 0 & 0 & NF \\
\hline 3334 & Primary Aluminum & - & 0 & w & w & 5.0 \\
\hline 3339 & Primary Nonferrous Metals, nec $\ldots \ldots \ldots \ldots \ldots \ldots \ldots \ldots$ & • & 0 & 2 & • & 1.8 \\
\hline 3353 & Aluminum Sheat, Plate, and Foll ....... & 1 & • & $\bar{w}$ & w & 1.0 \\
\hline 34 & Fabricated Metal Products ........ & 8 & 25 & 19 & 54 & 29.2 \\
\hline 35 & Industrial Machinery and Equipment ...... & 8 & 63 & 43 & 40 & 19.9 \\
\hline 357 & Computer and Office Equipment $\ldots \ldots \ldots \ldots \ldots \ldots \ldots$ & 0 & 0 & 1 & * & 29.4 \\
\hline 36 & Electronic and Other Electric Equipment $\ldots \ldots \ldots \ldots \ldots \ldots$ & 2 & 67 & 12 & 34 & 18.6 \\
\hline 37 & Transportation Equipment $\ldots \ldots \ldots \ldots \ldots \ldots \ldots \ldots \ldots$ & 25 & 128 & 36 & 74 & 6.9 \\
\hline 3711 & Motor Vehlcles and Car Bodies $\ldots \ldots \ldots \ldots \ldots \ldots \ldots \ldots$ & 12 & $\mathbf{w}$ & 10 & 8 & 5.1 \\
\hline 3714 & Motor Vehicle Parts and Accessories .... & 10 & 35 & 7 & 45 & 9.4 \\
\hline & Instruments and Related Products $\ldots \ldots \ldots \ldots \ldots \ldots \ldots \ldots$ & • & $\mathbf{Q}$ & 1 & 1 & 28.0 \\
\hline 3841 & Surgical and Medical instruments $\ldots \ldots \ldots \ldots \ldots \ldots \ldots$ & 0 & 0 & • & 1 & 27.3 \\
\hline 39 & Misc. Manufacturing Industries $\ldots \ldots \ldots \ldots \ldots \ldots \ldots \ldots$ & 1 & & 1 & & 33.3 \\
\hline & Total .... & 87 & 3,082 & 773 & 1,058 & 9.5 \\
\hline
\end{tabular}

See footnotes at end of table. 
Table A7. Shell Storage Capacity of Selected Petroleum Products by Census Region, Industry Group, and Selected Industries, 1991 (Continued) (Estimates in Thousand Barrels)

\begin{tabular}{|c|c|c|c|c|c|c|}
\hline $\begin{array}{c}\text { SiC } \\
\text { Code }\end{array}$ & $\begin{array}{c}\text { Industry Groups } \\
\text { and Industry }\end{array}$ & Motor Gasoline & Residual Fuel Oil & Diesel & $\begin{array}{c}\text { Other Distillate } \\
\text { Fuel Oil }\end{array}$ & $\begin{array}{c}\text { ASE } \\
\text { Row } \\
\text { Factors }\end{array}$ \\
\hline & & \multicolumn{4}{|c|}{ South Census Region } & \\
\hline & RSE Column Factors: & 1.1 & 0.9 & 1.0 & 1.0 & \\
\hline 20 & Food and Kindred Products $\ldots \ldots \ldots \ldots \ldots \ldots \ldots \ldots$ & 10 & 504 & 125 & 271 & 15.1 \\
\hline 2011 & 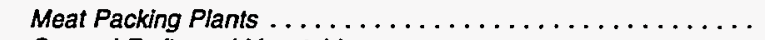 & 1 & 1 & 31 & 5 & 19.2 \\
\hline 2033 & Canned Fruits and Vegetables . . . . . . . . . . . . . & * & 9 & 2 & 1 & 25.1 \\
\hline 2037 & Frozen Fruits and Vegetables . . . . . . . . . . . . & $\cdot$ & 33 & 4 & 8 & 27.2 \\
\hline 2046 & 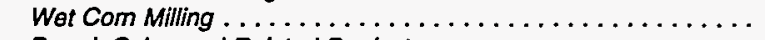 & $\cdot$ & 0 & Q & $\mathbf{Q}$ & 42.1 \\
\hline 2051 & Bread, Cake, and Related Products . . . . . . . . . . . & 1 & $\cdot$ & $\overline{2}$ & $W$ & 21.4 \\
\hline 2063 & 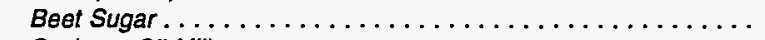 & * & 0 & $\cdot$ & 0 & 15.5 \\
\hline 2075 & 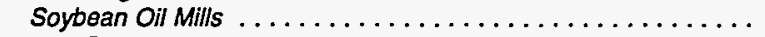 & $\bullet$ & W & W & W & 6.7 \\
\hline 2082 & 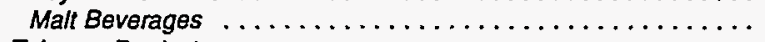 & - & 126 & $\cdot$ & W & 14.0 \\
\hline 21 & Tobacco Products $\ldots \ldots \ldots \ldots \ldots \ldots \ldots \ldots \ldots$ & 1 & 163 & 3 & 5 & 4.7 \\
\hline 22 & 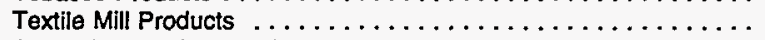 & 4 & 585 & 26 & 234 & 10.1 \\
\hline 23 & Apparel and Other Textile Products $\ldots \ldots \ldots \ldots \ldots \ldots$ & $\cdot$ & Q & 3 & 23 & 40.2 \\
\hline 24 & Lumber and Wood Products $\ldots \ldots \ldots \ldots \ldots \ldots$ & 11 & $\mathbf{Q}$ & 198 & 55 & 31.7 \\
\hline 25 & 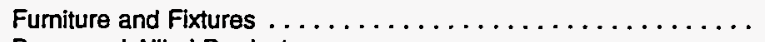 & 1 & 31 & 31 & 23 & 30.5 \\
\hline 26 & Paper and Allied Products $\ldots \ldots \ldots \ldots \ldots \ldots \ldots \ldots$ & 5 & 2,036 & 39 & 183 & 7.1 \\
\hline 2611 & 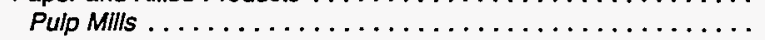 & * & 650 & 12 & 33 & 20.6 \\
\hline 2621 & Paper Mills . . . . . . . . . . . . . . . . . . & 2 & 521 & 13 & 52 & 3.5 \\
\hline 2631 & Paperboard Mills . . . . . . . . . . . . . . . . . & 3 & 855 & 12 & 21 & 6.9 \\
\hline 27 & Printing and Publishing $\ldots \ldots \ldots \ldots \ldots \ldots \ldots$ & $\mathbf{Q}$ & $\mathbf{Q}$ & $\mathbf{Q}$ & 17 & 36.1 \\
\hline 28 & Chemicals and Alled Products $\ldots \ldots \ldots \ldots \ldots \ldots \ldots$ & 15 & 3,142 & 106 & 390 & 8.2 \\
\hline 2812 & 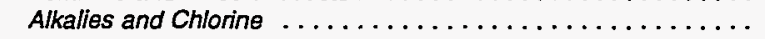 & * & 0 & W & 4 & 17.8 \\
\hline 2813 & 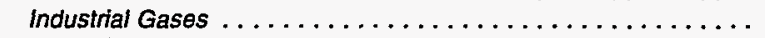 & 0 & 0 & $\bullet$ & * & 15.8 \\
\hline 2819 & Industrial Inorganic Chemicals, noc $\ldots \ldots \ldots \ldots \ldots \ldots$ & 1 & 94 & 33 & 82 & 11.0 \\
\hline 2821 & Plastics Materials and Resins $\ldots \ldots \ldots \ldots \ldots \ldots$ & 2 & 99 & 14 & 18 & 7.5 \\
\hline 2822 & 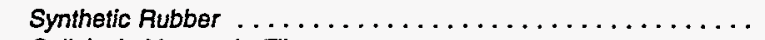 & 1 & W & 1 & $\cdot$ & 13.7 \\
\hline 2823 & Cellulosic Manmade Fibers . . . . . . . . . . . . . . . . & * & 0 & 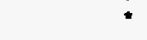 & 12 & 29.1 \\
\hline 2824 & Organic Fibers, Noncellulosic $\ldots \ldots \ldots \ldots \ldots \ldots$ & $\star$ & 488 & 4 & 5 & 3.9 \\
\hline 2865 & Cyclic Crudes and Intemediates $\ldots \ldots \ldots \ldots \ldots \ldots$ & 1 & 515 & $W$ & 8 & 11.7 \\
\hline 2869 & Industrial Organic Chemicals, nec $\ldots \ldots \ldots \ldots \ldots \ldots$ & 5 & 275 & 21 & 175 & 8.3 \\
\hline 2873 & Nitrogenous Fertilizers $\ldots \ldots \ldots \ldots \ldots \ldots \ldots \ldots$ & 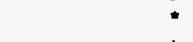 & 0 & 2 & 0 & 22.8 \\
\hline 2874 & Phosphatic Fertilizers . . . . . . . . . . . . . . . & 1 & 90 & $\bar{W}$ & 11 & 2.7 \\
\hline 29 & Petroleum and Coal Products $\ldots \ldots \ldots \ldots \ldots \ldots$ & 1 & 77 & 28 & 123 & 23.7 \\
\hline 2911 & Petroleum Refining $\ldots \ldots \ldots \ldots \ldots \ldots \ldots \ldots \ldots \ldots \ldots \ldots \ldots \ldots \ldots$ & * & W & 1 & W & 7.1 \\
\hline 30 & Rubber and Misc. Plastics Products $\ldots \ldots \ldots \ldots \ldots \ldots$ & 3 & 409 & $\mathbf{Q}$ & 71 & 13.4 \\
\hline 3011 & Tires and Inner Tubes . . . . . . . . . . . . . . . . . & 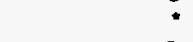 & 270 & 2 & 18 & 4.1 \\
\hline 308 & Miscellaneous Plastics Products, nec . . . . . . . . . . . & $\mathbf{Q}$ & 102 & $\vec{Q}$ & 44 & 17.4 \\
\hline 31 & Leather and Leather Products $\ldots \ldots \ldots \ldots \ldots \ldots \ldots$ & 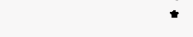 & 1 & 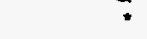 & 4 & 43.9 \\
\hline 32 & Stone, Clay and Glass Products $\ldots \ldots \ldots \ldots \ldots \ldots$ & 17 & 105 & 293 & 385 & 16.6 \\
\hline 3211 & 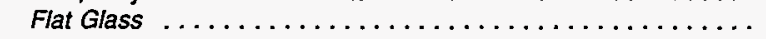 & $\bullet$ & 0 & 1 & W & 3.8 \\
\hline 3221 & 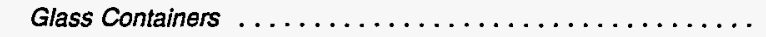 & $\star$ & $w$ & $\mathbf{W}$ & w & 15.8 \\
\hline 3229 & Pressed and Blown Glass, nec . . . . . . . . . . . . & - & W & W & W & 10.3 \\
\hline 3241 & Cement, Hydraulic . . . . . . . . . . . . . . . . & 1 & w & 22 & 11 & 22.3 \\
\hline 3274 & 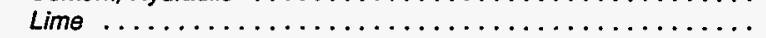 & 1 & 0 & 22 & 0 & 19.4 \\
\hline 3296 & 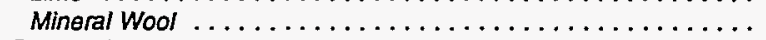 & : & * & $\bar{W}$ & W & 2.0 \\
\hline 33 & Primary Metal Industries $\ldots \ldots \ldots \ldots \ldots \ldots \ldots$ & 7 & w & 83 & 99 & 12.1 \\
\hline 3312 & Blast Fumaces and Steel Mills . . . . . . . . . . . . . & 1 & W & 51 & 14 & 7.3 \\
\hline 3313 & Electrometalurgical Products $\ldots \ldots \ldots \ldots \ldots \ldots \ldots$ & $\bullet$ & 0 & W & 0 & 9.7 \\
\hline 3321 & Gray and Ductile Iron Foundries $\ldots \ldots \ldots \ldots \ldots \ldots$ & 1 & * & 5 & 6 & 12.9 \\
\hline 3331 & 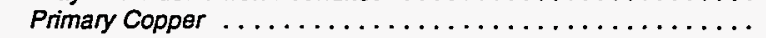 & 0 & 1 & $*$ & 1 & 1.0 \\
\hline 3334 & Primary Aluminum $\ldots \ldots \ldots \ldots \ldots \ldots \ldots \ldots$ & 2 & 0 & W & $\mathbf{w}$ & 4.2 \\
\hline 3339 & Primary Nonferrous Metals, nec . . . . . . . . . . . . . & * & 0 & 2 & $\mathbf{Q}$ & 50.4 \\
\hline 3353 & Aluminum Sheet, Plate, and Foil . . . . . . . . . . . . & 1 & 0 & 4 & $\bar{W}$ & 1.0 \\
\hline 34 & Fabricated Metal Products $\ldots \ldots \ldots \ldots \ldots \ldots \ldots \ldots \ldots$ & 15 & $\mathbf{Q}$ & 91 & 62 & 32.2 \\
\hline 35 & Industrial Machinery and Equipment $\ldots \ldots \ldots \ldots \ldots \ldots \ldots$ & 2 & 25 & 20 & 18 & 34.3 \\
\hline 357 & Computer and Office Equipment $\ldots \ldots \ldots \ldots \ldots \ldots$ & * & 1 & 1 & 6 & 23.5 \\
\hline 36 & Electronic and Other Electric Equipment . . . . . . . . . . & 1 & 55 & 27 & 25 & 21.6 \\
\hline 37 & Transportation Equipment $\ldots \ldots \ldots \ldots \ldots \ldots \ldots$ & 33 & W & $\mathbf{Q}$ & 68 & 18.6 \\
\hline 3711 & Motor Vehicles and Car Bodies $\ldots \ldots \ldots \ldots \ldots \ldots \ldots$ & 5 & 33 & 12 & $\mathbf{w}$ & 5.6 \\
\hline 3714 & 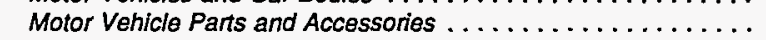 & * & 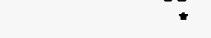 & 2 & 10 & 18.8 \\
\hline 38 & Instruments and Related Products . . . . . . . . . . . . . & 1 & 43 & 7 & 37 & 21.8 \\
\hline 3841 & Surgical and Medical Instruments $\ldots \ldots \ldots \ldots \ldots \ldots$ & 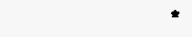 & 0 & 1 & 3 & 30.9 \\
\hline \multirow[t]{2}{*}{39} & Misc. Manufacturing Industries $\ldots \ldots \ldots \ldots \ldots \ldots \ldots$ & * & 3 & Q & 1 & 29.7 \\
\hline & 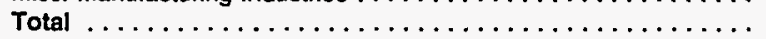 & 134 & 8,531 & 1,360 & 2,095 & 8.3 \\
\hline
\end{tabular}

See footnotes at end of table. 
Table A7. Shell Storage Capacity of Selected Petroleum Products by Census Region, Industry Group, and Selected Industries, 1991 (Continued) (Estimates in Thousand Barrels)

\begin{tabular}{|c|c|c|c|c|c|c|}
\hline $\begin{array}{c}\text { SIC } \\
\text { Code" }\end{array}$ & $\begin{array}{l}\text { Industry Groups } \\
\text { and Industry }\end{array}$ & Motor Gasoline & Residual Fuel Oil & Diesel & $\begin{array}{l}\text { Other Distillate } \\
\text { Fuel Oll }\end{array}$ & $\begin{array}{c}\text { RSE } \\
\text { Row } \\
\text { Factors }\end{array}$ \\
\hline & & \multicolumn{4}{|c|}{ West Census Reglon } & \\
\hline & RSE Column Factors: & 1.0 & 0.9 & 0.9 & 1.2 & \\
\hline 20 & Food and Kindred Products $\ldots \ldots \ldots \ldots \ldots \ldots \ldots \ldots \ldots$ & $\mathbf{Q}$ & 189 & 57 & 36 & 22.6 \\
\hline 2011 & Meat Packing Plants $\ldots \ldots \ldots \ldots \ldots \ldots \ldots \ldots \ldots \ldots \ldots$ & * & $\cdot$ & 1 & * & 19.6 \\
\hline 2033 & 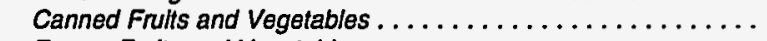 & 1 & 22 & 10 & * & 17.8 \\
\hline 2037 & Frozen Frults and Vegetables $\ldots \ldots \ldots \ldots \ldots \ldots \ldots \ldots$ & 1 & $\mathbf{Q}$ & $\mathbf{Q}$ & 1 & 27.8 \\
\hline 2046 & Wet Corn Milling $\ldots \ldots \ldots \ldots \ldots \ldots \ldots \ldots \ldots \ldots \ldots \ldots$ & 0 & 0 & . & 0 & 22.2 \\
\hline 2051 & Bread, Cake, and Related Products $\ldots \ldots \ldots \ldots \ldots \ldots \ldots$ & • & 0 & 1 & * & 37.0 \\
\hline 2063 & Beet Sugar $\ldots \ldots \ldots \ldots \ldots \ldots \ldots \ldots \ldots \ldots \ldots \ldots \ldots$ & 1 & w & 2 & w & 11.2 \\
\hline 2075 & 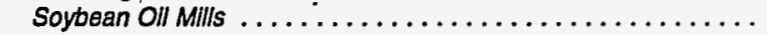 & 0 & 0 & 0 & 0 & NF \\
\hline 2082 & Malt Beverages $\ldots \ldots \ldots \ldots \ldots \ldots \ldots \ldots \ldots \ldots$ & 0 & w & 1 & W & 20.3 \\
\hline 21 & Tobacco Products ... & 0 & 0 & 0 & 0 & NF \\
\hline 22 & Textile Mill Products $\ldots \ldots \ldots \ldots \ldots \ldots \ldots \ldots \ldots \ldots \ldots$ & 0 & 0 & * & w & 23.7 \\
\hline 23 & Apparel and Other Textile Products $\ldots \ldots \ldots \ldots \ldots \ldots \ldots$ & NA & NA & NA & NA & NF \\
\hline 24 & Lumber and Wood Products $\ldots \ldots \ldots \ldots \ldots \ldots \ldots \ldots$ & 12 & 108 & 155 & 16 & 27.0 \\
\hline 25 & Furniture and Fixtures $[\ldots, \ldots, \ldots, \ldots, \ldots, \ldots, \ldots$ & 0 & 0 & " & $\mathbf{Q}$ & NF \\
\hline 26 & Paper and Alled Products $\ldots \ldots \ldots \ldots \ldots \ldots \ldots \ldots \ldots$ & 1 & 695 & 16 & 6 & 12.3 \\
\hline 2611 & Pulp Mills $\ldots \ldots \ldots \ldots \ldots \ldots \ldots \ldots \ldots \ldots \ldots \ldots \ldots \ldots$ & • & 214 & 5 & $\mathbf{0}$ & 20.8 \\
\hline 2621 & Paper Mills $\ldots \ldots \ldots \ldots \ldots \ldots \ldots \ldots \ldots \ldots \ldots \ldots \ldots$ & • & 167 & 6 & * & 5.6 \\
\hline 2631 & Paperboard MIlls $\ldots \ldots \ldots \ldots \ldots \ldots \ldots \ldots \ldots \ldots$ & 1 & 314 & 4 & 1 & 8.5 \\
\hline 27 & Printing and Publishing $\ldots \ldots \ldots \ldots \ldots \ldots \ldots \ldots \ldots \ldots$ & NA & NA & NA & NA & 48.6 \\
\hline 28 & Chemicals and Allied Products $\ldots \ldots \ldots \ldots \ldots \ldots \ldots \ldots$ & 9 & w & 147 & 250 & 15.0 \\
\hline 2812 & Alkalles and Chlorine $\ldots \ldots \ldots \ldots \ldots \ldots \ldots \ldots \ldots \ldots$ & * & 1 & - & • & 23.0 \\
\hline 2813 & Industrial Gases $\ldots \ldots \ldots \ldots \ldots \ldots \ldots \ldots \ldots \ldots$ & . & 0 & • & 0 & 20.3 \\
\hline 2819 & Industrial Inorganic Chemicals, nec $\ldots \ldots \ldots \ldots \ldots \ldots \ldots$ & 7 & w & 140 & w & 14.6 \\
\hline 2821 & Plastles Materials and Resins $\ldots \ldots \ldots \ldots \ldots \ldots \ldots \ldots$ & 0 & 0 & • & 0 & 11.1 \\
\hline 2822 & Synthetic Rubber $\ldots \ldots \ldots \ldots \ldots \ldots \ldots \ldots \ldots \ldots \ldots$ & 0 & 0 & * & 0 & 24.4 \\
\hline 2823 & Cellulosic Manmade Fibers $\ldots \ldots \ldots \ldots \ldots \ldots \ldots \ldots$ & 0 & 0 & 0 & 0 & $\mathrm{NF}$ \\
\hline 2824 & Organic Fibers, Noncellulosic $\ldots \ldots \ldots \ldots \ldots \ldots \ldots$ & 0 & 0 & 0 & 0 & NF \\
\hline 2865 & Cycllc Crudes and Intermedlates $\ldots \ldots \ldots \ldots \ldots \ldots \ldots \ldots$ & 0 & 0 & • & 0 & 25.5 \\
\hline 2869 & Industrial Organic Chemlcals, nec $\ldots \ldots \ldots \ldots \ldots \ldots \ldots$ & $\mathbf{Q}$ & 0 & * & $\mathbf{Q}$ & 49.9 \\
\hline 2873 & Nitrogenous Fertilizers $\ldots \ldots \ldots \ldots \ldots \ldots$. & • & 0 & 1 & 13 & 35.7 \\
\hline 2874 & Phosphatlc Fertllizers...$\ldots \ldots \ldots \ldots \ldots$ & • & 0 & W & 0 & 9.1 \\
\hline 29 & Petroleum and Coal Products ........ & Q & w & $\mathbf{Q}$ & $\mathbf{Q}$ & 5.3 \\
\hline 2911 & Pelroleum Refining $\ldots \ldots \ldots \ldots \ldots \ldots \ldots$ & 0 & w & 0 & 0 & 5.3 \\
\hline 30 & Rubber and Misc. Plastics Products $\ldots \ldots \ldots \ldots$ & 0 & • & 5 & 1 & 23.0 \\
\hline 3011 & Tires and Inner Tubes $\ldots \ldots \ldots \ldots \ldots \ldots \ldots \ldots \ldots \ldots$ & 0 & • & * & 0 & 7.6 \\
\hline 308 & Mlscellaneous Plastics Products, nec . . . . . . . . & 0 & 0 & $\mathbf{Q}$ & 0 & $\mathrm{NF}$ \\
\hline 31 & Leather and Leather Products $\ldots \ldots \ldots \ldots \ldots \ldots \ldots \ldots$ & 0 & 0 & $\mathbf{Q}$ & * & NF \\
\hline 32 & Stone, Clay and Glass Products . & 9 & 189 & 187 & 89 & 16.4 \\
\hline 3211 & Flat Glass $\ldots \ldots \ldots \ldots \ldots \ldots \ldots \ldots \ldots$ & • & w & w & w & 5.7 \\
\hline 3221 & Glass Contalners $\ldots \ldots \ldots \ldots \ldots \ldots$ & - & 1 & * & $\mathbf{w}$ & 15.0 \\
\hline 3229 & Pressed and Blown Glass, nec. & 0 & 0 & * & 0 & 14.4 \\
\hline 3241 & Cement, Hydraulic $\ldots \ldots \ldots \ldots \ldots \ldots \ldots$ & 2 & w & 21 & 2 & 24.4 \\
\hline 3274 & Lime ........... & - & w & w & 0 & 22.3 \\
\hline 3296 & Mineral Wool . ........... & 0 & 0 & w & 0 & 2.2 \\
\hline 33 & Primary Motal Industries . . . . . . . . . & 4 & w & 85 & 5 & 8.1 \\
\hline 3312 & Blast Fumaces and Steel Mllis $\ldots \ldots \ldots \ldots \ldots \ldots \ldots \ldots$ & 1 & w & 7 & • & 10.2 \\
\hline 3313 & Electrometalurglcal Products ..... & 0 & 0 & 0 & 0 & $\mathrm{NF}$ \\
\hline 3321 & Gray and Ductle Iron Foundries & . & 0 & 1 & 0 & 33.6 \\
\hline 3331 & 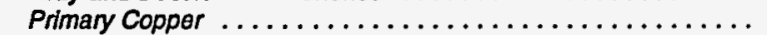 & 1 & w & $\mathbf{w}$ & 1 & 1.0 \\
\hline 3334 & Primary Aluminum & • & w & 2 & . & 4.6 \\
\hline 3339 & Primary Nonferrous Matals, nec.. & - & 0 & w & 1 & 1.0 \\
\hline 3353 & Aluminum Sheet, Plate, and Foil & - & 0 & w & 0 & 1.1 \\
\hline & Fabricated Metal Products . . . . . . & $\mathbf{Q}$ & w & 13 & $\mathbf{Q}$ & 35.8 \\
\hline 35 & Industrial Machinery and Equipment .... & $\mathbf{Q}$ & 0 & 5 & • & 25.5 \\
\hline 357 & Computer and Office Equipment & * & 0 & 1 & 0 & 31.3 \\
\hline 36 & Electronic and Other Electric Equipment . . . . . . . & $\mathbf{Q}$ & 0 & 4 & 2 & 38.0 \\
\hline
\end{tabular}

See footnotes at end of table. 
Table A7. Shell Storage Capacity of Selected Petroleum Products by Census Region, Industry Group, and Selected Industries, 1991 (Continued)

\section{(Estimates in Thousand Barrels)}

\begin{tabular}{|c|c|c|c|c|c|c|}
\hline \multirow[t]{3}{*}{$\begin{array}{c}\text { SIC } \\
\text { Code }^{*}\end{array}$} & $\begin{array}{l}\text { Industry Groups } \\
\text { and Industry }\end{array}$ & Motor Gasoline & Residual Fuel Oil & Diesel & $\begin{array}{c}\text { Other Distillate } \\
\text { Fuel Oil }\end{array}$ & \multirow[t]{3}{*}{$\begin{array}{c}\text { RSE } \\
\text { Row } \\
\text { Factors }\end{array}$} \\
\hline & & \multicolumn{4}{|c|}{ West Census Region } & \\
\hline & RSE Column Factors: & 1.0 & 0.9 & 0.9 & 1.2 & \\
\hline 37 & Transportation Equipment $\ldots \ldots \ldots \ldots \ldots \ldots \ldots \ldots$ & 9 & $w$ & 34 & 34 & 15.3 \\
\hline 3711 & Motor Vehicles and Car Bodies . . . . . . . . . . . . . . . . . & 1 & 0 & 3 & 0 & 7.8 \\
\hline 3714 & Motor Vehicle Parts and Accessories ................. & 0 & * & $\mathbf{Q}$ & 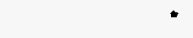 & NF \\
\hline 38 & Instruments and Related Products $\ldots \ldots \ldots \ldots \ldots \ldots \ldots$ & 4 & 1 & 4 & 2 & 22.6 \\
\hline 3841 & Surgical and Medical Instruments $\ldots \ldots \ldots \ldots \ldots \ldots \ldots \ldots$ & 0 & 0 & ? & 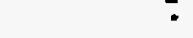 & 36.6 \\
\hline \multirow[t]{2}{*}{39} & Misc. Manufacturing Industries $\ldots \ldots \ldots \ldots \ldots \ldots \ldots \ldots$ & 0 & 0 & $\bullet$ & $\bullet$ & 34.8 \\
\hline & Total $\ldots \ldots \ldots \ldots \ldots \ldots \ldots \ldots \ldots \ldots \ldots \ldots$ & 79 & 1,464 & 732 & 460 & 8.9 \\
\hline
\end{tabular}

- See Appendices B and F for descriptions of the Standard Industrial Classitication system. NF=No applicable RSE row/column factor.

- Estimate less than 0.5. Data are included in higher level totals.

$W=$ Withheld to avoid disclosing data for individual establishments. Data are included in higher level totals.

$\mathrm{Q}=$ Withheld because Relative Standard Error is greater than 50 percent. Data are included in higher level totals.

$\mathrm{NA}=$ Not available. Data are included in higher level totals.

Notes: - To obtain a RSE percentage for any table cell, multiply the cell's corresponding RSE column and RSE row factors. - Totals may not equal sum of components because of independent rounding.

Source: Energy Information Administration, Office of Energy Markets and End Use, Energy End Use Division, Form ElA-846, "1991 Manufacturing Energy Consumption Survey:" 
Table A8. Selected Energy Operating Ratios for Total Energy Consumption for Heat, Power, and Electricity Generation by Census Region, Industry Group, and Selected Industries, 1991

\begin{tabular}{|c|c|c|c|c|c|c|c|}
\hline $\begin{array}{l}\text { SIC } \\
\text { Coden }\end{array}$ & $\begin{array}{l}\text { Industry Groups } \\
\text { and Industry }\end{array}$ & $\begin{array}{c}\text { Consumption per } \\
\text { Employee } \\
\text { (million Btu) }\end{array}$ & $\begin{array}{l}\text { Consumption per } \\
\text { Dollar of Value } \\
\text { Added } \\
\text { (thousand Btu) }\end{array}$ & $\begin{array}{l}\text { Consumption per } \\
\text { Dollar of Value of } \\
\text { Shipments } \\
\text { (thousand Btu) }\end{array}$ & $\begin{array}{c}\text { Major Byproducts } \\
\text { as a Percent of } \\
\text { Consumption } \\
\text { (percent) }\end{array}$ & $\begin{array}{c}\text { Fuel Oile as a } \\
\text { Percent of Natural } \\
\text { Gas } \\
\text { (percent) }\end{array}$ & $\begin{array}{c}\text { RSE } \\
\text { Row } \\
\text { Factors }\end{array}$ \\
\hline
\end{tabular}

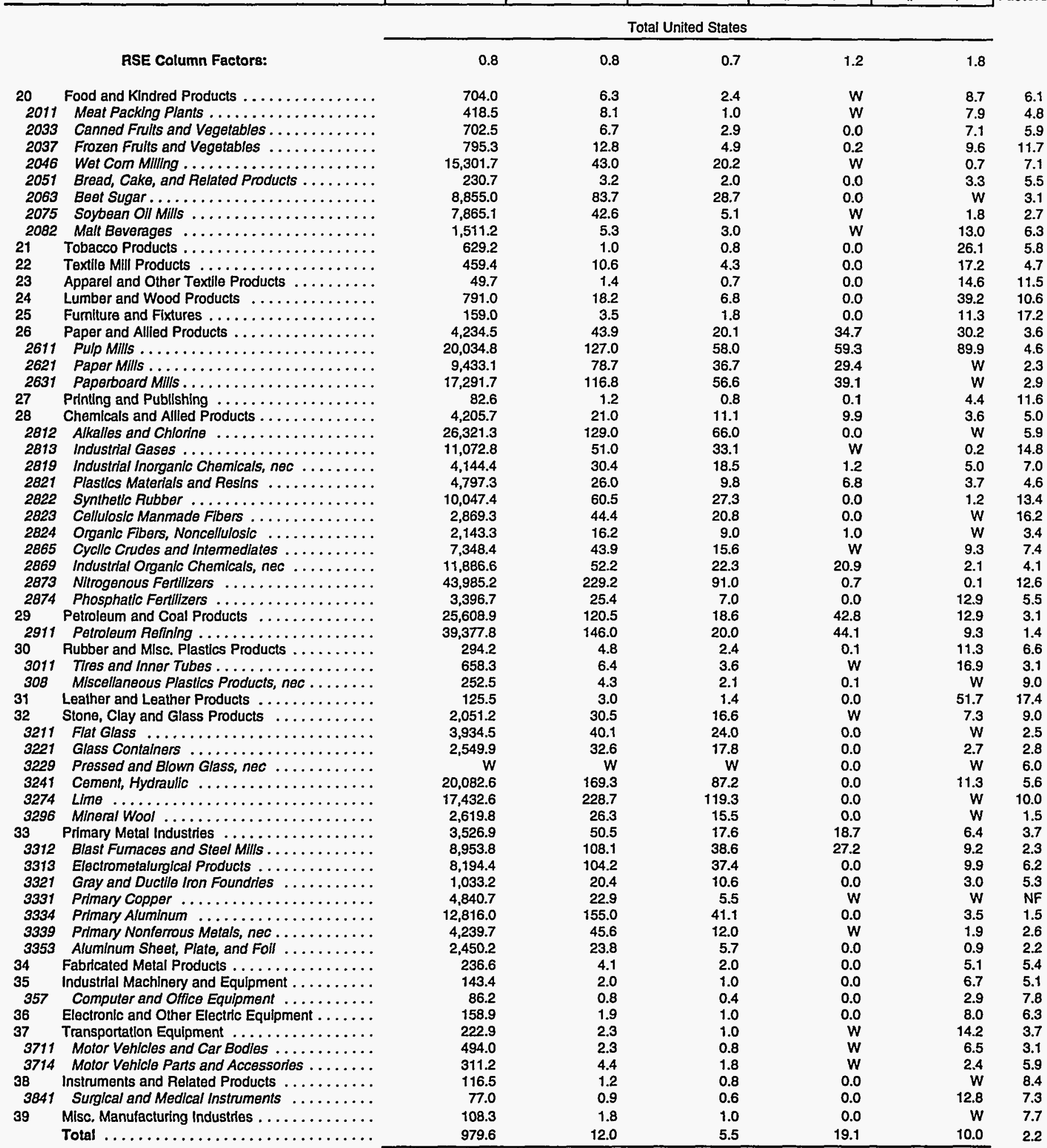

See footnotes at end of table. 
Table A8. Selected Energy Operating Ratios for Total Energy Consumption for Heat, Power, and Electricity Generation by Census Region, Industry Group, and Selected Industries, 1991 (Continued)

\begin{tabular}{|c|c|c|c|c|c|c|c|}
\hline \multirow[t]{3}{*}{$\begin{array}{l}\text { SIC } \\
\text { Code" }\end{array}$} & $\begin{array}{l}\text { Industry Groups } \\
\text { and Industry }\end{array}$ & $\begin{array}{c}\text { Consumption per } \\
\text { Employee } \\
\text { (million Btu) }\end{array}$ & $\begin{array}{c}\text { Consumption per } \\
\text { Dollar of Value } \\
\text { Added } \\
\text { (thousand Btu) }\end{array}$ & $\begin{array}{c}\text { Consumption per } \\
\text { Dollar of Value of } \\
\text { Shipments } \\
\text { (thousand Btu) } \\
\end{array}$ & $\begin{array}{c}\text { Major Byproducts } \\
\text { as a Percent of } \\
\text { Consumption } \\
\text { (percent) }\end{array}$ & $\begin{array}{c}\text { Fuel Oil as a } \\
\text { Percent of Natural } \\
\text { Gass } \\
\text { (percent) }\end{array}$ & $\begin{array}{l}\text { RSE } \\
\text { Row } \\
\text { Factors }\end{array}$ \\
\hline & & \multicolumn{6}{|c|}{ Northeast Census Region } \\
\hline & RSE Column Factors: & 0.8 & 0.9 & 0.8 & 1.1 & 1.5 & \\
\hline 20 & Food and Kindred Products & 473.6 & 3.9 & 1.7 & - & 30.0 & 10.0 \\
\hline 2011 & 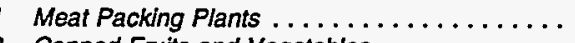 & 256.9 & 5.3 & 0.9 & w & w & 13.3 \\
\hline 2033 & 3 Canned Fruits and Vegetables . . . . . . . . . & 599.4 & 4.2 & 2.0 & 0.0 & 24.2 & 16.8 \\
\hline 2037 & Frozen Fruits and Vegetables . . . . . . . . . . & 621.2 & 7.3 & 3.0 & 0.0 & 592.9 & 23.7 \\
\hline 2046 & ; Wet Com Milling $\ldots \ldots \ldots \ldots \ldots \ldots \ldots$ & $1,175.1$ & 7.5 & 2.7 & 0.0 & Q & 14.9 \\
\hline 2051 & Bread, Cake, and Related Products . . . . . . . . & 284.7 & 3.0 & 2.1 & 0.0 & w & 10.5 \\
\hline 2063 & 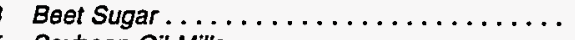 & 0.0 & 0.0 & 0.0 & 0.0 & 0.0 & NF \\
\hline 2075 & 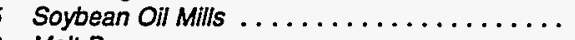 & 0.0 & 0.0 & 0.0 & 0.0 & 0.0 & NF \\
\hline 2082 & Malt Beverages $\ldots \ldots \ldots \ldots \ldots \ldots \ldots$ & $1,306.5$ & 5.4 & 3.0 & 0.0 & w & 5.6 \\
\hline 21 & Tobacco Products .. & NA & NA & NA & NA & NA & 21.4 \\
\hline 22 & $\ldots \ldots \ldots \ldots \ldots \ldots$ & 449.9 & 9.4 & 4.6 & 0.0 & 75.5 & 12.8 \\
\hline 23 & Apparel and Other Textile Products $\ldots \ldots \ldots \ldots$ & 37.4 & 1.0 & 0.5 & 0.0 & 25.3 & 17.4 \\
\hline 24 & Lumber and Wood Products $\ldots \ldots \ldots \ldots$ & NA & NA & NA & NA & NA & 36.4 \\
\hline 25 & Fumiture and Fixtures $\ldots \ldots \ldots \ldots \ldots \ldots$ & 153.6 & 2.7 & 1.4 & 0.0 & $\mathbf{Q}$ & 23.8 \\
\hline 26 & Paper and Allied Products $\ldots \ldots \ldots \ldots \ldots \ldots$ & $W$ & $w$ & $w$ & $w$ & 202.9 & 6.9 \\
\hline 2611 & Pulp Mills $\ldots \ldots \ldots \ldots \ldots \ldots \ldots \ldots \ldots$ & $13,002.3$ & 182.1 & 51.4 & 59.3 & 0.0 & 13.8 \\
\hline 2621 & Paper Mills ..... & $6,726.9$ & 68.5 & 29.9 & 22.2 & w & 2.9 \\
\hline 2631 & Paperboard Mills $\ldots \ldots \ldots \ldots \ldots \ldots \ldots \ldots$ & $6,067.5$ & 61.9 & 30.6 & 0.0 & W & 7.4 \\
\hline 27 & Printing and Publishing $\ldots \ldots \ldots \ldots \ldots$ & 91.0 & 1.4 & 0.9 & 0.0 & 16.9 & 17.4 \\
\hline 28 & Chemicals and Allied Products $\ldots \ldots \ldots \ldots \ldots$ & $1,041.6$ & 4.7 & 2.8 & $W$ & w & 10.1 \\
\hline 2812 & Alkalles and Chlorine $\ldots \ldots \ldots \ldots \ldots$ & $4,327.2$ & 53.6 & 17.9 & 0.0 & 0.0 & 4.9 \\
\hline 2813 & 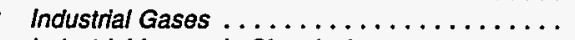 & $7,378.7$ & 35.2 & 23.9 & 0.0 & $w$ & 8.0 \\
\hline 2819 & Industrial Inorganic Chemicals, nec $\ldots \ldots \ldots$ & $1,945.1$ & 12.4 & 5.2 & 0.0 & 35.5 & 18.1 \\
\hline 2821 & Plastlos Materials and Resins $\ldots \ldots \ldots \ldots$ & $1,906.1$ & 16.5 & 6.3 & w & 49.7 & 7.7 \\
\hline 2822 & 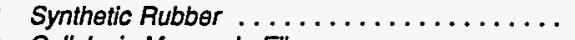 & w & $w$ & $w$ & 0.0 & w & 4.5 \\
\hline 2823 & Cellulosic Manmade Fibers & 0.0 & 0.0 & 0.0 & 0.0 & 0.0 & NF \\
\hline 2824 & Organic Fibers, Noncellulosic . . . . . . . . . . . & 357.1 & $\mathbf{Q}$ & $\mathbf{Q}$ & 0.0 & w & 14.8 \\
\hline 2865 & Cyclic Crudes and Intermediates . . . . . . . . . & $2,153.4$ & 15.7 & 6.7 & 0.0 & W & 12.3 \\
\hline 2869 & Industrial Organic Chemicals, nec . . . . . . . . & $1,951.7$ & 13.8 & 6.6 & w & W & 7.6 \\
\hline 2873 & Nitrogenous Fertilizers $\ldots \ldots \ldots \ldots \ldots$ & $1,825.6$ & 24.7 & 9.9 & 0.0 & Q & 44.7 \\
\hline 2874 & Phosphatic Fertilizers & 0.0 & 0.0 & 0.0 & 0.0 & 0.0 & NF \\
\hline 29 & Petroleum and Coal Products & $17,477.3$ & 87.0 & 15.3 & 36.3 & 190.7 & 7.3 \\
\hline 2911 & Petroleum Refining $\ldots \ldots \ldots \ldots \ldots \ldots \ldots$ & $29,648.9$ & 109.1 & 15.9 & 43.4 & W & 3.9 \\
\hline 30 & Rubber and Misc. Plastics Products $\ldots \ldots \ldots \ldots$ & 286.6 & 4.6 & 2.4 & W & w & 11.5 \\
\hline 3011 & Tires and Inner Tubes . . . . . . . . . . . . & 602.9 & 8.2 & 4.0 & 0.0 & 39.7 & 7.1 \\
\hline 308 & Miscellaneous Plastics Products, nec ........ & 277.7 & 4.4 & 2.2 & W & $w$ & 13.4 \\
\hline 31 & Leather and Leather Products $\ldots \ldots \ldots \ldots \ldots$ & 133.1 & 3.1 & 1.4 & 0.0 & 300.8 & 20.0 \\
\hline 32 & Stone, Clay and Glass Products .. & $1,894.5$ & 28.6 & 16.4 & 0.0 & 11.5 & 19.8 \\
\hline 3211 & Flat Glass & w & $w$ & w & 0.0 & * & 1.5 \\
\hline 3221 & Glass Containers $\ldots \ldots \ldots \ldots \ldots \ldots \ldots \ldots$ & 2,343.7 & 30.5 & 16.9 & 0.0 & 9.0 & 3.7 \\
\hline 3229 & Pressed and Blown Glass, nec $\ldots \ldots \ldots \ldots$ & w & w & W & 0.0 & w & 7.7 \\
\hline 3241 & Cement, Hydraulic . ................ & $19,686.4$ & 259.2 & 112.5 & 0.0 & w & 10.5 \\
\hline 3274 & Lime $\ldots \ldots \ldots \ldots \ldots$ & w & w & w & 0.0 & 311.2 & 8.9 \\
\hline 3296 & Mineral Wool ...... & $2,027.9$ & 18.5 & 12.0 & 0.0 & w & 1.1 \\
\hline 33 & Primary Metal Industries & $2,611.4$ & 39.4 & 13.4 & w & 6.9 & 8.0 \\
\hline 3312 & Blast Fumaces and Steel Mills ......... & $6,635.8$ & 88.3 & 30.8 & $w$ & $w$ & 5.1 \\
\hline 3313 & Electrometalungical Products ..... & W & W & W & 0.0 & $\mathbf{w}$ & 12.0 \\
\hline 3321 & Gray and Ductile Iron Foundries $\ldots \ldots \ldots \ldots$ & 867.5 & 17.7 & 9.3 & 0.0 & 3.6 & 11.0 \\
\hline 3331 & Primary Copper $\ldots \ldots \ldots \ldots \ldots \ldots$ & $2,623.2$ & 63.5 & 3.8 & 0.0 & w & NF \\
\hline 3334 & Primary Aluminum $\ldots \ldots \ldots \ldots$ & W & w & W & 0.0 & w & 1.7 \\
\hline 3339 & Primary Nonferrous Metals, nec... & w & w & w & 0.0 & w & 2.7 \\
\hline 3353 & Aluminum Sheet, Plate, and Foil ... & $w$ & w & w & 0.0 & w & 1.6 \\
\hline 34 & Fabricated Metal Products..$\ldots \ldots \ldots \ldots \ldots$ & 242.2 & 3.9 & 2.2 & 0.0 & 13.8 & 8.2 \\
\hline 35 & Industrial Machinery and Equipment .... & 118.7 & 1.6 & 0.9 & 0.0 & 29.1 & 9.0 \\
\hline 357 & Computer and Office Equipment $\ldots \ldots \ldots \ldots$ & 84.3 & 0.8 & 0.4 & 0.0 & 9.3 & 9.1 \\
\hline 36 & Electronic and Other Electric Equipment ....... & 149.4 & 1.7 & 1.0 & 0.0 & 34.8 & 10.7 \\
\hline 37 & Transportation Equipment $\ldots \ldots \ldots \ldots \ldots \ldots$ & w & $w$ & w & 0.0 & 90.1 & 5.2 \\
\hline 3711 & Motor Vehicles and Car Bodles . . & w & w & w & 0.0 & $w$ & 4.3 \\
\hline 3714 & Motor Vehicle Parts and Accessories . . . . . . . . & 272.1 & 4.2 & 1.6 & 0.0 & w & 9.5 \\
\hline 38 & Instruments and Related Products ..... & 172.6 & 1.7 & 1.1 & 0.0 & w & 13.0 \\
\hline 3841 & Surgical and Medical Instruments & 62.9 & 0.7 & 0.5 & 0.0 & 31.7 & 9.9 \\
\hline 39 & Misc. Manufacturing Industries $\ldots \ldots \ldots \ldots$ & W & $w$ & $w$ & 0.0 & $w$ & 11.8 \\
\hline & Total $\ldots .$. & 589.7 & 7.0 & 3.8 & w & 50.0 & 4.4 \\
\hline
\end{tabular}

See footnotes at end of table. 
Table A8. Selected Energy Operating Ratios for Total Energy Consumption for Heat,
Power, and Electricity Generation by Census Region, Industry Group, and
Selected Industries, 1991 (Continued)

\begin{tabular}{|c|c|c|c|c|c|c|c|}
\hline $\begin{array}{c}\text { SIC } \\
\text { Cod } \theta^{a}\end{array}$ & $\begin{array}{l}\text { Industry Groups } \\
\text { and Industry }\end{array}$ & $\begin{array}{c}\text { Consumption per } \\
\text { Employee } \\
\text { (million Btu) }\end{array}$ & $\begin{array}{l}\text { Consumption per } \\
\text { Dollar of Value } \\
\text { Added } \\
\text { (thousand Btu) }\end{array}$ & $\begin{array}{l}\text { Consumption per } \\
\text { Dollar of Value of } \\
\text { Shipments } \\
\text { (thousand Btu) }\end{array}$ & $\begin{array}{c}\text { Major Byproducts } \\
\text { as a Percent of } \\
\text { Consumption } \\
\text { (percent) }\end{array}$ & $\begin{array}{c}\text { Fuel Oile as a } \\
\text { Percent of Natural } \\
\text { Gas } \\
\text { (percent) }\end{array}$ & $\begin{array}{c}\text { RSE } \\
\text { Row } \\
\text { Factors }\end{array}$ \\
\hline
\end{tabular}

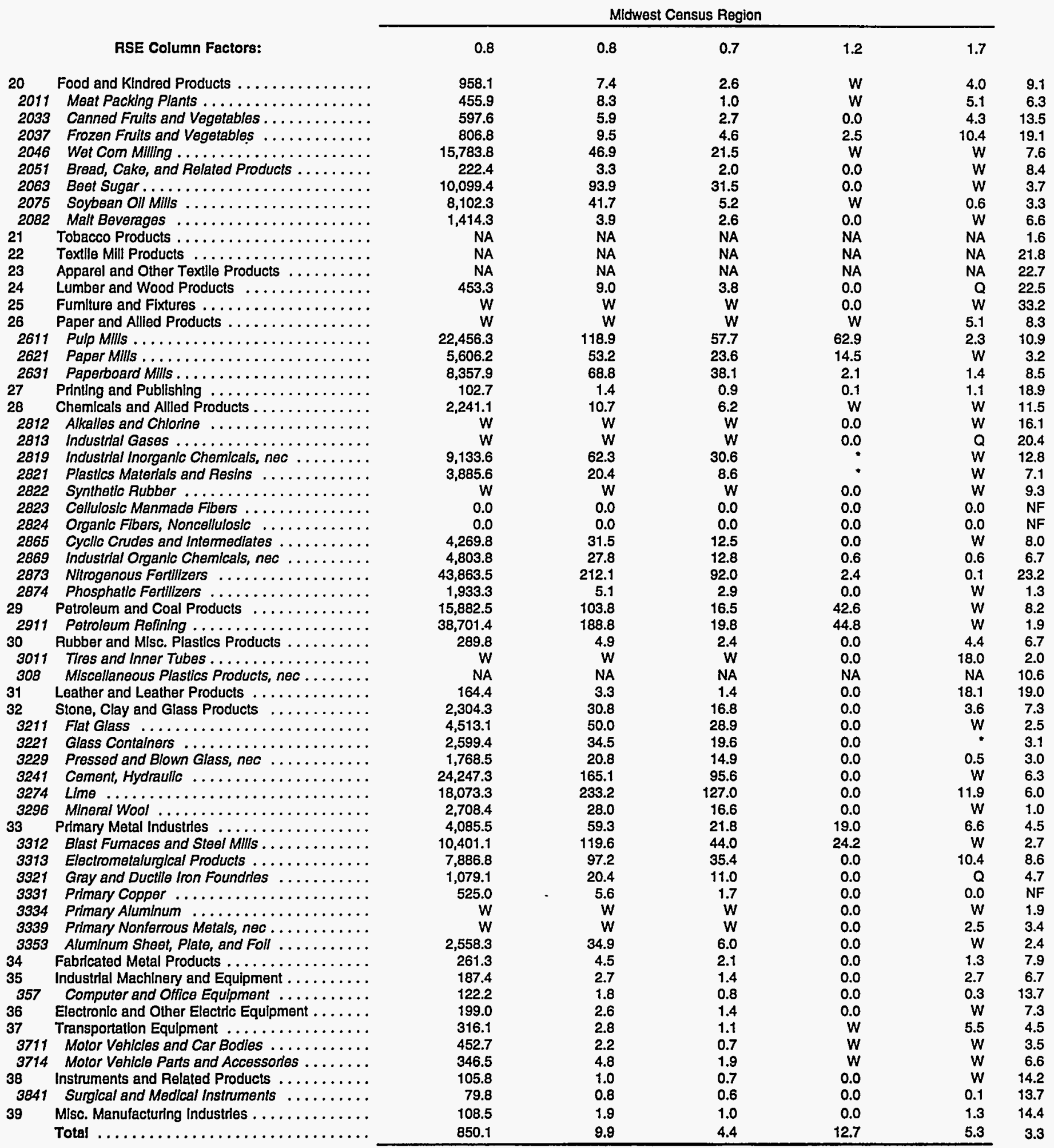

See footnotes at end of table. 


\section{Table A8. Selected Energy Operating Ratios for Total Energy Consumption for Heat, Power, and Electricity Generation by Census Region, Industry Group, and Selected Industries, 1991 (Continued)}

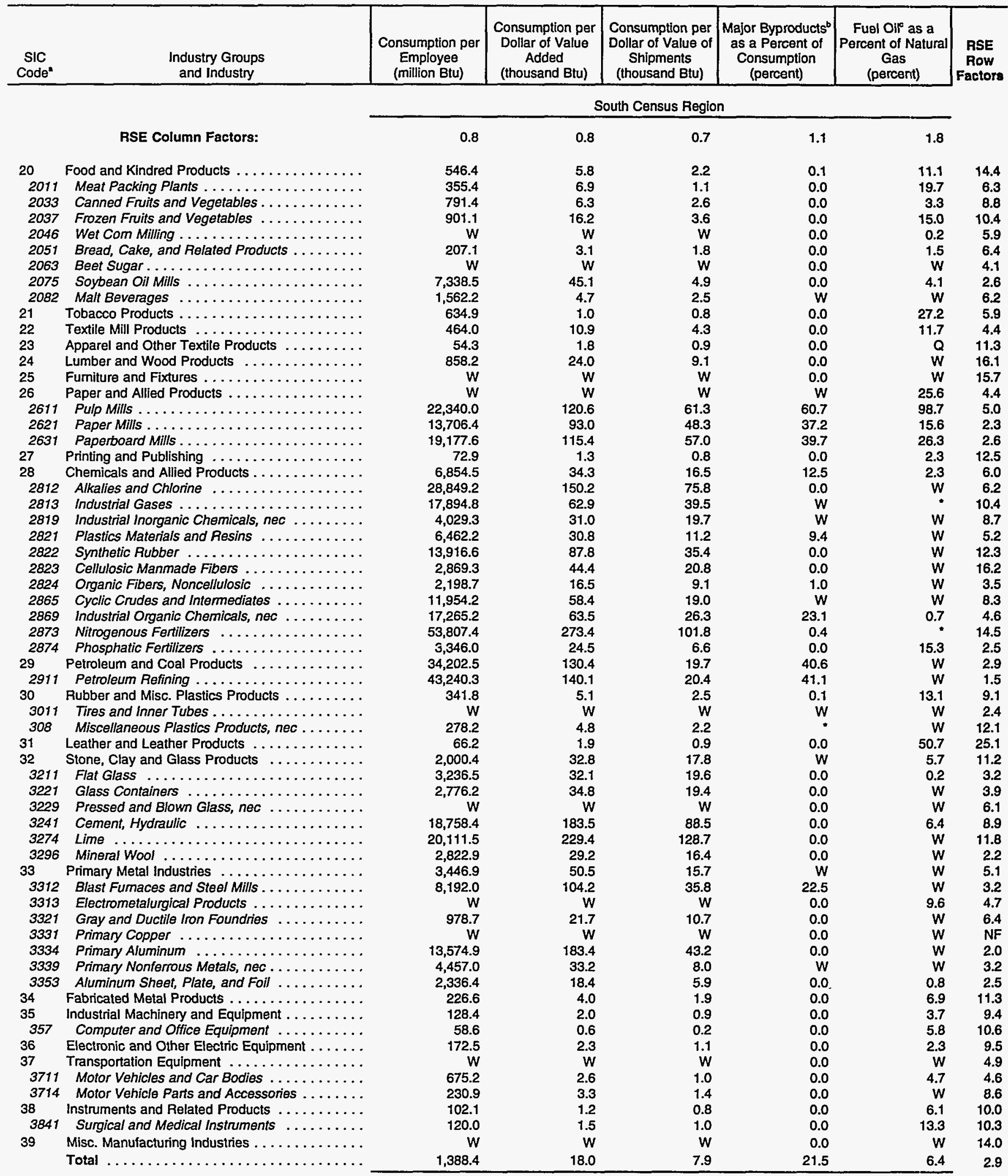

See footnotes at end of table. 
Table A8. Selected Energy Operating Ratios for Total Energy Consumption for Heat,
Power, and Electricity Generation by Census Region, Industry Group, and
Selected Industries, 1991 (Continued)

\begin{tabular}{cc|c|c|c|c|c}
\hline SIC & Industry Groups \\
Coda" & and Industry & $\begin{array}{c}\text { Consumption per } \\
\text { Employee } \\
\text { (million Btu) }\end{array}$ & $\begin{array}{c}\text { Consumption per } \\
\text { Dollar of Value } \\
\text { Added } \\
\text { (thousand Btu) }\end{array}$ & $\begin{array}{c}\text { Consumption per } \\
\text { Dollar of Value of } \\
\text { Shipments } \\
\text { (thousand Btu) }\end{array}$ & $\begin{array}{c}\text { Major Byproducts } \\
\text { as a Percent of } \\
\text { Consumption } \\
\text { (percent) }\end{array}$ & $\begin{array}{c}\text { Fuel Oif as a } \\
\text { Percent of Natural } \\
\text { Gas } \\
\text { (percent) }\end{array}$ \\
\hline
\end{tabular}

\begin{tabular}{|c|c|c|c|c|c|c|c|}
\hline & & & & Regio & & & \\
\hline & RSE Column Factors: & 0.8 & 0.9 & 0.8 & 1.1 & 1.5 & \\
\hline 20 & Food and Kindred Products $\ldots \ldots \ldots \ldots \ldots \ldots$ & 705.5 & 6.8 & 2.8 & $w$ & 6.9 & 10.6 \\
\hline 2033 & Canned Frults and Vegetables ............. & 785.0 & 9.3 & 3.9 & 0.0 & 5.7 & 6.9 \\
\hline 2037 & Frozen Fruils and Vegetables . ........... & 776.8 & 13.2 & 5.8 & 0.0 & 3.2 & 12.8 \\
\hline 2046 & 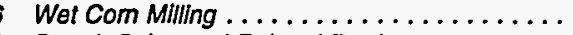 & w & w & w & 0.0 & w & 9.5 \\
\hline 2051 & Bread, Cake, and Related Products ......... & 241.3 & 3.2 & 2.0 & 0.0 & 0.2 & 12.5 \\
\hline 2082 & 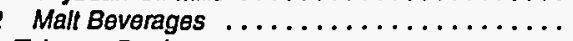 & $1,666.8$ & 7.7 & 4.0 & w & w & 8.3 \\
\hline 21 & Tobacco Products $\ldots \ldots \ldots \ldots \ldots \ldots \ldots$ & 0.0 & 0.0 & 0.0 & 0.0 & 0.0 & NF \\
\hline 22 & Textlie Mill Products $\ldots \ldots \ldots \ldots \ldots \ldots \ldots \ldots$ & 503.8 & 15.5 & 4.6 & 0.0 & 0.3 & 26.7 \\
\hline 23 & Apparel and Other Textlle Products $\ldots \ldots \ldots \ldots$ & NA & NA & NA & NA & NA & 21.5 \\
\hline 24 & Lumber and Wood Products $\ldots \ldots \ldots \ldots \ldots$ & $1,066.2$ & 22.9 & 7.1 & 0.0 & 75.3 & 15.7 \\
\hline 25 & Fumiture and Fixtures $\ldots \ldots \ldots \ldots \ldots \ldots \ldots$ & 49.5 & 1.0 & 0.5 & 0.0 & $\mathbf{Q}$ & 21.2 \\
\hline 26 & Paper and Allled Products $\ldots \ldots \ldots \ldots \ldots \ldots$ & $6,034.4$ & 60.8 & 25.1 & 42.0 & 10.2 & 9.3 \\
\hline 2812 & Alkalles and Chlorine $\ldots \ldots \ldots \ldots \ldots \ldots$ & w & w & w & 0.0 & w & 13.2 \\
\hline 2813 & Industrial Gases $\ldots \ldots \ldots \ldots \ldots \ldots \ldots \ldots$ & w & w & w & 0.0 & w & 7.3 \\
\hline 2819 & Industrial Inorganic Chemicals, nec . . . . . . . & $2,682.3$ & 18.8 & 13.6 & w & $w$ & 11.5 \\
\hline 2821 & Plastles Materials and Resins $\ldots \ldots \ldots \ldots$ & $1,080.9$ & 9.0 & 2.7 & 0.0 & 0.1 & 28.1 \\
\hline 2822 & Synthetic Rubber $\ldots \ldots \ldots \ldots \ldots \ldots \ldots$ & 72.6 & 0.7 & 0.2 & 0.0 & w & 2.5 \\
\hline 2823 & Cellulosic Manmade Fibers $\ldots \ldots \ldots \ldots \ldots$ & 0.0 & 0.0 & 0.0 & 0.0 & 0.0 & NF \\
\hline 2824 & Organlc Fibers, Noncellulosic $\ldots \ldots \ldots \ldots$ & 0.0 & 0.0 & 0.0 & 0.0 & 0.0 & NF \\
\hline 2865 & Cycllc Crudes and Intermedlates . . . . . . . . . & $\mathbf{Q}$ & $\mathbf{Q}$ & 1.9 & 0.0 & w & 21.6 \\
\hline 2869 & Industrial Organic Chemicals, nec .......... & $1,455.7$ & 8.9 & 4.9 & 0.0 & $\mathbf{Q}$ & 24.6 \\
\hline 2873 & Nitrogenous Fertilizers $\ldots \ldots \ldots \ldots \ldots \ldots$ & $27,666.8$ & 146.6 & 64.6 & 0.0 & * & 32.1 \\
\hline 2874 & Phosphatlc Fertllizers $\ldots \ldots \ldots \ldots \ldots \ldots$ & $3,740.7$ & 33.5 & 10.5 & 0.0 & 1.3 & 20.8 \\
\hline 29 & Petroleum and Coal Products $\ldots \ldots \ldots \ldots \ldots$ & $25,985.0$ & 130.7 & 19.2 & 51.1 & 13.3 & 4.4 \\
\hline 2911 & Potroleum Refining $\ldots \ldots \ldots \ldots \ldots \ldots$ & $35,323.9$ & 153.0 & 20.8 & 52.0 & 14.2 & 1.4 \\
\hline 30 & Rubber and Misc. Piastics Products . . . . . . . & 193.6 & 3.4 & 1.6 & 0.0 & $\mathbf{Q}$ & 15.4 \\
\hline 3011 & Tires and Inner Tubes $\ldots \ldots \ldots \ldots \ldots \ldots$ & 675.1 & 7.1 & 3.2 & 0.0 & $\mathbf{w}$ & 3.2 \\
\hline 3312 & Blast Fumaces and Steol Mills . . . . . . . . . & $8,445.4$ & 86.5 & 29.3 & W & w & 6.1 \\
\hline 3313 & Electrometalurgical Products . . . . . . . . . . . & 0.0 & 0.0 & 0.0 & 0.0 & 0.0 & NF \\
\hline 3321 & Gray and Ductile Iron Foundries ........ & $1,138.8$ & 16.5 & 8.7 & 0.0 & W & 17.8 \\
\hline 3331 & Primary Copper $\ldots \ldots \ldots \ldots \ldots \ldots \ldots$ & w & w & $w$ & w & W & NF \\
\hline 3334 & Primary Aluminum & $12,772.9$ & 135.0 & 44.2 & 0.0 & 1.7 & 1.6 \\
\hline 3339 & Primary Nonferrous Motals, nec . . . . . . . . . & $4,250.7$ & 72.5 & 21.1 & 0.0 & $\mathbf{w}$ & NF \\
\hline 3353 & Aluminum Sheet, Plate, and Foil & w & w & w & 0.0 & w & NF \\
\hline 34 & Fabricated Metal Products . . . . . . & 170.7 & 3.1 & 1.5 & 0.0 & 2.7 & 13.1 \\
\hline 35 & Industrial Machlnery and Equipment $\ldots \ldots \ldots \ldots$ & 92.5 & 1.1 & 0.6 & 0.0 & 1.0 & 14.5 \\
\hline 357 & Computer and Office Equipment ........ & 91.2 & 0.7 & 0.4 & 0.0 & 0.1 & 11.2 \\
\hline 36 & Electronic and Other Electric Equipment ....... & 105.7 & 1.1 & 0.6 & 0.0 & $\mathbf{Q}$ & 15.6 \\
\hline
\end{tabular}

See footnotes at end of table. 


\section{Table A8. Selected Energy Operating Ratios for Total Energy Consumption for Heat, Power, and Electricity Generation by Census Region, Industry Group, and Selected Industries, 1991 (Continued)}

\begin{tabular}{|c|c|c|c|c|c|c|c|}
\hline $\begin{array}{c}\text { SIC } \\
\text { Code }\end{array}$ & $\begin{array}{l}\text { Industry Groups } \\
\text { and Industry }\end{array}$ & $\begin{array}{c}\text { Consumption per } \\
\text { Employee } \\
\text { (million Btu) }\end{array}$ & $\begin{array}{c}\text { Consumption per } \\
\text { Dollar of Value } \\
\text { Added } \\
\text { (thousand Btu) }\end{array}$ & $\begin{array}{c}\text { Consumption per } \\
\text { Dollar of Value of } \\
\text { Shipments } \\
\text { (thousand Btu) }\end{array}$ & $\begin{array}{c}\text { Major Byproducts } \\
\text { as a Percent of } \\
\text { Consumption } \\
\text { (percent) }\end{array}$ & $\begin{array}{c}\text { Fuel Oif as a } \\
\text { Percent of Natural } \\
\text { Gas } \\
\text { (percent) }\end{array}$ & $\begin{array}{l}\text { RSE } \\
\text { Row } \\
\text { Factors }\end{array}$ \\
\hline & & \multicolumn{6}{|c|}{ West Census Region } \\
\hline \multicolumn{2}{|r|}{ RSE Column Factors: } & 0.8 & 0.9 & 0.8 & 1.1 & 1.5 & \\
\hline \multirow{7}{*}{$\begin{array}{c}37 \\
3711 \\
3714 \\
38 \\
3841 \\
39\end{array}$} & Transportation Equipment & 110.6 & 1.3 & 0.5 & 0.0 & $w$ & 5.9 \\
\hline & Motor Vehicles and Car Bodies & $W$ & $w$ & $w$ & 0.0 & $w$ & 3.6 \\
\hline & Motor Vehicle Parts and Accessories & 181.5 & 2.5 & 1.2 & 0.0 & 0.7 & 20.0 \\
\hline & Instruments and Related Products ... & 65.4 & 0.7 & 0.5 & 0.0 & 1.3 & 9.9 \\
\hline & Surgical and Medical Instruments & 62.3 & 0.7 & 0.4 & 0.0 & 4.5 & 17.1 \\
\hline & Misc. Manufacturing Industries . . . . . & 54.7 & 1.1 & 0.5 & 0.0 & 0.4 & 18.0 \\
\hline & Total $\ldots \ldots \ldots \ldots \ldots \ldots \ldots \ldots \ldots$ & 773.6 & 9.4 & 4.4 & w & 8.8 & 4.1 \\
\hline
\end{tabular}

\footnotetext{
- See Appendices B and F for descriptions of the Standard Industrial Classification system.

" "Major Byproduct" fuels include coke oven and blast furnace gas (produced primarily in the blast fumace industry, SIC 3312); still gas (produced primarily in refineries, SIC 2911); and pulping liquor (produced primarily in pulp and paper mills, SIC 2611 and 2621).

"Fuel Oil" includes distillate and residual.

NF=No applicable RSE row/column factor.

Estimate less than 0.5. Data are included in higher level totals.

$W=$ Withheld to avoid disclosing data for individual establishments. Data are included in higher level totals.

$\mathrm{Q}=$ Withheld because Relative Standard Error is greater than 50 percent. Data are included in higher level totals.

$N A=$ Not available. Data are included in higher level totals.

Notes: • To obtain a RSE percentage for any table cell, multiply the cell's corresponding RSE column and RSE row factors. • Totals may not equal sum of components because of independent rounding. - Operating ratios were calculated using the input energy estimates reported in Table A4.

Source: Energy Information Administration, Office of Energy Markets and End Use, Energy End Use and Integrated Statistics Division, Form EIA-846, "1991 Manufacturing Energy Consumption Survey," and Bureau of the Census, Industry Division, data files for the "1991 Annual Survey of Manufactures."
} 
Table A9. Total Primary Consumption of Energy for All Purposes by Census Region and Economic Characteristics of the Establishment, 1991

(Estimates in Btu or Physical Units)

\begin{tabular}{|c|c|c|c|c|c|c|c|c|c|c|}
\hline $\begin{array}{c}\text { Economic } \\
\text { Characteristics" }\end{array}$ & $\begin{array}{c}\text { Total } \\
\text { (trillion Btu) }\end{array}$ & $\begin{array}{c}\text { Net } \\
\text { Electricity } \\
\text { (million } \\
\text { kWh) }\end{array}$ & $\begin{array}{c}\text { Residual } \\
\text { Fuel Oil } \\
\text { (1000 bbls) }\end{array}$ & $\begin{array}{c}\text { Distillate } \\
\text { Fuel Oil }^{c} \\
\text { (1000 bbls) }\end{array}$ & $\begin{array}{l}\text { Natural Gas } \\
\text { (billion cu ft) }\end{array}$ & $\begin{array}{c}\text { LPG } \\
\text { (1000 bbls })\end{array}$ & $\begin{array}{c}\text { Coal } \\
\text { (1000 short } \\
\text { tons) }\end{array}$ & $\begin{array}{c}\text { Coke and } \\
\text { Breeze } \\
(1000 \text { short } \\
\text { tons })\end{array}$ & $\begin{array}{c}\text { Other } \\
\text { (trillion Btu) }\end{array}$ & $\begin{array}{c}\text { RSE } \\
\text { Row } \\
\text { Factors }\end{array}$ \\
\hline & \multicolumn{10}{|c|}{ Total United States } \\
\hline RSE Column Factors: & 0.6 & 0.5 & 1.4 & 1.3 & 0.8 & 1.2 & 1.3 & 1.5 & 0.9 & \\
\hline \multicolumn{11}{|l|}{$\begin{array}{l}\text { Value of Shipments and Recelpts } \\
\text { (million dollars) }\end{array}$} \\
\hline Under $20 \ldots \ldots \ldots \ldots$ & $\cdot 1,443$ & 110,693 & $w$ & 9,914 & 601 & $w$ & 5,203 & 354 & 207 & 9.8 \\
\hline $20-49 \ldots \ldots \ldots \ldots \ldots \ldots$ & 1,747 & 107,191 & 12,176 & 4,813 & 678 & 3,219 & 10,879 & 635 & 301 & 7.5 \\
\hline $50-99 \ldots \ldots \ldots \ldots \ldots \ldots$ & 1,619 & 91,770 & 8,633 & 2,390 & w & w & w & 467 & 273 & 5.6 \\
\hline $100-249 \ldots \ldots \ldots \ldots \ldots \ldots$ & 3,071 & 143,657 & 13,710 & 2,293 & 1,132 & 63,361 & w & $\mathbf{w}$ & 687 & 4.1 \\
\hline $250-499 \ldots \ldots \ldots \ldots \ldots$ & 3,081 & 113,398 & 15,370 & 2,393 & $w$ & 76,190 & $w$ & 915 & 964 & 3.8 \\
\hline 500 and $O v e r \ldots \ldots \ldots \ldots$ & 6,429 & 127,993 & w & 3,213 & 1,809 & 283,197 & 31,425 & w & 2,004 & 3.0 \\
\hline Not Ascertalned '...$\ldots \ldots$ & 2,868 & 0 & 0 & 0 & 0 & 0 & 0 & 0 & 2,868 & 1.4 \\
\hline Total $\ldots \ldots \ldots \ldots \ldots \ldots$ & 20,257 & 694,702 & 72,261 & 25,016 & 5,917 & 447,163 & 83,860 & 12,410 & 7,304 & 2.6 \\
\hline \multicolumn{11}{|l|}{ Employment Slze } \\
\hline Under $50 \ldots$. & 632 & 47,134 & $\mathbf{Q}$ & 5,777 & w & 2,188 & w & 38 & 69 & 13.8 \\
\hline $50-99 \ldots \ldots \ldots \ldots \ldots \ldots$ & 1,027 & 47,959 & 10,119 & $\mathbf{3 , 7 8 5}$ & 409 & 12,560 & 2,784 & 312 & 242 & 10.2 \\
\hline $100-249 \ldots \ldots \ldots \ldots \ldots \ldots$ & 2,807 & 123,214 & 10,051 & 5,196 & 1,142 & 68,412 & w & w & w & 6.6 \\
\hline $250-499 \ldots \ldots \ldots \ldots \ldots \ldots$ & 3,213 & 125,728 & 11,784 & 3,136 & 1,081 & 99,299 & 10,486 & 681 & 965 & 3.9 \\
\hline $500-999 \ldots \ldots \ldots \ldots \ldots$ & 3,615 & 152,257 & 13,040 & 2,705 & 1,121 & 104,843 & $w$ & w & $w$ & 3.7 \\
\hline 1,000 and Over $\ldots \ldots \ldots \ldots$ & 6,094 & 198,411 & $w$ & 4,419 & $w$ & 159,861 & 40,808 & 10,108 & 1,513 & 2.9 \\
\hline Not Ascertained ' $\ldots \ldots \ldots$. & 2,868 & & 0 & 0 & 0 & 0 & 0 & 0 & 2,868 & 1.4 \\
\hline \multirow[t]{2}{*}{ Total $\ldots \ldots \ldots \ldots \ldots$} & 20,257 & 694,702 & 72,261 & 25,016 & 5,917 & 447,163 & 83,860 & 12,410 & 7,304 & 2.6 \\
\hline & \multicolumn{10}{|c|}{ Northeast Census Reglon } \\
\hline RSE Column Factors: & 0.7 & 0.6 & 0.9 & 1.5 & 0.6 & 1.5 & 1.7 & 1.6 & 0.8 & \\
\hline \multicolumn{11}{|l|}{$\begin{array}{l}\text { Value of Shipments and Recelpts } \\
\text { (million dollars) }\end{array}$} \\
\hline Under $20 \ldots \ldots \ldots \ldots \ldots$ & 263 & 19,258 & 1,772 & 3,921 & $w$ & w & $\mathbf{Q}$ & w & 16 & 14.9 \\
\hline $20-49 \ldots \ldots \ldots \ldots \ldots \ldots$ & 229 & 14,952 & 3,740 & 1,440 & $w$ & 637 & 2,132 & $w$ & $w$ & 14.3 \\
\hline $50-99 \ldots \ldots \ldots \ldots \ldots \ldots$ & 164 & 11,177 & 3,365 & 695 & 71 & 334 & 538 & 55 & 13 & 8.3 \\
\hline $100-249 \ldots \ldots \ldots \ldots \ldots$ & 338 & 16,594 & 4,776 & 726 & 86 & w & w & w & w & 5.0 \\
\hline $250-499 \ldots \ldots \ldots \ldots \ldots$ & 220 & 11,762 & 6,948 & $\mathbf{w}$ & 49 & w & w & w & w & 5.5 \\
\hline 500 and Over $\ldots \ldots \ldots \ldots$ & 513 & 12,298 & 5,193 & w & 79 & W & w & 0 & 139 & 5.1 \\
\hline Not Ascertained ' .... & 285 & 0 & 0 & 0 & 0 & 0 & 0 & 0 & 285 & 1.9 \\
\hline Total.............. & 2,011 & 86,041 & 25,794 & 7,875 & 447 & 4,263 & w & $w$ & 588 & 5.0 \\
\hline \multicolumn{11}{|l|}{ Employment Size } \\
\hline Under $50 \ldots \ldots \ldots \ldots \ldots$ & $\mathbf{w}$ & 7,862 & 339 & $w$ & 37 & w & $\mathbf{w}$ & $\mathbf{Q}$ & 6 & 21.1 \\
\hline $50-99 \ldots \ldots \ldots \ldots \ldots$ & $W$ & 6,971 & 2,209 & $w$ & 40 & W & 152 & $w$ & $w$ & 16.0 \\
\hline $100-249 \ldots \ldots \ldots \ldots \ldots$ & 280 & 15,282 & 2,725 & 1,908 & 75 & w & 4,396 & 100 & $w$ & 15.5 \\
\hline $250-499 \ldots \ldots \ldots \ldots \ldots$ & 209 & 13,849 & 3,943 & 1,005 & 86 & 620 & $w$ & $w$ & 30 & 6.7 \\
\hline $500-999 \ldots \ldots \ldots \ldots \ldots \ldots$ & w & 21,944 & 6,854 & $w$ & 86 & $w$ & $w$ & $w$ & 136 & 4.9 \\
\hline 1,000 and Over $\ldots \ldots \ldots \ldots$ & $w$ & 20,132 & 9,725 & 1,057 & 123 & 403 & w & w & 107 & 5.3 \\
\hline Not Ascertained '..$\ldots \ldots \ldots$ & 285 & 0 & 0 & 0 & 0 & 0 & 0 & 0 & 285 & 1.9 \\
\hline Total $\ldots \ldots \ldots \ldots \ldots$ & 2,011 & 86,041 & 25,794 & 7,875 & 447 & 4,263 & $w$ & $w$ & 588 & 5.0 \\
\hline
\end{tabular}

See lootnotes at end of table. 
Table A9. Total Primary Consumption of Energy for All Purposes by Census Region and Economic Characteristics of the Establishment, 1991 (Continued)

(Estimates in Btu or Physical Units)

\begin{tabular}{|c|c|c|c|c|c|c|c|c|c|c|}
\hline $\begin{array}{c}\text { Economic } \\
\text { Characteristics }^{2}\end{array}$ & $\begin{array}{c}\text { Total } \\
\text { (trillion Btu) }\end{array}$ & $\begin{array}{c}\text { Net } \\
\text { Electricity } \\
\text { (million } \\
\text { kWh) }\end{array}$ & $\begin{array}{c}\text { Residual } \\
\text { Fuel Oil } \\
(1000 \text { bbls })\end{array}$ & $\begin{array}{c}\text { Distillate } \\
\text { Fuel Oilc } \\
\text { (1000 bbls) }\end{array}$ & $\begin{array}{l}\text { Natural Gas } \\
\text { (billion cu ft) }\end{array}$ & $\begin{array}{c}\text { LPG } \\
\text { (1000 bbis })\end{array}$ & $\begin{array}{c}\text { Coal } \\
\text { (1000 short } \\
\text { tons) }\end{array}$ & $\begin{array}{c}\text { Coke and } \\
\text { Breeze } \\
\text { (1000 short } \\
\text { tons) }\end{array}$ & $\begin{array}{c}\text { Othe }{ }^{\circ} \\
\text { (trillion Btu) }\end{array}$ & $\begin{array}{c}\text { RSE } \\
\text { Row } \\
\text { Factors }\end{array}$ \\
\hline & \multicolumn{9}{|c|}{ Midwest Census Region } & \\
\hline RSE Column Factors: & 0.6 & 0.6 & 1.5 & 1.7 & 0.6 & 1.5 & 0.9 & 1.4 & 0.9 & \\
\hline \multicolumn{11}{|l|}{$\begin{array}{l}\text { Value of Shipments and Receipts } \\
\text { (million dollars) }\end{array}$} \\
\hline Under $20 \ldots \ldots \ldots \ldots$ & w & 32,084 & 221 & $w$ & 222 & 1,033 & $w$ & 170 & $w$ & 13.6 \\
\hline $20-49 \ldots \ldots \ldots \ldots \ldots$ & 463 & 29,692 & 503 & $w$ & 237 & 732 & $w$ & W & 42 & 9.8 \\
\hline $50-99 \ldots \ldots \ldots \ldots \ldots$ & $w$ & 25,954 & 330 & 372 & $w$ & 359 & $w$ & 191 & 45 & 7.9 \\
\hline $100-249 \ldots \ldots \ldots \ldots \ldots$ & 520 & 29,626 & 990 & 340 & 204 & $W$ & $w$ & W & 81 & 5.5 \\
\hline $250-499 \ldots \ldots \ldots \ldots \ldots$ & 509 & 31,253 & 571 & 239 & W & $W$ & $w$ & $w$ & $w$ & 4.7 \\
\hline 500 and Over $\ldots \ldots \ldots \ldots$ & 1,560 & 56,492 & W & 998 & $W$ & $w$ & 15,932 & $w$ & 357 & 3.8 \\
\hline Not Ascertained $^{t} \ldots \ldots \ldots$ & 445 & 0 & 0 & 0 & 0 & 0 & 0 & 0 & 445 & 1.4 \\
\hline Total $\ldots \ldots \ldots \ldots \ldots$ & 4,385 & 205,102 & $W$ & 4,410 & 1,421 & W & 30,891 & 8,398 & 1,089 & 3.8 \\
\hline \multicolumn{11}{|l|}{ Employment Size } \\
\hline Under $50 \ldots \ldots \ldots \ldots$ & 216 & 13,381 & 128 & 1,371 & 120 & W & 172 & $w$ & $\mathbf{Q}$ & 20.9 \\
\hline $50-99 \ldots \ldots \ldots \ldots \ldots$ & W & 14,601 & $\mathbf{Q}$ & 503 & 118 & 439 & 1,162 & $w$ & $\bar{W}$ & 16.4 \\
\hline $100-249 \ldots \ldots \ldots \ldots \ldots$ & 630 & 35,608 & 873 & 642 & 284 & 833 & $w$ & 151 & $w$ & 8.4 \\
\hline $250-499 \ldots \ldots \ldots \ldots \ldots$ & 683 & 30,870 & 1,051 & 354 & $w$ & $w$ & $w$ & 247 & 147 & 5.2 \\
\hline $500-999 \ldots \ldots \ldots \ldots \ldots$ & $W$ & 29,186 & 962 & 404 & 197 & W & 3,246 & W & 201 & 5.2 \\
\hline 1,000 and Over $\ldots \ldots \ldots \ldots$ & 1,531 & 81,455 & W & 1,135 & W & $W$ & W & $w$ & 147 & 4.0 \\
\hline Not Ascertained ' $\ldots \ldots \ldots$. & 445 & 0 & 0 & 0 & 0 & 0 & 0 & 0 & 445 & 1.4 \\
\hline Total $\ldots \ldots \ldots \ldots \ldots$ & 4,385 & 205,102 & $W$ & 4,410 & 1,421 & W & 30,891 & 8,398 & 1,089 & 3.8 \\
\hline & \multicolumn{9}{|c|}{ South Census Region } & \\
\hline RSE Column Factors: & 0.6 & 0.5 & 1.7 & 1.3 & 0.9 & 1.0 & 0.9 & 1.7 & 1.0 & \\
\hline \multicolumn{11}{|l|}{$\begin{array}{l}\text { Value of Shipments and Receipts } \\
\text { (million dollars) }\end{array}$} \\
\hline Under $20 \ldots \ldots \ldots \ldots$ & 514 & 42,345 & $w$ & $w$ & $W$ & $w$ & 1,167 & W & 88 & 12.8 \\
\hline $20-49 \ldots \ldots \ldots \ldots \ldots$ & 763 & 49,656 & 7,224 & w & 286 & 1,447 & w & 291 & $w$ & 9.8 \\
\hline $50-99 \ldots \ldots \ldots \ldots \ldots \ldots$ & 753 & 41,663 & 3,783 & 815 & 290 & w & 3,457 & 188 & $W$ & 8.5 \\
\hline $100-249 \ldots \ldots \ldots \ldots \ldots$ & 1,664 & 63,521 & 6,905 & 866 & 644 & $w$ & $w$ & 157 & 351 & 5.6 \\
\hline $250-499 \ldots \ldots \ldots \ldots \ldots$ & 1,976 & 49,978 & 6,499 & 900 & 708 & W & W & W & 690 & 5.2 \\
\hline 500 and $O v e r . \ldots \ldots \ldots$ & 3,839 & 44,656 & 4,994 & 1,176 & $w$ & 271,076 & $w$ & w & 1,218 & 4.0 \\
\hline Not Ascertained ' $\ldots \ldots \ldots$ & 1,786 & 0 & 0 & 0 & 0 & 0 & 0 & 0 & 1,786 & 1.3 \\
\hline Total $\ldots \ldots \ldots \ldots \ldots \ldots$ & 11,296 & 291,819 & $\mathbf{W}$ & 8,481 & 3,368 & $w$ & 29,974 & 2,677 & 4,407 & 3.6 \\
\hline \multicolumn{11}{|l|}{ Employment Size } \\
\hline Under $50 \ldots \ldots \ldots \ldots$ & 233 & 17,812 & $\mathbf{Q}$ & $w$ & 121 & $w$ & $Q$ & 5 & 17 & 16.5 \\
\hline $50-99 \ldots \ldots \ldots \ldots \ldots$ & 517 & 18,313 & 7,200 & $w$ & 198 & $W$ & 477 & $w$ & $w$ & 14.7 \\
\hline $100-249 \ldots \ldots \ldots \ldots \ldots$ & 1,374 & 54,664 & 4,245 & 1,721 & 567 & $w$ & $W$ & $W$ & W & 7.6 \\
\hline $250-499 \ldots \ldots \ldots \ldots \ldots$ & 1,883 & 60,997 & 4,787 & 1,036 & 629 & $w$ & $w$ & 234 & 573 & 5.5 \\
\hline $500-999 \ldots \ldots \ldots \ldots \ldots$ & 2,004 & 69,714 & 4,824 & 1,092 & 675 & W & $w$ & 158 & 574 & 4.9 \\
\hline 1,000 and Over $\ldots \ldots \ldots \ldots$ & 3,499 & 70,319 & 9,440 & 1,916 & 1,178 & 155,633 & 13,523 & 1,914 & 1,071 & 4.2 \\
\hline Not Ascertained ${ }^{\prime} \ldots \ldots \ldots$ & 1,786 & 0 & 0 & 0 & 0 & 0 & 0 & 0 & 1,786 & 1.3 \\
\hline Total $\ldots \ldots \ldots \ldots \ldots \ldots$ & 11,296 & 291,819 & $w$ & 8,481 & 3,368 & $w$ & 29,974 & 2,677 & 4,407 & 3.6 \\
\hline
\end{tabular}

See footnotes at end of table. 
Table A9. Total Primary Consumption of Energy for All Purposes by Census Region and Economic Characteristics of the Establishment, 1991 (Continued) (Estimates in Btu or Physical Units)

\begin{tabular}{|c|c|c|c|c|c|c|c|c|c|c|}
\hline $\begin{array}{c}\text { Economic } \\
\text { Characteristics }\end{array}$ & $\begin{array}{c}\text { Total } \\
\text { (trillion Btu) }\end{array}$ & $\begin{array}{c}\text { Net } \\
\text { Electricity } \\
\text { (million } \\
\text { kWh) }\end{array}$ & $\begin{array}{c}\text { Residual } \\
\text { Fuel Oil } \\
\text { (1000 bbls) }\end{array}$ & $\begin{array}{c}\text { Distillate } \\
\text { Fuel Oil } \\
\text { (1000 bbls) }\end{array}$ & $\begin{array}{l}\text { Natural Gas } \\
\text { (billion cu } \mathrm{ft} \text { ) }\end{array}$ & $\begin{array}{c}\text { LPG } \\
(1000 \text { bbls })\end{array}$ & $\begin{array}{c}\text { Coal } \\
\text { (1000 short } \\
\text { tons) }\end{array}$ & $\begin{array}{c}\text { Coke and } \\
\text { Breeze } \\
(1000 \text { short } \\
\text { tons) }\end{array}$ & $\begin{array}{c}\text { Other" } \\
\text { (trillion Btu) }\end{array}$ & $\begin{array}{c}\text { RSE } \\
\text { Row } \\
\text { Factors }\end{array}$ \\
\hline & \multicolumn{10}{|c|}{ West Census Region } \\
\hline RSE Column Factors: & 0.7 & 0.6 & 1.1 & 1.3 & 0.9 & 0.8 & 1.8 & 1.4 & 1.0 & \\
\hline \multicolumn{11}{|l|}{$\begin{array}{l}\text { Value of Shipments and Receipts } \\
\text { (million dollars) }\end{array}$} \\
\hline Under $20 \ldots \ldots \ldots \ldots \ldots$ & w & 17,006 & 491 & 1,423 & $\mathbf{w}$ & 554 & w & 23 & $\mathbf{w}$ & 15.2 \\
\hline $20-49 \ldots \ldots \ldots \ldots \ldots \ldots$ & 292 & 12,892 & 710 & $w$ & w & 403 & $w$ & 120 & 108 & 12.6 \\
\hline $50-99 \ldots \ldots \ldots \ldots \ldots \ldots$ & $w$ & 12,976 & 1,155 & 507 & 107 & 472 & 807 & 33 & w & 8.6 \\
\hline $100-249 \ldots \ldots \ldots \ldots \ldots \ldots$ & 549 & 33,915 & 1,039 & 361 & 198 & 325 & $w$ & $w$ & $w$ & 8.3 \\
\hline $250-499 \ldots \ldots \ldots \ldots \ldots$ & 376 & 20,404 & 1,353 & $w$ & 87 & 1,238 & $w$ & w & 164 & 3.4 \\
\hline 500 and Over $\ldots \ldots \ldots \ldots$ & 518 & 14,547 & 596 & $w$ & $\mathbf{w}$ & 10,389 & $\mathbf{w}$ & 0 & 291 & 4.3 \\
\hline Not Ascertalned' $\ldots \ldots \ldots$. & 352 & 0 & 0 & 0 & 0 & 0 & 0 & 0 & 352 & 1.2 \\
\hline Total $\ldots \ldots \ldots \ldots \ldots \ldots \ldots$ & 2,565 & 111,741 & 5,344 & 4,250 & 681 & 13,381 & $w$ & $\mathbf{w}$ & 1,220 & 4.6 \\
\hline \multicolumn{11}{|l|}{ Employment Size } \\
\hline Under 50 & $w$ & 8,079 & $\mathbf{Q}$ & $w$ & $\mathbf{w}$ & 222 & w & w & $\mathbf{Q}$ & 10.9 \\
\hline $50-99 \ldots \ldots \ldots \ldots \ldots \ldots$ & 166 & 8,073 & 471 & w & 53 & $\mathbf{w}$ & 993 & 45 & 50 & 14.7 \\
\hline $100-249 \ldots \ldots \ldots \ldots \ldots$ & 524 & 17,660 & 2,209 & 924 & 216 & w & 1,666 & $w$ & 168 & 9.2 \\
\hline $250-499 \ldots \ldots \ldots \ldots \ldots$ & 437 & 20,012 & 2,003 & 741 & w & 1,391 & w & w & 214 & 6.1 \\
\hline $500-999 \ldots \ldots \ldots \ldots \ldots$ & 542 & 31,412 & 400 & $w$ & 162 & 4,464 & $w$ & $\mathbf{w}$ & $\mathbf{w}$ & 5.4 \\
\hline 1,000 and Over $\ldots \ldots \ldots \ldots$ & w & 26,505 & 242 & 310 & 104 & w & $w$ & $\mathbf{w}$ & 188 & 5.7 \\
\hline Not Ascertained ' $\ldots \ldots \ldots \ldots$ & 352 & 0 & 0 & 0 & 0 & 0 & 0 & 0 & 352 & 1.2 \\
\hline Total $\ldots \ldots \ldots \ldots \ldots \ldots$ & 2,565 & 111,741 & 5,344 & 4,250 & 681 & 13,381 & $\mathbf{w}$ & w & 1,220 & 4.6 \\
\hline
\end{tabular}

a Value of Shipments and Receipts and Employment Size were supplied by the Bureau of the Census. See Appendix B.

- "Net Electricity" is obtained by summing purchases, transfers in, and generation from noncombustible renewable resources, minus quantities sold and transferred out. It does not include electricity inputs from onsite cogeneration or generation from combustible fuels because that energy has already been included as generating fuel (Ior example, coal).

-Distlllate Fuel Oil" includes Nos. 1, 2, and 4 fuel oils and Nos. 1, 2, and 4 diesel fuels.

- "Natural Gas" includes natural gas obtained from utilities, transmission pipelines, and any other supplier(s) such as brokers and producers.

- "Other" includes net steam (the sum of purchases, generation from renewables, and net transfers), and other energy that respondents indicated was used to produce heat and power or as feedstock/raw material inputs. See also Footnote "f."

'The entry in the "Not Ascertained" row and the "Other" column consists of the feedstocks and raw material inputs that were consumed by petroleum refineries for the production of nonenergy products (i.e., asphalt, waxes, lubricants, and solvents), as well as feedstock consumption at adjoining petrochemical plants. That entry Includes all of those inputs, regardless of type. Those inputs that were converted to other energy products by petroleum refineries (e.g., crude oil converted to residual and distillate fuel oils) are excluded. The quantities of energy consumed by petroleum refineries for the production of heat and power are included in the appropriate "Value of Shipments and Receipts" or "Employment Size" rows. See Appendix B for more information.

$W=$ Withheld to avold disclosing data for individual establishments. Data are included in higher level totals.

$Q=$ Withheld because Relative Standard Error is greater than 50 percent. Data are included in higher level totals.

Notes: - To obtain a RSE percentage for any table cell, multiply the cell's corresponding RSE column and RSE row factors. - Totals may not equal sum of components because of independent rounding. - The derived estimates presented in this table are for the primary consumption of energy for heat and power and as feedstocks or raw materlal inputs. Primary consumption is defined as the consumption of the energy that was originally produced offsite or was produced onsite from input materials not classified as energy. Examples of the latter are hydrogen produced from the electrolysis of brine; the output of captive (onsite) mines or wells; woodchips, bark, and woodwaste from wood purchased as a raw material input; and waste materials such as wastepaper and packing materials. Primary consumption excludes quantifies of energy that are produced from other energy inputs and, therelore, avoids double counting.

Source: Energy Information Administration, Office of Energy Markets and End Use, Energy End Use and Integrated Statistics Division, Form ElA-846, "1991 Manulacturing Energy Consumption Survey," the Office of Oil and Gas, Petroleum Supply Division, Form ElA-810, "Monthly Refinery Report" for 1991, and the Bureau of the Census, Industry Division, data files for the "1991 Annual Survey of Manufactures." 
Table A10. Total Consumption of LPG, Distillate Fuel Oil, and Residual Fuel Oil for Selected Purposes by Census Region and Economic Characteristics off the Establishment, 1991

(Estimates in Barrels per Day)

\begin{tabular}{|c|c|c|c|c|c|c|c|c|c|c|}
\hline \multirow[b]{2}{*}{$\begin{array}{c}\text { Economic } \\
\text { Characteristics" }\end{array}$} & \multicolumn{3}{|c|}{ Primary Consumption for All Purposes } & \multicolumn{3}{|c|}{$\begin{array}{l}\text { Inputs for Heat, Power, and } \\
\text { Generation of Electricity }\end{array}$} & \multicolumn{3}{|c|}{$\begin{array}{l}\text { Primary Consumption for Nonfuel } \\
\text { Pumposes }\end{array}$} & \multirow{2}{*}{$\begin{array}{c}\text { RSE } \\
\text { Row } \\
\text { Factors }\end{array}$} \\
\hline & LPG & Distillate $^{b}$ & Residual & L.PG & Distillate ${ }^{b}$ & Residual & LPG & Distillate $^{b}$ & Residual & \\
\hline & \multicolumn{9}{|c|}{ Total United States } & \\
\hline RSE Column Factors: & 0.8 & 0.8 & 0.9 & 0.6 & 0.8 & 0.7 & 0.9 & 1.9 & 3.4 & \\
\hline \multicolumn{11}{|l|}{$\begin{array}{l}\text { Value of Shipments and Receipts } \\
\text { (million dollars) }\end{array}$} \\
\hline Under $20 \ldots \ldots \ldots \ldots \ldots$ & w & 27,161 & 15,301 & 11,206 & 25,451 & W & $\mathbf{W}$ & $\mathbf{Q}$ & $\mathbf{Q}$ & 14.8 \\
\hline $20-49 \ldots \ldots \ldots \ldots \ldots$ & 8,819 & 13,186 & 33,360 & 7,419 & 12,429 & 16,589 & 1,424 & 789 & 16,862 & 13.6 \\
\hline $50-99 \ldots \ldots \ldots \ldots \ldots \ldots$ & W & 6,548 & 23,652 & 5,188 & 6,482 & 28,319 & W & 188 & 4,787 & 9.0 \\
\hline $100-249 \ldots \ldots \ldots \ldots \ldots \ldots$ & 173,592 & 6,281 & 37,561 & 6,417 & 6,217 & 37,817 & 167,184 & 143 & 12 & 7.0 \\
\hline $250-499 \ldots \ldots \ldots \ldots \ldots \ldots$ & 208,740 & 6,557 & 42,111 & 5,653 & 6,348 & 42,111 & 203,274 & 207 & 0 & 4.4 \\
\hline 500 and Over . . . . . . . . . & 775,881 & 8,804 & 45,991 & 40,746 & 8,513 & $W$ & 735,308 & 291 & $W$ & 4.2 \\
\hline Total $\ldots \ldots \ldots \ldots \ldots \ldots$ & $1,225,103$ & 68,538 & 197,976 & 76,630 & 65,439 & 180,376 & $1,148,868$ & 3,330 & 27,456 & 6.0 \\
\hline \multicolumn{11}{|l|}{ Employment Size } \\
\hline Under $50 \ldots \ldots \ldots \ldots \ldots$ & 5,993 & 15,827 & $Q$ & 4,789 & 14,663 & 2,631 & 1,205 & $\mathbf{Q}$ & $\mathbf{Q}$ & 21.4 \\
\hline $50-99 \ldots \ldots \ldots \ldots \ldots \ldots$ & 34,411 & 10,370 & 27,722 & 4,540 & 9,302 & $w$ & 29,896 & 1,069 & W & 15.6 \\
\hline $100-249 \ldots \ldots \ldots \ldots \ldots \ldots$ & 187,430 & 14,234 & 27,538 & 19,971 & 13,867 & 32,231 & 167,460 & 398 & 4,787 & 8.1 \\
\hline $250-499 \ldots \ldots \ldots \ldots \ldots$ & 272,053 & 8,592 & 32,285 & 11,536 & 8,595 & 32,553 & 260,526 & 196 & 0 & 6.5 \\
\hline $500-999 \ldots \ldots \ldots \ldots \ldots$ & 287,241 & 7,410 & 35,727 & 18,794 & 7,298 & 35,758 & 268,447 & 113 & 12 & 6.8 \\
\hline 1,000 and Over $\ldots \ldots \ldots \ldots$ & 437,975 & 12,106 & 67,870 & 17,000 & 11,714 & W & 421,334 & 389 & $W$ & 4.4 \\
\hline Total $\ldots \ldots \ldots \ldots \ldots \ldots$ & $1,225,103$ & 68,538 & 197,976 & 76,630 & 65,439 & 180,376 & $1,148,868$ & 3,330 & 27,456 & 6.0 \\
\hline & \multicolumn{9}{|c|}{ Northeast Census Region } & \\
\hline RSE Column Factors: & 0.9 & 1.0 & 0.5 & 1.0 & 1.0 & 0.5 & 1.6 & 2.5 & NF & \\
\hline \multicolumn{11}{|l|}{$\begin{array}{l}\text { Value of Shipments and Receipts } \\
\text { (million dollars) }\end{array}$} \\
\hline Under $20 \ldots \ldots \ldots \ldots$ & $W$ & 10,744 & 4,854 & W & 10,557 & 4,854 & $\mathbf{W}$ & $\mathbf{Q}$ & 0 & 20.6 \\
\hline $20-49 \ldots \ldots \ldots \ldots \ldots \ldots$ & 1,745 & 3,946 & 10,246 & 1,736 & 3,941 & 10,246 & 9 & 5 & 0 & 21.7 \\
\hline $50-99 \ldots \ldots \ldots \ldots \ldots \ldots \ldots$ & 914 & 1,905 & 9,220 & 914 & 1,986 & 18,675 & - & 41 & 0 & 12.8 \\
\hline $100-249 \ldots \ldots \ldots \ldots \ldots \ldots$ & 2,143 & 1,989 & 13,084 & $W$ & 1,956 & 13,084 & W & 33 & 0 & 10.1 \\
\hline $250-499 \ldots \ldots \ldots \ldots \ldots$ & W & 912 & 19,036 & W & W & 19,036 & W & W & 0 & 8.2 \\
\hline 500 and Over $\ldots \ldots \ldots \ldots \ldots$ & 1,398 & 2,081 & 14,228 & 1,397 & W & 14,228 & - & W & 0 & 7.8 \\
\hline Total $\ldots \ldots \ldots \ldots \ldots \ldots$ & 11,681 & 21,576 & 70,668 & $W$ & 21,384 & 80,123 & $W$ & 315 & 0 & 9.3 \\
\hline \multicolumn{11}{|l|}{ Employment Size } \\
\hline Under $50 \ldots \ldots \ldots \ldots$ & 1,824 & 6,108 & 928 & W & 5,936 & 928 & $\mathbf{Q}$ & $\mathbf{Q}$ & 0 & 37.1 \\
\hline $50-99 \ldots \ldots \ldots \ldots \ldots \ldots \ldots$ & $W$ & 2,450 & 6,052 & 573 & $W$ & 6,052 & $\mathbf{W}$ & W & 0 & 20.7 \\
\hline $100-249 \ldots \ldots \ldots \ldots \ldots \ldots \ldots$ & 2,855 & 5,228 & 7,465 & $W$ & 5,212 & 16,920 & $W$ & 17 & 0 & 15.8 \\
\hline $250-499 \ldots \ldots \ldots \ldots \ldots$ & 1,700 & 2,754 & 10,802 & 1,683 & 2,864 & 10,802 & $Q$ & 10 & 0 & 11.5 \\
\hline $500-999 \ldots \ldots \ldots \ldots \ldots \ldots$ & 2,958 & 2,140 & 18,779 & $w$ & $W$ & 18,779 & W & $W$ & 0 & 8.0 \\
\hline 1,000 and Over $\ldots \ldots \ldots \ldots$ & W & 2,895 & 26,643 & $W$ & 2,812 & 26,643 & 33 & 83 & 0 & 9.0 \\
\hline Total $\ldots \ldots \ldots \ldots \ldots \ldots$ & 11,681 & 21,576 & 70,668 & $w$ & 21,384 & 80,123 & $w$ & 315 & 0 & 9.3 \\
\hline
\end{tabular}

See footnotes at end of table. 
Table A10. Total Consumption of LPG, Distillate Fuel Oil, and Residual Fuel Oil for Selected Purposes by Census Region and Economic Characteristics of the Establishment, 1991 (Continued) (Estimates in Barrels per Day)

\begin{tabular}{|c|c|c|c|c|c|c|c|c|c|c|}
\hline \multirow{2}{*}{$\begin{array}{c}\text { Economic } \\
\text { Characteristlcs }\end{array}$} & \multicolumn{3}{|c|}{ Primary Consumptlon for All Purposes } & \multicolumn{3}{|c|}{$\begin{array}{l}\text { Inputs for Heat, Power, and } \\
\text { Generation of Electricity }\end{array}$} & \multicolumn{3}{|c|}{$\begin{array}{l}\text { Primany Consumption for Nonfuel } \\
\text { Purposes }\end{array}$} & \multirow{2}{*}{$\begin{array}{c}\text { RSE } \\
\text { Row } \\
\text { Factors }\end{array}$} \\
\hline & LPG & Distillate ${ }^{b}$ & Residual & LPG & Distillate ${ }^{b}$ & Residual & LPG & Distillate ${ }^{b}$ & Residual & \\
\hline & \multicolumn{10}{|c|}{ Midwest Census Region } \\
\hline RSE Column Factors: & 0.8 & 0.9 & 0.8 & 0.7 & 0.8 & 0.8 & 1.3 & 1.4 & 2.5 & \\
\hline \multicolumn{11}{|l|}{$\begin{array}{l}\text { Value of Shipments and Recelpts } \\
\text { (million dollars) }\end{array}$} \\
\hline Under $20 \ldots \ldots \ldots \ldots \ldots$ & 2,831 & 4,766 & 606 & 2,713 & 3,847 & 589 & 118 & Q & Q & 27.1 \\
\hline $20-49 \ldots \ldots \ldots \ldots \ldots \ldots \ldots$ & 2,006 & 1,979 & 1,377 & 1,980 & 1,621 & 1,402 & 26 & $\mathbf{Q}$ & 0 & 25.6 \\
\hline $50-99 \ldots \ldots \ldots \ldots \ldots \ldots \ldots$ & 983 & 1,019 & 903 & 976 & 995 & 903 & 7 & 24 & 0 & 19.7 \\
\hline $100-249 \ldots \ldots \ldots \ldots \ldots \ldots \ldots$ & $w$ & 930 & w & $w$ & 973 & $w$ & $w$ & $\mathbf{Q}$ & 12 & 11.4 \\
\hline $250-499 \ldots \ldots \ldots \ldots \ldots \ldots \ldots$ & $w$ & 655 & 1,563 & 586 & 590 & 1,563 & $w$ & 62 & 0 & 7.3 \\
\hline 500 and $O v e r \ldots \ldots \ldots \ldots \ldots$ & 3,347 & 2,733 & 16,448 & $w$ & 2,618 & w & w & 115 & w & 5.8 \\
\hline Total $\ldots \ldots \ldots \ldots \ldots \ldots \ldots$ & $w$ & 12,083 & $w$ & 10,622 & 10,645 & 22,286 & $w$ & $\mathbf{Q}$ & $w$ & 7.7 \\
\hline \multicolumn{11}{|l|}{ Employment Size } \\
\hline Under $50 \ldots \ldots \ldots \ldots \ldots$ & 1,262 & 3,757 & 349 & 1,170 & 2,861 & 334 & 92 & $\mathbf{Q}$ & $\mathbf{Q}$ & 32.9 \\
\hline $50-99 \ldots \ldots \ldots \ldots \ldots \ldots \ldots$ & 1,204 & 1,379 & $\mathbf{Q}$ & 1,198 & w & $\mathbf{Q}$ & $\mathbf{Q}$ & $\mathbf{Q}$ & - & 34.5 \\
\hline $100-249 \ldots \ldots \ldots \ldots \ldots \ldots \ldots$ & 2,281 & 1,760 & w & w & 1,744 & w & w & 47 & 0 & 20.4 \\
\hline $250-499 \ldots \ldots \ldots \ldots \ldots \ldots \ldots$ & w & 970 & 2,879 & 2,884 & w & 3,147 & w & w & 0 & 9.3 \\
\hline $500-999 \ldots \ldots \ldots \ldots \ldots \ldots \ldots$ & w & 1,107 & 2,636 & 1,908 & 1,078 & 2,624 & w & 27 & 12 & 9.9 \\
\hline 1,000 and Over $\ldots \ldots \ldots \ldots \ldots$ & 1,233 & 3,111 & 14,699 & w & 2,947 & $\mathbf{w}$ & w & 161 & W & 6.2 \\
\hline \multirow[t]{2}{*}{ Total $\ldots \ldots \ldots \ldots \ldots \ldots \ldots$} & $W$ & 12,083 & w & 10,622 & 10,645 & 22,286 & w & $\mathbf{Q}$ & w & 7.7 \\
\hline & \multicolumn{10}{|c|}{ South Census Region } \\
\hline RSE Column Factors: & 0.7 & 0.9 & 1.2 & 0.8 & 0.9 & 0.9 & 0.7 & 1.4 & 2.4 & \\
\hline \multicolumn{11}{|l|}{$\begin{array}{l}\text { Value of Shipments and Recelpts } \\
\text { (million dollars) }\end{array}$} \\
\hline Under $20 \ldots \ldots \ldots \ldots \ldots \ldots$ & 6,475 & 7,753 & 8,496 & w & 7,152 & w & w & $\mathbf{Q}$ & $\mathbf{Q}$ & 18.9 \\
\hline $20-49 \ldots \ldots \ldots \ldots \ldots \ldots \ldots$ & 3,964 & 5,188 & 19,792 & 2,614 & 4,893 & 2,996 & 1,375 & $\mathbf{Q}$ & 16,862 & 15.0 \\
\hline $50-99 \ldots \ldots \ldots \ldots \ldots \ldots \ldots$ & w & 2,234 & w & 2,009 & 2,137 & w & w & 97 & 4,787 & 13.1 \\
\hline $100-249 \ldots \ldots \ldots \ldots \ldots \ldots \ldots$ & w & 2,373 & 18,919 & 2,968 & 2,320 & 18,919 & w & 54 & 0 & 6.2 \\
\hline $250-499 \ldots \ldots \ldots \ldots \ldots \ldots$ & w & 2,466 & 17,804 & 1,249 & 2,373 & 17,804 & w & 93 & 0 & 6.1 \\
\hline 500 and $O v e r . \ldots \ldots \ldots \ldots$ & W & 3,222 & 13,682 & 7,564 & 3,083 & 13,725 & $\mathbf{w}$ & 139 & 0 & 5.6 \\
\hline Total .................... & w & 23,236 & w & W & 21,957 & 63,327 & $w$ & 1,280 & $w$ & 7.5 \\
\hline \multicolumn{11}{|l|}{ Employment Size } \\
\hline Under $50 \ldots \ldots \ldots \ldots \ldots \ldots$ & 2,300 & 3,823 & $\mathbf{Q}$ & w & 3,725 & 1,320 & w & Q & Q & 23.9 \\
\hline $50-99 \ldots \ldots \ldots \ldots \ldots \ldots \ldots$ & w & 3,618 & 19,726 & 1,552 & 2,959 & $\mathbf{w}$ & w & $\mathbf{Q}$ & w & 20.3 \\
\hline $100-249 \ldots \ldots \ldots \ldots \ldots \ldots \ldots$ & 172,771 & 4,715 & $w$ & 6,002 & 4,471 & $\mathbf{w}$ & 166,769 & 244 & 4,787 & 10.8 \\
\hline $250-499 \ldots \ldots \ldots \ldots \ldots \ldots$ & w & 2,837 & 13,116 & 3,174 & 2,710 & 13,116 & w & 127 & 0 & 8.0 \\
\hline $500-999 \ldots \ldots \ldots \ldots \ldots \ldots$ & w & 2,992 & 13,217 & 2,537 & 2,956 & 13,260 & w & 35 & 0 & 8.9 \\
\hline 1,000 and Over $\ldots$ & 426,393 & 5,250 & 25,863 & 5,469 & 5,136 & 25,863 & 421,284 & 114 & 0 & 5.2 \\
\hline Total $\ldots \ldots \ldots \ldots$. & $\mathbf{w}$ & 23,236 & $w$ & w & 21,957 & 63,327 & $\mathbf{w}$ & 1,280 & w & 7.5 \\
\hline
\end{tabular}

See footnotes at end of table. 
Table A10. Total Consumption of LPG, Distillate Fuel Oil, and Residual Fuel Oil for Selected Purposes by Census Region and Economic Characteristics of the Establishment, 1991 (Continued)

(Estimates in Barrels per Day)

\begin{tabular}{|c|c|c|c|c|c|c|c|c|c|c|}
\hline \multirow[b]{2}{*}{$\begin{array}{c}\text { Economic } \\
\text { Characteristics" }\end{array}$} & \multicolumn{3}{|c|}{ Primary Consumption for All Purposes } & \multicolumn{3}{|c|}{$\begin{array}{l}\text { Inputs for Heat, Power, and } \\
\text { Generation of Electricity }\end{array}$} & \multicolumn{3}{|c|}{$\begin{array}{l}\text { Primary Consumption for Nonfuel } \\
\text { Purposes }\end{array}$} & \multirow{2}{*}{$\begin{array}{c}\text { RSE } \\
\text { Row } \\
\text { Factors }\end{array}$} \\
\hline & LPG & Distillate $^{b}$ & Residual & LPG & Distillate $^{b}$ & Residual & LPG & Distillate $^{b}$ & Residual & \\
\hline & \multicolumn{9}{|c|}{ West Census Region } & \\
\hline RSE Column Factors: & 0.6 & 0.9 & 0.8 & 0.6 & 0.9 & 0.8 & 3.2 & 2.1 & NF & \\
\hline \multicolumn{11}{|l|}{$\begin{array}{l}\text { Value of Shipments and Receipts } \\
\text { (million dollars) }\end{array}$} \\
\hline Under $20 \ldots \ldots \ldots \ldots$ & 1,518 & 3,898 & 1,345 & 1,456 & 3,895 & 1,345 & 64 & 4 & 0 & 20.9 \\
\hline $20-49 \ldots \ldots \ldots \ldots \ldots \ldots$ & 1,104 & 2,074 & 1,945 & 1,090 & 1,974 & 1,945 & 14 & 99 & 0 & 17.5 \\
\hline $50-99 \ldots \ldots \ldots \ldots \ldots \ldots$ & 1,293 & 1,390 & W & 1,290 & 1,363 & $W$ & 3 & 27 & 0 & 13.4 \\
\hline $100-249 \ldots \ldots \ldots \ldots \ldots \ldots$ & 890 & 988 & $\mathbf{W}$ & 872 & 968 & W & 18 & 20 & 0 & 12.3 \\
\hline $250-499 \ldots \ldots \ldots \ldots \ldots \ldots$ & $W$ & 2,525 & 3,707 & W & $w$ & 3,707 & 0 & $w$ & 0 & 4.6 \\
\hline 500 and Over ............ & W & 768 & 1,633 & $\mathbf{w}$ & $w$ & 1,633 & 1 & $\mathbf{w}$ & 0 & 6.5 \\
\hline Total $\ldots \ldots \ldots \ldots \ldots \ldots$ & 36,660 & 11,643 & 14,640 & 36,561 & 11,452 & 14,640 & 100 & 191 & 0 & 7.3 \\
\hline \multicolumn{11}{|l|}{ Employment Size } \\
\hline Under $50 \ldots \ldots \ldots \ldots$ & 607 & 2,140 & $\mathbf{Q}$ & 592 & 2,141 & $\mathbf{Q}$ & $Q$ & 0 & 0 & 33.4 \\
\hline $50-99 \ldots \ldots \ldots \ldots \ldots$ & 1,243 & 2,922 & 1,290 & 1,217 & 2,908 & 1,290 & $Q$ & a & 0 & 16.7 \\
\hline $100-249 \ldots \ldots \ldots \ldots \ldots \ldots$ & 9,523 & 2,531 & 6,051 & W & 2,440 & 6,051 & $W$ & $Q$ & 0 & 8.6 \\
\hline $250-499 \ldots \ldots \ldots \ldots \ldots$ & 3,811 & 2,030 & 5,489 & 3,794 & w & 5,489 & 17 & W & 0 & 9.2 \\
\hline $500-999 \ldots \ldots \ldots \ldots \ldots$ & W & 1,171 & 1,095 & $\mathbf{w}$ & $w$ & 1,095 & 0 & $W$ & 0 & 7.5 \\
\hline 1,000 and Over $\ldots \ldots \ldots \ldots$ & $W$ & 849 & 664 & W & 819 & 664 & W & 31 & 0 & 8.6 \\
\hline Total $\ldots \ldots \ldots \ldots \ldots \ldots$ & 36,660 & 11,643 & 14,640 & 36,561 & 11,452 & 14,640 & 100 & 191 & 0 & 7.3 \\
\hline
\end{tabular}

- Value of Shipments and Receipts and Employment Size were supplied by the Bureau of the Census. See Appendix B.

"Distillate" includes Nos. 1, 2, and 4 fuel oils and Nos. 1, 2, and 4 diesel fuels.

NF=No applicable RSE row/column factor.

- Estimate less than 0.5. Data are included in higher level totals.

$W=$ Withheld to avoid disclosing data for individual establishments. Data are included in higher level totais.

Q=Withheld because Relative Standard Error is greater than 50 percent. Data are included in higher level totals.

Notes: - To obtain a RSE percentage for any table cell, multiply the cell's corresponding RSE column and RSE row factors. • Totals may not equal suin of

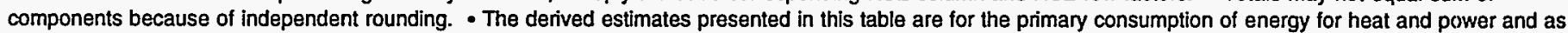

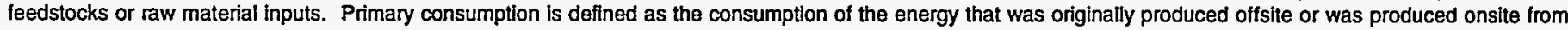

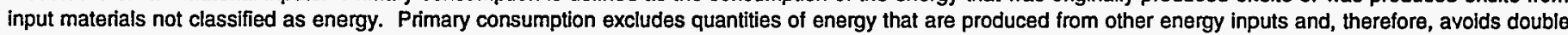
counting.

Source: Energy Information Administration, Office of Energy Markets and End Use, Energy End Use and Integrated Statistics Division, Form ElA-846, "1991 Manufacturing Energy Consumption Survey," and the Bureau of the Census, Industry Division, data files for the "1991 Annual Survey of Manufactures." 
Table A11. Total Primary Consumption of Combustible Energy for Nonfuel Purposes by Census Region and Economic Characteristics of the Establishment, 1991 (Estimates in Btu or Physical Units)

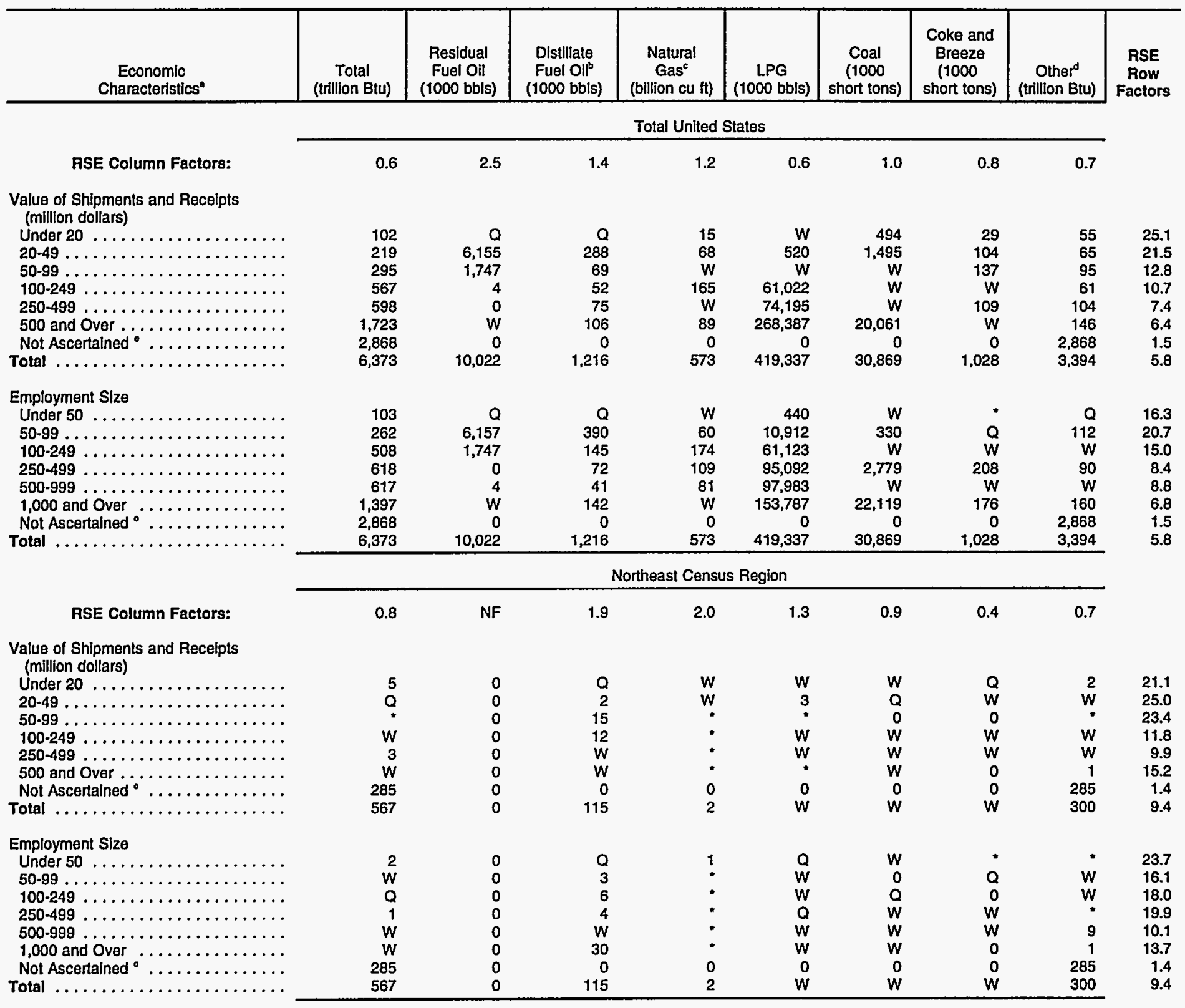

See tootnotes at end of table. 
Table A11. Total Primary Consumption of Combustible Energy for Nonfuel Purposes by Census Region and Economic Characteristics of the Establishment, 1991 (Continued) (Estimates in Btu or Physical Units)

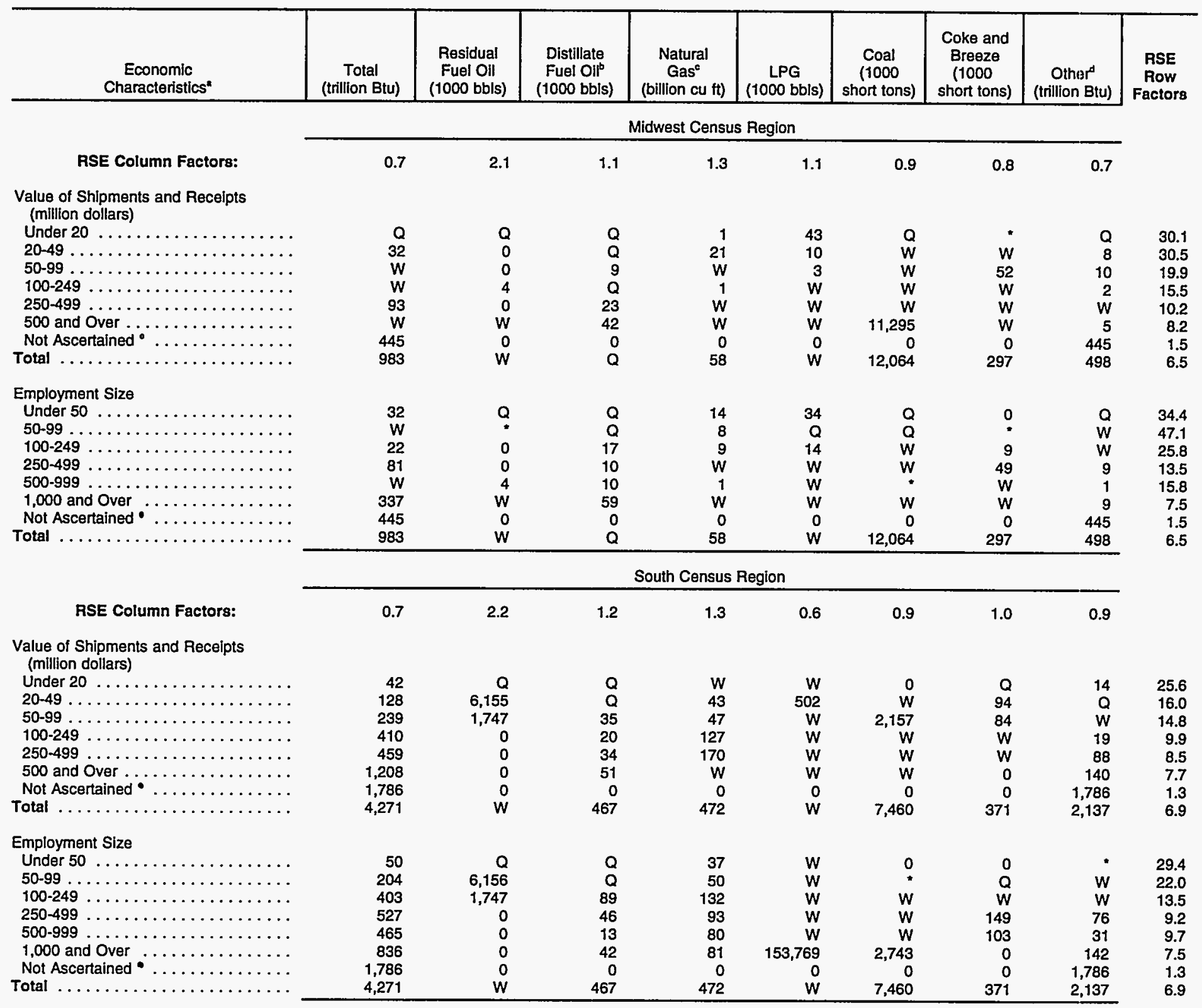

See footnotes at end of table. 
Table A11. Total Primary Consumption of Combustible Energy for Nonfuel Purposes by Census Region and Economic Characteristics of the Establishment, 1991 (Continued)

(Estimates in Btu or Physical Units)

\begin{tabular}{|c|c|c|c|c|c|c|c|c|c|}
\hline $\begin{array}{c}\text { Economic } \\
\text { Characteristles }\end{array}$ & $\begin{array}{c}\text { Total } \\
\text { (trillion Btu) } \\
\end{array}$ & $\begin{array}{c}\text { Residual } \\
\text { Fuel Oll } \\
(1000 \text { bbis }) \\
\end{array}$ & $\begin{array}{c}\text { Distillate } \\
\text { Fuel Oil } \\
\text { (1000 bbls) } \\
\end{array}$ & $\begin{array}{c}\begin{array}{c}\text { Natural } \\
\text { Gas }^{c} \\
\text { (billion cu } \mathrm{ft} \text { ) }\end{array} \\
\end{array}$ & $\begin{array}{c}\text { LPG } \\
\text { (1000 bbls) }\end{array}$ & $\begin{array}{c}\text { Coal } \\
(1000 \\
\text { short tons) }\end{array}$ & $\begin{array}{c}\text { Coke and } \\
\text { Breeze } \\
\text { (1000 } \\
\text { short tons) } \\
\end{array}$ & $\begin{array}{c}\text { Other } \\
\text { (trillion Btu) }\end{array}$ & $\begin{array}{c}\text { RSE } \\
\text { Row } \\
\text { Factors }\end{array}$ \\
\hline & \multicolumn{8}{|c|}{ West Census Region } & \\
\hline RSE Column Factors: & 0.7 & NF & 1.3 & 1.5 & 1.8 & 0.7 & 0.9 & 0.6 & \\
\hline \multicolumn{10}{|l|}{$\begin{array}{l}\text { Value of Shipments and Receipts } \\
\text { (million dollars) }\end{array}$} \\
\hline Under $20 \ldots \ldots \ldots \ldots \ldots \ldots \ldots$ & W & 0 & 2 & W & 23 & W & 0 & $\mathbf{Q}$ & 29.2 \\
\hline $20-49 \ldots \ldots \ldots \ldots \ldots \ldots$ & 33 & 0 & $\bar{W}$ & W & 5 & $w$ & * & 30 & 29.5 \\
\hline $50-99 \ldots \ldots \ldots \ldots \ldots \ldots$ & $W$ & 0 & 10 & 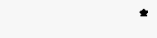 & 1 & 0 & 1 & W & 26.8 \\
\hline $100-249 \ldots \ldots \ldots \ldots \ldots \ldots$ & 74 & 0 & 7 & 36 & 7 & 2 & W & W & 14.8 \\
\hline $250-499 \ldots \ldots \ldots \ldots \ldots \ldots$ & 43 & 0 & W & 0 & 0 & w & $w$ & $W$ & 6.8 \\
\hline 500 and Over . . . . . . . . . . & $W$ & 0 & $W$ & W & $*$ & 0 & 0 & 1 & 20.1 \\
\hline Not Ascertained $\quad \ldots \ldots \ldots \ldots \ldots$ & 352 & 0 & 0 & 0 & 0 & 0 & 0 & 352 & 1.5 \\
\hline Total $\ldots \ldots \ldots \ldots \ldots \ldots \ldots$ & 552 & 0 & 70 & 41 & 36 & $w$ & W & 458 & 12.9 \\
\hline \multicolumn{10}{|l|}{ Employment Size } \\
\hline Under $50 \ldots \ldots \ldots \ldots \ldots$ & $Q$ & 0 & 0 & $w$ & $Q$ & $w$ & 0 & $Q$ & 13.1 \\
\hline $50-99 \ldots \ldots \ldots \ldots \ldots \ldots$ & 38 & 0 & $\mathbf{Q}$ & 3 & $\overline{\mathbf{Q}}$ & 325 & 0 & 26 & 29.1 \\
\hline $100-249 \ldots \ldots \ldots \ldots \ldots \ldots \ldots$ & 57 & 0 & $\mathbf{Q}$ & 33 & 14 & 0 & 1 & 23 & 34.8 \\
\hline $250-499 \ldots \ldots \ldots \ldots \ldots \ldots$ & 9 & 0 & 12 & W & 6 & W & W & 5 & 8.2 \\
\hline $500-999 \ldots \ldots \ldots \ldots \ldots \ldots$ & 35 & 0 & W & • & 0 & W & W & W & 9.3 \\
\hline 1,000 and $O v e r \ldots \ldots \ldots \ldots$ & $\mathbf{W}$ & 0 & 11 & 0 & $\mathbf{W}$ & W & $\mathbf{W}$ & 8 & 14.4 \\
\hline Not Ascertained ${ }^{\circ} \ldots \ldots \ldots \ldots$ & 352 & 0 & 0 & 0 & 0 & 0 & 0 & 352 & 1.5 \\
\hline Total $\ldots \ldots \ldots \ldots \ldots \ldots \ldots$ & 552 & 0 & 70 & 41 & 36 & W & W & 458 & 12.9 \\
\hline
\end{tabular}

- Value of Shipments and Receipts and Employment Size were supplied by the Bureau of the Census. See Appendix B.

"Distillate Fuel Oll" includes Nos. 1, 2, and 4 fuel oils and Nos. 1, 2, and 4 diesel fuels.

c "Natural Gas" includes natural gas obtained from utilities, transmission pipelines, and any other supplier(s) such as brokers and producers.

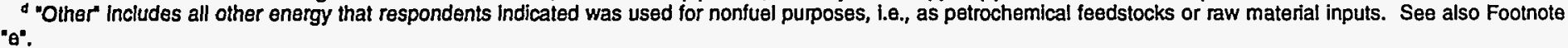

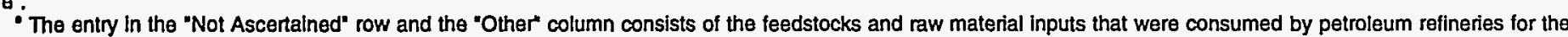

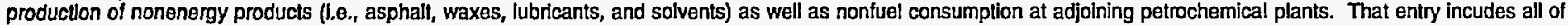

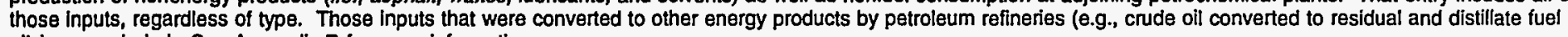
olls) are excluded. See Appendix B for more information.

NF $=$ No applicable RSE row/column factor.

- Estlmate less than 0.5. Data are included in higher level totals.

$W=W$ thheld to avold dlsclosing data for individual establishments. Data are included in higher level totals.

$\mathrm{Q}=$ Withheld because Relative Standard Error is greater than 50 percent. Data are included in higher level totals.

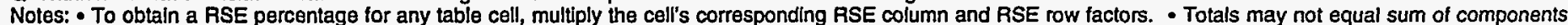
because of Independent rounding. - The derived estimates presented in this table are for the primary consumption of feedstocks or raw material inputs. Primary

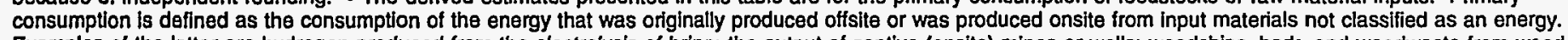

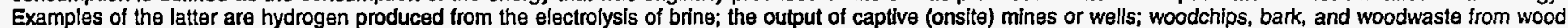

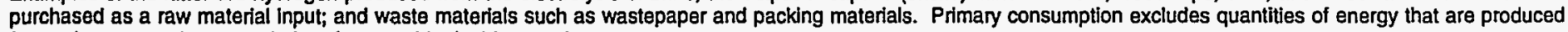
from other energy inputs and, therefore, avoids double counting.

Source: Energy Information Administration, Office of Energy Markets and End Use, Energy End Use and Integrated Statistics Division, Form EIA-846, "1991

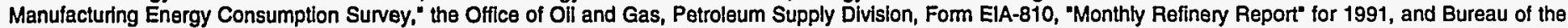
Census, Industry Division, data files for the "1991 Annual Survey of Manuiactures." 
Table A12. Total Inputs of Energy for Heat, Power, and Electricity Generation by Census Region and Economic Characteristics of the Establishment, 1991

(Estimates in Btu or Physical Units)

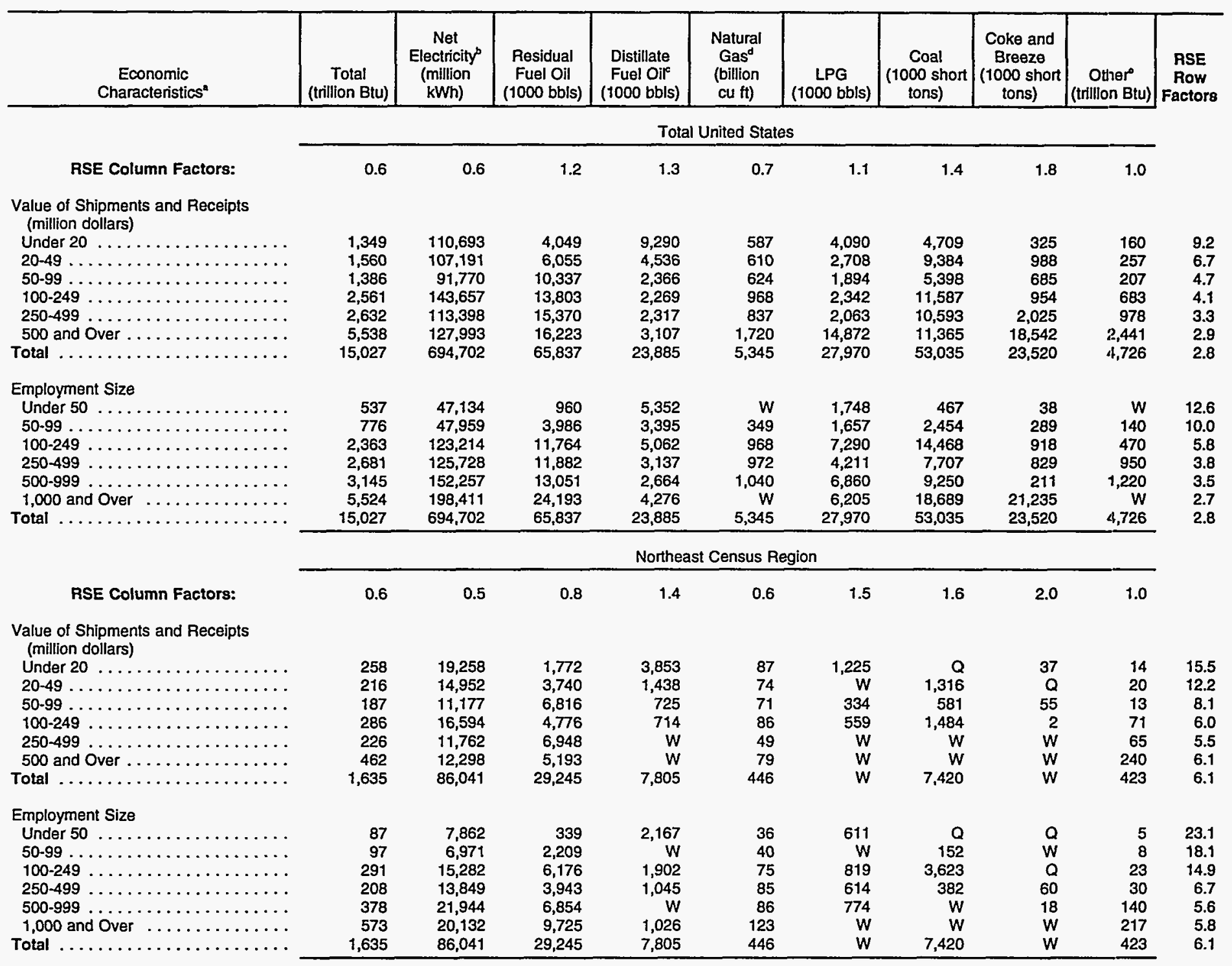

See footnotes at end of table. 
Table A12. Total Inputs of Energy for Heat, Power, and Electricity Generation by Census Region and Economic Characteristics of the Establishment, 1991 (Continued) (Estimates in Btu or Physical Units)

\begin{tabular}{|c|c|c|c|c|c|c|c|c|c|c|}
\hline $\begin{array}{c}\text { Economic } \\
\text { Characteristics }\end{array}$ & $\begin{array}{c}\text { Total } \\
\text { (trillion Btu) }\end{array}$ & $\begin{array}{c}\text { Net } \\
\text { Electricity } \\
\text { (million } \\
\text { kWh) } \\
\end{array}$ & $\begin{array}{c}\text { Residual } \\
\text { Fuel Oil } \\
\text { (1000 bbls })\end{array}$ & $\begin{array}{c}\text { Distillate } \\
\text { Fuel Olf } \\
\text { (1000 bbls) }\end{array}$ & $\begin{array}{c}\text { Natural } \\
\text { Gas }^{d} \\
\text { (billion } \\
\text { cu ft) } \\
\end{array}$ & $\begin{array}{c}\text { LPG } \\
(1000 \text { bb/s })\end{array}$ & $\begin{array}{c}\text { Coal } \\
(1000 \text { short } \\
\text { tons })\end{array}$ & $\begin{array}{c}\text { Coke and } \\
\text { Breeze } \\
(1000 \text { short } \\
\text { tons })\end{array}$ & $\begin{array}{c}\text { Other } \\
\text { (trílition Btu) }\end{array}$ & $\begin{array}{c}\text { RSE } \\
\text { Row } \\
\text { Factors }\end{array}$ \\
\hline & \multicolumn{9}{|c|}{ Midwest Census Region } & \\
\hline RSE Column Factors: & 0.5 & 0.6 & 1.6 & 1.6 & 0.6 & 1.3 & 1.0 & 1.6 & 1.0 & \\
\hline \multicolumn{11}{|l|}{$\begin{array}{l}\text { Value of Shipments and Recelpts } \\
\text { (million dollars) }\end{array}$} \\
\hline 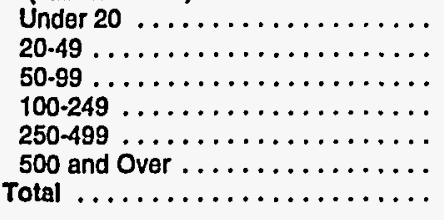 & $\begin{array}{r}424 \\
435 \\
422 \\
507 \\
429 \\
1,616 \\
3,833\end{array}$ & $\begin{array}{r}32,084 \\
29,692 \\
25,954 \\
29,626 \\
31,253 \\
56,492 \\
205,102\end{array}$ & $\begin{array}{r}215 \\
512 \\
330 \\
1,083 \\
571 \\
5,424 \\
8,134\end{array}$ & $\begin{array}{r}1,404 \\
592 \\
363 \\
355 \\
215 \\
956 \\
3,885\end{array}$ & $\begin{array}{r}221 \\
216 \\
203 \\
202 \\
163 \\
359 \\
1,363\end{array}$ & $\begin{array}{r}990 \\
723 \\
356 \\
381 \\
214 \\
1,213 \\
3,877\end{array}$ & $\begin{array}{r}1,211 \\
2,730 \\
2,710 \\
3,895 \\
3,644 \\
4,638 \\
18,828\end{array}$ & $\begin{array}{r}170 \\
103 \\
494 \\
740 \\
246 \\
14,036 \\
15,789\end{array}$ & $\begin{array}{r}43 \\
38 \\
46 \\
82 \\
61 \\
558 \\
829\end{array}$ & $\begin{array}{r}13.1 \\
9.2 \\
8.5 \\
5.5 \\
4.5 \\
4.1 \\
3.7\end{array}$ \\
\hline \multicolumn{11}{|l|}{ Employment Size } \\
\hline 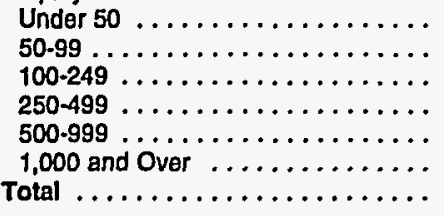 & $\begin{array}{r}185 \\
227 \\
612 \\
624 \\
593 \\
1,593 \\
3,833 \\
\end{array}$ & $\begin{array}{r}13,381 \\
14,601 \\
35,608 \\
30,870 \\
29,186 \\
81,455 \\
205,102 \\
\end{array}$ & $\begin{array}{r}122 \\
Q \\
882 \\
1,149 \\
958 \\
4,786 \\
8,134 \\
\end{array}$ & $\begin{array}{r}W \\
W \\
637 \\
374 \\
393 \\
1,076 \\
3,885 \\
\end{array}$ & $\begin{array}{r}106 \\
110 \\
275 \\
W \\
196 \\
W \\
1,363\end{array}$ & $\begin{array}{r}W \\
W \\
818 \\
1,053 \\
696 \\
445 \\
3,877 \\
\end{array}$ & $\begin{array}{r}172 \\
1,157 \\
5,342 \\
W \\
3,246 \\
W \\
18,828\end{array}$ & $\begin{array}{r}W \\
W \\
143 \\
553 \\
114 \\
14,876 \\
15,789\end{array}$ & $\begin{array}{r}W \\
30 \\
72 \\
150 \\
205 \\
W \\
829\end{array}$ & $\begin{array}{r}16.3 \\
16.0 \\
8.4 \\
5.1 \\
5.0 \\
4.0 \\
3.7\end{array}$ \\
\hline & \multicolumn{10}{|c|}{ South Census Region } \\
\hline RSE Column Factors: & 0.6 & 0.5 & 1.4 & 1.4 & 0.7 & 1.2 & 1.0 & 1.9 & 1.0 & \\
\hline \multicolumn{11}{|l|}{$\begin{array}{l}\text { Value of Shlpments and Recelpts } \\
\text { (million dollars) }\end{array}$} \\
\hline 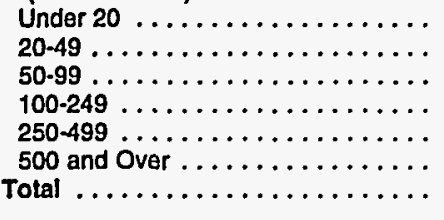 & $\begin{array}{r}480 \\
651 \\
532 \\
1,292 \\
1,609 \\
2,943 \\
7,507\end{array}$ & $\begin{array}{r}42,345 \\
49,656 \\
41,663 \\
63,521 \\
49,978 \\
44,656 \\
291,819\end{array}$ & $\begin{array}{r}1,571 \\
1,093 \\
2,036 \\
6,905 \\
6,499 \\
5,009 \\
23,114\end{array}$ & $\begin{array}{r}2,611 \\
1,786 \\
780 \\
847 \\
866 \\
1,125 \\
8,014\end{array}$ & $\begin{array}{r}190 \\
243 \\
242 \\
518 \\
538 \\
1,165 \\
2,896\end{array}$ & $\begin{array}{r}1,344 \\
954 \\
733 \\
1,083 \\
456 \\
W \\
W\end{array}$ & $\begin{array}{r}1,167 \\
3,420 \\
1,300 \\
W \\
W \\
W \\
22,514\end{array}$ & $\begin{array}{r}95 \\
572 \\
104 \\
W \\
W \\
W \\
W\end{array}$ & $\begin{array}{r}82 \\
120 \\
88 \\
370 \\
681 \\
1,352 \\
2,693\end{array}$ & $\begin{array}{r}12.4 \\
8.4 \\
6.9 \\
5.8 \\
5.2 \\
3.7 \\
3.3\end{array}$ \\
\hline \multicolumn{11}{|l|}{ Employment Size } \\
\hline 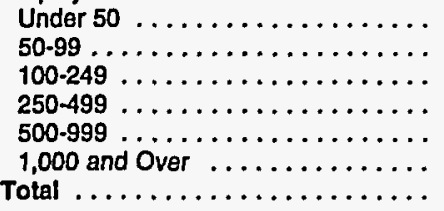 & $\begin{array}{r}190 \\
323 \\
994 \\
1,418 \\
1,666 \\
2,916 \\
7,507\end{array}$ & $\begin{array}{r}17,812 \\
18,313 \\
54,664 \\
60,997 \\
69,714 \\
70,319 \\
291,819\end{array}$ & $\begin{array}{r}482 \\
1,068 \\
2,497 \\
4,787 \\
4,840 \\
9,440 \\
23,114\end{array}$ & $\begin{array}{r}1,360 \\
1,080 \\
1,632 \\
989 \\
1,079 \\
1,875 \\
8,014\end{array}$ & $\begin{array}{r}84 \\
148 \\
435 \\
537 \\
595 \\
1,097 \\
2,896 \\
\end{array}$ & $\begin{array}{r}494 \\
566 \\
W \\
1,158 \\
926 \\
1,996 \\
W\end{array}$ & $\begin{array}{r}Q \\
477 \\
3,837 \\
2,544 \\
4,583 \\
10,780 \\
22,514\end{array}$ & $\begin{array}{r}5 \\
144 \\
W \\
85 \\
78 \\
3,860 \\
W\end{array}$ & $\begin{array}{r}24 \\
78 \\
229 \\
559 \\
671 \\
1,133 \\
2,693 \\
\end{array}$ & \begin{tabular}{r|}
16.5 \\
15.9 \\
7.0 \\
5.4 \\
4.7 \\
3.8 \\
3.3
\end{tabular} \\
\hline
\end{tabular}

See footnotes at end of table. 
Table A12. Total Inputs of Energy for Heat, Power, and Electricity Generation by Census Region and Economic Characteristics of the Establishment, 1991 (Continued) (Estimates in Btu or Physical Units)

\begin{tabular}{|c|c|c|c|c|c|c|c|c|c|c|}
\hline $\begin{array}{c}\text { Economic } \\
\text { Characteristlcs" }\end{array}$ & $\begin{array}{c}\text { Total } \\
\text { (trillion Btu) }\end{array}$ & $\begin{array}{c}\text { Net } \\
\text { Electricity } \\
\text { (million } \\
k W h)\end{array}$ & $\begin{array}{c}\text { Residual } \\
\text { Fuel Oil } \\
\text { (1000 bbls) }\end{array}$ & $\begin{array}{c}\text { Distillate } \\
\text { Fuel Oile } \\
(1000 \text { bbls })\end{array}$ & $\begin{array}{l}\text { Natural } \\
\text { Gas }^{d} \\
\text { (billion } \\
\text { cu ft) }\end{array}$ & $\begin{array}{c}\text { LPG } \\
(1000 \text { bbls })\end{array}$ & $\begin{array}{c}\text { Coal } \\
(1000 \text { short } \\
\text { tons })\end{array}$ & $\begin{array}{c}\text { Coke and } \\
\text { Breeze } \\
\left(\begin{array}{c}1000 \text { short } \\
\text { tons })\end{array}\right.\end{array}$ & $\begin{array}{c}\text { Other } \\
\text { (trililon Btu) }\end{array}$ & $\begin{array}{c}\text { RSE } \\
\text { Pow } \\
\text { Factors }\end{array}$ \\
\hline & \multicolumn{10}{|c|}{ West Census Region } \\
\hline RSE Column Factors: & 0.6 & 0.6 & 1.0 & 1.3 & 0.7 & 0.8 & 2.0 & 1.6 & 1.1 & \\
\hline \multicolumn{11}{|l|}{$\begin{array}{l}\text { Value of Shipments and Recelpts } \\
\text { (million dollars) }\end{array}$} \\
\hline Under $20 \ldots \ldots \ldots \ldots \ldots \ldots$ & 187 & 17,006 & 491 & 1,422 & 89 & 531 & 130 & 23 & 20 & 16.9 \\
\hline $20-49$ & 259 & 12,892 & 710 & 720 & 78 & $w$ & 1,918 & $w$ & 79 & 12.9 \\
\hline 50-99. & 245 & 12,976 & 1,155 & 497 & 107 & 471 & 807 & 32 & 60 & 8.9 \\
\hline $100-249 \ldots$ & 477 & 33,915 & 1,039 & 353 & 162 & 318 & w & $w$ & 161 & 7.7 \\
\hline $250-499 \ldots$ & 368 & 20,404 & 1,353 & $w$ & 87 & w & 32 & $w$ & 170 & 4.3 \\
\hline 500 and Over & 516 & 14,547 & 596 & w & 117 & 10,389 & w & 0 & 290 & 4.4 \\
\hline Total ................. & 2,052 & 111,741 & 5,344 & 4,180 & 640 & 13,345 & 4,274 & 1,053 & 780 & 4.9 \\
\hline \multicolumn{11}{|l|}{ Employment Size } \\
\hline Under 50 & 75 & 8,079 & $\mathbf{Q}$ & $w$ & w & w & 0 & w & Q & 11.5 \\
\hline $50-99 \ldots$ & 130 & 8,073 & 471 & 1,062 & 50 & 444 & 668 & 45 & 24 & 16.1 \\
\hline $100-249$ & 467 & 17,660 & 2,209 & 891 & 184 & $w$ & 1,666 & $w$ & 145 & 8.4 \\
\hline $250-499$ & 430 & 20,012 & 2,003 & 729 & $w$ & 1,385 & $w$ & 130 & 212 & 6.2 \\
\hline $500-999$ & 508 & 31,412 & 400 & $w$ & 162 & 4,464 & W & 0 & 204 & 5.2 \\
\hline 1,000 and Over. & 441 & 26,505 & 242 & 299 & 104 & w & $w$ & $w$ & 195 & 6.0 \\
\hline Total $\ldots \ldots \ldots \ldots$ & 2,052 & 111,741 & 5,344 & 4,180 & 640 & 13,345 & 4,274 & 1,053 & 780 & 4.9 \\
\hline
\end{tabular}

- Value of Shipments and Recelpts and Employment Size were supplied by the Bureau of the Census. See Appendix B.

- "Net Electricity" is obtained by summing purchases, transfers in, and generation from noncombustible renewable resources, minus quantitles sold and transierred out. It does not include electricity inputs from onsite cogeneration or generation from combustible fuels because that energy has already been included as generating fuel (for example, coal).

-Distillate Fuel Oil" includes Nos. 1, 2, and 4 fuel oils and Nos. 1, 2, and 4 diesel fuels.

- "Natural Gas" includes natural gas obtained from uttilties, transmission pipelines, and any other supplier(s) such as brokers and producers.

- "Other" includes net steam (the sum of purchases, generation from renewables, and net transfers), and other energy that respondents indicated was used to produce heat and power.

$W=$ Withheld to avoid disclosing data for individual establishments. Data are included in higher level totals.

$\mathrm{Q}=$ Withheld because Relative Standard Error is greater than 50 percent. Data are included in higher level totals.

Notes: - To obtain a RSE percentage for any table cell, multiply the cell's corresponding RSE column and RSE row factors. - Totals may not equal sum of components because of independent rounding. - The estimates presented in this table are for the total consumption of energy for the production of heat and power, regjardless of where the energy was produced. Specifically, the estimates include the quantities of energy that were originally produced offsite and purchased by or transferred to the establishment, plus those that were produced onsite from other energy or from input material not classified as energy, or were extracted from captive (onsite) mines or wells.

Source: Energy Information Administration, Office of Energy Markets and End Use, Energy End Use and Integrated Statistlcs Division, Form ElA-846, "1991

Manufacturing Energy Consumption Survey," and Bureau of the Census, Industry Division, data files for the "1991 Annual Survey of Manufactures." 
Table A13. Total Consumption of Offsite-Produced Energy for Heat, Power, and Electricity Generation by Census Region and Economic Characteristics of the Establishment, 1991

(Estimates in Btu or Physical Units)

\begin{tabular}{|c|c|c|c|c|c|c|c|c|c|c|}
\hline $\begin{array}{c}\text { Economic } \\
\text { Characteristlcs }^{a}\end{array}$ & $\begin{array}{c}\text { Total } \\
\text { (trillion Btu) }\end{array}$ & $\begin{array}{c}\text { Electricity" } \\
\text { (million kWh) }\end{array}$ & $\begin{array}{c}\text { Residual } \\
\text { Fuel Oll } \\
\text { (1000 bbls) }\end{array}$ & $\begin{array}{c}\text { Distillate } \\
\text { Fuel Oile } \\
\text { (1000 bbis) }\end{array}$ & 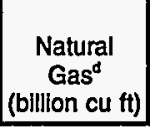 & $\begin{array}{c}\text { LPG } \\
\text { (1000 bbls) }\end{array}$ & $\begin{array}{c}\text { Coal } \\
(1000 \\
\text { short tons) }\end{array}$ & $\begin{array}{c}\text { Coke and } \\
\text { Breeze } \\
\text { (1000 } \\
\text { short tons) }\end{array}$ & $\begin{array}{c}\text { Other' } \\
\text { (trillion } \\
\text { Btu) }\end{array}$ & $\begin{array}{c}\text { RSE } \\
\text { Row } \\
\text { Factors }\end{array}$ \\
\hline & \multicolumn{9}{|c|}{ Total United States } & \\
\hline RSE Column Factors: & 0.6 & 0.6 & 1.2 & 1.3 & 0.6 & 1.2 & 1.4 & 1.7 & 1.2 & \\
\hline \multicolumn{11}{|l|}{$\begin{array}{l}\text { Value of Shipments and Recelpts } \\
\text { (million dollars) }\end{array}$} \\
\hline 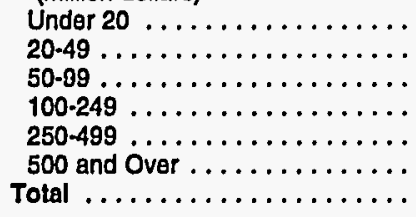 & $\begin{array}{r}1,232 \\
1,409 \\
1,238 \\
2,098 \\
1,854 \\
3,006 \\
10,837\end{array}$ & $\begin{array}{r}110,811 \\
109,423 \\
93,766 \\
148,565 \\
118,855 \\
137,060 \\
718,480\end{array}$ & $\begin{array}{r}3,985 \\
5,246 \\
6,848 \\
13,263 \\
14,549 \\
11,752 \\
55,643\end{array}$ & $\begin{array}{r}9,278 \\
4,509 \\
2,300 \\
2,199 \\
2,001 \\
2,816 \\
23,102\end{array}$ & $\begin{array}{r}587 \\
610 \\
623 \\
967 \\
837 \\
1,708 \\
5,332\end{array}$ & $\begin{array}{r}4,049 \\
2,699 \\
1,759 \\
2,288 \\
1,199 \\
6,177 \\
18,171\end{array}$ & $\begin{array}{r}4,521 \\
9,191 \\
5,398 \\
11,586 \\
10,593 \\
11,365 \\
52,653\end{array}$ & $\begin{array}{r}325 \\
531 \\
330 \\
931 \\
805 \\
8,459 \\
11,382\end{array}$ & $\begin{array}{r}47 \\
119 \\
84 \\
209 \\
223 \\
203 \\
884\end{array}$ & $\begin{array}{l}9.0 \\
6.6 \\
4.5 \\
3.9 \\
3.3 \\
3.2 \\
3.1\end{array}$ \\
\hline \multicolumn{11}{|l|}{ Employment Slze } \\
\hline 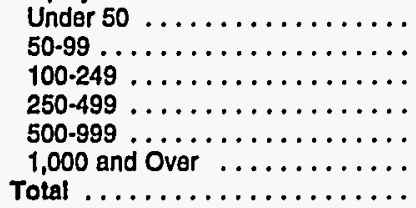 & $\begin{array}{r}511 \\
681 \\
2,003 \\
1,902 \\
2,120 \\
3,621 \\
10,837\end{array}$ & $\begin{array}{r}47,182 \\
48,502 \\
128,926 \\
129,141 \\
155,927 \\
208,803 \\
718,480\end{array}$ & $\begin{array}{r}897 \\
3,132 \\
6,919 \\
11,013 \\
11,012 \\
22,669 \\
55,643\end{array}$ & $\begin{array}{r}5,326 \\
3,135 \\
5,013 \\
2,966 \\
2,573 \\
4,090 \\
23,102\end{array}$ & $\begin{array}{r}W \\
349 \\
968 \\
964 \\
1,039 \\
W \\
5,332\end{array}$ & $\begin{array}{r}1,704 \\
1,516 \\
5,040 \\
3,061 \\
3,560 \\
3,290 \\
18,171\end{array}$ & $\begin{array}{r}467 \\
2,266 \\
14,276 \\
7,707 \\
9,249 \\
18,689 \\
52,653\end{array}$ & $\begin{array}{r}38 \\
289 \\
462 \\
472 \\
188 \\
9,932 \\
11,382\end{array}$ & $\begin{array}{r}W \\
54 \\
145 \\
186 \\
209 \\
W \\
884\end{array}$ & $\begin{array}{r}13.0 \\
10.6 \\
6.3 \\
3.9 \\
3.7 \\
3.0 \\
3.1\end{array}$ \\
\hline & \multicolumn{10}{|c|}{ Northeast Census Reglon } \\
\hline ASE Column Factors: & 0.6 & 0.5 & 0.7 & 1.4 & 0.5 & 1.6 & 1.6 & 2.3 & 1.0 & \\
\hline \multicolumn{11}{|l|}{$\begin{array}{l}\text { Value of Shipments and Recelpts } \\
\text { (milltion dollars) }\end{array}$} \\
\hline 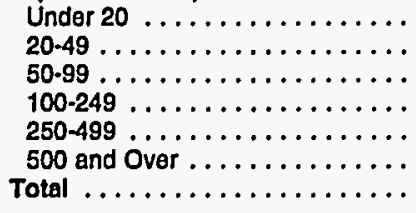 & $\begin{array}{r}253 \\
197 \\
163 \\
229 \\
185 \\
199 \\
1,226\end{array}$ & $\begin{array}{l}19,305 \\
15,043 \\
11,275 \\
16,470 \\
12,570 \\
13,188 \\
87,851\end{array}$ & $\begin{array}{r}1,772 \\
2,964 \\
3,365 \\
4,675 \\
6,948 \\
3,329 \\
23,054\end{array}$ & $\begin{array}{r}3,853 \\
1,438 \\
676 \\
696 \\
W \\
W \\
7,692\end{array}$ & $\begin{array}{r}87 \\
74 \\
71 \\
86 \\
49 \\
79 \\
445\end{array}$ & $\begin{array}{r}1,225 \\
W \\
334 \\
559 \\
W \\
W \\
3,168\end{array}$ & $\begin{array}{r}Q \\
1,316 \\
581 \\
1,484 \\
W \\
W \\
7,420\end{array}$ & $\begin{array}{r}37 \\
W \\
55 \\
2 \\
W \\
0 \\
680\end{array}$ & $\begin{array}{r}10 \\
8 \\
11 \\
15 \\
21 \\
18 \\
83\end{array}$ & $\begin{array}{r}15.1 \\
11.6 \\
8.3 \\
6.1 \\
5.6 \\
6.4 \\
6.0\end{array}$ \\
\hline \multicolumn{11}{|l|}{ Employment Size } \\
\hline 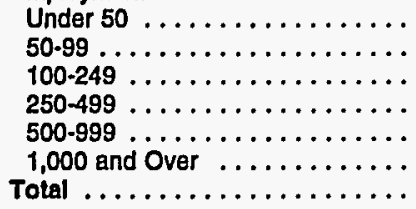 & $\begin{array}{r}85 \\
89 \\
255 \\
187 \\
254 \\
355 \\
1,226\end{array}$ & $\begin{array}{r}7,870 \\
7,026 \\
15,299 \\
14,158 \\
21,575 \\
21,923 \\
87,851\end{array}$ & $\begin{array}{r}339 \\
1,433 \\
2,725 \\
3,753 \\
5,343 \\
9,461 \\
23,054\end{array}$ & $\begin{array}{r}2,167 \\
W \\
1,898 \\
1,001 \\
W \\
1,025 \\
7,692\end{array}$ & $\begin{array}{r}36 \\
40 \\
75 \\
85 \\
86 \\
123 \\
445\end{array}$ & $\begin{array}{r}611 \\
W \\
819 \\
555 \\
599 \\
W \\
3,168\end{array}$ & $\begin{array}{r}Q \\
152 \\
3,623 \\
382 \\
W \\
W \\
7,420\end{array}$ & $\begin{array}{r}Q \\
W \\
100 \\
59 \\
18 \\
W \\
680\end{array}$ & $\begin{array}{r}4 \\
5 \\
11 \\
10 \\
28 \\
25 \\
83\end{array}$ & \begin{tabular}{r|}
22.8 \\
16.8 \\
16.1 \\
7.4 \\
5.8 \\
6.0 \\
6.0
\end{tabular} \\
\hline
\end{tabular}

See tootnotes at end of table. 
Table A13. Total Consumption of Offsite-Produced Energy for Heat, Power, and Electricity Generation by Census Region and Economic Characteristics of the Establishment, 1991 (Continued) (Estimates in Btu or Physical Units)

\begin{tabular}{|c|c|c|c|c|c|c|c|c|c|c|}
\hline $\begin{array}{c}\text { Economic } \\
\text { Characleristics }\end{array}$ & $\begin{array}{c}\text { Total } \\
\text { (trillion Btu) }\end{array}$ & $\mid \begin{array}{c}\text { Electricity } \\
\text { (million } \mathrm{kWh} \text { ) }\end{array}$ & $\begin{array}{c}\text { Residual } \\
\text { Fuel Oil } \\
\text { (1000 bbis) }\end{array}$ & $\begin{array}{c}\text { Distillate } \\
\text { Fuel Oit } \\
\text { (1000 bbls) }\end{array}$ & 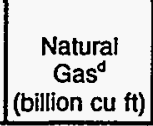 & $\begin{array}{c}\text { LPG } \\
\text { (1000 bbls) }\end{array}$ & $\begin{array}{c}\text { Coal } \\
(1000 \\
\text { short tons })\end{array}$ & $\begin{array}{c}\text { Coke and } \\
\text { Breeze } \\
\text { (1000 } \\
\text { short tons) }\end{array}$ & $\begin{array}{c}\text { Other } \\
\text { (trillion } \\
\text { Btu) }\end{array}$ & $\begin{array}{c}\text { RSE } \\
\text { Row } \\
\text { Factors }\end{array}$ \\
\hline & \multicolumn{10}{|c|}{ Midwest Census Region } \\
\hline RSE Column Factors: & 0.5 & 0.6 & 1.7 & 1.6 & 0.6 & 1.4 & 1.0 & 1.4 & 1.1 & \\
\hline \multicolumn{11}{|l|}{$\begin{array}{l}\text { Value of Shipments and Receipts } \\
\text { (million dollars) }\end{array}$} \\
\hline 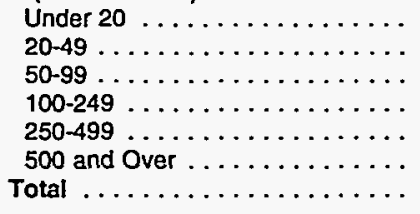 & $\begin{array}{r}390 \\
417 \\
393 \\
454 \\
390 \\
904 \\
2,948\end{array}$ & $\begin{array}{r}32,075 \\
29,764 \\
25,974 \\
29,843 \\
31,687 \\
57,759 \\
207,104\end{array}$ & $\begin{array}{r}177 \\
503 \\
330 \\
895 \\
543 \\
4,261 \\
6,709\end{array}$ & $\begin{array}{r}1,404 \\
580 \\
363 \\
326 \\
216 \\
916 \\
3,806\end{array}$ & $\begin{array}{r}221 \\
215 \\
203 \\
202 \\
163 \\
357 \\
1,362\end{array}$ & $\begin{array}{r}950 \\
723 \\
356 \\
370 \\
192 \\
597 \\
3,188\end{array}$ & $\begin{array}{r}1,022 \\
2,730 \\
2,710 \\
3,894 \\
3,644 \\
4,638 \\
18,639\end{array}$ & $\begin{array}{r}170 \\
103 \\
139 \\
740 \\
231 \\
6,718 \\
8,101\end{array}$ & $\begin{array}{r}13 \\
21 \\
26 \\
30 \\
21 \\
35 \\
147\end{array}$ & \begin{tabular}{r|}
13.3 \\
9.2 \\
8.1 \\
5.5 \\
4.8 \\
4.4 \\
3.9
\end{tabular} \\
\hline 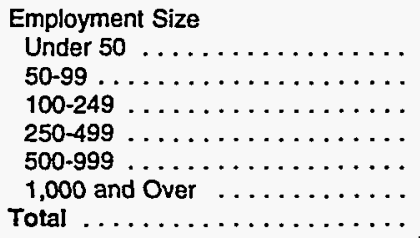 & $\begin{array}{r}172 \\
205 \\
565 \\
490 \\
413 \\
1,103 \\
2,948\end{array}$ & $\begin{array}{r}13,381 \\
14,593 \\
35,724 \\
31,054 \\
29,562 \\
82,790 \\
207,104\end{array}$ & $\begin{array}{r}Q \\
Q \\
651 \\
695 \\
635 \\
4,405 \\
6,709\end{array}$ & $\begin{array}{r}W \\
W \\
625 \\
341 \\
356 \\
1,077 \\
3,806\end{array}$ & $\begin{array}{r}106 \\
110 \\
274 \\
W \\
196 \\
W \\
1,362\end{array}$ & $\begin{array}{r}W \\
W \\
795 \\
695 \\
471 \\
403 \\
3,188\end{array}$ & $\begin{array}{r}172 \\
969 \\
5,342 \\
W \\
3,246 \\
W \\
18,639\end{array}$ & $\begin{array}{r}W \\
W \\
143 \\
198 \\
114 \\
7,542 \\
8,101\end{array}$ & $\begin{array}{r}W \\
13 \\
27 \\
28 \\
27 \\
W \\
147\end{array}$ & $\begin{array}{r}17.5 \\
15.2 \\
8.5 \\
5.4 \\
5.7 \\
4.1 \\
3.9\end{array}$ \\
\hline & \multicolumn{10}{|c|}{ South Census Region } \\
\hline RSE Column Factors: & 0.6 & 0.5 & 1.5 & 1.3 & 0.7 & 1.2 & 1.0 & 2.1 & 1.1 & \\
\hline \multicolumn{11}{|l|}{$\begin{array}{l}\text { Value of Shipments and Receipts } \\
\text { (million dollars) }\end{array}$} \\
\hline 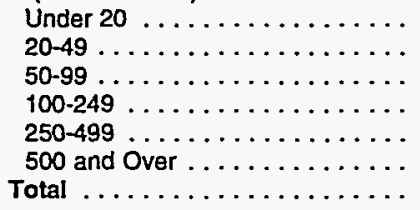 & $\begin{array}{r}418 \\
570 \\
474 \\
1,020 \\
1,092 \\
1,684 \\
5,258\end{array}$ & $\begin{array}{r}42,380 \\
49,899 \\
41,283 \\
67,150 \\
53,198 \\
51,217 \\
305,128\end{array}$ & $\begin{array}{r}1,545 \\
1,069 \\
2,036 \\
6,750 \\
6,479 \\
3,900 \\
21,779\end{array}$ & $\begin{array}{r}2,600 \\
1,783 \\
780 \\
824 \\
866 \\
929 \\
7,783\end{array}$ & $\begin{array}{r}190 \\
243 \\
242 \\
518 \\
538 \\
1,155 \\
2,885\end{array}$ & $\begin{array}{r}1,344 \\
945 \\
730 \\
1,080 \\
W \\
W \\
6,057\end{array}$ & $\begin{array}{r}1,167 \\
3,227 \\
1,300 \\
W \\
W \\
W \\
22,321\end{array}$ & $\begin{array}{r}95 \\
197 \\
104 \\
W \\
W \\
1,742 \\
2,306\end{array}$ & $\begin{array}{r}19 \\
53 \\
32 \\
87 \\
165 \\
130 \\
487\end{array}$ & $\begin{array}{r}12.0 \\
9.6 \\
6.9 \\
5.4 \\
5.3 \\
4.0 \\
3.5\end{array}$ \\
\hline \multicolumn{11}{|l|}{ Employment Size } \\
\hline 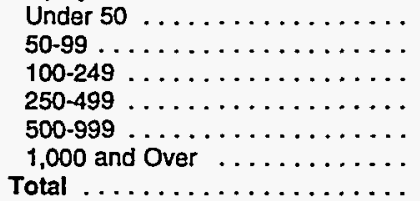 & $\begin{array}{r}178 \\
277 \\
823 \\
972 \\
1,120 \\
1,888 \\
5,258\end{array}$ & $\begin{array}{r}17,851 \\
18,515 \\
56,645 \\
62,945 \\
72,090 \\
77,082 \\
305,128\end{array}$ & $\begin{array}{r}456 \\
1,043 \\
2,269 \\
4,768 \\
4,664 \\
8,580 \\
21,779\end{array}$ & $\begin{array}{r}1,348 \\
1,079 \\
1,603 \\
985 \\
1,078 \\
1,689 \\
7,783\end{array}$ & $\begin{array}{r}84 \\
148 \\
435 \\
529 \\
595 \\
1,094 \\
2,885\end{array}$ & $\begin{array}{r}490 \\
557 \\
W \\
1,061 \\
894 \\
W \\
6,057\end{array}$ & $\begin{array}{r}Q \\
477 \\
3,644 \\
2,544 \\
4,583 \\
10,780 \\
22,321\end{array}$ & $\begin{array}{r}5 \\
144 \\
W \\
85 \\
55 \\
W \\
2,306\end{array}$ & $\begin{array}{r}11 \\
33 \\
69 \\
113 \\
119 \\
142 \\
487\end{array}$ & $\begin{array}{r}16.9 \\
16.6 \\
7.9 \\
5.5 \\
5.0 \\
4.3 \\
3.5\end{array}$ \\
\hline
\end{tabular}

See footnotes at end of table. 


\section{Table A13. Total Consumption of Offsite-Produced Energy for Heat, Power, and Electricity Generation by Census Region and Economic Characteristics of the Establishment, 1991 (Continued) (Estimates in Btu or Physical Units)}

\begin{tabular}{|c|c|c|c|c|c|c|c|c|c|c|}
\hline $\begin{array}{c}\text { Economic } \\
\text { Characteristics }\end{array}$ & $\begin{array}{c}\text { Total } \\
\text { (trillion Btu) }\end{array}$ & $\begin{array}{c}\text { Electricity } \\
\text { (million kWh) }\end{array}$ & $\begin{array}{c}\text { Residual } \\
\text { Fuel Oil } \\
\text { (1000 bbls) }\end{array}$ & $\begin{array}{c}\text { Distillate } \\
\text { Fuel Oil }^{e} \\
\text { (1000 bbls) }\end{array}$ & $\begin{array}{c}\text { Natural } \\
\text { Gas }^{d} \\
\text { (billion cu } \mathrm{ft} \text { ) }\end{array}$ & $\begin{array}{c}\text { LPG } \\
\text { (1000 bbls) }\end{array}$ & $\begin{array}{c}\text { Coal } \\
\text { (1000 } \\
\text { short tons) }\end{array}$ & $\begin{array}{c}\text { Coke and } \\
\text { Breeze } \\
\text { (1000 } \\
\text { short tons) }\end{array}$ & $\begin{array}{c}\text { Other } \\
\text { (trillion } \\
\text { Btu) }\end{array}$ & $\begin{array}{c}\text { RSE } \\
\text { Row } \\
\text { Factors }\end{array}$ \\
\hline & \multicolumn{9}{|c|}{ West Census Region } & \\
\hline RSE Column Factors: & 0.6 & 0.5 & 1.1 & 1.3 & 0.7 & 0.9 & 1.9 & 1.4 & 1.6 & \\
\hline \multicolumn{11}{|l|}{$\begin{array}{l}\text { Value of Shipments and Recelpts } \\
\text { (million dollars) }\end{array}$} \\
\hline Under $20 \ldots \ldots \ldots \ldots \ldots$ & 171 & 17,051 & 491 & 1,421 & 89 & 531 & 130 & 23 & 4 & 15.4 \\
\hline $20-49 \ldots \ldots \ldots \ldots \ldots$ & 224 & 14,717 & 710 & 707 & 78 & $W$ & 1,918 & $w$ & 38 & 12.7 \\
\hline $50-99 \ldots \ldots \ldots \ldots \ldots \ldots$ & 207 & 15,234 & 1,117 & 480 & 107 & 340 & 807 & 32 & 15 & 8.0 \\
\hline $100-249 \ldots \ldots \ldots \ldots \ldots \ldots$ & 395 & 35,101 & 943 & 353 & 162 & 279 & w & $w$ & 76 & 8.5 \\
\hline $250-499 \ldots \ldots \ldots \ldots \ldots$ & 187 & 21,400 & 579 & W & 87 & $w$ & 32 & 0 & 15 & 4.1 \\
\hline 500 and Over . . . . . . . . & 220 & 14,896 & 261 & $\mathbf{w}$ & 117 & 3,701 & $w$ & 0 & 20 & 4.6 \\
\hline Total $\ldots \ldots \ldots \ldots \ldots \ldots$ & 1,404 & 118,398 & 4,101 & 3,822 & 640 & 5,758 & 4,274 & 295 & 168 & 5.3 \\
\hline \multicolumn{11}{|l|}{ Employment Size } \\
\hline Under $50 \ldots \ldots \ldots \ldots$ & 76 & 8,081 & $\mathbf{Q}$ & $w$ & $W$ & $W$ & 0 & $w$ & 2 & 12.5 \\
\hline $50-99 \ldots \ldots \ldots \ldots \ldots$ & 109 & 8,367 & 418 & 820 & 50 & 312 & 668 & 45 & 4 & 16.2 \\
\hline $100-249 \ldots \ldots \ldots \ldots \ldots$ & 359 & 21,258 & 1,275 & 886 & 184 & $W$ & 1,666 & $W$ & 38 & 9.5 \\
\hline $250-499 \ldots \ldots \ldots \ldots \ldots$ & 253 & 20,984 & 1,797 & 640 & W & 750 & $W$ & 130 & 35 & 7.4 \\
\hline $500-999 \ldots \ldots \ldots \ldots \ldots$ & 332 & 32,700 & 372 & $w$ & 162 & 1,596 & $w$ & 0 & 34 & 6.0 \\
\hline 1,000 and Over $\ldots \ldots \ldots \ldots$ & 275 & 27,008 & 222 & 298 & 104 & W & W & 0 & 56 & 6.4 \\
\hline Total ... & 1,404 & 118,398 & 4,101 & 3,822 & 640 & 5,758 & 4,274 & 295 & 168 & 5.3 \\
\hline
\end{tabular}

- Value of Shipments and Recelpts and Employment Size were supplied by the Bureau of the Census. See Appendix B.

b "Electricity" consists of quantities of electricity that were purchased or transferred in, and is equivalent to "purchased electricity" in the Annual Survey of Manufactures.

"Distillate Fuel Oll" includes Nos. 1, 2, and 4 fuel oils and Nos. 1, 2, and 4 diesel fuels.

"Natural Gas" includes natural gas obtained from utilities, transmission pipelines, and any other supplier(s) such as brokers and producers.

- "Other" Includes all other energy that was purchased or transferred in and not shown elsewhere.

$W=W i t h$ held to avoid disclosing data for individual establishments. Data are included in higher level totals.

$\mathrm{Q}=$ Withheld because Relative Standard Error is greater than $\mathbf{5 0}$ percent. Data are included in higher level totals.

Notes: - To obtain a RSE percentage for any table cell, multiply the cell's corresponding RSE column and RSE row factors. - Totals may not equal sum of components because of Independent rounding. - The derived estimates presented in this table represent the consumption of energy originally produced offsite,

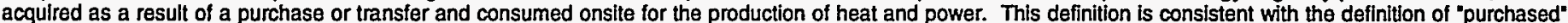

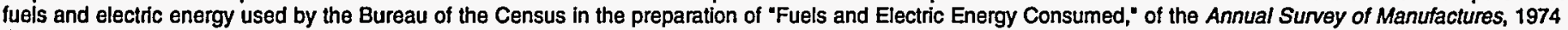
through 1981. See Appendix B.

Source: Energy Informatlon Administratlon, Office of Energy Markets and End Use, Energy End Use and Integrated Statistics Division, Form ElA-846, "1991 Manufacluring Energy Consumptlon Survey," and Bureau of the Census, Industry Division, data files for the "1991 Annual Survey of Manufactures." 


\section{Table A14. Shell Storage Capacity of Petroleum Products by Census Region and Economic Characteristics of the Establishment, 1991 (Estimates in Thousand Barrels)}

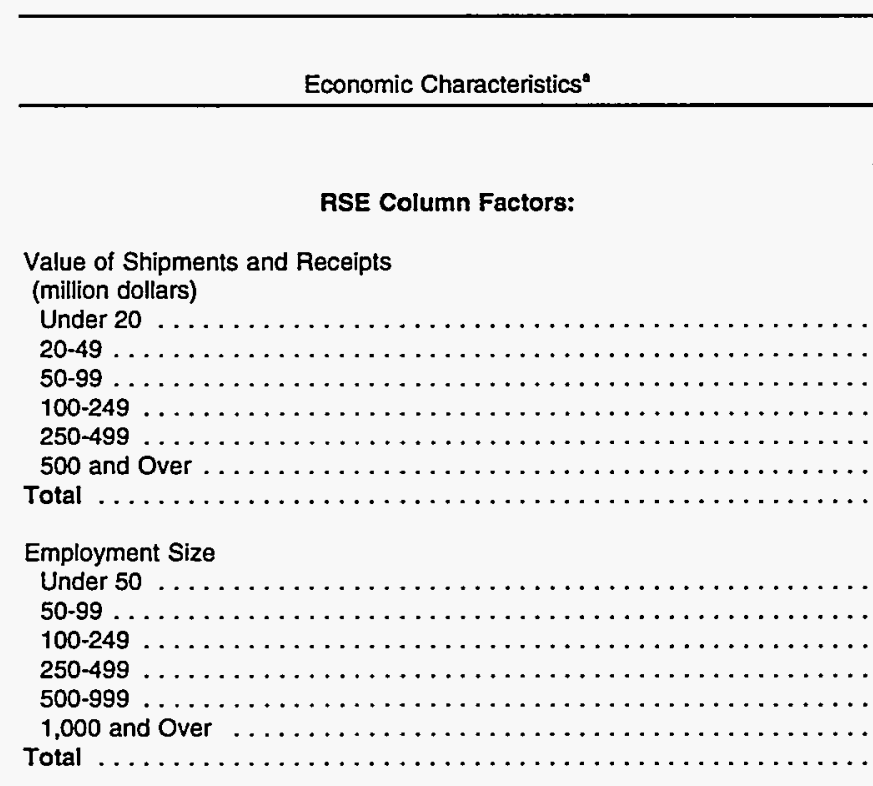

\section{RSE Column Factors:}

Motor Gasoline

Residual Fuel Oil

\begin{tabular}{|c|c|}
\hline Diesel & $\begin{array}{c}\text { Other Distillate } \\
\text { Fuel Oil }\end{array}$ \\
\hline
\end{tabular}

Total United States

\begin{tabular}{|c|c|c|c|c|}
\hline \multicolumn{4}{|c|}{ Total United States } & \\
\hline 1.2 & 0.8 & 1.3 & 0.8 & \\
\hline 101 & 944 & 1,416 & 1,107 & 14.9 \\
\hline 65 & 2,106 & 572 & 841 & 13.1 \\
\hline 42 & 2,901 & 335 & 757 & 9.5 \\
\hline 38 & 3,327 & 408 & 957 & 9.9 \\
\hline 30 & 3,525 & 333 & 954 & 5.3 \\
\hline 63 & 5,233 & 302 & 748 & 3.9 \\
\hline 339 & 18,036 & 3,365 & 5,364 & 5.3 \\
\hline 45 & 430 & 774 & 476 & 22.2 \\
\hline 37 & 1,460 & 446 & 510 & 22.2 \\
\hline 64 & 2,746 & 757 & 991 & 11.4 \\
\hline 53 & 2,665 & 484 & 833 & 10.0 \\
\hline 47 & 3,538 & 494 & 1,292 & 6.3 \\
\hline 94 & 7,197 & 410 & 1,262 & 3.5 \\
\hline 339 & 18,036 & 3,365 & 5,364 & 5.3 \\
\hline
\end{tabular}

Value of Shipments and Receipts

(million dollars)

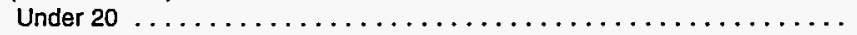

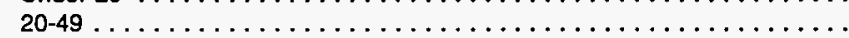

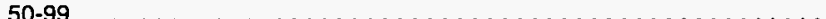

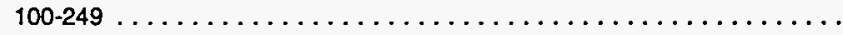

250-499.

500 and Over

Northeast Census Region

\begin{tabular}{|c|c|c|c|}
\hline 1.3 & 0.6 & 1.4 & 0.9 \\
\hline 11 & 266 & 234 & 527 \\
\hline 3 & 593 & 152 & 270 \\
\hline 5 & 1,198 & 23 & 330 \\
\hline 5 & 619 & 28 & 289 \\
\hline 5 & 994 & 42 & 124 \\
\hline 10 & 1,291 & 23 & 209 \\
\hline 39 & 4,959 & 500 & 1,751 \\
\hline 7 & 56 & 165 & 217 \\
\hline 2 & 381 & 33 & 146 \\
\hline 4 & 1,053 & 163 & 450 \\
\hline 4 & 597 & 49 & 207 \\
\hline 7 & 1,048 & 55 & 363 \\
\hline 15 & 1,825 & 36 & 368 \\
\hline 39 & 4,959 & 500 & 1,751 \\
\hline
\end{tabular}

Employment Size

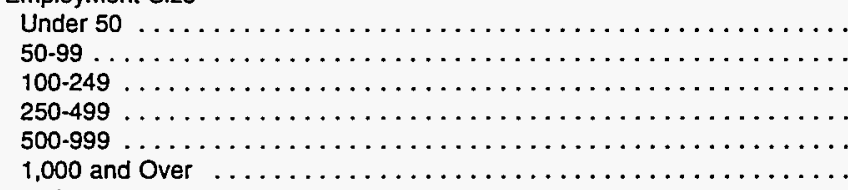

Total 


\section{Table A14. Shell Storage Capacity of Petroleum Products by Census Region and Economic Characteristics of the Establishment, 1991 (Continued) (Estimates in Thousand Barrels)}

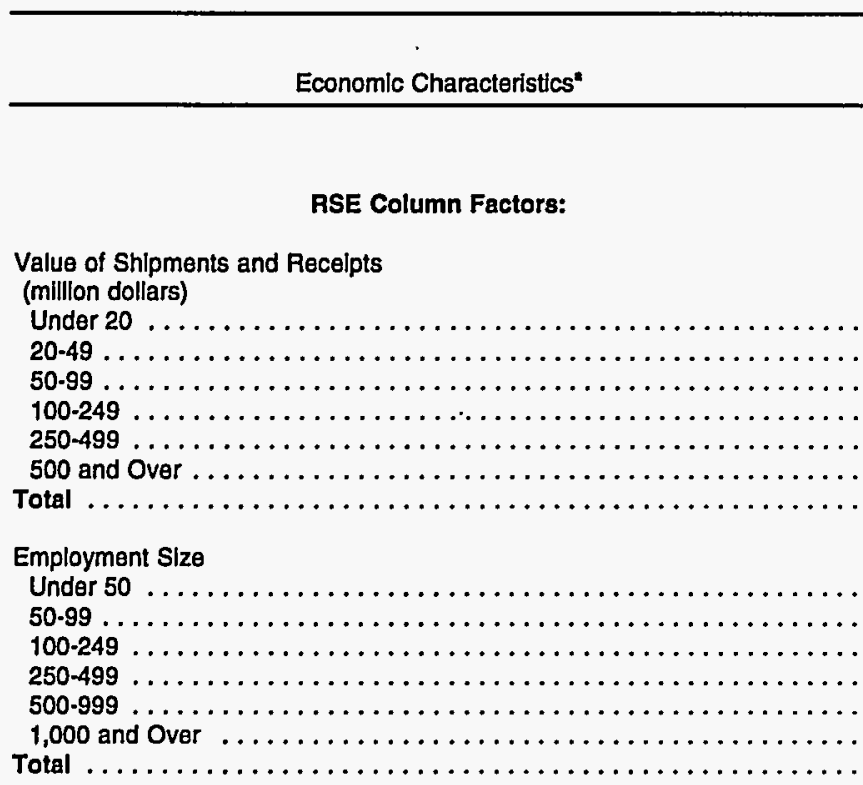

\begin{tabular}{c|c|c|c|} 
Motor Gasoline & Residual Fuel Oil & Dlesel & $\begin{array}{c}\text { Other Distillate } \\
\text { Fuel Oil }\end{array}$ \\
\hline \multicolumn{4}{c|}{ Midwest Census Region } \\
\hline 1.3 & 0.9 & 1.0 & 0.9
\end{tabular}

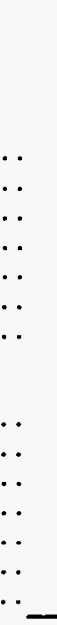

$\begin{array}{rr}22 & \\ 18 & 228 \\ 9 & 384 \\ 7 & 392 \\ 6 & 405 \\ 25 & 1,584 \\ 87 & 3,082\end{array}$

225
101
63
$Q$
49
137
773

$\begin{array}{rr} & \\ 235 & 24.9 \\ 236 & 18.9 \\ 107 & 17.6 \\ 127 & 9.2 \\ 129 & 8.3 \\ 222 & 6.1 \\ 1,058 & 9.5\end{array}$

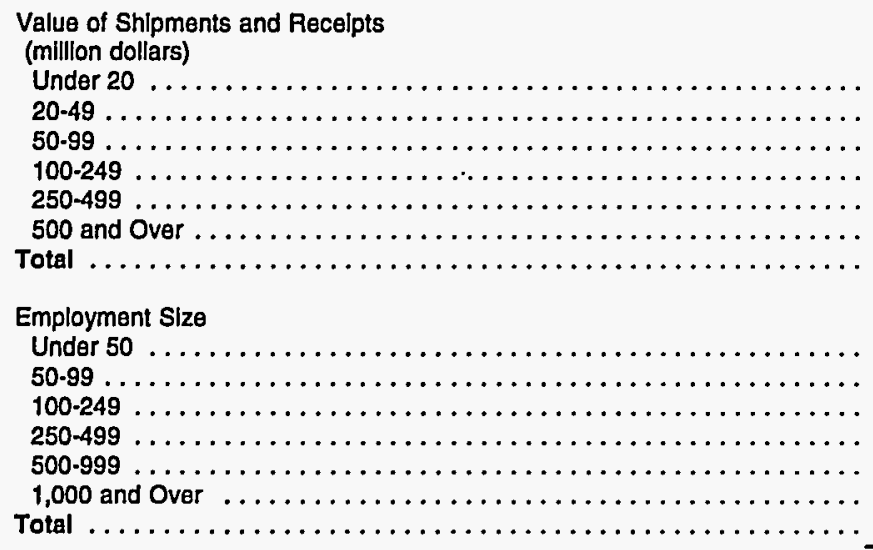 \\ RSE Column Factors:}

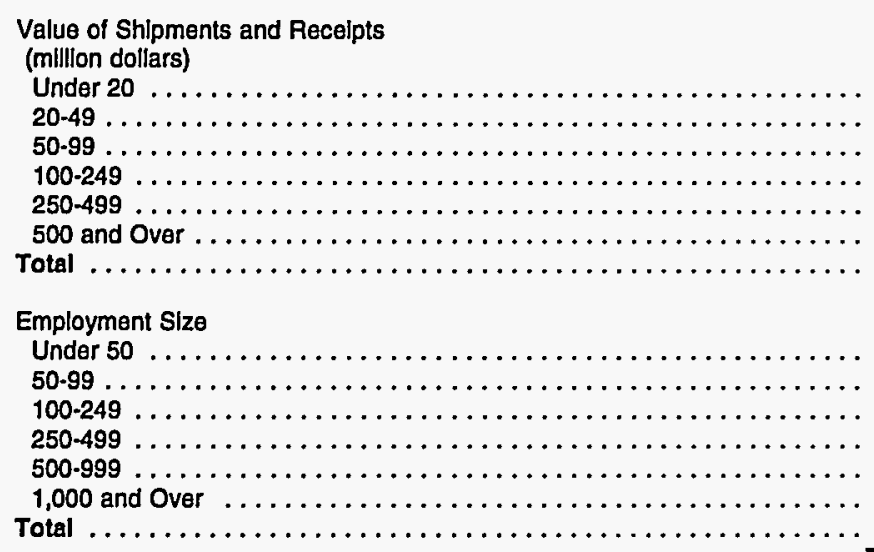

See footnotes at end of table.

\begin{tabular}{|c|c|c|c|c|}
\hline 8 & 47 & 143 & $w$ & 32.1 \\
\hline 6 & 111 & 85 & $w$ & 31.8 \\
\hline 15 & 410 & 98 & 170 & 17.0 \\
\hline 17 & 391 & $Q$ & 130 & 16.4 \\
\hline 8 & 344 & 62 & $w$ & 11.7 \\
\hline 33 & 1,779 & 184 & 332 & 5.0 \\
\hline 87 & 3,082 & 773 & 1,058 & 9.5 \\
\hline \multicolumn{4}{|c|}{ South Census Region } & \\
\hline 1.3 & 0.9 & 1.1 & 0.8 & \\
\hline 50 & 541 & 709 & 300 & 24.7 \\
\hline 22 & 1,087 & 191 & 315 & 17.2 \\
\hline 16 & 1,047 & 169 & 221 & 15.8 \\
\hline 16 & 1,841 & 133 & 512 & 8.0 \\
\hline 10 & 1,720 & 89 & 453 & 5.7 \\
\hline 19 & 2,295 & 70 & 295 & 6.0 \\
\hline 134 & 8,531 & 1,360 & 2,095 & 8.3 \\
\hline 24 & $Q$ & 325 & 77 & 27.8 \\
\hline$Q$ & 938 & 260 & 272 & 32.0 \\
\hline 20 & 987 & 336 & 345 & 16.1 \\
\hline 17 & 1,235 & 142 & 384 & 7.9 \\
\hline 18 & 1,693 & 155 & 507 & 9.7 \\
\hline 32 & 3,356 & 142 & 510 & 5.5 \\
\hline 134 & 8,531 & 1,360 & 2,095 & 8.3 \\
\hline
\end{tabular}




\section{Table A14. Shell Storage Capacity of Petroleum Products by Census Region and Economic Characteristics of the Establishment, 1991 (Continued) \\ (Estimates in Thousand Barrels)}

\begin{tabular}{|c|c|c|c|c|c|}
\hline Economic Characteristics" & Motor Gasoline & Residual Fuel Oil & Diesel & $\begin{array}{l}\text { Other Distillate } \\
\text { Fuel Oil }\end{array}$ & \multirow[t]{3}{*}{$\begin{array}{l}\text { ASE Row } \\
\text { Factorg }\end{array}$} \\
\hline \multirow[b]{2}{*}{ RSE Column Factors: } & \multicolumn{4}{|c|}{ West Census Region } & \\
\hline & 1.3 & 0.8 & 0.8 & 1.2 & \\
\hline \multicolumn{6}{|l|}{$\begin{array}{l}\text { Value of Shipments and Receipts } \\
\text { (million dollars) }\end{array}$} \\
\hline 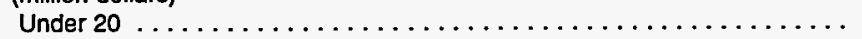 & 18 & 48 & 249 & 44 & 25.7 \\
\hline 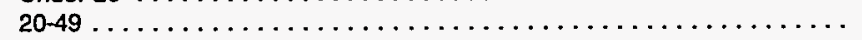 & 21 & 198 & 128 & 20 & 20.5 \\
\hline 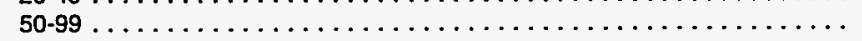 & 12 & 273 & 80 & 99 & 15.3 \\
\hline 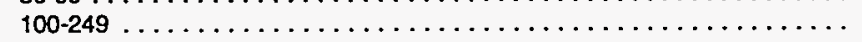 & 9 & 475 & 49 & 29 & 14.6 \\
\hline 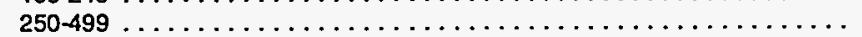 & 9 & 406 & 154 & 247 & 10.9 \\
\hline 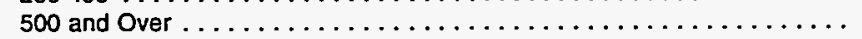 & 10 & 64 & 73 & 21 & 6.2 \\
\hline 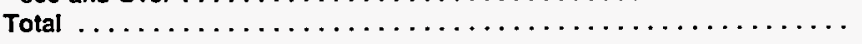 & 79 & 1,464 & 732 & 460 & 8.9 \\
\hline \multicolumn{6}{|l|}{ Employment Size } \\
\hline Under $50 \ldots \ldots$. & 6 & $\mathbf{Q}$ & 141 & $\mathbf{Q}$ & 32.9 \\
\hline 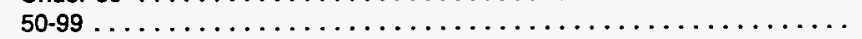 & 6 & 31 & 68 & $\mathbf{Q}$ & 29.7 \\
\hline 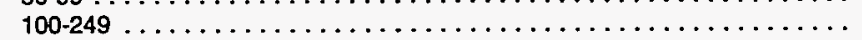 & 24 & 296 & 160 & 26 & 20.1 \\
\hline 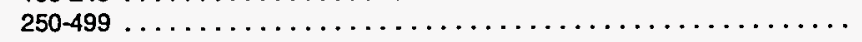 & 15 & 442 & 92 & 112 & 12.8 \\
\hline 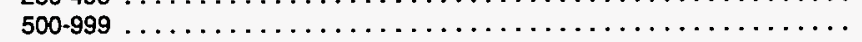 & 14 & 454 & 222 & W & 14.6 \\
\hline 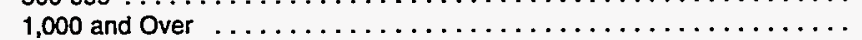 & 14 & 237 & 49 & 52 & 7.9 \\
\hline 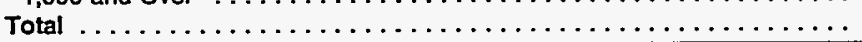 & 79 & 1,464 & 732 & 460 & 8.9 \\
\hline
\end{tabular}

\footnotetext{
- Value of Shipments and Receipts and Employment Size data were supplied by the Bureau of the Census. See Appendix B. $W=$ Withheld to avoid disclosing data for individual establishments. Data are included in higher level totals.

$\mathrm{Q}=$ Withheld because Relative Standard Error is greater than $\mathbf{5 0}$ percent. Data are included in higher level totals.

Notes: - To obtain a RSE percentage for any table cell, multiply the cell's corresponding RSE column and RSE row factors. - Totals may not equal sum of components because of independent rounding.

Source: Energy Information Administration, Office of Energy Markets and End Use, Energy End Use and Integrated Statistics Division, Form EIA-846, "1991 Manufacturing Energy Consumption Survey", and Bureau of the Census, Industry Division, data files for the "1991 Annual Survey of Manufactures."
} 
Table A15. Selected Energy Operating Ratios for Total Energy Consumption for Heat, Power, and Electricity Generation by Census Region and Economic Characteristics of the Establishment, 1991

\begin{tabular}{|c|c|c|c|c|c|c|}
\hline $\begin{array}{c}\text { Economic } \\
\text { Characteristics" }\end{array}$ & $\begin{array}{c}\text { Consumption per } \\
\text { Employee } \\
\text { (million Btu) }\end{array}$ & $\begin{array}{l}\text { Consumption per } \\
\text { Dollar of Value } \\
\text { Added } \\
\text { (thousand Btu) }\end{array}$ & $\begin{array}{l}\text { Consumption per } \\
\text { Dollar of Value of } \\
\text { Shipments } \\
\text { (thousand Btu) }\end{array}$ & $\begin{array}{c}\text { Major Byproducts } \\
\text { as a Percent of } \\
\text { Consumption } \\
\text { (percent) }\end{array}$ & $\begin{array}{c}\text { Fuel Oif as a } \\
\text { Percent of Natural } \\
\text { Gas } \\
\text { (percent) }\end{array}$ & $\begin{array}{c}\text { RSE } \\
\text { Row } \\
\text { Factors }\end{array}$ \\
\hline & \multicolumn{5}{|c|}{ Total United States } & \\
\hline ASE Column Factors: & 0.9 & 1.0 & 0.9 & 1.0 & 1.3 & \\
\hline \multicolumn{7}{|l|}{$\begin{array}{l}\text { Value of Shipments and Recelpts } \\
\text { (million dollars) }\end{array}$} \\
\hline Under $20 \ldots \ldots \ldots \ldots \ldots \ldots \ldots$ & 244.3 & 5.4 & 2.8 & W & 13.2 & 6.2 \\
\hline $20-49 \ldots \ldots \ldots \ldots \ldots \ldots \ldots \ldots$ & 531.0 & 8.3 & 3.7 & 0.6 & 10.3 & 6.3 \\
\hline $50-99 \ldots \ldots \ldots \ldots \ldots \ldots \ldots \ldots$ & 702.8 & 9.2 & 4.2 & 4.6 & 12.3 & 5.0 \\
\hline $100-249 \ldots \ldots \ldots \ldots \ldots \ldots \ldots \ldots \ldots$ & $1,365.5$ & 13.0 & 6.1 & 16.4 & 10.0 & 4.4 \\
\hline $250-499 \ldots \ldots \ldots \ldots \ldots \ldots \ldots \ldots$ & $2,680.8$ & 20.3 & 9.4 & 24.5 & 12.8 & 3.9 \\
\hline 500 and Over $\ldots \ldots \ldots \ldots \ldots \ldots \ldots$ & $2,702.9$ & 16.5 & 7.1 & 31.0 & 6.8 & 2.8 \\
\hline Total $\ldots \ldots \ldots \ldots \ldots \ldots \ldots \ldots \ldots$ & 979.6 & 12.0 & 5.5 & 19.1 & 10.0 & 2.2 \\
\hline \multicolumn{7}{|l|}{ Employment Size } \\
\hline Under $50 \ldots \ldots \ldots \ldots \ldots \ldots \ldots$ & 273.2 & 4.9 & 2.3 & 3.2 & 13.5 & 7.3 \\
\hline $50-99 \ldots \ldots \ldots \ldots \ldots \ldots \ldots \ldots \ldots$ & 494.5 & 7.8 & 3.4 & 2.4 & 12.5 & 7.8 \\
\hline $100-249 \ldots \ldots \ldots \ldots \ldots \ldots \ldots \ldots \ldots$ & 782.5 & 11.0 & 4.8 & 5.9 & 10.4 & 5.0 \\
\hline $250-499 \ldots \ldots \ldots \ldots \ldots \ldots \ldots \ldots$ & 977.6 & 12.9 & 5.9 & 19.7 & 9.3 & 3.9 \\
\hline $500-999 \ldots \ldots \ldots \ldots \ldots \ldots \ldots \ldots$ & $1,393.2$ & 15.7 & 7.4 & 24.0 & 9.1 & 3.3 \\
\hline 1,000 and Over $\ldots \ldots \ldots \ldots \ldots$ & $1,459.8$ & 13.1 & 6.2 & 25.5 & 9.8 & 2.6 \\
\hline \multirow[t]{2}{*}{ Total $\ldots \ldots \ldots \ldots \ldots \ldots \ldots \ldots \ldots \ldots$} & 979.6 & 12.0 & 5.5 & 19.1 & 10.0 & 2.2 \\
\hline & \multicolumn{6}{|c|}{ Northeast Census Region } \\
\hline ASE Column Factors: & 1.0 & 1.2 & 1.0 & 0.9 & 0.9 & \\
\hline \multicolumn{7}{|l|}{$\begin{array}{l}\text { Value of Shipments and Receipts } \\
\text { (million dollars) }\end{array}$} \\
\hline Under $20 \ldots \ldots \ldots \ldots \ldots \ldots \ldots$ & 275.6 & 5.6 & 2.9 & W & 4.2 & 15.4 \\
\hline $20-49 \ldots \ldots \ldots \ldots \ldots \ldots \ldots \ldots \ldots$ & 506.3 & 7.7 & 3.4 & $\mathbf{W}$ & 3.0 & 11.0 \\
\hline $50-99 \ldots \ldots \ldots \ldots \ldots \ldots \ldots \ldots$ & 756.5 & 8.9 & 4.2 & 3.0 & 2.0 & 6.8 \\
\hline $100-249 \ldots \ldots \ldots \ldots \ldots \ldots \ldots \ldots$ & 966.0 & 9.3 & 4.0 & 8.9 & 4.3 & 7.4 \\
\hline $250-499 \ldots \ldots \ldots \ldots \ldots \ldots \ldots \ldots$ & $1,250.7$ & 9.8 & 4.5 & 7.7 & 2.9 & 6.1 \\
\hline 500 and Over $\ldots \ldots \ldots \ldots \ldots \ldots \ldots$ & $2,356.2$ & 14.9 & 6.0 & 24.0 & 10.7 & 5.5 \\
\hline Total $\ldots \ldots \ldots \ldots \ldots \ldots \ldots \ldots \ldots$ & 850.1 & 9.9 & 4.4 & 12.7 & 5.3 & 4.4 \\
\hline \multicolumn{7}{|l|}{ Employment Size } \\
\hline Under $50 \ldots \ldots \ldots \ldots \ldots \ldots \ldots$ & 336.2 & 5.3 & 2.6 & W & 6.3 & 11.2 \\
\hline 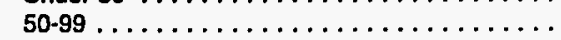 & 465.5 & 7.2 & 2.9 & 1.1 & 3.2 & 12.8 \\
\hline $100-249 \ldots \ldots \ldots \ldots \ldots \ldots \ldots \ldots$ & 656.6 & 9.2 & 4.0 & 2.9 & 3.3 & 12.8 \\
\hline $250-499 \ldots \ldots \ldots \ldots \ldots \ldots \ldots \ldots$ & 811.9 & 9.5 & 4.6 & 12.3 & 3.7 & 7.5 \\
\hline $500-999 \ldots \ldots \ldots \ldots \ldots \ldots \ldots \ldots \ldots$ & $1,000.7$ & 10.0 & 5.0 & 16.0 & 4.1 & 5.2 \\
\hline 1,000 and Over $\ldots \ldots \ldots \ldots \ldots \ldots$ & $1,349.9$ & 12.4 & 5.2 & 18.0 & 8.3 & 4.8 \\
\hline Total $\ldots \ldots \ldots \ldots \ldots \ldots \ldots \ldots$ & 850.1 & 9.9 & 4.4 & 12.7 & 5.3 & 4.4 \\
\hline
\end{tabular}

See footnotes at end of table. 
Table A15. Selected Energy Operating Ratios for Total Energy Consumption for Heat, Power, and Electricity Generation by Census Region and Economic Characteristics of the Establishment, 1991 (Continued)

\begin{tabular}{|c|c|c|c|c|c|c|}
\hline $\begin{array}{c}\text { Economic } \\
\text { Characteristics }\end{array}$ & $\begin{array}{c}\text { Consumption per } \\
\text { Employee } \\
\text { (million Btu) }\end{array}$ & $\begin{array}{l}\text { Consumption per } \\
\text { Dollar of Value } \\
\text { Added } \\
\text { (thousand Btu) }\end{array}$ & $\begin{array}{l}\text { Consumption per } \\
\text { Dollar of Value of } \\
\text { Shipments } \\
\text { (thousand Btu) }\end{array}$ & $\begin{array}{l}\text { Major Byproducts } \\
\text { as a Percent of } \\
\text { Consumption } \\
\text { (percent) }\end{array}$ & $\begin{array}{c}\text { Fuel Oife as a } \\
\text { Percent of Natural } \\
\text { Gas } \\
\text { (percent) }\end{array}$ & $\begin{array}{c}\text { RSE } \\
\text { Row } \\
\text { Factors }\end{array}$ \\
\hline & \multicolumn{5}{|c|}{ Midwest Census Region } & \\
\hline RSE Column Factors: & 0.9 & 1.0 & 0.9 & 0.9 & 1.4 & \\
\hline \multicolumn{7}{|l|}{$\begin{array}{l}\text { Value of Shipments and Receipts } \\
\text { (million dollars) }\end{array}$} \\
\hline Under $20 \ldots \ldots \ldots \ldots \ldots \ldots \ldots$ & 236.8 & 6.1 & 3.1 & $W$ & 12.8 & 8.1 \\
\hline $20-49 \ldots \ldots \ldots \ldots \ldots \ldots \ldots \ldots$ & 599.6 & 10.7 & 4.6 & 0.9 & 6.9 & 8.5 \\
\hline $50-99 \ldots \ldots \ldots \ldots \ldots \ldots \ldots \ldots \ldots$ & 719.1 & 10.5 & 4.5 & 4.3 & 6.9 & 10.4 \\
\hline $100-249 \ldots \ldots \ldots \ldots \ldots \ldots \ldots \ldots$ & $1,864.3$ & 19.0 & 8.4 & 16.9 & 9.1 & 6.4 \\
\hline $250-499 \ldots \ldots \ldots \ldots \ldots \ldots \ldots \ldots$ & $5,138.4$ & 36.2 & 15.8 & 27.4 & 8.3 & 5.2 \\
\hline 500 and Over $\ldots \ldots \ldots \ldots \ldots \ldots$ & $5,376.7$ & 25.9 & 10.7 & 31.3 & 3.2 & 3.6 \\
\hline Total $\ldots \ldots \ldots \ldots \ldots \ldots \ldots \ldots$ & $1,388.4$ & 18.0 & 7.9 & 21.5 & 6.4 & 3.3 \\
\hline \multicolumn{7}{|l|}{ Employment Size } \\
\hline Under $50 \ldots \ldots \ldots \ldots \ldots \ldots$ & 309.0 & 6.2 & 2.7 & $\mathbf{W}$ & 12.6 & 12.5 \\
\hline $50-99 \ldots \ldots \ldots \ldots \ldots \ldots \ldots$ & 647.1 & 11.3 & 4.7 & 3.6 & 8.5 & 12.7 \\
\hline $100-249 \ldots \ldots \ldots \ldots \ldots \ldots \ldots$ & 935.0 & 14.6 & 6.2 & 6.6 & 5.6 & 6.7 \\
\hline 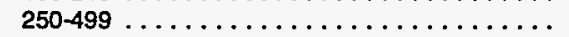 & $1,243.1$ & 19.5 & 8.1 & 21.6 & 6.5 & 5.6 \\
\hline $500-999 \ldots \ldots \ldots \ldots \ldots \ldots \ldots \ldots$ & $1,789.7$ & 21.6 & 9.5 & 26.2 & 6.0 & 4.6 \\
\hline 1,000 and Over $\ldots \ldots \ldots \ldots \ldots \ldots \ldots$ & $2,518.9$ & 21.0 & 9.9 & 27.0 & 6.2 & 3.3 \\
\hline \multirow[t]{2}{*}{ Total $\ldots \ldots \ldots \ldots \ldots \ldots \ldots$} & $1,388.4$ & 18.0 & 7.9 & 21.5 & 6.4 & 3.3 \\
\hline & \multicolumn{5}{|c|}{ South Census Region } & \\
\hline RSE Column Factors: & 0.9 & 1.0 & 0.9 & 0.9 & 1.4 & \\
\hline \multicolumn{7}{|l|}{$\begin{array}{l}\text { Value of Shipments and Receipts } \\
\text { (million dollars) }\end{array}$} \\
\hline Under $20 \ldots \ldots \ldots \ldots \ldots \ldots \ldots$ & 201.3 & 4.1 & 2.0 & 0.2 & 12.3 & 9.0 \\
\hline $20-49 \ldots \ldots \ldots \ldots \ldots \ldots \ldots \ldots$ & 575.9 & 7.8 & 3.4 & - & 10.8 & 8.8 \\
\hline 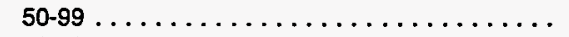 & 739.2 & 9.0 & 4.4 & 10.9 & 9.2 & 7.2 \\
\hline $100-249 \ldots \ldots \ldots \ldots \ldots \ldots \ldots \ldots$ & $1,457.6$ & 13.1 & 6.7 & 22.8 & 5.2 & 5.5 \\
\hline $250-499 \ldots \ldots \ldots \ldots \ldots \ldots \ldots$ & $2,390.4$ & 19.5 & 8.9 & 37.3 & 15.4 & 5.0 \\
\hline 500 and $O v e r . \ldots \ldots \ldots \ldots \ldots$ & $1,122.1$ & 9.2 & 4.0 & 43.8 & 4.4 & 3.6 \\
\hline Total $\ldots \ldots \ldots \ldots \ldots \ldots \ldots \ldots$ & 773.6 & 9.4 & 4.4 & W & 8.8 & 2.9 \\
\hline \multicolumn{7}{|l|}{ Employment Size } \\
\hline$\ldots \ldots \ldots \ldots \ldots \ldots$ & 184.8 & 3.4 & 1.6 & 0.2 & 11.4 & 12.5 \\
\hline $50-99 \ldots \ldots \ldots \ldots \ldots \ldots \ldots$ & 445.8 & 6.8 & 3.0 & 2.6 & 17.6 & 12.9 \\
\hline $100-249 \ldots \ldots \ldots \ldots \ldots \ldots \ldots \ldots$ & 934.8 & 11.4 & 4.9 & 11.8 & 10.1 & 6.8 \\
\hline $250-499 \ldots \ldots \ldots \ldots \ldots \ldots \ldots \ldots$ & $1,186.1$ & 15.0 & 6.6 & 29.8 & 16.3 & 5.2 \\
\hline $500-999 \ldots \ldots \ldots \ldots \ldots \ldots \ldots$ & $1,606.4$ & 17.5 & 8.5 & 27.7 & 3.0 & 5.0 \\
\hline 1,000 and Over $\ldots \ldots \ldots \ldots \ldots \ldots \ldots$ & 569.7 & 5.7 & 2.9 & 38.9 & 3.1 & 3.2 \\
\hline Total $\ldots \ldots \ldots \ldots \ldots \ldots \ldots$ & 773.6 & 9.4 & 4.4 & W & 8.8 & 2.9 \\
\hline
\end{tabular}

See footnotes at end of table. 


\section{Table A15. Selected Energy Operating Ratios for Total Energy Consumption for Heat, Power, and Electricity Generation by Census Region and Economic Characteristics of the Establishment, 1991 (Continued)}

\begin{tabular}{|c|c|c|c|c|c|c|}
\hline $\begin{array}{c}\text { Economic } \\
\text { Characteristlcs" }\end{array}$ & $\begin{array}{c}\text { Consumption per } \\
\text { Employee } \\
\text { (million Btu) }\end{array}$ & $\begin{array}{l}\text { Consumption per } \\
\text { Dollar of Value } \\
\text { Added } \\
\text { (thousand Btu) } \\
\end{array}$ & $\begin{array}{l}\text { Consumption per } \\
\text { Dollar of Value of } \\
\text { Shlpments } \\
\text { (thousand Btu) }\end{array}$ & $\begin{array}{l}\text { Major Byproducts } \\
\text { as a Percent of } \\
\text { Consumption } \\
\text { (percent) }\end{array}$ & $\begin{array}{c}\text { Fuel Olf as a } \\
\text { Percent of Natural } \\
\text { Gas } \\
\text { (percent) }\end{array}$ & $\begin{array}{l}\text { RSE } \\
\text { Row } \\
\text { Factors }\end{array}$ \\
\hline & \multicolumn{6}{|c|}{ West Census Reglon } \\
\hline RSE Column Factors: & 1.1 & 1.2 & 1.0 & 0.8 & 1.1 & \\
\hline \multicolumn{7}{|l|}{$\begin{array}{l}\text { Value of Shipments and Recelpts } \\
\text { (million dollars) }\end{array}$} \\
\hline Under $20 \ldots \ldots \ldots \ldots \ldots \ldots \ldots \ldots \ldots$ & 201 & 4 & 2 & 0 & 12 & 11.8 \\
\hline $20-49 \ldots \ldots \ldots \ldots \ldots \ldots \ldots \ldots \ldots \ldots \ldots$ & 576 & 8 & 3 & - & 11 & 12.9 \\
\hline 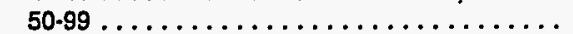 & 739 & 9 & 4 & 11 & 9 & 11.5 \\
\hline $100-249 \ldots \ldots \ldots \ldots \ldots \ldots \ldots \ldots \ldots \ldots$ & 1,458 & 13 & 7 & 23 & 5 & 9.7 \\
\hline $250-499 \ldots \ldots \ldots \ldots \ldots \ldots \ldots \ldots \ldots$ & 2,390 & 20 & 9 & 37 & 15 & 4.9 \\
\hline 500 and over $\ldots \ldots \ldots \ldots \ldots \ldots \ldots$ & 1,122 & 9 & 4 & 44 & 4 & 4.0 \\
\hline Total $\ldots \ldots \ldots \ldots \ldots \ldots \ldots \ldots \ldots$ & 774 & 9 & 4 & $w$ & 9 & 4.1 \\
\hline \multicolumn{7}{|l|}{ Employment Slze } \\
\hline Under $50 \ldots \ldots \ldots \ldots \ldots \ldots \ldots \ldots \ldots$ & 185 & 3 & 2 & 0 & 11 & 15.2 \\
\hline $50-99 \ldots \ldots \ldots \ldots \ldots \ldots \ldots \ldots \ldots$ & 446 & 7 & 3 & 3 & 18 & 12.5 \\
\hline 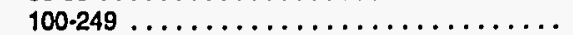 & 935 & 11 & 5 & 12 & 10 & 10.4 \\
\hline$\ldots \ldots \ldots \ldots \ldots \ldots \ldots$ & 1,186 & 15 & 7 & 30 & 16 & 7.9 \\
\hline $500-999 \ldots \ldots \ldots \ldots \ldots \ldots \ldots \ldots \ldots \ldots \ldots$ & 1,606 & 18 & 9 & 28 & 3 & 7.3 \\
\hline 1,000 and $0 v e r \quad \ldots \ldots \ldots \ldots \ldots \ldots \ldots$ & 570 & 6 & 3 & 39 & 3 & 4.5 \\
\hline Total ........ & 774 & 9 & 4 & W & 9 & 4.1 \\
\hline
\end{tabular}

- Value of Shlpments and Recelpts and Employment Size were supplied by the Bureau of the Census. See Appendix B.

b Major Byproduct" fuels include coke oven and blast furnace gas (produced primarily in the blast furnace industry, SIC 3312); still gas (produced primarily in refinerles, SIC 2911); and pulping llquor (produced primarily in pulp and paper mills, SIC 2611 and 2621).

"Fuel Oil" includes distillate and residual.

- Estlmate less than 0.5. Data are Included in higher level totals.

$W=$ Withheld to avold disclosing data for Individual establishments. Data are included in higher level totals.

Noles: - To obtain a ASE percentage for any table cell, multiply the cell's corresponding RSE column and RSE row factors. - Totals may not equal sum of components because of Independent rounding. - Operating ratios were calculated using the input energy estimates reported in Table A4.

Source: Energy Information Administration, Office of Energy Markets and End Use, Energy End Use and Integrated Statistics Division, Form ElA-846, "1991 Manufacturing Energy Consumption Survey," and Bureau of the Census, Industry Division, data files for the "1991 Annual Survey of Manufactures." 
Table A16. Components of Total Electricity Demand by Census Region, Industry Group, and Selected Industries, 1991 (Estimates in Million Kilowatthours)

\begin{tabular}{|c|c|c|c|c|c|c|c|}
\hline $\begin{array}{c}\text { SiC } \\
\text { Code }\end{array}$ & $\begin{array}{l}\text { Industry Groups } \\
\text { and Industry }\end{array}$ & Purchases & Transfers $\ln ^{b}$ & $\begin{array}{l}\text { Total Onsite } \\
\text { Generation }\end{array}$ & $\begin{array}{c}\text { Sales and/or } \\
\text { Transfers } \\
\text { Offsite }\end{array}$ & $\begin{array}{l}\text { Net Dernand } \\
\text { for Electricity }\end{array}$ & $\begin{array}{c}\text { ASE } \\
\text { Bow } \\
\text { Factors }\end{array}$ \\
\hline & & \multicolumn{5}{|c|}{ Total United States } & \\
\hline & RSE Column Factors: & 0.5 & 1.9 & 1.2 & 1.5 & 0.5 & \\
\hline 20 & Food and Kindred Products . & $w$ & W & 5,743 & 988 & 55,273 & 7.2 \\
\hline 2011 & Meat Packing Plants . . . . . . . . . . . . . . & 3,410 & 0 & 71 & 0 & 3,481 & 9.5 \\
\hline 2033 & Canned Fruits and Vegetables . . . . . . . . . . . . . & 1,415 & 0 & $w$ & $w$ & 1,724 & 14.0 \\
\hline 2037 & Frozen Fruits and Vegetables . . . . . . . . . . . . & 3,096 & $\mathbf{Q}$ & 135 & 27 & 3,205 & 17.8 \\
\hline 2046 & Wet Com Milling . . . . . . . . . . . . . . . & W & $\mathbf{W}$ & W & $\mathbf{W}$ & 5,820 & 11.8 \\
\hline 2051 & Bread, Cake, and Related Products . . . . . . . . . . . . & 2,240 & 0 & 33 & 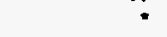 & 2,272 & 13.2 \\
\hline 2063 & Beet Sugar . . . . . . . . . . . . . . . . & 407 & 0 & W & $w$ & 848 & 6.9 \\
\hline 2075 & 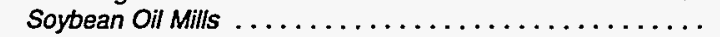 & W & W & $W$ & $\ddot{W}$ & 1,910 & 4.0 \\
\hline 2082 & Malt Beverages $\ldots \ldots \ldots \ldots \ldots \ldots \ldots \ldots \ldots$ & 2,371 & 0 & W & W & 2,886 & 11.2 \\
\hline 21 & Tobacco Products $\ldots \ldots \ldots \ldots \ldots \ldots \ldots \ldots \ldots$ & 1,468 & 0 & W & $W$ & 1,810 & 5.5 \\
\hline 22 & Textile Mill Products $\ldots \ldots \ldots \ldots \ldots \ldots \ldots \ldots$ & $W$ & W & W & $\mathbf{Q}$ & 29,866 & 7.9 \\
\hline 23 & Apparel and Other Textile Products $\ldots \ldots \ldots \ldots \ldots$ & 5,643 & $Q$ & 0 & 0 & 5,645 & 14.8 \\
\hline 24 & Lumber and Wood Products $\ldots \ldots \ldots \ldots \ldots \ldots$ & 19,209 & 366 & 2,950 & 1,976 & 20,549 & 17.4 \\
\hline 25 & Fumiture and Fixtures $\ldots \ldots \ldots \ldots \ldots \ldots \ldots \ldots$ & 4,913 & $Q$ & 33 & 1 & 4,948 & 17.3 \\
\hline 26 & Paper and Allied Products $\ldots \ldots \ldots \ldots \ldots \ldots \ldots$ & 63,744 & 1,308 & 53,831 & 9,012 & 109,871 & 3.7 \\
\hline 2611 & Pulp Mills . . . . . . . . . . . . . . . . . . & 2,871 & 6 & 6,138 & 462 & 8,553 & 15.1 \\
\hline 2621 & Paper Mils . . . . . . . . . . . . . . . . & W & W & 30,841 & 6,104 & 61,054 & 2.8 \\
\hline 2631 & Paperboard Mills . . . . . . . . . . . . . . . . & w & W & 16,739 & 2,378 & 26,971 & 5.2 \\
\hline 27 & Printing and Publishing $\ldots \ldots \ldots \ldots \ldots \ldots$ & 15,627 & $Q$ & $\mathbf{Q}$ & 0 & 15,641 & 11.1 \\
\hline 28 & Chemicals and Allied Products $\ldots \ldots \ldots \ldots \ldots \ldots$ & 131,858 & 7,201 & 41,428 & 9,967 & 170,520 & 4.4 \\
\hline 2812 & 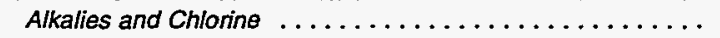 & 12,629 & 0 & W & W & 17,653 & 14.9 \\
\hline 2813 & 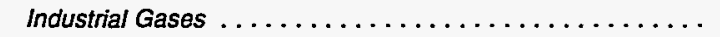 & W & $\mathbf{W}$ & w & W & 18,252 & 6.9 \\
\hline 2819 & Industrial Inorganic Chemicals, nec $\ldots \ldots \ldots \ldots \ldots$ & 38,026 & 150 & W & $W$ & 39,777 & 9.3 \\
\hline 2821 & Plastics Materials and Resins . . . . . . . . . . . . & W & W & 2,627 & 247 & 17,408 & 5.2 \\
\hline 2822 & Synthetic Rubber $\ldots \ldots \ldots \ldots \ldots \ldots \ldots \ldots \ldots \ldots \ldots \ldots \ldots$ & 1,946 & 0 & W & $\mathbf{W}$ & 1,877 & 14.0 \\
\hline 2823 & Cellulosic Manmade Fibers . . . . . . . . . . . . . . . & W & 0 & 961 & W & 1,170 & 26.3 \\
\hline 2824 & Organic Fibers, Noncellulosic $\ldots \ldots \ldots \ldots \ldots \ldots$ & 6,976 & 0 & 626 & 0 & 7,601 & 5.2 \\
\hline 2865 & 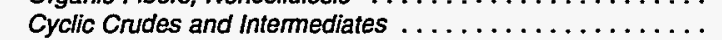 & 4,382 & 50 & 457 & 9 & 4,880 & 11.6 \\
\hline 2869 & Industrial Organic Chemicals, nec $\ldots \ldots \ldots \ldots \ldots \ldots$ & 18,588 & 1,555 & 21,661 & 5,040 & 36,764 & 5.4 \\
\hline 2873 & Nitrogenous Fertilizers $\ldots \ldots \ldots \ldots \ldots \ldots \ldots$ & 2,886 & 33 & 392 & 7 & 3,303 & 26.7 \\
\hline 2874 & Phosphatic Fertilizers . . . . . . . . . . . . . & 2,419 & 0 & 2,677 & 533 & 4,562 & 4.7 \\
\hline 29 & Petroleum and Coal Products $\ldots \ldots \ldots \ldots \ldots \ldots$ & 33,463 & 17 & 13,452 & 2,698 & 44,234 & 4.6 \\
\hline 2911 & 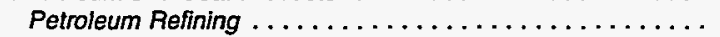 & W & W & 12,993 & 2,410 & 42,145 & 2.8 \\
\hline 30 & Rubber and Misc. Plastics Products $\ldots \ldots \ldots \ldots \ldots$ & 33,808 & $Q$ & 115 & 6 & 34,022 & 12.9 \\
\hline 3011 & Tires and Inner Tubes . . . . . . . . . . . . . . . . & W & W & $\star$ & 0 & 4,037 & 5.1 \\
\hline 308 & Miscellaneous Plastics Products, nec ........... & 25,514 & Q & $w$ & $\mathbf{Q}$ & 25,635 & 14.6 \\
\hline 31 & Leather and Leather Products $\ldots \ldots \ldots \ldots \ldots \ldots \ldots$ & 795 & $\cdot$ & 39 & $\cdot$ & 834 & 22.0 \\
\hline 32 & Stone, Clay and Glass Products $\ldots \ldots \ldots \ldots \ldots \ldots$ & 30,846 & 39 & 533 & 71 & 31,347 & 7.4 \\
\hline 3211 & 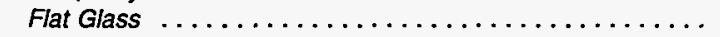 & 1,512 & 0 & W & W & 1,506 & 4.0 \\
\hline 3221 & 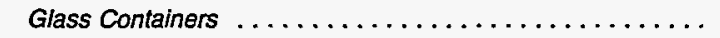 & 4,098 & 0 & 0 & 0 & 4,098 & 5.5 \\
\hline 3229 & Pressed and Blown Glass, nec . . . . . . . . . . & $W$ & W & * & $\star$ & 2,862 & 7.8 \\
\hline 3241 & 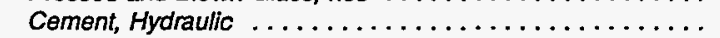 & 9,490 & 0 & w & W & 9,888 & 13.3 \\
\hline 3274 & 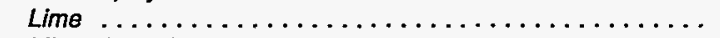 & 1,324 & 0 & 0 & 0 & 1,324 & 66.6 \\
\hline 3296 & Mineral Wool $\ldots \ldots \ldots \ldots \ldots \ldots \ldots \ldots \ldots \ldots$ & W & W & 0 & 0 & 2,821 & 1.5 \\
\hline 33 & Primary Metal Industries $\ldots \ldots \ldots \ldots \ldots \ldots \ldots$ & W & W & 8,370 & 1,949 & 153,499 & 3.0 \\
\hline 3312 & Blast Fumaces and Steel Mills . . . . . . . . . . . . . . & W & W & 6,235 & 1,297 & 44,417 & 4.5 \\
\hline 3313 & Electrometalurgical Products $\ldots \ldots \ldots \ldots \ldots \ldots \ldots \ldots$ & 3,796 & 0 & W & $W$ & 4,386 & 10.5 \\
\hline 3321 & Gray and Ductile Iron Foundries $\ldots \ldots \ldots \ldots \ldots$ & W & W & W & W & 6,413 & 12.1 \\
\hline 3331 & Primary Copper $\ldots \ldots \ldots \ldots \ldots \ldots \ldots \ldots$ & w & W & 279 & 0 & 1,525 & 1.1 \\
\hline 3334 & Primary Aluminum $\ldots \ldots \ldots \ldots \ldots \ldots \ldots \ldots \ldots \ldots \ldots \ldots$ & w & W & 0 & $w$ & 67,317 & 3.3 \\
\hline 3339 & Primary Nonferrous Metals, nec . . . . . . . . . . . . . & 3,784 & 0 & W & W & 4,433 & 1.8 \\
\hline 3353 & Aluminum Sheet, Plate, and Foil $\ldots \ldots \ldots \ldots \ldots \ldots$ & W & W & 0 & 0 & 4,261 & 1.2 \\
\hline 34 & Fabricated Metal Products $\ldots \ldots \ldots \ldots \ldots \ldots \ldots \ldots$ & 29,610 & 220 & W & W & 29,899 & 14.0 \\
\hline 35 & Industrial Machinery and Equipment $\ldots \ldots \ldots \ldots \ldots \ldots$ & 29,349 & 308 & 148 & 176 & 29,629 & 11.2 \\
\hline 357 & Computer and Office Equipment $\ldots \ldots \ldots \ldots \ldots$ & 4,369 & 29 & 17 & 9 & 4,405 & 16.7 \\
\hline 36 & Electronic and Other Electric Equipment . . . . . . . . . & 29,816 & 231 & $\mathbf{Q}$ & $\mathbf{Q}$ & 30,013 & 9.4 \\
\hline 37 & Transportation Equipment $\ldots \ldots \ldots \ldots \ldots \ldots \ldots$ & 35,160 & 241 & 714 & 761 & 35,355 & 5.6 \\
\hline 3711 & Motor Vehicles and Car Bodies . . . . . . . . . . . . . . & W & W & W & W & 7,705 & 4.8 \\
\hline 3714 & Motor Vehicle Parts and Accessories . . . . . . . . . . . . & W & $\mathbf{W}$ & W & W & 11,054 & 7.6 \\
\hline 38 & Instruments and Related Products $\ldots \ldots \ldots$ & $W$ & W & W & W & 13,673 & 15.2 \\
\hline 3841 & Surgical and Medical Instruments $\ldots \ldots \ldots \ldots \ldots$ & 1,161 & 0 & 0 & 0 & 1,161 & 11.1 \\
\hline \multirow[t]{2}{*}{39} & Misc. Manufacturing Industries $\ldots \ldots \ldots \ldots \ldots \ldots \ldots$ & 3,661 & 0 & * & 0 & 3,661 & 14.7 \\
\hline & 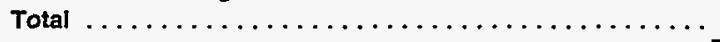 & 697,553 & 20,927 & 130,028 & 28,222 & 820,286 & 3.0 \\
\hline
\end{tabular}

See footnotes at end of table. 
Table A16. Components of Total Electricity Demand by Census Region, Industry Group, and Selected Industries, 1991 (Continued) (Estimates in Million Kilowatthours)

\begin{tabular}{|c|c|c|c|c|c|c|c|}
\hline $\begin{array}{c}\text { SIC } \\
\text { Coden }\end{array}$ & $\begin{array}{l}\text { Industry Groups } \\
\text { and Industry } \\
\end{array}$ & Purchases & Transfers In $^{b}$ & $\begin{array}{l}\text { Total Onsite } \\
\text { Generation }^{\circ} \\
\end{array}$ & $\begin{array}{c}\text { Sales and/or } \\
\text { Transfers } \\
\text { Offsite }\end{array}$ & $\begin{array}{l}\text { Net Demand } \\
\text { for Electricity }\end{array}$ & \multirow[t]{2}{*}{$\begin{array}{c}\text { RSE } \\
\text { Row } \\
\text { Factors }\end{array}$} \\
\hline & \multirow[b]{2}{*}{ RSE Column Factors: } & \multicolumn{5}{|c|}{ Northeast Census Region } & \\
\hline & & 0.7 & 1.4 & 1.2 & 1.3 & 0.7 & \\
\hline 20 & Food and Kindred Products $\ldots \ldots \ldots \ldots \ldots \ldots \ldots$ & 5,443 & 0 & 352 & 58 & 5,737 & 12.1 \\
\hline 2011 & Meat Packing Plants $\ldots \ldots \ldots \ldots \ldots \ldots \ldots \ldots \ldots$ & 141 & 0 & 0 & 0 & 141 & 25.5 \\
\hline 2033 & Canned Fruits and Vegetables $\ldots \ldots \ldots \ldots \ldots \ldots \ldots$ & 292 & 0 & 5 & 0 & 297 & 22.8 \\
\hline 2037 & 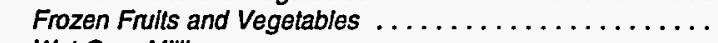 & 140 & 0 & 1 & 0 & 141 & 35.8 \\
\hline 2046 & Wet Com Milling $\ldots \ldots \ldots \ldots \ldots \ldots \ldots \ldots \ldots \ldots$ & 15 & 0 & 0 & $\mathbf{0}$ & 15 & 30.0 \\
\hline 2051 & Bread, Cake, and Related Products . . . . . . . . . & 382 & 0 & w & $\mathbf{0}$ & w & 16.6 \\
\hline 2063 & 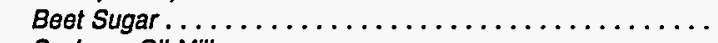 & 0 & 0 & 0 & 0 & 0 & NF \\
\hline 2075 & Soybean Oll Mills . . . . . . . . . . . . & 0 & 0 & 0 & $\mathbf{0}$ & 0 & NF \\
\hline 2082 & Malt Beverages .... & 521 & 0 & $W$ & W & 521 & 17.2 \\
\hline 21 & Tobacco Products . . & NA & NA & NA & NA & NA & 51.0 \\
\hline 22 & Textile Mill Products & 1,372 & 0 & w & $\mathbf{Q}$ & 1,404 & 21.8 \\
\hline 23 & Apparel and Other Textile Products ... & 495 & $\mathbf{Q}$ & 0 & 0 & 497 & 25.5 \\
\hline 24 & Lumber and Wood Products $\ldots \ldots \ldots \ldots \ldots \ldots \ldots$ & NA & NA & NA & NA & NA & 36.8 \\
\hline 25 & Fumiture and Fixtures $\ldots \ldots \ldots \ldots \ldots$ & 446 & Q & $\mathbf{Q}$ & $\mathbf{0}$ & 452 & 25.5 \\
\hline 26 & Paper and Alled Products . . . . . . . . . & 8,826 & 464 & 8,991 & 3,088 & 15,193 & 4.4 \\
\hline 2611 & 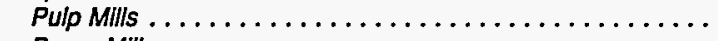 & $\mathbf{Q}$ & 0 & 384 & 15 & 420 & 34.6 \\
\hline 2621 & $\ldots \ldots \ldots \ldots \ldots$ & W & w & 8,253 & 2,990 & 11,141 & 3.9 \\
\hline 2631 & Paporboard Mills . . . . . . . . . . . . & W & w & W & w & 753 & 13.1 \\
\hline 27 & Printing and Publishing ${ }^{\prime} \ldots \ldots \ldots \ldots \ldots \ldots \ldots \ldots$ & 3,167 & 0 & $\mathbf{Q}$ & 0 & 3,178 & 21.0 \\
\hline 28 & Chemicals and Allied Products ..... & w & $\mathbf{w}$ & 780 & 207 & 10,084 & 8.7 \\
\hline 2812 & Alkalles and Chlorino & w & 0 & 0 & 0 & w & 39.0 \\
\hline 2813 & Industrial Gases $\ldots \ldots \ldots \ldots \ldots$ & w & w & 0 & w & w & 9.9 \\
\hline 2819 & Industrial Inorganic Chemicals, nec .. & w & w & w & 0 & w & 17.7 \\
\hline 2821 & Plastlos Materials and Resins $\ldots \ldots \ldots$ & w & w & w & w & 1,195 & 8.1 \\
\hline 2822 & Synthetic Rubber ........... & w & 0 & 0 & 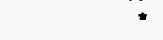 & $w$ & 31.5 \\
\hline 2823 & Cellulosic Manmade Fibers . . . . . . . & 0 & 0 & 0 & 0 & 0 & NF \\
\hline 2824 & Organic Fibers, Noncellulosic . . . . . . . & 95 & 0 & 0 & 0 & 95 & 30.0 \\
\hline 2865 & Cyclic Crudes and Intermediates & 406 & 0 & 0 & 0 & 406 & 22.5 \\
\hline 2869 & Industral Organic Chemicals, nec ... & w & w & $\mathbf{w}$ & $\mathbf{w}$ & 3,151 & 9.8 \\
\hline 2873 & Nitrogenous Fertilizers $\ldots \ldots \ldots \ldots$ & 29 & 0 & 0 & 0 & 29 & 61.5 \\
\hline 2874 & Phosphatic Fertilizers ............ & 0 & 0 & 0 & 0 & 0 & NF \\
\hline 29 & Petroleum and Coal Products $\ldots \ldots \ldots \ldots \ldots \ldots \ldots \ldots$ & 3,054 & 0 & $\mathbf{w}$ & $\mathbf{W}$ & 3,568 & 5.6 \\
\hline 2911 & Petroleum Refining . . . . . . . . . . & 2,515 & 0 & W & $\mathbf{w}$ & w & 4.2 \\
\hline 30 & Rubber and Misc. Plasties Products $\ldots \ldots \ldots \ldots \ldots \ldots$ & 5,484 & 0 & w & 6 & W & 19.9 \\
\hline 3011 & Tres and Inner Tubes $\ldots \ldots \ldots \ldots \ldots \ldots \ldots \ldots \ldots$ & 125 & 0 & 0 & 0 & 125 & 12.0 \\
\hline 308 & Miscellaneous Plastics Products, nec. & 4,810 & 0 & $\mathbf{w}$ & $\mathbf{Q}$ & 4,848 & 21.3 \\
\hline 31 & Leather and Leather Products $\ldots \ldots \ldots$ & 205 & * & 1 & * & 205 & 26.5 \\
\hline 32 & Stone, Clay and Glass Products $\ldots \ldots$ & 5,599 & 25 & $\mathbf{w}$ & $\mathbf{w}$ & 5,673 & 16.4 \\
\hline 3211 & Flat Glass $\ldots \ldots \ldots \ldots \ldots \ldots \ldots$ & w & 0 & 0 & 0 & W & 6.0 \\
\hline 3221 & Glass Containers $\ldots \ldots \ldots \ldots \ldots$ & 834 & 0 & 0 & 0 & 834 & 9.0 \\
\hline 3229 & Pressed and Blown Glass, nec ... & w & W & 0 & 0 & w & 8.7 \\
\hline 3241 & Cement, Hydraulic $\ldots \ldots \ldots \ldots \ldots$ & 1,334 & 0 & 0 & 0 & 1,334 & 21.0 \\
\hline 3274 & LIme $\ldots \ldots \ldots \ldots \ldots \ldots \ldots \ldots \ldots$ & Q & 0 & 0 & 0 & Q & NF \\
\hline 3296 & Mineral Wool & w & $w$ & 0 & 0 & 304 & 2.4 \\
\hline 33 & Primary Metal Industries & 17,771 & 0 & $\mathbf{w}$ & $\mathbf{w}$ & 18,832 & 5.3 \\
\hline 3312 & Blast Fumaces and Steel Mills . . . . . . . & 8,136 & 0 & w & $\mathbf{w}$ & 8,657 & 6.1 \\
\hline 3313 & Electrometalurgical Products ........ & W & 0 & 0 & 0 & W & 19.5 \\
\hline 3321 & Gray and Ductile Iron Foundries & 350 & 0 & 0 & 0 & 350 & 19.5 \\
\hline 3331 & Primary Copper $\ldots \ldots \ldots \ldots \ldots \ldots$ & w & 0 & 0 & 0 & $\mathbf{w}$ & 1.5 \\
\hline 3334 & Primary Aluminum $\ldots \ldots \ldots \ldots \ldots \ldots \ldots \ldots \ldots$ & w & 0 & 0 & 0 & w & 6.0 \\
\hline 3339 & Primary Nonferrous Metals, nec . . . . . . . . . . . . & 100 & 0 & w & $w$ & 640 & 1.5 \\
\hline 3353 & Aluminum Sheet, Plate, and Foil . & 451 & 0 & 0 & 0 & 451 & 3.0 \\
\hline 34 & Fabricated Metal Products $\ldots \ldots \ldots \ldots \ldots \ldots \ldots \ldots \ldots$ & 5,053 & $\mathbf{Q}$ & 83 & 0 & 5,158 & 16.4 \\
\hline 35 & Industrial Machinery and Equipment $\ldots \ldots \ldots \ldots \ldots \ldots$ & $\mathbf{w}$ & $\mathbf{w}$ & $\mathbf{w}$ & $\mathbf{Q}$ & w & 18.4 \\
\hline 357 & Computer and Office Equipment ...... & 819 & 0 & 0 & 0 & 819 & 25.5 \\
\hline 36 & Electronic and Other Electric Equipment . & 6,541 & $\mathbf{Q}$ & - & 11 & 6,544 & 17.0 \\
\hline 37 & Transportation Equipment & w & w & w & w & 3,464 & 10.7 \\
\hline 3711 & Motor Vehicles and Car Bodles ...... & w & 0 & 0 & 0 & w & 9.0 \\
\hline 3714 & Motor Vehicle Parts and Accessories . . . . . . . . . & 887 & 0 & 0 & 0 & 887 & 10.5 \\
\hline & Instruments and Related Products ...... & $\mathbf{w}$ & $\mathbf{Q}$ & w & w & w & 16.4 \\
\hline 3841 & Surglcal and Medical Instruments .... & 332 & 0 & 0 & 0 & 332 & 13.5 \\
\hline \multirow[t]{2}{*}{39} & Misc. Manufacturing Industries $\ldots \ldots \ldots \ldots \ldots \ldots \ldots$ & 1,187 & 0 & • & 0 & 1,187 & 17.9 \\
\hline & Total & 86,471 & 1,380 & 14,592 & 4,301 & 98,142 & 4.0 \\
\hline
\end{tabular}

See tootnotes at end of table. 
Table A16. Components of Total Electricity Demand by Census Region, Industry Group, and Selected Industries, 1991 (Continued)

(Estimates in Million Kilowatthours)

\begin{tabular}{|c|c|c|c|c|c|c|c|}
\hline $\begin{array}{c}\text { SIC } \\
\text { Code } \\
\end{array}$ & $\begin{array}{l}\text { Industry Groups } \\
\text { and Industry }\end{array}$ & Purchases & Transfers $1 n^{\circ}$ & $\begin{array}{l}\text { Total Onsite } \\
\text { Generation }\end{array}$ & $\begin{array}{c}\text { Sales and/or } \\
\text { Transfers } \\
\text { Offsite }\end{array}$ & $\begin{array}{l}\text { Net Demand } \\
\text { for Electricity }\end{array}$ & $\begin{array}{c}\text { RSE } \\
\text { Row } \\
\text { Factors }\end{array}$ \\
\hline & & \multicolumn{6}{|c|}{ Midwest Census Region } \\
\hline & RSE Column Factors: & 0.6 & 1.4 & 1.3 & 1.6 & 0.6 & \\
\hline 20 & Food and Kindred Products & w & w & 2,541 & 61 & 22,120 & 7.5 \\
\hline 2011 & Meat Packing Plants ......... & 2,065 & 0 & 71 & 0 & 2,137 & 10.1 \\
\hline 2033 & Canned Fruits and Vegetables. . & 375 & 0 & 0 & 0 & 375 & 11.5 \\
\hline 2037 & Frozen Fruits and Vegetables ... & 289 & 0 & 0 & 0 & 289 & 31.2 \\
\hline 2046 & Wet Com Milling . .......... & w & w & w & w & 4,809 & 12.7 \\
\hline 2051 & Bread, Cake, and Related Products. & 632 & 0 & 6 & 0 & 637 & 16.6 \\
\hline 2063 & Bett Sugar $\ldots \ldots \ldots \ldots \ldots \ldots \ldots \ldots$ & 234 & 0 & w & $\mathbf{w}$ & 399 & 7.7 \\
\hline 2075 & Soybean Oil Mills $\ldots \ldots \ldots \ldots \ldots$ & w & w & w & w & 1,344 & 4.9 \\
\hline 2082 & Malt Beverages. & 480 & 0 & w & 0 & W & 18.9 \\
\hline 21 & Tobacco Products & NA & NA & NA & NA & NA & 13.1 \\
\hline 22 & Textile Mill Products & NA & NA & NA & NA & NA & 34.5 \\
\hline 23 & Apparel and Other Textile Products & NA & NA & NA & NA & NA & 26.3 \\
\hline 24 & Lumber and Wood Products $\ldots \ldots \ldots \ldots \ldots$ & 2,834 & 0 & 68 & 0 & 2,902 & 24.6 \\
\hline 25 & Furniture and Fixtures $\ldots \ldots \ldots$ & 1,571 & 0 & 18 & 1 & 1,588 & 22.9 \\
\hline 26 & Paper and Allied Products ... & 14,038 & $\mathbf{Q}$ & 6,475 & 716 & 20,278 & 6.3 \\
\hline 2611 & 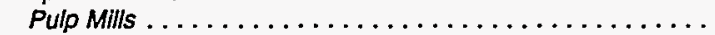 & 556 & 0 & 523 & 257 & 822 & 30.2 \\
\hline 2621 & Paper Mills .. & 7,730 & 0 & 4,777 & 438 & 12,069 & 5.4 \\
\hline 2631 & Paperboard Mills . & 2,059 & 0 & w & $\mathbf{w}$ & 3,192 & 10.9 \\
\hline 27 & Printing and Publishing $\ldots \ldots \ldots$ & 5,224 & 0 & * & 0 & 5,224 & 14.8 \\
\hline 28 & Chemicals and Allied Products. & w & w & 1,077 & 155 & 34,879 & 9.9 \\
\hline 2812 & Alkalies and Chlorine $\ldots \ldots \ldots$ & w & 0 & 0 & 0 & w & 39.4 \\
\hline 2813 & Industrial Gases . . . . . . . . . & 3,319 & w & 0 & 0 & w & 9.1 \\
\hline 2819 & Industrial Inorganic Chemicals, nec .. & W & w & w & 0 & $w$ & 19.6 \\
\hline 2821 & Plastics Materials and Resins ..... & 3,247 & 1 & w & w & 3,379 & 8.6 \\
\hline 2822 & Synthetic Rubber $\ldots \ldots \ldots \ldots \ldots$ & 241 & 0 & 0 & 0 & 241 & 26.3 \\
\hline 2823 & Cellulosic Manmade Fibers . . . . . . . & 0 & 0 & 0 & 0 & 0 & NF \\
\hline 2824 & Organic Fibers, Noncellulosic ...... & 0 & 0 & 0 & 0 & 0 & NF \\
\hline 2865 & Cyclic Crudes and Intermediates .... & 756 & 0 & w & 0 & w & 15.1 \\
\hline 2869 & Industrial Organic Chemicals, nec. & $w$ & w & w & w & 2,322 & 7.4 \\
\hline 2873 & Nitrogenous Fentilizers $\ldots \ldots \ldots \ldots$. & 556 & 0 & 4 & 0 & 560 & 35.5 \\
\hline 2874 & Phosphatic Fertilizers . ........... & 1 & 0 & 0 & 0 & 1 & 6.6 \\
\hline 29 & Petroleum and Coal Products ........ & W & $\mathbf{Q}$ & W & w & 7,547 & 7.0 \\
\hline 2911 & Petroleum Refining $\ldots \ldots \ldots \ldots \ldots \ldots \ldots \ldots$ & 6,168 & 0 & w & $\mathbf{w}$ & W & 3.4 \\
\hline 30 & Rubber and Misc. Plastics Products . . & 13,132 & $\mathbf{Q}$ & $\because$ & 0 & 13,237 & 13.4 \\
\hline 3011 & Tires and Inner Tubes . . . . . . . . . . & w & w & 0 & 0 & w & 5.0 \\
\hline 308 & Miscellaneous Plastics Products, nec . . & NA & NA & NA & NA & NA & 14.8 \\
\hline 31 & Leather and Leather Products . ........ & 262 & 0 & 8 & 0 & 270 & 25.4 \\
\hline 32 & Stone, Clay and Glass Products .. & w & w & w & w & 8,473 & 10.7 \\
\hline 3211 & Flat Glass $\ldots \ldots \ldots \ldots \ldots$ & $w$ & 0 & 0 & 0 & $W$ & 4.9 \\
\hline 3221 & Glass Containers $\ldots \ldots \ldots \ldots$ & 873 & 0 & 0 & 0 & 873 & 8.2 \\
\hline 3229 & Pressed and Blown Glass, nec. & 614 & 3 & 0 & 0 & 617 & 8.6 \\
\hline 3241 & Cement, Hydraulic . . . . . . . . . . & 2,247 & 0 & w & w & $w$ & 17.9 \\
\hline 3274 & $\ldots \ldots \ldots \ldots \ldots \ldots$ & 367 & 0 & 0 & 0 & 367 & 21.4 \\
\hline 3296 & Mineral Wool $\ldots \ldots \ldots \ldots \ldots \ldots \ldots$ & 1,251 & 0 & 0 & 0 & 1,251 & 1.6 \\
\hline 33 & Primary Metal Industries & $w$ & w & 3,377 & 776 & 50,133 & 4.7 \\
\hline 3312 & Blast Fumaces and Steel Mills. & w & $\mathbf{w}$ & 3,371 & 586 & 20,562 & 5.7 \\
\hline 3313 & Electrometalurgical Products ... & 2,378 & 0 & * & w & $w$ & 11.2 \\
\hline 3321 & Gray and Ductile Iron Foundries .. & 4,350 & 0 & $\mathbf{w}$ & $\mathbf{w}$ & 4,349 & 12.3 \\
\hline 3331 & Primary Copper $\ldots \ldots \ldots \ldots \ldots$ & w & 0 & 0 & 0 & w & 1.6 \\
\hline 3334 & Primary Aluminum & w & w & 0 & 0 & W & 5.7 \\
\hline 3339 & Primary Nonferrous Metals, nec... & 727 & 0 & 0 & w & w & 1.2 \\
\hline 3353 & Aluminum Sheet, Plate, and Foll & w & $\mathbf{w}$ & 0 & 0 & 1,453 & 2.0 \\
\hline 34 & Fabricated Metal Products . . . . . . & 12,749 & 198 & $\mathbf{Q}$ & W & 12,906 & 13.8 \\
\hline 35 & Industrial Machinery and Equipment ... & w & $\mathbf{Q}$ & w & w & 12,625 & 12.1 \\
\hline 357 & Computer and Office Equipment $\ldots \ldots \ldots \ldots \ldots \ldots \ldots$ & 796 & 23 & 0 & 0 & 820 & 21.7 \\
\hline 36 & Electronic and Other Electric Equipment ........... & 7,001 & 165 & $\mathbf{Q}$ & 7 & 7,176 & 14.3 \\
\hline 37 & Transportation Equipment $\ldots \ldots \ldots \ldots \ldots$ & w & w & $\bar{w}$ & w & 16,250 & 5.9 \\
\hline 3711 & Motor Vehicles and Car Bodies & w & w & w & w & 4,826 & 5.8 \\
\hline 3714 & Motor Vehicle Parts and Accessories. & w & w & w & w & 8,006 & 8.5 \\
\hline 38 & Instruments and Related Products $\ldots \ldots \ldots \ldots \ldots \ldots \ldots$ & 1,806 & 0 & 0 & 0 & 1,806 & 18.1 \\
\hline 3841 & Surgical and Medical Instruments .... & 276 & 0 & 0 & 0 & 276 & 19.7 \\
\hline \multirow[t]{2}{*}{39} & Misc. Manufacturing Industries . . . . . . & 929 & 0 & 0 & 0 & 929 & 18.1 \\
\hline & Total $\ldots \ldots \ldots \ldots \ldots \ldots \ldots$ & 198,408 & 8,696 & 15,097 & 2,707 & 219,493 & 4.2 \\
\hline
\end{tabular}

See footnotes at end of table. 
Table A16. Components of Total Electricity Demand by Census Region, Industry Group, and Selected Industries, 1991 (Continued)

(Estimates in Million Kilowatthours)

\begin{tabular}{|c|c|c|c|c|c|c|c|}
\hline $\begin{array}{c}\text { SIC } \\
\text { Code }\end{array}$ & $\begin{array}{l}\text { Industry Groups } \\
\text { and Industry }\end{array}$ & Purchases & Transfers $\ln ^{b}$ & $\begin{array}{l}\text { Total Onsite } \\
\text { Generation }^{c}\end{array}$ & $\begin{array}{c}\text { Sales and/or } \\
\text { Transfers } \\
\text { Offsite }\end{array}$ & $\begin{array}{l}\text { Net Demand } \\
\text { for Electricity }\end{array}$ & $\begin{array}{c}\text { RSE } \\
\text { Row } \\
\text { Factors }\end{array}$ \\
\hline
\end{tabular}

\begin{tabular}{|c|c|c|c|c|c|c|c|}
\hline & & & & ssus Regi & & & \\
\hline & RSE Column Factors: & 0.6 & 1.4 & 1.2 & 1.5 & 0.6 & \\
\hline 20 & Food and Kindred Products & w & $\mathbf{Q}$ & 1,365 & 245 & 17,302 & 11.9 \\
\hline 2011 & Meat Packing Plants $\ldots \ldots \ldots \ldots \ldots \ldots \ldots \ldots \ldots \ldots$ & 892 & 0 & 0 & 0 & 892 & 12.8 \\
\hline 2033 & Canned Frults and vegetables $\ldots \ldots \ldots \ldots \ldots \ldots \ldots$ & 200 & 0 & w & w & 495 & 24.9 \\
\hline 2037 & Frozen Frults and Vegetables $\ldots \ldots \ldots \ldots \ldots \ldots \ldots$ & 551 & 0 & 63 & 0 & 614 & 18.5 \\
\hline 2046 & Wet Com Milling $\ldots \ldots \ldots \ldots \ldots \ldots \ldots \ldots \ldots \ldots$ & 837 & 0 & w & W & w & 19.4 \\
\hline 2051 & Bread, Cako, and Related Products . . . . . . . . . . . . . & 838 & 0 & 3 & • & 841 & 16.8 \\
\hline 2063 & 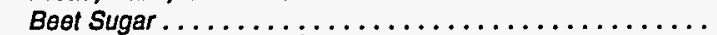 & w & 0 & w & 0 & w & 20.5 \\
\hline 2075 & Soybean Oll Mills $\ldots \ldots \ldots \ldots \ldots \ldots \ldots \ldots \ldots \ldots$ & 539 & 0 & W & w & 566 & 5.1 \\
\hline 2082 & $\ldots \ldots \ldots \ldots \ldots \ldots \ldots$ & 863 & 0 & W & 0 & W & 14.0 \\
\hline 21 & $\ldots \ldots \ldots \ldots \ldots \ldots \ldots$ & 1,452 & 0 & w & W & 1,794 & 5.2 \\
\hline 22 & Textile Mill Products $\ldots \ldots \ldots \ldots \ldots \ldots \ldots \ldots \ldots \ldots$ & W & W & w & W & 27,701 & 8.8 \\
\hline 23 & Apparel and Other Textlle Products $\ldots \ldots \ldots \ldots \ldots \ldots \ldots$ & 4,165 & 0 & 0 & 0 & 4,165 & 16.0 \\
\hline 24 & Lumber and Wood Products $\ldots \ldots \ldots \ldots \ldots \ldots \ldots \ldots$ & w & w & 33 & $\mathbf{Q}$ & W & 24.3 \\
\hline 25 & Furniture and Fixtures $\ldots \ldots \ldots \ldots \ldots \ldots \ldots \ldots \ldots \ldots$ & 2,592 & 0 & 12 & 0 & 2,604 & 18.8 \\
\hline 26 & Paper and Allied Products $\ldots \ldots \ldots \ldots \ldots \ldots \ldots \ldots \ldots$ & 27,095 & 260 & 32,331 & 2,253 & 57,432 & 5.6 \\
\hline 2611 & $\ldots \ldots \ldots \ldots \ldots \ldots \ldots$ & 1,421 & 0 & 4,319 & 172 & 5,568 & 15.1 \\
\hline 2621 & 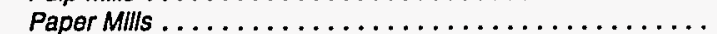 & w & $\mathbf{w}$ & 14,728 & 819 & 28,423 & 3.6 \\
\hline 2631 & Paperboard Mills $\ldots \ldots \ldots \ldots \ldots \ldots \ldots \ldots \ldots \ldots$ & 6,887 & 0 & 13,283 & 1,256 & 18,915 & 5.5 \\
\hline 27 & $\ldots \ldots \ldots \ldots \ldots \ldots \ldots$ & 4,797 & $\mathbf{Q}$ & 0 & 0 & 4,798 & 16.0 \\
\hline 28 & Chemicals and Allied Products $\ldots \ldots \ldots \ldots \ldots \ldots \ldots$ & 79,359 & 4,191 & 37,577 & 8,480 & 112,647 & 4.9 \\
\hline 2812 & Alkalles and Chlorine $\ldots \ldots \ldots \ldots \ldots \ldots \ldots \ldots \ldots$ & 10,289 & 0 & w & w & 15,313 & 15.1 \\
\hline 2813 & $\ldots \ldots \ldots \ldots \ldots \ldots \ldots \ldots$, & w & $\mathbf{w}$ & W & W & W & 8.7 \\
\hline 2819 & Industrlat Inorganic Chemicals, nec $\ldots \ldots \ldots \ldots \ldots \ldots \ldots$ & W & W & w & w & 18,122 & 12.6 \\
\hline 2821 & Plastlos Materials and Resins . . . . . . . . . . . . . . . & W & w & 2,409 & 232 & 12,622 & 6.2 \\
\hline 2822 & Synthetic Rubber $\ldots \ldots \ldots \ldots \ldots \ldots \ldots \ldots \ldots \ldots$ & w & 0 & w & W & w & 14.0 \\
\hline 2823 & Cellulosic Manmado Fibers $\ldots \ldots \ldots \ldots \ldots \ldots \ldots \ldots$ & w & 0 & 961 & W & 1,170 & 24.6 \\
\hline 2824 & Organic Fibers, Noncellulosic $\ldots \ldots \ldots \ldots \ldots \ldots \ldots$ & 6,881 & 0 & 626 & 0 & 7,507 & 4.7 \\
\hline 2865 & Cyclic Crudes and Intermediates . . . . . . . . . . . . . . & 3,209 & 50 & W & $\mathbf{9}$ & w & 12.7 \\
\hline 2869 & Industrlal Organlc Chemicals, nec .................. & 13,961 & 1,149 & 20,836 & 4,869 & 31,077 & 6.6 \\
\hline 2873 & Nitrogenous Fertllizers $\ldots \ldots \ldots \ldots \ldots \ldots \ldots \ldots \ldots$ & 1,872 & 0 & 233 & 7 & 2,097 & 31.3 \\
\hline 2874 & Phosphatic Fortillzers $\ldots \ldots \ldots \ldots \ldots \ldots \ldots \ldots$ & 1,908 & 0 & W & w & 3,998 & 2.4 \\
\hline 29 & Petroleum and Coal Products $\ldots \ldots \ldots \ldots \ldots \ldots \ldots$ & 15,699 & 0 & 8,781 & 1,821 & 22,658 & 2.9 \\
\hline 2911 & Petroleum Refining $\ldots \ldots \ldots \ldots \ldots \ldots \ldots \ldots \ldots \ldots$ & 15,216 & 0 & 8,780 & 1,821 & 22,175 & 2.9 \\
\hline 30 & Rubber and Misc. Plastics Products $\ldots \ldots \ldots \ldots \ldots \ldots \ldots$ & 12,328 & 0 & 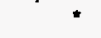 & 1 & 12,328 & 11.3 \\
\hline 3011 & 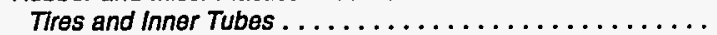 & 2,855 & 0 & • & 0 & 2,855 & 5.1 \\
\hline 308 & Miscellaneous Plastics Products, nec $\ldots \ldots \ldots \ldots \ldots \ldots$ & 7,801 & 0 & • & 0 & 7,801 & 14.4 \\
\hline 31 & Leather and Leather Products $\ldots \ldots \ldots \ldots \ldots \ldots \ldots \ldots$ & 258 & 0 & $\mathbf{Q}$ & 0 & 288 & 18.4 \\
\hline 32 & Stone, Clay and Glass Products $\ldots \ldots \ldots \ldots \ldots \ldots \ldots \ldots$ & w & w & W & W & 11,868 & 7.1 \\
\hline 3211 & 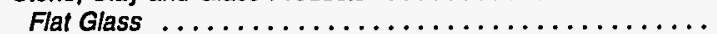 & 781 & 0 & 0 & W & w & 4.5 \\
\hline 3221 & Glass Containers $\ldots \ldots \ldots \ldots \ldots \ldots \ldots \ldots \ldots \ldots$ & 1,262 & 0 & 0 & 0 & 1,262 & 8.0 \\
\hline 3229 & Pressed and Blown Glass, nec $\ldots \ldots \ldots \ldots \ldots \ldots \ldots$ & 1,598 & 0 & • & * & 1,598 & 8.2 \\
\hline 3241 & Cement, Hydraulle $\ldots \ldots \ldots \ldots \ldots \ldots \ldots \ldots \ldots \ldots$ & 3,519 & 0 & 0 & $\mathbf{Q}$ & 3,518 & 14.4 \\
\hline 3274 & 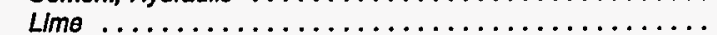 & 405 & 0 & 0 & 0 & 405 & 30.4 \\
\hline 3296 & Mineral Wool $\ldots \ldots \ldots \ldots \ldots \ldots \ldots \ldots \ldots \ldots \ldots$ & w & w & 0 & 0 & 936 & 1.5 \\
\hline 33 & Primary Metal Industries .... & w & $\mathbf{w}$ & 2,551 & 684 & 49,304 & 4.4 \\
\hline 3312 & Blast Fumaces and Steel Mills. & W & w & 1,785 & 293 & 12,529 & 6.5 \\
\hline 3313 & Electrometalurgical Products ... & W & 0 & w & w & 1,805 & 12.3 \\
\hline 3321 & Gray and Ductlle Iron Foundries & W & w & 0 & 0 & 1,622 & 15.8 \\
\hline 3331 & Primary Copper $\ldots \ldots \ldots \ldots \ldots$ & 200 & 0 & $\mathbf{w}$ & $\mathbf{0}$ & w & 1.3 \\
\hline 3334 & Prlmary Aluminum & w & $\mathbf{w}$ & 0 & w & 24,240 & 4.3 \\
\hline 3339 & Primary Nonferrous Metals, nec... & 1,694 & 0 & 0 & 0 & 1,694 & 6.4 \\
\hline 3353 & Aluminum Sheet, Plate, and Foll $\ldots \ldots \ldots \ldots \ldots \ldots$ & w & W & 0 & 0 & w & 1.9 \\
\hline 34 & Fabricated Metal Products $\ldots \ldots \ldots \ldots \ldots \ldots \ldots \ldots \ldots$ & 8,886 & 0 & 0 & 0 & 8,886 & 17.6 \\
\hline 35 & Industrlal Machinery and Equipment $\ldots \ldots \ldots \ldots \ldots \ldots \ldots$ & 8,138 & 0 & 17 & 9 & 8,146 & 16.6 \\
\hline 357 & Computer and Office Equipment ... & 809 & 0 & 17 & 9 & 816 & 20.8 \\
\hline 36 & Electronic and Other Electric Equipment $\ldots \ldots \ldots \ldots \ldots \ldots$ & 10,724 & $\mathbf{Q}$ & • & $\mathbf{Q}$ & 10,742 & 12.0 \\
\hline 37 & Transportation Equipment $\ldots \ldots \ldots \ldots \ldots \ldots \ldots \ldots \ldots$ & 8,542 & 0 & 1 & w & W & 8.0 \\
\hline 3711 & Motor Vehicles and Car Bodles & 2,375 & 0 & • & W & W & 6.0 \\
\hline 3714 & Motor Vehicle Parts and Accessories . . . . . . . . . . . . & 1,919 & 0 & 0 & 0 & 1,919 & 11.2 \\
\hline & Instruments and Related Products .... & 3,239 & $\mathbf{Q}$ & 0 & 0 & 3,285 & 14.4 \\
\hline 3841 & Surgical and Medical Instruments .. & 331 & 0 & 0 & 0 & 331 & 17.6 \\
\hline 39 & Mlsc. Manufacturing Industries . & 1,168 & 0 & 0 & 0 & 1,168 & 16.0 \\
\hline & 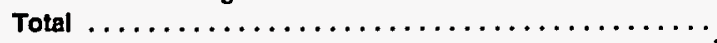 & 295,955 & 9,173 & 83,821 & 14,078 & 374,870 & 3.0 \\
\hline
\end{tabular}

See footnotes at end of table. 
Table A16. Components of Total Electricity Demand by Census Region, Industry Group, and Selected Industries, 1991 (Continued) (Estimates in Million Kilowatthours)

\begin{tabular}{|c|c|c|c|c|c|c|c|}
\hline $\begin{array}{l}\text { SIC } \\
\text { Code }\end{array}$ & $\begin{array}{l}\text { Industry Groups } \\
\text { and Industry }\end{array}$ & Purchases & Transfers $\ln ^{\mathrm{b}}$ & $\begin{array}{l}\text { Total Onsite } \\
\text { Generation }^{c}\end{array}$ & $\begin{array}{c}\text { Sales and/or } \\
\text { Transfers } \\
\text { Offsite }\end{array}$ & $\begin{array}{c}\text { Net Demand } \\
\text { for Electricity }\end{array}$ & $\begin{array}{c}\text { RSE } \\
\text { Row } \\
\text { Factors }\end{array}$ \\
\hline & & \multicolumn{6}{|c|}{ West Census Region } \\
\hline & RSE Column Factors: & 0.7 & 1.3 & 1.2 & 1.4 & 0.7 & \\
\hline 20 & Food and Kindred Products & 9,230 & Q & 1,485 & 624 & 10,114 & 11.0 \\
\hline 2011 & Meat Packing Plants .... & 311 & 0 & 0 & 0 & 311 & 14.8 \\
\hline 2033 & Canned Fruits and Vegetables . . . . . . . . . . . . . . & 547 & 0 & $\mathbf{w}$ & w & 556 & 15.2 \\
\hline 2037 & Frozen Fruits and Vegetables. . & 2,117 & $\mathbf{Q}$ & 71 & 27 & 2,162 & 20.5 \\
\hline 2046 & Wet Com Milling . . . . . . . . & 93 & 0 & $w$ & 0 & $w$ & 21.4 \\
\hline 2051 & Bread, Cake, and Related Products & 388 & 0 & w & 0 & w & 27.3 \\
\hline 2063 & Beet Sugar.. & w & 0 & 258 & 0 & w & 8.5 \\
\hline 2075 & Soybean Oil Mills $\ldots \ldots \ldots \ldots \ldots \ldots \ldots \ldots \ldots \ldots$ & 0 & 0 & 0 & 0 & 0 & NF \\
\hline 2082 & Malt Beverages $\ldots \ldots \ldots \ldots \ldots \ldots \ldots \ldots \ldots \ldots$ & 506 & 0 & w & w & 776 & 17.3 \\
\hline 21 & Tobacco Products . & 0 & 0 & 0 & 0 & 0 & $\mathrm{NF}$ \\
\hline 22 & Textile Mill Products & 274 & 0 & 0 & 0 & 274 & 35.5 \\
\hline 23 & Apparel and Other Textile Products $\ldots \ldots \ldots \ldots \ldots \ldots \ldots$ & NA & NA & NA & NA & NA & 31.0 \\
\hline 24 & Lumber and Wood Products $\ldots \ldots \ldots$ & w & W & 2,818 & w & $w$ & 20.9 \\
\hline 25 & Furniture and Fixtures $\ldots \ldots \ldots \ldots$. & 304 & 0 & 0 & 0 & 304 & 29.6 \\
\hline 26 & Paper and Allied Products . . . . . . . . & 13,785 & 103 & 6,035 & 2,955 & 16,968 & 6.8 \\
\hline 2611 & Pulp Mills . . . . . . . . . . . & 843 & 6 & 912 & 17 & 1,743 & 23.2 \\
\hline 2621 & Paper Mills $\ldots \ldots \ldots \ldots \ldots \ldots \ldots \ldots$ & 8,196 & 0 & 3,082 & 1,857 & 9,421 & 4.8 \\
\hline 2631 & Paperboard Mills .. & 3,155 & 0 & 2,037 & 1,081 & 4,111 & 7.2 \\
\hline 27 & Printing and Publishing & NA & NA & NA & NA & NA & 19.2 \\
\hline 28 & Chemicals and Allied Products. & 11,126 & 914 & 1,994 & 1,125 & 12,910 & 8.8 \\
\hline 2812 & Alkalies and Chlorine ........ & w & 0 & 0 & 0 & w & 20.7 \\
\hline 2813 & Industrial Gases $\ldots \ldots \ldots \ldots \ldots \ldots \ldots \ldots \ldots \ldots$ & 2,561 & w & w & 0 & 3,473 & 12.5 \\
\hline 2819 & Industrial Inorganic Chemicals, nec ... & 4,373 & 106 & W & w & w & 12.2 \\
\hline 2821 & Plastics Materials and Resins ...... & 189 & 23 & * & 0 & 212 & 16.6 \\
\hline 2822 & Synthetic Rubber . . . . . . . . . & * & 0 & 0 & 0 & * & 32.5 \\
\hline 2823 & Cellulosic Manmade Fibers .... & 0 & 0 & 0 & 0 & 0 & NF \\
\hline 2824 & Organic Fibers, Noncellulosic ..... & 0 & 0 & 0 & 0 & 0 & NF \\
\hline 2865 & Cyclic Crudes and Intermediates ... & 11 & 0 & 0 & 0 & 11 & 38.4 \\
\hline 2869 & Industrial Organic Chemicals, nec .. & $\mathbf{Q}$ & 2 & w & w & 214 & 21.3 \\
\hline 2873 & Nitrogenous Fentilizers $\ldots \ldots \ldots \ldots \ldots \ldots$ & 428 & 33 & 156 & 0 & 617 & 35.4 \\
\hline 2874 & Phosphatic Fertilizers . . . . . . . . . . & 510 & 0 & w & w & 562 & 11.7 \\
\hline 29 & Petroleum and Coal Products & w & w & 2,874 & 379 & 10,460 & 4.5 \\
\hline 2911 & Petroleum Refining.$\ldots \ldots \ldots$ & w & w & 2,423 & 133 & 9,952 & 3.0 \\
\hline 30 & Rubber and Misc. Plastics Products .... & 2,865 & 0 & w & 0 & w & 21.4 \\
\hline 3011 & Tires and Inner Tubes . . . . . . . . . & w & 0 & 0 & 0 & w & 8.9 \\
\hline 308 & Miscellaneous Plastics Products, nec . . . & 2,573 & 0 & - & 0 & 2,574 & 23.7 \\
\hline 31 & Leather and Leather Products ........ & 70 & 0 & 0 & 0 & 70 & 45.8 \\
\hline 32 & Stone, Clay and Glass Products & 5,156 & 0 & w & w & 5,333 & 14.4 \\
\hline 3211 & Flat Glass $\ldots \ldots \ldots \ldots \ldots \ldots \ldots$ & 148 & 0 & w & 0 & W & 4.7 \\
\hline 3221 & Glass Containers ........... & 1,129 & 0 & 0 & 0 & 1,129 & 8.9 \\
\hline 3229 & Pressed and Blown Glass, nec . . . & W & 0 & 0 & 0 & $w$ & 16.3 \\
\hline 3241 & Cement, Hydraulic & 2,390 & 0 & w & w & w & 21.5 \\
\hline 3274 & Lime & $w$ & 0 & 0 & 0 & w & 23.7 \\
\hline 3296 & Mineral Wool . . . . . . . . . . & 330 & 0 & 0 & 0 & 330 & 3.0 \\
\hline 33 & Primary Metal Industries & w & w & W & w & 35,230 & 3.8 \\
\hline 3312 & Blast Fumaces and Steel Mills .. & 2,531 & 0 & w & $w$ & 2,670 & 8.5 \\
\hline 3313 & Electrometalurgical Products ... & 0 & 0 & 0 & 0 & 0 & NF \\
\hline 3321 & Gray and Ductile Iron Foundries & 92 & 0 & 0 & 0 & 92 & 48.8 \\
\hline 3331 & Primary Copper & w & w & w & 0 & w & 1.1 \\
\hline 3334 & Primary Aluminum & 26,391 & 0 & 0 & 0 & 26,391 & 4.4 \\
\hline 3339 & Primary Nonferrous Metals, nec. & 1,263 & $\mathbf{0}$ & w & $\mathbf{0}$ & w & 1.2 \\
\hline 3353 & Aluminum Sheet, Plate, and Foil & w & 0 & 0 & 0 & $w$ & 1.5 \\
\hline 34 & Fabricated Metal Products . . . . . . & 2,922 & 0 & Q & 0 & $\therefore, 949$ & 14.8 \\
\hline 35 & Industrial Machinery and Equipment . & 3,614 & 6 & w & 0 & w & 20.6 \\
\hline 357 & Computer and Office Equipment & 1,945 & 6 & 0 & 0 & 1,950 & 16.4 \\
\hline 36 & Electronic and Other Electric Equipment . & 5,550 & 0 & - & 0 & 5,550 & 16.1 \\
\hline
\end{tabular}

See footnotes at end of table. 
Table A16. Components of Total Electricity Demand by Census Region, Industry Group, and Selected Industries, 1991 (Continued)

(Estimates in Million Kilowatthours)

\begin{tabular}{|c|c|c|c|c|c|c|c|}
\hline $\begin{array}{l}\text { SIC } \\
\text { Code }\end{array}$ & $\begin{array}{l}\text { Industry Groups } \\
\text { and Industry }\end{array}$ & Purchases & Transfers In $^{b}$ & $\begin{array}{l}\text { Total Onsite } \\
\text { Generatione }\end{array}$ & $\begin{array}{c}\text { Sales and/or } \\
\text { Transfers } \\
\text { Offsite }\end{array}$ & $\begin{array}{l}\text { Net Demand } \\
\text { for Electricity }\end{array}$ & $\begin{array}{c}\text { RSE } \\
\text { Row } \\
\text { Factors }\end{array}$ \\
\hline & & \multicolumn{5}{|c|}{ West Census Region } & \\
\hline & RSE Column Factors: & 0.7 & 1.3 & 1.2 & 1.4 & 0.7 & \\
\hline 37 & Transportation Equipment & 7,096 & 0 & W & 0 & W & 8.9 \\
\hline 3711 & Motor Vehicles and Car Bodles .................. & W & 0 & 0 & 0 & $\mathbf{W}$ & 8.9 \\
\hline 3714 & Motor Vehicle Parts and Accessories . . . . . . . . . . & 243 & 0 & 0 & 0 & 243 & 34.0 \\
\hline 38 & Instruments and Related Products $\ldots \ldots \ldots \ldots \ldots \ldots \ldots$ & 3,188 & $\mathbf{Q}$ & $w$ & - & $w$ & 16.0 \\
\hline 3841 & Surgical and Medical Instruments $\ldots \ldots \ldots \ldots \ldots \ldots$ & 222 & 0 & 0 & 0 & 222 & 25.1 \\
\hline \multirow[t]{2}{*}{39} & Misc. Manufacturing Industries $\ldots \ldots \ldots \ldots \ldots \ldots \ldots$ & 376 & 0 & 0 & 0 & 376 & 31.0 \\
\hline & Total $\ldots \ldots \ldots \ldots \ldots \ldots \ldots \ldots \ldots \ldots$ & 116,720 & 1,678 & 16,518 & 7,135 & 127,781 & 5.0 \\
\hline
\end{tabular}

- See Appendices B and F for descriptions of the Standard Industrial Classification system.

- "Transfers In" are the quantities purchased by a central purchasing agent or other establishment of the same company.

c "Onsite Generation" includes cogeneration, generation by renewable energy sources, and conventional generation by combustible fuels.

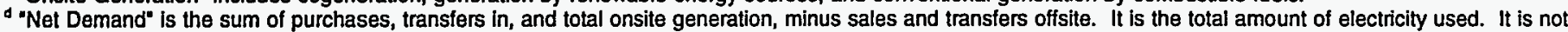
comparable to net electriclty which excludes electricity generated onsite by combustible energy sources.

NF=No applicable RSE row/column factor.

- Estimate less than 0.5. Data are included in higher level totals.

$W=$ Withheld to avoid disclosing data tor individual establishments. Data are included in higher level totals.

$\mathrm{Q}=$ Withheld because Relative Standard Error is greater than $\mathbf{5 0}$ percent. Data are included in higher level totals.

NA=Not avallable. Data are included In higher level totals.

Notes: - To obtain a RSE percentage for any table cell, multiply the cell's corresponding RSE column and RSE row factors. • Totals may not equal sum of components because of independent rounding.

Source: Energy Information Administration, Office of Energy Markets and End Use, Energy End Use and Integrated Statistics Division, Form ElA-846, "1991 Manufacturing Energy Consumption Survey." 


\section{Table A17. Components of Onsite Electricity Generation by Census Region, Industry Group, and Selected Industries, 1991}

(Estimates in Million Kilowatthours)

\begin{tabular}{|c|c|c|c|c|c|c|}
\hline $\begin{array}{c}\text { SIC } \\
\operatorname{Code}^{\circ} \\
\end{array}$ & $\begin{array}{c}\text { Industry Groups } \\
\text { and Industry }\end{array}$ & Total & Cogeneration & Renewables & Other & $\begin{array}{c}\text { RSE } \\
\text { Row } \\
\text { Factora }\end{array}$ \\
\hline & \multirow[b]{2}{*}{ RSE Column Factors: } & \multicolumn{4}{|c|}{ Total United States } & \\
\hline & & 0.8 & 0.8 & 1.4 & 1.2 & \\
\hline 20 & Food and Kindred Products $\ldots \ldots \ldots \ldots \ldots \ldots$ & 5,743 & 5,579 & 6 & 157 & 12.2 \\
\hline 2011 & Meat Packing Plants $\ldots \ldots \ldots \ldots \ldots \ldots \ldots$ & 71 & 71 & 0 & 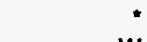 & 23.7 \\
\hline 2033 & Canned Fruits and Vegetables . . . . . . . . . . . . . & W & $w$ & 0 & W & 30.7 \\
\hline 2037 & Frozen Fruits and Vegetables $\ldots \ldots \ldots \ldots \ldots \ldots$ & 135 & 134 & 0 & 1 & 29.9 \\
\hline 2046 & 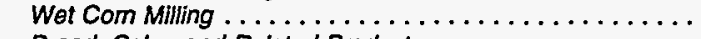 & W & $W$ & 0 & 0 & 12.6 \\
\hline 2051 & Bread, Cake, and Related Products . . . . . . . . . . . & 33 & 33 & 0 & * & 22.6 \\
\hline 2063 & 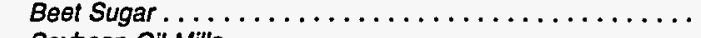 & W & 436 & 0 & W & 7.6 \\
\hline 2075 & Soybean Oll Mills . . . . . . . . . . . . . . . . . . & W & W & 0 & 0 & 6.3 \\
\hline 2082 & Malt Beverages $\ldots \ldots \ldots \ldots \ldots \ldots \ldots \ldots \ldots \ldots$ & W & W & 0 & 0 & 17.7 \\
\hline 21 & Tobacco Products $\ldots \ldots \ldots \ldots \ldots \ldots \ldots \ldots \ldots$ & W & W & 0 & 0 & 7.6 \\
\hline 22 & Textile Mill Products $\ldots \ldots \ldots \ldots \ldots \ldots \ldots \ldots$ & W & W & W & W & 19.6 \\
\hline 23 & Apparel and Other Textile Products $\ldots \ldots \ldots \ldots \ldots$ & 0 & 0 & 0 & 0 & NF \\
\hline 24 & Lumber and Wood Products $\ldots \ldots \ldots \ldots \ldots$ & 2,950 & 2,671 & $\mathbf{Q}$ & * & 41.9 \\
\hline 25 & Furniture and Fixtures $\ldots \ldots \ldots \ldots \ldots \ldots \ldots \ldots \ldots$ & 33 & 33 & 0 & 0 & 31.6 \\
\hline 26 & Paper and Allied Products $\ldots \ldots \ldots \ldots \ldots \ldots$ & 53,831 & 45,447 & 2,856 & 5,528 & 3.1 \\
\hline 2611 & 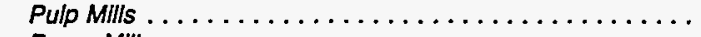 & 6,138 & 4,963 & W & W & 16.2 \\
\hline 2621 & 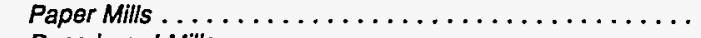 & 30,841 & 27,058 & 2,522 & 1,261 & 2.8 \\
\hline 2631 & Paperboard Mills . . . . . . . . . . . . . . . . & 16,739 & 13,361 & W & W & 6.0 \\
\hline 27 & Printing and Publishing $\ldots \ldots \ldots \ldots \ldots \ldots \ldots$ & Q & Q & 0 & • & NF \\
\hline 28 & Chemicals and Allied Products $\ldots \ldots \ldots \ldots \ldots \ldots$ & 41,428 & 38,348 & $\mathbf{Q}$ & 3,079 & 7.0 \\
\hline 2812 & Alkalies and Chlorine $\ldots \ldots \ldots \ldots \ldots \ldots \ldots$ & W & W & 0 & W & 20.4 \\
\hline 2813 & Industrial Gases $\ldots \ldots \ldots \ldots \ldots \ldots \ldots \ldots \ldots$ & $\mathbf{W}$ & w & 0 & $\mathbf{W}$ & 17.2 \\
\hline 2819 & Industrial Inorganic Chemicals, nec ............. & W & 2,654 & 0 & W & 11.0 \\
\hline 2821 & Plastics Materials and Resins $\ldots \ldots \ldots \ldots \ldots$ & 2,627 & 2,525 & 0 & 103 & 8.1 \\
\hline 2822 & Synthetic Rubber $\ldots \ldots \ldots \ldots \ldots \ldots \ldots \ldots \ldots \ldots$ & W & W & 0 & 0 & 24.0 \\
\hline 2823 & Cellulosic Manmade Fibers . . . . . . . . . . . . . & 961 & 961 & 0 & 0 & 24.0 \\
\hline 2824 & Organic Fibers, Noncellulosic $\ldots \ldots \ldots \ldots \ldots$ & 626 & 626 & 0 & 0 & 3.8 \\
\hline 2865 & Cyclic Crudes and Intermediates . . . . . . . . . . & 457 & W & 0 & $w$ & 19.8 \\
\hline 2869 & Industrial Organic Chemicals, nec . . . . . . . . . . & 21,661 & W & $\mathbf{Q}$ & W & 8.8 \\
\hline 2873 & Nitrogenous Fertilizers $\ldots \ldots \ldots \ldots \ldots \ldots \ldots$ & 392 & 391 & 0 & 1 & 40.1 \\
\hline 2874 & Phosphatic Fertilizers . . . . . . . . . . . . . . . & 2,677 & 2,677 & 0 & 0 & 2.5 \\
\hline 29 & Petroleum and Coal Products $\ldots \ldots \ldots \ldots \ldots \ldots$ & 13,452 & W & 0 & W & 2.5 \\
\hline 2911 & Petroleum Refining $\ldots \ldots \ldots \ldots \ldots \ldots \ldots \ldots$ & 12,993 & W & 0 & $W$ & 2.5 \\
\hline 30 & Rubber and Misc. Plastics Products $\ldots \ldots \ldots \ldots \ldots$ & 115 & W & W & - & 16.5 \\
\hline 3011 & Tires and Inner Tubes . . . . . . . . . . . . . . . & $\cdot$ & 0 & 0 & * & 7.3 \\
\hline 308 & Miscellaneous Plastics Products, noc . . . . . . . . . . . & W & W & 0 & - & 38.5 \\
\hline 31 & Leather and Leather Products $\ldots \ldots \ldots \ldots \ldots \ldots$ & 39 & 38 & 1 & 0 & 47.7 \\
\hline 32 & Stone, Clay and Glass Products $\ldots \ldots \ldots \ldots \ldots \ldots$ & 533 & 499 & 0 & $\mathbf{Q}$ & 20.8 \\
\hline 3211 & Flat Glass $\ldots \ldots \ldots \ldots \ldots \ldots \ldots \ldots \ldots \ldots \ldots \ldots \ldots$ & W & 0 & 0 & W & 6.3 \\
\hline 3221 & Glass Containers $\ldots \ldots \ldots \ldots \ldots \ldots \ldots \ldots \ldots$ & 0 & 0 & 0 & 0 & NF \\
\hline 3229 & Pressed and Blown Glass, nec . . . . . . . . . . & * & 0 & 0 & * & 10.4 \\
\hline 3241 & Cement, Hydraulic $\ldots \ldots \ldots \ldots \ldots \ldots \ldots \ldots$ & $\mathbf{W}$ & W & 0 & 0 & 24.0 \\
\hline 3274 & 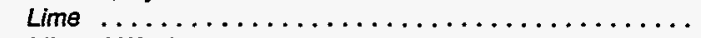 & 0 & 0 & 0 & 0 & NF \\
\hline 3296 & Mineral Wool $\ldots \ldots \ldots \ldots \ldots \ldots \ldots \ldots \ldots \ldots$ & 0 & 0 & 0 & 0 & NF \\
\hline 33 & Primary Metal Industries $\ldots \ldots \ldots \ldots \ldots \ldots \ldots$ & 8,370 & 4,956 & $W$ & W & 5.1 \\
\hline 3312 & Blast Fumaces and Steel Mills . . . . . . . . . . . . & 6,235 & 4,560 & 0 & 1,674 & 5.9 \\
\hline 3313 & Electrometalurgical Products . . . . . . . . . . . . & $W$ & W & W & - & 14.0 \\
\hline 3321 & Gray and Ductile Iron Foundries . . . . . . . . . . . & $w$ & W & 0 & - & 30.5 \\
\hline 3331 & Primary Copper $\ldots \ldots \ldots \ldots \ldots \ldots \ldots \ldots \ldots$ & 279 & W & 0 & W & 1.1 \\
\hline 3334 & Primany Aluminum $\ldots \ldots \ldots \ldots \ldots \ldots \ldots \ldots$ & 0 & 0 & 0 & 0 & NF \\
\hline 3339 & Primary Nonferrous Metals, nec . . . . . . . . . . . . . . & W & W & W & 0 & 1.1 \\
\hline 3353 & Aluminum Sheet, Plate, and Foil $\ldots \ldots \ldots \ldots \ldots$ & 0 & 0 & 0 & 0 & NF \\
\hline 34 & Fabricated Metal Products . . . . . . . . . . . . . & $w$ & W & * & - & 34.1 \\
\hline 35 & Industrial Machinery and Equipment $\ldots \ldots \ldots \ldots \ldots$ & 148 & 126 & $\mathbf{Q}$ & 19 & 18.2 \\
\hline 357 & Computer and Office Equipment $\ldots \ldots \ldots \ldots \ldots$ & 17 & 13 & 0 & 4 & 36.6 \\
\hline 36 & Electronic and Other Electric Equipment $\ldots \ldots \ldots \ldots \ldots$ & $\mathbf{Q}$ & $\mathbf{Q}$ & $\star$ & $\cdot$ & 19.6 \\
\hline 37 & Transportation Equipment $\ldots \ldots \ldots \ldots \ldots \ldots \ldots$ & 714 & 602 & W & W & 12.2 \\
\hline 3711 & Motor Vehicles and Car Bodles . . . . . . . . . . . . . & $\mathbf{W}$ & * & W & 0 & 9.8 \\
\hline 3714 & Motor Vehicle Parts and Accessories . . . . . . . . . . . & W & $w$ & 0 & 0 & 20.2 \\
\hline 38 & Instruments and Related Products $\ldots \ldots \ldots \ldots \ldots \ldots$ & W & W & $\mathbf{Q}$ & $\mathbf{Q}$ & 34.7 \\
\hline 3841 & Surgical and Medical Instruments $\ldots \ldots \ldots \ldots \ldots \ldots$ & 0 & 0 & 0 & 0 & NF \\
\hline 39 & Misc. Manufacturing Industries $\ldots \ldots \ldots \ldots \ldots \ldots$ & * & $*$ & 0 & $*$ & 40.1 \\
\hline & Total $\ldots \ldots \ldots \ldots \ldots \ldots \ldots \ldots \ldots \ldots \ldots \ldots$ & 130,028 & 113,912 & 4,444 & 11,672 & 4.0 \\
\hline
\end{tabular}

See footnotes at end of table. 

Table A17. Components of Onsite Electricity Generation by Census Region, Industry
Group, and Selected Industries, 1991 (Continued) (Estimates in Million Kilowatthours)

\begin{tabular}{|c|c|c|c|c|c|c|}
\hline \multirow[t]{3}{*}{$\begin{array}{l}\text { SIC } \\
\text { Codea }\end{array}$} & $\begin{array}{l}\text { Industry Groups } \\
\text { and Industry }\end{array}$ & Total & Cogeneration & Renowables & Other & \multirow[t]{2}{*}{$\begin{array}{c}\text { RSE } \\
\text { Row } \\
\text { Factors }\end{array}$} \\
\hline & \multirow[b]{2}{*}{ RSE Column Factors: } & \multicolumn{4}{|c|}{ Northeast Census Region } & \\
\hline & & 1.0 & 1.0 & 0.8 & 1.3 & \\
\hline 20 & Food and KIndred Products . & 352 & 339 & 0 & $Q$ & 17.1 \\
\hline 2011 & 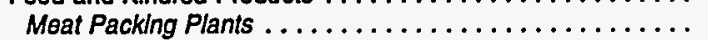 & 0 & 0 & 0 & 0 & NF \\
\hline 2033 & Canned Fruits and Vegetables . . . . . . . . . . . . . & 5 & 5 & 0 & 0 & 39.5 \\
\hline 2037 & Frozen Frults and Vegetables . . . . . . . . . . . . & 1 & 0 & 0 & 1 & 33.5 \\
\hline 2046 & 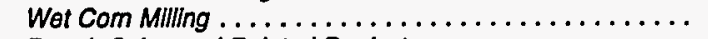 & 0 & 0 & 0 & 0 & NF \\
\hline 2051 & Bread, Cake, and Rolated Products . . . . . . . . . . . . & W & W & 0 & 0 & 26.0 \\
\hline 2063 & Beot Sugar . . . . . . . . . . . . . . . . . . . . . & 0 & 0 & 0 & 0 & NF \\
\hline 2075 & Soybean Oll Mllls . . . . . . . . . . . . . . . . & 0 & 0 & 0 & 0 & NF \\
\hline 2082 & Mall Beverages $\ldots \ldots \ldots \ldots \ldots \ldots \ldots \ldots \ldots \ldots$ & W & W & 0 & 0 & 23.9 \\
\hline 21 & Tobacco Products $\ldots \ldots \ldots \ldots \ldots \ldots \ldots \ldots$ & NA & NA & NA & NA & NF \\
\hline 22 & Textlle Mill Products $\ldots \ldots \ldots \ldots \ldots \ldots \ldots \ldots$ & w & W & 3 & 0 & 46.2 \\
\hline 23 & Apparel and Other Textlle Products $\ldots \ldots \ldots \ldots \ldots$ & 0 & 0 & 0 & 0 & NF \\
\hline 24 & Lumber and Wood Products $\ldots \ldots \ldots \ldots \ldots \ldots$ & NA & NA & NA & NA & NF \\
\hline 25 & Furniture and Fixtures $\ldots \ldots \ldots \ldots \ldots \ldots \ldots$ & Q & Q & 0 & 0 & NF \\
\hline 26 & Paper and Alled Products $\ldots \ldots \ldots \ldots \ldots \ldots \ldots$ & 8,991 & 6,829 & W & W & 4.2 \\
\hline 2611 & 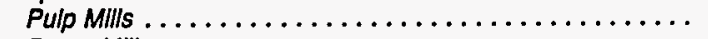 & 384 & W & W & 0 & 39.1 \\
\hline 2621 & Papor MIIls . . . . . . . . . . . . . . . . . . & 8,253 & 6,344 & 1,685 & 224 & 3.7 \\
\hline 2631 & 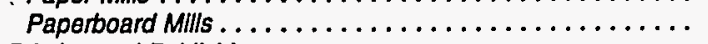 & $W$ & $W$ & 0 & W & 13.0 \\
\hline 27 & Printing and Publishing $\ldots \ldots \ldots \ldots \ldots \ldots \ldots$ & Q & $\mathbf{Q}$ & 0 & 0 & NF \\
\hline 28 & Chemlcals and Allied Products . . . . . . . . . . . . . & 780 & W & 0 & W & 11.3 \\
\hline 2812 & Alkalles and Chlorine $\ldots \ldots \ldots \ldots \ldots \ldots \ldots$ & 0 & 0 & 0 & 0 & NF \\
\hline 2813 & Industrial Gases . . . . . . . . . . . . . . . . & 0 & 0 & 0 & 0 & NF \\
\hline 2819 & Industrlal Inorganic Chemicals, nec . . . . . . . . . & $w$ & W & 0 & $\bullet$ & 15.7 \\
\hline 2821 & Plastles Materials and Resins . . . . . . . . . . . . & $W$ & W & 0 & $\bullet$ & 9.8 \\
\hline 2822 & 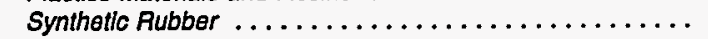 & 0 & 0 & 0 & 0 & NF \\
\hline 2823 & Collulosic Manmado Flbers . . . . . . . . . . . . . . . . . & 0 & 0 & $\mathbf{0}$ & 0 & NF \\
\hline 2824 & Organic Fibers, Noncellulosic $\ldots \ldots \ldots \ldots \ldots \ldots$ & 0 & 0 & 0 & 0 & NF \\
\hline 2865 & Cycllo Crudes and Intemediates . . . . . . . . . . . & 0 & 0 & 0 & 0 & NF \\
\hline 2869 & Industrial Organic Chemicals, nec .............. & W & $\mathbf{W}$ & $\mathbf{0}$ & W & 12.2 \\
\hline 2873 & Nitrogenous Fertllizers $\ldots \ldots \ldots \ldots \ldots \ldots \ldots$ & 0 & 0 & 0 & 0 & NF \\
\hline 2874 & Phosphatlc Fertilizers ................ & 0 & 0 & 0 & 0 & NF \\
\hline 29 & Petroleum and Coal Products $\ldots \ldots \ldots \ldots \ldots \ldots$ & $\mathbf{W}$ & 714 & 0 & W & 4.1 \\
\hline 2911 & 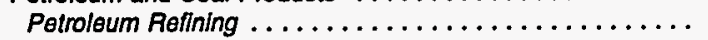 & $W$ & 714 & 0 & W & 4.1 \\
\hline 30 & Rubber and Misc. Plastics Products . . . . . . . . . . & W & 76 & W & • & 19.1 \\
\hline 3011 & Tires and Inner Tubos . . . . . . . . . . . . . . . . & 0 & 0 & 0 & 0 & NF \\
\hline 308 & Miscellaneous Plastlcs Products, nec . . . . . . . . . . & W & W & 0 & 0 & 32.2 \\
\hline 31 & Leather and Leather Products . . . . . . . . . . . . . & 1 & 0 & 1 & 0 & 50.3 \\
\hline 32 & Stone, Clay and Glass Products $\ldots \ldots \ldots \ldots \ldots \ldots$ & $w$ & $\mathbf{W}$ & 0 & $\mathbf{Q}$ & 26.1 \\
\hline 3211 & Flat Glass $\ldots \ldots \ldots \ldots \ldots \ldots \ldots \ldots \ldots \ldots \ldots \ldots \ldots$ & 0 & 0 & 0 & 0 & NF \\
\hline 3221 & Glass Containers $\ldots \ldots \ldots \ldots \ldots \ldots \ldots \ldots \ldots$ & 0 & 0 & 0 & 0 & NF \\
\hline 3229 & Pressed and Blown Glass, nec $\ldots \ldots \ldots \ldots \ldots \ldots$ & 0 & 0 & $\mathbf{0}$ & 0 & NF \\
\hline 3241 & Cement, Hydraullc ........................ & 0 & 0 & 0 & 0 & NF \\
\hline 3274 & 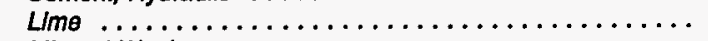 & 0 & 0 & 0 & 0 & NF \\
\hline 3296 & Mineral Wool $\ldots \ldots \ldots \ldots \ldots \ldots \ldots \ldots \ldots$ & 0 & 0 & 0 & 0 & NF \\
\hline 33 & Primary Metal industries $\ldots \ldots \ldots \ldots \ldots \ldots \ldots$ & $W$ & W & W & $W$ & 4.5 \\
\hline 3312 & Blast Fumaces and steel Mills . . . . . . . . . . . . . . & W & W & 0 & W & 8.1 \\
\hline 3313 & Electrometalurgical Products . . . . . . . . . . . . & 0 & 0 & 0 & 0 & NF \\
\hline 3321 & 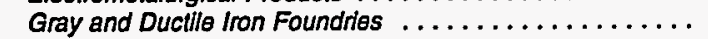 & 0 & 0 & 0 & 0 & NF \\
\hline 3331 & 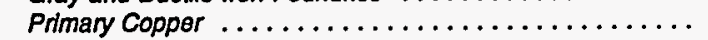 & 0 & 0 & 0 & 0 & NF \\
\hline 3334 & Primany Aluminum $\ldots \ldots \ldots \ldots \ldots \ldots \ldots \ldots \ldots$ & 0 & 0 & $\mathbf{0}$ & 0 & NF \\
\hline 3339 & Primary Nonferrous Metals, nec . . . . . . . . . . . . . . . . & W & $\mathbf{0}$ & W & 0 & 1.1 \\
\hline 3353 & Aluminum Shoet, Plate, and Foll . . . . . . . . . . . . & 0 & 0 & 0 & 0 & NF \\
\hline 34 & Fabricated Metal Products . . . . . . . . . . . . . . . & 83 & 83 & $*$ & 0 & 34.3 \\
\hline 35 & Industrial Machinery and Equipment ............. & W & W & $\mathbf{a}$ & $\mathbf{Q}$ & 44.2 \\
\hline 357 & Computer and Office Equipment $\ldots \ldots \ldots \ldots \ldots$ & 0 & 0 & 0 & 0 & NF \\
\hline 38 & Electronic and Other Electric Equipment $\ldots \ldots \ldots \ldots \ldots$ & 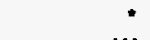 & 0 & 0 & * & 25.3 \\
\hline 37 & Transportation Equipment $\ldots \ldots \ldots \ldots \ldots \ldots \ldots$ & W & W & 0 & * & 15.8 \\
\hline 3711 & Motor Vehicles and Car Bodies . . . . . . . . . . . . . & 0 & 0 & 0 & 0 & NF \\
\hline 3714 & Motor Vehicle Parts and Accessories . . . . . . . . . . . & 0 & 0 & 0 & 0 & NF \\
\hline 38 & Instruments and Related Products $\ldots \ldots \ldots \ldots \ldots \ldots$ & W & W & $\mathbf{Q}$ & $\mathbf{Q}$ & 28.6 \\
\hline 3841 & Surglcal and Medlcal Instruments $\ldots \ldots \ldots \ldots \ldots$ & 0 & 0 & 0 & 0 & NF \\
\hline 39 & Misc. Manufacturing Industries $\ldots \ldots \ldots \ldots \ldots \ldots$ & - & - & 0 & $\bullet$ & 34.1 \\
\hline & Total $\ldots \ldots \ldots \ldots \ldots \ldots \ldots \ldots \ldots \ldots \ldots$ & 14,592 & 10,913 & 2,491 & 1,188 & 4.7 \\
\hline
\end{tabular}

Ses footnotes at end of table. 
Table A17. Components of Onsite Electricity Generation by Census Region, Industry
Group, and Selected Industries, 1991 (Continued) (Estimates in Million Kilowatthours)

\begin{tabular}{|c|c|c|c|c|c|c|}
\hline $\begin{array}{c}\text { SIC } \\
\text { Code }\end{array}$ & $\begin{array}{l}\text { Industry Groups } \\
\text { and Industry }\end{array}$ & Total & Cogeneration & Renewables & Other & \multirow[t]{3}{*}{$\begin{array}{c}\text { RSE } \\
\text { Row } \\
\text { Factors }\end{array}$} \\
\hline & \multirow[b]{2}{*}{ RSE Column Factors: } & \multicolumn{4}{|c|}{ Midwest Census Region } & \\
\hline & & 0.9 & 0.9 & 0.9 & 1.4 & \\
\hline 20 & Food and Kindred Products & 2,541 & 2,540 & 0 & - & 11.4 \\
\hline 2011 & Meat Packing Plants . . . . . . . . . . . & 71 & 71 & 0 & . & 20.3 \\
\hline 2033 & Canned Fruits and Vegetables . . . . . . . . . & 0 & 0 & 0 & 0 & NF \\
\hline 2037 & Frozen Fruits and Vegetables ..... & 0 & 0 & 0 & 0 & NF \\
\hline 2046 & Wet Corn Milling . ............ & $w$ & $w$ & 0 & 0 & 12.2 \\
\hline 2051 & Bread, Cake, and Related Products & 6 & 6 & 0 & • & 36.5 \\
\hline 2063 & Beet Sugar. & w & w & 0 & 0 & 7.7 \\
\hline 2075 & Soybean Oil Mills $\ldots \ldots \ldots \ldots \ldots$ & w & w & 0 & 0 & 5.5 \\
\hline 2082 & Malt Beverages $\ldots \ldots \ldots \ldots \ldots \ldots$ & w & w & 0 & 0 & 24.3 \\
\hline 21 & Tobacco Products $\ldots \ldots \ldots \ldots \ldots \ldots \ldots \ldots \ldots$ & NA & NA & NA & NA & NF \\
\hline 22 & Textile Mill Products $\ldots \ldots \ldots \ldots \ldots$ & NA & NA & NA & NA & NF \\
\hline 23 & Apparel and Other Textile Products & NA & NA & NA & NA & NF \\
\hline 24 & Lumber and Wood Products $\ldots . .$. & 68 & 68 & 0 & 0 & 38.7 \\
\hline 25 & Furniture and Fixtures $\ldots \ldots \ldots \ldots \ldots \ldots \ldots \ldots \ldots$ & 18 & 18 & 0 & 0 & 39.8 \\
\hline 26 & Paper and Allied Products $\ldots \ldots \ldots \ldots \ldots \ldots \ldots$ & 6,475 & 5,513 & w & $\mathbf{w}$ & 6.6 \\
\hline 2611 & Pulp Mills $\ldots \ldots \ldots \ldots \ldots \ldots$ & 523 & 523 & 0 & 0 & 28.8 \\
\hline 2621 & Paper Mills. & 4,777 & W & 475 & $w$ & 4.4 \\
\hline 2631 & Paperboard Mills . & w & 772 & w & 236 & 14.7 \\
\hline 27 & Printing and Publishing $\ldots \ldots \ldots \ldots$ & * & 0 & 0 & * & NF \\
\hline 28 & Chemicals and Allied Products ..... & 1,077 & 878 & 0 & 199 & 10.6 \\
\hline 2812 & Alkalies and Chlorine ....... & 0 & 0 & 0 & 0 & NF \\
\hline 2813 & Industrial Gases $\ldots \ldots \ldots \ldots \ldots$ & 0 & 0 & 0 & 0 & NF \\
\hline 2819 & Industrial Inorganic Chemicals, nec & w & w & 0 & 0 & 19.9 \\
\hline 2821 & Plastics Materials and Resins $\ldots \ldots \ldots \ldots$ & w & w & 0 & w & 12.0 \\
\hline 2822 & Synthetic Rubber $\ldots \ldots \ldots \ldots \ldots \ldots$ & 0 & 0 & 0 & 0 & NF \\
\hline 2823 & Cellulosic Manmade Fibers $\ldots \ldots \ldots \ldots$. & 0 & 0 & 0 & 0 & NF \\
\hline 2824 & Organic Fibers, Noncellulosic.$\ldots \ldots \ldots$. & 0 & 0 & 0 & 0 & NF \\
\hline 2865 & Cyclic Crudes and Intermediates & w & w & 0 & 0 & 17.7 \\
\hline 2869 & Industrial Organic Chemicals, nec . . . . . . & w & w & 0 & • & 9.2 \\
\hline 2873 & Nitrogenous Fertilizers $\ldots \ldots \ldots \ldots \ldots$ & 4 & 2 & 0 & 1 & 42.0 \\
\hline 2874 & Phosphatic Fertilizers $\ldots \ldots \ldots \ldots \ldots$ & 0 & 0 & 0 & 0 & NF \\
\hline 29 & Petroleum and Coal Products ...... & w & w & 0 & $\mathbf{w}$ & 5.0 \\
\hline 2911 & Petroleum Refining ..... & w & w & 0 & $w$ & 5.0 \\
\hline 30 & Rubber and Misc. Plastics Products & . & * & $\mathbf{0}$ & 0 & 25.4 \\
\hline 3011 & Tires and Inner Tubes . . . . . . . . . & 0 & 0 & 0 & 0 & NF \\
\hline 308 & Miscellaneous Plastics Products, nec . . . . . . . . . . & NA & NA & NA & NA & NF \\
\hline 31 & Leather and Leather Products . . . . . . & 8 & 8 & 0 & 0 & 49.8 \\
\hline 32 & Stone, Clay and Glass Products . . & w & w & 0 & 0 & 28.8 \\
\hline 3211 & Flat Glass $\ldots \ldots \ldots \ldots \ldots$ & 0 & 0 & 0 & 0 & NF \\
\hline 3221 & Glass Containers & 0 & 0 & $\mathbf{0}$ & 0 & NF \\
\hline 3229 & Pressed and Blown Glass, nec & 0 & 0 & $\mathbf{0}$ & 0 & NF \\
\hline 3241 & Cement, Hydraulic $\ldots \ldots \ldots \ldots \ldots \ldots \ldots \ldots \ldots \ldots$ & w & W & 0 & 0 & 28.8 \\
\hline 3274 & 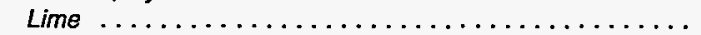 & 0 & 0 & 0 & 0 & NF \\
\hline 3296 & Mineral Wool & 0 & 0 & 0 & 0 & NF \\
\hline 33 & Primary Metal Industries & 3,377 & 2,573 & 0 & 804 & 7.2 \\
\hline 3312 & Blast Fumaces and Steel Mills. & 3,371 & 2,567 & 0 & 804 & 7.2 \\
\hline 3313 & Electrometalurgical Products. & • & 0 & 0 & • & 12.4 \\
\hline 3321 & Gray and Ductile Iron Foundries . & w & w & 0 & • & 26.1 \\
\hline 3331 & Primary Copper $\ldots \ldots \ldots \ldots \ldots \ldots \ldots \ldots \ldots$ & 0 & 0 & 0 & 0 & $\mathrm{NF}$ \\
\hline 3334 & Primary Aluminum & 0 & 0 & 0 & 0 & NF \\
\hline 3339 & Primary Nonferrous Metals, nec.... & 0 & 0 & 0 & 0 & NF \\
\hline 3353 & Aluminum Sheet, Plate, and Foil . . . . . . . & 0 & 0 & 0 & 0 & NF \\
\hline 34 & Fabricated Metal Products . . . . . . . & $\mathbf{Q}$ & $\mathbf{Q}$ & 0 & • & NF \\
\hline 35 & Industrial Machinery and Equipment & w & w & 0 & $w$ & 18.3 \\
\hline 357 & Computer and Office Equipment & 0 & 0 & 0 & 0 & NF \\
\hline 36 & Electronic and Other Electric Equipment & $\mathbf{Q}$ & $\mathbf{Q}$ & 0 & 0 & NF \\
\hline 37 & Transportation Equipment & w & $\mathbf{w}$ & w & w & 13.9 \\
\hline 3711 & Motor Vehicles and Car Bodies . . . . . & w & - & $\mathbf{w}$ & 0 & 11.3 \\
\hline 3714 & Motor Vehicle Parts and Accessories $\ldots \ldots \ldots \ldots \ldots$ & w & w & 0 & 0 & 17.7 \\
\hline 38 & Instruments and Related Products ...... & 0 & 0 & 0 & 0 & NF \\
\hline 3841 & Surgical and Medical Instruments ... & 0 & 0 & 0 & 0 & NF \\
\hline 39 & Misc. Manufacturing Industries ... & 0 & 0 & 0 & 0 & NF \\
\hline & 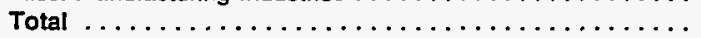 & 15,097 & 12,993 & 706 & 1,398 & 4.9 \\
\hline
\end{tabular}

See footnotes at end of table. 
Table A17. Components of Onsite Electricity Generation by Census Region, Industry Group, and Selected Industries, 1991 (Continued) (Estimates in Million Kilowatthours)

\begin{tabular}{|c|c|c|c|c|c|c|}
\hline $\begin{array}{c}\text { SIC } \\
\text { Code }^{a}\end{array}$ & $\begin{array}{l}\text { Industry Groups } \\
\text { and Industry }\end{array}$ & Total & Cogeneration & Renewables & Other & $\begin{array}{c}\text { RSE } \\
\text { Row } \\
\text { Factors }\end{array}$ \\
\hline & & \multicolumn{4}{|c|}{ South Census Region } & \\
\hline & RSE Column Factors: & 0.8 & 0.8 & 1.8 & 1.0 & \\
\hline 20 & Food and KIndred Products $\ldots \ldots \ldots \ldots \ldots \ldots$ & 1,365 & 1,283 & 0 & 83 & 28.2 \\
\hline 2011 & 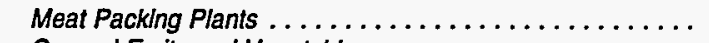 & 0 & 0 & 0 & 0 & NF \\
\hline 2033 & Canned Fruits and Vegetables . . . . . . . . . . . . . & w & w & 0 & 0 & 37.2 \\
\hline 2037 & Frozen Fruits and Vegetables $\ldots \ldots \ldots \ldots \ldots \ldots \ldots$ & 63 & 63 & $\mathbf{0}$ & 0 & 33.2 \\
\hline 2046 & 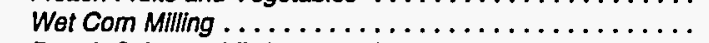 & W & W & $\mathbf{0}$ & 0 & 22.6 \\
\hline 2051 & Bread, Cake, and Related Products ............... & 3 & 3 & 0 & $\cdot$ & 45.7 \\
\hline 2063 & Beet Sugar $\ldots \ldots \ldots \ldots \ldots \ldots \ldots \ldots \ldots \ldots \ldots$ & w & w & 0 & 0 & 21.2 \\
\hline 2075 & 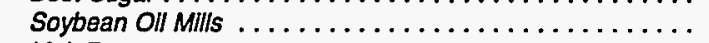 & W & w & 0 & 0 & 8.0 \\
\hline 2082 & 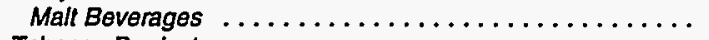 & W & w & 0 & 0 & 21.2 \\
\hline 21 & Tobacco Products $\ldots \ldots \ldots \ldots \ldots \ldots \ldots \ldots \ldots \ldots$ & W & w & 0 & 0 & 8.0 \\
\hline 22 & Textile Mill Products $\ldots \ldots \ldots \ldots \ldots \ldots \ldots \ldots \ldots$ & $\mathbf{w}$ & w & w & w & 19.7 \\
\hline 23 & Apparel and Other Textlle Products $\ldots \ldots \ldots \ldots \ldots \ldots$ & 0 & 0 & 0 & 0 & NF \\
\hline 24 & Lumber and Wood Products & 33 & 33 & 0 & • & 63.7 \\
\hline 25 & Fumiture and Flxtures $\ldots \ldots \ldots \ldots \ldots \ldots \ldots \ldots$ & 12 & 12 & 0 & 0 & 51.8 \\
\hline 26 & Paper and Allied Products $\ldots \ldots \ldots \ldots \ldots \ldots \ldots \ldots$ & 32,331 & 27,558 & w & w & 4.6 \\
\hline 2611 & Pulp Mills $\ldots \ldots \ldots \ldots \ldots \ldots \ldots \ldots \ldots \ldots \ldots$ & 4,319 & w & 0 & $w$ & 18.2 \\
\hline 2621 & 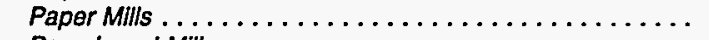 & 14,728 & 13,624 & $w$ & w & 3.6 \\
\hline 2631 & 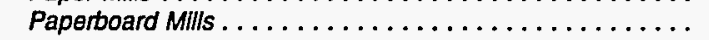 & 13,283 & W & $\ddot{w}$ & 2,787 & 6.6 \\
\hline 27 & Printing and Publishing $\ldots \ldots \ldots \ldots \ldots \ldots \ldots \ldots$ & 0 & 0 & 0 & 0 & NF \\
\hline 28 & Chemicals and Allied Products ................... & 37,577 & 34,780 & $\vec{Q}$ & 2,796 & 8.4 \\
\hline 2812 & Alkalles and Chlorine $\ldots \ldots \ldots \ldots$. & w & w & 0 & w & 22.3 \\
\hline 2813 & Industrial Gases $\ldots \ldots \ldots \ldots \ldots \ldots \ldots \ldots \ldots \ldots$ & w & w & 0 & 0 & 25.2 \\
\hline 2819 & Industrial Inorganic Chemicals, nec $\ldots \ldots \ldots \ldots \ldots \ldots$ & w & 1,918 & 0 & $w$ & 12.9 \\
\hline 2821 & Plastics Materials and Resins $\ldots \ldots \ldots \ldots \ldots$ & 2,409 & w & 0 & w & 9.2 \\
\hline 2822 & Synthetlc Rubber $\ldots \ldots \ldots \ldots \ldots \ldots \ldots \ldots \ldots \ldots \ldots$ & w & $\mathbf{w}$ & 0 & 0 & 25.2 \\
\hline 2823 & Cellulosic Manmade Fibers $\ldots \ldots \ldots \ldots \ldots \ldots \ldots \ldots$ & 961 & 961 & 0 & 0 & 25.2 \\
\hline 2824 & Organic Fibers, Noncellulosic $\ldots \ldots \ldots \ldots \ldots \ldots \ldots$ & 626 & 626 & 0 & 0 & 4.0 \\
\hline 2865 & Cycllc Crudes and Intermedlates $\ldots \ldots \ldots \ldots \ldots \ldots$ & w & w & 0 & w & 24.6 \\
\hline 2869 & Industrial Organic Chemicals, nec .... & 20,836 & W & $\mathbf{Q}$ & W & 10.0 \\
\hline 2873 & Nitrogenous Fortilizers $\ldots \ldots \ldots \ldots \ldots \ldots \ldots$ & 233 & 233 & 0 & 0 & 59.7 \\
\hline 2874 & Phosphatlc Fertilizers $\ldots \ldots \ldots \ldots \ldots \ldots \ldots$ & w & w & 0 & 0 & 2.7 \\
\hline 29 & Petroleum and Coal Products $\ldots \ldots \ldots \ldots \ldots \ldots \ldots$ & 8,781 & 8,580 & 0 & 201 & 4.0 \\
\hline 2911 & Petroleum Refining $\ldots \ldots \ldots \ldots \ldots \ldots \ldots \ldots \ldots$ & 8,780 & 8,580 & 0 & 199 & 4.0 \\
\hline 30 & Rubber and Mlsc. Plastics Products $\ldots \ldots \ldots \ldots \ldots \ldots$ & - & 0 & 0 & " & NF \\
\hline 3011 & Tres and Inner Tubes $\ldots \ldots \ldots \ldots \ldots \ldots \ldots \ldots$ & * & 0 & 0 & - & 8.1 \\
\hline 308 & Miscellaneous Plastics Products, nec . . . . . . . . . . & * & 0 & 0 & - & NF \\
\hline 31 & Leather and Leather Products . . . . . . . & Q & $\mathbf{Q}$ & 0 & 0 & NF \\
\hline 32 & Stone, Clay and Glass Products $\ldots . .$. & w & w & 0 & • & 24.3 \\
\hline 3211 & Flat Glass $\ldots \ldots \ldots \ldots \ldots \ldots \ldots \ldots \ldots \ldots$ & 0 & 0 & 0 & 0 & NF \\
\hline 3221 & Glass Containers $\ldots \ldots \ldots \ldots \ldots \ldots \ldots \ldots \ldots$ & 0 & 0 & 0 & 0 & NF \\
\hline 3229 & Pressed and Blown Glass, nec $\ldots \ldots \ldots \ldots \ldots \ldots$ & • & 0 & 0 & • & 11.5 \\
\hline 3241 & Cement, Hydraulic ........... & 0 & 0 & 0 & 0 & NF \\
\hline 3274 & $\operatorname{LIm} \boldsymbol{L}_{\ldots \ldots \ldots \ldots \ldots \ldots \ldots \ldots \ldots \ldots}$ & 0 & 0 & 0 & 0 & NF \\
\hline 3296 & Mineral Wool $\ldots \ldots \ldots \ldots \ldots$. & 0 & 0 & 0 & 0 & NF \\
\hline 33 & Primary Metal Industries & 2,551 & 1,673 & w & w & 8.9 \\
\hline 3312 & Blast Fumaces and Steel Mills $\ldots \ldots \ldots \ldots \ldots \ldots \ldots$ & 1,785 & w & 0 & w & 9.6 \\
\hline 3313 & Electrometalurgical Products ........ & w & W & w & 0 & 14.0 \\
\hline 3321 & Gray and Ductlle Iron Foundries ...... & 0 & 0 & 0 & 0 & NF \\
\hline 3331 & Primary Copper $\ldots \ldots \ldots \ldots \ldots \ldots \ldots \ldots \ldots \ldots$ & w & w & 0 & 0 & 1.3 \\
\hline 3334 & Primary Aluminum & 0 & 0 & $\mathbf{0}$ & 0 & NF \\
\hline 3339 & Primany Nonferrous Metals, nec...... & 0 & 0 & 0 & 0 & NF \\
\hline 3353 & Aluminum Sheet, Plate, and Foll $\ldots \ldots \ldots \ldots \ldots \ldots \ldots$ & 0 & 0 & 0 & 0 & NF \\
\hline 34 & Fabricated Metal Products $\ldots \ldots \ldots \ldots \ldots \ldots \ldots \ldots \ldots$ & 0 & 0 & 0 & 0 & NF \\
\hline 35 & Industrial Machinery and Equipment $\ldots \ldots \ldots \ldots \ldots \ldots$ & 17 & 13 & 0 & 4 & 39.9 \\
\hline 357 & Computer and Office Equipment $\ldots \ldots$ & 17 & 13 & 0 & 4 & 39.9 \\
\hline 36 & Electronic and Other Electric Equipment $\ldots \ldots \ldots \ldots \ldots \ldots$ & • & 0 & 0 & • & 26.5 \\
\hline 37 & Transportation Equipment & 1 & 1 & 0 & • & 20.2 \\
\hline 3711 & Motor Vehlcles and Car Bodies ..... & • & - & 0 & 0 & 13.3 \\
\hline 3714 & Motor Vehicle Parts and Accessories . . . . . . . . . . . . & 0 & 0 & 0 & 0 & NF \\
\hline 38 & Instruments and Related Products $\ldots \ldots \ldots \ldots \ldots \ldots \ldots$ & 0 & 0 & 0 & 0 & NF \\
\hline 3841 & Surgical and Medical Instruments $\ldots \ldots \ldots \ldots \ldots \ldots$ & 0 & 0 & 0 & 0 & NF \\
\hline 39 & Misc. Manufacturing Industries $\ldots \ldots \ldots \ldots \ldots \ldots \ldots$ & 0 & 0 & 0 & 0 & NF \\
\hline & Total .. & 83,821 & 75,037 & w & w & 4.8 \\
\hline
\end{tabular}

See footnotes at end of table. 
Table A17. Components of Onsite Electricity Generation by Census Region, Industry Group, and Selected Industries, 1991 (Continued) (Estimates in Million Kilowatthours)

\begin{tabular}{|c|c|c|c|c|c|c|}
\hline \multirow[t]{3}{*}{$\begin{array}{l}\text { SiC } \\
\text { Code }^{n}\end{array}$} & $\begin{array}{l}\text { Industry Groups } \\
\text { and Industry }\end{array}$ & Total & Cogeneration & Renewables & Other & \multirow[t]{3}{*}{$\begin{array}{c}\text { RSE } \\
\text { Row } \\
\text { Factors }\end{array}$} \\
\hline & & \multicolumn{4}{|c|}{ West Census Region } & \\
\hline & RSE Column Factors: & 0.8 & 0.8 & 1.4 & 1.1 & \\
\hline 20 & Food and Kindred Products & 1,485 & 1,417 & 6 & 62 & 16.0 \\
\hline 2011 & . $\ldots \ldots \ldots \ldots \ldots \ldots \ldots$, & 0 & 0 & 0 & 0 & NF \\
\hline 2033 & Canned Fruits and Vegetables . . . . . . . . . . . . & W & 0 & 0 & w & 31.7 \\
\hline 2037 & Frozen Fruits and Vegetables ... & 71 & 71 & 0 & 0 & 44.5 \\
\hline 2046 & Wet Com Milling $\ldots \ldots \ldots \ldots \ldots \ldots \ldots$ & w & $\mathbf{w}$ & 0 & 0 & 28.4 \\
\hline 2051 & Bread, Cake, and Related Products . & w & w & $\mathbf{0}$ & 0 & 40.8 \\
\hline 2063 & Beet Sugar $\ldots \ldots \ldots \ldots \ldots \ldots \ldots \ldots \ldots \ldots$ & 258 & w & 0 & w & 9.1 \\
\hline 2075 & Soybean Oil Mills & 0 & 0 & 0 & 0 & NF \\
\hline 2082 & Malt Beverages. & W & W & 0 & 0 & 28.4 \\
\hline 21 & Tobacco Products & 0 & 0 & 0 & 0 & NF \\
\hline 22 & Textile Mill Products & 0 & 0 & 0 & 0 & NF \\
\hline 23 & Apparel and Other Textile Products & NA & NA & NA & NA & NF \\
\hline 24 & Lumber and Wood Products ..... & 2,818 & 2,539 & $\mathbf{Q}$ & * & 40.4 \\
\hline 25 & Fumiture and Fixtures $\ldots \ldots \ldots \ldots \ldots \ldots$ & 0 & 0 & 0 & 0 & NF \\
\hline 26 & Paper and Allied Products $\ldots \ldots \ldots \ldots \ldots \ldots \ldots$ & 6,035 & 5,547 & w & w & 8.4 \\
\hline 2611 & Pulp Mills $\ldots \ldots \ldots \ldots \ldots \ldots \ldots \ldots \ldots$ & 912 & 721 & 0 & 190 & 24.3 \\
\hline 2621 & $\ldots \ldots \ldots \ldots$ & 3,082 & w & w & 0 & 7.2 \\
\hline 2631 & Paperboard Mills . & 2,037 & w & $\mathbf{0}$ & w & 9.3 \\
\hline 27 & Printing and Publishing & NA & NA & NA & NA & NF \\
\hline 28 & Chemicals and Allied Products $\ldots \ldots \ldots \ldots \ldots \ldots \ldots$ & 1,994 & w & 0 & w & 12.2 \\
\hline 2812 & Alkalies and Chlorine $\ldots \ldots \ldots \ldots \ldots \ldots \ldots \ldots$ & 0 & 0 & 0 & 0 & NF \\
\hline 2813 & $\ldots \ldots \ldots \ldots \ldots \ldots$ & w & w & 0 & w & 21.2 \\
\hline 2819 & Industrial Inorganic Chemicals, nec $\ldots \ldots \ldots \ldots$ & W & w & $\mathbf{0}$ & w & 18.5 \\
\hline 2821 & Plastics Materials and Resins $\ldots \ldots \ldots \ldots \ldots$ & * & 0 & 0 & - & 14.8 \\
\hline 2822 & Synthetic Rubber ............ & 0 & 0 & 0 & 0 & NF \\
\hline 2823 & Cellulosic Manmade Fibers. & 0 & 0 & 0 & 0 & NF \\
\hline 2824 & Organic Fibers, Noncellulosic & 0 & 0 & 0 & 0 & NF \\
\hline 2865 & Cyclic Crudes and Intermediates .... & 0 & 0 & 0 & 0 & NF \\
\hline 2869 & Industrial Organic Chemicals, nec .... & w & w & 0 & 0 & 17.3 \\
\hline 2873 & Nitrogenous Fertilizers $\ldots \ldots \ldots \ldots \ldots \ldots$ & 156 & 156 & 0 & 0 & 44.5 \\
\hline 2874 & Phosphatic Fentilizers & w & w & 0 & 0 & 3.7 \\
\hline 29 & Petroleum and Coal Products & 2,874 & W & 0 & w & 5.9 \\
\hline 2911 & Petroleum Refining $\ldots \ldots \ldots \ldots \ldots$ & 2,423 & W & 0 & W & 4.2 \\
\hline 30 & Rubber and Misc. Plastics Products . . . & w & W & 0 & 0 & 28.4 \\
\hline 3011 & 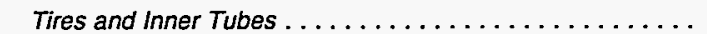 & 0 & 0 & 0 & 0 & $\mathrm{NF}$ \\
\hline 308 & Miscellaneous Plastics Products, nec. & - & . & 0 & 0 & NF \\
\hline 31 & Leather and Leather Products . . . . . . & 0 & $\mathbf{0}$ & 0 & 0 & NF \\
\hline 32 & Stone, Clay and Glass Products & W & w & 0 & Q & 29.0 \\
\hline 3211 & Flat Glass & w & 0 & 0 & w & 6.3 \\
\hline 3221 & Glass Containers & 0 & 0 & 0 & 0 & NF \\
\hline 3229 & Pressed and Blown Glass, nec ........ & 0 & 0 & 0 & 0 & NF \\
\hline 3241 & Cement, Hydraulic $\ldots \ldots \ldots \ldots \ldots \ldots \ldots$ & W & w & 0 & 0 & 32.1 \\
\hline 3274 & 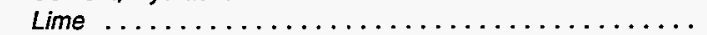 & 0 & 0 & 0 & 0 & NF \\
\hline 3296 & Mineral Wool & 0 & 0 & 0 & 0 & NF \\
\hline 33 & Primary Metal Industries $\ldots \ldots \ldots \ldots$ & w & w & 0 & w & 8.9 \\
\hline 3312 & Blast Fumaces and Steel Mills ... & W & w & 0 & 0 & 16.1 \\
\hline 3313 & Electrometalurgical Products .. & 0 & 0 & 0 & 0 & NF \\
\hline 3321 & Gray and Ductile Iron Foundries $\ldots \ldots \ldots \ldots \ldots \ldots$ & 0 & 0 & 0 & 0 & NF \\
\hline 3331 & Primary Copper $\ldots \ldots \ldots \ldots \ldots \ldots \ldots \ldots \ldots \ldots$ & W & W & 0 & w & 1.1 \\
\hline 3334 & Primary Aluminum $\ldots \ldots \ldots \ldots \ldots \ldots \ldots \ldots \ldots$ & 0 & 0 & 0 & 0 & NF \\
\hline 3339 & Primary Nonferrous Metals, nec . . . . . . . . . . . . & w & w & 0 & 0 & 1.2 \\
\hline 3353 & Aluminum Sheet, Plate, and Foil & 0 & 0 & 0 & 0 & NF \\
\hline 34 & Fabricated Metal Products . . . . . . & $\mathbf{Q}$ & $\mathbf{Q}$ & 0 & - & 29.8 \\
\hline 35 & Industrial Machinery and Equipment .... & $\bar{W}$ & $\overline{0}$ & 0 & w & 32.8 \\
\hline 357 & Computer and Office Equipment $\ldots \ldots \ldots \ldots \ldots \ldots$ & 0 & 0 & 0 & 0 & NF \\
\hline 36 & Electronic and Other Electric Equipment $\ldots \ldots \ldots \ldots \ldots \ldots$ & * & * & - & * & 33.1 \\
\hline
\end{tabular}

See footnotes at end of table. 


\section{Table A17. Components of Onsite Electricity Generation by Census Region, Industry Group, and Selected Industries, 1991 (Continued) \\ (Estimates in Million Kilowatthours)}

\begin{tabular}{|c|c|c|c|c|c|c|}
\hline $\begin{array}{c}\text { SIC } \\
\text { Codo" }\end{array}$ & $\begin{array}{l}\text { Industry Groups } \\
\text { and Industry }\end{array}$ & Total & Cogeneration & Renewables & Other & $\begin{array}{c}\text { RSE } \\
\text { Row } \\
\text { Factors }\end{array}$ \\
\hline & \multirow[b]{2}{*}{ RSE Column Factors: } & \multicolumn{4}{|c|}{ West Census Region } & \\
\hline & & 0.8 & 0.8 & 1.4 & 1.1 & \\
\hline 37 & Transportation Equipment & w & w & 0 & 1 & 17.1 \\
\hline 3711 & Motor Vehicles and Car Bodles . . . . . . . . . . . . . . & 0 & 0 & 0 & 0 & $\mathrm{NF}$ \\
\hline 3714 & Motor Vehicle Parts and Accessories . . . . . . . . . . . & 0 & 0 & 0 & 0 & NF \\
\hline & Instruments and Related Products $\ldots \ldots \ldots \ldots \ldots \ldots \ldots$ & w & w & 0 & 0 & 34.6 \\
\hline 3841 & Surglcal and Medical Instruments $\ldots \ldots \ldots \ldots \ldots \ldots$ & 0 & 0 & 0 & 0 & NF \\
\hline 39 & Misc. Manufacturing Industries $\ldots \ldots \ldots \ldots \ldots \ldots \ldots$ & 0 & 0 & 0 & 0 & NF \\
\hline & Total $\ldots \ldots \ldots \ldots \ldots \ldots \ldots \ldots \ldots \ldots \ldots \ldots$ & 16,518 & 14,969 & $\mathbf{w}$ & w & 12.1 \\
\hline
\end{tabular}

See Appendices B and F for descriptions of the Standard Industrial Classification system.

- Other" is that electricity obtained from a generator fueled by combustible energy sources such as diesel or other fuel oils.

$\mathrm{NF}=$ No applicable RSE row/column factor.

- Estimate less than 0.5. Data are included in higher level totals.

$W=$ Withheld to avold disclosing data for individual establishments. Data are included in higher level totals.

$\mathrm{Q}=$ Withheld because Relative Standard Error is greater than 50 percent. Data are included in higher level totals.

$N A=$ Not available. Data are included in higher level totals.

Notes: - To obtain a RSE percentage for any table cell, multiply the cell's corresponding RSE column and RSE row factors. - Totals may not equal sum of components because of independent rounding.

Source: Energy Information Administration, Office of Energy Markets and End Use, Energy End Use and Integrated Statistics Division, Form ElA-846, "1991 Manufacturing Energy Consumption Survey." 
Table A18. Quantity of Electricity Sold to Utility and Nonutility Purchasers by Census
Region, Industry Group, and Selected Industries, 1991 (Estimates in Million Kilowatthours)

\begin{tabular}{|c|c|c|c|c|c|}
\hline $\begin{array}{c}\text { SIC } \\
\text { Code }^{a}\end{array}$ & $\begin{array}{c}\text { Industry Groups } \\
\text { and Industry }\end{array}$ & Total Sold & Utility Purchaser & Nonutility Purchaser ${ }^{2}$ & $\begin{array}{c}\text { RSE } \\
\text { Row } \\
\text { Factors }\end{array}$ \\
\hline & \multirow[b]{2}{*}{ RSE Column Factors: } & \multicolumn{3}{|c|}{ Total United States } & \\
\hline & & 0.9 & 1.0 & 1.0 & \\
\hline 20 & Food and Kindred Products . . . . . . . & 988 & 940 & 48 & 16.2 \\
\hline 2011 & Meat Packing Plants . . .......... & 0 & 0 & 0 & NF \\
\hline 2033 & Canned Fruits and Vegetables . ................ & $w$ & w & w & 26.8 \\
\hline 2037 & Frozen Fruits and Vegetables ......... & 27 & 27 & 0 & 37.6 \\
\hline 2046 & Wet Com Milling ............... & w & w & 0 & 21.3 \\
\hline 2051 & Bread, Cake, and Related Products. & $\cdot$ & 0 & 0 & 26.8 \\
\hline 2063 & Beet Sugar.................. & w & w & $w$ & 14.5 \\
\hline 2075 & Soybean Oil Mills ................ & $\ddot{w}$ & W & 0 & 7.1 \\
\hline 2082 & Malt Beverages ................. & w & w & w & 20.8 \\
\hline 21 & 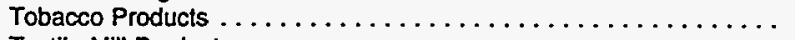 & w & w & $w$ & 7.3 \\
\hline 22 & Textile Mill Products $\ldots \ldots \ldots \ldots \ldots \ldots \ldots \ldots \ldots \ldots \ldots$ & $\mathbf{Q}$ & $\mathbf{Q}$ & w & 20.4 \\
\hline 23 & Apparel and Other Textile Products ... & 0 & 0 & 0 & NF \\
\hline 24 & Lumber and Wood Products $\ldots \ldots \ldots \ldots \ldots \ldots \ldots$ & 1,976 & 1,733 & $\mathbf{Q}$ & 44.6 \\
\hline 25 & Fumiture and Fixtures $\ldots \ldots \ldots \ldots \ldots \ldots \ldots \ldots$ & 1 & 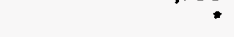 & 1 & 41.3 \\
\hline 26 & Paper and Allied Products . . . . . . . & 9,012 & 8,254 & 759 & 3.6 \\
\hline 2611 & 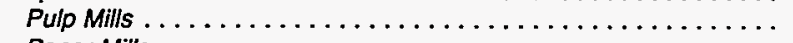 & 462 & 339 & 123 & 24.2 \\
\hline 2621 & 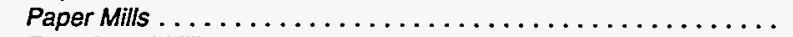 & 6,104 & 5,755 & 349 & 3.0 \\
\hline 2631 & 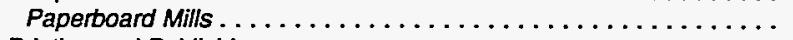 & 2,378 & 2,110 & 268 & 6.6 \\
\hline 27 & 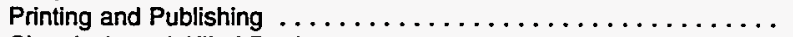 & 0 & 0 & 0 & NF \\
\hline 28 & Chemicals and Allied Products $\ldots \ldots \ldots \ldots \ldots \ldots \ldots$ & 9,967 & 7,269 & 2,698 & 7.2 \\
\hline 2812 & 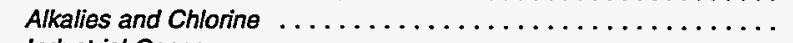 & w & w & 0 & 23.4 \\
\hline 2813 & Industrial Gases $\ldots \ldots \ldots \ldots \ldots \ldots \ldots \ldots \ldots \ldots \ldots \ldots \ldots$ & w & w & w & 19.0 \\
\hline 2819 & Industrial Inorganic Chemicals, nec ................. & w & w & w & 15.1 \\
\hline 2821 & 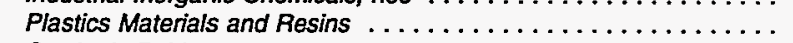 & 247 & $\ddot{w}$ & w & 8.5 \\
\hline 2822 & Synthetic Rubber $\ldots \ldots \ldots \ldots \ldots \ldots \ldots \ldots \ldots$ & w & 0 & w & 22.4 \\
\hline 2823 & Cellulosic Manmade Fibers ...................... & w & 18 & w & 34.0 \\
\hline 2824 & Organic Fibers, Noncellulosic . ......... & 0 & 0 & 0 & NF \\
\hline 2865 & Cyclic Crudes and Intermediates $\ldots \ldots \ldots \ldots \ldots \ldots \ldots \ldots$ & 9 & w & $w$ & 20.2 \\
\hline 2869 & Industral Organic Chemicals, nec $\ldots \ldots \ldots \ldots \ldots \ldots \ldots \ldots$ & 5,040 & 3,358 & 1,682 & 7.9 \\
\hline 2873 & Nitrogenous Fertilizers $\ldots \ldots \ldots \ldots \ldots \ldots \ldots \ldots$ & 7 & 7 & 0 & 49.8 \\
\hline 2874 & Phosphatic Fertilizers . .................... & 533 & w & w & 3.3 \\
\hline 29 & Petroleum and Coal Products & 2,698 & 1,276 & 1,422 & 5.9 \\
\hline 2911 & Petroleum Refining . . . . . & 2,410 & 1,029 & 1,381 & 4.3 \\
\hline 30 & Rubber and Misc. Plastics Products ... & 6 & 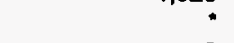 & 6 & 33.8 \\
\hline 3011 & 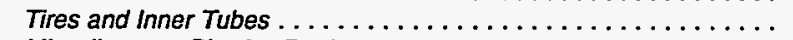 & 0 & 0 & 0 & NF \\
\hline 308 & Miscellaneous Plastics Products, nec . . . . . . . . . . . . & $\mathrm{Q}$ & 0 & $\mathbf{Q}$ & NF \\
\hline 31 & Leather and Leather Products ............. & • & * & 0 & 45.7 \\
\hline 32 & Stone, Clay and Glass Products $\ldots \ldots \ldots \ldots \ldots \ldots$ & 71 & w & $w$ & 12.7 \\
\hline 3211 & 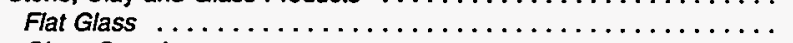 & $w$ & 0 & w & 6.1 \\
\hline 3221 & Glass Containers ... & 0 & 0 & 0 & NF \\
\hline 3229 & Pressed and Blown Glass, nec .............. & • & • & 0 & 13.2 \\
\hline 3241 & Cement, Hydraulic $\ldots \ldots \ldots \ldots \ldots \ldots \ldots \ldots$ & w & w & $\mathbf{Q}$ & 21.8 \\
\hline 3274 & 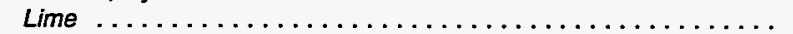 & 0 & $\mathbf{0}$ & 0 & $\mathrm{NF}$ \\
\hline 3296 & Mineral Wool ....... & 0 & 0 & 0 & NF \\
\hline 33 & Primary Metal Industries & 1,949 & 952 & 998 & 4.6 \\
\hline 3312 & Blast Fumaces and Steel Mills ... & 1,297 & 490 & 807 & 5.9 \\
\hline 3313 & Electrometalurgical Products . . . . . . . . & $w$ & 0 & w & 13.3 \\
\hline 3321 & Gray and Ductile Iron Foundries $\ldots \ldots \ldots \ldots \ldots$ & w & 0 & $\ddot{w}$ & 22.4 \\
\hline 3331 & 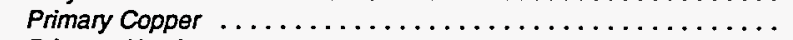 & 0 & 0 & 0 & NF \\
\hline 3334 & Primary Aluminum & w & w & 0 & 6.1 \\
\hline 3339 & Primary Nonferrous Metals, nec... & w & w & w & 1.0 \\
\hline 3353 & Aluminum Sheet, Plate, and Foil .. & 0 & 0 & 0 & NF \\
\hline 34 & Fabricated Metal Products . . . . . . . . . & w & 0 & w & 31.6 \\
\hline 35 & Industrial Machinery and Equipment ........... & 176 & 9 & 167 & 27.8 \\
\hline 357 & Computer and Office Equipment ...... & 9 & 9 & 0 & 33.5 \\
\hline 36 & Electronic and Other Electric Equipment .... & $\mathbf{Q}$ & 1 & Q & 32.7 \\
\hline 37 & Transportation Equipment . & 761 & w & w & 7.7 \\
\hline 3711 & Motor Vehicles and Car Bodies & w & $w$ & $\ddot{w}$ & 7.9 \\
\hline 3714 & Motor Vehicle Parts and Accessories . . . . . . . . . & w & w & w & 12.8 \\
\hline 38 & Instruments and Related Products $\ldots \ldots \ldots \ldots \ldots \ldots \ldots \ldots$ & w & w & $\cdot$ & 27.5 \\
\hline 3841 & Surgical and Medical Instruments $\ldots \ldots \ldots \ldots \ldots \ldots \ldots$ & 0 & 0 & 0 & NF \\
\hline 39 & Misc. Manufacturing Industries ... & 0 & 0 & 0 & NF \\
\hline & Total .. & 28,222 & 21,063 & 7,159 & 4.9 \\
\hline
\end{tabular}

See footnotes at end of table. 


\section{Table A18. Quantity of Electricity Sold to Utility and Nonutility Purchasers by Census Region, Industry Group, and Selected Industries, 1991 (Continued) (Estimates in Million Kilowatthours)}

\begin{tabular}{|c|c|c|c|c|c|}
\hline $\begin{array}{l}\text { SIC } \\
\text { Code" }\end{array}$ & $\begin{array}{c}\text { Industry Groups } \\
\text { and Industry }\end{array}$ & Total Sold & Utility Purchaser & Nonutility Purchaser & \multirow[t]{3}{*}{$\begin{array}{c}\text { RSE } \\
\text { Row } \\
\text { Factors }\end{array}$} \\
\hline & \multirow[b]{2}{*}{ RSE Column Factors: } & \multicolumn{3}{|c|}{ Northeast Census Region } & \\
\hline & & 0.9 & 1.0 & 1.1 & \\
\hline 20 & Food and Kindred Products $\ldots \ldots \ldots \ldots \ldots \ldots \ldots \ldots \ldots$ & 58 & 58 & 0 & 20.9 \\
\hline 2011 & Meat Packing Plants . . . . . . . . . . . . . . . . . & 0 & 0 & 0 & NF \\
\hline 2033 & Canned Fruits and Vegetables . . . . . . . . . . . . . . . . & 0 & \multirow{2}{*}{$\begin{array}{l}0 \\
0\end{array}$} & 0 & NF \\
\hline 2037 & 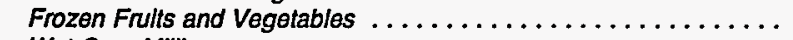 & 0 & & 0 & NF \\
\hline 2046 & 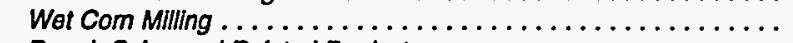 & 0 & 0 & 0 & $N F$ \\
\hline 2051 & Bread, Cake, and Related Products ........... & 0 & 0 & 0 & NF \\
\hline 2063 & 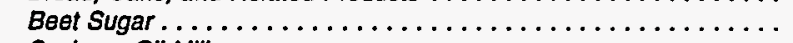 & 0 & \multirow{2}{*}{$\begin{array}{l}0 \\
0\end{array}$} & 0 & NF \\
\hline 2075 & 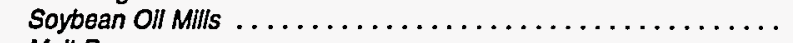 & 0 & & 0 & NF \\
\hline 2082 & Malt Beverages . ............... & w & $w$ & 0 & 24.0 \\
\hline 21 & 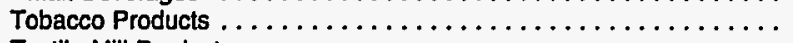 & NA & \multirow{2}{*}{$\begin{array}{r}\text { NA } \\
\mathrm{Q}\end{array}$} & NA & NF \\
\hline 22 & Textile Mill Products $\ldots \ldots \ldots \ldots \ldots \ldots \ldots \ldots \ldots \ldots \ldots$ & $\mathbf{Q}$ & & w & 25.7 \\
\hline 23 & Apparel and Other Textile Products $\ldots \ldots \ldots \ldots \ldots \ldots \ldots \ldots$ & 0 & 0 & 0 & $\mathrm{NF}$ \\
\hline 24 & 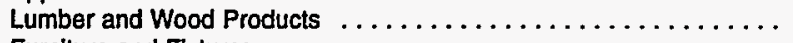 & NA & NA & NA & NF \\
\hline 25 & Fumlture and Fixtures $\ldots \ldots \ldots \ldots \ldots \ldots \ldots \ldots \ldots$ & 0 & 0 & 0 & NF \\
\hline 26 & Paper and Allied Products $\ldots \ldots \ldots \ldots \ldots \ldots \ldots \ldots \ldots$ & 3,088 & 2,961 & 127 & 4.6 \\
\hline 2611 & 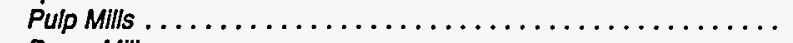 & 15 & 7 & 8 & 36.0 \\
\hline 2621 & $\ldots \ldots \ldots \ldots \cdots \cdots \cdots \cdots \cdots \cdots$ & 2,990 & 2,881 & 109 & 3.9 \\
\hline 2631 & Paperboard MIIls $\ldots \ldots \ldots \ldots \ldots \ldots \ldots \ldots \ldots \ldots \ldots \ldots$ & w & w & 0 & 17.7 \\
\hline 27 & Printing and Publishing $\ldots \ldots \ldots \ldots \ldots \ldots \ldots \ldots \ldots$ & 0 & 0 & 0 & $\mathrm{NF}$ \\
\hline 28 & Chemicals and Allied Products $\ldots \ldots \ldots \ldots$ & 207 & w & $\mathbf{w}$ & 20.9 \\
\hline 2812 & Alkalles and Chlorine ............... & 0 & 0 & 0 & NF \\
\hline 2813 & $\ldots \ldots \ldots \ldots \ldots \ldots \ldots \ldots \ldots \ldots \ldots$, & w & 0 & w & 18.7 \\
\hline 2819 & Industrial Inorganic Chemicals, nec $\ldots \ldots \ldots \ldots \ldots \ldots \ldots \ldots$ & 0 & 0 & 0 & NF \\
\hline 2821 & Plastlcs Materials and Resins $\ldots \ldots \ldots \ldots \ldots \ldots \ldots \ldots \ldots$ & W & - & w & 12.9 \\
\hline 2822 & Synthetic Rubber $\ldots \ldots \ldots \ldots \ldots \ldots \ldots \ldots \ldots \ldots$ & - & 0 & • & $\mathrm{NF}$ \\
\hline 2823 & Cellulosic Manmade Fibers $\ldots \ldots \ldots \ldots \ldots \ldots$ & 0 & 0 & 0 & NF \\
\hline 2824 & Organic Fibers, Noncellulosic . . . . . & 0 & 0 & 0 & NF \\
\hline 2865 & Cycllc Crudes and Intermediates .... & 0 & 0 & 0 & NF \\
\hline 2869 & Industrial Organic Chemicals, nec $\ldots \ldots \ldots \ldots \ldots \ldots \ldots \ldots$ & w & w & 0 & 13.6 \\
\hline 2873 & Nitrogenous Fertilizers $\ldots \ldots \ldots \ldots \ldots \ldots \ldots \ldots \ldots \ldots$ & 0 & 0 & 0 & NF \\
\hline 2874 & Phosphatlc Fertillzers .. & 0 & 0 & 0 & NF \\
\hline 29 & Petroleum and Coal Products $\ldots \ldots \ldots \ldots$ & W & W & w & 5.6 \\
\hline 2911 & Petroleum Refining . . . . . . . . . . . & w & w & W & 5.6 \\
\hline 30 & Rubber and Misc. Plastics Products .. & 6 & : & 5 & 36.0 \\
\hline 3011 & Tlres and Inner Tubes $\ldots \ldots \ldots \ldots \ldots \ldots \ldots \ldots \ldots \ldots$ & 0 & 0 & 0 & NF \\
\hline 308 & Miscellaneous Plastics Products, nec ........ & $\mathbf{Q}$ & 0 & $\mathbf{Q}$ & NF \\
\hline 31 & Leather and Leather Products $\ldots \ldots \ldots \ldots \ldots \ldots \ldots \ldots \ldots$ & . & * & 0 & 46.9 \\
\hline 32 & Stone, Clay and Glass Products $\ldots \ldots \ldots \ldots \ldots \ldots \ldots \ldots$ & w & w & w & 28.7 \\
\hline 3211 & 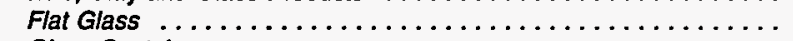 & 0 & 0 & 0 & NF \\
\hline 3221 & Glass Containers $\ldots \ldots \ldots \ldots \ldots \ldots$ & 0 & 0 & 0 & NF \\
\hline 3229 & Pressed and Blown Glass, nec ..... & 0 & 0 & 0 & NF \\
\hline 3241 & Cement, Hydraulle $\ldots \ldots \ldots \ldots \ldots \ldots \ldots \ldots \ldots \ldots \ldots \ldots$ & 0 & 0 & 0 & NF \\
\hline 3274 & 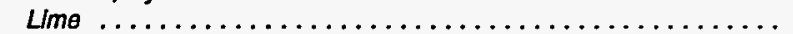 & 0 & 0 & 0 & NF \\
\hline 3296 & Mineral wool $\ldots \ldots \ldots \ldots \ldots \ldots \ldots \ldots \ldots \ldots \ldots \ldots$ & 0 & 0 & 0 & NF \\
\hline 33 & Primary Metal Industries $\ldots \ldots \ldots \ldots \ldots \ldots \ldots \ldots \ldots \ldots$ & w & w & w & 7.0 \\
\hline 3312 & Blast Fumaces and Steel Mills $\ldots \ldots \ldots \ldots \ldots \ldots \ldots \ldots$ & w & w & w & 8.0 \\
\hline 3313 & Electrometalurgical Products ..... & 0 & 0 & 0 & NF \\
\hline 3321 & Gray and Ductlle Iron Foundries ....... & 0 & 0 & 0 & NF \\
\hline 3331 & Primary Copper $\ldots \ldots \ldots \ldots \ldots \ldots \ldots \ldots \ldots \ldots \ldots \ldots \ldots$ & 0 & 0 & 0 & NF \\
\hline 3334 & Primary Aluminum $\ldots \ldots \ldots \ldots \ldots \ldots \ldots \ldots \ldots \ldots \ldots$ & 0 & 0 & 0 & NF \\
\hline 3339 & Primary Nonferrous Metals, nec $\ldots \ldots \ldots \ldots \ldots \ldots \ldots \ldots \ldots$ & w & w & 0 & 1.0 \\
\hline 3353 & Aluminum Sheet, Plate, and Foll $\ldots \ldots \ldots \ldots \ldots \ldots \ldots \ldots$ & 0 & 0 & 0 & NF \\
\hline 34 & Fabricated Metal Products $\ldots \ldots \ldots \ldots \ldots \ldots \ldots \ldots \ldots \ldots$ & 0 & 0 & 0 & NF \\
\hline 35 & Industrial Machinery and Equipment $\ldots \ldots \ldots \ldots \ldots \ldots \ldots$ & $\mathbf{Q}$ & 0 & $\mathbf{Q}$ & NF \\
\hline 357 & Computer and Office Equipment .......... & 0 & 0 & 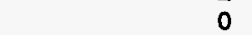 & NF \\
\hline 36 & Electronic and Other Electric Equipment .... & 11 & 0 & 11 & 33.5 \\
\hline 37 & Transportation Equipment & $\mathbf{w}$ & w & 10 & 17.5 \\
\hline 3711 & Motor Vehicles and Car Bodles $\ldots \ldots \ldots \ldots \ldots \ldots \ldots \ldots \ldots$ & 0 & 0 & 0 & NF \\
\hline 3714 & Motor Vehicle Parts and Accessories $\ldots \ldots \ldots \ldots \ldots \ldots \ldots$ & 0 & 0 & 0 & NF \\
\hline 38 & Instruments and Related Products ... & $\mathbf{w}$ & w & - & 30.7 \\
\hline 3841 & Surglcal and Modical Instruments. & 0 & 0 & 0 & $\mathrm{NF}$ \\
\hline 39 & Mlsc. Manufacturing Industries $\ldots \ldots \ldots \ldots \ldots \ldots \ldots \ldots$ & 0 & 0 & 0 & NF \\
\hline & Total & 4,301 & 3,987 & 314 & 3.8 \\
\hline
\end{tabular}

See footnotes at end of table. 
Table A18. Quantity of Electricity Sold to Utility and Nonutility Purchasers by Census Region, Industry Group, and Selected Industries, 1991 (Continued) (Estimates in Million Kilowatthours)

\begin{tabular}{|c|c|c|c|c|c|}
\hline \multirow[t]{3}{*}{$\begin{array}{c}\text { SIC } \\
\text { Code }^{*}\end{array}$} & $\begin{array}{l}\text { Industry Groups } \\
\text { and Industry }\end{array}$ & Total Sold & Utility Purchaser & Nonutility Purchaser & \multirow[t]{3}{*}{$\begin{array}{c}\text { RSE } \\
\text { Row } \\
\text { Factors }\end{array}$} \\
\hline & \multirow[b]{2}{*}{ RSE Column Factors: } & \multicolumn{3}{|c|}{ Midwest Census Region } & \\
\hline & & 1.0 & 1.0 & 1.0 & \\
\hline 20 & Food and Kindred Products $\ldots \ldots \ldots \ldots \ldots \ldots \ldots \ldots$ & 61 & 42 & 19 & 12.8 \\
\hline 2011 & Meat Packing Plants . . . . . . . . . . . . . . . . . . & 0 & 0 & 0 & NF \\
\hline 2033 & Canned Fruits and Vegetables . . . . . . . . . . . . . . . . & 0 & 0 & 0 & NF \\
\hline 2037 & Frozen Fruits and Vegetables $\ldots \ldots \ldots \ldots \ldots \ldots \ldots$ & 0 & 0 & 0 & NF \\
\hline 2046 & 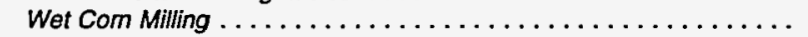 & W & W & 0 & 20.9 \\
\hline 2051 & Bread, Cake, and Related Products . . . . . . . . . . . . . & 0 & 0 & 0 & NF \\
\hline 2063 & Beot Sugar . . . . . . . . . . . . . . . . . . . . & W & W & W & 14.5 \\
\hline 2075 & Soybean Oil Mills . . . . . . . . . . . . . . . . . . & W & $\mathbf{w}$ & 0 & 9.0 \\
\hline 2082 & 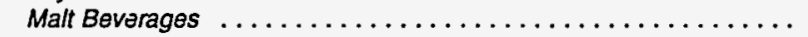 & 0 & 0 & 0 & NF \\
\hline 21 & Tobacco Products $\ldots \ldots \ldots \ldots \ldots \ldots \ldots \ldots \ldots \ldots \ldots$ & NA & NA & NA & NF \\
\hline 22 & Textile Mill Products $\ldots \ldots \ldots \ldots \ldots \ldots \ldots \ldots \ldots \ldots \ldots$ & NA & NA & NA & NF \\
\hline 23 & Apparel and Other Textile Products $\ldots \ldots \ldots \ldots \ldots \ldots \ldots$ & NA & NA & NA & NF \\
\hline 24 & Lumber and Wood Products $\ldots \ldots \ldots \ldots \ldots \ldots \ldots$ & 0 & 0 & 0 & NF \\
\hline 25 & 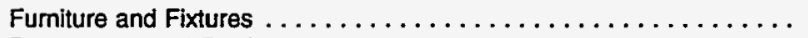 & 1 & * & 1 & 41.1 \\
\hline 26 & Paper and Allied Products $\ldots \ldots \ldots \ldots \ldots \ldots \ldots \ldots$ & 716 & W & $W$ & 10.4 \\
\hline 2611 & Pulp Mills . . . . . . . . . . . . . . . . . . . . & 257 & 257 & 0 & 35.9 \\
\hline 2621 & 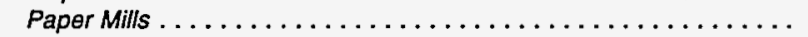 & 438 & W & W & 5.3 \\
\hline 2631 & 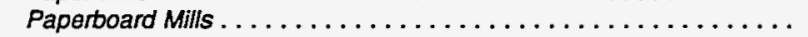 & W & $\cdot$ & W & 16.3 \\
\hline 27 & 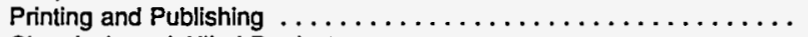 & 0 & 0 & 0 & NF \\
\hline 28 & Chemicals and Allied Products $\ldots \ldots \ldots \ldots \ldots \ldots \ldots$ & 155 & $\mathbf{W}$ & W & 16.0 \\
\hline 2812 & 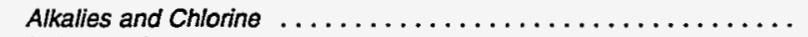 & 0 & 0 & 0 & NF \\
\hline 2813 & 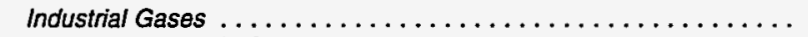 & 0 & 0 & 0 & NF \\
\hline 2819 & Industrial Inorganic Chemicals, noc $\ldots \ldots \ldots \ldots \ldots \ldots \ldots \ldots \ldots \ldots \ldots$ & 0 & 0 & 0 & NF \\
\hline 2821 & Plastics Materials and Resins $\ldots \ldots \ldots \ldots \ldots \ldots \ldots \ldots \ldots \ldots \ldots \ldots \ldots$ & W & $w$ & $W$ & 12.9 \\
\hline 2822 & 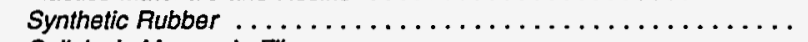 & 0 & 0 & 0 & NF \\
\hline 2823 & Cellulosic Manmado Fibers . . . . . . . . . . . . . . . & 0 & 0 & 0 & NF \\
\hline 2824 & Organic Fibers, Noncellulosic $\ldots \ldots \ldots \ldots \ldots \ldots$ & 0 & 0 & 0 & NF \\
\hline 2865 & Cyclic Crudes and Intermediates . . . . . . . . . . . . . . & 0 & 0 & 0 & NF \\
\hline 2869 & Industrial Organic Chemicals, nec $\ldots \ldots \ldots \ldots \ldots \ldots \ldots \ldots \ldots \ldots \ldots \ldots \ldots$ & W & W & $W$ & 10.4 \\
\hline 2873 & 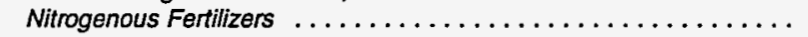 & 0 & 0 & 0 & NF \\
\hline 2874 & Phosphatic Fertilizers . . . . . . . . . . . . . . . & 0 & 0 & 0 & NF \\
\hline 29 & Petroleum and Coal Products $\ldots \ldots \ldots \ldots \ldots \ldots$ & W & $W$ & W & 21.4 \\
\hline 2911 & 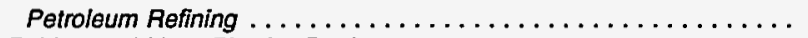 & W & W & W & 5.3 \\
\hline 30 & Rubber and Misc. Plastics Products $\ldots \ldots \ldots \ldots \ldots \ldots$ & 0 & 0 & 0 & NF \\
\hline 3011 & Tires and Inner Tubes . . . . . . . . . . . . . . . . . . & 0 & 0 & 0 & NF \\
\hline 308 & Miscellaneous Plastics Products, nec . . . . . . . . . . . . . & NA & NA & NA & NF \\
\hline 31 & Leather and Leather Products $\ldots \ldots \ldots \ldots \ldots \ldots \ldots$ & $\mathbf{0}$ & 0 & 0 & NF \\
\hline 32 & Stone, Clay and Glass Products $\ldots \ldots \ldots \ldots \ldots \ldots$ & W & W & 0 & 25.9 \\
\hline 3211 & 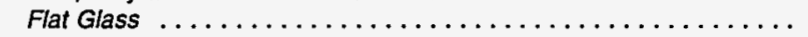 & 0 & 0 & 0 & NF \\
\hline 3221 & 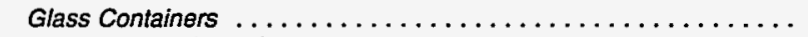 & 0 & 0 & 0 & NF \\
\hline 3229 & Pressed and Blown Glass, nec . . . . . . . . . . . . . & 0 & $\mathbf{0}$ & 0 & NF \\
\hline 3241 & 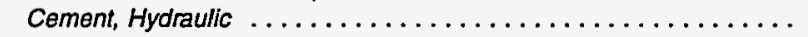 & W & W & 0 & 25.9 \\
\hline 3274 & 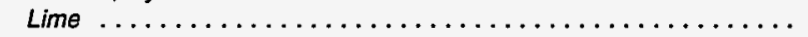 & 0 & 0 & 0 & NF \\
\hline 3296 & 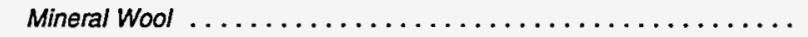 & 0 & 0 & 0 & NF \\
\hline 33 & 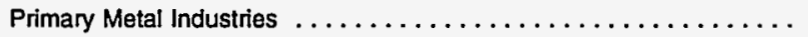 & 776 & 23 & 753 & 8.0 \\
\hline 3312 & Blast Fumaces and Steol Mills . . . . . . . . . . . . . . . & 586 & 22 & 563 & 8.3 \\
\hline 3313 & Electrometalurgical Products . . . . . . . . . . . . . . . . & W & 0 & W & 13.2 \\
\hline 3321 & Gray and Ductile Iron Foundries $\ldots \ldots \ldots \ldots \ldots \ldots$ & $\ddot{w}$ & 0 & $W$ & 22.4 \\
\hline 3331 & Primary Copper . . . . . . . . . . . . . . . . . & 0 & 0 & 0 & NF \\
\hline 3334 & Primary Aluminum $\ldots \ldots \ldots \ldots \ldots \ldots \ldots \ldots \ldots \ldots \ldots \ldots \ldots \ldots \ldots$ & 0 & 0 & 0 & NF \\
\hline 3339 & Primary Nonferrous Metals, nec . . . . . . . . . . . . . . . . & $\mathbf{W}$ & 0 & W & 1.0 \\
\hline 3353 & Aluminum Shest, Plate, and Foll . . . . . . . . . . . . & 0 & 0 & 0 & NF \\
\hline 34 & 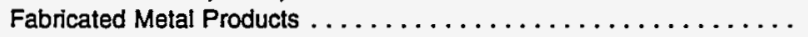 & W & 0 & W & 31.5 \\
\hline 35 & Industrial Machinery and Equipment $\ldots \ldots \ldots \ldots \ldots \ldots$ & W & 0 & W & 27.4 \\
\hline 357 & Computer and Office Equipment $\ldots \ldots \ldots \ldots \ldots \ldots \ldots$ & 0 & 0 & 0 & NF \\
\hline 36 & Electronic and Other Electric Equipment $\ldots \ldots \ldots \ldots \ldots \ldots$ & 7 & 1 & 6 & 33.0 \\
\hline 37 & Transportation Equipment $\ldots \ldots \ldots \ldots \ldots \ldots \ldots$ & $W$ & $\mathbf{W}$ & W & 7.6 \\
\hline 3711 & Motor Vehicles and Car Bodles . . . . . . . . . . . . . . & W & W & W & 7.9 \\
\hline 3714 & Motor Vehicle Parts and Accessories . . . . . . . . . . . . . . & $\mathbf{W}$ & $\mathbf{W}$ & W & 12.8 \\
\hline 38 & Instruments and Related Products $\ldots \ldots \ldots \ldots \ldots \ldots \ldots$ & 0 & 0 & 0 & NF \\
\hline 3841 & Surgical and Medical Instruments $\ldots \ldots \ldots \ldots \ldots \ldots$ & 0 & 0 & 0 & NF \\
\hline 39 & Misc. Manufacturing Industries $\ldots \ldots \ldots \ldots \ldots \ldots \ldots \ldots \ldots \ldots \ldots \ldots \ldots$ & 0 & 0 & 0 & NF \\
\hline & 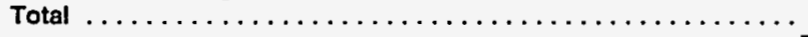 & 2,707 & 749 & 1,958 & 7.3 \\
\hline
\end{tabular}

See footnotes at end of table. 
Table A18. Quantity of Electricity Sold to Utility and Nonutility Purchasers by Census Region, Industry Group, and Selected Industries, 1991 (Continued) (Estimates in Million Kilowatthours)

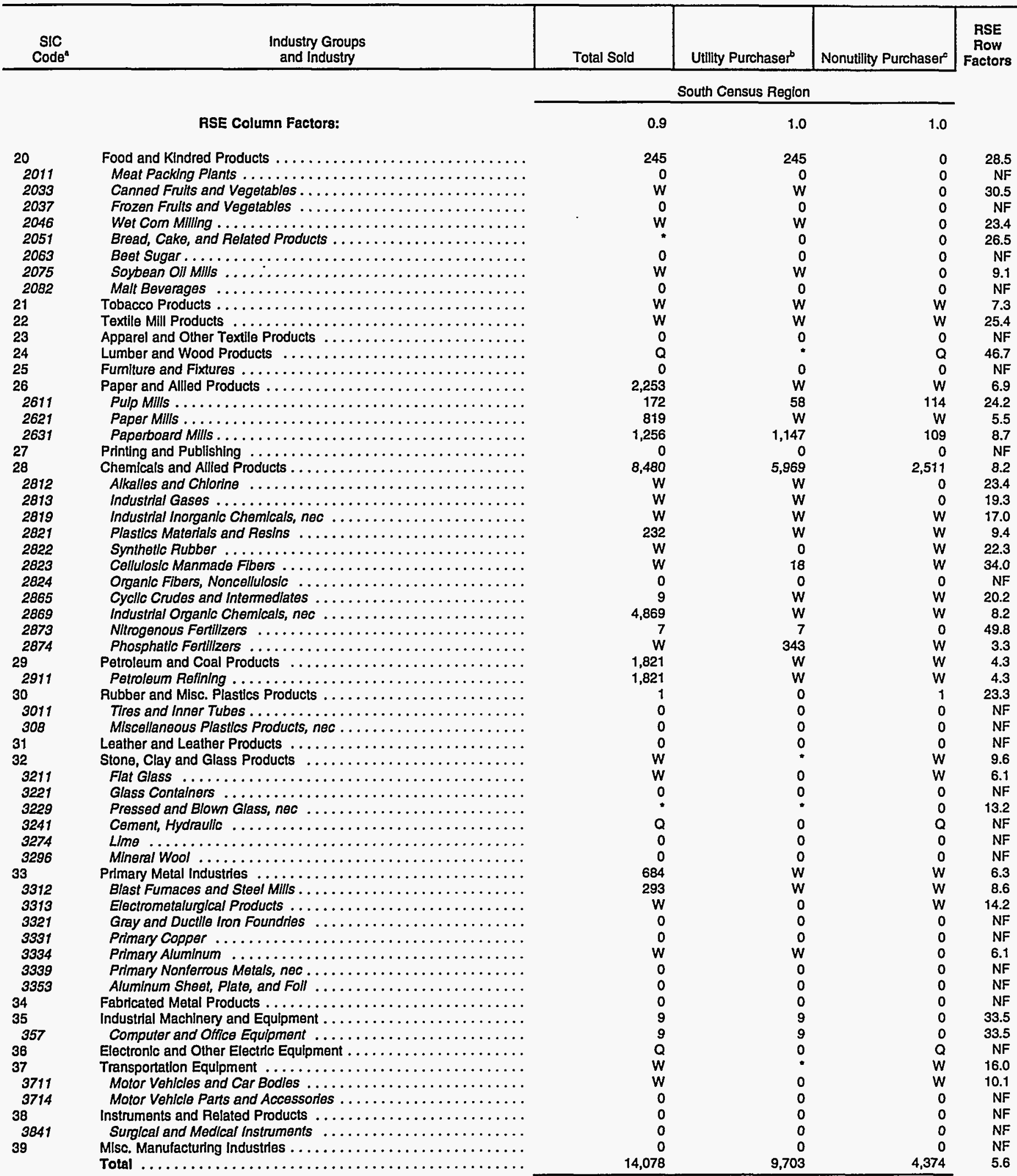

See footnotes at end of table. 


\section{Table A18. Quantity of Electricity Sold to Utility and Nonutility Purchasers by Census Region, Industry Group, and Selected Industries, 1991 (Continued) (Estimates in Million Kilowatthours)}

\begin{tabular}{|c|c|c|c|c|c|}
\hline $\begin{array}{c}\text { SIC } \\
\text { Code" }\end{array}$ & $\begin{array}{l}\text { Industry Groups } \\
\text { and Industry }\end{array}$ & Total Sold & Utility Purchaser & Nonutility Purchaser & $\begin{array}{c}\text { RSE } \\
\text { Row } \\
\text { Factors }\end{array}$ \\
\hline & & \multicolumn{3}{|c|}{ West Census Region } & \\
\hline & RSE Column Factors: & 1.0 & 1.0 & 1.1 & \\
\hline 20 & Food and Kindred Products $\ldots \ldots \ldots \ldots \ldots \ldots \ldots \ldots$ & 624 & 595 & 29 & 22.4 \\
\hline 2011 & 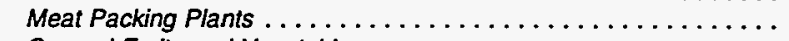 & 0 & 0 & 0 & NF \\
\hline 2033 & Canned Fruits and Vegetables $\ldots \ldots \ldots \ldots \ldots \ldots \ldots$ & W & 0 & W & 27.7 \\
\hline 2037 & Frozen Fruits and Vegetables $\ldots \ldots \ldots \ldots \ldots \ldots \ldots \ldots$ & 27 & 27 & 0 & 38.2 \\
\hline 2046 & Wet Com Milling . . . . . . . . . . . . . . . . . . & 0 & 0 & 0 & NF \\
\hline 2051 & Bread, Cake, and Related Products . . . . . . . . . . . . & 0 & $\mathbf{0}$ & 0 & NF \\
\hline 2063 & Beet Sugar . . . . . . . . . . . . . . . . . . . & 0 & 0 & 0 & NF \\
\hline 2075 & Soybeañ Oil Mills . . . . . . . . . . . . . . . . . . . . & 0 & 0 & 0 & NF \\
\hline 2082 & 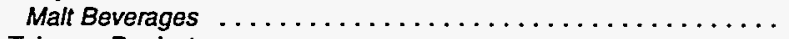 & 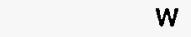 & 0 & $\mathbf{W}$ & 22.7 \\
\hline 21 & Tobacco Products $\ldots \ldots \ldots \ldots \ldots \ldots \ldots \ldots \ldots \ldots$ & 0 & 0 & 0 & NF \\
\hline 22 & Textile Mill Products $\ldots \ldots \ldots \ldots \ldots \ldots \ldots \ldots \ldots$ & 0 & 0 & 0 & NF \\
\hline 23 & Apparel and Other Textile Products $\ldots \ldots \ldots \ldots \ldots \ldots$ & NA & NA & NA & NF \\
\hline 24 & Lumber and wood Products $\ldots \ldots \ldots \ldots \ldots \ldots \ldots \ldots$ & $w$ & 1,723 & Q & 46.4 \\
\hline 25 & 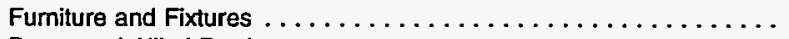 & 0 & 0 & $\overrightarrow{0}$ & NF \\
\hline 26 & Paper and Allied Products $\ldots \ldots \ldots \ldots \ldots \ldots \ldots \ldots$ & 2,955 & 2,802 & 153 & 6.5 \\
\hline 2611 & 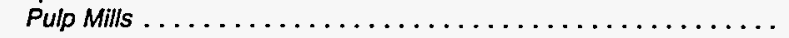 & 17 & 17 & 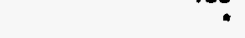 & 35.7 \\
\hline 2621 & Paper Mills . . . . . . . . . . . . . . . . . . . . & 1,857 & W & w & 5.6 \\
\hline 2631 & 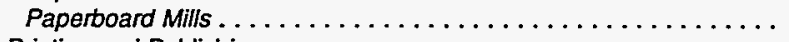 & 1,081 & W & $\mathbf{W}$ & 9.2 \\
\hline 27 & Printing and Publishing $\ldots \ldots \ldots \ldots \ldots \ldots \ldots \ldots \ldots \ldots \ldots \ldots \ldots \ldots \ldots$ & NA & NA & NA & NF \\
\hline 28 & Chemicals and Allied Products $\ldots \ldots \ldots \ldots \ldots \ldots$ & 1,125 & W & $\mathbf{W}$ & 12.6 \\
\hline 2812 & 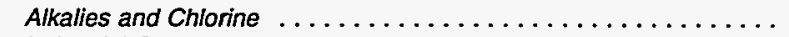 & 0 & 0 & 0 & NF \\
\hline 2813 & 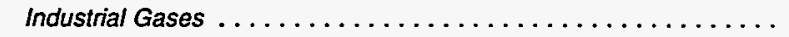 & 0 & 0 & 0 & NF \\
\hline 2819 & Industrial Inorganic Chemicals, nec $\ldots \ldots \ldots \ldots \ldots \ldots$ & $w$ & $w$ & $w$ & 15.9 \\
\hline 2821 & Plastics Materials and Resins $\ldots \ldots \ldots \ldots \ldots \ldots \ldots \ldots \ldots \ldots \ldots \ldots$ & 0 & 0 & 0 & NF \\
\hline 2822 & 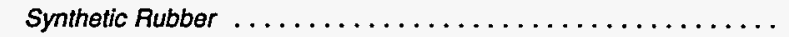 & 0 & 0 & 0 & NF \\
\hline 2823 & Cellulosic Manmade Fibers . . . . . . . . . . . . . . . . & 0 & 0 & 0 & NF \\
\hline 2824 & Organic Fibers, Noncellulosic $\ldots \ldots \ldots \ldots \ldots \ldots \ldots$ & 0 & 0 & 0 & NF \\
\hline 2865 & Cyclic Crudes and Intermediates . . . . . . . . . . . . . & 0 & 0 & 0 & NF \\
\hline 2869 & Industrial Organic Chemicals, nec . . . . . . . . . . . . . & $W$ & $\mathbf{W}$ & 0 & 14.4 \\
\hline 2873 & Nitrogenous Fertilizers $\ldots \ldots \ldots \ldots \ldots \ldots \ldots \ldots \ldots \ldots \ldots$ & 0 & 0 & 0 & NF \\
\hline 2874 & Phosphatic Fertilizers . . . . . . . . . . . . . . . & $\mathbf{w}$ & $W$ & 0 & 4.1 \\
\hline 29 & Petroleum and Coal Products $\ldots \ldots \ldots \ldots \ldots \ldots \ldots$ & 379 & $w$ & $w$ & 12.9 \\
\hline 2911 & 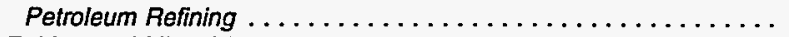 & 133 & $W$ & W & 4.6 \\
\hline 30 & Rubber and Misc. Plastics Products $\ldots \ldots \ldots \ldots \ldots \ldots \ldots \ldots$ & 0 & 0 & 0 & NF \\
\hline 3011 & Tires and Inner Tubes . . . . . . . . . . . . . . . . & 0 & 0 & 0 & NF \\
\hline 308 & Miscellaneous Plastics Products, nec . . . . . . . . . . . . & 0 & 0 & 0 & NF \\
\hline 31 & Leather and Leather Products $\ldots \ldots \ldots \ldots \ldots \ldots \ldots$ & 0 & 0 & 0 & NF \\
\hline 32 & Stone, Clay and Glass Products $\ldots \ldots \ldots \ldots \ldots \ldots \ldots \ldots$ & $w$ & $w$ & 0 & 22.7 \\
\hline 3211 & 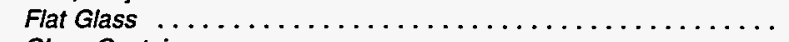 & 0 & 0 & 0 & NF \\
\hline 3221 & 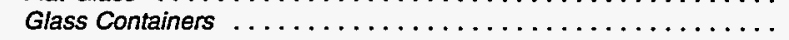 & 0 & 0 & 0 & NF \\
\hline 3229 & Pressed and Blown Glass, nec ................... & 0 & 0 & 0 & NF \\
\hline 3241 & Cement, Hydraulic . . . . . . . . . . . . . . . . . & $w$ & $w$ & 0 & 26.8 \\
\hline 3274 & 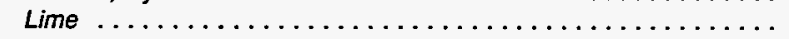 & 0 & 0 & 0 & NF \\
\hline 3296 & 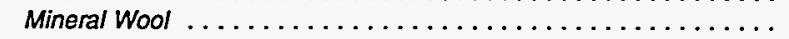 & 0 & 0 & 0 & NF \\
\hline 33 & Primary Metal Industries $\ldots \ldots \ldots \ldots \ldots \ldots \ldots \ldots$ & W & - & $\mathbf{w}$ & 12.3 \\
\hline 3312 & Blast Fumaces and Steel Mills . . . . . . . . . . . . . & W & $\cdot$ & $\mathbf{w}$ & 12.3 \\
\hline 3313 & Electrometalurgical Products $\ldots \ldots \ldots \ldots \ldots \ldots \ldots \ldots \ldots \ldots \ldots$ & 0 & 0 & 0 & NF \\
\hline 3321 & Gray and Ductile Iron Foundrios $\ldots \ldots \ldots \ldots \ldots \ldots$ & 0 & 0 & 0 & NF \\
\hline 3331 & Primary Copper $\ldots \ldots \ldots \ldots \ldots \ldots \ldots \ldots \ldots \ldots \ldots$ & 0 & 0 & 0 & NF \\
\hline 3334 & Primary Aluminum $\ldots \ldots \ldots \ldots \ldots \ldots \ldots \ldots \ldots \ldots \ldots \ldots \ldots \ldots \ldots$ & 0 & 0 & 0 & NF \\
\hline 3339 & Primary Nonferrous Metals, nec . . . . . . . . . . . . . . . & 0 & 0 & 0 & NF \\
\hline 3353 & 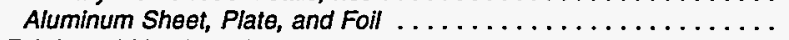 & 0 & 0 & 0 & NF \\
\hline 34 & Fabricated Metal Products $\ldots \ldots \ldots \ldots \ldots \ldots \ldots \ldots \ldots$ & 0 & 0 & 0 & NF \\
\hline 35 & Industrial Machinery and Equipment $\ldots \ldots \ldots \ldots \ldots \ldots$ & 0 & 0 & 0 & NF \\
\hline 357 & Computer and Office Equipment $\ldots \ldots \ldots \ldots \ldots \ldots$ & 0 & 0 & 0 & NF \\
\hline 36 & Electronic and Other Electric Equipment $\ldots \ldots \ldots \ldots \ldots \ldots$ & 0 & 0 & 0 & NF \\
\hline
\end{tabular}

See footnotes at end of table. 
Table A18. Quantity of Electricity Sold to Utility and Nonutility Purchasers by Census Region, Industry Group, and Selected Industries, 1991 (Continued) (Estimates in Million Kilowatthours)

\begin{tabular}{|c|c|c|c|c|c|}
\hline $\begin{array}{c}\text { SIC } \\
\text { Codea }\end{array}$ & $\begin{array}{l}\text { Industry Groups } \\
\text { and Industry }\end{array}$ & Total Sold & Utility Purchaser & Nonutility Purchaser & $\begin{array}{c}\text { RSE } \\
\text { Row } \\
\text { Factors }\end{array}$ \\
\hline & \multirow[b]{2}{*}{ RSE Column Factors: } & \multicolumn{3}{|c|}{ West Census Region } & \\
\hline & & 1.0 & 1.0 & 1.1 & \\
\hline 37 & Transportation Equipment & 0 & 0 & 0 & NF \\
\hline 3711 & Motor Vehicles and Car Bodies . . . . . . . . . . . . . & 0 & 0 & 0 & NF \\
\hline 3714 & Motor Vehiclo Parts and Accessories . . . . . . . . . . . . & 0 & 0 & 0 & NF \\
\hline 38 & Instruments and Related Products $\ldots \ldots \ldots \ldots \ldots$ & * & 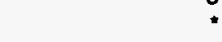 & 0 & 28.9 \\
\hline 3841 & Surgical and Medical Instruments $\ldots \ldots \ldots \ldots \ldots \ldots$ & 0 & 0 & 0 & NF \\
\hline 39 & Misc. Manufacturing Industries $\ldots \ldots \ldots \ldots \ldots \ldots \ldots$ & 0 & 0 & 0 & NF \\
\hline & Total $\ldots \ldots \ldots \ldots \ldots \ldots \ldots \ldots \ldots \ldots \ldots \ldots \ldots$ & 7,135 & 6,623 & 513 & 17.7 \\
\hline
\end{tabular}

- See Appendices B and F for descriptions of the Standard Industrial Classification system.

${ }^{b} \mathrm{~A}$ "Utility" is a company that produces and/or delivers electricity and/or natural gas, and is legally obligated to provide service to the public within its franchise area.

- Includes independent power producers, small power producers, and cogenerators not located at the establishment site.

NF=No applicable RSE row/column factor.

- Estimate less than 0.5. Data are included in higher level totals.

$\mathrm{W}=$ Withheld to avoid disclosing data for individual establishments. Data are included in higher level totals.

$Q=$ Withheld because Relative Standard Error is greater than 50 percent. Data are included in higher level totals.

$N A=$ Not available. Data are included in higher level totals.

Notes: - To obtain a RSE percentage for any table cell, multiply the cell's corresponding RSE column and RSE row factors. - Totals may not equal sum of components because of independent rounding.

Source: Energy Information Administration, Office of Energy Markets and End Use, Energy End Use and Integrated Statistics Division, Form ElA-846, "1991 Manufacturing Energy Consumption Survey." 


\section{Table A19. Components of Total Electricity Demand by Census Region and Economic Characteristics of the Establishment, 1991}

(Estimates in Million Kilowatthours)

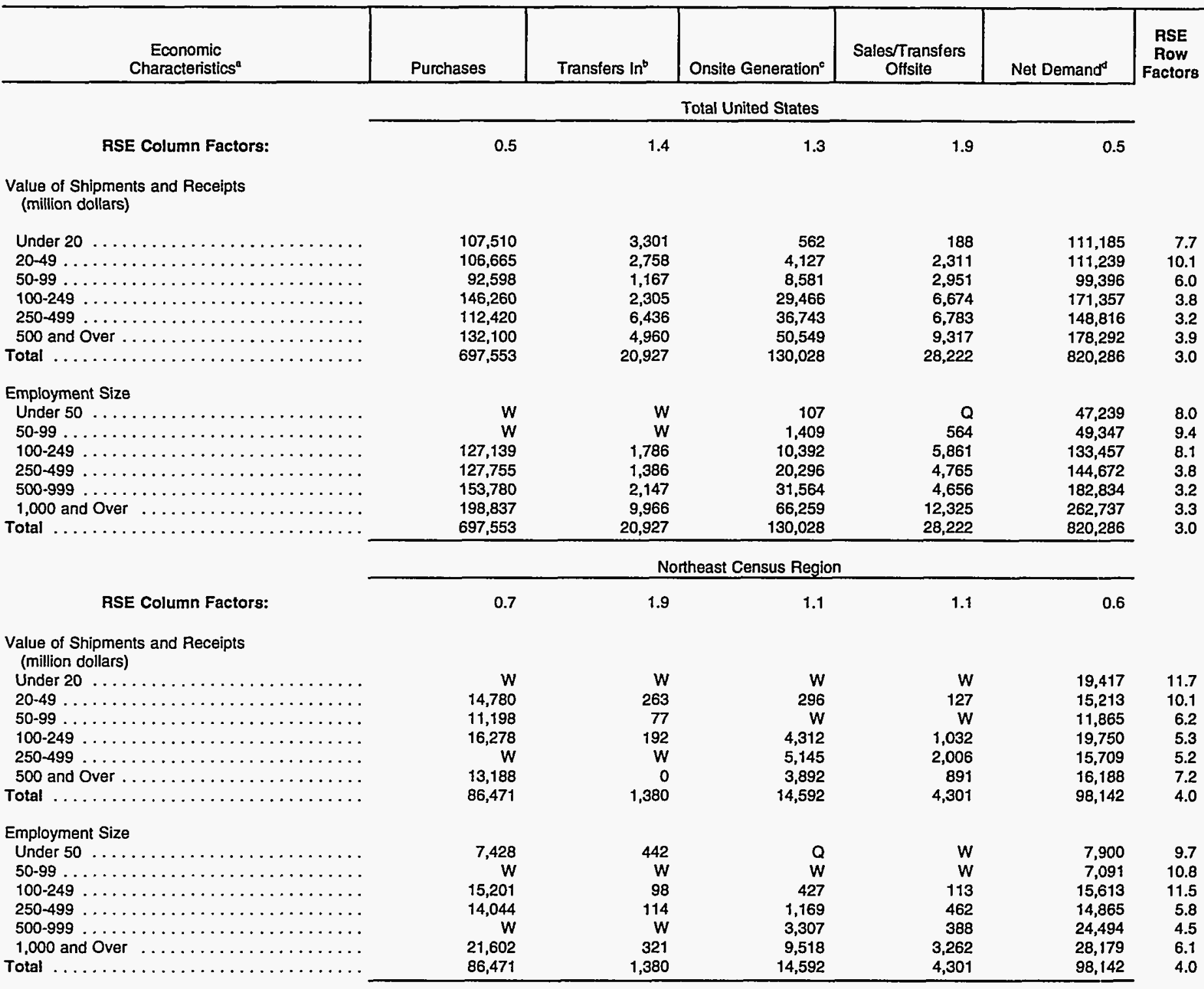

See footnotes at end of table. 


\section{Table A19. Components of Total Electricity Demand by Census Region and Economic Characteristics of the Establishment, 1991 (Continued) (Estimates in Million Kilowatthours)}

\begin{tabular}{|c|c|c|c|c|c|c|}
\hline $\begin{array}{c}\text { Economlc } \\
\text { Characteristles }\end{array}$ & Purchases & Transfers $\ln ^{b}$ & Onsite Generation ${ }^{c}$ & $\begin{array}{l}\text { Sales/Transfers } \\
\text { Offsite }\end{array}$ & Net Demand ${ }^{d}$ & $\begin{array}{c}\text { RSE } \\
\text { Row } \\
\text { Factors }\end{array}$ \\
\hline & \multicolumn{6}{|c|}{ Midwest Census Region } \\
\hline RSE Column Factors: & 0.5 & 1.8 & 1.2 & 1.6 & 0.5 & \\
\hline \multicolumn{7}{|l|}{$\begin{array}{l}\text { Value of Shipments and Receipts } \\
\text { (million dollars) }\end{array}$} \\
\hline Under $20 \ldots \ldots \ldots \ldots \ldots \ldots \ldots$ & $w$ & $w$ & 148 & * & 32,223 & 12.6 \\
\hline $20-49 \ldots \ldots \ldots \ldots \ldots \ldots \ldots \ldots \ldots \ldots$ & 28,358 & 1,407 & W & w & 30,245 & 12.6 \\
\hline $50-99 \ldots \ldots \ldots \ldots \ldots \ldots \ldots$ & 25,927 & 47 & W & w & 27,412 & 8.4 \\
\hline$\ldots \ldots \ldots \ldots \ldots \ldots \ldots \ldots \ldots$ & 29,584 & 259 & 3,241 & 644 & 32,440 & 6.4 \\
\hline $250-499 \ldots \ldots \ldots \ldots \ldots \ldots \ldots \ldots \ldots$ & w & w & 3,480 & 561 & 34,606 & 4.8 \\
\hline 500 and Over $\ldots \ldots \ldots \ldots \ldots \ldots \ldots \ldots$ & 56,635 & 1,124 & 6,154 & 1,347 & 62,566 & 6.2 \\
\hline 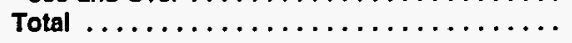 & 198,408 & 8,696 & 15,097 & 2,707 & 219,493 & 4.2 \\
\hline \multicolumn{7}{|l|}{ Employment Size } \\
\hline Under $50 \ldots .$. & w & w & 19 & 0 & 13,400 & 13.7 \\
\hline$\ldots \ldots \ldots$ & 14,492 & 101 & W & w & 14,735 & $\$ 1.3$ \\
\hline $100-249 \ldots \ldots \ldots \ldots \ldots \ldots \ldots \ldots \ldots$ & 34,762 & 962 & 1,767 & 145 & 37,346 & 10.7 \\
\hline $250-499 \ldots \ldots \ldots \ldots \ldots \ldots \ldots \ldots \ldots$ & 30,592 & 462 & 2,837 & 337 & 33,555 & 8.5 \\
\hline $500-999 \ldots \ldots \ldots \ldots \ldots \ldots \ldots \ldots \ldots$ & 29,448 & 114 & 2,740 & 598 & 31,703 & 6.4 \\
\hline 1,000 and Over & W & w & w & w & 88,754 & 4.7 \\
\hline \multirow[t]{2}{*}{ 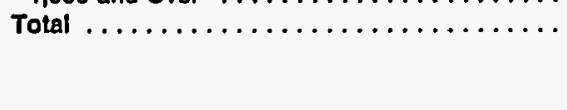 } & 198,408 & 8,696 & 15,097 & 2,707 & 219,493 & 4.2 \\
\hline & \multicolumn{6}{|c|}{ South Census Region } \\
\hline RSE Column Factors: & 0.6 & 1.5 & 1.2 & 1.7 & 0.6 & \\
\hline \multicolumn{7}{|l|}{$\begin{array}{l}\text { Value of Shipments and Receipts } \\
\text { (million dollars) }\end{array}$} \\
\hline Under $20 \ldots$. & 40,701 & 1,679 & $\mathbf{Q}$ & Q & 42,387 & 7.5 \\
\hline $20-49 \ldots \ldots$ & 49,075 & 824 & W & w & 50,163 & 10.6 \\
\hline 50-99. & 40,415 & 868 & $\{, 787$ & 182 & 42,888 & 5.9 \\
\hline $100-249 \ldots \ldots$ & 65,401 & 1,749 & 18,306 & 3,631 & 81,825 & 5.1 \\
\hline $250-499 \ldots \ldots$ & 52,982 & 216 & 25,939 & 3,220 & 75,917 & 5.7 \\
\hline 500 and Over. & 47,381 & 3,836 & 37,202 & 6,729 & 81,690 & 3.9 \\
\hline Total ....... & 295,955 & 9,173 & 83,821 & 14,078 & 374,870 & 3.0 \\
\hline \multicolumn{7}{|l|}{ Employment Size } \\
\hline Under $50 \ldots$ & w & w & 31 & Q & 17,842 & 7.6 \\
\hline $50-99 \ldots \ldots$ & 17,652 & 864 & W & Q & 18,646 & 14.3 \\
\hline$\ldots \ldots \ldots \ldots \ldots$ & 56,019 & 626 & W & w & 57,648 & 8.7 \\
\hline $250-499 \ldots$ & 62,432 & 513 & 12,849 & 2,532 & 73,262 & 4.5 \\
\hline 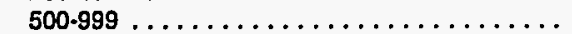 & 70,686 & 1,404 & 20,738 & 2,382 & 90,446 & 3.8 \\
\hline 1,000 and Over & w & w & 46,879 & 6,933 & 117,027 & 4.5 \\
\hline Total ........... & 295,955 & 9,173 & 83,821 & 14,078 & 374,870 & 3.0 \\
\hline
\end{tabular}

See footnotes at end of table. 

Table A19. Components of Total Electricity Demand by Census Region and Economic
Characteristics of the Establishment, 1991 (Continued)

(Estimates in Million Kilowatthours)

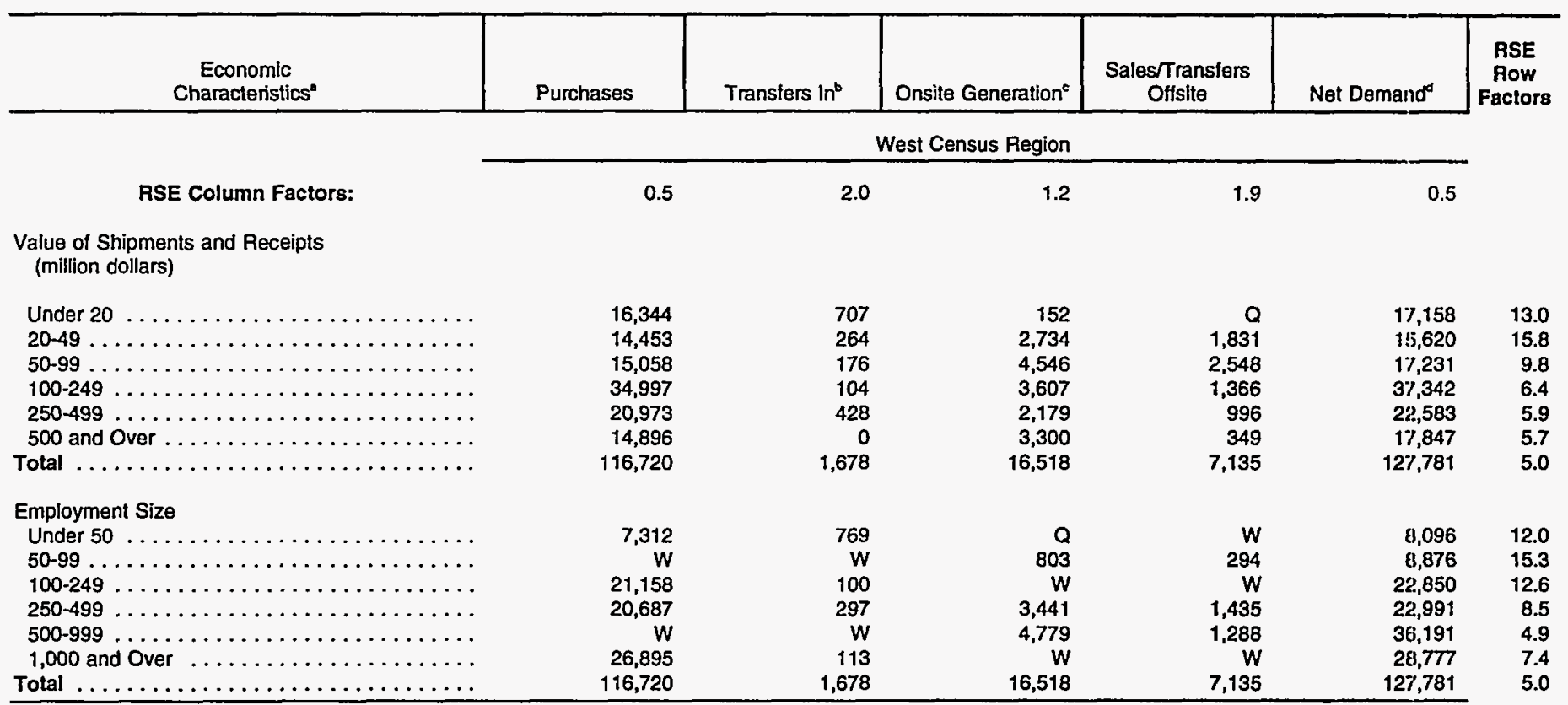

- Value of Shipments and Receipts and Employment Size were supplied by the Bureau of the Census. See Appendix B.

- "Transfers $\ln ^{\prime}$ are the quantities purchased by a central purchasing agent or other establishment of the same company.

c "Onsite Generation" includes cogeneration, generation by renewable energy sources, and conventional generation by combustible fuels.

"Net Demand" is the sum of purchases, transfers in, and total onsite generation, minus sales and transfers offsite. It is the total amount of electricity used. It is not comparable to net electricity which excludes electricity generated onsite by combustible energy sources.

- Estimate less than 0.5. Data are included in higher level totals.

$W=$ Withheld to avoid disclosing data for individual establishments. Data are included in higher level totals.

$\mathrm{Q}=$ Withheld because Relative Standard Error is greater than 50 percent. Data are included in higher level totals.

Notes: - To obtain a RSE percentage for any table cell, multiply the cell's corresponding RSE column and RSE row factors. - Totais may not equal stum of components because of independent rounding.

Source: Energy Information Administration, Office of Energy Markets and End Use, Energy End Use and Integrated Statistics Division, Form ElA-846, "1991 Manufacturing Energy Consumption Survey," and Bureau of the Census, Industry Division, data files for the "1991 Annual Survey of Manufactures." 
Table A20. Components of Onsite Electricity Generation by Census Region and Economic Characteristics of the Establishment, 1991

(Estimates in Million Kilowatthours)

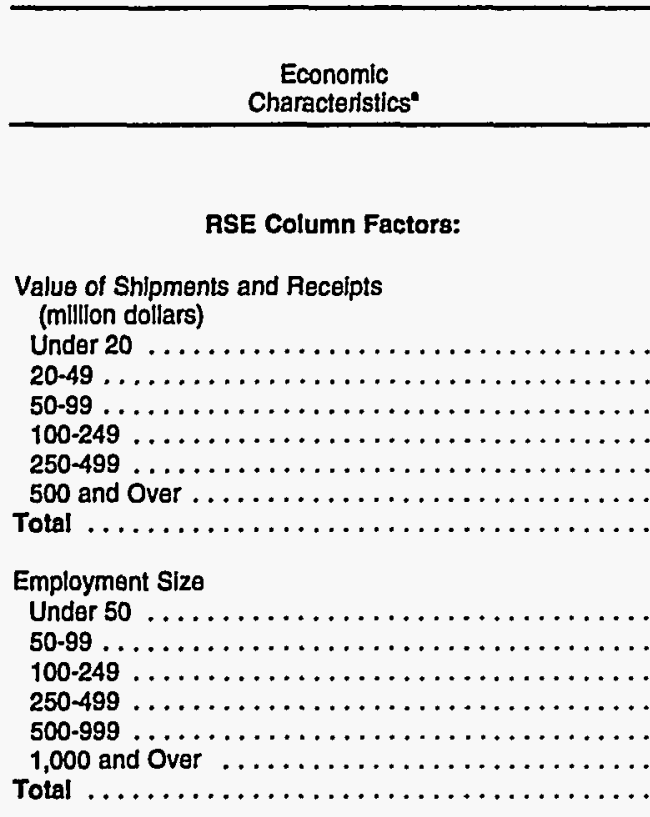

RSE Column Factors:

\begin{tabular}{|c|c|c|c|c|}
\hline 0.8 & 0.8 & 1.2 & 1.3 & \\
\hline $\begin{array}{r}562 \\
4,127 \\
8,581 \\
29,466 \\
36,743 \\
50,549 \\
130,028\end{array}$ & $\begin{array}{r}349 \\
3,917 \\
7,255 \\
25,688 \\
31,848 \\
44,855 \\
113,912\end{array}$ & $\begin{array}{r}W \\
79 \\
955 \\
1,766 \\
1,325 \\
W \\
4,444\end{array}$ & $\begin{array}{r}W \\
131 \\
371 \\
2,012 \\
3,571 \\
W \\
11,672\end{array}$ & $\begin{array}{r}23.0 \\
20.1 \\
10.0 \\
4.9 \\
4.2 \\
4.9 \\
4.0\end{array}$ \\
\hline $\begin{array}{r}107 \\
1,409 \\
10,392 \\
20,296 \\
31,564 \\
66,259 \\
130,028\end{array}$ & $\begin{array}{r}88 \\
1,303 \\
10,051 \\
17,889 \\
26,254 \\
58,328 \\
113,912\end{array}$ & $\begin{array}{r}Q \\
W \\
149 \\
1,352 \\
987 \\
1,933 \\
4,444\end{array}$ & $\begin{array}{r}Q \\
W \\
192 \\
1,056 \\
4,323 \\
5,999 \\
11,672\end{array}$ & $\begin{array}{r}36.1 \\
12.3 \\
15.0 \\
7.2 \\
4.1 \\
4.4 \\
4.0\end{array}$ \\
\hline \multicolumn{4}{|c|}{ Northeast Census Region } & \\
\hline 0.8 & 1.0 & 1.0 & 1.2 & \\
\hline $\begin{array}{r}W \\
296 \\
W \\
4,312 \\
5,145 \\
3,892 \\
14,592\end{array}$ & $\begin{array}{r}99 \\
174 \\
W \\
2,978 \\
3,804 \\
W \\
10,913\end{array}$ & $\begin{array}{r}W \\
36 \\
40 \\
1,156 \\
1,198 \\
W \\
2,491\end{array}$ & $\begin{array}{r}W \\
87 \\
W \\
178 \\
143 \\
625 \\
1,188\end{array}$ & $\begin{array}{r}29.4 \\
19.3 \\
6.2 \\
5.8 \\
5.1 \\
13.2 \\
4.7\end{array}$ \\
\hline $\begin{array}{r}Q \\
W \\
427 \\
1,169 \\
3,307 \\
9,518 \\
14,592\end{array}$ & $\begin{array}{r}Q \\
W \\
260 \\
850 \\
2,220 \\
7,505 \\
10,913\end{array}$ & $\begin{array}{r}W \\
W \\
96 \\
W \\
W \\
W \\
2,491\end{array}$ & $\begin{array}{r}Q \\
67 \\
70 \\
W \\
W \\
W \\
1,188\end{array}$ & $\begin{array}{r}8.0 \\
20.7 \\
16.9 \\
5.9 \\
6.0 \\
6.2 \\
4.7\end{array}$ \\
\hline
\end{tabular}

Total United States

See footnotes at end of table. 
Table A20. Components of Onsite Electricity Generation by Census Region and E:conomic Characteristics of the Establishment, 1991 (Continued)

(Estimates in Million Kilowatthours)

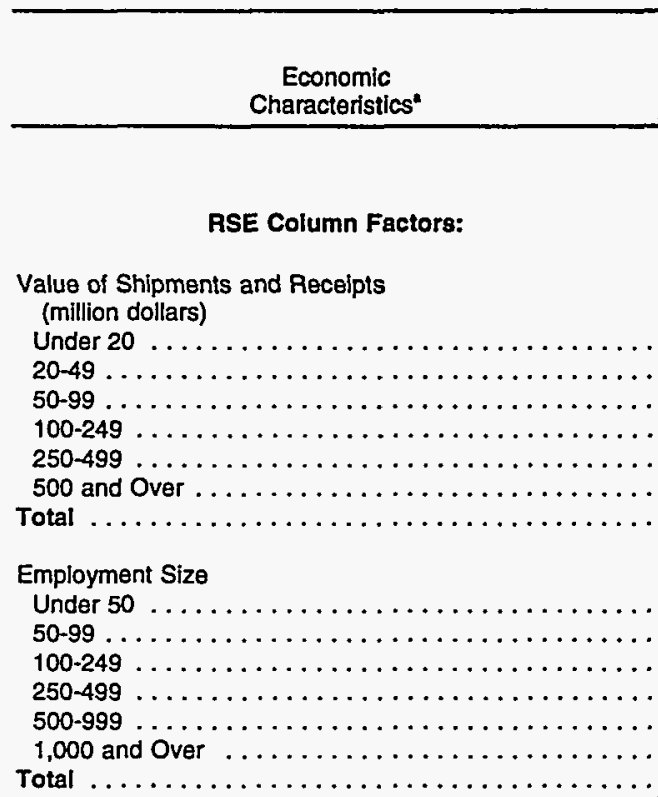

RSE Column Factors:

\begin{tabular}{|c|c|c|c|}
\hline Total & Cogeneration & Renewables & Other \\
\hline
\end{tabular}

Midwest Census Region

\begin{tabular}{lllll}
\multicolumn{1}{c}{ Mldwest Census Region } & & \\
\hline 0.9 & 0.9 & 0.8 & 1.4
\end{tabular}

1.4

Value of Shipments and Receipts

(million dollars)

$20-49$

$50-99$

500 and Over . . . . . . . . . . . . . . . . .

Total $\ldots \ldots \ldots \ldots \ldots \ldots$

\begin{tabular}{|c|c|c|c|c|}
\hline $\begin{array}{r}148 \\
W\end{array}$ & 67 & W & $Q$ & 21.1 \\
\hline$w$ & 1,308 & $w$ & W & 14.0 \\
\hline 3,241 & 2,765 & W & $w$ & 6.2 \\
\hline 3,480 & W & 127 & $w$ & 6.5 \\
\hline 6,154 & 5,053 & $W$ & $w$ & 6.7 \\
\hline 15,097 & 12,993 & 706 & 1,398 & 4.9 \\
\hline 19 & 19 & 0 & 0 & 50.4 \\
\hline$W$ & 126 & $\mathbf{W}$ & $Q$ & 11.9 \\
\hline 1,767 & 1,655 & 29 & $\mathbf{Q}$ & 12.4 \\
\hline 2,837 & 2,482 & W & $w$ & 11.0 \\
\hline 2,740 & 2,263 & $w$ & $w$ & 6.7 \\
\hline $\mathbf{W}$ & 6,448 & 292 & $w$ & 4.8 \\
\hline 15,097 & 12,993 & 706 & 1,398 & 4.9 \\
\hline \multicolumn{4}{|c|}{ South Census Region } & \\
\hline 0.7 & 0.7 & 1.8 & 1.2 & \\
\hline
\end{tabular}

\begin{tabular}{|c|c|c|c|c|}
\hline $\begin{array}{r}148 \\
W\end{array}$ & 67 & W & $Q$ & 21.1 \\
\hline$w$ & 1,308 & $w$ & W & 14.0 \\
\hline 3,241 & 2,765 & W & $w$ & 6.2 \\
\hline 3,480 & W & 127 & $w$ & 6.5 \\
\hline 6,154 & 5,053 & $W$ & $w$ & 6.7 \\
\hline 15,097 & 12,993 & 706 & 1,398 & 4.9 \\
\hline 19 & 19 & 0 & 0 & 50.4 \\
\hline$W$ & 126 & $\mathbf{W}$ & $Q$ & 11.9 \\
\hline 1,767 & 1,655 & 29 & $\mathbf{Q}$ & 12.4 \\
\hline 2,837 & 2,482 & W & $w$ & 11.0 \\
\hline 2,740 & 2,263 & $w$ & $w$ & 6.7 \\
\hline $\mathbf{W}$ & 6,448 & 292 & $w$ & 4.8 \\
\hline 15,097 & 12,993 & 706 & 1,398 & 4.9 \\
\hline \multicolumn{4}{|c|}{ South Census Region } & \\
\hline 0.7 & 0.7 & 1.8 & 1.2 & \\
\hline
\end{tabular}

\begin{tabular}{|c|c|c|c|c|}
\hline $\begin{array}{r}148 \\
W\end{array}$ & 67 & W & $Q$ & 21.1 \\
\hline$w$ & 1,308 & $w$ & W & 14.0 \\
\hline 3,241 & 2,765 & W & $w$ & 6.2 \\
\hline 3,480 & W & 127 & $w$ & 6.5 \\
\hline 6,154 & 5,053 & $W$ & $w$ & 6.7 \\
\hline 15,097 & 12,993 & 706 & 1,398 & 4.9 \\
\hline 19 & 19 & 0 & 0 & 50.4 \\
\hline$W$ & 126 & $\mathbf{W}$ & $Q$ & 11.9 \\
\hline 1,767 & 1,655 & 29 & $\mathbf{Q}$ & 12.4 \\
\hline 2,837 & 2,482 & W & $w$ & 11.0 \\
\hline 2,740 & 2,263 & $w$ & $w$ & 6.7 \\
\hline $\mathbf{W}$ & 6,448 & 292 & $w$ & 4.8 \\
\hline 15,097 & 12,993 & 706 & 1,398 & 4.9 \\
\hline \multicolumn{4}{|c|}{ South Census Region } & \\
\hline 0.7 & 0.7 & 1.8 & 1.2 & \\
\hline
\end{tabular}

Value of Shipments and Receipts

(million dollars)

Under 20

$20-49$

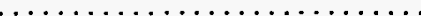

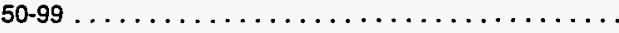

$100-249 \ldots \ldots \ldots \ldots \ldots \ldots \ldots \ldots \ldots \ldots \ldots \ldots \ldots \ldots$

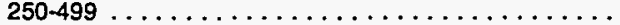

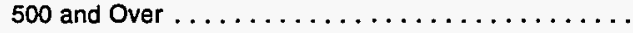

Total $\ldots \ldots \ldots \ldots \ldots \ldots \ldots \ldots \ldots \ldots$

$Q$
$W$
1,787
18,306
25,939
37,202
83,821

$Q$
$W$
$W$
16,837
22,733
33,784
75,037

$Q$
$W$
$W$
$W$
0
$W$
$W$

$Q$
$W$
82
$W$
3,207
$W$
$W$

NF

26.0

10.3

9.1

5.1

Employment Size

Under 50

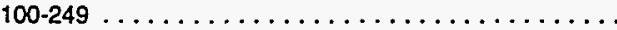

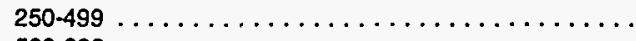

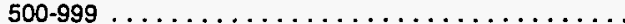

1,000 and Over $\ldots \ldots \ldots \ldots \ldots \ldots \ldots \ldots \ldots \ldots$

Total

$\ldots \ldots \ldots \ldots \ldots \ldots \ldots \ldots \ldots$

31
$W$
$W$
12,849
20,738
46,879
83,821

29
$W$
$W$
$W$
17,283
42,618
75,037

$Q$
$W$
$W$
$W$
$W$
$W$
$W$

See footnotes at end of table. 


\section{Table A20. Components of Onsite Electricity Generation by Census Region and Economic Characteristics of the Establishment, 1991 (Continued)}

(Estimates in Million Kilowatthours)

\begin{tabular}{|c|c|c|c|c|c|}
\hline $\begin{array}{l}\text { Economlc } \\
\text { Characteristles }^{\circ}\end{array}$ & Total & Cogeneration & Renewables & Other" & $\begin{array}{c}\text { RSE } \\
\text { Row } \\
\text { Factors }\end{array}$ \\
\hline & \multicolumn{4}{|c|}{ West Census Region } & \\
\hline RSE Column Factors: & 0.7 & 0.8 & 2.1 & 0.8 & \\
\hline \multicolumn{6}{|l|}{$\begin{array}{l}\text { Value of Shipments and Receipts } \\
\text { (million dollars) }\end{array}$} \\
\hline Under $20 \ldots \ldots \ldots \ldots \ldots \ldots \ldots \ldots \ldots \ldots$ & 152 & 142 & 0 & $\mathbf{Q}$ & 38.1 \\
\hline $20-49 \ldots \ldots \ldots \ldots \ldots \ldots \ldots \ldots \ldots \ldots \ldots \ldots$ & 2,734 & 2,726 & W & w & 25.4 \\
\hline $50.99 \ldots \ldots \ldots \ldots \ldots \ldots \ldots \ldots \ldots \ldots \ldots \ldots$ & 4,546 & 4,211 & $\mathbf{Q}$ & 44 & 16.7 \\
\hline $100-249 \ldots \ldots \ldots \ldots \ldots \ldots \ldots \ldots \ldots \ldots \ldots$ & 3,607 & 3,108 & W & W & 9.3 \\
\hline $250-499 \ldots \ldots \ldots \ldots \ldots \ldots \ldots \ldots \ldots \ldots \ldots \ldots$ & 2,179 & w & * & w & 6.8 \\
\hline 500 and Over $\ldots \ldots \ldots \ldots \ldots \ldots \ldots \ldots \ldots$ & 3,300 & w & 0 & W & 7.5 \\
\hline Total $\ldots \ldots \ldots \ldots \ldots \ldots \ldots \ldots \ldots \ldots \ldots \ldots \ldots \ldots \ldots \ldots \ldots \ldots \ldots$ & 16,518 & 14,969 & $\mathbf{W}$ & W & 12.1 \\
\hline \multicolumn{6}{|l|}{ Employment Size } \\
\hline ............. & $\mathbf{Q}$ & Q & 0 & $\mathbf{Q}$ & NF \\
\hline $50-99 \ldots \ldots \ldots \ldots \ldots \ldots \ldots \ldots \ldots \ldots \ldots \ldots$ & 803 & 796 & 0 & $\mathbf{Q}$ & 18.7 \\
\hline 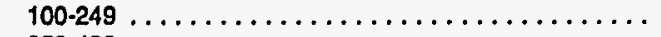 & W & W & 16 & W & 13.4 \\
\hline$\ldots \ldots \ldots \ldots \ldots$ & 3,441 & $\mathbf{w}$ & $\mathbf{Q}$ & 244 & 17.0 \\
\hline 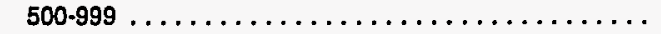 & 4,779 & 4,488 & - & 292 & 9.2 \\
\hline 1,000 and Over $\ldots$ & w & 1,757 & 0 & W & 10.0 \\
\hline Total $\ldots \ldots \ldots \ldots \ldots \ldots \ldots \ldots \ldots \ldots \ldots$ & 16,518 & 14,969 & w & w & 12.1 \\
\hline
\end{tabular}

\footnotetext{
- Value of Shipments and Receipts and Employment Size were supplied by the Bureau of the Census. See Appendix B.

- "Other" is that electricly obtalned from a generator fueled by combustible energy sources such as dlesel fuels or fuel oils.

NF=No applicable RSE row/column factor.

- Estimate less than 0.5. Data are included in higher level totals.

$W=$ Withheld to avoid disclosing data for individual establishments. Data are included in higher level totals.

$Q=$ Withheld because Relative Standard Error is greater than 50 percent. Data are included in higher level totals.

Notes: - To obtain a RSE percentage for any table cell, multiply the cell's corresponding RSE column and RSE row factors. - Totals may not equal sum of components because of Independent rounding.

Source: Energy Information Administration, Office of Energy Markets and End Use, Energy End Use and Integrated Statisties Division, Form ElA-846, "1991 Manulacturing Energy Consumption Survey," and Bureau of the Census, Industry Division, data files for the "1991 Annual Survey of Manufactures."
} 
Table A21. Quantity of Electricity Sold to Utility and Nonutility Purchasers by Census Region and Economic Characteristics of the Establishment, 1991

(Estimates in Million Kilowatthours)

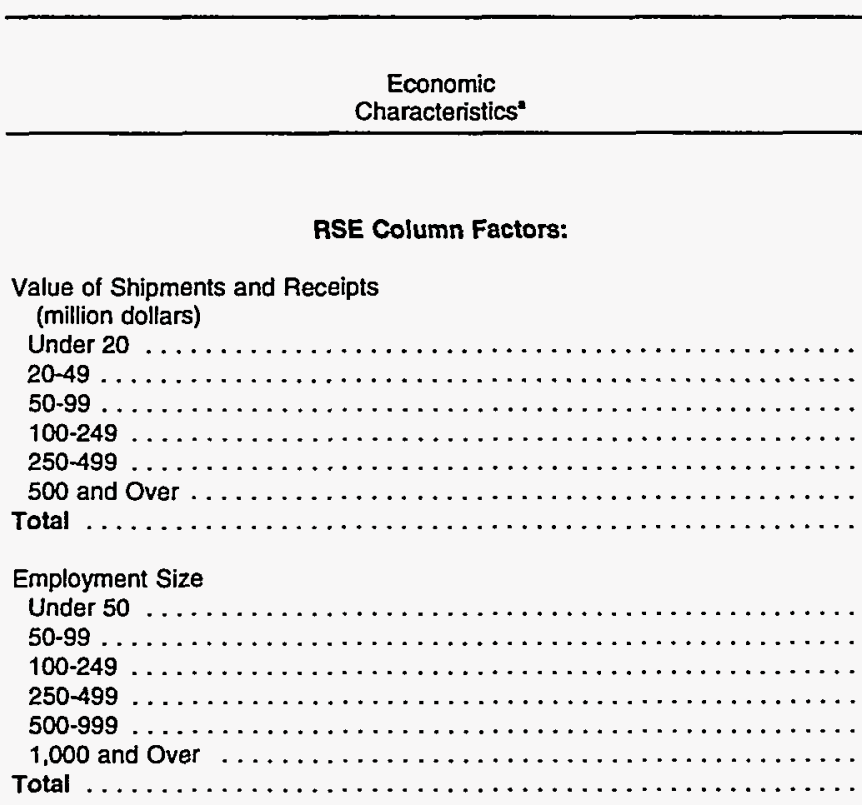

RSE Column Factors:

$[$ Total Sold

Utility Purchaser Nonutility Purchaser

Total United States

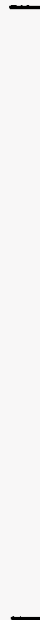

1.1

188
2,311
2,951
6,674
6,783
9,317

122

1,901
2,721

5,699

4,562

6,058

28,222

21,063

7,159

\begin{tabular}{rrrr}
$Q$ & $W$ & $Q$ & 7.4 \\
564 & $W$ & $W$ & 22.0 \\
5,861 & 5,444 & 417 & 14.2 \\
4,765 & 3,127 & 1,638 & 8.6 \\
4,656 & 3,471 & 1,186 & 6.1 \\
12,325 & 8,502 & 3,823 & 4.3 \\
28,222 & 21,063 & 7,159 & 4.9 \\
\hline
\end{tabular}

$\begin{array}{rr}66 & 35.6 \\ 410 & 39.5 \\ 230 & 9.6 \\ 974 & 7.1 \\ 2,220 & 4.8 \\ 3,259 & 4.9 \\ 7,159 & 4.9\end{array}$

Northeast Census Region

0.8

0.9

1.3

Value of Shipments and Receipts

(million dollars)

Under 20

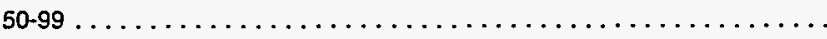

$100-249$

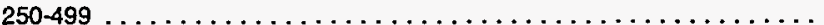

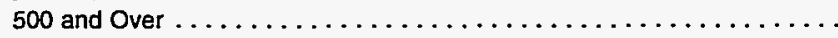

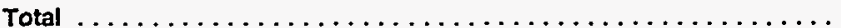

Employment Size

Under 50

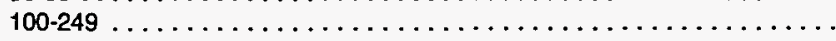

$250-499$

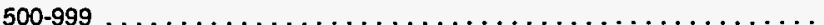

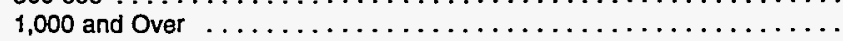

Total

.

$\begin{array}{rrr}W & W & 32.5 \\ 61 & 66 & 23.5 \\ W & 7 & 10.7 \\ 938 & 94 & 4.8 \\ W & W & 5.2 \\ W & W & 6.3 \\ 3,987 & 314 & 3.8\end{array}$

See footnotes at end of table. 
Table A21. Quantity of Electricity Sold to Utility and Nonutility Purchasers by Census Region and Economic Characteristics of the Establishment, 1991 (Continued) (Estimates in Million Kilowatthours)

\begin{tabular}{|c|c|c|c|c|}
\hline $\begin{array}{l}\text { Economic } \\
\text { Characteristles }\end{array}$ & Total Sold & Utility Purchaser & Nonutility Purchaser & $\begin{array}{c}\text { RSE } \\
\text { Row } \\
\text { Factors }\end{array}$ \\
\hline & \multicolumn{3}{|c|}{ Midwest Census Region } & \\
\hline RSE Column Factors: & 1.0 & 1.1 & 0.9 & \\
\hline \multicolumn{5}{|l|}{$\begin{array}{l}\text { Value of Shipments and Receipts } \\
\text { (million dollars) }\end{array}$} \\
\hline 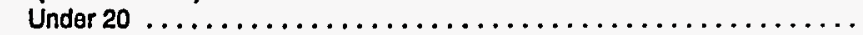 & - & - & 0 & NF \\
\hline 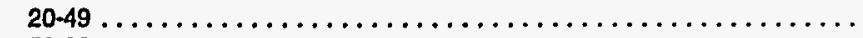 & w & 2 & w & 42.6 \\
\hline 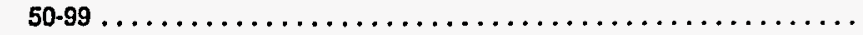 & w & w & w & 11.1 \\
\hline $100-249 \ldots \ldots \ldots \ldots \ldots \ldots \ldots \ldots \ldots \ldots \ldots \ldots \ldots \ldots \ldots \ldots \ldots$ & 644 & 336 & 309 & 16.5 \\
\hline 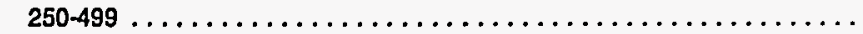 & 561 & w & $\mathbf{w}$ & 9.9 \\
\hline 500 and Over $\ldots \ldots \ldots \ldots \ldots \ldots \ldots$ & 1,347 & 47 & 1,300 & 6.3 \\
\hline 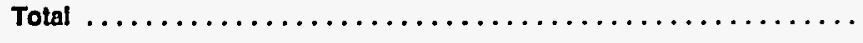 & 2,707 & 749 & 1,958 & 7.3 \\
\hline \multicolumn{5}{|l|}{$\begin{array}{l}\text { Employment Slze } \\
\text { Under } 50\end{array}$} \\
\hline Under $50 \ldots \ldots \ldots \ldots \ldots \ldots \ldots \ldots \ldots \ldots \ldots \ldots$ & 0 & 0 & 0 & NF \\
\hline $50-99 \ldots \ldots \ldots \ldots \ldots \ldots \ldots \ldots \ldots \ldots \ldots$ & $\mathbf{w}$ & w & 0 & 8.6 \\
\hline 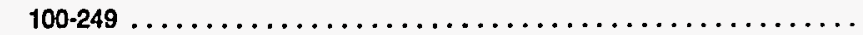 & 145 & 25 & 119 & 21.3 \\
\hline 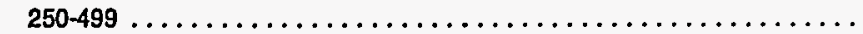 & 337 & 305 & 32 & 22.4. \\
\hline $500-999 \ldots \ldots \ldots \ldots$ & 598 & w & w & 7.9 \\
\hline 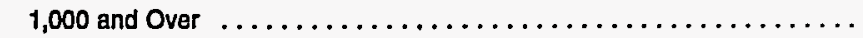 & w & w & w & 5.6 \\
\hline \multirow[t]{2}{*}{ 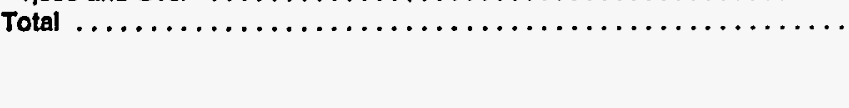 } & 2,707 & 749 & 1,958 & 7.3 \\
\hline & \multicolumn{3}{|c|}{ South Census Region } & \\
\hline RSE Column Factors: & 1.0 & 1.2 & 0.9 & \\
\hline \multicolumn{5}{|l|}{$\begin{array}{l}\text { Value of Shipments and Receipts } \\
\text { (million dollars) }\end{array}$} \\
\hline 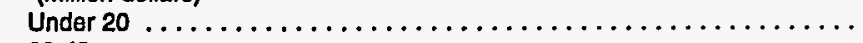 & $\mathbf{Q}$ & * & $\mathbf{Q}$ & 24.9 \\
\hline 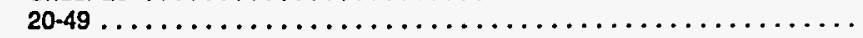 & $\mathbf{w}$ & 220 & w & 44.2 \\
\hline 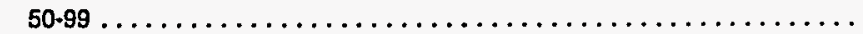 & 182 & 89 & 92 & 8.7 \\
\hline $100-249 \ldots \ldots \ldots \ldots \ldots \ldots \ldots \ldots \ldots \ldots \ldots \ldots \ldots \ldots \ldots \ldots$, & 3,631 & 3,137 & 494 & 11.1 \\
\hline $250-499 \ldots \ldots \ldots$ & 3,220 & 1,390 & 1,830 & 6.6 \\
\hline 500 and Over ....... & 6,729 & 4,868 & 1,861 & 5.9 \\
\hline 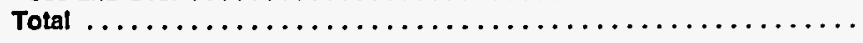 & 14,078 & 9,703 & 4,374 & 5.6 \\
\hline \multicolumn{5}{|l|}{ Employment Slze } \\
\hline Under $50 \ldots \ldots \ldots$ & $\mathbf{Q}$ & w & $\mathbf{Q}$ & 7.7 \\
\hline$\ldots \ldots \ldots \ldots \ldots \ldots \ldots \ldots \ldots \ldots$ & $\mathbf{Q}$ & $\mathbf{Q}$ & 6 & 12.5 \\
\hline $100-249 \ldots \ldots \ldots \ldots$ & w & w & w & 12.8 \\
\hline 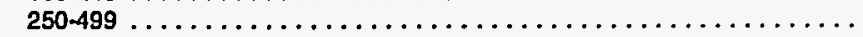 & 2,532 & 1,161 & 1,371 & 7.9 \\
\hline $500-999 \ldots \ldots \ldots$ & 2,382 & 1,643 & 739 & 8.0 \\
\hline 1,000 and Over $\ldots .$. & 6,933 & 4,887 & 2,046 & 5.5 \\
\hline Total $\ldots \ldots \ldots \ldots \ldots$ & 14,078 & 9,703 & 4,374 & 5.6 \\
\hline
\end{tabular}


Table A21. Quantity of Electricity Sold to Utility and Nonutility Purchasers by Census Region and Economic Characteristics of the Establishment, 1991 (Cortinued) (Estimates in Million Kilowatthours)

\begin{tabular}{|c|c|c|c|c|}
\hline $\begin{array}{c}\text { Economic } \\
\text { Characteristics" }\end{array}$ & Total Sold & Utility Purchaser & Nonutility Purchaser & $\begin{array}{c}\text { RSE } \\
\text { Row } \\
\text { Factors }\end{array}$ \\
\hline & \multicolumn{3}{|c|}{ West Census Region } & \\
\hline RSE Column Factors: & 0.9 & 1.0 & 1.1 & \\
\hline \multicolumn{5}{|l|}{$\begin{array}{l}\text { Value of Shipments and Receipts } \\
\text { (million dollars) }\end{array}$} \\
\hline Under $20 \ldots \ldots \ldots \ldots \ldots$ & $\mathbf{Q}$ & $\mathbf{Q}$ & w & 15.1 \\
\hline $20-49$ & 1,831 & 1,618 & $\mathbf{Q}$ & 48.2 \\
\hline $50-99$ & 2,548 & $w$ & w & 12.9 \\
\hline $100-249$ & 1,366 & 1,289 & 77 & 9.0 \\
\hline 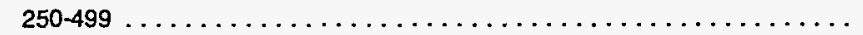 & 996 & w & w & 12.3 \\
\hline$\ldots \ldots \ldots \ldots \ldots \ldots \ldots \ldots \ldots \ldots \ldots \ldots$ & 349 & w & w & 16.3 \\
\hline Total ............. & 7,135 & 6,623 & 513 & 17.7 \\
\hline \multicolumn{5}{|l|}{ Employment Size } \\
\hline 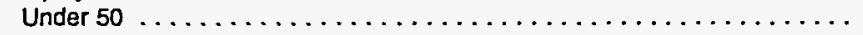 & w & 0 & w & 17.6 \\
\hline 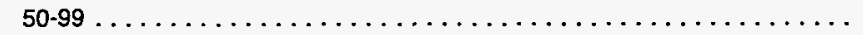 & 294 & 294 & $\cdot \dot{x}$ & 23.6 \\
\hline$\ldots \ldots \cdots \cdots \cdots \cdots$ & w & w & w & 19.8 \\
\hline $250-499$ & 1,435 & 1,214 & $\mathbf{Q}$ & 13.6 \\
\hline$\ldots \ldots \ldots \ldots \ldots$ & 1,288 & 1,211 & 78 & 10.9 \\
\hline 1,000 and Over & w & $w$ & w & 13.6 \\
\hline Total $\ldots \ldots \ldots \ldots$ & 7,135 & 6,623 & 513 & 17.7 \\
\hline
\end{tabular}

\footnotetext{
- Value of Shipments and Receipts and Employment Size were supplied by the Bureau of the Census. See Appendix B.

- A "Utility" is a company that produces and/or delivers electricity and/or natural gas, and is legally obligated to provide service to the public within its franchise area. - Includes independent power producers, small power producers, and cogenerators not located at the establishment site.

NF=No applicable RSE row/column factor.

- Estimate less than 0.5. Data are included in higher level totals.

$W=$ Withheld to avoid disclosing data for individual establishments. Data are included in higher level totals.

$Q=$ Withheld because Relative Standard Error is greater than 50 percent. Data are included in higher level totals.

Notes: - To obtain a RSE percentage for any table cell, multiply the cell's corresponding RSE column and RSE row factors. - Totals may not equal sum of components because of independent rounding.

Source: Energy Information Administration, Office of Energy Markets and End Use, Energy End Use and Integrated Statistics Division, Form ElA-846, "I991 Manufacturing Energy Consumption Survey," and Bureau of the Census, Industry Division, data files for the "1991 Annual Survey of Manufactures."
} 
Table A22. Total Quantity of Purchased Energy Sources by Census Region, Industry Group, and Selected Industries, 1991

(Estimates in Btu or Physical Units)

\begin{tabular}{|c|c|c|c|c|c|c|c|c|c|c|c|}
\hline$\underset{\text { Code }}{\text { SIC }}$ & $\begin{array}{l}\text { Industry Groups } \\
\text { and Industry }\end{array}$ & $\begin{array}{c}\text { Total } \\
\text { (Irillion } \\
\text { Btu) }\end{array}$ & $\begin{array}{l}\text { Electricity } \\
\text { (million } \\
\mathrm{kWh})\end{array}$ & $\begin{array}{l}\text { Residual } \\
\text { Fuel Oil } \\
(1000 \\
\text { bbls) }\end{array}$ & $\begin{array}{c}\text { Distillate } \\
\text { Fuel OilP } \\
(1000 \\
\text { bbls) }\end{array}$ & $\begin{array}{l}\text { Natural } \\
\text { Gas } \\
\text { (billion } \\
\text { cu ft) }\end{array}$ & $\begin{array}{l}\text { LPG } \\
(1000 \\
\text { bbls) }\end{array}$ & $\left|\begin{array}{c}\text { Coal } \\
(1000 \\
\text { short tons) }\end{array}\right|$ & $\begin{array}{c}\text { Coke and } \\
\text { Breeze } \\
(1000 \\
\text { short tons) }\end{array}$ & $\begin{array}{l}\text { Other" } \\
\text { (trillion } \\
\text { Btu) }\end{array}$ & $\begin{array}{c}\text { RSE } \\
\text { Row } \\
\text { Factors }\end{array}$ \\
\hline
\end{tabular}

Total United States

\begin{tabular}{|c|c|c|c|c|c|c|c|c|c|c|c|}
\hline & RSE Column Factors: & 0.6 & 0.6 & 1.3 & 1.3 & 0.7 & 1.2 & 1.2 & 1.5 & 1.2 & \\
\hline 20 & Food and Kindred Products & 918 & $w$ & 4,314 & 3,150 & $w$ & 1,425 & 6,918 & $w$ & 25 & 6.0 \\
\hline 2011 & Meat Packing Plants . . . . . . . . . . . . & 48 & 3,410 & 152 & 285 & 31 & 156 & 28 & 0 & * & 9.9 \\
\hline 2033 & Canned Frults and Vegotables . . . . . . . . & 44 & 1,415 & 290 & 130 & 35 & 126 & $\mathbf{Q}$ & 0 & * & 9.3 \\
\hline 2037 & Frozen Frults and Vegetables . . . . . . . & 40 & 3,096 & 322 & 76 & 25 & 41 & 0 & 0 & * & 14.8 \\
\hline 2046 & Wet Com Milling ........................ & 139 & $w$ & 29 & 31 & 51 & 1 & 3,058 & w & w & 11.7 \\
\hline 2051 & Bread, Cake, and Related Products .... & 32 & 2,240 & 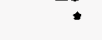 & 133 & 23 & 23 & 0 & 0 & • & 12.4 \\
\hline 2063 & Beet Sugar . . . . . . . . . . . . . . & 67 & 407 & w & 30 & 18 & 5 & 1,903 & w & • & 5.4 \\
\hline 2075 & Soyboan Oll Mills .............. & 47 & $w$ & 49 & 31 & 25 & 5 & 595 & 0 & w & 3.5 \\
\hline 2082 & Malt Boverages $\ldots \ldots \ldots \ldots \ldots \ldots$ & 50 & 2,371 & 411 & 58 & 22 & 8 & 705 & 0 & * & 11.2 \\
\hline 21 & Tobacco Products ................. & 26 & 1,468 & 133 & 41 & 4 & 24 & 681 & 0 & * & 6.2 \\
\hline 22 & Textlle Mill Products ............. & 267 & w & 1,953 & 1,071 & 105 & 634 & 1,259 & 0 & w & 7.2 \\
\hline 23 & Apparel and Other Textile Products ..... & 44 & 5,643 & $\mathbf{Q}$ & 142 & 18 & 169 & 88 & 0 & - & 17.9 \\
\hline 24 & Lumber and Wood Products .......... & 209 & 19,209 & 334 & 2,823 & 39 & 1,017 & 87 & 0 & 79 & 14.4 \\
\hline 25 & Furnlture and Fixtures $\ldots \ldots \ldots \ldots \ldots$ & 46 & 4,913 & 182 & 163 & 18 & 255 & 156 & 0 & 4 & 18.2 \\
\hline 26 & Paper and Allled Products . . . . . . . . . . & w & 63,744 & 24,543 & 1,600 & W & w & 12,878 & w & 264 & 4.0 \\
\hline 2611 & 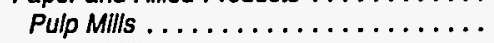 & $10 t$ & 2,871 & 4,183 & 162 & 32 & 143 & 330 & 0 & 23 & 14.8 \\
\hline 2621 & Paper Mills $\ldots \ldots \ldots \ldots \ldots \ldots \ldots$ & 768 & $\mathbf{w}$ & 13,529 & w & 252 & 617 & 8,562 & w & 103 & 2.9 \\
\hline 2631 & Paperboard Mills . . . . . . . . . . . . & 479 & w & w & w & w & w & w & 0 & 136 & 4.6 \\
\hline 27 & Printing and Publishing $\ldots \ldots \ldots \ldots \ldots$ & 108 & 15,627 & 50 & 319 & 47 & 179 & 0 & 0 & 4 & 12.3 \\
\hline 28 & Chemicals and Alled Products . . . . . . . . & 4,357 & 131,858 & w & 2,466 & 2,060 & 323,486 & w & 417 & 257 & 6.4 \\
\hline 2812 & Alkalles and Chlorine ............ & 159 & 12,629 & w & 43 & w & 2 & w & 0 & - & 16.0 \\
\hline 2813 & Industrial Gases ................. & $w$ & w & 0 & $\mathbf{Q}$ & 15 & w & 0 & 0 & 3 & 10.2 \\
\hline 2819 & Industrial Inorganic Chemicals, nec ..... & 317 & 38,026 & $w$ & W & W & 74 & w & 359 & 10 & 9.0 \\
\hline 2821 & Plastlcs Materlals and Resins ........ & $w$ & $w$ & 665 & 190 & 209 & w & 1,079 & 0 & w & 6.1 \\
\hline 2822 & Synthetlc Rubber .............. & 122 & 1,946 & 61 & 19 & w & 4,084 & w & 0 & w & 14.3 \\
\hline 2823 & Cellulosic Manmade Fibers ........... & 31 & w & 0 & 21 & $\mathbf{w}$ & 1 & 1,202 & 0 & * & 24.9 \\
\hline 2824 & Organic Fibers, Noncellulosic $\ldots \ldots \ldots$ & w & 6,976 & w & 54 & w & w & $\mathbf{w}$ & 0 & • & 3.9 \\
\hline 2865 & Cyclic Crudes and Intermedlates ...... & 202 & 4,382 & 1,164 & 84 & 87 & 18,127 & w & 0 & w & 12.3 \\
\hline 2869 & Industrial Organle Chemicals, nec ..... & $w$ & 18,588 & 2,002 & 480 & w & w & 3,782 & 0 & 129 & 6.6 \\
\hline 2873 & Nitrogenous Fertillzers ............. & 568 & 2,886 & 0 & 27 & 539 & 166 & 0 & 0 & $\mathbf{Q}$ & 23.4 \\
\hline 2874 & Phosphatic Fortllizers ............... & 66 & 2,419 & 250 & w & w & 1 & w & 0 & w & 4.8 \\
\hline 29 & Petroleum and Coal Products ... & 1,190 & 33,463 & $Q$ & 2,600 & 744 & 1,502 & w & w & 282 & 8.6 \\
\hline 2911 & Petroleum Refining . . . . . . . . . . & 970 & w & 0 & w & 700 & 857 & 135 & 0 & w & 4.4 \\
\hline 30 & Rubber and Misc. Plastics Products . . . . . & 237 & 33,808 & 1,259 & 531 & 93 & 844 & 302 & 0 & 5 & 9.1 \\
\hline 3011 & 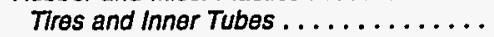 & w & w & 502 & 69 & 20 & 79 & w & 0 & w & 3.4 \\
\hline 308 & Miscellaneous Plastics Products, nec ... & 151 & 25,514 & 415 & w & 51 & 462 & 129 & 0 & w & 13.8 \\
\hline 31 & Leather and Leather Products . ........ & 12 & 795 & 225 & 221 & 5 & 45 & $\mathbf{Q}$ & 0 & $\mathbf{Q}$ & 24.9 \\
\hline 32 & Stone, Clay and Glass Products ....... & 879 & 30,846 & 1,379 & 3,582 & 369 & w & 13,238 & W & w & 7.6 \\
\hline 3211 & Flat Glass $\ldots \ldots \ldots \ldots \ldots \ldots$ & 49 & 1,512 & $w$ & 12 & 40 & 40 & * & 0 & w & 3.3 \\
\hline 3221 & 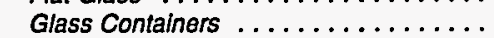 & 85 & 4,098 & 277 & 24 & 67 & 80 & 0 & 0 & • & 5.4 \\
\hline 3229 & Pressed and Blown Glass, nec . . . . . . & w & w & 81 & w & w & w & 0 & 0 & • & 8.1 \\
\hline 3241 & Cement, Hydraulls . . . . . . . . . . & 309 & 9,490 & 137 & 642 & 38 & 12 & 8,750 & 274 & 31 & 11.0 \\
\hline 3274 & 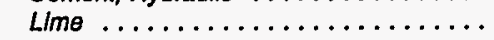 & 120 & 1,324 & $w$ & 244 & 8 & $\mathbf{Q}$ & 4,010 & w & 14 & 29.1 \\
\hline 3296 & 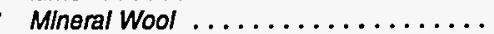 & 40 & w & w & w & 28 & w & * & W & • & 1.4 \\
\hline 33 & Primary Metal Industries . . . . . . . . & w & w & w & 1,845 & 674 & w & 28,045 & 8,129 & 68 & 3.7 \\
\hline 3312 & Blast Fumaces and Steel Mills . . . . . . . & 1,470 & w & w & W & 394 & 71 & 26,711 & 6,713 & 15 & 4.1 \\
\hline 3313 & Electrometalurgical Products .......... & 41 & 3,796 & 0 & 21 & 1 & w & 794 & w & W & 7.8 \\
\hline 3321 & Gray and Ductlle Iron Foundries ..... & w & w & 4 & 145 & 28 & 106 & w & w & 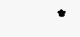 & 11.5 \\
\hline 3331 & Primary Copper .............. & 20 & $w$ & w & w & 15 & 3 & $w$ & w & * & 1.0 \\
\hline 3334 & Primary Aluminum & 268 & w & 1 & 129 & 20 & 42 & 40 & w & W & 3.1 \\
\hline 3339 & Primary Nonferrous Metals, nec . . . . . . . & $w$ & 3,784 & 1 & 53 & 16 & $\mathbf{w}$ & 346 & 243 & W & 1. \\
\hline 3353 & Aluminum Sheet, Plate, and Foll ....... & 58 & w & 0 & 75 & 41 & 61 & w & 0 & w & 1.5 \\
\hline 34 & Fabricated Metal Products..$\ldots \ldots \ldots \ldots$ & 306 & 29,610 & 500 & 1,020 & 170 & 1,234 & 255 & w & w & 11.3 \\
\hline 35 & Industrial Machinery and Equipment . . . . . & 235 & 29,349 & 476 & 770 & 106 & 680 & 483 & 24 & 4 & 11.6 \\
\hline 357 & Computer and Office Equipment ....... & 21 & 4,369 & 10 & 16 & 5 & 4 & 0 & 0 & * & 16.0 \\
\hline 36 & Electronlc and Other Electric Equipment . . & 211 & 29,816 & 607 & 421 & 77 & 404 & w & 44 & W & 10.3 \\
\hline 37 & Transportatlon Equipment $\ldots \ldots \ldots \ldots$ & 316 & 35,160 & 1,870 & 1,287 & 124 & 550 & w & W & 13 & 4. \\
\hline 3711 & Motor Vehlcles and Car Bodles ........ & 91 & w & 414 & 116 & 42 & 59 & w & $\ddot{w}$ & 5 & 3.3 \\
\hline 3714 & Motor Vehicle Parts and Accessories ... & 99 & w & 60 & W & 40 & 177 & W & w & w & 7. \\
\hline & Instruments and Related Products . . . . . . & 95 & w & 536 & w & 25 & $\mathbf{Q}$ & w & 0 & 1 & 12.4 \\
\hline 3841 & Surgical and Medlcal Instruments ..... & 6 & 1,161 & 9 & 30 & 2 & 8 & 0 & 0 & * & 15.1 \\
\hline 39 & Misc. Manufacturing Industries . . . . . & 33 & 3,661 & 114 & w & 14 & & & & $Q$ & 13.3 \\
\hline & Tota & 13,194 & 697,553 & 61,475 & 24,442 & 5,713 & 336,791 & 78,616 & 9,340 & 1,104 & \\
\hline
\end{tabular}

See footnotes at end of table. 
Table A22. Total Quantity of Purchased Energy Sources by Census Region, Industry Group, and Selected Industries, 1991 (Continued)

(Estimates in Btu or Physical Units)

\begin{tabular}{|c|c|c|c|c|c|c|c|c|c|c|c|}
\hline $\begin{array}{l}\text { SIC } \\
\text { Code* }\end{array}$ & $\begin{array}{l}\text { Industry Groups } \\
\text { and Industry }\end{array}$ & $\begin{array}{c}\text { Total } \\
\text { (trillion } \\
\text { Btu) }\end{array}$ & $\begin{array}{c}\text { Electricity } \\
\text { (million } \\
\text { kWh) }\end{array}$ & $\begin{array}{c}\text { Residual } \\
\text { Fuel Oil } \\
(1000 \\
\text { bbls })\end{array}$ & $\begin{array}{c}\text { Distillate } \\
\text { Fuel Oil } \\
(1000 \\
\text { bbls })\end{array}$ & $\begin{array}{c}\text { Natural } \\
\text { Gase }^{\mathrm{e}} \\
\text { (billion } \\
\text { cu ft) }\end{array}$ & $\begin{array}{l}\text { LPG } \\
(1000 \\
\text { bbls) }\end{array}$ & $\begin{array}{c}\text { Coal } \\
(1000 \\
\text { short tons })\end{array}$ & $\begin{array}{c}\text { Coke and } \\
\text { Breeze } \\
(1000 \\
\text { short tons })\end{array}$ & $\begin{array}{c}\text { Other' } \\
\text { (Itillion } \\
\text { Btu) }\end{array}$ & $\begin{array}{c}\text { RSE } \\
\text { Row } \\
\text { Factors }\end{array}$ \\
\hline & & \multicolumn{10}{|c|}{ Northeast Census Region } \\
\hline & RSE Column Factors: & 0.7 & 0.7 & 1.0 & 1.2 & 0.8 & 1.2 & 1.3 & 1.2 & 1.2 & \\
\hline 20 & Food and Kindred Products & 79 & 5,443 & 1,143 & 981 & 40 & 220 & 99 & 0 & a & 13.9 \\
\hline 2011 & Meat Packing Plants .... & 1 & 141 & w & 34 & 1 & Q & 0 & 0 & * & 23.6 \\
\hline 2033 & Canned Fruits and Vegetables . . . . . . . . & 6 & 292 & 146 & 22 & 4 & 13 & $\mathbf{Q}$ & 0 & • & 17.0 \\
\hline 2037 & Frozen Fruits and Vegetables . . . . . . & 1 & 140 & 128 & 3 & * & $\mathbf{Q}$ & 0 & 0 & * & 32.0 \\
\hline 2046 & Wet Com Milling . . . . . . & • & 15 & w & w & * & - & 0 & 0 & * & 23.1 \\
\hline 2051 & Bread, Cake, and Related Products . . . . & 7 & 382 & - & w & w & 8 & 0 & 0 & - & 18.7 \\
\hline 2063 & Beet Sugar & 0 & 0 & 0 & 0 & 0 & 0 & 0 & 0 & 0 & NF \\
\hline 2075 & Soybean Oil Mills & 0 & 0 & 0 & 0 & 0 & 0 & 0 & 0 & 0 & NF \\
\hline 2082 & Malt Beverages & 8 & 521 & w & 9 & 3 & 4 & w & 0 & 0 & 15.7 \\
\hline 21 & Tobacco Products. & NA & NA & NA & NA & NA & NA & NA & NA & NA & 24.8 \\
\hline 22 & Textile Mill Products & 27 & 1,372 & 777 & 557 & 10 & 164 & 17 & 0 & $\cdot$ & 19.1 \\
\hline 23 & Apparel and Other Textile Products ..... & 5 & 495 & 44 & 49 & 2 & a & $\mathbf{0}$ & 0 & - & 29.1 \\
\hline 24 & Lumber and Wood Products $\ldots \ldots \ldots \ldots$ & NA & NA & NA & NA & NA & NA & NA & NA & NA & 33.5 \\
\hline 25 & Furniture and Fixtures .... & 5 & 446 & $Q$ & 49 & 2 & 60 & 0 & 0 & 1 & 28.3 \\
\hline 26 & Paper and Allied Products & w & 8,826 & 11,431 & 624 & 36 & w & w & 0 & 38 & 6.0 \\
\hline 2611 & Pulp Mills $\ldots \ldots \ldots \ldots \ldots \ldots \ldots$ & 5 & Q & 290 & 7 & 0 & 13 & 0 & 0 & 3 & 33.2 \\
\hline 2621 & Paper Mills .... & 166 & w & 9,763 & $w$ & 19 & 284 & w & 0 & 30 & 4.3 \\
\hline 2631 & Paperboard Mills. & w & w & w & $\mathbf{Q}$ & 6 & 5 & w & 0 & $w$ & 15.1 \\
\hline 27 & Printing and Publishing & 23 & 3,167 & 36 & 241 & $\mathbf{9}$ & 31 & 0 & 0 & $\because$ & 25.2 \\
\hline 28 & Chemicals and Allied Products. & w & w & 3,254 & w & 58 & 862 & w & 0 & 14 & 8.5 \\
\hline 2812 & Alkalies and Chlorine ....... & - & w & 0 & 0 & - & 0 & 0 & 0 & 0 & 35.9 \\
\hline 2813 & Industrial Gases . . . . . . . & 5 & w & 0 & 1 & * & $\mathbf{Q}$ & 0 & 0 & 1 & 14.1 \\
\hline 2819 & Industrial Inorganic Chemicals, nec .... & 9 & w & $w$ & 81 & 6 & 14 & 0 & 0 & : & 21.4 \\
\hline 2821 & Plastics Materials and Resins & 21 & w & 476 & 108 & 8 & w & w & 0 & w & 9.9 \\
\hline 2822 & Synthetic Rubber ........ & w & w & w & * & w & * & 0 & 0 & : & 25.6 \\
\hline 2823 & Cellulosic Manmade Fibers & 0 & 0 & 0 & 0 & 0 & 0 & 0 & 0 & 0 & NF \\
\hline 2824 & Organic Fibers, Noncellulosic . . . . . . . & * & 95 & $Q$ & W & - & - & 0 & 0 & 0 & 14.6 \\
\hline 2865 & Cyclic Crudes and Intermediates . . . . . . & 12 & 406 & $\bar{w}$ & w & 7 & 2 & w & 0 & - & 20.7 \\
\hline 2869 & Industrial Organic Chemicals, nec . . . . & w & w & 1,601 & w & w & 258 & 0 & 0 & w & 9.0 \\
\hline 2873 & Nitrogenous Fertilizers $\ldots \ldots \ldots \ldots$. & 1 & 29 & 0 & 3 & 1 & 1 & 0 & 0 & • & 48.3 \\
\hline 2874 & Phosphatic Fertilizers & 0 & 0 & 0 & 0 & 0 & 0 & 0 & 0 & 0 & NF \\
\hline 29 & Petroleum and Coal Products & 59 & 3,054 & 0 & 1,290 & 28 & Q & w & 0 & w & 10.2 \\
\hline 2911 & Petroleum Refíning & 41 & 2,515 & 0 & 0 & 23 & 0 & w & 0 & w & 5.7 \\
\hline 30 & Rubber and Misc. Plasties Products & w & 5,484 & 455 & 206 & w & W & 86 & 0 & 1 & 19.6 \\
\hline 3011 & Tires and Inner Tubes . . . . . . . . . & 2 & 125 & 63 & Q & 1 & 5 & 0 & 0 & • & 12.5 \\
\hline 308 & Miscellaneous Plastics Products, nec... & 28 & 4,810 & 251 & w & 7 & 180 & w & 0 & w & 24.1 \\
\hline 31 & Leather and Leather Products $\ldots \ldots \ldots \ldots$ & 4 & 205 & 142 & 197 & 1 & 29 & $\mathbf{Q}$ & 0 & * & 27.3 \\
\hline 32 & Stone, Clay and Glass Products . & 170 & 5,599 & 434 & 734 & 57 & w & 3,505 & w & w & 17.8 \\
\hline 3211 & Flat Glass $\ldots \ldots \ldots \ldots \ldots \ldots$ & w & w & 0 & 1 & w & W & * & 0 & • & 4.5 \\
\hline 3221 & Glass Containers & 19 & 834 & 195 & 14 & 14 & 24 & 0 & 0 & • & 8.8 \\
\hline 3229 & Pressed and Blown Glass, nec .. & w & w & 80 & w & w & 8 & 0 & 0 & • & 10.8 \\
\hline 3241 & Cement, Hydraulic & 42 & 1,334 & 14 & w & - & 1 & 1,457 & w & w & 18.3 \\
\hline 3274 & Lime & $\mathbf{Q}$ & $Q$ & 0 & Q & - & $Q$ & Q & 0 & : & NF \\
\hline 3296 & Mineral Wool & 4 & w & 0 & 13 & w & w & * & w & - & 1.7 \\
\hline 33 & Primary Metal Industries & 466 & 17,771 & 770 & 331 & 102 & 308 & 9,969 & 691 & 10 & 9.4 \\
\hline 3312 & Blast Fumaces and Steel Mills . . . . . . . & 375 & 8,136 & 534 & 155 & 65 & 30 & w & 476 & $w$ & 7.2 \\
\hline 3313 & Electrometalurgical Products .... . & $w$ & w & 0 & 1 & - & ? & $w$ & w & - & 12.9 \\
\hline 3321 & Gray and Ductile Iron Foundries & 5 & 350 & 0 & 14 & 2 & 18 & 1 & 74 & - & 16.4 \\
\hline 3331 & Primary Copper ........... & * & w & 0 & w & " & * & 0 & $:$ & - & 1.0 \\
\hline 3334 & Primary Aluminum & w & w & 1 & w & w & w & w & 0 & w & 4.9 \\
\hline 3339 & Primary Nonferrous Metals, nec.. & w & 100 & 1 & W & 1 & 1 & W & w & - & 2.2 \\
\hline 3353 & Aluminum Sheet, Plate, and Foil & w & 451 & 0 & 12 & w & 22 & 0 & 0 & * & 1.5 \\
\hline 34 & Fabricated Metal Products . . . . . & 57 & 5,053 & 368 & 367 & 32 & 155 & 10 & 37 & 1 & 15.7 \\
\hline 35 & Industrial Machinery and Equipment & w & w & 396 & w & 15 & w & 0 & 0 & Q & 20.8 \\
\hline 357 & Computer and Office Equipment & 4 & 819 & 8 & 10 & 1 & 2 & 0 & 0 & * & 20.6 \\
\hline 36 & Electronic and Other Electric Equipment . & 43 & 6,541 & 503 & 292 & 14 & 167 & 4 & 2 & 1 & 16.1 \\
\hline 37 & Transportation Equipment & w & w & 1,077 & w & 10 & w & w & 0 & W & 10.9 \\
\hline 3711 & Motor Vehicles and Car Bodies ..... & w & w & w & w & 1 & 1 & 0 & 0 & - & 7.4 \\
\hline 3714 & Motor Vehicle Parts and Accessories. & 8 & 887 & w & 6 & w & w & w & 0 & . & 11.5 \\
\hline 38 & Instruments and Related Products & 50 & w & 513 & w & w & Q & w & 0 & 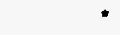 & 16.6 \\
\hline 3841 & Surgical and Medical Instruments & 2 & 332 & 9 & 16 & $\because$ & 2 & 0 & 0 & * & 18.3 \\
\hline 39 & Misc. Manufacturing Industries . & $\bar{w}$ & 1,187 & 84 & W & w & 40 & 22 & 0 & - & 17.8 \\
\hline & Total $\ldots \ldots \ldots \ldots \ldots \ldots$ & 1,482 & 86,471 & 21,586 & 7,849 & 445 & 3,803 & w & w & 90 & 5.4 \\
\hline
\end{tabular}

See footnotes at end of table. 
Table A22. Total Quantity of Purchased Energy Sources by Census Region, Industry Group, and Selected Industries, 1991 (Continued)

(Estimates in Btu or Physical Units)

\begin{tabular}{|c|c|c|c|c|c|c|c|c|c|c|c|}
\hline \multirow[t]{3}{*}{$\begin{array}{c}\text { SIC } \\
\text { Code" }\end{array}$} & $\begin{array}{l}\text { Industry Groups } \\
\text { and Industry }\end{array}$ & $\begin{array}{c}\text { Total } \\
\text { (trillion } \\
\text { Btu) }\end{array}$ & $\begin{array}{c}\text { Electricity } \\
\text { (million } \\
\text { kWh) }\end{array}$ & $\begin{array}{l}\text { Residual } \\
\text { Fuel Oil } \\
\text { (1000 } \\
\text { bbls) }\end{array}$ & $\begin{array}{c}\text { Distillate } \\
\text { Fuel Oil } \\
(1000 \\
\text { bbls })\end{array}$ & $\begin{array}{c}\text { Natural } \\
\text { Gas } \\
\text { (billion } \\
\text { cu ft) }\end{array}$ & $\begin{array}{l}\text { LPG } \\
(1000 \\
\text { bbls })\end{array}$ & $\begin{array}{c}\text { Coal } \\
(1000 \\
\text { short tons) }\end{array}$ & $\begin{array}{c}\text { Coke and } \\
\text { Breeze } \\
(1000 \\
\text { short tons) }\end{array}$ & $\begin{array}{l}\text { Other } \\
\text { (trillion } \\
\text { Btu) }\end{array}$ & \multirow[t]{3}{*}{$\begin{array}{c}\text { RSE } \\
\text { Row } \\
\text { Factors }\end{array}$} \\
\hline & & \multicolumn{9}{|c|}{ Midwest Census Region } & \\
\hline & RSE Column Factors: & 0.7 & 0.6 & 1.5 & 1.3 & 0.7 & 1.2 & 1.0 & 1.3 & 1.1 & \\
\hline 20 & Food and Kindred Products . . . . . . . . . & 414 & $w$ & 869 & 568 & W & 345 & 4,823 & $w$ & 10 & 8.8 \\
\hline 2011 & Meat Packing Plants . . . . . . . . . . . . & 32 & 2,065 & 132 & 59 & 22 & 14 & 28 & 0 & - & 9.7 \\
\hline 2033 & Canned Frults and Vegetables . . . . . . . . . & 10 & 375 & 0 & 62 & 8 & 39 & 0 & 0 & * & 17.0 \\
\hline 2037 & Frozen Fruits and Vegetables . . . . . . . . & 4 & 289 & 36 & 3 & 2 & 3 & 0 & 0 & * & 26.8 \\
\hline 2046 & 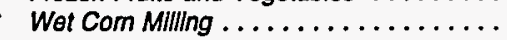 & 120 & W & w & 27 & 45 & * & 2,736 & W & $w$ & 13.4 \\
\hline 2051 & Bread, Cake, and Related Products . .... & 9 & 632 & 0 & $W$ & $w$ & - & 0 & 0 & * & 16.2 \\
\hline 2063 & 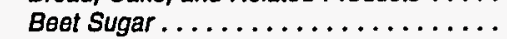 & 34 & 234 & W & 11 & 6 & 2 & 1,092 & $w$ & * & 6.6 \\
\hline 2075 & Soybean Oll Mills ................. & 32 & $w$ & 15 & 7 & 16 & w & $w$ & $\mathbf{0}$ & W & 4.3 \\
\hline 2082 & Malt Boverages $\ldots \ldots \ldots \ldots \ldots \ldots$ & 11 & 480 & 35 & 1 & $w$ & W & W & 0 & $\bullet$ & 17.3 \\
\hline 21 & Tobacco Products ................ & NA & NA & NA & NA & NA & NA & NA & NA & NA & 9.5 \\
\hline 22 & Textile Mill Products ............... & NA & NA & NA & NA & NA & NA & NA & NA & NA & 24.0 \\
\hline 23 & Apparel and Other Textlle Products ...... & NA & NA & NA & NA & NA & NA & NA & NA & NA & 30.9 \\
\hline 24 & Lumber and Wood Products .......... & 32 & 2,834 & $Q$ & 362 & 11 & 197 & 61 & 0 & 7 & 30.1 \\
\hline 25 & Fumiture and Fixtures $\ldots \ldots \ldots \ldots \ldots$ & $W$ & 1,571 & 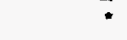 & 10 & $w$ & 45 & Q & 0 & $\cdot$ & 21.8 \\
\hline 26 & Paper and Allied Products . . . . . . . . . . & W & 14,038 & 799 & 160 & 112 & $W$ & 4,020 & $\mathbf{W}$ & 31 & 6.2 \\
\hline 2611 & Pulp MIIIs $\ldots \ldots \ldots \ldots \ldots \ldots \ldots$ & 10 & 556 & 0 & 14 & 3 & $\mathbf{Q}$ & 161 & 0 & 1 & 33.8 \\
\hline 2621 & Paper Mills . . . . . . . . . . . . . . . & 168 & 7,730 & W & 110 & 57 & 67 & 2,808 & $w$ & 15 & 5.4 \\
\hline 2631 & Paperboard Mills . . . . . . . . . . . . . . . & W & 2,059 & 39 & 12 & 23 & 20 & 969 & 0 & $W$ & 13.9 \\
\hline 27 & Printing and Publishing $\ldots \ldots \ldots \ldots \ldots$ & 42 & 5,224 & 10 & 28 & 22 & 60 & 0 & 0 & 1 & 16.8 \\
\hline 28 & Chemicals and Allied Products . . . . . . . . & $\bar{W}$ & $W$ & 321 & $\mathbf{Q}$ & 223 & W & 2,810 & 8 & W & 9.8 \\
\hline 2812 & Alkalles and Chlorine ............. & W & $W$ & 0 & $w$ & 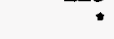 & 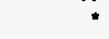 & 0 & 0 & $\bullet$ & 26.7 \\
\hline 2813 & Industrial Gases ................ & $\mathbf{W}$ & 3,319 & 0 & $\mathbf{Q}$ & $w$ & $\mathbf{Q}$ & 0 & 0 & - & 14.2 \\
\hline 2819 & Industrial Inorganic Chemicals, nec ..... & 71 & $W$ & $W$ & 3 & $w$ & 4 & $w$ & 1 & 1 & 12.8 \\
\hline 2821 & Plastics Materials and Resins ......... & w & 3,247 & W & $w$ & 24 & W & w & 0 & 8 & 9.1 \\
\hline 2822 & Synthotic Rubber ............... & $W$ & 241 & 0 & $W$ & $w$ & 1 & w & 0 & * & 18.8 \\
\hline 2823 & Cellulosic Manmade Fibers .......... & 0 & 0 & 0 & 0 & 0 & 0 & 0 & 0 & 0 & NF \\
\hline 2824 & Organic Fibers, Noncellulosic $\ldots \ldots \ldots$ & 0 & 0 & 0 & 0 & 0 & 0 & 0 & 0 & 0 & NF \\
\hline 2865 & Cyclic Crudes and Intermedlates ....... & $\mathbf{W}$ & 756 & W & $w$ & 14 & $W$ & $w$ & 0 & $w$ & 13.5 \\
\hline 2869 & Industrial Organic Chemicals, nec ...... & $\mathbf{W}$ & $W$ & 10 & $w$ & $w$ & 4,483 & 1,137 & 0 & 6 & 8.0 \\
\hline 2873 & Nitrogenous Fertilizers ........... & 74 & 556 & 0 & 5 & 69 & 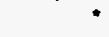 & 0 & 0 & * & 33.9 \\
\hline 2874 & Phosphatic Fertilizers $\ldots \ldots \ldots \ldots \ldots \ldots$ & 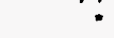 & 1 & 0 & - & 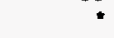 & - & 0 & 0 & - & 4.5 \\
\hline 29 & Petroleum and Coal Products $\ldots \ldots \ldots$ & 139 & $w$ & $\mathbf{Q}$ & 476 & 83 & Q & $w$ & 0 & $w$ & 12.8 \\
\hline 2911 & Petroleum Refining . . . . . . . . . . . . . & 93 & 6,168 & 0 & 0 & 67 & 0 & w & 0 & w & 4.6 \\
\hline 30 & Rubber and Misc. Plastics Products ...... & 94 & 13,132 & 269 & 33 & 41 & 173 & 134 & $\mathbf{0}$ & 1 & 11.6 \\
\hline 3011 & Tres and Inner Tubes .............. & $W$ & W & 186 & 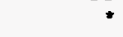 & 6 & W & $w$ & 0 & 1 & 4.0 \\
\hline 308 & Mlscellaneous Plastics Products, nec .... & NA & NA & NA & NA & NA & NA & NA & NA & NA & 19.5 \\
\hline 31 & Leather and Leather Products ........ & 4 & 262 & 58 & 5 & 2 & 11 & 0 & 0 & $\bullet$ & 25.6 \\
\hline 32 & Stone, Clay and Glass Products ....... & 251 & $w$ & 69 & $w$ & 105 & 123 & 3,488 & 83 & 30 & 10.2 \\
\hline 3211 & Flat Glass $\ldots \ldots \ldots \ldots \ldots \ldots$ & 13 & W & 0 & W & 11 & W & 0 & 0 & 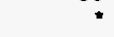 & 4.0 \\
\hline 3221 & Glass Containers .............. & 20 & 873 & 0 & 1 & 17 & 11 & 0 & 0 & * & 7.5 \\
\hline 3229 & Pressed and Blown Glass, nec ....... & 14 & 614 & $\cdot$ & 9 & 11 & 8 & 0 & 0 & $\cdot$ & 6.6 \\
\hline 3241 & Cement, Hydraullc ............. & 85 & 2,247 & w & $w$ & 6 & 5 & 2,366 & 0 & $w$ & 15.1 \\
\hline 3274 & 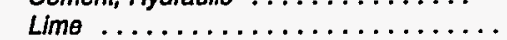 & 35 & 367 & 0 & 65 & 3 & 1 & 934 & $w$ & $w$ & 18.6 \\
\hline 3296 & 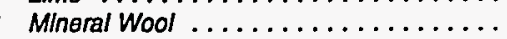 & 17 & 1,251 & $w$ & W & $\bar{W}$ & 14 & 0 & W & * & 1.1 \\
\hline 33 & Primary Metal Industries ............. & 936 & $W$ & w & 749 & w & 254 & 10,570 & 4,835 & $w$ & 5.2 \\
\hline 3312 & Blast Fumaces and Steel Mils . . . . . . . . & 687 & $w$ & W & $W$ & $w$ & 24 & 9,953 & 4,156 & 5 & 5.2 \\
\hline 3313 & Electrometalurgical Products .......... & 27 & 2,378 & 0 & 13 & 1 & $w$ & W & $w$ & w & 10.0 \\
\hline 3321 & Gray and Ductile Iron Foundries ..... & W & 4,350 & 4 & $\mathbf{Q}$ & $w$ & 38 & $w$ & $w$ & * & 12.1 \\
\hline 3331 & Primary Copper ............... & $\bullet$ & W & 0 & $\overline{0}$ & $\bullet$ & 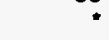 & 0 & 0 & 0 & 1.3 \\
\hline 3334 & Primary Aluminum ............. & $w$ & 7,782 & 0 & W & 3 & $W$ & $W$ & 0 & $\mathbf{W}$ & 5.0 \\
\hline 3339 & Primary Nonferrous Metals, nec . . . . . . . . & w & 727 & 0 & 11 & 3 & 7 & 0 & $w$ & $\cdot$ & 2.0 \\
\hline 3353 & Aluminum Sheot, Plate, and Foil ........ & 22 & $w$ & 0 & W & 16 & $W$ & 0 & 0 & $w$ & 1.3 \\
\hline 34 & Fabricated Metal Products . . . . . . . . . . . & W & 12,749 & 7 & w & 79 & W & 245 & $\mathbf{W}$ & w & 14.7 \\
\hline 35 & Industrial Machinery and Equipment ....... & 116 & W & 38 & $\mathbf{w}$ & 56 & $w$ & 483 & $\mathbf{Q}$ & $\mathbf{W}$ & 13.5 \\
\hline 357 & Computer and Office Equipment ....... & 4 & 796 & 0 & 1 & 2 & 1 & 0 & 0 & $\bullet$ & 24.0 \\
\hline 36 & Electronic and Other Electric Equipment ... . & 58 & 7,001 & 46 & W & 28 & 70 & W & 0 & W & 14.0 \\
\hline 37 & Transportation Equipment . . . . . . . . . & W & W & 391 & 311 & 70 & 216 & w & $\mathbf{W}$ & 6 & 6.1 \\
\hline 3711 & Motor Vehicles and Car Bodles . . . . . . . . & 63 & $w$ & W & $W$ & 29 & 26 & $W$ & $\mathbf{W}$ & $w$ & 4.6 \\
\hline 3714 & Motor Vehiclo Parts and Accessories .... & 75 & $\mathbf{W}$ & $\mathbf{W}$ & $\mathbf{W}$ & 29 & W & $W$ & $w$ & $w$ & 8.2 \\
\hline 38 & Instruments and Related Products . . . . . . & 11 & 1,806 & $\mathbf{Q}$ & $\mathbf{Q}$ & $w$ & 6 & $W$ & 0 & • & 22.4 \\
\hline 3841 & Surgical and Medical Instruments ...... & 1 & 276 & 0 & $*$ & $\because$ & 3 & 0 & 0 & - & 18.5 \\
\hline 39 & Mlsc. Manufacturing Industries . . . . . . . . . & 11 & 929 & 2 & 9 & 5 & 16 & 10 & 0 & $\mathbf{Q}$ & 23.6 \\
\hline & Total $\ldots \ldots \ldots \ldots \ldots \ldots$ & $\mathbf{W}$ & 198,408 & $W$ & 4,555 & 1,407 & W & 28,074 & 5,135 & 174 & 4.8 \\
\hline
\end{tabular}

Seo footnotes at end of table. 
Table A22. Total Quantity of Purchased Energy Sources by Census Region, Industry Group, and Selected Industries, 1991 (Continued)

(Estimates in Btu or Physical Units)

\begin{tabular}{|c|c|c|c|c|c|c|c|c|c|c|c|}
\hline \multirow[t]{3}{*}{$\begin{array}{l}\text { SIC } \\
\text { Code }\end{array}$} & $\begin{array}{l}\text { Industry Groups } \\
\text { and Industry }\end{array}$ & $\begin{array}{c}\text { Total } \\
\text { (trillion } \\
\text { Btu) }\end{array}$ & $\begin{array}{c}\text { Electricity } \\
\text { (million } \\
\text { kWh) }\end{array}$ & $\begin{array}{c}\text { Residual } \\
\text { Fuel Oil } \\
(1000 \\
\text { bbls })\end{array}$ & $\begin{array}{c}\text { Distillate } \\
\text { Fuel Oil } \\
(1000 \\
\text { bbls) }\end{array}$ & $\begin{array}{c}\text { Natural } \\
\text { Gas }^{\mathrm{c}} \\
\text { (billion } \\
\text { cu ft) } \\
\end{array}$ & $\begin{array}{c}\text { LPG } \\
(1000 \\
\text { bbls) }\end{array}$ & $\begin{array}{c}\text { Coal } \\
\text { (1000 } \\
\text { short tons) }\end{array}$ & $\begin{array}{c}\text { Coke and } \\
\text { Breeze } \\
(1000 \\
\text { short tons })\end{array}$ & $\begin{array}{l}\text { Otherd } \\
\text { (trillion } \\
\text { Btu) }\end{array}$ & $\begin{array}{c}\text { RSE } \\
\text { Row } \\
\text { Factors }\end{array}$ \\
\hline & & \multicolumn{10}{|c|}{ South Census Region } \\
\hline & RSE Column Factors: & 0.6 & 0.6 & 1.4 & 1.2 & 0.7 & 1.1 & 1.1 & 1.6 & 1.1 & \\
\hline 20 & Food and Kindred Products & 230 & w & 1,571 & 924 & 131 & 621 & 770 & w & w & 10.0 \\
\hline 2011 & Meat Packing Plants & 10 & 892 & 14 & 178 & 5 & 112 & 0 & 0 & - & 16.8 \\
\hline 2033 & Canned Fruits and Vegetables. & 7 & 200 & 26 & 9 & 6 & 14 & 0 & 0 & - & 19.3 \\
\hline 2037 & Frozen Fruits and Vegetables. & 8 & 551 & 123 & 12 & 5 & 6 & 0 & 0 & - & 17.8 \\
\hline 2046 & Wet Com Milling ... . & w & 837 & 0 & 2 & 4 & * & 322 & 0 & w & 22.5 \\
\hline 2051 & Bread, Cake, and Related Products . . . . . & 11 & 838 & 0 & 19 & 7 & 8 & 0 & 0 & - & 12.8 \\
\hline 2063 & Beet Sugar. & w & w & 0 & 1 & w & - & 0 & w & - & 17.0 \\
\hline 2075 & Soybean Oil Mills & 14 & 539 & 34 & 24 & 8 & w & $w$ & 0 & w & 4.9 \\
\hline 2082 & Malt Beverages .. & 14 & 863 & w & w & 8 & 1 & w & 0 & $\bullet$ & 13.9 \\
\hline 21 & Tobacco Products & 25 & 1,452 & 133 & 41 & 4 & 23 & 681 & 0 & - & 6.3 \\
\hline 22 & Textile Mill Products & 229 & w & 1,176 & 513 & 87 & 458 & 1,242 & 0 & w & 6.5 \\
\hline 23 & Apparel and Other Textile Products & 31 & 4,165 & Q & 71 & 12 & 134 & 83 & 0 & $\because$ & 22.8 \\
\hline 24 & Lumber and Wood Products ..... & 79 & $w$ & $\mathbf{Q}$ & w & 15 & 341 & 26 & 0 & 23 & 20.8 \\
\hline 25 & Furniture and Fixtures & w & 2,592 & 61 & 100 & w & 121 & $w$ & 0 & 3 & 18.8 \\
\hline 26 & Paper and Allied Products & 723 & 27,095 & 10,865 & 705 & w & 495 & 6,745 & 0 & w & 4.4 \\
\hline 2611 & Pulp Mills . . . . . . . . & 70 & 1,421 & 3,365 & 115 & 21 & 122 & 170 & 0 & 17 & 17.6 \\
\hline 2621 & Paper Mills . & 318 & w & 2,803 & w & 122 & 150 & 3,864 & 0 & 36 & 3.4 \\
\hline 2631 & Paperboard Mills . & $w$ & 6,887 & 4,609 & w & w & 39 & 2,674 & 0 & 79 & 5.2 \\
\hline 27 & Printing and Publishing & 28 & 4,797 & $\mathbf{Q}$ & 35 & 10 & 45 & 0 & 0 & 1 & 21.5 \\
\hline 28 & Chemicals and Allied Products. & w & 79,359 & w & 1,109 & 1,660 & w & w & 118 & 206 & 7.2 \\
\hline 2812 & Alkalies and Chlorine ....... & 147 & 10,289 & 0 & 35 & w & 1 & $w$ & 0 & 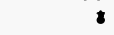 & 17.9 \\
\hline 2813 & Industrial Gases .. & w & w & 0 & * & 11 & w & 0 & 0 & 1 & 13.3 \\
\hline 2819 & Industrial Inorganic Chemicals, nec & w & w & w & w & $w$ & 49 & 527 & 68 & 6 & 12.1 \\
\hline 2821 & Plastics Materials and Resins . . . . & 425 & W & W & W & 176 & 50,420 & w & 0 & w & 5.5 \\
\hline 2822 & Synthetic Rubber $\ldots \ldots \ldots$ & 114 & w & W & w & w & 4,083 & 0 & 0 & w & 13.3 \\
\hline 2823 & Cellulosic Manmado Fibers & 31 & w & 0 & 21 & w & 1 & 1,202 & 0 & : & 25.2 \\
\hline 2824 & Organic Fibers, Noncellulosic & w & 6,881 & w & w & w & w & w & 0 & • & 3.9 \\
\hline 2865 & Cyclic Crudes and Intermediates & w & 3,209 & w & 16 & 66 & w & $w$ & 0 & 9 & 12.2 \\
\hline 2869 & Industrial Organic Chemicals, nec & w & 13,961 & 390 & w & w & w & 2,646 & 0 & 115 & 8.1 \\
\hline 2873 & Nitrogenous Fertilizers . ....... & 414 & 1,872 & 0 & 15 & 395 & 123 & 0 & 0 & - & 28.8 \\
\hline 2874 & Phosphatic Fertilizers & w & 1,908 & 250 & w & w & 1 & w & 0 & 21 & 3.3 \\
\hline 29 & Petroleum and Coal Products & 808 & 15,699 & $Q$ & w & 537 & 956 & w & w & $w$ & 7.9 \\
\hline 2911 & Petroleum Refining ....... & 714 & 15,216 & 0 & w & 524 & 857 & $w$ & 0 & $w$ & 4.1 \\
\hline 30 & Rubber and Misc. Plastics Products & 88 & 12,328 & 534 & 259 & 35 & 246 & 60 & 0 & 2 & 10.1 \\
\hline 3011 & Tires and Inner Tubes . . . . . . . . & w & 2,855 & w & $w$ & 13 & 53 & w & 0 & $\bar{w}$ & 4.1 \\
\hline 308 & Miscellaneous Plastics Products, nec & 46 & 7,801 & 158 & $w$ & 16 & 124 & W & 0 & • & 20.5 \\
\hline 31 & Leather and Leather Products & 2 & 258 & 26 & 9 & * & 3 & 0 & 0 & 0 & 24.7 \\
\hline 32 & Stone, Clay and Glass Products & 321 & W & 200 & W & 156 & W & 3,873 & 232 & 17 & 11.7 \\
\hline 3211 & Flat Glass $\ldots \ldots \ldots \ldots$ & 23 & 781 & 0 & 7 & 19 & 9 & 0 & 0 & . & 4.0 \\
\hline 3221 & Glass Containers & 29 & 1,262 & w & w & $w$ & 23 & 0 & 0 & - & 8.4 \\
\hline 3229 & Pressed and Blown Glass, nec & w & 1,598 & 1 & $w$ & $w$ & w & 0 & 0 & : & 9.1 \\
\hline 3241 & Cement, Hydraulic ......... & 104 & 3,519 & 62 & 191 & 21 & 4 & 2,555 & 190 & 7 & 16.5 \\
\hline 3274 & Lime & 37 & 405 & 0 & 69 & $w$ & : & 1,156 & w & w & 21.8 \\
\hline 3296 & Mineral Wool & 16 & w & w & 1 & 11 & 15 & 0 & w & - & 1.4 \\
\hline 33 & Primary Metal Industries & w & W & 1,202 & W & w & w & w & 2,484 & 15 & 4.5 \\
\hline 3312 & Blast Fumaces and Steol Mills. & 339 & w & 1,197 & w & w & 13 & 5,744 & 2,061 & w & 6.4 \\
\hline 3313 & Electrometalurgical Products .... & W & W & 0 & 7 & - & 0 & W & w & 1 & 11.2 \\
\hline 3321 & Gray and Ductile Iron Foundries & 21 & w & * & 56 & w & 46 & a & $\mathbf{w}$ & : & 11.6 \\
\hline 3331 & Primary Copper & w & 200 & w & 5 & w & 1 & 0 & 0 & - & 1.1 \\
\hline 3334 & Primary Aluminum & 94 & $w$ & 0 & w & $\ddot{w}$ & 9 & w & w & w & 4.2 \\
\hline 3339 & Primary Nonferrous Metals, nec. & w & 1,694 & 0 & 9 & $w$ & w & w & 0 & w & 3.9 \\
\hline 3353 & Aluminum Sheet, Plate, and Foil & 25 & W & 0 & 25 & 17 & 22 & w & 0 & - & 1.8 \\
\hline 34 & Fabricated Metal Products.$\ldots \ldots \ldots \ldots$. & W & 8,886 & $\mathbf{Q}$ & w & 42 & w & 0 & 23 & 3 & 21.4 \\
\hline 35 & Industrial Machinery and Equipment . & W & 8,138 & 42 & 126 & 26 & w & 1 & Q & w & 17.9 \\
\hline 357 & Computer and Office Equipment & 4 & 809 & 3 & $\mathbf{Q}$ & 1 & $\because$ & 0 & 0 & 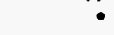 & 23.0 \\
\hline 36 & Electronic and Other Electric Equipment & 81 & 10,724 & 58 & 44 & 26 & 154 & $w$ & 41 & $w$ & 14.5 \\
\hline 37 & Transportation Equipment & 66 & 8,542 & w & 332 & 27 & 133 & w & 1 & 3 & 8.2 \\
\hline 3711 & Motor Vehicles and Car Bodies & 23 & 2,375 & 73 & 35 & 12 & 28 & w & 0 & w & 4.5 \\
\hline 3714 & Motor Vehicle Parts and Accessories & 13 & 1,919 & 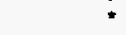 & 8 & W & 41 & $w$ & 1 & - & 11.9 \\
\hline 38 & Instruments and Related Products . & 17 & 3,239 & $\mathbf{Q}$ & 45 & 5 & 6 & 0 & 0 & 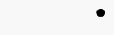 & 18.5 \\
\hline 3841 & Surgical and Medical instruments & 2 & 331 & 0 & 11 & * & 1 & 0 & 0 & 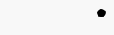 & 21.5 \\
\hline 39 & Misc. Manufacturing Industries. & w & 1,168 & 28 & & w & 25 & 0 & 0 & - & 21.1 \\
\hline & Total & w & 295,955 & w & 8,546 & 3,217 & $w$ & 27,864 & 2,911 & 628 & 4.0 \\
\hline
\end{tabular}

See footnotes at end of table. 
Table A22. Total Quantity of Purchased Energy Sources by Census Region, Industry Group, and Selected Industries, 1991 (Continued) (Estimates in Btu or Physical Units)

\begin{tabular}{|c|c|c|c|c|c|c|c|c|c|c|c|}
\hline $\begin{array}{l}\text { SIC } \\
\text { Code }\end{array}$ & $\begin{array}{l}\text { Industry Groups } \\
\text { and Industry }\end{array}$ & $\begin{array}{c}\text { Total } \\
\text { (trillion } \\
\text { Btu) }\end{array}$ & $\begin{array}{c}\text { Electricity } \\
\text { (million } \\
\text { kWh) }\end{array}$ & $\begin{array}{c}\text { Residual } \\
\text { Fuel Oll } \\
(1000 \\
\text { bbls) }\end{array}$ & $\begin{array}{c}\text { Distillate } \\
\text { Fuel Oi } \\
(1000 \\
\text { bbls })\end{array}$ & $\begin{array}{c}\text { Natural } \\
\text { Gase } \\
\text { (billion } \\
\text { cu ft) } \\
\end{array}$ & $\begin{array}{l}\text { LPG } \\
\text { (1000 } \\
\text { bbls) }\end{array}$ & $\begin{array}{c}\text { Coal } \\
(1000 \\
\text { short tons })\end{array}$ & $\begin{array}{c}\text { Coke and } \\
\text { Breeze } \\
(1000 \\
\text { short tons })\end{array}$ & $\begin{array}{c}\text { Other' } \\
\text { (trillion } \\
\text { Btu) }\end{array}$ & $\begin{array}{c}\text { RSE } \\
\text { Row } \\
\text { Factors }\end{array}$ \\
\hline & & \multicolumn{10}{|c|}{ West Census Reglon } \\
\hline & RSE Column Factors: & 0.8 & 0.7 & 1.2 & 1.1 & 0.8 & 1.1 & 1.2 & 1.2 & 1.2 & \\
\hline 20 & Food and Kindred Products & 194 & 9,230 & 731 & 676 & 116 & 239 & 1,226 & $w$ & $w$ & 9.9 \\
\hline 2011 & Meat Packing Plants .............. & 5 & 311 & w & 14 & 3 & $\mathbf{Q}$ & 0 & 0 & $\because$ & 17.1 \\
\hline 2033 & Canned Frulls and Vegotables . . . . . . . & 20 & 547 & 119 & 37 & 16 & 60 & 0 & 0 & • & 10.6 \\
\hline 2037 & Frozen Frults and Vegetables ......... & 26 & 2,117 & a & 57 & 17 & 31 & 0 & 0 & * & 16.9 \\
\hline 2046 & Wet Com Milling ................. & $w$ & 93 & 0 & w & 2 & - & 0 & 0 & w & 20.1 \\
\hline 2051 & Bread, Cake, and Related Products ..... & 6 & 388 & 0 & 2 & $\overline{5}$ & 7 & 0 & 0 & $\because$ & 25.8 \\
\hline 2063 & Beet Sugar. $\ldots \ldots \ldots \ldots \ldots \ldots \ldots$ & w & w & 39 & 18 & w & 3 & 811 & 52 & - & 8.4 \\
\hline 2075 & 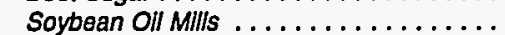 & 0 & 0 & 0 & 0 & 0 & 0 & 0 & 0 & 0 & NF \\
\hline 2082 & Malt Beverages $\ldots \ldots \ldots \ldots \ldots \ldots \ldots$ & 17 & 506 & $w$ & $w$ & $\mathbf{w}$ & $w$ & w & 0 & * & 16.3 \\
\hline 21 & Tobacco Products $\ldots \ldots \ldots \ldots \ldots \ldots \ldots$ & 0 & 0 & 0 & 0 & 0 & 0 & 0 & 0 & 0 & NF \\
\hline 22 & Textlle Mill Products ..... & 6 & 274 & 0 & 1 & 4 & 9 & 0 & 0 & " & 30.5 \\
\hline 23 & Apparel and Other Textile Products ...... & NA & NA & NA & NA & NA & NA & NA & NA & NA & 30.0 \\
\hline 24 & Lumber and Wood Products ........... & 87 & $w$ & 132 & w & 10 & 354 & 0 & 0 & 46 & 18.3 \\
\hline 25 & Fumiture and Fixtures $\ldots \ldots \ldots \ldots \ldots$ & 3 & 304 & 0 & $\mathbf{Q}$ & 1 & 28 & 0 & 0 & - & 32.6 \\
\hline 26 & Paper and Allied Products ............ & 242 & 13,785 & 1,449 & 110 & 108 & w & w & 0 & w & 6.3 \\
\hline 2611 & 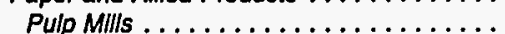 & 16 & 843 & 528 & 25 & 7 & 5 & 0 & 0 & 3 & 21.4 \\
\hline 2621 & Paper Mills. & 116 & 8,196 & w & 39 & 53 & 116 & w & 0 & 22 & 5.1 \\
\hline 2631 & Paperboard Mills . ... & 94 & 3,155 & w & w & 39 & w & w & 0 & 37 & 7.6 \\
\hline 27 & Printing and Publishing ... & NA & NA & NA & NA & NA & NA & NA & NA & NA & 21.6 \\
\hline 28 & Chemicals and Allied Products ... & w & 11,126 & W & 149 & 120 & w & w & 290 & w & 13.7 \\
\hline 2812 & Alkalles and Chlorine $\ldots \ldots \ldots \ldots \ldots$ & $w$ & w & $\ddot{w}$ & w & 4 & - & 0 & 0 & - & 20.1 \\
\hline 2813 & Industrial Gases .................. & $w$ & 2,561 & 0 & w & w & w & 0 & 0 & - & 15.7 \\
\hline 2819 & Industral Inorganic Chemicals, nec ...... & $\ddot{w}$ & 4,373 & w & 123 & w & $\mathbf{Q}$ & w & 290 & 3 & 11.5 \\
\hline 2821 & Plastles Materials and Resins ......... & 3 & 189 & 0 & " & 2 & 2 & 0 & 0 & . & 17.4 \\
\hline 2822 & Synthetlc Rubber.$\ldots \ldots \ldots \ldots$ & . & - & 0 & • & • & - & 0 & 0 & • & 24.0 \\
\hline 2823 & Cellulosic Manmade Fibers . . . . . . . . . & 0 & 0 & 0 & 0 & 0 & 0 & 0 & 0 & 0 & NF \\
\hline 2824 & Organic Fibers, Noncellulosic ......... & 0 & 0 & 0 & 0 & 0 & 0 & 0 & 0 & 0 & NF \\
\hline 2865 & Cyclic Crudes and Intermedlates... & : & 11 & 0 & : & : & 1 & 0 & 0 & • & 25.0 \\
\hline 2869 & Industrial Organic Chemicals, nec ...... & 5 & $\mathbf{Q}$ & 0 & a & w & $Q$ & 0 & 0 & w & 12.9 \\
\hline 2873 & Nitrogenous Fortllizers . . . . . . . . . & 79 & 428 & 0 & 4 & 74 & 42 & 0 & 0 & • & 40.8 \\
\hline 2874 & Phosphatlic Fertilizers ............. & $w$ & 510 & 0 & 7 & w & 0 & 0 & 0 & W & 21.6 \\
\hline 29 & Petroleum and Coal Products & 184 & w & 0 & $\mathbf{Q}$ & 96 & $\mathbf{Q}$ & 0 & 0 & w & 9.5 \\
\hline 2911 & Petroloum Refining . . . . . . . . & 122 & w & 0 & 0 & 85 & 0 & 0 & 0 & w & 2.8 \\
\hline 30 & Rubber and Mlsc. Piastics Products . . . . . & $w$ & 2,865 & 1 & $Q$ & w & w & $\mathbf{Q}$ & 0 & * & 14.9 \\
\hline 3011 & Tres and Inner Tubes .............. & $\because$ & $w$ & w & w & . & W & 0 & 0 & - & 7.0 \\
\hline 308 & MIscellanoous Plastlas Products, noc.... & 14 & 2,573 & 0 & $a$ & 5 & 37 & 0 & 0 & - & 21.9 \\
\hline 31 & Leather and Leather Products .......... & $\mathbf{Q}$ & 70 & 0 & $\vec{a}$ & $\mathbf{Q}$ & $\mathbf{Q}$ & 0 & 0 & - & 44.5 \\
\hline 32 & Stone, Clay and Glass Products & 138 & 5,156 & 677 & 678 & 51 & 125 & 2,373 & 48 & 5 & 15.2 \\
\hline 3211 & Flat Glass ............. & $w$ & 148 & w & w & w & w & 0 & 0 & - & 4.5 \\
\hline 3221 & Glass Containers ... & 18 & 1,129 & $w$ & $w$ & $w$ & 22 & 0 & 0 & - & 9.2 \\
\hline 3229 & Pressed and Blown Glass, nec & $w$ & $w$ & 0 & $\because$ & w & 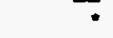 & 0 & 0 & - & 13.0 \\
\hline 3241 & Cement, Hydraullc ........... & 78 & 2,390 & w & 139 & 11 & 1 & 2,373 & $\mathbf{Q}$ & 3 & 21.3 \\
\hline 3274 & Lime .............. & $w$ & w & $w$ & W & w & - & 0 & 0 & * & 20.7 \\
\hline 3296 & Minoral Wool & 3 & 330 & 0 & w & $w$ & w & 0 & w & - & 2.1 \\
\hline 33 & Primary Metal Industries ... & 250 & $w$ & 90 & w & w & $w$ & w & 119 & w & 5.9 \\
\hline 3312 & Blast Fumaces and Steel Mills . . . . . . . & 69 & 2,531 & w & $w$ & w & 4 & w & 20 & 1 & 7.6 \\
\hline 3313 & Electrometalunglcal Products . . . . . . . . & 0 & 0 & 0 & 0 & 0 & 0 & 0 & 0 & 0 & NF \\
\hline 3321 & Gray and Ductlle Iron Foundries ..... & 3 & 92 & 0 & 3 & w & 3 & 0 & w & * & 37.3 \\
\hline 3331 & Primary Copper ............. & $w$ & W & $\mathbf{w}$ & w & $w$ & 1 & w & w & - & 1.0 \\
\hline & Primary Aluminum $\ldots \ldots \ldots \ldots \ldots$ & 118 & 26,391 & $\because$ & 18 & 6 & 28 & 6 & 0 & 22 & 3.5 \\
\hline 3339 & Primary Nonforrous Metals, nec.......... & $w$ & 1,263 & 0 & $w$ & $w$ & $w$ & w & w & 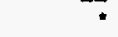 & 1.0 \\
\hline 3353 & Aluminum Sheot, Plate, and Foll .. & $w$ & $w$ & 0 & w & w & w & 0 & 0 & . & 1.1 \\
\hline & Fabricated Metal Products . . . . . . . . & 28 & 2,922 & : & 78 & 16 & 102 & 0 & 0 & 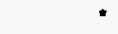 & 19.4 \\
\hline & Industrlal Machinery and Equipment ....... & 22 & 3,614 & 0 & 14 & 8 & 72 & 0 & 0 & " & 26.5 \\
\hline 357 & Computer and Olfice Equipment ... & 9 & 1,945 & 0 & . & 2 & $\mathbf{Q}$ & 0 & 0 & - & 16.0 \\
\hline 36 & Electronlc and Other Electric Equlpment .. & 29 & 5,550 & 0 & Q & 9 & 13 & 0 & 0 & $w$ & 17.5 \\
\hline
\end{tabular}

See footnotes at end of table. 
Table A22. Total Quantity of Purchased Energy Sources by Census Region, Industry Group, and Selected Industries, 1991 (Continued)

(Estimates in Btu or Physical Units)

\begin{tabular}{|c|c|c|c|c|c|c|c|c|c|c|c|}
\hline $\begin{array}{c}\text { SIC } \\
\text { Code" }\end{array}$ & $\begin{array}{l}\text { Industry Groups } \\
\text { and Industry }\end{array}$ & $\begin{array}{c}\text { Total } \\
\text { (trillion } \\
\text { Btu) }\end{array}$ & $\begin{array}{c}\text { Electricity } \\
\text { (million } \\
\text { kWh) }\end{array}$ & $\begin{array}{c}\text { Residual } \\
\text { Fuel Oil } \\
(1000 \\
\text { bbls })\end{array}$ & $\begin{array}{c}\text { Distillate } \\
\text { Fuel Oil } \\
(1000 \\
\text { bbls) }\end{array}$ & $\begin{array}{l}\text { Natural } \\
\text { Gas }^{\mathrm{e}} \\
\text { (billion } \\
\text { cu ft) }\end{array}$ & $\begin{array}{l}\text { LPG } \\
(1000 \\
\text { bbls) }\end{array}$ & $\begin{array}{c}\text { Coal } \\
\text { (1000 } \\
\text { short tons) }\end{array}$ & $\begin{array}{c}\text { Coke and } \\
\text { Breeze } \\
(1000 \\
\text { short tons })\end{array}$ & $\begin{array}{c}\text { Other } \\
\text { (trillion } \\
\text { Btu) }\end{array}$ & $\begin{array}{c}\text { RSE } \\
\text { Row } \\
\text { Factor8 }\end{array}$ \\
\hline & & \multicolumn{10}{|c|}{ West Census Region } \\
\hline \multicolumn{2}{|r|}{ RSE Column Factors: } & 0.8 & 0.7 & 1.2 & 1.1 & 0.8 & 1.1 & 1.2 & 1.2 & 1.2 & \\
\hline \multirow{7}{*}{$\begin{array}{c}37 \\
3711 \\
3714 \\
38 \\
3841 \\
39\end{array}$} & Transportation Equipment & w & 7,096 & w & w & 17 & w & 0 & 0 & $\mathbf{w}$ & 11.7 \\
\hline & Motor Vehicles and Car Bodies & w & w & 0 & w & 1 & 3 & 0 & 0 & • & 6.8 \\
\hline & Motor Vehicle Parts and Accessories .... . & 3 & 243 & $\mathbf{Q}$ & 2 & 2 & 7 & 0 & 0 & • & 21.5 \\
\hline & Instruments and Related Products . & 17 & 3,188 & 4 & 9 & 5 & 6 & 0 & 0 & • & 14.6 \\
\hline & Surgical and Medical Instruments ...... & 1 & 222 & 0 & 3 & - & 2 & 0 & 0 & • & 27.0 \\
\hline & Misc. Manufacturing Industries $\ldots \ldots \ldots \ldots$ & & 376 & 0 & 1 & 1 & 8 & 0 & 0 & - & 25.9 \\
\hline & Total $\ldots \ldots \ldots \ldots \ldots \ldots \ldots \ldots \ldots$ & 1,466 & 116,720 & 3,189 & 3,491 & 644 & 1,616 & $w$ & $w$ & 213 & 6.4 \\
\hline
\end{tabular}

- See Appendices $B$ and $F$ for descriptions of the Standard Industrial Classification system.

"Distillate Fuel Oil" includes Nos. 1, 2, and 4 fuel oils and Nos. 1, 2, and 4 diesel fuels.

"Natural Gas" includes natural gas obtained from utilities, transmission pipelines, and any other supplier(s) such as brokers and producers.

'Other energy sources include such combustible energy sources as wood waste, hydrogen, or waste oils and tars.

NF=No applicable RSE row/column factor.

* Estimate less than 0.5. Data are included in higher level totals.

$W=$ Withheld to avoid disclosing data for individual establishments. Data are included in higher level totals.

$\mathrm{Q}=$ Withheld because Relative Standard Error is greater than 50 percent. Data are included in higher level totals.

NA=Not available. Data are included in higher level totals.

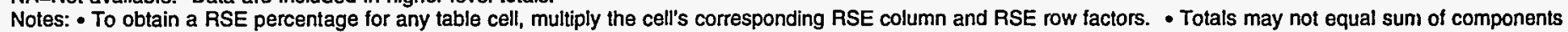

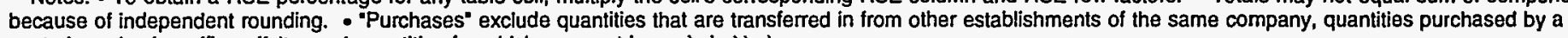
central purchasing office offsite, and quantities for which payment is made in-kind.

Source: Energy Information Administration, Office of Energy Markets and End Use, Energy End Use and Integrated Statistics Division, Form ElA-846, "1991 Manufacturing Energy Consumption Survey." 
Table A23. Quantity of Purchased Electricity, Steam, and Natural Gas by Type of Supplier, Census Region, Industry Group, and Selected Industries, 1991 (Estimates in Btu or Physical Units)

\begin{tabular}{|c|c|c|c|c|c|c|c|c|c|}
\hline \multirow[b]{2}{*}{$\begin{array}{c}\text { SIC } \\
\text { Code }\end{array}$} & \multirow[b]{2}{*}{$\begin{array}{l}\text { Industry Groups } \\
\text { and Industry }\end{array}$} & \multicolumn{2}{|c|}{$\begin{array}{c}\text { Electricity } \\
\text { (Million kWh) }\end{array}$} & \multicolumn{2}{|c|}{$\begin{array}{c}\text { Steam } \\
\text { (Billion Btu) }\end{array}$} & \multicolumn{3}{|c|}{$\begin{array}{l}\text { Natural Gas } \\
\text { (Billion cu } \mathrm{t} \text { ) }\end{array}$} & \multirow[b]{2}{*}{$\begin{array}{c}\text { RSE } \\
\text { Row } \\
\text { Factors }\end{array}$} \\
\hline & & $\begin{array}{c}\text { Utility } \\
\text { Supplier }^{b}\end{array}$ & $\begin{array}{l}\text { Nonutility } \\
\text { Supplier }\end{array}$ & $\begin{array}{c}\text { Utility } \\
\text { Supplier }\end{array}$ & $\begin{array}{l}\text { Nonutility } \\
\text { Supplier }\end{array}$ & $\begin{array}{c}\text { Utility } \\
\text { Supplier }\end{array}$ & $\begin{array}{c}\text { mission } \\
\text { Pipelines }\end{array}$ & $\begin{array}{l}\text { Other } \\
\text { Supplier }\end{array}$ & \\
\hline & & \multicolumn{7}{|c|}{ Total United States } & \\
\hline & RSE Coiumn Factors: & 0.5 & 1.9 & 1.5 & 1.3 & 0.7 & 0.9 & 0.8 & \\
\hline 20 & Food and Kindred Products . . . . . . . . . . & W & 195 & 9,879 & 8,308 & 269 & $\mathbf{w}$ & 145 & 7.3 \\
\hline 2011 & Meat Packing Plants . . . . . . . . . . . . & 3,375 & $\mathbf{Q}$ & W & $\mathbf{Q}$ & 17 & 7 & 7 & 9.3 \\
\hline 2033 & Canned Fruits and Vegetables . . . . . . . . . . & 1,414 & $*$ & 0 & 0 & 15 & 7 & 14 & 12.9 \\
\hline 2037 & Frozen Fruits and Vegetables . . . . . . . . . & 3,056 & 40 & 0 & 791 & 17 & 4 & 3 & 19.2 \\
\hline 2046 & 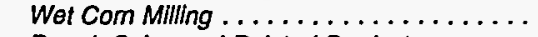 & W & W & W & W & W & $W$ & 23 & 14.5 \\
\hline 2051 & Bread, Cake, and Related Products . . . . . . & 2,226 & $\mathbf{Q}$ & W & 0 & 17 & 3 & 3 & 12.9 \\
\hline 2063 & Beet Sugar. . . . . . . . . . . . . . . & 407 & 0 & 0 & 0 & W & $W$ & 5 & 11.9 \\
\hline 2075 & Soybean Oll Mills ................. & $w$ & 0 & W & W & 7 & 7 & 11 & 4.5 \\
\hline 2082 & Malt Beverages $\ldots \ldots \ldots \ldots \ldots \ldots$ & 2,371 & 0 & $w$ & 0 & 4 & 6 & 12 & 12.6 \\
\hline 21 & Tobacco Products $\ldots \ldots \ldots \ldots \ldots \ldots \ldots$ & 1,468 & 0 & $W$ & 0 & 3 & - & 1 & 13.8 \\
\hline 22 & Textile Mill Products . . . . . . . . . . . . & $w$ & 167 & 2,094 & 4,791 & 74 & 15 & 16 & 10.1 \\
\hline 23 & Apparel and Other Textile Products ....... & 5,607 & 37 & 171 & 0 & 15 & 2 & 1 & 25.1 \\
\hline 24 & Lumber and Wood Products $\ldots \ldots \ldots \ldots$ & 19,042 & $\mathbf{Q}$ & Q & $\mathbf{Q}$ & 22 & 11 & 7 & 20.6 \\
\hline 25 & Furniture and Fixtures $\ldots \ldots \ldots \ldots \ldots$ & 4,889 & 25 & Q & 0 & 14 & 1 & 3 & 18.7 \\
\hline 26 & Paper and Alled Products . . . . . . . . . . & 63,403 & 341 & 8,371 & 10,661 & 222 & $w$ & 175 & 4.9 \\
\hline 2611 & Pulp MIlls $\ldots \ldots \ldots \ldots \ldots \ldots \ldots$ & 2,721 & 150 & 0 & $\mathbf{Q}$ & 18 & 9 & 4 & 21.9 \\
\hline 2621 & Paper MIIIs . . . . . . . . . . . & $w$ & W & 2,441 & 7,697 & 100 & 57 & 96 & 4.0 \\
\hline 2631 & Papertoard MIIIs . . . . . . . . . . . . . . & W & W & 5,877 & 2,343 & 58 & W & 61 & 7.4 \\
\hline 27 & Printing and Publishing $\ldots \ldots \ldots \ldots \ldots$ & 15,521 & $Q$ & 918 & 315 & 35 & 4 & 8 & 17.7 \\
\hline 28 & Chemicals and Allied Products . . . . . . . . & 119,332 & 12,526 & 29,967 & 75,597 & 533 & 927 & 600 & 6.8 \\
\hline 2812 & Alkalles and Chlorine $\ldots \ldots \ldots \ldots \ldots$ & W & W & 0 & W & $W$ & W & W & 19.1 \\
\hline 2813 & Industrial Gases $\ldots \ldots \ldots \ldots \ldots \ldots$ & W & 0 & 0 & W & $\mathbf{W}$ & W & W & 14.8 \\
\hline 2819 & Industrial Inorganic Chemicals, nec ...... & W & W & 0 & $-1,885$ & 49 & 57 & W & 10.7 \\
\hline 2821 & Plastlcs Materials and Resins . . . . . . . & $W$ & 300 & 3,998 & 9,206 & 37 & 120 & 52 & 5.9 \\
\hline 2822 & Synthetlo Rubber . . . . . . . . . . . & W & $W$ & $W$ & 7,690 & W & 69 & 3 & 14.2 \\
\hline 2823 & Cellulosic Manmade Fibers . . . . . . . . . & $w$ & 0 & 0 & 0 & W & 0 & 0 & 40.3 \\
\hline 2824 & Organic Fibers, Noncellulosic .......... & W & $w$ & $W$ & 137 & 13 & $w$ & W & 5.0 \\
\hline 2865 & Cyclic Crudes and Intermediates . . . . . . & W & W & W & 6,837 & 53 & 19 & 15 & 13.3 \\
\hline 2869 & Industrial Organic Chemicals, nec ....... & 17,931 & 656 & 15,524 & 32,722 & W & 376 & 186 & 5.8 \\
\hline 2873 & Nitrogenous Fertlizers $\ldots \ldots \ldots \ldots \ldots$ & 2,630 & 256 & Q & Q & 145 & 200 & 194 & 26.0 \\
\hline 2874 & Phosphatic Fertllizers . . . . . . . . . . & 2,419 & 0 & 0 & 0 & 7 & $w$ & $w$ & 10.9 \\
\hline 29 & Petroleum and Coal Products $\ldots \ldots \ldots \ldots$ & W & W & 24,535 & 14,625 & 170 & 449 & 124 & 3.1 \\
\hline 2911 & Petroleum Refining . . . . . . . . . . . . & W & W & $w$ & 14,490 & 141 & 445 & 113 & 2.8 \\
\hline 30 & Rubber and Misc. Plastics Products ........ & 33,688 & Q & $\mathbf{Q}$ & 1,438 & 61 & 6 & 27 & 9.5 \\
\hline 3011 & Tires and Inner Tubes .............. & W & 0 & 0 & W & 15 & 1 & 4 & 4.6 \\
\hline 308 & Miscellaneous Plastics Products, nec . . . . . . & 25,394 & Q & $\mathbf{Q}$ & 425 & 32 & 3 & 16 & 16.1 \\
\hline 31 & Leather and Leather Products . . . . . . . . . & 780 & 14 & 0 & 80 & 3 & - & 2 & 28.0 \\
\hline 32 & Stone, Clay and Glass Products $\ldots \ldots \ldots \ldots$ & 30,799 & 47 & $Q$ & 322 & 170 & 59 & 140 & 7.6 \\
\hline 3211 & Flat Glass $\ldots \ldots \ldots \ldots \ldots \ldots$ & 1,512 & 0 & $\mathbf{w}$ & $W$ & 16 & $W$ & $W$ & 4.0 \\
\hline 3221 & Glass Containers ............... & 4,098 & 0 & 0 & 0 & 23 & 9 & 35 & 6.4 \\
\hline 3229 & Pressed and Blown Glass, nec . . . . . . . . & $W$ & $w$ & 0 & W & 27 & $W$ & 9 & 7.1 \\
\hline 3241 & Cement, Hydraullc . . . . . . . . . . . & 9,490 & 0 & 0 & 0 & 16 & 10 & 12 & 24.2 \\
\hline 3274 & 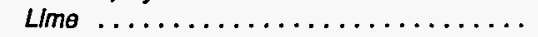 & 1,324 & 0 & 0 & 0 & $\mathbf{W}$ & 3 & $W$ & 27.6 \\
\hline 3296 & Mineral Wool . . . . . . . . . . . . . . & W & 0 & 0 & 0 & W & $W$ & 14 & 1.4 \\
\hline 33 & Primary Metal Industries . . . . . . . . . . . & W & W & $w$ & 1,772 & 216 & 86 & 371 & 4.8 \\
\hline 3312 & Blast Fumaces and Steol Mills . . . . . . . . & $W$ & $W$ & W & $w$ & 96 & 43 & 255 & 5.8 \\
\hline 3313 & Electrometalurgical Products . . . . . . . . . & W & W & W & 309 & * & 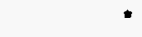 & 1 & 10.2 \\
\hline 3321 & Gray and Ductllo Iron Foundries ........ & W & $\cdot$ & 0 & $W$ & 16 & 1 & 10 & 11.9 \\
\hline 3331 & Primary Copper ............... & W & 0 & 0 & 0 & $\mathbf{W}$ & $w$ & $W$ & 1.4 \\
\hline 3334 & Primary Aluminum $\ldots \ldots \ldots \ldots \ldots$ & W & 0 & 0 & 0 & 9 & 6 & 5 & 4.1 \\
\hline 3339 & Primary Nonferrous Metals, nec . . . . . . . . & 3,784 & 0 & 0 & W & 4 & 4 & 9 & 2.9 \\
\hline 3353 & Aluminum Sheet, Plate, and Foll . . . . . . . & $W$ & 0 & $W$ & 0 & 9 & 7 & 26 & 1.7 \\
\hline 34 & Fabricated Metal Products . . . . . . . . . . & 29,463 & $Q$ & 701 & 3,756 & 106 & 20 & 44 & 13.0 \\
\hline 35 & Industrial Machinery and Equipment ........ & 29,348 & $\bar{Q}$ & 391 & 285 & 75 & 7 & 24 & 14.5 \\
\hline 357 & Computer and Offlce Equipment ......... & 4,368 & $\vec{Q}$ & 1 & 285 & 4 & * & 1 & 20.4 \\
\hline 36 & Electronic and Other Electric Equipment ..... & 29,764 & 51 & 1,386 & $\mathbf{Q}$ & 50 & 9 & 17 & 11.7 \\
\hline 37 & Transportation Equipment ............ & 34,634 & 526 & 1,541 & 3,282 & 53 & 16 & 56 & 5.8 \\
\hline 3711 & Motor Vehicles and Car Bodies .......... & $W$ & $W$ & 1,426 & W & 11 & 7 & 24 & 4.6 \\
\hline 3714 & Motor Vehicle Parts and Accessories ...... & W & $Q$ & $W$ & 2,157 & 14 & 6 & 20 & 8.0 \\
\hline 38 & Instruments and Related Products $\ldots \ldots \ldots$. & $w$ & Q & 29 & $\mathbf{W}$ & 16 & 4 & 5 & 15.2 \\
\hline 3841 & Surgical and Medical Instruments ........ & 1,157 & $\vec{Q}$ & $\mathbf{Q}$ & 0 & 2 & $\cdot$ & * & 17.6 \\
\hline 39 & Misc. Manufacturing Industries . . . . . . . . . . & 3,661 & 0 & $\mathbf{Q}$ & 69 & 12 & 1 & 2 & 19.0 \\
\hline & Total $\ldots \ldots \ldots \ldots \ldots \ldots \ldots$ & 681,538 & 16,015 & 84,438 & 134,033 & 2,117 & 1,829 & 1,767 & 4.0 \\
\hline
\end{tabular}


Table A23. Quantity of Purchased Electricity, Steam, and Natural Gas by Type of Supplier, Census Region, Industry Group, and Selected Industries, 1991 (Continued) (Estimates in Btu or Physical Units)

\begin{tabular}{|c|c|c|c|c|c|c|c|c|c|}
\hline \multirow[b]{2}{*}{$\begin{array}{c}\text { SIC } \\
\text { Code }^{a}\end{array}$} & \multirow[b]{2}{*}{$\begin{array}{l}\text { Industry Groups } \\
\text { and Industry }\end{array}$} & \multicolumn{2}{|c|}{$\begin{array}{c}\text { Electricity } \\
\text { (Million kWh) }\end{array}$} & \multicolumn{2}{|c|}{$\begin{array}{c}\text { Steam } \\
\text { (Billion Btu) }\end{array}$} & \multicolumn{3}{|c|}{$\begin{array}{l}\text { Natural Gas } \\
\text { (Billion cu } \mathrm{t} \text { ) }\end{array}$} & \multirow[b]{2}{*}{$\begin{array}{c}\text { RSE } \\
\text { Row } \\
\text { Factors }\end{array}$} \\
\hline & & $\begin{array}{c}\text { Utility } \\
\text { Supplier }\end{array}$ & $\begin{array}{l}\text { Nonutility } \\
\text { Supplier }\end{array}$ & $\begin{array}{l}\text { Utility } \\
\text { Supplier }\end{array}$ & $\begin{array}{l}\text { Nonutility } \\
\text { Supplier }\end{array}$ & $\begin{array}{c}\text { Utility } \\
\text { Supplier }\end{array}$ & $\begin{array}{l}\text { Trans- } \\
\text { mission } \\
\text { Pipelines }\end{array}$ & $\begin{array}{c}\text { Other } \\
\text { Supplier }\end{array}$ & \\
\hline & & \multicolumn{7}{|c|}{ Northeast Census Region } & \\
\hline & RSE Column Factors: & 0.6 & 1.5 & 1.2 & 1.2 & 0.8 & 1.0 & 0.9 & \\
\hline 20 & Food and Kindred Products $\ldots \ldots \ldots \ldots$ & 5,434 & $\mathbf{Q}$ & 1,512 & 875 & 23 & 6 & 11 & 19.5 \\
\hline 2011 & Meat Packing Plants . . . . . . . . . . & 141 & 0 & 0 & 0 & $\cdot$ & 0 & $\cdot$ & 25.6 \\
\hline 2033 & Canned Fruits and Vegetables . . . . . . . . . & 292 & 0 & 0 & 0 & W & $w$ & $\mathbf{Q}$ & 23.9 \\
\hline 2037 & Frozen Fruits and Vegetables . . . . . . . . & 140 & 0 & 0 & 36 & $\bullet$ & 0 & 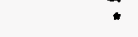 & 34.6 \\
\hline 2046 & Wet Com Milling . ................ & 15 & 0 & 0 & 0 & $*$ & 0 & 0 & 32.8 \\
\hline 2051 & Bread, Cake, and Related Products . . . . . . & $w$ & $\mathbf{Q}$ & 0 & 0 & 3 & 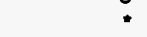 & $w$ & 22.1 \\
\hline 2063 & Beet Sugar . . . . . . . . . . . . . . & 0 & 0 & 0 & 0 & 0 & 0 & 0 & NF \\
\hline 2075 & Soybean Oil Mills .................. & 0 & 0 & 0 & 0 & 0 & 0 & 0 & NF \\
\hline 2082 & 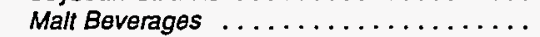 & 521 & 0 & 0 & 0 & $=$ & 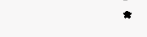 & $w$ & 23.5 \\
\hline 21 & Tobacco Products $\ldots \ldots \ldots \ldots \ldots \ldots$ & NA & NA & NA & NA & NA & NA & NA & 23.7 \\
\hline 22 & Textile Mill Products . . . . . . . . . . . . . . & 1,286 & 86 & $\mathbf{Q}$ & 1,906 & 8 & 1 & 1 & 23.9 \\
\hline 23 & Apparel and Other Textile Products ....... & 494 & $\mathbf{Q}$ & 171 & 0 & 2 & 0 & $\cdot$ & 32.1 \\
\hline 24 & Lumber and Wood Products ............ & NA & NA & NA & NA & NA & NA & NA & 39.9 \\
\hline 25 & Furniture and Fixtures $\ldots \ldots \ldots \ldots \ldots$ & 437 & $\mathbf{Q}$ & 0 & 0 & 1 & 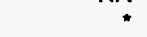 & $\because$ & 33.6 \\
\hline 26 & Paper and Allied Products . . . . . . . . . . . & W & $\bar{W}$ & 1,479 & $w$ & 18 & 3 & 15 & 10.4 \\
\hline 2611 & Pulp Mills $\ldots \ldots \ldots \ldots \ldots \ldots \ldots$ & 13 & Q & 0 & $\mathbf{Q}$ & 0 & 0 & 0 & 59.0 \\
\hline 2621 & Paper Mills . . . . . . . . . . . . . . . . & $W$ & $\mathbf{w}$ & W & 2,856 & 7 & * & 12 & 6.1 \\
\hline 2631 & Paperboard Mills . . . . . . . . . . . . . & $w$ & 0 & w & $W$ & 4 & $\mathbf{w}$ & $w$ & 20.0 \\
\hline 27 & Printing and Publishing $\ldots \ldots \ldots \ldots \ldots$ & 3,060 & $\mathbf{Q}$ & $\mathbf{Q}$ & 0 & 8 & 1 & 1 & 32.7 \\
\hline 28 & Chemicals and Allied Products . . . . . . . . . & W & 135 & $w$ & 6,413 & 29 & 7 & 22 & 10.3 \\
\hline 2812 & Alkalies and Chlorine ............. & w & 0 & 0 & 0 & 0 & $\cdot$ & 0 & 32.6 \\
\hline 2813 & Industrial Gases ................ & $w$ & 0 & 0 & 0 & 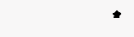 & 0 & 0 & 14.8 \\
\hline 2819 & Industrial Inorganic Chemicals, nec ....... & $w$ & 0 & 0 & $w$ & 3 & : & $Q$ & 21.3 \\
\hline 2821 & Plastics Materials and Resins ......... & $w$ & * & $w$ & W & 3 & 1 & 4 & 10.1 \\
\hline 2822 & Synthetic Rubber . . . . . . . . . & $w$ & 0 & 0 & w & 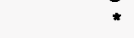 & 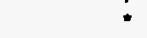 & $w$ & 24.9 \\
\hline 2823 & Cellulosic Manmade Fibers ............ & 0 & 0 & 0 & 0 & 0 & 0 & 0 & NF \\
\hline 2824 & Organic Fibers, Noncellulosic $\ldots \ldots \ldots \ldots$ & 95 & 0 & 0 & 0 & * & * & 0 & 14.9 \\
\hline 2865 & Cyclic Crudes and Intemediates ......... & $W$ & $w$ & 0 & $w$ & W & W & 1 & 20.3 \\
\hline 2869 & Industrial Organic Chemicals, nec $\ldots \ldots \ldots$ & w & w & $w$ & $w$ & 6 & $w$ & $w$ & 12.4 \\
\hline 2873 & Nitrogenous Fertilizers $\ldots \ldots \ldots \ldots \ldots$ & 5 & 25 & 0 & 7 & 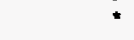 & 0 & 1 & 47.4 \\
\hline 2874 & Phosphatic Fertilizers . . . . . . . . . . . & 0 & 0 & 0 & 0 & 0 & 0 & 0 & NF \\
\hline 29 & Petroleum and Coal Products $\ldots . \ldots \ldots \ldots$ & 3,048 & $\mathbf{Q}$ & W & $\mathbf{Q}$ & 7 & 7 & 14 & 8.6 \\
\hline 2911 & Petroleum Refining $\ldots \ldots \ldots \ldots \ldots \ldots$ & 2,515 & 0 & $\ddot{w}$ & 0 & $w$ & 7 & $w$ & 5.3 \\
\hline 30 & Rubber and Misc. Plastics Products . . . . . . . & 5,484 & 0 & $\mathbf{Q}$ & $\mathbf{Q}$ & W & 1 & $w$ & 21.8 \\
\hline 3011 & Tires and Inner Tubes ............... & 125 & 0 & $\overline{0}$ & $\overline{0}$ & 1 & 0 & 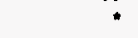 & 8.1 \\
\hline 308 & Miscellaneous Plastics Products, nec . . . . . & 4,810 & 0 & $\mathbf{Q}$ & $\mathbf{Q}$ & 5 & 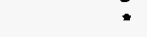 & 2 & 25.3 \\
\hline 31 & Leather and Leather Products . . . . . . . . . . & 205 & 0 & 0 & 0 & 1 & * & 0 & 29.1 \\
\hline 32 & Stone, Clay and Glass Products ......... & 5,558 & 40 & 0 & $w$ & 23 & 8 & 26 & 12.7 \\
\hline 3211 & Flat Glass $\ldots \ldots \ldots \ldots \ldots \ldots$ & W & 0 & 0 & 0 & 0 & $\mathbf{w}$ & $W$ & 5.6 \\
\hline 3221 & Glass Containers $\ldots \ldots \ldots \ldots \ldots \ldots$ & 834 & 0 & $\mathbf{0}$ & 0 & 3 & $w$ & W & 10.2 \\
\hline 3229 & Pressed and Blown Glass, nec . . . . . . . & $w$ & $w$ & 0 & $W$ & 8 & W & 2 & 8.9 \\
\hline 3241 & Cement, Hydraulic ............... & 1,334 & 0 & 0 & 0 & 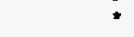 & 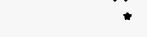 & $\overline{0}$ & 26.6 \\
\hline 3274 & Lime $\ldots \ldots \ldots \ldots \ldots \ldots \ldots$ & $Q$ & 0 & 0 & 0 & 0 & * & 0 & NF \\
\hline 3296 & Mineral Wool $\ldots \ldots \ldots \ldots \ldots \ldots \ldots$ & $\vec{W}$ & 0 & 0 & 0 & $\mathbf{W}$ & $\bullet$ & 2 & 2.5 \\
\hline 33 & Primary Metal Industries $\ldots \ldots \ldots \ldots \ldots \ldots$ & w & $w$ & 0 & 188 & 57 & 9 & 36 & 7.8 \\
\hline 3312 & Blast Fumaces and Steel Mills . . . . . . . . . . & 8,136 & 0 & 0 & W & 36 & 4 & 25 & 7.3 \\
\hline 3313 & Electrometalurgical Products . . . . . . . . . & $W$ & $w$ & 0 & W & 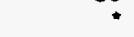 & 0 & 0 & 12.6 \\
\hline 3321 & Gray and Ductile Iron Foundries . . . . . . . & 350 & 0 & 0 & 0 & $w$ & $w$ & . & 23.5 \\
\hline 3331 & Primary Copper $\ldots \ldots \ldots \ldots \ldots \ldots$ & W & 0 & 0 & 0 & 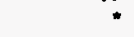 & 0 & $\bullet$ & 1.3 \\
\hline 3334 & Primary Aluminum $\ldots \ldots \ldots \ldots \ldots$ & $W$ & 0 & 0 & 0 & $w$ & 0 & 0 & 6.4 \\
\hline 3339 & Primary Nonferrous Metals, nec . . . . . . . . . & 100 & 0 & 0 & 0 & $\because$ & $\cdot$ & $\bullet$ & 3.0 \\
\hline 3353 & Aluminum Shest, Plate, and Foil ......... & 451 & 0 & 0 & 0 & $w$ & 0 & $w$ & 2.4 \\
\hline 34 & Fabricated Metal Products . . . . . . . . . . . . & 5,053 & 0 & $\mathbf{Q}$ & $W$ & 19 & 2 & 10 & 18.6 \\
\hline 35 & Industrial Machinery and Equipment $\ldots \ldots \ldots$ & $w$ & 0 & $\mathbf{0}$ & 285 & 9 & 1 & 5 & 21.5 \\
\hline 357 & Computer and Office Equipment $\ldots \ldots \ldots$ & 819 & 0 & 0 & 285 & 1 & $\star$ & 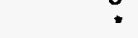 & 26.1 \\
\hline 36 & Electronic and Other Electric Equipment ..... & 6,541 & 0 & $W$ & 0 & 10 & 1 & 4 & 19.0 \\
\hline 37 & Transportation Equipment $\ldots \ldots \ldots \ldots \ldots$ & $W$ & $w$ & 0 & $w$ & 5 & 1 & 5 & 10.9 \\
\hline 3711 & Motor Vehicles and Car Bodies . . . . . . . . & W & 0 & 0 & 0 & - & 0 & W & 9.6 \\
\hline 3714 & Motor Vehicle Parts and Accessories . . . . . . & 887 & 0 & 0 & $w$ & $\mathbf{w}$ & 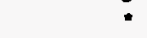 & 2 & 12.3 \\
\hline 38 & Instruments and Related Products ........ & W & $\mathbf{Q}$ & 0 & $w$ & 5 & 2 & $\vec{W}$ & 18.4 \\
\hline 3841 & Surgical and Medical Instruments ......... & 329 & $\mathbf{Q}$ & 0 & 0 & 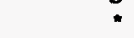 & 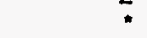 & 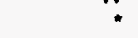 & 22.7 \\
\hline 39 & Misc. Manufacturing Industries . . . . . . . . . & 1,187 & 0 & 0 & 69 & $\mathbf{w}$ & $\cdot$ & 1 & 22.0 \\
\hline & Total $\ldots \ldots \ldots \ldots \ldots \ldots \ldots$ & 85,769 & 702 & 17,235 & 14,806 & 238 & 52 & 155 & 5.8 \\
\hline
\end{tabular}

See footnotes at end of table 
Table A23. Quantity of Purchased Electricity, Steam, and Natural Gas by Type of Supplier, Census Region, Industry Group, and Selected Industries, 1991 (Continued) (Estimates in Btu or Physical Units)

\begin{tabular}{|c|c|c|c|c|c|c|c|c|c|}
\hline \multirow[b]{2}{*}{$\begin{array}{c}\text { sic } \\
\text { Code" }\end{array}$} & \multirow[b]{2}{*}{$\begin{array}{l}\text { Industry Groups } \\
\text { and Industry }\end{array}$} & \multicolumn{2}{|c|}{$\begin{array}{c}\text { Electricity } \\
\text { (Million kWh) }\end{array}$} & \multicolumn{2}{|c|}{$\begin{array}{c}\text { Steam } \\
\text { (Billion Btu) }\end{array}$} & \multicolumn{3}{|c|}{$\begin{array}{l}\text { Natural Gas } \\
\text { (Billion cu ft) }\end{array}$} & \multirow[b]{2}{*}{$\begin{array}{c}\text { RSE } \\
\text { Row } \\
\text { Factors }\end{array}$} \\
\hline & & $\begin{array}{c}\text { Utility } \\
\text { Supplier }\end{array}$ & $\begin{array}{l}\text { Nonutility } \\
\text { Supplier }\end{array}$ & $\begin{array}{c}\text { Utility } \\
\text { Suppliet }\end{array}$ & $\begin{array}{c}\text { Nonutility } \\
\text { Supplier }\end{array}$ & $\begin{array}{c}\text { Utility } \\
\text { Supplier }\end{array}$ & $\begin{array}{c}\text { Trans- } \\
\text { mission } \\
\text { Pipelines }\end{array}$ & $\begin{array}{c}\text { Other } \\
\text { Supplier }\end{array}$ & \\
\hline & & \multicolumn{7}{|c|}{ Midwest Census Region } & \\
\hline & RSE Column Factors: & 0.5 & 1.6 & 1.3 & 1.5 & 0.8 & 1.0 & 0.8 & \\
\hline 20 & Food and Kindred Products $\ldots \ldots \ldots \ldots$ & W & 77 & 7,673 & 1,328 & 107 & W & 73 & 10.9 \\
\hline 2011 & Meat Packing Plants . . . . . . . . . . . & 2,065 & 0 & $W$ & 0 & 12 & 5 & 5 & 10.9 \\
\hline 2033 & Canned Fruits and Vegetables . . . . . . . . . & 375 & 0 & 0 & 0 & 4 & 2 & 2 & 16.8 \\
\hline 2037 & Frozen Fruits and Vegetables . . . . . . . . . & 289 & 0 & 0 & 0 & 1 & $\bullet$ & 1 & 38.7 \\
\hline 2046 & 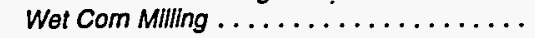 & W & 0 & $\mathbf{W}$ & 0 & 21 & W & W & 15.1 \\
\hline 2051 & Bread, Cake, and Related Products . . . . . . & $w$ & W & W & 0 & 4 & 1 & $w$ & 16.7 \\
\hline 2063 & Beet Sugar. . . . . . . . . . . . . . . & 234 & 0 & 0 & 0 & W & W & W & 13.5 \\
\hline 2075 & Soybean Oll Mll/s . . . . . . . . . . . . & W & 0 & W & 0 & 4 & 5 & 8 & 4.9 \\
\hline 2082 & 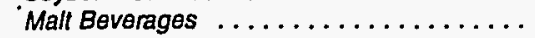 & 480 & 0 & W & 0 & 1 & w & W & 20.5 \\
\hline 21 & Tobacco Products $\ldots \ldots \ldots \ldots \ldots \ldots$ & NA & NA & NA & NA & NA & NA & NA & 12.5 \\
\hline 22 & Textlle Mill Products $\ldots \ldots \ldots \ldots \ldots \ldots$ & NA & NA & NA & NA & NA & NA & NA & 32.6 \\
\hline 23 & Apparel and Other Textile Products . . . . . . & NA & NA & NA & NA & NA & NA & NA & 35.9 \\
\hline 24 & Lumber and Wood Products ........... & 2,834 & 0 & 256 & 0 & 8 & $\mathbf{Q}$ & Q & 29.6 \\
\hline 25 & Furnlture and Fixtures $\ldots \ldots \ldots \ldots \ldots$ & 1,555 & 15 & 0 & 0 & W & 1 & 2 & 24.4 \\
\hline 26 & Paper and Allied Products . . . . . . . . . & $W$ & $w$ & W & W & 46 & 16 & 50 & 8.2 \\
\hline 2611 & Pulp Mills $\ldots \ldots \ldots \ldots \ldots \ldots \ldots$ & 556 & 0 & 0 & 0 & 2 & 0 & 1 & 45.7 \\
\hline 2621 & Paper MIIIs . . . . . . . . . . . . . & W & W & W & W & W & W & 34 & 7.0 \\
\hline 2631 & Paperboard Mills . . . . . . . . . . . . & 2,059 & 0 & w & 0 & 7 & $W$ & $w$ & 13.8 \\
\hline 27 & Printing and Publishing $\ldots \ldots \ldots \ldots \ldots$ & 5,224 & 0 & 470 & 315 & 15 & 3 & 5 & 21.3 \\
\hline 28 & Chemlcals and Allied Products . . . . . . . . . & W & W & $w$ & 8,921 & 61 & 72 & 89 & 11.7 \\
\hline 2812 & Alkalles and Chlorine .............. & W & 0 & 0 & 0 & 0 & 0 & 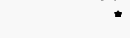 & 37.6 \\
\hline 2813 & 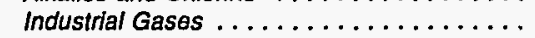 & 3,319 & 0 & 0 & W & $\cdot$ & 0 & $w$ & 15.4 \\
\hline 2819 & Industrial inorganic Chemicals, nec ...... & W & $W$ & 0 & W & 5 & 3 & $w$ & 12.9 \\
\hline 2821 & Plastles Materlals and Resins . . . . . . . & w & W & 2,479 & W & 6 & 1 & 17 & 8.5 \\
\hline 2822 & Symthetlc Rubber . . . . . . . . . . . & 241 & 0 & 0 & 0 & - & 0 & W & 22.9 \\
\hline 2823 & Cellulosic Manmado Fibors . . . . . . . . . . & 0 & 0 & 0 & 0 & 0 & 0 & 0 & NF \\
\hline 2824 & Organic Fibers, Noncellulosic .......... & 0 & 0 & 0 & 0 & 0 & 0 & 0 & NF \\
\hline 2865 & Cyclic Crudes and Intermediates . . . . . . . & 756 & 0 & 0 & $\mathbf{Q}$ & W & $w$ & 8 & 17.9 \\
\hline 2869 & Industrial Organic Chemicais, nec . . . . . . . & 1,120 & W & $w$ & $W$ & 14 & W & 7 & 9.9 \\
\hline 2873 & Nitrogenous Fertilizers . . . . . . . . . & 514 & $\mathbf{Q}$ & 0 & $\mathbf{Q}$ & 13 & 31 & 26 & 46.7 \\
\hline 2874 & Phosphatlc Fertilizers . . . . . . . . . . & 1 & 0 & 0 & 0 & $\bullet$ & 0 & * & 5.7 \\
\hline 29 & Petroleum and Coal Products $\ldots \ldots \ldots \ldots$ & $w$ & - & $\mathbf{Q}$ & W & 21 & 51 & 11 & 6.5 \\
\hline 2911 & Potroloum Refining . . . . . . . . . . . & 6,168 & * & 0 & W & $w$ & 49 & W & 3.9 \\
\hline 30 & Rubber and Misc. Plastics Products . . . . . . & 13,012 & $Q$ & 0 & 0 & 24 & 3 & 13 & 14.4 \\
\hline 3011 & Tres and inner Tubes . . . . . . . . . . . & W & 0 & 0 & 0 & $w$ & - & $w$ & 5.5 \\
\hline 308 & Mlscollaneous Plastics Products, nec . . . . . . & NA & NA & NA & NA & NA & NA & NA & 21.5 \\
\hline 31 & Leather and Leather Products . . . . . . . . . & 248 & 14 & 0 & 80 & 1 & $\bullet$ & 1 & 26.8 \\
\hline 32 & Stone, Clay and Glass Products $\ldots \ldots \ldots \ldots$ & $w$ & 0 & $\mathbf{Q}$ & $\mathbf{Q}$ & 41 & 14 & 49 & 8.7 \\
\hline 3211 & Flat Glass $\ldots \ldots \ldots \ldots \ldots \ldots$ & W & 0 & 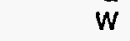 & 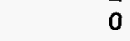 & 4 & 0 & 6 & 5.0 \\
\hline 3221 & Glass Containers .............. & 873 & 0 & 0 & 0 & $w$ & $w$ & 12 & 10.0 \\
\hline 3229 & Pressed and Blown Glass, nec ........ & 614 & 0 & 0 & 0 & 6 & $w$ & $W$ & 9.2 \\
\hline 3241 & Cement, Hydraullo . . . . . . . . . . . . & 2,247 & 0 & 0 & 0 & 3 & Q & 2 & 26.5 \\
\hline 3274 & 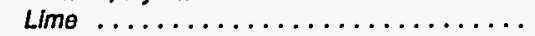 & 367 & 0 & 0 & 0 & $w$ & w & * & 29.7 \\
\hline 3296 & 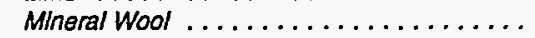 & 1,251 & 0 & 0 & 0 & 4 & w & 6 & 1.6 \\
\hline 33 & Primary Metal Industries . . . . . . . . . . & W & W & W & W & $w$ & 31 & 242 & 6.7 \\
\hline 3312 & Blast Fumaces and Steel MIlls . . . . . . . . . & w & W & W & W & $w$ & W & 183 & 7.4 \\
\hline 3313 & Electrometalurgical Products .......... & W & $W$ & W & $W$ & $\cdot$ & 0 & 1 & 11.4 \\
\hline 3321 & Gray and Ductile Iron Foundries ......... & 4,350 & * & 0 & W & W & W & 9 & 13.4 \\
\hline 3331 & 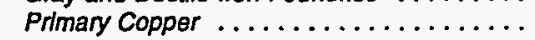 & $W$ & 0 & 0 & 0 & $\because$ & 0 & 0 & 1.6 \\
\hline 3334 & Primary Aluminum $\ldots \ldots \ldots \ldots$ & W & 0 & 0 & 0 & $W$ & 0 & W & 7.1 \\
\hline 3339 & Prlmary Nonferrous Metals, nec . . . . . . . . . & 727 & 0 & 0 & w & W & W & 1 & 1.8 \\
\hline 3353 & Aluminum Sheet, Plate, and Foil . . . . . . . . & $W$ & 0 & $w$ & 0 & $W$ & $w$ & 8 & 1.4 \\
\hline 34 & Fabricated Metal Products . . . . . . . . . & 12,640 & $\mathbf{Q}$ & 620 & 3,560 & 41 & W & W & 15.0 \\
\hline 35 & Industrial Machinery and Equipment ........ & $W$ & $Q$ & 391 & 0 & 35 & 4 & 17 & 15.6 \\
\hline 357 & Computer and Offlce Equipment $\ldots \ldots \ldots$ & 795 & $\mathbf{Q}$ & 1 & 0 & 1 & - & - & 26.9 \\
\hline 36 & Electronic and Other Electric Equipment ..... & 6,999 & $\bar{Q}$ & 884 & $\mathbf{Q}$ & 15 & 3 & 10 & 15.4 \\
\hline 37 & Transportation Equipment $\ldots \ldots \ldots \ldots \ldots$ & $W$ & 157 & W & 2,420 & 22 & 12 & 36 & 6.6 \\
\hline 3711 & Motor Vehicles and Car Bodles . . . . . . . . & W & $w$ & $W$ & $w$ & 6 & w & W & 5.4 \\
\hline 3714 & Motor Vehiclo Parts and Accessonles . . . . . . & W & $\mathbf{Q}$ & W & $w$ & 7 & 6 & 16 & 9.1 \\
\hline 38 & Instruments and Related Products $\ldots \ldots \ldots$. & 1,806 & 0 & $Q$ & 0 & 3 & $*$ & $W$ & 26.0 \\
\hline 3841 & Surgical and Medlcal Instruments ........ & 276 & 0 & $\mathbf{Q}$ & 0 & $\star$ & * & 0 & 18.8 \\
\hline 39 & Misc. Manufacturing Industries . . . . . . . . . & 929 & 0 & $\mathbf{Q}$ & 0 & 4 & • & 1 & 22.6 \\
\hline & Total $\ldots \ldots \ldots \ldots \ldots \ldots \ldots$ & 196,691 & 1,717 & 26,217 & 19,762 & 520 & 257 & 629 & 5.1 \\
\hline
\end{tabular}

See footnotes at end of table 
$\Rightarrow$ Table A23. Quantity of Purchased Electricity, Steam, and Natural Gas by Type of Supplier, Census Region, Industry Group, and Selected Industries, 1991 (Continued) (Estimates in Btu or Physical Units)

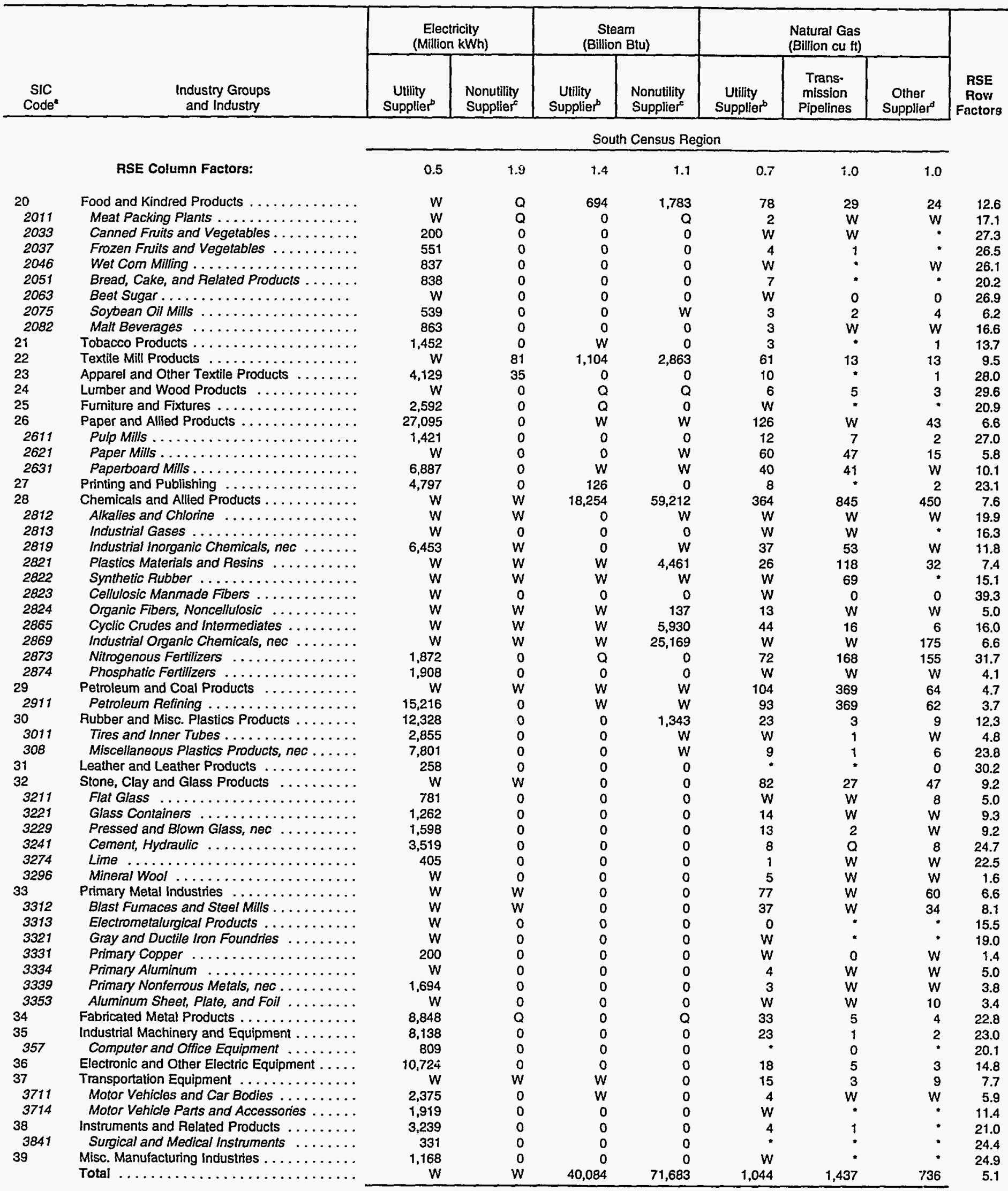

See footnotes at end of table 
Table A23. Quantity of Purchased Electricity, Steam, and Natural Gas by Type of Supplier, Census Region, Industry Group, and Selected Industries, 1991 (Continued) (Estimates in Btu or Physical Units)

\begin{tabular}{|c|c|c|c|c|c|c|c|c|c|}
\hline \multirow[b]{2}{*}{$\begin{array}{c}\text { SIC } \\
\text { Code }\end{array}$} & \multirow[b]{2}{*}{$\begin{array}{l}\text { Industry Groups } \\
\text { and Industry }\end{array}$} & \multicolumn{2}{|c|}{$\begin{array}{c}\text { Electricity } \\
\text { (Million kWh) }\end{array}$} & \multicolumn{2}{|c|}{$\begin{array}{c}\text { Steam } \\
\text { (Billion Btu) }\end{array}$} & \multicolumn{3}{|c|}{$\begin{array}{r}\text { Natural Gas } \\
\text { (Billion cu ft) }\end{array}$} & \multirow[b]{2}{*}{$\begin{array}{c}\text { RSE } \\
\text { Row } \\
\text { Factors }\end{array}$} \\
\hline & & $\begin{array}{c}\text { Utility } \\
\text { Supplier }\end{array}$ & $\begin{array}{l}\text { Nonutility } \\
\text { Supplier }\end{array}$ & $\begin{array}{c}\text { Utility } \\
\text { Supplier }\end{array}$ & $\begin{array}{l}\text { Nonutility } \\
\text { Supplier }\end{array}$ & $\begin{array}{c}\text { Utility } \\
\text { Supplier }\end{array}$ & $\begin{array}{c}\text { Trans- } \\
\text { mission } \\
\text { Pipelines }\end{array}$ & $\begin{array}{c}\text { Other } \\
\text { Supplier }\end{array}$ & \\
\hline & & \multicolumn{7}{|c|}{ West Census Region } & \\
\hline & RSE Column Factors: & 0.5 & 1.5 & 2.9 & 0.8 & 0.7 & 1.0 & 0.8 & \\
\hline 20 & Food and Kindred Products . . . . . . . . . . & 9,156 & 74 & 0 & 4,322 & 61 & 18 & 37 & 13.4 \\
\hline 2011 & Meat Packing Plants . . . . . . . . . . & W & W & 0 & 0 & 2 & $W$ & $W$ & 20.5 \\
\hline 2033 & Canned Fruits and Vegetables . . . . . . . . . & 547 & $\bullet$ & 0 & 0 & 5 & 1 & 10 & 15.5 \\
\hline 2037 & Frozen Fruits and Vegetables . . . . . . . . & 2,076 & 40 & 0 & 755 & 12 & 3 & 2 & 24.8 \\
\hline 2046 & Wet Com Milling . . . . . . . . . . . . . & W & $W$ & 0 & W & $\bullet$ & 0 & $w$ & 24.7 \\
\hline 2051 & Bread, Cake, and Related Products ....... & 388 & 0 & 0 & 0 & 4 & 1 & $\bullet$ & 27.3 \\
\hline 2063 & Beot Sugar . . . . . . . . . . . . & $w$ & 0 & 0 & 0 & W & 3 & $W$ & 17.3 \\
\hline 2075 & 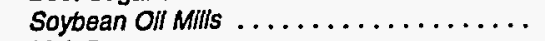 & 0 & 0 & 0 & 0 & 0 & 0 & 0 & NF \\
\hline 2082 & 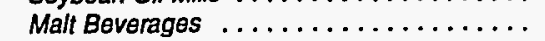 & 506 & 0 & 0 & 0 & $\cdot$ & $W$ & $w$ & 20.2 \\
\hline 21 & Tobacco Products $\ldots \ldots \ldots \ldots \ldots \ldots \ldots$ & 0 & 0 & 0 & 0 & 0 & 0 & 0 & NF \\
\hline 22 & Textlle Mill Products ............... & 274 & 0 & 0 & 0 & 3 & 0 & 1 & 37.9 \\
\hline 23 & Apparel and Other Textile Products $\ldots \ldots \ldots$ & NA & NA & NA & NA & NA & NA & NA & 40.5 \\
\hline 24 & Lumber and Wood Products ........... & $W$ & $\mathbf{Q}$ & 0 & $\mathbf{Q}$ & 5 & 3 & 1 & 31.7 \\
\hline 25 & Fumiture and Fixtures $\ldots \ldots \ldots \ldots \ldots$ & 304 & 0 & 0 & 0 & 1 & 0 & 0 & 39.5 \\
\hline 26 & Paper and Allied Products . . . . . . . . . . & 13,649 & 136 & $W$ & $w$ & 31 & 9 & 67 & 10.4 \\
\hline 2611 & Pulp Mills . . . . . . . . . . . . . . . & 732 & 111 & 0 & 0 & 3 & 2 & 1 & 36.2 \\
\hline 2621 & Paper Mills . . . . . . . . . . . . . . & 8,196 & 0 & 0 & 4,266 & $w$ & W & 34 & 8.5 \\
\hline 2631 & Paperboard Mills . . . . . . . . . . . . & $w$ & $W$ & $W$ & W & 8 & 0 & 31 & 12.1 \\
\hline 27 & Printing and Publishing $\ldots \ldots \ldots \ldots \ldots$ & NA & NA & NA & NA & NA & NA & NA & 26.3 \\
\hline 28 & Chemlcals and Allied Products . . . . . . . . & 10,934 & 192 & 0 & 1,051 & 79 & 2 & 39 & 22.6 \\
\hline 2812 & Alkalles and Chlorine $\ldots \ldots \ldots \ldots \ldots$ & W & 0 & 0 & W & W & 0 & $w$ & 29.9 \\
\hline 2813 & Industrial Gases . . . . . . . . . . . & 2,561 & 0 & 0 & 0 & * & 0 & $\mathbf{W}$ & 16.4 \\
\hline 2819 & Industrial Inorganic Chemicals, nec . . . . . . & 4,370 & 3 & 0 & 793 & 4 & 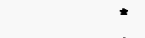 & $w$ & 14.8 \\
\hline 2821 & Plastlos Materials and Resins . . . . . . . . & 189 & 0 & 0 & W & 1 & $:$ & $:$ & 33.7 \\
\hline 2822 & Synthetic Rubber ................ & 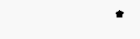 & 0 & 0 & 0 & • & 0 & 0 & 36.7 \\
\hline 2823 & Cellulosic Manmade Fibers . . . . . . . . . . & 0 & 0 & 0 & 0 & 0 & 0 & 0 & NF \\
\hline 2824 & Organic Flbers, Noncellulosic . . . . . . . . & 0 & 0 & 0 & 0 & 0 & 0 & 0 & NF \\
\hline 2865 & Cyclle Crudes and Intermediates ......... & 11 & 0 & 0 & 0 & * & 0 & 0 & 39.9 \\
\hline 2869 & Industrial Organic Chemicals, nec . . . . . . & $\mathbf{Q}$ & 0 & 0 & W & $W$ & 0 & W & 15.9 \\
\hline 2873 & Nitrogenous Fertilizers $\ldots \ldots \ldots \ldots$ & 239 & 189 & 0 & 28 & 60 & 1 & 13 & 49.0 \\
\hline 2874 & Phosphatic Fortilizers ............. & 510 & 0 & 0 & 0 & W & 0 & 0 & 52.1 \\
\hline 29 & Petroleum and Coal Products $\ldots \ldots \ldots \ldots$ & 7,057 & $w$ & 0 & 7,170 & 38 & 22 & 36 & 5.1 \\
\hline 2911 & Petroloum Rofining . . . . . . . . . . . & W & $W$ & 0 & 7,170 & 32 & 21 & 32 & 4.2 \\
\hline 30 & Rubber and Misc. Plastics Products . . . . . . . & 2,865 & 0 & 0 & W & $W$ & $\dot{*}$ & w & 21.0 \\
\hline 3011 & Tres and Inner Tubes . . . . . . . . . . . . . & W & 0 & 0 & W & $\cdot$ & 0 & 0 & 9.9 \\
\hline 308 & Miscellaneous Plastics Products, nec . . . . . & 2,573 & 0 & 0 & 0 & 4 & * & 1 & 35.3 \\
\hline 31 & Leather and Leather Products . . . . . . . . . & 70 & 0 & 0 & 0 & $\mathbf{Q}$ & 0 & $\bullet$ & 54.9 \\
\hline 32 & Stone, Clay and Glass Products $\ldots \ldots \ldots$ & W & $\mathbf{w}$ & 0 & $W$ & 24 & 10 & 18 & 15.4 \\
\hline 3211 & Flat Glass $\ldots \ldots \ldots \ldots \ldots \ldots$ & 148 & 0 & 0 & $w$ & $\mathbf{W}$ & $\cdot$ & $\cdot$ & 6.5 \\
\hline 3221 & Glass Containers .............. & 1,129 & 0 & 0 & 0 & W & W & 7 & 12.5 \\
\hline 3229 & Pressed and Blown Glass, nec . . . . . . . & $W$ & 0 & 0 & 0 & • & $\bullet$ & 0 & 17.5 \\
\hline 3241 & Coment, Hydraulic ............... & 2,390 & 0 & 0 & 0 & 6 & Q & 2 & 43.5 \\
\hline 3274 & 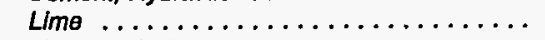 & W & 0 & 0 & 0 & $\bullet$ & $\mathbf{W}$ & - & 29.0 \\
\hline 3296 & 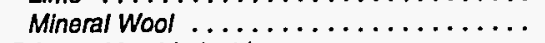 & 330 & 0 & 0 & 0 & $\cdot$ & 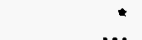 & $w$ & 2.8 \\
\hline 33 & Primary Metal Industries $\ldots \ldots \ldots \ldots \ldots$ & W & $w$ & 0 & W & $\mathbf{w}$ & $w$ & 33 & 10.3 \\
\hline 3312 & Blast Fumaces and Steel Mills . . . . . . . . . & 2,531 & 0 & 0 & 0 & $w$ & $w$ & 13 & 10.5 \\
\hline 3313 & Electrometalurglcal Products ........... & 0 & 0 & 0 & 0 & 0 & 0 & 0 & NF \\
\hline 3321 & Gray and Ductlle Iron Foundries ........ & 92 & 0 & 0 & 0 & $\mathbf{W}$ & 0 & 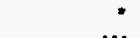 & 47.1 \\
\hline 3331 & Primary Copper ............... & $W$ & 0 & 0 & 0 & W & W & W & 1.4 \\
\hline 3334 & Primary Aluminum $\ldots \ldots \ldots \ldots \ldots$ & 26,391 & 0 & 0 & 0 & 2 & $w$ & W & 5.2 \\
\hline 3339 & Primary Nonferrous Metals, nec . . . . . . . . & 1,263 & 0 & 0 & 0 & $\bar{w}$ & $\cdot$ & W & 1.4 \\
\hline 3353 & Aluminum Sheet, Plate, and Foil . . . . . . & W & 0 & 0 & 0 & 0 & 0 & W & 1.6 \\
\hline 34 & Fabricaled Metal Products . . . . . . . . . . . & 2,922 & 0 & 0 & 0 & 13 & W & W & 23.3 \\
\hline 35 & Industrial Machlnery and Equipment . . . . . . & 3,614 & 0 & 0 & 0 & 7 & 1 & * & 35.5 \\
\hline 357 & Computer and Office Equipment ........ & 1,945 & 0 & 0 & 0 & 1 & $*$ & • & 23.1 \\
\hline 36 & Electronic and Other Electric Equipment ..... & 5,500 & 49 & $Q$ & 0 & 8 & $\bullet$ & 1 & 24.1 \\
\hline
\end{tabular}

See footnotes at end of table. 
Table A23. Quantity of Purchased Electricity, Steam, and Natural Gas by Type of Supplier, Census Region, Industry Group, and Selected Industries, 1991 (Continued) (Estimates in Btu or Physical Units)

\begin{tabular}{|c|c|c|c|c|c|c|c|c|c|}
\hline \multirow[b]{2}{*}{$\begin{array}{c}\text { SiC } \\
\text { Code }^{a}\end{array}$} & \multirow[b]{2}{*}{$\begin{array}{l}\text { Industry Groups } \\
\text { and industry }\end{array}$} & \multicolumn{2}{|c|}{$\begin{array}{c}\text { Electricity } \\
\text { (Million } k W h \text { ) }\end{array}$} & \multicolumn{2}{|c|}{$\begin{array}{c}\text { Steam } \\
\text { (Billion Btu) }\end{array}$} & \multicolumn{3}{|c|}{$\begin{array}{l}\text { Natural Gas } \\
\text { (Blllion cu t })\end{array}$} & \multirow[b]{2}{*}{$\begin{array}{c}\text { RSE } \\
\text { Row } \\
\text { Factors }\end{array}$} \\
\hline & & $\begin{array}{c}\text { Utility } \\
\text { Supplier }\end{array}$ & $\begin{array}{l}\text { Nonutility } \\
\text { Supplier }\end{array}$ & $\begin{array}{c}\text { Utility } \\
\text { Suppller }\end{array}$ & $\begin{array}{l}\text { Nonutlity } \\
\text { Supplier }\end{array}$ & $\begin{array}{c}\text { Unllity } \\
\text { Supplier }\end{array}$ & $\begin{array}{c}\text { Trans- } \\
\text { mission } \\
\text { Pipelines }\end{array}$ & $\begin{array}{c}\text { Other } \\
\text { Supplier }\end{array}$ & \\
\hline & \multirow[b]{2}{*}{ RSE Column Factors: } & \multicolumn{7}{|c|}{ West Census Region } & \\
\hline & & 0.5 & 1.5 & 2.9 & 0.8 & 0.7 & 1.0 & 0.8 & \\
\hline 37 & Transportation Equipment $\ldots \ldots \ldots \ldots \ldots$ & 6,924 & 172 & 0 & $w$ & 10 & 1 & 7 & 18.3 \\
\hline 3711 & Motor Vehicles and Car Bodies . . . . . . . . . & $W$ & 0 & 0 & 0 & 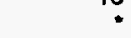 & 0 & ? & 9.6 \\
\hline 3714 & Motor Vehicle Parts and Accessories . . . . . . & 243 & 0 & 0 & 0 & 1 & 0 & 1 & 24.4 \\
\hline & Instruments and Related Products . . . . . . . & 3,188 & 0 & 24 & 0 & 4 & - & 2 & 19.4 \\
\hline 3841 & Surgical and Medical Instruments ........ & 222 & 0 & 0 & 0 & - & * & 0 & 28.8 \\
\hline 39 & Misc. Manufacturing Industries . . . . . . . . & 376 & 0 & 1 & 0 & 1 & $*$ & $\bullet$ & $\mathbf{3 1 . 7}$ \\
\hline & Total $\ldots \ldots \ldots \ldots \ldots \ldots \ldots \ldots \ldots$ & $w$ & $w$ & 903 & 27,783 & 315 & 83 & 246 & 8.8 \\
\hline
\end{tabular}

- See Appendices B and $F$ for descriptions of the Standard Industrial Classification system.

"A "Utility" is a company that produces and/or delivers electricity and/or natural gas, and is legally obligated to provide service to the public within its franchlse area.

"Includes independent power producers, small power producers, and cogenerators not located at the establishment site.

other suppliers of natural gas include such sources as brokers and producers.

NF $=$ No applicable RSE row/column factor.

- Estimate less than 0.5. Data are included in higher level totals.

$\mathrm{Q}=$ Withheld because Relative Standard Error is greater than 50 percent. Data are included in higher level totals.

$\mathrm{NA}=\mathrm{Not}$ available. Data are included in higher level totals.

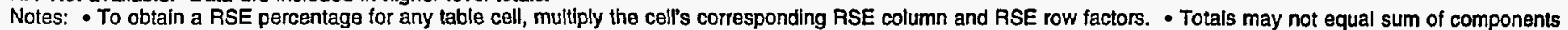
because of independent rounding. - "Purchases" exclude quantities that are transferred in from other establishments of the same company, quantities purchased by a central purchasing office offsite, and quantities for which payment is made in-kind.

Source: Energy Information Administration, Office of Energy Markets and End Use, Energy End Use and Integrated Statistics Division, Form ElA-846, "1991 Manufacturing Energy Consumption Survey." 
Table A24. Total Expenditures for Purchased Energy Sources by Census Region, Industry Group, and Selected Industries, 1991

(Estimates in Million Dollars)

\begin{tabular}{|c|c|c|c|c|c|c|c|c|c|c|c|}
\hline $\begin{array}{l}\text { SIC } \\
\text { Codea }\end{array}$ & $\begin{array}{l}\text { Industry Groups } \\
\text { and Industry }\end{array}$ & Total & Electricity & $\begin{array}{l}\text { Residual } \\
\text { Fuel Oll } \\
\end{array}$ & $\begin{array}{l}\text { Distillate } \\
\text { Fuel Oll } \\
\end{array}$ & $\begin{array}{c}\text { Natural } \\
\text { Gas }^{\circ}\end{array}$ & LPG & Coal & $\begin{array}{c}\text { Coke and } \\
\text { Breeze }\end{array}$ & Other & $\begin{array}{l}\text { RSE } \\
\text { Row } \\
\text { Factors }\end{array}$ \\
\hline & & \multicolumn{10}{|c|}{ Total United States } \\
\hline & RSE Column Factors: & 0.6 & 0.6 & 1.3 & 1.3 & 0.7 & 1.2 & 1.2 & 1.5 & 1.1 & \\
\hline 20 & Food and Kindred Products & 4,637 & $\mathbf{w}$ & 77 & 111 & w & 39 & 221 & w & 98 & 5.8 \\
\hline 2011 & Moat Packing Plants ................ & 254 & 160 & 2 & 10 & 75 & W & 1 & 0 & W & 9.7 \\
\hline 2033 & Canned Frults and Vegetables . . . . . . . . & 218 & 99 & 5 & 5 & 103 & 4 & - & 0 & 1 & 9.1 \\
\hline 2037 & Frozen Fruits and Vegetables . . . . . . . & 205 & 128 & W & 2 & 64 & 1 & 0 & 0 & $w$ & 13.9 \\
\hline 2046 & Wet Com Milling $\ldots \ldots \ldots \ldots \ldots$ & 357 & w & 1 & 1 & 119 & - & 90 & w & w & 11.6 \\
\hline 2051 & Bread, Cake, and Related Products .... & 228 & 138 & - & 5 & 82 & 1 & 0 & 0 & 3 & 13.0 \\
\hline 2063 & Beat Sugar $\ldots \ldots \ldots \ldots \ldots \ldots \ldots$ & 141 & 19 & w & 1 & 43 & * & 59 & w & 1 & 5.5 \\
\hline 2075 & Soyboan Oll Mills $\ldots \ldots \ldots \ldots \ldots \ldots$ & 144 & W & 1 & 1 & 54 & - & 19 & 0 & w & 3.4 \\
\hline 2082 & 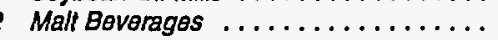 & 218 & 124 & w & $w$ & 58 & - & 25 & 0 & $w$ & 10.9 \\
\hline 21 & Tobacco Products .... & 123 & 73 & 2 & 1 & 14 & 1 & 32 & 0 & - & 6.7 \\
\hline 22 & Textlli Mill Products .. & 1,883 & W & 34 & 28 & 332 & 14 & 55 & 0 & w & 7.3 \\
\hline 23 & Apparel and Other Textlle Products ..... & 474 & 381 & $\mathbf{Q}$ & 6 & 71 & 5 & W & 0 & 2 & 16.9 \\
\hline 24 & Lumber and Wood Products $\ldots \ldots \ldots \ldots$ & 1,423 & 996 & w & 106 & 127 & 25 & w & 0 & 159 & 14.1 \\
\hline 25 & Furniture and Fixtures $\ldots \ldots \ldots \ldots \ldots$ & 421 & 322 & 4 & 6 & 69 & 8 & 7 & 0 & 6 & 16.6 \\
\hline 26 & Paper and Alled Products . . . . . . . . . & 5,385 & 2,655 & 389 & 43 & w & w & 538 & w & 492 & 4.0 \\
\hline 2611 & Pulp Mills $\ldots \ldots \ldots \ldots \ldots \ldots \ldots$ & 303 & 115 & 61 & 5 & 73 & 3 & 13 & 0 & 32 & 13.7 \\
\hline 2621 & Paper MIIIs . . . . . . . . . & 2,714 & w & 223 & $w$ & 566 & 14 & 351 & w & 199 & 3.2 \\
\hline 2631 & Paperboard MIIIs . . . . . . . . & 1,376 & W & w & w & w & W & w & 0 & 256 & 5.2 \\
\hline 27 & Printing and Publlshing ... & 1,274 & 1,055 & 1 & 11 & 173 & 6 & 0 & 0 & 27 & 12.7 \\
\hline 28 & Chemlcals and Allied Products . . . . . . . & 15,867 & 4,480 & w & 76 & 3,843 & 5,517 & W & 50 & 1,222 & 5.6 \\
\hline 2812 & Alkalles and Chlorine .............. & 492 & 305 & - & 1 & W & - & w & 0 & w & 14.9 \\
\hline 2813 & Industrial Gases $\ldots \ldots \ldots \ldots \ldots \ldots$ & 649 & w & 0 & - & 29 & w & 0 & 0 & w & 11.3 \\
\hline 2819 & Industrial Inorganic Chemicals, nec .... & 1,377 & 891 & W & w & W & 2 & W & 45 & 60 & 8.2 \\
\hline 2821 & Plastlos Materials and Resins ........ & 2,657 & w & 12 & 6 & 393 & w & 44 & 0 & w & 6.0 \\
\hline 2822 & Synthotlc Rubber $\ldots \ldots \ldots \ldots \ldots$ & 376 & 82 & w & 1 & w & $\mathbf{w}$ & w & 0 & w & 14.0 \\
\hline 2823 & Collulosic Manmado Fibers . . . . . . . . . & w & w & 0 & w & w & - & 46 & 0 & - & 24.7 \\
\hline 2824 & Organla Fibers, Noncellulosic . . . . . . . & w & 284 & w & w & w & w & w & 0 & 2 & 4.0 \\
\hline 2865 & Cycllc Crudes and Intermedlates . . . . . & 763 & 184 & 17 & 2 & 184 & w & w & 0 & w & 12.5 \\
\hline 2869 & Industrial Organic Chemicals, nec ..... & 5,896 & 660 & 35 & 16 & W & W & 123 & 0 & 512 & 6.3 \\
\hline 2873 & Nitrogenous Fertilizers $\ldots \ldots \ldots \ldots$ & 990 & 100 & 0 & 1 & 875 & W & 0 & 0 & w & 23.2 \\
\hline 2874 & Phosphatlc Fertilizers & 469 & 98 & 4 & w & w & - & W & 0 & w & 4.2 \\
\hline 29 & Petroleum and Coal Products .. & 3,757 & 1,441 & Q & 78 & 1,507 & 27 & w & • & 692 & 8.3 \\
\hline 2911 & Petroloum Refining ..... & 3,222 & w & 0 & w & 1,374 & $w$ & 5 & 0 & w & 4.4 \\
\hline 30 & Rubber and Mlsc. Plastics Products . & 2,367 & 1,960 & 20 & 17 & 301 & 24 & 15 & 0 & 31 & 9.2 \\
\hline 3011 & Tres and inner Tubes & $w$ & $w$ & 7 & $w$ & 49 & 2 & w & 0 & w & 4.2 \\
\hline 308 & Miscellaneous Plastlos Products, nec & 1,767 & 1,534 & 7 & W & 180 & 16 & 6 & 0 & w & 13.2 \\
\hline 31 & Leather and Leather Products . . . . . . . & 83 & 55 & w & 6 & 15 & 1 & $Q$ & 0 & w & 19.5 \\
\hline 32 & Stone, Clay and Glass Products ... & 3,218 & 1,516 & 27 & 125 & 961 & w & 468 & $w$ & $w$ & 6.9 \\
\hline 3211 & Flat Glass $\ldots \ldots \ldots \ldots$ & 179 & 68 & w & • & 99 & 1 & - & 0 & w & 3.2 \\
\hline 3221 & Glass Containers ............ & 375 & 197 & 6 & 1 & 169 & 2 & 0 & 0 & 1 & 5.2 \\
\hline 3229 & Pressed and Blown Glass, noc & w & w & $\mathbf{Q}$ & w & w & w & 0 & 0 & w & 6.2 \\
\hline 3241 & Coment, Hydraullc . . . . . . . . & 848 & 406 & 2 & 20 & 72 & - & 306 & 8 & 34 & 10.4 \\
\hline 3274 & LIme .............. & 243 & 59 & $\bar{w}$ & 8 & 18 & - & 141 & $w$ & 13 & 28.3 \\
\hline 3296 & Mineral Wool & 206 & w & • & w & 70 & w & * & w & - & 1.4 \\
\hline 33 & Primary Metal Industries & 9,358 & w & w & 63 & 1,753 & W & 1,396 & 942 & 318 & 3.6 \\
\hline 3312 & Blast Fumaces and Steel Mills. & 4,770 & $w$ & $w$ & $w$ & 947 & 2 & 1,330 & 736 & 40 & 4.1 \\
\hline 3313 & Electrometalurgical Products ..... & 158 & 95 & 0 & 1 & 4 & " & 32 & W & W & 7.5 \\
\hline 3321 & Gray and Ductlle Iron Foundries .. & $w$ & $w$ & - & 5 & 79 & 3 & $\mathbf{w}$ & $w$ & 5 & 11.4 \\
\hline 3331 & Primary Copper . .......... & 82 & w & - & w & 30 & - & • & w & 1 & 1.0 \\
\hline 3334 & 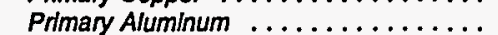 & 1,553 & $w$ & * & w & 54 & 1 & 10 & $w$ & w & 3.0 \\
\hline 3339 & Primary Nonferrous Metals, nec . . . & 211 & 110 & - & w & 36 & w & 17 & 25 & w & 2.0 \\
\hline 3353 & Aluminum Sheet, Plate, and Foll ... & 273 & w & 0 & 2 & 106 & 1 & W & 0 & w & 1.8 \\
\hline 34 & Fabricated Metal Products . . . . . . & 2,586 & 1,848 & 10 & 34 & 590 & 37 & 11 & $w$ & w & 11.1 \\
\hline 35 & Industrial Machinery and Equipment . & 2,303 & 1,817 & 9 & 26 & 377 & 19 & 17 & a & 35 & 10.2 \\
\hline 357 & Computer and Offlce Equipment .. & 278 & 256 & - & 1 & w & . & 0 & 0 & w & 15.6 \\
\hline 36 & Electronic and Other Electric Equlpment .. & 2,063 & 1,695 & 13 & 13 & 263 & 11 & $w$ & 4 & w & 9.7 \\
\hline 37 & Transportatlon Equlpment. & 2,571 & 1,942 & 36 & 40 & 397 & 14 & $\mathbf{w}$ & w & 72 & 5.0 \\
\hline 3711 & Motor Vehlcles and Car Bodles & 598 & $w$ & $w$ & 5 & 122 & 1 & w & w & 29 & 3.4 \\
\hline 3714 & Motor Vehicle Parts and Accessories ... & 770 & W & W & W & 126 & 4 & W & w & w & 6.3 \\
\hline & Instruments and Related Products ...... & 939 & w & 11 & $\ddot{w}$ & 90 & 2 & w & 0 & 5 & 12.5 \\
\hline 3841 & Surgical and Medlcal Instruments ..... & 84 & 75 & • & 1 & 8 & • & 0 & 0 & - & 13.9 \\
\hline 39 & Mlsc. Manufacturing Industries . . . . . & 328 & 246 & 2 & W & 58 & 3 & w & 0 & $\mathbf{Q}$ & 13.1 \\
\hline & Total. & 61,059 & 32,098 & 982 & 801 & 13,557 & 5,848 & 3,326 & 1,054 & 3,393 & 3.2 \\
\hline
\end{tabular}

See footnotes at end of table. 

Table A24. Total Expenditures for Purchased Energy Sources by Census Region, Industry
Group, and Selected Industries, 1991 (Continued) (Estimates in Million Dollars)

\begin{tabular}{|c|c|c|c|c|c|c|c|c|c|c|c|}
\hline $\begin{array}{c}\text { SIC } \\
\text { Code }\end{array}$ & $\begin{array}{l}\text { Industry Groups } \\
\text { and Industry }\end{array}$ & Total & Electricity & $\begin{array}{l}\text { Residual } \\
\text { Fuel Oil }\end{array}$ & $\begin{array}{l}\text { Distillate } \\
\text { Fuel Oip }\end{array}$ & $\begin{array}{c}\text { Natural } \\
\text { Gas }^{c}\end{array}$ & LPG & Coal & $\begin{array}{c}\text { Coke and } \\
\text { Breeze }\end{array}$ & Other & $\begin{array}{c}\text { RSE } \\
\text { Row } \\
\text { Factors }\end{array}$ \\
\hline & & \multicolumn{10}{|c|}{ Northeast Census Region } \\
\hline & RSE Column Factors: & 0.7 & 0.7 & 1.0 & 1.2 & 0.8 & 1.2 & 1.3 & 1.2 & 1.2 & \\
\hline 20 & Food and Kindred Products . . . . . . . . & 614 & 391 & 22 & 34 & 143 & 6 & 5 & 0 & $a$ & 14.1 \\
\hline 2011 & Meat Packing Plants .............. & 12 & 8 & 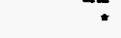 & 1 & 1 & 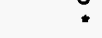 & 0 & $\mathbf{0}$ & 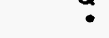 & 24.9 \\
\hline 2033 & Canned Fruits and Vegetables ........ & 42 & 22 & 3 & 1 & 16 & - & $\bullet$ & 0 & - & 17.1 \\
\hline 2037 & Frozen Fruits and Vegetables ........ & 13 & $\mathbf{W}$ & $\mathbf{w}$ & : & 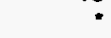 & * & 0 & 0 & $\cdot$ & 32.2 \\
\hline 2046 & Wet Com Milling . . . . . . . . . . . & 2 & 1 & $\cdot$ & * & * & - & 0 & 0 & - & 23.3 \\
\hline 2051 & Bread, Cake, and Related Products .... & 51 & 31 & - & $w$ & $w$ & * & 0 & 0 & • & 19.1 \\
\hline 2063 & Beet Sugar . . . . . . . . . . . . . & 0 & 0 & 0 & 0 & 0 & 0 & 0 & 0 & 0 & NF \\
\hline 2075 & Soybean Oil Mills ................ & 0 & 0 & 0 & 0 & 0 & 0 & 0 & 0 & 0 & NF \\
\hline 2082 & Malt Beverages ................ & 51 & 33 & $w$ & + & 10 & $*$ & $W$ & 0 & 0 & 15.4 \\
\hline 21 & Tobacco Products . . . . . . . . . . . & NA & NA & NA & NA & NA & NA & NA & NA & NA & 20.5 \\
\hline 22 & Textile Mill Products .............. & 198 & 108 & 14 & 15 & 43 & 4 & $W$ & 0 & $w$ & 18.7 \\
\hline 23 & Apparel and Other Textile Products ..... & 63 & 49 & 1 & 2 & 10 & $\mathbf{Q}$ & 0 & 0 & W & 28.7 \\
\hline 24 & Lumber and Wood Products . . . . . . . . & NA & NA & NA & NA & NA & NA & NA & NA & NA & 34.2 \\
\hline 25 & Fumiture and Fixtures $\ldots \ldots \ldots \ldots \ldots$ & 53 & 39 & Q & 2 & 8 & 1 & 0 & 0 & 1 & 27.9 \\
\hline 26 & Paper and Allied Products . . . . . . . . & 1,066 & 562 & 199 & 14 & 119 & $W$ & $w$ & 0 & 88 & 5.8 \\
\hline 2611 & Pulp Mills . . . . . . . . . . . . . . & W & $Q$ & W & $\because$ & 0 & 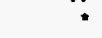 & 0 & 0 & $W$ & 33.6 \\
\hline 2621 & Paper Mills . . . . . . . . . . . . . . & 696 & 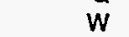 & 168 & W & 56 & 6 & $w$ & 0 & 64 & 4.2 \\
\hline 2631 & Paperboard Mills . . . . . . . . . . . . . & W & $w$ & W & $*$ & 17 & 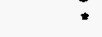 & $w$ & 0 & $w$ & 14.8 \\
\hline 27 & Printing and Publishing $\ldots \ldots \ldots \ldots$ & 328 & 268 & 1 & 8 & 43 & 1 & 0 & 0 & 6 & 24.0 \\
\hline 28 & Chemicals and Allied Products . . . . . . . . & W & W & 61 & W & 194 & 20 & $w$ & 0 & 70 & 8.6 \\
\hline 2812 & Alkalies and Chlorine ............ & $\bullet$ & * & 0 & 0 & $*$ & 0 & 0 & 0 & 0 & 35.0 \\
\hline 2813 & Industrial Gases . . . . . . . . . . . . & 47 & $w$ & 0 & * & $w$ & * & 0 & $\mathbf{0}$ & $w$ & 13.5 \\
\hline 2819 & Industrial Inorganic Chemicals, nec .... & 57 & w & $w$ & 3 & 19 & • & 0 & 0 & 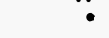 & 19.1 \\
\hline 2821 & Plastics Materials and Resins ........ & 131 & $w$ & $\mathbf{w}$ & $\mathbf{Q}$ & 25 & $w$ & $w$ & 0 & $w$ & 8.5 \\
\hline 2822 & 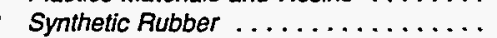 & $w$ & $w$ & w & 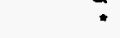 & $w$ & 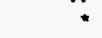 & 0 & 0 & $w$ & 25.0 \\
\hline 2823 & Cellulosic Manmade Fibers . . . . . . . . . & 0 & 0 & 0 & 0 & 0 & 0 & 0 & 0 & 0 & NF \\
\hline 2824 & Organic Fibers, Noncellulosic ......... & W & 6 & - & - & $w$ & 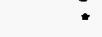 & 0 & 0 & 0 & 16.9 \\
\hline 2865 & Cyclic Crudes and Intermediates ....... & W & $w$ & W & $\mathbf{Q}$ & 22 & • & $w$ & 0 & $w$ & 19.1 \\
\hline 2869 & Industrial Organic Chemicals, nec ...... & $\mathbf{w}$ & $w$ & 29 & $\mathbf{W}$ & $\bar{w}$ & $w$ & 0 & 0 & $w$ & 8.6 \\
\hline 2873 & Nitrogenous Fertilizers $\ldots \ldots \ldots$ & W & 2 & 0 & $\star$ & $\ddot{w}$ & $\bullet$ & 0 & 0 & 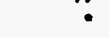 & 45.2 \\
\hline 2874 & Phosphatic Fentilizers . . . . . . . . . . & 0 & 0 & 0 & 0 & 0 & 0 & 0 & 0 & 0 & NF \\
\hline 29 & Petroleum and Coal Products ........ & 327 & 168 & 0 & 42 & 78 & $\mathbf{Q}$ & $w$ & 0 & $\mathbf{a}$ & 13.2 \\
\hline 2911 & Petroleum Refining ............... & 204 & 122 & 0 & 0 & $w$ & 0 & w & 0 & $\mathbf{W}$ & 5.5 \\
\hline 30 & Rubber and Misc. Plastics Products .... . & 485 & 410 & 8 & 6 & $W$ & $w$ & W & 0 & 5 & 20.4 \\
\hline 3011 & Tires and Inner Tubes $\ldots \ldots \ldots$ & 10 & 5 & $w$ & • & $\ddot{w}$ & $\bullet$ & 0 & 0 & $\bullet$ & 15.6 \\
\hline 308 & Miscellaneous Plastics Products, nec ... & 414 & 363 & 4 & $W$ & 31 & 5 & $w$ & 0 & $w$ & 24.6 \\
\hline 31 & Leather and Leather Products ......... & 31 & 18 & 2 & 5 & 3 & 1 & $\mathbf{Q}$ & $\mathbf{0}$ & $\because$ & 26.2 \\
\hline 32 & Stone, Clay and Glass Products ...... & 663 & 318 & 9 & 24 & 179 & $\mathbf{w}$ & 120 & $w$ & $w$ & 15.7 \\
\hline 3211 & Flat Glass ................ & $w$ & W & 0 & 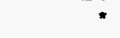 & W & 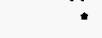 & 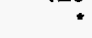 & 0 & $\bullet$ & 4.3 \\
\hline 3221 & Glass Containers ............... & 95 & 47 & 4 & - & 43 & 1 & 0 & 0 & $\cdot$ & 8.8 \\
\hline 3229 & Pressed and Blown Glass, nec ........ & $\mathbf{w}$ & W & $\mathbf{Q}$ & $w$ & $w$ & : & 0 & 0 & $w$ & 7.6 \\
\hline 3241 & Cement, Hydraulic ............. & 130 & 70 & * & $w$ & $\because$ & $*$ & 50 & $w$ & $w$ & 18.3 \\
\hline 3274 & Lime $\ldots \ldots \ldots \ldots$ & $\mathbf{Q}$ & $\mathbf{Q}$ & 0 & $\mathbf{Q}$ & $Q$ & * & $\mathbf{Q}$ & 0 & $Q$ & NF \\
\hline 3296 & 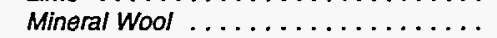 & 27 & $\vec{w}$ & 0 & W & $w$ & * & 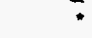 & 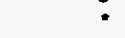 & 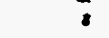 & 1.7 \\
\hline 33 & Primary Metal Industries . . . . . . . . . . & 1,750 & 795 & 13 & 12 & 324 & 8 & 493 & 69 & 36 & 10.3 \\
\hline 3312 & Blast Fumaces and Steol Mills ......... & 1,101 & 366 & 8 & 6 & 186 & 1 & $w$ & $W$ & $w$ & 6.9 \\
\hline 3313 & Electrometalurgical Products ......... & W & W & 0 & * & $\cdot$ & * & $w$ & 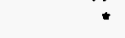 & $w$ & 12.2 \\
\hline 3321 & Gray and Ductile Iron Foundries ...... & 47 & 25 & 0 & * & 8 & 1 & $\because$ & 13 & 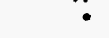 & 17.8 \\
\hline 3331 & Primary Copper . . . . . . . . . . . . & W & W & 0 & $*$ & $w$ & : & 0 & 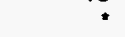 & - & 1.0 \\
\hline 3334 & Primary Aluminum $\ldots \ldots \ldots \ldots$ & $w$ & $W$ & 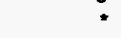 & $\bullet$ & $w$ & - & 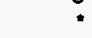 & 0 & $w$ & 4.9 \\
\hline 3339 & Primary Nonferrous Metals, nec . . . . . . . & W & 6 & * & * & 3 & $*$ & $w$ & $w$ & $\because$ & 2.3 \\
\hline 3353 & Aluminum Sheet, Plate, and Foil ...... & $W$ & 27 & 0 & $\bullet$ & $w$ & * & 0 & 0 & $\cdot$ & 1.5 \\
\hline 34 & Fabricated Metal Products . . . . . . . . . . & 544 & 381 & 7 & 12 & 128 & 5 & $w$ & $w$ & 4 & 15.3 \\
\hline 35 & Industrial Machinery and Equipment . . . . . & 496 & $w$ & 8 & $w$ & 67 & $w$ & 0 & 0 & 9 & 19.3 \\
\hline 357 & Computer and Office Equipment ...... & 63 & 57 & 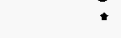 & 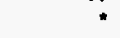 & $w$ & 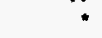 & 0 & 0 & $w$ & 19.5 \\
\hline 36 & Electronic and Other Electric Equipment .. & 537 & 450 & 12 & 9 & 58 & 4 & 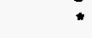 & 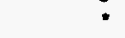 & 5 & 15.7 \\
\hline 37 & Transportation Equipment $\ldots \ldots \ldots \ldots$ & W & $W$ & 23 & W & 39 & $w$ & $w$ & 0 & $w$ & 10.6 \\
\hline 3711 & Motor Vehicles and Car Bodies ....... & W & $w$ & W & $*$ & $W$ & 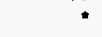 & 0 & 0 & 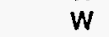 & 7.2 \\
\hline 3714 & Motor Vehicio Parts and Accessories ... & 81 & 63 & $*$ & * & $W$ & * & $w$ & 0 & $w$ & 12.4 \\
\hline 38 & Instruments and Related Products ...... & 400 & $W$ & 10 & $w$ & w & $\mathbf{Q}$ & $w$ & 0 & 1 & 14.7 \\
\hline 3841 & Surgical and Medical Instruments $\ldots \ldots$ & 29 & 26 & 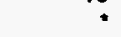 & 1 & 3 & $*$ & 0 & $\mathbf{0}$ & $\cdot$ & 18.2 \\
\hline 39 & Misc. Manufacturing Industries . . . . . . . . & $W$ & 94 & 2 & $w$ & $W$ & 1 & $w$ & 0 & $\bullet$ & 17.3 \\
\hline & Total $\ldots \ldots \ldots \ldots \ldots \ldots$ & 8,936 & 5,502 & 393 & 248 & 1,551 & 95 & W & W & 297 & 5.3 \\
\hline
\end{tabular}

See footnotes at end of table. 
Table A24. Total Expenditures for Purchased Energy Sources by Census Region, Industry Group, and Selected Industries, 1991 (Continued)

(Estimates in Million Dollars)

\begin{tabular}{cc|c|c|c|c|c|c|c|c|c|}
\hline $\begin{array}{c}\text { SIC } \\
\text { Codea }\end{array}$ & $\begin{array}{c}\text { Industry Groups } \\
\text { and Industry }\end{array}$ & Total & Electricity & $\begin{array}{l}\text { Residual } \\
\text { Fuel Oil }\end{array}$ & $\begin{array}{l}\text { Distillate } \\
\text { Fuel Oil }\end{array}$ & $\begin{array}{c}\text { Natural } \\
\text { Gas }^{\circ}\end{array}$ & LPG & $\begin{array}{c}\text { RSE } \\
\text { Row } \\
\text { Coal }\end{array}$ & $\begin{array}{c}\text { Coke and } \\
\text { Breeze }\end{array}$ & $\begin{array}{c}\text { Other } \\
\text { Factors }\end{array}$ \\
\hline
\end{tabular}

\begin{tabular}{|c|c|c|c|c|c|c|c|c|c|c|c|}
\hline & & & & & Midwe & ensus $\mathrm{F}$ & & & & & \\
\hline & RSE Column Factors: & 0.6 & 0.6 & 1.5 & 1.3 & 0.7 & 1.2 & 1.0 & 1.2 & 1.1 & \\
\hline 20 & Food and Kindred Products $\ldots \ldots \ldots \ldots$ & 1,674 & w & 12 & 19 & w & 9 & 146 & w & 44 & 7.8 \\
\hline 2011 & Meat Packing Plants ............ & 151 & 93 & 1 & 2 & 51 & * & 1 & 0 & 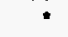 & 9.5 \\
\hline 2033 & Canned Fruils and Vegetables ......... & 48 & 22 & 0 & $\bar{w}$ & 22 & $w$ & 0 & 0 & 1 & 16.8 \\
\hline 2037 & Frozen Fruils and Vegetables . . . . . . . . & 22 & w & w & • & 6 & - & 0 & 0 & • & 26.9 \\
\hline 2046 & Wet Com Milling ................ & 289 & $w$ & $\because$ & 1 & 104 & - & 76 & w & w & 13.3 \\
\hline 2051 & Bread, Cake, and Related Products .... & 57 & 35 & 0 & $w$ & $w$ & $*$ & 0 & 0 & $\because$ & 16.6 \\
\hline 2063 & Beet Sugar $\ldots \ldots \ldots \ldots \ldots \ldots \ldots$ & 71 & $w$ & w & * & 13 & - & 36 & w & * & 6.6 \\
\hline 2075 & Soybean Oll MIlls ............... & 93 & w & - & * & 35 & • & w & 0 & w & 4.1 \\
\hline 2082 & Mall Beverages ..... & 42 & 21 & $\cdot$ & $\star$ & w & 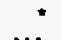 & $w$ & 0 & W & 17.1 \\
\hline 21 & Tobacco Products .... & NA & NA & NA & NA & NA & NA & NA & NA & NA & 9.5 \\
\hline 22 & Textlle Mill Products . . . . . . . . . . . . & NA & NA & NA & NA & NA & NA & NA & NA & NA & 24.8 \\
\hline 23 & Apparel and Other Textile Products . . . . & NA & NA & NA & NA & NA & NA & NA & NA & NA & 29.8 \\
\hline 24 & Lumber and Wood Products . .......... & 239 & 164 & Q & 14 & 40 & 4 & w & 0 & w & 29.4 \\
\hline 25 & Furniture and Fixtures $\ldots \ldots \ldots \ldots \ldots \ldots$ & $w$ & 92 & $*$ & $\because$ & W & 1 & Q & 0 & 1 & 21.3 \\
\hline 26 & Paper and Allied Products . . . . . . . . . . & 1,129 & 590 & 13 & 5 & 291 & w & 163 & w & 60 & 7.0 \\
\hline 2611 & 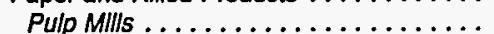 & $w$ & w & 0 & * & W & - & w & 0 & W & 33.3 \\
\hline 2621 & Paper MIIIs . . . . . . . . $\ldots \ldots \ldots \ldots$ & 582 & 287 & $w$ & 3 & 141 & 2 & 114 & $w$ & 24 & 5.4 \\
\hline 2631 & Paperboard Mills ................ & W & 84 & w & • & 55 & 1 & 41 & 0 & w & 14.3 \\
\hline 27 & Printing and Publishing..$\ldots \ldots \ldots \ldots$ & 381 & 299 & - & 1 & 71 & 2 & 0 & 0 & $\mathbf{Q}$ & 17.4 \\
\hline 28 & Chemlcals and Allied Products . .......... & 1,982 & $w$ & 5 & $\mathbf{Q}$ & 482 & $\bar{w}$ & 105 & $w$ & $\bar{w}$ & 9.1 \\
\hline 2812 & Alkallos and Chlorine $\ldots \ldots \ldots \ldots$ & w & w & 0 & * & w & - & 0 & 0 & • & 26.6 \\
\hline 2813 & Industrlal Gases .................. & W & W & 0 & - & w & • & 0 & 0 & w & 17.7 \\
\hline 2819 & Industral Inorganic Chemicals, nec .... & 366 & W & $w$ & $\cdot$ & $w$ & • & $w$ & * & 5 & 12.9 \\
\hline 2821 & Plastics Materials and Resins . . . . . . & 413 & 134 & " & * & 51 & w & $\mathbf{w}$ & 0 & w & 8.8 \\
\hline 2822 & Synthatic Rubber $\ldots \ldots \ldots \ldots \ldots$ & $w$ & 8 & 0 & $*$ & $w$ & $\because$ & $w$ & 0 & $\because$ & 17.9 \\
\hline 2823 & Cellulosic Manmade Fibers . . . . . . . . . & 0 & 0 & 0 & 0 & 0 & 0 & 0 & 0 & 0 & NF \\
\hline 2824 & Organic Fibers, Noncellulosic ........ & 0 & 0 & 0 & 0 & 0 & 0 & 0 & 0 & 0 & NF \\
\hline 2865 & Cyclic Crudes and Intermediates ....... & 130 & 34 & 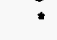 & : & 35 & w & $\mathbf{w}$ & 0 & w & 13.7 \\
\hline 2869 & Industrial Organic Chemicals, nec ..... & w & w & - & w & w & w & 37 & 0 & w & 7.4 \\
\hline 2873 & Nitrogenous Fertillzers ............ & w & 20 & 0 & $\because$ & $w$ & * & 0 & 0 & • & 32.7 \\
\hline 2874 & Phosphattc Fertillzers . . . . . . . . . & - & * & 0 & • & • & - & 0 & 0 & • & 4.5 \\
\hline 29 & Petroleum and Coal Products $\ldots \ldots \ldots \ldots$ & 502 & w & $\mathbf{Q}$ & $\mathbf{Q}$ & 185 & 3 & - & 0 & w & 11.1 \\
\hline 2911 & Petroleum Reflning ................ & 391 & 243 & 0 & 0 & w & 0 & $\cdot$ & 0 & w & 4.2 \\
\hline 30 & Rubber and Misc. Plastics Products . . . . . & 893 & 741 & 3 & 1 & 126 & 6 & 6 & 0 & 9 & 12.1 \\
\hline 3011 & Tres and Inner Tubes ............. & w & $w$ & 2 & : & 14 & : & w & 0 & w & 4.1 \\
\hline 308 & Miscellaneous Plastics Products, nec . . . & NA & NA & NA & NA & NA & NA & NA & NA & NA & 19.4 \\
\hline 31 & Leather and Leather Products . . . . . . . & 24 & 15 & w & • & w & • & 0 & 0 & • & 24.8 \\
\hline 32 & Stone, Clay and Glass Products ....... & 842 & w & w & w & 277 & 4 & 109 & 5 & з3 & 9.6 \\
\hline 3211 & Flat Glass $\ldots \ldots \ldots \ldots \ldots \ldots \ldots$ & 46 & $w$ & 0 & $*$ & 24 & - & 0 & 0 & w & 3.8 \\
\hline 3221 & Glass Containers ............. & 78 & 39 & 0 & • & 38 & - & 0 & 0 & * & 7.5 \\
\hline 3229 & Pressed and Blown Glass, noc ....... & 65 & 28 & • & * & 35 & - & 0 & 0 & 1 & 6.7 \\
\hline 3241 & Cement, Hydraulle .......... & 200 & 96 & - & w & 13 & - & 69 & 0 & w & 14.9 \\
\hline 3274 & Lime........... & 70 & 19 & 0 & 2 & 8 & • & 32 & $\mathbf{w}$ & $w$ & 18.2 \\
\hline 3296 & Mineral Wool ... & 80 & 47 & $\cdot$ & * & w & * & 0 & w & • & 1.1 \\
\hline 33 & Primary Metal Industries . . . . . . . . . & 3,856 & w & w & 27 & w & 7 & 534 & 571 & w & 5.4 \\
\hline 3312 & Blast Fumaces and Steol Mills . . . . . . . & 2,342 & w & w & $w$ & $w$ & 1 & 509 & 473 & 16 & 5.2 \\
\hline 3313 & Electrometalurgical Products ......... & 101 & 65 & 0 & $\bullet$ & w & - & w & $\mathbf{w}$ & w & 9.4 \\
\hline & Gray and Ductle Iron Foundries ....... & w & 215 & • & $\mathbf{Q}$ & w & 1 & w & w & 4 & 13.6 \\
\hline 3331 & Primary Copper ............ & - & • & 0 & 0 & • & • & 0 & 0 & 0 & 1.3 \\
\hline 3334 & Primary Aluminum & w & w & 0 & : & w & - & w & 0 & w & 5.0 \\
\hline 3339 & Primary Nonferrous Metals, nec ........... & $w$ & 26 & 0 & * & 6 & * & 0 & $w$ & $\because$ & 2.4 \\
\hline 3353 & Aluminum Sheet, Plate, and Foil ...... & 85 & w & 0 & $w$ & 37 & - & 0 & 0 & $\mathbf{w}$ & 1.5 \\
\hline 34 & Fabricated Metal Products . . . . . . . . . . . & 1,067 & 769 & * & w & 243 & w & $\mathbf{w}$ & $\mathbf{w}$ & w & 15.0 \\
\hline 35 & Industrial Machinery and Equipment ..... & 940 & w & $Q$ & $w$ & 186 & w & 17 & $\mathbf{Q}$ & w & 11.6 \\
\hline 357 & Computer and Offlce Equipment ....... & 43 & 38 & 0 & - & 5 & - & 0 & 0 & • & 23.1 \\
\hline 36 & Electronic and Other Electric Equipment .. & 460 & 350 & 1 & w & 88 & 2 & w & 0 & w & 13.9 \\
\hline & Transportation Equipment ......... & w & w & $w$ & 10 & 210 & 5 & w & w & 31 & 5.8 \\
\hline 3711 & Motor Vehicles and Car Bodles & 395 & w & $w$ & w & 80 & 1 & w & w & w & 4.7 \\
\hline 3714 & Motor Vehiclo Parts and Accessories ... & 547 & w & $w$ & w & 87 & w & w & w & $\mathbf{w}$ & 7.2 \\
\hline 38 & Instruments and Related Products ...... & 115 & 99 & $\cdot$ & 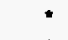 & $w$ & • & - & 0 & $\mathbf{w}$ & 22.8 \\
\hline 3841 & Surgical and Medical Instruments . & 16 & 13 & 0 & - & 2 & - & 0 & 0 & * & 21.1 \\
\hline 39 & MIsc. Manufacturing Industries . . . . & & 59 & - & " & 21 & 1 & w & 0 & $\mathbf{Q}$ & 22.3 \\
\hline & Total $\ldots \ldots \ldots \ldots \ldots \ldots \ldots \ldots$ & 15,598 & 9,135 & w & 152 & 3,657 & $\mathbf{w}$ & 1,161 & 597 & 474 & 4.1 \\
\hline
\end{tabular}

See footnotes at end of table. 
Table A24. Total Expenditures for Purchased Energy Sources by Census Region, Industry Group, and Selected Industries, 1991 (Continued)

(Estimates in Million Dollars)

\begin{tabular}{cc|c|c|c|c|c|c|c|c|c|}
\hline $\begin{array}{c}\text { SIC } \\
\text { Code }\end{array}$ & $\begin{array}{c}\text { Industry Groups } \\
\text { and Industry }\end{array}$ & Total & Electricity & $\begin{array}{c}\text { Residual } \\
\text { Fuel Oil }\end{array}$ & $\begin{array}{c}\text { Distillate } \\
\text { Fuel Oil }\end{array}$ & $\begin{array}{c}\text { Natural } \\
\text { Gas }^{\circ}\end{array}$ & $\begin{array}{c}\text { ASE } \\
\text { Row } \\
\text { LPG }\end{array}$ & $\begin{array}{c}\text { Coke and } \\
\text { Breeze }\end{array}$ & $\begin{array}{c}\text { Other } \\
\text { Factors }\end{array}$ \\
\hline
\end{tabular}

\begin{tabular}{|c|c|c|c|c|c|c|c|c|c|c|c|}
\hline & & & & & Soutt & ensus $\mathrm{R}$ & & & & & \\
\hline & RSE Column Factors: & 0.6 & 0.6 & 1.4 & 1.2 & 0.8 & 1.1 & 1.1 & 1.6 & 1.1 & \\
\hline 20 & Food and Kindred Products $\ldots \ldots \ldots \ldots$ & 1,336 & w & 28 & 32 & 368 & 17 & 34 & $w$ & w & 10.5 \\
\hline 2011 & Meat Packing Plants . . ........... & 69 & 45 & 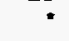 & 7 & 14 & w & 0 & 0 & w & 16.2 \\
\hline 2033 & Canned Fruits and Vegetables . . . . . . . . & 30 & 12 & * & * & 17 & * & 0 & 0 & * & 19.6 \\
\hline 2037 & Frozen Fruits and Vegetables & 52 & 31 & 3 & * & 18 & * & 0 & 0 & * & 17.0 \\
\hline 2046 & Wet Com Milling. & w & 31 & 0 & - & w & * & 14 & 0 & w & 22.6 \\
\hline 2051 & Bread, Cake, and Related Products .... & 73 & 45 & 0 & 1 & 27 & • & 0 & 0 & 1 & 13.2 \\
\hline 2063 & Beet Sugar . . . . . . . . & w & * & 0 & * & w & * & 0 & $w$ & : & 17.0 \\
\hline 2075 & Soybean Oil Mills & 51 & 23 & 1 & 1 & 19 & * & w & 0 & w & 4.9 \\
\hline 2082 & Malt Beverages ... & 67 & 41 & w & * & 20 & - & w & 0 & * & 13.8 \\
\hline 21 & Tobacco Products & 121 & 72 & 2 & 1 & 13 & 1 & 32 & 0 & - & 6.8 \\
\hline 22 & Textile Mill Products & 1,609 & w & 21 & 13 & 260 & 10 & w & 0 & w & 6.5 \\
\hline 23 & Apparel and Other Textile Products & 318 & 258 & $\mathbf{Q}$ & 3 & 44 & w & w & 0 & $w$ & 21.7 \\
\hline 24 & Lumber and Wood Products & 614 & w & $\mathbf{Q}$ & $w$ & 43 & 9 & $\mathbf{w}$ & 0 & 41 & 20.2 \\
\hline 25 & Furniture and Fixtures $\ldots \ldots \ldots \ldots \ldots$ & w & 160 & 1 & 3 & w & 4 & w & 0 & 4 & 18.7 \\
\hline 26 & Paper and Allied Products $\ldots \ldots \ldots \ldots$ & 2,223 & 1,039 & 152 & 20 & w & 13 & 281 & 0 & w & 4.4 \\
\hline 2611 & Pulp Mills $\ldots \ldots \ldots \ldots \ldots \ldots \ldots$ & 194 & 68 & w & 4 & $w$ & 2 & $w$ & 0 & 20 & 16.9 \\
\hline 2621 & Paper Mills & 998 & w & 39 & w & 242 & 3 & 156 & 0 & 54 & 3.8 \\
\hline 2631 & Paperboard Mills & w & 268 & 65 & w & $w$ & 1 & 117 & 0 & 86 & 5.3 \\
\hline 27 & Printing and Publishing .... & 327 & 284 & • & 1 & 33 & 2 & 0 & 0 & 7 & 21.1 \\
\hline 28 & Chemicals and Allied Products . & 12,374 & 2,677 & w & 35 & 2,942 & w & $w$ & 11 & 981 & 6.4 \\
\hline 2812 & Alkalies and Chlorine $\ldots \ldots \ldots \ldots$ & 428 & 251 & 0 & 1 & $w$ & - & w & 0 & w & 16.7 \\
\hline 2813 & Industrial Gases . . . . . . . . . . . & 388 & $w$ & 0 & * & 18 & w & 0 & 0 & w & 14.3 \\
\hline 2819 & Industrial Inorganic Chemicals, nec & $w$ & w & w & w & w & 1 & 28 & 6 & 38 & 10.2 \\
\hline 2821 & Plastics Materials and Resins .... & 2,090 & w & w & w & 308 & 1,340 & w & 0 & w & 5.2 \\
\hline 2822 & Synthetic Rubber $\ldots \ldots \ldots \ldots$ & 340 & w & • & * & w & w & 0 & 0 & w & 13.4 \\
\hline 2823 & Cellulosic Manmade Fibers & w & w & 0 & w & w & • & 46 & $\mathbf{0}$ & * & 25.1 \\
\hline 2824 & Organic Fibers, Noncellulosic $\ldots \ldots \ldots$ & w & 278 & W & W & w & w & w & 0 & 2 & 4.0 \\
\hline 2865 & Cyclic Crudes and Intermediates . . . . . . & 574 & 124 & W & 1 & 127 & $w$ & $\because$ & 0 & $\bar{w}$ & 12.8 \\
\hline 2869 & Industrial Organic Chemicals, nec . . . . . & 5,405 & 500 & 6 & W & W & w & 86 & 0 & 446 & 7.9 \\
\hline 2873 & Nitrogenous Fertilizers $\ldots \ldots \ldots \ldots$ & 728 & 68 & 0 & 1 & 656 & w & 0 & 0 & $w$ & 28.9 \\
\hline 2874 & Phosphatic Fertilizers . ........... & $w$ & w & 4 & w & w & • & w & 0 & 282 & 3.1 \\
\hline 29 & Petroleum and Coal Products & 2,149 & 581 & Q & W & 943 & w & w & * & w & 7.4 \\
\hline 2911 & Petroleum Refining $\ldots \ldots \ldots \ldots \ldots$ & 1,948 & 556 & 0 & $w$ & 911 & w & $w$ & 0 & w & 4.1 \\
\hline 30 & Rubber and Misc. Plastics Products . . . . & 746 & 599 & 9 & 8 & 104 & 7 & 3 & 0 & 16 & 10.7 \\
\hline 3011 & Tires and Inner Tubes & w & 121 & w & w & 31 & 1 & w & 0 & w & 4.0 \\
\hline 308 & Miscellaneous Plastics Products, nec... & 456 & 389 & 3 & w & 50 & 5 & w & 0 & $w$ & 19.7 \\
\hline 31 & Leather and Leather Products . . . . . . . & 21 & 17 & * & * & W & * & 0 & 0 & W & 23.2 \\
\hline 32 & Stone, Clay and Glass Products ....... & 1,145 & w & w & w & 364 & w & 149 & 11 & 30 & 10.6 \\
\hline 3211 & Flat Glass $\ldots \ldots \ldots \ldots \ldots$ & 76 & 31 & 0 & $\because$ & 43 & ? & 0 & 0 & 2 & 3.6 \\
\hline 3221 & Glass Containers & 105 & 54 & w & * & w & 1 & 0 & 0 & " & 8.3 \\
\hline 3229 & Pressed and Blown Glass, nec & w & 60 & * & - & w & $w$ & 0 & 0 & - & 10.6 \\
\hline 3241 & Cement, Hydraulic $\ldots \ldots \ldots \ldots \ldots$ & 284 & 130 & w & 6 & 36 & * & 97 & w & 9 & 15.7 \\
\hline 3274 & 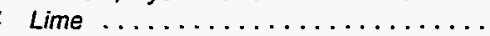 & 78 & 18 & 0 & 2 & w & * & 44 & 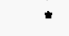 & w & 20.7 \\
\hline 3296 & Mineral Wool & 72 & W & * & * & 26 & * & 0 & w & $\because$ & 1.4 \\
\hline 33 & Primary Metal Industries & 2,503 & W & w & W & w & w & W & 284 & 70 & 4.7 \\
\hline 3312 & Blast Fumaces and Steel Mills . . . . & 1,112 & w & w & W & W & * & 272 & w & W & 5.9 \\
\hline 3313 & Electrometalurgical Products .......... & w & w & 0 & • & W & 0 & w & w & W & 11.0 \\
\hline 3321 & Gray and Ductile Iron Foundries ....... & 148 & w & - & 2 & w & 1 & $*$ & w & 1 & 12.1 \\
\hline 3331 & Primary Copper $\ldots \ldots \ldots \ldots \ldots \ldots$ & w & 9 & * & - & w & * & 0 & 0 & * & 1.1 \\
\hline 3334 & Primary Aluminum & 584 & w & 0 & w & w & • & $w$ & w & w & 4.0 \\
\hline 3339 & Primary Nonferrous Metals, nec . . . . . . & 91 & 52 & 0 & 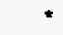 & w & w & W & 0 & $w$ & 5.6 \\
\hline 3353 & Aluminum Sheet, Plate, and Foil ........ & 123 & $W$ & 0 & $w$ & 46 & 1 & w & 0 & 2 & 1.8 \\
\hline 34 & Fabricated Metal Products $\ldots \ldots \ldots \ldots \ldots$ & 679 & 482 & Q & W & 150 & w & 0 & w & 17 & 20.3 \\
\hline 35 & Industrial Machinery and Equipment ..... & 561 & 447 & 1 & 4 & 90 & $w$ & * & * & w & 16.3 \\
\hline 357 & Computer and Office Equipment & 41 & 38 & * & - & 3 & • & 0 & 0 & $\because$ & 21.9 \\
\hline 36 & Electronic and Other Electric Equipment .. & 635 & 504 & 1 & w & 81 & 4 & $w$ & 4 & w & 14.5 \\
\hline 37 & Transportation Equipment & 541 & 415 & w & 10 & 85 & 3 & w & * & 19 & 8.5 \\
\hline 3711 & Motor Vehicles and Car Bodies & 153 & 103 & w & 1 & 35 & 1 & w & 0 & w & 4.4 \\
\hline 3714 & Motor Vehicle Parts and Accessories ... & 114 & 93 & $*$ & * & w & w & w & * & 1 & 12.1 \\
\hline & Instruments and Related Products . . . . . . & 190 & 169 & • & 1 & 18 & * & 0 & 0 & 1 & 18.3 \\
\hline 3841 & Surgical and Medical Instruments ..... & 20 & 18 & 0 & * & 2 & • & 0 & 0 & . & 21.3 \\
\hline 39 & Misc. Manufacturing Industries . . . . & w & 65 & • & * & $\bar{w}$ & 1 & 0 & 0 & Q & 19.9 \\
\hline & Total & 28,373 & 12,210 & w & 276 & 6,564 & $w$ & 1,166 & 315 & 2,030 & 3.9 \\
\hline
\end{tabular}

See footnotes at end of table. 
Table A24. Total Expenditures for Purchased Energy Sources by Census Region, Industry
Group, and Selected Industries, 1991 (Continued) (Estimates in Million Dollars)

\begin{tabular}{|c|c|c|c|c|c|c|c|c|c|c|c|}
\hline $\begin{array}{l}\text { SIC } \\
\text { Code" }\end{array}$ & $\begin{array}{l}\text { Industry Groups } \\
\text { and Industry }\end{array}$ & Total & Electricity & $\begin{array}{c}\text { Residual } \\
\text { Fuel Oil } \\
\end{array}$ & $\begin{array}{l}\text { Distillate } \\
\text { Fuel Oil } \\
\end{array}$ & $\begin{array}{c}\text { Natural } \\
\text { Gas }^{\circ}\end{array}$ & LPG & Coal & $\begin{array}{c}\text { Coke and } \\
\text { Breeze }\end{array}$ & Other & $\begin{array}{c}\text { RSE } \\
\text { Row } \\
\text { Factors }\end{array}$ \\
\hline & & \multicolumn{10}{|c|}{ West Census Region } \\
\hline & RSE Column Factors: & 0.7 & 0.7 & 1.3 & 1.1 & 0.8 & 1.1 & 1.2 & 1.2 & 1.0 & \\
\hline 20 & Food and Kindred Products & 1,013 & 556 & 15 & 25 & 343 & 8 & 36 & w & w & 10.4 \\
\hline 2011 & Meat Packing Plants .............. & 22 & 13 & $\cdot$ & 1 & 8 & $\cdot$ & 0 & 0 & * & 16.7 \\
\hline 2033 & Canned Frults and Vegetables......... & 97 & 43 & 2 & W & 48 & w & 0 & 0 & * & 10.1 \\
\hline 2037 & Frozen Frults and Vegetables ......... & 117 & 72 & $\mathbf{Q}$ & 2 & 39 & 1 & 0 & 0 & w & 16.8 \\
\hline 2046 & Wet Com Milling $\ldots \ldots \ldots \ldots \ldots \ldots$ & w & w & 0 & • & W & - & 0 & 0 & w & 20.9 \\
\hline 2051 & Bread, Cake, and Related Products .... & 47 & 28 & 0 & * & 18 & $\cdot$ & 0 & 0 & Q & 26.3 \\
\hline 2063 & Best Sugar $\ldots \ldots \ldots \ldots \ldots \ldots \ldots$ & $\mathbf{w}$ & $w$ & w & 1 & w & - & 22 & 8 & - & 8.1 \\
\hline 2075 & Soybean Oll Mills $\ldots \ldots \ldots \ldots \ldots \ldots$ & 0 & 0 & 0 & 0 & 0 & 0 & 0 & 0 & 0 & NF \\
\hline 2082 & Malt Beverages $\ldots \ldots \ldots \ldots \ldots \ldots$ & 58 & 29 & - & $w$ & w & • & w & 0 & - & 16.2 \\
\hline 21 & Tobacco Products $\ldots \ldots \ldots \ldots \ldots \ldots$ & 0 & 0 & 0 & 0 & 0 & 0 & 0 & 0 & 0 & $\mathrm{NF}$ \\
\hline 22 & Textile Mill Products $\ldots \ldots \ldots \ldots \ldots \ldots$ & 39 & 20 & 0 & * & 19 & • & 0 & 0 & - & 29.2 \\
\hline 23 & Apparel and Other Textile Products ..... & NA & NA & NA & NA & NA & NA & NA & NA & NA & 31.0 \\
\hline 24 & Lumber and Wood Products..$\ldots \ldots \ldots$. & 474 & $w$ & w & W & 27 & 9 & 0 & 0 & w & 18.6 \\
\hline 25 & Furniture and Fixtures $\ldots \ldots \ldots \ldots \ldots$ & 39 & 31 & 0 & * & 6 & 1 & 0 & 0 & - & 31.5 \\
\hline 26 & Paper and Allied Products .... & 967 & 464 & 26 & 4 & 267 & w & w & 0 & w & 7.4 \\
\hline 2611 & Pulp Mills $\ldots \ldots \ldots \ldots \ldots \ldots \ldots$ & 57 & 26 & 10 & 1 & 17 & - & 0 & 0 & $\mathbf{3}$ & 20.6 \\
\hline 2621 & Paper Mills $\ldots \ldots \ldots \ldots \ldots \ldots \ldots$ & 437 & 230 & w & 1 & 127 & 2 & w & 0 & 57 & 5.3 \\
\hline 2631 & Paperboard Mills . & 333 & 104 & w & w & 90 & w & w & 0 & 121 & 8.1 \\
\hline 27 & Printing and Publishing $\ldots .$. & NA & NA & NA & NA & NA & NA & NA & NA & NA & 23.1 \\
\hline 28 & Chemicals and Allied Products . & w & 349 & w & 5 & 224 & w & $w$ & 38 & w & 12.2 \\
\hline 2812 & Alkalles and Chlorine $\ldots \ldots \ldots \ldots \ldots$ & w & w & - & - & 8 & • & 0 & 0 & • & 19.5 \\
\hline 2813 & Industrial Gases ......... & w & 89 & 0 & • & w & * & 0 & 0 & * & 14.9 \\
\hline 2819 & Industrial Inorganic Chemicals, nec .... & W & 110 & w & 4 & w & * & w & 38 & 16 & 11.7 \\
\hline 2821 & Plastlos Materials and Resins . . . . . . . & 23 & 14 & 0 & • & 8 & • & 0 & 0 & • & 17.4 \\
\hline 2822 & Synthetle Rubber $\ldots \ldots \ldots \ldots \ldots \ldots$ & • & - & 0 & * & - & • & 0 & 0 & - & 24.2 \\
\hline 2823 & Collulosic Manmado Fibors . . . . . . . . . & 0 & 0 & 0 & 0 & 0 & 0 & 0 & 0 & 0 & $\mathrm{NF}$ \\
\hline 2824 & Organic Fibers, Noncellulosic . . . . . . . & 0 & 0 & 0 & 0 & 0 & 0 & 0 & 0 & 0 & NF \\
\hline 2865 & Cyclle Crudes and Intermediates ...... & w & W & 0 & • & • & * & 0 & 0 & w & 23.8 \\
\hline 2869 & Industrial Organic Chemicals, nec ..... & 21 & 7 & 0 & - & w & • & 0 & 0 & w & 20.5 \\
\hline 2873 & Nitrogenous Fertillzers $\ldots \ldots \ldots \ldots \ldots$ & w & 10 & 0 & • & w & w & 0 & 0 & $Q$ & 38.3 \\
\hline 2874 & Phosphatic Fertillzers $\ldots \ldots \ldots \ldots \ldots$ & w & w & 0 & - & w & 0 & 0 & 0 & W & 22.6 \\
\hline 29 & Petroleum and Coal Products & 779 & w & 0 & 2 & 302 & $\mathbf{Q}$ & 0 & 0 & w & 8.7 \\
\hline 2911 & Pelroleum Refining . & 679 & w & 0 & 0 & 263 & 0 & 0 & 0 & w & 3.4 \\
\hline 30 & Rubber and Misc. Plastles Products . . . . . & 243 & 209 & * & Q & w & w & $\mathbf{Q}$ & 0 & - & 18.2 \\
\hline 3011 & Tres and inner Tubes $\ldots \ldots \ldots \ldots \ldots$ & w & w & - & * & w & - & 0 & 0 & - & 6.7 \\
\hline 308 & Mlscellaneous Plastics Products, nec... & 212 & 187 & 0 & Q & 22 & 2 & 0 & 0 & - & 23.1 \\
\hline 31 & Leather and Leather Products . . . . . . . . & 8 & 4 & 0 & - & $\mathbf{Q}$ & - & 0 & 0 & - & 45.5 \\
\hline 32 & Stone, Clay and Glass Products & 567 & 282 & 13 & 23 & 141 & 3 & 90 & 2 & 13 & 13.8 \\
\hline 3211 & Flat Glass $\ldots \ldots \ldots \ldots \ldots \ldots \ldots$ & w & 10 & w & - & $w$ & • & 0 & 0 & 1 & 4.4 \\
\hline 3221 & Glass Contalners $\ldots \ldots \ldots \ldots$ & 96 & 56 & w & - & $w$ & • & 0 & 0 & - & 8.7 \\
\hline 3229 & Pressed and Blown Glass, nec . . . & w & w & 0 & • & w & • & 0 & 0 & - & 13.7 \\
\hline 3241 & Cement, Hydraullo $\ldots \ldots \ldots \ldots \ldots \ldots$ & 235 & 109 & w & 5 & 23 & •" & 90 & $\mathbf{Q}$ & 5 & 21.0 \\
\hline 3274 & 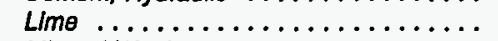 & $w$ & $w$ & w & 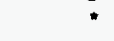 & $w$ & * & 0 & 0 & 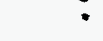 & 20.1 \\
\hline 3296 & Mineral Wool $\ldots \ldots \ldots \ldots \ldots \ldots \ldots$ & 27 & 20 & 0 & " & w & * & 0 & w & • & 2.1 \\
\hline 33 & Primary Metal Industries . & 1,248 & w & 1 & w & w & $w$ & w & 17 & w & 6.8 \\
\hline 3312 & Blast Fumaces and Steel Mills . . . . . . . & 215 & 89 & w & w & w & • & W & 1 & 4 & 7.6 \\
\hline 3313 & Electrometalurgical Products . . . . . . . & 0 & 0 & 0 & 0 & 0 & 0 & 0 & 0 & 0 & NF \\
\hline 3321 & Gray and Ductlle Iron Foundries ...... & 19 & 7 & 0 & " & w & • & 0 & w & - & 32.9 \\
\hline 3331 & Primary Copper ...... & w & w & * & w & w & " & • & w & 1 & 1.0 \\
\hline 3334 & Primary Aluminum & 651 & 515 & • & 1 & 14 & 1 & 2 & 0 & 119 & 3.5 \\
\hline 3339 & Primary Nonferrous Metals, nec . . . . . . . & $w$ & 28 & 0 & W & w & - & w & w & w & 1.0 \\
\hline 3353 & Aluminum Sheot, Plate, and Foll . . . . . . & w & w & 0 & - & w & - & 0 & 0 & - & 1.1 \\
\hline 34 & Fabricated Metal Products . . . . . . . . . & 296 & 216 & • & 3 & 69 & 3 & 0 & 0 & 4 & 19.1 \\
\hline 35 & Industrial Machinery and Equipment ..... . & 306 & 266 & 0 & w & 33 & 3 & 0 & 0 & Q & 27.7 \\
\hline 357 & Computer and Offlce Equipment . . & 130 & 122 & 0 & * & 7 & • & 0 & 0 & - & 14.7 \\
\hline 36 & Electronic and Other Electric Equipment .. & 432 & 391 & 0 & $\mathbf{Q}$ & 36 & 1 & 0 & 0 & w & 16.9 \\
\hline
\end{tabular}

See footnotes at end of table. 


\section{Table A24. Total Expenditures for Purchased Energy Sources by Census Region, Industry Group, and Selected Industries, 1991 (Continued) (Estimated in Million Dollars)}

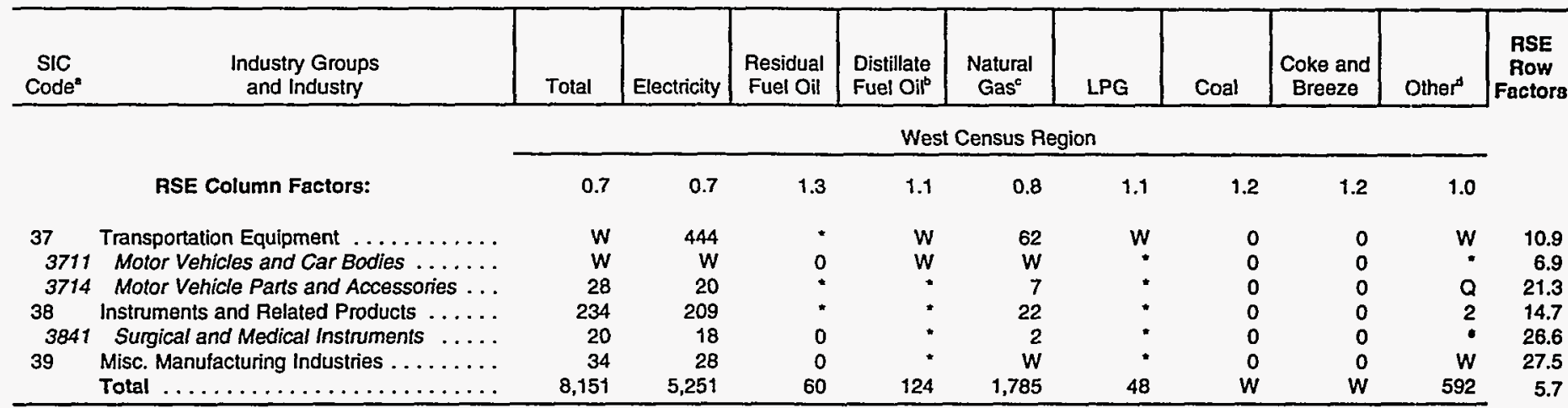

- See Appendices B and F for descriptions of the Standard Industrial Classification system.

"Distillate Fuel Oil" includes Nos. 1, 2, and 4 fuel oils and Nos. 1, 2, and 4 diesel fuels.

" "Natural Gas" inciudes natural gas obtained from utilities, transmission pipelines, and any other supplier(s) such as brokers and producers.

- Other energy sources include such combustible energy sources as wood waste, hydrogen, or waste oils and tars.

NF=No applicable RSE row/column factor.

Estimate less than 0.5. Data are included in higher level totals.

$W=$ Withheld to avoid disclosing data for individual establishments. Data are included in higher level totals.

$Q=$ Withheld because Relative Standard Error is greater than 50 percent. Data are included in higher level totals.

$\mathrm{NA}=$ Not available. Data are included in higher level totals.

Notes: - To obtain a RSE percentage for any table cell, multiply the cell's corresponding RSE column and RSE row factors. - Totals may not equal sum of components because of independent rounding. - To minimize respondent burden, quantities of petroleum based products (e.g., residual and distillate fuel oil and LPG) purchased, and associated expenditures, were not collected from the Refinery Industry, SIC 2911. These products are produced by petroleum refineries rather than purchased by them.

Source: Energy Information Administration, Office of Energy Markets and End Use, Energy End Use and Integrated Statistics Division, Form ElA-846, '1991 Manufacturing Energy Consumption Survey." 
Table A25. Average Prices of Selected Purchased Energy Sources by Census Region, Industry Group, and Selected Industries, 1991: Part 1

(Estimates in Dollars per Physical Unit)

\begin{tabular}{|c|c|c|c|c|c|c|c|c|}
\hline $\begin{array}{l}\text { SIC } \\
\operatorname{Cod}^{a}\end{array}$ & $\begin{array}{l}\text { Industry Groups } \\
\text { and Industry }\end{array}$ & $\begin{array}{l}\text { Electricity } \\
\text { (kWh) }\end{array}$ & $\begin{array}{c}\text { Residual Fuel } \\
\text { Oil } \\
\text { (gallon) }\end{array}$ & $\begin{array}{c}\text { Distillate Fuel } \\
\text { Oil } \\
\text { (gallon) }\end{array}$ & $\begin{array}{l}\text { Natural Gase } \\
(1000 \mathrm{cu} \mathrm{ft})\end{array}$ & $\begin{array}{l}\text { LPG } \\
\text { (gallon) }\end{array}$ & $\begin{array}{c}\text { Coal } \\
\text { (short ton) }\end{array}$ & $\begin{array}{c}\text { RSE } \\
\text { Row } \\
\text { Factors }\end{array}$ \\
\hline
\end{tabular}

Total United States

RSE Column Factors:

20

20 Food and Kindred Products . . . . . . . . .

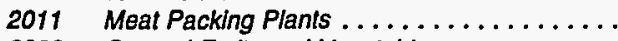

2033 Canned Frults and Vegetables ...........

2037 Frozen Frults and Vegetables ...........

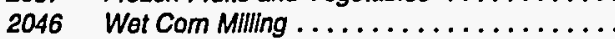

2051 Bread, Cake, and Related Products ........

2063 Beet Sugar....................

2075 Soybean Oll Mills $\ldots \ldots \ldots \ldots \ldots \ldots \ldots$

2082 Malt Beverages ....................

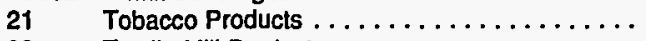

22 Textlle Mill Products $\ldots \ldots \ldots \ldots \ldots \ldots \ldots$

23 Apparel and Other Textile Products .........

24 Lumber and Wood Products ..............

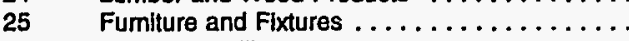

26 Paper and Allied Products . . . . . . . . . . .

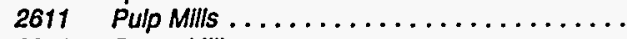

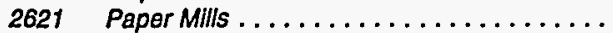

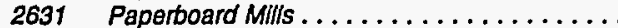

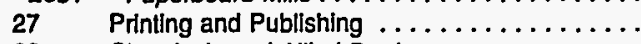

28 Chemicals and Allied Products ............

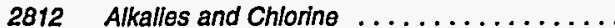

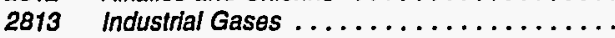

2819 Industrial Inorganic Chemicals, nec .......

2821 Plastics Materials and Resins ............

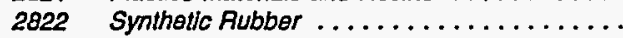

2823 Cellulosic Manmade Fibers ..............

2824 Organic Fibers, Noncellulosic ............

2865 Cyclic Crudes and Intermediates .............

2869 Industrial Organic Chemicals, nec ..........

2873 Nitrogenous Fertillzers . . . . . . . . . . .

2874 Phosphatic Fertilizers ................

29 Petroleum and Coal Products ............

2911 Petroloum Refining .................

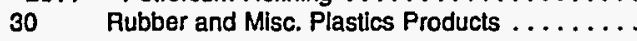

3011 Tres and Inner Tubes................

308 Miscellaneous Plastics Products, nec ........

31 Leather and Leather Products .............

32 Stone, Clay and Glass Products .............

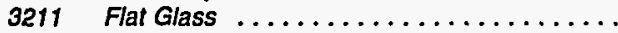

3221 Glass Contahners ...................

3229 Pressed and Blown Glass, nec ..........

3241 Cement, Hydraulle .................

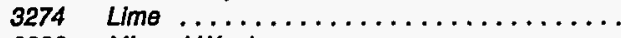

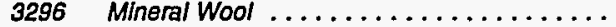

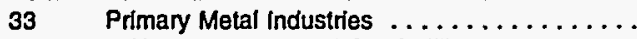

3312 Blast Fumaces and Steel Mills . . . . . . . . .

3313 Electrometalurgical Products ............

3321 Gray and Ductile Iron Foundries ..........

3331 Primary Copper ...................

3334 Primany Aluminum ${ }^{\circ} \ldots \ldots \ldots \ldots \ldots \ldots$

3339 Primary Nonferrous Metals, nec..........

3353 Aluminum Sheot, Plate, and Foll ..........

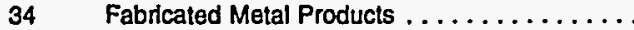

35 Industria! Machinery and Equipment .........

357 Computer and Office Equipment .............

36 Electronic and Other Electric Equlpment ......

37 Transportation Equipment ..............

3711 Motor Vehicles and Car Bodles ...........

3714 Motor Vehicle Parts and Accessories .......

38 Instruments and Related Products ..........

3841 Surglcal and Medical Instruments .........

39

Mlsc. Manufacturing Industries . . . . . . . . . . .

Total .........................

0.7

\section{7}

0.047

0.070

0.041

0.036

0.062

0.046

0.041

0.052

0.050

0.047

0.067

0.052

0.066

0.042

0.040

0.037

0.039

0.067

0.034

0.024

0.034

0.023

0.040

0.042

0.041

0.042

0.035

0.035

0.041

0.043

0.041

0.058

0.043

0.060

0.069

0.049

0.045

0.048

0.043

0.043

0.044

0.044

0.035

0.041

0.025

0.051

0.048

0.021

0.029

0.041

0.062

0.062

0.059

0.057

0.055

0.049

0.054

0.064

0.065

0.067

0.046

1.0

0.427

0.274

0.451

0.492

$W$
0.349

0.442

0.386

0.421

0.467

$W$
0.496

0.377

0.347

0.392

0.357

0.556

0.353

W
--
0.504

0.441
$W$

W

0.354

0.414

0.381

W

0.373

0.326

0.394

$W$
0.470

$W$
0.490

0.490
$W$

0.413

W

0.325

0.316

W

$W$
$W$

W

0.473

0.461
$W$

$W$
0.519

0.454

W

0.473

W

0.380

\subsection{1}

0.837

0.837

0.748

0.724

0.894

0.814

0.711

$W$
0.715

0.620

0.925

0.891

0.807

0.633

0.792

0.689

0.744

0.832

0.733

0.694

$W$
0.758

0.732
0.752

W

0.661

0.778

0.861

0.738

0.716

$W$
0.745

W

0.830

0.675

0.834

0.808

0.775

0.824

0.750

0.791

1.006

0.809

0.867

0.808

0.801

W

$W$
0.701

0.791

0.804

0.777

0.746

0.925

0.573

0.673

0.653

0.780

1.0

0.7

\subsection{8}

2.412

2.939

2.555

$$
\begin{array}{r}
2.348 \\
3.644
\end{array}
$$$$
2.309
$$$$
2.174
$$$$
2.630
$$$$
3.417
$$

$$
\begin{aligned}
& 3.417 \\
& 3.853
\end{aligned}
$$$$
\begin{aligned}
& 3.253 \\
& 3.229
\end{aligned}
$$$$
3.761
$$$$
2.360
$$$$
2.324
$$$$
2.248
$$$$
2.138
$$$$
\text { 3.719 }
$$$$
1.865
$$$$
\begin{array}{r}
W \\
1.914
\end{array}
$$$$
2.265
$$$$
1.880
$$$$
\begin{aligned}
& 1.880 \\
& 1.572
\end{aligned}
$$$$
\text { W }
$$$$
2.177
$$$$
2.113
$$$$
1.779
$$$$
1.623
$$$$
1.849
$$$$
2.026
$$$$
1.964
$$$$
3.229
$$$$
2.389
$$$$
3.497
$$$$
2.948
$$$$
2.608
$$$$
2.446
$$$$
2.519
$$$$
2.708
$$$$
1.874
$$ 
Table A25. Average Prices of Selected Purchased Energy Sources by Census Region, Industry Group, and Selected Industries, 1991: Part 1 (Continued) (Estimates in Dollars per Physical Unit)

\begin{tabular}{|c|c|c|c|c|c|c|c|c|}
\hline $\begin{array}{c}\text { SIC } \\
\text { Code }^{a} \\
\end{array}$ & $\begin{array}{c}\text { Industry Groups } \\
\text { and Industry }\end{array}$ & $\begin{array}{c}\text { Electricity } \\
(\mathrm{kWh})\end{array}$ & $\begin{array}{c}\text { Residual Fuel } \\
\text { Oil } \\
\text { (gallon) }\end{array}$ & $\begin{array}{c}\text { Distillate Fuel } \\
\text { Oil }^{b} \\
\text { (gallon) }\end{array}$ & $\begin{array}{l}\text { Natural Gas }{ }^{c} \\
(1000 \mathrm{cu} \mathrm{ft})\end{array}$ & $\begin{array}{c}\text { LPG } \\
\text { (gallon) }\end{array}$ & $\begin{array}{c}\text { Coal } \\
\text { (short ton) }\end{array}$ & $\begin{array}{c}\text { RSE } \\
\text { Row } \\
\text { Factors }\end{array}$ \\
\hline & & \multicolumn{7}{|c|}{ Northeast Census Region } \\
\hline & RSE Column Factors: & 0.8 & 0.6 & 0.9 & 3.2 & 1.1 & 0.6 & \\
\hline 20 & Food and Kindred Products . . . . . . . . . . & 0.072 & 0.466 & 0.837 & 3.525 & 0.609 & 47.499 & 4.2 \\
\hline 2011 & Meat Packing Plants . . . . . . . . . . . & 0.060 & W & 0.831 & 2.902 & 0.542 & $\ddot{2}$ & 4.6 \\
\hline 2033 & Canned Fruits and Vegetables . . . . . . . . . & 0.074 & 0.480 & 0.963 & 3.751 & 0.870 & W & 4.7 \\
\hline 2037 & Frozen Fruits and Vegetables . . . . . . . . . & W & W & W & 3.185 & W & -- & 5.6 \\
\hline 2046 & Wet Com Milling . . . . . . . . . . . . & 0.058 & $w$ & $w$ & W & W & $\cdots$ & 2.2 \\
\hline 2051 & Bread, Cake, and Related Products . . . . . . . & 0.080 & W & 0.857 & 3.609 & 0.653 & - & 3.6 \\
\hline 2063 & Beet Sugar . . . . . . . . . . . . . . . & -- & -- & -- & $\cdots$ & -- & -- & NF \\
\hline 2075 & 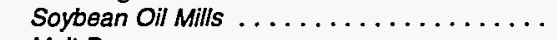 & -- & -- & -- & -- & 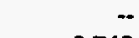 & $\therefore$ & NF \\
\hline 2082 & Malt Beverages $\ldots \ldots \ldots \ldots \ldots \ldots$ & 0.063 & W & 0.427 & 2.951 & 0.742 & W & 3.5 \\
\hline 21 & Tobacco Products $\ldots \ldots \ldots \ldots \ldots \ldots \ldots$ & NA & NA & NA & NA & NA & NA & 12.9 \\
\hline 22 & Textile Mill Products $\ldots \ldots \ldots \ldots \ldots \ldots \ldots$ & 0.079 & 0.422 & 0.628 & 4.169 & 0.509 & W & 5.3 \\
\hline 23 & Apparel and Other Textile Products $\ldots \ldots \ldots$ & 0.098 & 0.459 & 0.918 & 4.698 & 1.109 & -- & 7.2 \\
\hline 24 & Lumber and Wood Products $\ldots \ldots \ldots \ldots$ & NA & NA & NA & NA & NA & NA & 6.3 \\
\hline 25 & Furniture and Fixtures $\ldots \ldots \ldots \ldots \ldots$ & 0.087 & 0.485 & 0.887 & 4.326 & 0.522 & -- & 5.2 \\
\hline 26 & Paper and Allied Products . . . . . . . . . . . & 0.064 & 0.414 & 0.531 & 3.321 & 0.607 & 45.583 & 4.3 \\
\hline 2611 & Pulp Mills . . . . . . . . . . . . . . & W & W & W & -- & W & $\ddot{-}$ & 2.0 \\
\hline 2621 & Paper Mills . . . . . . . . . . . . . . . & 0.060 & 0.410 & 0.811 & 2.993 & 0.535 & 44.786 & 1.8 \\
\hline 2631 & Paperboard Mills . . . . . . . . . . . . & 0.068 & 0.447 & 0.678 & 2.873 & 0.770 & W & 3.6 \\
\hline 27 & Printing and Publishing $\ldots \ldots \ldots \ldots \ldots$ & 0.085 & 0.559 & 0.831 & 4.733 & 0.751 & - & 8.5 \\
\hline 28 & Chemicals and Allied Products . . . . . . . . & 0.052 & 0.448 & 0.711 & 3.361 & 0.559 & 43.314 & 3.5 \\
\hline 2812 & Alkalies and Chlorine $\ldots \ldots \ldots \ldots$ & $W$ & -- & -- & W & -- & -- & 3.1 \\
\hline 2813 & Industrial Gases . . . . . . . . . . . . . & 0.039 & - & W & $W$ & W & -- & 3.2 \\
\hline 2819 & Industrial Inorganic Chemicals, nec ....... & 0.062 & 0.494 & 0.739 & 3.358 & 0.517 & 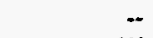 & 4.8 \\
\hline 2821 & Plastics Materials and Resins . . . . . . . . & 0.059 & W & 0.667 & 3.307 & W & $W$ & 2.8 \\
\hline 2822 & Synthetic Rubber $\ldots \ldots \ldots \ldots \ldots \ldots \ldots$ & W & W & W & W & W & - & 2.1 \\
\hline 2823 & Cellulosic Manmade Fibers . . . . . . . . . . . & -- & - & -- & -- & -- & - & NF \\
\hline 2824 & Organic Fibers, Noncellulosic . . . . . . . . . & 0.063 & W & $w$ & W & W & - & 4.0 \\
\hline 2865 & Cyclic Crudes and Intemediates ......... & W & 0.409 & 0.627 & 3.093 & 1.019 & W & 7.0 \\
\hline 2869 & Industrial Organic Chemicals, nec . . . . . . . & 0.029 & 0.426 & 0.798 & 3.386 & W & 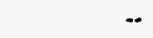 & 2.9 \\
\hline 2873 & Nitrogenous Fertilizers $\ldots \ldots \ldots \ldots \ldots$ & 0.061 & $\because$ & W & W & W & - & 7.6 \\
\hline 2874 & Phosphatic Fertilizers . . . . . . . . . . & -- & -- & -- & -- & -- & - & NF \\
\hline 29 & Petroleum and Coal Products $\ldots \ldots \ldots \ldots$ & 0.055 & - & 0.767 & 2.792 & 0.582 & 47.201 & 7.7 \\
\hline 2911 & Petroleum Refining . . . . . . . . . . . . . & 0.049 & -- & -- & W & -- & 36.953 & 3.3 \\
\hline 30 & Rubber and Misc. Plastics Products . . . . . . . & 0.075 & 0.421 & 0.716 & 4.345 & 0.584 & W & 5.6 \\
\hline 3011 & Tires and Inner Tubes . . . . . . . . . . . & 0.040 & W & $W$ & $W$ & 0.956 & - & 4.6 \\
\hline 308 & Miscellaneous Plastics Products, nec . . . . . . . & 0.076 & 0.399 & 0.733 & 4.404 & 0.671 & $\mathbf{W}$ & 5.4 \\
\hline 31 & Leather and Leather Products . . . . . . . . . & 0.087 & 0.398 & 0.650 & 4.772 & 0.703 & $\mathbf{W}$ & 7.4 \\
\hline 32 & Stone, Clay and Glass Products $\ldots \ldots \ldots \ldots$ & 0.057 & 0.476 & 0.789 & 3.150 & 0.743 & 34.202 & 5.2 \\
\hline 3211 & Flat Glass $\ldots \ldots \ldots \ldots \ldots \ldots \ldots \ldots$ & W & - & W & W & W & W & 1.5 \\
\hline 3221 & Glass Containers $\ldots \ldots \ldots \ldots \ldots \ldots$ & 0.057 & 0.478 & 0.699 & 3.093 & 0.542 & -- & 2.4 \\
\hline 3229 & Pressed and Blown Glass, nec . . . . . . . . & 0.053 & W & W & 2.751 & 0.776 & -- & 3.3 \\
\hline 3241 & Cement, Hydraulic . . . . . . . . . . . . & 0.052 & 0.561 & 0.727 & W & 1.187 & 34.495 & 5.6 \\
\hline 3274 & Lime $\ldots \ldots \ldots \ldots \ldots \ldots \ldots \ldots \ldots$ & 0.039 & -- & 0.844 & $W$ & W & 33.613 & 4.3 \\
\hline 3296 & Mineral wool . . . . . . . . . . . . & 0.058 & -- & W & 2.922 & 0.543 & W & 1.1 \\
\hline 33 & Primary Metal Industries & 0.045 & 0.398 & 0.879 & 3.170 & 0.615 & 49.467 & 3.8 \\
\hline 3312 & Blast Fumaces and Steol Mills . . . . . . . . . . & 0.045 & 0.343 & 0.926 & 2.883 & 0.668 & 49.604 & 1.9 \\
\hline 3313 & Electrometalurgical Products . . . . . . . . . . & W & -- & $W$ & 4.536 & $w$ & $\mathbf{W}$ & 2.3 \\
\hline 3321 & Gray and Ductile Iron Foundries ......... & 0.071 & $-\cdot$ & 0.831 & 3.666 & 0.687 & W & 5.9 \\
\hline 3331 & Primary Copper . . . . . . . . . . . . & W & -- & W & W & W &.- & NF \\
\hline 3334 & Primary Aluminum ${ }^{d} \ldots \ldots \ldots \ldots$ & $W$ & W & $W$ & W & W & W & 1.3 \\
\hline 3339 & Primary Nonferrous Metals, nec . . . . . . . . . & 0.055 & W & $W$ & 3.196 & 0.882 & W & 1.6 \\
\hline 3353 & Aluminum Sheet, Plate, and Foil . . . . . . . & 0.080 & 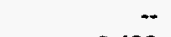 & W & 3.056 & 0.523 & -- & 0.6 \\
\hline 34 & Fabricated Metal Products . . . . . . . . . . & 0.075 & 0.438 & 0.809 & 4.022 & 0.783 & W & 5.5 \\
\hline 35 & Industrial Machinery and Equipment . . . . . . . . & 0.078 & 0.467 & 0.783 & 4.375 & 0.535 & -- & 7.4 \\
\hline 357 & Computer and Office Equipment . . . . . . . . & 0.070 & $W$ & 0.802 & $w$ & 0.883 & -- & 6.1 \\
\hline 36 & Electronic and Other Electric Equipment . . . . . & 0.069 & 0.545 & 0.708 & 4.129 & 0.551 & $W$ & 3.8 \\
\hline 37 & Transportation Equipment . . . . . . . . . . & 0.073 & 0.497 & 0.734 & 3.720 & 0.689 & W & 4.7 \\
\hline 3711 & Motor Vehicles and Car Bodies . . . . . . . . . & W & W & $W$ & $w$ & 0.617 & - & 1.6 \\
\hline 3714 & Motor Vehicle Parts and Accessories . . . . . . & 0.071 & W & 0.835 & 3.816 & 0.634 & $w$ & 3.9 \\
\hline 38 & Instruments and Related Products $\ldots \ldots \ldots \ldots$ & 0.078 & 0.472 & 0.670 & 3.870 & 0.558 & W & 4.2 \\
\hline 3841 & Surgical and Medical Instruments $\ldots \ldots \ldots$ & 0.077 & W & 0.760 & 5.509 & 0.745 & - & 3.4 \\
\hline \multirow[t]{2}{*}{39} & Misc. Manufacturing Industries . . . . . . . . . & 0.079 & 0.460 & 0.841 & 4.171 & 0.882 & W & 5.1 \\
\hline & Total $\ldots \ldots \ldots \ldots \ldots \ldots \ldots \ldots$ & 0.064 & 0.434 & 0.752 & 3.488 & 0.597 & 45.399 & 3.1 \\
\hline
\end{tabular}

See footnotes at end of table. 
Table A25. Average Prices of Selected Purchased Energy Sources by Census Region, Industry Group, and Selected Industries, 1991: Part 1 (Continued) (Estimates in Dollars per Physical Unit)

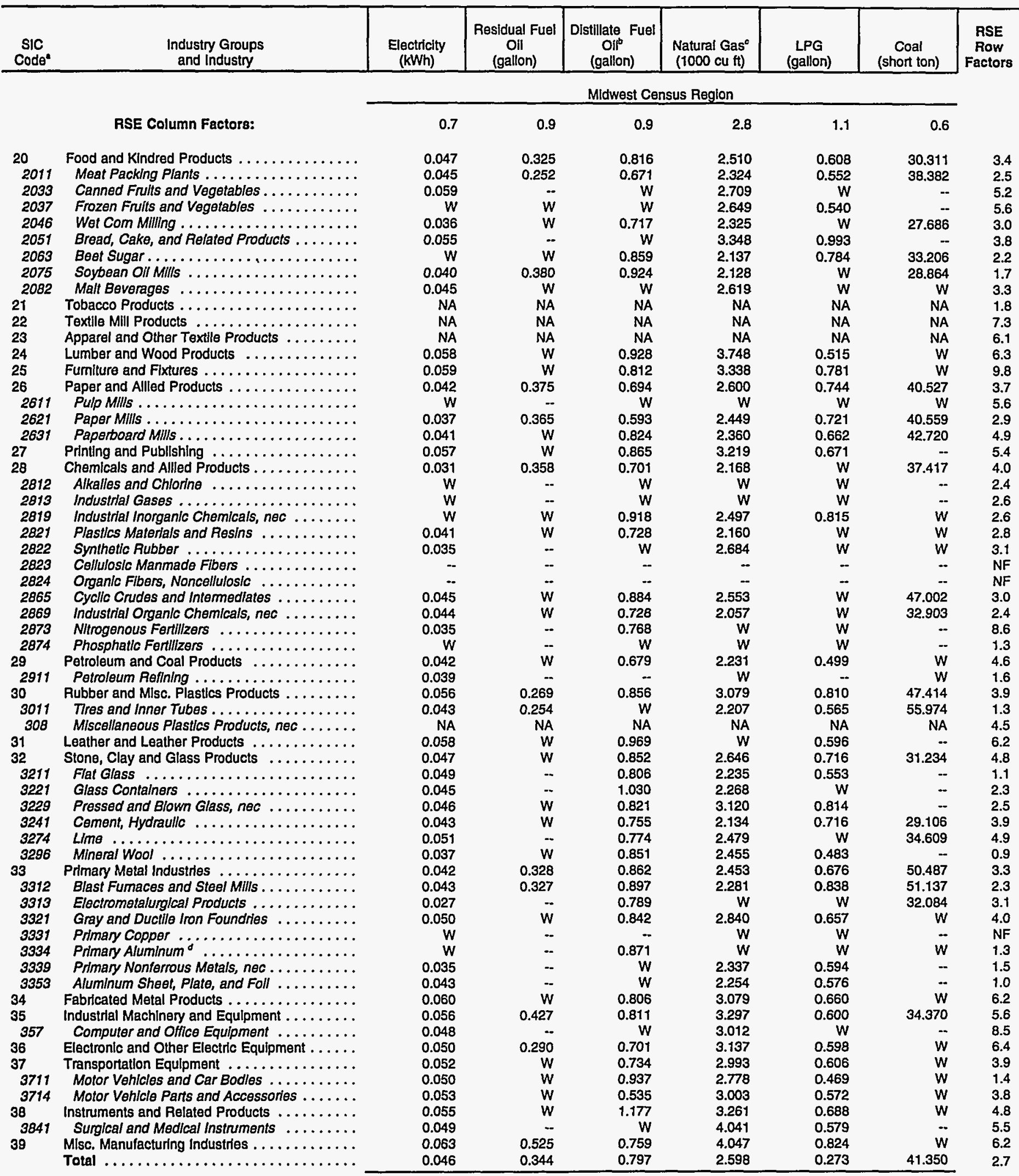

Seo footnotes at end of table. 


\section{Table A25. Average Prices of Selected Purchased Energy Sources by Census Region, Industry Group, and Selected Industries, 1991: Part 1 (Continued) \\ (Estimates in Dollars per Physical Unit)}

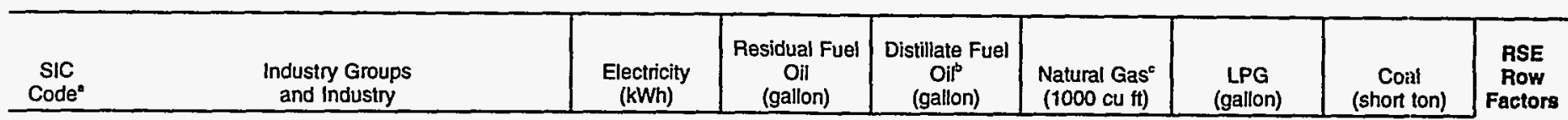

South Census Region

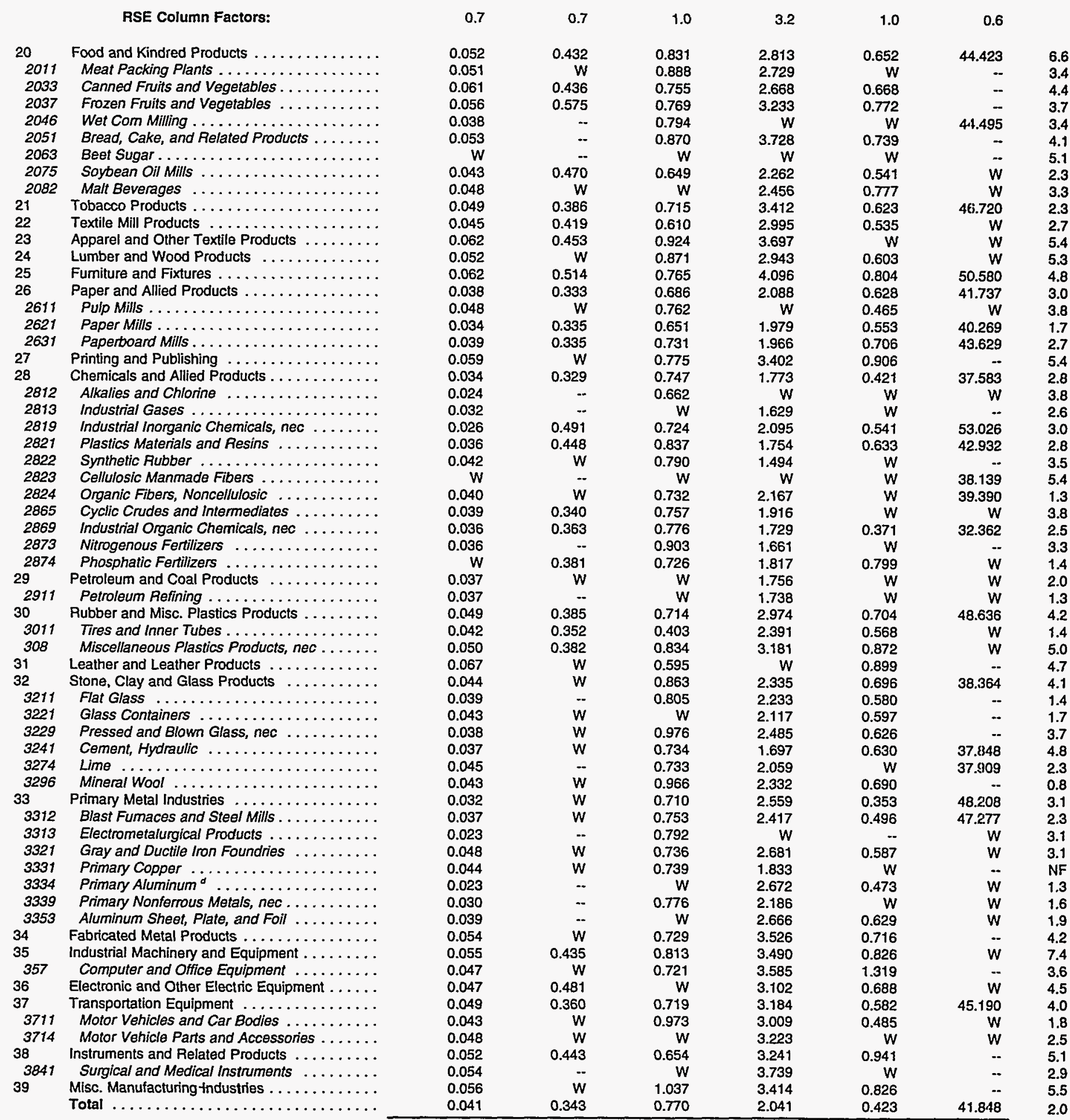

See footnotes at end of table. 
Table A25. Average Prices of Selected Purchased Energy Sources by Census Region, Industry Group, and Selected Industries, 1991: Part 1 (Continued)

(Estimates in Dollars per Physical Unit)

\begin{tabular}{|c|c|c|c|c|c|c|c|c|}
\hline \multirow[t]{3}{*}{$\underset{\text { Code }^{\text {SIC }}}{\text { SIC }}$} & $\begin{array}{l}\text { Industry Groups } \\
\text { and Industry }\end{array}$ & $\begin{array}{c}\text { Electricity } \\
\text { (kWh) }\end{array}$ & $\begin{array}{c}\text { Residual Fuel } \\
\text { Oil } \\
\text { (gallon) }\end{array}$ & $\begin{array}{c}\text { Distillate Fuel } \\
\text { Oil } \\
\text { (gallon) } \\
\end{array}$ & $\begin{array}{l}\text { Natural Gase } \\
(1000 \mathrm{cu} \mathrm{ft})\end{array}$ & $\begin{array}{c}\text { LPG } \\
\text { (gallon) }\end{array}$ & $\begin{array}{c}\text { Coal } \\
\text { (short ton) }\end{array}$ & \multirow[t]{2}{*}{$\begin{array}{l}\text { RSE } \\
\text { Row } \\
\text { Factors }\end{array}$} \\
\hline & & \multicolumn{6}{|c|}{ West Census Region } & \\
\hline & RSE Column Factors: & 1.0 & 0.7 & 0.7 & 3.5 & 0.9 & 0.7 & \\
\hline 20 & Food and Kindred Products & 0.060 & 0.478 & 0.881 & 2.968 & 0.764 & 29.436 & 4.0 \\
\hline 2011 & Meat Packing Plants . .... & 0.042 & $w$ & 0.904 & 2.424 & 0.636 & -- & 3.3 \\
\hline 2033 & Canned Fruils and Vegetables ..... . & 0.079 & 0.418 & w & 2.952 & w & -- & 2.8 \\
\hline 2037 & Frozen Frulls and Vegetables ...... & 0.034 & 0.582 & 0.722 & 2.320 & 0.621 & .. & 6.3 \\
\hline 2046 & Wet Com Milling . . . . . . . & W & -- & w & $\mathbf{w}$ & w & -• & 2.9 \\
\hline $205 y$ & Bread, Cake, and Related Products. & 0.071 & -- & 0.938 & 3.959 & 0.828 & -. & 6.3 \\
\hline 2063 & Beet Sugar.................. & 0.042 & w & 0.794 & 2.473 & 0.867 & 27.682 & 2.9 \\
\hline 2075 & Soybean Oll Mills ............... & .- & -- & -. & -- & -- & -. & NF \\
\hline 2082 & Malt Beverages ... & 0.057 & $w$ & $w$ & 2.696 & w & w & 2.8 \\
\hline 21 & Tobacco Products .... & -- & -- & - & $\cdot-$ & -- & - & NF \\
\hline 22 & Textlle Mill Products $\ldots \ldots \ldots \ldots \ldots$ & 0.072 & -- & w & 4.190 & 0.517 & -- & 9.5 \\
\hline 23 & Apparel and Other Textile Products ........ & NA & NA & NA & NA & NA & NA & 4.8 \\
\hline 24 & Lumber and wood Products ........ & 0.045 & w & 0.873 & 2.772 & 0.639 & -- & 7.3 \\
\hline 25 & Fumiture and Fixtures $\ldots \ldots \ldots \ldots \ldots$ & 0.102 & -. & 0.875 & 4.629 & 0.821 & -. & 7.0 \\
\hline 26 & Paper and Allied Products . . . . . . . . . & 0.034 & 0.427 & 0.786 & 2.465 & 0.646 & w & 3.8 \\
\hline 2611 & Pulp Mills $\ldots \ldots \ldots \ldots \ldots$ & 0.030 & 0.449 & 0.869 & 2.569 & 0.872 & -- & 4.4 \\
\hline 2621 & Paper Mills . . . . . . . . . . . & 0.028 & 0.396 & 0.699 & 2.388 & 0.468 & w & 2.1 \\
\hline 2631 & Paporboard Mills . . . . . . . . . & 0.033 & 0.423 & 0.778 & 2.345 & 0.658 & w & 2.1 \\
\hline 27 & Printling and Publishing & NA & NA & NA & NA & NA & NA & 6.6 \\
\hline 28 & Chemicals and Allied Products ... & 0.031 & w & 0.845 & 1.865 & 0.581 & 34.433 & 6.6 \\
\hline 2812 & Alkalles and Chlorine $\ldots \ldots \ldots \ldots$ & 0.022 & w & 0.806 & 2.024 & w & -. & 3.0 \\
\hline 2813 & Industrial Gases . . . . . . . . . . . . . & 0.035 & -- & w & w & w & -- & 2.4 \\
\hline 2819 & Industrlal Inorganic Chemicals, nec ........ & 0.025 & w & 0.828 & 2.882 & 0.620 & 34.433 & 5.4 \\
\hline 2821 & Plastlcs Materials and Resins ...... & 0.076 & - & 0.936 & 4.426 & 0.910 & - & 4.5 \\
\hline 2822 & Synthetic Rubber ............ & w & -- & w & w & w & - & 2.1 \\
\hline 2823 & Cellulosic Manmade Fibers . . . . . . . & - & -- & -. & -- & - & -. & NF \\
\hline 2824 & Organic Fibers, Noncellulosic ....... & .. & -- & -- & -- & -- & -- & NF \\
\hline 2865 & Cyclic Crudes and Intermediates .......... & w & -- & w & W & $\mathbf{w}$ & - & 5.5 \\
\hline 2869 & Industral Organic Chemicals, nec ... & 0.054 & -- & 0.838 & w & 1.244 & - & 13.8 \\
\hline 2873 & Nitrogenous Fertilizers .......... & 0.024 & .- & 0.810 & w & w & - & 6.8 \\
\hline 2874 & Phosphatic Fertilizers ........... & w & -. & 1.013 & $w$ & -- & - & 5.0 \\
\hline 29 & Petroleum and Coal Products ..... & 0.051 & -- & 0.589 & 3.137 & w & - & 3.0 \\
\hline 2911 & Petroleum Refining ............ & 0.050 & .- & - & 3.092 & - & - & 1.2 \\
\hline 30 & Rubber and Misc. Plastics Products ... & 0.073 & $w$ & 1.058 & 3.780 & 0.945 & w & 6.0 \\
\hline 3011 & Thres and Inner Tubes............ & w & $w$ & w & w & w & - & 2.1 \\
\hline 308 & Miscellaneous Plastics Products, nec. & 0.073 & -. & w & 4.353 & 0.975 & - & 4.8 \\
\hline 31 & Leather and Leather Products . ...... & 0.061 & -- & w & 1.692 & 1.089 & - & 5.3 \\
\hline 32 & Stone, Clay and Glass Products ..... & 0.055 & 0.460 & 0.800 & 2.758 & 0.612 & 38.055 & 4.6 \\
\hline 3211 & Flat Glass $\ldots \ldots \ldots \ldots \ldots \ldots \ldots$ & 0.068 & w & 0.797 & 3.356 & $\mathbf{w}$ & - & 1.2 \\
\hline 3221 & Glass Containers ............ & 0.050 & $w$ & W & 2.954 & 0.510 & -- & 2.3 \\
\hline 3229 & Pressed and Blown Glass, nec. & w & .. & w & w & w & - & 2.3 \\
\hline 3241 & Cement, Hydraulic ........... & 0.046 & w & 0.790 & 2.064 & 1.002 & 38.055 & 5.0 \\
\hline 3274 & Lime... & w & W & W & w & W & - & 3.1 \\
\hline 3296 & MIneral Wool & 0.062 & -. & w & 3.249 & 0.549 & - & 1.0 \\
\hline 33 & Primary Metal Industries & 0.025 & 0.348 & 0.758 & 2.606 & 0.737 & w & 2.8 \\
\hline 3312 & Blast Fumaces and Stoel Mills . . . & 0.035 & $w$ & 0.755 & 2.229 & 0.664 & w & 2.2 \\
\hline 3313 & Electrometalurgical Products ...... & -- & -- & -- & - & - & -- & NF \\
\hline 3321 & Gray and Ductile Iron Foundries . . . . . . . & 0.074 & -- & 0.875 & 3.122 & 0.822 & - & 7.6 \\
\hline 3331 & Primary Copper $\ldots \ldots \ldots \ldots \ldots \ldots \ldots$ & 0.048 & $w$ & w & 2.184 & w & w & NF \\
\hline 3334 & Primary Aluminum ${ }^{\prime}{ }^{\prime} \ldots \ldots \ldots \ldots \ldots \ldots \ldots$ & 0.020 & $w$ & 0.849 & 2.358 & 0.758 & 298.608 & 1.3 \\
\hline 3339 & Primary Nonforrous Motals, nec..... & 0.022 & - & w & 2.081 & w & w & NF \\
\hline 3353 & Aluminum Sheet, Plate, and Foll . & W & -- & w & w & w & - & NF \\
\hline & Fabricated Metal Products . . . . . . . & 0.074 & $w$ & 0.985 & 4.176 & 0.803 & - & 4.1 \\
\hline 35 & Industrial Machinery and Equipment . . . . . . . . & 0.074 & -. & w & 3.972 & 0.830 & - & 8.1 \\
\hline 357 & Computer and Office Equipment ......... & 0.063 & .. & w & 4.176 & 1.154 & - & 6.1 \\
\hline 36 & Electronic and Other Electric Equlpment & 0.070 & -- & 0.911 & 4.150 & 0.989 & - & 4.1 \\
\hline
\end{tabular}

See footnotes at end of table. 


\section{Table A25. Average Prices of Selected Purchased Energy Sources by Census Region, Industry Group, and Selected Industries, 1991: Part 1 (Continued) (Estimates in Dollars per Physical Unit)}

\begin{tabular}{|c|c|c|c|c|c|c|c|c|}
\hline \multirow[t]{3}{*}{$\begin{array}{c}\text { SIC } \\
\text { Code }\end{array}$} & $\begin{array}{l}\text { Industry Groups } \\
\text { and Industry }\end{array}$ & $\begin{array}{c}\text { Electricity } \\
(\mathrm{kWh})\end{array}$ & $\begin{array}{c}\text { Residual Fuel } \\
\text { Oil } \\
\text { (gallon) }\end{array}$ & $\begin{array}{c}\text { Distillate Fuel } \\
\text { Oif }^{\mathrm{B}} \\
\text { (gallon) }\end{array}$ & $\begin{array}{l}\text { Natural Gase } \\
(1000 \mathrm{cu} \mathrm{ft})\end{array}$ & $\begin{array}{c}\text { LPG } \\
\text { (gallon) }\end{array}$ & $\begin{array}{c}\text { Coal } \\
\text { (short ton) }\end{array}$ & \multirow[t]{3}{*}{$\begin{array}{c}\text { RSE } \\
\text { Row } \\
\text { Factors }\end{array}$} \\
\hline & & \multicolumn{6}{|c|}{ West Census Region } & \\
\hline & RSE Column Factors: & 1.0 & 0.7 & 0.7 & 3.5 & 0.9 & 0.7 & \\
\hline 37 & Transportation Equipment & 0.063 & W & 0.881 & 3.673 & 0.615 & -- & 5.6 \\
\hline 3711 & Motor Vehicles and Car Bodies . . . . . . . . & 0.062 & - & 0.878 & W & 0.729 & -- & 1.8 \\
\hline 3714 & Motor Vehicle Parts and Accessories . . . . . . . & 0.081 & w & W & 3.621 & 0.815 & -- & 7.0 \\
\hline & Instruments and Related Products $\ldots \ldots \ldots \ldots$ & 0.066 & $\mathbf{W}$ & 0.799 & 3.965 & 0.779 & -- & 5.8 \\
\hline 3841 & Surgical and Medical Instruments . . . . . . . . & 0.080 & -- & W & 4.716 & 0.758 & -- & 4.8 \\
\hline 39 & Misc. Manufacturing Industries . . . . . . . . . . & 0.074 & -- & 1.090 & W & 0.957 & -. & 5.1 \\
\hline & 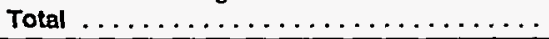 & 0.045 & 0.447 & 0.847 & 2.772 & 0.700 & 39.987 & 3.0 \\
\hline
\end{tabular}

- See Appendices B and F for descriptions of the Standard Industrial Classification system.

- "Distillate Fuel Oil" includes Nos. 1, 2, and 4 fuel oils and Nos. 1, 2, and 4 diesel fuels.

c "Natural Gas" includes natural gas obtained from utilities, transmission pipelines, and any other supplier(s) such as brokers and producers.

- The price estimates for coal for SIC 3334 include anthracite coal for the production of carbon anodes. Because of the high cost of transporting anthracite from the

East Coast to the West and South, the prices of coal in those regions are extremely high.

NF=No applicable RSE row/column factor.

$W=$ Withheld to avoid disclosing data for individual establishments. Data are included in higher level totals.

NA $=$ Not available. Data are included in higher level totals.

- Estimation of average price is not applicable.

Notes: - To obtain a RSE percentage for any table cell, multiply the cell's corresponding RSE column and RSE row factors. - Totals may not equal sum of components because of independent rounding.

Source: Energy Information Administration, Office of Energy Markets and End Use, Energy End Use and Integrated Statistics Division, Form ElA-846, "1991 Manufacturing Energy Consumption Survey:" 
Table A25 Average Prices of Selected Purchased Energy Sources by Census Region, Industry Group, and Selected Industries, 1991: Part 2

(Estimates in Dollars per Million Btu)

\begin{tabular}{|c|c|c|c|c|c|c|c|c|}
\hline $\begin{array}{c}\text { SIC } \\
\text { Code }\end{array}$ & $\begin{array}{l}\text { Industry Groups } \\
\text { and Industry }\end{array}$ & Electricity & $\begin{array}{c}\text { Residual Fuel } \\
\text { Oil }\end{array}$ & $\begin{array}{c}\text { Distillate Fuel } \\
\text { Oil }^{b}\end{array}$ & Natural Gas ${ }^{\mathrm{c}}$ & LPG & Coal & $\begin{array}{c}\text { RSE } \\
\text { Row } \\
\text { Factors }\end{array}$ \\
\hline & & \multicolumn{6}{|c|}{ Total United States } & \\
\hline & RSE Column Factors: & 0.7 & 0.8 & 1.0 & 2.8 & 1.0 & 0.7 & \\
\hline 20 & Food and Kindred Products . . . . . . . . . . & 15.789 & 2.854 & 6.064 & 2.697 & 7.596 & 1.433 & 4.5 \\
\hline 2011 & Meat Packing Plants . . . . . . . . . . . . & 13.726 & 1.831 & 6.035 & 2.342 & 5.782 & 1.723 & 2.9 \\
\hline 2033 & Canned Fruits and Vegetables . . . . . . . . . . & 20.591 & 3.013 & 6.032 & 2.853 & 7.937 & W & 2.9 \\
\hline 2037 & Frozen Frulls and Vegetables . . . . . . . . & 12.074 & 3.489 & 5.392 & 2.481 & 7.463 &.- & 5.1 \\
\hline 2046 & Wet Com Mlling . . . . . . . . . . . . . & 10.559 & 3.287 & 5.222 & 2.279 & 10.372 & 1.322 & 3.4 \\
\hline 2051 & Bread, Cake, and Related Products ........ & 18.046 & w & 6.446 & 3.538 & 8.617 & -- & 3.4 \\
\hline 2063 & Beet Sugar . . . . . . . . . . . . . & 13.413 & 2.334 & 5.868 & 2.242 & 9.345 & 1.379 & 2.1 \\
\hline 2075 & Soybean Oll Mills $\ldots \ldots \ldots \ldots \ldots \ldots \ldots$ & 12.041 & 2.953 & 5.127 & 2.111 & 7.733 & 1.443 & 1.8 \\
\hline 2082 & Malt Beverages $\ldots \ldots \ldots \ldots \ldots \ldots$ & 15.321 & W & 3.603 & 2.554 & 8.850 & 1.596 & 2.7 \\
\hline 21 & Tobacco Products . . . . . . . . . . . . . . & 14.588 & 2.577 & 5.153 & 3.317 & 7.251 & 2.097 & 2.3 \\
\hline 22 & Textile Mill Products $\ldots \ldots \ldots \ldots \ldots \ldots$ & 13.853 & 2.809 & 4.468 & 3.072 & 6.154 & 1.952 & 2.6 \\
\hline 23 & Apparel and Other Textile Products ........ & 19.773 & 3.121 & 6.667 & 3.741 & 7.860 & W & 5.2 \\
\hline 24 & Lumber and Wood Products $\ldots \ldots \ldots \ldots$ & 15.199 & 2.680 & 6.421 & 3.134 & 6.399 & W & 4.5 \\
\hline 25 & Fumlture and Fixtures $\ldots \ldots \ldots \ldots \ldots$ & 19.220 & 3.312 & 5.821 & 3.651 & 8.548 & 1.983 & 4.9 \\
\hline 26 & Paper and Allied Products . . . . . . . . . . . & 12.206 & 2.522 & 4.566 & 2.292 & 7.064 & 1.870 & 3.1 \\
\hline 2611 & Pulp Mills $\ldots \ldots \ldots \ldots \ldots \ldots \ldots \ldots$ & 11.776 & 2.316 & 5.708 & 2.256 & 5.388 & 1.727 & 3.8 \\
\hline 2621 & Paper MIlls . . . . . . . . . . . . . . . & 10.925 & 2.619 & 4.965 & 2.183 & 5.991 & 1.835 & 1.8 \\
\hline 2631 & Paperboard MIls . . . . . . . . . . . . . . & 11.385 & 2.388 & 5.363 & 2.076 & 7.472 & 1.971 & 2.8 \\
\hline 27 & Printlng and Publishing $\ldots \ldots \ldots \ldots \ldots$ & 19.778 & 3.717 & 6.002 & Q & 9.549 & - & 5.7 \\
\hline 28 & Chemicals and Allied Products . . . . . . . . . & 9.957 & 2.358 & 5.284 & 1.811 & 4.829 & 1.681 & 3.0 \\
\hline 2812 & 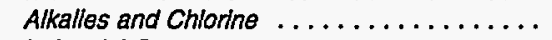 & 7.089 & W & 5.001 & W & 9.157 & W & 3.1 \\
\hline 2813 & Industrial Gases ................ & 9.935 & $-\cdot$ & W & 1.859 & W & $\overrightarrow{-}$ & 2.7 \\
\hline 2819 & Industrial Inorganic Chemicals, nec . . . . . . . & 6.866 & 3.367 & 5.468 & 2.199 & 6.125 & 2.090 & 3.7 \\
\hline 2821 & Plastlos Materials and Resins . . . . . . . . . & 11.609 & 2.943 & 5.278 & 1.826 & 6.102 & 1.809 & 2.4 \\
\hline 2822 & Synthetic Rubber . . . . . . . . . . . & 12.409 & W & 5.422 & 1.526 & W & W & 3.0 \\
\hline 2823 & Cellulosic Manmade Fibers . . . . . . . . . . . & W & -- & W & W & $W$ & 1.712 & 5.5 \\
\hline 2824 & Organic Fibers, Noncellulosic . . . . . . . . . & 11.937 & W & 5.208 & 2.114 & W & 1.743 & 1.3 \\
\hline 2865 & Cyclic Crudes and Intermedlates ......... & 12.307 & 2.365 & 4.768 & 2.051 & W & 2.021 & 4.4 \\
\hline 2869 & Industrial Organlc Chemicals, nec ........ & 10.404 & 2.768 & 5.611 & 1.727 & 4.427 & 1.460 & 2.5 \\
\hline 2873 & Nitrogenous Fortilizers $\ldots \ldots \ldots \ldots \ldots$ & 10.185 & -- & 6.211 & 1.576 & W & - & 3.7 \\
\hline 2874 & Phosphatlc Fertlizers . . . . . . . . . . & 11.925 & 2.547 & 5.324 & 1.795 & 8.753 & W & 2.1 \\
\hline 29 & Petroleum and Coal Products $\ldots \ldots \ldots \ldots$ & 12.617 & W & 5.164 & 1.967 & 4.603 & 2.010 & 4.5 \\
\hline 2911 & Petroleum Refining . . . . . . . . . . . . . . & 12.119 & -- & W & 1.907 & $W$ & 1.655 & 1.6 \\
\hline 30 & Rubber and Misc. Plastlcs Products . . . . . . . & 16.988 & 2.494 & 5.372 & 3.135 & 7.959 & 2.154 & 5.1 \\
\hline 3011 & Tires and Inner Tubos ................ & 12.565 & 2.180 & 3.070 & 2.319 & 6.911 & 2.417 & 2.0 \\
\hline 308 & Miscellanoous Plastlc Products, nec . . . . . . . . & 17.626 & 2.629 & 5.981 & 3.396 & 9.376 & 2.246 & 5.0 \\
\hline 31 & Leather and Leather Products . . . . . . . . . & 20.179 & 2.626 & 4.864 & 2.863 & 8.292 & w & 6.7 \\
\hline 32 & Stone, Clay and Glass Products $\ldots \ldots \ldots \ldots$ & 14.400 & 3.141 & 6.010 & 2.532 & 8.065 & 1.583 & 3.8 \\
\hline 3211 & Flat Glass $\ldots \ldots \ldots \ldots \ldots \ldots \ldots \ldots$ & 13,184 & W & 5.824 & 2.374 & 5.374 & w & 1.2 \\
\hline 3221 & Glass Containers . . . . . . . . . . . . & 14.077 & 3.273 & 5.587 & 2.446 & 6.758 & - & 2.0 \\
\hline 3229 & Pressed and Blown Glass, nec . . . . . . . . & 12.609 & W & 5.942 & 2.629 & 8.109 & - & 3.9 \\
\hline 3241 & Cement, Hydraulic . . . . . . . . . . . . & 12.532 & 2.759 & 5.409 & 1.820 & 8.735 & 1.570 & 4.6 \\
\hline 3274 & $\operatorname{Lime} \ldots \ldots \ldots \ldots \ldots \ldots \ldots \ldots \ldots$ & 13.032 & $W$ & 5.706 & 2.192 & 7.323 & 1.572 & 5.3 \\
\hline 3296 & 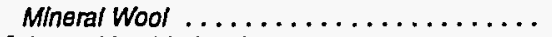 & 13.015 & $W$ & 7.250 & 2.434 & 6.685 & W & 1.1 \\
\hline 33 & Primary Metal Industries $\ldots \ldots \ldots \ldots \ldots$ & 10.178 & 2.172 & 5.835 & 2.527 & 5.230 & 1.878 & 2.6 \\
\hline 3312 & Blast Fumaces and Steel Mills . . . . . . . . . . & 12.114 & 2.111 & 6.254 & 2.336 & 7.518 & 1.868 & 2.4 \\
\hline 3313 & Electrometalurglcal Products . . . . . . . . & 7.339 & -- & 5.829 & 2.834 & $\mathbf{W}$ & 1.681 & 2.7 \\
\hline 3321 & Gray and Ductlle Iron Foundries $\ldots \ldots \ldots$ & 14.846 & $W$ & 5.774 & 2.785 & 6.955 & W & 4.1 \\
\hline 3331 & Primary Copper . . . . . . . . . . . . & 14.172 & W & $W$ & 2.021 & 8.257 & $W$ & NF \\
\hline 3334 & Primary Aluminum ${ }^{d} \ldots \ldots \ldots \ldots$ & 6.203 & W & 4.984 & 2.573 & 7.777 & 9.421 & 1.6 \\
\hline 3339 & Primary Nonferrous Motals, nec . . . . . . . . . . & 8.556 & $W$ & 5.332 & 2.164 & $w$ & 2.082 & 1.4 \\
\hline 3353 & Aluminum Sheet, Plate, and Foll ......... & 12.044 & -- & 5.052 & 2.478 & 6.655 & $w$ & 1.0 \\
\hline 34 & Fabricated Metal Products . . . . . . . . . . . & 18.293 & 3.159 & 5.706 & 3.373 & 8.192 & 1.993 & 5.3 \\
\hline 35 & Industrial Machinery and Equipment . . . . . . . . & 18.144 & 3.079 & 5.799 & 3.450 & 7.713 & 1.544 & 6.3 \\
\hline 357 & Computer and Office Equipment $\ldots \ldots \ldots$ & 17.146 & $w$ & 5.601 & 3.537 & 13.360 & 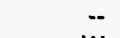 & 6.6 \\
\hline 36 & Electronic and Other Electric Equipment ...... & 16.661 & 3.470 & 5.245 & 3.320 & 7.279 & $W$ & 3.8 \\
\hline 37 & Transportation Equipment $\ldots . \ldots \ldots \ldots$ & 16.184 & 3.035 & 5.378 & 3.095 & 7.111 & 2.060 & 3.0 \\
\hline 3711 & Motor Vehicles and Car Bodles .......... & 14.497 & $W$ & 6.672 & 2.808 & 5.743 & 2.195 & 1.6 \\
\hline 3714 & Motor Vehicle Parts and Accessories . . . . . . . & 15.958 & 3.493 & 4.134 & 3.049 & 7.020 & W & 4.6 \\
\hline 38 & Instruments and Related Products ......... & 18.870 & 3.160 & 4.855 & 3.533 & 7.211 & W & 3.6 \\
\hline 3841 & Surglcal and Medical instruments $\ldots \ldots \ldots$ & 18.912 & $W$ & 4.712 & 4.336 & 8.523 & -- & 3.6 \\
\hline 39 & Misc. Manufacturing Industries . . . . . . . . . & 19.664 & 2.953 & 6.118 & 3.878 & 10.033 & $W$ & 3.9 \\
\hline & Total $\ldots . . . . . \ldots \ldots \ldots \ldots . . . .$. & 13.486 & 2.540 & 5.625 & 2.304 & 4.909 & 1.775 & 1.8 \\
\hline
\end{tabular}

See footnotes at end of table.

Energy Information Administration/Manufacturing Consumption of Energy 1991 
Table A25 Average Prices of Selected Purchased Energy Sources by Census Region, Industry Group, and Selected Industries, 1991: Part 2 (Continued)

(Estimates in Dollars per Million Btu)

\begin{tabular}{|c|c|c|c|c|c|c|c|c|}
\hline $\begin{array}{c}\text { SIC } \\
\text { Code }^{a}\end{array}$ & $\begin{array}{c}\text { Industry Groups } \\
\text { and Industry }\end{array}$ & Electricity & $\begin{array}{c}\text { Residual Fuel } \\
\text { Oil }\end{array}$ & $\begin{array}{c}\text { Distillate Fuel } \\
\text { Oip }\end{array}$ & Natural Gas ${ }^{c}$ & LPG & Coal & $\begin{array}{c}\text { RSE } \\
\text { Row } \\
\text { Factore }\end{array}$ \\
\hline & \multirow[b]{2}{*}{ RSE Column Factors: } & \multicolumn{6}{|c|}{ Northeast Census Region } & \\
\hline & & 0.8 & 0.6 & 0.9 & 3.2 & 1.1 & 0.6 & \\
\hline 20 & Food and Kindred Products . . . . . . . . . & 21.063 & 3.116 & 6.037 & 3.422 & 7.083 & 2.132 & 4.2 \\
\hline 2011 & Meat Packing Plants . . . . . . . . . . . & 17.566 & $W$ & 5.993 & 2.818 & 6.308 & -- & 4.6 \\
\hline 2033 & Canned Fruits and Vegetables . . . . . . . . . & 21.614 & 3.207 & 6.944 & 3.642 & 10.118 & $w$ & 4.7 \\
\hline 2037 & Frozen Fruits and Vegetables . . . . . . . . . & 20.503 & W & $w$ & 3.092 & $\mathbf{W}$ & -. & 5.6 \\
\hline 2046 & Wet Com Milling . . . . . . . . . . . . & 16.999 & $W$ & $\mathbf{W}$ & Q & $w$ & $\ddot{-\bullet}$ & 2.2 \\
\hline 2051 & Bread, Cake, and Related Products . . . . . . . & 23.445 & W & 6.178 & 3.504 & 7.595 & -- & 3.6 \\
\hline 2063 & Beet Sugar . . . . . . . . . . . . . . & - & - & - & -- & - & $\cdots$ & NF \\
\hline 2075 & 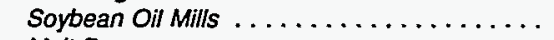 & - & -- & -- & - & $\cdots$ & 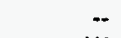 & NF \\
\hline 2082 & Malt Beverages . . . . . . . . . . . & 18.361 & W & 3.082 & 2.865 & 8.628 & $W$ & 3.5 \\
\hline 21 & Tobacco Products ................ & W & -- & - & W & w & - & 12.9 \\
\hline 22 & Textile Mill Products $\ldots \ldots \ldots \ldots \ldots \ldots$ & 23.068 & 2.822 & 4.529 & 4.048 & 5.918 & W & 5.3 \\
\hline 23 & Apparel and Other Textile Products ........ & 28.847 & 3.067 & 6.620 & 4.562 & 12.892 & $\cdots$ & 7.2 \\
\hline 24 & Lumber and Wood Products $\ldots \ldots \ldots \ldots$ & 22.589 & W & 7.677 & 3.829 & 5.518 & -- & 6.3 \\
\hline 25 & Fumiture and Fixtures $\ldots \ldots \ldots \ldots \ldots$ & 25.528 & 3.243 & 6.395 & 4.200 & 6.074 & -- & 5.2 \\
\hline 26 & Paper and Allied Products . . . . . . . . . . & 18.646 & 2.763 & 3.827 & 3.224 & 6.653 & 2.015 & 4.3 \\
\hline 2611 & Pulp Mills . . . . . . . . . . . . . . . . . & W & W & W & -- & W & -- & 2.0 \\
\hline 2621 & Paper Mills . . . . . . . . . . . . . . & 17.487 & 2.739 & 5.850 & 2.906 & 5.839 & 1.981 & 1.8 \\
\hline 2631 & Paperboard Mills . . . . . . . . . . . . . . & 19.813 & 2.987 & 4.889 & 2.789 & 8.474 & $w$ & 3.6 \\
\hline 27 & Printing and Publishing $\ldots \ldots \ldots \ldots \ldots$ & 24.811 & 3.736 & 5.991 & 4.595 & 8.717 & -- & 8.5 \\
\hline 28 & Chemicals and Allied Products . . . . . . . . . & 15.140 & 2.991 & 5.130 & 3.264 & 6.158 & 1.944 & 3.5 \\
\hline 2812 & Alkalies and Chlorine $\ldots \ldots \ldots \ldots \ldots$ & W & -- & - & W & -- & -. & 3.1 \\
\hline 2813 & Industrial Gases . . . . . . . . . . . . . . & 11.475 & - & $w$ & W & $w$ & - & 3.2 \\
\hline 2819 & Industrial Inorganic Chemicals, nec . . . . . . & 18.288 & 3.302 & 5.329 & 3.260 & 5.666 & - & 4.8 \\
\hline 2821 & Plastics Materials and Resins . . . . . . . . . & 17.275 & 2.969 & 4.807 & 3.211 & $W$ & w & 2.8 \\
\hline 2822 & Synthetic Rubber . . . . . . . . . . . . & W & W & W & $W$ & $w$ & -- & 2.1 \\
\hline 2823 & Cellulosic Manmade Fibers . . . . . . . . . . & -- & -- &.- & -- & - & $\cdots$ & NF \\
\hline 2824 & Organic Fibers, Noncellulosic $\ldots . . \ldots \ldots$ & 18.331 & $W$ & W & W & $W$ & - & 4.0 \\
\hline 2865 & Cyclic Crudes and Intermediates . . . . . . . . & 18.529 & 2.735 & 4.523 & 3.003 & 11.159 & W & 7.0 \\
\hline 2869 & Industrial Organic Chemicals, nec . . . . . . . & 8.524 & 2.845 & 5.756 & 3.287 & W & - & 2.9 \\
\hline 2873 & Nitrogenous Fertilizers $\ldots \ldots \ldots \ldots$ & 17.917 & - & W & W & $\mathbf{W}$ & $\cdots$ & 7.6 \\
\hline 2874 & Phosphatic Fertilizers . . . . . . . . . . . & -- & - & -- & $\cdots$ & - & -- & NF \\
\hline 29 & Petroleum and Coal Products $\ldots \ldots \ldots \ldots$ & 16.147 & -- & 5.531 & 2.711 & 6.376 & 2.057 & 7.7 \\
\hline 2911 & Petroleum Refining . . . . . . . . . . . & 14.259 & - & -- & 2.489 & - & 1.659 & 3.3 \\
\hline 30 & Rubber and Misc. Plastics Products . . . . . . . & 21.899 & 2.810 & 5.166 & 4.219 & 6.795 & W & 5.6 \\
\hline 3011 & Tires and Inner Tubes . . . . . . . . . . . . & 11.862 & W & W & 3.185 & 11.115 & - & 4.6 \\
\hline 308 & Miscellaneous Plastic Products, nec . . . . . . . & 22.129 & 2.663 & 5.282 & 4.276 & 7.806 & $w$ & 5.4 \\
\hline 31 & Leather and Leather Products . . . . . . . . . & 25.612 & 2.661 & 4.687 & 4.633 & 8.176 & W & 7.4 \\
\hline 32 & Stone, Clay and Glass Products $\ldots \ldots \ldots \ldots$ & 16.634 & 3.178 & 5.687 & 3.059 & 8.643 & 1.524 & 5.2 \\
\hline 3211 & Flat Glass $\ldots \ldots \ldots \ldots \ldots \ldots \ldots$ & W & -- & W & W & W & $w$ & 1.5 \\
\hline 3221 & Glass Containers . . . . . . . . . . . . & 16.623 & 3.195 & 5.043 & 3.002 & 6.308 & - & 2.4 \\
\hline 3229 & Pressed and Blown Glass, nec . . . . . . . . & 15.643 & W & W & 2.671 & 9.021 & -- & 3.3 \\
\hline 3241 & Cement, Hydraulic . . . . . . . . . . . . & 15,302 & 3.749 & 5.239 & W & 13.804 & 1.548 & 5.6 \\
\hline 3274 & Lime $\ldots \ldots \ldots \ldots \ldots \ldots \ldots \ldots$ & 11.456 & - & 6.083 & $\mathbf{Q}$ & W & 1.504 & 4.3 \\
\hline 3296 & 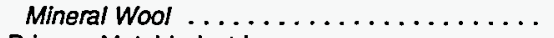 & 17.046 & - & W & 2.837 & 6.310 & W & 1.1 \\
\hline 33 & Primary Metal Industries . . . . . . . . . . . & 13.104 & 2.660 & 6.334 & 3.078 & 7.077 & 1.858 & 3.8 \\
\hline 3312 & Blast Fumaces and Steel Mills . . . . . . . . . & 13.192 & 2.292 & 6.678 & 2.799 & 7.308 & 1.853 & 1.9 \\
\hline 3313 & Electromotalurgical Products ........... & $W$ & -- & W & 4.404 & $W$ & W & 2.3 \\
\hline 3321 & Gray and Ductile Iron Foundries $\ldots \ldots \ldots$ & 20.940 & -- & 5.995 & 3.560 & 7.534 & $W$ & 5.9 \\
\hline 3331 & Primary Copper . . . . . . . . . . . & W & - & W & $W$ & $W$ & - & NF \\
\hline 3334 & Primary Aluminum ${ }^{\sigma} \ldots \ldots \ldots \ldots \ldots$ & $w$ & $W$ & W & W & $w$ & $\mathbf{W}$ & 1.3 \\
\hline 3339 & Primary Nonferrous Metals, nec . . . . . . . . . & 16.200 & $W$ & w & 3.103 & 9.664 & $w$ & 1.6 \\
\hline 3353 & Aluminum Sheet, Plate, and Foil . . . . . . . . & 17.443 & - & $W$ & 2.967 & 6.079 & - & 0.6 \\
\hline 34 & Fabricated Metal Products . . . . . . . . . . & 22.076 & 2.925 & 5.833 & 3.904 & 9.107 & W & 5.5 \\
\hline 35 & Industrial Machinery and Equipment . . . . . . . & 23.003 & 3.119 & 5.645 & 4.247 & 6.220 & - & 7.4 \\
\hline 357 & Computer and Office Equipment . . . . . . . & 20.564 & $w$ & 5.783 & 3.697 & 10.266 & - & 6.1 \\
\hline 36 & Electronic and Other Electric Equipment . . . . . & 20.168 & 3.641 & 5.107 & 4.009 & 6.412 & $w$ & 3.8 \\
\hline 37 & Transportation Equipment $\ldots \ldots \ldots \ldots \ldots$ & 21.540 & 3.323 & 5.295 & 3.612 & 8.014 & $\mathbf{w}$ & 4.7 \\
\hline 3711 & Motor Vehicles and Car Bodies . . . . . . . . & W & W & W & W & 7.170 & - & 1.6 \\
\hline 3714 & Motor Vehicle Parts and Accessories . . . . . . & 20.757 & $W$ & 6.023 & 3.705 & 7.373 & W & 3.9 \\
\hline 38 & Instruments and Related Products . . . . . . . & 22.821 & 3.153 & 4.828 & 3.757 & 6.485 & W & 4.2 \\
\hline 3841 & Surgical and Medical Instruments $\ldots \ldots \ldots$ & 22.616 & W & 5.481 & 5.348 & 8.661 & - & 3.4 \\
\hline 39 & Misc. Manufacturing Industries . . . . . . . . . & 23.084 & 3.075 & 6.063 & 4.050 & 10.254 & W & 5.1 \\
\hline & Total & 18.648 & 2.897 & 5.426 & 3.386 & 6.745 & 1.1322 & 3.1 \\
\hline
\end{tabular}

See footnotes at end of table. 
Table A25 Average Prices of Selected Purchased Energy Sources by Census Region, Industry Group, and Selected Industries, 1991: Part 2 (Continued) (Estimates in Dollars per Million Btu)

\begin{tabular}{|c|c|c|c|c|c|c|c|c|}
\hline \multirow[t]{3}{*}{$\begin{array}{c}\text { SIC } \\
\text { Code }\end{array}$} & $\begin{array}{l}\text { Industry Groups } \\
\text { and Industry }\end{array}$ & Electricity & $\begin{array}{c}\text { Residual Fuel } \\
\text { Oil }\end{array}$ & $\begin{array}{l}\text { Distillate } \\
\text { Fuel Oil }\end{array}$ & Natural Gas ${ }^{c}$ & LPG & Coal & $\begin{array}{c}\text { RSE } \\
\text { Row } \\
\text { Factors }\end{array}$ \\
\hline & & \multicolumn{6}{|c|}{ Midwest Census Region } & \\
\hline & RSE Column Factors: & 0.7 & 0.9 & 0.9 & 2.8 & 1.1 & 0.6 & \\
\hline 20 & Food and Kindred Products .. & 13.868 & 2.168 & 5.886 & 2.437 & 7.065 & 1.358 & 3.4 \\
\hline 2011 & Meat Packing Plants . . . . . . . . . . . & 13.138 & 1.683 & 4.836 & 2.256 & 6.417 & 1.723 & 2.5 \\
\hline 2033 & Canned Fruits and Vegetables . . . . . . . . . . & 17.272 & -- & 5.477 & 2.630 & 7.657 & - & 5.2 \\
\hline 2037 & Frozen Frults and Vegetables . . . . . . . . & 15.174 & W & $W$ & 2.572 & 6.281 & $\cdots$ & 5.6 \\
\hline 2046 & Wet Com Milling . . . . . . . . . . . . . . . . & 10.461 & W & 5.172 & 2.257 & W & 1.243 & 3.0 \\
\hline 2051 & Bread, Cake, and Related Products . . . . . . . & 16.236 & -- & W & 3.250 & 11.546 & $\cdots$ & 3.8 \\
\hline 2063 & Beot Sugar . . . . . . . . . . . . . & 14.018 & $W$ & 6.194 & 2.075 & 9.120 & 1.480 & 2.2 \\
\hline 2075 & 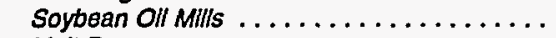 & 11.770 & 2.540 & 6.661 & 2.066 & W & 1.294 & 1.7 \\
\hline 2082 & Mall Beverages $\ldots \ldots \ldots \ldots \ldots \ldots$ & 13.105 & W & W & 2.543 & $\mathbf{W}$ & w & 3.3 \\
\hline 21 & Tobacco Products . . . . . . . . . . . . . . . & W & -- & W & W & -- & $\cdots$ & 1.8 \\
\hline 22 & Textlle Mill Products $\ldots \ldots \ldots \ldots \ldots \ldots \ldots$ & 16.553 & $W$ & W & 2.956 & 8.782 & -- & 7.3 \\
\hline 23 & Apparel and Other Textile Products $\ldots \ldots \ldots$. & 18.893 & $W$ & W & 3.362 & 6.271 & W & 6.1 \\
\hline 24 & Lumber and Wood Products $\ldots \ldots \ldots \ldots \ldots$ & 16.915 & $W$ & 6.691 & 3.638 & 5.617 & $W$ & 6.3 \\
\hline 25 & Fumiture and Fixtures $\ldots \ldots \ldots \ldots \ldots$ & 17.156 & $W$ & 5.853 & 3.241 & 9.081 & $w$ & 9.8 \\
\hline 26 & Paper and Alled Products . . . . . . . . . . . . & 12.316 & 2.506 & 5.003 & 2.524 & 8.180 & 1.819 & 3.7 \\
\hline 2611 & Pulp Mllis . . . . . . . . . . . . . . & W & - & W & W & W & W & 5.6 \\
\hline 2621 & Paper Mills . . . . . . . . . . . . & 10.895 & 2.440 & 4.277 & 2.378 & 7.909 & 1.820 & 2.9 \\
\hline 2631 & Paperboard MIIIs . . . . . . . . . . . . & 11.935 & W & 5.940 & 2.291 & 7.167 & 1.917 & 4.9 \\
\hline 27 & Printing and Publishing ... & 16.748 & W & 6.238 & 3.126 & 7.798 & $=$ & 5.4 \\
\hline 28 & Chemicals and Allied Products . . . . . . . . & 8.960 & 2.395 & 5.058 & 2.105 & W & 1.678 & 4.0 \\
\hline 2812 & Alkalles and Chlorine ............. & W & -- & W & W & W & -- & 2.4 \\
\hline 2813 & Industrial Gases . . . . . . . . . . . . & $W$ & - & w & W & $\mathbf{W}$ & - & 2.6 \\
\hline 2819 & Industrial Inorganic Chemicals, nec . . . . . . & W & $w$ & 6.619 & 2.425 & 8.932 & W & 2.6 \\
\hline 2821 & Plastios Materials and Resins . . . . . . . . & 12.113 & $w$ & 5.247 & 2.097 & W & $W$ & 2.8 \\
\hline 2822 & Synthetic Rubbar . . . . . . . . . . . . . & 10.140 & 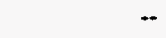 & W & 2.606 & W & W & 3.1 \\
\hline 2823 & Cellulosic Manmade Fibers . . . . . . . . . . . & -. & - & -- & - & - & -- & NF \\
\hline 2824 & Organic Fibers, Noncellulosic .......... & - & -- & -- & - & - & - & NF \\
\hline 2865 & Cycllc Crudes and Intermedlates ......... & 13.175 & W & 6.372 & 2.479 & W & 2.110 & 3.0 \\
\hline 2869 & Industrlal Organic Chemicals, nec . . . . . . . & 12.802 & $w$ & 5.250 & 1.997 & W & 1.477 & 2.4 \\
\hline 2873 & Nitrogenous Fertlizers $\ldots \ldots \ldots \ldots \ldots$ & 10.397 & -. & 5.537 & W & $W$ & - & 8.6 \\
\hline 2874 & Phosphatlc Fortilzers ............. & W & - & W & $W$ & $W$ & - & 1.3 \\
\hline 29 & Petroleum and Coal Products $\ldots \ldots \ldots \ldots$ & 12.226 & W & 4.895 & 2.166 & 5.469 & $w$ & 4.6 \\
\hline 2911 & Potroleum Rolining . . . . . . . . . . . . & 11.555 & - & - & 2.032 & - & $W$ & 1.6 \\
\hline 30 & Rubber and Misc. Plastics Products . . . . . . & 16.547 & 1.799 & 6.174 & 2.989 & 9.402 & 2.090 & 3.9 \\
\hline 3011 & Tires and Inner Tubes . . . . . . . . . . . . . & 12.615 & 1.699 & W & 2.143 & 6.575 & 2.488 & 1.3 \\
\hline 308 & Miscellaneous Plastic Products, nec....... & 16.870 & W & 6.492 & 3.166 & 10.346 & W & 4.5 \\
\hline 31 & Leather and Leather Products . . . . . . . . . . . & 17.086 & W & 6.989 & 3.087 & 6.927 & - & 6.2 \\
\hline 32 & Stone, Clay and Glass Products ......... & 13.827 & w & 6.140 & 2.569 & 8.328 & 1.402 & 4.8 \\
\hline 3211 & Flat Glass $\ldots \ldots \ldots \ldots \ldots \ldots \ldots$ & 14.435 & -- & 5.810 & 2.169 & 6.426 & - & 1.1 \\
\hline 3221 & Glass Containers $\ldots \ldots \ldots \ldots \ldots \ldots$ & 13.190 & -- & 7.428 & 2.202 & W & - & 2.3 \\
\hline 3229 & Pressed and Blown Glass, nec . . . . . . . . & 13.432 & $w$ & 5.917 & 3.029 & 9.467 & - & 2.5 \\
\hline 3241 & Cement, Hydraulic . . . . . . . . . . . . . & 12.578 & W & 5.446 & 2.072 & 8.320 & 1.306 & 3.9 \\
\hline 3274 & Lime $\ldots \ldots \ldots \ldots \ldots \ldots \ldots \ldots \ldots \ldots \ldots \ldots \ldots \ldots \ldots$ & 14.992 & -- & 5.583 & 2.407 & W & 1.552 & 4.9 \\
\hline 3296 & Mineral Wool . . . . . . . . . . . . . . . & 10.936 & $w$ & 6.137 & 2.384 & 5.612 & - & 0.9 \\
\hline 33 & Primary Metal Industries . . . . . . . . . . . & 12.197 & 2.188 & 6.218 & 2.381 & 7.688 & 1.915 & 3.3 \\
\hline 3312 & Blast Fumaces and Steol Mills . . . . . . . . . . & 12.708 & 2.187 & 6.466 & 2.214 & 9.176 & 1.926 & 2.3 \\
\hline 3313 & Electrometalurgical Products . . . . . . . . . & 7.968 & -- & 5.692 & 2.820 & W & 1.380 & 3.1 \\
\hline 3321 & Gray and Ductile Iron Foundries $\ldots \ldots \ldots$ & 14.509 & W & 6.074 & 2.757 & 7.153 & W & 4.0 \\
\hline 3331 & Primary Copper $\ldots \ldots \ldots \ldots \ldots \ldots$ & $W$ & - & 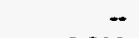 & W & W & 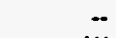 & NF \\
\hline 3334 & Primary Aluminum ${ }^{\circ} \ldots \ldots \ldots \ldots \ldots$ & $\mathbf{W}$ & - & 6.283 & W & W & W & 1.3 \\
\hline 3339 & Primary Nonferrous Metals, nec . . . . . . . . . & 10.289 & - & W & 2.269 & 6.512 & -- & 1.5 \\
\hline 3353 & Aluminum Sheet, Plate, and Foll ......... & 12.540 & - & W & 2.188 & 6.696 & -. & 1.0 \\
\hline 34 & Fabricated Metal Products . . . . . . . . . . . & 17.679 & w & 5.814 & 2.989 & 7.671 & 1.951 & 6.2 \\
\hline 35 & Industrial Machinery and Equipment . . . . . . . & 16.530 & 2.852 & 5.845 & 3.201 & 6.971 & 1.543 & 5.6 \\
\hline 357 & Computer and Office Equipment ......... & 14.040 & - & W & 2.924 & W & - & 8.5 \\
\hline 36 & Electronic and Other Electric Equipment . . . . . & 14.663 & 1.937 & 5.054 & 3.046 & 6.953 & $W$ & 6.4 \\
\hline 37 & Transportation Equipment $\ldots \ldots \ldots \ldots \ldots$ & 15.266 & 2.852 & 5.289 & 2.906 & 7.052 & $W$ & 3.9 \\
\hline 3711 & Motor Vehicles and Car Bodles . . . . . . . . . & 14.786 & W & 6.753 & 2.697 & 5.452 & W & 1.4 \\
\hline 3714 & Motor Vehicle Pants and Accessories . . . . . . . & 15.610 & W & 3.858 & 2.916 & 6.651 & $W$ & 3.8 \\
\hline 38 & Instruments and Related Products $\ldots \ldots \ldots \ldots$ & 16.084 & W & 8.487 & 3.166 & 8.004 & W & 4.8 \\
\hline 3841 & Surgical and Medlcal Instruments ........ & 14.297 & - & W & 3.923 & 6.730 & - & 5.5 \\
\hline 39 & Misc. Manufacturing Industries . . . . . . . . . & 18.547 & 3.510 & 5.472 & 3.929 & 9.581 & $w$ & 6.2 \\
\hline & Total $\ldots \ldots \ldots \ldots \ldots \ldots \ldots \ldots$ & 13.493 & 2.300 & 5.744 & 2.523 & 3.446 & 1.735 & 2.7 \\
\hline
\end{tabular}

See footnotes at end of table. 


\section{Table A25 Average Prices of Selected Purchased Energy Sources by Census Region, Industry Group, and Selected Industries, 1991: Part 2 (Continued) (Estimates in Dollars per Million Btu)}

\begin{tabular}{|c|c|c|c|c|c|c|c|}
\hline $\begin{array}{c}\text { SIC } \\
\text { Code* }\end{array}$ & $\begin{array}{l}\text { Industry Groups } \\
\text { and Industry }\end{array}$ & Electricity & $\begin{array}{c}\text { Residual Fuel } \\
\text { Oll }\end{array}$ & $\begin{array}{c}\text { Distillate Fuel } \\
\text { Oll } \\
\end{array}$ & Natural Gas & LPG & Coal \\
\hline
\end{tabular}

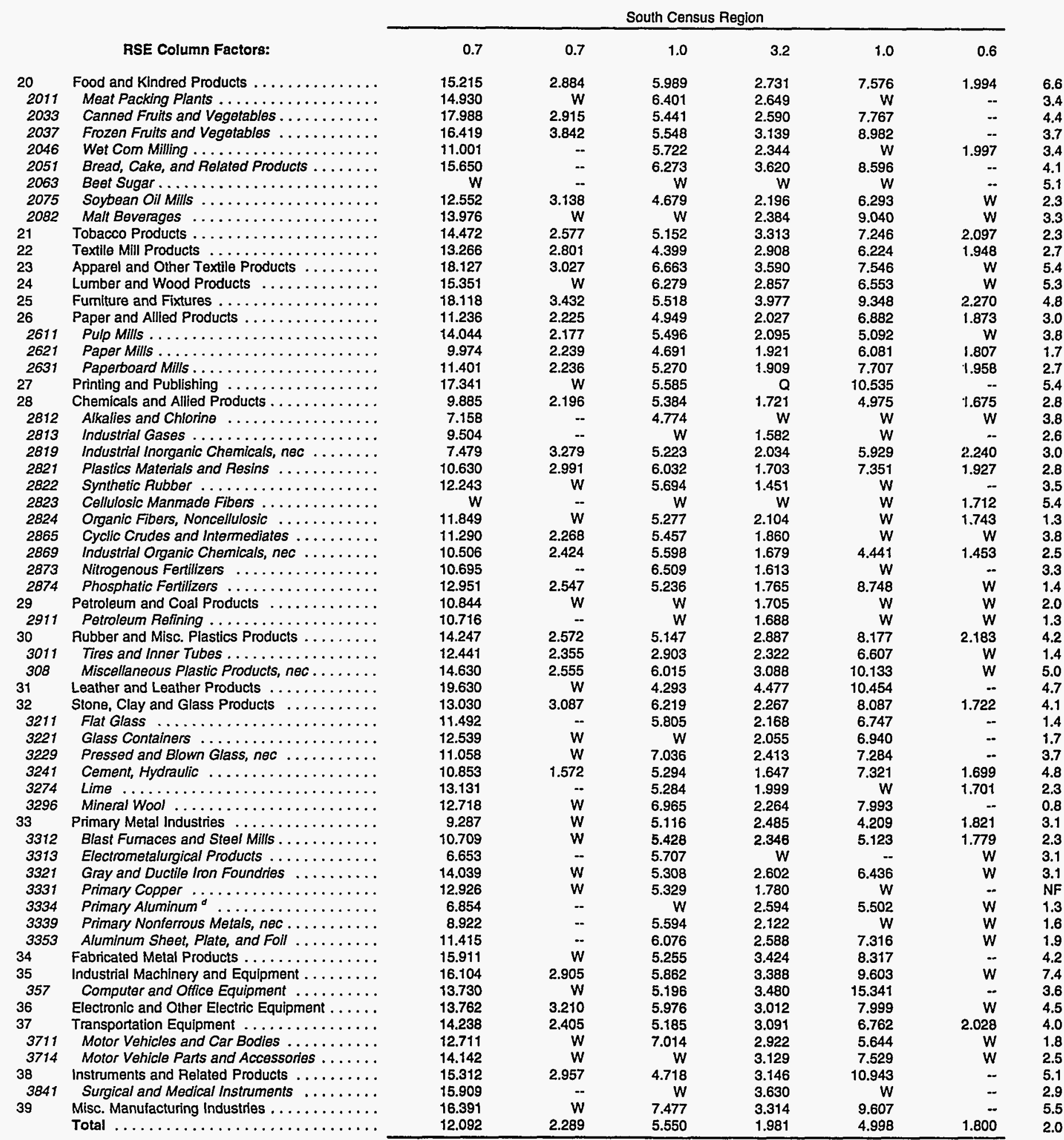

See footnotes at end of table. 
Table A25 Average Prices of Selected Purchased Energy Sources by Census Region, Industry Group, and Selected Industries, 1991: Part 2 (Continued)

(Estimates in Dollars per Million Btu)

\begin{tabular}{|c|c|c|c|c|c|c|c|c|}
\hline \multirow[t]{3}{*}{$\begin{array}{c}\text { SIC } \\
\text { Codea }\end{array}$} & $\begin{array}{l}\text { Industry Groups } \\
\text { and Industry }\end{array}$ & Electricity & $\begin{array}{c}\text { Residual Fuel } \\
\text { Oil }\end{array}$ & $\begin{array}{c}\text { Distillate Fuel } \\
\text { OIP }^{b}\end{array}$ & Natural Gas ${ }^{\circ}$ & LPG & Coal & \multirow[t]{3}{*}{$\begin{array}{c}\text { RSE } \\
\text { Row } \\
\text { Factors }\end{array}$} \\
\hline & & \multicolumn{6}{|c|}{ West Census Region } & \\
\hline & RSE Column Factors: & 1.0 & 0.7 & 0.7 & 3.5 & 0.9 & 0.7 & \\
\hline 20 & Food and KIndred Products . . . . . . . . . & 17.650 & 3.193 & 6.355 & $\mathbf{Q}$ & 8.889 & 1.321 & 4.0 \\
\hline 2011 & Meat Packing Plants . . . . . . . . . . . . & 12.440 & W & 6.517 & 2.354 & 7.395 & - & 3.3 \\
\hline 2033 & Canned Frults and Vegetables . . . . . . . . . . & 23.275 & 2.795 & W & 2.866 & 7.677 & - & 2.8 \\
\hline 2037 & Frozen Fruils and Vegetables . . . . . . . . . & 9.964 & 3.886 & 5.208 & 2.253 & 7.219 & - & 6.3 \\
\hline 2046 & Wet Com MIIIIng . . . . . . . . . . . . . & W & -. & W & W & W & - & 2.9 \\
\hline 2051 & Bread, Cake, and Related Products . . . . . . . & 20.845 & $\cdots$ & 6.761 & 3.844 & 9.630 & - & 6.3 \\
\hline 2063 & Beet Sugar . . . . . . . . . . . . . . & 12.429 & W & 5.723 & 2.401 & 10.080 & 1.242 & 2.9 \\
\hline 2075 & Soybean Oll MIlls $\ldots \ldots \ldots \ldots \ldots \ldots$ & -. & - & - & - & -. & - & NF \\
\hline 2082 & Mall Beverages ................ & 16.587 & W & W & 2.617 . & $w$ & $w$ & 2.8 \\
\hline 21 & Tobacco Products $\ldots \ldots \ldots \ldots \ldots \ldots \ldots$ & -. & - & - & $\therefore$ & - & -- & NF \\
\hline 22 & Textlle Mill Products $\ldots \ldots \ldots \ldots \ldots \ldots \ldots$ & 21.076 & -- & $W$ & 4.068 & 6.009 & - & 9.5 \\
\hline 23 & Apparel and Other Textlle Products $\ldots \ldots \ldots$. & 28.737 & $\mathbf{W}$ & $W$ & 5.259 & 16.485 & - & 4.8 \\
\hline 24 & Lumber and Wood Products $\ldots \ldots \ldots \ldots \ldots$ & 13.284 & 2.869 & 6.298 & 2.692 & 7.000 & $\cdots$ & 7.3 \\
\hline 25 & Fumiture and Fixtures $\ldots \ldots \ldots \ldots \ldots \ldots$ & 30.012 & - & 6.312 & 4.494 & 9.552 & - & 7.0 \\
\hline 26 & Paper and Allied Products $\ldots \ldots \ldots \ldots \ldots$ & 9.875 & 2.850 & 5.670 & 2.393 & 7.082 & $w$ & 3.8 \\
\hline 2611 & Pulp Mils $\ldots \ldots \ldots \ldots \ldots \ldots \ldots$ & 8.938 & 2.999 & 6.268 & 2.494 & 9.553 & $\cdots$ & 4.4 \\
\hline 2621 & Paper MIIIs . . . . . . . . . . . . . & 8.236 & 2.645 & 5.040 & 2.318 & 5.134 & 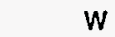 & 2.1 \\
\hline 2631 & Paperboard Mils .................. & 9.670 & 2.825 & 5.613 & 2.276 & 7.198 & W & 2.1 \\
\hline 27 & Printling and Publishing $\ldots \ldots \ldots \ldots \ldots$ & 24.524 & - & 6.750 & 4.457 & 11.538 & $\cdots$ & 6.6 \\
\hline 28 & Chemicals and Alled Products . . . . . . . . . & 9.182 & $\mathbf{W}$ & 6.096 & 1.810 & 5.897 & 1.545 & 6.6 \\
\hline 2812 & Alkalles and Chiorine $\ldots \ldots \ldots \ldots \ldots$ & 6.508 & $W$ & 5.813 & 1.966 & W & - & 3.0 \\
\hline 2813 & Indusirial Gases . . . . . . . . . . . . . & 10.185 & - & W & W & w & -- & 2.4 \\
\hline 2819 & Industral Inorganic Chemicals, nec . . . . . . . & 7.340 & $\mathbf{W}$ & 5.968 & 2.798 & 6.788 & 1.545 & 5.4 \\
\hline 2821 & Plastlos Materials and Resins .......... & 22.404 & - & 6.746 & 4.297 & 10.005 & - & 4.5 \\
\hline 2822 & 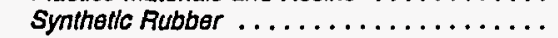 & W & - & W & W & W & -- & 2.1 \\
\hline 2823 & Cellulosic Manmade Fibers . . . . . . . . . . & $\cdots$ & -. & -- &.- & .. & $\cdots$ & NF \\
\hline 2824 & Organic Fibers, Noncellulosic . . . . . . . . . & -- & 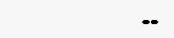 & -- & -. & - & $\cdots$ & NF \\
\hline 2865 & Cycllc Crudes and Intermediates . . . . . . . & W & - & $w$ & W & $w$ & $\cdots$ & 5.5 \\
\hline 2869 & Industrial Organle Chemicals, noc ........ & 15.702 & 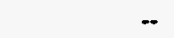 & 6.044 & $\ddot{W}$ & 13.084 & 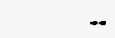 & 13.8 \\
\hline 2873 & Nitrogenous Fertilizers $\ldots \ldots \ldots \ldots \ldots$ & 7.151 & - & 5.839 & W & W & -- & 6.8 \\
\hline 2874 & Phosphatic Fortilizers ............. & W & 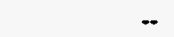 & 7.307 & W & .. & -- & 5.0 \\
\hline 29 & Petroleum and Coal Products $\ldots \ldots \ldots \ldots$ & 15.090 & - & 4.249 & 3.045 & $w$ & $\cdots$ & 3.0 \\
\hline 2911 & Petroleum Refining . . . . . . . . . . . & 14.658 & -. & -. & 3.002 & - & .. & 1.2 \\
\hline 30 & Rubber and Misc. Plastlcs Products . . . . . . . & 21.407 & $w$ & 7.628 & 3.670 & 10.991 & $w$ & 6.0 \\
\hline 3011 & Tres and Inner Tubes . . . . . . . . . . . . & $W$ & W & $W$ & $W$ & $w$ & .. & 2.1 \\
\hline 308 & Mlscellanoous Plastic Products, nec........ & 21.328 & - & $\mathbf{W}$ & 4.226 & 11.340 & .. & 4.8 \\
\hline 31 & Leather and Leather Products . . . . . . . . . . & 17.906 & - & $\mathbf{W}$ & $\mathbf{Q}$ & 12.659 & -- & 5.3 \\
\hline 32 & Stone, Clay and Glass Products $\ldots \ldots \ldots \ldots$ & 16.044 & 3.073 & 5.768 & 2.677 & 7.114 & 1.708 & 4.6 \\
\hline 3211 & Flat Glass $\ldots \ldots \ldots \ldots \ldots \ldots \ldots \ldots$ & 20.003 & $w$ & 5.747 & 3.258 & $W$ & -- & 1.2 \\
\hline 3221 & Glass Contalners ................ & 14.602 & $w$ & W & 2.868 & 5.932 & $\cdots$ & 2.3 \\
\hline 3229 & Pressed and Blown Glass, nec . . . . . . . . & W & $\cdots$ & W & $W$ & W & $\cdots$ & 2.3 \\
\hline 3241 & Cement, Hydraullc . . . . . . . . . . . . & 13.415 & $w$ & 5.695 & 2.004 & 11.656 & 1.708 & 5.0 \\
\hline 3274 & 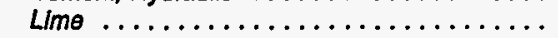 & W & $w$ & W & $W$ & $W$ & - & 3.1 \\
\hline 3296 & 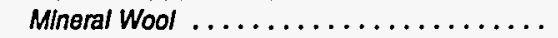 & 18.055 & -. & W & 3.155 & 6.383 & $-\bullet$ & 1.0 \\
\hline 33 & Primary Metal Industries . . . . . . . . . . . . . & 7.274 & 2.328 & 5.469 & 2.530 & 8.517 & $W$ & 2.8 \\
\hline 3312 & Blast Fumaces and Steel Mills . . . . . . . . . . & 10.352 & $w$ & 5.442 & 2.164 & 7.283 & $w$ & 2.2 \\
\hline 3313 & Electromotalurglcal Products . . . . . . . . . . & - &.- & - & - & -- & -- & NF \\
\hline 3321 & Gray and Ductle Iron Foundries ......... & 21.691 & $\ldots$ & 6.310 & 3.031 & 9.006 & $\cdots$ & 7.6 \\
\hline 3331 & Primary Copper $\ldots \ldots \ldots \ldots \ldots \ldots$ & 14.044 & $w$ & W & 2.120 & W & W & NF \\
\hline 3334 & Primary Aluminum ${ }^{\circ} \ldots \ldots \ldots \ldots$ & 5.717 & $W$ & 6.121 & 2.289 & 8.815 & 11.481 & 1.3 \\
\hline 3339 & Primary Nonferrous Motals, nec. . . . . . . . . . & 6.463 & $\ldots$ & $W$ & 2.020 & $W$ & W & NF \\
\hline 3353 & Aluminum Sheot, Plate, and Foll .......... & $W$ & $\cdots$ & w & $w$ & W & -- & NF \\
\hline 34 & Fabricated Metal Products . . . . . . . . . . . . . & 21.673 & W & 7.101 & 4.054 & 9.335 & -- & 4.1 \\
\hline 35 & Industrlal Machinery and Equlpment . . . . . . . . & 21.550 & - & $W$ & 3.856 & 9.650 & - & 8.1 \\
\hline 357 & Computer and Office Equipment .......... & 18.400 & $\cdots$ & $\mathbf{W}$ & 4.054 & 13.420 & -- & 6.1 \\
\hline 36 & Electronlc and Other Electric Equipment ...... & 20.652 & $\cdots$ & 6.565 & 4.029 & 11.497 & -- & 4.1 \\
\hline
\end{tabular}

See footnotes at end of table. 

Table A25. Average Prices of Selected Purchased Energy Sources by Census Region,
Industry Group, and Selected Industries, 1991: Part 2 (Continued) (Estimates in Dollars per Million Btu)

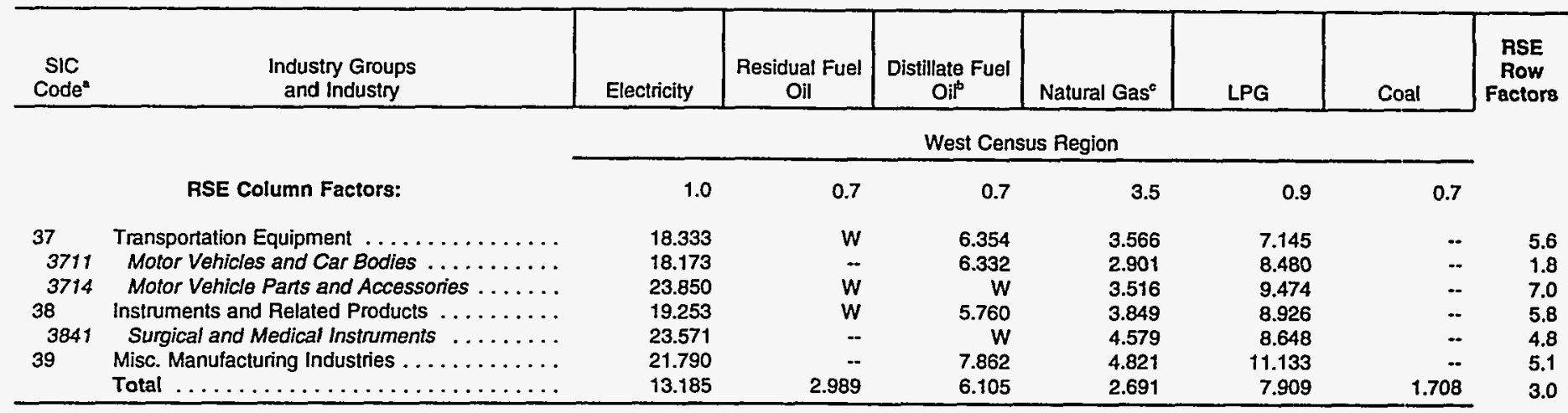

- See Appendices B and F for descriptions of the Standard Industrial Classification system.

- "Distillate Fuel Oil" includes Nos. 1, 2, and 4 fuel oils and Nos. 1, 2, and 4 diesel fuels.

- "Natural Gas" includes natural gas obtained from utilities, transmission pipelines, and any other supplier(s) such as brokers and producers.

- The price estimates for coal for SIC 3334 include anthracite coal for the production of carbon anodes. Because of the high cost of transporting anthracite from the

East Coast to the West and South, the prices of coal in those regions are extremely high.

NF=No applicable RSE row/column factor.

$W=$ Withheld to avoid disclosing data for individual establishments. Data are included in higher level totals.

$N A=$ Not available. Data are included in higher level totals.

- Estimation of average price is not applicable.

Notes: - To obtain a RSE percentage for any table cell, multiply the cell's corresponding RSE column and RSE row factors. - Totals may not equal sum of components because of independent rounding.

Source: Energy Information Administration, Otfice of Energy Markets and End Use, Energy End Use and Integrated Statistics Division, Form ElA-846, "1991 Manufacturing Energy Consumption Survey." 
Table A26. Total Quantity of Purchased Energy Sources by Census Region and Economic Characteristics of the Establishment, 1991 (Estimates in Btu or Physical Units)

\begin{tabular}{|c|c|c|c|c|c|c|c|c|c|c|}
\hline $\begin{array}{c}\text { Economic } \\
\text { Characteristics }\end{array}$ & $\begin{array}{c}\text { Total } \\
\text { (trillion Btu) }\end{array}$ & $\begin{array}{c}\text { Electricity } \\
\text { (million } \\
\text { kWh) }\end{array}$ & $\begin{array}{c}\text { Residual } \\
\text { Fuel Oil } \\
\text { (1000 bbls) }\end{array}$ & $\begin{array}{c}\text { Distillate } \\
\text { Fuel Oil } \\
\text { (1000 bbls) }\end{array}$ & $\begin{array}{c}\text { Natural } \\
\text { Gas }^{c} \\
\text { (billion } \\
\text { cu ft) } \\
\end{array}$ & $\begin{array}{c}\text { LPG } \\
\text { (1000 bbls) }\end{array}$ & $\begin{array}{c}\text { Coal } \\
\text { (1000 short } \\
\text { tons) }\end{array}$ & $\begin{array}{c}\text { Coke and } \\
\text { Breeze } \\
\text { (1000 short } \\
\text { tons) }\end{array}$ & $\begin{array}{c}\text { Other } \\
\text { (trillion Btu) }\end{array}$ & $\begin{array}{c}\text { RSE } \\
\text { Row } \\
\text { Factors }\end{array}$ \\
\hline & \multicolumn{10}{|c|}{ Total United States } \\
\hline RSE Column Factors: & 0.6 & 0.5 & 1.4 & 1.2 & 0.7 & 1.2 & 1.2 & 1.4 & 1.4 & \\
\hline \multicolumn{11}{|l|}{$\begin{array}{l}\text { Value of Shipments and Receipts } \\
\text { (million dollars) }\end{array}$} \\
\hline Under $20 \ldots \ldots \ldots \ldots \ldots \ldots$ & $W$ & 107,510 & W & 10,283 & 586 & W & 4,772 & 354 & 119 & 10.6 \\
\hline $20-49 \ldots \ldots \ldots \ldots \ldots \ldots \ldots$ & 1,568 & 106,665 & 10,892 & 4,840 & 675 & 3,301 & 10,599 & 666 & 139 & 7.8 \\
\hline $50-99 \ldots \ldots \ldots \ldots \ldots \ldots \ldots$ & W & 92,598 & 8,563 & 2,347 & W & W & $w$ & 456 & 178 & 6.2 \\
\hline $100-249 \ldots \ldots \ldots \ldots \ldots \ldots \ldots$ & 2,476 & 146,260 & 12,752 & 2,176 & 1,092 & 42,870 & W & $w$ & 212 & 4.1 \\
\hline $250-499 \ldots \ldots \ldots \ldots \ldots \ldots$ & 2,193 & 112,420 & 14,067 & 1,692 & W & 57,617 & $\mathbf{W}$ & 824 & 188 & 4.1 \\
\hline 500 and Over $\ldots \ldots \ldots \ldots \ldots \ldots$ & 4,137 & 132,100 & W & 3,105 & 1,704 & 213,145 & 27,672 & W & 269 & 3.4 \\
\hline Total $\ldots \ldots \ldots \ldots \ldots \ldots \ldots \ldots$ & 13,194 & 697,553 & 61,475 & 24,442 & 5,713 & 336,791 & 78,616 & 9,340 & 1,104 & 3.4 \\
\hline \multicolumn{11}{|l|}{ Employment Size } \\
\hline Under $50 \ldots \ldots \ldots \ldots \ldots$ & 586 & 42,680 & $\mathbf{Q}$ & 5,883 & $w$ & 2,111 & $\mathbf{W}$ & 33 & $\mathbf{Q}$ & 15.6 \\
\hline $50-99 \ldots \ldots \ldots \ldots \ldots \ldots \ldots$ & 912 & 47,362 & 8,824 & 3,327 & 400 & 13,005 & 2,270 & 339 & 159 & 11.4 \\
\hline $100-249 \ldots \ldots \ldots \ldots \ldots \ldots \ldots$ & 2,310 & 127,139 & 7,828 & 5,222 & 1,111 & 28,157 & $W$ & W & 175 & 6.8 \\
\hline $250-499 \ldots \ldots \ldots \ldots \ldots \ldots$ & 2,267 & 127,755 & 10,247 & 3,434 & 1,041 & 54,178 & 9,979 & 673 & 227 & 4.1 \\
\hline $500-999 \ldots \ldots \ldots \ldots \ldots \ldots \ldots$ & 2,613 & 153,780 & 9,893 & 2,565 & 1,110 & 87,235 & $W$ & W & 234 & 3.9 \\
\hline 1,000 and Over $\ldots \ldots \ldots \ldots \ldots$ & 4,507 & 198,837 & W & 4,010 & W & 152,106 & 36,818 & 7,030 & 254 & 3.3 \\
\hline \multirow[t]{2}{*}{ Total $\ldots \ldots \ldots \ldots \ldots \ldots \ldots$} & 13,194 & 697,553 & 61,475 & 24,442 & 5,713 & 336,791 & 78,616 & 9,340 & 1,104 & 3.4 \\
\hline & \multicolumn{10}{|c|}{ Northeast Census Region } \\
\hline RSE Column Factors: & 0.7 & 0.5 & 0.8 & 1.5 & 0.6 & 1.5 & 1.6 & 1.5 & 1.0 & \\
\hline \multicolumn{11}{|l|}{$\begin{array}{l}\text { Value of Shlpments and Receipts } \\
\text { (million dollars) }\end{array}$} \\
\hline Under $20 \ldots \ldots \ldots \ldots \ldots$ & W & 18,966 & 1,770 & 4,029 & $W$ & $w$ & $\mathbf{Q}$ & W & 11 & 14.4 \\
\hline $20-49 \ldots \ldots \ldots \ldots \ldots \ldots \ldots$ & 216 & 14,780 & 2,447 & 1,469 & $w$ & 639 & 2,087 & $\mathbf{W}$ & 11 & 13.4 \\
\hline $50-99 \ldots \ldots \ldots \ldots \ldots \ldots \ldots$ & 162 & 11,198 & 3,338 & 671 & 71 & 334 & 578 & 53 & 10 & 8.4 \\
\hline $100-249 \ldots \ldots \ldots \ldots \ldots \ldots \ldots$ & $w$ & 16,278 & 4,687 & 686 & 86 & $W$ & $W$ & $W$ & 20 & 5.1 \\
\hline $250-499 \ldots \ldots \ldots \ldots \ldots \ldots$ & 185 & 12,061 & 6,934 & w & 49 & 471 & w & W & 23 & 5.6 \\
\hline 500 and $O v a r . \ldots \ldots \ldots \ldots$ & 369 & 13,188 & 2,410 & W & 78 & 50 & $w$ & 0 & 14 & 6.2 \\
\hline Total $\ldots \ldots \ldots \ldots \ldots \ldots \ldots \ldots$ & 1,482 & 86,471 & 21,586 & 7,849 & 445 & 3,803 & W & W & 90 & 5.4 \\
\hline \multicolumn{11}{|l|}{ Employment Size } \\
\hline Under $50 \ldots \ldots \ldots \ldots \ldots \ldots$ & $w$ & 7,428 & 341 & $w$ & 37 & w & $w$ & $\mathbf{Q}$ & 4 & 20.8 \\
\hline $50-99 \ldots \ldots \ldots \ldots \ldots \ldots \ldots \ldots$ & W & 6,968 & 993 & W & 40 & $W$ & 145 & W & $w$ & 15.0 \\
\hline $100-249 \ldots \ldots \ldots \ldots \ldots \ldots \ldots$ & $W$ & 15,201 & 2,642 & 2,039 & 75 & $W$ & 4,454 & 104 & $W$ & 15.6 \\
\hline $250-499 \ldots \ldots \ldots \ldots \ldots \ldots$ & 184 & 14,044 & 3,644 & 990 & 85 & 538 & $W$ & $w$ & 8 & 7.6 \\
\hline $500-999 \ldots \ldots \ldots \ldots \ldots \ldots$ & $w$ & 21,229 & 4,480 & $W$ & 86 & 734 & W & $w$ & 30 & 5.5 \\
\hline 1,000 and Over $\ldots \ldots \ldots \ldots \ldots$ & W & 21,602 & 9,486 & 1,047 & 123 & 375 & $W$ & $W$ & 28 & 5.9 \\
\hline Total $\ldots \ldots \ldots \ldots \ldots \ldots \ldots$ & 1,482 & 86,471 & 21,586 & 7,849 & 445 & 3,803 & $W$ & $W$ & 90 & 5.4 \\
\hline
\end{tabular}

See footnotes at end of table. 
Table A26. Total Quantity of Purchased Energy Sources by Census Region and Economic Characteristics of the Establishment, 1991 (Continued)

(Estimates in Btu or Physical Units)

\begin{tabular}{|c|c|c|c|c|c|c|c|c|c|c|}
\hline $\begin{array}{c}\text { Economic } \\
\text { Characteristics }\end{array}$ & $\begin{array}{c}\text { Total } \\
\text { (trillion Btu) }\end{array}$ & $\begin{array}{c}\text { Electricity } \\
\text { (million } \\
\text { kWh) }\end{array}$ & $\begin{array}{c}\text { Residual } \\
\text { Fuel Oil } \\
\text { (1000 bbls) }\end{array}$ & $\begin{array}{c}\text { Distillate } \\
\text { Fuel Oil } \\
\text { (1000 bbls })\end{array}$ & $\begin{array}{c}\text { Natural } \\
\text { Gas }^{c} \\
\text { (billion } \\
\text { cu ft) } \\
\end{array}$ & $\begin{array}{c}\text { LPG } \\
\text { (1000 bbls) }\end{array}$ & $\begin{array}{c}\text { Coal } \\
(1000 \text { short } \\
\text { tons })\end{array}$ & $\begin{array}{c}\text { Coke and } \\
\text { Breeze } \\
\left(\begin{array}{c}1000 \text { short } \\
\text { tons })\end{array}\right.\end{array}$ & $\begin{array}{c}\text { Other } \\
\text { (trillion Btu) }\end{array}$ & $\begin{array}{c}\text { RSE } \\
\text { Row } \\
\text { Factors }\end{array}$ \\
\hline & \multicolumn{9}{|c|}{ Midwest Census Region } & \\
\hline RSE Column Factors: & 0.5 & 0.5 & 1.7 & 1.6 & 0.6 & 1.5 & 0.9 & 1.3 & 1.3 & \\
\hline \multicolumn{11}{|l|}{$\begin{array}{l}\text { Value of Shipments and Receipts } \\
\text { (million dollars) }\end{array}$} \\
\hline Under $20 \ldots \ldots \ldots \ldots \ldots \ldots$ & W & 31,500 & $\mathbf{Q}$ & $W$ & 221 & 957 & 1,030 & 169 & $w$ & 14.7 \\
\hline $20-49 \ldots \ldots \ldots \ldots \ldots \ldots$ & 445 & 28,358 & 511 & W & 237 & 819 & W & W & 29 & 10.0 \\
\hline $50-99 \ldots \ldots \ldots \ldots \ldots \ldots$ & W & 25,927 & 325 & 372 & $w$ & 351 & $W$ & 179 & 36 & 7.8 \\
\hline $100-249 \ldots \ldots \ldots \ldots \ldots \ldots \ldots$ & 466 & 29,584 & 852 & 338 & 200 & 4,753 & w & W & W & 5.4 \\
\hline $250-499 \ldots \ldots \ldots \ldots \ldots \ldots \ldots$ & W & 26,404 & W & 257 & $w$ & $W$ & $\mathbf{W}$ & w & 21 & 5.1 \\
\hline 500 and Over $\ldots \ldots \ldots \ldots \ldots$ & 1,050 & 56,635 & W & 962 & $w$ & 155 & 13,370 & 3,607 & 25 & 4.8 \\
\hline Total $\ldots \ldots \ldots \ldots \ldots \ldots \ldots$ & $W$ & 198,408 & W & 4,555 & 1,407 & $W$ & 28,074 & 5,135 & 174 & 4.8 \\
\hline \multicolumn{11}{|l|}{ Employment Size } \\
\hline Under $50 \ldots \ldots \ldots \ldots \ldots$ & $w$ & 12,049 & $\mathbf{Q}$ & 1,487 & 120 & $w$ & 172 & $w$ & 4 & 20.0 \\
\hline $50-99 \ldots \ldots \ldots \ldots \ldots \ldots \ldots \ldots \ldots \ldots \ldots \ldots$ & $w$ & 14,492 & $\mathbf{Q}$ & 552 & 116 & 439 & 973 & w & $w$ & 17.6 \\
\hline $100-249 \ldots \ldots \ldots \ldots \ldots \ldots \ldots$ & 582 & 34,762 & 529 & 645 & 282 & 874 & $W$ & 151 & W & 8.7 \\
\hline $250-499 \ldots \ldots \ldots \ldots \ldots \ldots$ & W & 30,592 & 491 & 344 & W & W & W & 237 & 32 & 6.2 \\
\hline $500-999 \ldots \ldots \ldots \ldots \ldots \ldots \ldots$ & $\mathbf{W}$ & 29,448 & 453 & 334 & 197 & $w$ & 3,254 & $w$ & 23 & 6.0 \\
\hline 1,000 and Over $\ldots \ldots \ldots \ldots$ & 1,247 & 77,065 & W & 1,194 & $W$ & $w$ & 13,870 & $w$ & 45 & 4.3 \\
\hline \multirow[t]{2}{*}{ Total $\ldots \ldots \ldots \ldots \ldots \ldots \ldots$} & W & 198,408 & W & 4,555 & 1,407 & w & 28,074 & 5,135 & 174 & 4.8 \\
\hline & \multicolumn{10}{|c|}{ South Census Region } \\
\hline RSE Column Factors: & 0.6 & 0.5 & 1.8 & 1.2 & 0.9 & 1.0 & 0.9 & 1.7 & 1.2 & \\
\hline \multicolumn{11}{|l|}{$\begin{array}{l}\text { Value of Shipments and Receipts } \\
\text { (million dollars) }\end{array}$} \\
\hline Under $20 \ldots \ldots \ldots \ldots \ldots$ & $w$ & 40,701 & W & W & $W$ & W & 1,160 & $w$ & 33 & 13.6 \\
\hline $20-49 \ldots \ldots \ldots \ldots \ldots \ldots$ & 678 & 49,075 & 7,221 & W & 285 & 1,441 & $W$ & 323 & 58 & 10.4 \\
\hline $50-99 \ldots \ldots \ldots \ldots \ldots \ldots$ & $w$ & 40,415 & 3,782 & 825 & 280 & $W$ & 3,038 & 190 & W & 9.1 \\
\hline $100-249 \ldots \ldots \ldots \ldots \ldots \ldots \ldots$ & W & 65,401 & 6,606 & 793 & 612 & W & $w$ & 148 & $w$ & 5.7 \\
\hline $250-499 \ldots \ldots \ldots \ldots \ldots \ldots$ & W & 52,982 & 6,432 & 895 & 675 & $w$ & $w$ & W & 124 & 5.5 \\
\hline 500 and Over . . . . . . . . . . & 2,555 & 47,381 & 3,128 & 1,227 & $W$ & 212,870 & 5,796 & W & 220 & 4.2 \\
\hline Total $\ldots \ldots \ldots \ldots \ldots \ldots$ & $w$ & 295,955 & W & 8,546 & 3,217 & $w$ & 27,864 & 2,911 & 628 & 4.0 \\
\hline \multicolumn{11}{|l|}{ Employment Size } \\
\hline Under $50 \ldots \ldots$ & W & 15,891 & $\mathbf{Q}$ & W & 107 & $w$ & $\mathbf{Q}$ & 5 & 10 & 16.9 \\
\hline $50-99 \ldots \ldots \ldots \ldots \ldots \ldots$ & W & 17,652 & 7,201 & w & 190 & W & 479 & $w$ & 98 & 16.0 \\
\hline$\ldots \ldots \ldots \ldots$ & W & 56,019 & 3,898 & 1,630 & 543 & W & $w$ & w & 83 & 8.6 \\
\hline$\ldots \ldots \ldots \ldots \ldots$ & $w$ & 62,432 & 4,736 & 1,543 & 587 & w & $W$ & 235 & 157 & 5.5 \\
\hline$\ldots \ldots \ldots \ldots \ldots$ & W & 70,686 & 4,530 & 1,116 & 664 & w & $\mathbf{W}$ & 150 & 118 & 4.9 \\
\hline 1,000 and Over $\ldots$ & 2,488 & 73,275 & 7,897 & 1,499 & 1,125 & 151,240 & 12,168 & 2,124 & 162 & 4.5 \\
\hline Total $\ldots \ldots \ldots \ldots \ldots \ldots \ldots$ & W & 295,955 & $w$ & 8,546 & 3,217 & W & 27,864 & 2,911 & 628 & 4.0 \\
\hline
\end{tabular}

See footnotes at end of table. 
Table A26. Total Quantity of Purchased Energy Sources by Census Region and Economic Characteristics of the Establishment, 1991 (Continued) (Estimates in Btu or Physical Units)

\begin{tabular}{|c|c|c|c|c|c|c|c|c|c|c|}
\hline $\begin{array}{c}\text { Economic } \\
\text { Characteristics }^{a}\end{array}$ & $\begin{array}{c}\text { Total } \\
\text { (trillion Btu) }\end{array}$ & $\begin{array}{c}\text { Electricity } \\
\text { (million } \\
\text { kWh) }\end{array}$ & $\begin{array}{c}\text { Residual } \\
\text { Fuel Oil } \\
\text { (1000 bbls) }\end{array}$ & $\begin{array}{c}\text { Distillate } \\
\text { Fuel Oib } \\
\text { (1000 bbls) }\end{array}$ & $\begin{array}{l}\text { Natural } \\
\text { Gas }^{c} \\
\text { (billion } \\
\text { cu ft) }\end{array}$ & $\begin{array}{c}\text { LPG } \\
\text { (1000 bbls) }\end{array}$ & $\begin{array}{c}\text { Coal } \\
\text { (1000 short } \\
\text { tons) }\end{array}$ & $\begin{array}{c}\text { Coke and } \\
\text { Breeze } \\
\text { (1000 short } \\
\text { tons) }\end{array}$ & $\begin{array}{c}\text { Other } \\
\text { (trillion Btu) }\end{array}$ & $\begin{array}{c}\text { RSE } \\
\text { Row } \\
\text { Factors }\end{array}$ \\
\hline & \multicolumn{9}{|c|}{ West Census Region } & \\
\hline RSE Column Factors: & 0.7 & 0.4 & 1.2 & 1.3 & 0.7 & 1.3 & 1.5 & 1.2 & 1.3 & \\
\hline \multicolumn{11}{|l|}{$\begin{array}{l}\text { Value of Shipments and Recelpts } \\
\text { (million dollars) }\end{array}$} \\
\hline 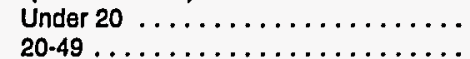 & $\begin{array}{r}W \\
229\end{array}$ & $\begin{array}{l}16,344 \\
14,453\end{array}$ & $\begin{array}{l}492 \\
712\end{array}$ & $\begin{array}{r}1,462 \\
W\end{array}$ & $\begin{array}{l}W \\
W\end{array}$ & $\begin{array}{l}556 \\
403\end{array}$ & $\begin{array}{r}295 \\
W\end{array}$ & $\begin{array}{r}23 \\
118\end{array}$ & $\begin{array}{l}Q \\
41\end{array}$ & $\begin{array}{l}15.5 \\
12.3\end{array}$ \\
\hline $50.99 \ldots \ldots \ldots \ldots \ldots \ldots \ldots \ldots$ & $W$ & 15,058 & 1,117 & 479 & 107 & 268 & 818 & 34 & $w$ & 9.0 \\
\hline $100-249 \ldots \ldots \ldots \ldots \ldots \ldots \ldots$ & 425 & 34,997 & 608 & 359 & 193 & 269 & $W$ & $w$ & 71 & 8.3 \\
\hline $250-499 \ldots \ldots \ldots \ldots \ldots \ldots \ldots$ & 217 & 20,973 & $w$ & W & 86 & 51 & W & $W$ & 20 & 5.0 \\
\hline 500 and Over $\ldots \ldots \ldots \ldots \ldots \ldots$ & 162 & 14,896 & $W$ & $W$ & 87 & 70 & W & 0 & 9 & 6.2 \\
\hline Total $\ldots \ldots \ldots \ldots \ldots \ldots \ldots \ldots$ & 1,466 & 116,720 & 3,189 & 3,491 & 644 & 1,616 & W & W & 213 & 6.4 \\
\hline \multicolumn{11}{|l|}{ Employment Size } \\
\hline Under $50 \ldots \ldots \ldots \ldots \ldots$ & W & 7,312 & $\mathbf{Q}$ & W & W & 222 & $W$ & $w$ & $\mathbf{Q}$ & 12.7 \\
\hline $50-99 \ldots \ldots \ldots \ldots \ldots \ldots \ldots$ & 127 & 8,251 & 390 & 567 & 53 & 245 & 673 & 45 & 21 & 17.6 \\
\hline $100-249 \ldots \ldots \ldots \ldots \ldots \ldots \ldots$ & $W$ & 21,158 & 759 & 909 & 211 & $W$ & 1,692 & W & 43 & 11.9 \\
\hline $250-499 \ldots \ldots \ldots \ldots \ldots \ldots \ldots$ & 247 & 20,687 & 1,377 & 557 & W & 309 & W & $w$ & 31 & 8.1 \\
\hline $500-999 \ldots \ldots \ldots \ldots \ldots \ldots$ & 360 & 32,418 & 430 & W & 163 & 155 & $W$ & $w$ & 62 & 6.8 \\
\hline 1,000 and Over $\ldots \ldots \ldots \ldots \ldots$ & W & 26,895 & 215 & 270 & 72 & $W$ & W & $W$ & 19 & 6.0 \\
\hline Total $\ldots \ldots \ldots \ldots$ & 1,466 & 116,720 & 3,189 & 3,491 & 644 & 1,616 & $w$ & $w$ & 213 & 6.4 \\
\hline
\end{tabular}

- Value of Shipments and Receipts and Employment Size were supplied by the Bureau of the Census. See Appendix B.

- "Distillate Fuel Oil" Inciudes Nos. 1, 2, and 4 fuel oils and Nos. 1, 2, and 4 diesel fuels.

- "Natural Gas" Includes natural gas obtalned from utilities, transmission pipelines, and any other supplier(s) such as brokers and producers.

"Other" energy sources include such combustible energy sources as wood waste, hydrogen, or waste oils and tars.

$W=$ Withheld to avold disclosing data for individual establishments. Data are included in higher level totals.

$\mathrm{Q}=$ Withheld because Relative Standard Error is greater than 50 percent. Data are included in higher level totals.

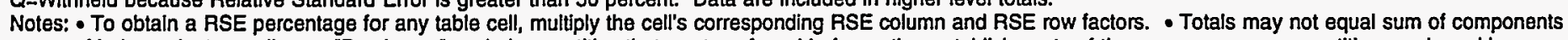

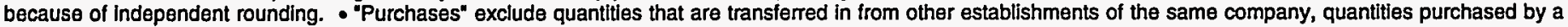
central purchasing offlce offsite, and quantities for which payment is made in-kind.

Source: Energy Information Administration, Office of Energy Markets and End Use, Energy End Use and Integrated Statistics Division, Form ElA-846, "1991 Manufacturing Energy Consumption Survey," and Bureau of the Census, Industry Division, data files for the "1991 Annual Survey of Manufactures." 
Table A27. Quantity of Purchased Electricity, Steam, and Natural Gas by Type of Supplier, Census Region, and Economic Characteristics of the Establishment, 1991 (Estimates in Btu or Physical Units)

\begin{tabular}{|c|c|c|c|c|c|c|c|c|}
\hline \multirow[b]{2}{*}{$\begin{array}{c}\text { Economic } \\
\text { Characteristics }\end{array}$} & \multicolumn{2}{|c|}{$\begin{array}{c}\text { Electricity } \\
\text { (Million kWh) }\end{array}$} & \multicolumn{2}{|c|}{$\begin{array}{c}\text { Steam } \\
\text { (Billion Btu) }\end{array}$} & \multicolumn{3}{|c|}{$\begin{array}{l}\text { Natural Gas } \\
\text { (Billion cu } \mathrm{ft} \text { ) }\end{array}$} & \multirow[b]{2}{*}{$\begin{array}{c}\text { RSE } \\
\text { Row } \\
\text { Factors }\end{array}$} \\
\hline & $\begin{array}{c}\text { Utility } \\
\text { Supplier }^{b}\end{array}$ & $\begin{array}{l}\text { Nonutility } \\
\text { Supplier }\end{array}$ & $\begin{array}{c}\text { Utility } \\
\text { Supplier }^{b}\end{array}$ & $\begin{array}{l}\text { Nonutility } \\
\text { Supplier }^{c}\end{array}$ & $\begin{array}{c}\text { Utility } \\
\text { Supplier }\end{array}$ & $\begin{array}{c}\text { Trans- } \\
\text { mission } \\
\text { Plpelines }\end{array}$ & $\begin{array}{c}\text { Other } \\
\text { Supplier }\end{array}$ & \\
\hline & \multicolumn{7}{|c|}{ Total United States } & \\
\hline RSE Column Factors: & 0.4 & 2.4 & 1.3 & 1.4 & 0.6 & 1.1 & 0.9 & \\
\hline \multicolumn{9}{|l|}{$\begin{array}{l}\text { Value of Shipments and Receipts } \\
\text { (million dollars) }\end{array}$} \\
\hline 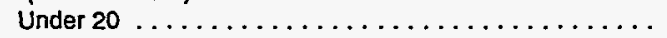 & 107,184 & 326 & 2,967 & 4,271 & 404 & 73 & 109 & 10.0 \\
\hline 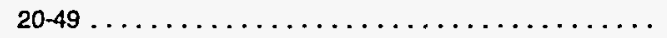 & 105,509 & 1,157 & 5,365 & 15,176 & 343 & 121 & 211 & 9.3 \\
\hline 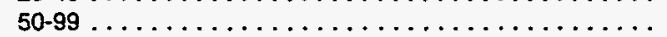 & 91,832 & 766 & 5,216 & 12,848 & 298 & 110 & $w$ & 7.8 \\
\hline 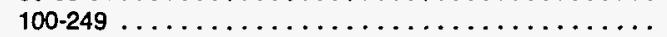 & 145,652 & 608 & 13,577 & 43,332 & 481 & 279 & 332 & 8.3 \\
\hline $250-499 \ldots \ldots \ldots \ldots \ldots \ldots \ldots \ldots \ldots$ & W & W & 20,676 & 19,144 & 285 & 435 & $W$ & 5.8 \\
\hline 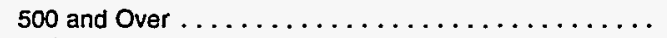 & $w$ & w & 36,636 & 39,262 & 306 & 811 & 587 & 3.8 \\
\hline Total $\ldots \ldots \ldots \ldots \ldots \ldots \ldots \ldots \ldots \ldots$ & 681,538 & 16,015 & 84,438 & 134,033 & 2,117 & 1,829 & 1,767 & 4.0 \\
\hline \multicolumn{9}{|l|}{ Employment Size } \\
\hline 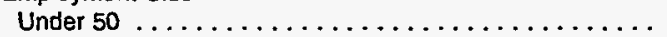 & $\mathbf{w}$ & 224 & 2,681 & 1,544 & 173 & 42 & $w$ & 15.0 \\
\hline 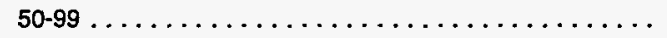 & $W$ & 448 & 2,869 & 11,325 & 221 & 58 & 121 & 11.2 \\
\hline $100-249 \ldots \ldots \ldots \ldots \ldots \ldots \ldots \ldots \ldots \ldots$ & 126,111 & 1,028 & 8,288 & 33,959 & 548 & 264 & 299 & 8.7 \\
\hline 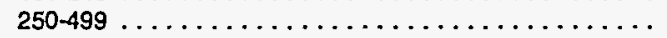 & 127,156 & 599 & 22,516 & 13,645 & 374 & 368 & 299 & 6.2 \\
\hline 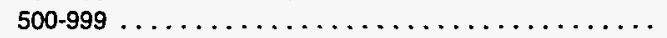 & 152,145 & 1,635 & 20,643 & 45,129 & 404 & 344 & 361 & 4.9 \\
\hline 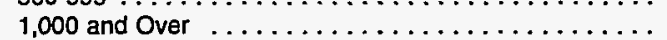 & 186,756 & 12,081 & 27,440 & 28,431 & 397 & 753 & $W$ & 4.6 \\
\hline Total $\ldots \ldots \ldots \ldots \ldots \ldots \ldots \ldots \ldots \ldots$ & 681,538 & 16,015 & 84,438 & 134,033 & 2,117 & 1,829 & 1,767 & 4.0 \\
\hline & \multicolumn{8}{|c|}{ Northeast Census Region } \\
\hline RSE Column Factors: & 0.4 & 2.1 & 1.7 & 1.4 & 0.6 & 1.1 & 0.7 & \\
\hline \multicolumn{9}{|l|}{$\begin{array}{l}\text { Value of Shipments and Receipts } \\
\text { (million dollars) }\end{array}$} \\
\hline Under $20 \ldots \ldots \ldots \ldots \ldots$ & $w$ & 128 & 1,387 & 1,766 & 62 & $W$ & $w$ & 18.9 \\
\hline 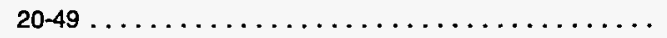 & 14,649 & $\mathbf{Q}$ & Q & 2,657 & 43 & $w$ & $W$ & 12.3 \\
\hline 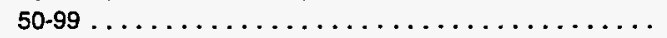 & 11,148 & 50 & 1,252 & W & 35 & 7 & 29 & 11.2 \\
\hline $100-249 \ldots \ldots \ldots \ldots \ldots \ldots \ldots \ldots \ldots \ldots$ & W & W & 1,183 & 5,793 & 42 & 15 & 29 & 7.0 \\
\hline 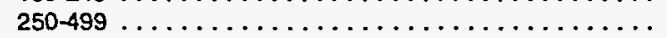 & W & 0 & 505 & 2,485 & 24 & 1 & 24 & 11.4 \\
\hline 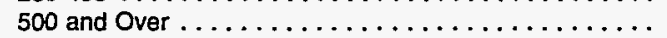 & $w$ & $w$ & W & $W$ & 32 & 9 & 37 & 8.0 \\
\hline Total $\ldots \ldots \ldots \ldots \ldots \ldots \ldots \ldots \ldots \ldots$ & 85,769 & 702 & 17,235 & 14,806 & 238 & 52 & 155 & 5.8 \\
\hline \multicolumn{9}{|l|}{ Employment Size } \\
\hline Under $50 \ldots \ldots \ldots \ldots \ldots \ldots \ldots \ldots \ldots$ & 7,349 & 79 & 0 & $\mathbf{Q}$ & 28 & 4 & 6 & 25.7 \\
\hline 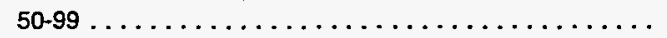 & $W$ & $w$ & $\mathbf{Q}$ & 2,539 & 24 & 5 & 10 & 18.5 \\
\hline 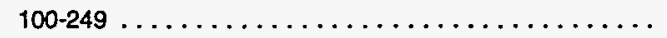 & 15,175 & 26 & 1,484 & 1,969 & 46 & 10 & 19 & 14.5 \\
\hline 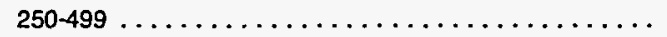 & 13,949 & $Q$ & $W$ & 424 & 44 & 14 & 27 & 10.0 \\
\hline 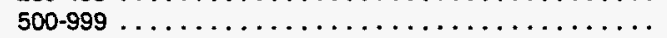 & W & 192 & w & 7,668 & 31 & 11 & 43 & 5.9 \\
\hline 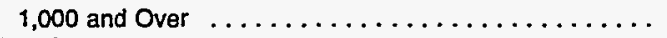 & $W$ & W & W & W & 64 & 8 & 51 & 7.9 \\
\hline Total $\ldots \ldots \ldots \ldots \ldots \ldots \ldots \ldots \ldots \ldots$ & 85,769 & 702 & 17,235 & 14,806 & 238 & 52 & 155 & 5.8 \\
\hline
\end{tabular}

See footnotes at end of table. 
Table A27. Quantity of Purchased Electricity, Steam, and Natural Gas by Type of Supplier, Census Region, and Economic Characteristics of the Establishment, 1991 (Continued) (Estimates in Btu or Physical Units)

\begin{tabular}{|c|c|c|c|c|c|c|c|c|}
\hline \multirow[b]{2}{*}{$\begin{array}{c}\text { Economic } \\
\text { Characteristics" }\end{array}$} & \multicolumn{2}{|c|}{$\begin{array}{c}\text { Electricity } \\
\text { (Million kWh) }\end{array}$} & \multicolumn{2}{|c|}{$\begin{array}{c}\text { Steam } \\
\text { (Billion Btu) }\end{array}$} & \multicolumn{3}{|c|}{$\begin{array}{l}\text { Natural Gas } \\
\text { (Billion cu tt) }\end{array}$} & \multirow[b]{2}{*}{$\begin{array}{c}\text { RSE } \\
\text { Row } \\
\text { Factors }\end{array}$} \\
\hline & $\begin{array}{l}\text { Utility } \\
\text { Suppllerb }^{b}\end{array}$ & $\begin{array}{l}\text { Nonutility } \\
\text { Supplier }\end{array}$ & $\begin{array}{l}\text { Utility } \\
\text { Supplier }\end{array}$ & $\begin{array}{l}\text { Nonutility } \\
\text { Supplier }\end{array}$ & $\begin{array}{l}\text { Utility } \\
\text { Supplier }\end{array}$ & $\begin{array}{c}\text { Trans- } \\
\text { mission } \\
\text { Pipelines }\end{array}$ & $\begin{array}{l}\text { Other } \\
\text { Supplier }\end{array}$ & \\
\hline & \multicolumn{7}{|c|}{ Midwest Census Region } & \\
\hline RSE Column Factors: & 0.4 & 2.5 & 1.3 & 2.0 & 0.6 & 0.9 & 0.7 & \\
\hline \multicolumn{9}{|l|}{$\begin{array}{l}\text { Valus of Shipments and Receipts } \\
\text { (million dollars) }\end{array}$} \\
\hline Under $20 \ldots \ldots \ldots \ldots \ldots \ldots \ldots \ldots \ldots$ & W & 161 & $\mathbf{Q}$ & 908 & 145 & 28 & 49 & 16.4 \\
\hline 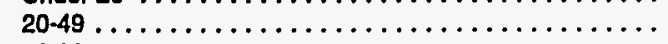 & 28,279 & 79 & 2,403 & 2,187 & 113 & 38 & 85 & 15.7 \\
\hline $50.99 \ldots \ldots \ldots \ldots \ldots \ldots \ldots \ldots \ldots \ldots \ldots$ & 25,687 & 239 & 3,476 & $w$ & 70 & 49 & $\mathbf{W}$ & 12.3 \\
\hline 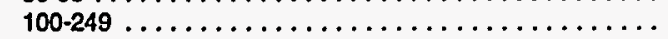 & 29,484 & 100 & 10,285 & 6,212 & 68 & 40 & 92 & 7.7 \\
\hline 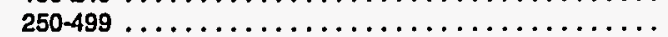 & W & 0 & 4,549 & W & W & W & $\mathbf{W}$ & 8.7 \\
\hline 500 and Over $\ldots \ldots \ldots \ldots \ldots \ldots \ldots \ldots \ldots$ & 55,498 & 1,138 & W & $w$ & W & W & 230 & 5.7 \\
\hline Total $\ldots \ldots \ldots \ldots \ldots \ldots \ldots \ldots \ldots \ldots \ldots$ & 196,691 & 1,717 & 26,217 & 19,762 & 520 & 257 & 629 & 5.1 \\
\hline \multicolumn{9}{|l|}{ Employment Size } \\
\hline Under $50 \ldots \ldots \ldots \ldots \ldots \ldots \ldots \ldots \ldots \ldots$ & W & $\mathbf{Q}$ & $\mathbf{Q}$ & $\mathbf{Q}$ & 69 & 26 & 25 & 22.6 \\
\hline $50-99 \ldots \ldots \ldots \ldots \ldots \ldots \ldots \ldots \ldots \ldots \ldots \ldots$ & 14,401 & 91 & $w$ & 2,061 & 68 & 15 & 33 & 15.8 \\
\hline $100-249 \ldots \ldots \ldots \ldots \ldots \ldots \ldots \ldots \ldots \ldots \ldots \ldots \ldots$ & 34,686 & 76 & 5,403 & 1,864 & 134 & 54 & 94 & 11.8 \\
\hline 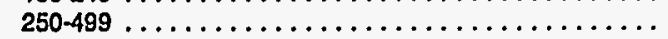 & 30,464 & 129 & 5,386 & 1,381 & 94 & W & 114 & 8.1 \\
\hline $500-999 \ldots \ldots \ldots \ldots \ldots \ldots \ldots \ldots \ldots \ldots \ldots$ & 29,314 & $\mathbf{Q}$ & 8,388 & W & 63 & 43 & 92 & 6.4 \\
\hline 1,000 and Over $\ldots \ldots \ldots \ldots \ldots \ldots \ldots \ldots \ldots$ & W & 1,177 & W & 10,558 & 93 & W & 270 & 5.6 \\
\hline \multirow[t]{2}{*}{ 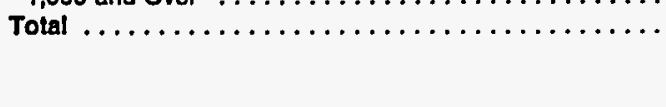 } & 196,691 & 1,717 & 26,217 & 19,762 & 520 & 257 & 629 & 5.1 \\
\hline & \multicolumn{7}{|c|}{ South Census Region } & \\
\hline RSE Column Factors: & 0.3 & 2.2 & 1.5 & 0.9 & 0.7 & 1.1 & 1.3 & \\
\hline \multicolumn{9}{|l|}{$\begin{array}{l}\text { Value of Shlpments and Recelpts } \\
\text { (million dollars) }\end{array}$} \\
\hline 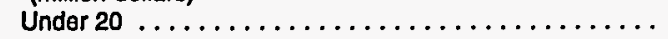 & 40,673 & 29 & 992 & 741 & 132 & W & 28 & 13.9 \\
\hline 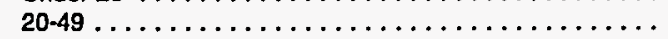 & 48,225 & 850 & 1,068 & 7,069 & 146 & 61 & 79 & 12.2 \\
\hline 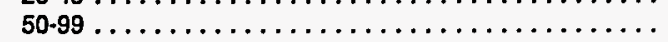 & 40,305 & 110 & 91 & 5,834 & 148 & 48 & 84 & 11.4 \\
\hline 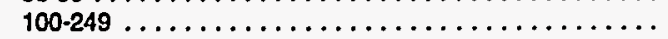 & W & W & 2,109 & 22,225 & 262 & 204 & 145 & 10.7 \\
\hline $250-499 \ldots \ldots \ldots \ldots \ldots \ldots \ldots \ldots \ldots$ & W & $\mathbf{W}$ & 15,623 & 12,073 & W & W & 113 & 7.4 \\
\hline 500 and $0 v e r \ldots \ldots \ldots \ldots \ldots \ldots \ldots \ldots \ldots$ & $W$ & $\mathbf{W}$ & 20,201 & 23,741 & $W$ & $w$ & 287 & 4.9 \\
\hline Total $\ldots \ldots \ldots \ldots \ldots \ldots \ldots \ldots \ldots \ldots$ & W & W & 40,084 & 71,683 & 1,044 & 1,437 & 736 & 5.1 \\
\hline \multicolumn{9}{|l|}{ Employment Size } \\
\hline$\ldots \ldots \ldots \ldots \ldots \ldots \ldots$ & W & 33 & 2,373 & 439 & 49 & 9 & 49 & 15.3 \\
\hline $50-99 \ldots \ldots \ldots \ldots \ldots \ldots \ldots \ldots \ldots \ldots$ & 17,442 & 210 & W & 4,481 & 96 & 34 & 60 & 12.1 \\
\hline 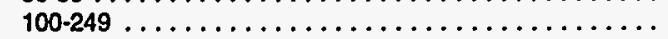 & W & W & 897 & 26,211 & 241 & 186 & 116 & 13.8 \\
\hline 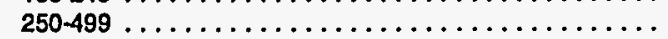 & 62,363 & 69 & W & 3,760 & 191 & 282 & 114 & 7.8 \\
\hline 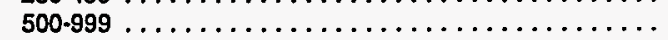 & 70,167 & 519 & W & 25,979 & 255 & 259 & 150 & 5.2 \\
\hline 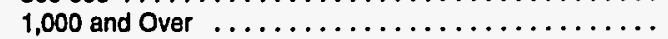 & W & $W$ & 16,875 & 10,812 & 211 & 667 & 247 & 5.1 \\
\hline 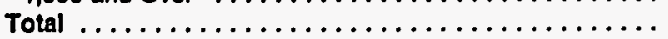 & $W$ & W & 40,084 & 71,683 & 1,044 & 1,437 & 736 & 5.1 \\
\hline
\end{tabular}

See footnotes at end of table. 


\section{Table A27. Quantity of Purchased Electricity, Steam, and Natural Gas by Type of Supplier, Census Region, and Economic Characteristics of the Establishment, 1991 (Continued) (Estimates in Btu or Physical Units)}

\begin{tabular}{|c|c|c|c|c|c|c|c|c|}
\hline \multirow[b]{2}{*}{$\begin{array}{c}\text { Economic } \\
\text { Characteristics" }\end{array}$} & \multicolumn{2}{|c|}{$\begin{array}{c}\text { Electricity } \\
\text { (Million kWh) }\end{array}$} & \multicolumn{2}{|c|}{$\begin{array}{c}\text { Steam } \\
\text { (Billion Btu) }\end{array}$} & \multicolumn{3}{|c|}{$\begin{array}{l}\text { Natural Gas } \\
\text { (Billion cu ft) }\end{array}$} & \multirow[b]{2}{*}{$\begin{array}{c}\text { RSE } \\
\text { Row } \\
\text { Factors }\end{array}$} \\
\hline & $\begin{array}{c}\text { Utility } \\
\text { Supplier }\end{array}$ & $\begin{array}{l}\text { Nonutility } \\
\text { Supplief }\end{array}$ & $\begin{array}{c}\text { Utility } \\
\text { Supplie? }\end{array}$ & $\begin{array}{l}\text { Nonutility } \\
\text { Supplier }\end{array}$ & $\begin{array}{c}\text { Utility } \\
\text { Supplier }\end{array}$ & $\begin{array}{c}\text { Trans- } \\
\text { mission } \\
\text { Plpelines }\end{array}$ & $\begin{array}{l}\text { Other } \\
\text { Suppller }\end{array}$ & \\
\hline & \multicolumn{7}{|c|}{ West Census Region } & \\
\hline RSE Column Factors: & 0.3 & 1.6 & 3.5 & 1.3 & 0.7 & 0.9 & 0.6 & \\
\hline \multicolumn{9}{|l|}{$\begin{array}{l}\text { Value of Shipments and Receipts } \\
\text { (million dollars) }\end{array}$} \\
\hline 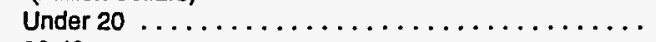 & 16,335 & 9 & 0 & 855 & 66 & $w$ & $w$ & 20.0 \\
\hline 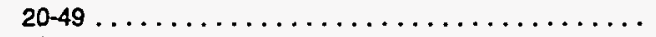 & 14,356 & $Q$ & 505 & 3,262 & 42 & W & $\mathbf{W}$ & 18.4 \\
\hline 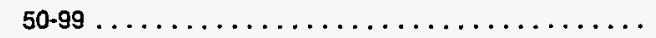 & 14,691 & 367 & $\mathbf{Q}$ & 4,218 & 44 & 6 & 57 & 13.6 \\
\hline $100-249 \ldots \ldots \ldots \ldots \ldots \ldots \ldots \ldots \ldots \ldots \ldots \ldots$ & 34,715 & Q & 1 & $\mathbf{Q}$ & 108 & 19 & 65 & 14.7 \\
\hline 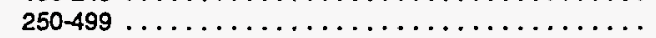 & 20,922 & 50 & 0 & $\bar{W}$ & 24 & 14 & 48 & 8.8 \\
\hline 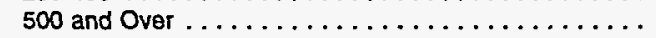 & 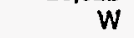 & W & 0 & 7,052 & 31 & 22 & 34 & 5.0 \\
\hline Total $\ldots \ldots \ldots \ldots \ldots \ldots \ldots \ldots \ldots \ldots \ldots$ & W & W & 903 & 27,783 & 315 & 83 & 246 & 8.8 \\
\hline \multicolumn{9}{|l|}{ Employment Size } \\
\hline 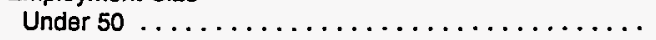 & 7,310 & 2 & 0 & $w$ & 28 & 3 & $w$ & 22.8 \\
\hline $50-99 \ldots \ldots \ldots \ldots \ldots \ldots \ldots \ldots \ldots \ldots \ldots$ & $W$ & $\vec{w}$ & 0 & 2,244 & 32 & 4 & 17 & 16.9 \\
\hline $100-249 \ldots \ldots \ldots \ldots \ldots \ldots \ldots \ldots \ldots \ldots$ & W & W & 505 & 3.915 & 127 & 15 & 70 & 18.7 \\
\hline 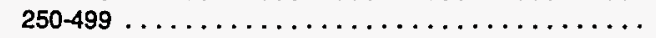 & 20,380 & 307 & $\mathbf{Q}$ & 8,080 & 45 & W & 43 & 11.0 \\
\hline 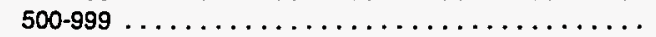 & W & 790 & 24 & $\mathbf{Q}$ & 55 & 31 & 77 & 8.6 \\
\hline 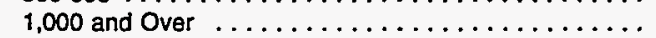 & $W$ & W & 0 & $\vec{W}$ & 29 & $w$ & w & 7.0 \\
\hline$\ldots \ldots \ldots \ldots \ldots$ & $W$ & $w$ & 903 & 27,783 & 315 & 83 & 246 & 8.8 \\
\hline
\end{tabular}

- Value of Shipments and Receipts and Employment Size were supplied by the Bureau of the Census. See Appendix 8.

' A "Utility" is a company that produces and/or delivers electricity and/or natural gas, and is legally obligated to provide service to the public within its franchise area.

' Includes independent power producers, small power producers, and cogenerators not located at the establishment site.

- Other suppliers of natural gas include such sources as brokers and producers.

$W=$ Withheld to avoid disclosing data for individual establishments. Data are included in higher level totals.

$\mathrm{Q}=$ Withheld because Relative Standard Error is greater than 50 percent. Data are included in higher level totals.

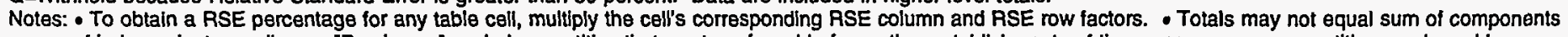

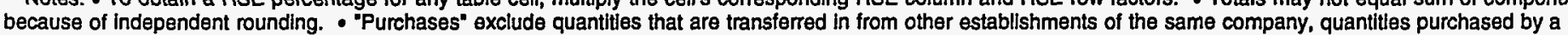
central purchasing office offsite, and quantities for which payment is made in-kind.

Source: Energy Information Administration, Office of Energy Markets and End Use, Energy End Use and Integrated Statistics Division, Form ElA-846, "1991 Manufacturing Energy Consumption Survey," and Bureau of the Census, Industry Division, data files for the "1991 Annual Survey of Manufactures." 
Table A28. Total Expenditures for Purchased Energy Sources by Census Region and Economic Characteristics of the Establishment, 1991 (Estimates in Million Dollars)

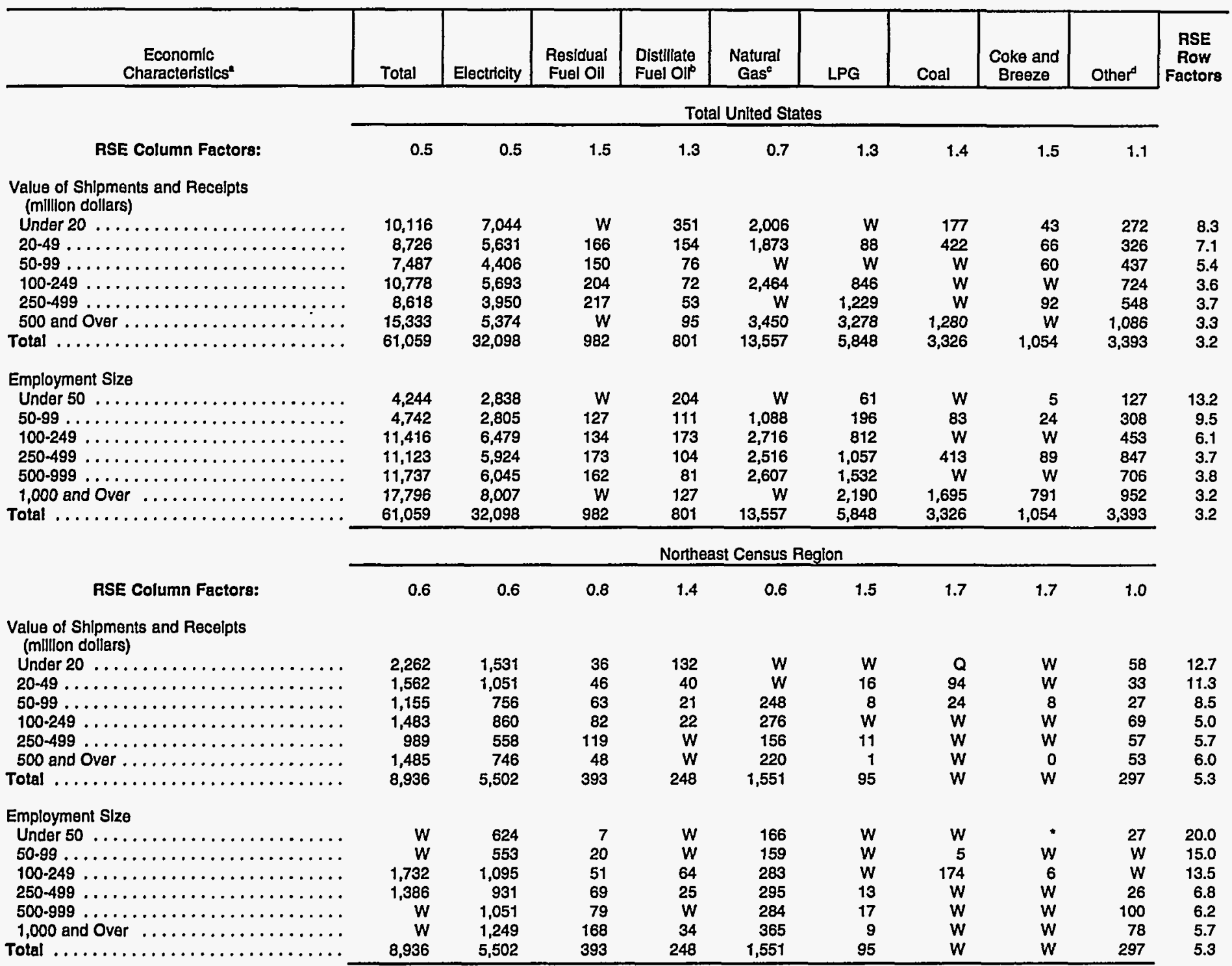

See footnotes at end of table. 
Table A28. Total Expenditures for Purchased Energy Sources by Census Region and Economic Characteristics of the Establishment, 1991 (Continued) (Estimates in Million Dollars)

\begin{tabular}{|c|c|c|c|c|c|c|c|c|c|c|}
\hline $\begin{array}{c}\text { Economic } \\
\text { Characteristics }^{a}\end{array}$ & Total & Electricity & $\begin{array}{c}\text { Residual } \\
\text { Fuel Oll }\end{array}$ & $\begin{array}{l}\text { Distillate } \\
\text { Fuel Oil }\end{array}$ & $\begin{array}{c}\text { Natural } \\
\text { Gas }^{c}\end{array}$ & LPG & Coal & $\begin{array}{c}\text { Coke and } \\
\text { Breeze }\end{array}$ & Other & $\begin{array}{c}\text { RSE } \\
\text { Row } \\
\text { Factore }\end{array}$ \\
\hline & \multicolumn{10}{|c|}{ Midwest Census Region } \\
\hline RSE Column Factors: & 0.5 & 0.5 & 1.7 & 1.7 & 0.6 & 1.4 & 1.0 & 1.4 & 1.1 & \\
\hline \multicolumn{11}{|l|}{$\begin{array}{l}\text { Value of Shipments and Receipts } \\
\text { (million dollars) }\end{array}$} \\
\hline Under $20 \ldots \ldots \ldots \ldots \ldots$ & $W$ & 2,002 & $\mathbf{Q}$ & W & 715 & 30 & 38 & 20 & $w$ & 11.6 \\
\hline 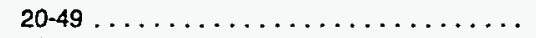 & 2,381 & 1,511 & 7 & $\mathbf{W}$ & 634 & 20 & $\mathbf{W}$ & W & 69 & 9.8 \\
\hline 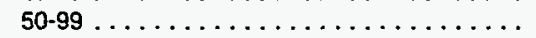 & W & 1,215 & 5 & 13 & $w$ & 10 & $w$ & 24 & 100 & 7.5 \\
\hline $100-249 \ldots \ldots \ldots \ldots \ldots \ldots \ldots$ & 2,258 & 1,313 & 13 & 11 & 533 & 62 & $w$ & $W$ & $w$ & 5.1 \\
\hline $250-499 \ldots \ldots \ldots \ldots \ldots \ldots$ & 1,883 & 1,032 & $W$ & 8 & w & $W$ & $w$ & W & 71 & 4.9 \\
\hline 500 and $O v e r \ldots \ldots \ldots \ldots \ldots$ & 4,121 & 2,062 & W & 31 & $w$ & 4 & 623 & 406 & 98 & 4.7 \\
\hline Total $\ldots \ldots \ldots \ldots \ldots \ldots \ldots \ldots$ & 15,598 & 9,135 & w & 152 & 3,657 & $w$ & 1,161 & 597 & 474 & 4.1 \\
\hline \multicolumn{11}{|l|}{ Employment Size } \\
\hline$\ldots \ldots \ldots \ldots$ & 1,240 & 785 & $\mathbf{Q}$ & 49 & 359 & W & 6 & $w$ & 23 & 18.3 \\
\hline 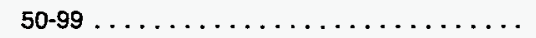 & $W$ & 848 & $\mathbf{Q}$ & 19 & 329 & 13 & 35 & W & $\mathbf{w}$ & 15.7 \\
\hline $100-249 \ldots \ldots \ldots \ldots \ldots \ldots \ldots \ldots$ & 2,941 & 1,826 & 8 & 23 & 754 & 23 & $\mathbf{W}$ & 23 & $\mathbf{w}$ & B.6 \\
\hline 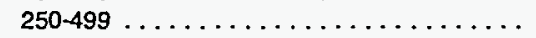 & 2,566 & 1,473 & 8 & 12 & $w$ & $W$ & $\mathbf{W}$ & 33 & 86 & 6.2 \\
\hline 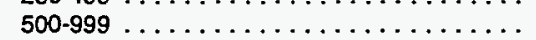 & W & 1,294 & 7 & 10 & 506 & W & 118 & $W$ & 66 & 5.8 \\
\hline 1,000 and Over $\ldots \ldots \ldots \ldots \ldots \ldots$ & 5,389 & 2,909 & $w$ & 39 & $w$ & w & 660 & $W$ & 151 & 4.2 \\
\hline \multirow[t]{2}{*}{ Total $\ldots \ldots \ldots \ldots \ldots \ldots \ldots \ldots$} & 15,598 & 9,135 & $w$ & 152 & 3,657 & $w$ & 1,161 & 597 & 474 & 4.1 \\
\hline & \multicolumn{10}{|c|}{ South Census Region } \\
\hline RSE Column Factors: & 0.5 & 0.5 & 1.8 & 1.4 & 0.8 & 1.1 & 0.9 & 1.7 & 1.1 & \\
\hline \multicolumn{11}{|l|}{$\begin{array}{l}\text { Value of Shipments and Receipts } \\
\text { (million dollars) }\end{array}$} \\
\hline Under $20 \ldots \ldots \ldots \ldots \ldots \ldots$ & 3,259 & 2,313 & $w$ & $w$ & $\mathbf{W}$ & $w$ & 47 & $w$ & 106 & 11.3 \\
\hline 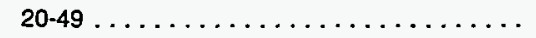 & 3,472 & 2,251 & 99 & $W$ & 710 & 40 & $\mathbf{W}$ & 25 & 129 & 9.5 \\
\hline $50-99 \ldots \ldots \ldots \ldots \ldots \ldots \ldots \ldots$ & 3,155 & 1,746 & 61 & 26 & 673 & $W$ & 147 & 23 & W & 7.8 \\
\hline $100-249 \ldots \ldots \ldots \ldots \ldots \ldots \ldots \ldots$ & 5,180 & 2,416 & 97 & 25 & 1,251 & W & $W$ & 20 & $w$ & 4.9 \\
\hline $250-499 \ldots \ldots \ldots \ldots \ldots \ldots$ & 4,768 & 1,740 & 89 & 27 & 1,239 & $W$ & W & $W$ & 344 & 4.7 \\
\hline 500 and $O v e r . \ldots \ldots \ldots \ldots$ & 8,540 & 1,744 & 45 & 35 & $W$ & 3,271 & 246 & $w$ & 890 & 4.3 \\
\hline Total $\ldots \ldots \ldots \ldots \ldots \ldots \ldots \ldots \ldots$ & 28,373 & 12,210 & $w$ & 276 & 6,564 & w & 1,166 & 315 & 2,030 & 3.9 \\
\hline \multicolumn{11}{|l|}{ Employment Size } \\
\hline Under $50 \ldots \ldots \ldots \ldots \ldots \ldots$ & 1,290 & 858 & $Q$ & $w$ & 272 & $w$ & $\mathbf{Q}$ & 1 & 46 & 13.7 \\
\hline $50-99 \ldots \ldots \ldots \ldots \ldots \ldots \ldots \ldots \ldots$ & 1,867 & 905 & 98 & W & 437 & $w$ & 20 & $w$ & 190 & 13.6 \\
\hline $100-249 \ldots \ldots \ldots \ldots \ldots \ldots$ & 4,873 & 2,466 & 61 & 54 & 1.165 & w & W & w & 190 & 8.3 \\
\hline $250-499 \ldots \ldots \ldots \ldots \ldots \ldots$ & 5,797 & 2,648 & 71 & 47 & 1,257 & w & w & 28 & 634 & 5.3 \\
\hline $500-999 \ldots \ldots \ldots \ldots \ldots \ldots$ & 6,110 & 2,682 & 68 & 35 & 1,344 & W & $w$ & 25 & 336 & 4.4 \\
\hline 1,000 and Over $\ldots \ldots \ldots \ldots \ldots$ & 8,436 & 2,651 & 112 & 45 & 2,088 & 2,168 & 505 & 232 & 634 & 4.4 \\
\hline Total $\ldots \ldots \ldots \ldots \ldots \ldots \ldots \ldots$ & 28,373 & 12,210 & $\bar{W}$ & 276 & 6,564 & $W$ & 1,166 & 315 & 2,030 & 3.9 \\
\hline
\end{tabular}

See footnotes at end of table. 
Table A28. Total Expenditures for Purchased Energy Sources by Census Region and Economic Characteristics of the Establishment, 1991 (Continued) (Estimates in Million Dollars)

\begin{tabular}{|c|c|c|c|c|c|c|c|c|c|c|}
\hline $\begin{array}{c}\text { Economic } \\
\text { Characteristlcs }\end{array}$ & Total & Electricity & $\begin{array}{l}\text { Residual } \\
\text { Fuel Oil }\end{array}$ & $\begin{array}{l}\text { Distillate } \\
\text { Fuel Oil }^{b}\end{array}$ & $\begin{array}{l}\text { Natural } \\
\text { Gas }^{\circ}\end{array}$ & LPG & Coal & $\begin{array}{c}\text { Coke and } \\
\text { Breeze }\end{array}$ & Other & $\begin{array}{c}\text { RSE } \\
\text { Row } \\
\text { Factors }\end{array}$ \\
\hline & \multicolumn{9}{|c|}{ West Census Region } & \\
\hline RSE Column Factors: & 0.5 & 0.6 & 1.3 & 1.4 & 0.7 & 1.4 & 1.6 & 1.2 & 1.0 & \\
\hline \multicolumn{11}{|l|}{$\begin{array}{l}\text { Value of Shipments and Receipts } \\
\text { (million dollars) }\end{array}$} \\
\hline Under $20 \ldots \ldots \ldots \ldots \ldots \ldots$ & W & 1,198 & 9 & 54 & $w$ & 18 & 12 & 2 & $W$ & 12.6 \\
\hline $20-49 \ldots \ldots \ldots \ldots \ldots \ldots \ldots \ldots$ & 1,311 & 818 & 14 & $W$ & W & 12 & $w$ & 16 & 95 & 11.5 \\
\hline $50-99 \ldots \ldots \ldots \ldots \ldots \ldots \ldots \ldots$ & W & 688 & 21 & 16 & 310 & 7 & 26 & 5 & $W$ & 8.9 \\
\hline $100-249 \ldots \ldots \ldots \ldots \ldots \ldots \ldots$ & 1,857 & 1,104 & 12 & 12 & 404 & 7 & $w$ & W & 244 & 7.3 \\
\hline $250-499 \ldots \ldots \ldots \ldots \ldots \ldots \ldots$ & 979 & 620 & W & $\mathbf{W}$ & 204 & 1 & W & $w$ & 75 & 4.8 \\
\hline 500 and $O v e r . \ldots \ldots \ldots \ldots \ldots \ldots$ & 1,187 & 822 & W & W & 292 & 2 & $W$ & 0 & 46 & 6.3 \\
\hline Total $\ldots \ldots \ldots \ldots \ldots \ldots \ldots \ldots$ & 8,151 & 5,251 & 60 & 124 & 1,785 & 48 & W & w & 592 & 5.7 \\
\hline \multicolumn{11}{|l|}{ Employment Size } \\
\hline Under $50 \ldots \ldots \ldots \ldots \ldots \ldots$ & W & 571 & $\mathbf{Q}$ & $w$ & $w$ & 7 & W & $W$ & 31 & 12.4 \\
\hline $50-99 \ldots \ldots \ldots \ldots \ldots \ldots \ldots \ldots$ & 772 & 499 & 7 & 22 & 163 & 8 & 22 & 2 & 48 & 16.0 \\
\hline $100-249 \ldots \ldots \ldots \ldots \ldots \ldots \ldots \ldots$ & 1,871 & 1,091 & 15 & 32 & 513 & $\mathbf{w}$ & 62 & W & 121 & 10.6 \\
\hline $250-499 \ldots \ldots \ldots \ldots \ldots \ldots \ldots$ & 1,374 & 873 & 25 & 20 & $w$ & 8 & $W$ & $\mathbf{W}$ & 100 & 7.7 \\
\hline $500-999 \ldots \ldots \ldots \ldots \ldots \ldots \ldots \ldots$ & 1,762 & 1,018 & 8 & $w$ & 473 & 4 & $w$ & $w$ & 204 & 6.6 \\
\hline 1,000 and Over $\ldots \ldots \ldots \ldots \ldots$ & W & 1,199 & 4 & 9 & 192 & W & $w$ & * & 88 & 5.8 \\
\hline Total $\ldots \ldots \ldots \ldots \ldots \ldots \ldots \ldots$ & 8,151 & 5,251 & 60 & 124 & 1,785 & 48 & $W$ & $w$ & 592 & 5.7 \\
\hline
\end{tabular}

- Value of Shipments and Recelpts and Employment Size were supplied by the Bureau of the Census. See Appendix B.

"Distillate Fuel Oll" includes Nos. 1, 2, and 4 fuel oils and Nos. 1, 2, and 4 diesel fuels.

- "Natural Gas" includes natural gas obtained from utilities, transmission pipelines, and any other supplier(s) such as brokers and producers.

"Other" energy sources include such combustible energy sources as wood waste, hydrogen, or waste oils and tars.

- Estimate less than 0.5. Data are Included in higher level totals.

$W=$ Withheld to avold disclosing data for individual establishments. Data are included in higher lovel totals.

$\mathrm{Q}=$ Withheld because Relative Standard Error is greater than 50 percent. Data are included in higher level totals.

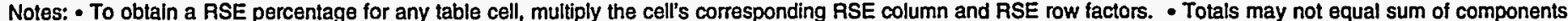

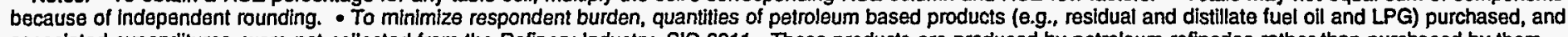

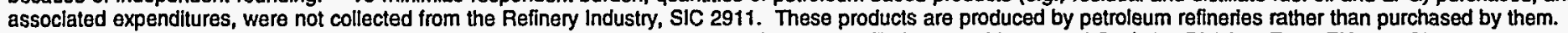

Source: Energy Information Administration, Office of Energy Markets and End Use, Energy End Use and Integrated Statistics Division, Form EIA-846, "1991

Manufacturing Energy Consumption Survey," and Bureau of the Census, Industry Division, data files for the "1991 Annual Survey of Manufactures." 
Table A29. Average Prices of Selected Purchased Energy Sources by Census Region and Economic Characteristics of the Establishment, 1991: Part 1 (Estimates in Dollars per Physical Unit)

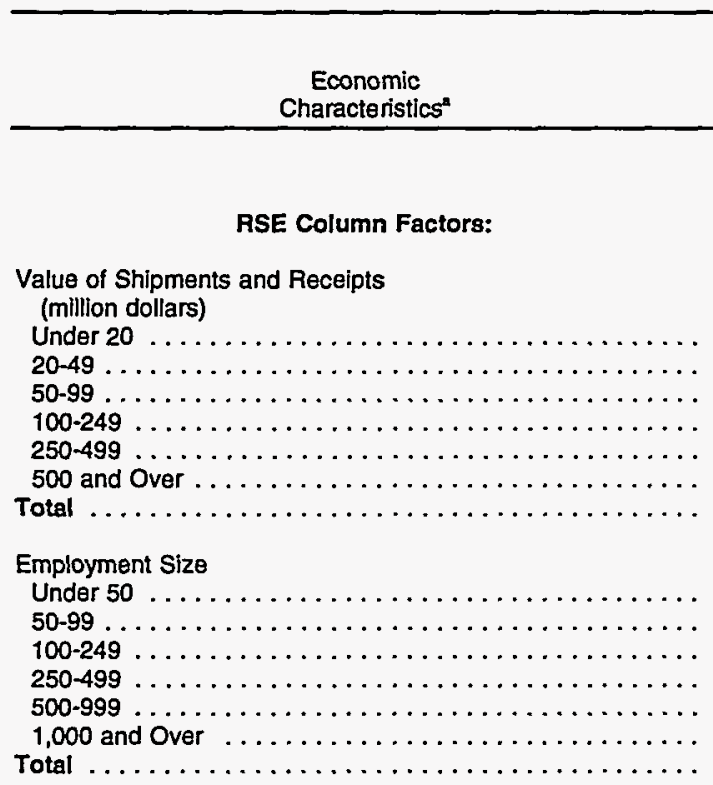

\section{RSE Column Factors:}

\begin{tabular}{c|c|c}
$\begin{array}{c}\text { Electricity } \\
\text { (kWh) }\end{array}$ & $\begin{array}{c}\text { Residual Fuel } \\
\text { Oil } \\
\text { (gallon) }\end{array}$ & D \\
\hline
\end{tabular}

Distillate Fuel
Oil
(gallon)

Natural Gas
$(1000 \mathrm{cu} \mathrm{ft})$

LPG
(gallon)

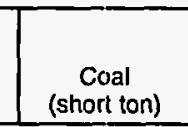

Total United States

\begin{tabular}{|c|c|c|c|c|c|c|}
\hline \multicolumn{6}{|c|}{ Total United States } & \\
\hline 0.7 & 1.2 & 1.1 & 0.8 & 1.2 & 1.0 & \\
\hline 0.066 & 0.404 & 0.813 & 3.422 & 0.705 & 37.024 & 3.4 \\
\hline 0.053 & 0.363 & 0.757 & 2.773 & 0.635 & 39.852 & 3.3 \\
\hline 0.048 & 0.416 & 0.768 & 2.626 & 0.426 & 41.897 & 2.3 \\
\hline 0.039 & 0.381 & 0.784 & 2.257 & 0.470 & 39.363 & 1.5 \\
\hline 0.035 & 0.368 & 0.752 & 2.035 & 0.508 & 41.518 & 1.9 \\
\hline 0.041 & 0.373 & 0.730 & 2.024 & 0.366 & 46.248 & 1.8 \\
\hline 0.046 & 0.380 & 0.780 & 2.373 & 0.413 & 42.305 & 1.6 \\
\hline 0.067 & 0.348 & 0.827 & 3.117 & 0.688 & 41.000 & 4.8 \\
\hline 0.059 & 0.344 & 0.797 & 2.724 & 0.359 & 36.523 & 3.4 \\
\hline 0.051 & 0.409 & 0.788 & 2.444 & 0.687 & 36.870 & 2.7 \\
\hline 0.046 & 0.401 & 0.722 & 2.418 & 0.465 & 41.419 & 2.6 \\
\hline 0.039 & 0.389 & 0.752 & 2.348 & 0.418 & 40.125 & 1.7 \\
\hline 0.040 & 0.375 & 0.754 & 2.107 & 0.343 & 46.036 & 1.4 \\
\hline 0.046 & 0.380 & 0.780 & 2.373 & 0.413 & 42.305 & 1.6 \\
\hline \multicolumn{7}{|c|}{ Northeast Census Region } \\
\hline 0.8 & 0.7 & 1.4 & 0.8 & 1.4 & 1.1 & \\
\hline 0.081 & 0.479 & 0.781 & 4.318 & 0.652 & 34.835 & 3.9 \\
\hline 0.071 & 0.448 & 0.651 & 3.731 & 0.591 & 45.177 & 4.7 \\
\hline 0.068 & 0.446 & 0.738 & 3.493 & 0.586 & 41.890 & 2.6 \\
\hline 0.053 & 0.416 & 0.775 & 3.198 & 0.527 & 41.970 & 1.7 \\
\hline 0.046 & 0.409 & 0.813 & 3.179 & 0.534 & 62.373 & 1.8 \\
\hline 0.057 & 0.472 & 0.765 & 2.836 & 0.706 & 49.040 & 2.0 \\
\hline 0.064 & 0.434 & 0.752 & 3.488 & 0.597 & 45.399 & 2.5 \\
\hline 0.084 & 0.455 & 0.820 & 4.477 & 0.678 & $w$ & 5.3 \\
\hline 0.079 & 0.473 & 0.732 & 3.989 & 0.705 & 35.449 & 4.3 \\
\hline 0.072 & 0.456 & 0.749 & 3.794 & 0.559 & 39.020 & 4.3 \\
\hline 0.066 & 0.448 & 0.600 & 3.462 & 0.560 & 48.043 & 3.2 \\
\hline 0.049 & 0.421 & 0.753 & 3.318 & 0.551 & 42.671 & 2.0 \\
\hline 0.058 & 0.423 & 0.775 & 2.976 & 0.568 & 49.335 & 2.0 \\
\hline 0.064 & 0.434 & 0.752 & 3.488 & 0.597 & 45.399 & 2.5 \\
\hline
\end{tabular}

Value of Shipments and Receipts

(million dollars)

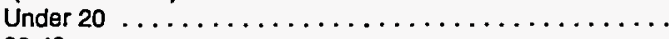

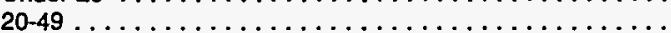

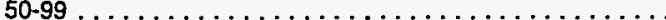

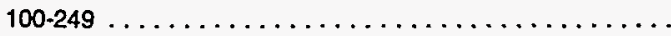

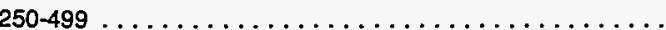

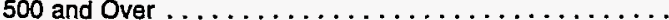

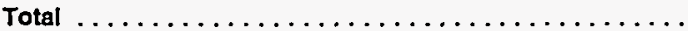

Employment Size

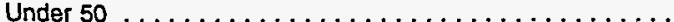

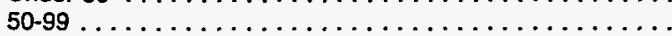

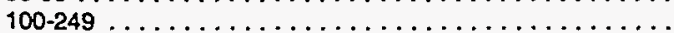

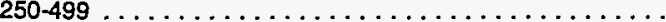

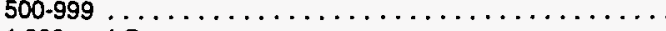

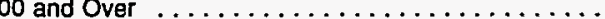

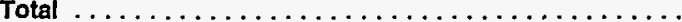

See footnotes at end of table. 
Table A29. Average Prices of Selected Purchased Energy Sources by Census Region and Economic Characteristics of the Establishment, 1991: Part 1 (Continued) (Estimates in Dollars per Physical Unit)

\begin{tabular}{|c|c|c|c|c|c|c|c|}
\hline $\begin{array}{c}\text { Economic } \\
\text { Characteristics }\end{array}$ & $\begin{array}{c}\text { Electricity } \\
(\mathrm{kWh})\end{array}$ & $\begin{array}{c}\text { Residual Fuel } \\
\text { Oil } \\
\text { (gallon) }\end{array}$ & $\begin{array}{c}\text { Distillate Fuel } \\
\text { Olib } \\
\text { (gallon) }\end{array}$ & $\begin{array}{l}\text { Natural Gas } \\
(1000 \mathrm{cu} \mathrm{ft})\end{array}$ & $\begin{array}{l}\text { LPG } \\
\text { (gallon) }\end{array}$ & $\begin{array}{c}\text { Coal } \\
\text { (short ton) }\end{array}$ & $\begin{array}{c}\text { RSE } \\
\text { Row } \\
\text { Factors }\end{array}$ \\
\hline & \multicolumn{6}{|c|}{ Midwest Census Region } & \\
\hline RSE Column Factors: & 0.8 & 1.2 & 1.2 & 0.8 & 1.3 & 0.9 & \\
\hline \multicolumn{8}{|l|}{$\begin{array}{l}\text { Value of Shipments and Recelpts } \\
\text { (million dollars) }\end{array}$} \\
\hline 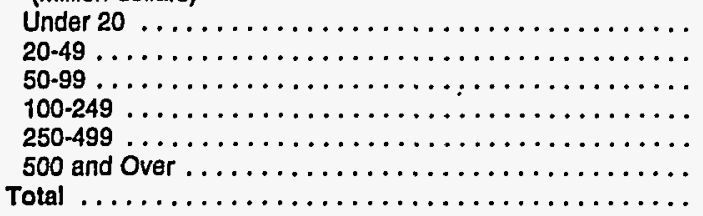 & $\begin{array}{l}0.064 \\
0.053 \\
0.047 \\
0.044 \\
0.039 \\
0.036 \\
0.046\end{array}$ & $\begin{array}{l}0.496 \\
0.319 \\
0.399 \\
0.371 \\
0.312 \\
0.336 \\
0.344\end{array}$ & $\begin{array}{l}0.808 \\
0.813 \\
0.809 \\
0.801 \\
0.746 \\
0.770 \\
0.797\end{array}$ & $\begin{array}{l}3.233 \\
2.680 \\
2.497 \\
2.659 \\
2.309 \\
2.323 \\
2.598\end{array}$ & $\begin{array}{r}0.752 \\
0.583 \\
0.680 \\
0.311 \\
W \\
0.612 \\
0.273\end{array}$ & $\begin{array}{l}37.029 \\
35.539 \\
38.557 \\
37.179 \\
34.955 \\
46.560 \\
41.350\end{array}$ & $\begin{array}{l}3.9 \\
3.5 \\
3.2 \\
2.5 \\
2.0 \\
2.2 \\
2.3\end{array}$ \\
\hline \\
\hline 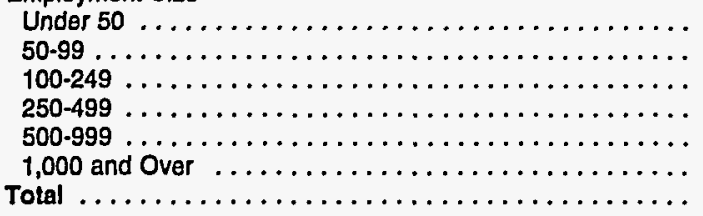 & $\begin{array}{l}0.065 \\
0.058 \\
0.053 \\
0.048 \\
0.044 \\
0.038 \\
0.046\end{array}$ & $\begin{array}{l}0.514 \\
0.319 \\
0.353 \\
0.395 \\
0.355 \\
0.336 \\
0.344\end{array}$ & $\begin{array}{l}0.783 \\
0.834 \\
0.832 \\
0.857 \\
0.735 \\
0.777 \\
0.797\end{array}$ & $\begin{array}{l}3.002 \\
2.831 \\
2.675 \\
2.537 \\
2.565 \\
2.425 \\
2.598\end{array}$ & $\begin{array}{r}0.782 \\
0.713 \\
0.619 \\
W \\
W \\
0.572 \\
0.273\end{array}$ & $\begin{array}{l}37.042 \\
36.212 \\
32.418 \\
37.811 \\
36.165 \\
47.601 \\
41.350\end{array}$ & $\begin{array}{l}6.1 \\
4.4 \\
3.6 \\
2.4 \\
2.4 \\
2.0 \\
2.3\end{array}$ \\
\hline Total $\ldots \ldots \ldots \ldots \ldots \ldots \ldots \ldots \ldots \ldots \ldots \ldots$ & \multicolumn{6}{|c|}{ South Census Region } & \\
\hline RSE Column Factors: & 0.7 & 1.4 & 1.2 & 0.9 & 1.1 & 0.8 & \\
\hline \multicolumn{8}{|l|}{$\begin{array}{l}\text { Value of Shlpments and Recelpts } \\
\text { (million dollars) }\end{array}$} \\
\hline 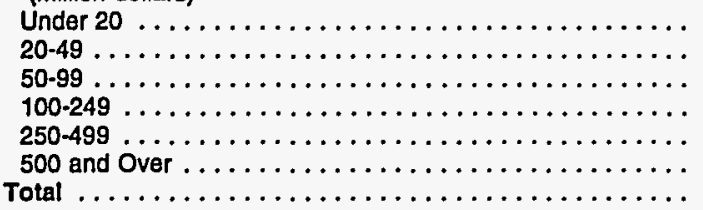 & $\begin{array}{l}0.057 \\
0.046 \\
0.043 \\
0.037 \\
0.033 \\
0.037 \\
0.041\end{array}$ & $\begin{array}{l}0.351 \\
0.325 \\
0.384 \\
0.350 \\
0.328 \\
0.340 \\
0.343\end{array}$ & $\begin{array}{l}0.828 \\
0.772 \\
0.759 \\
0.765 \\
0.718 \\
0.677 \\
0.770\end{array}$ & $\begin{array}{l}3.165 \\
2.491 \\
2.401 \\
2.045 \\
1.837 \\
1.781 \\
2.041\end{array}$ & $\begin{array}{r}0.696 \\
0.668 \\
W \\
0.488 \\
0.682 \\
0.366 \\
0.423\end{array}$ & $\begin{array}{l}40.353 \\
40.737 \\
48.399 \\
39.848 \\
41.303 \\
42.518 \\
41.848\end{array}$ & $\begin{array}{l}3.8 \\
3.2 \\
2.7 \\
2.0 \\
1.8 \\
1.6 \\
1.5\end{array}$ \\
\hline \multicolumn{8}{|l|}{ Employment Size } \\
\hline 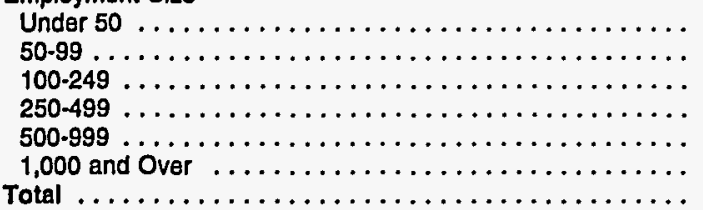 & $\begin{array}{l}0.054 \\
0.051 \\
0.044 \\
0.042 \\
0.038 \\
0.036 \\
0.041\end{array}$ & $\begin{array}{l}0.318 \\
0.323 \\
0.371 \\
0.355 \\
0.355 \\
0.339 \\
0.343\end{array}$ & $\begin{array}{l}0.881 \\
0.761 \\
0.787 \\
0.722 \\
0.751 \\
0.717 \\
0.770\end{array}$ & $\begin{array}{l}2.543 \\
2.296 \\
2.145 \\
2.143 \\
2.023 \\
1.856 \\
2.041\end{array}$ & $\begin{array}{l}0.637 \\
0.324 \\
0.694 \\
0.523 \\
0.454 \\
0.341 \\
0.423\end{array}$ & $\begin{array}{l}39.350 \\
42.045 \\
40.428 \\
45.524 \\
41.048 \\
41.522 \\
41.848\end{array}$ & $\begin{array}{l}5.4 \\
3.9 \\
2.8 \\
2.4 \\
2.0 \\
1.8 \\
1.5\end{array}$ \\
\hline
\end{tabular}

See footnotes at end of table. 


\section{Table A29. Average Prices of Selected Purchased Energy Sources by Census Region and Economic Characteristics of the Establishment, 1991: Part 1 (Continued) (Estimates in Dollars per Physical Unit)}

\begin{tabular}{|c|c|c|c|c|c|c|c|}
\hline $\begin{array}{c}\text { Economic } \\
\text { Characteristics }^{\circ}\end{array}$ & $\begin{array}{c}\text { Electricity } \\
(\mathrm{kWh})\end{array}$ & $\begin{array}{c}\begin{array}{c}\text { Residual Fuel } \\
\text { Oil } \\
\text { (gallon) }\end{array} \\
\end{array}$ & $\begin{array}{c}\text { Distillate Fuel } \\
\begin{array}{c}\text { Oif } \\
\text { (gallon) }\end{array} \\
\end{array}$ & $\begin{array}{c}\text { Natural Gas } \\
(1000 \mathrm{cu} \mathrm{ft}) \\
\end{array}$ & $\begin{array}{c}\text { LPG } \\
\text { (gallon) }\end{array}$ & $\begin{array}{c}\text { Coal } \\
\text { (short ton) }\end{array}$ & $\begin{array}{c}\text { RSE } \\
\text { Row } \\
\text { Factors }\end{array}$ \\
\hline & \multicolumn{7}{|c|}{ West Census Region } \\
\hline RSE Column Factors: & 0.8 & 0.9 & 1.1 & 1.0 & 1.1 & 1.1 & \\
\hline \multicolumn{8}{|l|}{$\begin{array}{l}\text { Value of Shipments and Receipts } \\
\text { (million dollars) }\end{array}$} \\
\hline 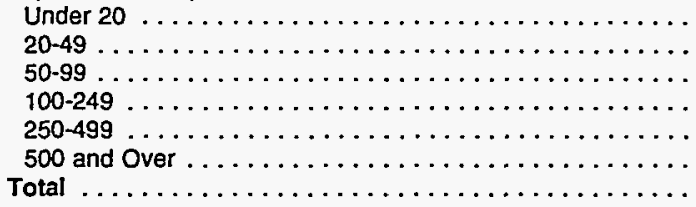 & $\begin{array}{l}0.073 \\
0.057 \\
0.046 \\
0.032 \\
0.030 \\
0.055 \\
0.045\end{array}$ & $\begin{array}{r}0.437 \\
0.477 \\
0.439 \\
0.456 \\
0.347 \\
W \\
0.447\end{array}$ & $\begin{array}{l}0.881 \\
0.873 \\
0.793 \\
0.828 \\
0.803 \\
0.740 \\
0.847\end{array}$ & $\begin{array}{l}3.550 \\
3.177 \\
2.895 \\
2.091 \\
2.372 \\
3.351 \\
2.772\end{array}$ & $\begin{array}{l}0.790 \\
0.695 \\
0.642 \\
0.588 \\
0.690 \\
0.676 \\
0.700\end{array}$ & $\begin{array}{r}40.892 \\
30.526 \\
31.367 \\
35.032 \\
W \\
W \\
39.987\end{array}$ & $\begin{array}{l}4.0 \\
4.2 \\
3.3 \\
2.8 \\
1.9 \\
2.2 \\
2.7\end{array}$ \\
\hline \multicolumn{8}{|l|}{ Employment Size } \\
\hline 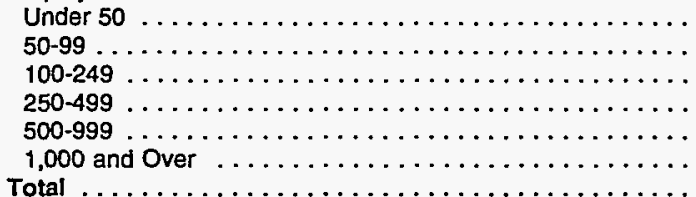 & $\begin{array}{l}0.078 \\
0.061 \\
0.052 \\
0.042 \\
0.031 \\
0.045 \\
0.045\end{array}$ & $\begin{array}{l}0.813 \\
0.414 \\
0.478 \\
0.438 \\
0.454 \\
0.422 \\
0.447\end{array}$ & $\begin{array}{l}0.834 \\
0.944 \\
0.848 \\
0.856 \\
0.768 \\
0.779 \\
0.847\end{array}$ & $\begin{array}{l}3.743 \\
3.075 \\
2.429 \\
2.813 \\
2.903 \\
2.661 \\
2.772\end{array}$ & $\begin{array}{l}0.745 \\
0.787 \\
0.705 \\
0.614 \\
0.658 \\
0.698 \\
0.700\end{array}$ & $\begin{array}{r}W \\
33.279 \\
36.658 \\
36.780 \\
W \\
W \\
39.987\end{array}$ & $\begin{array}{l}7.6 \\
5.5 \\
3.9 \\
3.4 \\
2.1 \\
2.6 \\
2.7\end{array}$ \\
\hline
\end{tabular}

- Value of Shipments and Receipts and Employment Size were supplied by the Bureau of the Census. See Appendix B.

"Distillate Fuel Oil" includes Nos. 1, 2, and 4 fuel oils and Nos. 1, 2, and 4 diesel fuels.

c "Natural Gas" includes natural gas obtained from utilities, transmission pipelines, and any other supplier(s) such as brokers and producers.

$W=$ Withheld to avoid disclosing data for individual establishments. Data are included in higher level totals.

Notes: - To obtain a RSE percentage for any table cell, multiply the cell's corresponding RSE column and RSE row factors. - Totals may not equal sum of components because of independent rounding.

Source: Energy Information Administration, Office of Energy Markets and End Use, Energy End Use and Integrated Statistics Division, Form ElA-846, "1991 Manufacturing Energy Consumption Survey," and Bureau of the Census, Industry Division, data files for the "1991 Annual Survey of Manufactures." 
Table A29. Average Prices of Selected Purchased Energy Sources by Census Region and Economic Characteristics of the Establishment, 1991: Part 2 (Estimates in Dollars per Million Btu)

\begin{tabular}{|c|c|c|c|c|c|c|c|}
\hline $\begin{array}{c}\text { Economic } \\
\text { Characteristics }\end{array}$ & Electricity & $\begin{array}{c}\text { Residual Fuel } \\
\text { Oil }\end{array}$ & $\begin{array}{c}\text { Distillate Fuel } \\
\text { Oil }^{6}\end{array}$ & Natural Gas ${ }^{e}$ & LPG & Coal & $\begin{array}{c}\text { RSE } \\
\text { Row } \\
\text { Factors }\end{array}$ \\
\hline & \multicolumn{6}{|c|}{ Total United States } & \\
\hline RSE Column Fectors: & 0.8 & 1.2 & 1.2 & 0.9 & 1.3 & 0.8 & \\
\hline \multicolumn{8}{|l|}{$\begin{array}{l}\text { Value of Shipments and Recelpts } \\
\text { (million dollars) }\end{array}$} \\
\hline 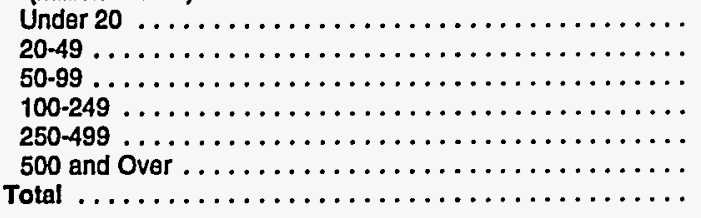 & $\begin{array}{l}19.202 \\
15.472 \\
13.944 \\
11.408 \\
10.298 \\
11.923 \\
13.486\end{array}$ & $\begin{array}{l}2.701 \\
2.422 \\
2.780 \\
2.543 \\
2.456 \\
2.489 \\
2.540\end{array}$ & $\begin{array}{l}5.863 \\
5.457 \\
5.537 \\
5.653 \\
5.419 \\
5.261 \\
5.625\end{array}$ & $\begin{array}{l}3.322 \\
2.693 \\
2.550 \\
2.191 \\
1.976 \\
1.965 \\
2.304\end{array}$ & $\begin{array}{l}8.021 \\
7.126 \\
4.683 \\
5.469 \\
6.236 \\
4.360 \\
4.909\end{array}$ & $\begin{array}{l}1.646 \\
1.735 \\
1.772 \\
1.676 \\
1.803 \\
1.851 \\
1.775\end{array}$ & $\begin{array}{l}3.4 \\
3.1 \\
2.3 \\
1.5 \\
1.7 \\
1.6 \\
1.4\end{array}$ \\
\hline \multirow[t]{2}{*}{ 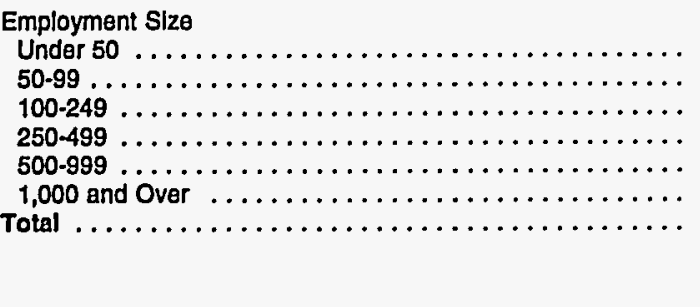 } & $\begin{array}{l}19.490 \\
17.355 \\
14.935 \\
13.590 \\
11.521 \\
11.802 \\
13.486\end{array}$ & $\begin{array}{l}2.322 \\
2.295 \\
2.729 \\
2.679 \\
2.601 \\
2.503 \\
2.540 \\
\end{array}$ & $\begin{array}{l}5.964 \\
5.746 \\
5.685 \\
5.205 \\
5.424 \\
5.439 \\
5.625 \\
\end{array}$ & $\begin{array}{l}3.026 \\
2.645 \\
2.373 \\
2.348 \\
2.280 \\
2.046 \\
2.304 \\
\end{array}$ & $\begin{array}{l}7.525 \\
4.322 \\
7.952 \\
5.346 \\
4.988 \\
4.125 \\
4.909 \\
\end{array}$ & $\begin{array}{l}1.744 \\
1.628 \\
1.622 \\
1.775 \\
1.694 \\
1.873 \\
1.775 \\
\end{array}$ & $\begin{array}{l}4.5 \\
3.4 \\
2.6 \\
2.6 \\
1.7 \\
1.4 \\
1.4\end{array}$ \\
\hline & \multicolumn{6}{|c|}{ Northeast Census Region } & \\
\hline RSE Column Factors: & 0.8 & 0.7 & 1.4 & 0.8 & 1.4 & 1.0 & \\
\hline \multicolumn{8}{|l|}{$\begin{array}{l}\text { Value of Shipments and Receipts } \\
\text { (million dollars) }\end{array}$} \\
\hline 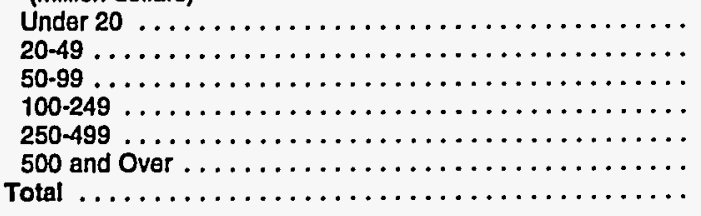 & $\begin{array}{l}23.652 \\
20.845 \\
19.788 \\
15.483 \\
13.557 \\
16.582 \\
18.648\end{array}$ & $\begin{array}{l}3.203 \\
2.995 \\
2.982 \\
2.781 \\
2.732 \\
3.154 \\
2.897\end{array}$ & $\begin{array}{l}5.630 \\
4.697 \\
5.319 \\
5.586 \\
5.861 \\
5.519 \\
5.426\end{array}$ & $\begin{array}{l}4.193 \\
3.622 \\
3.391 \\
3.105 \\
3.087 \\
2.754 \\
3.386\end{array}$ & $\begin{array}{l}7.415 \\
6.829 \\
6.740 \\
5.707 \\
6.123 \\
8.074 \\
6.745\end{array}$ & $\begin{array}{l}1.556 \\
1.869 \\
1.868 \\
1.674 \\
2.767 \\
1.879 \\
1.822\end{array}$ & $\begin{array}{l}3.9 \\
4.4 \\
2.6 \\
1.7 \\
2.0 \\
2.1 \\
2.4\end{array}$ \\
\hline \multicolumn{8}{|l|}{ Employment Size } \\
\hline 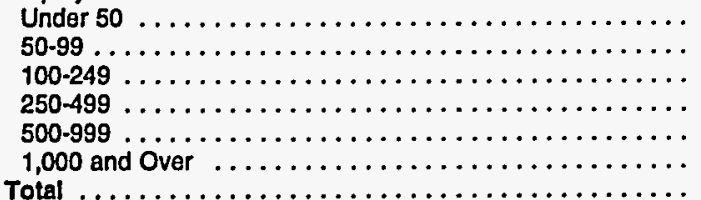 & $\begin{array}{l}24.605 \\
23.248 \\
21.118 \\
19.424 \\
14.506 \\
16.942 \\
18.648\end{array}$ & $\begin{array}{l}3.042 \\
3.157 \\
3.045 \\
2.995 \\
2.814 \\
2.825 \\
2.897\end{array}$ & $\begin{array}{l}5.914 \\
5.278 \\
5.401 \\
4.326 \\
5.432 \\
5.590 \\
5.426\end{array}$ & $\begin{array}{l}4.347 \\
3.873 \\
3.683 \\
3.361 \\
3.221 \\
2.890 \\
3.386\end{array}$ & $\begin{array}{l}7.588 \\
8.145 \\
6.183 \\
6.366 \\
6.333 \\
6.507 \\
6.745\end{array}$ & $\begin{array}{r}W \\
1.591 \\
1.684 \\
2.131 \\
1.676 \\
1.918 \\
1.822\end{array}$ & $\begin{array}{l}5.4 \\
4.3 \\
3.9 \\
3.2 \\
2.0 \\
2.0 \\
2.4\end{array}$ \\
\hline
\end{tabular}

See footnotes at end of table. 
Table A29. Average Prices of Selected Purchased Energy Sources by Census Region and Economic Characteristics of the Establishment, 1991: Part 2 (Continued) (Estimates in Dollars per Million Btu)

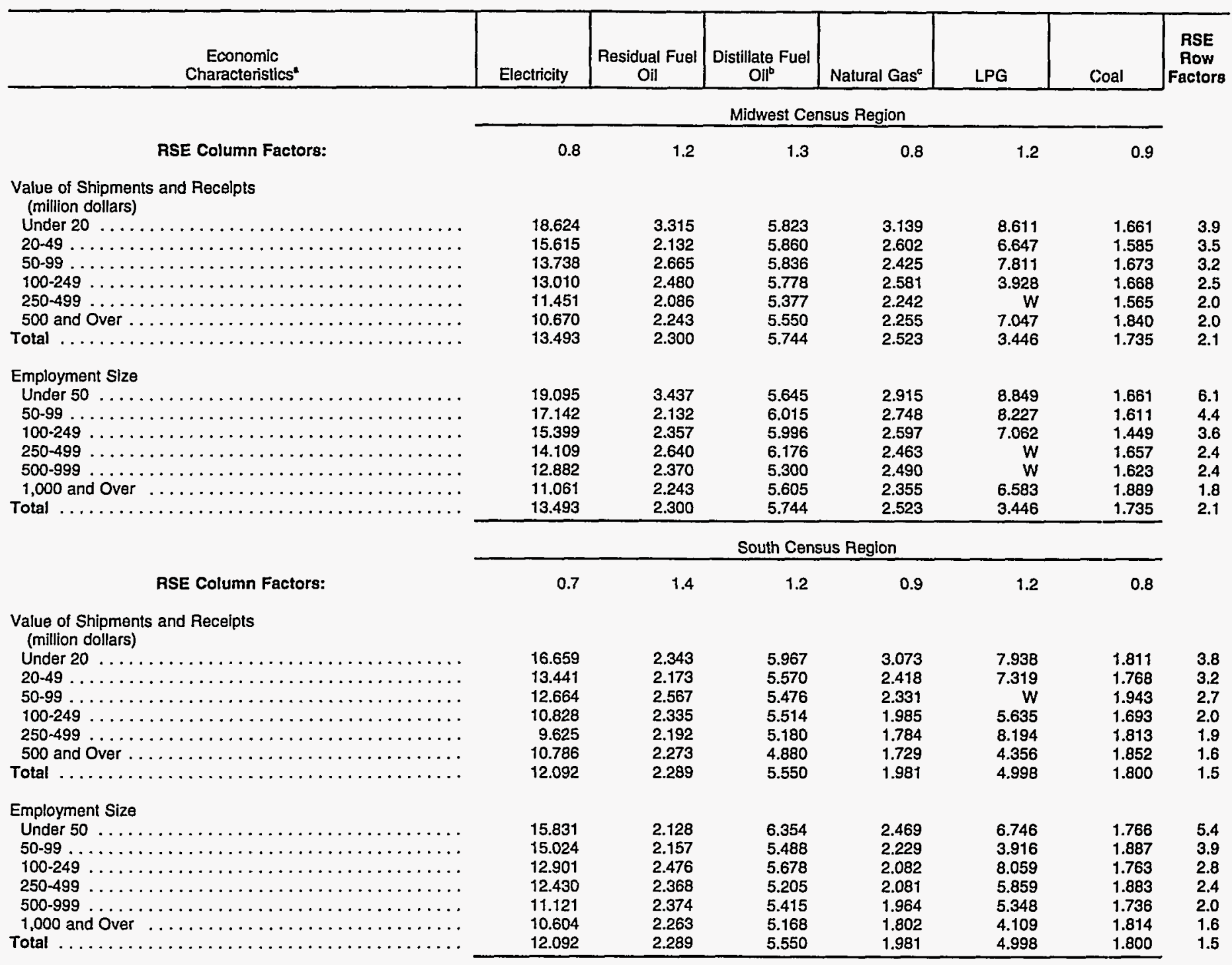

See footnotes at end of table. 


\section{Table A29. Average Prices of Selected Purchased Energy Sources by Census Region and Economic Characteristics of the Establishment, 1991: Part 2 (Continued) (Estimates in Dollars per Million Btu)}

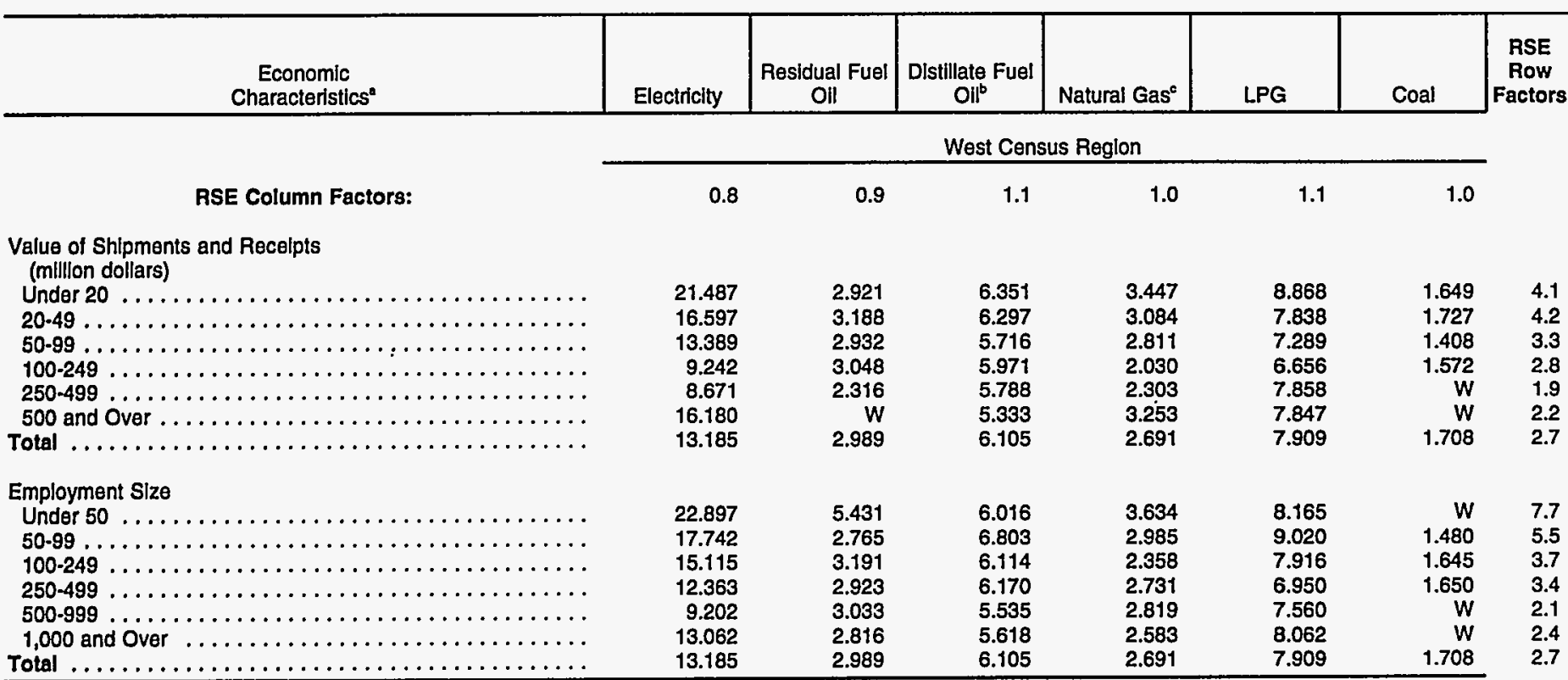

\footnotetext{
- Value of Shlpments and Receipts and Employment Size were supplied by the Bureau of the Census. See Appendix B.

- "Dlstillate Fuel Oll" includes Nos. 1, 2, and 4 fuel oils and Nos. 1, 2, and 4 diesel fuels.

- "Natural Gas" includes natural gas obtained from utilities, transmission pipelines, and any other supplier(s) such as brokers and producers.

$W=$ Withheld to avold disclosing data for individual establishments. Data are included in higher level totals.

Notes: - To obtain a RSE percentage for any table cell, multiply the cell's corresponding RSE column and RSE row factors. - Totals may not equal sum of components because of independent rounding.

Source: Energy Inlormation Administration, Office of Energy Markets and End Use, Energy End Use and Integrated Statistics Division, Form ElA-846, "1991 Manufacturing Energy Consumption Survey," and Bureau of the Census, Industry Division, data files for the "1991 Annual Survey of Manufactures."
} 
Table A30. Total Primary Consumption of Energy for All Purposes by Value of Shipment Categories, Industry Group, and Selected Industries, 1991

(Estimates in Trillion Btu)

\begin{tabular}{|c|c|c|c|c|c|c|c|c|c|}
\hline \multirow[b]{2}{*}{$\begin{array}{c}\text { SIC } \\
\text { Code }^{8}\end{array}$} & \multirow[b]{2}{*}{$\begin{array}{l}\text { Industry Groups } \\
\text { and Industry }\end{array}$} & \multirow[b]{2}{*}{ Total } & \multicolumn{6}{|c|}{$\begin{array}{l}\text { Value of Shipments and Receipts } \\
\text { (million dollars) }\end{array}$} & \multirow{3}{*}{$\begin{array}{c}\text { RSE } \\
\text { Row } \\
\text { Factora }\end{array}$} \\
\hline & & & Under 20 & $20-49$ & $50-99$ & $100-249$ & $250-499$ & $\begin{array}{l}500 \text { and } \\
\text { Over }\end{array}$ & \\
\hline & RSE Column Factors: & 0.6 & 1.6 & 1.3 & 1.0 & 0.8 & 1.0 & 1.1 & \\
\hline 20 & Food and Kindred Products. & 956 & 109 & 142 & 174 & 229 & 161 & 142 & 6.2 \\
\hline 2011 & Meat Packing Plants .... & 49 & 2 & 3 & 3 & 6 & 13 & 23 & 7.8 \\
\hline 2033 & Canned Fruits and Vegetables $\ldots \ldots \ldots \ldots \ldots \ldots$ & 44 & 9 & 10 & 8 & 12 & w & w & 11.8 \\
\hline 2037 & Frozen Fruits and Vegetables.... & 40 & 5 & 4 & 14 & 15 & 1 & 0 & 15.2 \\
\hline 2046 & Wet Com Milling $\ldots \ldots \ldots \ldots \ldots \ldots \ldots \ldots \ldots$ & 140 & - & w & w & 23 & 64 & w & 12.4 \\
\hline 2051 & Bread, Cake and Related Products . . . . . . . . . . . . & 32 & 10 & 13 & 6 & 3 & • & - & 10.5 \\
\hline 2063 & Beet Sugar $\ldots \ldots \ldots \ldots \ldots \ldots \ldots \ldots \ldots \ldots \ldots \ldots$ & 67 & 0 & 14 & 35 & 18 & 0 & 0 & 6.8 \\
\hline 2075 & Soybean Oil Mills . . . . . . . . . . . . . . . . . . . . . & 51 & * & • & $\mathbf{w}$ & 20 & 17 & w & 5.2 \\
\hline 2082 & Malt Beverages $\ldots \ldots \ldots \ldots \ldots \ldots \ldots \ldots \ldots$ & 50 & • & - & 2 & 5 & 7 & 36 & 11.6 \\
\hline 21 & Tobacco Manufactures $\ldots \ldots \ldots \ldots \ldots \ldots \ldots \ldots$ & 24 & * & 2 & 1 & w & w & 13 & 8.8 \\
\hline 22 & $\ldots \ldots \ldots \ldots \ldots \ldots \ldots$, & 274 & 54 & 81 & 63 & w & 22 & w & 7.2 \\
\hline 23 & Apparel and Other Textile Products $\ldots \ldots \ldots \ldots \ldots \ldots$ & 44 & 23 & 8 & 7 & 4 & 1 & 2 & 17.3 \\
\hline 24 & Lumber and Wood Products $\ldots \ldots \ldots \ldots \ldots \ldots \ldots$ & 451 & 161 & 206 & 60 & 23 & 0 & 1 & 17.0 \\
\hline 25 & Furniture and Fixtures $\ldots \ldots \ldots \ldots \ldots \ldots \ldots \ldots$ & 68 & 31 & 20 & 10 & 4 & • & 3 & 20.7 \\
\hline 26 & Paper and Allied Products . . . . . . . . . . . . . . & 2,506 & 69 & 107 & 234 & 917 & 1,060 & 119 & 4.2 \\
\hline 2611 & 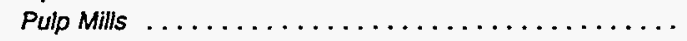 & 300 & 0 & 3 & 41 & 159 & 97 & 0 & 18.3 \\
\hline 2621 & $\ldots \ldots \ldots \ldots \ldots \ldots$ & 1,211 & 13 & 16 & 85 & 388 & 613 & 95 & 5.1 \\
\hline 2631 & $\ldots \ldots \ldots \ldots \ldots \ldots \ldots \ldots \ldots$, & 859 & $w$ & 33 & 83 & 358 & 340 & w & 7.0 \\
\hline 27 & Printing and Publishing $\ldots \ldots \ldots \ldots \ldots \ldots \ldots \ldots$ & 108 & 41 & 24 & 17 & 20 & 4 & 2 & 11.6 \\
\hline 28 & Chemicals and Allied Products $\ldots \ldots \ldots \ldots \ldots \ldots \ldots$ & 5,051 & 159 & 354 & 382 & 984 & 1,126 & 2,046 & 6.2 \\
\hline 2812 & Alkalies and Chlorine $\ldots \ldots \ldots \ldots \ldots \ldots \ldots \ldots$ & 160 & w & 8 & 22 & $\mathbf{w}$ & 0 & w & 15.7 \\
\hline 2813 & 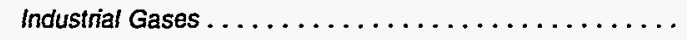 & $w$ & 36 & 19 & 62 & w & 0 & 0 & 7.9 \\
\hline 2819 & Industrial Inorganic Chemicals, nec . ............. & 325 & $w$ & 37 & 33 & 52 & 113 & W & 8.3 \\
\hline 2821 & Plastics Materials and Resins . ................ & 633 & 8 & 11 & 25 & 138 & 283 & 168 & 6.6 \\
\hline 2822 & Synthetic Rubber $\ldots \ldots \ldots \ldots \ldots \ldots \ldots \ldots \ldots$ & 119 & - & 1 & 11 & 10 & 97 & 0 & 15.0 \\
\hline 2823 & Cellulosic Manmade Fibers $\ldots \ldots \ldots \ldots \ldots \ldots \ldots \ldots$ & 31 & * & 0 & 4 & 10 & 12 & 6 & 32.1 \\
\hline 2824 & Organic Fibers, Noncellulosic . . . . . . . . . . . . . . . & $\mathbf{w}$ & $\mathbf{Q}$ & 1 & 2 & w & 35 & w & 3.7 \\
\hline 2865 & Cyclic Crudes and Intermediates $\ldots \ldots \ldots \ldots \ldots \ldots$ & 236 & 3 & w & 24 & 26 & 73 & w & 11.9 \\
\hline 2869 & Industrial Organic Chemicals, nec $\ldots \ldots \ldots \ldots \ldots \ldots$ & 2,289 & 32 & 41 & 28 & 291 & 359 & 1,538 & 7.0 \\
\hline 2873 & Nitrogenous Fertilizers $\ldots \ldots \ldots \ldots \ldots \ldots \ldots \ldots$ & 568 & 2 & 125 & 112 & 240 & 89 & 0 & 25.1 \\
\hline 2874 & Phosphatic Fertilizers $\ldots \ldots \ldots \ldots \ldots \ldots \ldots \ldots \ldots$ & 65 & 1 & - & w & 19 & 37 & w & 5.8 \\
\hline 29 & Petroleum and Coal Products & 5,967 & 104 & 60 & 80 & 77 & 220 & 2,557 & 5.6 \\
\hline 2911 & Petroleum Refining ${ }^{\circ}$ & 5,762 & 13 & 17 & 10 & 76 & 220 & 2,557 & 3.8 \\
\hline 30 & Rubber and Misc. Plastics Products $\ldots \ldots \ldots \ldots \ldots \ldots$ & 238 & 84 & 55 & 35 & 36 & w & w & 5.7 \\
\hline 3011 & Tres and Inner Tubes $\ldots \ldots \ldots \ldots \ldots \ldots \ldots \ldots \ldots$ & w & * & 3 & w & 10 & 19 & w & 3.7 \\
\hline 308 & Miscellaneous Plastics Products, nec & 151 & 63 & 40 & 25 & 21 & 2 & 0 & 10.4 \\
\hline 31 & Leather and Leather Products $\ldots \ldots \ldots \ldots \ldots \ldots \ldots$ & 12 & 5 & 4 & 2 & 2 & 0 & 0 & 22.1 \\
\hline 32 & Stone, Clay and Glass Products & 880 & 247 & 358 & 201 & 68 & $w$ & w & 7.8 \\
\hline 3211 & $\ldots \ldots \ldots \ldots \ldots$, & 49 & * & 8 & 24 & 16 & 0 & 0 & 3.1 \\
\hline 3221 & Glass Containers . . . . . . . . . . . . . . . . & 85 & * & w & 53 & $w$ & 0 & $\mathbf{0}$ & 6.4 \\
\hline 3229 & Pressed and Blown Glass, nec . . . . . . . . . . . . . . & $\mathbf{w}$ & 3 & 13 & w & 29 & 0 & 0 & 6.6 \\
\hline 3241 & Cement, Hydraulic . & 312 & 25 & 230 & 57 & D & 0 & 0 & 10.1 \\
\hline 3274 & Lime $\ldots \ldots \ldots \ldots \ldots$ & 117 & 83 & w & w & 0 & 0 & 0 & 28.7 \\
\hline 3296 & Mineral Wool . & 41 & 3 & 10 & 19 & w & w & 0 & 1.4 \\
\hline 33 & Primary Metal Industries & 2,467 & 99 & 143 & 182 & 474 & 358 & 1,210 & 4.8 \\
\hline 3312 & Blast Fumaces and Steel Mills & 1,673 & 6 & 49 & 93 & 226 & 179 & 1,122 & 7.6 \\
\hline 3313 & Electrometalurgical Products ..... & 41 & * & 10 & w & $\mathbf{w}$ & 0 & o & 9.8 \\
\hline 3321 & Gray and Ductile Iron Foundries $\ldots \ldots \ldots \ldots \ldots$ & w & W & 15 & 17 & 14 & w & 0 & 8.4 \\
\hline 3331 & Primary Copper $\ldots \ldots \ldots \ldots \ldots \ldots \ldots \ldots \ldots$ & 21 & W & 0 & * & w & 12 & $\mathbf{w}$ & 1.0 \\
\hline 3334 & Primary Aluminum ...... & 297 & - & • & 0 & 134 & w & W & 3.3 \\
\hline 3339 & Primary Nonferrous Metals, nec. & 52 & $w$ & 6 & 13 & 28 & 0 & w & 2.0 \\
\hline 3353 & Aluminum Sheet, Plate, and Foll . . & 61 & $\mathbf{Q}$ & 1 & 3 & 12 & 13 & 32 & 1.2 \\
\hline 34 & Fabricated Metal Products & 307 & 117 & 81 & 46 & 39 & 19 & 5 & 7.9 \\
\hline 35 & Industrial Machinery and Equipment ..... & 237 & 71 & 31 & 42 & 39 & 16 & 39 & 7.7 \\
\hline 357 & Computer and Office Equipment . . . . . . . . . . & 21 & 2 & 2 & 2 & 4 & 2 & 10 & 11.5 \\
\hline 36 & Electronic and Other Electric Equipment . . & 212 & 29 & 33 & 46 & 48 & 21 & 35 & 7.8 \\
\hline
\end{tabular}

See footnotes at end of table. 
Table A30. Total Primary Consumption of Energy for All Purposes by Value of Shipment Categories, Industry Group, and Selected Industries, 1991 (Continued) (Estimates in Trillion Btu)

\begin{tabular}{|c|c|c|c|c|c|c|c|c|c|}
\hline \multirow[b]{2}{*}{$\begin{array}{c}\text { SIC } \\
\text { Code }\end{array}$} & \multirow[b]{2}{*}{$\begin{array}{l}\text { Industry Groups } \\
\text { and Industry }\end{array}$} & \multirow[b]{2}{*}{ Total } & \multicolumn{6}{|c|}{$\begin{array}{l}\text { Value of Shipments and Receipts } \\
\text { (million dollars) }\end{array}$} & \multirow{3}{*}{$\begin{array}{c}\text { RSE } \\
\text { Row } \\
\text { Factors }\end{array}$} \\
\hline & & & Under 20 & $20-49$ & $50-99$ & $100-249$ & $250-499$ & $\begin{array}{l}500 \text { and } \\
\text { Over }\end{array}$ & \\
\hline & RSE Column Factors: & 0.6 & 1.6 & 1.3 & 1.0 & 0.8 & 1.0 & 1.1 & \\
\hline 37 & Transportation Equipment $\ldots \ldots \ldots \ldots \ldots \ldots$ & 323 & 18 & 21 & 21 & 26 & 32 & 205 & 5.3 \\
\hline 3711 & Motor Vehicles and Car Bodles . . . . . . . . . . . . . & 88 & $*$ & $*$ & $\cdot$ & - & 2 & 86 & 5.0 \\
\hline 3714 & Motor Vehiclo Parts and Accessories . . . . . . . . . . & 100 & 7 & 9 & 13 & 13 & 18 & 40 & 6.0 \\
\hline 38 & Instruments and Related Products $\ldots \ldots \ldots \ldots \ldots \ldots$ & 98 & 9 & 9 & 10 & 19 & 10 & 41 & 12.3 \\
\hline 3841 & Surgical and Medical Instruments . . . . . . . . . . . & 6 & 1 & 1 & 2 & 2 & * & - & 13.3 \\
\hline \multirow[t]{2}{*}{39} & Mlsc. Manufacturing Industries . . . . . . . . . . . . & 32 & 12 & 8 & 5 & 4 & 3 & 0 & 13.1 \\
\hline & Total $\ldots \ldots \ldots \ldots \ldots \ldots \ldots \ldots \ldots \ldots \ldots$ & 20,257 & 1,443 & 1,747 & 1,619 & 3,071 & 3,081 & 6,429 & 3.0 \\
\hline
\end{tabular}

"See Appendices $B$ and $F$ for descriptions of the Standard Industrial Classification system.

- Value of Shipments and Receipts were supplied by the Bureau of the Census.

'For the petroleum refining industry only, the feedstocks and raw material inputs for the production of nonenergy products (i.e., asphalt, waxes, lubricants, and solvents) and feedstock consumption at adjoining petrochemical plants are included in the "Total" column, regardless of type of energy. The remaining columns for the petroleum refining Industry include only energy that was consumed for the production of heat and power. See Appendix $B$ for more information.

- Estimate less than 0.5. Data are included in higher level totals.

$W=$ Withheld to avold disclosing data for individual establishments. Data are included in higher level totals.

$Q=$ Withheld because Relative Standard Error is greater than 50 percent. Data are included in higher level totals.

Notes: - To obtain a RSE percentage for any table cell, multiply the cell's corresponding RSE column and RSE row factors. - Totals may not equal sum of components because of Independent rounding. - The derived estimates presented in this table are for the primary consumption of energy for heat and power and as feedstocks or raw materlal inputs. Primary consumption is defined as the consumption of the energy that was originally produced offsite or was produced onsite from input materlals not classiffed as energy. Examples of the latter are hydrogen produced from the electrolysis of brine; the output of captive (onsite) mines or wells; woodchips, bark, and woodwaste from wood purchased as a raw material input; and waste materials such as wastepaper and packing materials. Primary consumption excludes quantitles of energy that are produced from other energy inputs and, therefore, avolds double counting.

Source: Energy Information Administration, Otfice of Energy Markets and End Use, Energy End Use and Integrated Statistics Divislon, Form ElA-846, "1991 Manulacturing Energy Consumption Survey," and Office of Oil and Gas, Petroleum Supply Division, Form EIA-810, "Monthly Refinery Report" for 1991, and the Bureau of the Census, Industry Division, data files for the "1991 Annual Survey of Manufactures." 
Table A31. Total Inputs of Energy for Heat, Power, and Electricity Generation by Value of Shipment Categories, Industry Group, and Selected Industries, 1991 (Estimates in Trillion Btu)

\begin{tabular}{|c|c|c|c|c|c|c|c|c|c|}
\hline \multirow[b]{2}{*}{$\begin{array}{c}\text { SIC } \\
\text { Code }^{a}\end{array}$} & \multirow[b]{2}{*}{$\begin{array}{l}\text { Industry Groups } \\
\text { and Industry }\end{array}$} & \multirow[b]{2}{*}{ Total } & \multicolumn{6}{|c|}{$\begin{array}{l}\text { Value of Shipments and Receipts } \\
\text { (million dollars) }\end{array}$} & \multirow{3}{*}{$\begin{array}{c}\text { RSE } \\
\text { Row } \\
\text { Factors }\end{array}$} \\
\hline & & & Under 20 & $20-49$ & $50-99$ & $100-249$ & $250-499$ & $\begin{array}{l}500 \text { and } \\
\text { Over }\end{array}$ & \\
\hline & RSE Column Factors: & 0.6 & 1.6 & 1.2 & 0.9 & 0.8 & 1.0 & 1.1 & \\
\hline 20 & Food and Kindred Products . . . . . . . . . . . . & 953 & 109 & 142 & 173 & 228 & 160 & 141 & 6.2 \\
\hline 2011 & 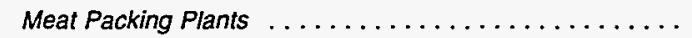 & 49 & 2 & 3 & 3 & 6 & 13 & 23 & 7.9 \\
\hline 2033 & Canned Fruits and Vegetables . . . . . . . . . . . & 44 & 9 & 10 & 8 & 12 & W & $w$ & 11.8 \\
\hline 2037 & Frozen Fruits and Vegetables . . . . . . . . . . . & 40 & 5 & 4 & 14 & 15 & 1 & 0 & 15.3 \\
\hline 2046 & Wet Com Milling $\ldots \ldots \ldots \ldots \ldots \ldots \ldots \ldots \ldots \ldots$ & 140 & - & W & $w$ & 23 & 64 & $w$ & 12.4 \\
\hline 2051 & Bread, Cake and Related Products . . . . . . . . . . & 32 & 10 & 13 & 6 & 3 & $\bullet$ & * & 10.5 \\
\hline 2063 & 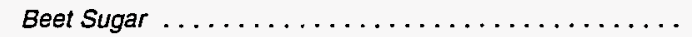 & 67 & 0 & 14 & 35 & 18 & 0 & 0 & 6.9 \\
\hline 2075 & Soybean Oil Mills . . . . . . . . . . . . . . . . . . & 50 & - & * & W & 20 & 17 & W & 5.2 \\
\hline 2082 & Malt Beverages . . . . . . . . . . . . . . & 50 & * & - & 2 & 5 & 7 & 36 & 11.7 \\
\hline 21 & Tobacco Manufactures $\ldots \ldots \ldots \ldots \ldots \ldots$ & 24 & * & 2 & 1 & W & $w$ & 13 & 8.8 \\
\hline 22 & Textile Mill Products $\ldots \ldots \ldots \ldots \ldots \ldots \ldots$ & 273 & 54 & 81 & 62 & W & 22 & W & 7.2 \\
\hline 23 & Apparel and Other Textile Products $\ldots \ldots \ldots \ldots$ & 44 & 23 & 8 & 7 & 4 & 1 & 2 & 17.3 \\
\hline 24 & Lumber and Wood Products $\ldots \ldots \ldots \ldots \ldots \ldots \ldots$ & 423 & 157 & 183 & 60 & 23 & 0 & 1 & 17.0 \\
\hline 25 & Furniture and Fixtures $\ldots \ldots \ldots \ldots \ldots \ldots$ & 67 & 30 & 20 & 10 & 4 & * & 3 & 20.8 \\
\hline 26 & Paper and Allied Products $\ldots \ldots \ldots \ldots \ldots \ldots \ldots$ & 2,472 & 67 & 104 & 227 & 900 & 1,056 & 118 & 4.3 \\
\hline 2611 & 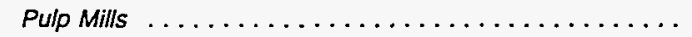 & 300 & 0 & 3 & 41 & 159 & 97 & 0 & 18.4 \\
\hline 2621 & Paper Mills ........................ & 1,204 & 13 & 16 & 82 & 388 & 609 & 95 & 5.1 \\
\hline 2631 & Paperboard Mills . . . . . . . . . . . . . . & 832 & $\mathbf{W}$ & 31 & 79 & 341 & 340 & W & 7.2 \\
\hline 27 & Printing and Publishing $\ldots \ldots \ldots \ldots \ldots \ldots$ & 108 & 41 & 24 & 17 & 20 & 4 & 2 & 11.6 \\
\hline 28 & Chemicals and Allied Products . . . . . . . . . . . . & 3,040 & 127 & 240 & 255 & 616 & 685 & 1,117 & 5.5 \\
\hline 2812 & Alkalies and Chlorine . . . . . . . . . . . . . . & 160 & W & 8 & 23 & $W$ & 0 & w & 15.7 \\
\hline 2813 & Industrial Gases . . . . . . . . . . . . . . . . & 91 & 34 & 18 & 17 & 21 & 0 & 0 & 6.5 \\
\hline 2819 & Industrial Inorganic Chemicals, nec . . . . . . . . . . & 311 & $\mathbf{W}$ & 35 & 28 & 50 & 110 & w & 8.0 \\
\hline 2821 & Plastics Materials and Resins . . . . . . . . . . . . & 288 & 7 & 11 & 18 & 59 & 115 & 77 & 6.4 \\
\hline 2822 & Synthetic Rubber . . . . . . . . . . . . . . & 112 & $\cdot$ & 1 & 10 & 10 & 91 & 0 & 14.8 \\
\hline 2823 & Cellulosic Manmade Fibers . . . . . . . . . . . . . . & 31 & * & 0 & 4 & 10 & 12 & 6 & 32.0 \\
\hline 2824 & Organic Fibers, Noncellulosic . . . . . . . . . . . . & 98 & $\mathbf{Q}$ & 1 & 2 & $W$ & 32 & W & 3.4 \\
\hline 2865 & Cyclic Crudes and Intermediates . . . . . . . . . . . & 159 & 3 & $\mathbf{W}$ & 17 & 23 & 56 & w & 11.6 \\
\hline 2869 & Industrial Organic Chemicals, nec . . . . . . . . . . & 1,191 & 25 & 38 & 27 & 163 & 181 & 756 & 7.0 \\
\hline 2873 & Nitrogenous Fertilizers . . . . . . . . . . . . . & 280 & 1 & 68 & 63 & 111 & 38 & 0 & 24.6 \\
\hline 2874 & Phosphatic Fertilizers . . . . . . . . . . . . . . & 34 & 1 & - & $\mathbf{Q}$ & 8 & 21 & W & 4.5 \\
\hline 29 & Petroleum and Coal Products $\ldots \ldots \ldots \ldots \ldots \ldots$ & 2,987 & 61 & 30 & 42 & 77 & 220 & 2,557 & 4.0 \\
\hline 2911 & Petroleum Refining $\ldots \ldots \ldots \ldots \ldots \ldots \ldots$ & 2,893 & 13 & 17 & 10 & 76 & 220 & 2,557 & 3.8 \\
\hline 30 & Rubber and Misc. Plastics Products $\ldots \ldots \ldots \ldots \ldots$ & 237 & 83 & 54 & 35 & 36 & $W$ & W & 5.8 \\
\hline 3011 & Tires and Inner Tubes . . . . . . . . . . . . . . & 42 & $\bullet$ & 3 & $\mathbf{w}$ & 10 & 18 & W & 3.8 \\
\hline 308 & Miscellaneous Plastics Products, nec . . . . . . . . . & 152 & 63 & 40 & 25 & 22 & 2 & 0 & 10.7 \\
\hline 31 & Leather and Leather Products $\ldots \ldots \ldots \ldots \ldots \ldots$ & 12 & 5 & 4 & 2 & 2 & 0 & 0 & 22.2 \\
\hline 32 & Stone, Clay and Glass Products $\ldots \ldots \ldots \ldots \ldots$ & 894 & 245 & 373 & 201 & 68 & $w$ & $w$ & 7.8 \\
\hline 3211 & 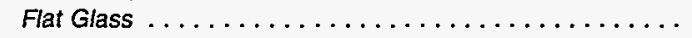 & 49 & * & 8 & 24 & 16 & 0 & 0 & 3.1 \\
\hline 3221 & Glass Containers $\ldots \ldots \ldots \ldots \ldots \ldots \ldots \ldots$ & 85 & * & W & 53 & $w$ & 0 & 0 & 6.4 \\
\hline 3229 & Pressed and Blown Glass, nec . . . . . . . . . . . & $\mathbf{W}$ & 3 & 13 & W & 29 & 0 & 0 & 6.7 \\
\hline 3241 & Cement, Hydraulic . . . . . . . . . . . . . . . & 329 & 25 & 245 & 58 & 0 & 0 & 0 & 10.1 \\
\hline 3274 & 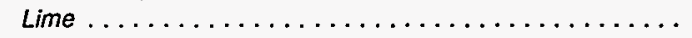 & 117 & 82 & W & W & 0 & 0 & 0 & 29.1 \\
\hline 3296 & Mineral Wool . . . . . . . . . . . . . . . . . . & 41 & 3 & 10 & 19 & W & W & 0 & 1.4 \\
\hline 33 & Primary Metal Industries . . . . . . . . . . . . . . & 2,292 & 94 & 114 & 134 & 355 & 355 & 1,240 & 4.3 \\
\hline 3312 & Blast Fumaces and Steel Mills . . . . . . . . . . . . & 1,569 & 2 & 25 & 51 & 139 & 197 & 1,154 & 6.9 \\
\hline 3313 & Electrometalurgical Products . . . . . . . . . . . . . & 31 & $\bullet$ & 7 & W & W & 0 & 0 & 9.9 \\
\hline 3321 & Gray and Ductile Iron Foundries $\ldots \ldots \ldots \ldots \ldots$ & 74 & W & 15 & 17 & 13 & w & 0 & 8.5 \\
\hline 3331 & Primary Copper . . . . . . . . . . . . . . . & 22 & W & 0 & $\bullet$ & $\mathbf{W}$ & 12 & W & 1.0 \\
\hline 3334 & Primary Aluminum $\ldots \ldots \ldots \ldots \ldots \ldots \ldots \ldots \ldots \ldots \ldots \ldots \ldots$ & 252 & * & * & 0 & 112 & W & $w$ & 3.4 \\
\hline 3339 & Primany Nonferrous Metals, nec . . . . . . . . . . . & 42 & W & 5 & 13 & 20 & 0 & W & 2.4 \\
\hline 3353 & Aluminum Sheet, Plate, and Foil . . . . . . . . . . . & 60 & $\mathbf{Q}$ & 1 & 3 & 12 & 13 & 32 & 1.2 \\
\hline 34 & Fabricated Metal Products $\ldots \ldots \ldots \ldots \ldots \ldots \ldots$ & 305 & 116 & 80 & 46 & 39 & 19 & 5 & 7.9 \\
\hline 35 & Industrial Machinery and Equipment $\ldots \ldots \ldots \ldots \ldots$ & 235 & 70 & 30 & 42 & 38 & 16 & 38 & 7.7 \\
\hline 357 & Computer and Office Equipment . . . . . . . . . . . . & 21 & 2 & 2 & 2 & 4 & 2 & 10 & 11.5 \\
\hline 36 & Electronic and Other Electric Equipment $\ldots \ldots \ldots \ldots \ldots$ & 196 & 28 & 33 & 35 & 45 & 21 & 35 & 7.3 \\
\hline
\end{tabular}

See footnotes at end of table. 
Table A31. Total Inputs of Energy for Heat, Power, and Electricity Generation by Value of Shipment Categories, Industry Group, and Selected Industries, 1991 (Continued) (Estimates in Trillion Btu)

\begin{tabular}{|c|c|c|c|c|c|c|c|c|c|}
\hline \multirow[b]{2}{*}{$\begin{array}{l}\text { SIC } \\
\text { Code* }\end{array}$} & \multirow[b]{2}{*}{$\begin{array}{l}\text { Industry Groups } \\
\text { and Industry }\end{array}$} & \multirow[b]{2}{*}{ Total } & \multicolumn{6}{|c|}{$\begin{array}{l}\text { Value of Shipments and Receipts } \\
\text { (million dollars) }\end{array}$} & \multirow{3}{*}{$\begin{array}{l}\text { RSE } \\
\text { Row } \\
\text { Factors }\end{array}$} \\
\hline & & & Under 20 & $20-49$ & $50-99$ & $100-249$ & $250-499$ & $\begin{array}{l}500 \text { and } \\
\text { Over }\end{array}$ & \\
\hline & RSE Column Factors: & 0.6 & 1.6 & 1.2 & 0.9 & 0.8 & 1.0 & 1.1 & \\
\hline 37 & Transportation Equipment ..... & 333 & 18 & 21 & 21 & 26 & 31 & 216 & 5.3 \\
\hline 3711 & Motor Vehicles and Car Bodles . . . . . . . . . . . . . . . & 105 & - & • & • & * & 2 & 102 & 5.6 \\
\hline 3714 & Motor Vehicle Parts and Accessories $\ldots \ldots \ldots \ldots \ldots$ & 99 & 7 & 9 & 13 & 13 & 18 & 39 & 6.0 \\
\hline 38 & Instruments and Related Products $\ldots \ldots \ldots \ldots \ldots \ldots$ & 98 & 9 & 9 & 10 & 19 & 10 & 41 & 12.3 \\
\hline 3841 & Surgical and Medical Instruments $\ldots \ldots \ldots \ldots \ldots \ldots$ & 6 & 1 & 1 & 2 & 2 & - & - & 13.3 \\
\hline \multirow[t]{2}{*}{39} & Misc. Manuiacturing Industries $\ldots \ldots \ldots \ldots \ldots \ldots$ & 31 & 12 & 7 & 5 & 4 & 3 & 0 & 12.7 \\
\hline & Total $\ldots \ldots \ldots \ldots \ldots \ldots \ldots \ldots \ldots \ldots \ldots \ldots \ldots \ldots \ldots \ldots \ldots \ldots$ & 15,027 & 1,349 & 1,560 & 1,386 & 2,561 & 2,632 & 5,538 & 2.7 \\
\hline
\end{tabular}

- See Appendices B and F for descriptions of the Standard Industrial Classification system.

'Value of Shipments and Recelpts were supplied by the Bureau of the Census.

- Estlmate less than 0.5. Data are included in higher level totals.

$W=$ Withheld to avold disciosing data for individual establlshments. Data are included in higher level totals.

$\mathrm{Q}=$ Withheld because Relative Standard Error is greater than 50 percent. Data are included in higher level totals.

Notes: - To obtaln a RSE percentage for any table cell, multiply the cell's corresponding RSE column and RSE row factors. - Totals may not equal sum of components because of independent rounding. - The estimates presented in this table are for the total consumption of energy for the production of heat and power, regardless of where the energy was produced. Specifically, the estimates include the quantities of energy that were originally produced offsite and purchased by or transferred to the establlshment, plus those that were produced onsite from other energy or input materials not classified as energy, or were extracted from captive (onsite) mines or wells.

Source: Energy Information Adminlstration, Office of Energy Markets and End Use, Energy End Use and Integrated Statistics Division, Form ElA-846, "1991 Manufacturing Energy Consumption Survey," and Bureau of the Census, Industry Division, data files for the "1991 Annual Survey of Manufactures." 


\section{Table A32. Total Consumption of Offsite-Produced Energy for Heat, Power, and Electricity Generation by Value of Shipment Categories, Industry Group, and Selected Industries, 1991 \\ (Estimates in Trillion Btu)}

\begin{tabular}{|c|c|c|c|c|c|c|c|c|c|}
\hline \multirow[b]{2}{*}{$\begin{array}{l}\text { SIC } \\
\text { Code }\end{array}$} & \multirow[b]{2}{*}{$\begin{array}{l}\text { Industry Groups } \\
\text { and Industry }\end{array}$} & \multirow[b]{2}{*}{ Total } & \multicolumn{6}{|c|}{$\begin{array}{l}\text { Value of Shipments and Receipts }{ }^{\mathrm{b}} \\
\text { (million dollars) }\end{array}$} & \multirow{3}{*}{$\begin{array}{c}\text { RSE } \\
\text { Row } \\
\text { Factors }\end{array}$} \\
\hline & & & Under 20 & $20-49$ & $50-99$ & $100-249$ & $250-499$ & $\begin{array}{l}500 \text { and } \\
\text { Over }\end{array}$ & \\
\hline & RSE Column Factors: & 0.6 & 1.6 & 1.2 & 0.9 & 0.8 & 1.0 & 1.1 & \\
\hline 20 & Food and Kindred Products . . . . & 922 & 109 & 135 & 174 & 203 & 161 & 141 & 5.2 \\
\hline 2011 & Meat Packing Plants $\ldots \ldots \ldots \ldots \ldots \ldots \ldots$ & 48 & 2 & 3 & 3 & 6 & 13 & 22 & 7.9 \\
\hline 2033 & Canned Fruits and Vegetables . . . . . . . . . . & 44 & 9 & 10 & 8 & 12 & $w$ & $W$ & 11.7 \\
\hline 2037 & Frozen Fruits and Vegetables . . . . . . . . . . . . . & 40 & 5 & 4 & 14 & 15 & 1 & 0 & 15.3 \\
\hline 2046 & Wet Com Milling $\ldots \ldots \ldots \ldots \ldots \ldots \ldots \ldots \ldots \ldots \ldots$ & 141 & * & W & W & 23 & 65 & $\mathbf{W}$ & 9.5 \\
\hline 2051 & Bread, Cake and Related Products . . . . . . . . . . . & 32 & 10 & 13 & 6 & 3 & • & * & 9.0 \\
\hline 2063 & 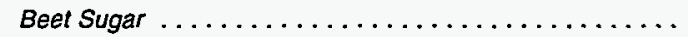 & 67 & 0 & 14 & 35 & 18 & 0 & 0 & 6.9 \\
\hline 2075 & Soybean Oil Mills . . . . . . . . . . . . . . . . & 50 & * & - & W & 20 & 17 & $W$ & 5.9 \\
\hline 2082 & Malt Beverages . . . . . . . . . . . . . . . & 50 & * & * & 2 & 5 & 7 & 36 & 11.7 \\
\hline 21 & Tobacco Manufactures $\ldots \ldots \ldots \ldots \ldots \ldots$ & 26 & $\cdot$ & 2 & 1 & $\mathbf{W}$ & $w$ & 15 & 8.8 \\
\hline 22 & Textile Mill Products $\ldots \ldots \ldots \ldots \ldots \ldots \ldots \ldots$ & 272 & 54 & 81 & 61 & W & 22 & 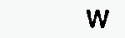 & 7.2 \\
\hline 23 & Apparel and Other Textile Products $\ldots \ldots \ldots \ldots \ldots$ & 44 & 23 & 8 & 7 & 4 & 1 & 2 & 17.3 \\
\hline 24 & Lumber and Wood Products $\ldots \ldots \ldots \ldots \ldots$ & 197 & 74 & 87 & 22 & 14 & 0 & 1 & 16.7 \\
\hline 25 & Furniture and Fixtures $\ldots \ldots \ldots \ldots \ldots \ldots$ & 46 & 16 & 15 & 8 & 3 & $\cdot$ & 3 & 16.8 \\
\hline 26 & Paper and Allied Products $\ldots \ldots \ldots \ldots \ldots \ldots$ & 1,540 & 63 & 104 & 187 & 555 & 566 & 65 & 4.4 \\
\hline 2611 & 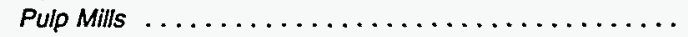 & 103 & 0 & 3 & 13 & 54 & 33 & 0 & 18.1 \\
\hline 2621 & Paper Mills . . . . . . . . . . . . . . . . . . & 774 & 12 & 16 & 74 & 282 & 346 & 43 & 5.1 \\
\hline 2631 & Paperboard Mills . . . . . . . . . . . . . . & 527 & W & 31 & 75 & 207 & 177 & W & 7.5 \\
\hline 27 & Printing and Publishing $\ldots \ldots \ldots \ldots \ldots \ldots$ & 108 & 41 & 24 & 17 & 20 & 4 & 2 & 11.6 \\
\hline 28 & Chemicals and Allied Products $\ldots \ldots \ldots \ldots \ldots \ldots$ & 2,674 & 119 & 233 & 242 & 594 & 625 & 861 & 5.5 \\
\hline 2812 & Alkalies and Chlorine $\ldots \ldots \ldots \ldots \ldots \ldots \ldots$ & 159 & W & 8 & 21 & W & 0 & W & 16.0 \\
\hline 2813 & Industrial Gases . . . . . . . . . . . . . . . & 86 & 34 & 18 & 13 & 21 & 0 & 0 & 6.2 \\
\hline 2819 & Industrial Inorganic Chemicals, nec ............ & 303 & W & 30 & 28 & 45 & 113 & $W$ & 7.8 \\
\hline 2821 & Plastics Materials and Resins . . . . . . . . . . . . & 262 & 7 & 11 & 15 & 56 & 108 & 65 & 6.3 \\
\hline 2822 & Synthetic Rubber . . . . . . . . . . . . . . . & 68 & * & 1 & 10 & 10 & 47 & 0 & 13.1 \\
\hline 2823 & Cellulosic Manmade Fibers . . . . . . . . . . . . . . . & 31 & $\bullet$ & 0 & 4 & 10 & 12 & 6 & 32.0 \\
\hline 2824 & Organic Fibers, Noncellulosic . . . . . . . . . . . . & 97 & $Q$ & 1 & 2 & W & 32 & $W$ & 3.4 \\
\hline 2865 & Cyclic Crudes and Intermediates . . . . . . . . . . . & 136 & 3 & W & 17 & 22 & 54 & W & 11.1 \\
\hline 2869 & Industrial Organic Chemicals, nec . . . . . . . . . . & 935 & 17 & 39 & 27 & 148 & 171 & 532 & 7.2 \\
\hline 2873 & Nitrogenous Fertilizers . . . . . . . . . . . . . & 278 & 1 & 68 & 63 & 109 & 38 & 0 & 24.5 \\
\hline 2874 & Phosphatic Fertilizers $\ldots \ldots \ldots \ldots \ldots \ldots$ & 36 & 1 & * & $\mathbf{Q}$ & 9 & 21 & $W$ & 4.6 \\
\hline 29 & Petroleum and Coal Products $\ldots \ldots \ldots \ldots \ldots$ & 1,138 & 53 & 22 & 18 & 42 & 60 & 943 & 4.5 \\
\hline 2911 & Petroleum Refining $\ldots \ldots \ldots \ldots \ldots \ldots \ldots \ldots \ldots \ldots \ldots \ldots$ & 1,065 & 5 & 9 & 7 & 41 & 60 & 943 & 4.4 \\
\hline 30 & Rubber and Misc. Plastics Products $\ldots \ldots \ldots \ldots \ldots \ldots$ & 235 & 83 & 54 & 35 & 34 & $w$ & $W$ & 5.7 \\
\hline 3011 & Tires and Inner Tubes $\ldots \ldots \ldots \ldots \ldots \ldots$ & 42 & * & 3 & $W$ & 10 & 18 & $w$ & 3.8 \\
\hline 308 & Miscellaneous Plastics Products, noc . . . . . . . . . & 150 & 63 & 40 & 25 & 21 & 2 & 0 & 10.5 \\
\hline 31 & Leather and Leather Products $\ldots \ldots \ldots \ldots \ldots$ & 12 & 5 & 4 & 2 & 2 & 0 & 0 & 22.1 \\
\hline 32 & Stone, Clay and Glass Products $\ldots \ldots \ldots \ldots \ldots \ldots$ & 877 & 245 & 358 & 200 & 68 & $W$ & $W$ & 7.8 \\
\hline 3211 & Flat Glass $\ldots \ldots \ldots \ldots \ldots \ldots \ldots \ldots \ldots \ldots \ldots \ldots \ldots \ldots$ & 49 & * & 8 & 24 & 16 & 0 & 0 & 3.1 \\
\hline 3221 & Glass Containers . . . . . . . . . . . . . . & 85 & • & W & 53 & W & 0 & 0 & 6.4 \\
\hline 3229 & Pressed and Blown Glass, nec . . . . . . . . . . & W & 3 & 13 & W & 29 & 0 & 0 & 6.6 \\
\hline 3241 & Cement, Hydraulic . . . . . . . . . . . . . . & 312 & 25 & 230 & 57 & 0 & 0 & 0 & 10.1 \\
\hline 3274 & 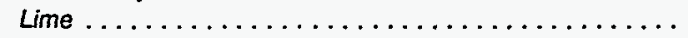 & 117 & 82 & W & W & 0 & 0 & 0 & 29.1 \\
\hline 3296 & Mineral Wool $\ldots \ldots \ldots \ldots \ldots \ldots \ldots \ldots \ldots \ldots$ & 40 & 3 & 10 & 19 & W & $W$ & 0 & 1.4 \\
\hline 33 & Primary Metal Industries $\ldots \ldots \ldots \ldots \ldots \ldots$ & 1,563 & 94 & 102 & 106 & 329 & 286 & 647 & 4.2 \\
\hline 3312 & Blast Fumaces and Steel Mills $\ldots \ldots \ldots \ldots \ldots \ldots$ & 842 & 2 & 12 & 25 & 115 & 128 & 560 & 6.7 \\
\hline 3313 & Electrometalurgical Products . . . . . . . . . . . . . . & 30 & - & 7 & W & W & 0 & 0 & 9.8 \\
\hline 3321 & Gray and Ductile Iron Foundries . . . . . . . . . . . & 74 & W & 15 & 17 & 13 & W & 0 & 8.5 \\
\hline 3331 & Primary Copper . . . . . . . . . . . . . . . . & 21 & W & 0 & $\bullet$ & W & 12 & W & 1.0 \\
\hline 3334 & Primary Aluminum $\ldots \ldots \ldots \ldots \ldots \ldots \ldots \ldots \ldots \ldots \ldots$ & 254 & * & • & 0 & 112 & $W$ & $w$ & 3.5 \\
\hline 3339 & Primary Nonferrous Metals, nec . . . . . . . . . . . & 40 & W & 5 & 13 & 18 & 0 & W & 2.4 \\
\hline 3353 & Aluminum Sheet, Plate, and Foil . . . . . . . . . . & 60 & $\mathbf{Q}$ & 1 & 3 & 12 & 13 & 32 & 1.2 \\
\hline 34 & Fabricated Metal Products $\ldots \ldots \ldots \ldots \ldots \ldots \ldots$ & 305 & 116 & 80 & 46 & 39 & 19 & 4 & 7.9 \\
\hline 35 & Industrial Machinery and Equipment $\ldots \ldots \ldots \ldots \ldots$ & 236 & 71 & 30 & 42 & 39 & 16 & 38 & 7.7 \\
\hline 357 & Computer and Office Equipment $\ldots \ldots \ldots \ldots \ldots \ldots$ & 21 & 2 & 2 & 2 & 4 & 2 & 10 & 11.5 \\
\hline 36 & Electronic and Other Electric Equipment $\ldots \ldots \ldots \ldots$ & 196 & 29 & 33 & 35 & 45 & 21 & 35 & 7.3 \\
\hline
\end{tabular}

See footnotes at end of table. 
Table A32. Total Consumption of Offsite-Produced Energy for Heat, Power, and Electricity Generation by Value of Shipment Categories, Industry Group, and Selected Industries, 1991 (Continued)

(Estimates in Trillion Btu)

\begin{tabular}{|c|c|c|c|c|c|c|c|c|c|}
\hline \multirow[b]{2}{*}{$\begin{array}{c}\text { SIC } \\
\text { Code }\end{array}$} & \multirow[b]{2}{*}{$\begin{array}{l}\text { Industry Groups } \\
\text { and Industry }\end{array}$} & \multirow[b]{2}{*}{ Total } & \multicolumn{6}{|c|}{$\begin{array}{l}\text { Value of Shipments and Receipts } \\
\text { (million dollars) }\end{array}$} & \multirow{3}{*}{$\begin{array}{c}\text { RSE } \\
\text { Row } \\
\text { Factors }\end{array}$} \\
\hline & & & Under 20 & $20-49$ & $50-99$ & $100-249$ & $250-499$ & $\begin{array}{l}500 \text { and } \\
\text { Over }\end{array}$ & \\
\hline & RSE Column Factors: & 0.6 & 1.6 & 1.2 & 0.9 & 0.8 & 1.0 & 1.1 & \\
\hline 37 & Transportatlon Equipment . . . . . & 318 & 18 & 21 & 21 & 26 & 31 & 201 & 5.3 \\
\hline 3711 & Motor Vehicles and Car Bodies . . . . . . . . . . . . . & 90 & $\cdot$ & 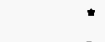 & 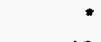 & $\bullet$ & 2 & 88 & 5.0 \\
\hline 3714 & Motor Vehicle Parts and Accessories $\ldots \ldots \ldots \ldots \ldots$ & 99 & 7 & 9 & 13 & 13 & 18 & 38 & 6.0 \\
\hline 38 & Instruments and Related Products $\ldots \ldots \ldots \ldots \ldots$ & 97 & 9 & 9 & 10 & 19 & 10 & 41 & 12.3 \\
\hline 3841 & Surglcal and Medlcal Instruments . . . . . . . . . . . & 6 & 1 & 1 & 2 & 2 & - & - & 13.3 \\
\hline \multirow[t]{2}{*}{39} & Misc. Manufacturing Industries . . . . . . . . . . . & 31 & 12 & 6 & 5 & 4 & 3 & 0 & 11.7 \\
\hline & Total $\ldots \ldots \ldots \ldots \ldots \ldots \ldots \ldots \ldots \ldots$ & 10,837 & 1,232 & 1,409 & 1,238 & 2,098 & 1,854 & 3,006 & 2.6 \\
\hline
\end{tabular}

- See Appendlces B and F for descriptions of the Standard Industrial Classification system.

- Value of Shlpments and Recelpts were supplied by the Bureau of the Census.

- Estimate less than 0.5. Data are included in higher level totals.

$W=W i t h$ held to avoid disclosing data for individual establishments. Data are included in higher level totals.

$\mathrm{Q}=$ Withheld because Relative Standard Error is greater than 50 percent. Data are included in higher level totals.

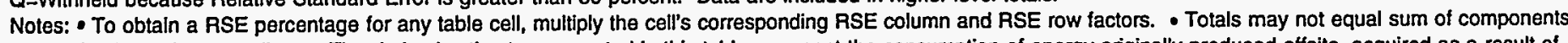

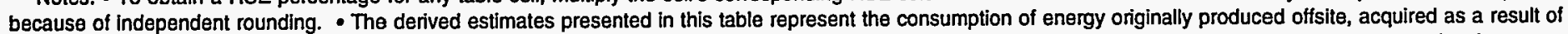

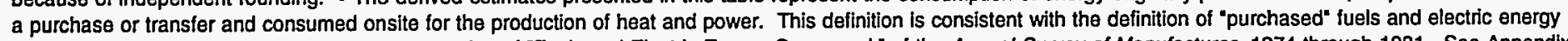

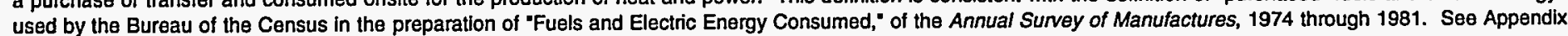
B.

Source: Energy Information Administration, Office of Energy Markets and End Use, Energy End Use and Integrated Statistics Division, Form ElA-846, "1991 Manufacturing Energy Consumption Survey," and Office of Oil and Gas, Petroleum Supply Division, Form EIA-810, "Monthly Refinery Report" for 1991. 
Table A33. Total Primary Consumption of Energy for All Purposes by Employment Size Categories, Industry Group, and Selected Industries, 1991

(Estimates in Trillion Btu)

\begin{tabular}{|c|c|c|c|c|c|c|c|c|c|}
\hline \multirow[b]{2}{*}{$\begin{array}{l}\text { SIC } \\
\text { Code }^{a}\end{array}$} & \multirow[b]{2}{*}{$\begin{array}{l}\text { Industry Groups } \\
\text { and Industry }\end{array}$} & \multirow[b]{2}{*}{ Total } & \multicolumn{6}{|c|}{ Employment Size ${ }^{b}$} & \multirow{3}{*}{$\begin{array}{c}\text { RSE } \\
\text { Row } \\
\text { Factors }\end{array}$} \\
\hline & & & Under 20 & $20-49$ & $50-99$ & $100-249$ & $250-499$ & $\begin{array}{l}500 \text { and } \\
\text { Over }\end{array}$ & \\
\hline & RSE Column Factors: & 0.6 & 2.0 & 1.6 & 1.1 & 0.8 & 0.8 & 0.8 & \\
\hline 20 & Food and Kindred Products & 956 & 68 & 99 & 246 & 262 & 152 & 128 & 6.3 \\
\hline 2011 & $\ldots \ldots \ldots \ldots \ldots \ldots$ & 49 & 1 & 2 & 5 & 5 & 8 & 29 & 8.8 \\
\hline 2033 & Canned Fruits and Vegetables & 44 & 3 & 4 & 12 & 12 & 9 & 5 & 13.3 \\
\hline 2037 & Frozen Fruits and Vegetables .... & 40 & 1 & 3 & 6 & 10 & 13 & 8 & 16.6 \\
\hline 2046 & $\ldots \ldots \ldots \ldots \ldots$ & 140 & * & W & 48 & 57 & 20 & W & 14.1 \\
\hline 2051 & Bread, Cake and Related Products . . . . . . . . . . . . . & 32 & 2 & 4 & 9 & 11 & 5 & 2 & 9.9 \\
\hline 2063 & . $\ldots \ldots \ldots \ldots \ldots \ldots \ldots$ & 67 & 0 & W & 34 & w & 0 & 0 & 6.8 \\
\hline 2075 & Soybean Oil Mills $\ldots \ldots \ldots \ldots \ldots \ldots \ldots \ldots \ldots \ldots$ & 51 & 8 & 18 & 10 & 16 & 0 & 0 & 2.8 \\
\hline 2082 & $\ldots \ldots \ldots \ldots \ldots \ldots \ldots$ & 50 & • & * & w & w & 14 & 31 & 11.8 \\
\hline 21 & Tobacco Manufactures $\ldots \ldots \ldots \ldots \ldots \ldots \ldots \ldots$ & 24 & * & • & 2 & 2 & 7 & 13 & 10.0 \\
\hline 22 & Textile Mill Products $\ldots \ldots \ldots \ldots \ldots \ldots \ldots \ldots$ & 274 & 4 & 11 & 52 & 82 & 83 & 42 & 7.4 \\
\hline 23 & Apparel and Other Textile Products $\ldots \ldots \ldots \ldots \ldots \ldots$ & 44 & 4 & 6 & 9 & 12 & 8 & 7 & 16.0 \\
\hline 24 & Lumber and Wood Products & 451 & 31 & 84 & 202 & 103 & 28 & 2 & 16.6 \\
\hline 25 & Furniture and Fixtures $\ldots \ldots \ldots \ldots \ldots \ldots \ldots \ldots$ & 68 & Q & Q & 8 & 17 & 12 & 10 & 19.3 \\
\hline 26 & Paper and Allied Products $\ldots \ldots \ldots \ldots \ldots \ldots \ldots \ldots$ & 2,506 & 11 & 56 & 188 & 448 & 882 & 922 & 5.0 \\
\hline 2611 & 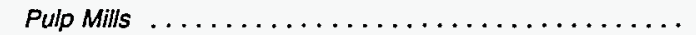 & 300 & - & Q & 17 & 83 & 147 & 51 & 20.2 \\
\hline 2621 & Paper Mills $\ldots \ldots \ldots \ldots \ldots \ldots \ldots \ldots \ldots \ldots \ldots$ & 1,211 & 1 & 12 & 57 & 125 & 328 & 689 & 5.1 \\
\hline 2631 & 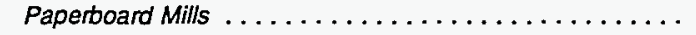 & 859 & w & w & 57 & 217 & 386 & 170 & 6.1 \\
\hline 27 & Printing and Publishing $[\ldots \ldots \ldots, \ldots \ldots \ldots \ldots$, & 108 & 20 & 10 & 22 & 22 & 19 & 16 & 11.6 \\
\hline 28 & Chemicals and Allied Products $\ldots \ldots \ldots \ldots \ldots \ldots \ldots$ & 5,051 & 193 & 320 & 977 & 1,001 & 931 & 1,630 & 5.7 \\
\hline 2812 & Alkalies and Chlorine. & 160 & • & $\mathbf{5}$ & 56 & w & w & w & 18.0 \\
\hline 2813 & Industrial Gases $\ldots \ldots \ldots \ldots \ldots \ldots \ldots \ldots \ldots \ldots$ & $\mathbf{w}$ & 44 & 30 & $\mathbf{w}$ & W & 0 & 0 & 7.9 \\
\hline 2819 & Industrial Inorganic Chemicals, nec $\ldots \ldots \ldots \ldots \ldots \ldots$ & 325 & 14 & 20 & 48 & 42 & 79 & 123 & 8.7 \\
\hline 2821 & Plastics Materials and Resins $\ldots \ldots \ldots \ldots \ldots \ldots \ldots$ & 633 & 6 & 27 & 108 & 262 & 149 & 80 & 7.0 \\
\hline 2822 & 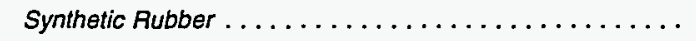 & 119 & * & * & 5 & w & 20 & w & 14.9 \\
\hline 2823 & Cellulosic Manmade Fibers . . . . . . . . . . . . . . . & 31 & 0 & * & 0 & 0 & 0 & 31 & 25.8 \\
\hline 2824 & Organic Fibers, Noncellulosic $\ldots \ldots \ldots \ldots \ldots \ldots \ldots$ & w & - & - & $\mathbf{Q}$ & 9 & 5 & w & 5.6 \\
\hline 2865 & Cyclic Crudes and Intermediates $\ldots \ldots \ldots \ldots \ldots \ldots$ & 236 & w & 22 & 42 & 31 & 128 & w & 12.2 \\
\hline 2869 & Industrial Organic Chemicals, nec ............... & 2,289 & 25 & 50 & 267 & 425 & 418 & 1,103 & 7.6 \\
\hline 2873 & Nitrogenous Fertilizers . . . . . . & 568 & 69 & 91 & 332 & 50 & 25 & 0 & 26.4 \\
\hline 2874 & 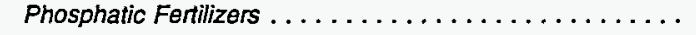 & 65 & * & 1 & $\mathbf{Q}$ & 36 & w & w & 4.0 \\
\hline 29 & Petroleum and Coal Products $\ldots \ldots \ldots \ldots \ldots \ldots$ & 5,967 & 97 & 154 & 262 & 671 & 700 & 1,215 & 3.7 \\
\hline 2911 & Petroleum Refining ${ }^{\circ} \ldots \ldots \ldots \ldots \ldots \ldots$ & 5,762 & 23 & 37 & 250 & 669 & 698 & 1,215 & 3.1 \\
\hline 30 & Rubber and Misc. Plastics Products .... & 238 & 25 & 32 & 55 & 44 & 40 & 43 & 7.0 \\
\hline 3011 & $\ldots \ldots \ldots \ldots \ldots \ldots \ldots \ldots \ldots$ & w & * & * & 2 & w & $\mathbf{W}$ & 34 & 4.4 \\
\hline 308 & Miscellaneous Plastics Products, nec $\ldots \ldots \ldots \ldots \ldots$ & 151 & 19 & 27 & 42 & 32 & 27 & 3 & 11.1 \\
\hline 31 & Leather and Leather Products & 12 & 1 & Q & 5 & 2 & 2 & 1 & 24.4 \\
\hline 32 & Stone, Clay and Glass Products $\ldots$ & 880 & 47 & 119 & 426 & 142 & 107 & 39 & 6.9 \\
\hline 3211 & $\ldots \ldots \ldots \ldots \ldots \ldots \ldots$ & 49 & * & * & W & 13 & 28 & w & 3.9 \\
\hline 3221 & Glass Containers $\ldots \ldots \ldots \ldots \ldots \ldots \ldots \ldots \ldots$ & 85 & 0 & 0 & 6 & 46 & 27 & 6 & 7.0 \\
\hline 3229 & Pressed and Blown Glass, nec $\ldots \ldots \ldots \ldots \ldots \ldots \ldots$ & w & * & Q & 8 & w & 19 & 21 & 7.8 \\
\hline 3241 & Cement, Hydraulic $\ldots \ldots \ldots \ldots \ldots \ldots \ldots \ldots \ldots$ & 312 & - & 36 & 250 & 26 & 0 & 0 & 13.3 \\
\hline 3274 & Lime . & 117 & $\mathbf{Q}$ & 21 & Q & 0 & w & 0 & 24.9 \\
\hline 3296 & Mineral Wool . . . . . . . . . . . . . . . . . . & 41 & $w$ & 2 & 8 & 20 & 7 & w & 1.5 \\
\hline 33 & Primary Metal Industries $\ldots \ldots \ldots \ldots \ldots$ & 2,467 & 37 & 43 & 167 & 225 & 473 & 1,521 & 6.0 \\
\hline 3312 & Blast Fumaces and Steel Mills & 1,673 & w & w & 56 & 112 & 181 & 1,318 & 7.8 \\
\hline 3313 & Electrometalurgical Products .... . & 41 & 0 & * & 14 & w & w & 0 & 10.8 \\
\hline 3321 & Gray and Ductile Iron Foundries . & W & 3 & 3 & W & W & 13 & 27 & 10.3 \\
\hline 3331 & Primary Copper $\ldots \ldots \ldots \ldots \ldots \ldots \ldots \ldots \ldots \ldots$ & 21 & - & 0 & W & W & 16 & 0 & 1.0 \\
\hline 3334 & Primary Aluminum $\ldots \ldots \ldots \ldots \ldots \ldots \ldots \ldots \ldots$ & 297 & * & - & * & W & 176 & w & 4.0 \\
\hline 3339 & Primary Nonferrous Metals, nec $\ldots \ldots \ldots \ldots \ldots \ldots$ & 52 & * & w & 7 & 9 & 23 & w & 2.9 \\
\hline 3353 & Aluminum Sheet, Plate, and Foil ............... & 61 & - & Q & 4 & 5 & 17 & 33 & 1.4 \\
\hline 34 & Fabricated Metal Products $\ldots \ldots \ldots \ldots \ldots \ldots \ldots \ldots$ & 307 & 42 & 42 & 91 & 57 & 31 & 44 & 7.7 \\
\hline 35 & Industrial Machinery and Equipment ........... & 237 & 33 & 16 & 33 & 37 & 45 & 74 & 8.8 \\
\hline 357 & Computer and Office Equipment ........ & 21 & 1 & 1 & 2 & 2 & 4 & 13 & 13.3 \\
\hline 36 & Electronic and Other Electric Equipment .. & 212 & 6 & 9 & 33 & 41 & 47 & 77 & 9.9 \\
\hline
\end{tabular}

See footnotes at end of table. 
Table A33. Total Primary Consumption of Energy for All Purposes by Employment Size Categories, Industry Group, and Selected Industries, 1991 (Continued) (Estimates in Trillion Btu)

\begin{tabular}{|c|c|c|c|c|c|c|c|c|c|}
\hline \multirow[b]{2}{*}{$\begin{array}{c}\text { SiC } \\
\text { Code }\end{array}$} & \multirow[b]{2}{*}{$\begin{array}{l}\text { Industry Groups } \\
\text { and Industry }\end{array}$} & \multirow[b]{2}{*}{ Total } & \multicolumn{6}{|c|}{ Employment Sizeb } & \multirow{3}{*}{$\begin{array}{c}\text { RSE } \\
\text { Row } \\
\text { Factors }\end{array}$} \\
\hline & & & \multirow{2}{*}{$\frac{\text { Under } 20}{2.0}$} & \multirow{2}{*}{$\frac{20-49}{1.6}$} & \multirow{2}{*}{$\frac{50-99}{1.1}$} & \multirow{2}{*}{$\frac{100-249}{0.8}$} & \multirow{2}{*}{$\frac{250-499}{0.8}$} & \multirow{2}{*}{$\frac{\begin{array}{c}500 \text { and } \\
\text { Over }\end{array}}{0.8}$} & \\
\hline & RSE Column Factors: & 0.6 & & & & & & & \\
\hline 37 & Transportation Equipment $\ldots \ldots \ldots \ldots \ldots \ldots$ & 323 & 3 & 5 & 17 & 25 & 28 & 246 & 7.5 \\
\hline 3711 & Motor Vehicles and Car Bodies . . . . . . . . . . . & 88 & * & $*$ & $\bullet$ & $*$ & 2 & 86 & 6.0 \\
\hline 3714 & Motor Vehicle Parts and Accessories . . . . . . . . . . & 100 & 2 & 1 & 9 & 13 & 14 & 62 & 8.2 \\
\hline 38 & Instruments and Related Products $\ldots \ldots \ldots \ldots \ldots$ & 98 & 2 & 3 & 6 & 11 & 17 & 59 & 15.4 \\
\hline 3841 & Surgical and Medlcal Instruments . . . . . . . . . . & 6 & $\bullet$ & * & 1 & 2 & 2 & 1 & 13.9 \\
\hline \multirow[t]{2}{*}{39} & Misc. Manufacturing Industries $\ldots \ldots \ldots \ldots \ldots$ & 32 & 4 & 3 & 8 & 9 & 4 & 4 & 14.2 \\
\hline & Total $\ldots \ldots \ldots \ldots \ldots \ldots \ldots \ldots \ldots \ldots \ldots \ldots \ldots \ldots \ldots$ & 20,257 & 632 & 1,027 & 2,807 & 3,213 & 3,615 & 6,094 & 3.0 \\
\hline
\end{tabular}

\footnotetext{
" See Appendices B and F for descriptions of the Standard Industrial Classification system.

'Employment Slze categories were supplied by the Bureau of the Census.

' For the petroleum refining industry only, the feedstocks and raw material inputs for the production of nonenergy products (i.e., asphalt, waxes, lubricants, and solvents) and feedstock consumption at adjoining petrochemical plants are included in the "Total" column, regardless of type of energy. The remaining columns for the petroleum refining industry Include only energy that was consumed for the production of heat and power. See Appendix B for more information.

- Estimate less than 0.5. Data are Included in higher level totals.

$W=$ Withheld to avold disclosing data for individual establishments. Data are included in higher level totals.

$Q=$ Withheld because Relative Standard Error is greater than 50 percent. Data are included in higher level totals.

Notes: - To obtain a RSE percentage for any table cell, multiply the cell's corresponding RSE column and RSE row factors. - Totals may not equal sum of components because of independent rounding. - The derived estimates presented in this table are for the primary consumption of energy for heat and power and as feedstocks or raw material inputs. Primary consumption is defined as the consumption of the energy that was originally produced offsite or was produced onsite from input materials not classlfied as energy. Examples of the latter are hydrogen produced from the electrolysis of brine; the output of captive (onsite) mines or wells; woodchips, bark, and woodwaste from wood purchased as a raw material input; and waste materials such as wastepaper and packing materials. Primary consumption excludes quantitles of energy that are produced from other energy inputs and, therefore, avolds double counting.

Source: Energy Informatlon Administration, Office of Energy Markets and End Use, Energy End Use and Integrated Statistics Division, Form ElA-846, "1991 Manufacturing Energy Consumptlon Survey," and Office of Oll and Gas, Petroleum Supply Division, Form EIA-810, "Monthly Refinery Report" for 1991, and Bureau of the Census, Industry Division, data files for the "1991 Annual Survey of Manufactures."
} 
Table A34. Total Inputs of Energy for Heat, Power, and Electricity Generation by Employment Size Categories, Industry Group, and Selected Industries, 1991 (Estimates in Trillion Btu)

\begin{tabular}{|c|c|c|c|c|c|c|c|c|c|}
\hline \multirow[b]{2}{*}{$\begin{array}{c}\text { SIC } \\
\text { Code }\end{array}$} & \multirow[b]{2}{*}{$\begin{array}{l}\text { Industry Groups } \\
\text { and Industry }\end{array}$} & \multirow[b]{2}{*}{ Total } & \multicolumn{6}{|c|}{ Employment Size ${ }^{b}$} & \multirow{3}{*}{$\begin{array}{c}\text { PSE } \\
\text { Row } \\
\text { Factors }\end{array}$} \\
\hline & & & Under 50 & $50-99$ & $100-249$ & $250-499$ & $500-999$ & $\begin{array}{c}1,000 \text { and } \\
\text { Over }\end{array}$ & \\
\hline & RSE Column Factors: & 0.6 & 2.0 & 1.6 & 1.1 & 0.8 & 0.8 & 0.8 & \\
\hline 20 & Food and Kindred Products $\ldots \ldots \ldots \ldots \ldots \ldots$ & 953 & 68 & 99 & 245 & 261 & 152 & 128 & 6.3 \\
\hline 2011 & Meat Packing Plants $\ldots \ldots \ldots \ldots \ldots \ldots$ & 49 & 1 & 2 & 4 & 5 & 8 & 29 & 9.0 \\
\hline 2033 & Canned Fruits and Vegetables . . . . . . . . . . . . & 44 & 3 & 4 & 12 & 12 & 9 & 5 & 13.3 \\
\hline 2037 & Frozen Fruits and Vegetables . . . . . . . . . . . & 40 & 1 & 3 & 6 & 10 & 13 & 8 & 16.6 \\
\hline 2046 & Wet Com Milling $\ldots \ldots \ldots \ldots \ldots \ldots \ldots$ & 140 & * & W & 48 & 57 & 20 & $w$ & 14.1 \\
\hline 2051 & Bread, Cake and Related Products . . . . . . . . . . . & 32 & 2 & 4 & 9 & 10 & 5 & 2 & 9.9 \\
\hline 2063 & 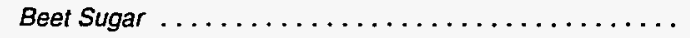 & 67 & 0 & W & 34 & W & 0 & 0 & 6.8 \\
\hline 2075 & Soybean Oil Mills . . . . . . . . . . . . . . . . . . & 50 & 8 & 18 & 9 & 15 & 0 & 0 & 2.8 \\
\hline 2082 & Malt Beverages . . . . . . . . . . . . . . & 50 & $\star$ & $\bullet$ & $w$ & W & 14 & 31 & 11.7 \\
\hline 21 & Tobacco Manufactures $\ldots \ldots \ldots \ldots \ldots \ldots \ldots$ & 24 & $\cdot$ & • & 2 & 2 & 7 & 13 & 10.0 \\
\hline 22 & Textile Mill Products $\ldots \ldots \ldots \ldots \ldots \ldots \ldots \ldots$ & 273 & 4 & 11 & 52 & 82 & 82 & 42 & 7.4 \\
\hline 23 & Apparel and Other Textile Products $\ldots \ldots \ldots \ldots \ldots$ & 44 & 4 & 6 & 9 & 12 & 8 & 7 & 16.0 \\
\hline 24 & Lumber and Wood Products $\ldots \ldots \ldots \ldots \ldots$ & 423 & 31 & 81 & 180 & 101 & 28 & 2 & 16.8 \\
\hline 25 & Fumiture and Fixtures $\ldots \ldots \ldots \ldots \ldots \ldots \ldots$ & 67 & $\mathbf{Q}$ & $\mathbf{Q}$ & 8 & 17 & 12 & 10 & 18.9 \\
\hline 26 & Paper and Allied Products $\ldots \ldots \ldots \ldots \ldots \ldots$ & 2,472 & 11 & 52 & 183 & 444 & 862 & 920 & 5.1 \\
\hline 2611 & Pulp Mills $\ldots \ldots \ldots \ldots \ldots \ldots \ldots \ldots \ldots \ldots$ & 300 & $\bullet$ & $\mathbf{Q}$ & 17 & 83 & 147 & 51 & 20.1 \\
\hline 2621 & 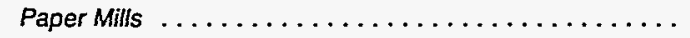 & 1,204 & 1 & 12 & 53 & 125 & 325 & 688 & 5.2 \\
\hline 2631 & 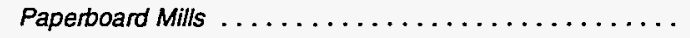 & 832 & W & W & 55 & 212 & 369 & 169 & 6.1 \\
\hline 27 & Printing and Publishing $\ldots \ldots \ldots \ldots \ldots \ldots$ & 108 & 20 & 10 & 22 & 22 & 19 & 16 & 11.6 \\
\hline 28 & Chemicals and Allied Products . . . . . . . . . . . . & 3,040 & 134 & 182 & 567 & 527 & 609 & 1,022 & 5.2 \\
\hline 2812 & Alkalies and Chlorine . . . . . . . . . . . . . . . & 160 & $*$ & 5 & 56 & W & W & $w$ & 17.9 \\
\hline 2813 & Industrial Gases . . . . . . . . . . . . . . . . . . & 91 & 42 & 23 & W & $W$ & 0 & 0 & 6.7 \\
\hline 2819 & Industrial Inorganic Chemicals, nec . . . . . . . . . . & 311 & 13 & 16 & 45 & 40 & 72 & 123 & 8.5 \\
\hline 2821 & Plastics Materials and Resins . . . . . . . . . . . . . & 288 & 6 & 16 & 38 & 109 & 68 & 49 & 6.1 \\
\hline 2822 & Synthetic Rubber . . . . . . . . . . . . . . . . & 112 & $\star$ & * & 5 & w & 21 & W & 14.7 \\
\hline 2823 & Cellulosic Manmade Fibers . . . . . . . . . . . . . & 31 & 0 & $*$ & 0 & 0 & 0 & 31 & 25.6 \\
\hline 2824 & Organic Fibers, Noncellulosic . . . . . . . . . . . . . & 98 & $\star$ & * & $\mathbf{Q}$ & 9 & 5 & 82 & 5.6 \\
\hline 2865 & Cyclic Crudes and Intermediates . . . . . . . . . . & 159 & $w$ & 10 & 32 & 30 & 76 & $W$ & 11.5 \\
\hline 2869 & Industrial Organic Chemicals, nec . . . . . . . . . . & 1,191 & 21 & 39 & 126 & 199 & 260 & 546 & 7.5 \\
\hline 2873 & Nitrogenous Fentilizers $\ldots \ldots \ldots \ldots \ldots \ldots \ldots$ & 280 & 29 & 42 & 176 & 22 & 11 & 0 & 26.2 \\
\hline 2874 & Phosphatic Fertilizers . . . . . . . . . . . . . . & 34 & $*$ & 1 & $\mathbf{Q}$ & 19 & W & 8 & 4.1 \\
\hline 29 & Petroleum and Coal Products $\ldots \ldots \ldots \ldots \ldots \ldots$ & 2,987 & 66 & 50 & 285 & 671 & 700 & 1,215 & 3.2 \\
\hline 2911 & Petroleum Refining $\ldots \ldots \ldots \ldots \ldots \ldots \ldots$ & 2,893 & 23 & 37 & 250 & 669 & 698 & 1,215 & 3.1 \\
\hline 30 & Rubber and Misc. Plastics Products $\ldots \ldots \ldots \ldots \ldots$ & 237 & 25 & 32 & 54 & 46 & 39 & 41 & 7.0 \\
\hline 3011 & Tires and inner Tubes . . . . . . . . . . . . . . . & 42 & - & * & 2 & $W$ & W & 33 & 4.4 \\
\hline 308 & Miscellaneous Plastics Products, nec . . . . . . . . . & 152 & 19 & 27 & 42 & 34 & 27 & 3 & 11.2 \\
\hline 31 & Leather and Leather Products $\ldots \ldots \ldots \ldots \ldots \ldots$ & 12 & 1 & Q & 5 & 2 & 2 & 1 & 24.2 \\
\hline 32 & Stone, Clay and Glass Products $\ldots \ldots \ldots \ldots \ldots \ldots$ & 894 & 47 & 119 & 439 & 142 & 107 & 39 & 6.9 \\
\hline 3211 & 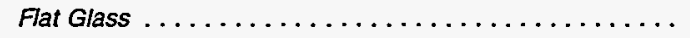 & 49 & - & $*$ & W & 12 & 28 & $W$ & 3.9 \\
\hline 3221 & Glass Containers . . . . . . . . . . . . . . . & 85 & 0 & 0 & 6 & 46 & 27 & 6 & 7.0 \\
\hline 3229 & Pressed and Blown Glass, nec . . . . . . . . . . . & $W$ & - & Q & 8 & W & 19 & 21 & 7.9 \\
\hline 3241 & Cement, Hydraulic . . . . . . . . . . . . . . . . & 329 & * & 36 & 266 & 27 & 0 & 0 & 13.3 \\
\hline 3274 & Lime . . . . . . . . . . . . . . . . . . & 117 & $\mathbf{Q}$ & 20 & Q & 0 & $w$ & 0 & 24.3 \\
\hline 3296 & Mineral Wool . . . . . . . . . . . . . . . . . . . . & 41 & $w$ & 2 & 8 & 20 & 7 & $w$ & 1.5 \\
\hline 33 & Primary Metal Industries $\ldots \ldots \ldots \ldots \ldots \ldots \ldots \ldots$ & 2,292 & 32 & 41 & 137 & 176 & 351 & 1,554 & 5.1 \\
\hline 3312 & Blast Fumaces and Steel Mills $\ldots \ldots \ldots \ldots \ldots \ldots$ & 1,569 & 1 & 1 & 33 & 70 & 92 & 1,372 & 5.6 \\
\hline 3313 & Electrometalurgical Products . . . . . . . . . . . . & 31 & 0 & * & 9 & W & $w$ & 0 & 10.8 \\
\hline 3321 & Gray and Ductile Iron Foundries $\ldots \ldots \ldots \ldots \ldots$ & 74 & 3 & 3 & W & W & 13 & 27 & 10.3 \\
\hline 3331 & Primary Copper . . . . . . . . . . . . . . . . & 22 & $\star$ & 0 & W & W & 16 & 0 & 1.1 \\
\hline 3334 & Primary Aluminum $\ldots \ldots \ldots \ldots \ldots \ldots \ldots \ldots$ & 252 & * & * & - & W & 148 & $W$ & 4.0 \\
\hline 3339 & Primary Nonferrous Metals, nec . . . . . . . . . . . . & 42 & * & W & 6 & 9 & 20 & $w$ & 3.1 \\
\hline 3353 & Aluminum Sheet, Plate, and Foil . . . . . . . . . . . & 60 & * & $\mathbf{Q}$ & 4 & 5 & 17 & 33 & 1.4 \\
\hline 34 & Fabricated Metal Products $\ldots \ldots \ldots \ldots \ldots \ldots \ldots$ & 305 & 41 & 42 & 91 & 56 & 31 & 44 & 7.7 \\
\hline 35 & Industrial Machinery and Equipment $\ldots \ldots \ldots \ldots \ldots$ & 235 & 32 & 16 & 33 & 37 & 44 & 73 & 8.8 \\
\hline 357 & Computer and Office Equipment . . . . . . . . . . . & 21 & 1 & 1 & 2 & 2 & 4 & 13 & 13.3 \\
\hline 36 & Electronic and Other Electric Equipment . . . . . . . . & 196 & 6 & 9 & 22 & 40 & 43 & 76 & 9.4 \\
\hline
\end{tabular}

See footnotes at end of table. 


\section{Table A34. Total Inputs of Energy for Heat, Power, and Electricity Generation by Employment Size Categories, Industry Group, and Selected Industries, 1991 (Continued) \\ (Estimates in Trillion Btu)}

\begin{tabular}{|c|c|c|c|c|c|c|c|c|c|}
\hline \multirow[b]{2}{*}{$\begin{array}{c}\text { SIC } \\
\text { Code }\end{array}$} & \multirow[b]{2}{*}{$\begin{array}{l}\text { Industry Groups } \\
\text { and Industry }\end{array}$} & \multirow[b]{2}{*}{ Total } & \multicolumn{6}{|c|}{ Employment Size } & \multirow{3}{*}{$\begin{array}{c}\text { RSE } \\
\text { Row } \\
\text { Factors }\end{array}$} \\
\hline & & & Under 50 & $50-99$ & $100-249$ & $250-499$ & $500-999$ & $\begin{array}{c}1,000 \text { and } \\
\text { Over }\end{array}$ & \\
\hline & RSE Column Factors: & 0.6 & 2.0 & 1.6 & 1.1 & 0.8 & 0.8 & 0.8 & \\
\hline 37 & Transportatlon Equipment $\ldots \ldots \ldots \ldots \ldots \ldots \ldots$ & 333 & 3 & 5 & 16 & 25 & 27 & 256 & 7.4 \\
\hline 3711 & Motor Vehicles and Car Bodies . . . . . . . . . . . . & 105 & $*$ & * & $\cdot$ & * & 2 & 102 & 6.9 \\
\hline 3714 & Motor Vehicle Pants and Accessories $\ldots \ldots \ldots \ldots \ldots$ & 99 & 2 & 1 & 8 & 13 & 14 & 61 & 8.2 \\
\hline 38 & Instruments and Related Products $\ldots \ldots \ldots \ldots \ldots \ldots$ & 98 & 2 & 3 & 6 & 11 & 17 & 59 & 15.4 \\
\hline 3841 & Surgical and Medical Instruments $\ldots \ldots \ldots \ldots \ldots \ldots$ & 6 & • & * & 1 & 2 & 2 & 1 & 13.9 \\
\hline \multirow[t]{2}{*}{39} & Misc. Manufacturing Industries $\ldots \ldots \ldots \ldots \ldots \ldots \ldots$ & 31 & 4 & 3 & 8 & 9 & 4 & 4 & 13.8 \\
\hline & Total $\ldots \ldots \ldots \ldots \ldots \ldots \ldots \ldots \ldots \ldots \ldots$ & 15,027 & 537 & 776 & 2,363 & 2,681 & 3,145 & 5,524 & 2.8 \\
\hline
\end{tabular}

- See Appendices B and $F$ for descriptions of the Standard Industrial Classification system.

${ }^{b}$ Employment Size categories were supplied by the Bureau of the Census.

- Estimate less than 0.5. Data are included in higher level totals.

$W=$ Withheld to avoid disclosing data for individual estabilshments. Data are included in higher level totals.

$\mathrm{Q}=$ Withheld because Relative Standard Error is greater than 50 percent. Data are included in higher level totals.

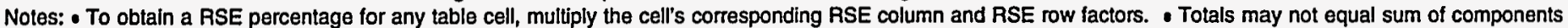

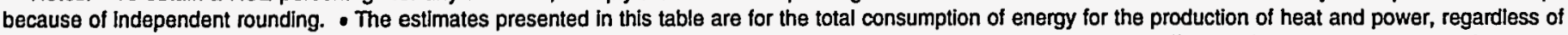

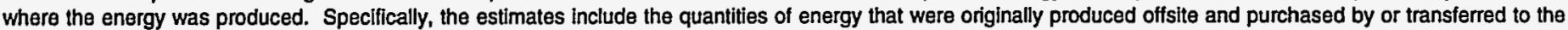

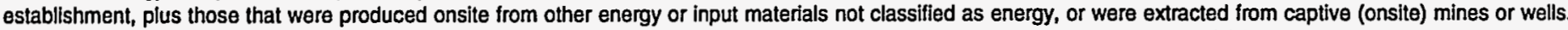

Source: Energy Information Administration, Office of Energy Markets and End Use, Energy End Use and Integrated Statistics Division, Form ElA-846, "1991 Manufacturing Energy Consumption Survey," and Bureau of the Census, Industry Division, data files for the "1991 Annual Survey of Manufactures." 

Generation by Employment Size Categories, Industry Group, and Selected Industries, 1991

(Estimates in Trillion Btu)

\begin{tabular}{|c|c|c|c|c|c|c|c|c|c|}
\hline \multirow[b]{2}{*}{$\begin{array}{l}\text { SIC } \\
\text { Code" }\end{array}$} & \multirow[b]{2}{*}{$\begin{array}{l}\text { Industry Groups } \\
\text { and Industry }\end{array}$} & \multirow[b]{2}{*}{ Total } & \multicolumn{6}{|c|}{ Employment Sizeb } & \multirow{3}{*}{$\begin{array}{c}\text { RSE } \\
\text { Row } \\
\text { Factors }\end{array}$} \\
\hline & & & Under 50 & $50-99$ & $100-249$ & $250-499$ & $500-999$ & $\begin{array}{c}1,000 \text { and } \\
\text { Over }\end{array}$ & \\
\hline & RSE Column Factors: & 0.6 & 2.0 & 1.6 & 1.1 & 0.8 & 0.8 & 0.8 & \\
\hline 20 & Food and Kindred Products & 922 & 68 & 99 & 243 & 234 & 152 & 128 & 5.3 \\
\hline 2011 & Meat Packing Plants $\ldots \ldots \ldots \ldots \ldots \ldots \ldots \ldots$ & 48 & 1 & 2 & 4 & 5 & 8 & 29 & 8.8 \\
\hline 2033 & Canned Fruits and Vegetables.$\ldots \ldots \ldots \ldots \ldots$ & 44 & 3 & 4 & 12 & 12 & 9 & 5 & 13.2 \\
\hline 2037 & 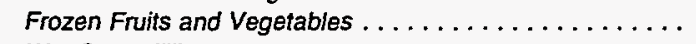 & 40 & 1 & 3 & 6 & 10 & 13 & 8 & 16.6 \\
\hline 2046 & Wet Com Milling & 141 & - & w & 49 & 58 & 20 & W & 14.1 \\
\hline 2051 & Bread, Cake and Related Products . . . . . . . . . . . . . & 32 & 2 & 4 & 9 & 10 & 5 & 2 & 9.9 \\
\hline 2063 & 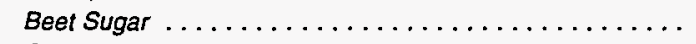 & 67 & 0 & w & 34 & $w$ & 0 & 0 & 6.8 \\
\hline 2075 & Soybean Oil Mills $\ldots \ldots \ldots \ldots \ldots \ldots \ldots \ldots \ldots$ & 50 & 8 & 18 & 9 & 15 & 0 & 0 & 2.8 \\
\hline 2082 & $\ldots \ldots \ldots \ldots \ldots \ldots$ & 50 & * & • & $w$ & $w$ & 14 & 31 & 11.8 \\
\hline 21 & $\ldots \ldots \ldots \ldots \ldots+$ & 26 & * & $\cdot$ & 2 & 2 & 7 & 15 & 10.0 \\
\hline 22 & Textile Mill Products & 272 & 4 & 11 & 52 & 82 & 81 & 42 & 7.4 \\
\hline 23 & Apparel and Other Textile Products & 44 & 4 & 6 & 9 & 12 & 7 & 7 & 16.0 \\
\hline 24 & Lumber and Wood Products $\ldots \ldots \ldots \ldots \ldots \ldots \ldots$ & 197 & 28 & 21 & 84 & 45 & 17 & 2 & 18.0 \\
\hline 25 & Fumiture and Fixtures $[\ldots, \cdots, \ldots, \ldots, \cdots, \cdots$ & 46 & 3 & 5 & 7 & 12 & 11 & 7 & 16.2 \\
\hline 26 & Paper and Allied Products $\ldots \ldots \ldots \ldots \ldots \ldots \ldots \ldots$ & 1,540 & 11 & 48 & 171 & 319 & 484 & 506 & 4.9 \\
\hline 2611 & $\ldots \ldots \ldots \ldots \ldots \ldots \ldots \ldots \ldots \ldots \ldots \ldots \ldots$ & 103 & * & $\mathbf{Q}$ & 6 & 27 & 48 & 22 & 19.5 \\
\hline 2621 & $\ldots \ldots \ldots \ldots \ldots \ldots \ldots \ldots \ldots \ldots \ldots \ldots \ldots$ & 774 & 1 & 11 & 53 & 116 & 232 & 361 & 4.9 \\
\hline 2631 & Paperboard Mills ..... & 527 & $\mathbf{w}$ & w & 57 & 153 & 183 & 111 & 6.3 \\
\hline 27 & Printing and Publishing $\ldots \ldots \ldots \ldots \ldots \ldots \ldots \ldots$ & 108 & 20 & 10 & 22 & 22 & 19 & 16 & 11.6 \\
\hline 28 & Chemicals and Allied Products $\ldots \ldots \ldots \ldots \ldots \ldots \ldots$ & 2,674 & 126 & 174 & 546 & 485 & 483 & 860 & 5.4 \\
\hline 2812 & Alkalies and Chlorine $\ldots \ldots \ldots \ldots \ldots \ldots \ldots \ldots$ & 159 & * & 5 & 59 & W & w & w & 18.5 \\
\hline 2813 & 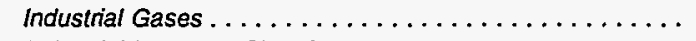 & 86 & 42 & 23 & w & W & 0 & 0 & 6.7 \\
\hline 2819 & Industrial Inorganic Chemicals, nec.............. & 303 & 13 & 16 & 40 & 35 & 75 & 123 & 8.4 \\
\hline 2821 & Plastics Materials and Resins $\ldots \ldots \ldots \ldots \ldots \ldots \ldots$ & 262 & 5 & 16 & 38 & 105 & 58 & 39 & 6.2 \\
\hline 2822 & 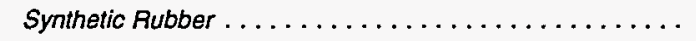 & 68 & • & * & 5 & W & 20 & $w$ & 13.8 \\
\hline 2823 & Cellulosic Manmade Fibers ................... & 31 & 0 & - & 0 & 0 & 0 & 31 & 25.8 \\
\hline 2824 & Organic Fibers, Noncellulosic . ................ & 97 & $\cdot$ & - & Q & 9 & 5 & w & 5.6 \\
\hline 2865 & Cyclic Crudes and Intermediates . . . . . . . . . . . . . & 136 & $\mathbf{w}$ & 9 & 30 & 29 & 56 & w & 11.2 \\
\hline 2869 & Industrial Organic Chemicals, nec . . . . . . . . . . . . . . . & 935 & 14 & 32 & 112 & 173 & 162 & 442 & 7.5 \\
\hline 2873 & Nitrogenous Fertilizers $\ldots \ldots \ldots \ldots \ldots \ldots \ldots \ldots$ & 278 & 28 & 43 & 175 & 21 & 11 & 0 & 26.0 \\
\hline 2874 & Phosphatic Fertilizers $\ldots \ldots \ldots \ldots \ldots \ldots \ldots \ldots \ldots$ & 36 & - & 1 & Q & 20 & w & w & 4.1 \\
\hline 29 & Petroleum and Coal Products $\ldots \ldots \ldots \ldots \ldots \ldots$ & 1,138 & 55 & 36 & 84 & 179 & 215 & 569 & 3.5 \\
\hline 2911 & Petroleum Refining $\ldots \ldots \ldots \ldots \ldots \ldots \ldots \ldots \ldots$ & 1,065 & 11 & 22 & 71 & 178 & 213 & 569 & 3.2 \\
\hline 30 & Rubber and Misc. Plastics Products $\ldots \ldots \ldots \ldots \ldots \ldots$ & 235 & 25 & 32 & 54 & 44 & 39 & 41 & 7.0 \\
\hline 3011 & $\ldots \ldots \ldots \ldots \ldots \ldots \ldots$ & 42 & * & * & 2 & w & w & 33 & 4.4 \\
\hline 308 & Miscellaneous Plastics Products, nec .... & 150 & 19 & 27 & 42 & 32 & 27 & 3 & 11.1 \\
\hline 31 & Leather and Leather Products $\ldots \ldots \ldots \ldots \ldots \ldots$ & 12 & 1 & $\mathbf{Q}$ & 5 & 2 & 2 & 1 & 24.3 \\
\hline 32 & Stone, Clay and Glass Products ... & 877 & 47 & 118 & 424 & 141 & 107 & 39 & 6.9 \\
\hline 3211 & Flat Glass & 49 & * & * & w & 12 & 28 & w & 3.9 \\
\hline 3221 & Glass Containers & 85 & 0 & 0 & 6 & 46 & 27 & 6 & 7.1 \\
\hline 3229 & Pressed and Blown Glass, nec . . . . . . . . . . . . . . & w & * & $\mathbf{Q}$ & 8 & w & 19 & 21 & 7.9 \\
\hline 3241 & Cement, Hydraulic $\ldots \ldots \ldots \ldots \ldots \ldots \ldots \ldots \ldots$ & 312 & - & 36 & 250 & 26 & 0 & 0 & 13.3 \\
\hline 3274 & $\operatorname{Lime} \ldots \ldots \ldots \ldots \ldots \ldots \ldots \ldots \ldots \ldots$ & 117 & $\mathbf{Q}$ & 20 & $\mathbf{Q}$ & 0 & w & 0 & 24.4 \\
\hline 3296 & Mineral Wool & 40 & $\mathbf{w}$ & 2 & 8 & 20 & 7 & W & 1.5 \\
\hline 33 & Primary Metal Industries . ....... & 1,563 & 32 & 41 & 124 & 147 & 328 & 889 & 4.8 \\
\hline 3312 & Blast Fumaces and Steel Mills .. & 842 & 1 & 1 & 20 & 43 & 71 & 706 & 4.9 \\
\hline 3313 & Electrometalurgical Products ..................... & 30 & 0 & * & 9 & w & w & 0 & 10.8 \\
\hline 3321 & Gray and Ductile Iron Foundries $\ldots \ldots \ldots \ldots \ldots \ldots$ & 74 & 3 & 3 & w & $w$ & 13 & 27 & 10.3 \\
\hline 3331 & Primary Copper $\ldots \ldots \ldots \ldots \ldots \ldots \ldots \ldots \ldots \ldots$ & 21 & • & 0 & w & w & 16 & 0 & 1.1 \\
\hline 3334 & Primary Aluminum & 254 & * & * & - & w & 148 & w & 4.0 \\
\hline 3339 & Primary Nonferrous Metals, nec . . & 40 & * & $w$ & 6 & 9 & 18 & $w$ & 3.1 \\
\hline 3353 & Aluminum Sheet, Plate, and Foil . & 60 & $*$ & Q & 4 & 5 & 17 & 33 & 1.4 \\
\hline 34 & Fabricated Metal Products ...... & 305 & 41 & 42 & 91 & 56 & 31 & 44 & 7.7 \\
\hline 35 & Industrial Machinery and Equipment $\ldots \ldots \ldots \ldots \ldots \ldots$ & 236 & 32 & 16 & 33 & 37 & 44 & 74 & 8.8 \\
\hline 357 & Computer and Office Equipment ........ & 21 & 1 & 1 & 2 & 2 & 4 & 13 & 13.3 \\
\hline 36 & Electronic and Other Electric Equipment ... & 196 & 6 & 9 & 22 & 40 & 43 & 76 & 9.4 \\
\hline
\end{tabular}

See footnotes at end of table. 
Table A35. Total Consumption of Offsite-Produced Energy for Heat, Power, and Electricity Generation by Employment Size Categories, Industry Group, and Selected Industries, 1991 (Continued)

(Estimates in Trillion Btu)

\begin{tabular}{|c|c|c|c|c|c|c|c|c|c|}
\hline \multirow[b]{2}{*}{$\begin{array}{l}\text { SIC } \\
\text { Code" }\end{array}$} & \multirow[b]{2}{*}{$\begin{array}{l}\text { Industry Groups } \\
\text { and Industry }\end{array}$} & \multirow[b]{2}{*}{ Total } & \multicolumn{6}{|c|}{ Employment Size $^{b}$} & \multirow{3}{*}{$\begin{array}{c}\text { RSE } \\
\text { Row } \\
\text { Factors }\end{array}$} \\
\hline & & & Under 50 & $50-99$ & $100-249$ & $250-499$ & $500-999$ & $\begin{array}{c}1,000 \text { and } \\
\text { Over }\end{array}$ & \\
\hline & RSE Column Factors: & 0.6 & 2.0 & 1.6 & 1.1 & 0.8 & 0.8 & 0.8 & \\
\hline 37 & Transportation Equipment & 318 & 3 & 5 & 16 & 25 & 27 & 242 & 7.4 \\
\hline 3711 & Motor Vehlcles and Car Bodles.. & 90 & • & • & $\bullet$ & - & 2 & 88 & 6.1 \\
\hline 3714 & Motor VehIcle Parts and Accessories & 99 & 2 & 1 & 8 & 13 & 14 & 60 & 8.2 \\
\hline 38 & Instruments and Related Products ........ & 97 & 2 & 3 & 6 & 11 & 17 & 59 & 15.4 \\
\hline 3841 & Surglcal and Medical Instrumenis . ............... & 6 & $*$ & * & 1 & 2 & 2 & 1 & 13.9 \\
\hline \multirow[t]{2}{*}{39} & Misc. Manufacturing Industries $\ldots \ldots \ldots \ldots$ & 31 & 4 & 3 & 8 & 8 & 4 & 4 & 13.0 \\
\hline & Total $\ldots \ldots \ldots \ldots \ldots \ldots \ldots \ldots \ldots \ldots \ldots \ldots \ldots \ldots \ldots$ & 10,837 & 511 & 681 & 2,003 & 1,902 & 2,120 & 3,621 & 2.8 \\
\hline
\end{tabular}

- See Appendices B and F for descriptions of the Standard Industrial Classification system.

'Employment Size categories were supplied by the Bureau of the Census.

- Estlmate less than 0.5. Data are included in higher level totals.

$W=$ Withheld to avoid disclosing data for individual establishments. Data are included in higher level totals.

$Q=$ Withheld because Relative Standard Error is greater than $\mathbf{5 0}$ percent. Data are included in higher level totals.

Notes: - To obtain a RSE percentage for any table cell, multiply the cell's corresponding RSE column and RSE row factors. - Totals may not equal sum of components because of independent rounding. - The derived estimates presented in this table represent the consumption of energy originally produced offsite, acquired as a result of a purchase or transfer and consumed onsite for the production of heat and power. This definition is consistent with the definition of "purchased" fuels and electric energy used by the Bureau of the Census in the preparation of "Fuels and Electric Energy Consumed," of the Annual Survey of Manufactures, 1974 through 1981. See Appendix B.

Source: Energy Information Administration, Office of Energy Markets and End Use, Energy End Use and Integrated Statistics Division, Form ElA-846, "1991 Manufacturing Energy Consumption Survey," and Office of Oil and Gas, Petroleum Supply Division, Form ElA-810, "Monthly Refinery Report" for 1991. 
Table A36. Total Inputs of Energy for Heat, Power, and Electricity Generation by Fuel Type, Industry Group, Selected Industries, and End Use, 1991: Part 1

(Estimates in Btu or Physical Units)

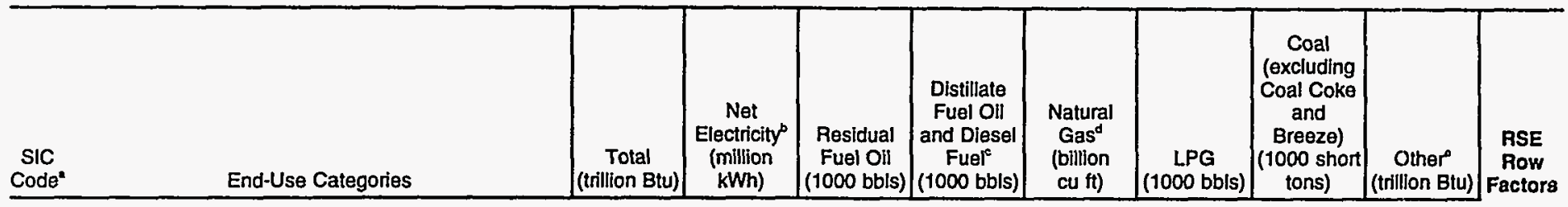

20-39 ALL INDUSTRY GROUPS

RSE Column Factors:

\begin{tabular}{|c|c|c|c|c|c|c|c|c|}
\hline NF & 0.4 & 1.6 & 1.5 & 0.7 & 1.0 & 1.6 & $\mathrm{NF}$ & \\
\hline 15,027 & 694,702 & 65,837 & 23,885 & 5,345 & 27,970 & 53,035 & 5,309 & 3.0 \\
\hline .. & $w$ & 47,009 & 6,850 & 2,037 & 4,928 & 38,473 & .. & 3.6 \\
\hline .. & 546,382 & 17,342 & 5,800 & 2,503 & 16,908 & 14,075 & -- & 4.1 \\
\hline .. & 68,853 & 16,959 & 3,177 & 2,312 & 12,704 & 14,075 & -- & 4.1 \\
\hline -. & 36,330 & 6 & 30 & 13 & 18 & 0 & .- & 12.4 \\
\hline - & 347,899 & 353 & 2,398 & 123 & 4,093 & 0 & .. & 8.4 \\
\hline .. & 89,005 & -- & -- & -- & - & -. & .. & 5.0 \\
\hline .. & 4,295 & 24 & 196 & 55 & 93 & * & .. & 14.0 \\
\hline- & 116,156 & 1,148 & 9,134 & 682 & 5,105 & $w$ & -- & 4.2 \\
\hline -- & 56,165 & 673 & 1,372 & 275 & 731 & 15 & .- & 6.8 \\
\hline .. & 47,309 & -- & -. & - & .. & -. & .. & 5.0 \\
\hline .. & 10,537 & $w$ & 81 & 22 & 62 & 0 & .- & 10.5 \\
\hline -. & 1,114 & - & 6,533 & - & 4,242 & .. & .. & 9.4 \\
\hline -- & - & 325 & 734 & 337 & 41 & $w$ & .- & 8.9 \\
\hline .. & 1,031 & W & 413 & 48 & 30 & 0 & .. & 11.2 \\
\hline 5,547 & $w$ & 339 & 01 & 124 & 8 & $w$ & 5.309 & 6 \\
\hline
\end{tabular}

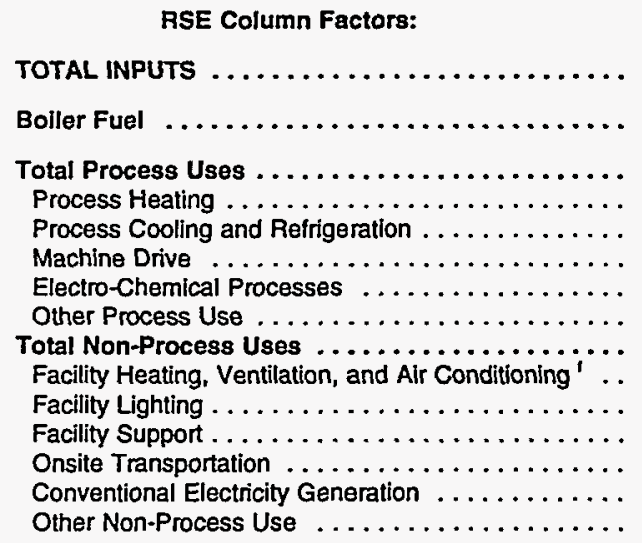

End Use Not Reported

5,547

FOOD and KINDAED PRODUCTS

RSE Column Factors:

$\begin{array}{rrrrr}\text { NF } & 0.5 & 1.5 & 1.5 & 0.7 \\ 953 & 49,536 & 4,317 & 2,966 & 497 \\ -- & 1,073 & 3,875 & 1,242 & 30 \\ . . & 38,445 & W & 270 & 140 \\ .- & 2,030 & 260 & 212 & 133 \\ . . & 12,711 & 0 & 15 & W \\ -- & 23,597 & Q & 35 & W \\ -. & 0 & -. & -. & \\ -. & 83 & 0 & 8 & \\ -- & 7,926 & W & 1,242 & 34 \\ -- & 3,430 & 26 & 128 & 20 \\ -- & 3,460 & -- & -. & \\ -- & 779 & Q & 23 & \\ -- & 163 & - & 812 & \\ -. & -- & 0 & 246 & 12 \\ -- & 94 & - & 33 & \\ 95 & 3,166 & 82 & 212 & 17\end{array}$

0.7
497
306
140
133
$W$
$W$
-
2
34
20
-
2
12
-
17

1.6
1,429
441
292
224
1
56
.-
11
598
50
--
14
533
$\vdots$
$Q$

0.9
6,913
6,414
$W$
$W$
0
0
-
0
$W$
$W$
-
0
-
$W$
0

NF

TOTAL INPUTS

Boller Fuel

Total Process Uses

Process Heating ...

Process Cooling and Refrigeration ............

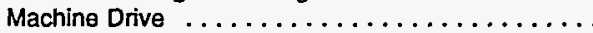

Electro-Chemical Processes $\ldots \ldots \ldots \ldots \ldots \ldots \ldots$

Other Process Use .....................

Total Non-Process Uses . . . . . . . . . . . .

Facility Heating, Ventilation, and Air Conditioning '

Facility Lighting.$\ldots \ldots \ldots \ldots \ldots \ldots \ldots$

Facility Support $\ldots \ldots \ldots \ldots \ldots \ldots \ldots \ldots \ldots$

Onsite Transportation $\ldots \ldots \ldots \ldots \ldots \ldots \ldots \ldots$

Conventional Electricity Generation..$\ldots \ldots \ldots \ldots$.

End Use Not Reported

Meat Packing Plants

RSE Column Factors:

TOTAL INPUTS

NF

49

$\begin{array}{rr}0.4 & 1.6 \\ 3,410 & 170 \\ 30 & 169 \\ 2,858 & : \\ W & 0 \\ 1,749 & 0 \\ 1,039 & * \\ 0 & -- \\ W & 0 \\ 403 & : \\ 169 & : \\ 195 & \ddot{0} \\ 37 & - \\ 2 & 0 \\ -. & 0 \\ 0 & * \\ 148 & \end{array}$

$\begin{array}{rr}1.0 & 0 . \\ 252 & 31 \\ 56 & 2 \\ 31 & \\ 19 & \\ Q & \\ 1 & \\ \because & \\ 132 & \\ Q & \\ \because & \\ 131 & \end{array}$

Total Process Uses

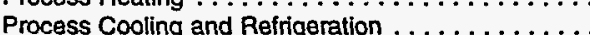

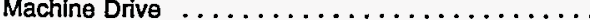

Electro-Chemical Processes $\ldots \ldots \ldots \ldots \ldots \ldots \ldots$

Other Process Use $\ldots \ldots \ldots \ldots \ldots \ldots \ldots \ldots \ldots$

Facility Heating, Ventilation, and Air Conditioning ' $\ldots$

Facility Lighting . . . . . . . . . . . . . . .

Facility Support . . . . . . . . . . . . . . . .

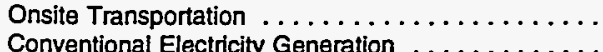

Other Non-Process Use

3

$\begin{array}{rrr}1.8 & \text { NF } & \\ 27 & 2 & 10.1 \\ 27 & . . & 12.9 \\ 0 & . . & 14.5 \\ 0 & -- & 17.0 \\ 0 & -- & 17.9 \\ 0 & -- & 19.8 \\ - & -- & \text { NF } \\ 0 & -- & 36.4 \\ 0 & -- & 13.2 \\ 0 & -. & 10.2 \\ - & -- & 14.1 \\ 0 & -- & 23.0 \\ -- & -- & 25.9 \\ 0 & -- & 36.1 \\ 0 & -- & 27.6 \\ 0 & 2 & 32.4\end{array}$

See footnotes at end of table. 
Table A36. Total Inputs of Energy for Heat, Power, and Electricity Generation by Fuel Type, Industry Group, Selected Industries, and End Use, 1991: Part 1 (Continued) (Estimates in Btu or Physical Units)

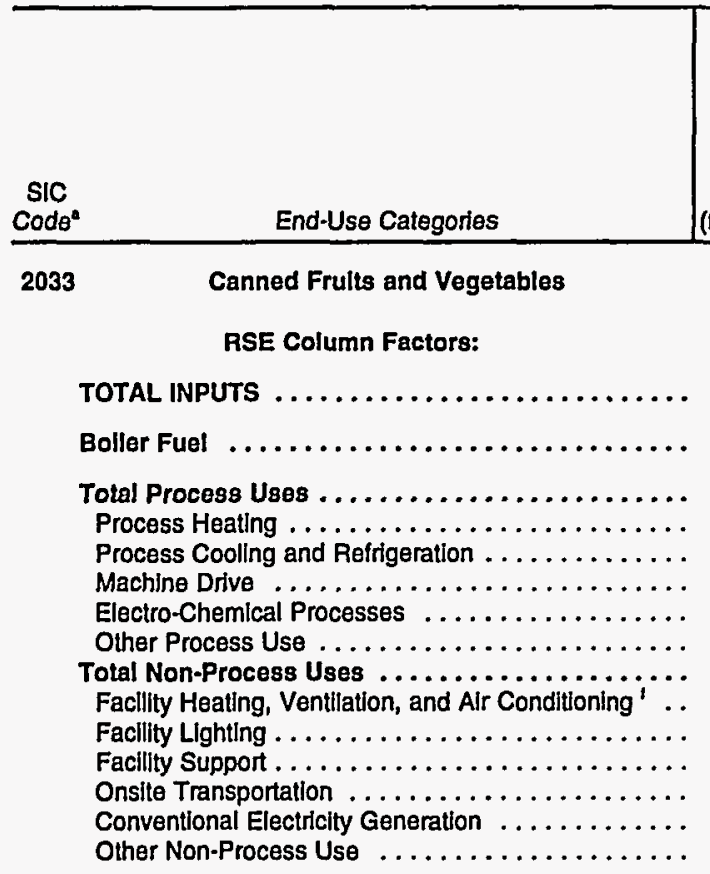

End Use Not Reported

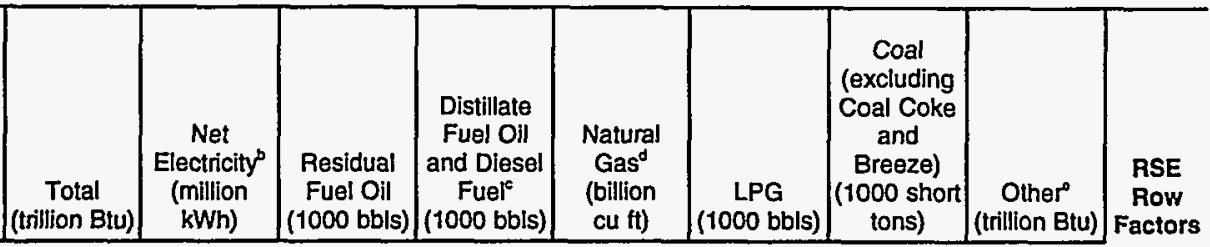

Frozen Frults and Vegetables

RSE Column Factors:

\begin{tabular}{rrr} 
NF & 0.6 & 1.2 \\
40 & 3,071 & 321 \\
.- & 248 & 259 \\
.- & 2,313 & 62 \\
-- & 150 & 62 \\
-- & 1,438 & 0 \\
-- & 716 & 0 \\
\hline- & 2 & - \\
.- & 7 & 0 \\
.- & 357 & 0 \\
- & 157 & 0 \\
-- & 165 & - \\
- & 25 & 0 \\
-- & 9 & - \\
.- &.- & 0 \\
-- & 1 & 0 \\
3 & 401 &.
\end{tabular}

$\begin{array}{crr}\text { NF } & 0.6 & 1.1 \\ 44 & 1,375 & 290 \\ - & 23 & 289 \\ -. & 1,053 & . \\ -- & 27 & 0 \\ - & 236 & 0 \\ -- & 785 & - \\ -- & 1 & - \\ -- & 4 & 0 \\ -- & 249 & 0 \\ -- & 84 & 0 \\ -- & 123 & - \\ -- & 32 & 0 \\ -- & 9 & - \\ -- & - & 0 \\ -. & 2 & 0 \\ 2 & 73 & 0\end{array}$

1.3
131
0
7
0
7
-
0
70
2
$\because-$
$W$
$W$
.
9

$\begin{array}{r}0.9 \\ 35 \\ 28 \\ 2 \\ 1 \\ \vdots \\ : \\ 4 \\ 1 \\ : \\ 0 \\ 3 \\ 0 \\ \hline\end{array}$

1.2
124
4
4
4
$:$
118
16
-
102
0
0
1

$N F$
$Q$
0
0
0
0
0
0
0
0
0
-
0
0
0

NF

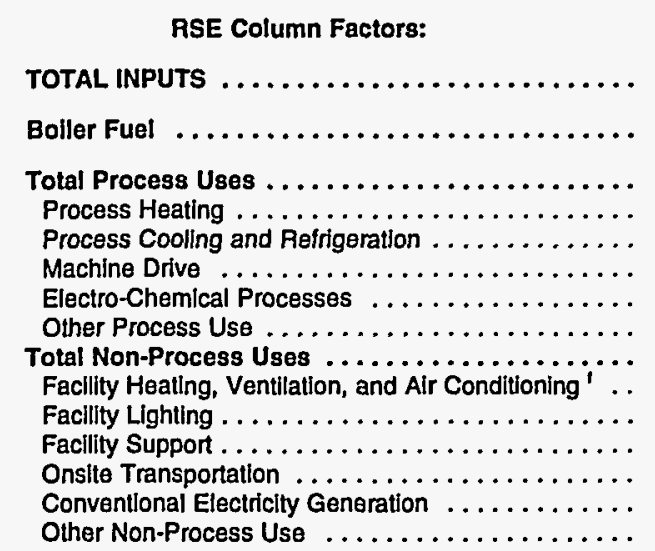

End Use Not Reported

Wet Corn Milling

RSE Column Factors:

NF

TOTAL INPUTS

140

$\begin{array}{rccc}0.6 & 1.1 & 1.1 & 0.8 \\ 4,054 & 29 & 30 & 51 \\ 142 & 29 & W & 25 \\ 3,783 & 0 & W & 24 \\ W & 0 & W & 24 \\ 29 & 0 & 0 & 0 \\ 3,721 & 0 & - & 0 \\ 0 & - & - & - \\ W & 0 & 0 & 0 \\ 129 & 0 & 3 & 1 \\ 51 & 0 & - & - \\ 53 & - & - & - \\ W & 0 & 0 & 0 \\ 1 & - & 3 & 1 \\ -- & 0 & : & 0 \\ W & 0 & - & 1 \\ 142 & 0 & 0 & \end{array}$

$\begin{array}{rrrr}1.3 & \text { NF } & \text { NF } & \\ 41 & 0 & 1 & 13.0 \\ 7 & 0 & - & 21.6 \\ 1 & 0 & - & 17.3 \\ . & 0 & - & 21.6 \\ 0 & 0 & - & 24.2 \\ 1 & 0 & -- & 24.3 \\ - & -. & - & 58.1 \\ 0 & 0 & -- & 46.9 \\ 32 & 0 & -- & 13.9 \\ - & 0 & - & 16.3 \\ -- & -. & - & 15.7 \\ - & 0 & - & 17.3 \\ 31 & -. & - & 16.1 \\ 0 & 0 & - & 27.2 \\ 0 & 0 & -- & 40.0 \\ 1 & 0 & 1 & 33.6\end{array}$

Boller Fuel

Total Process Uses . . . . . . . . . . . . . . .

Process Heating . . . . . . . . . . . . . . . . . . . .

Process Cooling and Refrigeration .............

Machine Drive ........................

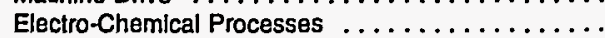

Other Process Use .......................

Total Non-Process Uses . . . . . . . . . . . . .

Faclity Heating, Ventilation, and Air Conditioning '..

Facility Llghtlng . . . . . . . . . . . . . . . . . .

Facillty Support . . . . . . . . . . . . . . . . . .

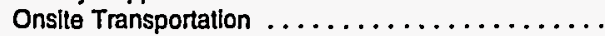

Conventional Electricity Generation $\ldots \ldots \ldots \ldots \ldots$

Other Non-Process Use $\ldots \ldots \ldots \ldots \ldots \ldots \ldots$

End Use Not Reported

142

1.2
76
28
$Q$
3
0
$Q$
-
0
30
$Q$
0
0
24
3
2
0

0.8
25
17
4
4
$\vdots$
-
2
1
-
0
1
0
2


Table A36. Total Inputs of Energy for Heat, Power, and Electricity Generation by Fuel Type, Industry Group, Selected Industries, and End Use, 1991: Part 1 (Continued) (Estimates in Btu or Physical Units)

\begin{tabular}{|c|c|c|c|c|c|c|c|c|c|c|}
\hline $\begin{array}{l}\text { SIC } \\
\text { Code* }\end{array}$ & End-Use Categories & $\begin{array}{c}\text { Total } \\
\text { (trillion Btu) }\end{array}$ & $\begin{array}{l}\text { Net } \\
\text { Electricity } \\
\text { (million } \\
\text { kWh) }\end{array}$ & $\begin{array}{c}\text { Residual } \\
\text { Fuel Oil } \\
(1000 \text { bbls })\end{array}$ & $\begin{array}{c}\text { Distillate } \\
\text { Fuel Oil } \\
\text { and Diesel } \\
\text { Fuel }^{\mathrm{c}} \\
\left(1000 \mathrm{bbls}^{2}\right)\end{array}$ & $\begin{array}{c}\text { Natural } \\
\text { Gas }^{d} \\
\text { (billion } \\
\text { cu ft) }\end{array}$ & $\begin{array}{c}\text { LPG } \\
\text { (1000 bbls) }\end{array}$ & $\begin{array}{c}\text { Coal } \\
\text { (excluding } \\
\text { Coal Coke } \\
\text { and } \\
\text { Breeze }) \\
\left(\begin{array}{c}1000 \text { short } \\
\text { tons) }\end{array}\right.\end{array}$ & $\begin{array}{c}\text { Other } \\
\text { (trillion Btu) }\end{array}$ & $\begin{array}{c}\text { RSE } \\
\text { Row } \\
\text { Factors }\end{array}$ \\
\hline
\end{tabular}

2051 Bread, Cake, and Related Products

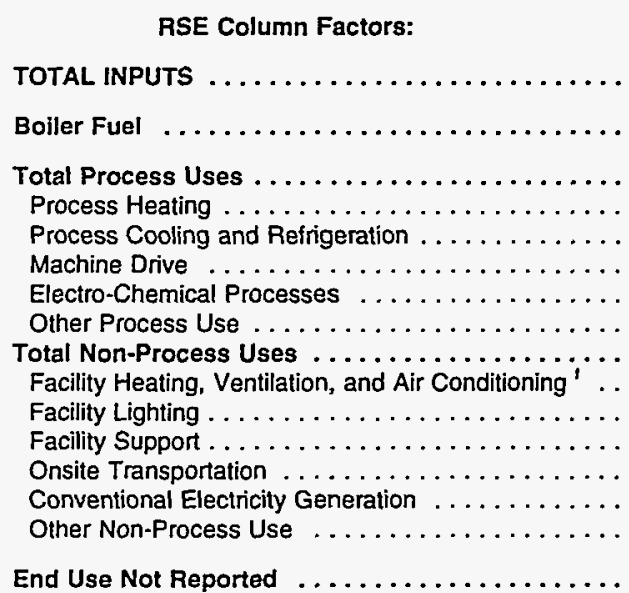

$\begin{array}{rr}\text { NF } & 0.5 \\ 32 & 2,240 \\ -- & 38 \\ -. & 1,577 \\ - & 143 \\ - & 415 \\ - & 1,017 \\ - & 0 \\ - & 2 \\ - & 521 \\ - & 207 \\ - & 241 \\ -- & 51 \\ - & Q \\ - & -- \\ - & 5 \\ 2 & 141\end{array}$

Beet Sugar

RSE Column Factors:

$\begin{array}{crr}\text { NF } & 1.1 & 1.5 \\ 67 & 386 & \\ -- & 7 & \\ -- & 343 & 104 \\ -- & 3 & 104 \\ -- & - & \\ -- & 339 & \\ -- & 0 & \\ -- & 0 & \\ - & 36 & \\ -- & 12 & \\ -- & 20 & \\ -- & 4 & \\ -- & - & \\ -- & -- & \\ -- & 0 & \\ \text { W } & 7\end{array}$

1.5
$W$
$W$
104
104
0
0
-
0
$W$
$W$
-
0
-
0
0
0

1.0
30
$W$
$W$
0
$W$
0
0
23
0
-
0
23
0
0
.

0.9
18
12
6
6
0
$\vdots$
0
$\vdots$
$:$
$\vdots$
0
0
0
0

$\begin{array}{rrrr}1.1 & 0.6 & \text { NF } & \\ 5 & 1,901 & \text { W } & 5.8 \\ 0 & 1,590 & -- & 10.2 \\ 1 & 311 & -- & 7.5 \\ . & 311 & -- & 12.3 \\ 0 & 0 & -. & 38.0 \\ 1 & 0 & -- & 8.3 \\ - & -. & -. & N F \\ 0 & 0 & -. & N F \\ 5 & 0 & -- & 6.6 \\ 1 & 0 & -- & 8.8 \\ - & - & - & 5.3 \\ 1 & 0 & -- & 9.7 \\ 2 & -. & -- & 8.2 \\ 0 & 0 & -- & N F \\ . & 0 & -- & 14.9 \\ . & 0 & W & 17.3\end{array}$

TOTAL INPUTS

Boiler Fuel

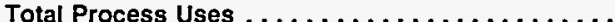

Process Heating $\ldots \ldots \ldots \ldots \ldots \ldots \ldots \ldots \ldots$

Process Cooling and Refrigeration . . . . . . . . . . .

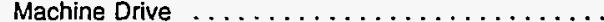

Electro-Chemical Processes ................

Other Process Use . . . . . . . . . . . . . . . . . . . .

Total Non-Process Uses . . . . . . . . . . . . . . .

Facility Heating, Ventilation, and Air Conditioning ' . .

Facility Lighting . . . . . . . . . . . . . . . . . . . . .

Facility Support . . . . . . . . . . . . . . . . . . .

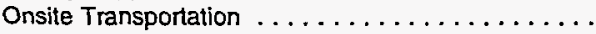

Conventional Electricity Generation ............

Other Non-Process Use $\ldots \ldots \ldots \ldots \ldots \ldots$

End Use Not Reported

0.7
1,616
111
1,388
22
23
1,342
0
0
96
43
46
6
.
--

1.5
42
42
$:$
0
0
$:-$
0
$:$
0
-
--
0
.
0

1.2
31
$W$
$W$
0
0
0

\begin{tabular}{|c|c|c|c|}
\hline 0.7 & 1.6 & 0.8 & $\mathrm{NF}$ \\
\hline 24 & 5 & 592 & 7 \\
\hline 17 & - & 592 & -. \\
\hline 5 & 2 & 0 & .. \\
\hline 5 & 2 & 0 & .. \\
\hline 0 & 0 & 0 & - \\
\hline 0 & * & 0 & -- \\
\hline- & - & $\ddot{*}$ & -. \\
\hline * & - & 0 & -. \\
\hline 1 & 3 & 0 & - \\
\hline * & 0 & 0 & - \\
\hline$\cdots$ & - & -. & $\cdots$ \\
\hline - & 0 & 0 & -. \\
\hline 0 & 3 & -. & -. \\
\hline 1 & 0 & 0 & - \\
\hline 0 & 0 & 0 & -. \\
\hline 0 & * & 0 & 7 \\
\hline
\end{tabular}

12.1

18.9

13.0

16.3

20.5

26.6

NF

27.1

13.3

14.2

11.2

23.1

21.5

35.0

39.9

27.3

See footnotes at end of table. 
Table A36. Total Inputs of Energy for Heat, Power, and Electricity Generation by Fuel Type, Industry Group, Selected Industries, and End Use, 1991: Part 1 (Continued) (Estimates in Btu or Physical Units)

\begin{tabular}{|c|c|c|c|c|c|c|c|c|c|c|}
\hline $\begin{array}{l}\text { SIC } \\
\text { Code" }\end{array}$ & End-Use Categories & $\begin{array}{c}\text { Total } \\
\text { (trillion Btu) }\end{array}$ & $\begin{array}{c}\text { Net } \\
\text { Electricity } \\
\text { (million } \\
\mathrm{kWh} \text { ) }\end{array}$ & $\begin{array}{c}\text { Residual } \\
\text { Fuel Oll } \\
\text { (1000 bbls) }\end{array}$ & $\begin{array}{c}\text { Distillate } \\
\text { Fuel Oil } \\
\text { and Diesel } \\
\text { Fuel }^{\mathrm{c}} \\
(1000 \mathrm{bbls})\end{array}$ & $\begin{array}{c}\text { Natura } \\
\text { Gass } \\
\text { (billion } \\
\text { cu ft) }\end{array}$ & $\begin{array}{c}\text { LPG } \\
\text { (1000 bbls) }\end{array}$ & $\begin{array}{c}\text { Coal } \\
\text { (excluding } \\
\text { Coal Coke } \\
\text { and } \\
\text { Breeze) } \\
\left(\begin{array}{c}1000 \text { short } \\
\text { tons) }\end{array}\right. \\
\end{array}$ & $\begin{array}{c}\text { Other } \\
\text { (trillion Btu) }\end{array}$ & $\begin{array}{c}\text { RSE } \\
\text { Row } \\
\text { Factors }\end{array}$ \\
\hline
\end{tabular}

2082 Malt Beverages

RSE Column Factors:

TOTAL INPUTS

NF

Boller Fuel

Total Process Uses . . . . . . . . . . . . . . . .

Process Heating $\ldots \ldots \ldots \ldots \ldots \ldots \ldots \ldots \ldots$

Process Cooling and Refrigeration .............

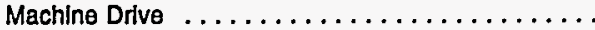

Electro-Chemical Processes ...............

Other Process Use $\ldots \ldots \ldots \ldots \ldots \ldots \ldots \ldots \ldots$

Total Non-Process Uses ................ Facility Heating, Ventilation, and Alr Conditioning ' . .

Facility Llghting . . . . . . . . . . . . . . . . . .

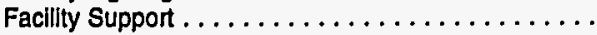

Onsite Transportation ....................

Conventlonal Electricity Generation ............

Other Non-Process Use

End Use Not Reported

RSE Column Factors:

TOTAL INPUTS

Boller Fuel

Total Process Uses . . . . . . . . . . . . . . . .

Process Heating $\ldots \ldots \ldots \ldots \ldots \ldots \ldots \ldots$

Process Coollng and Refrigeration .............

Machine Drive $\ldots \ldots \ldots \ldots \ldots \ldots \ldots \ldots$

Electro-Chemical Processes ...............

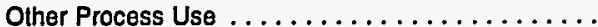

Total Non-Process Uses . . . . . . . . . . . . . Facilly Heating, Ventllation, and Air Conditioning ' ...

Facllty Lighting.$\ldots \ldots \ldots \ldots \ldots \ldots \ldots \ldots$

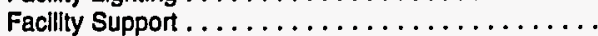

Onsite Transportation ....................

Conventional Electricity Generation ............

Other Non-Process Use .................

End Use Not Reported

RSE Column Factors:

TOTAL INPUTS

Boller Fuel

Total Process Uses . . . . . . . . . . . . . . .

Process Heatling $\ldots \ldots \ldots \ldots \ldots \ldots \ldots \ldots$

Process Coolling and Relrigeration .............

Machine Drive $\ldots \ldots \ldots \ldots \ldots \ldots \ldots \ldots$

Electro-Chemical Processes $\ldots \ldots \ldots \ldots \ldots \ldots$

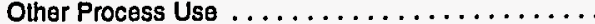

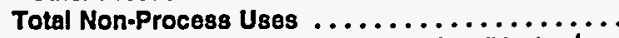
Facillty Heating, Ventllation, and Air Conditioning ' . .

Facllity Lighting.$\ldots \ldots \ldots \ldots \ldots \ldots \ldots \ldots$

Facillity Support .

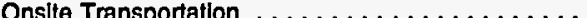

Conventional Electricity Generation . . . . . . . .

Other Non-Process Use $\ldots \ldots \ldots \ldots \ldots \ldots$.

End Use Not Reported

$\begin{array}{crr}\text { NF } & 0.5 & 1.3 \\ 50 & 2,328 & 419 \\ .- & 33 & 417 \\ -- & 1,772 & 0 \\ -- & 67 & 0 \\ -- & 769 & 0 \\ -- & 935 & 0 \\ -- & 0 & - \\ -- & 0 & 0 \\ -- & 460 & 2 \\ -- & 172 & 2 \\ -- & 193 & - \\ -- & 60 & 0 \\ -- & 34 & - \\ -- & -- & 0 \\ -- & 0 & 0 \\ 1 & 97 & 0\end{array}$

$$
1.4
$$

0.8
22
20
1
1
0
-
0
-
-
0
0
0
0

1.0
8
$\cdot$
0
0
0
0
0
0
$W$
$W$
-
0
4
0
0

$\begin{array}{rr}1.4 & \text { NF } \\ 706 & 1 \\ 706 & - \\ 0 & - \\ 0 & - \\ 0 & - \\ 0 & - \\ - & - \\ 0 & - \\ 1 & - \\ 1 & - \\ - & - \\ 0 & - \\ - & - \\ 0 & - \\ 0 & - \\ 0 & 1\end{array}$

$\begin{array}{rcc}1.1 & 0.6 & 0.9 \\ 1,002 & 135 & 40 \\ 5 & 135 & 39 \\ 633 & 0 & . \\ 22 & 0 & \cdot \\ W & 0 & 0 \\ 578 & 0 & - \\ 0 & -- & - \\ W & 0 & 0 \\ 364 & 0 & 2 \\ W & 0 & 0 \\ W & - & - \\ 19 & 0 & 0 \\ 2 & -- & 2 \\ -- & 0 & 0 \\ 0 & 0 & 0 \\ 5 & 0 & 0\end{array}$

$\begin{array}{rr}1.9 & 1.2 \\ 4 & 23 \\ 3 & W \\ 1 & W \\ 1 & W \\ : & 0 \\ - & 0 \\ 0 & 0 \\ : & 14 \\ : & : \\ : & 0 \\ 0 & 14 \\ 0 & 0 \\ 0 & : \\ 0 & .\end{array}$

$\begin{array}{rcc}0.6 & \text { NF } & \\ 692 & - & 6.3 \\ 692 & -- & 6.1 \\ 0 & -- & 5.2 \\ 0 & - & 8.6 \\ 0 & - & 6.2 \\ 0 & -. & 5.5 \\ - & - & \text { NF } \\ 0 & -- & 7.3 \\ 0 & - & 8.2 \\ 0 & - & 8.8 \\ -. & -- & 4.5 \\ 0 & -- & 4.4 \\ -. & - & 7.2 \\ 0 & - & \text { NF } \\ 0 & -- & 6.5 \\ 0 & . & 5.5\end{array}$

NF

$$
273
$$

$\begin{array}{rrr}0.4 & 1.3 & 1, \\ 29,532 & 1,966 & 1,064 \\ 174 & 1,706 & 811 \\ 21,335 & 99 & 3 \\ 1,011 & W & 37 \\ 2,160 & 0 & \\ 18,002 & W & \\ W & -. & \\ W & - & \\ 7,290 & 28 & 17 \\ 4,221 & 28 & \\ 2,652 & -- & \\ 369 & - & \\ 34 & -. & 34 \\ -. & 0 & \\ 14 & 0 & \\ 907 & 134 & \end{array}$

1.6

0.8

1.2

629

1.2

1,362

1,334

13

68

38

411

397

13

13

1
163

27

--

134 
Table A36. Total Inputs of Energy for Heat, Power, and Electricity Generation by Fuel Type, Industry Group, Selected Industries, and End Use, 1991: Part 1 (Continued) (Estimates in Btu or Physical Units)

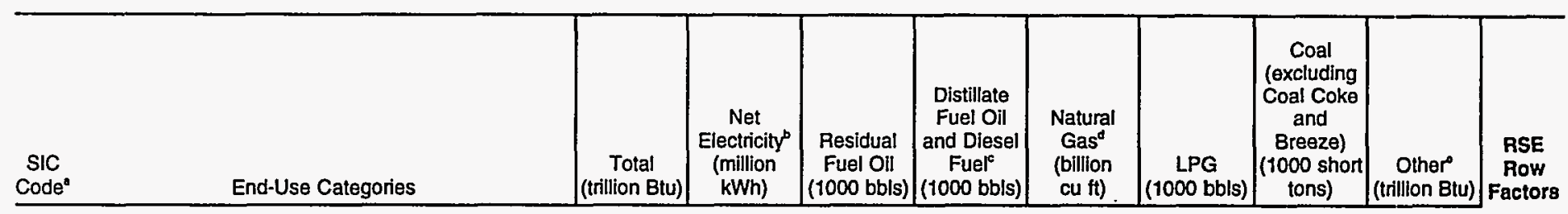

23 APPAREL and OTHER TEXTLLE PRODUCTS

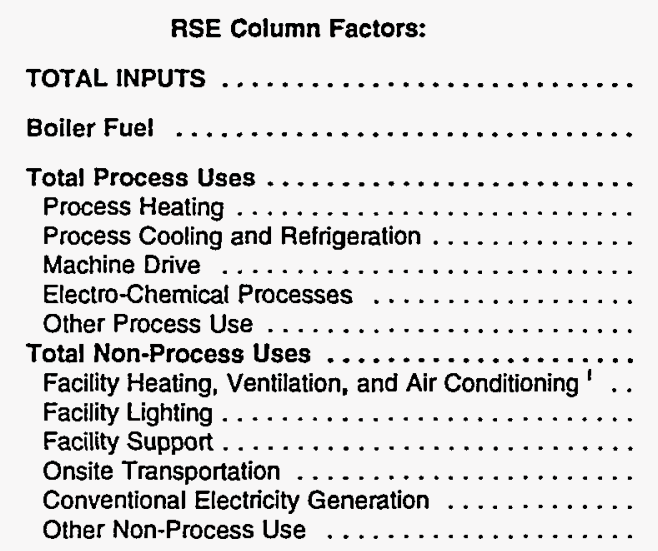

\begin{tabular}{|c|c|c|c|c|c|c|c|c|}
\hline NF & 0.5 & NF & 1.4 & 0.7 & 1.3 & 1.5 & NF & \\
\hline 44 & 5,645 & $Q$ & 142 & 18 & 158 & 88 & 1 & 16.8 \\
\hline .- & 56 & $Q$ & 76 & 6 & 42 & 88 & -- & 27.1 \\
\hline $\begin{array}{l}-- \\
--\end{array}$ & $\begin{array}{r}2,601 \\
194\end{array}$ & $\begin{array}{l}Q \\
0\end{array}$ & $\begin{array}{l}Q \\
Q\end{array}$ & $\begin{array}{l}6 \\
4\end{array}$ & $\begin{array}{l}56 \\
26\end{array}$ & $\begin{array}{l}0 \\
0\end{array}$ & $\ddot{-}$ & $\begin{array}{l}23.5 \\
30.3\end{array}$ \\
\hline .. & 90 & 0 & 0 & 0 & 0 & 0 & .. & 69.2 \\
\hline -- & 2,298 & $\mathbf{Q}$ & * & 1 & $Q$ & 0 & -- & 38.5 \\
\hline -- & 7 & - & - & -- & - & .. & -. & 85.0 \\
\hline -- & $Q$ & .0 & 0 & 0 & - & 0 & .. & 37.9 \\
\hline -. & 2,091 & $Q$ & 40 & 4 & 48 & 0 & -- & 19.1 \\
\hline -- & 1,133 & $Q$ & 31 & 4 & 35 & 0 & - & 22.8 \\
\hline -- & 829 & - & -- & -- & - & .. & -- & 17.8 \\
\hline- & 126 & 0 & " & . & 1 & 0 & -. & 36.1 \\
\hline-- & $\mathbf{Q}$ & -- & 10 & * & 12 & -- & .. & 19.9 \\
\hline -- & -. & 0 & 0 & * & 0 & 0 &. & NF \\
\hline- & 0 & 0 & - & 0 & 0 & 0 & -- & 33.4 \\
\hline 6 & 953 & $\mathbf{Q}$ & 14 & 2 & 13 & 0 & 1 & 29.6 \\
\hline
\end{tabular}

End Use Not Reported

LUMBER and WOOD PRODUCTS

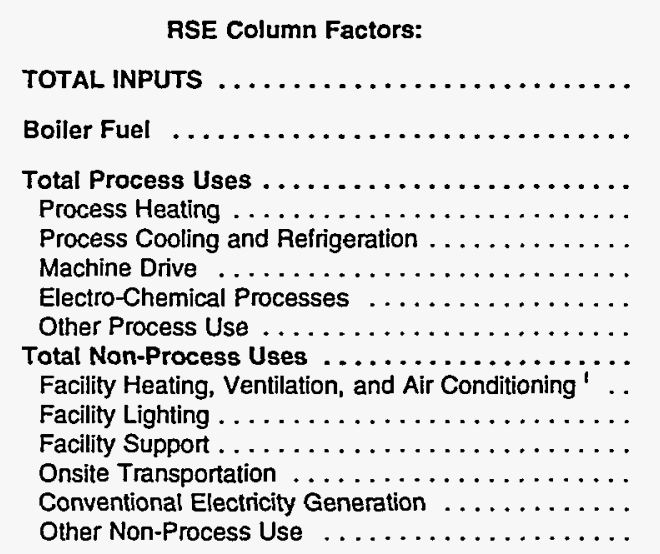

$\mathrm{NF}$

423
17,878

275

13,933

870

12,945

7

37
1,863

1,004

174

Q

2,082

\begin{tabular}{c}
1.3 \\
333 \\
295 \\
$Q$ \\
$Q$ \\
0 \\
0 \\
-- \\
0 \\
$Q$ \\
$Q$ \\
\hdashline- \\
0 \\
\hdashline- \\
0 \\
0
\end{tabular}

1.0
2,373
151
645
0
0
563
-
$Q$
1,016
$Q$
-
5
922
$Q$
2
561
1.1

1,000

63

450

338

Q

$\overrightarrow{\mathrm{Q}}$

455

Q

395

?

32

FURNITURE and FIXTURES

RSE Column Factors:

TOTAL INPUTS

Boller Fuel

Total Process Uses $\ldots \ldots \ldots \ldots \ldots \ldots \ldots \ldots \ldots \ldots$

Process Heating $\ldots \ldots \ldots \ldots \ldots \ldots \ldots \ldots \ldots$.

Process Cooling and Refrigeration ............

Machine Drive $\ldots \ldots \ldots \ldots \ldots \ldots \ldots \ldots . . . \ldots \ldots$

Electro-Chemical Processes ..................

Other Process Use . . . . . . . . . . . . . . . . .

Total Non-Process Uses . . . . . . . . . . . . .

Facility Heating, Ventilation, and Air Conditioning ' . .

Facility Lighting . . . . . . . . . . . . . . . . . .

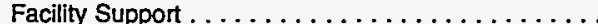

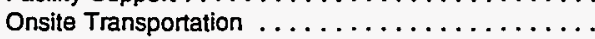

Conventional Electricity Generation . . . . . . . .

Other Non-Process Use $\ldots \ldots \ldots \ldots \ldots \ldots \ldots$.

End Use Not Reported

See footnotes at end of table. 
Table A36. Total Inputs of Energy for Heat, Power, and Electricity Generation by Fuel Type, Industry Group, Selected Industries, and End Use, 1991: Part 1 (Continued) (Estimates in Btu or Physical Units)

\begin{tabular}{|c|c|c|c|c|c|c|c|c|c|c|}
\hline $\begin{array}{c}\text { SIC } \\
\text { Code }\end{array}$ & End-Use Categories & $\begin{array}{c}\text { Total } \\
\text { (trillion Btu) }\end{array}$ & $\begin{array}{c}\text { Net } \\
\text { Electricity" } \\
\text { (million } \\
\mathrm{kWh}) \\
\end{array}$ & $\begin{array}{c}\text { Residual } \\
\text { Fuel Oil } \\
\text { (1000 bbls) }\end{array}$ & $\begin{array}{c}\text { Distillate } \\
\text { Fuel Oil } \\
\text { and Diesel } \\
\text { Fuel } \\
(1000 \mathrm{bbls})\end{array}$ & $\begin{array}{l}\text { Natural } \\
\text { Gas } \\
\text { (billion } \\
\text { cu fit) }\end{array}$ & $\begin{array}{c}\text { LPG } \\
\text { (1000 bbls) }\end{array}$ & $\begin{array}{c}\text { Coal } \\
\text { (excluding } \\
\text { Coal Coke } \\
\text { and } \\
\text { Breeze) } \\
\text { (1000 short } \\
\text { tons) }\end{array}$ & $\begin{array}{c}\text { Other } \\
\text { (trillion Btu) }\end{array}$ & $\mid \begin{array}{c}\text { RSE } \\
\text { Row } \\
\text { Factors }\end{array}$ \\
\hline
\end{tabular}

26

PAPER and ALLIED PRODUCTS

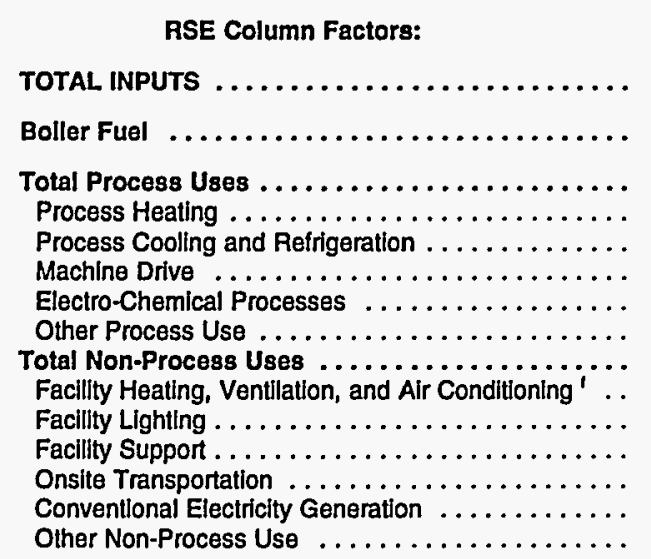

$\begin{array}{rrrr}\text { NF } & 0.8 & 0.8 & 1.7 \\ 2,472 & 58,896 & 24,883 & 1,566 \\ -- & 1,510 & 21,232 & 665 \\ -- & 50,222 & 3,311 & 391 \\ -- & 1,500 & 3,163 & 291 \\ -- & 792 & 0 & : \\ - & 46,857 & 148 & W \\ -- & 619 & - & - \\ - & 454 & 0 & W \\ - & 5,844 & 336 & 486 \\ - & 2,602 & W & 97 \\ -- & 591 & - & - \\ -- & 71 & -. & 377 \\ - & -. & W & 7 \\ - & 8 & 1 & Q \\ 1,272 & 2,830 & 3 & 24\end{array}$

0.8
532
350
120
103
11
$\ddot{6}$
52
$W$
-
$:$
37
$W$
9

1.4
$W$
$W$
426
368
$W$
44
13
624
$W$
-
609
$Q$
1
55

$\begin{array}{rcr}0.8 & N F & \\ 13,252 & W & 4.6 \\ 13,133 & -- & 5.8 \\ Q & - & 5.6 \\ Q & -- & 8.4 \\ 0 & - & 8.3 \\ 0 & -. & 10.0 \\ .- & -. & 7.9 \\ 0 & -. & 9.8 \\ . & - & 6.8 \\ . & - & 11.3 \\ - & - & 5.2 \\ 0 & -. & 10.5 \\ - & - & 6.3 \\ 0 & -- & 15.7 \\ 0 & -- & 21.1 \\ W & W & 19.3\end{array}$

2611

Pulp Mills

RSE Column Factors:

$\begin{array}{rrrr}\text { NF } & 0.8 & 1.0 & 0 \\ 300 & 2,537 & 4,500 & 155 \\ . . & 125 & 3,881 & 60 \\ - & 2,215 & 608 & 21 \\ -. & 16 & 604 & \\ -. & 18 & 0 & \\ -- & 2,105 & 4 & 17 \\ -. & 65 & - & \\ -. & Q & 0 & \\ -. & 197 & 7 & 7 \\ -. & 86 & 0 & \\ -. & 97 & -. & \\ -- & 13 & 0 & \\ -. & 1 & - & 74 \\ -. & 0 & 0 & \\ 222 & 125 & 3 & \end{array}$

0.9
155
60
21
3
0
17
.-
0
74
0
0
74
0
0
0

\begin{tabular}{l}
0.8 \\
32 \\
22 \\
9 \\
9 \\
0 \\
0 \\
0 \\
0 \\
$\vdots$ \\
\hline 0 \\
0 \\
0 \\
0
\end{tabular}

\begin{tabular}{r}
1.3 \\
141 \\
98 \\
19 \\
17 \\
0 \\
2 \\
0 \\
0 \\
22 \\
- \\
\hline 0 \\
22 \\
0 \\
0 \\
0
\end{tabular}

$\begin{array}{rrr}1.3 & \text { NF } & \\ 331 & 221 & 14.9 \\ 331 & - & 18.8 \\ 0 & - & 17.3 \\ 0 & - & 21.6 \\ 0 & - & 22.0 \\ 0 & - & 24.0 \\ . & - & 31.8 \\ 0 & - & \text { NF } \\ 0 & - & 18.8 \\ 0 & - & 22.6 \\ - & - & 15.9 \\ 0 & - & 22.0 \\ - & - & 18.9 \\ 0 & - & 40.5 \\ 0 & - & \text { NF } \\ 0 & 221 & 27.6\end{array}$

Paper Mills

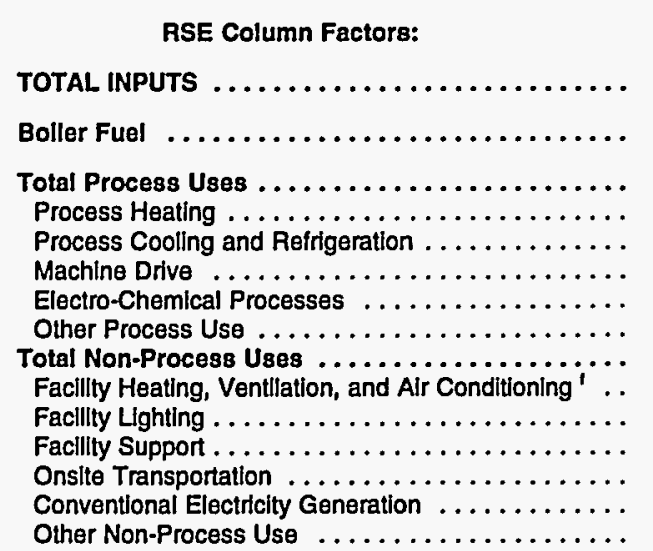

NF

1,204

0.9
32,735
553
29,462
407
274
28,205
499
77
2,223
1,054
905
240
$W$
--
$W$
1,050

$\begin{array}{rr}1.1 & 1.0 \\ 13,455 & W \\ 11,601 & 27 \\ 1,569 & \\ 1,569 & 141 \\ 0 & \\ - & 47 \\ 0 & \\ 284 & 19 \\ 258 & \\ -- & \\ 0 & \\ -\bar{W} & 190 \\ W & \\ 0 & \end{array}$

\begin{tabular}{rrrr}
1.0 & 1.1 & 1.0 & NF \\
252 & 613 & 8,634 & W \\
158 & $W$ & 8,634 & - \\
56 & 279 & 0 & - \\
47 & 263 & 0 & - \\
$W$ & 1 & 0 & - \\
$\bar{W}$ & 6 & 0 & - \\
37 & $\overline{-}$ & - & - \\
2 & $\mathbf{W}$ & $\vdots$ & - \\
- & - & - & - \\
\hline & - & - & - \\
34 & $W$ & - & - \\
0 & 0 & 0 & - \\
. & 1 & 0 & - \\
\hline
\end{tabular}

2.9 3.5 3.5

See footnotes at end of table. 
Table A36. Total Inputs of Energy for Heat, Power, and Electricity Generation by Fuel Type, Industry Group, Selected Industries, and End Use, 1991: Part 1 (Continued) (Estimates in Btu or Physical Units)

\begin{tabular}{|c|c|c|c|c|c|c|c|c|c|c|}
\hline $\begin{array}{c}\text { SIC } \\
\text { Code" }\end{array}$ & End-Use Categories & $\begin{array}{c}\text { Total } \\
\text { (trillion Btu) }\end{array}$ & $\begin{array}{l}\text { Net } \\
\text { Electricity } \\
\text { (million } \\
\text { kWh) }\end{array}$ & $\begin{array}{c}\text { Residual } \\
\text { Fuel Oil } \\
(1000 \text { bbls })\end{array}$ & $\begin{array}{c}\text { Distillate } \\
\text { Fuel Oil } \\
\text { and Diesel } \\
\text { Fuel } \\
(1000 \text { bbls })\end{array}$ & $\begin{array}{c}\text { Natural } \\
\text { Gas }^{\mathbf{d}} \\
\text { (billion } \\
\text { cu ft) }\end{array}$ & $\begin{array}{c}\mathrm{LPG} \\
(1000 \mathrm{bb} / \mathrm{s})\end{array}$ & $\begin{array}{c}\text { Coal } \\
\text { (excluding } \\
\text { Coal Coke } \\
\text { and } \\
\text { Breeze) } \\
\text { (1000 short } \\
\text { tons) }\end{array}$ & $\begin{array}{c}\text { Other } \\
\text { (trillion Btu) }\end{array}$ & $\begin{array}{c}\text { RSE } \\
\text { Row } \\
\text { Factors }\end{array}$ \\
\hline
\end{tabular}

2631 Paperboard Mills

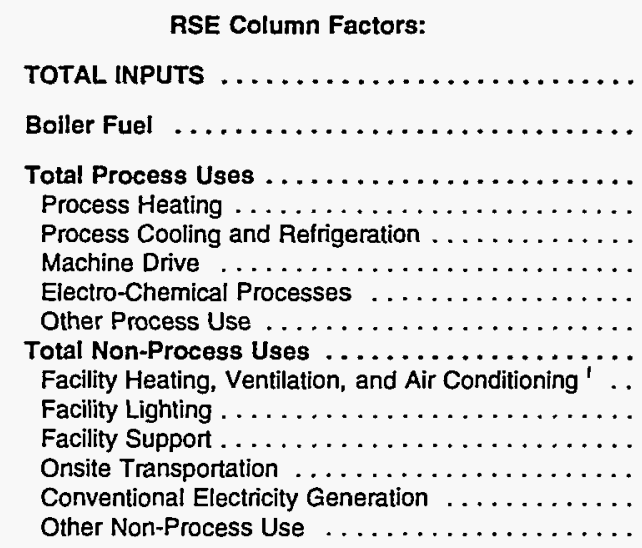

\begin{tabular}{|c|c|c|c|c|}
\hline NF & 0.8 & 1.5 & 1.0 & 0.8 \\
\hline 832 & 10,396 & W & 207 & 180 \\
\hline -- & 653 & 5,057 & 77 & 133 \\
\hline -- & 8,937 & w & $w$ & 36 \\
\hline-- & 412 & W & $w$ & w \\
\hline- & 58 & 0 & 0 & * \\
\hline -- & 8,083 & 0 & $w$ & w \\
\hline -- & W & 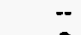 & $\ddot{*}$ & $\because$ \\
\hline -- & $w$ & 0 & W & 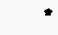 \\
\hline -. & 794 & 3 & 111 & w \\
\hline 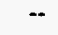 & 319 & 3 & 0 & 2 \\
\hline -- & 398 & -- & -- & -- \\
\hline-- & 76 & 0 & " & \\
\hline -. & $\mathbf{Q}$ & -- & 102 & 0 \\
\hline-- & $\because$ & 0 & 7 & W \\
\hline -- & * & 0 & $\mathbf{Q}$ & w \\
\hline 486 & 664 & $*$ & $w$ & $w$ \\
\hline
\end{tabular}

End Use Not Reported

\section{PRINTING and PUBLISHING}

RSE Column Factors:

NF

TOTAL INPUTS

Boiler Fuel

Total Process Uses ....................

Process Heating $\ldots \ldots \ldots \ldots \ldots \ldots \ldots \ldots \ldots$

Process Cooling and Refrigeration ..............

Machine Drive $\ldots \ldots \ldots \ldots \ldots \ldots \ldots \ldots \ldots$

Electro-Chemical Processes ...................

Other Process Use $\ldots \ldots \ldots \ldots \ldots \ldots \ldots \ldots$.

Total Non-Process Uses ................

Facility Heating, Ventilation, and Air Conditioning ${ }^{\prime}$...

Facility Lighting . . . . . . . . . . . . . . . . .

Facility Support . . . . . . . . . . . . . . .

Onsite Transportation ....................

Conventional Electricity Generation .............

Other Non-Process Use $\ldots \ldots \ldots \ldots \ldots \ldots \ldots$.

End Use Not Reported

\section{CHEMICALS and ALLIED PRODUCTS}

RSE Column Factors:

TOTAL INPUTS

Boiler Fuel

Total Process Uses

Process Cooling and Refrigeration ...............

Machine Drive ..........................

Electro-Chemical Processes..$\ldots \ldots \ldots \ldots \ldots$.

Other Process Use . . . . . . . . . . . . . . .

Total Non-Process Uses . . . . . . . . . . . . .

Facility Heating, Ventilation, and Air Conditioning ' ..

Facility Lighting $\ldots \ldots \ldots \ldots \ldots \ldots \ldots \ldots$

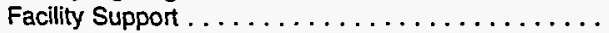

Onsite Transportation ...................

Conventional Electricity Generation .............

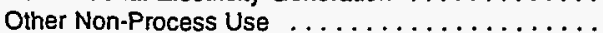

End Use Not Reported

0.5
15,629
108
7,599
190
551
6,674
76
109
5,480
2,928
1,954
536
50
-
$Q$
2,549

1.6

1.5

0.7

\begin{tabular}{r}
1.1 \\
179 \\
$W$ \\
$W$ \\
$W$ \\
20 \\
\hdashline \\
0 \\
134 \\
1 \\
- \\
$Q$ \\
131 \\
0 \\
$Q$ \\
$Q$
\end{tabular}

NF

$$
\begin{array}{r}
\text { NF } \\
0 \\
0
\end{array}
$$

NF

117

\begin{tabular}{rr}
$Q$ & 16 \\
$Q$ & 14 \\
0 & $\vdots$ \\
- & 1 \\
0 & $:$ \\
180 & 12 \\
109 & 11 \\
\hdashline- & - \\
2 & 1 \\
65 & 0 \\
$Q$ & 0 \\
$*$ & $*$ \\
8 & 5
\end{tabular}


Table A36. Total Inputs of Energy for Heat, Power, and Electricity Generation by Fuel Type, Industry Group, Selected Industries, and End Use, 1991: Part 1 (Continued) (Estimates in Btu or Physical Units)

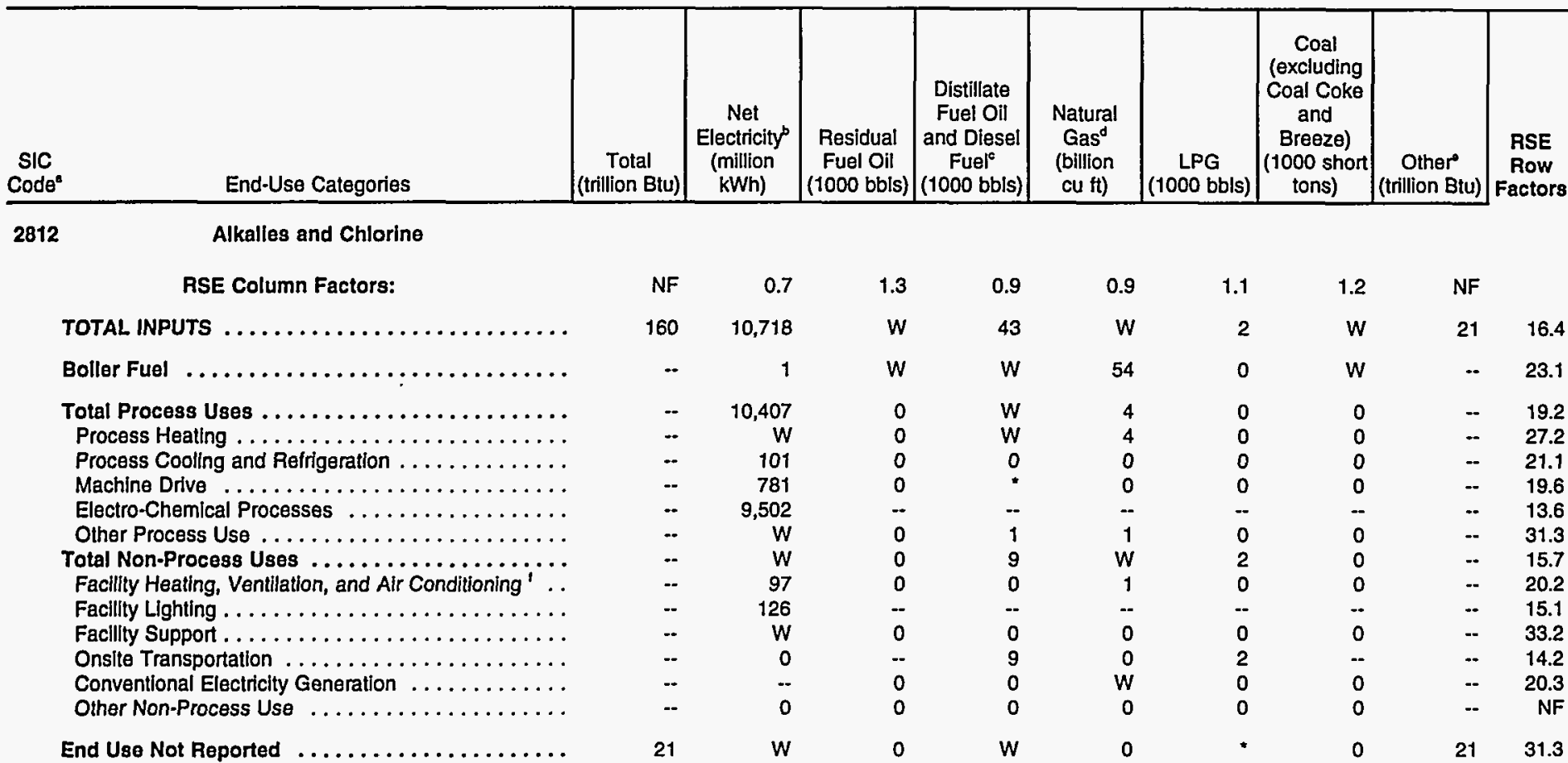

TOTAL INPUTS

Boller Fuel

Total Process Uses .....................

Process Heating $\ldots \ldots \ldots \ldots \ldots \ldots \ldots \ldots \ldots$

Process Cooling and Refrigeration $\ldots \ldots \ldots \ldots \ldots$

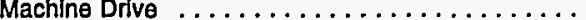

Electro-Chemical Processes $\ldots \ldots \ldots \ldots \ldots \ldots$.

Other Process Use . ..................

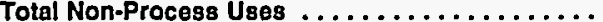

Facllity Heatling, Ventilation, and Air Conditioning ' .

Facllity Lightlng . . . . . . . . . . . . . . . . . .

Facility Support . . . . . $\ldots \ldots \ldots \ldots \ldots \ldots$

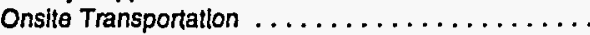

Conventional Electricity Generation . . . . . . . . . .

Other Non-Process Use . .................

End Use Not Reported

Industrial Inorganic Chemicals, nec

RSE Column Factors:

TOTAL INPUTS

Boller Fuel

Total Process Uses

Process Heating

Process Cooling and Refrigeration . . . . . . . . .

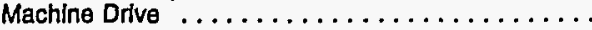

Electro-Chemlcal Processes . . ..............

Other Process Use $\ldots \ldots \ldots \ldots \ldots \ldots \ldots \ldots$

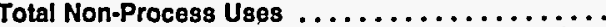

Facllity Heating, Ventilation, and Air Conditioning ' ${ }^{\prime}$.

Facllity Lighting $\ldots \ldots \ldots \ldots \ldots \ldots \ldots \ldots$

Facllty Support .......................

Onsite Transportation .....................

Conventional Electricity Generation .............

Other Non-Process Use $\ldots \ldots \ldots \ldots \ldots \ldots \ldots$

End Use Not Reported
NF

$$
9
$$

$\begin{array}{rcc}0.4 & \text { NF } & 1.3 \\ 17,854 & 0 & 7 \\ 0 & 0 & 1 \\ 16,733 & 0 & . \\ W & 0 & 0 \\ 98 & 0 & 0 \\ 16,432 & 0 & 0 \\ W & -. & -. \\ Q & 0 & . \\ W & 0 & 0 \\ 326 & 0 & 1 \\ 162 & . & . . \\ W & 0 & 0 \\ 0 & - & Q \\ - & 0 & 0 \\ 0 & 0 & 0 \\ W & 0 & .\end{array}$

$\begin{array}{rr}0.8 & 2.2 \\ 24 & Q \\ 8 & 0 \\ 11 & 0 \\ 11 & 0 \\ 0 & 0 \\ . & 0 \\ * & -. \\ 4 & 0 \\ . * & 0 \\ : & -. \\ 0 & 0 \\ 2 & 0 \\ 2 & 0 \\ . & 0\end{array}$

$$
\begin{array}{r}
\text { NF } \\
0 \\
0 \\
0 \\
0 \\
0 \\
0 \\
- \\
0 \\
0 \\
0 \\
\hdashline \\
0 \\
-0 \\
0
\end{array}
$$

NF NF

$5 \quad 14.2$

$-13.6$

$--\quad 11.2$

$-23.0$

-. $\quad 25.3$

-. $\quad 12.4$

-- $\quad 36.8$

- $\quad 34.3$

$-14.0$

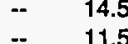

$--12.3$

$-\quad$ NF

.. 24.9

-. 24.9

$5 \quad 12.6$

\begin{tabular}{|c|c|c|c|c|c|c|}
\hline NF & 0.8 & 1.0 & 1.2 & 0.8 & 1.2 & 1.2 \\
\hline 311 & 37,077 & 691 & 456 & 136 & 75 & 743 \\
\hline -. & w & w & 187 & 69 & 23 & 403 \\
\hline .. & 35,008 & W & 78 & 53 & 33 & 304 \\
\hline .. & 2,151 & W & 45 & 52 & 33 & 304 \\
\hline-- & W & 0 & 0 & * & 0 & 0 \\
\hline -. & 28,380 & 0 & W & - & - & 0 \\
\hline -. & 4,088 & -- & -- & -. & -. & -- \\
\hline -- & W & 0 & W & " & • & 0 \\
\hline .. & 1,445 & 0 & 189 & 12 & 12 & w \\
\hline-- & 902 & 0 & 17 & W & 3 & w \\
\hline-- & 375 & -* & -- & $\cdots$ & .. & -. \\
\hline -. & 166 & 0 & W & - & - & 0 \\
\hline -. & W & -- & 157 & 0 & 8 & - \\
\hline .. & .- & 0 & W & W & 0 & 0 \\
\hline-- & W & 0 & t & • & - & 0 \\
\hline 25 & $w$ & 0 & 3 & 2 & $Q$ & $w$ \\
\hline
\end{tabular}

See footnotes at end of table. 
Table A36. Total Inputs of Energy for Heat, Power, and Electricity Generation by Fuel Type, Industry Group, Selected Industries, and End Use, 1991: Part 1 (Continued) (Estimates in Btu or Physical Units)

\begin{tabular}{|c|c|c|c|c|c|c|c|c|c|c|}
\hline $\begin{array}{l}\text { SIC } \\
\text { Code }^{a}\end{array}$ & End-Use Categories & $\begin{array}{c}\text { Total } \\
\text { (trillion Btu) }\end{array}$ & $\begin{array}{c}\text { Net } \\
\text { Electricity } \\
\text { (million } \\
\text { kWh) }\end{array}$ & $\begin{array}{c}\text { Residual } \\
\text { Fuel Oil } \\
\text { (1000 bbls) }\end{array}$ & $\begin{array}{c}\text { Distillate } \\
\text { Fuel Oil } \\
\text { and Diesel } \\
\text { Fuel } \\
\text { (1000 bbls) }\end{array}$ & $\begin{array}{c}\text { Natural } \\
\text { Gas }^{d} \\
\text { (billion } \\
\text { cu ft) }\end{array}$ & $\begin{array}{c}\text { LPG } \\
\text { (1000 bbls) }\end{array}$ & $\begin{array}{c}\text { Coal } \\
\text { (excluding } \\
\text { Coal Coke } \\
\text { and } \\
\text { Breeze) } \\
\text { (1000 short } \\
\text { tons) }\end{array}$ & $\begin{array}{c}\text { Other } \\
\text { (trillion Btu) }\end{array}$ & $\begin{array}{c}\text { RSE } \\
\text { Row } \\
\text { Factors }\end{array}$ \\
\hline
\end{tabular}

2821 Plastics Materials and Resins

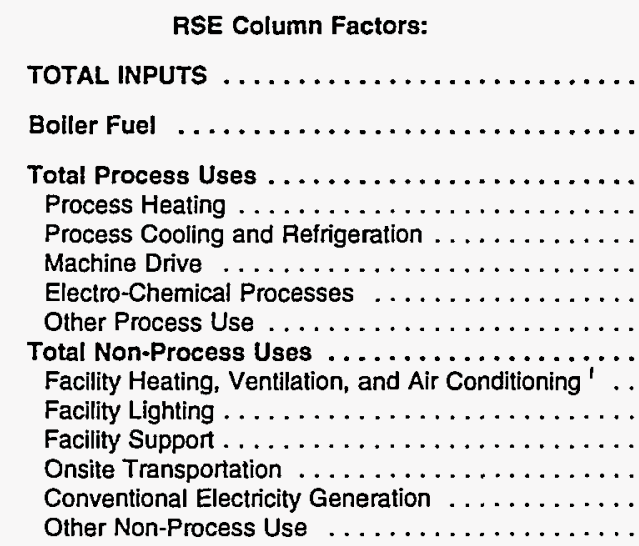

\begin{tabular}{|c|c|c|c|}
\hline NF & 0.6 & 1.3 & 1.3 \\
\hline 288 & 14,780 & 668 & 231 \\
\hline -- & 203 & $w$ & 142 \\
\hline -- & 12,909 & $w$ & 33 \\
\hline -- & $W$ & $w$ & 24 \\
\hline -- & 1,330 & 0 & 0 \\
\hline -- & 9,181 & 0 & 9 \\
\hline -. & 1,972 & .. & -. \\
\hline -- & W & 0 & - \\
\hline - & 1,396 & W & 55 \\
\hline -- & 675 & 0 & 3 \\
\hline -. & 549 & -- & -- \\
\hline -- & 164 & 0 & - \\
\hline -. & 7 & -. & 41 \\
\hline 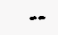 & -- & $w$ & 1 \\
\hline - & 1 & 0 & 9 \\
\hline 60 & 476 & 0 & 1 \\
\hline
\end{tabular}

End Use Not Reported

Synthetic Rubber

RSE Column Factors:

$\begin{array}{rrrr}\text { NF } & 0.6 & 1.4 & 1.0 \\ 112 & 1,794 & 64 & 18 \\ -- & W & 64 & W \\ -- & 1,549 & 0 & W \\ - & 19 & 0 & 0 \\ - & 223 & 0 & 0 \\ - & 1,305 & 0 & W \\ -- & 1 & -. & \\ -- & 1 & 0 & \\ -- & 237 & 0 & 6 \\ - & 120 & 0 & 0 \\ - & 111 & - & - \\ -- & 6 & 0 & \\ -. & * & -. & \\ - & -. & 0 & \\ - & 0 & 0 & \\ W & W & 0 & \end{array}$

TOTAL INPUTS

Boller Fuel

Total Process Uses

Process Heating ......

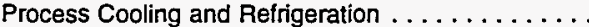

Machine Drive .....................

Electro-Chemical Processes $\ldots \ldots \ldots \ldots \ldots \ldots$

Other Process Use $\ldots \ldots \ldots \ldots \ldots \ldots \ldots \ldots \ldots$

Total Non-Process Uses . . . . . . . . . . . .

Facility Heating, Ventilation, and Air Conditioning ' ..

Facility Lighting.$\ldots \ldots \ldots \ldots \ldots \ldots \ldots$

Facility Support

Onsite Transportation $\ldots \ldots \ldots \ldots \ldots \ldots \ldots \ldots \ldots$

Conventional Electricity Generation . . . . . . . . .

Other Non-Process Use $\ldots \ldots \ldots \ldots \ldots \ldots$.

End Use Not Reported

\section{Cellulosic Manmade Fibers}

RSE Column Factors:

NF

TOTAL INPUTS

Boiler Fuel

Total Process Uses

Process Cooling and Reirigeration . . . . . . . . .

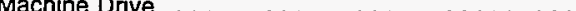

Electro-Chemical Processes $\ldots \ldots \ldots \ldots \ldots \ldots$

Other Process Use $\ldots \ldots \ldots \ldots \ldots \ldots \ldots \ldots$

Total Non-Process Uses . ..................

Facility Heating, Ventilation, and Air Conditioning ' . .

Facility Lighting . . .

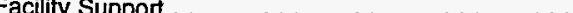

Onsite Transportation ....................

Conventional Electricity Generation $\ldots \ldots \ldots \ldots \ldots$. . .

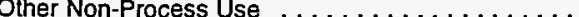

End Use Not Reported

See footnotes at end of table. 
Table A36. Total Inputs of Energy for Heat, Power, and Electricity Generation by Fuel Type, Industry Group, Selected Industries, and End Use, 1991: Part 1 (Continued) (Estimates in Btu or Physical Units)

\begin{tabular}{|c|c|c|c|c|c|c|c|c|c|c|}
\hline $\begin{array}{c}\text { SIC } \\
\text { Code }\end{array}$ & End-Use Categories & $\begin{array}{c}\text { Total } \\
\text { (trillion Btu) }\end{array}$ & $\begin{array}{l}\text { Net } \\
\text { Electricity } \\
\text { (million } \\
\text { kWh) }\end{array}$ & $\begin{array}{c}\text { Residual } \\
\text { Fuel Oil } \\
\text { (1000 bbls) }\end{array}$ & $\begin{array}{c}\text { Distillate } \\
\text { Fuel Oil } \\
\text { and Diesel } \\
\text { Fuel' } \\
\text { (1000 bbls) }\end{array}$ & $\begin{array}{l}\text { Natural } \\
\text { Gas }^{d} \\
\text { (billion } \\
\text { cu ft) }\end{array}$ & $\begin{array}{c}\text { LPG } \\
\text { (1000 bbls) }\end{array}$ & $\begin{array}{c}\text { Coal } \\
\text { (excluding } \\
\text { Coal Coke } \\
\text { and } \\
\text { Breeze) } \\
\left(\begin{array}{c}1000 \text { short } \\
\text { tons) }\end{array}\right.\end{array}$ & Other & $\begin{array}{c}\text { RSE } \\
\text { Row } \\
\text { Factors }\end{array}$ \\
\hline
\end{tabular}

2824 Organic Fibers, Noncellulosic

RSE Column Factors:

TOTAL INPUTS

Boller Fuel

Total Process Uses . . . . . . . . . . . . . . .

Process Heating $\ldots \ldots \ldots \ldots \ldots \ldots \ldots \ldots \ldots$

Process Cooling and Refrigeration .............

Machine Drive .......................

Electro-Chemical Processes ...............

Other Process Use ......................

Total Non-Process Uses ................. Facility Heatling, Ventilation, and Air Conditioning ' ...

Facillty Llighting . . . . . . . . . . . . . . . . . . . . .

Facillty Support ......................

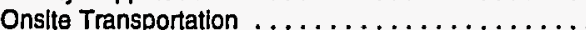

ConventIonal Electricity Generation . . . . . . . . . .

Other Non-Process Use $\ldots \ldots \ldots \ldots \ldots \ldots \ldots$

End Use Not Reported

$\begin{array}{rr}\text { NF } & 1.1 \\ 98 & 6,976 \\ -- & 68 \\ -. & 5,600 \\ -- & W \\ -- & 726 \\ -- & 3,894 \\ -- & W \\ -- & W \\ -- & 1,308 \\ -. & 703 \\ -. & 436 \\ -. & W \\ -. & 2 \\ -. & -. \\ -. & \text { W } \\ \text { W } & 68\end{array}$

2865

Cyclic Crudes and Intermediates

RSE Column Factors:

TOTAL INPUTS

Boiler Fuel

Total Process Uses

n...................

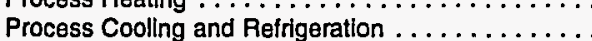

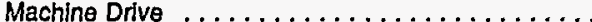

Electro-Chemical Processes ................

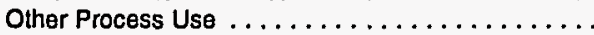

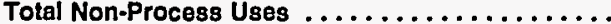

Facllity Heating, Ventillation, and Air Conditioning ' ..

Facllity Llghting . . . . . . . . . . . . . . . . . .

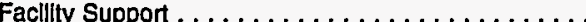

Onsile Transportation . ..................

Conventlonal Electricity Generation .............

Other Non-Process Use $\ldots \ldots \ldots \ldots \ldots \ldots$. . . . .

End Use Not Reported

Industrial Organle Chemlcals, nec

RSE Column Factors:

TOTAL INPUTS

Boller Fuel

Total Process Uses ....................

Process Heating .......................

Process Cooling and Refrigeration ............

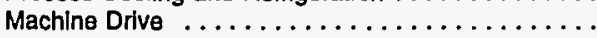

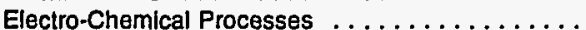

Other Process Use $\ldots \ldots \ldots \ldots \ldots \ldots \ldots \ldots \ldots$

Total Non-Process Uses . . . . . . . . . . . . . .

Facillty Heating, Ventilation, and Air Conditioning ${ }^{t}$..

Facllty Llghting . . . . . . . . . . . . . . . . . . . .

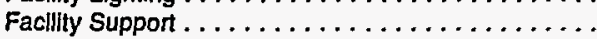

Onsite Transportation ...................

Conventional Electricity Generation ............

Other Non-Process Use $\ldots \ldots \ldots \ldots \ldots \ldots \ldots$.

End Use Not Reported

$\ldots \ldots \ldots \ldots \ldots$

3,894

W

703

36

2

68

$1.1 \quad 1.1$

w

1.0

53

0.9
$W$
23
$W$
$W$
$:$
$:$
$:$
$:$
$:$
0
0
0

1.1

$\begin{array}{rr}0.8 & N F \\ 1,558 & W \\ 1,558 & \end{array}$

3.5

$68 \quad 323$

34

2
1

w

(1)

(1)

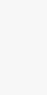

$\begin{array}{ll}- & 4.3 \\ - & 5.4 \\ - & 2.7\end{array}$

\begin{tabular}{r}
0.8 \\
94 \\
46 \\
37 \\
35 \\
0 \\
1 \\
\hdashline- \\
1 \\
$W$ \\
1 \\
-- \\
0 \\
$W$ \\
0 \\
$W$
\end{tabular}

$$
1.2
$$

79

w

41

\begin{tabular}{rr}
1.2 & 0.8 \\
439 & 625 \\
$Q$ & 2 \\
71 & 24 \\
41 & 19 \\
0 & \\
23 & \\
-- & \\
7 & \\
94 & \\
$Q$ & \\
$-\bar{W}$ & \\
64 & \\
\hline & \\
19 & \\
8 &
\end{tabular}

\begin{tabular}{r}
0.8 \\
625 \\
213 \\
241 \\
196 \\
2 \\
28 \\
\hdashline \\
16 \\
170 \\
3 \\
\hdashline \\
1 \\
0 \\
$W$ \\
$W$ \\
1
\end{tabular}


Table A36. Total Inputs of Energy for Heat, Power, and Electricity Generation by Fuel Type, Industry Group, Selected Industries, and End Use, 1991: Part 1 (Continued) (Estimates in Btu or Physical Units)

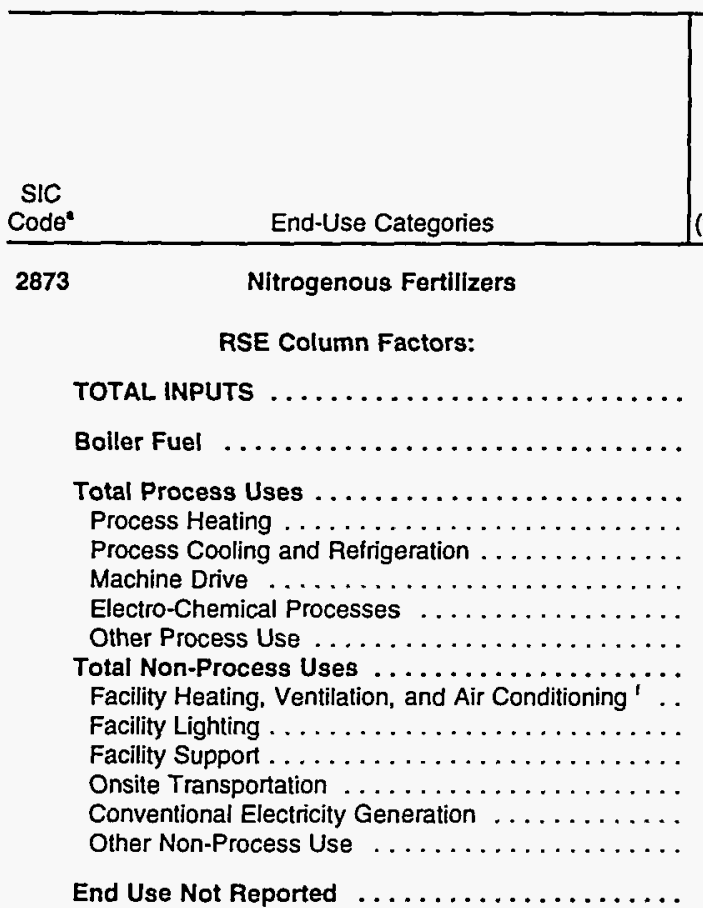
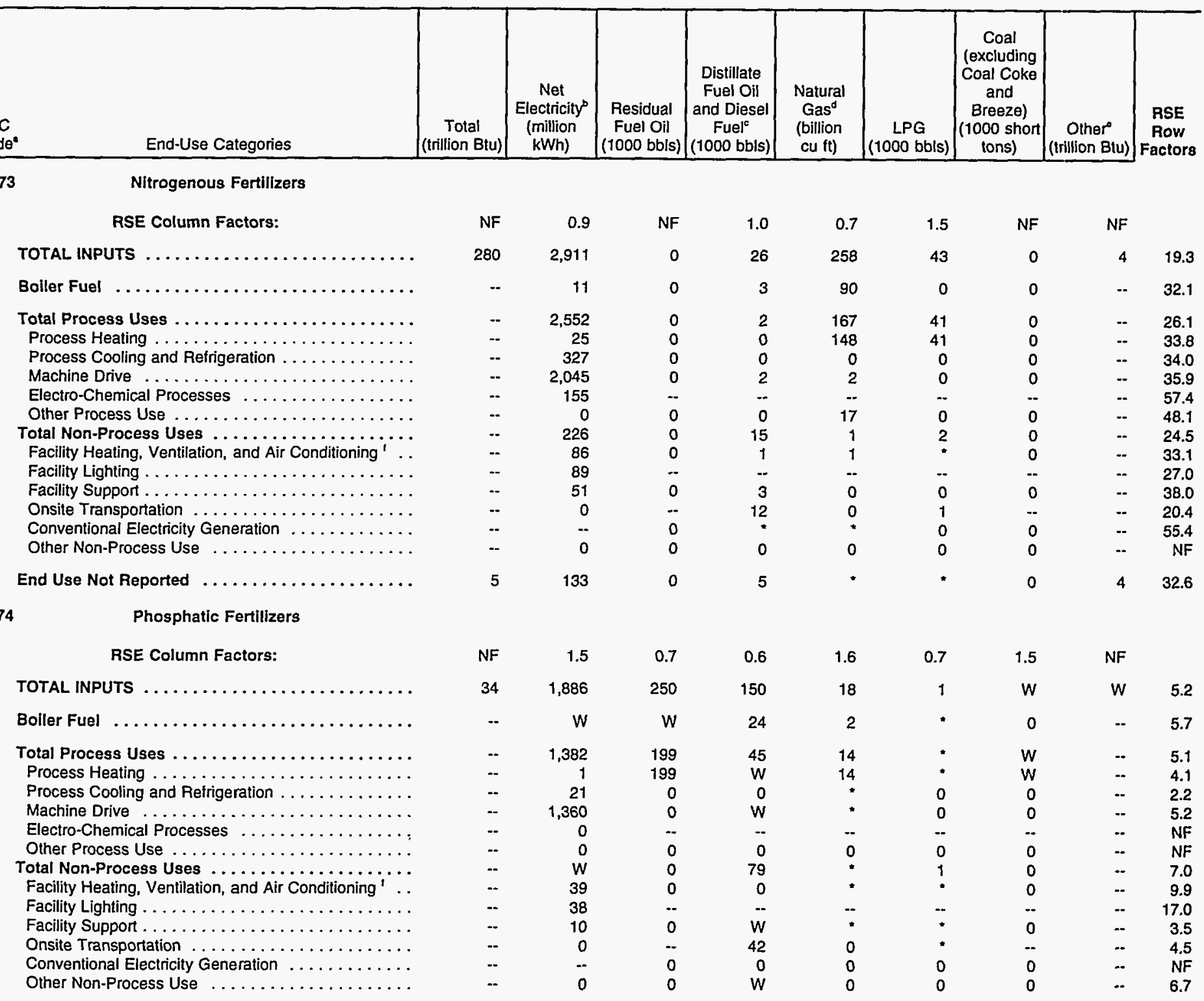

\begin{tabular}{rr} 
NF & 0.9 \\
280 & 2,911 \\
-- & 11 \\
-- & 2,552 \\
.- & 25 \\
-- & 327 \\
-- & 2,045 \\
.- & 155 \\
-- & 0 \\
-- & 226 \\
.- & 86 \\
-- & 89 \\
.- & 51 \\
-- & 0 \\
-- & -- \\
-- & 0 \\
\hline 5 & 133
\end{tabular}

NF
0
0
0
0
0
0
$-\%$
0
0
0
--
0
--
0
0
0

$\begin{array}{rr}1.0 & 0.7 \\ 26 & 258 \\ 3 & 90 \\ 2 & 167 \\ 0 & 148 \\ 0 & 0 \\ 2 & 2 \\ - & - \\ 0 & 17 \\ 15 & 1 \\ 1 & 1 \\ - & -- \\ 3 & 0 \\ 12 & 0 \\ - & \\ 0 & 0 \\ 5 & -\end{array}$

1.5
43
0
41
41
0
0
-
0
2
-
--
0
1
0
0
$*$

$\begin{array}{ccc}\text { NF } & \text { NF } & \\ 0 & 4 & 19.3 \\ 0 & -- & 32.1 \\ 0 & -- & 26.1 \\ 0 & -- & 33.8 \\ 0 & -- & 34.0 \\ 0 & -- & 35.9 \\ -- & -- & 57.4 \\ 0 & -- & 48.1 \\ 0 & -- & 24.5 \\ 0 & -- & 33.1 \\ -- & -- & 27.0 \\ 0 & -- & 38.0 \\ -- & -- & 20.4 \\ 0 & -- & 55.4 \\ 0 & -. & \text { NF } \\ 0 & 4 & 32.6\end{array}$

End Use Not Reported

$\begin{array}{crr}\text { NF } & 1.5 & 0.7 \\ 34 & 1,886 & 250 \\ -- & W & W \\ -. & 1,382 & 199 \\ -- & 1 & 199 \\ -- & 21 & 0 \\ -- & 1,360 & 0 \\ -- & 0 & -- \\ -- & 0 & 0 \\ -- & W & 0 \\ -- & 39 & 0 \\ -- & 38 & -. \\ -- & 10 & 0 \\ -- & 0 & -- \\ -- & -- & 0 \\ -- & 0 & 0 \\ W & W & W\end{array}$

0.6
150
24
45
$W$
0
$W$
-
0
79
0
-
$W$
42
0
$W$
$Q$

$\begin{array}{rrrrr}1.6 & 0.7 & 1.5 & N F & \\ 18 & 1 & W & W & 5.2 \\ 2 & * & 0 & -- & 5.7 \\ 14 & * & W & .- & 5.1 \\ 14 & * & W & -. & 4.1 \\ * & 0 & 0 & -- & 2.2 \\ * & 0 & 0 & -- & 5.2 \\ - & - & -. & -. & N F \\ 0 & 0 & 0 & -. & N F \\ * & 1 & 0 & -- & 7.0 \\ * & - & 0 & -- & 9.9 \\ - & - & -- & -- & 17.0 \\ * & * & 0 & -- & 3.5 \\ 0 & * & -. & -. & 4.5 \\ 0 & 0 & 0 & -. & N F \\ 0 & 0 & 0 & -- & 6.7 \\ 2 & * & 0 & W & 3.9\end{array}$

PETROLEUM and COAL PRODUCTS

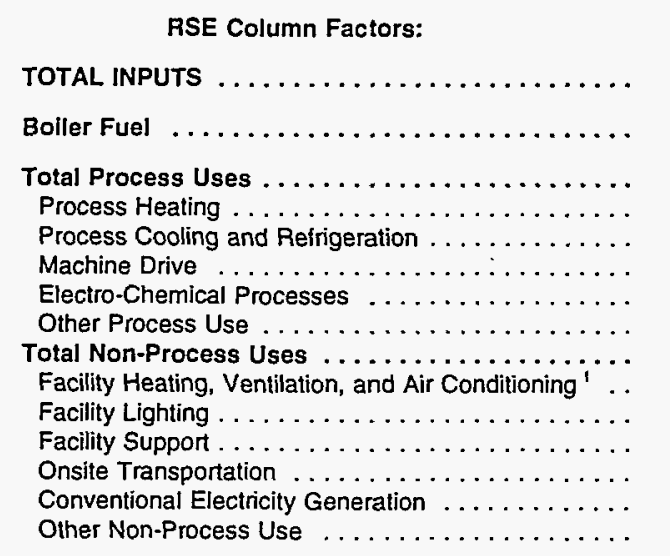


Table A36. Total Inputs of Energy for Heat, Power, and Electricity Generation by Fuel Type, Industry Group, Selected Industries, and End Use, 1991: Part 1 (Continued) (Estimates in Btu or Physical Units)

\begin{tabular}{|c|c|c|c|c|c|c|c|c|c|c|}
\hline$\underset{\text { Code }}{\text { SIC }}$ & End-Use Categories & $\begin{array}{c}\text { Total } \\
\text { (trillion Btu) }\end{array}$ & $\begin{array}{l}\text { Net } \\
\text { Electricity } \\
\text { (million } \\
\text { kWh) }\end{array}$ & $\begin{array}{c}\text { Residual } \\
\text { Fuel Oil } \\
\text { (1000 bbls) }\end{array}$ & $\begin{array}{c}\text { Distillate } \\
\text { Fuel Oil } \\
\text { and Diesel } \\
\text { Fuel }^{\circ} \\
\text { (1000 bbls })\end{array}$ & $\begin{array}{c}\text { Natural } \\
\text { Gas }^{d} \\
\text { (billion } \\
\text { cu } f t \text { ) }\end{array}$ & $\begin{array}{c}\text { LPG } \\
\text { (1000 bbls })\end{array}$ & $\begin{array}{c}\text { Coal } \\
\text { (excluding } \\
\text { Coal Coke } \\
\text { and } \\
\text { Breeze) } \\
\text { (1000 short } \\
\text { tons) }\end{array}$ & $\begin{array}{c}\text { Other } \\
\text { (trillion Btu) }\end{array}$ & $\begin{array}{c}\text { RSE } \\
\text { Row } \\
\text { Factors }\end{array}$ \\
\hline
\end{tabular}

$2911 \quad$ Petroleum Refining'

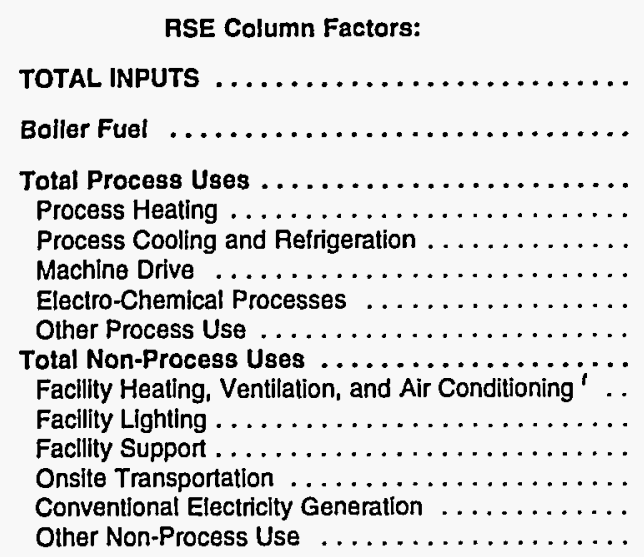

$\begin{array}{rrr}\text { NF } & 0.5 & \\ 2,893 & 29,152 & 10,2 \\ .- & W & 5,1 \\ .- & 26,325 & 5,0 \\ -. & 817 & 5,0 \\ -. & 1,528 & \\ -- & 23,888 & \\ -. & 82 & \\ -. & 9 \\ -. & 2,482 \\ -. & 1,173 \\ -. & 1,045 & \\ -. & 271 & \\ -. & (8) & \\ -. & -. & \\ 1,868 & 1 & \end{array}$

1.6
10,292
5,198
5,094
5,094
0
0
-
0
0
0
-
0
-
0
0
0

1.0
1,525
299
745
679
0
$W$
$-\bar{W}$
$W$
$:-$
449
$:$
$W$
$W$

0.5
769
246
416
380
$W$
$W$
-
$\div$
105
8
-
1
0
95
-
2

0.7
15,889
3,039
12,535
9,382
$W$
$W$
-
0
$W$
$W$
$\ddot{w}$
$\dot{W}$
.

3.6
134
134
0
0
0
0
0
0
0
0
-
0
--
0
0

NF

End Use Not Reported

w

0

1,864

TOTAL INPUTS RSE Column Factors:

Boiler Fuel

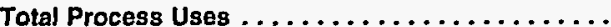

Process Heating $\ldots \ldots \ldots \ldots \ldots \ldots \ldots \ldots$. Process Cooling and Refrigeration .............

Machine Drive .......................

Electro-Chemical Processes ................

Other Process Use ....................

Total Non-Process Uses .................. Faclity Heating, Ventilation, and Air Conditioning' ... Facillity Lighting.$\ldots \ldots \ldots \ldots \ldots \ldots \ldots$

Facility Support .......................

Onsite Transportation ..................

Conventional Electricity Generation ............

Other Non-Process Use $\ldots \ldots \ldots \ldots \ldots \ldots$.

End Use Not Reported

NF

237

$$
0.5
$$

33,908

175

26,284

5,673

2,398

18,045

64
104

5,988

2,745

2,499

626

109

$\ddot{Q}$

1,636

1.1

1,253

1,084

$W \quad 28$

$\begin{array}{ll}W & 28\end{array}$

$\begin{array}{ll}W & W \\ 1 & 0\end{array}$

$\begin{array}{ll}1 & 0 \\ 0 & -\end{array}$

$\begin{array}{rr}0 & \\ W & 185\end{array}$

W 54

-

$\begin{array}{rr}-. & 123 \\ 0 & 2\end{array}$

0

Q

1.5
508
283
28
$W$
0
$Q$
-
-
185
54
-
1
123
2
$Q$
$Q$

$Q$

Total Process Uses . . . . . . . . . . . . . . .

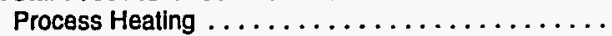

Process Cooling and Refrigeration .............

Machine Drive .....................

Electro-Chemical Processes . ..............

Other Process Use $\ldots \ldots \ldots \ldots \ldots \ldots \ldots \ldots \ldots \ldots$

Total Non-Process Uses ................ Facilly Heating, Ventllation, and Air Conditioning ' . .

Facllity Lighting . . . . . . . . . . . . . . . . . .

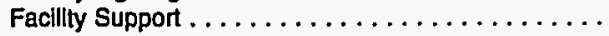

Onsite Transportation ....................

Conventional Electricity Generation . .............

Other Non-Process Use .................

End Use Not Reported 
Table A36. Total Inputs of Energy for Heat, Power, and Electricity Generation by Fuel Type, Industry Group, Selected Industries, and End Use, 1991: Part 1 (Continuled)

(Estimates in Btu or Physical Units)

\begin{tabular}{|c|c|c|c|c|c|c|c|c|c|c|}
\hline $\begin{array}{l}\text { SIC } \\
\text { Code }\end{array}$ & End-Use Calegories & $\begin{array}{c}\text { Total } \\
\text { (trillion Btu) }\end{array}$ & $\begin{array}{c}\text { Net } \\
\text { Electricity } \\
\text { (million } \\
\text { kWh) } \\
\end{array}$ & $\begin{array}{c}\text { Residual } \\
\text { Fuel Oil } \\
(1000 \text { bbls })\end{array}$ & $\begin{array}{c}\text { Distillate } \\
\text { Fuel Oil } \\
\text { and Diesel } \\
\text { Fuel }^{\circ} \\
\text { (1000 bbls) }\end{array}$ & $\begin{array}{c}\text { Natural } \\
\text { Gas }^{d} \\
\text { (billion } \\
\text { cu ft) }\end{array}$ & $\begin{array}{c}L P G \\
(1000 \text { bbls })\end{array}$ & $\begin{array}{c}\text { Coal } \\
\text { (excluding } \\
\text { Coal Coke } \\
\text { and } \\
\text { Breeze }) \\
(1000 \text { short } \\
\text { tons) }\end{array}$ & $\begin{array}{c}\text { Other } \\
\text { (Irillion Btu) }\end{array}$ & $\begin{array}{l}\text { RSE } \\
\text { Row } \\
\text { Factore }\end{array}$ \\
\hline
\end{tabular}

$308 \quad$ Miscellaneous Plastic Products, nec

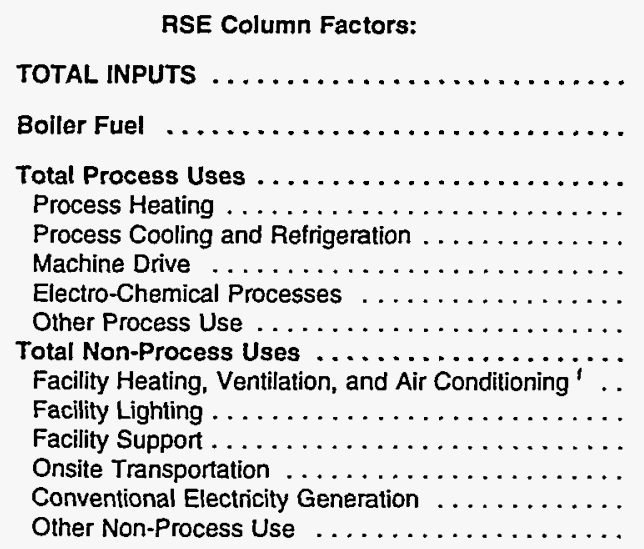

$\begin{array}{rrr}\text { NF } & 0.5 & 1.5 \\ 152 & 25,594 & 413 \\ -. & 103 & 316 \\ -- & 20,052 & 81 \\ -- & 4,953 & 81 \\ -- & 1,957 & 0 \\ -- & 12,994 & . \\ -- & 53 & - \\ -- & 94 & 0 \\ -. & 4,054 & 16 \\ -- & 1,871 & 16 \\ -- & 1,686 & - \\ -- & 427 & - \\ -- & 65 & - \\ -- & - & 0 \\ -- & Q & 0 \\ W & 1,488 & 0\end{array}$

$\begin{array}{rrr}1.7 & 0.7 & 1.0 \\ W & 51 & 396 \\ W & 24 & Q \\ 7 & 13 & 121 \\ 6 & 11 & 82 \\ 0 & 0 & 0 \\ Q & 1 & 39 \\ \because & \because & - \\ 146 & 12 & 230 \\ 33 & 11 & 27 \\ \because: & - & \div \\ 109 & 0 & 204 \\ Q & : & 0 \\ Q & : & : \\ Q & 3 & 18\end{array}$

\begin{tabular}{|c|c|c|}
\hline 1.1 & NF & \\
\hline 130 & $w$ & 13.3 \\
\hline 130 & -. & 21.4 \\
\hline 0 & .. & 17.9 \\
\hline 0 & -- & 21.6 \\
\hline 0 & - & 17.3 \\
\hline 0 & -- & 25.0 \\
\hline .- & -- & 65.5 \\
\hline 0 & -. & 45.0 \\
\hline 0 & -. & 16.0 \\
\hline 0 & .. & 24.0 \\
\hline -. & .- & 13.5 \\
\hline 0 & .- & 25.4 \\
\hline- & -. & 22.9 \\
\hline 0 & .. & NF \\
\hline 0 & -. & 44.9 \\
\hline 0 & $w$ & 33.2 \\
\hline
\end{tabular}

LEATHER and LEATHER PRODUCTS

RSE Column Factors:

TOTAL INPUTS

Boiler Fuel

Total Process Uses

Process Cooling and Refrigeration ...............

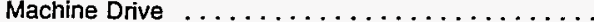

Electro-Chemical Processes $\ldots \ldots \ldots \ldots \ldots \ldots$

Other Process Use .......................

Total Non-Process Uses ..................

Facility Heating, Ventilation, and Air Conditioning ' ..

Facility Lighting .

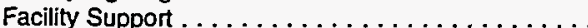

Onsite Transportation .................

Conventional Electricity Generation . . . . . . . . .

Other Non-Process Use

End Use Not Reported

STONE, CLAY and GLASS PRODUCTS

RSE Column Factors:

TOTAL INPUTS

Boiler Fuel

Total Process Uses

Process Cooling and Refrigeration ............

Machine Drive $\ldots \ldots \ldots \ldots \ldots \ldots \ldots \ldots \ldots$

Electro-Chemical Processes $\ldots \ldots \ldots \ldots \ldots \ldots$.

Other Process Use $\ldots \ldots \ldots \ldots \ldots \ldots \ldots \ldots \ldots$

Total Non-Process Uses $\ldots \ldots \ldots \ldots \ldots \ldots \ldots \ldots$

Facility Heating, Ventilation, and Air Conditioning ${ }^{\prime}$.. Facility Lighting .

Facility Support .........

Onsite Transportation ..................

Conventional Electricity Generation ............

Other Non-Process Use . . . . . . . . . . . . . . . . . .

End Use Not Reported

See footnotes at end of table. 
Table A36. Total Inputs of Energy for Heat, Power, and Electricity Generation by Fuel Type, Industry Group, Selected Industries, and End Use, 1991: Part 1 (Continued) (Estimates in Btu or Physical Units)

\begin{tabular}{|c|c|c|c|c|c|c|c|c|c|c|}
\hline$\underset{\operatorname{Cod} \theta^{\circ}}{\text { SIC }}$ & End-Use Categories & $\begin{array}{c}\text { Total } \\
\text { (trillion Btu) }\end{array}$ & $\begin{array}{l}\text { Net } \\
\text { Electricity } \\
\text { (million } \\
\text { kWh) }\end{array}$ & $\begin{array}{c}\text { Residual } \\
\text { Fuel Oil } \\
(1000 \text { bbls })\end{array}$ & $\begin{array}{c}\text { Distillate } \\
\text { Fuel Oil } \\
\text { and Dlesel } \\
\text { Fuel }^{\circ} \\
\text { (1000 bbls) }\end{array}$ & $\begin{array}{c}\text { Natural } \\
\text { Gas }^{d} \\
\text { (billion } \\
\text { cu ft) }\end{array}$ & $\begin{array}{c}\text { LPG } \\
\text { (1000 bbls) }\end{array}$ & $\begin{array}{c}\text { Coal } \\
\text { (excluding } \\
\text { Coal Coke } \\
\text { and } \\
\text { Breeze) } \\
(1000 \text { short } \\
\text { tons) }\end{array}$ & $\begin{array}{c}\text { Other } \\
\text { (trillion Btu) }\end{array}$ & $\begin{array}{c}\text { RSE } \\
\text { Row } \\
\text { Factors }\end{array}$ \\
\hline
\end{tabular}

3211

Flat Glass

RSE Column Factors:

$\begin{array}{rr}\text { NF } & 0.7 \\ 49 & 1,503 \\ -- & W \\ -- & 1,166 \\ -- & 652 \\ -- & 62 \\ -- & 453 \\ -- & 0 \\ -- & 0 \\ -- & W \\ -- & 151 \\ -- & W \\ -- & 15 \\ -- & 12 \\ -- & -. \\ -- & 0 \\ W & W\end{array}$

1.6
$W$
0
$W$
$W$
0
0
--
0
0
0
--
0
-
0
0
0

\begin{tabular}{rr}
0.9 & 0.6 \\
12 & 40 \\
0 & $W$ \\
$W$ & 35 \\
$W$ & 34 \\
. & 1 \\
- & $\cdot$ \\
\hline 0 & - \\
6 & 0 \\
0 & 2 \\
- & 1 \\
0 &.- \\
6 & 0 \\
$*$ & 0 \\
0 & 0 \\
$W$ & $W$
\end{tabular}

1.0
40
0
$W$
$W$
0
2
--
0
15
$*$
-
0
15
0
0
$W$

\begin{tabular}{rrr}
1.6 & NF & \\
- & W & 3.5 \\
0 & -- & 11.2 \\
$*$ & -- & 4.0 \\
0 & -- & 4.1 \\
0 & -- & 6.1 \\
0 & -- & 5.2 \\
-- & -- & NF \\
- & -- & 3.7 \\
0 & -- & 4.1 \\
0 & -- & 4.7 \\
-- & - & 4.3 \\
0 & -- & 7.4 \\
-- & -- & 5.6 \\
0 & -- & 4.4 \\
0 & -- & NF \\
\hline 0 & W & 5.6
\end{tabular}

Glass Containers

RSE Column Factors:

\begin{tabular}{|c|c|c|c|c|c|c|c|c|}
\hline NF & 0.7 & 1.5 & 1.3 & 0.6 & 1.2 & NF & NF & \\
\hline 85 & 4,098 & 276 & 23 & 67 & 82 & 0 & • & 4.8 \\
\hline-- & 1 & 1 & 5 & 1 & 0 & 0 & -- & 14.4 \\
\hline-- & 3,580 & 275 & 15 & 64 & 16 & 0 & -- & 6.0 \\
\hline - & 1,187 & 275 & 14 & 63 & $w$ & 0 & -- & 6.9 \\
\hline -. & $W$ & 0 & 0 & • & 0 & 0 & -- & 17.6 \\
\hline -- & 2,198 & 0 & 1 & • & $w$ & 0 & -- & 9.2 \\
\hline -- & W & -. & - & -- & -- & -- & -- & 27.3 \\
\hline- & $w$ & 0 & 0 & 1 & - & 0 & -. & 9.7 \\
\hline -- & 517 & " & 3 & 3 & 66 & 0 & -- & 6.0 \\
\hline -- & 196 & * & 1 & 2 & * & 0 & -- & 9.5 \\
\hline-- & 307 & -- & -- & -- & - & - & $\ldots$ & 6.1 \\
\hline -. & 14 & 0 & * & • & • & 0 & -. & 11.6 \\
\hline -- & 1 & - & 2 & 0 & 65 & -- & .. & 7.1 \\
\hline -. & -. & 0 & 0 & 0 & 0 & 0 & -- & NF \\
\hline$\cdots$ & 0 & 0 & 0 & 0 & 0 & 0 & -- & $\mathrm{NF}$ \\
\hline . & 1 & • & • & 0 & 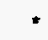 & 0 & * & 16.3 \\
\hline
\end{tabular}

TOTAL INPUTS

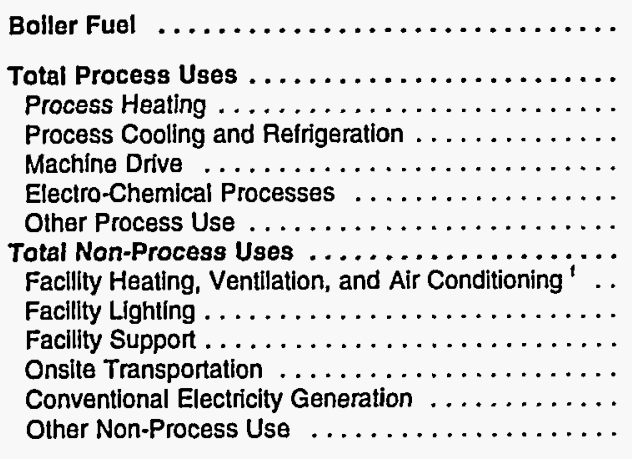

End Use Not Reported

Pressed and Blown Glass, nec.

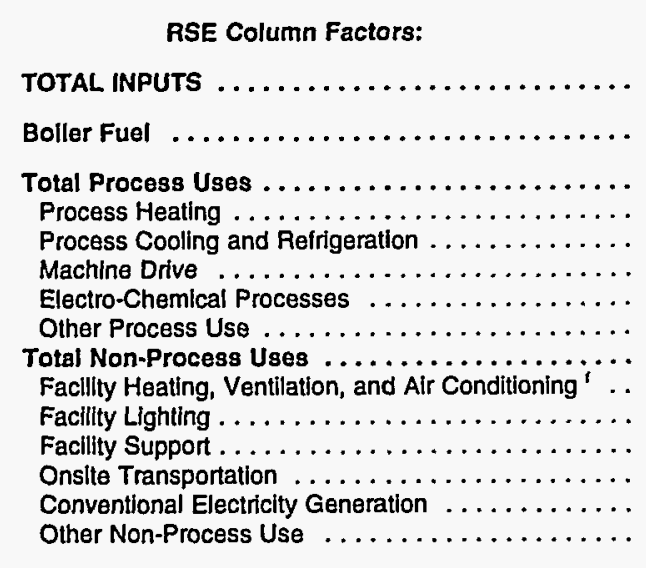

NF

W
0.6

2,862

1

2,315

1,279

W

869

1
$w$

W

293

178

35

7

w

w

\section{7}

81

1.0

0.6

1.0

NF

End Use Not Reported

w

See footnotes at end of table. 
Table A36. Total Inputs of Energy for Heat, Power, and Electricity Generation by Fuel Type, Industry Group, Selected Industries, and End Use, 1991: Part 1 (Continued) (Estimates in Btu or Physical Units)

\begin{tabular}{|c|c|c|c|c|c|c|c|c|c|c|}
\hline $\begin{array}{c}\text { SIC } \\
\text { Code" }\end{array}$ & End-Use Categories & $\begin{array}{c}\text { Total } \\
\text { (trillion Btu) }\end{array}$ & $\begin{array}{c}\text { Net } \\
\text { Electricity } \\
\text { (million } \\
\text { kWh) }\end{array}$ & $\begin{array}{c}\text { Residual } \\
\text { Fuel Oil } \\
\text { (1000 bbis) }\end{array}$ & $\begin{array}{c}\text { Distillate } \\
\text { Fuel Oil } \\
\text { and Diesel } \\
\text { Fuel } \\
\text { (1000 bbls) }\end{array}$ & $\begin{array}{c}\text { Natural } \\
\text { Gas }^{d} \\
\text { (billion } \\
\text { cu ft) }\end{array}$ & $\begin{array}{c}\text { LPG } \\
(1000 \mathrm{bb} / \mathrm{s})\end{array}$ & $\begin{array}{c}\text { Coal } \\
\text { (excluding } \\
\text { Coal Coke } \\
\text { and } \\
\text { Breeze) } \\
(1000 \text { short } \\
\text { tons) }\end{array}$ & $\begin{array}{c}\text { Other } \\
\text { (triltion Btu) }\end{array}$ & $\begin{array}{c}\text { RSE } \\
\text { Row } \\
\text { Factors }\end{array}$ \\
\hline
\end{tabular}

3241 Cement, Hydraulic

RSE Column Factors:

$\begin{array}{lr}\text { NF } & 0.9 \\ 329 & 9,455 \\ -- & \\ -. & 8,900 \\ -- & 1,640 \\ -- & 249 \\ -- & 6,979 \\ -- & \\ -. & \\ -. & 55 \\ -- & 264 \\ -- & 205 \\ -- & 84 \\ -- & \\ -. & \\ -. & \end{array}$

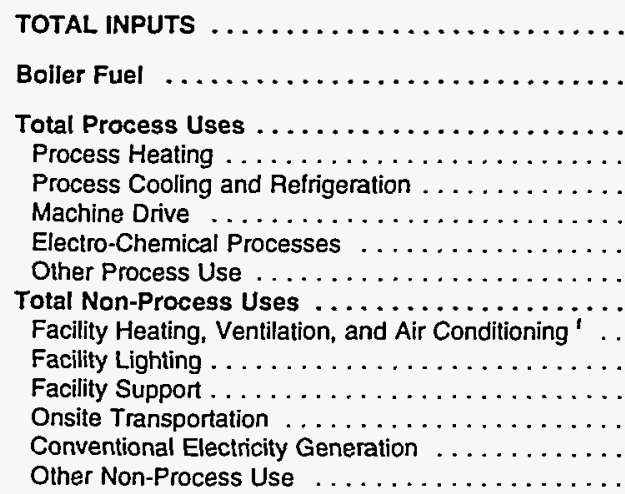

1.1

$\begin{array}{rrr}0.7 & \text { NF } & \\ 8,736 & 58 & 11.0 \\ 0 & -- & 26.2 \\ 8,736 & -- & 11.4 \\ 8,736 & -- & 12.7 \\ 0 & -- & 22.0 \\ 0 & -- & 13.5 \\ . . & - & \text { NF } \\ 0 & -. & 35.3 \\ 0 & - & 15.2 \\ 0 & -. & 20.9 \\ - & -- & 16.2 \\ 0 & -- & 21.8 \\ -. & -- & 17.1 \\ 0 & -. & 32.5 \\ 0 & -- & \text { NF }\end{array}$

End Use Not Reported

TOTAL INPUTS

End Use Not Reported

Total Process Uses . . . . . . . . . . . . . . . . .

Process Heating . . . . . . . . . . . . . . .

Process Cooling and Refrigeration ...........

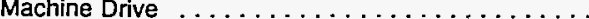

Electro-Chemical Processes $\ldots \ldots \ldots \ldots \ldots \ldots$ Other Process Use

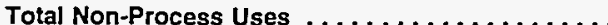

Facility Heating, Ventilation, and Air Conditioning ${ }^{\prime}$.

Facility Lighting

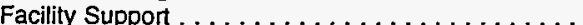

Onsite Transportation ....................

Conventional Electricity Generation $\ldots \ldots \ldots \ldots \ldots$

Other Non-Process Use

TOTAL INPUTS

Total Process Uses Process Cooling and Refrigeration . . . . . . . .

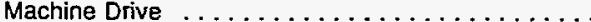

Electro-Chemical Processes .............

Other Process Use . . . . . . . . . . . . . . .

Total Non-Process Uses . . . . . . . . . . . . . . . . .

Facility Heating, Ventilation, and Air Conditioning ${ }^{\prime}$.. Facility Lighting .

Facility Support . . . .

Onsite Transportation . . . . . . . . . . . .

Conventional Electricity Generation

Other Non-Process Use

W

0 
Table A36. Total Inputs of Energy for Heat, Power, and Electricity Generation by Fuel Type, Industry Group, Selected Industries, and End Use, 1991: Part 1 (Continued) (Estimates in Btu or Physical Units)

\begin{tabular}{|c|c|c|c|c|c|c|c|c|c|c|}
\hline $\begin{array}{c}\operatorname{SIC} \\
\operatorname{Cod} \theta^{*}\end{array}$ & End-Use Categories & $\mid \begin{array}{c}\text { Total } \\
\text { (trillion Blu) }\end{array}$ & $\begin{array}{l}\text { Net } \\
\text { Electricity } \\
\text { (million } \\
\text { kWh) }\end{array}$ & $\begin{array}{c}\text { Residual } \\
\text { Fuel Oil } \\
(1000 \text { bbls })\end{array}$ & $\begin{array}{c}\text { Distillate } \\
\text { Fuel Oil } \\
\text { and Diesel } \\
\text { Fuel } \\
\text { (1000 bbls) }\end{array}$ & $\begin{array}{c}\text { Natural } \\
\text { Gas }^{d} \\
\text { (billion } \\
\text { cu ft) }\end{array}$ & $\begin{array}{c}\text { LPG } \\
\text { (1000 bbls) }\end{array}$ & $\begin{array}{c}\text { Coal } \\
\text { (excluding } \\
\text { Coal Coke } \\
\text { and } \\
\text { Breeze) } \\
(1000 \text { short } \\
\text { tons) }\end{array}$ & $\begin{array}{c}\text { Other } \\
\text { (trillion Btu) }\end{array}$ & \begin{tabular}{|c} 
RSE \\
Row \\
Factors
\end{tabular} \\
\hline
\end{tabular}

33 PRIMARY METAL INDUSTRIES

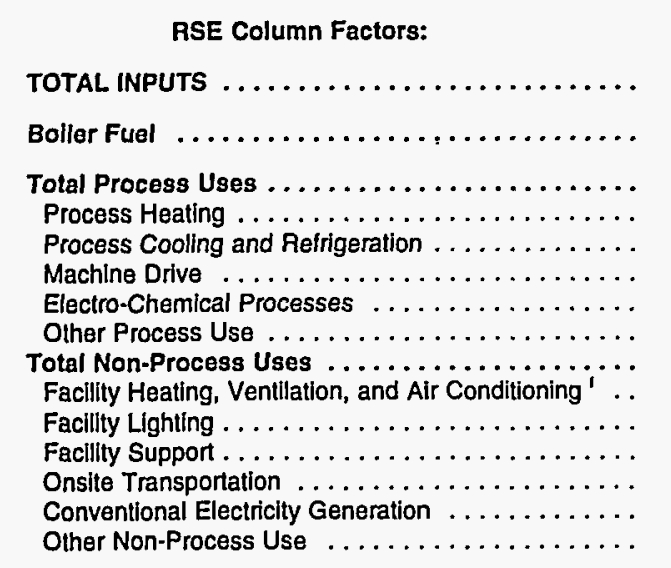

$\begin{array}{rrr}\text { NF } & 0.6 & 1.2 \\ 2,292 & 146,276 & 5,285 \\ -- & 341 & 3,963 \\ -- & 132,938 & 1,268 \\ -- & 29,435 & 1,261 \\ -- & 833 & 0 \\ -- & 34,482 & Q \\ -- & 66,954 & - \\ -- & 1,234 & 0 \\ -- & 10,552 & Q \\ -- & 4,640 & Q \\ -- & 4,715 & - \\ -- & 913 & Q \\ -- & 164 & - \\ -- & -. & 0 \\ -- & 120 & 0 \\ 1,041 & 2,786 & 1\end{array}$

1.2
1,806
88
444
347
0
96
--
1
1,176
58
--
1
1,094
$Q$
$W$
99

$\begin{array}{rr}0.7 & 2.2 \\ 666 & 888 \\ 90 & 38 \\ 507 & 298 \\ 501 & 239 \\ - & 0 \\ 4 & 54 \\ -- & - \\ 2 & 5 \\ 52 & 510 \\ 43 & 51 \\ -- & -- \\ 6 & 10 \\ * & 442 \\ 4 & \div \\ 1 & 7 \\ 17 & 41\end{array}$

$\begin{array}{rrr}0.8 & \text { NF } & \\ 2,054 & 1,014 & 4.6 \\ 1,690 & -- & 7.9 \\ W & -- & 7.2 \\ W & - & 8.0 \\ 0 & -- & 12.4 \\ 0 & -- & 7.5 \\ -- & - & 3.5 \\ 0 & -. & 11.4 \\ W & -. & 3.2 \\ 0 & - & 8.9 \\ -- & -- & 5.3 \\ 0 & -- & 9.1 \\ -. & -- & 4.3 \\ W & -- & 3.5 \\ 0 & -- & 13.9 \\ 1 & 1,014 & 14.5\end{array}$

3312

Blast Furnaces and Steel Mills

RSE Column Factors:

$\begin{array}{rrr}N F & 0.6 & 1.6 \\ 1,569 & 38,183 & 4,986 \\ -- & W & 3,810 \\ -- & 34,083 & 1,173 \\ -- & 15,635 & 1,173 \\ -- & 209 & 0 \\ -- & 16,602 & 0 \\ -- & 778 & - \\ -- & 859 & 0 \\ -- & 2,951 & 3 \\ -- & 1,212 & 3 \\ -- & 1,314 & - \\ -- & 332 & 0 \\ .- & W & -- \\ -- & -- & 0 \\ -- & W & 0 \\ 985 & W & 0\end{array}$

1.0
901
32
169
121
0
48
$\cdots$
658
$W$
-
0
610
$*$
$W$
42

0.7
387
61
303
301
0
1
--
1
21
16
--
4
0
$*$
1
2

1.0
74
$W$
22
19
0
2
-
2
37
5
-
6
26
$*$
1
$W$

$\begin{array}{rrr}1.6 & \text { NF } & \\ 1,075 & 978 & 4.1 \\ 1,056 & -. & 6.7 \\ 17 & -. & 4.8 \\ 17 & -. & 5.3 \\ 0 & -. & 9.8 \\ 0 & -. & 7.1 \\ -. & -. & 14.7 \\ 0 & -. & 14.0 \\ 0 & -. & 5.2 \\ 0 & -. & 7.5 \\ -- & -. & 4.9 \\ 0 & -- & 9.1 \\ -- & -- & 6.9 \\ 0 & -. & 15.0 \\ 0 & -. & 13.7 \\ 1 & 978 & 10.2\end{array}$

3313

Electrometalurglcal Products

RSE Column Factors:

\begin{tabular}{|c|c|c|c|c|c|c|c|}
\hline NF & 0.8 & NF & 0.9 & 0.9 & 1.3 & 1.3 & NF \\
\hline 31 & 4,222 & 0 & 20 & 1 & $w$ & $w$ & 3 \\
\hline -. & $w$ & 0 & 0 & • & 1 & $w$ & -- \\
\hline -. & 3,995 & 0 & $w$ & 1 & * & $w$ & -- \\
\hline-- & 3,024 & 0 & 0 & 1 & $\star$ & $w$ & -- \\
\hline -- & $w$ & 0 & 0 & 0 & 0 & 0 & $\ldots$ \\
\hline -- & 792 & 0 & $w$ & * & 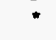 & 0 & .- \\
\hline -- & $w$ & -. & -- & -- & - & -- & -- \\
\hline- & $w$ & 0 & 0 & • & 0 & 0 & -. \\
\hline -- & $W$ & 0 & $w$ & • & $w$ & 0 & -- \\
\hline -- & 61 & 0 & 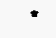 & • & • & 0 & .- \\
\hline-- & 105 & -. & -- & -- & - & - & -- \\
\hline -- & 20 & 0 & 0 & • & - & 0 & -- \\
\hline -- & W & - & $w$ & 0 & $w$ & - & -- \\
\hline -- & -- & 0 & . & 0 & 0 & 0 & -- \\
\hline- & 0 & 0 & 0 & 0 & 0 & 0 & .. \\
\hline 3 & $w$ & 0 & 0 & 0 & 0 & 0 & 3 \\
\hline
\end{tabular}

See footnotes at end of table. 
Table A36. Total Inputs of Energy for Heat, Power, and Electricity Generation by Fuel Type, Industry Group, Selected Industries, and End Use, 1991: Part 1 (Continued) (Estimates in Btu or Physical Units)

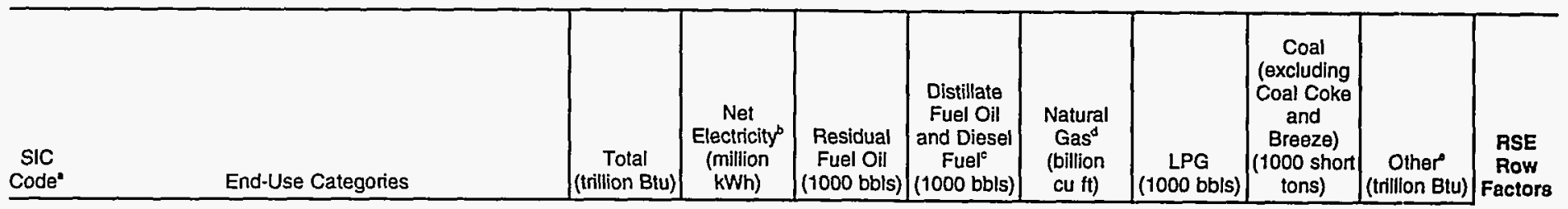

$3321 \quad$ Gray and Ductile Iron Foundries

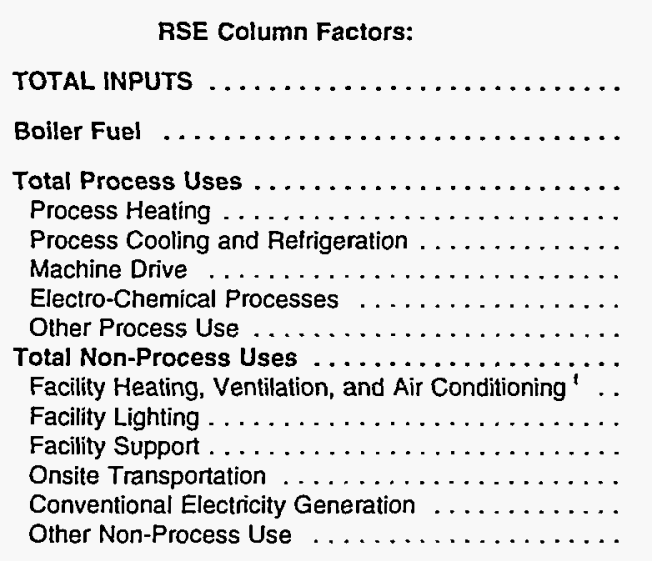

$\begin{array}{cr}\text { NF } & 0.6 \\ 74 & 6,412 \\ - & 24 \\ - & 5,138 \\ - & 16 \\ - & 2,694 \\ -- & 40 \\ -- & 2,393 \\ -- & W \\ -- & Q \\ -- & 1,048 \\ -- & 574 \\ -- & 414 \\ -- & 47 \\ -- & 12 \\ -- & - \\ -- & - \\ 24 & 226\end{array}$

End Use Not Reported $\ldots \ldots \ldots \ldots \ldots \ldots \ldots \ldots .24 \quad 246$

\begin{tabular}{cc}
1.6 \\
4 \\
$W$ \\
$Q$ \\
0 \\
0 \\
$Q$ \\
-- \\
0 \\
$:$ \\
. \\
.- \\
0 \\
\hline- \\
0 \\
0 \\
0
\end{tabular}

1.3
144
2
$Q$
$Q$
0
18
-
67
4
--
1
62
5
0
5

0.6
28
2
18
16
0
1
$:-$
7
6
-
$:$
0
$:$
1

\begin{tabular}{|c|c|c|}
\hline 1.3 & 1.2 & NF \\
\hline 105 & 5 & 22 \\
\hline • & 0 & -- \\
\hline 61 & 5 & -- \\
\hline 34 & 5 & -- \\
\hline 0 & 0 & -- \\
\hline 27 & 0 & -- \\
\hline 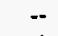 & -- & -- \\
\hline * & 0 & -- \\
\hline 37 & 0 & .. \\
\hline 8 & 0 & -. \\
\hline -- & - & -- \\
\hline 0 & 0 & -- \\
\hline 28 & -- & -- \\
\hline 0 & 0 & -- \\
\hline I & 0 & .. \\
\hline
\end{tabular}

3331

Primary Copper

RSE Column Factors:

$\begin{array}{rr}1.0 & 1 \\ 1,246 & \\ W & \\ 1,130 & \\ 86 & \\ W & \\ 638 & \\ 216 & \\ W & \\ 107 & \\ 43 & \\ 50 & \\ 14 & \\ 0 & \\ - & \\ 0 & \\ W & \end{array}$

1.0
$W$
$W$
$W$
$W$
0
0
.-
0
0
0
--
0
.-
0
0
0

$\begin{array}{cc}1.0 & 1.0 \\ W & 15 \\ 5 & 4 \\ W & 9 \\ W & 8 \\ 0 & 0 \\ 0 & 0 \\ -. & - \\ 0 & * \\ W & 2 \\ . & : \\ 0 & : \\ W & 0 \\ 1 & 2 \\ 0 & 0 \\ 0 & 0\end{array}$

1.0
3
0
$\vdots$
0
-
0
2
-
0
2
0
0
1

$\begin{array}{ccc}1.0 & N F & \\ W & 1 & 1.0 \\ 0 & -- & 1.0 \\ W & -- & 1.0 \\ W & -- & 1.0 \\ 0 & -- & 1.0 \\ 0 & - & 1.0 \\ - & - & 1.0 \\ 0 & -- & 1.0 \\ 0 & - & 1.0 \\ 0 & - & 1.0 \\ - & - & 1.0 \\ 0 & - & 1.0 \\ - & - & 1.0 \\ 0 & -- & 1.0 \\ 0 & -- & \text { NF } \\ 0 & 1 & 1.0\end{array}$

TOTAL INPUTS

Total Process Uses $\ldots \ldots \ldots \ldots \ldots \ldots \ldots \ldots \ldots$

Process Heating . . . . . . . . . . . . . . . .

Process Cooling and Refrigeration ............

Machine Drive.$\ldots \ldots \ldots \ldots \ldots \ldots \ldots \ldots$.

Electro-Chemical Processes ...............

Other Process Use . . . . . . . . . . . . . . .

Total Non-Process Uses . ................

Facility Heating, Ventilation, and Air Conditioning ' ..

Facility Lighting .

Facility Support

Onsite Transportation ................

Conventional Electricity Generation ...........

Other Non-Process Use

$\begin{array}{rc}W & * \\ 64,981 & 0 \\ W & 0 \\ W & 0 \\ 1,301 & 0 \\ 63,226 & - \\ W & 0 \\ W & \cdot \\ 1,207 & * \\ 822 & -. \\ 143 & 0 \\ W & -. \\ - & 0 \\ 0 & 0\end{array}$

W

End Use Not Reported

2

W

0

$\begin{array}{rr}W & 2 \\ W & 16 \\ W & 16 \\ 0 & 0 \\ 2 & \cdot \\ -- & -. \\ 0 & 0 \\ 87 & 1 \\ - & 1 \\ -- & -- \\ 0 & - \\ 87 & 0 \\ 0 & 0 \\ 0 & 0\end{array}$


Table A36. Total Inputs of Energy for Heat, Power, and Electricity Generation by Fuel Type, Industry Group, Selected Industries, and End Use, 1991: Part 1 (Continued) (Estimates in Btu or Physical Units)

\begin{tabular}{|c|c|c|c|c|c|c|c|c|c|c|}
\hline$\underset{\operatorname{Cod} \theta^{\circ}}{\text { SIC }}$ & End-Use Categories & $\begin{array}{c}\text { Total } \\
\text { (trillion Btu) }\end{array}$ & $\begin{array}{l}\text { Net } \\
\text { Electricity } \\
\text { (million } \\
\text { kWh) }\end{array}$ & $\begin{array}{c}\text { Residual } \\
\text { Fuel Oif } \\
\text { (1000 bbls) }\end{array}$ & $\begin{array}{c}\text { Distillate } \\
\text { Fuel Oil } \\
\text { and Diesel } \\
\text { Fuel }^{6} \\
\text { (1000 bbls) }\end{array}$ & $\begin{array}{c}\text { Natural } \\
\text { Gas }^{d} \\
\text { (billion } \\
\text { cu ft) }\end{array}$ & $\begin{array}{c}\text { LPG } \\
\text { (1000 bbls) }\end{array}$ & $\begin{array}{c}\text { Coal } \\
\text { (excluding } \\
\text { Coal Coke } \\
\text { and } \\
\text { Breeze) } \\
\text { (1000 short } \\
\text { tons) }\end{array}$ & $\begin{array}{c}\text { Other } \\
\text { (trillion Btu) }\end{array}$ & $\begin{array}{c}\text { RSE } \\
\text { Row } \\
\text { Factors }\end{array}$ \\
\hline
\end{tabular}

3339 Primary Nonferrous Metals, nec

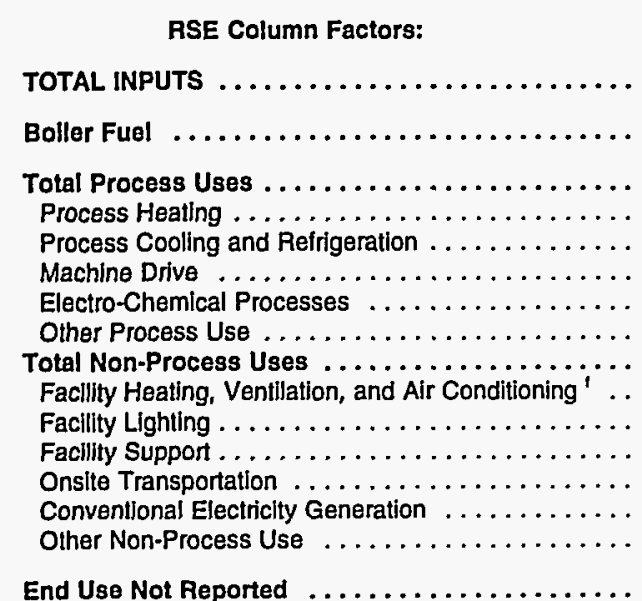

$\begin{array}{rrrr}\text { NF } & 1.3 & 0.4 & 1.6 \\ 42 & 4,312 & 1 & 53 \\ -- & W & 1 & \\ -- & 4,050 & 0 & 15 \\ -. & 1,378 & 0 & \\ -. & 19 & 0 & \\ -- & 651 & 0 & \\ - & 1,999 & -. & \\ -- & 2 & 0 & \\ -- & 230 & 0 & 34 \\ -- & 120 & 0 & \\ -- & 84 & - & \\ -. & 24 & 0 & \\ -- & -. & 0 & 39 \\ - & 0 & 0 & \\ 7 & W & 0\end{array}$

1.6
53
3
15
$W$
0
$W$
--
0
34
-
-
0
33
0
0
1

\begin{tabular}{|c|c|c|c|c|}
\hline 1.3 & 1.4 & 0.6 & NF & \\
\hline 16 & 19 & $w$ & $w$ & 1.9 \\
\hline 3 & 0 & $w$ & -- & 2.8 \\
\hline 7 & $w$ & 0 & .. & 1.6 \\
\hline 7 & W & 0 & -. & 1.3 \\
\hline 0 & 0 & 0 & - & 3.9 \\
\hline. & , & 0 & -- & 1.0 \\
\hline .- & -- & .- & . & 1.6 \\
\hline 0 & 0 & 0 & -- & 15.5 \\
\hline$w$ & $w$ & $w$ & -. & 1.5 \\
\hline 1 & $W$ & 0 & -- & 2. \\
\hline .. & .. & -. & . & 2.3 \\
\hline • & - & 0 & -- & 4.9 \\
\hline 0 & 11 & -- & .. & 1.4 \\
\hline$w$ & 0 & w & - & 1.1 \\
\hline 0 & 0 & 0 & - & NF \\
\hline$w$ & - & 0 & $w$ & 1.5 \\
\hline
\end{tabular}

3353

Aluminum Sheet, Plate, and Foil

RSE Column Factors:

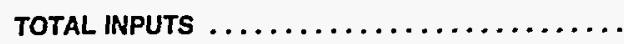

$\begin{array}{rr}1.5 & N F \\ 4,261 & 0 \\ W & 0 \\ 3,562 & 0 \\ 391 & 0 \\ W & 0 \\ 3,138 & 0 \\ 0 & - \\ W & 0 \\ 677 & 0 \\ 234 & 0 \\ 385 & - \\ 34 & 0 \\ 24 & - \\ -- & 0 \\ 1 & 0 \\ W & 0\end{array}$

0.8
67
$\cdot$
4
4
0
$\cdot$
--
0
57
$\cdot$
--
57
5
0
0
5

$\begin{array}{rr}1.2 & 0.8 \\ 41 & 62 \\ 2 & 2 \\ 37 & 19 \\ 37 & W \\ 0 & 0 \\ 0 & W \\ \because- & - \\ 2 & 0 \\ 2 & 42 \\ 2 & W \\ \because- & - \\ 0 & \text { W } \\ 0 & 0 \\ 0 & 0 \\ . & .\end{array}$

$\begin{array}{ccc}0.9 & \text { NF } & \\ W & W & 1.1 \\ W & -- & 1.0 \\ 0 & -- & 1.2 \\ 0 & - & 1.4 \\ 0 & - & 1.3 \\ 0 & - & 1.0 \\ - & - & \text { NF } \\ 0 & - & 0.7 \\ 0 & - & 1.4 \\ 0 & - & 1.4 \\ -- & - & 0.7 \\ 0 & - & 1.3 \\ -. & - & 1.0 \\ 0 & -. & \text { NF } \\ 0 & -- & 0.7 \\ 0 & W & 3.1\end{array}$

TOTAL INPUTS

NF

305

$\begin{array}{rr}0.5 & 1.7 \\ 29,772 & 501 \\ 241 & 35 \\ 20,603 & \\ 3,395 & 1 \\ 691 & \\ 15,105 & \\ 1,197 & \\ 215 & \\ 6,903 & \\ 2,892 & \\ 3,357 & \\ 529 & \\ 99 & \\ -- & \\ 25 & \end{array}$

\begin{tabular}{|c|c|c|c|}
\hline 1.6 & 0.5 & 1.1 & 1.4 \\
\hline 994 & 169 & 1,122 & 245 \\
\hline 248 & 36 & $Q$ & 238 \\
\hline $\begin{array}{l}241 \\
160\end{array}$ & 89 & 456 & $w$ \\
\hline $\begin{array}{r}160 \\
1\end{array}$ & 86 & w & $w$ \\
\hline 1 & * & • & 0 \\
\hline 71 & 1 & W & 0 \\
\hline- & -. & - & -- \\
\hline$Q$ & 1 & 5 & 0 \\
\hline 437 & 36 & 512 & 0 \\
\hline 113 & 33 & 44 & 0 \\
\hline 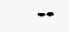 & -- & - & - \\
\hline 1 & 2 & $Q$ & 0 \\
\hline 320 & • & 464 & -. \\
\hline 1 & 1 & 0 & 0 \\
\hline$Q$ & * & • & 0 \\
\hline 68 & 8 & 136 & $Q$ \\
\hline
\end{tabular}

End Use Not Reported

$27 \quad 2,265$

\begin{tabular}{rr}
1.7 & 1 \\
501 & 994 \\
357 & 248 \\
$Q$ & 241 \\
14 & 160 \\
0 & \\
$Q$ & \\
-- & \\
$Q$ & \\
$Q$ & 437 \\
$Q$ & 119 \\
.- & \\
$Q$ & \\
-- & 320 \\
0 & \\
0 & \\
$Q$ & \\
\hline
\end{tabular}

See footnoles at end of table. 
Table A36. Total Inputs of Energy for Heat, Power, and Electricity Generation by Fuel Type, Industry Group, Selected Industries, and End Use, 1991: Part 1 (Continued)

(Estimates in Btu or Physical Units)

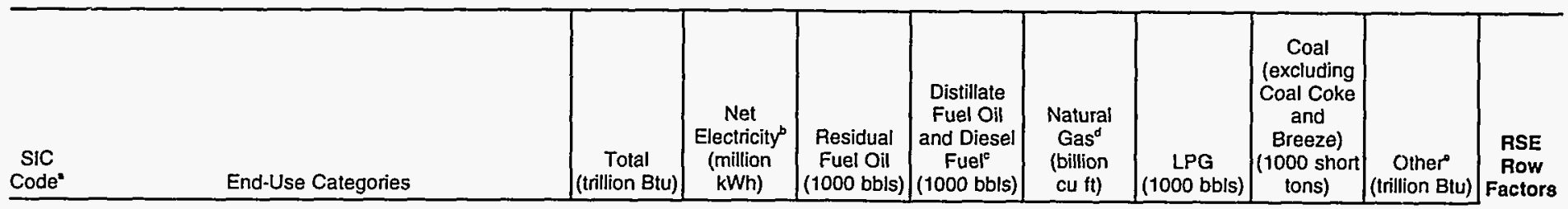

35 INDUSTRIAL MACHINERY and EQUIPMENT

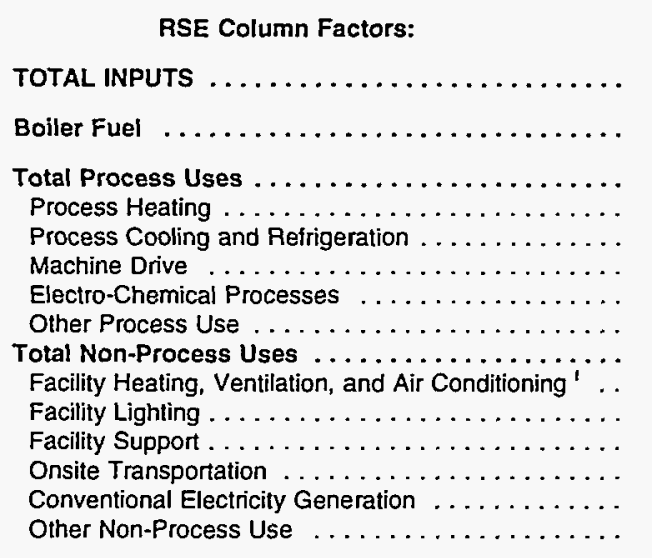

$\begin{array}{rrrr}\text { NF } & 0.4 & 2.0 & 1.2 \\ 235 & 29,484 & 490 & 718 \\ -- & 168 & 426 & 152 \\ -. & 16,411 & Q & 186 \\ -- & 2,261 & Q & 3 \\ -. & 980 & 0 & 0 \\ -- & 12,510 & * & 89 \\ -- & 268 & -- & - \\ -- & 392 & * & 94 \\ -- & 10,590 & 29 & 359 \\ -- & 4,934 & 28 & 222 \\ -- & 4,266 & -- & - \\ -- & 1,260 & Q & 4 \\ -- & 74 & -- & 76 \\ -- & -- & 0 & Q \\ -. & 56 & 0 & 46 \\ 25 & 2,483 & 20 & 20\end{array}$

End Use Not Reported $\ldots \ldots \ldots \ldots \ldots \ldots \ldots . \quad 25 \quad 2,483$

\section{Computer and Office Equipment}

RSE Column Factors: NF

TOTAL INPUTS

Boiler Fuel

Total Process Uses

Process Heating

Process Cooling and Refrigeration ..............

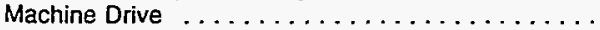

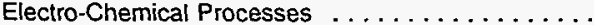

Other Process Use $\ldots \ldots \ldots \ldots \ldots \ldots \ldots \ldots$.

Total Non-Process Uses . ...............

Facility Heating, Ventilation, and Air Conditioning ' .

Facility Lighting . . .

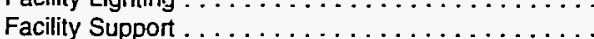

Onsite Transportation . ..................

Conventional Electricity Generation ............

Other Non-Process Use ...................

End Use Not Reported

...................

ELECTRONIC and OTHER ELECTRIC EQUIPMENT

\section{RSE Column Factors:}

TOTAL INPUTS

Boiler Fuel

Total Process Uses

Process Cooling and Refrigeration . . . . . . . . . .

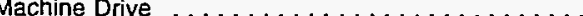

Electro-Chemical Processes . . . . . . . . . . . .

Other Process Use $\ldots \ldots \ldots \ldots \ldots \ldots \ldots \ldots \ldots$

Total Non-Process Uses . . . . . . . . . . . . . .

Facility Heating, Ventilation, and Air Conditioning ' .

Facility Lighting

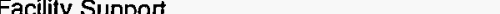

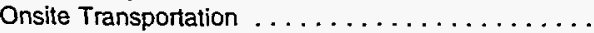

Conventional Electricity Generation ..............

Other Non-Process Use

End Use Not Reported

See footnotes at end of table. 
Table A36. Total Inputs of Energy for Heat, Power, and Electricity Generation by Fuel Type, Industry Group, Selected Industries, and End Use, 1991: Part 1 (Continued) (Estimates in Btu or Physical Units)

\begin{tabular}{|c|c|c|c|c|c|c|c|c|c|c|}
\hline$\underset{\operatorname{Cod} \theta^{\circ}}{\operatorname{SIC}}$ & End-Use Categories & $\begin{array}{c}\text { Total } \\
\text { (trillion Btu) }\end{array}$ & $\begin{array}{c}\text { Net } \\
\text { Electricity } \\
\text { (million } \\
\text { kWh) }\end{array}$ & $\begin{array}{c}\text { Residual } \\
\text { Fuel Oil } \\
\text { (1000 bbls) }\end{array}$ & $\begin{array}{c}\text { Distillate } \\
\text { Fuel Oil } \\
\text { and Diesel } \\
\text { Fuele } \\
\text { (1000 bbls })\end{array}$ & $\begin{array}{c}\text { Natural } \\
\text { Gas }^{d} \\
\text { (billion } \\
\text { cu ft) }\end{array}$ & $\begin{array}{c}\text { LPG } \\
\text { (1000 bbls) }\end{array}$ & $\begin{array}{c}\text { Coal } \\
\text { (excluding } \\
\text { Coal Coke } \\
\text { and } \\
\text { Breeze }) \\
(1000 \text { short } \\
\text { tons) }\end{array}$ & $\begin{array}{c}\text { Other } \\
\text { (trillion Btu) }\end{array}$ & $\begin{array}{c}\text { RSE } \\
\text { Row } \\
\text { Factors }\end{array}$ \\
\hline
\end{tabular}

37

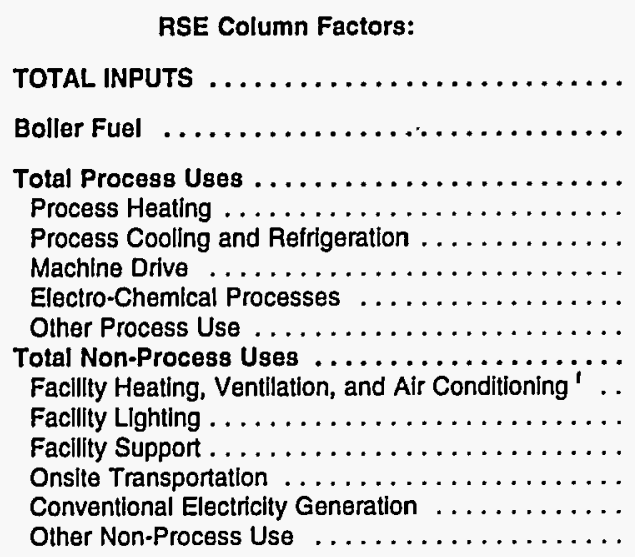

End Use Not Reported

1.5
526
36
154
97
0
0
$-\square$
0
315
69
--
2
241
$\vdots$
2
21

$\begin{array}{rrr}1.1 & \text { NF } & \\ 1,464 & 28 & 5.1 \\ W & -- & 7.0 \\ W & -- & 10.8 \\ W & -- & 8.3 \\ 0 & -- & 14.8 \\ 0 & -- & 14.3 \\ -. & -- & 10.7 \\ 0 & -- & 15.9 \\ 0 & -- & 7.1 \\ 0 & -- & 10.4 \\ -- & -- & 6.4 \\ 0 & -. & 8.3 \\ -- & -. & 8.1 \\ 0 & -- & 14.4 \\ 0 & -- & 15.2 \\ 0 & 28 & 12.6\end{array}$

3711

Motor Vehicles and Car Bodies

RSE Column Factors:

TOTAL INPUTS$$
\text { NF }
$$$$
105
$$

Boiler Fuel

Total Process Uses ...................

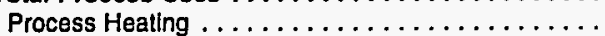

Process Cooling and Refrigeration .............

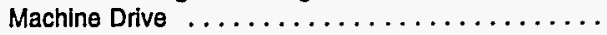

Electro-Chemical Processes ................

Other Process Use ......................

Total Non-Process Uses . ................

Facility Heating, Ventilation, and Air Conditioning ${ }^{i}$...

Facillty Lighting . . . . . . . . . . . . . . . . .

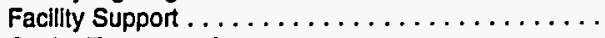

Onsite Transportation ...................

Conventional Electricity Generation . ...........

Other Non-Process Use .................

End Use Not Reported

Motor Vehicle Parts and Accessories

RSE Column Factors:

TOTAL INPUTS

Boller Fuel

Total Process Uses

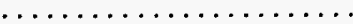

Process Cooling and Refrigeration .............

Machine Drive $\ldots \ldots \ldots \ldots \ldots \ldots \ldots \ldots$.

Electro-Chemical Processes ................

Other Process Use ......................

Total Non-Process Uses . . . . . . . . . . . . .

Facllity Heatlng, Ventilation, and Air Conditioning ' ...

Facllity Llghting .......................

Facllity Support $\ldots \ldots \ldots \ldots \ldots \ldots \ldots \ldots \ldots$

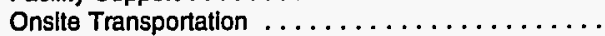

Conventlonal Electricity Generation ............

Olher Non-Process Use .................

End Use Not Reported

\begin{tabular}{|c|c|c|c|}
\hline NF & 0.5 & 1.4 & 1.3 \\
\hline 333 & 34,721 & 1,865 & 1,214 \\
\hline -- & 239 & $w$ & 261 \\
\hline .. & 19,990 & 18 & 167 \\
\hline -- & 3,010 & 13 & w \\
\hline -- & 1,529 & 0 & 0 \\
\hline -. & 14,409 & $\mathbf{Q}$ & 129 \\
\hline -. & 467 & -- & -. \\
\hline -- & 574 & * & w \\
\hline -. & 12,564 & W & 734 \\
\hline .. & 5,965 & 43 & W \\
\hline .. & 5,252 & -- & -- \\
\hline -. & 1,033 & w & 5 \\
\hline 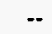 & 131 & -. & 310 \\
\hline .. & -- & w & W \\
\hline & 182 & $w$ & 180 \\
\hline & 2,166 & $\mathbf{Q}$ & 52 \\
\hline
\end{tabular}

0.7
129
47
51
48
$\vdots$
--
1
27
24
-2
2
1


3

1.8
408
$W$
$W$
$W$
0
0
.-
0
$W$
$W$
--
0
--
0
0
0

\begin{tabular}{rr}
1.2 & 0.7 \\
65 & 4 \\
6 & 1 \\
$W$ & 24 \\
$:$ & 23 \\
W & \\
\hdashline & \\
46 & \\
W & \\
W & \\
26 & \\
11 & \\
W &
\end{tabular}

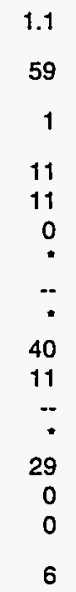

\begin{tabular}{|c|c|c|}
\hline 1.1 & NF & \\
\hline$w$ & $w$ & 3.0 \\
\hline$w$ & -. & 4.7 \\
\hline 0 & -. & 4.5 \\
\hline 0 & -. & 5.0 \\
\hline 0 & -. & 9.6 \\
\hline 0 & -- & 8.8 \\
\hline -. & -- & 7.4 \\
\hline 0 & -- & 9.7 \\
\hline 0 & .. & 3.8 \\
\hline 0 & -. & 5.6 \\
\hline .- & .. & 5.5 \\
\hline 0 & -- & 7.3 \\
\hline$\ldots$ & .. & 4.0 \\
\hline 0 & .. & 6.8 \\
\hline 0 & .- & 8.6 \\
\hline 0 & $w$ & 9.5 \\
\hline
\end{tabular}

See footnotes at end of table. 
Table A36. Total Inputs of Energy for Heat, Power, and Electricity Generation by Fuel Type, Industry Group, Selected Industries, and End Use, 1991: Part 1 (Contiriued) (Estimates in Btu or Physical Units)

\begin{tabular}{|c|c|c|c|c|c|c|c|c|c|c|}
\hline $\begin{array}{c}\text { SIC } \\
\text { Code* }\end{array}$ & End-Use Categories & $\begin{array}{c}\text { Total } \\
\text { (trillion Btu) }\end{array}$ & $\begin{array}{c}\text { Net } \\
\text { Electricity } \\
\text { (million } \\
\text { kWh) }\end{array}$ & $\begin{array}{c}\text { Residual } \\
\text { Fuel Oil } \\
\text { (1000 bbls) }\end{array}$ & $\begin{array}{c}\text { Distillate } \\
\text { Fuel Oil } \\
\text { and Diesel } \\
\text { Fuel }^{c} \\
\text { (1000 bbls })\end{array}$ & $\begin{array}{c}\text { Natural } \\
\text { Gas }^{d} \\
\text { (billion } \\
\text { cu ft) }\end{array}$ & $\begin{array}{c}\text { LPG } \\
\text { (1000 bbls })\end{array}$ & $\begin{array}{c}\text { Coal } \\
\text { (excluding } \\
\text { Coal Coke } \\
\text { and } \\
\text { Breeze }) \\
(1000 \text { short } \\
\text { tons })\end{array}$ & $\begin{array}{c}\text { Other } \\
\text { (trillion Btu) }\end{array}$ & $\begin{array}{c}\text { RSE } \\
\text { Row } \\
\text { Faclora }\end{array}$ \\
\hline
\end{tabular}

38 INSTRUMENTS and RELATED PRODUCTS

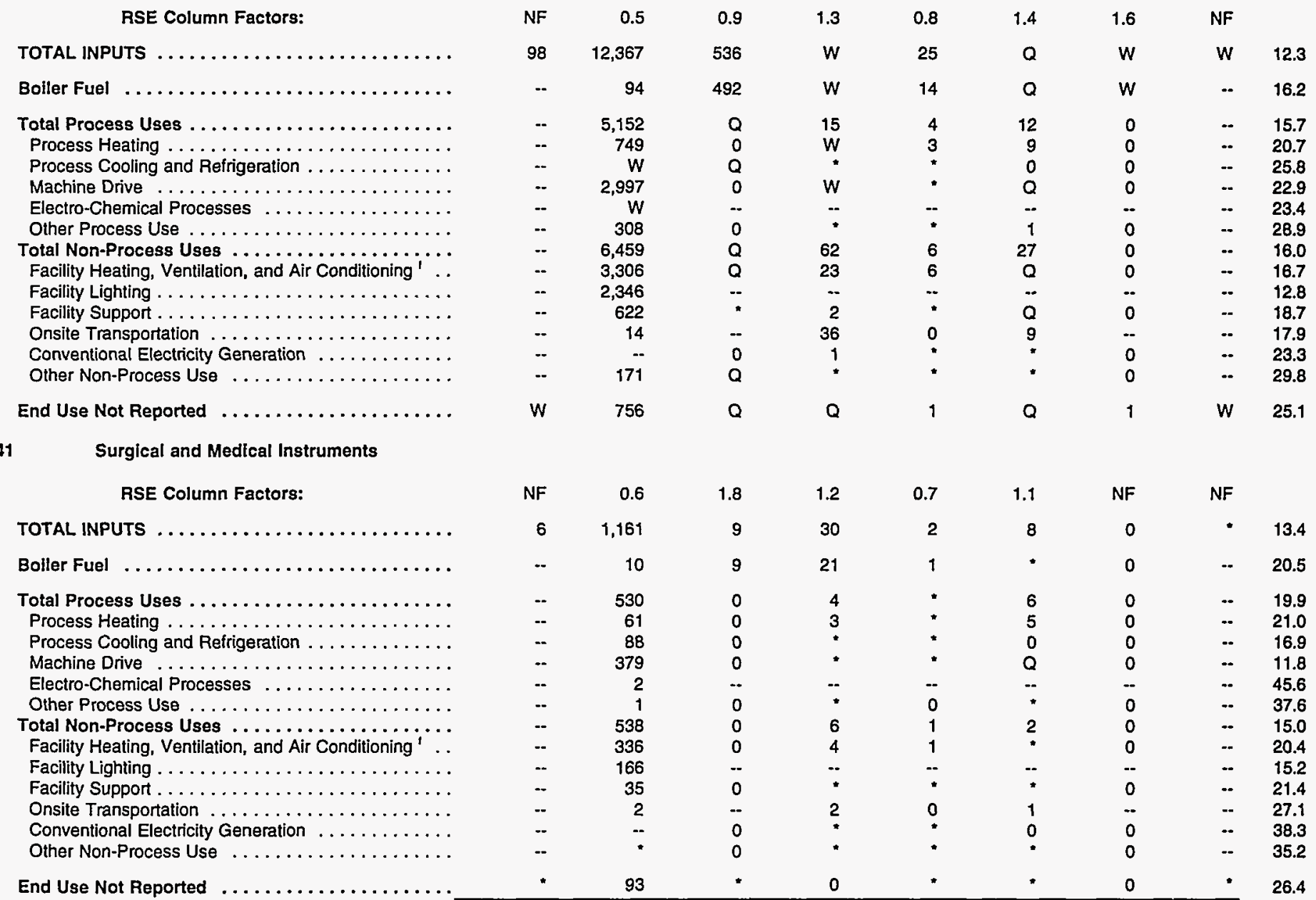

See footnotes at end of table. 
Table A36. Total Inputs of Energy for Heat, Power, and Electricity Generation by Fuel Type, Industry Group, Selected Industries, and End Use, 1991: Part 1 (Continued) (Estimates in Btu or Physical Units)

\begin{tabular}{|c|c|c|c|c|c|c|c|c|c|c|}
\hline $\begin{array}{c}\text { SiC } \\
\operatorname{Cod} \theta^{\circ}\end{array}$ & End-Use Categories & $\begin{array}{c}\text { Total } \\
\text { (trillion Btu) }\end{array}$ & $\begin{array}{c}\text { Net } \\
\text { Electricity } \\
\text { (million } \\
\mathrm{kWh})\end{array}$ & $\begin{array}{c}\text { Residual } \\
\text { Fuel Oil } \\
(1000 \text { bbls })\end{array}$ & $\begin{array}{c}\text { Distillate } \\
\text { Fuel Oil } \\
\text { and Diesel } \\
\text { Fuel }^{\circ} \\
\text { (1000 bbls) }\end{array}$ & $\begin{array}{l}\text { Natural } \\
\text { Gas }^{\circ} \\
\text { (billion } \\
\text { cu ft) }\end{array}$ & $\begin{array}{c}\text { LPG } \\
\text { (1000 bbls) }\end{array}$ & $\begin{array}{c}\text { Coal } \\
\text { (excluding } \\
\text { Coal Coke } \\
\text { and } \\
\text { Breeze }) \\
(1000 \text { short } \\
\text { tons) }\end{array}$ & $\begin{array}{c}\text { Other" } \\
\text { (trillion Btu) }\end{array}$ & $\begin{array}{c}\text { RSE } \\
\text { Row } \\
\text { Factors }\end{array}$ \\
\hline \multirow[t]{15}{*}{39} & \multicolumn{10}{|l|}{ MISC. MANUFACTURING INDUSTRIES } \\
\hline & RSE Column Factors: & NF & 0.5 & 1.2 & 1.3 & 0.7 & 1.0 & 1.8 & NF & \\
\hline & TOTAL INPUTS . . . . . . . . . . . . . . . . . & 31 & 3,661 & 115 & W & 14 & W & 32 & W & 13.3 \\
\hline & Boller Fuel $\ldots \ldots \ldots \ldots \ldots \ldots \ldots \ldots \ldots \ldots$ & -- & 29 & 99 & W & 5 & W & 30 & $\cdots$ & 20.9 \\
\hline & $\begin{array}{l}\text { Total Process Uses } \ldots \ldots \ldots \ldots \ldots \ldots \ldots \ldots \ldots \ldots \ldots \\
\text { Process Heating } \ldots \ldots \ldots \ldots \ldots \ldots \ldots \ldots \ldots \\
\text { Process Cooling and Refrigeration } \ldots \ldots \ldots \ldots \ldots \ldots\end{array}$ & $\ddot{-}$ & $\begin{array}{r}2,140 \\
503 \\
183\end{array}$ & $\begin{array}{l}Q \\
Q \\
0\end{array}$ & $\begin{array}{l}9 \\
Q \\
0\end{array}$ & $\begin{array}{l}5 \\
5 \\
-\end{array}$ & $\begin{array}{r}19 \\
8 \\
0\end{array}$ & $\begin{array}{l}0 \\
0 \\
0\end{array}$ & $\begin{array}{l}-- \\
--\end{array}$ & $\begin{array}{l}18.7 \\
20.0 \\
33.4\end{array}$ \\
\hline & Machlne Drive $\ldots \ldots \ldots \ldots \ldots \ldots \ldots \ldots \ldots \ldots \ldots \ldots \ldots \ldots \ldots$ & - & 1,399 & 0 & 1 & - & 10 & 0 & - & 29.7 \\
\hline & $\begin{array}{l}\text { Electro-Chemical Processes } \ldots \ldots \ldots \ldots \ldots \ldots \ldots \\
\text { Other Process Use } \ldots \ldots \ldots \ldots \ldots \ldots \ldots \ldots \ldots \ldots\end{array}$ & 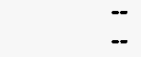 & 54 & $\overline{0}$ & $\ddot{*}$ & - & $\overline{1}$ & $\overline{0}$ & 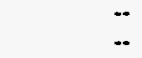 & $\begin{array}{l}68.1 \\
55.9\end{array}$ \\
\hline & Total Non-Process Uses .................... & $\cdots$ & 1,210 & 12 & 58 & 4 & 40 & Q & - & 20.5 \\
\hline & Facility Heating, Ventilation, and Air Conditioning ' ${ }^{\prime}$.. & -- & 592 & 12 & 39 & 3 & $\mathbf{Q}$ & Q & - & 23.6 \\
\hline & Facility Lightlng . . . . . . . . . . . . . . . . . & $\cdots$ & 484 & -- & - & -- & 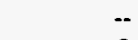 & 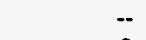 & - & 17.0 \\
\hline & Facility Support $\ldots \ldots \ldots \ldots \ldots \ldots \ldots \ldots$ & -- & 87 & $\bullet$ & - & * & 2 & 0 & - & 30.6 \\
\hline & Onsite Transportation $\ldots \ldots \ldots \ldots \ldots \ldots$ & $\rightarrow$ & 13 & -- & Q & * & 27 & -- & -- & 20.1 \\
\hline & Conventional Electricity Generation $\ldots \ldots \ldots \ldots$ & -- & -. & 0 & • & 0 & 0 & 0 & -. & 28.9 \\
\hline & Other Non-Process Use $\ldots \ldots \ldots \ldots \ldots \ldots \ldots$ & $\cdots$ & 34 & 0 & 2 & - & 2 & 0 & $\cdots$ & 39.6 \\
\hline & End Use Not Reported . . . . . . . . . . . . . & $W$ & 310 & 1 & 25 & 1 & 5 & 0 & $W$ & 36.7 \\
\hline
\end{tabular}

\footnotetext{
- See Appendlces B and F for descriptlons of the Standard Industrial Classification system.

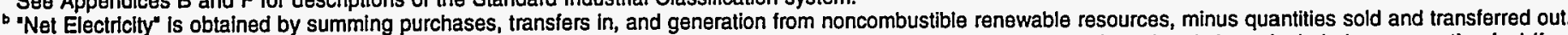

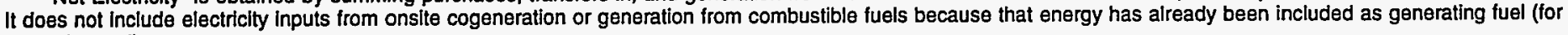
example, coal).

- Includes Nos. 1, 2, and 4 fuel olls and Nos. 1, 2, and 4 diesel fuels.

- "Natural Gas" includes natural gas obtained from utilities, transmission pipelines, and any other supplier(s) such as brokers and producers.

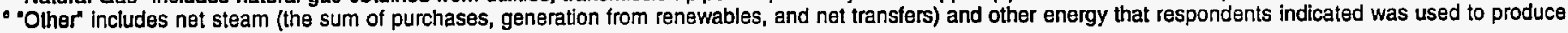
heat and power.

1 Excludes steam and hot water.

NF=No applicable RSE row/column factor.

- Estimate less than 0.5. Data are included in higher level totals.

$W=$ Withheld to avoid disclosing data for individual establishments. Data are included in higher level totals.

$\mathrm{Q}=$ Withheld because Relatlve Standard Error is greater than $\mathbf{5 0}$ percent. Data are included in higher level totals.

-- Estimation of energy input is not applicable.

$N A=$ Not avallable. Data are included in higher level totals.

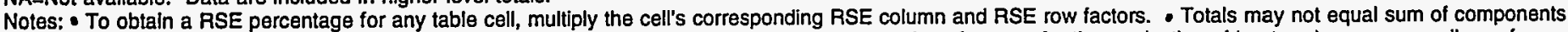

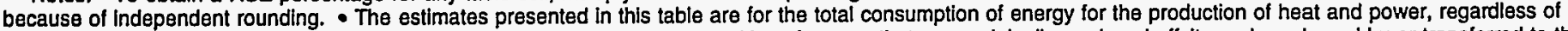

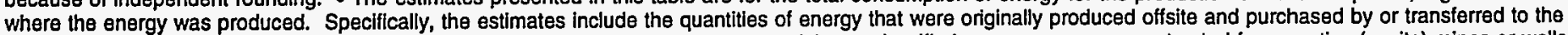

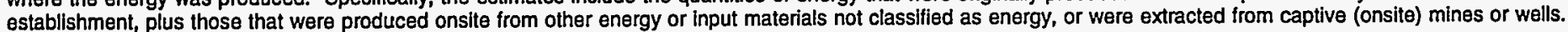

- Allocatlons to specific end uses are made on the basis of reasonable approximations by respondents. Manufacturing Energy Consumption Survey."
} 
Table A36. Total Inputs of Energy for Heat, Power, and Electricity Generation by Fuel Type, Industry Group, Selected Industries, and End Use, 1991: Part 2

(Estimates in Trillion Btu)

\begin{tabular}{|c|c|c|c|c|c|c|c|c|c|c|}
\hline $\begin{array}{c}\text { SIC } \\
\text { Code }\end{array}$ & End-Use Categories & Total & $\begin{array}{c}\text { Net } \\
\text { Electricity }\end{array}$ & $\begin{array}{l}\text { Residual } \\
\text { Fuel Oil }\end{array}$ & $\begin{array}{c}\text { Distillate } \\
\text { Fuel Oil } \\
\text { and Diesel } \\
\text { Fuel }\end{array}$ & $\begin{array}{l}\text { Natural } \\
\text { Gas }^{\delta}\end{array}$ & LPG & $\begin{array}{c}\text { Coal } \\
\text { (excluding } \\
\text { Coal Coke } \\
\text { and } \\
\text { Breeze) }\end{array}$ & Other & $\begin{array}{c}\text { RSE } \\
\text { Row } \\
\text { Factors }\end{array}$ \\
\hline
\end{tabular}

20-39

ALL INDUSTRY GROUPS

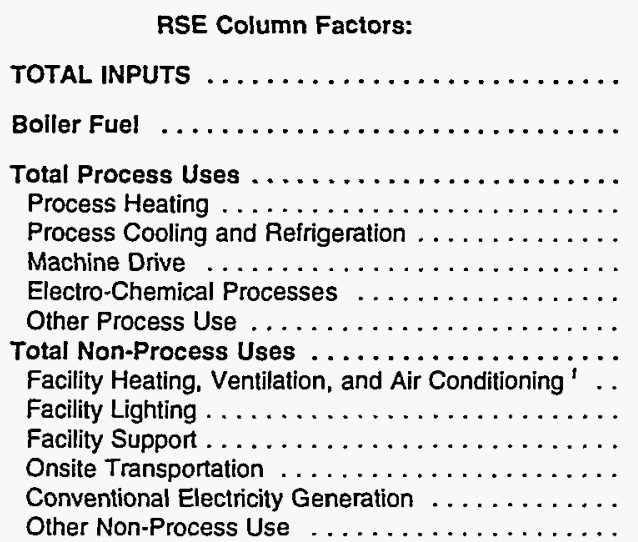

\begin{tabular}{|c|c|c|c|c|c|c|c|c|}
\hline $\mathrm{NF}$ & 0.4 & 1.6 & 1.5 & 0.7 & 1.0 & 1.6 & NF & \\
\hline 15,027 & 2,370 & 414 & 139 & 5,506 & 105 & 1,184 & 5,309 & 3.0 \\
\hline .. & w & 296 & 40 & 2,098 & 18 & 859 & -- & 3.6 \\
\hline -. & 1,864 & 109 & 34 & 2,578 & 64 & 314 & -- & 4.1 \\
\hline-- & 235 & 107 & 19 & 2,382 & 49 & 314 & - & 4.1 \\
\hline -. & 124 & * & * & 13 & • & 0 & -. & 12.4 \\
\hline$\cdots$ & 1,187 & 2 & 14 & 127 & 15 & 0 & -- & 8.4 \\
\hline -. & 304 & - & -. & -- & -- & -- & -- & 5.0 \\
\hline -. & 15 & * & 1 & 56 & * & • & -- & 14.0 \\
\hline-- & 396 & 7 & 53 & 702 & 19 & $w$ & -- & 4.2 \\
\hline -- & 192 & 4 & 8 & 283 & 3 & - & -- & 6.8 \\
\hline -- & 161 & -- & -- & - & -- & -- & .. & 5.0 \\
\hline-- & 36 & W & * & 23 & * & 0 & -- & 10.5 \\
\hline -. & 4 & -- & 38 & * & 16 & .. & .. & 9.4 \\
\hline -- & -- & 2 & 4 & 347 & * & W & -- & 8.9 \\
\hline -. & 4 & w & 2 & 49 & * & 0 & -- & 11.2 \\
\hline 5,547 & w & 2 & 12 & 128 & 4 & $w$ & 5,309 & 10.6 \\
\hline
\end{tabular}

End Use Not Reported

FOOD and KINDRED PRODUCTS

RSE Column Factors:

\begin{tabular}{r}
0.7 \\
512 \\
315 \\
144 \\
137 \\
$W$ \\
$W$ \\
\hdashline \\
2 \\
35 \\
20 \\
\hdashline \\
2 \\
12 \\
$:$ \\
17
\end{tabular}

TOTAL INPUTS

NF

Boiler Fuel

Total Process Uses

Process Cooling and Refrigeration ...........

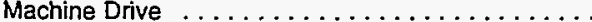

Electro-Chemical Processes...$\ldots \ldots \ldots \ldots \ldots$.

Other Process Use . . . . . . . . . . . . . . . .

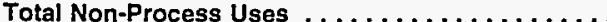

Facility Heating, Ventilation, and Air Conditioning ' . .

Facility Lighting.$\ldots \ldots \ldots \ldots \ldots \ldots \ldots \ldots \ldots$

Facility Support . . . . . . . . . . . . . . . . . .

Onsite Transportation $\ldots \ldots \ldots \ldots \ldots \ldots \ldots$.

Conventional Electricity Generation .............

Other Non-Process Use $\ldots \ldots \ldots \ldots \ldots \ldots$

End Use Not Reported

\begin{tabular}{|c|c|c|c|c|c|c|}
\hline 0.4 & 1.6 & 1.0 & 0.6 & 1.4 & 1.8 & NF \\
\hline 12 & 1 & 1 & 32 & 1 & 1 & 2 \\
\hline * & 1 & * & 22 & " & 1 & -. \\
\hline 10 & - & * & 6 & $\cdot$ & 0 & -. \\
\hline$w$ & 0 & * & 6 & * & 0 & -- \\
\hline 6 & 0 & $Q$ & . & * & 0 & .- \\
\hline 4 & * & $*$ & * & * & 0 & -- \\
\hline 0 & -- & -- & .. & -- & .- & .- \\
\hline$w$ & 0 & * & - & 0 & 0 & -- \\
\hline 1 & * & 1 & 3 & * & 0 & -- \\
\hline 1 & " & $Q$ & 2 & * & 0 & .. \\
\hline 1 & -. & -. & -. & -. & -- & -. \\
\hline$*$ & 0 & $*$ & - & $\mathrm{Q}$ & 0 & -- \\
\hline . & .. & 1 & * & * & -- & -- \\
\hline -. & 0 & * & 1 & 0 & 0 & -- \\
\hline 0 & 0 & * & 0 & 0 & 0 & -. \\
\hline$*$ & • & \pm & . & * & 0 & 2 \\
\hline
\end{tabular}


Table A36. Total Inputs of Energy for Heat, Power, and Electricity Generation by Fuel Type, Industry Group, Selected Industries, and End Use, 1991: Part 2 (Continued) (Estimates in Trillion Btu)

\begin{tabular}{|c|c|c|c|c|c|c|c|c|c|c|}
\hline $\begin{array}{l}\text { SIC } \\
\text { Code }\end{array}$ & End-Use Categories & Total & $\begin{array}{c}\text { Net } \\
\text { Electricity }\end{array}$ & $\begin{array}{l}\text { Residual } \\
\text { Fuel Oil }\end{array}$ & $\begin{array}{c}\text { Distillate } \\
\text { Fuel Oil } \\
\text { and Diesel } \\
\text { Fuel }^{c}\end{array}$ & $\begin{array}{l}\text { Natural } \\
\text { Gas }^{\circ}\end{array}$ & LPG & $\begin{array}{c}\text { Coal } \\
\text { (excluding } \\
\text { Coal Coke } \\
\text { and } \\
\text { Breeze) }\end{array}$ & Other & $\begin{array}{c}\text { RSE } \\
\text { Row } \\
\text { Factors }\end{array}$ \\
\hline
\end{tabular}

$2033 \quad$ Canned Fruits and Vegetables

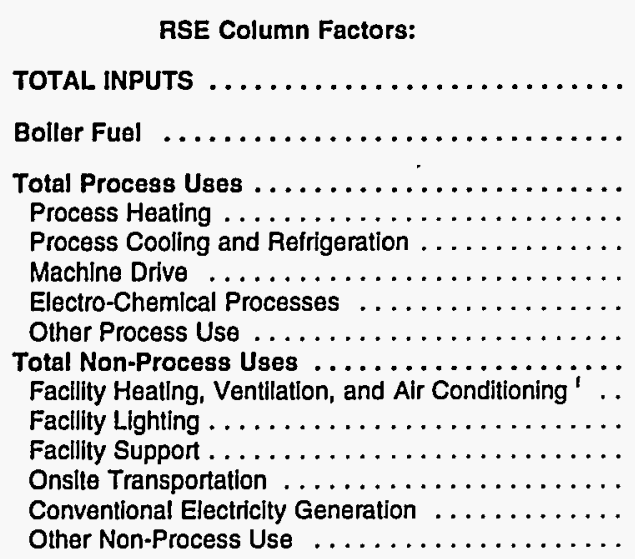

\begin{tabular}{|c|c|c|c|}
\hline NF & 0.6 & 1.1 & 1.3 \\
\hline 44 & 5 & 2 & 1 \\
\hline -- & * & 2 & $Q$ \\
\hline -- & 4 & • & * \\
\hline -. & " & 0 & 0 \\
\hline -- & 1 & 0 & * \\
\hline -- & 3 & • & * \\
\hline -- & " & -- & - \\
\hline -. & $*$ & 0 & 0 \\
\hline -- & 1 & 0 & • \\
\hline -- & * & 0 & * \\
\hline -- & • & -- & -- \\
\hline -- & * & 0 & * \\
\hline -- & * & -- & $w$ \\
\hline -- & -. & 0 & $w$ \\
\hline -- & * & 0 & " \\
\hline 2 & * & 0 & . \\
\hline
\end{tabular}

\begin{tabular}{|c|c|c|c|}
\hline 0.9 & 1.2 & NF & NF \\
\hline 36 & • & $\mathbf{Q}$ & - \\
\hline 29 & * & $\mathbf{Q}$ & -- \\
\hline 2 & • & 0 & -- \\
\hline 1 & * & 0 & -- \\
\hline * & * & 0 & -- \\
\hline * & * & 0 & -- \\
\hline -- & -- & -- & -- \\
\hline - & 0 & 0 & -- \\
\hline 4 & • & 0 & -. \\
\hline 1 & * & 0 & -. \\
\hline -- & -- & -- & -- \\
\hline • & * & 0 & -- \\
\hline 0 & * & -- & -. \\
\hline 3 & 0 & 0 & $\cdots$ \\
\hline 0 & 0 & 0 & -- \\
\hline 2 & * & 0 & • \\
\hline
\end{tabular}

2037

Frozen Frults and Vegetables

RSE Column Factors:

$\begin{array}{ll}\text { NF } & 0 \\ 40 & 10 \\ - & \\ - & \\ - & \\ - & \\ - & \\ - & \\ - & \\ - & \\ - & \\ - & \\ - & \\ - & \\ - & \\ 3 & \end{array}$

\begin{tabular}{|c|c|}
\hline 1.2 & 1.2 \\
\hline 2 & * \\
\hline 2 & * \\
\hline . & $\mathbf{Q}$ \\
\hline 0 & 0 \\
\hline 0 & $\mathbf{Q}$ \\
\hline -- & .. \\
\hline 0 & 0 \\
\hline 0 & t \\
\hline 0 & $\mathbf{Q}$ \\
\hline -. & -- \\
\hline 0 & 0 \\
\hline -. & • \\
\hline 0 & - \\
\hline 0 & * \\
\hline
\end{tabular}

0.8
26
18
4
4
$:$
-
2
1
-
0
1
0
2

1.3
$\cdot$
$\cdot$
$:$
0
$:$
$:-$
$\vdots$
$:$
$:$
$:$
0
0

NF

TOTAL INPUTS

Boller Fue

Total Process Uses

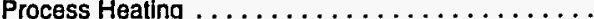

Machine Drive .........................

Electro-Chemical Processes ...............

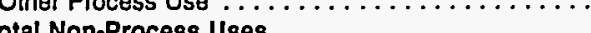

Facility Heating, Ventilation, and Air Conditioning ' .

Facillty Llghting . . .

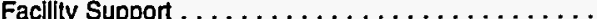

Onsite Transportation $\ldots \ldots \ldots \ldots \ldots \ldots \ldots \ldots$

Conventional Electricity Generation ..........

End Use Not Reported

Wet Corn Mililing

RSE Column Factors:

TOTAL INPUTS

$\begin{array}{cc}\text { NF } & 0 . \\ 140 & 14 \\ -- & \\ -- & \\ - & \\ - & \\ -- & \\ - & \\ -. & \\ -- & \\ - & \\ -- & \\ -- & \\ -- & \\ - & \\ -- & \\ 7 & \end{array}$

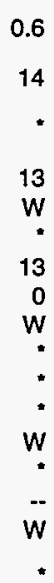

\begin{tabular}{cc}
1.1 & 1.1 \\
. & \\
& \\
0 & $W$ \\
0 & $W$ \\
0 & \\
0 & \\
\hline 0 & \\
0 & \\
0 & \\
-0 & \\
0 & \\
-0 & \\
0 & \\
0 & \\
0 &
\end{tabular}

\begin{tabular}{r}
1.1 \\
$W$ \\
$W$ \\
$W$ \\
0 \\
$:$ \\
0 \\
$:$ \\
$:$ \\
0 \\
$:$ \\
\hline \\
0
\end{tabular}

0.8
52
26
25
25
0
0
-
0
1
-
$\vdots$
0
1
0


Table A36. Total Inputs of Energy for Heat, Power, and Electricity Generation by Fuel Type, Industry Group, Selected Industries, and End Use, 1991: Part 2 (Continued) (Estimates in Trillion Btu)

\begin{tabular}{|c|c|c|c|c|c|c|c|c|c|}
\hline $\begin{array}{l}\text { SIC } \\
\text { Code: }\end{array}$ & End-Use Categories & Total & $\begin{array}{c}\text { Net } \\
\text { Electricity }\end{array}$ & $\begin{array}{l}\text { Residual } \\
\text { Fuel Oil } \\
\end{array}$ & $\begin{array}{c}\text { Distillate } \\
\text { Fuel Oil } \\
\text { and Diesel } \\
\text { Fuef }\end{array}$ & $\begin{array}{c}\text { Natural } \\
\text { Gas }^{\circ}\end{array}$ & LPG & $\begin{array}{c}\text { Coal } \\
\text { (excluding } \\
\text { Coal Coke } \\
\text { and } \\
\text { Breeze) } \\
\end{array}$ & Othere \\
\hline
\end{tabular}

2051 Bread, Cake, and Related Products

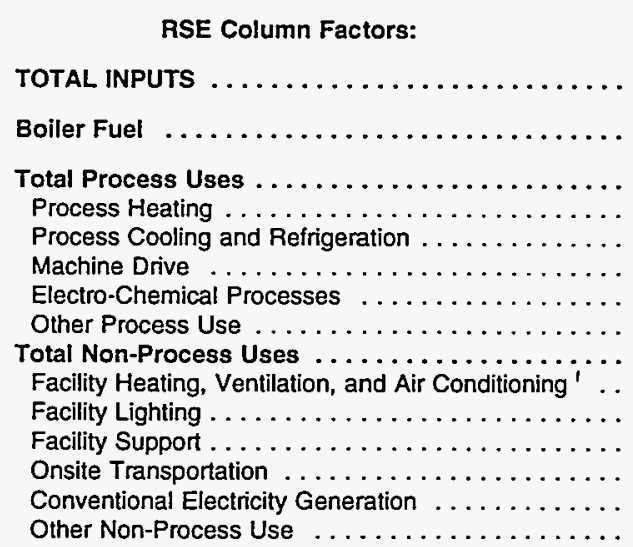

$\begin{array}{ll}\text { NF } & 0.5 \\ 32 & \\ -- \\ -- \\ -- \\ - \\ - \\ - \\ - \\ - \\ - \\ - \\ -- \\ - \\ - \\ -\end{array}$

End Use Not Reported

TOTAL INPUTS

Total Process Uses . . . . . . . . . . . . . . .

Process Heating $\ldots \ldots \ldots \ldots \ldots \ldots \ldots \ldots$

Process Cooling and Refrigeration .............

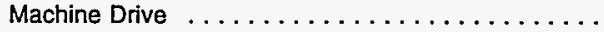

Electro-Chemical Processes . . . . . . . . . .

Other Process Use $\ldots \ldots \ldots \ldots \ldots \ldots \ldots \ldots \ldots$

Total Non-Process Uses . . . . . . . . . . . . . Facility Heating, Ventilation, and Air Conditioning ' ... Facility Lighting . . . . . . . . . . . . . . . . . . Facility Support

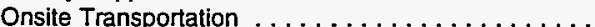

Conventional Electricity Generation . . . . . . . . . .

Other Non-Process Use $\ldots . \ldots \ldots \ldots \ldots \ldots \ldots$

End Use Not Reported

\section{Soybean Oil Mills}

RSE Column Factors:

TOTAL INPUTS

Boiler Fuel

Total Process Uses

Process Cooling and Refrigeration . . . . . . . . . .

Machine Drive ........................

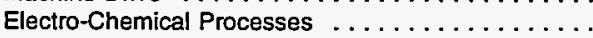

Other Process Use $\ldots \ldots \ldots \ldots \ldots \ldots \ldots \ldots \ldots$

Total Non-Process Uses . . . . . . . . . . . . .

Facility Heating, Ventilation, and Air Conditioning '

Facility Lighting . . . . . . . . . . . . . . . . . .

Facility Support $\ldots \ldots \ldots \ldots \ldots \ldots \ldots \ldots$

Onsite Transportation .................

Conventional Electricity Generation ............

Other Non-Process Use..$\ldots \ldots \ldots \ldots \ldots \ldots$

End Use Not Reported

\begin{tabular}{rr}
0.5 & 1.7 \\
8 & $\cdot$ \\
$*$ &. \\
5 & 0 \\
1 & 0 \\
3 & 0 \\
0 & 0 \\
$*$ & 0 \\
2 & 0 \\
1 & 0 \\
1 & - \\
\hline & 0 \\
Q & - \\
$-*$ & 0 \\
$*$ & 0 \\
$*$ & 0
\end{tabular}

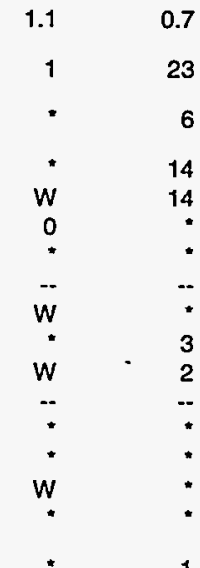

\begin{tabular}{|c|c|c|c|}
\hline 1.5 & NF & NF & \\
\hline * & 0 & • & 12.1 \\
\hline * & 0 & .. & 18.9 \\
\hline * & 0 & .- & 13.0 \\
\hline * & 0 & -. & 16.3 \\
\hline 0 & 0 & -- & 20.5 \\
\hline 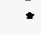 & 0 & -- & 26.6 \\
\hline -- & -- & .. & NF \\
\hline • & 0 & -- & 27.1 \\
\hline * & 0 & .. & 13.3 \\
\hline * & 0 & .. & 14.2 \\
\hline -. & -. & -- & 11.2 \\
\hline • & 0 & $\ldots$ & 23.1 \\
\hline * & -- & - & 21.5 \\
\hline * & 0 & -- & 35.0 \\
\hline 0 & 0 & -- & 39.9 \\
\hline - & 0 & - & 27.3 \\
\hline
\end{tabular}


Table A36. Total Inputs of Energy for Heat, Power, and Electricity Generation by Fuel Type, Industry Group, Selected Industries, and End Use, 1991: Part 2 (Continued) (Estimates in Trillion Btu)

\begin{tabular}{|c|c|c|c|c|c|c|c|c|c|c|}
\hline $\begin{array}{l}\text { SIC } \\
\text { Coden }^{a}\end{array}$ & End-Use Categories & Total & $\begin{array}{c}\text { Net } \\
\text { Electricity }\end{array}$ & $\begin{array}{l}\text { Residual } \\
\text { Fuel Oil }\end{array}$ & $\begin{array}{c}\text { Distillate } \\
\text { Fuel Oil } \\
\text { and Diesel } \\
\text { Fuel }^{6}\end{array}$ & $\begin{array}{l}\text { Natural } \\
\text { Gas }^{d}\end{array}$ & LPG & $\begin{array}{c}\text { Coal } \\
\text { (excluding } \\
\text { Coal Coke } \\
\text { and } \\
\text { Breeze) }\end{array}$ & Other & $\begin{array}{c}\text { RSE } \\
\text { Row } \\
\text { Factors }\end{array}$ \\
\hline
\end{tabular}

2082

Malt Beverages

RSE Column Factors:

\begin{tabular}{|c|c|}
\hline NF & 0.5 \\
\hline 50 & 8 \\
\hline .. & . \\
\hline .. & 6 \\
\hline -- & • \\
\hline -- & 3 \\
\hline -- & 3 \\
\hline -. & 0 \\
\hline .. & 0 \\
\hline -. & 2 \\
\hline -. & 1 \\
\hline .. & 1 \\
\hline *. & * \\
\hline -- & * \\
\hline -. & -- \\
\hline .. & 0 \\
\hline 1 & * \\
\hline
\end{tabular}

1.3
3
3
0
0
0
0
-
0
$:$
-
-
-
0
0
0

\begin{tabular}{|c|c|}
\hline 1.4 & 0.8 \\
\hline * & 23 \\
\hline$w$ & 21 \\
\hline - & 1 \\
\hline 0 & 1 \\
\hline 0 & \\
\hline 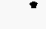 & 0 \\
\hline-- & -- \\
\hline 0 & 0 \\
\hline w & \\
\hline 0 & * \\
\hline- & $\because$ \\
\hline . & 0 \\
\hline W & 0 \\
\hline 0 & 0 \\
\hline 0 & \\
\hline & \\
\hline
\end{tabular}

\begin{tabular}{c}
1.0 \\
$\cdot$ \\
$\cdot$ \\
0 \\
0 \\
0 \\
0 \\
\hdashline \\
0 \\
$w$ \\
$W$ \\
-- \\
0 \\
0 \\
0 \\
0 \\
$W$
\end{tabular}

1.4
16
16
0
0
0
0
-
0
.
-
0
-
0
0
0

NF

TOTAL INPUTS

Boller Fuel

Total Process Uses $\ldots \ldots \ldots \ldots \ldots \ldots$

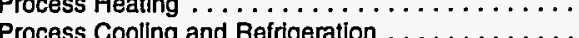

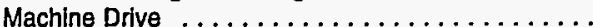

Electro-Chemical Processes $\ldots \ldots \ldots \ldots \ldots \ldots$

Other Process Use ......................

Total Non-Process Uses $\ldots \ldots \ldots \ldots \ldots \ldots \ldots \ldots$

Facility Lighting . . . . . . . . . . . . . . . . . .

Facllity Support $\ldots \ldots \ldots \ldots \ldots \ldots \ldots \ldots$

Onsite Transportation $\ldots \ldots \ldots \ldots \ldots \ldots \ldots$

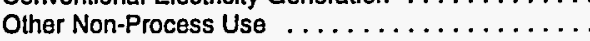

0.9
$\cdot$
.
$:$
0
$\cdot-$
0
0
$-\cdot$
0
0
0
0

$$
1.9
$$$$
1.2 \quad 0.6
$$

NF

TOTAL INPUTS

Boiler Fuel

Total Process Uses . .

Process Heating $\ldots \ldots \ldots \ldots \ldots \ldots \ldots \ldots \ldots$

Process Coolling and Refrigeration . . . . . . . . . .

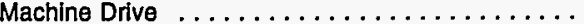

Electro-Chemical Processes ...............

Other Process Use $\ldots \ldots \ldots \ldots \ldots \ldots \ldots \ldots \ldots$

Total Non-Process Uses . . . . . . . . . . . . . .

Facility Heating, Ventilation, and Air Conditioning ' .

Facillty Lightling . . . . . . . . . . . . . . . . . .

Facillty Support $\ldots \ldots \ldots \ldots \ldots \ldots \ldots \ldots$

Onsite Transportation $\ldots \ldots \ldots \ldots \ldots \ldots \ldots \ldots$

Conventional Electricity Generation . . . . . . . . . .

Other Non-Process Use $\ldots \ldots \ldots \ldots \ldots \ldots$.

End Use Not Reported

\section{TEXTILE MILL PRODUCTS}

RSE Column Factors:

\begin{tabular}{|c|c|c|c|c|c|c|c|}
\hline NF & 0.4 & 1.3 & 1.6 & 0.8 & 1.2 & 1.2 & NF \\
\hline 273 & 101 & 12 & 6 & 108 & 2 & 31 & 13 \\
\hline -. & 1 & 11 & 5 & 70 & • & 30 & -- \\
\hline -. & 73 & 1 & • & 31 & 1 & $w$ & -- \\
\hline -- & 3 & $w$ & • & 28 & 1 & $w$ & .. \\
\hline -. & 7 & 0 & * & • & • & 0 & -- \\
\hline -- & 61 & $w$ & • & 2 & • & 0 & -- \\
\hline -. & $w$ & -: & -- & -- & -. & -- & -- \\
\hline -. & $w$ & * & " & 1 & * & 0 & .• \\
\hline -- & 25 & " & 1 & 4 & 1 & $*$ & •. \\
\hline -. & 14 & * & $w$ & 4 & * & • & -- \\
\hline -- & 9 & -. & -- & - & -- & - & -- \\
\hline- & 1 & • & - & • & • & 0 & + \\
\hline -- & $*$ & -- & • & 0 & " & -- & -- \\
\hline -- & -- & 0 & $\mathbf{Q}$ & • & * & 0 & -. \\
\hline-- & " & 0 & • & * & * & 0 & -- \\
\hline 20 & 2 & 1 & $\mathbf{Q}$ & 3 & * & $w$ & 13 \\
\hline
\end{tabular}

TOTAL INPUTS

TS

Boller Fuel

Total Process Uses $\ldots \ldots \ldots \ldots \ldots \ldots \ldots \ldots \ldots$

Process Heating $\ldots \ldots \ldots \ldots \ldots \ldots \ldots \ldots$

Process Cooling and Refrigeration . . . . . . . . .

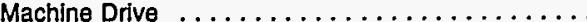

Electro-Chemlcal Processes ................

Other Process Use $\ldots \ldots \ldots \ldots \ldots \ldots \ldots \ldots$

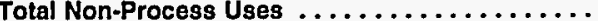

Facilly Heating, Ventilation, and Air Conditioning ' . .

Facillty Lighting . . . . . . . . . . . . . . . . . .

Facillity Support ......................

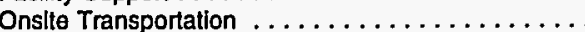

ConventIonal Electricity Generation ............

Other Non-Process Use

End Use Not Reported 
Table A36. Total Inputs of Energy for Heat, Power, and Electricity Generation by Fuel Type, Industry Group, Selected Industries, and End Use, 1991: Part 2 (Continued) (Estimates in Trillion Btu)

\begin{tabular}{|c|c|c|c|c|c|c|c|c|c|c|}
\hline $\begin{array}{c}\text { SIC } \\
\text { Code" }\end{array}$ & End-Use Categories & Total & $\begin{array}{c}\mathrm{Net} \\
\text { Electricity" }\end{array}$ & $\begin{array}{l}\text { Residual } \\
\text { Fuel Oil }\end{array}$ & $\begin{array}{c}\text { Distillate } \\
\text { Fuel Oil } \\
\text { and Diesel } \\
\text { Fuel" }\end{array}$ & $\begin{array}{l}\text { Natural } \\
\text { Gas }^{\circ}\end{array}$ & LPG & $\begin{array}{c}\text { Coal } \\
\text { (excluding } \\
\text { Coal Coke } \\
\text { and } \\
\text { Breaze) }\end{array}$ & Other & \begin{tabular}{|} 
RSE \\
Row \\
Factors
\end{tabular} \\
\hline
\end{tabular}

APPAREL and OTHER TEXTILE PRODUCTS

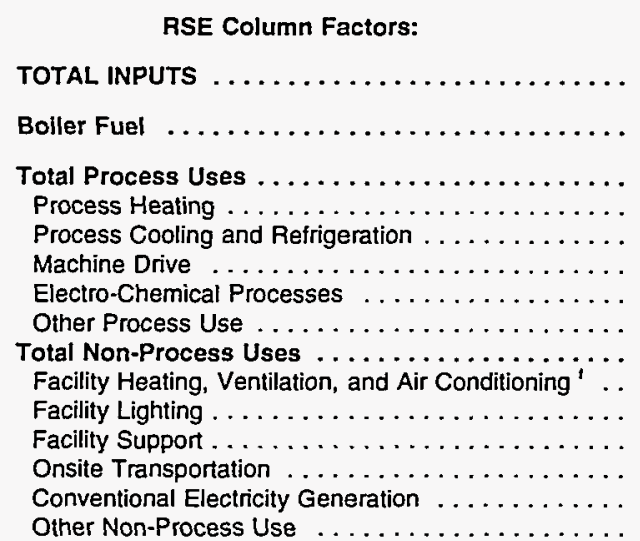

$\begin{array}{ccc}\text { NF } & 0.5 & \text { NF } \\ 44 & 19 & \mathrm{Q} \\ -- & . & \mathrm{Q} \\ -- & 9 & \mathrm{Q} \\ -- & 1 & 0 \\ -- & - & 0 \\ -- & 8 & \mathrm{Q} \\ -- & * & - \\ -- & \mathrm{Q} & 0 \\ - & 7 & \mathrm{Q} \\ -- & 4 & \mathrm{Q} \\ -- & 3 & - \\ -- & - & 0 \\ -- & \mathrm{Q} & -- \\ -- & -- & 0 \\ -- & 0 & 0 \\ 6 & 3 & 0\end{array}$

End Use Not Reported

LUMBER and WOOD PRODUCTS

RSE Column Factors:

TOTAL INPUTS

Boiler Fuel

Total Process Uses $\ldots \ldots \ldots \ldots \ldots \ldots \ldots \ldots$

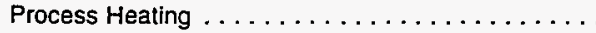

Process Cooling and Refrigeration .............

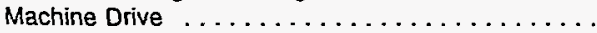

Electro-Chemical Processes $\ldots . . \ldots \ldots \ldots \ldots \ldots$.

Other Process Use . . .

Total Non-Process Uses .................

Facility Heating, Ventilation, and Air Conditioning ${ }^{i}$..

Facility Lighting

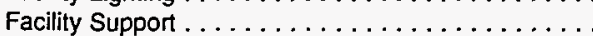

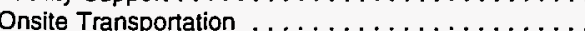

Conventional Electricity Generation ............

Other Non-Process Use $\ldots \ldots \ldots \ldots \ldots \ldots \ldots$.

End Use Not Reported $\ldots \ldots \ldots \ldots \ldots \ldots \ldots . \quad 314$

FURNITURE and FIXTURES

RSE Column Factors:

TOTAL INPUTS

Boiler Fue

Total Process Uses

Process Heating

Process Cooling and Refrigeration ...........

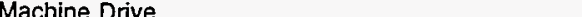

Electro-Chemical Processes $\ldots \ldots \ldots \ldots \ldots \ldots$.

Other Process Use . . . . . . . . . . . . . . .

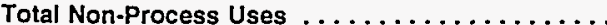

Facility Heating, Ventilation, and Air Conditioning '

Facility Lighting . . .

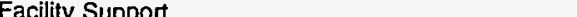

Onsite Transportation $\ldots \ldots \ldots \ldots \ldots \ldots \ldots \ldots$

Conventional Electricity Generation $\ldots \ldots \ldots \ldots \ldots$

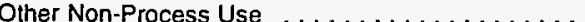

End Use Not Reported

NF

\begin{tabular}{|c|c|c|}
\hline NF & 0.6 & 1.3 \\
\hline 423 & 61 & 2 \\
\hline .- & 1 & 2 \\
\hline .- & 48 & $Q$ \\
\hline .- & 3 & $\mathrm{Q}$ \\
\hline -. & * & 0 \\
\hline .. & 44 & 0 \\
\hline- & $*$ & -- \\
\hline -- & $*$ & 0 \\
\hline -. & 6 & $Q$ \\
\hline -- & 2 & $\mathbf{Q}$ \\
\hline .. & 3 & .. \\
\hline .. & 1 & 0 \\
\hline -- & $Q$ & .- \\
\hline -. & .- & 0 \\
\hline-- & • & 0 \\
\hline
\end{tabular}

NF
67

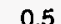

2.0

1.1

0.6

NF

$1 \quad 19$

3

25

17.9

28.5

19.0

25.6

36.5

24.1

40.6

55.1

22.1

26.8

18.3

34.4

23.4

NF

59.5

25

5.4

8.1

2.5

7.9

18.4

9.8

5.8

NF

32.6 
Table A36. Total Inputs of Energy for Heat, Power, and Electricity Generation by Fuel Type, Industry Group, Selected Industries, and End Use, 1991: Part 2 (Continued) (Estimates in Trillion Btu)

\begin{tabular}{|c|c|c|c|c|c|c|c|c|c|c|}
\hline $\begin{array}{l}\text { SIC } \\
\text { Code }\end{array}$ & End-Use Categories & Total & $\begin{array}{c}\text { Net } \\
\text { Electricity }\end{array}$ & $\begin{array}{l}\text { Residual } \\
\text { Fuel Oil }\end{array}$ & $\begin{array}{c}\text { Distillate } \\
\text { Fuel Oil } \\
\text { and Diesel } \\
\text { Fuef }\end{array}$ & $\begin{array}{l}\text { Natural } \\
\text { Gas }^{d}\end{array}$ & LPG & $\begin{array}{c}\text { Coal } \\
\text { (excluding } \\
\text { Coal Coke } \\
\text { and } \\
\text { Breeze) }\end{array}$ & Other & $\begin{array}{c}\text { RSE } \\
\text { Row } \\
\text { Factors }\end{array}$ \\
\hline
\end{tabular}

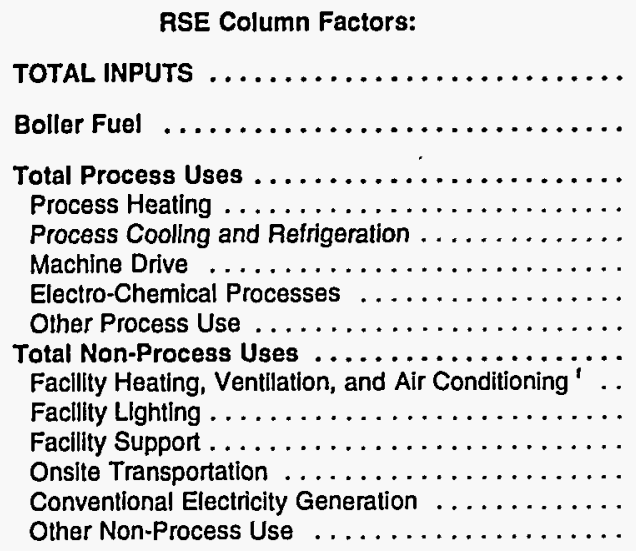

\begin{tabular}{|c|c|}
\hline NF & 0.8 \\
\hline 2,472 & 201 \\
\hline -- & 5 \\
\hline -- & 171 \\
\hline -- & 5 \\
\hline -. & 3 \\
\hline -. & 160 \\
\hline -- & 2 \\
\hline -- & 2 \\
\hline -- & 20 \\
\hline -- & 9 \\
\hline -- & 9 \\
\hline -. & 2 \\
\hline -- & " \\
\hline -- & -- \\
\hline -- & • \\
\hline 1,272 & 5 \\
\hline
\end{tabular}

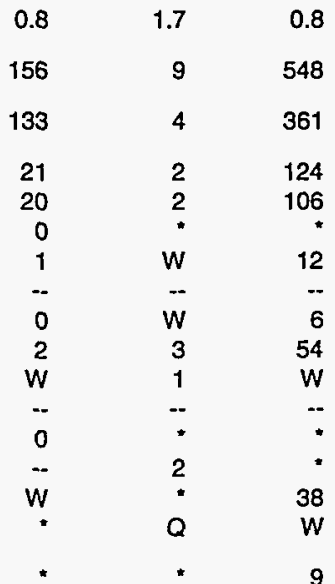

End Use Not Reported

Pulp Mills

RSE Column Factors:

$\begin{array}{ccc}\text { NF } & 0.8 & 1 \\ 300 & 9 & 28 \\ -- & * & 24 \\ -- & 8 \\ - & : \\ -- & 7 \\ - & : \\ - & 0 \\ - & 1 \\ - & : \\ -- & : \\ - & : \\ -- & 0 \\ 222 & -\end{array}$

$$
\begin{array}{r}
1.0 \\
28 \\
24 \\
4
\end{array}
$$

\section{4}

0.8

1.3

TOTAL INPUTS

$\begin{array}{rr}0.9 & 0.8 \\ 1 & 32 \\ - & 23\end{array}$

1.3

NF

Boiler Fuel

Total Process Uses

Process Heating $\ldots \ldots \ldots \ldots \ldots \ldots \ldots \ldots \ldots$

Process Cooling and Refrigeration ............

Machine Drive .......................

Electro-Chemical Processes ................

Other Process Use $\ldots \ldots \ldots \ldots \ldots \ldots \ldots \ldots$

Total Non-Process Uses . ..................

Facility Heating, Ventilation, and Air Conditioning ' . .

Facility Lighting

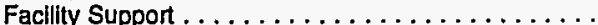

Onsite Transportation ...................

Conventional Electricity Generation .............

Other Non-Process Use

End Use Not Reported

Paper Mills

RSE Column Factors:

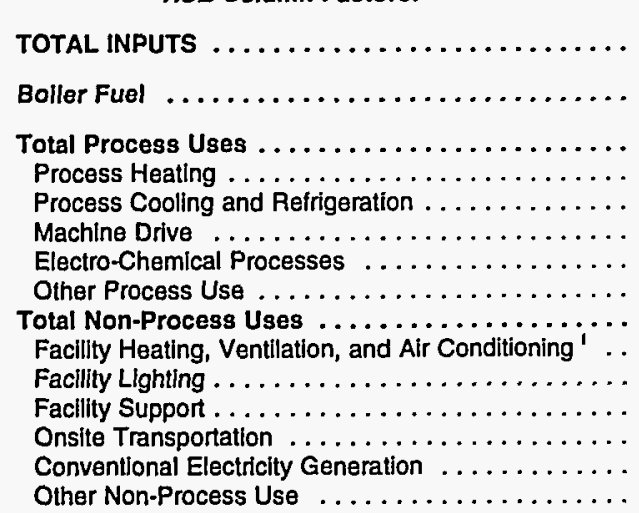

NF

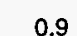

$\begin{array}{ll}1.1 & 1.0 \\ 85 & W\end{array}$

1.0

1.1

1.0

NF

1,204

$112 \quad 85$

260

2

193

W

14.9

18.8

17.3

21.6

22.0

End Use Not Reported 
Table A36. Total Inputs of Energy for Heat, Power, and Electricity Generation by Fuel Type, Industry Group, Selected Industries, and End Use, 1991: Part 2 (Continued) (Estimates in Trillion Btu)

\begin{tabular}{|c|c|c|c|c|c|c|c|c|c|c|}
\hline $\begin{array}{c}\text { SIC } \\
\text { Code" }\end{array}$ & End-Use Categories & Total & $\begin{array}{c}\text { Net } \\
\text { Electricity }\end{array}$ & $\begin{array}{l}\text { Residual } \\
\text { Fuel Oil }\end{array}$ & $\begin{array}{c}\text { Distillate } \\
\text { Fuel Oil } \\
\text { and Diesel } \\
\text { Fuel }^{c}\end{array}$ & $\begin{array}{c}\text { Natural } \\
\text { Gas }^{d}\end{array}$ & LPG & $\begin{array}{c}\text { Coal } \\
\text { (excluding } \\
\text { Coal Coke } \\
\text { and } \\
\text { Breeze) }\end{array}$ & Other & $\begin{array}{c}\text { RSE } \\
\text { Row } \\
\text { Factors }\end{array}$ \\
\hline
\end{tabular}

2631

Paperboard Mills

RSE Column Factors:

TOTAL INPUTS

832

Boiler Fuel

Total Process Uses

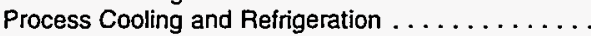

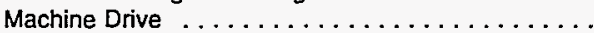

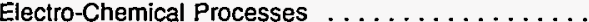

Other Process Use . . . . . . . . . . . . . .

Total Non-Process Uses . . . . . . . . . . . . . .

Facility Heating, Ventilation, and Air Conditioning ${ }^{\prime}$..

Facility Lighting

Facility Support .

Conventional Electricity Generation ..........

Other Non-Process Use . . . . . . . . . . . .

End Use Not Reported

\section{PRINTING and PUBLISHING}

RSE Column Factors:

TOTAL INPUTS

Boiler Fuel

Total Process Uses

Process Cooling and Refrigeration . . . . . . . . .

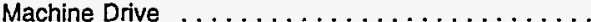

Electro-Chemical Processes ..............

Other Process Use . . . . . . . . . . . . . .

Total Non-Process Uses . . . . . . . . . . . . . . . . .

Facility Heating, Ventilation, and Air Conditioning ${ }^{\prime} \ldots$

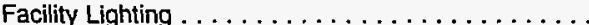

Facility Support . . . . . . . . . . . . . . . . . .

Onsite Transportation $\ldots \ldots \ldots \ldots \ldots \ldots \ldots \ldots \ldots$

Conventional Electricity Generation ...........

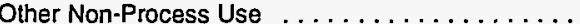

End Use Not Reported

CHEMICALS and ALLIED PRODUCTS

RSE Column Factors:

TOTAL INPUTS

Boiler Fuel

Total Process Uses

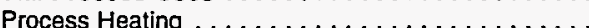

Process Cooling and Refrigeration . . . . . . . .

Machine Drive

Electro-Chemical Processes $\ldots \ldots \ldots \ldots$

Other Process Use . . . . . . . . . . . . . . . . . .

Total Non-Process Uses $\ldots \ldots \ldots \ldots \ldots \ldots \ldots \ldots$

Facility Heating, Ventilation, and Air Conditioning ${ }^{i} \ldots$

Facility Lighting

Facility Support

Onsite Transportation .

Conventional Electricity Genere

Other Non-Process Use . . . . . . . . . . . .

End Use Not Reported

486

NF

108

NF

3,040

W
NF $\quad 0.8$

-.

$-28$

$\cdots \quad W$

$\begin{array}{ll}- & W \\ -- & 3\end{array}$

$\begin{array}{llll} & & 1 & W \\ - & 1 & 0 & 0\end{array}$

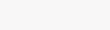

$\cdots$

0.5

53

,

26

$\begin{array}{ll}-. & 1 \\ - & 2\end{array}$

$--\quad 23$

-.

-- 19

$\begin{array}{ll}- & 10 \\ - & 7\end{array}$

$\begin{array}{ll}- & 2 \\ - & \end{array}$

$-$

$18 \quad 8$

440

0.9

0.8

1.5

1.3

NF

.- W

, 669

4

253

614

244

673

-- $\quad 387$

$\begin{array}{ll}-. & 14 \\ -. & 27\end{array}$

- $\quad 286$

-. 60

$\begin{array}{rr}-- & 1 \\ -- & 41\end{array}$

-. 21

-. 15

$\begin{array}{ll}-. & 4 \\ -. & 4\end{array}$

$\ddot{*} \quad$

w$$
28
$$$$
2
$$$$
\begin{aligned}
& 2 \\
& 1
\end{aligned}
$$$$
\begin{array}{r}
2 \\
55
\end{array}
$$$$
55
$$$$
39
$$$$
256
$$$$
\text { * W }
$$$$
4
$$$$
\begin{array}{r}
0 \\
191 \\
W
\end{array}
$$

21 
Table A36. Total Inputs of Energy for Heat, Power, and Electricity Generation by Fuel Type, Industry Group, Selected Industries, and End Use, 1991: Part 2 (Continued) (Estimates in Trillion Btu)

\begin{tabular}{|c|c|c|c|c|c|c|c|c|c|c|}
\hline $\begin{array}{l}\text { SIC } \\
\text { Code }\end{array}$ & End-Use Categories & Total & $\begin{array}{c}\text { Net } \\
\text { Electricity }\end{array}$ & $\begin{array}{l}\text { Residual } \\
\text { Fuel Oil }\end{array}$ & $\begin{array}{c}\text { Distillate } \\
\text { Fuel Oil } \\
\text { and Diese! } \\
\text { Fuel }^{c}\end{array}$ & $\begin{array}{c}\text { Natural } \\
\text { Gas }^{d}\end{array}$ & LPG & $\begin{array}{c}\text { Coal } \\
\text { (excluding } \\
\text { Coal Coke } \\
\text { and } \\
\text { Breeze) }\end{array}$ & Other & $\begin{array}{c}\text { RSE } \\
\text { Row } \\
\text { Factors }\end{array}$ \\
\hline
\end{tabular}

2812

Alkalles and Chlorine

RSE Column Factors:

\begin{tabular}{|c|c|c|}
\hline NF & 0.7 & 1.3 \\
\hline 160 & 37 & $w$ \\
\hline -- & " & $w$ \\
\hline -. & 36 & 0 \\
\hline -- & $\mathbf{W}$ & 0 \\
\hline -- & • & 0 \\
\hline .. & 3 & 0 \\
\hline -- & 32 & - \\
\hline -- & $w$ & 0 \\
\hline -- & $W$ & 0 \\
\hline -- & * & 0 \\
\hline -- & • & -- \\
\hline -- & $W$ & 0 \\
\hline-- & 0 & - \\
\hline -. & - & 0 \\
\hline -- & 0 & 0 \\
\hline
\end{tabular}

$\begin{array}{rr}0.9 & 0.9 \\ \text { W } & W \\ W & 56 \\ W & 4 \\ 0 & 4 \\ - & 0 \\ - & 0 \\ : & - \\ 0 & 1 \\ - & 1 \\ 0 & - \\ : & 0 \\ 0 & 0 \\ 0 & 0 \\ W & 0\end{array}$

\begin{tabular}{cc}
1.1 & 1.2 \\
$*$ & $W$ \\
0 & $W$ \\
0 & 0 \\
0 & 0 \\
0 & 0 \\
0 & 0 \\
-- & -- \\
0 & 0 \\
$*$ & 0 \\
0 & 0 \\
-- & -- \\
0 & 0 \\
0 & -- \\
0 & 0 \\
\hline & 0
\end{tabular}

NF

TOTAL INPUTS

Boller Fuel

Total Process Uses

Process Heating .

Process Coollng and Relrigeration . . . . . . . . .

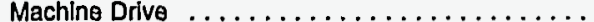

Electro-Chemical Processes $\ldots \ldots \ldots \ldots \ldots \ldots$

Other Process Use . . . . . . . . . . . . .

Total Non-Process Uses . . . . . . . . . . . . . . . . .

Facillty Heating, Ventllation, and Air Conditioning ${ }^{\prime}$..

Facilly Lighting .

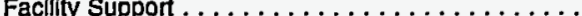

Onsite Transportation $\ldots \ldots \ldots \ldots \ldots \ldots \ldots \ldots$

Conventional Electriclty Generation ............

End Use Not Reported

RSE Column Factors:

NF

TOTAL INPUTS

g

Boller Fuel

Total Process Uses . . . . . . . . . . . . . . . . .

Process Heating

Process Cooling and Retrigere

Electro-Chemical Processes . . . . . . . . . . .

Other Process Use . . . . . . . . . . . . . . . . .

Total Non-Process Uses . . . . . . . . . . . . . . . . .

Facillty Heating, Ventllation, and Air Conditioning

Facility Llghting . . . . . . . . . . . . . . . . . . .

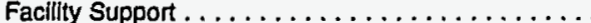

Onsite Transportation ..................

Conventional Electricity Generation . ............

Other Non-Process Use

End Use Not Reported

......................

Industrial Inorganlc Chemicals, nec

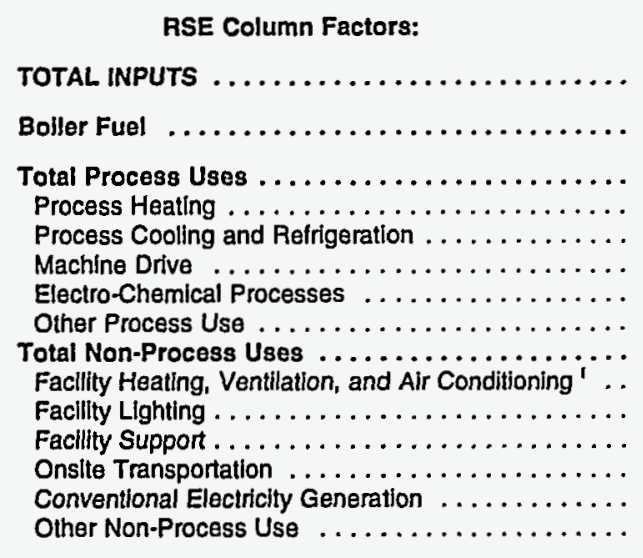

NF

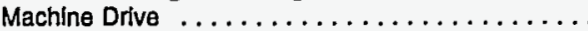

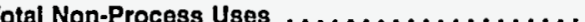
Facllity Heating, Ventilation, and Air Conditioning ' .

Facility Support $\ldots \ldots \ldots \ldots \ldots \ldots \ldots \ldots \ldots \ldots \ldots$

Conventional Electricity Generation ............

End Use Not Reported 
Table A36. Total Inputs of Energy for Heat, Power, and Electricity Generation by Fuel Type, Industry Group, Selected Industries, and End Use, 1991: Part 2 (Continued)

(Estimates in Trillion Btu)

\begin{tabular}{|c|c|c|c|c|c|c|c|c|c|c|}
\hline $\begin{array}{c}\text { SIC } \\
\text { Code" }\end{array}$ & End-Use Categories & Total & $\begin{array}{c}\text { Net } \\
\text { Electricity" }\end{array}$ & $\begin{array}{l}\text { Residual } \\
\text { Fuel Oil }\end{array}$ & $\begin{array}{c}\text { Distillate } \\
\text { Fuel Oil } \\
\text { and Diesel } \\
\text { Fuel }^{\mathrm{e}}\end{array}$ & $\begin{array}{l}\text { Natural } \\
\text { Gas }^{d}\end{array}$ & LPG & $\begin{array}{c}\text { Coal } \\
\text { (excluding } \\
\text { Coal Coke } \\
\text { and } \\
\text { Breeze) }\end{array}$ & Other & $\begin{array}{l}\text { RSE } \\
\text { Row } \\
\text { Factors }\end{array}$ \\
\hline
\end{tabular}

$2821 \quad$ Plastics Materials and Resins

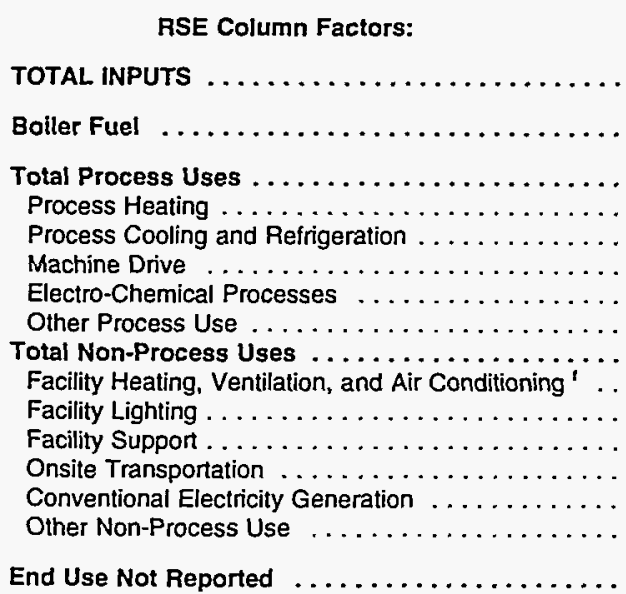

$\begin{array}{crr}\text { NF } & 0.6 & 1.3 \\ 288 & 50 & \\ -- & 1 & \\ -. & 44 & W \\ - & W & W \\ - & 5 & \\ - & 31 & \\ - & 7 & \\ - & W & \\ - & 5 & \\ -- & 2 & \\ -- & 2 & \\ -- & 1 & \\ -- & - & \\ -. & -- & W \\ -- & - & \\ 60 & 1 & \end{array}$

1.3
4
$W$
$W$
$W$
0
0
--
0
$W$
0
-
0
.-
$W$
0

$\begin{array}{rr}1.3 & 0.9 \\ 1 & 151 \\ 1 & 81 \\ * & 53 \\ * & 43 \\ 0 & : \\ : & W \\ * & w \\ * & 15 \\ * & 2 \\ \because & - \\ : & 1 \\ : & 0 \\ . & \\ * & \end{array}$

0.9
151
81
53
43
$\dot{W}$
$-\ddot{W}$
15
2
-1
1
0
13
-
2

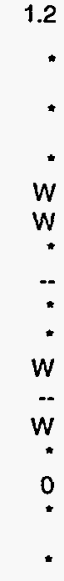

$$
0.9
$$

NF

Synthetic Rubber

RSE Column Factors:

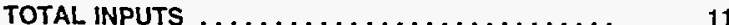

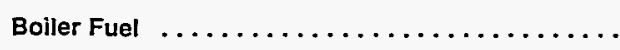

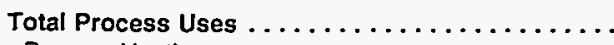

Process Heating $\ldots \ldots \ldots \ldots \ldots \ldots \ldots \ldots \ldots$

Process Cooling and Refrigeration .............

Machine Drive .........................

Electro-Chemical Processes $\ldots \ldots \ldots \ldots \ldots \ldots$.

Other Process Use $\ldots \ldots \ldots \ldots \ldots \ldots \ldots \ldots$.

Total Non-Process Uses .................

Facility Heating, Ventilation, and Air Conditioning ' .

Facility Lighting..$\ldots \ldots \ldots \ldots \ldots \ldots \ldots$

Facility Support . . . . . . . . . . . . . .

Onsite Transportation ...................

Conventional Electricity Generation ............

Other Non-Process Use . . . . . . . . . . . . . .

End Use Not Reported

\begin{tabular}{|c|c|c|}
\hline$N F$ & 0.6 & 1.4 \\
\hline 112 & 6 & * \\
\hline .. & $w$ & * \\
\hline .. & 5 & 0 \\
\hline -. & $*$ & 0 \\
\hline .- & 1 & 0 \\
\hline- & 4 & 0 \\
\hline-- & $\cdot$ & - \\
\hline -- & * & 0 \\
\hline -- & 1 & 0 \\
\hline-- & $\cdot$ & 0 \\
\hline -- & * & - \\
\hline & * & 0 \\
\hline 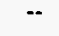 & $*$ & $\ddot{-}$ \\
\hline & -. & 0 \\
\hline- & 0 & 0 \\
\hline$w$ & $w$ & 0 \\
\hline
\end{tabular}

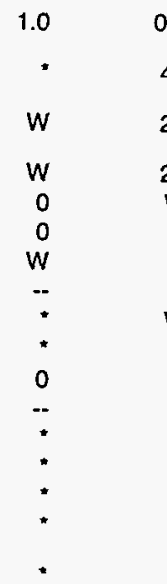

0.9
44
24
20
W
$:$
$:$
W
$:$
$:$
$:$
$:$

1.0
.
0
.
0
0
--
0
$W$
$*$
0
$W$
0
0
$w$

$$
\begin{gathered}
1.4 \\
W \\
W \\
0 \\
0 \\
0 \\
0 \\
- \\
0 \\
0 \\
0 \\
- \\
0 \\
- \\
0 \\
0
\end{gathered}
$$

NF

W

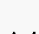

\section{NF}

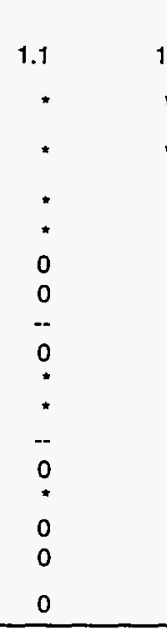

w

22.7

TOTAL INPUTS

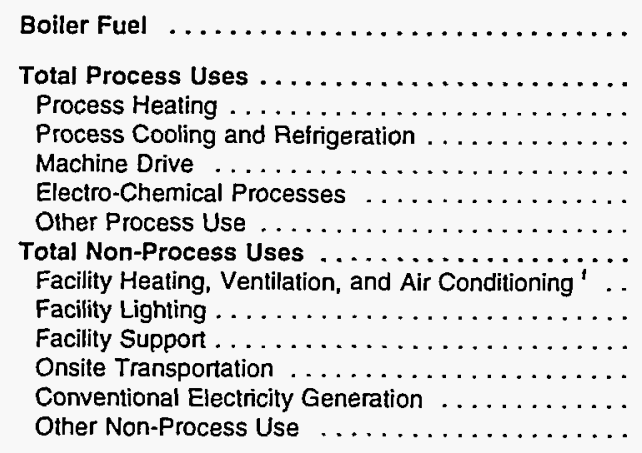

End Use Not Reported

$\begin{array}{cc}1.1 & \text { NF } \\ W & 0 \\ W & 0 \\ W & 0 \\ W & 0 \\ W & 0 \\ W & 0 \\ 0 & - \\ * & 0 \\ W & 0 \\ W & 0 \\ W & - \\ W & 0 \\ * & - \\ . . & 0 \\ 0 & 0 \\ W & 0\end{array}$

\begin{tabular}{c}
1.1 \\
$w$ \\
$W$ \\
2 \\
2 \\
0 \\
0 \\
\hdashline \\
0 \\
0 \\
\hdashline \\
0 \\
0 \\
0 \\
0
\end{tabular}

NF 
Table A36. Total Inputs of Energy for Heat, Power, and Electricity Generation by Fuel Type, Industry Group, Selected Industries, and End Use, 1991: Part 2 (Continued) (Estimates in Trillion Btu)

\begin{tabular}{|c|c|c|c|c|c|c|c|c|c|c|}
\hline $\begin{array}{c}\text { SIC } \\
\text { Cod } \theta^{*}\end{array}$ & End-Use Categories & Total & $\begin{array}{c}\text { Net } \\
\text { Electricity }\end{array}$ & $\begin{array}{l}\text { Residual } \\
\text { Fuel Oil }\end{array}$ & $\begin{array}{c}\text { Distillate } \\
\text { Fuel Oil } \\
\text { and Diesel } \\
\text { Fuel }\end{array}$ & $\begin{array}{c}\text { Natural } \\
\text { Gas }^{\circ}\end{array}$ & LPG & $\begin{array}{c}\text { Coal } \\
\text { (excluding } \\
\text { Coal Coke } \\
\text { and } \\
\text { Breeze) }\end{array}$ & Other & $\begin{array}{c}\text { RSE } \\
\text { Row } \\
\text { Factors }\end{array}$ \\
\hline
\end{tabular}

2824

Organic Fibers, Noncellulosic

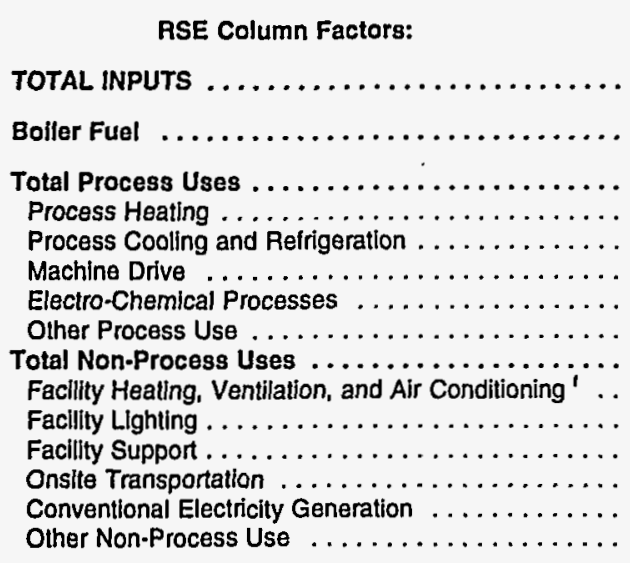

NF

98

End Use Not Reported

Cyclic Crudes and Intermediates

\begin{tabular}{|c|c|c|}
\hline NF & 1.1 & 1.1 \\
\hline 98 & 24 & $w$ \\
\hline -- & * & 2 \\
\hline- & 19 & $W$ \\
\hline -- & $w$ & $W$ \\
\hline -- & 2 & 0 \\
\hline .. & 13 & 0 \\
\hline -- & W & -- \\
\hline -. & W & 0 \\
\hline -- & 4 & W \\
\hline -- & 2 & $W$ \\
\hline -- & 1 & -- \\
\hline -. & $W$ & 0 \\
\hline -- & " & - \\
\hline -- & -- & 0 \\
\hline- & $W$ & 0 \\
\hline$W$ & * & $w$ \\
\hline
\end{tabular}

$\begin{array}{ll}1.0 & 0.9\end{array}$

w

23

W

W

NF

159

TOTAL INPUTS

Boller Fuel

Total Process Uses

$\ldots \cdots \cdots \cdots \cdots$

Process Cooling and Reirigeration ...........

Machine Drive .......................

Electro-Chemlcal Processes $\ldots \ldots \ldots \ldots \ldots \ldots \ldots$

Other Process Use . . . . . . . . . . . . . . . .

Total Non-Process Uses . . . . . . . . . . . . . . . . .

Facility Heating, Ventilation, and Air Conditioning ' .

Facillty Lighting . . . . . . . . . . . . . .

Facilly Support.

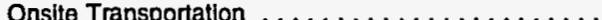

Conventlonal Electricity Generation . . . . . . . . .

Other Non-Process Use . . . . . . . . . . . . .

End Use Not Reported

\section{7}

$$
15
$$

2869

Industrial Organlc Chem/cals, nec

RSE Column Factors:

TOTAL INPUTS

Boller Fuel

Total Process Uses

Process Heating $\ldots \ldots \ldots \ldots \ldots \ldots \ldots \ldots \ldots$

Process Cooling and Refrigeration ...........

Machine Drive ..................

Electro-Chemical Processes ...............

Other Process Use . . . . . . . . . . . . . . . .

Total Non-Process Uses .................

Faclity Heating, Ventllation, and Air Conditioning ' ..

Facillty Lighting . . . . . . . . . . . . . . . .

Facillty Support

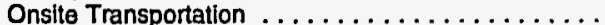

Conventlonal Electricity Generation ...........

Other Non-Process Use . . . . . . . . . . . . . .

End Use Not Reported 
Table A36. Total Inputs of Energy for Heat, Power, and Electricity Generation by Fuel Type, Industry Group, Selected Industries, and End Use, 1991: Part 2 (Continued) (Estimates in Trillion Btu)

\begin{tabular}{|c|c|c|c|c|c|c|c|c|c|c|}
\hline $\begin{array}{l}\text { SIC } \\
\text { Code }\end{array}$ & End-Use Categories & Total & $\begin{array}{c}\text { Net } \\
\text { Electricity }\end{array}$ & $\begin{array}{l}\text { Residual } \\
\text { Fuel Oil }\end{array}$ & $\begin{array}{c}\text { Distillate } \\
\text { Fuel Oil } \\
\text { and Diesel } \\
\text { Fuel }^{c}\end{array}$ & $\begin{array}{l}\text { Natural } \\
\text { Gas }^{\mathrm{d}}\end{array}$ & LPG & $\begin{array}{c}\text { Coal } \\
\text { (excluding } \\
\text { Coal Coke } \\
\text { and } \\
\text { Breeze) }\end{array}$ & Other & $\begin{array}{c}\text { RSE } \\
\text { Row } \\
\text { Factors }\end{array}$ \\
\hline
\end{tabular}

2873

Nitrogenous Fertilizers

RSE Column Factors:

TOTAL INPUTS

TS ......................................

Boiler Fuel $\ldots \ldots \ldots \ldots \ldots \ldots \ldots \ldots$.

Total Process Uses $\ldots \ldots \ldots \ldots \ldots \ldots \ldots \ldots$

Process Heating $\ldots \ldots \ldots \ldots \ldots \ldots \ldots \ldots \ldots$

Process Cooling and Refrigeration ............

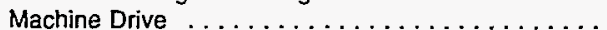

Electro-Chemical Processes $\ldots \ldots \ldots \ldots \ldots \ldots \ldots$

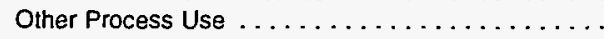

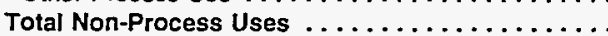

Facility Heating, Ventilation, and Air Conditioning ' ..

Facility Lighting $\ldots \ldots \ldots \ldots \ldots \ldots \ldots \ldots$

Facility Support .

Onsite Transportation

Conventional Electricity Generation ............

Other Non-Process Use...$\ldots \ldots \ldots \ldots \ldots \ldots$.

End Use Not Reported

\begin{tabular}{|c|c|c|c|c|}
\hline NF & 0.9 & NF & 1.0 & 0.7 \\
\hline 280 & 10 & 0 & - & 266 \\
\hline -- & • & 0 & * & 93 \\
\hline -- & 9 & 0 & * & 172 \\
\hline -. & • & 0 & 0 & 153 \\
\hline .. & 1 & 0 & 0 & 0 \\
\hline -. & 7 & 0 & * & 2 \\
\hline .. & 1 & -. & -- & - \\
\hline -. & 0 & 0 & 0 & 18 \\
\hline -. & 1 & 0 & * & 1 \\
\hline -- & * & 0 & * & 1 \\
\hline -. & 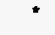 & -- & -- & -. \\
\hline -- & * & 0 & * & 0 \\
\hline -- & 0 & -- & " & 0 \\
\hline -- & -- & 0 & * & 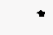 \\
\hline -- & 0 & 0 & 0 & 0 \\
\hline 5 & $*$ & 0 & * & \\
\hline
\end{tabular}

\begin{tabular}{c}
1.5 \\
. \\
0 \\
$:$ \\
$\vdots$ \\
0 \\
\hdashline \\
0 \\
$:$ \\
-- \\
0 \\
$:$ \\
0 \\
0 \\
\hline
\end{tabular}

$\begin{array}{ccc}\text { NF } & \text { NF } & \\ 0 & 4 & 19.3 \\ 0 & -. & 32.1 \\ 0 & -. & 26.1 \\ 0 & -. & 33.8 \\ 0 & -. & 34.0 \\ 0 & -. & 35.9 \\ - & - & 57.4 \\ 0 & -- & 48.1 \\ 0 & -. & 24.5 \\ 0 & -. & 33.1 \\ - & -. & 27.0 \\ 0 & -. & 38.0 \\ - & -. & 20.4 \\ 0 & -. & 55.4 \\ 0 & -. & \text { NF } \\ 0 & 4 & 32.6\end{array}$

2874

Phosphatic Fertilizers

RSE Column Factors:

\begin{tabular}{|c|c|c|c|}
\hline NF & 1.5 & 0.7 & 0.6 \\
\hline 34 & 6 & 2 & 1 \\
\hline -- & $w$ & $w$ & * \\
\hline .- & 5 & 1 & * \\
\hline -. & 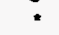 & 1 & $w$ \\
\hline .- & * & 0 & 0 \\
\hline .- & 5 & 0 & $w$ \\
\hline .. & 0 & .- & -- \\
\hline .. & 0 & 0 & 0 \\
\hline -. & $w$ & 0 & * \\
\hline .. & $*$ & 0 & 0 \\
\hline .. & $*$ &. & $\ldots$ \\
\hline .. & $\star$ & 0 & w \\
\hline .. & 0 & - & $\because$ \\
\hline -. & .. & 0 & 0 \\
\hline -- & 0 & 0 & W \\
\hline
\end{tabular}

TOTAL INPUTS $\ldots \ldots \ldots \ldots \ldots \ldots \ldots \ldots \ldots$

Boiler Fuel $\ldots \ldots \ldots \ldots \ldots \ldots \ldots \ldots \ldots$

Total Process Uses . . . . . . . . . . . . . . . .

Process Heating.$\ldots \ldots \ldots \ldots \ldots \ldots \ldots \ldots \ldots$

Process Cooling and Refrigeration ...........

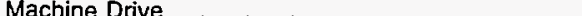

Electro-Chemical Processes $\ldots \ldots \ldots \ldots \ldots \ldots \ldots$

Other Process Use ....................

Total Non-Process Uses . . . . . . . . . . . . . .

Facility Heating, Ventilation, and Air Conditioning' .

Facility Lighting . . . . . . . . . . . . . . . .

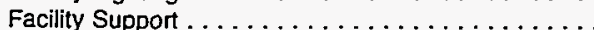

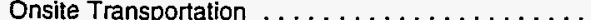

Conventional Electricity Generation .............

Other Non-Process Use ...................

End Use Not Reported

W w

W

Q

$\begin{array}{r}1.6 \\ 19 \\ 2 \\ 15 \\ 14 \\ : \\ : \\ 0 \\ : \\ :- \\ : \\ 0 \\ 0 \\ \hline\end{array}$

0.7

PETROLEUM and COAL PRODUCTS

RSE Column Factors:

\begin{tabular}{|c|c|c|}
\hline NF & 0.5 & 0.9 \\
\hline 2,987 & 105 & 87 \\
\hline -- & 1 & 38 \\
\hline -- & 94 & 49 \\
\hline -- & 3 & 49 \\
\hline -- & 5 & 0 \\
\hline -- & 85 & 0 \\
\hline -. & 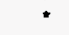 & -- \\
\hline -- & " & 0 \\
\hline -. & 9 & 0 \\
\hline- & 4 & 0 \\
\hline -. & 4 & -. \\
\hline -. & 1 & 0 \\
\hline 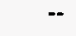 & 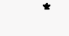 & -- \\
\hline- & -- & 0 \\
\hline -. & * & 0 \\
\hline
\end{tabular}

\begin{tabular}{|c|c|c|c|c|}
\hline 0.5 & 0.8 & 2.8 & NF & \\
\hline 838 & 63 & $w$ & W & 4.6 \\
\hline 263 & 12 & $w$ & .- & 6.2 \\
\hline 460 & 49 & $w$ & -- & 6.1 \\
\hline 422 & 38 & $w$ & - & 7.4 \\
\hline w & $w$ & 0 & .. & 8.2 \\
\hline w & $w$ & 0 & -- & 8.2 \\
\hline 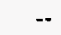 & .- & -- & -- & 8.8 \\
\hline 1 & 0 & 0 & -- & 11.2 \\
\hline 110 & * & 0 & -. & 9.2 \\
\hline 10 & $w$ & 0 & -. & 10.3 \\
\hline -. & - & -- & -. & 6.6 \\
\hline 2 & $w$ & 0 & -- & 10.1 \\
\hline & * & - & -- & 11.5 \\
\hline 98 & $w$ & 0 & -- & 7.5 \\
\hline & 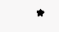 & 0 & .- & 17.0 \\
\hline
\end{tabular}

1,877

1

0

5

w

38.9

See footnotes at end of table. 
Table A36. Total Inputs of Energy for Heat, Power, and Electricity Generation by Fuel Type, Industry Group, Selected Industries, and End Use, 1991: Part 2 (Continued) (Estimates in Trillion Btu)

\begin{tabular}{|c|c|c|c|c|c|c|c|c|c|c|}
\hline $\begin{array}{l}\text { SIC } \\
\text { Code }^{\circ}\end{array}$ & End-Use Categories & Total & $\begin{array}{c}\mathrm{Net} \\
\text { Electricitys }\end{array}$ & $\begin{array}{l}\text { Residual } \\
\text { Fuel Oil }\end{array}$ & $\begin{array}{c}\text { Distillate } \\
\text { Fuel Oil } \\
\text { and Diesel } \\
\text { Fuel }\end{array}$ & $\begin{array}{c}\text { Natural } \\
\mathrm{Gas}^{\mathrm{d}}\end{array}$ & LPG & $\begin{array}{c}\text { Coal } \\
\text { (excluding } \\
\text { Coal Coke } \\
\text { and } \\
\text { Breeze) } \\
\end{array}$ & Other & $\begin{array}{c}\text { RSE } \\
\text { Row } \\
\text { Factors }\end{array}$ \\
\hline
\end{tabular}

2911

Petroleum Refining

RSE Column Factors:

TOTAL INPUTS

$\begin{array}{rrr}\text { NF } & 0.5 & 1.6 \\ 2,893 & 99 & 65 \\ -- & W & 33 \\ -. & 90 & 32 \\ -- & 3 & 32 \\ -- & 5 & 0 \\ -- & 82 & 0 \\ -- & * & - \\ - & * & 0 \\ -- & 8 & 0 \\ -- & 4 & 0 \\ -- & 1 & - \\ -- & * & 0 \\ -- & -. & 0 \\ 1,868 & W & 0\end{array}$

1.0
9
2
4
4
0
$W$
$\ddot{W}$
$W$
$:-$
$:$
3
$W$
$W$

0.5
792
253
429
392
$W$
$W$
-
108
8
-
1
0
98
-
2

0.7
60
11
48
36
$w$
$w$
-
0
$w$
$w$
--
$w$
$w$
.
$w$

NF

Boller Fuel

Total Process Uses

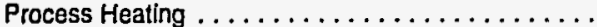

Process Cooling and Refrigeration ...............

Machine Drive ........................

Electro-Chemical Processes ................

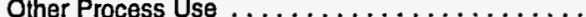

Total Non-Process Uses . ...............

Facllity Heating, Ventilation, and Air Conditioning' .

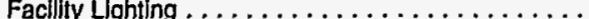

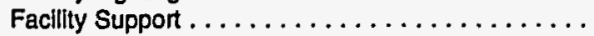

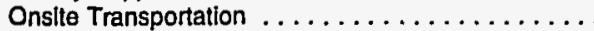

Conventional Electricity Generation $\ldots \ldots \ldots \ldots \ldots$

End Use Not Reported

RUBBER and MISC. PLASTICS PRODUCTS

RSE Column Factors:

\begin{tabular}{|c|c|c|c|}
\hline NF & 0.5 & 1.1 & 1.5 \\
\hline 237 & 116 & 8 & 3 \\
\hline -- & 1 & 7 & 2 \\
\hline -- & 90 & $w$ & * \\
\hline -- & 19 & $w$ & $w$ \\
\hline -- & 8 & • & 0 \\
\hline .- & 62 & * & $Q$ \\
\hline -. & * & - & $\cdots$ \\
\hline -- & • & 0 & * \\
\hline -- & 20 & $w$ & 1 \\
\hline .. & 9 & w & * \\
\hline .. & 9 & -. & -- \\
\hline -- & 2 & - & • \\
\hline -- & 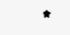 & -- & 1 \\
\hline -- & -. & 0 & * \\
\hline .. & $Q$ & 0 & Q \\
\hline 14 & 5 & $Q$ & $Q$ \\
\hline
\end{tabular}

Boller Fuel

Total Process Uses

Process Cooling and Refrigeration ..............

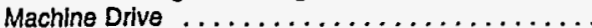

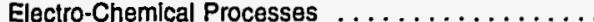

Other Process Use ...................

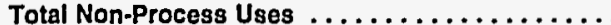

Faclity Heating, Ventilation, and Air Conditioning ' .

Facllity Lighting.$\ldots \ldots \ldots \ldots \ldots \ldots \ldots$

Facility Support . . . . . . . . . . . . . . . . . .

Onsite Transportation .....................

Conventional Electricity Generation .............

Other Non-Process Use . . . . . . . . . . . . . . .

End Use Not Reported

Tires and Inner Tubes

RSE Column Factors:

TOTAL INPUTS

NF

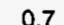

1.5

0.9

$\begin{array}{rrrr}1.2 & 1.2 & \text { NF } & \\ 3 & 7 & 5 & 9.8 \\ Q & 7 & -- & 11.7 \\ 1 & 0 & - & 17.1 \\ 1 & 0 & - & 19.3 \\ 0 & 0 & - & 20.2 \\ - & 0 & - & 20.5 \\ - & - & - & 55.5 \\ . & 0 & - & 32.4 \\ 1 & 0 & - & 11.9 \\ - & 0 & - & 18.1 \\ -- & - & - & 9.6 \\ W & 0 & -- & 12.1 \\ 1 & -- & -- & 22.8 \\ 0 & 0 & - & 20.0 \\ W & 0 & -. & 14.7\end{array}$

Boller Fuel

42

Total Process Uses .....................

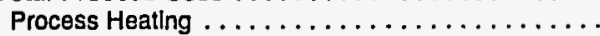

Process Cooling and Relrigeration .............

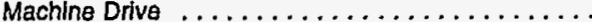

Electro-Chemical Processes ................

Other Process Use . . . . . . . . . . . . . . . .

Total Non-Process Uses ...................

Facility Heatlng, Ventilation, and Air Conditioning ' .. Facilly Lighting . .

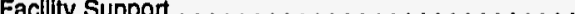

Onsite Transportation ..................

ConventIonai Electricity Generation ............

Other Non-Process Use

End Use Not Reported

...................

.....................

1

w

\begin{tabular}{|c|c|c|c|}
\hline 0.8 & 1.1 & NF & \\
\hline - & 2 & 1 & 3.6 \\
\hline 0 & 2 & -. & 3.4 \\
\hline w & 0 & -- & 5.8 \\
\hline 0 & 0 & .- & 16.0 \\
\hline 0 & 0 & .- & 4.5 \\
\hline$w$ & 0 & -- & 5.5 \\
\hline -- & -- & -- & $N F$ \\
\hline 0 & 0 & -. & 6.2 \\
\hline W & 0 & -. & 7.4 \\
\hline$Q$ & 0 & -. & 6.5 \\
\hline- & .- & -- & 3.0 \\
\hline$w$ & 0 & - & 6.0 \\
\hline & .. & -. & 6.6 \\
\hline U & 0 & -- & 3.3 \\
\hline w & 0 & .. & 6.5 \\
\hline • & 0 & 1 & 6.6 \\
\hline
\end{tabular}

See footnotes at end of table. 
Table A36. Total Inputs of Energy for Heat, Power, and Electricity Generation by Fuel Type, Industry Group, Selected Industries, and End Use, 1991: Part 2 (Continued) (Estimates in Trillion Btu)

\begin{tabular}{|c|c|c|c|c|c|c|c|c|c|c|}
\hline $\begin{array}{c}\text { SIC } \\
\text { Code }^{a}\end{array}$ & End-Use Categories & Total & $\begin{array}{c}\text { Net } \\
\text { Electricity }\end{array}$ & $\begin{array}{l}\text { Residual } \\
\text { Fuel Oil }\end{array}$ & $\begin{array}{c}\text { Distillate } \\
\text { Fuel Oil } \\
\text { and Diesel } \\
\text { Fuel }^{c}\end{array}$ & $\begin{array}{c}\text { Natural } \\
\text { Gas }^{d}\end{array}$ & LPG & $\begin{array}{c}\text { Coal } \\
\text { (excluding } \\
\text { Coal Coke } \\
\text { and } \\
\text { Breeze) }\end{array}$ & Other & $\begin{array}{c}\text { RSE } \\
\text { Row } \\
\text { Factors }\end{array}$ \\
\hline
\end{tabular}

308

Miscellaneous Plastic Products, nec

RSE Column Factors:

TOTAL INPUTS

Boiler Fuel

Total Process Uses

Process Cooling and Refrigeration $\ldots \ldots \ldots \ldots \ldots \ldots$

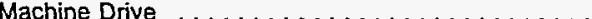

Electro-Chemical Processes . . . . . . . . . . . .

Other Process Use . . . . . . . . . . . . . .

Total Non-Process Uses . . . . . . . . . . . . . . . . .

Facility Heating, Ventilation, and Air Conditioning ' .

Facility Lighting

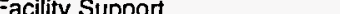

Onsite Transportation . . . . . . . . . . . . . .

Conventional Electricity Generation

Other Non-Process Use . ..................

End Use Not Reported

\begin{tabular}{|c|c|c|}
\hline NF & 0.5 & 1.5 \\
\hline 152 & 87 & 3 \\
\hline+- & * & 2 \\
\hline -- & 68 & 1 \\
\hline-- & 17 & 1 \\
\hline -- & 7 & 0 \\
\hline-- & 44 & . \\
\hline -- & * & - \\
\hline-- & " & 0 \\
\hline -- & 14 & * \\
\hline -- & 6 & " \\
\hline- & 6 & -- \\
\hline -. & 1 & * \\
\hline- & * & .. \\
\hline -- & -- & 0 \\
\hline -- & $\mathbf{Q}$ & 0 \\
\hline
\end{tabular}

W

LEATHER and LEATHER PRODUCTS

ASE Column Factors:

NF

TOTAL INPUTS

Boiler Fuel

Total Process Uses

Process Heating .

Process Cooling and Refrigeration ............

Machine Drive

Electro-Chemical Processes .............

Other Process Use . . . . . . . . . . . . . .

Total Non-Process Uses . . . . . . . . . . . . . . . .

Facility Heating, Ventilation, and Air Conditioning $i$...

Facility Lighting . . . . . . . . . . . . . . . . . . .

Facility Support

Onsite Transportation $\ldots \ldots \ldots \ldots \ldots \ldots \ldots \ldots \ldots$

Conventional Electricity Generation . . . . . . . . . .

Other Non-Process Use

End Use Not Reported

STONE, CLAY and GLASS PRODUCTS

RSE Column Factors:

TOTAL INPUTS

Boiler Fuel

Total Process Uses . . . . . . . . . . . . . . . .

Process Heating . . . . . . . . . . . . . . . . . . . . . . . . . . . .

Process Cooling and Refrigeration .............

Machine Drive . . . . . . . . . . . . . . . . . . .

Electro-Chemical Processes . . . . . . . . . . .

Other Process Use

Total Non-Process Uses . . . . . . . . . . . . . . . . . Facility Heating, Ventilation, and Air Conditioning '

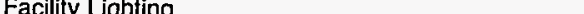

Facility Support . . . . . . . . . . . . . . . . . .

Onsite Transportation

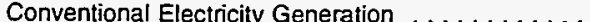

Other Non-Process Use

End Use Not Reported

See footnotes at end of table. 
Table A36. Total Inputs of Energy for Heat, Power, and Electricity Generation by Fuel Type, Industry Group, Selected Industries, and End Use, 1991: Part 2 (Continued) (Estimates in Trillion Btu)

\begin{tabular}{|c|c|c|c|c|c|c|c|c|c|c|}
\hline $\begin{array}{c}\text { SIC } \\
\text { Code }\end{array}$ & End-Use Categories & Total & $\begin{array}{c}\text { Net } \\
\text { Electricity }\end{array}$ & $\begin{array}{l}\text { Residual } \\
\text { Fuel Oil }\end{array}$ & $\begin{array}{c}\text { Distillate } \\
\text { Fuel Oil } \\
\text { and Diesel } \\
\text { Fuef }\end{array}$ & $\begin{array}{c}\text { Natural } \\
\text { Gas }^{d}\end{array}$ & LPG & $\begin{array}{c}\text { Coal } \\
\text { (excluding } \\
\text { Coal Coke } \\
\text { and } \\
\text { Breeze) }\end{array}$ & Other & $\begin{array}{c}\text { RSE } \\
\text { Row } \\
\text { Factors }\end{array}$ \\
\hline
\end{tabular}

3211

Flat Glass

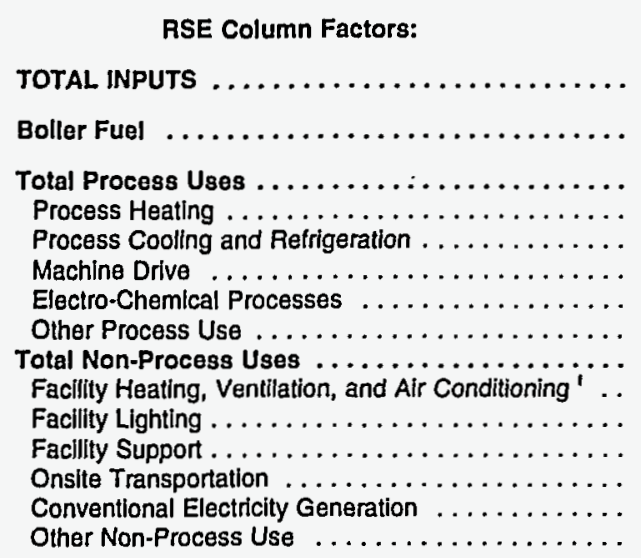

$$
49
$$

End Use Not Reported

\section{Glass Containers}

RSE Column Factors:

TOTAL INPUTS

Boller Fuel

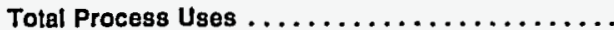

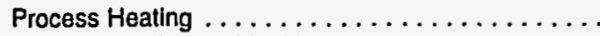

Process Cooling and Refrigeration .............

Machine Drive .....................

Electro-Chemical Processes ................

Other Process Use .....................

Total Non-Process Uses . . . . . . . . . . . . . .

Faclity Heating, Ventilation, and Air Conditioning ${ }^{i}$.. Facllity Llghting .

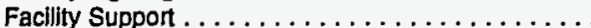

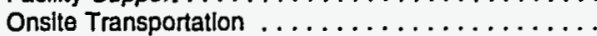

Conventional Electricity Generation . . . . . . . . . .

Other Non-Process Use

End Use Not Reported

Pressed and Blown Glass, nec.

RSE Column Factors:

TOTAL INPUTS

Boller Fuel

Total Process Uses $\ldots \ldots \ldots \ldots \ldots \ldots \ldots \ldots$.

Process Heating.$\ldots \ldots \ldots \ldots \ldots \ldots \ldots \ldots$

Process Cooling and Refrigeration . . . . . . . . .

Machlne Drive .....................

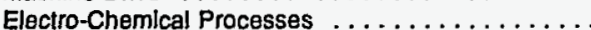

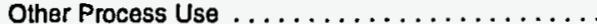

Total Non-Process Uses $\ldots \ldots \ldots \ldots \ldots \ldots \ldots$ Facllity Heating, Ventilation, and Air Conditioning' '.

Facillty Lighting

Facllity Support . . . . . . . . . . . . . . .

Onsite Transportation ...................

Conventional Electricity Generation ..............

Other Non-Process Use $\ldots \ldots \ldots \ldots \ldots \ldots \ldots$

End Use Not Reported

W $\quad 10$

$\begin{array}{cc}0.9 & 0 . \\ 0 & \\ w & \\ W & \\ \vdots \\ - \\ 0 \\ 0 \\ - \\ 0 \\ :\end{array}$

\begin{tabular}{r}
0.6 \\
42 \\
$w$ \\
36 \\
35 \\
1 \\
\hdashline \\
0 \\
2 \\
1 \\
\hdashline- \\
0 \\
0 \\
0 \\
$W$
\end{tabular}

$$
\begin{array}{r}
1.0 \\
. \\
0 \\
W \\
W \\
0 \\
\vdots \\
0 \\
\vdots \\
- \\
0 \\
\vdots \\
0 \\
0 \\
W
\end{array}
$$

$1.6 \quad$ NF

-. $w$

W

NF

85

$\begin{array}{rrrr}0.7 & 1.5 & 1.3 & 0.6 \\ 14 & 2 & * & 69\end{array}$

1.2

NF
0

NF

\section{1}

$$
0
$$

0

66

w

0
$W$

0
0
0
0

-
0
0

0

o

$\ddot{0}$

0

- 4.8

-. 14.4

-. 6.0

$\begin{array}{rr}- & 6.0 \\ - & 6.9 \\ - & 17.6\end{array}$ 
Table A36. Total Inputs of Energy for Heat, Power, and Electricity Generation by Fuel Type, Industry Group, Selected Industries, and End Use, 1991: Part 2 (Continued) (Estimates in Trillion Btu)

\begin{tabular}{|c|c|c|c|c|c|c|c|c|c|c|}
\hline $\begin{array}{c}\text { SIC } \\
\text { Code" }\end{array}$ & End-Use Categories & Total & $\begin{array}{c}\text { Net } \\
\text { Electricity" }\end{array}$ & $\begin{array}{c}\text { Residual } \\
\text { Fuel Oil }\end{array}$ & $\begin{array}{c}\text { Distillate } \\
\text { Fuel Oil } \\
\text { and Diesel } \\
\text { Fuel }^{c}\end{array}$ & $\begin{array}{c}\text { Natural } \\
\text { Gas }^{d}\end{array}$ & LPG & $\begin{array}{c}\text { Coal } \\
\text { (excluding } \\
\text { Coal Coke } \\
\text { and } \\
\text { Breeze) }\end{array}$ & Other & $\begin{array}{c}\text { RSE } \\
\text { Row } \\
\text { Factors }\end{array}$ \\
\hline
\end{tabular}

3241

Cement, Hydraulic

RSE Column Factors: NF

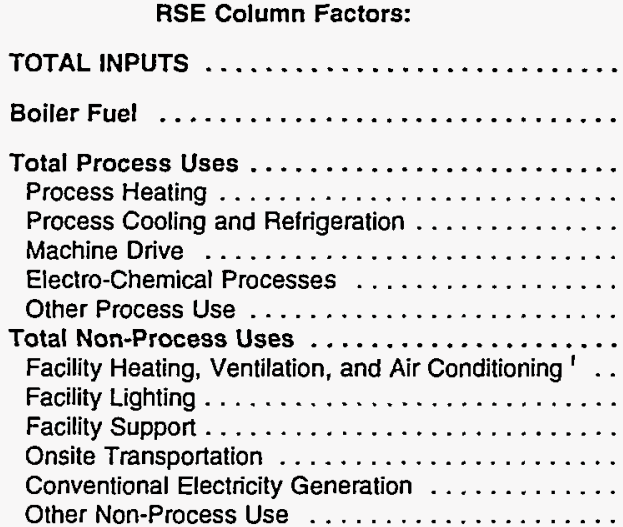

NF $\quad 0.9$

329

1.7
1
0
1
$W$
0
$W$
-
0
0
0
-
0
--
0
0

\begin{tabular}{r}
0.8 \\
4 \\
\\
1 \\
1 \\
0 \\
W \\
-- \\
W \\
2 \\
- \\
- \\
2 \\
\hline \\
0
\end{tabular}

\begin{tabular}{r}
1.2 \\
39 \\
\\
38 \\
38 \\
0 \\
- \\
\hdashline \\
0 \\
1 \\
1 \\
-- \\
$\vdots$ \\
0 \\
0 \\
0
\end{tabular}

End Use Not Reported

TOTAL INPUTS

\begin{tabular}{|c|c|c|c|c|c|c|}
\hline 0.9 & 0.9 & 0.6 & 0.8 & 1.8 & $N F$ & \\
\hline W & 1 & 8 & $\mathbf{Q}$ & 88 & w & 25.9 \\
\hline 0 & $Q$ & * & $Q$ & 0 & -- & $\mathrm{NF}$ \\
\hline$w$ & •" & 8 & - & 88 & -- & 22.7 \\
\hline w & $Q$ & 8 & 0 & 88 & 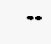 & 22.0 \\
\hline 0 & ? & ? & ? & 0 & 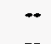 & $\begin{array}{l}12.7 \\
0\end{array}$ \\
\hline 0 & " & " & • & 0 & -- & 27.9 \\
\hline 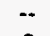 & - & - & -- & & -- & $N F$ \\
\hline 0 & w & 0 & 0 & 0 & 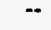 & 24.7 \\
\hline 0 & 1 & * & $*$ & 0 & 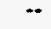 & 31.3 \\
\hline 0 & * & * & * & 0 & -- & 23.8 \\
\hline & $\ddot{\ddot{n}}$ & & 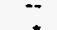 & & - & 23.8 \\
\hline .. & 1 & 0 & . & 0 & $\overline{-}$ & $\begin{array}{r}8.2 \\
34\end{array}$ \\
\hline 0 & 0 & 0 & 0 & 0 & -- & $\mathrm{NF}$ \\
\hline 0 & 0 & 0 & 0 & 0 & - & $\mathrm{NF}$ \\
\hline 0 & Q & 0 & $*$ & 0 & $w$ & $\mathrm{NF}$ \\
\hline
\end{tabular}

Boiler Fuel

Total Process Uses

Process Heating

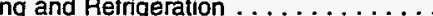

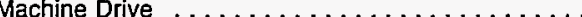

Electro-Chemical Processes . . . . . . . . . .

Other Process Use . . . . . . . . . . . . . . . .

Total Non-Process Uses ... . . . . . . . . . . . . . .

Facility Heating, Ventilation, and Air Conditioning ${ }^{i} \ldots$

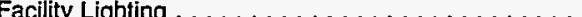

Facility Support . . . . . . . . . . . . . . . . . .

Onsite Transportation $\ldots \ldots \ldots \ldots \ldots \ldots \ldots \ldots$

Conventional Electricity Generation ............

Other Non-Process Use . . . . . . . . . . . . . . .

End Use Not Reported

Mineral Wool

RSE Column Factors:

\begin{tabular}{|c|c|c|c|c|c|c|c|}
\hline NF & 0.7 & 1.2 & 1.1 & 0.8 & 1.1 & 1.2 & NF \\
\hline 41 & 10 & $w$ & * & 29 & • & • & $w$ \\
\hline- & $w$ & $w$ & - & 1 & * & • & .. \\
\hline -- & 8 & 0 & " & 23 & $w$ & 0 & -. \\
\hline -. & 4 & 0 & • & 22 & * & 0 & -. \\
\hline .. & $w$ & 0 & 0 & * & 0 & 0 & -. \\
\hline- & 4 & 0 & * & * & $w$ & 0 & + \\
\hline -- & 0 & -. & - & -- & -- & -. & -. \\
\hline -- & $w$ & 0 & 0 & * & 0 & 0 & -- \\
\hline -- & $w$ & 0 & * & 2 & • & 0 & -- \\
\hline -- & * & 0 & $w$ & 2 & * & 0 & $\cdots$ \\
\hline- & * & -- & -- & -- & $=$ & -- & -- \\
\hline$=$ & * & 0 & $w$ & * & 0 & 0 & - \\
\hline 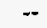 & $w$ & -- & * & 0 & * & - & -. \\
\hline -- & 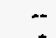 & 0 & $\star$ & 0 & 0 & 0 & - \\
\hline- & * & 0 & • & 0 & 0 & 0 & - \\
\hline$W$ & $w$ & 0 & * & 2 & $w$ & 0 & $w$ \\
\hline
\end{tabular}

Boiler Fuel

Total Process Uses

Process Heating

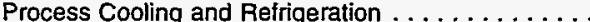

Machine Drive ....................

Electro-Chemical Processes $\ldots \ldots \ldots \ldots \ldots \ldots$. . . .

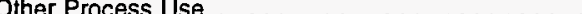

Total Non-Process Uses . . . . . . . . . . . . .

Facility Heating, Ventilation, and Air Conditioning ${ }^{\prime}$..

Facility Lighting

Facility Support . . . . . . . . . . . . . . . .

Onsite Transportation $\ldots \ldots \ldots \ldots \ldots \ldots \ldots \ldots$

Conventional Electricity Generation ...........

Other Non-Process Use . . . . . . . . . . . . . .

End Use Not Reported 
Table A36. Total Inputs of Energy for Heat, Power, and Electricity Generation by Fuel Type, Industry Group, Selected Industries, and End Use, 1991: Part 2 (Continued) (Estimates in Trillion Btu)

\begin{tabular}{cc|c|c|c|c|c|c|c|c}
\hline SIC & End-Use Categories & Total & $\begin{array}{c}\text { Net } \\
\text { Electricity }\end{array}$ & $\begin{array}{c}\text { Coal } \\
\text { Residual } \\
\text { Fuel Oil }\end{array}$ & $\begin{array}{c}\text { Distillate } \\
\text { Fuel Oil } \\
\text { and Diesel } \\
\text { Fuer }^{\circ}\end{array}$ & $\begin{array}{c}\text { Natural } \\
\text { Gas }^{\circ}\end{array}$ & $\begin{array}{c}\text { RSE } \\
\text { Coal Coke } \\
\text { and } \\
\text { Breeze) }\end{array}$ & $\begin{array}{c}\text { Row } \\
\text { LFG }\end{array}$ \\
\hline
\end{tabular}

33

PRIMARY METAL INDUSTRIES

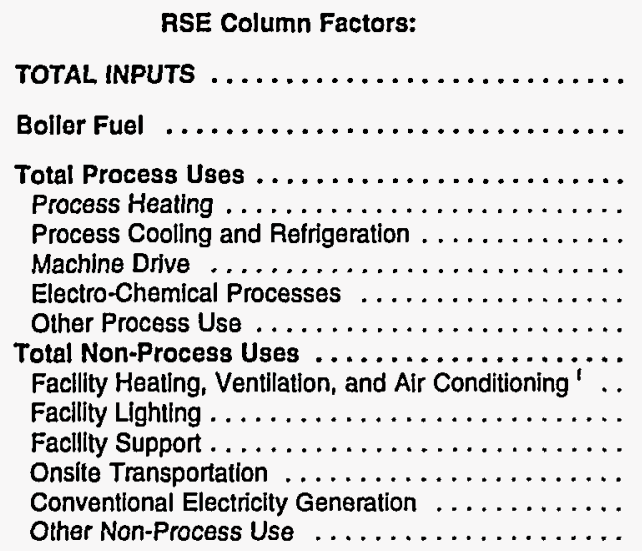

NF
2,292
..
.-
--
--
--
--
--
--
--
--
--
--
--

End Use Not Reported $\ldots \ldots \ldots \ldots \ldots \ldots \ldots \ldots$. 1,041

$\begin{array}{rr}0.6 & 1.2 \\ 499 & 33 \\ 1 & 25 \\ 454 & 8 \\ 100 & 8 \\ 3 & 0 \\ 118 & Q \\ 228 & -- \\ 4 & 0 \\ 36 & Q \\ 16 & 0 \\ 16 & -. \\ 3 & Q \\ 1 & -- \\ -- & 0 \\ - & 0 \\ & \end{array}$

$\begin{array}{rrr}1.2 & 0.7 \\ 11 & 686 \\ 1 & 93 \\ 3 & 522 \\ 2 & 516 \\ 0 & \\ 1 & \\ -\cdot & \\ 7 & \\ \cdot & \\ - & \\ \cdot & \\ 6 & \\ Q & \\ W & \\ 1 & \end{array}$

\begin{tabular}{r}
0.7 \\
686 \\
93 \\
522 \\
516 \\
- \\
4 \\
- \\
2 \\
54 \\
44 \\
- \\
6 \\
\hline \\
1 \\
17
\end{tabular}

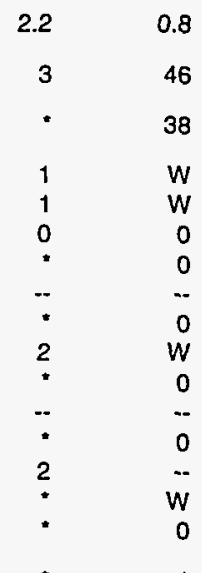

NF

Blast Furnaces and Steel Mills

RSE Column Factors:

\begin{tabular}{|c|c|c|c|}
\hline NF & 0.6 & 1.6 & 1.0 \\
\hline 1,569 & 130 & 31 & 5 \\
\hline -- & $w$ & 24 & * \\
\hline$\cdots$ & 116 & 7 & 1 \\
\hline .. & 53 & 7 & 1 \\
\hline-- & 1 & 0 & 0 \\
\hline -- & 57 & 0 & - \\
\hline- & 3 & -- & - \\
\hline -- & 3 & 0 & " \\
\hline -- & 10 & - & 4 \\
\hline -- & 4 & - & $w$ \\
\hline-- & 4 & -. & - \\
\hline -- & 1 & 0 & 0 \\
\hline -- & $w$ & -- & 4 \\
\hline -- & -- & 0 & - \\
\hline -- & $w$ & 0 & $w$ \\
\hline & & 0 & * \\
\hline
\end{tabular}

TOTAL INPUTS

Boiler Fuel

Total Process Uses . . . . . . . . . . . . .

Process Heating . . . . . . . . . . . . . . . . . . .

Process Cooling and Refrigeration ...........

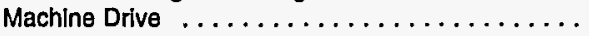

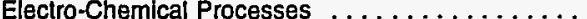

Other Process Use ......................

Total Non-Process Uses . . . . . . . . . . . . . . . .

Facllity Heating, Ventllation, and Air Conditioning '

Facility Lighting . . . . . . . . . . . . . . .

Facillty Support . . . . . . . . . . . . . .

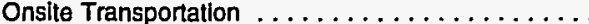

Conventional Electricity Generation ...........

Other Non-Process Use ................

End Use Not Reported . . . . . . . . . . . . .

$\begin{array}{rrrrr}0.7 & 1.0 & 1.6 & \text { NF } & \\ 399 & * & 24 & 978 & 4.1 \\ 63 & W & 24 & -- & 6.7 \\ 312 & * & - & -. & 4.8 \\ 310 & * & - & -- & 5.3 \\ 0 & 0 & 0 & - & 9.8 \\ 1 & * & 0 & -- & 7.1 \\ -* & -* & -- & -- & 14.7 \\ 1 & * & 0 & -. & 14.0 \\ 22 & * & 0 & -- & 5.2 \\ 17 & * & 0 & -- & 7.5 \\ -- & - & - & -- & 4.9 \\ 4 & * & 0 & -- & 9.1 \\ 0 & * & -- & - & 6.9 \\ - & * & 0 & -- & 15.0 \\ 1 & * & 0 & -- & 13.7 \\ 2 & W & * & 978 & 10.2\end{array}$

3313

Electrometalurgical Products

RSE Column Factors:

$\begin{array}{ll}\text { NF } & 0.8 \\ 31 \\ -- \\ -- \\ -- \\ -- \\ -- \\ -- \\ -- \\ -- \\ -- \\ -- \\ -- \\ -- \\ -- \\ .-\end{array}$

$\begin{array}{cc}0.8 & \text { NF } \\ 14 & 0 \\ W & 0 \\ 14 & \\ 10 & 0 \\ W & 0 \\ 3 & 0 \\ W & \\ W & 0 \\ W & 0 \\ * & 0 \\ * & - \\ W & 0 \\ * & 0 \\ 0 & 0\end{array}$

0.9

w

End Use Not Reported

3

W

o

o

o

3

8.4

12.6

9.5

9.3

18.4

12.3

15.8

14.1
10.1

11.8

7.9

10.5

11.8

15.5
NF

18.4

See footnotes at end of table. 
Table A36. Total Inputs of Energy for Heat, Power, and Electricity Generation by Fuel Type, Industry Group, Selected Industries, and End Use, 1991: Part 2 (Continued)

(Estimates in Trillion Btu)

\begin{tabular}{|c|c|c|c|c|c|c|c|c|c|c|}
\hline $\begin{array}{l}\text { SIC } \\
\text { Code }^{A}\end{array}$ & End-Use Categories & Total & $\begin{array}{c}\text { Net } \\
\text { Electricity" }\end{array}$ & $\begin{array}{l}\text { Residual } \\
\text { Fuel Oil }\end{array}$ & \begin{tabular}{|c} 
Distillate \\
Fuel Oil \\
and Diese \\
Fuer
\end{tabular} & $\begin{array}{c}\text { Natura } \\
\text { Gas }^{d}\end{array}$ & LPG & $\begin{array}{c}\text { Coal } \\
\text { (excluding } \\
\text { Coal Coke } \\
\text { and } \\
\text { Breeze) }\end{array}$ & Other & $\begin{array}{c}\text { RSE } \\
\text { Row } \\
\text { Factor }\end{array}$ \\
\hline
\end{tabular}

$3321 \quad$ Gray and Ductile Iron Foundries

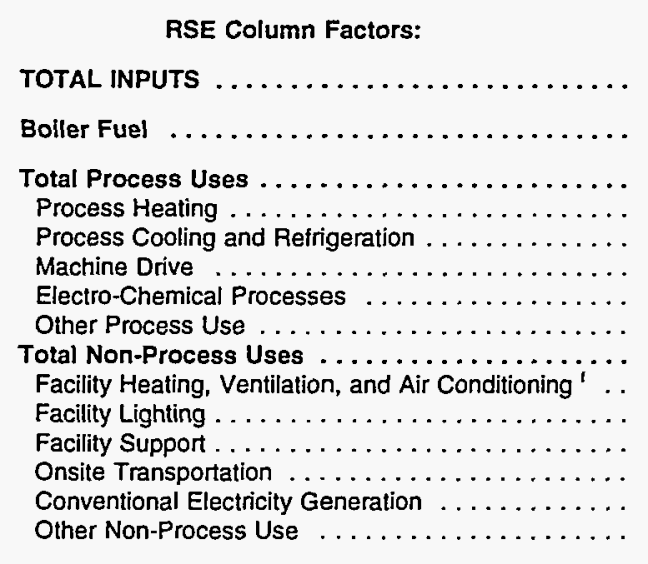

\begin{tabular}{|c|c|c|c|c|c|c|c|c|}
\hline$N F$ & 0.6 & 1.6 & 1.3 & 0.6 & 1.3 & 1.2 & $N F$ & \\
\hline 74 & 22 & * & 1 & 28 & * & - & 22 & 14.0 \\
\hline .. & . & $w$ & * & 2 & • & 0 & -. & 18.9 \\
\hline -. & 18 & $Q$ & Q & 18 & * & - & -- & 13.7 \\
\hline -- & 9 & 0 & 0 & 17 & • & • & -. & 16.4 \\
\hline -. & " & 0 & 0 & 0 & 0 & 0 & -. & 16.3 \\
\hline -- & 8 & $Q$ & * & 1 & * & 0 & -. & 11.5 \\
\hline -- & $w$ & - & -- & -. & -- & -. & -- & 39.9 \\
\hline -- & $Q$ & 0 & • & * & - & 0 & -. & 19.4 \\
\hline -- & 4 & * & * & 7 & * & 0 & -- & 11.0 \\
\hline .- & 2 & * & . & 7 & * & 0 & .- & 20.0 \\
\hline -. & $\overline{1}$ & -- & -- & -- & -. & .. & .- & 10.9 \\
\hline -- & * & 0 & * & * & 0 & 0 & .- & 12.2 \\
\hline -. & • & -- & • & * & • & -- & -- & 17.7 \\
\hline -- & -- & 0 & * & 0 & 0 & 0 & -- & 13.6 \\
\hline -. & • & 0 & 0 & * & * & 0 & - & 37.5 \\
\hline 24 & 1 & 0 & * & 1 & . & * & 22 & 29.1 \\
\hline
\end{tabular}

End Use Not Reported

Primary Copper

RSE Column Factors:

TOTAL INPUTS

\begin{tabular}{|c|c|c|c|c|c|c|c|}
\hline NF & 1.0 & 1.0 & 1.0 & 1.0 & 1.0 & 1.0 & NF \\
\hline 22 & 4 & W & $w$ & 15 & " & $w$ & 1 \\
\hline -. & $w$ & w & * & 4 & 0 & 0 & -- \\
\hline -- & 4 & $w$ & $w$ & 9 & • & $w$ & -- \\
\hline - - & * & $w$ & $w$ & 8 & * & $w$ & -- \\
\hline -- & $w$ & 0 & 0 & 0 & 0 & 0 & -- \\
\hline .. & 2 & 0 & 0 & 0 & * & 0 & -- \\
\hline -- & 1 & -- & -- & - & -- & -- & -. \\
\hline -- & $w$ & 0 & 0 & • & 0 & 0 & *. \\
\hline -- & 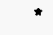 & 0 & $w$ & 2 & • & 0 & -. \\
\hline -- & • & 0 & * & * & * & 0 & -- \\
\hline -- & • & -. & - & - & - & -- & $*$ \\
\hline -- & * & 0 & 0 & * & 0 & 0 & $\cdots$ \\
\hline -. & 0 & -. & W & 0 & * & -- & -. \\
\hline -- & .. & 0 & * & 2 & 0 & 0 & -- \\
\hline -- & 0 & 0 & 0 & 0 & 0 & D & -. \\
\hline 1 & $w$ & 0 & 0 & * & * & 0 & 1 \\
\hline
\end{tabular}

Boiler Fuel

s $\ldots \ldots \ldots \ldots \ldots \ldots \ldots \ldots$

Total Process Uses $\ldots \ldots \ldots \ldots \ldots \ldots \ldots \ldots \ldots$

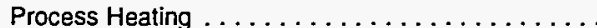

Process Cooling and Refrigeration ............

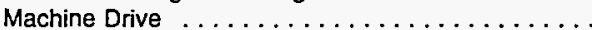

Electro-Chemical Processes $\ldots \ldots \ldots \ldots \ldots \ldots$

Other Process Use $\ldots \ldots \ldots \ldots \ldots \ldots \ldots \ldots$

Total Non-Process Uses . . . . . . . . . . . . .

Facility Heating, Ventilation, and Air Conditioning '

Facility Lighting $\ldots \ldots \ldots \ldots \ldots \ldots \ldots$

Facility Support . . . . . . . . . . . . . .

Onsite Transportation ....................

Conventional Electricity Generation ............

Other Non-Process Use . . . . . . . . . . . . . . . . . .

End Use Not Reported

Primary Aluminum

RSE Column Factors:

TOTAL INPUTS

$\mathrm{NF}$

Boiler Fuel

$\ldots \ldots \ldots \ldots \ldots \ldots \ldots \ldots$

$\begin{array}{rcc}0.8 & 1.4 & 1 . \\ 230 & * & \\ W & * & W \\ 222 & 0 & W \\ W & 0 & W \\ W & 0 & \\ 4 & 0 & \\ 216 & - & \\ W & 0 & \\ W & : & \\ 4 & * & \\ 3 & - & \\ W & 0 & \\ W & - & \\ - & 0 & 0 \\ 0 & 0 & \end{array}$

Total Process Uses . . . . . . . . . . . . . . . .

Process Heating . . . . . . . . . . . . . . .

Process Cooling and Refrigeration . . . . . . . . . .

Machine Drive

Electro-Chemical Processes $\ldots \ldots \ldots \ldots \ldots \ldots \ldots$

Other Process Use . . . . . . . . . . . . . . . . . .

Total Non-Process Uses $\ldots \ldots \ldots \ldots \ldots \ldots \ldots$

Facility Heating, Ventilation, and Air Conditioning' .

Facility Lighting

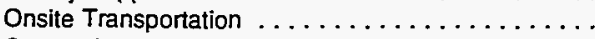

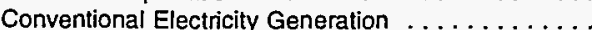

Other Non-Process Use

End Use Not Reported

$\ldots \ldots \ldots \ldots \ldots \ldots$

w

\begin{tabular}{|c|c|c|c|c|}
\hline 1.1 & 0.8 & 1.1 & NF & NF \\
\hline 1 & 21 & * & 0 & 1 \\
\hline W & 2 & $w$ & 0 & -- \\
\hline$w$ & 17 & * & 0 & -- \\
\hline W & 17 & * & 0 & -. \\
\hline 0 & 0 & 0 & 0 & $\ldots$ \\
\hline * & * & * & 0 & -- \\
\hline -- & -- & -- & -- & -- \\
\hline 0 & 0 & 0 & 0 & -. \\
\hline 1 & 1 & - & 0 & -- \\
\hline & 1 & * & 0 & -- \\
\hline$\cdots$ & - & -- & -- & .. \\
\hline 0 & * & 0 & 0 & -- \\
\hline 1 & 0 & * & -- & -. \\
\hline 0 & 0 & 0 & 0 & -- \\
\hline 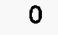 & 0 & • & 0 & -- \\
\hline * & 1 & $w$ & 0 & 1 \\
\hline
\end{tabular}


Table A36. Total Inputs of Energy for Heat, Power, and Electricity Generation by Fuel Type, Industry Group, Selected Industries, and End Use, 1991: Part 2 (Continued) (Estimates in Trillion Btu)

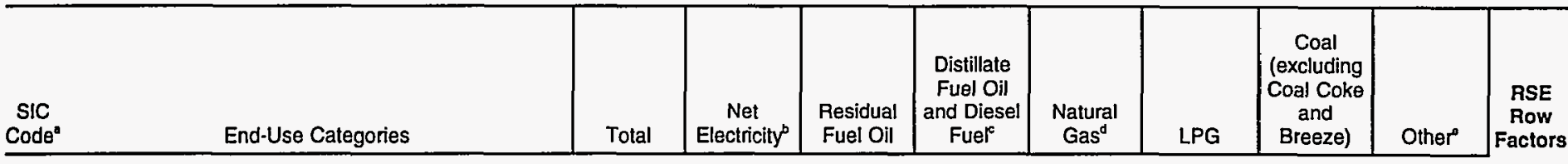

3339

\section{Primary Nonferrous Metals, nec}

RSE Column Factors:

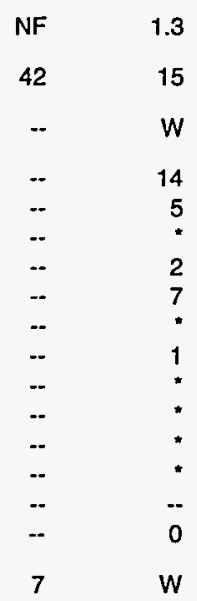

0.4
$*$
.
0
0
0
0
-
0
0
0
-
0
-
0
0
0

\begin{tabular}{|c|c|}
\hline 1.6 & 1.3 \\
\hline - & 17 \\
\hline • & 3 \\
\hline - & 8 \\
\hline$w$ & 8 \\
\hline 0 & 0 \\
\hline$w$ & * \\
\hline -- & -- \\
\hline 0 & 0 \\
\hline . & w \\
\hline 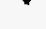 & 1 \\
\hline$\ddot{-}$ & - \\
\hline 0 & \\
\hline 0 & $w$ \\
\hline 0 & 0 \\
\hline • & $w$ \\
\hline
\end{tabular}

1.4
$*$
0
$W$
$W$
0
$:-$
0
$W$
$W$
$:-$
$:$
0
0
.

$\begin{array}{rrr}0.6 & \text { NF } & \\ \text { W } & \text { W } & 1.9 \\ W & -- & 2.8 \\ 0 & - & 1.6 \\ 0 & - & 1.3 \\ 0 & - & 3.9 \\ 0 & - & 1.0 \\ - & - & 1.6 \\ 0 & - & 15.5 \\ W & -- & 1.5 \\ 0 & -- & 2.5 \\ - & - & 2.3 \\ 0 & - & 4.9 \\ -- & - & 1.4 \\ \text { W } & -- & 1.1 \\ 0 & -- & \text { NF } \\ 0 & \text { W } & 1.5\end{array}$

Aluminum Sheet, Plate, and Foil

RSE Column Factors:

\begin{tabular}{|c|c|}
\hline NF & 1.5 \\
\hline 60 & 15 \\
\hline -- & $w$ \\
\hline .- & 12 \\
\hline -- & 1 \\
\hline -. & $w$ \\
\hline .- & 11 \\
\hline -. & 0 \\
\hline -. & w \\
\hline -- & 2 \\
\hline -- & 1 \\
\hline -. & 1 \\
\hline -- & - \\
\hline .- & • \\
\hline -- & -- \\
\hline - & . \\
\hline$w$ & $w$ \\
\hline
\end{tabular}

$\begin{array}{rr}0.8 & 1.2 \\ * & 43 \\ * & 2 \\ * & 38 \\ . & 38 \\ 0 & 0 \\ . & 0 \\ 0 & : \\ : & 2 \\ . & 2 \\ : & : \\ : & 0 \\ 0 & 0 \\ 0 & 0 \\ . & .\end{array}$

\begin{tabular}{|c|c|c|c|}
\hline 0.8 & 0.9 & NF & \\
\hline * & $w$ & w & 1.1 \\
\hline * & $w$ & -- & 1.0 \\
\hline * & 0 & -- & 1.2 \\
\hline w & 0 & .. & 1.4 \\
\hline 0 & 0 & -. & 1.3 \\
\hline$w$ & 0 & -- & 1.0 \\
\hline -- & $\cdots$ & -. & NF \\
\hline 0 & 0 & - & 0.7 \\
\hline - & 0 & - & 1.4 \\
\hline w & 0 & -- & 1.4 \\
\hline 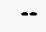 & -- & -- & 0.7 \\
\hline * & 0 & .- & 1.3 \\
\hline$w$ & -- & -. & 1.0 \\
\hline 0 & 0 & -- & NF \\
\hline 0 & 0 & -- & 0.7 \\
\hline * & 0 & $w$ & 3.1 \\
\hline
\end{tabular}

\section{RSE Column Factors:}

TOTAL INPUTS

Boller Fuel

Total Process Uses $\ldots \ldots \ldots \ldots \ldots \ldots \ldots \ldots \ldots$

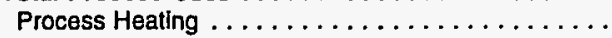

Process Cooling and Refrigeration ............

Machine Drive...$\ldots \ldots \ldots \ldots \ldots \ldots \ldots \ldots$

Electro-Chemical Processes ..................

Other Process Use .....................

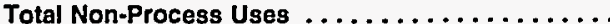
Facility Heating, Ventilation, and Air Conditioning '.. Facility Lighting . . . . . . . . . . . . . . . . .

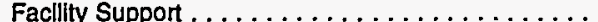

Onsile Transportation ................... Conventlonal Electricity Generation ............

Other Non-Process Use $\ldots \ldots \ldots \ldots \ldots \ldots \ldots$

End Use Not Reported

See footnotes at end of table. 
Table A36. Total Inputs of Energy for Heat, Power, and Electricity Generation by Fuel Type, Industry Group, Selected Industries, and End Use, 1991: Part 2 (Continued) (Estimates in Trillion Btu)

\begin{tabular}{|c|c|c|c|c|c|c|c|c|c|c|}
\hline $\begin{array}{c}\text { SIC } \\
\text { Code }\end{array}$ & End-Use Categories & Total & $\begin{array}{c}\mathrm{Net} \\
\text { Electricity }\end{array}$ & $\begin{array}{l}\text { Residual } \\
\text { Fuel Oil }\end{array}$ & $\begin{array}{c}\text { Distillate } \\
\text { Fuel Oit } \\
\text { and Diesel } \\
\text { Fuel }^{e}\end{array}$ & $\begin{array}{c}\text { Natural } \\
\text { Gas }^{d}\end{array}$ & LPG & $\begin{array}{c}\text { Coal } \\
\text { (excluding } \\
\text { Coal Coke } \\
\text { and } \\
\text { Breeze) }\end{array}$ & Other & $\begin{array}{c}\text { RSE } \\
\text { Row } \\
\text { Factors }\end{array}$ \\
\hline
\end{tabular}

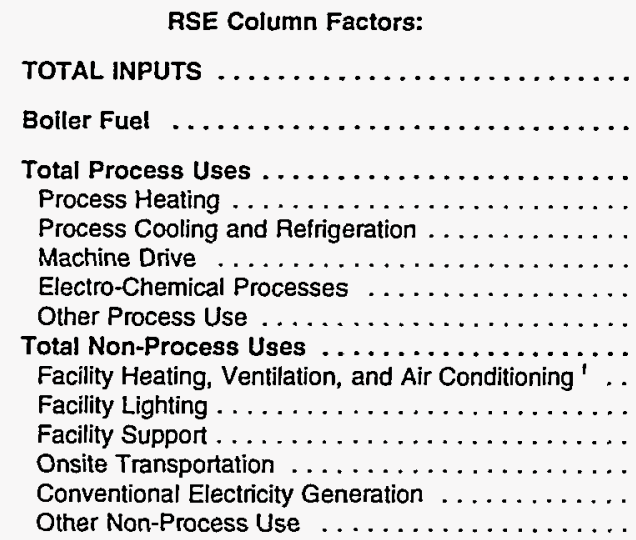

\begin{tabular}{|c|c|}
\hline NF & 0.4 \\
\hline 235 & 101 \\
\hline- & 1 \\
\hline -. & 56 \\
\hline -- & 8 \\
\hline-- & 3 \\
\hline- & 43 \\
\hline-- & 1 \\
\hline$\cdots$ & 1 \\
\hline- & 36 \\
\hline -. & 17 \\
\hline-- & 15 \\
\hline-- & 4 \\
\hline -- & * \\
\hline -- & - \\
\hline-- & - \\
\hline 25 & \\
\hline
\end{tabular}

\begin{tabular}{c}
2.0 \\
3 \\
3 \\
$\mathrm{Q}$ \\
$\mathrm{Q}$ \\
0 \\
$\vdots$ \\
$\vdots$ \\
$\vdots$ \\
\hdashline \\
$\mathrm{Q}$ \\
\hdashline 0 \\
0 \\
.
\end{tabular}

\begin{tabular}{rr}
1.2 & 0.7 \\
4 & 109 \\
1 & 28 \\
1 & 37 \\
$:$ & 32 \\
0 & $:$ \\
1 & 3 \\
\hdashline- & - \\
1 & 1 \\
2 & 33 \\
1 & 31 \\
-- & - \\
$:$ & 1 \\
0 & 0 \\
$:$ & $:$
\end{tabular}

\begin{tabular}{r}
1.4 \\
2 \\
. \\
1 \\
0 \\
$Q$ \\
$\because-$ \\
1 \\
- \\
\hline \\
1 \\
$Q$ \\
$Q$ \\
$Q$
\end{tabular}

End Use Not Reported

11

NF

RSE Column Factors:

TOTAL INPUTS

Boiler Fuel

Total Process Uses

Process Cooling and Refrigeration . . . . . . . . .

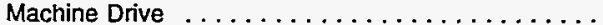

Electro-Chemical Processes $\ldots \ldots \ldots \ldots \ldots \ldots \ldots$

Other Process Use . . . . . . . . . . . . . . . . . .

Total Non-Process Uses . . . . . . . . . . . . . . . . . . Facility Heating, Ventilation, and Air Conditioning ' $\ldots$

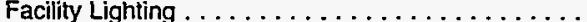

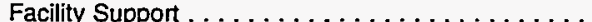
Onsite Transportation . . . . . . . . . . . . Conventional Electricity Generation . . . . . . . . . .

Other Non-Process Use

End Use Not Reported

ELECTRONIC and OTHER ELECTRIC EQUIPMENT

\footnotetext{
TOTAL INPUTS

Column Factors:

Boiler Fuel

Total Process Uses . . . . . . . . . . . . . . . . .

Process Heating . . . . . . . . . . . . . . .

Process Cooling and Refrigeration ............

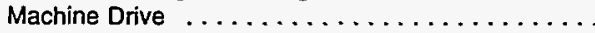

Electro-Chemical Processes ..............

Other Process Use

Total Non-Process Uses . . . . . . . . . . . . . . Facility Heating, Ventilation, and Air Conditioning '

Facility Lighting

Facility Support . . . . . . . . . . . . . . .

Onsite Transportation

Other Non-Process Use . . . . . . . . . . . . . .

End Use Not Reported
} 
Table A36. Total Inputs of Energy for Heat, Power, and Electricity Generation by Fuel Type, Industry Group, Selected Industries, and End Use, 1991: Part 2 (Continued) (Estimates in Trillion Btu)

\begin{tabular}{|c|c|c|c|c|c|c|c|c|c|c|}
\hline $\begin{array}{l}\text { SIC } \\
\text { Code }^{\circ}\end{array}$ & End-Use Categories & Total & $\begin{array}{c}\text { Net } \\
\text { Electricity" }\end{array}$ & $\begin{array}{l}\text { Residual } \\
\text { Fuel Oil }\end{array}$ & $\begin{array}{c}\text { Distillate } \\
\text { Fuel Oil } \\
\text { and Diesel } \\
\text { Fuel }^{\circ}\end{array}$ & $\begin{array}{l}\text { Natural } \\
\text { Gas }^{d}\end{array}$ & LPG & $\begin{array}{l}\text { Coal } \\
\text { (excluding } \\
\text { Coal Coke } \\
\text { and } \\
\text { Breeze) }\end{array}$ & Othere & $\begin{array}{c}\text { RSE } \\
\text { Row } \\
\text { Factor }\end{array}$ \\
\hline
\end{tabular}

37

TRANSPORTATION EQUIPMENT

RSE Column Factors:

TOTAL INPUTS

Boller Fuel

Total Process Uses . . . . . . . . . . . . . . .

Process Heating ......................

Process Cooling and Refrigeration .............

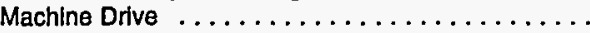

Electro-Chemical Processes $\ldots \ldots \ldots \ldots \ldots \ldots$

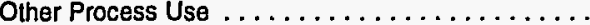

Total Non-Process Uses .................

Facllity Heating, Ventilation, and Air Conditioning ' ..

Facility Llghting $\ldots \ldots \ldots \ldots \ldots \ldots \ldots \ldots \ldots$

Faciltty Support. .

Conventional Electricity Generation ............

Olher Non-Process Use ...................

End Use Not Reported

333

68

10

49

2

$\begin{array}{rr}-. & 2 \\ -- & 43\end{array}$

20

$-$

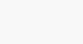

39

Motor Vehicles and Car Bodies

RSE Column Factors:

TOTAL INPUTS

Boiler Fuel

NF

105

Total Process Uses . . . . . . . . . . . . . . . . . .

Process Heating $\ldots \ldots \ldots \ldots \ldots \ldots \ldots \ldots$

Process Cooling and Refrigeration . . . . . . . . . .

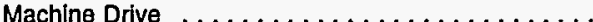

Electro-Chemical Processes..$\ldots \ldots \ldots \ldots \ldots \ldots$.

Other Process Use ....................

Total Non-Process Uses . ..................

Facilly Heating, Ventilation, and Air Conditioning ' . .

Facllity Lighting $\ldots \ldots \ldots \ldots \ldots \ldots \ldots \ldots \ldots \ldots$

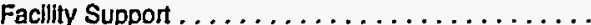

Onsite Transportation $\ldots \ldots \ldots \ldots \ldots \ldots \ldots$.

Conventional Electricity Generation .............

Other Non-Process Use

End Use Not Reported

Motor Vehicle Parts and Accessories

RSE Column Factors:

TOTAL INPUTS

Boller Fuel

Total Process Uses

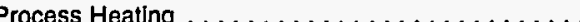

Process Cooling and Refrigeration ............

Machine Drive ..........................

Electro-Chemical Processes $\ldots \ldots \ldots \ldots \ldots \ldots$

Other Process Use . . . . . . . . . . . . . . . .

Total Non-Process Uses .................

Facility Heating, Ventilation, and Air Conditioning ' . .

Facillty Lighting $\ldots \ldots \ldots \ldots \ldots \ldots \ldots \ldots$

Facillty Support

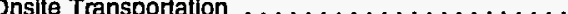

Conventional Electricity Generation .............

Other Non-Process Use ....................

End Use Not Reported$$
7
$$

$\begin{array}{r}0.5 \\ 118 \\ 1 \\ 68 \\ 10 \\ 5 \\ 49 \\ 2 \\ 2 \\ 43 \\ 20 \\ 18 \\ 4 \\ - \\ \hline 1\end{array}$

\begin{tabular}{c}
1.4 \\
12 \\
$W$ \\
$\vdots$ \\
0 \\
$Q$ \\
\hdashline \\
$W$ \\
\hline- \\
$W$ \\
- \\
$W$ \\
$W$ \\
$Q$
\end{tabular}

1.3
7
2
1
$W$
0
1
-
$W$
4
$W$
$\because-$
2
$W$
1
.

0.7

$\begin{array}{rrr}1.5 & 1.1 & \text { NF } \\ 2 & 33 & 28\end{array}$

5.1

48

53

49

2

$\overline{1}$

28

25

2

-

3

- $w$

$-\quad 7.0$$$
\overline{\mathrm{Q}}
$$$$
\begin{array}{rrrr}
0 & 0 & . . & 7.9 \\
. & 0 & \ldots & 10.4 \\
. . & 0 & . . & 6.4
\end{array}
$$$$
\begin{array}{llll}
. & . . & 6.4 \\
* & 0 & . . & 8.3
\end{array}
$$$$
\begin{array}{llll} 
& 0 & .- & 8.3 \\
1 & - & . . & 8.1
\end{array}
$$$$
\begin{array}{rrrr}
- & 0 & -- & 14.4 \\
- & 0 & -- & 15.2
\end{array}
$$

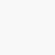

\begin{tabular}{|c|c|c|c|}
\hline 1.1 & 1.1 & $\mathrm{NF}$ & \\
\hline * & $w$ & $w$ & 3.0 \\
\hline • & $w$ & -- & 4.7 \\
\hline • & 0 & -- & 4.5 \\
\hline - & 0 & -- & 5.0 \\
\hline 0 & 0 & -. & 9.6 \\
\hline • & 0 & -- & 8.8 \\
\hline -- & -- & -- & 7.4 \\
\hline • & 0 & -- & 9.7 \\
\hline • & 0 & -- & 3.8 \\
\hline$*$ & 0 & -- & 5.6 \\
\hline -- & -. & .. & 5.5 \\
\hline$*$ & 0 & -. & 7.3 \\
\hline * & -. & .. & 4.0 \\
\hline 0 & 0 & .. & 6.8 \\
\hline 0 & 0 & .- & 8.6 \\
\hline * & 0 & $w$ & 9.5 \\
\hline
\end{tabular}

$\begin{array}{ccc}1.8 & 1.2 & 0.7 \\ 3 & * & 45 \\ W & * & 11 \\ W & W & 24 \\ W & : & 23 \\ 0 & 0 & \vdots \\ 0 & W & 1 \\ - & \because & : \\ 0 & : & 9 \\ W & : & 8 \\ W & W & - \\ - & - & 1 \\ 0 & W & 0 \\ -- & : & 0 \\ 0 & : & \\ 0 & * & \\ 0 & W & \end{array}$

\begin{tabular}{|c|c|c|c|c|c|c|c|}
\hline NF & 0.4 & 2.0 & 1.2 & 0.7 & 1.5 & 1.0 & $\mathrm{NF}$ \\
\hline 99 & 37 & * & 1 & 41 & 1 & $w$ & $w$ \\
\hline -- & - & * & $w$ & 12 & $w$ & $w$ & -- \\
\hline .- & 25 & * & * & 19 & . & $w$ & -- \\
\hline -- & 3 & - & $w$ & 18 & * & $w$ & -- \\
\hline .- & 2 & 0 & 0 & • & 0 & 0 & -. \\
\hline -. & 20 & $Q$ & $w$ & • & • & 0 & -- \\
\hline .- & . & - & -- & -- & -. & - & -. \\
\hline -- & - & $=$ & $Q$ & * & 0 & 0 & -. \\
\hline .- & 10 & * & 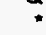 & 9 & 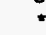 & 0 & .. \\
\hline -. & 5 & • & $w$ & 9 & $Q$ & 0 & -- \\
\hline .- & 4 & -- & .- & -- & .. & -. & -. \\
\hline .. & 1 & 0 & * & - & * & 0 & -- \\
\hline -- & $w$ & -- & $w$ & * & * & - & -. \\
\hline -- & -. & 0 & $\because$ & • & 0 & 0 & .. \\
\hline -- & $w$ & * & • & • & $\star$ & 0 & -. \\
\hline$w$ & 1 & . & $w$ & 1 & $w$ & 0 & $w$ \\
\hline
\end{tabular}


Table A36. Total Inputs of Energy for Heat, Power, and Electricity Generation by Fuel Type, Industry Group, Selected Industries, and End Use, 1991: Part 2 (Continued)

(Estimates in Trillion Btu)

\begin{tabular}{|c|c|c|c|c|c|c|c|c|c|c|}
\hline $\begin{array}{l}\text { SIC } \\
\text { Code }\end{array}$ & End-Use Categories & Total & $\begin{array}{c}\text { Net } \\
\text { Electricity }\end{array}$ & $\begin{array}{l}\text { Residual } \\
\text { Fuel Oil }\end{array}$ & $\begin{array}{c}\text { Distillate } \\
\text { Fuel Oil } \\
\text { and Diesel } \\
\text { Fuel }^{\circ}\end{array}$ & $\begin{array}{c}\text { Natural } \\
\text { Gas }^{d}\end{array}$ & LPG & $\begin{array}{c}\text { Coal } \\
\text { (excluding } \\
\text { Coal Coke } \\
\text { and } \\
\text { Breeze) }\end{array}$ & Other & $\begin{array}{l}\text { RSE } \\
\text { Row } \\
\text { Factors }\end{array}$ \\
\hline
\end{tabular}

38 INSTRUMENTS and RELATED PRODUCTS

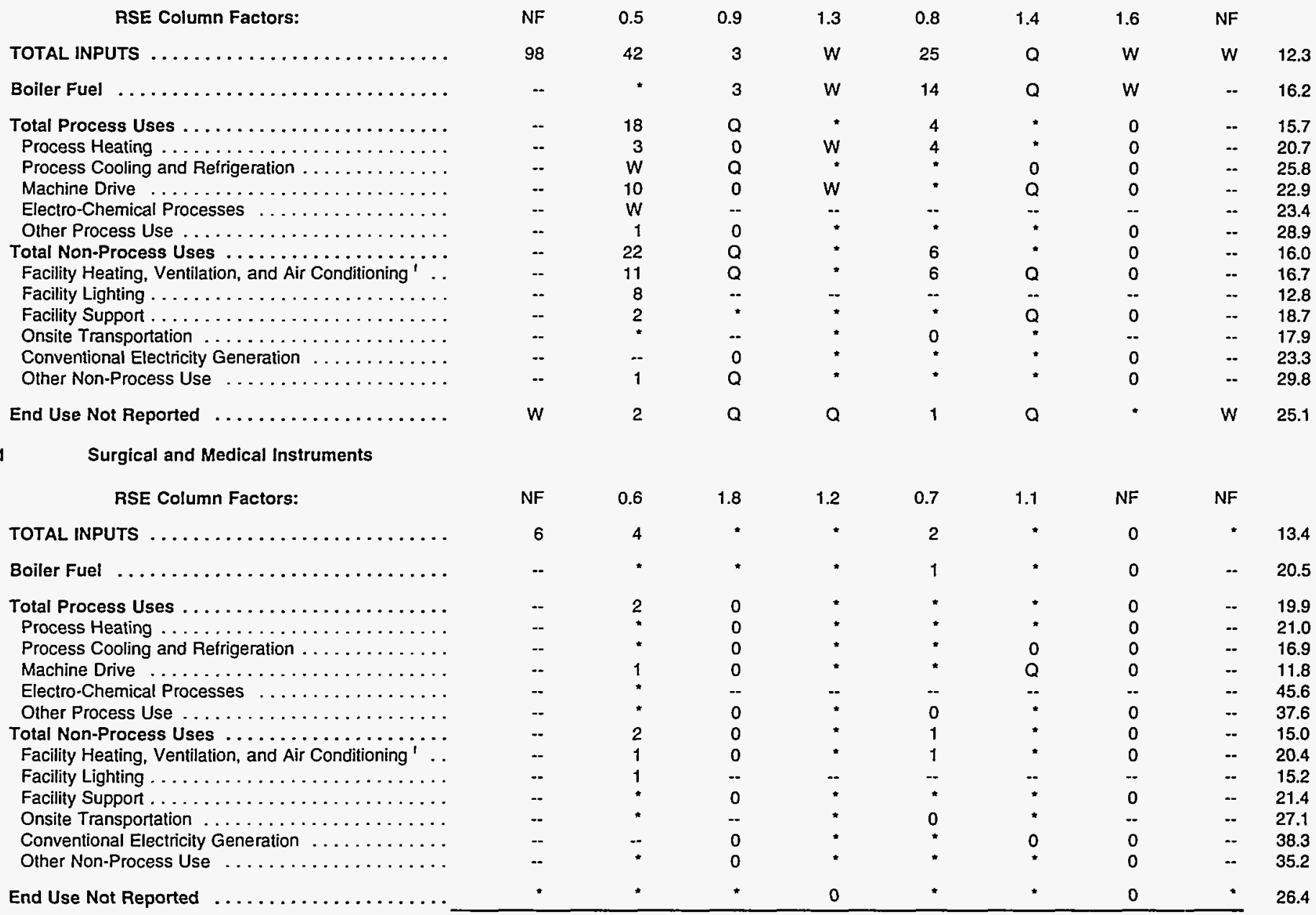

See footnotes at end of table. 
Table A36. Total Inputs of Energy for Heat, Power, and Electricity Generation by Fuel Type, Industry Group, Selected Industries, and End Use, 1991: Part 2 (Continued) (Estimates in Trillion Btu)

\begin{tabular}{|c|c|c|c|c|c|c|c|c|c|c|}
\hline $\begin{array}{c}\text { SIC } \\
\text { Code* }\end{array}$ & End-Use Categories & Total & $\begin{array}{c}\text { Net } \\
\text { Electricityb }\end{array}$ & $\begin{array}{l}\text { Residual } \\
\text { Fuel Oil }\end{array}$ & $\begin{array}{c}\text { Distillate } \\
\text { Fuel Oil } \\
\text { and Diesel } \\
\text { Fuef }\end{array}$ & $\begin{array}{c}\text { Natural } \\
\text { Gas }^{d}\end{array}$ & LPG & $\begin{array}{c}\text { Coal } \\
\text { (excluding } \\
\text { Coal Coke } \\
\text { and } \\
\text { Breeze) }\end{array}$ & Other & $\begin{array}{c}\text { RSE } \\
\text { Row } \\
\text { Factors }\end{array}$ \\
\hline
\end{tabular}

RSE Column Factors:

TOTAL INPUTS

Boller Fuel

Total Process Uses $\ldots \ldots \ldots \ldots \ldots \ldots \ldots \ldots \ldots$

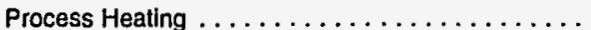

Process Cooling and Refrigeration .............

Machine Drive $\ldots \ldots \ldots \ldots \ldots \ldots \ldots \ldots \ldots$

Electro-Chemical Processes $\ldots \ldots \ldots \ldots \ldots \ldots$

Other Process Use ....................

Total Non-Process Uses ................. Facility Heating, Ventilation, and Air Conditioning '

Facility Lighting . . . . . . . . . . . . . . . . . . .

Facility Support .......................

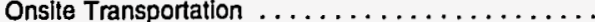

Conventional Electricity Generation $\ldots \ldots \ldots \ldots$

Other Non-Process Use .................

End Use Not Reported

\begin{tabular}{|c|c|}
\hline NF & 0.5 \\
\hline 31 & 12 \\
\hline -. & * \\
\hline .. & 7 \\
\hline -- & 2 \\
\hline -- & 1 \\
\hline -- & 5 \\
\hline -- & • \\
\hline -- & * \\
\hline -- & 4 \\
\hline -- & 2 \\
\hline -- & 2 \\
\hline -- & * \\
\hline-- & " \\
\hline -. & -- \\
\hline-- & * \\
\hline$w$ & 1 \\
\hline
\end{tabular}

1.3
W
W
Q
0
$:$
$:$
$:$
:
$:$

$\begin{array}{cc}0.7 & 1.0 \\ 15 & \\ 5 & \\ 5 & \\ 5 & \\ : & \\ - & \\ 4 & \\ 3 & \\ : & \\ : & \\ : & \\ 0 & \\ 1 & \end{array}$

\begin{tabular}{|c|c|}
\hline 1.0 & 1.8 \\
\hline$w$ & 1 \\
\hline$w$ & 1 \\
\hline * & 0 \\
\hline * & 0 \\
\hline 0 & 0 \\
\hline & 0 \\
\hline-- & -- \\
\hline & 0 \\
\hline " & $Q$ \\
\hline$Q$ & $Q$ \\
\hline$\overline{-}$ & -- \\
\hline & 0 \\
\hline 0 & 0 \\
\hline 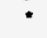 & 0 \\
\hline * & 0 \\
\hline
\end{tabular}

$\begin{array}{ll}\text { NF } & \\ W & 13.3 \\ -- & 20.9 \\ -. & 18.7 \\ - & 20.0 \\ - & 33.4 \\ - & 29.7 \\ - & 68.1 \\ - & 55.9 \\ - & 20.5 \\ - & 23.6 \\ - & 17.0 \\ - & 30.6 \\ - & 20.1 \\ - & 28.9 \\ - & 39.6 \\ W & 36.7\end{array}$

- See Appendices B and F for descriptions of the Standard Industrial Classification system.

b "Net Electricity" is obtained by summing purchases, transfers in, and generation from noncombustible renewable resources, minus quantities sold and transferred out.

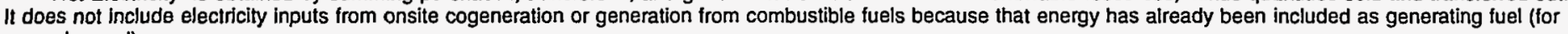
example, coal).

Includes Nos. 1,2, and 4 fuel oils and Nos. 1,2, and 4 diesel fuels.

"Natural Gas" includes natural gas obtained from utilities, transmission pipelines, and any other supplier(s) such as brokers and producers.

- "Other" includes net steam (the sum of purchases, generation from renewables, and net transfers) and other energy that respondents indicated was used to produce heat and power.

'Excludes steam and hot water.

NF $=$ No applicable RSE row/column factor.

- Estimate less than 0.5. Data are included in higher level totals.

$W=$ Withheld to avoid disclosing data for individual establishments. Data are included in higher level totals.

$\mathrm{Q}=$ Withheld because Relative Standard Error is greater than 50 percent. Data are included in higher level totals.

.- Estimation of energy input is not applicable.

$N A=N o t$ avallable. Data are included in higher level totals.

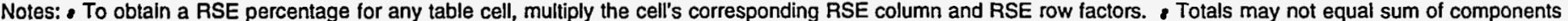
because of independent rounding. - The estimates presented in this table are for the total consumption of energy for the production of heat and power, regardless of

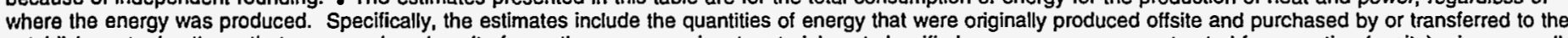

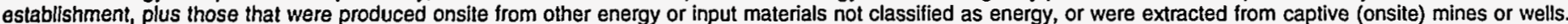
- Allocations to specific end uses are made on the basis of reasonable approximations by respondents.

Source: Energy Information Administration, Otfice of Energy Markets and End Use, Energy End Use and Integrated Statistics Division, Form ElA-846, "1991 Manufacturing Energy Consumption Survey." 
Table A37. Total Inputs of Energy for Heat, Power, and Electricity Generation by Fuel Type, Census Region, and End Use, 1991: Part 1

(Estimates in Btu or Physical Units)

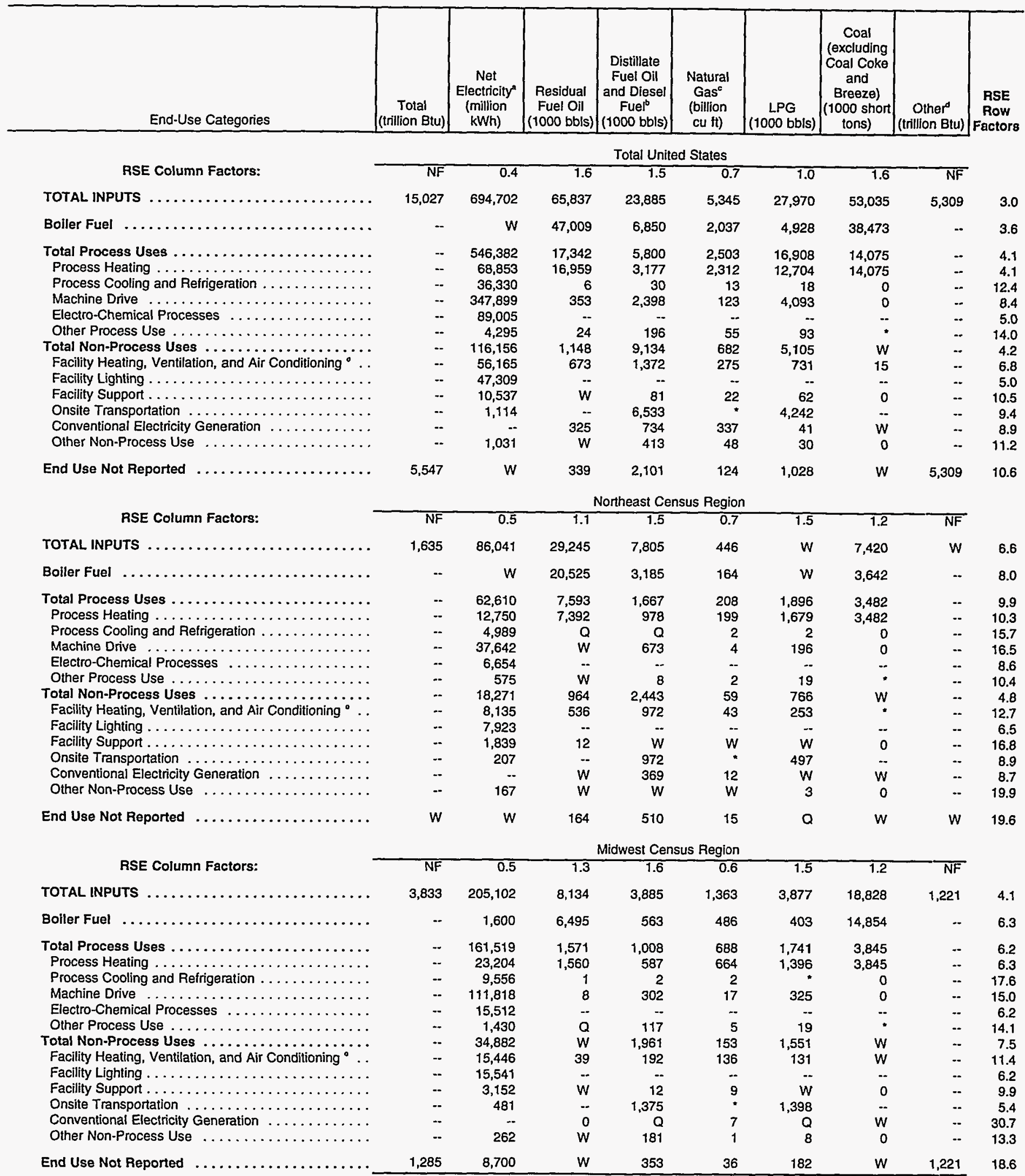

See footnotes at end of table. 
Table A37. Total Inputs of Energy for Heat, Power, and Electricity Generation by Fuel Type, Census Region, and End Use, 1991: Part 1 (Continued)

(Estimates in Btu or Physical Units)

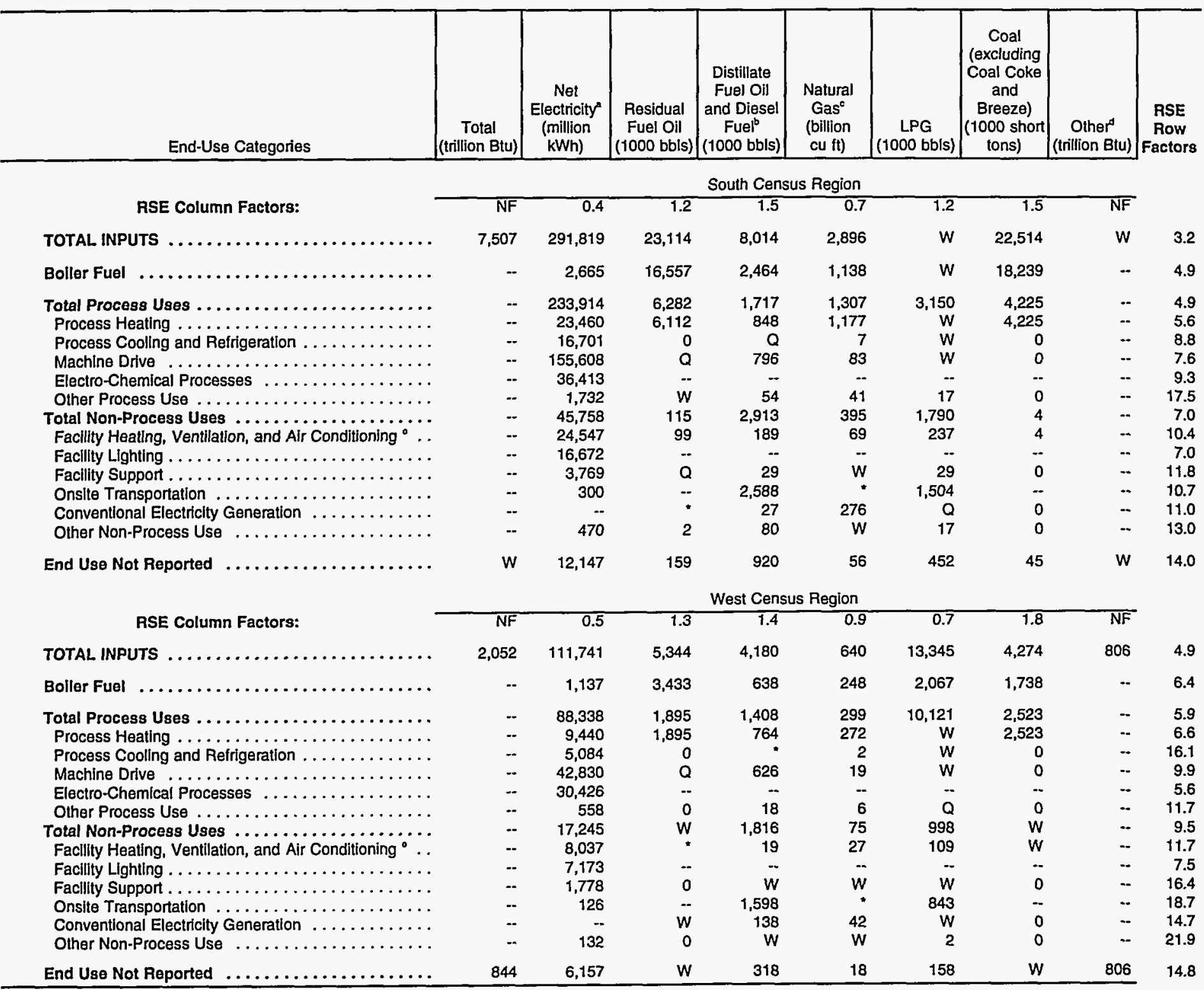

- "Net Electricity" is obtained by summing purchases, transfers in, and generation from noncombustible renewable resources, minus quantities sold and transferred out.

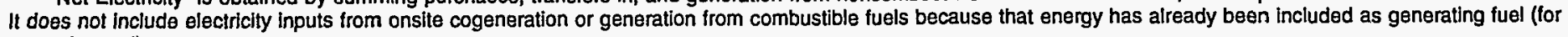
example, coal).

Includes Nos. 1, 2, and 4 fuel oils and Nos. 1, 2, and 4 diesel fuels.

c "Natural Gas" includes natural gas obtained from utilities, transmission pipelines, and any other supplier(s) such as brokers and producers.

a "Other" includes net steam (the sum of purchases, generation from renewables, and net transfers) and other energy that respondents indicated was used to produce heat and power.

- Excludes steam and hot water.

NF=No appllcable RSE row/column factor.

Estimate less than 0.5 . Data are included in higher level totals.

$W=W i t h h e l d$ to avold disclosing data for individual establishments. Data are included in higher level totals.

$Q=$ Withheld because Relative Standard Error is greater than 50 percent. Data are included in higher level totals.

- Estlmatlon of energy input is not applicable.

$\mathrm{NA}=$ Not available. Data are included in higher level totals.

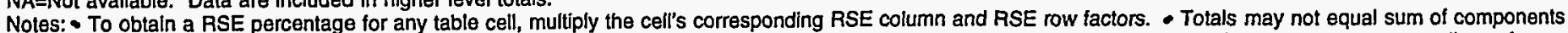
because of independent rounding. The estimates presented in this table are for the total consumption of energy for the production of heat and power, regardless of

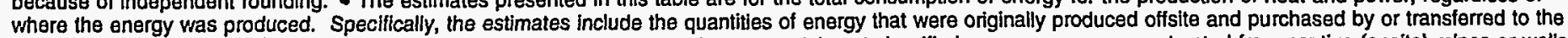

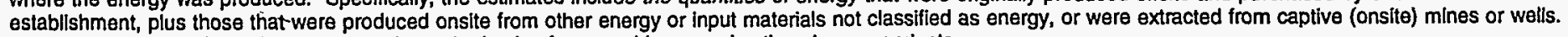
- Allocatlons to specific end uses are made on the basis of reasonable approximations by respondents.

Source: Energy Information Administration, Office of Energy Markets and End Use, Energy End Use and Integrated Statistics Division, Form ElA-846. "1991 Manufacturing Energy Consumption Survey." 
Table A37. Total Inputs of Energy for Heat, Power, and Electricity Generation by Fuel Type, Census Region, and End Use, 1991: Part 2 (Estimates in Trillion Btu)

\begin{tabular}{|c|c|c|c|c|c|c|c|c|c|}
\hline End-Use Categories & Total & $\begin{array}{c}\text { Net } \\
\text { Electricity" }\end{array}$ & $\begin{array}{l}\text { Residual } \\
\text { Fuel Oil }\end{array}$ & $\begin{array}{c}\text { Distillate } \\
\text { Fuel Oil } \\
\text { and Diesel } \\
\text { Fuel }^{\mathrm{b}}\end{array}$ & $\begin{array}{c}\text { Natural } \\
\text { Gas }^{\circ}\end{array}$ & LPG & $\begin{array}{c}\text { Coal } \\
\text { (excluding } \\
\text { Coal Coke } \\
\text { and } \\
\text { Breeze) } \\
\end{array}$ & Other & $\begin{array}{c}\text { RSE } \\
\text { Row } \\
\text { Factors }\end{array}$ \\
\hline & \multicolumn{9}{|c|}{ Total United States } \\
\hline RSE Column Factors: & NF & 0.4 & 1.6 & 1.5 & 0.7 & 1.0 & $\overline{1.6}$ & NF & \\
\hline TOTAL INPUTS $\ldots \ldots \ldots \ldots \ldots$ & 15,027 & 2,370 & 414 & 139 & 5,506 & 105 & 1,184 & 5,309 & 3.0 \\
\hline Boiler Fuel $\ldots \ldots \ldots$ & $-\cdot$ & w & 296 & 40 & 2,098 & 18 & 859 & -- & 3.6 \\
\hline Total Process Uses. & -- & 1,864 & 109 & 34 & 2,578 & 64 & 314 & -- & 4.1 \\
\hline Process Heating ... & $-\cdot$ & 235 & 107 & 19 & 2,382 & 49 & 314 & -. & 4.1 \\
\hline Process Cooling and Refrigeration & -- & 124 & * & $\therefore$ & 13 & 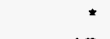 & 0 & -- & 12.4 \\
\hline Machine Drive $\ldots \ldots \ldots \ldots \ldots$ & -. & 1,187 & 2 & 14 & 127 & 15 & 0 & -- & 8.4 \\
\hline Electro-Chemical Processes ... & -- & 304 & -- & -- & -- & -- & -- & -- & 5.0 \\
\hline Other Process Use . . . . . . . & -- & 15 & * & 1 & 56 & * & • & -. & 14.0 \\
\hline Total Non-Process Uses & -- & 396 & 7 & 53 & 702 & 19 & w & -- & 4.2 \\
\hline Facility Heating, Ventilation, and Air Conditioning ${ }^{\circ}$ & $-\cdot$ & 192 & 4 & 8 & 283 & 3 & $\cdot$ & -- & 6.8 \\
\hline Facility Lighting $\ldots \ldots \ldots \ldots \ldots \ldots \ldots \ldots$ & -- & 161 & -- & -. & - & -- & -. & -- & 5.0 \\
\hline Facility Support $\ldots \ldots \ldots \ldots \ldots \ldots \ldots \ldots \ldots$ & -. & 36 & w & ” & 23 & * & 0 & -- & 10.5 \\
\hline Onsite Transportation $\ldots \ldots \ldots \ldots \ldots \ldots \ldots \ldots$ & -. & 4 & -. & 38 & • & 16 & -- & - & 9.4 \\
\hline Conventional Electricity Generation ......... & -- & -. & 2 & 4 & 347 & $\cdot$ & w & - & 8.9 \\
\hline Other Non-Process Use $\ldots$ & - & 4 & w & 2 & 49 & * & 0 & -- & 11.2 \\
\hline \multirow[t]{2}{*}{ End Use Not Reported . } & 5,547 & $w$ & 2 & 12 & 128 & 4 & $w$ & 5,309 & 10.6 \\
\hline & \multicolumn{9}{|c|}{ Northeast Census Region } \\
\hline RSE Column Factors: & $\overline{N F}$ & 0.5 & 1.1 & 1.5 & 0.7 & 1.5 & 1.2 & $\overline{\mathrm{NF}}$ & \\
\hline TOTAL INPUTS $\ldots . . . \ldots$ & 1,635 & 294 & 184 & 45 & 459 & $w$ & 167 & $w$ & 6.6 \\
\hline Boiler Fuel...$\ldots$. & - & $w$ & 129 & 19 & 169 & $w$ & 82 & - & 8.0 \\
\hline Total Process Uses. & -- & 214 & 48 & 10 & 214 & 7 & 78 & - & 9.9 \\
\hline Process Heating . & -- & 44 & 46 & 6 & 205 & 6 & 78 & -. & 10.3 \\
\hline Process Cooling and Refrigeration & -. & 17 & Q & $\mathbf{Q}$ & 2 & * & 0 & .. & 15.7 \\
\hline Machine Drive $\ldots \ldots \ldots \ldots$ & -- & 128 & W & 4 & 5 & 1 & 0 & -- & 16.5 \\
\hline Electro-Chemical Processes $\ldots$ & -. & 23 & $-\cdot$ & -- & -. & -. & -- & -- & 8.6 \\
\hline Other Process Use $\ldots \ldots \ldots \ldots \ldots \ldots \ldots \ldots$ & - & 2 & W & * & 2 & • & * & - & 10.4 \\
\hline Total Non-Process Uses $\ldots \ldots \ldots \ldots \ldots \ldots$ & -. & 62 & 6 & 14 & 60 & 3 & w & -- & 4.8 \\
\hline Facility Heating, Ventilation, and Air Conditioning ${ }^{\circ} \ldots$ & - & 28 & 3 & 6 & 45 & 1 & * & -- & 12.7 \\
\hline Facility Lighting $\ldots \ldots \ldots \ldots \ldots \ldots \ldots \ldots$ & -- & 27 & $-\cdot$ & $\ddot{-}$ & $\ddot{-}$ & -- & - & -- & 6.5 \\
\hline Facility Support .... & -- & 6 & • & W & w & w & 0 & $-\cdot$ & 16.8 \\
\hline Onsite Transportation & -- & 1 & -. & 6 & $*$ & 2 & $\ddot{-}$ & -. & 8.9 \\
\hline Conventional Electricity Generation . & -- & -. & w & 2 & 12 & w & w & -. & 8.7 \\
\hline Other Non-Process Use $\ldots \ldots \ldots$ & -- & 1 & $w$ & W & w & * & 0 & -. & 19.9 \\
\hline \multirow[t]{2}{*}{ End Use Not Reported } & w & $w$ & 2 & 3 & 15 & $\mathbf{Q}$ & $w$ & $w$ & 19.6 \\
\hline & \multicolumn{9}{|c|}{ Midwest Census Region } \\
\hline RSE Column Factors: & NF & 0.5 & 1.3 & 1.6 & 0.6 & 1.5 & 1.2 & $\overline{\mathrm{NF}}$ & \\
\hline TOTAL INPUTS . & 3,833 & 700 & 51 & 23 & 1,404 & 14 & 420 & 1,221 & 4.1 \\
\hline Boiler Fuel $\quad \ldots \ldots \ldots \ldots \ldots \ldots \ldots \ldots \ldots$ & -- & 5 & 41 & 3 & 501 & 1 & 331 & -- & 6.3 \\
\hline Total Process Uses . . . . . . . . . . . & -- & 551 & 10 & 6 & 709 & 7 & 86 & - & 6.2 \\
\hline Process Heating $\ldots \ldots \ldots \ldots \ldots \ldots$ & -- & 79 & 10 & 3 & 684 & 5 & 86 & -- & 6.3 \\
\hline Process Cooling and Refrigeration . & -. & 33 & $*$ & * & 2 & : & 0 & -- & 17.6 \\
\hline Machine Drive $\ldots \ldots \ldots \ldots \ldots$ & -- & 382 & * & 2 & 17 & 1 & 0 & -- & 15.0 \\
\hline Electro-Chemical Processes & -- & 53 & $\ddot{\theta}$ & -- & $-\cdot$ & - & -- & -- & 6.2 \\
\hline Other Process Use .. & -- & 5 & Q & 1 & 6 & * & * & -. & 14.1 \\
\hline Total Non-Process Uses $\ldots \ldots \ldots \ldots \ldots \ldots$ & - & 119 & w & 11 & 158 & 6 & W & -. & 7.5 \\
\hline Facility Heating, Ventilation, and Air Conditioning ${ }^{\circ} \ldots$ & -- & 53 & * & 1 & 140 & * & w & -. & 11.4 \\
\hline Facility Lighting $\ldots \ldots \ldots \ldots \ldots \ldots \ldots \ldots$ & -- & 53 & -. & -- & -- & -- & - & -- & 6.2 \\
\hline Facility Support . & -- & 11 & W & * & 9 & w & 0 & -- & 9.9 \\
\hline Onsite Transportation $\ldots \ldots \ldots \ldots \ldots \ldots$ & - & 2 & -. & 8 & * & 5 & - & -- & 5.4 \\
\hline Conventional Electricity Generation .. & - & -- & 0 & Q & 7 & $\mathbf{Q}$ & w & -- & 30.7 \\
\hline Other Non-Process Use $\ldots \ldots \ldots \ldots$ & -- & 1 & w & 1 & 1 & * & 0 & -. & 13.3 \\
\hline End Use Not Reported & 1,285 & 24 & W & 2 & 37 & 1 & w & 1,221 & 18.6 \\
\hline
\end{tabular}

See footnotes at end of table. 
Table A37. Total Inputs of Energy for Heat, Power, and Electricity Generation by Fuel Type, Census Region, and End Use, 1991: Part 2 (Continued) (Estimates in Trillion Btu)

\begin{tabular}{|c|c|c|c|c|c|c|c|c|c|}
\hline End-Use Categories & Total & $\begin{array}{c}\text { Net } \\
\text { Electricity }\end{array}$ & $\begin{array}{c}\text { Residual } \\
\text { Fuel Oil }\end{array}$ & $\begin{array}{c}\text { Distillate } \\
\text { Fuel Oil } \\
\text { and Diesel } \\
\text { Fuel }^{b}\end{array}$ & $\begin{array}{c}\text { Natural } \\
\text { Gas }^{\circ}\end{array}$ & LPG & $\begin{array}{c}\text { Coal } \\
\text { (excluding } \\
\text { Coal Coke } \\
\text { and } \\
\text { Breeze) } \\
\end{array}$ & Other & $\begin{array}{c}\text { RSE } \\
\text { Row } \\
\text { Factors }\end{array}$ \\
\hline & \multicolumn{9}{|c|}{ South Census Region } \\
\hline RSE Column Factors: & $\mathrm{NF}$ & $\overline{0.4}$ & 1.2 & 1.5 & 0.7 & 1.2 & 1.5 & NF & \\
\hline TOTAL INPUTS $\ldots \ldots \ldots \ldots \ldots \ldots \ldots \ldots \ldots$ & 7,507 & 996 & 145 & 47 & 2,983 & $w$ & 502 & w & 3.2 \\
\hline Boiler Fuel $\ldots \ldots \ldots \ldots \ldots \ldots \ldots \ldots \ldots \ldots$ &.- & 9 & 104 & 14 & 1,172 & W & 407 & -. & 4.9 \\
\hline Total Process Uses . . . . . . . . . . . . . . . & .. & 798 & 39 & 10 & 1,346 & 11 & 94 & .. & 4.9 \\
\hline Process Heating.... & -. & 80 & 38 & 5 & 1,212 & w & 94 & -- & 5.6 \\
\hline Process Cooling and Refrigeration & -. & 57 & 0 & $\mathbf{Q}$ & 8 & W & 0 & .-. & 8.8 \\
\hline Machine Drive $\ldots \ldots \ldots \ldots \ldots$ & -- & 531 & $\mathbf{Q}$ & 5 & 85 & w & 0 & -- & 7.6 \\
\hline Electro-Chemical Processes $\ldots \ldots \ldots \ldots \ldots \ldots$ & -- & 124 & -- & - & -. & - & -. & .. & 9.3 \\
\hline Other Process Use $\ldots \ldots \ldots \ldots \ldots \ldots \ldots \ldots \ldots$ & -. & 6 & w & - & 42 & - & 0 & -- & 17.5 \\
\hline Total Non-Process Uses $\ldots \ldots \ldots \ldots \ldots \ldots \ldots \ldots$ & -. & 156 & 1 & 17 & 407 & 7 & * & -- & 7.0 \\
\hline Facillty Heatling, Ventilation, and Air Conditioning ${ }^{\circ}$. & -. & 84 & 1 & 1 & 71 & 1 & - & -. & 10.4 \\
\hline Facllity Lighting $\ldots \ldots \ldots \ldots \ldots \ldots \ldots \ldots \ldots$ & -. & 57 & -- & -. & -. & -. & -- & - & 7.0 \\
\hline Facility Support $\ldots \ldots \ldots \ldots \ldots \ldots \ldots \ldots$ & -- & 13 & $\mathbf{Q}$ & * & w & - & 0 & -. & 11.8 \\
\hline Onsite Transportation $\ldots \ldots \ldots \ldots \ldots \ldots \ldots \ldots$ & -- & 1 & -- & 15 & * & 6 & -- & .. & 10.7 \\
\hline Conventlonal Electricity Generation & -- & - & - & - & 284 & $\mathbf{Q}$ & 0 & -. & 11.0 \\
\hline Other Non-Process Use $\ldots \ldots \ldots$. & - & 2 & * & - & $w$ & 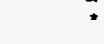 & 0 & .. & 13.0 \\
\hline \multirow[t]{2}{*}{ End Use Not Reported } & $w$ & 32 & 1 & 5 & 58 & 2 & 1 & $w$ & 14.0 \\
\hline & \multicolumn{9}{|c|}{ West Census Region } \\
\hline RSE Column Factors: & $\mathrm{NF}$ & 0.5 & 1.3 & 1.4 & 0.9 & 0.7 & 1.8 & $\overline{N F}$ & \\
\hline TOTAL INPUTS $\ldots \ldots \ldots \ldots \ldots \ldots \ldots \ldots \ldots$ & 2,052 & 381 & 34 & 24 & 659 & 51 & 95 & 806 & 4.9 \\
\hline Boller Fuel $\ldots . \ldots \ldots$ & -. & 4 & 22 & 4 & 256 & 8 & 39 & -- & 6.4 \\
\hline Total Process Uses. & -- & 301 & 12 & 8 & 308 & 39 & 56 & -- & 5.9 \\
\hline Process Heating . . . & .- & 32 & 12 & 4 & 280 & $w$ & 56 & -- & 6.6 \\
\hline Process Coolling and Refrigeration & .- & 17 & 0 & : & 2 & w & 0 & -. & 16.1 \\
\hline Machine Drive...$\ldots \ldots \ldots$. & -- & 146 & $\mathbf{Q}$ & 4 & 20 & w & 0 & -- & 9.9 \\
\hline Electro-Chemical Processes .... & -- & 104 & -. & -- & -- & -- & -. & -- & 5.6 \\
\hline Other Process Use. & -. & 2 & 0 & * & 6 & Q & 0 & .. & 11.7 \\
\hline Total Non-Process Uses . . . . . . . . . & -- & 59 & w & 11 & 77 & 4 & w & .- & 9.5 \\
\hline Facilty Heating, Ventilation, and Air Conditioning ${ }^{\circ}$. & .. & 27 & $\because$ & $\because$ & 28 & : & w & -. & 11.7 \\
\hline Facility Lighting $\ldots \ldots \ldots \ldots \ldots \ldots \ldots \ldots$ & .- & 24 & - & .- & -- & .- & .. & -- & 7.5 \\
\hline Facility Support. . & .- & 6 & 0 & w & w & w & 0 & -- & 16.4 \\
\hline Onsite Transportation & .- & : & -. & 9 & • & 3 & .. & -- & 18.7 \\
\hline Conventional Electricity Generation . . & -- & -- & W & 1 & 43 & w & 0 & -. & 14.7 \\
\hline Other Non-Process Use & -- & * & 0 & $w$ & w & • & 0 & -- & 21.9 \\
\hline End Use Not Reported $\ldots \ldots \ldots \ldots \ldots \ldots \ldots$ & 844 & 17 & $w$ & 2 & 18 & 1 & w & 806 & 14.8 \\
\hline
\end{tabular}

- "Net Electricity" is obtained by summing purchases, transfers in, and generation from noncombustible renewable resources, minus quantities sold and transferred out.

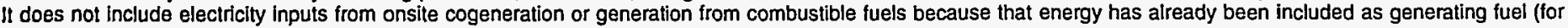
example, coal).

Includes Nos. 1, 2, and 4 fuel oils and Nos. 1, 2, and 4 diesel fuels.

c "Natural Gas" includes natural gas obtained from utilities, transmission pipelines, and any other supplier(s) such as brokers and producers.

- "Other includes net steam (the sum of purchases, generation from renewables, and net transfers) and other energy that respondents indicated was used to produce heat and power.

- Excludes steam and hot water.

NF=No appllcable RSE row/column factor.

- Estlmate less than 0.5. Data are included in higher level totals.

$W=W i t h h e l d$ to avoid disclosing data for individual establishments. Data are included in higher level totals.

$Q=$ Withheld because Relative Standard Error is greater than 50 percent. Data are included in higher level totals.

- Estlmation of energy Input is not applicable.

$N A=$ Not available. Data are included in higher level totals.

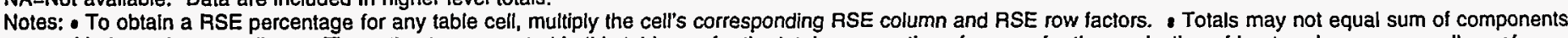
because of Independent rounding. - The estimates presented in this table are for the total consumption of energy for the production of heat and power, regardless of where the energy was produced. Specifically, the estimates inciude the quantities of energy that were originally produced offsite and purchased by or transferred to the

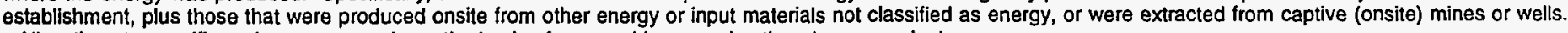
- Allocations to specific end uses are made on the basis of reasonable approximations by respondents.

Source: Energy Information Administration, Office of Energy Markets and End Use, Energy End Use and Integrated Statistics Division, Form ElA-846, "1991 Manufacturing Energy Consumption Survey." 
Table A38. Selected Combustible Inputs of Energy for Heat, Power, and Electricity Cieneration and Net Demand for Electricity by Fuel Type and End Use, 1991: Part 1

(Estimates in Btu or Physical Units)

\begin{tabular}{|c|c|c|c|c|c|c|c|c|}
\hline $\begin{array}{l}\text { SIC } \\
\text { Code }\end{array}$ & End-Use Categories & $\begin{array}{l}\text { Net Demand } \\
\text { for Electricity } \\
\text { (million kWh) }\end{array}$ & $\begin{array}{c}\text { Residual Fuel } \\
\text { Oil } \\
\text { (1000 bbls })\end{array}$ & $\begin{array}{c}\text { Distillate Fuel } \\
\text { Oil and Diesel } \\
\text { Fuel } \\
\text { (1000 bbls })\end{array}$ & $\begin{array}{l}\text { Natural Gas } \\
\text { (billion cu } \mathrm{ft} \text { ) }\end{array}$ & $\begin{array}{c}\text { LPG } \\
(1000 \mathrm{bbls})\end{array}$ & $\begin{array}{c}\text { Coal } \\
\text { (excluding } \\
\text { Coal Coke } \\
\text { and Breeze) } \\
\text { (1000 short } \\
\text { toris) }\end{array}$ & $\begin{array}{c}\text { RSE } \\
\text { Row } \\
\text { Factor8 }\end{array}$ \\
\hline
\end{tabular}

20-39 ALL. INDUSTRY GROUPS

RSE Column Factors:

TOTAL INPUTS

Boiler Fuel

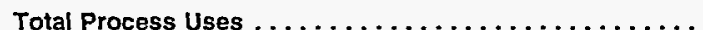

Process Heating .

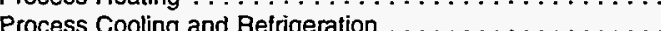

Machine Drive $\ldots \ldots \ldots \ldots \ldots \ldots \ldots$

Electro-Chemical Processes

Other Process Use

Total Non-Process Uses ......................

Facility Heating, Ventilation, and Air Conditioning ${ }^{\circ} \ldots \ldots$.

Facility Lighting . . . . . . . . . . . . . . . . . . . .

Facility Support . . . . . . . . . . . . . . . . .

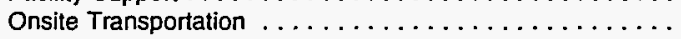

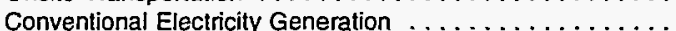

Other Non-Process Use $\ldots \ldots \ldots \ldots \ldots \ldots \ldots \ldots$

End Use Not Reported

FOOD and KINDRED PRODUCTS

RSE Column Factors:

TOTAL INPUTS

.........................

Boiler Fuel $\ldots$

Total Process Uses $\ldots \ldots \ldots \ldots \ldots \ldots \ldots \ldots \ldots \ldots$

Process Heating . .......................

Process Cooling and Refrigeration ...............

Machine Drive $\ldots \ldots \ldots \ldots \ldots \ldots \ldots \ldots \ldots$.

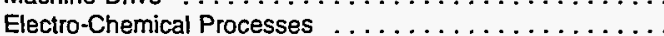

Other Process Use $\ldots \ldots \ldots \ldots \ldots \ldots \ldots \ldots \ldots \ldots \ldots$

Total Non-Process Uses . . . . . . . . . . . . . . . .

Facility Heating, Ventilation, and Air Conditioning ${ }^{\circ} . \ldots \ldots$.

Facility Lighting . . . . . . . . . . . . . . . . . . . . .

Facility Support . . . . . . . . . . . . . . . . .

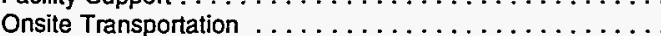

Conventional Electricity Generation . . . . . . . . . . . .

Other Non-Process Use $\ldots \ldots \ldots \ldots \ldots \ldots \ldots \ldots$

End Use Not Reported...$\ldots \ldots \ldots \ldots \ldots \ldots$

0.4
820,286
9,245
657,659
71,658
40,987
434,349
105,663
5,001
125,751
60,301
51,443
11,522
1,298
-
1,187
27,631

$\begin{array}{rr}1.7 & 1.5 \\ 65,837 & 23,885 \\ 47,009 & 6,850 \\ 17,342 & 5,800 \\ 16,959 & 3,177 \\ 6 & 30 \\ 353 & 2,398 \\ -- & -- \\ 24 & 196 \\ 1,148 & 9,134 \\ 673 & 1,372 \\ -. & - \\ W & 81 \\ -. & 6,533 \\ 325 & 734 \\ W & 413 \\ 339 & 2,101\end{array}$

0.7
5,345
2,037
2,503
2,312
13
123
-
55
682
275
.-
22
337
48
124

1.0
27,970
4,928
16,908
12,704
18
4,093
.-
93
5,105
731
--
62
4,242
41
30
1,028

1.6

$53,035 \quad 3.0$

38,473

14,075

14,075

$0 \quad 12.4$

\begin{tabular}{rr}
0 & 8.4 \\
.- & 7.7 \\
\hline
\end{tabular}

$-\quad 14.0$
$W$

W

15

4.2
6.8

5.1

9.6

9.4

8.8

10.9

10.6

$\begin{array}{rrr}0.5 & 1.5 & 1.5 \\ 55,273 & 4,317 & 2,966 \\ 1,392 & 3,875 & 1,242 \\ 43,198 & W & 270 \\ 2,141 & 260 & 212 \\ 13,366 & 0 & 15 \\ 27,580 & Q & 35 \\ Q & - & -- \\ 87 & 0 & 8 \\ 8,590 & W & 1,242 \\ 3,672 & 26 & 128 \\ 3,731 & -- & - \\ 849 & Q & 23 \\ 177 & -. & 812 \\ -. & 0 & 246 \\ 162 & - & 33 \\ 2,093 & 82 & 212\end{array}$

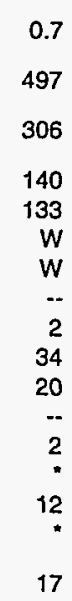

1.6
1,429
441
292
224
1
56
-0
11
598
50
-
14
533
.
0
$Q$

0.9

6,913

5.9

5.414

8.4

W 9.4

W $\quad 11.8$

17.6

17.8
NF

$\mathrm{NF}$

33.2

11.4

16.1

8.5

17.5

10.5

22.6

22.3

24.5

2011

Meat Packing Plants

RSE Column Factors:

$\begin{array}{rr}0.4 & 1.6 \\ 3,481 & 170 \\ 30 & 169 \\ 2,916 & \cdot \\ W & 0 \\ 1,774 & 0 \\ 1,072 & - \\ 0 & - \\ W & 0 \\ 417 & : \\ 174 & - \\ 201 & - \\ 41 & 0 \\ 2 & -- \\ -. & 0 \\ 0 & 0 \\ 118 & -\end{array}$

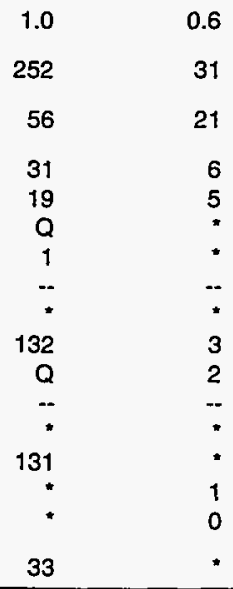

\begin{tabular}{rr}
1.4 & 1.8 \\
157 & 27 \\
91 & 27 \\
47 & 0 \\
44 & 0 \\
1 & 0 \\
2 & 0 \\
-- & - \\
0 & 0 \\
10 & 0 \\
3 & 0 \\
-- & - \\
$\mathrm{Q}$ & 0 \\
5 & - \\
0 & 0 \\
0 & 0 \\
8 & 0 \\
\hline
\end{tabular}

10.1

12.9

14.5

17.0

17.8

19.8

NF

36.3

13.2

10.2

14.0

22.9

25.9

36.2

27.6

33.4 
Table A38. Selected Combustible Inputs of Energy for Heat, Power, and Electricity Generation and Net Demand for Electricity by Fuel Type and End Use, 1991: Part 1 (Continued) (Estimates in Btu or Physical Units)

\begin{tabular}{|c|c|c|c|c|c|c|c|}
\hline $\begin{array}{c}\text { SIC } \\
\text { Code" }\end{array}$ & End-Use Categories & $\begin{array}{c}\text { Net Demand } \\
\text { for Electricity } \\
\text { (million kWh) }\end{array}$ & $\begin{array}{c}\text { Residual Fuel } \\
\text { Oil } \\
\text { (1000 bbls) }\end{array}$ & $\begin{array}{c}\text { Distillate Fuel } \\
\text { Oil and Diesel } \\
\text { Fuef } \\
(1000 \mathrm{bbls})\end{array}$ & $\begin{array}{l}\text { Natural Gas } \\
\text { (billion cu ft) }\end{array}$ & $\begin{array}{c}\text { LPG } \\
\text { (1000 bbls) }\end{array}$ & $\begin{array}{c}\text { Coal } \\
\text { (excluding } \\
\text { Coal Coke } \\
\text { and Breeze) } \\
\text { (1000 short } \\
\text { tons) }\end{array}$ \\
\hline
\end{tabular}

2033 Canned Frults and Vegetables

2037

RSE Column Factors: $\quad 0.7$

TOTALIINPUTS

Boller Fuel $\ldots \ldots \ldots \ldots \ldots \ldots \ldots \ldots \ldots \ldots \ldots \ldots$

Total Process Uses . . . . . . . . . . . . . . . . . . . .

Process Heating $\ldots \ldots \ldots \ldots \ldots \ldots \ldots \ldots \ldots \ldots \ldots$

Process Cooling and Refrigeration .............

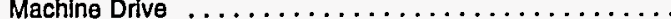

Electro-Chemical Processes . . . . . . . . . . . .

Other Process Use . . . . . . . . . . . . . . . . . . .

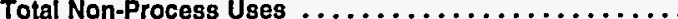

Facility Heating, Ventilation, and Air Conditioning $\bullet . . .$.

Facillty Lighting . . . . . . . . . . . . . . . . . . .

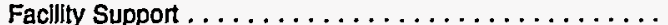

Onsite Transportation $\ldots \ldots \ldots \ldots \ldots \ldots \ldots \ldots \ldots$

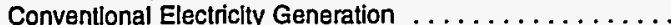

Other Non-Process Use $\ldots \ldots \ldots \ldots \ldots \ldots \ldots \ldots$

Frozen Fruits and Vegetables

RSE Column Factors:

TOTAL INPUTS

Boiler Fuel $\ldots . \ldots \ldots \ldots \ldots \ldots \ldots \ldots \ldots \ldots$

Total Process Uses . . . . . . . . . . . . . . . . .

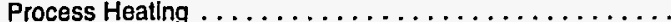

Process Cooling and Refrigeration . . . . . . . . . . . .

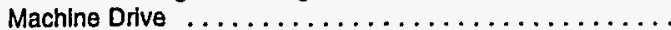

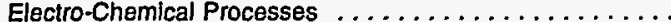

Other Process Use $\ldots \ldots \ldots \ldots \ldots \ldots \ldots \ldots \ldots$

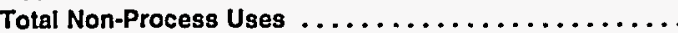

Facility Heating, Ventilation, and Air Conditioning ${ }^{\circ} . \ldots \ldots$.

Facllty Llghting . . . . . . . . . . . . . . . . . . .

Facility Suppont.

Onsite Transportation .............

Conventional Electricity Generation ................

Other Non-Process Use . . . . . . . . . . . . . . . . .

End Use Not Reported
0.7
1,724

25

1,369

28

415

921

1
4

280

101

132

9

50

$\begin{array}{rr}0.7 & 1 \\ 3,205 & 321 \\ 248 & 25 \\ 2,430 & 62 \\ 151 & 6 \\ 1,509 & \\ 760 & \\ 2 & \\ 7 & \\ 375 & \\ 165 & \\ 171 & \\ 28 & \\ 9 & \\ - & \\ 1 & \\ 153 & \end{array}$

289
1.1

290

0

$\begin{array}{ll}0 & 0 \\ 0 & \end{array}$

- 7

$\begin{array}{ll}0 & 0\end{array}$

$\begin{array}{rr}0 & 70 \\ 0 & 2\end{array}$

o

- W

0

Q

1.2

321

259

62

62

0

Q

$0 \quad 0$

$0 \quad 30$

$\begin{array}{llll}0 & - & - & 1 \\ 0 & 0 & -\end{array}$

- 24

02

Q

Wet Corn Milling

RSE Column Factors:

0.7

1.1

1.1

0.8

1.4

1.1

TOTAL INPUTS

5,820

29

30

3,051

11.1

261

29

W

12.5

Total Process Uses

Process Heating $\ldots \ldots \ldots \ldots \ldots \ldots \ldots \ldots \ldots \ldots \ldots$

Process Cooling and Refrigeration . . . . . . . . . . .

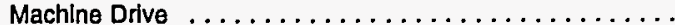

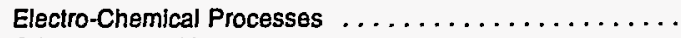

Other Process Use . . . . . . . . . . . . . . . . . . .

Total Non-Process Uses .....................

Facillty Heating, Ventliation, and Air Conditioning ${ }^{\circ} . . . \ldots$.

Facllity Llghting . . . . . . . . . . . . . . . . . . . . . .

Facility Support .

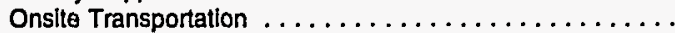

Conventlonal Electricity Generation .................

Other Non-Process Use

5,383

$W$
34

5,307

$\begin{gathered}0 \\ W\end{gathered}$

$W$
176

72

69

W

$\bar{w}$

0

End Use Not Reported

0

0 
Table A38. Selected Combustible Inputs of Energy for Heat, Power, and Electricity Generation and Net Demand for Electricity by Fuel Type and End Use, 1991: Part 1 (Continued) (Estimates in Btu or Physical Units)

\begin{tabular}{|c|c|c|c|c|c|c|c|c|}
\hline $\begin{array}{c}\text { SIC } \\
\text { Code" }\end{array}$ & End-Use Categories & $\begin{array}{l}\text { Net Demand } \\
\text { for Electricity } \\
\text { (million kWh) }\end{array}$ & $\begin{array}{c}\text { Residual Fuel } \\
\text { Oil } \\
(1000 \text { bbls })\end{array}$ & $\begin{array}{c}\text { Distillate Fuel } \\
\text { Oil and Diesel } \\
\text { Fue }{ }^{\circ} \\
(1000 \text { bbls })\end{array}$ & $\begin{array}{l}\text { Natural Gas } \\
\text { (billion cu ft) }\end{array}$ & $\begin{array}{c}\text { LPG } \\
(1000 \mathrm{bbls})\end{array}$ & $\begin{array}{c}\text { Coal } \\
\text { (excluding } \\
\text { Coal Coke } \\
\text { and Breeze) } \\
\text { (1000 short } \\
\text { tons) }\end{array}$ & $\begin{array}{c}\text { RSE } \\
\text { Row } \\
\text { Factors }\end{array}$ \\
\hline
\end{tabular}

2051 Bread, Cake, and Related Products

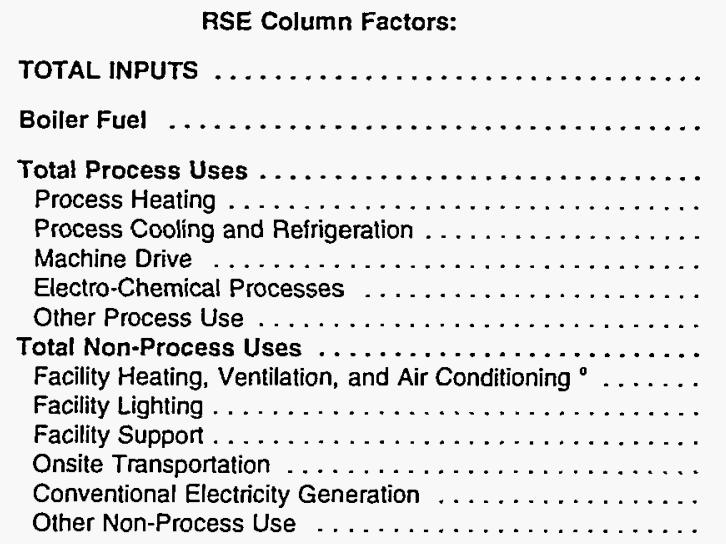

$\begin{array}{rrr}0.5 & 1.7 & 1 . \\ 2,272 & * & 13 \\ 38 & * & 4 \\ 1,602 & 0 & 4 \\ 150 & 0 & W \\ 421 & 0 & \\ 1,028 & 0 & \\ 0 & -- & - \\ 2 & 0 & W \\ 529 & 0 & 38 \\ 210 & 0 & W \\ 245 & -. & - \\ 53 & 0 & 20 \\ 0 & -. & W \\ - & 0 & 9 \\ 5 & 0 & \end{array}$

\begin{tabular}{|c|c|c|c|}
\hline 1.1 & 0.7 & 1.5 & NF \\
\hline 131 & 22 & 23 & 0 \\
\hline 41 & 5 & 3 & 0 \\
\hline 44 & 14 & 10 & 0 \\
\hline$w$ & 13 & 10 & 0 \\
\hline 0 & * & 0 & 0 \\
\hline * & " & $*$ & 0 \\
\hline -- & -. & -. & -- \\
\hline$w$ & * & * & 0 \\
\hline 38 & 2 & 9 & 0 \\
\hline$w$ & 2 & 3 & 0 \\
\hline- & -- & -- & -- \\
\hline • & * & • & 0 \\
\hline 20 & * & 6 & -- \\
\hline$w$ & $\star$ & * & 0 \\
\hline • & * & 0 & 0 \\
\hline 9 & 1 & 2 & 0 \\
\hline
\end{tabular}

$\begin{array}{rr}0.9 & 1.6 \\ 848 & W \\ 13 & W \\ 744 & 104 \\ W & 104 \\ 1 & 0 \\ W & 0 \\ 0 & -- \\ 0 & 0 \\ 90 & W \\ 33 & W \\ 48 & -- \\ 9 & 0 \\ * & - \\ -- & 0 \\ 0 & 0 \\ 0 & 0\end{array}$

TOTAL INPUTS

$\begin{array}{rr}1.1 & 0 \\ 30 & 1 \\ W & 1 \\ W & \\ 0 & \\ W & \\ -- \\ 0 \\ 23 \\ 0 \\ -. \\ 0 \\ 23 \\ 0 \\ 0 \\ .\end{array}$

$\begin{array}{rrrr}0.9 & 1.1 & 0.7 & \\ 18 & 5 & 1,901 & 5.6 \\ 12 & 0 & 1,590 & 9.6 \\ 6 & 1 & 311 & 7.2 \\ 6 & * & 311 & 10.2 \\ 0 & 0 & 0 & 34.6 \\ * & 1 & 0 & 8.1 \\ -* & -- & -- & N F \\ 0 & 0 & 0 & N F \\ * & 5 & 0 & 6.5 \\ * & 1 & 0 & 8.7 \\ -- & -- & -. & 5.8 \\ * & 1 & 0 & 9.9 \\ 0 & 2 & -. & 8.7 \\ 0 & 0 & 0 & N F \\ 0 & * & 0 & 14.5 \\ 0 & * & 0 & 15.2\end{array}$

2075

Soybean Oil Mills

RSE Column Factors:

TOTAL INPUTS

$\begin{array}{rr}0.8 & 1 \\ 1,910 & 4 \\ W & 4 \\ 1,605 & \\ 22 & \\ 27 & \\ 1,555 & \\ 0 & \\ 0 & \\ 117 & \\ 50 & \\ 61 & \\ 7 & \\ * & \\ 0 & \end{array}$

\begin{tabular}{c}
1.5 \\
42 \\
42 \\
$\cdot$ \\
0 \\
0 \\
- \\
0 \\
0 \\
$-\cdot$ \\
0 \\
\hdashline \\
0 \\
$*$ \\
0
\end{tabular}

$\begin{array}{ll}1.1 & 0.7 \\ \text { W1 } & 24 \\ W & 17 \\ \text { W } & \\ - & \\ 0 & \\ 8 & \\ 0 & \\ - & \\ 8 & \\ 0 & \\ *\end{array}$

0.8

592

Boiler Fuel

Total Process Uses . . . . . . . . . . . . . . . . .

Process Heating . . . . . . . . . . . . . . . . . . . .

Process Cooling and Refrigeration ...............

Machine Drive

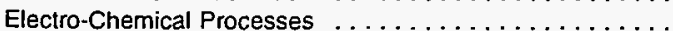

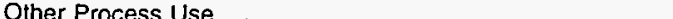

Total Non-Process Uses . . . . . . . . . . . . . . .

Facility Heating, Ventilation, and Air Conditioning ${ }^{\circ} . \ldots \ldots$

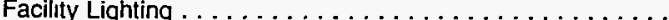

Facility Support $\ldots \ldots \ldots \ldots \ldots \ldots \ldots \ldots \ldots \ldots$

Onsite Transportation $\ldots \ldots \ldots \ldots \ldots \ldots \ldots \ldots \ldots$

Conventional Electricity Generation $\ldots \ldots \ldots \ldots \ldots$

End Use Not Reported

$\ldots \ldots \ldots \ldots \ldots \ldots \ldots$ 
Table A38. Selected Combustible Inputs of Energy for Heat, Power, and Electricity Generation and Net Demand for Electricity by Fuel Type and End Use, 1991: Part 1 (Continued) (Estimates in Btu or Physical Units)

\begin{tabular}{|c|c|c|c|c|c|c|c|}
\hline $\begin{array}{c}\text { SIC } \\
\text { Code* }\end{array}$ & End-Use Categories & $\begin{array}{l}\text { Net Demand } \\
\text { for Electricity } \\
\text { (million } \mathrm{kWh} \text { ) }\end{array}$ & $\begin{array}{c}\text { Residual Fuel } \\
\text { Oil } \\
\text { (1000 bbls) }\end{array}$ & $\begin{array}{c}\text { Distillate Fuel } \\
\text { Oil and Diesel } \\
\text { Fuef } \\
\text { (1000 bbls) }\end{array}$ & $\begin{array}{l}\text { Natural Gas } \\
\text { (billion } \mathrm{cu} f \mathrm{ft}\end{array}$ & $\begin{array}{c}\text { LPG } \\
\text { (1000 bbls) }\end{array}$ & $\begin{array}{c}\text { Coal } \\
\text { (excluding } \\
\text { Coal Coke } \\
\text { and Breeze) } \\
\text { (1000 short } \\
\text { tons) }\end{array}$ \\
\hline
\end{tabular}

$2082 \quad$ Malt Beverages

RSE Column Factors:

TOTAL INPUTS

$\begin{array}{rrr}0.6 & 1.3 & 1.4 \\ 2,886 & 419 & 58 \\ W & 417 & W \\ 2,196 & 0 & * \\ 70 & 0 & 0 \\ 950 & 0 & 0 \\ 1,176 & 0 & * \\ 0 & -. & - \\ 0 & 0 & 0 \\ 581 & 2 & W \\ 216 & 2 & 0 \\ 247 & - & - \\ 77 & 0 & * \\ 42 & -- & W \\ - & 0 & 0 \\ 0 & 0 & *\end{array}$

\begin{tabular}{rrrr}
0.8 & 1.0 & 1.4 & \\
22 & 8 & 706 & 10.1 \\
20 & $*$ & 706 & 13.2 \\
1 & 0 & 0 & 13.8 \\
1 & 0 & 0 & 21.8 \\
$*$ & 0 & 0 & 19.4 \\
0 & 0 & 0 & 14.5 \\
-- & -- & -- & $N F$ \\
0 & 0 & 0 & $N F$ \\
$*$ & $W$ & 1 & 14.0 \\
- & $W$ & 1 & 16.4 \\
\hline 0 & -- & -- & 10.6 \\
0 & 0 & 0 & 16.4 \\
0 & 4 & -- & 14.0 \\
0 & 0 & 0 & $N F$ \\
$*$ & 0 & 0 & $N F$ \\
& $W$ & 0 & 25.6
\end{tabular}

TOBACCO PRODUCTS

RSE Column Factors:

$\begin{array}{rr}0.9 & 0.7 \\ 1,810 & 135 \\ 5 & 135 \\ 996 & 0 \\ 22 & 0 \\ W & 0 \\ 941 & 0 \\ 0 & - \\ W & 0 \\ 809 & 0 \\ 576 & 0 \\ 213 & - \\ 19 & 0 \\ 2 & - \\ -. & 0 \\ 0 & 0 \\ 0 & 0\end{array}$

1.0
40
39
.
0
.
0
2
0
$\therefore$
0
2
0
0
0

$\begin{array}{rr}2.0 & 1.3 \\ 4 & 23 \\ 3 & W \\ 1 & W \\ 1 & W \\ . & 0 \\ . & 0 \\ 0 & - \\ \cdot & 0 \\ - & 14 \\ - & - \\ 0 & 0 \\ 0 & 14 \\ 0 & 0 \\ 0 & .\end{array}$

$\begin{array}{cc}0.7 & \\ 692 & 5.8 \\ 692 & 6.1 \\ 0 & 5.0 \\ 0 & 8.8 \\ 0 & 6.7 \\ 0 & 5.3 \\ .- & \text { NF } \\ 0 & 9.1 \\ 0 & 6.8 \\ 0 & 7.0 \\ -- & 3.4 \\ 0 & 4.3 \\ -- & 7.5 \\ 0 & \text { NF } \\ 0 & 6.3 \\ 0 & 6.3\end{array}$

TOTAL INPUTS

$\begin{array}{rrr}0.4 & 1.3 & 1.6 \\ 29,866 & 1,966 & 1,064 \\ 177 & 1,706 & 811 \\ 21,621 & 99 & 38 \\ 1,019 & W & 37 \\ 2,176 & 0 & \cdot \\ 18,260 & W & 1 \\ W & -- & - \\ W & \div & - \\ 7,336 & 28 & 173 \\ 4,238 & 28 & W \\ 2,679 & - & -- \\ 370 & - & - \\ 34 & -- & 34 \\ -- & 0 & Q \\ 14 & 0 & 1 \\ 733 & 134 & Q\end{array}$

\begin{tabular}{|c|c|c|}
\hline 0.8 & 1.2 & 1.2 \\
\hline 105 & 629 & 1,362 \\
\hline 68 & 38 & 1,334 \\
\hline 30 & 411 & $W$ \\
\hline 27 & 397 & $W$ \\
\hline - & - & 0 \\
\hline 2 & 13 & 0 \\
\hline -- & -- & -- \\
\hline 1 & 1 & 0 \\
\hline 4 & 163 & 2 \\
\hline 4 & 27 & 2 \\
\hline -- & - & -- \\
\hline • & * & 0 \\
\hline 0 & 134 & -. \\
\hline " & 1 & 0 \\
\hline " & * & 0 \\
\hline 3 & 17 & $w$ \\
\hline
\end{tabular}

Total Process Uses . . . . . . . . . . . . . . . . .

Process Heating . . . . . . . . . . . . . . . . . .

Process Cooling and Refrigeration ..............

Machine Drive ......................

Electro-Chemical Processes . . . . . . . . . . . . .

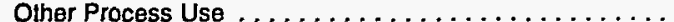

Total Non-Process Uses . . . . . . . . . . . . . . . . .

Facllity Heating, Ventilation, and Air Conditioning ${ }^{\circ} \ldots \ldots$

Facility Lighting . . . . . . . . . . . . . . . . . .

Facility Support . . . . . . . . . . . . . . . .

Onsite Transportation $\ldots \ldots \ldots \ldots \ldots \ldots \ldots \ldots$

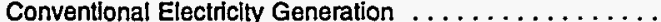

Other Non-Process Use ...................

End Use Not Reported

733 

and Net Demand for Electricity by Fuel Type and End Use, 1991: Part 1 (Continued) (Estimates in Btu or Physical Units)

\begin{tabular}{|c|c|c|c|c|c|c|c|c|}
\hline $\begin{array}{c}\text { SIC } \\
\text { Code }\end{array}$ & End-Use Categories & $\begin{array}{l}\text { Net Demand } \\
\text { tor Electricity } \\
\text { (million kWh) }\end{array}$ & $\begin{array}{c}\text { Residual Fuel } \\
\text { Oil } \\
(1000 \text { bbls })\end{array}$ & $\begin{array}{c}\text { Distillate Fuel } \\
\text { Oil and Diesel } \\
\text { Fuef } \\
\text { (1000 bbls) }\end{array}$ & $\begin{array}{l}\text { Natural Gas } \\
\text { (billion cu } \mathrm{ft} \text { ) }\end{array}$ & $\begin{array}{c}\text { LPG } \\
\text { (1000 bbls) }\end{array}$ & $\begin{array}{c}\text { Coal } \\
\text { (excluding } \\
\text { Coal Coke } \\
\text { and Breeze) } \\
\text { (1000 short } \\
\text { tons) }\end{array}$ & $\begin{array}{c}\text { RSE } \\
\text { Row } \\
\text { Faclors }\end{array}$ \\
\hline
\end{tabular}

23 APPAREL and OTHER TEXTILE PRODUCTS

RSE Column Factors:

TOTAL INPUTS

Boiler Fuel

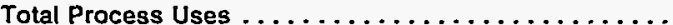

Process Heating $\ldots \ldots \ldots \ldots \ldots \ldots \ldots \ldots \ldots$

Process Cooling and Refrigeration . . . . . . . . . . .

Machine Drive

Electro-Chemical Processes . . . . . . . . . . . .

Other Process Use . . . . . . . . . . . . . . . . . . . . .

Total Non-Process Uses . . . . . . . . . . . . . . . . . . .

Facility Heating, Ventilation, and Air Conditioning ${ }^{\circ} \ldots \ldots$.

Facility Lighting . . . . . . . . . . . . . . . .

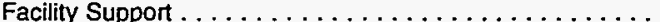

Onsite Transportation . . . . . . . . . . . . . . .

Conventional Electricity Generation $\ldots \ldots \ldots \ldots \ldots \ldots$

Other Non-Process Use

End Use Not Reported

\section{LUMBER and WOOD PRODUCTS}

RSE Column Factors:

TOTAL INPUTS

Boiler Fuel

Total Process Uses . . . . . . . . . . . . . . . . . . .

Process Heating . . . . . . . . . . . . . . . .

Process Cooling and Refrigeration . . . . . . . . . .

Machine Drive . . . . . . . . . . . . . . . .

Electro-Chemical Processes $\ldots \ldots \ldots \ldots \ldots \ldots \ldots \ldots \ldots$

Other Process Use . . . . . . . . . . . . . . . . . . .

Total Non-Process Uses . . . . . . . . . . . . . . .

Facility Heating, Ventilation, and Air Conditioning ${ }^{\circ} \ldots \ldots$.

Facility Lighting . .....................

Facility Support .....................

Onsite Transportation $\ldots \ldots \ldots \ldots \ldots \ldots \ldots \ldots \ldots \ldots$

Conventional Electricity Generation $\ldots \ldots \ldots \ldots \ldots \ldots$

Other Non-Process Use . . . . . . . . . . . . . .

End Use Not Reported

\section{FURNTUUE and FIXTURES}

RSE Column Factors:

TOTAL INPUTS

Boiler Fuel

Total Process Uses

$\ldots \ldots \ldots \ldots \ldots \ldots \ldots \ldots$

Process Cooling and Refrigeration . . . . . . . . . .

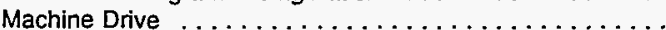

Electro-Chemical Processes ................

Other Process Use . . . . . . . . . . . . . . . . .

Total Non-Process Uses . . . . . . . . . . . . . . . .

Facility Heating, Ventilation, and Air Conditioning ${ }^{\circ} \ldots \ldots$.

Facility Lighting . . . . . . . . . . . . . . . . . .

Facility Support

Conventional Electricity Generation . . . . . . . . . .

Other Non-Process Use $\ldots \ldots \ldots \ldots \ldots \ldots \ldots \ldots \ldots \ldots$

End Use Not Reported

1.4
142
76
$Q$
$Q$
0
-
0
40
31
--
10
0
$*$
14

14

$\begin{array}{rr}0.5 & 1.3 \\ 20,549 & 333 \\ 456 & 295 \\ 16,145 & \\ 978 & 0 \\ 99 & 0 \\ 15,016 & 0 \\ 7 & - \\ 44 & 0 \\ 2,074 & 0 \\ 728 & 0 \\ 1,130 & - \\ 186 & 0 \\ 28 & - \\ -- & 0 \\ Q & 0 \\ 1,875 & 0\end{array}$

$\begin{array}{rrr}0.5 & 1.9 & 1 \\ 4,948 & 184 & 16 \\ 82 & Q & 50 \\ 3,104 & * \\ 175 & 0 \\ W & 0 & \\ 2,846 & - & \\ 22 & 0 & \\ W & 0 & \\ 1,435 & - & \\ 595 & - & \\ 699 & - & \\ 115 & 0 & \\ W & - & \end{array}$

0.7

$\begin{array}{rrr}1.3 & 1.5 & \\ 158 & 88 & 16.8 \\ 42 & 88 & 27.1 \\ 56 & 0 & 23.5 \\ 26 & 0 & 30.2 \\ 0 & 0 & 68.8 \\ Q & 0 & 38.4 \\ -- & -. & 84.5 \\ - & 0 & 38.0 \\ 48 & 0 & 19.1 \\ 35 & 0 & 22.8 \\ -- & - & 17.7 \\ 1 & 0 & 36.0 \\ 12 & - & 20.0 \\ 0 & 0 & N F \\ 0 & 0 & 33.5 \\ 13 & 0 & 30.1\end{array}$

1 
Table A38. Selected Combustible Inputs of Energy for Heat, Power, and Electricity Generation and Net Demand for Electricity by Fuel Type and End Use, 1991: Part 1 (Continued) (Estimates in Btu or Physical Units)

\begin{tabular}{|c|c|c|c|c|c|c|c|c|}
\hline$\underset{\text { Code }}{\text { SIC }}$ & End-Use Categories & $\begin{array}{l}\text { Net Demand } \\
\text { for Electricity } \\
\text { (million } \mathrm{kWh} \text { ) }\end{array}$ & $\begin{array}{c}\text { Residual Fuel } \\
\text { Oil } \\
\text { (1000 bbls) }\end{array}$ & $\begin{array}{c}\text { Distillate Fuel } \\
\text { Oil and Diesel } \\
\text { Fuel } \\
\text { (1000 bbls) }\end{array}$ & $\begin{array}{l}\text { Natural Gas } \\
\text { (billion cu ft) }\end{array}$ & $\begin{array}{c}\text { LPG } \\
\text { (1000 bb/s) }\end{array}$ & $\begin{array}{c}\text { Coal } \\
\text { (excluding } \\
\text { Coal Coke } \\
\text { and Breeze) } \\
\text { (1000 short } \\
\text { tons) }\end{array}$ & \begin{tabular}{|c} 
RSE \\
Row \\
Factors
\end{tabular} \\
\hline
\end{tabular}

RSE Column Factors:

TOTAL INPUTS

Boller Fuel

Total Process Uses . . . . . . . . . . . . . . . .

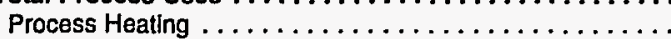

Process Cooling and Refrigeration ...............

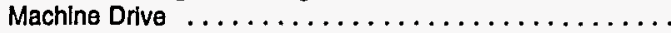

Electro-Chemical Processes $\ldots \ldots \ldots \ldots \ldots \ldots \ldots \ldots \ldots$

Other Process Use . . . . . . . . . . . . . . . . . . . . . . . .

Total Non-Process Uses . . . . . . . . . . . . . . . . .

Facllity Heating, Ventilation, and Air Conditioning ${ }^{\circ} \ldots . .$.

Facllity Lighting . . . . . . . . . . . . . . . . .

Facility Support

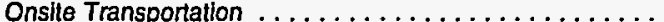

Conventional Electricity Generation $\ldots \ldots \ldots \ldots \ldots \ldots$

Other Non-Process Use ....................

End Use Not Reported

Pulp Milis

RSE Column Factors:

TOTAL INPUTS

Boller Fuel

Total Process Uses

Process Heating $\ldots \ldots \ldots \ldots \ldots \ldots \ldots \ldots \ldots \ldots$

Process Cooling and Refrigeration . . . . . . . . . . .

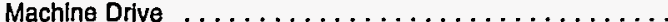

Electro-Chemical Processes $\ldots \ldots \ldots \ldots \ldots \ldots \ldots \ldots$

Other Process Use $\ldots \ldots \ldots \ldots \ldots \ldots \ldots \ldots \ldots$

Total Non-Process Uses . . . . . . . . . . . . . . . . . . .

Facillty Heating, Ventilation, and Air Conditioning ${ }^{\circ} \ldots \ldots$. . .

Facllity Lighting . . . . . . . . . . . . . . . . .

Facility Support . . . . . . . . . . . . . . . . . . .

Onsite Transportation $\ldots \ldots \ldots \ldots \ldots \ldots \ldots \ldots \ldots \ldots$

Conventlonal Electricity Generation . . . . . . . . . . .

Other Non-Process Use . . . . . . . . . . . . . . . . . .

End Use Not Reported $\ldots \ldots \ldots \ldots \ldots \ldots \ldots$

Paper Mills

RSE Column Factors:

TOTAL INPUTS

Boiler Fuel

Total Process Uses

Process Heating

Process Cooling and Refrigeration . . . . . . . . . .

Machine Drive ........................

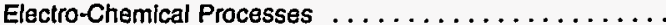

Other Process Use . . . . . . . . . . . . . . . .

Total Non-Process Uses . . . . . . . . . . . . . . . . . . . .

Facillty Heating, Ventilatlon, and Air Conditioning ${ }^{\circ}$.......

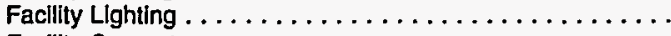

Facility Support . . . . . . . . . . . . . . . . . .

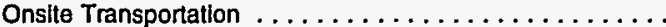

Conventional Electricity Generation . . . . . . . . . .

Other Non-Process Use ....................

End Use Not Reported

$\begin{array}{rr}0.9 & 1.8 \\ 24,883 & 1,566 \\ 21,232 & 665 \\ 3,311 & 391 \\ 3,163 & 291 \\ 0 & - \\ 148 & W \\ -\ddot{0} & - \\ 336 & W \\ W & 486 \\ -- & 97 \\ 0 & - \\ -- & 2 \\ W & 377 \\ 1 & 7 \\ 3 & Q\end{array}$

8,553

285

7,699

26
88

7,158

273

154

569

237

292

39

2

0

$\begin{array}{rr}1.1 & 1.0 \\ 4,500 & 155 \\ 3,881 & 60 \\ 608 & 21 \\ 604 & 3 \\ 0 & 0 \\ 4 & 17 \\ -- & - \\ 0 & 0 \\ 7 & 74 \\ 0 & - \\ -- & - \\ 0 & 0 \\ -- & 74 \\ 7 & 0 \\ 0 & 0 \\ 3 & 0\end{array}$

0.7

1.1

$61,054 \quad 13,455$

1,544

53,957

1,135

683
51,313

582

245

4,130

1,815

1,800

462

w

1,422
11,601

1,569

1,569

0

$\overline{0}$

0
284
$W$

W

o

w
1.0

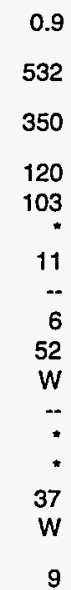

9

0.8

32

22

9

9
0$$
\text { . }
$$$$
\text { (a) }
$$$$
0
$$$$
0
$$$$
\text { o }
$$$$
0
$$

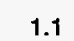

1.1

1.1

8,634

2.7

$\begin{array}{rr}W & 252 \\ 275 & 158\end{array}$

613

8,634

3.3

3.3

4.1

7.1

4.3

7.2

9.6

4.9

4.2

2.9

3.9

8.5

8.3

6.1 
Table A38. Selected Combustible Inputs of Energy for Heat, Power, and Electricity Generation and Net Demand for Electricity by Fuel Type and End Use, 1991: Part 1 (Continued) (Estimates in Btu or Physical Units)

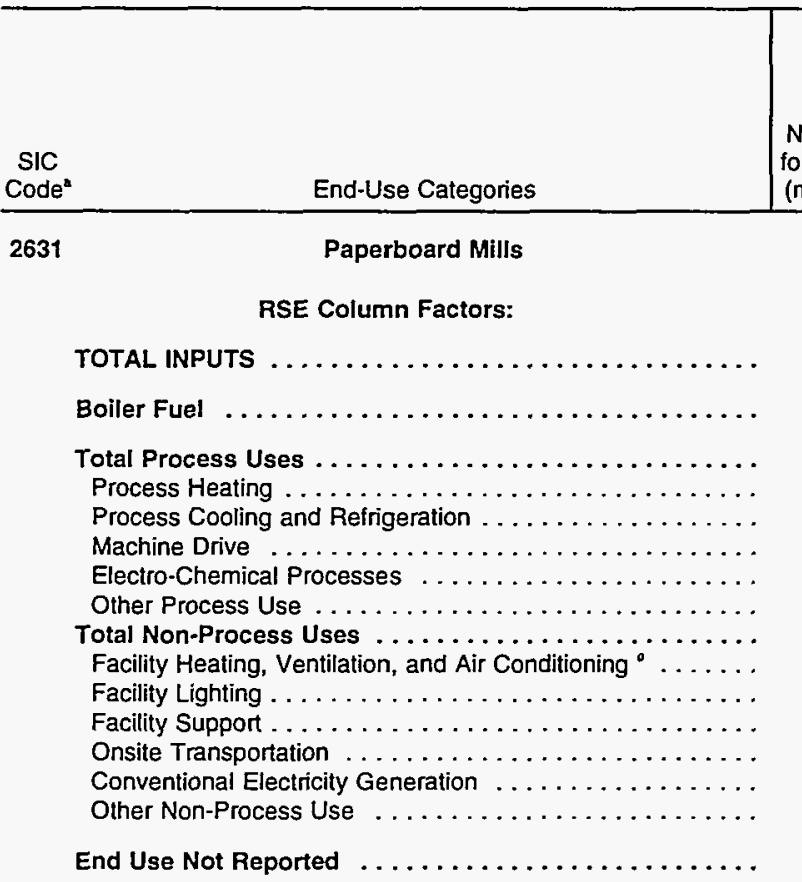

\begin{tabular}{|c|c|c|c|c|c|c|}
\hline $\begin{array}{l}\text { Net Demand } \\
\text { for Electricity } \\
\text { (million kWh) }\end{array}$ & $\begin{array}{c}\text { Residual Fuel } \\
\text { Oil } \\
\text { (1000 bbls) }\end{array}$ & $\begin{array}{c}\text { Distillate Fuel } \\
\text { Oil and Diesel } \\
\text { Fuel } \\
\text { (1000 bbls) }\end{array}$ & $\begin{array}{l}\text { Natural Gas } \\
\text { (billion } \mathrm{cu} \mathrm{ft} \text { ) }\end{array}$ & $\begin{array}{c}\text { LPG } \\
\text { (1000 bbls) }\end{array}$ & $\begin{array}{c}\text { Coal } \\
\text { (excluding } \\
\text { Coal Coke } \\
\text { and Breeze) } \\
\text { (1000 short } \\
\text { tons) }\end{array}$ & $\begin{array}{c}\text { RSE } \\
\text { Row } \\
\text { Factors }\end{array}$ \\
\hline
\end{tabular}

$\begin{array}{rrr}0.6 & 1.5 & \\ 26,971 & W & 207 \\ 1,560 & 5,057 & \\ 23,663 & W & \\ 593 & W & \\ W & 0 & \\ 22,343 & 0 & \\ 167 & - & \\ W & 0 & \\ 1,672 & 3 & 1 \\ 715 & 3 & \\ 793 & - & \\ 160 & 0 & \\ Q & -. & 102 \\ - & 0 & \\ 2 & 0 & \\ Q & * & \end{array}$

\begin{tabular}{rr}
1.1 & 0.8 \\
207 & 180 \\
77 & 133 \\
$W$ & 36 \\
$W$ & $W$ \\
0 & \\
$W$ & \\
\hdashline- & \\
2 & \\
111 & \\
0 & \\
$-:$ & \\
102 & \\
7 & \\
$Q$ & $W$ \\
$W$ & $W$
\end{tabular}

0.8

$\begin{array}{rrr}1.2 & 0.9 & \\ 93 & W & 4.4 \\ 1 & 4.048 & 7.0 \\ 5 & 0 & 5.3 \\ 3 & 0 & 9.4 \\ 0 & 0 & 13.7 \\ 2 & 0 & 9.4 \\ -- & -. & 20.1 \\ 0 & 0 & 19.1 \\ 84 & 0 & 8.7 \\ . & 0 & 13.3 \\ -- & -. & 9.3 \\ 0 & 0 & 13.8 \\ 84 & -. & 4.7 \\ 0 & 0 & 26.1 \\ 0 & 0 & 23.0 \\ 2 & 0 & 14.5\end{array}$

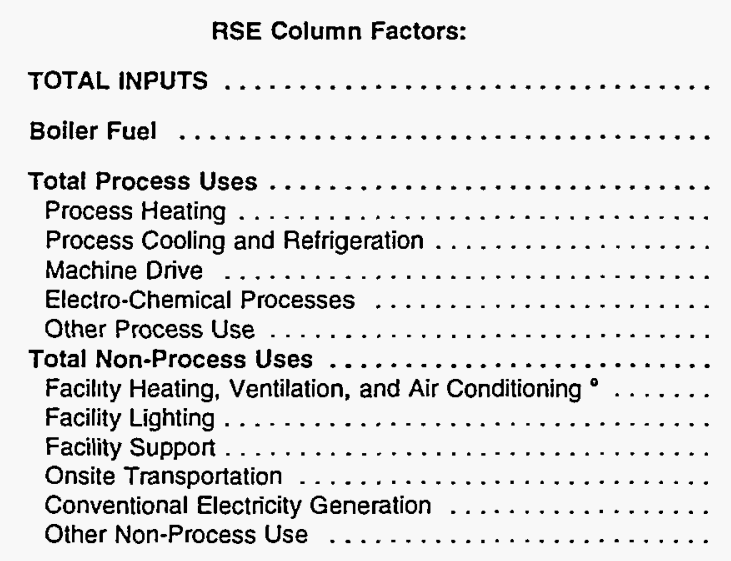

15,641

$\begin{array}{rr}1.5 & 0.7 \\ 312 & 47 \\ 117 & 1 \\ Q & 1 \\ Q & 14 \\ 0 & \\ * & \\ -- & \\ 0 & \\ 180 & \\ 109 & 1 \\ -- & \\ 2 & \\ 65 & \\ Q & \\ * & \\ 8 & \end{array}$

0.7
47
14
16
14
$\vdots$
1
-
12
11
-
1
0
0
$\vdots$
5

$\begin{array}{rrr}1.1 & \text { NF } & \\ 179 & 0 & 12.6 \\ W & 0 & 26.7 \\ W & 0 & 15.9 \\ W & 0 & 23.2 \\ * & 0 & 31.9 \\ 20 & 0 & 26.4 \\ -- & -. & 79.8 \\ 0 & 0 & 60.4 \\ 134 & 0 & 18.7 \\ 1 & 0 & 23.3 \\ -- & -- & 16.0 \\ Q & 0 & 27.9 \\ 131 & -. & 24.1 \\ 0 & 0 & N F \\ Q & 0 & 18.9 \\ O & 0 & 25.4\end{array}$

Total Process Uses $\ldots \ldots \ldots \ldots \ldots \ldots \ldots \ldots \ldots \ldots$

Process Heating . . . . . . . . . . . . . . . . . .

Process Cooling and Refrigeration ................

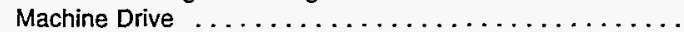

Electro-Chemical Processes $\ldots \ldots \ldots \ldots \ldots \ldots \ldots$.

Other Process Use . . . . . . . . . . . . . . . . . .

Total Non-Process Uses . . . . . . . . . . . . . . .

Facilty Heating, Ventilation, and Air Conditioning ${ }^{\circ} \ldots \ldots$.

Facility Lighting . . . . . . . . . . . . . . . . . . . .

Facility Support . . . . . . . . . . . . . . . .

Onsite Transportation . . . . . . . . . . . . . . . .

Conventional Electricity Generation $\ldots \ldots \ldots \ldots \ldots \ldots \ldots$

Other Non-Process Use . . . . . . . . . . . . . . . .

151,906

4,756

9,921

103,402

33,485 
Table A38. Selected Combustible Inputs of Energy for Heat, Power, and Electricity Generation and Net Demand for Electricity by Fuel Type and End Use, 1991: Part 1 (Continued) (Estimates in Btu or Physical Units)

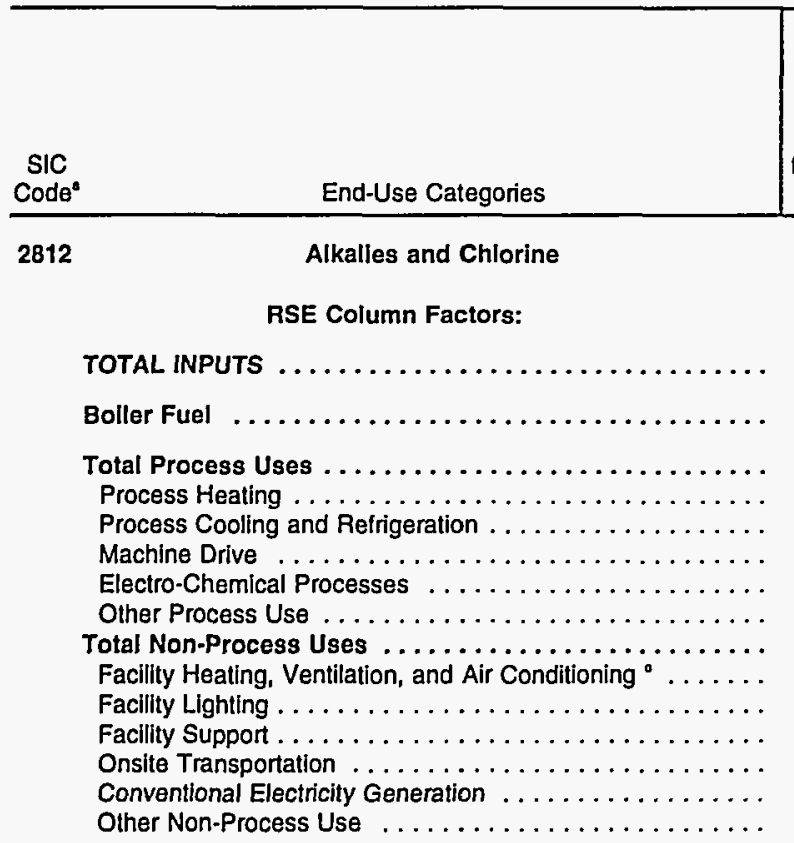

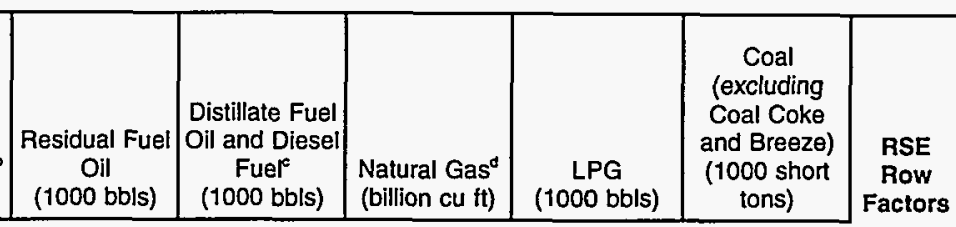

End Use Not Reported

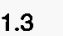

W

0.9
43
$W$
$W$
$W$
0
$*$
-
1
9
0
--
0
9
0
0
$W$

\begin{tabular}{c}
0.9 \\
$W$ \\
\hline 54 \\
4 \\
4 \\
0 \\
0 \\
$\cdots$ \\
1 \\
$W$ \\
1 \\
- \\
0 \\
0 \\
$W$ \\
0 \\
0 \\
0
\end{tabular}

$\begin{array}{rcc}0.4 & N F & 1.3 \\ 18,252 & 0 & 7 \\ 0 & 0 & 1 \\ 17,127 & 0 & * \\ W & 0 & 0 \\ 98 & 0 & 0 \\ 16,826 & 0 & 0 \\ W & -- & - \\ Q & 0 & * \\ W & 0 & 0 \\ 328 & 0 & 1 \\ 164 & -- & - \\ W & 0 & 0 \\ 0 & -- & Q \\ - & 0 & 0 \\ 0 & 0 & 0 \\ W & 0 & *\end{array}$

0.8
24
8
11
11
0
-
-
4
-
-
0
2
2

End Use Not Reported

Industrial Inorganic Chemicals, nec

$$
\text { RSE Column Factors: }
$$

TOTAL INPUTS

Boller Fuel

Total Process Uses $\ldots \ldots \ldots \ldots \ldots \ldots \ldots \ldots \ldots \ldots$

Process Heating . . . . . . . . . . . . . . . . . .

Process Cooling and Refrigeration .............

Machine Drive ....................

Electro-Chemical Processes $\ldots \ldots \ldots \ldots \ldots \ldots \ldots \ldots$

Other Process Use . . . . . . . . . . . . . . . . . .

Total Non-Process Uses . . . . . . . . . . . . . . . .

Facility Heating, Ventilation, and Air Conditioning ${ }^{\circ} \ldots \ldots$

Facility Lighting . . . . . . . . . . . . . . . . . .

Facillty Support . . . . . . . . . . . . . . . . .

Onsite Transportation . . . . . . . . . . . . . . .

Conventional Electricity Generation $\ldots \ldots \ldots \ldots \ldots \ldots$

Other Non-Process Use ..................

End Use Not Reported

$\ldots \ldots \ldots \ldots \ldots \ldots \ldots$

\begin{tabular}{|c|c|c|c|c|c|c|}
\hline 0.8 & 1.0 & 1.2 & 0.8 & 1.2 & 1.2 & \\
\hline 39,777 & 691 & 456 & 136 & 75 & 743 & 8.8 \\
\hline w & w & 187 & 69 & 23 & 403 & 10.1 \\
\hline 37,381 & w & 78 & 53 & 33 & 304 & 11.7 \\
\hline 2,258 & w & 45 & 52 & 33 & 304 & 13.8 \\
\hline w & 0 & 0 & • & 0 & 0 & 12.9 \\
\hline 30,588 & 0 & $w$ & - & * & 0 & 12.9 \\
\hline 4,120 & -- & -- & -- & - & - & 14.4 \\
\hline$w$ & 0 & $w$ & * & * & 0 & 16.8 \\
\hline 1,713 & 0 & 189 & 12 & 12 & W & 10.0 \\
\hline 1,023 & 0 & 17 & W & 3 & W & 9.6 \\
\hline 435 & -- & -. & -- & - & -- & 6.0 \\
\hline 208 & 0 & w & • & - & 0 & 11.7 \\
\hline w & - & 157 & 0 & 8 & $-\cdot$ & 10.4 \\
\hline-- & 0 & $w$ & w & 0 & 0 & 16.7 \\
\hline W & 0 & * & " & • & 0 & 15.4 \\
\hline W & 0 & 3 & 2 & Q & w & 14.3 \\
\hline
\end{tabular}

See footnotes at end of table. 
Table A38. Selected Combustible Inputs of Energy for Heat, Power, and Electricity Cieneration and Net Demand for Electricity by Fuel Type and End Use, 1991: Part 1 (Continued) (Estimates in Btu or Physical Units)

\begin{tabular}{|c|c|c|c|c|c|c|c|}
\hline $\begin{array}{c}\text { SIC } \\
\text { Code }^{a}\end{array}$ & End-Use Categories & $\begin{array}{l}\text { Net Demand } \\
\text { for Electricity } \\
\text { (million kWh) }\end{array}$ & $\begin{array}{c}\text { Pesidual Fuel } \\
\text { Oll } \\
\text { (1000 bbls) }\end{array}$ & $\begin{array}{c}\text { Distillate Fuel } \\
\text { Oil and Diesel } \\
\text { Fuel } \\
\text { (1000 bbls) }\end{array}$ & $\begin{array}{l}\text { Natural Gas } \\
\text { (billion cu ft) }\end{array}$ & $\begin{array}{c}\text { LPG } \\
(1000 \mathrm{bbls})\end{array}$ & $\begin{array}{c}\text { Coal } \\
\text { (excluding } \\
\text { Coal Coke } \\
\text { and Breeze) } \\
\text { (1000 short } \\
\text { tons) }\end{array}$ \\
\hline
\end{tabular}

2821 Plastics Materials and Resins

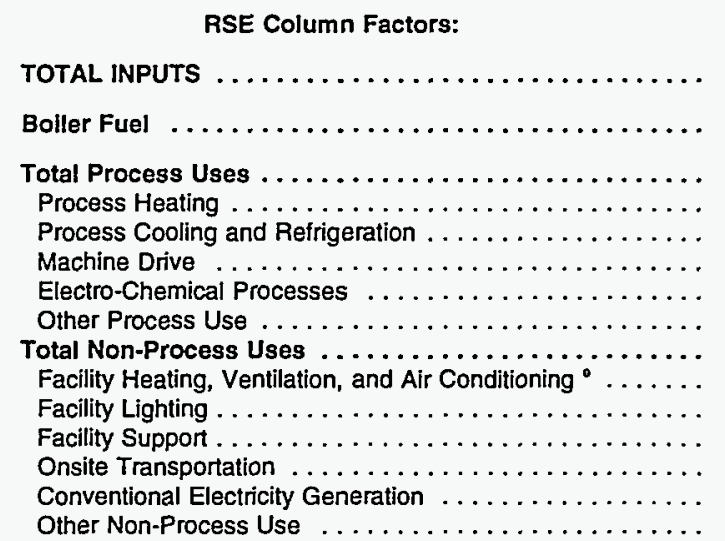

0.6
17,408
218
15,382
$W$
1,759
11,197
1,975
$W$
1,535
756
594
174
10
.-
1
273

1.3
668
$W$
$W$
$W$
0
0
-0
0
$W$
0
.-
0
-
$W$
0
0

End Use Not Reported

1.3
231
142
33
24
0
9
+
55
3
-
4
41
1
9
1

0.9
146
78
51
42
$W$
$W$
--
$W$
15
1
--
1
0
12
-
2

1.2
54
1
21
$W$
$W$
$*$
-
30
$W$
--
$W$
20
0

$\begin{array}{rr}0.9 & \\ 1,074 & 6.1 \\ 1,074 & 7.9 \\ 0 & 5.9 \\ 0 & 6.8 \\ 0 & 10.2 \\ 0 & 7.5 \\ - & 15.7 \\ 0 & 10.3 \\ 0 & 6.7 \\ 0 & 7.2 \\ -- & 4.7 \\ 0 & 8.2 \\ -0 & 6.2 \\ 0 & 8.7 \\ 0 & 16.2 \\ 0 & 14.5\end{array}$

2822

Synthetic Rubber

RSE Column Factors:

TOTAL INPUTS

0.6
1,87

Boiler Fuel $\ldots \ldots \ldots \ldots \ldots \ldots \ldots \ldots \ldots \ldots \ldots \ldots$

Total Process Uses . . . . . . . . . . . . . . . . . . .

Process Heating . . . . . . . . . . . . . . . . .

Process Cooling and Refrigeration . . . . . . . . . . .

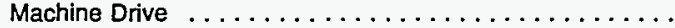

Electro-Chemical Processes $\ldots \ldots \ldots \ldots \ldots \ldots \ldots \ldots$

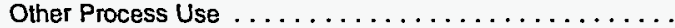

Total Non-Process Uses . . . . . . . . . . . . . . .

Facility Heating, Ventilation, and Air Conditioning ${ }^{\circ} \ldots \ldots$

Facility Lighting . . . . . . . . . . . . . . . . . . .

Facility Support . . . . . . . . . . . . . . . . . .

Onsite Transportation . ..................

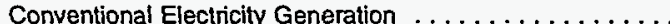

Other Non-Process Use $\ldots \ldots \ldots \ldots \ldots \ldots \ldots \ldots$

End Use Not Reported

0.6
1,877
$W$
1,62
19
22
1,37

$W$
12
113
$W$

$$
1.0
$$

$\begin{array}{lrrr}0.9 & 1.0 & 1.4 & \\ 43 & 10 & W & 12.4 \\ 23 & 0 & W & 15.2\end{array}$

W

19

1,378

$\begin{array}{rrrrrrr}378 & 0 & W & * & * & 0 & 17.0 \\ 1 & -- & -- & - & - & 36.0\end{array}$

$\begin{array}{lllllll}1 & - & - & - & - & 36.0 \\ W & 0 & - & W & 0 & 0 & 25.9\end{array}$

12.8

17.1

14.7

16.9

17.0

23.6

23.6

Cellulosic Manmade Fibers

RSE Column Factors:

\begin{tabular}{|c|c|c|c|c|c|}
\hline 0.9 & NF & 1.2 & 1.1 & 1.1 & 0.8 \\
\hline 1,170 & 0 & 21 & $w$ & 1 & 1,202 \\
\hline 101 & 0 & 17 & $w$ & " & 1,202 \\
\hline 834 & 0 & 1 & 2 & 0 & 0 \\
\hline 16 & 0 & 1 & 2 & 0 & 0 \\
\hline 159 & 0 & 0 & 0 & 0 & 0 \\
\hline 657 & 0 & 0 & 0 & 0 & 0 \\
\hline 0 & -- & -. & -- & -- & $\ldots$ \\
\hline 2 & 0 & 0 & 0 & 0 & 0 \\
\hline 234 & 0 & 4 & • & 1 & 0 \\
\hline 101 & 0 & 4 & 0 & $\star$ & 0 \\
\hline 107 & -. & -. & - & -. & $\ldots$ \\
\hline 10 & 0 & 0 & • & 0 & 0 \\
\hline 17 & $\cdots$ & * & 0 & 1 & . \\
\hline- & 0 & 0 & 0 & 0 & 0 \\
\hline 0 & 0 & 0 & 0 & 0 & 0 \\
\hline 0 & 0 & 0 & 0 & • & 0 \\
\hline
\end{tabular}

TOTAL INPUTS

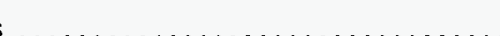

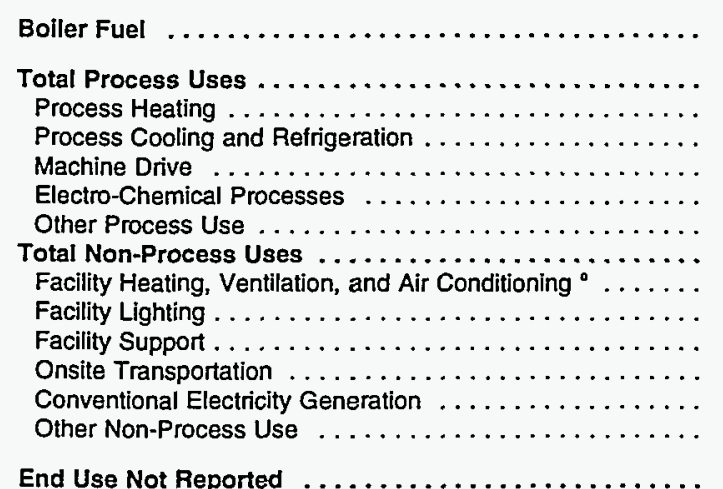

End Use Not Reported 
Table A38. Selected Combustible Inputs of Energy for Heat, Power, and Electricity Generation and Net Demand for Electricity by Fuel Type and End Use, 1991: Part 1 (Continued) (Estimates in Btu or Physical Units)

\begin{tabular}{|c|c|c|c|c|c|c|c|c|}
\hline $\begin{array}{c}\text { SIC } \\
\text { Code" }\end{array}$ & End-Use Categories & $\begin{array}{l}\text { Net Demand } \\
\text { for Electricity } \\
\text { (million kWh) }\end{array}$ & $\begin{array}{c}\text { Residual Fuel } \\
\text { Oil } \\
\text { (1000 bbls })\end{array}$ & $\begin{array}{c}\text { Distillate Fuel } \\
\text { Oil and Diesel } \\
\text { Fuel } \\
(1000 \text { bbls })\end{array}$ & $\begin{array}{l}\text { Natural Gas } \\
\text { (billion cu ft) }\end{array}$ & $\begin{array}{c}\text { LPG } \\
\text { (1000 bbls) }\end{array}$ & $\begin{array}{c}\text { Coal } \\
\text { (excluding } \\
\text { Coal Coke } \\
\text { and Breeze) } \\
\text { (1000 short } \\
\text { tons) }\end{array}$ & $\begin{array}{c}\text { RSE } \\
\text { Row } \\
\text { Factors }\end{array}$ \\
\hline
\end{tabular}

2824

Organle Fibers, Noncellulosic

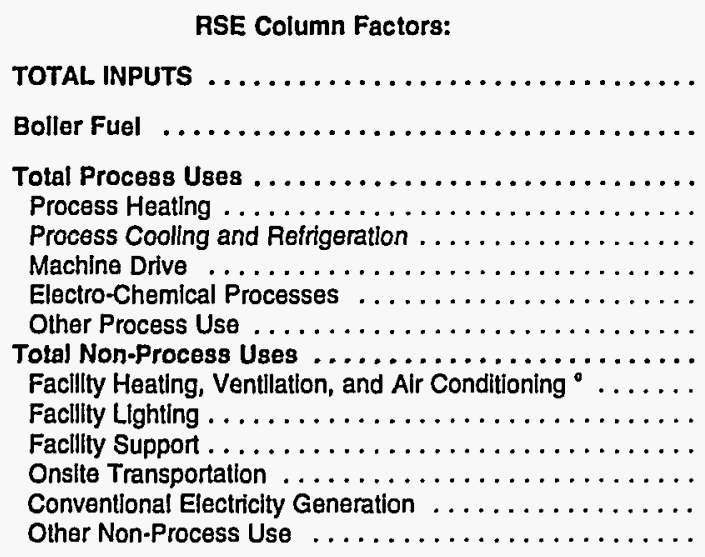

1.1
7,601
79
6,047
$W$
790
4,244
$W$
$W$
1,475
789
494
$W$
2
--
$W$
0

$\begin{array}{rr}1.1 & 1.0 \\ W & 53 \\ 323 & 34 \\ W & 2 \\ W & 1 \\ 0 & 0 \\ 0 & \cdot \\ - & - \\ 0 & 0 \\ W & 17 \\ W & \cdot \\ -- & - \\ 0 & 15 \\ -- & 0 \\ 0 & 1 \\ 1 & \cdot \\ W & \end{array}$

\begin{tabular}{|c|c|c|c|}
\hline 0.9 & 1.1 & 0.8 & \\
\hline$w$ & 38 & 1,558 & 3.5 \\
\hline 23 & $w$ & 1,558 & 3.8 \\
\hline$w$ & $w$ & 0 & 4.0 \\
\hline W & $w$ & 0 & 5.3 \\
\hline * & 0 & 0 & 1.8 \\
\hline " & • & 0 & 5.6 \\
\hline 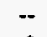 & -- & -- & 6.3 \\
\hline$*$ & 0 & 0 & 5.4 \\
\hline 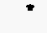 & 18 & 0 & 4.8 \\
\hline •" & 2 & 0 & 6.4 \\
\hline-- & -- & -- & 5.4 \\
\hline • & • & 0 & 6.3 \\
\hline 0 & 16 & $\cdots$ & 4.5 \\
\hline 0 & 0 & 0 & NF \\
\hline 0 & • & 0 & 5.8 \\
\hline 0 & • & 0 & 6.6 \\
\hline
\end{tabular}

\begin{tabular}{rr}
1.2 & 1.2 \\
$W$ & 136 \\
$W$ & 104 \\
$W$ & 13 \\
0 & $:$ \\
0 & 12 \\
- & $:-$ \\
0 & $*$ \\
$:$ & 19 \\
- & $:$ \\
\hline 0 & 19 \\
-- & 1 \\
0 & 0 \\
0 & 0 \\
0 &
\end{tabular}

$\begin{array}{rrrr}0.8 & 1.2 & 1.0 & \\ 94 & 79 & W & 11.6 \\ 46 & W & W & 13.4 \\ 37 & 41 & 0 & 12.6 \\ 35 & W & 0 & 17.9 \\ 0 & 0 & 0 & 17.5 \\ 1 & W & 0 & 15.5 \\ -. & -- & -. & N F \\ 1 & W & 0 & 23.1 \\ W & W & 0 & 13.7 \\ 1 & - & 0 & 17.9 \\ -- & - & -- & 9.0 \\ - & W & 0 & 17.9 \\ 0 & 0 & -- & 15.9 \\ W & 0 & 0 & 22.5 \\ 0 & 1 & 0 & 32.9 \\ W & & 1 & 22.4\end{array}$

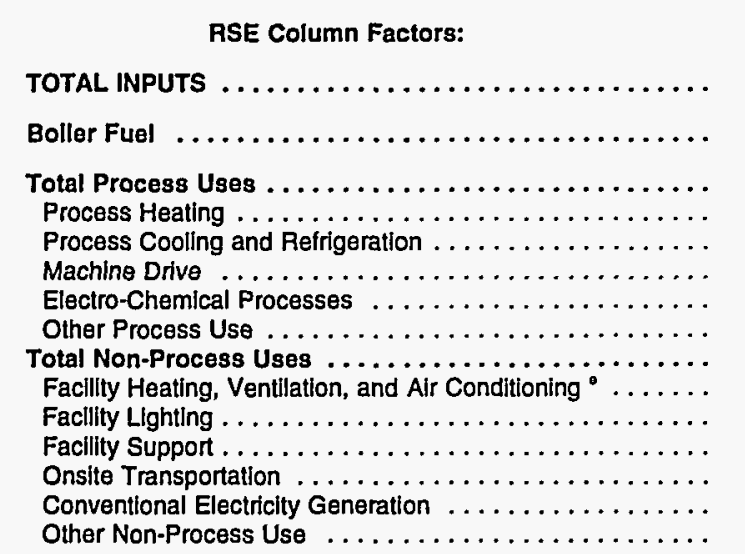

\begin{tabular}{|c|c|c|c|c|c|}
\hline 0.6 & 1.5 & 1.2 & 0.8 & 1.4 & 0.8 \\
\hline 36,764 & 1,747 & 439 & 625 & 825 & 3,819 \\
\hline 190 & 1,595 & $\mathbf{Q}$ & 213 & $w$ & 3,819 \\
\hline 33,715 & 149 & 71 & 241 & $w$ & 0 \\
\hline 498 & $w$ & 41 & 196 & $W$ & 0 \\
\hline 2,903 & 0 & 0 & 2 & 2 & 0 \\
\hline 19,547 & 0 & 23 & 28 & 3 & 0 \\
\hline 10,626 & -- & $\cdots$ & -- & $\cdots$ & -- \\
\hline 142 & $w$ & 7 & 16 & 7 & 0 \\
\hline 2,570 & 1 & 94 & 170 & 33 & 0 \\
\hline 1,080 & 1 & $Q$ & 3 & $W$ & 0 \\
\hline 982 & -- & -- & $\cdots$ & - & -. \\
\hline 369 & 0 & $w$ & 1 & 1 & 0 \\
\hline 14 & $\cdots$ & 64 & 0 & $W$ & - \\
\hline-- & 0 & • & $W$ & • & 0 \\
\hline 126 & 0 & 19 & $w$ & • & 0 \\
\hline 289 & 1 & 8 & 1 & • & 0 \\
\hline
\end{tabular}

See footnotes at end of table. 
Table A38. Selected Combustible Inputs of Energy for Heat, Power, and Electricity Generation and Net Demand for Electricity by Fuel Type and End Use, 1991: Part 1 (Continued) (Estimates in Btu or Physical Units)

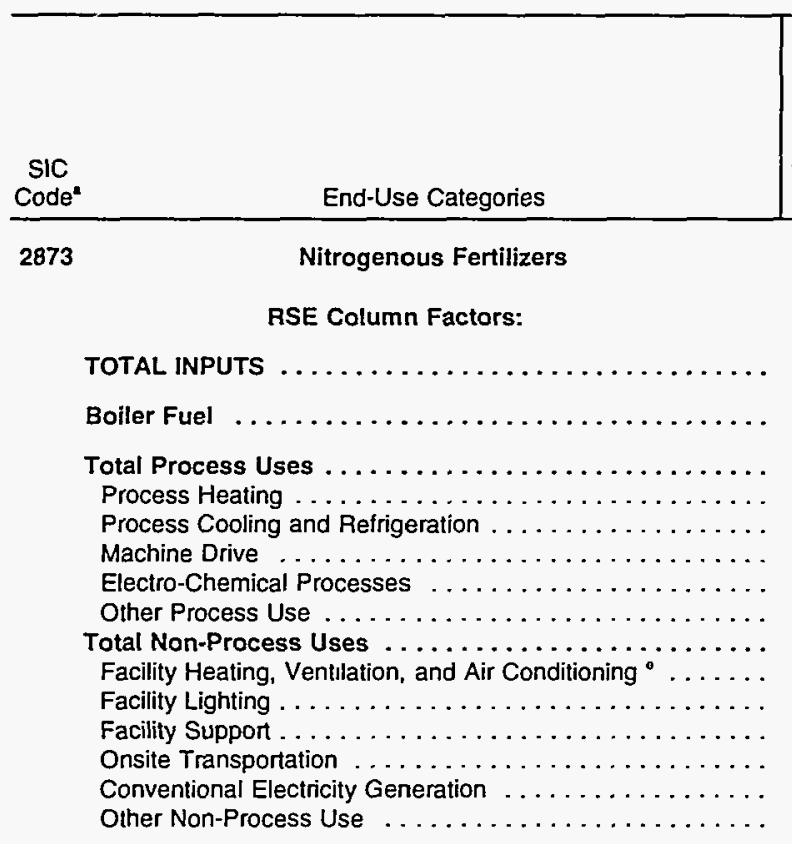
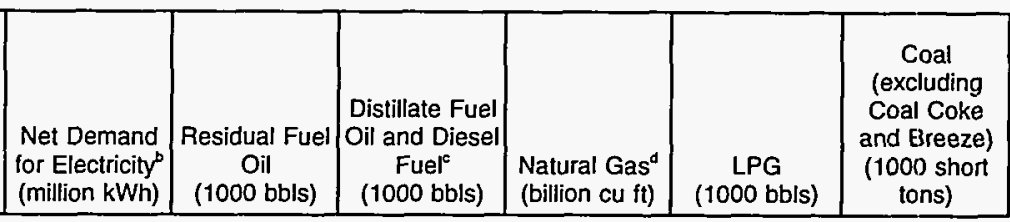

RSE
Row
Factors

End Use Not Reported $\ldots \ldots \ldots \ldots \ldots \ldots \ldots \ldots \ldots$

$\begin{array}{rr}0.8 \\ 3,303 \\ 11 \\ 2,920 \\ 25 \\ 327 \\ 2,414 \\ 155 \\ 0 \\ 249 \\ 96 \\ 99 \\ 54 \\ 0 \\ -. \\ 0 & \\ 123 & \end{array}$

$\begin{array}{rrr}\text { NF } & 1.1 & 0.8 \\ 0 & 26 & 258 \\ 0 & 3 & 90 \\ 0 & 2 & 167 \\ 0 & 0 & 148 \\ 0 & 0 & 0 \\ 0 & 2 & 2 \\ - & -. & -. \\ 0 & 0 & 17 \\ 0 & 15 & 1 \\ 0 & 1 & 1 \\ - & - & - \\ 0 & 3 & 0 \\ -- & 12 & 0 \\ 0 & + & . \\ 0 & 0 & 0 \\ 0 & 5 & .\end{array}$

$\begin{array}{rr}0.8 & 1.5 \\ 258 & \\ 90 & 43 \\ 167 & \\ 148 & \\ 0 & \\ 2 & \\ . & \\ 17 & \\ 1 & \\ 1 & \\ - & \\ 0 & \\ 0 & \\ : & \end{array}$

1.5
43
0
41
41
0
0
--
0
2
.
-
0
1
0
0

NF

TOTAL INPUTS

Boiler Fuel . .

W

Total Process Uses

Process Heating . . . . . . . . . . . . . . . . . .

Process Cooling and Refrigeration ................

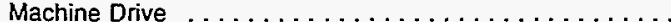

Electro-Chemical Processes $\ldots \ldots \ldots \ldots \ldots \ldots \ldots$

Other Process Use . . . . . . . . . . . . . . . . . .

Total Non-Process Uses . . . . . . . . . . . . . . . . . .

Facility Heating, Ventilation, and Air Conditioning ${ }^{\circ} . \ldots \ldots$

Facility Lighting.$\ldots \ldots \ldots \ldots \ldots \ldots \ldots \ldots \ldots$

Facility Support . . . . . . . . . . . . . . . . . . .

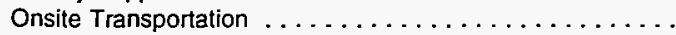

Conventional Electricity Generation ..............

Other Non-Process Use $\ldots . . . \ldots \ldots \ldots \ldots \ldots$.

End Use Not Reported

\begin{tabular}{|c|}
\hline 199 \\
\hline 199 \\
\hline 0 \\
\hline 0 \\
\hline \\
\hline 0 \\
\hline 0 \\
\hline 0 \\
\hline -- \\
\hline 0 \\
\hline \\
\hline 0 \\
\hline
\end{tabular}

\section{PETROLEUM and COAL PRODUCTS}

RSE Column Factors:

TOTAL INPUTS

$\begin{array}{rr}0.4 & 0.9 \\ 44,234 & 13,862 \\ 393 & 6,086 \\ 39,976 & 7,776 \\ 1,210 & 7,776 \\ 2,432 & 0 \\ 36,219 & 0 \\ W & - \\ W & 0 \\ 3,637 & 0 \\ 1,667 & 0 \\ 1,499 & - \\ 375 & 0 \\ W & - \\ - & 0 \\ W & 0 \\ 229 & 0\end{array}$

2.0
3,599
614
1,870
1,241
$Q$
597
-
$W$
873
12
--
$Q$
658
$Q$
$W$
$Q$

\begin{tabular}{rrr}
0.5 & 0.8 & 2.8 \\
813 & 16,528 & $W$ \\
256 & 3,122 & $W$ \\
446 & 12,880 & $W$ \\
410 & 9,724 & $W$ \\
$W$ & $W$ & 0 \\
$W$ & $W$ & 0 \\
.- & - & - \\
1 & 0 & 0 \\
107 & 114 & 0 \\
10 & $W$ & 0 \\
-- & -- & - \\
2 & $W$ & 0 \\
$-*$ & 40 & - \\
95 & $W$ & 0 \\
$*$ & $*$ & 0 \\
5 & 412 & 0 \\
\hline
\end{tabular}

4.6
6.0
6.1
7.2
7.3
8.3
9.3
11.3
8.4
10.4
4.7
9.3
12.2
7.5
16.0
51.3 
Table A38. Selected Combustible Inputs of Energy for Heat, Power, and Electricity Generation and Net Demand for Electricity by Fuel Type and End Use, 1991: Part 1 (Continued) (Estimates in Btu or Physical Units)

\begin{tabular}{|c|c|c|c|c|c|c|c|}
\hline $\begin{array}{c}\text { SIC } \\
\operatorname{Cod\theta ^{*}}\end{array}$ & End-Use Categories & $\begin{array}{c}\text { Net Demand } \\
\text { for Electricity } \\
\text { (million } \mathrm{kWh} \text { ) }\end{array}$ & $\begin{array}{c}\text { Residual Fuel } \\
\text { Oil } \\
(1000 \text { bbls })\end{array}$ & $\begin{array}{c}\text { Distillate Fuel } \\
\text { Oil and Diesel } \\
\text { Fuel" } \\
\text { (1000 bbls) }\end{array}$ & $\begin{array}{l}\text { Natural Gas } \\
\text { (billion cu ft) }\end{array}$ & $\begin{array}{c}\text { LPG } \\
\text { (1000 bbls) }\end{array}$ & $\begin{array}{c}\text { Coal } \\
\text { (excluding } \\
\text { Coal Coke } \\
\text { and Breeze) } \\
\text { (1000 short } \\
\text { tons) }\end{array}$ \\
\hline
\end{tabular}

2911 Petroleum Refining*

RSE Column Factors:

$\begin{array}{rrrr}0.5 & 1.6 & 0.9 & 0 \\ 42,145 & 10,292 & 1,525 & 769 \\ W & 5,198 & 299 & 246 \\ 38,355 & 5,094 & 745 & 416 \\ 1,025 & 5,094 & 679 & 38 \\ 2,403 & 0 & 0 & \\ 34,814 & 0 & W & \\ W & -- & -- & \\ W & 0 & W & \\ 3,369 & 0 & W & \\ 1,551 & 0 & -- & \\ 1,387 & -- & * & \\ 346 & 0 & 449 & \\ W & -- & \text {. } & \\ -- & 0 & W & \end{array}$

$\begin{array}{rrrr}0.5 & 0.7 & 3.6 & \\ 769 & 15,889 & 134 & 3.9 \\ 246 & 3,039 & 134 & 3.3 \\ 416 & 12,535 & 0 & 4.7 \\ 380 & 9,382 & 0 & 5.6 \\ W & W & 0 & 7.2 \\ W & W & 0 & 5.3 \\ -. & -- & - & 8.2 \\ * & 0 & 0 & 8.0 \\ 105 & W & 0 & 5.8 \\ 8 & W & 0 & 6.3 \\ -- & - & -. & 4.1 \\ 1 & W & 0 & 7.2 \\ 0 & * & -. & 8.2 \\ 95 & W & 0 & 14.6 \\ * & * & 0 & 8.8 \\ & W & 0 & 8.1\end{array}$

TOTAL INPUTS

Boiler Fuel

S.

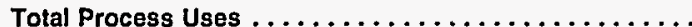

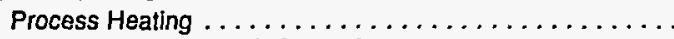

Process Cooling and Refrigeration ...............

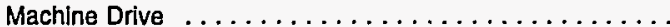

Electro-Chemical Processes $\ldots \ldots \ldots \ldots \ldots \ldots \ldots \ldots$

Other Process Use ........................

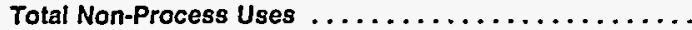

Facility Heating, Ventilation, and Air Conditioning ${ }^{\circ}$..........

Facility Lighting . . . . . . . . . . . . . . . . . . . .

Facility Support . . . . . . . . . . . . . . . . . .

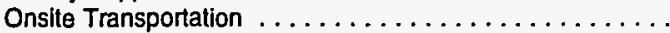

Conventional Electricity Generation . . . . . . . . . . . .

Other Non-Process Use . . . . . . . . . . . . . . . .

End Use Not Reported

RUBBER and MISC. PLASTICS PRODUCTS

RSE Column Factors:

$\begin{array}{rr}0.5 & 1.1 \\ 34,022 & 1,253 \\ 175 & 1,084 \\ 26,368 & W \\ 5,682 & W \\ 2,406 & 1 \\ 18,111 & . \\ 64 & - \\ 104 & 0 \\ 6,018 & W \\ 2,758 & W \\ 2,516 & - \\ 626 & * \\ 109 & - \\ -. & 0 \\ Q & 0 \\ 1,461 & 0\end{array}$

1.5
508
283
28
$W$
0
$Q$
--
185
54
.-
1
123
2
$Q$
0

$$
\begin{array}{r}
0.9 \\
93 \\
55 \\
19 \\
16 \\
3 \\
: \\
16 \\
15 \\
: \\
: \\
:
\end{array}
$$

$\begin{array}{rrr}1.2 & 1.2 & \\ 786 & 295 & 9.8 \\ Q & 295 & 11.7 \\ 263 & 0 & 17.1 \\ 216 & 0 & 19.3 \\ 0 & 0 & 20.2 \\ 46 & 0 & 20.5 \\ - & - & 55.3 \\ 1 & 0 & 32.4 \\ 364 & 0 & 11.9 \\ 37 & 0 & 18.1 \\ - & -- & 9.5 \\ W & 0 & 12.1 \\ 316 & - & 22.8 \\ 0 & 0 & 20.0 \\ W & 0 & 14.7 \\ 21 & 0 & 27.3\end{array}$

3011

Tires and Inner Tubes

RSE Column Factors:

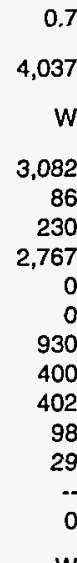

\section{1}

$$
1.5
$$

1.0

0.8

79

Boiler Fuel

$\ldots . .$.

Total Process Uses $\ldots \ldots \ldots \ldots \ldots \ldots \ldots \ldots \ldots \ldots$

Process Heating .

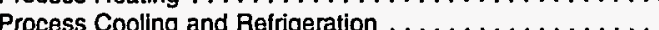

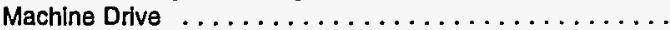

Electro-Chemical Processes ...................

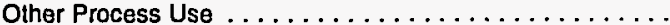

Total Non-Process Uses . . . . . . . . . . . . . . .

Facillty Heating, Ventilation, and Air Conditioning ${ }^{\circ} \ldots \ldots$.

Facllity Lighting . . . . . . . . . . . . . . . . . . .

Facility support $\ldots \ldots \ldots \ldots \ldots \ldots \ldots \ldots \ldots \ldots$

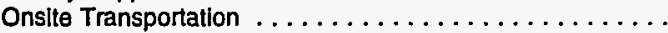

Conventional Electricity Generation . . . . . . . . . . . .

Other Non-Process Use $\ldots \ldots \ldots \ldots \ldots \ldots \ldots \ldots$.

End Use Not Reported

$\begin{array}{rrr}4,037 & 506 & 68 \\ W & 479 & 59 \\ 3,082 & Q & 4 \\ 86 & Q & 0 \\ 230 & 0 & 0 \\ 2,767 & 0 & 4 \\ 0 & - & - \\ 0 & 0 & 0 \\ 930 & 2 & 5 \\ 400 & 2 & Q \\ 402 & - & - \\ 98 & * & 1 \\ 29 & - & 1 \\ - & 0 & \div \\ 0 & 0 & 1 \\ W & * & *\end{array}$


Table A38. Selected Combustible Inputs of Energy for Heat, Power, and Electricity Ceneration and Net Demand for Electricity by Fuel Type and End Use, 1991: Part 1 (Continued) (Estimates in Btu or Physical Units)

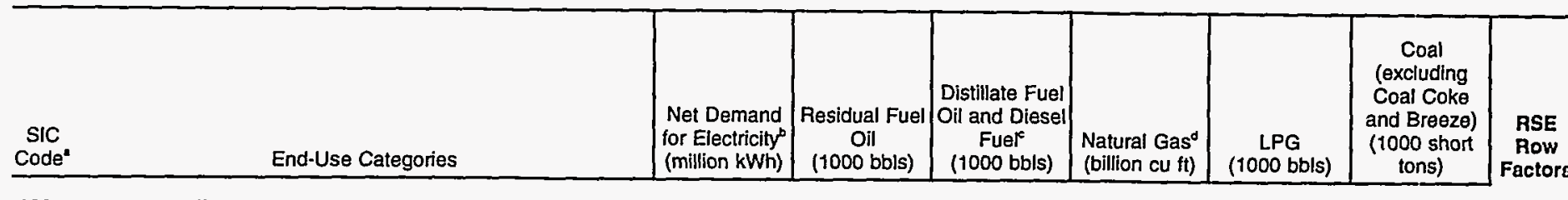

$308 \quad$ Miscellaneous Plastic Products, nec

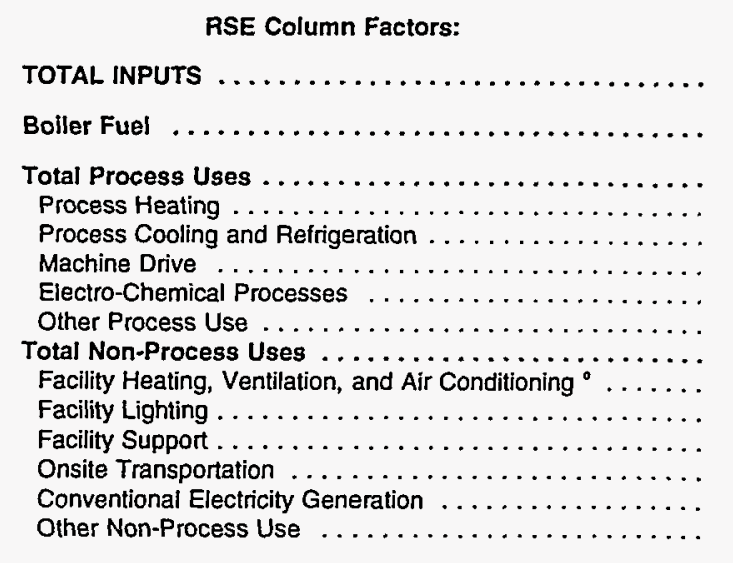

$\begin{array}{rr}0.5 & 1.5 \\ 25,635 & 413 \\ 103 & 316 \\ 20,085 & 81 \\ 4,953 & 81 \\ 1,962 & 0 \\ 13,023 & - \\ 53 & - \\ 94 & 0 \\ 4,061 & 16 \\ 1,875 & 16 \\ 1,689 & - \\ 428 & - \\ 65 & - \\ - & 0 \\ 0 & 0 \\ 1,385 & 0\end{array}$

1.7
$W$
$W$
7
6
0
$Q$
-
146
33
$\because-$
109
$Q$
$Q$
$Q$

0.7
51
24
13
11
0
1
-
12
11
-
0
$:$
3

$\begin{array}{rrr}1.0 & 1.1 & \\ 396 & 130 & 13.3 \\ 0 & 130 & 21.4 \\ 121 & 0 & 17.9 \\ 82 & 0 & 21.6 \\ 0 & 0 & 17.3 \\ 39 & 0 & 25.0 \\ -. & -. & 65.3 \\ 230 & 0 & 45.0 \\ 27 & 0 & 16.0 \\ -. & 0 & 24.0 \\ 204 & -. & 13.4 \\ 0 & 0 & 25.4 \\ . & 0 & 22.9 \\ 18 & 0 & \text { NF } \\ & 0 & 45.0 \\ & 0 & 33.8\end{array}$

TOTAL INPUTS

Boiler Fuel

Total Process Uses

Process Heating .

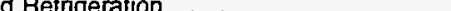

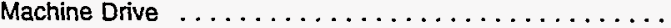

Electro-Chemical Processes $\ldots \ldots \ldots \ldots \ldots \ldots \ldots$.

Other Process Use $\ldots \ldots \ldots \ldots \ldots \ldots \ldots \ldots \ldots \ldots$

Total Non-Process Uses . . . . . . . . . . . . . . . .

Facility Heating, Ventilation, and Air Conditioning ${ }^{\circ} \ldots \ldots$.

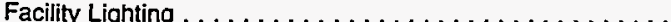

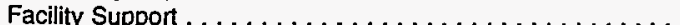

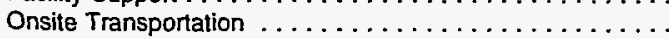

Conventional Electricity Generation . . . . . . . . . . . . . .

Other Non-Process Use $\ldots \ldots \ldots \ldots \ldots \ldots \ldots \ldots$

End Use Not Reported $\ldots \ldots \ldots \ldots \ldots \ldots \ldots \ldots \ldots$

\begin{tabular}{|c|c|c|c|c|}
\hline 1.3 & 0.9 & 1.1 & 1.6 & \\
\hline 220 & 5 & 44 & $Q$ & 24.0 \\
\hline 155 & 2 & 2 & 0 & 31.0 \\
\hline 11 & 1 & 26 & 0 & 28.5 \\
\hline 11 & 1 & w & 0 & 36.1 \\
\hline 0 & • & 0 & 0 & 58.6 \\
\hline 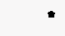 & * & $Q$ & 0 & 28.2 \\
\hline -- & -- & - & -. & 105.5 \\
\hline 0 & • & * & 0 & 51.0 \\
\hline 41 & $Q$ & 16 & . & 25.8 \\
\hline 16 & $a$ & 6 & * & 29.7 \\
\hline -. & -- & -. &.- & 23.4 \\
\hline * & 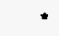 & - & 0 & 35.9 \\
\hline 16 & 0 & 9 & -- & 36.0 \\
\hline 0 & 0 & 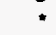 & 0 & 40.1 \\
\hline$Q$ & 0 & 0 & 0 & 105.5 \\
\hline 13 & - & • & 0 & 33.5 \\
\hline
\end{tabular}

STONE, CLAY and GLASS PRODUCTS

RSE Column Factors:

$\begin{array}{rr}0.5 & 1.1 \\ 31,347 & 1,345 \\ 122 & 208 \\ 26,631 & 1,122 \\ 8,025 & W \\ 804 & 0 \\ 17,604 & W \\ 20 & - \\ 178 & 0 \\ 3,622 & 0 \\ 1,637 & 0 \\ 1,614 & - \\ 301 & 0 \\ 42 & - \\ -- & 0 \\ 27 & 0 \\ 972 & *\end{array}$

$\begin{array}{rr}1.5 & 0.6 \\ 3,312 & 369 \\ 365 & 16 \\ 1,020 & 323 \\ W & 317 \\ * & 2 \\ 616 & 3 \\ -. & -. \\ W & 1 \\ 1,341 & 18 \\ 79 & 17 \\ -. & - \\ 7 & 1 \\ 1,192 & : \\ Q & : \\ 27 & 0 \\ 586 & 12\end{array}$

$\begin{array}{rrr}0.6 & 1.6 & 1.2 \\ 369 & 577 & 13,127 \\ 16 & 9 & W \\ 323 & 221 & 13,060 \\ 317 & 154 & 13,060 \\ 2 & * & 0 \\ 3 & 67 & 0 \\ - & - & - \\ 1 & 1 & : \\ 18 & 300 & 0 \\ 17 & 29 & 0 \\ -. & - & - \\ 1 & 5 & 0 \\ * & 265 & - \\ * & \vdots & 0 \\ 0 & : & 0 \\ 12 & 48 & W\end{array}$

Total Process Uses

Process Heating

Process Cooling and Refrigeration $\ldots \ldots \ldots \ldots \ldots \ldots \ldots \ldots$

Machine Drive $\ldots \ldots \ldots \ldots \ldots \ldots \ldots \ldots \ldots \ldots$

Electro-Chemical Processes $\ldots \ldots \ldots \ldots \ldots \ldots \ldots$

Other Process Use ............................

Facility Heating, Ventilation, and Air Conditioning $\ldots \ldots \ldots$

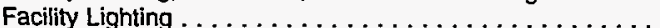

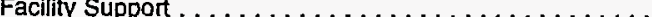

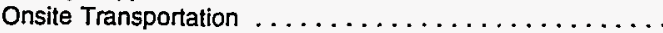

Conventional Electricity Generation $\ldots \ldots \ldots \ldots \ldots \ldots \ldots$

End Use Not Reported 
Table A38. Selected Combustible Inputs of Energy for Heat, Power, and Electricity Generation and Net Demand for Electricity by Fuel Type and End Use, 1991: Part 1 (Continued) (Estimates in Btu or Physical Units)

\begin{tabular}{|c|c|c|c|c|c|c|c|c|}
\hline $\begin{array}{c}\text { SIC } \\
\text { Code }\end{array}$ & End-Use Categories & $\begin{array}{l}\text { Net Demand } \\
\text { for Electricity } \\
\text { (million } k W h \text { ) }\end{array}$ & $\begin{array}{c}\text { Residual Fuel } \\
\text { Oil } \\
\text { (1000 bbls) }\end{array}$ & $\begin{array}{c}\text { Distillate Fuel } \\
\text { Oil and Diesel } \\
\text { Fuel } \\
\text { (1000 bbls })\end{array}$ & $\begin{array}{l}\text { Natural Gas } \\
\text { (billion cu } t \text { ) }\end{array}$ & $\begin{array}{c}\text { LPG } \\
(1000 \text { bbls })\end{array}$ & $\begin{array}{c}\text { Coal } \\
\text { (excluding } \\
\text { Coal Coke } \\
\text { and Breeze) } \\
\text { (1000 short } \\
\text { tons) }\end{array}$ & $\begin{array}{c}\text { RSE } \\
\text { Row } \\
\text { Factors }\end{array}$ \\
\hline
\end{tabular}

3211

Flat Glass

RSE Column Factors:

$\begin{array}{rr}0.7 & 1.6 \\ 1,506 & W \\ W & 0 \\ 1,168 & W \\ 653 & W \\ 62 & 0 \\ 454 & 0 \\ 0 & - \\ 0 & 0 \\ W & 0 \\ 152 & 0 \\ W & - \\ 15 & 0 \\ 12 & - \\ -. & 0 \\ 0 & 0 \\ W & 0\end{array}$

\begin{tabular}{r}
0.9 \\
12 \\
0 \\
$W$ \\
$W$ \\
$\vdots$ \\
$:-$ \\
0 \\
6 \\
0 \\
\hdashline \\
0 \\
6 \\
0 \\
$W$
\end{tabular}

\begin{tabular}{r}
0.6 \\
40 \\
$W$ \\
35 \\
34 \\
1 \\
-- \\
0 \\
2 \\
1 \\
\hdashline- \\
0 \\
0 \\
0 \\
$W$
\end{tabular}

$\begin{array}{rrr}1.0 & 1.6 & \\ 40 & \cdot & 3.5 \\ 0 & 0 & 11.2 \\ W & . & 4.0 \\ W & 0 & 4.1 \\ 0 & 0 & 6.1 \\ 2 & 0 & 5.2 \\ -- & -. & N F \\ 0 & - & 3.7 \\ 15 & 0 & 4.1 \\ - & 0 & 4.7 \\ - & -. & 4.3 \\ 0 & 0 & 7.4 \\ 15 & -. & 5.6 \\ 0 & 0 & 4.4 \\ 0 & 0 & N F \\ W & 0 & 5.6\end{array}$

$\begin{array}{rrr}0.6 & 1.6 & 1.4 \\ 4,098 & 276 & 23 \\ 1 & 1 & 5 \\ 3,580 & 275 & 15 \\ 1,187 & 275 & 14 \\ W & 0 & 0 \\ 2,198 & 0 & 1 \\ W & - & - \\ W & 0 & 0 \\ 517 & : & 3 \\ 196 & - & 1 \\ 307 & -. & - \\ 14 & 0 & : \\ 1 & -. & 2 \\ - & 0 & 0 \\ 0 & 0 & 0 \\ 0 & & \end{array}$

$\begin{array}{rrrr}0.6 & 1.2 & N F & \\ 67 & 82 & 0 & 4.8 \\ 1 & 0 & 0 & 14.3 \\ 64 & 16 & 0 & 6.0 \\ 63 & W & 0 & 6.9 \\ : & 0 & 0 & 18.2 \\ - & W & 0 & 9.3 \\ - & -- & - & 28.8 \\ 1 & - & 0 & 9.9 \\ 3 & 66 & 0 & 6.0 \\ 2 & - & 0 & 9.5 \\ - & -- & - & 6.4 \\ 0 & - & 0 & 11.7 \\ 0 & 65 & -- & 7.2 \\ 0 & 0 & 0 & N F \\ 0 & 0 & 0 & N F\end{array}$

End Use Not Reported

TOTAL INPUTS

Boller Fuel

Total Process Uses .......................

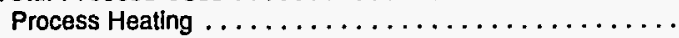

Process Cooling and Refrigeration ..............

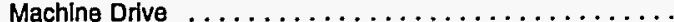

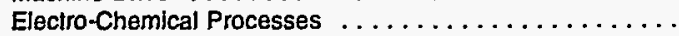

Other Process Use ..........................

Total Non-Process Uses . ......................

Facllity Heating, Ventilation, and Air Conditioning ${ }^{\circ} . \ldots$. .

Facillty Lighting . . . . . . . . . . . . . . . . . . .

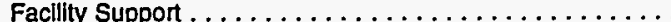

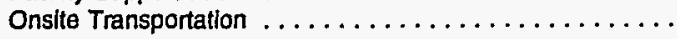

Conventional Electricity Generation . . . . . . . . . . .

Other Non-Process Use ....................

Boller Fuel

Total Process Uses . . . . . . . . . . . . . . . . . .

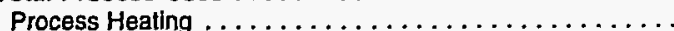

Process Coollng and Refrigeration . . . . . . . . . . . .

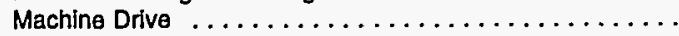

Electro-Chemical Processes ...................

Other Process Use . . . . . . . . . . . . . . . . . . . . .

Total Non-Process Uses . ...................

Facillty Heatling, Ventilation, and Air Conditioning ${ }^{\circ} . . . .$.

Facillty Lighting . . . . . . . . . . . . . . . . . . . .

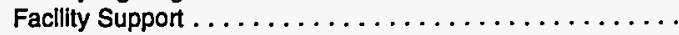

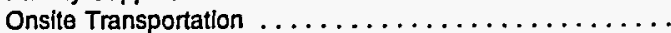

Conventlonal Electricity Generation ................

Other Non-Process Use . . . . . . . . . . . . . . .

End Use Not Reported 
Table A38. Selected Combustible Inputs of Energy for Heat, Power, and Electricity Generation and Net Demand for Electricity by Fuel Type and End Use, 1991: Part 1 (Continued) (Estimates in Btu or Physical Units)

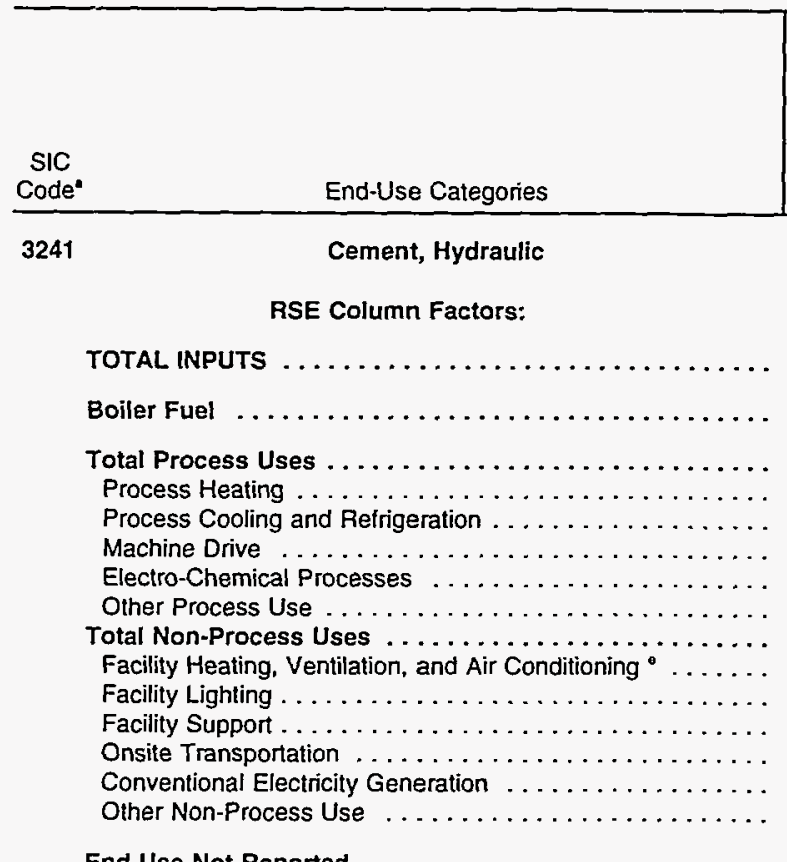

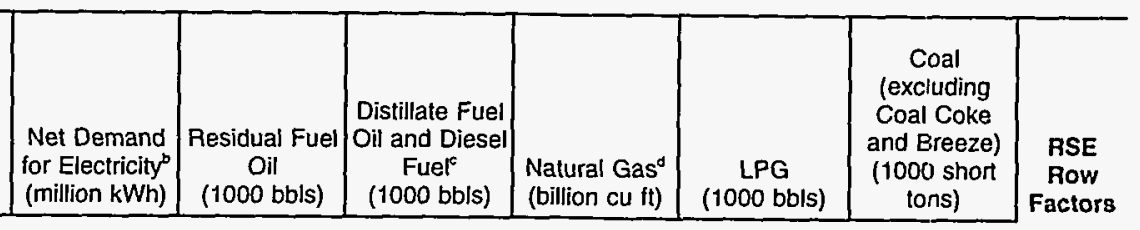

End Use Not Reported .

\begin{tabular}{|c|c|c|c|c|c|c|}
\hline 0.8 & 1.7 & 0.8 & 1.2 & 1.1 & 0.7 & \\
\hline 9,888 & 138 & 616 & 38 & 12 & 8,736 & 10.7 \\
\hline$Q$ & 0 & 18 & * & 0 & 0 & 26.1 \\
\hline 9,305 & 138 & 248 & 37 & 6 & 8,736 & 11.4 \\
\hline 1,640 & w & 162 & 37 & 5 & 8,736 & 12.7 \\
\hline 249 & 0 & 0 & 0 & 0 & 0 & 22.7 \\
\hline 7,385 & $w$ & $w$ & * & " & 0 & 13.6 \\
\hline 0 & -- & -- & -. & -- & $\because$ & NF \\
\hline 31 & 0 & w & 0 & * & 0 & 35.8 \\
\hline 581 & 0 & 322 & 1 & 6 & 0 & 15.0 \\
\hline 273 & 0 & 4 & 1 & 3 & 0 & 20.7 \\
\hline 222 & -- & -- & -- & -- & -. & 15.5 \\
\hline 85 & 0 & 4 & * & * & 0 & 21.7 \\
\hline 1 & -- & 314 & 0 & 2 & -. & 17.3 \\
\hline -. & 0 & * & 0 & 0 & 0 & 32.4 \\
\hline 0 & 0 & 0 & 0 & " & 0 & NF \\
\hline$Q$ & " & 28 & 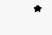 & * & 0 & 30.1 \\
\hline
\end{tabular}

3274

Lime

RSE Column Factors:

\begin{tabular}{|c|c|c|c|c|c|c|}
\hline 1.3 & 0.9 & 0.9 & 0.6 & 0.8 & 1.8 & \\
\hline 1,324 & $w$ & 240 & 8 & $a$ & 3,926 & 25.9 \\
\hline$Q$ & 0 & $Q$ & * & $Q$ & 0 & NF \\
\hline 1,180 & $w$ & 55 & 8 & * & 3,926 & 22.7 \\
\hline 280 & $w$ & $Q$ & 8 & 0 & 3,926 & 22.0 \\
\hline 1 & 0 & 0 & 0 & 0 & 0 & 12.7 \\
\hline 899 & 0 & 30 & * & $\star$ & 0 & 27.9 \\
\hline 0 & -- & -- & -- & -. & -. & NF \\
\hline 0 & 0 & $W$ & 0 & 0 & 0 & 24.7 \\
\hline 91 & 0 & 159 & 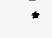 & 1 & 0 & 31.3 \\
\hline 43 & 0 & 2 & • & 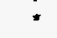 & 0 & 23.8 \\
\hline 45 & -- & -- & .. & -- & -- & 23.8 \\
\hline 3 & 0 & 0 & 0 & * & 0 & 8.2 \\
\hline 0 & - & 158 & 0 & 1 & - & 34.6 \\
\hline -- & 0 & 0 & 0 & 0 & 0 & NF \\
\hline 0 & 0 & 0 & 0 & 0 & 0 & NF \\
\hline$Q$ & 0 & $\mathrm{Q}$ & 0 & 0 & 0 & NF \\
\hline
\end{tabular}

TOTAL INPUTS

.

Boiler Fuel

Total Process Uses . . . . . . . . . . . . . . . .

Process Heating . . . . . . . . . . . . . . . . .

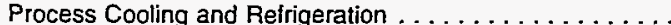

Machine Drive .....................

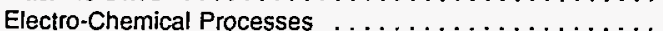

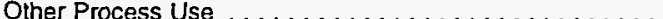

Total Non-Process Uses . . . . . . . . . . . . . . . .

Facility Heating, Ventilation, and Air Conditioning ${ }^{\circ} \ldots .$.

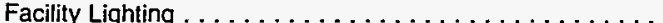

Facility Support . . . . . . . . . . . . . . . .

Onsite Transportation ....................

Conventional Electricity Generation ................

Other Non-Process Use $\ldots \ldots \ldots \ldots \ldots \ldots \ldots \ldots \ldots$

End Use Not Reported

Mineral Wool

RSE Column Factors:

TOTAL INPUTS

Boiler Fuel $\ldots \ldots \ldots \ldots \ldots \ldots \ldots \ldots \ldots \ldots \ldots$

$\begin{array}{rc}0.7 & 1.2 \\ 2,821 & W \\ W & W \\ 2,489 & 0 \\ 1,242 & 0 \\ W & 0 \\ 1,183 & 0 \\ 0 & -- \\ W & 0 \\ W & 0 \\ 120 & 0 \\ 109 & -- \\ 24 & 0 \\ W & -- \\ -- & 0 \\ 2 & 0 \\ W & 0\end{array}$

$$
\begin{array}{r}
1.1 \\
12 \\
* \\
* \\
0 \\
+ \\
\hline 0 \\
11 \\
W \\
-- \\
W \\
3 \\
* \\
\\
*
\end{array}
$$

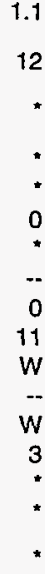$$
0.8
$$$$
1.1
$$$$
1.2
$$

Total Process Uses . . . . . . . . . . . . . . . .

Process Heating . . . . . . . . . . . . . . . . . .

Process Cooling and Refrigeration . . . . . . . . . .

Machine Drive

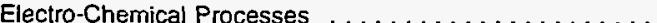

Other Process Use . . . . . . . . . . . . . . . . . . . .

Total Non-Process Uses $\ldots \ldots \ldots \ldots \ldots \ldots \ldots \ldots \ldots \ldots \ldots$

Facility Heating, Ventilation, and Air Conditioning ${ }^{\circ} \ldots$. . .

Facility Lighting . . . . . . . . . . . . . . . . . .

Facility Support

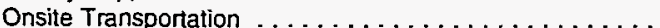

Conventional Electricity Generation . . . . . . . . . . . . .

Other Non-Process Use $\ldots \ldots \ldots \ldots \ldots \ldots \ldots \ldots \ldots \ldots$

End Use Not Reported 
Table A38. Selected Combustible Inputs of Energy for Heat, Power, and Electricity Generation and Net Demand for Electricity by Fuel Type and End Use, 1991: Part 1 (Continued) (Estimates in Btu or Physical Units)

\begin{tabular}{|c|c|c|c|c|c|c|c|c|}
\hline $\begin{array}{c}\text { SIC } \\
\text { Code }\end{array}$ & End-Use Categories & $\begin{array}{l}\text { Net Demand } \\
\text { for Electricity } \\
\text { (million kWh) }\end{array}$ & $\begin{array}{c}\text { Residual Fuel } \\
\text { Oil } \\
(1000 \text { bbis })\end{array}$ & $\begin{array}{c}\text { Distillate Fuel } \\
\text { Oil and Diesel } \\
\text { Fuel }{ }^{c} \\
(1000 \mathrm{bbls})\end{array}$ & $\begin{array}{l}\text { Natural Gas } \\
\text { (billion cu ft) }\end{array}$ & $\begin{array}{c}\text { LPG } \\
\text { (1000 bbls) }\end{array}$ & $\begin{array}{c}\text { Coal } \\
\text { (excluding } \\
\text { Coal Coke } \\
\text { and Breeze) } \\
\begin{array}{c}(1000 \text { short } \\
\text { tons) }\end{array} \\
\end{array}$ & $\begin{array}{c}\text { RSE } \\
\text { Row } \\
\text { Factors }\end{array}$ \\
\hline
\end{tabular}

33 PRIMARY METAL INDUSTRIES

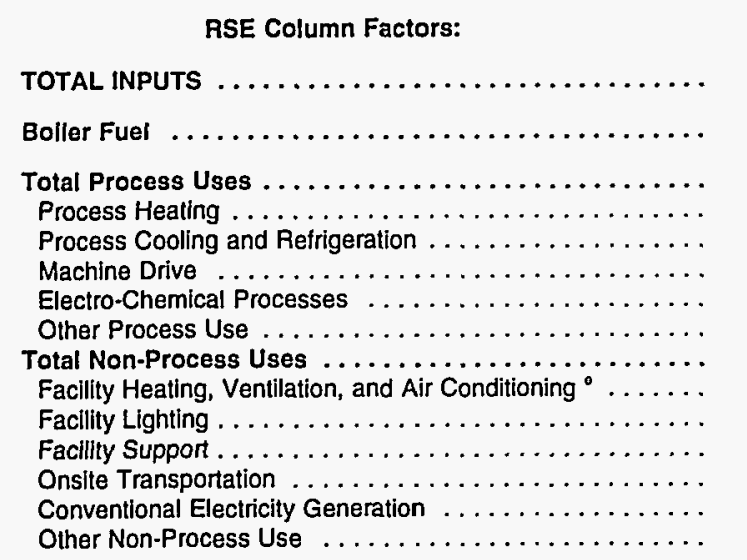

$\begin{array}{rr}0.6 & 1.2 \\ 153,499 & 5,285 \\ 416 & 3,963 \\ 139,042 & 1,268 \\ 30,085 & 1,261 \\ 966 & 0 \\ 39,262 & Q \\ 67,189 & - \\ 1,541 & 0 \\ 11,596 & Q \\ 4,971 & 0 \\ 5,232 & - \\ 1,071 & 0 \\ 173 & - \\ -- & 0 \\ 148 & 0 \\ 2,445 & 1\end{array}$

1.3
1,806
88
444
347
0
96
-
1
1,176
58
-
1
1,094
$Q$
$W$
99

0.7
666
90
507
501
$\vdots$
-
2
52
43
-
6
4
1
17

$\begin{array}{rrr}2.2 & 0.8 & \\ 888 & 2,054 & 4.6 \\ 38 & 1,690 & 7.6 \\ 298 & W & 7.2 \\ 239 & W & 8.0 \\ 0 & 0 & 12.7 \\ 54 & 0 & 7.5 \\ - & -- & 3.6 \\ 5 & 0 & 11.1 \\ 510 & W & 3.2 \\ 51 & 0 & 8.9 \\ -. & - & 5.4 \\ 10 & 0 & 9.1 \\ 442 & -- & 4.3 \\ * & W & 3.5 \\ 7 & 0 & 13.4 \\ 41 & 1 & 14.8\end{array}$

3312

Blast Furnaces and Steel Mills

RSE Column Factors:

$\begin{array}{rr}0.7 & 1.6 \\ 44,417 & 4,986 \\ 189 & 3,810 \\ 39,279 & 1,173 \\ 16,078 & 1,173 \\ 316 & 0 \\ 20,973 & 0 \\ 796 & - \\ 1,115 & 0 \\ 3,922 & 3 \\ 1,513 & 3 \\ 1,795 & -- \\ 484 & 0 \\ W & - \\ -- & 0 \\ W & 0 \\ 1,027 & 0\end{array}$

0.9
901
32
169
121
0
48
--
658
$W$
-
0
610
$:$
$W$
42

$\begin{array}{rr}0.7 & 1 \\ 387 & 74 \\ 61 & \\ 303 & \\ 301 & 19 \\ 0 & \\ 1 & \\ -- & \\ 1 & \\ 21 & \\ 16 & \\ -- & \\ 4 & \\ 0 & \\ * & \\ 1 & \\ 2 & \end{array}$

$\begin{array}{rrr}1.0 & 1.5 & \\ 74 & 1,075 & 4.4 \\ W & 1,056 & 6.7 \\ 22 & 17 & 5.2 \\ 19 & 17 & 5.3 \\ 0 & 0 & 9.2 \\ 2 & 0 & 7.0 \\ - & - & 13.8 \\ 2 & 0 & 13.4 \\ 37 & 0 & 5.2 \\ 5 & 0 & 7.4 \\ - & - & 4.6 \\ 6 & 0 & 8.9 \\ 26 & -- & 7.0 \\ . & 0 & 15.0 \\ 1 & 0 & 13.2 \\ W & 1 & 10.4\end{array}$

TOTAL INPUTS

$\begin{array}{rr}0.8 & N F \\ 4,386 & 0 \\ W & 0 \\ 4,153 & 0 \\ 3,158 & 0 \\ W & 0 \\ 814 & 0 \\ W & - \\ W & 0 \\ W & 0 \\ 61 & 0 \\ 108 & - \\ 20 & 0 \\ W & - \\ -- & 0 \\ 0 & 0 \\ 0 & 0\end{array}$

\begin{tabular}{|c|c|c|c|}
\hline 0.9 & 0.9 & 1.3 & 1.3 \\
\hline 20 & 1 & $w$ & $w$ \\
\hline 0 & • & 1 & $w$ \\
\hline$w$ & 1 & * & $w$ \\
\hline 0 & 1 & * & w \\
\hline 0 & 0 & 0 & 0 \\
\hline$w$ & . & * & 0 \\
\hline -- & -- & -- & -- \\
\hline 0 & - & 0 & 0 \\
\hline w & * & $w$ & 0 \\
\hline . & * & * & 0 \\
\hline -- & -- & .. & - \\
\hline 0 & • & * & 0 \\
\hline W & 0 & $w$ & - \\
\hline . & 0 & 0 & 0 \\
\hline 0 & 0 & 0 & 0 \\
\hline 0 & 0 & 0 & 0 \\
\hline
\end{tabular}

End Use Not Reported $\ldots \ldots \ldots \ldots \ldots \ldots \ldots \ldots$

Electrometalurgical Products

RSE Column Factors:

TOTAL INPUTS

Boiler Fuel $\ldots \ldots \ldots \ldots \ldots \ldots \ldots \ldots \ldots \ldots \ldots \ldots$

Total Process Uses $\ldots \ldots \ldots \ldots \ldots \ldots \ldots \ldots \ldots \ldots$

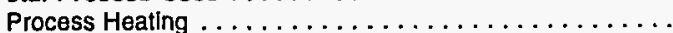

Process Cooling and Refrigeration .................

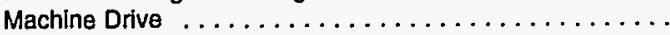

Electro-Chemical Processes ....................

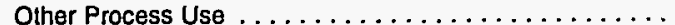

Total Non-Process Uses $\ldots \ldots \ldots \ldots \ldots \ldots \ldots \ldots$.

Facillity Heating, Ventilation, and Air Conditioning ${ }^{\circ} \ldots \ldots$.

Facility Lighting . . . . . . . . . . . . . . . . . . . . .

Facllity Suppont $\ldots \ldots \ldots \ldots \ldots \ldots \ldots \ldots \ldots \ldots$

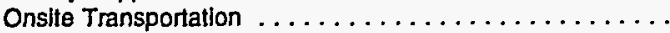

Conventional Electricity Generation .................

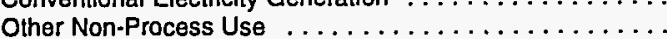

See footnotes at end of table. 
Table A38. Selected Combustible Inputs of Energy for Heat, Power, and Electricity Generation and Net Demand for Electricity by Fuel Type and End Use, 1991: Part 1 (Ciontinued) (Estimates in Btu or Physical Units)

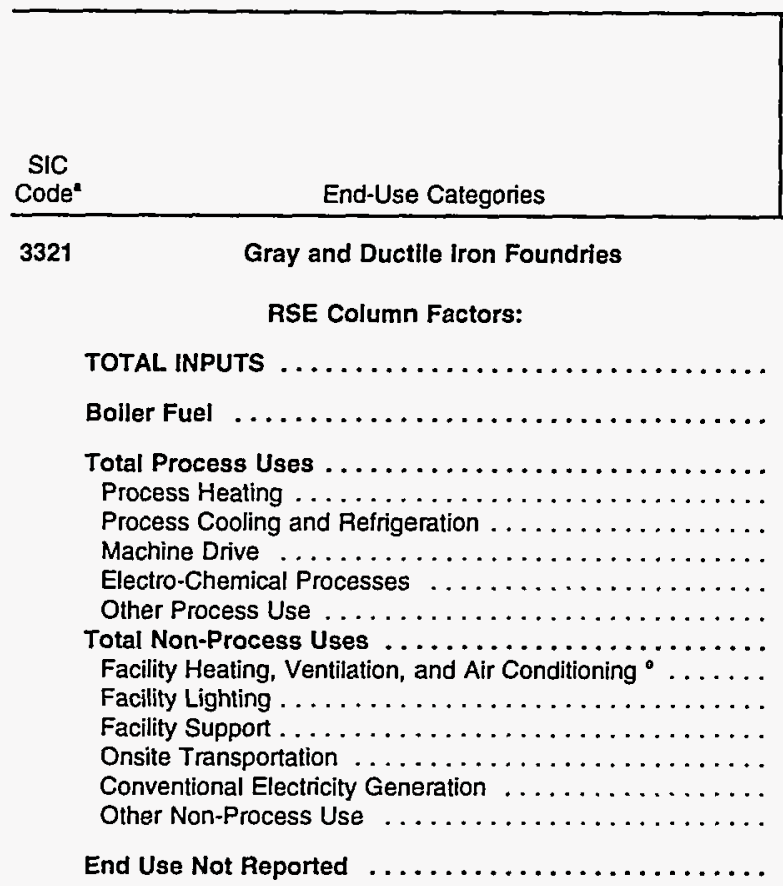

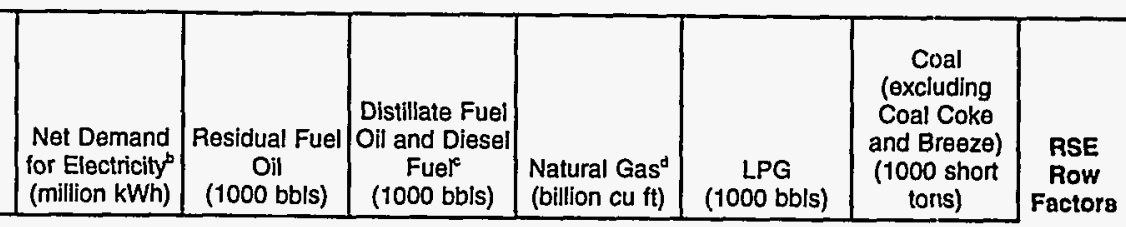

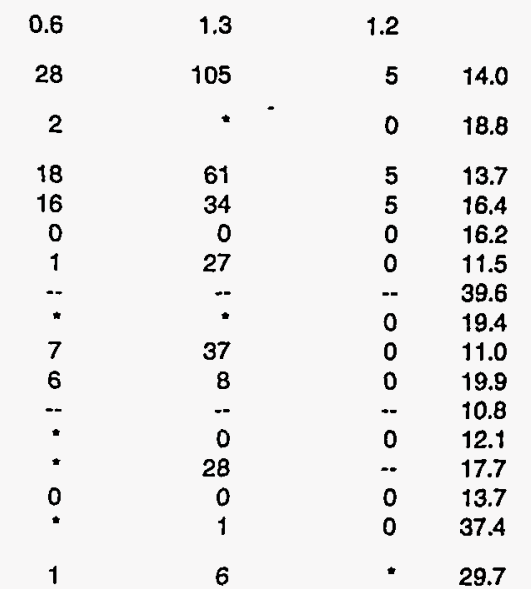

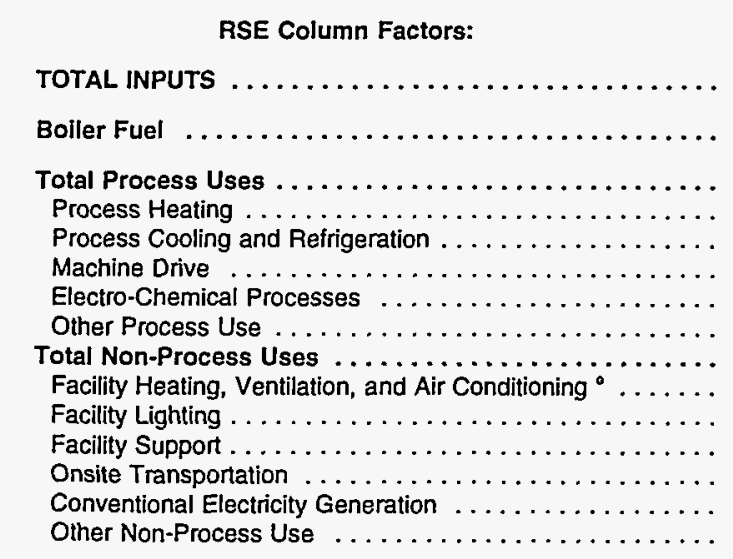

\begin{tabular}{|c|c|c|c|c|c|c|}
\hline 1.0 & 1.0 & 1.0 & 1.0 & 1.0 & 1.0 & \\
\hline 1,525 & $w$ & $w$ & 15 & 3 & $w$ & 1.0 \\
\hline$w$ & $w$ & 5 & 4 & 0 & 0 & 1.0 \\
\hline 1,385 & $w$ & W & 9 & . & $w$ & 1.0 \\
\hline 112 & $w$ & $w$ & 8 & * & $w$ & 1.0 \\
\hline$w$ & 0 & 0 & 0 & 0 & 0 & 1.0 \\
\hline 741 & 0 & 0 & 0 & • & 0 & 1.0 \\
\hline 267 & .. & .- & .. & -- & .. & 1.0 \\
\hline W & 0 & 0 & • & 0 & 0 & 1.0 \\
\hline W & 0 & $w$ & 2 & 2 & 0 & 1.0 \\
\hline 52 & 0 & : & 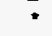 & . & 0 & 1.0 \\
\hline 59 & -. & -. & .- & .- & .. & 1.0 \\
\hline$w$ & 0 & 0 & * & 0 & 0 & 1.0 \\
\hline 0 & -- & $w$ & 0 & 2 & .. & 1.0 \\
\hline -- & 0 & 1 & 2 & 0 & 0 & 1.0 \\
\hline 0 & 0 & 0 & 0 & 0 & 0 & $\mathrm{NF}$ \\
\hline 0 & 0 & 0 & 0 & 1 & 0 & \\
\hline
\end{tabular}

$\begin{array}{rrrr}1.1 & 0.8 & 1.1 & N F \\ 127 & 20 & 42 & 0 \\ W & 2 & W & 0 \\ W & 16 & 18 & 0 \\ W & 16 & 18 & 0 \\ 0 & 0 & 0 & 0 \\ 2 & - & - & 0 \\ -. & 0 & 0 & 0 \\ 0 & 0 & 15 & 0 \\ 87 & 1 & 1 & 0 \\ . & 1 & - & - \\ -0 & -. & 0 & 0 \\ 0 & * & 14 & - \\ 87 & 0 & 0 & 0 \\ 0 & 0 & 1 & 0 \\ 0 & 0 & W & 0 \\ 2 & 1 & W\end{array}$


Table A38. Selected Combustible Inputs of Energy for Heat, Power, and Electricity Generation and Net Demand for Electricity by Fuel Type and End Use, 1991: Part 1 (Continued) (Estimates in Btu or Physical Units)

\begin{tabular}{|c|c|c|c|c|c|c|c|c|}
\hline $\begin{array}{c}\text { Sic } \\
\text { Coden }\end{array}$ & End-Use Categories & $\begin{array}{l}\text { Net Demand } \\
\text { for Electricity } \\
\text { (million kWh) }\end{array}$ & $\begin{array}{c}\text { Residual Fuel } \\
\text { Oil } \\
\text { (1000 bbls) }\end{array}$ & $\begin{array}{c}\text { Distillate Fuel } \\
\text { Oil and Diesel } \\
\text { Fuef } \\
\text { (1000 bbls) }\end{array}$ & $\begin{array}{l}\text { Natural Gas } \\
\text { (billion cu ft) }\end{array}$ & $\begin{array}{c}\text { LPG } \\
\text { (1000 bbls) }\end{array}$ & $\begin{array}{c}\text { Coal } \\
\text { (excluding } \\
\text { Coal Coke } \\
\text { and Breeze) } \\
\text { (1000 short } \\
\text { tons) }\end{array}$ & $\begin{array}{c}\text { RSE } \\
\text { Row } \\
\text { Factors }\end{array}$ \\
\hline
\end{tabular}

3339 Primary Nonferrous Metals, nec

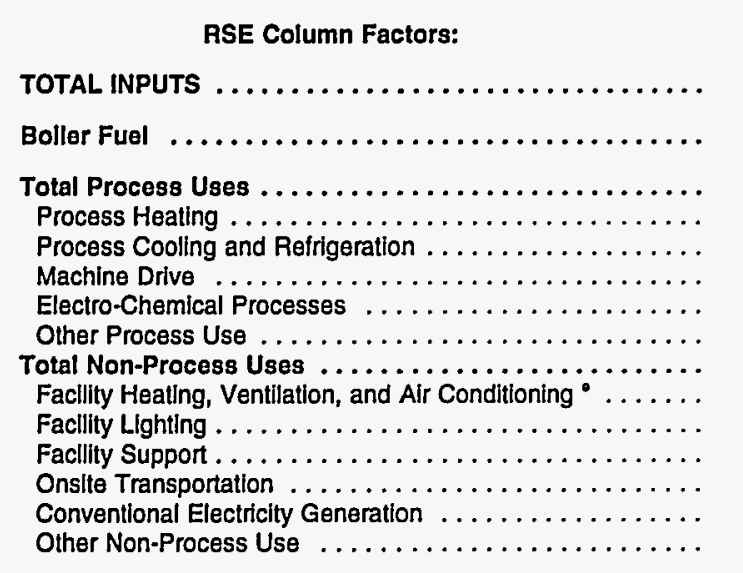

$\begin{array}{rrrrrrr}1.3 & 0.4 & 1.6 & 1.3 & 1.4 & 0.6 & \\ 4,433 & 1 & 53 & 16 & 19 & W & 1.9 \\ W & 1 & 3 & 3 & 0 & W & 2.8 \\ 4,170 & 0 & 15 & 7 & W & 0 & 1.6 \\ 1,378 & 0 & W & 7 & W & 0 & 1.3 \\ 19 & 0 & 0 & 0 & 0 & 0 & 3.7 \\ 668 & 0 & W & - & - & 0 & 1.0 \\ 2,102 & - & - & - & - & - & 1.5 \\ 2 & 0 & 0 & 0 & 0 & 0 & 15.0 \\ 232 & 0 & 34 & W & W & W & 1.5 \\ 120 & 0 & - & 1 & W & 0 & 2.5 \\ 85 & - & - & - & - & - & 2.2 \\ 24 & 0 & 0 & - & - & - & 4.9 \\ 2 & - & 33 & 0 & 11 & -. & 1.4 \\ - & 0 & 0 & W & 0 & W & 1.2 \\ 0 & 0 & 0 & 0 & 0 & 0 & \text { NF } \\ W & 0 & 1 & W & - & 0 & 1.8\end{array}$

End Use Not Reported

Aluminum Sheet, Plate, and Foll

RSE Column Factors:

\begin{tabular}{|c|c|c|c|c|c|c|}
\hline 1.2 & $\mathrm{NF}$ & 0.8 & 1.3 & 0.8 & 0.9 & \\
\hline 4,261 & 0 & 67 & 41 & 62 & $w$ & 1.1 \\
\hline$w$ & 0 & $*$ & 2 & 2 & w & 1.0 \\
\hline 3,562 & 0 & 4 & 37 & 19 & 0 & 1.2 \\
\hline 391 & 0 & 4 & 37 & $w$ & 0 & 1.4 \\
\hline$w$ & 0 & 0 & 0 & 0 & 0 & 1.7 \\
\hline 3,138 & 0 & - & 0 & $w$ & 0 & 1.1 \\
\hline 0 & .. & -- & - & - & -- & NF \\
\hline w & 0 & 0 & - & 0 & 0 & 0.8 \\
\hline 677 & 0 & 57 & 2 & 42 & 0 & 1.4 \\
\hline 234 & 0 & * & 2 & w & 0 & 1.4 \\
\hline 385 & -- & -. & .- & -. & -- & 0.8 \\
\hline 34 & 0 & • & - & - & 0 & 1.3 \\
\hline 24 & -- & 57 & 0 & $w$ & -- & 1.1 \\
\hline -. & 0 & 0 & 0 & 0 & 0 & NF \\
\hline 1 & 0 & 0 & 0 & 0 & 0 & 0.8 \\
\hline$Q$ & 0 & 5 & • & - & 0 & 1.3 \\
\hline
\end{tabular}

TOTAL INPUTS

............................

Boller Fuel $\ldots \ldots \ldots \ldots \ldots \ldots \ldots \ldots \ldots \ldots \ldots \ldots \ldots \ldots \ldots \ldots \ldots$

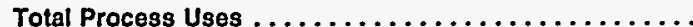

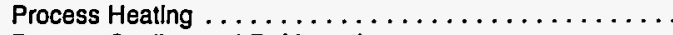

Process Cooling and Refrigeration $\ldots \ldots \ldots \ldots \ldots \ldots$

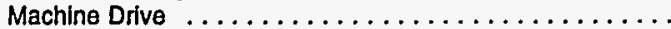

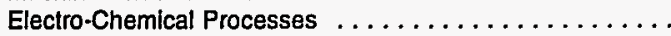

Other Process Use . . . . . . . . . . . . . . . . .

Total Non-Process Uses ....................

Facillity Heating, Ventllation, and Air Conditioning ${ }^{\circ} . \ldots \ldots$.

Facillty LIghting $\ldots \ldots \ldots \ldots \ldots \ldots \ldots \ldots \ldots$

Facility Support . . . . . . . . . . . . . . . . .

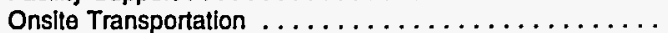

Conventional Electricity Generation $\ldots \ldots \ldots \ldots \ldots \ldots$

Other Non-Process Use $\ldots \ldots \ldots \ldots \ldots \ldots \ldots \ldots$

End Use Not Reported

\section{FABRICATED METAL PRODUCTS}

RSE Column Factors:

\begin{tabular}{|c|c|c|c|c|c|}
\hline 0.5 & 1.7 & 1.6 & 0.5 & 1.1 & 1.4 \\
\hline 29,899 & 501 & 994 & 169 & 1,122 & 245 \\
\hline 244 & 357 & 248 & 36 & $Q$ & 238 \\
\hline $\begin{array}{r}20,689 \\
3,399\end{array}$ & $\begin{array}{r}Q \\
14\end{array}$ & $\begin{array}{l}241 \\
160\end{array}$ & $\begin{array}{l}89 \\
86\end{array}$ & $\begin{array}{r}456 \\
W\end{array}$ & $\begin{array}{l}w \\
w\end{array}$ \\
\hline 697 & 0 & 1 & $\because$ & 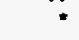 & 0 \\
\hline 15,139 & $\mathbf{Q}$ & 71 & 1 & $w$ & 0 \\
\hline 1,240 & -- & -- & -- & -- & -- \\
\hline 215 & $Q$ & $Q$ & 1 & 5 & 0 \\
\hline 6,939 & $\bar{Q}$ & 437 & 36 & 512 & 0 \\
\hline 2,905 & $\mathbf{Q}$ & 113 & 33 & 44 & 0 \\
\hline 3,376 & -- & - & -- & - & -. \\
\hline 533 & $Q$ & 1 & 2 & $Q$ & 0 \\
\hline 99 & -- & 320 & - & 464 & -- \\
\hline -. & 0 & 1 & 1 & 0 & 0 \\
\hline 25 & 0 & $Q$ & - & 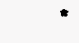 & 0 \\
\hline 2,027 & $Q$ & 68 & 8 & 136 & Q \\
\hline
\end{tabular}

TOTAL INPUTS

Boller Fuel $\ldots \ldots \ldots \ldots \ldots \ldots \ldots \ldots \ldots \ldots$

Total Process Uses . . . . . . . . . . . . . . . . . . .

Process Heating $\ldots \ldots \ldots \ldots \ldots \ldots \ldots \ldots \ldots \ldots$

Process Cooling and Refrigeration $\ldots \ldots \ldots \ldots \ldots \ldots$

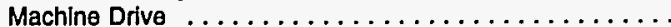

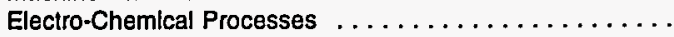

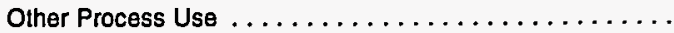

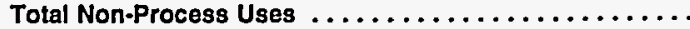

Facllity Heating, Ventilation, and Air Conditioning ${ }^{\circ} \ldots \ldots$.

Facllity Llghting . . . . . . . . . . . . . . . . . . .

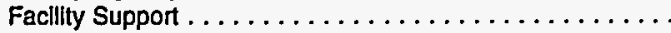

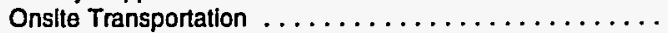

Conventional Electricity Generation . . . . . . . . . . .

Other Non-Process Use $\ldots \ldots \ldots \ldots \ldots \ldots \ldots \ldots$

End Use Not Reported

2,027 

and Net Demand for Electricity by Fuel Type and End Use, 1991: Part 1 (Ciontinued) (Estimates in Btu or Physical Units)

\begin{tabular}{|c|c|c|c|c|c|c|c|c|}
\hline $\begin{array}{c}\text { SIC } \\
\text { Code" }\end{array}$ & End-Use Categories & $\begin{array}{l}\text { Net Demand } \\
\text { for Electricity } \\
\text { (million kWh) }\end{array}$ & $\begin{array}{c}\text { Residual Fuel } \\
\text { Oil } \\
\text { (1000 bbls) }\end{array}$ & $\begin{array}{c}\text { Distillate Fuel } \\
\text { Oil and Diesel } \\
\text { Fuel } \\
(1000 \text { bbls })\end{array}$ & $\begin{array}{l}\text { Natural Gas } \\
\text { (billion cu ft) }\end{array}$ & $\begin{array}{c}\text { LPG } \\
\text { (1000 bbls) }\end{array}$ & $\begin{array}{c}\text { Coal } \\
\text { (excluding } \\
\text { Coal Coke } \\
\text { and Breeze) } \\
\text { (1000 short } \\
\text { tons) }\end{array}$ & $\begin{array}{c}\text { RSE } \\
\text { Row } \\
\text { Factorg }\end{array}$ \\
\hline
\end{tabular}

35 INDUSTAIAL MACHINERY and EQUIPMENT

RSE Column Factors:

TOTAL INPUTS

Boiler Fuel

Total Process Uses

Process Heating $\ldots \ldots \ldots \ldots \ldots \ldots \ldots \ldots \ldots \ldots$

Process Cooling and Reirigeration . . . . . . . . . . . .

Machine Drive

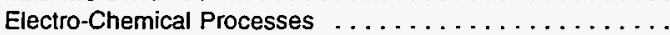

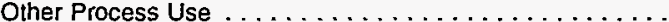

Total Non-Process Uses . . . . . . . . . . . . . . . . . . .

Facility Heating, Ventilation, and Air Conditioning ${ }^{\circ} \ldots \ldots$.

Facility Lighting . . . . . . . . . . . . . . . . . . . .

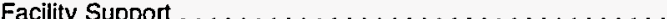

Onsite Transportation . . . . . . . . . . . . . . . .

Conventional Electricity Generation ...............

Other Non-Process Use . . . . . . . . . . . . . . . . . . .

End Use Not Reported

Computer and Office Equipment

RSE Column Factors:

TOTAL INPUTS

Boiler Fuel

Total Process Uses . . . . . . . . . . . . . . . . . .

Process Heating . . . . . . . . . . . . . . . . .

Process Cooling and Refrigeration . . . . . . . . . . . .

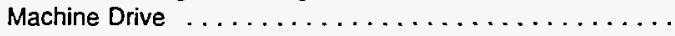

Electro-Chemical Processes .................

Other Process Use . . . . . . . . . . . . . . . . . . . .

Total Non-Process Uses . . . . . . . . . . . . . . .

Facility Heating, Ventilation, and Air Conditioning ${ }^{\circ} \ldots \ldots$.

Facility Lighting . .......................

Facility Support . . . . . . . . . . . . . . . . .

Onsite Transportation .....................

Conventional Electricity Generation ..............

Other Non-Process Use $\ldots \ldots \ldots \ldots \ldots \ldots \ldots \ldots \ldots$

End Use Not Reported

$\begin{array}{rr}0.4 & 2.0 \\ 29,629 & 490 \\ 168 & 426 \\ 16,489 & Q \\ 2,266 & 0 \\ 983 & 0 \\ 12,581 & * \\ 268 & - \\ 392 & * \\ 10,657 & 29 \\ 4,961 & 28 \\ 4,291 & - \\ 1,274 & 0 \\ 74 & - \\ -- & 0 \\ 56 & 0 \\ 2,315 & 20\end{array}$

1.2
718
152
186
3
0
89
--
94
359
222
-
4
76
$\mathrm{Q}$
46
20

0.7
106
27
36
31
$*$
3
.-
1
32
30
$-*$
1
0
$*$
$*$
11

1.4
651
35
230
94
0
$Q$
--
6
337
82
.-
$Q$
245
$Q$
$Q$

50

\begin{tabular}{|c|c|c|c|}
\hline 0.7 & 1.6 & NF & \\
\hline 5 & 4 & 0 & 15.0 \\
\hline 4 & $\star$ & 0 & 20.9 \\
\hline$\star$ & • & 0 & 19.9 \\
\hline$\star$ & • & 0 & 19.2 \\
\hline$\star$ & 0 & 0 & 31.5 \\
\hline$\star$ & • & 0 & 20.4 \\
\hline -- & - & -. & 24.6 \\
\hline * & 0 & 0 & 36.8 \\
\hline 1 & 4 & 0 & 18.5 \\
\hline 1 & 3 & 0 & 21.3 \\
\hline -- & -- & -- & 12.3 \\
\hline$\star$ & $*$ & 0 & 24.1 \\
\hline 0 & 1 & -- & 24.3 \\
\hline " & • & 0 & 25.2 \\
\hline$*$ & 0 & 0 & 43.6 \\
\hline . & * & 0 & 24.0 \\
\hline
\end{tabular}

ELECTRONIC and OTHER ELECTRIC EQUIPMENT

RSE Column Factors:

TOTAL INPUTS . ....................

Boiler Fuel $\ldots \ldots \ldots \ldots \ldots \ldots \ldots \ldots \ldots \ldots \ldots$

Total Process Uses $\ldots \ldots \ldots \ldots \ldots \ldots \ldots \ldots \ldots \ldots \ldots$

Process Heating . . . . . . . . . . . . . . . . . . . .

Process Cooling and Refrigeration . . . . . . . . . .

Machine Drive .....................

Electro-Chemical Processes . . . . . . . . . . . . . .

Other Process Use . . . . . . . . . . . . . . . . .

Total Non-Process Uses . . . . . . . . . . . . . . . .

Facility Heating, Ventilation, and Air Conditioning ${ }^{\circ} \ldots \ldots$.

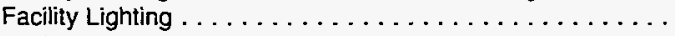

Facilty Support . . . . . . . . . . . . . . . . . . . . .

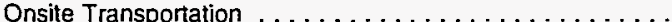

Conventional Electricity Generation . . . . . . . . . . . .

Other Non-Process Use . . . . . . . . . . . . . . .

End Use Not Reported

$\begin{array}{rr}0.5 & 1.5 \\ 4,405 & 11 \\ 17 & 8 \\ 1,579 & 0 \\ 184 & 0 \\ 428 & 0 \\ 680 & 0 \\ 54 & - \\ 233 & 0 \\ 2,364 & 3 \\ 1,149 & 3 \\ 734 & - \\ 429 & 0 \\ 4 & - \\ -- & 0 \\ 48 & 0 \\ 446 & 1\end{array}$

$\begin{array}{rr}1.2 & 0.7 \\ 16 & 5 \\ 5 & 4 \\ * & * \\ 0 & * \\ 0 & * \\ * & * \\ 0 & * \\ 11 & * \\ -- & 1 \\ 1 & * \\ 1 & * \\ * & * \\ Q & *\end{array}$

15.0

20.9

19.2

31.5

24.6

36.8

21.3

12.3

24.3

25.2

24.0

\begin{tabular}{|c|c|c|c|c|c|}
\hline 0.4 & 1.6 & 1.2 & 0.6 & 1.1 & 1.7 \\
\hline 30,013 & 612 & 416 & 76 & 396 & $w$ \\
\hline 171 & 571 & 233 & 29 & 22 & $w$ \\
\hline 17,387 & $Q$ & 27 & 31 & 180 & 6 \\
\hline 4,662 & $a$ & 22 & 29 & 159 & 6 \\
\hline 2,180 & 1 & $\star$ & $Q$ & * & 0 \\
\hline 9,003 & $*$ & 3 & 1 & 13 & 0 \\
\hline 1,319 & - & -- & -- & -. & -- \\
\hline 222 & 0 & 1 & $*$ & 8 & 0 \\
\hline 11,114 & 36 & 110 & 15 & 177 & 1 \\
\hline 6,100 & 36 & 77 & 14 & 55 & 1 \\
\hline 3,825 & - & - & -. & - & $\ldots$ \\
\hline 1,059 & 0 & $Q$ & 1 & $\mathbf{Q}$ & 0 \\
\hline 76 & -- & 24 & 0 & 119 & -- \\
\hline -- & 0 & 2 & * & $\star$ & 0 \\
\hline 53 & 0 & 3 & * & $Q$ & 0 \\
\hline 1,341 & * & 47 & 2 & 16 & 0 \\
\hline
\end{tabular}


Table A38. Selected Combustible Inputs of Energy for Heat, Power, and Electricity Generation and Net Demand for Electricity by Fuel Type and End Use, 1991: Part 1 (Continued) (Estimates in Btu or Physical Units)

\begin{tabular}{|c|c|c|c|c|c|c|c|c|}
\hline $\begin{array}{c}\text { SIC } \\
\text { Code' }\end{array}$ & End-Use Categories & $\begin{array}{l}\text { Net Demand } \\
\text { for Electricity } \\
\text { (million } \mathrm{kWh} \text { ) }\end{array}$ & $\begin{array}{c}\text { Residual Fuel } \\
\text { Oif } \\
\text { (1000 bbls) }\end{array}$ & $\begin{array}{c}\text { Distillate Fuel } \\
\text { Oil and Diesel } \\
\text { Fuef } \\
(1000 \text { bbls })\end{array}$ & $\begin{array}{l}\text { Natural Gas } \\
\text { (billion cu ft) }\end{array}$ & $\begin{array}{c}\text { LPG } \\
\text { (1000 bbls) }\end{array}$ & $\begin{array}{c}\text { Coal } \\
\text { (excluding } \\
\text { Coal Coke } \\
\text { and Breeze) } \\
\text { (1000 short } \\
\text { tons) }\end{array}$ & $\begin{array}{c}\text { RSE } \\
\text { Row } \\
\text { Factors }\end{array}$ \\
\hline
\end{tabular}

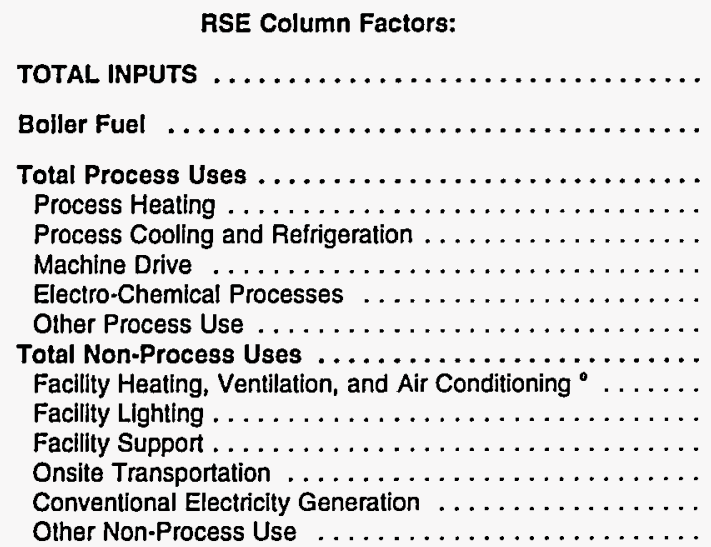

$\begin{array}{rr}0.5 & 1.4 \\ 35,355 & 1,865 \\ 241 & W \\ 20,391 & 18 \\ 3,071 & 13 \\ 1,558 & 0 \\ 14,718 & Q \\ 469 & -- \\ 574 & - \\ 12,796 & W \\ 6,071 & 43 \\ 5,362 & -- \\ 1,049 & W \\ 132 & - \\ -- & W \\ 182 & W \\ 1,927 & O\end{array}$

1.3
1,214
261
167
$W$
0
129
--
$W$
734
$W$
--
5
310
$W$
180
52

0.7
129
47
51
48
2
--
1
27
24
-
2
$\vdots$
1
3

$\begin{array}{rrr}1.5 & 1.1 & \\ 526 & 1.464 & 5.1 \\ 36 & W & 7.0 \\ 154 & W & 10.8 \\ 97 & W & 8.3 \\ 0 & 0 & 14.7 \\ Q & 0 & 14.3 \\ -- & -. & 10.6 \\ Q & 0 & 15.9 \\ 315 & 0 & 7.1 \\ 69 & 0 & 10.4 \\ -. & -. & 6.4 \\ 2 & 0 & 8.3 \\ 241 & -. & 8.1 \\ . & 0 & 14.4 \\ 2 & 0 & 15.2 \\ 21 & 0 & 13.0\end{array}$

3711 Motor Vehicles and Car Bodies

RSE Column Factors:

$\begin{array}{rr}0.6 & 1 \\ 7,705 & 408 \\ W & \\ 4,960 & \\ 538 & \\ 470 & \\ 3,580 & \\ W & \\ W & \\ 2,602 & \\ 1,448 & \\ 925 & \\ 182 & \\ W & \\ -. & \\ W & \\ W & \end{array}$

TOTAL INPUTS

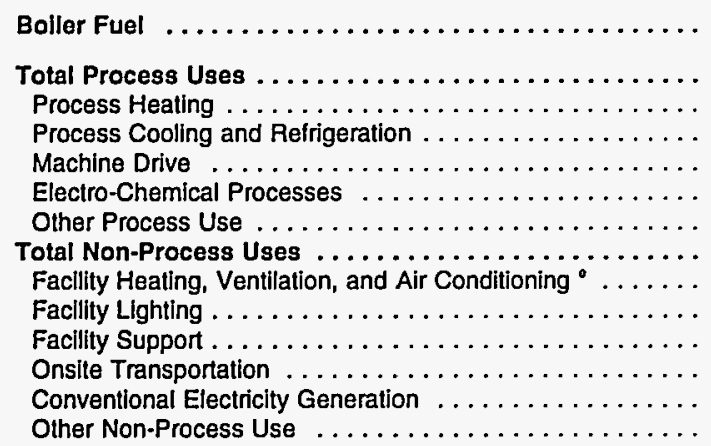

End Use Not Reported

$\begin{array}{rr}1.8 & 1.2 \\ 408 & 65 \\ W & 6 \\ W & W \\ W & : \\ 0 & 0 \\ 0 & W \\ -- & \div \\ 0 & : \\ W & 46 \\ W & W \\ -- & - \\ 0 & W \\ -- & 26 \\ 0 & : \\ 0 & 11 \\ 0 & W\end{array}$

\begin{tabular}{c}
0.7 \\
44 \\
11 \\
24 \\
23 \\
$\vdots$ \\
\hdashline \\
9 \\
8 \\
- \\
1 \\
0 \\
0 \\
$:$ \\
.
\end{tabular}

$\begin{array}{rrr}1.1 & 1.1 & \\ 59 & W & 3.0 \\ 1 & W & 4.7 \\ 11 & 0 & 4.5 \\ 11 & 0 & 5.0 \\ 0 & 0 & 9.5 \\ . & 0 & 8.8 \\ -. & -- & 7.2 \\ 40 & 0 & 9.7 \\ 11 & 0 & 3.8 \\ -- & 0 & 5.6 \\ 29 & -- & 5.4 \\ 0 & 0 & 7.3 \\ 0 & -- & 4.0 \\ 6 & 0 & 6.9 \\ 6 & 0 & 8.6 \\ & 0 & 10.7\end{array}$

3714

Motor Vehicle Parts and Accessories

RSE Column Factors:

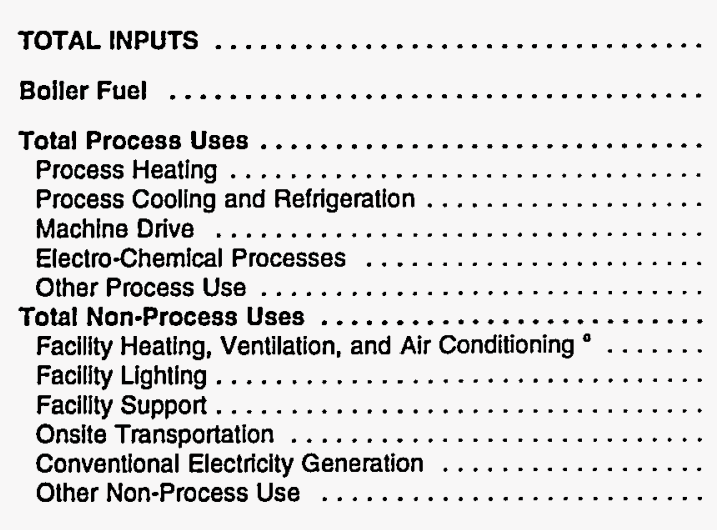

End Use Not Reported

$\begin{array}{rr}0.4 & 2.0 \\ 11,054 & 60 \\ 47 & 55 \\ 7,497 & 3 \\ 927 & 1 \\ 502 & 0 \\ 5,854 & 0 \\ 144 & -- \\ 69 & - \\ 3,087 & 2 \\ 1,526 & 2 \\ 1,231 & -- \\ 253 & 0 \\ W & -. \\ -- & 0 \\ W & - \\ 424 & *\end{array}$

\begin{tabular}{rc}
1.5 & 1.0 \\
168 & $W$ \\
$W$ & $W$ \\
32 & $W$ \\
22 & $W$ \\
0 & 0 \\
10 & 0 \\
-- & -- \\
0 & 0 \\
120 & 0 \\
$Q$ & 0 \\
-- & - \\
\hline & 0 \\
107 & -- \\
0 & 0 \\
$*$ & 0 \\
$W$ & 0 \\
\hline
\end{tabular}


Table A38. Selected Combustible Inputs of Energy for Heat, Power, and Electricity Generation and Net Demand for Electricity by Fuel Type and End Use, 1991: Part 1 (Continued) (Estimates in Btu or Physical Units)

\begin{tabular}{|c|c|c|c|c|c|c|c|c|}
\hline $\begin{array}{l}\text { SIC } \\
\text { Code" } \\
\end{array}$ & End-Use Categories & $\begin{array}{l}\text { Net Demand } \\
\text { for Electricity } \\
\text { (million kWh) }\end{array}$ & $\begin{array}{c}\text { Residual Fuel } \\
\text { Oil } \\
(1000 \text { bbls })\end{array}$ & $\begin{array}{c}\text { Distillate Fuel } \\
\text { Oil and Diesel } \\
\text { Fuel } \\
(1000 \text { bbls })\end{array}$ & $\begin{array}{c}\text { Natural Gas } \\
\text { (billion cu ft) }\end{array}$ & $\begin{array}{c}\text { LPG } \\
\text { (1000 bbls) }\end{array}$ & $\begin{array}{c}\text { Coal } \\
\text { (excluding } \\
\text { Coal Coke } \\
\text { and Breaze) } \\
\text { (1000 short } \\
\text { tons) }\end{array}$ & $\begin{array}{c}\text { RSE } \\
\text { Row } \\
\text { Factors }\end{array}$ \\
\hline
\end{tabular}

38 INSTRUMENTS and RELATED PRODUCTS

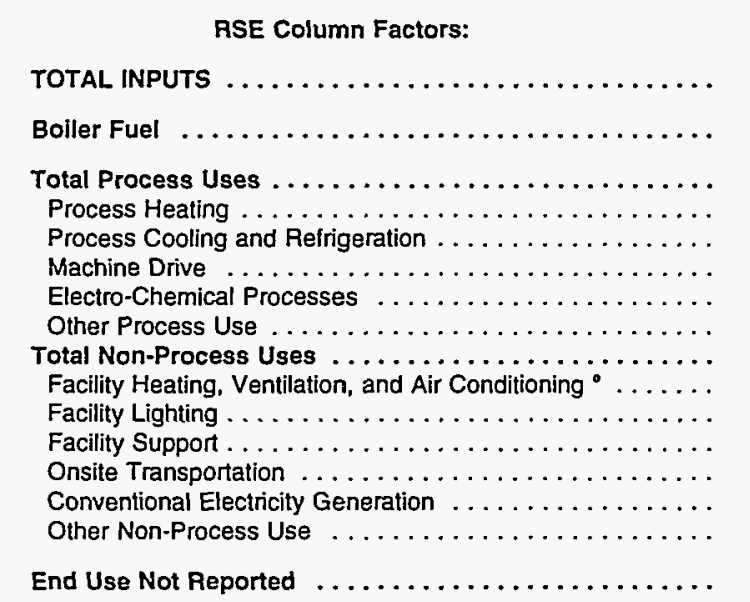

\begin{tabular}{|c|c|c|c|c|c|c|}
\hline 0.5 & 0.8 & 1.3 & 0.8 & 1.4 & 1.5 & \\
\hline 13,673 & 536 & w & 25 & $Q$ & $w$ & 12.8 \\
\hline 285 & 492 & $w$ & 14 & $Q$ & $w$ & 17.7 \\
\hline $\begin{array}{r}5,878 \\
800\end{array}$ & $\begin{array}{l}Q \\
0\end{array}$ & $\begin{array}{l}15 \\
W\end{array}$ & $\begin{array}{l}4 \\
3\end{array}$ & $\begin{array}{r}12 \\
9\end{array}$ & $\begin{array}{l}0 \\
0\end{array}$ & $\begin{array}{l}15.9 \\
20.1\end{array}$ \\
\hline 1,085 & $Q$ & $\because$ & : & 0 & 0 & 26.4 \\
\hline 3,523 & 0 & w & - & $Q$ & 0 & 23.4 \\
\hline 161 & -- & -- & .. & .- & .. & 21.4 \\
\hline 308 & 0 & - & * & 1 & 0 & 27.8 \\
\hline 6,845 & $Q$ & 62 & 6 & 27 & 0 & 15.5 \\
\hline 3,499 & 0 & 23 & 6 & $Q$ & 0 & 16.2 \\
\hline 2,539 & - & -. & -- & .- & -. & 11.7 \\
\hline 622 & * & 2 & $\cdot$ & $Q$ & 0 & 18.1 \\
\hline 14 & -- & 36 & 0 & 9 & .- & 17.2 \\
\hline .. & 0 & 1 & * & 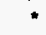 & 0 & 23.1 \\
\hline 171 & $Q$ & * & * & * & 0 & 29.0 \\
\hline 665 & $Q$ & $\mathbf{Q}$ & 1 & $Q$ & 1 & 25.7 \\
\hline
\end{tabular}

\section{Surgical and Medical instruments}

RSE Column Factors:

\begin{tabular}{|c|c|c|c|c|c|}
\hline 0.6 & 1.8 & 1.2 & 0.7 & 1.1 & NF \\
\hline 1,161 & 9 & 30 & 2 & 8 & 0 \\
\hline 10 & 9 & 21 & 1 & * & 0 \\
\hline $\begin{array}{r}530 \\
61 \\
88\end{array}$ & $\begin{array}{l}0 \\
0 \\
0\end{array}$ & $\begin{array}{l}4 \\
3 \\
\end{array}$ & $\dot{*}$ & $\begin{array}{l}6 \\
5 \\
0\end{array}$ & $\begin{array}{l}0 \\
0 \\
0\end{array}$ \\
\hline 379 & 0 & " & • & Q & 0 \\
\hline 2 & -- & -. & .. & $=$ & -. \\
\hline 1 & 0 & * & 0 & " & 0 \\
\hline 538 & 0 & 6 & 1 & 2 & 0 \\
\hline 336 & 0 & 4 & 1 & . & 0 \\
\hline 166 & -- & -- & -- & -- & .. \\
\hline 35 & 0 & * & . & * & 0 \\
\hline 2 & -- & 2 & 0 & 1 & -- \\
\hline -. & 0 & 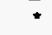 & : & 0 & 0 \\
\hline " & 0 & - & * & 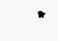 & 0 \\
\hline 83 & • & 0 & * & • & 0 \\
\hline
\end{tabular}

TOTAL INPUTS

Boiler Fuel

$\ldots \ldots \ldots \ldots \ldots \ldots \ldots \ldots \ldots \ldots$

Total Process Uses

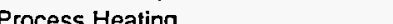

Process Cooling and Refrigeration ...............

Machine Drive

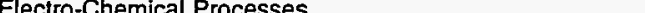

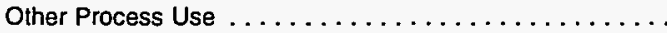

Total Non-Process Uses . . . . . . . . . . . . . . .

Facility Heating, Ventilation, and Air Conditioning ${ }^{\circ} \ldots \ldots$.

Facility Lighting $\ldots \ldots \ldots \ldots \ldots \ldots \ldots \ldots \ldots \ldots$

Facility Support . . .

Onsite Transportation

Other Non-Process Use $\ldots \ldots \ldots \ldots \ldots \ldots \ldots \ldots \ldots$

End Use Not Reported

$\ldots \ldots \ldots \ldots \ldots \ldots \ldots \ldots$

See footnotes at end of table. 
Table A38. Selected Combustible Inputs of Energy for Heat, Power, and Electricity Generation and Net Demand for Electricity by Fuel Type and End Use, 1991: Part 1 (Continued) (Estimates in Btu or Physical Units)

\begin{tabular}{|c|c|c|c|c|c|c|c|c|}
\hline $\begin{array}{l}\text { SIC } \\
\text { Code }\end{array}$ & End-Use Categories & $\begin{array}{l}\text { Net Demand } \\
\text { for Electricity } \\
\text { (million kWh) }\end{array}$ & $\begin{array}{c}\text { Residual Fuel } \\
\text { Oil } \\
(1000 \text { bbls })\end{array}$ & $\begin{array}{c}\text { Distillate Fuel } \\
\text { Oil and Diesel } \\
\text { Fuel } \\
\text { (1000 bbls })\end{array}$ & $\begin{array}{l}\text { Natural Gas } \\
\text { (billion cu ft) }\end{array}$ & $\begin{array}{c}\text { LPG } \\
(1000 \text { bbis })\end{array}$ & $\begin{array}{c}\text { Coal } \\
\text { (excluding } \\
\text { Coal Coke } \\
\text { and Breeze) } \\
\text { (1000 short } \\
\text { tons) }\end{array}$ & $\begin{array}{l}\text { RSE } \\
\text { Row } \\
\text { Factors }\end{array}$ \\
\hline \multirow[t]{16}{*}{39} & \multicolumn{8}{|l|}{ MISC. MANUFACTURING INDUSTRIES } \\
\hline & RSE Column Factors: & 0.5 & 1.2 & 1.3 & 0.7 & 1.0 & 1.8 & \\
\hline & TOTAL INPUTS $\ldots \ldots \ldots \ldots \ldots \ldots \ldots \ldots \ldots \ldots$ & 3,661 & 115 & $w$ & 14 & w & 32 & 13.3 \\
\hline & Boller Fuel $\ldots \ldots \ldots \ldots \ldots \ldots \ldots$ & 29 & 99 & w & 5 & $w$ & 30 & 20.9 \\
\hline & $\begin{array}{l}\text { Total Process Uses } \ldots \ldots \ldots \ldots \ldots \ldots \ldots \ldots \ldots \ldots \ldots \ldots \\
\text { Process Heating } \ldots \ldots \ldots \ldots \ldots \ldots \ldots \ldots \ldots \ldots \ldots \\
\text { Process Cooling and Refrigeration } \ldots \ldots \ldots \ldots \ldots \ldots \ldots\end{array}$ & $\begin{array}{r}2,140 \\
503 \\
183\end{array}$ & $\begin{array}{l}Q \\
Q \\
0\end{array}$ & $\begin{array}{l}9 \\
Q \\
0\end{array}$ & $\begin{array}{l}5 \\
5\end{array}$ & $\begin{array}{r}19 \\
8 \\
0\end{array}$ & $\begin{array}{l}0 \\
0 \\
0\end{array}$ & $\begin{array}{l}18.7 \\
19.9 \\
33.3\end{array}$ \\
\hline & Machine Drive & 1,399 & 0 & 1 & * & 10 & 0 & 29.6 \\
\hline & Electro-Chemical Processes ... & 54 & -- & -- & -- & -. & -- & 67.6 \\
\hline & Other Process Use . . . . . . . . & - & 0 & * & - & 1 & 0 & 55.7 \\
\hline & Total Non-Process Uses $\ldots \ldots \ldots \ldots \ldots \ldots \ldots \ldots \ldots$ & 1,210 & 12 & 58 & 4 & 40 & Q & 20.5 \\
\hline & Facility Heating, Ventilation, and Air Conditioning ${ }^{\circ}$ & 592 & 12 & 39 & 3 & $\mathbf{Q}$ & Q & 23.6 \\
\hline & Facllity Lighting $\ldots \ldots \ldots \ldots \ldots \ldots \ldots \ldots \ldots$ & 484 & -- & -- & -- & -- & -- & 16.9 \\
\hline & Facllity Support ..... & 87 & - & * & * & 2 & O & 30.6 \\
\hline & Onslte Transportation & 13 & -- & $\mathbf{Q}$ & * & 27 & -. & 20.0 \\
\hline & Conventional Electricity Generation & .- & 0 & - & 0 & 0 & 0 & 29.0 \\
\hline & Other Non-Process Use .......... & 34 & 0 & 2 & : & 2 & 0 & 39.5 \\
\hline & End Use Not Reported $\ldots \ldots \ldots \ldots \ldots \ldots \ldots \ldots \ldots$ & 281 & 1 & 25 & 1 & 5 & 0 & 37.5 \\
\hline
\end{tabular}

\footnotetext{
" See Appendices $B$ and $F$ for descriptions of the Standard Industrial Classification system.

b "Net Demand for Electricity" is the sum of purchases, transfers in, and total onsite electricity generation, minus sales and transfers offsite. It is the total amount of electricity used by establlshments. "Net Demand for Electricity" is not directly comparable with "Net Electricity" which specifically excludes electricity generated onsite by combustible energy sources.

Includes Nos. 1, 2, and 4 fuel oils and Nos. 1, 2, and 4 diesel fuels.

- "Natural Gas" Includes natural gas obtained from utilities, transmission pipelines, and any other supplier(s) such as brokers and producers.

- Excludes steam and hot water.

NF=No applicable RSE row/column factor.

- Estimate less than 0.5. Data are included in higher level totals.

$W=$ Withheld to avoid disclosing data for individual establishments. Data are included in higher level totals.

$Q=$ Withheld because Relative Standard Error is greater than 50 percent. Data are included in higher level totals.

- Estimation of energy input is not applicable.

$N A=$ Not available. Data are included in higher level totals.

Notes: • To obtain a RSE percentage for any table cell, multiply the cell's corresponding RSE column and RSE row factors. - Totals may not equal sum of components because of independent rounding. - The estimates presented in this table are for the total consumption of energy for the production of heat and power, regardless of where the energy was produced. Specifically, the estimates include the quantities of energy that were originally produced offsite and purchased by or transferred to the establishment, plus those that were produced onsite from other energy or input materials not classified as energy, or were extracted from captive (onsite) mines or wells. - Allocations to specific end uses are made on the basis of reasonable approximations by respondents.

Source: Energy Information Administration, Office of Energy Markets and End Use, Energy End Use and Integrated Statistics Division, Form ElA-846, "1991 Manulacturing Energy Consumption Survey."
} 
Table A38. Selected Combustible Inputs of Energy for Heat, Power, and Electricity Generation and Net Demand for Electricity by Fuel Type and End Use, 1991: Part 2 (Estimates in Trillion Btu)

\begin{tabular}{|c|c|c|c|c|c|c|c|c|}
\hline $\begin{array}{c}\text { SIC } \\
\text { Code }\end{array}$ & End-Use Categories & $\begin{array}{l}\text { Net Demand } \\
\text { for Electricity }\end{array}$ & $\begin{array}{c}\text { Residual Fuel } \\
\text { Oil }\end{array}$ & $\begin{array}{c}\text { Distillate Fuel } \\
\text { Oil and Diesel } \\
\text { Fuel }^{c}\end{array}$ & Natural Gas ${ }^{\mathrm{d}}$ & LPG & $\begin{array}{c}\text { Coal } \\
\text { (excluding } \\
\text { Coall Coke } \\
\text { and Breeze) }\end{array}$ & $\begin{array}{c}\text { RSE } \\
\text { Row } \\
\text { Factors }\end{array}$ \\
\hline
\end{tabular}

20-39 ALL INDUSTRY GROUPS

RSE Column Factors:

TOTAL INPUTS

Boiler Fuel

Total Process Uses

$\ldots \ldots \ldots \ldots \ldots \ldots$

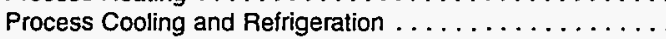

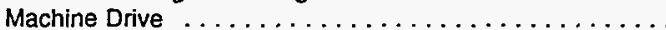

Electro-Chemical Processes $\ldots \ldots \ldots \ldots \ldots \ldots \ldots$

Other Process Use . . . . . . . . . . . . . . . .

Total Non-Process Uses . . . . . . . . . . . . . . .

Facility Heating, Ventilation, and Air Conditioning ${ }^{\circ}$.......

Facility Lighting . . . . . . . . . . . . . . . . .

Facility Support . . . . . . . . . . . . . . . . .

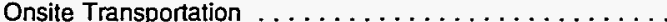

Conventional Electricity Generation . . . . . . . . . .

Other Non-Process Use $\ldots . . \ldots \ldots \ldots \ldots \ldots \ldots . . .$.

End Use Not Reported

$\begin{array}{rr}0.4 & 1.7 \\ 2,799 & 414 \\ 32 & 296 \\ 2,244 & 109 \\ 244 & 107 \\ 140 & - \\ 1,482 & 2 \\ 361 & - \\ 17 & - \\ 429 & 7 \\ 206 & 4 \\ 176 & -- \\ 39 & W \\ 4 & - \\ -- & 2 \\ 4 & W \\ 94 & 2\end{array}$

FOOD and KINDRED PRODUCTS

RSE Column Factors:

TOTAL INPUTS

Boiler Fuel

Total Process Uses

Process Heating $\ldots \ldots \ldots \ldots \ldots \ldots \ldots \ldots \ldots$

Process Cooling and Refrigeration . .............

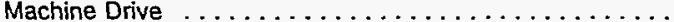

Electro-Chemical Processes $\ldots \ldots \ldots \ldots \ldots \ldots \ldots$

Other Process Use . . . . . . . . . . . . . . . . .

Total Non-Process Uses . . . . . . . . . . . . . . . . . .

Facility Heating, Ventilation, and Air Conditioning ${ }^{\circ} \ldots \ldots \ldots$

Facility Lighting . . . . . . . . . . . . . . .

Facility Support . . . . . . . . . . . . . . . . .

Onsite Transportation $\ldots \ldots \ldots \ldots \ldots \ldots \ldots \ldots \ldots \ldots$

Conventional Electricity Generation . . . . . . . . . . .

Other Non-Process Use $\ldots \ldots \ldots \ldots \ldots \ldots$

End Use Not Reported

Meat Packing Plants

RSE Column Factors:

TOTAL INPUTS

Boiler Fue

Total Process Uses . . . . . . . . . . . . . . . . .

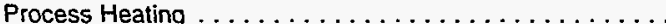

Process Cooling and Refrigeration . . . . . . . . . .

Machine Drive .........................

Electro-Chemical Processes . . . . . . . . . . . . . .

Other Process Use . . . . . . . . . . . . . . . .

Total Non-Process Uses . . . . . . . . . . . . . . . .

Facility Heating, Ventilation, and Air Conditioning ${ }^{\circ} \ldots \ldots$.

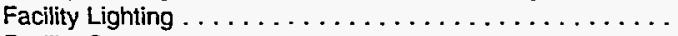

Facility Support . . . . . . . . . . . . . . . . .

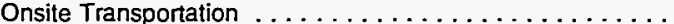

Conventional Electricity Generation . . . . . . . . . .

Other Non-Process Use . . . . . . . . . . . . . . . .

End Use Not Reported
189

189

5

147

7

94

29
13

13
13

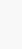

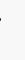

7

0.4

12

1.6

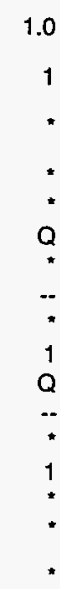

0.7
5,506
2,098
2,578
2,381
13
127
--
56
702
283
--
23
$*$
347
49
128

1.0

1.6

$1,184 \quad 3.0$

$859 \quad 3.6$

$314 \quad 4.1$

$314 \quad 4.1$

12.4

8.4

7.7

14.0

4.2

6.8

5.1

9.6

9.4

8.8
10.9

10.6

128

w

0.7
512
315
144
137
$W$
$W$
--
2
35
20
$-\because$
2
12
$*$
17

0.9

154

5.9

143

8.4

9.4

11.8

17.6

17.8

NF

33.2

11.4

16.1

8.5

17.5
10.5

22.6

22.3

24.5

1

17

Q

\begin{tabular}{ccc}
0.6 & 1.4 & 1.8 \\
32 & 1 & 1 \\
22 & $*$ & 1 \\
6 & $*$ & 0 \\
6 & $*$ & 0 \\
$*$ & $*$ & 0 \\
$-*$ & $-\cdot$ & 0 \\
3 & 0 & 0 \\
2 & $*$ & 0 \\
$-*$ & 0 & 0 \\
$*$ & $*$ & 0 \\
$*$ & 0 & - \\
1 & 0 & 0 \\
0 & $*$ & 0 \\
$*$ & 0 & 0 \\
\hline
\end{tabular}

10.1

12.9

14.5

17.0

17.8

19.8

36.3

13.2

10.2

14.0

22.9

25.9

36.2

27.6

33.4

See footnotes at end of table. 
Table A38. Selected Combustible Inputs of Energy for Heat, Power, and Electricity Generation and Net Demand for Electricity by Fuel Type and End Use, 1991: Part 2 (Continued) (Estimates in Trillion Btu)

\begin{tabular}{|c|c|c|c|c|c|c|c|c|}
\hline $\begin{array}{c}\text { SIC } \\
\text { Code* }\end{array}$ & End-Use Categories & $\begin{array}{l}\text { Net Demand } \\
\text { for Electricity" }\end{array}$ & $\begin{array}{c}\text { Residual Fuel } \\
\text { Oil }\end{array}$ & $\begin{array}{c}\text { Distillate Fuel } \\
\text { Oil and Diesel } \\
\text { Fuele }\end{array}$ & Natural Gas ${ }^{d}$ & LPG & $\begin{array}{c}\text { Coal } \\
\text { (excluding } \\
\text { Coal Coke } \\
\text { and Breeze) }\end{array}$ & $\begin{array}{c}\text { RSE } \\
\text { Row } \\
\text { Factors }\end{array}$ \\
\hline
\end{tabular}

2033

Canned Fruits and Vegetables

RSE Column Factors:

0.7

TOTAL INPUTS

Boiler Fuel

Total Process Uses

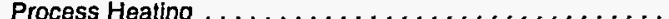

Process Cooling and Refrigeration . . . . . . . . . . . .

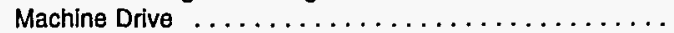

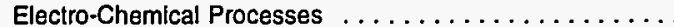

Other Process Use . . . . . . . . . . . . . . . . .

Total Non-Process Uses .....................

Facility Heating, Ventilation, and Air Conditioning ${ }^{\circ} . . . \ldots$.

Facillty Lighting $\ldots \ldots \ldots \ldots \ldots \ldots \ldots \ldots \ldots$

Facilly Support . . . . . . . . . . . . . . . . . .

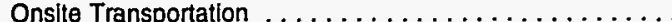

Conventional Electricity Generation $\ldots \ldots \ldots \ldots \ldots \ldots$

Other Non-Process Use $\ldots \ldots \ldots \ldots \ldots \ldots \ldots \ldots$

End Use Not Reported

2037

Frozen Fruits and Vegetables

RSE Column Factors:

TOTAL INPUTS

Boiler Fuel

Total Process Uses . . . . . . . . . . . . . . . . . .

Process Heating . . . . . . . . . . . . . . . . . .

Process Cooling and Refrigeration . . . . . . . . . . .

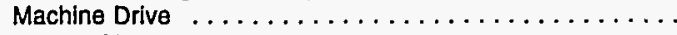

Electro-Chemical Processes $\ldots \ldots \ldots \ldots \ldots \ldots \ldots$

Other Process Use $\ldots \ldots \ldots \ldots \ldots \ldots \ldots \ldots \ldots \ldots \ldots$

Total Non-Process Uses . ..................

Facility Heating, Ventilation, and Air Conditioning ${ }^{\circ} . \ldots \ldots$.

Facllity Lighting $\ldots \ldots \ldots \ldots \ldots \ldots \ldots \ldots \ldots \ldots \ldots$

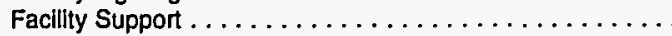

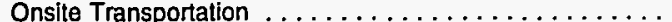

Conventional Electricity Generation . . . . . . . . . . .

Other Non-Process Use $\ldots \ldots \ldots \ldots \ldots \ldots \ldots \ldots \ldots$

End Use Not Reported

Wet Corn Milling

RSE Column Factors:

TOTAL INPUTS

Boller Fuel

Total Process Uses

Process Heating.$\ldots \ldots \ldots \ldots \ldots \ldots \ldots \ldots \ldots \ldots$

Process Cooling and Refrigeration . . . . . . . . . . .

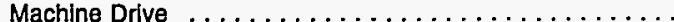

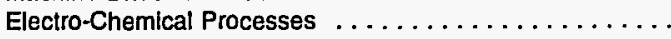

Other Process Use ........................

Total Non-Process Uses ......................

Facility Heating, Ventilation, and Air Conditioning ${ }^{\circ} \ldots \ldots$.

Facllity Llghting . . . . . . . . . . . . . . . . . . . . .

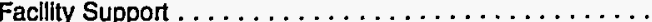

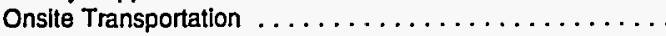

Conventional Electricity Generation ................

Other Non-Process Use $\ldots \ldots \ldots \ldots \ldots \ldots \ldots \ldots$

End Use Not Reported

See footnotes at end of table.
0.7

11

9.7
13.2
15.6
24.9
20.3
17.8
34.5
34.4
11.4
14.3
7.2
22.1
10.9
26.5
21.8
23.7

13.0

21.6

17.4

21.6

23.8

24.2

46.1

13.8

16.2

15.1

18.1

27.

39.0

38.6

0.7

20

1

18

W

18

0

w

-

W

w

0
11.1

12.5

13.6

16.0

14.8

15.2

NF

34.1

16.2

10.4

22.0

15.4

21.6

22.3

22.1 
Table A38. Selected Combustible Inputs of Energy for Heat, Power, and Electricity Generation and Net Demand for Electricity by Fuel Type and End Use, 1991: Part 2 (Continued) (Estimates in Trillion Btu)

\begin{tabular}{|c|c|c|c|c|c|c|c|c|}
\hline $\begin{array}{c}\text { SIC } \\
\text { Code }\end{array}$ & End-Use Categories & $\begin{array}{l}\text { Net Demand } \\
\text { for Electricity }\end{array}$ & $\begin{array}{c}\text { Residual Fuel } \\
\text { Oil }\end{array}$ & $\begin{array}{c}\text { Distillate Fuel } \\
\text { Oil and Diesel } \\
\text { Fuer }\end{array}$ & Natural Gas ${ }^{\circ}$ & LPG & $\begin{array}{c}\text { Coal } \\
\text { (excluding } \\
\text { Coal Coke } \\
\text { and Breeze) }\end{array}$ & $\begin{array}{c}\text { RSE } \\
\text { Row } \\
\text { Factors }\end{array}$ \\
\hline
\end{tabular}

2051 Bread, Cake, and Related Products

RSE Column Factors:

$\begin{array}{ll}0.5 & 1.7 \\ 8 & \\ * \\ 5 \\ 1 \\ 1 \\ 4 \\ 0 \\ 2 \\ 1 \\ 1 \\ 0 \\ - \\ *\end{array}$

1.7
$*$
$*$
0
0
0
0
-
0
0
0
$\ddot{0}$
$\ddot{0}$
0
0

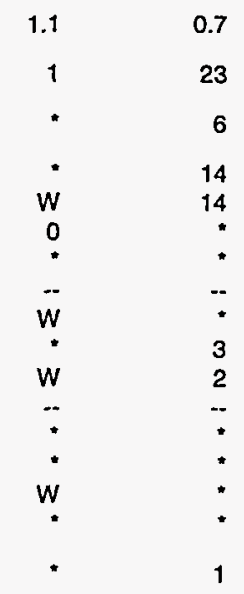

12.1

TOTAL INPUTS

Boiler Fuel

Total Process Uses . . . . . . . . . . . . . . . .

Process Heating . . . . . . . . . . . . . . . .

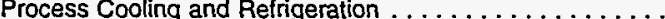

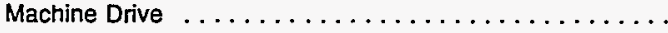

Electro-Chemical Processes $\ldots \ldots \ldots \ldots \ldots \ldots \ldots \ldots \ldots$

Other Process Use . . . . . . . . . . . . . . . . . . . . .

Total Non-Process Uses . . . . . . . . . . . . . . .

Facility Heating, Ventilation, and Air Conditioning ${ }^{\circ} \ldots \ldots$.

Facility Lighting

Facility support . . . . . . . . . . . . . . . .

Onsite Transportation . . . . . . . . . . . . . .

Conventional Electricity Generation . . . . . . . . . . .

Other Non-Process Use $\ldots \ldots \ldots \ldots \ldots \ldots \ldots \ldots \ldots$

End Use Not Reported

Beet Sugar

RSE Column Factors:

0.9

TOTAL INPUTS

1.6

1.1
$w$
$w$
$\dot{0}$
$w$
-
0
0
-
0
0
0

0.9

1.1

0.7

Boiler Fuel

Total Process Uses $\ldots \ldots \ldots \ldots \ldots \ldots \ldots \ldots \ldots \ldots$

Process Heating.$\ldots \ldots \ldots \ldots \ldots \ldots \ldots \ldots \ldots \ldots$

Process Cooling and Refrigeration . . . . . . . . . . .

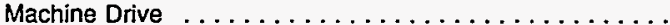

Electro-Chemical Processes . . . . . . . . . . . . . . . .

Other Process Use $\ldots \ldots \ldots \ldots \ldots \ldots \ldots \ldots \ldots \ldots$

Total Non-Process Uses . . . . . . . . . . . . . . .

Facility Heating, Ventilation, and Air Conditioning ${ }^{\circ} . \ldots \ldots$.

Facility Lighting.$\ldots \ldots \ldots \ldots \ldots \ldots \ldots \ldots \ldots$

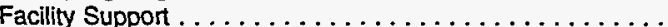

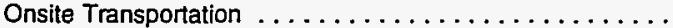

Conventional Electricity Generation ..............

Other Non-Process Use $\ldots \ldots \ldots \ldots \ldots \ldots \ldots \ldots \ldots$

End Use Not Reported

TOTAL INPUTS

Boiler Fuel

$\ldots \ldots \ldots \ldots \ldots \ldots \ldots$

Total Process Uses . . . . . . . . . . . . . . . . . . .

Process Heating $\ldots \ldots \ldots \ldots \ldots \ldots \ldots \ldots \ldots \ldots$

Process Cooling and Refrigeration ..............

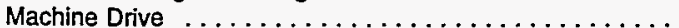

Electro-Chemical Processes $\ldots \ldots \ldots \ldots \ldots \ldots \ldots \ldots$

Other Process Use ......................

Total Non-Process Uses . . . . . . . . . . . . . . . .

Facility Heating, Ventilation, and Air Conditioning ${ }^{\circ} \ldots \ldots \ldots$

Facility Lighting $\ldots \ldots \ldots \ldots \ldots \ldots \ldots \ldots \ldots$

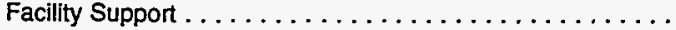

Onsite Transportation $\ldots \ldots \ldots \ldots \ldots \ldots \ldots \ldots \ldots \ldots \ldots$

Conventional Electricity Generation ..............

Other Non-Process Use $\ldots \ldots \ldots \ldots \ldots \ldots \ldots \ldots$

End Use Not Reported

$\ldots \ldots \ldots \ldots \ldots \ldots \ldots \ldots \ldots \ldots \ldots$

\begin{tabular}{|c|c|c|c|c|c|}
\hline 0.8 & 1.5 & 1.1 & 0.7 & 1.5 & 0.8 \\
\hline 7 & - & * & 24 & * & 13 \\
\hline$w$ & * & $w$ & 18 & * & 13 \\
\hline 5 & - & $w$ & 6 & * & 0 \\
\hline * & 0 & w & 5 & " & 0 \\
\hline * & 0 & 0 & 0 & 0 & \\
\hline 5 & * & • & 0 & • & 0 \\
\hline 0 & -- & -- & -- & -. & .. \\
\hline 0 & 0 & 0 & * & " & 促 \\
\hline & * & * & 1 & * & 0 \\
\hline " & 0 & 0 & * & 0 & 0 \\
\hline . & $\ddot{-}$ & 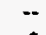 & - & -- & \\
\hline • & 0 & * & * & 0 & 0 \\
\hline * & 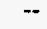 & • & 0 & * & \\
\hline$\ddot{0}$ & 0 & : & 1 & 0 & 0 \\
\hline 0 & • & 0 & 0 & 0 & 0 \\
\hline$w$ & 0 & " & 0 & * & 0 \\
\hline
\end{tabular}


Table A38. Selected Combustible Inputs of Energy for Heat, Power, and Electricity Generation and Net Demand for Electricity by Fuel Type and End Use, 1991: Part 2 (Continued) (Estimates in Trillion Btu)

\begin{tabular}{|c|c|c|c|c|c|c|c|c|}
\hline $\begin{array}{l}\text { SIC } \\
\text { Codea }\end{array}$ & End-Use Categories & $\begin{array}{c}\text { Net Demand } \\
\text { for Electricity }\end{array}$ & $\begin{array}{c}\text { Residual Fuel } \\
\text { Oil }\end{array}$ & \begin{tabular}{|c|} 
Distillate Fuel \\
Oil and Diesel \\
Fuel $^{\circ}$
\end{tabular} & Natural Gas ${ }^{d}$ & LPG & $\begin{array}{c}\text { Coal } \\
\text { (excluding } \\
\text { Coal Coke } \\
\text { and Breeze) }\end{array}$ & $\begin{array}{c}\text { RSE } \\
\text { Row } \\
\text { Factors }\end{array}$ \\
\hline
\end{tabular}

$$
0.6
$$

TOTAL INPUTS

Boller Fuel

Total Process Uses ......................

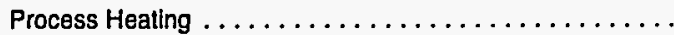

Process Cooling and Refrigeration ..............

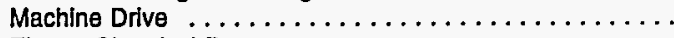

Electro-Chemical Processes ...................

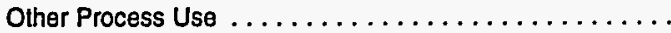

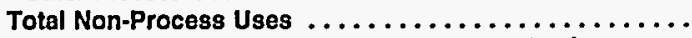

Facillty Heating, Ventilation, and Air Conditioning ${ }^{\circ} \ldots \ldots$.

Facillty Lighting

Facillty Support.

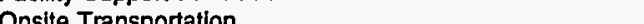

Conventlonal Electricity Generation $\ldots \ldots \ldots \ldots \ldots \ldots$

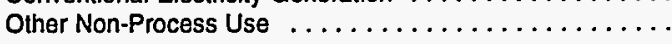

End Use Not Reported

TOBACCO PRODUCTS

TOTAL INPUTS

Boller Fuel

Total Process Uses . . . . . . . . . . . . . . . . . .

Process Heating $\ldots \ldots \ldots \ldots \ldots \ldots \ldots \ldots \ldots \ldots$

Process Cooling and Refrigeration . . . . . . . . . . . .

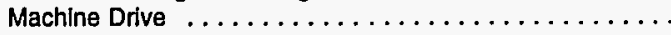

Electro-Chemical Processes $\ldots \ldots \ldots \ldots \ldots \ldots \ldots$.

Other Process Use . . . . . . . . . . . . . . . . .

Total Non-Process Uses ...................

Facillty Heating, Ventilation, and Air Conditioning ${ }^{\circ}$

Facillty Lighting

Facillty Support . . . . . . . . . . . . . . . . . .

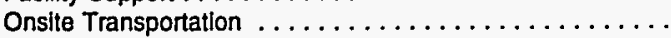

Conventional Electricity Generation $\ldots \ldots \ldots \ldots \ldots \ldots$

Other Non-Process Use $\ldots \ldots \ldots \ldots \ldots \ldots \ldots \ldots$.

End Use Not Reported

TEXTILE MILL PRODUCTS

RSE Column Factors:

TOTAL INPUTS

Boiler fuel

Total Process Uses . . . . . . . . . . . . . . . . .

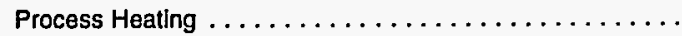

Process Cooling and Refrigeration ...............

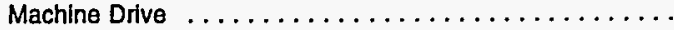

Electro-Chemical Processes..$\ldots \ldots \ldots \ldots \ldots \ldots$.

Other Process Use .........................

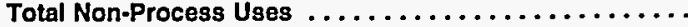

Facllity Heating, Ventilation, and Air Conditioning ${ }^{\circ}$.......

Facilly Lighting . . . . . . . . . . . . . . . . . . . .

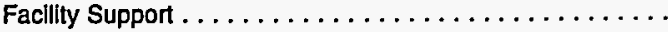

Onsite Transportation $\ldots \ldots \ldots \ldots \ldots \ldots \ldots \ldots \ldots$

Conventional Electricity Generation

Other Non-Process Use

End Use Not Reported

See footnotes at end of table.

10.1

\begin{tabular}{|c|c|c|c|c|c|}
\hline 0.4 & 1.3 & 1.6 & 0.8 & 1.2 & 1.2 \\
\hline 102 & 12 & 6 & 108 & 2 & 31 \\
\hline 1 & 11 & 5 & 70 & • & 30 \\
\hline 74 & 1 & * & 31 & 1 & w \\
\hline 3 & $w$ & • & 28 & 1 & $w$ \\
\hline 7 & 0 & : & * & : & 0 \\
\hline 62 & w & • & 2 & 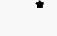 & 0 \\
\hline w & 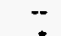 & $\because$ & 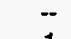 & - & $\overline{-}$ \\
\hline$w$ & & : & 1 & & ? \\
\hline 25 & 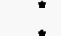 & 1 & 4 & 1 & \\
\hline 14 & " & w & 4 & 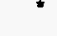 & .. \\
\hline $\begin{array}{l}9 \\
1\end{array}$ & $\because$ & 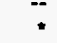 & 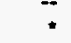 & $\because$ & 0 \\
\hline & -- & * & 0 & * & \\
\hline 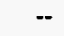 & 0 & $\mathbf{Q}$ & " & : & 0 \\
\hline & 0 & & & & \\
\hline 2 & 1 & $\mathbf{Q}$ & 3 & • & $w$ \\
\hline
\end{tabular}


Table A38. Selected Combustible Inputs of Energy for Heat, Power, and Electricity Cieneration and Net Demand for Electricity by Fuel Type and End Use, 1991: Part 2 (Continued) (Estimates in Trillion Btu)

\begin{tabular}{|c|c|c|c|c|c|c|c|c|}
\hline $\begin{array}{c}\text { SIC } \\
\text { Code }^{a}\end{array}$ & End-Use Categories & $\begin{array}{c}\text { Net Demand } \\
\text { for Electricity }\end{array}$ & $\begin{array}{c}\text { Residual Fuel } \\
\text { Oil }\end{array}$ & $\begin{array}{c}\text { Distillate Fuel } \\
\text { Oil and Diese! } \\
\text { Fuel }\end{array}$ & Natural Gas ${ }^{\circ}$ & LPG & $\begin{array}{c}\text { Coal } \\
\text { (excluding } \\
\text { Coal Coke } \\
\text { and Elreeze) }\end{array}$ & $\begin{array}{c}\text { RSE } \\
\text { Row } \\
\text { Factors }\end{array}$ \\
\hline
\end{tabular}

RSE Column Factors:

TOTAL INPUTS

Boiler Fuel

Total Process Uses

Process Heating

Process Cooling and Refrigeration ...............

Machine Drive

Electro-Chemical Processes

Other Process Use ..........................

Total Non-Process Uses $\ldots \ldots \ldots \ldots \ldots \ldots \ldots \ldots \ldots$

Facility Heating, Ventilation, and Air Conditioning ${ }^{\circ} \ldots .$.

Facility Lighting

Facility Support

Onsite Transportation .............

Conventional Electricity Generation .............

Other Non-Process Use $\ldots \ldots \ldots \ldots \ldots \ldots \ldots \ldots \ldots$

End Use Not Reported

\section{LUMBER and WOOD PRODUCTS}

RSE Column Factors:

TOTAL INPUTS

Boiler Fuel

Total Process Uses

Process Cooling and Refrigeration ..............

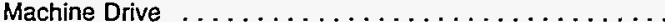

Electro-Chemical Processes $\ldots \ldots \ldots \ldots \ldots \ldots \ldots \ldots$

Other Process Use . . . . . . . . . . . . . . . .

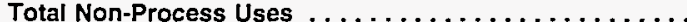

Facility Heating, Ventilation, and Air Conditioning ${ }^{\circ} \ldots \ldots$.

Facility Lighting . . . . . . . . . . . . . . . . . . .

Facility Support

Onsite Transportation $\ldots \ldots \ldots \ldots \ldots \ldots \ldots \ldots \ldots$

Conventional Electricity Generation $\ldots \ldots \ldots \ldots \ldots \ldots \ldots$

Other Non-Process Use ..........

End Use Not Reported

FURNITURE and FIXTURES

RSE Column Factors:

TOTAL INPUTS

Boiler Fuel

Total Process Uses

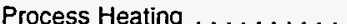

Process Cooling and Refrigeration ...............

Machine Drive

Electro-Chemical Processes $\ldots \ldots \ldots \ldots \ldots \ldots \ldots \ldots \ldots$

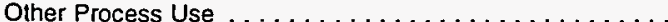

Total Non-Process Uses $\ldots \ldots \ldots \ldots \ldots \ldots \ldots \ldots \ldots \ldots$

Facility Heating, Ventilation, and Air Conditioning ${ }^{\circ} \ldots . .$.

Facility Lighting

Facility Support

Onsite Transportation

Conventional Electricity Generation . . . . . . . . . . .

Other Non-Process Use $\ldots \ldots \ldots \ldots \ldots \ldots \ldots \ldots \ldots \ldots$

End Use Not Reported

\begin{tabular}{r}
0.5 \\
19 \\
$*$ \\
9 \\
1 \\
8 \\
+ \\
$Q$ \\
7 \\
4 \\
3 \\
$\vdots$ \\
$Q$ \\
\hdashline \\
0 \\
3
\end{tabular}

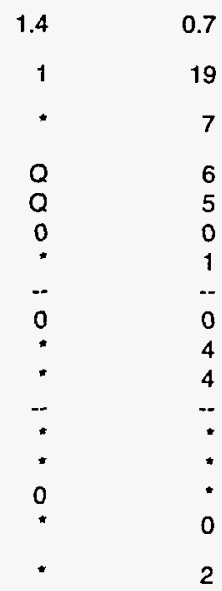

16.8

27.1

23.5

30.2

68.8

38.4

84.5

38.0

19.1

22.8

17.7

36.0

20.0

NF
33.5

30.1

$$
0.5
$$

1.3

1.0

1.0

14.8

24.6

17.1

22.3

59.5

15.5

72.5

87.3

18.5

26.8

18.6

35.7

24.7

NF

46.7

34.2

6

Q

3

4

17.9

28.1

18.9

25.0

36.2

24.0

40.2

55.0

22.0

26.7

18.1

34.3

23.3

NF

59.1

36.6

See footnotes at end of table. 
Table A38. Selected Combustible Inputs of Energy for Heat, Power, and Electricity Generation and Net Demand for Electricity by Fuel Type and End Use, 1991: Part 2 (Continued) (Estimates in Trillion Btu)

\begin{tabular}{|c|c|c|c|c|c|c|c|c|}
\hline $\begin{array}{l}\text { SIC } \\
\text { Code" }\end{array}$ & End-Use Categories & $\begin{array}{l}\text { Net Demand } \\
\text { for Electricity }\end{array}$ & $\begin{array}{c}\text { Residual Fuel } \\
\text { Oil }\end{array}$ & $\begin{array}{c}\text { Distillate Fuel } \\
\text { Oil and Diesel } \\
\text { Fuel }\end{array}$ & Natural Gas ${ }^{\circ}$ & LPG & $\begin{array}{c}\text { Coal } \\
\text { (excluding } \\
\text { Coal Coke } \\
\text { and Breeze) }\end{array}$ & $\begin{array}{c}\text { RSE } \\
\text { Row } \\
\text { Factors }\end{array}$ \\
\hline
\end{tabular}

$\begin{array}{rrr}1.5 & 0.8 & \\ W & 296 & 4.3 \\ W & 293 & 5.8 \\ 2 & 0 & 5.2 \\ 1 & 0 & 7.7 \\ * & 0 & 7.7 \\ * & 0 & 9.3 \\ \because * & - & 13.7 \\ 2 & 0 & 9.6 \\ W & : & 6.5 \\ - & * & 10.8 \\ * & -- & 5.1 \\ 2 & 0 & 9.9 \\ Q & -. & 6.3 \\ * & 0 & 14.8 \\ * & 0 & 16.6 \\ * & W & 19.6\end{array}$

TOTAL INPUTS

Total Process Uses . . . . . . . . . . . . . . . . .

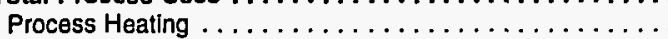

Process Cooling and Refrigeration

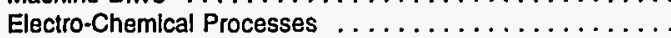

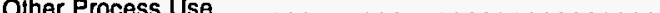

Total Non-Process Uses . ...................

Facllity Heating, Ventilation, and Air Conditioning ${ }^{\circ} \ldots \ldots$.

Facllity Lighting . . . . . . . . . . . . . . . . . . . . .

Facility Support $\ldots \ldots \ldots \ldots \ldots \ldots \ldots \ldots \ldots$

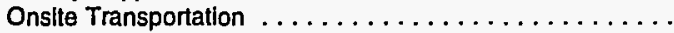

Conventional Electricity Generation

Other Non-Process Use $\ldots \ldots \ldots \ldots \ldots \ldots \ldots \ldots$

End Use Not Reported

Paper Mills

RSE Column Factors:

TOTAL INPUTS

Boiler Fuel $\ldots \ldots \ldots \ldots \ldots \ldots \ldots \ldots \ldots \ldots$

Total Process Uses . . . . . . . . . . . . . . . . .

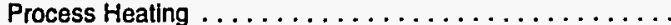

Process Cooling and Refrigeration ................

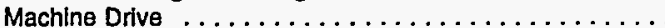

Electro-Chemical Processes ...................

Other Process Use . . . . . . . . . . . . . . . . .

Total Non-Process Uses . . . . . . . . . . . . . . . .

Facllity Heating, Ventilation, and Air Conditioning ${ }^{\bullet} \ldots \ldots$.

Facillty Lighting . . . . . . . . . . . . . . . . . . . . .

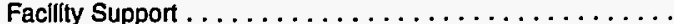

Onslte Transportation .........................

Conventlonal Electricity Generation .................

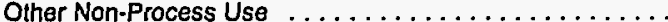

End Use Not Reported

\begin{tabular}{|c|c|c|c|c|c|}
\hline 0.7 & 1.1 & 1.0 & 1.1 & 1.1 & 1.1 \\
\hline 208 & 85 & $w$ & 260 & 2 & 193 \\
\hline 5 & 73 & 2 & 163 & $w$ & 193 \\
\hline 184 & 10 & W & 58 & 1 & 0 \\
\hline 4 & 10 & 1 & 48 & 1 & 0 \\
\hline 2 & 0 & • & * & . & 0 \\
\hline 175 & * & * & W & • & 0 \\
\hline 2 & -- & -. & - & -- & -- \\
\hline 1 & 0 & $w$ & $w$ & - & 0 \\
\hline 14 & 2 & 1 & 38 & $w$ & * \\
\hline 6 & W & * & 2 & * & - \\
\hline 6 & -- & -- & -- & -- & -. \\
\hline 2 & 0 & * & • & * & 0 \\
\hline W & -. & 1 & 0 & w & - \\
\hline -- & W & - & 36 & 0 & 0 \\
\hline W & * & - & 0 & * & 0 \\
\hline 5 & 0 & - & * & * & 0 \\
\hline
\end{tabular}

See footnotes at end of table. 
Table A38. Selected Combustible Inputs of Energy for Heat, Power, and Electricity Cieneration and Net Demand for Electricity by Fuel Type and End Use, 1991: Part 2 (Continued) (Estimates in Trillion Btu)

\begin{tabular}{|c|c|c|c|c|c|c|c|c|}
\hline $\begin{array}{c}\text { SIC } \\
\text { Code" }\end{array}$ & End-Use Categories & $\begin{array}{c}\text { Net Demand } \\
\text { for Electricity }\end{array}$ & $\begin{array}{c}\text { Residual Fuel } \\
\text { Oil }\end{array}$ & $\begin{array}{c}\text { Distillate Fuel } \\
\text { OII and Dlesel } \\
\text { Fuel }^{c}\end{array}$ & Natural Gas & LPG & $\begin{array}{c}\text { Coal } \\
\text { (excluding } \\
\text { Coal Coke } \\
\text { and Breaze) }\end{array}$ & $\begin{array}{c}\text { RSE } \\
\text { Row } \\
\text { Factoro }\end{array}$ \\
\hline
\end{tabular}

2631

Paperboard Mills

RSE Column Factors:

TOTAL INPUTS

Boller Fuel

Total Process Uses

Process Cooling and Refrigeration $\ldots \ldots \ldots \ldots \ldots \ldots \ldots$

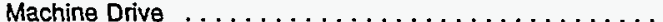

Electro-Chemical Processes $\ldots \ldots \ldots \ldots \ldots \ldots \ldots \ldots$

Other Process Use ..........................

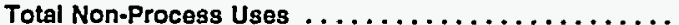

Faclity Heating, Ventilation, and Air Conditioning...$\ldots$.

Facility Lighting.$\ldots \ldots \ldots \ldots \ldots \ldots \ldots \ldots \ldots \ldots \ldots$

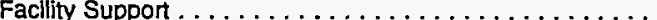

Onsite Transportation $\ldots \ldots \ldots \ldots \ldots \ldots \ldots \ldots \ldots$

Conventional Electricity Generation . . . . . . . . . . . . . .

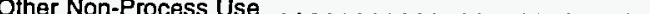

End Use Not Reported

27

\section{PRINTING and PUBLISHING}

RSE Column Factors:

TOTAL INPUTS

Boiler Fuel

Total Process Uses $\ldots \ldots \ldots \ldots \ldots \ldots \ldots \ldots \ldots \ldots$

Process Heating $\ldots \ldots \ldots \ldots \ldots \ldots \ldots \ldots \ldots \ldots$

Process Cooling and Refrigeration ..................

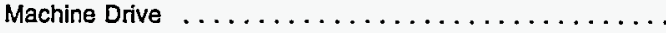

Electro-Chemical Processes ...................

Other Process Use ...........................

Total Non-Process Uses . . . . . . . . . . . . . . . . .

Facility Heating, Ventilation, and Air Conditioning ${ }^{\circ} \ldots \ldots$.

Facility Lighting . .

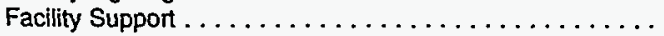

Onsite Transportation .......................

Conventional Electricity Generation . . . . . . . . . . . . .

Other Non-Process Use $\ldots \ldots \ldots \ldots \ldots \ldots \ldots$

End Use Not Reported

CHEMICALS and ALLIED PRODUCTS

RSE Column Factors:

TOTAL INPUTS

Boller Fuel

Total Process Uses

Process Heating ...

Process Cooling and Refrige.

Machine Drive

Electro-Chemical Processes $\ldots \ldots \ldots \ldots \ldots \ldots \ldots \ldots$

Other Process Use . ........................

Total Non-Process Uses . . . . . . . . . . . . . . .

Facility Heating, Ventilation, and Air Conditioning ${ }^{\circ} . \ldots \ldots$

Facility Lighting . . .

Facility Support

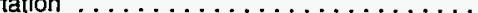

Conventional Electricity Generation . . . . . . . . . . . .

Other Non-Process Use..$\ldots \ldots \ldots \ldots \ldots \ldots \ldots \ldots$

End Use Not Reported

$\begin{array}{rr}0.6 & 1.5 \\ 92 & W \\ 5 & 32 \\ 81 & W \\ 2 & W \\ W & 0 \\ 76 & 0 \\ 1 & - \\ W & 0 \\ 6 & \vdots \\ 2 & \cdot \\ 3 & -- \\ 1 & 0 \\ Q & -- \\ -- & 0 \\ - & 0 \\ 0 & -\end{array}$

0.5

53

*

26

1
2
23
5
19
10
7
2
-
0
8

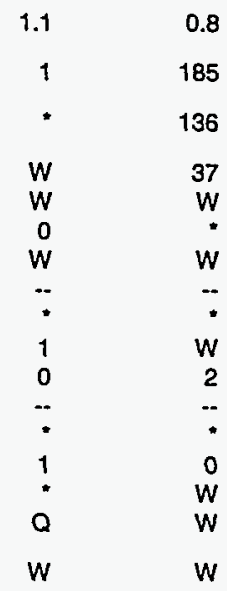

0.8
185
136
37
$w$
$w$
$:$
$w$
2
$-:$
0
$w$
$w$
$w$

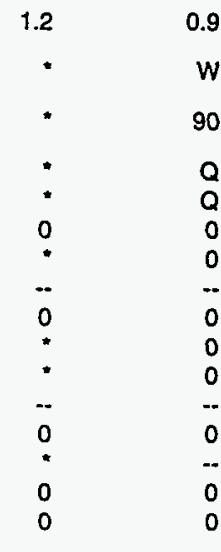

4.4

7.0

5.3

9.4

9.7

9.4

20.1

19.1

8.7

13.3

9.3

13.8

4.7

26.1

23.0

14.5

$\begin{array}{rcc}0.5 & 1.6 & 1.5 \\ 53 & * \\ * & * \\ 26 & 0 \\ 1 & 0 \\ 2 & 0 \\ 23 & 0 \\ * & 0 \\ 19 & : \\ 10 & : \\ 7 & - \\ 2 & - \\ - & 0 \\ -0 & 0 \\ 8 & 0\end{array}$

1.5
2
1
$Q$
$Q$
0
$:-$
0
1
1
$:$
$*$
$Q$
$*$
.

$$
\begin{array}{r}
0.7 \\
48 \\
14 \\
17 \\
15 \\
: \\
2 \\
\cdots \\
12 \\
11 \\
-- \\
1 \\
0 \\
0 \\
-
\end{array}
$$

$\begin{array}{rrr}1.1 & \text { NF } & \\ 1 & 0 & 12.6\end{array}$

$\begin{array}{ccc}1 & 0 & 12.6 \\ W & 0 & 26.7\end{array}$

$\begin{array}{lll}W & 0 & 15.9\end{array}$

$W \quad 0 \quad 23.2$

31.9

26.4

79.8

60.4

18.7

23.3

16.0

27.9

24.1

$\mathrm{NF}$

18.9

25.4

\begin{tabular}{|c|c|c|c|c|c|}
\hline 0.6 & 0.9 & 1.2 & 0.8 & 1.6 & 1.3 \\
\hline 582 & 48 & 12 & 1,669 & 4 & 253 \\
\hline 4 & 28 & 6 & 719 & 2 & 244 \\
\hline $\begin{array}{r}518 \\
16\end{array}$ & $\begin{array}{c}W \\
19\end{array}$ & $\begin{array}{l}2 \\
2\end{array}$ & $\begin{array}{l}673 \\
577\end{array}$ & $\begin{array}{l}2 \\
1\end{array}$ & $\begin{array}{l}8 \\
8\end{array}$ \\
\hline 34 & 0 & 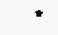 & 2 & $w$ & 0 \\
\hline 353 & 0 & 1 & 55 & • & 0 \\
\hline 114 & $\ddot{-}$ & -- & $\ldots$ & .. & -. \\
\hline 1 & $w$ & * & 39 & $w$ & 0 \\
\hline 49 & W & 3 & 256 & 1 & $w$ \\
\hline 25 & $w$ & * & w & : & $w$ \\
\hline 18 & -- & -. & -- & -- & -- \\
\hline 5 & 0 & * & 4 & * & 0 \\
\hline * & -- & 2 & 0 & 1 & .. \\
\hline -- & W & " & 191 & : & 0 \\
\hline 1 & . & " & $w$ & • & 0 \\
\hline 11 & - & * & 21 & . & $w$ \\
\hline
\end{tabular}

5.0

6.6

7.2

9.1

9.4

7.4

9.1

12.1

6.7

10.1

7.3
10.5

7.5

11.8

10.7

14.9

See footnotes at end of table. 
Table A38. Selected Combustible Inputs of Energy for Heat, Power, and Electricity Generation and Net Demand for Electricity by Fuel Type and End Use, 1991: Part 2 (Continued) (Estimates in Trillion Btu)

\begin{tabular}{|c|c|c|c|c|c|c|c|c|}
\hline $\begin{array}{l}\text { SIC } \\
\text { Codoa }\end{array}$ & - End-Use Categories & $\begin{array}{c}\text { Net Demand } \\
\text { for Electricity }\end{array}$ & $\begin{array}{c}\text { Residual Fuel } \\
\text { Oil }\end{array}$ & $\begin{array}{c}\text { Distillate Fuel } \\
\text { Oil and Dlesel } \\
\text { Fuer }\end{array}$ & Natural Gas ${ }^{d}$ & LPG & $\begin{array}{c}\text { Coal } \\
\text { (excluding } \\
\text { Coal Coke }\end{array}$ & $\begin{array}{c}\text { RSE } \\
\text { Row } \\
\text { Factors }\end{array}$ \\
\hline
\end{tabular}

2812

Alkalles and Chlorine

RSE Column Factors:

0.7
60
$W$
59
$W$
$W$
5
53
$W$
1
$W$
1
$W$
0
.-
0
$W$

1.3
$W$
$w$
0
0
0
0
-
0
0
0
-
0
-
0
0
0

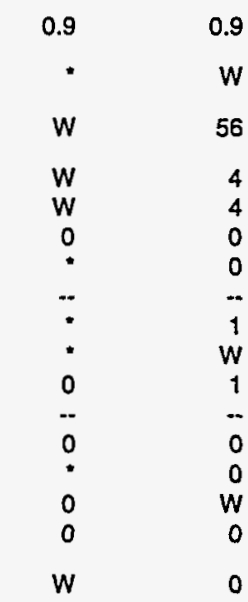

$\begin{array}{rrr}1.1 & 1.2 & \\ * & W & 16.4 \\ 0 & W & 23.0 \\ 0 & 0 & 19.2 \\ 0 & 0 & 27.5 \\ 0 & 0 & 19.3 \\ 0 & 0 & 19.5 \\ - & .- & 13.3 \\ 0 & 0 & 31.2 \\ . & 0 & 16.1 \\ 0 & 0 & 20.1 \\ -- & -- & 14.8 \\ 0 & 0 & 31.1 \\ \dot{0} & - & 14.3 \\ 0 & 0 & 20.4 \\ 0 & 0 & \text { NF } \\ . & 0 & 31.8\end{array}$

2813

Industrial Gases

RSE Column Factors:

$\begin{array}{rr}0.4 & N \\ 62 & \\ 0 & \\ 58 & \\ W & \\ 57 & \\ W & \\ Q & \\ W & \\ 1 & \\ 1 & \\ W & \\ 0 & \\ - & \\ 0 & 0 \\ W & 0 \\ W & \end{array}$

2819

TOTAL INPUTS

Boller Fuel

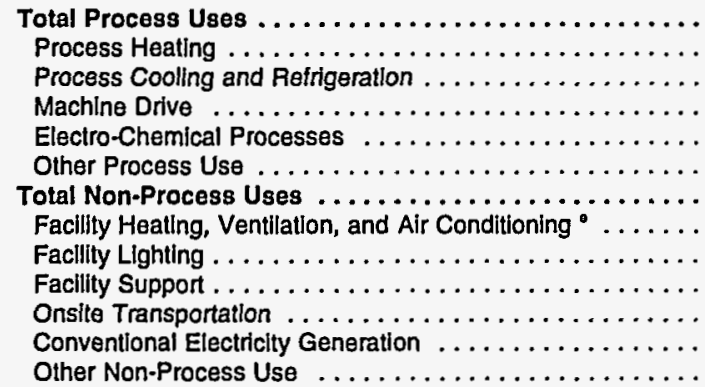

End Use Not Reported

Industrial Inorganic Chemicals, nec

RSE Column Factors:

\begin{tabular}{|c|c|c|c|c|c|}
\hline 0.8 & 1.0 & 1.2 & 0.8 & 1.2 & 1.2 \\
\hline 136 & 4 & 3 & 140 & * & 17 \\
\hline$w$ & $w$ & 1 & 71 & * & 9 \\
\hline 128 & $w$ & - & 54 & • & 7 \\
\hline 8 & $w$ & - & 54 & - & 7 \\
\hline W & 0 & 0 & • & 0 & 0 \\
\hline 104 & 0 & $w$ & • & * & 0 \\
\hline 14 & .. & -- & -- & -- & -- \\
\hline$w$ & 0 & $w$ & - & - & 0 \\
\hline 6 & 0 & 1 & 12 & * & $w$ \\
\hline 3 & 0 & - & W & - & $w$ \\
\hline 1 & -. & -- & -- & -- & -- \\
\hline 1 & 0 & $w$ & * & * & 0 \\
\hline w & -. & 1 & 0 & * & -. \\
\hline & 0 & w & $w$ & 0 & 0 \\
\hline$w$ & 0 & * & - & - & 0 \\
\hline$w$ & 0 & - & 2 & $Q$ & $w$ \\
\hline
\end{tabular}

8.8

TOTAL INPUTS

Boiler Fuel

Total Process Uses ........................

Process Heating $\ldots \ldots \ldots \ldots \ldots \ldots \ldots \ldots \ldots \ldots$.

Process Cooling and Refrigeration ..................

Machine Drive $\ldots \ldots \ldots \ldots \ldots \ldots \ldots \ldots \ldots$.

Electro-Chemical Processes

Other Process Use .....

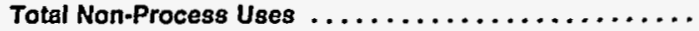

Facility Heating, Ventilation, and Air Conditioning ${ }^{2} \ldots \ldots$. .

Facility Lighting . . . . . . . . . . . . . . . . . . . . . .

Facllity Support $\ldots \ldots \ldots \ldots \ldots \ldots \ldots \ldots \ldots \ldots$

Onsite Transportation.$\ldots \ldots \ldots \ldots \ldots \ldots \ldots \ldots$

Conventional Electricity Generation $\ldots \ldots \ldots \ldots \ldots \ldots$

Other Non-Process Use $\ldots \ldots \ldots \ldots \ldots \ldots \ldots \ldots$

End Use Not Reported

\begin{tabular}{|c|c|c|c|c|}
\hline 1.3 & 0.8 & 2.2 & NF & \\
\hline • & 25 & $Q$ & 0 & 14.2 \\
\hline - & 8 & 0 & 0 & 13.6 \\
\hline " & 12 & 0 & 0 & 11.2 \\
\hline 0 & 11 & 0 & 0 & 23.0 \\
\hline 0 & 0 & 0 & 0 & 25.3 \\
\hline 0 & • & 0 & 0 & 12.4 \\
\hline -. & .- & .- & .- & 36.8 \\
\hline$*$ & • & 0 & 0 & 34.3 \\
\hline $\mathbf{Q}$ & 4 & $Q$ & 0 & 14.0 \\
\hline & 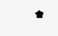 & $\overrightarrow{0}$ & 0 & 14.5 \\
\hline- & .. & -- & -. & 11.5 \\
\hline 0 & • & 0 & 0 & 12.3 \\
\hline$Q$ & 0 & $Q$ & -. & NF \\
\hline 0 & 2 & 0 & 0 & 24.9 \\
\hline 0 & 2 & 0 & 0 & 24.9 \\
\hline 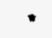 & • & • & 0 & 12.6 \\
\hline
\end{tabular}


Table A38. Selected Combustible Inputs of Energy for Heat, Power, and Electricity Generation and Net Demand for Electricity by Fuel Type and End Use, 1991: Part 2 (Ciontinued) (Estimates in Trillion Btu)

\begin{tabular}{|c|c|c|c|c|c|c|c|c|}
\hline $\begin{array}{c}\text { SIC } \\
\text { Code" }\end{array}$ & End-Use Categories & $\begin{array}{l}\text { Net Demand } \\
\text { for Electricity" }\end{array}$ & $\begin{array}{c}\text { Residual Fuel } \\
\text { Oil }\end{array}$ & $\begin{array}{c}\text { Distillate Fuel } \\
\text { Oil and Diesel } \\
\text { Fuel }^{\mathrm{c}}\end{array}$ & Natural Gas & LPG & $\begin{array}{c}\text { Coal } \\
\text { (excluding } \\
\text { Coal Coke } \\
\text { and Breeze) }\end{array}$ & $\begin{array}{c}\text { RSE } \\
\text { Bow } \\
\text { Factors }\end{array}$ \\
\hline
\end{tabular}

2821

Plastics Materials and Resins

RSE Column Factors:

TOTAL INPUTS

$\begin{array}{rr}0.6 & 1.3 \\ 59 & 4 \\ 1 & W \\ 52 & W \\ W & W \\ 6 & 0 \\ 38 & 0 \\ 7 & -- \\ W & 0 \\ 5 & W \\ 3 & 0 \\ 2 & -- \\ 1 & 0 \\ -- & - \\ * & 0 \\ 1 & 0\end{array}$

Synthetic Rubber

RSE Column Factors:

TOTAL INPUTS

Boiler Fuel

Total Process Uses $\ldots \ldots \ldots \ldots \ldots \ldots \ldots \ldots \ldots \ldots$

Process Heating $\ldots \ldots \ldots \ldots \ldots \ldots \ldots \ldots \ldots \ldots \ldots$

Process Cooling and Refrigeration . . . . . . . . . . . .

Machine Drive

Electro-Chemical Processes $\ldots \ldots \ldots \ldots \ldots$

Total Non-Process Uses . . . . . . . . . . . . . . .

Facility Heating, Ventilation, and Air Conditioning ${ }^{\circ} \ldots \ldots \ldots$

Facility Lighting . .

Facility Support . . . . . . . . . . . . . . . .

Onsite Transportation $\ldots \ldots \ldots \ldots \ldots \ldots \ldots \ldots \ldots \ldots \ldots$

Conventional Electricity Generation ..............

Other Non-Process Use . . . . . . . . . . . . . .

End Use Not Reported

Cellulosic Manmade Fibers

$$
0 .
$$

$\begin{array}{rrrrr}1.0 & 0.9 & 1.0 & 1.4 & \\ * & 44 & * & W & 12.4 \\ W & 24 & 0 & W & 15.2 \\ W & 20 & * & 0 & 17.2 \\ 0 & W & 0 & 0 & 19.3 \\ 0 & * & 0 & 0 & 19.2 \\ W & * & - & 0 & 17.0 \\ * & - & 0 & -- & 36.0 \\ * & W & W & 0 & 25.9 \\ * & * & -- & 0 & 12.8 \\ 0 & * & 0 & 0 & 17.1 \\ -* & 0 & W & 0 & 14.7 \\ * & * & 0 & -. & 17.0 \\ * & * & 0 & 0 & 23.6 \\ * & * & W & 0 & 23.6 \\ * & * & * & 0 & 23.2\end{array}$

\section{RSE Column Factors:}

TOTAL INPUTS

Boiler Fuel

Total Process Uses

Process Heating

(n)

Machine Drive

Electro-Chemical Processes $\ldots \ldots \ldots \ldots \ldots$

Other Process Use . . . . . . . . . . . . . . . . . . .

Total Non-Process Uses $\ldots \ldots \ldots \ldots \ldots \ldots \ldots \ldots \ldots \ldots \ldots \ldots \ldots$

Facility Heating, Ventilation, and Air Conditioning ${ }^{\circ} \ldots \ldots$.

Facility Lighting . . . . . . . . . . . . . . . . .

Facility Support .

Onsite Transportation . . . . . . . . . .

Conventional Electricity Generation . . . . . . . . . . .

Other Non-Process Use $\ldots \ldots \ldots \ldots \ldots \ldots \ldots \ldots \ldots$

End Use Not Reported

$\ldots \ldots \ldots \ldots \ldots \ldots \ldots \ldots \ldots$

\begin{tabular}{|c|c|c|c|c|c|}
\hline 0.9 & NF & 1.2 & 1.1 & 1.1 & 0.8 \\
\hline 4 & 0 & * & $w$ & • & 27 \\
\hline . & 0 & * & W & * & 27 \\
\hline 3 & 0 & * & 2 & 0 & 0 \\
\hline * & 0 & * & 2 & 0 & 0 \\
\hline 1 & 0 & 0 & 0 & 0 & 0 \\
\hline 2 & 0 & 0 & 0 & 0 & 0 \\
\hline 0 & -- & -- & -- & .. & -. \\
\hline 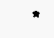 & 0 & 0 & 0 & 0 & 0 \\
\hline 1 & 0 & * & 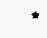 & $\star$ & 0 \\
\hline : & 0 & * & 0 & " & 0 \\
\hline * & - & -- & 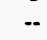 & -- & -. \\
\hline$\star$ & 0 & 0 & * & 0 & 0 \\
\hline$*$ & -. & 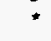 & 0 & 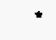 & $=$ \\
\hline-- & 0 & 0 & 0 & 0 & 0 \\
\hline 0 & 0 & 0 & 0 & 0 & 0 \\
\hline 0 & 0 & 0 & 0 & * & 0 \\
\hline
\end{tabular}

See footnotes at end of table. 
Table A38. Selected Combustible Inputs of Energy for Heat, Power, and Electricity Generation and Net Demand for Electricity by Fuel Type and End Use, 1991: Part 2 (Continued) (Estimates in Trillion Btu)

\begin{tabular}{|c|c|c|c|c|c|c|c|c|}
\hline $\begin{array}{l}\text { SIC } \\
\text { Code }^{a}\end{array}$ & End-Use Categories & $\begin{array}{l}\text { Net Demand } \\
\text { for Electricity }\end{array}$ & $\begin{array}{c}\text { Residual Fuel } \\
\text { Oil }\end{array}$ & $\begin{array}{c}\text { Distillate Fuel } \\
\text { Oil and Diesel } \\
\text { Fuel }\end{array}$ & Natural Gas ${ }^{d}$ & LPG & $\begin{array}{c}\text { Coal } \\
\text { (excluding } \\
\text { Coal Coke } \\
\text { and Breeze) }\end{array}$ & $\begin{array}{c}\text { RSE } \\
\text { Row } \\
\text { Factors }\end{array}$ \\
\hline
\end{tabular}

2824

Organic Fibers, Noncellulosic

RSE Column Factors:

\begin{tabular}{|c|c|c|}
\hline 1.1 & 1.1 & 1.0 \\
\hline 26 & $w$ & • \\
\hline * & 2 & * \\
\hline 21 & $w$ & - \\
\hline$w$ & $w$ & . \\
\hline 3 & 0 & 0 \\
\hline 14 & 0 & * \\
\hline$w$ & -- & -- \\
\hline$w$ & 0 & 0 \\
\hline 5 & $w$ & - \\
\hline 3 & $w$ & * \\
\hline 2 & -- & -. \\
\hline$w$ & 0 & • \\
\hline & -- & • \\
\hline 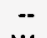 & 0 & 0 \\
\hline$w$ & * & * \\
\hline 0 & W & * \\
\hline
\end{tabular}

\begin{tabular}{ccc}
1.1 & 0.8 & \\
$*$ & 35 & 3.5 \\
$W$ & 35 & 3.8 \\
$W$ & 0 & 4.0 \\
$W$ & 0 & 5.3 \\
0 & 0 & 1.8 \\
$*$ & 0 & 5.6 \\
\hline- & -- & 6.3 \\
. & 0 & 5.4 \\
$*$ & 0 & 4.8 \\
- & 0 & 6.4 \\
$*$ & -- & 5.4 \\
$*$ & 0 & 6.3 \\
0 & -- & 4.5 \\
$*$ & 0 & NF \\
$*$ & 0 & 5.8 \\
\hline & 0 & 6.6
\end{tabular}

2865

TOTAL INPUTS

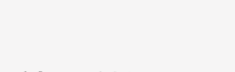

Boller Fuel

Total Process Uses . . . . . . . . . . . . . . . . .

Process Heating $\ldots \ldots \ldots \ldots \ldots \ldots \ldots \ldots \ldots \ldots \ldots$

Process Cooling and Refrigeration ..............

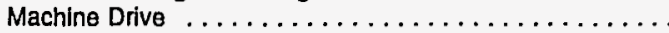

Electro-Chemical Processes $\ldots \ldots \ldots \ldots \ldots \ldots \ldots \ldots \ldots$

Other Process Use . . . . . . . . . . . . . . . . . . . .

Total Non-Process Uses . . . . . . . . . . . . . . . . . .

Facility Healing, Ventilation, and Air Conditioning ${ }^{\circ} \ldots \ldots$.

Facility Lighting . . . . . . . . . . . . . . . . .

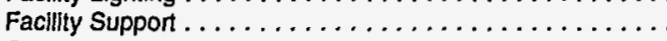

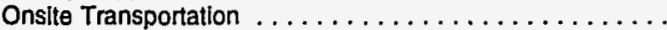

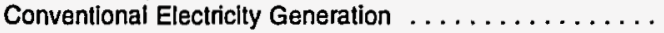

Other Non-Process Use $\ldots \ldots \ldots \ldots \ldots \ldots \ldots \ldots$

End Use Not Reported $\ldots \ldots \ldots \ldots \ldots \ldots \ldots \ldots \ldots$

Cyclic Crudes and Intermediates

RSE Column Factors:

TOTAL INPUTS

Boller Fuel

$\ldots \ldots \ldots \ldots \ldots \ldots \ldots \ldots \ldots$

1.2

1.2

1.2

1.0

Total Process Uses . . . . . . . . . . . . . . . . . . .

Process Heating . . . . . . . . . . . . . . . . .

Process Cooling and Refrigeration . . . . . . . . . . .

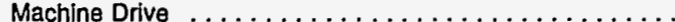

Electro-Chemical Processes $\ldots \ldots \ldots \ldots \ldots \ldots \ldots \ldots$

Other Process Use . . . . . . . . . . . . . . . . .

Total Non-Process Uses . . . . . . . . . . . . . . . . . . .

Facility Heating, Ventilation, and Air Conditioning ${ }^{\bullet} \ldots \ldots \ldots$

Facility Lighting

Onsite Transportation . . . . . . . . . . . . . . .

Conventional Electricity Generation ..............

Other Non-Process Use . . . . . . . . . . . . . . . . .

End Use Not Reported

W

TOTAL INPUTS

Boiler Fuel

Total Process Uses . . . . . . . . . . . . . . . .

Process Heating.

Process Cooling and Refrigeration $\ldots \ldots \ldots \ldots \ldots \ldots \ldots \ldots$

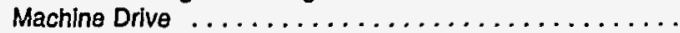

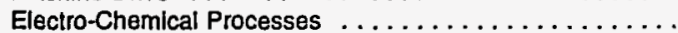

Other Process Use $\ldots \ldots \ldots \ldots \ldots \ldots \ldots \ldots \ldots \ldots$

Total Non-Process Uses ...................

Facility Heating, Ventilation, and Air Conditioning

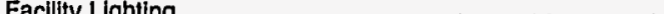

Facility Support . . . . . . . . . . . . . . . .

Onsite Transportation

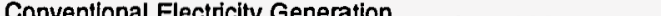

Other Non-Process Use . . . . . . . . . . . . . . . 

Table A38. Selected Combustible Inputs of Energy for Heat, Power, and Electricity Generation
and Net Demand for Electricity by Fuel Type and End Use, 1991: Part 2 (Continued) (Estimates in Trillion Btu)

\begin{tabular}{|c|c|c|c|c|c|c|c|c|}
\hline $\begin{array}{c}\text { SIC } \\
\text { Code" } \\
\end{array}$ & End-Use Categories & $\begin{array}{l}\text { Net Demand } \\
\text { for Electricity }\end{array}$ & $\begin{array}{c}\text { Residual Fuel } \\
\text { Oil }\end{array}$ & $\mid \begin{array}{c}\text { Distillate Fue! } \\
\text { Oil and Diesel } \\
\text { Fuer }\end{array}$ & Natural Gas ${ }^{\circ}$ & LPG & $\begin{array}{c}\text { Coal } \\
\text { (excluding } \\
\text { Coal Coke }\end{array}$ & \begin{tabular}{|c} 
RSE \\
Row \\
Factors
\end{tabular} \\
\hline
\end{tabular}

2873

Nitrogenous Fertilizers

RSE Column Factors:

TOTAL INPUTS

Boiler Fuel

Total Process Uses

Process Heating

Process Cooling and Refrigeration . . . . . . . . . . . .

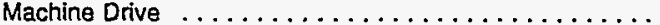

Electro-Chemical Processes .................

Other Process Use . . . . . . . . . . . . . . . .

Total Non-Process Uses . . . . . . . . . . . . . . . . . . .

Facility Heating, Ventilation, and Air Conditioning ${ }^{\circ} \ldots \ldots$

Facility Lighting . . . . . . . . . . . . . . . . .

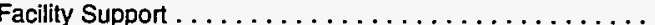

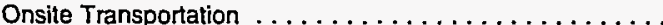

Conventional Electricity Generation $\ldots \ldots \ldots \ldots \ldots \ldots \ldots$

Other Non-Process Use $\ldots \ldots \ldots \ldots \ldots \ldots \ldots \ldots$

End Use Not Reported

Phosphatic Fertilizers

RSE Column Factors:

TOTAL INPUTS

Boiler Fuel

Total Process Uses

Process Heating $\ldots \ldots \ldots \ldots \ldots \ldots \ldots \ldots \ldots \ldots$

Process Cooling and Refrigeration . . . . . . . . .

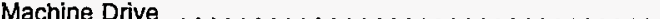

Electro-Chemical Processes $\ldots \ldots \ldots \ldots \ldots \ldots \ldots \ldots$

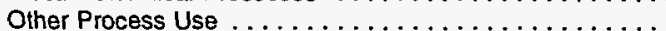

Total Non-Process Uses . . . . . . . . . . . . . . . . . . . .

Facility Heating, Ventilation, and Air Conditioning ${ }^{\circ} \ldots \ldots$.

Facility Lighting . . . . . . . . . . . . . . . . . . . . .

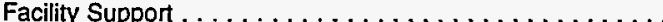

Onsite Transportation . . . . . . . . . . . . . . .

Conventional Electricity Generation . . . . . . . . . . .

Other Non-Process Use

End Use Not Reported

PETROLEUM and COAL PRODUCTS

RSE Column Factors:

TOTAL INPUTS

Boiler Fuel

Total Process Uses . . . . . . . . . . . . . . . . . .

Process Heating . . . . . . . . . . . . . . . .

Process Cooling and Refrigeration . . . . . . . . . .

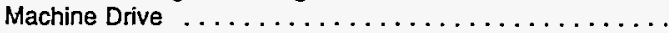

Electro-Chemical Processes $\ldots \ldots \ldots \ldots \ldots \ldots \ldots \ldots \ldots$

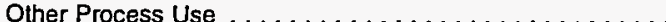

Total Non-Process Uses . . . . . . . . . . . . . . . . . .

Facility Heating, Ventilation, and Air Conditioning ${ }^{\circ} \ldots \ldots \ldots$

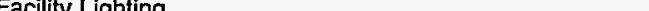

Facility Suppont . . . . . . . . . . . . . . . .

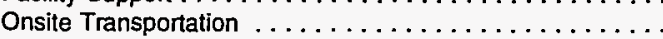

Conventional Electricity Generation . . . . . . . . . . .

Other Non-Process Use $\ldots \ldots \ldots \ldots \ldots \ldots \ldots \ldots$

End Use Not Reported
0.8

11

10

$$
\begin{aligned}
& 1 \\
& 8
\end{aligned}
$$

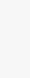$$
1
$$

(


Table A38. Selected Combustible Inputs of Energy for Heat, Power, and Electricity Generation and Net Demand for Electricity by Fuel Type and End Use, 1991: Part 2 (Continued) (Estimates in Trillion Btu)

\begin{tabular}{|c|c|c|c|c|c|c|c|c|}
\hline $\begin{array}{c}\text { SIC } \\
\text { Code" }\end{array}$ & End-Use Categories & $\begin{array}{l}\text { Net Demand } \\
\text { for Electricity }\end{array}$ & $\begin{array}{c}\text { Residual Fuel } \\
\text { Oil }\end{array}$ & $\begin{array}{c}\text { Distillate Fuel } \\
\text { Oil and Diesel } \\
\text { Fuel }^{c}\end{array}$ & Natural Gas ${ }^{d}$ & LPG & $\begin{array}{c}\text { Coal } \\
\text { (excluding } \\
\text { Coal Coke } \\
\text { and Breeze) }\end{array}$ & $\begin{array}{c}\text { RSE } \\
\text { Row } \\
\text { Factors }\end{array}$ \\
\hline
\end{tabular}

2911

Petroleum Refining'

RSE Column Factors:

$\begin{array}{rr}0.5 & 1.6 \\ 144 & 65 \\ W & 33 \\ 131 & 32 \\ 3 & 32 \\ 8 & 0 \\ 119 & 0 \\ W & - \\ W & 0 \\ 11 & 0 \\ 5 & 0 \\ 5 & - \\ 1 & 0 \\ W & - \\ -- & 0 \\ W & 0 \\ W & 0\end{array}$

\begin{tabular}{|c|c|}
\hline 0.9 & 0.5 \\
\hline 9 & 792 \\
\hline 2 & 253 \\
\hline 4 & 429 \\
\hline 4 & 392 \\
\hline 0 & $w$ \\
\hline$W$ & $w$ \\
\hline-- & .. \\
\hline$w$ & • \\
\hline W & 108 \\
\hline & 8 \\
\hline$\overline{-}$ & $\ddot{*}$ \\
\hline 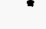 & 1 \\
\hline 3 & 0 \\
\hline * & 98 \\
\hline$w$ & * \\
\hline$w$ & 2 \\
\hline
\end{tabular}

$\begin{array}{rrr}0.7 & 3.6 & \\ 60 & 3 & 3.9 \\ 11 & 3 & 3.3 \\ 48 & 0 & 4.7 \\ 36 & 0 & 5.6 \\ \text { W } & 0 & 7.2 \\ \text { W } & 0 & 5.3 \\ - & - & 8.2 \\ 0 & 0 & 8.0 \\ \text { W } & 0 & 5.8 \\ \text { W } & 0 & 6.3 \\ -- & -- & 4.1 \\ \text { W } & 0 & 7.2 \\ . & -- & 8.2 \\ \text { W } & 0 & 14.6 \\ * & 0 & 8.8 \\ \text { W } & 0 & 8.1\end{array}$

RUBBER and MISC. PLASTICS PRODUCTS

RSE Column Factors:

TOTAL INPUTS

Boller Fuel

Total Process Uses . . . . . . . . . . . . . . . . . .

Process Heating . . . . . . . . . . . . . . . . . .

Process Cooling and Refrigeration .............

Machine Drive $\ldots \ldots \ldots \ldots \ldots \ldots \ldots \ldots \ldots \ldots$

Electro-Chemical Processes . . . . . . . . . . . .

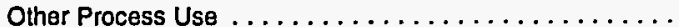

Total Non-Process Uses ....................

Facility Heating, Ventilation, and Air Conditioning ${ }^{\circ} \ldots \ldots$.

Facllity Llghting . . . . . . . . . . . . . . . . . .

Facillty Support . . . . . . . . . . . . . . . .

Onsite Transportation $\ldots \ldots \ldots \ldots \ldots \ldots \ldots \ldots \ldots \ldots$

Conventional Electricity Generation $\ldots \ldots \ldots \ldots \ldots \ldots$

Other Non-Process Use $\ldots \ldots \ldots \ldots \ldots \ldots \ldots \ldots$

End Use Not Reported

$\begin{array}{r}1.1 \\ 8 \\ 7 \\ W \\ W \\ \hline \\ \hline \\ \text { W } \\ W \\ - \\ \hline \\ \hline 0 \\ 0 \\ \hline\end{array}$

Total Process Uses $\ldots \ldots \ldots \ldots \ldots \ldots \ldots \ldots \ldots \ldots \ldots$

Process Heating $\ldots \ldots \ldots \ldots \ldots \ldots \ldots \ldots \ldots \ldots$

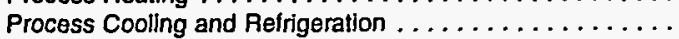

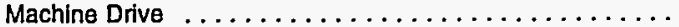

Electro-Chemical Processes $\ldots \ldots \ldots \ldots \ldots \ldots \ldots \ldots$

Other Process Use . . . . . . . . . . . . . . . . .

Total Non-Process Uses . . . . . . . . . . . . . . . .

Facility Heating, Ventilation, and Air Conditioning ${ }^{\circ} \ldots \ldots$.

Facllity Lighting $\ldots \ldots \ldots \ldots \ldots \ldots \ldots \ldots \ldots \ldots$

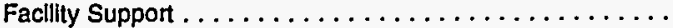

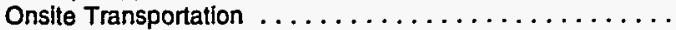

Conventlonal Electricity Generation $\ldots \ldots \ldots \ldots \ldots \ldots$

Other Non-Process Use

End Use Not Reported

$\ldots \ldots \ldots \ldots \ldots \ldots \ldots \ldots \ldots \ldots \ldots \ldots \ldots \ldots \ldots$ 
Table A38. Selected Combustible Inputs of Energy for Heat, Power, and Electricity Generation and Net Demand for Electricity by Fuel Type and End Use, 1991: Part 2 (Continued) (Estimates in Trillion Btu)

\begin{tabular}{|c|c|c|c|c|c|c|c|c|}
\hline $\begin{array}{c}\text { SIC } \\
\text { Code" }\end{array}$ & End-Use Categories & $\begin{array}{l}\text { Net Demand } \\
\text { for Electricity }\end{array}$ & $\begin{array}{c}\text { Residual Fuel } \\
\text { Oil }\end{array}$ & $\begin{array}{c}\text { Distillate Fuel } \\
\text { Oil and Diesel } \\
\text { Fuel }\end{array}$ & Natural Gas ${ }^{d}$ & LPG & $\begin{array}{c}\text { Coal } \\
\text { (excluding } \\
\text { Coal Coke } \\
\text { and Breeze) }\end{array}$ & $\begin{array}{c}\text { RSE } \\
\text { Row } \\
\text { Factors }\end{array}$ \\
\hline
\end{tabular}

308

Miscellaneous Plastic Products, nec

RSE Column Factors:

\begin{tabular}{rr}
0.5 & 1.5 \\
87 & 3 \\
$\cdot$ & 2 \\
69 & 1 \\
17 & 1 \\
7 & 0 \\
44 & $:$ \\
$\vdots$ & 0 \\
14 & $:$ \\
6 & $:$ \\
6 & $:$ \\
1 & - \\
$:$ & 0 \\
\hline- & 0 \\
5 & 0
\end{tabular}

1.7
$w$
$w$
$\vdots$
0
$Q$
$:$
$\vdots$
$:$
$:$
1
0
$Q$
$Q$

0.7
53
24
13
12
0
1
-
12
12
-
-
0
$*$

3

13.3

TOTAL INPUTS

Boiler Fuel

Total Process Uses

Process Heating . . . . . . . . . . . . . . . . . . . . . . . .

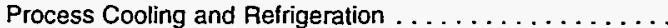

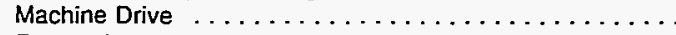

Electro-Chemical Processes $\ldots \ldots \ldots \ldots \ldots \ldots \ldots \ldots$

Other Process Use . . . . . . . . . . . . . . . . . . . . . .

Total Non-Process Uses . . . . . . . . . . . . . .

Facility Heating, Ventilation, and Air Conditioning ${ }^{\circ} \ldots \ldots$.

Facility Lighting

Facility Support . . . . . . . . . . . . . . . . .

Onsite Transportation . . . . . . . . . . . . . .

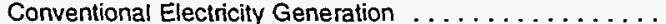

End Use Not Reported

LEATHER and LEATHER PRODUCTS

RSE Column Factors:

0.4

TOTAL INPUTS

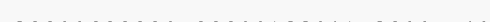

Boiler Fuel $\ldots \ldots \ldots \ldots \ldots \ldots \ldots \ldots \ldots \ldots \ldots \ldots$

Total Process Uses $\ldots \ldots \ldots \ldots \ldots \ldots \ldots \ldots \ldots \ldots$

Process Heating . . . . . . . . . . . . . . . . . .

Process Cooling and Refrigeration $\ldots \ldots \ldots \ldots \ldots \ldots \ldots$

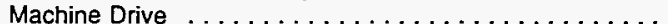

Electro-Chemical Processes . . . . . . . . . . . .

Other Process Use . . . . . . . . . . . . . . .

Total Non-Process Uses . . . . . . . . . . . . . . . .

Facility Heating, Ventilation, and Air Conditioning ${ }^{\circ} \ldots \ldots$

Facility Lighting $\ldots \ldots \ldots \ldots \ldots \ldots \ldots \ldots$

Facility Support . . . . . . . . . . . . . . . . . . .

Onsite Transportation . . . . . . . . . . . . . .

Conventional Electricity Generation . . . . . . . . . . .

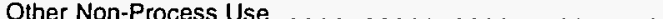

End Use Not Reported

STONE, CLAY and GLASS PRODUCTS

RSE Column Factors:

TOTAL INPUTS

107

1.1

1.5

0.6

1.6

1.2

Boiler Fuel

...........................

Total Process Uses . . . . . . . . . . . . . . . . .

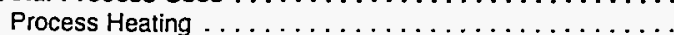

Process Cooling and Refrigeration . . . . . . . . . . . .

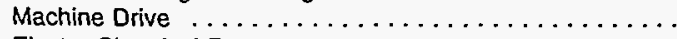

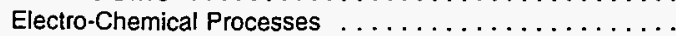

Other Process Use . . . . . . . . . . . . . . . . . .

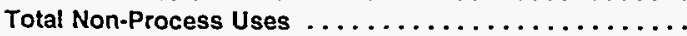

Facility Heating, Ventilation, and Air Conditioning ${ }^{\circ} \ldots \ldots$.

Facility Lighting . . .

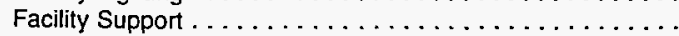

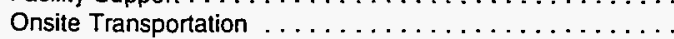

Conventional Electricity Generation

Other Non-Process Use $\ldots \ldots \ldots \ldots \ldots \ldots \ldots \ldots \ldots$

End Use Not Reported

$\ldots \ldots \ldots \ldots \ldots \ldots \ldots \ldots+\cdots$ 
Table A38. Selected Combustible Inputs of Energy for Heat, Power, and Electricity Generation and Net Demand for Electricity by Fuel Type and End Use, 1991: Part 2 (Continued) (Estimates in Trillion Btu)

\begin{tabular}{|c|c|c|c|c|c|c|c|c|}
\hline $\begin{array}{l}\text { SIC } \\
\text { Code' }\end{array}$ & End-Use Categories & $\begin{array}{l}\text { Net Demand } \\
\text { for Electricity }\end{array}$ & $\begin{array}{c}\text { Residual Fuel } \\
\text { Oil }\end{array}$ & $\begin{array}{c}\text { Distillate Fuel } \\
\text { Oil and Diesel } \\
\text { Fuef }\end{array}$ & Natural Gas ${ }^{d}$ & LPG & $\begin{array}{c}\text { Coal } \\
\text { (excluding } \\
\text { Coal Coke } \\
\text { and Breeze) }\end{array}$ & \begin{tabular}{|c} 
RSE \\
Row \\
Factors
\end{tabular} \\
\hline
\end{tabular}

3211

Flat Glass

RSE Column Factors:

\begin{tabular}{rr}
0.7 & 1.6 \\
5 & $W$ \\
$W$ & 0 \\
4 & $W$ \\
2 & $W$ \\
$:$ & 0 \\
2 & 0 \\
0 & - \\
0 & 0 \\
$W$ & 0 \\
1 & 0 \\
$W$ & - \\
$:$ & 0 \\
$*$ & - \\
\hline 0 & 0 \\
$W$ & 0
\end{tabular}

TOTAL INPUTS

Boiler Fuel

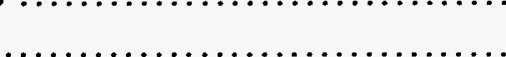

Total Process Uses

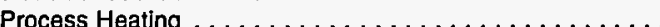

Process Cooling and Refrigeration $\ldots \ldots \ldots \ldots \ldots \ldots$

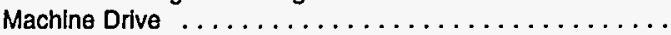

Electro-Chemical Processes ..................

Other Process Use .......................

Total Non-Process Uses . . . . . . . . . . . . . . . . .

Facllity Heating, Ventilation, and Air Conditioning ${ }^{\circ} . \ldots \ldots$.

Facility Lighting.$\ldots \ldots \ldots \ldots \ldots \ldots \ldots \ldots \ldots$

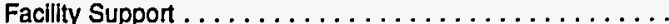

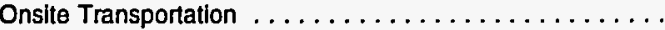

Conventional Electricity Generation.$\ldots \ldots \ldots \ldots \ldots$

Other Non-Process Use $\ldots \ldots \ldots \ldots \ldots \ldots \ldots \ldots$

End Use Not Reported

Glass Containers

RSE Column Factors:

0.6
14
.
12
4
$W$
7
$W$
$W$
2
1
1
$:$
..
0
0

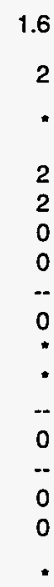

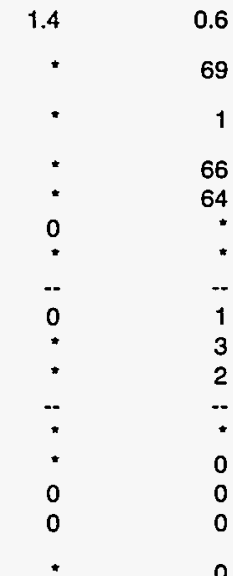

\begin{tabular}{rr}
0.9 & 0.6 \\
$*$ & 42 \\
0 & $W$ \\
$W$ & 36 \\
$W$ & 35 \\
$*$ & 1 \\
$*$ & - \\
-- & 0 \\
0 & 2 \\
\hline & 1 \\
\hline- & $\div$ \\
0 & 0 \\
$*$ & 0 \\
$*$ & 0 \\
0 & $W$
\end{tabular}

\begin{tabular}{rrr}
1.0 & 1.6 & \\
. & $*$ & 3.5 \\
0 & 0 & 11.2 \\
$W$ &. & 4.0 \\
$W$ & 0 & 4.1 \\
0 & 0 & 6.1 \\
. & 0 & 5.2 \\
.- & - & NF \\
0 & $*$ & 3.7 \\
. & 0 & 4.1 \\
- & 0 & 4.7 \\
\hline 0 & -- & 4.3 \\
. & 0 & 7.4 \\
0 &.- & 5.6 \\
0 & 0 & 4.4 \\
W & 0 & NF \\
\hline & 0 & 5.6
\end{tabular}

TOTAL INPUTS

Boller Fuel

Total Process Uses

Process Heating ......

Process Cooling and Refrigeration . . . . . . . . . . .

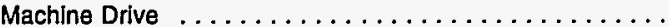

Electro-Chemical Processes $\ldots \ldots \ldots \ldots \ldots \ldots \ldots \ldots$

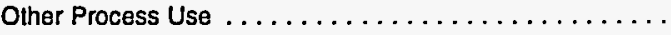

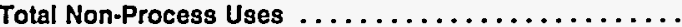

Facility Heating, Ventilation, and Air Conditioning ${ }^{\circ} \ldots \ldots$.

Facility Lighting . .

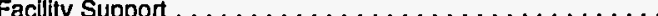

Onsite Transportation $\ldots \ldots \ldots \ldots \ldots \ldots \ldots \ldots$

Conventional Electricity Generation ..............

Other Non-Process Use

End Use Not Reported

Pressed and Blown Glass, nec.

RSE Column Factors:

\begin{tabular}{|c|c|c|c|c|c|c|}
\hline 0.6 & 2.7 & 1.0 & 0.6 & 1.0 & NF & \\
\hline 10 & 1 & * & $w$ & • & 0 & 8.8 \\
\hline - & W & • & $w$ & 0 & 0 & 9.8 \\
\hline 8 & $Q$ & W & 37 & : & 0 & 10.2 \\
\hline 4 & $\vec{Q}$ & $w$ & 36 & . & 0 & 11.2 \\
\hline$w$ & 0 & 0 & 0 & 0 & 0 & 8.1 \\
\hline 3 & : & * & . & * & 0 & 8.0 \\
\hline . & -- & .. & -. & -- & -- & 11.3 \\
\hline$w$ & 0 & • & 0 & - & 0 & 16.0 \\
\hline$w$ & . & $w$ & 2 & • & 0 & 5.9 \\
\hline 1 & • & * & 2 & • & 0 & 7.2 \\
\hline 1 & .. & .- & - & .- & .- & 8.1 \\
\hline . & 0 & * & - & 0 & 0 & 9.9 \\
\hline - & -- & - & 0 & • & - & 6.0 \\
\hline -. & 0 & $w$ & 0 & 0 & 0 & 11.8 \\
\hline$w$ & 0 & • & 0 & 0 & 0 & 13.7 \\
\hline$w$ & 0 & * & w & $Q$ & 0 & 14.1 \\
\hline
\end{tabular}

See footnotes at end of table. 
Table A38. Selected Combustible Inputs of Energy for Heat, Power, and Electricity Gieneration and Net Demand for Electricity by Fuel Type and End Use, 1991: Part 2 (Ciontinued) (Estimates in Trillion Btu)

\begin{tabular}{|c|c|c|c|c|c|c|c|c|}
\hline $\begin{array}{c}\text { SIC } \\
\text { Code }\end{array}$ & End-Use Categories & $\begin{array}{l}\text { Net Demand } \\
\text { for Electricity }\end{array}$ & $\begin{array}{c}\text { Residual Fuel } \\
\text { Oil }\end{array}$ & $\begin{array}{c}\text { Distillate Fuel } \\
\text { Oil and Diesel } \\
\text { Fuef }\end{array}$ & Natural Gas ${ }^{d}$ & LPG & $\begin{array}{c}\text { Coal } \\
\text { (excluding } \\
\text { Coal Coke } \\
\text { and Braeza) }\end{array}$ & $\begin{array}{c}\text { RSE } \\
\text { Row } \\
\text { Factorg }\end{array}$ \\
\hline
\end{tabular}

3241

Cement, Hydraulic

RSE Column Factors:

TOTAL INPUTS

0.8
34
$Q$
32
6
1
25
0
2
2
1
1
.
.-
0
$Q$

1.7
1
0
1
$W$
0
$W$
--
0
0
0
-
0
-
0
0
.

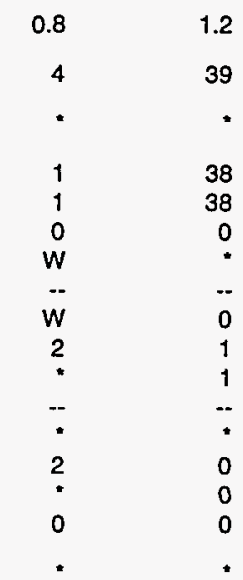

$\begin{array}{rr}1.1 & 0.7 \\ * & 195 \\ 0 & 0 \\ . & 195 \\ 0 & 195 \\ . & 0 \\ . & 0 \\ : & 0 \\ . & 0 \\ : & 0 \\ : & 0 \\ 0 & - \\ . & 0 \\ . & 0\end{array}$

10.7

Boiler Fuel

Total Process Uses

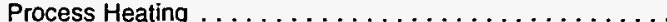

Process Cooling and Refrigeration . . . . . . . . .

Machine Drive $\ldots \ldots \ldots \ldots \ldots \ldots \ldots \ldots \ldots \ldots \ldots$

Other Process Use . . . . . . . . . . . . . . . . .

Total Non-Process Uses $\ldots \ldots \ldots \ldots \ldots \ldots \ldots \ldots \ldots \ldots$

Facility Heating, Ventilation, and Air Conditioning ${ }^{\circ} \ldots \ldots$.

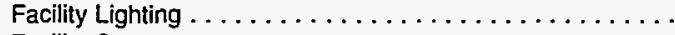

Facility Support

Onsite Transportation $\ldots \ldots \ldots \ldots$

Conventional Electricity Generation $\ldots \ldots \ldots \ldots \ldots \ldots \ldots$

End Use Not Reported

Lime

RSE Column Factors:

1.3

TOTAL INPUTS $\ldots \ldots \ldots \ldots \ldots \ldots \ldots \ldots \ldots \ldots$

Boller Fuel $\ldots \ldots \ldots \ldots \ldots \ldots \ldots \ldots \ldots \ldots \ldots \ldots \ldots$

Total Process Uses . . . . . . . . . . . . . . . . . . .

Process Heating $\ldots \ldots \ldots \ldots \ldots \ldots \ldots \ldots \ldots \ldots$

Process Cooling and Refrigeration . . . . . . . . . . .

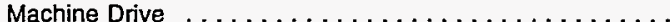

Electro-Chemical Processes . . . . . . . . . . . .

Other Process Use . . . . . . . . . . . . . . . . . .

Total Non-Process Uses . . . . . . . . . . . . . . . .

Facility Heating, Ventilation, and Air Conditioning ${ }^{\circ} \ldots \ldots \ldots$

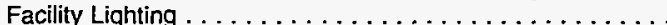

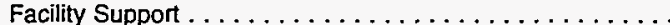

Onsite Transportation . . . . . . . . . . . . . .

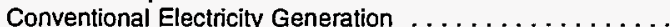

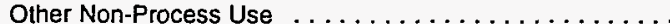

End Use Not Reported

3296

Mineral Wool

RSE Column Factors:

TOTAL INPUTS

Boiler Fuel $\ldots \ldots \ldots \ldots \ldots \ldots \ldots \ldots \ldots \ldots \ldots \ldots$

Total Process Uses

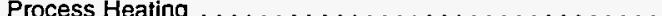

Process Cooling and Refrigeration . . . . . . . . . .

Machine Drive $\ldots \ldots \ldots \ldots \ldots \ldots \ldots \ldots \ldots \ldots \ldots \ldots \ldots$

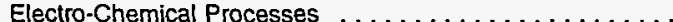

Other Process Use . . . . . . . . . . . . . . . . .

Total Non-Process Uses $\ldots \ldots \ldots \ldots \ldots \ldots \ldots \ldots \ldots \ldots$

Facility Heating, Ventilation, and Air Conditioning

Facility Lighting . . . . . . . . . . . . . . . . . .

Facility Support.

Onsite Transportation

Conventional Electricity Generation . . . . . . . . . . .

Other Non-Process Use $\ldots \ldots \ldots \ldots \ldots \ldots \ldots \ldots \ldots$

End Use Not Reported

5

Q

0

$$
\text { . }
$$

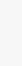$$
0
$$

Q

\begin{tabular}{c}
0.9 \\
$W$ \\
0 \\
$W$ \\
$W$ \\
0 \\
0 \\
- \\
0 \\
0 \\
0 \\
\hdashline \\
0 \\
- \\
0 \\
0 \\
0
\end{tabular}

\begin{tabular}{|c|c|}
\hline 0.9 & 0.6 \\
\hline 1 & 8 \\
\hline 0 & * \\
\hline * & 8 \\
\hline $\begin{array}{l}Q \\
0\end{array}$ & $\begin{array}{l}8 \\
0\end{array}$ \\
\hline -- & -- \\
\hline w & 0 \\
\hline 1 & : \\
\hline .- & .. \\
\hline 0 & 0 \\
\hline 1 & $\begin{array}{l}0 \\
0\end{array}$ \\
\hline o & 0 \\
\hline
\end{tabular}

$\begin{array}{ll}0.8 & 1.8 \\ Q & 8 \\ 0 & \end{array}$

25.9

NF

22.7

22.0

12.7

27.9

NF

24.7

31.3

23.8

23.8

8.2

34.6

NF

NF

NF

\begin{tabular}{|c|c|c|c|c|c|}
\hline 0.7 & 1.2 & 1.1 & 0.8 & 1.1 & 1.2 \\
\hline 10 & $w$ & • & 29 & • & * \\
\hline$w$ & $w$ & • & 1 & • & * \\
\hline 8 & 0 & * & 23 & $w$ & 0 \\
\hline 4 & 0 & * & 22 & - & 0 \\
\hline$w$ & 0 & 0 & $\star$ & 0 & 0 \\
\hline 4 & 0 & * & $\star$ & $w$ & 0 \\
\hline 0 & -- & -. & -. & -. & .. \\
\hline$w$ & 0 & 0 & • & 0 & 0 \\
\hline$w$ & 0 & " & 2 & 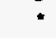 & 0 \\
\hline 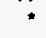 & 0 & $w$ & 2 & • & 0 \\
\hline * & - & -. & -. & -. & $\ldots$ \\
\hline * & 0 & $w$ & $\star$ & 0 & 0 \\
\hline$W$ & -- & $*$ & 0 & * & .. \\
\hline- & 0 & * & 0 & 0 & 0 \\
\hline " & 0 & * & 0 & 0 & 0 \\
\hline W & 0 & * & 2 & $w$ & 0 \\
\hline
\end{tabular}

1.6

1.8

1.3

1.5

1.8 
Table A38. Selected Combustible Inputs of Energy for Heat, Power, and Electricity Generation and Net Demand for Electricity by Fuel Type and End Use, 1991: Part 2 (Continued) (Estimates in Trillion Btu)

\begin{tabular}{|c|c|c|c|c|c|c|c|c|}
\hline $\begin{array}{l}\operatorname{SIC} \\
\operatorname{Code}^{a}\end{array}$ & End-Use Categories & $\begin{array}{l}\text { Net Demand } \\
\text { for Electricity }\end{array}$ & $\begin{array}{c}\text { Residual Fue! } \\
\text { Oil }\end{array}$ & $\begin{array}{c}\text { Distillate Fuel } \\
\text { Oil and Diesel } \\
\text { Fuer }\end{array}$ & Natural Gas ${ }^{d}$ & LPG & $\begin{array}{c}\text { Coal } \\
\text { (excluding } \\
\text { Coal Coke } \\
\text { and Breeze) }\end{array}$ & $\begin{array}{c}\text { RSE } \\
\text { Row } \\
\text { Factors }\end{array}$ \\
\hline
\end{tabular}

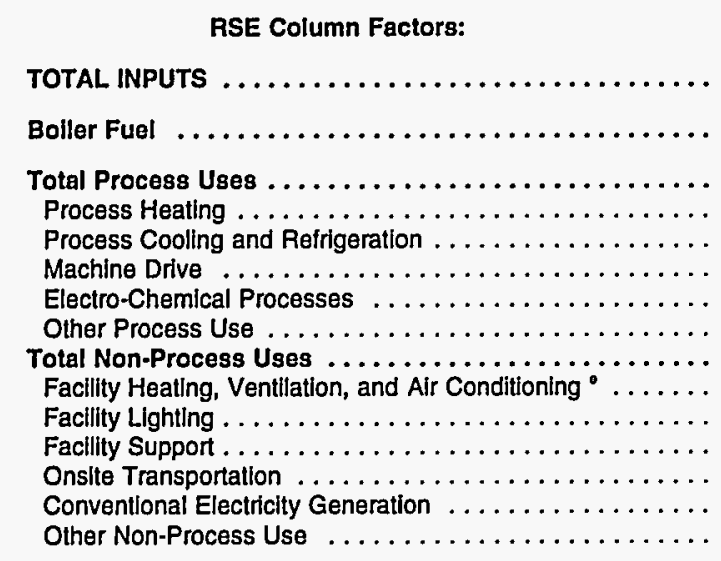

0.6
524
1
474
103
3
134
229
5
40
17
18
4
1
-
1
8

$\begin{array}{ll}1.2 & 1.3 \\ 33 & \\ 25 & \\ 8 & \\ 8 & \\ 0 & \\ Q & \\ -- & \\ 0 & \\ Q & \\ Q & \\ -- & \\ Q & \\ -- & \\ 0 & \\ 0 & \\ \cdot & \end{array}$

1.3
11
1
3
2
0
1
$\because$
7
$:$
$:$
6
$Q$
$W$
1

\begin{tabular}{|c|c|c|c|}
\hline 0.7 & 2.2 & 0.8 & \\
\hline 686 & 3 & 46 & 4.6 \\
\hline 93 & - & 38 & 7.6 \\
\hline 522 & 1 & $w$ & 7.2 \\
\hline 516 & 1 & $w$ & 8.0 \\
\hline • & 0 & 0 & 12.7 \\
\hline 4 & . & 0 & 7.5 \\
\hline -- & -- & -- & 3.6 \\
\hline 2 & • & 0 & 11.1 \\
\hline 54 & 2 & $w$ & 3.2 \\
\hline 44 & • & 0 & 8.9 \\
\hline -- & -- & .. & 5.4 \\
\hline 6 & - & 0 & 9.1 \\
\hline * & 2 & .. & 4.3 \\
\hline 4 & * & $w$ & 3.5 \\
\hline 1 & * & 0 & 13.4 \\
\hline 17 & * & • & 14.8 \\
\hline
\end{tabular}

3312 Blast Furnaces and Steel Mills

\section{RSE Column Factors:}

TOTAL INPUTS

152

Boller Fuel $\ldots \ldots \ldots \ldots \ldots \ldots \ldots \ldots \ldots \ldots \ldots$

Total Process Uses . . . . . . . . . . . . . . . . . .

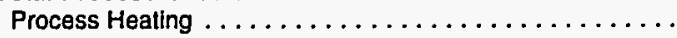

Process Cooling and Refrigeration ...............

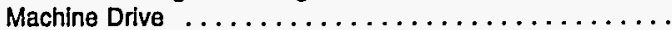

Electro-Chemical Processes ....................

Other Process Use . . . . . . . . . . . . . . . .

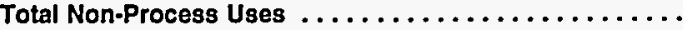

Facility Heating, Ventilation, and Air Conditioning ${ }^{\circ} . \ldots \ldots$.

Facility Lighting . . . . . . . . . . . . . . . . . . .

Facility Support . . . . . . . . . . . . . . . .

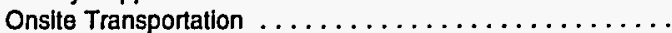

Conventional Electricity Generation ...............

Other Non-Process Use $\ldots \ldots \ldots \ldots \ldots \ldots \ldots$.

End Use Not Reported

134

5

24

63

312

310

$\begin{array}{lll}7 & 1 & 310 \\ 7 & 0 & \end{array}$

72

4
13

5

W

w

Electrometalurgical Products

TOTAL INPUTS

15

0

Boller Fuel

W

Total Process Uses $\ldots \ldots \ldots \ldots \ldots \ldots \ldots \ldots \ldots \ldots \ldots$

Process Heating $\ldots \ldots \ldots \ldots \ldots \ldots \ldots \ldots \ldots \ldots$

Process Cooling and Refrigeration . . . . . . . . . . .

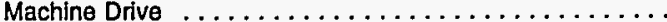

Electro-Chemical Processes . . . . . . . . . . . . . .

Other Process Use $\ldots \ldots \ldots \ldots \ldots \ldots \ldots \ldots \ldots$

Total Non-Process Uses . ....................

Facillty Heating, Ventilation, and Air Conditioning ${ }^{\circ} . \ldots \ldots$.

Facility Lighting . . . . . . . . . . . . . . . . . .

Facility Support

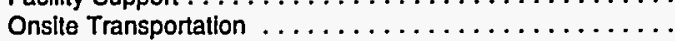

Conventional Electricity Generation . . . . . . . . . . .

Other Non-Process Use

End Use Not Reported

$\begin{array}{cc}W & 0 \\ 14 & 0 \\ 11 & 0 \\ W & 0 \\ 3 & 0 \\ W & \div \\ W & 0 \\ W & 0 \\ : & 0 \\ W & 0 \\ -- & 0 \\ 0 & 0\end{array}$

$$
\text { - }
$$


Table A38. Selected Combustible Inputs of Energy for Heat, Power, and Electricity Generation and Net Demand for Electricity by Fuel Type and End Use, 1991: Part 2 (Continued) (Estimates in Trillion Btu)

\begin{tabular}{|c|c|c|c|c|c|c|c|c|}
\hline $\begin{array}{c}\text { SIC } \\
\text { Code }\end{array}$ & End-Use Categories & $\begin{array}{l}\text { Net Demand } \\
\text { for Electricity }\end{array}$ & $\begin{array}{c}\text { Residual Fuel } \\
\text { Oil }\end{array}$ & $\begin{array}{c}\text { Distillate Fuel } \\
\text { Oil and Diesel } \\
\text { Fuel }^{c}\end{array}$ & Natural Gas ${ }^{d}$ & LPG & $\begin{array}{c}\text { Coal } \\
\text { (excluding } \\
\text { Coal Coke } \\
\text { and Rreeze) }\end{array}$ & $\begin{array}{c}\text { RSE } \\
\text { Row } \\
\text { Factors }\end{array}$ \\
\hline
\end{tabular}

3321

Gray and Ductile Iron Foundries

RSE Column Factors:

$\begin{array}{rr}0.6 & 1 \\ 22 & \\ * & \\ 18 & \\ 9 & \\ 8 & \\ W & \\ Q & \\ 4 \\ 2 \\ 1 \\ : \\ -: \\ : \\ 1\end{array}$

$\begin{array}{rr}1.6 & 1.3 \\ * & 1 \\ W & * \\ Q & 0 \\ 0 & Q \\ 0 & 0 \\ Q & : \\ - & : \\ 0 & : \\ : & : \\ - & : \\ 0 & : \\ -0 & 0 \\ 0 & \end{array}$

0.6
28
2
18
17
0
1
$\because$
7
7
$\because$
$\vdots$
0
$:$
1

\begin{tabular}{|c|c|c|}
\hline 1.3 & 1.2 & \\
\hline . & * & 14.0 \\
\hline * & 0 & 18.8 \\
\hline * & - & 13.7 \\
\hline * & - & 16.4 \\
\hline 0 & 0 & 16.2 \\
\hline 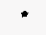 & 0 & 11.5 \\
\hline- & -- & 39.6 \\
\hline . & 0 & 19.4 \\
\hline . & 0 & 11.0 \\
\hline • & 0 & 19.9 \\
\hline .. & .. & 10.8 \\
\hline 0 & 0 & 12.1 \\
\hline 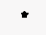 & -. & 17.7 \\
\hline 0 & 0 & 13.7 \\
\hline • & 0 & 37.4 \\
\hline " & - & 29.7 \\
\hline
\end{tabular}

3331

Primary Copper

RSE Column Factors:

TOTAL INPUTS

1.0
5
$W$
5
$W$
3
1
$W$
$W$
$:$
$:$
$W$
0
--
0
0

\begin{tabular}{c}
1.0 \\
$W$ \\
$W$ \\
$W$ \\
$W$ \\
0 \\
0 \\
\hline- \\
0 \\
0 \\
0 \\
- \\
0 \\
- \\
0 \\
0 \\
0
\end{tabular}

\begin{tabular}{r}
1.0 \\
$W$ \\
\\
$W$ \\
$W$ \\
0 \\
0 \\
- \\
0 \\
$W$ \\
$*$ \\
\hdashline \\
0 \\
$W$ \\
$\vdots$ \\
0 \\
0
\end{tabular}

1.0
15
4
9
8
0
0
-
2
-
-
0
2
0
0

$\begin{array}{ccc}1.0 & 1.0 & \\ . & W & 1.0 \\ 0 & 0 & 1.0 \\ * & W & 1.0 \\ * & W & 1.0 \\ 0 & 0 & 1.0 \\ - & 0 & 1.0 \\ 0 & -. & 1.0 \\ * & 0 & 1.0 \\ * & 0 & 1.0 \\ - & 0 & 1.0 \\ 0 & -- & 1.0 \\ * & 0 & 1.0 \\ 0 & -. & 1.0 \\ 0 & 0 & 1.0 \\ * & 0 & \text { NF } \\ & 0 & 1.0\end{array}$

Primary Aluminum

RSE Column Factors:

TOTAL INPUTS

$\begin{array}{rrr}0.8 & 1.4 \\ 230 & \cdot \\ W & * \\ 222 & 0 & 1.1 \\ W & 0 & \\ W & 0 & \\ 4 & 0 & \\ 216 & - \\ W & - \\ W & - \\ 4 & 0 \\ 3 & - \\ W & 0 \\ - & 0 \\ 0 & 0\end{array}$

Boiler Fuel $\ldots \ldots \ldots \ldots \ldots \ldots \ldots \ldots \ldots \ldots \ldots \ldots$

Total Process Uses

Process Heating .

Process Cooling and Refrigeration $\ldots \ldots \ldots \ldots \ldots$

Machine Drive

Electro-Chemical Processes $\ldots \ldots \ldots \ldots \ldots \ldots \ldots \ldots \ldots$

Other Process Use . . . . . . . . . . . . . . .

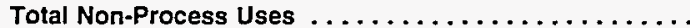

Facility Heating, Ventilation, and Air Conditioning ${ }^{\circ} \ldots \ldots$

Facility Lighting

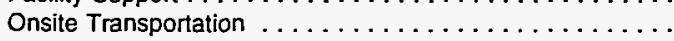

Conventional Electricity Generation $\ldots \ldots \ldots \ldots \ldots \ldots$

Other Non-Process Use . . . . . . . . . . . . . . . .

End Use Not Reported

\begin{tabular}{|c|c|c|c|}
\hline 1.1 & 0.8 & 1.1 & NF \\
\hline 1 & 21 & * & 0 \\
\hline$W$ & 2 & $w$ & 0 \\
\hline$w$ & 17 & • & 0 \\
\hline$w$ & 17 & - & 0 \\
\hline 0 & 0 & 0 & 0 \\
\hline * & • & * & 0 \\
\hline -- & -- & -- & - \\
\hline 0 & 0 & 0 & 0 \\
\hline 1 & 1 & * & 0 \\
\hline * & 1 & * & 0 \\
\hline -- & -- & -- & -- \\
\hline 0 & * & 0 & 0 \\
\hline 1 & 0 & " & $\cdots$ \\
\hline 0 & 0 & 0 & 0 \\
\hline 0 & 0 & * & 0 \\
\hline * & 1 & $w$ & 0 \\
\hline
\end{tabular}


Table A38. Selected Combustible Inputs of Energy for Heat, Power, and Electricity Generation and Net Demand for Electricity by Fuel Type and End Use, 1991: Part 2 (Continued) (Estimates in Trillion Btu)

\begin{tabular}{|c|c|c|c|c|c|c|c|c|}
\hline $\begin{array}{c}\text { SIC } \\
\text { Code" }\end{array}$ & End-Use Categories & $\begin{array}{c}\text { Net Demand } \\
\text { for Electricity }\end{array}$ & $\begin{array}{c}\begin{array}{c}\text { Residual Fuel } \\
\text { Oil }\end{array} \\
\end{array}$ & $\begin{array}{c}\text { Distillate Fuel } \\
\text { Oil and Diesel } \\
\text { Fuef }^{\circ}\end{array}$ & Natural Gas ${ }^{d}$ & LPG & $\begin{array}{c}\text { Coal } \\
\text { (excluding } \\
\text { Coal Coke } \\
\text { and Breeze) }\end{array}$ & $\begin{array}{c}\text { RSE } \\
\text { Row } \\
\text { Factors }\end{array}$ \\
\hline
\end{tabular}

3339

Primary Nonferrous Metals, nec

RSE Column Factors:

TOTAL INPUTS

Boiler Fuel

Total Process Uses . . . . . . . . . . . . . . . . . . . .

Process Heating $\ldots \ldots \ldots \ldots \ldots \ldots \ldots \ldots \ldots \ldots \ldots$

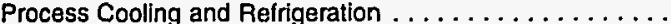

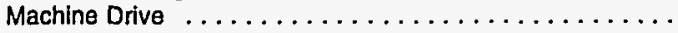

Electro-Chemical Processes ................

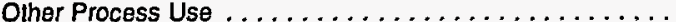

Total Non-Process Uses . . . . . . . . . . . . . . . .

Facility Heating, Ventilation, and Air Conditioning ${ }^{\circ} \ldots \ldots$.

Facility Lighting . . . . . . . . . . . . . . . . .

Facility Support .

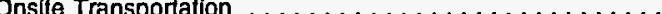

Conventional Electricity Generation ............

Other Non-Process Use $\ldots \ldots \ldots \ldots \ldots \ldots \ldots \ldots$

End Use Not Reported

Aluminum Sheet, Flate, and Foll

RSE Column Factors:

TOTAL INPUTS

Boller Fuel

Total Process Uses . . . . . . . . . . . . . . . . . .

Process Heating . . . . . . . . . . . . . . . . .

Process Cooling and Refrigeration .............

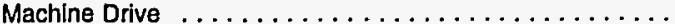

Electro-Chemical Processes $\ldots \ldots \ldots \ldots \ldots \ldots \ldots \ldots$

Other Process Use . . . . . . . . . . . . . . . . . . .

Total Non-Process Uses . . . . . . . . . . . . . . . . .

Facility Heating, Ventilation, and Air Conditioning ${ }^{\circ} . . . .$.

Facility Lighting . . . . . . . . . . . . . . . . . . . .

Facility Support . . . . . . . . . . . . . . . . .

Onsite Transportation . . . . . . . . . . . . .

Conventional Electricity Generation ...............

Other Non-Process Use $\ldots \ldots \ldots \ldots \ldots \ldots \ldots \ldots$

End Use Not Reported

\section{FABRICATED METAL PRODUCTS}

RSE Column Factors:

TOTAL INPUTS

Boller Fuel

Total Process Uses

Process Cooling and Refrigeration . . . . . . . . . . .

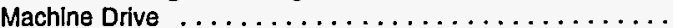

Electro-Chemical Processes . . . . . . . . . . . . .

Other Process Use $\ldots \ldots \ldots \ldots \ldots \ldots \ldots \ldots \ldots \ldots$

Total Non-Process Uses . . . . . . . . . . . . . . . . . .

Facllity Heating, Ventilation, and Air Conditioning ${ }^{\circ} \ldots \ldots$.

Facllity Lighting $\ldots \ldots \ldots \ldots \ldots \ldots \ldots \ldots$

Facility Support . . . . . . . . . . . . . . . . . .

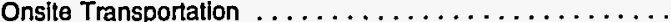

Conventional Electricity Generation ................

Other Non-Process Use $\ldots \ldots \ldots \ldots \ldots \ldots \ldots \ldots$

End Use Not Reported
1.3

15

W

14

5

$\begin{array}{cc}: & 0 \\ : & 0 \\ : & 0 \\ 0 & 0 \\ w & 0\end{array}$

1.2

NF

0.8

1.3

15

w

12

W

11
0

,

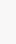

\begin{tabular}{c}
0.4 \\
$\cdot$ \\
\\
0 \\
0 \\
0 \\
0 \\
-- \\
0 \\
0 \\
0 \\
\hdashline 0 \\
0 \\
\hline 0 \\
0 \\
0
\end{tabular}

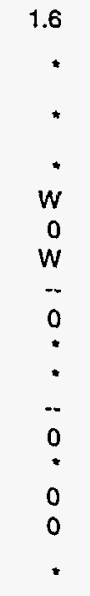

\begin{tabular}{c}
1.3 \\
17 \\
3 \\
8 \\
8 \\
0 \\
$\vdots$ \\
0 \\
$W$ \\
1 \\
\hdashline- \\
0 \\
$W$ \\
0 \\
$W$
\end{tabular}

1.4
0
0
$W$
$W$
0
-
-
$W$
$W$
$:-$
$\vdots$
0
0
.

0.6

\begin{tabular}{cr}
$W$ & 1.9 \\
$W$ & 2.8 \\
0 & 1.6 \\
0 & 1.3 \\
0 & 3.7 \\
0 & 1.0 \\
\hline- & 1.5 \\
0 & 15.0 \\
$W$ & 1.5 \\
0 & 2.5 \\
\hline- & 2.2 \\
0 & 4.9 \\
-- & 1.4 \\
$W$ & 1.2 \\
0 & $\mathrm{NF}$ \\
0 & 1.8
\end{tabular}

\begin{tabular}{|c|c|c|c|c|c|c|}
\hline 0.5 & 1.7 & 1.6 & 0.5 & 1.1 & 1.4 & \\
\hline 102 & 3 & 6 & 174 & 4 & 5 & 11.4 \\
\hline 1 & 2 & 1 & 37 & Q & 5 & 15.7 \\
\hline 71 & $\mathbf{Q}$ & 1 & 91 & 2 & $w$ & 16.7 \\
\hline 12 & * & 1 & 89 & W & W & 19.5 \\
\hline 2 & 0 & * & * & * & 0 & 21.6 \\
\hline 52 & $Q$ & • & 1 & W & 0 & 25.7 \\
\hline 4 & -. & -. & -. & - & .. & 24.3 \\
\hline 1 & $\mathbf{Q}$ & $Q$ & 1 & * & 0 & 50.1 \\
\hline 24 & $Q$ & 3 & 37 & 2 & 0 & 14.2 \\
\hline 10 & $Q$ & 1 & 34 & . & 0 & 19.5 \\
\hline 12 & .- & -- & -- & .. & -. & 15.5 \\
\hline 2 & $\mathbf{Q}$ & . & 2 & $Q$ & 0 & 24.3 \\
\hline 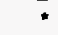 & .- & 2 & * & 2 & -- & 17.9 \\
\hline .- & 0 & * & 1 & 0 & 0 & 36.2 \\
\hline * & 0 & $\mathbf{Q}$ & * & . & 0 & 64.8 \\
\hline 7 & $Q$ & • & 8 & * & $\mathbf{Q}$ & 33.6 \\
\hline
\end{tabular}

See footnotes at end of table. 
Table A38. Selected Combustible Inputs of Energy for Heat, Power, and Electricity Gieneration and Net Demand for Electricity by Fuel Type and End Use, 1991: Part 2 (Continued) (Estimates in Trillion Btu)

\begin{tabular}{|c|c|c|c|c|c|c|c|c|}
\hline $\begin{array}{c}\text { SIC } \\
\text { Code" }\end{array}$ & End-Use Categories & $\begin{array}{c}\text { Net Demand } \\
\text { for Electricity }\end{array}$ & $\begin{array}{c}\text { Residual Fuel } \\
\text { Oil }\end{array}$ & $\mid \begin{array}{c}\text { Distillate Fuel } \\
\text { Oil and Diesel } \\
\text { Fuel }\end{array}$ & Natural Gas ${ }^{d}$ & LFG & $\begin{array}{c}\text { Coal } \\
\text { (excluding } \\
\text { Coal Coke }\end{array}$ & $\begin{array}{c}\text { RSE } \\
\text { Row } \\
\text { Factors }\end{array}$ \\
\hline
\end{tabular}

TOTAL INPUTS

Boiler Fuel $\ldots \ldots \ldots \ldots \ldots \ldots \ldots \ldots \ldots \ldots \ldots$

Total Process Uses $\ldots \ldots \ldots \ldots \ldots \ldots \ldots \ldots \ldots \ldots$

Process Heating . . . . . . . . . . . . . . . . . . .

Process Cooling and Refrigeration . . . . . . . . . .

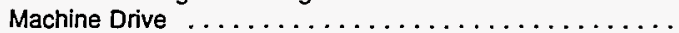

Electro-Chemical Processes ..................

Other Process Use . . . . . . . . . . . . . . . .

Total Non-Process Uses . . . . . . . . . . . . . . . .

Facility Heating, Ventilation, and Air Conditioning ${ }^{\circ} \ldots \ldots$.

Facility Lighting . . . . . . . . . . . . . . . .

Facility Support

Onsite Transportation $\ldots \ldots \ldots \ldots \ldots \ldots \ldots \ldots$

Conventional Electricity Generation . . . . . . . . . .

Other Non-Process Use $\ldots \ldots \ldots \ldots \ldots \ldots \ldots \ldots$

End Use Not Reported

TOTAL INPUTS

Boiler Fuel

Total Process Uses

.......................

Process Cooling and Refrigeration ....................

Machine Drive..$\ldots \ldots \ldots \ldots \ldots \ldots \ldots \ldots \ldots \ldots$

Electro-Chemical Processes . . . . . . . . . . . . .

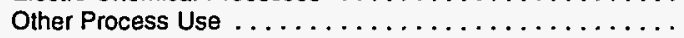

Total Non-Process Uses . . . . . . . . . . . . . . .

Facility Heating, Ventilation, and Air Conditioning ${ }^{\circ} \ldots \ldots$.

Facility Lighting $\ldots \ldots \ldots \ldots \ldots \ldots \ldots \ldots$

Facility Support . . . . . . . . . . . . . . . . .

Onsite Transportation $\ldots \ldots \ldots \ldots \ldots \ldots \ldots \ldots \ldots$

Conventional Electricity Generation ..............

Other Non-Process Use $\ldots \ldots \ldots \ldots \ldots \ldots \ldots \ldots \ldots . . . . . .$.

End Use Not Reported

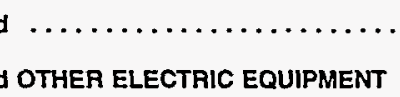

RSE Golumn Factors:

TOTAL INPUTS

Boiler Fuel

Total Process Uses

.........................

Process Cooling and Refrigeration ...............

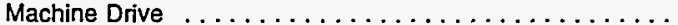

Electro-Chemical Processes . . . . . . . . . . . . . .

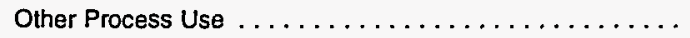

Total Non-Process Uses . ...................

Facility Heating, Ventilation, and Air Conditioning ${ }^{\circ} \ldots \ldots$. Facility Lighting . .

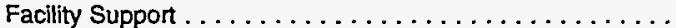

Onsite Transportation.$\ldots \ldots \ldots \ldots \ldots \ldots \ldots \ldots$.

Conventional Electricity Generation

Other Non-Process Use

............................

End Use Not Reported

$$
5
$$

*

\begin{tabular}{|c|c|c|c|c|c|}
\hline 0.4 & 1.6 & 1.2 & 0.6 & 1.1 & 1.7 \\
\hline 102 & 4 & 2 & 79 & 1 & $w$ \\
\hline 1 & 4 & 1 & 29 & * & w \\
\hline 59 & $Q$ & * & 32 & 1 & • \\
\hline 16 & $Q$ & * & 30 & 1 & \\
\hline 7 & " & * & $Q$ & : & 0 \\
\hline 31 & * & - & 1 & * & 0 \\
\hline 5 & -- & - & -. & -- & - \\
\hline 1 & 0 & • & * & * & 0 \\
\hline 38 & * & 1 & 15 & 1 & * \\
\hline 21 & * & * & 15 & $*$ & " \\
\hline 13 & -- & - & -- & -. & -- \\
\hline 4 & 0 & $Q$ & 1 & $Q$ & 0 \\
\hline * & -- & * & 0 & • & -- \\
\hline 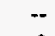 & 0 & * & * & * & 0 \\
\hline - & 0 & * & * & $Q$ & 0 \\
\hline 5 & * & * & 2 & . & 0 \\
\hline
\end{tabular}


Table A38. Selected Combustible Inputs of Energy for Heat, Power, and Electricity Generation and Net Demand for Electricity by Fuel Type and End Use, 1991: Part 2 (Continued) (Estimates in Trillion Btu)

\begin{tabular}{|c|c|c|c|c|c|c|c|c|}
\hline $\begin{array}{c}\text { SIC } \\
\text { Coden }\end{array}$ & End-Use Categories & $\begin{array}{l}\text { Net Demand } \\
\text { for Electricity }\end{array}$ & $\begin{array}{c}\text { Residual Fuel } \\
\text { Oil }\end{array}$ & $\begin{array}{c}\text { Distillate Fuel } \\
\text { Oil and Diesel } \\
\text { Fuel }^{\circ}\end{array}$ & Natural Gas & LPG & $\begin{array}{c}\text { Coal } \\
\text { (excluding } \\
\text { Coal Coke } \\
\text { and Breeze) }\end{array}$ & $\begin{array}{c}\text { RSE } \\
\text { Row } \\
\text { Factors }\end{array}$ \\
\hline
\end{tabular}

TOTAL INPUTS

Boller Fuel

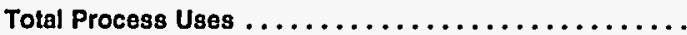

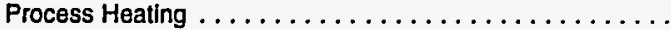

Process Cooling and Refrigeration . . . . . . . . . . . .

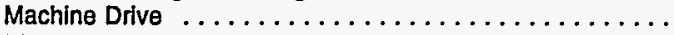

Electro-Chemical Processes . . . . . . . . . . . . .

Other Process Use $\ldots \ldots \ldots \ldots \ldots \ldots \ldots \ldots \ldots \ldots$

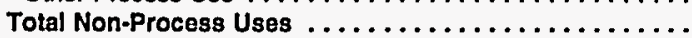

Facility Heating, Ventilation, and Air Conditioning $\bullet . . \ldots$.

Facillty Lighting..$\ldots \ldots \ldots \ldots \ldots \ldots \ldots \ldots$

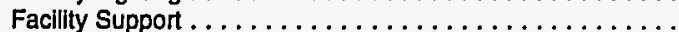

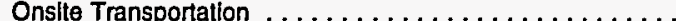

Conventional Electricity Generation .................

Other Non-Process Use $\ldots \ldots \ldots \ldots \ldots \ldots \ldots \ldots \ldots$

End Use Not Reported

\section{2}

w

70

10

5
50

50
2

2
44

44
21

18

18

$\ddot{1}$

7

3711

TOTAL INPUTS

Boller Fuel

Total Process Uses $\ldots \ldots \ldots \ldots \ldots \ldots \ldots \ldots \ldots \ldots$

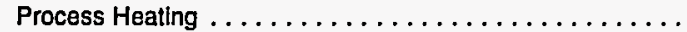

Process Cooling and Refrigeration . . . . . . . . . . .

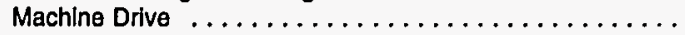

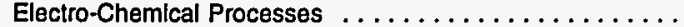

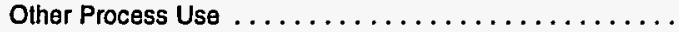

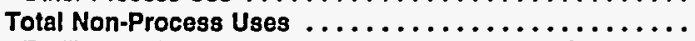

Facility Heating, Ventilation, and Air Conditioning ${ }^{\circ} \ldots \ldots$.

Facllity Llghting $\ldots \ldots \ldots \ldots \ldots \ldots \ldots \ldots \ldots$

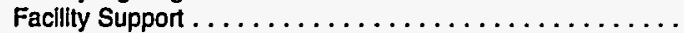

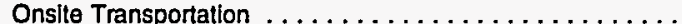

Conventional Electricity Generation ...............

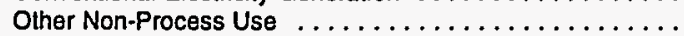

End Use Not Reported

$W$
17
2
2
12
$W$
$W$
9
5
3
1
$W$
--
$W$
$W$

Motor Vehicle Parts and Accessories

RSE Column Factors:

TOTAL INPUTS

0.4

38

Boller Fuel

Total Process Uses

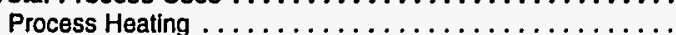

Process Cooling and Refrigeration . . . . . . . . . . .

Machine Drive $\ldots \ldots \ldots \ldots \ldots \ldots \ldots \ldots \ldots$

Electro-Chemlcal Processes .................

Other Process Use $\ldots \ldots \ldots \ldots \ldots \ldots \ldots \ldots \ldots \ldots \ldots$

Total Non-Process Uses . . . . . . . . . . . . . . .

Facility Heating, Ventilation, and Air Conditioning ${ }^{\circ} . \ldots \ldots$.

Facility Lighting . . . . . . . . . . . . . . . . . . .

Facility Support .

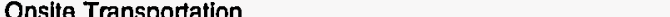

Conventional Electricity Generation $\ldots \ldots \ldots \ldots \ldots \ldots$

Other Non-Process Use $\ldots \ldots \ldots \ldots \ldots \ldots \ldots \ldots \ldots$

End Use Not Reported

See footnotes at end of table. 
Table A38. Selected Combustible Inputs of Energy for Heat, Power, and Electricity Generation and Net Demand for Electricity by Fuel Type and End Use, 1991: Part 2 (Continued) (Estimates in Trillion Btu)

\begin{tabular}{|c|c|c|c|c|c|c|c|c|}
\hline $\begin{array}{c}\text { SIC } \\
\text { Code" }\end{array}$ & End-Use Categories & $\begin{array}{l}\text { Net Demand } \\
\text { for Electricity }\end{array}$ & $\begin{array}{c}\text { Residual Fuel } \\
\text { Oil }\end{array}$ & $\begin{array}{c}\text { Distillate Fuel } \\
\text { Oil and Diesel } \\
\text { Fuef }\end{array}$ & Natural Gas ${ }^{d}$ & LPG & $\begin{array}{c}\text { Coal } \\
\text { (excluding } \\
\text { Coal Coke }\end{array}$ & $\begin{array}{c}\text { RSE } \\
\text { Row } \\
\text { Factors }\end{array}$ \\
\hline
\end{tabular}

RSE Column Factors:

TOTAL INPUTS

Boiler Fuel

Total Process Uses

$\ldots \ldots \ldots \ldots$

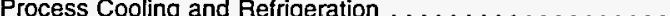

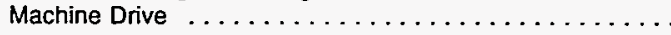

Electro-Chemical Processes $\ldots \ldots \ldots \ldots \ldots \ldots \ldots$

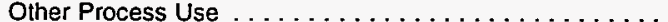

Total Non-Process Uses . . . . . . . . . . . . .

Facility Heating, Ventilation, and Air Conditioning ${ }^{\circ} \ldots \ldots$

Facility Lighting .

Facility Support

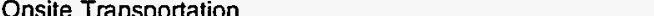

Conventional Electricity Generation . . . . . . . . . .

Other Non-Process Use $\ldots \ldots \ldots \ldots \ldots \ldots \ldots$

End Use Not Reported

Surgical and Medical Instruments

RSE Column Factors:

TOTAL INPUTS

Boiler Fuel

Total Process Uses

Process Cooling and Refrigeration $\ldots \ldots \ldots \ldots \ldots$

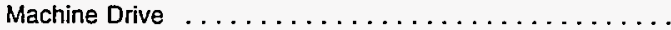

Electro-Chemical Processes $\ldots \ldots \ldots \ldots \ldots \ldots \ldots \ldots \ldots$

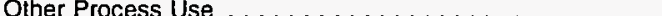

Total Non-Process Uses . . . . . . . . . . . . . . . . .

Facility Heating, Ventilation, and Air Conditioning ${ }^{\circ} \ldots \ldots$

Facility Lighting

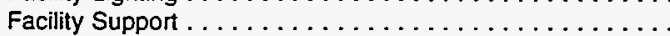

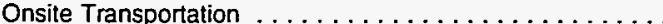

Conventional Electricity Generation . . . . . . . . . .

Other Non-Process Use

End Use Not Reported

$\begin{array}{rr}0.5 & 0.8 \\ 47 & \\ 1 & \\ 20 & \\ 3 & \\ 4 & \\ 12 & \\ 1 & \\ 1 & \\ 23 & \\ 12 & \\ 9 & \\ 2 & \\ - & \\ -- & \\ 1 & \\ 2 & \end{array}$

$$
\begin{aligned}
& 0.8 \\
& 3 \\
& 3 \\
& Q \\
& 0 \\
& Q \\
& 0 \\
& - \\
& 0 \\
& Q \\
& Q \\
& -- \\
& - \\
& -- \\
& 0 \\
& Q \\
& Q
\end{aligned}
$$

3

\begin{tabular}{cc}
1.3 & 0.8 \\
$W$ & 25 \\
$W$ & 14 \\
$*$ & 4 \\
$W$ & 4 \\
$W$ & $*$ \\
$\because *$ & $\div$ \\
$*$ & $*$ \\
$*$ & 6 \\
$*$ & $\div$ \\
$*$ & $*$ \\
\hline & $*$
\end{tabular}

1.4

12.8

3

Q

\begin{tabular}{|c|c|c|c|}
\hline 1.2 & 0.7 & 1.1 & NF \\
\hline * & 2 & " & 0 \\
\hline . & 1 & . & 0 \\
\hline * & - & • & 0 \\
\hline * & • & * & 0 \\
\hline • & • & 0 & 0 \\
\hline 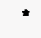 & * & $\mathbf{Q}$ & 0 \\
\hline -- & $=$ & -- & - \\
\hline - & 0 & $\star$ & 0 \\
\hline • & 1 & $*$ & 0 \\
\hline - & 1 & $*$ & 0 \\
\hline - & -- & -. & -- \\
\hline * & * & . & 0 \\
\hline • & 0 & " & - \\
\hline & $\star$ & 0 & 0 \\
\hline * & * & $\star$ & 0 \\
\hline 0 & * & * & 0 \\
\hline
\end{tabular}

0

0.6

1.8

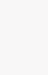

See footnotes at end of table. 
Table A38. Selected Combustible Inputs of Energy for Heat, Power, and Electricity Generation and Net Demand for Electricity by Fuel Type and End Use, 1991: Part 2 (Continued) (Estimates in Trillion Btu)

\begin{tabular}{|c|c|c|c|c|c|c|c|c|}
\hline $\begin{array}{c}\text { SIC } \\
\text { Code }\end{array}$ & End-Use Categories & $\begin{array}{l}\text { Net Demand } \\
\text { for Electricity }\end{array}$ & $\begin{array}{c}\text { Residual Fuel } \\
\text { Oil }\end{array}$ & $\begin{array}{c}\text { Distillate Fuel } \\
\text { Oil and Diesel } \\
\text { Fuel }\end{array}$ & Natural Gas ${ }^{d}$ & LPG & $\begin{array}{c}\text { Coal } \\
\text { (excluding } \\
\text { Coal Coke } \\
\text { and Breeze) }\end{array}$ & $\begin{array}{c}\text { RSE } \\
\text { Row } \\
\text { Factors }\end{array}$ \\
\hline
\end{tabular}

RSE Column Factors:

TOTAL INPUTS

Boller Fuel

Total Process Uses

Process Heating ........

Process Cooling and Refrigeration $\ldots \ldots \ldots \ldots \ldots \ldots \ldots$

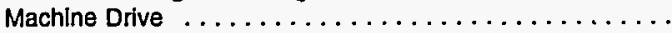

Electro-Chemical Processes . . . . . . . . . . . . .

Other Process Use . . . . . . . . . . . . . . . .

Total Non.Process Uses . . . . . . . . . . . . . . . . .

Facility Heating, Ventilation, and Air Conditioning ${ }^{\circ} \ldots \ldots$.

Facility Lighting $\ldots \ldots \ldots \ldots \ldots \ldots \ldots \ldots \ldots$

Facillty Support . . . . . . . . . . . . . . . . . .

Onslie Transportation $\ldots \ldots \ldots \ldots \ldots \ldots \ldots \ldots$

Conventional Electricity Generation $\ldots \ldots \ldots \ldots \ldots \ldots$

Other Non-Process Use $\ldots \ldots \ldots \ldots \ldots \ldots \ldots \ldots$

End Use Not Reported

$\ldots \ldots \ldots \ldots \ldots \ldots \ldots$

\begin{tabular}{|c|c|c|c|c|c|}
\hline 0.5 & 1.2 & 1.3 & 0.7 & 1.0 & 1.8 \\
\hline 12 & 1 & $w$ & 15 & $W$ & 1 \\
\hline * & 1 & $w$ & 5 & $w$ & 1 \\
\hline 7 & $Q$ & * & 5 & $\star$ & 0 \\
\hline 2 & $Q$ & $Q$ & 5 & $\bullet$ & 0 \\
\hline 1 & 0 & 0 & $*$ & 0 & 0 \\
\hline 5 & 0 & * & " & * & 0 \\
\hline * & -- & - & $\cdots$ & -. & - \\
\hline * & 0 & * & • & * & 0 \\
\hline 4 & " & * & 4 & " & $Q$ \\
\hline 2 & • & * & 3 & $\mathbf{Q}$ & Q \\
\hline 2 & -- & - & -- & - & $\overline{-}$ \\
\hline 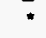 & • & • & * & " & 0 \\
\hline * & -. & $Q$ & • & $\star$ & $\cdots$ \\
\hline .- & 0 & * & 0 & 0 & 0 \\
\hline * & 0 & * & $*$ & * & 0 \\
\hline 1 & • & " & 1 & * & 0 \\
\hline
\end{tabular}

See Appendices $B$ and $F$ for descriptions of the Standard Industrial Classification system.

b "Net Demand for Electricity" is the sum of purchases, transfers in, and total onsite electricity generation, minus sales and transfers offsite. It is the total amount of

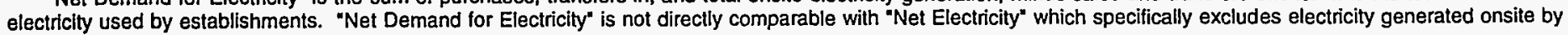
combustible energy sources.

'Includes Nos. 1, 2, and 4 fuel oils and Nos. 1, 2, and 4 diesel fuels.

d "Natural Gas" includes natural gas obtained from utilities, transmission pipelines, and any other supplier(s) such as brokers and producers.

- Excludes steam and hot water.

NF=No applicable RSE row/column factor.

- Estimate less than 0.5. Data are included in higher level totals.

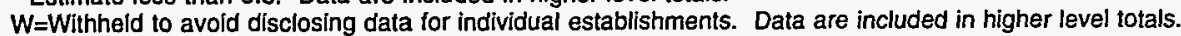

$\mathrm{Q}=$ Withheld because Relative Standard Error is greater than 50 percent. Data are included in higher level totals.

-- Estimation of energy input is not applicable.

NA=Not avallable. Data are included in higher level totals.

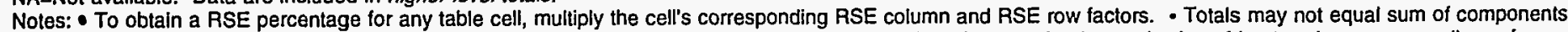
because of independent rounding. - The estimates presented in this table are for the total consumption of energy for the production of heat and power, regardless of

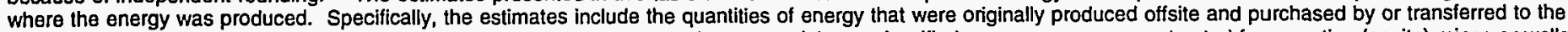

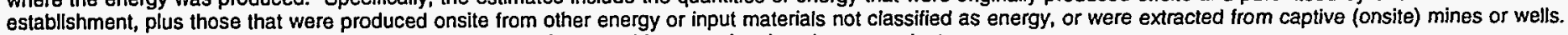

- Allocatlons to specific end uses are made on the basis of reasonable approximations by respondents.

Source: Energy Information Administration, Office of Energy Markets and End Use, Energy End Use and Integrated Statistics Division, Form ElA-846, "1991 Manufacturing Energy Consumption Survey:" 
Table A39. Selected Combustible Inputs of Energy for Heat, Power, and Electricity Generation and Net Demand for Electricity by Fuel Type, Census Region, and End Use, 1991: Part 1

(Estimates in Btu or Physical Units)

\begin{tabular}{|c|c|c|c|c|c|c|c|}
\hline End-Use Categories & $\begin{array}{l}\text { Net Demand } \\
\text { for Electricity } \\
\text { (million kWh) } \\
\end{array}$ & $\begin{array}{c}\text { Residual Fuel } \\
\text { Oil } \\
\text { (1000 bbls })\end{array}$ & $\begin{array}{c}\text { Distillate Fuel } \\
\text { Oil and Diesel } \\
\text { Fuel }^{b} \\
(1000 \text { bbls })\end{array}$ & $\begin{array}{l}\text { Natural Gas } \\
\text { (billion cu ft) }\end{array}$ & $\begin{array}{c}L P G \\
(1000 \mathrm{bb} / \mathrm{s})\end{array}$ & $\begin{array}{c}\text { Coal } \\
\text { (excluding } \\
\text { Coal Coke } \\
\text { and Breeze) } \\
\text { (1000 short } \\
\text { tons) }\end{array}$ & $\begin{array}{c}\text { RSE } \\
\text { Row } \\
\text { Factors }\end{array}$ \\
\hline & \multicolumn{7}{|c|}{ Total United States } \\
\hline RSE Column Factors: & 0.4 & $\overline{1.7}$ & 1.5 & 0.7 & 1.0 & $\overline{1.6}$ & \\
\hline TOTAL INPUTS ..... & 820,286 & 65,837 & 23,885 & 5,345 & 27,970 & 53,035 & 3.0 \\
\hline Boiler Fuel $\ldots \ldots \ldots \ldots \ldots \ldots \ldots \ldots$ & 9,245 & 47,009 & 6,850 & 2,037 & 4,928 & 38,473 & 3.6 \\
\hline & 657,659 & 17,342 & 5,800 & 2,503 & 16,908 & 14,075 & 4.1 \\
\hline $\begin{array}{l}\text { Process Heating } \ldots \ldots \ldots \ldots \ldots \ldots \ldots \ldots \ldots \ldots \\
\text { Process Cooling and Refrigeration }\end{array}$ & 71,658 & 16,959 & 3,177 & 2,312 & 12,704 & 14,075 & 4.1 \\
\hline $\begin{array}{l}\text { Process Cooling and Refrigeration . } \\
\text { Machine Drive }\end{array}$ & 40,987 & 6 & 30 & 13 & 18 & 0 & 12.4 \\
\hline Machine Drive $\ldots \ldots \ldots \ldots \ldots$ & 434,349 & 353 & 2,398 & 123 & 4,093 & 0 & 8.4 \\
\hline Electro-Chemical Processes .... & 105,663 & -- & -. & - & -. & -. & 7.7 \\
\hline Other Process Use ....... & 5,001 & 24 & 196 & 55 & 93 & - & 14.0 \\
\hline Total Non-Process Uses $\ldots \ldots \ldots \ldots \ldots \ldots \ldots \ldots$ & 125,751 & 1,148 & 9,134 & 682 & 5,105 & w & 4.2 \\
\hline Facility Heating, Ventilation, and Air Conditioning ${ }^{d} \ldots \ldots \ldots$ & 60,301 & 673 & 1,372 & 275 & 731 & 15 & 6.8 \\
\hline Facility Lighting $\ldots \ldots \ldots \ldots \ldots \ldots \ldots \ldots \ldots \ldots$ & 51,443 & - & - & -- & -. & .. & 5.1 \\
\hline Facility Support $\ldots \ldots \ldots \ldots \ldots \ldots \ldots \ldots \ldots \ldots$ & 11,522 & w & 81 & 22 & 62 & 0 & 9.6 \\
\hline Onsite Transportation & 1,298 & - & 6,533 & • & 4,242 & -. & 9.4 \\
\hline Conventional Electricity Generation & - & 325 & 734 & 337 & 41 & $w$ & 8.8 \\
\hline Other Non-Process Use $\ldots \ldots \ldots$ & 1,187 & W & 413 & 48 & 30 & 0 & 10.9 \\
\hline \multirow[t]{2}{*}{ End Use Not Reported } & 27,631 & 339 & 2,101 & 124 & 1,028 & $w$ & 10.6 \\
\hline & \multicolumn{7}{|c|}{ Northeast Census Region } \\
\hline RSE Column Factors: & 0.5 & 1.1 & 1.5 & 0.7 & 1.6 & $\overline{1.2}$ & \\
\hline TOTAL INPUTS & 98,142 & 29,245 & 7,805 & 446 & $w$ & 7,420 & 6.6 \\
\hline Boiler Fuel $\ldots \ldots \ldots \ldots \ldots$ & 1,244 & 20,525 & 3,185 & 164 & $w$ & 3,642 & 7.7 \\
\hline Total Process Uses . . & 72,047 & 7,593 & 1,667 & 208 & 1,896 & 3,482 & 9.9 \\
\hline Process Heating ... & 13,038 & 7,392 & 978 & 199 & 1,679 & 3,482 & 10.3 \\
\hline Process Cooling and Refrigeration . & 5,506 & $Q$ & $Q$ & 2 & 2 & 0 & 15.8 \\
\hline Machine Drive $\ldots \ldots \ldots \ldots \ldots$ & 46,197 & $w$ & 673 & 4 & 196 & 0 & 16.5 \\
\hline Electro-Chemical Processes .... & 6,708 & - & - & -- & - & .. & 8.8 \\
\hline Other Process Use & 598 & w & 8 & 2 & 19 & • & 10.4 \\
\hline Total Non-Process Uses $\ldots \ldots \ldots \ldots \ldots \ldots \ldots$ & 20,209 & 964 & 2,443 & 59 & 766 & w & 4.8 \\
\hline Facility Heating, Ventilation, and Air Conditioning ${ }^{d}$ & 8,932 & 536 & 972 & 43 & 253 & $\because$ & 12.7 \\
\hline Facility Lighting $\ldots \ldots \ldots \ldots \ldots \ldots \ldots \ldots$ & 8,865 & -- & - & -- & - & -- & 6.6 \\
\hline Facility Support $\ldots \ldots \ldots \ldots \ldots \ldots \ldots \ldots \ldots \ldots \ldots$ & 1,980 & 12 & W & w & w & 0 & 16.8 \\
\hline Onsite Transportation $\ldots \ldots \ldots \ldots$ & 219 & -- & 972 & • & 497 & -. & 8.6 \\
\hline Conventional Electricity Generation .. & -- & $w$ & 369 & 12 & $w$ & w & 8.7 \\
\hline Other Non-Process Use $\ldots \ldots \ldots \ldots$ & 213 & $w$ & $w$ & $\bar{w}$ & 3 & 0 & 19.1 \\
\hline \multirow[t]{2}{*}{ End Use Not Reported } & 4,641 & 164 & 510 & 15 & a & $w$ & 20.2 \\
\hline & \multicolumn{7}{|c|}{ Midwest Census Region } \\
\hline RSE Column Factors: & 0.5 & 1.3 & 1.6 & 0.6 & 1.5 & $\overline{1.2}$ & \\
\hline TOTAL INPUTS ... & 219,493 & 8,134 & 3,885 & 1,363 & 3,877 & 18,828 & 4.1 \\
\hline Boiler Fuel $\ldots \ldots \ldots \ldots \ldots \ldots \ldots \ldots \ldots \ldots \ldots \ldots \ldots \ldots$ & 2,040 & 6,495 & 563 & 486 & 403 & 14,854 & 6.1 \\
\hline Total Process Uses. & 173,687 & 1,571 & 1,008 & 688 & 1,741 & 3,845 & 6.2 \\
\hline Process Heating $\ldots \ldots \ldots \ldots \ldots$ & 23,742 & 1,560 & 587 & 664 & 1,396 & 3,845 & 6.3 \\
\hline Process Cooling and Refrigeration & 9,951 & 1 & 2 & 2 & $\cdot$ & 0 & 17.6 \\
\hline Machine Drive ...... & 122,728 & 8 & 302 & 17 & 325 & 0 & 14.1 \\
\hline Electro-Chemical Processes. & 15,570 & -- & - & -- & - & .. & 6.5 \\
\hline Other Process Use . . . . . & 1,697 & $\mathbf{Q}$ & 117 & 5 & 19 & * & 13.7 \\
\hline Total Non-Process Uses $\ldots \ldots \ldots \ldots \ldots \ldots \ldots$ & 36,513 & $w$ & 1,961 & 153 & 1,551 & w & 7.5 \\
\hline Facility Heating, Ventilation, and Air Conditioning ${ }^{d} \ldots \ldots$. & 16,099 & 39 & 192 & 136 & 131 & $w$ & 11.4 \\
\hline Facility Lighting $\ldots \ldots \ldots \ldots \ldots \ldots \ldots \ldots \ldots$ & 16,275 & -. & -- & -. & -. & -- & 6.5 \\
\hline Facility Support $\ldots \ldots \ldots \ldots \ldots \ldots \ldots \ldots \ldots \ldots \ldots$ & 3,356 & w & 12 & 9 & W & 0 & 9.9 \\
\hline Onsite Transportation $\ldots \ldots \ldots \ldots \ldots \ldots \ldots \ldots \ldots$ & 487 & -- & 1,375 & $*$ & 1,398 & .. & 5.4 \\
\hline Conventional Electricity Generation & -- & 0 & $Q$ & 7 & $\mathbf{Q}$ & $w$ & 30.5 \\
\hline Other Non-Process Use $\ldots \ldots \ldots$ & 296 & $w$ & 181 & 1 & 8 & 0 & 12.9 \\
\hline End Use Not Reported & 7,253 & w & 353 & 36 & 182 & $w$ & 19.0 \\
\hline
\end{tabular}

See footnotes at end of table. 
Table A39. Selected Combustible Inputs of Energy for Heat, Power, and Electricity Generation and Net Demand for Electricity by Fuel Type, Census Region, and End Use, 1991: Part 1 (Continued)

(Estimates in Btu or Physical Units)

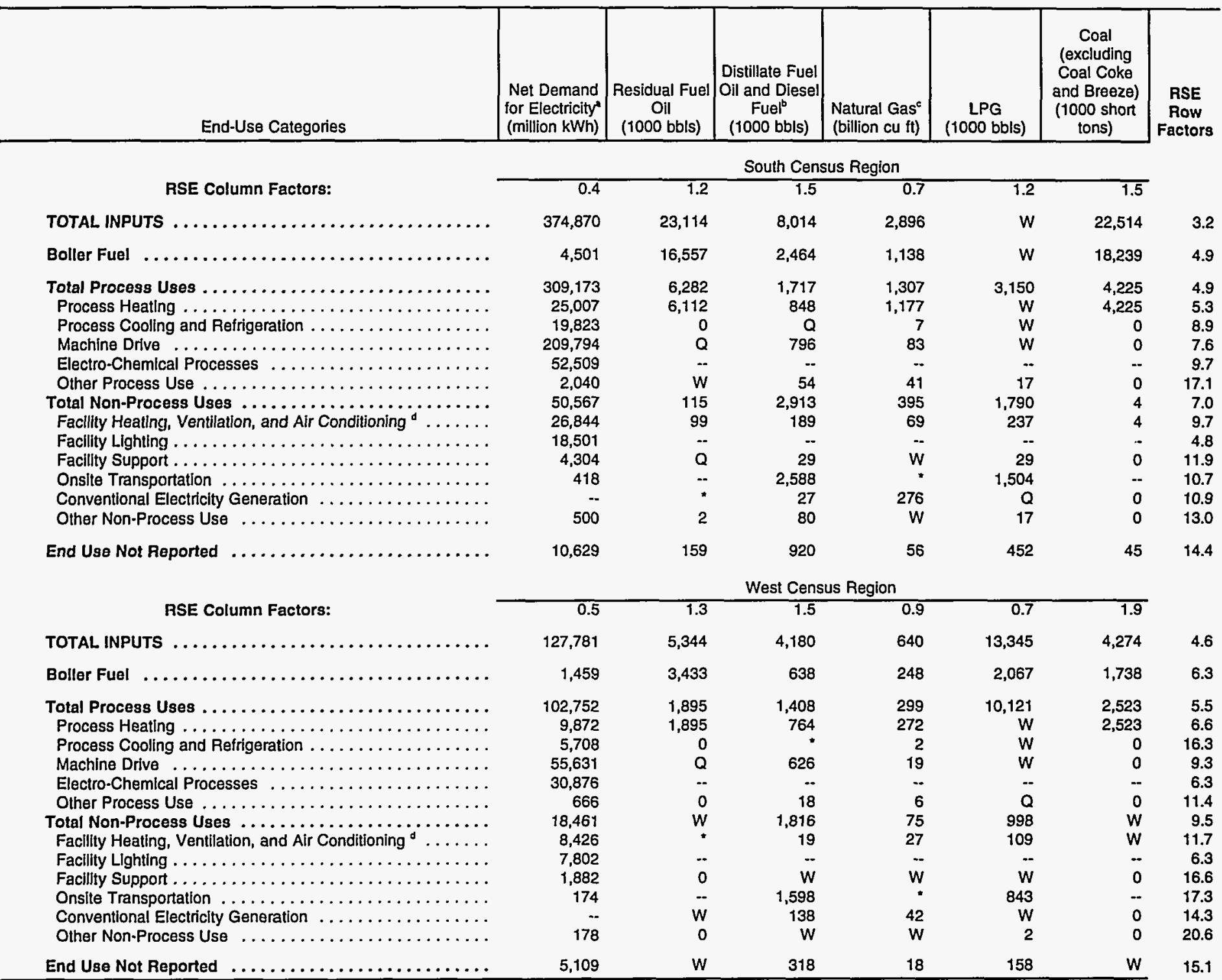

"Net Demand for Electricity" is the sum of purchases, transfers in, and total onsite electricity generation, minus sales and transfers offsite. It is the total amount of

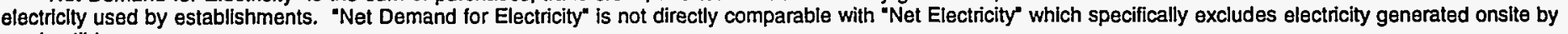
combustible energy sources.

Includes Nos. 1,2, and 4 fuel oils and Nos, 1,2, and 4 diesel fuels.

" "Natural Gas" Includes natural gas obtained from utilities, transmission pipelines, and any other supplier(s) such as brokers and producers.

- Excludes steam and hot water.

NF=No applicable RSE row/column factor.

- Estimate less than 0.5. Data are included in higher level totals.

$W=$ Withheld to avoid disclosing data for individual establishments. Data are included in higher level totals.

$\mathrm{Q}=$ Withheld because Relative Standard Error is greater than 50 percent. Data are included in higher level totals.

-- Estimation of energy input is not applicable.

NA=Not available. Data are Included in higher level totals.

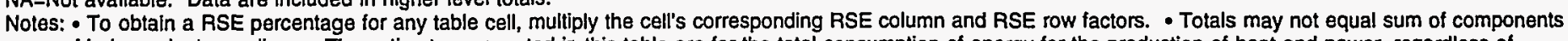

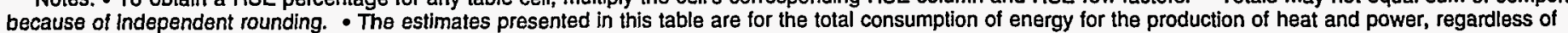

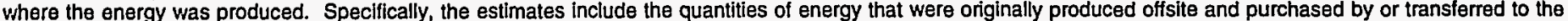

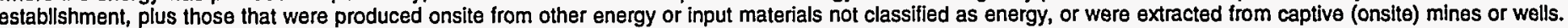
- Allocatlons to specific end uses are made on the basis of reasonable approximations by respondents.

Source: Energy Information Administration, Office of Energy Markets and End Use, Energy End Use and Integrated Statistics Division, Form EIA-846, "1991 Manufacturing Energy Consumption Survey." 
Table A39. Selected Combustible Inputs of Energy for Heat, Power, and Electricity Generation and Net Demand for Electricity by Fuel Type, Census Region, and End Use, 1991: Part 2

(Estimates in Trillion Btu)

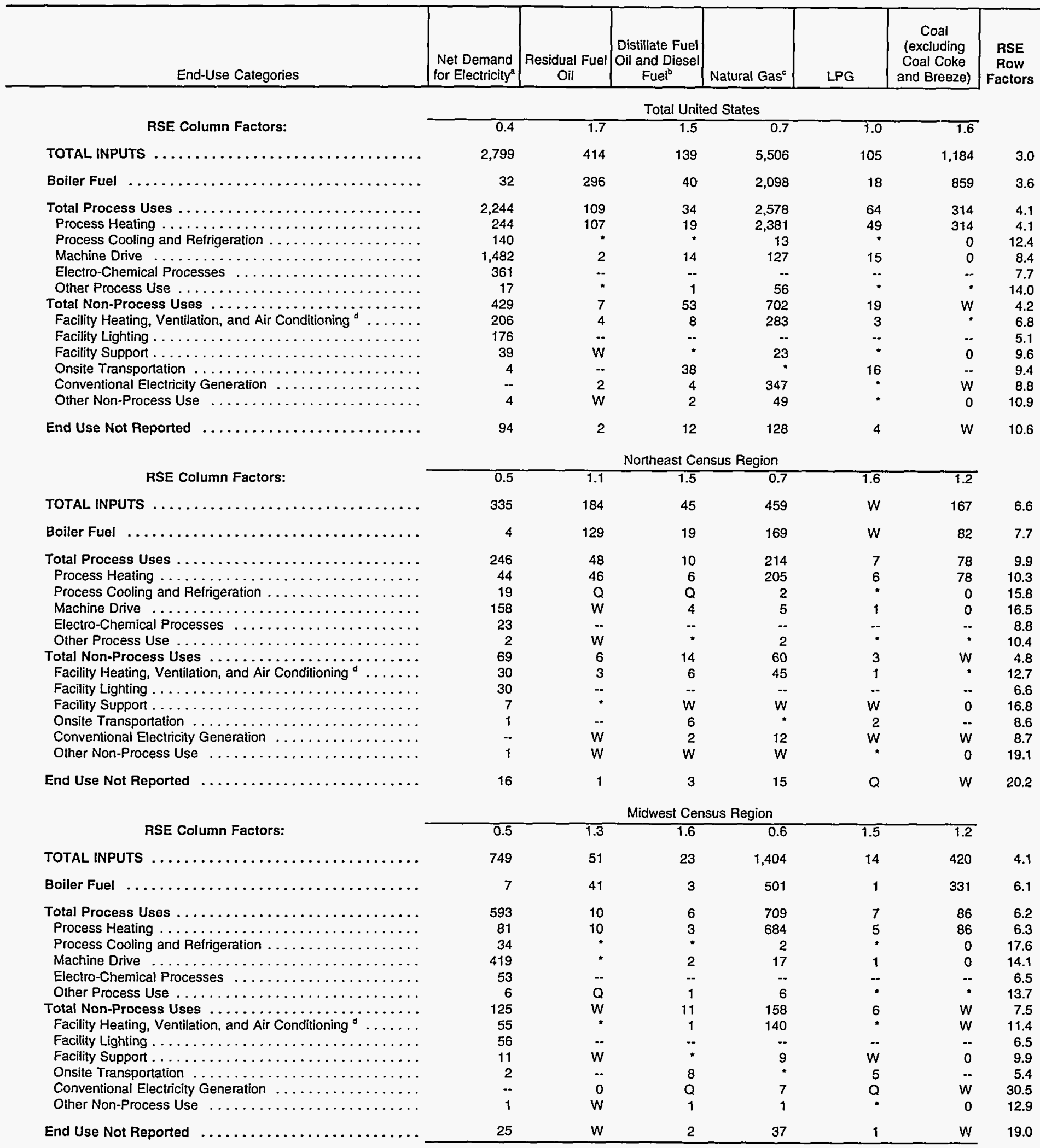

See footnotes at end of table. 
Table A39. Selected Combustible Inputs of Energy for Heat, Power, and Electricity Generation and Net Demand for Electricity by Fuel Type, Census Region, and End Use, 1991: Part 2 (Continued)

(Estimates in Trillion Btu)

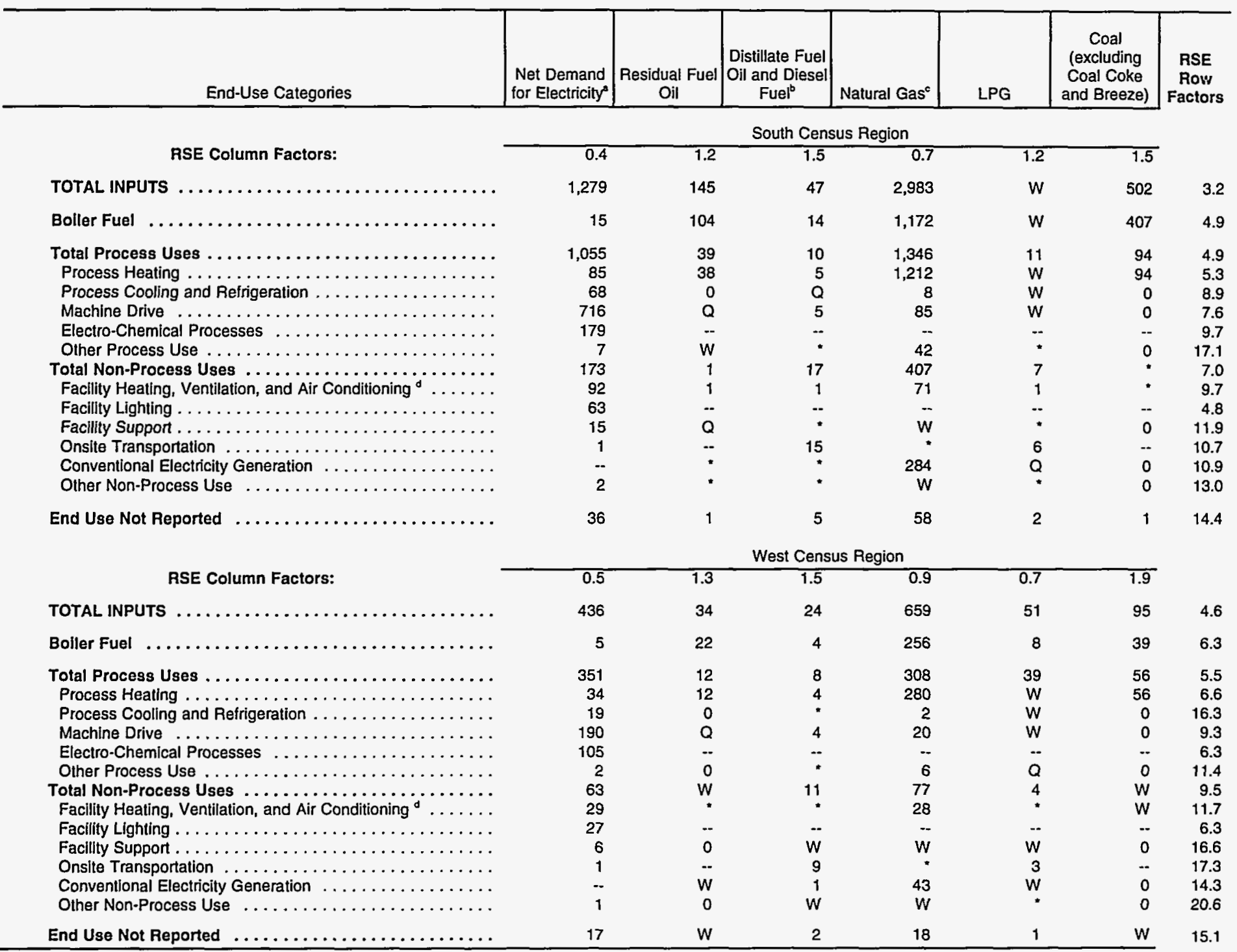

- "Net Demand for Electricity" is the sum of purchases, transfers in, and total onsite electricity generation, minus sales and transfers offsite. It is the total amount of

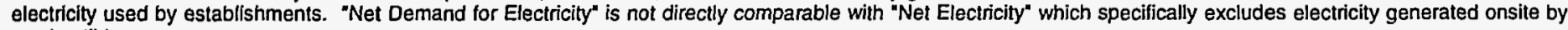
combustible energy sources.

Includes Nos. 1, 2, and 4 fuel oils and Nos. 1, 2, and 4 diesel fuels.

c "Natural Gas" includes natural gas obtained from utilities, transmission pipelines, and any other supplier(s) such as brokers and producers.

'Excludes steam and hot water.

NF=No applicable RSE row/column factor.

- Estimate less than 0.5. Data are included in higher level totals.

$W=$ Withheld to avold disclosing data for individual establishments. Data are included in higher level totals

$\mathrm{Q}=$ Withheld because Relative Standard Error is greater than 50 percent. Data are included in higher level totals.

-- Estimation of energy input is not applicable.

$N A=$ Not available. Data are included in higher level totals.

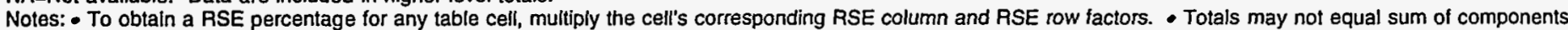

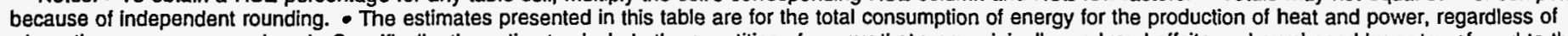

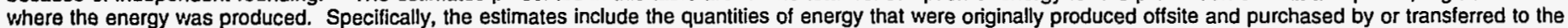

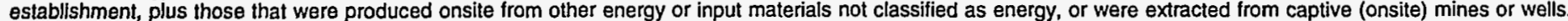
- Allocations to specific end uses are made on the basis of reasonable approximations by respondents.

Source: Energy Information Administration, Office of Energy Markets and End Use, Energy End Use and Integrated Statistics Division, Form ElA-846, "1991 Manufacturing Energy Consumption Survey." 
Table A40. Total Inputs of Energy for Heat, Power, and Electricity Generation by Energy Management Program Sponsorship, Industry Group, Selected Industries, and Type of Energy Management Program, 1991

(Estimates in Trillion Btu)

\begin{tabular}{|c|c|c|c|c|c|c|c|}
\hline \multirow[b]{2}{*}{$\begin{array}{l}\text { SIC } \\
\text { Code" }\end{array}$} & \multirow[b]{2}{*}{$\begin{array}{l}\text { Industry Groups } \\
\text { and Industry }\end{array}$} & \multirow[b]{2}{*}{$\begin{array}{c}\text { No Energy } \\
\text { Management } \\
\text { Program }\end{array}$} & \multicolumn{4}{|c|}{ Type of Sponsorship of Management Programs (1989 through 1991) } & \multirow{3}{*}{$\begin{array}{c}\text { RSE } \\
\text { Row } \\
\text { Factors }\end{array}$} \\
\hline & & & $\begin{array}{l}\text { Any Type of } \\
\text { Sponsorship }\end{array}$ & $\begin{array}{l}\text { Utility/Supplier } \\
\text { Sponsored } \\
\text { Involvement }\end{array}$ & $\begin{array}{c}\text { Only Own or } \\
\text { Other Third Party } \\
\text { Sponsorship }\end{array}$ & $\begin{array}{l}\text { Both Types of } \\
\text { Sponsorship }\end{array}$ & \\
\hline & olumn Factors: & 0.6 & 0.8 & 1.4 & 1.0 & 1.5 & \\
\hline
\end{tabular}

20-39

ALL INDUSTRY GROUPS

Participation in One or More of the Following Types of

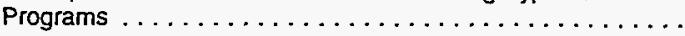

Energy Audits $\ldots \ldots \ldots \ldots \ldots \ldots \ldots \ldots \ldots \ldots$

Direct Electricity Load Control $\ldots \ldots \ldots \ldots \ldots \ldots \ldots \ldots$

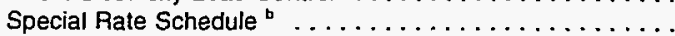

Standby Generation Program . . . . . . . . . . . . . . .

Equipment Rebate(s) . . . . . . . . . . . . . . . .

Equipment Installation or Retrofit for the Primary Purpose of Improving Energy Efficiency Affecting:

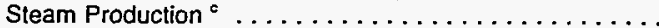

Direct/Indirect Process Heating . . . . . . . . . . . .

Direct Process Cooling/Refrigeration . . . . . . . . . . .

Direct Machine Drive ${ }^{d} \ldots \ldots \ldots \ldots \ldots \ldots \ldots \ldots$

Facility Heating, Ventilation, and Air Conditioning $\ldots \ldots \ldots$

Facility Lighting . . . . . . . . . . . . . . . . .

Equipment Installation/Retrofit for the Primary Purpose of

Using a Different Energy Source ${ }^{\circ} \ldots \ldots \ldots \ldots \ldots \ldots \ldots$

Other:

$\begin{array}{rr}4,284 & 10,743 \\ 7,944 & 7,083 \\ 9,941 & 5,086 \\ 8,509 & 6,517 \\ 13,894 & 1,133 \\ 14,244 & 783 \\ & \\ 10,269 & 4,757 \\ 9,881 & 5,146 \\ 12,815 & 2,212 \\ 9,672 & 5,354 \\ 11,603 & 3,424 \\ 9,622 & 5,405 \\ & \\ 13,333 & 1,693 \\ 14,651 & 376\end{array}$

FOOD and KINDRED PRODUCTS

20

Participation in One or More of the Following Types of

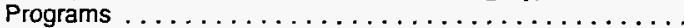

Energy Audits $\ldots \ldots \ldots \ldots \ldots \ldots \ldots \ldots \ldots \ldots \ldots$

Direct Electricity Load Control $\ldots \ldots \ldots \ldots \ldots \ldots \ldots \ldots$

Special Rate Schedule ${ }^{\circ} \ldots . . . \ldots \ldots \ldots$

Standby Generation Program . . . . . . . . . . . .

Equipment Rebate(s)

Equipment Installation or Retrofit for the Primary Purpose of

Improving Energy Efficiency Affecting:

Steam Production ${ }^{c}$

Direct/lndirect Process Heating . . .

Direct Process Cooling/Refrigeration . . . . . . . . . .

Direct Machine Drive ${ }^{\mathrm{d}} \ldots \ldots \ldots \ldots \ldots \ldots \ldots \ldots \ldots$

Facility Heating, Ventilation, and Air Conditioning .......

Facility Lighting . . . . . . . . . . . . . . . . .

Equipment Installation/Retrofit for the Primary Purpose of

Using a Different Energy Source ${ }^{\circ} \ldots \ldots \ldots \ldots \ldots \ldots$

Other'

Meat Packing Plants

2011

Participation in One or More of the Following Types of

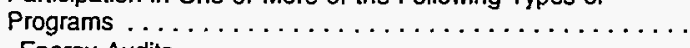

Energy Audits . . . . . . . . . . . . . . . .

Direct Electricity Load Control . . . . . . . . . . . . . . .

Special Rate Schedule ${ }^{b} \ldots \ldots \ldots \ldots \ldots \ldots \ldots \ldots \ldots$

Standby Generation Program . . . . . . . . . . . . . .

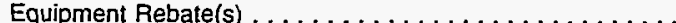

Equipment Installation or Retrofit for the Primary Purpose of Improving Energy Efficiency Affecting:

Steam Production ${ }^{\circ} \ldots \ldots \ldots \ldots \ldots \ldots \ldots \ldots \ldots$

Direct/ndirect Process Heating . . . . . . . . . . . .

Direct Process Cooling/Refrigeration . . . . . . . . . .

Direct Machine Drive ${ }^{d} \ldots \ldots \ldots \ldots \ldots \ldots \ldots \ldots \ldots \ldots$

Facility Heating. Ventilation, and Air Conditioning $\ldots \ldots$.

Facility Lighting . . . . . . . . . . . . . . . .

Equipment Installation/Retrofit for the Primary Purpose of

Using a Different Energy Source ${ }^{\circ} \ldots \ldots \ldots \ldots \ldots \ldots \ldots$

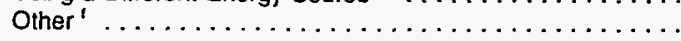

348

604

739

654

893

840

666

660

787

594

755

643

895

942

1,184
532
565
3,939
176
582


200
209
69
351
175
518

49
56

184

565

170

582

200

209

351

175

8

56

606

349

215

299

61

113

288

294

167

359

198

58

11

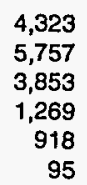

$\begin{array}{rr}5,236 & 2.3 \\ 793 & 2.4 \\ 668 & 2.9 \\ 1,310 & 2.5 \\ 38 & 4.6 \\ 106 & 3.9\end{array}$

4,360

4,517

2,059

4,511

3,061

4,285

1,525

271
$197 \quad 2.9$

$419 \quad 3.0$

$84 \quad 3.8$

$493 \quad 2.8$

$187 \quad 2.8$

$602 \quad 2.5$

$120 \quad 4.2$

3.9

See footnotes at end of table. 
Table A40. Total Inputs of Energy for Heat, Power, and Electricity Generation by Energy Management Program Sponsorship, Industry Group, Selected Industries, and Type of Energy Management Program, 1991: (Continued)

(Estimates in Trillion Btu)

\begin{tabular}{|c|c|c|c|c|c|c|c|}
\hline \multirow[b]{2}{*}{$\begin{array}{l}\text { SIC } \\
\text { Code }^{a}\end{array}$} & \multirow[b]{2}{*}{$\begin{array}{l}\text { Industry Groups } \\
\text { and Industry }\end{array}$} & \multirow[b]{2}{*}{$\begin{array}{l}\text { No Energy } \\
\text { Management } \\
\text { Program }\end{array}$} & \multicolumn{4}{|c|}{ Type of Sponsorship of Management Programs (1989 through 1991) } & \multirow{3}{*}{$\begin{array}{l}\text { RSE } \\
\text { Row } \\
\text { Factors }\end{array}$} \\
\hline & & & $\begin{array}{l}\text { Any Type of } \\
\text { Sponsorship }\end{array}$ & $\begin{array}{c}\text { Utility/Supplier } \\
\text { Sponsored } \\
\text { Involvement }\end{array}$ & $\begin{array}{c}\text { Only Own or } \\
\text { Other Third Party } \\
\text { Sponsorship } \\
\end{array}$ & $\begin{array}{l}\text { Both Types of } \\
\text { Sponsorship }\end{array}$ & \\
\hline & RSE Column Factors: & 0.6 & 0.8 & 1.4 & 1.0 & 1.5 & \\
\hline
\end{tabular}

2033

Canned Fruits and Vegetables

Participation in One or More of the Following Types of

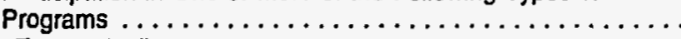

Engrgy Audits $\ldots \ldots \ldots \ldots \ldots \ldots \ldots \ldots \ldots \ldots \ldots \ldots$

Direct Electricity Load Control $\ldots \ldots \ldots \ldots \ldots \ldots \ldots$

Special Rate Schedule ${ }^{b}$

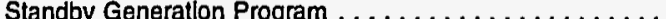

Equipment Rebate(s) . . . . . . . . . . . . . . . . .

Equlpment Installation or Retrofit for the Primary Purpose of Improving Energy Efficiency Affecting:

Steam Production ${ }^{\mathrm{c}}$. . . . . . . . . . . . . . . . . .

Dlrect/Indlrect Process Heating ..................

Direct Process Cooling/Refrigeration . . . . . . . . . . . . .

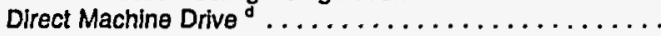

Facllty Heating, Ventilation, and Air Conditioning .........

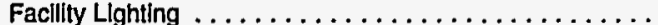

Equipment Installation/Retrofit for the Primary Purpose of

Using a Dlfferent Energy Source ${ }^{\circ} \ldots \ldots \ldots \ldots \ldots \ldots$.

Other ${ }^{1} \ldots \ldots \ldots \ldots \ldots \ldots \ldots \ldots \ldots \ldots \ldots$

Frozen Fruits and Vegetables

Particlpation in One or More of the Following Types of

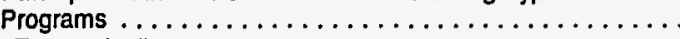

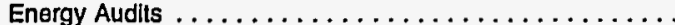

Direct Electricity Load Control $\ldots \ldots \ldots \ldots \ldots \ldots \ldots$

Special Rate Schedule ${ }^{\circ}$

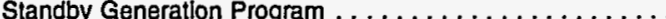

Equipment Rebate(s) ......................

Equlpment Installation or Retrofit for the Primary Purpose of Improving Energy Efficiency Affecting:

Steam Production ${ }^{\circ} \ldots \ldots \ldots \ldots \ldots \ldots$

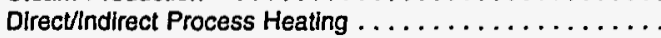

Direct Process Cooling/Refrigeration . . . . . . . . . . .

Direct Machine Drive ${ }^{\mathrm{d}} \ldots \ldots \ldots \ldots \ldots \ldots \ldots \ldots \ldots$

Facllity Heating, Ventilation, and Air Conditioning ..........

Faclity Lighting.$\ldots \ldots \ldots \ldots \ldots \ldots \ldots \ldots$

Equipment Installation/Retrofit for the Primary Purpose of

Using a Different Energy Source ${ }^{\circ} \ldots \ldots \ldots \ldots \ldots \ldots \ldots$

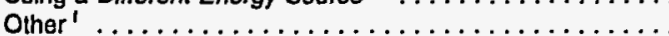

Wet Corn Miling

Participation in One or More of the Following Types of

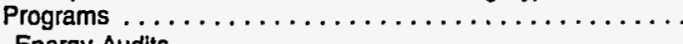

Energy Audits $\ldots \ldots \ldots \ldots \ldots \ldots \ldots \ldots \ldots \ldots \ldots$

Direct Electricity Load Control $\ldots \ldots \ldots \ldots \ldots \ldots \ldots$

Speclal Rate Schedule ${ }^{b} \ldots \ldots \ldots \ldots \ldots \ldots \ldots \ldots$

Standby Generation Program . . . . . . . . . . . . . . .

Equipment Rebate(s)

Equipment Installation or Retrofit for the Primary Pupose of

Improving Energy Efficiency Affecting:

Steam Production ${ }^{2} \ldots \ldots \ldots \ldots \ldots \ldots \ldots \ldots \ldots$

Direct/lndirect Process Heating . .................

Direct Process Cooling/Refrigeration . . . . . . . . . . . .

Direct Machine Drive ${ }^{d}$......................

Facillity Heating, Ventilation, and Air Conditioning ......

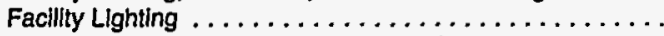

Equipment Installation/Retrofit for the Primary Purpose of

Using a Different Energy Source ${ }^{\star} \ldots \ldots \ldots \ldots \ldots \ldots$

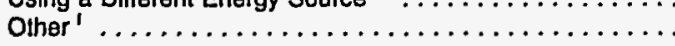

\begin{tabular}{|c|c|c|c|c|c|}
\hline 16 & 28 & 9 & 9 & 9 & 10.2 \\
\hline 29 & 15 & 5 & 9 & 1 & 11.5 \\
\hline 37 & 8 & 3 & 4 & 1 & 11.0 \\
\hline 35 & 10 & 8 & 1 & 1 & 10.9 \\
\hline 40 & 5 & 1 & 4 & : & 19.5 \\
\hline 35 & 9 & 9 & 0 & • & 12.4 \\
\hline 30 & 14 & 5 & 9 & • & 12.7 \\
\hline 37 & 8 & w & 5 & $Q$ & 14.2 \\
\hline 35 & 9 & 1 & 7 & 0 & 15.8 \\
\hline 27 & 18 & 7 & 8 & 2 & 13 \\
\hline 34 & 11 & 2 & 9 & • & 13.4 \\
\hline 28 & 16 & 8 & 6 & 2 & 12.2 \\
\hline 40 & 4 & 0 & 4 & - & 21.3 \\
\hline 43 & 1 & . & 1 & • & 20 \\
\hline
\end{tabular}

See footnotes at end of table. 
Table A40. Total Inputs of Energy for Heat, Power, and Electricity Generation by Energy. Management Program Sponsorship, Industry Group, Selected Industries, and Type of Energy Management Program, 1991: (Continued)

(Estimates in Trillion Btu)

\begin{tabular}{|c|c|c|c|c|c|c|c|}
\hline \multirow[b]{2}{*}{$\begin{array}{c}\text { SIC } \\
\text { Code }\end{array}$} & \multirow[b]{2}{*}{$\begin{array}{l}\text { Industry Groups } \\
\text { and Industry }\end{array}$} & \multirow[b]{2}{*}{$\begin{array}{l}\text { No Energy } \\
\text { Management } \\
\text { Program }\end{array}$} & \multicolumn{4}{|c|}{ Type of Sponsorship of Management Programs (1989 through 1991) } & \multirow{3}{*}{$\begin{array}{c}\text { RSE } \\
\text { Row } \\
\text { Factors }\end{array}$} \\
\hline & & & $\begin{array}{l}\text { Any Type of } \\
\text { Sponsorship }\end{array}$ & $\begin{array}{l}\text { Utility/Supplier } \\
\text { Sponsored } \\
\text { Involvement }\end{array}$ & $\begin{array}{c}\text { Only Own or } \\
\text { Other Third Party } \\
\text { Sponsorship }\end{array}$ & $\begin{array}{l}\text { Both Types of } \\
\text { Sporisorship }\end{array}$ & \\
\hline & RSE Column Factors: & 0.6 & 0.8 & 1.4 & 1.0 & 1.5 & \\
\hline
\end{tabular}

2051

Bread, Cake, and Related Products

Participation in One or More of the Following Types of

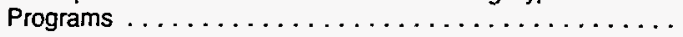

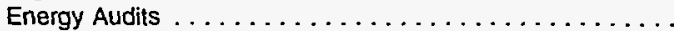

Direct Electricity Load Control . . . . . . . . . . . . .

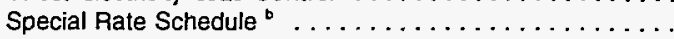

Standby Generation Program . . . . . . . . . . . . . .

Equipment Rebate(s) . . . . . . . . . . . . . . . . . . .

Equipment Installation or Retrofit for the Primary Purpose of Improving Energy Efficiency Affecting:

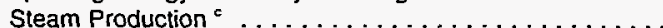

Direct/Indirect Process Heating . . . . . . . . . . . . .

Direct Process Cooling/Refrigeration . . . . . . . . . . .

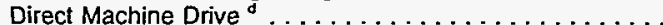

Facility Heating, Ventilation, and Air Conditioning ......

Facility Lighting . . . . . . . . . . . . . . . . . .

Equipment Installation/Retrofit for the Primary Purpose of

Using a Different Energy Source ${ }^{\circ} \ldots \ldots \ldots \ldots \ldots \ldots \ldots$

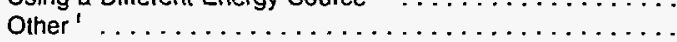

Beet Sugar

2063

Participation in One or More of the Following Types of

Programs . . . . . . . . . . . . . . . . . .

Energy Audits . . . . . . . . . . . . . . . .

Direct Electricity Load Control $\ldots \ldots \ldots \ldots \ldots \ldots \ldots \ldots$

Special Rate Schedule ${ }^{b} \ldots \ldots \ldots \ldots$

Standby Generation Program . . . . . . . . . . . . . . .

Equipment Rebate(s) . . . . . . . . . . . . . . . .

Equipment installation or Retrofit for the Primary Purpose of Improving Energy Efficiency Affecting:

Steam Production ${ }^{c} \ldots \ldots \ldots \ldots \ldots \ldots \ldots \ldots$

Directlndirect Process Heating . . . . . . . . . . . . .

Direct Process Cooling/Réfrigeration . . . . . . . . . . .

Direct Machine Drive ${ }^{d} \ldots \ldots \ldots \ldots \ldots \ldots \ldots \ldots$

Facility Heating, Ventilation, and Air Conditioning

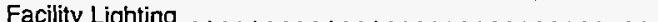

Equipment Installation/Retrofit for the Primary Purpose of

Using a Different Energy Source ${ }^{0} \ldots \ldots \ldots \ldots \ldots \ldots \ldots$

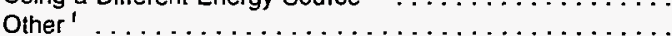

\section{Soybean Oil Mills}

Participation in One or More of the Following Types of

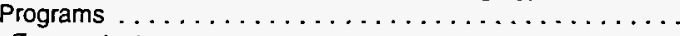

Energy Audıts . . . . . . . . . . . . . . . . . .

Direct Electricity Load Control . . . . . . . . . . . . . .

Special Rate Schedule ${ }^{b} \ldots . \ldots \ldots \ldots \ldots \ldots \ldots \ldots$

Standby Generation Program $\ldots \ldots \ldots \ldots \ldots \ldots \ldots \ldots \ldots \ldots$

Equipment Rebate(s)

Equipment Installation or Retrofit for the Primary Purpose of Improving Energy Efficiency Affecting:

Steam Production ${ }^{c} \ldots \ldots \ldots \ldots \ldots \ldots \ldots \ldots \ldots \ldots \ldots$

Direct/lndirect Process Heating . . . . . . . . . . . . . . .

Direct Process Cooling/Refrigeration . . . . . . . . . . . .

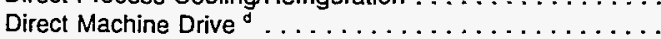

Facility Heating, Ventilation, and Air Conditioning ......

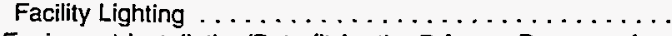

Equipment Installation/Retrofit for the Primary Purpose of

Using a Different Energy Source ${ }^{\circ} \ldots \ldots \ldots \ldots \ldots \ldots$.

Other'

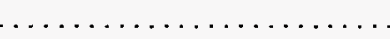

See footnotes at end of table. 
Table A40. Total Inputs of Energy for Heat, Power, and Electricity Generation by Energy Management Program Sponsorship, Industry Group, Selected Industries, and Type of Energy Management Program, 1991: (Continued)

(Estimates in Trillion Btu)

\begin{tabular}{|c|c|c|c|c|c|c|c|}
\hline \multirow[b]{2}{*}{$\begin{array}{l}\text { SIC } \\
\text { Code }^{a}\end{array}$} & \multirow[b]{2}{*}{$\begin{array}{l}\text { Industry Groups } \\
\text { and Industry }\end{array}$} & \multirow[b]{2}{*}{$\begin{array}{c}\text { No Energy } \\
\text { Management } \\
\text { Program }\end{array}$} & \multicolumn{4}{|c|}{ Type of Sponsorship of Management Programs (1989 through 1991) } & \multirow{3}{*}{$\begin{array}{l}\text { RSE } \\
\text { Row } \\
\text { Factors }\end{array}$} \\
\hline & & & $\begin{array}{l}\text { Any Type of } \\
\text { Sponsorship }\end{array}$ & $\begin{array}{l}\text { Utility/Supplier } \\
\text { Sponsored } \\
\text { Involvement }\end{array}$ & $\begin{array}{c}\text { Only Own or } \\
\text { Other Third Party } \\
\text { Sponsorship }\end{array}$ & $\begin{array}{l}\text { Both Types of } \\
\text { Sponsorship }\end{array}$ & \\
\hline & RSE Column Factors: & 0.6 & 0.8 & 1.4 & 1.0 & 1.5 & \\
\hline
\end{tabular}

2082

Malt Beverages

Participation in One or More of the Following Types of

Programs . . . . . . . . . . . . . . . .

Energy Audits . . . . . . . . . . . . . . . . . .

Direct Electricity Load Control . . . . . . . . . . . .

Special Rate Schedule ${ }^{b} \ldots \ldots \ldots \ldots \ldots \ldots \ldots \ldots \ldots$

Standby Generation Program . . . . . . . . . . . .

Equipment Rebate(s) . . . . . . . . . . . . . . . .

Equipment Installation or Retrofit for the Primary Purpose of Improving Energy Efficiency Affecting:

Steam Production ${ }^{c} \ldots \ldots \ldots \ldots \ldots \ldots \ldots \ldots$

Direct/Indirect Process Heating . . . . . . . . . . . . . .

Direct Process Cooling/Refrigeration . . . . . . . . .

Direct Machlne Drive ${ }^{d} \ldots \ldots \ldots \ldots \ldots \ldots \ldots \ldots . . . \ldots$

Facility Heating, Ventilation, and Air Conditioning ......

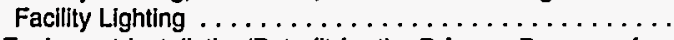

Equipment Installation/Retrofit for the Primary Purpose of

Using a Different Energy Source ${ }^{\circ}$. . . . . . . . . . . .

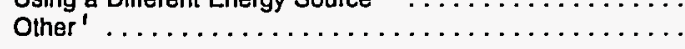

TOBACCO PRODUCTS

Participation in One or More of the Following Types of

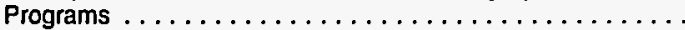

Energy Audits . . . . . . . . . . . . . . . .

Direct Electricity Load Control $\ldots \ldots \ldots \ldots \ldots \ldots \ldots$

Special Rate Schedule ${ }^{b} \ldots \ldots \ldots \ldots \ldots \ldots \ldots \ldots \ldots$

Standby Generation Program . . . . . . . . . . . . .

Equipment Rebate(s) . . . . . . . . . . . . . . . . . . .

Equipment Installation or Retrofit for the Primary Purpose of

Improving Energy Efficiency Affecting:

Steam Production ${ }^{c} \ldots \ldots \ldots \ldots \ldots \ldots \ldots \ldots \ldots$

Direct/lndirect Process Heating . . . . . . . . . . . .

Direct Process Cooling/Refrigeration . . . . . . . . . .

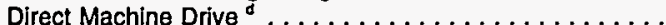

Facillty Heating, Ventilation, and Air Conditioning . . . . .

Facility Lighting . . . . . .................

Equipment Installation/Retrofit for the Primary Purpose of

Using a Different Energy Source ${ }^{\bullet} \ldots \ldots \ldots \ldots \ldots \ldots \ldots$

Other'

TEXTILE MILL PRODUCTS

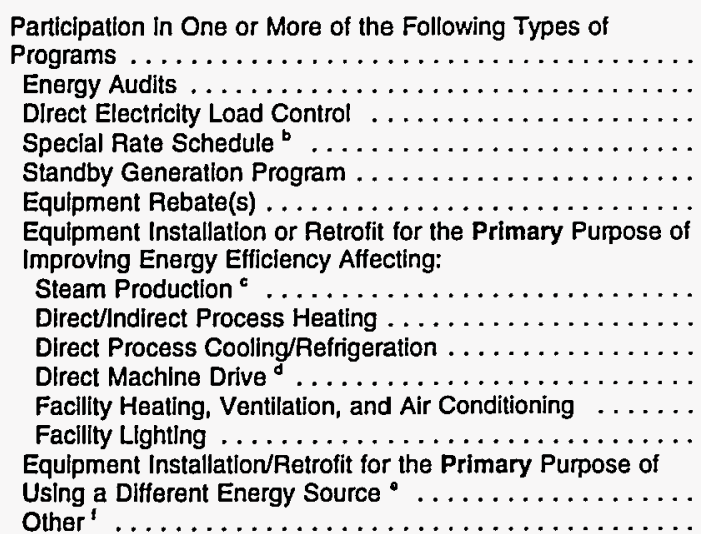

78

143

211

142

269

267

211

233

247

195

209
165

265

268

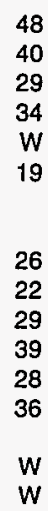

40

29
34

W

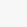

4

9

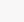

2

W

W

$W$
12
3
15

0

19
33
26
$W$
$W$
0

11.8

11.1

13.1

11.4

17.2

12.5

11.8

11.2

13.5

10.5

13.8

11.5

15.0

17.9

See footnotes at end of table.

Energy Information Administration/Manufacturing Consumption of Energy 1991 
Table A40. Total Inputs of Energy for Heat, Power, and Electricity Generation by Energy Management Program Sponsorship, Industry Group, Selected Industries, and Type of Energy Management Program, 1991: (Continued)

(Estimates in Trillion Btu)

\begin{tabular}{|c|c|c|c|c|c|c|c|}
\hline \multirow[b]{2}{*}{$\begin{array}{c}\text { SIC } \\
\text { Code" } \\
\end{array}$} & \multirow[b]{2}{*}{$\begin{array}{l}\text { Industry Groups } \\
\text { and Industry }\end{array}$} & \multirow[b]{2}{*}{$\begin{array}{l}\text { No Energy } \\
\text { Management } \\
\text { Program }\end{array}$} & \multicolumn{4}{|c|}{ Type of Sponsorship of Management Programs (1989 through 1991) } & \multirow{3}{*}{$\begin{array}{c}\text { RSE } \\
\text { Row } \\
\text { Factors }\end{array}$} \\
\hline & & & $\begin{array}{l}\text { Any Type of } \\
\text { Sponsorship } \\
\end{array}$ & $\begin{array}{c}\text { Utility/Supplier } \\
\text { Sponsored } \\
\text { Involvement }\end{array}$ & \begin{tabular}{|c} 
Only Own or \\
Other Third Party \\
Sponsorship \\
\end{tabular} & $\begin{array}{c}\text { Both Types of } \\
\text { Sponsorship }\end{array}$ & \\
\hline & RSE Column Factors: & 0.6 & 0.8 & 1.4 & 1.0 & 1.5 & \\
\hline
\end{tabular}

23 APPAREL and OTHER TEXTILE PRODUCTS

Participation in One or More of the Following Types of

Programs ......................

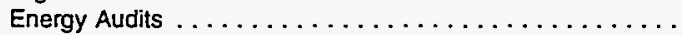

Direct Electricity Load Control $\ldots \ldots \ldots \ldots \ldots \ldots \ldots \ldots$

Special Rate Schedule ${ }^{b} \ldots \ldots \ldots \ldots \ldots$

Standby Generation Program . . . . . . . . . . . . . .

Equipment Rebate(s) . . . . . . . . . . . . . . . . .

Equipment Installation or Retrofit for the Primary Purpose of Improving Energy Efficiency Affecting:

Steam Production ${ }^{c} \ldots \ldots \ldots \ldots \ldots \ldots \ldots \ldots \ldots$

Direct/Indirect Process Heating . ................

Direct Process Cooling/Refrigeration . . . . . . . . . .

Direct Machine Drive ${ }^{\text {a }} \ldots \ldots \ldots \ldots \ldots \ldots \ldots \ldots \ldots \ldots$

Facility Heating, Ventilation, and Air Conditioning .......

Facility Lighting $\ldots \ldots \ldots \ldots \ldots \ldots \ldots \ldots$

Equipment Installation/Retrofit for the Primary Purpose of

Using a Different Energy Source ${ }^{\circ} \ldots \ldots \ldots \ldots \ldots \ldots$

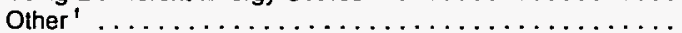

LUMBER and WOOD PRODUCTS

Participation in One or More of the Following Types of

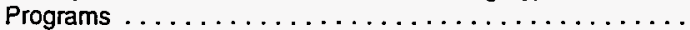

Energy Audits . . . . . . . . . . . . . . . .

Direct Electricity Load Control . . . . . . . . . . . . . .

Special Rate Schedule ${ }^{b} \ldots \ldots \ldots \ldots \ldots \ldots \ldots \ldots$

Standby Generation Program . . . . . . . . . . . .

Equipment Rebate(s) $\ldots \ldots \ldots \ldots \ldots \ldots \ldots \ldots \ldots \ldots$

Equipment Installation or Retrolit for the Primary Purpose of Improving Energy Efficiency Affecting:

Steam Production ${ }^{\mathrm{a}} \ldots \ldots \ldots \ldots \ldots \ldots \ldots \ldots \ldots$

Direct/Indirect Process Heating . . . . . . . . . . . . .

Direct Process Cooling/Refrigeration . . . . . . . . . .

Direct Machine Drive ${ }^{\circ} \ldots \ldots \ldots \ldots \ldots \ldots \ldots \ldots \ldots$

Facility Heating, Ventilation, and Air Conditioning ......

Facility Lighting . . . . . . . . . . . . . . . .

Equipment Installation/Retrofit for the Primary Purpose of

Using a Different Energy Source ${ }^{\circ}$................

Other $^{1} \ldots \ldots \ldots \ldots \ldots \ldots \ldots \ldots \ldots \ldots \ldots$

FURNTURE and FIXTURES

Participation in One or More of the Following Types of

Programs . . . . . . . . . . . . . . . . .

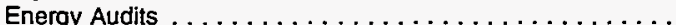

Direct Electricity Load Control $\ldots \ldots \ldots \ldots \ldots \ldots \ldots \ldots$

Special Rate Schedule ${ }^{\circ} \ldots \ldots \ldots \ldots \ldots \ldots \ldots \ldots \ldots$

Standby Generation Program . . . . . . . . . . . . . . .

Equipment Rebate(s) . . . . . . . . . . . . . . . .

Equipment Installation or Retrofit for the Primary Purpose of Improving Energy Efficiency Affecting:

Steam Production ${ }^{c} \ldots \ldots \ldots \ldots \ldots \ldots \ldots \ldots \ldots$

Direct/Indirect Process Heating . . . . . . . . . . . . .

Direct Process Cooling/Refrigeration . . . . . . . . . . .

Direct Machine Drive ${ }^{d} \ldots \ldots \ldots \ldots \ldots \ldots \ldots \ldots$

Facility Heating, Ventilation, and Air Conditioning $\ldots \ldots \ldots$

Facility Lighting . . . . . . . . . . . . . . . . . . . . . . .

Equipment Installation/Retrofit for the Primary Purpose of

Using a Different Energy Source ${ }^{\bullet} \ldots \ldots \ldots \ldots \ldots \ldots \ldots$

Other ${ }^{\prime}$

.................

\begin{tabular}{|c|c|c|c|c|c|}
\hline 29 & 16 & 3 & 5 & 7 & 15.7 \\
\hline 34 & 10 & 3 & 6 & 1 & 18.5 \\
\hline 38 & 6 & 2 & 4 & : & 20.7 \\
\hline 38 & 6 & 4 & . & 2 & 19.8 \\
\hline 44 & - & 0 & • & 0 & 14.5 \\
\hline 43 & 1 & 1 & 0 & - & 27.8 \\
\hline 42 & 2 & • & 2 & - & 26.9 \\
\hline 40 & 4 & • & 4 & 0 & 25.5 \\
\hline 43 & 1 & • & 1 & 0 & 28.8 \\
\hline 37 & 7 & 2 & 3 & 1 & 24.8 \\
\hline 37 & 7 & 1 & 6 & 1 & 20.7 \\
\hline 35 & 9 & 1 & 7 & 1 & 20.2 \\
\hline 43 & 1 & 1 & • & 0 & 25.6 \\
\hline 44 & 0 & 0 & 0 & 0 & 14.5 \\
\hline
\end{tabular}

See footnotes at end of table. 
Table A40. Total Inputs of Energy for Heat, Power, and Electricity Generation by Energy Management Program Sponsorship, Industry Group, Selected Industries, and Type of Energy Management Program, 1991: (Continued) (Estimates in Trillion Btu)

\begin{tabular}{|c|c|c|c|c|c|c|c|}
\hline \multirow[b]{2}{*}{$\underset{\text { Code }}{\text { SIC }}$} & \multirow[b]{2}{*}{$\begin{array}{l}\text { Industry Groups } \\
\text { and Industry }\end{array}$} & \multirow[b]{2}{*}{$\begin{array}{c}\text { No Energy } \\
\text { Management } \\
\text { Program }\end{array}$} & \multicolumn{4}{|c|}{ Type of Sponsorship of Management Programs (1989 through 1991) } & \multirow{3}{*}{\begin{tabular}{|c} 
RSE \\
Row \\
Factors
\end{tabular}} \\
\hline & & & $\begin{array}{l}\text { Any Type of } \\
\text { Sponsorship }\end{array}$ & $\begin{array}{l}\text { Utility/Supplier } \\
\text { Sponsored } \\
\text { Involvement }\end{array}$ & $\begin{array}{c}\text { Only Own or } \\
\text { Other Third Party } \\
\text { Sponsorship }\end{array}$ & $\begin{array}{l}\text { Both Types of } \\
\text { Sponsorship }\end{array}$ & \\
\hline & Column Factors: & 0.6 & 0.8 & 1.4 & 1.0 & 1.5 & \\
\hline
\end{tabular}

Particlpation In One or More of the Following Types of

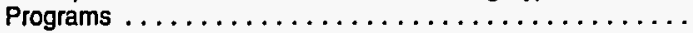

Energy Audits $\ldots \ldots \ldots \ldots \ldots \ldots \ldots \ldots \ldots \ldots$

Direct Electricity Load Control $\ldots \ldots \ldots \ldots \ldots \ldots \ldots \ldots$

Speclal Rate Schedule ${ }^{b} \ldots \ldots \ldots \ldots \ldots \ldots \ldots \ldots \ldots \ldots \ldots$

Standby Generation Program . . . . . . . . . . . . . .

Equipment Rebate(s) . . . . . . . . . . . . . . . .

Equipment Installatlon or Retrofit for the Primary Purpose of Improving Energy Efficiency Affecting:

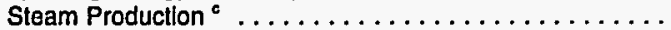

Direct/ndirect Process Heating . . . . . . . . . . .

Direct Process Cooling/Refrigeration . . . . . . . . . .

Direct Machine Drive ${ }^{d} \ldots \ldots \ldots \ldots \ldots \ldots \ldots \ldots \ldots \ldots$

Facllity Heating, Ventilation, and Air Conditioning $\ldots \ldots$.

Facillty Llghting .......................

Equipment Installation/Retrofit for the Primary Purpose of

Using a Different Energy Source ${ }^{\circ} \ldots \ldots \ldots \ldots \ldots \ldots \ldots$

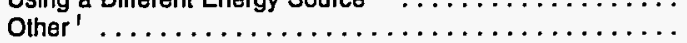

2611

Pulp Mills

Participation in One or More of the Following Types of

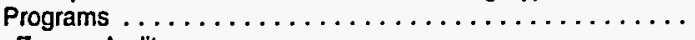

Energy Audits $\ldots \ldots \ldots \ldots \ldots \ldots \ldots \ldots \ldots \ldots \ldots \ldots$

Direct Electricity Load Control . . . . . . . . . . . .

Special Rate Schedule ${ }^{b} \ldots \ldots \ldots \ldots \ldots \ldots \ldots \ldots \ldots$

Standby Generation Program . . . . . . . . . . . . .

Equipment Rebate(s) . . . . . . . . . . . . . . . . .

Equipment Installation or Retrofit for the Primary Purpose of Improving Energy Efficiency Affecting:

Steam Production ${ }^{c} \ldots \ldots \ldots \ldots \ldots \ldots \ldots \ldots \ldots \ldots$

Directlndirect Process Heating . . . . . . . . . . . .

Direct Process Cooling/Refrigeration . . . . . . . . . .

Direct Machlne Drive ${ }^{d} \ldots \ldots \ldots \ldots \ldots \ldots \ldots \ldots \ldots$

Facility Heating, Ventilation, and Air Conditioning ......

Facility Lighting . . . . . . . . . . . . . . . . . . . . .

Equipment Installation/Retrofit for the Primary Purpose of

Using a Different Energy Source ${ }^{\circ} \ldots \ldots \ldots \ldots \ldots \ldots \ldots$

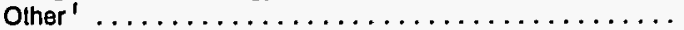

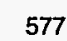

1,242

1,317

1,161

2,252

2,259

1,502

1,670

2,172

1,388

1,893

1,660

2,081

2,431

$\begin{array}{rr}1,895 & 185 \\ 1,230 & 120 \\ 1,155 & 169 \\ 1,311 & 929 \\ 221 & 67 \\ 213 & 152\end{array}$

970

802

301
1,084

580

812

391

42
53

113

149

106

257

280

182

225

266

173

265

189

266

300

Paper MIIls

Participation in One or More of the Following Types of

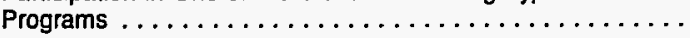

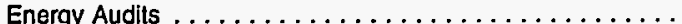

Direct Electricity Load Control $\ldots \ldots \ldots \ldots \ldots \ldots \ldots \ldots$

Special Rate Schedule ${ }^{b} \ldots \ldots \ldots \ldots \ldots \ldots \ldots \ldots \ldots$

Standby Generation Program . . . . . . . . . . . . .

Equlpment Rebate(s) . . . . . . . . . . . . . . . .

Equipment Installation or Retrofit for the Primary Purpose of

Improving Energy Efficiency Affecting:

Steam Production $^{c} \ldots \ldots \ldots \ldots \ldots \ldots \ldots \ldots \ldots$

Direct/Indirect Process Heating . . . . . . . . . . . . .

Direct Process Cooling/Refrigeration . . . . . . . . . .

Direct Machine Drive ${ }^{\mathrm{d}} \ldots \ldots \ldots \ldots \ldots \ldots \ldots \ldots$

Facility Heating, Ventilation, and Air Conditioning $\ldots \ldots \ldots$

Facillty Lighting . . . . . . . . . . . . . . . .

Equipment Installation/Retrofit for the Primary Purpose of

Using a Different Energy Source ${ }^{\star} \ldots \ldots \ldots \ldots \ldots \ldots \ldots$

Other $^{\prime} \ldots \ldots \ldots \ldots \ldots \ldots \ldots \ldots \ldots \ldots \ldots \ldots \ldots$

\begin{tabular}{rrrrrr}
230 & 974 & 54 & 274 & 646 & 2.9 \\
521 & 683 & 92 & 497 & 94 & 3.2 \\
574 & 630 & $W$ & 430 & $W$ & 2.9 \\
537 & 667 & 469 & 88 & 110 & 2.9 \\
1,073 & 131 & $W$ & $W$ & 0 & 4.0 \\
1,048 & 156 & 119 & $W$ & $W$ & 4.1 \\
& & & & & \\
754 & 450 & $W$ & 394 & $W$ & 3.6 \\
768 & 435 & $W$ & 394 & $W$ & 3.9 \\
1,060 & 144 & $W$ & 106 & $W$ & 4.3 \\
622 & 582 & $W$ & 477 & 65 & 3.5 \\
869 & 335 & 69 & 300 & $W$ & 4.0 \\
767 & 437 & & 314 & 54 & 3.1 \\
& & $W$ & 190 & $W$ & 4.0 \\
974 & 230 & $W$ & $W$ & $W$ & 6.0 \\
$W$ & $W$ & 0 & & & \\
\hline
\end{tabular}

See footnotes at end of table. 
Table A40. Total Inputs of Energy for Heat, Power, and Electricity Generation by Energy Management Program Sponsorship, Industry Group, Selected Industries, and Type of Energy Management Program, 1991: (Continued)

(Estimates in Trillion Btu)

\begin{tabular}{|c|c|c|c|c|c|c|c|}
\hline \multirow[b]{2}{*}{$\begin{array}{l}\text { SIC } \\
\text { Code }\end{array}$} & \multirow[b]{2}{*}{$\begin{array}{l}\text { Industry Groups } \\
\text { and Industry }\end{array}$} & \multirow[b]{2}{*}{$\begin{array}{l}\text { No Energy } \\
\text { Management } \\
\text { Program }\end{array}$} & \multicolumn{4}{|c|}{ Type of Sponsorship of Management Programs (1989 through 1991) } & \multirow{3}{*}{$\begin{array}{l}\text { RSE } \\
\text { Row } \\
\text { Factors }\end{array}$} \\
\hline & & & $\begin{array}{l}\text { Any Type of } \\
\text { Sponsorship }\end{array}$ & $\begin{array}{l}\text { Utility/Supplier } \\
\text { Sponsored } \\
\text { Involvement }\end{array}$ & $\begin{array}{l}\text { Only Own or } \\
\text { Other Third Party } \\
\text { Sponsorship }\end{array}$ & $\begin{array}{l}\text { Both Types of } \\
\text { Sponsorship }\end{array}$ & \\
\hline & RSE Column Factors: & 0.6 & 0.8 & 1.4 & 1.0 & 1.5 & \\
\hline
\end{tabular}

2631

Paperboard Mills

Participation in One or More of the Following Types of

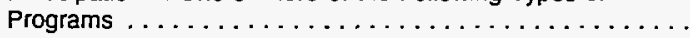

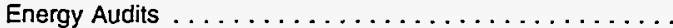

Direct Electricity Load Control . . . . . . . . . . .

Special Rate Schedule ${ }^{b} \ldots \ldots \ldots \ldots \ldots \ldots \ldots \ldots \ldots \ldots$

Standby Generation Program . . . . . . . . . . . .

Equipment Rebate(s) . . . . . . . . . . . . . . . .

Equipment Installation or Retrofit for the Primary Purpose of

Improving Energy Efficiency Affecting:

Steam Production ${ }^{\circ} \ldots \ldots \ldots \ldots \ldots \ldots \ldots \ldots$

Direct/lndirect Process Heating . . . . . . . . . . . .

Direct Process Cooling/Refrigeration . . . . . . . . . .

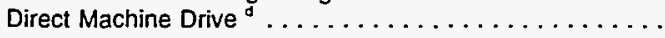

Facility Heating, Ventilation, and Air Conditioning .......

Facillty Lighting . . . . . . . . . . . . . . . . . . . .

Equipment installation/Retrofit for the Primary Purpose of

Using a Different Energy Source ${ }^{\circ} \ldots \ldots \ldots \ldots \ldots \ldots \ldots$

Other'

\section{PRINTING and PUBLISHING}

Participation in One or More of the Following Types of

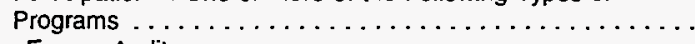

Energy Audits . . ......................

Direct Electricity Load Control $\ldots \ldots \ldots \ldots \ldots \ldots \ldots \ldots$

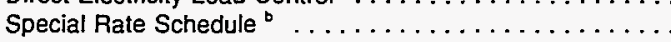

Standby Generation Program . . . . . . . . . . . . . . . .

Equipment Rebate(s) . . . . . . . . . . . . . . . . . .

Equipment Installation or Retrofit for the Primary Pupose of Improving Energy Efficiency Affecting:

Steam Production ${ }^{c} \ldots \ldots \ldots \ldots \ldots \ldots \ldots \ldots \ldots \ldots$

Direct/lndirect Process Heating $\ldots \ldots \ldots \ldots \ldots \ldots \ldots \ldots \ldots$

Direct Process Cooling/Refrigeration . . . . . . . . . . .

Direct Machine Drive ${ }^{d} \ldots \ldots \ldots \ldots \ldots \ldots \ldots \ldots$

Facillty Heating, Ventilation, and Air Conditioning .......

Facility Lighting . . . . . . . . . . . . . . . . .

Equipment Installation/Retrofit for the Primary Purpose of

Using a Different Energy Source ${ }^{\circ} \ldots \ldots \ldots \ldots \ldots \ldots \ldots$

Other' $\ldots \ldots \ldots \ldots \ldots \ldots \ldots \ldots \ldots \ldots \ldots \ldots$

$\begin{array}{rr}229 & 603 \\ 511 & 321 \\ 488 & 344 \\ 425 & 407 \\ 787 & 45 \\ 803 & 29 \\ & \\ & \\ 446 & 386 \\ 559 & 273 \\ 724 & 108 \\ 485 & 347 \\ 642 & 190 \\ 604 & 228 \\ 708 & 124 \\ W & W\end{array}$

96
11
$W$
306
$W$
21

$W$
$W$
0
4
$W$
6
$W$

0

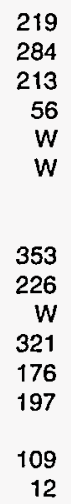

$\begin{array}{rr}287 & 5.4 \\ 26 & 6.9 \\ W & 5.9 \\ 44 & 6.5 \\ 0 & 9.4 \\ W & 10.7\end{array}$

CHEMICALS and ALLIED PRODUCTS

Participation in One or More of the Following Types of

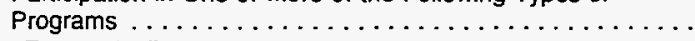

Energy Audits $\ldots \ldots \ldots \ldots \ldots \ldots \ldots \ldots \ldots \ldots$

Direct Electricity Load Control $\ldots \ldots \ldots \ldots \ldots \ldots \ldots \ldots$

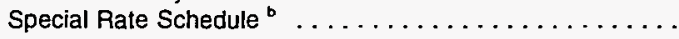

Standby Generation Program . . . . . . . . . . . . .

Equipment Rebate(s) . . . . . . . . . . . . . . . .

Equipment installation or Retrofit for the Primary Purpose of Improving Energy Efficiency Affecting:

Steam Production ${ }^{c} \ldots \ldots \ldots \ldots \ldots \ldots \ldots \ldots \ldots$

Direct/lndirect Process Heating . . . . . . . . . . . .

Direct Process Cooling/Refrigeration . . . . . . . . . .

Direct Machine Drive ${ }^{d} \ldots \ldots \ldots \ldots \ldots \ldots \ldots \ldots$

Facility Heating, Ventilation, and Air Conditioning $\ldots \ldots \ldots$

Facility Lighting . . . . . . . . . . . . . . . .

Equipment Installation/Retrofit for the Primary Purpose of

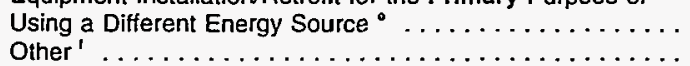

$\ldots \ldots \ldots \ldots \ldots \ldots$

See footnotes at end of table.

$\begin{array}{rr}900 & 2,140 \\ 1,573 & 1,467 \\ 2,056 & 985 \\ 1,836 & 1,204 \\ 2,871 & 170 \\ 2,973 & 67 \\ & \\ 2,198 & 843 \\ 2,025 & 1,015 \\ 2,289 & 75 \\ 1,928 & 1,112 \\ 2,427 & 614 \\ 2,239 & 801 \\ & \\ 2,793 & 247 \\ 2,935 & 105\end{array}$

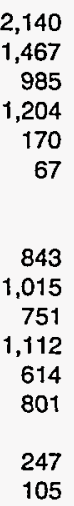

105

174
28
64
741
58
23

26
18
$W$
29
8
38
6
$W$

$\begin{array}{rrr}1,111 & 855 & 4.9 \\ 1,349 & 90 & 5.3 \\ 840 & 80 & 6.2 \\ 276 & 187 & 5.2 \\ 92 & 19 & 8.6 \\ 34 & 11 & 9.5\end{array}$

$\begin{array}{lll}806 & 11 & 6.4\end{array}$

$990 \quad 7 \quad 6.1$

$\begin{array}{lll}732 & \text { W } & 7.7\end{array}$

$\begin{array}{lll}1.067 & 16 & 5.3\end{array}$

$10 \quad 7.3$

$\begin{array}{lll}708 & 56 & 4.7\end{array}$

$\begin{array}{rrr}230 & 10 & 7.9 \\ 99 & W & 5.8\end{array}$ 
Table A40. Total Inputs of Energy for Heat, Power, and Electricity Generation by Energy Management Program Sponsorship, Industry Group, Selected Industries, and Type of Energy Management Program, 1991: (Continued)

(Estimates in Trillion Btu)

\begin{tabular}{|c|c|c|c|c|c|c|c|}
\hline \multirow[b]{2}{*}{$\begin{array}{c}\text { SIC } \\
\text { Code }^{a}\end{array}$} & \multirow[b]{2}{*}{$\begin{array}{l}\text { Industry Groups } \\
\text { and Industry }\end{array}$} & \multirow[b]{2}{*}{$\begin{array}{c}\text { No Energy } \\
\text { Management } \\
\text { Program }\end{array}$} & \multicolumn{4}{|c|}{ Type of Sponsorship of Management Programs (1989 through 1991) } & \multirow{3}{*}{$\begin{array}{c}\text { RSE } \\
\text { Row } \\
\text { Factors }\end{array}$} \\
\hline & & & $\begin{array}{l}\text { Any Type of } \\
\text { Sponsorship }\end{array}$ & $\begin{array}{c}\text { Only } \\
\text { Utility/Supplier } \\
\text { Sponsored } \\
\text { Involvement }\end{array}$ & $\begin{array}{c}\text { Only Own or } \\
\text { Other Third Party } \\
\text { Sponsorship }\end{array}$ & $\begin{array}{l}\text { Both Types of } \\
\text { Sponsorship }\end{array}$ & \\
\hline & olumn Factors: & 0.6 & 0.8 & 1.4 & 1.0 & 1.5 & \\
\hline
\end{tabular}

2812

Alkalies and Chlorine

Particlpation in One or More of the Following Types of

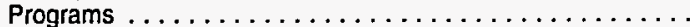

Energy Audits $\ldots \ldots \ldots \ldots \ldots \ldots \ldots \ldots \ldots \ldots$

Direct Electricity Load Control $\ldots \ldots \ldots \ldots \ldots \ldots \ldots \ldots$

Special Rate Schedule ${ }^{\prime} \ldots \ldots \ldots \ldots \ldots \ldots \ldots \ldots \ldots$

Standby Generation Program . . . . . . . . . . . . .

Equipment Rebate(s) . . . . . . . . . . . . . . . . . . .

Equipment Installation or Retrofit for the Primary Purpose of Improving Energy Efficiency Affecting:

Steam Production ${ }^{c} \ldots \ldots \ldots \ldots \ldots \ldots \ldots \ldots$

Direct/ndirect Process Heating $\ldots \ldots \ldots \ldots \ldots \ldots \ldots$

Direct Process Cooling/Refrigeration . . . . . . . . . .

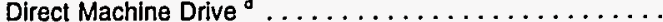

Facility Heating, Ventilation, and Air Conditioning ......

Facllity Lighting.$\ldots \ldots \ldots \ldots \ldots \ldots \ldots \ldots$

Equipment Installation/Retrofit for the Primary Purpose of

Using a Different Energy Source ${ }^{\circ} \ldots \ldots \ldots \ldots \ldots \ldots \ldots$

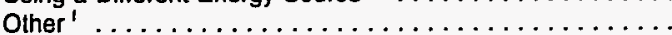

$\begin{array}{lr}23 & 137 \\ W & \\ W & \\ 26 & 134 \\ W & \end{array}$

137
$W$
$W$
134
$W$
$W$

$$
\begin{array}{r}
W \\
W \\
W \\
66 \\
0 \\
0
\end{array}
$$

$\begin{array}{rrr}\text { W } & 121 & 16.0 \\ W & W & 18.6 \\ W & 0 & 17.5 \\ W & W & 16.3 \\ \text { W } & 0 & 25.4 \\ \text { W } & 0 & 25.4\end{array}$

2813

Industrial Gases

Participation in One or More of the Following Types of

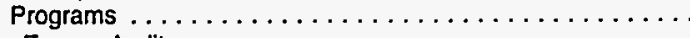

Energy Audits $\ldots \ldots \ldots \ldots \ldots \ldots \ldots \ldots \ldots \ldots$

Direct Electricity Load Control $\ldots \ldots \ldots \ldots \ldots \ldots \ldots$

Speclal Rate Schedule ${ }^{b} \ldots \ldots \ldots \ldots \ldots \ldots \ldots \ldots \ldots$

Standby Generation Program . . . . . . . . . . . . .

Equipment Rebate(s)

Equipment Installation or Retrofit for the Primary Purpose of Improving Energy Efficiency Affecting:

Steam Production ${ }^{c} \ldots \ldots \ldots \ldots \ldots \ldots \ldots \ldots \ldots$

Direct/Indirect Process Heating . . . . . . . . . . . .

Direct Process Cooling/Refrigeration . . . . . . . . . .

Direct Machine Drive ${ }^{d} \ldots \ldots \ldots \ldots \ldots \ldots \ldots \ldots \ldots$

Facility Heating, Ventilation, and Air Conditioning ......

Facillty Lighting . . . . . . . . . . . . . . . . . . . .

Equipment Installation/Retrofit for the Primary Purpose of

Using a Different Energy Source ${ }^{\circ} \ldots \ldots \ldots \ldots \ldots \ldots \ldots$

Other ${ }^{\prime} \ldots \ldots \ldots \ldots \ldots \ldots \ldots \ldots \ldots \ldots \ldots$

$\begin{array}{rr}86 & 74 \\ W & \\ 110 & \\ W & \\ W & 5 \\ W & \\ W & \end{array}$

74
$W$
$W$
59
$W$
$W$
$W$
$W$

57
$W$
$W$
61
$W$
90

W

89

W

90

90

89

90

2819

Industrial Inorganic Chemicals, nec

Participation in One or More of the Following Types of

Programs . . . . . . . . . . . . . . . . . . . . . . . .

Energy Audits $\ldots \ldots \ldots \ldots \ldots \ldots \ldots \ldots \ldots \ldots$

Direct Electricity Load Control $\ldots \ldots \ldots \ldots \ldots \ldots \ldots \ldots$

Special Rate Schedule ${ }^{b} \ldots \ldots \ldots \ldots \ldots \ldots \ldots \ldots$

Standby Generation Program . . . . . . . . . . . . .

Equipment Rebate(s) . . . . . . . . . . . . . . . .

Equipment Installation or Retrofit for the Primary Pupose of Improving Energy Efficiency Affecting:

Steam Production ${ }^{c} \ldots \ldots \ldots \ldots \ldots \ldots \ldots \ldots \ldots$

Dlrect/lndirect Process Heating . . . . . . . . . . . .

Direct Process Cooling/Refrigeration . . . . . . . . . .

Direct Machine Drive ${ }^{\mathrm{d}} \ldots \ldots \ldots \ldots \ldots \ldots \ldots \ldots \ldots$

Facllity Heating, Ventilation, and Air Conditioning ......

Facillty Lighting . . . . . . . . . . . . . . . . .

Equipment Installation/Retrofit for the Primary Purpose of

Using a Different Energy Source

Other'

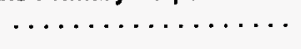

See footnotes at end of table.

Energy Information Administration/Manufacturing Consumption of Energy 1991 
Table A40. Total Inputs of Energy for Heat, Power, and Electricity Generation by Energy Management Program Sponsorship, Industry Group, Selected Industries, and Type of Energy Management Program, 1991: (Continued) (Estimates in Trillion Btu)

\begin{tabular}{|c|c|c|c|c|c|c|c|}
\hline \multirow[b]{2}{*}{$\begin{array}{l}\text { SIC } \\
\text { Code }^{\circ}\end{array}$} & \multirow[b]{2}{*}{$\begin{array}{l}\text { Industry Groups } \\
\text { and Industry }\end{array}$} & \multirow[b]{2}{*}{$\begin{array}{c}\text { No Energy } \\
\text { Management } \\
\text { Program }\end{array}$} & \multicolumn{4}{|c|}{ Type of Sponsorship of Management Programs (1989 through 1991) } & \multirow{3}{*}{$\begin{array}{c}\text { RSE } \\
\text { Row } \\
\text { Factore }\end{array}$} \\
\hline & & & $\begin{array}{l}\text { Any Type of } \\
\text { Sponsorship }\end{array}$ & $\begin{array}{c}\text { Utility/Supplier } \\
\text { Sponsored } \\
\text { Involvement }\end{array}$ & $\begin{array}{c}\text { Only Own or } \\
\text { Other Third Party } \\
\text { Sponsorship } \\
\end{array}$ & $\begin{array}{c}\text { Both Types of } \\
\text { Sponsorship }\end{array}$ & \\
\hline & RSE Column Factors: & 0.6 & 0.8 & 1.4 & 1.0 & 1.5 & \\
\hline
\end{tabular}

2821

Plastics Materials and Resins

Participation in One or More of the Following Types of

Programs . . . . . . . . . . . . . . . . . . . .

Energy Audits $\ldots \ldots \ldots \ldots \ldots \ldots \ldots \ldots \ldots \ldots \ldots \ldots$

Direct Electricity Load Control . . . . . . . . . . . . .

Special Rate Schedule $^{\natural} \ldots \ldots \ldots \ldots \ldots \ldots \ldots \ldots \ldots$

Standby Generation Program . . . . . . . . . . . . .

Equipment Rebate(s) . . . . . . . . . . . . . . . . . .

Equipment Installation or Retrofit for the Primary Purpose of

Improving Energy Efficiency Affecting:

Steam Production ${ }^{c} \ldots \ldots \ldots \ldots \ldots \ldots \ldots \ldots$

Direct/lndirect Process Heating . . . . . . . . . . . . .

Direct Process Cooling/Refrigeration . . . . . . . . . .

Direct Machine Drive ${ }^{d} \ldots \ldots \ldots \ldots \ldots \ldots \ldots \ldots \ldots \ldots$

Facility Heating, Ventilation, and Air Conditioning ......

Facility Lighting . . . . . . . . . . . . . . .

Equipment Installation/Retrofit for the Primary Purpose of

Using a Different Energy Source ${ }^{\circ} \ldots \ldots \ldots \ldots \ldots \ldots \ldots$

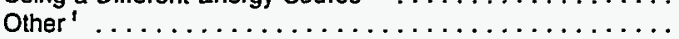

Synthetic Rubber

Participation in One or More of the Following Types of

Programs .....................

Energy Audits $\ldots \ldots \ldots \ldots \ldots \ldots \ldots \ldots \ldots \ldots$

Direct Electricity Load Control $\ldots \ldots \ldots \ldots \ldots \ldots \ldots \ldots$

Special Rate Schedule ${ }^{b} \ldots \ldots \ldots \ldots \ldots \ldots \ldots \ldots \ldots$

Standby Generation Program . . . . . . . . . . . . .

Equipment Rebate(s) . . . . . . . . . . . . . . . . .

Equipment Installation or Retrofit for the Primary Purpose of

Improving Energy Efticiency Affecting:

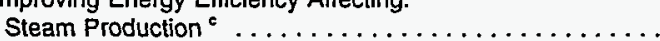

Direct/lndirect Process Heating . . . . . . . . . . . . . .

Direct Process Cooling/Refrigeration . . . . . . . . . .

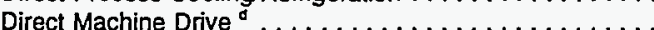

Facility Heating, Ventilation, and Air Conditioning ......

Facility Lighting . . . . . . . . . . . . . . .

Equipment Installation/Retrofit for the Primary Purpose of

Using a Different Energy Source ${ }^{\circ} \ldots \ldots \ldots \ldots \ldots \ldots \ldots$

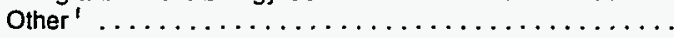

105

163

216

171

263

285

229

236

242

190

243

217

w

262

16
19
$W$
$W$
112
112

96
93
$W$
$W$
0

109

W

$W$
92

98
100

112

112

183

125

72

117

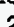

46

70

W

26

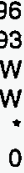

1
$w$
6
0

85
85
$W$
$W$
0
0

$\begin{array}{rr}0 & 3 \\ 0 & W \\ W & W \\ 0 & W \\ W & 14 \\ 0 & W \\ & \end{array}$

61
104
49
$W$
$W$

58

51
46

97

44
$W$

W

26

10.6
8.3

Cellulosic Manmade Fibers

Participation in One or More of the Following Types of

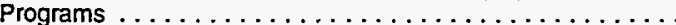

Energy Audits $\ldots \ldots \ldots \ldots \ldots \ldots \ldots \ldots \ldots \ldots$

Direct Electricity Load Control $\ldots \ldots \ldots \ldots \ldots \ldots \ldots \ldots$

Special Rate Schedule ${ }^{b} \ldots \ldots \ldots \ldots \ldots$

Standby Generation Program . . . . . . . . . . . . .

Equipment Rebate(s) . . . . . . . . . . . . . . . .

Equipment Installation or Retrofit for the Primary Purpose of Improving Energy Efficiency Affecting:

Steam Production ${ }^{c} \ldots \ldots \ldots \ldots \ldots \ldots \ldots \ldots \ldots \ldots$

Direct/lndirect Process Heating . . . . . . . . . . . . . .

Direct Process Cooling/Refrigeration . . . . . . . . . .

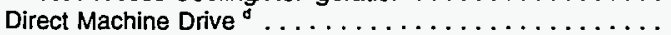

Facility Heating, Ventilation, and Air Conditioning . . . . . .

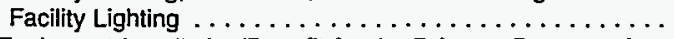

Equipment Installation/Retrofit for the Primary Purpose of

Using a Different Energy Source ${ }^{\circ} \ldots \ldots \ldots \ldots \ldots \ldots$.

Other $^{\prime} \ldots \ldots \ldots \ldots \ldots \ldots \ldots \ldots \ldots \ldots \ldots \ldots$

\begin{tabular}{rrrrrr}
$*$ & 31 & 0 & 28 & 4 & 28.5 \\
16 & 15 & 0 & 15 & 0 & 35.1 \\
4 & 28 & 0 & 28 & 0 & 32.1 \\
25 & 6 & 0 & 6 & 0 & 38.3 \\
28 & 4 & 4 & 0 & 0 & 33.2 \\
31 & 0 & 0 & 0 & 0 & 34.4 \\
& & & & & \\
10 & 21 & 0 & 21 & 0 & 33.0 \\
25 & 6 & 0 & 6 & 0 & 38.3 \\
25 & 6 & 0 & 6 & 0 & 38.3 \\
10 & 22 & 0 & 22 & 0 & 33.1 \\
21 & 10 & 0 & 10 & 0 & 33.4 \\
10 & 22 & 0 & 22 & 0 & 34.4 \\
31 & & 0 & 0 & 0 & 34.4 \\
31 & 0 & 0 & 0 & 0 & 0 \\
\hline
\end{tabular}

See footnotes at end of table. 
Table A40. Total Inputs of Energy for Heat, Power, and Electricity Generation by Energy Management Program Sponsorship, Industry Group, Selected Industries, and Type of Energy Management Program, 1991: (Continued) (Estimates in Trillion Btu)

\begin{tabular}{|c|c|c|c|c|c|c|c|}
\hline \multirow[b]{2}{*}{$\begin{array}{l}\text { SIC } \\
\text { Code" }\end{array}$} & \multirow[b]{2}{*}{$\begin{array}{l}\text { Industry Groups } \\
\text { and Industry }\end{array}$} & \multirow[b]{2}{*}{$\begin{array}{c}\text { No Energy } \\
\text { Management } \\
\text { Program }\end{array}$} & \multicolumn{4}{|c|}{ Type of Sponsorship of Management Programs (1989 through 1991) } & \multirow[b]{2}{*}{$\begin{array}{l}\text { RSE } \\
\text { Row } \\
\text { Factors }\end{array}$} \\
\hline & & & $\begin{array}{l}\text { Any Type of } \\
\text { Sponsorship }\end{array}$ & $\begin{array}{l}\text { Utility/Supplier } \\
\text { Sponsored } \\
\text { Involvement }\end{array}$ & $\begin{array}{c}\text { Only Own or } \\
\text { Other Third Party } \\
\text { Sponsorship }\end{array}$ & $\begin{array}{l}\text { Both Types of } \\
\text { Sponsorship }\end{array}$ & \\
\hline & Column Factors: & 0.6 & 0.8 & 1.4 & 1.0 & 1.5 & \\
\hline
\end{tabular}

2824

Organic Flbers, Noncellulosic

Participation in One or More of the Following Types of

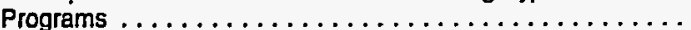

Energy Audlts $\ldots \ldots \ldots \ldots \ldots \ldots \ldots \ldots \ldots$

Dlrect Electriclty Load Control . . . . . . . . . . . . . . .

Speclal Rate Schedule ${ }^{b} \ldots \ldots \ldots \ldots \ldots \ldots \ldots \ldots$

Standby Generation Program . . . . . . . . . . . .

Equipment Rebate(s) . . . . . . . . . . . . . . . .

Equipment Installation or Retrofit for the Primary Purpose of

Improving Energy Efficlency Affecting:

Steam Production ${ }^{c} \ldots \ldots \ldots \ldots \ldots \ldots \ldots \ldots$

Dlrect/Indlrect Process Heating . . . . . . . . . . . .

DIrect Process Cooling/Refrigeration . . . . . . . . . .

Dlrect Machlne Drive ${ }^{d} \ldots \ldots \ldots \ldots \ldots \ldots \ldots$

Facility Heating, Ventllation, and Air Conditioning ......

Facility Lighting . . . . . . . . . . . . . . . .

Equipment Installation/Retrofit for the Primary Purpose of

Using a Different Energy Source ${ }^{\circ} \ldots \ldots \ldots \ldots \ldots \ldots \ldots$

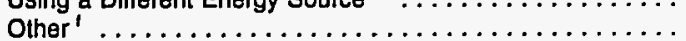

2865

Cyclic Crudes and Intermediates

Particlpation in One or More of the Following Types of

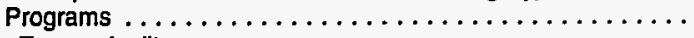

Energy Audits $\ldots \ldots \ldots \ldots \ldots \ldots \ldots \ldots \ldots \ldots$

Dlrect Electricity Load Control . . . . . . . . . . . . . .

Special Rate Schedule $^{b} \ldots \ldots \ldots \ldots \ldots \ldots \ldots \ldots$

Standby Generation Program . . . . . . . . . . . . . .

Equlpment Rebate(s) ......................

Equipment Installation or Retrofit for the Primary Purpose of Improving Energy Efficlency Affecting:

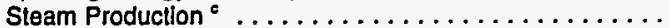

Direct/lndirect Process Heating . . . . . . . . . . . .

Direct Process Cooling/Retrigeration . . . . . . . . . .

Direct Machine Drive ${ }^{d} \ldots \ldots \ldots \ldots \ldots \ldots \ldots \ldots$

Facllity Heatling, Ventilation, and Alr Conditioning ......

Facility Llghting . . . . . . . . . . . . . . . . . . . . . . .

Equlpment Installation/Retrofit for the Primary Purpose of

Using a Different Energy Source $\ldots \ldots \ldots \ldots \ldots \ldots \ldots$

Other $^{\prime} \ldots \ldots \ldots \ldots \ldots \ldots \ldots \ldots \ldots \ldots \ldots \ldots$

Industrial Organic Chemicals, nec

Participation in One or More of the Following Types of

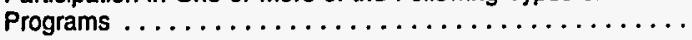

Energy Audits $\ldots \ldots \ldots \ldots \ldots \ldots \ldots \ldots \ldots \ldots \ldots$

Direct Electricity Load Control $\ldots \ldots \ldots \ldots \ldots \ldots \ldots \ldots$

Special Rate Schedule ${ }^{b} \ldots \ldots \ldots \ldots \ldots \ldots \ldots \ldots$

Standby Generation Program . . . . . . . . . . . .

Equlpment Rebate(s) . . . . . . . . . . . . . . .

Equlpment Installation or Retrofit for the Primary Pupose of Improving Energy Efficiency Affecting:

Steam Production ${ }^{c} \ldots \ldots \ldots \ldots \ldots \ldots \ldots \ldots \ldots$

Direct/Indirect Process Heating . . . . . . . . . . . . .

Direct Process Cooling/Refrigeration . . . . . . . . .

Direct Machine Drive ${ }^{d} \ldots \ldots \ldots \ldots \ldots \ldots \ldots \ldots \ldots$

Facility Heating, Ventilation, and Air Conditioning ......

Facillty Lighting . . . . . . . . . . . . . . . . . . .

Equlpment Installation/Retrofit for the Primary Purpose of

Using a Different Energy Source ${ }^{\bullet} \ldots \ldots \ldots \ldots \ldots \ldots \ldots$

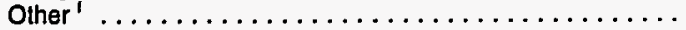

See footnotes at end of table. w

$\begin{array}{rr}848 & 8 \\ 671 & 3 \\ 353 & 1 \\ 415 & 305 \\ 30 & W \\ 2 & 2\end{array}$

494

\begin{tabular}{|c|c|c|c|c|}
\hline 815 & 375 & * & $w$ & w \\
\hline 650 & 540 & * & 540 & 0 \\
\hline 759 & 432 & $w$ & $w$ & * \\
\hline 625 & 566 & 1 & 559 & 5 \\
\hline 966 & 225 & 1 & 224 & • \\
\hline 925 & 266 & 8 & 257 & 1 \\
\hline 1,046 & 145 & 1 & 144 & 0 \\
\hline 1,137 & 53 & 0 & $w$ & $w$ \\
\hline
\end{tabular}


Table A40. Total Inputs of Energy for Heat, Power, and Electricity Generation by Energy Management Program Sponsorship, Industry Group, Selected Industries, and Type of Energy Management Program, 1991: (Continued)

(Estimates in Trillion Btu)

\begin{tabular}{|c|c|c|c|c|c|c|c|}
\hline \multirow[b]{2}{*}{$\begin{array}{l}\text { SIC } \\
\text { Code }\end{array}$} & \multirow[b]{2}{*}{$\begin{array}{l}\text { Industry Groups } \\
\text { and Industry }\end{array}$} & \multirow[b]{2}{*}{$\begin{array}{l}\text { No Energy } \\
\text { Management } \\
\text { Program }\end{array}$} & \multicolumn{4}{|c|}{ Type of Sponsorship of Management Programs (1989 through 1991) } & \multirow[b]{2}{*}{$\begin{array}{c}\text { RSE } \\
\text { Row } \\
\text { Factors }\end{array}$} \\
\hline & & & $\begin{array}{l}\text { Any Type of } \\
\text { Sponsorship }\end{array}$ & $\begin{array}{l}\text { Utility/Supplier } \\
\text { Sponsored } \\
\text { Involvement }\end{array}$ & $\begin{array}{c}\text { Only Own or } \\
\text { Other Third Party } \\
\text { Sponsorship }\end{array}$ & $\begin{array}{l}\text { Both Types of } \\
\text { Sponsorship }\end{array}$ & \\
\hline & Column Factors: & 0.6 & 0.8 & 1.4 & 1.0 & 1.5 & \\
\hline
\end{tabular}

2873

Nitrogenous Fertilizers

Participation in One or More of the Following Types of

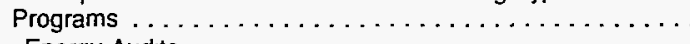

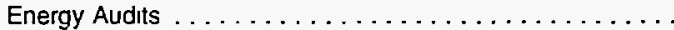

Direct Electricity Load Control $\ldots \ldots \ldots \ldots \ldots \ldots \ldots$

Special Rate Schedule ${ }^{b}$

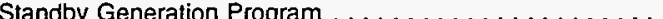

Equipment Rebate(s) . . . . . . . . . . . . . . .

Equipment Installation or Retrofit for the Primary Purpose of Improving Energy Efficiency Affecting:

Steam Production ${ }^{c} \ldots \ldots \ldots \ldots \ldots \ldots \ldots \ldots$

Direct/lndirect Process Heating . . . . . . . . . . . . . .

Direct Process Cooling/Refrigeration . . . . . . . . . . . . .

Direct Machine Drive a ....................

Facility Heating, Ventilation, and Air Conditioning $\ldots \ldots$.

Facility Lighting . . . . . . . . . . . . . . . . . .

Equipment Installation/Retrofit for the Primary Purpose of

Using a Different Energy Source ${ }^{\circ} \ldots \ldots \ldots \ldots \ldots \ldots$

Other ${ }^{1}$

Phosphatic Fertilizers

Participation in One or More of the Following Types of

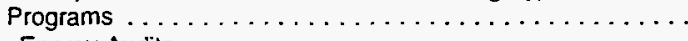

Energy Audits.$\ldots \ldots \ldots \ldots \ldots \ldots \ldots \ldots \ldots$

Direct Electricity Load Control $\ldots \ldots \ldots \ldots \ldots \ldots$

Special Rate Schedule ${ }^{b}$

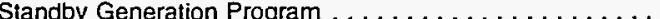

Equipment Rebate(s) . . . . . . . . . . . . . . . .

Equipment Installation or Retrofit for the Primary Purpose of Improving Energy Efficiency Affecting:

Steam Production ${ }^{c} \ldots \ldots \ldots \ldots \ldots \ldots \ldots \ldots$

Direct/Indirect Process Heating . . . . . . . . . . . . . .

Direct Process Cooling/Refrigeration . . . . . . . . . . . .

Direct Machine Drive ${ }^{\circ} \ldots \ldots \ldots \ldots \ldots \ldots \ldots \ldots$

Facility Heating, Ventilation, and Air Conditioning $\ldots \ldots$.

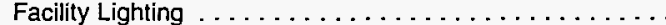

Equipment Installation/Retrofit for the Primary Purpose of

Using a Different Energy Source ${ }^{\circ} \ldots \ldots \ldots \ldots \ldots \ldots$

Other'

\section{PETROLEUM and COAL PRODUCTS}

Participation in One or More of the Following Types of

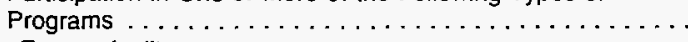

Energy Audits . . . . . . . . . . . . . . . . .

Direct Electricity Load Control . . . . . . . . . . . . . .

Special Rate Schedule ${ }^{\mathrm{B}}$

Standby Generation Program . . . . . . . . . . . . . . . . .

Equipment Rebate(s) . . . . . . . . . . . . . . . .

Equipment Installation or Retrofit for the Primary Purpose of Improving Energy Efficiency Affecting:

Steam Production ${ }^{c} \ldots \ldots \ldots \ldots \ldots \ldots \ldots \ldots$

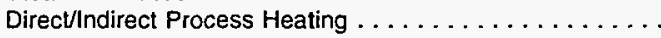

Direct Process Cooling/Refrigeration . . . . . . . . . . . .

Direct Machine Drive ${ }^{\circ} \ldots \ldots \ldots \ldots \ldots \ldots \ldots \ldots$

Facility Heating, Ventilation, and Air Conditioning $\ldots \ldots$.

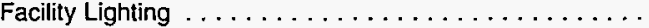

Equipment Installation/Retrofit for the Primary Purpose of

Using a Different Energy Source ${ }^{\circ} \ldots \ldots \ldots \ldots \ldots \ldots \ldots$

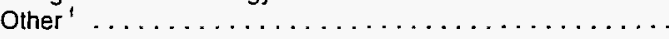

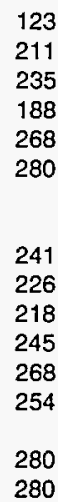

158
69
45
93
$Q$
0


39
55
62
36
12
26

0
0

82
1
22
80
0
0

1
0
0
1
0
1

0
0

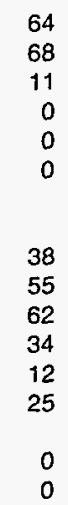

$\begin{array}{ll}\mathrm{Q} & 24.1 \\ 0 & 28.0 \\ \mathrm{Q} & 35.1 \\ \mathrm{Q} & 26.3 \\ \mathrm{Q} & 25.3 \\ 0 & 25.3 \\ & \\ & \\ 0 & 29.9 \\ 0 & 25.8 \\ 0 & 27.3 \\ 0 & 31.5 \\ 0 & 33.6 \\ 0 & 30.4 \\ & \\ 0 & 25.3 \\ 0 & 25.3\end{array}$

See footnotes at end of table.

$\begin{array}{rrrrrr}736 & 2,251 & 190 & 1,164 & 897 & 2.4 \\ 1,517 & 1,470 & 18 & 1,326 & 126 & 3.2 \\ 2,311 & 675 & 2 & W & W & 5.2 \\ 1,915 & 1,072 & 639 & 274 & 159 & 2.6 \\ 2,765 & 222 & W & W & 0 & 4.3 \\ 2,878 & 109 & 109 & * & 0 & 6.1 \\ & & & & & \\ 1,758 & 1,228 & W & 1,141 & W & 3.1 \\ 1,758 & 1,229 & W & 1,130 & W & 3.0 \\ 2,557 & 430 & 0 & 429 & 1 & 2.9 \\ 1,899 & 1,088 & W & 913 & W & 2.9 \\ 2,487 & 500 & Q & 440 & 60 & 3.2 \\ 2,023 & 964 & 75 & 800 & 90 & 2.8 \\ & & & & & \\ 2,677 & 310 & * & 310 & 0 & 3.4 \\ 2,918 & 69 & 0 & W & W & 4.1\end{array}$


Table A40. Total Inputs of Energy for Heat, Power, and Electricity Generation by Energy Management Program Sponsorship, Industry Group, Selected Industries, and Type of Energy Management Program, 1991: (Continued)

(Estimates in Trillion Btu)

\begin{tabular}{|c|c|c|c|c|c|c|c|}
\hline \multirow[b]{2}{*}{$\begin{array}{c}\text { SIC } \\
\text { Coda" }\end{array}$} & \multirow[b]{2}{*}{$\begin{array}{l}\text { Industry Groups } \\
\text { and Industry }\end{array}$} & \multirow[b]{2}{*}{$\begin{array}{c}\text { No Energy } \\
\text { Management } \\
\text { Program }\end{array}$} & \multicolumn{4}{|c|}{ Type of Sponsorship of Management Programs (1989 through 1991) } & \multirow{3}{*}{$\begin{array}{l}\text { RSE } \\
\text { Row } \\
\text { Factors }\end{array}$} \\
\hline & & & $\begin{array}{l}\text { Any Type of } \\
\text { Sponsorship }\end{array}$ & $\begin{array}{l}\text { Utility/Supplier } \\
\text { Sponsored } \\
\text { Involvement }\end{array}$ & $\begin{array}{c}\text { Only Own or } \\
\text { Other Third Party } \\
\text { Sponsorship }\end{array}$ & $\begin{array}{l}\text { Both Types of } \\
\text { Sponsorship }\end{array}$ & \\
\hline & RSE Column Factors: & 0.6 & 0.8 & 1.4 & 1.0 & 1.5 & \\
\hline
\end{tabular}

2911

Petroleum Refining*

Participation in One or More of the Following Types of

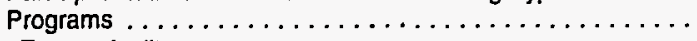

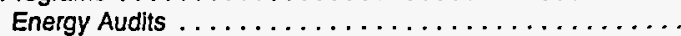

Direct Electricity Load Control $\ldots \ldots \ldots \ldots \ldots \ldots \ldots$

Special Rate Schedule ${ }^{b} \ldots \ldots \ldots \ldots \ldots \ldots \ldots \ldots \ldots$

Standby Generation Program . . . . . . . . . . . . . . . .

Equipment Rebate(s) . . . . . . . . . . . . . . . . .

Equipment Installation or Retrofit for the Primary Purpose of

Improving Energy Efficlency Affecting:

Steam Production ${ }^{\circ} \ldots \ldots \ldots \ldots \ldots \ldots \ldots \ldots \ldots$

Direct/ndirect Process Heating . . . . . . . . . . . . . .

Direct Process Cooling/Refrigeration . . . . . . . . . . . .

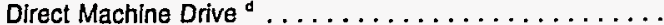

Facility Heating, Ventilation, and Air Conditioning ......

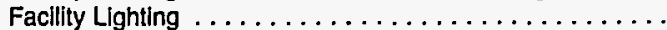

Equipment Installation/Retrofit for the Primary Purpose of

Using a Different Energy Source ${ }^{\circ} \ldots \ldots \ldots \ldots \ldots \ldots$.

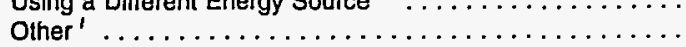

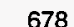

1,448

2,230

1,837

2,674

2,786

1,677

1,678

2,464

1,818

2,402

1,937

2,588

2,824

RUBBER and MISC. PLASTICS PRODUCTS

Participation in One or More of the Following Types of

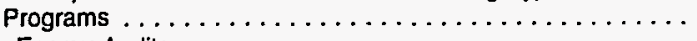

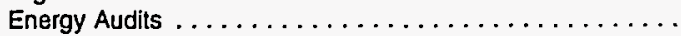

Direct Electricity Load Control . . . . . . . . . . . . .

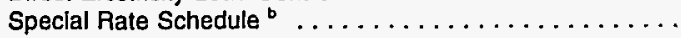

Standby Generation Program . . . . . . . . . . . . . .

Equipment Rebate(s) . .....................

Equipment Installation or Retrofit for the Primary Purpose of Improving Energy Efficiency Affecting:

Steam Production ${ }^{c} \ldots \ldots \ldots \ldots \ldots \ldots \ldots \ldots \ldots$

Dlrect/Indirect Process Heating . . . . . . . . . . . . . .

Direct Process Cooling/Refrígeration . . . . . . . . . . .

Direct Machine Drive ${ }^{d} \ldots \ldots \ldots \ldots \ldots \ldots \ldots \ldots$. . . . . . . . . .

Facllity Heating, Ventilation, and Air Conditioning .......

Facillty Lighting . ......................

Equipment Installation/Retrofit for the Primary Purpose of

Using a Different Energy Source ${ }^{\circ} \ldots \ldots \ldots \ldots \ldots \ldots$

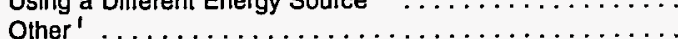

Particlpation in One or More of the Following Types of

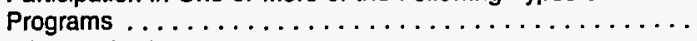

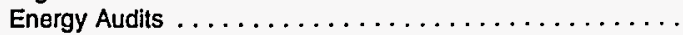

Direct Electricity Load Control . . . . . . . . . . . . . .

Special Rate Schedule ${ }^{b}$.....................

Standby Generation Program . . . . . . . . . . . . .

Equipment Rebate(s) . . . . . . . . . . . . . . . . .

Equipment Installation or Retrofit for the Primary Pupose of Improving Energy Efficiency Affecting:

Steam Production ${ }^{c} \ldots \ldots \ldots \ldots \ldots \ldots \ldots \ldots \ldots$

Direct/Indirect Process Heating . . . . . . . . . . . . .

Direct Process Cooling/Refrigeration . . . . . . . . . . . .

Direct Machine Drive ${ }^{d} \ldots \ldots \ldots \ldots \ldots \ldots \ldots \ldots$

Facllity Healing, Ventilation, and Air Conditioning $\ldots \ldots$.

Facllity Lighting . . . . . . . . . . . . . . . . .

Equipment Installation/Retrofit for the Primary Purpose of

Using a Different Energy Source ${ }^{\circ} \ldots \ldots \ldots \ldots \ldots \ldots$

Other

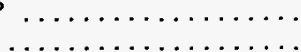

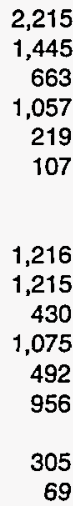

1,445

663

057

107

1,216

1,215

430

492

956

69

14
0
628
$W$
107

1,308

271

$W$
0

1,130

1,117

429

902

432

794

305

W

$888 \quad 2.4$

$124 \quad 2.6$

W 2.9

$\begin{array}{ll}58 & 2.4 \\ 0 & 4.2\end{array}$

$\begin{array}{ll}0 & 4.2 \\ 0 & 3.6\end{array}$

W $\quad 3.0$

W 2.9

$1 \quad 2.9$

W 2.9

$60 \quad 3.2$

$\begin{array}{ll}90 & 2.8\end{array}$

$\begin{array}{ll}0 & 3.4\end{array}$

W 4.1

See lootnotes at end of table.

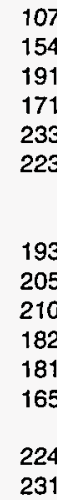

154

191

171

223

193

205

210

182

165

224

231

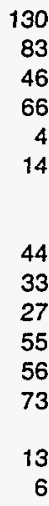

130

83

4

14

44

33

6


Table A40. Total Inputs of Energy for Heat, Power, and Electricity Generation by Energy Management Program Sponsorship, Industry Group, Selected Industries, and Type of Energy Management Program, 1991: (Continued)

(Estimates in Trillion Btu)

\begin{tabular}{|c|c|c|c|c|c|c|c|}
\hline \multirow[b]{2}{*}{$\begin{array}{l}\text { SIC } \\
\text { Code }\end{array}$} & \multirow[b]{2}{*}{$\begin{array}{l}\text { Industry Groups } \\
\text { and Industry }\end{array}$} & \multirow[b]{2}{*}{$\begin{array}{c}\text { No Energy } \\
\text { Management } \\
\text { Program }\end{array}$} & \multicolumn{4}{|c|}{ Type of Sponsorship of Management Programs (1989 through 1991) } & \multirow{3}{*}{$\begin{array}{l}\text { RSE } \\
\text { Row } \\
\text { Factors }\end{array}$} \\
\hline & & & $\begin{array}{l}\text { Any Type of } \\
\text { Sponsorship }\end{array}$ & $\begin{array}{c}\text { Utility/Supplier } \\
\text { Sponsored } \\
\text { Involvement }\end{array}$ & $\begin{array}{c}\text { Only Own or } \\
\text { Other Third Party } \\
\text { Sponsorship }\end{array}$ & $\begin{array}{l}\text { Both Types of } \\
\text { Sponsorship }\end{array}$ & \\
\hline & RSE Column Factors: & 0.6 & 0.8 & 1.4 & 1.0 & 1.5 & \\
\hline
\end{tabular}

308

Miscellaneous Plastic Products, nec

Participation in One or More of the Following Types of

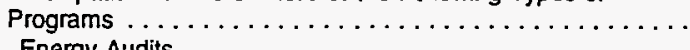

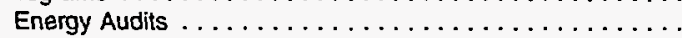

Direct Electricity Load Control $\ldots \ldots \ldots \ldots \ldots \ldots \ldots$

Special Rate Schedule ${ }^{b} \ldots \ldots \ldots \ldots \ldots \ldots \ldots \ldots \ldots$

Standby Generation Program $\ldots \ldots \ldots \ldots \ldots \ldots \ldots \ldots$

Equipment Rebate(s)

Equipment Installation or Retrofit for the Primary Purpose of Improving Energy Efficiency Affecting:

Steam Production ${ }^{\circ} \ldots \ldots \ldots \ldots \ldots \ldots \ldots \ldots \ldots \ldots$

Direct/lndirect Process Heating . . . . . . . . . . . . .

Direct Process Cooling/Refrigeration $\ldots \ldots \ldots \ldots \ldots \ldots \ldots$

Direct Machine Drive ${ }^{d} \ldots \ldots \ldots \ldots \ldots \ldots \ldots \ldots$

Facility Heating, Ventilation, and Air Conditioning $\ldots \ldots$.

Facility Lighting . .......................

Equipment InstallationRetrofit for the Primary Purpose of

Using a Different Energy Source ${ }^{\circ} \ldots \ldots \ldots \ldots \ldots \ldots \ldots$

Other"

\section{LEATHER and LEATHER PRODUCTS}

Participation in One or More of the Following Types of

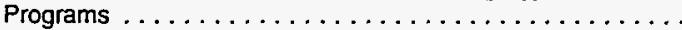

Energy Audits . . . . . . . . . . . . . . . . .

Direct Electricity Load Control . . . . . . . . . . . . .

Special Rate Schedule ${ }^{b} \ldots \ldots \ldots \ldots \ldots \ldots \ldots \ldots$

Standby Generation Program . . . . . . . . . . . . .

Equipment Rebate(s) . . . . . . . . . . . . . . . . .

Equipment Installation or Retrofit for the Primary Purpose of

Improving Energy Efficiency Affecting:

Steam Production ${ }^{\circ} \ldots \ldots \ldots \ldots \ldots \ldots \ldots \ldots \ldots \ldots \ldots$

Direct/lndirect Process Heating . . . . . . . . . . . . . .

Direct Process Cooling/Refrigeration . . . . . . . . . .

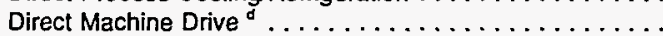

Facility Heating, Ventilation, and Air Conditioning . ......

Facility Lighting $\ldots \ldots \ldots \ldots \ldots \ldots \ldots \ldots \ldots$

Equipment Installation/Retrofit for the Primary Purpose of

Using a Different Energy Source ${ }^{\circ} \ldots \ldots \ldots \ldots \ldots \ldots$

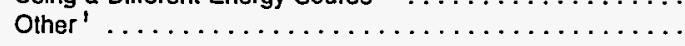

81

115

130

120

144

142

137

138

124

127
115

149

147

$\begin{array}{rr}71 & 15 \\ 37 & 1 \\ 22 & \\ 32 & \\ 1 & \\ 8 & \end{array}$

15
10
7
24
$?$
6

28

21

21
13

5

Q

10

15

13

28

25
37

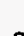

5

6

9

12

13

22

20
26

3

2

Q

$\begin{array}{ll}0 & 24.8 \\ 2 & 27.4\end{array}$

STONE, CLAY and GLASS PRODUCTS

Participation in One or More of the Following Types of

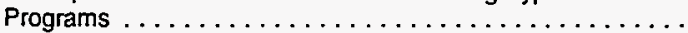

Energy Audits . . . . . . . . . . . . . . . . .

Direct Electricity Load Control $\ldots \ldots \ldots \ldots \ldots \ldots \ldots \ldots$

Special Rate Schedule ${ }^{b} \ldots \ldots \ldots \ldots \ldots$

Standby Generation Program . . . . . . . . . . . . . .

Equipment Rebate(s) . . . . . . . . . . . . . . . . .

Equipment Installation or Retrofit for the Primary Purpose of

Improving Energy Efficiency Affecting:

Steam Production ${ }^{c} \ldots \ldots \ldots \ldots \ldots \ldots \ldots \ldots \ldots$

Direct/Indirect Process Heating . . . . . . . . . . . .

Direct Process Cooling/Refrigeration . . . . . . . . . . .

Direct Machine Drive ${ }^{\mathrm{d}} \ldots \ldots \ldots \ldots \ldots \ldots \ldots \ldots \ldots$

Facility Heating, Ventilation, and Air Conditioning .......

Facility Lighting . . . . . . . . . . . . . . . . .

Equipment Installation/Retrofit for the Primary Purpose of

Using a Different Energy Source ${ }^{\circ} \ldots \ldots \ldots \ldots \ldots \ldots \ldots$

Other $^{\prime}$

.........................

See footnotes at end of table. 
Table A40. Total Inputs of Energy for Heat, Power, and Electricity Generation by Energy Management Program Sponsorship, Industry Group, Selected Industries, and Type of Energy Management Program, 1991: (Continued)

(Estimates in Trillion Btu)

\begin{tabular}{|c|c|c|c|c|c|c|c|}
\hline \multirow[b]{2}{*}{$\begin{array}{l}\text { SIC } \\
\text { Code }^{a}\end{array}$} & \multirow[b]{2}{*}{$\begin{array}{l}\text { Industry Groups } \\
\text { and Industry }\end{array}$} & \multirow[b]{2}{*}{$\begin{array}{l}\text { No Energy } \\
\text { Management } \\
\text { Program }\end{array}$} & \multicolumn{4}{|c|}{ Type of Sponsorship of Management Programs (1989 through 1991) } & \multirow{3}{*}{$\begin{array}{c}\text { RSE } \\
\text { Row } \\
\text { Factors }\end{array}$} \\
\hline & & & $\begin{array}{l}\text { Any Type of } \\
\text { Sponsorship }\end{array}$ & $\begin{array}{l}\text { Utility/Supplier } \\
\text { Sponsored } \\
\text { Involvement }\end{array}$ & $\begin{array}{c}\text { Only Own or } \\
\text { Other Third Party } \\
\text { Sponsorship }\end{array}$ & $\begin{array}{c}\text { Both Types of } \\
\text { Sponsorship }\end{array}$ & \\
\hline & RSE Column Factors: & 0.6 & 0.8 & 1.4 & 1.0 & 1.5 & \\
\hline
\end{tabular}

3211

Flat Glass

Participation in One or More of the Following Types of

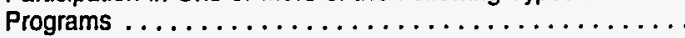

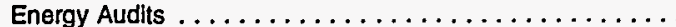

Direct Electricity Load Control $\ldots \ldots \ldots \ldots \ldots \ldots \ldots$

Special Rate Schedule ${ }^{b} \ldots \ldots \ldots \ldots \ldots \ldots \ldots \ldots$

Standby Generation Program . . . . . . . . . . . . .

Equipment Rebate(s) . . . . . . . . . . . . . . . .

Equipment Installation or Retrofit for the Primary Purpose of

Improving Energy Efficiency Affecting:

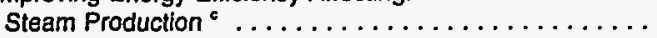

Direct/ndlrect Process Heating ...................

Direct Process Coollng/Refrigeration . . . . . . . . . . .

Direct Machine Drive ${ }^{\mathrm{d}} \ldots \ldots \ldots \ldots \ldots \ldots \ldots \ldots \ldots$

Facllity Heating, Ventllation, and Air Conditioning ......

Facillty Lighting $\ldots \ldots \ldots \ldots \ldots \ldots \ldots \ldots \ldots$

Equipment Installation/Retrofit for the Primary Purpose of

Using a Different Energy Source ${ }^{\circ} \ldots \ldots \ldots \ldots \ldots \ldots$.

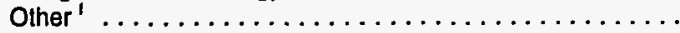

Glass Containers

Participation in One or More of the Following Types of

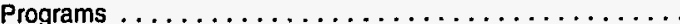

Energy Audits $\ldots \ldots \ldots \ldots \ldots \ldots \ldots \ldots \ldots \ldots$

Direct Electricity Load Control $\ldots \ldots \ldots \ldots \ldots \ldots$

Special Rate Schedule ${ }^{\circ} \ldots \ldots \ldots \ldots \ldots \ldots \ldots \ldots$

Standby Generation Program . . . . . . . . . . . . . .

Equipment Rebate(s) . .....................

Equipment Installation or Retrofit for the Primary Purpose of

Improving Energy Efficiency Affecting:

Steam Production ${ }^{\circ}$. . . . . . . . . . . . . . . . .

Directlindirect Process Heating ....................

Direct Process Cooling/Refrigeration . . . . . . . . . . .

Direct Machine Drive ${ }^{d} \ldots \ldots \ldots \ldots \ldots \ldots \ldots \ldots$

Facility Heating, Ventilation, and Air Conditioning ........

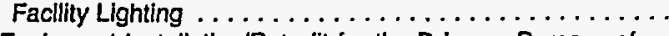

Equipment Installation/Retrofit for the Primary Purpose of

Using a Different Energy Source ${ }^{\circ} \ldots \ldots \ldots \ldots \ldots \ldots$

Other ${ }^{\prime}$. . . . . . . . . . . . . . . . . . . . . . .

Pressed and Blown Glass, nec.

Participation in One or More of the Following Types of

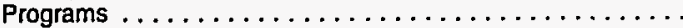

Energy Audits $\ldots \ldots \ldots \ldots \ldots \ldots \ldots \ldots \ldots \ldots \ldots$

Direct Electricity Load Control $\ldots \ldots \ldots \ldots \ldots \ldots \ldots$

Special Rate Schedule ${ }^{b} \ldots \ldots \ldots \ldots \ldots \ldots \ldots \ldots$

Standby Generation Program . . . . . . . . . . . . .

Equipment Rebate(s) .....................

Equipment Installation or Retrotit for the Primary Purpose of Improving Energy Efficlency Affecling:

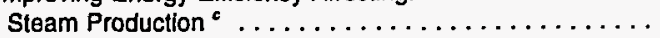

Direct/ndirect Process Heating .....................

Direct Process Cooling/Aefrigeration . . . . . . . . . . .

Direct Machine Drive ${ }^{d} \ldots \ldots \ldots \ldots \ldots \ldots \ldots \ldots$.

Facility Heating, Ventilation, and Air Conditioning .......

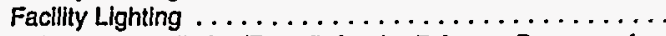

Equipment Installation/Retrofit for the Primary Purpose of

Using a Different Energy Source ${ }^{\circ} \ldots \ldots \ldots \ldots \ldots \ldots$

Other'

$\begin{array}{cr}W & W \\ 28 & 20 \\ 34 & 14 \\ 15 & 34 \\ 42 & 7 \\ W & W \\ & \\ W & W \\ 34 & 15 \\ W & W \\ 33 & 16 \\ 39 & 9 \\ 41 & 8 \\ W & W \\ & \\ 48 & 1\end{array}$

$W$
20
14
34
7
$W$

$W$
15
$W$
16
9
8
$W$
1

$\begin{array}{ll}16 & 16 \\ W & 15 \\ W & W \\ 20 & W \\ W & W \\ W & 0\end{array}$

$16 \quad W \quad 2.9$

$\begin{array}{lll}W & 0 & 3.2\end{array}$

$\begin{array}{lll}0 & 3.1 \\ 0 & 4.4\end{array}$

4.4
4.2

footnotes at end of table.

\begin{tabular}{|c|c|c|c|c|c|}
\hline 23 & 62 & 19 & 15 & 28 & 4.9 \\
\hline 61 & 24 & 11 & 12 & 1 & 6.9 \\
\hline 79 & 6 & $w$ & W & 0 & 9.3 \\
\hline 43 & 42 & 41 & . & 1 & 7.3 \\
\hline$w$ & $w$ & 0 & w & 0 & 9.3 \\
\hline W & $w$ & $w$ & 0 & 0 & 9.6 \\
\hline$w$ & $w$ & $w$ & $w$ & 0 & 11.8 \\
\hline 53 & 33 & W & 27 & $w$ & 7.2 \\
\hline W & $w$ & $w$ & $w$ & 0 & 7.3 \\
\hline 53 & 32 & $w$ & $w$ & 0 & 6.1 \\
\hline W & W & w & $w$ & 0 & 10.0 \\
\hline 67 & 18 & 9 & 8 & 1 & 7.7 \\
\hline$w$ & $w$ & $w$ & $w$ & w & 11.4 \\
\hline 84 & 1 & 1 & 1 & 0 & 11.8 \\
\hline
\end{tabular}

See footnotes at end of table. 
Table A40. Total Inputs of Energy for Heat, Power, and Electricity Generation by Energy Management Program Sponsorship, Industry Group, Selected Industries, and Type of Energy Management Program, 1991: (Continued)

(Estimates in Trillion Btu)

\begin{tabular}{|c|c|c|c|c|c|c|c|}
\hline \multirow[b]{2}{*}{$\begin{array}{l}\text { SIC } \\
\text { Code: }\end{array}$} & \multirow[b]{2}{*}{$\begin{array}{l}\text { Industry Groups } \\
\text { and industry }\end{array}$} & \multirow[b]{2}{*}{$\begin{array}{c}\text { No Energy } \\
\text { Management } \\
\text { Program }\end{array}$} & \multicolumn{4}{|c|}{ Type of Sponsorship of Management Programs (1989 through 1991) } & \multirow{3}{*}{$\begin{array}{l}\text { RSE } \\
\text { Row } \\
\text { Factors }\end{array}$} \\
\hline & & & $\begin{array}{l}\text { Any Type of } \\
\text { Sponsorship }\end{array}$ & $\begin{array}{l}\text { Utility/Supplier } \\
\text { Sponsored } \\
\text { Involvement }\end{array}$ & $\begin{array}{c}\text { Only Own or } \\
\text { Other Third Party } \\
\text { Sponsorship }\end{array}$ & $\begin{array}{l}\text { Both Types of } \\
\text { Sponsorship }\end{array}$ & \\
\hline & RSE Column Factors: & 0.6 & 0.8 & 1.4 & 1.0 & 1.5 & \\
\hline
\end{tabular}

3241

Cement, Hydraulic

Participation in One or More of the Following Types of

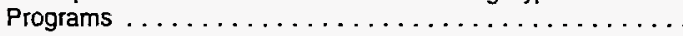

Energy Audits . . . . . . . . . . . . . . . .

Direct Electricity Load Control . . . . . . . . . . . .

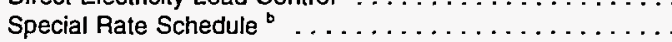

Standby Generation Program . . . . . . . . . . . .

Equipment Rebate(s) . . . . . . . . . . . . . . . .

Equipment Installation or Retrofit for the Primary Purpose of Improving Energy Efficiency Affecting:

Steam Production ${ }^{\circ} \ldots \ldots \ldots \ldots \ldots \ldots \ldots \ldots \ldots$

Direct/lndirect Process Heating . . . . . . . . . . . . . .

Direct Process Cooling/Refrigeration . . . . . . . . . . . .

Direct Machine Drive ${ }^{d} . \ldots \ldots \ldots \ldots \ldots \ldots \ldots \ldots \ldots$

Facility Heating, Ventilation, and Air Conditioning .......

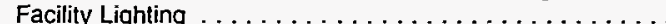

Equipment Installation/Retrofit for the Primary Purpose of

Using a Different Energy Source ${ }^{\circ} \ldots \ldots \ldots \ldots \ldots \ldots \ldots$

Other'

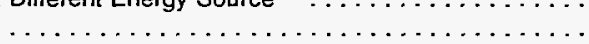

Lime

3274

Participation in One or More of the Following Types of

Programs . . . . . . . . . . . . . . . . . .

Energy Audits . . . . . . . . . . . . . . . . .

Direct Electricity Load Control $\ldots \ldots \ldots \ldots \ldots \ldots \ldots \ldots$

Special Rate Schedule ${ }^{b} \ldots \ldots \ldots \ldots \ldots \ldots \ldots \ldots \ldots$

Standby Generation Program . . . . . . . . . . . . . .

Equipment Rebate(s)

Equipment Installation or Retrofit for the Primary Purpose of

Improving Energy Efficiency Affecting:

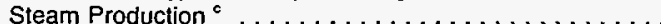

Direct/ndirect Process Heating . . . . . . . . . . . .

Direct Process Cooling/Refrigeration . . . . . . . . . .

Direct Machine Drive ${ }^{d} \ldots \ldots \ldots \ldots \ldots \ldots \ldots$. . . . . .

Facility Heating, Ventilation, and Air Conditioning $\ldots \ldots$.

Facilıty Lughting . . . . . . . . . . . . . . . . .

Equipment Installation/Retroft for the Primary Purpose of

Using a Different Energy Source ${ }^{\bullet} \ldots \ldots \ldots \ldots \ldots \ldots$

Other:

. $\ldots \ldots \ldots \ldots$

Mineral Wool

Participation in One or More of the Following Types of

Programs . . . . . . . . . . . . . . . . . . .

Energy Audits $\ldots \ldots \ldots \ldots \ldots \ldots \ldots \ldots \ldots \ldots \ldots$

Direct Electricity Load Control $\ldots \ldots \ldots \ldots \ldots \ldots \ldots \ldots$

Special Rate Schedule ${ }^{b} \ldots \ldots \ldots \ldots \ldots \ldots \ldots \ldots$

Standby Generation Program . . . . . . . . . . . . . . .

Equipment Rebate(s)

Equipment Installation or Retrofit for the Primary Pumpose of

Improving Energy Efficiency Affecting:

Steam Production ${ }^{c}$

Direct/Indirect Process Heating $\ldots \ldots \ldots \ldots \ldots \ldots$

Direct Process Cooling/Refrigeration . . . . . . . . . . .

Direct Machine Drive ${ }^{d} \ldots \ldots \ldots \ldots \ldots \ldots \ldots \ldots$

Facility Heating, Ventilation, and Air Conditioning ......

Facility Lighting . . . . . . . . . . . . . . . . . . . . .

Equipment Installation/Retrofit for the Primary Purpose of

Using a Different Energy Source ${ }^{\circ} \ldots \ldots \ldots \ldots \ldots \ldots$.

Other'

$\ldots \ldots \ldots \ldots \ldots \ldots \ldots$

$\begin{array}{rr}86 & 243 \\ 201 & 128 \\ 185 & 144 \\ 139 & 189 \\ 317 & 12 \\ 292 & 37 \\ & \\ 311 & \\ 291 & 18 \\ 326 & 38 \\ 175 & \mathrm{Q} \\ 275 & 153 \\ 200 & 53 \\ & 129 \\ 284 & \\ W & 45 \\ & W\end{array}$

52
26
29
138
0
26

$W$
$W$
0
0
18
$W$
17

0
0

48

90

100

21

Q

12.5

12.7

19.7

13.4

$\begin{array}{rr}44 & 0 \\ 101 & 16 \\ 74 & 0 \\ 105 & 12 \\ 117 & 0 \\ W & W\end{array}$

3
4
$W$

Q

12

16

12
0

W

18.9

38

Q

104
$W$

101

15.8

12.7
12.3

17.6

12.4

45
$W$

$0 \quad 20.1$

See footnotes at end of table. 
Table A40. Total Inputs of Energy for Heat, Power, and Electricity Generation by Energy Management Program Sponsorship, Industry Group, Selected Industries, and Type of Energy Management Program, 1991: (Continued)

(Estimates in Trillion Btu)

\begin{tabular}{|c|c|c|c|c|c|c|c|}
\hline \multirow[b]{2}{*}{$\begin{array}{l}\operatorname{SIC} \\
\operatorname{Cod\theta ^{\circ }}\end{array}$} & \multirow[b]{2}{*}{$\begin{array}{l}\text { Industry Groups } \\
\text { and Industry }\end{array}$} & \multirow[b]{2}{*}{$\begin{array}{c}\text { No Energy } \\
\text { Management } \\
\text { Program }\end{array}$} & \multicolumn{4}{|c|}{ Type of Sponsorship of Management Programs (1989 through 1991) } & \multirow{3}{*}{$\begin{array}{l}\text { RSE } \\
\text { Row } \\
\text { Factors }\end{array}$} \\
\hline & & & $\begin{array}{l}\text { Any Type of } \\
\text { Sponsorship }\end{array}$ & $\begin{array}{l}\text { Only } \\
\text { Utility/Supplier } \\
\text { Sponsored } \\
\text { Involvement }\end{array}$ & $\begin{array}{c}\text { Only Own or } \\
\text { Other Third Party } \\
\text { Sponsorship }\end{array}$ & $\begin{array}{l}\text { Both Types of } \\
\text { Sponsorship }\end{array}$ & \\
\hline & ;olumn Factors: & 0.6 & 0.8 & 1.4 & 1.0 & 1.5 & \\
\hline
\end{tabular}

33

PRIMARY METAL INDUSTRIES

Participation in One or More of the Following Types of

Programs . . . . . . . . . . . . . . . . . .

Energy Audits . . . . . . . . . . . . . . . . .

Direct Electricity Load Control $\ldots \ldots \ldots \ldots \ldots \ldots \ldots \ldots$

Special Rate Schedule ${ }^{\mathrm{b}} \ldots \ldots \ldots \ldots \ldots \ldots \ldots \ldots \ldots$

Standby Generation Program . . . . . . . . . . . . .

Equipment Rebate(s) . . . . . . . . . . . . . . . . . .

Equipment Installation or Retrofit for the Primary Purpose of Improving Energy Efficiency Affecting:

Steam Production ${ }^{c} \ldots \ldots \ldots \ldots \ldots \ldots \ldots \ldots \ldots$

Direct/lndirect Process Heating . . . . . . . . . . . .

Direct Process Cooling/Refrigeration . . . . . . . . . .

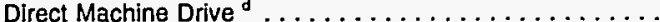

Facility Heating, Ventilation, and Air Conditioning $\ldots \ldots$.

Facility Lighting $\ldots \ldots \ldots \ldots \ldots \ldots \ldots \ldots \ldots$

Equipment Installation/Retrofit for the Primary Purpose of

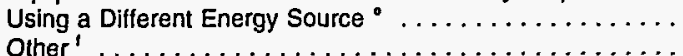

0.6

0.8

$\begin{array}{rr}351 & 1,941 \\ 914 & 1,378 \\ 1,028 & 1,264 \\ 804 & 1,488 \\ 1,987 & 305 \\ 2,249 & 43 \\ & \\ 1,323 & 969 \\ 1,018 & 1,274 \\ 2,018 & 274 \\ 1,474 & 818 \\ 1,514 & 779 \\ 926 & 1,366 \\ & \\ 1,861 & 431 \\ 2,205 & 88\end{array}$
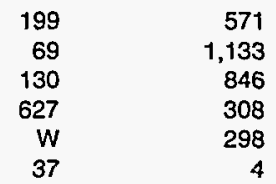

1,171

176

288

553

308
298
4

$\mathrm{Q} \quad 9.0$

3312

Blast Furnaces and Steel Mills

Participation in One or More of the Following Types of

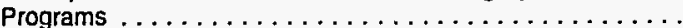

Energy Audits . . . . . . . . . . . . . . . . .

Direct Electricity Load Control $\ldots \ldots \ldots \ldots \ldots \ldots \ldots \ldots$

Special Rate Schedule ${ }^{\circ} \ldots \ldots \ldots \ldots \ldots \ldots \ldots \ldots$

Standby Generation Program . . . . . . . . . . . . . .

Equipment Rebate(s) $\ldots \ldots \ldots \ldots \ldots \ldots \ldots \ldots \ldots$

Equipment Installation or Retrofit for the Primary Purpose of

Improving Energy Efficiency Affecting:

Steam Production ${ }^{e} \ldots \ldots \ldots \ldots \ldots \ldots \ldots \ldots \ldots$

Dlrect/Indirect Process Heating . . . . . . . . . . . . .

Direct Process Cooling/Refrigeration . . . . . . . . .

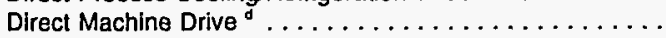

Facillty Heating, Ventilation, and Air Conditioning ......

Facility Lighting . . . . . . . . . . . . . . . .

Equipment Installation/Retrofit for the Primary Purpose of

Using a Different Energy Source ${ }^{\circ} \ldots \ldots \ldots \ldots \ldots \ldots \ldots$

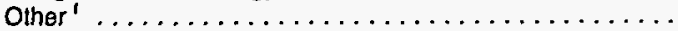

Electrometalurgical Products

Participation in One or More of the Following Types of

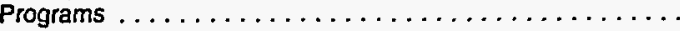

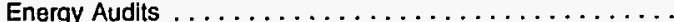

Direct Electricity Load Control . . . . . . . . . . . .

Special Rate Schedule ${ }^{b} \ldots \ldots \ldots \ldots \ldots \ldots \ldots \ldots$

Standby Generation Program . . . . . . . . . . . . . . .

Equipment Rebate(s) . . . . . . . . . . . . . . . . .

Equipment Installation or Retrofit for the Primary Purpose of Improving Energy Efficiency Affecting:

Steam Production $^{c} \ldots \ldots \ldots \ldots \ldots \ldots \ldots \ldots$

Direct/lndlect Process Heating . . . . . . . . . . . .

Direct Process Cooling/Refrigeration . . . . . . . . . . .

Dlrect Machine Drive ${ }^{\circ} \ldots \ldots \ldots \ldots \ldots \ldots \ldots \ldots \ldots$

Facility Heating, Ventilation, and Air Conditioning ......

Facility Lighting . . . . . . . . . . . . . . . . . . . . .

Equipment Installation/Retrofit for the Primary Purpose of

Using a Different Energy Source ${ }^{\circ} \ldots \ldots \ldots \ldots \ldots \ldots \ldots$

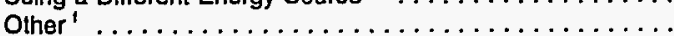

1,279

1,560

$$
\begin{array}{r}
1,444 \\
1,109 \\
1,032 \\
1,169 \\
290 \\
8
\end{array}
$$

662

489

$W$
893

854

392

1,172

1,172
$W$

907
1,080
$W$
676
715
1,177
396
$W$

893
944
273
633
714
1,124

$W$
52

See footnotes at end of table. 


\section{Table A40. Total Inputs of Energy for Heat, Power, and Electricity Generation by Energy Management Program Sponsorship, Industry Group, Selected Industries, and Type of Energy Management Program, 1991: (Continued)}

(Estimates in Trillion Btu)

\begin{tabular}{|c|c|c|c|c|c|c|c|}
\hline \multirow[b]{2}{*}{$\begin{array}{c}\text { SIC } \\
\text { Code }^{*} \\
\end{array}$} & \multirow[b]{2}{*}{$\begin{array}{l}\text { Industry Groups } \\
\text { and Industry }\end{array}$} & \multirow[b]{2}{*}{$\begin{array}{c}\text { No Energy } \\
\text { Management } \\
\text { Program }\end{array}$} & \multicolumn{4}{|c|}{ Type of Sponsorship of Management Programs (1989 through 1991) } & \multirow{3}{*}{$\begin{array}{c}\text { RSE } \\
\text { Row } \\
\text { Factors }\end{array}$} \\
\hline & & & $\begin{array}{l}\text { Any Type of } \\
\text { Sponsorship }\end{array}$ & $\begin{array}{c}\text { Only } \\
\text { Utility/Supplier } \\
\text { Sponsored } \\
\text { Involvement } \\
\end{array}$ & $\begin{array}{c}\text { Only Own or } \\
\text { Other Third Party } \\
\text { Sponsorship }\end{array}$ & $\begin{array}{l}\text { Both Types of } \\
\text { Sponsorship }\end{array}$ & \\
\hline & RSE Column Factors: & 0.6 & 0.8 & 1.4 & 1.0 & 1.5 & \\
\hline
\end{tabular}

3321

Gray and Ductile Iron Foundries

Participation in One or More of the Following Types of

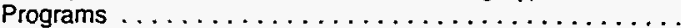

Energy Audits $\ldots \ldots \ldots \ldots \ldots \ldots \ldots \ldots \ldots \ldots \ldots$

Direct Electricity Load Control $\ldots \ldots \ldots \ldots \ldots \ldots \ldots$.

Special Rate Schedule ${ }^{b} \ldots \ldots \ldots \ldots \ldots \ldots \ldots \ldots$.

Standby Generation Program . . . . . . . . . . . . . . . .

Equipment Rebate(s) . . . . . . . . . . . . . . . . . . . . .

Equipment Installation or Retrofit for the Primary Purpose of Improving Energy Efficiency Affecting:

Steam Production ${ }^{\circ}$...

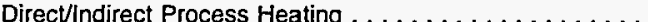

Direct Process Cooling/Refrigeration ...............

Direct Machine Drive d .....................

Facility Heating, Ventilation, and Air Conditioning .......

Facility Lighting $\ldots \ldots \ldots \ldots \ldots \ldots \ldots \ldots$

Equipment Installation/Retrofit for the Primary Purpose of

Using a Different Energy Source ${ }^{\circ} \ldots \ldots \ldots \ldots \ldots \ldots$

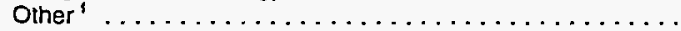

\section{Primary Copper}

Participation in One or More of the Following Types of

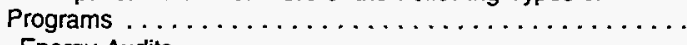

Energy Audits . . . . . . . . . . . . . . . . .

Direct Electricity Load Control $\ldots \ldots \ldots \ldots \ldots \ldots \ldots$

Special Rate Schedule ${ }^{b} \ldots \ldots \ldots \ldots \ldots \ldots \ldots \ldots$.

Standby Generation Program . . . . . . . . . . . . . . .

Equipment Rebate(s) . ....................

Equipment installation or Retrofit for the Primary Purpose of Improving Energy Efficiency Affecting:

Steam Production ${ }^{\circ} \ldots \ldots \ldots \ldots \ldots \ldots \ldots \ldots \ldots \ldots$

Direct/Indirect Process Heating ...................

Direct Process Cooling/Rerrigeration . . . . . . . . . . . . .

Direct Machine Drive ${ }^{d} . \ldots \ldots \ldots \ldots \ldots \ldots \ldots \ldots$

Facility Heating, Ventilation, and Air Conditioning .......

Facility Lighting . . . . . . . . . . . . . . . . . . . .

Equipment Installation/Retrofit for the Primary Purpose of

Using a Different Energy Source ${ }^{\circ} \ldots \ldots \ldots \ldots \ldots \ldots$.

Other ${ }^{\prime}$

16
31
37
33
70
69

73
63
$W$
52
60
43
62
68

$\begin{array}{rr}58 & 11 \\ 43 & 6 \\ 37 & 9 \\ 41 & 32 \\ 4 & 1 \\ 5 & 5 \\ & \\ 1 & 0 \\ 11 & 1 \\ W & 0 \\ 22 & 2 \\ 14 & 1 \\ 32 & W \\ 12 & \\ 6 & \end{array}$

Primary Aluminum

Participation in One or More of the Following Types of

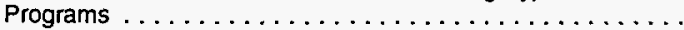

Energy Audits . . . . . . . . . . . . . . . . .

Direct Electricity Load Control ...................

Special Rate Schedule ${ }^{b} \ldots \ldots \ldots \ldots \ldots \ldots \ldots \ldots \ldots$

Standby Generation Program . . . . . . . . . . . . .

Equipment Rebate(s) . . . . . . . . . . . . . . . .

Equipment Installation or Retrofit for the Primary Purpose of

Improving Energy Efficiency Affecting:

Steam Production ${ }^{\circ}$

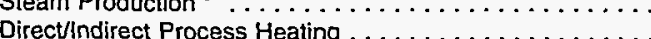

Direct Process Cooling/Refrigeration . . . . . . . . . . . .

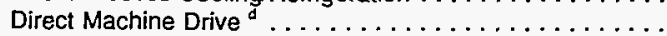

Facility Heating, ventilation, and Air Conditioning ..........

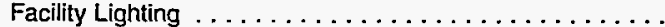

Equipment Installation/Retrofit for the Primary Purpose of

Using a Different Energy Source ${ }^{\circ} \ldots \ldots \ldots \ldots \ldots \ldots$.

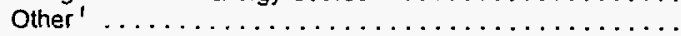

\begin{tabular}{rrrrrr}
$W$ & $W$ & $W$ & 94 & 72 & 2.9 \\
169 & 84 & $W$ & 49 & $W$ & 3.7 \\
153 & 99 & $W$ & 76 & $W$ & 3.7 \\
126 & 127 & 83 & 43 & 0 & 3.6 \\
252 & 0 & 0 & 0 & 0 & 3.6 \\
252 & 0 & 0 & 0 & 0 & 3.6 \\
& & & & & \\
$W$ & $W$ & 0 & $W$ & 0 & 4.8 \\
143 & 110 & $W$ & 89 & $W$ & 3.7 \\
$W$ & $W$ & 0 & $W$ & 0 & 5.4 \\
209 & 43 & 0 & 43 & 0 & 4.1 \\
252 & 0 & 0 & 0 & 0 & 3.6 \\
203 & 49 & 0 & $W$ & $W$ & 3.8 \\
& & 0 & & & \\
252 & 0 & 0 & 0 & 0 & 3.6 \\
196 & 56 & $W$ & $W$ & 0 & 3.5 \\
\hline
\end{tabular}


Table A40. Total Inputs of Energy for Heat, Power, and Electricity Generation by Energy Management Program Sponsorship, Industry Group, Selected Industries, and Type of Energy Management Program, 1991: (Continued)

(Estimates in Trillion Btu)

\begin{tabular}{|c|c|c|c|c|c|c|c|}
\hline \multirow[b]{2}{*}{$\begin{array}{l}\text { SIC } \\
\text { Code" }\end{array}$} & \multirow[b]{2}{*}{$\begin{array}{l}\text { Industry Groups } \\
\text { and Industry }\end{array}$} & \multirow[b]{2}{*}{$\begin{array}{c}\text { No Energy } \\
\text { Management } \\
\text { Program }\end{array}$} & \multicolumn{4}{|c|}{ Type of Sponsorship of Management Programs (1989 through 1991) } & \multirow{3}{*}{$\begin{array}{l}\text { RSE } \\
\text { Row } \\
\text { Factors }\end{array}$} \\
\hline & & & $\begin{array}{l}\text { Any Type of } \\
\text { Sponsorship }\end{array}$ & $\begin{array}{l}\text { Utility/Supplier } \\
\text { Sponsored } \\
\text { Involvement }\end{array}$ & $\begin{array}{c}\text { Only Own or } \\
\text { Other Third Party } \\
\text { Sponsorship }\end{array}$ & $\begin{array}{l}\text { Both Types of } \\
\text { Sponsorship }\end{array}$ & \\
\hline & Column Factors: & 0.6 & 0.8 & 1.4 & 1.0 & 1.5 & \\
\hline
\end{tabular}

3339

Primary Nonferrous Metals, nec

Participation in One or More of the Following Types of

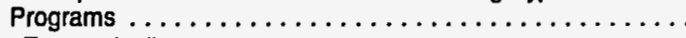

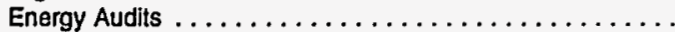

Direct Electricity Load Control $\ldots \ldots \ldots \ldots \ldots \ldots \ldots$

Special Rate Schedule ${ }^{\triangleright} \ldots \ldots \ldots \ldots \ldots \ldots \ldots \ldots \ldots$

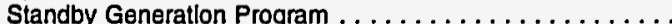

Equlpment Rebate(s) . . . . . . . . . . . . . . . .

Equipment Installatlon or Retrofit for the Primary Purpose of

Improving Energy Efficiency Affecting:

Steam Productlon ${ }^{2} \ldots \ldots \ldots \ldots \ldots \ldots \ldots \ldots \ldots$

Dlrect/indlrect Process Heating . . . . . . . . . . . . . . .

Dlrect Process Cooling/Retrigeration . . . . . . . . . . .

Dlrect Machine Drive ${ }^{d}$......................

Facility Heating, Ventilation, and Air Conditioning ......

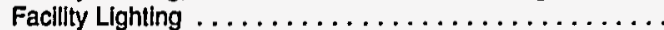

Equipment Installation/Retrofit for the Primary Purpose of

Using a Dlfierent Energy Source ${ }^{\circ} \ldots \ldots \ldots \ldots \ldots \ldots$

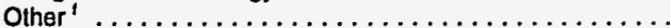

Aluminum Sheet, Plate, and Foll

Particlpation in One or More of the Following Types of

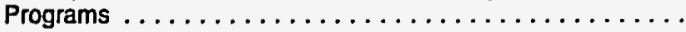

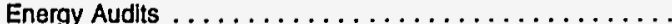

Direct Electricily Load Control . .................

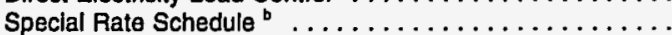

Standby Generation Program . . . . . . . . . . . . . . . .

Equlpment Rebate(s) .......................

Equipment Installation or Retrofit for the Primary Purpose of Improving Energy Efficiency Affecting:

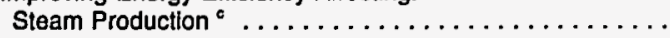

Direct/ndirect Process Heating . . . . . . . . . . . . . .

Direct Process Cooling/Refrigeration . . . . . . . . . . . . .

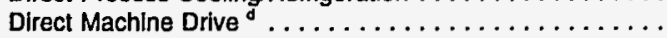

Facility Heating, Ventllation, and Air Conditioning ......

Faclity Lighting . . . . . . . . . . . . . . . . . . .

Equipment Installation/Retrofit for the Primary Purpose of

Using a Different Energy Source ${ }^{\circ}$. . . . . . . . . . . .

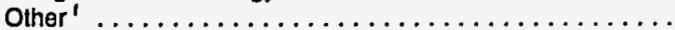

\section{FABRICATED METAL PRODUCTS}

Participation in One or More of the Following Types of

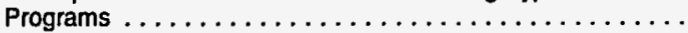

Energy Audits . . . . . . . . . . . . . . . . . .

Direct Electricity Load Control . . . . . . . . . . . . . . .

Speclal Rate Schedule ${ }^{b} \ldots \ldots \ldots \ldots \ldots \ldots \ldots \ldots \ldots \ldots$

Standby Generation Program . . . . . . . . . . . . .

Equipment Rebate(s) . . . . . . . . . . . . . . . . .

Equipment Installation or Retrofit for the Primary Purpose of

Improving Energy Efficiency Affecting:

Steam Production ${ }^{c} \ldots \ldots \ldots \ldots \ldots \ldots \ldots \ldots \ldots$

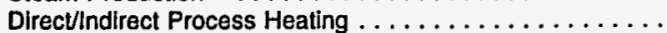

Direct Process Cooling/Refrigeration . . . . . . . . . . .

Direct Machine Drive ${ }^{\mathrm{J}} \ldots \ldots \ldots \ldots \ldots \ldots \ldots \ldots$.

Facility Heating, Ventilation, and Air Conditioning ......

Facility Lighting $\ldots \ldots \ldots \ldots \ldots \ldots \ldots \ldots \ldots$

Equipment Installation/Retrofit for the Primary Purpose of

Using a Different Energy Source ${ }^{\circ} \ldots \ldots \ldots \ldots \ldots \ldots \ldots$

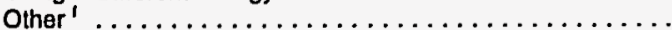

$\begin{array}{lr}26 & 16 \\ W & W \\ 40 & \\ 32 & 10 \\ 42 & \\ 42 & \end{array}$

16
$W$
2
10
0
t

W

38

42

41

w

42

42

$\begin{array}{rrr}11 & W & 3.6 \\ W & \vdots & 4.8 \\ 2 & \vdots & 4.2 \\ W & 0 & 4.3 \\ 0 & 0 & 5.4 \\ 0 & 0 & 16.3\end{array}$

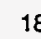

32

53

$\begin{array}{rrrrrr}156 & 148 & 29 & 55 & 65 & 7.0 \\ 203 & 101 & 29 & 50 & 22 & 7.7 \\ 250 & 55 & 15 & 35 & 5 & 9.3 \\ 231 & 73 & 48 & 13 & 12 & 8.3 \\ 296 & 9 & Q & 7 & 0 & 14.2 \\ 287 & 18 & 15 & 1 & 1 & 15.6 \\ & & & & & \\ 270 & 34 & 4 & 29 & 2 & 12.1 \\ 268 & 37 & 7 & 29 & 1 & 11.4 \\ 287 & 18 & 2 & 15 & 1 & 16.3 \\ 251 & 54 & 10 & 40 & 4 & 9.7 \\ 243 & 62 & 10 & 50 & 3 & 10.9 \\ 214 & 91 & 19 & 59 & 12 & 8.3 \\ 289 & 16 & 3 & 10 & 3 & 14.1 \\ 300 & 4 & - & 4 & - & 17.2\end{array}$

See footnotes at end of table.
} 
Table A40. Total Inputs of Energy for Heat, Power, and Electricity Generation by Energy Management Program Sponsorship, Industry Group, Selected Industries, and Type of Energy Management Program, 1991: (Continued)

(Estimates in Trillion Btu)

\begin{tabular}{|c|c|c|c|c|c|c|c|}
\hline \multirow[b]{2}{*}{$\begin{array}{l}\text { SIC } \\
\text { Code }\end{array}$} & \multirow[b]{2}{*}{$\begin{array}{l}\text { Industry Groups } \\
\text { and Industry }\end{array}$} & \multirow[b]{2}{*}{$\begin{array}{l}\text { No Energy } \\
\text { Management } \\
\text { Program }\end{array}$} & \multicolumn{4}{|c|}{ Type of Sponsorship of Management Programs (1989 through 1991) } & \multirow{3}{*}{$\begin{array}{l}\text { RSE } \\
\text { Row } \\
\text { Factors }\end{array}$} \\
\hline & & & $\begin{array}{l}\text { Any Type of } \\
\text { Sponsorship }\end{array}$ & $\begin{array}{l}\text { Utility/Supplier } \\
\text { Sponsored } \\
\text { Involvement }\end{array}$ & $\begin{array}{c}\text { Only Own or } \\
\text { Other Third Party } \\
\text { Sponsorship }\end{array}$ & $\begin{array}{l}\text { Both Types of } \\
\text { Sponsorship }\end{array}$ & \\
\hline & RSE Column Factors: & 0.6 & 0.8 & 1.4 & 1.0 & 1.5 & \\
\hline
\end{tabular}

Participation in One or More of the Following Types of

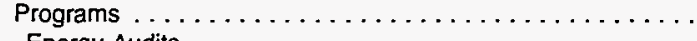

Energy Audits . . . . . . . . . . . . . . . . . .

Direct Electricity Load Control . . . . . . . . . . . . . .

Special Rate Schedule ${ }^{\circ} \ldots \ldots \ldots \ldots \ldots \ldots \ldots \ldots$

Standby Generation Program . . . . . . . . . . . . . .

Equipment Rebate(s) . . . . . . . . . . . . . . . . . . . . .

Equipment Installation or Retrofit for the Primary Purpose of

Improving Energy Efficiency Affecting:

Steam Production ${ }^{c} \ldots \ldots \ldots \ldots \ldots \ldots \ldots \ldots \ldots \ldots$

Direct/ndirect Process Heating . . . . . . . . . . . . . . .

Direct Process Cooling/Refrigeration . . . . . . . . . . .

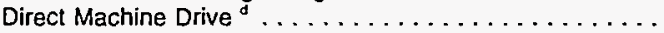

Facility Heating, Ventilation, and Air Conditioning ......

Facility Lighting $\ldots \ldots \ldots \ldots \ldots \ldots \ldots \ldots \ldots$

Equipment Installation/Retrofit for the Primary Purpose of

Using a Different Energy Source ${ }^{\circ} \ldots \ldots \ldots \ldots \ldots \ldots \ldots$

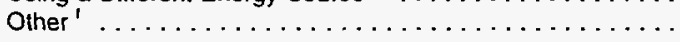

Computer and Office Equipment

Participation in One or More of the Following Types of

Programs . . . . . . . . . . . . . . . . .

Energy Audits . . . . . . . . . . . . . . . .

Direct Electricity Load Control $\ldots \ldots \ldots \ldots \ldots \ldots \ldots \ldots$

Special Rate Schedule ${ }^{\circ} \ldots \ldots \ldots \ldots \ldots \ldots \ldots \ldots \ldots$

Standby Generation Program . . . . . . . . . . . . .

Equipment Rebate(s) . . . . . . . . . . . . . . . .

Equipment Installation or Retrofit for the Primary Purpose of

Improving Energy Effıciency Alfecting:

Sieam Production ${ }^{c} \ldots \ldots \ldots \ldots \ldots \ldots \ldots \ldots$

Direct/Indirect Process Heating . . . . . . . . . . . . . .

Direct Process Cooling/Refrigeration . . . . . . . . . . .

Direct Machine Drive ${ }^{d} \ldots \ldots \ldots \ldots \ldots \ldots \ldots \ldots \ldots$

Facility Heating, Ventilation, and Air Conditioning . . . . .

Facility Lighting . . . . . . . . . . . . . . . . . . . . . . . .

Equipment Installation/Retrofit for the Primary Purpose of

Using a Different Energy Source ${ }^{\circ} \ldots \ldots \ldots \ldots \ldots \ldots \ldots$

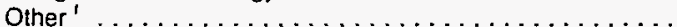

ELECTRONIC and OTHER ELECTRIC EQUIPMENT

Participation in One or More of the Following Types of

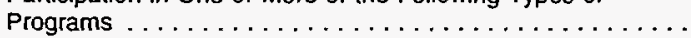

Energy Audits . . . . . . . . . . . . . . . . .

Direct Electricity Load Control $\ldots \ldots \ldots \ldots \ldots \ldots \ldots$

Special Rate Schedule ${ }^{\circ} \ldots \ldots \ldots \ldots \ldots \ldots \ldots \ldots \ldots$

Standby Generation Program . . . . . . . . . . . . .

Equipment Rebate(s) . . . . . . . . . . . . . . . . . .

Equipment Installation or Retrofit for the Primary Purpose of Improving Energy Efficiency Affecting:

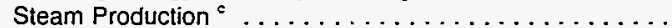

Direct/lndirect Process Heating . . . . . . . . . . . . .

Direct Process Cooling/Refrigeration . . . . . . . . . . . .

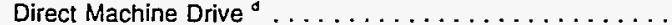

Facility Heating, Ventilation, and Air Conditioning ......

Facility Lighting $\ldots \ldots \ldots \ldots \ldots \ldots \ldots \ldots$

Equipment Installation/Retrofit for the Primary Purpose of

Using a Different Energy Source ${ }^{\circ} \ldots \ldots \ldots \ldots \ldots \ldots \ldots$

Other'

$\ldots \ldots \ldots \ldots \ldots$

107
149
176
164
219
214

See footnotes at end of table. 
Table A40. Total Inputs of Energy for Heat, Power, and Electricity Generation by Energy Management Program Sponsorship, Industry Group, Selected Industries, and Type of Energy Management Program, 1991: (Continued)

(Estimates in Trillion Btu)

\begin{tabular}{|c|c|c|c|c|c|c|c|}
\hline \multirow[b]{2}{*}{$\begin{array}{c}\text { SIC } \\
\text { Code }^{\circ}\end{array}$} & \multirow[b]{2}{*}{$\begin{array}{l}\text { Industry Groups } \\
\text { and Industry }\end{array}$} & \multirow[b]{2}{*}{$\begin{array}{c}\text { No Energy } \\
\text { Management } \\
\text { Program }\end{array}$} & \multicolumn{4}{|c|}{ Type of Sponsorship of Management Programs (1989 through 1991) } & \multirow{3}{*}{$\begin{array}{c}\text { RSE } \\
\text { Row } \\
\text { Factors }\end{array}$} \\
\hline & & & $\begin{array}{l}\text { Any Type of } \\
\text { Sponsorship }\end{array}$ & $\begin{array}{l}\text { Utility/Supplier } \\
\text { Sponsored } \\
\text { Involvement }\end{array}$ & \begin{tabular}{|c|} 
Only Own or \\
Other Third Party \\
Sponsorship
\end{tabular} & $\begin{array}{c}\text { Both Types of } \\
\text { Sponsorship }\end{array}$ & \\
\hline & RSE Column Factors: & 0.6 & 0.8 & 1.4 & 1.0 & 1.5 & \\
\hline
\end{tabular}

37

TRANSPORTATION EQUIPMENT

Participation in One or More of the Following Types of

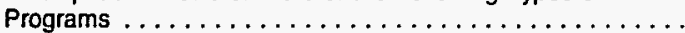

Energy Audits $\ldots \ldots \ldots \ldots \ldots \ldots \ldots \ldots \ldots \ldots$

Direct Electricity Load Control $\ldots \ldots \ldots \ldots \ldots \ldots \ldots \ldots$

Special Rate Schedule ${ }^{\circ} \ldots \ldots \ldots \ldots \ldots \ldots \ldots \ldots \ldots$

Standby Generation Program . . . . . . . . . . . . .

Equipment Rebate(s)

Equipment Installation or Retrofit for the Primary Purpose of

Improving Energy Efficiency Affecting:

Steam Production ${ }^{c} \ldots \ldots \ldots \ldots \ldots \ldots \ldots \ldots \ldots$

Direct/Indirect Process Heating . . . . . . . . . . . .

Direct Process Cooling/Refrigeration . . . . . . . . . .

Direct Machine Drive ${ }^{d}$. . . . . . . . . . . . . . . . . . .

Facillty Heating, Ventilation, and Air Conditioning ......

Facility Lighting . . . . . . . . . . . . . . .

Equipment Installation/Retrofit for the Primary Purpose of

Using a Different Energy Source ${ }^{0} \ldots \ldots \ldots \ldots \ldots \ldots \ldots$

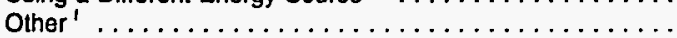

Motor Vehicles and Car Bodies

3711

One or More of the Following Types of

Participation in One or More of the Following Types of
Programs $\ldots \ldots \ldots \ldots \ldots \ldots \ldots \ldots \ldots \ldots \ldots \ldots \ldots \ldots \ldots$

Energy Audits . . . . . . . . . . . . . . . . . .

Direct Electricity Load Control . . . . . . . . . . . .

Special Rate Schedule ${ }^{b} \ldots \ldots \ldots \ldots \ldots \ldots \ldots \ldots \ldots$

Standby Generation Program . . . . . . . . . . . . . .

Equipment Rebate(s) . . . . . . . . . . . . . . . . . .

Equipment Installation or Retrofit for the Primary Pupose of Improving Energy Efficiency Affecting:

Steam Production $^{c} \ldots \ldots \ldots \ldots \ldots \ldots \ldots \ldots \ldots$

Direct/lndirect Process Heating . . . . . . . . . . . . . .

Direct Process Cooling/Refrigeration ..............

Direct Machine Drive ${ }^{\mathrm{d}} \ldots \ldots \ldots \ldots \ldots \ldots \ldots \ldots$

Facility Heating, Ventilation, and Air Conditioning $\ldots \ldots \ldots$

Facility Lighting . . . . . . . . . . . . . . . . . .

Equipment Installation/Retrofit for the Primary Purpose of

Using a Different Energy Source ${ }^{\bullet} \ldots \ldots \ldots \ldots \ldots \ldots \ldots$

Other ${ }^{\prime}$

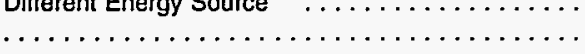

3714

Motor Vehicle Parts and Accessories

Particlpation in One or More of the Following Types of

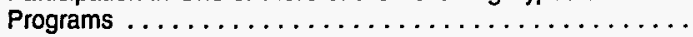

Energy Audits .........................

Direct Electricity Load Control $\ldots \ldots \ldots \ldots \ldots \ldots \ldots \ldots$

Speclal Rate Schedule ${ }^{b}, \ldots \ldots \ldots \ldots \ldots \ldots \ldots \ldots \ldots \ldots$

Standby Generation Program . . . . . . . . . . . . . . . .

Equipment Rebate(s) . . . . . . . . . . . . . . . . . .

Equipment Installation or Retrofit for the Primary Purpose of

Improving Energy Efficlency Affecting:

Steam Production ${ }^{c} \ldots \ldots \ldots \ldots \ldots \ldots \ldots \ldots$

DirectIndirect Process Heating . ...............

Direct Process Cooling/Refrigeration . . . . . . . . . .

Direct Machine Drive ${ }^{d} \ldots \ldots \ldots \ldots \ldots \ldots \ldots \ldots \ldots$

Facillty Heating, Ventilation, and Air Conditioning .......

Facillty Llghting . . . . . . . . . . . . . . . .

Equipment Installation/Retrofit for the Primary Purpose of

Using a Different Energy Source ${ }^{\circ} \ldots \ldots \ldots \ldots \ldots \ldots \ldots$.

Other $^{\prime} \ldots \ldots \ldots \ldots \ldots \ldots \ldots \ldots \ldots \ldots \ldots$

64

120

213

158

313

283

230

243

267

213

172

114

283

319
268

213

119

175

20

50

90
65

161

219

50

14

4

30

66

43

100

99

65

73

W

64

31

90

97

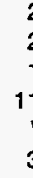

22

15

W

31

2

9
5

10

10

W

77
135
$W$
31
14
$W$


96
79
60
103
137
149

41
$W$

See footnotes at end of table. 
Table A40. Total Inputs of Energy for Heat, Power, and Electricity Generation by Energy Management Program Sponsorship, Industry Group, Selected Industries, and Type of Energy Management Program, 1991: (Continued)

(Estimates in Trillion Btu)

\begin{tabular}{|c|c|c|c|c|c|c|c|}
\hline \multirow[b]{2}{*}{$\begin{array}{l}\text { SIC } \\
\text { Code" }\end{array}$} & \multirow[b]{2}{*}{$\begin{array}{l}\text { Industry Groups } \\
\text { and Industry }\end{array}$} & \multirow[b]{2}{*}{$\begin{array}{c}\text { No Energy } \\
\text { Management } \\
\text { Program }\end{array}$} & \multicolumn{4}{|c|}{ Type of Sponsorship of Management Programs (1989 through 1991) } & \multirow{3}{*}{$\begin{array}{c}\text { RSE } \\
\text { Row } \\
\text { Factors }\end{array}$} \\
\hline & & & $\begin{array}{l}\text { Any Type of } \\
\text { Sponsorship }\end{array}$ & $\begin{array}{l}\text { Utility/Supplier } \\
\text { Sponsored } \\
\text { Involvement }\end{array}$ & \begin{tabular}{|c|} 
Only Own or \\
Other Third Party \\
Sponsorship
\end{tabular} & $\begin{array}{l}\text { Both Types of } \\
\text { Sponsorship }\end{array}$ & \\
\hline & RSE Column Factors: & 0.6 & 0.8 & 1.4 & 1.0 & 1.5 & \\
\hline
\end{tabular}

38

INSTRUMENTS and RELATED PRODUCTS

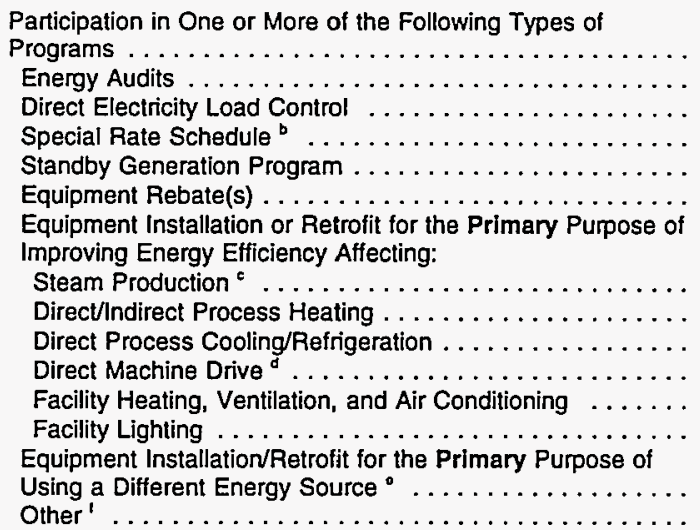

\section{Surgical and Medical Instruments}

Participation in One or More of the Following Types of

Programs . . . . . . . . . . . . . . . . . .

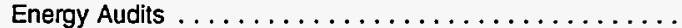

Direct Electricity Load Control $\ldots \ldots \ldots \ldots \ldots \ldots \ldots \ldots$

Special Rate Schedule ${ }^{b} \ldots \ldots \ldots \ldots \ldots \ldots \ldots \ldots \ldots \ldots$

Standby Generation Program . . . . . . . . . . . . . .

Equipment Rebate(s) . . . . . . . . . . . . . . .

Equipment Installation or Retrofit for the Primary Pupose of Improving Energy Efficiency Affecting:

Steam Production ${ }^{c} \ldots \ldots \ldots \ldots \ldots \ldots \ldots \ldots \ldots \ldots$

Direct/lndirect Process Heating . . . . . . . . . . . . .

Direct Process Cooling/Refrigeration . . . . . . . . . .

Direct Machine Drive ${ }^{\text {a }} . \ldots \ldots \ldots \ldots \ldots \ldots \ldots \ldots$

Facility Heating, Ventilation, and Air Conditioning $\ldots \ldots \ldots$

Facility Lighting . . . . . . . . . . . . . . . . . . . . . .

Equipment Installation/Retrofit for the Primary Purpose of

Using a Different Energy Source ${ }^{\circ}$

Other'

Different Energy Source ${ }^{\circ} \ldots \ldots \ldots \ldots \ldots \ldots$

See footnotes at end of table. 
Table A40. Total Inputs of Energy for Heat, Power, and Electricity Generation by Energy Management Program Sponsorship, Industry Group, Selected Industries, and Type of Energy Management Program, 1991: (Continued)

(Estimates in Trillion Btu)

\begin{tabular}{|c|c|c|c|c|c|c|c|}
\hline \multirow[b]{2}{*}{$\begin{array}{c}\operatorname{SIC} \\
\operatorname{Cod} \theta^{a}\end{array}$} & \multirow[b]{2}{*}{$\begin{array}{l}\text { Industry Groups } \\
\text { and Industry }\end{array}$} & \multirow[b]{2}{*}{$\begin{array}{l}\text { No Energy } \\
\text { Management } \\
\text { Program }\end{array}$} & \multicolumn{4}{|c|}{ Type of Sponsorship of Management Programs (1989 through 1991) } & \multirow{3}{*}{\begin{tabular}{|l} 
RSE \\
Row \\
Factors
\end{tabular}} \\
\hline & & & $\begin{array}{l}\text { Any Type of } \\
\text { Sponsorship }\end{array}$ & $\begin{array}{l}\text { Utility/Supplier } \\
\text { Sponsored } \\
\text { Involvement }\end{array}$ & $\begin{array}{c}\text { Only Own or } \\
\text { Other Third Party } \\
\text { Sponsorship }\end{array}$ & $\begin{array}{c}\text { Both Types of } \\
\text { Sponsorship }\end{array}$ & \\
\hline & RSE Column Factors: & 0.6 & 0.8 & 1.4 & 1.0 & 1.5 & \\
\hline
\end{tabular}

39

MISC. MANUFACTURING INDUSTRIES

17
22
27
25
31
30

29
29
28
28
23
22
30
31

$\begin{array}{rr}14 & 3 \\ 10 & 2 \\ 5 & \\ 6 & 5 \\ 2 & \end{array}$

3
2
1
5
2

$\begin{array}{rrr}4 & 7 & 11.2 \\ W & W & 12.3 \\ 3 & 1 & 15.2 \\ W & W & 14.6 \\ * & 0 & 24.3 \\ * & * & 19.7\end{array}$

Equlpment Rebate(s) $\ldots \ldots \ldots \ldots \ldots \ldots \ldots \ldots \ldots \ldots \ldots$ Improving Energy Efficiency Affecting:

Steam Production ${ }^{\circ} \ldots \ldots \ldots \ldots \ldots \ldots \ldots \ldots \ldots \ldots$

Dlrect/lndirect Process Heating ...................

Direct Process Cooling/Refrigeration ...............

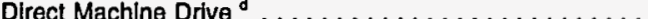

Facility Heating, Ventilation, and Air Conditioning $\ldots \ldots$.

Facllity Lighting ........................

Equipment Installation/Retrofit for the Primary Purpose of

Using a Different Energy Source ${ }^{\circ} \ldots \ldots \ldots \ldots \ldots \ldots$

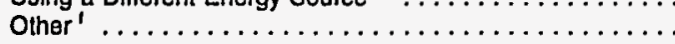

\begin{tabular}{llll}
3 & $*$ & 2 & \\
2 & $:$ & 2 & \\
4 & 1 & 3 & \\
4 & 1 & 6 & 1 \\
8 & 3 & 5 & 2 \\
9 & & 2 & 0 \\
2 & 0 & 1 & 0 \\
1 & & & \\
\hline
\end{tabular}

- See Appendices B and F for descriptions of the Standard Industrial Classification system.

- For example, interruptible or time-of-use rates.

- For example, boilers, burners, and nozzles.

¿ For example, adjustable speed drives, motors, and pumps.

- For example, electrification of a subset of the manufacturing operation.

' Included are power factor corrections, improvements in operating procedures, and other energy management programs reported by respondents.

- Estimate less than 0.5. Data are included in higher level totals.

$W=$ Withheld to avoid disclosing data for individual establishments. Data are included in higher level totals.

$\mathrm{Q}=$ Withheld because Relative Standard Error is greater than 50 percent. Data are included in higher level totals.

Notes: - To obtain a RSE percentage for any table cell, multiply the cell's corresponding RSE column and RSE row factors. - Totals may not equal sum of components because of Independent rounding. - The estimates presented in this table are for the total consumption of energy for the production of heat and power, regardless of where the energy was produced. Specifically, the estimates include the quantities of energy that were originally produced offisite and purchased by or transferred to the establishment, plus those that were produced onsite from other energy or input materials not classified as energy, or were extracted from captive (onsite) mines or wells.

- "Sponsorship" is determined by the respondent.

Source: Energy Information Adminlstration, Office of Energy Markets and End Use, Energy End Use and Integrated Statistics Division, Form ElA-846, "1991 Manufacturing Energy Consumption Survey." 
Table A41. Total Inputs of Energy for Heat, Power, and Electricity Generation by Census Region, Industry Group, Selected Industries, and Type of Energy Management Program, 1991

(Estimates in Trillion Btu)

\begin{tabular}{|c|c|c|c|c|c|c|c|}
\hline \multirow{2}{*}{$\begin{array}{l}\text { SIC } \\
\text { Code" }\end{array}$} & \multirow{2}{*}{$\begin{array}{l}\text { Industry Groups } \\
\text { and Industry }\end{array}$} & \multirow[b]{2}{*}{ Total } & \multicolumn{4}{|c|}{ Census Region } & \multirow{3}{*}{$\begin{array}{c}\text { RSE } \\
\text { Row } \\
\text { Factors }\end{array}$} \\
\hline & & & Northeast & Midwest & South & West & \\
\hline & RSE Column Factors: & 0.7 & 1.3 & 1.0 & 0.9 & 1.2 & \\
\hline
\end{tabular}

$20-39$

ALL INDUSTRY GROUPS

Participation in One or More of the Following Types of

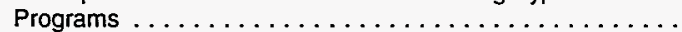

Energy Audits $\ldots \ldots \ldots \ldots \ldots \ldots \ldots \ldots \ldots \ldots \ldots$

Direct Electricity Load Control . . . . . . . . . . . .

Special Rate Schedule ${ }^{b} \ldots \ldots \ldots \ldots \ldots \ldots \ldots \ldots$

Standby Generation Program . . . . . . . . . . . . . . .

Equipment Rebate(s) . . . . . . . . . . . . . . . . . . .

Equipment Installation or Retrofit for the Primary Purpose of Improving Energy Efficiency Affecting:

Steam Production $^{c} \ldots \ldots \ldots \ldots \ldots \ldots \ldots \ldots \ldots$

Direct/lndirect Process Heating . . . . . . . . . . . . . . . . .

Direct Process Cooling/Refrigeration . . . . . . . . . . .

Direct Machine Drive ${ }^{a} \ldots \ldots \ldots \ldots \ldots \ldots \ldots \ldots \ldots$

Facility Heating, Ventilation, and Air Conditioning $\ldots .$.

Facility Lighting . . . . . . . . . . . . . . .

Equipment Installation/Retrofit for the Primary Purpose of

Using a Different Energy Source ${ }^{\circ} \ldots \ldots \ldots \ldots \ldots \ldots \ldots$

Other'

FOOD and KINDRED PRODUCTS

Participation in One or More of the Following Types of

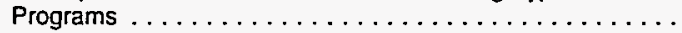

Energy Audits $\ldots \ldots \ldots \ldots \ldots \ldots \ldots \ldots \ldots \ldots \ldots . . . \ldots$

Direct Electricity Load Control $\ldots \ldots \ldots \ldots \ldots \ldots \ldots \ldots$

Special Rate Schedule ${ }^{\circ} \ldots \ldots \ldots \ldots \ldots \ldots \ldots \ldots$

Standby Generation Program $\ldots \ldots \ldots \ldots \ldots \ldots \ldots \ldots$

Equipment Rebate(s) . . . . . . . . . . . . . . . . . .

Equipment Installation or Retrofit for the Primary Purpose of

Improving Energy Efficiency Affecting:

Steam Production $^{\circ} \ldots \ldots \ldots \ldots \ldots \ldots \ldots$

Direct/lndirect Process Heating . . . . . . . . . . . .

Direct Process Cooling/Refrigeration . . . . . . . . .

Direct Machine Drive ${ }^{d}$... . . . . . . . . . . . . . . . .

Facility Heating, Ventilation, and Air Conditioning . . . . .

Facility Lighting .

Equipment Installation/Retrofit for the Primary Purpose of

Using a Different Energy Source ${ }^{\circ} \ldots \ldots \ldots \ldots \ldots \ldots \ldots$

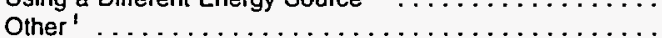

\section{0,743 \\ 7,083 \\ 5,086 \\ 6,517 \\ 1,133 \\ 783 \\ 4,757 \\ 5,146 \\ 2,212 \\ 5,354 \\ 3,424 \\ 5,405 \\ 1,693 \\ 376}

606

349

215

299

61

113

288

294

167

359

198

311

58

11

Meat Packing Plants

Participation in One or More of the Following Types of

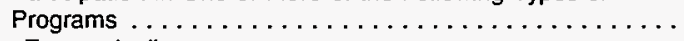

Energy Audits $\ldots \ldots \ldots \ldots \ldots \ldots \ldots \ldots \ldots$

Direct Electricity Load Control $\ldots \ldots \ldots \ldots \ldots \ldots \ldots \ldots$

Special Rate Schedule ${ }^{b} \ldots \ldots \ldots \ldots \ldots \ldots \ldots \ldots \ldots$

Standby Generation Program . . . . . . . . . . . . .

Equipment Rebate(s)

Equipment Installation or Retrofit for the Primary Purpose of Improving Energy Efficiency Affecting:

Steam Production ${ }^{c} \ldots \ldots \ldots \ldots \ldots \ldots \ldots \ldots$

Direct/lndirect Process Heating . . . . . . . . . . . . .

Direct Process Cooling/Refrigeration . . . . . . . . . .

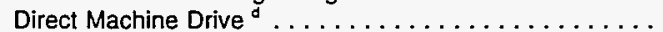

Facility Heating, Ventilation, and Air Conditioning .....

Facility Lighting . . . . . . . . . . . . . . . . . .

Equipment Installation/Retrofit for the Primary Purpose of

Using a Different Energy Source ${ }^{\circ} \ldots \ldots \ldots \ldots \ldots \ldots$

Other'

$\ldots \ldots \ldots \ldots \ldots \ldots \ldots$

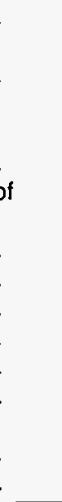

.

\begin{tabular}{|c|c|c|c|c|c|}
\hline 28 & * & 23 & 3 & 2 & 10.5 \\
\hline 20 & $*$ & 16 & 2 & 1 & 12.0 \\
\hline 9 & \pm & 7 & 2 & 1 & 14.8 \\
\hline 18 & * & 15 & 1 & 1 & 11.9 \\
\hline 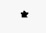 & 0 & • & 0 & 0 & 24.0 \\
\hline 4 & • & 3 & 1 & 0 & 20.1 \\
\hline 5 & • & 5 & * & - & 15.5 \\
\hline 10 & • & 10 & * & • & 14.1 \\
\hline 13 & * & 11 & 1 & * & 12.9 \\
\hline 12 & • & 10 & 1 & - & 15.8 \\
\hline 4 & • & 3 & * & - & 19.6 \\
\hline 10 & * & 7 & 1 & 1 & 13.9 \\
\hline 2 & 0 & 2 & 0 & 0 & 19.2 \\
\hline 1 & 0 & 1 & 0 & 0 & 32.5 \\
\hline
\end{tabular}

See footnotes at end of table. 
Table A41. Total Inputs of Energy for Heat, Power, and Electricity Generation by Census Region, Industry Group, Selected Industries, and Type of Energy Management Program, 1991 (Continued)

(Estimates in Trillion Btu)

\begin{tabular}{|c|c|c|c|c|c|c|c|}
\hline \multirow{2}{*}{$\begin{array}{c}\text { SIC } \\
\operatorname{Cod} \theta^{\circ}\end{array}$} & \multirow{2}{*}{$\begin{array}{l}\text { Industry Groups } \\
\text { and Industry }\end{array}$} & \multirow[b]{2}{*}{ Total } & \multicolumn{4}{|c|}{ Census Region } & \multirow{3}{*}{$\begin{array}{c}\text { RSE } \\
\text { Row } \\
\text { Factors }\end{array}$} \\
\hline & & & Northeast & Midwest & South & West & \\
\hline & Column Factors: & 0.7 & 1.3 & 1.0 & 0.9 & 1.2 & \\
\hline
\end{tabular}

2033

Canned Fruits and Vegetables

Participation in One or More of the Following Types of

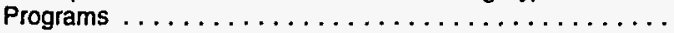

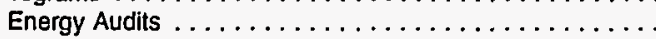

Direct Electricity Load Control $\ldots \ldots \ldots \ldots \ldots \ldots \ldots$

Special Rate Schedule ${ }^{b} \ldots \ldots \ldots \ldots \ldots \ldots \ldots \ldots . . . \ldots \ldots$

Standby Generation Program . . . . . . . . . . . . . .

Equipment Rebate(s)

Equipment Installation or Retrofit for the Primary Purpose of Improving Energy Efficiency Affecting:

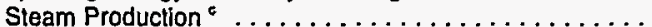

Direct/lndirect Process Heating . ...............

Direct Process Cooling/Refrigeration . . . . . . . . . . .

Direct Machine Drive o .....................

Facility Heating, Ventilation, and Air Conditioning ......

Facility Lighting . . . . . . . . . . . . . . . .

Equipment Installation/Retrofit for the Primary Purpose of

Using a Different Energy Source ${ }^{\circ}$.................

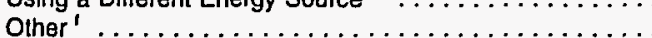

2037

Frozen Fruits and Vegetables

Particlpation in One or More of the Following Types of

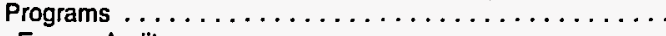

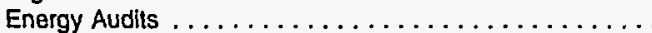

Direct Electricity Load Control . . . . . . . . . . . . . .

Special Rate Schedule ${ }^{b} \ldots \ldots \ldots \ldots \ldots \ldots \ldots \ldots$

Standby Generation Program . . . . . . . . . . . . . . . . .

Equipment Rebate(s) . .

Equipment Installation or Retrofit for the Primary Purpose of Improving Energy Efficiency Affecting:

Steam Production ${ }^{c} \ldots \ldots \ldots \ldots \ldots \ldots \ldots$

Direct/Indirect Process Heating ..................

Direct Process Cooling/Refrigeration . . . . . . . . . . .

Direct Machine Drive ${ }^{d}$.....................

Facility Heating, Ventilation, and Air Conditioning ......

Facillty Llghting . . . . . . . . . . . . . . . . . .

Equipment Installation/Retrofit for the Primary Purpose of

Using a Different Energy Source ${ }^{\circ} \ldots \ldots \ldots \ldots \ldots \ldots$

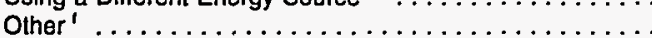

Wet Corn Milling

Participation in One or More of the Following Types of

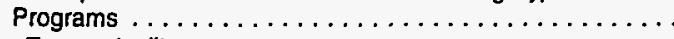

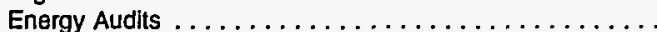

Direct Electricity Load Control . .................

Special fate Schedule ${ }^{b} \ldots \ldots \ldots \ldots \ldots \ldots \ldots$.

Standby Generatton Program . . . . . . . . . . . . . . . . . .

Equipment Rebate(s)

Equipment Installation or Retrofit for the Primary Purpose of Improving Energy Efficlency Affecting:

Steam Production ${ }^{c} \ldots \ldots \ldots \ldots \ldots \ldots \ldots \ldots \ldots$

DIrect/Indirect Process Heating .................

Direct Process Cooling/Refrigeration . . . . . . . . . . . .

Direct Machine Drive ${ }^{\circ} . \ldots \ldots \ldots \ldots \ldots \ldots \ldots . . . \ldots$.

Facilly Heating, Ventilation, and Air Conditioning .....

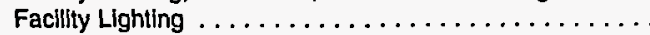

Equipment Installation/Retrofit for the Primary Purpose of

Using a Different Energy Source ${ }^{\circ} \ldots \ldots \ldots \ldots \ldots \ldots$

Other ${ }^{\prime}$

$\ldots \ldots \ldots \ldots \ldots \ldots \ldots$

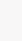

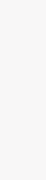

5
2
1
2
$W$

See footnotes at end of table. 
Table A41. Total Inputs of Energy for Heat, Power, and Electricity Generation by Census Region, Industry Group, Selected Industries, and Type of Energy Management Program, 1991 (Continued)

(Estimates in Trillion Btu)

\begin{tabular}{|c|c|c|c|c|c|c|c|}
\hline \multirow{2}{*}{$\begin{array}{l}\text { SIC } \\
\text { Code" }\end{array}$} & \multirow{2}{*}{$\begin{array}{l}\text { Industry Groups } \\
\text { and Industry }\end{array}$} & \multirow[b]{2}{*}{ Total } & \multicolumn{4}{|c|}{ Census Region } & \multirow{3}{*}{$\begin{array}{c}\text { RSE } \\
\text { Row } \\
\text { Factore }\end{array}$} \\
\hline & & & Northeast & Midwest & South & West & \\
\hline & RSE Column Factors: & 0.7 & 1.3 & 1.0 & 0.9 & 1.2 & \\
\hline
\end{tabular}

2051

Bread, Cake, and Related Products

Participation in One or More of the Following Types of

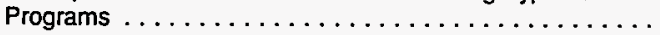

Energy Audits $\ldots \ldots \ldots \ldots \ldots \ldots \ldots \ldots \ldots$

Direct Electricity Load Control..$\ldots \ldots \ldots \ldots \ldots \ldots$

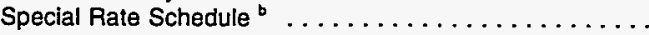

Standby Generation Program . . . . . . . . . . . . . . . . .

Equipment Rebate(s) . . . . . . . . . . . . . . .

Equipment Installation or Retrofit for the Primary Purpose of Improving Energy Efficiency Affecting:

Steam Production ${ }^{c} \ldots \ldots \ldots \ldots \ldots \ldots \ldots \ldots \ldots$

Direct/Indirect Process Heating . . . . . . . . . . . . . . .

Direct Process Cooling/Refrigeration . . . . . . . . . . .

Direct Machine Drive ${ }^{\circ} . \ldots \ldots \ldots \ldots \ldots \ldots \ldots$.

Facility Heating, Ventilation, and Air Conditioning ......

Facility Lighting . . . . . . . . . . . . . . . . .

Equipment Installation/Retrofit for the Primary Purpose of

Using a Different Energy Source ${ }^{\circ} \ldots \ldots \ldots \ldots \ldots \ldots$

Other'

Beet Sugar

2063

Participation in One or More of the Following Types of

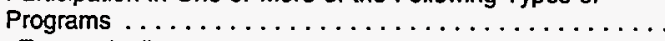

Energy Audits . . . . . . . . . . . . . . . . . .

Direct Electricity Load Control $\ldots \ldots \ldots \ldots \ldots \ldots \ldots$

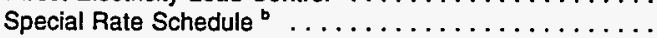

Standby Generation Program . . . . . . . . . . . . . . .

Equipment Rebate(s) $\ldots \ldots \ldots \ldots \ldots \ldots \ldots \ldots \ldots$

Equipment Installation or Retrofit for the Primary Purpose of Improving Energy Efficiency Affecting:

Steam Production ${ }^{\mathrm{C}}$

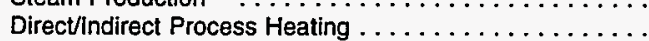

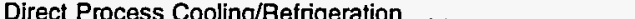

Direct Machine Drive o....................

Facility Heating, Ventilation, and Air Conditioning .......

Facility Lighting . .........................

Equipment Installation/Retrofit for the Primary Purpose of

Using a Different Energy Source ${ }^{\circ} \ldots \ldots \ldots \ldots \ldots \ldots$.

Other'

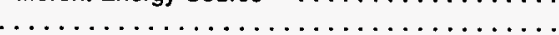

Soybean Oil Mills

Participation in One or More of the Following Types of

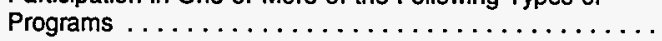

Energy Audits $\ldots \ldots \ldots \ldots \ldots \ldots \ldots \ldots \ldots \ldots \ldots$

Direct Electricity Load Control $\ldots \ldots \ldots \ldots \ldots \ldots \ldots$

Special Rate Schedule ${ }^{b} \ldots \ldots \ldots \ldots \ldots \ldots \ldots \ldots$

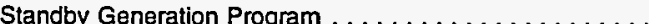

Equipment Rebate(s) . . . . . . . . . . . . . . .

Equipment Installation or Retrofit for the Primary Purpose of Improving Energy Efficiency Affecting:

Steam Production ${ }^{c} \ldots \ldots \ldots \ldots \ldots \ldots \ldots \ldots$

Direct/ndirect Process Heating . . . . . . . . . . . . . . .

Direct Process Cooling/Refrigeration . . . . . . . . . . .

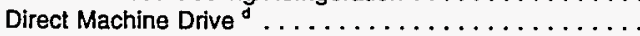

Facility Heating, Ventilation, and Air Conditioning .......

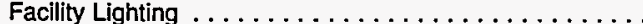

Equipment Installation/Retrofit for the Primary Purpose of

Using a Different Energy Source ${ }^{\circ} \ldots \ldots \ldots \ldots \ldots \ldots$.

Other:

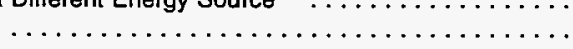

\begin{tabular}{|c|c|c|c|c|c|}
\hline 15 & 5 & 3 & 3 & 3 & 12.3 \\
\hline 11 & 4 & 2 & 2 & 3 & 15.0 \\
\hline 4 & 1 & 1 & 1 & 1 & 18.4 \\
\hline 9 & 2 & 1 & 3 & 2 & 15.2 \\
\hline 3 & 1 & * & 1 & $\mathbf{Q}$ & 29.8 \\
\hline 4 & 3 & * & * & 1 & 29.6 \\
\hline 3 & 1 & * & 1 & 1 & 20.2 \\
\hline 2 & • & * & • & Q & 23.3 \\
\hline 4 & 2 & * & 1 & 1 & 23.2 \\
\hline 7 & 3 & 1 & 2 & 2 & 18.2 \\
\hline 4 & 1 & - & 1 & 1 & 21.9 \\
\hline 8 & 3 & 1 & 2 & 1 & 16.2 \\
\hline 3 & 1 & * & * & $Q$ & 30.0 \\
\hline 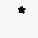 & 0 & • & 0 & 0 & 0.1 \\
\hline
\end{tabular}

5.0

15.2

9.8

20.2

18.2

30.0

30.1

See footnotes at end of table. 
Table A41. Total Inputs of Energy for Heat, Power, and Electricity Generation by Census Region, Industry Group, Selected Industries, and Type of Energy Management Program, 1991 (Continued)

(Estimates in Trillion Btu)

\begin{tabular}{|c|c|c|c|c|c|c|c|}
\hline \multirow{2}{*}{$\begin{array}{c}\text { SIC } \\
\text { Codea } \\
\end{array}$} & \multirow{2}{*}{$\begin{array}{l}\text { Industry Groups } \\
\text { and Industry }\end{array}$} & \multirow[b]{2}{*}{ Total } & \multicolumn{4}{|c|}{ Census Region } & \multirow{3}{*}{$\begin{array}{c}\text { RSE } \\
\text { Row } \\
\text { Factors }\end{array}$} \\
\hline & & & Northeast & Midwest & South & West & \\
\hline & RSE Column Factors: & 0.7 & 1.3 & 1.0 & 0.9 & 1.2 & \\
\hline
\end{tabular}

2082

Malt Beverages

Participatlon In One or More of the Following Types of

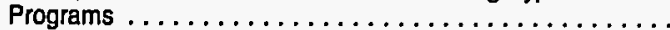

Energy Audits $\ldots \ldots \ldots \ldots \ldots \ldots \ldots \ldots \ldots \ldots \ldots$

Dlrect Electricity Load Control $\ldots \ldots \ldots \ldots \ldots \ldots \ldots$

Special Rate Schedule ${ }^{b} \ldots \ldots \ldots \ldots \ldots \ldots \ldots \ldots \ldots$

Standby Generatlon Program . . . . . . . . . . . . . .

Equlpment Rebate(s) . .......................

Equipment Installatlon or Retrofit for the Primary Purpose of Improving Energy Efficlency Affecting:

Steam Production ${ }^{\circ} \ldots \ldots \ldots \ldots \ldots \ldots \ldots \ldots \ldots$

Direct/lndirect Process Heating . . . . . . . . . . . . . .

Direct Process Coollng/Refrigeration ...............

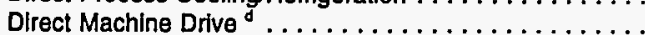

Facillty Heating, Ventilation, and Air Conditloning ......

Facllity Llghting . ......................

Equipment Installation/Retrofit for the Primary Purpose of

Using a Different Energy Source ${ }^{\circ} \ldots \ldots \ldots \ldots \ldots \ldots$.

Other'

\section{TOBACCO PRODUCTS}

Participation in One or More of the Following Types of

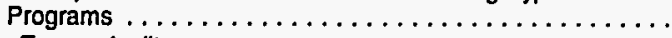

Energy Audits.$\ldots \ldots \ldots \ldots \ldots \ldots \ldots \ldots \ldots \ldots \ldots \ldots \ldots \ldots \ldots$

Direct Electricity Load Control $\ldots \ldots \ldots \ldots \ldots \ldots \ldots$

Speclal Rate Schedule ${ }^{b} \ldots \ldots \ldots \ldots \ldots \ldots \ldots$.

Standby Generation Program . . . . . . . . . . . . . . .

Equlpment Rebate(s)

Equipment Installation or Retrofit for the Primary Purpose of

Improving Energy Efficlency Affecting:

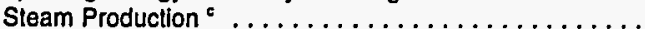

Direct/lndirect Process Heating . .................

Direct Process Cooling/Refrigeration . . . . . . . . . . . .

Direct Machine Drive o .........................

Facillty Heating, Ventilation, and Air Conditioning ......

Faclity Lighting . . .........................

Equipment Installation/Retrofit for the Primary Purpose of

Using a Different Energy Source ${ }^{-} \ldots \ldots \ldots \ldots \ldots \ldots$.

Other'

TEXTILE MILL PRODUCTS

Participation in One or More of the Following Types of

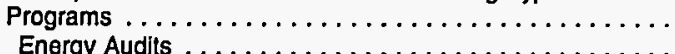

Direct Electrity Load Control $\ldots \ldots \ldots \ldots \ldots \ldots$

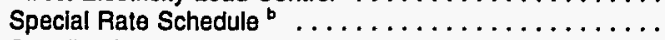

Standby Generation Program . . . . . . . . . . . . . . . . .

Equlpment Rebate(s) ......................

Equipment Installation or Retrofit for the Primary Purpose of Improving Energy Efficlency Affecting:

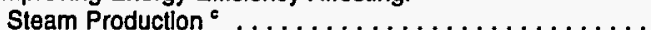

Direct//ndirect Process Heating ..................

Direct Process Cooling/Refrigeration ..............

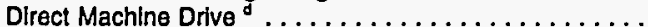

Facility Heating, Ventilation, and Air Conditioning .......

Facllity Llghting . .....................

Equipment Installation/Retrofit for the Primary Purpose of

Using a Different Energy Source ${ }^{-} \ldots \ldots \ldots \ldots \ldots \ldots$.

Other'

See footnotes at end of table. 
Table A41. Total Inputs of Energy for Heat, Power, and Electricity Generation by Census Region, Industry Group, Selected Industries, and Type of Energy Management Program, 1991 (Continued)

(Estimates in Trillion Btu)

\begin{tabular}{|c|c|c|c|c|c|c|c|}
\hline \multirow{2}{*}{$\begin{array}{c}\text { SIC } \\
\text { Code* }\end{array}$} & \multirow{2}{*}{$\begin{array}{l}\text { Industry Groups } \\
\text { and Industry }\end{array}$} & \multirow[b]{2}{*}{ Total } & \multicolumn{4}{|c|}{ Census Region } & \multirow{3}{*}{$\begin{array}{c}\text { RSE } \\
\text { Row } \\
\text { Factors }\end{array}$} \\
\hline & & & Northeast & Midwest & South & West & \\
\hline & RSE Column Factors: & 0.7 & 1.3 & 1.0 & 0.9 & 1.2 & \\
\hline
\end{tabular}

Participation in One or More of the Following Types of

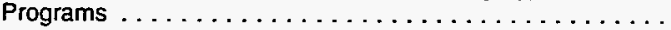

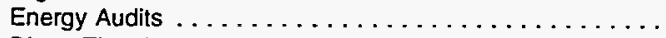

Direct Electricity Load Control . . . . . . . . . . . . . .

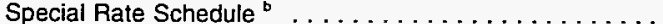

Standby Generation Program . . . . . . . . . . . .

Equipment Rebate(s) ..........................

Equipment Installation or Retrofit for the Primary Pumose of Improving Energy Efficiency Affecting:

Steam Production ${ }^{\circ}$.

Direct Process Cooling/Refrigeration . . . . . . . . . . . . .

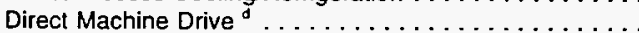

Facility Heating, Ventilation, and Air Conditioning ......

Facility Lighting . . . . . . . . . . . . . . . .

Equipment Installation/Retrofit for the Primary Purpose of

Using a Different Energy Source ${ }^{\circ} \ldots \ldots \ldots \ldots \ldots \ldots$.

Other ${ }^{\prime} \ldots \ldots \ldots \ldots \ldots \ldots \ldots \ldots \ldots \ldots \ldots \ldots$

LUMBER and WOOD PRODUCTS

Participation in One or More of the Following Types of

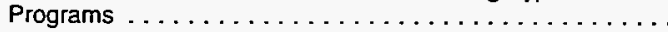

Energy Audits . . . . . . . . . . . . . . . .

Direct Electricity Load Control $\ldots \ldots \ldots \ldots \ldots \ldots \ldots$

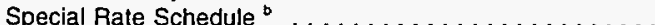

Standby Generation Program . . . . . . . . . . . . .

Equipment Rebate(s) ........................

Equipment Installation or Retrofit for the Primary Purpose of Improving Energy Efficiency Affecting:

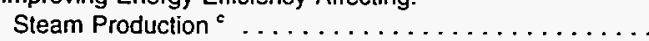

Direct/Indirect Process Heating . . . . . . . . . . . . .

Direct Process Cooling/Refrigeration . . . . . . . . . .

Direct Machine Drive d. . . . . . . . . . . . . . . .

Facilty Heating, Ventilatıon, and Air Conditioning ......

Facility Lighting . . . . . . . . . . . . . . . .

Equipment Installation/Retrofit for the Primary Purpose of

Using a Different Energy Source ${ }^{\circ} \ldots \ldots \ldots \ldots \ldots \ldots$

Other "

FURNTURE and FIXTURES

Participation in One or More of the Following Types of

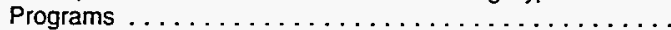

Energy Audits.$\ldots \ldots \ldots \ldots \ldots \ldots \ldots \ldots \ldots$

Direct Electricity Load Control $\ldots \ldots \ldots \ldots \ldots \ldots \ldots \ldots$

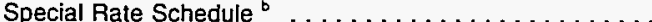

Standby Generation Program . . . . . . . . . . . . . . .

Equipment Rebate(s) . . . . . . . . . . . . . . . . . . . .

Equipment Installation or Retrofit for the Primary Purpose of Improving Energy Efficiency Affecting:

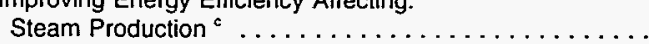

Direct/lndirect Process Heating . . . . . . . . . . . . . . .

Direct Process Cooling/Refrigeration . . . . . . . . . .

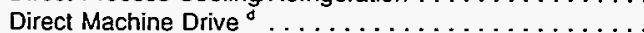

Facility Heating, Ventilation, and Air Conditioning ......

Faciltty Lighting . . . . . . . . . . . . . . . . . .

Equipment Installation/Retrofit for the Primary Purpose of

Using a Different Energy Source ${ }^{\circ} \ldots \ldots \ldots \ldots . . . .$.

Other '

.....................

\begin{tabular}{|c|c|c|c|c|c|}
\hline 22 & 4 & 9 & 8 & - & 19.7 \\
\hline 12 & 3 & 6 & 3 & * & 24.8 \\
\hline 9 & 1 & 5 & 3 & - & 27.0 \\
\hline 8 & 1 & 5 & 3 & 0 & 25.4 \\
\hline 1 & * & * & * & 0 & 46.1 \\
\hline 6 & $Q$ & 4 & * & 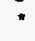 & 37.2 \\
\hline 8 & $Q$ & 5 & 2 & * & 29.0 \\
\hline 5 & 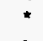 & 5 & 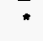 & 0 & 33.2 \\
\hline 4 & 0 & 4 & $Q$ & 0 & 39.6 \\
\hline 8 & 1 & 5 & 2 & : & 28.0 \\
\hline 11 & $\mathbf{Q}$ & 6 & 3 & - & 24.2 \\
\hline 12 & 2 & 7 & 3 & ' & 20.0 \\
\hline 5 & $\mathbf{Q}$ & 3 & * & 0 & 40.0 \\
\hline $\mathbf{Q}$ & 0 & 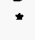 & * & 0 & NF \\
\hline
\end{tabular}

See footnotes at end of table. 
Table A41. Total Inputs of Energy for Heat, Power, and Electricity Generation by Census Region, Industry Group, Selected Industries, and Type of Energy Management Program, 1991 (Continued)

(Estimates in Trillion Btu)

\begin{tabular}{|c|c|c|c|c|c|c|c|}
\hline \multirow[b]{2}{*}{$\begin{array}{l}\text { SIC } \\
\text { Code }\end{array}$} & \multirow[b]{2}{*}{$\begin{array}{l}\text { Industry Groups } \\
\text { and Industry }\end{array}$} & \multirow[b]{2}{*}{ Total } & \multicolumn{4}{|c|}{ Census Region } & \multirow{3}{*}{$\begin{array}{c}\text { RSE } \\
\text { Row } \\
\text { Factors }\end{array}$} \\
\hline & & & Northeast & Midwest & South & West & \\
\hline & olumn Factors: & 0.7 & $1.3^{\circ}$ & 1.0 & 0.9 & 1.2 & \\
\hline
\end{tabular}

Participation in One or More of the Following Types of

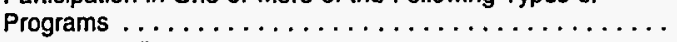

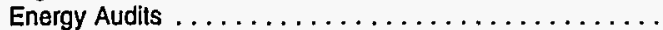

Direct Electricity Load Control $\ldots \ldots \ldots \ldots \ldots \ldots \ldots$

Special Rate Schedule ${ }^{\circ} \ldots \ldots \ldots \ldots \ldots \ldots \ldots \ldots \ldots$

Standby Generation Program . . . . . . . . . . . . .

Equipment Rebate(s) $\ldots \ldots \ldots \ldots \ldots \ldots \ldots \ldots \ldots \ldots$
Equipment Installation or Retrofit tor the Primary Purpose of Improving Energy Efficiency Affecting:

Steam Production ${ }^{c} \ldots \ldots \ldots \ldots$

Direct/lndirect Process Heating . . . . . . . . . . . .

Direct Process Cooling/Refrigeration . . . . . . . . . .

Direct Machine Drive ${ }^{d} \ldots \ldots \ldots \ldots \ldots \ldots \ldots \ldots$

Facility Heating, Ventilation, and Air Conditioning ......

Facility LIghting . . . . . . . . . . . . . . . . . . . .

Equipment Installation/Retrofit for the Primary Purpose of

Using a Different Energy Source ${ }^{\circ} \ldots \ldots \ldots \ldots \ldots \ldots$

Other'

$\ldots \ldots \ldots \ldots \ldots \ldots \ldots \ldots \ldots$

$\begin{array}{rr}1,895 & 219 \\ 1,230 & 170 \\ 1,155 & 145 \\ 1,311 & 147 \\ 221 & W \\ 213 & 72 \\ & \\ 970 & 122 \\ 802 & 137 \\ 301 & 30 \\ 1,084 & 181 \\ 580 & 132 \\ 812 & 165 \\ 391 & \\ 42 & 56 \\ & 1\end{array}$

Pulp Mills

Participation in One or More of the Following Types of

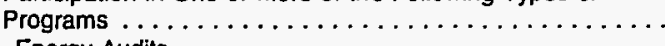

Energy Audits $\ldots \ldots \ldots \ldots \ldots \ldots \ldots \ldots \ldots$

Direct Electricity Load Control $\ldots \ldots \ldots \ldots \ldots \ldots \ldots$

Special Rate Schedule ${ }^{\circ} \ldots \ldots \ldots \ldots \ldots \ldots \ldots \ldots$

Standby Generation Program . . . . . . . . . . . . . .

Equipment Rebate(s) . . . . . . . . . . . . . . . . .

Equipment Installation or Retrofit for the Primary Purpose of Improving Energy Efficiency Affecting:

Steam Production ${ }^{c} \ldots \ldots \ldots \ldots$

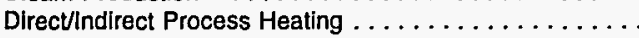

Direct Process Cooling/Refrigeration . . . . . . . . . .

Direct Machine Drive ${ }^{d}$. . . . . . . . . . . . . . . . . . .

Facility Heating, Ventilation, and Air Conditioning $\ldots \ldots$.

Facillty Lighting . . . . . . . . . . . . . . . . . . .

Equipment Installation/Retrofit for the Primary Purpose of

Using a Different Energy Source ${ }^{\circ} \ldots \ldots \ldots \ldots \ldots \ldots$.

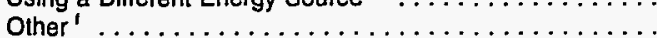

\section{Paper Mills}

Participation in One or More of the Following Types of

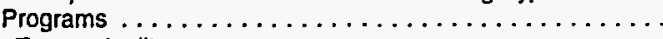

Energy Audits $\ldots \ldots \ldots \ldots \ldots \ldots \ldots \ldots \ldots \ldots$

Direct Electricity Load Control $\ldots \ldots \ldots \ldots \ldots \ldots \ldots$

Special Rate Schedule ${ }^{b} \ldots \ldots \ldots \ldots \ldots \ldots \ldots$

Standby Generation Program . . . . . . . . . . . . .

Equipment Rebate(s)

Equipment Installation or Retrolit for the Primary Purpose of

Improving Energy Efficiency Affecting:

Steam Production ${ }^{c} \ldots \ldots \ldots \ldots \ldots \ldots \ldots$

Direct/Indirect Process Heating . . . . . . . . . . . .

Direct Process Cooling/Refrigeration . . . . . . . . . .

Direct Machine Drive d ....................

Facility Heating, Ventllation, and Air Conditioning $\ldots .$.

Facility Lighting $\ldots \ldots \ldots \ldots \ldots \ldots \ldots \ldots \ldots$

Equipment Installation/Retrofit for the Primary Purpose of

Using a Different Energy Source ${ }^{\circ} \ldots \ldots \ldots \ldots \ldots \ldots \ldots$

Other'

See footnotes at end of table. 
Table A41. Total Inputs of Energy for Heat, Power, and Electricity Generation by Census Region, Industry Group, Selected Industries, and Type of Energy Management Program, 1991 (Continued)

(Estimates in Trillion Btu)

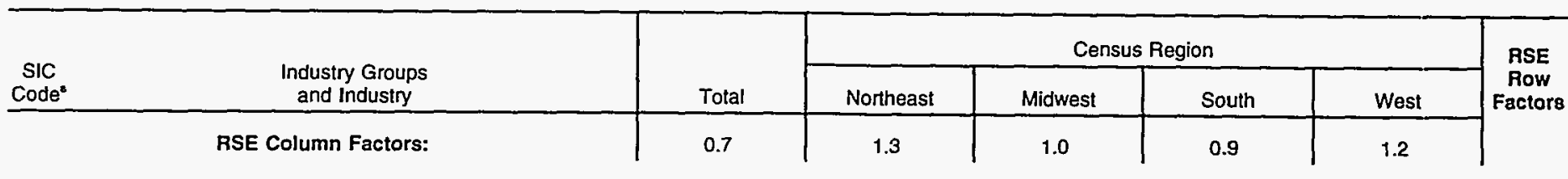

2631

Paperboard Mills

Participation in One or More of the Following Types of

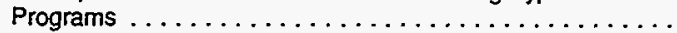

Energy Audits . . . . . . . . . . . . . . . .

Direct Electricity Load Control $\ldots \ldots \ldots \ldots \ldots \ldots \ldots \ldots$

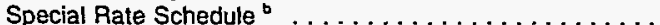

Standby Generation Program . . . . . . . . . . . .

Equipment Rebate(s) .

Equipment Installation or Retrofit for the Primary Purpose of

Improving Energy Efficiency Affecting:

Steam Production ${ }^{c} \ldots \ldots \ldots \ldots \ldots \ldots \ldots \ldots \ldots \ldots$

Direct/indirect Process Heating . . . . . . . . . . . .

Direct Process Cooling/Refrigeration . . . . . . . . . . . . .

Direct Machine Drive ${ }^{d} \ldots \ldots \ldots \ldots \ldots \ldots \ldots \ldots \ldots$

Facility Heating, Ventilation, and Air Conditioning .....

Facility Lighting . . . . . . . . . . . . . . . . . . . . . .

Equipment Installation/Retrofit for the Primary Purpose of

Using a Different Energy Source ${ }^{\circ} \ldots \ldots \ldots \ldots \ldots \ldots \ldots$

Other $^{\prime}$

\section{PRINTING and PUBLISHING}

Participation in One or More of the Following Types of

Programs $\ldots \ldots \ldots \ldots \ldots \ldots \ldots \ldots \ldots \ldots \ldots . . . \ldots \ldots \ldots$

Energy Audits $\ldots \ldots \ldots \ldots \ldots \ldots \ldots \ldots \ldots \ldots$

Direct Electricity Load Control ..................

Special Rate Schedule ${ }^{b} \ldots \ldots \ldots \ldots \ldots \ldots \ldots \ldots$

Standby Generation Program . . . . . . . . . . . .

Equipment Rebate(s) .

Equipment Installation or Retrofit for the Primary Purpose of

Improving Energy Efficiency Affecting:

Steam Production $^{c} \ldots \ldots \ldots \ldots \ldots \ldots \ldots \ldots$

Direct/ndirect Process Heating . . . . . . . . . . . . . .

Direct Process Cooling/Refrigeration . . . . . . . . . .

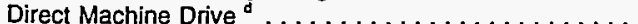

Facility Heating, Ventilation, and Air Conditioning ......

Facility Lighting . . . . . . . . . . . . . . . .

Equipment Installation/Retrofit for the Primary Purpose of

Using a Different Energy Source ${ }^{\circ} \ldots \ldots \ldots \ldots \ldots \ldots$

Other'

Participation in One or More of the Following Types of

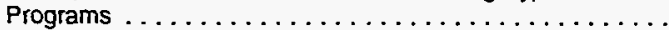

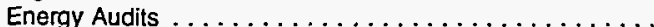

Direct Electricity Load Control $\ldots \ldots \ldots \ldots \ldots \ldots \ldots \ldots \ldots$

Special Rate Schedule ${ }^{b} \ldots \ldots \ldots \ldots \ldots \ldots \ldots \ldots \ldots \ldots$

Standby Generation Program . . . . . . . . . . . . . . .

Equipment Rebate(s) . .

Equipment Installation or Retrofit for the Primary Pumo. . Improving Energy Efficiency Affecting:

Steam Production ${ }^{c} \ldots \ldots \ldots \ldots \ldots \ldots \ldots \ldots \ldots$

Direct/lndirect Process Heating . . . . . . . . . . . .

Direct Process Cooling/Refrigeration . . . . . . . . . . .

Direct Machine Drive ${ }^{d} \ldots \ldots \ldots \ldots \ldots \ldots \ldots \ldots \ldots$

Facility Heating, Ventilation, and Air Conditioning

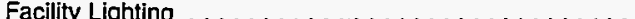

Equipment Installation/Retrofit for the Primary Purpose of

Using a Different Energy Source ${ }^{\circ} \ldots \ldots \ldots \ldots \ldots \ldots \ldots$

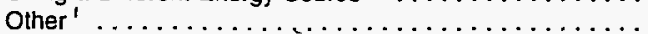

$\begin{array}{rrr}603 & 9 & 40 \\ 321 & 2 & 18 \\ 344 & 1 & 23 \\ 45 & \text { W } & 20 \\ 29 & 0 & \text { Q }\end{array}$

40
18
23
20
$W$
2

$\begin{array}{rr}4 & 6 \\ W & 3 \\ 1 & 0 \\ 4 & 23 \\ 1 & 9 \\ W & 15 \\ Q & 3 \\ 0 & W\end{array}$

\begin{tabular}{|c|c|c|c|c|}
\hline 2,140 & 85 & 290 & 1,697 & 68 \\
\hline 1,467 & 60 & 194 & 1,185 & 29 \\
\hline 985 & 38 & 147 & 767 & 32 \\
\hline 1,204 & 56 & 102 & 998 & 47 \\
\hline 170 & 14 & 13 & 134 & 9 \\
\hline 67 & 19 & 12 & $w$ & $w$ \\
\hline 843 & 38 & 157 & 610 & 37 \\
\hline 1,015 & 18 & 99 & 862 & 36 \\
\hline 751 & 24 & 93 & 621 & 13 \\
\hline 1,112 & 58 & 115 & 903 & 36 \\
\hline 614 & 31 & 144 & 413 & 25 \\
\hline 801 & 55 & 152 & 569 & 25 \\
\hline 247 & 11 & 24 & 197 & 15 \\
\hline 105 & 8 & $w$ & 89 & $W$ \\
\hline
\end{tabular}

See footnotes at end of table. 
Table A41. Total Inputs of Energy for Heat, Power, and Electricity Generation by Census Region, Industry Group, Selected Industries, and Type of Energy Management Program, 1991 (Continued)

(Estimates in Trillion Btu)

\begin{tabular}{|c|c|c|c|c|c|c|c|}
\hline \multirow{2}{*}{$\begin{array}{c}\text { SIC } \\
\text { Codea }\end{array}$} & \multirow{2}{*}{$\begin{array}{l}\text { Industry Groups } \\
\text { and Industry }\end{array}$} & \multirow[b]{2}{*}{ Total } & \multicolumn{4}{|c|}{ Census Region } & \multirow{3}{*}{$\begin{array}{c}\text { RSE } \\
\text { Row } \\
\text { Factors }\end{array}$} \\
\hline & & & Northeast & Midwest & South & West & \\
\hline & RSE Column Factors: & 0.7 & 1.3 & 1.0 & 0.9 & 1.2 & \\
\hline
\end{tabular}

2812

Alkalies and Chlorine

Particlpation in One or More of the Following Types of

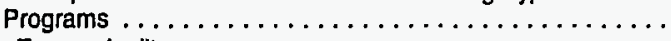

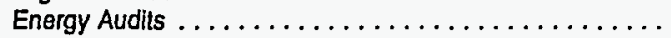

Direct Electricity Load Control $\ldots \ldots \ldots \ldots \ldots \ldots \ldots$.

Special Rate Schedule ${ }^{b}$......................

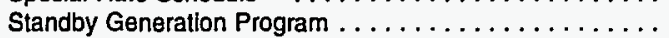

Equlpment Rebate(s) .....................

Equlpment Installation or Retrofit for the Primary Purpose of Improving Energy Efficiency Affecting:

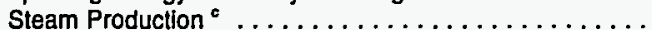

Direct/lndirect Process Heating ................

Direct Process Cooling/Refrigeration ...............

Direct Machine Drive...$\ldots \ldots \ldots \ldots \ldots \ldots \ldots$

Facllity Heating, Ventilation, and Air Conditioning ......

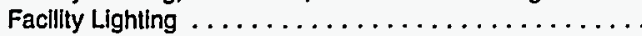

Equipment Installation/Retrofit for the Primary Purpose of

Using a Different Energy Source ${ }^{\circ} \ldots \ldots \ldots \ldots \ldots \ldots$.

Other'

\section{Industrial Gases}

Participation in One or More of the Following Types of

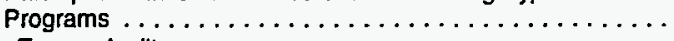

Energy Audits $\ldots \ldots \ldots \ldots \ldots \ldots \ldots \ldots \ldots$

Direct Electricity Load Control . . . . . . . . . . . . . .

Speclal Rate Schedule ${ }^{\circ} \ldots \ldots \ldots \ldots \ldots \ldots \ldots$.

Standby Generation Program . . . . . . . . . . . . . . . . . .

Equipment Rebate(s) .

Equipment Installatlon or Retrofit for the Primary Purpose of

Improving Energy Efficiency Affecting:

Steam Production ${ }^{c}$

Direct/lndirect Process Heating . . . . . . . . . . . . .

Direct Process Cooling/Refrigeration . . . . . . . . . . .

Direct Machine Drive ${ }^{\circ} \ldots \ldots \ldots \ldots \ldots \ldots \ldots \ldots$

Facillty Heating, Ventilation, and Air Conditioning .......

Facility Lighting . ......................

Equipment Installation/Retrofit for the Primary Purpose of

Using a Different Energy Source ${ }^{\circ} \ldots \ldots \ldots \ldots \ldots \ldots$

Other!

Industrial Inorganic Chemicals, nec

Participation in One or More of the Following Types of

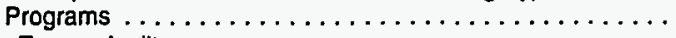

Energy Audits . . . . . . . . . . . . . . . . . .

Dlrect Electricity Load Control $\ldots \ldots \ldots \ldots \ldots \ldots \ldots$

Special Rate Schedule ${ }^{\circ} . \ldots \ldots \ldots \ldots \ldots \ldots \ldots$.

Standby Generation Program . . . . . . . . . . . . . . .

Equipment Rebate(s)

Equipment Installation or Retrofit for the Primary Purpose of Improving Energy Efficiency Affecting:

Steam Production ${ }^{\circ} \ldots \ldots \ldots \ldots \ldots \ldots \ldots \ldots \ldots$

Direct/ndirect Process Heating . .................

Direct Process Cooling/Refrigeration . . . . . . . . . . .

Direct Machine Drive ${ }^{d} \ldots . . \ldots \ldots \ldots \ldots \ldots \ldots$

Facility Heating, Ventilation, and Air Conditioning ......

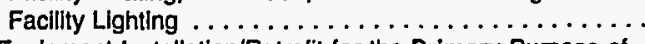

Equlpment Installation/Retrofit for the Primary Purpose of

Using a Different Energy Source ${ }^{\circ}$..................

Other'

$\begin{array}{rr}137 & 0 \\ W & 0 \\ W & 0 \\ 134 & 0 \\ W & 0 \\ W & 0 \\ & \\ 74 & 0 \\ W & 0 \\ W & 0 \\ 59 & 0 \\ W & 0 \\ W & 0 \\ W & 0 \\ W & 0\end{array}$

0
0
0
0
0
0

127
$W$
$W$
$W$
0

$\begin{array}{ll}11 & 13.9 \\ W & 18.9 \\ W & 18.9 \\ W & 15.0 \\ W & 28.0 \\ W & 28.0\end{array}$

28.0

20.1

25.4

27.2

22.5

27.2

27.2

28.0

See footnotes at end of table. 
Table A41. Total Inputs of Energy for Heat, Power, and Electricity Generation by Census Region, Industry Group, Selected Industries, and Type of Energy Management Program, 1991 (Continued)

(Estimates in Trillion Btu)

\begin{tabular}{|c|c|c|c|c|c|c|c|}
\hline \multirow{2}{*}{$\begin{array}{l}\text { SIC } \\
\text { Code" }\end{array}$} & \multirow{2}{*}{$\begin{array}{l}\text { Industry Groups } \\
\text { and Industry }\end{array}$} & \multirow[b]{2}{*}{ Total } & \multicolumn{4}{|c|}{ Census Region } & \multirow{3}{*}{$\begin{array}{c}\text { RSE } \\
\text { Row } \\
\text { Factors }\end{array}$} \\
\hline & & & Northeast & Midwest & South & West & \\
\hline & RSE Column Factors: & 0.7 & 1.3 & 1.0 & 0.9 & 1.2 & \\
\hline
\end{tabular}

2821

Plastics Materials and Resins

Participation in One or More of the Following Types of

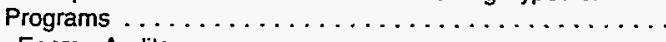

Energy Audits $\ldots \ldots \ldots \ldots \ldots \ldots \ldots \ldots \ldots \ldots \ldots$

Direct Electricity Load Control . . . . . . . . . . . . . .

Special Rate Schedule ${ }^{b} \ldots \ldots \ldots \ldots \ldots \ldots \ldots \ldots \ldots$

Standby Generation Program . . . . . . . . . . . .

Equipment Rebate(s) . . . . . . . . . . . . . . . . . . . . .

Equipment Installation or Retrofit for the Primary Purpose of Improving Energy Efficiency Affecting:

Steam Production ${ }^{c} \ldots \ldots \ldots \ldots \ldots \ldots \ldots \ldots$

Direct Indirect Process Heating . . . . . . . . . . . . . . . .

Direct Process Cooling/Refrigeration . . . . . . . . . . .

Direct Machine Drive ${ }^{\circ} \ldots \ldots \ldots \ldots \ldots \ldots \ldots \ldots$

Facility Heating, Ventilation, and Air Conditioning .....

Facility Lighting $\ldots \ldots \ldots \ldots \ldots \ldots \ldots \ldots \ldots$

Equipment Installation/Retrofit for the Primary Purpose of

Using a Different Energy Source ${ }^{\circ} \ldots \ldots \ldots \ldots \ldots \ldots \ldots$

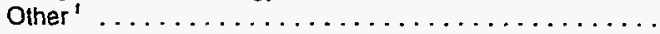

Synthetic Rubber

Participation in One or More of the Following Types of

Programs . . . . . . . . . . . . . . . . . .

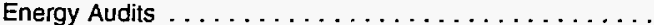

Direct Electricity Load Control . . . . . . . . . . . .

Special Rate Schedule ${ }^{\circ} \ldots \ldots \ldots \ldots \ldots \ldots \ldots \ldots \ldots$

Standby Generation Program . . . . . . . . . . . . . . .

Equipment Rebate(s) . . . . . . . . . . . . . . .

Equipment Installation or Retrofit for the Primary Purpose of Improving Energy Efficiency Affecting:

Steam Production ${ }^{c} \ldots \ldots \ldots \ldots \ldots \ldots \ldots \ldots \ldots$

Direct/Indirect Process Heating . . . . . . . . . . . .

Direct Process Cooling/Refrigeration . . . . . . . . .

Direct Machine Drive ${ }^{d} \ldots \ldots \ldots \ldots \ldots \ldots \ldots$

Facility Heating, Ventilation, and Air Conditioning $\ldots .$.

Facility Lighting . . . . . . . . . . . . . . . .

Equipment Instaliation/Retrofit for the Primary Purpose of

Using a Different Energy Source ${ }^{\circ} \ldots \ldots \ldots \ldots \ldots \ldots \ldots$

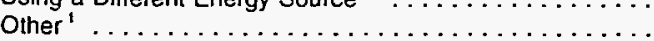

Cellulosic Manmade Fibers

Participation in One or More of the Following Types of

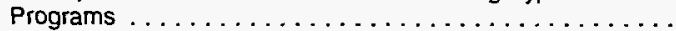

Energy Audits . . . . . . . . . . . . . . . . .

Direct Electricity Load Control $\ldots \ldots \ldots \ldots \ldots \ldots \ldots$

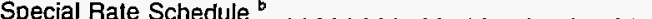

Standby Generation Program . . . . . . . . . . . . . . .

Equipment Rebate(s) . . . . . . . . . . . . . . . . . . . .

Equipment Installation or Retrofit for the Primary Purpose of Improving Energy Efficiency Affecting:

Steam Production ${ }^{c} \ldots \ldots \ldots \ldots \ldots \ldots \ldots \ldots$

Direct/lndirect Process Heating . . . . . . . . . . . . . .

Direct Process Cooling/Refrigeration . . . . . . . . . .

Direct Machine Drive ${ }^{\mathrm{d}} \ldots \ldots \ldots \ldots \ldots \ldots \ldots \ldots$

Facility Heating, Ventilation, and Air Conditioning .....

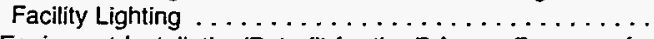

Equipment Installation/Retrofit for the Primary Purpose of

Using a Different Energy Source ${ }^{\circ} \ldots \ldots \ldots \ldots \ldots \ldots \ldots$

Other"

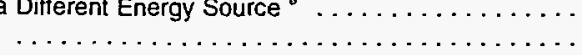

183
125
72
117
25
2


58
52
46
98
45
70
$W$
26

$\begin{array}{rr}12 \\ 9 \\ W \\ 11 \\ W \\ 1 \\ \\ W & \\ 1 & \\ 1 & \\ 9 & \\ W & \\ 3 & \\ W & \\ W & \end{array}$

$\begin{array}{rr}45 & 124 \\ 34 & 82 \\ W & 42 \\ 17 & 88 \\ W & W\end{array}$

124
82
42
88
$W$

8.0

7.0

9.0

10.3

10.7

W

30

w

$\begin{array}{rrr}33 & Q & 8.7 \\ 21 & Q & 8.2 \\ 16 & Q & 8.8 \\ 49 & Q & 9.1 \\ 13 & Q & 8.3 \\ 52 & 1 & 11.6 \\ W & & \\ W & 0 & 13.2 \\ W & 0 & 11.1\end{array}$

93

W

W

W

W

20

14
12

$W$
$W$
0
0
0
0

92
89
$W$
$W$
$*$
0 
Table A41. Total Inputs of Energy for Heat, Power, and Electricity Generation by Census Region, Industry Group, Selected Industries, and Type of Energy Management Program, 1991 (Continued)

(Estimates in Trillion Btu)

\begin{tabular}{|c|c|c|c|c|c|c|c|}
\hline \multirow[b]{2}{*}{$\begin{array}{l}\text { SIC } \\
\text { Code }\end{array}$} & \multirow[b]{2}{*}{$\begin{array}{l}\text { Industry Groups } \\
\text { and Industry }\end{array}$} & \multirow[b]{2}{*}{ Total } & \multicolumn{4}{|c|}{ Census Region } & \multirow{3}{*}{$\begin{array}{c}\text { RSE } \\
\text { Row } \\
\text { Factors }\end{array}$} \\
\hline & & & Northeast & Midwest & South & West & \\
\hline & Column Factors: & 0.7 & 1.3 & 1.0 & 0.9 & 1.2 & \\
\hline
\end{tabular}

2824

Organic Fibers, Noncellulosic

Participation in One or More of the Following Types of

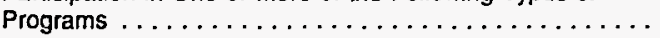

Energy Audits . . . . . . . . . . . . . . . . .

Direct Electricity Load Control .................

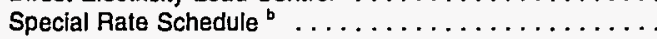

Standby Generation Program . . . . . . . . . . . . . .

Equipment Rebate(s) . . . . . . . . . . . . . . . . .

Equipment Installation or Retrofit for the Primary Purpose of Improving Energy Efficiency Affecting:

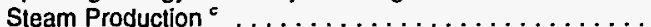

Direct/lndirect Process Heating . . . . . . . . . . .

Direct Process Cooling/Refrigeration . . . . . . . . . . . .

Direct Machine Drive ${ }^{\circ} \ldots \ldots \ldots \ldots \ldots \ldots \ldots . \ldots$

Facility Heating, Ventilation, and Air Conditioning .....

Facility Lighting . . . . . . . . . . . . . . . . . . . . . .

Equipment Installation/Retrofit for the Primary Purpose of

Using a Different Energy Source ${ }^{\circ} \ldots \ldots \ldots \ldots \ldots \ldots$

Other'

\section{Cyclic Crudes and Intermediates}

Participation in One or More of the Following Types of

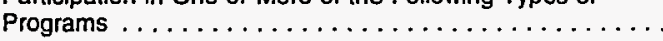

Energy Audits . . . . . . . . . . . . . . . . .

Direct Electricity Load Control . . . . . . . . . . . . . .

Special Rate Schedule ${ }^{b} \ldots \ldots \ldots \ldots \ldots \ldots \ldots \ldots \ldots$

Standby Generation Program . . . . . . . . . . . . . . .

Equipment Rebate(s)

Equipment Installation or Retrofit for the Primary Purpose of Improving Energy Efficiency Affecting:

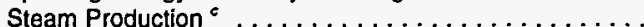

Direct/lndirect Process Heating . . . . . . . . . . .

Direct Process Cooling/Refrigeration . . . . . . . . . .

Direct Machine Drive d......................

Facility Heating, Ventilation, and Air Conditioning .....

Facility Lighting . . . . . . . . . . . . . . . . . . . . .

Equipment Installation/Retrofit for the Primary Purpose of

Using a Different Energy Source ${ }^{\circ} \ldots \ldots \ldots \ldots \ldots \ldots$

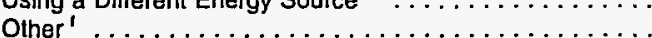

Industrial Organic Chemicals, nec

Participation in One or More of the Following Types of

Programs . . . . . . . . . . . . . . . . . .

Energy Audits . . . . . . . . . . . . . . . . . . .

Direct Electricity Load Control . . . . . . . . . . . . .

Special Rate Schedule ${ }^{\circ} \ldots \ldots \ldots \ldots \ldots \ldots \ldots \ldots$

Standby Generation Program . . . . . . . . . . . . . .

Equipment Rebate(s) .

Equipment Installation or Retrofit for the Primary Purpose of Improving Energy Efficiency Affecting:

Steam Production ${ }^{c} \ldots \ldots \ldots \ldots \ldots \ldots \ldots \ldots$

Direct/Indirect Process Heating . . . . . . . . . . .

Direct Process Cooling/Refrigeration . . . . . . . . . .

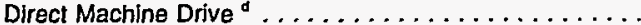

Facility Heating, Ventilation, and Air Conditioning .....

Facillty Lighting . . . . . . . . . . . . . . .

Equipment Installation/Retrofit for the Primary Purpose of

Using a Different Energy Source ${ }^{\circ} \ldots \ldots \ldots \ldots \ldots \ldots$

Other'

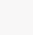



\section{9}

49

28

52

*

25

31

31
20

32

5
35

W

W

$\begin{array}{rr}848 & W \\ 671 & 19 \\ 353 & 15 \\ 415 & 18 \\ 30 & W \\ 2 & \end{array}$

$W$
19
15
18
$W$
1

45

W

775

640

322

377

26

14.3

14.1

16.6

14.7

24.3

17.1

16.1

17.0

22.5

19.3

28.8

23.1

28.5

25.8

$\begin{array}{rrrrrr}375 & W & 34 & 328 & W & 7.5 \\ 540 & W & 24 & 504 & W & 7.9 \\ 432 & W & 8 & 415 & W & 10.3 \\ 566 & W & 27 & 521 & W & 7.1 \\ 225 & 9 & 12 & 202 & 1 & 10.2 \\ 266 & 17 & W & 231 & W & 8.3 \\ 145 & & 2 & 142 & 0 & 11.7 \\ 53 & 1 & 1 & 51 & 0 & 10.5\end{array}$

See footnotes at end of table. 
Table A41. Total Inputs of Energy for Heat, Power, and Electricity Generation by Census Region, Industry Group, Selected Industries, and Type of Energy Management Program, 1991 (Continued)

(Estimates in Trillion Btu)

\begin{tabular}{|c|c|c|c|c|c|c|c|}
\hline \multirow{2}{*}{$\begin{array}{c}\text { SIC } \\
\text { Code }\end{array}$} & \multirow{2}{*}{$\begin{array}{l}\text { Industry Groups } \\
\text { and Industry }\end{array}$} & \multirow[b]{2}{*}{ Total } & \multicolumn{4}{|c|}{ Census Region } & \multirow{3}{*}{$\begin{array}{c}\text { RSE } \\
\text { Row } \\
\text { Factorg }\end{array}$} \\
\hline & & & Northeast & Midwest & South & West & \\
\hline & RSE Column Factors: & 0.7 & 1.3 & 1.0 & 0.9 & 1.2 & \\
\hline
\end{tabular}

2873

Nitrogenous Fertilizers

Participation in One or More of the Following Types of

Programs . . . . . . . . . . . . . . . . . .

Energy Audits . . . . . . . . . . . . . . . . .

Direct Electricity Load Control $\ldots \ldots \ldots \ldots \ldots \ldots \ldots$

Special Rate Schedule ${ }^{b} \ldots \ldots \ldots \ldots \ldots \ldots \ldots \ldots$

Standby Generation Program . . . . . . . . . . . . .

Equipment Rebate(s) . . . . . . . . . . . . . . . . . . . .

Equipment Installation or Retrofit for the Primary Pumpose of Improving Energy Efficiency Affecting:

Steam Production ${ }^{c} \ldots \ldots \ldots \ldots \ldots \ldots \ldots \ldots \ldots$

Direct/Indirect Process Heating . . . . . . . . . . . .

Direct Process Cooling/Refrigeration . . . . . . . . . .

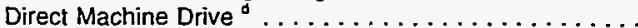

Facility Heating, Ventilation, and Air Conditioning $\ldots \ldots$.

Facility Lighting . . . . . . . . . . . . . . . .

Equipment Installation/Retrofit for the Primary Purpose of

Using a Different Energy Source ${ }^{\circ} \ldots \ldots \ldots \ldots \ldots \ldots$

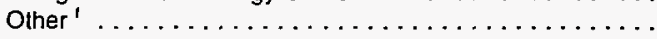

Phosphatic Fertilizers

Participation in One or More of the Following Types of

Programs ..........................

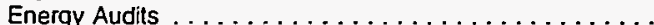

Direct Electricity Load Control $\ldots \ldots \ldots \ldots \ldots \ldots \ldots \ldots$

Special Rate Schedule ${ }^{\circ} \ldots \ldots \ldots \ldots \ldots \ldots \ldots \ldots$

Standby Generation Program . . . . . . . . . . . . . . . .

Equipment Rebate(s) . . . . . . . . . . . . . . . . .

Equipment Installation or Retrofit for the Primary Purpose of

Improving Energy Efficiency Affecting:

Steam Production ${ }^{c} \ldots \ldots \ldots \ldots \ldots \ldots \ldots \ldots \ldots \ldots$

Direct/lndirect Process Heating . . . . . . . . . . . .

Direct Process Cooling/Refrigeration . . . . . . . . . . .

Direct Machine Drive ${ }^{\mathbf{d}} \ldots \ldots \ldots \ldots \ldots \ldots \ldots \ldots$

Facility Heating, Ventilation, and Air Conditioning $\ldots \ldots$

Facility Lighting . . . . . . . . . . . . . . . . . . . .

Equipment Installation/Retrofit for the Primary Purpose of

Using a Different Energy Source ${ }^{\circ} \ldots \ldots \ldots \ldots \ldots \ldots$.

Other' $^{\prime} \ldots \ldots \ldots \ldots \ldots \ldots \ldots \ldots \ldots \ldots \ldots \ldots$

PETROLEUM and COAL PRODUCTS

Participation in One or More of the Following Types of

Programs . . . . . . . . . . . . . . . . . . . .

Energy Audits $\ldots \ldots \ldots \ldots \ldots \ldots \ldots \ldots \ldots \ldots \ldots$

Direct Electricity Load Control . . . . . . . . . . . . .

Special Rate Schedule ${ }^{b} \ldots \ldots \ldots \ldots \ldots \ldots \ldots \ldots$

Standby Generation Program . . . . . . . . . . . .

Equipment Rebate(s)

Equipment Installation or Retrofit for the Primary Pupose of

Improving Energy Efficiency Affecting:

Steam Production ${ }^{c} \ldots \ldots \ldots \ldots \ldots \ldots \ldots \ldots$

Direct/lndirect Process Heating . . . . . . . . . . . .

Direct Process Cooling/Refrigeration . . . . . . . . .

Direct Machine Drive ${ }^{d} \ldots \ldots \ldots \ldots \ldots \ldots \ldots \ldots \ldots$

Facility Heating, Ventilation, and Air Conditioning ......

Facility Lighting $\ldots \ldots \ldots \ldots \ldots \ldots \ldots \ldots$

Equipment Installation/Retrofit for the Primary Purpose of

Using a Different Energy Source ${ }^{\circ} \ldots \ldots \ldots \ldots \ldots \ldots$

Other'

$\begin{array}{rr}158 & 0 \\ 69 & 0 \\ 45 & 0 \\ 93 & 0 \\ 0 & 0 \\ 0 & 0 \\ & \\ 39 & \\ 55 & 0 \\ 62 & 0 \\ 36 & 0 \\ 12 & 0 \\ 26 & 0 \\ 0 & 0 \\ 0 & 0\end{array}$

33
33
7
0
0
0

13
12
28
0
12
12
0
0

123
34
38
93
$Q$
0

25
43
34
34
0
12
0
0

30.0

34.0

43.5

33.4

NF

37.4

31.4

35.2

37.6

42.1

39.5

NF

NF

See footnotes at end of table. 
Table A41. Total Inputs of Energy for Heat, Power, and Electricity Generation by Census Region, Industry Group, Selected Industries, and Type of Energy Management Program, 1991 (Continued)

(Estimates in Trillion Btu)

\begin{tabular}{|c|c|c|c|c|c|c|c|}
\hline \multirow[b]{2}{*}{$\begin{array}{l}\text { SIC } \\
\text { Code* }\end{array}$} & \multirow[b]{2}{*}{$\begin{array}{l}\text { Industry Groups } \\
\text { and Industry }\end{array}$} & \multirow[b]{2}{*}{ Total } & \multicolumn{4}{|c|}{ Census Region } & \multirow{3}{*}{$\begin{array}{l}\text { RSE } \\
\text { Row } \\
\text { Factors }\end{array}$} \\
\hline & & & Northeast & Midwest & South & West & \\
\hline & Column Factors: & 0.7 & 1.3 & 1.0 & 0.9 & 1.2 & \\
\hline
\end{tabular}

2911

Petroleum Refining

Participation In One or More of the Following Types of

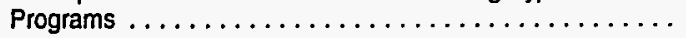

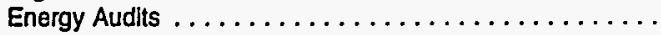

Direct Electricity Load Control . . . . . . . . . . . . .

Speclal Rate Schedule ${ }^{b} \ldots \ldots \ldots \ldots \ldots \ldots \ldots \ldots \ldots$

Standby Generation Program . . . . . . . . . . . . .

Equipment Rebate(s) . . . . . . . . . . . . . . . . . . .

Equipment Installation or Retrofit for the Primary Purpose of Improving Energy Efficiency Affecting:

Steam Production ${ }^{c} \ldots \ldots \ldots \ldots \ldots \ldots \ldots \ldots \ldots$

Dlrect/lndirect Process Heating . . . . . . . . . . .

Direct Process Cooling/Refrigeration . . . . . . . . .

Direct Machine Drive ${ }^{d} \ldots \ldots \ldots \ldots \ldots \ldots \ldots \ldots$

Facillty Heating, Ventilation, and Air Conditioning .....

Facillty Lighting . . . . . . . . . . . . . . . .

Equipment Installation/Retrofit for the Primary Purpose of

Using a Different Energy Source ${ }^{\circ} \ldots \ldots \ldots \ldots \ldots \ldots \ldots$

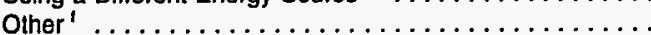

$\begin{array}{rr}2,215 & 143 \\ 1,445 & \\ 663 & \\ 1,057 & 138 \\ 219 & \\ 107 & \end{array}$

$\begin{array}{rr}143 & 3 \\ W & \\ W & \\ W & 185 \\ 0 & \end{array}$

$\begin{array}{rrrr}359 & 1,179 & 534 & 2.4 \\ W & 787 & 306 & 2.8 \\ W & 310 & 155 & 3.4 \\ 185 & 526 & 208 & 2.8 \\ 0 & 191 & W & 4.9 \\ W & 0 & W & 4.8\end{array}$

RUBBER and MISC. PLASTICS PRODUCTS

Participation in One or More of the Following Types of

Programs . . . . . . . . . . . . . . . . . .

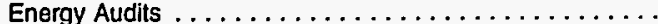

Dlrect Electricity Load Control $\ldots \ldots \ldots \ldots \ldots \ldots \ldots$

Special Rate Schedule ${ }^{b} \ldots \ldots \ldots \ldots \ldots \ldots \ldots \ldots$

Standby Generation Program . . . . . . . . . . . . . .

Equipment Rebate(s) . . . . . . . . . . . . . . . .

Equipment Installation or Retrofit for the Primary Purpose of Improving Energy Efficiency Affecting:

Steam Production ${ }^{c} \ldots \ldots \ldots \ldots \ldots \ldots \ldots \ldots \ldots$

Direct/lndirect Process Heating . . . . . . . . . . . .

Direct Process Cooling/Refrigeration . . . . . . . . .

Direct Machine Drive ${ }^{2} . . \ldots \ldots \ldots \ldots \ldots \ldots \ldots \ldots$

Facllity Heating, Ventilation, and Air Conditioning .....

Facllity Llghting . . . . . . . . . . . . . . . . . . . . .

Equipment Installatlon/Retrofit for the Primary Purpose of

Using a Different Energy Source ${ }^{\circ} \ldots \ldots \ldots \ldots \ldots \ldots \ldots$

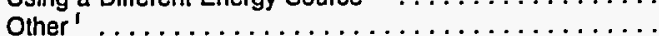

$\begin{array}{rr}1,216 & W \\ 1,215 & W \\ 430 & W \\ 1,075 & W \\ 492 & W \\ 956 & W \\ 305 & \\ 69 & 0\end{array}$

$w$
$w$
$w$
$w$
$w$
$w$
42
$w$

$\begin{array}{rrr}676 & 269 & 3.1 \\ 586 & 368 & 3.1 \\ W & 175 & 3.2 \\ 532 & 317 & 2.9 \\ 207 & 184 & 4.0 \\ 459 & 330 & 3.1 \\ & & \\ 96 & 167 & 3.9 \\ 23 & 0 & 5.5\end{array}$

3011

Tires and Inner Tubes

Participation in One or More of the Following Types of

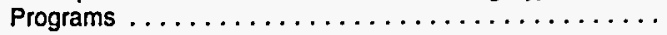

Energy Audits . . . . . . . . . . . . . . . . .

Dlrect Electricity Load Control $\ldots \ldots \ldots \ldots \ldots \ldots \ldots$

Special Rate Schedule ${ }^{b} \ldots \ldots \ldots \ldots \ldots \ldots \ldots \ldots \ldots$

Standby Generation Program . . . . . . . . . . . . .

Equlpment Rebate(s) . . . . . . . . . . . . . . .

Equipment Installation or Retrofit for the Primary Purpose of Improving Energy Efficiency Affecting:

Steam Production ${ }^{\circ} \ldots \ldots \ldots \ldots \ldots \ldots \ldots \ldots \ldots$

Direct/lndlrect Process Heating . . . . . . . . . . . . .

Direct Process Cooling/Refrigeration . . . . . . . . . .

Direct Machine Drive ${ }^{d} \ldots \ldots \ldots \ldots \ldots \ldots \ldots$

Facility Heating, Ventilation, and Air Conditioning ......

Facllity Lighting . . . . . . . . . . . . . . .

Equipment Installation/Retrofit for the Primary Purpose of

Using a Different Energy Source ${ }^{\circ} \ldots \ldots \ldots \ldots \ldots \ldots \ldots$

Other'

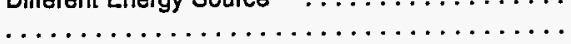

\begin{tabular}{|c|c|c|c|c|}
\hline 37 & 2 & 11 & 24 & - \\
\hline 32 & 1 & 8 & 22 & • \\
\hline 16 & $W$ & $w$ & 11 & 0 \\
\hline 25 & 1 & 6 & 18 & 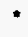 \\
\hline$W$ & 0 & $w$ & • & 0 \\
\hline$w$ & - & W & 0 & • \\
\hline 28 & 1 & 7 & 20 & * \\
\hline 14 & 1 & 4 & 9 & $\star$ \\
\hline 11 & $w$ & $W$ & 8 & 0 \\
\hline 20 & 1 & 6 & 13 & 0 \\
\hline 22 & 1 & 7 & 14 & 0 \\
\hline 24 & 1 & 8 & 14 & • \\
\hline 8 & $w$ & $w$ & 4 & 0 \\
\hline 1 & 0 & 0 & 1 & • \\
\hline
\end{tabular}

See footnotes at end of table.

Energy Information Administration/Manufacturing Consumption of Energy 1991 
Table A41. Total Inputs of Energy for Heat, Power, and Electricity Generation by Census Region, Industry Group, Selected Industries, and Type of Energy Management Program, 1991 (Continued)

(Estimates in Trillion Btu)

\begin{tabular}{|c|c|c|c|c|c|c|c|}
\hline \multirow{2}{*}{$\begin{array}{l}\text { SIC } \\
\text { Code }\end{array}$} & \multirow{2}{*}{$\begin{array}{l}\text { Industry Groups } \\
\text { and Industry }\end{array}$} & \multirow[b]{2}{*}{ Total } & \multicolumn{4}{|c|}{ Census Region } & \multirow{3}{*}{$\begin{array}{c}\text { RSE } \\
\text { Row } \\
\text { Factors }\end{array}$} \\
\hline & & & Northeast & Midwest & South & West & \\
\hline & RSE Column Factors: & 0.7 & 1.3 & 1.0 & 0.9 & 1.2 & \\
\hline
\end{tabular}

308

Miscellaneous Plastic Products, nec

Participation in One or More of the Following Types of

Programs

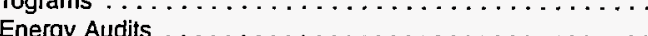

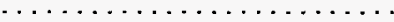

Special Rate Schedule ${ }^{b} \ldots \ldots \ldots \ldots \ldots \ldots \ldots \ldots \ldots \ldots$

Standby Generation Program . . . . . . . . . . . . . . . .

Equipment Rebate(s) . . . . . . . . . . . . . . . . . . . . .

Equipment Installation or Retrofit for the Primary Purpose of Improving Energy Efficiency Affecting:

Steam Production ${ }^{c}$

Direct/lndirect Process Heating . . . . . . . . . . . . .

Direct Process Cooling/Refrigeration . . . . . . . . . . . . .

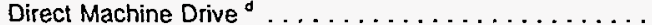

Facility Heating, Ventilation, and Air Conditioning .....

Facility Lighting . . . . . . . . . . . . . . . .

Equipment Installation/Retrofit for the Primary Purpose of

Using a Different Energy Source ${ }^{\circ} \ldots \ldots \ldots \ldots \ldots \ldots$

Other"

\section{LEATHER and LEATHER PRODUCTS}

Participation in One or More of the Following Types of

\section{Programs}

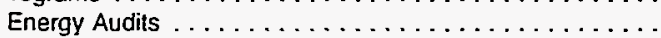

Direct Electricity Load Control . . . . . . . . . . . .

Special Rate Schedule ${ }^{\circ} . . \ldots \ldots \ldots \ldots \ldots \ldots \ldots$

Standby Generatıon Program . . . . . . . . . . . . . .

Equipment Rebate(s) . . . . . . . . . . . . . . . .

Equipment Installation or Retroft for the Primary Purpose of Improving Energy Efficiency Affecting:

Steam Production ${ }^{c}$

Direct/Indirect Process Heating .......

Direct Process Cooling/Refrigeration . . . . . . . . . .

Direct Machine Drive ${ }^{a} \ldots \ldots \ldots \ldots \ldots \ldots \ldots \ldots$

Facility Heating, Ventilation, and Air Conditioning . . . . .

Facility Lighting . . . . . . . . . . . . . . . . .

Equipment Installation/Retrofit for the Primary Purpose of

Using a Different Energy Source ${ }^{\circ} \ldots \ldots \ldots \ldots \ldots \ldots \ldots$

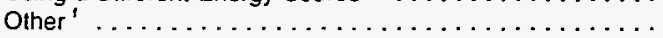

STONE, CLAY and GLASS PRODUCTS

Participation in One or More of the Following Types of

Programs . . . . . . . . . . . . . . . . . . .

Energy Audits . . . . . . . . . . . . . . . . . . .

Direct Electricity Load Control . . . . . . . . . . .

Special Rate Schedule ${ }^{b} \ldots \ldots \ldots \ldots \ldots \ldots \ldots \ldots \ldots$

Standby Generation Program . . . . . . . . . . . . . . .

Equipment Rebate(s) . . . . . . . . . . . . . . . .

Equipment Installation or Retrofit for the Primary Purpose of Improving Energy Efficiency Affecting:

Steam Production ${ }^{c} \ldots \ldots \ldots \ldots \ldots \ldots \ldots \ldots$

Direct/Indirect Process Heating . . . . . . . . . . . . .

Direct Process Cooling/Refrigeration . . . . . . . . . . .

Direct Machine Drive ${ }^{\circ} \ldots \ldots \ldots \ldots \ldots \ldots \ldots \ldots$

Facilty Heating, Ventulation, and Air Conditioning . . . . .

Facility Lighting . . . . . . . . . . . . . . . . . . .

Equipment Installatıon/Retrofit for the Primary Purpose of

Using a Different Energy Source ${ }^{\circ} \ldots \ldots \ldots \ldots \ldots \ldots \ldots$

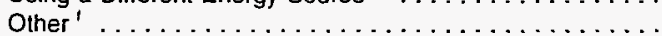

\begin{tabular}{|c|c|c|c|c|c|}
\hline 71 & 14 & 29 & 21 & 7 & 12.0 \\
\hline 37 & 6 & 15 & 14 & 3 & 15.9 \\
\hline 22 & 2 & 11 & 7 & 1 & 23.2 \\
\hline 32 & 4 & 11 & 13 & 4 & 15.9 \\
\hline 1 & 0 & * & 1 & • & 52.3 \\
\hline 8 & 1 & 3 & 1 & 2 & 28.1 \\
\hline 10 & 3 & 4 & 3 & 0 & 25.1 \\
\hline 15 & 3 & 9 & 3 & 0 & 26.2 \\
\hline 13 & 3 & 5 & 4 & 1 & 25.0 \\
\hline 28 & 7 & 9 & 9 & 3 & 17.0 \\
\hline 25 & 4 & 12 & 8 & 1 & 19.1 \\
\hline 37 & 7 & 14 & 13 & 2 & 15.3 \\
\hline 3 & * & 2 & $\mathbf{Q}$ & " & 45.3 \\
\hline 5 & $Q$ & 2 & $Q$ & 0 & 42.0 \\
\hline
\end{tabular}

ee footnotes at end of table. 
Table A41. Total Inputs of Energy for Heat, Power, and Electricity Generation by Census Region, Industry Group, Selected Industries, and Type of Energy Management Program, 1991 (Continued)

(Estimates in Trillion Btu)

\begin{tabular}{|c|c|c|c|c|c|c|c|}
\hline \multirow{2}{*}{$\begin{array}{c}\text { SIC } \\
\text { Code }\end{array}$} & \multirow{2}{*}{$\begin{array}{l}\text { Industry Groups } \\
\text { and Industry }\end{array}$} & \multirow[b]{2}{*}{ Total } & \multicolumn{4}{|c|}{ Census Region } & \multirow{3}{*}{$\begin{array}{c}\text { RSE } \\
\text { Row } \\
\text { Factors }\end{array}$} \\
\hline & & & Northeast & Midwest & South & West & \\
\hline & RSE Column Factors: & 0.7 & 1.3 & 1.0 & 0.9 & 1.2 & \\
\hline
\end{tabular}

3211

Flat Glass

Participation in One or More of the Following Types of

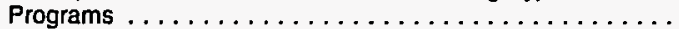

Energy Audits $\ldots \ldots \ldots \ldots \ldots \ldots \ldots \ldots \ldots \ldots \ldots$

Direct Electricity Load Control $\ldots \ldots \ldots \ldots \ldots \ldots \ldots$

Special Rate Schedule ${ }^{b} \ldots \ldots \ldots \ldots \ldots \ldots \ldots \ldots . . . \ldots$

Standby Generation Program . . . . . . . . . . . . .

Equipment Rebate(s) ......................

Equipment Installation or Retrofit for the Primary Purpose of Improving Energy Efficiency Affecting:

Steam Production ${ }^{c} \ldots \ldots \ldots \ldots \ldots \ldots \ldots \ldots$

Direct/Indirect Process Heating . . . . . . . . . . . .

Direct Process Cooling/Refrigeration . . . . . . . . . .

Direct Machine Drive ${ }^{d}$............................

Facility Heating, Ventilation, and Air Conditioning $\ldots \ldots$.

Facility Lighting . . . . . . . . . . . . . . . . . . .

Equipment Installation/Retrofit for the Primary Purpose of

Using a Different Energy Source ${ }^{\circ} \ldots \ldots \ldots \ldots \ldots \ldots$

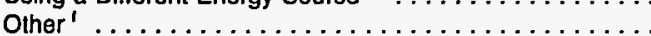

20

14

7
$w$

W

15

W

16

9

W

Glass Containers

Participation in One or More of the Following Types of

Programs . . . . . . . . . . . . . . . . . . . . .

Energy Audits $\ldots \ldots \ldots \ldots \ldots \ldots \ldots \ldots \ldots$

Direct Electricity Load Control $\ldots \ldots \ldots \ldots \ldots \ldots \ldots$

Special Rate Schedule ${ }^{b} \ldots \ldots \ldots \ldots \ldots \ldots \ldots \ldots \ldots$

Standby Generation Program . . . . . . . . . . . . . .

Equipment Rebate(s) . . . . . . . . . . . . . . .

Equipment Installation or Retrofit for the Primary Purpose of Improving Energy Efficiency Affecting:

Steam Production ${ }^{c} \ldots \ldots \ldots \ldots \ldots \ldots \ldots \ldots \ldots$

Direct/indirect Process Heating . . . . . . . . . . . . .

Direct Process Cooling/Refrigeration . . . . . . . . . . . .

Direct Machine Drive ${ }^{d} \ldots \ldots \ldots \ldots \ldots \ldots \ldots \ldots \ldots$

Facility Heating, Ventilation, and Air Conditioning ......

Facility Lighting . . . . . . . . . . . . . . . . . . . . . . . .

Equipment Installation/Retrofit for the Primary Purpose of

Using a Dlfferent Energy Source ${ }^{\bullet} \ldots \ldots \ldots \ldots \ldots \ldots \ldots$

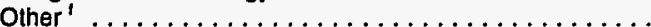

Pressed and Blown Glass, nec.

Participation in One or More of the Following Types of

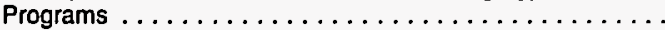

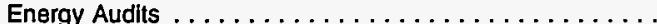

Direct Electricity Load Control . . . . . . . . . . . .

Special Rate Schedule ${ }^{b} \ldots \ldots \ldots \ldots \ldots \ldots \ldots \ldots \ldots \ldots$

Standby Generation Program . . . . . . . . . . . . . . .

Equlpment Rebate(s) . . . . . . . . . . . . . . . . .

Equipment Installation or Retrofit for the Primary Purpose of Improving Energy Efficiency Affecting:

Steam Production $^{c} \ldots \ldots \ldots \ldots \ldots \ldots \ldots \ldots \ldots$

Direct/lndirect Process Heating . . . . . . . . . . . . .

Direct Process Cooling/Refrigeration . . . . . . . . .

Direct Machine Drive ${ }^{d} \ldots \ldots \ldots \ldots \ldots \ldots \ldots \ldots \ldots$

Facllity Heating, Ventilation, and Air Conditioning ......

Facility Lighting . . . . . . . . . . . . . . .

Equipment Installation/Retrofit for the Primary Purpose of

Using a Different Energy Source ${ }^{\circ} \ldots \ldots \ldots \ldots \ldots \ldots$.

Other'

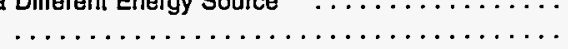

See footnotes at end of table. 
Table A41. Total Inputs of Energy for Heat, Power, and Electricity Generation by Census Region, Industry Group, Selected Industries, and Type of Energy Management Program, 1991 (Continued)

(Estimates in Trillion Btu)

\begin{tabular}{|c|c|c|c|c|c|c|c|}
\hline \multirow{2}{*}{$\begin{array}{l}\text { SIC } \\
\text { Code }\end{array}$} & \multirow{2}{*}{$\begin{array}{l}\text { Industry Groups } \\
\text { and Industry }\end{array}$} & \multirow[b]{2}{*}{ Total } & \multicolumn{4}{|c|}{ Census Region } & \multirow{3}{*}{$\begin{array}{c}\text { RSE } \\
\text { Row } \\
\text { Factors }\end{array}$} \\
\hline & & & Northeast & Midwest & South & West & \\
\hline & RSE Column Factors: & 0.7 & 1.3 & 1.0 & 0.9 & 1.2 & \\
\hline
\end{tabular}

3241

Cement, Hydraulic

Participation in One or More of the Following Types of

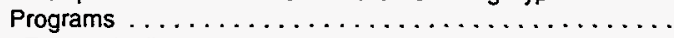

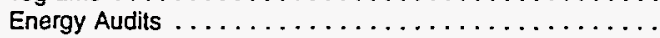

Direct Electricity Load Control $\ldots \ldots \ldots \ldots \ldots \ldots \ldots$.

Special Rate Schedule ${ }^{\mathrm{b}} \ldots \ldots \ldots \ldots \ldots \ldots \ldots \ldots$

Standby Generation Program . . . . . . . . . . . .

Equipment Rebate(s) .....................

Equipment Installation or Retrofit for the Primary Purpose of Improving Energy Efficiency Affecting:

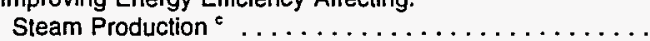

Direct/ndirect Process Heating . . . . . . . . . . . . .

Direct Process Cooling/Refrigeration ...............

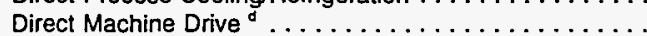

Facility Heating, Ventilation, and Air Conditioning ......

Facility Lighting $\ldots \ldots \ldots \ldots \ldots \ldots \ldots \ldots \ldots$

Equipment Installation/Retrofit for the Primary Purpose of

Using a Different Energy Source ${ }^{\bullet} \ldots \ldots \ldots \ldots \ldots \ldots$.

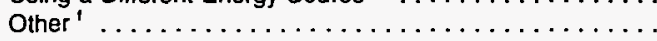

Lime

Participation in One or More of the Following Types of

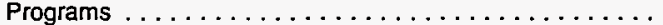

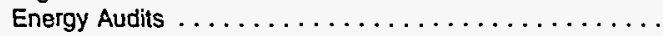

Direct Electricity Load Control . ................

Special Rate Schedule ${ }^{b} \ldots \ldots \ldots \ldots \ldots \ldots \ldots \ldots \ldots$

Standby Generation Program . . . . . . . . . . . . . .

Equipment Rebate(s) .......................

Equipment Installation or Retrofit for the Primary Purpose of Improving Energy Eficiency Affecting:

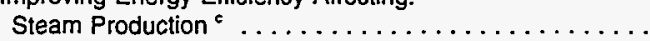

Direct/Indirect Process Heating . ................

Direct Process Cooling/Refrigeration . . . . . . . . . .

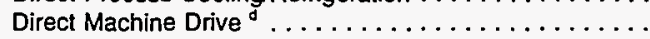

Facility Heating, Ventilation, and Air Conditioning ......

Facility Lighting . . . . . . . . . . . . . . . .

Equipment Installation/Retrofit for the Primary Purpose of

Using a Different Energy Source ${ }^{\circ}$................

Other ${ }^{\prime} \ldots \ldots \ldots \ldots \ldots \ldots \ldots \ldots \ldots$.

Mineral Wool

Participation in One or More of the Following Types of

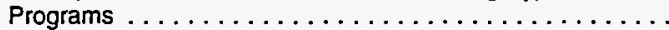

Energy Audits . . . . . . . . . . . . . . . . . .

Direct Electricity Load Control $\ldots \ldots \ldots \ldots \ldots \ldots \ldots \ldots$

Special Rate Schedule ${ }^{\circ} \ldots \ldots \ldots \ldots \ldots \ldots \ldots \ldots$

Standby Generation Program . . . . . . . . . . . . . .

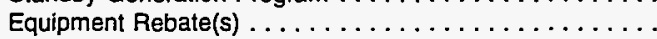

Equipment installation or Retrofit for the Primary Purpose of

Improving Energy Efficiency Affecting:

Steam Production ${ }^{c} \ldots \ldots \ldots \ldots \ldots \ldots \ldots$.

Direct/lndirect Process Heating . . . . . . . . . . . . . . . . .

Direct Process Cooling/Refrigeration . . . . . . . . .

Direct Machine Drive ${ }^{\circ} \ldots \ldots \ldots \ldots \ldots \ldots \ldots$.

Facility Heating, Ventilation, and Air Conditioning ......

Facility Lighting $\ldots \ldots \ldots \ldots \ldots \ldots \ldots \ldots$

Equipment Installation/Retrofit for the Primary Purpose of

Using a Different Energy Source ${ }^{\circ} \ldots \ldots \ldots \ldots \ldots \ldots$

Other'

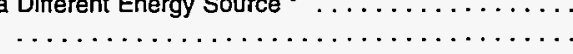

$\begin{array}{rrrrrr}243 & 26 & 65 & 79 & 71 & 12.3 \\ 128 & \mathrm{Q} & 35 & 43 & 45 & 19.8 \\ 144 & 15 & 31 & 55 & 42 & 17.0 \\ 189 & 24 & 57 & 65 & 43 & 13.5 \\ 12 & 0 & 0 & Q & W & 21.0 \\ 37 & 19 & 0 & Q & 15 & 17.3 \\ & & & & & \\ 18 & W & 0 & Q & W & 22.4 \\ 38 & 0 & 20 & 9 & 9 & 22.7 \\ Q & 0 & 0 & Q & 0 & \text { NF } \\ 153 & 24 & 37 & 36 & 56 & 14.0 \\ 53 & W & W & 11 & 30 & 22.8 \\ 129 & 21 & 31 & 28 & 49 & 15.5 \\ & & & & & \\ 45 & 0 & 14 & 15 & 0 & 28.7 \\ W & 0 & W & 0 & 0 & 31.3\end{array}$

See footnotes at end of table. 
Table A41. Total Inputs of Energy for Heat, Power, and Electricity Generation by Census Region, Industry Group, Selected Industries, and Type of Energy Management Program, 1991 (Continued)

(Estimates in Trillion Btu)

\begin{tabular}{|c|c|c|c|c|c|c|c|}
\hline \multirow{2}{*}{$\begin{array}{l}\text { SIC } \\
\text { Code }\end{array}$} & \multirow{2}{*}{$\begin{array}{l}\text { Industry Groups } \\
\text { and Industry }\end{array}$} & \multirow[b]{2}{*}{ Total } & \multicolumn{4}{|c|}{ Census Region } & \multirow{3}{*}{$\begin{array}{c}\text { RSE } \\
\text { Row } \\
\text { Factors }\end{array}$} \\
\hline & & & Northeast & Midwest & South & West & \\
\hline & RSE Column Factors: & 0.7 & 1.3 & 1.0 & 0.9 & 1.2 & \\
\hline
\end{tabular}

33

PRIMARY METAL INDUSTRIES

Partlcipatlon in One or More of the Following Types of

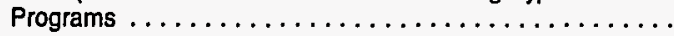

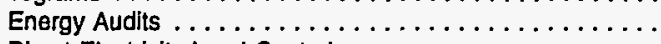

Direct Electrlcity Load Control $\ldots \ldots \ldots \ldots \ldots \ldots \ldots$

Special Rate Schedule ${ }^{\circ} \ldots \ldots \ldots \ldots \ldots \ldots \ldots \ldots$.

Standby Generation Program ...................

Equlpment Rebate(s) ......................

Equipment Installation or Retrofit for the Primary Purpose of Improving Energy Efficiency Affecting:

Steam Productlon ${ }^{c} \ldots \ldots \ldots \ldots$

Direct/Indirect Process Heating . ................

Dlrect Process Cooling/Refrigeration . . . . . . . . . . .

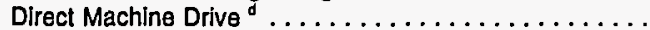

Facllity Heating, Ventllation, and Alr Conditioning ......

Facilliy Llghting . .....................

Equipment Installation/Retrofit for the Primary Purpose of

Using a Different Energy Source ${ }^{\circ} \ldots \ldots \ldots \ldots \ldots \ldots$

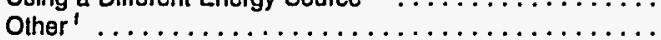

$\begin{array}{rrrrrr}1,941 & 277 & 1,021 & 446 & 198 & 4.1 \\ 1,378 & 102 & 910 & 279 & 87 & 4.3 \\ 1,264 & 221 & 733 & 251 & 59 & 4.7 \\ 1,488 & 256 & 792 & 335 & 106 & 4.4 \\ 305 & W & 291 & 7 & W & 9.2 \\ 43 & 11 & 19 & 3 & 11 & 14.7 \\ & & & & & \\ 969 & 22 & 679 & 216 & 53 & 6.4 \\ 1,274 & 174 & 721 & 313 & 65 & 5.1 \\ 274 & W & W & W & 3 & 10.7 \\ 818 & 30 & 561 & 189 & 38 & 5.5 \\ 779 & 32 & 667 & 72 & 7 & 7.5 \\ 1,366 & 230 & 876 & 224 & 37 & 5.3 \\ & & & & & \\ 431 & 48 & 355 & 25 & 2 & 9.9 \\ 88 & W & 31 & W & 34 & 6.3\end{array}$

3312

Blast Furnaces and Steel Mills

Partlclpation in One or More of the Following Types of

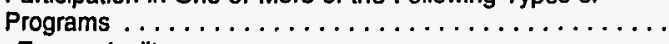

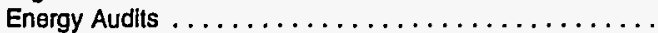

Dlrect Electricity Load Control . . . . . . . . . . . . . .

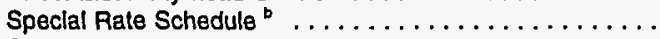

Standby Generation Program . . . . . . . . . . . . . . .

Equipment Rebate(s) . . . . . . . . . . . . . . . .

Equipment Installation or Retrofit for the Primary Purpose of Improving Energy Efficiency Affecting:

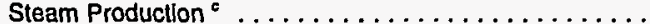

Direct/Indirect Process Heating . ..................

Direct Process Cooling/Refrigeration ...................

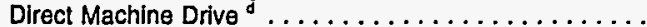

Facillty Heating, Ventllation, and Air Conditioning ......

Facllity Lighting . . ....................

Equipment Installation/Retrofit for the Primary Purpose of

Using a Different Energy Source ${ }^{\circ}$.................

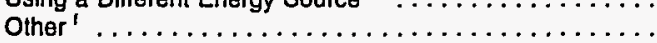

88

W

1,444

1,109

1,032

1,169

290

907

1,080

W

676

715

1,177

396

W

Electrometalurgical Products in One or More of the Following Types of

Particlpation in One or More of the Following Types of

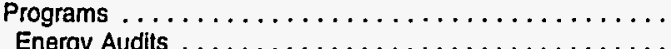

Direct Electricity Load Control ................

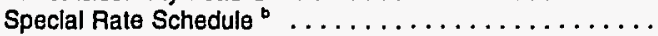

Standby Generation Program . . . . . . . . . . . . . . . . .

Equlpment Rebate(s) . ....................

Equipment Installation or Retrofit for the Primary Purpose of Improving Energy Efficiency Affecting:

Steam Production ${ }^{c} \ldots \ldots \ldots \ldots \ldots \ldots \ldots \ldots \ldots$.

Direct/Indirect Process Heating . ................

Direct Process Cooling/Refrigeration . . . . . . . . . . . .

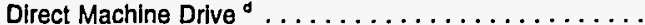

Facility Heating, Ventilation, and Air Conditioning .....

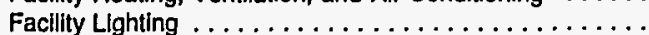

Equipment Installatlon/Retrofit for the Primary Purpose of

Using a Different Energy Source ${ }^{\circ} \ldots \ldots \ldots \ldots \ldots \ldots$

Other'

\begin{tabular}{|c|c|c|c|c|}
\hline W & * & 6 & $w$ & 0 \\
\hline W & • & $w$ & $w$ & 0 \\
\hline 5 & • & 5 & 0 & 0 \\
\hline W & 0 & 3 & $w$ & 0 \\
\hline " & 0 & " & 0 & 0 \\
\hline W & 0 & $w$ & 0 & 0 \\
\hline W & 0 & 0 & w & 0 \\
\hline$w$ & 0 & $w$ & 0 & 0 \\
\hline 0 & 0 & 0 & 0 & 0 \\
\hline$w$ & * & $w$ & 0 & 0 \\
\hline$w$ & * & $w$ & 0 & 0 \\
\hline W & * & $w$ & $w$ & 0 \\
\hline$w$ & 0 & 0 & $w$ & 0 \\
\hline 0 & 0 & 0 & 0 & 0 \\
\hline
\end{tabular}

9.7

13.9

10.9

13.0

16.8

16.8

17.3

16.8

NF

12.9

14.3

13.4

17.3 NF

See footnotes at end of table. 
Table A41. Total Inputs of Energy for Heat, Power, and Electricity Generation by Census Region, Industry Group, Selected Industries, and Type of Energy Management Program, 1991 (Continued)

(Estimates in Trillion Btu)

\begin{tabular}{|c|c|c|c|c|c|c|c|}
\hline \multirow{2}{*}{$\begin{array}{l}\text { SIC } \\
\text { Code: }\end{array}$} & \multirow[b]{2}{*}{$\begin{array}{l}\text { Industry Groups } \\
\text { and Industry }\end{array}$} & \multirow[b]{2}{*}{ Total } & \multicolumn{4}{|c|}{ Census Region } & \multirow{3}{*}{$\begin{array}{c}\text { RSE } \\
\text { Row } \\
\text { Factors }\end{array}$} \\
\hline & & & Northeast & Midwest & South & West & \\
\hline & Column Factors: & 0.7 & 1.3 & 1.0 & 0.9 & 1.2 & \\
\hline
\end{tabular}

3321

Gray and Ductile Iron Foundries

Participation in One or More of the Following Types of

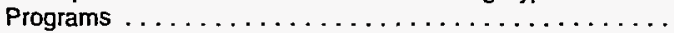

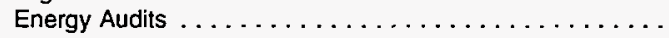

Direct Electricity Load Control $\ldots \ldots \ldots \ldots \ldots \ldots \ldots$

Special Rate Schedule ${ }^{b} \ldots . . \ldots \ldots \ldots \ldots \ldots \ldots$.

Standby Generation Program . . . . . . . . . . . . . .

Equipment Rebate(s) . . . . . . . . . . . . . . . . . . .

Equipment Installation or Retrofit for the Primary Purpose of

Improving Energy Efficiency Affecting:

Steam Production ${ }^{c} \ldots \ldots \ldots \ldots \ldots \ldots \ldots \ldots \ldots$

Direct/lndirect Process Heating . . . . . . . . . . . . . .

Direct Process Cooling/Refrigeration . . . . . . . . . .

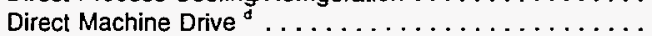

Facility Heating, Ventilation, and Air Conditioning ......

Facility Lighting . . . . . . . . . . . . . . . . . .

Equipment Installation/Retrofit for the Primary Purpose of

Using a Different Energy Source ${ }^{\circ} \ldots \ldots \ldots \ldots \ldots \ldots$

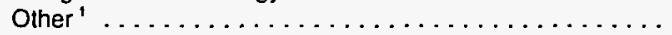

Primary Copper

Participation in One or More of the Following Types of

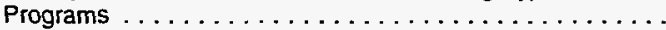

Energy Audits . . . . . . . . . . . . . . . .

Direct Electricity Load Control . . . . . . . . . . . . . . .

Special Rate Schedule ${ }^{b} \ldots \ldots \ldots \ldots \ldots \ldots \ldots \ldots$

Standby Generation Program . . . . . . . . . . . . . .

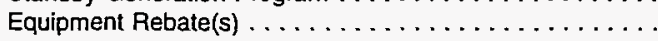

Equipment Installation or Retrofit for the Primary Purpose of Improving Energy Efficiency Affecting:

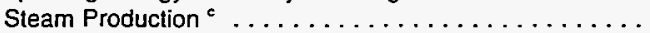

Direct/indirect Process Heating . . . . . . . . . . . . . .

Direct Process Cooling/Refrigeration . . . . . . . . . . . . .

Direct Machine Drive o . . . . . . . . . . . . . . . .

Facility Heating, Ventilation, and Air Conditioning .....

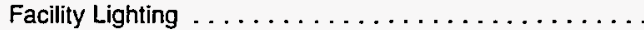

Equipment Installation/Retrofit for the Primary Purpose of

Using a Different Energy Source ${ }^{\circ} \ldots \ldots \ldots \ldots \ldots \ldots$

Other ${ }^{\prime}$. . . . . . . . . . . . . . . . . . . . . . . .

Primary Aluminum

Participation in One or More of the Following Types of

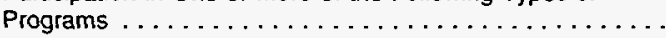

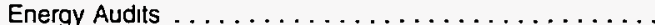

Direct Electricity Load Control $\ldots \ldots \ldots \ldots \ldots \ldots$.

Special Rate Schedule ${ }^{\circ} \ldots \ldots \ldots \ldots \ldots \ldots \ldots$.

Standby Generation Program . . . . . . . . . . . . . . .

Equipment Rebate(s) . . . . . . . . . . . . . . .

Equipment Installation or Retrofit for the Primary Purpose of

Improving Energy Efficiency Affecting:

Steam Production ${ }^{\circ} \ldots \ldots \ldots \ldots \ldots \ldots \ldots \ldots$.

Direct/Indirect Process Heating . . . . . . . . . . . . . . . .

Direct Process Cooling/Refrigeration . . . . . . . . . . .

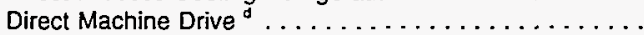

Facility Heating, Ventilation, and Air Conditioning .....

Facility Lighting . . . . . . . . . . . . . . . . . .

Equipment Installation/Retrofit for the Primary Purpose of

Using a Different Energy Source ${ }^{\circ} \ldots \ldots \ldots \ldots \ldots \ldots$

Other"

..............................

\begin{tabular}{|c|c|c|c|c|c|}
\hline 58 & 2 & 37 & 17 & 1 & 9.6 \\
\hline 43 & 2 & 29 & 11 & 1 & 11.3 \\
\hline 37 & 1 & 26 & 10 & . & 13.4 \\
\hline 41 & 2 & 27 & 12 & 1 & 11.7 \\
\hline 4 & * & 3 & 1 & 0 & 16.0 \\
\hline 5 & * & 4 & 0 & 1 & 14.8 \\
\hline 1 & * & 1 & 0 & 0 & 19.0 \\
\hline 11 & 1 & 3 & $w$ & $w$ & 15.7 \\
\hline$w$ & 0 & • & w & 0 & 31.6 \\
\hline 22 & 1 & 12 & 8 & 1 & 13.8 \\
\hline 14 & 1 & 7 & 6 & 0 & 15.3 \\
\hline 32 & 1 & 22 & 8 & 1 & 13.1 \\
\hline 12 & * & $w$ & $w$ & 0 & 23.8 \\
\hline 6 & 0 & W & $w$ & 0 & 19.7 \\
\hline
\end{tabular}

See footnotes at end of table. 
Table A41. Total Inputs of Energy for Heat, Power, and Electricity Generation by Census Region, Industry Group, Selected Industries, and Type of Energy Management Program, 1991 (Continued)

(Estimates in Trillion Btu)

\begin{tabular}{|c|c|c|c|c|c|c|c|}
\hline \multirow[b]{2}{*}{$\begin{array}{l}\text { SIC } \\
\text { Code" }\end{array}$} & \multirow{2}{*}{$\begin{array}{l}\text { Industry Groups } \\
\text { and Industry }\end{array}$} & \multirow[b]{2}{*}{ Total } & \multicolumn{4}{|c|}{ Census Region } & \multirow{3}{*}{$\begin{array}{c}\text { RSE } \\
\text { Row } \\
\text { Factors }\end{array}$} \\
\hline & & & Northeast & Midwest & South & West & \\
\hline & RSE Column Factors: & 0.7 & 1.3 & 1.0 & 0.9 & 1.2 & \\
\hline
\end{tabular}

3339

Primary Nonferrous Metals, nec

Participation in One or More of the Following Types of

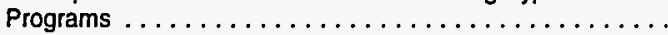

Energy Audits ...........................

Direct Electricity Load Control .................

Speclal Rate Schedule ${ }^{b} \ldots \ldots \ldots \ldots \ldots \ldots \ldots \ldots \ldots$.

Standby Generation Program . . . . . . . . . . . . .

Equipment Rebate(s) .....................

Equipment Installation or Retrofit for the Primary Purpose of Improving Energy Efficiency Affecting:

Steam Production ${ }^{\circ}$.

Direct/ndirect Process Heating ..........

Direct Process Cooling/Refrigeration ..............

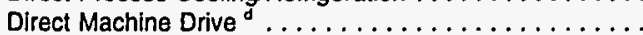

Facility Heating, Ventilation, and Air Conditioning ......

Facllity Llghting . . . . . . . . . . . . . . . . .

Equipment Installation/Retrofit for the Primary Purpose of

Using a Different Energy Source ${ }^{\bullet} \ldots \ldots \ldots \ldots \ldots \ldots$.

Other'

Aluminum Sheet, Plate, and Foil

Participation in One or More of the Following Types of

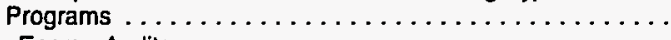

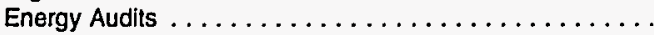

Direct Electricity Load Control . . . . . . . . . . . . . . .

Special Rate Schedule ${ }^{b} \ldots \ldots \ldots \ldots \ldots \ldots \ldots$.

Standby Generation Program . . . . . . . . . . . . . .

Equjpment Rebate(s) . . . . . . . . . . . . . . . . . .

Equipment Installation or Retrofit for the Primary Purpose of Improving Energy Efficiency Affecting:

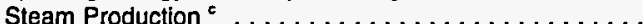

Direct/ndirect Process Heating . . . . . . . . . . . . .

Direct Process Cooling/Refrigeration . . . . . . . . . . . .

Direct Machine Drive d .....................

Facility Heating, Ventilation, and Air Conditioning $\ldots .$.

Facility Lighting . . . . . . . . . . . . . . . . . .

Equipment Installation/Retrofit for the Primary Purpose of

Using a Different Energy Source ${ }^{\circ} \ldots \ldots \ldots \ldots \ldots \ldots$.

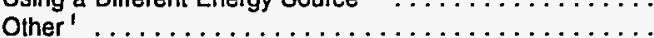

\section{FABRICATED METAL PRODUCTS}

Participation in One or More of the Following Types of

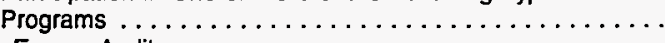

Energy Audits . . . . . . . . . . . . . . . . . .

Direct Electricity Load Control . . . . . . . . . . . . .

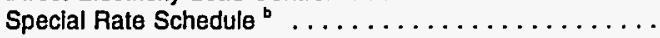

Standby Generation Program . . . . . . . . . . . . . .

Equipment Rebate(s) $\ldots \ldots \ldots \ldots \ldots \ldots \ldots \ldots$

Equipment Installation or Retrofit for the Primary Purpose of Improving Energy Efficiency Affecting:

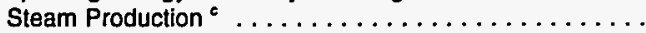

Direct/indirect Process Heating ....................

Direct Process Cooling/Refrigeration . . . . . . . . . . . .

Direct Machine Drive ${ }^{\circ} . \ldots \ldots \ldots \ldots \ldots \ldots \ldots$.

Facllity Heating, Ventilation, and Air Conditioning ......

Facllity Lighting . . . . . . . . . . . . . . . . .

Equipment Installation/Retrofit for the Primary Purpose of

Using a Different Energy Source ${ }^{\circ} \ldots \ldots \ldots \ldots \ldots \ldots \ldots$

Other'

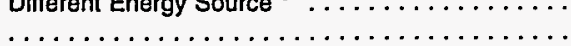

See foolnotes at end of table. 
Table A41. Total Inputs of Energy for Heat, Power, and Electricity Generation by Census Region, Industry Group, Selected Industries, and Type of Energy Management Program, 1991 (Continued)

(Estimates in Trillion Btu)

\begin{tabular}{|c|c|c|c|c|c|c|c|}
\hline \multirow{2}{*}{$\begin{array}{c}\text { SIC } \\
\text { Code" }\end{array}$} & \multirow{2}{*}{$\begin{array}{c}\text { Industry Groups } \\
\text { and Industry }\end{array}$} & \multirow[b]{2}{*}{ Total } & \multicolumn{4}{|c|}{ Census Region } & \multirow{3}{*}{$\begin{array}{c}\text { RSE } \\
\text { Row } \\
\text { Factors }\end{array}$} \\
\hline & & & Northeast & Midwest & South & West & \\
\hline & RSE Column Factors: & 0.7 & 1.3 & 1.0 & 0.9 & 1.2 & \\
\hline
\end{tabular}

35 INDUSTRIAL MACHINERY and EQUIPMENT

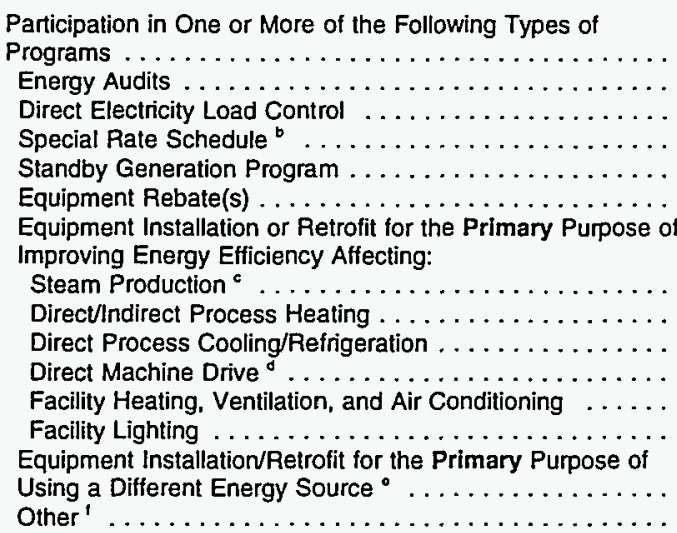

Computer and Office Equipment

Participation in One or More of the Following Types of

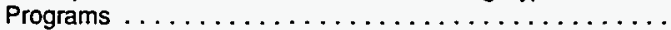

Energy Audits $\ldots \ldots \ldots \ldots \ldots \ldots \ldots \ldots \ldots \ldots$

Direct Electricity Load Control $\ldots \ldots \ldots \ldots \ldots \ldots \ldots \ldots$

Special Rate Schedule ${ }^{b} \ldots \ldots \ldots \ldots \ldots \ldots \ldots \ldots \ldots$

Standby Generation Program . . . . . . . . . . . .

Equipment Rebate(s) ......................

Equipment Installation or Retrofit for the Primary Purpose of Improving Energy Efficiency Affecting:

Steam Production ${ }^{\circ} \ldots \ldots \ldots \ldots \ldots \ldots \ldots \ldots \ldots$

Direct/Indirect Process Heating ..................

Direct Process Cooling/Refrigeration . . . . . . . . .

Direct Machine Drive ${ }^{4} \ldots \ldots \ldots \ldots \ldots \ldots \ldots \ldots$

Facility Heating, Ventilation, and Air Conditioning . . . . .

Facility Lighting $\ldots \ldots \ldots \ldots \ldots \ldots \ldots \ldots$

Equipment Installation/Retrofit for the Primary Purpose of

Using a Different Energy Source ${ }^{\circ} \ldots \ldots \ldots \ldots \ldots \ldots$

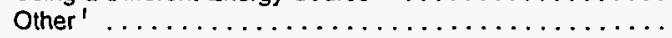

ELECTRONIC and OTHER ELECTRIC EQUIPMENT

Participation in One or More of the Following Types of

Programs . . . . . . . . . . . . . . . . .

Energy Audits . . . . . . . . . . . . . . . . .

Direct Electricity Load Control $\ldots \ldots \ldots \ldots \ldots \ldots \ldots \ldots \ldots$

Special Rate Schedule ${ }^{b} \ldots \ldots \ldots \ldots \ldots \ldots \ldots \ldots \ldots \ldots$

Standby Generation Program . . . . . . . . . . . .

Equipment Rebate(s) . . . . . . . . . . . . . . . .

Equipment Installation or Retrofit for the Primary Purpose of Improving Energy Efficiency Affecting:

Steam Production ${ }^{2} \ldots \ldots \ldots \ldots \ldots \ldots \ldots \ldots$

Direct/lndirect Process Heating . . . . . . . . . . . . . .

Direct Process Cooling/Refrigeration . . . . . . . . . .

Direct Machine Drive ${ }^{\mathrm{d}} \ldots \ldots \ldots \ldots \ldots \ldots \ldots . \ldots . \ldots$

Facility Heating, Ventilation, and Air Conditioning . .....

Facility Lighting $\ldots \ldots \ldots \ldots \ldots \ldots \ldots \ldots \ldots$

Equipment Installation/Retrofit for the Primary Purpose of

Using a Different Energy Source ${ }^{\circ} \ldots \ldots \ldots \ldots \ldots \ldots$.

Other'

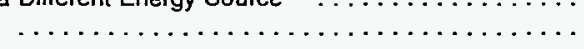

128
86
59
71
16
21

28

19

14

Q

35

26

23

58

78
91

11

3

16
13
10
11
4
5

$$
3
$$$$
0
$$

65
43
26
38
9
11

26
17
16
14
2
1

9.3

11.1

12.9

10.7

16.0

15.6

17.8

17.5

15.2

13.3

11.4

10.2

26.8

30.0

13.4
15.4
18.7
16.7
26.4
21.6

22.1
22.8
20.5
18.4
16.1
15.0
29.3
33.6

See footnotes at end of table. 
Table A41. Total Inputs of Energy for Heat, Power, and Electricity Generation by Census Region, Industry Group, Selected Industries, and Type of Energy Management Program, 1991 (Continued) (Estimates in Trillion Btu)

\begin{tabular}{|c|c|c|c|c|c|c|c|}
\hline \multirow{2}{*}{$\begin{array}{l}\text { SIC } \\
\text { Code" }\end{array}$} & \multirow{2}{*}{$\begin{array}{l}\text { Industry Groups } \\
\text { and Industry }\end{array}$} & \multirow[b]{2}{*}{ Total } & \multicolumn{4}{|c|}{ Census Region } & \multirow{3}{*}{$\begin{array}{c}\text { RSE } \\
\text { Row } \\
\text { Factors }\end{array}$} \\
\hline & & & Northeast & Midwest & South & West & \\
\hline & RSE Column Factors: & 0.7 & 1.3 & 1.0 & 0.9 & 1.2 & \\
\hline
\end{tabular}

37

TRANSPORTATION EQUIPMENT

Participation in One or More of the Following Types of

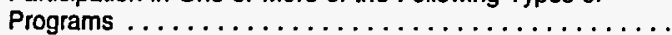

Energy Audits . . . . . . . . . . . . . . . . .

Direct Electricity Load Control . . . . . . . . . . .

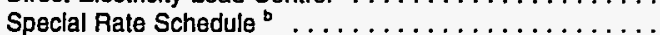

Standby Generation Program . . . . . . . . . . . .

Equlpment Rebate(s) . . . . . . . . . . . . . . . .

Equipment Installation or Retrofit for the Primary Purpose of

Improving Energy Efficiency Affecting:

Steam Production $^{c} \ldots \ldots \ldots \ldots \ldots \ldots \ldots \ldots \ldots \ldots$

DIrect/Indirect Process Heating .................

Direct Process Cooling/Refrigeration . . . . . . . . . .

Direct Machine Drive ${ }^{\mathrm{d}} \ldots \ldots \ldots \ldots \ldots \ldots \ldots \ldots \ldots \ldots \ldots \ldots$

Facillty Heating, Ventilation, and Air Conditioning . . . . .

Facility Lighting . ....................

Equipment Installation/Retrofit for the Primary Purpose of

Using a Different Energy Source ${ }^{\circ} \ldots \ldots \ldots \ldots \ldots \ldots$

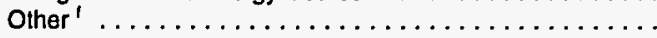

3711

Motor Vehicles and Car Bodies

Participation in One or More of the Following Types of

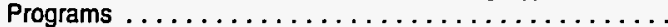

Energy Audits . . . . . . . . . . . . . . . . .

Direct Electricity Load Control $\ldots \ldots \ldots \ldots \ldots \ldots \ldots$

Special Rate Schedule ${ }^{b} \ldots \ldots \ldots \ldots \ldots \ldots \ldots \ldots \ldots$

Standby Generation Program . . . . . . . . . . . . .

Equlpment Rebate(s) . . . . . . . . . . . . . . . .

Equlpment Installation or Retrofit for the Primary Purpose of Improving Energy Efficiency Affecting:

Steam Production ${ }^{c} \ldots \ldots \ldots \ldots \ldots \ldots \ldots \ldots$

Direct/lndirect Process Heating . . . . . . . . . . .

Direct Process Cooling/Refrigeration . . . . . . . . . .

Direct Machine Drive ${ }^{d} \ldots . . \ldots \ldots$. . . . . . . . . . . . .

Facility Heating, Ventilation, and Air Conditioning .....

Facility Lighting . . . . . . . . . . . . . . . . . .

Equipment Installation/Retrofit for the Primary Purpose of

Using a Different Energy Source ${ }^{\circ} \ldots \ldots \ldots \ldots \ldots \ldots \ldots$

Other'

Motor Vehicle Parts and Accessories

Participation in One or More of the Following Types of

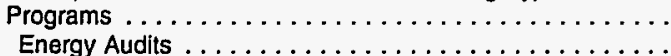

Direct Electricity Load Control . . . . . . . . . . . .

Special Rate Schedule ${ }^{b} \ldots \ldots \ldots \ldots \ldots \ldots \ldots \ldots \ldots$

Standby Generation Program . . . . . . . . . . . . .

Equlpment Rebate(s)

Equipment Installation or Retrofit for the Primary Purpose of Improving Energy Efficiency Affecting:

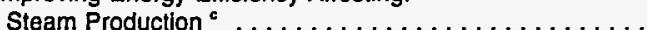

Direct/Indirect Process Heating . . . . . . . . . . .

Direct Process Cooling/Refrigeration $\ldots \ldots \ldots \ldots \ldots \ldots$

Direct Machine Drive $\ldots . . \ldots \ldots \ldots \ldots \ldots$

Facility Heating, Ventilation, and Air Conditioning .....

Facillty Lighting . ..................

Equipment Installation/Retrofit for the Primary Purpose of

Using a Different Energy Source ${ }^{\circ} \ldots \ldots \ldots \ldots \ldots \ldots \ldots$

Other'

$\ldots \ldots \ldots \ldots \ldots \ldots \ldots \ldots$

\begin{tabular}{|c|c|c|c|c|c|}
\hline 268 & 34 & 139 & 59 & 36 & 3.7 \\
\hline 213 & 29 & 112 & 44 & 29 & 4.4 \\
\hline 119 & 18 & 56 & 29 & 16 & 4.9 \\
\hline 175 & 17 & 91 & 44 & 23 & 4.8 \\
\hline 20 & W & 11 & 4 & W & 7.9 \\
\hline 50 & 17 & 7 & 6 & 20 & 7.4 \\
\hline 103 & 17 & 57 & 11 & 18 & 5.1 \\
\hline 90 & 12 & 51 & 11 & 16 & 5.4 \\
\hline 65 & 13 & 27 & 8 & 17 & 6.0 \\
\hline 119 & 19 & 63 & 13 & 24 & 4.8 \\
\hline 161 & 21 & 86 & 29 & 25 & 4.6 \\
\hline 219 & 31 & 108 & 47 & 32 & 4.2 \\
\hline 50 & 6 & 20 & 8 & 15 & 6.2 \\
\hline 14 & " & 13 & • & * & 14.0 \\
\hline
\end{tabular}

See footnotes at end of table. 
Table A41. Total Inputs of Energy for Heat, Power, and Electricity Generation by Census Region, Industry Group, Selected Industries, and Type of Energy Management Program, 1991 (Continued)

(Estimates in Trillion Btu)

\begin{tabular}{|c|c|c|c|c|c|c|c|}
\hline \multirow[b]{2}{*}{$\begin{array}{c}\text { SIC } \\
\text { Code" }\end{array}$} & \multirow{2}{*}{$\begin{array}{l}\text { Industry Groups } \\
\text { and Industry }\end{array}$} & \multirow[b]{2}{*}{ Total } & \multicolumn{4}{|c|}{ Census Region } & \multirow{3}{*}{$\begin{array}{c}\text { RSE } \\
\text { Row } \\
\text { Factors }\end{array}$} \\
\hline & & & Northeast & Midwest & South & West & \\
\hline & RSE Column Factors: & 0.7 & 1.3 & 1.0 & 0.9 & 1.2 & \\
\hline
\end{tabular}

38

INSTRUMENTS and RELATED PRODUCTS

Participation in One or More of the Following Types of

Programs . . . . . . . . . . . . . . . . .

Energy Audits . . . . . . . . . . . . . . . . .

Direct Electricity Load Control . . . . . . . . . . . .

Special Rate Schedule ${ }^{b} \ldots \ldots \ldots \ldots \ldots \ldots \ldots \ldots$

Standby Generation Program . . . . . . . . . . . .

Equipment Rebate(s) . . . . . . . . . . . . . . . . .

Equipment Installation or Retrofit for the Primary Purpose of

Improving Energy Efficiency Affecting:

Steam Production ${ }^{c} \ldots \ldots \ldots \ldots \ldots \ldots \ldots \ldots . . \ldots$

Direct/lndirect Process Heating . . . . . . . . . . . . .

Direct Process Cooling/Refrigeration . . . . . . . . .

Direct Machine Drive ${ }^{\mathrm{a}} \ldots \ldots \ldots \ldots \ldots \ldots \ldots . . . \ldots \ldots$

Facility Heating, Ventilation, and Air Conditioning . . . . . .

Facility Lighting..$\ldots \ldots \ldots \ldots \ldots \ldots \ldots$

Equipment Installation/Retrofit for the Primary Purpose of

Using a Different Energy Source ${ }^{\circ} \ldots \ldots \ldots \ldots \ldots \ldots \ldots$

Other'

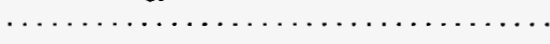

Surgical and Medical Instruments

Participation in One or More of the Following Types of

Programs . . . . . . . . . . . . . . . . . .

Energy Audits ........................

Direct Electricity Load Control . . . . . . . . . . . . .

Special Rate Schedule ${ }^{b} \ldots \ldots \ldots \ldots \ldots \ldots \ldots \ldots$

Standby Generation Program . . . . . . . . . . . . .

Equipment Rebate(s) . . . . . . . . . . . . . . . .

Equipment Installation or Retrofit for the Primary Purpose of

Improving Energy Efficiency Affecting:

Steam Production ${ }^{c} \ldots \ldots \ldots \ldots \ldots \ldots \ldots \ldots . . . \ldots$

Direct/lndirect Process Heating . . . . . . . . . . .

Direct Process Cooling/Refrigeration . . . . . . . . .

Direct Machine Drive ${ }^{d} \ldots \ldots \ldots \ldots \ldots . . \ldots \ldots . . .$.

Facility Heating, Ventilation, and Air Conditioning

Facility Lighting $\ldots \ldots \ldots \ldots \ldots \ldots \ldots \ldots$

Equipment Installation/Retrofit for the Primary Purpose of

Using a Different Energy Source ${ }^{\circ} \ldots \ldots \ldots \ldots \ldots \ldots \ldots$

Other ${ }^{\prime}$

$\begin{array}{rr}77 & \\ 66 & \\ 44 & 41 \\ 50 & 31 \\ 32 & \\ 15 & \\ & 33 \\ 39 & \\ 33 & \\ 38 & 31 \\ 46 & 26 \\ 62 & 30 \\ 67 & 33 \\ 31 & \\ 1 & \end{array}$

$\begin{array}{rr}44 & 8 \\ 41 & 5 \\ 31 & 3 \\ 33 & 4 \\ 26 & \\ 7 & 1\end{array}$

$\begin{array}{rrr}14 & 12 & 11.3 \\ 10 & 10 & 13.2 \\ 4 & 7 & 15.7 \\ 6 & 7 & 14.2 \\ 2 & 4 & 23.4 \\ 1 & 6 & 16.7 \\ & & \\ 3 & & \\ 2 & 5 & 18.6 \\ 3 & 4 & 23.6 \\ 4 & 4 & 20.2 \\ 9 & 6 & 15.4 \\ 8 & 9 & 13.2 \\ 3 & 11 & 12.2 \\ . & & \\ & . & 25.8 \\ & 1 & 26.0\end{array}$

See footnotes at end of table. 
Table A41. Total Inputs of Energy for Heat, Power, and Electricity Generation by Census Region, Industry Group, Selected Industries, and Type of Energy Management Program, 1991 (Continued)

(Estimates in Trillion Btu)

\begin{tabular}{|c|c|c|c|c|c|c|c|}
\hline \multirow{2}{*}{$\begin{array}{l}\text { Sic } \\
\text { Code" }\end{array}$} & \multirow[b]{2}{*}{$\begin{array}{c}\text { Industry Groups } \\
\text { and Industry }\end{array}$} & \multirow[b]{2}{*}{ Total } & \multicolumn{4}{|c|}{ Census Region } & \multirow{3}{*}{$\begin{array}{c}\text { RSE } \\
\text { Row } \\
\text { Factors }\end{array}$} \\
\hline & & & Northeast & Midwest & South & West & \\
\hline & olumn Factors: & 0.7 & 1.3 & 1.0 & 0.9 & 1.2 & \\
\hline
\end{tabular}

39

MISC. MANUFACTURING INDUSTRIES

Participation in One or More of the Following Types of

\begin{tabular}{|c|c|c|c|c|c|}
\hline Programs $\ldots \ldots \ldots \ldots \ldots \ldots \ldots \ldots \ldots \ldots$ & 14 & 6 & 3 & 4 & 1 \\
\hline Energy Audits $\ldots \ldots \ldots \ldots \ldots \ldots \ldots \ldots \ldots$ & 10 & 4 & 1 & 4 & 1 \\
\hline Direct Electricity Load Control $\ldots \ldots \ldots$. . . . . . . . . & 5 & 2 & 1 & 2 & $\bullet$ \\
\hline Special Rate Schedule ${ }^{b} \ldots \ldots \ldots \ldots \ldots$ & 6 & 3 & 1 & 2 & 1 \\
\hline Standby Generation Program . . . . . . . . . . . . & $\star$ & • & 0 & 0 & $*$ \\
\hline $\begin{array}{l}\text { Equipment Rebate(s) } \ldots \ldots \ldots \ldots \ldots \ldots \ldots \ldots \ldots \text {. } \ldots \ldots \ldots \text {. } \\
\text { Equipment Installation or Retrofit for the Primary Pumose of } \\
\text { Improving Energy Efticiency Affecting: }\end{array}$ & 2 & 1 & $*$ & 0 & * \\
\hline Steam Production ${ }^{c} \ldots \ldots \ldots \ldots \ldots \ldots \ldots \ldots$ & 3 & 1 & 1 & 1 & $*$ \\
\hline Direct/Indirect Process Heating $\ldots \ldots \ldots \ldots \ldots \ldots$ & 2 & 1 & 1 & 1 & $=$ \\
\hline Direct Process Cooling/Refrigeration . . . . . . . . . . & 4 & 2 & 1 & 1 & 1 \\
\hline 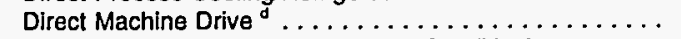 & 4 & 2 & 1 & 1 & $*$ \\
\hline Facility Heating, Ventilation, and Air Conditioning $\ldots \ldots$ & 8 & 3 & 2 & 3 & 1 \\
\hline 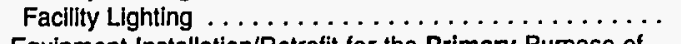 & 9 & 5 & 1 & 2 & 1 \\
\hline $\begin{array}{l}\text { Equipment Installation/Retrofit for the Primary Purpose of } \\
\text { Using a Different Energy Source }{ }^{\circ} \ldots \ldots \ldots \ldots \ldots \ldots \ldots\end{array}$ & 2 & 1 & 0 & 1 & • \\
\hline Other $^{\prime} \ldots \ldots \ldots \ldots \ldots \ldots \ldots \ldots \ldots \ldots \ldots$ & 1 & * & * & * & 0 \\
\hline
\end{tabular}

- See Appendices B and F for descriptions of the Standard Industrial Classification system.

- For example, interruptible or time-of-use rates.

c For example, bollers, burners, and nozzles.

For example, adjustable speed drives, motors, and pumps.

- For example, electrification of a subset of the manufacturing operation.

$'$ Included are power factor corrections, improvements in operating procedures, and other energy management programs reported by respondents.

NF=No applicable RSE row/column factor.

Estimate less than 0.5. Data are included in higher level totals.

$W=$ Withheld to avold disclosing data for individual establishments. Data are included in higher level totals.

$\mathrm{Q}=$ Withheld because Relative Standard Error is greater than $\mathbf{5 0}$ percent. Data are included in higher level totals.

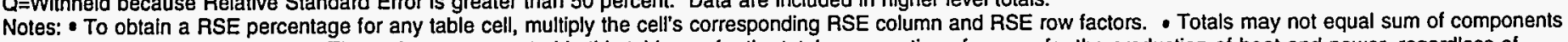

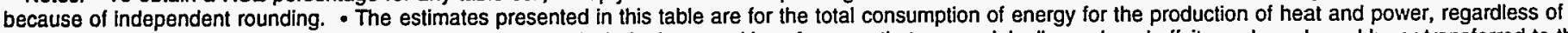

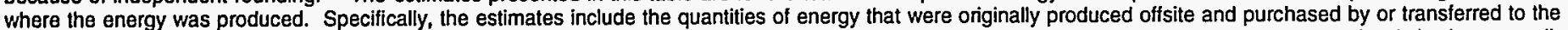

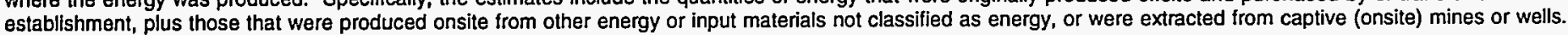

" "Sponsorship" is determined by the respondent.

Source: Energy Information Administration, Office of Energy Markets and End Use, Energy End Use and Integrated Statistics Division, Form ElA-846, "1991 Manufacturing Energy Consumption Survey." 
Table A42. Total Inputs of Energy for Heat, Power, and Electricity Generation by Employment Size and Presence of General Technologies, 1991 (Estimates in Trillion Btu)

\begin{tabular}{|c|c|c|c|c|c|c|c|c|}
\hline \multirow[b]{2}{*}{ General Technologies } & \multirow[b]{2}{*}{ Total } & \multicolumn{6}{|c|}{ Employment Size" } & \multirow{3}{*}{$\begin{array}{c}\text { RSE } \\
\text { Row } \\
\text { Factors }\end{array}$} \\
\hline & & Under 50 & $50-99$ & $100-249$ & $250-499$ & $500-999$ & $\begin{array}{c}1,000 \text { and } \\
\text { Over }\end{array}$ & \\
\hline RSE Column Factors: & 0.6 & 2.1 & 2.2 & 1.2 & 0.7 & 0.6 & 0.7 & \\
\hline One or More General Technologies Present & 12,510 & 262 & 513 & 1,716 & 2,192 & 2,783 & 5,044 & 2.9 \\
\hline Computer Control of Building Environment ${ }^{b} \ldots \ldots \ldots \ldots \ldots$ & 3,081 & 48 & 95 & 204 & 454 & 701 & 1,579 & 4.5 \\
\hline Computer Control of Processes or Major Energy-Using Equipment ${ }^{c} \ldots$ & 10,300 & 122 & 314 & 1,191 & 1,676 & 2,433 & 4,564 & 3.3 \\
\hline Waste Heat Recovery . . . . . . . . . . . . . . . . & 9,158 & 95 & 226 & 1,012 & 1,480 & 2,141 & 4,204 & 3.5 \\
\hline Adjustable-Speed Motors $\ldots \ldots \ldots \ldots \ldots \ldots \ldots \ldots \ldots$ & 8,623 & 134 & 279 & 1,085 & 1,502 & 1,951 & 3,672 & 3.3 \\
\hline 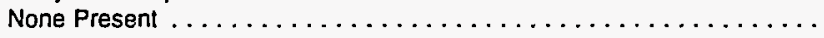 & 2,516 & 275 & 263 & 648 & 489 & 363 & 479 & 4.8 \\
\hline Total $\ldots \ldots \ldots \ldots \ldots \ldots \ldots \ldots \ldots \ldots \ldots \ldots \ldots \ldots$ & 15,027 & 537 & 776 & 2,365 & 2,681 & 3,145 & 5,524 & 2.8 \\
\hline
\end{tabular}

\footnotetext{
- Employment Size categories were supplied by the Bureau of the Census. See Appendix B.

- For example, space heating or cooling and lighting.

For example, boilers or furnaces.

Notes: - To obtain a RSE percentage for any table cell, multiply the cell's corresponding RSE column and RSE row factors. - Totals may not equal sum of components because of independent rounding. - The estimates presented in this table are for the total consumption of energy for the production of heat and power, regardless of where the energy was produced. Specifically, the estimates include the quantities of energy that were originally produced offsite and purchased by or transferred to the establishment, plus those that were produced onsite from other energy or input materials not classified as energy, or were extracted from captive (onsite) mines or wells.

Source: Energy Information Administration, Office of Energy Markets and End Use, Energy End Use and Integrated Statistics Division, Form ElA-846, "1991

Manufacturing Energy Consumption Survey", and Bureau of the Census, Industry Division, data files for the "1991 Annual Survey of Manufactures."
}

\section{Table A43. Total Inputs of Energy for Heat, Power, and Electricity Generation by Value of Shipments and Presence of General Technologies, 1991 (Estimates in Trillion Btu)}

\begin{tabular}{|c|c|c|c|c|c|c|c|c|}
\hline \multirow[b]{2}{*}{ General Technologies } & \multirow[b]{2}{*}{ Total } & \multicolumn{6}{|c|}{$\begin{array}{l}\text { Value of Shipments and Receipts" } \\
\text { (million dollars) }\end{array}$} & \multirow{3}{*}{$\begin{array}{c}\text { RSE } \\
\text { Row } \\
\text { Factors }\end{array}$} \\
\hline & & Under 20 & $20-49$ & $50-99$ & $100-249$ & $250-499$ & $\begin{array}{c}500 \text { and } \\
\text { Over }\end{array}$ & \\
\hline RSE Column Factors: & 0.6 & 2.0 & 1.3 & 1.1 & 1.0 & 0.9 & 0.7 & \\
\hline One or More General Technologies Present & 12,510 & 683 & 1,071 & 1,117 & 2,240 & 2,241 & 5,158 & 2.8 \\
\hline Computer Control of Building Environment ${ }^{b} \ldots \ldots \ldots \ldots \ldots$ & 3,081 & 138 & 151 & 187 & 516 & 630 & 1,460 & 3.9 \\
\hline Computer Control of Processes or Major Energy-Using Equipment ${ }^{c} \ldots$ & 10,300 & 324 & 695 & 760 & 1,896 & 2,072 & 4,553 & 3.2 \\
\hline 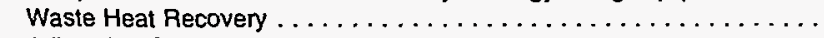 & 9,158 & 222 & 392 & 571 & 1,514 & 1,827 & 4,633 & 3.3 \\
\hline Adjustable-Speed Motors $\ldots \ldots \ldots \ldots \ldots \ldots \ldots \ldots \ldots \ldots \ldots$ & 8,623 & 413 & 702 & 801 & 1,687 & 1,592 & 3,428 & 3.2 \\
\hline None Present $\ldots \ldots \ldots \ldots \ldots \ldots \ldots \ldots \ldots \ldots \ldots \ldots$ & 2,516 & 666 & 490 & 268 & 321 & 391 & 380 & 4.3 \\
\hline 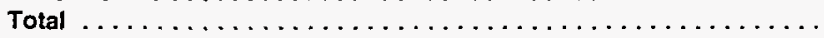 & 15,027 & 1,349 & 1,560 & 1,386 & 2,561 & 2,632 & 5,538 & 2.7 \\
\hline
\end{tabular}

- Value of Shipments and Receipts were supplied by the Bureau of the Census. See Appendix B.

${ }^{\circ}$ For example, space heating or cooling and lighting.

For example, boilers or furnaces.

Notes: - To obtain a RSE percentage for any table cell, mulliply the cell's corresponding RSE column and RSE row factors. - Totals may not equal sum of components because of independent rounding. - The estimates presented in this table are for the total consumption of energy for the production of heat and power, regardless of where the energy was produced. Specifically, the estimates include the quantities of energy that were originally produced offsite and purchased by or transferred to the establishment, plus those that were produced onsite from other energy or input materials not classified as energy, or were extracted from captive (onsite) mines or wells.

Source: Energy Information Administration, Office of Energy Markets and End Use, Energy End Use and Integrated Statistics Division, Form EIA-846, "1991

Manufacturing Energy Consumption Survey", and Bureau of the Census, Industry Division, data files for the "1991 Annual Survey of Manufactures." 
Table A44. Total Inputs of Energy for Heat, Power, and Electricity Generation by Census Region, Industry Group, Selected Industries, Presence of General Technologies, and Industry-Specific Technologies for Selected Industries, 1991 (Estimates in Trillion Btu)

\begin{tabular}{|c|c|c|c|c|c|c|c|}
\hline \multirow{2}{*}{$\begin{array}{c}\text { SIC } \\
\text { Cod } \theta^{4} \\
\end{array}$} & \multirow[b]{2}{*}{$\begin{array}{l}\text { Industry Groups } \\
\text { and Industry }\end{array}$} & \multirow[b]{2}{*}{ Total } & \multicolumn{4}{|c|}{ Census Region } & \multirow{3}{*}{$\begin{array}{c}\text { RSE } \\
\text { Row } \\
\text { Factors }\end{array}$} \\
\hline & & & Northeast & Midwest & South & West & \\
\hline & RSE Column Factors: & 0.7 & 1.3 & 1.0 & 0.9 & 1.3 & \\
\hline
\end{tabular}

20-39

\section{ALL INDUSTRY GROUPS}

General Energy-Related Technologies

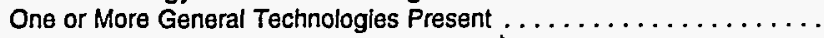
Computer Control of Bullding Environment ${ }^{b} \ldots \ldots \ldots \ldots \ldots \ldots \ldots$ Computer Control of Processes or Major Energy-Using Equipment ${ }^{c} \ldots$

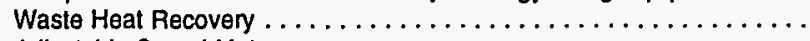

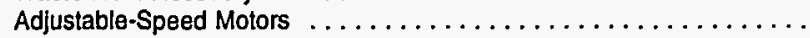

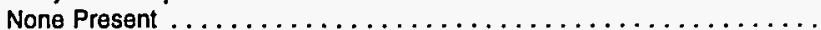

$\begin{array}{rr}12,510 & 1,282 \\ 3,081 & 473 \\ 10,300 & 1,033 \\ 9,158 & 828 \\ 8,623 & 791 \\ 2,516 & 352\end{array}$

3,130
942
2,570
2,219
2,356
703

\section{FOOD and KINDRED PRODUCTS}

General Energy-Related Technologies

One or More General Technologies Present $\ldots \ldots \ldots \ldots \ldots \ldots \ldots$

Computer Control of Bullding Environment ${ }^{\circ} \ldots \ldots \ldots \ldots \ldots \ldots$

Computer Control of Processes or Major Energy-Using Equipment ${ }^{c} \ldots$

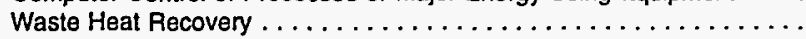

Adjustable-Speed Motors $\ldots \ldots \ldots \ldots \ldots \ldots \ldots \ldots \ldots \ldots \ldots$

None Present $\ldots \ldots \ldots \ldots \ldots \ldots \ldots \ldots \ldots \ldots \ldots \ldots \ldots$.

Industry-Specific Technologies

One or More Industry-Specific Technologies Present ..............

Infrared Heating . . . . . . . . . . . . . . . . . . . . . . .

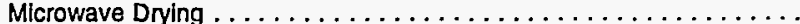

Closed-Cycle Heat Pump System Used to Recover Heat ..........

Open-Cycle Heat Pump System Used to Produce steam ..........

Gas-Driven Rotary Engines and/or Turbines . . . . . . . . . . . .

Membrane Separation..$\ldots \ldots \ldots \ldots \ldots \ldots \ldots \ldots \ldots \ldots$.

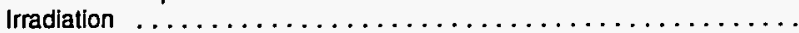

Freeze Concentration . . . . . . . . . . . . . . . . . . . . .

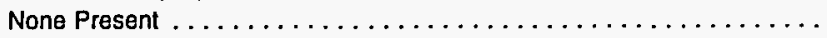

73
252
230
273

$\begin{array}{rrr}196 & 167 & 5.8 \\ 46 & 29 & 8.2 \\ 103 & 108 & 5.3 \\ 118 & 80 & 8.4 \\ 137 & 132 & 5.1 \\ 58 & 35 & 8.8\end{array}$

\section{Meat Packing Plants}

General Energy-Related Technologies

One or More General Technologies Present .................

Computer Control of Building Environment ${ }^{b}$.................

Computer Control of Processes or Major Energy-Using Equipment ${ }^{c} \ldots$

Waste Heat Recovery . . . . . . . . . . . . . . . . . . . .

Adjustable-Speed Motors $\ldots \ldots \ldots \ldots \ldots \ldots \ldots \ldots \ldots \ldots$

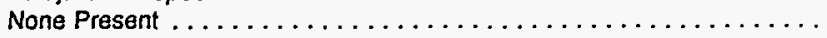

Industry-Specific Technologies

One or More Industry-Specific Technologies Present . .............

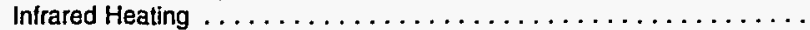

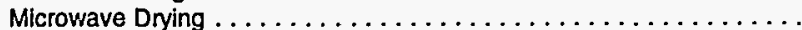

Closed-Cycle Heat Pump System Used to Recover Heat ..........

Open-Cycle Heat Pump System Used to Produce steam ..........

Gas-Driven Rotary Engines and/or Turbines ...............

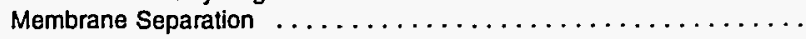

Irradiation $\ldots \ldots \ldots \ldots \ldots \ldots \ldots \ldots \ldots \ldots \ldots \ldots \ldots \ldots \ldots$.

Freeze Concentration $\ldots \ldots \ldots \ldots \ldots \ldots \ldots \ldots \ldots \ldots \ldots$

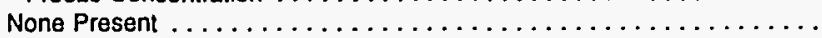

\begin{tabular}{|c|c|c|c|c|}
\hline 40 & 1 & 29 & 6 & 4 \\
\hline 15 & $w$ & 9 & 3 & $w$ \\
\hline 34 & 1 & 25 & 5 & 3 \\
\hline 32 & 1 & 24 & 3 & 3 \\
\hline 34 & 1 & 24 & 5 & 4 \\
\hline 9 & * & 4 & 4 & 1 \\
\hline 23 & 1 & 15 & 4 & 3 \\
\hline 1 & - & * & • & * \\
\hline 0 & 0 & 0 & 0 & 0 \\
\hline 7 & - & 5 & * & 1 \\
\hline 3 & 0 & 2 & * & 1 \\
\hline 4 & - & 4 & . & 0 \\
\hline$w$ & 0 & $w$ & 1 & • \\
\hline 0 & 0 & 0 & 0 & 0 \\
\hline 5 & 0 & 4 & 1 & - \\
\hline 26 & 1 & 18 & 6 & 2 \\
\hline
\end{tabular}


Table A44. Total Inputs of Energy for Heat, Power, and Electricity Generation by Census Region, Industry Group, Selected Industries, Presence of General Technologies, and Industry-Specific Technologies for Selected Industries, 1991 (Continued) (Estimates in Trillion Btu)

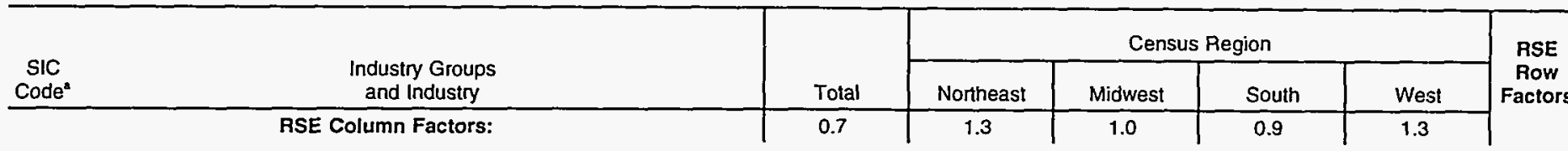

2033

Canned Fruits and Vegetables

General Energy-Related Technologies

One or More General Technologies Present

Computer Control of Building Environm

Computer Control of Processes or Major Energy-Using Equipment ${ }^{c} \ldots$

Waste Heat Recovery . . . . . . . . . . . . . . . . . . . .

Adjustable-Speed Motors $\ldots \ldots \ldots \ldots \ldots \ldots \ldots \ldots \ldots \ldots \ldots \ldots$

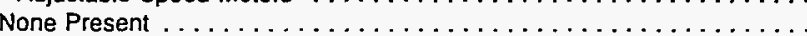

Industry-Specific Technologies

One or More Industry-Specific Technologies Present . . . . . . . . .

Infrared Heating $\ldots \ldots \ldots \ldots \ldots \ldots \ldots \ldots \ldots \ldots \ldots \ldots \ldots \ldots$

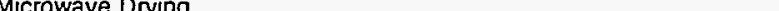

Closed-Cycle Heat Pump System Used to Recover Heat . . . . . . . .

Open-Cycle Heat Pump System Used to Produce steam . . . . . . . . .

Gas-Driven Rotary Engines and/or Turbines . . . . . . . . . . . . . . .

Membrane Separation.$\ldots \ldots \ldots \ldots \ldots \ldots \ldots \ldots \ldots \ldots \ldots \ldots$

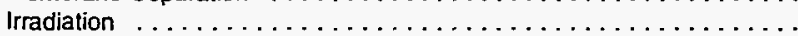

Freeze Concentration . . . . . . . . . . . . . . . . . . . . .

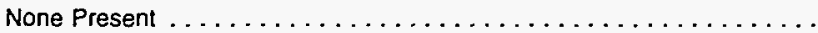

2037

Frozen Fruits and Vegetables

General Energy-Related Technologies

One or More General Technologies Present

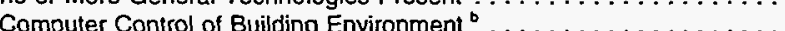

Computer Control of Processes or Major Energy-Using Equipment ${ }^{\circ}$...

Waste Heat Recovery . . . . . . . . . . . . . . . . . . . . .

Adjustable-Speed Motors $\ldots \ldots \ldots \ldots \ldots \ldots \ldots \ldots \ldots \ldots \ldots$

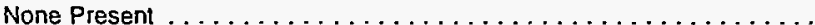

Industry-Specific Technologies

One or More Industry-Specific Technologies Present . . . . . . . . . . . .

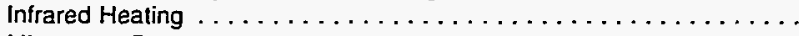

Microwave Drying . . . . . . . . . . . . . . . . . . .

Closed-Cycle Heat Pump System Used to Recover Heat . . . . . . . . . . .

Open-Cycle Heat Pump System Used to Produce steam ..........

Gas-Driven Rotary Engines and/or Turbines . . . . . . . . . . . . . .

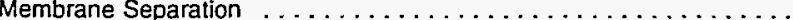

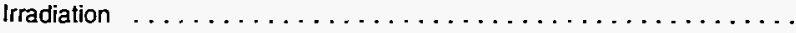

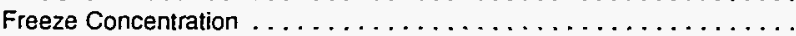

None Present

Wet Corn Milling

2046

General Energy-Related Technologies

One or More General Technologies Present

Computer Control of Building Environment ${ }^{0} \ldots \ldots \ldots \ldots \ldots \ldots \ldots \ldots$ Computer Control of Processes or Major Energy-Using Equipment ${ }^{c} \ldots$

Waste Heat Recovery . . . . . . . . . . . . . . . . . . . . . . .

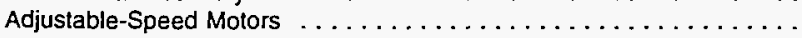

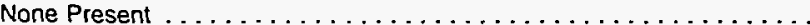

Industry-Specific Technologies

One or More Industry-Specific Technologies Present . . . . . . . . . .

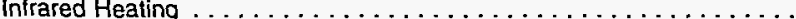

Microwave Drying . . . . . . . . . . . . . . . . . . . . . .

Closed-Cycle Heat Pump System Used to Recover Heat . . . . . . . .

Open-Cycle Heat Pump System Used to Produce steam . . . . . . . . .

Gas-Driven Rotary Engines and/or Turbines . . . . . . . . . . . . .

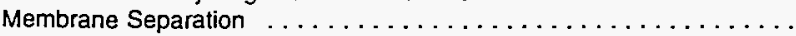

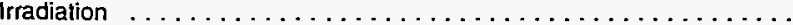

Freeze Concentration .........................

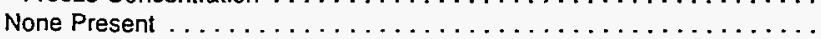

34
4
19
20
27
11

4

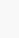

2

3

$\begin{array}{rr}14 & 1 \\ 3 & \vdots \\ 4 & 0 \\ 1 & 1 \\ 6 & 0 \\ 6 & 0 \\ \vdots & 0 \\ 1 & : \\ 30 & 6\end{array}$

2
$*$
$:$
0
0
0
$\vdots$
9

5
0
0
1
$w$
$w$
$:$
2

15.6

$\begin{array}{rrrrrr}12 & 1 & 1 & 5 & 5 & 17.6 \\ * & 0 & * & * & 0 & 41.0 \\ 0 & 0 & 0 & 0 & 0 & \mathrm{NF} \\ 4 & 0 & 0 & 1 & 2 & 21.2 \\ 1 & 1 & 0 & 0 & 0 & 39.3 \\ 4 & 0 & 5 & 2 & 2 & 29.0 \\ 2 & 0 & 0 & 0 & 2 & 26.5 \\ 0 & 0 & 0 & 0 & 0 & \text { NF } \\ 7 & 1 & * & 4 & 1 & 24.5 \\ 27 & * & 3 & 3 & 21 & 14.6\end{array}$

See footnotes at end of table. 
Table A44. Total Inputs of Energy for Heat, Power, and Electricity Generation by Census Region, Industry Group, Selected Industries, Presence of General Technologies, and Industry-Specific Technologies for Selected Industries, 1991 (Continued) (Estimates in Trillion Btu)

\begin{tabular}{cc|c|c|c|c|c|}
\hline \multirow{2}{*}{$\begin{array}{c}\text { SIC } \\
\text { Industry Groups } \\
\text { and Industry }\end{array}$} & & & \multicolumn{3}{|c|}{ Census Region } \\
\cline { 4 - 7 } & RSE Column Factors: & Total & Northeast & Midwest & South & West \\
\hline Factors
\end{tabular}

2051

Bread, Cake, and Related Products

General Energy-Related Technologies

One or More General Technologies Present ...

Computer Control of Building Environment ${ }^{\circ} \ldots \ldots \ldots \ldots \ldots \ldots \ldots \ldots$

Computer Control of Processes or Major Energy-Using Equipment ${ }^{c}$...

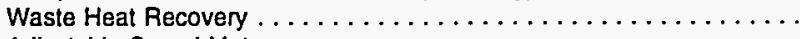

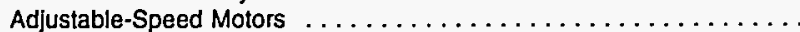

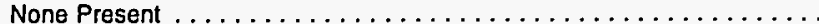

Industry-Specific Technologies

One or More Industry-Specific Technologies Present ..............

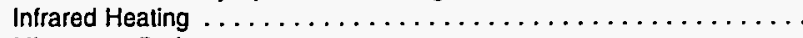

Microwave Drying .

Closed-Cycle Heat Pump System Used to Recover Heat ...

Open-Cycle Heat Pump System Used to Produce steam ..........

Gas-Driven Rotary Engines and/or Turbines . . . . . . . . . . . . .

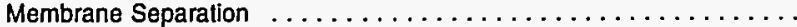

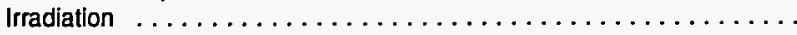

Freeze Concentration . . . . . . . . . . . . . . . . . . . . .

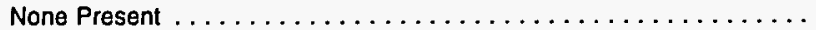

\section{Beet Sugar}

General Energy-Related Technologies

One or More General Technologies Present

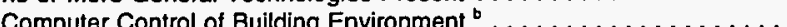

Computer Control of Processes or Major Energy-Using Equipment ${ }^{c}$...

Waste Heat Recovery . . . . . . . . . . . . . . . . . . . . . .

Adjustable-Speed Motors $\ldots \ldots \ldots \ldots \ldots \ldots \ldots \ldots \ldots \ldots \ldots$

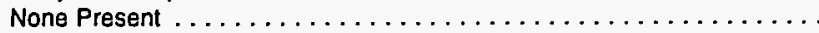

Industry-Specific Technologies

One or More Industry-Specific Technologies Present .............

Infrared Heating . . . . . . . . . . . . . . . . . . . . . . . .

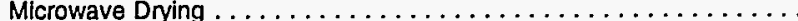

Closed-Cycle Heat Pump System Used to Recover Heat...$\ldots \ldots$.

Open-Cycle Heat Pump System Used to Produce steam ..........

Gas-Driven Rotary Engines and/or Turbines . . . . . . . . . . . . .

Membrane Separation $\ldots \ldots \ldots \ldots \ldots \ldots \ldots \ldots \ldots \ldots \ldots$.

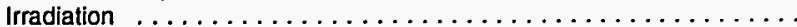

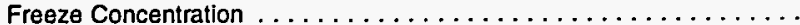

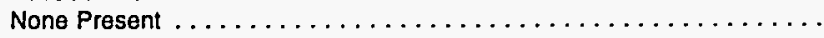

Soybean Oil Mills

General Energy-Related Technologies

One or More General Technologies Present .

Computer Control of Building Environment ".

Computer Control of Processes or Major Energy-Using Equipment ${ }^{\circ} \ldots$

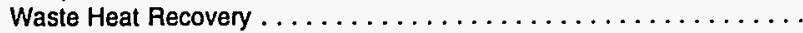

Adjustable-Speed Motors $\ldots \ldots \ldots \ldots \ldots \ldots \ldots \ldots \ldots \ldots \ldots$

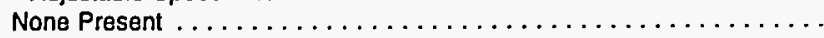

Industry-Specific Technologies

One or More Industry-Specific Technologies Present . . . . . . . . . .

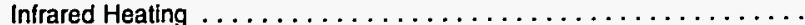

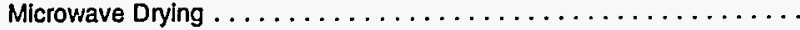

Closed-Cycle Heat Pump System Used to Recover Heat . . . . . . . .

Open-Cycle Heat Pump System Used to Produce steam . . . . . . . . . .

Gas-Driven Rotary Engines and/or Turbines . . . . . . . . . . . . .

Membrane Separation.$\ldots \ldots \ldots \ldots \ldots \ldots \ldots \ldots \ldots \ldots \ldots$

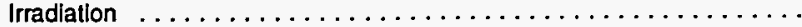

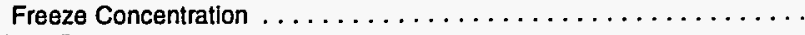

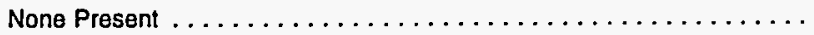

$\begin{array}{rr}19 & 5 \\ 4 & 2 \\ 10 & 2 \\ 3 & 2 \\ 17 & 5 \\ 13 & 2\end{array}$

5
1
3
1
4
3

5
2
3
5
5
6

$\begin{array}{rr}8 & 2 \\ 1 & 0 \\ 0 & 0 \\ 1 & \vdots \\ 1 & \\ 2 & 1 \\ * & 0 \\ 0 & 0 \\ 24 & 5\end{array}$

2
1
0
$\vdots$
1
$\vdots$
7

57
0
51
21
46
10

0
0
0
0
0
0

$\begin{array}{rrrrrr}W & 0 & W & 0 & W & 15.3 \\ W & 0 & W & 0 & 0 & 19.4 \\ 0 & 0 & 0 & 0 & 0 & N F \\ W & 0 & 0 & 0 & W & 17.0 \\ 0 & 0 & 0 & 0 & 0 & \text { NF } \\ 0 & 0 & 0 & 0 & 0 & \text { NF } \\ W & 0 & W & 0 & W & 11.4 \\ 0 & 0 & 0 & 0 & 0 & \text { NF } \\ 0 & 0 & 0 & 0 & 0 & \text { NF } \\ W & 0 & W & W & W & 7.7\end{array}$

See footnotes at end of table. 
Table A44. Total Inputs of Energy for Heat, Power, and Electricity Generation by Census Region, Industry Group, Selected Industries, Presence of General Technologies, and Industry-Specific Technologies for Selected Industries, 1991 (Continued) (Estimates in Trillion Btu)

\begin{tabular}{|c|c|c|c|c|c|c|c|}
\hline \multirow[b]{2}{*}{$\begin{array}{l}\text { SIC } \\
\text { Code" }\end{array}$} & \multirow{2}{*}{$\begin{array}{l}\text { Industry Groups } \\
\text { and Industry }\end{array}$} & \multirow[b]{2}{*}{ Total } & \multicolumn{4}{|c|}{ Census Region } & \multirow{3}{*}{$\begin{array}{c}\text { RSE } \\
\text { Row } \\
\text { Factors }\end{array}$} \\
\hline & & & Northeast & Midwest & South & West & \\
\hline & RSE Column Factors: & 0.7 & 1.3 & 1.0 & 0.9 & 1.3 & \\
\hline
\end{tabular}

2082

Malt Beverages

General Energy-Related Technologies

One or More General Technologies Present

Computer Control of Building Environment ${ }^{b} \ldots \ldots \ldots \ldots \ldots \ldots$

Computer Control of Processes or Major Energy-U. . . . . . . . . . .

Waste Heat Recovery . . . . . . . . . . . . . . . . . . .

Adjustable-Speed Motors . . . . . . . . . . . . . . . . .

None Present .

Industry-Specific Technologies

One or More Industry-Specific Technologies Present . . . . . . . . . . . . . .

Infrared Heating . . . . . . . . . . . . . . . . . . . . .

Microwave Drying . . . . . . . . . . . . . . . . . . . .

Closed-Cycle Heat Pump System Used to Recover Heat . . . . . . . . .

Open-Cycle Heat Pump System Used to Produce steam .........

Gas-Driven Rotary Engines and/or Turbines . . . . . . . . . . . .

Membrane Separation . . . . . . . . . . . . . . . . . . . .

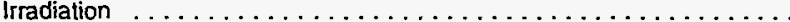

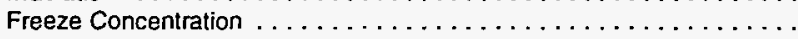

None Present . . . . . . . . . . . . . . . . . . . . . . .

TOBACCO PRODUCTS

General Energy-Related Technologies

One or More General Technologies Present .

Computer Control of Processes or Major Energy-Using Equipment ${ }^{c}$...

Waste Heat Recovery . . . . . . . . . . . . . . . . . . . . . .

Adjustable-Speed Motors $\ldots \ldots \ldots \ldots \ldots \ldots \ldots \ldots \ldots \ldots \ldots$

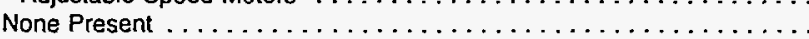

TEXTILE MILL PRODUCTS

General Energy-Related Technologies

One or More General Technologies Present

Computer Control of Build

Computer Control of Processes or Major Energy-Using Equipment ${ }^{c} \ldots$

Waste Heat Recovery . . . . . . . . . . . . . . . . . . . . . . . .

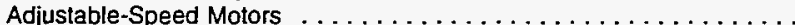

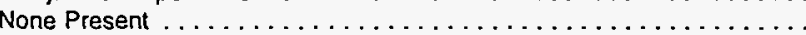

50
29
35
27
50
0

8
7
7
8
8

11
$W$
10
10
11
0

$\begin{array}{rrr}14 & 17 & 10.6 \\ 4 & W & 16.1 \\ 12 & 6 & 10.2 \\ 6 & 4 & 11.5 \\ 14 & 17 & 10.6 \\ 0 & 0 & \text { NF }\end{array}$

Industry-Specific Technologies

One or More Industry-Specific Technologies Present . . . . . . . . .

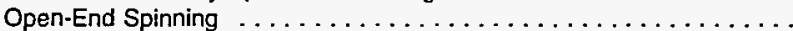

Water-Jet Weaving . . . . . . . . . . . . . . . . . . . . .

Projectile Weaving $\ldots \ldots \ldots \ldots \ldots \ldots \ldots \ldots \ldots \ldots \ldots \ldots$

Wet-on-Wet Dyeing and Finishing . . . . . . . . . . . . .

Indirect Steam Heating of Dye . . . . . . . . . . . . . . . . . . .

Dye Bath Reuse . . . . . . . . . . . . . . . . . . . . .

Foam Dyeing .

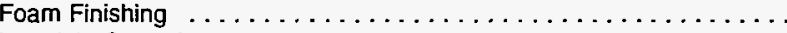

Low-Add-On Finishing

35
1
0
$W$
$W$
16
0
1
1
15

$\begin{array}{rr}W & \\ 1 & \\ 0 & \\ 0 & \\ 0 & \\ 1 & \\ 0 & \\ 0 & \\ 0 & \\ W & \end{array}$

13.5
20.4
NF
19.7
22.3
19.5
NF
24.4
28.9
12.5

$\begin{array}{rrrrrr}13 & * & * & 13 & 0 & 9.3 \\ 10 & 0 & * & 10 & 0 & 5.9 \\ 12 & * & * & 11 & 0 & 6.0 \\ W & 0 & * & W & 0 & 6.3 \\ 13 & * & * & 13 & 0 & 9.3 \\ 11 & 0 & 0 & 11 & 0 & 11.3\end{array}$

APPAREL and OTHER TEXTILE PRODUCTS

General Energy-Related Technologies

One or More General Technologies Present

Computer Control of Building Environment ${ }^{\circ} \ldots \ldots \ldots \ldots \ldots \ldots \ldots \ldots \ldots \ldots$ Computer Control of Processes or Major Energy-Using Equipment ${ }^{c}$...

Waste Heat Recovery

Adjustable-Speed Motors

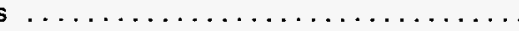

.

$\begin{array}{rrrrrr}184 & 16 & 3 & 163 & 2 & 9.1 \\ 59 & 2 & * & 56 & 1 & 13.9 \\ 92 & 5 & 1 & 85 & * & 12.0 \\ 104 & 11 & 2 & 90 & 1 & 11.1 \\ 138 & 13 & 2 & 122 & 2 & 9.3 \\ 89 & 11 & 3 & 72 & 3 & 15.0\end{array}$

See footnotes at end of table. 
Table A44. Total Inputs of Energy for Heat, Power, and Electricity Generation by Census Region, Industry Group, Selected Industries, Presence of General Technologies, and Industry-Specific Technologies for Selected Industries, 1991 (Continued) (Estimates in Trillion Btu)

\begin{tabular}{cc|c|c|c|c|c|}
\hline \multirow{2}{*}{$\begin{array}{c}\text { Industry Groups } \\
\text { and Industry }\end{array}$} & & \multicolumn{3}{|c}{ Census Region } \\
\cline { 3 - 7 } Code" & RSE Column Factors: & Total & Northeast & Midwest & South & Wow \\
\hline & RSE Coctors \\
\hline
\end{tabular}

General Energy-Related Technologies

One or More General Technologies Present

Computer Control of Bullding Environment ${ }^{b} \ldots \ldots \ldots \ldots \ldots \ldots \ldots \ldots \ldots \ldots$ Computer Control of Processes or Major Energy-Using Equipment ${ }^{c}$...

Waste Heat Recovery . . . . . . . . . . . . . . . . . . . .

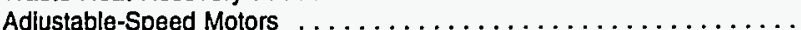

None Present .

\section{PAPER and ALLIED PRODUCTS}

General Energy-Related Technologies

One or More General Technologies Present $\ldots \ldots \ldots \ldots \ldots \ldots$

Computer Control of Building Environment ${ }^{\circ} \ldots \ldots \ldots \ldots \ldots \ldots \ldots$

Computer Control of Processes or Major Energy-Using Equipment ${ }^{c}$...

Waste Heat Recovery . . . . . . . . . . . . . . . . .

Adjustable-Speed Motors $\ldots \ldots \ldots \ldots \ldots \ldots \ldots \ldots \ldots \ldots$

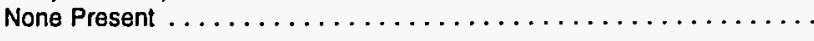

Industry-Speciflc Technologies

One or More Industry-Specific Technologies Present . . . . . . . . . .

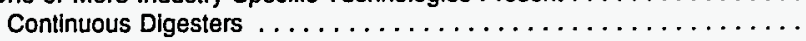

Displacement Bleaching Process . . . . . . . . . . . . . . .

Top-Wire (Hybrid) Paper Forming $\ldots \ldots \ldots \ldots \ldots \ldots \ldots \ldots$

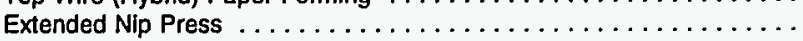

Higher Nip Pressures . . . . . . . . . . . . . . . . . . . . .

Extended Deliquefaction Displacement Heating Processes . . . . . . . .

Multi-Effect Falling-Film Evaporators for Black Liquor Evaporation and

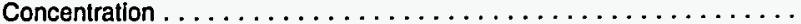

Vapor Recompression Evaporation of Black Liquor . . . . . . . . . .

Waste-Heat Recovery Technologies in Lime Kilns $\ldots \ldots \ldots \ldots \ldots \ldots$

Improved Filtration Techniques Allowing Flexibility in the Selection of

Fuel Other Then Natural Gas and Distillate Fuel Oil for Lime

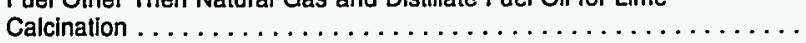

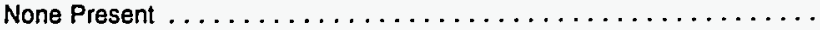

$\begin{array}{rr}2,240 & 259 \\ 412 & 5 \\ 2,037 & 23 \\ 1,837 & 197 \\ 1,992 & 220 \\ 232 & 27 \\ & \\ 2,005 & 18 \\ 1,232 & 114 \\ 350 & 6 \\ 1,004 & 111 \\ 557 & 60 \\ 747 & 81 \\ 225 & 20 \\ 1,192 & 113 \\ 217 & 28 \\ 579 & \\ & \\ 218 & \\ 468 & 9\end{array}$

5
2
4
2
5
11

12
8
7
6
9
0

$\begin{array}{rll}17 & 1 & 17.0 \\ 3 & 1 & 22.7 \\ 4 & : & 26.2 \\ 5 & : & 25.1 \\ 12 & : & 18.9 \\ 14 & 2 & 27.5\end{array}$

$\begin{array}{rrrrr}259 & 301 & 1,323 & 358 & 3.9 \\ 52 & 71 & 249 & 40 & 5.9 \\ 232 & 255 & 1,258 & 293 & 3.7 \\ 197 & 222 & 1,098 & 320 & 4.1 \\ 220 & 253 & 1,170 & 349 & 3.9 \\ 27 & 54 & 120 & 31 & 6.5\end{array}$

$\begin{array}{llll}27 & 54 & 120 & 31\end{array}$

See footnotes at end of table. 
Table A44. Total Inputs of Energy for Heat, Power, and Electricity Generation by Census Region, Industry Group, Selected Industries, Presence of General Technologies, and Industry-Specific Technologies for Selected Industries, 1991 (Continued) (Estimates in Trillion Btu)

\begin{tabular}{|c|c|c|c|c|c|c|c|}
\hline \multirow{2}{*}{$\begin{array}{c}\text { SIC } \\
\text { Code }^{2}\end{array}$} & \multirow{2}{*}{$\begin{array}{l}\text { Industry Groups } \\
\text { and Industry }\end{array}$} & \multirow[b]{2}{*}{ Total } & \multicolumn{4}{|c|}{ Census Region } & \multirow{3}{*}{$\begin{array}{r}\text { RSE } \\
\text { Row } \\
\text { Factor }\end{array}$} \\
\hline & & & Northeast & Midwest & South & West & \\
\hline & RSE Column Factors: & 0.7 & 1.3 & 1.0 & 0.9 & 1.3 & \\
\hline
\end{tabular}

2611

General Energy-Related Technologies

One or More General Technologies Present

Computer Control of Building Environment ${ }^{b} \ldots \ldots \ldots \ldots \ldots \ldots \ldots \ldots$

Computer Control of Processes or Major Energy-Using Equipment ${ }^{e}$...

Waste Heat Recovery . . . . . . . . . . . . . . . . . . . .

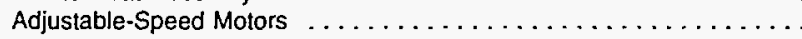

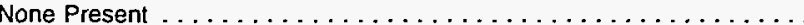

Industry-Specific Technologies

One or More Industry-Specific Technologies Present . . . . . . . . . .

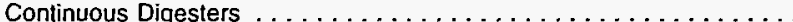

Displacement Bleaching Process . . . . . . . . . . . . . . .

Top-Wire (Hybrid) Paper Forming $\ldots \ldots \ldots \ldots \ldots \ldots \ldots \ldots$

Extended Nip Press . . . . . . . . . . . . . . . . . . . . . . .

Higher Nip Pressures . . . . . . . . . . . . . . . . . . .

Extended Deliquefaction Displacement Heating Processes . . . . . . . .

Multi-Efiect Falling-Film Evaporators for Black Liquor Evaporation and

Concentration . . . . . . . . . . . . . . . . . . . . . .

Vapor Recompression Evaporation of Black Liquor . . . . . . . . . . .

Waste-Heat Recovery Technologies in Lime Kilns . . . . . . . . . . . .

Improved Filtration Techniques Allowing Flexibility in the Selection of

Fuel Other Then Natural Gas and Distillate Fuel Oil for Lime

Calcination . . . . . . . . . . . . . . . . . . . . . . .

None Present . . . . . . . . . . . . . . . . . . . . . .

34
270
270
254
12

12

1

\section{Paper Mills}

General Energy-Related Technologies

One or More General Technologies Present . . . . . . . . . . . . .

Computer Control of Building Environment ${ }^{\circ} \ldots \ldots \ldots \ldots \ldots \ldots$ Computer Control of Processes or Major Energy-Using Equipment ${ }^{c}$...

Waste Heat Recovery . . . . . . . . . . . . . . . . . . .

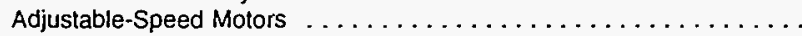

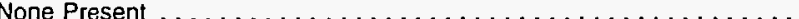

Industry-Specific Technologies

One or More Industry-Specific Technologies Present . . . . . . . . . . . . .

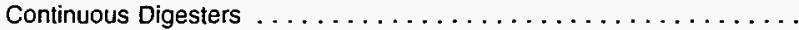

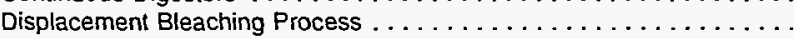

Top-Wire (Hybnd) Paper Forming . . . . . . . . . . . . . . . . . .

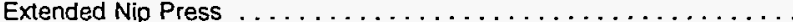

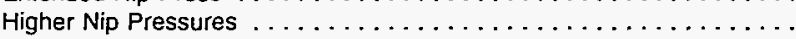

Extended Deliquefaction Displacement Heating Processes . . . . . . . .

Multi-Effect Falling-Film Evaporators for Black Liquor Evaporation and

Concentration . . . . . . . . . . . . . . . . . . . . .

Vapor Recompression Evaporation of Black Liquor . . . . . . . . . . .

Waste-Heat Recovery Technologies in Lime Kilns . . . . . . . . . . . .

Improved Filtration Techniques Allowing Flexibility in the Selection of

Fuel Other Then Natural Gas and Distillate Fuel Oil for Lime

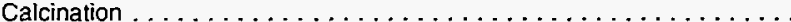

None Present $\ldots \ldots \ldots \ldots \ldots \ldots \ldots \ldots \ldots \ldots \ldots \ldots$

$$
\text { ... }
$$

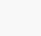


Table A44. Total Inputs of Energy for Heat, Power, and Electricity Generation by Census Region, Industry Group, Selected Industries, Presence of General Technologies, and Industry-Specific Technologies for Selected Industries, 1991 (Continued) (Estimates in Trillion Btu)

\begin{tabular}{|c|c|c|c|c|c|c|c|}
\hline \multirow[b]{2}{*}{$\begin{array}{l}\text { SIC } \\
\text { Code }\end{array}$} & \multirow{2}{*}{$\begin{array}{l}\text { Industry Groups } \\
\text { and Industry }\end{array}$} & \multirow[b]{2}{*}{ Total } & \multicolumn{4}{|c|}{ Census Region } & \multirow{3}{*}{$\begin{array}{c}\text { RSE } \\
\text { Row } \\
\text { Factors }\end{array}$} \\
\hline & & & Northeast & Midwest & South & West & \\
\hline & RSE Column Factors: & 0.7 & 1.3 & 1.0 & 0.9 & 1.3 & \\
\hline
\end{tabular}

2631

Paperboard Mills

General Energy-Related Technologies

One or More General Technologies Present

Waste Heat Recoveny

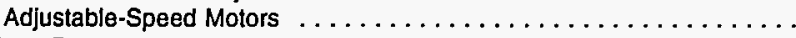

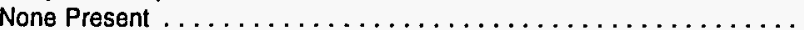

Industry-Specific Technologies

One or More Industry-Specific Technologies Present ..............

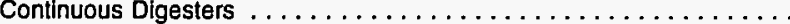

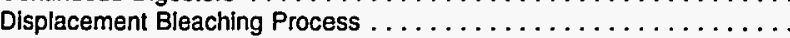

Top-Wire (Hybrid) Paper Forming $\ldots \ldots \ldots \ldots \ldots \ldots \ldots \ldots \ldots$

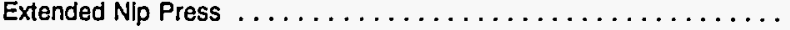

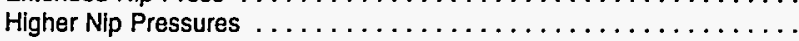

Extended Deliquefaction Displacement Heating Processes .........

Multi-Efiect Falling-Film Evaporators for Black Liquor Evaporation and

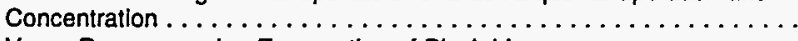

Vapor Recompression Evaporation of Black Liquor . . . . . . . . . . . .

Waste-Heat Recovery Technologies in Lime Kilns .............

Improved Filtration Techniques Allowing Flexibility in the Selection of

Fuel Other Then Natural Gas and Distillate Fuel Oil for Lime

Calcination . . . . . . . . . . . . . . . . . . . . . .

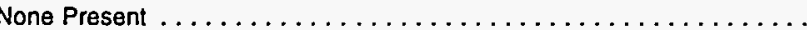

PRINTING and PUBLISHING

General Energy-Related Technologies

One or More General Technologies Present

Computer Control of Processes or Major Energy-Using Equipment ${ }^{\mathrm{c}} \ldots \ldots$

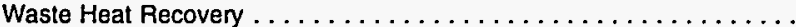

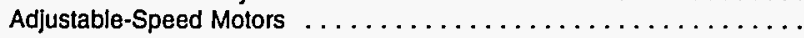

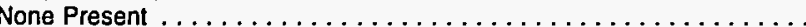

$\begin{array}{rr}760 & 11 \\ 101 & 0 \\ 718 & 8 \\ 591 & 5 \\ 678 & 7 \\ 72 & 5\end{array}$

11
0
8
5
7
5

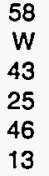

523
72
513
399
459
53

$\begin{array}{rr}168 & 6.2 \\ W & 11.4 \\ 154 & 6.9 \\ 161 & 7.2 \\ 165 & 7.2 \\ 2 & 13.2\end{array}$

$\begin{array}{rrr}526 & 161 & 7.2 \\ 397 & 90 & 6.0 \\ 54 & 0 & 12.6 \\ 289 & 25 & 9.1 \\ 252 & 32 & 8.2 \\ 268 & 34 & 9.4 \\ 56 & 0 & 11.1 \\ & & \\ 318 & 56 & 7.1 \\ 35 & 91 & 12.0 \\ W & W & 9.7\end{array}$

One or More General Technologies Present ...

Computer Control of Building Environment ${ }^{b} \ldots \ldots \ldots \ldots \ldots \ldots \ldots \ldots$ Computer Control of Processes or Major Energy-Using Equipment ${ }^{c} \ldots$

Waste Heat Recovery $\ldots \ldots \ldots \ldots \ldots \ldots \ldots \ldots \ldots \ldots \ldots$

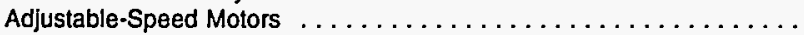

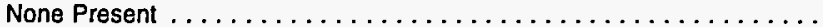

Industry-Specilic Technologies

One or More Industry-Specific Technologies Present ..............

1,893

Replacement of Electrically Heated Platens in the Thermoset Molding

Process with a Gas-Fired Central Thermal Fluid System ...........

Processing Residuals as Alternative Feedstocks . . . . . . . . . . . . .

Biomass Materials Used as Altemative Feedstocks ...........

Bloprocessing of Petroleum, Natural Gas, Coal or Other Fossil-Based

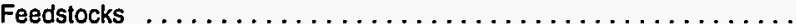

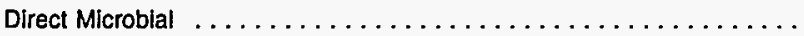

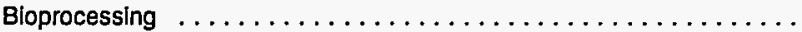

Gaslfication of Biomass Feedstocks $\ldots \ldots \ldots \ldots \ldots \ldots \ldots \ldots$

Fast Pyrolysis of Biomass Feedstocks $\ldots \ldots \ldots \ldots \ldots \ldots \ldots \ldots$

Immobilized Enzyme Processes $\ldots \ldots \ldots \ldots \ldots \ldots \ldots \ldots \ldots \ldots$

Innovalive Catalytic Processes $\ldots \ldots \ldots \ldots \ldots \ldots \ldots \ldots \ldots \ldots$

Recycling of Materials . . . . . . . . . . . . . . . . . . .

Hydrolysis of Biomass Materials . ...................

Enhanced Bloprocessing with Genetically Engineered Feedstocks or

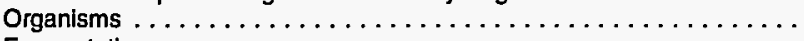

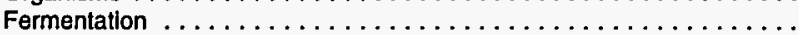

Fractionation of Blomass . . . . . . . . . . . . . . . . . . . .

Distlllation Process Improvements $\ldots \ldots \ldots \ldots \ldots \ldots \ldots \ldots \ldots \ldots$

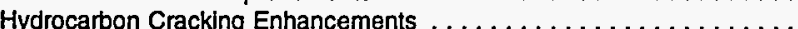

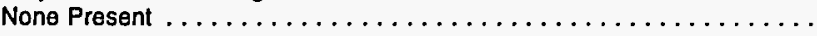

5
900

34

$0 \quad W$

351

126

224

282
242

47

2,020
340
1,715
1,721
1,397
348

5.1

6.4

5.0

6.0

5.0

8.7 
Table A44. Total Inputs of Energy for Heat, Power, and Electricity Generation by Census Region, Industry Group, Selected Industries, Presence of General Technologies, and Industry-Specific Technologies for Selected Industries, 1991 (Continued) (Estimates in Trillion Btu)

\begin{tabular}{|c|c|c|c|c|c|c|c|}
\hline \multirow{2}{*}{$\begin{array}{l}\text { SIC } \\
\text { Code }\end{array}$} & \multirow{2}{*}{$\begin{array}{l}\text { Industry Groups } \\
\text { and Industry }\end{array}$} & \multirow[b]{2}{*}{ Total } & \multicolumn{4}{|c|}{ Census Region } & \multirow{3}{*}{$\begin{array}{c}\text { RSE } \\
\text { Row } \\
\text { Factor8 }\end{array}$} \\
\hline & & & Northeast & Midwest & South & West & \\
\hline & Jlumn Factors: & 0.7 & 1.3 & 1.0 & 0.9 & 1.3 & \\
\hline
\end{tabular}

2812

Alkalies and Chlorine

General Energy-Related Technologies

One or More General Technologies Present

Computer Control of Building Environment ${ }^{3} \ldots \ldots \ldots \ldots \ldots \ldots \ldots$ Computer Control of Processes or Major Energy-Using Equipment ${ } \ldots$...

Waste Heat Recovery . . . . . . . . . . . . . . . . .

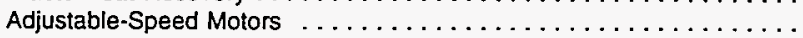

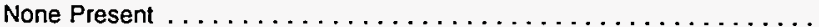

Industry-Specific Technologies

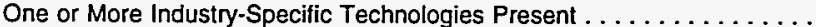

Replacement of Electrically Heated Platens in the Thermoset Molding

Process with a Gas-Fired Central Thermal Fluid System . . . . . . . . .

Processing Residuals as Alternative Feedstocks . . ............

Biomass Materials Used as Altemative Feedstocks . . . . . . . . .

Bioprocessing of Petroleum, Natural Gas, Coal or Other Fossil-Based

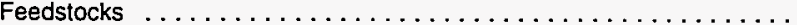

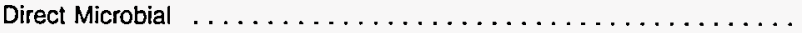

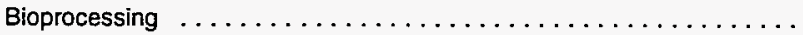

Gasification of Biomass Feedstocks . . . . . . . . . . . . . . .

Fast Pyrolysis of Biomass Feedstocks $\ldots \ldots \ldots \ldots \ldots \ldots \ldots \ldots$

Immobilized Enzyme Processes ...................

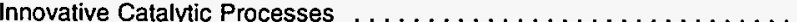

Recycling of Materials $\ldots \ldots \ldots \ldots \ldots \ldots \ldots \ldots \ldots \ldots \ldots$

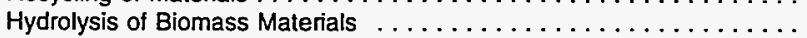

Enhanced Bioprocessing with Genetically Engineered Feedstocks or

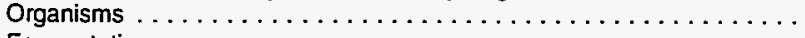

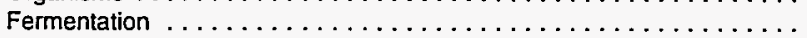

Fractionation of Biomass . . . . . . . . . . . . . . . .

Distillation Process Improvements . . . . . . . . . . . . .

Hydrocarbon Cracking Enhancements . . . . . . . . . . . . .

None Present

$$
\ldots \ldots \ldots \ldots \ldots \ldots
$$

General Energy-Related Technologies

One or More General Technologies Present

Computer Control of Building Environment ${ }^{b} \ldots \ldots \ldots \ldots \ldots \ldots \ldots$ Computer Control of Processes or Major Energy-Using Equipment ${ }^{\circ}$....

Waste Heat Recovery . . . . . . . . . . . . . . . . . . . .

Adjustable-Speed Motors $\ldots \ldots \ldots \ldots \ldots \ldots \ldots \ldots \ldots \ldots \ldots$

None Present $\ldots \ldots \ldots \ldots \ldots \ldots \ldots \ldots \ldots \ldots \ldots \ldots$

Industry-Specific Technologies

One or More Industry-Specific Technologies Present .............

Replacement of Electrically Heated Platens in the Thermoset Molding

Process with a Gas-Fired Central Thermal Fiuid System . . . . . . . . .

Processing Residuals as Alternative Feedstocks $\ldots \ldots \ldots \ldots \ldots \ldots$

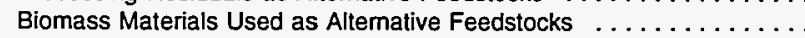

Bioprocessing of Petroleum, Natural Gas, Coal or Other Fossil-Based

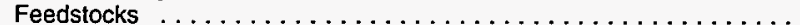

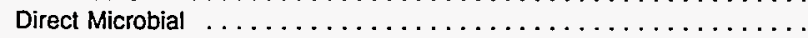

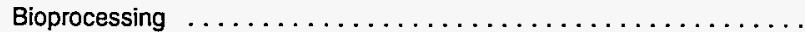

Gasification of Biomass Feedstocks $\ldots \ldots \ldots \ldots \ldots \ldots \ldots \ldots \ldots$

Fast Pyrolysis of Biomass Feedstocks $\ldots \ldots \ldots \ldots \ldots \ldots \ldots \ldots$

Immobilized Enzyme Processes $\ldots \ldots \ldots \ldots \ldots \ldots \ldots \ldots \ldots$

Innovative Catalytic Processes $\ldots \ldots \ldots \ldots \ldots \ldots \ldots \ldots \ldots$

Recycling of Materials . . . . . . . . . . . . . . . . . . . . .

Hydrolysis of Biomass Materials $\ldots \ldots \ldots \ldots \ldots \ldots \ldots \ldots \ldots$.

Enhanced Bioprocessing with Genetically Engineered Feedstocks or

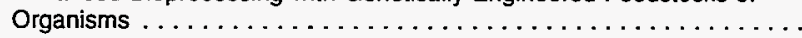

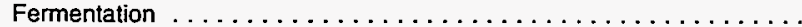

Fractionation of Biomass . . . . . . . . . . . . . . . . . . .

Distillation Process Improvements . . . . . . . . . . . . . . .

Hydrocarbon Cracking Enhancements $\ldots \ldots \ldots \ldots \ldots \ldots \ldots \ldots$

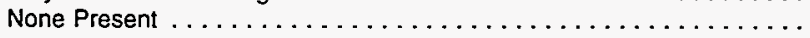

137
$W$
121
103
107
$W$

$\begin{array}{rr}0 & 124 \\ 0 & W \\ 0 & 11 \\ 0 & W \\ 0 & 10 \\ W & W\end{array}$

$\begin{array}{rrr}124 & 12 & 14.7 \\ W & W & 22.2 \\ 112 & 9 & 14.6 \\ W & W & 17.0 \\ 102 & 5 & 17.0 \\ W & 0 & 27.6\end{array}$

86

$\begin{array}{ccc}W & W & 20.6 \\ 0 & 0 & N F \\ W & W & 25.1 \\ 0 & 0 & N F\end{array}$

0

$$
0
$$$$
\text { NF }
$$

$\stackrel{0}{w}$

0

$$
\begin{array}{r}
0 \\
0 \\
0 \\
W \\
0
\end{array}
$$

0
0
0
0
0
0
75

$\mathrm{NF}$
$\mathrm{NF}$
32.6
$\mathrm{NF}$
$\mathrm{NF}$
$\mathrm{NF}$
$\mathrm{NF}$
23.0
$\mathrm{NF}$
$\mathrm{NF}$
$\mathrm{NF}$
$\mathrm{NF}$
$\mathrm{NF}$
$\mathrm{NF}$
17.4

$\begin{array}{rrrrrr}W & 4 & 13 & W & 14 & 7.2 \\ W & 1 & 1 & W & W & 10.1 \\ 63 & W & W & 32 & 14 & 7.2 \\ 21 & 0 & W & W & W & 14.0 \\ W & 0 & 5 & W & 1 & 18.0 \\ W & 1 & 5 & W & 2 & 9.5\end{array}$

$\begin{array}{rrrrrr}W & 1 & 1 & \text { W } & \text { W } & 9.5 \\ 0 & 0 & 0 & 0 & 0 & \text { NF } \\ 0 & 0 & 0 & * & 0 & 23.8 \\ 0 & 0 & 0 & 0 & 0 & \text { NF }\end{array}$

$\begin{array}{rl}0 & 0 \\ 0 & 0 \\ 0 & 0 \\ 0 & 0 \\ 0 & 0 \\ W & 4\end{array}$

0
0
0
0
0
0
0
1
0
0
0
0
0
0
17

\begin{tabular}{rr}
0 & 0 \\
0 & 0 \\
0 & 0 \\
0 & 0 \\
0 & 0 \\
0 & 0 \\
0 & 0 \\
$W$ & $W$ \\
0 & 0 \\
0 & 0 \\
0 & 0 \\
0 & 0 \\
0 & 0 \\
0 & 0 \\
$W$ & $W$ \\
\hline
\end{tabular}


Table A44. Total Inputs of Energy for Heat, Power, and Electricity Generation by Census Region, Industry Group, Selected Industries, Presence of General Technologies, and Industry-Specific Technologies for Selected Industries, 1991 (Continued) (Estimates in Trillion Btu)

\begin{tabular}{|c|c|c|c|c|c|c|c|}
\hline \multirow[b]{2}{*}{$\underset{\text { Code" }}{\text { SIC }}$} & \multirow[b]{2}{*}{$\begin{array}{l}\text { Industry Groups } \\
\text { and Industry }\end{array}$} & \multirow[b]{2}{*}{ Total } & \multicolumn{4}{|c|}{ Census Region } & \multirow{3}{*}{$\begin{array}{c}\text { RSE } \\
\text { Row } \\
\text { Factors }\end{array}$} \\
\hline & & & Northeast & Midwest & South & West & \\
\hline & RSE Column Factors: & 0.7 & 1.3 & 1.0 & 0.9 & 1.3 & \\
\hline
\end{tabular}

2819

Industrlal Inorganic Chemicals, nec

General Energy-Related Technologies

One or More General Technologies Present

Computer Control of Bullding Environment ".

Computer Control of Processes or Major Energy-Using Equipment ${ }^{\circ} \ldots$

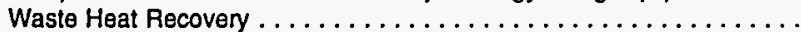

Adjustable-Speed Motors $\ldots \ldots \ldots \ldots \ldots \ldots \ldots \ldots \ldots \ldots$

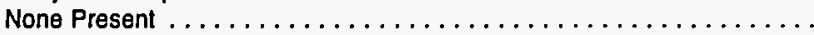

Industry-Specific Technologles

One or More Industry-Specific Technologies Present . .............

Replacement of Electrically Heated Platens in the Thermoset Molding

Process with a Gas-Flred Central Thermal Fluid System . . . . . . . . . .

Processing Residuals as Allernative Feedstocks $\ldots \ldots \ldots \ldots \ldots \ldots$

Blomass Materials Used as Alternative Feedstocks .............

Bloprocessing of Petroleum, Natural Gas, Coal or Other Fossit-Based

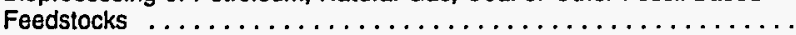

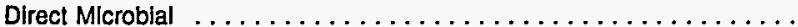

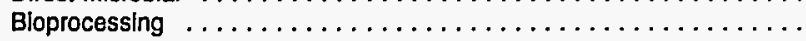

Gasification of Blomass Feedstocks . . . . . . . . . . . . . . . .

Fast Pyrolysis of Biomass Feedstocks . . . . . . . . . . . . . .

Immobillzed Enzyme Processes $\ldots \ldots \ldots \ldots \ldots \ldots \ldots \ldots \ldots$

Innovative Catalytlc Processes $\ldots \ldots \ldots \ldots \ldots \ldots \ldots \ldots \ldots \ldots$

Recycling of Materials $\ldots \ldots \ldots \ldots \ldots \ldots \ldots \ldots \ldots \ldots \ldots$

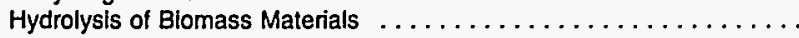

Enhanced Bloprocessing with Genetically Engineered Feedstocks or

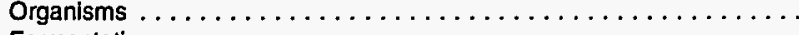

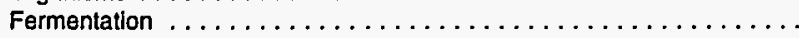

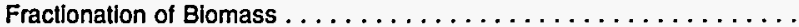

Distillation Process Improvements $\ldots \ldots \ldots \ldots \ldots \ldots \ldots \ldots$.

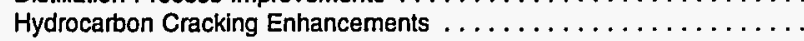

None Present . . . . . . . . . . . . . . . . . . . . . . . .

\begin{tabular}{|c|c|c|c|c|c|}
\hline 242 & 8 & 66 & 131 & 36 & 9.9 \\
\hline 106 & 3 & $w$ & $w$ & 23 & 11.5 \\
\hline 167 & 5 & 9 & 122 & 31 & 9.6 \\
\hline 194 & 7 & 62 & 93 & 32 & 11.1 \\
\hline 151 & 5 & 11 & 112 & 23 & 8.0 \\
\hline 69 & 2 & 4 & 58 & 5 & 13.6 \\
\hline 149 & 1 & $w$ & 57 & $w$ & 10.1 \\
\hline 1 & 0 & . & 1 & • & 15.9 \\
\hline 26 & . & $w$ & 16 & $w$ & 11.9 \\
\hline 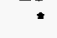 & 0 & . & 0 & 0 & 21.9 \\
\hline 6 & 0 & • & $w$ & $w_{i}$ & 16.8 \\
\hline+ & 0 & * & 0 & 0 & 21.9 \\
\hline$w$ & 0 & $w$ & $w$ & 0 & 20.3 \\
\hline . & 0 & " & 0 & 0 & 21.9 \\
\hline . & 0 & - & 0 & 0 & 21.9 \\
\hline * & 0 & * & 0 & • & 21.3 \\
\hline 7 & 0 & - & 5 & 1 & 12.9 \\
\hline 130 & 1 & $w$ & 49 & $w$ & 11.0 \\
\hline & 0 & . & 0 & 0 & 21.9 \\
\hline${ }^{*}$ & 0 & . & 0 & 0 & 21.9 \\
\hline • & 0 & * & 0 & 0 & 21.9 \\
\hline * & 0 & - & 0 & 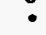 & 21.3 \\
\hline 0 & 0 & 0 & 0 & 0 & NF \\
\hline$w$ & 0 & . & $w$ & 0 & 20.7 \\
\hline 161 & 8 & $w$ & 132 & $w$ & 8.8 \\
\hline
\end{tabular}

Plastics Materials and Resins

General Energy-Related Technologies

One or More General Technologies Present

Computer Control of Building Environment ${ }^{\circ} \ldots \ldots \ldots \ldots \ldots \ldots \ldots$

Computer Control of Processes or Major Energy-Using Equipment ${ }^{\circ}$...

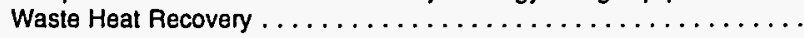

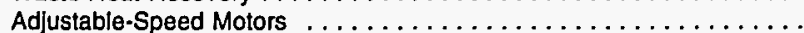

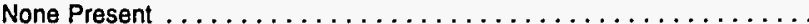

Industry-Specific Technologies

One or More Industry-Specific Technologies Present ............

Replacement of Electrically Heated Platens in the Thermoset Molding

Process with a Gas-Fired Central Thermal Fluid System . . . . . . . . .

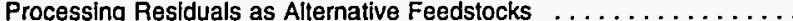

Blomass Materials Used as Altemative Feedstocks ................

Bloprocessing of Petroleum, Natural Gas, Coal or Other Fossil-Based

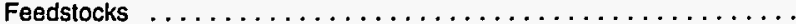

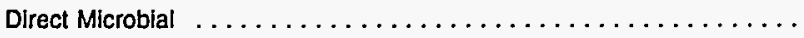

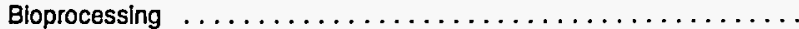

Gasification of Blomass Feedstocks . . . . . . . . . . . . . . . .

Fast Pyrolysis of Biomass Feedstocks . . . . . . . . . . . . . .

Immobilized Enzyme Processes $\ldots \ldots \ldots \ldots \ldots \ldots \ldots \ldots \ldots$

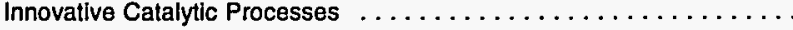

Recycling of Materials $\ldots \ldots \ldots \ldots \ldots \ldots \ldots \ldots \ldots \ldots \ldots \ldots$

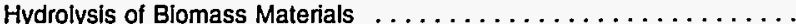

Enhanced Bloprocessing with Genetically Engineered Feedstocks or

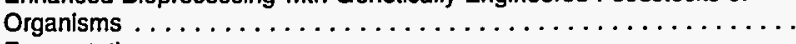

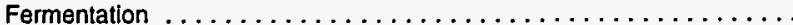

Fractionation of Blomass $\ldots \ldots \ldots \ldots \ldots \ldots \ldots \ldots \ldots \ldots \ldots \ldots$

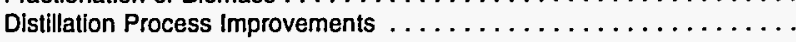

Hydrocarbon Cracking Enhancements . . . . . . . . . . . . . .

None Present . . . . . . . . . . . . . . . . . . . . . . .

\begin{tabular}{|c|c|c|c|c|c|}
\hline 264 & 16 & 53 & 194 & 2 & 7.4 \\
\hline 90 & $w$ & $w$ & 67 & * & 7.7 \\
\hline 222 & 12 & 51 & 157 & $Q$ & 5.7 \\
\hline 206 & 10 & 41 & 155 & 1 & 6.0 \\
\hline 174 & 13 & 50 & 110 & 1 & 7.7 \\
\hline 23 & 4 & 6 & 13 & 1 & 9.3 \\
\hline
\end{tabular}

\begin{tabular}{|c|c|c|c|c|c|}
\hline 198 & 11 & 44 & 143 & 1 & 5.8 \\
\hline * & 0 & - & - & 0 & 19.4 \\
\hline 48 & 1 & W & w & 0 & 10.1 \\
\hline 1 & 0 & 0 & 1 & 0 & 17.6 \\
\hline$w$ & 0 & 0 & $w$ & 0 & 17.6 \\
\hline 19 & 0 & 2 & 16 & • & 11.5 \\
\hline$w$ & * & 0 & $w$ & 0 & 15.0 \\
\hline$w$ & 0 & 0 & $w$ & 0 & 17.6 \\
\hline 1 & 1 & 0 & 0 & 0 & 14.9 \\
\hline 0 & 0 & 0 & 0 & 0 & NF \\
\hline 9 & W & 1 & w & • & 11.3 \\
\hline 116 & 11 & 23 & 83 & 1 & 6.6 \\
\hline 0 & 0 & 0 & 0 & 0 & NF \\
\hline$w$ & 0 & 0 & $w$ & 0 & 17.6 \\
\hline 0 & 0 & 0 & 0 & 0 & $N F$ \\
\hline 0 & 0 & 0 & 0 & 0 & NF \\
\hline 0 & 0 & 0 & 0 & 0 & NF \\
\hline$w$ & 0 & $w$ & $w$ & 0 & 12.7 \\
\hline 89 & 9 & 15 & 64 & 2 & 8.0 \\
\hline
\end{tabular}

See footnotes at end of table. 
Table A44. Total Inputs of Energy for Heat, Power, and Electricity Generation by Census Region, Industry Group, Selected Industries, Presence of General Teclinologies, and Industry-Specific Technologies for Selected Industries, 1991 (Continued) (Estimates in Trillion Btu)

\begin{tabular}{|c|c|c|c|c|c|c|c|}
\hline \multirow{2}{*}{$\begin{array}{c}\text { SIC } \\
\text { Code }\end{array}$} & \multirow{2}{*}{$\begin{array}{l}\text { Industry Groups } \\
\text { and Industry }\end{array}$} & \multirow[b]{2}{*}{ Total } & \multicolumn{4}{|c|}{ Census Region } & \multirow{3}{*}{$\begin{array}{c}\text { RSE } \\
\text { Row } \\
\text { Factors }\end{array}$} \\
\hline & & & Northeast & Midwest & South & West & \\
\hline & RSE Column Factors: & 0.7 & 1.3 & 1.0 & 0.9 & 1.3 & \\
\hline
\end{tabular}

2822

General Energy-Related Technologies

One or More General Technologies Present

Computer Control of Building Environment ${ }^{b}$.......

Computer Control of Processes or Major Energy-Using Equipment ${ }^{c} \ldots$

Waste Heat Recovery . . . . . . . . . . . . . . . . . . . . .

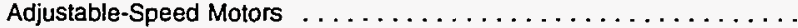

None Present . . . . . . . . . . . . . . . . . . . . .

Industry-Specific Technologies

One or More Industry-Specific Technologies Present . . . . . . . . . .

Replacement of Electrically Heated Platens in the Thermoset Molding

Process with a Gas-Fired Central Thermal Fluid System . . . . . . . . . .

Processing Residuals as Alternative Feedstocks $\ldots \ldots \ldots \ldots \ldots \ldots$

Biomass Materials Used as Altemative Feedstocks $\ldots \ldots \ldots \ldots \ldots \ldots$

Bioprocessing of Petroleum, Natural Gas, Coal or Other Fossil-Based

Feedstocks

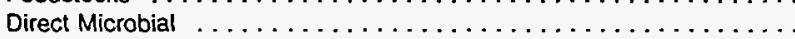

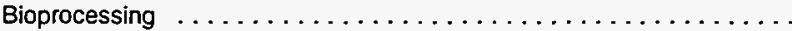

Gasification of Biomass Feedstocks . . . . . . . . . . . . . . .

Fast Pyrolysis of Biomass Feedstocks $\ldots \ldots \ldots \ldots \ldots \ldots \ldots$

Immobilized Enzyme Processes $\ldots \ldots \ldots \ldots \ldots \ldots \ldots \ldots \ldots \ldots$

Innovative Catalytic Processes $\ldots \ldots \ldots \ldots \ldots \ldots \ldots \ldots \ldots$

Recycling of Materials . . . . . . . . . . . . . . . . . .

Hydrolysis of Biomass Materials $\ldots \ldots \ldots \ldots \ldots \ldots \ldots \ldots \ldots$

Enhanced Bioprocessing with Genetically Engineered Feedstocks or

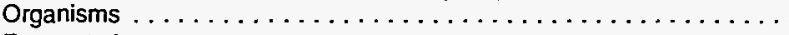

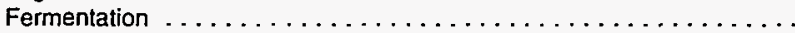

Fractionation of Biomass . . . . . . . . . . . . . . . . . .

Distillation Process Improvements . . . . . . . . . . . . . .

Hydrocarbon Cracking Enhancements . . . . . . . . . . . .

None Present . . . . . . . . . . . . . . . . . . . . . . . . . . . .

General Energy-Related Technologies

One or More General Technologies Present . . . . . . . . . . . .

Computer Control of Building Environment ${ }^{\mathrm{n}} \ldots \ldots \ldots \ldots \ldots \ldots \ldots \ldots \ldots \ldots$ Computer Control of Processes or Major Energy-Using Equipment ${ }^{c} \ldots$

Waste Heat Recovery . . . . . . . . . . . . . . . . . .

Adjustable-Speed Motors . . . . . . . . . . . . . . . . . . .

Industry-Specific Technologies

One or More Industry-Specific Technologies Present . . . . . . . . . . . . . .

Replacement of Electrically Heated Platens in the Thermoset Molding

Process with a Gas-Fired Central Thermal Fluid System . . . . . . . . . .

Processing Residuals as Alternative Feedstocks ..............

Biomass Materials Used as Alternative Feedstocks . . . . . . . . . . .

Bioprocessing of Petroleum, Natural Gas, Coal or Other Fossil-Based

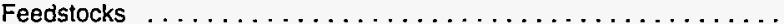

Direct Microbial . . . . . . . . . . . . . . . . . . .

Bioprocessing . . . . . . . . . . . . . . . . . . . .

Gasification of Biomass Feedstocks ....................

Fast Pyrolysis of Biomass Feedstocks $\ldots \ldots \ldots \ldots \ldots \ldots \ldots \ldots$

Immobilized Enzyme Processes $\ldots \ldots \ldots \ldots \ldots \ldots \ldots \ldots \ldots$

Innovative Catalytic Processes $\ldots \ldots \ldots \ldots \ldots \ldots \ldots \ldots \ldots$

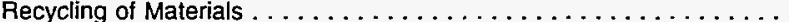

Hydrolysis of Biomass Materials . . . . . . . . . . . . . . . . Enhanced Bioprocessing with Genetically Engineered Feedstocks or

Organisms

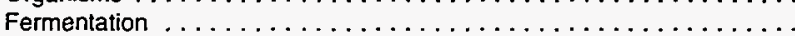

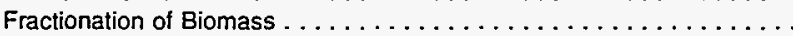

Distillation Process Improvements ...................

Hydrocarbon Cracking Enhancements $\ldots \ldots \ldots \ldots \ldots \ldots \ldots \ldots \ldots$

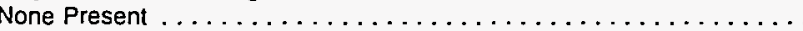

$\begin{array}{ll}W & W \\ W & \\ 39 & \\ 29 & W \\ 36 & W \\ W & W\end{array}$

$W$
0
$W$
$W$
$W$

$W$
0
$W$
$W$
$W$

103

W

W

W

0

0

0
$W$

0

0

W

28
0

0

0
0

0
0

10

$W$

0

0
0

0
0

0

0
0

0
0

0

0
W

\section{0}

0

0
0
0
0
$W$

13.9

21.3

13.6

15.2

14.6

21.2

19.4

NF
20.1
NF

NF

NF

NF
26.4

26.4

NF

NF

27.6

15.5

NF

NF

NF

NF

NF

NF

14.3

23.8

31.4

23.8

26.4

23.8

42.7

0

31.4

NF

42.7

NF

NF

NF

NF

NF

NF

NF

NF

42.7
NF

NF

NF

NF

NF

NF

See footnotes at end of table. 
Table A44. Total Inputs of Energy for Heat, Power, and Electricity Generation by Census Region, Industry Group, Selected Industries, Presence of General Technologies, and Industry-Specific Technologies for Selected Industries, 1991 (Continued) (Estimates in Trillion Btu)

\begin{tabular}{|c|c|c|c|c|c|c|c|}
\hline \multirow{2}{*}{$\begin{array}{l}\text { SIC } \\
\text { Code" }\end{array}$} & \multirow[b]{2}{*}{$\begin{array}{l}\text { Industry Groups } \\
\text { and Industry }\end{array}$} & \multirow[b]{2}{*}{ Total } & \multicolumn{4}{|c|}{ Census Region } & \multirow{3}{*}{$\begin{array}{c}\text { RSE } \\
\text { Row } \\
\text { Factors }\end{array}$} \\
\hline & & & Northeast & Midwest & South & West & \\
\hline & RSE Column Factors: & 0.7 & 1.3 & 1.0 & 0.9 & 1.3 & \\
\hline
\end{tabular}

2824

Organic Fibers, Noncellulosic

General Energy-Related Technologies

One or More General Technologies Present $\ldots \ldots \ldots \ldots \ldots \ldots \ldots$

Computer Control of Building Environment ${ }^{\circ} \ldots \ldots \ldots \ldots \ldots \ldots \ldots$ Computer Control of Processes or Major Energy-Using Equipment ${ }^{c} \ldots$

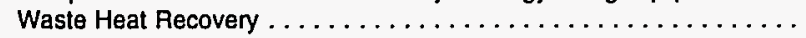

Adjustable-Speed Motors $\ldots \ldots \ldots \ldots \ldots \ldots \ldots \ldots \ldots \ldots$

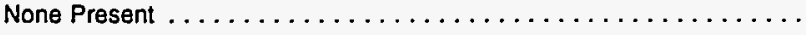

Industry-Specific Technologies

One or More Industry-Specific Technologies Present ............

Replacement of Electrically Heated Platens in the Thermoset Molding

Process with a Gas-Fired Central Thermal Fluid System . . . . . . . . .

Processing Residuals as Alternative Feedstocks $\ldots \ldots \ldots \ldots \ldots$

Biomass Materials Used as Alternative Feedstocks $\ldots \ldots \ldots \ldots \ldots$

Bioprocessing of Petroleum, Natural Gas, Coal or Other Fossil-Based

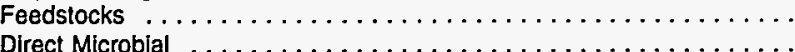

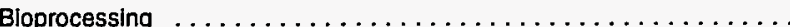

Gasification of Biomass Feedstocks . . . . . . . . . . . . . . .

Fast Pyrolysis of Biomass Feedstocks . . . . . . . . . . . . . .

Immobillzed Enzyme Processes $\ldots \ldots \ldots \ldots \ldots \ldots \ldots \ldots \ldots \ldots$

Innovative Catalytic Processes $\ldots \ldots \ldots \ldots \ldots \ldots \ldots \ldots \ldots$

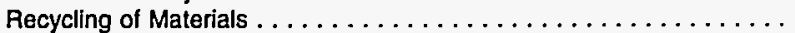

Hydrolysis of Biomass Materials $\ldots \ldots \ldots \ldots \ldots \ldots \ldots \ldots \ldots$

Enhanced Bioprocessing with Genetically Engineered Feedstocks or

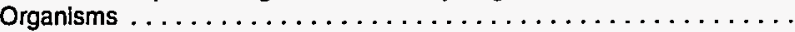

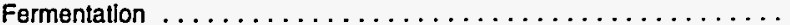

Fractionation of Biomass . . . . . . . . . . . . . . . . . .

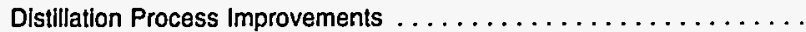

Hydrocarbon Cracking Enhancements $\ldots \ldots \ldots \ldots \ldots \ldots \ldots \ldots$

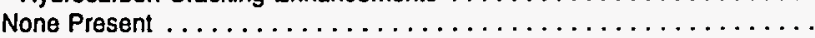

$\begin{array}{ll}84 & * \\ 25 & 0 \\ 82 & 0 \\ 65 & 0 \\ 69 & :\end{array}$

55

55

3.8

0

17

0

17
0

NF

6.3

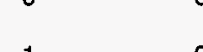

NF

8.8

8.8

8.8

NF

NF

NF

3.8
NF

8.8

NF

NF

NF

6.8

General Energy-Related Technologies

One or More General Technologies Present

Computer Control of Building Environment.

Computer Control of Processes or Major Energy-Using Equipment ${ }^{c} \ldots$

Waste Heat Recovery . . . . . . . . . . . . . . . . . . . . . . . .

Adjustable-Speed Motors $\ldots \ldots \ldots \ldots \ldots \ldots \ldots \ldots \ldots \ldots$

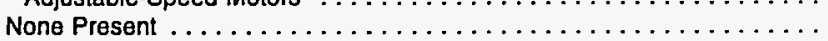

Industry-Speciflc Technologies

One or More Industry-Specific Technologies Present ............

Replacement of Electrically Heated Platens in the Thermoset Molding

Process with a Gas-Fired Central Thermal Fluid System . . . . . . . . . .

Processing Residuals as Alternative Feedstocks $\ldots \ldots \ldots \ldots \ldots \ldots$

Biomass Materials Used as Altemative Feedstocks . . . . . . . . . .

Bioprocessing of Petroleum, Natural Gas, Coal or Other Fossil-Based

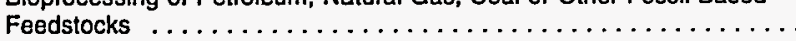

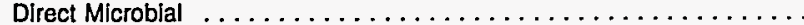

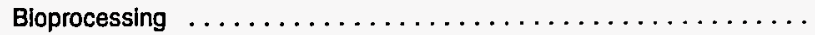

Gasification of Biomass Feedstocks $\ldots \ldots \ldots \ldots \ldots \ldots \ldots \ldots \ldots \ldots$

Fast Pyrolysis of Biomass Feedstocks $\ldots \ldots \ldots \ldots \ldots \ldots \ldots \ldots \ldots$

Immobilized Enzyme Processes $\ldots \ldots \ldots \ldots \ldots \ldots \ldots \ldots \ldots$

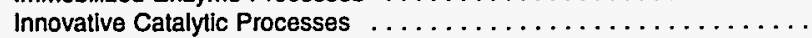

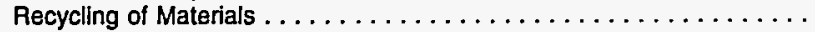

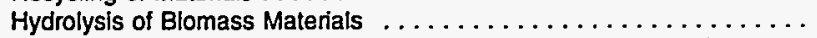

Enhanced Bloprocessing with Genetically Engineered Feedstocks or

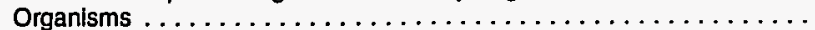

Fermentation . . . . . . . . . . . . . . . . . . . . . . .

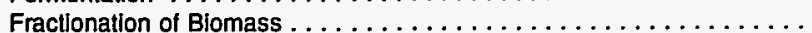

Distillation Process Improvements . . . . . . . . . . . . . . . .

Hydrocarbon Cracking Enhancements $\ldots \ldots \ldots \ldots \ldots \ldots \ldots \ldots$

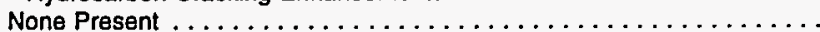


Table A44. Total Inputs of Energy for Heat, Power, and Electricity Generation by Census Region, Industry Group, Selected Industries, Presence of General Technologies, and Industry-Specific Technologies for Selected Industries, 1991 (Continued) (Estimates in Trillion Btu)

\begin{tabular}{|c|c|c|c|c|c|c|c|}
\hline \multirow{2}{*}{$\begin{array}{l}\text { SIC } \\
\text { Code }^{\circ}\end{array}$} & \multirow{2}{*}{$\begin{array}{l}\text { Industry Groups } \\
\text { and Industry }\end{array}$} & \multirow[b]{2}{*}{ Total } & \multicolumn{4}{|c|}{ Census Region } & \multirow{3}{*}{$\begin{array}{c}\text { RSE } \\
\text { Row } \\
\text { Factore }\end{array}$} \\
\hline & & & Northeast & Midwest & South & West & \\
\hline & RSE Column Factors: & 0.7 & 1.3 & 1.0 & 0.9 & 1.3 & \\
\hline
\end{tabular}

2869

Industrial Organic Chemicals, nec

General Energy-Related Technologies

One or More General Technologies Present

Computer Control of Building Environment ${ }^{\circ} \ldots \ldots \ldots \ldots \ldots \ldots \ldots$

Computer Control of Processes or Major Energy-Using Equipment ${ }^{c} \ldots$

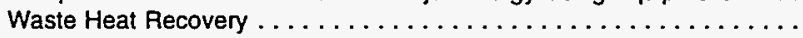

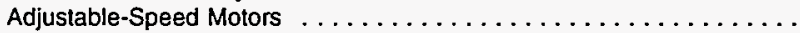

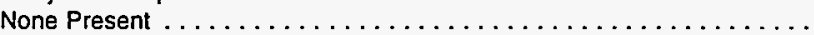

1,095
149
914
1,007
836
95

Industry-Specific Technologies

One or More Industry-Specific Technologies Present ............

Replacement of Electrically Heated Platens in the Thermoset Molding

Process with a Gas-Fired Central Thermal Fluid System . . . . . . . . . .

Processing Residuals as Alternative Feedstocks $\ldots \ldots \ldots \ldots \ldots \ldots$.

Biomass Materials Used as Altemative Feedstocks ................

Bioprocessing of Petroleum, Natural Gas, Coal or Other Fossil-Based

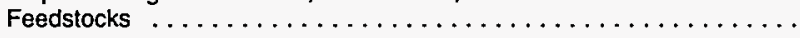

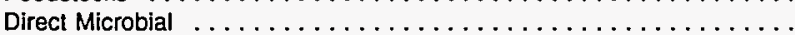

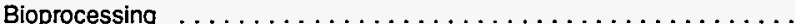

Gasification of Biomass Feedstocks . . . . . . . . . . . . .

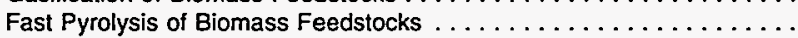

Immobilized Enzyme Processes $\ldots \ldots \ldots \ldots \ldots \ldots \ldots \ldots \ldots \ldots$

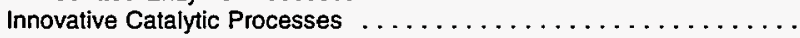

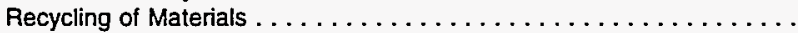

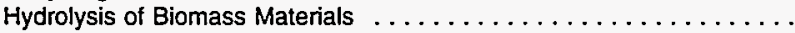

Enhanced Bioprocessing with Genetically Engineered Feedstocks or

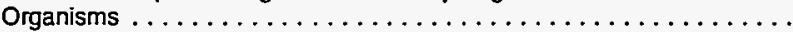

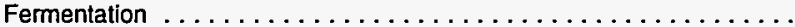

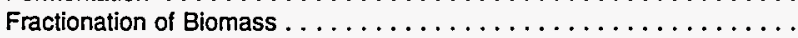

Distillation Process Improvements . . . . . . . . . . . . . . .

Hydrocarbon Cracking Enhancements . . . . . . . . . . . . . .

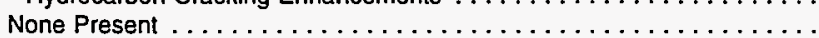

General Energy-Related Technologies

One or More General Technologies Present

Computer Control of Building Environment ${ }^{b} \ldots \ldots \ldots \ldots \ldots \ldots$.

Computer Control of Processes or Major Energy-Using Equipment ${ }^{e} \ldots$

Waste Heat Recovery . . . . . . . . . . . . . . . . . . . . . .

Adjustable-Speed Motors $\ldots \ldots \ldots \ldots \ldots \ldots \ldots \ldots \ldots \ldots$

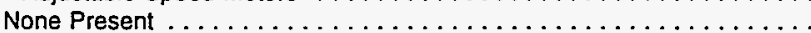

Industry-Specific Technologies

One or More Industry-Specific Technologies Present .............

Replacement of Electrically Heated Platens in the Thermoset Molding

Process with a Gas-Fired Central Thermal Fluid System ...........

Processing Residuals as Alternative Feedstocks $\ldots \ldots \ldots \ldots \ldots \ldots$.

Biomass Materials Used as Altemative Feedstocks . . . . . . . . . . .

Bioprocessing of Petroleum, Natural Gas, Coal or Other Fossil-Based

Feedstocks . . . . . . . . . . . . . . . . . . . . . .

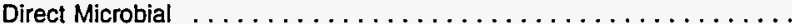

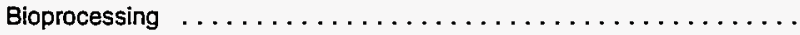

Gasification of Biomass Feedstocks . . . . . . . . . . . . . . . . .

Fast Pyrolysis of Biomass Feedstocks . . . . . . . . . . . . . .

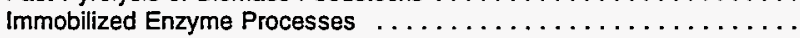

Innovative Catalytic Processes $\ldots \ldots \ldots \ldots \ldots \ldots \ldots \ldots \ldots$.

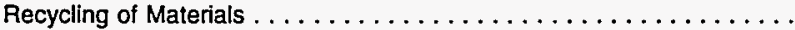

Hydrolysis of Biomass Materials . ....................

Enhanced Bioprocessing with Genetically Engineered Feedstocks or

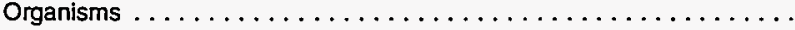

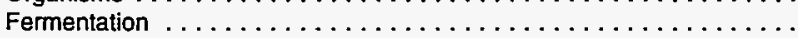

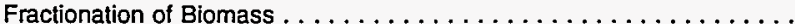

Distillation Process Improvements $\ldots \ldots \ldots \ldots \ldots \ldots \ldots \ldots \ldots$

Hydrocarbon Cracking Enhancements $\ldots \ldots \ldots \ldots \ldots \ldots \ldots \ldots$

None Present . . . . . . . . . . . . . . . . . . . . . . .
620

30

0

22

175

w

0
434

710

Q

w

17

0

537

313

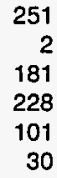

251
2
181
228
101
30

162

* 31

89

29.7

$\begin{array}{rrrr}0 & 0 & 0 & 0 \\ 92 & 0 & 0 & 53\end{array}$

0

0

NF

31.1

$\begin{array}{llllll}0 & 0 & 0 & 0 & 0 & \text { NF }\end{array}$

$\begin{array}{llllll}0 & 0 & 0 & 0 & 0 & \text { NF } \\ 0 & 0 & 0 & 0 & 0 & \text { NF }\end{array}$

$\begin{array}{lll}0 & 0 \\ 0 & 0\end{array}$

$\begin{array}{ll}0 & 0 \\ 0 & 0\end{array}$

0

0
49

78

0

0
0
0
0
14
118

5.7

8.1

6.2 
Table A44. Total Inputs of Energy for Heat, Power, and Electricity Generation by Census Region, Industry Group, Selected Industries, Presence of General Technologies, and Industry-Specific Technologies for Selected Industries, 1991 (Continued) (Estimates in Trillion Btu)

\begin{tabular}{|c|c|c|c|c|c|c|c|}
\hline \multirow{2}{*}{$\begin{array}{l}\text { SIC } \\
\text { Code* }\end{array}$} & \multirow{2}{*}{$\begin{array}{l}\text { Industry Groups } \\
\text { and Industry }\end{array}$} & \multirow[b]{2}{*}{ Total } & \multicolumn{4}{|c|}{ Census Region } & \multirow{3}{*}{$\begin{array}{c}\text { RSE } \\
\text { Row } \\
\text { Factors }\end{array}$} \\
\hline & & & Northeast & Midwest & South & West & \\
\hline & RSE Column Factors: & 0.7 & 1.3 & 1.0 & 0.9 & 1.3 & \\
\hline
\end{tabular}

2874

General Energy-Related Technologies

One or More General Technologies Present

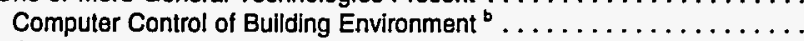

Computer Control of Processes or Major Energy-Using Equipment ${ }^{c} \ldots$

Waste Heat Recovery . . . . . . . . . . . . . . . . . . . .

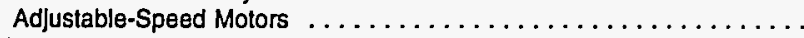

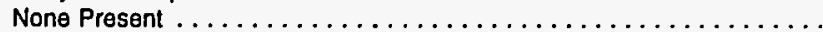

Industry-Specific Technologles

One or More Industry-Specific Technologies Present . . . . . . . . . . .

Replacement of Electrically Heated Platens in the Thermoset Molding

Process with a Gas-Fired Central Thermal Fluid System ...........

Processing Residuals as Alternative Feedstocks . ...............

Blomass Materials Used as Altemative Feedstocks . ............

Bioprocessing of Petroleum, Natural Gas, Coal or Other Fossil-Based

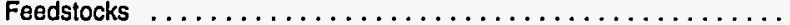

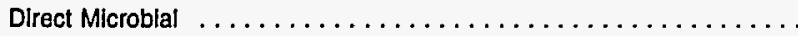

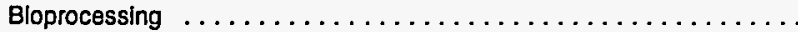

Gaslfication of Biomass Feedstocks $\ldots \ldots \ldots \ldots \ldots \ldots \ldots \ldots \ldots \ldots$

Fast Pyrolysis of Biomass Feedstocks $\ldots \ldots \ldots \ldots \ldots \ldots \ldots \ldots$

Immobilized Enzyme Processes $\ldots \ldots \ldots \ldots \ldots \ldots \ldots \ldots \ldots$

Innovative Catalytic Processes $\ldots \ldots \ldots \ldots \ldots \ldots \ldots \ldots \ldots \ldots$

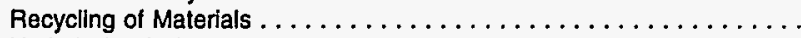

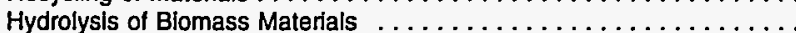

Enhanced Bloprocessing with Genetically Engineered Feedstocks or

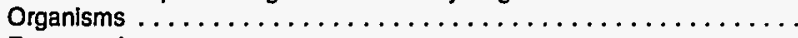

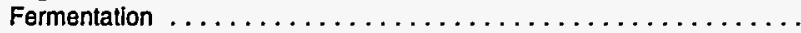

Fractionation of Blomass $\ldots \ldots \ldots \ldots \ldots \ldots \ldots \ldots \ldots \ldots \ldots \ldots$

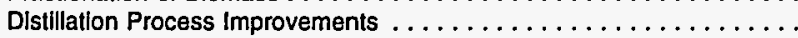

Hydrocarbon Cracking Enhancements $\ldots \ldots \ldots \ldots \ldots \ldots \ldots \ldots$

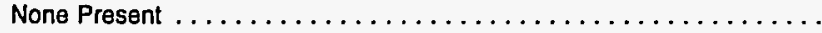

PETROLEUM and COAL PRODUCTS

General Energy-Related Technologies

One or More General Technologies Present .

Computer Control of Bullding Environment ${ }^{\circ} \ldots \ldots \ldots \ldots \ldots \ldots \ldots$

Computer Control of Processes or Major Energy-Using Equipment ${ }^{c}$...

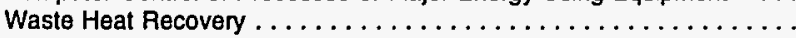

Adjustable-Speed Motors . . . . . . . . . . . . . . . . . .

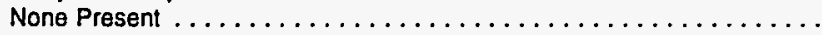

Petroleum Refining'

2911

General Energy-Related Technologies

One or More General Technologies Present ............... 2,636

Computer Control of Building Environment ${ }^{b} \ldots \ldots \ldots \ldots \ldots \ldots \ldots \ldots . . \ldots \ldots$

Computer Control of Processes or Major Energy-Using Equipment ${ }^{c} \ldots \quad \quad 2,271$

Waste Heat Recovery . . . . . . . . . . . . . . . . . . . . . . . . . . $\quad 2,398$

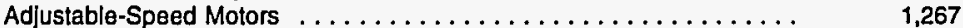

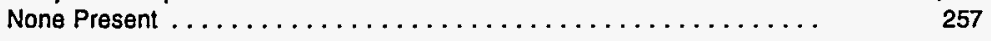

$\begin{array}{rrrrrr}2,703 & 196 & 456 & 1,534 & 518 & 2.4 \\ 651 & W & 187 & 273 & W & 3.4 \\ 2,306 & 169 & 415 & 1,377 & 343 & 2.7 \\ 2,435 & 133 & 396 & 1,412 & 494 & 2.4 \\ 1,295 & 9 & 260 & 763 & 264 & 3.4 \\ 284 & 42 & 46 & 92 & 104 & 5.8\end{array}$

RUBBER and MISC. PLASTICS PRODUCTS

General Energy-Related Technologies

One or More General Technologies Present ................. 161

Computer Control of Building Environment ${ }^{b} \ldots \ldots \ldots \ldots \ldots \ldots \ldots$

Computer Control of Processes or Major Energy-Using Equipment ${ }^{c} \ldots$

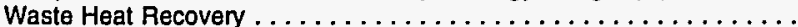

Adjustable-Speed Motors $\ldots \ldots \ldots \ldots \ldots \ldots \ldots \ldots \ldots \ldots$

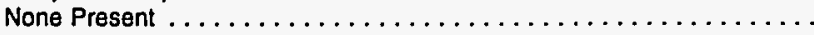

$\begin{array}{rrrrrr}31 & 0 & * & 26 & Q & 5.6 \\ 0 & 0 & 0 & 0 & 0 & N F \\ 26 & 0 & 0 & W & W & 3.5 \\ 30 & 0 & 0 & 26 & 0 & 5.6 \\ 19 & 0 & 0 & 14 & Q & 8.1 \\ 3 & 0 & 0 & 3 & & 3.5\end{array}$

$19 \quad 0 \quad * \quad 19$

3.9

NF

5.0 
Table A44. Total Inputs of Energy for Heat, Power, and Electricity Generation by Ciensus Region, Industry Group, Selected Industries, Presence of General Technologies, and Industry-Specific Technologies for Selected Industries, 1991 (Continued) (Estimates in Trillion Btu)

\begin{tabular}{|c|c|c|c|c|c|c|c|}
\hline \multirow{2}{*}{$\begin{array}{c}\text { SIC } \\
\text { Code" }\end{array}$} & \multirow[b]{2}{*}{$\begin{array}{l}\text { Industry Groups } \\
\text { and Industry }\end{array}$} & \multirow[b]{2}{*}{ Total } & \multicolumn{4}{|c|}{ Census Region } & \multirow{3}{*}{$\begin{array}{c}\text { RSE } \\
\text { Row } \\
\text { Factors }\end{array}$} \\
\hline & & & Northeast & Midwest & South & West & \\
\hline & RSE Column Factors: & 0.7 & 1.3 & 1.0 & 0.9 & 1.3 & \\
\hline
\end{tabular}

3011

Tires and Inner Tubes

General Energy-Related Technologies

One or More General Technologies Present

Computer Control of Building Environment ${ }^{b} \ldots \ldots \ldots \ldots \ldots \ldots \ldots \ldots$

Computer Control of Processes or Major Energy-Using Equipment ${ }^{c} \ldots$

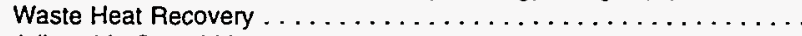

Adjustable-Speed Motors

None Present $\ldots$

Miscellaneous Plastic Products, nec

General Energy-Related Technologies

One or More General Technologies Present

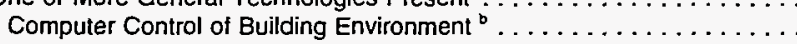

Computer Control of Processes or Major Energy-Using Equipment ${ }^{c} \ldots$

Waste Heat Recovery . . . . . . . . . . . . . . . . . . . . . .

Adjustable-Speed Motors $\ldots \ldots \ldots \ldots \ldots \ldots \ldots \ldots \ldots \ldots \ldots$

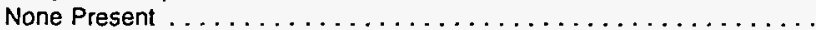

LEATHER and LEATHER PRODUCTS

31

General Energy-Related Technologies

One or More General Technologies Present ...

Computer Control of Building Environment ${ }^{\circ} \ldots \ldots \ldots \ldots \ldots \ldots \ldots \ldots \ldots$.

Computer Control of Processes or Major Energy-Using Equipment ${ }^{c} \ldots$

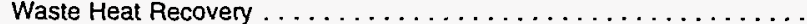

Adjustable-Speed Motors $\ldots \ldots \ldots \ldots \ldots \ldots \ldots \ldots \ldots \ldots$

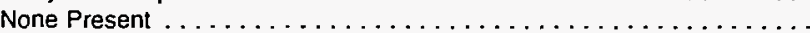

STONE, CLAY and GLASS PRODUCTS

General Energy-Related Technologies

One or More General Technologies Present $\ldots \ldots \ldots \ldots \ldots \ldots$

Computer Control of Building Environment ${ }^{b} \ldots \ldots \ldots \ldots \ldots \ldots$.

Computer Control of Processes or Major Energy-Using Equipment ${ }^{c} \ldots$

Waste Heat Recovery . . . . . . . . . . . . . . . . . . . . .

Adjustable-Speed Motors $\ldots \ldots \ldots \ldots \ldots \ldots \ldots \ldots \ldots \ldots \ldots$

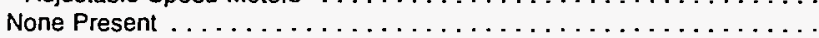

Industry-Specific Technologies

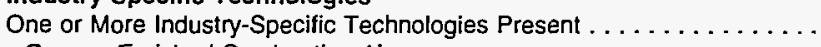

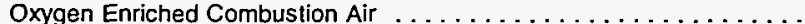

Forehearth Designed for Independently Applied Heating and Cooling

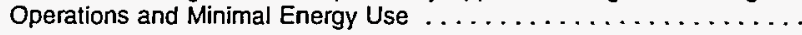

Forehearth Designed to Eliminate Side to Middle Temperature

Gradients with Improved Temperature Stability . . . . . . . . . . . .

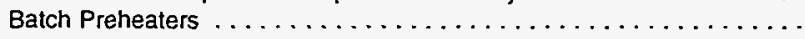

Cogeneration System Which Uses Waste Heat Rejected in Furmace

Exhaust to Generate Steam in Waste Heat Boilers . . . . . . . . . . . . .

Advanced Glass Refiner.$\ldots \ldots \ldots \ldots \ldots \ldots \ldots \ldots \ldots \ldots$

High-Efficiency Classifiers in Closed-Circuit Grinding Plants .........

Improved Grinding Media and Linings, Wear-Resistant Materials such

as High Chrome Alloys, and Classifying Liners ................

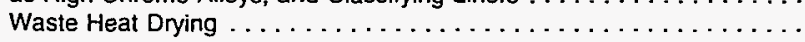

Dry-Suspension Preheater Kilns $\ldots \ldots \ldots \ldots \ldots \ldots \ldots \ldots \ldots$

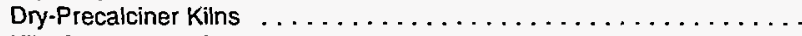

Kiln Combustion System Improvement such as Semi-Direct/ndirect

Coal Firing, Optimal Oxygen Levels and Advanced Burners Matched

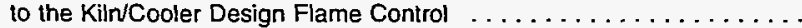

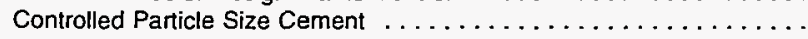

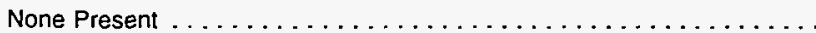

35
13
25
23
24
6

1
$w$
1
1
1

9
$W$
5
7
8
3

$\begin{array}{rll}36 & 7 & 11.2 \\ 6 & 1 & 21.4 \\ 20 & 4 & 14.4 \\ 13 & 3 & 20.0 \\ 31 & 6 & 12.9 \\ 11 & 7 & 14.8\end{array}$

$\begin{array}{llllll}6 & 3 & 2 & 1 & : & 27.3 \\ 1 & : & : & : & : & 20.4 \\ 3 & 1 & 1 & Q & \dot{0} & 35.4 \\ 2 & \mathrm{Q} & 1 & \mathrm{Q} & 0 & 51.7 \\ 4 & 2 & 1 & 1 & \dot{0} & 33.5 \\ 6 & 1 & 2 & 1 & \mathrm{Q} & 22.7\end{array}$

$\begin{array}{rrrrrr}644 & 107 & 187 & 248 & 102 & 6.6 \\ 69 & 15 & 28 & 23 & 4 & 10.0 \\ 497 & 82 & 162 & 175 & 77 & 6.2 \\ 257 & 39 & 91 & 87 & 41 & 7.9 \\ 454 & 68 & 139 & 170 & 78 & 6.8 \\ 250 & Q & 66 & 75 & 35 & 13.8\end{array}$

See footnotes at end of table. 
Table A44. Total Inputs of Energy for Heat, Power, and Electricity Generation by Census Region, Industry Group, Selected Industries, Presence of General Technologies, and Industry-Specific Technologies for Selected Industries, 1991 (Continued) (Estimates in Trillion Btu)

\begin{tabular}{|c|c|c|c|c|c|c|c|}
\hline \multirow[b]{2}{*}{$\begin{array}{l}\text { SIC } \\
\text { Code }\end{array}$} & \multirow[b]{2}{*}{$\begin{array}{l}\text { Industry Groups } \\
\text { and Industry }\end{array}$} & \multirow[b]{2}{*}{ Total } & \multicolumn{4}{|c|}{ Census Region } & \multirow{3}{*}{$\begin{array}{c}\text { RSE } \\
\text { Row } \\
\text { Factors }\end{array}$} \\
\hline & & & Northeast & Midwest & South & West & \\
\hline & RSE Column Factors: & 0.7 & 1.3 & 1.0 & 0.9 & 1.3 & \\
\hline
\end{tabular}

3211

General Energy-Related Techn

One or More General Technologies Present.

Computer Control of Bullding Environment ${ }^{\circ}$....

Computer Control of Processes or Major Energy-Using Equipment ${ }^{\circ} \ldots$

Waste Heat Recovery . . . . . . . . . . . . . . . . . . . .

Adjustable-Speed Motors $\ldots \ldots \ldots \ldots \ldots \ldots \ldots \ldots \ldots \ldots \ldots$

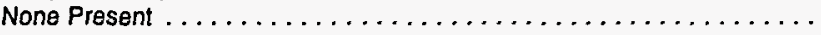

Industry-Specific Technologies

One or More Industry-Specific Technologies Present .............

Oxygen Enriched Combustion Air . ....................

Forehearth Designed for Independently Applied Heating and Cooling

Operations and Minimal Energy Use . ....................

Forehearth Designed to Eliminate Side to Middle Temperature

Gradients with Improved Temperature Stability . . . . . . . . . . . .

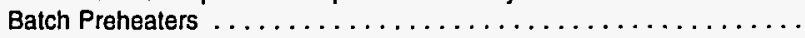

Cogeneration System Which Uses Waste Heat Rejected in Fumace

Exhaust to Generate Steam in Waste Heat Boilers ..............

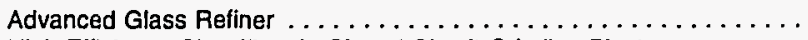

High-Efficiency Classifiers in Closed-Circuit Grinding Plants .........

Improved Grinding Media and Linings, Wear-Resistant Materials such

as High Chrome Alloys, and Classifying Liners ................

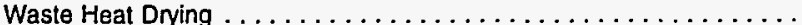

Dry-Suspension Preheater Kilns $\ldots \ldots \ldots \ldots \ldots \ldots \ldots \ldots \ldots$

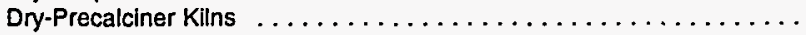

Kiln Combustion System Improvement such as Semi-Direct/lndirect

Coal Firing, Optimal Oxygen Levels and Advanced Burners Matched

to the Kllin/Cooler Design Flame Control $\ldots \ldots \ldots \ldots \ldots \ldots \ldots$

Controlled Particle Size Cement .........................

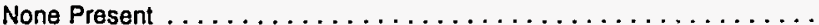

Glass Containers

General Energy-Related Technologies

One or More General Technologies Present

Computer Control of Building Environment ${ }^{b} \ldots \ldots \ldots \ldots \ldots \ldots \ldots$.

Computer Control of Processes or Major Energy-Using Equipment ${ }^{\circ}$...

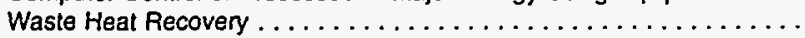

Adjustable-Speed Motors $\ldots \ldots \ldots \ldots \ldots \ldots \ldots \ldots \ldots \ldots$

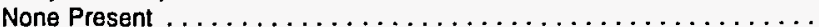

Industry-Specific Technologies

One or More Industry-Specific Technologies Present ..............

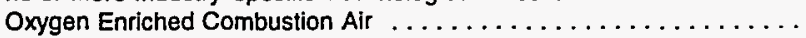

Forehearth Designed for Independently Applied Heating and Cooling

Operations and Minimal Energy Use ...................

Forehearth Designed to Eliminate Side to Middle Temperature

Gradlents with Improved Temperature Stability . . . . . . . . . . . . . .

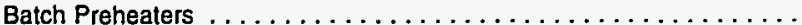

Cogeneration System Which Uses Waste Heat Rejected in Fumace

Exhaust to Generate Steam in Waste Heat Boilers . . . . . . . . . . .

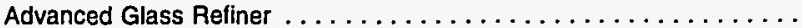

High-Elficiency Classifiers in Closed-Circuit Grinding Plants ........ Improved Grinding Media and Linings, Wear-Resistant Materials such

as High Chrome Alloys, and Classifying Liners . . . . . . . . . . . .

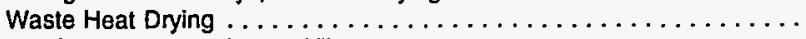

Dry-Suspension Preheater Kilns $\ldots \ldots \ldots \ldots \ldots \ldots \ldots \ldots \ldots$

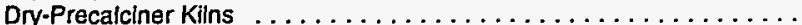

Kiln Combustion System Improvement such as Semi-Direct/Indirect

Coal Firing, Optimal Oxygen Levels and Advanced Burners Matched

to the Kiln/Cooler Design Flame Control $\ldots \ldots \ldots \ldots \ldots \ldots \ldots$.

Controlled Particle Size Cement $\ldots \ldots \ldots \ldots \ldots \ldots \ldots \ldots \ldots$

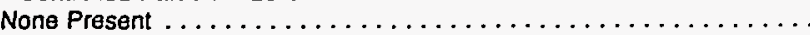

$W$
$W$
41
13
23
$W$

$w$
0
$w$
0
$w$
0

13
1
13
5
8
0

$\begin{array}{ccc}W & W & 3.1 \\ W & 1 & 4.8 \\ 19 & W & 3.1 \\ W & W & 4.4 \\ 10 & W & 4.1 \\ W & W & 5.3\end{array}$

12

6

$\begin{array}{rrr}14 & W & 4.2 \\ 9 & 1 & 4.8\end{array}$

1

0

7.0

9

W

5.5

$\begin{array}{rrrrrr}80 & 18 & 20 & 24 & 18 & 5.0 \\ 12 & W & 7 & * & W & 11.6 \\ 80 & 18 & 20 & 24 & 18 & 5.0 \\ 61 & 11 & 14 & 22 & 15 & 5.7 \\ W & 0 & W & 1 & 0 & 18.2 \\ & & & & & \\ 0 & 0 & 0 & 0 & 0 & N F \\ 37 & 12 & W & 12 & W & 6.6 \\ 0 & 0 & 0 & 0 & 0 & \text { NF } \\ & & 0 & 0 & 0 & \text { NF } \\ 0 & 0 & 0 & 0 & 0 & \text { NF } \\ 0 & 0 & 0 & 0 & 0 & N F \\ 0 & 0 & 0 & 0 & 0 & \text { NF } \\ 0 & 0 & 0 & 0 & 0\end{array}$

See footnotes at end of table. 
Table A44. Total Inputs of Energy for Heat, Power, and Electricity Generation by Census Region, Industry Group, Selected Industries, Presence of General Technologies, and Industry-Specific Technologies for Selected Industries, 1991 (Continued) (Estimates in Trillion Btu)

\begin{tabular}{|c|c|c|c|c|c|c|c|}
\hline & RSE Column Factors: & 0.7 & 1.3 & 1.0 & 0.9 & 1.3 & $\begin{array}{c}\text { RSE } \\
\text { Row } \\
\text { Factors }\end{array}$ \\
\hline
\end{tabular}

3229

General Energy-Related Technologies

One or More General Technologies Present .

Computer Control of Processes or Major Energy-Using Equipment ${ }^{\circ} \ldots$

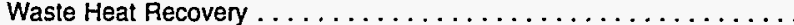

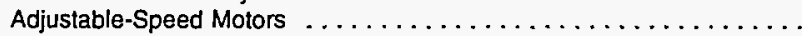

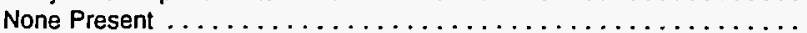

Industry-Specific Technologies

One or More Industry-Specific Technologies Present .............

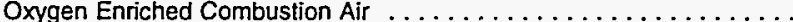

Forehearth Designed for Independently Applied Heating and Cooling

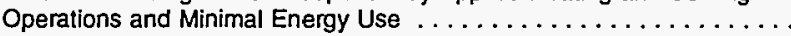

Forehearth Designed to Eliminate Side to Middle Temperature

Gradients with improved Temperature Stability . . . . . . . . . . .

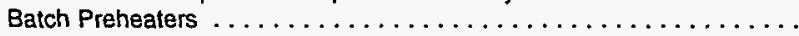

Cogeneration System Which Uses Waste Heat Rejected in Fumace

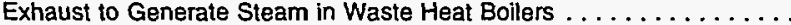

Advanced Glass Refiner . . . . . . . . . . . . . . . . . . .

High-Efficiency Classifiers in Closed-Circuit Grinding Plants ..........

Improved Grinding Media and Linings, Wear-Resistant Materials such

as High Chrome Alloys, and Classitying Liners . . . . . . . . . . . .

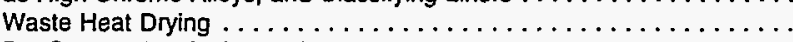

Dry-Suspension Preheater Kilns $\ldots \ldots \ldots \ldots \ldots \ldots \ldots \ldots$

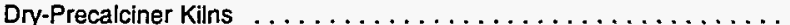

Kiln Combustion System Improvement such as Semi-Direct/lndirect

Coal Firing, Optimal Oxygen Levels and Advanced Burners Matched

to the Kilr/Cooler Design Flame Control $\ldots \ldots \ldots \ldots \ldots \ldots \ldots \ldots$

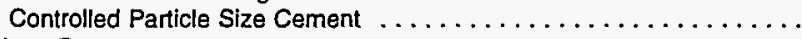

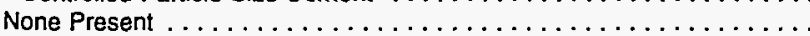

\section{Cement, Hydraulic}

General Energy-Related Technologles

One or More General Technologies Present

Computer Control of Building Environment ${ }^{\circ} \ldots \ldots \ldots \ldots \ldots \ldots$

Computer Control of Processes or Major Energy-Using Equipment ${ }^{\circ} \ldots$

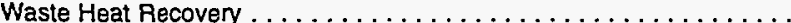

Adjustable-Speed Motors $\ldots \ldots \ldots \ldots \ldots \ldots \ldots \ldots \ldots \ldots$

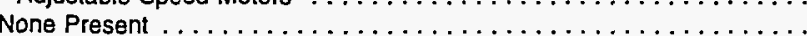

Industry-Specific Technologies

One or More Industry-Specific Technologies Present . ............

Oxygen Enriched Combustion Air ......................

Forehearth Designed for Independently Applied Heating and Cooling

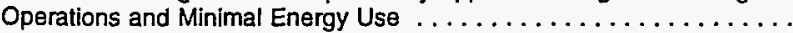

Forehearth Designed to Eliminate Side to Middle Temperature

Gradients with Improved Temperature Stability . . . . . . . . . . . .

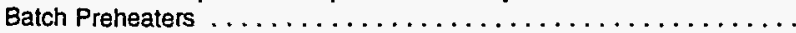

Cogeneration System Which Uses Waste Heat Rejected in Fumace

Exhaust to Generate Steam in Waste Heat Boilers . . . . . . . . . . . .

Advanced Glass Refiner . . . . . . . . . . . . . . . . . . .

High-Efficiency Classifiers in Closed-Circuit Grinding Plants .........

Improved Grinding Media and Linings, Wear-Resistant Materials such

as High Chrome Alloys, and Classifying Liners . ...............

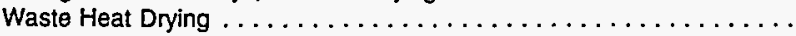

Dry-Suspension Preheater Kilns $\ldots \ldots \ldots \ldots \ldots \ldots \ldots \ldots \ldots$

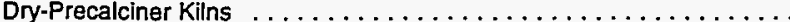

Kiln Combustion System Improvement such as Semi-Direct/lndirect

Coal Firing, Optimal Oxygen Levels and Advanced Burners Matched

to the Kiln'Cooler Design Flame Control . . . . . . . . . . . . . . .

Controlled Particle Size Cement $\ldots \ldots \ldots \ldots \ldots \ldots \ldots \ldots \ldots$

None Present

$\begin{array}{ll}W & W \\ 11 & \\ 41 & \\ 15 & \\ 27 & \\ W & \end{array}$

$W$
$W$
11
4
9
$W$

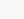


Table A44. Total Inputs of Energy for Heat, Power, and Electricity Generation by Census Region, Industry Group, Selected Industries, Presence of General Technologies, and Industry-Specific Technologies for Selected Industries, 1991 (Continued) (Estimates in Trillion Btu)

\begin{tabular}{|c|c|c|c|c|c|c|c|}
\hline \multirow[b]{2}{*}{$\begin{array}{l}\text { SIC } \\
\operatorname{Cod}^{a}\end{array}$} & \multirow[b]{2}{*}{$\begin{array}{l}\text { Industry Groups } \\
\text { and Industry }\end{array}$} & \multirow[b]{2}{*}{ Total } & \multicolumn{4}{|c|}{ Census Region } & \multirow{3}{*}{$\begin{array}{c}\text { RSE } \\
\text { Row } \\
\text { Factors }\end{array}$} \\
\hline & & & Northeast & Midwest & South & West & \\
\hline & RSE Column Factors: & 0.7 & 1.3 & 1.0 & 0.9 & 1.3 & \\
\hline
\end{tabular}

3274

\section{Lime}

General Energy-Related Technologies

One or More General Technologies Present

Computer Control of Bullding Environment ${ }^{6} \ldots \ldots \ldots \ldots \ldots \ldots \ldots \ldots$

Computer Control of Processes or Major Energy-Using Equipment ${ }^{\circ} \ldots$

Waste Heat Recovery . . . . . . . . . . . . . . . . . . . .

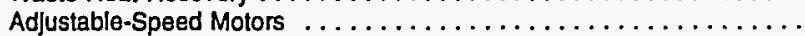

None Present

Industry-Specific Technologles

$\begin{array}{rl}61 & W \\ W & \\ 43 & \\ 19 & \\ 46 & 0 \\ Q & \end{array}$

$\begin{array}{rr}W & 27 \\ 1 & W \\ W & 26 \\ 0 & W \\ Q & W\end{array}$

$\begin{array}{cc}27 & 31 \\ W & \\ 26 & \\ W & W \\ W & W \\ 8 & \end{array}$

$\begin{array}{rrr}31 & W & 18.5 \\ 0 & 0 & 15.2 \\ 15 & W & 17.9 \\ W & 0 & 20.1 \\ W & 1 & 24.4 \\ 5 & 0 & 28.8\end{array}$

One or More Industry-Speclfic Technologies Present ..............

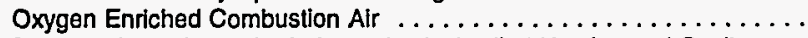

Forehearth Designed for Independently Applled Heating and Cooling

Operatlons and Minimal Energy Use ....................

Forehearth Designed to Ellminate Side to Middle Temperature

Gradlents with Improved Temperature Stability . . . . . . . . . . .

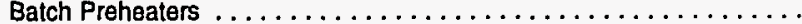

Cogeneration System Which Uses Waste Heat Rejected in Furnace

Exhaust to Generate Steam in Waste Heat Boilers ...............

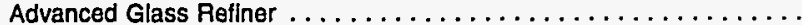

High-Efflclency Classiflers in Closed-Circuit Grinding Plants ........

improved Grinding Media and Linings, Wear-Resistant Materials such

as High Chrome Alloys, and Classifying Liners ..............

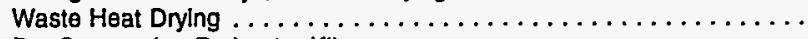

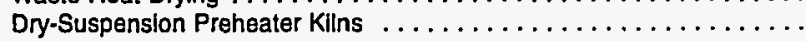

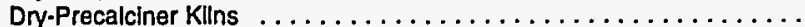

Kilin Combustion System Improvement such as Semi-Direct/ndirect

Coal Firing, Optimal Oxygen Levels and Advanced Bumers Matched

to the Klin/Cooler Design Flame Control $\ldots \ldots \ldots \ldots \ldots \ldots \ldots$.

Controlled Partlcle Size Cement $\ldots \ldots \ldots \ldots \ldots \ldots \ldots \ldots \ldots$

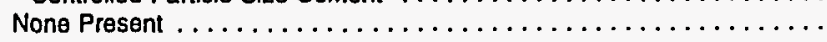

Mineral Wool

General Energy-Related Technologies

One or More General Technologles Present ..................

Computer Control of Bullding Environment ${ }^{b} \ldots \ldots \ldots \ldots \ldots \ldots \ldots$

Computer Control of Processes or Major Energy-Using Equipment ${ }^{\circ}$....

Waste Heat Recovery $\ldots \ldots \ldots \ldots \ldots \ldots \ldots \ldots \ldots \ldots \ldots$

Adjustable-Speed Motors $\ldots \ldots \ldots \ldots \ldots \ldots \ldots \ldots \ldots \ldots$

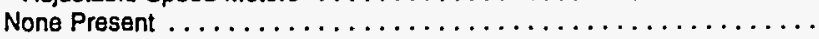

51

0

0

0

6

10

W4

Q

Industry-Specific Technologie

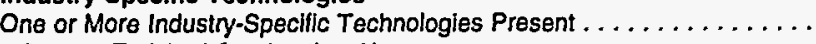

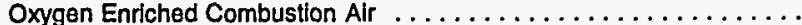

Forehearth Designed for Independently Applied Heating and Cooling

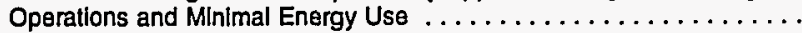

Forehearth Designed to Eliminate Side to Middle Temperature

Gradients with Improved Temperature Stability ...............

Batch Preheaters . . . . . . . . . . . . . . . . . . . . . . .

Cogeneration System Which Uses Waste Heat Rejected in Fumace

Exhaust to Generate Steam In Waste Heat Boilers . . . . . . . . . . .

Advanced Glass Rellner . . . . . . . . . . . . . . . . . . .

High-Effliciency Classifiers in Closed-Clrcuit Grinding Plants ........ Improved Grindling Media and Linings, Wear-Resistant Materials such

as High Chrome Alloys, and Classifying Liners $\ldots \ldots \ldots \ldots \ldots \ldots$.

Waste Heat Drying . . . . . . . . . . . . . . . . . . . .

Dry-Suspension Preheater Kllns $\ldots \ldots \ldots \ldots \ldots \ldots \ldots \ldots \ldots$

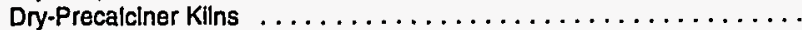

Kiln Combustion System Improvement such as Semi-Direct/ndirect

Coal Firing, Optimal Oxygen Levels and Advanced Bumers Matched

to the Kiln/Cooler Design Flame Control $\ldots \ldots \ldots \ldots \ldots \ldots \ldots$.

Controlled Partlcle Size Cement $\ldots \ldots \ldots \ldots \ldots \ldots \ldots \ldots \ldots \ldots$

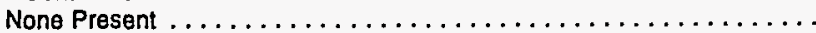

1$$
0
$$

0

31

31
0

$\begin{array}{rrr}17 & W & 17.6 \\ 0 & 0 & \text { NF } \\ 0 & 0 & \text { NF } \\ 0 & 0 & \text { NF } \\ 0 & 0 & \text { NF }\end{array}$

$\begin{array}{rrrrrr}24 & W & 13 & 7 & W & 1.3 \\ 16 & 2 & 9 & 4 & 1 & 1.7 \\ 13 & W & 7 & 3 & W & 1.5 \\ 11 & 1 & 7 & 2 & 0 & 1.8 \\ 0 & 0 & 0 & 0 & 0 & N F \\ . & 0 & - & 0 & 0 & 2.4 \\ W & 0 & 0 & W & 0 & 2.3 \\ 0 & 0 & 0 & 0 & 0 & N F \\ W & 0 & W & 0 & 0 & 2.4 \\ 0 & 0 & 0 & 0 & 0 & N F \\ 0 & 0 & 0 & 0 & 0 & N F \\ 0 & 0 & 0 & 0 & 0 & N F\end{array}$

See footnotes at end of table. 
Table A44. Total Inputs of Energy for Heat, Power, and Electricity Generation by Census Region, Industry Group, Selected Industries, Presence of General Technologies, and Industry-Specific Technologies for Selected Industries, 1991 (Continued) (Estimates in Trillion Btu)

\begin{tabular}{|c|c|c|c|c|c|c|c|}
\hline \multirow{2}{*}{$\begin{array}{l}\text { SIC } \\
\text { Code" }\end{array}$} & \multirow{2}{*}{$\begin{array}{l}\text { Industry Groups } \\
\text { and Industry }\end{array}$} & \multirow[b]{2}{*}{ Total } & \multicolumn{4}{|c|}{ Census Region } & \multirow{3}{*}{$\begin{array}{c}\text { RSE } \\
\text { Row } \\
\text { Factors }\end{array}$} \\
\hline & & & Northeast & Midwest & South & West & \\
\hline & RSE Column Factors: & 0.7 & 1.3 & 1.0 & 0.9 & 1.3 & \\
\hline
\end{tabular}

PRIMARY METAL INDUSTRIES

General Energy-Related Technologies

One or More General Technologies Present

Computer Control of Building Environment ${ }^{\circ} \ldots \ldots \ldots \ldots \ldots \ldots \ldots$

Computer Control of Processes or Major Energy-Using Equipment ${ }^{\circ} \ldots$

Waste Heat Recovery . . . . . . . . . . . . . . . . . . . .

Adjustable-Speed Motors $\ldots \ldots \ldots \ldots \ldots \ldots \ldots \ldots \ldots \ldots$

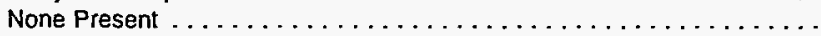

1,989
557
1,884
1,433
1,415
303

323
158
301
266
196
34

$\begin{array}{rrrr}1,025 & 464 & 177 & 4.1 \\ 208 & 112 & 78 & 7.4 \\ 978 & 443 & 162 & 4.3 \\ 828 & 260 & 79 & 5.7 \\ 854 & 282 & 82 & 5.0 \\ 143 & 79 & 47 & 5.9\end{array}$

Industry-Specific Technologies

One or More Industry-Specific Technologies Present . . . . . . . . . . .

Dry Quenching During the Coking Process ..............

External Desulfurization of the Charge for Ironmaking . . . . . . . . . . .

Hydrocarbon Injection to Maintain Blast Furnace Temperatures . . . . . . .

Direct Reduction Ironmaking - Sponge Iron Produced Directly from

Iron Ore . . . . . . . . . . . . . . . . . . . . . . .

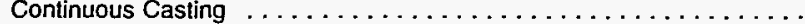

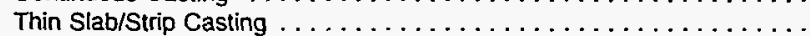

Waste Heat Boilers/Heat Exchangers in Combination with Reheat

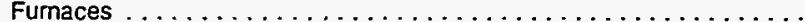

Evaporative Cooling of Skid Rails . . . . . . . . . . . . .

Electric Induction Reheat Fumaces . . . . . . . . . . . . . . .

Hot Changing - Moving Steel Directly from the Caster to the Reheat

Furnaces . . . . . . . . . . . . . . . . . . . .

Direct Rolling Required no Reheating $\ldots \ldots \ldots \ldots \ldots \ldots \ldots \ldots \ldots \ldots$

Plasmasmelt Smelting of Partially Reduced Iron Powder with

Pulverized Coal . . . . . . . . . . . . . . . . . . . .

Cold Bonding (COBO) Pelletizing Technique $\ldots \ldots \ldots \ldots \ldots \ldots$

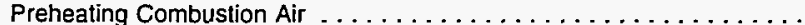

Preheating Raw Materials . . . . . . . . . . . . . . . .

Top Gas Pressure Recovery from the Blast Furnace $\ldots \ldots \ldots \ldots \ldots$

Slab Heat Recovery . . . . . . . . . . . . . . . . . . . .

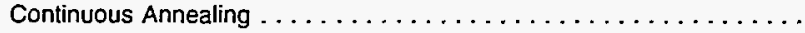

Continuous Cold Rolling $\ldots \ldots \ldots \ldots \ldots \ldots \ldots \ldots \ldots \ldots \ldots$

Bottom Tap Vessels . . . . . . . . . . . . . . . . . . . . . .

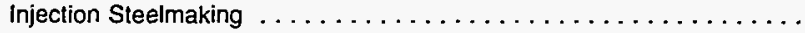

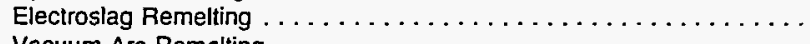

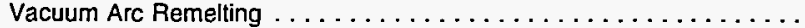

Oxygen Injection to Blast Fumace $\ldots \ldots \ldots \ldots \ldots \ldots \ldots \ldots \ldots$

Coal Injection to Blast Fumace . . . . . . . . . . . . . . .

Steel Ladle Metallurgy with Reheat Furnace ..............

None Present . . . . . . . . . . . . . . . . . . . . .

1,580

W

710

1,142

W

1,168

W

926

W

78

986

196

0
0

1,283

444

287

255
983

618

257

330

7
$W$

1,023

, 023
$W$

867

867
713

\section{6}

0

W

921
0
514

514

756

$W$
874

874
$W$

661

W

792

W

0

0

799

364

176

254

625

316

218

210

1
$W$

763

1
716

716

247

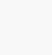

322
$W$

$W$
191

W

249
$W$

130

130
3

55

145

W

0
0

0
242

72
$w$

W

1
$w$
$w$

W

30

W

162

162
$W$

140

221
7.4

5.7

5.0
5.9

5.1

13.9

9.0

14.4

14.4

5.6

11.4

6.5

6.5
11.2

8.1

6.6
8.3

NF

NF

5.7

6.9

9.2

11.0

6.9

8.6

9.0

9.8

5.9

8.7

7.0

17.0

6.5

3.7

See footnotes at end of table. 
Table A44. Total Inputs of Energy for Heat, Power, and Electricity Generation by Census Region, Industry Group, Selected Industries, Presence of General Technologies, and Industry-Specific Technologies for Selected Industries, 1991 (Continued) (Estimates in Trillion Btu)

\begin{tabular}{cc|c|c|c|c|c|c|}
\hline \multirow{2}{*}{$\begin{array}{c}\text { Industry Groups } \\
\text { and Industry }\end{array}$} & & \multicolumn{3}{|c}{ Census Region } \\
\cline { 3 - 7 } Code & RSE Column Factors: & Total & Northeast & Midwest & South & Wow \\
\hline & Factors \\
\hline
\end{tabular}

3312

General Energy-Related Technologies

One or More General Technologies Present

$\ldots \ldots \ldots$

Computer Control of Building Environment ${ }^{b} \ldots \ldots \ldots \ldots \ldots \ldots \ldots$

Computer Control of Processes or Major Energy-Using Equipment ${ }^{c} \ldots$

Waste Heat Recovery . . . . . . . . . . . . . . . . . . . .

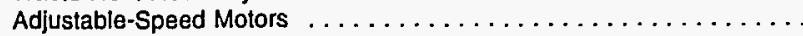

None Present $\ldots \ldots \ldots \ldots \ldots \ldots \ldots \ldots \ldots \ldots \ldots \ldots \ldots \ldots \ldots$

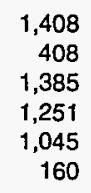

408

408
1,385

1,251

160

Industry-Speciflc Technologies

One or More Industry-Specific Technologies Present $\ldots \ldots \ldots \ldots \ldots \ldots$

Dry Quenching During the Coking Process ...............

External Desulfurization of the Charge for Ironmaking . . . . . . . . . .

Hydrocarbon Injection to Maintain Blast Fumace Temperatures . . . . . .

Direct Reduction Ironmaking - Sponge Iron Produced Directly from

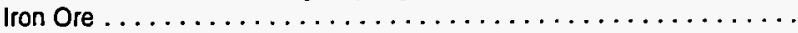

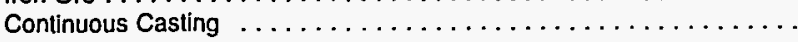

Thin Slab/Strip Casting . . . . . . . . . . . . . . . . . . . .

Waste Heat Boilers/Heat Exchangers in Combination with Reheat

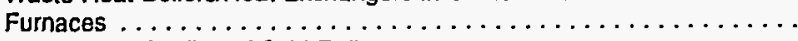

Evaporative Cooling of Skid Rails $\ldots \ldots \ldots \ldots \ldots \ldots \ldots \ldots$

Electric Induction Reheat Fumaces $\ldots \ldots \ldots \ldots \ldots \ldots \ldots \ldots \ldots$

Hot Changing - Moving Steel Directly from the Caster to the Reheat

Furnaces . . . . . . . . . . . . . . . . . . . .

Direct Rolling Required no Reheating $\ldots \ldots \ldots \ldots \ldots \ldots \ldots \ldots$

Plasmasmelt Smelting of Partially Reduced Iron Powder with

Pulverized Coal

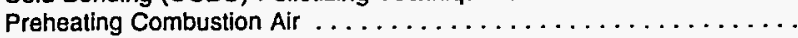

Preheating Raw Materials . . . . . . . . . . . . . . . . .

Top Gas Pressure Recovery from the Blast Furnace $\ldots \ldots \ldots \ldots \ldots$

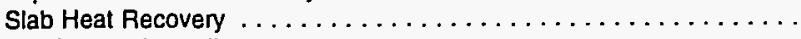

Continuous Annealing . . . . . . . . . . . . . . . . . . .

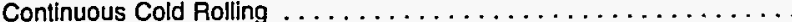

Bottom Tap Vessels . . . . . . . . . . . . . . . . . . .

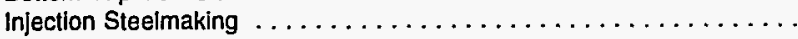

Electroslag Remelting . . . . . . . . . . . . . . . . . . .

Vacuum Arc Remelting .

Oxygen Injection to Blast Furnace $\ldots \ldots \ldots \ldots \ldots \ldots \ldots \ldots \ldots \ldots \ldots$

Coal Injection to Blast Fumace $\ldots \ldots \ldots \ldots \ldots \ldots \ldots \ldots \ldots \ldots$

Steel Ladle Metallurgy with Reheat Furnace . . . . . . . . . . . .

None Present
1,491

W

685

1,142

W

1,152

W

902

W

55

985

193

0
0

1,238

1,238
410

283

255

968

614

243

330

7

W

997

W

867

25

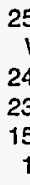

254

$W$
247

233

154
15

\section{W}

$W$
0
$W$

W

W

0
31

0

N

$W$
$W$
$W$

43

43
47

$\begin{array}{rr}0 & 0 \\ 0 & 0 \\ 223 & 778 \\ W & W \\ W & 176 \\ 0 & 254 \\ 174 & 621 \\ W & 314 \\ W & 213 \\ W & 210 \\ 6 & 1 \\ 7 & W \\ W & 752 \\ W & 0 \\ W & 716 \\ W & 31\end{array}$

726
96

886

0
499

499
756

W

866
$W$

658

9

6.1

9.8

6.1

6.5

6.8
6.7

W

157
41

293

W

$W$
190

W

242
0

121

3
$W$

W

144
1

0

230

230
$W$

W 
Table A44. Total Inputs of Energy for Heat, Power, and Electricity Generation by Census Region, Industry Group, Selected Industries, Presence of General Technologies, and Industry-Specific Technologies for Selected Industries, 1991 (Continued) (Estimates in Trillion Btu)

\begin{tabular}{cc|c|c|c|c|c|c|}
\hline \multirow{2}{*}{$\begin{array}{c}\text { Industry Groups } \\
\text { and Industy }\end{array}$} & & \multicolumn{3}{|c|}{ Census Region } \\
\cline { 3 - 7 } Code" & RSE Column Factors: & Total & Northeast & Midwest & South & Wost \\
\hline & Factors \\
\hline
\end{tabular}

3313

Electrometalurgical Products

General Energy-Related Technologies

One or More General Technologies Present ...

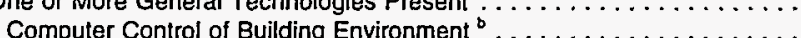

Computer Control of Processes or Major Energy-Using Equipment ${ }^{a} \ldots$

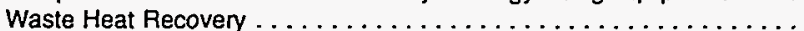

Adjustable-Speed Motors . . . . . . . . . . . . . . . . .

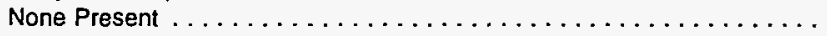

Industry-Speciłic Technologies

One or More Industry-Specific Technologies Present . ............

Dry Quenching During the Coking Process

Extemal Desulfurization of the Charge for Ironmaking ....................

Hydrocarbon Injection to Maintain Blast Fumace Temperatures ........

Direct Reduction Ironmaking - Sponge Iron Produced Directly from

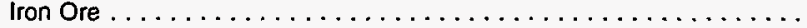

Continuous Casting $\ldots \ldots \ldots \ldots \ldots \ldots \ldots \ldots \ldots \ldots \ldots \ldots \ldots$

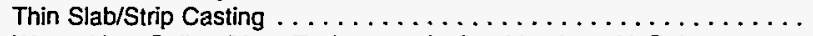

Waste Heat Boilers/Heat Exchangers in Combination with Reheat

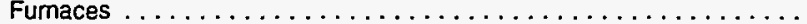

Evaporative Cooling of Skid Rails $\ldots \ldots \ldots \ldots \ldots \ldots \ldots \ldots$

Electric Induction Reheat Fumaces $\ldots \ldots \ldots \ldots \ldots \ldots \ldots \ldots$.

Hot Changing - Moving Steel Directly from the Caster to the Reheat

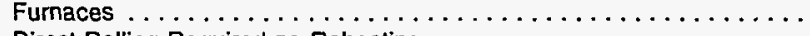

Direct Rolling Required no Reheating $\ldots \ldots \ldots \ldots \ldots \ldots \ldots \ldots$

Plasmasmelt Smeiting of Partially Reduced Iron Powder with

Pulverized Coal

Cold Bonding (COBO) Pelletizing Technique $\ldots \ldots \ldots \ldots \ldots \ldots \ldots$

Preheating Combustion Air.$\ldots \ldots \ldots \ldots \ldots \ldots \ldots \ldots \ldots \ldots$.

Preheating Raw Materials $\ldots \ldots \ldots \ldots \ldots \ldots \ldots \ldots \ldots \ldots$

Top Gas Pressure Recovery from the Blast Furnace $\ldots \ldots \ldots \ldots \ldots$

Slab Heat Recovery . . . . . . . . . . . . . . . . . . . . . . . . .

Continuous Annealing $\ldots \ldots \ldots \ldots \ldots \ldots \ldots \ldots \ldots \ldots \ldots \ldots$

Continuous Cold Rolling $\ldots \ldots \ldots \ldots \ldots \ldots \ldots \ldots \ldots \ldots$

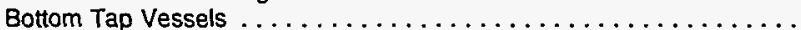

Injection Steelmaking . . . . . . . . . . . . . . . . . . . .

Electroslag Remelting $\ldots \ldots \ldots \ldots \ldots \ldots \ldots \ldots \ldots \ldots \ldots$

Vacuum Arc Remelting .

Oxygen Injection to Blast Fumace.

Coal Injection to Blast Furnace $\ldots \ldots \ldots \ldots \ldots \ldots \ldots \ldots \ldots \ldots \ldots \ldots \ldots$.

Steel Ladle Metallurgy with Reheat Furnace $\ldots \ldots \ldots \ldots \ldots \ldots \ldots \ldots$

None Present ...

$\begin{array}{rr}W & W \\ 0 & 0 \\ 29 & W \\ W & 0 \\ W & : \\ W & \end{array}$

$\begin{array}{rr}W & 19 \\ 0 & 0 \\ W & 19 \\ 0 & W \\ * & W \\ * & 0\end{array}$

$W$
0
$W$
0
0
$W$

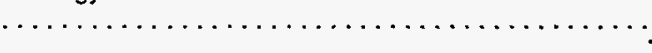

\begin{tabular}{|c|c|c|c|c|}
\hline 1 & 0 & 1 & 0 & 0 \\
\hline 0 & 0 & 0 & 0 & 0 \\
\hline 0 & 0 & 0 & 0 & 0 \\
\hline 0 & 0 & 0 & 0 & 0 \\
\hline 0 & 0 & 0 & 0 & 0 \\
\hline 0 & 0 & 0 & 0 & 0 \\
\hline 1 & 0 & 1 & 0 & 0 \\
\hline 0 & 0 & 0 & 0 & 0 \\
\hline 0 & 0 & 0 & 0 & 0 \\
\hline 0 & 0 & 0 & 0 & 0 \\
\hline 0 & 0 & 0 & 0 & 0 \\
\hline 1 & 0 & 1 & 0 & 0 \\
\hline 0 & 0 & 0 & 0 & 0 \\
\hline 0 & 0 & 0 & 0 & 0 \\
\hline 0 & 0 & 0 & 0 & 0 \\
\hline 1 & 0 & 1 & 0 & 0 \\
\hline 0 & 0 & 0 & 0 & 0 \\
\hline 0 & 0 & 0 & 0 & 0 \\
\hline 1 & 0 & 1 & 0 & 0 \\
\hline 1 & 0 & 1 & 0 & 0 \\
\hline 0 & 0 & 0 & 0 & 0 \\
\hline 0 & 0 & 0 & 0 & 0 \\
\hline 0 & 0 & 0 & 0 & 0 \\
\hline 0 & 0 & 0 & 0 & 0 \\
\hline 0 & 0 & 0 & 0 & 0 \\
\hline 0 & 0 & 0 & 0 & 0 \\
\hline 0 & 0 & 0 & 0 & 0 \\
\hline 30 & $W$ & 18 & $W$ & 0 \\
\hline
\end{tabular}


Table A44. Total Inputs of Energy for Heat, Power, and Electricity Generation by Census Region, Industry Group, Selected Industries, Presence of General Technologies, and Industry-Specific Technologies for Selected Industries, 1991 (Continued) (Estimates in Trillion Btu)

\begin{tabular}{|c|c|c|c|c|c|c|c|}
\hline \multirow{2}{*}{$\underset{\text { Code }}{\text { SIC }}$} & \multirow{2}{*}{$\begin{array}{l}\text { Industry Groups } \\
\text { and Industry }\end{array}$} & \multirow[b]{2}{*}{ Total } & \multicolumn{4}{|c|}{ Census Region } & \multirow{3}{*}{$\begin{array}{c}\text { RSE } \\
\text { Row } \\
\text { Factors }\end{array}$} \\
\hline & & & Northeast & Midwest & South & West & \\
\hline & RSE Column Factors: & 0.7 & 1.3 & 1.0 & 0.9 & 1.3 & \\
\hline
\end{tabular}

3321

Gray and Ductile Iron Foundries

General Energy-Related Technologies

One or More General Technologies Present .

Computer Control of Building Environment ${ }^{b} \ldots \ldots \ldots \ldots \ldots \ldots \ldots$

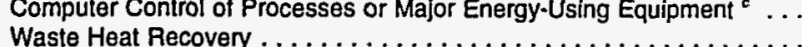

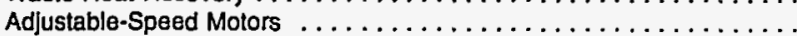

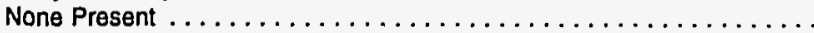

Industry-Specific Technologies

One or More Industry-Specific Technologies Present . . . . . . . . . .

Dry Quenching During the Coking Process

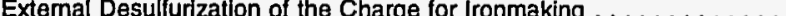

Hydrocarbon Injection to Maintain Blast Fumace Temperatures . . . . . . .

Direct Reduction Ironmaking - Sponge Iron Produced Directly Irom

Iron Ore . . . . . . . . . . . . . . . . . . . . . . . . .

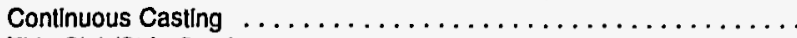

Thin Slab/Strip Casting

Waste Heat Bollers/Heat Exchangers in Combination with Reheat

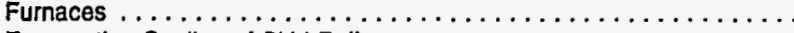

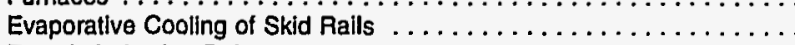

Electric Induction Reheat Fumaces . . . . . . . . . . . . . . . . . .

Hot Changing - Moving Steel Direclly from the Caster to the Reheat

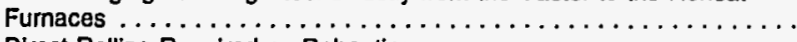

Dlrect Rolling Required no Reheating $\ldots \ldots \ldots \ldots \ldots \ldots \ldots \ldots \ldots \ldots$

Plasmasmelt Smeiling of Partially Reduced Iron Powder with

Pulverlzed Coal . . . . . . . . . . . . . . . . . . . .

Cold Bonding (COBO) Pellellzing Technique $\ldots \ldots \ldots \ldots \ldots \ldots \ldots$

Preheating Combustion Air . . . . . . . . . . . . . . . . . .

Preheating Raw Materials . . . . . . . . . . . . . . . . .

Top Gas Pressure Recovery from the Blast Furnace

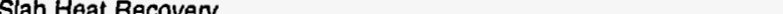

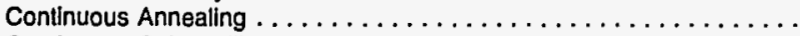

Contlnuous Cold Rolling

Bottom Tap Vessels $\ldots . . \cdots$

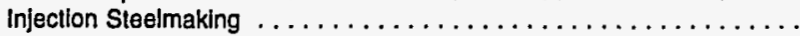

Electroslag Remelting.

Vacuum Arc Remelting

Steel Ladle Metallurgy with Reheat Furnace.

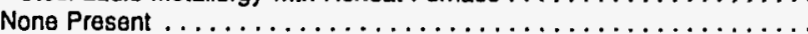

55
11
48
19
24
19

4
3
2
3
2

34
10
28
10
13
11

15

11.2

19.8

9.7

11.5

10.2

14.7

55

5

26

4

3

1

29

11.1

NF

12.2

27.6

NF

NF.0
NF 
Table A44. Total Inputs of Energy for Heat, Power, and Electricity Generation by Census Region, Industry Group, Selected Industries, Presence of General Technologies, and Industry-Specific Technologies for Selected Industries, 1991 (Continued) (Estimates in Trillion Btu)

\begin{tabular}{|c|c|c|c|c|c|c|c|}
\hline & RSE Column Factors: & 0.7 & 1.3 & 1.0 & 0.9 & 1.3 & $\begin{array}{c}\text { RSE } \\
\text { Row } \\
\text { Factor }\end{array}$ \\
\hline
\end{tabular}

3331

Primary Copper

General Energy-Related Technologies

One or More General Technologies Present

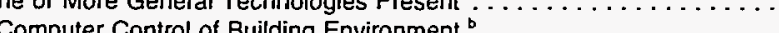

Computer Control of Processes or Major Energy-Using Equipment ${ }^{\circ} \ldots$

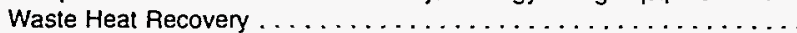

Adjustable-Speed Motors . . . . . . . . . . . . . . . . . . .

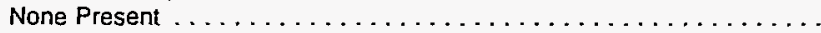

Industry-Specitic Technologies

One or More Industry-Specific Technologies Present . . . . . . . . . . .

Dry Quenching During the Coking Process $\ldots \ldots \ldots \ldots \ldots \ldots$

External Desulfurization of the Charge for Ironmaking . . . . . . . . . . .

Hydrocarbon Injection to Maintain Blast Fumace Temperatures . . . . . .

Direct Reduction Ironmaking - Sponge Iron Produced Directly from

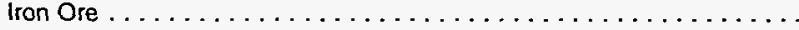

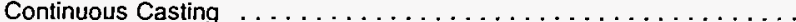

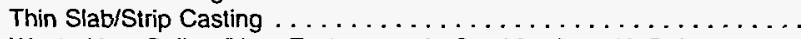

Waste Heat Boilers/Heat Exchangers in Combination with Reheat

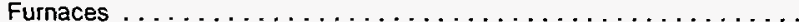

Evaporative Cooling of Skid Rails $\ldots \ldots \ldots \ldots \ldots \ldots \ldots \ldots$

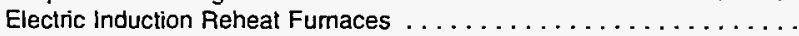

Hot Changing - Moving Steel Directly from the Caster to the Reheat

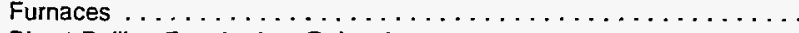

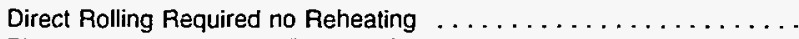

Plasmasmelt Smelting of Partially Reduced Iron Powder with

Pulverized Coal

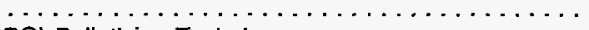

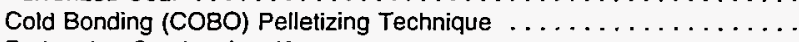

Preheating Combustion Air $\ldots \ldots \ldots \ldots \ldots \ldots \ldots \ldots \ldots \ldots$

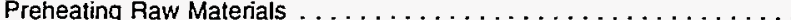

Top Gas Pressure Recovery from the Blast Furnace $\ldots \ldots \ldots \ldots$. . . .

Slab Heat Recovery

Continuous Cold Rolling $\ldots \ldots \ldots \ldots \ldots \ldots \ldots \ldots \ldots \ldots$

Bottom Tap Vessels .

Injection Steelmaking ....

Electroslag Remelting

Vacuum Arc Remelting

年

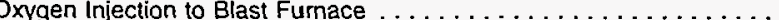

Coal Injection to Blast Fumace $\ldots \ldots \ldots \ldots \ldots \ldots \ldots \ldots \ldots$

Steel Ladle Metallurgy with Reheat Furnace $\ldots \ldots \ldots \ldots \ldots \ldots \ldots$

None Present ...

$\begin{array}{lll}W & : & 0 \\ W & 0 & 0 \\ 21 & \vdots & 0 \\ 12 & \vdots & 0 \\ 16 & \vdots & 0 \\ W & 0 & *\end{array}$

$\begin{array}{ccc}W & W & 1.0 \\ W & 0 & 1.3 \\ W & W & 1.0 \\ W & W & 1.0 \\ W & W & 1.0 \\ * & W & 1.1\end{array}$

\begin{tabular}{|c|c|c|c|c|}
\hline W & * & 0 & $w$ & $w$ \\
\hline 0 & 0 & 0 & 0 & 0 \\
\hline 0 & 0 & 0 & 0 & 0 \\
\hline 0 & 0 & 0 & 0 & 0 \\
\hline 0 & 0 & 0 & 0 & 0 \\
\hline W & * & 0 & $w$ & * \\
\hline 0 & 0 & 0 & 0 & 0 \\
\hline 14 & 0 & 0 & $w$ & $w$ \\
\hline 0 & 0 & 0 & 0 & 0 \\
\hline 0 & 0 & 0 & 0 & 0 \\
\hline 0 & 0 & 0 & 0 & 0 \\
\hline 0 & 0 & 0 & 0 & 0 \\
\hline 0 & 0 & 0 & 0 & 0 \\
\hline 0 & 0 & 0 & 0 & 0 \\
\hline$W$ & 0 & 0 & * & $w$ \\
\hline W & 0 & 0 & $w$ & 0 \\
\hline$w$ & 0 & 0 & $w$ & 0 \\
\hline 0 & 0 & 0 & 0 & 0 \\
\hline 0 & 0 & 0 & 0 & 0 \\
\hline 0 & 0 & 0 & 0 & 0 \\
\hline 0 & 0 & 0 & 0 & 0 \\
\hline 0 & 0 & 0 & 0 & 0 \\
\hline 0 & 0 & 0 & 0 & 0 \\
\hline 0 & 0 & 0 & 0 & 0 \\
\hline 1 & 0 & 0 & 0 & 1 \\
\hline 0 & 0 & 0 & 0 & 0 \\
\hline 0 & 0 & 0 & 0 & 0 \\
\hline$w$ & * & * & * & $w$ \\
\hline
\end{tabular}

See footnotes at end of table. 
Table A44. Total Inputs of Energy for Heat, Power, and Electricity Generation by Census Region, Industry Group, Selected Industries, Presence of General Technologies, and Industry-Specific Technologies for Selected Industries, 1991 (Continued) (Estimates in Trillion Btu)

\begin{tabular}{|c|c|c|c|c|c|c|c|}
\hline \multirow[b]{2}{*}{$\begin{array}{l}\text { SIC } \\
\text { Code" }\end{array}$} & \multirow[b]{2}{*}{$\begin{array}{l}\text { Industry Groups } \\
\text { and Industry }\end{array}$} & \multirow[b]{2}{*}{ Total } & \multicolumn{4}{|c|}{ Census Region } & \multirow{3}{*}{$\begin{array}{r}\text { RSE } \\
\text { Row } \\
\text { Factor }\end{array}$} \\
\hline & & & Northeast & Midwest & South & West & \\
\hline & RSE Column Factors: & 0.7 & 1.3 & 1.0 & 0.9 & 1.3 & \\
\hline
\end{tabular}

3334

Primary Aluminum

General Energy-Related Technologies

One or More General Technologies Present ................ 215

Computer Control of Building Environment ${ }^{\circ} \ldots \ldots \ldots \ldots \ldots \ldots$. Computer Control of Processes or Major Energy-Using Equipment ${ }^{*} \ldots$

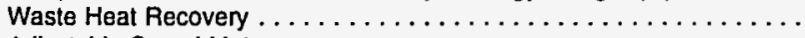

Adjustable-Speed Motors

None Present. .

Industry-Specific Technologies

One or More Industry-Specific Technologies Present . . . . . . . . . . .

Dry Quenching During the Coking Process $\ldots \ldots \ldots \ldots \ldots \ldots$

External Desulfurization of the Charge for Ironmaking . . . . . . . . .

Hydrocarbon Injection to Maintain Blast Furnace Temperatures ....... .

Direct Reduction Ironmaking - Sponge Iron Produced Directly from

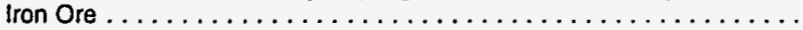

Continuous Casting $\ldots \ldots \ldots \ldots \ldots \ldots \ldots \ldots \ldots \ldots \ldots \ldots \ldots \ldots$

Thin Slab/Strip Casting . . . . . . . . . . . . . . . . . .

Waste Heat Boilers/Heat Exchangers in Combination with Reheat

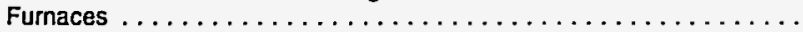

Evaporative Cooling of Skid Rails $\ldots \ldots \ldots \ldots \ldots \ldots \ldots \ldots \ldots \ldots$

Electric Induction Reheat Fumaces ...................

Hot Changing - Moving Steel Directly from the Caster to the Reheat

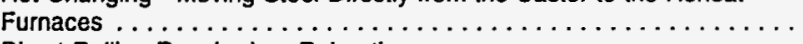

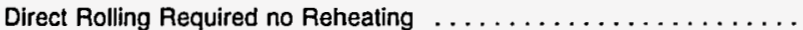

Plasmasmelt Smelting of Partially Reduced Iron Powder with

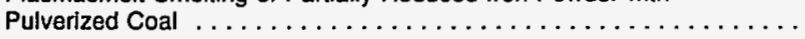

Cold Bonding (COBO) Pelletizing Technique $\ldots \ldots \ldots \ldots \ldots \ldots$

Preheating Combustion Air . . . . . . . . . . . . . . . . . . .

Preheating Raw Materials $\ldots \ldots \ldots \ldots \ldots \ldots \ldots \ldots \ldots \ldots$

Top Gas Pressure Recovery from the Blast Furnace $\ldots \ldots \ldots \ldots$.

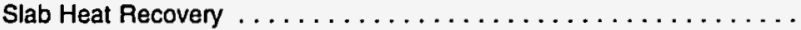

Continuous Annealing . . . . . . . . . . . . . . . . . .

Continuous Cold Rolling $\ldots \ldots \ldots \ldots \ldots \ldots \ldots \ldots \ldots \ldots \ldots$

Bottom Tap Vesseis $\ldots \ldots \ldots \ldots \ldots \ldots \ldots \ldots \ldots \ldots \ldots \ldots$

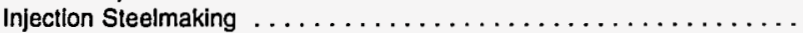

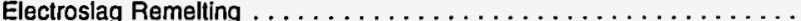

Vacuum Arc Remelting .

Oxygen Injection to Blast Furnace

Coal Injection to Blast Furnace

Steel Ladle Metallurgy with Reheat Furnace ................

None Present . .

$\begin{array}{rr}47 & \\ 0 & \\ W & 87 \\ W & \\ W & 5 \\ * & \end{array}$

$\begin{array}{lll}W & W & 3.1 \\ 47 & W & 3.8 \\ 84 & 68 & 3.1 \\ W & W & 4.8 \\ 53 & 42 & 4.2 \\ W & W & 5.2\end{array}$

3.1

See footnotes at end of table. 
Table A44. Total Inputs of Energy for Heat, Power, and Electricity Generation by Census Region, Industry Group, Selected Industries, Presence of General Technologies, and Industry-Specific Technologies for Selected Industries, 1991 (Continued) (Estimates in Trillion Btu)

\begin{tabular}{|c|c|c|c|c|c|c|c|}
\hline \multirow{2}{*}{$\begin{array}{l}\text { SIC } \\
\text { Code }\end{array}$} & \multirow{2}{*}{$\begin{array}{l}\text { Industry Groups } \\
\text { and Industry }\end{array}$} & \multirow[b]{2}{*}{ Total } & \multicolumn{4}{|c|}{ Census Region } & \multirow{3}{*}{$\begin{array}{c}\text { RSE } \\
\text { Row } \\
\text { Factors }\end{array}$} \\
\hline & & & Northeast & Midwest & South & West & \\
\hline & RSE Column Factors: & 0.7 & 1.3 & 1.0 & 0.9 & 1.3 & \\
\hline
\end{tabular}

3339

General Energy-Related Technologies

One or More General Technologies Present .

Computer Control of Building Environment ${ }^{0} \ldots \ldots \ldots \ldots \ldots \ldots \ldots \ldots$

Computer Control of Processes or Major Energy-Using Equipme

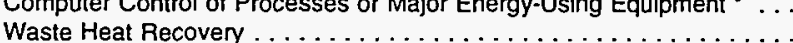

Adjustable-Speed Motors $\ldots \ldots \ldots \ldots \ldots \ldots \ldots \ldots \ldots \ldots$

None Present . . . . . . . . . . . . . . . . . . . . .

Industry-Specific Technologies

One or More Industry-Specific Technologies Present . . . . . . . . . .

Dry Quenching During the Coking Process . . . . . . . . . . .

External Desulfurization of the Charge for Ironmaking . . . . . . . . . . . .

Hydrocarbon Injection to Maintain Blast Fumace Temperatures . . . . . . .

Direct Reduction Ironmaking - Sponge Iron Produced Directly from

Iron Ore . . . . . . . . . . . . . . . . . . . . . .

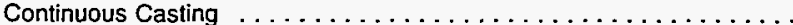

Thin Slab/Strip Casting . . . . . . . . . . . . . . . . . .

Waste Heat Boilers/Heat Exchangers in Combination with Reheat

Furnaces . . . . . . . . . . . . . . . . . . . .

Evaporative Cooling of Skid Rails $\ldots \ldots \ldots \ldots \ldots \ldots \ldots \ldots$

Electric Induction Reheat Fumaces . . . . . . . . . . . . . .

Hot Changing - Moving Steel Directly from the Caster to the Reheat

Furnaces . . . . . . . . . . . . . . . . . . .

Direct Rolling Required no Reheating $\ldots \ldots \ldots \ldots \ldots \ldots \ldots \ldots \ldots$

Plasmasmelt Smelting of Partially Reduced Iron Powder with

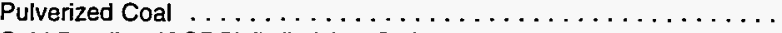

Cold Bonding (COBO) Pelletizing Technique $\ldots \ldots \ldots \ldots \ldots \ldots \ldots$

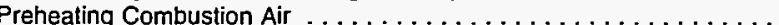

Preheating Raw Materials $\ldots \ldots \ldots \ldots \ldots \ldots \ldots \ldots \ldots$

Top Gas Pressure Recovery from the Blast Furnace $\ldots \ldots \ldots \ldots \ldots$

Slab Heat Recovery . . . . . . . . . . . . . . . . . . .

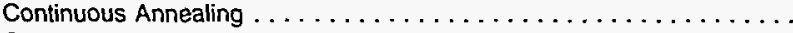

Continuous Cold Rolling

Injection Steelmaking

Electroslag Remelting

Oxygen Injection to Blast Fumace . . . . . . . . . . . . . . .

Coal injection to Blast Fumace . . . . . . . . . . . . . . .

Steel Ladle Metallurgy with Reheat Furnace

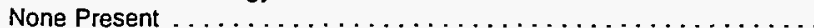

$\begin{array}{rrrrrr}36 & W & 7 & W & W & 1.8 \\ W & W & 0 & 0 & * & 1.0 \\ 21 & W & 4 & 5 & W & 2.3 \\ 13 & 0 & W & W & W & 1.1 \\ 26 & W & 6 & 8 & W & 1.9 \\ 6 & * & * & W & W & 5.5\end{array}$


Table A44. Total Inputs of Energy for Heat, Power, and Electricity Generation by Census Region, Industry Group, Selected Industries, Presence of General Technologies, and Industry-Specific Technologies for Selected Industries, 1991 (Continued) (Estimates in Trillion Btu)

\begin{tabular}{|c|c|c|c|c|c|c|c|}
\hline \multirow[b]{2}{*}{$\underset{\operatorname{Cod\theta }^{\mathrm{S}}}{\operatorname{SIC}}$} & \multirow[b]{2}{*}{$\begin{array}{l}\text { Industry Groups } \\
\text { and Industry }\end{array}$} & \multirow[b]{2}{*}{ Total } & \multicolumn{4}{|c|}{ Census Region } & \multirow{3}{*}{$\begin{array}{c}\text { RSE } \\
\text { Row } \\
\text { Factors }\end{array}$} \\
\hline & & & Northeast & Midwest & South & West & \\
\hline & RSE Column Factors: & 0.7 & 1.3 & 1.0 & 0.9 & 1.3 & \\
\hline
\end{tabular}

3353

Aluminum Sheet, Plate, and Foil

General Energy-Related Technologies

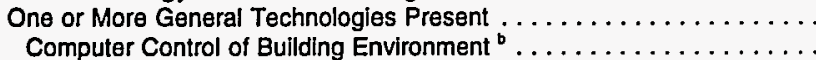

Computer Control of Processes or Major Energy-Using Equipment ${ }^{\circ} \ldots$

Waste Heat Recovery . . . . . . . . . . . . . . . . . . .

Adjustable-Speed Motors $\ldots \ldots \ldots \ldots \ldots \ldots \ldots \ldots \ldots \ldots \ldots \ldots$

None Present

Industry-Speclfle Technologies

One or More Industry-Specific Technologies Present . . . . . . . . . .

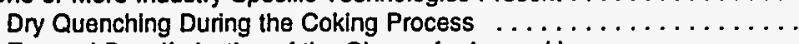

Extemal Desulfurization of the Charge for Ironmaking . . . . . . . . .

Hydrocarbon Injection to Maintain Blast Fumace Temperatures . . . . . . .

Oirect Reduction Ironmaking - Sponge Iron Produced Directly from

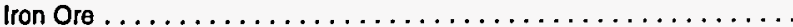

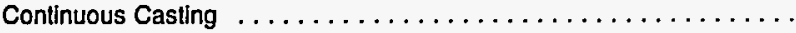

Thin Slab/Strip Casting . . . . . . . . . . . . . . . . . . . . .

Waste Heat Boilers/Heat Exchangers in Combination with Reheat

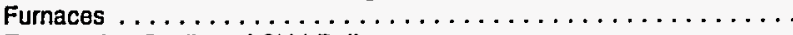

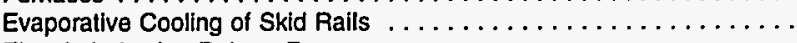

Electric Induction Reheat Fumaces ...................

Hot Changing - Moving Steel Directly from the Caster to the Reheat

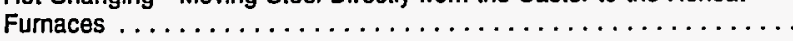

Direct Rolling Required no Reheating $\ldots \ldots \ldots \ldots \ldots \ldots \ldots \ldots$

Plasmasmelt Smelting of Partially Reduced Iron Powder with

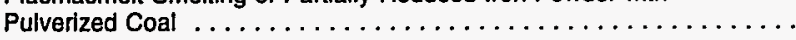

Cold Bonding (COBO) Pelletizing Technique . . . . . . . . . . .

Preheating Combustion Air . . . . . . . . . . . . . . . . .

Preheating Raw Materials . . . . . . . . . . . . . . . . .

Top Gas Pressure Recovery from the Blast Furnace . . . . . . . . . . .

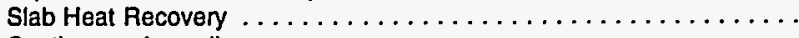

Continuous Annealing . . . . . . . . . . . . . . . . . . .

Contlnuous Cold Rolling . . . . . . . . . . . . . . . . . . .

Bottom Tap Vessels . . . . . . . . . . . . . . . . . . . . .

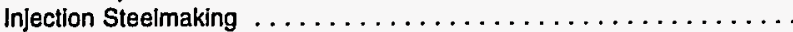

Electroslag Remelting . . . . . . . . . . . . . . . . . . . . .

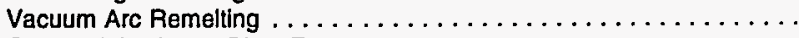

Oxygen Injection to Blast Fumace $\ldots \ldots \ldots \ldots \ldots \ldots \ldots \ldots \ldots$

Coal Injection to Blast Fumace $\ldots \ldots \ldots \ldots \ldots \ldots \ldots \ldots \ldots$

Steel Ladle Metallurgy with Reheat Furnace . . . . . . . . . . . . .

None Present .............................

FABRICATED METAL PRODUCTS

General Energy-Related Technologies

One or More General Technologies Present

Computer Control of Building Environment ${ }^{\circ} \ldots \ldots \ldots \ldots \ldots \ldots \ldots \ldots$

Computer Control of Processes or Major Energy-Using Equipment ${ }^{\mathrm{e}} \ldots$

Waste Heat Recovery . . . . . . . . . . . . . . . . . .

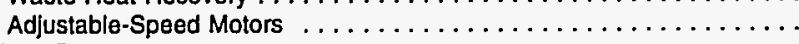

None Present . . . . . . . . . . . . . . . . . . . . . . . . . .

INDUSTRIAL MACHINERY and EQUIPMENT

General Energy-Related Technologies

One or More General Technologies Present . . . . . . . . . . . .

Computer Control of Building Environment ${ }^{b} \ldots \ldots \ldots \ldots \ldots \ldots \ldots$

Computer Control of Processes or Major Energy-Using Equipment ${ }^{c}$...

Waste Heat Recovery . . . . . . . . . . . . . . . . . . .

Adjustable-Speed Motors $\ldots \ldots \ldots \ldots \ldots \ldots \ldots \ldots \ldots \ldots \ldots \ldots$

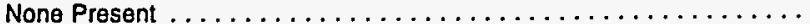

$\begin{array}{rrrrrr}58 & W & 24 & 23 & W & 1.5 \\ 7 & W & W & W & 0 & 1.1 \\ 57 & W & 24 & 22 & W & 1.0 \\ 24 & W & W & 10 & 1 & 1.8 \\ 50 & W & 23 & 18 & W & 1.5 \\ 2 & 0 & - & 2 & 0 & 2.2\end{array}$

1.5
1.1
1.0
1.8
1.5

NF

NF

NF

NF

NF

NF

NF

NF

NF

NF

NF

NF

NF

NF

NF

NF

NF

NF

NF

NF

NF

NF

NF

1.4

See footnotes at end of table. 
Table A44. Total Inputs of Energy for Heat, Power, and Electricity Generation by Census Region, Industry Group, Selected Industries, Presence of General Technologies, and Industry-Specific Technologies for Selected Industries, 1991 (Continued) (Estimates in Trillion Btu)

\begin{tabular}{|c|c|c|c|c|c|c|c|}
\hline \multirow{2}{*}{$\begin{array}{l}\text { SIC } \\
\text { Code }^{\circ}\end{array}$} & \multirow{2}{*}{$\begin{array}{l}\text { Industry Groups } \\
\text { and Industry }\end{array}$} & \multirow[b]{2}{*}{ Total } & \multicolumn{4}{|c|}{ Census Region } & \multirow{2}{*}{$\begin{array}{c}\text { RSE } \\
\text { Row } \\
\text { Factors }\end{array}$} \\
\hline & & & Northeast & Midwest & South & West & \\
\hline & RSE Column Factors: & 0.7 & 1.3 & 1.0 & 0.9 & 1.3 & \\
\hline
\end{tabular}

357

Computer and Office Equipment

General Energy-Related Technologies

One or More General Technologies Present

Computer Control of Building Environn

Computer Control of Processes or Major Energy-Using Equipment ${ }^{\circ} \ldots$

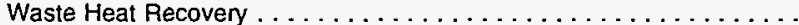

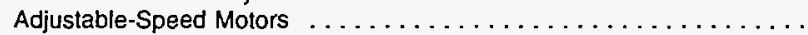

None Present . .

ELECTRONIC and OTHER ELECTRIC EQUIPMENT

36

General Energy-Related Technologies

One or More General Technologies Present

Computer Control of Building Environment ${ }^{b} \ldots \ldots \ldots \ldots \ldots \ldots . . . . . . . .$.

Computer Control of Processes or Major Energy-Using Equipment ${ }^{c} \ldots$

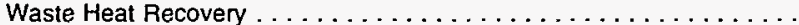

Adjustable-Speed Motors $\ldots \ldots \ldots \ldots \ldots \ldots \ldots \ldots \ldots \ldots \ldots$

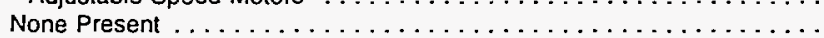

TRANSPORTATION EQUIPMENT

37

General Energy-Related Technologies

One or More General Technologies Present . . . . . . . . . . . . . . . . . 25

Computer Control of Building Environment ${ }^{b} \ldots \ldots \ldots \ldots \ldots \ldots$.

Computer Control of Processes or Major Energy-Using Equipment ${ }^{\circ} \ldots$

Waste Heat Recovery . . . . . . . . . . . . . . . . . . . . . . .

Adjustable-Speed Motors $\ldots \ldots \ldots \ldots \ldots \ldots \ldots \ldots \ldots \ldots \ldots$

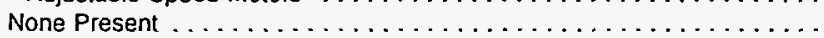

Motor Vehicles and Car Bodies

General Energy-Related Technologies

One or More General Technologies Present . . . . . . . . . . . . .

Computer Control of Building Environment ${ }^{b} \ldots \ldots \ldots \ldots \ldots \ldots \ldots$

Computer Control of Processes or Major Energy-Using Equipment ${ }^{\circ}$...

Waste Heat Recovery . . . . . . . . . . . . . . . . . . .

Adjustable-Speed Motors $\ldots \ldots \ldots \ldots \ldots \ldots \ldots \ldots \ldots \ldots \ldots$

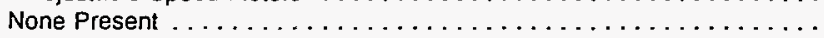

Motor Vehicle Parts and Accessories

General Energy-Related Technologies

One or More General Technologies Present ...

Computer Control of Building Environment ${ }^{b}$...

Computer Control of Processes or Major Energy-Using Equipment ${ }^{\circ} \ldots$

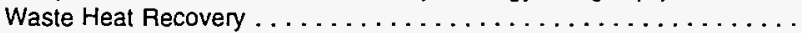

Adjustable-Speed Motors $\ldots \ldots \ldots \ldots \ldots \ldots \ldots \ldots \ldots \ldots \ldots$

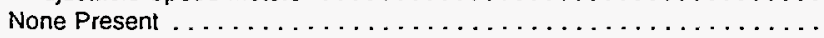

INSTRUMENTS and RELATED PRODUCTS

General Energy-Related Technologies

One or More General Technologies Present

Computer Control of Building Environment ${ }^{\circ}$.........

Computer Control of Processes or Major Energy-Using Equipment ${ }^{\circ}$....

Waste Heat Recovery . . . . . . . . . . . . . . . . . . . . . . .

Adjustable-Speed Motors $\ldots \ldots \ldots \ldots \ldots \ldots \ldots \ldots \ldots \ldots \ldots$

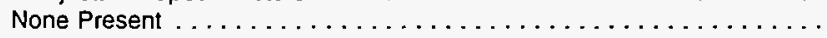

Surgical and Medical Instruments

General Energy-Related Technologies

One or More General Technologies Present . .

Computer Control of Processes or Major Energy-Using Equipment ${ }^{\circ} \ldots$

Waste Heat Recovery . . . . . . . . . . . . . . . . . . . . . . . .

Adjustable-Speed Motors $\ldots \ldots \ldots \ldots \ldots \ldots \ldots \ldots \ldots \ldots \ldots$

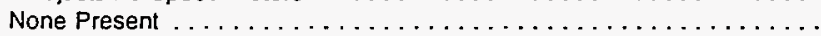

1

$\begin{array}{rrrrrr}W & W & W & 33 & 1 & 4.4 \\ 55 & 1 & 38 & 16 & * & 4.8 \\ 59 & W & 37 & 18 & W & 4.0 \\ 39 & 1 & 16 & 21 & 1 & 6.2 \\ 71 & W & 35 & 32 & W & 4.5 \\ W & w & W & 3 & 1 & 6.8\end{array}$


Table A44. Total Inputs of Energy for Heat, Power, and Electricity Generation by Census Region, Industry Group, Selected Industries, Presence of General Technologies, and Industry-Specific Technologies for Selected Industries, 1991 (Continued) (Estimates in Trillion Btu)

\begin{tabular}{|c|c|c|c|c|c|c|c|}
\hline \multirow{2}{*}{$\begin{array}{l}\text { SIC } \\
\text { Code }\end{array}$} & \multirow{2}{*}{$\begin{array}{l}\text { Industry Groups } \\
\text { and Industry }\end{array}$} & \multirow[b]{2}{*}{ Total } & \multicolumn{4}{|c|}{ Census Region } & \multirow{3}{*}{$\begin{array}{c}\text { RSE } \\
\text { Row } \\
\text { Factors }\end{array}$} \\
\hline & & & Northeast & Midwest & South & West & \\
\hline & RSE Column Factors: & 0.7 & 1.3 & 1.0 & 0.9 & 1.3 & \\
\hline
\end{tabular}

39

MISC. MANUFACTURING INDUSTRIES

General Energy-Related Technologies

One or More General Technologies Present ................

Computer Control of Building Environment ${ }^{b}$

Computer Control of Processes or Major Energy-Using Equipment ${ }^{c}$...

Waste Heat Recovery. .

Adjustable-Speed Motors

$\ldots \ldots \ldots \ldots$
$\ldots \ldots \ldots \ldots \ldots \ldots \ldots \ldots \ldots \ldots$

- See Appendices B and F for descriptions of the Standard Industrial Classification system.

bor example, space heating or cooling and lighting.

'For example, boilers or furnaces.

NF=No applicable RSE row/column factor.

- Estimate less than 0.5. Data are included in higher level totals.

$W=$ Withheld to avoid disclosing data for individual establishments. Data are included in higher level totals.

$\mathrm{Q}=$ Withheld because Relative Standard Error is greater than 50 percent. Data are included in higher level totals.

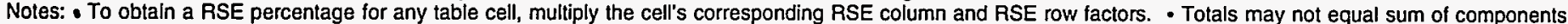

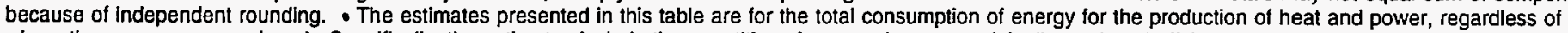

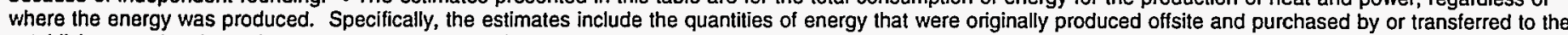

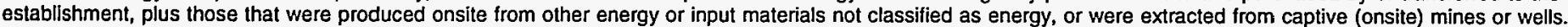

Source: Energy Information Administration, Office of Energy Markets and End Use, Energy End Use and Integrated Statistics Division, Form EIA-846, "1991

Manufacturing Energy Consumption Survey." 
Table A45. Total Inputs of Energy for Heat, Power, and Electricity Generation by Enclosed Floorspace, Percent Conditioned Floorspace, and Presence of Computer Controls for Building Environment, 1991

(Estimates in Trillion Btu)

\begin{tabular}{c|c|c|c|}
\hline \multirow{2}{*}{$\begin{array}{c}\text { Enclosed Floorspace and Percent Conditioned } \\
\text { Floorspace }\end{array}$} & & \multicolumn{2}{|c|}{ Presence of Computer Controls for Building Environment } \\
\cline { 3 - 4 } & Total & Present & Not Present \\
\hline RSE Column Factors: & 0.8 & 1.3 & Factors \\
\hline
\end{tabular}

ALL SQUARE FEET CATEGORIES

Approximate Conditioned Floorspace

(Percent Heated or Cooled)

Total . . . . . . . . . . . . . . . . . . . .

100 Percent $\ldots \ldots \ldots \ldots \ldots \ldots \ldots \ldots \ldots$

About 75 Percent . . . . . . . . . . . .

About 50 Percent . . . . . . . . . . . . .

About 25 Percent $\ldots \ldots \ldots \ldots \ldots \ldots \ldots \ldots$

None $\ldots \ldots \ldots \ldots \ldots \ldots \ldots \ldots \ldots \ldots$

Don't Know . . . . . . . . . . . . . .

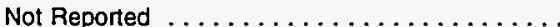

15,027

W

W

W

3,451

871

1,967

366
3,061

$W$

W

W

484

29

278

41

$\begin{array}{rr}11,945 & 2.0 \\ W & 2.6 \\ W & 2.3 \\ 1,307 & 3.8 \\ 2,967 & 3.3 \\ 841 & 9.4 \\ 1,689 & 3.6 \\ 325 & 5.6\end{array}$

25,000 OR LESS SQUARE FEET OF FLOOORSPACE

Approximate Conditioned Floorspace

(Percent Heated or Cooled)

Total . . . . . . . . . . . . . . . . . .

100 Percent $\ldots \ldots \ldots \ldots \ldots \ldots \ldots \ldots \ldots$

About 75 Percent . . . . . . . . . . . . .

About 50 Percent ................

About 25 Percent $\ldots \ldots \ldots \ldots \ldots \ldots \ldots \ldots$

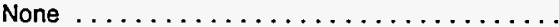

Don't know ...................

Not Reported.$\ldots \ldots \ldots \ldots \ldots \ldots \ldots \ldots$

25,001-100,000 SQUARE FEET OF FLOORSPACE Approximate Conditioned Floorspace

(Percent Heated or Cooled)

Total . . . . . . . . . . . . . . . . . . . .

100 Percent $\ldots \ldots \ldots \ldots \ldots \ldots \ldots \ldots \ldots \ldots$

About 75 Percent $\ldots \ldots \ldots \ldots \ldots \ldots \ldots \ldots$

About 50 Percent . . . . . . . . . . . . .

About 25 Percent $\ldots \ldots \ldots \ldots \ldots \ldots \ldots \ldots$

None .......................

Don't Know . . . . . . . . . . . . . .

Not Reported

$100,001-200,000$ SQUARE FEET OF FLOORSPACE

Approximate Conditioned Floorspace

(Percent Heated or Cooled)

Total . . . . . . . . . . . . . . . . . . .

100 Percent $\ldots \ldots \ldots \ldots \ldots \ldots \ldots \ldots \ldots$

About 75 Percent . . . . . . . . . . . . .

About 50 Percent $\ldots \ldots \ldots \ldots \ldots \ldots \ldots$

About 25 Percent $\ldots \ldots \ldots \ldots \ldots \ldots \ldots \ldots$

None . . . . . . . . . . . . . . . . . .

Don't know ...................

Not Reported

$\ldots \ldots \ldots \ldots \ldots \ldots \ldots \ldots$

1,440

445

350

217

273

99

47

10
424

144

61
28

71

56

5

54
27
9
$W$
$W$
$\cdot$
$Q$
$Q$

See footnotes at end of table. 
Table A45. Total Inputs of Energy for Heat, Power, and Electricity Generation by Enclosed Floorspace, Percent Conditioned Floorspace, and Presence of Computer Controls for Building Environment, 1991 (Continued)

(Estimates in Trillion Btu)

\begin{tabular}{c|c|c|c|}
\hline \multirow{2}{*}{$\begin{array}{c}\text { Enclosed Floorspace and Percent Conditioned } \\
\text { Floorspace }\end{array}$} & & \multicolumn{2}{|c|}{ Presence of Computer Controls tor Building Environment } \\
\cline { 3 - 4 } & Total & Present & Not Present \\
\hline RSE Column Factors: & 0.8 & 1.3 & Factors \\
\hline
\end{tabular}

200,001-500,000 SQUARE FEET OF FLOORSPACE Approximate Conditioned Floorspace

(Percent Heated or Cooled)

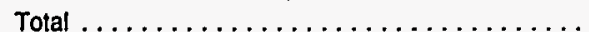

100 Percent $\ldots \ldots \ldots \ldots \ldots \ldots \ldots \ldots \ldots \ldots$

About 75 Percent $\ldots \ldots \ldots \ldots \ldots \ldots \ldots \ldots$

About 50 Percent $\ldots \ldots \ldots \ldots \ldots \ldots \ldots \ldots$

About 25 Percent $\ldots \ldots \ldots \ldots \ldots \ldots \ldots \ldots$

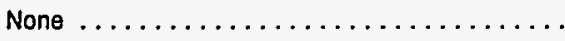

Don't know $\ldots \ldots \ldots \ldots \ldots \ldots \ldots \ldots \ldots$

Not Reported $\ldots \ldots \ldots \ldots \ldots \ldots \ldots \ldots \ldots$

2,967
674
920
367
718
166
88
34

557
200
223
41
86
$W$
$W$
-

$2,410 \quad 3.0$

674

920

367

718

88

34

$474 \quad 4.3$

$697 \quad 3.3$

$326 \quad 5.2$

$632 \quad 6.1$

W 21.4

W $\quad 7.0$

500,001-750,000 SQUARE FEET OF FLOORSPACE

Approximate Conditioned Floorspace

(Percent Heated or Cooled)

Total . . . . . . . . . . . . . . . . .

100 Percent.$\ldots \ldots \ldots \ldots \ldots \ldots \ldots \ldots \ldots$

About 75 Percent $\ldots \ldots \ldots \ldots \ldots \ldots \ldots$

About 50 Percent.$\ldots \ldots \ldots \ldots \ldots \ldots \ldots$.

About 25 Percent $\ldots \ldots \ldots \ldots \ldots \ldots \ldots \ldots$

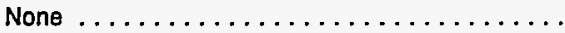

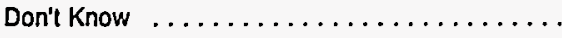

Not Reported $\ldots \ldots \ldots \ldots \ldots \ldots \ldots \ldots . . . . .$.

1,589

459

435

131

371

110

79

339
158
80
20
77
$W$
$W$
0

750,001-1,000,000 SQUARE FEET OF FLOORSPACE Approximate Conditioned Floorspace (Percent Heated or Cooled)

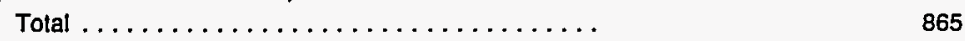

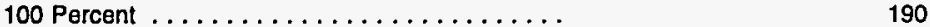

About 75 Percent ................... 174

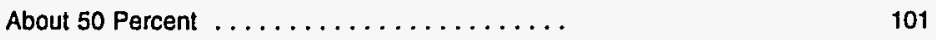

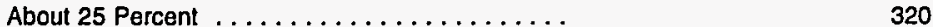

None $\ldots \ldots \ldots \ldots \ldots \ldots \ldots \ldots \ldots \ldots \ldots \ldots$ w

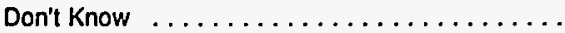

Not Reported $\ldots \ldots \ldots \ldots \ldots \ldots \ldots \ldots \ldots \ldots \ldots$

$1,000,001-5,000,000$ SQUARE FEET OF FLOORSPACE

Approximate Conditioned Floorspace

(Percent Heated or Cooled)

Total .............................

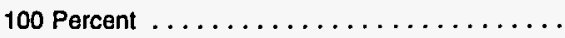

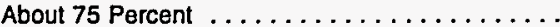

About 50 Percent .....................

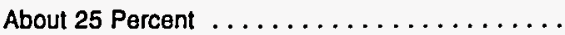

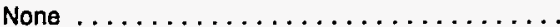

Don't Know

Not Reported

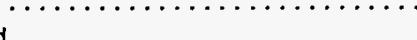

34

w

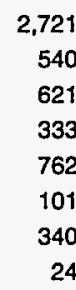

$\begin{array}{rrr}162 & 702 & 3.6 \\ 81 & 109 & 4.9 \\ 27 & 147 & 4.6 \\ 22 & 79 & 7.3 \\ 13 & 307 & 7.3 \\ W & W & 14.7 \\ \cdot & 34 & 9.3 \\ W & W & 12.4\end{array}$

See footnotes at end of table. 
Table A45. Total Inputs of Energy for Heat, Power, and Electricity Generation by Enclosed Floorspace, Percent Conditioned Floorspace, and Presence of Computer Controls for Building Environment, 1991 (Continued) (Estimates in Trillion Btu)

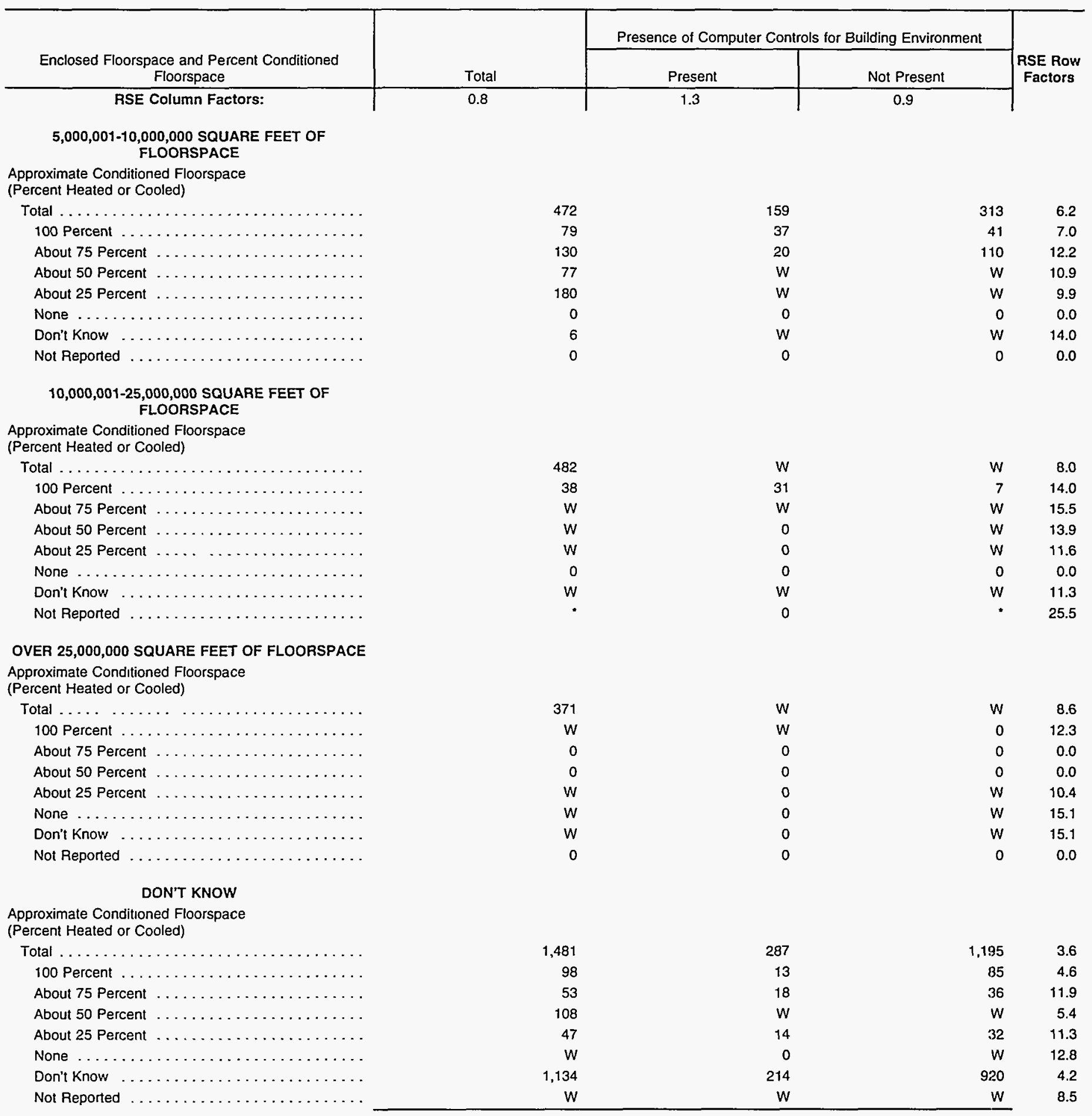


Table A45. Total Inputs of Energy for Heat, Power, and Electricity Generation by Enclosed Floorspace, Percent Conditioned Floorspace, and Presence of Computer Controls for Building Environment, 1991 (Continued) (Estimates in Trillion Btu)

\begin{tabular}{|c|c|c|c|c|c|c|c|}
\hline \multirow{2}{*}{$\begin{array}{c}\text { Enclosed Floorspace and Percent Conditioned } \\
\text { Floorspace }\end{array}$} & \multirow[b]{2}{*}{ Total } & & \multicolumn{4}{|c|}{ Presence of Computer Controls for Building Environment } & \multirow{3}{*}{$\begin{array}{l}\text { RSE Row } \\
\text { Factors }\end{array}$} \\
\hline & & & \multicolumn{2}{|l|}{ Present } & \multicolumn{2}{|l|}{ Not Present } & \\
\hline RSE Column Factors: & 0.8 & & 1.3 & & 0.9 & & \\
\hline \multicolumn{8}{|l|}{$\begin{array}{l}\text { Approximate Conditioned Floorspace } \\
\text { (Percent Heated or Cooled) }\end{array}$} \\
\hline Total $\ldots \ldots \ldots \ldots \ldots \ldots \ldots \ldots \ldots \ldots$ & & 323 & & 38 & & 285 & 6.6 \\
\hline 100 Percent $\ldots \ldots \ldots \ldots \ldots \ldots \ldots \ldots \ldots$ & & 16 & & W & & W & 11.8 \\
\hline About 75 Percent $\ldots \ldots \ldots \ldots \ldots \ldots$ & & 22 & & 17 & & 5 & 16.1 \\
\hline About 50 Percent $\ldots \ldots \ldots \ldots \ldots \ldots$ & & 7 & & 0 & & 7 & 20.9 \\
\hline About 25 Percent $\ldots \ldots \ldots \ldots \ldots \ldots$ & & 31 & & W & & W & 15.9 \\
\hline None $\ldots \ldots \ldots \ldots \ldots \ldots \ldots \ldots \ldots$ & & 7 & & 0 & & 7 & 20.9 \\
\hline Don't Know . . . . . . . . . . . . . . . & & 12 & & 0 & & 12 & 40.6 \\
\hline Not Reported $\ldots \ldots \ldots \ldots \ldots \ldots \ldots \ldots$ & & 229 & & 7 & & 222 & 9.6 \\
\hline
\end{tabular}

- Estimate less than 0.5. Data are included in higher level totals.

$W=$ Withheld to avold disclosing data for individual establishments. Data are included in higher level totals.

$\mathrm{Q}=$ Withheld because Relative Standard Error is greater than 50 percent. Data are included in higher level totals.

Notes: - To obtain a RSE percentage for any table cell, multiply the cell's corresponding RSE column and RSE row factors. • Totals may not equal sum of components because of independent rounding. - The estimates presented in this table are for the total consumption of energy for the production of heat and power, regardless of where the energy was produced. Specifically, the estimates include the quantities of energy that were originally produced offsite and purchased by or transferred to the establishment, plus those that were produced onsite from other energy or input materials not classified as energy, or were extracted from captive (onsite) mines or wells.

Source: Energy Information Administration, Office of Energy Markets and End Use, Energy End Use and Integrated Statistics Division, Form ElA-846, "1991 Manufacturing Energy Consumption Survey." 
Table A46. Total Expenditures for Purchased Electricity, Steam, and Natural Gas by Type of Supplier, Census Region, Industry Group, and Selected Industries, 1991 (Estimates in Million Dollars)

\begin{tabular}{|c|c|c|c|c|c|c|c|c|c|}
\hline \multirow[b]{2}{*}{$\begin{array}{c}\text { SIC } \\
\text { Code }\end{array}$} & \multirow[b]{2}{*}{$\begin{array}{l}\text { Industry Groups } \\
\text { and Industry }\end{array}$} & \multicolumn{2}{|c|}{ Electricity } & \multicolumn{2}{|c|}{ Steam } & \multicolumn{3}{|c|}{ Natural Gas } & \multirow{2}{*}{$\begin{array}{c}\text { RSE } \\
\text { Row } \\
\text { Factors }\end{array}$} \\
\hline & & $\begin{array}{c}\text { Utility } \\
\text { Supplier }\end{array}$ & $\begin{array}{l}\text { Nonutility } \\
\text { Supplier }\end{array}$ & $\begin{array}{l}\text { Utility } \\
\text { Supplier }\end{array}$ & $\begin{array}{l}\text { Nonutility } \\
\text { Supplier }\end{array}$ & $\begin{array}{l}\text { Utility } \\
\text { Supplier }\end{array}$ & $\begin{array}{c}\text { Transmission } \\
\text { Pipelines }\end{array}$ & $\begin{array}{l}\text { Other } \\
\text { Supplier }\end{array}$ & \\
\hline & & \multicolumn{7}{|c|}{ Total United States } & \\
\hline & RSE Column Factors: & 0.5 & 2.0 & 1.5 & 1.3 & 0.7 & 0.9 & 0.8 & \\
\hline 20 & Food and Kindred Products $\ldots \ldots \ldots \ldots$ & W & 11 & 35 & 30 & 803 & $w$ & 371 & 7.7 \\
\hline 2011 & Meat Packing Plants . . . . . . . . . . . . & 158 & $\mathbf{Q}$ & $\mathbf{W}$ & * & 43 & 16 & 16 & 9.2 \\
\hline 2033 & Canned Fruits and Vegetables . . . . . . . . . . & 99 & $*$ & 0 & 0 & 46 & 18 & 39 & 13.2 \\
\hline 2037 & Frozen Fruits and Vegetables. & W & W & 0 & $W$ & 46 & 10 & 8 & 18.8 \\
\hline 2046 & 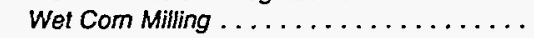 & W & $w$ & W & W & W & W & 52 & 14.4 \\
\hline 2051 & Bread, Cake, and Related Products . . . . . . & W & $w$ & $\cdot$ & 0 & 64 & 8 & 9 & 15.2 \\
\hline 2063 & Beet Sugar . . . . . . . . . . . . . & 19 & 0 & 0 & 0 & $w$ & $w$ & 15 & 12.7 \\
\hline 2075 & Soybean Oil Mills . . . . . . . . . . . . & W & 0 & $w$ & w & 15 & 13 & 26 & 4.4 \\
\hline 2082 & Malt Beverages ... & 124 & 0 & $W$ & 0 & W & $W$ & 31 & 12.9 \\
\hline 21 & Tobacco Products $\ldots \ldots \ldots \ldots \ldots \ldots \ldots \ldots$ & 73 & 0 & - & 0 & 11 & $W$ & $W$ & 14.8 \\
\hline 22 & Textile Mill Products $\ldots \ldots \ldots \ldots \ldots \ldots$ & W & $w$ & 9 & 22 & 248 & 43 & 41 & 10.7 \\
\hline 23 & Apparel and Other Textile Products . . . . . . & 378 & 3 & $\cdot$ & 0 & 61 & W & W & 23.6 \\
\hline 24 & Lumber and Wood Products ........... & $W$ & $\mathbf{Q}$ & $\mathbf{Q}$ & 19 & 79 & 29 & 18 & 24.2 \\
\hline 25 & Furniture and Fixtures $\ldots \ldots \ldots \ldots \ldots$ & 320 & $\mathbf{Q}$ & $\bullet$ & 0 & 57 & 4 & 8 & 17.8 \\
\hline 26 & Paper and Allied Products . . . . . . . . . . . & 2,639 & 16 & 25 & 34 & 557 & $\mathbf{w}$ & 421 & 5.1 \\
\hline 2611 & Pulp Mills . . . . . . . . . . . . . . & W & W & 0 & Q & 44 & W & W & 21.1 \\
\hline 2621 & Paper Mills $\ldots \ldots \ldots \ldots \ldots \ldots \ldots \ldots \ldots$ & W & $w$ & 7 & 28 & 216 & 114 & 237 & 4.2 \\
\hline 2631 & Paperboard Mills . . . . . . . . . . . . . & W & $w$ & 18 & W & 132 & W & 134 & 7.5 \\
\hline 27 & Printing and Publishing $\ldots \ldots \ldots \ldots \ldots$ & 1,041 & $Q$ & 6 & $w$ & 138 & 13 & 22 & 18.9 \\
\hline 28 & Chemicals and Allied Products . . . . . . . . . & 4,222 & 258 & 90 & 204 & 1,113 & 1,606 & 1,124 & 6.1 \\
\hline 2812 & Alkalies and Chlorine $\ldots \ldots \ldots \ldots \ldots$ & $W$ & $w$ & 0 & $w$ & W & W & W & 18.6 \\
\hline 2813 & Industrial Gases . . . . . . . . . . . . . & W & 0 & 0 & $\bullet$ & $w$ & W & $w$ & 14.7 \\
\hline 2819 & Industrial Inorganic Chemicals, nec . . . . . & W & $w$ & 0 & 8 & 114 & 122 & W & 9.9 \\
\hline 2821 & Plastics Materials and Resins ......... & $w$ & 12 & 15 & 32 & 88 & 204 & 100 & 5.9 \\
\hline 2822 & Synthetic Rubber $\ldots \ldots \ldots \ldots \ldots \ldots$ & $W$ & $w$ & W & 23 & $w$ & 102 & 10 & 14.2 \\
\hline 2823 & Cellulosic Manmade Fibers . . . . . . . . . . & W & 0 & 0 & 0 & $w$ & 0 & 0 & 40.0 \\
\hline 2824 & Organic Fibers, Noncellulosic . . . . . . . . & W & $w$ & $\cdot$ & 1 & 34 & $W$ & $w$ & 5.0 \\
\hline 2865 & Cyclic Crudes and Intermediates . . . . . . . . & W & w & W & 21 & 106 & 38 & 40 & 12.7 \\
\hline 2869 & Industrial Organic Chemicals, nec ....... & 641 & 18 & 45 & 85 & W & 638 & 327 & 5.7 \\
\hline 2873 & Nitrogenous Fertilizers $\ldots \ldots \ldots \ldots \ldots$ & 95 & 5 & $\mathbf{Q}$ & $\mathbf{Q}$ & 221 & 338 & 317 & 24.8 \\
\hline 2874 & Phosphatic Fentilizers . . . . . . . . . . . & 98 & 0 & 0 & 0 & 15 & $w$ & w & 10.1 \\
\hline 29 & Petroleum and Coal Products $\ldots \ldots \ldots \ldots$ & W & W & $w$ & 48 & 433 & 803 & 270 & 3.4 \\
\hline 2911 & Petroleum Refining $\ldots \ldots \ldots \ldots \ldots$ & W & $w$ & W & 47 & 342 & 791 & 240 & 3.0 \\
\hline 30 & Rubber and Misc. Plastics Products . . . . . . . & 1,953 & $\mathbf{Q}$ & $\mathbf{Q}$ & 7 & 212 & 19 & 70 & 9.8 \\
\hline 3011 & Tires and Inner Tubes . . . . . . . . . . . . & $W$ & 0 & $\overline{0}$ & $w$ & 37 & 3 & 9 & 4.7 \\
\hline 308 & Miscellaneous Plastic Products, nec . . . . . . & 1,528 & $Q$ & Q & $W$ & 125 & 11 & 44 & 15.8 \\
\hline 31 & Leather and Leather Products .......... & W & $\bar{W}$ & $\hat{0}$ & $\because$ & 10 & 1 & 4 & 27.9 \\
\hline 32 & Stone, Clay and Glass Products $\ldots \ldots \ldots$ & W & $w$ & $\mathbf{Q}$ & $w$ & 470 & 140 & 351 & 7.8 \\
\hline 3211 & Flat Glass $\ldots \ldots \ldots \ldots \ldots \ldots$ & 68 & 0 & W & $w$ & 43 & W & $W$ & 3.9 \\
\hline 3221 & Glass Containers . . . . . . . . . . . . & 197 & 0 & 0 & 0 & 59 & 20 & 89 & 6.1 \\
\hline 3229 & Pressed and Blown Glass, nec ......... & W & W & 0 & - & 70 & W & 29 & 7.1 \\
\hline 3241 & Cement, Hydraulic . . . . . . . . . . . . & 406 & 0 & 0 & 0 & 33 & 17 & 22 & 22.3 \\
\hline 3274 & Lime $\ldots \ldots \ldots \ldots \ldots \ldots \ldots$ & 59 & 0 & 0 & 0 & W & 7 & 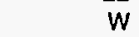 & 27.7 \\
\hline 3296 & Mineral wool $\ldots \ldots \ldots \ldots \ldots \ldots$ & W & 0 & 0 & 0 & $w$ & $w$ & 34 & 1.4 \\
\hline 33 & Primary Metal Industries . . . . . . . . . . & $\mathbf{W}$ & $w$ & $W$ & 8 & 664 & 209 & 881 & 5.3 \\
\hline 3312 & Blast Fumaces and Steel Mills . . . . . . . . . & W & W & W & $w$ & 261 & 100 & 586 & 6.2 \\
\hline 3313 & Electrometalurgical Products . . . . . . . . . & W & $w$ & $\mathbf{W}$ & $w$ & $w$ & . & $W$ & 10.0 \\
\hline 3321 & Gray and Ductile Iron Foundries ........ & W & 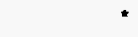 & 0 & W & 49 & 5 & 26 & 11.7 \\
\hline 3331 & Primary Copper .............. & W & 0 & 0 & 0 & $W$ & W & W & 1.4 \\
\hline 3334 & Primary Aluminum $\ldots \ldots \ldots \ldots \ldots \ldots$ & W & 0 & 0 & 0 & 27 & 13 & 14 & 4.1 \\
\hline 3339 & Primary Nonferrous Metals, nec . . . . . . . & 110 & 0 & 0 & " & 10 & 9 & 18 & 3.2 \\
\hline 3353 & Aluminum Sheet, Plate, and Foil . . . . . . & W & 0 & $W$ & 0 & 27 & 13 & 65 & 2.1 \\
\hline 34 & Fabricated Metal Products . . . . . . . . . . . . & $W$ & W & $W$ & $w$ & 408 & 60 & 122 & 14.0 \\
\hline 35 & Industrial Machinery and Equipment . . . . . . . & 1,817 & * & W & W & 282 & 23 & 72 & 15.1 \\
\hline 357 & Computer and Office Equipment . . . . . . . & 255 & - & 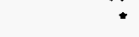 & $\ddot{w}$ & 16 & $W$ & $w$ & 18.8 \\
\hline 36 & Electronic and Other Electric Equipment . . . . . & W & $w$ & W & $\cdot$ & 188 & 25 & 50 & 12.3 \\
\hline 37 & Transportation Equipment $\ldots \ldots \ldots \ldots \ldots$ & 1,906 & 36 & 6 & 10 & 193 & 44 & 159 & 5.5 \\
\hline 3711 & Motor Vehicles and Car Bodies & $W$ & $w$ & 5 & $w$ & 37 & 21 & 64 & 4.6 \\
\hline 3714 & Motor Vehicle Parts and Accessories ...... & W & $Q$ & W & $\mathbf{W}$ & 51 & 18 & 58 & 7.9 \\
\hline 38 & Instruments and Related Products . . . . . . . & W & 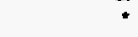 & 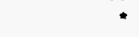 & 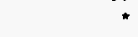 & 62 & 12 & 16 & 15.9 \\
\hline 3841 & Surgical and Medical instruments $\ldots \ldots \ldots$ & 75 & . & * & 0 & 7 & 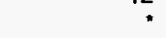 & 1 & 16.8 \\
\hline 39 & Misc. Manufacturing Industries . . . . . . . . . . & 246 & 0 & • & . & 48 & 3 & 6 & 20.3 \\
\hline & Total $\ldots \ldots \ldots \ldots \ldots \ldots$ & 31,636 & 461 & 267 & 390 & 6,037 & 3,507 & 4,013 & 3.4 \\
\hline
\end{tabular}

See footnotes at end of table. 
Table A46. Total Expenditures for Purchased Electricity, Steam, and Natural Gas by Type of Supplier, Census Region, Industry Group, and Selected Industries, 1991 (Continued) (Estimates in Million Dollars)

\begin{tabular}{|c|c|c|c|c|c|c|c|c|c|}
\hline \multirow[b]{2}{*}{$\begin{array}{l}\text { SIC } \\
\text { Code* }\end{array}$} & \multirow[b]{2}{*}{$\begin{array}{l}\text { Industry Groups } \\
\text { and Industry }\end{array}$} & \multicolumn{2}{|c|}{ Electricity } & \multicolumn{2}{|c|}{ Steam } & \multicolumn{3}{|c|}{ Natural Gas } & \multirow[b]{2}{*}{$\begin{array}{c}\text { RSE } \\
\text { Row } \\
\text { Factors }\end{array}$} \\
\hline & & $\begin{array}{c}\text { Utility } \\
\text { Supplier }\end{array}$ & $\begin{array}{l}\text { Nonutility } \\
\text { Supplier }\end{array}$ & $\begin{array}{c}\text { Utility } \\
\text { Supplier }\end{array}$ & $\begin{array}{l}\text { Nonutility } \\
\text { Supplier }\end{array}$ & $\begin{array}{c}\text { Utility } \\
\text { Supplier }\end{array}$ & $\begin{array}{c}\text { Transmission } \\
\text { Pipelines }\end{array}$ & $\begin{array}{l}\text { Other } \\
\text { Supplier }\end{array}$ & \\
\hline & & \multicolumn{7}{|c|}{ Northeast Census Region } & \\
\hline & RSE Column Factors: & 0.6 & 1.6 & 1.2 & 1.2 & 0.8 & 1.0 & 0.9 & \\
\hline $\begin{array}{l}20 \\
2011\end{array}$ & $\begin{array}{l}\text { Food and Kindred Products } \ldots \ldots \ldots \ldots \ldots \\
\text { Meat Packing Plants }\end{array}$ & $\begin{array}{r}391 \\
8\end{array}$ & Q & $\begin{array}{r}W \\
0\end{array}$ & $Q$ & 87 & 22 & 34 & 17.4 \\
\hline $\begin{array}{l}2011 \\
2033\end{array}$ & 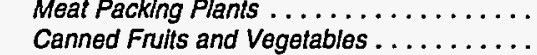 & $\begin{array}{r}8 \\
22\end{array}$ & $\begin{array}{l}0 \\
0\end{array}$ & $\begin{array}{l}0 \\
0\end{array}$ & $\begin{array}{l}0 \\
0\end{array}$ & $\begin{array}{l}W \\
W\end{array}$ & $\stackrel{0}{w}$ & $\begin{array}{c}W \\
Q\end{array}$ & $\begin{array}{l}25.2 \\
23.0\end{array}$ \\
\hline 2037 & - Frozen Fruilts and Vegetables .... & $w$ & 0 & 0 & : & $\because$ & 0 & ? & 35.3 \\
\hline 2046 & Wet Com Milling . . . . . . . . . . & 1 & 0 & 0 & 0 & * & 0 & 0 & 36.9 \\
\hline 2051 & Bread, Cake, and Related Products & w & Q & 0 & 0 & 11 & 2 & w & 21.7 \\
\hline 2063 & Beot Sugar $\ldots \ldots \ldots \ldots \ldots \ldots$ & 0 & 0 & 0 & 0 & 0 & 0 & 0 & NF \\
\hline 2075 & Soyboan Oll Mills $\ldots \ldots \ldots \ldots$. & 0 & 0 & 0 & 0 & 0 & 0 & 0 & NF \\
\hline 2082 & Malt Beverages. & 33 & 0 & 0 & 0 & $\mathbf{Q}$ & w & $w$ & 17.3 \\
\hline 21 & Tobacco Products . . . . . . & NA & NA & NA & NA & NA & NA & NA & 26.4 \\
\hline 22 & Textile Mill Products & w & w & $Q$ & 10 & 36 & 4 & 3 & 23.1 \\
\hline 23 & Apparel and Other Textlle Products . & 49 & * & • & 0 & 10 & 0 & 0 & 31.5 \\
\hline 24 & Lumber and Wood Products..$\ldots \ldots \ldots \ldots$ & NA & NA & NA & NA & NA & NA & NA & 40.9 \\
\hline 25 & Furniture and Fixtures $\ldots \ldots \ldots \ldots$ & $w$ & $Q$ & 0 & 0 & 6 & $\mathbf{Q}$ & 1 & 31.0 \\
\hline 26 & Paper and Allied Products & w & w & 6 & w & w & w & 45 & 10.7 \\
\hline 2611 & Pulp MIlls . . . . . . . & w & $\mathbf{Q}$ & 0 & Q & 0 & 0 & 0 & 57.8 \\
\hline 2621 & Paper MIIIs & w & $w$ & w & 8 & 21 & * & 35 & 6.1 \\
\hline 2631 & Paperboard Mills ... & w & 0 & w & : & 11 & w & w & 20.4 \\
\hline 27 & Printing and Publishing $\ldots \ldots \ldots \ldots$ & 254 & $\mathbf{Q}$ & a & 0 & 38 & $w$ & $w$ & 29.7 \\
\hline 28 & Chemicals and Allied Products . . . . . . . . & w & 8 & $\mathbf{w}$ & 39 & 102 & 21 & 71 & 10.7 \\
\hline 2812 & Alkalles and Chlorine ............. & $\because$ & 0 & 0 & 0 & 0 & $\because$ & 0 & 32.3 \\
\hline 2813 & Industrial Gases . . . . . . . . . . & w & 0 & 0 & 0 & w & 0 & 0 & 14.3 \\
\hline 2819 & Industrial Inorganic Chemicals, nec & $w$ & 0 & 0 & - & 11 & 1 & 6 & 25.6 \\
\hline 2821 & Plastlcs Materials and Resins . . . . . & w & * & w & w & 10 & 3 & 12 & 10.5 \\
\hline 2822 & Synthetic Rubber . . . . . . . . . & $w$ & 0 & 0 & w & w & • & w & 24.7 \\
\hline 2823 & Cellulosic Manmade Fibers $\ldots \ldots \ldots \ldots \ldots$ & 0 & 0 & 0 & 0 & 0 & 0 & 0 & NF \\
\hline 2824 & Organic Fibers, Noncellulosic $\ldots \ldots \ldots \ldots$. & 6 & 0 & 0 & 0 & - & - & 0 & 17.1 \\
\hline 2865 & Cyclic Crudes and Intermediates .... & w & w & 0 & w & w & w & w & 21.2 \\
\hline 2869 & Industrial Organlc Chemicals, nec ........ & $w$ & w & w & w & 20 & w & w & 12.3 \\
\hline 2873 & Nitrogenous Fertilizers $\ldots \ldots \ldots \ldots \ldots \ldots$ & $w$ & $w$ & 0 & $\because$ & 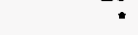 & 0 & $w$ & 46.7 \\
\hline 2874 & Phosphatlc Fertilizers . . . . . . . . . . & 0 & 0 & 0 & 0 & 0 & 0 & 0 & NF \\
\hline 29 & Petroleum and Coal Products & 168 & $\mathbf{Q}$ & w & * & 23 & w & w & 10.4 \\
\hline 2911 & Petroleum Refining . . . . . . . & 122 & 0 & w & 0 & w & 19 & w & 5.3 \\
\hline 30 & Rubber and Misc. Plastics Products .. & 410 & 0 & $\ddot{Q}$ & : & $w$ & 2 & w & 24.0 \\
\hline 3011 & Tlros and Inner Tubes . . . . . . . . . & 5 & 0 & 0 & 0 & 3 & 0 & $\bullet$ & 10.0 \\
\hline 308 & Miscellaneous Plastic Products, nec. & 363 & 0 & $\mathbf{Q}$ & * & 22 & $w$ & w & 27.9 \\
\hline 31 & Leather and Leather Products . ...... & 18 & 0 & 0 & 0 & 3 & $\because$ & 0 & 28.7 \\
\hline 32 & Stone, Clay and Glass Products & w & w & 0 & - & 77 & 25 & 77 & 13.2 \\
\hline 3211 & Flat Glass $\ldots \ldots \ldots \ldots \ldots \ldots \ldots \ldots$ & w & 0 & 0 & 0 & 0 & w & w & 5.5 \\
\hline 3221 & Glass Containers.$\ldots \ldots \ldots \ldots \ldots \ldots$ & 47 & 0 & 0 & 0 & 11 & W & $w$ & 10.2 \\
\hline 3229 & Pressed and Blown Glass, nec .... & $\ddot{w}$ & w & 0 & : & 21 & w & 4 & 8.7 \\
\hline 3241 & Cement, Hydraulic.$\ldots \ldots \ldots \ldots$ & 70 & 0 & 0 & 0 & • & $\because$ & 0 & 26.7 \\
\hline 3274 & Limo $\ldots \ldots \ldots \ldots \ldots \ldots \ldots$ & $\mathbf{Q}$ & 0 & 0 & 0 & 0 & $\mathbf{Q}$ & 0 & NF \\
\hline 3296 & Mineral Wool ............... & $w$ & 0 & 0 & 0 & $w$ & * & 5 & 2.5 \\
\hline 33 & Primary Metal Industries ...... & w & w & 0 & 1 & 184 & 32 & 107 & 8.4 \\
\hline 3312 & Blast Fumaces and Steel Mills .... & 366 & 0 & 0 & - & 104 & 11 & 71 & 7.1 \\
\hline 3313 & Electromelalurgical Products . . . . . & w & w & 0 & - & . & 0 & 0 & 12.0 \\
\hline 3321 & Gray and Ductlle Iron Foundries... & 25 & 0 & 0 & 0 & w & w & $\mathbf{Q}$ & 20.1 \\
\hline 3331 & Primary Copper & w & 0 & 0 & 0 & * & 0 & * & 1.3 \\
\hline 3334 & Primary Aluminum & $w$ & 0 & 0 & 0 & $w$ & 0 & 0 & 6.5 \\
\hline 3339 & Primary Nonferrous Metals, nec... & 6 & 0 & 0 & 0 & • & w & w & 3.9 \\
\hline 3353 & Aluminum Sheet, Plate, and Foil .... & 27 & 0 & 0 & 0 & $w$ & 0 & $\ddot{w}$ & 2.4 \\
\hline 34 & Fabricated Metal Products ... & 381 & 0 & • & w & 85 & 12 & 31 & 18.5 \\
\hline 35 & Industrial Machinery and Equipment . & $w$ & 0 & $\mathbf{0}$ & w & 46 & 5 & 16 & 21.4 \\
\hline 357 & Computer and Office Equipment & 57 & 0 & 0 & w & 4 & * & w & 24.8 \\
\hline 36 & Electronic and Other Electric Equipment & 450 & 0 & w & 0 & 43 & 2 & 12 & 19.7 \\
\hline 37 & Transportation Equipment ... & $w$ & w & 0 & w & 20 & $\bar{w}$ & w & 11.1 \\
\hline 3711 & Motor Vehicles and Car Bodles & w & 0 & 0 & 0 & w & 0 & w & 9.6 \\
\hline 3714 & Motor Vehicle Parts and Accessories & 63 & 0 & 0 & w & $w$ & w & 8 & 12.9 \\
\hline 38 & Instruments and Related Products ... & w & * & 0 & $\because$ & 22 & 6 & w & 18.2 \\
\hline 3841 & Surglcal and Medical Instruments & 25 & * & 0 & 0 & 2 & * & * & 21.9 \\
\hline 39 & Misc. Manufacturing Industries . . . . . & 94 & 0 & 0 & * & w & $\mathbf{Q}$ & w & 21.8 \\
\hline & Total $\ldots \ldots \ldots \ldots \ldots \ldots$ & 5,447 & 55 & w & 70 & 902 & 172 & 477 & 6.6 \\
\hline
\end{tabular}

See footnotes at end of table. 
Table A46. Total Expenditures for Purchased Electricity, Steam, and Natural Gas by Type of Supplier, Census Region, Industry Group, and Selected Industries, 1991 (Continued)

(Estimates in Million Dollars)

\begin{tabular}{|c|c|c|c|c|c|c|c|c|c|}
\hline \multirow[b]{2}{*}{$\begin{array}{c}\text { SiC } \\
\text { Code }\end{array}$} & \multirow[b]{2}{*}{$\begin{array}{l}\text { Industry Groups } \\
\text { and Industry }\end{array}$} & \multicolumn{2}{|c|}{ Electricity } & \multicolumn{2}{|c|}{ Steam } & \multicolumn{3}{|c|}{ Natural Gas } & \multirow[b]{2}{*}{$\begin{array}{c}\text { RSE } \\
\text { Row } \\
\text { Factors }\end{array}$} \\
\hline & & $\begin{array}{c}\text { Utility } \\
\text { Supplier }\end{array}$ & $\begin{array}{l}\text { Nonutility } \\
\text { Supplier }\end{array}$ & $\begin{array}{c}\text { Utility } \\
\text { Supplier }\end{array}$ & $\begin{array}{l}\text { Nonutility } \\
\text { Supplier }\end{array}$ & $\begin{array}{l}\text { Utility } \\
\text { Supplier }^{b}\end{array}$ & $\begin{array}{c}\text { Transmission } \\
\text { Pipelines }\end{array}$ & $\begin{array}{c}\text { Other } \\
\text { Supplier }\end{array}$ & \\
\hline & & \multicolumn{7}{|c|}{ Midwest Census Region } & \\
\hline & RSE Column Factors: & 0.5 & 1.6 & 1.3 & 1.4 & 0.8 & 0.9 & 0.8 & \\
\hline 20 & Food and Kindred Products . & W & 5 & 30 & 4 & 280 & $w$ & 176 & 10.8 \\
\hline 2011 & Meat Packing Plants . . . . . . . . . . . . & 93 & 0 & W & 0 & 30 & 10 & 11 & 10.5 \\
\hline 2033 & Canned Fruits and Vegetables . . . . . . . . . & 22 & 0 & 0 & 0 & 13 & 5 & 5 & 18.0 \\
\hline 2037 & Frozen Fruits and Vegetables . . . . . . . . . & W & 0 & 0 & 0 & 3 & $\cdot$ & 3 & 39.9 \\
\hline 2046 & Wet Corn Milling . . . . . . . . . . . . & W & 0 & W & 0 & w & $w$ & $w$ & 15.0 \\
\hline 2051 & Bread, Cake, and Related Products . . . . . . & W & $w$ & $\cdot$ & 0 & 14 & 4 & $w$ & 17.3 \\
\hline 2063 & Beet Sugar . . . . . . . . . . . . . . & W & 0 & 0 & 0 & $w$ & $W$ & $w$ & 13.5 \\
\hline 2075 & Soybean Oil Mills . . . . . . . . . . . . . . & W & 0 & W & 0 & $W$ & $w$ & 18 & 4.7 \\
\hline 2082 & Malt Beverages . . . . . . . . . . . & 21 & 0 & W & 0 & 2 & W & $w$ & 20.9 \\
\hline 21 & Tobacco Products . . . . . . . . . . . . . & NA & NA & NA & NA & NA & NA & NA & 12.4 \\
\hline 22 & Textile Mill Products $\ldots \ldots \ldots \ldots \ldots \ldots$ & NA & NA & NA & NA & NA & NA & NA & 34.7 \\
\hline 23 & Apparel and Other Textile Products ...... & NA & NA & NA & NA & NA & NA & NA & 35.6 \\
\hline 24 & Lumber and Wood Products $\ldots . . . \ldots \ldots$ & 164 & 0 & W & 0 & 33 & Q & $Q$ & 31.2 \\
\hline 25 & Furniture and Fixtures $\ldots \ldots \ldots \ldots \ldots$ & $W$ & W & 0 & 0 & $W$ & 2 & 6 & 23.7 \\
\hline 26 & Paper and Allied Products . . . . . . . . . . . & W & W & W & W & 130 & 40 & 121 & 8.7 \\
\hline 2611 & Pulp Mills . . . . . . . . . . . . . . . & W & 0 & 0 & 0 & W & 0 & W & 45.5 \\
\hline 2621 & Paper Mills . . . . . . . . . . . . . . & $w$ & $w$ & $w$ & $w$ & w & $w$ & 83 & 7.1 \\
\hline 2631 & Paperboard Mlls . . . . . . . . . . . . & 84 & 0 & W & 0 & 16 & $W$ & $W$ & 14.8 \\
\hline 27 & Printing and Publishing $\ldots \ldots \ldots \ldots$ & 299 & 0 & 3 & W & 52 & 6 & 13 & 21.8 \\
\hline 28 & Chemicals and Allied Products . . . . . . . & W & W & W & 31 & 152 & 137 & 194 & 10.7 \\
\hline 2812 & Alkalies and Chlorine $\ldots \ldots \ldots \ldots \ldots$ & W & 0 & 0 & 0 & 0 & 0 & $W$ & 36.7 \\
\hline 2813 & Industrial Gases . . . . . . . . . . . . & W & 0 & 0 & - & W & 0 & W & 16.0 \\
\hline 2819 & Industrial Inorganic Chemicals, nec . . . . . & W & $w$ & 0 & * & 14 & 8 & $w$ & 12.8 \\
\hline 2821 & Plastics Materials and Resins . . . . . . . . & W & W & 9 & W & 16 & 3 & 32 & 7.9 \\
\hline 2822 & Synthetic Rubber . . . . . . . . . . . . . & 8 & 0 & 0 & 0 & 1 & 0 & $W$ & 21.3 \\
\hline 2823 & Cellulosic Manmade Fibers . . . . . . . . . . & 0 & 0 & 0 & 0 & 0 & 0 & 0 & NF \\
\hline 2824 & Organic Fibers, Noncellulosic . . . . . . . . & 0 & 0 & 0 & 0 & 0 & 0 & 0 & NF \\
\hline 2865 & Cyclic Crudes and intemediates . . . . . . & 34 & 0 & 0 & $Q$ & W & W & 20 & 18.2 \\
\hline 2869 & Industrial Organic Chemicals, nec . . . . . . & 53 & W & $w$ & $\mathbf{W}$ & 33 & $W$ & 17 & 10.3 \\
\hline 2873 & Nitrogenous Fertilizers $\ldots \ldots \ldots \ldots \ldots$ & W & $Q$ & 0 & $Q$ & $W$ & $W$ & $w$ & 46.2 \\
\hline 2874 & Phosphatic Fertilizers . . . . . . . . . . & $\cdot$ & 0 & 0 & 0 & * & 0 & $\cdot$ & 5.7 \\
\hline 29 & Petroleum and Coal Products $\ldots \ldots \ldots \ldots$ & W & * & Q & W & 60 & W & W & 7.0 \\
\hline 2911 & Petroleum Refining . . . . . . . . . . . & 243 & * & 0 & W & W & 94 & W & 3.9 \\
\hline 30 & Rubber and Misc. Plastics Products . . . . . . . & 735 & $\mathrm{Q}$ & 0 & 0 & 80 & 9 & 37 & 15.4 \\
\hline 3011 & Tires and Inner Tubes . . . . . . . . . . . . . & W & 0 & 0 & 0 & W & $W$ & W & 5.5 \\
\hline 308 & Miscellaneous Plastic Products, nec. . . . . . . & NA & NA & NA & NA & NA & NA & NA & 21.4 \\
\hline 31 & Leather and Leather Products . . . . . . . . & $W$ & W & 0 & $*$ & 3 & * & W & 27.4 \\
\hline 32 & Stone, Clay and Glass Products $\ldots . \ldots \ldots$ & W & 0 & $Q$ & * & 121 & 37 & 120 & 9.7 \\
\hline 3211 & Flat Glass $\ldots \ldots \ldots \ldots \ldots \ldots$ & W & 0 & W & 0 & 11 & 0 & 14 & 5.0 \\
\hline 3221 & Glass Containers . . . . . . . . . . . & 39 & 0 & 0 & 0 & W & W & 25 & 9.8 \\
\hline 3229 & Pressed and Blown Glass, nec . . . . . . . . & 28 & 0 & 0 & 0 & 16 & $w$ & $w$ & 9.2 \\
\hline 3241 & Cement, Hydraulic . . . . . . . . . . . . & 96 & 0 & 0 & 0 & 6 & $Q$ & W & 24.5 \\
\hline 3274 & 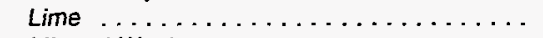 & 19 & 0 & 0 & 0 & W & $W$ & $\cdot$ & 28.5 \\
\hline 3296 & 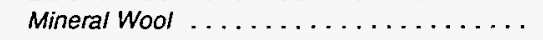 & 47 & 0 & 0 & 0 & 12 & W & 13 & 1.9 \\
\hline 33 & Primary Metal Industries . . . . . . . . . . & W & $W$ & W & $W$ & W & 71 & 554 & 7.6 \\
\hline 3312 & Blast Fumaces and Steel Mills . . . . . . . . . & W & $w$ & W & W & W & w & 409 & 7.4 \\
\hline 3313 & Electrometalurgical Products . . . . . . . . & $W$ & $w$ & $w$ & $W$ & w & 0 & 2 & 10.7 \\
\hline 3321 & Gray and Ductile Iron Foundries ........ & 215 & * & 0 & W & W & $w$ & 24 & 13.3 \\
\hline 3331 & Primary Copper . . . . . . . . . . . . . & " & 0 & 0 & 0 & \pm & 0 & 0 & 1.5 \\
\hline 3334 & Primary Aluminum $\ldots \ldots \ldots \ldots \ldots$ & W & 0 & 0 & 0 & W & 0 & W & 7.0 \\
\hline 3339 & Primary Nonferrous Metals, nec . . . . . . . . . & 26 & 0 & 0 & * & W & W & 3 & 2.4 \\
\hline 3353 & Aluminum Sheet, Plate, and Foil . . . . . . . & W & 0 & $w$ & 0 & $w$ & $w$ & 17 & 1.5 \\
\hline 34 & Fabricated Metal Products . . . . . . . . . . . & 763 & $\mathrm{Q}$ & W & W & 142 & $w$ & $W$ & 15.0 \\
\hline 35 & Industral Machinery and Equipment . . . . . . . & W & * & W & 0 & 125 & 12 & 50 & 15.4 \\
\hline 357 & Computer and Office Equipment ........ & 38 & * & * & 0 & 4 & $\mathbf{Q}$ & $\star$ & 27.5 \\
\hline 36 & Electronic and Other Electric Equipment .... . & 350 & * & W & * & 53 & 7 & 28 & 15.9 \\
\hline 37 & Transportation Equipment $\ldots \ldots \ldots \ldots \ldots$ & W & W & W & 5 & 79 & 33 & 98 & 6.8 \\
\hline 3711 & Motor Vehicles and Car Bodies . . . . . . . . & W & w & $w$ & W & 20 & W & $W$ & 5.4 \\
\hline 3714 & Motor Vehicle Parts and Accessories . . . . . . & $W$ & $\mathrm{Q}$ & W & W & 25 & 15 & 46 & 9.3 \\
\hline 38 & Instruments and Related Products . . . . . . & 99 & 0 & * & 0 & 11 & $\mathbf{Q}$ & $W$ & 26.9 \\
\hline 3841 & Surgical and Medical Instruments ....... & 13 & 0 & * & 0 & 2 & " & 0 & 18.6 \\
\hline 39 & Misc. Manufacturing Industries . . . . . . . . . & 59 & 0 & * & 0 & W & 2 & W & 23.2 \\
\hline & Total $\ldots \ldots \ldots \ldots \ldots \ldots \ldots \ldots$ & 9,048 & 87 & 97 & 56 & 1,575 & 572 & 1,510 & 4.8 \\
\hline
\end{tabular}

See footnotes at end of table. 
Table A46. Total Expenditures for Purchased Electricity, Steam, and Natural Gas by Type of Supplier, Census Region, Industry Group, and Selected Industries, 1991 (Continued)

(Estimates in Million Dollars)

\begin{tabular}{|c|c|c|c|c|c|c|c|c|c|}
\hline \multirow[b]{2}{*}{$\begin{array}{l}\text { SIC } \\
\text { Code }^{\circ}\end{array}$} & \multirow[b]{2}{*}{$\begin{array}{l}\text { Industry Groups } \\
\text { and Industry }\end{array}$} & \multicolumn{2}{|c|}{ Electricity } & \multicolumn{2}{|c|}{ Steam } & \multicolumn{3}{|c|}{ Natural Gas } & \multirow{2}{*}{$\begin{array}{c}\text { RSE } \\
\text { Row } \\
\text { Factors }\end{array}$} \\
\hline & & $\begin{array}{c}\text { Utility } \\
\text { Supplier }\end{array}$ & $\begin{array}{l}\text { Nonutility } \\
\text { Supplier }\end{array}$ & $\begin{array}{c}\text { Utility } \\
\text { Supplier }\end{array}$ & $\begin{array}{l}\text { Nonutility } \\
\text { Supplier }\end{array}$ & $\begin{array}{c}\text { Utility } \\
\text { Supplier }\end{array}$ & \begin{tabular}{|c|} 
Transmission \\
Pipelines
\end{tabular} & $\begin{array}{c}\text { Other } \\
\text { Supplier" }\end{array}$ & \\
\hline & & \multicolumn{7}{|c|}{ South Census Region } & \\
\hline & RSE Column Factors: & 0.5 & 1.9 & 1.5 & 1.1 & 0.7 & 0.9 & 1.0 & \\
\hline 20 & $\begin{array}{l}\text { Food and Kindred Products } \ldots \\
\text { Meat Packing Plants } \ldots . . .\end{array}$ & $\begin{array}{l}w \\
W\end{array}$ & $\begin{array}{l}Q \\
Q\end{array}$ & $\begin{array}{c}W \\
0\end{array}$ & W & $\begin{array}{r}244 \\
9\end{array}$ & $\stackrel{68}{W}$ & $\begin{array}{l}56 \\
W\end{array}$ & $\begin{array}{l}12.9 \\
16.8\end{array}$ \\
\hline 2033 & Canned Fruits and Vegetables. & 12 & 0 & 0 & 0 & W & W & 1 & 29.7 \\
\hline 2037 & Frozen Fruils and Vegetables & 31 & 0 & 0 & 0 & 13 & w & w & 26.8 \\
\hline 2046 & Wet Com Milling . . . . . . . . & 31 & 0 & 0 & 0 & w & - & w & 26.2 \\
\hline 2051 & Bread, Cake, and Related Products . & 45 & 0 & 0 & 0 & 25 & 1 & 1 & 21.6 \\
\hline 2063 & 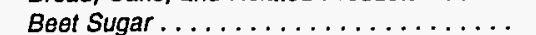 & * & 0 & 0 & 0 & w & 0 & 0 & 26.9 \\
\hline 2075 & Soybean Oil Mills & 23 & 0 & 0 & w & w & w & 8 & 6.2 \\
\hline 2082 & Malt Beverages & 41 & 0 & 0 & 0 & 7 & w & w & 17.1 \\
\hline 21 & Tobacco Products . & 72 & 0 & * & 0 & 10 & W & w & 14.6 \\
\hline 22 & Textile Mill Products & W & W & 5 & 12 & 191 & 37 & 32 & 9.2 \\
\hline 23 & Apparel and Other Textile Products & 255 & 2 & 0 & 0 & 40 & W & w & 26.2 \\
\hline 24 & Lumber and Wood Products .... & W & 0 & Q & Q & 21 & 15 & 7 & 31.9 \\
\hline 25 & Furniture and Fixtures .... & 160 & 0 & * & 0 & w & Q & 1 & 21.8 \\
\hline 26 & Paper and Allied Products & 1,039 & 0 & w & w & 282 & w & 90 & 6.8 \\
\hline 2611 & Pulp Mills .......... & 68 & 0 & 0 & 0 & 30 & w & w & 26.9 \\
\hline 2621 & Paper Mills . & w & 0 & 0 & w & 116 & 95 & 31 & 6.9 \\
\hline 2631 & Paperboard Mills . & 268 & 0 & w & - & 86 & 72 & w & 9.8 \\
\hline 27 & Printing and Publishing & 284 & 0 & * & 0 & 27 & $\mathbf{Q}$ & w & 22.5 \\
\hline 28 & Chemicals and Allied Products . & w & $w$ & 54 & 130 & 726 & 1,441 & 775 & 6.8 \\
\hline 2812 & Alkalles and Chlorine ..... & w & w & 0 & w & w & W & w & 19.5 \\
\hline 2813 & Industrial Gases .... . & w & 0 & 0 & 0 & w & w & w & 15.7 \\
\hline 2819 & Industrial Inorganic Chemicals, nec & 231 & w & 0 & w & 78 & 111 & w & 11.2 \\
\hline 2821 & Plastics Materials and Resins .... & $w$ & w & $w$ & 15 & 54 & 199 & 55 & 7.4 \\
\hline 2822 & Synthetlc Rubber ........ & w & w & w & w & w & 101 & w & 14.8 \\
\hline 2823 & Cellulosic Manmade Fibers & w & 0 & 0 & 0 & W & 0 & 0 & 38.5 \\
\hline 2824 & Organic Fibers, Noncellulosic & w & w & * & 1 & 33 & w & $w$ & 5.0 \\
\hline 2865 & Cyclic Crudes and Intermediates & w & W & w & 16 & 81 & w & W & 15.0 \\
\hline 2869 & Industrial Organic Chemicals, nec & W & w & w & 46 & w & w & 297 & 6.3 \\
\hline 2873 & Nitrogenous Fertilizers . . . . . . & 68 & 0 & $\mathbf{Q}$ & 0 & w & w & 251 & 31.5 \\
\hline 2874 & Phosphatlc Fertilizers ... & w & 0 & 0 & 0 & $w$ & w & w & 3.7 \\
\hline 29 & Petroleum and Coal Products & w & $w$ & w & w & 198 & 629 & 115 & 4.9 \\
\hline 2911 & Petroleum Refining ...... & 556 & 0 & w & w & 172 & 627 & 112 & 3.7 \\
\hline 30 & Rubber and Misc. Plastics Products & 599 & 0 & 0 & 6 & 76 & 8 & 21 & 14.0 \\
\hline 3011 & Tires and Inner Tubes. . . . . . . . & 121 & 0 & 0 & w & w & w & w & 5.1 \\
\hline 308 & Miscellaneous Plastic Products, nec. & 389 & 0 & 0 & w & 33 & 5 & 13 & 24.5 \\
\hline 31 & Leather and Leather Products . & 17 & 0 & 0 & 0 & w & • & 0 & 30.3 \\
\hline 32 & Stone, Clay and Glass Products & $w$ & $w$ & 0 & 0 & 203 & 56 & 104 & 8.7 \\
\hline 3211 & Flat Glass $\ldots \ldots \ldots \ldots \ldots$ & 31 & 0 & 0 & 0 & w & W & 18 & 5.1 \\
\hline 3221 & Glass Containers & 54 & 0 & 0 & 0 & 29 & W & w & 9.2 \\
\hline 3229 & Pressed and Blown Glass, nec & 60 & 0 & 0 & 0 & 33 & W & w & 9.9 \\
\hline 3241 & Cement, Hydraulic ......... & 130 & 0 & 0 & 0 & 14 & Q & 13 & 24.1 \\
\hline 3274 & Lime & 18 & 0 & 0 & 0 & 4 & w & $w$ & 23.0 \\
\hline 3296 & Mineral Wool & $w$ & 0 & 0 & 0 & 10 & $w$ & $w$ & 2.2 \\
\hline 33 & Primary Metal Industries & w & w & 0 & 0 & 217 & W & 148 & 6.9 \\
\hline 3312 & Blast Fumaces and Steel Mills & $w$ & w & 0 & 0 & 95 & w & 81 & 7.9 \\
\hline 3313 & Electrometalurgical Products & $w$ & 0 & 0 & 0 & 0 & * & $w$ & 15.7 \\
\hline 3321 & Gray and Ductlle Iron Foundries & $w$ & 0 & 0 & 0 & w & • & 1 & 19.7 \\
\hline 3331 & Primary Copper.$\ldots \ldots \ldots \ldots$ & 9 & 0 & 0 & $\mathbf{0}$ & w & 0 & w & 1.4 \\
\hline 3334 & Primary Aluminum & $w$ & 0 & 0 & 0 & 11 & $w$ & w & 5.1 \\
\hline 3339 & Primary Nonferrous Metals, nec. & 52 & 0 & 0 & 0 & W & W & W & 4.8 \\
\hline 3353 & Aluminum Sheet, Plate, and Foil & $w$ & 0 & 0 & 0 & $w$ & $w$ & 27 & 3.7 \\
\hline 34 & Fabricated Metal Products . . & $w$ & Q & 0 & Q & 124 & 13 & 13 & 22.9 \\
\hline 35 & Industrial Machinery and Equipment ........ & 447 & 0 & 0 & 0 & 82 & w & W & 22.5 \\
\hline 357 & Computer and Office Equipment & 38 & 0 & 0 & 0 & 2 & 0 & 1 & 20.2 \\
\hline 36 & Electronic and Other Electric Equipment & 504 & 0 & 0 & 0 & 59 & 14 & 8 & 14.5 \\
\hline 37 & Transportation Equipment.$\ldots \ldots \ldots \ldots$ & w & w & w & 0 & 52 & 8 & 25 & 7.8 \\
\hline 3711 & Motor Vehicles and Car Bodies .... & 103 & 0 & w & 0 & 14 & w & w & 5.9 \\
\hline 3714 & Motor Vehicle Parts and Accessories. & 93 & 0 & 0 & 0 & w & w & 1 & 11.3 \\
\hline 38 & Instruments and Related Products..$\ldots \ldots \ldots$ & 169 & 0 & 0 & 0 & 12 & 5 & 1 & 21.5 \\
\hline 3841 & Surglcal and Medical Instruments & 18 & 0 & 0 & 0 & 1 & • & * & 23.7 \\
\hline 39 & Misc. Manufacturing Industries $\ldots \ldots \ldots \ldots$ & 65 & 0 & 0 & 0 & w & $Q$ & • & 24.4 \\
\hline & Total $\ldots \ldots \ldots \ldots \ldots \ldots \ldots$ & w & w & 112 & 171 & 2,599 & 2,553 & 1,412 & 4.6 \\
\hline
\end{tabular}

See footnotes at end of table. 
Table A46. Total Expenditures for Purchased Electricity, Steam, and Natural Gas by Type of Supplier, Census Region, Industry Group, and Selected Industries, 1991 (Continued)

(Estimates in Million Dollars)

\begin{tabular}{|c|c|c|c|c|c|c|c|c|c|}
\hline \multirow[b]{2}{*}{$\begin{array}{l}\text { SIC } \\
\text { Code }\end{array}$} & \multirow[b]{2}{*}{$\begin{array}{l}\text { Industry Groups } \\
\text { and Industry }\end{array}$} & \multicolumn{2}{|c|}{ Electricity } & \multicolumn{2}{|c|}{ Steam } & \multicolumn{3}{|c|}{ Natural Gas } & \multirow{2}{*}{$\begin{array}{c}\text { RSE } \\
\text { Row } \\
\text { Factors }\end{array}$} \\
\hline & & $\begin{array}{c}\text { Utility } \\
\text { Supplier }\end{array}$ & $\begin{array}{l}\text { Nonutility } \\
\text { Supplier }\end{array}$ & $\begin{array}{c}\text { Utility } \\
\text { Supplier }\end{array}$ & $\begin{array}{l}\text { Nonutility } \\
\text { Supplier }\end{array}$ & $\begin{array}{c}\text { Utility } \\
\text { Supplierb }\end{array}$ & \begin{tabular}{|c|} 
Transmission \\
Pipelines
\end{tabular} & $\begin{array}{c}\text { Other } \\
\text { Supplier }\end{array}$ & \\
\hline & & \multicolumn{7}{|c|}{ West Census Region } & \\
\hline & RSE Column Factors: & 0.5 & 1.6 & 3.0 & 0.8 & 0.7 & 1.0 & 0.8 & \\
\hline 20 & Food and Kindred Products & $w$ & $w$ & 0 & 17 & 192 & 47 & 104 & 15.5 \\
\hline 2011 & Meat Packing Plants . . . . . . . . & w & w & 0 & 0 & w & w & w & 19.1 \\
\hline 2033 & 3 Canned Fruits and Vegetables. & 43 & * & 0 & 0 & 17 & 3 & 28 & 14.4 \\
\hline 2037 & Frozen Fruits and Vegetables. & w & w & 0 & w & 29 & w & $w$ & 24.9 \\
\hline 2046 & Wet Com Milling .......... & $w$ & W & 0 & w & w & 0 & $w$ & 24.6 \\
\hline 2051 & Bread, Cake, and Related Products & 28 & 0 & 0 & 0 & 15 & 2 & $\because$ & 31.5 \\
\hline 2063 & $B$ Beet Sugar $\ldots \ldots \ldots \ldots \ldots \ldots$ & w & 0 & 0 & 0 & w & $\bar{w}$ & w & 16.7 \\
\hline 2075 & Soybean Oil Mills .......... & 0 & 0 & 0 & 0 & 0 & 0 & 0 & NF \\
\hline 2082 & Malt Beverages .......... & 29 & 0 & 0 & 0 & w & w & w & 20.4 \\
\hline 21 & Tobacco Products .... & 0 & 0 & 0 & 0 & 0 & 0 & 0 & NF \\
\hline 22 & Textile Mill Products.$\ldots \ldots \ldots$ & 20 & 0 & 0 & 0 & 16 & 0 & 3 & 37.8 \\
\hline 23 & Apparel and Other Textile Products & NA & NA & NA & NA & NA & NA & NA & 42.3 \\
\hline 24 & Lumber and Wood Products $\ldots .$. & w & $\mathbf{Q}$ & 0 & 17 & 17 & $w$ & w & 37.4 \\
\hline 25 & Furniture and Fixtures .... & 31 & 0 & 0 & 0 & 6 & 0 & 0 & 40.6 \\
\hline 26 & Paper and Allied Products... & w & w & w & w & w & w & 166 & 11.6 \\
\hline 2611 & Pulp Mills . . . . . . . & w & w & 0 & 0 & w & w & w & 34.7 \\
\hline 2621 & Paper Mills. & 230 & 0 & 0 & 17 & w & w & 88 & 8.4 \\
\hline 2631 & Paperhoard Mills & w & $w$ & $w$ & w & 19 & 0 & 72 & 11.8 \\
\hline 27 & Printing and Publishing $\ldots \ldots \ldots$ & NA & NA & NA & NA & NA & NA & NA & 36.7 \\
\hline 28 & Chemicals and Allied Products . . . & w & w & 0 & 4 & 133 & 7 & 84 & 19.9 \\
\hline 2812 & Alkalies and Chlorine ..... & w & 0 & 0 & : & w & 0 & w & 29.7 \\
\hline 2813 & 3 Industrial Gases .... & 89 & 0 & 0 & 0 & $\because$ & 0 & $w$ & 15.1 \\
\hline 2819 & Industrial Inorganic Chemicals, nec & 109 & * & 0 & w & 10 & $\mathrm{Q}$ & $w$ & 14.7 \\
\hline 2821 & Plastics Materials and Resins . ... & 14 & 0 & 0 & * & Q & • & $\mathbf{Q}$ & 24.9 \\
\hline 2822 & Synthetic Rubber $\ldots \ldots \ldots \ldots \ldots$ & $\because$ & 0 & 0 & 0 & - & 0 & 0 & 36.8 \\
\hline 2823 & Cellulosic Manmade Fibers ..... & 0 & 0 & 0 & 0 & 0 & 0 & 0 & NF \\
\hline 2824 & Organic Fibers, Noncellulosic ...... & 0 & 0 & 0 & 0 & 0 & 0 & 0 & NF \\
\hline 2865 & Cyclic Crudes and Intermediates.. & w & 0 & 0 & w & - & 0 & 0 & 33.5 \\
\hline 2869 & Industrial Organic Chemicals, nec & 7 & 0 & 0 & : & w & 0 & $w$ & 24.6 \\
\hline 2873 & Nitrogenous Fertilizers & w & w & 0 & * & w & w & w & 48.7 \\
\hline 2874 & Phosphatic Fertilizers & w & 0 & 0 & 0 & w & 0 & 0 & 57.4 \\
\hline 29 & Petroleum and Coal Products & 364 & w & 0 & w & 152 & 54 & 96 & 5.8 \\
\hline 2911 & Petroleum Refining ...... & w & w & 0 & $w$ & 129 & 51 & 83 & 4.4 \\
\hline 30 & Rubber and Misc. Plastics Products & 209 & 0 & 0 & $\because$ & W & Q & w & 21.2 \\
\hline 3011 & Tires and Inner Tubes. . . . . . . . & w & 0 & 0 & - & w & 0 & 0 & 10.1 \\
\hline 308 & Miscellaneous Plastic Products, nec & 187 & 0 & 0 & 0 & 18 & $Q$ & $w$ & 36.2 \\
\hline 31 & Leather and Leather Products ... & 4 & 0 & 0 & 0 & Q & 0 & w & 49.0 \\
\hline 32 & Stone, Clay and Glass Products & w & w & 0 & w & 69 & 22 & 50 & 14.1 \\
\hline 3211 & $\ldots \ldots \ldots \ldots \ldots \ldots$ & 10 & 0 & 0 & w & w & $\bar{W}$ & w & 6.6 \\
\hline 3221 & Glass Containers ............. & 56 & 0 & 0 & 0 & $w$ & $\ddot{w}$ & 20 & 12.6 \\
\hline 3229 & Pressed and Blown Glass, nec & w & 0 & 0 & 0 & $\because$ & $\ddot{w}$ & 0 & 17.9 \\
\hline 3241 & Cement, Hydraulic .. & 109 & 0 & 0 & 0 & 13 & $\mathrm{a}$ & w & 38.7 \\
\hline 3274 & Lime & w & 0 & 0 & 0 & * & $\bar{w}$ & $\because$ & 28.6 \\
\hline 3296 & Mineral Wool & 20 & 0 & 0 & 0 & w & w & w & 2.8 \\
\hline 33 & Primary Metal Industries & w & w & 0 & w & w & w & 71 & 11.3 \\
\hline 3312 & Blast Fumaces and Steel Mills . . & 89 & 0 & 0 & 0 & $w$ & $w$ & 25 & 10.5 \\
\hline 3313 & Electrometalurgical Products ... & 0 & 0 & 0 & 0 & 0 & 0 & 0 & NF \\
\hline 3321 & Gray and Ductile Iron Foundries & 7 & 0 & 0 & 0 & w & 0 & : & 41.8 \\
\hline 3331 & Primary Copper & w & $\mathbf{0}$ & 0 & 0 & w & $w$ & w & 1.4 \\
\hline 3334 & 4 Primary Aluminum & 515 & 0 & 0 & 0 & 5 & $w$ & w & 5.5 \\
\hline 3339 & Primary Nonferrous Metals, nec & 28 & 0 & 0 & 0 & w & $\because$ & w & 1.4 \\
\hline 3353 & 3 Aluminum Sheet, Plate, and Foil & w & 0 & 0 & 0 & 0 & 0 & W & 1.6 \\
\hline 34 & Fabricated Metal Products ...... . & 216 & 0 & 0 & 0 & 57 & w & w & 24.2 \\
\hline 35 & Industrial Machinery and Equipment & 266 & 0 & 0 & 0 & 30 & $w$ & w & 38.6 \\
\hline 357 & Computer and Office Equipment & 122 & 0 & 0 & 0 & 6 & $w$ & w & 22.8 \\
\hline 36 & Electronic and Other Electric Equipment & $w$ & $\mathbf{Q}$ & $Q$ & 0 & 33 & 1 & 2 & 25.0 \\
\hline
\end{tabular}

See footnotes at end of table. 
Table A46. Total Expenditures for Purchased Electricity, Steam, and Natural Gas by Type of Supplier, Census Region, Industry Group, and Selected Industries, 1991 (Continued)

(Estimates in Million Dollars)

\begin{tabular}{|c|c|c|c|c|c|c|c|c|c|}
\hline \multirow[b]{2}{*}{$\begin{array}{c}\text { SIC } \\
\text { Code* } \\
\end{array}$} & \multirow[b]{2}{*}{$\begin{array}{l}\text { Industry Groups } \\
\text { and Industry }\end{array}$} & \multicolumn{2}{|c|}{ Electricity } & \multicolumn{2}{|c|}{ Steam } & \multicolumn{3}{|c|}{ Natural Gas } & \multirow[b]{2}{*}{$\begin{array}{c}\text { RSE } \\
\text { Row } \\
\text { Factors }\end{array}$} \\
\hline & & $\begin{array}{c}\text { Utility } \\
\text { Supplier }\end{array}$ & $\begin{array}{l}\text { Nonutility } \\
\text { Supplier }\end{array}$ & $\begin{array}{c}\text { Utility } \\
\text { Supplier }\end{array}$ & $\begin{array}{l}\text { Nonutility } \\
\text { Supplier }\end{array}$ & $\begin{array}{c}\text { Utility } \\
\text { Supplier }\end{array}$ & $\begin{array}{c}\text { Transmission } \\
\text { Pipelines }\end{array}$ & $\begin{array}{c}\text { Other } \\
\text { Supplier }\end{array}$ & \\
\hline & & \multicolumn{7}{|c|}{ West Census Region } & \\
\hline & ASE Column Factors: & 0.5 & 1.6 & 3.0 & 0.8 & 0.7 & 1.0 & 0.8 & \\
\hline 37 & Transportation Equipment & W & $w$ & 0 & w & 42 & W & W & 19.3 \\
\hline 3711 & Motor Vehicles and Car Bodies & W & 0 & 0 & 0 & W & 0 & W & 10.0 \\
\hline 3714 & Motor Vehicle Parts and Accessories . . . . . . & 20 & 0 & 0 & 0 & 5 & 0 & 2 & 26.1 \\
\hline 38 & Instruments and Related Products $\ldots \ldots \ldots$. & 209 & 0 & • & 0 & 17 & W & W & 19.1 \\
\hline 3841 & Surgical and Medlcal Instruments ....... & 18 & 0 & 0 & 0 & 2 & $*$ & 0 & 28.1 \\
\hline \multirow[t]{2}{*}{39} & Misc. Manufacturing Industries . . . . . . . . & 28 & 0 & $\bullet$ & 0 & W & " & $\cdot$ & 32.6 \\
\hline & Total $\ldots \ldots \ldots \ldots \ldots \ldots \ldots \ldots$ & W & W & W & 94 & 961 & 210 & 615 & 7.7 \\
\hline
\end{tabular}

\footnotetext{
- See Appendices B and F for descriptions of the Standard Industrial Classification system.

"A "Utility" is a company that produces and/or delivers electricity and/or natural gas, and is legally obligated to provide service to the public within its franchise area.

Includes independent power producers, small power producers, and cogenerators not located at the establishment site.

- Other suppllers of natural gas include such sources as brokers and producers.

NF=No applicable RSE row/column factor.

- Estlmate less than 0.5. Data are included in higher level totals.

$W=$ Withheld to avoid disciosing data for individual establishments. Data are included in higher level totals.

$\mathrm{Q}=$ Withheld because Relative Standard Error is greater than 50 percent. Data are included in higher level totals.

$N A=N o t$ avallable. Data are included in higher level tables.

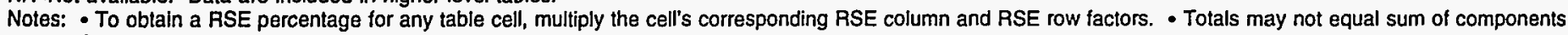
because of Independent rounding.

Source: Energy Information Administration, Office of Energy Markets and End Use, Energy End Use and Integrated Statistics Division, Form ElA-846, "1991

Manufacturing Energy Consumption Survey."
} 
Table A47. Average Prices of Purchased Electricity, Steam, and Natural Gas by Type of Supplier, Census Region, Industry Group, and Selected Industries, 1991 (Estimates in Dollars per Physical Units)

\begin{tabular}{|c|c|c|c|c|c|c|c|c|c|}
\hline \multirow[b]{2}{*}{$\begin{array}{c}\text { SIC } \\
\text { Code }^{\circ}\end{array}$} & \multirow[b]{2}{*}{$\begin{array}{l}\text { Industry Groups } \\
\text { and Industry }\end{array}$} & \multicolumn{2}{|c|}{$\begin{array}{c}\text { Electricity } \\
\text { (Million kWh) }\end{array}$} & \multicolumn{2}{|c|}{$\begin{array}{c}\text { Steam } \\
\text { (Billion Btu) }\end{array}$} & \multicolumn{3}{|c|}{$\begin{array}{l}\text { Natural Gas } \\
(1000 \mathrm{cu} \mathrm{ft})\end{array}$} & \multirow{2}{*}{$\begin{array}{c}\text { RSE } \\
\text { Row } \\
\text { Factors }\end{array}$} \\
\hline & & $\begin{array}{c}\text { Utility } \\
\text { Supplier }\end{array}$ & $\begin{array}{l}\text { Nonutility } \\
\text { Supplier }\end{array}$ & $\begin{array}{c}\text { Utility } \\
\text { Supplier }\end{array}$ & $\begin{array}{l}\text { Nonutility } \\
\text { Supplier }\end{array}$ & $\begin{array}{c}\text { Utility } \\
\text { Suppliers }\end{array}$ & $\begin{array}{c}\text { Transmission } \\
\text { Pipelines }\end{array}$ & $\begin{array}{l}\text { Other } \\
\text { Supplier }^{\text {t }}\end{array}$ & \\
\hline
\end{tabular}

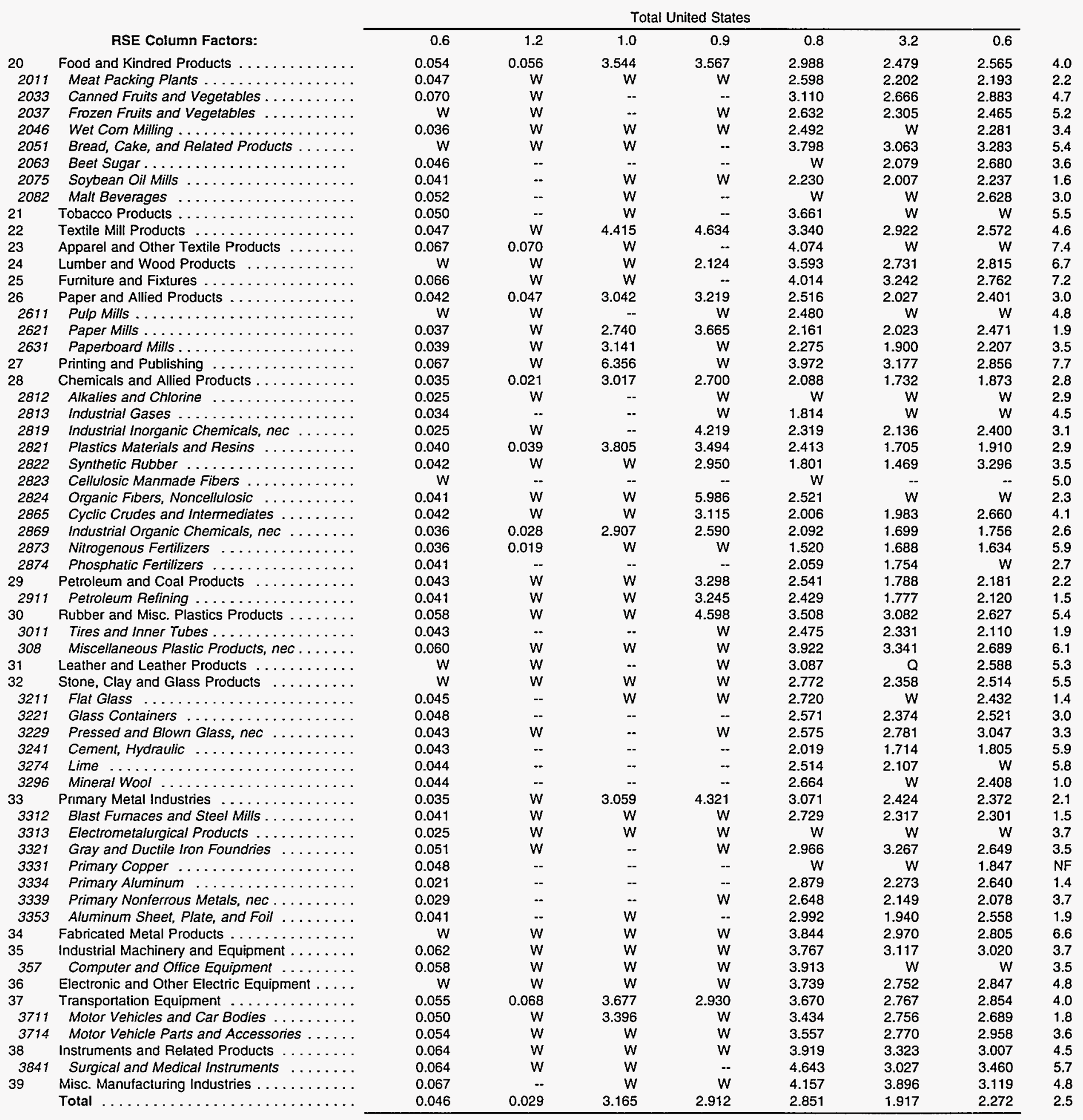


Table A47. Average Prices of Purchased Electricity, Steam, and Natural Gas by Type of Supplier, Census Region, Industry Group, and Selected Industries, 1991 (Continued)

(Estimates in Dollars per Physical Units)

\begin{tabular}{|c|c|c|c|c|c|c|c|c|c|}
\hline \multirow[b]{2}{*}{$\begin{array}{l}\text { SIC } \\
\text { Code" }\end{array}$} & \multirow[b]{2}{*}{$\begin{array}{l}\text { Industry Groups } \\
\text { and Industry }\end{array}$} & \multicolumn{2}{|c|}{$\begin{array}{c}\text { Electricity } \\
\text { (Million kWh) }\end{array}$} & \multicolumn{2}{|c|}{$\begin{array}{c}\text { Steam } \\
\text { (Billion Btu) }\end{array}$} & \multicolumn{3}{|c|}{$\begin{array}{l}\text { Natural Gas } \\
(1000 \mathrm{cu} \mathrm{ft})\end{array}$} & \multirow{2}{*}{$\begin{array}{c}\text { RSE } \\
\text { Row } \\
\text { Factors }\end{array}$} \\
\hline & & $\begin{array}{c}\text { Utility } \\
\text { Supplier }\end{array}$ & $\begin{array}{l}\text { Nonutility } \\
\text { Supplier }\end{array}$ & $\begin{array}{l}\text { Utility } \\
\text { Supplierb }\end{array}$ & $\begin{array}{l}\text { Nonutility } \\
\text { Supplier }\end{array}$ & $\begin{array}{l}\text { Utility } \\
\text { Supplierb }\end{array}$ & $\begin{array}{c}\text { Transmission } \\
\text { Pipelines }\end{array}$ & $\begin{array}{l}\text { Other } \\
\text { Supplier }\end{array}$ & \\
\hline & & \multicolumn{7}{|c|}{ Northeast Census Region } & \\
\hline & RSE Column Factor: & 0.9 & 0.7 & 0.8 & 0.6 & 0.9 & 4.4 & $\overline{0.8}$ & \\
\hline 20 & Food and Kindred Products & 0.072 & $w$ & w & $w$ & 3.745 & 3.372 & 3.149 & 8.5 \\
\hline 2011 & Meat Packing Plants .... & 0.060 & -. & -- & -. & $w$ & -- & W & 4.6 \\
\hline 2033 & Canned Fruits and Vegetables. & 0.074 & -- & -- & -- & 3.700 & 3.918 & 3.753 & 5.5 \\
\hline 2037 & Frozen Fruits and Vegetables & w & -- & .- & w & 3.225 & - & w & 4.3 \\
\hline 2046 & Wet Com Milling . . . . . . . . & 0.058 & .. & -- & -- & w & -. & -. & 2.7 \\
\hline 2051 & Bread, Cake, and Related Products & 0.080 & w & .- & .. & 3.543 & 3.251 & w & 4.3 \\
\hline 2063 & Boet Sugar . . . . . . . . . . . . . & -. & .. & -- & -- & -. & -. & -. & NF \\
\hline 2075 & Soybean Oll Mills $\ldots \ldots \ldots \ldots$ & -- & -- & -- & -. & -- & -. & - & NF \\
\hline 2082 & Mall Beverages. & 0.063 & -. & -- & -- & w & w & w & 4.3 \\
\hline 21 & Tobacco Products & NA & NA & NA & NA & NA & NA & NA & 10.4 \\
\hline 22 & Textile Mill Products & w & w & 3.864 & 5.060 & 4.505 & 3.045 & 3.167 & 9.5 \\
\hline 23 & Apparel and Other Textile Products & 0.098 & W & w & -- & 4.765 & $\cdots$ & w & 4.3 \\
\hline 24 & Lumber and Wood Products & NA & NA & NA & NA & NA & NA & NA & 8.5 \\
\hline 25 & Furniture and Fixtures .... & w & w & -- & -- & 4.659 & w & 3.637 & 5.3 \\
\hline 26 & Paper and Allied Products . & 0.064 & w & 4.083 & 2.446 & w & $w$ & 3.001 & 4.0 \\
\hline 2611 & Pulp MIIIs $\ldots \ldots \ldots \ldots \ldots$ & w & W & -- & w & -- & - & -. & 1.2 \\
\hline 2621 & Paper Mills . & 0.060 & w & 3.495 & 2.825 & 3.130 & w & 2.917 & 1.9 \\
\hline 2631 & Paperboard Mills . . . . . . . . & 0.068 & -. & W & W & 2.730 & 2.882 & w & 4.3 \\
\hline 27 & Printing and Publishing.$\ldots \ldots \ldots$. & 0.083 & W & 8.938 & -. & 4.809 & W & w & 7.8 \\
\hline 28 & Chemicals and Allied Products .. & 0.052 & 0.056 & w & 6.053 & 3.566 & 2.998 & 3.211 & 3.7 \\
\hline 2812 & Alkalles and Chlorine ...... & w & .. & -. & -. & .. & w & -. & 2.6 \\
\hline 2813 & Industrial Gases . . . . . . & 0.039 & -- & -- & -- & w & .. & - & 2.7 \\
\hline 2819 & Industrial Inorganic Chemicals, nec & 0.062 & .. & .- & w & 3.829 & 3.645 & 2.702 & 4.6 \\
\hline 2821 & Plastlcs Materials and Resins . . . . & 0.059 & w & w & 3.901 & 3.401 & 3.043 & 3.297 & 2.5 \\
\hline 2822 & Synthetic Rubber ....... & w & -- & -- & w & w & w & w & 2.3 \\
\hline 2823 & Cellulosic Manmade Fibers & -. & -- & .- & .. & -- & . & -. & $\mathrm{NF}$ \\
\hline 2824 & Organic Fibers, Noncellulosic & 0.063 & -- & -- & -- & w & w & ... & 5.8 \\
\hline 2865 & Cyclic Crudes and Intermediates ... & 0.064 & w & .- & w & w & 2.993 & $w$ & 4.9 \\
\hline 2869 & Industrial Organic Chemicals, nec . . . . . . & 0.029 & w & w & $w$ & 3.486 & 3.688 & 3.065 & 2.0 \\
\hline 2873 & Nitrogenous Fertilizers $\ldots \ldots$ & w & w & -- & w & w & $\cdots$ & w & 3.0 \\
\hline 2874 & Phosphatic Fertilizers & -. & -- & .. & -- & -- & $\ldots$ & - & NF \\
\hline 29 & Petroleum and Coal Products & 0.055 & w & w & w & 3.387 & w & w & 2.9 \\
\hline 2911 & Petroleum Relining $\ldots \ldots \ldots \ldots$ & 0.049 & -- & w & -. & 2.907 & 2.735 & w & 1.3 \\
\hline 30 & Rubber and Misc. Plastics Products .. & 0.075 & .- & $w$ & W & 4.655 & 3.688 & 3.517 & 6.8 \\
\hline 3011 & Tres and Inner Tubes . . . . . . . . . & 0.040 & .- & -. & -- & 3.294 & - & w & 3.3 \\
\hline 308 & Miscellaneous Plastic Products, nec. & 0.076 & -- & w & w & 4.881 & w & w & 7.5 \\
\hline 31 & Leather and Leather Products ... & 0.087 & -- & -- & -- & 4.974 & $Q$ & - & 4.4 \\
\hline 32 & Stone, Clay and Glass Products & w & w & -. & w & 3.344 & 3.097 & 2.994 & 5.4 \\
\hline 3211 & Flat Glass $\ldots \ldots \ldots \ldots \ldots \ldots$ & w & .. & .- & -. & - & w & w & 1.3 \\
\hline 3221 & Glass Containers & 0.057 & -. & .. & -- & 3.647 & w & 2.997 & 2.5 \\
\hline 3229 & Pressed and Blown Glass, nec. & 0.054 & w & .- & w & 2.596 & 3.247 & 2.968 & 2.4 \\
\hline 3241 & Cement, Hydraulic . . . . . . . & 0.052 & -- & -- & -- & W & w & -. & 3.1 \\
\hline 3274 & 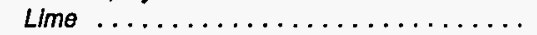 & 0.039 & -- & -- & -- & -- & $Q$ & -- & 5.7 \\
\hline 3296 & Mineral Wool & 0.058 & -. & -- & -- & 4.658 & W & 2.519 & 0.9 \\
\hline 33 & Primary Metal Industries $\ldots \ldots \ldots$ & 0.045 & w & -- & 5.251 & 3.255 & 3.376 & 2.982 & 3.2 \\
\hline 3312 & Blast Fumaces and Steel Mills. & 0.045 & -. & -- & w & 2.850 & 3.158 & 2.890 & 2.0 \\
\hline 3313 & Electrometalurgical Products .. & w & w & .. & w & 4.536 & - & -. & 3.1 \\
\hline 3321 & Gray and Ducllle Iron Foundries $\ldots \ldots \ldots$ & 0.071 & -- & -- & -- & 3.832 & w & 3.440 & 6.1 \\
\hline 3331 & Primary Copper $\ldots \ldots \ldots \ldots \ldots \ldots \ldots$ & w & -- & -- & -- & w & - & w & NF \\
\hline 3334 & Primary Aluminum ... & w & -. & -- & -- & w & - & -- & 1.1 \\
\hline 3339 & Primary Nonferrous Metals, nec. & 0.055 & -- & .- & -- & w & w & w & 2.1 \\
\hline 3353 & Aluminum Sheet, Plate, and Foil & 0.060 & -- & -- & -. & w & -- & w & 1.6 \\
\hline 34 & Fabricated Metal Products . . . . . . & 0.075 & .- & w & w & 4.388 & 4.771 & 3.124 & 5.3 \\
\hline 35 & Industrlal Machinery and Equipment . . . . . . . & 0.078 & -- & -- & w & 4.957 & 4.188 & 3.331 & 4.4 \\
\hline 357 & Computer and Office Equipment ...... & 0.070 & -- & -. & w & 4.186 & w & W & 3.1 \\
\hline 36 & Electronic and Other Electric Equipment . & 0.069 & -- & w & -- & 4.475 & 3.072 & 3.388 & 4.5 \\
\hline 37 & Transportation Equipment .... & 0.073 & w & -. & w & 3.804 & w & w & 3.5 \\
\hline 3711 & Motor Vehicles and Car Bodies & W & -- & -- & -- & w & $\ldots$ & w & 1.5 \\
\hline 3714 & Motor Vehicle Pants and Accessories & 0.071 & -- & -- & w & 4.020 & w & 3.834 & 2.8 \\
\hline 38 & Instruments and Related Products & 0.078 & w & -- & w & 4.099 & 3.342 & 3.730 & 4.6 \\
\hline 3841 & Surgical and Medical Instruments & 0.077 & w & .. & -. & 5.622 & w & w & 5.2 \\
\hline 39 & Mlsc. Manufacturing Industries . & 0.079 & -- & -. & w & 4.265 & $Q$ & w & 3.5 \\
\hline & Total $\ldots \ldots \ldots \ldots \ldots \ldots$ & 0.064 & 0.079 & w & 4.736 & 3.798 & 3.317 & 3.070 & 4.1 \\
\hline
\end{tabular}

See footnotes at end of table. 


\section{Table A47. Average Prices of Purchased Electricity, Steam, and Natural Gas by Type of Supplier, Census Region, Industry Group, and Selected Industries, 1991 (Continued)}

(Estimates in Dollars per Physical Units)

\begin{tabular}{|c|c|c|c|c|c|c|c|c|c|}
\hline \multirow[b]{2}{*}{$\begin{array}{l}\text { SIC } \\
\text { Code* }\end{array}$} & \multirow[b]{2}{*}{$\begin{array}{l}\text { Industry Groups } \\
\text { and Industry }\end{array}$} & \multicolumn{2}{|c|}{$\begin{array}{c}\text { Electricity } \\
\text { (Million kWh) }\end{array}$} & \multicolumn{2}{|c|}{$\begin{array}{c}\text { Steam } \\
\text { (Billion Btu) }\end{array}$} & \multicolumn{3}{|c|}{$\begin{array}{l}\text { Natural Gas } \\
(1000 \mathrm{cu} \mathrm{ft})\end{array}$} & \multirow[b]{2}{*}{$\begin{array}{c}\text { RSE } \\
\text { Row } \\
\text { Factors }\end{array}$} \\
\hline & & $\begin{array}{c}\text { Utility } \\
\text { Supplier }\end{array}$ & $\begin{array}{l}\text { Nonutility } \\
\text { Supplier }\end{array}$ & $\begin{array}{c}\text { Utility } \\
\text { Supplierb }\end{array}$ & $\begin{array}{l}\text { Nonutility } \\
\text { Supplier }\end{array}$ & $\begin{array}{c}\text { Utility } \\
\text { Supplier }\end{array}$ & $\begin{array}{c}\text { Transmission } \\
\text { Pipelines }\end{array}$ & $\begin{array}{c}\text { Other } \\
\text { Supplier }\end{array}$ & \\
\hline & & \multicolumn{7}{|c|}{ Midwest Census Region } & \\
\hline & RSE Column Factors: & 0.7 & 1.0 & 0.8 & 0.8 & 0.8 & 3.6 & 0.7 & \\
\hline 20 & Food and Kindred Products ... & 0.047 & 0.065 & 3.970 & 3.137 & 2.623 & 2.336 & 2.422 & 4.7 \\
\hline 2011 & Meat Packing Plants . . . . . . . & 0.045 & - & w & -- & 2.380 & 2.250 & 2.254 & 2.8 \\
\hline 2033 & Canned Fruits and Vegetables... & 0.059 & -. & - & - & 2.847 & 2.627 & 2.479 & 4.6 \\
\hline 2037 & Frozen Fruits and Vegetables & $w$ & -- & -- & -- & 2.686 & w & 2.624 & 7.3 \\
\hline 2046 & Wet Com Milling .......... & 0.036 & -- & $w$ & - & $w$ & $w$ & 2.269 & 3.3 \\
\hline 2051 & Bread, Cake, and Related Products & 0.055 & $w$ & w & -. & 3.625 & 2.984 & 2.870 & 4.8 \\
\hline 2063 & 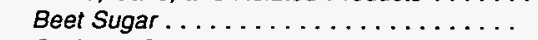 & w & -- & -- & .. & $w$ & w & w & 2.3 \\
\hline 2075 & Soybean Oil Mills ........... & 0.040 & -- & $w$ & .. & $w$ & $w$ & 2.328 & 1.9 \\
\hline 2082 & Malt Beverages & 0.045 & - & w & -. & 2.660 & w & w & 4.1 \\
\hline 21 & Tobacco Products & NA & NA & NA & NA & NA & NA & NA & 1.4 \\
\hline 22 & Textile Mill Products & NA & NA & NA & NA & NA & NA & NA & 3.8 \\
\hline 23 & Apparel and Other Textile Products & NA & NA & NA & NA & NA & NA & NA & 8.6 \\
\hline 24 & Lumber and Wood Products ...... & 0.058 & .. & w & -. & 3.910 & $Q$ & $w$ & 9.1 \\
\hline 25 & Furniture and Fixtures $\ldots \ldots \ldots \ldots$ & w & $w$ &.- & -- & 3.640 & 3.006 & 2.586 & 5.6 \\
\hline 26 & Paper and Allied Products & 0.042 & $w$ & 2.919 & $w$ & 2.808 & 2.520 & 2.431 & 2.7 \\
\hline 2611 & Pulp Mills . . . . . . . . & w & - & .- & -. & w & -. & w & 2.1 \\
\hline 2621 & Paper Mills . & 0.037 & $w$ & w & w & 2.482 & 2.506 & 2.424 & 1.7 \\
\hline 2631 & Paperboard Mills. & 0.041 & - & W & - & 2.480 & 2.406 & 2.199 & 3.5 \\
\hline 27 & Printing and Publishing $\ldots \ldots$ & 0.057 & -- & 5.349 & $w$ & 3.559 & 2.443 & 2.608 & 8.8 \\
\hline 28 & Chemicals and Allied Products. & 0.031 & $w$ & 3.249 & 3.518 & 2.486 & 1.891 & 2.177 & 4.8 \\
\hline 2812 & Alkalies and Chlorine ...... & w & - & -- & -. & .. & .. & w & 1.4 \\
\hline 2813 & Industrial Gases ....... & w & -. & - & w & w & .. & w & 3.3 \\
\hline 2819 & Industrial Inorganic Chemicals, nec & W & $w$ &.- & $w$ & 2.601 & 2.308 & 2.525 & 2.4 \\
\hline 2821 & Plastics Materials and Resins . ... & 0.042 & w & 3.594 & 3.298 & 2.600 & 2.990 & 1.950 & 2.8 \\
\hline 2822 & Synthetic Rubber . ........ & 0.035 & -- & -- & -- & 2.613 & -- & $w$ & 2.6 \\
\hline 2823 & Cellulosic Manmade Fibers & .. & -- & .. & -- & .. & -. & -. & NF \\
\hline 2824 & Organic Fibers, Noncellulosic .... & - & .. & .. & -. & -- & - & -- & $\mathrm{NF}$ \\
\hline 2865 & Cyclic Crudes and Intermediates & 0.045 & -- & -- & $w$ & 2.576 & $w$ & 2.570 & 4.4 \\
\hline 2869 & Industrial Organic Chemicals, nec & 0.047 & $w$ & w & w & 2.331 & w & 2.507 & 3.7 \\
\hline 2873 & Nitrogenous Fertilizers . . . . . . & w & $w$ & -- & w & W & w & w & 4.0 \\
\hline 2874 & Phosphatic Fertilizers & w & - & -- & -- & w & - & $w$ & 1.4 \\
\hline 29 & Petroleum and Coal Products & 0.042 & $w$ & $w$ & $w$ & 2.818 & $w$ & $\ddot{w}$ & 2.7 \\
\hline 2911 & Petroleum Refining . . . . . . . & 0.039 & $w$ & -- & $w$ & 2.643 & 1.940 & 2.217 & 1.4 \\
\hline 30 & Rubber and Misc. Plastics Products & 0.056 & w & -- & -. & 3.280 & 3.016 & 2.729 & 5.3 \\
\hline 3011 & Tires and Inner Tubes . . . . . . . . . & 0.043 & $-\cdot$ & -- & -- & 2.283 & w & w & 1.6 \\
\hline 308 & Miscellaneous Plastic Products, nec. & NA & NA & NA & NA & NA & NA & NA & 7.3 \\
\hline 31 & Leather and Leather Products . . . . . & w & w & .. & w & 4.039 & Q & $w$ & 4.7 \\
\hline 32 & Stone, Clay and Glass Products & 0.047 & -. & w & W & 2.925 & 2.575 & 2.435 & 3.5 \\
\hline 3211 & Flat Glass $\ldots \ldots \ldots \ldots$. & 0.049 & $\cdots$ & w & .. & 2.382 & -- & 2.132 & 1.3 \\
\hline 3221 & Glass Containers . & 0.045 & -- & -- & -- & 3.656 & $w$ & 2.063 & 3.4 \\
\hline 3229 & Pressed and Blown Glass, nec & 0.046 & -- & -- & -- & 2.919 & w & 3.405 & 2.4 \\
\hline 3241 & Cement, Hydraulic ........ & 0.043 & - &.- & -. & 2.328 & $Q$ & $w$ & 5.9 \\
\hline 3274 & Lime...$\ldots \ldots \ldots \ldots$ & 0.051 & -- & -- & .. & 2.542 & W & w & 5.0 \\
\hline 3296 & Mineral Wool ............. & 0.037 & -- & -- & -- & 2.831 & $w$ & 2.297 & 1.1 \\
\hline 33 & Primary Metal Industries . . . . . . & 0.041 & w & 3.059 & 4.593 & 3.113 & 2.325 & 2.291 & 2.1 \\
\hline 3312 & Blast Fumaces and Steel Mills. & 0.043 & W & $w$ & W & 2.823 & 2.136 & 2.231 & 1.4 \\
\hline 3313 & Electrometalurgical Products & 0.027 & w & w & w & w & .. & 2.789 & 2.4 \\
\hline 3321 & Gray and Ductile Iron Foundries & 0.050 & w & -- & w & 3.092 & 3.583 & 2.620 & 3.9 \\
\hline 3331 & Primary Copper...$\ldots \ldots \ldots$ & w & - & .. & -. & w & -- & -- & NF \\
\hline 3334 & Primary Aluminum & w & -- & -- & -. & $w$ & -. & $w$ & 1.3 \\
\hline 3339 & Primary Nonferrous Metals, nec. & 0.035 & -- & .. & w & $w$ & 2.447 & 2.318 & 2.1 \\
\hline 3353 & Aluminum Sheet, Plate, and Foil & 0.043 & -- & $w$ & -. & w & $w$ & 2.172 & 1.8 \\
\hline 34 & Fabricated Metal Products . . . . . . & 0.060 & w & w & w & 3.492 & 2.667 & 2.632 & 7.7 \\
\hline 35 & Industrial Machinery and Equipment & 0.056 & w & w & -- & 3.531 & 2.678 & 2.965 & 4.8 \\
\hline 357 & Computer and Office Equipment & 0.048 & w & w & -- & 3.117 & a & w & 5.0 \\
\hline 36 & Electronic and Other Electric Equipment & 0.050 & w & w & w & 3.402 & 2.744 & 2.825 & 3.7 \\
\hline 37 & Transportation Equipment ..... & 0.052 & W & W & 1.962 & 3.542 & 2.700 & 2.751 & 4.2 \\
\hline 3711 & Motor Vehicles and Car Bodies & 0.051 & w & W & W & 3.524 & 2.665 & 2.559 & 1.6 \\
\hline 3714 & Motor Vehicle Parts and Accessories & 0.053 & w & w & w & 3.606 & 2.728 & 2.839 & 4.0 \\
\hline 38 & Instruments and Related Products $\ldots \ldots \ldots$. & 0.055 & -- & w & -- & 3.503 & Q & 2.706 & 6.5 \\
\hline 3841 & Surgical and Medical Instruments & 0.049 & - & w & -- & 4.051 & Q & -- & 4.1 \\
\hline 39 & Misc. Manufacturing Industries $\ldots \ldots \ldots \ldots$ & 0.063 & -- & w & -- & w & 3.860 & w & 7.0 \\
\hline & Total $\ldots \ldots \ldots \ldots \ldots \ldots$ & 0.046 & 0.051 & 3.696 & 2.835 & 3.026 & 2.223 & 2.398 & 3.2 \\
\hline
\end{tabular}

See footnotes at end of table. 
Table A47. Average Prices of Purchased Electricity, Steam, and Natural Gas by Type of Supplier, Census Region, Industry Group, and Selected Industries, 1991 (Continued)

(Estimates in Dollars per Physical Units)

\begin{tabular}{|c|c|c|c|c|c|c|c|c|c|}
\hline \multirow[b]{2}{*}{$\begin{array}{l}\text { SIC } \\
\text { Code }^{\circ}\end{array}$} & \multirow[b]{2}{*}{$\begin{array}{l}\text { Industry Groups } \\
\text { and Industry }\end{array}$} & \multicolumn{2}{|c|}{$\begin{array}{c}\text { Electricity } \\
\text { (Million } k W h \text { ) }\end{array}$} & \multicolumn{2}{|c|}{$\begin{array}{c}\text { Steam } \\
\text { (Billion Btu) }\end{array}$} & \multicolumn{3}{|c|}{$\begin{array}{l}\text { Natural Gas } \\
(1000 \mathrm{cu} \mathrm{ft})\end{array}$} & \multirow[b]{2}{*}{$\begin{array}{c}\text { RSE } \\
\text { Row } \\
\text { Factors }\end{array}$} \\
\hline & & $\begin{array}{c}\text { Utility } \\
\text { Supplier }\end{array}$ & $\begin{array}{l}\text { Nonutility } \\
\text { Supplier }\end{array}$ & $\begin{array}{c}\text { Utility } \\
\text { Supplier }\end{array}$ & $\begin{array}{l}\text { Nonutility } \\
\text { Supplier }\end{array}$ & $\begin{array}{c}\text { Utillty } \\
\text { Supplier }\end{array}$ & $\begin{array}{c}\text { Transmission } \\
\text { Pipelines } \\
\end{array}$ & $\begin{array}{c}\text { Other } \\
\text { Supplier }\end{array}$ & \\
\hline & & \multicolumn{7}{|c|}{ South Census Region } & \\
\hline & RSE Column Factors: & 0.7 & 0.8 & 0.6 & 0.9 & 0.9 & 4.4 & 0.9 & \\
\hline 20 & Food and Kindred Products .. & 0.052 & $w$ & $w$ & $w$ & 3.126 & 2.341 & 2.365 & 2.9 \\
\hline 2011 & Meat Packing Plants . & 0.051 & w & -- & w & 3.711 & 1.977 & 1.884 & 3.5 \\
\hline 2033 & Canned Fruits and Vegetables. & 0.061 & - & - & -- & 3.055 & W & 2.555 & 5.8 \\
\hline 2037 & Frozen Fruits and Vegetables ......... & 0.056 & -. & -- & -. & 3.425 & w & W & 3.8 \\
\hline 2046 & Wot Com Milling . .......... & 0.038 & .. & -- & .. & w & $\mathrm{Q}$ & $w$ & 1.9 \\
\hline 2051 & Bread, Cake, and Related Products ....... & 0.053 & -- & -- & -- & 3.788 & 3.227 & 3.104 & 4.5 \\
\hline 2063 & 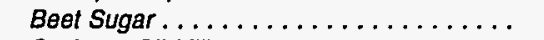 & W & -- & -- & -. & W & -- & - & 1.1 \\
\hline 2075 & Soybean Oll Mills . . . . . . . . . . . . & 0.043 & -- & -. & w & w & w & 2.062 & 1.4 \\
\hline 2082 & Malt Beverages ....................... & 0.048 & -- & -- & -- & 2.440 & w & 1.791 & 4.4 \\
\hline 21 & Tobacco Products $\ldots \ldots \ldots \ldots \ldots \ldots \ldots$ & 0.049 & -- & w & -. & 3.676 & w & w & 3.9 \\
\hline 22 & Textile Mill Products ................ & 0.045 & $w$ & 4.862 & 4.343 & 3.121 & 2.913 & 2.480 & 4.1 \\
\hline 23 & Apparel and Other Textle Products ......... & 0.062 & 0.069 & -. & -- & 3.865 & W & w & 7.5 \\
\hline 24 & Lumber and Wood Products $\ldots \ldots \ldots \ldots \ldots$ & 0.052 & .. & w & w & 3.280 & 2.898 & 2.295 & 5.0 \\
\hline 25 & Furnlture and Fixtures $\ldots \ldots \ldots \ldots \ldots \ldots$ & 0.062 & .- & w & -- & 4.181 & $\mathrm{a}$ & 3.045 & 4.4 \\
\hline 26 & Paper and Alled Products ............. & 0.038 & -. & $w$ & w & 2.243 & 1.891 & 2.074 & 2.2 \\
\hline 2611 & Pulp Mills ... & 0.048 & -- & -- & -- & 2.429 & w & w & 3.7 \\
\hline 2621 & Paper Mills . & 0.034 & -- & -- & w & 1.930 & 2.026 & 2.029 & 1.9 \\
\hline 2631 & Paperboard Mills.... & 0.039 & - & w & w & 2.167 & 1.767 & 1.975 & 2.5 \\
\hline 27 & Printing and Publishing $\ldots \ldots \ldots \ldots$ & 0.059 & - & $w$ & -. & 3.516 & $Q$ & $w$ & 4.4 \\
\hline 28 & Chemicals and Allied Products . ....... & 0.036 & 0.020 & 2.944 & 2.196 & 1.994 & 1.705 & 1.720 & 2.6 \\
\hline 2812 & Alkalies and Chlorine $\ldots . .$. & w & w & .. & w & w & w & w & 2.1 \\
\hline 2813 & Industrial Gases $\ldots \ldots \ldots \ldots \ldots \ldots$ & 0.032 & -. & -- & -- & w & w & $w$ & 3.3 \\
\hline 2819 & Industrial Inorganic Chemicals, nec & 0.036 & w & -- & w & 2.100 & 2.106 & 2.050 & 3.2 \\
\hline 2821 & Plastics Materials and Resins . . . . . & 0.036 & w & w & 3.383 & 2.097 & 1.684 & 1.736 & 2.6 \\
\hline 2822 & Synthetlc Rubber ........... & 0.041 & W & w & 2.906 & 1.709 & 1.464 & w & 3.6 \\
\hline 2823 & Cellulosic Manmade Fibers & $w$ & -- & -- & -- & w & -. & -. & 4.3 \\
\hline 2824 & Organic Fibers, Noncellulosic . . . . . . . . & 0.040 & W & w & 5.986 & 2.513 & w & $w$ & 1.9 \\
\hline 2865 & Cycllc Crudes and Intermedlates .......... & 0.039 & w & w & 2.639 & 1.823 & w & $w$ & 2.9 \\
\hline 2869 & Industrial Organic Chemicals, nec & 0.036 & w & 3.114 & 1.847 & 1.944 & 1.690 & 1.699 & 2.8 \\
\hline 2873 & Nitrogenous Fertilizers $\ldots \ldots \ldots$ & 0.036 & -- & w & -. & w & w & 1.620 & 4.3 \\
\hline 2874 & Phosphatlc Fertilizers & w & -- & -- & -. & 3.176 & 1.754 & w & 1.1 \\
\hline 29 & Petroleum and Coal Products .. & 0.037 & $w$ & $w$ & 2.401 & 1.907 & 1.702 & 1.818 & 2.2 \\
\hline 2911 & Petroleum Refining . . . . . . . . & 0.037 & -- & W & 2.301 & 1.838 & 1.701 & 1.811 & 1.3 \\
\hline 30 & Rubber and Misc. Plastics Products & 0.049 & -- & -- & 4.789 & 3.232 & 2.880 & 2.324 & 4.3 \\
\hline 3011 & Tires and Inner Tubes . . . . . . . . . . & 0.042 & -- & -- & W & 2.450 & W & 2.242 & 1.7 \\
\hline 308 & Miscellaneous Plastic Products, nec...... . & 0.050 & .- & -- & w & 3.765 & 3.424 & 2.248 & 4.9 \\
\hline 31 & Leather and Leather Products & 0.067 & -. & -- & -. & w & w & -- & 6.8 \\
\hline 32 & Stone, Clay and Glass Products & 0.044 & w & .. & -. & 2.489 & 2.066 & 2.224 & 2.8 \\
\hline 3211 & Flat Glass .... & 0.039 & .. & .. & -- & 2.650 & w & 2.269 & 1.3 \\
\hline 3221 & Glass Containers & 0.043 & .- & -- & .. & 2.022 & $w$ & 2.349 & 3.1 \\
\hline 3229 & Pressed and Blown Glass, nec . ..... & 0.038 & -- & -- & -. & 2.470 & w & 2.621 & 2.4 \\
\hline 3241 & Coment, Hydraullc $\ldots \ldots \ldots \ldots \ldots \ldots$ & 0.037 & -- & -- & -- & 1.750 & $\mathbf{Q}$ & 1.615 & 2.4 \\
\hline 3274 & Lime $\ldots \ldots \ldots \ldots \ldots \ldots \ldots \ldots$ & 0.045 & -. & -- & -. & 2.408 & $\mathbf{w}$ & w & 3.8 \\
\hline 3296 & Mineral Wool & 0.043 & -- & -- & -- & 2.231 & $w$ & w & 1.0 \\
\hline 33 & Primary Metal Industries & 0.032 & w & -- & .- & 2.807 & 2.141 & 2.462 & 2.2 \\
\hline 3312 & Blast Fumaces and Steel Mills . . . . . . . . . . & 0.037 & w & -- & -. & 2.570 & 2.085 & 2.400 & 1.7 \\
\hline 3313 & Electromelalurgical Products . . . . . & 0.023 & -- & -- & -. & -- & w & W & 3.0 \\
\hline 3321 & Gray and Ductile Iron Foundries ... & 0.048 & -. & .. & -- & 2.705 & W & 2.591 & 4.2 \\
\hline 3331 & Primary Copper .......... & 0.044 & -. & -. & .. & w & -- & w & NF \\
\hline 3334 & Primary Aluminum & 0.023 & -- & -- & -- & 2.775 & w & 2.953 & 2.1 \\
\hline 3339 & Primary Nonferrous Metals, nec . . . . . . . . . & 0.030 & -. & -. & -. & W & 2.006 & w & 6.4 \\
\hline 3353 & Aluminum Sheet, Plate, and Foil & 0.039 & -. & -- & .- & 3.120 & 2.055 & 2.633 & 2.8 \\
\hline 34 & Fabricated Metal Products . . . . . . & w & w & - & w & 3.730 & 2.507 & 3.168 & 4.4 \\
\hline 35 & Industrial Machinery and Equipment . & 0.055 & -- & -- & -- & 3.563 & w & w & 6.2 \\
\hline 357 & Computer and Office Equipment & 0.047 & -- & -- & -- & 4.138 & -- & 2.849 & 4.4 \\
\hline 36 & Electronic and Other Electric Equipment ..... & 0.047 & -. & .. & -. & 3.377 & 2.690 & 2.325 & 4.2 \\
\hline 37 & Transportation Equipment ...... & 0.049 & $w$ & $w$ & -. & 3.432 & 3.003 & 2.815 & 2.7 \\
\hline 3711 & Motor Vehicles and Car Bodies ... & 0.043 & -. & W & -. & 3.231 & w & w & 2.1 \\
\hline 3714 & Motor Vehicle Parts and Accessories. & 0.048 & -. & -- & -. & 3.250 & w & 3.011 & 3.0 \\
\hline 38 & Instruments and Related Products . & 0.052 & -- & -- & -- & 3.383 & 3.169 & 2.226 & 5.4 \\
\hline 3841 & Surgical and Medical Instruments $\ldots \ldots \ldots$ & 0.054 & .. & -- & .. & 4.170 & 2.977 & w & 3.7 \\
\hline 39 & Misc. Manufacturing Industries $\ldots \ldots \ldots \ldots$ & 0.056 & -- & -- & -- & 3.480 & $\mathbf{Q}$ & w & 6.1 \\
\hline & Total & 0.042 & 0.020 & 2.790 & 2.380 & 2.490 & 1.776 & 1.919 & 2.2 \\
\hline
\end{tabular}

See footnotes at end of table. 


\section{Table A47. Average Prices of Purchased Electricity, Steam, and Natural Gas by Type of Supplier, Census Region, Industry Group, and Selected Industries, 1991 (Continued)}

(Estimates in Dollars per Physical Units)

\begin{tabular}{|c|c|c|c|c|c|c|c|c|c|}
\hline \multirow[b]{2}{*}{$\begin{array}{c}\text { SIC } \\
\text { Code" }\end{array}$} & \multirow[b]{2}{*}{$\begin{array}{l}\text { Industry Groups } \\
\text { and Industry }\end{array}$} & \multicolumn{2}{|c|}{$\begin{array}{c}\text { Electricity } \\
\text { (Million kWh) }\end{array}$} & \multicolumn{2}{|c|}{$\begin{array}{c}\text { Steam } \\
\text { (Billion Btu) }\end{array}$} & \multicolumn{3}{|c|}{$\begin{array}{l}\text { Natural Gas } \\
(1000 \mathrm{cu} \mathrm{ft})\end{array}$} & \multirow[b]{2}{*}{$\begin{array}{c}\text { RSE } \\
\text { Row } \\
\text { Factors }\end{array}$} \\
\hline & & $\begin{array}{c}\text { Utility } \\
\text { Supplier }\end{array}$ & $\begin{array}{l}\text { Nonutility } \\
\text { Supplier }\end{array}$ & $\begin{array}{c}\text { Utility } \\
\text { Supplier }^{b}\end{array}$ & $\begin{array}{l}\text { Nonutility } \\
\text { Supplier }\end{array}$ & $\begin{array}{c}\text { Utility } \\
\text { Supplier }\end{array}$ & $\begin{array}{c}\text { Transmission } \\
\text { Pipelines }\end{array}$ & $\begin{array}{c}\text { Other } \\
\text { Supplier }\end{array}$ & \\
\hline & & \multicolumn{7}{|c|}{ West Census Region } & \\
\hline & RSE Column Factors: & 0.9 & 0.8 & 0.4 & 0.8 & 1.0 & 4.6 & 0.8 & \\
\hline 20 & Food and Kindred Products & W & w & -- & 3.860 & 3.165 & 2.643 & 2.800 & 5.5 \\
\hline 2011 & Meat Packing Plants . . & 0.042 & W & -- & -- & W & W & W & 4.1 \\
\hline 2033 & Canned Fruits and Vegetables . . . . . . . . . & 0.079 & $w$ & -- & -- & 3.116 & 2.967 & 2.862 & 3.7 \\
\hline 2037 & Frozen Fruits and Vegetables . . . . . . . . & W & W & -. & W & 2.382 & W & W & 4.8 \\
\hline 2046 & Wet Com Milling . . . . . . . . . . . . . & W & W & - & W & W & .. & W & 1.8 \\
\hline 2051 & Bread, Cake, and Related Products . . . . . . & 0.071 & -- & -- & -. & 4.220 & 3.037 & 2.741 & 6.7 \\
\hline 2063 & Beet Sugar . . . . . . . . . . . . . . & 0.042 & -- & -- & $-\cdot$ & W & W & W & 3.4 \\
\hline 2075 & Soybean Oil Mills . . . . . . . . . . . . & -- & $\ldots$ & -- & 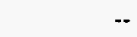 & -- & -. & -- & NF \\
\hline 2082 & Mált Beverages ................. & 0.057 & -- & -- & -- & $w$ & W & 2.933 & 2.7 \\
\hline 21 & Tobacco Products . . . . . . . . . . . . & -- &.- &.- & -- & -- &.$\cdot$ & -- & NF \\
\hline 22 & Textile Mill Products . . . . . . . . . . . . & 0.072 & 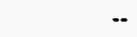 & -. & -- & 4.479 & -- & 3.136 & 5.2 \\
\hline 23 & Apparel and Other Textile Products ....... & NA & NA & NA & NA & NA & NA & NA & 10.0 \\
\hline 24 & Lumber and Wood Products $\ldots \ldots \ldots \ldots$ & 0.045 & W & -- & 2.079 & 3.282 & W & $W$ & 8.5 \\
\hline 25 & Furniture and Fixtures $\ldots \ldots \ldots \ldots \ldots$ & 0.102 & -. & -- & - & 4.629 & $\cdots$ & -. & 9.4 \\
\hline 26 & Paper and Allied Products . . . . . . . . . . . & W & $W$ & $w$ & 3.576 & W & $W$ & 2.456 & 2.6 \\
\hline 2611 & Pulp Mills . . . . . . . . . . . . . . . . & $W$ & W & -- & -- & W & $\mathbf{W}$ & W & 4.0 \\
\hline 2621 & Paper Mills . . . . . . . . . . . . . & 0.028 & -- & -- & 4.059 & 2.245 & W & 2.561 & 2.1 \\
\hline 2631 & Paperboard Mills . . . . . . . . . . . . . & 0.033 & $W$ & $w$ & 2.364 & 2.425 & -- & 2.325 & 3.0 \\
\hline 27 & Printing and Publishing $\ldots \ldots \ldots \ldots$ & NA & NA & NA & NA & NA & NA & NA & 8.9 \\
\hline 28 & Chemicals and Allied Products . . . . . . . . & W & W & $-\cdot$ & 3.655 & 1.682 & 2.776 & 2.183 & 6.9 \\
\hline 2812 & Alkalies and Chlorine . . . . . . . . . . & 0.022 & -- & -- & W & W & - & W & 2.1 \\
\hline 2813 & Industrial Gases . . . . . . . . . . . . . & 0.035 & -- & .. & -- & W &.- & W & 3.5 \\
\hline 2819 & Industrial Inorganic Chemicals, nec ...... & 0.025 & 0.089 &.- & W & 2.910 & $Q$ & $W$ & 3.1 \\
\hline 2821 & Plastics Materials and Resins . . . . . . . . & 0.076 & - & -- & W & 5.197 & $w$ & 1.904 & 5.8 \\
\hline 2822 & Synthetic Rubber . . . . . . . . . . & W & $\cdots$ & -- & -- & W & -- & -. & NF \\
\hline 2823 & Cellulosic Manmade Fibers . . . . . . . . . . & -- & $-\cdot$ & -. & -- & -- & -- & $-\cdot$ & NF \\
\hline 2824 & Organic Fibers, Noncellulosic . . . . . . . . & -- & -- & -- & -- & -- & -- & -- & NF \\
\hline 2865 & Cyclic Crudes and Intermediates . . . . . . . & W &.- & -- & W & W & -- & -. & 9.1 \\
\hline 2869 & Industrial Organic Chemicals, nec . . . . . . & 0.054 & $\cdots$ & -. & W & W & $-\cdot$ & W & 5.6 \\
\hline 2873 & Nitrogenous Fertilizers . . . . . . . . . . & $W$ & $W$ & -- & $W$ & $W$ & $W$ & $w$ & 3.6 \\
\hline 2874 & Phosphatic Fertilizers . . . . . . . . . & W & -- & -- & -- & W &.- & $\cdots$ & 3.4 \\
\hline 29 & Petroleum and Coal Products $\ldots \ldots \ldots \ldots$ & 0.052 & $W$ & -- & W & 3.955 & 2.491 & 2.649 & 2.0 \\
\hline 2911 & Petroleum Refining . . . . . . . . . . . . . & 0.050 & $W$ & -- & $W$ & 4.011 & 2.431 & 2.602 & 1.6 \\
\hline 30 & Rubber and Misc. Plastics Products . . . . . . & 0.073 & -- &.- & $w$ & 4.215 & Q & 2.376 & 4.9 \\
\hline 3011 & Tires and inner Tubes . . . . . . . . . . . . & W & - & -- & W & W & - & - & 1.4 \\
\hline 308 & Miscellaneous Plastic Products, nec . . . . . . & 0.073 & - & -- & -. & 4.593 & $Q$ & $w$ & 6.7 \\
\hline 31 & Leather and Leather Products $\ldots \ldots \ldots \ldots$ & 0.061 & -- & -- & -. & W &. & W & 7.8 \\
\hline 32 & Stone, Clay and Glass Products $\ldots . . \ldots$. & 0.055 & $W$ & -- & W & 2.929 & 2.251 & 2.811 & 3.6 \\
\hline 3211 & Flat Glass $\ldots \ldots \ldots \ldots \ldots \ldots$ & 0.068 & - & -- & W & 3.386 & W & W & 1.2 \\
\hline 3221 & Glass Containers . . . . . . . . . . . & 0.050 & - & -. & -- & $w$ & W & 2.850 & 2.7 \\
\hline 3229 & Pressed and Blown Glass, nec . . . . . . . & W & -- & -- & -- & $w$ & W & - & 2.5 \\
\hline 3241 & Cement, Hydraulic . . . . . . . . . . . & 0.046 & -- & -- & -. & 2.259 & $Q$ & W & 8.3 \\
\hline 3274 & Lime . . . . . . . . . . . . . & W & -- & -- & -- & W & $\vec{w}$ & W & 4.1 \\
\hline 3296 & 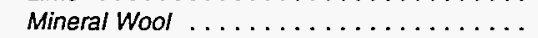 & 0.062 & - & -- & -- & W & W & 3.303 & 0.9 \\
\hline 33 & Primary Metal Industries $\ldots \ldots \ldots \ldots \ldots$ & 0.025 & W & -. & W & 3.492 & 2.643 & 2.132 & 2.1 \\
\hline 3312 & Blast Fumaces and Steel Mills . . . . . . . . . . & 0.035 & -- & -- & -- & W & W & 1.909 & 2.2 \\
\hline 3313 & Electrometalurgical Products . . . . . . . . & -- & -- & -. & -- & -- & -- & -. & NF \\
\hline 3321 & Gray and Ductile Iron Foundries $\ldots \ldots \ldots$ & 0.074 & -- & -- & -- & 3.094 & -- & $w$ & 11.2 \\
\hline 3331 & Primary Copper . . . . . . . . . . . . & 0.048 & -- & -- & $\cdots$ & W & W & $W$ & NF \\
\hline 3334 & Primary Aluminum $\ldots \ldots \ldots \ldots \ldots$ & 0.020 & -- &.- & -- & 2.463 & 2.178 & W & 1.3 \\
\hline 3339 & Primary Nonferrous Metals, nec . . . . . . . . & 0.022 & -- & -- & -- & $w$ & W & W & NF \\
\hline 3353 & Aluminum Sheet, Plate, and Foil ........ & W & - & -- & -- & -- & -- & W & NF \\
\hline 34 & Fabricated Metal Products . . . . . . . . & 0.074 & -- & -- & -. & 4.430 & 3.664 & 2.909 & 6.1 \\
\hline 35 & Industrial Machinery and Equipment . . . . . . & 0.074 &.- & -- & -- & 4.042 & W & W & 7.9 \\
\hline 357 & Computer and Office Equipment ....... & 0.063 & 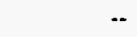 & $\cdots$ & -- & 4.458 & $w$ & W & 3.4 \\
\hline 36 & Electronic and Other Electric Equipment . . . . . & W & $W$ & W & -- & 4.320 & 3.092 & 2.927 & 6.0 \\
\hline
\end{tabular}

See footnotes at end of table. 


\section{Table A47. Average Prices of Purchased Electricity, Steam, and Natural Gas by Type of Supplier, Census Region, Industry Group, and Selected Industries, 1991 (Continued) (Estimates in Dollars per Physical Units)}

\begin{tabular}{|c|c|c|c|c|c|c|c|c|c|}
\hline \multirow[b]{2}{*}{$\begin{array}{c}\text { SIC } \\
\text { Code* }\end{array}$} & \multirow[b]{2}{*}{$\begin{array}{l}\text { Industry Groups } \\
\text { and Industry }\end{array}$} & \multicolumn{2}{|c|}{$\begin{array}{c}\text { Electricity } \\
\text { (Million kWh) }\end{array}$} & \multicolumn{2}{|c|}{$\begin{array}{c}\text { Steam } \\
\text { (Billion Btu) }\end{array}$} & \multicolumn{3}{|c|}{$\begin{array}{l}\text { Natural Gas } \\
(1000 \mathrm{cu} \mathrm{ft})\end{array}$} & \multirow[b]{2}{*}{$\begin{array}{c}\text { RSE } \\
\text { Row } \\
\text { Factors }\end{array}$} \\
\hline & & $\begin{array}{c}\text { Utility } \\
\text { Supplier }\end{array}$ & $\begin{array}{l}\text { Nonutility } \\
\text { Supplier }\end{array}$ & $\begin{array}{c}\text { Utility } \\
\text { Supplier }\end{array}$ & $\begin{array}{l}\text { Nonutility } \\
\text { Supplier }\end{array}$ & $\begin{array}{c}\text { Utility } \\
\text { Supplier }^{b}\end{array}$ & $\begin{array}{c}\text { Transmission } \\
\text { Pipelines }\end{array}$ & $\begin{array}{c}\text { Other } \\
\text { Supplier }\end{array}$ & \\
\hline & & \multicolumn{8}{|c|}{ West Census Region } \\
\hline & RSE Column Factors: & 0.9 & 0.8 & 0.4 & 0.8 & 1.0 & 4.6 & 0.8 & \\
\hline 37 & Transportation Equipment & W & W & -- & W & 4.255 & 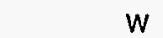 & W & 5.9 \\
\hline 3711 & Motor Vehicles and Car Bodies . . . . . . . . & 0.062 & -. & - & - & W & - & $w$ & 2.3 \\
\hline 3714 & Motor Vehicle Parts and Accessories . . . . . . & 0.081 & -- & $\cdots$ & -- & 4.036 &.- & 2.921 & 6.8 \\
\hline & Instruments and Related Products $\ldots \ldots \ldots$ & 0.066 & - & W & - & 4.531 & W & W & 4.1 \\
\hline 3841 & Surglcal and Medical Instruments ....... & 0.080 &.- & -- & - & 4.714 & $W$ & $\cdots$ & 5.6 \\
\hline \multirow[t]{2}{*}{39} & MIsc. Manufacturing Industries $\ldots \ldots \ldots \ldots$ & 0.074 & -- & W & -- & W & Q & W & 4.0 \\
\hline & Total $\ldots \ldots \ldots \ldots \ldots \ldots \ldots$ & 0.045 & 0.045 & $W$ & 3.366 & 3.045 & 2.539 & 2.499 & 5.4 \\
\hline
\end{tabular}

\footnotetext{
" See Appendices B and F for descriptions of the Standard Industrial Classification system.

"A "Utility" is a company that produces and/or delivers electricity and/or natural gas, and is legally obligated to provide service to the public within its franchise area.

- Includes independent power producers, small power producers, and cogenerators not located at the establishment site.

'Other suppliers of natural gas include such sources as brokers and producers.

NF=No applicable RSE row/column factor.

$W=$ Withheld to avoid disclosing data for individual establishments. Data are included in higher level totals.

$\mathrm{Q}=$ Withheld because Relative Standard Error is greater than 50 percent. Data are included in higher level totals.

NA=Not avallable. Data are included in higher level totals.

- Estimation of average price is not applicable.

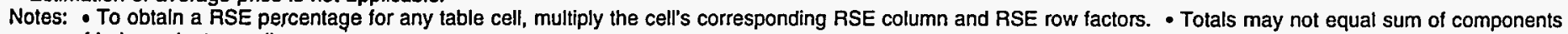
because of independent rounding.

Source: Energy Information Administration, Office of Energy Markets and End Use, Energy End Use and Integrated Statistics Division, Form ElA-846, "1991 Manufacturing Energy Consumption Survey."
} 
Table A48. Total Expenditures for Purchased Electricity, Steam, and Natural Gas by Type of Supplier, Census Region, and Economic Characteristics of the Establishment, 1991

(Estimates in Million Dollars)

\begin{tabular}{|c|c|c|c|c|c|c|c|c|}
\hline \multirow[b]{2}{*}{$\begin{array}{c}\text { Economic } \\
\text { Characteristics }\end{array}$} & \multicolumn{2}{|c|}{ Electricity } & \multicolumn{2}{|c|}{ Steam } & \multicolumn{3}{|c|}{ Natural Gas } & \multirow{2}{*}{$\begin{array}{c}\text { RSE } \\
\text { Row } \\
\text { Factore }\end{array}$} \\
\hline & Utility Supplier & $\begin{array}{l}\text { Nonutility } \\
\text { Supplier }\end{array}$ & $\begin{array}{c}\text { Utility } \\
\text { Supplier }\end{array}$ & $\begin{array}{l}\text { Nonutility } \\
\text { Supplier }\end{array}$ & $\begin{array}{c}\text { Utility } \\
\text { Supplier }\end{array}$ & $\begin{array}{c}\text { Transmission } \\
\text { Pipelines }\end{array}$ & $\begin{array}{c}\text { Other } \\
\text { Suppller }\end{array}$ & \\
\hline & \multicolumn{7}{|c|}{ Total United States } & \\
\hline RSE Column Factors: & 0.4 & 2.7 & 1.5 & 1.3 & 0.6 & 1.0 & 0.8 & \\
\hline \multicolumn{9}{|l|}{$\begin{array}{l}\text { Value of Shipments and Receipts } \\
\text { (million dollars) }\end{array}$} \\
\hline Under $20 \ldots \ldots \ldots \ldots \ldots \ldots$ & 7,020 & 24 & 11 & 21 & 1,502 & 209 & 295 & 10.6 \\
\hline 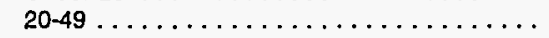 & 5,591 & 40 & 16 & 47 & 1,100 & 276 & 497 & 9.0 \\
\hline $50-99 \ldots \ldots \ldots \ldots \ldots \ldots \ldots$ & 4,379 & 26 & 20 & 54 & 878 & 253 & $W$ & 7.5 \\
\hline $100-249 \ldots \ldots \ldots \ldots \ldots \ldots \ldots$ & 5,660 & 33 & 52 & 114 & 1,142 & 553 & 769 & 6.8 \\
\hline $250-499 \ldots \ldots \ldots \ldots \ldots \ldots$ & $W$ & W & 62 & 52 & 655 & 770 & $W$ & 5.7 \\
\hline 500 and $O v e r . \ldots \ldots \ldots \ldots \ldots$ & W & W & 107 & 102 & 762 & 1,446 & 1,243 & 3.4 \\
\hline Total $\ldots \ldots \ldots \ldots \ldots \ldots \ldots \ldots$ & 31,636 & 461 & 267 & 390 & 6,037 & 3,507 & 4,013 & 3.4 \\
\hline \multicolumn{9}{|l|}{ Employment Size } \\
\hline$\ldots \ldots \ldots \ldots \ldots$ & W & $W$ & 8 & 9 & 650 & 109 & W & 15.3 \\
\hline $50-99 \ldots \ldots \ldots \ldots \ldots \ldots \ldots \ldots$ & $W$ & W & 8 & 44 & 679 & 139 & 271 & 11.2 \\
\hline $100-249 \ldots \ldots \ldots \ldots \ldots \ldots \ldots \ldots$ & 6,453 & 26 & 35 & 84 & 1,504 & 522 & 690 & 7.9 \\
\hline 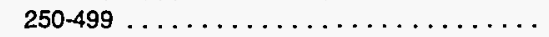 & 5,895 & 29 & 64 & 50 & 1,112 & 695 & 709 & 6.2 \\
\hline $500-999 \ldots \ldots \ldots \ldots \ldots \ldots \ldots$ & 5,971 & 74 & 63 & 117 & 1,079 & 697 & 831 & 4.4 \\
\hline 1,000 and Over $\ldots \ldots \ldots \ldots \ldots$ & 7,712 & 295 & 89 & 86 & 1,013 & 1,345 & W & 3.7 \\
\hline Total $\ldots \ldots \ldots \ldots \ldots \ldots \ldots \ldots$ & 31,636 & 461 & 267 & 390 & 6,037 & 3,507 & 4,013 & 3.4 \\
\hline & \multicolumn{8}{|c|}{ Northeast Census Region } \\
\hline RSE Column Factors: & 0.4 & 2.2 & 1.8 & 1.5 & 0.6 & 1.0 & 0.7 & \\
\hline \multicolumn{9}{|l|}{$\begin{array}{l}\text { Value of Shipments and Receipts } \\
\text { (million dollars) }\end{array}$} \\
\hline Under $20 \ldots \ldots \ldots \ldots \ldots \ldots$ & W & W & Q & 11 & 286 & W & W & 19.7 \\
\hline $20-49 \ldots \ldots \ldots \ldots \ldots \ldots \ldots$ & 1,039 & $\mathbf{Q}$ & 5 & 6 & 172 & $W$ & W & 14.0 \\
\hline 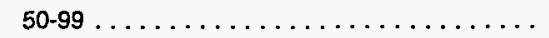 & 754 & 2 & 6 & W & 135 & 22 & 91 & 11.5 \\
\hline $100-249 \ldots \ldots \ldots \ldots \ldots \ldots \ldots$ & W & W & $W$ & 35 & 141 & 48 & 87 & 7.8 \\
\hline $250-499 \ldots \ldots \ldots \ldots \ldots \ldots \ldots$ & 558 & 0 & W & 10 & 79 & 5 & 72 & 11.8 \\
\hline 500 and Over . . . . . . . . . . . . . & W & W & W & $W$ & 90 & 25 & 106 & 8.3 \\
\hline Total $\ldots \ldots \ldots \ldots \ldots \ldots$ & 5,447 & 55 & W & 70 & 902 & 172 & 477 & 6.6 \\
\hline \multicolumn{9}{|l|}{ Employment Size } \\
\hline$\ldots \ldots \ldots \ldots$ & 616 & $\mathbf{Q}$ & 0 & Q & 132 & 17 & 17 & 27.8 \\
\hline 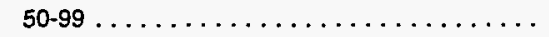 & $W$ & W & $Q$ & 9 & 106 & 19 & 35 & 20.3 \\
\hline $100-249 \ldots \ldots \ldots \ldots \ldots \ldots \ldots$ & 1,094 & $\mathbf{Q}$ & 8 & 6 & 192 & 32 & 59 & 15.6 \\
\hline $250-499 \ldots \ldots \ldots \ldots \ldots \ldots \ldots$ & 920 & $\vec{Q}$ & W & 2 & 163 & 45 & 88 & 9.8 \\
\hline $500-999 \ldots \ldots \ldots \ldots \ldots \ldots \ldots$ & W & $w$ & W & 42 & 118 & 37 & 129 & 6.7 \\
\hline 1,000 and $O v e r \ldots \ldots \ldots \ldots$ & $W$ & W & W & W & 193 & 22 & 150 & 8.1 \\
\hline Total $\ldots \ldots \ldots \ldots \ldots \ldots \ldots$ & 5,447 & 55 & W & 70 & 902 & 172 & 477 & 6.6 \\
\hline
\end{tabular}

See footnotes at end of table. 
Table A48. Total Expenditures for Purchased Electricity, Steam, and Natural Gas by Type of Supplier, Census Region, and Economic Characteristics of the Establishment, 1991 (Continued)

(Estimates in Million Dollars)

\begin{tabular}{|c|c|c|c|c|c|c|c|c|}
\hline \multirow[b]{2}{*}{$\begin{array}{c}\text { Economic } \\
\text { Characteristics } \\
\end{array}$} & \multicolumn{2}{|c|}{ Electricity } & \multicolumn{2}{|c|}{ Steam } & \multicolumn{3}{|c|}{ Natural Gas } & \multirow[b]{2}{*}{$\begin{array}{c}\text { RSE } \\
\text { Row } \\
\text { Factors }\end{array}$} \\
\hline & Utility Supplier| & $\begin{array}{l}\text { Nonutility } \\
\text { Supplier }\end{array}$ & $\begin{array}{c}\text { Utility } \\
\text { Supplier }\end{array}$ & $\begin{array}{l}\text { Nonutility } \\
\text { Supplier }\end{array}$ & $\begin{array}{c}\text { Utility } \\
\text { Supplier }\end{array}$ & $\begin{array}{c}\text { Transmission } \\
\text { Pipelines } \\
\end{array}$ & $\begin{array}{c}\text { Other } \\
\text { Suppller }\end{array}$ & \\
\hline & \multicolumn{8}{|c|}{ Midwest Census Region } \\
\hline RSE Column Factors: & 0.4 & 2.7 & 1.5 & 2.0 & 0.6 & 0.9 & 0.6 & \\
\hline \multicolumn{9}{|l|}{$\begin{array}{l}\text { Value of Shipments and Receipts } \\
\text { (milliton dollars) }\end{array}$} \\
\hline Under $20 \ldots$ & w & w & $Q$ & 5 & 506 & 77 & 133 & 16.7 \\
\hline $20-49$ & 1,507 & 3 & W & 9 & 336 & 87 & 211 & 14.9 \\
\hline$\ldots \ldots \ldots \ldots \ldots \ldots \ldots \ldots$ & 1,204 & 11 & 13 & w & 209 & 106 & w & 11.6 \\
\hline $100-249 \ldots \ldots \ldots \ldots \ldots \ldots \ldots \ldots \ldots$ & 1,307 & 6 & 34 & 11 & 197 & 95 & 241 & 6.7 \\
\hline $250-499 \ldots \ldots \ldots \ldots \ldots \ldots \ldots \ldots \ldots$ & 1,032 & 0 & W & W & w & W & w & 8.3 \\
\hline 500 and Over. & W & W & w & w. & W & W & 522 & 5.8 \\
\hline Total $\ldots \ldots \ldots \ldots \ldots \ldots \ldots \ldots \ldots \ldots \ldots$ & 9,048 & 87 & 97 & 56 & 1,575 & 572 & 1,510 & 4.8 \\
\hline \multicolumn{9}{|l|}{ Employment Size } \\
\hline Under $50 \ldots \ldots \ldots \ldots \ldots \ldots \ldots \ldots$ & w & $\mathbf{Q}$ & a & $Q$ & 241 & 58 & 61 & 21.5 \\
\hline$\ldots \ldots \ldots \ldots \ldots \ldots \ldots \ldots$ & 844 & 4 & w & w & 206 & 37 & 86 & 15.8 \\
\hline $100-249 \ldots \ldots \ldots \ldots \ldots \ldots \ldots \ldots \ldots$ & w & w & 20 & 7 & 404 & 118 & 232 & 11.6 \\
\hline $250-499 \ldots \ldots \ldots \ldots \ldots \ldots \ldots \ldots \ldots$ & w & w & 16 & 8 & 273 & W & 277 & 8.0 \\
\hline $500-999$ & 1,287 & $\mathbf{Q}$ & 30 & $\mathbf{w}$ & 192 & 94 & 220 & 7.2 \\
\hline 1,000 and $O v e r \quad \ldots \ldots \ldots \ldots$ & w & W & w & 23 & 259 & $\mathbf{w}$ & 634 & 5.1 \\
\hline Total $\ldots \ldots \ldots \ldots \ldots \ldots \ldots$ & 9,048 & 87 & 97 & 56 & 1,575 & 572 & 1,510 & 4.8 \\
\hline & \multicolumn{8}{|c|}{ South Census Region } \\
\hline RSE Column Factors: & 0.4 & 2.5 & 1.4 & 0.9 & 0.6 & 1.2 & 1.2 & \\
\hline \multicolumn{9}{|l|}{$\begin{array}{l}\text { Value of Shipments and Recelpts } \\
\text { (millton dollars) }\end{array}$} \\
\hline Under $20 \ldots \ldots \ldots \ldots \ldots \ldots \ldots \ldots$ & 2,312 & 2 & Q & w & 460 & w & 65 & 12.5 \\
\hline $20-49 \ldots \ldots \ldots \ldots \ldots \ldots \ldots \ldots \ldots \ldots$ & 2,231 & 20 & w & 21 & 436 & 122 & 152 & 11.2 \\
\hline $50-99 \ldots \ldots \ldots \ldots \ldots \ldots \ldots \ldots \ldots \ldots$ & 1,741 & 5 & • & w & 393 & 107 & 172 & 10.7 \\
\hline $100-249 \ldots \ldots \ldots \ldots \ldots \ldots \ldots \ldots \ldots \ldots$ & w & w & w & 50 & 594 & 365 & 292 & 9.6 \\
\hline $250-499 \ldots \ldots \ldots \ldots \ldots$ & w & w & 42 & 27 & w & w & 212 & 7.0 \\
\hline 500 and 0 ver $\ldots \ldots \ldots \ldots \ldots \ldots$ & W & w & 53 & 47 & W & $\mathbf{w}$ & 519 & 5.0 \\
\hline 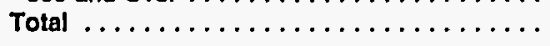 & w & w & 112 & 171 & 2,599 & 2,553 & 1,412 & 4.6 \\
\hline \multicolumn{9}{|l|}{ Employment Size } \\
\hline Under $50 \ldots \ldots$ & w & w & 7 & 1 & 165 & w & w & 17.6 \\
\hline $50-99 \ldots \ldots \ldots$ & 895 & 10 & w & 17 & 256 & $\mathbf{w}$ & w & 11.9 \\
\hline $100-249 \ldots \ldots \ldots \ldots \ldots$ & w & w & w & 56 & 605 & 336 & 225 & 11.3 \\
\hline $250-499 \ldots$ & w & w & w & 15 & 532 & 495 & 230 & 8.0 \\
\hline $500-999 \ldots \ldots \ldots \ldots \ldots \ldots$ & 2,665 & 17 & w & 51 & 569 & 483 & 292 & 5.3 \\
\hline 1,000 and Over $\ldots \ldots \ldots \ldots \ldots \ldots \ldots \ldots$ & w & w & w & 30 & 473 & 1,146 & 470 & 5.0 \\
\hline Total $\ldots \ldots \ldots \ldots \ldots$ & w & w & 112 & 171 & 2,599 & 2,553 & 1,412 & 4.6 \\
\hline
\end{tabular}

See tootnotes at end of table. 
Table A48. Total Expenditures for Purchased Electricity, Steam, and Natural Gas by Type of Supplier, Census Region, and Economic Characteristics of the Establishment, 1991 (Continued) (Estimates in Million Dollars)

\begin{tabular}{|c|c|c|c|c|c|c|c|c|}
\hline \multirow[b]{2}{*}{$\begin{array}{c}\text { Economic } \\
\text { Characteristics }\end{array}$} & \multicolumn{2}{|c|}{ Electricity } & \multicolumn{2}{|c|}{ Steam } & \multicolumn{3}{|c|}{ Natural Gas } & \multirow[b]{2}{*}{$\begin{array}{c}\text { RSE } \\
\text { Row } \\
\text { Factors }\end{array}$} \\
\hline & Utility Supplier & $\begin{array}{l}\text { Nonutility } \\
\text { Supplier }\end{array}$ & $\begin{array}{c}\text { Utility } \\
\text { Supplier }\end{array}$ & $\begin{array}{l}\text { Nonutility } \\
\text { Supplier }\end{array}$ & $\begin{array}{c}\text { Utility } \\
\text { Supplier }\end{array}$ & $\begin{array}{c}\text { Transmission } \\
\text { Pipelines } \\
\end{array}$ & $\begin{array}{c}\text { Other } \\
\text { Supplier }\end{array}$ & \\
\hline & \multicolumn{7}{|c|}{ West Census Region } & \\
\hline RSE Column Factors: & 0.4 & 1.4 & 3.7 & 1.3 & 0.6 & 0.9 & 0.6 & \\
\hline \multicolumn{9}{|l|}{$\begin{array}{l}\text { Value of Shipments and Receipts } \\
\text { (million dollars) }\end{array}$} \\
\hline Under $20 \ldots \ldots \ldots \ldots \ldots \ldots \ldots$ & 1,198 & * & 0 & $\mathbf{Q}$ & 250 & $W$ & $w$ & 17.6 \\
\hline $20-49 \ldots \ldots \ldots \ldots \ldots \ldots$ & 813 & Q & W & 11 & 156 & $W$ & W & 16.2 \\
\hline 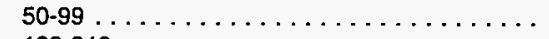 & 680 & 7 & Q & 19 & 141 & 18 & 152 & 14.1 \\
\hline $100-249 \ldots \ldots \ldots \ldots \ldots \ldots$ & 1,089 & $Q$ & $\star$ & 18 & 209 & 45 & 150 & 15.5 \\
\hline $250-499 \ldots \ldots \ldots \ldots \ldots \ldots \ldots$ & W & W & 0 & W & 64 & 33 & 107 & 9.4 \\
\hline 500 and Over $\ldots \ldots \ldots \ldots \ldots$ & W & W & 0 & 32 & 140 & 56 & 96 & 5.4 \\
\hline Total $\ldots \ldots \ldots \ldots \ldots \ldots \ldots$ & W & $w$ & W & 94 & 961 & 210 & 615 & 7.7 \\
\hline \multicolumn{9}{|l|}{ Employment Size } \\
\hline Under $50 \ldots \ldots \ldots \ldots \ldots \ldots$ & 571 & * & 0 & W & 113 & $w$ & W & 25.0 \\
\hline $50-99 \ldots \ldots \ldots \ldots \ldots \ldots \ldots$ & W & W & 0 & $w$ & 111 & W & W & 17.4 \\
\hline $100-249 \ldots \ldots \ldots \ldots \ldots \ldots \ldots \ldots$ & W & $\mathbf{Q}$ & $w$ & 15 & 303 & 36 & 175 & 15.8 \\
\hline $250-499 \ldots \ldots \ldots \ldots \ldots \ldots$ & 864 & $\mathbf{Q}$ & $\mathbf{Q}$ & 26 & 144 & $W$ & 115 & 10.3 \\
\hline $500-999 \ldots \ldots \ldots \ldots \ldots \ldots$ & W & W & - & W & 200 & 84 & 189 & 10.1 \\
\hline 1,000 and Over $\ldots \ldots \ldots \ldots \ldots$ & W & W & 0 & W & 88 & $W$ & W & 6.9 \\
\hline Total $\ldots \ldots \ldots \ldots \ldots \ldots \ldots \ldots$ & W & W & W & 94 & 961 & 210 & 615 & 7.7 \\
\hline
\end{tabular}

- Value of Shipments and Receipts and Employment Size categories were supplied by the Bureau of the Census. See Appendix B.

'A "Utility" is a company that produces and/or delivers electricity and/or natural gas, and is legally obligated to provide service to the public within its franchise area.

- Includes independent power producers, small power producers, and cogenerators not located at the establishment site.

- Other suppliers of natural gas include such sources as brokers and producers.

- Estimate less than 0.5. Data are included in higher level totals.

$\mathrm{Q}=$ Withheld because Relative Standard Error is greater than $\mathbf{5 0}$ percent. Data are included in higher level totals.

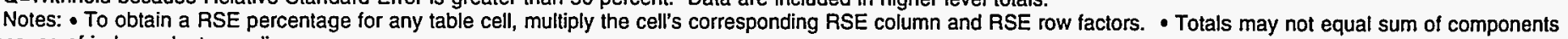
because of independent rounding.

Source: Energy Information Administration, Office of Energy Markets and End Use, Energy End Use and Integrated Statistics Division, Form ElA-846, "1991 Manufacturing Energy Consumption Survey," and Bureau of the Census, Industry Division, data files for the "1991 Annual Survey of Manufactures." 
Table A49. Average Prices of Purchased Electricity, Steam, and Natural Gas by Type of Supplier, Census Region, and Economic Characteristics of the Establishment, 1991 (Estimates in Dollars per Physical Units)

\begin{tabular}{|c|c|c|c|c|c|c|c|c|}
\hline \multirow[b]{2}{*}{$\begin{array}{c}\text { Economic } \\
\text { Characteristics }\end{array}$} & \multicolumn{2}{|c|}{$\begin{array}{c}\text { Electricity } \\
\text { (Million } \mathrm{kWh} \text { ) }\end{array}$} & \multicolumn{2}{|c|}{$\begin{array}{c}\text { Steam } \\
\text { (Billion Btu) }\end{array}$} & \multicolumn{3}{|c|}{$\begin{array}{l}\text { Natural Gas } \\
(1000 \mathrm{cu} \mathrm{ft})\end{array}$} & \multirow[b]{2}{*}{$\begin{array}{c}\text { RSE } \\
\text { Row } \\
\text { Factors }\end{array}$} \\
\hline & Utility Supplier & $\begin{array}{l}\text { Nonutility } \\
\text { Supplier }\end{array}$ & $\begin{array}{l}\text { Utility } \\
\text { Supplier }\end{array}$ & $\begin{array}{l}\text { Nonutility } \\
\text { Supplier }\end{array}$ & $\begin{array}{c}\text { Utility } \\
\text { Supplier }\end{array}$ & $\begin{array}{c}\text { Transmission } \\
\text { Pipelines }\end{array}$ & $\begin{array}{l}\text { Other } \\
\text { Supplier }\end{array}$ & \\
\hline & \multicolumn{7}{|c|}{ Total United States } & \\
\hline RSE Column Factors: & 0.4 & 1.9 & 1.0 & 1.2 & 0.6 & 2.9 & 0.7 & \\
\hline \multicolumn{9}{|l|}{$\begin{array}{l}\text { Value of Shipments and Recejpts } \\
\text { (million dollars) }\end{array}$} \\
\hline Under $20 \ldots \ldots \ldots \ldots \ldots \ldots$ & 0.065 & 0.075 & 3.764 & 4.933 & 3.715 & 2.868 & 2.707 & 5.6 \\
\hline $20-49 \ldots \ldots \ldots \ldots \ldots \ldots \ldots$ & 0.053 & 0.035 & 2.939 & 3.120 & 3.204 & 2.270 & 2.361 & 5.6 \\
\hline $50-99 \ldots \ldots \ldots \ldots \ldots \ldots \ldots \ldots$ & 0.048 & 0.034 & 3.797 & 4.179 & 2.945 & 2.306 & 2.397 & 4.8 \\
\hline $100-249 \ldots \ldots \ldots \ldots \ldots \ldots \ldots$ & 0.039 & 0.055 & 3.834 & 2.624 & 2.376 & 1.981 & 2.317 & 4.5 \\
\hline $250-499 \ldots \ldots \ldots \ldots \ldots \ldots \ldots \ldots$ & 0.037 & W & 2.987 & 2.728 & 2.298 & 1.771 & 2.185 & 2.5 \\
\hline 500 and $O v e r \ldots \ldots \ldots \ldots \ldots \ldots$ & 0.040 & 0.050 & 2.913 & 2.604 & 2.486 & 1.783 & 2.117 & 2.6 \\
\hline Total $\ldots \ldots \ldots \ldots \ldots \ldots \ldots \ldots$ & 0.046 & 0.029 & 3.165 & 2.912 & 2.851 & 1.917 & 2.272 & 2.5 \\
\hline \multicolumn{9}{|l|}{ Employment Size } \\
\hline Under $50 \ldots \ldots \ldots \ldots \ldots \ldots$ & 0.066 & $W$ & 3.145 & 5.910 & 3.757 & 2.600 & 2.108 & 7.0 \\
\hline $50-99 \ldots \ldots \ldots \ldots \ldots \ldots \ldots$ & 0.059 & W & 2.930 & 3.897 & 3.066 & 2.403 & 2.250 & 6.2 \\
\hline $100-249 \ldots \ldots \ldots \ldots \ldots \ldots \ldots$ & 0.051 & 0.025 & 4.197 & 2.476 & 2.743 & 1.974 & 2.311 & 5.2 \\
\hline 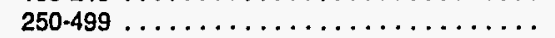 & 0.046 & 0.048 & 2.830 & 3.680 & 2.975 & 1.890 & 2.373 & 4.3 \\
\hline $500-999 \ldots \ldots \ldots \ldots \ldots \ldots \ldots$ & 0.039 & 0.046 & 3.066 & 2.583 & 2.669 & 2.025 & 2.298 & 3.4 \\
\hline 1,000 and Over $\ldots \ldots \ldots \ldots \ldots \ldots$ & 0.041 & 0.024 & 3.230 & 3.029 & 2.554 & 1.787 & 2.214 & 2.7 \\
\hline Total $\ldots \ldots \ldots \ldots \ldots \ldots \ldots \ldots \ldots$ & 0.046 & 0.029 & 3.165 & 2.912 & 2.851 & 1.917 & 2.272 & 2.5 \\
\hline & \multicolumn{8}{|c|}{ Northeast Census Region } \\
\hline RSE Column Factors: & 0.5 & 1.7 & 1.2 & 1.1 & 0.6 & 2.9 & 0.5 & \\
\hline \multicolumn{9}{|l|}{$\begin{array}{l}\text { Value of Shipments and Receipts } \\
\text { (million dollars) }\end{array}$} \\
\hline Under $20 \ldots \ldots \ldots \ldots \ldots \ldots$ & 0.081 & W & 4.831 & 5.981 & 4.644 & 3.996 & 3.265 & 7.9 \\
\hline 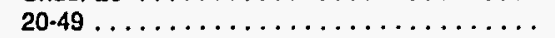 & 0.071 & 0.093 & 3.285 & 2.373 & 4.032 & 3.386 & 3.275 & 7.2 \\
\hline 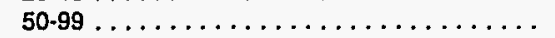 & 0.068 & 0.049 & 4.407 & 4.420 & 3.806 & 3.384 & 3.137 & 4.7 \\
\hline $100-249 \ldots \ldots \ldots \ldots \ldots \ldots \ldots \ldots \ldots \ldots \ldots \ldots \ldots$ & 0.053 & 0.065 & W & 5.971 & 3.381 & 3.070 & 3.004 & 3.4 \\
\hline $250-499 \ldots \ldots \ldots \ldots \ldots \ldots \ldots$ & $w$ & - & $w$ & 3.860 & 3.273 & 3.609 & 3.060 & 3.1 \\
\hline 500 and $O v e r \ldots \ldots \ldots \ldots \ldots$ & 0.056 & $W$ & W & 4.221 & 2.798 & 2.825 & 2.873 & 2.7 \\
\hline Total $\ldots \ldots \ldots \ldots \ldots \ldots \ldots \ldots$ & 0.064 & 0.079 & 3.273 & 4.736 & 3.798 & 3.317 & 3.070 & 4.1 \\
\hline \multicolumn{9}{|l|}{ Employment Size } \\
\hline Under $50 \ldots$. & 0.084 & 0.101 & - & W & 4.776 & 4.379 & 3.054 & 8.7 \\
\hline 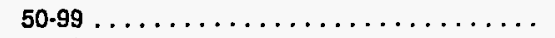 & 0.079 & 0.071 & W & 3.709 & 4.351 & 3.474 & 3.399 & 5.8 \\
\hline $100-249 \ldots \ldots \ldots \ldots \ldots \ldots \ldots$ & 0.072 & 0.049 & 5.080 & 3.112 & 4.149 & 3.343 & 3.146 & 7.6 \\
\hline $250-499 \ldots \ldots \ldots \ldots \ldots \ldots \ldots$ & 0.066 & 0.115 & 3.846 & 4.186 & 3.688 & 3.233 & 3.212 & 5.5 \\
\hline $500-999 \ldots \ldots \ldots \ldots \ldots \ldots \ldots$ & 0.049 & W & W & 5.445 & 3.780 & 3.214 & 3.010 & 2.7 \\
\hline 1,000 and Over $\ldots \ldots \ldots \ldots \ldots \ldots$ & 0.058 & W & $w$ & 4.248 & 3.001 & 2.937 & 2.951 & 3.2 \\
\hline Total $\ldots \ldots \ldots \ldots \ldots \ldots \ldots \ldots$ & 0.064 & 0.079 & 3.273 & 4.736 & 3.798 & 3.317 & 3.070 & 4.1 \\
\hline
\end{tabular}

See footnotes at end of table. 
Table A49. Average Prices of Purchased Electricity, Steam, and Natural Gas by Type of Supplier, Census Region, and Economic Characteristics of the Establishment, 1991 (Continued)

(Estimates in Dollars per Physical Units)

\begin{tabular}{|c|c|c|c|c|c|c|c|c|}
\hline \multirow[b]{2}{*}{$\begin{array}{c}\text { Economic } \\
\text { Characteristics" }\end{array}$} & \multicolumn{2}{|c|}{$\begin{array}{c}\text { Electricity } \\
\text { (Million kWh) }\end{array}$} & \multicolumn{2}{|c|}{$\begin{array}{l}\text { Steam } \\
\text { (Billion Btu) }\end{array}$} & \multicolumn{3}{|c|}{$\begin{array}{l}\text { Natural Gas } \\
(1000 \mathrm{cu} \mathrm{ft})\end{array}$} & \multirow[b]{2}{*}{$\begin{array}{c}\text { RSE } \\
\text { Row } \\
\text { Factors }\end{array}$} \\
\hline & Utility Supplier & $\begin{array}{l}\text { Nonutility } \\
\text { Supplier }\end{array}$ & $\begin{array}{l}\text { Utility } \\
\text { Supplier }\end{array}$ & $\begin{array}{l}\text { Nonutility } \\
\text { Supplier }\end{array}$ & $\begin{array}{l}\text { Utility } \\
\text { Supplier }\end{array}$ & $\begin{array}{l}\text { Transmission } \\
\text { Pipelines }\end{array}$ & $\begin{array}{l}\text { Other } \\
\text { Supplier }\end{array}$ & \\
\hline & \multicolumn{7}{|c|}{ Midwest Census Region } & \\
\hline RSE Column Factors: & 0.5 & 1.3 & 1.2 & 1.8 & 0.6 & 2.7 & 0.5 & \\
\hline \multicolumn{9}{|l|}{$\begin{array}{l}\text { Value of Shipments and Receipts } \\
\text { (million dollars) }\end{array}$} \\
\hline Under $20 \ldots \ldots \ldots \ldots \ldots \ldots \ldots$ & 0.064 & w & 3.004 & 5.394 & 3.501 & 2.761 & 2.711 & 6.5 \\
\hline 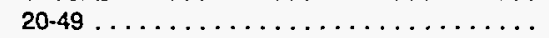 & 0.053 & 0.043 & W & 4.012 & 2.960 & 2.310 & 2.474 & 6.4 \\
\hline $50-99 \ldots \ldots \ldots \ldots \ldots \ldots \ldots \ldots$ & 0.047 & 0.047 & 3.787 & 4.468 & 2.972 & 2.148 & 2.320 & 4.4 \\
\hline 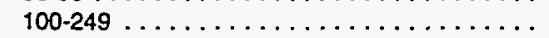 & 0.044 & 0.059 & 3.307 & 1.695 & 2.902 & 2.360 & 2.611 & 4.0 \\
\hline $250-499 \ldots \ldots \ldots \ldots \ldots \ldots$ & $w$ & 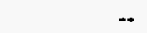 & W & W & 2.583 & 1.848 & 2.333 & 3.7 \\
\hline 500 and Over . . . . . . . . . . . . . & $w$ & W & 4.484 & 2.610 & 2.674 & 2.131 & 2.273 & 3.5 \\
\hline Total $\ldots \ldots \ldots \ldots \ldots \ldots \ldots$ & 0.046 & 0.051 & 3.696 & 2.835 & 3.026 & 2.223 & 2.398 & 3.2 \\
\hline \multicolumn{9}{|l|}{ Employment Size } \\
\hline Under $50 \ldots \ldots \ldots \ldots \ldots \ldots$ & 0.065 & $w$ & $W$ & 6.021 & 3.511 & 2.247 & 2.391 & 10.2 \\
\hline $50-99 \ldots \ldots \ldots \ldots \ldots \ldots \ldots$ & 0.059 & 0.042 & $w$ & w & 3.008 & 2.563 & 2.583 & 5.9 \\
\hline $100-249 \ldots \ldots \ldots \ldots \ldots \ldots \ldots$ & W & W & 3.681 & 3.807 & 3.014 & 2.202 & 2.463 & 5.9 \\
\hline $250-499 \ldots \ldots \ldots \ldots \ldots \ldots$ & $w$ & W & 3.021 & 5.762 & 2.899 & 2.158 & 2.423 & 3.2 \\
\hline $500-999 \ldots \ldots \ldots \ldots \ldots \ldots$ & 0.044 & 0.053 & 3.520 & W & 3.064 & 2.193 & 2.397 & 3.9 \\
\hline 1,000 and $O v e r \ldots \ldots \ldots \ldots \ldots \ldots$ & 0.038 & W & 4.785 & 2.216 & 2.800 & 2.230 & 2.344 & 4.0 \\
\hline \multirow[t]{2}{*}{ Total $\ldots \ldots \ldots \ldots \ldots \ldots \ldots \ldots \ldots \ldots$} & 0.046 & 0.051 & 3.696 & 2.835 & 3.026 & 2.223 & 2.398 & 3.2 \\
\hline & \multicolumn{8}{|c|}{ South Census Region } \\
\hline RSE Column Factors: & 0.4 & 1.1 & 1.0 & 1.0 & 0.7 & 3.9 & 0.8 & \\
\hline \multicolumn{9}{|l|}{$\begin{array}{l}\text { Value of Shipments and Receipts } \\
\text { (million dollars) }\end{array}$} \\
\hline Under $20 \ldots \ldots \ldots \ldots \ldots \ldots$ & 0.057 & 0.054 & 2.724 & $W$ & 3.480 & 2.447 & 2.377 & 6.0 \\
\hline $20-49 \ldots \ldots \ldots \ldots \ldots \ldots \ldots$ & 0.046 & 0.023 & $w$ & 3.016 & 2.996 & 2.023 & 1.919 & 6.3 \\
\hline 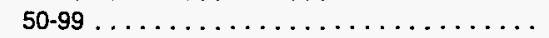 & 0.043 & 0.046 & $W$ & $W$ & 2.655 & 2.247 & 2.043 & 4.9 \\
\hline $100-249 \ldots \ldots \ldots \ldots \ldots \ldots \ldots \ldots \ldots \ldots$ & 0.037 & W & $W$ & 2.263 & 2.263 & 1.793 & 2.004 & 3.9 \\
\hline $250-499 \ldots \ldots \ldots \ldots \ldots \ldots$ & 0.036 & w & 2.671 & 2.272 & 2.028 & 1.734 & 1.883 & 2.4 \\
\hline 500 and Over . . . . . . . . . . . & 0.037 & W & 2.623 & 1.962 & 1.999 & 1.717 & 1.810 & 2.6 \\
\hline Total $\ldots \ldots \ldots \ldots \ldots \ldots \ldots \ldots \ldots$ & 0.042 & 0.020 & 2.790 & 2.380 & 2.490 & 1.776 & 1.919 & 2.2 \\
\hline \multicolumn{9}{|l|}{ Employment Size } \\
\hline$\ldots \ldots \ldots \ldots \ldots$ & 0.054 & W & 3.135 & 2.933 & 3.363 & $w$ & W & 6.6 \\
\hline 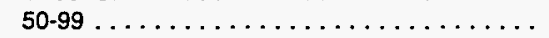 & 0.051 & 0.049 & $W$ & 3.849 & 2.656 & W & W & 4.8 \\
\hline $100-249 \ldots \ldots \ldots \ldots \ldots \ldots \ldots$ & 0.044 & W & W & 2.146 & 2.508 & 1.804 & 1.937 & 5.7 \\
\hline $250-499 \ldots \ldots \ldots \ldots \ldots \ldots$ & W & W & W & 3.969 & 2.787 & 1.758 & 2.017 & 3.2 \\
\hline $500-999 \ldots \ldots \ldots \ldots \ldots \ldots$ & 0.038 & 0.032 & 2.349 & 1.954 & 2.229 & 1.863 & 1.949 & 3.6 \\
\hline 1,000 and $O v e r \ldots \ldots \ldots \ldots \ldots \ldots$ & 0.039 & W & W & 2.786 & 2.238 & 1.718 & 1.903 & 2.0 \\
\hline Total $\ldots \ldots \ldots \ldots \ldots \ldots \ldots \ldots$ & 0.042 & 0.020 & 2.790 & 2.380 & 2.490 & 1.776 & 1.919 & 2.2 \\
\hline
\end{tabular}

See footnotes at end of table. 
Table A50. Selected Energy Operating Ratios for Total Energy Consumption for Heat, Power, and Electricity Generation by Industry Group, Selected Industries, and Economic Characteristics of the Establishment, 1991

\begin{tabular}{|c|c|c|c|c|c|c|c|}
\hline $\begin{array}{l}\text { SIC } \\
\text { Code }\end{array}$ & $\begin{array}{c}\text { Economic } \\
\text { Characteristics }^{b}\end{array}$ & $\begin{array}{c}\text { Consumption per } \\
\text { Employee } \\
\text { (million Btu) }\end{array}$ & $\begin{array}{c}\text { Consumption per } \\
\text { Dollar of Value } \\
\text { Added } \\
\text { (thousand Btu) }\end{array}$ & $\begin{array}{l}\text { Consumption per } \\
\text { Dollar of Value of } \\
\text { Shipments } \\
\text { (thousand Btu) }\end{array}$ & $\begin{array}{l}\text { Major Byproducts } \\
\text { as a Percent of } \\
\text { Consumption } \\
\text { (percent) }\end{array}$ & $\begin{array}{c}\text { Fuel Oil as a } \\
\text { Percent of Natural } \\
\text { Gas } \\
\text { (percent) }\end{array}$ & \multirow[t]{2}{*}{$\begin{array}{c}\text { RSE } \\
\text { Row } \\
\text { Factors }\end{array}$} \\
\hline & RSE Column Factors: & 0.8 & 0.8 & 0.8 & 1.3 & 1.7 & \\
\hline
\end{tabular}

20-39 ALL INDUSTRY GROUPS

Value of Shipments and Receipts

(million dollars)

Under $20 \ldots \ldots \ldots \ldots \ldots \ldots \ldots \ldots$

$20.49 \ldots \ldots \ldots \ldots \ldots \ldots \ldots \ldots \ldots$

$50-99 \ldots \ldots \ldots \ldots \ldots \ldots \ldots \ldots$

$100-249 \ldots \ldots \ldots \ldots \ldots \ldots \ldots \ldots \ldots$

$250-499 \ldots \ldots \ldots \ldots \ldots \ldots \ldots \ldots \ldots \quad 2,680.8$

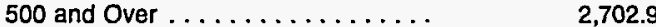

Total ............... 979.6

16.5
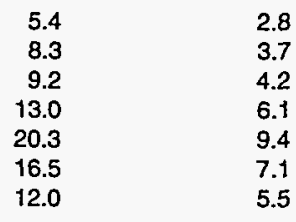

2.8
3.7

4.2

6.1
9.4

7.1

FOOD and KINDRED PRODUCTS

20

Value of Shipments and Receipts

(million dollars)

Under $20 \ldots \ldots \ldots \ldots \ldots \ldots \ldots$

$20-49 \ldots \ldots \ldots \ldots \ldots \ldots . . . \ldots$

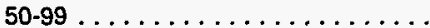

$100-249 \ldots \ldots \ldots \ldots \ldots \ldots \ldots$

$250-499 \ldots \ldots \ldots \ldots \ldots \ldots \ldots$

500 and Over . . . . . . . . . . . .

Total ................

Meat Packing Plants

Value of Shipments and Receipts (million dollars)

Under $20 \ldots \ldots \ldots \ldots \ldots \ldots . . . \ldots$

$20-49 \ldots \ldots \ldots \ldots \ldots \ldots \ldots \ldots$

$50-99 \ldots \ldots \ldots \ldots \ldots \ldots \ldots$

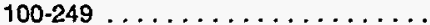

250-499 . . . . . . . . . . . .

500 and Over ................

Total

Canned Fruits and Vegetables

Value of Shipments and Receipts

(million dollars)

Under $20 \ldots \ldots \ldots \ldots \ldots . . .$.

20-49 . . . . . . . . . . . .

$50-99 \ldots \ldots \ldots \ldots \ldots \ldots \ldots \ldots$.

$100-249 \ldots \ldots \ldots \ldots \ldots \ldots \ldots$

250-499 .................

500 and Over $\ldots \ldots \ldots \ldots \ldots \ldots \ldots$

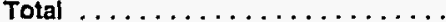

212.0

350.4

340.7

467.8

478.3

438.1

418.5

689.1

655.0

552.3

792.9

W

702.5
6.7

8.6

7.6

8.3

8.4
8.2

8.1
3.2
2.0

2.2

2.9

2.2
13.2

10.3

12.3

10.0
12.8

6.8
10.0

2037

Frozen Fruits and Vegetables

Value of Shipments and Receipts (million dollars)

Under $20 \ldots \ldots \ldots \ldots \ldots \ldots$

$20-49 \ldots \ldots \ldots \ldots \ldots \ldots \ldots$

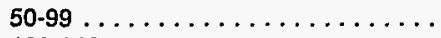

$100-249 \ldots \ldots \ldots \ldots \ldots \ldots \ldots \ldots \ldots \ldots$

$250-499 \ldots \ldots \ldots \ldots \ldots \ldots \ldots$

500 and Over $\ldots \ldots \ldots \ldots \ldots \ldots$

Total

$\ldots \ldots \ldots \ldots \ldots \ldots \ldots$

\begin{tabular}{rrrrrr}
439.8 & 9.7 & 4.3 & 0.0 & $Q$ & 29.1 \\
506.9 & 7.8 & 3.3 & 0.0 & 8.0 & 21.1 \\
854.9 & 15.0 & 5.3 & 0.0 & 14.8 & 8.5 \\
$1,179.9$ & 14.9 & 5.6 & 0.6 & 3.3 & 10.3 \\
881.3 & 10.8 & 2.7 & 0.0 & 1.0 & 4.2 \\
0.0 & 0.0 & 0.0 & 0.0 & 0.0 & NF \\
795.3 & 12.8 & 4.9 & 0.2 & 9.6 & 11.7 \\
\hline
\end{tabular}

See footnotes at end of table. 
Table A49. Average Prices of Purchased Electricity, Steam, and Natural Gas by Type of Supplier, Census Region, and Economic Characteristics of the Establishment, 1991 (Continued) (Estimates in Dollars per Physical Units)

\begin{tabular}{|c|c|c|c|c|c|c|c|c|}
\hline \multirow[b]{2}{*}{$\begin{array}{c}\text { Economic } \\
\text { Characteristics }\end{array}$} & \multicolumn{2}{|c|}{$\begin{array}{c}\text { Electricity } \\
\text { (Million kWh) }\end{array}$} & \multicolumn{2}{|c|}{$\begin{array}{c}\text { Steam } \\
\text { (Billion Btu) }\end{array}$} & \multicolumn{3}{|c|}{$\begin{array}{l}\text { Natural Gas } \\
(1000 \mathrm{cu} \mathrm{ft})\end{array}$} & \multirow[b]{2}{*}{$\begin{array}{c}\text { RSE } \\
\text { Row } \\
\text { Factors }\end{array}$} \\
\hline & Utility Supplier & $\begin{array}{l}\text { Nonutlity } \\
\text { Supplier }\end{array}$ & $\begin{array}{c}\text { Uillity } \\
\text { Supplier }\end{array}$ & $\begin{array}{l}\text { Nonutility } \\
\text { Supplier }\end{array}$ & $\begin{array}{c}\text { Utility } \\
\text { Supplier }\end{array}$ & $\begin{array}{c}\text { Transmission } \\
\text { Pipelines } \\
\end{array}$ & $\begin{array}{c}\text { Other } \\
\text { Supplier }\end{array}$ & \\
\hline & \multicolumn{8}{|c|}{ West Census Region } \\
\hline RSE Column Factors: & 0.6 & 1.7 & 0.6 & 1.5 & 0.9 & 2.5 & 0.6 & \\
\hline \multicolumn{9}{|l|}{$\begin{array}{l}\text { Value of Shipments and Receipts } \\
\text { (million dollars) }\end{array}$} \\
\hline Under 20 & 0.073 & 0.043 & -. & w & 3.787 & 3.317 & 2.708 & 7.7 \\
\hline $20-49 \ldots$ & 0.057 & w & W & 3.353 & 3.750 & 2.462 & 2.609 & 6.0 \\
\hline $50-99$ & 0.046 & 0.020 & w & 4.494 & 3.186 & 2.888 & 2.670 & 4.6 \\
\hline$\ldots \ldots$ & 0.031 & 0.052 & W & 2.008 & 1.931 & 2.300 & 2.292 & 6.8 \\
\hline$\ldots \ldots$ & w & W & -- & w & 2.672 & 2.423 & 2.208 & 3.2 \\
\hline 500 and $O v e r \ldots \ldots \ldots \ldots \ldots \ldots \ldots$ & 0.055 & w & -- & 4.498 & 4.498 & 2.506 & 2.845 & 1.6 \\
\hline Total $\ldots \ldots \ldots \ldots \ldots \ldots \ldots \ldots \ldots \ldots$ & 0.045 & 0.045 & $w$ & 3.366 & 3.045 & 2.539 & 2.499 & 5.4 \\
\hline \multicolumn{9}{|l|}{ Employment Size } \\
\hline Under $50 \ldots$. & 0.078 & w & -- & w & 4.047 & w & 2.737 & 6.8 \\
\hline $50-99$ & 0.061 & $w$ & -- & w & 3.443 & w & w & 7.5 \\
\hline $100-249$ & 0.052 & w & $w$ & 3.731 & 2.390 & 2.399 & 2.505 & 7.8 \\
\hline $250-499$ & 0.042 & 0.028 & w & 3.163 & 3.235 & 2.110 & 2.647 & 5.0 \\
\hline $500-999$ & 0.031 & w & w & w & 3.630 & 2.698 & 2.463 & 4.4 \\
\hline$\cdots \cdots, \cdots,{ }_{1}$ & 0.044 & w & -- & w & 3.092 & 2.438 & 2.354 & 2.4 \\
\hline Total $\ldots \ldots \ldots \ldots \ldots$ & 0.045 & 0.045 & w & 3.366 & 3.045 & 2.539 & 2.499 & 5.4 \\
\hline
\end{tabular}

- Value of Shipments and Receipts and Employment Size categories were supplied by the Bureau of the Census. See Appendix B.

- A "Utility" is a company that produces and/or delivers electricity and/or natural gas, and is legally obligated to provide service to the public within its franchise area.

Includes independent power producers, small power producers, and cogenerators not located at the establishment site.

- Other suppliers of natural gas include such sources as brokers and producers.

- Estimate less than 0.5. Data are included in higher level totals.

$W=$ Withheld to avoid disclosing data for individual establishments. Data are included in higher level totals.

$\mathrm{Q}=$ Withheld because Relative Standard Error is greater than 50 percent. Data are included in higher level totals.

- Estimation of average price is not applicable.

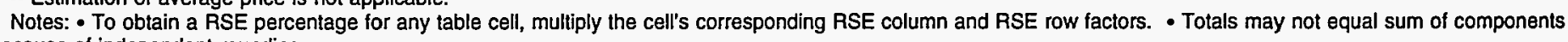
because of independent rounding.

Source: Energy Information Administration, Office of Energy Markets and End Use, Energy End Use and Integrated Statistics Division, Form ElA-846, "1991 Manufacturing Energy Consumption Survey," and Bureau of the Census, Industry Division, data files for the "1991 Annual Survey of Manufactures." 
Table A50. Selected Energy Operating Ratios for Total Energy Consumption for Heat, Power, and Electricity Generation by Industry Group, Selected Industries, and Economic Characteristics of the Establishment, 1991 (Continued)

\begin{tabular}{|c|c|c|c|c|c|c|c|}
\hline $\begin{array}{l}\text { SIC } \\
\text { Code }\end{array}$ & $\begin{array}{c}\text { Economic } \\
\text { Characteristics }^{b}\end{array}$ & $\begin{array}{l}\text { Consumption per } \\
\text { Employee } \\
\text { (million Btu) }\end{array}$ & $\begin{array}{l}\text { Consumption per } \\
\text { Dollar of Value } \\
\text { Added } \\
\text { (thousand Btu) }\end{array}$ & $\begin{array}{l}\text { Consumption per } \\
\text { Dollar of Value of } \\
\text { Shipments } \\
\text { (thousand Btu) }\end{array}$ & $\begin{array}{l}\text { Major Byproducts } \\
\text { as a Percent of } \\
\text { Consumption } \\
\text { (percent) }\end{array}$ & $\begin{array}{c}\text { Fuel Oild as a } \\
\text { Percent of Natural } \\
\text { Gas } \\
\text { (percent) }\end{array}$ & \multirow[t]{2}{*}{$\begin{array}{l}\text { RSE } \\
\text { Row } \\
\text { Factors }\end{array}$} \\
\hline & mn Factors: & 0.8 & 0.8 & 0.8 & 1.3 & 1.7 & \\
\hline
\end{tabular}

2046

Wet Corn Milling

Value of Shipments and Receipts

(million dollars)

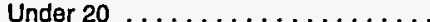

$20-49 \ldots \ldots \ldots \ldots \ldots \ldots \ldots$

$50-99 \ldots \ldots \ldots \ldots \ldots \ldots \ldots$

$100-249 \ldots \ldots \ldots \ldots \ldots \ldots \ldots \ldots$

250-499 ................

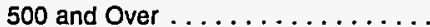

Total ................

2051 Bread, Cake, and Related Products

Value of Shipments and Receipts

(million dollars)

Under $20 \ldots \ldots \ldots \ldots \ldots \ldots \ldots$. . . . .

$20-49 \ldots \ldots \ldots \ldots \ldots \ldots \ldots \ldots$

$50-99 \ldots \ldots \ldots \ldots \ldots \ldots \ldots \ldots$

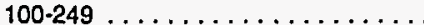

$250-499 \ldots \ldots \ldots \ldots \ldots \ldots \ldots$

500 and Over . . . . . . . . . .

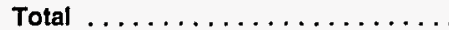

$\begin{array}{rr}W & W \\ W & W \\ W & W \\ 11,854.7 & 43.0 \\ 12,325.9 & 37.9 \\ W & W \\ 15,301.7 & 43.0\end{array}$

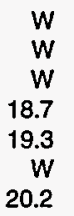

0.0
0.0
0.0
0.0
$W$
0.0
$W$

2063

Beet Sugar

Value of Shipments and Receipts (million dollars)

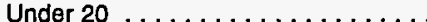

$20-49 \ldots \ldots \ldots \ldots \ldots \ldots \ldots \ldots$

$50-99 \ldots \ldots \ldots \ldots \ldots \ldots \ldots \ldots$

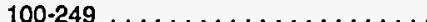

$250-499 \ldots \ldots \ldots \ldots \ldots \ldots \ldots$

500 and Over . . . . . . . . . .

Total

\section{Soybean Oil Mills}

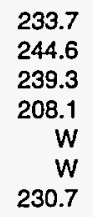

5.3
3.5
2.5
1.7
$W$
$W$
3.2

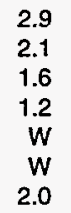

0.0

2075

Value of Shipments and Receipts

(million dollars)

Under $20 \ldots \ldots \ldots \ldots \ldots \ldots \ldots$. . . .

$20-49 \ldots \ldots \ldots \ldots \ldots \ldots \ldots \ldots$

$50-99 \ldots \ldots \ldots \ldots \ldots \ldots \ldots$

100-249.

250-499 ................

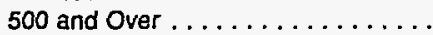

Total

\section{Malt Beverages}

Value of Shipments and Receipts

(million dollars)

Under $20 \ldots \ldots \ldots \ldots \ldots \ldots$. . . .

$20-49 \ldots \ldots \ldots \ldots \ldots \ldots \ldots$

$50-99 \ldots \ldots \ldots \ldots \ldots \ldots \ldots \ldots$

$100-249 \ldots \ldots \ldots \ldots \ldots \ldots \ldots$

$250-499 \ldots \ldots \ldots \ldots \ldots \ldots \ldots$.

500 and Over . . . . . . . . . .

Total ...................

$\begin{array}{rr}2,560.1 & 41.8 \\ 2,781.1 & 13.3 \\ W & W \\ 7,894.3 & 48.6 \\ 8,593.2 & 44.3 \\ W & W \\ 7,865.1 & 42.6\end{array}$

0.0
98.9
81.7
77.7
0.0
0.0
83.7

0.0
33.2
27.2
28.7
0.0
0.0
28.7

$8,855.0$

83.7

9.7
4.9
$W$
4.7
5.1
$W$
5.1

0.0
0.0
0.0
0.0
0.0
$W$
$W$

See footnotes at end of table. 
Table A50. Selected Energy Operating Ratios for Total Energy Consumption for Heat, Power, and Electricity Generation by Industry Group, Selected Industries, and Economic Characteristics of the Establishment, 1991 (Continued)

\begin{tabular}{|c|c|c|c|c|c|c|c|}
\hline $\begin{array}{c}\text { SIC } \\
\text { Code" }\end{array}$ & $\begin{array}{c}\text { Economic } \\
\text { Characteristics }^{b}\end{array}$ & $\begin{array}{c}\text { Consumption per } \\
\text { Employee } \\
\text { (million Btu) }\end{array}$ & $\begin{array}{c}\text { Consumption per } \\
\text { Dollar of Value } \\
\text { Added } \\
\text { (thousand Btu) }\end{array}$ & $\begin{array}{c}\text { Consumption per } \\
\text { Dollar of Value of } \\
\text { Shipments } \\
\text { (thousand Btu) }\end{array}$ & $\begin{array}{l}\text { Major Byproducts } \\
\text { as a Percent of } \\
\text { Consumption } \\
\text { (percent) }\end{array}$ & $\begin{array}{c}\text { Fuel OId as a } \\
\text { Percent of Natural } \\
\text { Gas } \\
\text { (percent) }\end{array}$ & \multirow[t]{2}{*}{$\begin{array}{c}\text { RSE } \\
\text { Row } \\
\text { Factors }\end{array}$} \\
\hline & mn Factors: & 0.8 & 0.8 & 0.8 & 1.3 & 1.7 & \\
\hline
\end{tabular}

21

TOBACCO PRODUCTS

Value of Shipments and Receipts (million dollars)

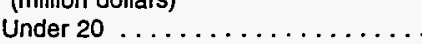

$20-49 \ldots \ldots \ldots \ldots \ldots \ldots \ldots \ldots$

$50-99 \ldots \ldots \ldots \ldots \ldots \ldots \ldots . . . \ldots$

$100-249 \ldots \ldots \ldots \ldots \ldots \ldots \ldots . \ldots \ldots$

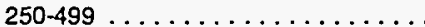

500 and Over .............

Total

$\begin{array}{rr}226.2 & 4.0 \\ 696.6 & 7.4 \\ 347.3 & 2.7 \\ W & W \\ W & W \\ 538.1 & 0.6 \\ 629.2 & 1.0\end{array}$

2.3
3.8
1.2
$W$
$W$
0.5
0.8

0.0

0.0

0.0

0.0

0.0

0.0
0.0

19.6
29.8
13.0
4.9
3.3
3.4
5.8

22

TEXTILE MILL PRODUCTS

Value of Shipments and Receipts

(million dollars)

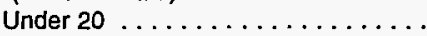

$20-49 \ldots \ldots \ldots \ldots \ldots \ldots \ldots \ldots . . . \ldots$

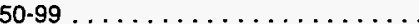

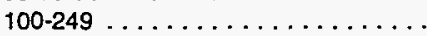

$250-499 \ldots \ldots \ldots \ldots \ldots \ldots \ldots$

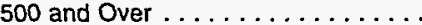

Total ...................

283.4

454.8

559.6

$W$
853.2

W

459.4

\section{APPAREL and OTHER TEXTILE} PRODUCTS

Value of Shipments and Receipts (million dollars)

Under $20 \ldots \ldots \ldots \ldots \ldots \ldots$

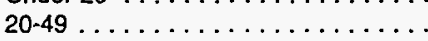

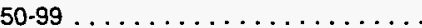

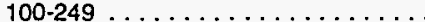

$250-499 \ldots \ldots \ldots \ldots \ldots \ldots$

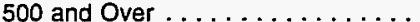

Total ..................

24

LUMBER and WOOD PRODUCTS

Value of Shipments and Receipts

(million dollars)

Under $20 \ldots \ldots \ldots \ldots . . . . .$.

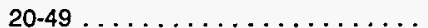

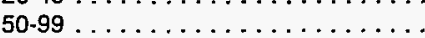

$100-249 \ldots \ldots \ldots \ldots \ldots \ldots \ldots \ldots . \ldots \ldots \ldots$

$250-499$. . . . . . . . . . . .

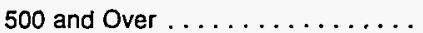

Total ....................

\section{FURNTURE and FIXTURES}

Value of Shipments and Receipts

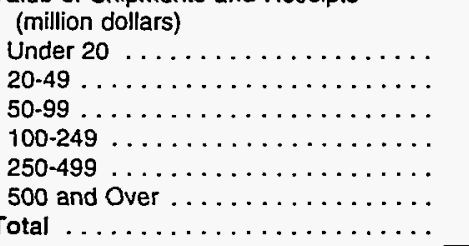

8.7
11.3
11.3
$W$
14.8
$W$
10.6
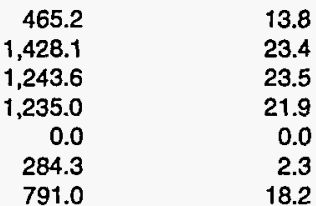

1.6
1.2
1.3
1.6
0.6
$Q$
1.4

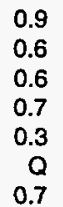

0.0
0.0
0.0
0.0
0.0
0.0
0.0

12.5

16.8

17.9

30.2

21.7

12.2

11.5

\begin{tabular}{|c|c|c|c|c|}
\hline 165.7 & 4.1 & 2.0 & 0.0 & 19.1 \\
\hline 139.2 & 3.2 & 1.6 & 0.0 & 11.5 \\
\hline 173.8 & 3.9 & 2.0 & 0.0 & 1.7 \\
\hline 149.3 & 2.1 & 1.0 & 0.0 & 8.0 \\
\hline 96.0 & 1.1 & 0.6 & 0.0 & 3.0 \\
\hline 277.2 & 3.7 & 2.4 & 0.0 & 1.2 \\
\hline 159.0 & 3.5 & 1.8 & 0.0 & 11.3 \\
\hline
\end{tabular}

See footnotes at end of table. 
Table A50. Selected Energy Operating Ratios for Total Energy Consumption for Heat, Power, and Electricity Generation by Industry Group, Selected Industries, and Economic Characteristics of the Establishment, 1991 (Continued)

\begin{tabular}{|c|c|c|c|c|c|c|c|}
\hline $\begin{array}{c}\text { SIC } \\
\text { Codes }\end{array}$ & $\begin{array}{c}\text { Economic } \\
\text { Characteristles }^{\circ}\end{array}$ & $\begin{array}{c}\text { Consumption per } \\
\text { Employee } \\
\text { (million Btu) }\end{array}$ & $\begin{array}{c}\text { Consumption per } \\
\text { Dollar of Value } \\
\text { Added } \\
\text { (thousand Btu) }\end{array}$ & $\begin{array}{c}\text { Consumption per } \\
\text { Dollar of Value of } \\
\text { Shipments } \\
\text { (thousand Btu) } \\
\end{array}$ & $\begin{array}{l}\text { Major Byproducts } \\
\text { as a Percent of } \\
\text { Consumption } \\
\text { (percent) }\end{array}$ & $\begin{array}{c}\text { Fuel Oil as a } \\
\text { Percent of Natural } \\
\text { Gas } \\
\text { (percent) }\end{array}$ & \multirow[t]{2}{*}{$\begin{array}{c}\text { RSE } \\
\text { Row } \\
\text { Factors }\end{array}$} \\
\hline & mn Factors: & 0.8 & 0.8 & 0.8 & 1.3 & 1.7 & \\
\hline
\end{tabular}

26

PAPER and ALLIED PRODUCTS

Value of Shlpments and Receipts

(million dollars)

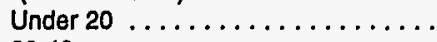

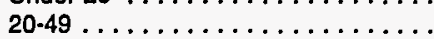

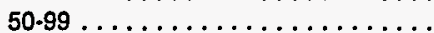

$100-249 \ldots \ldots \ldots \ldots \ldots \ldots \ldots \ldots$

250-499 $\ldots \ldots \ldots \ldots \ldots \ldots \ldots$

500 and Over .................

Total .....................

443.6

679.8

$2,927.4$

$9,468.0$

$12,181.4$

$5,900.1$

$4,234.5$

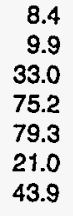

Pulp Mills

Value of Shipments and Recelpts

(million dollars)

Under $20 \ldots \ldots \ldots \ldots \ldots \ldots$.

$20.49 \ldots \ldots \ldots \ldots \ldots \ldots \ldots$.

$50-99 \ldots \ldots \ldots \ldots \ldots \ldots \ldots$.

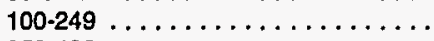

250-499 ..................

500 and $O v e r . \ldots \ldots \ldots \ldots \ldots$.

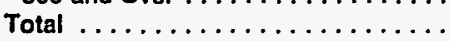

2621

Paper Mills

Value of Shipments and Receipts

(million dollars)

Under $20 \ldots \ldots \ldots \ldots \ldots \ldots$.

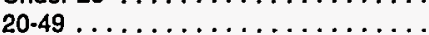

$50.99 \ldots \ldots \ldots \ldots \ldots \ldots \ldots \ldots$

$100-249 \ldots \ldots \ldots \ldots \ldots \ldots \ldots$.

250-499 .....................

500 and Over ...............

Total ......................

2631

Paperboard Mills

Value of Shipments and Receipts

(million dollars)

Under $20 \ldots \ldots \ldots \ldots \ldots .$.

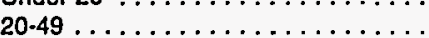

$50-99 \ldots \ldots \ldots \ldots \ldots \ldots \ldots$.

$100-249 \ldots \ldots \ldots \ldots \ldots \ldots$.

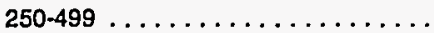

500 and Over ................

Total ....................

PRINTING and PUBLISHING

27

Value of Shipments and Receipts

(million dollars)

Under $20 \ldots \ldots \ldots \ldots \ldots \ldots$.

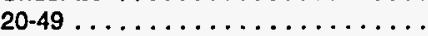

$50-99 \ldots \ldots \ldots \ldots \ldots \ldots \ldots$.

100-249 ..................

$250-499 \ldots \ldots \ldots \ldots \ldots \ldots \ldots$.

500 and Over ................

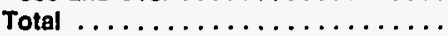

0.0

$4,725.7$

$15,957.9$

$21,823.3$

$21,254.2$

$20,034.8$

3.7
3.8
14.2
35.0
39.5
12.7
20.1

3.7

3.8

35.0

39.5

20.1
0.0

0.0
17.0

38.2

41.3

32.8

34.7

0.0
127.0

62.7
58.3

61.0

0.0

59.3

$\begin{array}{rr}0.0 & \text { NF } \\ 1.9 & 23.2 \\ 50.6 & 9.6 \\ 85.8 & 5.5 \\ 158.9 & 5.6 \\ 0.0 & \text { NF } \\ 89.9 & 4.6\end{array}$

$3,489.0$

$2,818.2$

$5,691.5$

$8,669.1$

$11,994.8$

$11,611.3$

$9,433.1$

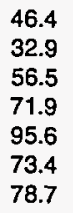

46.4
32.9

56.5

71.9
95.6

73.4

78.7
19.3

14.5

24.3

32.8

45.2

41.7

36.7
0.0

10.0

22.0

36.4

40.3

29.4 
Table A50. Selected Energy Operating Ratios for Total Energy Consumption for Heat, Power, and Electricity Generation by Industry Group, Selected Industries, and Economic Characteristics of the Establishment, 1991 (Continued)

\begin{tabular}{|c|c|c|c|c|c|c|c|}
\hline $\begin{array}{l}\text { SiC } \\
\text { Code" }\end{array}$ & $\begin{array}{c}\text { Economic } \\
\text { Characteristics }^{\circ}\end{array}$ & $\begin{array}{l}\text { Consumption per } \\
\text { Employee } \\
\text { (million Btu) }\end{array}$ & $\begin{array}{c}\text { Consumption per } \\
\text { Dollar of Value } \\
\text { Added } \\
\text { (thousand Btu) }\end{array}$ & $\begin{array}{l}\text { Consumption per } \\
\text { Dollar of Value of } \\
\text { Shipments } \\
\text { (thousand Btu) }\end{array}$ & $\begin{array}{l}\text { Major Byproducts } \\
\text { as a Percent of } \\
\text { Consumption } \\
\text { (percent) }\end{array}$ & $\begin{array}{c}\text { Fuel Oil as a } \\
\text { Percent of Natural } \\
\text { Gas } \\
\text { (percent) }\end{array}$ & \multirow[t]{2}{*}{$\begin{array}{c}\text { RSE } \\
\text { Row } \\
\text { Factors }\end{array}$} \\
\hline & Imn Factors: & 0.8 & 0.8 & 0.8 & 1.3 & 1.7 & \\
\hline
\end{tabular}

28

CHEMICALS and ALLIED PRODUCTS

Value of Shipments and Receipts

(million dollars)

Under $20 \ldots \ldots \ldots \ldots \ldots . . . .$.

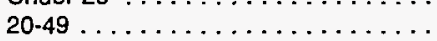

$50-99 \ldots \ldots \ldots \ldots \ldots \ldots \ldots \ldots$

$100-249 \ldots \ldots \ldots \ldots \ldots \ldots \ldots$.

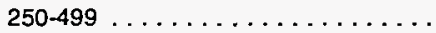

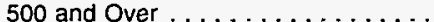

Total ..................
$1,100.1$

$3,454.2$

$3,568.4$

$4,064.3$

$5,815.1$

$5,670.1$

$4,205.7$

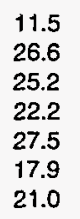

2812

Alkalies and Chlorine

Value of Shipments and Receipts

(million dollars)

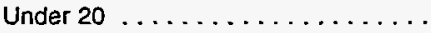

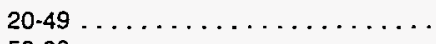

$50-99 \ldots \ldots \ldots \ldots \ldots \ldots$

$100-249 \ldots \ldots \ldots \ldots \ldots \ldots \ldots$

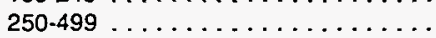

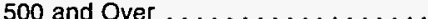

Total ...........

2813

Industrial Gases

Value of Shipments and Receipts

(million dollars)

Under $20 \ldots . . . \ldots . . . . .$.

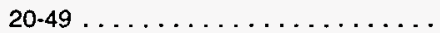

$50-99 \ldots \ldots \ldots \ldots \ldots \ldots$.

$100-249 \ldots \ldots \ldots \ldots \ldots \ldots \ldots$

250-499 ................

500 and Over ...............

Total ........

Industrial Inorganic Chemicals, nec

Value of Shipments and Receipts

(million dollars)

Under $20 \ldots \ldots \ldots \ldots \ldots . . . .$.

$20-49 \ldots \ldots \ldots \ldots \ldots \ldots \ldots . \ldots$

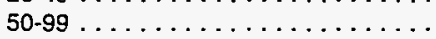

$100-249 \ldots \ldots \ldots \ldots \ldots \ldots \ldots$

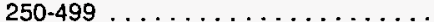

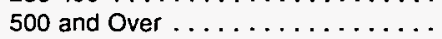

Total $\ldots \ldots \ldots \ldots \ldots \ldots \ldots \ldots$

Plastics Materials and Resins

Value of Shipments and Receipts

(million dollars)

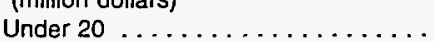

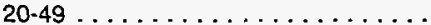

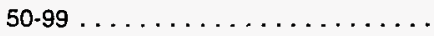

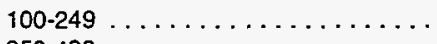

$250-499 \ldots \ldots \ldots \ldots \ldots \ldots \ldots$

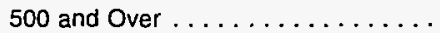

Total
$5,310.2$

$24,315.7$

$26,119.6$

$55,761.1$

0.0

$11,072.8$
$W$
157.6
88.1
$W$
0.0
$W$
129.0

39.4
44.5
87.6
68.9
0.0
0.0
51.0
282
W

4,349.5

$3,334.4$

$8,143.2$

$9,827.6$

$W$
$4,144.4$

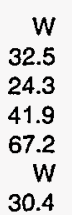

$W$
32.5
24.3
41.9
67.2
$W$
30.4
5.3

11.4

11.4

11.3

13.9

11.0

11.1

$W$
1.2
1.8
4.4
6.4
19.2
9.9

$6.3 \quad 15.7$

W 18.1

$7.7 \quad 12.9$

$\begin{array}{ll}W & 9.9 \\ 4.4 & 8.7\end{array}$

1.9

$\begin{array}{ll}1.9 & 6.5 \\ 3.6 & 5.0\end{array}$

24.5

30.9

48.9

51.3

0.0

0.0

33.1

$W$
57.9
41.6
$W$
0.0
$W$
66.0

0.0

0.0

0.0

0.0
0.0

0.0

0.0

$\begin{array}{rr}0.0 & 8.6 \\ W & 15.6 \\ W & 7.8 \\ W & 9.7 \\ 0.0 & N F \\ W & 2.5 \\ W & 5.9\end{array}$

5.9

See footnotes at end of table. 
Table A50. Selected Energy Operating Ratios for Total Energy Consumption for Heat, Power, and Electricity Generation by Industry Group, Selected Industries, and Economic Characteristics of the Establishment, 1991 (Continued)

\begin{tabular}{|c|c|c|c|c|c|c|c|}
\hline $\begin{array}{l}\text { SIC } \\
\text { Code }\end{array}$ & $\begin{array}{c}\text { Economic } \\
\text { Characteristics }^{b}\end{array}$ & $\begin{array}{l}\text { Consumption per } \\
\text { Employee } \\
\text { (million Btu) }\end{array}$ & $\begin{array}{l}\text { Consumption per } \\
\text { Dollar of Value } \\
\text { Added } \\
\text { (thousand Btu) }\end{array}$ & $\begin{array}{l}\text { Consumption per } \\
\text { Dollar of Value of } \\
\text { Shipments } \\
\text { (thousand Btu) }\end{array}$ & $\begin{array}{l}\text { Major Byproducts } \\
\text { as a Percent of } \\
\text { Consumption } \\
\text { (percent) }\end{array}$ & $\begin{array}{c}\text { Fuel Oild as a } \\
\text { Percent of Natural } \\
\text { Gas } \\
\text { (percent) }\end{array}$ & \multirow[t]{2}{*}{$\begin{array}{c}\text { RSE } \\
\text { Row } \\
\text { Factors }\end{array}$} \\
\hline & umn Factors: & 0.8 & 0.8 & 0.8 & 1.3 & 1.7 & \\
\hline
\end{tabular}

2822

Synthetic Rubber Value of Shipments and Receipts
(million dollars)

Under $20 \ldots \ldots \ldots \ldots \ldots \ldots \ldots \ldots$. . . . . . . . . . . . . . .

$20-49 \ldots \ldots \ldots \ldots \ldots \ldots \ldots \ldots \ldots$

$50-99 \ldots \ldots \ldots \ldots \ldots \ldots \ldots \ldots \ldots \quad 4,0.2$

$100-249 \ldots \ldots \ldots \ldots \ldots \ldots \ldots . \ldots \ldots$

$250-499 \ldots \ldots \ldots \ldots \ldots \ldots \ldots \ldots \quad 18,099.4$

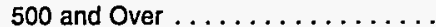

$8,099.4$
0.0

$10,047.4$

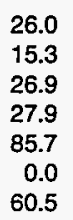

6.2
5.7
10.8
12.5
41.6
0.0
27.3

0.0
0.0
0.0
0.0
0.0
0.0
0.0

20.4

Cellulosic Manmade Fibers

Value of Shlpments and Receipts (million dollars)

Under $20 \ldots \ldots \ldots \ldots \ldots \ldots \ldots$. . . . .

$20-49 \ldots \ldots \ldots \ldots \ldots \ldots \ldots \ldots \ldots \ldots$

$50-99 \ldots \ldots \ldots \ldots \ldots \ldots \ldots \ldots$

$100-249 \ldots \ldots \ldots \ldots \ldots \ldots \ldots \ldots$

$250-499 \ldots \ldots \ldots \ldots \ldots \ldots . . . \ldots$

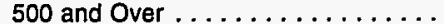

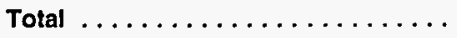

341.1
0.0
$2,362.4$
$5,319.5$
$5,110.1$
$1,211.4$
$2,869.3$

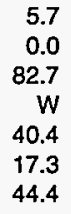

1.6
0.0
49.2
69.9
25.4
7.8
20.8

0.0
0.0
0.0
0.0
0.0
0.0
0.0

$\begin{array}{rr}0.0 & 23.4 \\ 0.0 & N F \\ 79.5 & 4.0 \\ W & 4.0 \\ 1.3 & 2.2 \\ 14.9 & 5.7 \\ W & 16.2\end{array}$

2824

Organic Flbers, Noncellulosic

Value of Shipments and Receipts

(million dollars)

Under $20 \ldots \ldots \ldots \ldots \ldots . . . \ldots$

$20.49 \ldots \ldots \ldots \ldots \ldots \ldots$

$50-99 \ldots \ldots \ldots \ldots \ldots \ldots \ldots$

$100-249 \ldots \ldots \ldots \ldots \ldots \ldots \ldots$

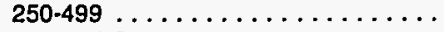

500 and Over $\ldots \ldots \ldots \ldots \ldots \ldots \ldots$

Total $\ldots \ldots \ldots \ldots \ldots \ldots \ldots . . . .$.

Cyclic Crudes and Intermediates

Value of Shipments and Receipts

(million dollars)

Under $20 \ldots \ldots \ldots \ldots \ldots \ldots \ldots$

$20-49 \ldots \ldots \ldots \ldots \ldots \ldots \ldots \ldots$

$50-99 \ldots \ldots \ldots \ldots \ldots \ldots \ldots \ldots$

$100-249 \ldots \ldots \ldots \ldots \ldots \ldots \ldots \ldots$

$250-499 \ldots \ldots \ldots \ldots \ldots \ldots$

500 and Over ...............

Total $\ldots \ldots \ldots \ldots \ldots \ldots$

Industrial Organic Chemicals, nec

Value of Shipments and Receipts

(million dollars)

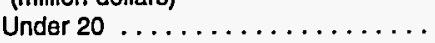

$20-49 \ldots \ldots \ldots \ldots \ldots \ldots \ldots$.

$50-99 \ldots \ldots \ldots \ldots \ldots \ldots \ldots$.

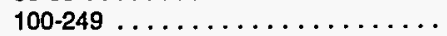

$250-499 \ldots \ldots \ldots \ldots \ldots \ldots \ldots$

500 and Over . . . . . . . . . . .

Total .
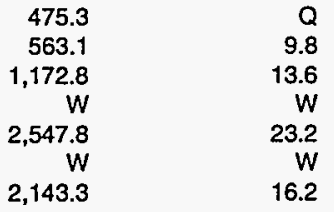

$1,453.7$
$W$
$3,680.9$
$3,510.1$
$16,280.6$
$W$
$7,348.4$

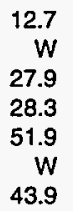

$2,489.9$

$4,414.5$

$3,143.9$

$9,356.0$

$10,590.6$

$19,805.2$

$11,886.6$

\begin{tabular}{lr}
27.3 & 12.2 \\
28.2 & 13.1 \\
17.2 & 7.3 \\
44.5 & 20.0 \\
48.2 & 19.7 \\
65.5 & 27.8 \\
52.2 & 22.3 \\
\hline
\end{tabular}

0.0
0.0
0.0
0.0
0.0
$W$
1.0

$\begin{array}{rr}Q & 44.3 \\ W & 2.8 \\ W & 3.1 \\ W & 2.6 \\ 8.0 & 2.6 \\ W & 3.2 \\ W & 3.4\end{array}$

3.9
5.5
5.8
$W$
10.8
$W$
9.0

5.7
$W$
11.5
10.3
17.2
$W$
15.6

$W$
0.0
$W$
0.0
$W$
$W$
$W$

$\begin{array}{rr}Q & 29.9 \\ W & 9.9 \\ W & 8.6 \\ 16.8 & 8.0 \\ W & 8.8 \\ W & 8.9 \\ 9.3 & 7.4\end{array}$

See footnotes at end of table. 
Table A50. Selected Energy Operating Ratios for Total Energy Consumption for Heat, Power, and Electricity Generation by Industry Group, Selected Industries, and Economic Characteristics of the Establishment, 1991 (Continued)

\begin{tabular}{|c|c|c|c|c|c|c|c|}
\hline $\begin{array}{l}\text { SIC } \\
\text { Code }\end{array}$ & $\begin{array}{c}\text { Economic } \\
\text { Characteristics } \\
\end{array}$ & $\begin{array}{c}\text { Consumption per } \\
\text { Employee } \\
\text { (million Btu) } \\
\end{array}$ & $\begin{array}{c}\text { Consumption per } \\
\text { Dollar of Value } \\
\text { Added } \\
\text { (thousand Btu) }\end{array}$ & $\begin{array}{l}\text { Consumption per } \\
\text { Dollar of Value of } \\
\text { Shipments } \\
\text { (thousand Btu) }\end{array}$ & $\begin{array}{l}\text { Major Byproducts } \\
\text { as a Percent of } \\
\text { Consumption } \\
\text { (percent) }\end{array}$ & $\begin{array}{c}\text { Fuel Olld as a } \\
\text { Percent of Natural } \\
\text { Gas } \\
\text { (percent) }\end{array}$ & \multirow[t]{2}{*}{$\begin{array}{c}\text { RSE } \\
\text { Row } \\
\text { Factore }\end{array}$} \\
\hline & nn Factors: & 0.8 & 0.8 & 0.8 & 1.3 & 1.7 & \\
\hline
\end{tabular}

$2873 \quad$ Nitrogenous Fertllizers

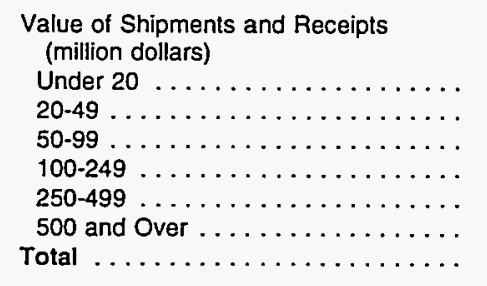

$\begin{array}{rr}973.9 & 12.0 \\ 39,593.0 & 255.7 \\ 43,614.8 & 209.7 \\ 59,577.3 & 283.6 \\ 124,935.2 & 207.7 \\ 0.0 & 0.0 \\ 43,985.2 & 229.2\end{array}$

2874

Phosphatic Fertilizers

Value of Shipments and Receipts (million dollars)

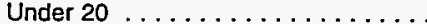

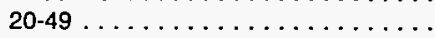

$50-99 \ldots \ldots \ldots \ldots \ldots \ldots \ldots \ldots$

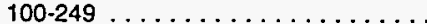

$250-499 \ldots \ldots \ldots \ldots \ldots \ldots \ldots \ldots \ldots$

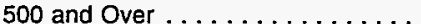

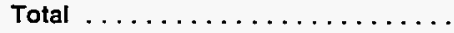

$\begin{array}{rr}1,525.6 & 22.6 \\ W & W \\ 2,917.1 & 46.6 \\ W & W \\ 4,534.3 & 30.2 \\ W & W \\ 3,396.7 & 25.4\end{array}$

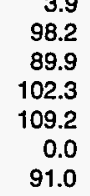

3.9
98.2
89.9
02.3
09.2
0.0
91.0

PETROLEUM and COAL PRODUCTS

Value of Shipments and Receipts (million dollars)

Under $20 \ldots \ldots \ldots \ldots \ldots \ldots$. . . . . . .

20-49 . . . . . . . . . . . .

$50-99 \ldots \ldots \ldots \ldots \ldots \ldots \ldots$

$100-249 \ldots \ldots \ldots \ldots \ldots \ldots \ldots$

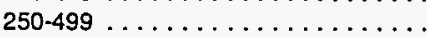

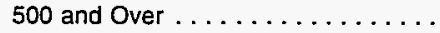

Total ...................

2911

Petroleum Refining

Value of Shipments and Receipts (million dollars)

Under $20 \ldots \ldots \ldots \ldots \ldots \ldots \ldots$

$20-49 \ldots \ldots \ldots \ldots \ldots \ldots \ldots$

$50-99 \ldots \ldots \ldots \ldots \ldots \ldots \ldots \ldots$

$100-249 \ldots \ldots \ldots \ldots \ldots \ldots$

250-499 .................

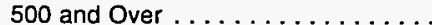

Total $\ldots \ldots \ldots \ldots \ldots \ldots \ldots \ldots$
$26,582.5$

$28,571.9$

$7,731.8$

$20,313.9$

$30,983.5$

$42,403.1$

$39,377.8$
32.6

16.7

37.4

63.6

135.7

148.9

120.5

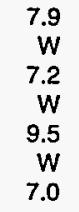

11.5

4.5

10.4

13.0

17.6

20.3

18.6
7.9
$W$
7.2
$W$
9.5
$W$
7.0

0.0
1.1
0.6
0.7
0.0
0.0
0.7

0.0
0.0
0.0
0.0
0.0
0.0
0.0

$\begin{array}{rr}13.4 & 14.4 \\ W & 1.6 \\ Q & 19.2 \\ W & 3.2 \\ W & 2.7 \\ W & 2.2 \\ 12.9 & 5.5\end{array}$

30

RUBBER and MISC. PLASTICS PRODUCTS

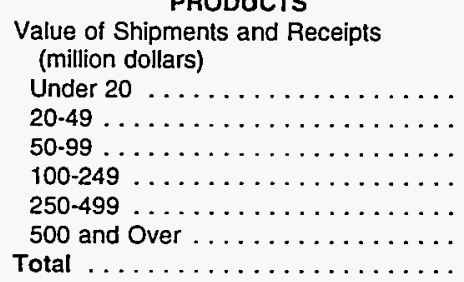

647.7

129.7

57.2

107.7

135.7

148.9

146.0
98.8

28.5

9.2

17.5

17.6

20.3

20.0

$\begin{array}{rr}W & 17.8 \\ 53.7 & 29.8 \\ W & 16.1 \\ W & 7.7 \\ W & 1.6 \\ W & 1.4 \\ 12.9 & 3.1\end{array}$

286.4

321.8

679.6

W

294.2

\begin{tabular}{rrrr}
4.2 & 2.1 & 0.0 & 6.3 \\
4.3 & 2.1 & 0.1 & 14.6 \\
5.0 & 2.4 & $W$ & $W$ \\
7.4 & 3.8 & $W$ & $W$ \\
$W$ & $W$ & $W$ & $W$ \\
$W$ & $W$ & 0.0 & 11.3 \\
4.8 & 2.4 & 0.1 & $W$ \\
\hline
\end{tabular}

See footnotes at end of table. 
Table A50. Selected Energy Operating Ratios for Total Energy Consumption for Heat, Power, and Electricity Generation by Industry Group, Selected Industries, and Economic Characteristics of the Establishment, 1991 (Continued)

\begin{tabular}{|c|c|c|c|c|c|c|c|}
\hline $\begin{array}{c}\text { SIC } \\
\text { Code" }\end{array}$ & $\begin{array}{c}\text { Economic } \\
\text { Characteristics }^{\circ}\end{array}$ & $\begin{array}{c}\text { Consumption per } \\
\text { Employee } \\
\text { (million Btu) }\end{array}$ & $\begin{array}{c}\text { Consumption per } \\
\text { Dollar of Value } \\
\text { Added } \\
\text { (thousand Btu) }\end{array}$ & $\begin{array}{c}\text { Consumption per } \\
\text { Dollar of Value of } \\
\text { Shipments } \\
\text { (thousand Btu) }\end{array}$ & $\begin{array}{l}\text { Major Byproducts } \\
\text { as a Percent of } \\
\text { Consumption } \\
\text { (percent) }\end{array}$ & $\begin{array}{c}\text { Fuel Oil as a } \\
\text { Percent of Natural } \\
\text { Gas } \\
\text { (percent) }\end{array}$ & \multirow[t]{2}{*}{$\begin{array}{c}\text { RSE } \\
\text { Row } \\
\text { Factors }\end{array}$} \\
\hline & mn Factors: & 0.8 & 0.8 & 0.8 & 1.3 & 1.7 & \\
\hline
\end{tabular}

3221

Glass Containers

Value of Shipments and Receipts

(million dollars)

Under $20 \ldots \ldots \ldots \ldots \ldots \ldots$

$20-49 \ldots \ldots \ldots \ldots \ldots \ldots \ldots \ldots$

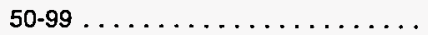

$100-249 \ldots \ldots \ldots \ldots \ldots \ldots \ldots \ldots$

$250-499 \ldots \ldots \ldots \ldots \ldots \ldots \ldots \ldots$

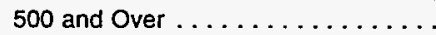

Total $\ldots \ldots \ldots \ldots \ldots$

Pressed and Blown Glass, nec.

Value of Shipments and Receipts (million dollars)

Under $20 \ldots \ldots \ldots \ldots \ldots \ldots \ldots . \ldots . \ldots$

$20-49 \ldots \ldots \ldots \ldots \ldots \ldots \ldots \ldots \ldots$

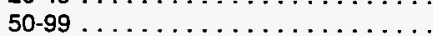

$100-249 \ldots \ldots \ldots \ldots \ldots \ldots \ldots$

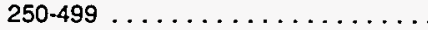

500 and Over . . . . . . . . .

Total .....................

$\begin{array}{rr}W & W \\ W & W \\ 2,709.3 & 32.3 \\ W & W \\ 0.0 & 0.0 \\ 0.0 & 0.0 \\ 2,549.9 & 32.6\end{array}$

$W$
$W$
17.6
$W$
0.0
0.0
17.8

0.0

0.0

0.0

0.0

0.0

32.6

17.8

0.0

$\begin{array}{rr}0.0 & 5.9 \\ W & 3.4 \\ W & 3.0 \\ W & 5.7 \\ 0.0 & \text { NF } \\ 0.0 & \text { NF } \\ 2.7 & 2.8\end{array}$

3241

Cement, Hydraulic

Value of Shipments and Receipts (million dollars)

Under $20 \ldots \ldots \ldots \ldots$. . . . . . $11,578.2$

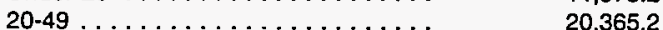

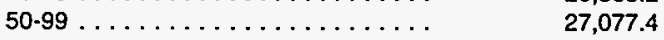

$100-249 \ldots \ldots \ldots \ldots \ldots \ldots \ldots$

$250-499 \ldots \ldots \ldots \ldots \ldots \ldots \ldots$

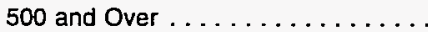

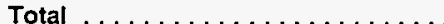

0.0

$W$
2,1523

0.0

0.0

19.2
$W$

26.3

0.0

0.0

13.7
$W$

.7
$W .7$

16.1

0.0

W

0.0
0.0
0.0

Lime

3274

Value of Shipments and Receipts

(million dollars)

Under $20 \ldots \ldots \ldots \ldots \ldots \ldots$

$20-49 \ldots \ldots \ldots \ldots \ldots \ldots \ldots$.

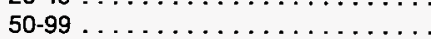

$100-249$

500 and Over . . . . . . . . . . .

Total

Mineral Wool
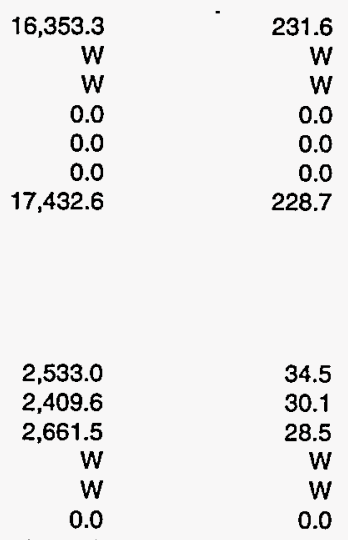

$2,619.8$

146.0
181.5
139.5
0.0
0.0
0.0
169.3

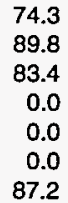

0.0

0.0

0.0

0.0

0.0

0.0
0.0
0.0
0.0
0.0
0.0
0.0

33.9

5.4

3.5

3.4

NF

6.0

3296

Value of Shipments and Receipts

(million dollars)

Under $20 \ldots . . . \ldots \ldots \ldots . . .$.

$20-49 \ldots \ldots \ldots \ldots \ldots \ldots \ldots \ldots$

$50-99 \ldots \ldots \ldots \ldots \ldots \ldots \ldots \ldots$

100-249.

$250-499 \ldots \ldots \ldots \ldots \ldots \ldots \ldots$

500 and Over $\ldots \ldots \ldots \ldots \ldots \ldots$

Total

...................

See footnotes at end of table. 
Table A50. Selected Energy Operating Ratios for Total Energy Consumption for Heat, Power, and Electricity Generation by Industry Group, Selected Industries, and Economic Characteristics of the Establishment, 1991 (Continued)

\begin{tabular}{cc|c|c|c|c|c|c|}
\hline $\begin{array}{c}\text { SIC } \\
\text { Code" }\end{array}$ & $\begin{array}{c}\text { Economic } \\
\text { Characteristics }\end{array}$ & $\begin{array}{c}\text { Consumption per } \\
\text { Employee } \\
\text { (million Btu) }\end{array}$ & $\begin{array}{c}\text { Consumption per } \\
\text { Dollar of Value } \\
\text { Added } \\
\text { (thousand Btu) }\end{array}$ & $\begin{array}{c}\text { Consumption per } \\
\text { Dollar of Value of } \\
\text { Shipments } \\
\text { (thousand Btu) }\end{array}$ & $\begin{array}{c}\text { Major Byproducts } \\
\text { as a Percent of } \\
\text { Consumption } \\
\text { (percent) }\end{array}$ & $\begin{array}{c}\text { Fuel Oild as a } \\
\text { Percent of Natural } \\
\text { Gas } \\
\text { (percent) }\end{array}$ & $\begin{array}{c}\text { RSE } \\
\text { Row } \\
\text { Factors }\end{array}$ \\
\hline RSE Column Factors: & 0.8 & 0.8 & 0.8 & 1.3 & 1.7
\end{tabular}

33

PRIMARY METAL INDUSTRIES

\begin{tabular}{|c|c|}
\hline \multicolumn{2}{|l|}{$\begin{array}{l}\text { Value of Shipments and Receipts } \\
\text { (million dollars) }\end{array}$} \\
\hline Under $20 \ldots \ldots$ & 581.3 \\
\hline $20-49 \ldots$ & 983.3 \\
\hline $50-99 \ldots \ldots \ldots$ & $1,636.5$ \\
\hline $100-249 \ldots \ldots \ldots \ldots \ldots \ldots \ldots$ & $3,531.5$ \\
\hline $250-499 \ldots \ldots \ldots \ldots \ldots \ldots$ & $5,155.5$ \\
\hline 500 and Over $\ldots \ldots \ldots$ & $10,264.8$ \\
\hline Total $\ldots . . \ldots$ & $3,526.9$ \\
\hline
\end{tabular}

3312 Blast Furnaces and Steel Mills

\begin{tabular}{|c|c|}
\hline \multicolumn{2}{|l|}{$\begin{array}{l}\text { Value of Shipments and Receipts } \\
\text { (million dollars) }\end{array}$} \\
\hline Under $20 \ldots \ldots \ldots \ldots$ & 771 \\
\hline $20-49 \ldots$ & 4,659 \\
\hline $50-99 \ldots \ldots \ldots \ldots \ldots \ldots$ & 5,641 \\
\hline $100-249 \ldots \ldots \ldots \ldots \ldots \ldots$ & 4,609 \\
\hline $250-499 \ldots \ldots \ldots \ldots \ldots \ldots$ & 6,306 \\
\hline 500 and Over $\ldots \ldots \ldots \ldots \ldots$ & 11,909 \\
\hline Total $\ldots \ldots \ldots \ldots \ldots \ldots \ldots$ & 8,953 \\
\hline
\end{tabular}

3313

Electrómetalurgical Products

Value of Shipments and Receipts (million dollars)

Under $20 \ldots \ldots \ldots \ldots \ldots \ldots \ldots$

$20-49 \ldots \ldots \ldots \ldots \ldots \ldots \ldots \ldots$

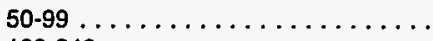

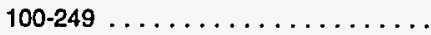

$250-499 \ldots \ldots \ldots \ldots \ldots \ldots \ldots$

500 and Over $\ldots \ldots \ldots \ldots \ldots \ldots \ldots$

Total .....................

.659 .3

$5,641.4$

$11,909.7$

$8,953.8$

12.8
16.7
23.5
48.2
63.1
99.2
50.5

5.9

6.1

7.9

14.3

19.8

34.2

17.6

$w$
$w$
13.5
5.6
12.2
27.8
18.7

$\begin{array}{rr}W & 13.6 \\ W & 11.5 \\ 2.4 & 8.7 \\ 1.8 & 7.0 \\ 3.3 & 4.4 \\ 12.1 & 3.1 \\ 6.4 & 3.7\end{array}$

Gray and Ductile Iron Foundries

3321

Value of Shipments and Receipts

(million dollars)

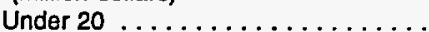

20-49 ......................

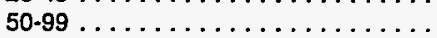

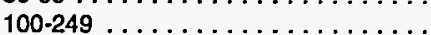

250-499 ..................

500 and Over . . . . . . . . . . . .

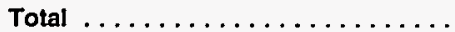

3331

\section{Primary Copper}

Value of Shipments and Receipts
(million dollars)

Under $20 \ldots \ldots \ldots \ldots \ldots . . . . .$.

$20.49 \ldots \ldots \ldots \ldots \ldots \ldots \ldots$.

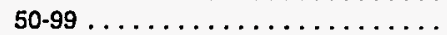

100-249 .

250-499 .....................

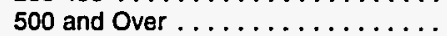

Total

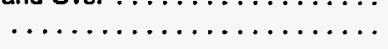

$\begin{array}{rr}W & W \\ 7,149.9 & 120.5 \\ W & W \\ W & W \\ 0.0 & 0.0 \\ 0.0 & 0.0 \\ 8,194.4 & 104.2\end{array}$

12.1
78.9
76.1
74.6
85.0
125.6
108.1

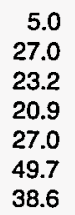

0.0
$W$
35.1
13.8
21.8
29.8
27.2

$\begin{array}{rr}W & 33.2 \\ W & 13.9 \\ W & 5.8 \\ W & 4.0 \\ W & 4.2 \\ W & 2.6 \\ 9.2 & 2.3\end{array}$

$\begin{array}{rr}W & W \\ 959.7 & 18.8 \\ 1,313.5 & 20.4 \\ 1,217.1 & 25.1 \\ W & W \\ 0.0 & 0.0 \\ 1,033.2 & 20.4\end{array}$

$W$
10.1
10.5
12.8
$W$
0.0
10.6

0.0
0.0
0.0
0.0
0.0
0.0
0.0

$\begin{array}{rr}W & 7.2 \\ 17.5 & 5.8 \\ W & 6.3 \\ W & 12.2 \\ 0.0 & N F \\ 0.0 & N F \\ 9.9 & 6.2\end{array}$

See footnotes at end of table.

\begin{tabular}{ll}
0.0 & $W$ \\
0.0 & 0.0 \\
0.0 & $W$ \\
0.0 & $W$ \\
$W$ & $W$ \\
0.0 & $W$ \\
$W$ & $W$ \\
\hline
\end{tabular}


Table A50. Selected Energy Operating Ratios for Total Energy Consumption for Heat, Power, and Electricity Generation by Industry Group, Selected Industries, and Economic Characteristics of the Establishment, 1991 (Continued)

\begin{tabular}{|c|c|c|c|c|c|c|c|}
\hline $\begin{array}{c}\text { SIC } \\
\text { Code* }\end{array}$ & $\begin{array}{c}\text { Economic } \\
\text { Characteristics }^{b}\end{array}$ & $\begin{array}{c}\text { Consumption per } \\
\text { Employee } \\
\text { (million Btu) }\end{array}$ & $\begin{array}{c}\text { Consumption per } \\
\text { Dollar of Value } \\
\text { Added } \\
\text { (thousand Btu) }\end{array}$ & $\begin{array}{c}\text { Consumption per } \\
\text { Dollar of Value of } \\
\text { Shipments } \\
\text { (thousand Btu) }\end{array}$ & $\begin{array}{c}\text { Major Byproducts } \\
\text { as a Percent of } \\
\text { Consumption } \\
\text { (percent) }\end{array}$ & $\begin{array}{c}\text { Fuel Oild as a } \\
\text { Percent of Natural } \\
\text { Gas } \\
\text { (percent) }\end{array}$ & \multirow[t]{2}{*}{$\begin{array}{c}\text { RSE } \\
\text { Row } \\
\text { Factors }\end{array}$} \\
\hline & Imn Factors: & 0.8 & 0.8 & 0.8 & 1.3 & 1.7 & \\
\hline
\end{tabular}

3334

Primary Aluminum

Value of Shipments and Receipts (million dollars)

Under $20 \ldots \ldots \ldots \ldots \ldots \ldots \ldots . \ldots . \ldots$

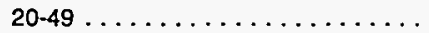

$50-99 \ldots \ldots \ldots \ldots \ldots \ldots \ldots$

100-249.

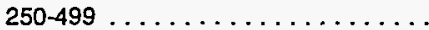

500 and Over . . . . . . . . . . . .

Total ......................

$\begin{array}{rr}W & Q \\ W & W \\ 0.0 & 0.0 \\ 12,787.4 & 162.2 \\ W & W \\ W & W \\ 12,816.0 & 155.0\end{array}$

$W$
$W$
0.0
46.1
$W$
$W$
41.1

0.0
0.0
0.0
0.0
0.0
0.0
0.0

$\begin{array}{rr}0.0 & 3.6 \\ 0.0 & 2.0 \\ 0.0 & \text { NF } \\ 1.5 & 1.5 \\ W & 1.6 \\ W & 2.1 \\ 3.5 & 1.5\end{array}$

3339

Primary Nonferrous Metals, nec

Value of Shipments and Receipts (million dollars)

Under $20 \ldots \ldots \ldots \ldots \ldots \ldots \ldots$. . . . . .

$20-49 \ldots \ldots \ldots \ldots \ldots \ldots \ldots \ldots$

$50-99 \ldots \ldots \ldots \ldots \ldots \ldots \ldots \ldots \ldots$

$100-249 \ldots \ldots \ldots \ldots \ldots \ldots \ldots$

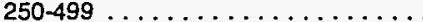

500 and Over . . . . . . . . . .

Total $\ldots \ldots \ldots \ldots \ldots \ldots$

Aluminum Sheet, Plate, and Foil

Value of Shipments and Receipts

(million dollars)

Under $20 \ldots \ldots \ldots \ldots \ldots \ldots \ldots$

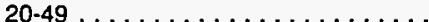

$50-99 \ldots \ldots \ldots \ldots \ldots \ldots \ldots \ldots$

$100-249 \ldots \ldots \ldots \ldots \ldots \ldots \ldots \ldots$

$250-499 \ldots \ldots \ldots \ldots \ldots \ldots \ldots$

500 and Over $\ldots \ldots \ldots \ldots \ldots \ldots$

Total $\ldots \ldots \ldots \ldots \ldots \ldots \ldots \ldots$

$\begin{array}{rr}W & W \\ 2,723.8 & 25.9 \\ 5,860.1 & 82.5 \\ 4,523.3 & 61.4 \\ 0.0 & 0.0 \\ W & W \\ 4,239.7 & 45.6\end{array}$

$W$
10.8
25.5
17.2
0.0
$W$
12.0

0.0
0.0
0.0
$W$
0.0
0.0
$W$

$\begin{array}{rr}4.5 & 9.6 \\ W & 9.7 \\ 2.9 & \text { NF } \\ 1.2 & \text { NF } \\ 0.0 & \text { NF } \\ \text { W } & \text { NF } \\ 1.9 & 2.6\end{array}$

FABRICATED METAL PRODUCTS

Value of Shipments and Receipts

(million dollars)

Under $20 \ldots \ldots \ldots \ldots \ldots \ldots \ldots$

$20-49$

$50-99 \ldots \ldots \ldots \ldots \ldots \ldots \ldots \ldots \ldots$

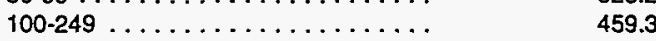

$250-499 \ldots \ldots . \ldots \ldots$

500 and Over . . . . . . . . . . . . 259.0

Total $\ldots \ldots \ldots \ldots \ldots \ldots \ldots$

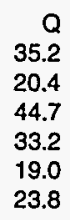

Q

2,554.3

$2,450.2$

INDUSTRIAL MACHINERY and EQUIPMENT

Value of Shipments and Receipts

(million dollars)

Under $20 \ldots \ldots \ldots \ldots \ldots \ldots \ldots$

20-49 ...................

$50-99 \ldots \ldots \ldots \ldots \ldots \ldots \ldots \ldots$

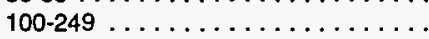

$250-499 \ldots \ldots \ldots \ldots \ldots \ldots \ldots$

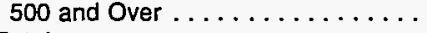

3.8
3.9
4.5
5.5
4.3
2.2
4.1

2.0
1.8
2.1
2.5
1.8
1.4
2.0

0.0

0.0

0.0

0.0

0.0

0.0
0.0

$\begin{array}{rr}7.7 & 9.0 \\ 2.7 & 8.6 \\ 2.0 & 10.6 \\ W & 8.8 \\ 6.1 & 10.6 \\ W & 11.3 \\ 5.1 & 5.4\end{array}$

Total

97.3
134.7
173.6
193.9
254.3
201.9
143.4

1.9
2.1
2.6
2.4
2.4
1.5
2.0

1.1
1.1
1.2
1.1
1.0
0.7
1.0

0.0
0.0
0.0
0.0
0.0
0.0
0.0

9.3 
Table A50. Selected Energy Operating Ratios for Total Energy Consumption for Heat, Power, and Electricity Generation by Industry Group, Selected Industries, and Economic Characteristics of the Establishment, 1991 (Continued)

\begin{tabular}{cc|c|c|c|c|c}
\hline SIC & $\begin{array}{c}\text { Economic } \\
\text { Characteristics }\end{array}$ & $\begin{array}{c}\text { Consumption per } \\
\text { Employee } \\
\text { (million Btu) }\end{array}$ & $\begin{array}{c}\text { Consumption per } \\
\text { Dollar of Value } \\
\text { Added } \\
\text { (thousand Btu) }\end{array}$ & $\begin{array}{c}\text { Consumption per } \\
\text { Dollar of Value of } \\
\text { Shipments } \\
\text { (thousand Btu) }\end{array}$ & $\begin{array}{c}\text { Major Byproducts } \\
\text { as a Percent of } \\
\text { Consumption } \\
\text { (percent) }\end{array}$ & $\begin{array}{c}\text { Fuel Oild as a } \\
\text { Percent of Natural } \\
\text { Gas } \\
\text { (percent) }\end{array}$ \\
\hline RSE Column Factors: & 0.8 & 0.8 & 0.8 & 1.3 & $\begin{array}{c}\text { RSE } \\
\text { Row } \\
\text { Factors }\end{array}$ \\
\hline
\end{tabular}

$357 \quad$ Computer and Office Equipment

\begin{tabular}{|c|c|c|c|c|c|c|}
\hline \multicolumn{7}{|l|}{$\begin{array}{l}\text { Value of Shipments and Receipts } \\
\text { (million dollars) }\end{array}$} \\
\hline Under $20 \ldots \ldots \ldots \ldots \ldots$ & 77.0 & 1.5 & 0.8 & 0.0 & 11.1 & 18.5 \\
\hline $20-49 \ldots \ldots \ldots$ & 78.9 & 1.0 & 0.6 & 0.0 & $\mathbf{Q}$ & 26.9 \\
\hline $50-99 \ldots \ldots \ldots \ldots \ldots \ldots \ldots$ & 87.7 & 1.4 & 0.6 & 0.0 & 1.3 & 11.5 \\
\hline $100-249 \ldots \ldots \ldots \ldots \ldots \ldots \ldots \ldots$ & 82.6 & 1.1 & 0.5 & 0.0 & 1.0 & 12.6 \\
\hline $250-499$ & 128.5 & 0.8 & 0.3 & 0.0 & 4.2 & 11.4 \\
\hline 500 and $O v e r \ldots \ldots \ldots \ldots \ldots$ & 86.0 & 0.6 & 0.3 & 0.0 & 2.3 & 10.9 \\
\hline Total $\ldots \ldots \ldots \ldots \ldots \ldots \ldots \ldots$ & 86.2 & 0.8 & 0.4 & 0.0 & 2.9 & 7.8 \\
\hline
\end{tabular}

36 ELECTRONIC and OTHER ELECTRIC EQUIPMENT

Value of Shipments and Receipts

(million dollars)

Under $20 \ldots \ldots \ldots \ldots \ldots \ldots \ldots$

$20-49 \ldots \ldots \ldots \ldots \ldots \ldots \ldots \ldots \ldots$

$50-99 \ldots \ldots \ldots \ldots \ldots \ldots \ldots \ldots \ldots+19.4 \quad 198.4$

$100-249 \ldots \ldots \ldots \ldots \ldots \ldots \ldots \ldots \ldots+19.2$

$250-499 \ldots \ldots \ldots \ldots \ldots \ldots \ldots . . . \ldots$

500 and Over .............. $\quad 208.9$

Total ................. 158.9

$\begin{array}{lll}1.8 & 0.9 \\ 1.9 & 1.6 & 0.8\end{array}$

TRANSPORTATION EQUIPMENT

37

Value of Shipments and Receipts

(million dollars)

Under $20 \ldots \ldots \ldots \ldots \ldots \ldots \ldots$. 122.9

$20-49 \ldots \ldots \ldots \ldots \ldots \ldots \ldots \ldots . \ldots \ldots$

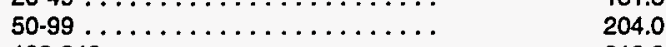

$100-249 \ldots \ldots \ldots \ldots \ldots \ldots \ldots .213 .2$

$250-499 \ldots \ldots \ldots \ldots \ldots \ldots \ldots \ldots$

500 and Over ................

Total $\ldots \ldots \ldots \ldots \ldots \ldots \ldots \ldots . . . .222 .9$

$\begin{array}{ll}1.9 & 1.1 \\ 2.1 & 1.0 \\ 2.6 & 1.4 \\ 1.9 & 1.1 \\ 1.8 & 0.9 \\ 1.6 & 0.8 \\ 1.9 & 1.0\end{array}$

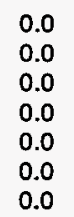

$\begin{array}{rr}3.2 & 18.2 \\ 8.2 & 17.8 \\ 7.7 & 9.8 \\ 6.5 & 10.6 \\ 2.7 & 9.9 \\ 20.7 & 8.5 \\ 8.0 & 6.3\end{array}$

3711

Motor Vehicles and Car Bodies

Value of Shipments and Receipts (million dollars)

Under $20 \ldots \ldots \ldots \ldots \ldots \ldots \ldots$

$20.49 \ldots \ldots \ldots \ldots \ldots \ldots \ldots \ldots$

$50-99 \ldots \ldots \ldots \ldots \ldots \ldots \ldots \ldots$

$100-249 \ldots \ldots \ldots \ldots \ldots \ldots \ldots \ldots$

250-499 .................

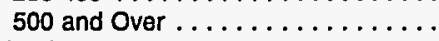

Total ......................

3714

Motor Vehicle Parts and Accessories

Value of Shipments and Receipts (million dollars)

Under $20 \ldots \ldots \ldots \ldots \ldots \ldots .$.

$20-49 \ldots \ldots \ldots \ldots \ldots \ldots \ldots$

$50-99 \ldots \ldots \ldots \ldots \ldots \ldots \ldots$.

$100-249 \ldots \ldots \ldots \ldots \ldots \ldots \ldots \ldots$

250-499 ..................

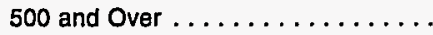

Total

.........................

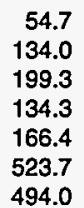

1.2
2.8
8.8
1.3
2.4
2.3
2.3

2.6
3.3
3.2
2.6
2.9
2.1
2.3

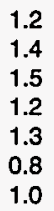

0.0
0.0
$W$
0.0
0.0
$W$
$W$

See footnotes at end of table. 
Table A50. Selected Energy Operating Ratios for Total Energy Consumption for Heat, Power, and Electricity Generation by Industry Group, Selected Industries, and Economic Characteristics of the Establishment, 1991 (Continued)

\begin{tabular}{|c|c|c|c|c|c|c|c|}
\hline $\begin{array}{l}\text { SIC } \\
\text { Code }\end{array}$ & $\begin{array}{c}\text { Economic } \\
\text { Characteristics }^{b}\end{array}$ & $\begin{array}{c}\text { Consumption per } \\
\text { Employee } \\
\text { (million Btu) }\end{array}$ & $\begin{array}{c}\text { Consumption per } \\
\text { Dollar of Value } \\
\text { Added } \\
\text { (thousand Btu) }\end{array}$ & $\begin{array}{c}\text { Consumption per } \\
\text { Dollar of Value of } \\
\text { Shipments } \\
\text { (thousand Btu) }\end{array}$ & $\begin{array}{l}\text { Major Byproducts } \\
\text { as a Percent of } \\
\text { Consumption } \\
\text { (percent) }\end{array}$ & $\begin{array}{c}\text { Fuel Oil }{ }^{d} \text { as a } \\
\text { Percent of Natural } \\
\text { Gas } \\
\text { (percent) }\end{array}$ & \multirow[t]{2}{*}{$\begin{array}{c}\text { RSE } \\
\text { Row } \\
\text { Factors }\end{array}$} \\
\hline & umn Factors: & 0.8 & 0.8 & 0.8 & 1.3 & 1.7 & \\
\hline
\end{tabular}

38

INSTRUMENTS and RELATED PRODUCTS

Value of Shipments and Receipts

(million dollars)

Under $20 \ldots \ldots \ldots$

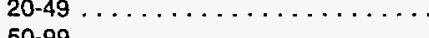

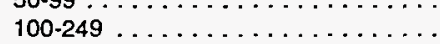

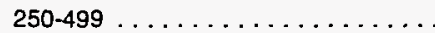

500 and Over . . . . . . . . . .

Total . . . . . . . . . . . . .

60.7
66.3
87.8
103.6
128.3
220.9
116.5

1.0
1.0
1.2
1.1
1.0
1.6
1.2

0.7

0.6

0.7

0.7
0.7

0.8

0.0
0.0
0.0
0.0
0.0
0.0
0.0

0.0
0.0
0.0
0.0
0.0
0.0
0.0

$\begin{array}{rr}\text { W } & 22.1 \\ W & 17.3 \\ 23.7 & 13.7 \\ W & 10.3 \\ W & 11.4 \\ W & 15.1 \\ W & 8.4\end{array}$

$3841 \quad$ Surgical and Medical Instruments

Value of Shipments and Receipts (million dollars)

Under $20 \ldots \ldots \ldots \ldots \ldots \ldots$

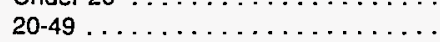

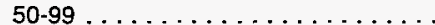

$100-249 \ldots \ldots \ldots \ldots \ldots \ldots \ldots$

$250-499 \ldots \ldots \ldots \ldots \ldots \ldots \ldots$

500 and Over $\ldots \ldots \ldots \ldots \ldots \ldots$

Total ...................

MISC. MANUFACTURING INDUSTRIES

39

Value of Shipments and Receipts

(million dollars)

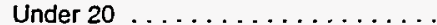

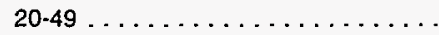

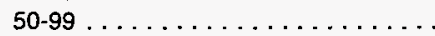

$100-249$

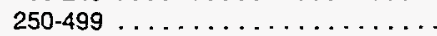

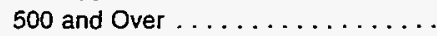

Total ....................

\begin{tabular}{|c|c|c|c|c|}
\hline 70.6 & 1.7 & 0.9 & 0.0 & $w$ \\
\hline 118.7 & 1.8 & 1.0 & 0.0 & $w$ \\
\hline 206.6 & 2.3 & 1.2 & 0.0 & W \\
\hline 159.0 & 1.5 & 0.8 & 0.0 & 14.0 \\
\hline 282.9 & 2.3 & 1.4 & 0.0 & 6.3 \\
\hline 0.0 & 0.0 & 0.0 & 0.0 & 0.0 \\
\hline 108.3 & 1.8 & 1.0 & 0.0 & W \\
\hline
\end{tabular}

- See Appendices B and F for descriptions of the Standard Industrial Classification system.

- Value of Shipments and Receipts were supplied by the Bureau of the Census. See Appendix B.

- Major Byproduct" fuels include coke oven gas and blast furnace gas (produced primarily in the blast fumace industry, SIC 3312 ); still gas (produced primarily in petroleum refineries, SIC 2911); and pulping liquor (produced primarily in pulp and paper mills, SIC 2611 and 2621).

"Fuel Oil" includes distillate and residual fuel oils.

NF=No applicable RSE row/column factor.

- Estimate less than 0.5. Data are included in higher level totals.

$W=$ Withheld to avoid disclosing data for individual establishments. Data are included in higher level totals.

Notes: - To obtain a RSE percentage for any table cell, multiply the cell's corresponding RSE column and RSE row factors. - Totals may not equal sum of components because of independent rounding. - Operating ratios were calculated using the input energy estimates reported in Table A4.

Source: Energy Information Administration, Office of Energy Markets and End Use, Energy End Use and Integrated Statistics Division, Form EIA-846, "1991 Manufacturing Energy Consumption Survey," and Bureau of the Census, Industry Division, data files for the "1991 Annual Survey of Manufactures." 
Table A51. Selected Energy Operating Ratios for Total Energy Consumption for Heat, Power, and Electricity Generation by Census Region and Economic Characteristics of the Establishment, 1991

\begin{tabular}{|c|c|c|c|c|c|c|c|}
\hline $\begin{array}{c}\text { SIC } \\
\text { Code" }\end{array}$ & $\begin{array}{c}\text { Economic } \\
\text { Characteristics }^{\circ}\end{array}$ & $\begin{array}{l}\text { Consumption per } \\
\text { Employee } \\
\text { (million Btu) }\end{array}$ & $\begin{array}{l}\text { Consumption per } \\
\text { Dollar of Value } \\
\text { Added } \\
\text { (thousand Btu) }\end{array}$ & $\begin{array}{c}\text { Consumption per } \\
\text { Dollar of Value of } \\
\text { Shipments } \\
\text { (thousand Btu) }\end{array}$ & $\begin{array}{l}\text { Major Byproducts } \\
\text { as a Percent of } \\
\text { Consumption } \\
\text { (percent) }\end{array}$ & $\begin{array}{c}\text { Fuel Oid as a } \\
\text { Percent of Natural } \\
\text { Gas } \\
\text { (percent) }\end{array}$ & \multirow[t]{2}{*}{$\begin{array}{l}\text { RSE } \\
\text { Row } \\
\text { Factors }\end{array}$} \\
\hline & umn Factors: & 0.8 & 0.8 & 0.7 & 1.1 & 1.7 & \\
\hline
\end{tabular}

$20-39$

ALL INDUSTRY GROUPS
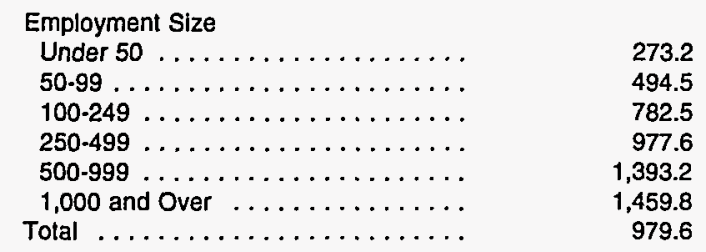

4.9
7.8
11.0
12.9
15.7
13.1
12.0

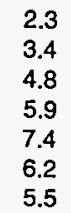

3.2
2.4
5.9
19.7
24.0
25.5
19.1

$\begin{array}{rr}W & 7.3 \\ 12.5 & 7.8 \\ 10.4 & 5.0 \\ 9.3 & 3.9 \\ 9.1 & 3.3 \\ W & 2.6 \\ 10.0 & 2.2\end{array}$

20

FOOD and KINDRED PRODUCTS

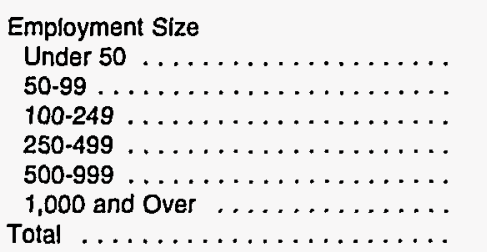

699.9
735.4
774.0
847.4
592.8
531.0
704.0

9.0
5.9
6.6
7.2
5.5
5.2
6.3

2.3
1.8
2.5
3.2
2.5
1.9
2.4

169.4

249.2

368.1

412.8

513.3

437.6

418.5

3.9
6.1
8.7
7.4
11.2
7.9
8.1

820.2

667.3

701.6

606.8

766.7

892.7

702.5

6.5
11.2
8.2
5.8
5.1
8.1
6.7

2037

Frozen Fruits and Vegetables

\begin{tabular}{|c|c|}
\hline \multicolumn{2}{|l|}{ Employment Size } \\
\hline$\ldots \ldots \ldots \ldots$ & $\mathbf{Q}$ \\
\hline $50-99$. & 762.6 \\
\hline$\ldots \ldots \ldots \ldots \ldots$ & 655.2 \\
\hline $250-499 \ldots \ldots \ldots \ldots \ldots \ldots$ & 803.1 \\
\hline $500-999 \ldots \ldots \ldots \ldots \ldots \ldots$ & 928.3 \\
\hline 1,000 and Over .. & 801.8 \\
\hline Total & 795.3 \\
\hline
\end{tabular}

Total ................... 795.3

0.0
0.0
0.1
$W$
0.2
0.7
$W$

$\begin{array}{rr}10.8 & 15.1 \\ W & 13.7 \\ 7.4 & 12.9 \\ 8.0 & 10.7 \\ W & 7.9 \\ 8.5 & 7.0 \\ 8.7 & 6.1\end{array}$

2046 Wet Corn Milling

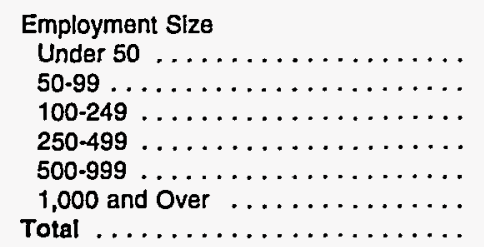

$\begin{array}{rr}W & W \\ W & W \\ 27,943.7 & 44.8 \\ 18,316.7 & 39.7 \\ 9,842.6 & 49.6 \\ W & W \\ 15,301.7 & 43.0\end{array}$

$Q$
4.1
3.7
4.8
6.2
5.7
4.9

0.0
0.0
0.0
0.0
0.7
0.0
0.2

$\begin{array}{rr}0.0 & 40.2 \\ Q & 41.7 \\ 15.1 & 15.8 \\ 13.5 & 13.0 \\ 4.3 & 11.1 \\ 0.2 & 12.0 \\ 9.6 & 11.7\end{array}$

See footnotes at end of table. 
Table A51. Selected Energy Operating Ratios for Total Energy Consumption for Heat,
Power, and Electricity Generation by Census Region and Economic
Characteristics of the Establishment, 1991 (Continued)

\begin{tabular}{|c|c|c|c|c|c|c|c|}
\hline $\begin{array}{l}\text { SIC } \\
\text { Code }^{a}\end{array}$ & $\begin{array}{c}\text { Economic } \\
\text { Characteristics }\end{array}$ & $\begin{array}{l}\text { Consumption per } \\
\text { Employee } \\
\text { (million Btu) }\end{array}$ & $\begin{array}{l}\text { Consumption per } \\
\text { Dollar of Value } \\
\text { Added } \\
\text { (thousand Btu) }\end{array}$ & $\begin{array}{l}\text { Consumption per } \\
\text { Dollar of Value of } \\
\text { Shipments } \\
\text { (thousand Btu) }\end{array}$ & $\begin{array}{l}\text { Major Byproducts } \\
\text { as a Percent of } \\
\text { Consumption } \\
\text { (percent) }\end{array}$ & $\begin{array}{c}\text { Fuel Olf as a } \\
\text { Percent of Natural } \\
\text { Gas } \\
\text { (percent) }\end{array}$ & \multirow[t]{2}{*}{$\begin{array}{l}\text { RSE } \\
\text { Row } \\
\text { Factors }\end{array}$} \\
\hline & umn Factors: & 0.8 & 0.8 & 0.7 & 1.1 & 1.7 & \\
\hline
\end{tabular}

2051 Bread, Cake, and Related Products

Employment Size

Under $50 \ldots \ldots \ldots \ldots \ldots \ldots \ldots \ldots . \ldots \ldots$

$50-99 \ldots \ldots \ldots \ldots \ldots \ldots \ldots \ldots \ldots$

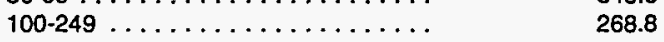

$250-499 \ldots \ldots \ldots \ldots \ldots \ldots \ldots \ldots$

$500-999 \ldots \ldots \ldots \ldots \ldots \ldots \ldots \ldots$

1,000 and Over $\ldots \ldots \ldots \ldots \ldots \ldots \ldots . \ldots \ldots$

Total ................ 230.7

Beet Sugar

2063

Employment Size

Under $50 \ldots \ldots \ldots \ldots \ldots \ldots$

$2075 \quad$ Soybean Oll Mills

Employment Size

2082

Employment Size

50-99.

3.8
5.8
3.7
3.0
2.5
1.7
3.2

2.2
3.3
2.2
1.9
1.6
1.2
2.0

0.0

0.0

0.0

0.0

0.0

0.0

$\begin{array}{rr}0.0 & 21.7 \\ Q & 15.7 \\ 2.7 & 11.9 \\ 5.3 & 4.5 \\ W & 6.3 \\ W & 7.1 \\ 3.3 & 5.5\end{array}$

1.7

5.7

4.5

6.3

7.1

Employment Size

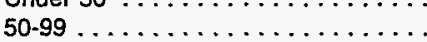

$100-249 \ldots \ldots \ldots \ldots \ldots \ldots \ldots$

$250-499 \ldots \ldots \ldots \ldots \ldots \ldots$.

$500-999 \ldots \ldots \ldots \ldots \ldots \ldots \ldots$

1,000 and Over $\ldots \ldots \ldots \ldots \ldots$

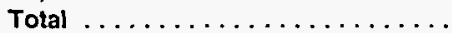

Under $50 \ldots \ldots \ldots \ldots \ldots \ldots$

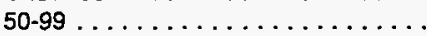

$100-249 \ldots \ldots \ldots \ldots \ldots \ldots \ldots$

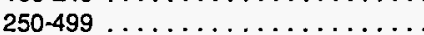

$500-999 \ldots \ldots \ldots \ldots \ldots \ldots \ldots$

1,000 and Over $\ldots \ldots \ldots \ldots \ldots$

Total $\ldots \ldots \ldots \ldots \ldots \ldots \ldots$

\section{Malt Beverages}

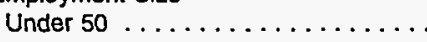

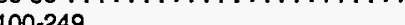

$250-499 \ldots \ldots \ldots \ldots \ldots \ldots \ldots \ldots \ldots \ldots$

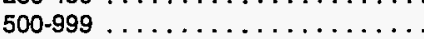

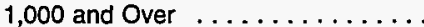

Total ..................

$\begin{array}{rr}0.0 & 0.0 \\ W & W \\ 8,939.4 & 87.3 \\ W & W \\ 0.0 & 0.0 \\ 0.0 & 0.0 \\ 8,855.0 & 83.7\end{array}$

\section{TOBACCO PRODUCTS}

Under $50 \ldots \ldots \ldots \ldots \ldots \ldots \ldots$

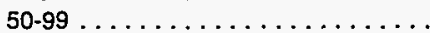

$100-249 \ldots \ldots \ldots \ldots \ldots \ldots \ldots \ldots \ldots$

$250-499 \ldots \ldots \ldots \ldots \ldots \ldots \ldots$

$500-999 \ldots \ldots \ldots \ldots \ldots \ldots \ldots$

1,000 and Over $\ldots \ldots \ldots \ldots \ldots \ldots$.

Total $\ldots \ldots \ldots \ldots \ldots \ldots \ldots \ldots$
$8,405.5$

$8,821.1$

$7,834.3$

$6,772.1$

0.0

0.0
$7,865.1$

441.7

$W$
$W$
$W$
$1,484.5$

$1,589.6$

$1,511.2$
50.8

37.8

38.2

49.5

0.0

0.0
42.6

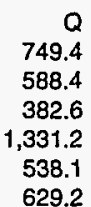

TEXTILE MILL PRODUCTS

7.0
$W$
$W$
$W$
5.2
5.1
5.3

Employment Size

Under $50 \ldots \ldots \ldots \ldots \ldots \ldots \ldots . \ldots$

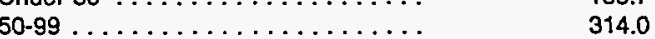

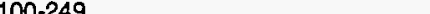

$250-499 \ldots \ldots \ldots \ldots \ldots \ldots \ldots \ldots$

$500-999 \ldots \ldots \ldots \ldots \ldots \ldots \ldots$

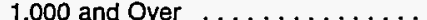

448.4
565.9

461.6

Total $\ldots \ldots \ldots \ldots \ldots \ldots \ldots \ldots$

459.4

0.0
$W$
29.8
$W$
0.0
0.0
28.7

0.0
0.0
0.0
0.0
0.0
0.0

$\begin{array}{rr}0.0 & N F \\ W & 6.3 \\ 4.1 & 3.8 \\ W & 4.0 \\ 0.0 & N F \\ 0.0 & \text { NF } \\ W & 3.1\end{array}$

3.1

22

See footnotes at end of table. 
Table A51. Selected Energy Operating Ratios for Total Energy Consumption for Heat, Power, and Electricity Generation by Census Region and Economic Characteristics of the Establishment, 1991 (Continued)

\begin{tabular}{|c|c|c|c|c|c|c|c|}
\hline $\begin{array}{l}\text { SIC } \\
\text { Code }\end{array}$ & $\begin{array}{c}\text { Economic } \\
\text { Characteristics }\end{array}$ & $\begin{array}{l}\text { Consumption per } \\
\text { Employee } \\
\text { (million Btu) }\end{array}$ & $\begin{array}{l}\text { Consumption per } \\
\text { Dollar of Value } \\
\text { Added } \\
\text { (thousand Btu) }\end{array}$ & $\begin{array}{l}\text { Consumption per } \\
\text { Dollar of Value of } \\
\text { Shipments } \\
\text { (thousand Btu) }\end{array}$ & $\begin{array}{l}\text { Major Byproducts } \\
\text { as a Percent of } \\
\text { Consumption } \\
\text { (percent) }\end{array}$ & $\begin{array}{c}\text { Fuel Oil as a } \\
\text { Percent of Natural } \\
\text { Gas } \\
\text { (percent) }\end{array}$ & \multirow[t]{2}{*}{$\begin{array}{c}\text { RSE } \\
\text { Row } \\
\text { Factors }\end{array}$} \\
\hline & umn Factors: & 0.8 & 0.8 & 0.7 & 1.1 & 1.7 & \\
\hline
\end{tabular}

23

$$
\begin{aligned}
& \text { APPAREL and OTHER TEXTILE } \\
& \text { PRODUCTS }
\end{aligned}
$$

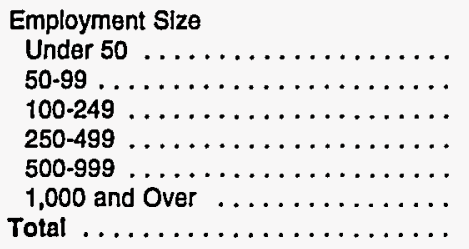

$\begin{array}{rr}32.0 & 0.9 \\ 54.0 & 2.1 \\ 39.2 & 1.2 \\ 43.5 & 1.3 \\ 60.0 & 1.4 \\ 145.1 & 3.1 \\ 49.7 & 1.4\end{array}$

$\begin{array}{ll}0.9 & 0.5 \\ 2.1 & 1.2 \\ 1.2 & 0.7 \\ 1.3 & 0.6 \\ 1.4 & 0.8 \\ 3.1 & 1.8 \\ 1.4 & 0.7\end{array}$

$\begin{array}{ll}0.5 & 0.0 \\ 1.2 & 0.0 \\ 0.7 & 0.0 \\ 0.6 & 0.0 \\ 0.8 & 0.0 \\ 1.8 & 0.0 \\ 0.7 & 0.0\end{array}$

$\begin{array}{rr}\mathrm{Q} & 27.6 \\ \mathrm{Q} & \mathbf{3 1 . 8} \\ 9.9 & 15.6 \\ 6.7 & 17.4 \\ 3.8 & 18.7 \\ 9.9 & 18.5 \\ 14.6 & 11.5\end{array}$

24

LUMBER and WOOD PRODUCTS

Employment Size

Under $50 \ldots \ldots \ldots \ldots \ldots \ldots \ldots \ldots . \ldots \ldots \ldots$

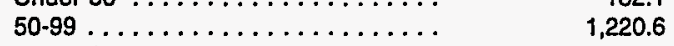

$100-249 \ldots \ldots \ldots \ldots \ldots \ldots \ldots . \ldots \ldots 1.2$

$250-499 \ldots \ldots \ldots \ldots \ldots \ldots \ldots \ldots \quad 1,378.2$

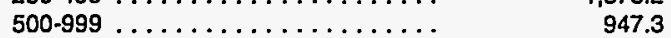

1,000 and Over ............. 160.7

Total . . . . . . . $79 \ldots \ldots \ldots$

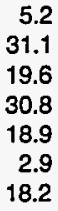

1.9

11.4

7.5

11.6

7.9

6.8

1.8 7.4 18.7 18.5

Employment Size

Under $50 \ldots \ldots \ldots \ldots \ldots \ldots$

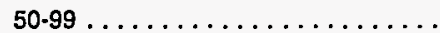

$100-249 \ldots \ldots \ldots \ldots \ldots \ldots \ldots$

$250-499 \ldots \ldots \ldots \ldots \ldots \ldots \ldots$.

$500-999 \ldots \ldots \ldots \ldots \ldots \ldots \ldots$

1,000 and Over $\ldots \ldots \ldots \ldots \ldots \ldots$

Total ...................

$\begin{array}{rr}Q & Q \\ Q & Q \\ 108.4 & 2.3 \\ 140.7 & 3.1 \\ 169.1 & 3.6 \\ 179.7 & 3.7 \\ 159.0 & 3.5\end{array}$

PAPER and ALLIED PRODUCTS

Employment Size

Under $50 \ldots \ldots \ldots \ldots \ldots \ldots \ldots$

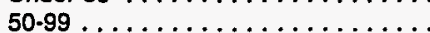

$100-249 \ldots \ldots \ldots \ldots \ldots \ldots \ldots$

$250-499 \ldots \ldots \ldots \ldots \ldots \ldots \ldots \ldots$

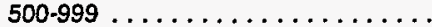

1,000 and Over .............

Total ..................

2611

\section{Pulp MIJls}

Employment Size

$$
\text { Under } 50 \ldots \ldots \ldots \ldots \ldots \ldots \ldots
$$

$50.99 \ldots \ldots \ldots \ldots \ldots \ldots \ldots \ldots$

$100-249 \ldots \ldots \ldots \ldots \ldots \ldots \ldots \ldots$

$250-499 \ldots \ldots \ldots \ldots \ldots \ldots \ldots$

$500-999 \ldots \ldots \ldots \ldots \ldots \ldots \ldots$

1,000 and Over $\ldots \ldots \ldots \ldots \ldots$

Total ....................

Paper Mills

2621

256.5
772.5
$1,084.8$
$3,990.8$
$8,860.1$
$9,604.8$
$4,234.5$

4.2
12.6
15.1
43.1
62.7
69.4
43.9

0.0
0.0
0.0
0.0
0.0
0.0
0.0

Employment Size

Under $50 \ldots \ldots \ldots \ldots \ldots \ldots$

$50-99 \ldots \ldots \ldots \ldots \ldots \ldots \ldots$

$100-249 \ldots \ldots \ldots \ldots \ldots \ldots \ldots$.

$250-499 \ldots \ldots \ldots \ldots \ldots \ldots \ldots \ldots$

$500-999 \ldots \ldots \ldots \ldots \ldots \ldots . \ldots$

1,000 and Over $\ldots \ldots \ldots \ldots \ldots$

Total $\ldots \ldots \ldots \ldots \ldots \ldots \ldots$

$\begin{array}{rl}225.5 & 49.5 \\ 43.0 & 16.7 \\ 49.7 & 21.7 \\ 44.4 & 21.1 \\ 82.5 & 35.2 \\ 95.6 & 47.7 \\ 78.7 & 36.7\end{array}$


Table A51. Selected Energy Operating Ratios for Total Energy Consumption for Heat, Power, and Electricity Generation by Census Region and Economic Characteristics of the Establishment, 1991 (Continued)

\begin{tabular}{|c|c|c|c|c|c|c|c|}
\hline $\begin{array}{c}\text { SIC } \\
\text { Code }\end{array}$ & $\begin{array}{c}\text { Economic } \\
\text { Characteristics }^{\circ} \\
\end{array}$ & $\begin{array}{c}\text { Consumption per } \\
\text { Employee } \\
\text { (million Btu) }\end{array}$ & $\begin{array}{c}\text { Consumption per } \\
\text { Dollar of Value } \\
\text { Added } \\
\text { (thousand Btu) }\end{array}$ & $\begin{array}{c}\text { Consumption per } \\
\text { Dollar of Value of } \\
\text { Shipments } \\
\text { (thousand Btu) }\end{array}$ & $\begin{array}{c}\text { Major Byproducts } \\
\text { as a Percent of } \\
\text { Consumption } \\
\text { (percent) }\end{array}$ & $\begin{array}{c}\text { Fuel Oir as a } \\
\text { Percent of Natural } \\
\text { Gas } \\
\text { (percent) }\end{array}$ & $\begin{array}{c}\text { RSE } \\
\text { Row } \\
\text { Factors }\end{array}$ \\
\hline & mn Factors: & 0.8 & 0.8 & 0.7 & 1.1 & 1.7 & \\
\hline
\end{tabular}

2631

Paperboard Mills

Employment Size

Under $50 \ldots \ldots \ldots \ldots \ldots \ldots \ldots$

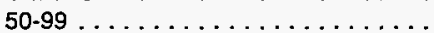

$100-249 \ldots \ldots \ldots \ldots \ldots \ldots \ldots$

$250-499 \ldots \ldots \ldots \ldots \ldots \ldots \ldots \ldots$

$500-999 \ldots \ldots \ldots \ldots \ldots \ldots \ldots$

1,000 and Over $\ldots \ldots \ldots \ldots \ldots$

Total $\ldots \ldots \ldots \ldots \ldots \ldots$

$\begin{array}{rr}W & W \\ W & W \\ 8,603.0 & 65.3 \\ 16,662.8 & 104.2 \\ 23,337.8 & 143.6 \\ 19,253.5 & 138.6 \\ 17,291.7 & 116.8\end{array}$

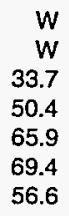

0.0
0.0
$W$
28.0
47.9
52.8
39.1

$\begin{array}{rr}W & 8.5 \\ W & 10.8 \\ 15.7 & 7.4 \\ 25.5 & 3.4 \\ W & 2.6 \\ W & 5.8 \\ W & 2.9\end{array}$

27

PRINTING and PUBLISHING

Employment Size

Under $50 \ldots \ldots \ldots \ldots \ldots \ldots \ldots$

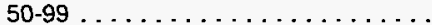

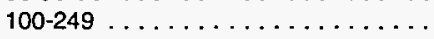

$250-499 \ldots \ldots \ldots \ldots \ldots \ldots$

$500-999 \ldots \ldots \ldots \ldots \ldots \ldots \ldots$

1,000 and Over . . . . . . . . . .

Total .................

60.0

63.4

104.4

97.3

100.4

78.9

82.6

28

CHEMICALS and ALLIED PRODUCTS

Employment Size

Under $50 \ldots \ldots \ldots \ldots \ldots \ldots$

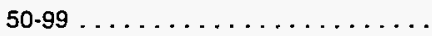

$100-249 \ldots \ldots \ldots \ldots \ldots . \ldots . \ldots$

$250-499 \ldots \ldots \ldots \ldots \ldots \ldots \ldots$

$500-999 \ldots \ldots \ldots \ldots \ldots \ldots . . \ldots \ldots$

1,000 and Over . . . . . . . . .

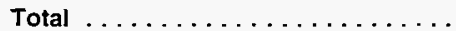

2812

Alkalies and Chlorine

Employment Size

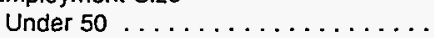

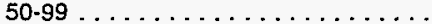

$100-249 \ldots \ldots \ldots \ldots \ldots \ldots \ldots$

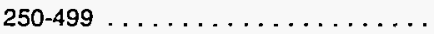

$500-999 \ldots \ldots \ldots \ldots \ldots \ldots$

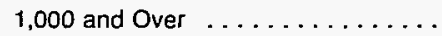

Total .................

Industrial Gases

2813

Employment Size

Under $50 \ldots \ldots \ldots \ldots \ldots \ldots \ldots$

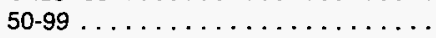

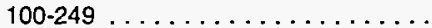

$250-499 \ldots \ldots \ldots \ldots \ldots \ldots$

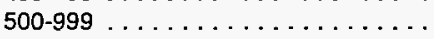

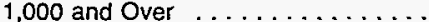

Total ....................

Industrial Inorganic Chemicals, nec

Employment Size

Under $50 \ldots \ldots \ldots \ldots \ldots \ldots$

$50-99 \ldots \ldots \ldots \ldots \ldots \ldots$

$100-249 \ldots \ldots \ldots \ldots \ldots \ldots$

$250-499 \ldots \ldots \ldots \ldots \ldots \ldots \ldots \ldots$

$500-999 \ldots \ldots \ldots \ldots \ldots \ldots \ldots$

1,000 and Over .............

Total
$2,195.2$

$3,150.4$

$5,075.0$

$5,013.0$

$5,652.9$

$3,654.9$

$4,205.7$
$W$
$16,131.7$
$28,655.0$
$W$
$W$
$W$

$26,321.3$

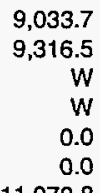

$11,072.8$
14.9

18.7

28.0

21.6

20.4

19.7

21.0

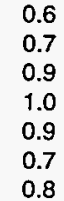

0.6

0.7
0.9

1.0

0.9
0.7

0.8

0.0
0.0
0.0
$W$
0.0
0.3
0.1

23.7

17.4

12.5

21.2

17.8

13.7

11.6

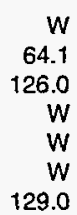

39.6

52.2

W

0.0

0.0

51.0
6.8
8.3
12.4
11.0
11.4
12.1
11.1
0.0

0.0

0.0
0.0

0.0

0.0

0.0
0.0
0.0
$W$
0.0
0.0
$W$

Q

25.6

35.0

$W$
0.0

0.0

33.1

\begin{tabular}{|c|c|c|c|c|}
\hline $3,565.8$ & 24.1 & 9.9 & * & 3.7 \\
\hline $3,141.5$ & 24.2 & 10.0 & 0.0 & $w$ \\
\hline $4,611.3$ & 27.8 & 12.7 & $w$ & 17.7 \\
\hline $7,163.3$ & 43.8 & 25.6 & $w$ & $W$ \\
\hline $8,680.9$ & 71.7 & 34.1 & $w$ & $w$ \\
\hline $2,917.5$ & 22.7 & 18.8 & 0.0 & $w$ \\
\hline $4,144.4$ & 30.4 & 18.5 & 1.2 & 5.0 \\
\hline
\end{tabular}

See footnotes at end of table. 
Table A51. Selected Energy Operating Ratios for Total Energy Consumption for Heat, Power, and Electricity Generation by Census Region and Economic Characteristics of the Establishment, 1991 (Continued)

\begin{tabular}{|c|c|c|c|c|c|c|c|}
\hline $\begin{array}{l}\text { SIC } \\
\text { Code" }\end{array}$ & $\begin{array}{c}\text { Economic } \\
\text { Characteristics }\end{array}$ & $\begin{array}{l}\text { Consumplion per } \\
\text { Employee } \\
\text { (million Blu) }\end{array}$ & $\begin{array}{l}\text { Consumption per } \\
\text { Dollar of Value } \\
\text { Added } \\
\text { (thousand Btu) }\end{array}$ & $\begin{array}{l}\text { Consumption per } \\
\text { Dollar of Value of } \\
\text { Shipments } \\
\text { (thousand Btu) }\end{array}$ & $\begin{array}{l}\text { Major Byproducts } \\
\text { as a Percent of } \\
\text { Consumption } \\
\text { (percent) }\end{array}$ & $\begin{array}{c}\text { Fuel } \mathrm{Oil}^{\mathrm{d}} \text { as a } \\
\text { Percent of Natural } \\
\text { Gas } \\
\text { (percent) }\end{array}$ & $\begin{array}{l}\text { RSE } \\
\text { Row } \\
\text { Factors }\end{array}$ \\
\hline
\end{tabular}

2821

Plastics Materials and Resins

Employment Size

Under $50 \ldots \ldots \ldots \ldots \ldots \ldots \ldots$

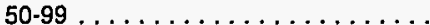

$100-249 \ldots \ldots \ldots \ldots \ldots \ldots \ldots \ldots$

$250-499 \ldots \ldots \ldots \ldots \ldots \ldots \ldots \ldots \ldots$

$500-999 \ldots \ldots \ldots \ldots \ldots \ldots \ldots$

1,000 and Over .............

Total ...................

$3,361.3$

$8,943.2$

$6,024.6$

$3,577.7$

$4,797.3$

12.1
12.7
17.4
48.6
30.8
18.8
26.0

3.7
4.2
5.4
16.7
12.8
9.8
9.8

$W$
$W$
0.8
$W$
2.0
$W$
6.8

$\begin{array}{rr}Q & 21.5 \\ 10.6 & 6.6 \\ W & 6.9 \\ W & 6.0 \\ W & 4.8 \\ W & 6.0 \\ 3.7 & 4.6\end{array}$

2822

Synthetic Rubber

Employment Size

Under $50 \ldots \ldots \ldots \ldots \ldots \ldots$. . . .

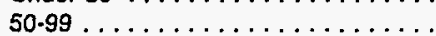

$100-249 \ldots \ldots \ldots \ldots \ldots \ldots \ldots$

$250-499 \ldots \ldots \ldots \ldots \ldots \ldots$

$500-999 \ldots \ldots \ldots \ldots \ldots \ldots$

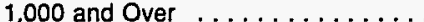

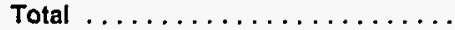

W

$1,052.6$

$3,087.3$

$4,486.4$

W

$10,047.4$

Cellulosic Manmade Fibers

Employment Size

Under $50 \ldots \ldots \ldots \ldots \ldots \ldots$

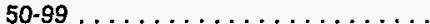

$100-249 \ldots \ldots \ldots \ldots \ldots \ldots \ldots$

$250-499 \ldots \ldots \ldots \ldots \ldots \ldots \ldots$

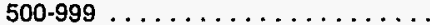

1,000 and Over $\ldots \ldots \ldots \ldots \ldots$

Total ................

Organic Fibers, Noncellulosic

2824

Employment Size

Under $50 \ldots \ldots \ldots \ldots \ldots \ldots$.

$50-99 \ldots \ldots \ldots \ldots \ldots \ldots \ldots$

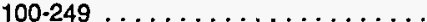

$250-499 \ldots \ldots \ldots \ldots \ldots \ldots \ldots$

$500-999 \ldots \ldots \ldots \ldots \ldots \ldots$

1,000 and Over ..............

Total ..................

Cyclic Crudes and Intermediates

Employment Size

Under $50 \ldots \ldots \ldots \ldots \ldots \ldots$

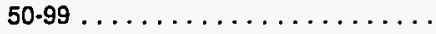

$100-249 \ldots \ldots \ldots \ldots \ldots \ldots \ldots$

$250-499 \ldots \ldots \ldots \ldots \ldots \ldots$

$500-999 \ldots \ldots \ldots \ldots \ldots \ldots \ldots$

1,000 and over $\ldots \ldots \ldots \ldots \ldots \ldots$

Total

Industrial Organic Chemicals, nec

Employment Size

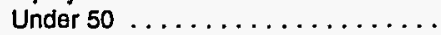

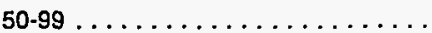

$100-249 \ldots \ldots \ldots \ldots \ldots \ldots \ldots$

$250-499 \ldots \ldots \ldots \ldots \ldots \ldots \ldots$.

$500-999 \ldots \ldots \ldots \ldots \ldots \ldots \ldots$

1,000 and Over $\ldots \ldots \ldots \ldots \ldots$.

Total $\ldots \ldots \ldots \ldots \ldots \ldots \ldots$
W

$6,631.3$

$5,722.6$

$4,856.5$

$12,735.8$

$W$
$7,348.4$

$3,910.5$

$5,866.3$

$7,236.5$

$14,973.2$

$15,452,3$

$13,409.6$

$11,886.6$

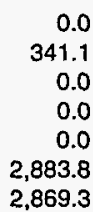

0.0

5.7

0.0

0.0

44.6

44.4

$Q$
$W$
11.3
21.2
11.9
16.5
16.2

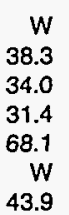

43.9

$W$
2.6
7.8
$W$
12.1
$W$
27.3

0.0

0.0

0.0

0.0

0.0

0.0

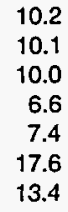

10.1

0.0

6.6
7.4

17.6

13.4 
Table A51. Selected Energy Operating Ratios for Total Energy Consumption for Heat, Power, and Electricity Generation by Census Region and Economic Characteristics of the Establishment, 1991 (Continued)

\begin{tabular}{|c|c|c|c|c|c|c|c|}
\hline $\begin{array}{l}\text { SIC } \\
\text { Code }\end{array}$ & $\begin{array}{c}\text { Economic } \\
\text { Characteristics }^{b}\end{array}$ & $\begin{array}{c}\text { Consumption per } \\
\text { Employee } \\
\text { (million Blu) }\end{array}$ & $\begin{array}{c}\text { Consumption per } \\
\text { Dollar of Value } \\
\text { Added } \\
\text { (thousand Btu) }\end{array}$ & $\begin{array}{l}\text { Consumption per } \\
\text { Dollar of Value of } \\
\text { Shipments } \\
\text { (thousand Btu) }\end{array}$ & $\begin{array}{l}\text { Major Byproducts } \\
\text { as a Percent of } \\
\text { Consumption } \\
\text { (percent) }\end{array}$ & $\begin{array}{c}\text { Fuel Oil as a } \\
\text { Percent of Natural } \\
\text { Gas } \\
\text { (percent) }\end{array}$ & \multirow[t]{2}{*}{$\begin{array}{l}\text { RSE } \\
\text { Row } \\
\text { Factors }\end{array}$} \\
\hline & mn Factors: & 0.8 & 0.8 & 0.7 & 1.1 & 1.7 & \\
\hline
\end{tabular}

2873

Nitrogenous Fertilizers

Employment Size

Under $50 \ldots \ldots \ldots \ldots \ldots \ldots \ldots$

$50-99 \ldots \ldots \ldots \ldots \ldots \ldots \ldots . . . \ldots$

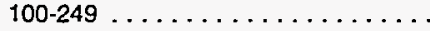

$250-499 \ldots \ldots \ldots \ldots \ldots \ldots \ldots$

$500-999 \ldots \ldots \ldots \ldots \ldots \ldots \ldots$

1,000 and Over . . . . . . . . . . .

Total $\ldots \ldots \ldots \ldots \ldots \ldots . . . . . .$.

$40,343.8$
$27,465.0$
$67,395.9$
$26,185.6$
$16,595.5$
0.0
$43,985.2$

Phosphatic Fertilizers

2874

Employment Size

Under $50 \ldots \ldots \ldots \ldots \ldots \ldots$. . . . . .

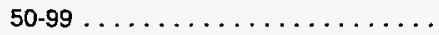

$100-249 \ldots \ldots \ldots \ldots \ldots \ldots \ldots$

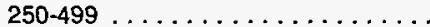

$500-999 \ldots \ldots \ldots \ldots \ldots \ldots \ldots$.

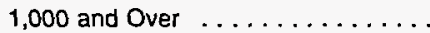

Total .....................

$$
\begin{array}{r}
1,067.3 \\
2,125.3 \\
W \\
8,275.7 \\
W \\
1,967.9 \\
3,396.7
\end{array}
$$

PETROLEUM and COAL PRODUCTS

Employment Size

Under $50 \ldots \ldots \ldots \ldots \ldots \ldots$

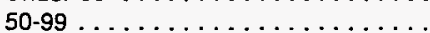

$100-249 \ldots \ldots \ldots \ldots \ldots \ldots$

$250-499 \ldots \ldots \ldots \ldots \ldots \ldots \ldots$

$500-999 \ldots \ldots \ldots \ldots \ldots \ldots$.

1,000 and Over . . . . . . . . .

Total ................

Petroleum Refining

2911

Employment Size

Under $50 \ldots \ldots \ldots \ldots . . . \ldots$

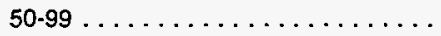

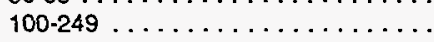

$250-499 \ldots \ldots \ldots \ldots \ldots \ldots \ldots$

$500-999 \ldots \ldots \ldots \ldots \ldots \ldots$.

1,000 and Over $\ldots \ldots \ldots \ldots \ldots$.

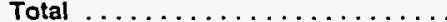

RUBBER and MISC. PLASTICS PRODUCTS

Employment Size

Under $50 \ldots \ldots \ldots \ldots \ldots \ldots \ldots$

$50-99 \ldots \ldots \ldots \ldots \ldots \ldots$

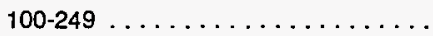

$250-499 \ldots \ldots \ldots \ldots \ldots \ldots \ldots$

$500-999 \ldots \ldots \ldots \ldots \ldots \ldots$

1,000 and Over . . . . . . . . .

Total .................

Tires and Inner Tubes

3011

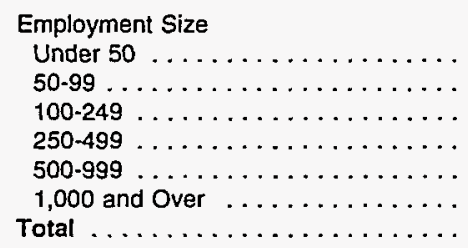

$25,239.7$

$28,796.5$

$29,281.7$

$43,536.0$

$36,062.6$

$43,413.9$

$39,377.8$
6.8
19.8
$W$
39.4
$W$
13.3
25.4

187.9
175.4
285.9
206.7
104.3
0.0
229.2

229.2

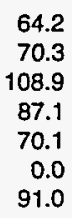

2.7
2.4
$W$
13.2
$W$
5.3
7.0

2.6
0.0
0.7
0.0
0.0
0.0
0.7

0.0
0.0
0.0
0.0
0.0
0.0
0.0

0.0

$W$
18.8
37.0
48.2
44.2
43.0
42.8

$\begin{array}{rr}W & 19.2 \\ 55.5 & 18.3 \\ 74.3 & 7.8 \\ W & 1.8 \\ W & 1.9 \\ W & 1.5 \\ 12.9 & 3.1\end{array}$

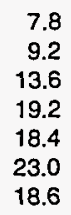

21.9

166.6

83.4

108.3

163.3

133.7

159.9

146.0

$\begin{array}{ll}3.8 & 1.7 \\ 3.6 & 1.8 \\ 4.4 & 2.2 \\ 5.5 & 2.8 \\ 6.1 & 3.1 \\ 5.6 & 3.1 \\ 4.8 & 2.4\end{array}$

0.0
0.0
0.1
$W$
$W$
0.1

$\begin{array}{rrr}479.1 & Q & 9.1 \\ W & W & W \\ 535.2 & 5.5 & 2.3 \\ W & W & W \\ W & W & W \\ 657.7 & 6.0 & 3.5 \\ 658.3 & 6.4 & 3.6\end{array}$

\begin{tabular}{lr}
0.0 & 0.0 \\
0.0 & $W$ \\
0.0 & $Q$ \\
0.0 & $W$ \\
0.0 & $W$ \\
$W$ & $W$ \\
$W$ & 16.9 \\
\hline
\end{tabular}


Table A51. Selected Energy Operating Ratios for Total Energy Consumption for Heat, Power, and Electricity Generation by Census Region and Economic Characteristics of the Establishment, 1991 (Continued)

\begin{tabular}{|c|c|c|c|c|c|c|c|}
\hline $\begin{array}{c}\text { SIC } \\
\text { Code" }\end{array}$ & $\begin{array}{c}\text { Economic } \\
\text { Characteristlcs }^{b}\end{array}$ & $\begin{array}{c}\text { Consumption per } \\
\text { Employee } \\
\text { (million Btu) }\end{array}$ & $\begin{array}{c}\text { Consumption per } \\
\text { Dollar of Value } \\
\text { Added } \\
\text { (thousand Btu) }\end{array}$ & $\begin{array}{l}\text { Consumption per } \\
\text { Dollar of Value of } \\
\text { Shipments } \\
\text { (thousand Btu) }\end{array}$ & $\begin{array}{l}\text { Major Byproducts } \\
\text { as a Percent of } \\
\text { Consumption } \\
\text { (percent) }\end{array}$ & $\begin{array}{c}\text { Fuel } \mathrm{Oid}^{\mathrm{d}} \text { as a } \\
\text { Percent of Natural } \\
\text { Gas } \\
\text { (percent) }\end{array}$ & \multirow[t]{2}{*}{$\begin{array}{l}\text { RSE } \\
\text { Row } \\
\text { Factors }\end{array}$} \\
\hline & umn Factors: & 0.8 & 0.8 & 0.7 & 1.1 & 1.7 & \\
\hline
\end{tabular}

308 Miscellaneous Plastic Products, nec

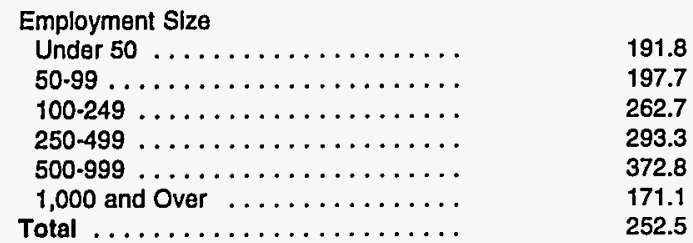

$\begin{array}{ll}191.8 & 3.4 \\ 197.7 & 3.5 \\ 262.7 & 4.2 \\ 293.3 & 5.2 \\ 372.8 & 5.9 \\ 171.1 & 2.5 \\ 252.5 & 4.3\end{array}$

LEATHER and LEATHER PRODUCTS

31

Employment Size

Under $50 \ldots \ldots \ldots \ldots \ldots \ldots$

$50-99 \ldots \ldots \ldots \ldots \ldots \ldots \ldots \ldots . . . \ldots \ldots$

100-249

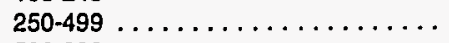

$500-999 \ldots \ldots \ldots \ldots \ldots \ldots \ldots$

1,000 and Over $\ldots \ldots \ldots \ldots \ldots$

Total ....................

69.1

175.6
64.7

130.2

104.9

125.5

1.5
1.7
2.0
2.6
2.9
1.5
2.1

0.0
0.0
$W$
$W$
0.0
0.1

$\begin{array}{rr}Q & 15.1 \\ W & 13.8 \\ W & 14.9 \\ W & 15.8 \\ W & 14.5 \\ Q & 33.1 \\ W & 9.0\end{array}$

32

STONE, CLAY and GLASS PRODUCTS

Employment Size

Under $50 \ldots \ldots \ldots \ldots \ldots \ldots \ldots \ldots$

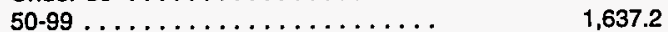

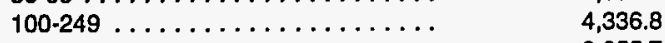

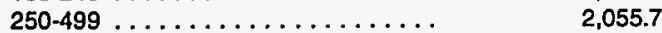

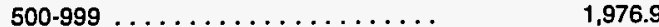

$\begin{array}{lr}1,000 \text { and Over } \ldots \ldots \ldots \ldots \ldots \ldots & 1,353.5 \\ \text { Total } & 2,051.2\end{array}$

Total $\ldots \ldots \ldots \ldots \ldots$

Flat Glass

3211

Employment Size

Under $50 \ldots \ldots \ldots \ldots \ldots \ldots \ldots$

$50-99 \ldots \ldots \ldots \ldots \ldots \ldots \ldots \ldots$.

$100-249 \ldots \ldots \ldots \ldots \ldots \ldots \ldots$

$250-499 \ldots \ldots \ldots \ldots \ldots \ldots \ldots$

$500-999 \ldots \ldots \ldots \ldots \ldots \ldots \ldots$

1,000 and Over $\ldots \ldots \ldots \ldots \ldots$.

Total ..................

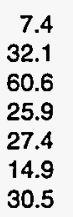

3.6

16.3

32.5

15.4

16.3

9.6
16.6

0.8
2.1
2.2
0.8
1.2
0.7
1.4

0.0

0.0

0.0

0.0

0.0

0.0
0.0

41.6

61.2

22.9

21.8

17.5

$\begin{array}{rr}Q & 23.6 \\ 51.7 & 17.4\end{array}$

3221

Glass Containers

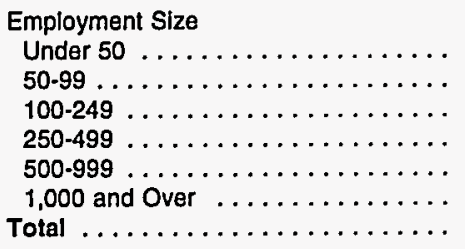

0.0
0.0
$2,604.0$
$2,736.5$
$2,589.3$
$1,573.0$
$2,549.9$

$W$
$W$
$W$
$3,627.3$
$4,834.2$
$W$
$3,934.5$

$W$
$W$
$W$
36.1
47.5
$W$
40.1

$W$
$W$
$W$
23.0
27.5
$W$
24.0

0.0
0.0
$W$
0.0
0.0
0.0
$W$

$\begin{array}{rr}44.3 & 19.0 \\ 9.4 & 12.9 \\ W & 14.4 \\ 3.1 & 5.3 \\ W & 6.0 \\ W & 6.5 \\ 7.3 & 9.0\end{array}$

Pressed and Blown Glass, nec.

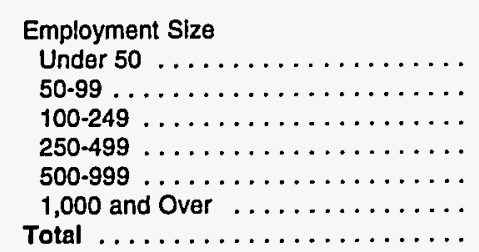

\begin{tabular}{|c|c|c|c|c|c|}
\hline 107.0 & 1.6 & 1.0 & 0.0 & 0.0 & 25.6 \\
\hline 0 & $Q$ & $Q$ & 0.0 & $\mathbf{Q}$ & NF \\
\hline $1,767.0$ & 18.0 & 11.6 & 0.0 & - & 14.5 \\
\hline$W$ & $w$ & w & 0.0 & $w$ & 4.2 \\
\hline $1,722.7$ & 24.9 & 16.1 & 0.0 & w & 3.2 \\
\hline $2,084.6$ & 26.4 & 16.2 & 0.0 & 0.9 & 4.2 \\
\hline$W$ & W & $w$ & 0.0 & w & 6.0 \\
\hline
\end{tabular}

0.0
0.0
28.2
30.6
38.6
31.2
32.6

0.0
0.0
16.2
17.4
19.3
15.8
17.8

$$
\begin{aligned}
& 0.0 \\
& 0.0 \\
& 0.0 \\
& 0.0 \\
& 0.0 \\
& 0.0 \\
& 0.0
\end{aligned}
$$

$\begin{array}{cc}0.0 & N F \\ 0.0 & N F \\ W & 4.3 \\ W & 3.0 \\ W & 4.4 \\ W & 6.0 \\ 2.7 & 2.8\end{array}$

See footnotes at end of table. 
Table A51. Selected Energy Operating Ratios for Total Energy Consumption for Heat, Power, and Electricity Generation by Census Region and Economic Characteristics of the Establishment, 1991 (Continued)

\begin{tabular}{|c|c|c|c|c|c|c|c|}
\hline $\begin{array}{l}\text { SIC } \\
\text { Code" }\end{array}$ & $\begin{array}{c}\text { Economic } \\
\text { Characteristics }^{\circ}\end{array}$ & $\begin{array}{c}\text { Consumption per } \\
\text { Employee } \\
\text { (million Btu) }\end{array}$ & $\begin{array}{c}\text { Consumption per } \\
\text { Dollar of Value } \\
\text { Added } \\
\text { (thousand Btu) }\end{array}$ & $\begin{array}{l}\text { Consumption per } \\
\text { Dollar of Value of } \\
\text { Shipments } \\
\text { (thousand Btu) }\end{array}$ & $\begin{array}{l}\text { Major Byproducts } \\
\text { as a Percent of } \\
\text { Consumption } \\
\text { (percent) }\end{array}$ & $\begin{array}{c}\text { Fuel Oid as a } \\
\text { Percent of Natural } \\
\text { Gas } \\
\text { (percent) }\end{array}$ & \multirow[t]{2}{*}{$\begin{array}{c}\text { RSE } \\
\text { Row } \\
\text { Factors }\end{array}$} \\
\hline & umn Factors: & 0.8 & 0.8 & 0.7 & 1.1 & 1.7 & \\
\hline
\end{tabular}

3241

Cement, Hydraulic

Employment Size

Under $50 \ldots \ldots \ldots \ldots \ldots \ldots \ldots \ldots$

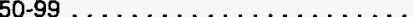

$100-249 \ldots \ldots \ldots \ldots \ldots \ldots \ldots$

$250-499 \ldots \ldots \ldots \ldots \ldots \ldots \ldots \ldots$

$500-999 \ldots \ldots \ldots \ldots \ldots \ldots \ldots$

1,000 and Over $\ldots \ldots \ldots \ldots \ldots$

Total

Lime

3274

Employment Size

Under $50 \ldots \ldots \ldots \ldots \ldots \ldots \ldots$

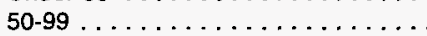

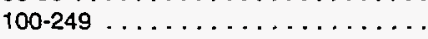

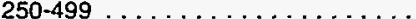

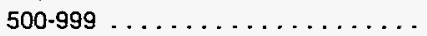

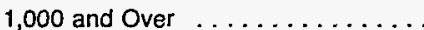

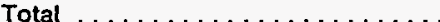

Mineral Wool

3296

Employment Size

Under $50 \ldots \ldots \ldots \ldots \ldots \ldots$.

$50-99 \ldots \ldots \ldots \ldots \ldots \ldots \ldots$

$100-249 \ldots \ldots \ldots \ldots \ldots \ldots \ldots$

$250-499 \ldots \ldots \ldots \ldots \ldots \ldots . . . \ldots$

$500-999 \ldots \ldots \ldots \ldots \ldots \ldots$

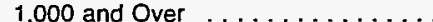

Total $\ldots \ldots \ldots \ldots \ldots \ldots \ldots$

PRIMARY METAL INDUSTRIES

33

Employment Size

Under $50 \ldots \ldots \ldots \ldots . . \ldots$.

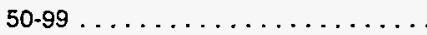

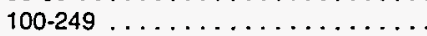

$250-499 \ldots \ldots \ldots \ldots \ldots$

$500-999 \ldots \ldots \ldots \ldots \ldots \ldots \ldots$

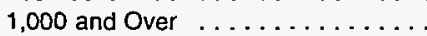

Total ..................

\section{Blast Furnaces and Steel Mills}

Employment Size

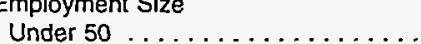

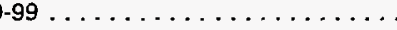

$100-249 \ldots \ldots \ldots \ldots \ldots \ldots \ldots . . . \ldots \ldots$

$250-499 \ldots \ldots \ldots \ldots \ldots \ldots \ldots$

$500-999 \ldots \ldots \ldots \ldots \ldots \ldots \ldots$

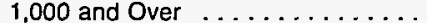

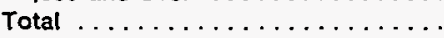

Electrometalurgical Products

3313
$20,288.0$

$21,001.0$

$22,270.9$

0.0

0.0
$20,082.6$

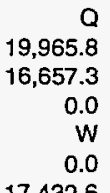

$17,432.6$
606.8

719.2

$1,080.2$

$1,617.1$

$3,545.9$

$7,597.2$

3.526 .9
2.6

137.5

184.2

206.9

0.0

0.0
169.3

1.7
70.3
92.8
116.1
0.0
0.0
87.2

$W$
111.7
122.2
0.0
$W$
0.0

119.3

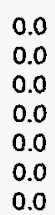

0.0

$\begin{array}{rr}0 & 34.1 \\ 2.1 & 13.4 \\ 13.9 & 5.2 \\ 16.5 & 7.7 \\ 0.0 & \text { NF } \\ 0.0 & \text { NF } \\ 11.3 & 5.6\end{array}$

$\begin{array}{rr}W & W \\ 3,039.6 & 24.4 \\ 2,991.7 & 32.6 \\ 2,576.0 & 25.5 \\ 2,512.3 & 32.4 \\ W & W \\ 2,619.8 & 26.3\end{array}$

$W$
10.9
18.7
15.3
17.7
$W$
15.5
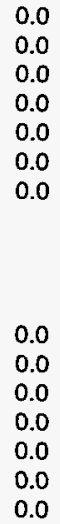

$\begin{array}{rr}W & 31.4 \\ W & 9.2 \\ 27.5 & 7.1 \\ 0.0 & \text { NF } \\ W & 20.1 \\ 0.0 & \text { NF } \\ W & 10.0\end{array}$

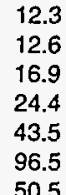

12.3
12.6
16.9
24.4
43.5
96.5
50.5
5.0

4.2

5.7

8.4

13.9

35.4

17.6
0.0
0.0
$W$
10.3
$W$
25.1
18.7

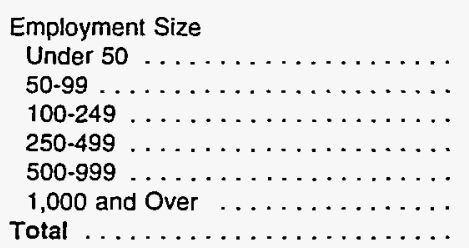


Table A51. Selected Energy Operating Ratios for Total Energy Consumption for Heat, Power, and Electricity Generation by Census Region and Economic Characteristics of the Establishment, 1991 (Continued)

\begin{tabular}{|c|c|c|c|c|c|c|c|}
\hline$\underset{\text { Code }}{\text { SIC }}$ & $\begin{array}{c}\text { Economic } \\
\text { Characteristics }^{\circ}\end{array}$ & $\begin{array}{l}\text { Consumption per } \\
\text { Employee } \\
\text { (million Btu) }\end{array}$ & $\begin{array}{l}\text { Consumption per } \\
\text { Dollar of Value } \\
\text { Added } \\
\text { (thousand Biu) }\end{array}$ & $\begin{array}{l}\text { Consumption per } \\
\text { Dollar of Value of } \\
\text { Shipments } \\
\text { (thousand Btu) }\end{array}$ & $\begin{array}{l}\text { Major Byproducts } \\
\text { as a Percent of } \\
\text { Consumption } \\
\text { (percent) }\end{array}$ & $\begin{array}{c}\text { Fuel Oif as a } \\
\text { Percent of Natural } \\
\text { Gas } \\
\text { (percent) }\end{array}$ & \multirow[t]{2}{*}{$\begin{array}{c}\text { RSE } \\
\text { Row } \\
\text { Factors }\end{array}$} \\
\hline & Imn Factors: & 0.8 & 0.8 & 0.7 & 1.1 & 1.7 & \\
\hline
\end{tabular}

$3321 \quad$ Gray and Ductlle Iron Foundries

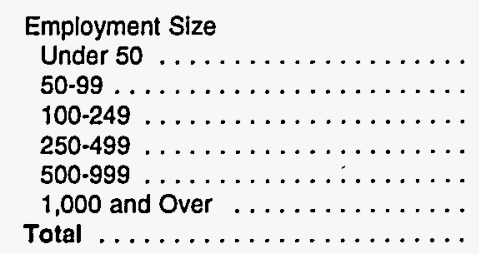

3331

\section{Primary Copper}

576.1
644.6
$W$
$W$
$1,025.1$
$1,378.8$
$1,033.2$

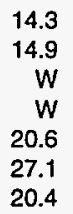

$\begin{array}{rr}W & W \\ 0.0 & 0.0 \\ W & W \\ W & W \\ 6,062.6 & 30.2 \\ 0.0 & 0.0 \\ 4,840.7 & 22.9\end{array}$

3334

Primary Aluminum

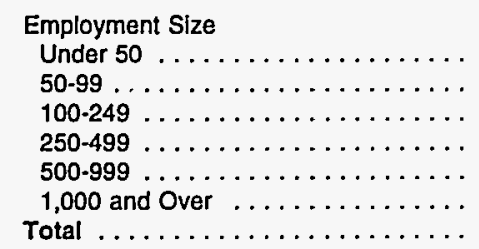

3339

Primary Nonferrous Metals, nec

Employment Size

Under $50 \ldots \ldots \ldots \ldots \ldots \ldots$.

$50-99 \ldots \ldots \ldots \ldots \ldots \ldots \ldots \ldots$

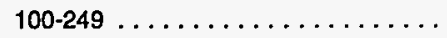

$250-499 \ldots \ldots \ldots \ldots \ldots \ldots \ldots$

$500-999 \ldots \ldots \ldots \ldots \ldots \ldots \ldots$

1,000 and Over $\ldots \ldots \ldots \ldots \ldots \ldots \ldots$

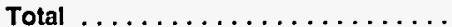

Aluminum Sheet, Plate, and Foil

Employment Size

Under $50 \ldots \ldots \ldots \ldots \ldots . . . .$.

$50-99 \ldots \ldots \ldots \ldots \ldots \ldots \ldots \ldots$

100-249 .................

250-499 ...............

$500-999 \ldots \ldots \ldots \ldots \ldots \ldots \ldots \ldots$

1,000 and Over $\ldots \ldots \ldots \ldots \ldots \ldots$

Total

FABRICATED METAL PRODUCTS

Employment Size

Under $50 \ldots \ldots \ldots \ldots \ldots \ldots$

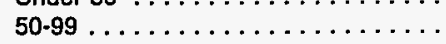

100-249 . . . . . . . . . . . .

$250-499 \ldots \ldots \ldots \ldots \ldots \ldots \ldots \ldots$

$500-999 \ldots \ldots \ldots \ldots \ldots \ldots . . . \ldots$

1,000 and Over ..............

Total $\ldots \ldots \ldots \ldots \ldots \ldots \ldots$

$\begin{array}{rr}92.7 & 1.1 \\ 1,297.4 & 14.0 \\ 2,750.4 & 30.2 \\ 1,885.5 & 31.6 \\ 3,055.9 & 25.5 \\ 2,409.6 & 22.6 \\ 2,450.2 & 23.8\end{array}$

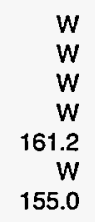

$\begin{array}{rr}W & W \\ W & W \\ W & W \\ W & W \\ 13,272.0 & 161.2 \\ W & W \\ 12,816.0 & 155.0\end{array}$

8.0
8.3
$W$
$W$
10.5
13.7
10.6

0.0
0.0
0.0
0.0
0.0
0.0
0.0

$\begin{array}{rr}Q & 25.6 \\ Q & 15.9 \\ W & 9.2 \\ W & 3.8 \\ 2.9 & 4.9 \\ W & 5.1 \\ 3.0 & 5.3\end{array}$

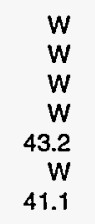

0.0
0.0
0.0
0.0
0.0
0.0
0.0

8.0

$W$
0.0
$W$
$W$
7.7
0.0
5.5

0.0
0.0
0.0
0.0
$W$
0.0
$W$

$\begin{array}{rrrrrr}1,578.5 & 18.4 & 3.1 & 0.0 & 1.5 & 27.6 \\ W & W & W & 0.0 & W & 6.3 \\ 3,170.0 & 35.3 & 9.7 & 0.0 & W & 5.3 \\ 3,631.1 & 45.7 & 12.3 & 0.0 & W & N F \\ 8,424.6 & 69.8 & 12.9 & 0.0 & W & N F \\ W & W & W & W & 0.0 & N F \\ 4,239.7 & 45.6 & 12.0 & W & 1.9 & 2.6\end{array}$

See footnotes at end of table.

Energy Information Administration/Manufacturing Consumption of Energy 1991 
Table A51. Selected Energy Operating Ratios for Total Energy Consumption for Heat, Power, and Electricity Generation by Census Region and Economic Characteristics of the Establishment, 1991 (Continued)

\begin{tabular}{|c|c|c|c|c|c|c|c|}
\hline $\begin{array}{c}\text { SIC } \\
\text { Code" }\end{array}$ & $\begin{array}{c}\text { Economic } \\
\text { Characteristics }^{b}\end{array}$ & $\begin{array}{l}\text { Consumption per } \\
\text { Employee } \\
\text { (million Btu) }\end{array}$ & $\begin{array}{c}\text { Consumption per } \\
\text { Dollar of Value } \\
\text { Added } \\
\text { (thousand Btu) }\end{array}$ & $\begin{array}{l}\text { Consumption per } \\
\text { Dollar of Value of } \\
\text { Shipments } \\
\text { (thousand Btu) }\end{array}$ & $\begin{array}{l}\text { Major Byproducts } \\
\text { as a Percent of } \\
\text { Consumption } \\
\text { (percent) }\end{array}$ & $\begin{array}{c}\text { Fuel Oild as a } \\
\text { Percent of Natural } \\
\text { Gas } \\
\text { (percent) }\end{array}$ & \multirow[t]{2}{*}{$\begin{array}{c}\text { RSE } \\
\text { Row } \\
\text { Factors }\end{array}$} \\
\hline & Imn Factors: & 0.8 & 0.8 & 0.7 & 1.1 & 1.7 & \\
\hline
\end{tabular}

INDUSTRIAL MACHINERY and EQUIPMENT

Employment Size

Under $50 \ldots \ldots \ldots \ldots \ldots \ldots \ldots \ldots$

$50-99 \ldots \ldots \ldots \ldots \ldots \ldots \ldots . \ldots \ldots$

$100-249 \ldots \ldots \ldots \ldots \ldots \ldots \ldots \ldots \ldots$

$250-499 \ldots \ldots \ldots \ldots \ldots \ldots \ldots \ldots \ldots$

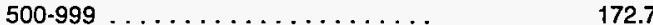

1,000 and Over ............. 201.5

Total .................

Computer and Office Equipment

357

Employment Size

Under $50 \ldots \ldots \ldots \ldots \ldots \ldots \ldots$

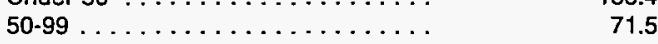

$100-249 \ldots \ldots \ldots \ldots \ldots \ldots \ldots$

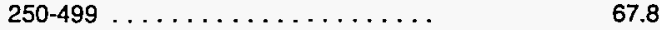

$500-999 \ldots \ldots \ldots \ldots \ldots \ldots \ldots \ldots$

1,000 and over . . . . . . . 85.5

Total ................ 86.2

ELECTRONIC and OTHER ELECTRIC EQUIPMENT

Employment Size

Under $50 \ldots \ldots \ldots \ldots \ldots \ldots \ldots$

$50-99 \ldots \ldots \ldots \ldots \ldots \ldots \ldots$

$100-249 \ldots \ldots \ldots \ldots \ldots \ldots \ldots \ldots$

$250-499 \ldots \ldots \ldots \ldots \ldots \ldots \ldots$

$500-999 \ldots \ldots \ldots \ldots \ldots \ldots \ldots \ldots$

1,000 and Over $\ldots \ldots \ldots \ldots \ldots$

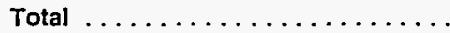

TRANSPORTATION EQUIPMENT

37

Employment Size

Under $50 \ldots \ldots \ldots \ldots \ldots \ldots \ldots \ldots$

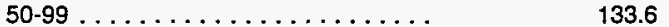

$100-249 \ldots \ldots \ldots \ldots \ldots \ldots \ldots \ldots$

$250-499 \ldots \ldots \ldots \ldots \ldots \ldots \ldots \ldots$

$500-999 \ldots \ldots \ldots \ldots \ldots \ldots \ldots \ldots \ldots$

1,000 and Over $\ldots \ldots \ldots \ldots \ldots \ldots . \ldots \ldots$

Total ................. 222.9

3711 Motor Vehicles and Car Bodies

Employment Size

Under $50 \ldots \ldots \ldots \ldots \ldots \ldots \ldots . \ldots \ldots$

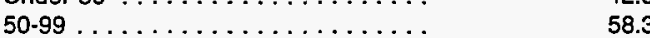

$100-249 \ldots \ldots \ldots \ldots \ldots \ldots \ldots \ldots$

$250-499 \ldots \ldots \ldots \ldots \ldots \ldots \ldots \ldots$

$500-999 \ldots \ldots \ldots \ldots \ldots \ldots \ldots \ldots . \ldots \ldots$

1,000 and Over .............. 511.0

Total .................. 494.0

Motor Vehicie Parts and Accessories

Employment Size

Under $50 \ldots \ldots \ldots \ldots \ldots \ldots \ldots \ldots$

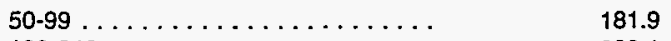

$100-249 \ldots \ldots \ldots \ldots \ldots \ldots \ldots \ldots$

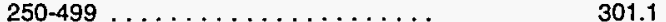

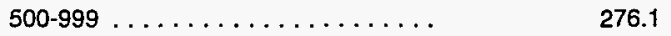

1,000 and Over $\ldots \ldots \ldots \ldots \ldots \ldots . \ldots$

Total ....................

84.8

97.8

80.9

93.1

311.2

1.9
1.5
1.8
2.6
2.5
1.9
2.0

1.1
0.9
0.9
1.2
1.2
0.9
1.0

0.0

0.0
0.0
0.0
0.0
0.0
0.0

$\begin{array}{rr}6.1 & 14.9 \\ 4.6 & 14.9 \\ 4.9 & 12.4 \\ 3.1 & 12.8 \\ 10.3 & 8.5 \\ 8.4 & 6.6 \\ 6.7 & 5.1\end{array}$

2.6
0.5
0.9
1.1
1.2
0.7
0.8

1.4
0.3
0.5
0.4
0.5
0.3
0.4

0.0

0.0

0.0

0.0
0.0

0.0

0.0

$\begin{array}{rr}* & 29.3 \\ 13.8 & 25.7 \\ \mathrm{Q} & 26.1 \\ \mathrm{Q} & 14.6 \\ 3.3 & 7.9 \\ 1.9 & 9.6 \\ 2.9 & 7.8\end{array}$

1.2
1.6
1.5
2.3
2.4
1.9
1.9

0.0
0.0
0.0
0.0
0.0
0.0
0.0

31.5

24.5

22.4

14.4

9.0

5.9

$8.0 \quad 6.3$

See footnotes at end of table. 
Table A51. Selected Energy Operating Ratios for Total Energy Consumption for Heat, Power, and Electricity Generation by Census Region and Economic Characteristics of the Establishment, 1991 (Continued)

\begin{tabular}{|c|c|c|c|c|c|c|c|}
\hline $\begin{array}{l}\text { SIC } \\
\text { Code }\end{array}$ & $\begin{array}{c}\text { Economic } \\
\text { Characteristics }^{\mathrm{b}}\end{array}$ & $\begin{array}{c}\text { Consumption per } \\
\text { Employee } \\
\text { (million Btu) }\end{array}$ & $\begin{array}{c}\text { Consumption per } \\
\text { Dollar of Value } \\
\text { Added } \\
\text { (thousand Btu) }\end{array}$ & $\begin{array}{c}\text { Consumption per } \\
\text { Dollar of Value of } \\
\text { Shipments } \\
\text { (thousand Btu) }\end{array}$ & $\begin{array}{c}\text { Major Byproducts } \\
\text { as a Percent of } \\
\text { Consumption } \\
\text { (percent) }\end{array}$ & $\begin{array}{c}\text { Fuel Oil as a } \\
\text { Percent of Natural } \\
\text { Gas } \\
\text { (percent) }\end{array}$ & \multirow[t]{2}{*}{$\begin{array}{c}\text { RSE } \\
\text { Row } \\
\text { Factors }\end{array}$} \\
\hline & mn Factors: & 0.8 & 0.8 & 0.7 & 1.1 & 1.7 & \\
\hline
\end{tabular}

38 INSTRUMENTS and RELATED PRODUCTS

\begin{tabular}{|c|c|}
\hline \multicolumn{2}{|l|}{ Employment Size } \\
\hline Under 50 & 114.0 \\
\hline $50-99 \ldots \ldots \ldots \ldots \ldots \ldots$ & 76.5 \\
\hline $100-249 \ldots \ldots \ldots \ldots \ldots \ldots$ & 53.8 \\
\hline $250-499 \ldots \ldots \ldots \ldots \ldots \ldots$ & 81.1 \\
\hline $500-999 \ldots \ldots \ldots \ldots \ldots$ & 113.3 \\
\hline 1,000 and Over $\ldots \ldots \ldots \ldots$ & 149.6 \\
\hline Total . . . . . . . & 116.5 \\
\hline
\end{tabular}

$\begin{array}{rrrrrr}104.6 & 1.9 & 1.1 & 0.0 & 0 & 50.4 \\ 50.1 & 0.6 & 0.4 & 0.0 & 0.0 & 21.7 \\ 63.6 & 0.9 & 0.6 & 0.0 & 9.2 & 13.5 \\ 101.6 & 1.3 & 0.8 & 0.0 & 26.5 & 9.7 \\ 80.9 & 0.8 & 0.6 & 0.0 & 13.1 & 10.8 \\ 62.5 & 0.6 & 0.5 & 0.0 & 0.8 & 14.3 \\ 77.0 & 0.9 & 0.6 & 0.0 & 12.8 & 7.3\end{array}$

39

Surglcal and Medical Instruments

\section{Employment Size}

Under $50 \ldots \ldots \ldots \ldots \ldots \ldots \ldots$

$50-99 \ldots \ldots \ldots \ldots \ldots \ldots \ldots$

$100-249 \ldots \ldots \ldots \ldots \ldots \ldots \ldots \ldots$

$250-499 \ldots \ldots \ldots \ldots \ldots \ldots \ldots$

$500-999 \ldots \ldots \ldots \ldots \ldots \ldots \ldots$

1,000 and Over $\ldots \ldots \ldots \ldots \ldots \ldots$

Total .................

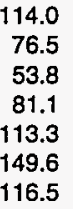

$\begin{array}{ll}1.5 & 1.0 \\ 1.0 & 0.6 \\ 0.8 & 0.5 \\ 0.9 & 0.6 \\ 1.2 & 0.8 \\ 1.4 & 1.0 \\ 1.2 & 0.8\end{array}$

$\begin{array}{rrr}60.4 & 1.5 & 0.8 \\ 67.1 & 1.7 & 0.8 \\ 85.6 & 1.5 & 0.8 \\ 175.4 & 2.5 & 1.3 \\ 163.0 & 1.8 & 1.1 \\ 201.2 & 2.1 & 1.2 \\ 108.3 & 1.8 & 1.0\end{array}$

\begin{tabular}{lrr}
0.8 & 0.0 & $Q$ \\
0.8 & 0.0 & 16.8 \\
0.8 & 0.0 & 5.8 \\
1.3 & 0.0 & 11.9 \\
1.1 & 0.0 & 14.2 \\
1.2 & 0.0 & $W$ \\
1.0 & 0.0 & $W$ \\
\hline
\end{tabular}

See Appendices B and F for descriptions of the Standard Industrial Classification system.

- Employment Size categories were supplied by the Bureau of the Census. See Appendix B.

- "Major Byproduct" fuels include coke oven gas and blast fumace gas (produced primarily in the blast fumace industry, SIC 3312 ); still gas (produced primarily in petroleum refineries, SIC 2911); and pulping liquor (produced primarily in pulp and paper mills, SIC 2611 and 2621).

"Fuel Oll" Includes dlstillate and residual fuel oils.

NF=No appllcable RSE row/column factor.

- Estlmate less than 0.5. Data are included in higher level totals.

$W=$ Withheld to avoid disclosing data for individual establishments. Data are included in higher level totals.

$\mathrm{Q}=$ Withheld because Relative Standard Error is greater than 50 percent. Data are included in higher level totals.

Notes: - To obtain a RSE percentage for any table cell, multiply the cell's corresponding RSE column and RSE row factors. - Totals may not equal sum of components because of independent rounding. - Operating ratios were calculated using the input energy estimates reported in Table A4.

Source: Energy Information Administration, Office of Energy Markets and End Use, Energy End Use and Integrated Statistics Division, Form ElA-846, "1991 Manulacturing Energy Consumption Survey," and Bureau of the Census, Industry Division, data files for the "1991 Annual Survey of Manufactures." 


\section{Table A52. Nonswitchable Minimum Requirements and Maximum Consumption Potential by Census Region, 1991}

(Estimates in Physical Units)

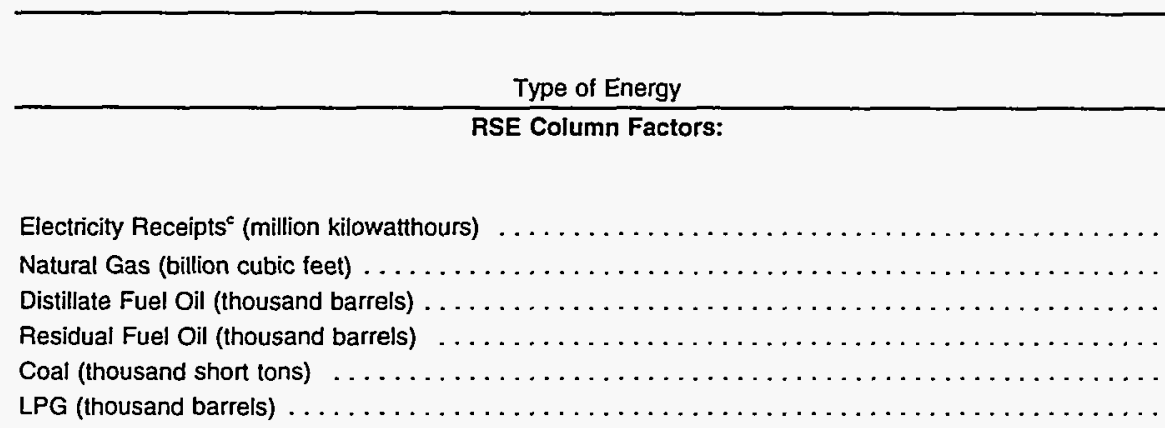

Electricity Receipts (million kilowatthours)

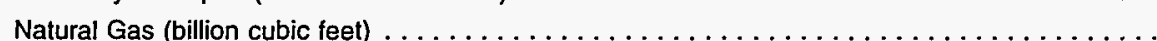

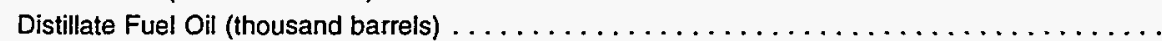

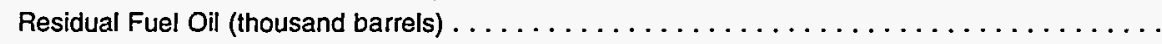

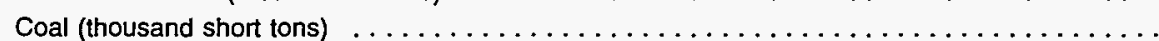

LPG (thousand barrels)

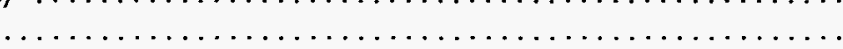

\begin{tabular}{|c|c|c|c}
$\begin{array}{c}\text { Actual } \\
\text { Consumption }\end{array}$ & $\begin{array}{c}\text { Minimum } \\
\text { Consumption }\end{array}$ & $\begin{array}{c}\text { Maximum } \\
\text { Consumplion }\end{array}$ & $\begin{array}{c}\text { RSE Row } \\
\text { Factors }\end{array}$ \\
\hline 1.0 & 1.2 & 0.8 &
\end{tabular}

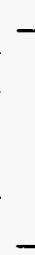

\begin{tabular}{rrr}
\multicolumn{3}{c}{ Total United States } \\
\hline 718,480 & 701,478 & 766,887 \\
5,345 & 3,485 & 5,887 \\
23,885 & 19,113 & 201,081 \\
65,837 & 36,488 & 201,921 \\
53,035 & 29,425 & 58,996 \\
27,970 & 14,689 & 236,983 \\
\hline
\end{tabular}

Northeast Census Region

\begin{tabular}{rrrr}
87,851 & 85,980 & 92,709 & 3.0 \\
446 & 240 & 519 & 3.0 \\
7,805 & 6,109 & 30,640 & 7.1 \\
29,245 & 17,562 & 47,504 & 3.6 \\
7,420 & 5,258 & 7,685 & 25.7 \\
$W$ & 2,235 & 22,767 & 8.3 \\
\hline
\end{tabular}

Electricity Receipts ${ }^{c}$ (million kilowatthours)

\begin{tabular}{lrrrr}
\multicolumn{4}{c}{ Midwest Census Region } & \\
$\ldots$ & 207,104 & 202,036 & 220,317 & 3.0 \\
$\ldots$ & 1,363 & 839 & 1,542 & 2.3 \\
$\ldots$ & 3,885 & 3,305 & 59,433 & 7.1 \\
$\ldots$ & 8,134 & 3,504 & 39,914 & 4.7 \\
$\ldots$ & 18,828 & 10,643 & 21,332 & 3.3 \\
$\ldots$ & 3,877 & 2,274 & 59,563 & 5.8 \\
\hline
\end{tabular}

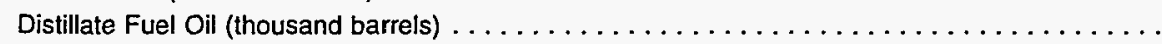

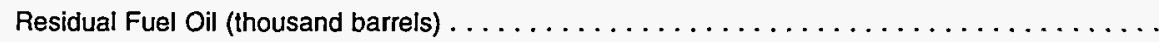

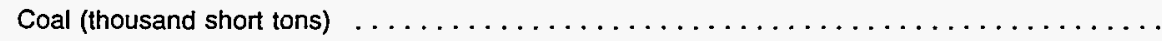

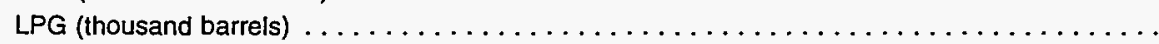

3,877

South Census Region

Electricity Receipts ${ }^{c}$ (million kilowatthours)

\begin{tabular}{lrrrr}
\multicolumn{4}{c}{ South Census Region } & \\
\cline { 2 - 4 } & 305,128 & 297,691 & 328,832 & 2.0 \\
$\ldots$ & 2,896 & 2,053 & 3,095 & 2.3 \\
$\ldots$ & 8,014 & 6,371 & 80,904 & 5.0 \\
$\ldots$ & 23,114 & 13,511 & 86,548 & 4.2 \\
$\ldots$ & 22,514 & 12,155 & 25,094 & 3.3 \\
$\cdots$ & $W$ & 4,019 & 105,597 & 4.2 \\
\hline
\end{tabular}

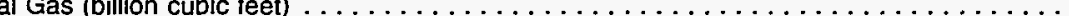

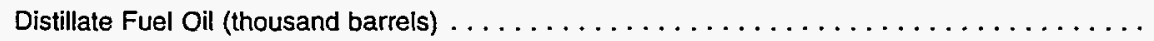

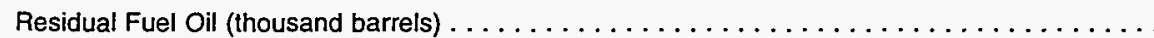

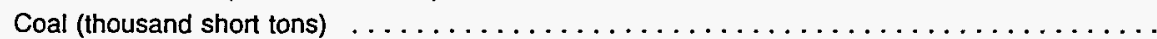

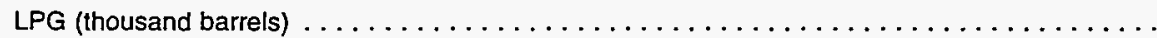

W

\begin{tabular}{rrr}
\multicolumn{3}{c}{ West Census Region } \\
\hline 118,398 & 115,771 & 125,029 \\
640 & 353 & 730 \\
4,180 & 3,328 & 30,104 \\
5,344 & 1,911 & 27,956 \\
4,274 & 1,370 & 4,884 \\
13,345 & 6,161 & 49,056 \\
\hline
\end{tabular}

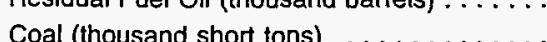

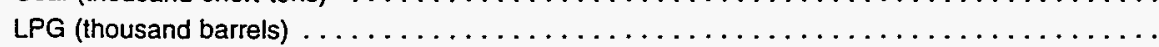

4,274
13,345

6,161

\footnotetext{
- Minimum consumption represents actual 1991 consumption decreased by the quantity of the designated type of energy that would no longer have been required if all ascertained switching from that type of energy had occurred. The minimum value includes the quantity of 1991 consumption for which switching capability was not ascertained.

Maximum consumption represents actual 1991 consumption increased by the quantity of the designated type of energy that would have been required if all ascertained switching into that type of energy had occurred. This value assumes that all indicated substitutions were possible simultaneously and the substitutable amount consists of the sum of all possible switches to the designated type of energy. The estimate assumes that 1991 output remained constant.

"Electricity Receipts" represents those quantities of electricity generated off the manufacturing establishment site and available at the site for consumplion. it includes those quantities for which payment was made, quantities transferred in, quantities purchased and paid for by a central purchasing entity, and quantities for

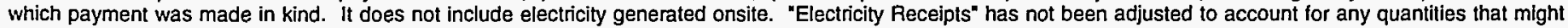

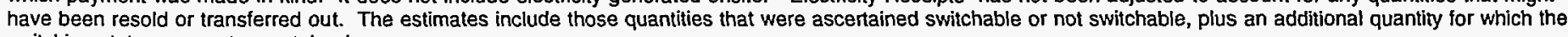
switching status was not ascertained.

Notes: - To obtain a RSE percentage for any table cell, multiply the cell's corresponding RSE column and RSE row factors. • Totals may not equal sum of components because of independent rounding.

Source: Energy Information Administration, Office of Energy Markets and End Use, Energy End Use Division, Form ElA-846, "1991 Manufacturing Energy Consumption Survey."
} 
Table A53. Capability to Switch from Electricity to Alternative Energy Sources by Industry Group, Selected Industries, and Selected Characteristics, 1991

(Estimates in Million Kilowatthours)

\begin{tabular}{|c|c|c|c|c|c|c|c|c|c|c|c|c|}
\hline \multirow[b]{2}{*}{$\begin{array}{l}\text { SIC } \\
\text { Code" }\end{array}$} & \multirow[b]{2}{*}{$\begin{array}{l}\text { Industry Groups } \\
\text { and Industry }\end{array}$} & \multicolumn{3}{|c|}{ Electricity Receipts } & \multicolumn{7}{|c|}{ Altemative Types of Energy } & \multirow[b]{2}{*}{$\begin{array}{c}\text { RSE } \\
\text { Row } \\
\text { Factors }\end{array}$} \\
\hline & & $\begin{array}{c}\text { Total } \\
\text { Receipts }\end{array}$ & Switchable & $\begin{array}{c}\text { Not } \\
\text { Switchable }\end{array}$ & $\begin{array}{c}\text { Natural } \\
\text { Gas }\end{array}$ & $\begin{array}{l}\text { Distillate } \\
\text { Fuel Oil }\end{array}$ & $\begin{array}{l}\text { Residual } \\
\text { Fuel Oil }\end{array}$ & Coal & LPG & $\begin{array}{c}\text { Coal Coke } \\
\text { and } \\
\text { Breeze }\end{array}$ & Other & \\
\hline & RSE Column Factors: & 0.4 & 0.9 & 0.4 & 1.0 & 1.2 & 1.2 & 1.2 & 2.1 & 1.6 & 1.5 & \\
\hline 20 & Food and Kindred Products $\ldots \ldots \ldots \ldots$ & 50,518 & 1,305 & 45,174 & 325 & 378 & 193 & 244 & 15 & 183 & $\mathbf{Q}$ & 12.7 \\
\hline 2011 & Meat Packing Plants $\ldots \ldots \ldots \ldots \ldots$ & 3,410 & 64 & 3,051 & w & w & 0 & 0 & 0 & 0 & 0 & 16.1 \\
\hline 2033 & Canned Fruits and Vegetables . . . . . . . & 1,415 & 14 & 1,316 & 0 & 1 & 0 & $Q$ & 0 & 0 & 0 & 20.5 \\
\hline 2037 & Frozen Frults and Vegetables . . . . . . . . & 3,097 & 102 & 2,798 & 99 & 85 & 67 & 0 & 0 & 69 & 0 & 25.4 \\
\hline 2046 & Wet Com Milling $\ldots \ldots \ldots \ldots \ldots \ldots$ & 4,143 & $w$ & $w$ & 0 & 0 & 0 & W & 0 & 0 & 0 & 20.3 \\
\hline 2051 & Bread, Cake and Related Products . . . . . & 2,240 & 53 & 1,971 & 27 & $\mathbf{Q}$ & * & 0 & 0 & 22 & 0 & 21.6 \\
\hline 2063 & Bett Sugar $\ldots \ldots \ldots \ldots \ldots \ldots \ldots$ & 407 & 0 & 390 & 0 & 0 & 0 & 0 & 0 & 0 & 0 & 13.9 \\
\hline 2075 & Soyboan Oll Mills . . . . . . . . . . . . . & 1,632 & 34 & 1,527 & $w$ & $W$ & $w$ & $w$ & 0 & W & 0 & 5.9 \\
\hline 2082 & Malt Boverages . . . . . . . . . . . . . & 2,371 & 67 & 2,287 & $w$ & $W$ & $w$ & $w$ & 0 & 0 & 0 & 17.0 \\
\hline 21 & Tobacco Manufactures $\ldots \ldots \ldots \ldots \ldots$ & 1,468 & $W$ & 1,452 & $w$ & 0 & 0 & W & 0 & 0 & 0 & 8.9 \\
\hline 22 & Textile Mill Products $\ldots \ldots \ldots \ldots \ldots$ & 29,522 & 266 & 28,232 & 0 & $\mathbf{Q}$ & 0 & $\mathbf{Q}$ & 0 & 0 & 0 & 11.9 \\
\hline 23 & Apparel and Other Textile Products .... . & 5,645 & 187 & 4,730 & Q & $\mathbf{Q}$ & 0 & $Q$ & 0 & 0 & 0 & 29.0 \\
\hline 24 & Lumber and Wood Products ........ & 19,575 & 348 & 17,791 & 74 & $\mathbf{Q}$ & $*$ & 0 & 0 & $\mathbf{Q}$ & 31 & 29.2 \\
\hline 25 & Fumiture and Fixtures $\ldots \ldots \ldots \ldots \ldots$ & 4,916 & 122 & 4,452 & Q & 21 & 0 & 0 & 0 & 20 & Q & 25.0 \\
\hline 26 & Paper and Allied Products . . . . . . . . . & 65,052 & 3,181 & 59,815 & 1,406 & 576 & 649 & 859 & 128 & 47 & 230 & 9.3 \\
\hline 2611 & Pulp Mills $\ldots \ldots \ldots \ldots \ldots \ldots \ldots$ & 2,877 & 60 & 2,817 & 60 & 3 & 60 & 0 & 0 & 0 & 0 & 31.8 \\
\hline 2621 & Paper Mills ................ & 36,317 & 1,766 & 33,741 & 457 & 236 & 155 & 357 & $w$ & 0 & $\mathbf{W}$ & 4.3 \\
\hline 2631 & Paporboard Mils ............... & 12,611 & 899 & 11,582 & 647 & W & 393 & 327 & 0 & 0 & $\mathbf{w}$ & 10.1 \\
\hline 27 & Printing and Publishing $\ldots \ldots \ldots \ldots \ldots$ & 15,629 & 728 & 13,064 & 341 & 187 & $Q$ & 0 & $\mathbf{Q}$ & $\mathbf{Q}$ & $\mathbf{Q}$ & 25.8 \\
\hline 28 & Chemicals and Allied Products . . . . . . . & 139,059 & 2,377 & 130,168 & 1,414 & 532 & 156 & 322 & 0 & 225 & 259 & 8.2 \\
\hline 2812 & Alkalles and Chlorine ............ & 12,629 & 0 & 11,569 & 0 & 0 & 0 & 0 & 0 & 0 & 0 & 23.6 \\
\hline 2813 & Industrial Gases . . . . . . . . . . . . . & 17,894 & 260 & 17,220 & $w$ & 0 & 0 & 0 & 0 & 0 & 0 & 11.0 \\
\hline 2819 & Industrlal inorganic Chemicals, nec ..... & 38,176 & 92 & 37,303 & 61 & 23 & $w$ & $w$ & 0 & $w$ & 5 & 13.4 \\
\hline 2821 & Plastics Materials and Resins ......... & 15,027 & 283 & 13,373 & $w$ & 141 & 0 & 0 & 0 & W & $w$ & 8.9 \\
\hline 2822 & Symthetlc Rubber ............... & 1,946 & W & 1,896 & 0 & W & 0 & 0 & 0 & 0 & 0 & 18.7 \\
\hline 2823 & Cellulosic Manmado Fibers . . . . . . . . . . & W & 23 & W & 0 & 1 & 0 & 23 & 0 & 0 & 0 & 39.1 \\
\hline 2824 & Organle Fibers, Noncellulosic . . . . . . . . & 6,976 & $w$ & 6,418 & $w$ & 0 & $w$ & $w$ & 0 & 0 & 0 & 6.8 \\
\hline 2865 & Cyclic Crudes and Intermediates . . . . . . & 4,432 & 91 & 4,262 & $w$ & $w$ & 0 & 0 & 0 & 0 & $w$ & 18.8 \\
\hline 2869 & Industrial Organic Chemicals, noc ...... & 20,143 & 1,058 & 18,375 & 698 & 242 & $w$ & $w$ & 0 & w & $w$ & 7.4 \\
\hline 2873 & Nitrogenous Fertilizers . . . . . . . . . . & 2,918 & 83 & 2,807 & 83 & 0 & 0 & 0 & 0 & 0 & 0 & 35.4 \\
\hline 2874 & Phosphatic Fertilizers . . . . . . . . . . & 2,419 & W & W & 0 & $w$ & 0 & 0 & 0 & W & 0 & 7.5 \\
\hline 29 & Petroleum and Coal Products $\ldots \ldots \ldots$ & 33,480 & 1,437 & 31,336 & 1,130 & 451 & 576 & $w$ & 0 & 563 & 241 & 3.6 \\
\hline 2911 & Petroleum Refining ............. & 31,562 & 1,360 & 29,664 & 1,122 & 376 & 574 & W & 0 & 556 & 233 & 3.0 \\
\hline 30 & Rubber and Misc. Plastics Products ..... & 33,913 & 877 & 30,592 & 106 & 164 & 27 & 28 & 0 & 73 & 77 & 21.5 \\
\hline 3011 & Tires and Inner Tubes . . . . . . . . . & 4,037 & 53 & 3,876 & 0 & W & 0 & W & 0 & 0 & 0 & 6.4 \\
\hline 308 & Miscellaneous Plastic Products, nec .... & 25,597 & 669 & 22,954 & 64 & 79 & $\mathbf{Q}$ & $Q$ & 0 & $\mathbf{Q}$ & $\mathbf{Q}$ & 26.7 \\
\hline 31 & Leather and Leather Products ......... & 795 & W & $w$ & Q & 0 & $\mathbf{0}$ & 0 & 0 & 0 & 0 & 25.7 \\
\hline 32 & Stone, Clay and Glass Products . . . . . . . & 30,885 & 568 & 28,665 & 161 & 273 & 37 & 97 & w & 96 & 67 & 12.3 \\
\hline 3211 & Flat Glass ................. & 1,512 & W & 1,485 & 0 & w & 0 & 0 & 0 & 0 & $w$ & 5.3 \\
\hline 3221 & Glass Containers .............. & 4,098 & 142 & 3,634 & 50 & 90 & $w$ & 0 & 0 & $w$ & 0 & 9.5 \\
\hline 3229 & Pressed and Blown Glass, nec . . . . . . . & 2,862 & 118 & 2,578 & 58 & 73 & w & 0 & 0 & W & $w$ & 8.4 \\
\hline 3241 & Cement, Hydraulic ............... & 9,490 & 10 & 9,288 & 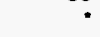 & 10 & $\because$ & : & 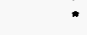 & $\cdot$ & 0 & 27.5 \\
\hline 3274 & 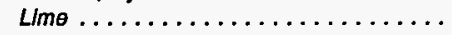 & 1,324 & W & 1,243 & 0 & 0 & 0 & $w$ & $w$ & 0 & 0 & 35.1 \\
\hline 3296 & 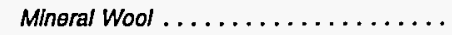 & 2,821 & $w$ & 2,560 & 0 & w & $w$ & 0 & 0 & 0 & 0 & 2.2 \\
\hline 33 & Prtmary Metal Industries . . . . . . . . . . & 147,078 & 2,111 & 139,395 & 1,898 & 254 & 1,650 & * & $w$ & $Q$ & 182 & 9.4 \\
\hline 3312 & Blast Fumaces and Steol Mills ....... & 39,480 & 1,716 & 36,545 & 1,708 & W & 1,640 & 0 & 0 & W & $w$ & 7.4 \\
\hline 3313 & Electrometalurgical Products . . . . . . . . . & 3,796 & W & $w$ & 0 & $w$ & 0 & 0 & 0 & 0 & 0 & 14.9 \\
\hline 3321 & Gray and Ductilo Iron Foundries . . . . . . . & 6,414 & 63 & 6,148 & $\mathbf{Q}$ & $\mathbf{Q}$ & $Q$ & $\cdot$ & $w$ & $\mathbf{Q}$ & 11 & 17.1 \\
\hline 3331 & Primary Copper . . . . . . . . . . . & 1,246 & W & 929 & $\vec{w}$ & $\overline{0}$ & $\overline{0}$ & 0 & 0 & $\overline{0}$ & $w$ & 1.4 \\
\hline 3334 & Primary Aluminum $\ldots \ldots \ldots \ldots \ldots \ldots$ & 67,707 & $w$ & 66,272 & 0 & $w$ & 0 & 0 & 0 & 0 & 0 & 5.6 \\
\hline 3339 & Primary Nonterrous Metals, nec . . . . . . & 3,784 & 1 & 3,469 & $\cdot$ & $\because$ & . & 0 & 0 & 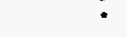 & $\cdot$ & 1.5 \\
\hline 3353 & Aluminum Sheet, Plate, and Foll ........ & 4,261 & 1 & 4,064 & 1 & 0 & 0 & 0 & 0 & 0 & 0 & 1.7 \\
\hline 34 & Fabricated Metal Products . . . . . . . . . . . & 29,830 & 623 & 26,132 & 207 & 195 & 26 & $\mathbf{Q}$ & 7 & 52 & 86 & 23.0 \\
\hline 35 & Industrial Machinery and Equipment ...... & 29,658 & 876 & 25,615 & 231 & 174 & $Q$ & $\vec{w}$ & - & 177 & a & 18.7 \\
\hline 357 & Computer and Office Equipment ........ & 4,398 & 75 & 3,713 & 29 & 29 & 24 & 0 & $\cdot$ & 11 & $\cdot$ & 17.3 \\
\hline 36 & Electronic and Other Electric Equipment .. & 30,046 & 840 & 26,318 & 165 & 227 & 62 & $Q$ & 0 & 69 & 13 & 20.1 \\
\hline 37 & Transportation Equipment $\ldots \ldots \ldots \ldots$. & 35,401 & 493 & 32,696 & 148 & 209 & $\mathbf{Q}$ & $\overrightarrow{0}$ & 0 & 39 & $\cdot$ & 11.9 \\
\hline 3711 & Motor Vehicles and Car Bodies ........ & 8,285 & W & 7,603 & 0 & W & $\mathbf{w}$ & 0 & 0 & 0 & 0 & 6.7 \\
\hline 3714 & Motor Vehicle Parts and Accessories ... & 10,918 & 203 & 9,891 & 30 & 73 & $\bullet$ & 0 & 0 & $\mathbf{Q}$ & • & 11.5 \\
\hline 38 & Instruments and Related Products ...... & 12,349 & 506 & 10,704 & 31 & 54 & 114 & $w$ & 0 & 14 & 43 & 20.3 \\
\hline 3841 & Surglcal and Medical Instruments ...... & 1,161 & 2 & 1,000 & 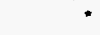 & 1 & $\bullet$ & 0 & 0 & 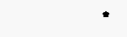 & $\cdot$ & 23.5 \\
\hline 39 & Misc. Manufacturing Industries . . . . . . . . & 3,661 & 122 & 3,216 & Q & 13 & 0 & $\mathbf{Q}$ & 0 & Q & $\mathbf{Q}$ & 24.1 \\
\hline & Total $\ldots \ldots \ldots \ldots \ldots \ldots \ldots \ldots$ & 718,480 & 17,003 & 660,279 & 7,730 & 3,827 & 3,604 & 1,738 & 255 & 1,648 & 1,455 & 5.7 \\
\hline
\end{tabular}

See footnotes at end of table. 
Table A53. Capability to Switch from Electricity to Alternative Energy Sources by Industry Group, Selected Industries, and Selected Characteristics, 1991 (Continued) (Estimates in Million Kilowatthours)

\begin{tabular}{|c|c|c|c|c|c|c|c|c|c|c|c|}
\hline \multirow[b]{2}{*}{ Selected Characteristics } & \multicolumn{3}{|c|}{ Electricity Receipts } & \multicolumn{7}{|c|}{ Altemative Types of Energy" } & \multirow[b]{2}{*}{$\begin{array}{c}\text { RSE } \\
\text { Row } \\
\text { Factors }\end{array}$} \\
\hline & $\begin{array}{c}\text { Total } \\
\text { Receipts }^{e}\end{array}$ & Switchable & $\begin{array}{c}\text { Not } \\
\text { Switchable }\end{array}$ & $\begin{array}{c}\text { Natural } \\
\text { Gas }\end{array}$ & $\begin{array}{l}\text { Distillate } \\
\text { Fuel Oil } \\
\end{array}$ & $\begin{array}{c}\text { Residual } \\
\text { Fuel Oil }\end{array}$ & Coal & LPG & $\begin{array}{c}\text { Coal Coke } \\
\text { and } \\
\text { Breeze }\end{array}$ & Other' & \\
\hline RSE Column Factors: & 0.3 & 0.8 & 0.3 & 0.9 & 1.2 & 1.1 & 1.6 & 3.6 & 1.4 & 1.7 & \\
\hline \multicolumn{12}{|l|}{ Census Region } \\
\hline Northeast $\ldots \ldots \ldots \ldots \ldots \ldots \ldots$ & 87,851 & 1,871 & 79,702 & 447 & 321 & 408 & 216 & $Q$ & 219 & 342 & 12.5 \\
\hline$\ldots \ldots \ldots \ldots \ldots \ldots \ldots$ & 207,104 & 5,068 & 191,373 & 2,279 & 1,421 & 1,291 & 605 & $\bar{Q}$ & 447 & 260 & 9.1 \\
\hline 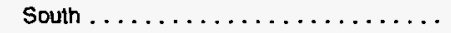 & 305,128 & 7,436 & 282,152 & 3,738 & 1,260 & 1,484 & 820 & 165 & 505 & 435 & 7.2 \\
\hline West $\ldots \ldots \ldots \ldots \ldots \ldots \ldots \ldots \ldots$ & 118,398 & 2,628 & 107,052 & 1,266 & 824 & 421 & 97 & 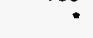 & 1478 & 418 & 10.0 \\
\hline Total $\ldots \ldots \ldots \ldots \ldots \ldots \ldots$ & 718,480 & 17,003 & 660,279 & 7,730 & 3,827 & 3,604 & 1,738 & 255 & 1,648 & 1,455 & 5.7 \\
\hline \multicolumn{12}{|l|}{$\begin{array}{l}\text { Value of Shipments and Receipts } \\
\text { (million dollars) }\end{array}$} \\
\hline Under $20 \ldots \ldots \ldots \ldots \ldots$ & 110,811 & 3,008 & 95,098 & 600 & 654 & 147 & 84 & $Q$ & 425 & 332 & 17.3 \\
\hline $20-49 \ldots \ldots$ & 109,423 & 2,407 & 98,977 & 479 & 521 & 45 & 154 & 54 & 130 & 337 & 14.0 \\
\hline 50-99. & 93,766 & 1,700 & 85,511 & 509 & 513 & 213 & 104 & $w$ & 193 & 131 & 11.7 \\
\hline $100-249$ & 148,565 & 2,793 & 138,126 & 1,398 & 781 & 325 & 421 & 113 & 168 & 227 & 9.2 \\
\hline $250-499$ & 118,855 & 2,375 & 113,597 & 853 & 397 & 552 & 486 & $w$ & 164 & 270 & 6.9 \\
\hline 500 and Over. & 137,060 & 4,720 & 128,971 & 3,891 & 961 & 2,322 & 489 & 15 & 569 & 158 & 5.3 \\
\hline Total $\ldots \ldots \ldots \ldots \ldots \ldots \ldots$ & 718,480 & 17,003 & 660,279 & 7,730 & 3,827 & 3,604 & 1,738 & 255 & 1,648 & 1,455 & 5.7 \\
\hline \multicolumn{12}{|l|}{ Employment Size • } \\
\hline Under 50 & 47,182 & 1,168 & 40,297 & 302 & 265 & 116 & Q & a & 260 & 138 & 22.0 \\
\hline$\ldots \ldots \ldots \ldots \ldots \ldots$ & 48,502 & 1,043 & 43,440 & 344 & 215 & 48 & $\mathbf{Q}$ & 0 & 138 & 45 & 19.5 \\
\hline $100-249 \ldots \ldots \ldots \ldots \ldots \ldots \ldots$ & 128,926 & 2,910 & 113,966 & 794 & 728 & 123 & 113 & $\mathbf{Q}$ & 286 & 517 & 12.3 \\
\hline $250-499 \ldots \ldots \ldots \ldots \ldots \ldots \ldots$ & 129,141 & 2,896 & 118,305 & 1,023 & 627 & 468 & 174 & 60 & 417 & 278 & 8.4 \\
\hline $500-999 \ldots \ldots \ldots \ldots \ldots \ldots \ldots$ & 155,927 & 3,010 & 147,267 & 1,523 & 895 & 653 & 647 & a & 161 & 323 & 6.2 \\
\hline 1,000 and Over & 208,803 & 5,975 & 197,004 & 3,744 & 1,096 & 2,196 & 704 & W & 388 & 153 & 7.0 \\
\hline Total $\ldots \ldots \ldots \ldots \ldots \ldots \ldots$ & 718,480 & 17,003 & 660,279 & 7,730 & 3,827 & 3,604 & 1,738 & 255 & 1,648 & 1,455 & 5.7 \\
\hline
\end{tabular}

- See Appendices B and F for descriptions of the Standard Industrial Classification system.

b "Altemative Types of Energy" consist of those energy sources that could have been substituted for electricity receipts during 1991 . The quantities are expressed In millions of kilowatthours, and therefore represent the quantity of electricity that could have been displaced by the given alternative type of energy.

"Total Receipts" represents those quantities of electricity generated off the manufacturing establishment site and available at the site for consumption. It includes those quantities for which payment was made, quantities transferred in, quantities purchased and paid for by a central purchasing entity, and quantities for which payment was made in kind. It does not include electricity generated onsite. "Electricity Receipts" has not been adjusted to account for any quantitles that might have been resold or transferred out. The estimates include those quantities that were ascertained switchable or not switchable, plus an additional quantity for which the switching status was not ascertained.

"Other includes all other types of energy not already identified that respondents indicated could have been consumed in place of electricity.

- Value of Shipments and Receipts and Employment Size categories were supplied by the Bureau of the Census. See Appendix B.

- Estimate less than 0.5. Data are included in higher level totals.

$W=$ Withheld to avoid disclosing data for individual establishments. Data are included in higher level totals.

$Q=$ Withneld because Relative Standard Error is greater than 50 percent. Data are included in higher level totals.

Notes: - To obtain a RSE percentage for any table cell, multiply the cell's corresponding RSE column and RSE row factors. - Totals may not equal sum of components because of independent rounding.

Source: Energy Information Administration, Office of Energy Markets and End Use, Energy End Use Division, Form ElA-846, "1991 Manufacturing Energy Consumption Survey." 
Table A54. Capability to Switch from Natural Gas to Alternative Energy Sources by Industry Group, Selected Industries, and Selected Characteristics, 1991 (Estimates in Billion Cubic Feet)

\begin{tabular}{|c|c|c|c|c|c|c|c|c|c|c|c|c|}
\hline \multirow[b]{2}{*}{$\begin{array}{l}\text { SIC } \\
\text { Code }\end{array}$} & \multirow[b]{2}{*}{$\begin{array}{l}\text { Industry Groups } \\
\text { and Industry }\end{array}$} & \multicolumn{3}{|c|}{ Natural Gas } & \multicolumn{7}{|c|}{ Alternative Types of Energy } & \multirow[b]{2}{*}{$\begin{array}{c}\text { RSE } \\
\text { Row } \\
\text { Factors }\end{array}$} \\
\hline & & $\begin{array}{c}\text { Total } \\
\text { Consumed }^{\epsilon}\end{array}$ & Switchable & $\begin{array}{c}\text { Not } \\
\text { Switchable }\end{array}$ & $\begin{array}{l}\text { Electricity } \\
\text { Receipts } \\
\end{array}$ & $\begin{array}{l}\text { Distillate } \\
\text { Fuel Oil }\end{array}$ & $\begin{array}{l}\text { Residual } \\
\text { Fuel Oil }\end{array}$ & Coal & LPG & $\begin{array}{c}\text { Coal Coke } \\
\text { and } \\
\text { Breeze }\end{array}$ & Other & \\
\hline & RSE Column Factors: & 0.5 & 0.6 & 0.7 & 1.6 & 0.8 & 0.8 & 1.3 & 2.2 & 0.9 & 1.5 & \\
\hline 20 & Food and Kindred Products . . . . . . . . . & 497 & 261 & 206 & 17 & 128 & 92 & 16 & $w$ & 73 & 10 & 7.1 \\
\hline 2011 & Moat Packing Plants . . . . . . . . . & 31 & 18 & 11 & $*$ & 10 & 4 & 1 & 0 & 6 & - & 10.4 \\
\hline 2033 & Canned Fuilts and Vegetables . . . . . . . & 35 & 19 & 14 & $w$ & 8 & 8 & • & 0 & 3 & 1 & 13.8 \\
\hline 2037 & Frozen Fruits and Vegotables . . . . . . . & 25 & 11 & 12 & 1 & 6 & 5 & • & 0 & 2 & 1 & 17.2 \\
\hline 2046 & 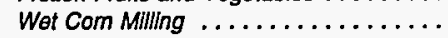 & 51 & 27 & 24 & $w$ & 18 & 5 & w & $w$ & 4 & * & 13.8 \\
\hline 2051 & Bread, Cake and Related Products ..... & 22 & 8 & 12 & 1 & 2 & $\cdot$ & 0 & 0 & 5 & * & 14.9 \\
\hline 2063 & 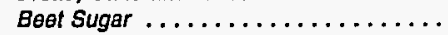 & 18 & 8 & 10 & $\cdot$ & 1 & 7 & 1 & $\cdot$ & • & $\cdot$ & 12.5 \\
\hline 2075 & 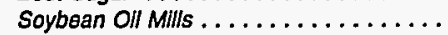 & 24 & 17 & 6 & * & 12 & 6 & 1 & 0 & 3 & 0 & 4.5 \\
\hline 2082 & 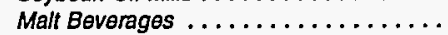 & 22 & 18 & 4 & 1 & 5 & 16 & 2 & 0 & $w$ & $w$ & 12.6 \\
\hline 21 & Tobacco Manufactures $\ldots \ldots \ldots \ldots$ & 4 & 3 & 1 & 0 & 1 & 2 & 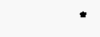 & 0 & 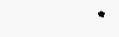 & 0 & 18.2 \\
\hline 22 & Textlle Mill Products $\ldots \ldots \ldots \ldots \ldots \ldots$ & 105 & 72 & 24 & 1 & 28 & 32 & 7 & $\cdot$ & 21 & 3 & 8.9 \\
\hline 23 & Apparel and Other Textlie Products ..... & 18 & 5 & 11 & $\cdot$ & 2 & 2 & $\cdot$ & $\cdot$ & 1 & - & 24.3 \\
\hline 24 & Lumber and Wood Products .......... & 39 & 14 & 18 & 2 & 5 & 2 & * & 0 & 7 & 1 & 24.1 \\
\hline 25 & Fumiture and Fixtures ............ & 18 & 5 & 10 & 1 & 1 & 1 & • & $\mathbf{0}$ & 3 & - & 21.4 \\
\hline 26 & Paper and Allied Products ........... & 532 & 273 & 246 & 20 & 91 & 159 & 21 & 1 & 23 & 14 & 5.8 \\
\hline 2611 & 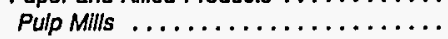 & 32 & 12 & 19 & 1 & 5 & 7 & 0 & 0 & 3 & $\because$ & 21.6 \\
\hline 2621 & Paper Mills $\ldots \ldots \ldots \ldots \ldots \ldots$ & 252 & 123 & 126 & 16 & 48 & 59 & 13 & 1 & 5 & 8 & 6.0 \\
\hline 2631 & Paperboard Mills ............... & 180 & 111 & 67 & 3 & 20 & 86 & 8 & 0 & 8 & 5 & 7.6 \\
\hline 27 & Printing and Publishing ........... & 47 & 11 & 28 & 1 & 5 & 3 & • & $\bullet$ & 5 & - & 19.1 \\
\hline 28 & Chemlcals and Allied Products . . . . . . . . . & 1,620 & 331 & 1,267 & 31 & 143 & 98 & 20 & $W$ & 88 & 30 & 5.8 \\
\hline 2812 & Alkallos and Chlorine .............. & $w$ & 3 & W & 0 & $\cdot$ & 3 & 0 & $\mathbf{0}$ & 0 & 0 & 25.0 \\
\hline 2813 & Industral Gasos . . . . . . . . . . . . . & 24 & 3 & 20 & $w$ & 0 & 0 & 0 & 0 & $\cdot$ & 1 & 14.7 \\
\hline 2819 & Industrial Inorganle Chemicals, nec ..... & 136 & 26 & 107 & 3 & 15 & 2 & 1 & 0 & 7 & 2 & 9.3 \\
\hline 2821 & Plastlos Materials and Resins . . . . . . . . & 146 & 27 & 116 & 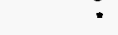 & 10 & $\overrightarrow{9}$ & 1 & 0 & 10 & 1 & 8.7 \\
\hline 2822 & Symthetlc Rubber ............... & 43 & 7 & 36 & 0 & 3 & 3 & $\bullet$ & 0 & $t$ & 1 & 18.2 \\
\hline 2823 & Celluloslc Manmade Fibers . . . . . . . . . . & $w$ & 1 & $w$ & 0 & 1 & 0 & 0 & 0 & $\cdot$ & 0 & 44.7 \\
\hline 2824 & Organle Fibers, Noncellulosic . . . . . . . . & $w$ & 18 & $w$ & 1 & 5 & 13 & 1 & 0 & 1 & 0 & 5.6 \\
\hline 2865 & Cycllc Crudes and Intermediates . . . . . . . & 94 & 26 & 67 & 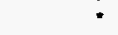 & 15 & 15 & 1 & 0 & 1 & 2 & 13.9 \\
\hline 2869 & Industrial Organlc Chemicals, noc ...... & 625 & 139 & 482 & 23 & 44 & 21 & $w$ & $w$ & 52 & 16 & 6.8 \\
\hline 2873 & Nitrogenous Fentilizers ............. & 258 & 4 & 254 & 0 & 1 & 1 & 0 & 0 & 2 & 0 & 39.9 \\
\hline 2874 & Phosphatle Fertllizers ............. & 18 & 2 & 16 & - & 2 & 1 & 0 & 0 & 1 & $\cdot$ & 4.6 \\
\hline 29 & Petroleum and Coal Products $\ldots \ldots \ldots$ & 813 & 270 & 536 & 11 & 92 & 74 & $w$ & 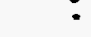 & 195 & 9 & 4.7 \\
\hline 2911 & Petroloum Reflning ............... & 769 & 249 & 516 & 10 & 74 & 71 & w & 0 & 190 & 8 & 3.1 \\
\hline 30 & Rubber and Misc. Plastics Products ...... & 93 & 51 & 32 & 2 & 29 & 24 & 1 & 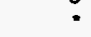 & 9 & 1 & 10.5 \\
\hline 3011 & 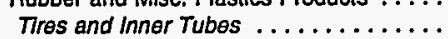 & 21 & 18 & 2 & 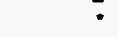 & 10 & 15 & 1 & 0 & 1 & : & 4.3 \\
\hline 308 & Mlscellaneous Plastlc Products, nec .... & 51 & 23 & 22 & 2 & 13 & 7 & : & $*$ & 6 & - & 18.0 \\
\hline 31 & Leather and Leather Products . . . . . . . . & 5 & 2 & 3 & 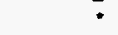 & 1 & 1 & 0 & 0 & $\cdot$ & - & 34.9 \\
\hline 32 & Stone, Clay and Glass Products . . . . . . . & 369 & 194 & 162 & 1 & 92 & 24 & 25 & 5 & 77 & 13 & 7.2 \\
\hline 3211 & Flat Glass $\ldots \ldots \ldots \ldots \ldots \ldots \ldots$ & 40 & 20 & 20 & 0 & $W$ & 0 & 0 & 0 & $w$ & $w$ & 3.7 \\
\hline 3221 & Glass Containers $\ldots \ldots \ldots \ldots \ldots \ldots \ldots$ & 67 & 42 & 24 & $\cdot$ & 18 & 7 & 0 & 0 & 24 & 1 & 6.7 \\
\hline 3229 & Pressed and Blown Glass, nec . . . . . . . . & $w$ & 15 & $w$ & • & 7 & $\dot{w}$ & 0 & 0 & 7 & i & 6.9 \\
\hline 3241 & Cement, Hydraulfe ................ & 38 & 32 & 6 & • & 6 & 2 & 22 & 5 & 2 & 3 & 20.8 \\
\hline 3274 & Lime $\ldots \ldots \ldots \ldots \ldots \ldots \ldots \ldots$ & 8 & 3 & 5 & 0 & 2 & 1 & 1 & $=$ & $\cdot$ & 1 & 16.9 \\
\hline 3296 & Mineral Wool $\ldots \ldots \ldots \ldots \ldots \ldots$ & 28 & 12 & 14 & 0 & 3 & 2 & 0 & 0 & 10 & - & 1.5 \\
\hline 33 & Primary Motal Industries . . . . . . . . . . & 666 & 187 & 460 & 8 & 56 & 69 & $w$ & 30 & 66 & 2 & 5.7 \\
\hline 3312 & Blast Fumaces and Stool Mills ....... & 387 & 99 & 285 & $w$ & 21 & 56 & $w$ & 30 & 12 & 2 & 5.5 \\
\hline 3313 & Electrometalurgical Products . . . . . . . . . & 1 & 1 & 1 & $\because$ & $\cdot$ & 0 & 0 & 0 & • & 0 & 13.4 \\
\hline 3321 & Gray and Ductllo Iron Foundrias ....... & 28 & 8 & 18 & * & 3 & 1 & 0 & 0 & 5 & : & 13.0 \\
\hline 3331 & Primary Copper ................. & 15 & 7 & 7 & 0 & $w$ & $w$ & $\cdot$ & 0 & W & 0 & 1.3 \\
\hline 3334 & Primany Aluminum ............. & 20 & 10 & 10 & 0 & 3 & 2 & 0 & 0 & 8 & 0 & 3.6 \\
\hline 3339 & Primary Nonferrous Metals, nec . . . . . . . & 16 & 7 & 8 & $\cdot$ & 6 & $\cdot$ & 0 & 0 & 1 & * & 2.9 \\
\hline 3353 & Aluminum Sheet, Plate, and Foll ........ & 41 & 8 & 33 & - & 2 & • & 0 & 0 & 6 & 0 & 1.6 \\
\hline 34 & Fabricated Metal Products . . . . . . . . . . & 169 & 48 & 106 & 5 & 14 & 7 & 2 & $\mathbf{Q}$ & 26 & 5 & 12.7 \\
\hline 35 & Industrial Machinery and Equipment ..... & 106 & 33 & 60 & 3 & 13 & 8 & 1 & $\cdot$ & 13 & 2 & 13.4 \\
\hline 357 & Computer and Office Equipment ........ & 5 & 2 & 2 & $\cdot$ & 1 & 1 & 0 & 0 & * & $\cdot$ & 17.7 \\
\hline 36 & Electronlc and Other Electric Equipment .. & 76 & 36 & 35 & 2 & 15 & 9 & 1 & - & 16 & 2 & 11.9 \\
\hline 37 & Transportation Equipment ........... & 129 & 50 & 74 & 2 & 18 & 13 & 5 & * & 17 & 4 & 6.1 \\
\hline 3711 & Motor Vehicles and Car Bodles . . . . . . . & 44 & 16 & 27 & * & 3 & 2 & $w$ & 0 & 9 & 1 & 4.7 \\
\hline 3714 & Motor Vehicle Parts and Accessories ... & 40 & 15 & 24 & 1 & 5 & 4 & 1 & 0 & 6 & 1 & 7.3 \\
\hline 38 & Instruments and Related Products ...... & 25 & 10 & 11 & 1 & 7 & 3 & - & 0 & 1 & 1 & 14.5 \\
\hline 3841 & Surglcal and Medical Instruments .. & 2 & $\cdot$ & 1 & : & * & - & 0 & 0 & - & $\cdot$ & 20.8 \\
\hline \multirow[t]{2}{*}{39} & Misc. Manufacturing Industries . . . . . . . . & 14 & 4 & 8 & $\cdot$ & 2 & 1 & - & 0 & 2 & $\bullet$ & 18.2 \\
\hline & Total $\ldots \ldots \ldots \ldots \ldots \ldots \ldots$ & 5,345 & 1,860 & 3,299 & 109 & 745 & 625 & 107 & 55 & 645 & 98 & 2.9 \\
\hline
\end{tabular}

See footnotes at end of table. 


\section{Table A54. Capability to Switch from Natural Gas to Alternative Energy Sources by Industry Group, Selected Industries, and Selected Characteristics, 1991 (Continued) (Estimates in Billion Cubic Feet)}

\begin{tabular}{|c|c|c|c|c|c|c|c|c|c|c|c|}
\hline \multirow[b]{2}{*}{ Selected Characteristics } & \multicolumn{3}{|c|}{ Natural Gas } & \multicolumn{7}{|c|}{ Altemative Types of Energy ${ }^{b}$} & \multirow[b]{2}{*}{$\begin{array}{c}\text { RSE } \\
\text { Row } \\
\text { Factors }\end{array}$} \\
\hline & $\begin{array}{c}\text { Total } \\
\text { Consumed }\end{array}$ & Switchable & $\begin{array}{c}\text { Not } \\
\text { Switchable }\end{array}$ & $\begin{array}{l}\text { Electricity } \\
\text { Receipts }^{d}\end{array}$ & $\begin{array}{l}\text { Distıllate } \\
\text { Fuel Oil }\end{array}$ & $\begin{array}{c}\text { Residual } \\
\text { Fuel Oil }\end{array}$ & Coal & LPG & $\begin{array}{c}\text { Coal Coke } \\
\text { and } \\
\text { Breeze }\end{array}$ & Other" & \\
\hline RSE Column Factors: & 0.5 & 0.5 & 0.7 & 1.7 & 0.7 & 0.7 & 1.7 & 2.7 & 0.7 & 1.6 & \\
\hline \multicolumn{12}{|l|}{ Census Region } \\
\hline Northeast .... & 446 & 206 & 211 & 5 & 95 & 88 & 3 & $\cdot$ & 49 & 13 & 6.1 \\
\hline Midwest $\ldots \ldots \ldots \ldots \ldots \ldots \ldots$ & 1,363 & 525 & 780 & 30 & 226 & 167 & 48 & 45 & 172 & 21 & 4.6 \\
\hline South $\ldots \ldots \ldots \ldots \ldots \ldots \ldots$ & 2,896 & 843 & 1,984 & 57 & 313 & 264 & 43 & 8 & 310 & 51 & 4.0 \\
\hline West $\ldots \ldots \ldots \ldots \ldots \ldots \ldots$ & 640 & 287 & 323 & 17 & 111 & 107 & 13 & 3 & 114 & 14 & 7.1 \\
\hline$\ldots \ldots \ldots \ldots \ldots$ & 5,345 & 1,860 & 3,299 & 109 & 745 & 625 & 107 & 55 & 645 & 98 & 2.9 \\
\hline \multicolumn{12}{|l|}{$\begin{array}{l}\text { Value of Shipments and Receipts } \\
\text { (million dollars) }\end{array}$} \\
\hline Under $20 \ldots \ldots \ldots \ldots \ldots \ldots$ & 587 & 180 & 313 & 17 & 87 & 41 & 9 & 3 & 69 & 11 & 10.1 \\
\hline $20-49 \ldots \ldots \ldots \ldots \ldots \ldots \ldots \ldots$ & 610 & 249 & 324 & 9 & 121 & 61 & 27 & 7 & 79 & 16 & 7.6 \\
\hline $50-99 \ldots \ldots \ldots \ldots \ldots \ldots \ldots$ & 624 & 284 & 318 & 11 & 130 & 85 & 10 & $w$ & 98 & 12 & 6.9 \\
\hline $100-249 \ldots \ldots \ldots \ldots \ldots \ldots$ & 968 & 365 & 578 & 22 & 151 & 128 & 14 & $*$ & 109 & 22 & 4.6 \\
\hline $250-499 \ldots \ldots \ldots \ldots \ldots \ldots \ldots$ & 837 & 272 & 560 & 13 & 104 & 136 & 20 & $w$ & 62 & 10 & 4.7 \\
\hline 500 and $0 v e r \ldots \ldots \ldots \ldots \ldots \ldots$ & 1,720 & 510 & 1,205 & 37 & 151 & 174 & 28 & 37 & 229 & 27 & 3.7 \\
\hline Total $\ldots \ldots \ldots \ldots \ldots \ldots \ldots$ & 5,345 & 1,860 & 3,299 & 109 & 745 & 625 & 107 & 55 & 645 & 98 & 2.9 \\
\hline \multicolumn{12}{|l|}{ Employment Size' } \\
\hline Under $50 \ldots \ldots \ldots \ldots \ldots \ldots$ & $w$ & 67 & $w$ & 8 & 39 & 17 & 1 & * & 24 & 3 & 15.2 \\
\hline $50-99 \ldots \ldots \ldots \ldots \ldots \ldots$ & 349 & 119 & 200 & 7 & 64 & 36 & 10 & $w$ & 33 & 5 & 11.6 \\
\hline $100-249 \ldots \ldots \ldots \ldots \ldots \ldots \ldots$ & 968 & 335 & 580 & 21 & 165 & 80 & 30 & 13 & 107 & 28 & 7.2 \\
\hline $250-499 \ldots \ldots \ldots \ldots \ldots \ldots \ldots$ & 972 & 396 & 549 & 28 & 159 & 126 & 20 & $w$ & 157 & 17 & 5.0 \\
\hline $500-999 \ldots \ldots \ldots \ldots \ldots \ldots$ & 1,040 & 431 & 592 & 10 & 160 & 152 & 16 & 3 & 165 & 30 & 4.5 \\
\hline 1,000 and Over $\ldots \ldots \ldots \ldots \ldots \ldots$ & W & 513 & $w$ & 36 & 158 & 215 & 30 & 31 & 159 & 16 & 3.4 \\
\hline Total $\ldots \ldots \ldots \ldots \ldots \ldots$ & 5,345 & 1,860 & 3,299 & 109 & 745 & 625 & 107 & 55 & 645 & 98 & 2.9 \\
\hline
\end{tabular}

- See Appendices B and F for descriptions of the Standard Industrial Classification system.

b "Altemative Types of Energy" consist of those energy sources that could have been substituted for natural gas during 1991. The quantities are expressed in billions of cubic feet, and therefore represent the quantity of natural gas that could have been displaced by the given altemative type of energy.

"Total Consumed" represents those quantities of natural gas that were ascertained switchable or not switchable, plus an additional quantity for which the switching status was not ascertained.

"Electricity Receipts" represents those quantities of electricity generated off the manufacturing establishment site and available at the site for consumption. It includes those quantities for which payment was made, quantities transferred in, quantities purchased and paid for by a central purchasing entity, and quantities for which payment was made in kind. It does not include electricity generated onsite. "Electricity Receipts" has not been adjusted to account for any quantities that might

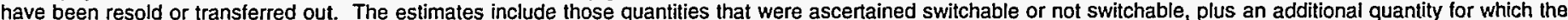
switching status was not ascertained.

- "Other" includes all other types of energy not already identified that respondents indicated could have been consumed in place of natural gas.

' Value of Shipments and Receipts and Employment Size categories were supplied by the Bureau of the Census. See Appendix B.

- Estimate less than 0.5. Data are included in higher level totals.

$W=$ Withheld to avoid disclosing data for individual establishments. Data are included in higher level totals.

$\mathrm{Q}=$ Withheld because Relative Standard Error is greater than 50 percent. Data are included in higher level totals.

Notes: - To obtain a RSE percentage for any table cell, multiply the cell's corresponding RSE column and RSE row factors. - Totals may not equal sum of components because of independent rounding.

Source: Energy Information Administration, Office of Energy Markets and End Use, Energy End Use Division, Form ElA-846, "1991 Manufacturing Energy Consumption Survey." 
Table A55. Capability to Switch from Distillate Fuel Oil to Alternative Energy Sources by Industry Group, Selected Industries, and Selected Characteristics, 1991 (Estimates in Thousand Barrels)

\begin{tabular}{|c|c|c|c|c|c|c|c|c|c|c|c|c|}
\hline \multirow[b]{2}{*}{$\begin{array}{l}\text { Sic } \\
\operatorname{Cod} \theta^{\circ}\end{array}$} & \multirow[b]{2}{*}{$\begin{array}{l}\text { Industry Groups } \\
\text { and Industry }\end{array}$} & \multicolumn{3}{|c|}{ Distillate Fuel Oil } & \multicolumn{7}{|c|}{ Altemative Types of Energy ${ }^{b}$} & \multirow[b]{2}{*}{$\begin{array}{c}\text { RSE } \\
\text { Row } \\
\text { Factors }\end{array}$} \\
\hline & & $\begin{array}{c}\text { Total } \\
\text { Consumed }\end{array}$ & Switchable & $\begin{array}{c}\text { Not } \\
\text { Switchable }\end{array}$ & $\begin{array}{l}\text { Electricity } \\
\text { Receipts }\end{array}$ & $\begin{array}{c}\text { Natural } \\
\text { Gas }\end{array}$ & $\begin{array}{l}\text { Residual } \\
\text { Fuel Oil } \\
\end{array}$ & Coal & LPG & $\begin{array}{c}\text { Coal Coke } \\
\text { and } \\
\text { Breeze }\end{array}$ & Other" & \\
\hline & RSE Column Factors: & 0.7 & 0.9 & 0.7 & 1.1 & 0.9 & 1.2 & 0.9 & 1.6 & 1.1 & 1.2 & \\
\hline 20 & Food and Kindred Products . . . . . . . & 2,966 & 662 & 1,822 & $Q$ & 569 & $\mathbf{Q}$ & - & * & 109 & 40 & 20.3 \\
\hline 2011 & Meat Packing Plants . . . . . . . . . . & 252 & 18 & 101 & $*$ & 15 & 1 & 0 & 0 & 0 & 0 & 17.3 \\
\hline 2033 & Canned Fruits and Vegetables ....... & 131 & a & 89 & 0 & 8 & 2 & 0 & 0 & $\cdot$ & 0 & 22.0 \\
\hline 2037 & Frozen Fruits and Vegetables . . . . . . . & 76 & 40 & 33 & 2 & 8 & 0 & 0 & 0 & 24 & 0 & 30.5 \\
\hline 2046 & Wot Com Milling $\ldots \ldots \ldots \ldots \ldots \ldots$ & 30 & 1 & $w$ & 1 & * & 0 & 0 & 0 & $\cdot$ & 0 & 24.1 \\
\hline 2051 & Bread, Cako and Related Products ... . & 131 & 36 & 85 & $\cdot$ & 36 & 0 & 0 & 0 & 9 & 0 & 31.8 \\
\hline 2063 & Beot Sugar ................ & 30 & 2 & 26 & 0 & 0 & 0 & 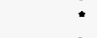 & 0 & - & : & 11.7 \\
\hline 2075 & Soybean Oll Mills . . . . . . . . . . . & 31 & 22 & 7 & 0 & 22 & $w$ & 0 & 0 & $w$ & $w$ & 7.0 \\
\hline 2082 & Malt Beverages . . . . . . . . . . . & 58 & $w$ & $w$ & $\cdot$ & W & 0 & 0 & 0 & $\bullet$ & $w$ & 20.3 \\
\hline 21 & Tobacco Manufactures $\ldots \ldots \ldots \ldots$ & 40 & $w$ & $w$ & 0 & $w$ & 0 & 0 & 0 & • & 0 & 7.6 \\
\hline 22 & Textlie Mill Products . . . . . . . . . . & 1,064 & 245 & 477 & 27 & 207 & $Q$ & 9 & 0 & 37 & $\mathbf{Q}$ & 26.2 \\
\hline 23 & Apparel and Other Textile Products .... & 142 & Q & W & $Q$ & 7 & $\bar{a}$ & $\mathbf{Q}$ & $Q$ & $Q$ & $\mathrm{Q}$ & 38.9 \\
\hline 24 & Lumber and Wood Products ......... & 2,373 & 140 & 1,466 & $\mathbf{Q}$ & Q & $Q$ & 0 & $\bar{Q}$ & $\overline{0}$ & 31 & 24.9 \\
\hline 25 & Fumiture and Fixtures $\ldots \ldots \ldots \ldots$. & 162 & $Q$ & 138 & 0 & $Q$ & $*$ & 0 & $\overrightarrow{0}$ & 1 & $Q$ & 35.3 \\
\hline 26 & Paper and Allied Products . . . . . . . . & 1,566 & 383 & 1,065 & 29 & 245 & 129 & 63 & $w$ & 47 & $\bar{Q}$ & 13.2 \\
\hline 2611 & Pulp Mills $\ldots \ldots \ldots \ldots \ldots \ldots \ldots$ & 155 & 38 & 113 & 0 & 37 & 1 & 0 & 0 & 0 & $\hat{0}$ & 25.9 \\
\hline 2621 & Paper Mills . . . . . . . . . . . . & W & 190 & w & 27 & 74 & 90 & 53 & $w$ & $w$ & $w$ & 6.2 \\
\hline 2631 & Paperboard MIIls ............. & 207 & 27 & 171 & 0 & 11 & 5 & W & 0 & W & $\bullet$ & 9.9 \\
\hline 27 & Printing and Publishing .......... & 312 & 125 & 137 & $\mathbf{Q}$ & 121 & 26 & 0 & 0 & a & * & 41.4 \\
\hline 28 & Chemicals and Allied Products . . . . . . . & 2,083 & 488 & 1,313 & 13 & 331 & 112 & 9 & 0 & 169 & $Q$ & 16.5 \\
\hline 2812 & Alkalles and Chlorine ............ & 43 & W & w & 0 & $w$ & 0 & 0 & 0 & 0 & 0 & 24.4 \\
\hline 2813 & 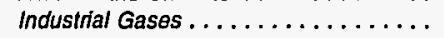 & 7 & 0 & 7 & 0 & 0 & 0 & 0 & 0 & 0 & 0 & 68.9 \\
\hline 2819 & Industral Inorganic Chemicals, nec . . . . & 456 & 62 & 295 & - & 48 & $w$ & 0 & 0 & 3 & 1 & 14.9 \\
\hline 2821 & Plastles Materials and Resins . . . . . . . & 231 & 90 & 131 & * & 81 & 8 & 0 & $\mathbf{0}$ & $w$ & 0 & 15.4 \\
\hline 2822 & Synthetlc Rubber .............. & 18 & $w$ & 13 & 0 & 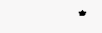 & 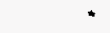 & 0 & 0 & $w$ & 0 & 19.5 \\
\hline 2823 & Cellulosic Manmado Fibers . . . . . . . . & 21 & 1 & 20 & 0 & 0 & 0 & 1 & 0 & 0 & 0 & 34.0 \\
\hline 2824 & Organic Fibers, Noncellulosic . . . . . . . & 53 & 13 & 38 & 0 & 13 & 1 & $w$ & 0 & 0 & 0 & 5.2 \\
\hline 2865 & Cyclle Crudes and intermediates . . . . . & 136 & 73 & $Q$ & 1 & 71 & $w$ & 0 & 0 & 3 & 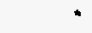 & 22.4 \\
\hline 2869 & Industrial Organic Chemicals, nec . . . . . & 439 & 40 & 359 & 8 & 23 & 17 & 0 & $\mathbf{0}$ & 17 & $\mathbf{Q}$ & 24.1 \\
\hline 2873 & Nitrogenous Fertilizers . .......... & 26 & 2 & 15 & $\cdot$ & 1 & 0 & 0 & $\mathbf{0}$ & $Q$ & $\mathbf{Q}$ & 36.2 \\
\hline 2874 & Phosphatlc Fertilizers . . . . . . . . . . & 150 & 20 & 107 & - & $w$ & 0 & 0 & $\mathbf{0}$ & 20 & $\cdot$ & 3.3 \\
\hline 29 & Petroleum and Coal Products $\ldots \ldots \ldots$ & 3,599 & 1,325 & 1,465 & $w$ & 700 & 138 & w & 0 & 96 & 118 & 17.0 \\
\hline 2911 & Petroloum Refining $\ldots \ldots \ldots \ldots \ldots$ & 1,525 & 961 & 546 & $w$ & 403 & 114 & $w$ & 0 & 72 & $w$ & 6.6 \\
\hline 30 & Rubber and Misc. Plastics Products ... . & 508 & 148 & 295 & 10 & 123 & 62 & 13 & $Q$ & 27 & 13 & 27.6 \\
\hline 3011 & Tires and Inner Tubes . . . . . . . . . & 68 & 62 & 6 & $w$ & $w$ & $W$ & $w$ & 0 & 0 & 0 & 7.3 \\
\hline 308 & Mlscellaneous Plastlc Products, nec ... & $w$ & 35 & 190 & $\mathbf{Q}$ & 27 & 13 & $Q$ & $Q$ & 13 & $Q$ & 37.8 \\
\hline 31 & Leather and Leather Products . . . . . . . & 220 & 7 & 193 & Q & $Q$ & 0 & $\cdot$ & 0 & 0 & $Q$ & 48.6 \\
\hline 32 & Stone, Clay and Glass Products . . . . . . & 3,312 & 289 & 2,640 & 16 & 113 & $\mathbf{Q}$ & $\mathbf{Q}$ & • & 67 & 46 & 20.0 \\
\hline 3211 & Flat Glass $\ldots \ldots \ldots \ldots \ldots \ldots \ldots$ & 12 & $W$ & $w$ & 0 & W & 0 & 0 & $\mathbf{0}$ & 0 & 0 & 5.4 \\
\hline 3221 & Glass Containers . . . . . . . . . & 23 & 17 & 5 & 1 & 17 & 1 & 0 & 0 & $w$ & 0 & 13.0 \\
\hline 3229 & Pressed and Blown Glass, nec . . . . . . . & 38 & $w$ & $W$ & $w$ & W & * & 0 & 0 & $W$ & 0 & 12.5 \\
\hline 3241 & Cement, Hydraulic . . . . . . . . . . . & 616 & 84 & 510 & $w$ & 9 & $\mathbf{Q}$ & Q & 0 & $w$ & $w$ & 18.0 \\
\hline 3274 & $\operatorname{Lim} \theta \ldots \ldots \ldots \ldots \ldots \ldots \ldots$ & 240 & • & 219 & 0 & $\cdot$ & 0 & 0 & 0 & 0 & 0 & 38.0 \\
\hline 3296 & Mineral Wool . . . . . . . . . . . . & 12 & $*$ & 12 & 0 & $\cdot$ & 0 & 0 & 0 & $\cdot$ & 0 & 2.4 \\
\hline 33 & Primary Metal Industries $\ldots \ldots \ldots \ldots$ & 1,806 & 303 & 1,267 & $\mathbf{Q}$ & 246 & 30 & 0 & 0 & 66 & w & 8.4 \\
\hline 3312 & Blast Fumaces and Steol Mills . . . . . . & 901 & 83 & 740 & $\cdot$ & 36 & $w$ & 0 & 0 & W & $w$ & 9.2 \\
\hline 3313 & Electrometalurgical Products . . . . . . . . & 20 & 0 & 17 & 0 & 0 & 0 & 0 & 0 & 0 & 0 & 10.2 \\
\hline 3321 & Gray and Ductile Iron Foundries ...... & 144 & 8 & 128 & $\cdot$ & 7 & 0 & 0 & 0 & 1 & 1 & 26.0 \\
\hline 3331 & Primary Copper . ............. & $w$ & $w$ & 25 & 1 & $w$ & $w$ & 0 & 0 & w & 0 & 1.1 \\
\hline 3334 & Primary Aluminum $\ldots \ldots \ldots \ldots \ldots$ & 127 & 0 & 123 & 0 & 0 & 0 & 0 & 0 & 0 & 0 & 5.8 \\
\hline 3339 & Primary Nonferrous Motals, nec . . . . . & 53 & $\cdot$ & 43 & 0 & $*$ & 0 & 0 & 0 & $\cdot$ & 0 & 1.9 \\
\hline 3353 & Aluminum Sheet, Plate, and Foil . . . . . . & 67 & $w$ & 55 & 0 & $W$ & 0 & 0 & 0 & 1 & 0 & 1.2 \\
\hline 34 & Fabricated Metal Products . . . . . . . . & 994 & 188 & 632 & $\mathbf{Q}$ & 151 & 28 & $\cdot$ & $\cdot$ & $Q$ & $Q$ & 31.8 \\
\hline 35 & Industrial Machinery and Equipment .... & 718 & 43 & 535 & $\mathbf{Q}$ & 22 & 2 & 0 & $Q$ & $\mathbf{Q}$ & Q & 24.1 \\
\hline 357 & Computer and Office Equipment . . . . . . & 16 & 6 & 6 & 1 & 6 & $\bullet$ & 0 & 0 & 1 & $\cdot$ & 32.0 \\
\hline 36 & Electronlc and Other Electric Equipment . & 416 & 138 & 183 & 19 & 132 & $\bullet$ & 0 & 0 & 18 & • & 24.4 \\
\hline 37 & Transportation Equlpment $\ldots \ldots \ldots \ldots$ & 1,214 & 132 & 893 & 29 & 93 & 60 & 6 & 0 & 7 & 2 & 14.1 \\
\hline 3711 & Motor Vehicles and Car Bodies . . . . . . . & 65 & 3 & 50 & $\bullet$ & 3 & $*$ & 0 & 0 & - & 1 & 6.7 \\
\hline 3714 & Motor Vehicle Parts and Accessorles .. & 104 & $w$ & 41 & $Q$ & $w$ & W & $Q$ & 0 & 1 & 1 & 17.1 \\
\hline 38 & Instruments and Related Products ..... & $w$ & 73 & 134 & $Q$ & 60 & 28 & Q & 0 & 32 & 9 & 31.7 \\
\hline 3841 & Surglcal and Modical Instruments ..... & 30 & 1 & 22 & $\cdot$ & * & 0 & $\cdot$ & 0 & $*$ & 0 & 32.1 \\
\hline 39 & Misc. Manufacturing Industries . . . . . . . & w & 30 & 63 & $Q$ & 27 & 2 & 0 & 0 & 13 & $Q$ & 28.3 \\
\hline & Total $\ldots \ldots \ldots \ldots \ldots \ldots \ldots$ & 23,885 & 4,772 & 14,821 & 327 & 3,186 & 903 & 159 & 19 & 824 & 406 & 10.5 \\
\hline
\end{tabular}

See footnotes at end of table. 


\section{Table A55. Capability to Switch from Distillate Fuel Oil to Alternative Energy Sources by Industry Group, Selected Industries, and Selected Characteristics, 1991 (Continued) (Estimates in Thousand Barrels)}

\begin{tabular}{|c|c|c|c|c|c|c|c|c|c|c|c|}
\hline \multirow[b]{2}{*}{ Selected Characteristics } & \multicolumn{3}{|c|}{ Distillate Fuel Oil } & \multicolumn{7}{|c|}{ Alternative Types of Energy" } & \multirow[b]{2}{*}{$\begin{array}{c}\text { RSE } \\
\text { Row } \\
\text { Factors }\end{array}$} \\
\hline & $\begin{array}{c}\text { Total } \\
\text { Consumed }\end{array}$ & Switchable & $\begin{array}{c}\text { Not } \\
\text { Switchable }\end{array}$ & $\begin{array}{l}\text { Electricity } \\
\text { Receipts }^{d}\end{array}$ & $\begin{array}{c}\text { Natural } \\
\text { Gas }\end{array}$ & $\begin{array}{l}\text { Residual } \\
\text { Fuel Oil } \\
\end{array}$ & Coal & LPG & $\begin{array}{c}\text { Coal Coke } \\
\text { and } \\
\text { Breeze }\end{array}$ & Other" & \\
\hline RSE Column Factors: & 0.5 & 0.6 & 0.5 & 1.8 & 0.8 & 1.2 & 0.9 & 2.2 & 1.3 & 1.5 & \\
\hline \multicolumn{12}{|l|}{ Census Region } \\
\hline Northeast $\ldots \ldots \ldots \ldots \ldots \ldots$ & 7,805 & 1,696 & 4,444 & 185 & 1,414 & 353 & 17 & 0 & 313 & 120 & 23.0 \\
\hline$\ldots \ldots \ldots \ldots \ldots$ & 3,885 & 581 & 2,538 & 31 & 356 & 110 & 43 & $w$ & 109 & 90 & 11.9 \\
\hline South $\ldots \ldots \ldots \ldots \ldots \ldots \ldots$ & 8,014 & 1,644 & 5,019 & 94 & 813 & 325 & 88 & $\cdot$ & 329 & 63 & 11.6 \\
\hline West $\ldots \ldots \ldots \ldots \ldots \ldots \ldots$ & 4,180 & 852 & 2,820 & 17 & 602 & 115 & 12 & Q & 74 & 133 & 16.2 \\
\hline Total $\ldots \ldots \ldots \ldots \ldots \ldots \ldots$ & 23,885 & 4,772 & 14,821 & 327 & 3,186 & 903 & 159 & 19 & 824 & 406 & 10.5 \\
\hline \multicolumn{12}{|l|}{$\begin{array}{l}\text { Value of Shipments and Receipts ' } \\
\text { (million dollars) }\end{array}$} \\
\hline Under $20 \ldots \ldots \ldots \ldots \ldots \ldots$ & 9,290 & 1,188 & 5,440 & Q & 828 & 354 & $Q$ & a & 257 & 131 & 26.2 \\
\hline $20-49 \ldots \ldots \ldots \ldots \ldots \ldots \ldots$ & 4,536 & 872 & 3,186 & 35 & 650 & 162 & 25 & $Q$ & 178 & 86 & 18.0 \\
\hline $50-99 \ldots \ldots \ldots \ldots \ldots \ldots \ldots$ & 2,366 & 591 & 1,464 & 79 & 419 & 96 & $w$ & Q & 125 & 30 & 13.8 \\
\hline $100-249 \ldots \ldots \ldots \ldots \ldots \ldots \ldots$ & 2,269 & 486 & 1,504 & 16 & 441 & 104 & 54 & $\cdot$ & 63 & 23 & 10.0 \\
\hline $250-499 \ldots \ldots \ldots \ldots \ldots \ldots \ldots$ & 2,317 & 597 & 1,450 & 54 & 379 & 111 & 27 & $w$ & 47 & $w$ & 7.3 \\
\hline 500 and Over $\ldots \ldots \ldots \ldots \ldots$ & 3,107 & 1,038 & 1,776 & 39 & 469 & 77 & $w$ & 0 & 154 & $w$ & 8.6 \\
\hline Total $\ldots \ldots \ldots \ldots \ldots \ldots \ldots \ldots$ & 23,885 & 4,772 & 14,821 & 327 & 3,186 & 903 & 159 & 19 & 824 & 406 & 10.5 \\
\hline \multicolumn{12}{|l|}{ Employment Size ' } \\
\hline Under $50 \ldots \ldots \ldots \ldots \ldots \ldots$ & 5,352 & 642 & 3,235 & Q & 426 & 158 & $Q$ & 0 & 120 & 59 & 34.0 \\
\hline $50-99 \ldots \ldots \ldots \ldots \ldots \ldots \ldots \ldots$ & 3,395 & 657 & 2,117 & Q & 427 & 211 & $Q$ & 0 & 104 & $W$ & 20.8 \\
\hline $100-249 \ldots \ldots \ldots \ldots \ldots \ldots \ldots$ & 5,062 & 872 & 3,185 & 15 & 653 & 177 & $w$ & $Q$ & 212 & 124 & 18.4 \\
\hline $250-499 \ldots \ldots \ldots \ldots \ldots \ldots$ & 3,137 & 821 & 1,976 & 65 & 708 & 131 & 18 & 14 & 129 & 38 & 17.6 \\
\hline $500-999 \ldots \ldots \ldots \ldots \ldots \ldots \ldots$ & 2,664 & 797 & 1,530 & 108 & 584 & 54 & 34 & w & 161 & W & 11.8 \\
\hline 1,000 and Over $\ldots \ldots \ldots \ldots \ldots$ & 4,276 & 983 & 2,776 & 40 & 388 & 172 & 55 & $*$ & 97 & 33 & 7.7 \\
\hline Total $\ldots \ldots \ldots \ldots \ldots \ldots \ldots$ & 23,885 & 4,772 & 14,821 & 327 & 3,186 & 903 & 159 & 19 & 824 & 406 & 10.5 \\
\hline
\end{tabular}

- See Appendices $B$ and $F$ for descriptions of the Standard Industrial Classification system.

- "Altemative Types of Energy" consist of those energy sources that could have been subslituted for distillate fuel oil during 1991. The quantities are expressed in thousands of barrels, and therefore represent the quantity of distillate fuel oil that could have been displaced by the given alternative type of energy.

"Total Consumed" represents those quantities of distillate fuel oil that were ascertained switchable or not switchable, plus an additional quantity for which the switching status was not ascertained.

"Electricity Receipts" represents those quantities of electricity generated off the manufacturing establishment site and available at the site for consumption. $I t$ includes those quantities for which payment was made, quantities transferred in, quantities purchased and paid for by a central purchasing entity, and quanlities for which payment was made in kind. It does not include electricity generated onsite. "Electricity Receipts" has not been adjusted to account for any quantities that might have been resold or transferred out. The estimates include those quantities that were ascertained switchable or not switchable, plus an additional quantity for which the switching status was not ascertained.

- "Other" includes all other types of energy not already identified that respondents indicated could have been consumed in place of distillate fuel oil.

'Value of Shipments and Receipts and Employment Size categories were supplied by the Bureau of the Census. See Appendix B.

- Estimate less than 0.5. Data are included in higher level totals.

$W=$ Withheld to avoid disclosing data for individual establishments. Data are included in higher level totals.

$Q=$ Withheld because Relative Standard Error is greater than 50 percent. Data are included in higher level totals.

Notes: - To obtain a RSE percentage for any table cell, multiply the cell's corresponding RSE column and RSE row factors. - Totals may not equal sum of components because of independent rounding.

Source: Energy Information Administration, Office of Energy Markets and End Use, Energy End Use Division, Form ElA-846, "1991 Manufacturing Energy Consumption Survey." 
Table A56. Capability to Switch from Residual Fuel Oil to Alternative Energy Sources by Industry Group, Selected Industries, and Selected Characteristics, 1991 (Estimates in Thousand Barrels)

\begin{tabular}{|c|c|c|c|c|c|c|c|c|c|c|c|c|}
\hline \multirow[b]{2}{*}{$\begin{array}{c}\text { SIC } \\
\text { Code" }\end{array}$} & \multirow[b]{2}{*}{$\begin{array}{l}\text { Industry Groups } \\
\text { and Industry }\end{array}$} & \multicolumn{3}{|c|}{ Residual Fuel Oil } & \multicolumn{7}{|c|}{ Alternative Types of Energy } & \multirow[b]{2}{*}{$\begin{array}{c}\text { RSE } \\
\text { Row } \\
\text { Factors }\end{array}$} \\
\hline & & $\begin{array}{c}\text { Total } \\
\text { Consumed }\end{array}$ & Switchable & $\begin{array}{c}\text { Not } \\
\text { Switchable }\end{array}$ & $\begin{array}{l}\text { Electricity } \\
\text { Receipts }\end{array}$ & $\begin{array}{c}\text { Natural } \\
\text { Gas }\end{array}$ & $\begin{array}{l}\text { Distillate } \\
\text { Fuel Oil }\end{array}$ & Coal & LPG & $\begin{array}{c}\text { Coal Coke } \\
\text { and } \\
\text { Breeze }\end{array}$ & Other & \\
\hline & RSE Column Factors: & 0.7 & 0.7 & 0.9 & 1.3 & 0.7 & 1.0 & 1.3 & 1.3 & 1.2 & 1.2 & \\
\hline 20 & Food and Kindred Products . . . . . . . & 4,317 & 2,138 & 1,477 & $\mathbf{Q}$ & 1,583 & 996 & $Q$ & $w$ & 321 & 209 & 17.1 \\
\hline 2011 & Meat Packing Plants ............. & 170 & 169 & 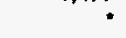 & $*$ & 164 & 92 & 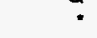 & 0 & $w$ & $w$ & 21.8 \\
\hline 2033 & Canned Fnils and Vogetables . . . . . . . & 290 & 177 & 114 & 0 & 166 & 50 & 0 & 0 & $w$ & 0 & 16.6 \\
\hline 2037 & Frozen Frults and Vegetables ........ & 321 & 93 & 201 & 3 & 23 & $\mathbf{Q}$ & 3 & 0 & $\mathbf{Q}$ & 36 & 31.6 \\
\hline 2046 & Wet Com Milling ............... & 29 & 26 & 0 & $w$ & 26 & $\overline{0}$ & $w$ & $w$ & $\vec{w}$ & 0 & 18.4 \\
\hline 2051 & Bread, Cake and Relafed Products ..... & 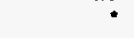 & 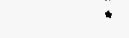 & 0 & 0 & 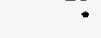 & 0 & 0 & 0 & 0 & 0 & 35.5 \\
\hline 2063 & Boot Sugar . . . . . . . . . . . . . & $w$ & $w$ & $w$ & 0 & $w$ & 0 & 0 & 0 & 0 & 0 & 15.6 \\
\hline 2075 & Soybean Oil Mils ............... & 42 & 19 & $w$ & 0 & 18 & W & - & 0 & $w$ & 0 & 7.5 \\
\hline 2082 & Malt Boveragos ................ & 419 & 53 & $w$ & 0 & 50 & w & • & 0 & 0 & 1 & 18.3 \\
\hline 21 & Tobacco Manufactures ........... & 135 & 75 & 59 & 0 & $w$ & $w$ & $w$ & 0 & 0 & 0 & 6.6 \\
\hline 22 & Textile Mill Products ............... & 1,966 & 1,021 & 737 & 1 & 716 & 369 & 117 & $w$ & 192 & 2 & 13.0 \\
\hline 23 & Apparel and Other Textile Products ..... & $Q$ & 73 & Q & 0 & 73 & 46 & 27 & 27 & 27 & 44 & 39.7 \\
\hline 24 & Lumber and Wood Products .......... & 333 & 157 & 91 & 0 & 157 & 100 & 0 & 0 & Q & 0 & 38.8 \\
\hline 25 & Furniture and Fixtures $\ldots \ldots \ldots \ldots \ldots$ & 184 & 51 & $\mathbf{Q}$ & $\mathbf{0}$ & Q & 26 & 0 & 0 & $\bar{a}$ & $Q$ & 50.6 \\
\hline 26 & Paper and Allied Products . . . . . . . . & 24,883 & 7,778 & 16,492 & 270 & 3,809 & 3,627 & 810 & $w$ & 260 & 238 & 8.2 \\
\hline 2611 & Pulp MIIIs $\ldots \ldots \ldots \ldots \ldots \ldots \ldots$ & 4,500 & 1,179 & 2,880 & 24 & 808 & 596 & 0 & 0 & 15 & 115 & 23.5 \\
\hline 2621 & Paper Milis . . . . . . . . . . . . . & 13,455 & 3,640 & 9,775 & $W$ & 1,542 & 1,760 & $w$ & $w$ & $w$ & 18 & 5.5 \\
\hline 2631 & Paperboard Mills .............. & W & 2,737 & $w$ & w & 1,298 & 1,181 & w & 0 & $\mathbf{Q}$ & 97 & 10.6 \\
\hline 27 & Printing and Publishing $\ldots \ldots \ldots \ldots \ldots$ & 50 & 31 & $Q$ & 0 & 31 & 0 & 0 & 0 & 0 & 0 & 32.2 \\
\hline 28 & ChemIcals and Alljed Products . . . . . . . & 7,573 & 2,546 & 4,914 & $w$ & 1,909 & 536 & 311 & 50 & 321 & 225 & 7.6 \\
\hline 2812 & Alkalles and Chlorine ............ & $w$ & $w$ & 0 & 0 & w & 0 & 0 & 0 & 0 & 0 & 36.9 \\
\hline 2813 & Industrial Gases . . . . . . . . . . . . . & 0 & 0 & 0 & 0 & 0 & 0 & 0 & 0 & 0 & 0 & 0.0 \\
\hline 2819 & Industrial Inorganic Chemicals, nec . . . . . & 691 & 339 & 351 & 0 & 322 & 95 & $w$ & 0 & $w$ & $w$ & 13.0 \\
\hline 2821 & Plastics Materials and Resins . . . . . . . . & 668 & 167 & 500 & $w$ & 158 & 19 & 0 & $w$ & $w$ & $W$ & 9.5 \\
\hline 2822 & Synthetic Rubber . . . . . . . . . . . . . . & 64 & 64 & 0 & 0 & 64 & $w$ & 0 & 0 & $w$ & 0 & 23.2 \\
\hline 2823 & Cellulosic Manmado Fibors . . . . . . . . . & 0 & 0 & 0 & 0 & 0 & 0 & 0 & 0 & 0 & 0 & 0.0 \\
\hline 2824 & Organic Fibers, Noncellulosic . . . . . . . . . & $w$ & 447 & $w$ & $w$ & 163 & 67 & $w$ & 0 & $w$ & 0 & 4.6 \\
\hline 2865 & Cyclic Crudes and Intermedlates ....... & 1,299 & $w$ & $w$ & 0 & 453 & 118 & $w$ & $w$ & w & $w$ & 16.2 \\
\hline 2869 & Industrial Organic Chemicals, nec ...... & 1,747 & 396 & 1,332 & $w$ & 279 & 71 & 0 & 0 & $w$ & 41 & 9.1 \\
\hline 2873 & Nitrogenous Fertilizers ............ & 0 & 0 & 0 & 0 & 0 & 0 & 0 & 0 & 0 & 0 & 0.0 \\
\hline 2874 & Phosphatic Fertlizers . . . . . . . . . . & 250 & $w$ & $w$ & 0 & w & w & $w$ & $w$ & $w$ & 0 & 3.9 \\
\hline 29 & Petroleum and Coal Products $\ldots \ldots \ldots$. & 13,862 & 8,883 & 4,724 & 222 & 6,201 & 2,844 & 183 & $w$ & 3,876 & 1,228 & 5.2 \\
\hline 2911 & Petroleum Relining ............... & 10,292 & 6,175 & 3,861 & 221 & 4,259 & 2,725 & 183 & 0 & 3,875 & 1,166 & 5.2 \\
\hline 30 & Rubber and Misc. Plastics Products ..... & 1,253 & 1,005 & 219 & $w$ & 829 & 316 & 46 & 0 & 90 & 97 & 14.3 \\
\hline 3011 & Tires and inner Tubes .............. & 506 & $w$ & $w$ & $w$ & 455 & 63 & 0 & 0 & $w$ & 0 & 7.3 \\
\hline 308 & Miscellaneous Plastic Products, nec .... & 413 & 273 & 122 & 0 & 252 & 46 & 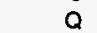 & 0 & 0 & 97 & 27.5 \\
\hline 31 & Leather and Leather Products .... . . . . . & 225 & 138 & 85 & 2 & 66 & 72 & 0 & 0 & 0 & 0 & 39.7 \\
\hline 32 & Stone, Clay and Glass Products . . . . . . . . & 1,345 & 896 & 447 & 20 & 809 & 65 & 95 & $w$ & 124 & $W$ & 16.0 \\
\hline 3211 & Flat Glass ................. & $w$ & 0 & $w$ & 0 & 0 & 0 & 0 & 0 & 0 & 0 & 7.6 \\
\hline 3221 & Glass Containors . . . . . . . . . . . & 276 & 276 & $\because$ & : & 276 & $w$ & 0 & 0 & 0 & 0 & 14.0 \\
\hline 3229 & Pressed and Blown Glass, nec . . . . . . . . & 81 & $w$ & $Q$ & 0 & $w$ & 0 & 0 & 0 & $w$ & $W$ & 18.3 \\
\hline 3241 & Cement, Hydraullc . . . . . . . . . . . . & 138 & 89 & 49 & $w$ & 11 & 0 & 67 & 0 & 0 & 0 & 26.3 \\
\hline 3274 & $\operatorname{LIm}{ }^{\prime} \ldots \ldots \ldots \ldots \ldots \ldots \ldots \ldots$ & $w$ & $w$ & 0 & 0 & $w$ & $w$ & $w$ & $w$ & 0 & 0 & 24.5 \\
\hline 3296 & 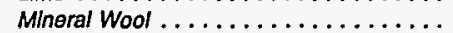 & $w$ & $w$ & 0 & 0 & w & 0 & 0 & 0 & 0 & 0 & 2.8 \\
\hline 33 & Primary Metal Industries . . . . . . . . . . . & 5,285 & 2,572 & 2,624 & $w$ & 1,703 & 180 & $w$ & $w$ & $w$ & $w$ & 8.9 \\
\hline 3312 & Blast Fumacos and Steel Mills ........ & 4,986 & 2,455 & 2,531 & $w$ & 1,586 & $w$ & $\ddot{w}$ & $\dddot{w}$ & w & $w$ & 9.4 \\
\hline 3313 & Electrometalurglcal Products ........... & 0 & 0 & 0 & 0 & 0 & 0 & 0 & 0 & 0 & 0 & 0.0 \\
\hline 3321 & Gray and Ductile Iron Foundries ....... & 4 & $w$ & $Q$ & 0 & $w$ & 0 & 0 & 0 & 0 & 0 & 34.2 \\
\hline 3331 & Primary Copper ................ & $w$ & $w$ & 0 & 0 & $w$ & $w$ & 0 & 0 & 0 & $w$ & 1.2 \\
\hline 3334 & Primary Aluminum . . . . . . . . . . . . & $\because$ & 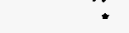 & 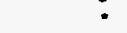 & 0 & 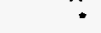 & 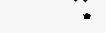 & 0 & 0 & 0 & 0 & 7.6 \\
\hline 3339 & Primary Nonferrous Metals, nec ....... & 1 & 1 & 0 & 0 & 1 & 0 & 0 & 0 & 0 & 0 & 1.4 \\
\hline 3353 & Aluminum Sheot, Plato, and Foll ....... & 0 & 0 & 0 & 0 & 0 & 0 & 0 & 0 & 0 & 0 & 0.0 \\
\hline 34 & Fabricated Metal Products . . . . . . . . . . & 501 & 205 & 175 & $w$ & 195 & $w$ & 0 & 0 & $\mathbf{Q}$ & 0 & 32.8 \\
\hline 35 & Industrial Machinery and Equipment ...... & 490 & 191 & 218 & 0 & 148 & 60 & 0 & 0 & 0 & 0 & 27.8 \\
\hline 357 & Computer and Office Equipment ........ & 11 & 6 & $Q$ & 0 & 6 & 0 & 0 & 0 & 0 & 0 & 37.8 \\
\hline 36 & Electronic and Other Electric Equipment .. & 612 & 368 & 183 & 0 & 326 & 63 & 16 & $\mathbf{Q}$ & 30 & 0 & 24.3 \\
\hline 37 & Transportation Equipment . . . . . . . . . & 1,865 & 728 & 978 & 29 & 636 & 56 & $w$ & $\overline{0}$ & 58 & $\vec{Q}$ & 16.5 \\
\hline 3711 & Motor Vehicles and Car Bodjes ......... & 408 & $w$ & $w$ & $w$ & 81 & 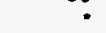 & 0 & 0 & $w$ & 0 & 6.6 \\
\hline 3714 & Motor Vehiclo Parts and Accessories ... & 60 & $w$ & w & 0 & $w$ & $w$ & $w$ & 0 & $w$ & 0 & 15.0 \\
\hline 38 & Instruments and Related Products ...... & 536 & 404 & 101 & $w$ & 144 & 285 & $w$ & 0 & $Q$ & 10 & 22.3 \\
\hline 3841 & Surglcal and Medical Instruments ...... & 9 & 9 & 0 & 0 & 0 & 9 & 0 & 0 & $\overline{0}$ & 9 & 40.6 \\
\hline 39 & Misc. Manufacturing Industries . . . . . . . . . & 115 & 89 & 18 & 0 & 88 & 44 & 10 & 0 & 10 & 0 & 26.5 \\
\hline & Total $\ldots \ldots \ldots \ldots \ldots \ldots \ldots \ldots$ & 65,837 & 29,349 & 33,913 & 1,267 & 19,511 & 9,716 & 2,565 & 1,756 & 5,625 & 2,227 & 4.3 \\
\hline
\end{tabular}

See footnotes at end of table. 
Table A56. Capability to Switch from Residual Fuel Oil to Alternative Energy Sources by Industry Group, Selected Industries, and Selected Characteristics, 1991 (Continued)

\section{(Estimates in Thousand Barrels)}

\begin{tabular}{|c|c|c|c|c|c|c|c|c|c|c|c|}
\hline \multirow[b]{2}{*}{ Selected Characteristics } & \multicolumn{3}{|c|}{ Residual Fuel Oai } & \multicolumn{7}{|c|}{ Altemative Types of Energy } & \multirow[b]{2}{*}{$\begin{array}{c}\text { ASE } \\
\text { Row } \\
\text { Factors }\end{array}$} \\
\hline & $\begin{array}{c}\text { Total } \\
\text { Consumedc }\end{array}$ & Switchable & $\begin{array}{c}\text { Not } \\
\text { Switchable }\end{array}$ & $\begin{array}{l}\text { Electricity } \\
\text { Receipts }^{\circ}\end{array}$ & $\begin{array}{c}\text { Natural } \\
\text { Gas }\end{array}$ & $\begin{array}{l}\text { Distıllate } \\
\text { Fuel Oil }\end{array}$ & Coal & LPG & $\begin{array}{c}\text { Coal Coke } \\
\text { and } \\
\text { Breeze }\end{array}$ & Other & \\
\hline RSE Column Factors: & 0.6 & 0.6 & 0.9 & 1.7 & 0.7 & 1.0 & 1.6 & 1.5 & 0.9 & 1.3 & \\
\hline \multicolumn{12}{|l|}{ Census Region } \\
\hline Northeast $\ldots \ldots \ldots \ldots \ldots \ldots \ldots$ & 29,245 & 11,683 & 16,692 & 397 & 8,103 & 2,689 & $w$ & $w$ & 2,574 & 1,106 & 6.4 \\
\hline Midwest & 8,134 & 4,630 & 2,923 & $w$ & 3,694 & 1,144 & $w$ & $w$ & 1,039 & 249 & 6.9 \\
\hline South $\ldots \ldots \ldots \ldots \ldots \ldots \ldots \ldots$ & 23,114 & 9,603 & 12,408 & 632 & 5,747 & 3,853 & 1,547 & 280 & 896 & 446 & 7.2 \\
\hline West $\ldots \ldots \ldots \ldots \ldots \ldots \ldots \ldots$ & 5,344 & 3,432 & 1,890 & $w$ & 1,968 & 2,030 & 22 & w & 1,115 & 426 & 7.5 \\
\hline Total $\ldots \ldots \ldots \ldots \ldots \ldots \ldots \ldots$ & 65,837 & 29,349 & 33,913 & 1,267 & 19,511 & 9,716 & 2,565 & 1,756 & 5,625 & 2,227 & 4.3 \\
\hline \multicolumn{12}{|l|}{$\begin{array}{l}\text { Value of Shipments and Receipts ' } \\
\text { (million dollars) }\end{array}$} \\
\hline Under $20 \ldots \ldots \ldots \ldots \ldots$ & 4,049 & 1,994 & 1,531 & $Q$ & 1,398 & 1,021 & $W$ & $w$ & 468 & Q & 20.7 \\
\hline $20-49 \ldots \ldots \ldots \ldots \ldots \ldots$ & 6,055 & 2,420 & 3,181 & 28 & 1,749 & 830 & 323 & 80 & 340 & 292 & 16.4 \\
\hline $50-99 \ldots \ldots$ & 10,337 & 5,030 & 4,935 & 20 & 3,483 & 1,014 & $w$ & $w$ & 305 & 116 & 9.1 \\
\hline $100-249 \ldots$ & 13,803 & 5,862 & 7,349 & 28 & 3,519 & 2,752 & 636 & 29 & 619 & 404 & 8.7 \\
\hline $250-499 \ldots \ldots \ldots$ & 15,370 & 5,681 & 9,665 & 403 & 3,506 & 2,232 & 487 & $w$ & 1,222 & 388 & 4.9 \\
\hline 500 and Over ... & 16,223 & 8,363 & 7,252 & 621 & 5,855 & 1,867 & 991 & W & 2,671 & 931 & 4.8 \\
\hline Total $\ldots \ldots \ldots \ldots \ldots \ldots \ldots$ & 65,837 & 29,349 & 33,913 & 1,267 & 19,511 & 9,716 & 2,565 & 1,756 & 5,625 & 2,227 & 4.3 \\
\hline \multicolumn{12}{|l|}{ Employment Size ${ }^{t}$} \\
\hline Under $50 \ldots \ldots$ & 960 & 589 & 167 & $Q$ & 320 & 376 & $\mathbf{0}$ & 0 & 191 & $Q$ & 37.7 \\
\hline $50-99 \ldots \ldots \ldots$ & 3,986 & 1,731 & 1,994 & 16 & 1,316 & 644 & 190 & $w$ & 436 & 35 & 24.7 \\
\hline $100-249 \ldots \ldots \ldots \ldots \ldots \ldots \ldots$ & 11,764 & 6,333 & 4,749 & $w$ & 4,488 & 1,904 & 293 & $w$ & 1,162 & 464 & 5.8 \\
\hline $250-499 \ldots \ldots \ldots \ldots \ldots \ldots$ & 11,882 & 5,768 & 5,271 & 151 & 3,274 & 2,594 & 497 & 75 & 869 & 385 & 7.7 \\
\hline $500-999 \ldots \ldots \ldots \ldots \ldots \ldots \ldots$ & 13,051 & 5,997 & 6,898 & 58 & 4,032 & 2,246 & 86 & $W$ & 1,535 & 1,094 & 6.5 \\
\hline 1,000 and Over $\ldots \ldots \ldots \ldots \ldots$ & 24,193 & 8,930 & 14,834 & 824 & 6,082 & 1,952 & 1,498 & $w$ & 1,433 & 187 & 4.9 \\
\hline Total $\ldots \ldots \ldots \ldots \ldots \ldots \ldots$ & 65,837 & 29,349 & 33,913 & 1,267 & 19,511 & 9,716 & 2,565 & 1,756 & 5,625 & 2,227 & 4.3 \\
\hline
\end{tabular}

\footnotetext{
- See Appendices B and $F$ for descriptions of the Standard Industrial Classification system.

-Altemative Types of Energy" consist of those energy sources that could have been substituted for residual fuel oil during 1991. The quantities are expressed in thousands of barrels, and therefore represent the quantity of residual fuel oil that could have been displaced by the given alternative type of energy.

c "Total Consumed" represents those quantities of residual fuel oil that were ascertained switchable or not switchable, plus an additional quantity for which the switching status was not ascertained.

- Electricity Receipts" represents those quantities of electricity generated off the manufacturing establishment site and available at the site for consumption. It includes those quantities for which payment was made, quantities transferred in, quantities purchased and paid for by a central purchasing entity, and quantities for

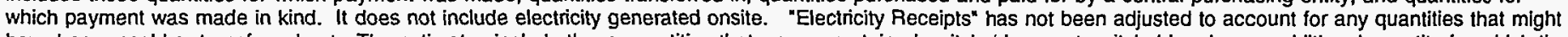

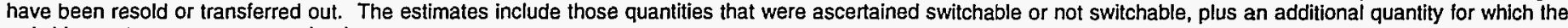
switching status was not ascertained.

- "Other" includes all other types of energy not already identified that respondents indicated could have been consumed in place of residual fuel oil.

'Value of Shipments and Receipts and Employment Size categories were supplied by the Bureau of the Census. See Appendix B.

- Estimate less than 0.5. Data are included in higher level totals.

$W=$ Withheld to avoid disclosing data for individual establishments. Data are included in higher level totals.

$\mathrm{Q}=$ Withheld because Relative Standard Error is greater than 50 percent. Data are included in higher level totals.

Notes: - To obtain a RSE percentage for any table cell, multiply the cell's corresponding RSE column and RSE row factors. - Totals may not equal sum of components because of independent rounding.

Source: Energy Information Administration, Office of Energy Markets and End Use, Energy End Use Division, Form ElA-846, "1991 Manufacturing Energy Consumption Survey."
} 
Table A57. Capability to Switch from Coal to Alternative Energy Sources by Industry
Group, Selected Industries, and Selected Characteristics, 1991 (Estimates in Thousand Short Tons)

\begin{tabular}{|c|c|c|c|c|c|c|c|c|c|c|c|}
\hline \multirow[b]{2}{*}{$\begin{array}{c}\text { SIC } \\
\text { Code* }\end{array}$} & \multirow[b]{2}{*}{$\begin{array}{c}\text { Industry Groups } \\
\text { and Industry }\end{array}$} & \multicolumn{3}{|c|}{ Coal } & \multicolumn{6}{|c|}{ Allemative Types of Energy" } & \multirow[b]{2}{*}{$\begin{array}{c}\text { RSE } \\
\text { Row } \\
\text { Factors }\end{array}$} \\
\hline & & $\begin{array}{c}\text { Total } \\
\text { Consumed }\end{array}$ & Switchable & $\begin{array}{c}\text { Not } \\
\text { Switchable }\end{array}$ & $\begin{array}{l}\text { Electricity } \\
\text { Receipts }^{d}\end{array}$ & $\begin{array}{c}\text { Natural } \\
\text { Gas }\end{array}$ & $\begin{array}{l}\text { Distillate } \\
\text { Fuel Oil }\end{array}$ & $\begin{array}{l}\text { Residual } \\
\text { Fuel Oil }\end{array}$ & LPG & Other & \\
\hline & RSE Column Factors: & 0.8 & 0.8 & 0.9 & 1.1 & 0.9 & 1.1 & 1.0 & 1.2 & 1.4 & \\
\hline 20 & Food and Kindred Products & 6,913 & 3,728 & 2,882 & 576 & 2,683 & 2,066 & 642 & $w$ & * & 10.3 \\
\hline 2011 & Moat Packing Plants $\ldots \ldots \ldots \ldots \ldots$ & 27 & W & $w$ & 1 & $w$ & 0 & 0 & 1 & 0 & 25.5 \\
\hline 2033 & Canned Frults and Vegetables . . . . . . . & $Q$ & 0 & $\mathbf{Q}$ & 0 & 0 & 0 & 0 & 0 & 0 & 0.0 \\
\hline 2037 & Frozen Fruits and Vegetables . . . . . . . . . & 0 & 0 & 0 & 0 & 0 & 0 & 0 & 0 & 0 & 0.0 \\
\hline 2046 & Wet Com Miling $\ldots \ldots \ldots \ldots \ldots \ldots$ & 3,051 & 2,145 & 810 & W & 1,871 & w & 0 & 0 & - & 14.1 \\
\hline 2051 & Bread, Cake and Related Products . . . . . . . & 0 & 0 & 0 & 0 & 0 & 0 & 0 & 0 & 0 & 0.0 \\
\hline 2063 & Beet Sugar $\ldots \ldots \ldots \ldots \ldots \ldots \ldots$ & 1,901 & W & 1,394 & $w$ & $w$ & $w$ & 0 & $w$ & 0 & 10.2 \\
\hline 2075 & Soybran Oll Mills . . . . . . . . . . . . . . & 592 & 241 & 351 & W & 149 & 136 & 0 & 0 & 0 & 5.7 \\
\hline 2082 & Malt Boverages . . . . . . . . . . . . & 706 & 681 & 18 & $w$ & W & w & $w$ & 0 & 0 & 18.2 \\
\hline 21 & Tobacco Manufactures $\ldots \ldots \ldots \ldots \ldots$ & 692 & 242 & $w$ & $w$ & $w$ & w & $w$ & 0 & 0 & 6.7 \\
\hline 22 & Textile Mill Products $\ldots \ldots \ldots \ldots \ldots \ldots$ & 1,362 & 827 & 445 & $\mathbf{Q}$ & 684 & 408 & 310 & 125 & $w$ & 11.0 \\
\hline 23 & Apparel and Other Textile Products ....... & 88 & w & 80 & $\overline{0}$ & 0 & 0 & W & 0 & 0 & 35.6 \\
\hline 24 & Lumber and Wood Products .......... & 92 & 8 & 83 & 0 & 0 & 0 & $\because$ & 0 & 8 & 36.8 \\
\hline 25 & Fumiture and Fixtures $\ldots \ldots \ldots \ldots \ldots$ & 157 & $Q$ & 54 & 0 & $\mathbf{Q}$ & $\mathbf{Q}$ & Q & $Q$ & $\mathbf{Q}$ & 34.9 \\
\hline 26 & Paper and Allied Products . . . . . . . . . . & 13,252 & 6,148 & 6,844 & 701 & 2,734 & 2,027 & 3,207 & 215 & 429 & 4.2 \\
\hline 2611 & Pulp Mills $\ldots \ldots \ldots \ldots \ldots \ldots \ldots$ & 331 & 171 & 161 & 10 & 15 & 62 & 171 & 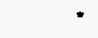 & 18 & 30.3 \\
\hline 2621 & 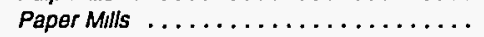 & 8,634 & 3,929 & 4,562 & 583 & 2,041 & 1,522 & 1,739 & 0 & $w$ & 3.0 \\
\hline 2631 & 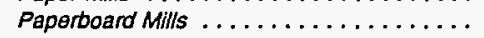 & $w$ & 1,938 & W & W & 636 & 425 & 1,289 & 215 & 170 & 7.5 \\
\hline 27 & Printing and Publishing $\ldots \ldots \ldots \ldots \ldots$ & 0 & 0 & 0 & 0 & 0 & 0 & 0 & 0 & 0 & 0.0 \\
\hline 28 & Chemicals and Allied Products .......... & 11,345 & 3,702 & 7,176 & 213 & 2,590 & 1,825 & 1,380 & 749 & $w$ & 6.0 \\
\hline 2812 & Alkalies and Chlorino ............. & W & w & w & 0 & w & 0 & 0 & 0 & 0 & 29.3 \\
\hline 2813 & Industral Gases . . . . . . . . . . . . . & 0 & 0 & 0 & 0 & 0 & 0 & 0 & 0 & 0 & 0.0 \\
\hline 2819 & Industrial Inorganic Chemicals, nec ...... & 743 & 113 & 408 & $w$ & 79 & 54 & $w$ & $w$ & $w$ & 11.5 \\
\hline 2821 & Plastics Materials and Resins . . . . . . . . . & 1,074 & 354 & 719 & 0 & 313 & 0 & 185 & 0 & 0 & 8.2 \\
\hline 2822 & 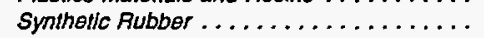 & $w$ & $w$ & 1 & 0 & w & $w$ & 0 & 0 & 0 & 22.7 \\
\hline 2823 & Cellulosic Manmade Fibers . . . . . . . . . . & 1,202 & 0 & 1,202 & 0 & 0 & 0 & 0 & 0 & 0 & 23.2 \\
\hline 2824 & Organic Fibers, Noncellulosic .......... & 1,558 & $w$ & 759 & $w$ & w & $w$ & $w$ & 0 & 0 & 4.3 \\
\hline 2865 & Cyclic Crudes and Intermediates ......... & $W$ & 41 & $w$ & 0 & $w$ & $w$ & 0 & 0 & $w$ & 18.1 \\
\hline 2869 & Industrial Organic Chemicals, nec ........ & 3,819 & 1,758 & 2,048 & 66 & 1,591 & 1,115 & $w$ & 737 & 0 & 8.0 \\
\hline 2873 & Nitrogenous Fentllizers .............. & 0 & 0 & 0 & 0 & 0 & 0 & 0 & 0 & 0 & 0.0 \\
\hline 2874 & Phosphatic Fertllizers . . . . . . . . . . . & w & $w$ & 0 & 0 & 0 & $w$ & $w$ & 0 & 0 & 6.7 \\
\hline 29 & Petroleum and Coal Products $\ldots \ldots \ldots \ldots$ & $w$ & $w$ & $w$ & $W$ & 97 & 88 & 124 & $w$ & 0 & 8.7 \\
\hline 2911 & Petroleum Refining $\ldots \ldots \ldots \ldots \ldots$ & 134 & 116 & 17 & 1 & 91 & 88 & 98 & $w$ & 0 & 11.5 \\
\hline 30 & Rubber and Misc. Plastics Products ....... & 295 & 128 & 130 & 0 & 102 & 37 & 35 & 0 & $\mathbf{Q}$ & 15.9 \\
\hline 3011 & Tres and Inner Tubes . . . . . . . . . . & 75 & w & $w$ & 0 & 23 & $w$ & $w$ & 0 & 1 & 5.9 \\
\hline 308 & Miscellaneous Plastic Products, nec . . . . . & 130 & 75 & 33 & 0 & $W$ & 0 & 26 & 0 & $\mathbf{Q}$ & 29.6 \\
\hline 31 & Leather and Leather Products . . . . . . . . & a & 0 & $Q$ & 0 & 0 & 0 & 0 & 0 & 0 & 0.0 \\
\hline 32 & Stone, Clay and Glass Products . . . . . . . . & 13,127 & 6,129 & 6,767 & 0 & 5,276 & 1,241 & 1,786 & 671 & 822 & 12.2 \\
\hline 3211 & Flat Glass $\ldots \ldots \ldots \ldots \ldots \ldots \ldots$ & $\bullet$ & 0 & $*$ & 0 & 0 & 0 & 0 & 0 & 0 & 7.3 \\
\hline 3221 & Glass Containers . . . . . . . . . . & 0 & 0 & 0 & 0 & 0 & 0 & 0 & 0 & 0 & 0.0 \\
\hline 3229 & Pressed and Blown Glass, nec . . . . . . . . & 0 & 0 & 0 & 0 & 0 & 0 & 0 & 0 & 0 & 0.0 \\
\hline 3241 & Cement, Hydraullc . . . . . . . . . . . & 8,736 & 4,631 & 4,016 & 0 & 3,871 & 1,085 & 1,679 & 569 & 775 & 11.0 \\
\hline 3274 & LIme $\ldots \ldots \ldots \ldots \ldots \ldots \ldots \ldots$ & 3,926 & 1,359 & $Q$ & 0 & 1,359 & 128 & $w$ & $w$ & $w$ & 20.2 \\
\hline 3296 & Mineral Wool . . . . . . . . . . . . . & - & 0 & * & 0 & 0 & 0 & 0 & 0 & 0 & 2.4 \\
\hline 33 & Primary Metal Industries $\ldots \ldots \ldots \ldots \ldots$ & 2,054 & 1,236 & 790 & $W$ & $w$ & 10 & $w$ & 0 & $w$ & 8.1 \\
\hline 3312 & Blast Fumaces and Stoel Mills . . . & 1,075 & $w$ & $w$ & $w$ & $w$ & 8 & $w$ & 0 & $w$ & 12.0 \\
\hline 3313 & Electrometalurglcal Products . . . . . . . . . & $w$ & 0 & $w$ & 0 & 0 & 0 & 0 & 0 & 0 & 13.4 \\
\hline 3321 & Gray and Ductlle Iron Foundries ......... & 5 & 4 & 1 & 0 & 0 & 0 & 0 & 0 & w & 22.4 \\
\hline 3331 & 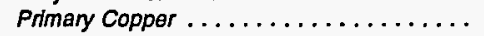 & W & 0 & 0 & 0 & 0 & 0 & 0 & 0 & 0 & 1.3 \\
\hline 3334 & Primary Aluminum . . . . . . . . . . & 0 & 0 & 0 & 0 & 0 & 0 & $\mathbf{0}$ & 0 & 0 & 0.0 \\
\hline 3339 & Primany Nonferrous Metals, nec ........ & $w$ & $w$ & $w$ & $w$ & 0 & 0 & 0 & 0 & 0 & 1.1 \\
\hline 3353 & Aluminum Shoot, Plate, and Foil . . . . . . . & $w$ & $w$ & 0 & 0 & $w$ & 0 & 0 & 0 & 0 & 1.2 \\
\hline 34 & Fabricaled Metal Products . . . . . . . . . . & 245 & 54 & 189 & $W$ & 51 & 46 & $w$ & 0 & 0 & 22.9 \\
\hline 35 & Industrial Machinery and Equipment . . . . . . & 480 & 178 & 302 & 0 & 178 & W & W & $w$ & 0 & 18.7 \\
\hline 357 & Computer and Office Equipment . . . . . . . . & 0 & 0 & 0 & 0 & 0 & 0 & 0 & 0 & 0 & 0.0 \\
\hline 36 & Electronic and Other Electric Equipment . . . . & $w$ & 37 & $w$ & 0 & 15 & 3 & 4 & 0 & 0 & 25.1 \\
\hline 37 & Transportation Equipment ............ & 1,464 & 277 & 1,074 & $\mathbf{Q}$ & 239 & 36 & $w$ & W & $w$ & 7.6 \\
\hline 3711 & Motor Vehlcles and Car Bodles . . . . . . . . & W & $w$ & 330 & 0 & $w$ & 0 & 0 & $w$ & $w$ & 6.4 \\
\hline 3714 & Motor Vehicle Parts and Accessories ..... & $w$ & $w$ & $w$ & $\mathbf{Q}$ & $w$ & 13 & w & $w$ & w & 10.1 \\
\hline 38 & Instruments and Related Products . . . . . . & $w$ & $w$ & $w$ & $W$ & 0 & $w$ & $w$ & 0 & 0 & 29.0 \\
\hline 3841 & Surglcal and Medical Instruments . . . . . . . & 0 & 0 & 0 & 0 & 0 & 0 & 0 & 0 & 0 & 0.0 \\
\hline \multirow[t]{2}{*}{39} & Misc. Manufacturing Industries . . . . . . . . & 32 & 32 & 0 & 0 & 32 & $Q$ & 22 & 20 & 0 & 35.6 \\
\hline & Total $\ldots \ldots \ldots \ldots \ldots \ldots \ldots \ldots$ & 53,035 & 23,610 & 27,779 & 1,821 & 15,656 & 8,129 & 8,352 & 2,003 & 1,456 & 5.1 \\
\hline
\end{tabular}

See footnotes at end of table. 
Table A57. Capability to Switch from Coal to Alternative Energy Sources by Industry Group, Selected Industries, and Selected Characteristics, 1991 (Continued) (Estimates in Thousand Short Tons)

\begin{tabular}{|c|c|c|c|c|c|c|c|c|c|c|}
\hline \multirow[b]{2}{*}{ Selected Characteristics } & \multicolumn{3}{|c|}{ Coal } & \multicolumn{6}{|c|}{ Altemative Types of Energy ${ }^{b}$} & \multirow[b]{2}{*}{$\begin{array}{c}\text { RSE } \\
\text { Row } \\
\text { Factors }\end{array}$} \\
\hline & $\begin{array}{c}\text { Total } \\
\text { Consumed }\end{array}$ & Switchable & $\begin{array}{c}\text { Not } \\
\text { Switchable }\end{array}$ & $\begin{array}{l}\text { Electricity } \\
\text { Receipts }^{\circ}\end{array}$ & $\begin{array}{c}\begin{array}{c}\text { Natural } \\
\text { Gas }\end{array} \\
\end{array}$ & $\begin{array}{l}\text { Distillate } \\
\text { Fuel Oil } \\
\end{array}$ & $\begin{array}{l}\text { Residual } \\
\text { Fuel Oil } \\
\end{array}$ & LPG & Olher" & \\
\hline RSE Column Factors: & 0.7 & 0.8 & 0.9 & 1.0 & 0.9 & 1.0 & 0.9 & 1.4 & 1.7 & \\
\hline \multicolumn{11}{|l|}{ Census Region } \\
\hline Northeast & 7,420 & 2,162 & 5,007 & $w$ & 572 & 629 & 886 & $w$ & 0 & 14.7 \\
\hline$\ldots \ldots \ldots \ldots \ldots \ldots \ldots$ & 18,828 & 8,185 & 10,119 & 564 & 6,597 & 3,398 & 909 & 691 & 554 & 7.0 \\
\hline South $\ldots \ldots \ldots \ldots \ldots \ldots \ldots \ldots \ldots$ & 22,514 & 10,359 & 11,402 & 759 & 6,328 & 3,245 & 5,341 & 1,164 & 871 & 4.9 \\
\hline West $\ldots \ldots \ldots \ldots \ldots \ldots \ldots \ldots \ldots$ & 4,274 & 2,904 & 1,251 & $w$ & 2,160 & 859 & 1,216 & $w$ & 30 & 14.6 \\
\hline Total $\ldots \ldots \ldots \ldots \ldots \ldots \ldots \ldots$ & 53,035 & 23,610 & 27,779 & 1,821 & 15,656 & 8,129 & 8,352 & 2,003 & 1,456 & 5.1 \\
\hline \multicolumn{11}{|l|}{$\begin{array}{l}\text { Value of Shipments and Receipts ' } \\
\text { (million dollars) }\end{array}$} \\
\hline Under $20 \ldots \ldots \ldots \ldots \ldots \ldots$ & 4,709 & 1,497 & $\mathbf{Q}$ & $\mathbf{Q}$ & 1,250 & 495 & 181 & 185 & 172 & 18.8 \\
\hline $20-49 \ldots \ldots \ldots \ldots \ldots \ldots \ldots \ldots$ & 9,384 & 4,177 & 4,712 & W & 3,478 & 970 & 1,453 & 632 & 679 & 11.0 \\
\hline $50-99 \ldots \ldots \ldots \ldots \ldots \ldots \ldots \ldots$ & 5,398 & 2,302 & 2,938 & 31 & 1,597 & 533 & 872 & $w$ & $w$ & 8.9 \\
\hline $100-249 \ldots \ldots \ldots \ldots \ldots \ldots \ldots \ldots$ & 11,587 & 4,962 & 6,371 & 564 & 3,197 & 2,065 & 1,538 & 788 & 141 & 4.9 \\
\hline $250-499 \ldots \ldots \ldots \ldots \ldots \ldots \ldots \ldots$ & 10,593 & 5,356 & 4,987 & 584 & 2,802 & 1,627 & 2,591 & $W$ & $w$ & 5.5 \\
\hline 500 and Over $\ldots \ldots \ldots \ldots \ldots \ldots$ & 11,365 & 5,316 & 5,736 & 598 & 3,332 & 2,438 & 1,717 & 307 & $w$ & 8.1 \\
\hline Total $\ldots \ldots \ldots \ldots \ldots \ldots \ldots$ & 53,035 & 23,610 & 27,779 & 1,821 & 15,656 & 8,129 & 8,352 & 2,003 & 1,456 & 5.1 \\
\hline \multicolumn{11}{|l|}{ Employment Size ' } \\
\hline Under $50 \ldots \ldots \ldots \ldots \ldots \ldots$ & 467 & Q & 126 & 0 & $\mathbf{Q}$ & 0 & $\mathbf{Q}$ & 0 & 0 & 52.2 \\
\hline $50-99 \ldots \ldots \ldots \ldots \ldots \ldots \ldots \ldots \ldots$ & 2,454 & 1,222 & 1,113 & W & 933 & 451 & 153 & $\mathbf{Q}$ & 157 & 23.3 \\
\hline $100-249 \ldots \ldots \ldots \ldots \ldots \ldots \ldots \ldots$ & 14,468 & 5,587 & 8,249 & w & 4,627 & 2,303 & 1,347 & 653 & 809 & 11.2 \\
\hline $250-499 \ldots \ldots \ldots \ldots \ldots \ldots \ldots \ldots$ & 7,707 & 4,283 & 3,197 & $W$ & 3,358 & 1,715 & 1,696 & 330 & 18 & 7.5 \\
\hline $500-999$ & 9,250 & 4,403 & 4,742 & 550 & 2,305 & 1,382 & 1,376 & 391 & 150 & 5.8 \\
\hline 1,000 and Over $\ldots \ldots \ldots \ldots \ldots \ldots$ & 18,689 & 7,789 & 10,351 & 666 & 4,107 & 2,277 & 3,768 & 548 & 322 & 5.0 \\
\hline Total $\ldots \ldots \ldots \ldots \ldots \ldots \ldots \ldots$ & 53,035 & 23,610 & 27,779 & 1,821 & 15,656 & 8,129 & 8,352 & 2,003 & 1,456 & 5.1 \\
\hline
\end{tabular}

- See Appendices B and F for descriptions of the Standard industrial Classification system.

b "Altemative Types of Energy" consist of those energy sources that could have been substituted for coal during 1991 . The quantities are expressed in thousands of short tons, and therefore represent the quantity of coal that could have been displaced by the given alternative type of energy.

"Total Consumed" represents those quantities of coal that were ascertained switchable or not switchable, plus an additional quantity for which the switching status was not ascertained.

" "Electricity Peceipts" represents those quantities of electricity generated off the manufacturing establishment site and available at the site for consumptlon. It includes those quantities for which payment was made, quantities transferred in, quantities purchased and paid for by a central purchasing entity, and quantitles for which payment was made in kind. It does not include electricity generated onsite. "Electricity Receipts" has not been adjusted to account for any quantities that might have been resoid or transferred out. The estimates include those quantities that were ascertained switchable or not switchable, plus an additional quantity for which the switching status was not ascertained.

- Other includes all other types of energy not already identified that respondents indicated could have been consumed in place of coal.

'Value of Shipments and Receipts and Employment Size categories were supplied by the Bureau of the Census. See Appendix B.

- Estimate less than 0.5. Data are included in higher level totals.

$W=$ Withheld to avoid disclosing data for individual establishments. Data are included in higher level totals.

$Q=$ Withheld because Relative Standard Error is greater than 50 percent. Data are included in higher level totals.

Notes: - To obtain a RSE percentage for any table cell, multiply the cell's corresponding RSE column and RSE row factors. - Totals may not equal sum of components because of independent rounding.

Source: Energy Information Administration, Office of Energy Markets and End Use, Energy End Use Division, Form ElA-846, "1991 Manufacturing Energy Consumption Survey." 
Table A58. Capability to Switch from LPG to Alternative Energy Sources by Industry Group, Selected Industries, and Selected Characteristics, 1991

(Estimates in Thousand Barrels)

\begin{tabular}{|c|c|c|c|c|c|c|c|c|c|c|c|c|}
\hline \multirow[b]{2}{*}{$\begin{array}{c}\text { SIC } \\
\text { Code" }\end{array}$} & \multirow[b]{2}{*}{$\begin{array}{l}\text { Industry Groups } \\
\text { and Industry }\end{array}$} & \multicolumn{3}{|c|}{ LPG } & \multicolumn{7}{|c|}{ Alternative Types of Energy" } & \multirow[b]{2}{*}{$\begin{array}{c}\text { RSE } \\
\text { How } \\
\text { Factors }\end{array}$} \\
\hline & & $\mid \begin{array}{c}\text { Total } \\
\text { Consumed }\end{array}$ & Switchable & $\begin{array}{c}\text { Not } \\
\text { Switchable }\end{array}$ & $\begin{array}{l}\text { Electricity } \\
\text { Receipts }\end{array}$ & $\begin{array}{c}\text { Natural } \\
\text { Gas }\end{array}$ & $\begin{array}{l}\text { Distillate } \\
\text { Fuel Oil }\end{array}$ & $\begin{array}{l}\text { Residual } \\
\text { Fuel Oil }\end{array}$ & Coal & $\begin{array}{c}\text { Coal } \\
\text { Coke and } \\
\text { Breeze }\end{array}$ & Other & \\
\hline & RSE Column Factors: & 0.6 & 0.8 & 0.7 & 1.2 & 0.9 & 1.1 & 1.0 & 1.3 & 1.7 & 1.2 & \\
\hline 20 & Food and KIndred Products ...... & 1,429 & 404 & 745 & 31 & 214 & 233 & 140 & . & 0 & $w$ & 19.8 \\
\hline 2011 & Meat Packing Plants & 157 & 124 & 25 & $\because$ & 103 & 122 & 102 & 0 & 0 & w & 28.9 \\
\hline 2033 & Canned Frults and Vegetables .. & 124 & 15 & 89 & w & 10 & $w$ & 0 & 0 & 0 & 2 & 19.1 \\
\hline 2037 & Frozen Fruits and Vegetables... & 41 & 15 & 18 & 1 & 1 & 11 & 1 & 0 & 0 & 2 & 26.8 \\
\hline 2046 & Wet Com Milling ........... & 1 & 1 & $\because$ & 1 & * & $\because$ & 0 & 0 & 0 & 0 & 32.4 \\
\hline 2051 & Bread, Cake and Related Products & 23 & 13 & 8 & : & 9 & $\uparrow$ & : & 0 & 0 & $w$ & 24.5 \\
\hline 2063 & Boot Sugar . . . . . . . . . . . & 5 & 1 & 4 & 0 & 1 & 0 & 0 & . & 0 & 0 & 10.9 \\
\hline 2075 & Soybean Oil Mills ............. & 5 & w & w & • & w & $w$ & $w$ & 0 & 0 & $w$ & 7.9 \\
\hline 2082 & Malt Beverages ............. & 8 & 1 & 7 & • & 0 & 0 & 0 & 0 & 0 & 1 & 17.5 \\
\hline 21 & Tobacco Manufactures ... & 23 & $w$ & w & 0 & w & 0 & 0 & 0 & 0 & $w$ & 8.2 \\
\hline 22 & Textlle Mill Products ..... & 629 & 139 & 452 & 12 & 110 & 38 & 4 & * & $w$ & 12 & 21.8 \\
\hline 23 & Apparef and Other Textile Products & 158 & 43 & 81 & Q & $\mathbf{Q}$ & Q & Q & $\mathrm{Q}$ & 0 & $Q$ & 44.1 \\
\hline 24 & Lumber and Wood Products ..... & 1,000 & 170 & 759 & $\mathrm{Q}$ & 44 & 88 & $\vec{a}$ & 0 & 0 & 41 & 28.3 \\
\hline 25 & Furniture and Fixtures.$\ldots \ldots \ldots$ & 255 & 72 & 150 & $\vec{Q}$ & 12 & Q & $\vec{Q}$ & 0 & 0 & a & 29.2 \\
\hline 26 & Paper and Alled Products . & $w$ & 307 & $w$ & 64 & 129 & 95 & 14 & 0 & 0 & 27 & 15.6 \\
\hline 2611 & Pulp MIIls $\ldots \ldots \ldots \ldots \ldots \ldots$ & 141 & 74 & 63 & 0 & 74 & 2 & 2 & 0 & 0 & 0 & 34.6 \\
\hline 2621 & Paper Mills $\ldots \ldots \ldots \ldots \ldots \ldots$ & 613 & 104 & 491 & $w$ & 11 & $w$ & 0 & 0 & 0 & w & 6.6 \\
\hline 2631 & Paperboard Mills ....... & 93 & 11 & 72 & 0 & 7 & 0 & 0 & 0 & 0 & 3 & 12.8 \\
\hline 27 & Printling and Publlshing . ........ & 179 & 18 & 137 & 4 & 14 & 9 & * & 0 & 0 & a & 25.0 \\
\hline 28 & Chemlcals and Allied Products .... & 1,263 & 971 & 235 & 5 & 931 & 12 & 3 & $w$ & 0 & 10 & 11.5 \\
\hline 2812 & Alkalles and Chlorine ........ & 2 & 0 & 1 & 0 & 0 & 0 & 0 & 0 & 0 & 0 & 28.6 \\
\hline 2813 & Industrial Gases ......... & $\bar{Q}$ & 0 & $a$ & 0 & 0 & 0 & 0 & 0 & 0 & 0 & 0.0 \\
\hline 2819 & Industrial Inorganic Chomicals, nec ..... & 75 & 45 & 27 & $\mathrm{Q}$ & 42 & 5 & • & 0 & 0 & Q & 19.6 \\
\hline 2821 & Plastics Materials and Resins ..... & 54 & 17 & 35 & 1 & 15 & 1 & 1 & 0 & 0 & 1 & 9.3 \\
\hline 2822 & Synthetic Rubber ............ & 10 & $\because$ & 7 & 0 & 0 & 0 & 0 & 0 & 0 & : & 16.9 \\
\hline 2823 & Cellulosic Manmado Fibors.... & 1 & - & $i$ & 0 & 0 & : & 0 & 0 & 0 & 0 & 37.4 \\
\hline 2824 & Organic Fibers, Noncellulosic .... & 38 & 4 & 34 & 0 & w & * & w & 0 & 0 & 0 & 6.6 \\
\hline 2865 & Cycllc Crudes and Intermediates... & 79 & $w$ & $w$ & • & $w$ & - & 0 & 0 & 0 & 0 & 20.2 \\
\hline 2869 & Industrial Organic Chemicals, nec... & 825 & 789 & 23 & w & 788 & * & * & w & 0 & w & 11.6 \\
\hline 2873 & Nilrogenous Fortilizers . . . . . . . . . . & 43 & 42 & 1 & 0 & 42 & 0 & 0 & 0 & 0 & 0 & 60.4 \\
\hline 2874 & Phosphatli Fentilzers ........... & 1 & * & $i$ & . & 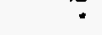 & 0 & 0 & 0 & 0 & 0 & 4.0 \\
\hline 29 & Petroleum and Coal Products & 16,528 & 10,119 & 6,139 & 272 & 9,260 & 1,712 & 1,929 & 0 & 0 & w & 4.7 \\
\hline 2911 & Petroleum Refining ........ & 15,889 & 9,790 & 6,099 & 272 & 9,260 & 1,383 & 1,912 & 0 & 0 & $w$ & 3.7 \\
\hline 30 & Rubber and Misc. Plastics Products . & 786 & 63 & 678 & 22 & 14 & 26 & Q & $\mathrm{Q}$ & Q & 15 & 29.7 \\
\hline 3011 & Tres and Inner Tubes .............. & 79 & 10 & 68 & 1 & $\because$ & . & 0 & 0 & 0 & 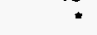 & 7.0 \\
\hline 308 & MIscellanoous Plastic Products, noc .... & 396 & 40 & 320 & 19 & 11 & 14 & $Q$ & $\mathbf{a}$ & $a$ & 9 & 29.7 \\
\hline 31 & Leather and Leather Products ..... & 44 & 2 & 35 & • & 1 & * & • & 0 & 0 & - & 38.0 \\
\hline 32 & Stone, Clay and Glass Products . & 577 & 130 & 384 & 15 & 90 & 62 & w & 0 & 0 & 9 & 15.8 \\
\hline 3211 & Flat Glass $\ldots \ldots \ldots \ldots \ldots \ldots \ldots$ & 40 & w & w & 1 & w & W & 0 & 0 & 0 & • & 7.0 \\
\hline 3221 & Glass Containars .......... & 82 & 23 & 54 & 1 & 20 & w & • & 0 & 0 & 1 & 11.5 \\
\hline 3229 & Pressed and Blown Glass, nec. & 31 & 6 & 22 & : & 6 & 0 & $w$ & 0 & 0 & 0 & 12.6 \\
\hline 3241 & Cement, Hydraulic .......... & 12 & 5 & 7 & 1 & 4 & - & 0 & 0 & 0 & 1 & 19.7 \\
\hline 3274 & Lime $\ldots \ldots \ldots \ldots \ldots \ldots$ & $a$ & : & $a$ & : & 0 & - & 0 & 0 & 0 & 0 & 21.4 \\
\hline 3296 & Mineral Wool .......... & 41 & 14 & 27 & w & 12 & 5 & 0 & 0 & 0 & • & 1.8 \\
\hline 33 & Primary Metal Industries & 888 & 299 & 519 & 34 & 209 & 47 & 21 & : & 0 & 21 & 22.0 \\
\hline 3312 & Blast Fumaces and Steol Mills ... & 74 & 13 & 43 & $\because$ & 11 & 1 & $w$ & 0 & 0 & 1 & 8.0 \\
\hline 3313 & Eloctrometalurgical Products... & w & 1 & $w$ & 0 & 1 & 0 & 0 & 0 & 0 & 0 & 18.5 \\
\hline 3321 & Gray and Ductlle Iron Foundries & 105 & 9 & 86 & 0 & 5 & 0 & 0 & - & 0 & 2 & 30.7 \\
\hline 3331 & Primary Copper ............ & 3 & 1 & 1 & 0 & 1 & 0 & 0 & 0 & 0 & 0 & 1.4 \\
\hline 3334 & Primary Aluminum & 42 & 10 & 29 & 0 & 10 & w & 0 & 0 & 0 & 0 & 4.7 \\
\hline 3339 & Primary Nonferrous Metals, nec .. & 19 & 1 & 16 & 0 & • & 1 & 0 & 0 & 0 & 0 & 2.0 \\
\hline 3353 & Aluminum Sheot, Plate, and Foll ... & 62 & 21 & 41 & 0 & w & $w$ & 0 & 0 & 0 & . & 1.2 \\
\hline 34 & Fabricated Melal Products $\ldots \ldots \ldots \ldots \ldots$ & 1,122 & 213 & 725 & 31 & 123 & $\mathrm{Q}$ & 3 & * & w & $Q$ & 25.5 \\
\hline 35 & Industrial Machinery and Equipment . & 651 & 127 & 431 & 22 & 72 & 8 & 2 & 0 & 0 & 17 & 31.3 \\
\hline 357 & Computer and Office Equipment .... & 4 & $Q$ & 1 & $\mathbf{Q}$ & $\mathbf{Q}$ & . & 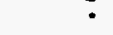 & 0 & 0 & $\because$ & 32.5 \\
\hline 36 & Electronic and Other Electric Equipment .. & 396 & 78 & 262 & 15 & 56 & 8 & 0 & 0 & 0 & 4 & 22.9 \\
\hline 37 & Transportation Equipment $\ldots \ldots \ldots \ldots \ldots$ & 526 & 110 & 375 & 31 & 76 & 33 & 4 & $\mathrm{Q}$ & 0 & 7 & 16.3 \\
\hline 3711 & Motor Vehicles and Car Bodies ......... & 59 & 23 & 30 & 1 & 21 & 0 & $\mathbf{Q}$ & 0 & 0 & - & 6.5 \\
\hline 3714 & Motor Vehicle Pants and Accessories ... & 168 & 57 & 102 & 25 & 34 & 28 & 0 & a & 0 & 4 & 17.7 \\
\hline 38 & Instruments and Related Products ...... & Q & $w$ & 0 & $\because$ & 2 & 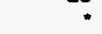 & 0 & * & 0 & : & 24.9 \\
\hline 3841 & Surgical and Medical Instruments & 8 & 1 & 6 & - & 1 & 0 & 0 & 0 & 0 & 0 & 31.2 \\
\hline 39 & Misc. Manufacturing Industries ... & w & 10 & $w$ & - & & & 0 & 0 & 0 & & 30.1 \\
\hline & Total .. & 27,970 & 13,281 & 13,158 & 637 & 11,405 & 2,454 & 2,135 & 30 & 6 & 1,539 & 6.2 \\
\hline
\end{tabular}

See footnoles at end of table. 
Table A58. Capability to Switch from LPG to Alternative Energy Sources by Industry Group, Selected Industries, and Selected Characteristics, 1991 (Continued) (Estimates in Thousand Barrels)

\begin{tabular}{|c|c|c|c|c|c|c|c|c|c|c|c|}
\hline \multirow[b]{2}{*}{ Selected Characteristics } & \multicolumn{3}{|c|}{ LPG } & \multicolumn{7}{|c|}{ Alternative Types of Energy } & \multirow[b]{2}{*}{$\begin{array}{l}\text { RES } \\
\text { Row } \\
\text { Factors }\end{array}$} \\
\hline & $\begin{array}{c}\text { Total } \\
\text { Consumed }^{c}\end{array}$ & Switchable & $\begin{array}{c}\text { Not } \\
\text { Switchable }\end{array}$ & $\begin{array}{l}\text { Electricity } \\
\text { Receipts }\end{array}$ & $\begin{array}{c}\text { Natural } \\
\text { Gas } \\
\end{array}$ & $\begin{array}{l}\text { Distillate } \\
\text { Fuel Oil }\end{array}$ & $\begin{array}{r}\text { Residual } \\
\text { Fuel Oil }\end{array}$ & Coal & $\begin{array}{c}\text { Coal } \\
\text { Coke and } \\
\text { Breeze }\end{array}$ & Other & \\
\hline RSE Column Factors: & 0.5 & 0.6 & 0.6 & 1.1 & 0.6 & 1.1 & 0.7 & 2.6 & 3.5 & 1.4 & \\
\hline \multicolumn{12}{|l|}{ Census Aegion } \\
\hline Northeast $\ldots \ldots \ldots \ldots \ldots \ldots \ldots$ & $w$ & 1,183 & 1,872 & 78 & 483 & 778 & 356 & - & 0 & 32 & 18.8 \\
\hline Midwest $\ldots \ldots \ldots \ldots \ldots \ldots \ldots$ & 3,877 & 1,603 & 2,041 & 270 & 1,198 & 660 & 773 & Q & $w$ & 102 & 9.4 \\
\hline South ... & w & 3,312 & 3,263 & 139 & 2,908 & 426 & 212 & 27 & $w$ & 1,345 & 9.6 \\
\hline$\ldots \ldots$ & 13,345 & 7,183 & 5,982 & 149 & 6,816 & 590 & 795 & $Q$ & $Q$ & 60 & 6.5 \\
\hline Total $\ldots \ldots \ldots \ldots \ldots \ldots \ldots$ & 27,970 & 13,281 & 13,158 & 637 & 11,405 & 2,454 & 2,135 & 30 & 6 & 1,539 & 6.2 \\
\hline \multicolumn{12}{|l|}{$\begin{array}{l}\text { Value of Shipments and Receipts' } \\
\text { (million dollars) }\end{array}$} \\
\hline Under $20 \ldots \ldots \ldots \ldots \ldots \ldots$ & 4,090 & 1,097 & 2,140 & 241 & 421 & 629 & 142 & Q & 0 & 88 & 22.9 \\
\hline$\ldots \ldots \ldots \ldots \ldots$ & 2,708 & 584 & 1,861 & 104 & 293 & 179 & $w$ & $\vec{Q}$ & $Q$ & 90 & 22.4 \\
\hline $50-99 \ldots \ldots \ldots \ldots \ldots \ldots \ldots \ldots$ & 1,894 & 613 & 1,121 & 30 & 497 & 249 & $w$ & $\cdot$ & 0 & $w$ & 9.9 \\
\hline $100-249 \ldots \ldots \ldots \ldots \ldots \ldots \ldots$ & 2,342 & 946 & 1,241 & 52 & 762 & 149 & 2 & • & $w$ & 30 & 12.4 \\
\hline $250-499 \ldots \ldots \ldots \ldots \ldots \ldots \ldots$ & 2,063 & 1,004 & 1.016 & 103 & 814 & 341 & 671 & $\cdot$ & $\cdot$ & $w$ & 7.9 \\
\hline 500 and $0 v e r . \ldots \ldots \ldots \ldots \ldots . . . \ldots$ & 14,872 & 9,037 & 5,779 & 107 & 8,618 & 907 & 1,100 & w & $*$ & $w$ & 5.3 \\
\hline Total $\ldots \ldots \ldots \ldots \ldots \ldots \ldots$ & 27,970 & 13,281 & 13.158 & 637 & 11,405 & 2,454 & 2,135 & 30 & 6 & 1,539 & 6.2 \\
\hline \multicolumn{12}{|l|}{ Employment Size ' } \\
\hline Under $50 \ldots \ldots \ldots \ldots \ldots \ldots$ & 1,748 & 671 & 639 & 177 & 202 & 475 & 110 & $\mathbf{Q}$ & 0 & 47 & 29.0 \\
\hline $50-99 \ldots \ldots \ldots$ & 1,657 & 543 & 903 & 50 & 349 & 267 & 205 & 0 & 0 & 35 & 20.7 \\
\hline $100-249 \ldots \ldots \ldots \ldots \ldots \ldots \ldots$ & 7,290 & 2,142 & 4,734 & 129 & 1,764 & 276 & 332 & $\mathbf{Q}$ & 0 & $w$ & 9.5 \\
\hline $250-499 \ldots \ldots \ldots \ldots \ldots \ldots \ldots$ & 4,211 & 1,839 & 2,184 & 202 & 1,472 & 823 & 821 & $Q$ & $Q$ & $W$ & 6.8 \\
\hline $500-999 \ldots \ldots \ldots \ldots \ldots \ldots$ & 6,860 & 3,747 & 2,950 & 42 & 3,344 & 590 & $w$ & 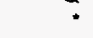 & $w$ & 102 & 9.0 \\
\hline 1,000 and Over $\ldots \ldots \ldots \ldots \ldots$ & 6,205 & 4,339 & 1,749 & 37 & 4,275 & 22 & $w$ & $w$ & W & $w$ & 7.5 \\
\hline Total $\ldots \ldots \ldots \ldots \ldots \ldots$ & 27,970 & 13,281 & 13,158 & 637 & 11,405 & 2,454 & 2,135 & 30 & 6 & 1,539 & 6.2 \\
\hline
\end{tabular}

\footnotetext{
See Appendices B and F for descriptions of the Standard Industrial Classification system.

- Altemative Types of Energy" consist of those energy sources that could have been substituted for LPG during 1991. The quantities are expressed in thousands of barrels, and therefore represent the quantity of LPG that could have been displaced by the given alternative type of energy.

"Total Consumed" represents those quantities of LPG that were ascertained switchable or not switchable, plus an additional quantity for which the switching status was not ascertained.

"Electricity Receipts" represents those quantities of electricity generated off the manufacturing establishment site and available at the site for consumption. It includes those quantities for which payment was made, quantities transferred in, quantities purchased and paid for by a central purchasing entity, and quantities for which payment was made in kind. It does not include electricity generated onsite. "Electricity Receipts" has not been adjusted to account for any quantities that might have been resold or transferred out. The estimates include those quantities that were ascertained switchable or not switchable, plus an additional quantity for which the switching status was not ascertained.

- "Other" includes all other types of energy not already identified that respondents indicated could have been consumed in place of LPG.

' Value of Shipments and Receipts and Employment Size categories were supplied by the Bureau of the Census. See Appendix B.

- Estimate less than 0.5. Data are included in higher level totals.

$W=$ Withheld to avoid disclosing data for individual establishments. Data are included in higher level totals.

$Q=$ Withheld because Relative Standard Error is greater than 50 percent. Data are included in higher level totals.

Notes: - To obtain a RSE percentage for any table cell, multiply the cell's corresponding RSE column and RSE row factors. - Totals may not equal sum of components because of independent rounding.

Source: Energy Information Administration, Office of Energy Markets and End Use, Energy End Use Division, Form ElA-846, "1991 Manufacturing Energy Consumption Survey."
} 


\section{Appendix B}

\section{Survey Design, Implementation, and Estimates}


. 


\section{Appendix B Survey Design, Implementation, and Estimates}

\section{Introduction}

The 1991 Manufacturing Energy Consumption Survey (MECS) has been designed by the Energy Information Administration (EIA) to provide information related to energy consumption in the manufacturing sector of the U.S economy. It is the third such survey to be completed. The MECS is an ongoing survey that is conducted every 3 years. Beginning in 1994, the MECS will be conducted every 2 years to determine the trends in manufacturing energy use more accurately.

The basic unit of data collection for this survey is the manufacturing establishment. A nationally representative sample of these establishments supplied the information through mailed questionnaires. The Industry Division of the Bureau of the Census, in consultation with EIA, selected the MECS sample, conducted fieldwork, and processed the data.

This appendix presents a summary of the design and implementation procedures for the survey, highlights differences between reporting periods, and describes the types of estimates included in this report. For more detailed design, methodology, and background information refer to the EIA publication Manufacturing Energy Consumption Survey: Methodological Report, DOE/EIA-0514 (Washington, DC, 1988).

\section{Description of the Manufacturing Sector}

The manufacturing sector consists of all manufacturing establishments in the 50 States and the District of Columbia. The working definition of a manufacturing establishment is the definition stated in the Office of Management and Budget's Standard Industrial Classification (SIC) Manual:

[Manufacturing establishments are] ... engaged in the mechanical or chemical transformation of materials or substances into new products. These establishments are usually described as plants, factories, or mills and characteristically use power driven machines and materials handling equipment. Establishments engaged in assembling component parts of manufactured products are also considered manufacturing if the new product is neither a structure nor other fixed improvement. Also included is the blending of materials such as lubricating oil, plastics, resins, or liquors. ${ }^{20}$

\section{Overview of Changes from the 1988 Survey}

Sample Design. The designed sample size was increased from approximately 12,000 establishments to 16,000 . Sample cases were not automatically retained from the previous survey cycle; the sample was selected directly from the 1991 Annual Survey of Manufactures (ASM) file.

Coverage. New frame updating procedures instituted by the Bureau of the Census allowed for consistent coverage of the manufacturing sector mail surveys. For that reason, the coverage in the 1991 MECS is 98 percent of the manufacturing population as measured in total payroll. The sampling process itself provided that level of coverage, and no special adjustments were used to increase it. The 1988 survey covered 100 percent of the population but special adjustments to the survey estimates were required to achieve complete coverage. Therefore, many of the potential sources of bias that pertained to the 1988 survey estimates are not relevant to the 1991 survey. Because of the difference in coverage between the two years, care must be exercised when comparing absolute quantities. Small differences might be correctly attributable to coverage rather than real change.

Industry Estimates. The increase in sample size allowed for separate estimates for 42 industries and industry groups in addition to the 20 larger major groups. In 1988, there were only 10 industries for which separate estimates were publishable beyond the 20 major groups.

(Continued on Next Page) 
The SIC Manual contains a hierarchial classification system that groups establishments according to their primary economic activities. This system divides the manufacturing sector (referred to as "manufacturing division" in the SIC Manual) into 20 major industrial groups that are relatively homogeneous with respect to primary output. Each of these major industrial groups is assigned a two-digit code. The two-digit codes for the manufacturing sector range from SIC 20, Food and Kindred Products, through SIC 39, Miscellaneous Manufacturing Industries. Each major group is subdivided into three-digit groups, which are further divided into four-digit industries. For example, SIC 20 includes SIC 201, Meat Products, which, in turn, is subdivided into SIC 2011, Meat Packing Plants; SIC 2012, Sausages and Other Prepared Meat Products; SIC 2016, Poultry Dressing Plants; and SIC 2017, Poultry and Egg Processing.

The SIC category is the single most important classification variable in the MECS data system, both for selecting the MECS sample and analyzing the MECS data. The categories of primary interest for the MECS are the 20 major industrial groups (SIC 20 through 39) and the 42 three- and four-digit industries that consumed the most energy, demonstrated high growth, or had a special programmatic interest. A description of these 20 major industrial groups and 42 industry groups and industries appears in Appendix $\mathrm{G}$ of this report.

The 1988 and 1991 MECS used the SIC classification system presented in the 1987 edition of SIC manual. The 1985 MECS was based on the 1972 SIC Manual. For the most part, the revisions were minor and had a negligible effect on the MECS estimates. However, there were some revisions that would affect comparisons between 1985 and subsequent years. Among the more significant revisions was the one concerning the way certain petrochemical plants were classified for the 1988 and $1991 \mathrm{MECS}$, as opposed to the 1985 MECS. If the primary product of a petrochemical plant in 1985 was an LPG, it was classified in SIC 2911, Petroleum Refining, regardless of how the LPG was produced. For the later survey years, the establishment was classified in SIC 2911 only if the LPG was produced by a refinery process. If the LPG was produced by a chemical process, the establishment was classified in organic chemicals (SIC 2865 or 2869). Thus, when comparing the estimates for SIC's 2911, 2865, and 2869 between 1985 and later MECS reports, the reader is cautioned to take the classification differences into account. ${ }^{21}$

\footnotetext{
${ }^{21}$ An effort was made to account for the SIC revisions by reclassifying the 1988 MECS estimates according to the 1972 SIC codes. The revised consumption estimates were used to form energy efficiency change estimates. See Energy Information Administration, Manufacturing Energy Consumption Survey: Changes in Energy Intensity in the Manufacturing Sector, 1980-1988, DOE/EIA-0552(80-88) (Washington, DC, 1991). For future publications of this kind, the 1985 MECS estimates will be reclassified according to the 1987 SIC codes.
}

Additional Data Items for Improved Estimation. EIA has recognized a potential for overestimating energy source quantities in industries that produce and sell energy sources. This will occur when an establishment in such an industry uses an energy source as an input to a process (i.e, feedstock), produces another energy source as a result of that process, and then sells or transfers the produced energy source to another establishment. The quantity of the receipt in the second establishment would duplicate the feedstock use in the first. Part of the solution has been to estimate consumption in petroleum refineries differently from other industries (see section entitled "Feedstocks and Offsite-Produced Fuel at Petroleum Refineries" in this appendix). To correct the problem for other industries, the MECS now collects shipments offsite of energy sources produced onsite (see "Development of the Data File" in this appendix).

A second problem arose in estimating consumption specifically for liquefied petroleum gases (LPG) and petroleum coke. There are different types of those energy sources and one type may be used in the production of another. The collection of just the broad categories hindered the proper identification and estimation of the internal consumption-production flow. To address this problem, the MECS now collects three categories of petroleum coke and four categories of LPG.

New Data Collection Sections. The survey forms were expanded to include sections on end-use energy consumption, estimated square footage of building floorspace, energy management activities (including utility/supplier demand-side management), and energysaving technologies.

Discontinued Data Items. Two data items concerning onsite electricity generation were discontinued. These were interconnection status with an electric utility and whether the establishment was a Qualifying Facility under the Public Utility Regulatory Policies Act of 1978. These items were no longer required because they are collected as part of a new survey, Form ElA-867, "Annual Nonutility Power Producer Report." Within the fuelswitching section, the data items concerning time required to make a switch were discontinued. The MECS estimates of fuel-switching capability are limited to a 30-day window for eligibility. The additional respondent burden necessary to further categorize this time was not justified by user interest. 


\section{The Sampling Frame and Its Relationship to the Manufacturing Sector}

As mentioned in the Introduction to this appendix, the Census Bureau serves as the collecting and compiling agent for the MECS. A major responsibility of the Industry Division of the Census Bureau is to conduct the Census of Manufactures (CM) and the Annual Survey of Manufactures (ASM).

\section{Census of Manufactures}

The CM is conducted for years ending in "2" or "7" (for example, 1987), and obtains economic data for the complete universe of approximately 350,000 manufacturing establishments in the United States. For the purposes of data collection, the CM universe is divided into two major subsets as follows.

1. Small Single-Establishment Companies Not Sent a Report Form. Generally, companies with less than five employees are excused from filing a CM report. Those with 5 through 20 employees are excused or sent a report form based on the magnitude of their annual payroll and shipments data. Approximately 125,000 establishments are excused due to this criterion.

2. Establishments Sent a Report Form. All companies with 20 or more employees are mailed a CM report form.

\section{Annual Survey of Manufactures}

The ASM is conducted during non-CM years to provide estimates of economic characteristics for the universe of manufacturing establishments. As with the CM, the ASM contains two components. The first component is the mail portion, a probability sample of manufacturing establishments selected from the list of establishments that are sent the CM report form (see above). Those establishments are weighted so that they represent the mail portion of the $\mathrm{CM}$ universe. The second component of the ASM is the nonmail portion of the CM. These small establishments are not sent an ASM questionnaire, but their contribution to economic statistics is estimated based on selected information obtained annually from other Federal agencies.

The 1991 MECS sample was drawn directly from the 1987 CM with appropriate updates. That approach made the coverage equivalent to the 1991 ASM mail file. ${ }^{22}$

\section{Coverage Differences Between MECS 1985, 1988, and 1991}

Due to differences among the 1985, 1988, and 199I survey designs, the coverage of manufacturing establishments varied slightly from survey to survey. Therefore, comparisons of estimates produced from the surveys and analysis of trends must be done with caution.

The 1991 MECS covers 98 percent of the manufacturing sector as measured by total payroll. The two percent of the payroll not covered is known to be represented by a population of relatively small but numerous manufacturing establishments. When taken as a whole, they account for roughly two percent of a number of different economic measures, including energy consumption. The cost and difficulty of accurately surveying this population necessitated the decision to exclude it from the estimates presented in this report.

\footnotetext{
${ }^{22}$ Establishments that were first eligible for the MECS in calendar year 1991 were not mailed a questionnaire. Rather, their contribution was accounted for in the nonresponse adjustment. See section entitled "Estimation Process" in this appendix.
} 
The 1988 MECS estimates covered the entire manufacturing sector. The small establishments excluded from the 1991 survey were "included" by means of a population adjustment factor. That adjustment factor was necessitated because of normal degradation of the sample coupled with the desire to retain the active portion of the 1985 sample, rather than entirely reselect the sample. This method meant that establishments had to be classified according to very specific definitions. For example, establishments that ceased operation since 1985 had to be distinguished from those that merely underwent a change of ownership. To counteract the operational errors that might have occurred while maintaining a sample built in this way, a ratio adjustment to the $1987 \mathrm{CM}$ was chosen. That adjustment by necessity also included the portion of the manufacturing sector that was not originally intended for estimation, the two percent of certain economic measures represented by the smallest establishments. ${ }^{23}$ The 1991 survey did not use an adjustment factor because: (1) there was no readily available population adjustment for 1991 estimates, and (2) the relative simplicity of the sample design yielded fewer operational errors.

The 1985 estimates excluded the smallest establishments from coverage. This would tend to make 1985 coverage comparable with 1991. Due to Census updating procedures that were in place during the 1985 survey, the coverage might have been somewhat less than the 98 percent of the 1991 survey, but exact estimates of coverage are difficult to estimate. Therefore, caution must be exercised by readers who wish to compare 1985, 1988, and 1991 estimates. $^{24}$

\section{Sample Design}

\section{Sample Size}

The designed size of the MECS sample was set at 16,000. Previous data have shown that this size sample optimally allocated to the various strata would lead to estimates having desired sampling error. The targeted sampling errors in terms of relative standard errors (RSE) for previous MECS samples were:

- No more than two percent for total energy consumption in energy-intensive SIC major groups or specific industries;

- No more than five percent for total energy consumption in the other SIC groups; and

- No more than ten percent for the four major fuel categories (electricity, natural gas, coal, and the aggregate of all remaining energy sources) in any SIC that contributes more than one percent of the total national consumption of that fuel.

The 1991 MECS sample was designed using the experience of the 1985 and 1988 sample designs and estimated sampling errors for those surveys. That experience and knowledge of the current ASM populations allowed the sample sizes to be allocated so that the RSE targets could be met for $1991 .{ }^{25}$

\footnotetext{
${ }^{23}$ For a more detailed explanation of the population adjustment factor, see Appendix A, Energy Information Administration, Manufacturing Energy Consumption Survey: Consumption of Energy, 1988, DOE/EIA-0512(88), op. cit.

${ }^{24}$ Comparisons among the survey years can be more appropriately made using internal ratios (e.g., quantity of offsite-produced energy per value of shipments) because the coverage differences will appear affect both energy consumption and value of shipments equivalently in a given year. Indeed, for that reason the adjustment factor in the 1988 survey was not a factor in comparing energy intensity change estimates. See Energy Information Administration, Manufacturing Energy Consumption Survey: Changes in Energy Intensity in the Manufacturing Sector, 19801988, DOE/EIA-0552 (80-88).

${ }^{25}$ The RSE targets were not input directly into formulas and algorithms usually used in allocating sample cases according to probabilityproportional-to-size sampling. Sample was allocated according to what was expected to achieve the specified targets. If tests on achieved RSE's of correlated ASM measures were not obtained, samples would have been re-allocated. This proved unnecessary.
} 
The major purpose of increasing the sample size was to be able to produce separate energy estimates for more industries than before. The previous two surveys published estimates for the 20 major groups that comprise manufacturing and 10 four-digit industries. Those 10 four-digit industries were the most energy-consuming in manufacturing. The $1991 \mathrm{MECS}$ has 40 four-digit industries, 2 three-digit industry groups, and 20 major groups. The industry additions come from three groups: (1) industries not in the top 10, but with high energy consumption; (2) certain high-growth industries such as computers and medical instruments; and (3) industries for which there are identifiable policy interests or conservation opportunities.

The increase in sample size also allowed for greater reliability of existing estimates, especially among the major groups (two-digit SIC's). For example, SIC 20 in the current sample design includes eight four-digit industries for which estimates were publishable. Previously, in the 1985 and 1988 designs, no four-digit industries in SIC 20 were published separately. The addition of the eight industries in 1991 yielded greater reliability of the SIC 20 major group estimates by having more sample cases in the publishable four-digit industries that comprise SIC 20. Thus, overall reliability in SIC 20 was improved without adding sample cases for that express purpose.

\section{Sampling Methodology}

The selection of the MECS sample was a two-stage selection process, with the first stage being the selection of the ASM mail sample from the CM frame. The second stage was the subselection of the MECS sample from the ASM mail sample. Thus, a MECS sample establishment is selected conditionally upon it having been selected into the ASM mail sample, which means that its probability of selection from the ASM sample is conditional. Therefore, the overall probability of selection into the MECS sample is represented by the product of this conditional probability and its ASM selection probability.

The probabilities for selection into the MECS sample are proportional to an energy measure of size (MOS). Calculations of the MOS are different from previous years, although closely related. Actually, two MOS's were computed for each establishment. The first was based on the 1990 ASM purchased electricity quantity. The other was based on the ASM cost of purchased fuels (excluding electricity). The probability of selection would be the maximum of the two probabilities computed using the two different MOS's.

The energy MOS for the 1985 and 1988 surveys was formed by taking a previous total Btu measure per cost of fuels and electric energy at the establishment updated by multiplying that ratio by a more current measure of average cost of fuels and electric energy. If the establishment had come into existence since the time of the last energy data available, industry averages would be employed for the ratio of Btu to cost of fuels. Neither MOS in the 1991 MECS is as highly correlated with energy consumption as the MOS used in the 1985 and 1988 sample designs. However, one advantage of the 1991 method is the fact that ASM data are used directly. Thus, each establishment has its own MOS and industry averages are no longer needed. ${ }^{26}$

The MECS sample for the 42 separately published three- and four-digit industries included 100 percent of the corresponding ASM mail sample, with four exceptions. This was done to maximize reliability (i.e, minimize the standard errors) for those important energy-intensive and growth industries. However, four of those industries had such a large ASM sample it was impractical to include all of the establishments. Those industries were SIC 2051, "Bread, Cake, and Related Products"; SIC 2813, "Industrial Gases"; SIC 308, "Miscellaneous Plastic Products, not otherwise classified"; and SIC 3714, "Motor Vehicles Parts and Accessories." The number of cases included in the MECS sample was large enough to ensure sampling errors of less than 5 percent as measured on certain ASM variables. $^{27}$

\footnotetext{
${ }^{26} \mathrm{By}$ using two different probabilities of selection, the expected sample size would be larger than would be expected by using only one. The sample allocations for each stratum were adjusted as necessary to stay close to the EIA targeted sample sizes.

${ }^{27}$ The MECS sample design was tested using 1990 ASM data. After the sample was selected, relative standard errors (RSE's) were computed with the MECS sample using energy related measures such as cost of fuels and purchased electricity quantity.
} 
The remaining establishments were sampled from the 20 two-digit groups in a pattern designed to keep sampling errors within pre-established bounds for estimates of total consumption and consumption of four major types of energy: electricity, natural gas, coal, and the aggregate of all other types. The procedure for subselecting ASM sample establishments into the MECS sample were such that their overall probabilities of selection for the MECS were proportional to an estimated energy MOS. The overall probabilities for selection of the MECS sample establishments ranged from 0.002 to 1.000 .

The total sample size actually selected was 16,054 . Of these 305 establishments were determined to be out-of-scope or no longer in business prior to the MECS mailing, leaving a mail sample of 15,749 . At the final closing, 14,299 questionnaires were received, a response rate of 91 percent.

\section{Fieldwork, Editing, and Quality Control}

The 1991 MECS continued the method that was started with the 1988 survey of using customized questionnaires for specific industries. The three questionnaires were:

- Form EIA-846(A).-This questionnaire was sent to the majority of the sample and collected the basic consumption, expenditure, fuel-switching, end-use, and technology information.

- Form EIA-846(B).--This questionnaire was sent exclusively to establishments in the Petroleum Refining Industry (SIC 2911). The design of the questionnaire took advantage of the fact that other EIA surveys collect certain consumption and expenditure data from the refinery population. Thus, the EIA-846(B) did not require respondents to report on particular data items.

- Form EIA-846(C).- This questionnaire was sent to producers of Chemicals and Allied Products (SIC 28), producers of Petroleum and Coal Products other than Petroleum Refining (SIC 29 excluding SIC 2911), Lumber and Wood Products (SIC 24), Paper and Allied Products (SIC 26), and selected Primary Metals Industries (in SIC 33). It is similar to the EIA-846(A) except that it collects additional information on shipments of energy sources produced onsite and a different set of specific technologies related to energy efficiency.

The questionnaires were mailed to the in-scope MECS sample establishments in two groups. The first group consisted of those companies that only have establishments receiving the EIA-846(A). They were mailed the questionnaires on February 24, 1992. All other companies were mailed the questionnaires on March 17, 1992. ${ }^{28}$ Returned questionnaires were subjected to initial screening procedures for completeness, and incomplete forms or responses with obvious inconsistencies were set aside for review by industry specialists. Valid returned questionnaires were forwarded directly to check-in and then to data entry.

All forms that were incomplete or failed the initial screening procedures were carefully reviewed by the industry specialists from the Census Bureau and EIA. These specialists retrieved missing data and verified questionable items by telephone contact with the individual who completed the questionnaire. Once the forms were completed and verified, they were forwarded to check-in and to data entry.

The resulting MECS data file was then subjected to a series of computer edits. Those edits included consistency checks among data items from different parts of the MECS and between the MECS and the 1991 ASM, as well as checks for outliers in the distribution of individual variables. Records with failed edits were reviewed and followed up by industry specialists.

\footnotetext{
${ }^{28}$ The MECS sample is selected according to establishment characteristics. However, the central administrative offices of multi-establishment companies were the addressees of the questionnaires and were responsible for distributing them to their establishments.
} 


\section{Development of the Data File}

The estimates in this report were developed from a data file consisting of both directly reported values and more complex items derived from a combination of directly reported values. Reported values consist of responses to the 1991 MECS questionnaires (Appendix F). Those values were supplemented by estimates of energy consumption for nonfuel purposes and offsite-produced fuel consumption at petroleum refineries from another EIA questionnaire. ${ }^{29}$ Additionally, the responses to the questionnaire for each responding establishment were supplemented by the following economic data:

- Value of shipments and receipts

- Value added by manufacturing

- Total employment.

These economic data were not collected by the 1991 MECS but were provided by the Census Bureau by linking the 1991 ASM economic data and MECS energy data at the establishment level.

The reported energy values were used to construct several derived values, which, in turn, were used to prepare the estimates appearing in selected tables in this report (See "Survey Estimates" section in this appendix.) These derived values are defined as follows:

1. Energy produced offsite and consumed as a fuel. This derived value represents onsite consumption of fuels that were originally produced offsite. That is, they arrived at the establishment as the result of a purchase, or were transferred to the establishment from outside sources. As such, this derived value is equivalent to "consumption of purchased" fuels as reported by the Census Bureau for the years 1974-1981. The Census Bureau defines "purchased" fuels to include those actually purchased plus those transferred in from other establishments. ${ }^{30}$

2. Energy produced offsite and consumed for nonfuel purposes. This derived value also represents energy that was originally produced offsite. This energy was used at the establishment site as raw material inputs and feedstocks.

3. Energy produced onsite from nonenergy inputs and consumed onsite as a fuel. This derived value covers materials such as wood chips, bark, and wood waste, and pulping liquor. These fuels are produced primarily in pulp and paper mills as a byproduct of wood used in the pulping process. Wood for pulping is not classified as energy in the MECS, and, therefore, would not have been included as an input. This derived value also covers waste materials, biomass, and hydrogen that was produced from the electrolysis of brine. Energy sources such as petroleum and coal that were consumed as fuel and originated onsite from captive mines or wells (an unusual occurrence) are included here also.

4. Energy produced onsite from nonenergy inputs and consumed for nonfuel purposes. Most onsite-produced energy that is used for nonfuel purposes is derived from other types of energy. The major exception is hydrogen that is produced from the electrolysis of brine. Hydrogen produced in this manner and used for nonfuel purposes is the major occurrence of this derived value. Energy sources such as petroleum and coal that were consumed as a nonfuel and originated onsite from captive mines or wells are included here also.

5. Energy produced onsite from energy inputs and consumed as a fuel. This derived value covers a wide range of fuels consumed onsite that are produced onsite as direct products or byproducts of other types of energy.

\footnotetext{
${ }^{29}$ The calculations for these quantities are discussed in the sections of this appendix titled, "Consumption for Nonfuel Purposes at Refineries" and "Offsite-Produced Fuel Use at Refineries."

${ }^{30}$ U.S. Department of Commerce, Bureau of the Census, Annual Survey (Census) of Manufactures, "Fuels and Electric Energy Consumed," 1974-1982 (Washington, DC).
} 
6. Energy produced onsite from energy inputs and consumed onsite for nonfuel purposes. This derived value includes all petrochemical feedstocks and other raw material inputs that were produced onsite from existing energy or from other onsite-produced energy.

7. Energy produced onsite from energy inputs and shipped to other establishments. This derived value is new to the 1991 MECS. Data are now collected for certain industries that produce and sell energy sources to other establishments. Most notably, these industries include Blast Furnaces and Steel Mills (SIC 3312) and various industries in Chemicals and Allied Products (SIC 28). If an establishment converts an energy source into a fuel and then ships it offsite to another establishment, the total Btu quantity among the producing and receiving establishments would be duplicative and thus overstated. By deducting this derived value from the producing establishments, the amount consumed at the receiving establishments would not be duplicative.

The first four of those derived values represent an addition to the energy consumed onsite, and are described in this publication as primary consumption (that is, either they were produced offsite or were produced onsite from nonenergy inputs). The fifth derived value described above does not represent an addition because it was produced onsite from energy that is already reported as input. Such energy represents duplicate counting of the input energy content. It is, however, a useful measure of onsite-produced fuel consumption and is not duplicative with respect to an estimate of total fuel consumption. The sixth derived value is duplicative with respect to the consumption of energy for nonfuel purposes, and, therefore, was not used to prepare estimates. It was included only for computational purposes and completeness. The seventh derived value appears as a special table in this appendix and is used to adjust primary consumption. The adjustment was excluded from the detailed statistical tables (e.g., Table A1) so that continuity would be maintained with previous estimates of primary consumption.

\section{Assumptions Underlying Derived Values}

Two basic assumptions are necessary to produce the derived values from the data reported on the MECS questionnaire. First, it is assumed that any energy produced onsite is disposed of as it is produced. That is, it is burned as a fuel and/or consumed as an input or feedstock; any excess is flared, dumped, transferred-out, sold, or is placed into inventory. For the purpose of computing the derived values, a quantity of an energy source produced onsite and placed into inventory during the previous year is not considered onsite production in the reporting year. A corollary of this assumption is that any energy source that was consumed onsite and originated offsite was acquired only if there was not sufficient onsite production to meet the establishment's needs of the energy source in the current year. Second, it is assumed that the priority use of onsite production is first as a shipment (if applicable), then as an input or feedstock, and last as a fuel. These assumptions are believed to reflect the energy use patterns at the vast majority of, but not all, establishments. The assumptions do provide a consistent method of determining an establishment's nonduplicative total energy consumption and its reliance on outside providers to supply it.

\section{The Estimation Process}

Estimates in this report represented 98 percent of the of manufacturing payroll and shipments in the CM universe. Coverage was equivalent to the 1991 ASM mail file. The two percent not covered are the smallest manufacturing establishments that were not sent an ASM form. ASM imputes those establishments' data for publications by using industry averages. As discussed previously, the MECS no longer covers the small establishments either directly or through a ratio adjustment.

Population representation is accomplished by veighting the data from the establishment records in the consumption data file. Weighting is the process of multiplying the reported or derived values by a case-specific constant designed to inflate the data from each sample case to that portion of the population that it represents. The first, basic component in the MECS weights is the sampling weight. The sampling weight for a MECS sample case is the reciprocal of its overall probability of selection into the ASM and subsequent selection for the MECS. 
The second component of the MECS weights is an adjustment for nonresponse. Adjustment factors to account for nonresponse were calculated by using the known energy measures of size of the respondents and the total sample. Because an establishment is selected into the MECS sample with a probability proportional to the establishment's energy measure of size, that measure can be viewed as an establishment's estimated contribution to energy consumption in 1991. A separate adjustment factor was computed for each of the 62 sampling strata ${ }^{31}$ and took the form:

$$
a_{s}=\frac{\sum_{j}^{\text {Sample }} \operatorname{MOS}_{s, j}}{\sum_{i}^{\text {Resp. }} \operatorname{MOS}_{s, i}},
$$

where $M O S_{s, j}$ is the measure of size for MECS sample establishment $j$ in stratum $s$, and $M O S_{s, i}$ is the measure of size for MECS respondent $i$ in stratum $s^{32}$ The adjustment factor was then multiplied by the sampling weight to produce the final MECS weight.

\section{Feedstocks and Offsite-Produced Fuel at Petroleum Refineries}

The basic function of a petroleum refinery (SIC 2911) is to manufacture a wide variety of petroleum products from crude oil and other liquid hydrocarbon inputs. Those products can be grouped into three classes of use. The largest portion of refinery output is in the form of fuels that are ultimately consumed strictly for their energy content (e.g., motor gasoline, kerosene, and diesel oil). Many refinery products, however, are consumed, not for their energy content, but for their chemical properties. This class of energy products is generally known as petrochemical feedstocks. Finally, a third class of products consists of finished materials that are consumed for specific physical properties, rather than for their energy content or chemical properties. Those finished materials include asphalt, lubricants, waxes, and solvents, and are referred to as nonenergy products. ${ }^{33}$

The MECS was specifically designed to collect information on the consumption of energy for heat, power, and electricity generation, and as petrochemical feedstocks and other raw material inputs. The consumption of energy was reported directly by the establishments in the MECS sample, and the estimates in this report reflect that consumption. For most industries, the end result of energy inputs is manufactured products that are not considered energy products. However, fuels and some petrochemical feedstocks produced from refinery inputs are treated as energy products by their subsequent users ${ }^{34}$, and are reported not only in other manufacturing industries, but also in EIA surveys of consumption in other end-use sectors (residential households, residential vehicles, and commercial buildings). In that sense, refineries do not "use up" the majority of their inputs. They merely convert them from one form of energy (for example, crude oil) to another more usable form (for example, motor gasoline). Therefore, classifying refinery inputs that go into fuels and certain petrochemical feedstocks as refinery consumption would have resulted in massive double counting of total energy consumption, both within the manufacturing sector, and across other energy-consuming sectors in the U.S. economy.

\footnotetext{
${ }^{31}$ For the $1985 \mathrm{MECS}$, adjustment cells were defined by cross-classifying sampling stratum with levels of employment size category. Employment size proved not to be worthwhile in using as an adjustment factor and was discontinued in later surveys.

${ }^{32}$ Although there were two measures of size used, it was necessary to select only one measure to form the nonresponse adjustment. The measure chosen was one that combined the two: it was the 1991 ASM cost of combustible fuels plus the cost of electricity.

${ }^{33}$ Certain petroleum products can be classified according to the end user of the product. For example, propane might be a fuel or a feedstock depending on the needs of the receiving establishment.

${ }^{34}$ Whether a respondent reports a petrochemical feedstock as an energy source receipt often depends on the type of feedstock received. If the feedstock received is commonly used as a fuel, such as distillate fuel oil or ethane, then it is assumed that respondents will report it as an energy source receipt. If the refinery product received for petrochemical feedstock use is not normally considered a fuel, the assumption is made that respondents would not report it as an energy source receipt.
} 
The third class of refinery products, nonenergy products, must be treated differently. The creation of those products by the refinery also requires energy inputs, primarily crude oil. The products are combustible and have a known heat content expressed in British thermal units (Btu). Asphalt, for example, contains 6.636 million Btu per 42-gallon barrel. However, the products are not recognized as energy by their subsequent consumers, and no provision was made for collecting data on their consumption from the MECS respondents. Therefore, the transformation of energy inputs to nonenergy products must be counted as refinery consumption, or it will never be accounted for anywhere in EIA's consumption surveys.

One characteristic of petroleum refineries is that, except for losses caused by spills, contamination, etc., the Btu content of the energy inputs exactly equals the Btu content of the outputs. Therefore, one only needs to know the quantities of those nonenergy products that were shipped by a refinery in order to know the quantity of energy inputs that was used to produce them. EIA produces such information for all refinery products from the "Monthly Refinery Report," Form EIA-810. This form collects information on the monthly shipments from the universe of refineries in the United States. These data were the basis for estimating the input energy requirements for the nonenergy products.

The shipment quantities of the nonenergy products and certain classifications of petrochemical feedstocks, as reported on the "Monthly Refinery Report," were converted to Btu and summed to produce a monthly refinery total. Those totals were then summed across refineries and months to produce the total Btu value of refinery shipments of nonenergy products for 1991. That total was used to represent the total Btu value of the inputs used to produce the nonenergy products, and was inserted directly into the appropriate tables of this report to represent nonfuel consumption in refineries. (See "Survey Estimates" in this appendix.) Because the individual energy inputs corresponding to these shipments were not identified, the Btu value was entered in the "other" column.

The "Monthly Refinery Report" covers only the refinery part of an establishment while the MECS Forms EIA-846(A) through $(C)$ cover energy use at the entire site, as defined by the Bureau of the Census. This difference affects MECS estimation only for cases in which a MECS report reflects energy use at both a refinery and a co-located petrochemical plant. For these cases, establishment nonfuel use is not completely estimated by shipments of refinery nonenergy products as measured by the EIA-810. The format of the MECS refining report, Form EIA-846(B) (see Appendix F), allows respondents to report energy-related data from a petrochemical plant co-located with the refinery. Form EIA-846(B) collected nonfuel use at and shipments of energy sources from the co-located petrochemical plant (Columns 9 and 10 of Section II). The total Btu of the consumption as a nonfuel minus the petrochemical plant shipments of energy sources is added across energy sources and establishments to the previously discussed refinery shipments of nonenergy sources. Note that for the petrochemical plant, estimation of nonfuel use is measured directly, as the majority of that usage does not appear in products that will later be converted to fuel use by other manufacturing plants. The additional nonfuel use estimated for the adjoining petrochemical plants proved to be small relative to the refinery usage because the majority of petrochemical plants report separately on the MECS. Because the resulting quantities was unreliable ${ }^{35}$ and quite small compared to refinery shipments of nonenergy sources, they were excluded from the total refinery nonfuel estimates.

The EIA- 810 data are also used to calculate the offsite-produced fuel use at the refinery establishment. (See "Derived Values" in this appendix.) Because Version A of Section II of Form EIA-846(B) collects only total fuel use of petroleum products (regardless of their origin), it was necessary to use the EIA-810 data to calculate the offsite-produced fuel ratio for those products. Estimation of the ratio utilized the same assumptions described in the section on "Assumptions Underlying Derived Values" except that EIA- 810 data were used instead. This ratio is then applied to the MECS estimated value of total fuel. ${ }^{36}$ The estimator takes the form:

$$
O_{p, M E C S}=\left(\frac{O_{p, E L \Lambda-810}}{F_{p, E H \Lambda-810}}\right) \cdot F_{p, M E C S}
$$

\footnotetext{
${ }^{35}$ Examination of the MECS refinery reports showed evidence that reporting for the adjoining petrochemical operations in the last two columns caused considerable respondent confusion.

${ }^{36}$ The MECS value for total fuel would also include the amount used at the adjoining petrochemical plant if one were present. Using a ratio based on refinery-only data from the EIA-810 on that portion of the establishment is a source of error. However, refinery fuel use will usually dominate the petrochemical fuel use especially for petroleum products.
} 
where $O_{p, M E C S}$ is the MECS estimate of the amount of petroleum product $p$ produced offsite and consumed as a fuel, $O_{p, E I A-8 I 0}$ is the EIA-810 estimate of the amount of petroleum product $p$ produced offsite and consumed as a fuel, $F_{p, M E C S}$ is the MECS estimate of total fuel use of petroleum product $p$, and $F_{p, E A-8 I 0}$ is the ELA-810 estimate of the total fuel use of petroleum product $p$.

Estimates of the contribution to fuel consumption of offsite-produced nonpetroleum products are calculated directly from MECS data, using the same method employed in other SIC's.

\section{Shipments of Energy Sources Produced Onsite}

Manufacturers who produce energy sources do so not only for their own consumption but often sell or transfer the products to other establishments. The most notable example in manufacturing is petroleum refineries. Energy consumption for those establishments is estimated using a special method as has been explained in an earlier section. The principal products of petroleum refineries are energy sources. Primary consumption in petroleum refineries, by virtue of the special method already described, does not need to account for outgoing energy products since it excludes incoming energy sources used for raw materials. Yet there are other types of manufacturers that produce and sell energy sources as secondary products. If the energy content of the sold energy source materials from these are counted at the producing establishment, there would be double counting when the energy source is counted at the receiving establishment. Primary consumption, as currently defined, avoids double counting of intraestablishment use of an energy source which results from an onsite transformation from another energy source. However, it may include double counting of inter-establishment use of such transformed energy sources. The 1991 MECS can further adjust primary consumption by deducting the amount of sold energy sources that were produced onsite.

The example that has the greatest effect on total energy consumption is coal to make coke. A steel mill processes coal to make coke for later use in the steel making process. Primary consumption counts the quantity of coal as the original nonfuel input. Any onsite consumption of coke is not included in primary consumption as it duplicates the coal use. It the steel mill sells and ships some of the coke to another establishment, it will show up as a shipment of an offsite-produced energy source in the second establishment and will be included in primary consumption. That would result in double counting. The double counting can be eliminated by subtracting the energy equivalent of coke shipments from primary consumption.

Table B1. presents these shipment adjustments by SIC. The total shipments adjustment is 560.1 trillion Btu. That means a better estimate of total primary consumption is 19,797 trillion Btu. However, if the purpose is to be comparable with previous years, the original estimate of 20,257 trillion Btu should be used.

Table B1. Total Shipments of Energy Sources Produced Onsite from the Nonfuel Use of Other Energy Sources, by Industry Group and Selected Industries, 1991 (Estimates in Trillion Btu)

\begin{tabular}{|c|c|c|}
\hline $\begin{array}{l}\text { SIC } \\
\text { Code }\end{array}$ & Industry Groups and Industry & Quantity Shipped \\
\hline & & Total United States \\
\hline 28 & Chemicais and Allied Products & 233 \\
\hline 2813 & Industrial Gases ....... & W \\
\hline 2821 & 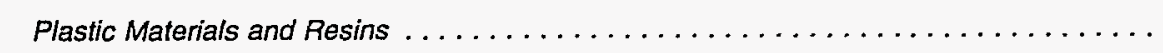 & 3 \\
\hline 2824 & 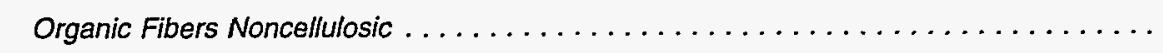 & W \\
\hline 2865 & 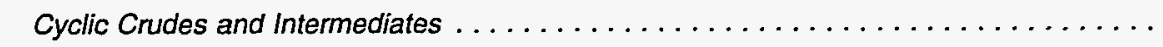 & W \\
\hline 2869 & 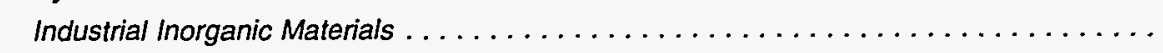 & 221 \\
\hline 29 & Petroleum and Coal Products (excluding Refining) $\ldots \ldots \ldots \ldots \ldots$ & 79 \\
\hline \multirow[t]{2}{*}{3312} & Blast Furnaces and Steel Mills & 248 \\
\hline & Total $\ldots \ldots \ldots \ldots \ldots \ldots \ldots \ldots \ldots \ldots \ldots \ldots \ldots \ldots \ldots \ldots \ldots \ldots \ldots$ & 560 \\
\hline
\end{tabular}

Source: Energy Information Administration, Office of Energy Markets and End Use, Energy End Use and Integrated Statistics Division, 1991 Manufacturing Energy Consumption Survey. 


\section{Concept of Fuel-Switching Capability}

EIA continues to employ the concept of fuel-switching that was developed prior to the 1985 survey. After extensive consultation with potential data users and data providers for the 1985 survey, EIA developed a tightly-specified concept of fuel-switching capability based on the following set of principles:

- Switching data would cover consumption of energy for heat, power, and onsite electricity generation only. Switching of energy consumed as feedstock or raw material inputs would not be considered.

- Switching data would focus on capability (what could be done) rather than actual performance (what was, or is being done) or future possibilities (what might be possible).

- Switching capability would be collected for a closed historical reference period, rather than the present, or some future reference period.

- Switching capability would be collected for the one-year reference period used for MECS consumption data, to tie in with the consumption data and avoid seasonal bias.

- The survey would measure short-term response capability; that is, actions that could have taken place within 30 days of a decision to switch.

- Switching capability would reflect the total flexibility provided by an establishment's equipment configuration. Both multiple-fired equipment and redundant or backup equipment could contribute to capability.

- The survey would measure in-place capability; that is, capability provided by equipment that was already installed, or was available at the establishment for installation during the reference period. Major modifications to the design capabilities of equipment and major capital expenditures were not to be considered in assessing capability.

- Switching capability would be valid only if, following the switch from one type of energy to another, the establishment would have been able to maintain its actual production schedule during the reference period.

- Switching capability provided by an establishment's equipment configuration could be limited or negated by legal or practical constraints such as binding supply contracts, interruptible service, environmental regulations, or unavailability of supply or delivery systems for a potential alternative.

- Economic considerations were not to be considered a practical constraint in evaluating switching capability. The survey was designed to measure potential response to changes in economics or supply patterns.

The MECS obtained fuel-switching data by asking respondents to determine the amounts of 1991 input energy consumption of six major types of energy that could have been switched to one or more alternatives in accordance with the previously listed principles. The six types of energy were purchased electricity, natural gas, distillate oil, residual oil, coal (excluding coke), ${ }^{37}$ and LPG. Respondents were directed to provide the quantities of switchable consumption by subtracting the quantities that were not switchable from the quantities that were actually consumed during 1991. Such an approach is clear and saves burden because it starts with a previously-reported quantity and allows the respondent to subtract quantities known to be nonswitchable because of any one of the various conditions discussed above. The alternative would be to force the respondent to add up quantities for all energy uses for which all aspects of the concept are satisfied. Once the total switchable quantities had been determined, the remaining task was to determine how much of each switchable quantity could have been replaced by specific alternatives.

\footnotetext{
${ }^{37}$ The exclusion of coke from the coal was a change from previous MECS years. It was excluded because coke was found to be virtually nonswitchable in its most common use, the production of steel.
} 


\section{How To Measure Discretionary Fuel Use}

One of the more interesting summary statistics that can be developed from the estimates of actual consumption, minimum consumption, and maximum consumption is the discretionary-use rate. The discretionary use rate is a measure, in percent, of the extent to which manufacturers elected to consume discretionary quantities of a given energy source.

The discretionary-use rate is calculated as:

$$
U S E=\frac{A C T-M I N}{M A X-M I N} \times 100
$$

where USE is the discretionary use rate of a given energy source,

ACT is the actual consumption of that energy source,

MIN is the minimum consumption, which would have been achieved if all ascertained switching from that type of energy had occurred.

and MAX is the maximum consumption, which would have been achieved if all ascertained switching into that type of energy had occurred.

Thus, the discretionary-use rate is a measure of the depth, in percent, into the discretionary range of consumption to which manufacturers chose to go, given their fuel-switching capabilities and production levels of 1991 .

If manufacturers had chosen to minimize their consumption of a given energy source by using alternative energy sources whenever possible, then $\mathrm{ACT}=\mathrm{MIN}$, and the discretionary-use rate would be 0 percent. At the other extreme, if manufacturers had chosen to maximize the consumption of a given energy source by using that energy source whenever possible, then ACT = MAX, and the discretionary-use rate would be 100 percent.

Note that (ACT - MIN) is equivalent to the "switchable" amount of the given energy source that was consumed, that is, the amount of the energy source that was consumed even though it could have been switched to another energy source.

\section{Survey Estimates Presented in Appendix A}

Except for some estimates of energy consumption for nonfuel purposes at petroleum refineries, all energy consumption and energy-related statistics produced from MECS data are calculated by inflating the data collected from the responding establishments with the adjusted sampling weights. These weights establish the relationship between the responding establishments and the manufacturing population as defined for the MECS. Two types of statistics are shown in this report: aggregates (for example, total natural gas consumption in the hydraulic cement industry), and ratios (for example, the amount of fuel consumed per dollar of value added in the manufacturing sector). These statistics are based on the originally reported values, or the derived values discussed earlier, and appear in Tables A1 through A58.

\section{Primary Consumption for All Purposes}

Tables A1 and A9 present estimates of the total primary consumption of energy for all purposes by the manufacturing sector. This measure is intended to represent total demand for energy by manufactures. ${ }^{38}$ Except for petroleum refineries, the estimates in Tables $\mathrm{A} 1$ and $\mathrm{A} 9$ are based on the following derived values:

\footnotetext{
${ }^{38}$ Note that the word "primary" in the MECS usage refers to the first use of energy at a manufacturing site. It has nothing to do with accounting for energy losses in generating and transmitting electricity. That usage is embodied in the term "primary energy," commonly used in EIA to indicate energy measures that take such losses into account. All energy estimates in MECS that include electricity consumption use its end-use conversion value, $3412 \mathrm{Btu}$ per $\mathrm{kWh}$.
} 
- Energy produced offsite and consumed as a fuel

- Energy produced offsite and consumed for nonfuel purposes

- Energy produced onsite from nonenergy inputs and consumed as a fuel

- Energy produced onsite from nonenergy inputs and consumed for nonfuel purposes.

They also include estimates of net electricity and steam consumption; that is, purchases plus transfers in and generation from noncombustible renewable resources, minus quantities sold and transferred out. Primary consumption excludes quantities of energy that were produced from other energy inputs and, therefore, avoids intraestablishment double-counting.

The estimates shown in the petroleum refinery row of Table A1 are conceptually different from the estimates in the other rows of that table. For all industries except petroleum refineries, each cell represents the total primary consumption of energy for all purposes. In the petroleum refinery row, the cell entries for "net electricity" through "coke and breeze" represent only the quantities of given type of energy that was consumed as a fuel. The "other" cell of the petroleum refinery row includes other energy that was consumed as a fuel plus the quantity of energy (mostly crude oil) that was consumed for the production of nonenergy products, as estimated by the Btu value of the shipments. Note that although the estimates shown in the refinery row are computed differently, the total Btu does represent a nonduplicative measure of primary consumption. (For more information, refer to "Feedstocks and Offsite-Produce Fuel at Petroleum Refineries" in this appendix.)

Table A9 shows primary consumption for all purposes by economic characteristics of the establishment. In that table, the cell in the row entitled "not ascertainable" and the column labeled "other" contains the total quantity of energy consumed for the production of nonenergy products by refineries. The quantities of energy consumed for the production of heat and power in refineries are included throughout the remainder of the table, depending on the value of shipments or employment size of the responding establishment.

\section{Primary Consumption for Nonfuel Purposes}

Tables A3 and A11 present the total primary consumption of combustible energy for nonfuel purposes. These tables are based upon aggregates of the derived values of energy produced offsite plus those produced onsite from nonenergy inputs, and consumed onsite for nonfuel purposes. Tables A3 and A11 present the nonfuel primary consumption component of Tables A1 and A9. The entry in the "other" column of the petroleum refinery row of Table 2 represents the total inputs (mostly crude oil) for the production of nonenergy products. The other cells in the petroleum refinery row contain zeros because refinery inputs are available in aggregate form only.

Except for petroleum refineries (see "Feedstocks and Offsite-Produce Fuel at Petroleum Refineries" in this appendix), the estimates in Tables $\mathrm{A} 3$ and $\mathrm{A} 11$ are based on the following derived values:

- Energy produced offsite and consumed for nonfuel purposes

- Energy produced onsite from nonenergy inputs and consumed for nonfuel purposes.

\section{Input Energy for Heat, Power, and Electricity Generation}

Tables A4 and A12 present estimates of input energy for the production of heat, power, and electricity generation. For combustible energy, the estimates are based upon the reported MECS questionnaire responses to "Quantity consumed onsite as a fuel" (see Appendix F). That reported value is exactly equal to the sum of the following derived values: 
- Energy produced offsite and consumed as a fuel

- Energy produced onsite from nonenergy inputs and consumed as a fuel

- Energy produced onsite from energy products and consumed as a fuel.

Thus, the estimates of combustible energy in Tables A4 and A12 represent total consumption as a fuel, regardless of where the energy was produced.

The consumption estimates for fuel use are not duplicative. There is clearly no duplication for quantities that were produced offsite as well as for those produced onsite from nonenergy sources. Quantities produced onsite from other energy inputs result from consumption of an energy source as a feedstock or raw material input. They do not result from the consumption of an energy as a fuel.

Examples of energy produced onsite from other energy sources include,

- Coke oven gas produced as a byproduct of the destructive distillation of coal to produce coke

- Petroleum coke produced in refineries as a result of the high temperature treatment of petroleum fractions

- Still gas produced in refineries as a result of distillation, cracking, reforming, and other processes.

From those examples, it is clear that the input energy was not consumed as a fuel and would not have been included elsewhere in Tables A4 and A12.

The estimates of electricity and steam (note that steam is included in the "other" energy category) must conform to the same criteria as combustible energy. That is, they must represent inputs to produce heat and power, and to generate electricity that do not duplicate energy content represented elsewhere in Tables A4 and A12.

In the case of electricity, the quantities generated onsite by conventional generation or cogeneration must be excluded because the input fuels to produce the electricity (coal, for example) are already counted elsewhere in the table. Thus, the nonduplicative measure of electricity input for Tables A4 and A12 is the same net electricity estimate that appeared in Tables A1 and A9. The same rationale applies to steam. Onsite production is excluded because the input fuel would be counted elsewhere. Thus, the allocation of energy to the various sources shown in Tables A4 and $\mathrm{A} 12$ is consistent with a concept of "first use" of energy for heat, power, and electricity generation.

\section{Other Topics}

Tables A5 and A13 present the total consumption of offsite-produced energy sources as a fuel. As noted, these estimates are approximately definitionally equivalent to the Census Bureau's "purchased" fuels.

The estimates in Tables A5 and A13 are based on the derived value, energy produced offsite and consumed onsite as a fuel.

Tables A2 and A10 present consumption estimates of selected petroleum products expressed in barrels per day rather than in barrels. Included are three estimates of consumption that have been described previously: (1) primary consumption (Table A1), (2) total input energy (Table A4), and (3) consumption for nonfuel purposes (Table A3). These estimates are presented for the convenience of the data user and were derived simply by taking the annual consumption estimate and dividing by 365 .

Table A6 presents quantities of total inputs of byproduct and "other" energy sources used for heat, power, and electricity generation. These estimates are components of the estimates of combustible energy sources found in the last column of Tables A4 and A12. Net steam (see explanation for Tables A4 and A12) is not included in Table A6 but is included in the "Other" column in Tables A4 and A12. 
Tables A7 and A14 present total shell storage capacity of residual oil, distillate oil and motor gasoline. Shell storage capacity includes all onsite capacity, including that which is dedicated or leased for storage of energy owned by other establishments.

Tables A 8 and A15 present estimates of several energy-related operating ratios. These estimates are computed from energy data reported by the MECS responding establishments and economic data reported on the ASM for the same establishments. The consumption values used in the formation of these ratios appear in Tables A4 and A12. It is not possible to exactly reconstruct the 1991 ASM estimates of economic variables by dividing MECS consumption by corresponding ratios of consumption per economic unit. Due to different purposes of the MECS and ASM, the size and weighting scheme of the MECS and ASM samples are different. Therefore, a MECS estimate for an economic variable would be expected to be slightly different due to sampling error, especially for the entries representing a relatively small number of establishments.

Tables A16 and A19 present components of electricity demand. These quantities are calculated directly from responses to the MECS questionnaire. Note that the quantity "net demand for electricity" is not equivalent to "net electricity" shown in Tables A1, A4, A9, and A12. The latter quantity excludes onsite generation by combustible energy sources.

Tables A17 and A20 present components of onsite electricity generation. These components are cogeneration, generation using renewable energy sources, and conventional generation using combustible energy sources. These data are weighted totals of reported responses.

Tables A18 and A21 present quantities of electricity sold to utility and nonutility purchasers. These data are weighted totals of reported responses.

Tables A22 through A29 present purchases, expenditures, and average prices for energy sources. The purchased quantities shown in Tables A22 and A26 are not values of consumption. These data are the amounts actually purchased in the open market regardless of their later disposition. Quantities received through transfers or from a central purchasing office are excluded. The prices shown in Tables A25 and A29 are the results of simple division of the expenditures presented in Tables A25 and A28 by the purchased quantities in Tables A22 and A26. Prices are shown in both dollars per physical unit and dollars per million Btu. Both the expenditures and quantities purchased were values estimated directly from responses to the MECS questionnaires.

Tables A23, A27, and A46 through A49 present purchases, expenditure, and price tables for electricity, natural gas, and steam. These tables break down the gross purchases for these energy sources by the type of supplier. Electricity and steam suppliers are either utilities or nonutilities. The classifications of the natural gas suppliers are utilities, transmission pipelines, and other suppliers.

The increase in sample size yielded other benefits besides increases in separately publishable SIC's and data reliability. There are now enough sample cases to support two-way categorizations of data that were not possible previously. Tables A30 through A32 present three different measures of energy consumption by SIC and value of shipments category. Tables A33 through A35 present the same consumption measures by SIC and categories of total employment.

Tables A36 through A39 are presentations of results from questionnaire items new to the 1991 survey. Respondents were asked to assign their total input energy consumption of selected major energy sources to various end uses in the establishment. The energy consumption measures used as a baseline for each combustible energy source are found in Tables A4 and A12. They are shown in Tables A36 through A39 as the line item, "Total Inputs." Electricity end-use data were collected on the MECS questionnaire as net demand for electricity (purchases plus transfers in plus onsite generation minus sales and transfers out). Those estimates first appear in Tables A16 and A19 and were collected for end-use data because quantities of net demand represent the actual amount available for use at the establishment. Tables 38 and 39 show the results using that measure of electricity consumption. 
Net demand for electricity duplicates the fuel consumption of combustible energy sources used in the process of electricity generation. Tables A36 and A37 show the end-use estimates using the concept of net electricity. Net electricity, the concept used in conjunction with "Primary Consumption" and "Total Inputs of Energy For Heat Power and Electricity Generation" is defined as the sum of purchases and transfers in plus onsite generation from noncombustible renewable resources minus sales and transfers out. Unlike net demand for electricity, net electricity excludes onsite generation of electricity from combustible energy sources. Thus, it does not double-count the energy content of combustible energy sources used to generate electricity. End-use consumption in terms of net electricity was calculated by forming ratios of net demand for electricity for each end-use to total net demand for electricity at the establishment, and multiplying those ratios by the quantity of net electricity at the establishment.

The total inputs row in tables A36 and A37 include a category "Other" to show how much of the total input energy is not accounted for by major energy sources. For some SIC's it is a substantial amount. For example, coal coke and refinery off-gas are significant contributors to boiler fuel and process heat. Data are not available to break down the "other" category by end-use. Further, steam (the major output from boilers) is excluded from these tables. Consequently, total input energy for any end-use category other than boiler fuel would be underestimated by the amount of steam that contributes to that end-use. ${ }^{39}$ Therefore, summing consumption over the end-use categories for which data are available would give a misleading indication of the energy actually used.

For any individual energy source, the estimates in the end-use categories represent direct use. When electricity is considered independently of the combustible energy sources, the more meaningful amount would be in terms of net demand (Tables A38 and A39) rather than net electricity (Tables A36 and A37).

Tables A40 and A41 present estimates of participation by establishments in energy management activities. Total input energy is the measure of interest. If an establishment indicates participation in an activity, its energy consumption is counted in the appropriate category. If not, it is counted in "None Identified." shows subcategories of participation: utility/supplier sponsorship (often referred to as Demand-Side Management), own or third-party sponsorship, or a mixture of both.

Tables A42 through A44 present estimates of total input energy consumption broken down by SIC, establishment size, and presence of selected energy-saving technologies. These technologies are known to save energy but may not have been installed for that purpose.

Tables A45 presents estimates of total input energy consumption by floorspace square-footage category, percent of heated and/or cooled space, and presence or absence of computer controls for building environment. All three of these items are new to the 1991 MECS.

Tables A50 and A51 are new to the 1991 MECS. They present the operating ratios shown in Tables A8 and A15 but broken down further into both SIC and category of value of shipments (Table A50) or category of total employment (Table A51). These tables are now possible due the increased MECS sample size.

Table A52 presents estimates of nonswitchable minimum requirements and the maximum consumption potential of the different types of energy covered specifically by the fuel-switching section of the MECS. An estimate of the actual consumption of each type of energy is provided as a reference point. That consumption estimate represents the quantity that was consumed onsite for the production of heat, power, and the generation of electricity in 1991. The estimates are identical to the ones found in Table 4, except for estimates of electricity. For fuel-switching, the electricity quantity considered is electricity receipts found in Table 5. (The reasons for using electricity receipts are described below in the discussion of Tables A53 through A58.)

\footnotetext{
${ }^{39}$ In the case of cogeneration, the underestimation could be expressed in terms of an unknown amount of steam and electricity.

${ }^{40}$ The MECS sampling method efficiently estimates energy consumption but not establishment counts. Therefore, energy consumption was chosen as the measure of participation rather than number of participating establishments.
} 
One of the purposes of Table A52 is to provide an estimate of the smallest possible quantity of a given type of energy that would have been required in 1991 (keeping production constant), if all possible ascertained switching away from that type of energy had taken place. The quantities given in the minimum consumption column of Table A52 are likely to be higher than the true minimum energy requirements because they include the quantity of 1991 consumption for which switching capability was not ascertained. Some unknown proportion of this latter quantity could likely have been replaced.

Table A52 also provides estimates of the maximum energy consumption that would have been possible if all ascertained switching to that type of energy had occurred. The estimates assume that all indicated substitutions were simultaneously possible and the substitutable amount consists of the sum of all possible switches to the designated type of energy. An assumption of this kind is necessary because there is no specified limit to a respondent's ability to switch into an energy source. Note that there is a given limit to a respondent's ability to switch out of an energy source provided by the third row of the fuel-switching section of the MECS questionnaire (see Appendix F, Manufacturing Energy Consumption Survey Forms).

Tables A53 through A58 present estimates of the capability of substituting specific alternative types of energy for those actually consumed, holding production constant. Each table contains information for the specific type of energy that was actually consumed for the production of heat, power, and generated electricity in 1991. It should be noted that the first column of Table A53 refers to "total receipts" of electricity, while the first column of Tables A54 through A58 refers to "total consumed" natural gas, distillate fuel oil, residual fuel oil, coal, and LPG, respectively.

Thus, the quantities of electricity generated onsite are excluded as are the quantities of electricity leaving the establishment site. When considering fuel-switching capabilities, total electricity receipts is a more meaningful quantity than total electricity consumption. A respondent who has onsite generation of electricity has, more than likely, used an additional amount of a combustible energy source to operate the generator. It is a valid question to ask, "How much of that self-generation is replaceable by electricity receipts?" However, it is more reasonable and of greater interest to collect the fuel-switching data for the fuel used to generate the electricity by asking respondents to show the quantity of electricity receipts that could replace the combustible fuel.

In Tables A53 through A58, the estimates provided in each column under "alternative types of energy" should be considered independently because respondents were instructed to enter the maximum amount of the quantity of the energy actually consumed which could have been replaced by a given alternative. For example, Table A53 shows that for Paper and Allied Products (SIC 26), a total of 3,181 million kilowatthours (kWh) of electricity receipts was switchable. Natural gas could have replaced 1,406 million $\mathrm{kWh}$ of that quantity. The other replacement quantities were distillate fuel oil, 576 million $\mathrm{kWh}$; residual fuel oil, 649 million $\mathrm{kWh}$; coal 859 million $\mathrm{kWh}$; LPG, 128 million $\mathrm{kWh}$; coal coke and breeze, 47 million $\mathrm{kWh}$; and other fuels, 230 million $\mathrm{kWh}$. Because each value represents the maximum quantity of electricity receipts that could have been replaced, their sum exceeds the total quantity of electricity that was ascertained as switchable. That difference indicates that some establishments had more than one type of energy that could have been substituted for electricity usage during 1991.

\section{The Heat Content of Energy Sources}

Many of the estimates of individual energy sources in this report are presented in physical units (kilowatthours, barrels, and short tons). Row totals and combinations of types of energy are presented in Btu. Tables A1 through A5 are presented in physical units and Btu in Parts 1 and 2, respectively.

A Btu is the quantity of heat required to raise the temperature of 1 pound of water by 1 degree Fahrenheit. Thus, converting physical units of a given type of energy to Btu is a means of expressing the heat content of that energy source. All Btu quantities are in terms of higher heating value, with no regard for efficiency of use. Because no energy consumption process is 100 percent efficient (although some are considerably more energy efficient than others), Btu figures must be considered as the maximum available heat content. The following table presents the Btu conversion factors of major types of energy. 


\begin{tabular}{|c|c|}
\hline Type of Energy & $\begin{array}{c}\text { British Thermal Units } \\
\text { (thousands) }\end{array}$ \\
\hline 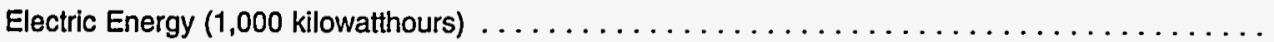 & 3,412 \\
\hline 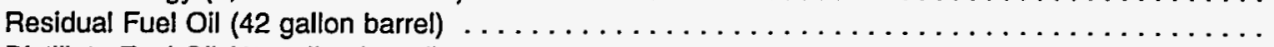 & 6,287 \\
\hline 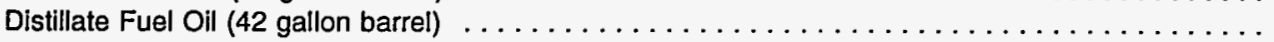 & 5,825 \\
\hline 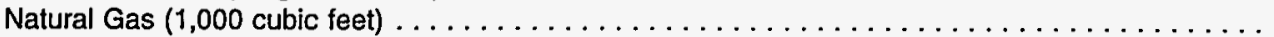 & 1,030 \\
\hline Liquefied Petroleum Gas (42 gallon barrel) . & 3,614 \\
\hline 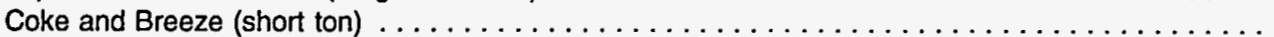 & 24,800 \\
\hline 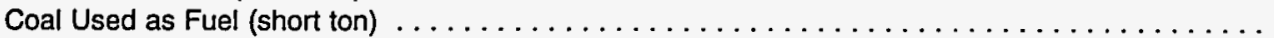 & 22,276 \\
\hline 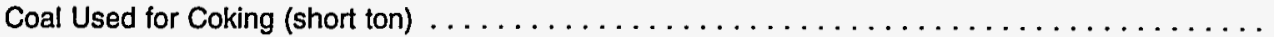 & 26,800 \\
\hline
\end{tabular}

Source: Energy Information Administration, Monthly Energy Review (August 1992), pp. 147-151.

\section{Revisions to 1988 MECS Estimates}

An error was discovered after the 1988 MECS data were published. The nonenergy source shipments at petroleum refineries (see "Feedstocks and Offsite-Produced Fuel at Petroleum Refineries" in this appendix) was miscalculated and, as a result, overestimated. The following corrections should be made to the relevant 1988 MECS tables:

Table B3. Revisions to 1988 MECS Tables

(Estimates in Trillion Btu)

1988 MECS Publication Table 1 (Parts 1 and 2)

\begin{tabular}{|c|c|c|c|c|c|}
\hline \multirow{2}{*}{$\begin{array}{c}\text { SIC } \\
\text { Code }\end{array}$} & \multirow[b]{2}{*}{ Industry Groups and Industry } & \multicolumn{2}{|c|}{ As Published } & \multicolumn{2}{|c|}{ Revised } \\
\hline & & Total & Other & Total & Other \\
\hline & & \multicolumn{4}{|c|}{ Northeast } \\
\hline 2911 & Petroleum Refining $\ldots \ldots \ldots \ldots$ & 583 & $w$ & 546 & $w$ \\
\hline \multirow[t]{3}{*}{29} & Petroleum and Coal Products & 598 & 523 & 560 & 485 \\
\hline & Total & 2,301 & 675 & 2,263 & 637 \\
\hline & & \multicolumn{4}{|c|}{ Midwest } \\
\hline 2911 & Petroleum Refining $\ldots \ldots \ldots \ldots$ & 948 & 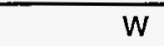 & 894 & $\bar{W}$ \\
\hline \multirow[t]{3}{*}{29} & Petroleum and Coal Products .... & 976 & 827 & 923 & 773 \\
\hline & Total & 4,778 & 1,011 & 4,724 & 958 \\
\hline & & \multicolumn{4}{|c|}{ South } \\
\hline 2911 & Petroleum Refining $\ldots \ldots \ldots \ldots$ & 3,657 & 3,069 & 3,381 & 2,793 \\
\hline \multirow[t]{3}{*}{29} & Petroleum and Coal Products & 3,703 & 3,090 & 3,427 & 2,813 \\
\hline & Total & 10,839 & 4,622 & 10,563 & 4,345 \\
\hline & & \multicolumn{4}{|c|}{ West } \\
\hline 2911 & Petroleum Refining . . . . . . & 1,121 & W & 1,073 & $\bar{W}$ \\
\hline \multirow[t]{3}{*}{29} & Petroleum and Coal Products & 1,134 & 953 & 1,086 & 905 \\
\hline & Total $\ldots \ldots \ldots \ldots \ldots$ & 2,616 & 1,373 & 2,568 & 1,325 \\
\hline & & \multicolumn{4}{|c|}{ United States } \\
\hline 2911 & Petroleum Refining & 6,310 & 5,364 & 5,893 & 4,947 \\
\hline \multirow[t]{2}{*}{29} & Petroleum and Coal Products .. & 6,411 & 5,393 & 5,995 & 4,976 \\
\hline & Total & 20,534 & 7,682 & 20,118 & 7,265 \\
\hline
\end{tabular}


Table B3. Revisions to 1988 MECS Tables (Continued)

1988 MECS Publication Table 2 (Parts 1 or 2)

\begin{tabular}{|c|c|c|c|c|c|}
\hline \multirow{2}{*}{$\begin{array}{l}\text { SIC } \\
\text { Code }\end{array}$} & \multirow[b]{2}{*}{ Industry Groups and Industry } & \multicolumn{2}{|c|}{ As Published } & \multicolumn{2}{|c|}{ Revised } \\
\hline & & Total & Other & Total & Other \\
\hline & & \multicolumn{4}{|c|}{ Northeast } \\
\hline 2911 & Petroleum Refining $\ldots \ldots \ldots \ldots$ & 367 & 367 & 329 & 329 \\
\hline \multirow[t]{3}{*}{29} & Petroleum and Coal Products & 368 & Q & 330 & Q \\
\hline & Total $\ldots \ldots \ldots \ldots \ldots \ldots$ & 699 & w & 661 & W \\
\hline & & \multicolumn{4}{|c|}{ Midwest } \\
\hline 2911 & Petroleum Refining $\ldots \ldots \ldots \ldots$ & 482 & 482 & 429 & 429 \\
\hline \multirow[t]{3}{*}{29} & Petroleum and Coal Products . & 486 & $\mathbf{Q}$ & 432 & $Q$ \\
\hline & Total $\ldots \ldots \ldots \ldots \ldots \ldots$ & 1,163 & W & 1,109 & W \\
\hline & & \multicolumn{4}{|c|}{ South } \\
\hline 2911 & Petroleum Refining $\ldots \ldots \ldots \ldots$ & 2,005 & 2,005 & 1,729 & 1,729 \\
\hline \multirow[t]{3}{*}{29} & Petroleum and Coal Products .... & $Q$ & Q & $Q$ & $\mathrm{Q}$ \\
\hline & Total $\ldots \ldots \ldots \ldots \ldots$ & 3,779 & W & 3,503 & W \\
\hline & & \multicolumn{4}{|c|}{ West } \\
\hline 2911 & Petroleum Refining . . & 403 & 403 & 355 & 355 \\
\hline \multirow[t]{3}{*}{29} & Petroleum and Coal Products & 410 & W & 361 & W \\
\hline & Total $\ldots \ldots \ldots \ldots \ldots$ & 491 & W & 443 & W \\
\hline & & \multicolumn{4}{|c|}{ United States } \\
\hline 2911 & Petroleum Refining $\ldots \ldots \ldots \ldots$ & 3,258 & 3,258 & 2,841 & 2,841 \\
\hline \multirow[t]{2}{*}{29} & Petroleum and Coal Products & 3,290 & 3,285 & 2,874 & 2,868 \\
\hline & Total & 6,132 & 3,531 & 5,716 & 3,114 \\
\hline
\end{tabular}

1988 MECS Publication Table 7

(Primary Consumption by Economic Characteristic)

\begin{tabular}{l|r|r|r|r}
\hline \multirow{2}{*}{ Characteristic } & \multicolumn{2}{|c|}{ As Published } & \multicolumn{3}{c}{ Revised } \\
\cline { 2 - 6 } & \multicolumn{1}{c|}{ Total } & \multicolumn{1}{c}{ Other } & \multicolumn{1}{c}{ Total } & Other \\
\hline Not Ascertained $\ldots \ldots \ldots \ldots \ldots \ldots \ldots \ldots$ & 3,258 & 3,258 & 2,841 & 2841 \\
Total $\ldots \ldots \ldots \ldots \ldots$ & 20,534 & 7,682 & 20,118 & 7265 \\
\hline
\end{tabular}

1988 MECS Publication Table 8

(Primary Consumption for Nonfuel Use by Economic Characteristic)

\begin{tabular}{|c|c|c|c|c|}
\hline \multirow[b]{2}{*}{ Characteristic } & \multicolumn{2}{|c|}{ As Published } & \multicolumn{2}{|c|}{ Revised } \\
\hline & Total & Other & Total & Other \\
\hline Not Ascertained & 3,258 & 3,258 & 2,841 & 2,841 \\
\hline Total $\ldots \ldots \ldots \ldots \ldots \ldots \ldots \ldots$ & 6,132 & 3,531 & 5,716 & 3,114 \\
\hline
\end{tabular}




\section{Appendix C Quality of the Data}





\section{Appendix C \\ Quality of the Data}

\section{Introduction}

All data collection activities and the population estimates produced from them are subject to a variety of errors. These errors may be broadly classified under two general types, sampling and nonsampling errors.

Sampling errors are defined as the variability in a survey estimator that arises because data used to estimate population values are collected from a sample of units rather than completely enumerating the entire population. Each possible sample produces different estimates of population values, depending on the set of respondents that are selected. Consider, for example, a sample of two units from a population comprised of three units. In this example, there exists three possible sample sets of respondents, each of which produces different estimate of the population total. The difference between the estimate calculated from one of the samples and the population total is referred to as the sampling error. Nonsampling errors, on the other hand, occur in any data collection activity, whether a sample survey or a complete enumeration of the population. Nonsampling errors may be associated with any part of a survey process except sampling and can include both random and systematic (biasing) errors. Commonly recognized sources of nonsampling error include undercoverage, random and systematic response errors, unit and item nonresponse, data processing errors, and tabulation errors. This appendix describes the effect of both sampling and nonsampling errors on data from the MECS. In addition, the measure for sampling errors of the population estimates are given. More details are presented in the methodological report for the MECS. ${ }^{41}$

\section{Sampling Error}

The estimated values appearing in this report were developed from a sample of the universe of manufacturing establishments and, as a result, will differ from true population values that would be obtained from a complete enumeration of the manufacturing universe. This is because the MECS sample is only one of a very large number of samples that could have been selected under the same sampling specifications. Each possible sample would yield its own unique estimates of the true population values, with the differences attributable to the particular set of establishments selected into each sample.

One measure of variability due to sampling is the square root of the average of the squared differences between the estimates that would be produced by all possible samples and the mean value of those estimates. This type of measure is commonly known as sampling error. Estimates of the magnitude of these sampling errors based on data from a single sample are provided by a statistic known as the standard error of an estimate. Standard errors for MECS estimates are directly computed from the reported data using the formula:

$$
S_{P}=\sqrt{\sum_{i=1}^{n} y_{i}^{2}\left(W_{i}\right)\left(W_{i}-1\right)},
$$

where $\hat{Y}=\Sigma\left(y_{i} W_{i}\right)$ is the MECS survey estimator, $y_{i}$ is the reported value of characteristic $Y$ for the $i^{\text {th }}$ MECS sample establishment, $W_{i}$ is the final adjusted weight used to inflate the sample data to population estimates, and $n$ is the number of MECS respondents. Justification for this formula is found in the MECS methodological report.

Estimates of standard errors have been computed from the MECS sample data for the estimated aggregate values and selected ratios appearing in this report. In the 1985 and 1988 MECS reports, measures of precision were presented separately in the form of relative standard errors (RSE), that is, the standard error divided by the estimated value to which it refers. In this report, computed RSE's for Table 1 through Table 52 are approximated in a twofactor model and are imbedded into each table as "row and column factors."

\footnotetext{
"Energy Information Administration, Manufacturing Energy Consumption Survey: Methodological Report, 1985. Although this report describes data quality in the 1985 MECS, much of the discussion still holds for the 1991 MECS.
} 


\section{Sampling Error from Generalized Variances}

The RSE's computed using standard errors from Equation (3) may be efficiently modeled by a Generalized Variance procedure, which has been successfully used in several complex sample surveys conducted by EIA. This procedure provides a comprehensive means of reporting generalized relative standard errors, which minimizes the publishing space required to present standard errors, and eases reader's use of precision measures. Actual RSE's (by Equation 3) are used for statistical tests and confidence intervals presented in the text, and for determining if a population estimate is too imprecise to publish (RSE greater than 50 percent).

The estimator used to approximate RSE's is based on a two-factor model. This model-based estimator is given as

$$
R \hat{S} E_{i, j}=R_{i} \circ C_{j},
$$

where $R_{i}$ is the row factor for the $i^{\text {th }}$ row and $C_{j}$ is the column factor for the $j^{\text {th }}$ column used to compute the generalized RSE of the sample estimate at the intersection of the $i^{\text {th }}$ row and $j^{\text {th }}$ column. Since RSE's calculated by this Generalized Variance technique are approximates, confidence intervals and statistical tests of significance must also be regarded as only approximate. See Figure $\mathrm{C} 1$ for a specific example of computing an approximate RSE.

\section{Derivation of Row and Column Factors}

Row and column factors are derived by an analysis of variance procedure with the table of RSE's. Although analysis of variance is used to derive row and column effects from which row and column factors are computed, this Generalized Variance procedure can not be considered an analysis of variance because the primary concern here is to determine model fit rather than to analyze the effects of row and column variables on the RSE's. The two-way model is fit separately for each log transformed RSE table and is consistent for every table in this report. Because of this consistency over all tables, the model can be written in general format as

$$
\log \left(R S E_{i, j}\right)=m+r_{i}+c_{j}+e_{i, j}
$$

where $m$ is the grand mean of $\log \left(R S E_{i, j}\right)$ of a "balanced" table composed of $I$ non-zero rows and $J$ non-zero columns, $r_{i}$ is the effect of the $i^{i h}$ row, $c_{j}$ is the effect of the $j^{\text {th }}$ row, and $e_{i, j}$ is the error term. Model parameters are fit by the standard formulas for Ordinary Least Squares given by Cochran and Cox. ${ }^{42}$ For a given table of log(RSE) estimates, point estimators of model parameters are given as

$$
\begin{aligned}
& \hat{m}=\frac{\sum_{i=1}^{I} \sum_{j=1}^{J} \log \left(R S E_{i, j}\right)}{I \circ J}=\overline{\log \left(R S E_{\circ, 0}\right)} \\
& \hat{r}_{i}=\frac{\sum_{j=1}^{J} \log \left(R S E_{i, j}\right)}{J}-\hat{m}=\overline{\log \left(R S E_{i, \bullet}\right)}-\hat{m} \\
& \hat{c}_{j}=\frac{\sum_{i=1}^{I} \log \left(R S E_{i, j}\right)}{I}-\hat{m}=\overline{\log \left(R S E_{\bullet, j}\right)}-\hat{m} .
\end{aligned}
$$

\footnotetext{
${ }^{42}$ Cochran, William G., and Cox, Gertrude M. (1957), Experimental Design (2nd ed.), New York: John Wiley \& Sons, Inc.
} 
Figure C1. Calculation of Generalized Relative Standard Error (RSE)

Table 1. Total Primary Consumption, 1991

\begin{tabular}{|c|c|c|c|c|c|c|c|c|c|c|c|}
\hline $\begin{array}{l}\text { SIC } \\
\text { Code }\end{array}$ & $\begin{array}{l}\text { Industry Groups } \\
\text { and Industry }\end{array}$ & $\begin{array}{c}\text { Total } \\
\text { (trillion } \\
\text { Btu) }\end{array}$ & $\begin{array}{c}\text { Net } \\
\text { Electricity } \\
\text { (million } \\
\text { kWh) }\end{array}$ & $\begin{array}{c}\text { Residual } \\
\text { Fuel Oil } \\
(1000 \\
\text { bbls })\end{array}$ & \begin{tabular}{|c|} 
Distillate \\
Fuel Oil \\
$(1000$ \\
bbls $)$
\end{tabular} & $\begin{array}{l}\text { Natural } \\
\text { Gas } \\
\text { (billion } \\
\text { cu ft) }\end{array}$ & $\begin{array}{c}\text { LPG } \\
\text { (1000 } \\
\text { bbls) }\end{array}$ & $\begin{array}{c}\text { Coal } \\
(1000 \\
\text { short } \\
\text { tons })\end{array}$ & $\begin{array}{c}\text { Coke } \\
\text { and } \\
\text { Breeze } \\
\text { (1000 } \\
\text { short } \\
\text { tons) }\end{array}$ & $\begin{array}{c}\text { Other } \\
\text { (trillion } \\
\text { Btu) }\end{array}$ & $\begin{array}{c}\text { RSE } \\
\text { Row } \\
\text { Factor }\end{array}$ \\
\hline & RSE Column Factors: & 0.6 & 0.6 & 1.3 & 1.3 & 0.7 & 1.1 & 1.2 & 1.5 & 1.1 & \\
\hline & & \multicolumn{10}{|c|}{ Total United States } \\
\hline 20 & Food and Kindred Products & 956 & 49,536 & 4,317 & 2,968 & W & 1,433 & 6,913 & w & w & 7.2 \\
\hline 2011 & Meat Packing Plants . $\therefore \ldots$. & 49 & 3,410 & 170 & 252 & 31 & 157 & 27 & 0 & 2 & 9.9 \\
\hline 2033 & Canned Fruits and Vegetables. & 44 & 1,375 & 290 & 131 & 35 & 126 & Q & 0 & - & 10.4 \\
\hline 2037 & Frozen Fruits and Vegetables & 40 & 3,071 & 321 & 76 & 25 & 41 & 0 & 0 & 1 & 14.9 \\
\hline 2046 & Wet Com Milling . & 140 & 4,054 & 29 & 31 & 51 & 1 & 3,051 & w & w & 11.8 \\
\hline 2051 & Bread, Cake, and Related Products ... & 32 & 2,240 & * & 131 & 23 & 23 & 0 & 0 & - & 12.4 \\
\hline 2063 & Beet Sugar. . . . . . . . . . . . . . & 67 & 386 & W & 30 & 18 & 5 & 1,901 & w & * & 5.4 \\
\hline 2075 & Soybean Oil Mills $\ldots \ldots \ldots \ldots \ldots \ldots$ & 51 & 1,616 & 42 & 31 & 25 & 5 & 592 & 0 & 7 & 3.5 \\
\hline 2082 & Mall Beverages & 50 & 2,328 & 419 & 58 & 22 & 8 & 706 & 0 & 1 & 10.8 \\
\hline 21 & Tobacco Products $\ldots \ldots \ldots \ldots \ldots \ldots$ & 24 & 1,002 & 135 & 40 & 4 & 23 & 692 & 0 & • & 6.6 \\
\hline 22 & Textile Mill Products & 274 & 29,532 & 1,966 & 1,064 & 105 & 629 & 1,362 & 0 & 13 & 7.0 \\
\hline 23 & Apparel and Other Textile Products & 44 & 5,645 & $\mathbf{Q}$ & 142 & $18 i$ & 159 & 88 & 0 & 1 & 17.9 \\
\hline 24 & Lumber and Wood Products $\ldots \ldots \ldots$ & 451 & 17,878 & 333 & 2,753 & 39 & 1,009 & 92 & 0 & 325 & 14.3 \\
\hline 25 & Furniture and Fixtures & 68 & 4,915 & 184 & 163 & 18 & 255 & 157 & 0 & 26 & 20.1 \\
\hline 26 & Paper and Allied Products & 2,506 & 58,896 & 24,883 & 1,593 & W & 1,379 & 13,252 & w & w & 4.2 \\
\hline 2611 & Pulp Mills $\ldots \ldots \ldots \ldots \ldots \ldots \ldots$ & 300 & 2,537 & 4,500 & 162 & 32 & 141 & 331 & 0 & 221 & 14.2 \\
\hline 2621 & $\ldots \ldots \ldots \ldots \ldots \ldots$ & 1,211 & 32,735 & 13,455 & W I & 252 & 616 & 8,634 & W & 555 & 3.0 \\
\hline 2631 & Paperboard Mills . & 859 & 10,396 & w & w & $w$ & W & w & $\mathbf{0}$ & 505 & 4.5 \\
\hline 27 & Printing and Publishing $\ldots \ldots \ldots \ldots \ldots$ & 108 & 15,629 & 50 & 318 & 47 & 181 & 0 & 0 & 4 & 12.6 \\
\hline 28 & Chemicals and Allied Products $\ldots \ldots \ldots$ & 5,486 & 129,093 & w & 2,412 & 2,163 & $\bar{w}$ & $\bar{w}$ & 423 & $\overline{1.019}$ & 6.1 \\
\hline 2812 & Alkalies and Chlorine & 160 & 10,718 & w & 43 & W & $\overline{2}$ & W & $\overline{0}$ & $\overline{21}{ }^{-}$ & 15.6 \\
\hline 2813 & $\ldots \ldots \ldots \ldots \ldots$ & w & 17,854 & 0 & 7 & w & w & 0 & 0 & 3 & 13.8 \\
\hline 2819 & Industrial Inorganic Chemicals, nec & 325 & 37,077 & W & w & w & 75 & w & 362 & 17 & 8.5 \\
\hline 2821 & Plastics Materials and Resins ....... & 636 & 14,780 & 668 & 192 & 210 & $w$ & 1,074 & 0 & w & 6.4 \\
\hline 2822 & Synthetic Rubber & 119 & 1,794 & 64 & 19 & W & 4,084 & w & 0 & w & 14.1 \\
\hline 2823 & Cellulosic Manmade Fibers . . . . . . . & 31 & w & 0 & 21 & w & 1 & 1,202 & 0 & $*$ & 25.3 \\
\hline 2824 & Organic Fibers, Noncellulosic ... & w & 6,976 & W & 53 & W & W & W & 0 & 1 & 4.0 \\
\hline 2865 & Cyclic Crudes and Intermediates & 251 & 4,423 & 1,153 & 96 & 102 & 20,942 & W & 0 & w & 12.1 \\
\hline 2869 & Industrial Organic Chemicals, nec & 2,705 & 15,104 & 1,747 & 502 & W & w & 3,819 & 0 & 784 & 7.0 \\
\hline 2873 & Nitrogenous Fertillzers & 568 & 2,911 & 0 & 26 & 539 & 166 & 0 & 0 & 2 & 23.1 \\
\hline 2874 & Phosphatic Fertilizers & 65 & 1,886 & 250 & w & $w$ & 1 & W & 0 & w & 4.9 \\
\hline
\end{tabular}

Source: Energy Information Administration, Office of Energy Markets and End Use, Energy End Use and Integrated Statistics Division, 1991 Manufacturing Energy Consumption Survey. 
The row and column factors are then computed by back-transforming the estimated model parameters; that is, by taking the $\log ^{-1}$ of the model effects. This transformation yields

$$
\begin{aligned}
& R_{i}=\log ^{-1}\left(\hat{m}+\hat{r}_{i}\right)=\log ^{-1}\left(\overline{\log \left(R S E_{i, .}\right)}\right) \\
& C_{j}=\log ^{-1}\left(\hat{c}_{j}\right)=\log ^{-1}\left(\overline{\log \left(R S E_{\cdot, j}\right)}-\overline{\log (R S E)}\right)
\end{aligned}
$$

For ease of presentation, the row factor includes the grand mean, $m$. Because of this factoring, the row factor for the $i^{\text {th }}$ row can alternately be expressed as the geometric mean of $i^{\text {th }}$ row:

$$
R_{i}=\left(\prod_{j=1}^{J} R S E_{i, j}\right)^{\frac{1}{J}}
$$

And, column factors, $C$, for a given table have a geometric mean equal to 1.0 .

Since the MECS report presents a variety of energy-related estimates that are unique to certain industries, measures of the precision of population estimates are sometimes equal to zero or withheld from publication. When an RSE table contains a zero or withheld RSE, the table of RSE's is considered for generalization purposes to be "unbalanced". When the condition of an "unbalanced" table arises, substitute RSE estimates are inserted for these missing elements of the RSE table. Substitution of missing RSE's elements is based on an iterative procedure developed by Cochran and Cox. ${ }^{43}$ A detailed description of the automated procedure used to produce the row and column factors appearing in this report can be found in Gargiullo and Goldberg. ${ }^{44}$

\section{Sampling Error of Proportions}

The estimates in this report can be used to produce proportion statistics based on the ratio of various estimates reported in the tables. Proportions are not given in the "Detailed Statistics Tables" but can be used to clarify the analysis. A proportion is the statistic of the form

$$
\hat{P}=\frac{\hat{Y}}{\hat{X}},
$$

where $\hat{Y}$ and $\hat{X}$ are survey-based estimates of aggregate parameters $Y$ and $X$, respectively, and characteristic $X$ "encompasses" characteristic $Y(\mathrm{Y} \subset \mathrm{X})$. That is, each population element (and, thus, each sample case) that contributes to $Y$ also contributes to $X$, and the value of $X$ for each element is greater than or equal to the value of $Y$.

From standard errors given by Equation (3) that are then generalized by Equation (4), the approximate RSE's of aggregate statistics can be used to produce an upper-bound on the approximate errors for proportions. The straightforward additive error formula shown in Equation (3) gives rise to a similarly straightforward upper-bound approximation to the error of an estimated proportion. The approximation can be expressed in terms of the generalized RSE's of the aggregate statistics entering into the proportion as

$R \hat{S E}(\hat{P}) \leq \sqrt{\left[R \hat{S} E^{2}(\hat{Y}) \cdot(1-2 \cdot \hat{P})\right]+R \hat{S} E^{2}(\hat{X})}$

Justification for this formula is found in the MECS methodological report.

\footnotetext{
${ }^{43}$ Ibid.

${ }^{44}$ Gargiullo, P.M., and Goldberg, M.L. "A Modified Table Producing Language (TPL) for Producing Tables of Survey Statistics with Variances" Proceeding.s of the Bureau of the Census Fifth Annual Research Conference. (1989.)
} 


\section{Nonsampling Errors and Bias}

Nonsampling errors that affect MECS sample units can be divided into four major categories:

- Operational errors, including editing, coding, and tabulation errors.

- Errors of measurement, including a lack of precision by the respondent, failure of the respondent to understand instructions, etc..

- Errors of estimation, including the assumptions underlying the derived values.

- Errors of nonobservation, including nonresponse and noncoverage.

These errors are collectively referred to as nonsampling errors because they are not related to the sampling process, and, thus, would be equally likely to occur in a complete census or a sample survey.

It is felt that operational errors are not a major concern for the estimates included in this report. The quality control procedures that were employed for check-in, editing, coding and keying the returned questionnaires (Appendix B) are standard procedures that are in place at the Bureau of the Census and have withstood the test of time. Data tabulations were independently verified by comparing marginal totals in tables generated from files supplied to EIA with corresponding totals generated directly from microdata files maintained at the Census Bureau.

Errors of measurement are a concern in any data collection activity. The survey results for the MECS were subjected to extensive computer editing procedures which were specifically designed to detect errors of measurement. Responses that failed these tests for reasonableness and consistency were recalled by analysts familiar with manufacturing processes and energy use. Major errors, including omissions and misreporting by orders of magnitude, were corrected. No editing procedure is capable of identifying all measurement errors, however, and some small errors will remain. To the extent that these errors are due to random, rather than systematic misjudgments, they are compensating in the aggregate totals presented in this report, and it is believed that there are few large systematic biases that result from them.

Errors of estimation of energy consumption could have resulted from the assumptions that underlie the derived values (see Appendix B), and the estimates of the consumption of onsite- and offsite-produced fuels and raw material inputs could be biased as a result of such errors. For example, the derivation logic makes the assumption that energy produced onsite at a manufacturing establishment is considered first as a shipped product, second as a feedstock, and lastly as a fuel. If that logic does not hold, derived estimate values will be misapportioned. However, considering the mechanisms required to produce energy onsite, it is highly probable that this logic accurately represents manufactures. These nonsampling errors, if present, are relevant only for tables in this report that are based on derived values. Estimates based upon reported values would not be subject to this potential source of bias.

Errors of estimation of energy consumption based on fuel-switching data could have resulted from the assumptions that underlie the formation of the maximum consumption estimates. Implicit in these estimates is the assumption that all potential switches at an establishment can be made simultaneously. For example, if a respondent indicates that natural gas could substitute for both distillate fuel oil and coal, the two quantities are summed together (after converting to like units) to contribute to the maximum consumption of natural gas. To the extent that one or more substitutions are constrained by the performance of another, the published maximum consumption quantities presented in Table A52 would overestimate the "true" value.

Finally, several potential sources of nonsampling error and bias result from errors of nonobservation. As described in Appendix B, the 1991 MECS represents, in terms of sampling coverage, the mail frame of the 1991 ASM or 98 percent of the manufacturing universe. Even though the MECS is a legislatively mandated survey and sampled establishments are given sufficient opportunity and time to respond, nonresponse occurs in the MECS and is accounted for in a nonresponse adjustment of sampling weights presented in Appendix B. Clearly, had these adjustments not been performed, the estimates produced from only the responding establishments would not have been representative of the target universe for the MECS. Such estimates would have been biased. Adjusting the sampling weights to reflect the target universe is an attempt to mitigate the potential effects of such a bias. 
Adjustment factors are calculated for each of the 62 published strata to account for the variation of nonresponse between strata. Each stratum represents a relatively homogeneous subgrouping of establishments with respect to primary product output and level of fuel consumption. It is theorized that the MECS sampling procedure - selecting establishments based upon their relative amount of purchased electricity or fuel expenditures - would be reflected in adjustment factors using total energy costs (sum of fuel and electricity expenditures) as the control variable rather than using either purchased electricity or fuel expenditures.

Implicit in that procedure is the assumption that primary product output and level of fuel consumption are highly correlated with energy expenditure patterns, so that the establishments within a stratum would also be homogeneous with respect to the quantities, types, and shares of energy consumed as fuels and for nonfuel purposes. Also, the weight adjustment method assumes that the relationship between survey variables of interest and the control variable used for constructing the adjusted sample weight is the same for the population covered by MECS respondents within an adjustment stratum as it is for the rest of the population within that stratum.

To the extent that the nonresponding establishments within the adjustment stratum share the energy expenditure patterns of the responding establishments within the strata, the resulting adjustments to the MECS estimates will tend to be minimally biased. If, on the other hand, the energy expenditure patterns of the responding and nonresponding establishments differ substantially, the resulting adjustments are potentially biased, and the overall estimates may not accurately represent the originally targeted MECS universe.

More detailed information on sources of nonsampling error in the MECS can be found in the methodological report. 
Appendix D

Comparability of MECS Estimates with Other Series 



\section{Appendix D \\ Comparability of MECS Estimates with Other Series}

The Energy Information Administration (EIA) collects data from two distinct sources that, in their entirety, provide a comprehensive picture of energy production, marketing, and consumption in the United States. ${ }^{45}$ One set of surveys is directed to the suppliers and marketers of specific fuels (including electricity). The second group of surveys collects comprehensive energy consumption and related data directly from end-use consumers.

Because there is a seeming correspondence between energy supplied and energy consumed, it is tempting to compare or merge their results. However, there are important differences between the supplier and end-user surveys that need to be taken into account in doing such comparisons or other analyses. This appendix discusses the relationship of the supplier surveys and the Manufacturing Energy Consumption Survey (MECS).

\section{An Overview of EIA Surveys}

\section{The End-User Surveys}

The overall purpose of the end-user surveys is to provide comprehensive baseline data on energy consumption and related characteristics for major sectors of the U.S. economy. Accordingly, the end-user surveys are conducted for the manufacturing sector, commercial buildings, residential households, and residential transportation. These surveys collect data directly from samples of the energy-consuming units comprising those sectors. The results of these enduser surveys are available in a variety of EIA publications (see Appendix I). The end-user surveys are:

- Form ELA-457A/G, Residential Energy Consumption Survey (RECS)-The RECS collects information on energy consumption, energy expenditures, and housing and demographic characteristics for residential households in the United States. The survey is conducted triennially using a complex area sample of residential housing units.

- Form EIA-846A/D, Manufacturing Energy Consumption Survey (MECS)-The MECS collects information on energy consumption, end-uses of energy, fuel-switching capabilities, energy management activities, and technology penetration for manufacturing establishments in the United States. The survey was conducted triennially beginning in 1985, and will become a biennial survey beginning with data year 1994 . The MECS uses complex list sampling techniques to develop its sample of manufacturing establishments.

- Form EIA-871A/H, Commercial Buildings Energy Consumption Survey (CBECS)--The CBECS provides comprehensive information on the consumption of energy, energy expenditures, and energyconsuming characteristics of the commercial buildings in the United States. The survey is conducted triennially using a complex area sample of commercial buildings.

- Form EIA-876A/E, Residential Transportation Energy Consumption Survey (RTECS)—The RTECS collects information on the number and types of vehicles per household, annual vehicle miles traveled, Vehicle Identification Number (VIN), and vehicle characteristics. Fuel consumption, expenditures, and fuel efficiency are estimated using data from the Environmental Protection Agency, Bureau of Labor Statistics, and Lundberg Survey, Inc. The survey is conducted triennially, as a companion survey to the RECS.

\footnotetext{
${ }^{45}$ Descriptions of all EIA data collection activities are included in Energy Information Administration, Directory of Energy Data Collection Forms, DOE/EIA-0449(90) (Washington, DC, January 1991).
} 


\section{The Supplier Surveys}

The EIA conducts numerous supplier surveys. The overall purpose of these surveys is to measure the quantity of a specific fuel produced and/or supplied to the market, along with other information related to the fuel's production and supply. The results of these surveys are published in several EIA reports. ${ }^{46}$ Among the supplier surveys ${ }^{47}$ are:

- Form EIA-3, Quarterly Coal Consumption Report, Manufacturing Plants-This form collects information about coal consumption, stocks, and receipts (quantity and price) directly from manufacturing establishments and could be classified as an end-user survey. Because it collects information only on coal consumption and does not collect characteristics data, it is typically viewed as a supplier survey.

- Form EIA-5, Quarterly Coke Plant Report-This form provides information on the production, transfers, consumption, sales, and stocks of coal, coke, and breeze. Respondents include all establishments operating coke plants.

- Form EIA-6, Coal Distribution Report-Form EIA-6 surveys all U.S. companies (producers and/or distributors) that own or purchase and distribute more than 50,000 short tons of coal annually. Quarterly data are collected on coal production and purchases, distribution by consumer category, and method of transportation. At present, there are approximately 1,300 respondents to the EIA-6. The data are collected on a quarterly basis.

- Form EIA-176, Annual Report of Natural and Supplemental Gas Supply and Disposition-Form EIA176 provides annual data on the consumption of natural gas as reported by natural gas and synthetic gas producers, processors, distributors, and pipeline operators. Data are collected on the consumption, disposition, movement, and supply of natural and synthetic gas.

- Form EIA-810, Monthly Refinery Report-Form EIA-810 provides information regarding the balance between supply (beginning stocks, receipts, and production) and disposition (input, shipments, fuel use and losses, and ending stocks) of refined petroleum products. Data are provided by all refineries and blending plants.

- Form EIA-821, Annual Fuel Oil and Kerosene Sales Report-Form EIA-821 provides annual data on the sales by petroleum distributors of distillate and residual fuel oil and kerosene to end-use sectors and State of destination. The survey is sent to a sample of fuel oil dealers in the 50 States and the District of Columbia.

- Form EIA-861, Annual Electric Utility Report-Form EIA-861 is used to survey all electric utilities in the United States. The survey collects annual data on power production and sales of electricity from approximately 3,250 electric utilities.

- Form EIA-867, Annual Nonutility Power Producer Report-Form EIA-867 collects annual data from nonutility power producers who own or plan to install electric generation equipment with a total capacity of five megawatts or more at an existing or proposed site. This survey collects information from the nonutility power producer on electricity generation, installed capacity, and energy consumption devoted to power production.

\footnotetext{
${ }^{46}$ For a complete list of publications see Energy Information Administration, EIA Publications Directory 1992, DOE/EIA-0149(92) (Washington, DC, June 1993).

${ }^{47}$ In order to be consistent with the 1991 MECS, the descriptions of the supplier surveys are of the 1991 versions.
} 


\section{Combined Results of the Supplier Surveys}

In addition to supporting fuel-specific publications of EIA, the results of the supplier surveys are combined to produce estimates of total energy consumption by consuming sector. The consuming sectors consist of the commercial, residential, industrial, transportation, and electric utilities sectors. The resulting combined estimates are published by EIA in the Monthly Energy Review (MER), the State Energy Data Report (SEDR), and the Annual Energy Review (AER). Table D1 presents the 1991 combined industrial estimates as they appear in the MER and SEDR.

Table D1. Combined Industrial Energy Consumption Estimates and Sources of Information, 1991

\begin{tabular}{|c|c|c|c|}
\hline \multirow[b]{2}{*}{ Description of Energy Source } & \multicolumn{2}{|c|}{$\begin{array}{l}\text { Combined Estimates } \\
\text { (Trillion British Thermal Units) }\end{array}$} & \multirow[b]{2}{*}{ Relevant Supplier Surveys, Publications, and Notes } \\
\hline & SEDR & MER & \\
\hline Coal ........... & $2,600.4$ & 2,601 & Form ElA-3, "Quarterly Coal Consumption Report, Manufac- \\
\hline Bituminous Coal and Lignite. & $2,592.3$ & NP & furing Plants"; Form-ElA-5, "Quarterly Coke Plant Report"; \\
\hline Anthracite $\ldots \ldots \ldots \ldots$ & 8.1 & NP & Form EIA-6, "Coal Distribution Report" \\
\hline Natural Gas . . & $8,657.1$ & 8,641 & $\begin{array}{l}\text { Form ElA-176, "Annual Report of Natural and Supplemental } \\
\text { Gas Supply and Disposition" }\end{array}$ \\
\hline Petroleum & $8,057.7$ & 8,057 & Petroleum Supply Annual 1991, DOE/EIA-0340 \\
\hline Asphalt and Road Oil & $1,076.5$ & NP & Petroleum Supply Annual 1991, DOE/EIA-0340 \\
\hline Distillate Fuel Oil ... & $1,139.2$ & NP & Fuel Oil and Kerosene Sales, 1991, DOE/EIA-0535(91) \\
\hline Kerosene ...... & 11.4 & NP & Fuel Oil and Kerosene Sales, 1991, DOE/EIA-0535(91) \\
\hline LPG . . . . . & $1,749.2$ & NP & $\begin{array}{l}\text { American Petroleum Institute, } 1991 \text { Sales of Natural Gas } \\
\text { Liquids and Liquefied Refinery Gases }\end{array}$ \\
\hline Lubricants . & 166.7 & NP & $\begin{array}{l}\text { Bureau of the Census, Current Industrial Reports, "Sales of } \\
\text { Lubricating and industrial Oils and Greases, } 1977^{4}\end{array}$ \\
\hline Motor Gasoline & 193.3 & NP & Federal Highway Administration, Highway Statistics \\
\hline Residual Fuel Oil & 335.9 & NP & Fuel Oil and Kerosene Sales, 1991, DOE/EIA-0535(91) \\
\hline Other .......... & $3,385.5$ & NP & Petroleum Supply Annual 1991, DOE/EIA-0340 \\
\hline Industrial Hydropower & 32.7 & 33 & $\begin{array}{l}\text { Estimates of hydroelectric power represent the average } \\
\text { generation over the 6-year period of 1974-1979, the last period } \\
\text { for which data are available. }\end{array}$ \\
\hline Net Imports of Coal Coke .... & 8.9 & 9 & $\begin{array}{l}\text { Bureau of the Census, U.S. Department of Commerce, } \\
\text { "Monthly Report IM 145." }\end{array}$ \\
\hline Electricity & $3,229.7$ & 3,230 & Form EIA-861, "Annual Electric Utility Report" \\
\hline Net Energy . & $22,586.6$ & 22,570 & Total estimated end-use energy consumption. \\
\hline \multicolumn{4}{|l|}{ Electrical System } \\
\hline Energy Losses. & $7,014.1$ & 7,022 & $\begin{array}{l}\text { Electrical system energy losses represent the amount of } \\
\text { energy lost during generation, transmission, and distribution of } \\
\text { electricity. These losses are calculated as the difference } \\
\text { between the total heat content of energy input at electric utili- } \\
\text { ties and the total heat content of electricity sold to end-use } \\
\text { consumers. }\end{array}$ \\
\hline \multirow[t]{2}{*}{ Total Consumption } & $29,600.7$ & 29,592 & \\
\hline & & & Total consumption including electrical system energy losses. \\
\hline
\end{tabular}

$\mathrm{NP}=$ Not published. Estimate is included in higher-level totals.

Note: Slight differences between the SEDR and MER estimates are attributable to rounding differences and to the fact that the SEDR Btu estimates are developed from State-level Btu conversion factors while MER Btu estimates are developed from national-level Btu conversion factors.

Sources: Energy Information Administration, State Energy Data Report 1991, DOE/EIA-0214(91) (Washington, DC, May 1993), Table 15 and Appendices A and C, and Monthly Energy Review September 1993, DOE/EIA-0035(93/09) (Washington, DC, September 1993), Table 2.4 and pp. 40-45. 


\section{Defining the Industrial Sector}

In general, the "industrial sector" is defined as consisting of manufacturing, mining, construction, agriculture, fisheries, and forestry. The approximate SIC equivalent of the industrial sector includes major group codes 01 through $39 .^{48}$ There are a few definitional irregularities, however, that preclude a perfect mapping of the supplier surveys to that range of SIC codes. As pointed out in the MER,

although end-use allocations are made according to [the sector definitions] as closely as possible, some data are collected by using different classifications. For example, data on agricultural use of natural gas are collected and reported in the commercial sector rather than the industrial sector. Since agricultural use of natural gas cannot be identified separately, it is included in the commercial sector.... [rather than the industrial sector. $]^{49}$

The allocations to the industrial sector will be discussed in more detail in the subsequent sections on individual energy sources.

\section{Comparing the MECS and Industrial Sector Estimates}

The MECS produces four separate estimates of manufacturing energy consumption, which are: (1) Total Primary Consumption of Energy for All Purposes (Table A1), (2) Total Primary Consumption of Energy for Nonfuel Purposes (Table A3), (3) Total Inputs of Energy for Heat, Power, and Electricity Generation (Table A4), and (4) Total Consumption of Offsite-Produced Energy for Heat, Power, and Electricity Generation (Table A5). The differences among those estimates are discussed in detail elsewhere in Appendix B of this report.

The combined estimates for the industrial sector published in SEDR are conceptually similar to the MECS estimates of Total Primary Consumption of Energy For All Purposes, because both series measure energy consumption as a fuel and as a raw material or feedstock. Table D2 presents a comparison of those MECS estimates and the combined industrial estimates appearing in SEDR.

\section{Coal Consumption}

The 1991 estimate of coal consumption from the MECS is 2,006 trillion British thermal units (Btu) (Table A1, Part 2) and the combined estimate published in SEDR for the industrial sector is 2,600 trillion Btu (Table D1). It is tempting to attribute that difference to the disparate coverage of the manufacturing and industrial sectors and to conclude that the difference of approximately 594 trillion Btu is due to additional consumption in the construction, mining, agriculture, forestry, and fisheries sectors. That interpretation is only partially correct, however.

Table D2 shows that the SEDR estimate of coal consumption consists of two basic components-coal consumption by coke plants, 907 trillion Btu and coal consumption by other industrial, 1,694 trillion Btu. The consumption by coke plants can be further disaggregated into consumption by furnace coke plants, 787 trillion Btu, and consumption by merchant coke plants, 120 trillion Btu. ${ }^{50}$

The inclusion of merchant coke plants represents a major departure from the MECS. A merchant coke plant is one whose coke is produced for sale on the commercial market. According to the SIC Manual, these coke plants are classified in SIC 4925, "Mixed, Manufactured, or Liquefied Petroleum Gas Production and/or Distribution." They are classified in that industry because they produce coke oven gas as a primary product and coke as a byproduct. Since the MECS covers only the manufacturing sector (SIC 20 - 39), the merchant coke plants are excluded from the estimates of coal consumption. Deducting the quantity of coal consumed by those plants from the SEDR estimate

\footnotetext{
${ }^{48}$ See Office of Management and Budget, Standard Industrial Classification Manual 1987, (Washington, DC, 1987).

${ }^{49}$ Energy Information Administration, Monthly Energy Review September 1993, p. 40.

${ }^{50}$ Energy Information Administration, Quarterly Coal Report October-December 1992, DOE/EIA-0121(92/4Q), (Washington, DC, May 1993), Table 48. Short tons converted to British thermal units using standard EIA conversion rates.
} 
Table D2. A Comparison of the Components of MECS and SEDR Estimates of Energy Consumption (Trillion British Thermal Units)

\begin{tabular}{|c|c|c|c|}
\hline \multicolumn{2}{|c|}{$\begin{array}{l}1991 \text { Manufacturing Energy Consumption Survey } \\
\text { Total Primary Consumption of Energy } \\
\text { for All Purposes }\end{array}$} & \multicolumn{2}{|l|}{$\begin{array}{l}1991 \text { State Energy Data Report } \\
\text { Industrial Sector Energy Consumption Estimates }\end{array}$} \\
\hline Energy Source Description & Estimate & Energy Source Description & Estimate \\
\hline \multirow[t]{3}{*}{ Coal } & 2,006 & Coal $\ldots \ldots \ldots \ldots \ldots \ldots \ldots \ldots \ldots$ & $2,600.4$ \\
\hline & & Coke Plants $\ldots \ldots \ldots \ldots \ldots \ldots \ldots$ & 907.3 \\
\hline & & Other Industrial . . . . . . . . . . . . . & $1,693.2$ \\
\hline \multirow[t]{3}{*}{ Natural Gas . . . . . . . . . . . . . . . . . } & 6,095 & Natural Gas $\ldots \ldots \ldots \ldots \ldots \ldots \ldots \ldots \ldots$ & $8,657.1$ \\
\hline & & Industrial $\ldots \ldots \ldots \ldots \ldots \ldots \ldots \ldots$ & $7,470.6$ \\
\hline & & Lease and Plant Fuel . . . . . . . . . . . . . & $1,186.5$ \\
\hline Net Electricity $\ldots \ldots \ldots \ldots \ldots \ldots \ldots \ldots$ & 2,370 & Electricity $\ldots \ldots \ldots \ldots \ldots \ldots \ldots \ldots \ldots$ & $3,229.7$ \\
\hline Purchased Electricity $\ldots \ldots \ldots \ldots \ldots$ & 2,380 & Electricity Sales & $3,229.7$ \\
\hline Transfers $I n \ldots \ldots \ldots \ldots \ldots \ldots$ & 71 & & \\
\hline \multicolumn{4}{|l|}{ Generation from Nonrenewable } \\
\hline Combustible Resources . . . . . . . . . . . & 15 & & \\
\hline Electricity Sales/Transfers Out . . . . . . . . . & -96 & & \\
\hline \multirow[t]{3}{*}{ Coke and Breeze $\ldots \ldots \ldots \ldots \ldots \ldots \ldots \ldots$} & 308 & Net Imports of Coal Coke $\ldots \ldots \ldots \ldots \ldots \ldots \ldots$ & 8.9 \\
\hline & & Coke imports . . . . . . . . . . . . . & 27.3 \\
\hline & & Coke Exports $\ldots \ldots \ldots \ldots \ldots \ldots \ldots \ldots$ & 18.4 \\
\hline Residual Fuel Oll $\ldots \ldots \ldots \ldots \ldots \ldots \ldots \ldots$ & 454 & Residual Fuel Oil $\ldots \ldots \ldots \ldots \ldots \ldots \ldots \ldots$ & 335.9 \\
\hline 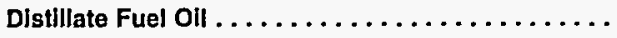 & 146 & Distillate Fuel Oil $\ldots \ldots \ldots \ldots \ldots \ldots \ldots \ldots$ & $1,139.2$ \\
\hline Llquefled Petroleum Gases . . . . . . . . . . . . & 1,574 & Liquefied Petroleum Gases $\ldots \ldots \ldots \ldots \ldots \ldots$ & $1,749.2$ \\
\hline other $\ldots \ldots \ldots \ldots \ldots \ldots \ldots \ldots \ldots \ldots$ & 7,304 & & \\
\hline Asphalt and Road Oil . . . . . . . . . . . . & 1,078 & Asphalt and Road Oil $\ldots \ldots \ldots \ldots \ldots \ldots$ & $1,076.5$ \\
\hline Lubricants $\ldots \ldots \ldots \ldots \ldots \ldots \ldots$ & 380 & Lubricants $\ldots \ldots \ldots \ldots \ldots \ldots \ldots \ldots \ldots$ & 166.7 \\
\hline Kerosene . . . . . . . . . . . . . . . . & 48 & Kerosene $\ldots \ldots \ldots \ldots \ldots \ldots \ldots \ldots \ldots$ & 11.4 \\
\hline \multirow[t]{2}{*}{ Finished Motor Gas $\ldots \ldots \ldots \ldots \ldots \ldots$} & 81 & Finished Motor Gas . . . . . . . . . . . . . . . & 193.3 \\
\hline & & Other Petroleum $\ldots \ldots \ldots \ldots \ldots \ldots \ldots \ldots$ & $3,385.5$ \\
\hline Naphtha $<401$ Degrees . . . . . . . . . . . & 299 & Naphtha $<401$ Degrees . . . . . . . . . . . . & 298.9 \\
\hline Other Oils $\geq 401$ Degrees . . . . . . . . . . . & 795 & Other Oils $\geq 401$ Degrees . . . . . . . . . . . & 827.3 \\
\hline Speclal Naphthas $\ldots \ldots \ldots \ldots \ldots \ldots$ & 134 & Special Naphthas . . . . . . . . . . . . . & 88.0 \\
\hline 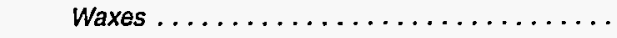 & 41 & 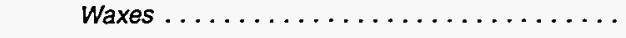 & 35.1 \\
\hline Miscellaneous Nontuel Products $\ldots \ldots \ldots \ldots$ & 141 & Miscellaneous Fuel \& Nonfuel Products . . . . . . . & 152.6 \\
\hline Crude OII $\ldots \ldots \ldots \ldots \ldots \ldots \ldots \ldots$ & 0 & Crude Oil ...................... & 38.9 \\
\hline 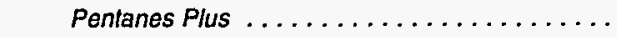 & -- & Pentanes Plus $\ldots \ldots \ldots \ldots \ldots \ldots$ & 294.0 \\
\hline Unfinished Olls . . . . . . . . . . . . . . & -- & Unfinished Oils $\ldots \ldots \ldots \ldots \ldots \ldots \ldots$ & -450.2 \\
\hline Motor Gas Blending Compounds . . . . . . . . . & -- & Motor Gas Blending Compounds . . . . . . . . & -25.9 \\
\hline Aviation Gas Blending Compounds . . . . . . . . & - & Aviation Gas Blending Compounds ........ & -0.1 \\
\hline Petroleum Coke . . . . . . . . . . . . . . & 617 & Petroleum Coke . . . . . . . . . . . . . & 700.3 \\
\hline Still Gas Waste Gas . . . . . . . . . . . . . & 1,399 & Still Gas . . . . . . . . . . . . . . . . & $1,426.6$ \\
\hline Pulping Liquor $\ldots \ldots \ldots \ldots \ldots \ldots \ldots$ & 857 & Pulping Liquor $\ldots \ldots \ldots \ldots \ldots \ldots \ldots \ldots$ & -- \\
\hline Wood Chips, Bark, Wood Waste . . . . . . . . & 666 & Wood Chips, Bark, Wood Waste $\ldots \ldots \ldots \ldots \ldots$ & -- \\
\hline Net Steam/Hot Water $\ldots \ldots \ldots \ldots \ldots \ldots \ldots$ & 239 & Net Steam/Hot Water $\ldots \ldots \ldots \ldots \ldots \ldots \ldots$ & $\cdots$ \\
\hline Miscellaneous $\ldots \ldots \ldots \ldots \ldots \ldots$ & 529 & Miscellaneous $\ldots \ldots \ldots \ldots \ldots \ldots \ldots \ldots$ & $\cdots$ \\
\hline \multirow[t]{3}{*}{ Total } & 20,257 & Net Energy' $\ldots \ldots \ldots \ldots \ldots \ldots \ldots \ldots \ldots \ldots \ldots$ & $22,553.9$ \\
\hline & & Electrical System Energy Losses $\ldots \ldots \ldots \ldots \ldots$ & $7,014.1$ \\
\hline & & Total $^{1} \ldots \ldots \ldots \ldots \ldots \ldots \ldots \ldots \ldots \ldots$ & $29,568.0$ \\
\hline
\end{tabular}

'Excludes Industrial Hydropower.

$\rightarrow=$ Not applicable. Energy source is not included in series.

Note: Totals may not equal sum of components due to independent rounding.

Sources: The MECS estimates of major energy sources (boldface) are from Table A1 of this report. The components of the MECS estimates (italics) are from Tables A6, A16, and unpublished sources. The SEDR estimates of major energy sources (boldface) are from Energy information Administration, State Energy Data Report 1991, DOE/EIA-0214(91) (Washington, DC, May 1993), Table 15. The components are from Quarterly Coal Report OctoberDecember 1992, DOE/EIA-0121(92/4Q) (Washington, DC, October 1992), Tables 2, 48, and 49; Natural Gas Annual 1991, DOEJEIA-0131(91) (Washington, DC, October 1992), Tables 16 and 17; and Petroleum Supply Annual, Volume 1, DOEJEIA-0340(92)/1 (Washington, DC, May 1993), Table 2. Where necessary, physical units were converted to British thermal units using the thermal conversion factors in Energy information Administration, Monthly Energy Review September 1993, DOE/EIA-0035(93/09) (Washington, DC, September 1993), Appendix B. 
yields 2,480 trillion Btu. The SEDR estimate of coal consumption by other industrial plants, 1,694 trillion Btu, can also be further disaggregated. Of that total quantity, 1,494 trillion Btu was consumed by manufacturing plants ${ }^{51}$ and 199 trillion Btu by the nonmanufacturing portion of the industrial sector. The nonmanufacturing portion of the industrial sector is, of course, excluded from the MECS estimate. Subtracting that quantity from the adjusted SEDR estimate results in 2,281 trillion Btu.

The remaining difference between the MECS and SEDR estimates of coal consumption (275 trillion Btu) can be accounted for by two factors. First, Form EIA-3, "Quarterly Coal Consumption Report, Manufacturing Plants," collects coal consumption information from coal gasification plants and classifies those plants in SIC 29, "Petroleum and Coal Products." The coal gasification plants are excluded from the MECS sample. ${ }^{52}$ Second, Form EIA-3 collects coal consumption information from electricity generating plants that are owned by manufacturing companies but are not co-located with a manufacturing establishment. Those generating facilities are defined as being a part of the manufacturing sector by Form EIA-3, but are excluded from the MECS because, according to the SIC Manual, they should be classified in SIC 4911, "Electric Services." For reasons of confidentiality, the exact values included in the SEDR estimates of coal consumption for the coal gasification plants and electricity generating plants not colocated with a manufacturing establishment cannot be shown. Suffice it to say, however, that the total of these values account for virtually all of the remaining difference of 275 trillion Btu between the MECS estimate and the adjusted SEDR estimate.

\section{Natural Gas}

The 1991 estimates for natural gas consumption for the MECS and the combined industrial estimates published in SEDR are, respectively, 6,095 and 8,657 trillion Btu. Since the SEDR estimates of natural gas come directly from estimates produced from Form EIA-176, "Annual Report of Natural and Supplemental Gas Supply and Distribution," most of that discrepancy can be accounted for by the differences in the coverage of the industrial sector as defined for the EIA-176 and manufacturing sector as defined for the MECS.

- Both the 1988 and 1991 MECS samples excluded very small establishments. While it was possible to adjust the 1988 MECS estimates for that exclusion, such a procedure was not feasible for the 1991 MECS (see Appendix B). However, the 1988 adjustments indicate that the excluded small establishments would account for roughly 200 trillion Btu of natural gas consumption that was excluded from the 1991 MECS. Deducting that quantity from the SEDR estimate leaves 8,457 trillion Btu.

- The estimates of the industrial consumption included in SEDR include lease and plant fuel. Lease and plant fuel is defined as "Natural gas used in well, field, and lease operations (such as gas used in drilling operations, heaters, dehydrators, and field compressors) and as fuel in natural gas processing plants. ${ }^{153}$ The SIC Manual classifies those establishments in the mining sector and, as a result, they are excluded from the MECS. Lease and plant fuel consumption accounted for 1,187 trillion Btu in 1991 (Table D2), and is selfprovided (i.e., not purchased). The SEDR estimate, excluding lease and plant fuel, is 7,271 trillion Btu.

- The SEDR estimate of industrial natural gas consumption also includes purchased natural gas by the mining sector. The 1987 Census of Mineral Industries ${ }^{54}$ indicates that the mining sector consumed 459 trillion Btu of purchased natural gas. As noted, the mining sector is excluded from the MECS. Excluding purchased natural gas by the mining sector from the SEDR estimate results in 6,812 trillion Btu.

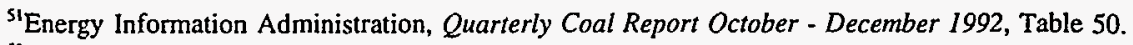

${ }^{52}$ There is some question about the appropriate SIC code for coal gasification plants. According to the SIC Manual, coal gasification plants located at the mine site should be classified in SIC 1311, crude petroleum and natural gas. There is no SIC code for coal gasification plants located at sites other than the mine site. The most logical classification for such plants would be SIC 2999, products of petroleum and coal, not elsewhere classified, although a case could be made for classifying them in SIC 4925 , mixed, manufactured, or liquefied petroleum gas production and/or distribution.

${ }^{53}$ Energy Information Administration, Natural Gas Annual 1991, DOE/EIA-0131(91) (Washington, DC, October 1992), p. 250.

${ }^{54}$ U.S. Bureau of the Census, 1987 Census of Mineral Industries, Fuels and Electric Energy Consumed, MIC87-S-2, (Washington, D.C. December 1990), Table 2. The Census of Mineral Industries is conducted only every five years. The estimates for 1992 are not yet available.
} 
The remaining difference of 717 trillion Btu between the MECS and the adjusted SEDR estimate of industrial natural gas consumption is more difficult to explain. The difference cannot be attributed to the agriculture, forestry, and fishing division (SIC 01 through 09) because those industries are excluded from both the MECS and the SEDR estimates of natural gas consumption. Specifically,

Industrial consumers are establishments engaged in a process which creates or changes raw or unfinished materials into another form or product. Generation of electricity, other than by electric utilities, is included. In general, industrial establishments would be those in Standard Industrial Classification major group codes 10 through $39 . .^{55}$

The SEDR estimate of industrial natural gas consumption does include the construction industries (SIC 15 through 17), and these estimates are excluded from the MECS. The Bureau of the Census estimates that, in 1987, the total cost of natural gas and manufactured gas for the construction division was only $\$ 303.5$ million, ${ }^{56}$ or the equivalent of approximately 100 trillion Btu. Excluding the construction industries from the SEDR estimates leaves 6,712 trillion Btu.

Finally, as noted in the above definition, the SEDR estimate (as taken from Form EIA-176) also includes the natural gas consumed in the generation of electricity by generating facilities other than electric utilities. Some of those generating facilities are co-located with manufacturing plants, others are owned by manufacturing operations, but not co-located with manufacturing plants, and still others are totally independent of manufacturing. In general, these generating facilities are known as nonutility power producers (NPP), and EIA collects electricity generation and related information from them using Form EIA-867, "Annual Nonutility Power Producer Report."

Many of the NPP's use natural gas as an input fuel to generate electricity. According to the results of the EIA-867, all NPP's generated 131 billion $\mathrm{kWh}^{57}$ of electricity using 1,617 trillion Btu of natural gas as an input fuel ${ }^{58}$ in 1991, for an efficiency rate of 28 percent. ${ }^{59}$ All of that natural gas consumption would be included in the SEDR estimate of industrial natural gas consumption. However, the MECS includes only the natural gas consumed by those NPP's co-located with manufacturing plants. Thus, to check the comparability of the MECS and SEDR estimates of natural gas consumption, it is necessary to deduct from the SEDR estimate the quantity of natural gas consumed as a generating fuel by independent NPP's and those NPP's owned by manufacturers but not co-located with a manufacturing plant.

In 1991, manufacturers generated 125,584 million $\mathrm{kWh}$ of electricity from nonrenewable energy sources (Table A16). The MECS does not provide information on the quantities of input fuels consumed to generate that electricity, so it cannot be determined precisely how much of the natural gas-produced electricity reported for NPP's originated in the manufacturing sector. However, some speculation is possible.

Manufacturers consumed 3,311 trillion Btu of selected energy sources as a boiler fuel in 1991, of which 2,098 trillion Btu (63 percent) was natural gas (Table A36, Part 2). Clearly, not all of that boiler output was used to generate electricity. However, if electricity was generated in proportion to the quantities of boiler fuel, then natural gas would have accounted for 63 percent of the electricity generated, or approximately 79,800 million $\mathrm{kWh}$ (272 trillion Btu). That quantity of electricity would have required 982 trillion Btu of natural gas as an input fuel (assuming an efficiency rate of 28 percent). Thus, of the 1,617 trillion Btu of natural gas input for nonutiliy power generation reported by the EIA-867 and captured in the SEDR, 982 trillion would be accounted for by facilities covered by the MECS. The remaining 635 trillion Btu would have been consumed in facilities outside the scope of the MECS. Deducting that amount from the adjusted SEDR estimate of 6,712 trillion Btu yields 6,077 trillion Btu of natural gas, an estimate that is quite close to the MECS estimate of 6,095 trillion Btu.

\footnotetext{
${ }^{55}$ Form EIA-176, "Annual Report of Natural and Supplemental Gas Supply and Disposition," p. 7.

${ }^{56}$ U.S. Bureau of the Census, 1987 Census of Construction Industries.

${ }^{37}$ Energy Information Administration, Electric Power Annual 1991, DOE/EIA-0348(91), (Washington, DC, February 1993), Table 70.

${ }^{58}$ Unpublished 1991 estimate of natural gas consumption obtained by telephone from EIA's Office of Coal, Nuclear, Electric and Alternate

${ }^{59}$ In a perfect world, the heating value of the quantity of energy consumed to generate electricity would exactly equal the heating value of the quantity of electricity produced. Such a relationship would be 100 percent efficient. In fact, however, electricity generation is not 100 percent efficient. For example, in 1991, electric utilities consumed 29.70 quadrillion Btu of input fuels to produce 10.14 quadrillion Btu of electricity. The difference of 19.56 quadrillion Btu represents the conversion loss due to the inefficiencies inherent in the generation process. Thus, the efficiency rate for utilities is approximately 35 percent. See Energy Information Administration, Annual energy Review 1991, DOE/EIA-0384(91), (Washington, DC, June 1992). p. 207.
} Fuels. 


\section{Net Electricity}

The MECS provides an estimate of "net electricity," defined as the sum of purchases (2,380 trillion Btu), transfers in (71 trillion Btu), and generation from noncombustible renewable resources (15 trillion Btu), minus quantities of electricity sold or transferred out (96 trillion Btu). Thus, the MECS estimate of net electricity is 2,370 trillion Btu (Table D2). ${ }^{60}$

The combined estimate of industrial electricity consumption published in SEDR is based on industrial sales data as reported on Form EIA-861, "Annual Electric Utility Report." The SEDR estimate is 3,230 trillion Btu. Assuming that sales by utilities equal consumption by customers, the appropriate MECS measure for comparative purposes is purchased electricity, 2,380 trillion Btu (Table A22, converted to British thermal units). Thus, the initial difference between the two estimates of purchased electricity is 850 trillion Btu.

Most of that difference can be explained by the differences in the definitions of the manufacturing and industrial sectors. Specifically, the estimate included in SEDR represents electricity sales to SIC 01 through 39, while the MECS estimate of purchased electricity is for SIC 20 through 39 only.

- The agriculture, forestry, and fishing division is represented by SIC's 01 through 09 . Energy consumption estimates are not available for the entire division, but the U.S. Department of Agriculture does collect farm expenditure data for electricity. For 1991, total expenditures for electricity for agricultural production (SIC's 01 and 02 ) were $\$ 2,567$ million, ${ }^{61}$ or roughly 190 trillion Btu. ${ }^{62}$ Deducting that quantity from the SEDR estimate yields 3,040 trillion Btu of electricity.

- The mining division is represented by SIC's 10 through 14. According to the 1987 Census of Mineral Industries, the mining division purchased 68,176 million $\mathrm{kWh}$ (233 trillion Btu) of electricity in 1987, the latest year for which data are available. Subtracting that quantity from the SEDR estimate of electricity results in 2,807 trillion Btu of electricity.

- The construction division is represented by SIC's 15 through 17. According to the 1987 Census of Construction Industries, total expenditures for electricity were $\$ 1,089$ million, ${ }^{63}$ or approximately 80 trillion Btu. Deducting that quantity from the SEDR estimate yields 2,727 trillion Btu.

Thus, the final difference between the MECS estimate of 2,380 trillion Btu of electricity consumption and the adjusted SEDR estimate of 2,727 trillion Btu is 347 trillion Btu. It is reasonable to hypothesize that most of that remaining difference could be accounted for by: (1) the remaining SIC's in the agriculture, forestry and fishing division for which estimates are not available, and (2) increased consumption in the mining and construction divisions between 1987 and 1991. Moreover, the estimates of electricity sales to the industrial sector do not strictly follow SIC classification criteria. The instructions for Form EIA-861 provide the following definition of the industrial sector:

The industrial sector is generally defined as manufacturing, construction, mining, agriculture, fishing, and forestry establishments, Standard Industrial Classification (SIC) codes 01-39. [For the purpose of reporting, the] utility may classify industrial service using the SIC codes or based on demand or annual usage exceeding some specified limit. The limit may be set by the utility based on the rate schedule of the utility. ${ }^{64}$

\footnotetext{
${ }^{60}$ The MECS uses "net electricity" as a measure of electricity consumption in order to avoid double counting. See Appendix X.

${ }^{61}$ Unpublished estimate obtained by telephone from U.S. Department of Agriculture, National Agricultural Statistics Service, Agricultural Statistics Board.

${ }^{62}$ Conversion based on $\$ 13.486$ per million Btu. See Table A25.

${ }^{63}$ Cost information obtained by telephone.

${ }^{64}$ Form EIA-861, "Annual Electric Utility Report for the Reporting Period 1991," p. xi.
} 
This situation is potentially troublesome when making comparisons between the MECS and SEDR industrial estimates of electricity. The extent to which the respondents to Form EIA-861 classify their industrial customers based on SIC codes or their industrial rate schedules is unknown. Moreover, because the industrial rate schedule may be established by the utility, the criteria are likely to be inconsistent from utility to utility. Therefore, a customer receiving an industrial rate from a utility does not guarantee that the customer is, in fact, an industrial facility. Many commercial buildings are sufficiently large to qualify for an industrial rate, and, conversely, many small industrial facilities, while not large enough to qualify for an industrial rate, would qualify for a commercial rate. Unfortunately, there is no way to quantify the impact of these alternative methods of classifying industrial customers.

\section{Coke and Breeze}

Coke and breeze are produced by heating bituminous coal in the absence of air. That process drives off the volatile constituents of the coal and produces a porous residue consisting of carbon and mineral ash, known as coal coke. Breeze is the residue from the fine screenings of coke. Coke and breeze are used primarily as a fuel in blast furnaces.

The MECS reports that 308 trillion Btu of coke and breeze was consumed by manufacturers during 1991. The SEDR combined estimate for the industrial sector reports that the net imports of coal coke (imports minus exports) was 8.9 trillion Btu.

Both of these estimates represent attempts to avoid double counting energy sources. The MECS estimates of the primary consumption of energy and the SEDR industrial estimates include the quantity of bituminous coal used to produce the coke and breeze. Therefore, including both the coal consumed as a raw material to produce coke and the resulting coke and breeze would result in double counting. Accordingly, the SEDR industrial estimates include only net imports of coal coke.

The MECS approach also attempts to avoid the double counting that would result from including coke and breeze and the bituminous coal used to produce them. The MECS consumption estimate of 308 trillion Btu of coke and breeze excludes the quantities of those energy sources that were produced and consumed on the establishment site, and, therefore, the estimates are free of intraestablishment double counting. ${ }^{65}$ However, the MECS estimate still includes all offsite-produced (purchased and transferred in) coke and breeze, whether produced domestically or imported. Because of these different approaches, the MECS and SEDR estimates of the consumption of coke and breeze are totally incomparable.

The MECS estimate of coke and breeze consumption can be verified by reference to other EIA series. The Quarterly Coal Report includes estimates of the quantity of coke and breeze sold by coke plants. In 1991, total commercial sales of coke and breeze were 9,503 thousand short tons. ${ }^{66}$ Coke and breeze are heavy-duty energy sources, and would thus be expected to be consumed primarily within the industrial sector, by manufacturers. The MECS reports the quantity of coke and breeze that was purchased by manufacturers. In 1991, those total purchases were 9,340 thousand short tons (Table A22), a value that is quite close to the sales estimate.

\section{Residual Fuel Oil}

The MECS estimate of the primary consumption of residual fuel oil for 1991 is 454 trillion Btu. The estimate appearing in SEDR for the industrial sector is $\mathbf{3 3 5 . 9}$ trillion Btu. The difference in these two is not only substantial, it is in the opposite direction from what would be expected. The reason for that difference is not understood.

\footnotetext{
${ }^{65}$ For more details on removing interestablishment duplication from the estimates in Table Al, see Appendix B.

${ }^{66}$ Energy Information Administration, Quarterly Coal Report, January - March 1991, April - June 1991, July - September 1991, October December 1991, Table A6.
} 


\section{Distillate Fuel Oil}

The MECS estimate of the primary consumption of distillate fuel oil for 1991 is 146 trillion Btu. The estimate for the industrial sector published in SEDR was 1,139 trillion Btu. It is reasonable to attribute the difference between the estimates ( 993 trillion Btu) to the additional consumption in the construction, mining, agriculture, forestry, and fisheries sectors. For example, agricultural production (SIC's 01 and 02) accounted for approximately 400 trillion Btu of diesel fuel in $1991^{67}$, and the mining division (SIC's 10 - 14) accounted for approximately 170 trillion Btu in $1987^{68}$. Accounting for these sectors reduces the difference between the estimates to 423 trillion Btu. The remaining discrepancy could be accounted for by the construction sector, the remainder of the agricultural sector, and growth in the mining sector between 1987 and 1991.

\section{Liquefied Petroleum Gases (LPG)}

The 1991 MECS estimate of the primary consumption of LPG was 1,574 trillion Btu. The estimate published in SEDR was 1,749 trillion Btu. In the case of LPG, the difference of approximately 175 trillion Btu reasonably may be attributed to additional consumption in the construction, mining, agriculture, forestry, and fisheries sectors.

\section{Nonenergy Products}

The MECS estimates of primary consumption include an estimate of the quantity of energy consumed to produce nonenergy products at refineries. Those products are asphalt and road oil, lubricants, naphtha $<401$ degrees, other oils $\geq 401$ degrees, special naphthas, waxes, and miscellaneous nonfuel products. (See Appendix B for a discussion of the rationale for including estimates of nonenergy products in the MECS.) The total energy consumption to produce those products included the MECS in 1991 was 2,868 trillion Btu. That estimate was taken from an annual summary of shipments data adjusted for inventory change as reported by petroleum refineries on Form EIA-810, "Monthly Refinery Report." The estimate appearing in SEDR for these products is 2,640 trillion Btu, or 222 trillion Btu less than the MECS estimate. That estimate is taken directly from Petroleum Supply Annual 1991.

The difference in the estimates is attributable to slightly different estimating approaches between the MECS and those employed to derive the estimate that appears in the Petroleum Supply Annual. Specifically, the MECS estimate, as noted, represents sales of the products adjusted for inventory change. These estimates are derived to show only the quantities of the nonenergy products produced and shipped from petroleum refineries. The estimates in the Petroleum Supply Annual, however, are specifically designed to estimate the quantities of these products supplied to the market, regardless of their origin. Thus, the estimates are derived as refinery production, plus imports, minus stock change, minus exports. Except for net imports, the estimates of nonenergy products reported in the Petroleum Supply Annual and those included in the MECS are comparable.

\section{Kerosene}

The MECS estimate of the primary consumption of kerosene is 48 trillion Btu, and the estimate published in SEDR is 11.4 trillion Btu. Reasons for this difference are unknown.

\section{Finished Motor Gasoline}

The MECS estimate of the primary consumption of finished motor gasoline is 81 trillion Btu and the estimate published in SEDR is 193 trillion Btu. That substantial difference may be attributable to the additional consumption in the construction, mining, agriculture, forestry, and fisheries sectors, which are excluded from the MECS estimates.

\footnotetext{
${ }^{67}$ Unpublished estimate obtained by telephone from U.S. Department of Agriculture, National Agricultural Statistics Service, Agricultural Statistics Board.

${ }^{8}$ U.S. Bureau of the Census, Census of Mineral Industries, Fuels and Electric Energy Consumed, Table 2.
} 


\section{Crude Oil}

Crude oil inputs to refineries are excluded from the MECS except when that crude oil is consumed as a fuel. In general, the consumption of crude oil as a fuel is an extremely rare occurrence, and the MECS sample was too small to provide a reliable estimate. Accordingly, the MECS estimate of the primary consumption of crude oil is given as zero. The 1991 estimate appearing in SEDR for crude oil is 39 trillion Btu. Therefore, the two series are not comparable, but the difference is so small that it is inconsequential.

\section{Pentanes Plus, Unfinished Oils, Gasoline Blending Compounds}

In general, the MECS excludes all inputs to the refinery process in order to avoid double counting. Pentanes plus, unfinished oils, and motor gas/aviation gas blending compounds are among those inputs that are excluded. The estimates appearing in SEDR are taken directly from the Petroleum Supply Annual, which is specifically designed to reflect petroleum balance. Unfinished oils and blending compounds appear as negatives in the estimates included in SEDR because these products "... have entered the primary supply channels with their production not having been reported [elsewhere]. ${ }^{69}$

\section{Petroleum Coke and Still Gas/Waste Gas}

The MECS estimates of the primary consumption of petroleum coke and still gas/waste gas for 1991 were 617 and 1,399 trillion Btu, respectively. The estimates appearing in SEDR for these energy sources were 700 and 1,427 trillion Btu. Thus, the MECS and SEDR estimates of these energy sources are quite close and judged to be comparable.

\section{Pulping Liquor; Wood Chips, Bark, Wood Waste; Net Steam and Hot Water; and Miscellaneous Energy Sources}

All of these energy sources are included in the MECS, but excluded from the industrial estimates appearing in SEDR. In the MECS, these energy sources account for 2,291 trillion Btu. These estimates are excluded from the SEDR because of a lack of consistent historical data.

\section{Electrical System Energy Losses}

The heat content of a kilowatthour of electricity, regardless of the generation process, is 3,412 Btu. That quantity represents the amount of useful energy contained in a kilowatthour of electricity, and is used as a conversion factor to produce the MECS estimates of end-user consumption.

Electricity production, on the other hand, is typically measured as the heat value of the energy sources that were consumed by utilities to produce electricity. On the average, fossil-fueled generating plants require about 10,352 Btu of energy to produce one kilowatthour of electricity. Nuclear steam generating plants require $10,760 \mathrm{Btu}$ per kilowatthour, and geothermal generating plants require 20,997 Btu per kilowatthour. ${ }^{70}$ These values vary from one utility to another.

Electrical system energy losses include all losses incurred in the generation, transmission, and distribution of electricity, including plant use and unaccounted for quantities. These losses are estimated in SEDR as the difference between the total of all energy input at electric utilities and the total electricity sold to end users. ${ }^{71}$

\footnotetext{
${ }^{69}$ Energy Information Administration, Petroleum Supply Annual 1991 Volume 1, DOE/EIA-0340(91)1 (Washington, DC, June 1992 ), p. 140.

${ }^{70}$ Energy Information Administration, State Energy Data Report 1991, Appendix D, p. 475.

${ }^{71}$ Energy Information Administration, State Energy Data Report 1991, Appendix A, p. 396.
} 
The 1991 industrial estimates, as reported in SEDR, include electrical system energy losses of 7,014 trillion Btu. End-use consumption for the industrial sector was estimated as 3,230 trillion Btu. Thus, electric utilities consumed $10,244(7,014+3,230)$ trillion Btu of energy to produce the 3,230 trillion Btu of electricity consumed by the industrial sector.

The MECS does not include, nor does it require an estimate of electrical system energy loses because it is designed to produce estimates of end-use consumption. However, electrical system energy losses that would be associated with manufacturing end-use consumption can easily be estimated for the MECS by multiplying the end-use consumption of purchased electricity (in Btu) by 2.0023 , i.e., $(10,244-3,412) / 3,412=2.0023$. Thus, electrical system energy losses associated with the consumption of purchased electricity by the manufacturing sector would be 4,765 trillion Btu, i.e., $2.0023 \times 2,380=4,765$.

\section{A Final Observation}

This appendix has shown that many of the substantial differences between the MECS estimates of the primary consumption of energy and the combined estimates resulting from the supplier surveys can be reconciled by carefully reviewing the coverage and definitions of the data series involved. It should be emphasized that the differences are not an indication of the relative strengths or weaknesses of either series. Rather, the differences in the estimates simply reflect the differences in the intents of the end-user surveys and the supplier surveys. The overall purpose of the end-user surveys is to provide baseline energy consumption and related characteristics data for various groups of end users (manufacturers, residential housing and transportation, and commercial buildings). The overall purpose of the supplier surveys, on the other hand, is to provide baseline data on the production and supply of various fuels. To reiterate, data users should be extremely wary of attempting to compare or combine the results of the end-user and supplier surveys without careful attention to the origins and purposes of the different estimates. 


\section{Appendix E}

MECS Estimates by

International Standard

Industrial Codes 



\section{Appendix E}

\section{MECS Estimates by International Standard Industrial Codes}

The consumer demand for MECS estimates published on an alternate classification system resulted in a study on the capability of the 1991 MECS sample to produce reliable energy estimates based on the International Standard Industrial Coding system (ISIC). ${ }^{72}$

In some respects, the ISIC and SIC systems classifications are similar. Each divides the manufacturing sector by economic activities. The ISIC system has 9 major groups (two-digit) that are composed from 70 industries (fourdigit), while the SIC system that has been traditionally used by EIA has 20 major groups (two-digit) and over 400 industries (four-digit). The matching, at the four-digit level, of these ISIC and SIC activities enables the MECS to produce ISIC based estimates of energy consumption.

The ability to publish ISIC tables is defined by two factors: the industries publishable under the sample design of the MECS and the disclosure patterns of the current MECS tables. ISIC based energy estimates are primarily restricted for publication to those SIC estimates that are explicitly planned for under the MECS sampling plan. The ISIC system, for example, excludes SIC 2411 (Logging) from the manufacturing sector. As a result of that exclusion, the 1991 MECS estimate of consumption in the manufacturing sector must exclude SIC 2411. However, the MECS was not designed to reliably produce an independent estimate for SIC 2411, rather SIC 2411 was grouped within the major group SIC 24 (Lumber and Wood Products). Specifically, the 1991 MECS was designed to provide precise energy related estimates for 62 categories of Standard Industrial Codes (SIC):

- forty selected industries (4-digit);

- two industry groups (3-digit); and,

- twenty major industry groups (2-digit) of the manufacturing division. ${ }^{73}$

To the extent that these SIC groupings match the ISIC system at the two-digit level, the MECS estimates have been tabulated on the ISIC system (See Table E1). In order for a ISIC estimate to be presented, each ISIC estimate must be comprised of a minimum proportion of the SIC estimates that were explicitly designed for the MECS, as measured by energy consumed for purposes of Heat, Power, and Electricity Generation (Table A4). That minimum proportion was 90 percent for the 1991 MECS; however, most ISIC estimates exceeded that proportion. For example, ISIC major group 31 (Food Processing, Beverages, and Tobacco) is 100 percent comprised by combining SIC 20 (Food and Kindred Products) and SIC 21 (Tobacco Products).

Disclosure analysis represents a secondary problem in developing ISIC based energy estimates. That analysis is based on a disclosure study and is mandatory under the confidentiality legislation of Title 13 . Withheld estimates are a major concern when ISIC estimates are produced for public review. ${ }^{74}$ Due to the ISIC exclusion of SIC 2411, for example, the ISIC manufacturing total has been withheld for confidentiality purposes.

\footnotetext{
${ }^{72}$ Since Version 3 of the International Standard Industrial Classification system was incomplete at the time of this study, Version 2 was provided by the U.S. Bureau of the Census. Taylor Murphy, industry analyst at the Bureau of the Census, acted as the liaison for EIA.

${ }^{73}$ For additional information on the sample design for the 1991 MECS, see the Energy Information Administration report "Development of the 1991 Manufacturing Energy Consumption Survey", DOE/EIA - 0555(92)/2.

74"Withheld estimates" pertain to disclosures and to estimates that do not meet EIA publication standards. Disclosure will be conducted at the primary and secondary levels of the MECS.
} 
Table E1. Manufacturing Consumption of Energy for Purposes of Heat, Power, and Electricity Generation by International Standard Industrial Codes, 1991

\begin{tabular}{|c|c|c|}
\hline $\begin{array}{l}\text { ISIC } \\
\text { Code }\end{array}$ & International Industry Groups & $\begin{array}{c}\text { Total } \\
\text { (trillion Btu) }\end{array}$ \\
\hline 31 & Food Processing, Beverages, and Tobacco & 978 \\
\hline 32 & 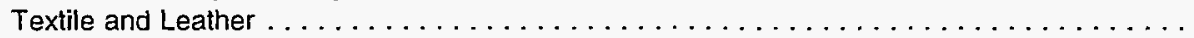 & 335 \\
\hline 33 & Wood and Wood Products, other than Pulp and Paper $\ldots \ldots \ldots \ldots \ldots \ldots$ & 465 \\
\hline 34 & 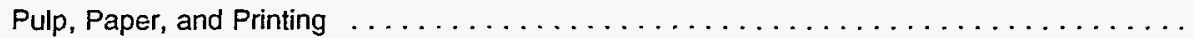 & 2,573 \\
\hline 35 & 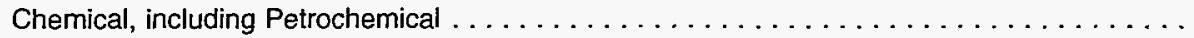 & 6,276 \\
\hline 36 & 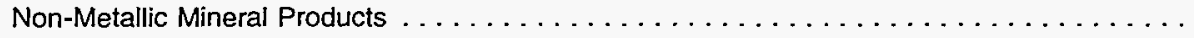 & 899 \\
\hline 37 & 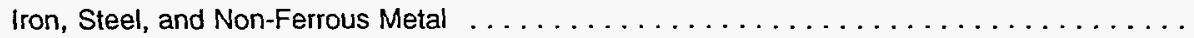 & 2,282 \\
\hline 38 & 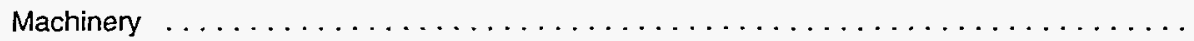 & W \\
\hline \multirow[t]{2}{*}{39} & Miscellaneous Manufacturing Industries $\ldots \ldots \ldots \ldots \ldots \ldots \ldots \ldots$ & W \\
\hline & 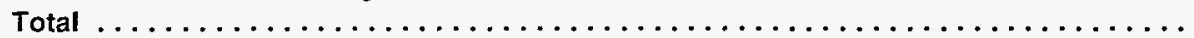 & W \\
\hline
\end{tabular}

Source: Energy Information Administration, Office of Energy Markets and End Use, Energy End Use and Integrated Statistics Division, 1991 Manulacturing Energy Consumption Survey.

$W=$ Withheld to avoid disclosing data for individual establishments or due to lack of industry-level representation in sample design. 


\section{Appendix F \\ Manufacturing Energy \\ Consumption Survey \\ Forms}





\section{Manufacturing Energy Consumption Survey Form EIA-846A}

OMB No. 1905-0169: Approval Expires 11/3094

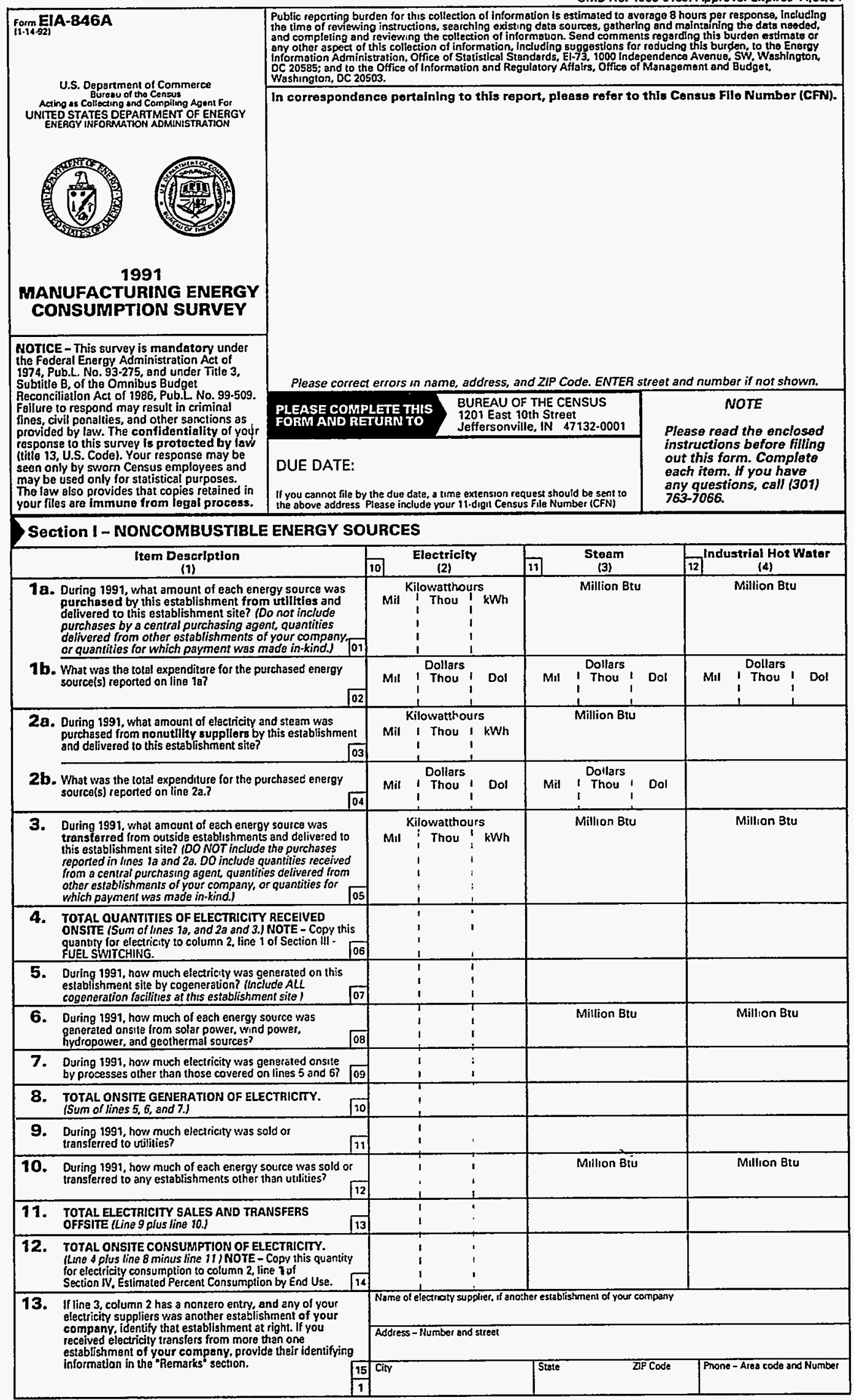




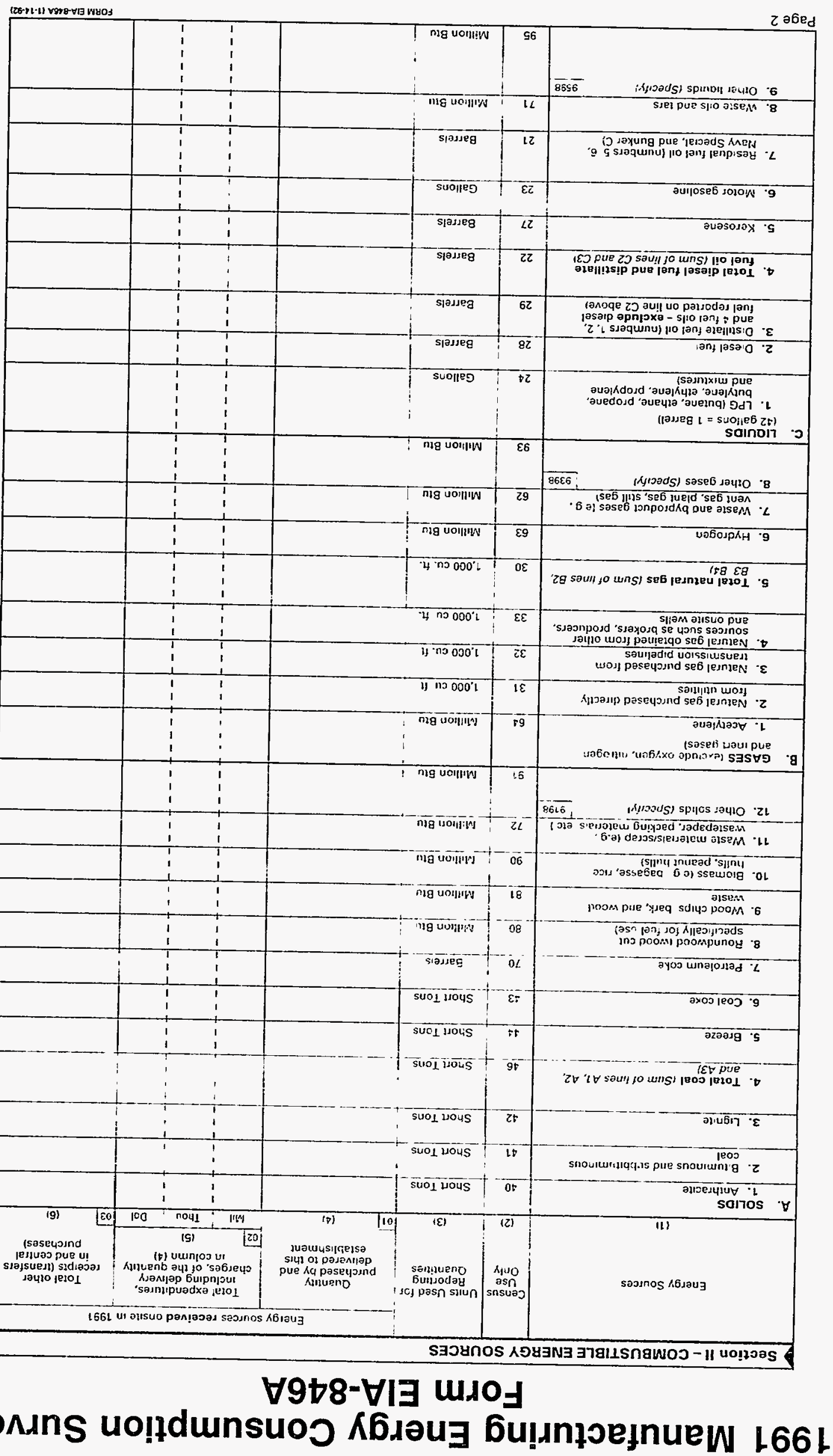


1991 Manufacturing Energy Consumption Survey Form EIA-846A

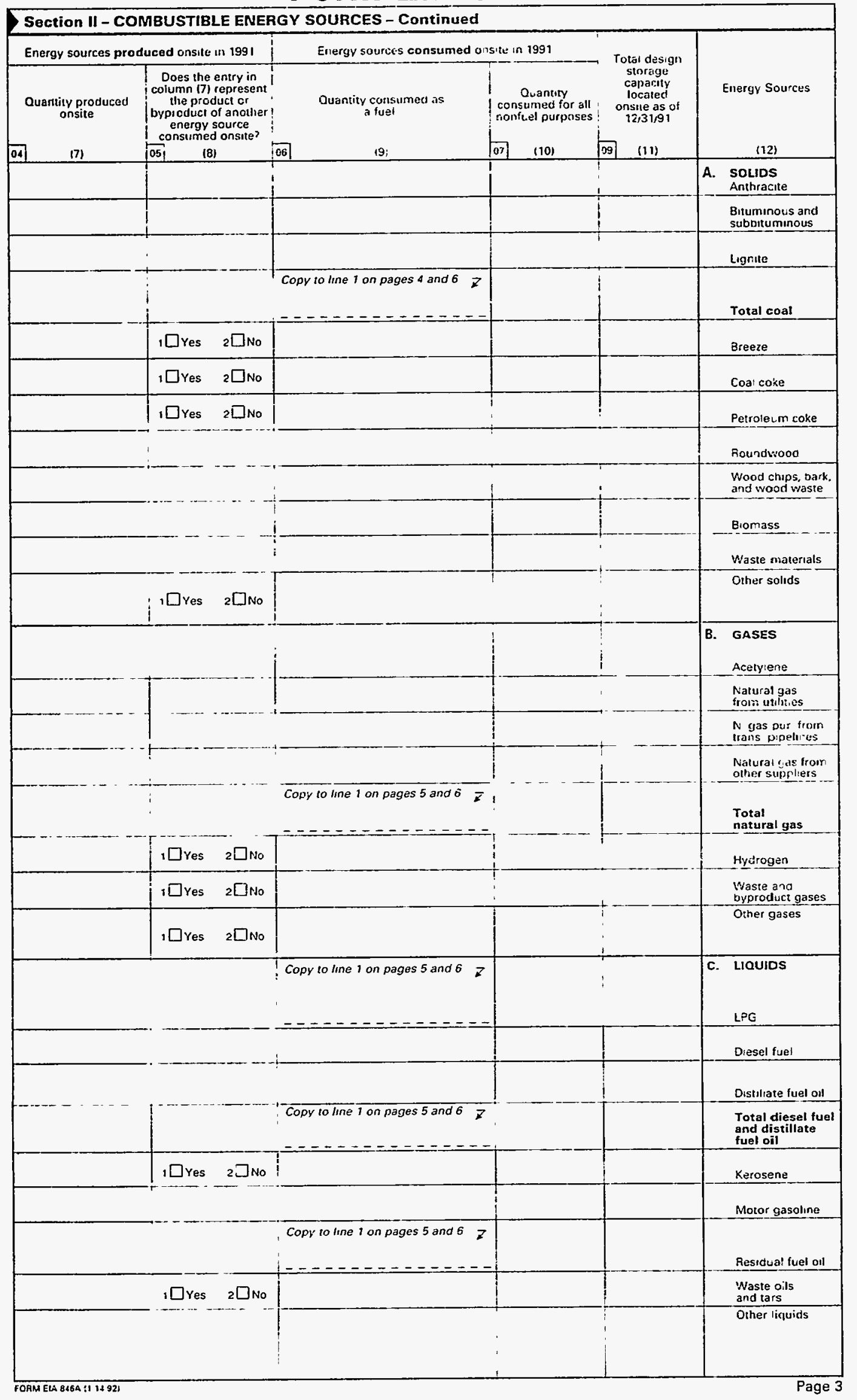




\section{Manufacturing Energy Consumption Survey Form EIA-846A}

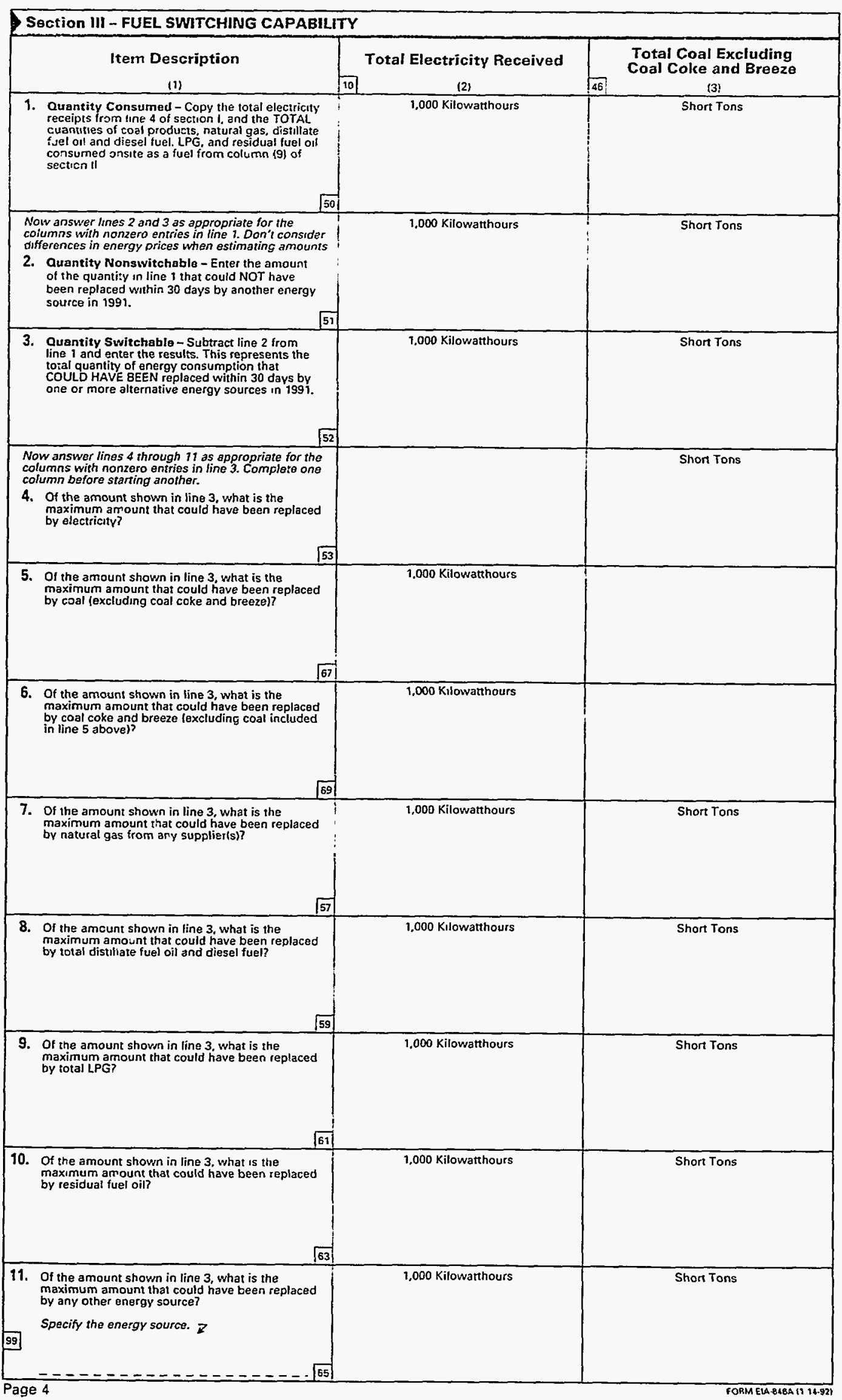



Form EIA-846A

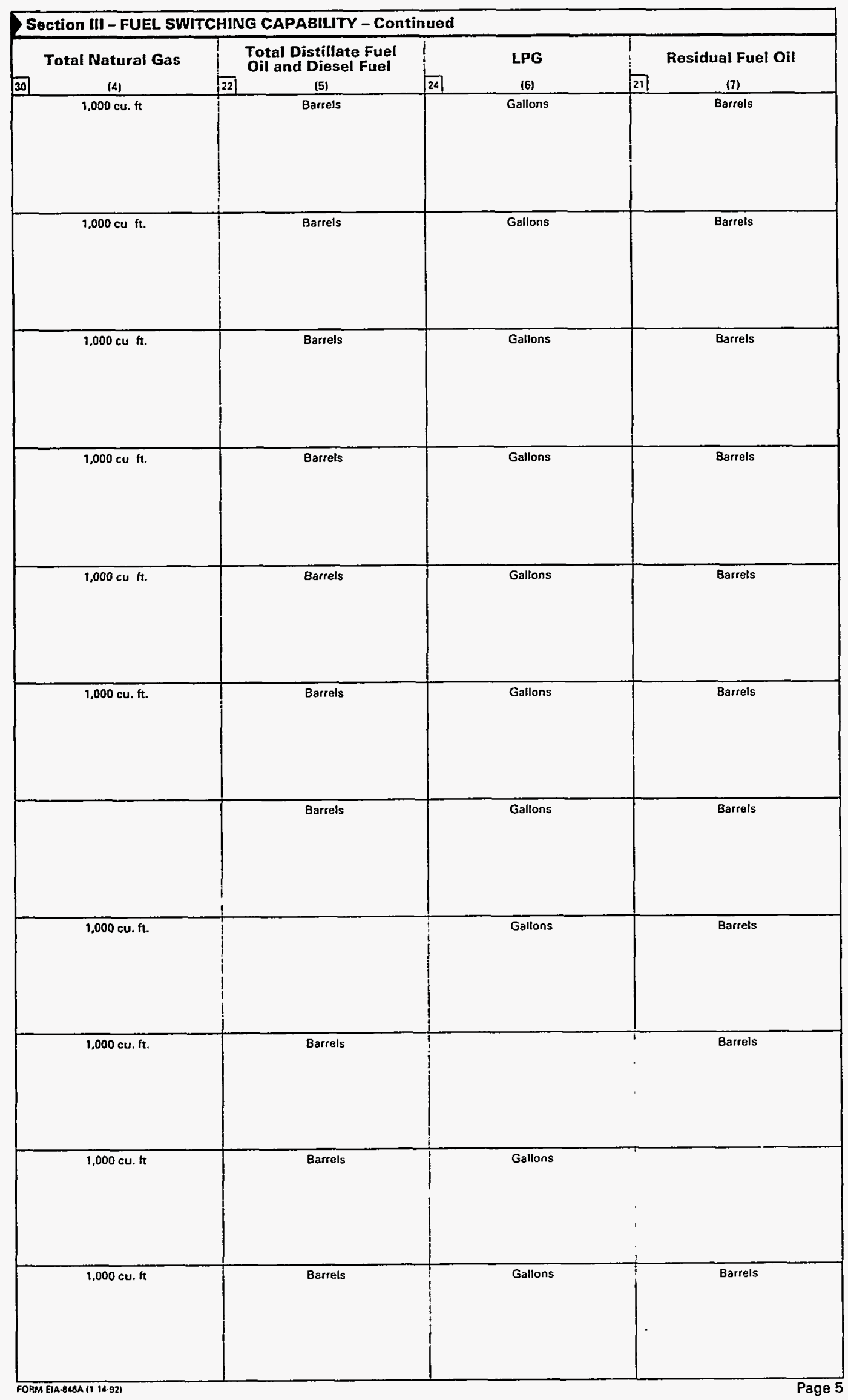




\section{Manufacturing Energy Consumption Survey Form EIA-846A}

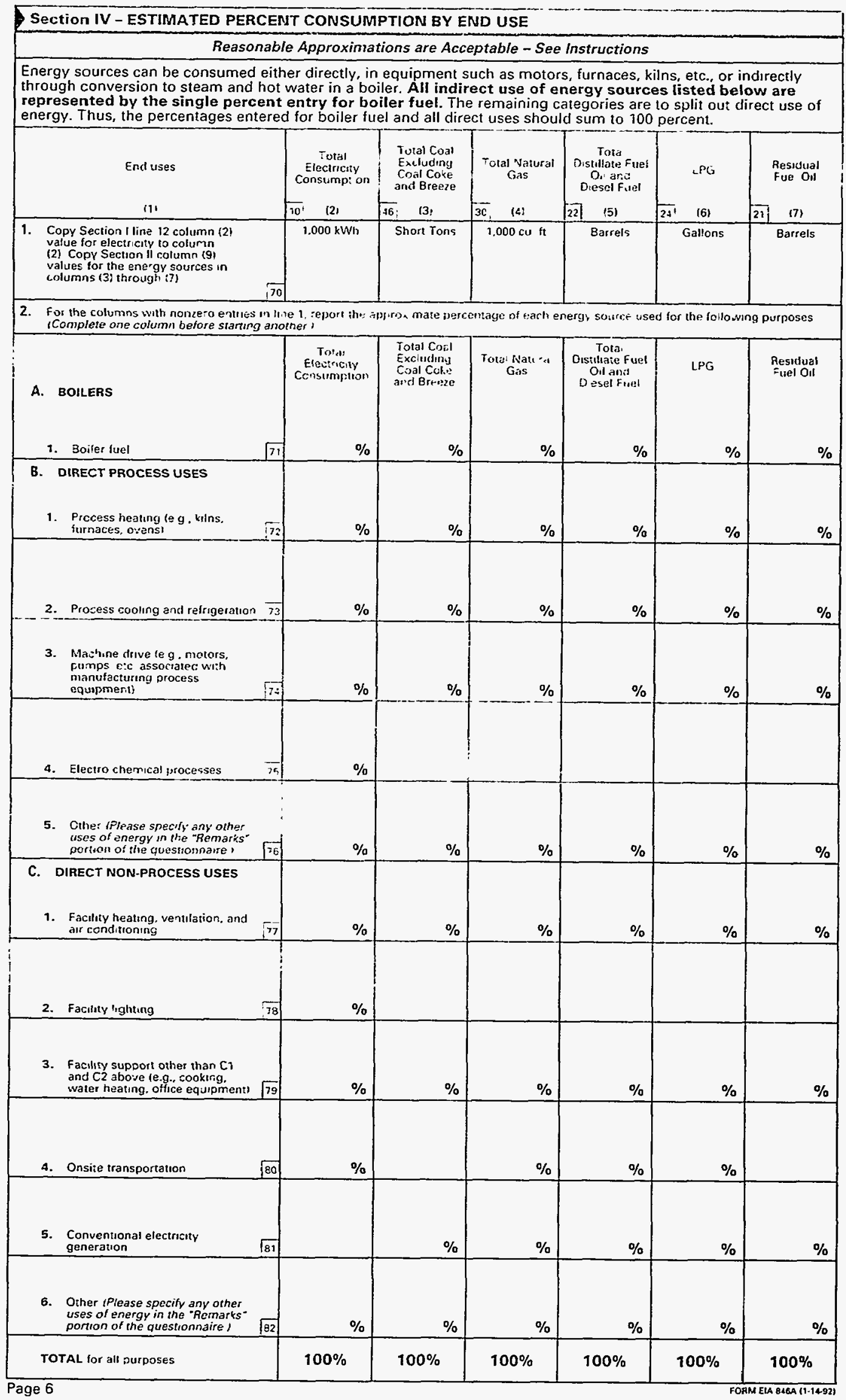




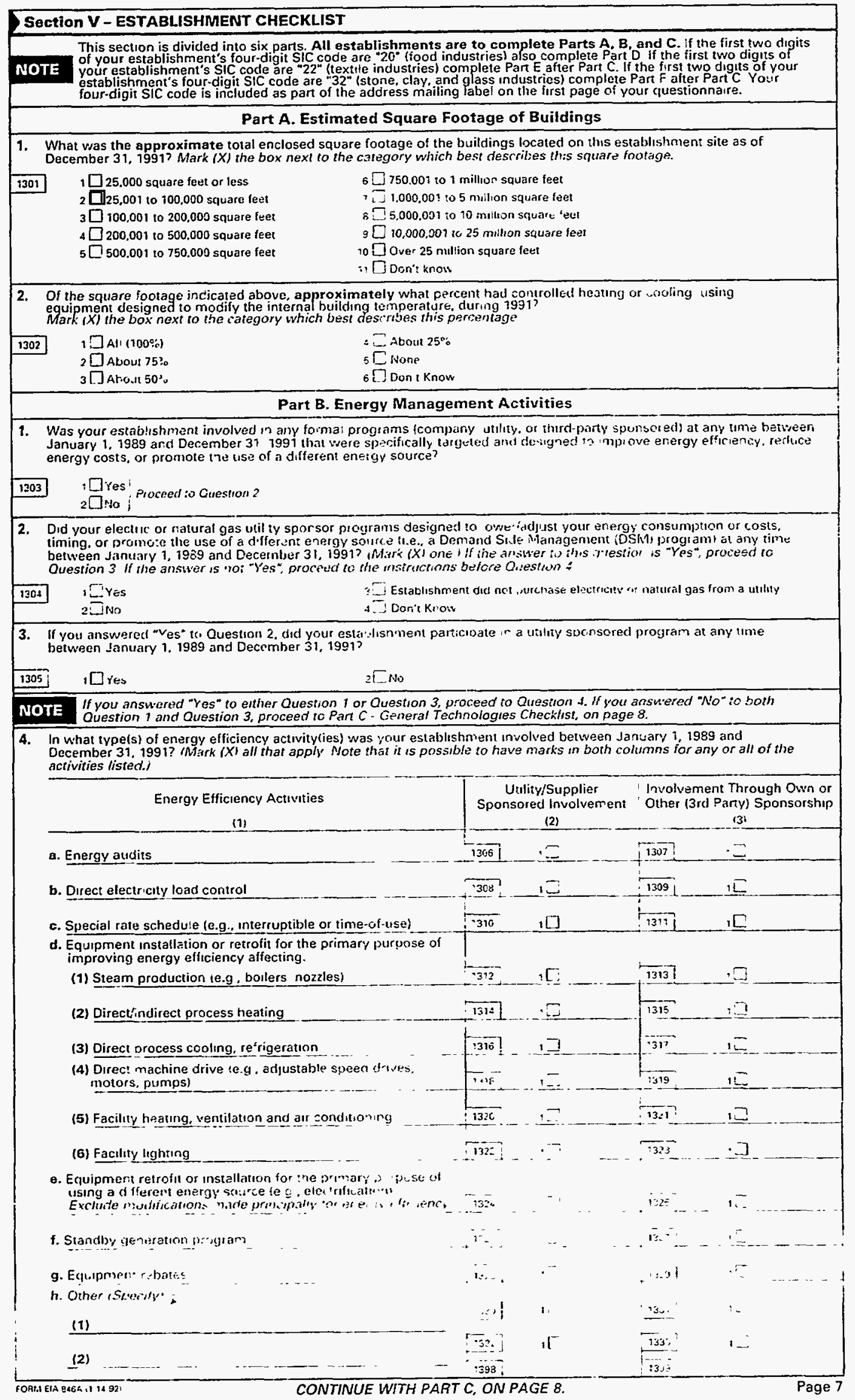




\section{Manufacturing Energy Consumption Survey Form EIA-846A}

Section V-ESTABLISHMENT CHECKLIST - Continued

All establishments should complete Part $C$. Mark $(X)$ the box next to each technology that was in place at your

establishment during 1997. Mark (X) all technologies that apply. If your establishment has a two-digit SIC code other than 20 22, cr 32, proceed to Section Vi-Remarks. Establishments in SiC 20 should complete Part D-Technology Checklist for Food in SIC 32 should complete ParT F - Technology Checklist for Stone, Clay and Glass industries before proceeding to Section VI.

Part C. - General Technologies

$1401,[$ Computer control of bulding environment fe.g., space heating or cooling equipment, lights)

1402 , $\square$ computer control of processes or major energy-using equipment (e.g., boilers, furnaces, conveyers) used in the manufacturing process 1403 1. Waste heat recovery

1406], [ Adjustab e-speed motors

Part D. - SIC 20 Food Industries: Specific Technologies

\begin{tabular}{|c|c|c|c|}
\hline & $1=\ln$ & 1409 & tary engines and/or turt \\
\hline 1405 & 二 Microwave drying & 1430 & $1 \square$ Membrane separation \\
\hline $40 ?$ & i- Clcsed-cycle heat pump system used to recover $t$ & 1411 & rradiation \\
\hline $08^{\circ}$ & Open-cycle reat pump system used to produ & 1412 & Freeze concentration \\
\hline
\end{tabular}

14081 Z Open-cycle neat pump system used to produce steam

Part E. - SiC 22 Textile Industries: Specific Technologies

\begin{tabular}{|c|c|c|}
\hline $1413: Z$ Open-end spinming & 1418 & $1 \square$ Dye bath reuse \\
\hline $1 \$ 14 \quad 1 \ldots$ Water-jar weaving & 1429 & $1 \square$ Foam dyeing \\
\hline $3+15$ VProjectile weaving & 1420 & $1 \square$ Foam prınting \\
\hline 1416 i [ Wet-on-wet dyeing and finishung & 1421 & $1 \square$ foam finishing \\
\hline 1212, Cindirect steam heating of dye & 1422 & $1 \square$ Low-add-on finishing \\
\hline
\end{tabular}

Part F. - SIC 32 Stone, Clay, and Glass Industries: Specific Technologies

Glass related

:223: Doxygen enriched combustion air

$\because 24, \square$ Forehearth designed for independently applied heatung and cacting operations and minimal energy use

$425, \square$ Foreheanh designed to eliminate side to middie temperature gradients with improved temperature

1428 1DBatch preheaters

$-\$ 27, \square$ Ccgenerasion system which uses waste heat rejected in furnace exhaust to generate steam in waste heat boilers

i428 : $\square$ Advanced glass refiner

Section V1 - REMARKS

Please use this space or atlach a separate sheet for any explanations that may be essential in understanding your reported data. Be sure tc include the name, address, and telephone number of power generating establishments of your company that transferred or de'ivered electricity or steam to your establishment in 1991 if you did not have enough room in Section 1. i599.

Section VII-CERTIFICATION

Name of person to contact regarding this report - Print or type

1429 High-efficiency classifiers in closed-circult grunding plants Improved grinding media and linings, wear resistant materials such as high chrome alloys, and classifying liners

$14311 \square$ Waste heat drying

1432 1 Dry-suspension prehester kilns

143310 Dry-precalcìner kilns

1434 1 $\square$ Kiln combustion system improvement such as semi-directindirect coal firing, optimal oxygen levels and ananced burners matched to the kiln/cooler design flame

1435] $1 \square$ Controlled particle size cement

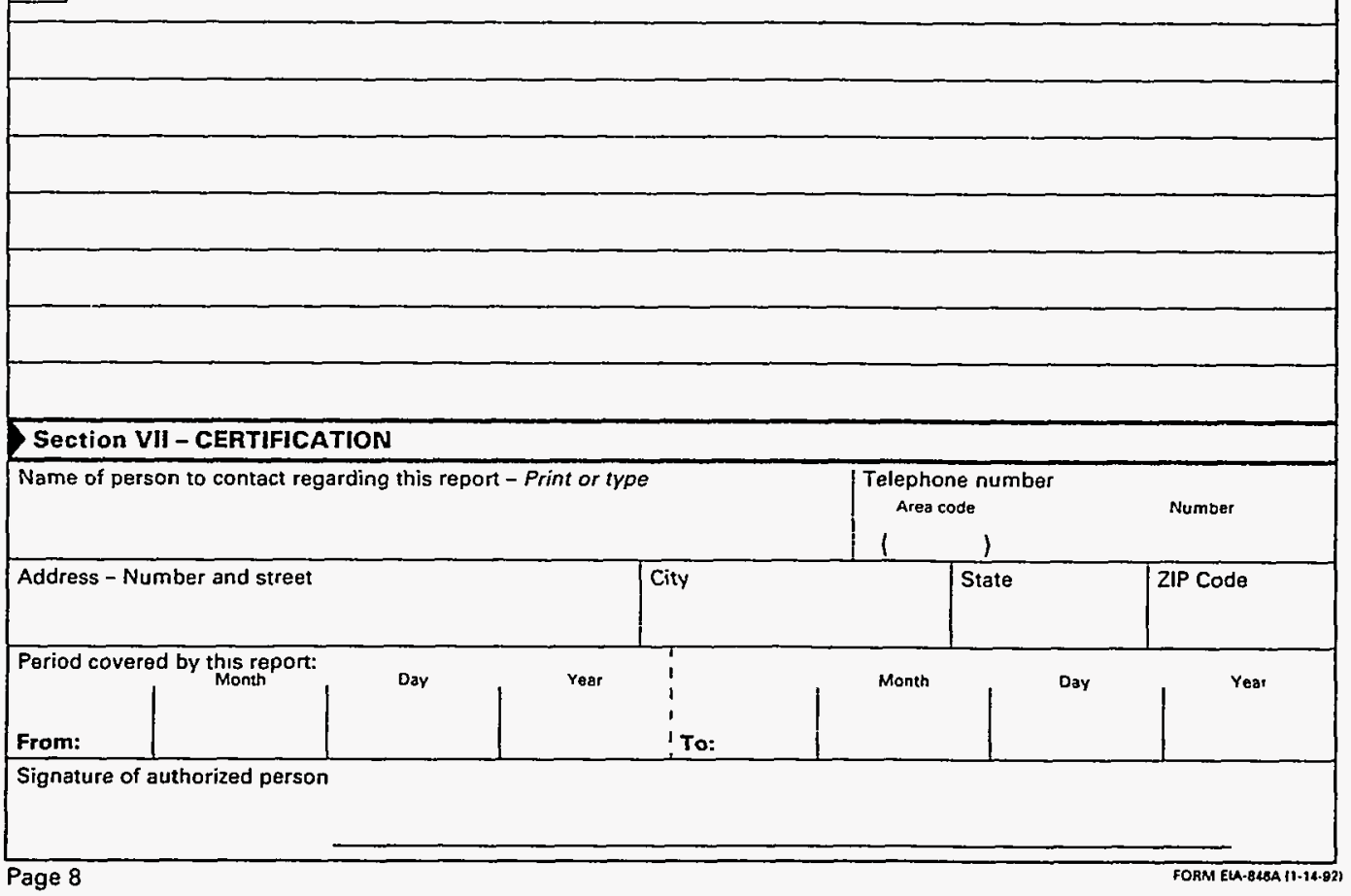




\section{Manufacturing Energy Consumption Survey Form EIA-846B}

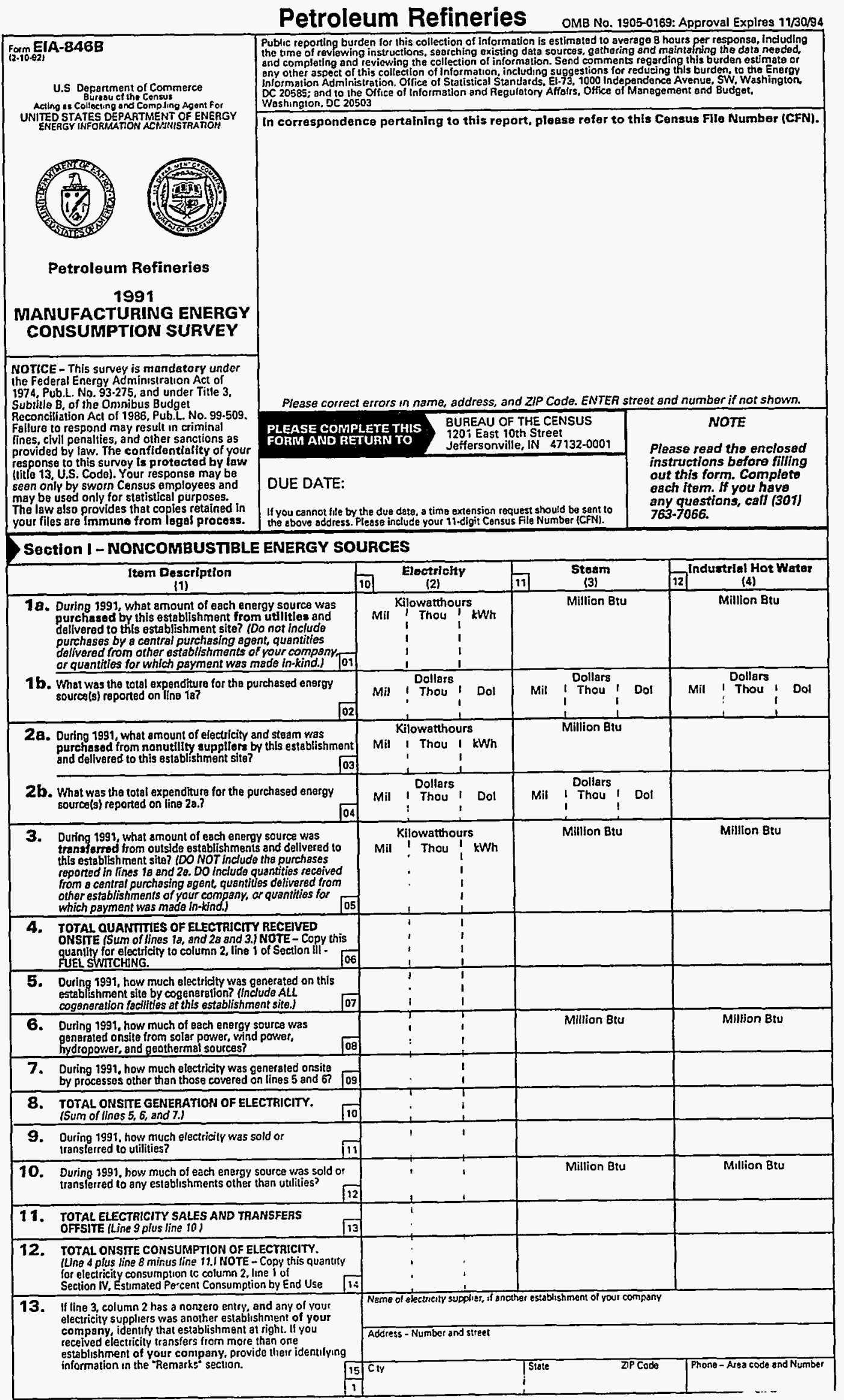




\section{Manufacturing Energy Consumption Survey Form EIA-846B}

\section{Petroleum Refineries}

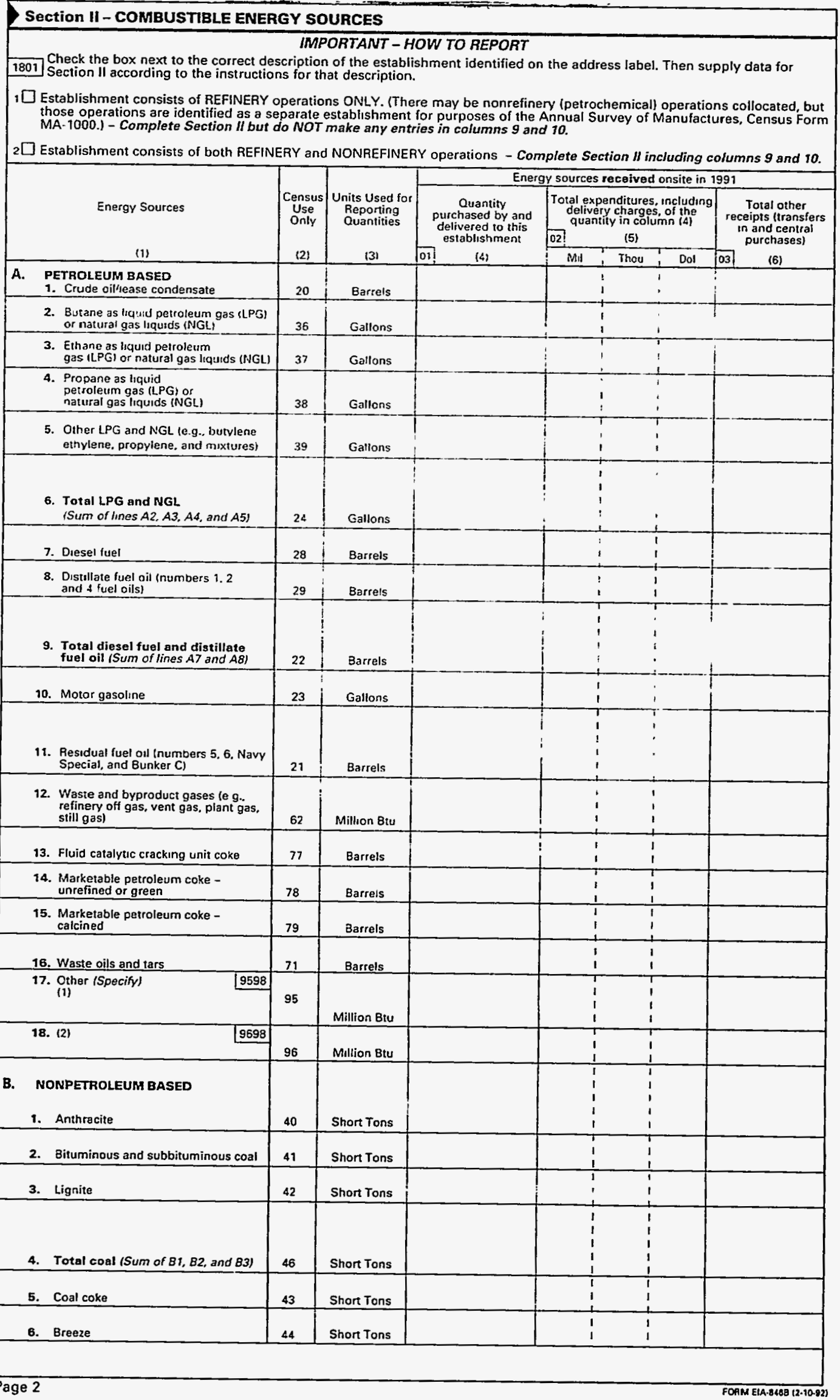




\section{Manufacturing Energy Consumption Survey Form EIA-846B \\ Petroleum Refineries}

Section II - COMBUSTIBLE ENERGY SOURCES - Continued

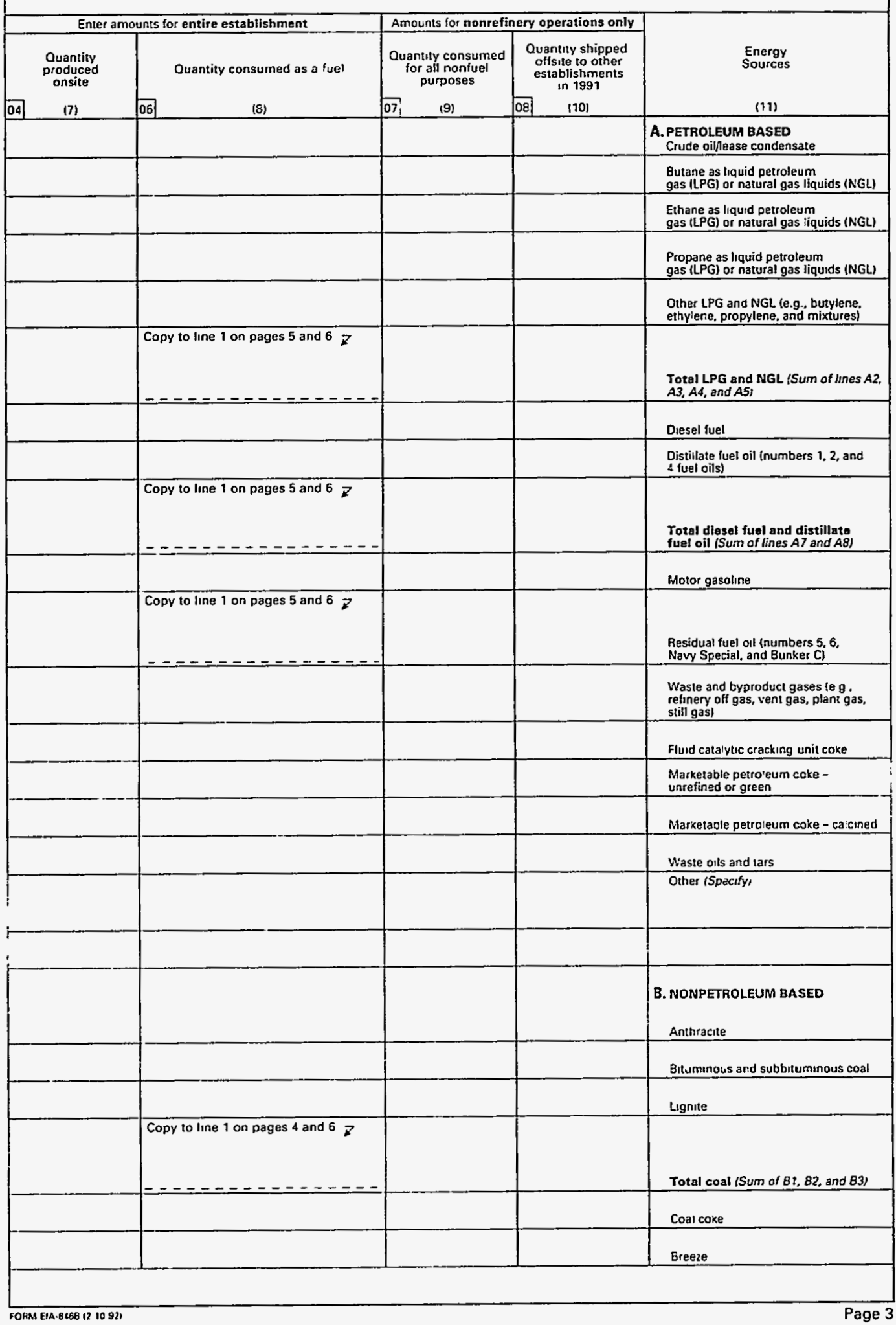




\section{Manufacturing Energy Consumption Survey Form EIA-846B}

\section{Petroleum Refineries}

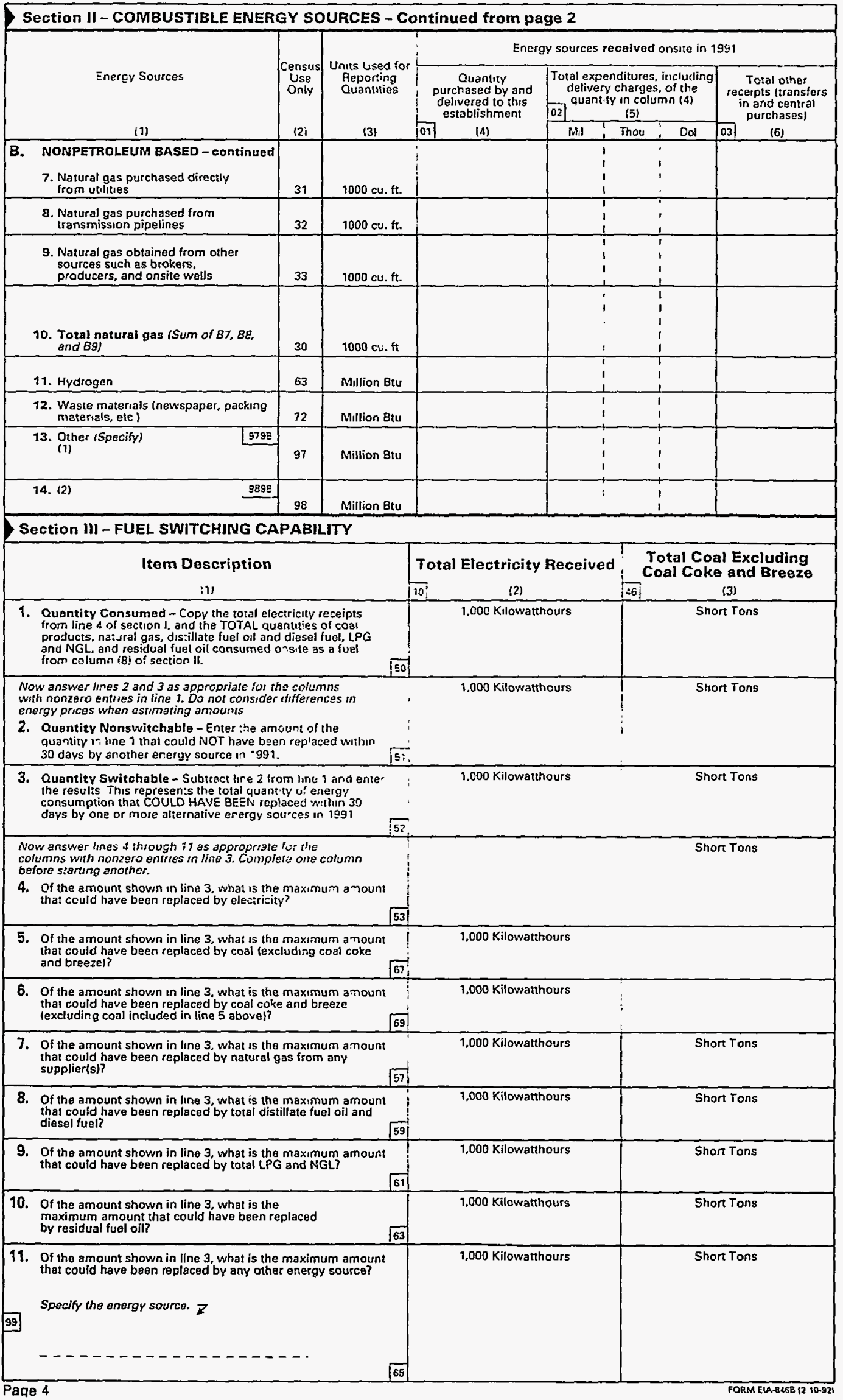




\section{Manufacturing Energy Consumption Survey Form EIA-846B}

Petroleum Refineries

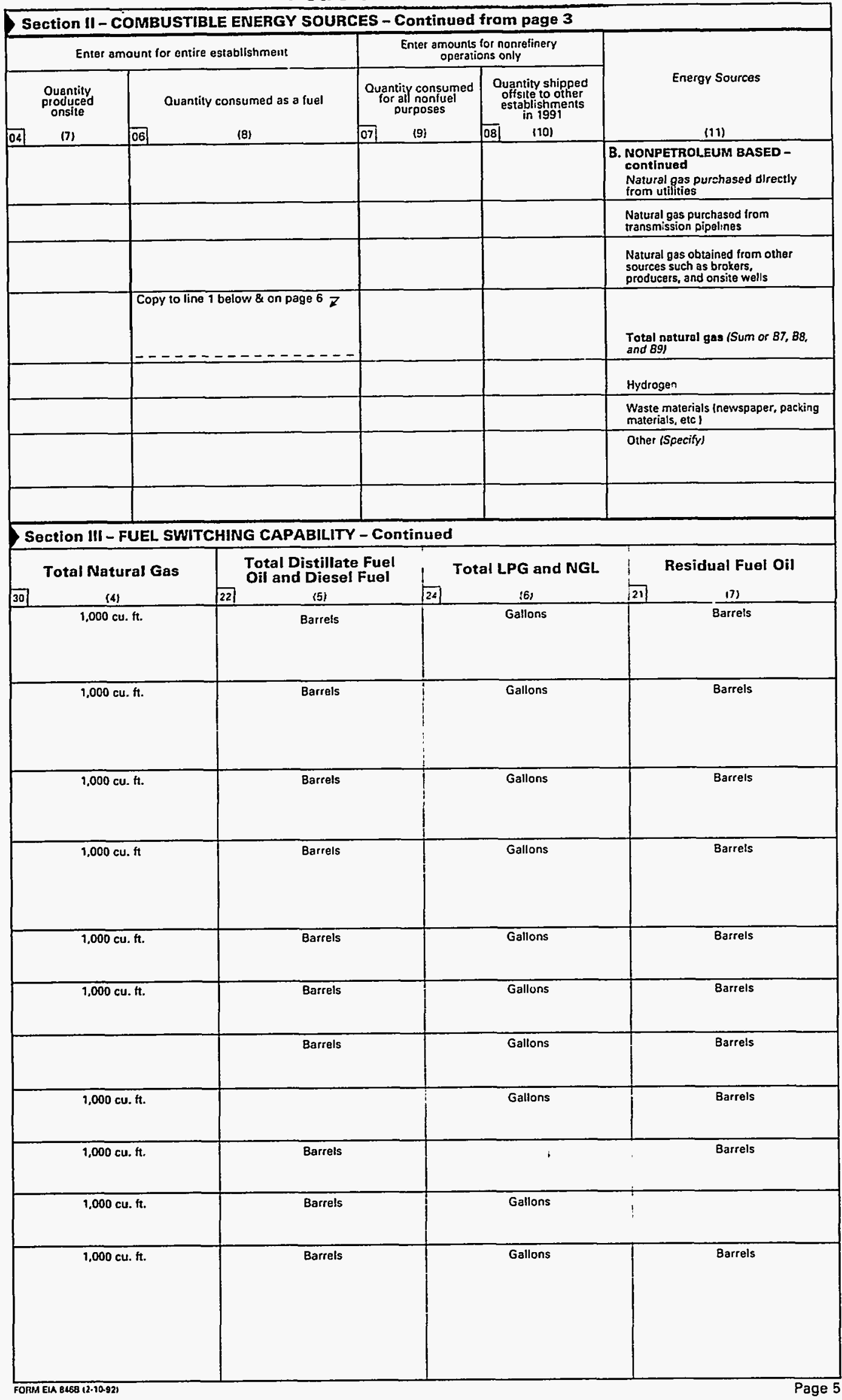




\section{Manufacturing Energy Consumption Survey Form EIA-846B}

\section{Petroleum Refineries}

\begin{tabular}{|c|c|c|c|c|c|c|}
\hline \multicolumn{7}{|c|}{ Section IV - ESTIMATED PERCENT CONSUMPTION BY END USE } \\
\hline \multicolumn{7}{|c|}{ Reasonable Approximations are Acceptable - See Instructions } \\
\hline \multicolumn{7}{|c|}{$\begin{array}{l}\text { Energy sources can be consumed either directly, in equipment such as motors, furnaces, kilns, etc., or indirectly } \\
\text { through conversion to steam and hot water in a boiler. All indirect use of energy sources listed below are } \\
\text { represented by the single percent entry for boiler fuel. The remaining categories are to split oul direct use of } \\
\text { energy. Thus, the percentages entered for boiler fuel and all direct uses should sum to } 100 \text { percent. }\end{array}$} \\
\hline End uses & $\begin{array}{l}\text { Total } \\
\text { Electruiv } \\
\text { Consumpiner }\end{array}$ & 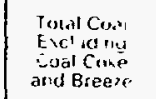 & 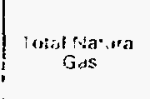 & 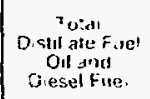 & $\begin{array}{l}\text { Total } L P G \\
\text { andingl }\end{array}$ & $\begin{array}{l}\text { Fonstrduat } \\
\text { Fuet On! }\end{array}$ \\
\hline (1) & 10: 21 & $46 \quad 31$ & $30]$ & $\because 2 \quad$ (b) & $24 \quad .6$ & $21 ! \quad(7)$ \\
\hline $\begin{array}{l}\text { 1. Copy Section I line } 12 \text { columin } 12 \text { ) } \\
\text { value for electricity to coltumn } \\
\text { (21) Copy Section } 11 \text { column (8) } \\
\text { values for the energy sources in } \\
\text { columns ( } 3 \text { ) through }(7)\end{array}$ & $1,000 \mathrm{kWh}$ & Short Tons & $1,000 \mathrm{cu} . \mathrm{ft}$ & Barrels & Gallons & Barrels \\
\hline \multicolumn{7}{|c|}{$\begin{array}{l}\text { 2. For the coiumns with nonzero entries in line } 1 \text {, report the approximate percentage of eacr erergy source used for the following purposes } \\
\text { iComplete one column before starting another., }\end{array}$} \\
\hline A. BOILERS & $\begin{array}{c}\text { Total } \\
\text { Electricity } \\
\text { Consumption }\end{array}$ & $\begin{array}{l}\text { Total Coat } \\
\text { Excluding } \\
\text { Coal Coke } \\
\text { and Breeze }\end{array}$ & $\begin{array}{l}\text { Total Vaiural } \\
\text { Gas }\end{array}$ & $\begin{array}{l}\text { Toial } \\
\text { Distiliate Fue! } \\
\text { Ol anc } \\
\text { Diesel Fuel }\end{array}$ & $\begin{array}{l}\text { Tolal LPG } \\
\text { and } \mathrm{NGL}\end{array}$ & $\begin{array}{l}\text { Residual } \\
\text { fuel Oul }\end{array}$ \\
\hline 1. Boiler fuel & $\%$ & $\%$ & $\%$ & $\%$ & $\%$ & $\%$ \\
\hline B. DIRECT PRUCESS USES & & & & & & \\
\hline $\begin{array}{l}\text { 1. Process heating (e.g, kilns, } \\
\text { furnaces. ovens) }\end{array}$ & $\%$ & $\%$ & $\%$ & $\%$ & $\%$ & $\%$ \\
\hline 2. Process cooling and retrigeration 73 & $\%$ & $\%$ & $\%$ & $\%$ & $\%$ & $\%$ \\
\hline $\begin{array}{l}\text { 3. Machine drive le.g., motors, } \\
\text { pumps, etc. associated with } \\
\text { manuacicuring process } \\
\text { equipment) }\end{array}$ & $\%$ & $\%$ & $\%$ & $\%$ & $\%$ & $\%$ \\
\hline 4. Electro-chemical processes & $\%$ & & & & & \\
\hline $\begin{array}{l}\text { 5. Other (Please specify any other } \\
\text { uses of energy in ihe "Remarks" } \\
\text { portion of the questionnare.) }\end{array}$ & $\%$ & $\%$ & $\%$ & $\%$ & $\%$ & $\%$ \\
\hline $\begin{array}{l}\text { C. DIRECT NON-PROCESS USES } \\
\text { 1. Facility hiea:ng ventilation, and } \\
\text { airconditioring }\end{array}$ & $\%$ & $\%$ & $\%$ & $\%$ & $\%$ & $\%$ \\
\hline 2. Facslity lightung & $\%$ & & & & & \\
\hline $\begin{array}{l}\text { 3. Facl iy support other than } C_{i} \\
\text { and C2 above (e.g.c cooktng. } \\
\text { water heating, office equiprient } \\
79\end{array}$ & $\%$ & $\%$ & $\%$ & $\%$ & $\%$ & $\%$ \\
\hline 4. Orsite transportation & $\%$ & & $\%$ & $\%$ & $\%$ & \\
\hline $\begin{array}{l}\text { 5. Conventional electrritity } \\
\text { generation }\end{array}$ & & $\%$ & $\%$ & $\%$ & $\%$ & $\%$ \\
\hline $\begin{array}{l}\text { 6. Other (Please specify any other } \\
\text { uses of ene:gy in the "Remarks" } \\
\text { portion of the questionnaire., }\end{array}$ & $\%$ & $\%$ & $\%$ & $\%$ & $\%$ & $\%$ \\
\hline TOTAL for all purposes & $100 \%$ & $100 \%$ & $100 \%$ & $100 \%$ & $100 \%$ & $100 \%$ \\
\hline
\end{tabular}




\section{Manufacturing Energy Consumption Survey Form EIA-846B}

\section{Petroleum Refineries}

\begin{tabular}{|c|c|c|c|c|c|}
\hline \multicolumn{6}{|c|}{ Section V - ESTABLISHMENT CHECKLIST } \\
\hline \multicolumn{6}{|c|}{ All establishments are to complete Parts A, B, and C. } \\
\hline \multicolumn{6}{|c|}{ Part A. Estimated Square Footage of Buildings } \\
\hline \multicolumn{6}{|c|}{$\begin{array}{l}\text { What was the opproximate total enclosed square footage of the buildings located on this establishment site as of } \\
\text { December } 31,1991 \text { ? Mark }(X) \text { the box next to the category which best describes this square footage. }\end{array}$} \\
\hline & $\begin{array}{l}1 \square 25,000 \text { square feet or less } \\
2 \square 25,001 \text { to } 100,000 \text { square feet } \\
3 \square 100,001 \text { to } 200.000 \text { square feet } \\
6 \square 200.001 \text { to } 500,000 \text { square feet } \\
5 \square 500,001 \text { to } 750,000 \text { square feet }\end{array}$ & \multicolumn{4}{|c|}{$\begin{array}{l}6 \square 750,001 \text { to } 1 \text { mithon square feet } \\
7 \square 1,000,001 \text { to } 5 \text { million square feet } \\
8 \square 5.000,001 \text { to } 10 \text { million square feet } \\
9 \square 70,000,001 \text { to } 25 \text { million square feet } \\
10 \square \text { Over } 25 \text { million square feet } \\
11 \square \text { Dor't knows }\end{array}$} \\
\hline \multicolumn{6}{|c|}{$\begin{array}{l}\text { Of the square footage indicated above, approximately what percent had controlled heating or cooling, using } \\
\text { equipment designed to modify the internal building temperature, durng } 1991 \text { ? } \\
\text { Mark }(X) \text { the box next to the category whits pest describes this percentage }\end{array}$} \\
\hline 1302 & $\begin{array}{l}, \square \text { All (100\%) } \\
2 \square \text { About } 75 \% \\
3 \square \text { About } 50 \%\end{array}$ & \multicolumn{4}{|c|}{$\begin{array}{l}\text { About } 25^{\circ} \text { v } \\
5 \text { Jone } \\
\text { Dont Know }\end{array}$} \\
\hline \multicolumn{6}{|c|}{ Part B. Energy Management Activities } \\
\hline \multicolumn{6}{|c|}{$\begin{array}{l}\text { 1. Was your establishment involved in ariy formal programs (company, uility, or third-panty sponsoredl at any time between } \\
\text { January } 2,1989 \text { and December } 31 \text {, } 1991 \text { that were specifically targeted and designed to improve energy efficiency, reduce } \\
\text { energy costs, or promote the use of a different energy source? }\end{array}$} \\
\hline 1303 & \multicolumn{5}{|l|}{$\begin{array}{l}\text { 1[IYes Proceed to Quesum } 2 \\
2 \square \text { wo }\end{array}$} \\
\hline \multicolumn{6}{|c|}{ 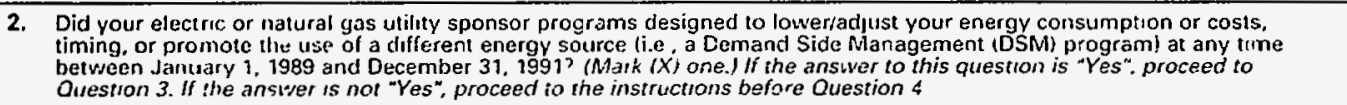 } \\
\hline 1304 & $\begin{array}{l}\text { [IYes } \\
2[] \text { roo }\end{array}$ & \multicolumn{4}{|c|}{ 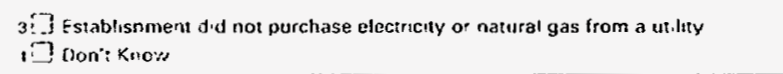 } \\
\hline \multicolumn{6}{|c|}{$\begin{array}{l}\text { 3. If you answered "Yes" to Question 2, did yous estabish nent participate in a utility sponsored program at any tume } \\
\text { between January 1, } 1989 \text { and December } 31 \text { 19912 }\end{array}$} \\
\hline \multirow{2}{*}{1305} & ${ }_{1}^{\square} \square \mathrm{yes}$ & \multicolumn{4}{|l|}{${ }_{2}[\mathrm{No}$} \\
\hline & \multicolumn{5}{|c|}{ NOTE } \\
\hline \multicolumn{6}{|c|}{$\begin{array}{l}\text { 4. In what type(s) of energy efficiency activity(ies) was your establishment involved between January } 1,1989 \text { and } \\
\text { December } 31 \text {, } 1991 \text { ? (Mark (X) all that apply. Note that it is possible to have marks in both columns for any or all of the } \\
\text { activities listed.) }\end{array}$} \\
\hline & $\begin{array}{c}\text { Energy Efficiency Ac } \\
\text { (1) } \\
\end{array}$ & & $\begin{array}{l}\text { Utility/Supplier } \\
\text { Sponsored Involvement } \\
\text { (2) }\end{array}$ & $\begin{array}{l}\text { Invol } \\
\text { Othe: } \\
\end{array}$ & $\begin{array}{l}\text { Through Own or } \\
\text { Party) Sponsorship } \\
\text { (3) }\end{array}$ \\
\hline \multicolumn{3}{|c|}{ a. Energy audits } & 1305 & 1307 & $\sqrt{\square}$ \\
\hline \multicolumn{3}{|c|}{ b. Direct electricity load control } & .1308 & 1309 & 1 \\
\hline \multicolumn{3}{|c|}{ c. Special rate schedule (e.g., interruptible or time-of-use) } & 1310 & 1311 & $\square$ \\
\hline \multicolumn{6}{|c|}{$\begin{array}{l}\text { d. Equipment installation or retrofit for the primary purpose of } \\
\text { improving energy efficiency affecting: }\end{array}$} \\
\hline \multicolumn{3}{|c|}{ (2) Directindirect process heating } & 1314 & 1315 & $1 \square$ \\
\hline \multicolumn{3}{|c|}{ (3) Direct process cooling, refrigeration } & 1316 & 1317 & ${ }_{1} \square$ \\
\hline \multicolumn{3}{|c|}{$\begin{array}{l}\text { (4) Direct machine drive (e.g., adjustable speed drives, } \\
\text { motors, pumps) }\end{array}$} & 1318 & 1319 & $1 ㅁ$ \\
\hline \multicolumn{3}{|c|}{ (5) Facility heating, ventilation and air conditioning } & 1320 & 1321 & 미 \\
\hline \multicolumn{3}{|c|}{ (6) Facility lighting } & \begin{tabular}{|r|r|}
322 \\
\end{tabular} & 7323 & 1 미 \\
\hline \multicolumn{3}{|c|}{$\begin{array}{l}\text { 0. Equipment retrofit or installation for the primary purpose of } \\
\text { using a different energy source (e.g.e electrification). } \\
\text { Exclude modifications made principally for energy etficiency }\end{array}$} & 1324 & 1325 & $1 \mathrm{D}$ \\
\hline \multicolumn{3}{|c|}{ f. Standby generation program } & 1326 & 1327 &,$\square$ \\
\hline \multicolumn{3}{|c|}{ g. Equipment rebates } & 1328 & \begin{tabular}{|l|}
1329 \\
\end{tabular} &,$\square$ \\
\hline & (1) & & 1330 & 1331 & ${ }_{1} \square$ \\
\hline & (2) & & 1332 & 1333 & $1 \square$ \\
\hline
\end{tabular}




\section{Manufacturing Energy Consumption Survey Form EIA-846B}

\section{Petroleum Refineries}

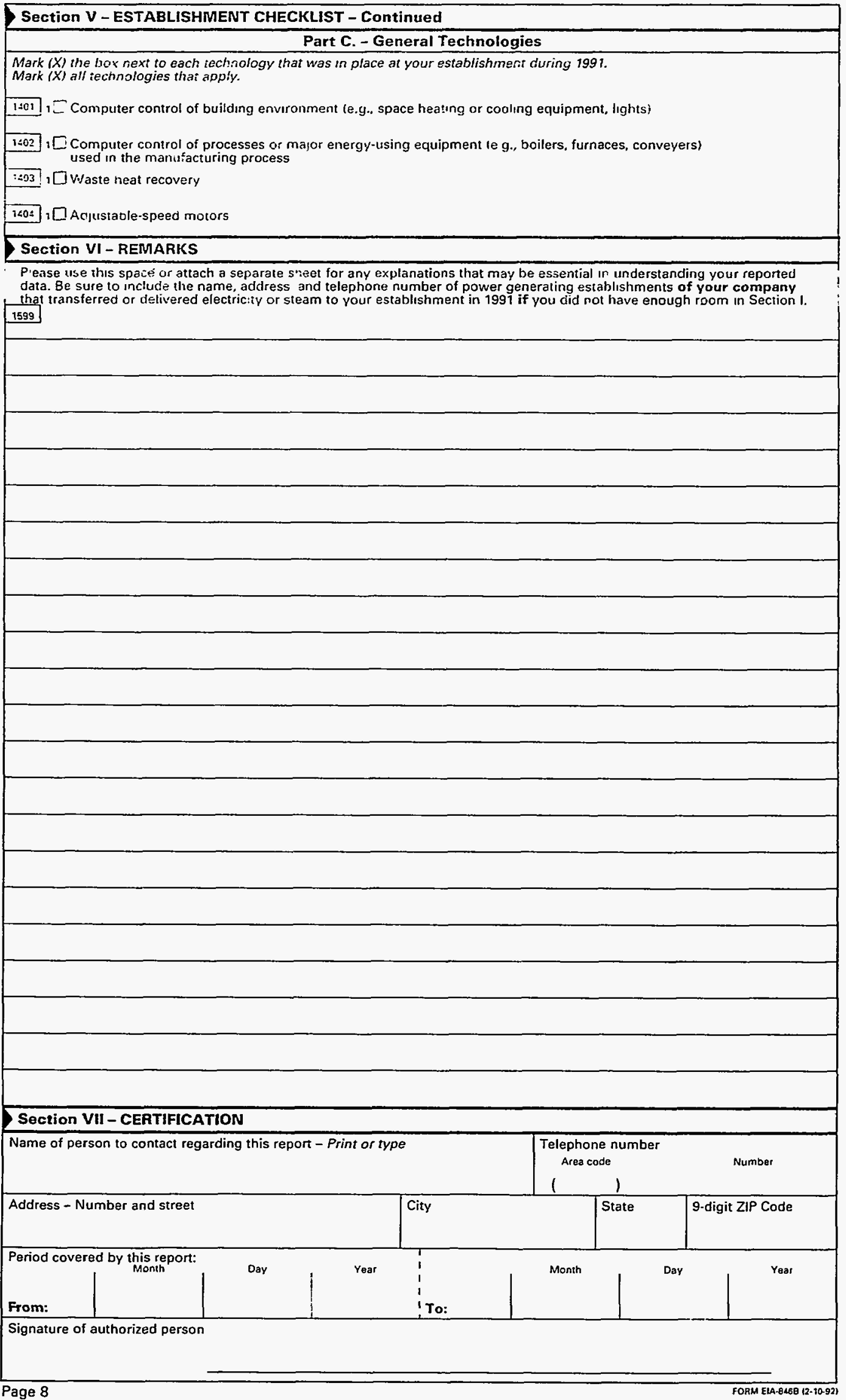




\section{Manufacturing Energy Consumption Survey Form EIA-846C}

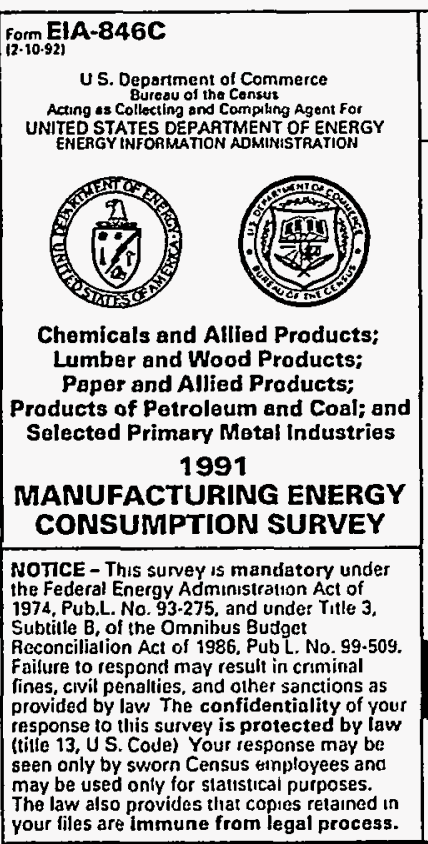

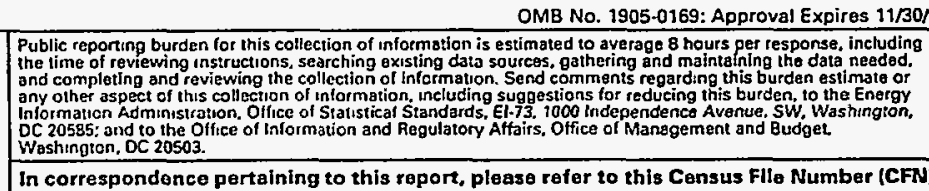

Please correct errors in Ilame, address, and ZIP Code. ENTER street and number if not shovm. PLEASE COMPLETE THIS
FOAMANDAU OF THE CENSUS
$\begin{aligned} & \text { 1201 East 10th Street } \\ & \text { Jeffersonville. IN 47132-0001 }\end{aligned}$

DUE DATE:

"Y vou cannot fite by the Gue date. a time extension request should te sent 10
the above address Please indude your 11 .digit Census file Number (CFN)

NOTE

Please read the enciosed instructions before filling out this form. Complete each item If you have any questions, call (301) any question

Section I - NONCOMBUSTIBLE ENERGY SOURCES

\begin{tabular}{|c|c|c|c|}
\hline $\begin{array}{l}\text { Item Description } \\
\text { (1) }\end{array}$ & $\begin{array}{c}\text { Electricisy } \\
(2 ;\end{array}$ & $\begin{array}{c}\text { Steam } \\
\text { (3) }\end{array}$ & \begin{tabular}{|l|l|}
$12 !$ & Industrial Hot Water \\
\end{tabular} \\
\hline 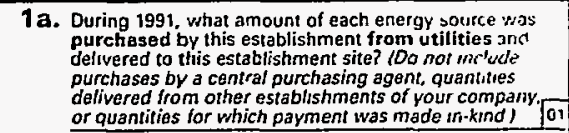 & $\begin{array}{c}\text { Kilowathours } \\
\text { Mol Thou } \\
\vdots \\
\end{array}$ & Miltion Btu & Milion Btu \\
\hline $\begin{array}{l}\text { 1b. What was the lotal expenditure for the putcnased encrgy } \\
\text { sourcelsi reponed on line ta? }\end{array}$ & Mil : Thou : Dol & Mil $\begin{array}{c}\text { Dollars } \\
\text { Thou } \\
\end{array}$ & Mil : Thou : Dol \\
\hline $\begin{array}{l}\text { 2a. During 1991, what amount of electrictly and steam vias } \\
\text { purehased from nonutility suppliers by this } \\
\text { establishment and delivered to this establistirt tni site? }\end{array}$ & $\begin{array}{l}\text { KIlowathours } \\
\text { MII I Thou: kWh } \\
\text { I }\end{array}$ & Million Btu & \\
\hline $\begin{array}{l}\text { 2b. What was the total expenditure for the purchased saergy } \\
\text { source(s) reconed on line } 2 a \text { ? }\end{array}$ & Mil I Thou : Dol & Mil I Thou ! Dol & \\
\hline 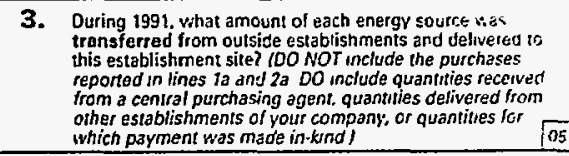 & Mil $\begin{array}{c}\text { Kiowatthours } \\
1 \\
1 \\
\end{array}$ & Million Btu & Million Btu \\
\hline 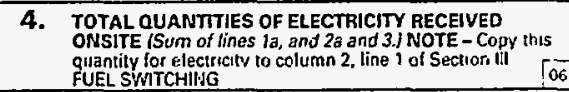 & $\begin{array}{l}1 \\
1 \\
1 \\
\end{array}$ & & \\
\hline $\begin{array}{l}\text { 5. Durng } 1991 \text { how much electuctly was generated on this } \\
\text { establishment ste by cogeneration? (include All } \\
\text { cugenerotion facthries at this establisnment ste, }\end{array}$ & i & & \\
\hline $\begin{array}{l}\text { 6. During 199i, how much of each erresgy source was } \\
\text { generated onsite from solar power, wind power } \\
\text { hydropovrer, and geothermat sources? }\end{array}$ & $\begin{array}{l}T \\
1 \\
1\end{array}$ & Mitlion Btu & Miltion Btu \\
\hline $\begin{array}{l}\text { 7. During } 1591 \text {, how mucts electricty was generated onsite } \\
\text { by processes other than those covered on lines } 5 \text { and } 57\end{array}$ & $i$ & & \\
\hline $\begin{array}{l}\text { 8. TOTAL ONSITE GENERATION OF ELECTRICITY. } \\
\text { iSun of tines } 5,6, \text { and } 7 \text {, }\end{array}$ & i & & \\
\hline $\begin{array}{l}\text { 9. Durng 1991, how much electricitv was sold or } \\
\text { uansferred io utulties? }\end{array}$ & $\vdots$ & & \\
\hline $\begin{array}{l}\text { 10. Durng } 1991 \text {, how much of each energy seurce was sillo " } \\
\text { transierred to any establishmenis other than ututhis }\end{array}$ & i & Million Ztu & Milion Btu \\
\hline $\begin{array}{l}\text { 11. TOTAL ELECTRICITY SALES AND TRANSFERS } \\
\text { OFFSITE (LIME } 9 \text { pius tine } 10 \text {. }\end{array}$ & i & & \\
\hline 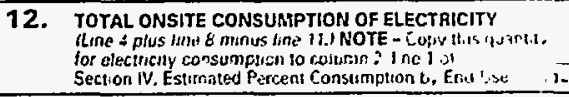 & 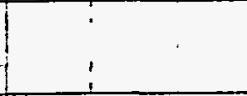 & & \\
\hline \multirow[t]{3}{*}{13.} & \multicolumn{3}{|c|}{ Damt of e ectisity supplar if ansiher establishment of your company } \\
\hline & \multicolumn{3}{|l|}{ indress - Itumber and stretet } \\
\hline & Sny & ZIP Cade & Phone-Area code and Number \\
\hline
\end{tabular}




\section{Manufacturing Energy Consumption Survey Form EIA-846C}

\begin{tabular}{|c|c|c|c|c|c|c|}
\hline \multicolumn{7}{|c|}{ Section II - COMBUSTIBLE ENERGY SOURCES } \\
\hline \multirow{4}{*}{ Energy Scurces } & \multirow{4}{*}{$\begin{array}{c}\text { Census } \\
\text { Use } \\
\text { Orily } \\
\\
\\
2, \\
\end{array}$} & \multirow{4}{*}{$\begin{array}{c}\text { Units Used for } \\
\text { Reponing } \\
\text { Quantities } \\
\\
\end{array}$} & \multicolumn{4}{|c|}{ Energy sources received onsite in ;991 } \\
\hline & & & \multirow{3}{*}{ 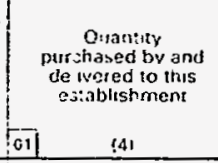 } & \multirow{2}{*}{\multicolumn{2}{|c|}{$\begin{array}{c}\text { Total expenditures. } \\
\text { mcluding delivery } \\
\text { charges, of the quantity } \\
\text { in column (4) }\end{array}$}} & \multirow{2}{*}{$\begin{array}{c}\text { Ouantity of other } \\
\text { seceppls (tronsfers } \\
\text { in and central } \\
\text { purchases) }\end{array}$} \\
\hline & & & & & & \\
\hline & & & & M.1. & rhou Dol & $03 \quad(6)$ \\
\hline $\begin{array}{l}\text { A. SOLIDS } \\
\text { 1. Anthracite } \\
\end{array}$ & 40 & Shor: Tons & & & : & \\
\hline $\begin{array}{l}\text { 2. Bituminous and subbituminous } \\
\text { coal }\end{array}$ & 41 & Short Tons & & & & \\
\hline 3. Lignite & 42 & Shont Tons & & & $\vdots$ & \\
\hline $\begin{array}{l}\text { 4. Total coal (Sum of limes } A 1, A 2 \text {, } \\
\text { and A3) }\end{array}$ & 46 & Shent Tons & & & i & \\
\hline 5. Breeze & 44 & Short Tons & & & 1 & \\
\hline 6. Coat coke & 43. & Short Tons & & & i & \\
\hline 7. Flud catalyuc cracking unit coke & & Barrels & & & 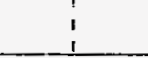 & \\
\hline $\begin{array}{l}\text { 8. Marketable petroleum coke - } \\
\text { unrefined or green }\end{array}$ & 78 & Barrels & & & $\frac{1}{1}$ & \\
\hline $\begin{array}{l}\text { 9. Marketable petroleum coke - } \\
\text { calcined }\end{array}$ & & Barrels & & & i & \\
\hline $\begin{array}{l}\text { 10. Roundwood twood cut } \\
\text { specifically for luel uses }\end{array}$ & 80 & Millor Bru & & & 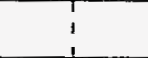 & \\
\hline 11. Wood chips, vark, and woou & 81 & Mitlon Btu & & & 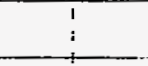 & \\
\hline $\begin{array}{l}\text { 12. Bromass te o , bagasse, rice } \\
\text { hulls. peanut hulls: }\end{array}$ & 90 & Mintuon Btu & & & $i$ & \\
\hline $\begin{array}{l}\text { 13. Waste materniss'sc'ap le g. } \\
\text { wastepioper. packing materials, etc }\end{array}$ & 72 & Milion Btu & & & 1 & \\
\hline 14. Otner solids iSpectify & & Multion Btu & & & i & \\
\hline $\begin{array}{l}\text { B. GASES fexclude oxygen, ntrogen, } \\
\text { and Ineri gasesi } \\
\text { 1. Natural gass purchased drecily } \\
\text { from uttuties }\end{array}$ & 31 & $1,000 \mathrm{cos}$ it. & & & $\begin{array}{c}1 \\
\vdots \\
1 \\
1\end{array}$ & \\
\hline $\begin{array}{l}\text { 2. Natural gas purchased from } \\
\text { transmission pipelines }\end{array}$ & 32 & $i, 030 \mathrm{cu} . \mathrm{At}$ & & & $\begin{array}{r}1 \\
1 \\
1\end{array}$ & \\
\hline $\begin{array}{l}\text { 3. Natural gas obained from } \\
\text { other sources such as brokers, } \\
\text { ploducers, and onsite wells }\end{array}$ & 33 & $1,000 \mathrm{cu}$. ff. & & & 1 & \\
\hline $\begin{array}{l}\text { 4. Total natural gas ISum of lines } B \text { i, } \\
82 \text {, and } B 3 \text { I }\end{array}$ & 30 & $1,000 \mathrm{cu} \mathrm{fit}$. & & & $\begin{array}{l}1 \\
1 \\
1\end{array}$ & \\
\hline 5. Acetyiene & 64 & Mullion Btu & & & 1 & \\
\hline 6. Blast furnace gas & 60 & Million Bru & & & $i$ & \\
\hline 7. Coke oven gas & 51 & Million Btu & & & 1 & \\
\hline 8. Hydrogen & 63 & Million Btu & & & 1 & \\
\hline $\begin{array}{l}\text { 9. Waste and byproduct gases ie.g., } \\
\text { refinery gas, vent gas, plant gas. } \\
\text { still gas) }\end{array}$ & 62 & Million Btu & & & $i$ & \\
\hline 10. Other gases (Spectif) & 93 & Million Btu & & & $\begin{array}{l}1 \\
1 \\
1 \\
1\end{array}$ & \\
\hline $\begin{array}{l}\text { c. LIQUIDS } \\
\text { 142 gallons = } 1 \text { Barrel) } \\
\text { 1. Butane as liquid petroleum gas } \\
\text { (LPG) or natural gas liquids (NGL) }\end{array}$ & 36 & Gallons & & & $\begin{array}{l}1 \\
1 \\
1 \\
1\end{array}$ & \\
\hline $\begin{array}{l}\text { 2. Etnane as liquid petroleum gas (LPG) } \\
\text { or natural gas liquids (NGL) }\end{array}$ & 37 & Gallons & & & $i$ & \\
\hline $\begin{array}{l}\text { 3. Propane as liquid petroteum gas } \\
\text { (LPG) or natural gas liquids (NGL) }\end{array}$ & 38 & Gallons & & & $\begin{array}{c}1 \\
1 \\
1\end{array}$ & \\
\hline $\begin{array}{l}\text { 4. Other (LPG) and INGL) (e g.o butylene, } \\
\text { ethylene. propylene, and mixtures) }\end{array}$ & 39 & Gallons & & & $\begin{array}{l}1 \\
1 \\
\end{array}$ & \\
\hline $\begin{array}{l}\text { 5. Toral (LPG) and (NGL) } \\
\text { (Sum of lines } C 1, C 2, C 3 \text {, and } C 4 \text { ) }\end{array}$ & 24 & Gallons & & & $\begin{array}{l}1 \\
1 \\
1\end{array}$ & \\
\hline
\end{tabular}




\section{Form EIA-846C}

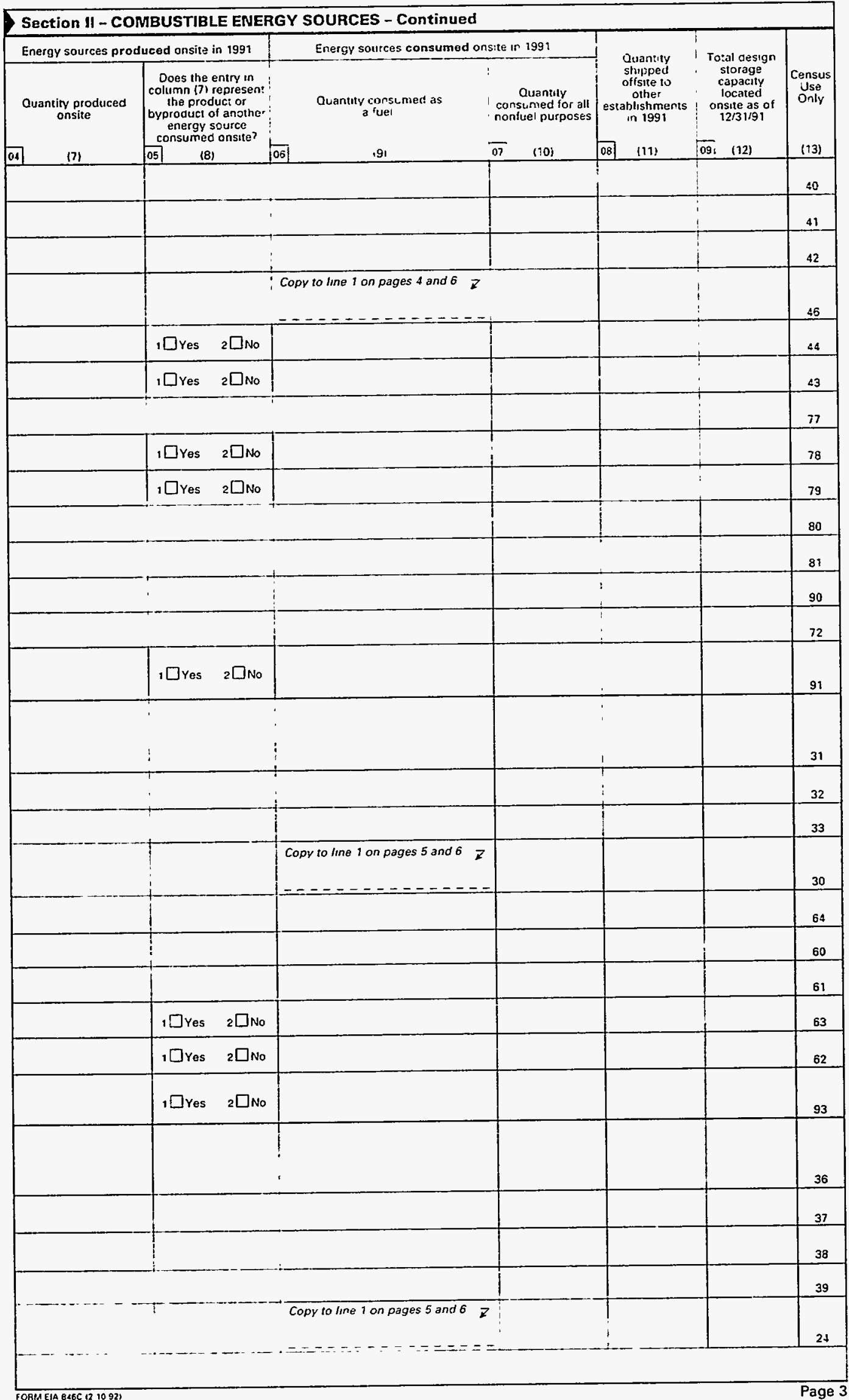




\section{Manufacturing Energy Consumption Survey Form EIA-846C}

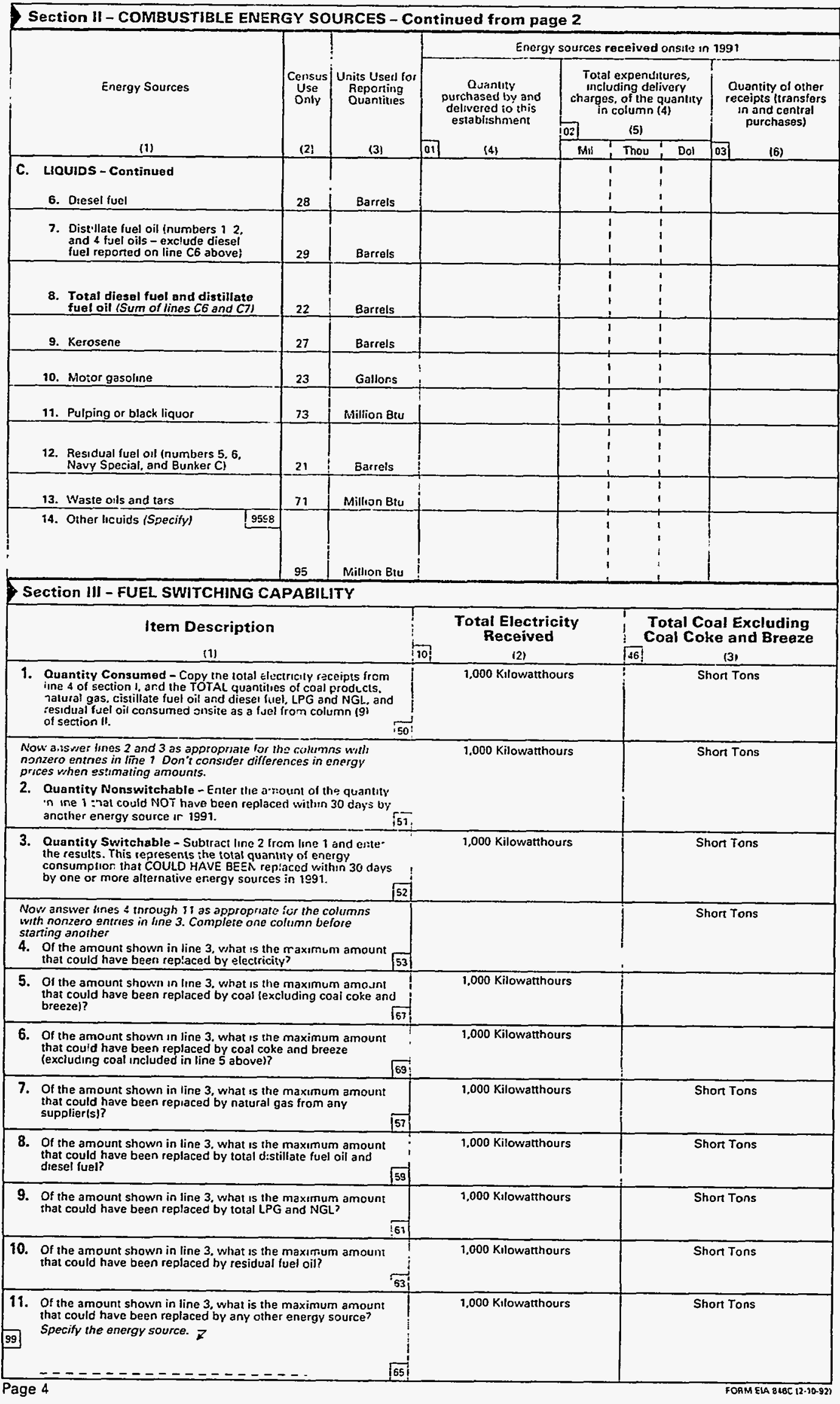



Form EIA-846C

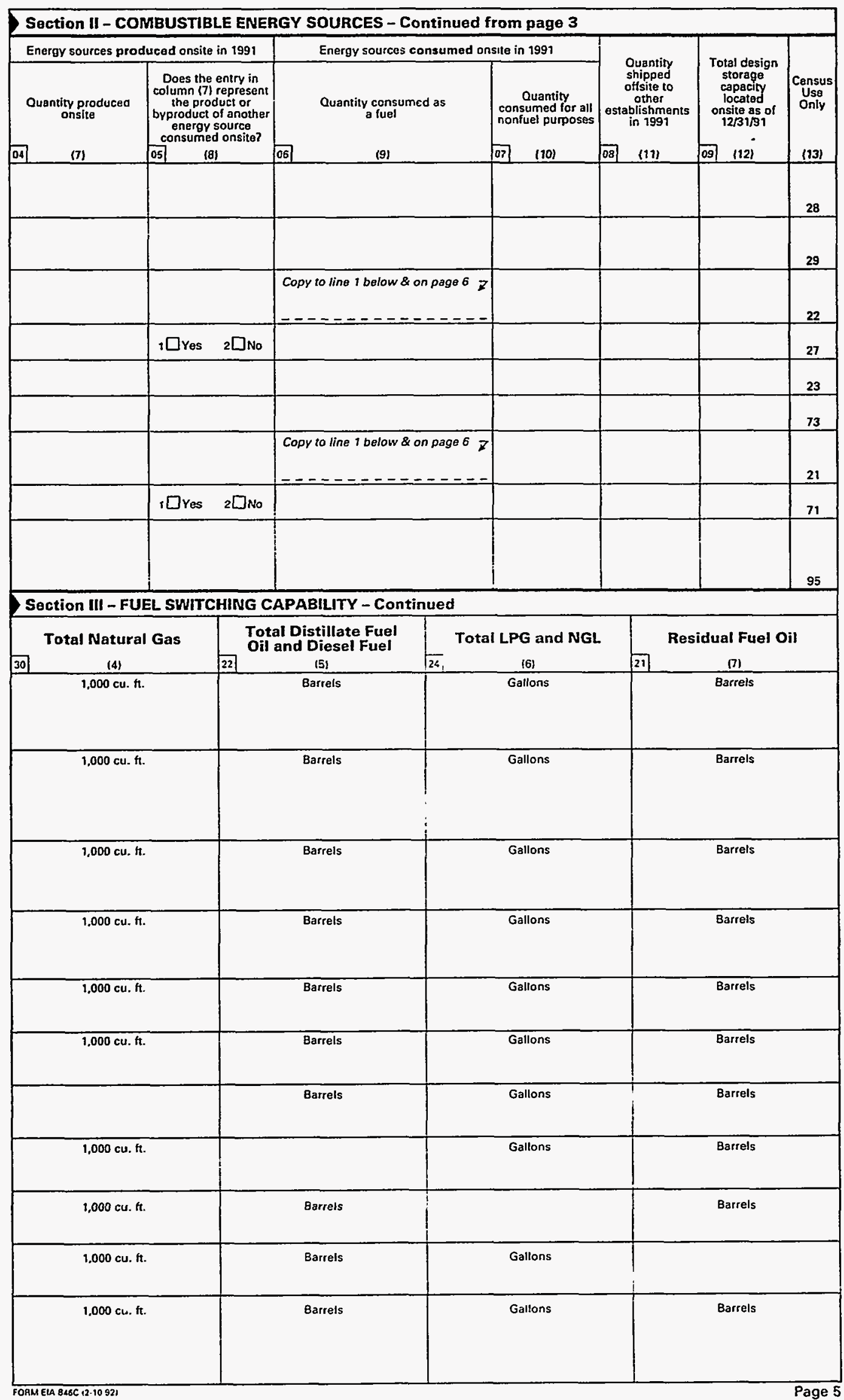



Form EIA-846C

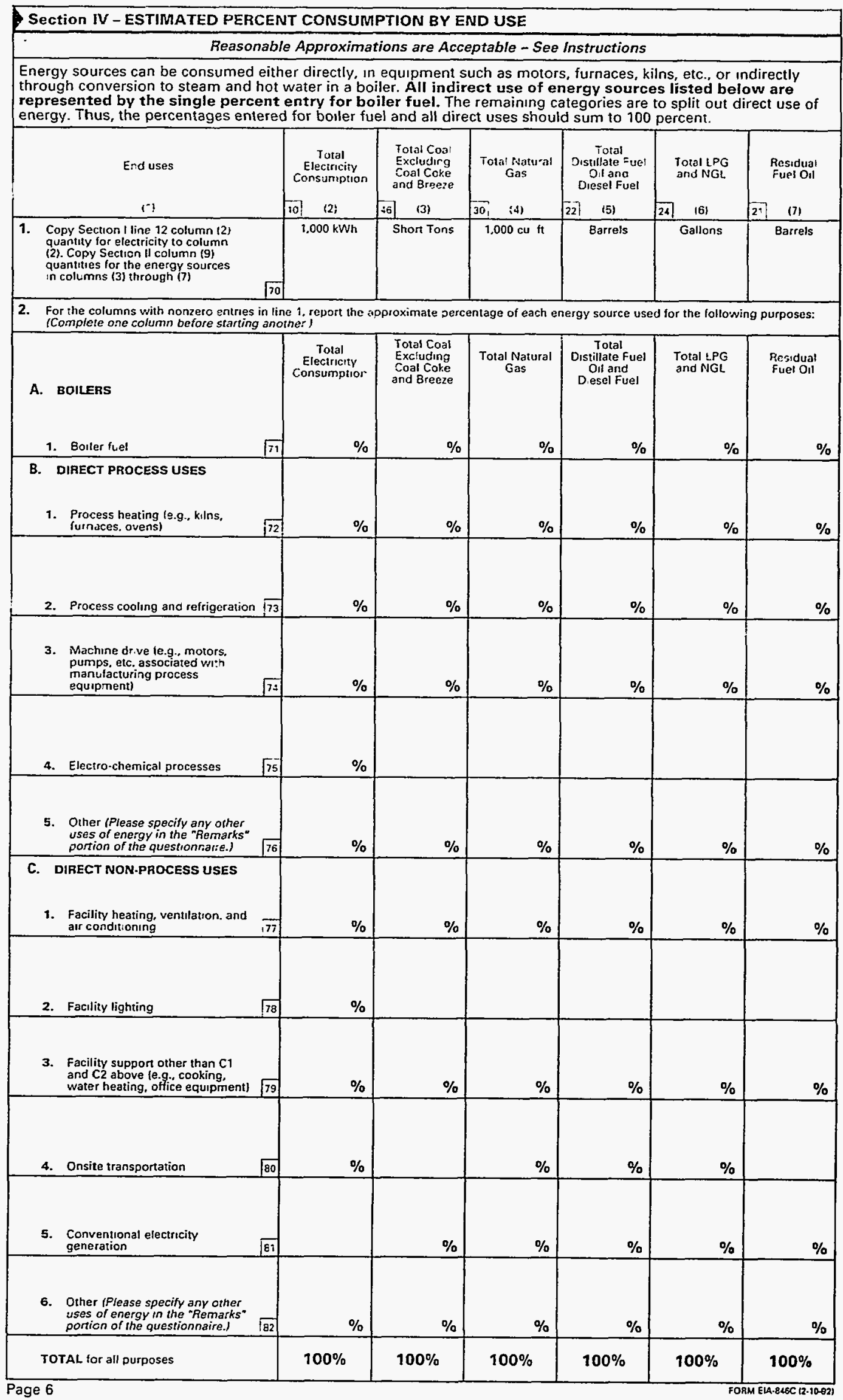


Section V-ESTABLISHMENT CHECKLIST

This section is divided into six parts. All establishments are to complete Parts $A, B$, and C. If the first two digits NOTE digits of your establishment's SIC code are "28" Ichemicals and allied products) complete Pant E after Part C. If the firs Your four-digit SIC code is included as part of the address mailing label on the first page of your questionnaire

\section{Part A. Estimated Square Footage of Buildings}

1. What was the approximate total enclosed square footage of the buildings located on this establishment site as of December 31,19917 Mark $(X)$ the box next to the category which best describes this square faotage.

$1301: \square 25,000$ square feet or less $2 \square 25,001$ to 100,000 square feet $6 \square 750,001$ to 1 million square feat

$3 \square 100,001$ to 200,000 square feet 1. 1,000,001 to 5 million square fee: 4 口 200,001 to 500,000 square feet 8 5,000,001 to 10 million square feet $4 \square 200,001$ to 500,000 square feet
500,001 to 750,000 square feet 9 10,000,001 to $25 \mathrm{~m}$ 'llition square feet $10 \square$ Over 25 million square feet $11 \square$ Don't know

2. Of the square footage indicated above, approximately what percent had controlled heating or cooling, using Mark $(X)$ the box next to the category which best describes this percentage.

$1302 \quad 1 \square$ All $(100 \%)$ $2 \square$ About 75\%

$4 \square$ About 25\% $3 \square$ About $50 \%$ $5 \square$ None 6 Don't Know

Part B. Energy Management Activities

1. Was your establishment involved in any formal programs (company, ut,lity, or third-party sponsored) at any time between January 1,1989 and December 31,1991 that were specifically targeted and designed to improve energy efficiency. reduce energy costs, or promote the use of a different energy source?

$1303 \quad 1 \square$ Yes 1 Proceed to Question 2

2. Did your electric or natural gas utility sponsor orograms designed to lowerfadjust your energy consumption or costs, timing, or promote the use of a different energy source (i.e., a Demand Side Management (DSM) program) at any tim between January 1,1989 and December 31.1991 ?

$1304 \quad 3[$ Establishment did not purchase electricity or natural gas from a utitity. 1304 2Divo 4L Don't Know

3. If you answered "Yes" to Question 2, did your establishment participate in a uthlity sponsored program at any time beiweer January 1. 1989 and December 31 1991?

1305. 1DYes 2 C No

NOTE

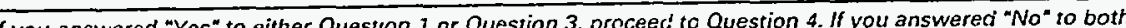
you answered "Yes" to either Questron 1 or Question 3 , proceec' to Question 4 . If you answe
Ouestion 1 and Question 3 , proceed to Part $C$. General Technologies Checklist, on page 8.

4. In what type(s) of energy efficiency activity(ies) was your establishment involved between January 1, 1989 and activities listed.

\begin{tabular}{|c|c|c|c|c|}
\hline $\begin{array}{l}\text { Energy Efficiency Activities } \\
\text { (1) }\end{array}$ & \multicolumn{2}{|c|}{$\begin{array}{l}\text { Uti: ty/Supplier } \\
\text { Sponsored Involvement } \\
\text { (2) }\end{array}$} & \multicolumn{2}{|c|}{$\begin{array}{l}\text { Involvement Through Own or } \\
\text { Other (3rd Party) Sponsorship }\end{array}$} \\
\hline D. Energy audits & 1306 & $1 \square$ & 1307 & 10 \\
\hline b. Direct electricity load contral & 1308 & $1 \square$ & 1309 & $\square$ \\
\hline c. Special rate schedule (e.g., interruptible or time-of-use) & 1310 & $1 \square$ & 1311 & $1 \square$ \\
\hline $\begin{array}{l}\text { d. Equipment installation or retrofit for the primary purpose of } \\
\text { improving energy efficiency affecting: } \\
\text { (1) Steam production (e.g., boilers, nozzles) }\end{array}$ & 1312 & $1 \square$ & 1373 & 10 \\
\hline (2) Direct/indirect process heating & 1314 & $1 \square$ & 1315 & $1 \square$ \\
\hline (3) Direct process cooling, refrigeration & 1316 & $\square$ & 1317 & $1 \square$ \\
\hline $\begin{array}{l}\text { (4) Direct machine drive (e.g., adjustable speed drives, } \\
\text { motors, pumps) }\end{array}$ & 1318 & $1 \square$ & \begin{tabular}{|l|l|}
1319 \\
\end{tabular} & 1[ \\
\hline (5) Facility heating, ventilation and air conditioning & 1320 & $\square$ & 1321 & $1 \square$ \\
\hline (6) Facility lighting & 1322 & $1 \square$ & 1323 & $1 \square$ \\
\hline $\begin{array}{l}\text { e. Equipment retrofit or installation for the primary purpose of } \\
\text { using a different energy source (e.g.. electrification). } \\
\text { Exclude modifications made principally for energy efficiency }\end{array}$ & 1324 & $1 \square$ & 1325 & $1 \square$ \\
\hline f. Standby generation program & 1326 & 1 & 1327 & $1 \square$ \\
\hline g. Equipment rebates & 1328 & $1 \square$ & 1329 & $1 \square$ \\
\hline $\begin{array}{l}\text { h. Other (Specify) ₹ } \\
\text { (1) }\end{array}$ & 1330 & 10 & 1331 & $1 \square$ \\
\hline (2) & $\frac{1332}{1398}$ & $1 \square$ & 1333 & 1 \\
\hline
\end{tabular}




\section{Manufacturing Energy Consumption Survey Form EIA-846C}

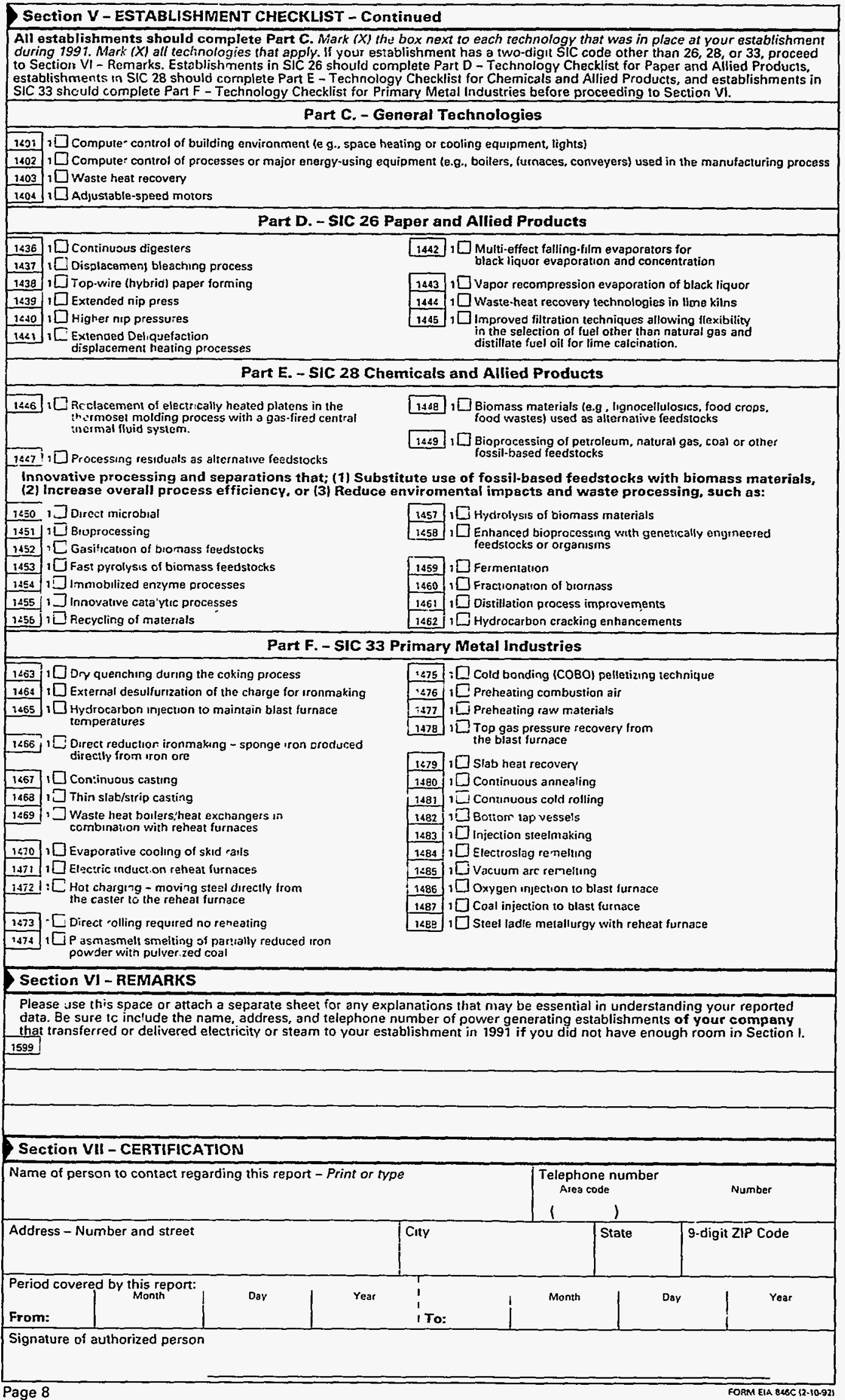




\section{Appendix G Descriptions of Major Industrial Groups and Selected Industries}





\section{Appendix G}

\section{Descriptions of Major Industrial Groups and Selected Industries}

This appendix contains descriptions of industrial groups and selected industries taken from the Standard Industrial Classification Manual, 1987 (SIC). ${ }^{75}$ This appendix includes descriptions of the 30 groups that comprise the strata of the Manufacturing Energy Consumption Survey. These are the 20 major industrial groups (two-digit SIC) and the 10 major energy-consuming industries (four-digit SIC). The Standard Industrial Classification system is described in Appendix B.

SIC 20-Food and Kindred Products: This major group includes establishments manufacturing foods and beverages for human consumption and certain related products such as manufactured ice, chewing gum, vegetable and animal fats and oils, and prepared feeds for animals and fowls.

SIC 2011-Meat Packing Plants: Establishments primarily engaged in the slaughtering, for their own account or on a contract basis for the trade, of cattle, hogs, sheep, lambs, and calves for meat to be sold or to be used on the same premises in canning, cooking, curing, and freezing, and in making sausage, lard, and other products.

SIC 2033-Canned Fruits and Vegetables: Establishments primarily engaged in canning fruits, vegetables, and fruit and vegetable juices: and in manufacturing catsup and similar tomato sauces or natural and imitation preserves, jams, and jellies.

SIC 2037-Frozen Fruits and Vegetables: Establishments primarily engaged in freezing fruits, fruit juices, and vegetables. These establishments also produce important byproducts such as fresh or dried citrus pulp.

SIC 2046 Wet Corn Milling: Establishments primarily engaged in milling corn or sorghum grain (milo) by the wet process, and producing starch, syrup, oil, sugar, and byproducts such as gluten feed and meal. Also included in this industry are establishments primarily engaged in manufacturing starch from other vegetable sources (e.g., potatoes, wheat).

SIC 2051-Bread, Cake and Related Products: Establishments primarily engaged in manufacturing fresh or frozen bread and bread-type rolls and fresh cakes, pies, pastries and other similar "perishable" bakery products.

SIC 2062-Cane Sugar Refining: Establishments primarily engaged in refining purchased raw cane sugar and sugar syrup.

SIC 2063-Beet Sugar: Establishments primarily engaged in manufacturing sugar from sugar beets.

SIC 2075-Soybean Oil Mills: Establishments primarily engaged in manufacturing soybean oil, cake, and meal, and soybean protein isolates and concentrates or in processing purchased soybean oil other than into edible cooking oils.

SIC 21-Tobacco Products: This major group includes establishments engaged in manufacturing cigarettes, cigars, smoking and chewing tobacco, snuff, and reconstituted tobacco and in stemming and redrying tobacco.

SIC 22-Textile Mill Products: This major group includes establishments engaged in performing any of the following operations: (1) preparation of fiber and subsequent manufacturing of yarn, thread, braids, twine, and cordage; (2) manufacturing broadwoven fabrics, narrow woven fabrics, knit fabrics, and carpets and rugs from yarn; (3) dyeing and finishing fiber, yarn, fabrics, and knit apparel; (4) coating, waterproofing, or otherwise treating fabrics;

(5) the integrated manufacture of knit apparel and other finished articles from yarn; and (6) the manufacture of felt goods, lace goods, nonwoven fabrics, and miscellaneous textiles.

${ }^{75}$ Executive Office of the President, Office of Management and Budget, Standard Industrial Classification Manual, 1987, pp. 67-263. 
SIC 23-Apparel and Other Textile Products: This major group, known as the cutting-up and needle trades, includes establishments producing clothing and fabricating products by cutting and sewing purchased woven or knit textile fabrics and related materials, such as leather, rubberized fabrics, plastics, and furs.

SIC 24-Lumber and Wood Products: This major group includes establishments engaged in cutting timber and pulpwood; merchant sawmills, lath mills, shingle mills, cooperage stock mills, planing mills, and plywood and veneer mills engaged in producing lumber and wood basic materials; and establishments engaged in manufacturing finished articles made entirely or mainly of wood or related materials.

SIC 25-Furniture and Fixtures: This major group includes establishments engaged in manufacturing household, office, public building, and restaurant furniture; and office and store fixtures.

SIC 26-Paper and Allied Products: This major group includes establishments primarily engaged in the manufacture of pulps from wood and other cellulose fibers, and from rags; the manufacture of paper and paper board; and the manufacture of paper and paperboard into converted products, such as paper coated off the paper machine, paper bags, paper boxes, and envelopes.

SIC 2611-Pulp Mills: Establishments primarily engaged in manufacturing pulp from wood or from other materials, such as rags, linters, wastepaper, and straw.

SIC 2621-Paper Mills: Establishments primarily engaged in manufacturing paper from wood pulp and other fiber pulp, and which may also manufacture converted paper products.

SIC 2631-Paperboard Mills: Establishments primarily engaged in manufacturing paperboard, including paperboard coated on the paperboard machine, from wood pulp and other fiber pulp.

SIC 27-Printing and Publishing: This major group includes establishments engaged in printing by one or more common processes, such as letterpress, lithography (including offset), gravure, or screen; and those establishments which perform services for the printing trade, such as bookbinding and platemaking.

SIC 28-Chemicals and Allied Products: This major group includes establishments producing basic chemicals, and establishments manufacturing products by predominantly chemical processes. Establishments classified in this major group manufacture three general classes of products: (1) basic chemicals, such as acids, alkalies, salts, and organic chemicals; (2) chemical products to be used in further manufacture, such as synthetic fibers, plastics materials, dry colors, and pigments; and (3) finished chemical products to be used for ultimate consumption, such as drugs, cosmetics, and soaps; or to be used as materials or supplies in other industries, such as paints, fertilizers, and explosives.

SIC 2812-Alkalies and Chlorine: Establishments primarily engaged in manufacturing alkalies and chlorine.

SIC 2813-Industrial Gases: Establishments primarily engaged in manufacturing industrial gases (including organic) for sale in compressed, liquid, and solid forms.

SIC 2819-Industrial Inorganic Chemicals, Not Elsewhere Classified: Establishments primarily engaged in manufacturing industrial organic chemicals, excluding alkalies and chlorine, industrial gases, and inorganic pigments.

SIC 2821-Plastics Materials and Resins: Establishments primarily engaged in manufacturing synthetic resins, plastics materials, and nonvulcanizable elastomers.

SIC 2822-Synthetic Rubber: Establishments primarily engaged in manufacturing synthetic rubber by polymerization or copolymerization. An elastomer for the purpose of this classification is a rubber-like material capable of vulcanization, such as copolymers of butadiene and styrene, or butadiene and acrylonitrile, polybutadienes, chloroprene rubbers, and isobutylene-isoprene copolymers. 
SIC 2823-Cellulosic Manmade Fibers: Establishments primarily engaged in manufacturing cellulosic fibers (including cellulose acetate and regenerated cellulose such as rayon by the viscose or cuprammonium process) in the from of monofilament, yarn, staple, or tow suitable for further manufacturing on spindles, looms, knitting machines, or other textile processing equipment.

SIC 2824-Organic Fibers, Noncellulosic: Establishments primarily engaged in manufacturing manmade organic fibers, except cellulosic (including those of regenerated proteins, and of polymers or copolymers of such components as vinyl chloride, vinylidene chloride, linear esters, vinyl alcohols, acrylonitrile, ethylenes, amides, and related polymeric materials), in the form of monofilament, yarn, staple, or tow suitable for further manufacturing on spindles, looms, knitting machines, or other textile processing equipment.

SIC 2865-Cyclic Crudes and Intermediates: Establishments primarily engaged in manufacturing cyclic organic crudes and intermediates, and organic dyes and pigments. Important products of this industry include: (1) aromatic chemicals, such as benzene, toluene, mixed xylenes naphthalene; (2) synthetic organic dyes; and (3) synthetic organic pigments.

SIC 2869-Industrial Organic Chemicals, Not Elsewhere Classified: Establishments primarily engaged in manufacturing industrial organic chemicals, excluding gum and wood chemicals, and cyclic organic crudes and intermediates, and organic dyes and pigments.

SIC 2873-Nitrogenous Fertilizers: Establishments primarily engaged in manufacturing nitrogenous fertilizer materials or mixed fertilizers from nitrogenous materials produced in the same establishment.

SIC 2874—Phosphatic Fertilizers: Establishments primarily engaged in manufacturing phosphatic fertilizer materials, or mixed fertilizers from phosphatic materials produced in the same establishment.

SIC 29-Petroleum Refining and Related Industries: This major group includes establishments primarily engaged in petroleum refining, manufacturing paving and roofing materials, and compounding lubricating oils and greases from purchased materials.

SIC 2911-Petroleum Refining: Establishments primarily engaged in producing gasoline, kerosene, distillate fuel oils, residual fuel oils, and lubricants, through fractionation or straight distillation of crude oil, redistillation of unfinished petroleum derivatives, cracking or other processes.

SIC 30-Rubber and Miscellaneous Plastics Products: This major group includes establishments manufacturing products, not elsewhere classified, from plastics, resins, and from natural, synthetic, or reclaimed rubber, gutta percha, balata, or gutta siak.

SIC 3011-Tires and Inner Tubes: Establishments primarily engaged in manufacturing pneumatic casings, inner tubes, and solid and cushion tires for all types of vehicles, airplanes, farm equipment, and children's vehicles; tiring; camelback; and tire repair and retreading materials.

SIC 308-Miscellaneous Plastics Products Not Elsewhere Classified: Establishments primarily engaged in manufacturing (1) unsupported plastics film and sheet from purchased resins or from resins produced in the same plant; (2) unsupported plastics profiles, rods, tubes, and other shapes; (3) laminated plastics plate, sheet, profiles, rods, and tubes; (4) plastics pipe; (5) Plastics bottles; (6) custom compounding of purchased plastics resins; (7) plastics plumbing fixtures; and (8) plastics products not elsewhere classified.

SIC 31-Leather and Leather Products: This major group includes establishments engaged in tanning, currying, and finishing hides and skins, leather converters, and establishments manufacturing finished leather and artificial leather products and some similar products made of other materials.

SIC 32-Stone, Clay, Glass, and Concrete Products: This major group includes establishments manufacturing flat glass and other glass products, cement, structural clay products, pottery, concrete and gypsum products, cut stone, abrasive and asbestos products, and other products from materials taken principally from the earth in the form of stone, clay, and sand. 
SIC 3211-Flat Glass: Establishments primarily engaged in manufacturing flat glass. This industry also produces laminated glass, but establishments primarily engaged in manufacturing laminated glass from purchased flat glass are not included.

SIC 3221-Class Containers: Establishments primarily engaged in manufacturing glass containers for commercial packing and bottling, and for home canning.

SIC 3229-Pressed and Blown Glass and Glassware, Not Elsewhere Classified: Establishments primarily engaged in manufacturing glass and glassware, not elsewhere classified, pressed, blown, or shaped from glass produced in the same establishment. Establishments primarily engaged in manufacturing textile glass fibers are also included in this industry. Establishments primarily engaged in the production of pressed lenses for vehicular lighting, beacons, and lanterns are also included in this industry.

SIC 3241-Cement, Hydraulic: Establishments primarily engaged in manufacturing hydraulic cement, including portland, natural, masonry, and pozzolana cements.

SIC 3274-Lime: Establishments primarily engaged in manufacturing quicklime, hydrated lime, and "dead-burned" dolomite from limestone, dolomite shells, or other substances.

SIC 3296-Mineral Wool: Establishments primarily engaged in manufacturing mineral wool and mineral wool insulation products made of such siliceous materials as rock, slag and glass, or combinations thereof.

SIC 33-Primary Metal Industries: This major group includes establishments engaged in smelting and refining ferrous and nonferrous metals from ore, pig, or scrap; in rolling, drawing, and alloying metals; in manufacturing castings and other basic metal products; and in manufacturing nails, spikes, and insulated wire and cable.

SIC 3312-Steel Works, Blast Furnaces (Including Coke Ovens), and Rolling Mills: Establishments primarily engaged in manufacturing hot metal, pig iron, and silvery pig iron from iron ore and iron and steel scrap; converting pig iron, scrap iron, and scrap steel into steel; and in hot-rolling iron and steel into basic shapes, such as plates, sheets, strips, rods, bars, and tubing.

SIC 3313-Electrometallurgical Products: Establishments primarily engaged in manufacturing ferrous and nonferrous metal additive alloys by electrometallurgical or metallothermic processes, including high percentage ferroalloys and high percentage nonferrous additive alloys.

SIC 3321-Gray and Ductile Iron Foundries: Establishments primarily engaged in manufacturing gray and ductile iron castings, including cast iron pressure and soil pipes and fittings.

SIC 3331-Primary Copper: Establishments primarily engaged in smelting copper from the ore, and in refining copper by electrolytic or other processes.

SIC 3334-Primary Production of Aluminum: Establishments primarily engaged in producing aluminum from alumina and in refining aluminum by any process.

SIC 3339-Primary Nonferrous Metals, Not Elsewhere Classified: Establishments primarily engaged in smelting and refining nonferrous metals, except copper and aluminum.

SIC 3353-Aluminum Sheet, Plate, and Foil: Establishments primarily engaged in flat rolling aluminum and aluminum-base alloy basic shapes, such as sheet, plate, an foil, including establishments producing welded tube.

SIC 34-Fabricated Metal Products: This major group includes establishments engaged in fabricating ferrous and nonferrous metal products such as metal cans, tinware, handtools, cutlery, general hardware, nonelectric heating apparatus, fabricated structural metal products, metal forgings, metal stampings, ordnance (except vehicles and guided missiles), and a variety of metal and wire products, not elsewhere classified. 
SIC 35-Industrial Machinery and Equipment: This major group includes establishments engaged in manufacturing industrial and commercial machinery and equipment and computers.

SIC 357-Computer and Office Equipment: Establishments primarily engaged in manufacturing electronic computers; computer storage devices; computer terminals; point-of-sale devices; funds transfer devices and other calculating and accounting machines; and office machines and devices not elsewhere classified, including typewriters and word processing equipment.

SIC 36-Electronic and Other Electric Equipment: This major group includes establishments engaged in manufacturing machinery, apparatus, and supplies for the generation, storage, transmission, transformation, and utilization of electrical energy.

SIC 37-Transportation Equipment: This major group includes establishments engaged in manufacturing equipment for transportation of passengers and cargo by land, air, and water.

SIC 3711-Motor Vehicles and Car Bodies: Establishments primarily engaged in manufacturing or assembling complete passenger automobiles, trucks, commercial cars and buses, and special purpose motor vehicles which are for highway use. This industry also includes establishments primarily engaged in manufacturing chassis and passenger car bodies.

SIC 3714-Motor Vehicle Parts and Accessories: Establishments primarily engaged in manufacturing motor vehicle parts ad accessories, but not engaged in manufacturing complete motor vehicles or passenger car bodies.

SIC 38-Instruments and Related Products: This major group includes establishments engaged in manufacturing instruments (including professional and scientific) for measuring, testing, analyzing, and controlling, and their associated sensors and accessories; optical instruments and lenses; surveying and drafting instruments; hydrological, hydrographic, meteorological, and geophysical equipment; search, detection, navigation, and guidance systems and equipment; surgical, medical, and dental instruments, equipment and supplies; ophthalmic goods; photographic equipment and supplies; and watches and clocks.

SIC 39-Miscellaneous Manufacturing Industries: This major group includes establishments primarily engaged in manufacturing products not classified in any other major group. 
Appendix $\mathbf{H}$

Map of U.S.

Census Regions 



\section{Appendix H}

\section{Map of U.S. Census Regions}

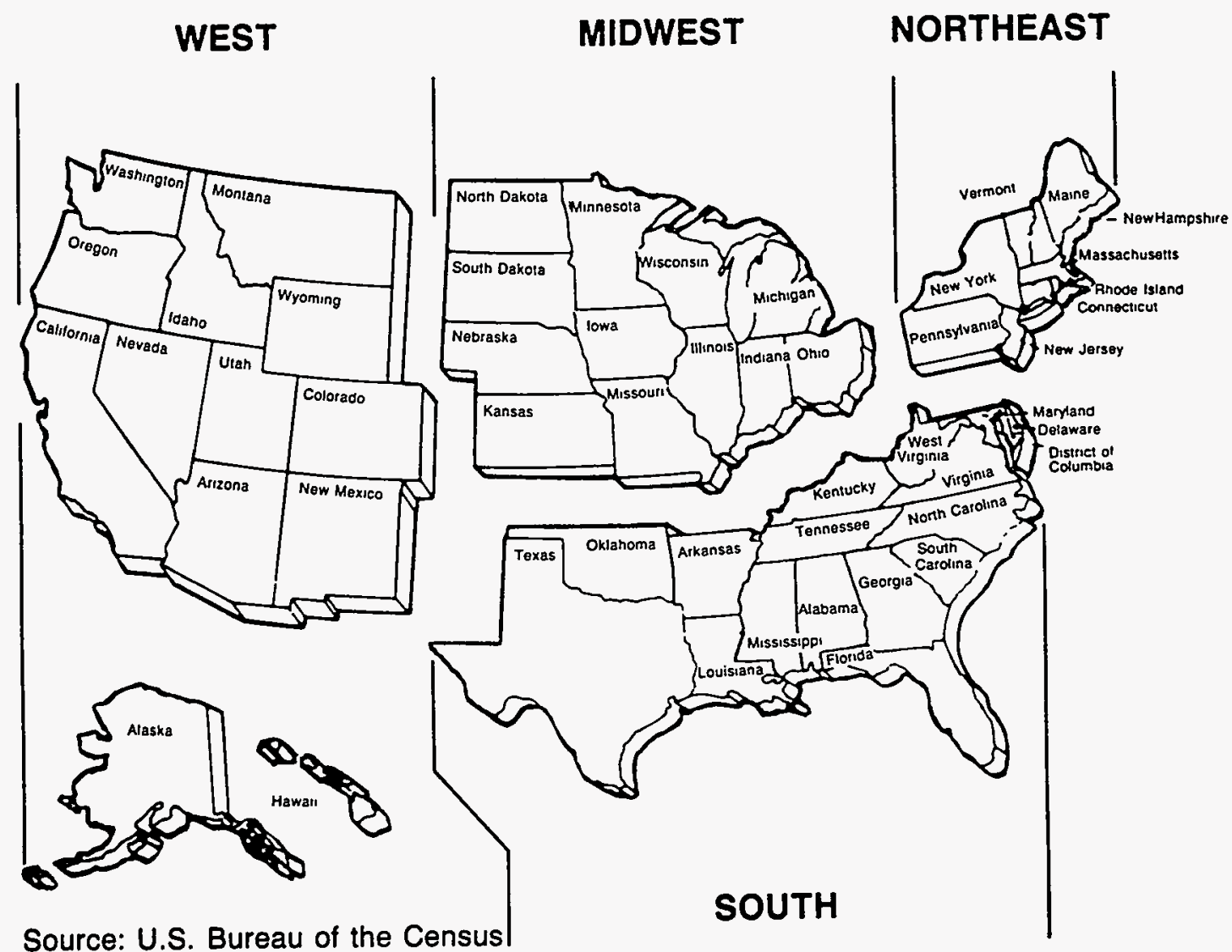




\section{Appendix I \\ Metric Conversion \\ Factors}




\section{Appendix I}

\section{Metric Conversion Factors}

Data in the Energy Information Administration publications are expressed in units, such as British thermal units, barrels, cubic feet, and short tons, that historically have been used in the United States. However, because U.S. activities involve foreign nations, most of which use metric units of measure, the United States is committed to making the transition to the metric system. The metric conversion factors presented in Table I1 can be used to calculate the metric-unit equivalents of values expressed in U.S. units. For example, 500 short tons are the equivalent of 453.6 metric tons ( 500 short tons $\times 0.9071847$ metric tons/short tons $=453.6$ metric tons).

Table I1. Metric Conversion Factors

\begin{tabular}{|c|c|c|c|c|}
\hline Type of Unit & U.S. Unit & & Conversion Factor & Metric Unit \\
\hline Mass & $\begin{array}{l}\text { Short Tons } \\
\text { Short Tons Uranium Oxide }\left(\mathrm{U}_{3} \mathrm{O}_{8}\right) \\
\text { Short Tons Uranium Fluoride }\left(\mathrm{UF}_{6}\right) \\
\text { Long Tons } \\
\text { Pounds }(\mathrm{lb}) \\
\text { Pounds Uranium Oxide }\left(\mathrm{lb} \mathrm{U}_{3} \mathrm{O}_{8}\right) \\
\text { Ounces, Avoirdupois }(\mathrm{oz})\end{array}$ & $\begin{array}{l}x \\
x \\
x \\
x \\
x \\
x \\
x\end{array}$ & $\begin{array}{l}0.9071847 \\
0.769 \\
0.613 \\
1.016 \\
0.45359237^{\mathrm{a}} \\
0.384645^{\mathrm{b}} \\
28.34952\end{array}$ & $\begin{array}{l}=\text { Metric Tons }(\mathrm{t}) \\
=\text { Metric Tons Uranium }(\mathrm{U}) \\
=\text { Metric Tons Uranium }(\mathrm{U}) \\
=\text { Metric Tons }(\mathrm{t}) \\
=\text { Kilograms }(\mathrm{kg}) \\
=\text { Kilograms }(\mathrm{Kg}) \\
=\text { Grams }(\mathrm{g})\end{array}$ \\
\hline Volume & 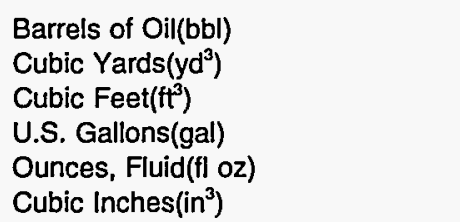 & $\begin{array}{l}x \\
x \\
x \\
x \\
x \\
x\end{array}$ & $\begin{array}{l}0.1589873 \\
0.765555 \\
0.02831685 \\
3.785412 \\
29.57353 \\
16.38706\end{array}$ & $\begin{array}{l}=\text { Cubic Meters }\left(\mathrm{m}^{3}\right) \\
=\text { Cubic Meters }\left(\mathrm{m}^{3}\right) \\
=\text { Cubic Meters }\left(\mathrm{m}^{3}\right) \\
=\text { Liter }(\mathrm{L}) \\
=\text { Milliliters }(\mathrm{ml}) \\
=\text { Milliliters }(\mathrm{ml})\end{array}$ \\
\hline Length & $\begin{array}{l}\text { Miles (mi) } \\
\text { Yards (yd) } \\
\text { Feet (ft) } \\
\text { Inches (in) }\end{array}$ & $\begin{array}{l}x \\
x \\
x \\
x\end{array}$ & $\begin{array}{l}1,609344^{a} \\
0.9144^{a} \\
0.3048^{a} \\
2.54^{a}\end{array}$ & $\begin{array}{l}=\text { Kilometers }(\mathrm{km}) \\
=\text { Meters }(\mathrm{m}) \\
=\text { Meters }(\mathrm{m}) \\
=\text { Centimeters }(\mathrm{cm})\end{array}$ \\
\hline Area & $\begin{array}{l}\text { Acres } \\
\text { Square Miles }\left(\mathrm{mi}^{2}\right) \\
\text { Square Yards }\left(\mathrm{yd}^{2}\right) \\
\text { Square Feet }\left(\mathrm{ft}^{2}\right) \\
\text { Square Inches }\left(\mathrm{in}^{2}\right)\end{array}$ & $\begin{array}{l}x \\
x \\
x \\
x \\
x\end{array}$ & $\begin{array}{l}0.40469 \\
2,589988 \\
0.8361274 \\
0.09290304^{a} \\
6.45616^{\mathrm{a}}\end{array}$ & $\begin{array}{l}=\text { Hectares }(\text { ha) } \\
=\text { Square Kilometers }\left(\mathrm{km}^{2}\right) \\
=\text { Square Meters }\left(\mathrm{m}^{2}\right) \\
=\text { Square Meters }\left(\mathrm{m}^{2}\right) \\
=\text { Square Centimeters }\left(\mathrm{cm}^{2}\right)\end{array}$ \\
\hline Temperature & Degrees Fahrenheit ${ }^{\mathrm{C}}\left({ }^{\circ} \mathrm{F}\right)$ & $x$ & $5 / 9{\text { (after subtracting } 32)^{a}}^{a}$ & $=$ Degrees Celsius $\left({ }^{\circ} \mathrm{C}\right)$ \\
\hline Energy & $\begin{array}{l}\text { British thermal units (Btu) } \\
\text { Calories (cal) } \\
\text { Kilowatthours (kWh) }\end{array}$ & $\begin{array}{l}x \\
x \\
x\end{array}$ & $\begin{array}{l}1,055.056 \\
4.1868 \\
3.6\end{array}$ & $\begin{array}{l}=\text { Joules }(\mathrm{J}) \\
=\text { Joules }(\mathrm{J}) \\
=\text { Megajoules }(\mathrm{MJ})\end{array}$ \\
\hline
\end{tabular}

"Exact Conversion.

'Calculated by the Energy information Administration.

'To convert degrees Celșius $\left({ }^{\circ} \mathrm{C}\right)$ to degrees Fahrenheit $\left({ }^{\circ} \mathrm{F}\right)$ multiply by $9 / 5$, then add 32 .

Sources: -General Services Administration, Federal Standard 376B, Preferred Metric Units for General Use by the Federal Govemment (Washington, DC, January 27, 1993), pp. 9-11, 13, and 16. - National Institute of Standards and Technology, Special Publications 330, 811, and 814. •American National Standards Institute/Institute of Electrical and Electronic Engineers, ANS/EEE Std.268-1982, pp 28 and 29. •Energy Information Administration/Monthly Energy Review August 1993, Appendix B, pp 161. 
Appendix $\mathrm{J}$

Related EIA

Publications on

Energy Consumption 



\section{Appendix J}

\section{Related EIA Publications on Energy Consumption}

For information about how to obtain these publications, see the inside cover of this report. Please note that the prices quoted here are subject to change.

In addition to the reports listed below, public use data tapes and data diskettes for the residential, residential transportation, and commercial sectors are available from the National Technical Information Service (NTIS). To obtain information on how to order the tapes/diskettes, you may call NTIS at 703-487-4807, FAX number 703-321-8547. Data diskettes can also be obtained from the Office of Scientific and Technical Information (OSTI). For OSTI ordering information, call 615-576-8401.

\section{Industrial Sector}

"Energy Preview: Manufacturing Energy Consumption Survey Preliminary Estimates, 1991," Monthly Energy Review, September 1993, DOE/EIA0035(93/01).

"Energy Efficiency in the Manufacturing Sector," Monthly Energy Review (Article), p.1, December 1992.

Manufacturing Energy Consumption Survey: Changes in Energy Intensity in the Manufacturing Sector 1980-1988, December 1991, DOE/EIA-0552(80-88). GPO Stock No. 061-003-00734-1, \$4.75.

Manufacturing Energy Consumption Survey: Manufacturing Fuel-Switching Capability 1988; September 1991, DOE/EIA-0515(88), GPO Stock No. 061-00300720-1, $\$ 9.00$.

Manufacturing Energy Consumption Survey: Consumption of Energy, 1988; May 1991, DOE/EIA0512(88), GPO Stock No. 061-003-00703-8, \$11.00.

Manufacturing Energy Consumption Survey: Energy Efficiency in Manufacturing, 1985; January 1990, DOE/EIA-0516(85), GPO Stock No. 061-003-006507, \$4.25.
Manufacturing Energy Consumption Survey: FuelSwitching Capability, 1985; December 1988, DOE/EIA-0515(85), GPO Stock No. 061-003$00601-9, \$ 3.50$.

Manufacturing Energy Consumption Survey: Methodological Report, 1985; November 1988, DOE/ELA0514(85), GPO Stock No. 061-003-00595-1, \$6.00.

Manufacturing Energy Consumption Survey: Consumption of Energy, 1985; November 1988, DOE/EIA-0512(85), GPO Stock No. 061-003-00594-2, $\$ 6.00$.

"Manufacturing Sector Energy Consumption 1985 Provisional Estimates," Monthly Energy Review (Article), pp. vii-x, January 1987, DOE/EIA-0035(87/01).

Report on the 1980 Manufacturing Industries' Energy Consumption Study and Survey of Large Combustors; February 1983, DOE/EIA-0358, GPO Stock No. 061-003-00293-5, \$5.00.

Industrial Energy Consumption, Survey of Large Combustors: Report on Alternate Fuel-BurningCapabilities of Large Boilers in 1979; February 1982, DOE/EIA-0304, GPO Stock No. 061-003-0233-1, $\$ 2.50$.

Methodological Report of the 1980 Manufacturing Industries Survey of Large Combustors (EIA-463); March 1982, DOE/EIA-0306 (no GPO Stock No.).

\section{Other Publications on the Industry Sector}

Energy Consumption Series--Derived Annual Estimates of Manufacturing Energy Consumption 19741988, August 1992, DOE/EIA-0555(92)/3, GPO Stock No. 061-003-00766-0, $\$ 7.00$.

Energy Consumption Series--Development of the 1991 Manufacturing Energy Consumption Survey, May 1992, DOE/EIA-0555(92)/2, GPO Stock No. 061-00300757-1, \$5.50. 


\section{Commercial Sector}

Note: The name of the Nonresidential Buildings Energy Consumption Survey was changed to the Commercial Buildings Energy Consumption Survey, beginning with the 1989 survey. The survey name was also dropped from the report title at that time and subsequently.

\section{Characteristics of Buildings}

Commercial Buildings Characteristics 1992; April 1994, DOE/EIA-0246(92), GPO Stock No. 061-00300850-0, $\$ 28.00$.

"Commercial Buildings Characteristics 1992," Monthly Energy Review, January 1994, DOE/EIA0035(94/01).

Commercial Buildings Characteristics 1989; June 1991, DOE/EIA-0246(89), GPO Stock No. 061-00300699-0, \$18.00.

Nonresidential Buildings Energy Consumption Survey: Characteristics of Commercial Buildings, 1986; September 1988, DOE/EIA-0246(86), GPO Stock No. 061-003-00580-2, \$16.00.

Nonresidential Buildings Energy Consumption Survey: Characteristics of Commercial Buildings, 1983; July 1985, DOE/EIA-0246(83), GPO Stock No. 061-00300439-3, \$7.50.

Nonresidential Buildings Energy Consumption Survey: Characteristics of Commercial Buildings, 1983; A Supplemental Reference, DOE/EIA-M008, \$22.95. Available from the NTIS, Order No. DE-85015581.

Nonresidential Buildings Energy Consumption Survey: Fuel Characteristics and Conservation Practices; June 1981, DOE/EIA-0278, GPO Stock No. 061-00300200-5, $\$ 9.00$.

Nonresidential Buildings Energy Consumption Survey: Building Characteristics; March 1981, DOE/EIA-0246, GPO Stock No. 061-003-00171-8, \$6.50.

\section{Consumption and Expenditures}

Commercial Buildings Consumption and Expenditures 1989; April 1992, DOE/EIA-0318(89), GPO Stock No. 061-003-00753-8, \$25.00.
Nonresidential Buildings Energy Consumption Survey: Commercial Buildings Consumption and Expenditures 1986; May 1989, DOE/EIA-0318(86), GPO Stock No. 061-003-00613-2, \$19.00.

Nonresidential Buildings Energy C̣onsumption Survey: Commercial Buildings, Consumption and Expenditures 1983; September 1986, DOE/EIA-0318(83), GPO Stock No. 061-003-00496-2, \$13.00.

Nonresidential Buildings Energy Consumption Survey: 1979 Consumption and Expenditures, Part 1: Natural Gas and Electricity; March 1983, DOE/EIA-0318/1, GPO Stock No. 061-003-00298-6, \$9.50.

Nonresidential Buildings Energy Consumption Survey: 1979 Consumption and Expenditures, Part 2: Steam, Coal, Fuel Oil, LPG, and Total Fuels; December 1983, DOE/EIA-0318(79)/2, GPO Stock No. 061-003-00366-4, $\$ 6.00$.

\section{Other Publications on the Commercial Sector}

"Assessment of Energy Use in Multibuilding Facilities," Monthly Energy Review, December 1993, DOE/EIA-0035(93/12).

Energy Consumption Series--Assessment of Energy Use in Multibuilding Facilities, August 1993, DOE/EIA-0555(93)/1, GPO Stock No. 061-003$00817-8, \$ 7.50$.

Energy Consumption Series--User-Needs Study for the 1992 Commercial Buildings Energy Consumption Survey, September 1992, DOE/EIA-0555(92)/4, GPO Stock No. 061-003-00770-8, \$8.50.

Energy Consumption Series-Lighting in Commercial Buildings; March 1992, DOE/EIA-0555(92)/1, GPO Stock No. 061-003-00749-0, \$6.50.

\section{Residential Sector}

\section{Housing Characteristics}

Note: The survey name was dropped from the beginning of the report title starting with the 1987 data reports.

Housing Characteristics 1990; May 1992, DOE/EIA0314(90), GPO Stock No. 061-003-00754-6, \$23.00.

Housing Characteristics 1987; May 1989, DOE/EIA0314(87), GPO Stock No. 061-003-00619-1, \$13.00. 
Residential Energy Consumption Survey: Housing Characteristics 1984; October 1986, DOE/EIA-0314(84), GPO Stock No. 061-003-00499-7, $\$ 12.00$.

Residential Energy Consumption Survey: Housing Characteristics, 1982; August 1984, DOE/EIA0314(82), GPO Stock No. 061-003-00393-1, \$7.00.

Residential Energy Consumption Survey Housing Characteristics, 1981; August 1983, DOE/EIA0314(81), GPO Stock No. 061-003-00330-3, \$6.50.

Residential Energy Consumption Survey: Housing Characteristics, 1980; June 1982, DOE/ELA-0314, GPO Stock No. 061-003-00256-1, \$11.00.

Residential Energy Consumption Survey: Characteristics of the Housing Stock and Households, 1978; February 1980, DOE/EIA-0207/2, GPO Stock No. 061-003-00093-2, $\$ 4.25$.

Residential Energy Consumption Survey: Conservation; February 1980, DOE/EIA-0207/3, GPO Stock No. 061-003-00087-8, $\$ 6.00$.

Preliminary Conservation Tables from the National Interim Energy Consumption Survey; August 1979, DOE/EIA-0193/P (no GPO Stock No.).

Characteristics of the Housing Stock and Households: Preliminary Findings from the National Interim Energy Consumption Survey; October 1979, DOEEIA-0199/P (no GPO Stock No. available).

\section{Consumption and Expenditures}

Note: The survey name was dropped from the beginning of the report title starting with the 1987 data reports. The titles were changed to Household Energy Consumption and Expenditures 1987, Part 1: National and Part 2: Regional.

"Household Energy Consumption and Expenditures 1990," Monthly Energy Review, August 1993, DOE/EIA-0035(93/08).

Household Energy Consumption and Expenditures 1990; February 1993, DOE/EIA-0321/1(90), GPO Stock No. 061-003-00795-3, \$22.00.

Household Energy Consumption and Expenditures 1990 S; DOE/EIA-0321/2(90), GPO Stock No. 061003-00796-1, \$21.00.
Household Energy Consumption and Expenditures 1987, Part 1: National Data; October 1989, DOE/EIA-0321/1(87), GPO Stock No. 061-00300635-3, \$15.00. Note: Energy end-use data are included in this report.

Household Energy Consumption and Expenditures 1987, Part 2: Regional Data; DOE/EIA-0321/2(87) (no GPO Stock No. available), $\$ 16.00$.

Residential Energy Consumption Survey: Consumption and Expenditures, April 1984 Through March 1985, Part 1: National Data; March 1987, DOE/EIA-0321/1(84), GPO Stock No. 061-003$00519-5, \$ 9.50$.

Residential Energy Consumption Survey: Consumption and Expenditures, April 1984 Through March 1985, Part 2: Regional Data; May 1987, DOE/EIA0321/2(84), GPO Stock No. 061-003-00528-4, \$17.00. Note: Energy end-use data are included in this report.

Residential Energy Consumption Survey: Consumption and Expenditures, April 1982 Through March 1983, Part 1: National Data; November 1984, DOE/EIA-0321/1(82), GPO Stock No. 061-003-0041$1-3, \$ 7.00$.

Residential Energy Consumption Survey: Consumption and Expenditures, April 1982 Through March 1983, Part 2: Regional Data; December 1984, DOE/EIA-0321/2(82), GPO Stock No. 061-00300414-8, $\$ 9.50$.

Residential Energy Consumption Survey: Consumption and Expenditures, April 1981 Through March 1982, Part 1: National Data; September 1983, DOE/EIA-0321/1(81), GPO Stock No. 061-003$00340-1, \$ 6.00$.

Residential Energy Consumption Survey: Consumption and Expenditures, April 1981 Through March 1982, Part 2: Regional Data; October 1983, DOE/EIA-0321/2(81), GPO Stock No. 061-003$00357-5, \$ 8.00$.

Residential Energy Consumption Survey: Consumption and Expenditures, April 1980 Through March 1981, Part 1: National Data; September 1982, DOE/EIA-0321/1(80), GPO Stock No. 061-003-0027$8-1, \$ 7.50$. 
Residential Energy Consumption Survey: Consumption and Expenditures, April 1980 Through March 1981, Part 2: Regional Data; June 1983, DOE/EIA-0321/2(80), GPO Stock No. 061-003$00319-2, \$ 7.00$.

Residential Energy Consumption Survey: 1979-1980 Consumption and Expenditures, Part 1: National Data (Including Conservation); April 1981, DOE/EIA-0262/1, GPO Stock No. 061-003-00191-2, $\$ 6.50$.

Residential Energy Consumption Survey: 1979-1980 Consumption and Expenditures, Part II: Regional Data; May 1981, DOE/EIA-0262/2, GPO Stock No. 061-003-00189-1, \$8.50.

Residential Energy Consumption Survey: Consumption and Expenditures, April 1978 Through March 1979; July 1980, DOE/EIA-0207/5, GPO Stock No. 061-003-00131-9, \$7.50.

Single-Family Households: Fuel Oil Inventories and Expenditures: National Interim Energy Consumption Survey; December 1979, DOE/EIA-0207/1, GPO Stock No. 061-003-00075-4, \$3.50.

\section{Other Publications on the Residential Sector}

Energy Consumption Series--User-Needs Study of the 1993 Residential Energy Consumption Survey, September 1993, DOE/EIA-0555(93)/2, GPO 061-003$00819-4, \$ 13.00$.

"End-Use Consumption of Residential Energy" Monthly Energy Review (Article), pp. vii-xiv, July 1987, DOE/EIA-0035(87/07).

Residential Energy Consumption Survey: Trends in Consumption and Expenditures 1978-1984 June 1987, DOE/EIA-0482, GPO Stock No. 061-003-00535-7, $\$ 12.00$.

Residential Conservation Measures; July 1986, SR/EEUD/86/01 (no GPO Stock No.).

An Economic Evaluation of Energy Conservation and Renewable Energy Tax Credits; October 1985, Service Report (no GPO Stock No.).

Residential Energy Consumption and Expenditures by End Use for 1978, 1980, and 1981; December 1984, DOE/EIA-0458, GPO Stock No. 061-003-00415-6, $\$ 4.50$.
Weatherization Program Evaluation, SR-EEUD84-1; August 1984 (available from the Office of the Assistant Secretary for Conservation and Renewable Energy, Department of Energy).

Residential Energy Consumption Survey: Regression Analysis of Energy Consumption by End Use; October 1983, DOE/EIA-0431, GPO Stock No. 061-00300$347-8, \$ 5.00$.

National Interim Energy Consumption Survey: Exploring the Variability In Energy Consumption; July 1981, DOE/EIA-0272, GPO Stock No. 061-003-00205-6, $\$ 5.00$.

National Interim Energy Consumption Survey: Exploring the Variability in Energy Consumption--A Supplement; October 1981, DOE/EIA-0272/S, GPO Stock No. 061-003-00217-0, \$4.50.

Energy Use by U.S. Households; November 1980, DOE/EIA-0248 (brochure, no GPO Stock No.).

\section{Residential Transportation Sector}

Note: The survey name was dropped from the beginning of the report title starting with the 1988 data report, and the report title changed to Household Vehicles Energy Consumption 1988.

Household Vehicles Energy Consumption 1991; December 1993, DOE/EIA-0464(91), GPO Stock No. 061-003-00652-3, \$14.00.

"Energy Preview: Residential Transportation Energy Consumption Survey Preliminary Estimates, 1991," Monthly Energy Review, January 1993, DOE/EIA0035(93/01).

Household Vehicles Energy Consumption 1988; February 1990, DOE/EIA-0464(88), GPO Stock No. 061-003-00652-3, \$11.00.

Residential Transportation Energy Consumption Survey: Consumption Patterns of Household Vehicles 1985; April 1987, DOE/EIA-0464(85), GPO Stock No. 061-003-00521-7, $\$ 8.50$.

Residential Transportation Energy Consumption Survey: Consumption Patterns of Household Vehicles, 1983; January 1985, DOE/EIA-0464(83), GPO Stock No. 061-003-00420-2, \$4.50. 
Residential Energy Consumption Survey: Consumption Patterns of Household Vehicles, Supplement: January 1981 to September 1981; February 1983, DOE/EIA-0328, GPO Stock No. 061-003-00297-8, $\$ 4.75$.

Residential Energy Consumption Survey: Consumption Patterns of Household Vehicles, June 1979 to December 1980; April 1982, DOE/EIA-0319 (no GPO Stock No.).

\section{Cross-Sector}

Energy Consumption by End-Use Sector: A Comparison of Measures by Consumption and Supply Surveys; April 6, 1990, DOE/EIA-0533 (no GPO Stock No. available), $\$ 2.50$.

Natural Gas: Use and Expenditures; April 1983, DOE/EIA-0382, GPO Stock No. 061-003-00307-9, $\$ 5.50$.

\section{Public Use Tapes}

Note: All tapes are available through the NTIS.

\section{Residential and Residential Transportation Sectors}

Residential Energy Consumption Survey: 1987 and Residential Transportation Energy Consumption Survey, 1988, Order No. PB90-501461, \$220.

Residential Energy Consumption Survey: 1984 and Residential Transportation Energy Consumption Survey, 1985; Order No. PB87-186540, \$220.

Residential Energy Consumption Survey: 1982 and Residential Transportation Energy Consumption Survey, 1983; Order No. PB85-221760, \$220.

Residential Energy Consumption Survey: Consumption and Expenditures, 1980-1981; Monthly Billing Data; Order No. PB84-166230, \$220.

Residential Energy Consumption Survey: Housing Characteristics, 1981; Consumption and Expenditures, 1981-1982; Monthly Billing Data; Order No. PB84-120476, $\$ 220$.

Residential Energy Consumption Survey: Housing Characteristics, Annualized Consumption and Expenditures, 1980-1981; Order No. PB83-199554, $\$ 220$.
Residential Energy Consumption Survey: Household Transportation Panel Monthly Gas Purchases and Vehicle and Household Characteristics, 6/79-9/81; Order No. PB84-162452, \$220.

Residential Energy Consumption Survey: Household Screener Survey, 1979-1980; Order No. PB82-114877, $\$ 220$.

Residential Energy Consumption Survey: Household Monthly Energy Consumption and Expenditures, 1978-1979; Order No. PB82-114901, \$220.

National Interim Energy Consumption Survey (Residential), 1978; Order No. PB81-108714, \$220.

\section{Commercial Sector}

Nonresidential Buildings Energy Consumption Survey: 1986 Data; Order No. PB90-500034, \$220.

Nonresidential Buildings Energy Consumption Survey: 1979 and 1983 Data; Order No. PB88-245162, \$220.

\section{Public Use Diskettes}

Note: Diskettes are available through the OSTI and NTIS.

Commercial Buildings Consumption and Expenditures, 1992 Data, OSTI - ASCII or dBASE format, order by title, $\$ 10$ per diskette or $\$ 40.00$ set of four. NTIS-ASCI or dBASE format, order by title, call for prices.

Commercial Buildings Characteristics 1992 Data, OSTI - ASCII or dBASE format, order by title, $\$ 10$ per diskette or $\$ 40.00$ set of four. NTIS - ASCII or dBASE format, order No. PB-94-504305, call for prices.

Residential Energy Consumption Survey 1987 Data, OSTI - ASCII or dBASE format, order by title, $\$ 10$ per diskette, $\$ 40$ set of four. NTIS - ASCII format: Order No. PB-91-505115, $\$ 130$, and dBASE format: Order No. PB-91-505107, \$130.

Commercial Buildings Energy Consumption Survey 1989 data, OSTI - ASCI format, order by title, $\$ 10$ per diskette, $\$ 40$ set of four. NTIS - ASCII format: Order No. PB92-504232, \$140. 
Nonresidential Buildings Energy Consumption Survey 1986 Data, NTIS - ASCII format: Order No. PB91$506808, \$ 130$.

Residential Transportation Energy Consumption Survey 1988 Data, OTSI - ASCII or dBASE format, order by title, $\$ 10$ per diskette, $\$ 40$ set of four.

NTIS - ASCII format: Order No. PB91-507269,

dBASE format: Order No. PB91-507277, \$50 each.

\section{Planned Publications}

Sample Design for the Residential Energy Consumption Survey (Energy Consumption Series); planned for September 1994.
Commercial End-Use Intensities (Energy Consumption Series); planned for October 1994.

Measuring Energy Efficiency in the U.S. Economy (Energy Consumption Series); planned for January 1995.

Buildings and Energy in the 1980's (Energy Consumption Series); planned for December 1994.

Commercial Buildings Energy Consumption and Expenditures 1992; planned for early 1995.

Housing Characteristics 1993; planned for early 1995.

Note: The Energy Information Administration also publishes annually the State Energy Data Report, Consumption Estimates, DOE/EIA-0214 and the State Energy Price and Expenditures Report, DOE/EIA-0376; and the Monthly Energy Review, DOE/EIA-0035. These reports contain annual and monthly consumption information derived from EIA supply surveys. 


\section{Glossary}

Anthracite: A hard, black, lustrous coal containing a high percentage of fixed carbon and a low percentage of volatile matter. It is often referred to as hard coal.

Barrel: A volumetric unit of measure equivalent to 42 U.S. gallons.

Biomass: Organic (animal waste), nonfossil plant material constituting an exploitable energy source.

Bituminous Coal: A soft coal (the most common solid fossil fuel), which is high in carbonaceous matter, with a volatility greater than anthracite.

Blast Furnace: A shaft furnace in which solid fuel is burned with an air blast to smelt ore in a continuous operation.

Blast Furnace Gas: The waste combustible gas generated in a blast furnace when iron ore is being reduced with coke to metallic iron. It is commonly used as a fuel within the steel works.

Breeze: The residue from the fine screenings of crushed coke.

British Thermal Unit (Btu): The amount of energy required to raise the temperature of one pound of water one degree Fahrenheit.

Butane $\left(\mathrm{C}_{4} \mathrm{H}_{10}\right)$ : A normally gaseous, paraffinic hydrocarbon extracted from natural gas or refinery gas streams. It includes isobutane (a branch-chain configuration) and isobutane (a straight-chain configuration). It is used primarily for blending into highoctane gasoline, for residential and commercial heating, and for industrial uses, especially the manufacture of chemicals and rubber.

Butylene $\left(\mathrm{C}_{4} \mathbf{H}_{8}\right)$ : A normally gaseous, olefinic hydrocarbon recovered from the refinery processes, and converted to alkylate, a high-octane gasoline blending component.

Byproduct: A secondary or additional product resulting from the feedstock use of energy or the processing of nonenergy materials. For example, the more common byproducts of coke ovens are coal gas, tar, and a mixture of benzene, toluene, and xylenes (BTX).
Census Region: A geographic area defined by the Bureau of the Census, consisting of various States selected according to population size and physical location. The States are grouped into four regions:

1. Northeast: Connecticut, Maine, Massachusetts, New Hampshire, New Jersey, New York, Pennsylvania, Rhode Island, and Vermont.

2. South: Alabama, Arkansas, Delaware, District of Columbia, Florida, Georgia, Kentucky, Louisiana, Maryland, Mississippi, North Carolina, Oklahoma, South Carolina, Tennessee, Texas, Virginia, and West Virginia.

3. Midwest: Illinois, Indiana, Iowa, Kansas, Michigan, Minnesota, Missouri, Nebraska, North Dakota, Ohio, South Dakota, and Wisconsin.

4. West: Alaska, Arizona, California, Colorado, Hawaii, Idaho, Montana, Nevada, New Mexico, Oregon, Utah, Washington, and Wyoming.

Coal Coke: The strong, porous residue, consisting of carbon and mineral ash, which is formed when the volatile constituents of bituminous coal are driven off by heat in the absence of or with a limited supply of air. Coal coke is used primarily in blast furnaces.

Cogeneration: The production of electrical energy and another form of useful energy (such as heat or steam) through the sequential use of energy.

Coke Oven Gas: The mixture of permanent gases produced by the carbonization of coal in a coke oven at temperatures in excess of 1,000 degrees Celsius.

Consumption: The use of energy as a source of heat or power, or as an input to the manufacturing process.

Conversion Factor: A number that translates units of one system into corresponding values of another system. Conversion factors are used to translate physical units of measure for various energy sources into their Btu equivalents.

Crude Oil: A mixture of hydrocarbons that exists in a liquid state in natural underground reservoirs and remains liquid at atmospheric pressure after passing through surface separating facilities. Crude oil is reported as liquid equivalents at the surface (excluding basic sediment and water), measured in terms of stock tank barrels of 42 U.S. gallons at atmospheric pressure, and corrected to 60 degrees Fahrenheit. 
Distillate Fuel Oil: A general classification for light fuel oils distilled during the refining process. The classification includes products known as Nos. 1, 2, and 4 fuel oils; and Nos. 1, 2, and 4 diesel fuels. Distillate fuel oil is used primarily for space heating, on-and-off highway engine fuel, and electric power generation.

\section{Embodied Energy for Electricity: See Primary Energy.}

Energy: The capacity for doing work as measured in the capability of doing work (potential energy) or the conversion of this capability to motion (kinetic energy).

Energy Source: A substance such as natural gas, coal, or electricity that supplies heat or power.

Establishment: As defined by the 1987 Standard Industrial Classification Manual, "...an economic unit, generally at a single physical location, where business is conducted or where services or industrial operations are performed." (See Manufacturing Establishment).

Ethane $\left(\mathrm{C}_{2} \mathrm{H}_{6}\right)$ : A colorless, odorless, gaseous hydrocarbon extracted from natural gas or refinery gas streams. Ethane is used primarily as a petrochemical feedstock for the production of chemicals and plastic materials.

Ethylene $\left(\mathrm{C}_{2} \mathrm{H}_{4}\right)$ : A colorless, flammable, gaseous olefinic hydrocarbon recovered from natural gas and petroleum. Ethylene is used primarily as a petrochemical feedstock for numerous chemical applications and the production of consumer goods.

Expenditures: Funds spent for energy purchased and paid for, or delivered to a manufacturer during a calendar year. For the purposes of the MECS, the expenditure dollar includes State and local taxes and delivery charges.

Fossil Fuel: Any naturally occurring organic fuel, such as coal crude oil, and natural gas.

Fuel: Any substance that can be burned to produce heat.

Fuel Use (of Energy): Use of energy in the production of heat, steam, power, or the generation of electricity.
Generation: The process of producing steam or electrical energy by transforming other forms of energy.

Geothermal Energy: Hot water or steam, extracted from reservoirs in the earth's crust, which is generally supplied to steam turbines that drive generators to produce electricity.

Hydroelectric Power: Electricity generated by a turbine driven by falling water.

Hydrogen $\left(\mathrm{H}_{2}\right)$ : A colorless, odorless, highly flammable gaseous element; the lightest of all gases and the most abundant element in the universe.

Industrial Sector: A subdivision of U.S. economic activity defined by the Energy Information Administration to include manufacturing, construction, mining, agriculture, fishing, and forestry establishments.

Kilowatthour ( $\mathrm{kWh}$ ): A unit of work or energy, measured as 1,000 watts (1 kilowatt) of power expended for 1 hour. Once generated, one $\mathrm{kWh}$ is equivalent to 3,412 Btu.

Liquefied Petroleum Gases (LPG): Ethane, ethylene, propane, propylene, normal butane, butylene, ethane-propane mixtures, propane-butane mixtures, and isobutane produced at refineries or natural gas processing plants, including plants that fractionate raw natural gas plant liquids.

Lease Condensate: A natural gas liquid recovered from gas well gas (associated and nonassociated) in lease separators or field facilities. Lease condensate consists primarily of pentanes and heavier hydrocarbons. Volumes are reported in terms of barrels of 42 U.S. gallons, at atmospheric pressure, and corrected to 60 degrees Fahrenheit.

Lease Separator: A facility located at the surface for the purposes of (1) separating casinghead gas from produced crude oil and water at the temperature and pressure conditions of the separator; and (2) separating gas from that portion of associated gas and nonassociated gas which liquefies at temperature and pressure conditions of the separator.

Lignite: A brownish-black coal of low rand with a high percentage of inherent moisture and volatile matter content. It is also referred to as brown coal. 
Manufacturing Division: One of 10 fields of economic activity defined by the Standard Industrial Classification Manual. The manufacturing division includes all establishments engaged in the mechanical or chemical transformation of materials or substances into new products. Other divisions of the U.S. economy are agriculture, forestry, fishing, hunting, and trapping; mining; construction; transportation, communications, electric, gas, and sanitary services; wholesale trade; retail trade; finance, insurance, and real estate; personal, business, professional, repair, recreation, and other services; and public administration. The establishments in the manufacturing division constitute the universe for the MECS.

Manufacturing Establishment: An economic unit at a single physical location where mechanical or chemical transformation of materials or substances into new products are performed. These operations are generally conducted in facilities described as plants, factories, or mills, and characteristically use power-driven machines and materials-handling equipment. In addition, the assembly of components of manufactured products is considered manufacturing, as in the blending of materials such as lubricating oils, plastics, resins, or liquors. (See Establishment).

Motor Gasoline: A complex mixture of relatively volatile hydrocarbons, with or without small quantities of additives, obtained by blending appropriate refinery streams to form a fuel suitable for use in sparkignition engines. Motor gasoline includes both leaded and unleaded grades of finished motor gasoline, blending components, and gasohol.

Natural Gas: A mixture of hydrocarbon compounds and small quantities of various nonhydrocarbons existing in the gaseous phase or in solution with oil in natural underground reservoirs at reservoir conditions. Natural gas may be subclassified as:

1. Associated Gas: Free natural gas, commonly known as gas-cap gas, which overlies and is in contact with crude oil in the reservoir.

2. Dissolved Gas: Natural gas which is in solution with crude oil in the reservoir at reservoir conditions.

3. Nonassociated Gas: Free natural gas not in contact with crude oil in the reservoir.

All natural gas volumes are reported in cubic feet at a pressure base of 14.73 psia, at 60 degrees Fahrenheit.
Nonfuel Use (of Energy): Use of energy as a feedstock or raw material input.

Petroleum Coke: A solid residue, high in carbon content and low in hydrogen, which is the final product of thermal decomposition in the condensation process in cracking crude oil. Petroleum coke can yield almost pure carbon or artificial graphite suitable for the production of carbon or graphite electrodes, structural graphite, motor brushes, dry cells, and similar products.

Petrochemical Feedstock: Chemical feedstocks derived from petroleum, and used principally for the manufacture of chemicals, synthetic rubber, and a variety of plastics.

Plant: Commonly used as a synonym for an establishment. However, the term can also be used to refer to a particular process within an establishment.

Primary Energy: All energy consumed by end users, excluding electricity but including the energy consumed at electric utilities to generate electricity.

Propane $\left(\mathrm{C}_{3} \mathrm{H}_{8}\right)$ : A colorless, gaseous hydrocarbon extracted from natural gas or refinery gas streams. It is used primarily for residential and commercial heating and cooling, and also as a fuel for transportation. Industrial applications include use as a petrochemical feedstock.

Propylene $\left(\mathrm{C}_{3} \mathbf{H}_{6}\right)$ : A gaseous hydrocarbon recovered from refinery processes. Propylene is used primarily as a petrochemical feedstock.

Pulping Liquor (Black Liquor): The alkaline spent liquor removed from the digesters in the process of chemically pulping wood. After evaporation, the liquor is burned as a fuel in a recovery furnace that permits the recovery of certain basic chemicals.

Quadrillion Btu: Equivalent to $10^{15}$ Btu.

Refinery: A plant, device, or process which heats crude oil so that it separates into chemical components, which are then distilled off as more usable substances.

Relative Standard Error (RSE): A percentage measure of the precision of a survey statistic. The RSE is defined as the standard error of a survey estimate divided by the survey estimate and multiplied by 100 . The standard error is the square root of the variance. 
Residual Fuel Oil: The general classification for the heavier oils that remain after the distillate fuel oils and lighter hydrocarbons are distilled away in refinery operations. The classification includes No. 5 (light and heavy), No. 6 (including heavy-grade, so called Bunker C oil), and Navy Special fuel oil.

Roundwood: Wood cut specifically for use as a fuel.

Short Ton: A unit of weight equal to 2,000 pounds.

Solar Energy: The radiant energy of the sun, which can be converted into other forms of energy, such as heat or electricity.

Standard Industrial Classification (SIC): A classification scheme developed by the Office of Management and Budget, which categorizes establishments into groups with similar economic activities.

Still Gas (Refinery Gas): Any form or mixture of gas produced in refineries by distillation, cracking, reforming, and other processes, the principal constituents of which are methane, hydrogen, ethane, ethylene, propane, propylene, butanes, butylene, etc. Still gas is used as a petrochemical feedstock and as a fuel in refineries.

Storage Capacity: For the purposes of the MECS, storage capacity includes any volumetric capacity (including tank tops and tank bottoms) that is on the establishment site even it is dedicated or leased for the storage of an energy source by other establishments.
Subbituminous Coal: A dull, black coal of intermediate rank between lignite and bituminous coal. Subbituminous coal, like bituminous coal, is used as a fuel.

Turbine: A machine for generating rotary mechanical power from an energy stream (such as water, steam, or hot gas). Turbines convert kinetic energy to mechanical energy through the principles of impulse and reaction, or a mixture of the two.

Waste Materials: Otherwise discarded combustible materials which, when burned, produce energy for such purposes as space heating and electric power generation. The size of the waste may be reduced by shredders, grinders, or hammermills. Noncombustible materials, if any, may be removed. The waste may be dried and then burned, either alone or in combination with fossil fuels.

Waste Oils and Tar: Petroleum-based materials that are worthless for any purpose other than fuel use.

Wind Energy: Energy present in wind motion that can be converted to mechanical energy for driving pumps, mills, and electric power generators. Wind pushes against sails, vanes, or blades radiating from a central rotating shaft.

Wood Waste: Wood byproducts used as a fuel. Included are limb wood, wood chips, bark, sawdust, forest residues, charcoal, and pulp waste. 